\title{
ACTA ACUSTICA
} UNITED WITH

ACUSTICA

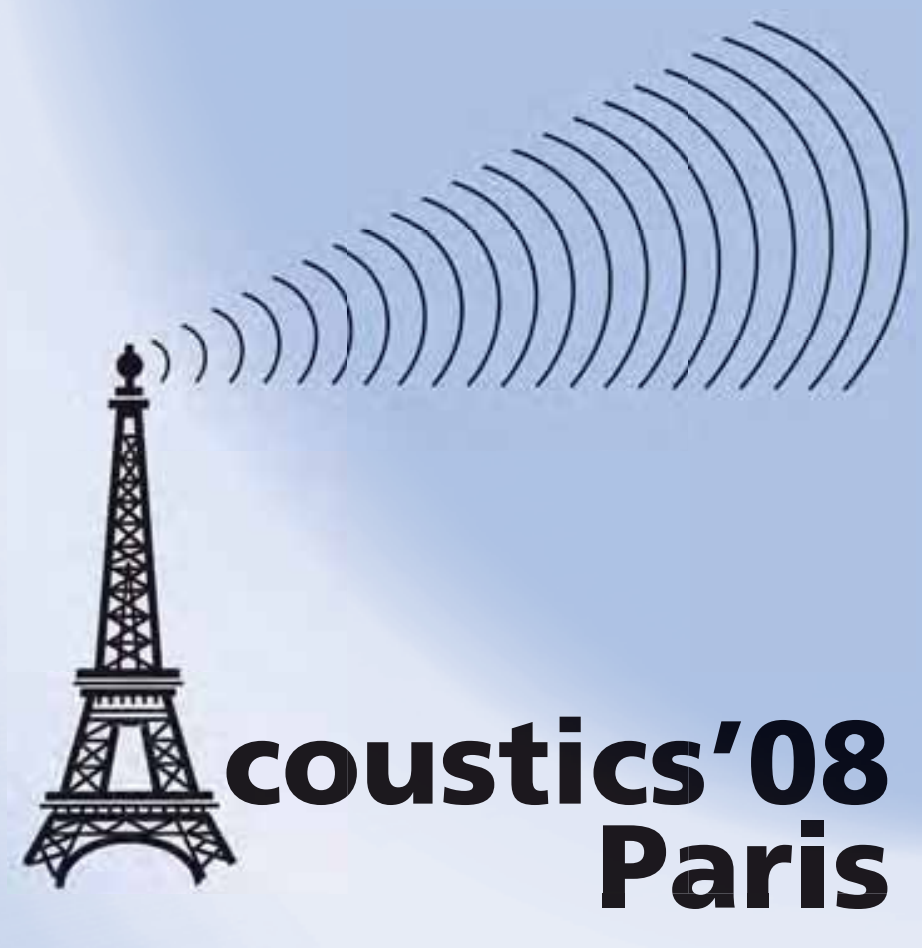

June 29 - July 4, 2008

5th Forum Acusticum

155th ASA Meeting

ge Congrès Français d'Acoustique

integrating

60th anniversary of the "Société Française d'Acoustique"

and two major European Conferences:

7th European conference on noise control (euronoise)

9th European Conference on Underwater Acoustics (ecua)

Abstracts 


\section{Acta Acustica UNITED WITH ACUSTICA}

The Journal of the European Acoustics Association (EAA) - International Journal on Acoustics

\section{Editor-in-Chief}

Prof. Dr. ir. Dick Botteldooren

Ghent University

Department of Information Technology

Acoustics Group

St. Pietersnieuwstraat 41

B-9000 Gent, Belgium

Tel.: $\quad+3292649968$

Fax: $\quad+3292649969$

E-mail: aa.a@intec.ugent.be

\section{Associate Editors}

Prof. K. Attenborough, GB-Hull

Dr. Pierre Badin, F-Grenoble

Prof. Murray Campbell, GB-Edinburgh

Dr. K. Yves Cazals, F-Marseille

Prof. Jean-Marc Conoir, F-Le Havre

Dr. François Coulouvrat, F-Paris

Prof. Trevor J. Cox, GB-Salford

Prof. Dr. Danièle Dubois, F-Paris

Prof. S. J. Elliott, GB-Southampton

Prof. Juan A. Gallego-Juárez, E-Madrid

Prof. Amando García, E-Valencia

Prof. Jean-Louis Guyader, F-Villeurbane

Dr. Kurt Heutschi, CH-Duebendorf

Prof. Daniel Juvé, F-Ecully

Prof. Jian Kang, UK-Sheffield

Dr. Chris de Korte, NL-Nijmegen

Prof. Wolfgang Kropp, S-Göteborg

Dr. Denis Lafarge, F-Le Mans

Prof. Walter Lauriks, B-Leuven

Jun.-Prof. Dr. Steffen Marburg, D-Dresden

Dr. Bernard Richardson, GB-Cardiff

Prof. Dr. Gerhard M. Sessler, D-Darmstadt

Prof. Andrzej Stepnowski, PL-Gdansk

Prof. Peter Svensson, NO-Trondheim

Prof. Michael Taroudakis, GR-Heraklion

Jun.-Prof. Dr. Jesko L. Verhey, D-Oldenburg

Prof. Gerrit Vermeir, B-Leuven

\section{Abstracted/Indexed in:}

Cambridge Scientific Abstracts / Electronics and Communication Abstracts;

Current Contents / Physical, Chemical and Earth Sciences;

Current Contents / Engineering,

Computing \& Technology;

Ei Compendex Plus (Engineering Information); FRANCIS (CNRS); INSPEC;

Research Alert; SciSearch; Science Citation Index. Recognized by the European Physical Society
Acta Acustica united with Acustica,

the journal of the European Acoustics

Association (EAA), is an international journal

on acoustics. Manuscripts of articles and letters should be submitted to the Editor-in-Chief. They will be forwarded to the Associate Editor respon sible for the subject area of the paper. A detailed list of Associate Editors and Instructions for Authors are given on the last pages of each issue. Manuscripts are refereed by two experts and reviewed by the Associate Editor and Editor-in-Chief before acceptance. No honorarium is paid. The first author of an article will receive 25 offprints free-of-charge. Further offprints may be ordered at extra costs. A price list is available from the publisher. All articles published in this journal represent the opinions of the authors and do not necessarily reflect the opinions of the editors. The publisher cannot be held responsible for unsolicited manuscripts. Submission of a manuscript implies that the work has not been published before and that it is not under consideration elsewhere.

With the permission to publish in this journal the author agrees to exclusive transfer of the copyright to the publisher until expiry of the copyright. The transfer of the copyright explicitly includes the right to produce electronic versions and the right for electronic storage in databases as well as the right to reproduce and publish such versions online and offline without any extra remuneration.

The journal and all articles and illustrations published herein are protected by copyright. Beyond the narrow copyright restrictions and without the express written permission of the publisher, no part of this journal may be translated into a foreign and/or machine readable language, reproduced, stored in a retrieval system, or transmitted, in any form or by any means, electronic, mechanical, photocopying, microfilming, recording or otherwise

The use of general descriptive names, trade names, trademarks, etc. in a publication, even if not specifically identified, does not imply that these names are not protected by the relevant laws and regulations.

\section{Internet}

http://www.Acta-Acustica-united-with-Acustica.com http://www.ingentaconnect.com/content/dav/aaua http://eaa-fenestra.org/acta

\section{Subscription Information}

Acta Acustica united with Acustica is published bimonthly. Annual institutional subscription rate (Vol. 94, 2008, No 1-6): $€ 846.00$ plus shipping \& handling costs (Europe: $€ 27.00$; rest of the world: $€ 48.00)$. Airmail costs upon request. Price for a single copy: $€ 202.00$ plus shipping \& handling costs. Institutional subscriptions include online access to the full text via

http://www.ingenta.connect.com provided a licence agreement is concluded with the publisher. No online availability for additional supplement issues (congress abstracts issues) which are published in irregular intervals. Every print issue includes a CD-ROM with the contents of the current issue. The CD is not sold separately.

Members of a national acoustical member-society of the European Acoustics Association (EAA) receive the journal from their society.

Subscriptions are made on a continuation basis unless ordered for a limited period. Cancellations may only be made for a complete annual volume, they must be received by the publisher by

15 November of the preceding year at the latest.

Publisher

S. Hirzel Verlag $\mathrm{GmbH} \& \mathrm{Co}$.

Birkenwaldstrasse 44

D-70191 Stuttgart, Germany

Tel.: +49 711 2582-0, Fax: +49 711 2582-290

E-mail: ActaAcustica@Hirzel.de

Production Manager

Dr. Johann Wempen, Daucher Strasse 98

D-85053 Ingolstadt, Germany

Tel.: +49 841975852

E-mail: wempen@t-online.de

Responsible for Text Material

Prof. Dr. Dick Botteldooren, Acoustics Group,

Department of Information Technology,

Ghent University, St. Pietersnieuwstraat 41 ,

B-9000 Gent, Belgium.

Responsible for Advertisements:

S. Hirzel Verlag, Birkenwaldstrasse 44

D-70191 Stuttgart, Germany

Tel.: +49 71125 82-245, Fax: +49 71125 82-252

E-mail: anzeigen@hirzel.de.

Present effective rate card: October 1, 2007.

Printed by W. Kohlhammer Druckerei GmbH + Co. KG, Augsburger Strasse 722, 70329 Stuttgart

(C) 2008 S. Hirzel Verlag GmbH \& Co., Stuttgart; European Acoustics Association,

Calle Serrano 144, E-28006 Madrid, Spain

ISSN 1610-1928 (Print), ISSN 1861-9959 (Online)

Acta Acustica united with Acustica

Volume 94 Supplement 1

(Acta Acustica Vol. 16 Suppl. 1,

Acustica Vol. 94 Suppl. 1) 


\section{Acta Acustica suntro wry ACUSTICA}

The Journal of the European Acoustics Association (EAA) - International Journal on Acoustics

\section{Table of Contents}

Acoustics' 08 - Paris · June 29 - July 4, 2008 page

Maps of Meeting Rooms at Palais des Congrés ............................ A 2

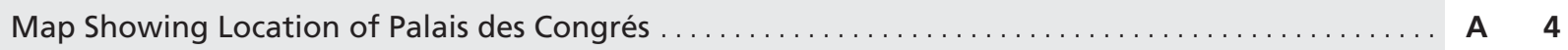

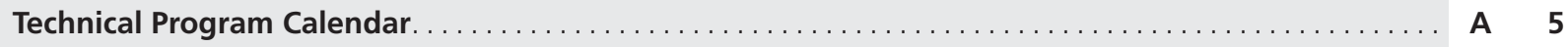

Schedule of Committee Meetings and Other Events $\ldots \ldots \ldots \ldots \ldots \ldots \ldots \ldots \ldots \ldots \ldots \ldots \ldots \ldots$ A 19

Meeting Information $\ldots \ldots \ldots \ldots \ldots \ldots \ldots \ldots \ldots \ldots \ldots \ldots \ldots \ldots \ldots \ldots \ldots \ldots \ldots \ldots \ldots \ldots \ldots$

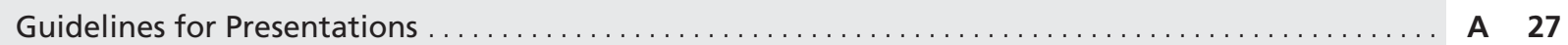

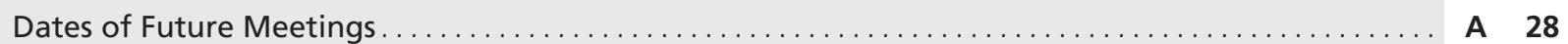

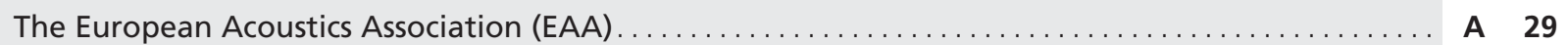

The EAA Young Researcher Grants for East European Students . . . . . . . . . . . . . . . . . . . A 31

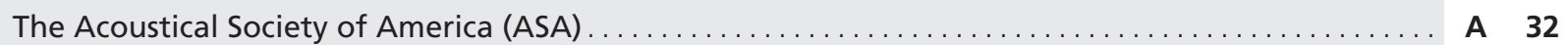

The French Acoustical Society (Société Française d'Acoustique, SFA) . . . . . . . . . . . . ... A 34

Opening Ceremony $\left(1 a_{\_}\right)$, Monday Morning $\ldots \ldots \ldots \ldots \ldots \ldots \ldots \ldots \ldots \ldots \ldots \ldots \ldots \ldots \ldots$ S 1

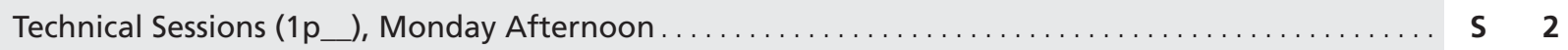

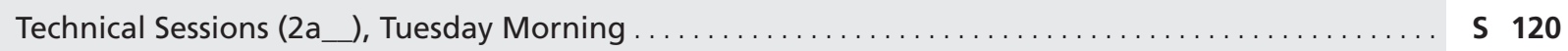

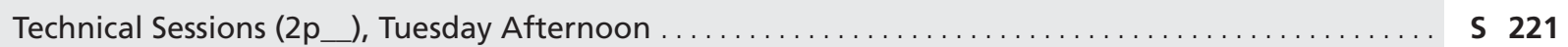

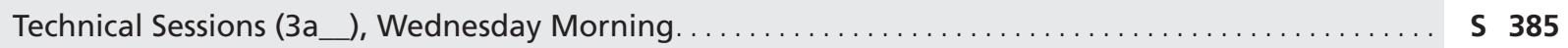

Technical Sessions $\left(3 p \_\right)$, Wednesday Afternoon. . . . . . . . . . . . . . . . . . . . . . . . . . . S 474

Awards Ceremony and Plenary Session, Wednesday Afternoon ...................... S 500

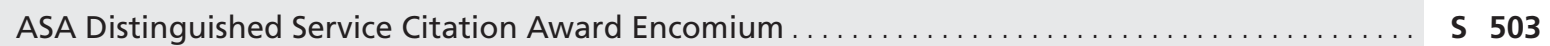

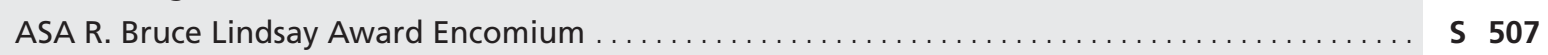

ASA Helmholtz-Rayleigh Interdisciplinary Silver Medal Award Encomium . . . . . . . . . . . . S 511

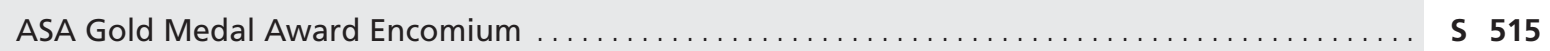

EAA Lifetime Achievements in Acoustics Award Encomium. . . . . . . . . . . . . . . . . . . . S 519

EAA Contributions to the Promotion of Acoustics in Europe Award Encomium ........... S 521

EAA Outstanding Recent Scientific Results Published in

Acta Acustica united with Acustica Award Encomium . . . . . . . . . . . . . . . . . . . . . . . S 522

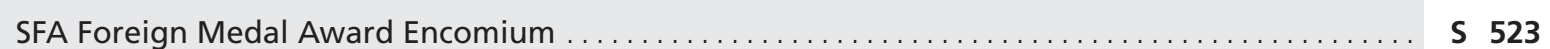

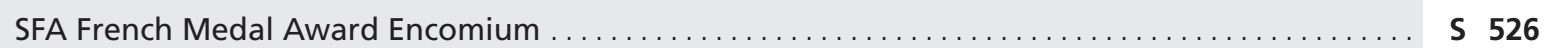

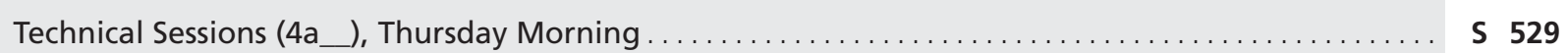

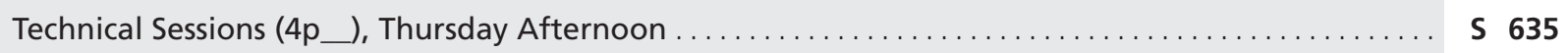

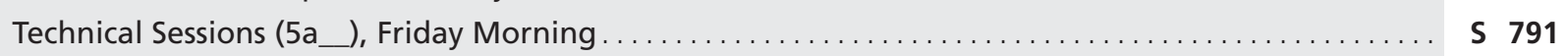

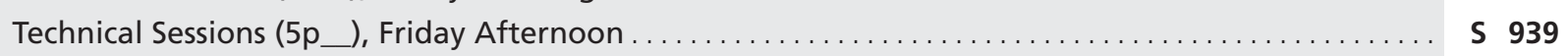

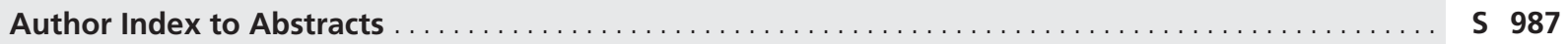



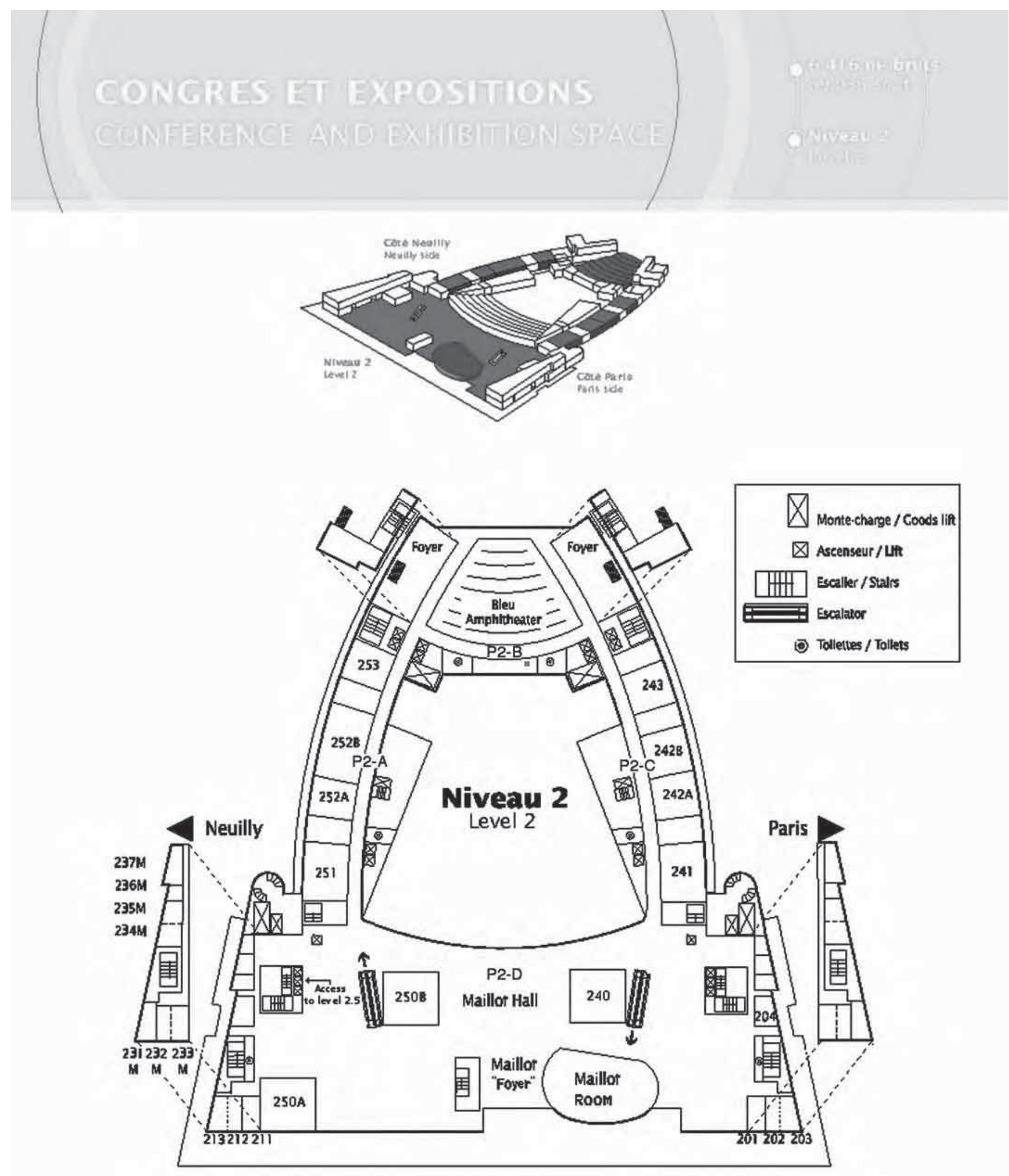

P2-A, P2-B, P2-C, P2-D, denote locations of postersessions as follows:

AA-P2-B; MU-P2-D; NS-P2-A; PP-P2-C; SA-P2-B; SC-P2-D 


\section{(9) CONGRES ET EXPOSITIONS CONFERENCE AND EXHIBITION SPACE}
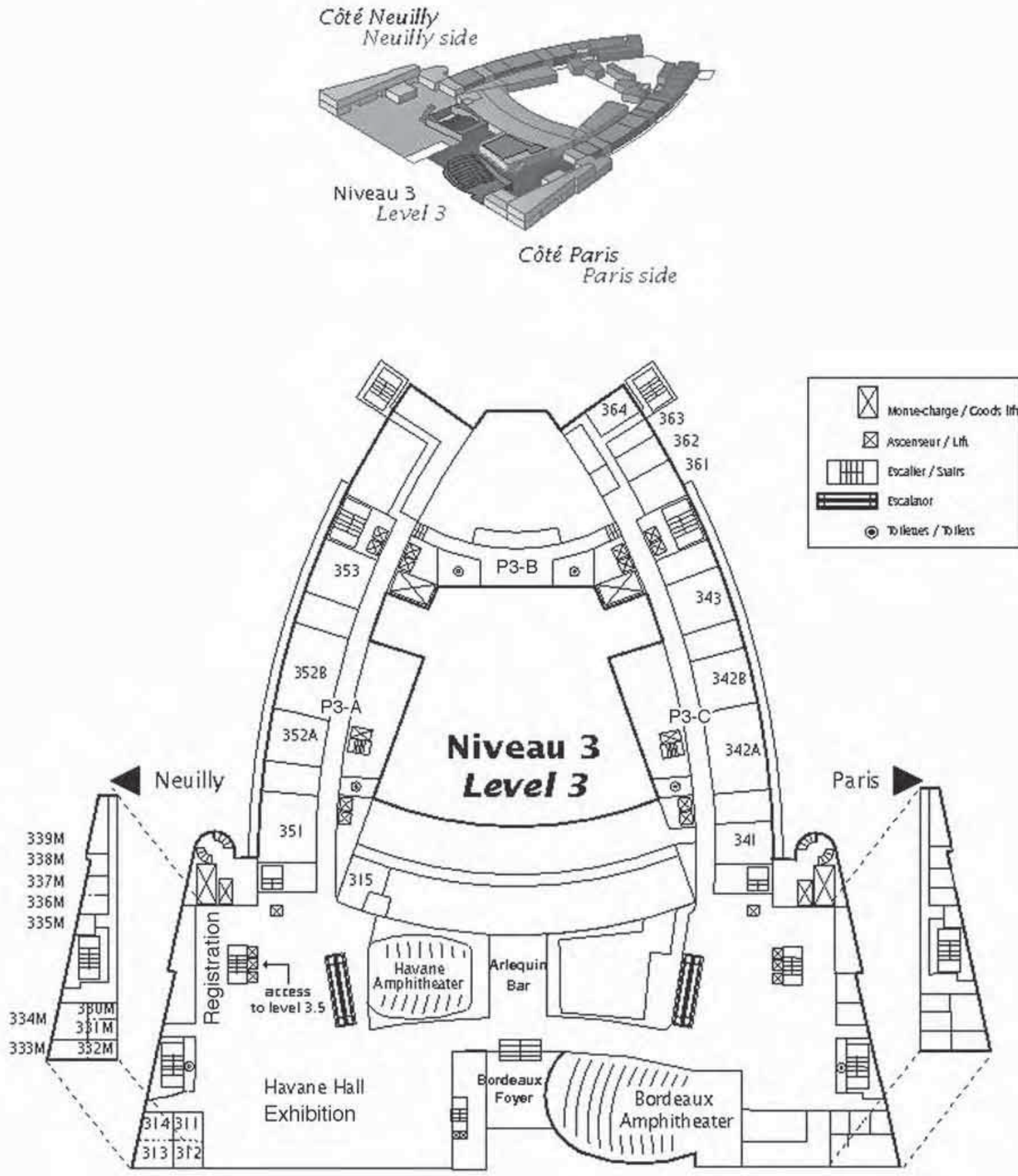

P3-A, P3-B, and P3-C denote locations of poster sessions as follows:

AB, AO-P3-C; BB-P3-B; EA-P3-C; PA-P3-A; SP, UW-P3-C 


\section{LOCATION OF CONFERENCE CENTER}
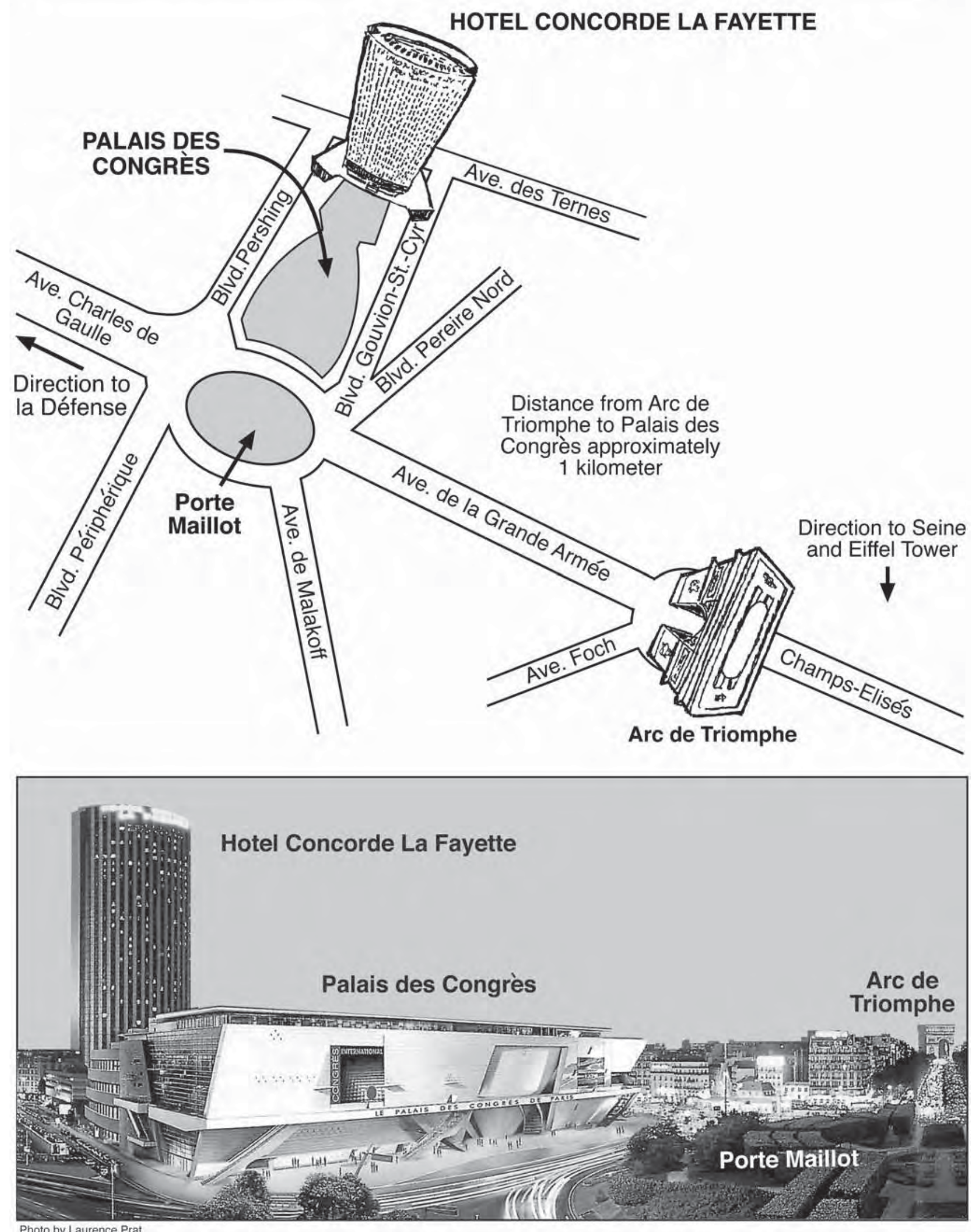

Photo by Laurence Prat 


\section{TECHNICAL PROGRAM CALENDAR \\ Acoustics'08 Paris \\ 30 June-4 July 2008}

\section{MONDAY MORNING}

8:40 1aID Opening Ceremony: Amphi Grand

\section{MONDAY AFTERNOON}

1:00 1pAAa Architectural Acoustics and Noise: Acoustics of Open-Plan Spaces I. Room 202/203

1:00 1pAAb Architectural Acoustics: Acoustics of Concert Halls I. Room 252B

1:00 1pAAc Architectural Acoustics and Psychological and Physiological Acoustics: Speech Segregation in Rooms I. Room 253

4:40 1pAAd Architectural Acoustics and Musical Acoustics: Surround Sound Acoustics I. Room 202/203

5:00 1pAAe Architectural Acoustics and Noise: Low Frequency Absorption: Mechanisms, Measurement Methods, and Application I. Room 253

1:00 1pAB Animal Bioacoustics, Noise, and ECUA: Anthropogenic Noise Effects on Animals I. Room 342B

1:00 1pAOa Acoustical Oceanography and ECUA: Acoustical Oceanography of Polar Environments I. Room 342A

4:20 1pAOb Acoustical Oceanography and ECUA: Marine Ecosystem Acoustics I. Room $342 \mathrm{~A}$

1:00 $1 \mathrm{pBBa} \quad$ Biomedical Ultrasound/Bioresponse to Vibration: High-Intensity Focused Ultrasound I. Room 352B

$1: 00 \quad 1 \mathrm{pBBb} \quad$ Biomedical Ultrasound/Bioresponse to Vibration: Quantitative Ultrasound Methods for Diagnosis and Therapy I. Room 362/363

5:40 $1 \mathrm{pBBc} \quad$ Biomedical Ultrasound/Bioresponse to Vibration and Engineering Acoustics: High-Intensity Focused Ultrasound Metrology and Standards I. Room 352B

1:00 1pEAa Engineering Acoustics, Underwater Acoustics, Signal Processing in Acoustics, and ECUA: Sensor Technologies for Autonomous Acoustic Sensing Systems I. Room 341

1:00 1pEAb Engineering Acoustics and Signal Processing in Acoustics: Microphone Array Signal Processing I. Room 353

4:20 1pEAc $\quad$ Engineering Acoustics and Psychological and Physiological Acoustics: Hearing Aid Engineering I. Room 353
5:40 1pEAd

$1: 00$

Transducer Design and Modeling I.

Room 341

\section{1pMUa \\ Musical Acoustics: Acoustic \\ Measurements on Wind Instruments I. Amphi Maillot \\ 4:20 1pMUb Musical Acoustics: Edge Tone and Flue Pipes I. Amphi Maillot \\ 6:00 1pMUc Musical Acoustics and Psychological and Physiological Acoustics: Acoustics and Psychoacoustics of Pipe Organs. Amphi Maillot \\ 1:00 1pNSa Noise, Physical Acoustics, and EURONOISE: Aeroacoustics I. Room $250 \mathrm{~A}$ \\ 1:00 1pNSb Noise and EURONOISE: Action Planning and Global Solutions for Urban Noise I. Room 251 \\ 1:00 1pNSc Noise and EURONOISE: Noise Mapping Techniques and Uncertainties I. Room 252A}

5:40 1pNSd Noise and EURONOISE: Environmental Noise Mapping I. Room $252 \mathrm{~A}$

1:00 1pPAa Physical Acoustics: Acoustics of Porous Media I. Amphi Havane

1:00 1pPAb Physical Acoustics: Phononic Crystals I. Room 351

1:00 1pPAc $\quad$ Physical Acoustics and Signal Processing in Acoustics: Acoustic Landmine Detection I. Room 352A

4:00 1pPAd Physical Acoustics: Sonic, Ultrasonic, and Megasonic Cleaning I. Room 352A

7:00 1pPAe Physical Acoustics: Mathematical and Numerical Methods I. Room 351

1:00 $\quad 1 \mathrm{pPPa} \quad$ Psychological and Physiological Acoustics: Integrated Approaches to Auditory Scene Analysis I. Room 241

$1: 00 \quad 1 \mathrm{pPPb} \quad$ Psychological and Physiological Acoustics: Cochlear Implants: Going Beyond the Envelope I. Room 242A

1:00 1pSAa Structural Acoustics and Vibration and EURONOISE: Vibration and Radiation from Complex Structural Systems I. Room 242B

1:00 1pSAb Structural Acoustics and Vibration and EURONOISE: General Topics in Structural Acoustics and Vibration I. Room 243

1:00 1pSCa
Speech Communication: Speech Technology I. Room 240 
1:00 1pSCb Speech Communication: Measurement of Sociophonetic Variation in Speech. Room 250B

4:20 1pSCc Speech Communication: General Topics in Speech Communication I (Poster Session). P2-D, Level 2

1:00 1pSPa Signal Processing in Acoustics: Sound Reproduction and Source Separation. Room 343

4:00 $\quad 1 \mathrm{pSPb} \quad$ Signal Processing in Acoustics: Acoustical Nondestructive Evaluation, Ultrasonics, and Imaging I. Room 343

1:00 1pUW Underwater Acoustics, Acoustical Oceanography, and ECUA: Seabed and Sea Surface Interaction Measurements and Modeling. Amphi Bourdeaux

\section{TUESDAY MORNING}

8:00 2aAAa Architectural Acoustics: Acoustics of Concert Halls II. Room 252B

8:00 $2 \mathrm{aAAb}$ Architectural Acoustics and Noise:

Low Frequency Absorption:

Mechanisms, Measurement Methods and Application II. Room 253

8:20 2aAAc Architectural Acoustics and Engineering Acoustics: Acoustics and Electroacoustics of Small Rooms. Room 202/203

9:20 2aAAd Architectural Acoustics and Noise: Acoustics and Privacy in Healthcare Facilities I: Emerging Policy Around the World. Room 253

10:20 2aAAe Architectural Acoustics, Musical Acoustics, Physical Acoustics, and Noise: Acoustics of Opera Houses I. Room 252B

8:00 $2 \mathrm{aAB}$ Animal Bioacoustics and ECUA: Animal Bioacoustic Censusing I. Room 342B

8:00 2aAOa Acoustical Oceanography and ECUA: Marine Ecosystem Acoustics II. Room $342 \mathrm{~A}$

10:40 2aAOb Acoustical Oceanography, Underwater Acoustics, Signal Processing in Acoustics, and ECUA: Geoacoustic Characterization of the Ocean Bottom and Geoacoustic Inversion I. Room $342 \mathrm{~A}$

8:00 2aBB Biomedical Ultrasound/Bioresponse to Vibration and Physical Acoustics: Ultrasound Contrast Agents for Imaging I. Room 352B

8:00 2aEAa Engineering Acoustics, Underwater Acoustics, and ECUA: Sonar Transducer Design and Modeling II. Room 341
8:00 2aEAb Engineering Acoustics: Acoustic Evaluation I. Room 353

8:00 2aMUa Musical Acoustics and Physical Acoustics: Brass Instrument Acoustics I. Amphi Maillot

11:00 2aMUb Musical Acoustics: Interaction Between Instrument and Instrumentalist I. Amphi Maillot

8:00 2aNSa Noise, Physical Acoustics, and EURONOISE: Aeroacoustics II. Room $250 \mathrm{~A}$

8:00 2aNSb Noise, Biomedical Ultrasound/ Bioresponse to Vibration, ASA Committee on Standards, and EURONOISE: Session in Honor of Henning von Gierke. Room 251

8:00 2aNSc Noise and EURONOISE: Physical and Psychophysical Evaluation of Vehicle Exterior Noise I. Room 252A

10:20 2aNSd Noise and EURONOISE: Environmental Noise Mapping II. Room 251

11:00 2aNSe Noise and EURONOISE: General Topics in Noise I. Room 252A

8:00 2aPAa Physical Acoustics: Acoustics of Porous Media II. Amphi Havane

8:00 2aPAb Physical Acoustics: Diffraction of Waves on Periodical Structures:

Acoustic, Ultrasonic and AcoustoOptical Diffraction Phenomena I. Room 351

8:00 2aPAc Physical Acoustics: Outdoor Sound Propagation and Uncertainties I. Room 352A

8:00 2aPAd Physical Acoustics: Photoacoustics I. Room 362/363

8:00 2aPPa Psychological and Physiological Acoustics and ASA Committee on Standards: Applications of Psychoacoustics I. Room 241

8:00 $2 \mathrm{aPPb} \quad$ Psychological and Physiological Acoustics: Auditory Perception and Signal Processing by Prostheses I. Room 242A

11:20 2aPPc Psychological and Physiological Acoustics: Binaural Perception by Hearing-Aid Wearers. Room 242A

8:00 2aSAa Structural Acoustics and Vibration and EURONOISE: Vibration and Radiation from Complex Structural Systems II. Room 242B

8:00 $2 \mathrm{aSAb} \quad$ Structural Acoustics and Vibration and EURONOISE: General Topics in Structural Acoustics and Vibration II. Room 243

11:00 2aSAc Structural Acoustics and Vibration and EURONOISE: Source Characterization in Structure Borne Noise Problems I. Room 242B 


\begin{tabular}{|c|c|c|}
\hline $8: 20$ & $2 \mathrm{aSCa}$ & $\begin{array}{l}\text { Speech Communication: Speech } \\
\text { Technology II. Room } 240\end{array}$ \\
\hline $8: 20$ & $2 \mathrm{aSCb}$ & $\begin{array}{l}\text { Speech Communication: How do } \\
\text { Physical and Motor Knowledge Matter } \\
\text { to Speech Perception? Room 250B }\end{array}$ \\
\hline $10: 40$ & $2 \mathrm{aSCc}$ & $\begin{array}{l}\text { Speech Communication: Speech } \\
\text { Recognition in Noisy Environments. } \\
\text { Room 250B }\end{array}$ \\
\hline $11: 00$ & $2 \mathrm{aSCd}$ & $\begin{array}{l}\text { Speech Communication: Speech } \\
\text { Perception I. Room } 240\end{array}$ \\
\hline 8:00 & $2 \mathrm{aSPa}$ & $\begin{array}{l}\text { Signal Processing in Acoustics, } \\
\text { Physical Acoustics, Biomedical } \\
\text { Ultrasound/Bioresponse to Vibration, } \\
\text { and Underwater Acoustics: Overview } \\
\text { of Time Reversal in Acoustics I. Room } \\
343\end{array}$ \\
\hline $10: 40$ & $2 \mathrm{aSPb}$ & $\begin{array}{l}\text { Signal Processing in Acoustics, } \\
\text { Biomedical Ultrasound/Bioresponse to } \\
\text { Vibration, and Underwater Acoustics: } \\
\text { Time Reversal Methods for Array } \\
\text { Imaging and Signal Processing I. Room } \\
343\end{array}$ \\
\hline $8: 40$ & 2aUW & $\begin{array}{l}\text { Underwater Acoustics and ECUA: } \\
\text { Fifty Years of Progress in Sonar } \\
\text { Acoustic Research: The Role of } \\
\text { NURC/SACLANTCEN. Amphi } \\
\text { Bordeaux }\end{array}$ \\
\hline
\end{tabular}

\section{TUESDAY MORNING SESSIONS CONTINUED}

$\begin{array}{lll}2: 00 & 2 \mathrm{aAOb} & \text { Room 342A } \\ \text { 1:40 } & 2 \mathrm{aMUb} & \text { Amphi Maillot } \\ \text { 2:00 } & 2 \mathrm{aNSa} & \text { Room 250A } \\ \text { 2:00 } & 2 \mathrm{aNSd} & \text { Room 251 } \\ 2: 00 & 2 \mathrm{aNSe} & \text { Room 252A } \\ 2: 00 & 2 \mathrm{aPAb} & \text { Room 351 } \\ 2: 00 & 2 \mathrm{aPAc} & \text { Room 352A } \\ 2: 00 & 2 \mathrm{aPPa} & \text { Room 241 } \\ 2: 00 & 2 \mathrm{aPPc} & \text { Room 242A }\end{array}$

\section{TUESDAY AFTERNOON}

2:00 2pAAa Architectural Acoustics and ASA Committee on Standards: Comparison of US and European Standards in Building/Room Acoustics I. Room 202/203

2:00 2pAAb Architectural Acoustics, Musical Acoustics, Physical Acoustics, and Noise: Acoustics of Opera Houses II. Room 242B

2:00 2pAAc Architectural Acoustics and Noise: Acoustics and Privacy in Healthcare Facilities II: Emerging Research Around the World I. Room 243

3:40 2pAAd Architectural Acoustics and Noise: Acoustics of Open-Plan Spaces II (Poster Session). P2-B, Level 2
3:40 2pAAf Architectural Acoustics, Musical Acoustics, Physical Acoustics, and Noise: Acoustics of Opera Houses III (Poster Session). P2-B, Level 2

3:40 2pAAg Architectural Acoustics: Acoustics of Concert Halls II (Poster Session). P2-B, Level 2

3:40 2pAAh Architectural Acoustics and Noise: Low Frequency Absorption: Mechanisms, Measurement Methods and Application III (Poster Session). P2-B, Level 2

3:40 2pAAi Architectural Acoustics and ASA Committee on Standards: Comparison of US and European Standards in Building/Room Acoustics II (Poster Session ). P2-B, Level 2

3:40 2pAAj Architectural Acoustics and Musical Acoustics: Surround Sound Acoustics II (Poster Session). P2-B, Level 2

5:20 2pAAk Architectural Acoustics: Case Studies and Design Approaches. Room 242B

2:20 2pABa Animal Bioacoustics, Underwater Acoustics, Acoustical Oceanography, Signal Processing in Acoustics, and ECUA: Animal Sonar Systems I. Room 342B

3:40 2pABb Animal Bioacoustics and ECUA: Animal Bioacoustic Censusing II (Poster Session). P3-C, Level 3

3:40 2pABc Animal Bioacoustics, Noise, and ECUA: Anthropogenic Noise Effects on Animals II (Poster Session). P3-C, Level 3

3:40 2pABd Animal Bioacoustics, Underwater Acoustics, Acoustical Oceanography, Signal Processing in Acoustics, and ECUA: Animal Sonar Systems II (Poster Session). P3-C, Level 3

3:40 2pAOa Acoustical Oceanography and ECUA: Marine Ecosystem Acoustics III (Poster Session). P3-C, Level 3

3:40 2pAOb Acoustical Oceanography and ECUA: Acoustical Oceanography of Polar Environments II (Poster Session). P3-C, Level 3

3:40 2pAOc Acoustical Oceanography, Underwater Acoustics, Signal Processing in Acoustics, and ECUA: Geoacoustic Characterization of the Ocean Bottom and Geoacoustic Inversion II (Poster Session). P3-C, Level 3

$2: 40 \quad 2 \mathrm{pBBa} \quad$ Biomedical Ultrasound/Bioresponse to Vibration: Ultrasound Contrast Agents for Therapy I. Room 352B

3:40 $2 \mathrm{pBBb} \quad$ Biomedical Ultrasound/Bioresponse to Vibration: Ultrasound Contrast Agents for Therapy II (Poster Session). P3-B, Level 3 
3:40

$2 \mathrm{pBBc}$

Biomedical Ultrasound/Bioresponse to Vibration and Physical Acoustics:

Ultrasound Contrast Agents for Imaging II (Poster Session). P3-B, Level 3

$3: 40 \quad 2 \mathrm{pBBd} \quad$ Biomedical Ultrasound/Bioresponse to Vibration and Engineering Acoustics: High-Intensity Focused Ultrasound Metrology and Standards II (Poster Session). P3-B, Level 3

$3: 40 \quad 2 \mathrm{pBBe}$

Biomedical Ultrasound/Bioresponse to Vibration: High-Intensity Focused Ultrasound II (Poster Session). P3-B, Level 3

3:40 2pBBf Biomedical Ultrasound/Bioresponse to Vibration: Quantitative Ultrasound Methods for Diagnosis and Therapy II (Poster Session). P3-B, Level 3

2:00 2pEAa Engineering Acoustics: Silicon Microphones. Room 353

3:40 $2 \mathrm{pEAb} \quad$ Engineering Acoustics and Signal Processing in Acoustics: Microphone Array Si gnal Processing II (Poster Session). P3-C, Level 3

3:40 2pEAc Engineering Acoustics: Acoustic Evaluation II (Poster Session). P3-C, Level 3

3:40 2pEAe Engineering Acoustics, Underwater Acoustics, Signal Processing in Acoustics, and ECUA: Sensor Technologies for Autonomous Acoustic Sensing Systems II (Poster Session). P3-C, Level 3

\section{3:40 2pEAf Engineering Acoustics and} Psychological and Physiological Acoustics: Hearing Aid Engineering II (Poster Session). P3-C, Level 3

3:40 2pEAg Engineering Acoustics, Underwater Acoustics, and ECUA: Sonar Transducer Design and Modeling III (Poster Session). P3-C, Level 3

3:40 2pMUa Musical Acoustics and Physical Acoustics: Brass Instrument Acoustics II (Poster Session). P2-D, Level 2

3:40 2pMUb Musical Acoustics: Edge Tone and Flue Pipes II (Poster Session). P2-D, Level 2

3:40 2pMUc Musical Acoustics: Acoustic Measurements on Wind Instruments II (Poster Session). P2-D, Level 2

3:40 2pMUd Musical Acoustics: Interaction Between Instrument and Instrumentalist II (Poster Session). P2-D, Level 2

5:20 2pMUe Musical Acoustics and Speech Communication: Singing Voice and Source-Filter Interaction. Amphi Maillot

$2: 00 \quad 2 \mathrm{pNSa}$ and EURONOISE: Sound Quality Tools and Applications I. Room 253

3:40 2pNSb Noise, Physical Acoustics, and EURONOISE: Aeroacoustics III (Poster Session). P2-A, Level 2

3:40 2pNSc Noise, ASA Committee on Standards, and EURONOISE: Comparing Noise Regulations and Codes in USA and Europe (Poster Session). P2-A, Level 2

3:40 2pNSd Noise, ASA Committee on Standards, and EURONOISE: Sound Quality Tools and Applications II (Poster Session). P2-A, Level 2

3:40 2pNSe Noise and EURONO ISE: General Topics in Noise II (Poster Session). P2-A, Level 2

3:40 2pNSf Noise, Computational Acoustics, and EURONOISE: Time-Domain Modeling Methods in Acoustics I (Poster Session). P2-A, Level 2

3:40 2pNSg Noise and EURONOISE: EU Projects for Aircraft Noise Reduction I (Poster Session). P2-A, Level 2

3:40 2pNSh Noise and EURONOISE: Action Planning and Global Solutions for Urban Noise II (Poster Session). P2-A, Level 2

3:40 2pNSi Noise and EURONOISE: Railway Noise and Vibration I (Poster Session). P2-A, Level 2

3:40 2pNSj Noise and EURONOISE: Noise, Structure Borne Noise from Building Technical Equipment, and Ground Borne Noise from Railways I (Poster Session). P2-A, Level 2

3:40 2pNSk Noise and EURONOISE: Physical and Psychophysical Evaluation of Vehicle Exterior Noise II (Poster Session). P2-A, Level 2

3:40 2pNSl Noise and EURONOISE: Environmental Noise Mapping III (Poster Session). P2-A, Level 2

3:40 2pNSm Noise and EURONOISE: Noise Mapping Techniques and Uncertainties II (Poster Session). P2-A, Level 2

5:20 2pNSn Noise and EURONOISE: Railway Noise and Vibration II. Room 251

5:20 2pNSo Noise and EURONOISE: Noise, Structure Borne Noise from Building Technical Equipment, and Ground Borne Noise from Railways II. Room 252A

2:00 2pPAa Physical Acoustics: Nonlinear Acoustics in Earthquake Processes and Other Earth Processes I. Amphi Havane

2:20 2pPAb Physical Acoustics: Nonlinear Acoustics of Unconsolidated Granular Media I. Room 362/363 
Physical Acoustics: Diffraction of Waves on Periodical Structures:

Acoustic, Ultrasonic and Acousto-Optical Diffraction Phenomena II (Poster Session). P3-A, Level 3

3:40 2pPAd Physical Acoustics: Outdoor Sound Propagation and Uncertainties II (Poster Session). P3-A, Level 3

3:40 2pPAe Physical Acoustics: Phononic Crystals II (Poster Session). P3-A, Level 3

3:40 2pPAf Physical Acoustics: Acoustics of Porous Media III (Poster Session). P3-A, Level 3

3:40 2pPAg Physical Acoustics: Photoacoustics II (Poster Session). P3-A, Level 3

3:40 2pPAh Physical Acoustics and Signal Processing in Acoustics: Acoustic Landmine Detection II (Poster Session). P3-A, Level 3

3:40 2pPAi Physical Acoustics: Nonlinear Acoustics of Unconsolidated Granular Media II (Poster Session). P3-A, Level 3

3:40 2pPAj Physical Acoustics: Nonlinear Acoustics in Earthquake Processes and Other Earth Processes II (Poster Session). P3-A, Level 3

3:40 2pPAk Physical Acoustics: Sonic, Ultrasonic, and Megasonic Cleaning II (Poster Session). P3-A, Level 3

3:40 2pPAl Physical Acoustics: Mathematical and Numerical Methods II (Poster Session). P3-A, Level 3

3:40 $2 \mathrm{pPPa} \quad$ Psychological and Physiological Acoustics: General Topics in Psychological and Physiological Acoustics I (Poster Session). P2-C, Level 2

3:40 $2 \mathrm{pPPb} \quad$ Psychological and Physiological Acoustics and ASA Committee on Standards: Applications of Psychoacoustics II (Poster Session). P2-C, Level 2

3:40 2pPPc Psychological and Physiological Acoustics: Loudness, from Controlled Stimuli to Environmental Sounds I (Poster Session). P2-C, Level 2

3:40 2pPPd Psychological and Physiological Acoustics: Integrated Approaches to Auditory Scene Analysis II (Poster Session). P2-C, Level 2

3:40 2pPPe Psychological and Physiological Acoustics: Cochlear Implants: Going Beyond the Envelope II (Poster Session). P2-C, Level 2

\section{3:40 2pPPf}

$5: 20 \quad 2 p P P g$

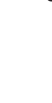

2:00 2pSAa

$3: 40 \quad 2 \mathrm{pSAb}$

in Confined Space I. Room 252B

(1)
Structural Acoustics and Vibration and EURONOISE: Source

Characterization in Structure Borne

Noise Problems II (Poster Session).

P2-B, Level 2

3:40 2pSAc

Structural Acoustics and Vibration and EURONOISE: Acoustic Imaging in Confined Space II (Poster Session). P2-B, Level 2

3:40 2pSAd Structural Acoustics and Vibration and EURONOISE: Vibration and Radiation from Complex Structural Systems III (Poster Session). P2-B, Level 2

3:40 2pSAe Structural Acoustics and Vibration and EURONOISE: General Topics in Structural Acoustics and Vibration III (Poster Session). P2-B, Level 2

2:00 2pSCa Speech Communication: Speech Perception II. Room 240

2:00 2pSCb Speech Communication: Speech Articulation I. Room 250B

3:40 2pSCc Speech Communication: General Topics in Speech Communication II (Poster Session). P2-D, Level 2

2:00 $2 \mathrm{pSPa} \quad$ Signal Processing in Acoustics: Beamforming, Localization, and Tracking I. Room 343

3:40 $2 \mathrm{pSPb}$ Signal Processing in Acoustics: Beamforming, Localization, and Tracking II (Poster Session). P3-C, Level 3

3:40 2pSPc Signal Processing in Acoustics: Acoustical Nondestructive Evaluation, Ultrasonics, and Imaging II (Poster Session). P3-C, Level 3

3:40 2pSPd Signal Processing in Acoustics and Physical Acoustics: Biomedical Applications of Time-Reversal II (Poster Session). P3-C, Level 3

3:40 2pSPe Signal Processing in Acoustics, Acoustical Oceanography, and ECUA: Bayesian Signal Processing I (Poster Session). P3-C, Level 3 
$3: 40 \quad 2 p S P f$

Signal Processing in Acoustics, Biomedical Ultrasound/Bioresponse to Vibration, and Underwater Acoustics: Time Reversal Methods for Array Ima ging and Signal Processing II (Poster Session). P3-C, Level 3

3:40 2pSPg Signal Processing in Acoustics, Physical Acoustics, Biomedical Ultrasound/Bioresponse to Vibration, and Underwater Acoustics: Overview of Time Reversal in Acoustics II (Poster Session). P3-C, Level 3

5:00 2pSPh Signal Processing in Acoustics:

Advances in Acoustic Sensors and

Networks for Defense Applications I.

Room 343

2:00 2pUWa Underwater Acoustics and ECUA

Auralization of Sonar Signals. Amphi Bordeaux

2:00 $2 \mathrm{pUWb}$ Underwater Acoustics and ECUA:

Sonar System and Transducer

Calibration Methodology I. Room 341

3:40 2pUWd Underwater Acoustics and ECUA:

Sonar System and Transducer

Calibration Methodology II (Poster

Session). P3-C, Level 3

3:40 2pUWe Underwater Acoustics and ECUA:

Geoacoustic Sediment Modeling I

(Poster Session). P3-C, Level 3

3:40 2pUWf Underwater Acoustics, Signal

Processing in Acoustics and ECUA:

Acoustic Vector Fields and Sensor

Processing I (Poster Session). P3-C, Level 3

3:40 2pUWg Underwater Acoustics and ECUA:

High Frequency Variability I (Poster

Session). P3-C, Level 3

\section{WEDNESDAY MORNING}

8:00 3aAAa Architectural Acoustics: Case Studies and Design Approaches I. Room 242B

8:00 $3 \mathrm{aAAb}$ Architectural Acoustics: Prediction Methods in Building Acoustics I. Room 243

8:00 3aABa Animal Bioacoustics, Underwater Acoustics, Acoustical Oceanography, Signal Processing in Acoustics, and ECUA: Animal Sonar Systems III. Room 342B

10:20 $3 \mathrm{aABb}$ Animal Bioacoustics and ECUA: Odontocete Acoustics I. Room 342B
8:00 3aAO Acoustical Oceanography, Underwater Acoustics, Signal Processing in Acoustics, and ECUA: Geoacoustic Characterization of the Ocean Bottom and Geoacoustic Inversion III. Room $342 \mathrm{~A}$

8:00 $3 \mathrm{aBBa} \quad$ Biomedical Ultrasound/Bioresponse to Vibration and Physical Acoustics:

Shock Waves in Medicine. Room 352B

11:00 $3 \mathrm{aBBb} \quad$ Biomedical Ultrasound/Bioresponse to Vibration and Physical Acoustics:

Light and Sound for Medical Imaging and Therapy I. Room 352B

8:00 3aEAa Engineering Acoustics and Signal Processing in Acoustics: Transducers and Signal Processing for the Oil and Gas Industry I. Room 353

10:20 $3 \mathrm{aEAb} \quad$ Engineering Acoustics: Ultrasonic Acoustics MEMS I. Room 353

8:00 3aMUa Musical Acoustics and Speech Communication: Interdisciplinary Research on the Science of Singing: A Tribute to Johan Sundberg. Amphi Maillot

11:00 3aMUb Musical Acoustics: Plucked Stringed Instruments I. Amphi Maillot

8:00 3aNSa Noise, Physical Acoustics, and EURONOISE: Aeroacoustics IV. Room 250A

8:00 3aNSb Noise and EURONOISE: Railway Noise and Vibration III. Room 251

8:00 3aNSc Noise, Computational Acoustics, and EURONOISE: Source Identification in Radiation and Scattering I. Room 252A

8:00 3aNSd Noise and EURONOISE: Tire-Road Noise from the Road Perspective I. Room 253

10:00 3aNSe Noise, Structural Acoustics and Vibration, Signal Processing in Acoustics, and EURONOISE:

Airframe Noise Measurement, Prediction, and Control I. Room 250A

10:40 3aNSf Noise and EURONOISE: Soundscape in the Heritage of Urban and Natural Areas I. Room 251

8:00 3aPAa Physical Acoustics: Nonlinear Acoustics of Consolidated Materials and Non Destructive Testing I. Amphi Havane

8:00 3aPAb Physical Acoustics: Acoustic Probes of Planetary Environments I. Room 351

8:00 3aPAc Physical Acoustics and Computational Acoustics: Combustion Noise and Thermo-Acoustics I. Room 352A

8:00 3aPAd Physical Acoustics: Ultrasonics: Material Characterization I. Room $362 / 363$ 
9:20 3aPAe Physical Acoustics: Ultrasonics: NDT and Layered Systems I. Room 362/363

11:20 3aPAf Physical Acoustics: Ultrasonics: Industrial NDT I. Room 351

8:00 3aPPa Psychological and Physiological Acoustics and ASA Committee on Standards: Applications of Psychoacoustics III. Room 241

8:00 $3 \mathrm{aPPb} \quad$ Psychological and Physiological Acoustics: Auditory Perception of Sound Source Properties I. Room 242A

9:00 3aPPc Psychological and Physiological Acoustics, Architectural Acoustics, Noise, and Signal Processing in Acoustics: Jens Blauert and His Contributions. Room 241

8:00 3aSAa Structural Acoustics and Vibration, Computational Acoustics, and EURONOISE: Efficient Boundary Element Methods I. Room 252B

10:40 3aSAb Structural Acoustics and Vibration, Computational Acoustics, and EURONOISE: Fluid-Structure Interaction I. Room 252B

8:00 3aSCa Speech Communication: Speech Prosody and How it Relates to Segmental Aspects of Speech. Room 250B

8:40 3aSCb Speech Communication: Speech Perception III. Room 240

10:40 3aSCc Speech Communication: Speech Articulation II. Room 240

10:40 3aSCd Speech Communication: Prosody I. Room 250B

8:00 $\quad 3 \mathrm{aSPa} \quad$ Signal Processing in Acoustics and Physical Acoustics: Biomedical Applications of Time-Reversal II. Room 343

11:00 3aSPb Signal Processing in Acoustics: Filter Design, Detection and Estimation I. Room 343

8:00 3aUWa Underwater Acoustics, Acoustical Oceanography, and ECUA: Impact of Environmental Variability on Mid-Frequency Sonar Performance. Amphi Bordeaux

8:00 3aUWb Underwater Acoustics and ECUA: Sonar System and Transducer Calibration Methodology III. Room 341

10:40 3aUWc Underwater Acoustics, Signal Processing in Acoustics, and ECUA: Acoustic Vector Fields and Sensor Processing II. Room 341

11:20 3aUWd Underwater Acoustics and ECUA: Geoacoustic Sediment Modeling II. Room 342A
WEDNESDAY MORNING SESSIONS CONTINUED

1:40 3aAAb Room 243

$1: 40 \quad 3 \mathrm{aBBb} \quad$ Room 352B

2:00 3aEAb Room 353

1:40 3aPAa Amphi Havane

2:00 3aSAb Room 252B

2:00 3aUWd Room 342A

\section{WEDNESDAY AFTERNOON}

2:00 3pAA Architectural Acoustics: Architectural Acoustics Potpourri I. Room 242B

2:00 3pAB Animal Bioacoustics, Psychological and Physiological Acoustics, and ECUA: Auditory Brainstem Response and Behavior Correlation I. Room 342B

1:40 3pMUa Musical Acoustics: Bowed and Keyboard Stringed Instruments I. Amphi Maillot

1:40 3pMUb Musical Acoustics: Wind Instruments I. Room 202/203

1:40 3pNSa Noise, ASA Committee on Standards, and EURONOISE: Sleep Disturbances and Other Health Effects. Room 252A

$2: 00 \quad 3 p N S b \quad$ Noise, ASA Committee on Standards, and EURONOISE: Prominent Discrete Tones I. Room 251

2:20 3pNSc Noise and EURONOISE: Car Acoustics I. Room 253

1:40 3pPAa Physical Acoustics: Quantum Acoustics I. Room 351

2:00 3pPAb Physical Acoustics: General Topics in Nonlinear Acoustics I. Room 352A

2:00 $\quad 3 \mathrm{pPPa} \quad$ Psychological and Physiological Acoustics: General Topics in Psychological and Physiological Acoustics II. Room 241

2:00 $\quad 3 \mathrm{pPPb} \quad$ Psychological and Physiological Acoustics: General Topics in Psychological and Physiological Acoustics III. Room 242A

2:00 3pSCa Speech Communication: Phonetics. Room 240

2:00 3pSCb Speech Communication: Prosody II. Room 250B

2:00 3pSP Signal Processing in Acoustics, Acoustical Oceanography, and ECUA: Model-Based Signal Processing I. Room 343

2:00 3pUWa Underwater Acoustics and ECUA: Fluctuations and Statistics. Amphi Bordeaux

2:00 3pUWb Underwater Acoustics and ECUA: Array Processing. Room 341 
2:00 3pUWc Underwater Acoustics and ECUA:

Objects Scattering and Detection. Room 362/363

$3: 40$

Awards Ceremony: Amphi Bleu/Amphi Bordeaux

\section{WEDNESDAY EVENING}

$5: 40$

$5: 40$

$5: 40$

Plenary Lectures: Amphi Bordeaux

Acoustical Oceanography Prize

Lecture: Amphi Havane

60th Anniversary Celebration, SFA:

Amphi Bleu

\section{THURSDAY MORNING}

\begin{tabular}{|c|c|c|}
\hline $8: 00$ & 4aAAa & $\begin{array}{l}\text { Architectural Acoustics: Architectural } \\
\text { Acoustics Potpourri II. Room 242B }\end{array}$ \\
\hline 8:00 & $4 \mathrm{aAAb}$ & $\begin{array}{l}\text { Architectural Acoustics: Prediction } \\
\text { Methods in Building Acoustics II. Room } \\
243\end{array}$ \\
\hline $10: 00$ & $4 \mathrm{aAAc}$ & $\begin{array}{l}\text { Architectural Acoustics: Measuring } \\
\text { Methods and Uncertainty in Building } \\
\text { Acoustics I. Room } 243\end{array}$ \\
\hline 8:00 & $4 \mathrm{aABa}$ & $\begin{array}{l}\text { Animal Bioacoustics, Psychological } \\
\text { and Physiological Acoustics, and } \\
\text { ECUA: Auditory Brainstem Response } \\
\text { and Behavior Correlation II. Room } \\
\text { 342B }\end{array}$ \\
\hline $10: 40$ & $4 a A B b$ & $\begin{array}{l}\text { Animal Bioacoustics: Sound Production } \\
\text { and Reception in Amphibious Marine } \\
\text { Mammals. Room 342B }\end{array}$ \\
\hline $11: 00$ & $4 \mathrm{aAO}$ & $\begin{array}{l}\text { Acoustical Oceanography, Signal } \\
\text { Processing in Acoustics, and ECUA: } \\
\text { Adjoint Modeling for Geoacoustic } \\
\text { Inversion. Room } 342 \mathrm{~A}\end{array}$ \\
\hline $8: 00$ & $4 \mathrm{aBB}$ & $\begin{array}{l}\text { Biomedical Ultrasound/Bioresponse to } \\
\text { Vibration: Theoretical and } \\
\text { Computational Models of Ultrasonic } \\
\text { Propagation in Bones I. Room 352B }\end{array}$ \\
\hline $8: 00$ & $4 \mathrm{aEA}$ & $\begin{array}{l}\text { Engineering Acoustics: Sound Fields I. } \\
\text { Room } 353\end{array}$ \\
\hline $8: 00$ & 4aEDa & $\begin{array}{l}\text { Education in Acoustics: Acoustics in } \\
\text { the Public School Science Classrooms. } \\
\text { Room 202/203 }\end{array}$ \\
\hline $11: 20$ & $4 \mathrm{aEDb}$ & $\begin{array}{l}\text { Education in Acoustics: Take 5's. } \\
\text { Room 202/203 }\end{array}$ \\
\hline 8:00 & 4aMU & $\begin{array}{l}\text { Musical Acoustics: Virtual Musical } \\
\text { Instruments I. Amphi Maillot }\end{array}$ \\
\hline $8: 00$ & 4aNSa & $\begin{array}{l}\text { Noise and EURONOISE: EU Projects } \\
\text { for Aircraft Noise Reduction II. Room } \\
250 \mathrm{~A}\end{array}$ \\
\hline $8: 00$ & $4 \mathrm{aNSb}$ & $\begin{array}{l}\text { Noise, ASA Committee on Standards } \\
\text { and EURONOISE: Measurement of } \\
\text { Occupational Noise Exposure I. Room } \\
251\end{array}$ \\
\hline
\end{tabular}

8:00 4aNSc Noise, Computational Acoustics, and EURONOISE: Time-Domain Modeling Methods in Acoustics II. Room 252A

8:00 4aNSd Noise and EURONOISE: Car Acoustics II. Room 253

10:20 4aNSe Noise and EURONOISE: Noise from Wind Power Projects I. Room 250A

10:40 4aNSf Noise and EURONOISE: Fan Noise and Low-Mach Number Rotating Blade Noise I. Room 252A

8:00 4aPAa Physical Acoustics: Thermoacoustics I. Amphi Havane

8:00 4aPAb Physical Acoustics: Ultrafast Acoustics I. Room 351

8:00 4aPAc Physical Acoustics, Acoustical Oceanography, and Biomedical Ultrasound/Bioresponse to Vibration: Acoustically Activated Bubble Dynamics and Applications I. Room 352A

8:00 $\quad 4 \mathrm{aPPa} \quad$ Psychological and Physiological Acoustics: General Topics in Psychological and Physiological Acoustics IV. Room 241

8:40 $4 \mathrm{aPPb} \quad$ Psychological and Physiological Acoustics: General Topics in Psychological and Physiological Acoustics V. Room 242A

8:00 4aSAa Structural Acoustics and Vibration, Computational Acoustics, and EURONOISE: Fluid-Structure Interaction II. Room 252B

11:40 4aSAb Structural Acoustics and Vibration and EURONOISE: Distributed Active Noise and Vibration Control I. Room 252B

8:40 4aSCa Speech Communication: Acoustics of Speech Production: Aeroacoustics and Phonation. Room 240

8:40 4aSCb Speech Communication: Neurobiology of Speech Perception. Room 250B

8:00 4aSPa Signal Processing in Acoustics, Acoustical Oceanography, and ECUA: Model-Based Signal Processing II. Room 343

10:40 4aSPb Signal Processing in Acoustics, Acoustical Oceanography, and ECUA: Bayesian Signal Processing II. Room 343.

8:00 4aUWa Underwater Acoustics, Acoustical Oceanography, and ECUA: Impact of Internal Waves on Shallow Water Propagation. Amphi Bordeaux

8:00 $4 \mathrm{aUWb}$ Underwater Acoustics and ECUA: Determination of Acoustic Properties of Materials for Sonar Applications I. Room 341 
4aUWc Underwater Acoustics and ECUA: Geoacoustic Sediment Modeling III. Room 342A

8:00 4aUWd Underwater Acoustics and ECUA: Propagation and Reverberation. Room 362/363

11:40 4aUWe Underwater Acoustics and ECUA: Tank Experiments I. Room 341

\section{THURSDAY MORNING SESSION CONTINUED}

$\begin{array}{lll}\text { 1:40 } & 4 \mathrm{aAAc} & \text { Room 243 } \\ \text { 2:00 } & 4 \mathrm{aNSf} & \text { Room 252A } \\ \text { 1:40 } & 4 \mathrm{aPAa} & \text { Amphi Havane } \\ \text { 1:40 } & 4 \mathrm{aPAb} & \text { Room 351 } \\ \text { 1:40 } & 4 \mathrm{aPAc} & \text { Room 352A } \\ 2: 00 & 4 \mathrm{aPPb} & \text { Room 242A } \\ 2: 00 & 4 \mathrm{aSAb} & \text { Room 252B } \\ 2: 00 & 4 \mathrm{aSCa} & \text { Room 240 } \\ 2: 00 & 4 \mathrm{aSPb} & \text { Room 343 } \\ 1: 40 & 4 \mathrm{aUWa} & \text { Amphi Bordeaux } \\ 2: 00 & 4 \mathrm{aUWe} & \text { Room 341 }\end{array}$

\section{THURSDAY AFTERNOON}

1:20 4pAAa Architectural Acoustics: Archeological Acoustics I. Room 242B

3:40 4pAAb Architectural Acoustics: Archeological Acoustics II (Poster Session). P2-B, Level 2

3:40 4pAAc Architectural Acoustics and Psychological and Physiological Acoustics: Speech Segregation in Rooms II (Poster Session). P2-B, Level 2

3:40 4pAAd Architectural Acoustics: Architectural Acoustics Potpourri III (Poster Session). P2-B, Level 2

3:40 4pAAe Architectural Acoustics and Noise: Acoustics and Privacy in Healthcare Facilities II: Emerging Research Around the World II (Poster Session). P2-B, Level 2

3:40 4pAAf Architectural Acoustics: Case Studies and Design Approaches II (Poster Session). P2-B, Level 2

3:40 4pAAg Architectural Acoustics: Prediction Methods in Building Acoustics III (Poster Session). P2-B, Level 2

3:40 4pAAh Architectural Acoustics: Measuring Methods and Uncertainty in Building Acoustics II (Poster Session). P2-B, Level 2

3:40 4pAAi Architectural Acoustics and the National Council of Acoustical Consultants: Student Design Competition. P2-B, Level 2
$3: 40 \quad 4 p A B b$

Animal Bioacoustics, Psychological and Physiological Acoustics, and ECUA: Auditory Brainstem Response and Behavior Correlation III (Poster Session). P3-C, Level 3

3:40 4pABc Animal Bioacoustics and ECUA: Odontocete Acoustics II (Poster Session). P3-C, Level 3

5:20 4pABd Animal Bioacoustics: General Topics in Animal Bioacoustics I. Room 343

2:00 4pAOa Acoustical Oceanography, Underwater Acoustics, and ECUA: Rapid

Environmental Assessment. Room 342A

3:40 4pAOb Acoustical Oceanography, Signal Processing in Acoustics, and ECUA: Passive Acoustic Tomography I (Poster Session). P3-C, Level 3

3:40 4pAOc Acoustical Oceanography and ECUA: General Topics in Acoustical Oceanography I (Poster Session). P3-C, Level 3

3:40 4pAOd Acoustical Oceanography and ECUA: Acoustic Characterization of Sea Floor Habitats I (Poster Session). P3-C, Level 3

5:20 4pAOg Acoustical Oceanography and ECUA: Acoustic Characterization of Sea Floor Habitats II. Room 342A

5:40 4pAOh Acoustical Oceanography, Signal Processing in Acoustics, and ECUA: Passive Acoustic Tomography II. Amphi Bordeaux

2:00 $\quad 4 \mathrm{pBBa} \quad$ Biomedical Ultrasound/Bioresponse to Vibration: Ultrasonic Characterization of Bone I. Room 352B

3:40 $4 \mathrm{pBBb} \quad$ Biomedical Ultrasound/Bioresponse to Vibration: Ultrasonic Characterization of Bone II (Poster Session). P3-B, Level 3

3:40 $4 \mathrm{pBBc} \quad$ Biomedical Ultrasound/Bioresponse to Vibration: Theoretical and Computational Models of Ultrasonic Propagation in Bones II (Poster Session). P3-B, Level 3

3:40 $4 \mathrm{pBBd} \quad$ Biomedical Ultrasound/Bioresponse to Vibration and Physical Acoustics:

Light and Sound for Medical Imaging and Therapy II (Poster Session). P3-B, Level 3

2:00 4pEAa Engineering Acoustics: Transducers I. Room 353

2:20 4pEAb Engineering Acoustics, Underwater Acoustics, Acoustical Oceanography, and ECUA: Acoustics in Marine Archeology. Room 342B

3:40 4pEAc Engineering Acoustics: Ultrasonic Acoustic MEMS II (Poster Session). P3-C, Level 3 
Processing in Acoustics: Transducers and Signal Processing for the Oil and Gas Industry II (Poster Session). P3-C, Level 3

3:40 4pEAe Engineering Acoustics: Transducers II (Poster Session). P3-C, Level 3

3:40 4pEAf Engineering Acoustics: Sound Fields II (Poster Session). P3-C, Level 3

2:20 4pED Education in Acoustics: Acoustics Education Software. Room 202/203

1:40 4pMUa Musical Acoustics: Control of Natural and Synthetic Musical Sounds I. Amphi Maillot

3:40 4pMUb Musical Acoustics: Bowed and Keyboard Stringed Instruments II (Poster Session). P2-D, Level 2

3:40 4pMUc Musical Acoustics: Plucked Stringed Instruments II (Poster Session). P2-D, Level 2

3:40 4pMUd Musical Acoustics: Wind Instruments II (Poster Session). P2-D, Level 2

3:40 4pMUe Musical Acoustics: Control of Natural and Synthetic Musical Sounds II (Poster Session). P2-D, Level 2

3:40 4pMUf Musical Acoustics: Virtual Musical Instruments II (Poster Session). P2-D, Level 2

2:00 4pNSa Noise and EURONOISE: Vibration Perception I. Room 250A

2:00 $4 \mathrm{pNSb} \quad$ Noise, Architectural Acoustics, and EURONOISE: Noise, Vibration and Acoustics for Medical and Research Facilities and Their Occupants I. Room 251

2:00 4pNSc Noise and EURONOISE: Potential to Reduce Tire/Road Noise I. Room 253

3:40 4pNSd Noise and EURONOISE: Cultural Variations in Sound/Noise Assessment I (Poster Session). P2-A, Level 2

3:40 4pNSf Noise and EURONOISE: Car Acoustics III (Poster Session). P2-A, Level 2

$3: 40 \quad 4 \mathrm{pNSg} \quad$ Noise, Architectural Acoustics, and EURONOISE: Noise, Vibration and Acoustics for Medical and Research Facilities and Their Occupants II (Pos ter Session). P2-A, Level 2

3:40 4pNSh Noise, ASA Committee on Standards, and EURONOISE: Measurement of Occupational Noise Exposure II (Poster Session). P2-A, Level 2

3:40 4pNSi Noise, Structural Acoustics and Vibration, Signal Processing in Acoustics, and EURONOISE: Airframe Noise Measurement, Prediction, and Control II (Poster Session). P2-A, Level 2
3:40 4pNSj Noise and EURONOISE: Soundscape in the Heritage of Urban and Natural Areas II (Poster Session). P2-A, Level 2

3:40 4pNSk Noise and EURONOISE: Potential to Reduce Tire/Road Noise II (Poster Session). P2-A, Level 2

3:40 4pNS1 Noise and EURONOISE: Noise from Wind Power Projects II (Poster Session). P2-A, Level 2

3:40 4pNSm Noise and EURONOISE: Vibration Perception II (Poster Session). P2-A, Level 2

3:40 4pNSn Noise, Computational Acoustics, and EURONOISE: Source Identification in Radiation and Scattering II (Poster Session). P2-A, Level 2

3:40 4pNSo Noise, ASA Committee on Standards, and EURONOISE: Prominent Discrete Tones II (Poster Session). P2-A, Level 2

3:40 4pNSp Noise and EURONOISE: Tire-Road Noise from the Road Perspective II (Poster Session). P2-A, Level 2

$3: 40 \quad 4 p N S q$ Noise and EURONOISE: Fan Noise and Low-Mach Number Rotating Blade Noise II (Poster Session). P2-A, Level 2

5:20 4pNSr Noise and EURONOISE: Cultural Variations in Sound/Noise Assessment II. Room 250A

3:40 4pPAa Physical Acoustics: Ultrasonics: Material Characterization II (Poster Session). P3-A, Level 3

3:40 $\quad 4 \mathrm{pPAb} \quad$ Physical Acoustics and Computational Acoustics: Combustion Noise and Thermo-Acoustics II (Poster Session). P3-A, Level 3

4:00 4pPAc Physical Acoustics: Quantum Acoustics II (Poster Session). P3-A, Level 3

3:40 4pPAd Physical Acoustics: General Topics in Nonlinear Acoustics II (Poster Session). P3-A, Level 3

3:40 4pPAe Physical Acoustics: Ultrasonics: NDT and Layered Systems II (Poster Session). P3-A, Level 3

3:40 4pPAf Physical Acoustics: Ultrasonics: Industrial NDT II (Poster Session). P3-A, Level 3

3:40 4pPAg Physical Acoustics: Nonlinear Acoustics of Consolidated Materials and Non Destructive Testing II (Poster Session). P3-A, Level 3

3:40 4pPAh Physical Acoustics: Acoustic Probes of Planetary Environments II (Poster Session). P3-A, Level 3

3:40 4pPAi Physical Acoustics, Acoustical Oceanography, and Biomedical Ultrasound/Bioreponse to Vibration: Acoustically Activated Bubble Dynamics and Applications II (Poster Session). P3-A, Level 3 


\begin{tabular}{|c|c|c|}
\hline $3: 40$ & $4 \mathrm{pPAj}$ & $\begin{array}{l}\text { Physical Acoustics: Ultrafast Acoustics } \\
\text { II (Poster Session). P3-A, Level } 3\end{array}$ \\
\hline $3: 40$ & 4pPAk & $\begin{array}{l}\text { Physical Acoustics: Thermoacoustics II } \\
\text { (Poster Session). P3-A, Level } 3\end{array}$ \\
\hline 2:00 & $4 \mathrm{pPPa}$ & $\begin{array}{l}\text { Psychological and Physiological } \\
\text { Acoustics: Role of Temporal Fine } \\
\text { Structure in Speech and Non-Speech } \\
\text { Perception for Normal and } \\
\text { Hearing-Impaired People I. Room } 241\end{array}$ \\
\hline $3 \cdot 40$ & $4 \mathrm{pPPb}$ & $\begin{array}{l}\text { Psychological and Physiological } \\
\text { Acoustics: Role of Temporal Fine } \\
\text { Structure in Speech and Non-Speech } \\
\text { Perception for Normal and } \\
\text { Hearing-Impaired People II (Poster } \\
\text { Session). P2-C, Level } 2\end{array}$ \\
\hline 3.40 & $4 \mathrm{pPPc}$ & $\begin{array}{l}\text { Psychological and Physiological } \\
\text { Acoustics: General Topics in } \\
\text { Psychological and Physiological } \\
\text { Acoustics VI (Poster Session). P2-C, } \\
\text { Level } 2\end{array}$ \\
\hline 3.40 & $4 \mathrm{pPPd}$ & $\begin{array}{l}\text { Psychological and Physiological } \\
\text { Acoustics: Auditory Perception of } \\
\text { Sound Source Properties II (Poster } \\
\text { Session). P2-C, Level } 2\end{array}$ \\
\hline $3: 40$ & $4 \mathrm{pSAa}$ & $\begin{array}{l}\text { Structural Acoustics and Vibration, } \\
\text { Computational Acoustics, and } \\
\text { EURONOISE: Efficient Boundary } \\
\text { Element Methods II (Poster Session). } \\
\text { P2-B, Level } 2\end{array}$ \\
\hline & $4 \mathrm{pSAb}$ & Structural Acoustics and Vibration \\
\hline
\end{tabular}
and EURONOISE: Distributed Active Noise and Vibration Control II (Poster Session). P2-B, Level 2

3:40 4pSAc Structural Acoustics and Vibration, Computational Acoustics, and EURONOISE: Fluid-Structure Interaction III (Poster Session). P2-B, Level 2

2:00 4pSCa Speech Communication: Articulatory Modeling and Control of Speech and Singing Organs. Room 250B

3:40 4pSCb Speech Communication: General Topics in Speech Communication III (Poster Session). P2-D, Level 2

3:40 4pSPa Signal Processing in Acoustics, Acoustical Oceanography, and ECUA: Model-Based Signal Processing III (Poster Session). P3-C, Level 3

3:40 $4 \mathrm{pSPb} \quad$ Signal Processing in Acoustics: Advances in Acoustic Sensors and Networks for Defense Applications II (Poster Session). P3-C, Level 3

3:40 4pSPc Signal Processing in Acoustics: Filter Design, Detection, and Estimation II (Poster Session). P3-C, Level 3

2:00 4pUWa Underwater Acoustics and ECUA: Image and Signal Processing. Room $362 / 363$
3:00 4pUWb Underwater Acoustics and ECUA: High Frequency Variability II. Amphi Bordeaux

3:40 4pUWc Underwater Acoustics and ECUA: Tank Experiments II (Poster Session). P3-C, Level 3

3:40 4pUWd Underwater Acoustics and ECUA: Determination of Acoustic Properties of Materials for Sonar Applications II (Poster Session). P3-C, Level 3

3:40 4pUWe Underwater Acoustics and ECUA: General Topics in Underwater Acoustics (Poster Session). P3-C, Level 3

3:40 4pUWf Underwater Acoustics and ECUA: Sound Propagation in 3-Dimensional Environments I (Poster Session). P3-C, Level 3

5:20 4pUWg Underwater Acoustics and ECUA: Nonlinear Acoustic Methods in Searching for Buried Objects. Room 342B

5:20 4pUWh Underwater Acoustics, Computational Acoustics, and ECUA: Numerical Methods in Underwater Scattering. Room 362/363

\section{FRIDAY MORNING}

8:00 5aAAa Architectural Acoustics: New Frontiers in Room Acoustical Modeling I. Room 242B

8:00 5aAAb Architectural Acoustics and Noise: Airborne and Impact Sound Insulation I. Room 243

9:40 5aAAc Architectural Acoustics and Noise: Airborne and Impact Sound Insulation II (Poster Session). P2-B, Level 2

9:40 5aAAd Architectural Acoustics: Coupled Volume Acoustics I (Poster Session). P2-B, Level 2

9:40 5aAAe Architectural Acoustics: New Frontiers in Room Acoustical Modeling II (Poster Session). P2-B, Level 2

9:40 5aAAf Architectural Acoustics and Musical Acoustics: New Measurement Parameters in Performing Arts Spaces I (Poster Session). P2-B, Level 2

8:00 5aABa Animal Bioacoustics: General Topics in Animal Bioacoustics II. Amphi Bleu

9:40 5aABb Animal Bioacoustics: General Topics in Animal Bioacoustics III (Poster Session). P3-C, Level 3

11:00 5aAO Acoustical Oceanography and ECUA: General Topics in Acoustical Oceanography II. Room 343 
8:00

$5 \mathrm{aBBa}$ Biomedical Ultrasound/Bioresponse to Vibration and Engineering Acoustics: Transducers for Medical Imaging and Therapy I. Room 353

$8: 20 \quad 5 \mathrm{aBBb} \quad$ Biomedical Ultrasound/Bioresponse to Vibration: Ultrasonic Characterization of Bone III. Room 352B

9:40 $5 \mathrm{aBBc}$ Biomedical Ultrasound/Bioresponse to Vibration and Engineering Acoustics: Transducers for Medical Imaging and Therapy II (Poster Session). P3-B, Level 3

9:40 5aBBd Biomedical Ultrasound/Bioresponse to Vibration: General Topics in

Biomedical Ultrasound/Bioresponse to Vibration (Poster Session). P3-B, Level 3

9:40 $5 \mathrm{aBBe} \quad$ Biomedical Ultrasound/Bioresponse to Vibration: Biomedical Applications of Acoustic Radiation Force I (Poster Session). P3-B, Level 3

11:00 5aBBf Biomedical Ultrasound/Bioresponse to Vibration: Biomedical Applications of Acoustic Radiation Force II. Room 352B

8:00 5aED Education in Acoustics: General Topics in Education in Acoustics. Room 202/203

8:00 5aMUa Musical Acoustics: Control of Natural and Synthetic Musical Sounds III. Amphi Maillot

9:40 5aMUb Musical Acoustics: General (Mode Conversion, Radiation, and Perception of Musical Sound) (Poster Session). P2-D, Level 2

9:40 5aMUc Musical Acoustics: Plucked and Struck Idiophones I (Poster Session). P2-D, Level 2

9:40 5aMUd Musical Acoustics and Signal Processing in Acoustics: Signal Representations and Models of Musical Sounds I (Poster Session). P2-D, Level 2

11:00 5aMUe Musical Acoustics and Signal Processing in Acoustics: Signal Representations and Models of Musical Sounds II. Amphi Maillot

11:00 5aMUf Musical Acoustics: Plucked and Struck Idiophones II. Room 202/203

8:00 5aNSa Noise and EURONOISE: Soundscape \& Community Noise I. Room 250A

8:00 5aNSb Noise, Architectural Acoustics, and EURONOISE: Noise, Vibration and Acoustics for Medical and Research Facilities and Their Occupants III. Room 251
8:00 5aNSc Noise and EURONOISE: Acoustic Performance of Energy Efficient Building Products I. Room 252A
9:40 5aNSd Noise, ASA Committee on Standards, Architectural Acoustics, and EURONOISE: Classroom Acoustics I (Poster Session). P2-A, Level 2
9:40 5aNSe Noise and EURONOISE: Examples of Noise Control I (Poster Session). P2-A, Level 2
9:40 5aNSf Noise and EURONOISE: Soundscape \& Community Noise II (Poster Session). P2-A, Level 2
9:40 5aNSg Noise and EURONOISE: Propagation and Urban Noise I (Poster Session). P2-A, Level 2
9:40 5aNSh Noise, Structural Acoustics and Vibration, Physical Acoustics, and EURONOISE: Sound and Vibration from Explosions in Air I (Poster Session). P2-A, Level 2
$9: 40 \quad 5 \mathrm{aNSi}$
Noise and EURONOISE: Acoustic Performance of Energy Efficient Building Products II (Poster Session). P2-A, Level 2

11:00 5aNSj Noise and EURONOISE: Examples of Noise Control II. Room 251

8:00 5aPAa Physical Acoustics: Infrasound I. Amphi Havane

8:00 5aPAb Physical Acoustics: Time Reversal Acoustics for Nonlinear Imaging I. Room 351

8:00 5aPAc Physical Acoustics: Vibrations in Plates I. Room 352A

9:40 5aPAd Physical Acoustics: Time Reversal Acoustics for Nonlinear Imaging II (Poster Session). P3-A, Level 3

9:40 5aPAe Physical Acoustics: Ultrasonics: Transducers and Instruments I (Poster Session). P3-A, Level 3

9:40 5aPAf Physical Acoustics: Infrasound II (Poster Session). P3-A, Level 3

9:40 5aPAg Physical Acoustics: Ultrasonics Under Extreme Conditions I (Poster Session). P3-A, Level 3

9:40 5aPAh Physical Acoustics: Ducts and Waveguides I (Poster Session). P3-A, Level 3

9:40 5aPAi Physical Acoustics: General Topics in Physical Acoustics I (Poster Session) P3-A, Level 3

9:40 5aPAj Physical Acoustics: Scattering and Diffraction I (Poster Session). P3-A, Level 3

9:40 5aPAk Physical Acoustics: Vibrations in Plates II (Poster Session). P3-A, Level 3 
11:20 5aPAl Physical Acoustics: Ultrasonics:

Transducers and Instruments II. Room $352 \mathrm{~A}$

8:00 5aPPa Psychological and Physiological

Acoustics: Cross-Spectral Auditory

Integration: Physiological,

Psychophysical, and Clinical Evidence I.

Room 241

8:00 $\quad 5 \mathrm{aPP} \quad$ Psychological and Physiological

Acoustics: Otoacoustic Emissions, from

Cochlear Modeling to Experimental

Techniques and Back I. Room 242A

9:40 5aPPc Psychological and Physiological

Acoustics: Otoacoustic Emissions, from

Cochlear Modeling to Experimental

Techniques and Back II (Poster

Session). P2-C, Level 2

9:40 5aPPd Psychological and Physiological

Acoustics: General Topics in

Psychological and Physiological

Acoustics VII (Poster Session). P2-C,

Level 2

9:40 5aPPe Psychological and Physiological

Acoustics and Computational

Acoustics: Computational Auralization I

(Poster Session). P2-C, Level 2

9:40 5aPPf Psychological and Physiological

Acoustics and Speech

Communication: Acoustic Features and

Speech Perception I (Poster Session).

P2-C, Level 2

9:40 5aPPg Psychological and Physiological

Acoustics: Cross-Spectral Auditory

Integration: Physiological,

Psychophysical, and Clinical Evidence II

(Poster Session). P2-C, Level 2

8:00 5aSAa Structural Acoustics and Vibration, ASA Committee on Standards, and EURONOISE: Ground Vehicle Noise and Vibration I. Room 252B

8:00 5aSAb Structural Acoustics and Vibration and EURONOISE: Active Noise

Control: New Strategies and Innovative

Concepts I. Room 253

9:40 5aSAc Structural Acoustics and Vibration and EURONOISE: Active Noise

Control: New Strategies and Innovative

Concepts II (Poster Session). P2-B,

Level 2

9:40 5aSAd Structural Acoustics and Vibration, ASA Committee on Standards, and EURONOISE: Ground Vehicle Noise and Vibration II (Poster Session). P2-B, Level 2

8:00 5aSCa Speech Communication: Speaker Identification by Machine. Room 240
8:40 5aSCb Speech Communication:

Cross-Language Speech Perception and

Production. Room 250B

10:20 5aSCc $\quad$ Speech Communication: General Topics in Speech Communication IV

(Poster Session). P2-D, Level 2

8:00 5aUWa Underwater Acoustics, Signal

Processing in Acoustics, and ECUA:

Broadband Underwater Communications

I. Amphi Bordeaux

8:00 5aUWb Underwater Acoustics and ECUA:

Environmental Impact on Propagation.

Room 341

8:00 5aUWc Underwater Acoustics and ECUA:

High Frequency Scattering I. Room $342 \mathrm{~A}$

8:00 5aUWd Underwater Acoustics and ECUA:

Low-Frequency and High-Frequency

Synthetic Aperture Sonar. Room 342B

8:00 5aUWe Underwater Acoustics and ECUA:

Monitoring Systems and Ambient Noise. Room 362/363

9:40 5aUWf Underwater Acoustics, Signal

Processing in Acoustics, and ECUA:

Broadband Underwater Communications II (Poster Session). P3-C, Level 3

9:40 5aUWg Underwater Acoustics and ECUA:

High Frequency Scattering II (Poster

Session). P3-C, Level 3

11:00 5aUWh Underwater Acoustics and ECUA:

Sensor Coalition. Room 341

11:40 5aUWi Underwater Acoustics and ECUA: Synthetic Aperture Sonar and Radar Convergences. Room 342B

\section{FRIDAY MORNING SESSIONS CONTINUED}

$\begin{array}{lll}\text { 2:00 } & \text { 5aAAa } & \text { Room 242B } \\ \text { 1:40 } & \text { 5aAAb } & \text { Room 243 } \\ \text { 1:40 } & 5 \mathrm{aABa} & \text { Amphi Bleu } \\ \text { 2:00 } & 5 \mathrm{aBBf} & \text { Room 352B } \\ 1: 40 & 5 \mathrm{aMUe} & \text { Amphi Maillot } \\ 2: 00 & 5 \mathrm{aNSa} & \text { Room 250A } \\ \text { 2:00 } & 5 \mathrm{aNSj} & \text { Room 251 } \\ 1: 40 & 5 \mathrm{aPAa} & \text { Amphi Havane } \\ 2: 00 & 5 \mathrm{aPAl} & \text { Room 352A } \\ 2: 00 & 5 \mathrm{aSAb} & \text { Room 253 }\end{array}$

\section{FRIDAY AFTERNOON}

3:20 5pAAa Architectural Acoustics and Musical Acoustics: New Measurement Parameters in Performing Arts Spaces II. Room 242B

3:40 5pAAb Architectural Acoustics: Coupled Volume Acoustics II. Room 243 
1:40 5pAO Acoustical Oceanography and ECUA: Acoustical Tomography and Long Range Propagation. Room 343

2:00 5pBB Biomedical Ultrasound/Bioresponse to Vibration: General Topics in Biomedical Ultrasound/Bioresponse to Vibration II. Room 353

2:00 5pNSa Noise, ASA Committee on Standards, Architectural Acoustics, and EURONOISE: Classroom Acoustics II. Room 252A

2:00 $5 \mathrm{pNSb} \quad$ Noise, Structural Acoustics and Vibration, Physical Acoustics, and EURONOISE: Sound and Vibration from Explosions in Air II. Room 252B

4:20 5pNSc Noise and EURONOISE: Propagation and Urban Noise II. Room 251

2:00 5pPAa Physical Acoustics: Ducts and Waveguides II. Room 351

3:20 5pPAb Physical Acoustics: Ultrasonics Under Extreme Conditions II. Amphi Havane

4:00 5pPAc Physical Acoustics: General Topics in Physical Acoustics II. Room 352A

5:00 5pPAd Physical Acoustics: Scattering and Diffraction II. Room 351
2:00 $5 \mathrm{pPPa} \quad$ Psychological and Physiological Acoustics and Speech

Communication: Acoustic Features and Speech Perception II. Room 241

2:00 $\quad 5 \mathrm{pPPb} \quad$ Psychological and Physiological Acoustics and Computational Acoustics: Computational Auralization II. Room 242A

2:00 5pSC Speech Communication: Multimodal Speech Technology. Room 240

1:40 5pUWa Underwater Acoustics and ECUA: Sound Propagation in 3-Dimensional Environments II. Amphi Bordeaux

1:40 5pUWb Underwater Acoustics and ECUA: Scattering From Objects Near Boundaries. Room 342A

2:00 5pUWc Underwater Acoustics and ECUA: Automatic Target Recognition, Sensors, and Algorithms. Room 341

2:20 5pUWd Underwater Acoustics and ECUA: Acoustic Data Fusion. Room 342B

4:00 5pUWe Underwater Acoustics and ECUA: Noise Suppression, Robust Direction of Arrival, and Target Strength Estimation. Room 342B

4:40 5pUWf Underwater Acoustics and ECUA: Sound Generation and Attenuation. Room 343 


\section{SCHEDULE OF COMMITTEE MEETINGS AND OTHER EVENTS}

\begin{tabular}{|c|c|c|}
\hline Sun, 29 Jun, 10:00 a.m. & Executive Council & $336-337 \mathrm{M}$ \\
\hline Mon, 30 Jun, 3:30 p.m. & Technical Council & $336-337 \mathrm{M}$ \\
\hline Mon, 30 June, 7:00 p.m. & Editorial Board AA.A & $242 \mathrm{~A}$ \\
\hline Tue, 1 Jul, 7:30 a.m. & Public Policy & $338 \mathrm{M}$ \\
\hline Tue, 1 Jul, 11:45 a.m. & Editorial Board ASA & See website \\
\hline Tue, 1 Jul, 12:00 noon & Audit Committee & $336-337 \mathrm{M}$ \\
\hline Tue, 1 Jul, 12:40 p.m. & EAA Student Meeting & 353 \\
\hline Tue, 1 Jul, 1:30 p.m. & Meetings Committee & $334 \mathrm{M}$ \\
\hline Tue, 1 Jul, 6:40 p.m. & Education in Acoustics & $202 / 203$ \\
\hline Wed, 2 Jul, 7:00 a.m. & Publication Policy & $336-337 \mathrm{M}$ \\
\hline Wed, 2 Jul, 11:00 a.m. & Medals and Awards & $336-337 \mathrm{M}$ \\
\hline Wed, 2 Jul, 11:30 a.m. & Public Relations & $338 \mathrm{M}$ \\
\hline Wed, 2 Jul, 12:00 noon & Membership & $334 \mathrm{M}$ \\
\hline Thu, 3 Jul, 7:30 a.m. & Tutorials & $336-337 \mathrm{M}$ \\
\hline Thu, 3 Jul, 12:00 noon & External Affairs & $336-337 \mathrm{M}$ \\
\hline Fri, 4 Jul, 7:00 a.m. & Technical Council & $336-337 \mathrm{M}$ \\
\hline Fri, 4 Jul, 9:30 a.m. & Executive Council & $336-337 \mathrm{M}$ \\
\hline
\end{tabular}

Wed, 2 Jul, 5:30 p.m.

S1/WG20 Groun

Impedance

\section{MEETING SERVICES, SPECIAL EVENTS AND SOCIAL} FUNCTIONS

Sun, 29 Jun,

2:00 p.m.-9:00 p.m.

Mon-Tue, 30 Jun-1 Jul,

Thu, 3 Jul, 7:00 p.m. (ASA/EAA)

Noise (ASA/EAA) 251

Psychological and 241

Physiological Acoustics

(ASA/EAA)

Thu, 3 Jul, 7:00 p.m.

Structural Acoustics and 252B

Thu. 3 Jul, 7.00 p.m

Vibration (ASA)

Speech Communication 250B

(ASA)

\section{STANDARDS COMMITTEES AND WORKING GROUP MEETINGS}

Mon, 30 Jun, 7:00 p.m. ASACOS Steering and 334M ASACOS
Mon, 30 Jun,

7:00 a.m.-6:00 p.m.

Tue-Fri, 1-4 Jul,

7:45 a.m.-4:00 p.m.

Mon, 30 Jun,

12 noon-6:00 p.m

Tue.-Fri, 1-4 Jul,

8:00 a.m.-6:00 p.m.

Tue-Fri, 1-4 Jul,

9:40 a.m.-11:20 a.m.

3:20 p.m-5: 00 p.m.

Thu-Fri, 3-4 Jul,

3:20 p.m.-5:00 p.m.

Mon, 30 Jun,

12 noon-6:00 p.m.

Tue-Fri, 1-4 Jul,

8:00 a.m.-6:00 p.m.

Mon-Fri, 30 Jun-4 Jul,

9:00 a.m.-5:00 p.m.

Mon, 30 Jun, 8:40 a.m.

Mon, 30 Jun, 10:40 a.m.

Mon-Thu, 1-3 Jul,

8:00 a.m.-6:00 p.m.

Fri, 4 Jul,

8:00 a.m.-4:00 p.m.

Mon, 30 Jun, 11:40 a.m.

Tue,-Fri, 1-4 Jul,

8:00 a.m.-11:00 a.m.

Tue, 1 Jul, 12:40 p.m.

Tue, 1 Jul

Wed, 2 Jul, 12:40 p.m.

Wed, 2 Jul, 3:40 p.m.

Registration

Registration

A/V Preview

Coffee Breaks

Havane and Maillot Halls

Internet and Email

237M (level 2.5) and 315

Gallery of Acoustics

Havane Hall

Opening Ceremony

Grand Amphitheater

Plenary Lectures

Exhibition

Grand Amphitheater

Havane Hall

Exhibit Opening Reception Exhibition Area

(Havane Hall)

Accompanying persons Maillot Foyer

Women in Acoustics

Maillot Hall

Lunch

Social Reception

Students Luncheon

Medals and Awards

Ceremony (live)

Medals and Awards

Wed, 2 Jul, 5:40 p.m.

Plenary Lectures

SFA 60th Anniversary

meeting

Wed, 2 Jul, 5:40 p.m.

Acoustical Oceanography

Prize Lecture

Wed, 2 Jul, 8:00 p.m.

Fri, 4 Jul, 6:20 p.m.

Banquet Dinner

Closing Ceremony

See meeting website

Maillot Hall

Bleu Amphitheater

Bordeaux Amphitheater

Bordeaux Amphitheater

Bleu Amphitheater

Havane Amphitheater

See meeting website

Bleu Amphitheater

Bleu Amphitheater
Ceremony (video) 


\author{
$155^{\text {th }}$ Meeting of the Acoustical Society of America \\ 5th Forum Acusticum of the European Acoustics Association \\ 9th Congrès Français d'Acoustique of the French Acoustical Society \\ integrating the 7th EUROpean conference on NOISE control (euronoise), \\ the 9th European Conference on Underwater Acoustics (ecua) \\ and the 60th Anniversary of the French Acoustical Society
}

Acoustics'08 Paris will be held Monday through Friday, 30 June-4 July 2008, at the Palais des Congrès in Paris, France. This meeting incorporates the 155th Meeting of the Acoustical Society of America (ASA), the 5th Forum Acusticum of the European Acoustics Association (EAA), and the 9th Congrès Français d'Acoustique of the French Acoustical Society (SFA) integrating the 7th EUROpean conference on NOISE control (euronoise), the 9th European Conference on Underwater Acoustics (ecua) and the 60th Anniversary of the SFA.

The on-site registration desk will open on Sunday, 29 June, from 2:00 p.m. to 9:00 p.m. in Havane Hall on level 0 of the Palais des Congrès. It will be open from 7:00 a.m. to 6:00 p.m. on Monday, 30 June, and 7:45 a.m. to 4:00 p.m. Tuesday through Friday. Technical sessions will be held Monday through Friday.

\section{TABLE OF CONTENTS}

1. HOTEL AND DORMITORY INFORMATION

2. TRANSPORTATION AND TRAVEL DIRECTIONS

3. STUDENT TRANSPORTATION SUBSIDIES

4. MESSAGES FOR ATTENDEES

5. REGISTRATION

6. TECHNICAL SESSIONS

7. TECHNICAL SESSION DESIGNATIONS

8. PLENARY LECTURES

9. OPENING CEREMONY

10. CLOSING CEREMONY

11. BEST STUDENT PAPER AWARDS

12. STUDENT DESIGN COMPETITION

13. GALLERY OF ACOUSTICS

14. TECHNICAL TOURS

15. TECHNICAL COMMITTEE OPEN MEETINGS

16. PLENARY SESSION AND AWARDS CEREMONY

17. MEDWIN PRIZE IN ACOUSTICAL OCEANOGRAPHY AND ACOUSTICAL OCEANOGRAPHY PRIZE LECTURE

18. 60th ANNIVERSARY OF THE FRENCH ACOUSTICAL SOCIETY

19. EXHIBITION AND OPENING RECEPTION

20. MEETING CD ROM

21. ECUA PROCEEDINGS

22. SPONSORS

23. COFFEE BREAKS

24. A/V PREVIEW ROOM

25. E-MAIL AND INTERNET ACCESS

26. BUFFET SOCIAL

27. BANQUET

28. STUDENTS LUNCHEON

29. COMMITTEE ON WOMEN IN ACOUSTICS LUNCHEON

30. CHILD CARE

31. ACCOMPANYING PERSONS PROGRAM

32. PRE- AND POST-CONFERENCE TOURS

33. PARIS CITY INFORMATION

34. MEETING ORGANIZING COMMITTEE

35. TECHNICAL ORGANIZING COMMITTEE

36. TECHNICAL PROGRAM ORGANIZING COMMITTEE

37. PHOTOGRAPHING AND RECORDING

38. NOTE TO SMOKERS

39. ABSTRACT ERRATA
40. GUIDELINES FOR ORAL PRESENTATIONS

41. SUGGESTIONS FOR EFFECTIVE POSTER PRESENTATIONS

42. DATES OF FUTURE MEETINGS

\section{HOTEL AND DORMITORY INFORMATION}

A variety of options for accommodations are described on the meeting website at www.acoustics08-paris.org, including hotels, dormitories, and making reservations through a travel agency.

The Concorde Lafayette Hotel is located in the same building as the Palais des Congrès and provides access for handicapped persons. The cut-off date for making reservations at the reduced rate was Wednesday, 30 April. Please contact the hotel for information on room availability: Hotel Concorde Lafayette, 3, Place du Général Koenig, 75017 Paris, France; +33 (0)1 40685068 (T); +33 (0)1 40 685043 (F); 1-800-888-4747 (US reservations).

The organizers have commissioned a travel agency to handle hotel reservations which offers all price ranges for hotels. To book through this agency, please visit the following website: www.acoustics08-paris.org/venue/ accommodation/travel-agency/

The meeting secretariat has booked and paid for a limited number of low-cost rooms. These rooms are located in several university residences in Paris that are within easy travel distance to the meeting site by public transportation. Rooms will be booked on a "first-come first-served" basis. For dormitory descriptions and reservations, please visit the following website: www.acoustics08-paris.org/students/ dormitories

\section{TRANSPORTATION AND TRAVEL DIRECTIONS}

Air: Two main airports serve Paris: Paris-Charles de Gaulle International Airport (Airport code: CDG) and ParisOrly International Airport (Airport code: ORY). They are served by most international airlines. Please note your departure airport and terminal since there are two drop off points for ground transportation. Further information is available at www.aeroportsdeparis.fr/ADP/en-GB/Passangers.

Ground Transportation: Paris-Charles de Gaulle International Airport is approximately 18.5 miles $(30 \mathrm{~km})$ from the Palais des Congrès (Porte Maillot).

Taxis: A taxi costs around $€ 50.00$ from the airport to Paris city center or to Palais des Congrès. An extra charge of about 15\% applies at night from 7:00 p.m. to 7:00 a.m. and on Sundays and public holidays. Taxis are available upon exiting the baggage claim area. Terminal 1 exit 20 at arrivals level; terminals $2 \mathrm{~A}$ and $2 \mathrm{C}$ exit 6 ; terminals $2 \mathrm{~B}$ and $2 \mathrm{D}$ exit 7 ; terminals $2 \mathrm{E}$ and $2 \mathrm{~F}$ exit 1 . Approximate travel time: 35 minutes.

Air France Shuttle: Direct shuttle service is available from the airport to Palais des Congrès for $€ 13.00$ per person, one way or $€ 18.00$ per person, round trip. Follow the "Air 
France Shuttle" sign at the terminal. Line 2 shuttle stops at "Porte Maillot" 100 meters from the meeting center entrance (boulevard Gouvion St-Cyr opposite the Méridien hotel). Shuttles are available from 5:50 a.m. to 11:00 p.m. Approximate travel time: 45 minutes.

Public transportation: Follow "RER" signs at the terminal. Take RER B towards "Robinson" or "Saint Rémy les Chevreuse" and exit at "St. Michel Notre Dame." At "St. Michel Notre Dame" station follow "RER C" signs. Take RER C towards "Pontoise" or "Argenteuil" and exit at "Porte Maillot" station. RER B is available from 5:00 a.m. to 11:50 p.m. Approximate travel time: 1 hour. Ticket machines are located in the RER station and valid tickets are required to enter and exit the RER part of Metro. A one-way ticket costs $€ 8.20$. When you return to the airport, check to be sure that you are on the line to CDG 1 and 2 .

Paris-Orly International Airport is approximately 14.3 miles $(23 \mathrm{~km})$ from the Palais des Congrès (Porte Maillot).

Taxis: A taxi costs around $€ 50.00$ from the airport to Paris city center or to Palais des Congrès. An extra charge of about $15 \%$ applies at night from 7:00 p.m. to 7:00 a.m. and on Sundays and public holidays. Taxis are available at terminal Paris-Orly Sud exit L and terminal Paris-Orly Ouest exit I at the arrivals level. Approximate travel time: 30 minutes.

Air France Shuttle: Direct shuttle service is available from the airport to Invalides for $€ 8.00$ (per person, one way) or $€ 12.00$ (per person, round trip). Follow the "Air France Shuttle" sign at the terminal. Line 1 shuttle stops at "Invalides." Then purchase a Metro ticket (cost €1.30) and take RER C towards "Pontoise" or "Argenteuil" and exit at "Porte Maillot" station. Shuttles are available from 6:00 a.m. to 11:00 p.m. Approximate travel time: 45 minutes.

Public transportation: Follow "Orlyval" signs at the terminal. Take Orlyval metro towards "Antony" and exit at "Antony" station. Take RER B towards "Aéroport Charles de Gaulle" or "Mitry Claye" and exit at St. Michel Notre Dame. At "St. Michel Notre Dame" station, follow "RER C" signs. Take RER C towards "Pontoise" or "Argenteuil" and exit at "Porte Maillot" station. "Orlyval" is available from 6:00 a.m. to 11:00 p.m. Approximate travel time: 1 hour. Ticket machines are located in the RER station. A one-way ticket costs $€ 9.30$.

Car rental: Car rental agencies have offices at both airports, although having a car in Paris is usually not advantageous. Underground parking is available at the Palais des Congrès. The 24-hour rate is €27.00. Detailed driving directions from both airports can be found at www.acoustics08-paris.org/venue/access-transport/

\section{STUDENT TRANSPORTATION SUBSIDIES}

To encourage student participation, travel grants have been provided by ASA, EAA, and SFA to students and young scientists presenting papers to partially defray transportation expenses to the meeting. The deadline for the present meeting has passed.

\section{MESSAGES FOR ATTENDEES}

Messages for attendees may be left by calling +33 (0)637883979 during the meeting. The message board will be located in Havane Hall level 3 near the registration desk. This board may also be used by attendees who wish to contact one another. Members or others who wish to communicate with the Acoustical Society of America should visit Exhibition booth \#63 in Havane Hall on level 3.

\section{REGISTRATION}

Registration is required for all attendees and accompanying persons to pass through security. Registration badges must be worn in order to participate in technical sessions and other meeting activities.

On-site registration will be possible but advance registration is highly recommended. The on-site registration desk will open on level 0 of the Palais des Congrès on Sunday, 29 June from 2:00 p.m. to 9:00 p.m. and in Havane Hall on level 3 on Monday, 30 June, from 7:00 a.m. to 6:00 p.m. and Tuesday to Friday, 1-4 July, from 7:45 a.m. to 4:00 p.m.

Credit card is the preferred mode of payment. The secure online registration system accepts the following cards: Visa, Mastercard, and American Express. Checks drawn on French banks and travelers checks in Euros will also be accepted. Meeting attendees who have pre-registered may pick up their badges and registration materials at the pre-registration desk.

The registration fees in Euros $(€)$ are $€ 550$ for members of the ASA, EAA, and SFA; $€ 600$ for nonmembers; $€ 250$ for Emeritus members (emeritus status must be pre-approved); $€ 250$ for student members of ASA, EAA, and SFA (with current student ID cards); $€ 300$ for student nonmembers (with current student ID cards); and €200 for accompanying persons (entitles participation in Accompanying Persons Program and the hospitality room but does not include access to meeting rooms, except during opening and closing ceremonies). No tax will be added [the SFA is not subject to VAT (sales) tax]. NOTE: No reimbursement will be made for registration cancellation received after 8 April 2008.

\section{TECHNICAL SESSIONS}

The technical program includes 428 sessions, with 3588 papers scheduled for presentation during the meeting. Note that many sessions are scheduled in two parts and some in multiple parts, that is, I, II, III, etc.

A floor plan of the Palais des Congrès appears on pages A8 and A9. All oral presentations, both invited and contributed, have been allotted 16 minutes plus 4 minutes for questions and for participants to move to other sessions. An audio signal will be emitted to signify the start of each presentation, the end of the presentation, and the end of discussion.

Session Chairs have been instructed to adhere strictly to the printed time schedule, both to be fair to all speakers and to permit attendees to schedule moving from one session to another to hear specific papers. If an author is not present to deliver a lecture-style paper, the Session Chairs have been instructed either to call for additional discussion of papers already given or to declare a short recess so that subsequent papers are not given ahead of the designated times.

General poster sessions will be held on Tuesday, 1 July (3:40 p.m to 5:20 p.m.), Thursday, 3 July (3:40 p.m to 5:20 
p.m.) and Friday, 4 July (9:40 a.m to 11:20 a.m.). Lecture sessions will recess during General poster sessions. Speech Communication poster sessions will be held on Monday, 30 June (4:20 p.m. to 7:20 p.m.), Tuesday, 1 July (3:40 p.m to 6:40 p.m.), Thursday, 3 July (3:40 p.m to 6:40 p.m.) and Friday, 4 July (10:20 a.m to 12:40 p.m.). Poster sessions will be located in several areas according to the primary Technical Committee that organized the session: P2-A for NS, P2-B for AA and SA, P2-C for PP, P2-D for MU and $\mathrm{SC}$, P3-A for PA, P3-B for BB, P3-C for AB, AO, EA, SP and UW. Poster areas appear on the map on pages A8 and A9.

\section{TECHNICAL SESSION DESIGNATIONS}

The first character is a number indicating the day the session will be held as follows:

1-Monday, 30 June

2-Tuesday, 1 July

3-Wednesday, 2 July

4-Thursday, 3 July

5-Friday, 4 July

The second character is a lower case "a" for a.m., "p" for p.m., or "e" for evening corresponding to the time of day the session will take place. The third and fourth characters are capital letters indicating the primary Technical Committee that organized the session using the following abbreviations or codes:

AA Architectural Acoustics

AB Animal Bioacoustics

AO Acoustical Oceanography

BB Biomedical Ultrasound/Bioresponse to Vibration

EA Engineering Acoustics

ED Education in Acoustics

ID Interdisciplinary

MU Musical Acoustics

NS Noise

PA Physical Acoustics

PP Psychological and Physiological Acoustics

SA Structural Acoustics and Vibration

SC Speech Communication

SP Signal Processing in Acoustics

UW Underwater Acoustics

Sessions that are cosponsored by the Computational Acoustics technical committee of the European Acoustics Association, ECUA (European Conference on Underwater Acoustics), or Euronoise (European Conference on Noise Control) are so noted in the session titles.

In sessions where the same group is the primary organizer of more than one session scheduled in the same morning or afternoon, a fifth character (a lower-case letter) is used to distinguish the sessions, e.g., "a," "b," "c," etc. Each paper within a session is identified by a paper number following the session-designating characters, in a conventional manner. As hypothetical examples: paper 2pEA3 would be the third paper in a session on Tuesday afternoon organized by the Engineering Acoustics Technical Committee; 3 pSAb5 would be the fifth paper in the second of two sessions on Wednesday afternoon sponsored by the Structural Acoustics and Vibration Technical Committee.
Note that technical sessions are listed both in the calendar and the body of the program in the numerical and alphabetical order of the session designations rather than the order of their starting times. For example, session 3aAA would be listed ahead of session $3 \mathrm{aAO}$ even if the latter session began earlier in the same morning.

\section{PLENARY LECTURES}

Four plenary lectures will be given at Acoustics'08 Paris: Two at the Opening Ceremony on Monday morning, 30 June, and two others during the ASA/EAA/SFA plenary session on Wednesday afternoon, 2 July. See sections 9 and 16 for details about these lectures.

\section{OPENING CEREMONY}

The meeting will begin with an opening ceremony on Monday, 30 June, at 8:40 a.m. in the "Grand Amphitheater" of Palais des Congrès (entrance located on level 0).

The ceremony will start with a special welcome from the vice-president of the Ile de France regional council and addresses by the presidents of ASA, EAA and SFA. Musical interludes will be given and two plenary lectures will be presented: "How sound from human activities affects marine mammals," by Peter Tyack of the Woods Hole Oceanographic Institution, MA, USA and "New trends in aeroacoustics: From acoustic analogies to direct numerical simulations," by Daniel Juvé of Ecole Centrale de Lyon, France. Abstracts of these lectures can be found in Session 1aID.

\section{CLOSING CEREMONY}

The meeting will end with a closing ceremony on Friday, 4 July, at 6:20 p.m. in the Bleu Amphitheater (level 2).

\section{BEST STUDENT PAPER AWARDS}

A "Best Paper" Awards competition for students and young presenters has been organized by each Technical Committee at the Acoustics'08 Paris meeting. Each Technical Area will appoint a subcommittee to review the papers or posters at the meeting and to select the Best Papers based both on content and presentation. Following the meeting winners will be announced in ECHOES (ASA's newsletter), in the EAA and SFA newsletters, and on the ASA Student Zone (http://www.acosoc.org/student/) and EAA (www.eaa-fenestra.org) websites. Winners (up to two per Technical Committee) will receive cash prizes.

\section{STUDENT DESIGN COMPETITION}

The Acoustical Society of America's Technical Committee on Architectural Acoustics, with support from the Robert Bradford Newman Student Award Fund and the National Council of Acoustical Consultants, is sponsoring a student design competition to be judged and displayed at Acoustics'08 Paris. It will be held in session 4pAAi on Thursday, 3 July, at 3:40 p.m. The Student Design Competition is intended to encourage students in the disciplines of architecture, engineering, physics, and other curricula that involve building design and/or acoustics to express their knowledge of architectural acoustics and noise control in the design of a facility in which acoustical 
considerations are of significant importance. The 2008 competition involves the conceptual design for the inclusion of a theater in a university building project.

The submitted poster presentations will be displayed at the meeting in Maillot Hall and judged by a panel of practicing design professionals. The judges will include acoustics consultants and may include architects and theater consultants. Entries will be evaluated on technical merit, design vision, adherence to the design scenario, and effectiveness of presentation. An award of USD \$1,250 will be made to the individual or team with the entry chosen as "First Honors." Commendation awards of USD\$700 will be made to four individuals or teams of other outstanding entries. Awards are funded through a grant from the Wenger Foundation by the Newman Student Award Fund.

\section{GALLERY OF ACOUSTICS}

The Technical Committee on Signal Processing in Acoustics will sponsor its tenth Gallery of Acoustics at Acoustics'08 Paris. The objective of the Gallery is to enhance meetings by providing a compact and free-format setting for researchers to display their work to all meeting attendees in a forum emphasizing the diversity and interdisciplinary nature of acoustics. The Gallery of Acoustics provides a means by which we can all share and appreciate the natural beauty and aesthetic appeal of acoustical phenomena. The Gallery will be held in Havane Hall between exhibition booths 20 and 21 in the Palais des Congrès, Monday to Friday, 30 June-4 July, and will include posters, videos, and audio clips of images and/or sounds generated by acoustic processes or resulting from signal processing of acoustic data. Meeting attendees are asked to vote on the entries on the basis of aesthetic/artistic appeal, ability to convey and exchange information, and originality. A cash prize of USD $\$ 1000$ will be awarded to the winning entry.

\section{TECHNICAL TOURS}

Several technical tours are planned during the meeting. There is no charge to participate in technical tours but participants must register on the meeting website at www.acoustics08-paris.org/program/technical-tours by 23 May. The number of participants is limited for each tour and registration can only be accepted on a space-available basis. For technical tours taking place in Paris, participants must arrange their own transportation to and from the tour site (maps and instructions for each tour will be available at the registration desk in Havane Hall). For technical tours outside Paris, bus transportation will be provided and participants will meet at level 0 of Palais des Congrès. Additional information on technical tours will be available on the meeting website.

- LABE, European Acoustics Laboratory in the Building Industry (Tuesday, 1 July, 2:00 p.m. to 9:00 p.m., earlier return is possible by using ground transportation; limited to 100 participants; bus transportation provided). For the 10th anniversary of the LABE and within the context of the Acoustics'08 Paris conference, the CSTB plans a session of acoustic tests and innovative acoustic approaches. Demonstrations will include: measurement of level intensity in rainfall noise (according to the standard EN ISO 140-18); measurement of airborne and structure borne sound from building equipment with demonstration of a whirlpool bath (according to draft standard pr EN 15657-1); measurement of walking noise on floor coverings (according to the standard NF S 31-057 and European draft WG1/AHG7 of 25/11/2005); presentation of virtual models produced on the basis of physical models (acoustics, lighting, etc.); presentation of an active window (research program TERIA); demonstration of the software ACOUBAT Sound V5.0 (2008) and of its new functions (offices, roof, acoustic correction, etc.). Based on the European standards of simulations (EN 12354-1 to 3 and 6), this software makes it possible to assess the acoustic performance of a building based on the performance of its constituent products; simulation of acoustic performance in products (partition wall and floating screen); road traffic noise reduction dives (CE marking, simplified implementation, R\&D). This event will end with a brunch offered by LABE.

- LAM: Musical Acoustics Laboratory (Tuesday, 1 July, and Wednesday, 2 July, at 2:30 p.m.; duration-1 and one-half hours; limited to 80 participants; meet at LAM): demonstrations in musical acoustics, visualization of streaming in wind instruments, use of new materials in stringed instruments, research on optical supports (CD, DVD) for sound records, enclosures with nozzles for audio-engineering, synthesis by gesture control, www.lam.jussieu.fr

- Musée des Arts et Métiers (Tuesday, 1 July, at 2:00 p.m. and 4:00 p.m.; duration-2 hours; limited to 100 participants; meet at museum): visit to the museum of science and technology including Blériot airplane, Lavoisier laboratory, www.arts-et-metiers.net

- IAT Aerotechnical Institute (Wednesday, 2 July, at 12:15 p.m.; duration-3 and one-half hours including transportation; limited to 40 participants; bus transportation provided): Wind tunnels for car testing and real and reduced (2/5th) scales (test room, test stream, fan, etc.) and of the associated measurement facilities (aerodynamic force measurement balance, acoustical measurements, set-up, etc.), www.cnam.fr/instituts/IAT

- ONERA/CEPR French Aerospace Laboratory Thruster Test Center (Wednesday, 2 July, 2:00 p.m.); duration-2 and one-half hours including travel; limited to 25 participants; bus transportation provided): Anechoic wind tunnel, RACE tests facility (fans and turbojets), www.onera.fr/english. For this tour, participants must complete and return the form available on the meeting website to the Conference Secretariat at acoustics08@laposte.com prior to the meeting. Participants must also bring their photo ID (identify cards for UE citizens; passports for citizens of other countries) with them on the tour.

- IRCAM (Institut de Recherche et Coordination Acoustique/Musique) (Thursday, 3 July at 3:00 p.m. and Friday, 4 July, at 10:00 a.m.; duration-1 and one-half hours; limited to 100 participants; meet at IRCAM): 
demonstrations in musical acoustics, sound spatialization, sound design, synthesis methods and sound treatment, www.ircam.fr

- Guided tour "Pipe organs of Paris" (Thursday, 3 July, at 2:00 p.m.; duration-3 hours; limited to 200 participants; meet at Sainte Elisabeth church). Paris is the location of probably the largest number of pipe organs in the world with more than 250 instruments in public buildings, mainly churches. The oldest pipes and organ cases in the city date back to the late 16 th and early 17 th centuries, as well as other magnificent instruments dating back to the 18th, 19th, 20th, and even 21st centuries. These instruments are often associated with French composers of the past such as Du Caurroy, Rameau, Couperin, Daquin, Clerambault, Franck, Saint Saens, Bizet, Gounod, Widor, Vierne, Dupré, Messiaen, just to name a few, who were organists in Paris. Nowadays, almost all major instruments can be heard several times a week during church services or concerts, and are served today as in the past by distinguished musicians. The tour will begin with a short introduction to the Parisian organ landscape, presenting the history of organ building in Paris, and the most important instruments of the city. Following this introduction, a detailed technical and musical visit to the historical organ of Sainte Elisabeth will be offered. The tour will continue with visits to one or more interesting instruments in the center of Paris, depending on attendance and availability.

- UGC cinema facility in Paris La Défense (Friday, 4 July, at 10:00 a.m.; duration-one hour; limited to 60 participants; meet at UGC la défense. The UGC cinema of Paris La Defense features 16 projection rooms, one of them a former Imax theatre. It was outfitted in the former automobile museum facility in 2006. Due to the severe weight restrictions applicable in this building, the partitioning of the projection rooms was performed using gypsum board walls supported by beams, while the flooring was made of wooden panels. The visit will begin with a brief explaination of how a cinema facility operates. Next, the types of walls (with a height ranging up to $14 \mathrm{~m}$ ) and floors used in such a construction will be described, as well as the absorptive treatment applied. Lastly, the former Imax facility will be visited and its transformation into a regular projection room explained.

\section{TECHNICAL COMMITTEE OPEN MEETINGS}

Technical Committees of the Acoustical Society of America and of the European Acoustics Association will hold joint open meetings the in Palais des Congrès. These are working, collegial meetings and much of the work of the Societies is accomplished by actions that originate and are taken in these meetings. All meeting participants are cordially invited to attend these meetings and to participate actively in the discussions. Please refer to the schedule on page A34 for the times and locations of these Committee meetings.

\section{PLENARY SESSION AND AWARDS CEREMONY}

A joint ASA/EAA/SFA plenary session will be held Wednesday, 2 July, starting at 3:40 p.m. in the Bleu
Amphitheater (level 2). The program will include presentation of awards, recognition of newly-elected fellows and plenary lectures. Video retransmission will be available in the Bordeaux Amphitheater (level 3) only for presentation of awards and recognition of fellows.

The session will begin with recognition of new fellows of the Acoustical Society of America, followed by the presentation of medals and awards by the Acoustical Society of America, the European Acoustics Association, and the French Acoustical Society. The medals and awards ceremony will be followed by two plenary lectures in the Bordeaux Amphitheater at 5:40 p.m. The plenary lectures are: "Binaural hearing and systems for sound reproduction," by Philip Nelson of the University of Southampton, UK and "Optoacoustics: Can ultrasonic imaging become the preferred modality for molecular imaging?" by Matthew O'Donnell of University of Washington, WA, USA. Abstracts of these lectures can be found on page 3469 .

The Acoustical Society of America will present the following awards: the 2008 Medwin Prize in Acoustical Oceanography to Mark V. Trevorrow, the Distinguished Service Citation to Charles E. Schmid, the R. Bruce Lindsay Award to Tyrone M. Porter, the Helmholtz-Rayleigh Interdisciplinary Silver Medal to James V. Candy, and the Gold Medal to Patricia K. Kuhl. Mary K. Fagan, recipient of the Research Grant in Speech Science of the American Speech-Language-Hearing Foundation will be introduced.

The European Acoustics Association will present the following awards: the EAA Award for "Lifetime Achievements in Acoustics" to Gunnar Rasmussen, the EAA Award for "Contributing to Promotion of Acoustics in Europe" to the Federation of Acoustical Societies of Europe (FASE), and the EAA Award for "Outstanding Scientific Results Published Recently in Acta Acustica united with Acustica" to Catherine Guastavino, Brian F.G. Katz, JeanDominique Polack, Daniel J. Levitin, Danielé Dubois for the paper Ecological Validity of Soundscape Reproduction.

The French Acoustical Society will present the following awards: The French Medal to Vitalyi Gusev and the Foreign Medal to Walter Lauriks.

After the awards ceremony there will be a celebration of the 60th anniversary of the Société Française d'Acoustique in the Bleu Amphitheater at 5:40 p.m. and the ASA's Acoustical Oceanography Prize Lecture will be presented in the Havane Amphitheater.

\section{MEDWIN PRIZE IN ACOUSTICAL OCEANOGRAPHY AND ACOUSTICAL OCEANOGRAPHY PRIZE LECTURE}

The 2008 Medwin Prize in Acoustical Oceanography will be awarded to Mark V. Trevorrow, Defence R\&D Canada Atlantic, Dartmouth, NS, Canada, at the Plenary Session on Wednesday, 2 July. Mark Trevorrow will present the Acoustical Oceanography Prize Lecture titled "The use of moored inverted echosounders for monitoring near-surface processes" on Wednesday, 2 July at 5:40 p.m. in the Havane Amphitheater. 


\section{6OTH ANNIVERSARY OF THE FRENCH ACOUSTICAL SOCIETY}

The session celebrating the 60th anniversary of the French Acoustical Society will be held Wednesday, 2 July, starting at 5:40 p.m. in the Bleu Amphitheater (level 3). This session will be held in French.

\section{EXHIBITION AND OPENING RECEPTION}

The meeting will be highlighted by an exhibition covering all areas of acoustics (instruments, equipment, software, services, etc.).

The exhibition is conveniently located in Havane Hall (level 3) near the registration area and meeting rooms. In order to facilitate contact between participants and exhibitors, both morning and afternoon coffee breaks as well as the opening reception will be held in the exhibition area.

The exhibition will open on Monday, 30 June, at 11:40 a.m. with an opening reception from 11:50 a.m. to 1:00 p.m. The exhibition schedule is: Monday, 30 June, 8:00 a.m. to 6:00 p.m., Tuesday, 1 July through Thursday, 3 July, 8:00 a.m. to 6:00 p.m., and Friday, 4 July, 8:00 a.m. to 4:00 p.m. The exhibition will close on Friday, 4 July, at 4:00 p.m.

A 5\% discount is given to companies or institutions that are sustaining members of ASA, SFA, or any National Acoustical Society of the EAA. Detailed information about the exhibition is available on the meeting website at http:// www.acoustics08-paris.org/exhibition and in the exhibition catalog.

\section{MEETING CD ROM}

Proceedings of the Acoustics'08 Paris meeting will be published and distributed at the meeting to all participants. It will consist in three separate CDs: one general, one euronoise, and one ecua. The three CDs are included in the registration fees.

\section{ECUA PROCEEDINGS}

Paper proceedings will be available for the ecua part of the meeting and will be sold separately at production cost.

\section{SPONSORS}

The following institutions and companies are sponsors of the Acoustics'08 Paris meeting:

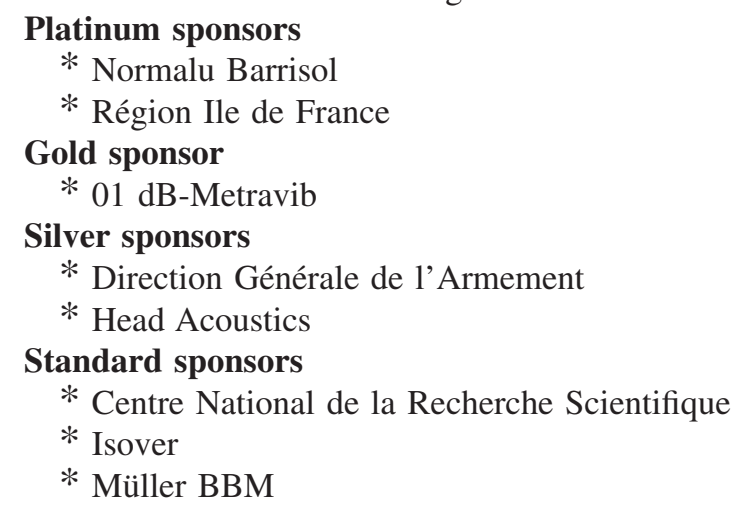

\section{COFFEE BREAKS}

Coffee breaks will be held in Maillot Hall A (level 2) and in Havane Hall (level 3). Morning coffee breaks will be held between 9:40 a.m. and 11:20 a.m. on Tuesday, Wednesday, Thursday, and Friday. Afternoon coffee breaks will be held between 3:20 p.m. and 5:00 p.m. on Monday, Tuesday, Thursday, and Friday. An afternoon coffee break will not be scheduled on Wednesday.

A cash bar and snacks will be available at the Arlequin bar (level 3) during the meeting from 8:00 a.m. to 6:30 p.m.

\section{A/V PREVIEW ROOM}

Rooms 234M and 235M (level 2.5) will be set up as A/V preview rooms for authors' convenience, and will be available on Monday from 12:00 noon to 6:00 p.m. and Tuesday through Friday, 8:00 a.m. to 6:00 p.m.

\section{E-MAIL AND INTERNET ACCESS}

Areas providing internet access will be available in rooms 237M (level 2.5) and 315 (level 3) on Monday from 12:00 noon to 6:00 p.m. and Tuesday through Friday from 8:00 a.m. to 6:00 p.m. The internet areas will provide desktop computers and ethernet connections for attendees laptop computers. Information about WiFi access will be available at the meeting.

\section{BUFFET SOCIAL}

A reception will be held on Tuesday evening, 1 July. Further information will be given on the meeting website.

\section{BANQUET}

The banquet will be held on Wednesday evening, 2 July. Further information will be provided on the meeting website. Those who wish to attend must make reservations on the meeting website at www.acoustics08-paris.org/registration/ before Friday, 23 May. After 23 May, reservations can only be accepted on a space-available basis.

\section{STUDENTS LUNCHEON}

The students luncheon will be held at 12:40 p.m. on Wednesday 2 July. The location will be provided on the meeting website and on the luncheon ticket. This reception will provide an opportunity for students to meet informally with fellow students and other meeting participants. All students are encouraged to attend, especially students who are first time attendees or those from smaller universities. The luncheon is free for all students but requires prior reservation. Those who wish to attend must make reservations on the meeting website at www.acoustics08paris.org/registration/ before Friday, 23 May. After 23 May, reservations can only be accepted on a space-available basis.

\section{COMMITTEE ON WOMEN IN ACOUSTICS LUNCHEON}

The Women in Acoustics luncheon will be held at 12:40 p.m. on Tuesday, 1 July, in Maillot Hall (level 2). After 8 March, the fee is $€ 20$ (€10 for students). Those who wish to attend must make reservations on the meeting website at www.acoustics08-paris.org/registration/ before Friday, 23 May. After 23 May, reservations can only be accepted on a space-available basis.

\section{CHILD CARE}

Information concerning child care will be added to the meeting website at www.acoustics08-paris.org/venue/childcare when details become available. 


\section{ACCOMPANYING PERSONS PROGRAM}

Spouses and other visitors are welcome at Acoustics'08 Paris. The on-site registration fee for accompanying persons is $€ 200$ and entitles participation in the Accompanying Persons Program, but does not include access to meeting rooms (except Opening, Closing, and Awards Ceremonies).

A hospitality room for accompanying persons will be open in Maillot Foyer (level 2) at the Palais des Congrès from 8:00 a.m. to 11:00 a.m. Tuesday through Friday.

Further information on the accompanying persons program is available on the conference website at www.acoustics08-paris.org/accompanying-persons.

\section{PRE- AND POST-CONFERENCE TOURS}

Information concerning pre- and post-conference tours will be added to the meeting website when details become available.

\section{PARIS CITY INFORMATION}

Weather-Paris in July is usually sunny but of course showers may occur. Temperatures during the day range from an average high of 77 degrees $F$ ( 25 degrees $\mathrm{C}$ ) to an average low of 59 degrees (15 degrees $\mathrm{C}$ ).

Local Time-Standard time zone (GMT $+1 \mathrm{hr})$ and daylight saving time $(+1 \mathrm{hr})$ give a time zone offset of GMT +2 hours.

Currency and foreign exchange-The local currency is EURO (€). Currency can be exchanged at some banks and preferably at "Bureau de change" that can be found all around the "Palais de Congrès," in large department stores, main railway stations, airports and near tourist attractions. Please note: Although the exchange rate is fixed, commission rates are not. They have to be clearly displayed.

Banking and credit cards-Banks are open generally from 9:00 a.m. to 5:00 p.m. with an optional break at lunch time, from Monday to Friday or from Tuesday to Saturday. Automatic Teller Machines (cash dispensers) can be found almost everywhere and accept most international cards (Visa, Eurocard, and Mastercard). In shops and restaurants, mainly Visa, Eurocard, and Mastercard are accepted. American Express cards are also accepted in several places (but not all, please check signs at the entrance).

Electricity-The power supply is $220 \mathrm{~V}, 50 \mathrm{~Hz}$. Round European style two-pin plugs are used. Appliances designed to operate on $110 / 120 \mathrm{~V}$ require a voltage converter and a plug adapter.

Restaurants-Paris offers a large variety of restaurants and cafés. Prices for a full menu (3 courses) range from about 20 to 40 Euros. Typical lunch time is $12: 30$ p.m. and dinner 8:00 p.m.

Useful links-For the latest information about the city of Paris, please visit the following links:

Paris Convention and Visitors Bureau website: http:// en.parisinfo.com

General info about Paris: http://www.paris.org, http:// www.v1.paris.fr/en/

Pariscope magazine, what's up in Paris (in French): http:// www.pariscope.fr

Tickets for all shows, concerts, and events in Paris: http:// www.ticketnet.fr/shop/en/accueil.asp

Metro interactive map: http:/www.ratp.fr

Find a place or itinerary in Paris: http://www.mappy.fr

\section{MEETING ORGANIZING COMMITTEE}

Bertrand Dubus (SFA), Michael Vorländer (EAA), William Yost (ASA), and Manell Zakharia (SFA), Cochairs; Didier Cassereau, Information System Manager; Catherine Lavandier, Secretary; Francine Luppé and Jean-Dominique Polack, Publications; Manuel Melon and Guillaume Pellerin, Webmasters; Bastian Epp, EAA Student Representative; Brian Monson, Michael Canney, ASA Student Representatives; Franck Teston, Treasurer; ASA: Elaine Moran (ASA Office Manager), Charles Schmid (ASA Executive Director); SFA: Evelyne Dewayse (SFA Secretary), Armelle Guilloux (Conference Secretary).

\section{TECHNICAL ORGANIZING COMMITTEE}

Philippe Blanc-Benon (SFA), Dick Botteldooren (EAA), William Hartmann (ASA), Pascal Laugier (SFA), Technical Program Cochairs; Architectural Acoustics: Lily Wang and Brandon Tinianov (ASA), Jens Holger Rindel (EAA); Animal Bioacoustics: Richard Fay (ASA), Michel André (EAA); Biomedical Ultrasound/Bioresponse to Vibration: Michael Bailey (ASA), Oleg Sapozhnikov (EAA); Engineering Acoustics: Daniel Warren (ASA), Gerhard Sessler (EAA); Musical Acoustics: James Beauchamp (ASA), Murray Campbell (EAA); Noise: Brigitte SchulteFortkamp (ASA), Pierre-Etienne Gautier (euronoise); Physical Acoustics: Murray Korman (ASA), Walter Lauriks (EAA); Psychological and Physiological Acoustics: Elizabeth Strickland (ASA), Armin Kohlrausch (EAA); Signal Processing in Acoustics: David Chambers (ASA), Stephen Elliott (EAA); Speech Communication: Maureen Stone and Carol Espy-Wilson (ASA), Pierre Badin (EAA), Xavier Pelorson, AFCP (Association Française de Communication Parlée); Structural Acoustics and Vibration: Sean Wu (ASA), Wolfgang Kropp (EAA); Underwater Acoustics and Acoustical Oceanography: James Miller and Kevin LePage (ASA), Yann Stéphan and David Bradley (ecua).

ECUA Committee: David Bradley (ASA), Yann Stéphan (SFA), Cochairs; ASA Liaisons: Michael Buckingham, Kenneth Foote, Oleg Godin, William Kuperman, James Lynch, Henrik Schmidt, Eric Thorsos, Ross Chapman, James Miller, Kevin LePage; ECUA: Leif Bjørnø, Philippe Blondel, Giovanni Cannelli, Jean-Pierre Hermand, Sergio Jesus, Even Borten Lunde, Nicholas Pace, John Papadakis, Dick Simons, Andrzej Stepnowski, Manell Zakharia, EAA TC Hydroacoustics: Eugeniusz Kozaczka (TC chair), Bodo Nolte, Michael Taroudakis.

Euronoise Committee: Brigitte Schulte-Fortkamp (ASA), Pierre-Étienne Gautier (SFA), Cochairs; ASA Liaisons: Bennett Brooks, Anthony Hoover, Luc Mongeau, James Nelson, Michael Stinson; Euronoise Liaisons: Bernard Berry, Luigi Maffei, Joachim Scheuren, Jean Tourret; EAA TC Noise Liaisons: Keith Attenborough, Michel Bérengier, Giovanni Brambilla, Geert Degrande, Klaus Genuit, Barbara Griefahn, Michael Griffin, Jean-Louis Guyader, Fabrice Junker, Sabine Meunier, Anna Preis. 


\section{TECHNICAL PROGRAM ORGANIZING COMMITTEE}

David Adams, Jens Rindel, Architectural Acoustics; Andone Lavery, Acoustical Oceanography; Michel André, Animal Bioacoustics; Neil Owen, Emmanuel Bossy, Biomedical Ultrasound/Bioresponse to Vibration; Martin Ochmann, Computational Acoustics; Daniel Warren, Engineering Acoustics; Antoine Chaigne, Murray Campbell, Musical Acoustics; Brigitte Schulte-Fortkamp, Keith Attenborough, Pierre Etienne Gautier, Noise; Peter Rogers, Walter Lauriks, Physical Acoustics; Alain de Cheveigne, Armin Kohlrausch, Psychological and Physiological Acoustics; Jean-Pierre Hermand, Steven Elliott, Signal Processing in Acoustics; Paul Iverson, Pierre Badin, Speech Communication; Yves Berthelot, Wolfgang Kropp, Structural Acoustics and Vibration; David Bradley, Kevin LePage, Yann Stéphan, Underwater Acoustics.

\section{PHOTOGRAPHING AND RECORDING}

Photographing and recording during regular sessions are not permitted without prior permission from the Acoustics'08 Paris Organizing Committee.

\section{NOTE TO SMOKERS}

Smoking is prohibited indoors.

\section{ABSTRACT ERRATA}

This meeting program is Part 2 of the May 2008 issue of The Journal of the Acoustical Society of America. Corrections, for printer's errors only, may be submitted for publication in the Errata section of the Journal.

\section{GUIDELINES FOR ORAL PRESENTATIONS}

\section{A. Preparation of Visual Aids}

1. Allow at least one minute of your talk for each slide or other visual aid, e.g., no more than 12 slides for a 15minute talk.

2. Minimize the number of lines of text and the number of curves shown on one visual aid. More than 12 lines of text or 5 curves are too many to be comprehended within one minute. Too little is better than too much.

3. Make symbols no less than $1 / 3$ the height of a capital letter. Break away any line that would otherwise pass through a character or symbol.

4. Black lines on clear background or white lines on black background are more legible than colored lines. Use color only if required to distinguish different curves or elements.

\section{B. Presentation}

1. Organize your talk with introduction, body, and summary or conclusion. Include only facts or concepts that can be explained adequately in the allotted time. Rehearse talk so you can confidently deliver it in that time.

2. An audible signal will be broadcast to note the start and end of your paper. Session Chairs have been instructed to adhere to the time schedule and to stop your presentation if you run over.

3. An $\mathrm{A} / \mathrm{V}$ preview room will be available for viewing computer presentations, transparencies, or videotapes before your session starts.
4. Arrive early enough so that you can meet the session chair, load your computer presentation onto the computer provided, and familiarize yourself with the microphone, laser pointer, and other equipment.

5. Every time you display a graphic the audience needs time to interpret it. Although you are familiar with the data, the audience may not be. Describe the abscissa, ordinate, units, and the legend for each curve. If the shape of a curve or some other feature is important, tell the audience what they should observe in order to grasp the point. They won't have time to figure it out for themselves.

\section{References}

1. Hubbard, H.H. "Guidelines for the planning and preparation of illustrated technical talks," J. Acoust. Soc. Am. 60, 995-998 (1976).

2. Young, R.W. “On presenting a technical paper,” J. Acoust. Soc. Am. 61, 1086-1087 (1977).

\section{SUGGESTIONS FOR EFFECTIVE POSTER PRESENTATIONS}

A board approximately 37 in. (width) $\times 63$ in. (height) $(0.94 \times 1.6 \mathrm{~m})$ in portrait layout will be provided for the display of each poster paper. Supplies will be available for attaching the poster material to the display boards. Each board will be marked with the board location number. Note that the board location number may not correspond with the abstract number. Authors should furnish, as part of their poster presentation, a sign giving the abstract number, paper title and author(s) name, in lettering approximately $1-1 / 2$ inch $(3.8 \mathrm{~cm})$ high. Free-hand lettering with a felt pen is entirely adequate.

A poster paper should be able to "stand alone," that is, be understandable even if the author is not present to explain, discuss, and answer questions. This is highly desirable because the author may not be present for the entire time the poster papers are on display, and when the author is engaged in discussion with one person, others may want to study the display without interrupting an ongoing dialogue. To meet the "stand alone" criteria, it is suggested that authors consider the following elements for a poster. Each element can be arranged on one or more standard letter-size sheets. Where typing is needed, large font type is very effective. Careful hand lettering, at least $1 / 4$ in. $(0.6 \mathrm{~cm})$ high, is also acceptable. Obviously, it may not be appropriate for any given paper to include all of the "elements" listed below. These are merely suggestions:

1. Objective, purpose, goal, etc.

2. Background information. Prior work.

3. Assumptions, etc.

4. Experimental arrangements, block diagram, sketch, photos, parameters.

5. Outline of the theoretical development.

6. Data, graphs, tables, etc.

Graphs and photographs should be a minimum of $8 \times 10$ inches $(20 \times 25 \mathrm{~cm})$. Figure captions are essential. Avoid glossy paper. Display objects, such as transducer elements or materials, demonstration tapes, etc., are excellent supplements to a poster. Since the poster paper is not archival, feel free to bring incompletely reduced data for discussion. 


\section{DATES OF FUTURE MEETINGS}

For further information on any ASA meeting, or to obtain instructions for the preparation and submission of meeting abstracts, contact the Acoustical Society of America, Suite 1NO1, 2 Huntington Quadrangle, Melville, NY 11747-4502; Telephone: 516-576-2360; Fax: 516-576-2377; E-mail: asa@aip.org ; Web: http://asa.aip.org 156th Meeting, Miami, Florida, 10-14 November 2008 157th Meeting, Portland, Oregon, 18-22 May 2009

For further information on any EAA meeting, please refer to EAA website at www.eaa- fenestra.org/Events

6th Forum Acusticum, Aalborg, Denmark, 2011 


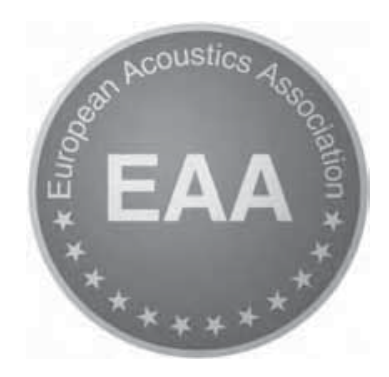

\section{Serving 30 societies with more than 9000 members www.eaa-fenestra.org www.european-acoustics.net}

The European Acoustics Association (EAA) is a non-profit entity established in 1992 that includes in its membership societies in European countries interested in the objectives of the EAA. The objectives of EAA are as follows:

- To promote and spread the science of acoustics, its technologies and applications, throughout Europe and the entire world.

- To interface with associations whose activities are related to acoustics, in order to promote development and progress of acoustics in its different aspects and applications.

- To establish contacts across member associations from each country, with public and private organisations and enterprises, with associations, science institutions, universities, professional organisations, etc., to assist them in reaching their goals.

- To promote the formation of national acoustical societies in European countries where these do not exist, and to support and strengthen activities of national associations in those countries where they do exist, respecting the principle of subsidiarity.

- To promote acoustic research and application of corresponding technologies. To publish a European journal on acoustics, in printed as well as in electronic format.

- To organize and promote congresses, to publish books and monographs, and to engage in all those activities that are connected with the diffusion, promotion and development of acoustics.

- To establish agreements for collaboration with European and international entities in order to better serve the objectives of EAA.

- To foster the exchange of knowledge, experience and initiatives present in any one of the member countries, for better development of and progress in acoustics.

- To stimulate education activities and platforms in acoustics at all educational levels, both academic and professional.

- To promote and divulge the establishment and implementation of norms and recommendations in the various fields of acoustics, especially in the area of environmental acoustics, for a better quality of life.

The EAA gathers 30 acoustical societies and serves more than 9000 individual members all over Europe.

National Acoustical societies from Austria, Belgium, Bulgaria, Croatia, Czech Rep., Denmark, Finland, France, Germany, Great Britain, Greece, Hungary, Iceland, Italy, Latvia, Lithuania, Netherlands, Norway, Poland, Portugal, Romania, Russia, Serbia \& Montenegro, Slovakia, Slovenia, Spain, Sweden, Switzerland, Turkey, and Ukraine are members of EAA. EAA is democratically organized (one vote per country) with an assembly, a board and an executive council.

EAA offers a range of products:

ACTA ACUSTICA united with ACUSTICA

Product Manager and Editor in Chief: Dick Botteldooren

Acta Acustica united with Acustica is an international, peer-reviewed journal on acoustics and is the journal of the EAA. It is published by Hirzel Verlag.

The journal reports on original scientific research in all subjects in the field of acoustics and on engineering applications. The journal includes scientific papers, technical and applied papers, book reviews, short communications, doctoral thesis abstracts, etc. In irregular intervals special issues and review articles are also published. See www.acta-acustica-united-with-acustica.com for more information.

Members of the National Acoustical Societies belonging to EAA and corresponding members of the EAA receive Acta Acustica united with Acustica as part of their membership or can subscribe to the journal at a special rate. The journal is available online via Ingenta Connect: www.ingentaconnect.com/content/dav/aaua.

\section{NUNTIUS ACUSTICUS}

Product Manager: Brigitte Schulte-Fortkamp

Nuntius Acusticus is the "acoustic messenger" of EAA to vitalize communication between and in the European acoustical societies on a variety of topics. It is published bimonthly in electronic format and distributed together with Acta Acustica united with Acustica. The Nuntius Acusticus provides news of acoustics: information on EU activities, on noise control policy or on other political topics, education in acoustics, education and research guides and news or publications from the Technical Committees, new products, instrumentation and materials. It also contains national journals or newsletters of the European acoustical societies.

\section{EAA INDEX}

\section{Product Manager: Tino Bucak}

(former Product Manager: Amando Garcia)

The EAA Index, published in electronic format every 3 years, is a collection of useful information for all Societies' members and for the entire community of European acousticians.

The EAA Index contains general information concerning each Society member (addresses, history, profile), an individual membership directory (data of all individual members of each Society including main field of interest) and a directory of Sustaining Members, institutions and companies with brief activity descriptions. It also contains information, such as that related to EAA products (Acta Acustica united with Acustica, Fenestra, Nuntius Acusticus, etc.), the EAA Statutes, past and present EAA Officers, the PACS classification scheme, and a section titled Cyber@coustics, where a significant number of most useful Internet addresses closely related with acoustics are collected.

\section{DOCUMENTA ACUSTICA}

\section{Product Manager: Sergio Luzzi}

(former Product Manager: Jean Dominique Polack)

Documenta Acustica is the literature distribution system of the EAA. It distributes conference and symposia proceedings as well as books, reports and theses. It helps to distribute information that is otherwise confined to European acoustical societies, laboratories or companies.

Information on items in stock and order forms are available on www.eaafenestra.org. Documenta Acustica was handled by the French Acoustical Society (SFA) from January 1999 to January 2008 at which time it was transferred to the Acoustical Society of Italy (AIA) for handling. 


\section{FENESTRA}

Product Manager: Malte Kob

Fenestra Acustica, the website of EAA, is available at: www.europeanacoustics.net - www.eaa-fenestra.org - www.european-acoustics.org www.euracoustics.org

Fenestra provides information on the association and its members (products, technical committees, organisational structure and policies, contact information), up-to-date news, upcoming events, links to other non-profit organisations in acoustics and much more. A special section - EAA job market - offers information about vacant positions or scholarships in acoustics at universities, research centres, and firms.

\section{FORUM ACUSTICUM}

Contact persons: Luigi Maffei, Peter Svensson

Forum Acusticum is the triennial international convention organised by a national acoustical society on behalf of the European Acoustics Association (EAA). It is, in effect, a forum comprising a variety of different activities: a) high-quality scientific congress with invited plenary lectures, structured sessions, invited and contributed papers, b) a special focus on European topics, such as standardisation, regulations, $\mathrm{R} \& \mathrm{D}$ programs, professional certification, education in acoustics, c) an exhibition that includes commercial firms, laboratories and agencies, d) a social meeting of acousticians with receptions, visits, awards.

1996: Antwerp, Belgium; 1999: Berlin, Germany (jointly with the Acoustical Society of America); 2002: Seville, Spain; 2005: Budapest, Hungary; 2008: Paris, France (jointly with Acoustical Society of America); 2011: Aalborg, Denmark

\section{EURONOISE}

\section{Contact person: Joachim Scheuren}

Euronoise is the European Conference and Exhibition on Noise Control, coordinated by the EAA Technical Committee Noise and organised by a national acoustical society on behalf of the European Acoustics Association (EAA).

1992: London, Great Britain; 1995: Lyon, France; 1998: Munich, Germany; 2001: Patras, Greece; 2003: Naples, Italy ; 2006: Tampere, Finland; 2008: Paris, France (included in Forum Acusticum); 2009: Edinburgh, Scotland, UK

\section{EAA SYMPOSIA}

Contact person: Kristian Jambrošić

EAA symposia are scientific meetings under the aegis of the EAA with a focus on specialised fields. They are typically organized by one or more member societies of EAA in conjunction with the Technical Committees of EAA. Other prerequisites are special registration fees for members of EAA member societies, English as one of the official languages of the meeting, availability of proceedings for Documenta Acustica. EAA supports the symposia through announcement in its official media and offering an electronic system for the symposium and proceedings management.

\section{SCHOLA}

Product manager: Malte Kob

The new product Schola is an online platform for education in acoustics in Europe: www.european-acoustics.net/schola.

Through Fenestra, it offers information on university acoustics courses in Europe. Schola supports joint education initiatives of European universities on acoustics at the different levels (Bachelor, Master, Ph.D.) Since 2005 EAA has launched a policy in favour of young researchers. EAA Young Researcher Grants have been awarded to students in East European countries for participation in international conferences on acoustics. Recently, through Schola, EAA has started to support the EAA Student and Young Researchers Council whose purposes are, among others, to connect young acousticians across Europe to share problem solutions and exchange ideas via the Internet and at acoustics conferences.

\section{TECHNICAL COMMITTEES (TCs)}

\section{Contact person: Michael Vorländer}

EAA has 7 technical committees which, at different levels, are in charge of organizing specific activities (technical reports, round robin tests, structured session organization at congresses, symposia, etc.). Technical committees are open to all individual members of EAA member societies who are interested in the specific topic and each technical committee is coordinated by a Chairman.

TCs: Computational Acoustics, Musical Acoustics, Hydroacoustics, Noise, Psychological and Physiological Acoustics, Room and Building Acoustics, Ultrasound.

\section{BOARD 2007-2009}

President: Luigi Maffei

Vice President: Michael Vorländer

Vice President: Peter Svensson

General Secretary: Kristian Jambrošić

Treasurer: J. Salvador Santiago

\section{EAA Office:}

Antonio Perez-Lopéz

SEA, Serrano 1444, 28006 Madrid, Spain

Past Boards

EAA 2004-2006

Michael Vorländer (president), Eugeniusz Kozaczka (vice president), JeanDominique Polack (vice president), Luigi Maffei (general secretary), J. Salvador Santiago (treasurer)

EAA 2001-2003

Volker Mellert (president), Truls Gjestland (vice president), Andrea Paoloni (vice president), Etienne Parizet (general secretary), J. Salvador Santiago (treasurer)

EAA (EEIG) 1998-2000

Adrian Houtsma (chairman), Peter Wheeler (executive director), Antonio Perez-Lopéz (financial controller)

EAA (EEIG) 1995-1997

Jens Blauert (chairman of the board), Manell Zakharia (managing director), Mark Tatham (controller)

EAA (EEIG) 1992-1996

Jens Blauert (chairman of the board), Armand Dancer (managing director), Peter Wheeler (controller) 


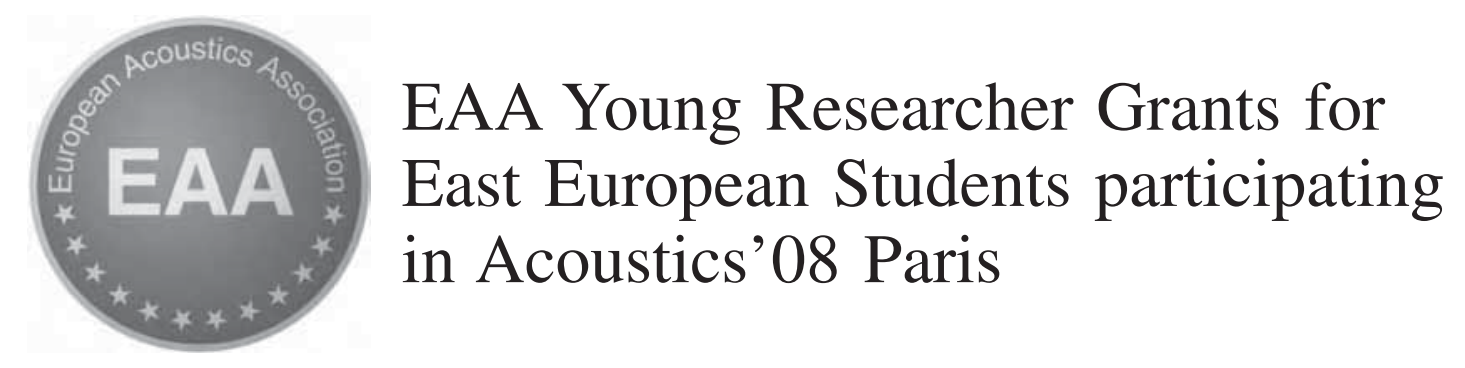

Following the announcement of the student grants in FENESTRA (www.eaa-fenestra.org) and by email to the national EAA member societies, in total 19 applications were submitted from Croatia, Czech Republic, Hungary, Lithuania, Poland, Slovakia and Russia.

EAA is grateful for the excellent applications and the interesting proposals and abstracts for Acoustics'08 Paris. The jury had to make a hard decision about the 11 winners. The main criteria were quality of the application and the abstract.

The winners are (in alphabetical order):

\begin{tabular}{lllll} 
Name & First Name & Country & Abstract & Page \\
\hline Bőhm & Tamás & Hungary & $5 a S C c 27$ & S918 \\
Faranosov & Georgy A. & Russia & 2pNSb8 & S281 \\
Havranek & Zdenek & Czech Rep. & 2pSAa9 & S342 \\
Kazdailis & Paulius & Lithuania & 2pPAc4 & S307 \\
Keprt & Jiri & Czech Rep. & 2pSAe10 & S349 \\
Lapka & Wojciech & Poland & & \\
Petošić & Antonio & Croatia & $4 p$ Ad7 & S728 \\
Shurup & Andrey S. & Russia & $4 p U W h 7$ & S790 \\
Tomoriová & Beáta & Slovakia & $4 p P P c 17$ & S750 \\
Yuldashev & Petr V. & Russia & 2pNSb1 & S280 \\
Zhitlukhina & Yulia V. & Russia & $4 p P A e 4$ & S730 \\
\hline
\end{tabular}

With congratulations,

for the jury,

Luigi Maffei

President of EAA 


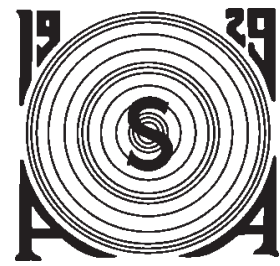

\section{Acoustical Society of America http://asa.aip.org}

\section{What we are:}

The Acoustical Society of America (ASA) is the world's premier society devoted to the science and technology of acoustics. ASA was organized in 1929, and now:

- has over 7,500 members worldwide

- publishes the world's leading journal on acoustics

- holds semi-annual week-long meetings advancing all fields of acoustics

- publishes ANSI and international standards

- keeps members informed on all aspects of acoustics

\section{Who we are and what we do:}

ASA membership includes men and women from over 70 countries: engineers, physicists, speech and hearing scientists, psychologists, biologists, and scientists in many other disciplines. ASA meetings feature exceptional congeniality and convenient access to the broad spectrum of acoustics. Whether it is the songs of humpback whales, advances in speech and hearing science, the design of concert halls or musical instruments, or medical applications of ultrasound, you will hear about it in the ASA. The ASA offers a variety of membership options including full Membership, Associate Membership, Corresponding Associate Membership, and Student Membership. All members receive a subscription to The Journal of the Acoustical Society of America. ASA offers students and recent graduates opportunities for fellowships, scholarships, and travel funds. (It provided travel support for over 100 students for Acoustics'08 Paris). Its Committee on Women in Acoustics and its Student Council promote activities for women and students within the Society.

\section{Benefits of membership:}

- The Journal of the Acoustical Society of America (JASA) (over 7,000 pages per year)

- JASA Express Letters (open access journal)

- Acoustics Today magazine (soon to be published online)

- ECHOES newsletter on current issues

- Proceedings of Meetings on Acoustics (online open access publication)

- Access to ASA's Digital Library, including JASA archives back to 1929

- Reduced meeting registration fees

- Hardcover and Online Books and CD ROM discounts (see next page)

- Free standards

- Job postings in acoustics

- Electronic announcements of important information on acoustics

- Recognition of outstanding acousticians through medals, awards, and prizes

- Promotion of acoustics to the general public

- Acoustics.org containing resources on acoustics for the general public

- Educational opportunities for young students

Details about membership and how to join can be found at $<$ http://asa.aip.org/map_membership.html $>$. 


\section{Upcoming ASA meetings and ASA cosponsored symposia:}

2008

- Aug. 19-22 - Acoustic Communication by Animals, Corvallis, Oregon

- Nov. 10-14 - 156 $6^{\text {th }}$ ASA meeting, Miami, Florida

2009

- May 19-23 - $157^{\text {th }}$ ASA meeting, Portland, Oregon

- May 23-24 - Second Language Speech Learning, Portland, Oregon

- Sep. 25-26 - Pacific Rim Underwater Acoustics Conference, Xían, China

- Oct. 26-30 - $158^{\text {th }}$ ASA meeting, San Antonio, Texas

\section{ASA Books, CDs, and Videos:}

The ASA has published over 50 titles covering a wide variety of topics on acoustics. These include reprints of classic books, poster session collections, translations, and reprint collections in hard cover, online, and CD ROM formats. Following are examples of the titles available from ASA. The full catalog, with tables of contents and prefaces, can be found online at $<$ http://asa.aip.org/publications.html $>$. Online books and acoustical standards can be purchased for immediate download from the ASA Store at $<\mathrm{http}$ //asastore.aip.org/> where you will find a complete listing of available publications.

\section{Hardcover titles:}

Acoustics by Leo L. Beranek

Acoustics-An Introduction to Its Physical Principles and Applications by Allan D. Pierce

Experiments in Hearing by George von Békésy

Handbook of Acoustical Measurements and Noise Control edited by Cyril M. Harris

Halls for Music Performance edited by Ian Hoffman, Christopher Storch, and Timothy Foulkes

Vibration of Plates and Vibration of Shells by Arthur Leissa

\section{Online titles:}

Acoustics by W. Donkin

Thermoacoustics by Gregory W. Swift

Hearing in Vertebrates: A Psychophysical Handbook edited by Richard R. Fay

\section{CD titles:}

Scientific Papers by Lord Rayleigh 1869-1919

Auditory Demonstrations

\section{Videos:}

Measuring Speech Production - demonstrations for teaching courses on speech acoustics, physiology, and instrumentation produced by Maureen Stone

Speech Perception produced by Patricia K. Kuhl

\section{Standards:}

American National Standards developed by Accredited Standards Committees S1-Acoustics, S2-Mechanical Vibration and Shock, S3-Bioacoustics, and S12-Noise

ISO Standards 


\section{Société Française d'Acoustique http://www.sfa.asso.fr}

The French Acoustical Society (Société Française d'Acoustique, SFA) is a non-profit association uniting the French and French-speaking acoustician community. Initially created in 1948 as the "Groupement des Acousticiens de Langue Française" (GALF), it exists in its present form since 1986. Its aim is to promote acoustics in all its aspects: research, applications, and teaching.

About a thousand members belong to the SFA, representing a variety of professional activities and nations (about twenty countries). They include engineers, university teachers, researchers, physicians, and audiologists, covering a wide range from ultrasonics to speech and hearing. SFA members are structured according to their specialities and regions of origin. These groups and sections are active in organising both high-level scientific meetings as well as popularizing events.

The SFA is active at the national, the European and the international levels. It organizes regular scientific meetings, such as the Congrès Français d'Acoustique (CFA). It also co-operates with other French scientific societies such as the Société Française de Physique (SFP), the Société des Électriciens et Électroniciens, the Société Hydrotechnique de France (SHF) and the Société Française des Mécaniciens (SFM), in organising trans-academic congresses. Finally, as one of the main founding members of the European Acoustics Association (EAA), SFA takes part in international committees or associations on acoustics such as the International Committee on Acoustics (ICA) and the International Institute for Noise Control Engineering (I-INCE).

The SFA has representatives in several national authorities such as the Conseil National du Bruit (the National Council on Noise, created under the aegis of the French Department of Environment) and cooperates with official organisms such as the Centre d'Information et de Documentation sur le Bruit (CIDB).

The association offers four grades of membership:

- Active Members,

- Benefactor Members - individuals or institutions, private or public

- Honorary Members,

- Founding Members.

Its actions are supported both by public organisations (research laboratories, State institutions) and private companies (manufacturers or service providers).

The SFA distributes a bimonthly journal to its members: the "Courrier de la SFA", and a weekly eletter "Echo Hebdo". It also publishes, jointly with the Centre d'Information et de Documentation sur le Bruit (CIDB), a bimonthly journal "Acoustique et Techniques". It contributes, along with the other European Societies of acoustics, to the publication of "Acta Acustica united with Acustica". 
The SFA created, in partnership with the French publisher HERMES, a series of books in "Collection d'Acoustique". Several works have already been published, such as "Petite Histoire de l'Acoustique", by Pierre Liénard, "Acoustique Industrielle et Aéroacoustique" by Serge Léwy, and "Manuel d'Acoustique Fondamentale" by Michel Bruneau.

More information can be found on the SFA website: http://www.sfa.asso.fr

During Acoustics'08 Paris, the 60th anniversary of the French Acoustical Society will be celebrated, on Wednesday 2nd July. The celebration will be focused on the presentation of the "White Book" of acoustics in France, and the publishing of a DVD including the main part of the French journals in acoustics since 1934 . 
Order your acoustic brochure now, write an e-mail to

andrew.koster@saint-gobain.com

The ISOVER Acoustic Comfort Classes.

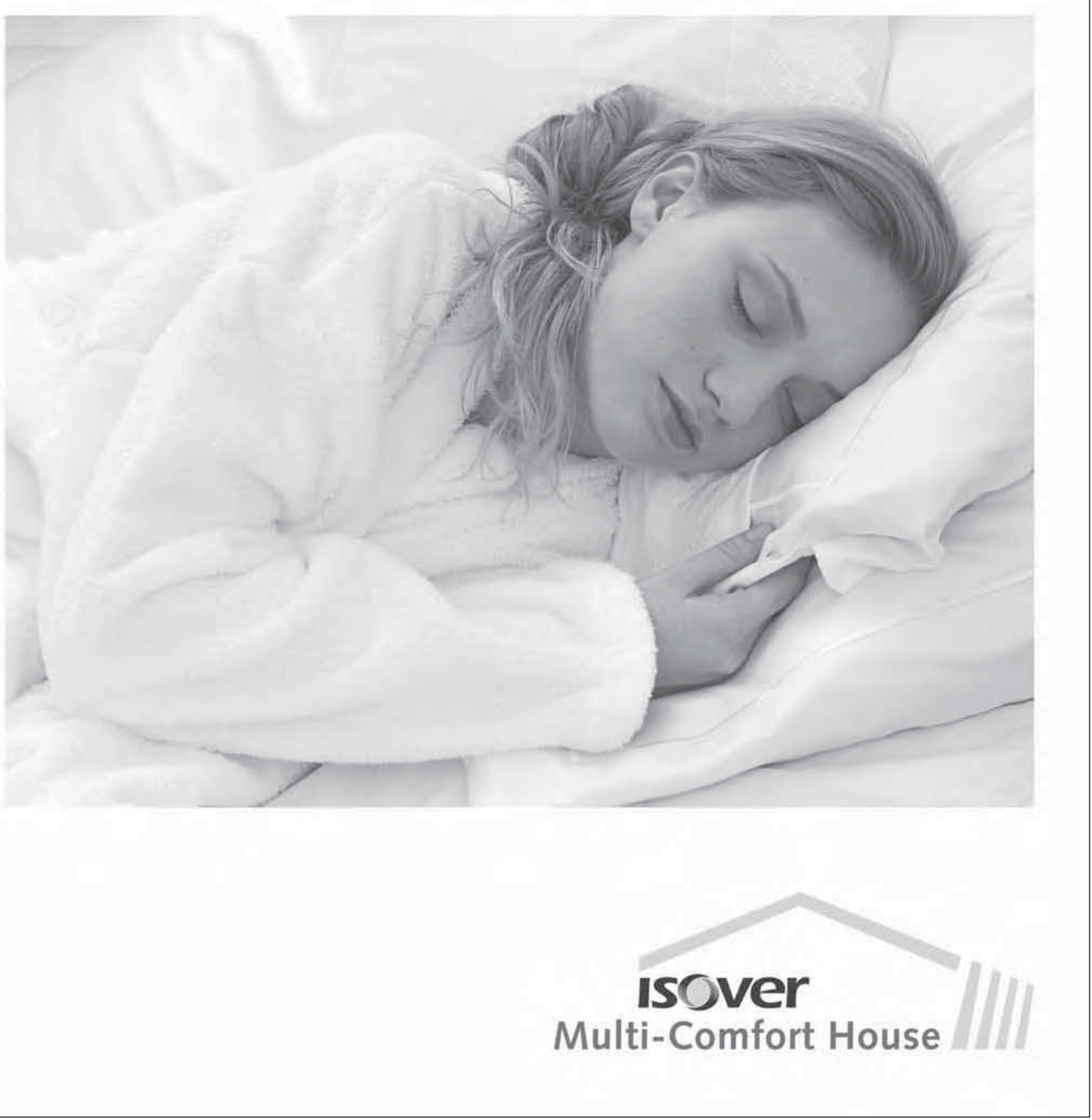




\section{Session 1aID}

\section{Opening Ceremony}

The Opening Ceremony will include a special welcome from the Vice-President of Ile de France Regional Council, addresses by National sponsors, and addresses by the Presidents of the Acoustical Society of America, the European Acoustics Association, and the French Acoustical Society. Musical interludes will be given and two plenary lectures will be presented.

\section{Plenary Lectures}

Introduced by George Frisk, Florida Atlantic University, USA

10:30

1aID1. How sound from human activities affects marine mammals. Peter Tyack (Woods Hole Oceanographic Institution, Applied Ocean Physics \& Engineering Dept., Woods Hole, MA 02543, USA, ptyack@whoi.edu)

Effects of sound on marine mammals have traditionally been defined either as injury or disruption of behavior. The earliest concern about behavioral disruption was that elevated noise could reduce the range of communication by masking faint signals. Few studies have documented this effect, but recent work emphasizes mechanisms animals use to compensate for elevated noise. Many studies have documented changes in behavior as a function of exposure, but it has proven difficult to relate these to effects on the welfare of individual animals or on the status of populations. Promising approaches to interpreting effects include avoidance of habitat, energetics of foraging, and applying models of anti-predator behavior to human disturbance. During the 1990s acoustic criteria for injury were designated based upon temporary hearing loss. Accumulating evidence of atypical mass strandings of beaked whales coincident with naval sonar exercises suggest that injury or death may result from behavioral responses of some species at lower exposure levels in some settings. A tagged beaked whale showed similar but weaker responses to experimental playback of a mid-frequency sonar sound compared to calls of killer whales, suggesting that anti-predator response may harm animals after exposure to levels of sound very unlikely to cause injury directly.

Introduced by Luigi Maffei, Second University of Naples, Italy

11:10

1aID2. New Trends in Aeroacoustics: From acoustic analogies to direct numerical simulations. Daniel Juvé (Ecole centrale de Lyon, 36 avenue Guy de Collongue, 69134 Ecully cedex, France, daniel.juve@ec-lyon.fr)

Modern aeroacoustics started in the early 1950's when Lighthill developed his famous acoustic analogy in an attempt to understand, and reduce, the terrifying noise generated by jet aircrafts. For nearly 50 years the subject of aerodynamic sound was dominated by approaches based upon this analogy or variants of it. Recently, the availability of powerful computing facilities combined with the development of numerical algorithms specially designed to simulate sound propagation over large distances has paved the way for "a second golden age of aeroacoustics" (to quote Lighthill himself). In this talk we will first give an overview of this evolution from acoustic analogies to computational aeroacoustics (CAA). Typical illustrations of the CAA approach will then be presented and applications for transportation systems will be discussed. 


\title{
Session 1pAAa
}

\section{Architectural Acoustics and Noise: Acoustics of Open-Plan Spaces I}

\author{
Kenneth P. Roy, Cochair \\ Armstrong Building Products, 2500 Columbia Ave, Lancaster, PA 17603, USA
}

Valtteri O. Hongisto, Cochair

Finnish Institute of Occupational Health, Lemminkäisenkatu 14-18 B, Turku, 20520, Finland

\author{
Stephen Sinclair, Cochair \\ Centre for Interdisciplinary Research in Music Media \& Technology, Schulich School of Music, McGill Univ., \\ Montreal H3A IE3, QC, Canada
}

\section{Invited Papers}

\author{
1:00
}

1pAAa1. Spatial decay, behaviour and space planning models European and International standards on open office acoustics. Pierre Chigot (Saint Gobain Ecophon SA, box 30030, 60291 Rantigny, France, pierre.chigot@saint-gobain.com)

Recent standardisation work on open plan offices acoustics is characterized by a shift in the way room acoustics is handled. Spatial decay (expressed through rate of spatial decay per doubling of distance, DL2) is preferred to temporal decay (expressed through reverberation time, RT). The complexity of open plan office acoustic design results from the fundamental contradiction of communication and concentration. DL2 reflects better the challenges of sound control in such rooms, which is basically sound propagation control. Beyond, this contradiction, specific space analysis models and tools can help to visualize the acoustic interactions between workstations, teams and departments accommodated in the same room. These models are now ready to be included in standardization work. International technical standard for field measurements of DL2 integrates new descriptive models for open plan office acoustics, taking into account geometric proportions, presence of screens and furniture as well as group behaviour and speech characteristics, such as increased energy contents at low frequencies, voice levels, raised hearing sensitivity at high frequencies. Also, normative guidelines from Netherlands and France integrating this approach will be presented.

1pAAa2. Acoustics and sustainable design in exposed structures. Kenneth P. Roy (Armstrong Building Products, 2500 Columbia Ave, Lancaster, PA 17603, USA, kproy@armstrong.com), Anita L. Snader (Armstrong World Industries, 2500 Columbia Ave, R\&D Bldg. 5, Lancaster, PA 17604, USA, alsnader@armstrong.com)

Two very important emerging architectural trends in the USA are to 1) 'open up' the space design as exposed structures, and 2) incorporate sustainable design into building interiors. We as acoustic designers would like to know the consequences of both on acoustics performance and occupant satisfaction and work performance. The first concern is addressed in a study by CISCA evaluating the effects of ceiling plenum vs. exposed structures in both office and retail spaces. And the second issue of compatibility of sustainable design objectives with acoustic performance is addressed by a pertinent field survey by the Center for the Built Environment (CBE at Univ. of CA, Berkeley) on occupant satisfaction and performance. The issue of the availability and use of 'green' acoustic building materials that not only meet sustainability and energy goals, but also serve to ensure that our buildings actually work for their intended purposes will be addressed. Finally, the increased awareness of the acoustic impact of 'green' design on occupant satisfaction is being seen in the evolution of LEED and other 'green' rating systems.

\section{$1: 40$}

1pAAa3. Differences in perception of noise and privacy in different office types. Christina Bodin Danielsson (The Royal Institute of Technology, KTH, Roslagsgatan 37, 11354 Stockholm, Sweden, c.danielsson@comhem.se)

Differences between office types with regard to their architectural and functional features may have an impact on the employees' disturbance by noise and perception of privacy. These aspects may in its turn have an impact on employees' satisfaction and psychological responses to the office environment. In a study 469 employees in 26 different companies have rated their satisfaction with the office environment in seven different office-types: cell-office, shared room office, small open plan office, medium open plan office, large open plan office, flex-office and combi-office. This paper discusses the results concerning the employees' perception of noise and privacy and put the results in relation to the two different aspects of privacy, visual and acoustic privacy, and the role of personal control. In the statistical analysis adjustments for potential confounders as age, gender, job rank and line of business were done, which are known to have an influence on perception and satisfaction. Substantial differences between employees in different office-types were found. The fact that there were such differences between different types of offices that mean sharing of workspace and work facilities was a surprise. Architectural and functional features of the offices are discussed as the main exploratory factors for these results. 


\section{Contributed Paper}

2:00

1pAAa4. Effect of sound masking on workers in an open office. Annu Haapakangas (Finnish Institute of Occupational Health, Lemminkäisenkatu 14-18 B, 20520 Turku, Finland, annu.haapakangas@ttl.fi), Valtteri O. Hongisto (Finnish Institute of Occupational Health, Lemminkäisenkatu 14-18 B, 20520 Turku, Finland, valtteri.hongisto@ttl.fi)

Appropriate masking sound is necessary for reaching acceptable speech privacy in open offices. However, the electronic masking systems have not become very popular although the importance of masking is emphasized in design guidelines worldwide. One reason may be that very few scientific field experiments have been published in this area. The aim of this pilot study was to investigate the effects of artificial masking sound on workers in a small department of 15 workers. Measurements and questionnaires were conducted before and after launching the system. Masking sound $44 \mathrm{dBA}$ was produced using centralized pink noise generator and ceiling loudspeakers. The spectrum reminded ventilation noise. Initial background noise level was $36 \mathrm{dBA}$. Acoustic measurements showed that masking sound reduced the radius of distraction $\mathrm{rD}$ from $15 \mathrm{~m}$ to $7 \mathrm{~m}$, i.e. acoustic privacy improved significantly. The questionnaire revealed several positive changes attributable to masking. Distraction caused by speech and other varying office sounds was reduced. Noise-related stress was reduced. Evaluations of acoustic environment, speech privacy and self-rated work efficiency were improved. The results showed no adverse effects of masking on workers.

\section{Invited Papers}

1pAAa5. Speech Privacy Options for the Open Ceiling Office Environment. Dana S. Hougland (Shen Milsom Wilke, Inc., 1822 Blake Street, Suite 2A, Denver, CO 80202, USA, dhougland@ smwinc.com)

The popularity of the open ceiling and loft office environment continues to grow. The design and effectiveness of sound masking systems in an open office environment are well established for traditional office spaces with acoustically absorptive ceilings systems. Aesthetic preferences for open ceilings with minimal ceiling treatment popular in the creative reuse of older buildings and more minimalist industrial spaces limit the sound masking options available to achieve an acceptable level of speech privacy in an open office environment. Traditional sound masking systems are often subjectively viewed as harsh, unpleasant or visually inappropriate. Several options for alternative designs are evaluated as to the evenness of coverage, effectiveness in achieving an appropriate level of speech privacy, aesthetic integration into the environment and subjective impression of the occupants.

2:40

1pAAa6. Effective sound masking for speech privacy in open plan offices. Joel Lewitz (Rosen Goldberg Der \& Lewitz, Inc., 1100 Larkspur Landing Circle, Suite 354, Larkspur, CA 94939, USA, jlewitz@RGDLacoustics.com)

For over 30 years acousticians have studied, measured and evaluated open plan offices to improve speech privacy and freedom from distraction. This paper addresses the relative importance of the many factors affecting speech privacy in open plan offices. Adjusting the background noise level at the receiver has proven to be a very powerful and relatively inexpensive method to optimize the acoustical environment in open plan offices. However, sound masking systems are not always properly tuned to achieve the maximum benefit. In this paper, the importance of the shape, smoothness and level of the masking sound spectrum are discussed and demonstrated with case studies. Recent legislation in the U.S., such as the Healthcare Insurance Portability and Accountability Act of 1996, also known as HIPAA, has broadened speech privacy concerns from open plan to closed plan. Sound masking is an attractive option for both configurations. Differences in sound masking design and tuning between open plan and closed plan are explored in this paper.

\section{3:00}

1pAAa7. Room acoustical measures for open plan spaces. Erling Nilsson (Saint-Gobain Ecophon AB, Box 500, SE-260 61 Hyllinge, Sweden, erling.nilsson@ecophon.se), Björn Hellström (ÅF-Ingemansson AB, Box 47321, SE-100 74 Stockholm, Sweden, Bjorn.Hellstrom@afconsult.com), Björn Berthelsen (Saint-Gobain Ecophon AB, Box 500, SE-260 61 Hyllinge, Sweden, bjorn .berthelsen@ecophon.se)

In an ongoing Nordic cooperation project the acoustical conditions in open-plan spaces are investigated. Of special interest is to find suitable acoustical parameters that reveal the acoustical conditions in these spaces and also are of importance for the subjective perceived acoustics. Measurements have so far been carried out in five open plan offices accompanied with an inquiry gathering the subjective judgments by the staff. A program for the acoustical measurements was designed specifying how to perform the measurements, which type of parameters to measure and how to report the results. The acoustical parameters included in the measurements are Reverberation time T20, Early Decay Time (EDT), Deutlichkeit (D50), Speech transmission index (STI), Speech intelligibility index (SII), Privacy Index (PI), Rate of spatial decay of sound pressure levels per distance doubling (DL2), Excess of sound pressure level with respect to a reference curve (DLf), background noise levels in occupied and unoccupied offices. The behavior of these parameters will be discussed and suitable parameters for evaluation of open plan spaces will be suggested.

\section{3:20-3:40 Break}

$$
3: 40
$$

1pAAa8. Characterization of acoustics in open offices - four case studies. Jukka S. Keränen (Finnish Institute of Occupational Health, Lemminkäisenkatu 14-18 B, 20520 Turku, Finland, jukka.keranen@ttl.fi), Petra Virjonen (Finnish Institute of Occupational Health, Lemminkäisenkatu 14-18 B, 20520 Turku, Finland, petra.virjonen@gmail.com), Valtteri O. Hongisto (Finnish Institute of Occupational Health, Lemminkäisenkatu 14-18 B, 20520 Turku, Finland, valtteri.hongisto@ttl.fi)

Acoustic design in open offices aims to reduction of distractions and improvement of speech privacy. Room acoustical design includes high room absorption, high and absorptive screens and appropriate masking sound level. The aim of this study is to show, how these individual design components can affect on room acoustics, using modern room acoustical descriptors. Individual acoustical remedies were studied in four case studies independently. The implemented room acoustical changes were increased ceiling absorption, 
sound-absorptive screens, curtains between workers and increased masking sound level. Radius of distraction, $\mathrm{r}_{\mathrm{D}}$, spatial decay rate of speech, $\mathrm{DL}_{2}$, and speech level at $4 \mathrm{~m}$ from speaker, $\mathrm{L}_{\mathrm{p}, \mathrm{S}, 4 \mathrm{~m}}$, were determined before and after the room acoustical change. These three simple and robust single-number parameters are recommended to characterize the acoustic conditions of open offices because they react logically to room acoustical changes.

\title{
Contributed Papers
}

\section{4:00}

1pAAa9. Possibilities for acoustic improvement of the far field in open-plan offices. Catrin Humer (TGM Federal Institute of Technology, Wexstrasse 19-23, 1200 Vienna, Austria, catrin.humer@tgm.ac.at), Herbert Muellner (TGM Federal Institute of Technology, Wexstrasse 19-23, 1200 Vienna, Austria, herbert.muellner@tgm.ac.at)

An increase in open-plan offices has occurred recently. This makes it necessary to examine and, if necessary, to improve the acoustic conditions in the planning phase. With acoustic simulations it is possible to verify the acoustic quality of offices without much increase in money and time. In order to get the needed absorption area of the room to reduce the reverberation time and the sound level, open-plan offices are usually partitioned with screens. To improve the acoustics in the close range, special conditions, which mainly concern the height, have to be met. Also the absorption and the height of the ceiling have a strong influence on the acoustic quality of the neighbouring workstations. In the far field additional planning bases apply - particularly to raise the horizontal absorption and to get the optimal screening effect. This report highlights the possibilities of the acoustic improvements in the far field in open-plan offices and shows examples of simulated and improved options.

\section{4:20}

1pAAa10. Modeling the sound field of open spaces using a diffusion equation model. Yun Jing (Rensselaer Polytechnic Institute, Greene Building, School of Architecture, 110 8th Street, Troy, NY 12180, USA, jingy@rpi.edu), Ning Xiang (Rensselaer Polytechnic Institute, Greene Building, School of Architecture, 110 8th Street, Troy, NY 12180, USA, xiangn@rpi.edu)

Steady-state and transient sound field modeling of open spaces, such as urban squares, courtyard-type traditional Chinese theaters, is discussed in this paper. It is well known that the application of the statistical roomacoustic theory is strongly limited in this case, because of the high nonuniformity of the absorption coefficient. In this study, a diffusion equation along with a recently proposed modified boundary condition [Y. Jing and N. Xiang, J. Acoust. Soc. Am., 123, 2008, 145-153] is used to predict the acoustic characteristics of open spaces, in terms of both sound pressure level distributions and reverberation times. Three different types of open spaces are presented, including urban squares, quasi-cubic open rooms, and urban streets. This paper will compare the simulation results with the experimental results conducted in all these three cases (scale model), to verify the validity and efficiency of the diffusion equation model.

\section{Session 1pAAb}

\section{Architectural Acoustics: Acoustics of Concert Halls I}

\author{
Takayuki Hidaka, Cochair \\ Takenaka R\&D Institute, 1-5-1, Otsuka, Inzai, Chiba, 270-1395, Japan \\ Daniel E. Commins, Cochair \\ Commins Acoustics Workshop, 15 rue Laurence Savart, Paris, 75020, France
}

Invited Papers

1:00

1pAAb1. From backward integration to number-theoretic diffusors. Manfred Schroeder (University of Goettingen, Rieswartenweg 8, 37077 Goettingen, Germany, mrs17@aol.com)

As a student of physics at Göttingen I was interested in the statistics of electromagnetic resonances in metallic cavities when Erwin Meyer offered me a scholarship in concert-hall acoustics. So we compromised and my thesis eventually appeared under the double heading "The statistical parameters of the frequency responses of large enclosures. Experiments with electromagnetic waves".-After moving to Bell Laboratories in New Jersey, I continued to explore, with Heiner Kuttruft, frequency responses in concert halls by digital simulation, confirming my earlier theory that, above a critical frequency, these responses are just "noise" in the frequency domain. Later I dabbled in anti-feedback circuits and explored, with Ben Logan, artificial reverberation that spawned a minor industry-now dubbed sound scapes (first realized by John Chowning) and virtual reality. Then came the work (with Gerhard Sessler, Jim West, Bishnu Atal and Carol Bird) on Philharmonic Hall in New York, which lead to a new method of measuring reverberation time ("backward" integration) and new surface structures, based on number theory, for better sound diffusion. (The idea came to me during a talk by André Weil on "Gauss Sums" during the celebration of the 200th anniversary of Gauss' birth.) 
1pAAb2. Echo suppression effect and coloration of periodic-type diffusers. Masahiro Toyoda (Pioneering Research Unit, B104, Kyoto University Katsura, Nishikyo-ku, 615-8530 Kyoto, Japan, masahiro.toyoda@kupru.iae.kyoto-u.ac.jp), Tomohiro Furukawa (Kajima Corporation, Akasaka 6-5-30, Minato-ku, 107-8502 Tokyo, Japan, furuktom@kajima.com), Daiji Takahashi (Pioneering Research Unit, B104, Kyoto University Katsura, Nishikyo-ku, 615-8530 Kyoto, Japan, tkhs@ archi.kyoto-u.ac.jp)

Walls and ceilings are often designed to suppress undesirable echoes. For that purpose, walls and ceilings are given absorptive surfaces which absorb sound energy or uneven surfaces which provide wave diffusion. While it is not difficult to predict the degree of echo suppression effects in the case of absorptive surfaces, it is generally difficult to do that in the case of uneven surfaces. In addition, if the surfaces have periodicity, unintended effects called coloration can distort tonal characteristics of the sound field. Thus, many acoustic designers would hesitate to employ this kind of periodic-type diffusers. However, there are some cases where it is necessary to suppress echoes without any energy loss. Periodic-type diffusers have the advantages of easy estimation of diffusion properties, simple method of design, and ready availability. In this study, a subjective experiment with simulated stimuli was carried out to clarify the echo suppression effect of the periodic-type diffusers from the viewpoint of reflected energy level. Although coloration interfered in subjective judgments in some cases of the experiment, it is shown that the periodic-type diffusers have echo suppression effect if coloration does not occur. This study is also devoted to discussion about the cause of the coloration.

\section{$1: 40$}

1pAAb3. Experimental analysis of the acoustical behaviour of Musikverein in concert and ballet configurations. Angelo Farina (University of Parma, Via delle Scienze 181/A, Industrial Engineering Dept., 43100 Parma, Italy, farina@ unipr.it), Daniel E. Commins (Commins Acoustics Workshop, 15 rue Laurence Savart, 75020 Paris, France, d.commins@comminsacoustics.com), Nicola Prodi (Engineering Dept. - Univ. of Ferrara, Via Saragat, 1, 44100 Ferrara, Italy, nicola.prodi@unife.it)

Musikverein is among the best concert halls in the world. But the hall is also employed for open-to-the-public ballets. All seats in main stalls are removed, and packed under the wooden floor thanks to a large opening. Detailed acoustical measurements have been performed, with the aim of evaluating the different acoustical behavior with and without the seats. Furthermore, it was attempted to assess the contribution of the air volume under the floor, as a possible "resonant chamber", with volume and absorption significantly different when it is filled with packed seats. The results did show that the main acoustical parameters vary significantly between the "concert" setup and the "public ballet" setup, which is more reverberant, with lesser clarity and more envelopment. Regarding the hypothesis that the volume under the floor has some effect on the acoustical behaviour of the room, the experimental results excluded that this is possible. Furthermore, measurements with accelerometers and pressure/velocity probes did not reveal any difference between the behaviour of the "openable" part of the floor and the surrounding "fixed" floor. So it was concluded that the large difference in acoustical behaviour is simply caused by the removal of the seats, and by changes in fittings of the stage.

\section{2:00}

1pAAb4. Acoustical considerations in the design for 'La Philharmonie de Paris'. Harold Marshall (Marshall Day Acoustics LTD, P O Box 5811, Wellesley St., 1000 Auckland, New Zealand, harold.marshall@marshallday.co.nz), Joanne O. Valentine (Marshall Day Acoustics LTD, P O Box 5811, Wellesley St., 1000 Auckland, New Zealand, joanne.valentine@marshallday.co.nz), Thomas Scelo (Marshall Day Acoustics LTD, P O Box 5811, Wellesley St., 1000 Auckland, New Zealand, thomas.scelo@marshallday.co.nz)

The 2006 competition instructions for "La Philharmonie de Paris" included a detailed acoustical specification and the general requirement that the design should be a "new typology". The early and late sound fields respectively should be controlled independently. This paper gives an account of the acoustical aspect of the winning design, its relationship to the architecture at the competition phase, and the methodology for developing the design in subsequent phases. The new typology developed here is called the "bicameral adaptable concert hall". There are partial precedents for the concepts advanced and these will be identified. Reference is also made to a related paper (at this conference) which discusses the issue of coupling between the acoustical spaces.

\section{$2: 20$}

1pAAb5. Acoustical design of Shenzhen Concert Hall, Shenzhen China. Keiji Oguchi (Nagata Acoustics Inc., 2130 Sawtelle Blvd., Suite 307A, Los Angeles, CA 90025, USA, oguchi@nagata.co.jp), Yasuhisa Toyota (Nagata Acoustics Inc., 2130 Sawtelle Blvd., Suite 307A, Los Angeles, CA 90025, USA, toyota@nagata.co.jp)

The Shenzhen Concert Hall complex opened in Shenzhen, China on October 12, 2007. The complex contains the Symphony Hall with 1,800 audience seats, the Theater Studio with $400-580$ seats and some rooms for rehearsing. The Symphony Hall has a vineyard configuration with a steep audience block arrangement, called "canyon terrace". At the widest point of the audience seating, the room spans $45 \mathrm{~m}$, is $60 \mathrm{~m}$ long and has a ceiling height of $25 \mathrm{~m}$. The polycarbonate ensemble reflector panels are suspended at the height of approx.15m above the stage. The acoustical design and characteristics of the Concert Hall are reported.

\section{2:40}

1pAAb6. A comparison between shoebox and non-shoebox halls based on objective measurements in actual halls. Takayuki Hidaka (Takenaka R\&D Institute, 1-5-1, Otsuka, 270-1395 Inzai, Chiba, Japan, hidaka.takayuki@takenaka.co.jp), Leo L. Beranek (975 Memorial Drive, Suite 804, Cambridge, MA 02138, USA, beranekleo@ieee.org), Noriko Nishihara (Takenaka R\&D Institute, 1-5-1, Otsuka, 270-1395 Inzai, Chiba, Japan, nishihara.noriko@takenaka.co.jp)

In recent papers, the sound absorption by chairs and audiences were determined for shoebox (SB) and non-shoebox (NSB) types of concert halls (Beranek, JASA 120, 1399 (2006); Hidaka, Proc. ICA2007). The purpose of this paper is to examine objective differences in the sound fields in these two hall types. This investigation follows reports of music goers that the average subjective sound quality 
in NSB and SB halls differs appreciably and that there are greater subjective differences in sound quality from one seating location to another in NSB halls. Special attention is given to the temporal and amplitude structure in the early part (before $80 \mathrm{msec}$ ) of the impulse responses measured at various seat positions in six well-known halls (three SB and three NSB). To the extent the data permit, the usual objective parameters are also analyzed, both averaged and at various seat locations, in a total of 12 halls: reverberation time, early decay time, C80 (3-band), interaural cross-correlation coefficient (3-band middle frequency early before $80 \mathrm{~ms}$ ), initial-time-delay-gap, bass ratio, and strength factors at low- and mid-frequencies. The differences found are discussed with reference to the subjective reports.

\section{3:00}

1pAAb7. Changing seating arrangements of the orchestras - a challenge for the concert hall design. Jürgen Meyer (Bergiusstr. 2a, D-38116 Braunschweig, Germany, juergen.meyer-bs@t-online.de)

For the future of concert hall design, two aspects will play an increasing role: the search for new shapes created by the architects and changing seating arrangements preferred by the conductors. Whereas in the last decades, acoustic consultants could assume that e.g. celli and double basses were positioned on the right side of the stage, they will to have consider now that more and more conductors - particularly those of high international reputation - go back to the classic seating arrangement (celli in the center, 2nd violins on the right side. Among others, this distinct tendency leads to the following questions: Which are the tonal (acoustic) advantages of the German seating arrangement and by which roomacoustical means can they been supported? In which directions - seen from the player - exist important reflection areas for timbre, clarity and spaciousness? How should risers on the stage be designed for improving the listeners' sound impression and supporting the musicians' mutual hearing? Do celli and basses need podium areas capable of vibrating? Furthermore, where are critical areas and delay times for generating echos or erroneous localization of French horns or other winds? These questions shall be discussed from physical and psychoacoustic aspects.

1pAAb8. Acoustical analysis of a variable roof configuration concert hall: The São Paulo Hall. Roberto A. Tenenbaum (Instituto Politecnico, Universidade do Estado do Rio de Janeiro, Rua Alberto Rangel, S/N, 28630-050 Nova Friburgo, Brazil, tenenbaum@iprj.uerj.br), Lenine V. Oliveira (Universidade Federal do Rio de Janeiro, Av. Marechal Trompowsky S/N, 21970-000 Rio de Janeiro, Brazil, leninevas@ig.com.br), Swen Müller (Instituto Nacional de Metrologia e Controle da Qualidade, Av. N.S. das Graças, S/N, 21000-000 Xerem, Brazil, smuller@inmetro.gov.br)

The São Paulo Hall (SPH) is recognized by musicians and musical critics in general by its good acoustical quality. It has, as an important feature, variable acoustics due to its movable roof, which is employed by musicians to tune the room according to the musical style. This work addresses the acoustical quality of SPH and its dependence on the roof configuration. Impulse responses for one source, eight microphone and two dummy head positions, using the sweep-sine technique with pre-emphasis, were obtained for seven roof configurations. Some details of the measurement procedure to ensure good accuracy are discussed. The measurement results were then used to compute the main room acoustical quality parameters, for each octave band between 63 and $8000 \mathrm{~Hz}$. The spatial average and deviation of these data are presented as a function of the frequency band. The influence of the roof configurations on the obtained acoustical parameters is also discussed. Furthermore, comparative plots among SSP at the chosen roof configurations and two famous and representative halls from the classical and romantic periods are reported and discussed. It is concluded that the movable roof is very effective in modifying the room acoustics and that the musician's choices are quite good.

\section{$3: 40$}

1pAAb9. The acoustic design of the Elisabeth Murdoch Hall, Melbourne, Australia. Raf Orlowski (Arup Acoustics, St Giles Hall Pound Hill, CB3 0AE Cambridge, UK, Raf.Orlowski@arup.com)

The Elisabeth Murdoch Hall is a 1000 seat recital space which will be completed in the latter half of 2008 . The overall geometry is based on a classical shoebox shape to provide acoustic excellence although the internal appearance is wholly contemporary. The walls and ceiling of the hall are clad in timber panelling which is stepped in and out and also modulated to provide diffusion at mid and low frequencies. In addition, the surface layer of the panels is grooved or cut away to form an abstract pattern which is both decorative and diffusing at high frequencies. A notable aspect of the hall is that it has no orchestral reflector. The important early reflections for good orchestral ensemble are provided by articulating and shaping the surfaces around the stage. Although the hall is primarily for recitals of classical music, provision is made for amplified music by introducing acoustic drapes.

\section{4:00-4:20 Break}

1pAAb10. Trends in preference, programming and design of concert halls for symphonic music. Anders C. Gade (Dept. of Acoustic Technology, Technical University of Denmark, Building 352, DK 2800 Lyngby, Denmark, acg@ oersted.dtu.dk)

This paper discusses the evolution in taste regarding concert hall acoustics and how this can be reflected in the new halls being built today. The clients' and listener's preferences are not only based on listening in existing halls; but also on listening to reproduced music recorded with microphones close to the orchestra and with artificial reverberation added. The result may be a desire for higher clarity as well as a more full reverberation than what is found in most existing halls. Without being very specific in the brief regarding geometrical detailing - which is not desirable as it will limit architectural freedom and evolution - we can only specify a desire for acoustic conditions in this direction by setting targets for standardized objective room acoustic parameters. In this paper, measured ISO 3382 data are used to illustrate typical differences between "live" and recorded concert experiences, and it is seen how visionary hall designs over the last four decades tend to move the acoustics of halls in the same direction. Finally, it is suggested how target values for ISO 3382 parameters - in spite of their limitations - can be set up to drive the design in such a direction. 
1pAAb11. Describing measurement uncertainties in room acoustics with the concept of GUM. Ingo Witew (Institute of Technical Acoustics, Templergraben 55, 52056 Aachen, Germany, Ingo.Witew@akustik.rwth-aachen.de), Renzo Vitale (Institute of Technical Acoustics, Templergraben 55, 52056 Aachen, Germany, renzo.vitale@akustik.rwth-aachen.de)

Considering the uncertainties in room acoustical measurements is a crucial step towards quality evaluation, comparability between different measurements methods and instrumentation. In the preceding discussion it was shown that the concepts specified in the "Guide to the expression of Uncertainty in Measurements" (GUM) can well be applied for room acoustical measurement tasks. In this initiatory step a detailed analytical formulation of a model function was avoided for reasons of simplicity and practicability. The waiving of complex modeling, however, requires comprehensive series of measurements to assess the measurement uncertainties. In this paper it is discussed how modeling can reduce such requirements. A special focus is placed on the influence of the directional radiation properties of sound sources.

\section{5:00}

1pAAb12. Simplified Room Acoustic Measurements. Tor Halmrast (Statsbygg, Biskop Gunnerus gt 6, pb 8106, Dep, NO-0032 Oslo, Norway, th@statsbygg.no), Anders Buen (Brekke \& Strand akustikk as, Hovfaret 17, 0275 Oslo, Norway, Anders.Buen@bsakustikk.no)

Standardised room acoustic measurements are needed for concert halls etc., but they imply heavy equipment. Thus, many interesting rooms are not measured. Most musicians (and even some acousticians) find that clapping and shouting in a room/hall might give an overview of the room acoustics. In the literature we even find that acoustic parameters are derived from scratchy recordings of music. Modern handheld high quality recording technology and possibilities of analysing sound files gives that one should investigate simpler (non standardised) measurements for simpler rooms/halls. This paper gives analysis of Impulse-Responses recorded from balloons, paper bags, clapping, compared with measurements taken with more academic measurement equipment.

\section{Contributed Papers}

$5: 20$

1pAAb13. Acoustics of concert halls with organs in Russia: trends and problems. Pavel Kravchun (Moscow State University, Faculty of Physics, Department of Acoustics, Box 15, 125130 Moscow, Russian Federation, gedackt@mail.ru)

Over the past decades the organ music has become one of the most popular kinds of classical music in Russia. About 30 new organs were built in concert halls, and several old instruments were restored or rebuilt. Acoustical properties of 15 well-known Russian concert halls with organs (Moscow, St. Petersburg, Perm, Kazan, Astrakhan, etc.) are presented and considered in the paper. In many cases acoustics in the concert halls is not so good for the solo organ music. The reasons are discussed. Acoustics of the Russian concert halls with adjustable acoustics is considered (Moscow, Kazan). It was noted that the "optimum" reverberation time recommended by different acousticians for organ music had a tendency to be longer and longer in the 20th century. Values of the average sound absorption coefficient for organ front were estimated. Some problems in organ-building connected with the concert hall acoustics are discussed.

\section{5:40}

1pAAb14. Dexterity and Spirit: The Peter Kiewit Concert Hall at the Holland Performing Arts Center. Dawn Schuette (Threshold Acoustics, 53 W. Jackson Blvd, Suite 1734, Chicago, IL 60604, USA, dschuette @ thresholdacoustics.com)

Bathed in natural light, the Peter Kiewit Concert Hall is the focal point of the architecturally innovative Holland Performing Arts Center. The space features cast plaster panels for acoustic diffusion, a tunable canopy over stage for acoustic support, and natural felt banners for reverberation and reflection control. The space transforms easily from an orchestral concert setting to an environment friendly to amplified sound, allowing orchestral music one night to seem as naturally suited to the room as amplified music the following evening. The world-class concert hall acoustics provided by this venue meld seamlessly with the amplified use as well resulting in a unique venue for the music community. This hall is an excellent starting point for a continued discussion about how to successfully incorporate variability in high quality acoustic venues. [Work completed while author was employed as a Principal Consultant at Kirkegaard Associates.]

\section{6:00}

1pAAb15. A new symphonic hall, Musikhuset Aarhus, Denmark. Carsten Lemvigh Fog (COWI A/S, Parallelvej 2, DK 2800 Kgs. Lyngby, Denmark, clf@ cowi.dk), Richard M. Ballinger (COWI A/S, Odensevej 95, DK 5260 Odense S, Denmark, rmb@cowi.dk)

During the past 3 years Musikhuset Aarhus in Denmark has been enlarged by an area of approximately $17.000 \mathrm{~m}^{2}$ to a new total area of approximately $34.000 \mathrm{~m}^{2}$. The new part of the complex contains a symphonic concert hall for Aarhus Symphonic Orchestra with 1200 seats, a hall for rythmic contemporary music with 600 seats and a hall for classical music with 160 seats. The complex also houses the new premises for Aarhus Academy of Music. Important design considerations will be highlighted in the paper and the results of the preliminary and final computer modeling of room acoustics will be presented using examples of auralisation. In conclusion the room acoustic parameters (measured according to ISO 3382 standard) will be discussed, also in respect of the initial reaction from ochestral musicians.

\section{6:20}

1pAAb16. Qualitative Standard for Symphonic Concert Environment. Christopher Jaffe (167 East Rocks Road, Norwalk, CT 06851, USA, ADK117@GMAIL.COM)

All acousticians strive to obtain a symphonic concert environment that will be well received by musicians, professional music critics and the general public. Currently, this environment corresponds to the reflective energy measurements found in what might be designated as the rectangular European or American Concert Hall of the late nineteenth century, such as the Grosser Musikvereinssaal in Vienna and Symphony Hall in Boston Massachusetts. It is well known that these measurements can be correlated to subjective musical judgments and that halls incorporating these measurements have been universally designated as having excellent environments for symphonic concerts. But how did these specific reflections become an acoustical standard. This paper presents a theory of how the rectangular European and American style concert hall became an acoustical standard at the beginning of the twentieth century and poses the question as to whether this standard is still applicable in the twenty first century? 


\section{$6: 40$}

1pAAb17. Acoustical characterization of orchestra platforms. Maria Giovannini (Politecnico di Torino, Corso Duca degli Abruzzi, 24, 10129 Turin, Italy, maria.giovannini@polito.it), Arianna Astolfi (Politecnico di Torino, Department of Energetics, Corso Duca degli Abruzzi, 24, 10129 Turin, Italy, arianna.astolfi@polito.it), Giulio Barbato (Politecnico di Torino, Corso Duca degli Abruzzi, 24, 10129 Turin, Italy, giulio.barbato @ polito.it)

In concert halls and other performance spaces it is important that acoustic conditions allow the musicians to hear each other and that there is a sufficient response from the room. Acoustical characterization of spaces for musicians, such as orchestra platforms, orchestra pits, and rehearsal rooms requires the measurements of the Early and Late Support, and other acoustical parameters described in the ISO/DC 3382-1 and in Literature. This work shows results from measurements carried out on five orchestra platforms, different in shape, size and materials of the boundary surfaces. Up to eight source positions were chosen in order to represent the main instrumental sections of the orchestra playing on the platform. Measurements were carried out without musicians, in conditions of empty stage and stage equipped as in concert configuration with stands, chairs, and risers. Some metrological problems connected with the reproducibility of results are considered as the directivity of the source and the small movements of the source and the microphone. The correlations between objective data show that the measured parameters are not always independent, but some groups of correlated measures, not completely separated, are found. The comparison of results allows to draw some connections with the main stage architectural features.
7:00

1pAAb18. On the Reverberation time of Gamelan Bali Concert Hall based on the Physio-Acoustic Responses. I Gde Nyoman Merthayasa (Institute of Technology Bandung, Dept. of Engineering Physics - ITB, Jln. Ganesha no. 10, 40132 Bandung, Indonesia, ignmerth@tf.itb .ac.id), Farida Idealistina Muchtadi (Institute of Technology Bandung, Dept. of Engineering Physics - ITB, Jln. Ganesha no. 10, 40132 Bandung, Indonesia, lies@tf.itb.ac.id)

Based on the fourth orthogonal acoustic factors of a concert hall design theory, it is possible to calculate the acoustical quality at any position in a proposed Gamelan Bali concert hall. And, it is well known that the value of preferred sound fields depends upon the characteristics of the sound source signal. Previous research has reported the most preferred value of the four orthogonal factors of Gamelan Bali sound fields based on the subjective response. It would be important to obtain the objective response, based on the physio-acoustics measurement using Electroechepalograph (EEG), when the subjects were excited by a certain variation of sound fields while other parameters were kept constant. In this research, the subjects were excited by a serial variation of the subsequent reverberation time of Gamelan Bali music. The brain activities were measured using EEG in the temporal areas. The result shows that the maximum power spectral of Alpha Waves of the subjects occurred when the reverberation time of Gamelan Bali were between $750 \mathrm{mS}$ to $1750 \mathrm{mS}$.

\title{
Session 1pAAc
}

\section{Architectural Acoustics and Psychological and Physiological Acoustics: Speech Segregation in Rooms I}

\author{
Barbara Shinn-Cunningham, Cochair \\ Boston University Hearing Research Center, 677 Beacon Street, Boston, MA 02215, USA \\ Mathieu Lavandier, Cochair \\ Cardiff University, School of Psychology, Tower Building, Park Place, Cardiff, CF10 3AT, UK
}

\section{Invited Papers}

1pAAc1. Issues in the perception of multiple speech sources under reverberant listening conditions. Chris J. Darwin (University of Sussex, Psychology, School of Life Sciences, BN1 9QG Brighton, UK, cjd@sussex.ac.uk)

This paper will review the changes that room acoustics can make to the cues available for speech perception and those available for the localisation of sound sources, and give an overview of the perceptual mechanisms underlying the causes of the reduced intelligibility that reverberant room acoustics can make to the intelligibility of speech that is masked by additional sources of speech. Major factors that contribute to the interference of one source of speech by another are the obscuring of primary speech cues either by masking or by the failure of simultaneous grouping mechanisms, and the failure to track the sounds of an individual talker across time. The role of spatial localisation mechanisms to both these factors will be discussed.

\section{$1: 20$}

1pAAc2. Room-acoustic factors in attentional tracking. Anthony J. Watkins (Reading University, Department of Psychology, RG6 6AL Reading, UK, syswatkn@rdg.ac.uk), Simon J. Makin (Reading University, Department of Psychology, RG6 6AL Reading, UK, S.J.Makin@reading.ac.uk), Andrew Raimond (Reading University, Department of Psychology, RG6 6AL Reading, UK, a.raimond@ reading.ac.uk)

In a "busy" auditory environment listeners can selectively attend to one of several simultaneous messages by tracking one listener's voice characteristics. Here we ask how well other cues compete for attention with such characteristics, using variations in the spatial position of sound sources in a (virtual) seminar room. Listeners decided which of two simultaneous target words belonged in an attended "context" phrase when it was played with a simultaneous "distracter" context that had a different wording. Talker difference was 
in competition with a position difference, so that the target-word chosen indicates which cue-type the listener was tracking. The main findings are that room-acoustic factors provide some tracking cues, whose salience increases with distance separation. This increase is more prominent in diotic conditions, indicating that these cues are largely monaural. The room-acoustic factors might therefore be the spectral- and temporal-envelope effects of reverberation on the timbre of speech. By contrast, the salience of cues associated with differences in sounds' bearings tends to decrease with distance, and these cues are more effective in dichotic conditions. In other conditions, where a distance and a bearing difference cooperate, they can completely override a talker difference at various distances.

\section{$1: 40$}

1pAAc3. Effects of reverberation on spatial release from masking. Richard L. Freyman (Univ. of Massachusetts, Dept. of Communication Disorders, 358 N. Pleasant St., Amherst, MA 01003, USA, rlf@ comdis.umass.edu), Patrick M. Zurek (Sensimetrics Corp, 48 Grove St., Somerville, MA 02144, USA, pat@ sens.com)

When sounds that we want to listen to (targets) and interfering sounds (maskers) are separated spatially, several advantages are observed relative to the baseline condition in which target and masker come from the same location. This paper will discuss how and to what extent these advantages are disrupted by room reverberation, which reduces better-ear signal-to-noise ratios and interaural intensity differences, and obscures interaural time differences. Statistical analysis of room acoustics combined with a model of binaural release from masking can, with the articulation index, help quantify expected reductions in speech recognition benefits of target/masker spatial separation in reverberation. This paper will also discuss some advantages of target/masker spatial separation that appear to be well preserved in reverberant rooms. Specifically, reverberation does not eliminate the perceptual differences between target and masker that arise from their different physical locations. The advantages of perceived spatial differences are seen when target and masking sounds share similar characteristics and are confusable with one another. In such cases spatial differences appear to resolve the confusion. This paper will describe research indicating that barely discriminable spatial differences between target and masker are sufficient for spatial release from masking in these circumstances. [Work supported by NIH DC-01625]

\section{2:00}

1pAAc4. Some Temporal and Spatial Effects of Room Acoustics on Speech Privacy. John S. Bradley (National Research Council, 1200 Montreal Rd, Ottawa, ON K1A 0R6, Canada, john.bradley@nrc-cnrc.gc.ca), Marina Apfel (National Research Council, 1200 Montreal Rd, Ottawa, ON K1A 0R6, Canada, Marina.Apfel@gmx.de), Brad N. Gover (National Research Council, 1200 Montreal Rd, Ottawa, ON K1A 0R6, Canada, Brad.Gover@nrc-cnrc.gc.ca)

The speech privacy of meeting rooms relates to the possibility of eavesdroppers outside the room being able to understand speech from within the meeting room. Although the speech privacy of meeting rooms is usually assumed to relate to the level of the speech transmitted from the room relative to the ambient noise outside the room, the intelligibility of the transmitted speech is also influenced by room acoustics. However, the audibility of speech is only influenced by the levels of the transmitted speech and ambient noise. This paper reports on a series of speech intelligibility tests in which the components of the problem were systematically added. As expected, adding early reflections of speech from various directions increases intelligibility but later-arriving speech sounds decrease intelligibility. While a spatial separation of speech and noise sources in free field conditions increases speech intelligibility (spatial release from masking), in more realistic conditions, representing a diffuse ambient noise field, the effects are much smaller. Conditions are further complicated by the sever filtering of the speech on transmission through typical walls. The combined effects can be estimated and are significant. If ignored costly over-design of the sound isolation of the meeting room could occur.

\section{$2: 20$}

1pAAc5. Binaural room acoustics - cross-correlation. William M. Hartmann (Michigan State University, Dept. of Physics and Astronomy, East Lansing, MI 48824, USA, hartmann@pa.msu.edu), Neil L. Aaronson (Michigan State University, Dept. of Physics and Astronomy, East Lansing, MI 48824, USA, AARONSON@PA.MSU.EDU)

The subject of "Binaural room acoustics" combines the disciplines of room acoustics with facts and models of human binaural physiology and psychology. It considers the linear effects of rooms on the inputs to the two ears of a listener, and it considers the nonlinear effects of binaural hearing processes on perceptually relevant acoustical characterizations of rooms. Linear effects of interest are binaural differences in amplitude and phase spectra, which are combined in the cross-correlation function (CCF). A psychophysically relevant CCF is computed over frequency bands approximately corresponding to auditory filters. Because the bands are narrow, the effects on the CCF of frequency-dependent interaural parameters, most importantly dispersion, can be modeled analytically. The roles of amplitude incoherence and dispersion can also be independently modelled. A systematic experimental approach begins with the dependence of the CCF on head related transfer functions (HRTF) as measured at different azimuths in a free field. Although there are notable individual differences, the effects of HRTFs appear to be almost entirely below detection threshold. An empirical relationship between the CCF for a waveform and for its envelope has been established numerically and in extensive room experiments. [Work supported by the NIDCD, grant DC00181.]

\section{2:40}

1pAAc6. Speech segregation in rooms: Importance of the interferer interaural coherence. Mathieu Lavandier (Cardiff University, School of Psychology, Tower Building, Park Place, CF10 3AT Cardiff, UK, lavandiermn@ @ardiff.ac.uk), John F. Culling (Cardiff University, School of Psychology, Tower Building, Park Place, CF10 3AT Cardiff, UK, CullingJ@cardiff.ac.uk)

When an interferer is present, reverberation degrades speech intelligibility not only by its direct effect on the target, but also by affecting the interferer [Lavandier \& Culling, J. Acoust. Soc. Am., 122 (3), 2007]. This latter effect occurs at lower levels of reverberation than the former intrinsic degradation of the target, and can be explained by a binaural mechanism. We show here that the interaural coherence of the interferer is the principal parameter predicting the resulting loss of intelligibility. Speech reception thresholds were measured under headphones, using running speech targets and speech-shaped noise interferers. The stimuli were created by con- 
sidering a listener and a spatially-separated target and interferer in virtual rooms. The reverberation was varied by modifying the absorption coefficient of every room surfaces simultaneously. Different absorption coefficients were used for target and interferer. With the target anechoic, the interferer was tested in several configurations, in rooms with different sizes and absorptions, at different distances and azimuths from the listener. Several computations of coherence were compared, in order to reveal the one allowing the best prediction of intelligibility.

1pAAc7. Effects of pitch and spatial separation on selective attention in anechoic and reverberant environments. Scott Bressler (Boston University Hearing Research Center, 677 Beacon Street, Boston, MA 02215, USA, SCBressler@verizon.net), Barbara Shinn-Cunningham (Boston University Hearing Research Center, 677 Beacon Street, Boston, MA 02215, USA, shinn@cns.bu.edu)

Subjects identified a random, spoken sequence of five monotonized digits $\left(\mathrm{F}_{0}=100 \mathrm{~Hz}\right)$ presented from 0 azimuth. A monotonized masking sentence $\left(\mathrm{F}_{0}=84,89,94,100,106,112\right.$, or $\left.119 \mathrm{~Hz}\right)$ was presented simultaneously from either $0^{\circ}$ and $+90^{\circ}$ azimuth $($ chosen randomly on each trial). The same talker recorded both target and masker speech. KEMAR-derived transfer functions simulated either a reverberant or anechoic environment. In contrast to previous studies, in the anechoic condition, differences in pitch provided little benefit and differences in location gave improvements explainable by improvements in the target-to- masker ratio at the acoustically better ear. In reverberant conditions, differences in target and masker location improved performance more than differences in pitch; however, performance was best when there were differences in both location and pitch. Results suggest that when a target utterance is easy to segregate and select (such as in anechoic space when the target is a digit sequence embedded in a competing sentence), highlevel attributes such as pitch and location do not improve the ability to selectively attend in a speech-on-speech task. However, in more challenging, reverberant conditions, location and pitch cues can aid segregation and/or selection.

\section{3:20}

1pAAc8. Effects of reverberation on perceptual segregation of competing voices by difference in fundamental frequency. Mickael Deroche (Cardiff University, School of Psychology, Tower Building, Park Place, CF10 3AT Cardiff, UK, derochem@cf.ac.uk), John F. Culling (Cardiff University, School of Psychology, Tower Building, Park Place, CF10 3AT Cardiff, UK, CullingJ@cardiff.ac.uk)

Three experiments investigated the effect of reverberation on listeners' ability to use the difference in fundamental frequency (F0) between a target and an interfering voice to perceptually separate them. Speech reception thresholds (SRTs) were measured for a monotonized or frequency-modulated male voice against one or two other monotonized or frequency-modulated male voices or against a speech-shaped, pulse train within a virtual room with controlled reverberation. The sources of targets and interferers were always co-located both straight ahead of the listener. In the two first experiments, the beneficial effect of a F0 difference was reduced in reverberation when the target and interferer's F0 were frequency-modulated at $5 \mathrm{~Hz}$. Experiment 3 investigated this interaction between reverberation, a F0 difference and the target and/or interferer F0's modulation: the results highlighted the relevance of the interferer F0's modulation alone in the presence of reverberation. This finding is consistent with the idea that the effect of F0 difference is mediated by a harmonic-cancellation process. The cancellation of the interferer is disrupted by the reverberation only when the interferer's F0 is frequency-modulated, irrespective of the modulation of the target's F0. A frequency-overlap between the F0 of the target and the maskers does not seem a cause of worse impairment in intelligibility.

\section{3:40-4:00 Break}

\section{4:00}

1pAAc9. The effects of fluctuating interaural cues on the segregation of speech in rooms: Revisited. Douglas S. Brungart (AFRL, 2610 Seventh Street, WPAFB, OH 45433, USA, douglas.brungart@ wpafb.af.mil), Nandini Iyer (AFRL, 2610 Seventh Street, WPAFB, OH 45433, USA, nandini.iyer@wpafb.af.mil)

Spatial separation is known to improve the segregation of talkers in anechoic environments, but spatial auditory cues can be severely degraded in reverberant rooms. One might expect the random disruptions in the interaural time and level differences (ILDs and ITDs) that occur in reverberant environments to eliminate many of the intelligibility benefits that normally occur for spatially separated speech. However, the precedence effect often leads to a robust perception of spatial location even in extremely echoic environments. This can result in an apparent separation between talkers that may facilitate selective attention to the location of the target speech even in a highly reverberant room. Also, random fluctuations in ITD and ILD may lead to differences in the apparent source widths of the target and masking sounds, which might serve as a segregation cue. In this talk, we examine the effects fluctuating interaural difference cues have on the segregation of target speech from competing speech or noise. These results, originally scheduled for presentation at the $152 \mathrm{nd}$ meeting, suggest that differences in apparent source width can be used to segregate competing speech signals even when the target and masking signals appear to originate from the same direction relative to the listener.

\section{$4: 20$}

1pAAc10. A reverberation-robust automatic speech recognition system based on temporal masking. Guy J. Brown (University of Sheffield, Dept. of Computer Science, Regent Court, 211 Portobello Street, S1 4DP Sheffield, UK, g.brown@dcs.shef.ac.uk), Kalle J. Palomäki (Adaptive Informatics Research Centre, Helsinki University of Technology, P.O. Box 5400, 02015 Espoo, Finland, kalle .palomaki@tkk.fi)

Previously, we have proposed a reverberation-robust system for automatic speech recognition (ASR) based on a temporal masking principle. In the first pathway of this system, speech is analysed by a bank of auditory filters, in order to provide acoustic features for the recogniser. In the second pathway, a bandpass modulation filter $(1.5 \mathrm{~Hz}-8.2 \mathrm{~Hz})$ detects regions of the envelope in each filter 
channel that contain strong speech energy. Regions of the modulation filter output that exceed a threshold are labelled as reliable evidence for the speech in a time-frequency mask; regions that fall below the threshold are dominated by reverberation and labelled as unreliable. The acoustic features and time-frequency mask are then decoded by a "missing data" ASR system. Here we describe modifications of this system that bring it into closer agreement with purported mechanisms of human perceptual compensation for reverberation, as determined by psychophysical studies [Watkins \& Makin, JASA 121, 257-266]. Specifically, we use a metric for identifying regions of reliable speech that gives greater emphasis to reverberation "tails," and is computed independently for each frequency band. The performance of the system is evaluated on a corpus of spoken digits convolved with recorded room impulse responses.

$$
\text { 4:40 }
$$

1pAAc11. Monaural segregation of reverberant speech. Zhaozhang Jin (Ohio State University, Department of Computer Science \& Engineering, 2015 Neil Ave., Columbus, OH 43210, USA, jinzh@cse.ohio-state.edu), Deliang Wang (Ohio State University, Department of Computer Science \& Engineering, 2015 Neil Ave., Columbus, OH 43210, USA, dwang@cse.ohio-state.edu)

A major source of signal degradation in realistic environments is room reverberation. Monaural speech segregation in reverberant environments is a particularly challenging problem. Although inverse filtering has been proposed to partially restore the harmonicity of reverberant speech before segregation, this approach is sensitive to different room configurations. In this study, we investigate monaural segregation of reverberant speech by employing a supervised learning approach to map from a set of pitch-based auditory features to a grouping cue, which encodes the posterior probability of a time-frequency unit being target dominant given observed features. We devise a novel objective function for the learning process, which relates to the goal of maximizing SNR directly. The models trained using this new objective function yield significantly better results for unit labeling. In our segregation system, a segmentation and grouping framework is utilized in order to capture segments reliably under reverberant conditions and organize them into streams. Systematic evaluations show that our approach produces very promising results.

MONDAY AFTERNOON, 30 JUNE 2008

ROOM 202/203, 4:40 TO 7:20 P.M.

\title{
Session 1pAAd
}

\section{Architectural Acoustics and Musical Acoustics: Surround Sound Acoustics I}

\author{
Anthony Hoover, Cochair \\ McKay Conant Hoover Inc., 5655 Lindero Canyon Rd, Suite 325, Westlake Village, CA 91362, USA
}

Alexander Case, Cochair

Fermata Audio \& Acoustics, PO Box 1161, Portsmouth, NH 03802, USA

Etienne Corteel, Cochair

Sonic Emotion, Eichweg, 6, Oberglatt, CH-8154, Switzerland

\section{Contributed Papers}

\section{$4: 40$}

1pAAd1. Virtual acoustic environments for music performance, rehearsal, and recording. Wieslaw Woszczyk (McGill University, Schulich School of Music, 555 Sherbrooke Street West, Montreal, QC H3A 1E3, Canada, wieslaw@music.mcgill.ca), Doyuen Ko (McGill University, Schulich School of Music, 555 Sherbrooke Street West, Montreal, QC H3A 1E3, Canada, doyuen.ko@mail.mcgill.ca), Tom Beghin (McGill University, Schulich School of Music, 555 Sherbrooke Street West, Montreal, QC H3A 1E3, Canada, tom.beghin@mcgill.ca), Martha De Francisco (McGill University, Schulich School of Music, 555 Sherbrooke Street West, Montreal, QC H3A 1E3, Canada, martha.defrancisco@mcgill.ca), Jeremy Tusz (McGill University, Schulich School of Music, 555 Sherbrooke Street West, Montreal, QC H3A 1E3, Canada, tusz@mac.com), Ryan Miller (McGill University, Schulich School of Music, 555 Sherbrooke Street West, Montreal, QC H3A 1E3, Canada, ryan.miller@mail.mcgill.ca)

A comprehensive 24-channel sound field rendering system was built at McGill University's CIRMMT Centre to immerse scholar/performer Tom Beghin in virtual concert spaces where Haydn composed and performed his keyboard sonatas, or expected them to be performed. The virtual rooms were reconstructed from detailed measurements made in Haydn's historical rooms in Europe using a high-resolution 24 bit/96 kHz impulse-response measuring system. In an acoustically treated laboratory, 24 custom loudspeaker arrays were arranged on a surface of a hemisphere surrounding the performer. Multiple fast DSP engines convolved live signals with 24 im- pulse responses allowing the performer to rehearse and record in each room with low-latency virtual acoustics. This paper describes the details of system design, the method of measurement, and discusses various aspects of recording and performing in virtual acoustic environments. The method has also been tested in a large room during public performance by having virtualroom responses and high-definition photo images projected from the stage. Surround sound recordings of Haydn's complete solo keyboard music made in several virtual rooms and matched to specific historical instruments will be released in a collection of 13 commercial SA-CD's by the end of 2008 .

\section{5:00}

1pAAd2. Prediction of spatial perceptual attributes of reproduced sound across the listening area. Philip J. Jackson (University of Surrey, Centre for Vision, Speech and Signal Processing, GU2 7XH Guildford, UK, p.jackson@surrey.ac.uk), Martin Dewhirst (University of Surrey, Institute of Sound Recording, GU2 7XH Guildford, UK, m.dewhirst@surrey.ac.uk), Slawomir Zielinski (University of Surrey, Institute of Sound Recording, GU2 7XH Guildford, UK, s.zielinski@surrey.ac.uk), Francis J. Rumsey (University of Surrey, Institute of Sound Recording, GU2 7XH Guildford, UK, f.rumsey@surrey.ac.uk), Robert Conetta (University of Surrey, Institute of 
Sound Recording, GU2 7XH Guildford, UK, r.conetta@surrey.ac.uk),David Meares (DJM Consultancy, Winthrift, Nuthurst, RH13 6RG Horsham, UK, meares_david@yahoo.co.uk), Søren Bech (Bang \& Olufsen A/S, Peter Bangsvej 15, DK-7600 Struer, Denmark, sbe@bang-olufsen.dk)

Audio systems and recordings are optimized for listening at the "sweet spot," but how well do they work elsewhere? An acoustic-perceptual model has been developed that simulates sound reproduction in a variety of formats, including mono, two-channel stereo, five-channel surround and wave- field synthesis. A virtual listener placed anywhere in the listening area is used to extract binaural signals, and hence interaural cues to the spatial attributes of the soundfield. Using subjectively-validated models of spatial sound perception, we can predict the way that human listeners would perceive these attributes, such as the direction (azimuth) and width of a phantom source. Results will be presented across the listening area for different source signals, sound scenes and reproduction systems, illustrating their spatial fidelity in perceptual terms. Future work investigates the effects of typical reproduction degradations.

\section{Invited Papers}

\section{$5: 20$}

1pAAd3. Measuring perceived spatial quality changes in surround sound reproduction. Francis J. Rumsey (University of Surrey, Institute of Sound Recording, GU2 7XH Guildford, UK, f.rumsey@surrey.ac.uk), Slawomir Zielinski (University of Surrey, Institute of Sound Recording, GU2 7XH Guildford, UK, s.zielinski@surrey.ac.uk), Philip J. Jackson (University of Surrey, Centre for Vision, Speech and Signal Processing, GU2 7XH Guildford, UK, p.jackson@ surrey.ac.uk), Martin Dewhirst (University of Surrey, Institute of Sound Recording, GU2 7XH Guildford, UK, m.dewhirst@ surrey.ac.uk), Robert Conetta (University of Surrey, Institute of Sound Recording, GU2 7XH Guildford, UK, r.conetta@surrey.ac.uk), Søren Bech (Bang \& Olufsen A/S, Peter Bangsvej 15, DK-7600 Struer, Denmark, sbe@bang-olufsen.dk), David Meares (DJM Consultancy, Winthrift, Nuthurst, RH13 6RG Horsham, UK, meares _david@yahoo.co.uk)

The spatial quality of audio content delivery systems is becoming increasingly important as service providers attempt to deliver enhanced experiences of spatial immersion and naturalness in audio-visual applications. Examples are virtual reality, telepresence, home cinema, games and communications products. The QESTRAL project is developing an artificial listener that will compare the perceived quality of a spatial audio reproduction to a reference reproduction. The model is calibrated using data from listening tests, and utilises a range of metrics to predict the resulting spatial sound quality ratings. Potential application areas for the model are outlined, together with exemplary results obtained from some of its component parts.

1pAAd4. Dynamic sound image creation by un-directional sound beam reproduction toward an interior surface with a DSP controlled line array speaker for Surround Sound Composition. Yasushi Shimizu (Center for Advanced Sound Technologies, Yamaha, 203 Matsunokijima, 4380192 Iwata, Shizuoka, Japan, yasushi_shimizu@gmx.yamaha.com), Masato Hata (Yamaha, 203 Matsunokijima, 4380192 Iwata, Shizuoka, Japan, hata@beat.yamaha.co.jp), Atsuko Ito (Center for Advanced Sound Technologies, Yamaha, 203 Matsunokijima, 4380192 Iwata, Shizuoka, Japan, atsuko1_ito@gmx.yamaha.com), Akira Miki (Yamaha, 203 Matsunokijima, 4380192 Iwata, Shizuoka, Japan, akira_miki@gmx.yamaha.com), Masato Hatanaka (Hashimoto Bldg, 301, 4-1-25, Komazawa, Setagaya, 1540012 Tokyo, Japan, info@hatanakamasato.net)

Surround Sound Composition in an indoor space is frequently provided with spatial sounds, giving both a sound localization and surround sound effect. Systems have been installed in public spaces such as a museum and retail shops. However, technical improvements are required to provide a small sweet spot and to address architectural designs for speaker installation, because multiple speakers are required for surround sound effect. Recently, we have been applying DSP beam-forming technology with a line-array speaker to create a 3D sound image by scanning a focused sound beam onto an interior surface. The DSP array speaker is capable of providing static and dynamic control in beam steering and sound focusing. Therefore, dynamic spatial sound effect on the interior surface is possible, wherein un-directional sound reproduction is analogous to un-directional lighting. We present three case studies; 1) Virtual 3D Surround Sound in multi-media improvisation, 2) 3D Spatial Sound Synthesis in sound installation and 3) Sound Installation with a Dynamic Sound Beam Scanning.

\section{6:00}

1pAAd5. How does spatial auditory perception impact how we enjoy surround sound? Barbara Shinn-Cunningham (Boston University Hearing Research Center, 677 Beacon Street, Boston, MA 02215, USA, shinn@cns.bu.edu), K Anthony Hoover (McKay Conant Hoover, Inc., 5655 Lindero Canyon Road, Suite 325, Westlake Village, CA 91362, USA, thoover@mchinc.com)

Surround sound has become an essential element of enormously popular home entertainment systems, with consumers becoming increasingly eager to invest in high-quality audio systems. While even a cursory web search can uncover numerous criteria, standards, and blogs describing how to select and set up surround-audio equipment, architectural effects are typically ignored or, at best, treated naively. However, architectural acoustics profoundly affect the perceptual experience of a surround-sound listener. Similarly, sound engineers, producers, and artists agonize over every aspect of soundtrack design and production, including the spatial cues embedded in a surround-sound recording, but they have no control over the acoustic environments in which consumers experience their art. How do the acoustics of surround-listening spaces influence the perceptual experiences of the ordinary listener? Is accurate sound localization a proper, desirable, or achievable goal? Would more loudspeakers or higher fidelity improve the surround experience of a typical consumer? These issues will be discussed, taking into account basic psychophysical issues as well as disheartening truths about how ordinary consumers experience sound from their expensive home entertainment systems. 
1pAAd6. Acoustics of a large immersive environment, The Allosphere. David Conant (McKay Conant Hoover, Inc., 5655 Lindero Canyon Road, Suite 325, Westlake Village, CA 91362, USA, dconant@mchinc.com), William Chu (McKay Conant Hoover, Inc., 5655 Lindero Canyon Road, Suite 325, Westlake Village, CA 91362, USA, wchu@mchinc.com), Thomas McNally (McKay Conant Hoover, Inc., 5655 Lindero Canyon Road, Suite 325, Westlake Village, CA 91362, USA, tmcnally@ mchinc.com), K Anthony Hoover (McKay Conant Hoover, Inc., 5655 Lindero Canyon Road, Suite 325, Westlake Village, CA 91362, USA, thoover@mchinc.com), Stephen Pope (Center for Research in Electronic Art Technology, U. C. Santa Barbara, 220 Santa Anita Road, Santa Barbara, CA 93105, USA, Stephen@HeavenEverywhere.com)

The Allosphere is a new audio/visual immersion space for the California Nanosystems Institute at The University of California, Santa Barbara, used for both scientific and performing-arts studies. This three-story sphere with central-axis catwalk permits an unusually large experiential region. The huge perforated-metal visual-projection sphere, with its principle listening locations centered inside the sphere, introduces multiple considerations and compromises, especially since the ideal acoustical environment is anechoic. Video projection requires opaque light reflectivity of the concave projection surface, while audio solicits extreme sound transmissibility of the screen plus full-range sound absorptivity outside the sphere. The design requires high-fidelity spatialization of a large number of simulated sound sources over a large region near the core, and support of vector-based amplitude panning, Ambisonic playback, and wavefield synthesis. This paper discusses considerations that both conform to, and lie outside of, traditional acoustical analysis methodologies, and briefly reviews the electroacoustic systems design.

\section{$6: 40$}

1pAAd7. Case study on assessing audibility by "Perspicuity" for sounds added to factory noise, using different types of speaker in directional performance. Yasushi Shimizu (Center for Advanced Sound Technologies, Yamaha, 203 Matsunokijima, 4380192 Iwata, Shizuoka, Japan, yasushi_shimizu@gmx.yamaha.com), Mai Fujiwara (Center for Advanced Sound Technologies, Yamaha, 203 Matsunokijima, 4380192 Iwata, Shizuoka, Japan, mai_fujiwara@gmx.yamaha.com), Atsuko Ito (Center for Advanced Sound Technologies, Yamaha, 203 Matsunokijima, 4380192 Iwata, Shizuoka, Japan, atsuko1_ito@gmx.yamaha.com)

Music and other sounds have been played to provide a pleasant sound environment for workers in a factory. In terms of "Sound Perspicuity" this case study was conducted to assess audibility of spatial sounds, which were adjusted in level without increasing total sound pressure level (SPL) at the workstation. Furthermore, the added sounds were not audible at other workstations. Three speaker systems, including line-array speaker $(60 \mathrm{~cm}$ in length), were used for the case study. The ratio of added sound (Signal) to factory noise (Noise) was analyzed at every measuring point on a grid of $50 \mathrm{~cm}$ by $50 \mathrm{~cm}$. Audibility listening tests were performed for the added sounds, such as natural-environment sounds and musical instrument tones, with Signal to Noise ratios analyzed from three types of speaker. As a result, a sound is recognized as "Kiwadachi" by a Japanese adjective word even when the factory noise has higher levels than the added sounds. Audibility test results for each added sound are discussed with different Signal to Noise ratios.

\section{Contributed Paper}

\section{7:00}

1pAAd8. Spatial Audio Reproduction Using Distributed Mode Loudspeakers. Joshua Atkins (Johns Hopkins University, 853 West 35th Street, Baltimore, MD 21211, USA, joshatkins@jhu.edu), James West (Johns Hopkins University, Department of Electrical Engineering, 3400 North Charles Street, Baltimore, MD 21218, USA, jimwest@jhu.edu)

For future telecommunication systems to become more pervasive in society they must have the ability to produce high quality surround sound audio for multiple listeners. Current 5.1 audio systems lack the capacity to handle large group telecommunications because of their limited optimal listening positions ("sweet spots") and their preference for sound generated in front of the listeners. This is due in part to the non-optimal position of the loudspeakers and also to the fact that very directional speakers are commonly used. Recently, a new form of speaker has been developed called a distributed mode loudspeaker (DML). These speakers use bending waves in a panel to radiate sound. Consequently, they can be very good omnidirectional sources throughout nearly all of the human hearing range. Experiments using a higher order ambisonics (HOA) approach to sound field capture and generation will be presented to show how conventional electrodynamic speaker arrays compare to equivalent DML arrays for spatial sound reproduction. This work is funded by NSF Grant IIS-0534221. 


\title{
Session 1pAAe
}

\section{Architectural Acoustics and Noise: Low Frequency Absorption: Mechanisms, Measurement Methods, and Application I}

\author{
Peter D'Antonio, Cochair \\ RPG Diffusor Systems, Inc., 651-C Commerce Drive, Upper Marlboro, MD 20774, USA \\ Christian Nocke, Cochair \\ Akustikbüro Oldenburg, Katharinenstr. 10, Oldenburg, 26121, Germany
}

\section{Invited Papers}

\section{5:00}

1pAAe1. Overview of low frequency control options in rooms. Peter D'Antonio (RPG Diffusor Systems, Inc., 651-C Commerce Drive, Upper Marlboro, MD 20774, USA, pdantonio@rpginc.com)

Low frequency control of modes and speaker boundary interference in rooms has been a persistent problem that affects the sound field in rooms. However, many innovative techniques have been developed to address these problems. This presentation will be an overview of approaches that address the optimization of room dimensions to provide the flattest room response and speaker/listener placement to minimize speaker-boundary interference; the mechanism and design of passive absorption devices based on Helmholtz resonators, diaphragmatic limp mass membranes, plate resonators and microperforated panels will be described and characterized; as well as electro-acoustic approaches, using equalization, active absorbers and the optimal placement of multiple in-phase subwoofers.

\section{$5: 20$}

1pAAe2. Low frequency sound absorption measurements - limits and ideas. Christian Nocke (Akustikbüro Oldenburg, Katharinenstr. 10, 26121 Oldenburg, Germany, nocke@akustikbuero-oldenburg.de)

Many (standardised) measurement procedures for sound absorption rely on geometrical approximations or statistical approaches. They fail whenever the limit of geometrical acoustics is reached. Also many room acoustic modeling procedures assume geometrical spreading of sound and neglect the wave characteristics of sound waves. In this paper various approaches towards the measurement of sound absorption are briefly reviewed and discussed in the light of low frequency applications. Approaches towards analytic descriptions for sound fields in room at low frequencies from the early 1930s are reviewed. The approaches might be the basis for future developments on new procedures for measurement techniques. Finally measurement results on the deduction of sound absorption at low frequencies are presented.

\section{$5: 40$}

1pAAe3. Enhancing bass absorbers using activated carbon. Fouad Bechwati (University of Salford, Acoustics Research Centre, Newton Building, M5 4WT Salford, UK, f.bechwati@pgr.salford.ac.uk), Trevor J. Cox (University of Salford, Acoustics Research Centre, Newton Building, M5 4WT Salford, UK, t.j.cox@salford.ac.uk), Olga Umnova (University of Salford, Acoustics Research Centre, Newton Building, M5 4WT Salford, UK, o.umnova@salford.ac.uk), Mark R. Avis (University of Salford, Acoustics Research Centre, Newton Building, M5 4WT Salford, UK, m.r.avis@salford.ac.uk)

Bass absorbers, either Helmholtz or membrane devices, are commonly used in small rooms to reduce the effects of standing wave modes. Previously, it has been shown that activated carbon can be used to change the compliance of loudspeaker enclosures. It has also been shown that activated carbon displays excess absorption at low frequencies. It has been suggested that the change in compliance and absorption is due to adsorption and desorption of air molecules on the surface of the carbon, caused by the compressions and rarefactions of sound waves. This paper investigates the extent of these effects to and their significance for resonant absorbers. The results show that activated carbon can lower the resonant frequency of a Helmholtz absorber, and also increases the quality factor of the resonance. The paper will discuss what the results mean in terms of the physical interaction between activated carbon and sound, as well as the practical application of the material to bass absorbers.

\section{6:00}

1pAAe4. An improved low frequency radiation model for finite sound reflectors. Jonathan Rathsam (Univ. of Nebraska - Lincoln, Architectural Eng. Program, 1110 S. 67th St., Omaha, NE 68182-0681, USA, jrathsam@mail.unomaha.edu), Lily M. Wang (University of Nebraska - Lincoln, 1110 S. 67th St., Omaha, NE 68182-0681, USA, LWang4@ UNL.edu), Jens Holger Rindel (Odeon A/S, Scion DTU, Diplomvej Buildling 381, DK-2800 Lyngby, Denmark, jhr@ odeon.dk), Claus Lynge Christensen (Odeon A/S, Scion DTU, Diplomvej Buildling 381, DK-2800 Lyngby, Denmark, clc@odeon.dk)

Geometric computer models for room acoustics, such as ODEON, predict sound fields most reliably at high frequencies. At low frequencies, algorithms must be modified to account for deviations from geometrical acoustics caused by wave phenomena. For finite reflectors, a common low frequency model is based on the Kirchhoff-Fresnel Diffraction Approximation, which predicts a uniform $6 \mathrm{~dB}$ 
per octave slope below a reflector's geometrical limiting frequency. As discussed in this paper, highly accurate Boundary Element Method simulations, not subject to the Kirchhoff Approximation, suggest the use of an additional, lower limiting frequency and slope of $12 \mathrm{~dB}$ per octave to represent the reflector's response at the lowest frequencies. This second limiting frequency and $12 \mathrm{~dB}$ per octave slope, referred to as the dipole limiting frequency, are presented in a form suitable for insertion into a geometric computer model. [Work supported by the National Science Foundation.]

\section{Contributed Papers}

6:20

1pAAe5. Control of low-frequency sound to improve spatial uniformity in music production spaces. Ronald Eligator (Acoustic Dimensions, 145 Huguenot Street, New Rochelle, NY 10801, USA, religator @acousticdimensions.com)

Commercial music post-production spaces are often less than $300 \mathrm{ft}^{2}(28$ $\mathrm{m}^{2}$ ) and $2700 \mathrm{ft}^{3}\left(76.5 \mathrm{~m}^{3}\right)$, and it is not uncommon for them to be one-third to one-half this area. Even with careful room design, creating spatiallyuniform sound fields, especially at low-frequencies, can be thwarted by the physical restraints imposed by the room's dimensions, boundary conditions and restraints imposed by other functional requirements and budget. This paper presents successful, and not so successful, approaches to managing these challenges through speaker selection, placement and mounting, as well as room shaping and finishes and furniture design. Results of design analysis, room acoustic testing, and listening tests are compared.

\section{6:40}

1pAAe6. The Perforated Panel Resonator with Flexible Tube Bundles and its Appliations. Yadong Lu (Institute of Acoustics, Chinese Academy of Sciences, P. O. Box 2712, 21 Beisihuanxilu St., 100080 Beijing, China, yadong@mail.ioa.ac.cn), Huide Tang (Tianjin Hearing-aid Factory, Chenglinzhuang Rd. No. 93, 300161 Tianjin, China, tanghuide@eyou.com), Qiang Wang (Jinyusun Ventilation and Airconditioning Equipment Ltd., Golden-delta Development Region. Tongzhou Dist., 101112 Beijing, China, jinyushun2003@yahoo.com.cn), Jing Tian (Institute of Acoustics, Chinese Academy of Sciences, P. O. Box 2712, 21 Beisihuanxilu St., 100080 Beijing, China, tian@mail.ioa.ac.cn), Youli Qu (Beijing Electrical Designing Institute, 15 Guananmenxijie St., 100055 Beijing, China, thgink@tom.com), Bo Qiu (Institute of Acoustics, Chinese Academy of Sciences, P. O. Box 2712, 21 Beisihuanxilu St., 100080 Beijing, China, nkcubert@yahoo.com.cn)

The sound absorptive mechanism and properties of the perforated panel resonator with flexible tube bundles are presented. Based upon that, some practical applications of the sound absorbing structure are introduced as follows. First of all, the perforated panel resonator with flexible tube bundles is used in designing a highly sound absorptive chamber, which is used as an audio-video laboratory. The acoustical performances of the chamber are presented. Second, the perforated panel resonator with flexible tube bundles is used in highly efficient duct silencer. The total noise level of axial fan source is attenuated to background noise level. Not only middle and high frequency noise is reduced, but also low frequency noise is suppressed considerably. Third, the perforated panel resonator with flexible tube bundles is used in a small-type centrifugal fan's casing treatments. The cutoff region of the centrifugal fan's casing is also acoustically treated. After the acoustical treatments, total sound power level of the fan is reduced obviously. Fourth, the perforated panel resonator with flexible tube bundles is used in designing the exhaust ducts and sound absorptive channel walls for an electrical transformer substation. The noise emitted from the transformer substation is reduced to surrounding background noise level.

\section{7:00}

1pAAe7. Shape-optimization of several multi-modal resonators accounting for room/resonator acoustical coupling. Octávio Inácio (Musical Acoustics Laboratory - ESMAE-IPP, Rua da Alegria, 503, 4000-045 Porto, Portugal, OctavioInacio@esmae-ipp.pt), José Antunes (Applied Dynamics Laboratory, Instituto Tecnológico e Nuclear, Estrada Nacional 10, 2686-953 Sacavém, Portugal, jantunes@itn.pt)

Helmholtz resonators are often applied for the sound equalisation of control rooms, through adequate leveling of the low frequency acoustic modal room responses. In several recent papers we proposed to improve the efficiency of such devices by, instead of using basic Helmholtz resonators with uniform cross-section, develop shape optimized multi-modal resonators in order to cope with a larger number of intrusive room modes. We thus showed the feasibility of resonator shape-optimization, in order to obtain a target set of acoustic eigenvalues, within imposed physical and/or geometrical constraints. More recently, we developed an efficient substructure theoretical approach to compute the coupled acoustical modes of rooms fitted with several multi-mode resonators, later also including viscous boundary layer absorption effects at the room/resonator interfaces. In the present paper we extend a further step these results by applying the previously developed optimization techniques to the fully coupled room/resonator model. We thus obtain truly representative results for the optimized complex acoustical problem, which highlight the potential of the proposed corrective methodology. 


\title{
Session 1pAB
}

\section{Animal Bioacoustics, Noise, and ECUA: Anthropogenic Noise Effects on Animals I}

\author{
Michael Stocker, Cochair \\ Ocean Conservation Research, P.O. Box 559, Lagunitas, CA 94938, USA \\ Jonathan Gordon, Cochair \\ Sea Mammal Research Unit, Gatty Marine Laboratory, University of St Andrews, St Andrews, KY16 8LB, UK
}

\section{Contributed Papers}

\section{1:00}

1pAB1. Automated classification of odontocetes in open ocean environments using a novel multiclass suport vector machine. Susan Jarvis (Worcester Polytechnic Institute, 100 Institute Rd, Worcester, MA 01609, USA, sjarvis@ece.wpi.edu), Nancy A. Dimarzio (Naval Undersea Warfare Center Division Newport, 1176 Howell Street, Bldg 1351, 2nd Floor, Newport, RI 02841, USA, dimarziona@npt.nuwc.navy.mil), Ronald P. Morrissey (Naval Undersea Warfare Center Division Newport, 1176 Howell Street, Bldg 1351, 2nd Floor, Newport, RI 02841, USA, morrisseyrp@npt.nuwc.navy.mil), Jessica Ward (NAVSEA, Newport Undersea Warfare Center, Newport, RI RI 02841, USA, wardja@npt.nuwc.navy.mil), David Moretti (NAVSEA, Newport Undersea Warfare Center, Newport, RI RI 02841, USA, MorettiDJ@npt.nuwc.navy mil)

Mid-frequency active sonar has recently been implicated in several marine mammal stranding events in various geographic locations. This has caused increased interest in monitoring and mitigating the effects of anthropogenic noise on marine mammals. Some species can be more susceptible sonar than others. For example, beaked whales (particularly Mesoplodon densirostris) have been the predominant species involved in most of the strandings. Key to effective monitoring and mitigation is the ability to automatically detect and identify the animals present, especially the species of high interest like beaked whales. This paper presents a novel class-specific support vector machine (CS-SVM) methodology for automated, specieslevel classification of small odontocetes. A CS-SVM has been developed and trained to identify click vocalization from several species of odontocetes including Mesoplodon densirostris. The algorithm processes time series data in a fully automated fashion first detecting and then classifying click vocalizations. The current CS-SVM configuration correctly identifies clicks from M. densirostris over $95 \%$ of time. Further results from the application of this automated classifier to data recorded at the AUTEC and SCORE undersea range facilities will be presented.
$1: 20$

1pAB2. Effect of vessels and swimmers on the behavior of spinner dolphins (Stenella longirostris) off the Big Island of Hawai'i. Carmen Bazúa Durán (UNAM, Facultad de Ciencias, Depto. Física, Lab Acústica, Circuito exterior s/n, Cd. Universitaria, 04510 Mexico, D.F., Mexico, bazua@ servidor.unam.mx), Nina V. Luna Valiente (UAM-Xochimilco, División de Ciencias Biológicas y de la Salud, 04960 Mexico, D.F., Mexico, bazua@servidor.unam.mx)

Many cetaceans live in coastal waters that are accessible to humans. In Kealakekua Bay, Hawai'i human activities occur during morning hours, when spinner dolphins rest and socialize (feeding occurs at night). Five human activities (swimmers, kayaks, motorized-boats, and narrow- or broadband engine noises) within $200 \mathrm{~m}$ of the dolphins were monitored to investigate their effect on dolphin behavior. Dolphin behavior was measured as aerial behavior (high, medium, low energy), acoustic behavior (whistles, burst-pulses, echoclick-trains), herd size, herd coordination (high, medium, low), and diving interval ( $\mathrm{DI}<1 \mathrm{~min}, 1 \mathrm{~min}<\mathrm{DI}<2 \mathrm{~min}, \mathrm{DI}>2 \mathrm{~min})$ in the presence or absence of each human activity. Interactions between human activities and dolphins occurred $71 \%$ of the time, having simultaneous presence of several human activities. When only one human activity was present, motorboats and broad-noise increased herd-coordination and number of aerial behaviors; motorboats and both engine-noises reduced number of acoustic behaviors; kayaks and narrow-noise increased herd-coordination, DI and some acoustic behaviors; swimmers reduced herd-coordination and increased number of acoustic behaviors. Therefore, all five human activities studied changed dolphin behavior and changed it differently. Spinner dolphins probably can distinguish efficiently between human activities and may be interrupting their rest cycle to warn each other, which could have effects on this species welfare.

\section{Invited Papers}

\section{$1: 40$}

1pAB3. Effects of sound on the behavior of toothed whales. Peter Tyack (Woods Hole Oceanographic Institution, Applied Ocean Physics \& Engineering Dept., Woods Hole, MA 02543, USA, ptyack@whoi.edu), Ian Boyd (Sea Mammal Research Unit, Gatty Marine Laboratory, University of St Andrews, KY16 8LB St Andrews, UK, ilb@st-andrews.ac.uk), Diane Claridge (Bahamas Marine Mammal Research Organisation, P.O. Box AB-20714, Abaco, 0 Marsh Harbour, Bahamas, bmms@oii.net), Christopher W. Clark (Cornell University Laboratory of Ornithology, Bioacoustics Research Program, 159 Sapsucker Woods Road, Ithaca, NY 14850, USA, cwc2@cornell.edu), David Moretti (NAVSEA, Newport Undersea Warfare Center, Newport, RI RI 02841, USA, MorettiDJ@npt.nuwc.navy.mil), Brandon Southall (National Marine Fisheries Service, 1315 East West Hwy, SSMC III \#12539, Silver Spring, MD 20910-6233, USA, Brandon.Southall@noaa.gov)

We report initial results from a study on behavioral responses of beaked and other whales to sonar and other sounds. This research is designed to provide new science-based approaches for mitigating the risk of sonar to beaked and other whales. The study was conducted at the Atlantic Undersea Test and Evaluation Center (AUTEC) range near Andros Island in the Bahamas, where Blainville's beaked whales (Mesoplodon densirostris) can regularly be detected using passive acoustic monitoring of their echolocation clicks. Tags recorded sound at the whale and behavior of the whale. Data were collected from 10 tag deployments, 6 on Blainville's beaked whales and 4 on pilot whales. 109 hours of data were collected from tags, 74 hours from beaked whales and 34 hours from pilot whales. 
Playbacks of mid-frequency sonar and killer whale calls were performed on 3 of the tagged whales, 1 beaked whale and 2 pilot whales. The tagged beaked whale responded to both sonar and killer whale sounds by premature cessation of clicking during foraging dives (RL $=\sim 117 \mathrm{~dB}$ re $1 \mu \mathrm{Pa}$ for the killer whale sound, $\sim 145 \mathrm{~dB}$ for the sonar), and an unusually slow and long ascent.

1pAB4. Acoustic flexibility in singing birds under noisy urban conditions. Hans Slabbekoorn (Leiden University, Kaiserstraat 63, 2311 GP Leiden, Netherlands, H.W.Slabbekoorn@Biology.LeidenUniv.NL)

Birds rely on song for defending a territory and attracting a mate. More and more breeding areas are affected by human activities and anthropogenic noise has become almost omnipresent. Consequently, song function in and around cities, and alongside highways, train tracks and airports, is increasingly hampered by noise interference. Several counter strategies in producing sounds, related to changes in loudness, pitch, and timing, can help out under these noisy urban conditions. Observational data have yielded several interesting patterns which show remarkable singing flexibility in different species that are typically common in cities. Here I present data for a new species, the chiffchaff (Phylloscopus collybita) showing spectral and temporal adaptation which reduces masking alongside a highway in the Netherlands. Furthermore, experimental field data reveal for the first time that this species is able to adjust immediately to exposure to highway noise via artificial playback in quiet territories. Acoustic flexibility may be key to efficient use of the 'left-over acoustic space' and may determine whether individual birds can maintain their territory and breed successfully. Variation in flexibility among species may explain which birds will remain widespread in our noisy world and which will be pushed back into 'silent' habitat pockets.

\section{Contributed Papers}

\section{2:20}

1pAB5. Comodulation masking release in the bottlenose dolphin (Tursiops truncatus). Brian K. Branstetter (US Navy Marine Mammal Program, Space and Naval Warfare Systems Center, 53560 Hull St., Code 71510, San Diego, CA 92152, USA, branstet@hawaii.edu), James J. Finneran (US Navy Marine Mammal Program, Space and Naval Warfare Systems Center, 53560 Hull St., Code 71510, San Diego, CA 92152, USA, james.finneran@navy.mil), Linda S. Green (Science Applications International Corporation, 4065 Hancock St., San Diego, CA 92110, USA, branstet@hawaii.edu), Erik E. Robinson (Science Applications International Corporation, 4065 Hancock St., San Diego, CA 92110, USA, branstet@hawaii.edu), Megan N. Tormey (Science Applications International Corporation, 4065 Hancock St., San Diego, CA 92110, USA, branstet@hawaii.edu), Randall L. Dear (Science Applications International Corporation, 4065 Hancock St., San Diego, CA 92110, USA, RANDALL.L .DEAR@saic.com)

The power spectrum model of masking, derived from critical band or critical ratio measurements is often cited and used to extrapolate the effects of masking noise on the signal detection abilities of marine mammals. However, the power spectrum model may not generalize well to environmental noise that is often coherently amplitude-modulated across frequency regions. The current study demonstrates a pattern of masking where temporally fluctuating comodulated noise produces lower masked thresholds (up to a $17 \mathrm{~dB}$ difference) compared to Gaussian noise of the same spectral density level. Noise possessing wide bandwidths, low temporal modulation rates, and across-frequency temporal envelope coherency resulted in lower masked thresholds, a phenomenon known as comodulation masking release. The results are consistent with a model where dolphins compare temporal envelope information across auditory filters to aid in signal detection. Furthermore, results suggest conventional models of masking derived from experiments using random Gaussian noise may not generalize well to environmental noise that dolphins actually encounter.

\section{2:40}

1pAB6. Investigating noise effects on the call amplitude of endangered Southern Resident killer whales (Orcinus orca). Marla Holt (NOAA Northwest Fisheries Science Center, Marine Mammal Program, 2725 Montlake Blvd East, Seattle, WA 98112, USA, Marla.Holt@noaa.gov), Val Veirs (Colorado College, Department of Physics, Colorado Springs, CO 80903, USA, vveirs@coloradocollege.edu), Scott Veirs (Beam Reach Marine Science and Sustainability School, 7044 17th Ave NE, Seattle, WA 98115, USA, scott@beamreach.org)

Anthropogenic sound exposure has been identified as a potential threat to endangered Southern Resident killer whales (SRKWs). Such exposure can mask important biological sounds including those used for social functions. Vocal animals might compensate for increased background noise by calling louder (the Lombard effect). In this study, amplitude compensation was investigated in SRKW communication calls recorded off of San Juan Island, WA, U.S.A. Data were obtained from two calibrated recording systems, a shore-based hydrophone array [V. Veirs, J. Acoust. Soc. Am., 116, 2615 (2004)] and a boat-based vertical array. Calls were localized using time of arrival differences to estimate range and apparent source levels after background levels were subtracted from the signal. Call levels were calculated assuming spreading loss functions determined from sound propagation observations made in situ. Results demonstrated a positive relationship between call levels and background levels. Costs of such vocal flexibility are unknown, but may include changes in energetic demand and/or communicative functionality. Within the data ranges measured in this study, killer whales communicatively adjusted for fluctuating background noise levels. However, other sounds detected during passive listening (e.g. prey sounds) might be more susceptible to masking effects [Work supported by NOAA and Colorado College.]

\section{Invited Papers}

\section{3:00}

1pAB7. Effects of noise on hearing in odontocetes. James J. Finneran (US Navy Marine Mammal Program, Space and Naval Warfare Systems Center, 53560 Hull St., Code 71510, San Diego, CA 92152, USA, james.finneran@navy.mil), Carolyn E. Schlundt (EDO Professional Services, 3276 Rosecrans St., San Diego, CA 92110, USA, carolyn.melka@edocorp.com), Sam H. Ridgway (University of California, San Diego, Dept. of Pathology, School of Medicine, 9500 Gilman Drive, La Jolla, CA 92093, USA, ridgway @ spawar.navy.mil)

Increased public concern and regulation of activities involving anthropogenic sound have resulted in a pressing need for specific information regarding safe limits for marine mammals exposed to underwater noise. Since many marine mammals have sensitive hearing and rely upon underwater sound for communicating, foraging, and navigating, the potential effects of noise on their hearing is of particular concern. One of the most familiar consequences of noise exposure is an increase in threshold that persists after the cessation of the noise, called a temporary thresholds shift (TTS) or permanent threshold shift (PTS). Although there are no PTS data for marine 
mammals, there have been TTS measurements in a number of species, including bottlenose dolphins and belugas. These studies compare hearing thresholds before and after subjects are exposed to intense sounds. The results are analogous to data from terrestrial mammals, where TTS depends on the exposure frequency, sound pressure, duration, and temporal pattern. This talk reviews the major findings related to the growth of and recovery from TTS in bottlenose dolphins and belugas and discusses the application of these data to acoustic exposure guidelines.

\section{$3: 20$}

1pAB8. Noise-induced permanent threshold shift in a harbor seal. David Kastak (UCSC Institute of Marine Sciences, Long Marine Lab - University of California, 100 Shaffer Road, Santa Cruz, CA 95060, USA, kastak@ucsc.edu), Jason Mulsow (UCSC Institute of Marine Sciences, Long Marine Lab - University of California, 100 Shaffer Road, Santa Cruz, CA 95060, USA, jmulsow@ucsc.edu), Asila Ghoul (UCSC Institute of Marine Sciences, Long Marine Lab - University of California, 100 Shaffer Road, Santa Cruz, CA 95060, USA, asila@ucsc.edu), Colleen Reichmuth (UCSC Institute of Marine Sciences, Long Marine Lab - University of California, 100 Shaffer Road, Santa Cruz, CA 95060, USA, coll@ucsc.edu)

Investigation of auditory temporary threshold shift (TTS) in marine mammals has provided a means of predicting the harmful effects of underwater anthropogenic noise. As complete recovery of hearing is requisite in these studies, they have been considered appropriate in light of subject availability and ethical considerations. In our psychophysical studies of TTS in pinnipeds, we have employed methods designed to safely titrate from sound levels of noise inducing no TTS to levels of significant but completely recoverable hearing loss. In the present study, these methods were used with a harbor seal (Phoca vitulina) exposed to an underwater $4.1 \mathrm{kHz}$ pure tone fatiguing stimulus. Sound levels and durations were gradually increased to a maximum received sound pressure of $184 \mathrm{~dB}$ re $1 \mu \mathrm{Pa}$ with a duration of $60 \mathrm{~s}\left(\mathrm{SEL}=202 \mathrm{~dB}\right.$ re $\left.1 \mu \mathrm{Pa}^{2} \mathrm{~s}\right)$. Upon the second exposure to this fatiguing stimulus, an initial threshold shift in excess of $50 \mathrm{~dB}$ was estimated at a test frequency of $5.8 \mathrm{kHz}$, a half-octave above the fatiguing tone. Recovery from this unexpectedly large shift occurred at a rate of $-10 \mathrm{~dB}$ per $\log (\mathrm{min})$, with an apparently permanent threshold shift of 7 to $10 \mathrm{~dB}$ evident after more than two months following exposure.

\section{3:40-4:00 Break}

\section{4:00}

1pAB9. Anthropogenic sounds - Potential effects on fish. Arthur Popper (Dept. of Biology, University of Maryland, College Park, MD 20742, USA, apopper@umd.edu), Svein Løkkeborg (Fish Capture Division, Institute of Marine Research, P.O. Box 1870, Nordnes N-5817 Bergen, Norway, svein.loekkeborg@imr.no), Robert McCauley (Curtin University, Centre for Marine Science and Technology, GPO Box U 1987, 6845 Perth, Australia, r.mccauley@cmst.curtin.edu.au)

There is concern that human-generated sounds may have deleterious effects on fish. This paper will review some of what is currently known about these effects, and consider the questions that have to be answered before developing models to enable "prediction" of sound effects on particular fish species. A major restriction is that there are few peer-reviewed data on effects of anthropogenic sources on fish. Extrapolation from these results is further confounded since experiments differ in many ways, each of which may alter the resultant impact on fish. For example, studies vary in sounds types tested (e.g., pile driving vs. ship noise), signal parameters (intensity, number of repetitions), species used, fish age, etc. Moreover, a singularly important issue is that while many of the issues and impact mechanisms are potentially amenable to experimental lab study, the ultimate questions regarding the effects of sound on fish behavior need to field based and require long-term observations where behaviour of wild fish is not constrained. Only by observing fish in the wild will we ultimately understand if, and how, anthropogenic sounds impact fish both during exposure and, more importantly, for extended periods after the termination of the sound.

1pAB10. Characterizing the relative contributions of large vessels to total ocean noise fields: a case study using the Gerry E. Studds Stellwagen Bank National Marine Sanctuary. Leila T. Hatch (US NOAA, Stellwagen Bank National Marine Sanctuary, 175 Edward Foster Road, Scituate, MA 02066, USA, leila.hatch@noaa.gov), Christopher W. Clark (Cornell University Laboratory of Ornithology, Bioacoustics Research Program, 159 Sapsucker Woods Road, Ithaca, NY 14850, USA, cwc2@cornell.edu), Sofie Van Parijs (US NOAA, Northeast Fisheries Science Center, 166 Water Street, Woods Hole, MA 02543, USA, sofie.vanparijs@noaa.gov), Richard Merrick (US NOAA, Northeast Fisheries Science Center, 166 Water Street, Woods Hole, MA 02543, USA, richard.merrick@noaa.gov), Dimitri Ponirakis (Cornell University Laboratory of Ornithology, Bioacoustics Research Program, 159 Sapsucker Woods Road, Ithaca, NY 14850, USA, dwp22@ cornell.edu), Kurt Schwehr (University of New Hampshire, Center for Coastal and Ocean Mapping Joint Hydrographic Center, 24 Colovos Road, Durham, NH 03824, USA, kurt@ccom.unh.edu), Michael A. Thompson (US NOAA, Stellwagen Bank National Marine Sanctuary, 175 Edward Foster Road, Scituate, MA 02066, USA, michael.a.thompson@noaa.gov), David Wiley (US NOAA, Stellwagen Bank National Marine Sanctuary, 175 Edward Foster Road, Scituate, MA 02066, USA, david.wiley@noaa.gov)

Understanding and mitigating the effects of underwater noise on marine species requires substantial information regarding acoustic contributions from shipping. In 2006, we used the U.S. Coast Guard's Automatic Identification System (AIS) to describe patterns of large commercial ship traffic within a U.S. National Marine Sanctuary. AIS data were combined with low-frequency acoustic data from an array of nine-ten autonomous recording units deployed throughout 2006. Analysis of received sound levels (10-1000 Hz, root-mean squared decibels re $1 \mu$ Pascal \pm standard error) averaged $119.5 \pm 0.3$ at high traffic locations. High traffic locations experienced double the acoustic power of less trafficked locations for the majority of the time period analyzed. Average source level estimates (71-141 $\mathrm{Hz}$, root-mean squared decibels re $1 \mu \mathrm{Pascal} \pm$ standard error) for individual vessels ranged from $158 \pm 2$ (research vessel) to $186 \pm 2$ (oil tanker). Tankers were estimated to contribute two times more acoustic power to the region annually than cargo ships, and over one 
hundred times more than research vessels. Our results indicate that noise produced by large commercial traffic was at levels and within frequencies that warrant concern among managers regarding the ability of endangered whales to maintain acoustic contact within greater sanctuary waters.

\section{Contributed Papers}

4:40

1pAB11. Estimates of chronic SNR exposure levels of sperm whales (Physeter macrocephalus) to airguns in the Gulf of Mexico. Aaron Thode (Marine Physical Laboratory, Scripps Institution of Oceanography, 9500 Gilman Dr, MC 0238, La Jolla, CA 92093-0238, USA, athode@ucsd.edu), Martha Winsor (Hatfield Marine Science Center, Oregon State University, Newport, CA 97365, USA, martha.winsor@oregonstate.edu), Bruce Mate (Hatfield Marine Science Center, Oregon State University, Newport, CA 97365, USA, bruce.mate@oregonstate.edu), Matt Howard (Dept. of Oceanography, Texas A\&M University, 3146 TAMU, College Station, TX 77843, USA, mkhoward@tamu.edu), Katherine Kim (HLS Research, Inc., 3366 N. Torrey Pines Ct., Ste. 310, La Jolla, CA 92037, USA, Katherine.Kim@HLSResearch.com), John_Diebold (Lamont-Doherty Earth Observatory of Columbia University, 61 Route 9W, Palisades, NY 10964-8000, USA, johnd@1deo.columbia.edu), Maya Tolstoy (Lamont-Doherty Earth Observatory of Columbia University, 61 Route 9W, Palisades, NY 10964-8000, USA, tolstoy@1deo.columbia.edu)

A synthesis effort by the Sperm Whale Seismic Study (SWSS) has estimated chronic levels of low-level, long-term acoustic exposures of sperm whales in the Gulf of Mexico due to seismic airgun activity. Between 2002 and 2005, satellite-tags were attached to 53 whales of both sexes in the northern Gulf, with an average tag viability of 187 days. Data provided by the International Association of Geophysical Contractors (IAGC) permitted the range estimation of active seismic vessels to each whale location. The ray-tracing model BELLHOP was used to estimate received levels from each seismic vessel for every space-time data point at frequencies between 10 to $1000 \mathrm{~Hz}$, using simulated source signatures from a 3590 in 3 seismic array. Winds speeds from numeric models at each space-time location were converted into ambient noise levels, and shipping noise levels were crudely estimated. The preliminary synthesis using a simple spherical spreading transmission loss model suggests that over $95 \%$ of the whale positions reported during times of airgun activity occurred in circumstances where the received airgun pulses had $0 \mathrm{~dB}$ signal-to-noise ratio (SNR) or greater compared to ambient levels, and between $25-60 \%$ of all locations have received SNRs of $20 \mathrm{~dB}$ or greater, depending on the SNR definition used. [Sponsored by US Minerals Management Service].

\section{5:00}

1pAB12. Underwater radiated noise due to the piling for the Q7 Offshore Wind Park. Christ A.f. De Jong (TNO Science and Industry, P.O. Box 155, 2600 AD Delft, Netherlands, christ.dejong@tno.nl), Michael A. Ainslie (TNO Defence, Security and Safety, Oude Waalsdorperweg 63, 2597 AK The Hague, Netherlands, michael.ainslie@tno.nl)

The Q7 is the second offshore wind farm in the Dutch sector of the North Sea and, at $23 \mathrm{~km}$ off the Dutch coast, the world's first to be located outside the 12-mile limit. To support the wind turbines, monopiles (54 metres long steel pipes with a diameter of 4 metres) are hammered into the seabed using a pile-driver. The underwater radiated noise during the impulsive hammering of 9 out of 61 monopiles was monitored. Although there is a wide concern about the impact of piling noise on the marine life in the area, there are no criteria for the maximum acceptable noise levels. A quantitative comparison of the results of various studies in which the underwater noise from pile driving has been measured and reported is difficult, due to the lack of standardization in the level definitions and data processing. The Q7 data have been analyzed in terms of a broadband sound exposure level, peak pressure and pulse duration and a 1/3-octave band frequency spectrum of the sound pressure at different hydrophone locations, for each hammer stroke that has been recorded. The results are discussed in relation to the stroke energy and the hydrophone distance and depth.
5:20

1pAB13. Monitoring the temporal and spatial characteristics of the noise radiated from marine piling. Paul A. Lepper (Loughborough University, Electronic \& Electrical Engineering, LE11 3TU Leicestershire, UK, p.a.lepper@lboro.ac.uk), Stephen P. Robinson (National Physical Laboratory, Hampton Road, TW11 OLW Teddington, UK, Stephen.Robinson@npl .co.uk)

Noise is often an unintended by-product of offshore activities, and a significant source of impulsive underwater noise is marine piling, where a pile is driven into the sea-bed using a hydraulic hammer. This paper describes work undertaken to monitor the underwater radiated noise during offshore marine piling. The measurements made include full characterisation of the temporal variation of the resulting sound field, including frequency content of the pulses and the increasing amplitude typically observed during the soft start period. Results are also shown for the spatial variation of the field, including the effect on propagation of significant bathymetry changes (for example, due to the presence of a sand bank). The energy within each acoustic pulse is an appropriate measure of the sound field, having the advantage that it is relatively straightforward to add the contributions from each pulse in order to derive an overall sound exposure level (SEL) in a manner analogous to methods familiar from air acoustics. An example is then given of how the measured data and predictive modeling may be used to calculate an overall exposure for an animal in the vicinity, using various assumptions about the location and mobility of the animal.

\section{5:40}

1pAB14. Monitoring, measuring and describing ocean noise over ecologically viable scales with applications to impacts on large whales. Christopher W. Clark (Cornell University Laboratory of Ornithology, Bioacoustics Research Program, 159 Sapsucker Woods Road, Ithaca, NY 14850, USA, cwc2@cornell.edu), Chris Tremblay (Cornell University Laboratory of Ornithology, Bioacoustics Research Program, 159 Sapsucker Woods Road, Ithaca, NY 14850, USA, cjt35@cornell.edu), Melissa Fowler (Cornell University Laboratory of Ornithology, Bioacoustics Research Program, 159 Sapsucker Woods Road, Ithaca, NY 14850, USA mcf3@cornell.edu), Christi Diamond (Cornell University Laboratory of Ornithology, Bioacoustics Research Program, 159 Sapsucker Woods Road, Ithaca, NY 14850, USA, cd254@cornell.edu), Dimitri Ponirakis (Cornell University Laboratory of Ornithology, Bioacoustics Research Program, 159 Sapsucker Woods Road, Ithaca, NY 14850, USA, dwp22@cornell.edu), Clara McCarthy (Cornell University Laboratory of Ornithology, Bioacoustics Research Program, 159 Sapsucker Woods Road, Ithaca, NY 14850, USA, cmm255@cornell.edu), Janelle Morano (Cornell University Laboratory of Ornithology, Bioacoustics Research Program, 159 Sapsucker Woods Road, Ithaca, NY 14850, USA, j1m394@cornell.edu), Charles Muirhead (Cornell University Laboratory of Ornithology, Bioacoustics Research Program, 159 Sapsucker Woods Road, Ithaca, NY 14850, USA, cam236@cornell.edu), Anita Murray (Cornell University Laboratory of Ornithology, Bioacoustics Research Program, 159 Sapsucker Woods Road, Ithaca, NY 14850, USA, am678@cornell.edu), Liz Rowland (Cornell University Laboratory of Ornithology, Bioacoustics Research Program, 159 Sapsucker Woods Road, Ithaca, NY 14850, USA, edr6@cornell.edu), Catherine Vannicola (Cornell University Laboratory of Ornithology, Bioacoustics Research Program, 159 Sapsucker Woods Road, Ithaca, NY 14850, USA, cmv44@cornell.edu), Ann Warde (Cornell University Laboratory of Ornithology, Bioacoustics Research Program, 159 Sapsucker Woods Road, Ithaca, NY 14850, USA, amw38@cornell.edu)

Large whales communicate primarily in the low $(<1000 \mathrm{~Hz})$ frequency bands; the same frequency range within which anthropogenic ocean noise has been increasing over the last half century at approximately 3-5 $\mathrm{dB} /$ decade. The working assumption and hypothesis hold that rising ambient noise levels negatively impact whales by interfering with communication, navigation and predator detection, and that over the long-term such effects 
could reduce breeding success and population size. There is a lack of empirical data on the spatial-temporal dynamics of ambient noise by which to assess potential impacts. One year of continuous acoustic data from an ongoing distributed array of 19 continuous seafloor recorders covering a 1000 $\mathrm{km}^{2}$ area in Massachusetts Bay, USA were analyzed for ambient noise statistics and the occurrences, locations and vocal behaviors of fin, humpback and right whales. Noise levels were greater than $120 \mathrm{~dB}$ rms re $1 \mu \mathrm{Pa}$ in the $10-1000 \mathrm{~Hz}$ band throughout the $1000 \mathrm{~km}^{2}$ area for $\geqslant 50 \%$ of the season when whales were present. If animals are relying on acoustic cues to coordinate feeding and social interactions, these high noise levels impose a significant loss of acoustic habitat during the whales' residency in the area.

\section{6:00}

1pAB15. Environmental Risk Management Capability: Advice on minimising the impact of sonar on marine mammals. Colin Schofield (BAE Systems Integrated System Technologies, Unit D1, Marabout Industrial Estate, DT1 1YA Dorchester, UK, colin.schofield @ baesystems.com)

The Environmental Risk Management Capability - Sonar (ERMC(S)) has now been accepted into service by the UK Royal Navy. ERMC(S) is the first operational software package that provides a comprehensive risk assessment of the common concern of the potential impact of sonar usage on marine mammals. The algorithms include allowance for extended periods of use and advice on achievable mitigation options. Now that the system has achieved this significant milestone, this paper reviews the elements of the system that have made progress to this stage so successful: -The techniques and data used by the algorithms. -The inclusive development approach that allowed all the concerned stakeholders to be satisfied with the processes utilised. -The annual review cycle that will enable the tool to maintain its links with forefront research in this area. -The application of the system to non-military sound sources as the benchmark approach. This paper will show how ERMC(S) can be develop into a tool for both commercial and military sound sources using proving and established techniques.

\section{$6: 20$}

1pAB16. Proposed Marine Mammal Noise Exposure Criteria: Current Data Base, Limitations, and Research Needs. Darlene R. Ketten (Woods Hole Oceanographic Institution, Applied Ocean Physics \& Engineering Dept., Woods Hole, MA 02543, USA, dketten@whoi.edu), Ann E. Bowles (Hubbs-Sea World Research Institute, 2595 Ingraham Street, San Diego, CA 92109, USA, abowles@hswri.com), William T. Ellison (Marine Acoustics, Inc, 809 Aquidneck Ave, Middletown, RI 02842, USA, WEMAI@aol.com), James J. Finneran (US Navy Marine Mammal Program, Space and Naval Warfare Systems Center, 53560 Hull St., Code 71510, San Diego, CA 92152, USA, james.finneran@navy.mil), Roger L. Gentry (ProScience Consulting, LLC, P.O. Box 177, Dickerson, MD 20842-0177, USA, roger.gentry@comcast.net), Charles R. Greene (Greeneridge Sciences, Inc., 4512 Via Huerto, Santa Barbara, CA 93110, USA, cgreene@greeneridge.com), David Kastak (UCSC Institute of Marine Sciences, Long Marine Lab - University of California, 100 Shaffer Road, Santa Cruz, CA 95060, USA, kastak@ucsc.edu), James H.
Miller(University of Rhode Island, Department of Ocean Engineering, Narragansett Bay Campus, Narragansett, RI 02882, USA, miller@egr.uri.edu), Paul E. Nachtigall (University of Hawaii, Hawaii Institute of Marine Biology, P.O. Box 1106, Kailua, HI 96734, USA, nachtiga@hawaii.edu), W J. Richardson (LGL Ltd., Environmental Research Associates, P.O. Box 280, 22 Fisher St, King City, AB L7B 1A6, Canada,wjr@lgl.com), Brandon Southall (National Marine Fisheries Service, 1315 East West Hwy, SSMC III \#12539, Silver Spring, MD 209106233, USA, Brandon.Southall@noaa.gov), Jeanette A. Thomas (Western Illinois University Quad Cities, Department of Biological Sciences, 3561 60th St., Moline, IL 61265, USA, j-thomas@wiu.edu), Peter Tyack (Woods Hole Oceanographic Institution, Applied Ocean Physics \& Engineering Dept., Woods Hole, MA 02543, USA, ptyack@whoi.edu)

This paper presents the findings of an inter-disciplinary expert panel based on comprehensive review of hearing and noise effects data for marine and land mammals. The principal motivation for the review was to assist formulation of data-based noise exposure criteria in light of rapidly evolving research advances in marine mammal hearing and underwater noise effects. Levels estimated to induce permanent hearing loss were determined for single exposure events for cetaceans (in water) and pinnipeds (in air and water) for each of 15 sound type/animal group combinations. These recommendations represent a current best estimate only and are modular, with modifiable key variables; e.g., source and exposure, to facilitate revision as data improve. In some cases, relatively explicit injury limits are proposed, e.g., $186 \mathrm{~dB}$ re: $1 \mu \mathrm{Pa}^{2}$-s (frequency-weighted sound exposure level) and 218 $\mathrm{dB}$ re: $1 \mu \mathrm{Pa}_{\text {peak }}$ (unweighted peak sound pressure level) for pinnipeds in water exposed to multiple sound pulses. In others, particularly for behavioural effects of multiple-pulse and non-pulse exposures, response severity and significance are quantitatively scored, but the data do not allow identification of specific broadly-applicable disturbance thresholds. These findings are a current best effort and include a discussion of limitations and recommended research needed to address data gaps.

\section{$6: 40$}

1pAB17. A simple acoustical exposure metric based on biological thresholds and integrating a temporal characteristics exposure axis. Michael Stocker (Ocean Conservation Research, P.O. Box 559, Lagunitas, CA 94938, USA, mstocker@OCR.org), Tom Reuterdahl (505 LaVerne Ave, Mill Valley, CA 94941, USA, euler1@sbcglobal.net), Libbie Horn (975 Black Mountain Rd., Hillsborough, CA 94-1-, USA, libbie.horn@gmail.com), Gail Hurley (768 Lovell Ave., Mill Valley, CA 94941, USA, hurleyitaly @ hotmail.com)

Anthropogenic noise is compromising habitat for marine mammals, fish and potentially other marine organisms. Determining acceptable exposure thresholds is confounded by the fact that marine animals have adapted to some exceedingly loud naturally occurring sounds, while exposure to certain anthropogenic noises at equivalent or lower amplitudes causes harm. It is clear that mitigation levels can not be established by signal amplitude alone and that other signal characteristics are significant factors in biological responses to noise exposure. This proposed metric continues ongoing work on a simple exposure metric based on broadband frequency and amplitude representation of a subject noise with the time domain represented in the $\mathrm{Z}$ axis. 


\title{
Session 1pAOa
}

\section{Acoustical Oceanography and ECUA: Acoustical Oceanography of Polar Environments I}

\author{
Peter N. Mikhalevsky, Cochair \\ Science Applications International Corporation, 4001 N. Fairfax Dr. Suite 850, Arlington, VA 22203, USA \\ Hanne Sagen, Cochair \\ Nansen Environmental and Remote Sensing Center, Thormфlensgt. 47, Bergen, 5006, Norway
}

\section{Invited Papers}

\section{1:00}

1pAOa1. Acoustic communication and navigation in the Arctic Ocean. Arthur B. Baggeroer (MIT, Department of Mathematics, 77 Massachusetts Avenue, Cambridge, MA 02139, USA, abb@boreas.mit.edu), Lee Freitag (Woods Hole Oceanographic Inst., 266 Woods Hole Road, MS 18, Woods Hole, MA 02543, USA, lfreitag@whoi.edu), James E. Priesig (Woods Hole Oceanographic Inst., 266 Woods Hole Road, MS 18, Woods Hole, MA 02543, USA, jreisig@whoi.edu), Daniel Nagle (Naval Undersea Systems Center, 1176 Howell Street, Newport, RI 02841, USA, nagledt@npt.nuwc.navy.mil)

Acoustic communication and navigation in the Arctic usually implies surface interaction because of the upward refracting profile. While open water leeds and polyna exist, sensors must also be deployed beneath the ice. Nominal roughness of several meters plus ubiquitous ice keels all lead to complicated multipath propagation typically of durations from 0.1 to 10 seconds. Pack ice drifts at scales of 0.1-0.2 nm/s leading to doppler shifts/spreads of $2-8 \mathrm{~Hz}$ at $10-20 \mathrm{kHz}$ carriers. These lead to highly spread channels with lots of ISI. Wise signal and code designs coupled with equalizers are critical to successful performance of acomms system even at low rates. Navigation such as by long base line systems encounter the same multipath. Path detectors and trackers are easily confused in determining interactions with the underside of the ice cover. Often, first arrivals are weak and do not trigger detectors. This leads to "lane" skipping by tracking algorthms and sychonization errors for acomms. This implies clever "track association" algorithms to identify the arrival patterns and then transform them to locations which move with ice drift. This presentation outlines acoustic problems which communicaton and navigation systems must overcome using data take from several Arctic experiments.

$1: 20$

1pAOa2. Propagation, scattering and reverberation in an ice-covered Arctic ocean. Henrik Schmidt (MIT, 77 Mass Ave, 5-204, Cambridge, MA 02139, USA, henrik@mit.edu), Kevin D. Lepage (Naval Research Laboratory, 4555 Overlook Ave SW, Washington, DC 20375, USA, kevin.lepage@nrl.navy.mil)

The Arctic Ocean is a unique acoustic environment due to the ice cover and the strongly upward refracting sound speed profile. A large amount of theoretical and experimental research has focused on the significance of the scattering of sound by the rough ice cover. In spite of this, a strong anomali persisted until the early 1990s in the propagation of low-frequency sound in particular, with prevailing rough-surface scattering theories incapable of explaining the frequency dependence of transmission loss observed experimentally. More recent modeling was cabable of reproducing the observed transmission losses by combining the effects of ice elasticity, rough interface scattering, and waveguide propagation [JASA, 96:1783-1795,1994]. Thus, it was demonstrated that incoherent scattering into Lamb waves in the ice provided a significant loss mechanism. This mechanism itself did not explain the frequency dependence, but when incorporating the bilinear sound speed profile of the arctic ocean, it was found that the lower-order modes become disproportionately attenuated, explaining the high attenuation observed at low frequencies. The finding was later confirmed by succesfully modeling the modal losses observed in 1994 Trans-Arctic Propagation experiments, and the ability of the same model to accurately predict observed, long-range reverberation measurements [JASA, 111:747-760,2001]. [Work supported by ONR.]

\section{$1: 40$}

1pAOa3. Long-term variations of ice breaking noise in Antarctica. Alexander Gavrilov (Curtin University of Technology, Centre for Marine Sci \& Tech, GPO Box U1987, 6845 Perth, WA, Australia, a.gavrilov@cmst.curtin.edu.au), Binghui Li (Curtin University of Technology, Centre for Marine Sci \& Tech, GPO Box U1987, 6845 Perth, WA, Australia, binghui.li@postgrad.curtin.edu.au)

Numerous low frequency transient signals arriving from Antarctica have been detected in five-year sea noise recordings in 20022006 at the hydroacoustic listening station deployed off Cape Leeuwin in Western Australia as part of the International Monitoring System of the Comprehensive Nuclear-Test-Ban Treaty. The majority of these signals consist of a single arrival of mode 1 which dominates the higher modes and undergoes strong frequency dispersion due to long-range propagation in the polar ocean environment. These signals are believed to be emitted primarily by ice rifting and breaking events on the Antarctic shelf. Several regions along the Antarctic coast associated with certain glaciers and ice tongues were found to persistently be much more active in generating ice noise than other 
parts of the Antarctic shelf. An analysis of long-term changes in the frequency of occurrence of ice events showed noticeable seasonal cycles, but did not reveal any significant interannual variations against the background of short-term fluctuations. Some of the ice events detected at the Cape Leeuwin station were also identified in sea noise recordings made on autonomous acoustic loggers deployed on the Antarctic shelf, which allowed us to investigate characteristics of the acoustic signals from ice events near their origins.

\section{Contributed Papers}

\section{2:00}

1pAOa4. Localization of Antarctic ice breaking events by frequency dispersion of the signals received at a single hydroacoustic station in the Indian Ocean. Binghui Li (Curtin University of Technology, Centre for Marine Sci \& Tech, GPO Box U1987, 6845 Perth, WA, Australia, binghui.li@postgrad.curtin.edu.au), Alexander Gavrilov (Curtin University of Technology, Centre for Marine Sci \& Tech, GPO Box U1987, 6845 Perth, WA, Australia, a.gavrilov@cmst.curtin.edu.au)

Transient acoustic signals from Antarctic ice cracking and breaking events, featuring significant frequency dispersion, were observed at the hydroacoustic stations deployed in the Indian Ocean as part of the International Monitoring System (IMS) of the Comprehensive Nuclear-Test-Ban Treaty. Based on a comparison with numerical predictions, the measured dispersion characteristics were used to estimate the range between ice events and the receiver. Combined with the bearing capability of the IMS stations, these estimates allow us to locate ice events from a single hydroacoustic station. An analysis of range estimation errors due to uncertainty of the measured time-frequency structure of signal arrivals and due to variations of the sound speed profiles was also conducted. The analysis showed that the location accuracy from a single hydroacoustic station for the typical ice events was of the same order, as that determined from an intersection of bearings from two remote stations, if the signal frequency bandwidth is as wide as at least $5 \mathrm{~Hz}$ and lies within $5 \mathrm{~Hz}$ to $35 \mathrm{~Hz}$ frequency range. This localization method was examined by analysing several ice events detected at both the Cape Leeuwin IMS station and a sea noise logger deployed off the Antarctic shelf.
2:20

1pAOa5. Year-round acoustic monitoring of large whales in polar environments: a comparison of Davis and Bransfield Straits. Kathleen Stafford (University of Washington Applied Physics Lab, 1013 40th St NE, Seattle, WA 98105, USA, stafford@apl.washington.edu), Malene J. Simon (University of Aarhus, Institute of Biology, C.F. Mollers Alle 1131, 8000 Aarhus C, Denmark, MaSi@natur.gl), Robert Dziak (Oregon State Univ. and NOAA, 2030 SE Marine Science Dr., Newport, OR 97365, USA, robert.p.dziak@noaa.gov), David K. Mellinger (Oregon State Univ. and NOAA, 2030 SE Marine Science Dr., Newport, OR 97365, USA, David .Mellinger@oregonstate.edu)

Both Davis Strait, in the Arctic between Canada and Greenland, and Bransfield Strait, in the Antarctic between the Antarctic Peninsula and the South Shetland Islands, are areas that experience extreme inter-seasonal differences in temperature, ice cover and productivity. The seasonally productive nature of both of these ice-influenced areas provides habitat for foraging large whales such as blue (Balaenoptera musculus) and fin (B. physalus) whales. In order to monitor and compare the seasonal occurrence of these species at high latitude regions at opposite ends of the globe, autonomous underwater hydrophone packages were deployed for one year in each Strait. Three instruments were deployed and recovered in Davis Strait from October 2006-07 and five in Bransfield Strait from November 2006-07. Blue and fin whales were recorded seasonally in both regions and exhibited the geographic acoustic distinctions between northern and southern hemisphere populations. Comparisons of the seasonal occurrence and the influence of ice cover on this occurrence will be compared and contrasted between north and south.

\section{Invited Papers}

\section{2:40}

1pAOa6. Acoustic navigation and communications for high latitude ocean research (ANCHOR). Craig M. Lee (Applied Physics Laboratory, University of Washington, 1013 NE 40th St, Seattle, WA 98105-6698, USA, craig@apl.washington.edu), Jason I. Gobat (Applied Physics Laboratory, University of Washington, 1013 NE 40th St, Seattle, WA 98105-6698, USA, jgobat@apl .washington.edu)

Recent community reports on autonomous and Lagrangian platforms and Arctic observing identify the development of under-ice navigation and telemetry technologies as one of the critical factors limiting the scope of autonomous (e.g. floats, AUVs and gliders) high-latitude measurement efforts. These platforms could provide persistent, high-resolution, basin-wide sampling in ice-covered regions and collect measurements near the critical ice-water interface. Motivated by the dramatic advances in temporal and spatial reach promised by autonomous sampling and by the need to coordinate nascent efforts to develop navigation and communication system components, an international group of acousticians, platform developers, high-latitude oceanographers and marine mammal researchers gathered in Seattle, U.S.A. from 27 February - 1 March for an NSF Office of Polar Programs sponsored Acoustic Navigation and Communication for High-latitude Ocean Research workshop. Workshop participants summarized the current state of knowledge concerning Arctic acoustics, navigation and communications, developed an overarching system specification to guide community-wide engineering efforts and established an active community and steering group to guide long-term efforts and ensure interoperability between elements developed by disparate teams. This presentation will summarize workshop findings and provide an update on recent developments stemming from the EU DAMOCLES and US NSF Arctic Observing Network programs.

\section{3:00}

1pAOa7. Sound fixing and ranging beneath sea-ice. Jean-Claude Gascard (University Pierre \& Marie Curie, 4 place Jussieu, Locean tour 45-46, 5e, 75005 Paris, France, jga@locean-ipsl.upmc.fr)

Sound fixing and ranging techniques, the so-called SOFAR technique, is widely used in the field of oceanography for tracking neutrally buoyant floats underwater over large distances (several thousands of kilometres) and for long periods of time (several years). The range propagation depends largely on the existence or not of the SOFAR sound channel. Twenty years ago we investigated SOFAR acoustic propagation under sea-ice over large distances and at various frequencies from $80 \mathrm{~Hz}$ up to $1560 \mathrm{~Hz}$. We discovered the range for acoustic propagation was much reduced (100 kms to $150 \mathrm{kms})$ no matter the frequency used. This was due to large scattering induced by under sea-ice topography since there is no way to avoid acoustic rays to bounce back to the surface towards sea-ice and create multiple reflections. More recently during the Tara Damocles transpolar drift across the Arctic Ocean, we reinvestigated the 
SOFAR acoustic range propagation under sea-ice for longer periods of time in various conditions (summer and winter conditions). Our results confirmed those obtained 20 years ago and indicated a high sensitivity to the ambient conditions. We are presenting the results obtained during the late fall- early winter months of the Tara transpolar drift across the Arctic Ocean.

\title{
Contributed Papers
}

3:20

1pAOa8. The Fram Strait acoustic tomography system. Hanne Sagen (Nansen Environmental and Remote Sensing Center, Thormølensgt. 47, 5006 Bergen, Norway, hanne.sagen@nersc.no), Stein Sandven (Nansen Environmental and Remote Sensing Center, Thormølensgt. 47, 5006 Bergen, Norway, stein.sandven@nersc.no), Peter Worcester (MPL, Scripps Institution of Oceanography, University of California, San Diego, La Jolla, CA 92093-0238, USA, pworcester@ucsd.edu), Matthew Dzieciuch (MPL Scripps Institution of Oceanography, University of California, San Diego, La Jolla, CA 92093-0238, USA, mad@ucsd.edu), Emmanuel Skarsoulis (FORTH/IACM, N. Plastira 100, Vasilika Voutes, GR-70013 Heraklion, Greece, eskars@iacm.forth.gr)

The deep and wide Fram Strait between Greenland and Spitzbergen is the main influx and efflux gate to the Arctic Basin. Although major resources are invested in measurements of current and temperature here (http: //asof.npoar.no), the flux estimates still have significant deficiencies and errors. Our objective is to build, test, validate and use an innovated integrated observing and modeling system, including acoustic tomography, for improved monitoring of volume, heat and freshwater transports in the Fram Strait. As part of the DAMOCLES-IP, (=Developing Arctic Modeling and Observing Capabilities for Long-term Environmental Studies - Integrated Project) a first step acoustic tomography system is to be installed in the Fram Strait between East Greenland and West Spitzbergen in August 2008. The first step tomography system consists of one acoustic source near the Svalbard shelf and one receiver array in the middle of the Fram Strait. An extended acoustic system serving both tomography and glider navigation is planned implemented in 2009 within the recently funded project "Acoustic technology for observing the interior of the Arctic Ocean" (ACOBAR). We will present the specification of the tomography systems; our experimental plans, and plans for data analysis, including data assimilation.
3:40

1pAOa9. Ocean acoustic tomography: Travel-time inversion in the eastern Fram Strait. Emmanuel Skarsoulis (FORTH/IACM, N. Plastira 100, Vasilika Voutes, GR-70013 Heraklion, Greece, eskars@iacm.forth.gr), George Piperakis (FORTH/IACM, N. Plastira 100, Vasilika Voutes, GR-70013 Heraklion, Greece, piperak@iacm.forth.gr), Michael Kalogerakis (TEI Crete/FORTH/IACM, Vassilika Vouton, GR-711 10 Heraklion, Crete, Greece, mixalis@iacm.forth.gr), Hanne Sagen (Nansen Environmental and Remote Sensing Center, Thormølensgt. 47, 5006 Bergen, Norway, hanne.sagen @ nersc.no)

The Fram Strait is the main passage through which the mass and heat exchange between the Atlantic and the Arctic Ocean takes place. In the framework of DAMOCLES project (EU/FP6) a tomography experiment is planned to be conducted at latitude of 78N50 between 1E and 7E. A nearreal-time discrete inversion scheme has been developed relying on the matched-peak inversion approach seeking to maximize agreement between theoretical and observed peaks. The model relations between model parameters (temperature distributions) and travel times are implemented using a ray-tracing code accounting for the effects of range dependence. The parameter space is discretized and the corresponding model relations are stored in a database for later use - stored model relations greatly accelerate the inversion process. In the absence of real measurements the performance of the inversion scheme was tested using synthetic data relying on 3-year long time series of temperature data. The particular propagation conditions give rise to different inversion errors at different depths, with the minimum obtained at $250 \mathrm{~m}$ depth, in the core of the northward flowing Atlantic water. For the implementation of the tomographic analysis a software system called SMTAS has been developed running in a dedicated server connected to the internet.

\section{Session 1pAOb}

\section{Acoustical Oceanography and ECUA: Marine Ecosystem Acoustics I}

\author{
Kenneth G. Foote, Cochair \\ Woods Hole Oceanographic Institution, Woods Hole, MA 02543, USA \\ Olav R. Godoe, Cochair \\ Institute of Marine Research, PO Box 1870, Bergen, 5817, Norway
}

\section{Invited Papers}

\begin{abstract}
$4: 20$
1pAOb1. Ecosystem acoustics: meeting management needs. Olav Rune Godoe (Institute of Marine Research, C. Sundtsgt 64, Nordnes, 5817 Bergen, Norway, olavrune@imr.no)

Marine ecosystems and their exploited resources are often monitored through quasi-synoptic scientific surveys that produce estimates of population characteristics at periodic intervals. Spatial and temporal variability is an integral part of ecosystem function but is often ignored in traditional assessment methodology due to the inability of conventional surveys to resolve and quantify such effects. This confounds estimates of the state of the ecosystem and its main populations and obscures understanding of its dynamics and function. The concept and approach of ecosystem acoustics, which are elaborated and illustrated in this presentation, exploit both active and passive acoustics over their full bandwidth from a range of platforms giving temporal and spatial resolution sufficient for assessing
\end{abstract}


the state of ecosystems including their main component organisms. Application of new platforms and sensors, as well as combinations of these, is enhancing the power of the approach. Ecosystem acoustics enables observations of marine life to be made on spatial and temporal scales relevant to understanding ecosystem function and assessment of system dynamics and state.

\section{4:40}

1pAOb2. Acoustical methods that provide an integrated view of the marine ecosystem. Anne Lebourges-Dhaussy (IRD, Technopole de la Pointe du Diable, BP 70, 29280 Plouzane, France, Anne.Lebourges.Dhaussy@ird.fr), Erwan Josse (IRD, Technopole de la Pointe du Diable, BP 70, 29280 Plouzane, France, Erwan.Josse@ird.fr)

The fish stock assessment are not anymore the sole purpose of the "fisheries acoustics" as it appears clearly that the fish belongs to a whole ecosystem, of which functioning mechanisms must be understood to better assess the fish populations' health state. The biotic factors, as prey availability, are of great importance and can be studied also by acoustic tools. The multifrequency systems (echosounders or profilers), increase the size range of the organisms that can be detected; new multibeam sonars provide a high resolution allowing studies close to boundaries. The results of the SIMFAMI European program are presented. It aimed at marine organism classification through multifrequency information, including fish/plankton separation and classification of meso/macroplankton. It provided new methods to classify and describe the various biotic components of the ecosystem from fish to plankton. A link between the use of these new methods and the former micro/mesoplankton classification tools as well as passive acoustic tools allows to reach the classification of a huge range of organisms and to study their interactions. Results of such experiences done on various upwelling ecosystems are shown. Additionally for complex fields, new multibeam sonars can advantageously help in the description of fish distributions.

\section{5:00}

1pAOb3. Three-dimensional structure of thin zooplankton layers is impacted by foraging fish. Kelly Benoit-Bird (Oregon State University, 104 COAS Admin Bldg, Corvallis, OR 97333, USA, kbenoit@ coas.oregonstate.edu)

The role of predation by fish in the formation and persistence of zooplankton thin layers was assessed from three-dimensional observations using a multibeam sonar attached to a mechanical rotator. Zooplankton in Monterey Bay, CA, USA were found in intense layers with vertical scales of $0.5-2.5 \mathrm{~m}$ at night. These features comprised more than $90 \%$ of the zooplankton biomass and had volume scattering strengths of up to $-35 \mathrm{~dB}$. These thin zooplankton layers showed complex structure with significant though gradual undulations in their depth, thickness, and intensity. Fish spent significantly more time within zooplankton layers than was expected by chance, suggesting concentrated foraging activity. Sonar tracks of individual fish showed them diving through zooplankton layers. These dives were correlated with a decrease in the intensity of zooplankton scattering at the scale of tens of centimeters, resulting in the appearance of holes in the layer. Continued observation of layers revealed that these voids slowly filled in with zooplankton after the fish's departure. At the level of foraging observed, fish can have a significant effect on the distribution of zooplankton layers, however, the layers are resilient to apparent foraging by fish with reformation of the distinct layer often occurring post-predation.

\section{$5: 20$}

1pAOb4. Acoustic assessment of trophic dominance in a marine ecosystem. Richard E. Thorne (Prince William Sound Science Center, P.O. Box 705, Cordova, AK 99574, USA, rthorne@pwssc.org), Gary L. Thomas (University of Miami, RSMAS, 4600 Rickenbacker Causeway, Miami, FL 33149, USA, gthomas@rsmas.miami.edu)

High-frequency acoustic surveys over the past 15 years show that the trophic structure in Prince William Sound (PWS), Alaska, functions as a wasp-waist ecosystem. Three dominant biomasses in PWS are: (1) Pacific herring (2) walleye pollock and (3) the largebodied copepods (Neocalanus spps.) that dominate the spring zooplankton assemblage. The acoustic surveys and associated ecosystem observations suggest that the relative dominance of herring and pollock affects the composition of the apex predators. The near-shore and near-surface distribution of herring provides access by surface-oriented marine mammal and seabird predators, such as sea lions, seals, murres and cormorants, to a crucial winter-period food source. In contrast, the deep, off-shore distribution of the pollock favors large benthic predators, such as demersal sharks, halibut, and flounder. The acoustic surveys also demonstrated that the abundance of large-bodied copepods in PWS is critical to ecosystem productivity, including survival of juvenile pink salmon, and may affect the relative dominance of herring and pollock. An additional outcome of the long-term database on Pacific herring was the discovery of linkages between the 1989 Exxon Valdez oil spill and a subsequent collapse of the herring along with associated predators.

\section{$5: 40$}

1pAOb5. Saguenay fjord entrance whale feeding ground: Acoustic study of sill dynamics and tidal aggregation of forage fish. Yvan Simard (Fisheries and Oceans Canada \& ISMER-UQAR, 850 route de la Mer, P.O. Box 1000, Mont-Joli, QC G5H-3Z4, Canada, simardy@dfo-mpo.gc.ca), Nathalie Roy (Fisheries and Oceans Canada \& ISMER-UQAR, 850 route de la Mer, P.O. Box 1000, MontJoli, QC G5H-3Z4, Canada, royn@dfo-mpo.gc.ca), François Saucier (Marine Sciences Institute, University of Québec, 310 Allée des Ursulines, P.O. Box. 3300, Rimouski, QC G5L-3A1, Canada, francois_saucier@uqar.qc.ca), Jacques Gagné (Fisheries and Oceans Canada, 850 route de la Mer, P.O. Box 1000, Mont-Joli, QC G5H-3Z4, Canada, gagneja@dfo-mpo.gc.ca), Samuel Giard (Fisheries and Oceans Canada, 850 route de la Mer, P.O. Box 1000, Mont-Joli, QC G5H-3Z4, Canada, giards@dfo-mpo.gc.ca)

The Saguenay fjord entrance in the St. Lawrence Estuary at Tadoussac is a world famous site where beluga and minke whales can be regularly observed from the coast. Strong tidal upwelling over the shallow sill of the fjord controls the exchanges with the St. Lawrence. In May 2005, an intensive oceanographic and acoustic survey was conducted to understand how the complex 3D hydrodynamics may contribute to concentrating whale preys. High time-space resolution acoustics (38 and $120 \mathrm{kHz}$ split-beam), ADCP (acoustic Doppler current profilers), CTD profiles, plankton and micronekton sampling were used to track the 3D movements of water masses, zooplankton and forage fish. During flood, the dense cold waters that jump over the sill block the Saguenay outflow and subduct into the fjord with their fish and zooplankton content. This complex 3D circulation generates fronts and convergence zones where 
biomass is concentrating until current reversal occurs during ebb. These concentrations are then advected and dispersed downstream over the sill with the Saguenay surface plume. This whale prey tidal concentrating process depends on the existence of sufficient forage fish densities in the mesoscale neighbourhood. It is likely at the basis of the persistent high frequentation of this area by whales.

6:00

1pAOb6. Multibeam sonar and echosounder to study schooling behaviour and predator-prey interactions of marine animals. Leif Nøttestad (Institute of Marine Research, Distribution and Trophic Interactions, P.O. Box 1870 Nordnes, N-5817 Bergen, Norway, leif.nottestad@imr.no)

In the past the technological developments and improved spatial and temporal resolution in hydro-acoustics, have made it possible to study schooling and aggregation behaviour of fish and predator-prey interactions in considerable detail. Hydro-acoustic methodology have thus revealed new and interesting scientific findings on animal behaviour and ecology previously difficult to observe and document from the marine environment. A fundamental challenge is still to be able to track individual fish within a school or animal aggregation at the same time as we observe the collective movements. The spatial resolution of hydro-acoustics is usually not sufficient enough to track individual fish within dense aggregations. Underlying mechanisms and motivations of individual animals swimming in aggregations are needed, in order to improve our understanding and predictability of collective behaviour. Improved post-processing programs for quantitative analysis of multi-beam sonar data are also needed. High-resolution underwater cameras, Crittercam-technology and individual tagging will offer complementary data sources to our understanding of detailed behaviour of marine animals. I will present synoptic ecological studies using multi-beam sonar and echosounder on schooling behaviour and predator-prey interactions including fish, seabirds and marine mammals.

6:20

1pAOb7. Acoustic characteristics of vertically migrating and non-migrating organisms observed in the North Pacific Ocean. Kouichi Sawada (Nat. Res. Inst. Fish. Engine., FRA, 7620-7 Hasaki, 314-0408 Kamisu, Japan, ksawada@fra.affrc.go.jp), Hiroki Yasuma (Hokkaido Univ., 3-1-1 Minato-machi, 041-8611 Hakodate, Japan, ANB52615@ nifty.com), Taro Ichii (Nat. Res. Inst. Fish. Sci., FRA, 2-12-4 Fukuura, Kanazawa, 235-8648 Yokohama, Japan, ichii@fra.affrc.go.jp), Hideyuki Takahashi (Nat. Res. Inst. Fish. Engine., FRA, 7620-7 Hasaki, 314-0408 Kamisu, Japan, hideyuki@fra.affrc.go.jp), Koki Abe (Nat. Res. Inst. Fish. Engine., FRA, 7620-7 Hasaki, 314-0408 Kamisu, Japan, abec@ fra.affrc.go.jp)

Acoustic characteristics of vertically migrating and non-migrating organisms in a day were investigated for species identification. In July 27-30, 2004, both organisms were observed using ship echosounders ( $38 \mathrm{kHz}$ and $120 \mathrm{kHz}$ ) and a tethered acoustic-optical composite system (J-QUEST) from several hours before sunset to several hours after the sunrise, drifting a research vessel, Shunyo-maru, in the North Pacific Ocean. The J-QUEST is an instrumental package composed of an split-beam echosounder (70 kHz) and a stereo TV camera system. The J-QUEST depth was changed from $220 \mathrm{~m}$ to $25 \mathrm{~m}$ to observe vertical migration. Boreopacific gonate squids were observed by the stereo TV camera and they are considered to conduct diurnal vertical migration to feed migrating organisms. Average TS of the ascending layer and Boreopacific squid were measured in situ using the J-QUEST. Average area scattering strength (SA) values of the ascending layer, non-migrating layer, descending layer, aggregation of Boreo pacific squids, and that of Japanee anchovy were measured at $38 \mathrm{kHz}$ and $120 \mathrm{kHz}$, There was a constant and different SA difference between the two frequencies at each layer except migrating layer. The ascending and the descending layer have almost the same SA differences. It indicates that the both layers composed of the same organisms.

1pAOb8. Acoustic GIS-based monitoring of Atlantic cod ecosystems in coastal Newfoundland. George A. Rose (Memorial Univ. of Newfoundland, Marine Inst., 155 Ridge Rd., St. John’s, NL A1C5R3, Canada, grose@mi.mun.ca)

Several bays in Newfoundland hold the largest extent groups of overwintering and spawning Atlantic cod (Gadus morhua), and are spawning areas for capelin (Mallotus villosus), the most important forage species. These species co-exist in coastal ecosystems whose physical features and ecological sensitivities restrict monitoring using conventional fisheries methods. Active and passive acoustic methods, acoustic telemetry, oceanographic instrumentation and ROV video have been used to monitor overwintering and spawning distributions and abundance of cod, using mobile and fixed platforms. Cod behaviour is complex and features high mobility both horizontally and vertically, especially during migratory and spawning periods. Overwintering cod have school packing densities $>>1$ fish. $\mathrm{m}^{-3}$. Spawning features increased mobility and vertical structures or "columns" of individual fish and sound production captured using stationary hydrophones. Acoustic returns from aquatic vegetation and bottom types have been used to map juvenile habitat. Acoustic telemetry has established the movement patterns of male and female fish during spawning and the homing characteristics of cod as the basis of their stock structure. The movements and spawning behaviour of capelin can also be monitored as can interactions between predators and prey. An experiment using real-time and 3D location telemetry in a comprehensive GIS system will be described. 
1pAOb9. Using multifrequency acoustic scattering techniques to study mixed zooplankton populations. Andone Lavery (Woods Hole Oceanographic Institution, Applied Ocean Physics \& Engineering Department, 98 Water Street, MS \#11, Woods Hole, MA 02543, USA, alavery@whoi.edu), Peter Wiebe (Woods Hole Oceanographic Institution, Biology Department, 45 Water Street, MS \#33, Woods Hole, MA 02543, USA, pwiebe@whoi.edu), Timothy K. Stanton (Woods Hole Oceanographic Institution, Applied Ocean Physics \& Engineering Department, 98 Water Street, MS \#11, Woods Hole, MA 02543, USA, tstanton@whoi.edu), Gareth Lawson (Stanford University, Hopkins Marine Station, 120 Oceanview Blvd, Pacific Grove, CA 93950, USA, glawson@stanford.edu), Mark Benfield (Louisiana State University, Department of Oceanography \& Coastal Sciences, 2179 Energy, Coast \& Environment Building, Baton Rouge, LA 70803, USA, mbenfie@1su.edu), Nancy Copley (Woods Hole Oceanographic Institution, Biology Department, 45 Water Street, MS \#33, Woods Hole, MA 02543, USA, ncopley@whoi.edu)

Zooplankton populations can be highly heterogeneous and variable on spatial scales of centimeters to kilometers and temporal scales of minutes to years. High-frequency acoustic scattering techniques allow a synoptic view of zooplankton distribution and abundance on these scales. However, there are well-known complications in the interpretation of acoustic scattering returns, often limiting the inference of quantitative parameters (such as size or abundance) to restricted locations and conditions. Multifrequency and broadband acoustic scattering techniques, combined with sophisticated instrument platforms that combine multiple sensors allowing acoustical, optical, and other environmental measurements, reduce the ambiguities typically associated to the interpretation of acoustic scattering at single frequencies and expand the conditions under which inference of quantitative biological parameters is possible. The focus of this talk is a decade-long study aimed at understanding the distribution and abundance of mixed zooplankton populations in the Gulf of Maine. Simultaneous multifrequency acoustic, optical, environmental, and net measurements were performed. Insights gained from this study are presented together with recent measurements involving broadband acoustic scattering from zooplankton in the presence of nonlinear internal solitary waves. These studies highlight the importance of an integrated approach to understanding heterogeneous zooplankton populations on relevant spatial and temporal scales.

\title{
Session 1pBBa
}

\section{Biomedical Ultrasound/Bioresponse to Vibration: High-Intensity Focused Ultrasound I}

\author{
Lawrence A. Crum, Cochair \\ Center for Industrial and Medical Ultrasound, Applied Physics Lab., University of Washington, 1013 NE 40th St., \\ Seattle, WA 98105, USA \\ Jean Yves Chapelon, Cochair \\ INSERM, U556, 151 Cours Albert Thomas, Lyon, 69003, France
}

\section{Invited Papers}

\section{1:00}

1pBBa1. The feasibility of local harmonic motion imaging for the guidance and control of focused ultrasound surgery. Kullervo Hynynen (University of Toronto, Sunnybrook Health Sciences Centre, Suite S6 65b, 2075 Bayview Ave, Toronto, ON M4N 3M5, Canada, khynynen@sri.utoronto.ca), Laura Curiel (University of Toronto, Sunnybrook Health Sciences Centre, Suite S6 65b, 2075 Bayview Ave, Toronto, ON M4N 3M5, Canada, 1curiel@sri.utoronto.ca), Rajiv Chopra (University of Toronto, Sunnybrook Health Sciences Centre, Suite S6 65b, 2075 Bayview Ave, Toronto, ON M4N 3M5, Canada, chopra@ sri.utoronto.ca)

A local harmonic motion (LHM) can be generated within the tissues by the periodic induction of radiation force using a focused ultrasound (FUS) transducer. Tissue motion can then be tracked by collecting RF signals during the excitation using a separate transducer. Finally, displacement estimates can be obtained by cross-correlating the collected RF signals. The characteristics of the induced LHM depend on the local elastic properties of the tissues making it an attractive tool for imaging and therapy control applications. LHM measurements have been obtained in vivo on rabbit muscle and it was observed that the amplitude of the motion was significantly reduced after coagulation. LHM was successfully used to spatially detect the presence of the coagulation lesions within the tissues as a drop in LHM amplitude. It was also possible to detect the location of an implanted VX2 tumor when a spatial scan was performed as the LHM amplitude was lower inside the tumor because of an increased stiffness. Measurements of LHM during tissue heating using FUS reflected the changes in stiffness and revealed the apparition of coagulation showing the potential of these measurements as an alternative control for the FUS exposure. [Work supported by NIH GrantR33 CA102884 and CRC.] 
1pBBa2. Clinical experiences with extracorporeal Ultrasound-guided high-intensity focused ultrasound treatment for cancer patients. Feng Wu (Chongqing Medical University, Institute of Ultrasound Engineering in Medicine \& Clinical Centre for Tumour Therapy, 1 Medical College Road, 400016 Chongqing, China, mfengwu@yahoo.com)

Noninvasive, image-guided tumour thermal ablation with extracorporeal high-intensity focused ultrasound (HIFU) has received increasing interest in the treatment of patients with solid tumours. Since December 1997, an extracorporeal ultrasound-guided HIFU system (Mode-JC, Haifu Technology Co. Ltd., Chongqing, China) has been used to treat approximately 10,000 patients with solid tumours in China, including those of liver, breast, bone, kidney, pancreas, soft tissue, and uterus. The same device has been recently introduced into the UK, Italy, Japan, and South Korea, and so far, more than 1,000 patients have received HIFU treatment outside China. The purpose of this article is to introduce our clinical experiences using extracorporeal, ultrasound-guided HIFU ablation for solid tumours. Five-year follow-up data are observed in patients with primary liver cancer, breast cancer, and osteosarcoma. Among patients treated with HIFU, an extremely low major complication rate is observed. In conclusion, our clinical studies indicate that HIFU treatment is a safe, effective, and feasible modality in the treatment of cancer patients.

\section{$1: 40$}

1pBBa3. Adaptive focusing for bone and motion artifacts corrections in High Intensity Focused Ultrasound Therapy. Mickael Tanter (Laboratoire Ondes et Acoustique, ESPCI, Université Paris 7, CNRS, 10 rue Vauquelin, 75005 Paris, France, michael.tanter@espci.fr), Mathieu Pernot (Laboratoire Ondes et Acoustique, ESPCI, Université Paris 7, CNRS, 10 rue Vauquelin, 75005 Paris, France, mathieu.pernot@espci.fr), Jean-François Aubry (Laboratoire Ondes et Acoustique, ESPCI, Université Paris 7, CNRS, 10 rue Vauquelin, 75005 Paris, France, jean-francois.aubry@espci.fr), Mathias Fink (Laboratoire Ondes et Acoustique, ESPCI, Université Paris 7, CNRS, 10 rue Vauquelin, 75005 Paris, France, mathias.fink@espci.fr)

Despite extensive and fast progress of HIFU clinical applications, many issues still need to be addressed. Distortions caused by defocusing obstacles, such as the skull or ribs, on the ultrasonic therapeutic beam are still being investigated. Multi-element transducer technology must be used in order to achieve such transcranial or transcostal adaptive focusing. Second, the problem of motion artifacts, a key component in the treatment of abdominal lesions, has been shown to significantly influence the efficacy and treatment time. Though many methods have been proposed for the detection of organ motion, little work has been done to develop a comprehensive solution including motion tracking and feedback correction in real time. This paper is a review of the work achieved by authors in transcranial HIFU, transcostal HIFU, and motion compensated HIFU. For these three issues, the optimal solution can be reached using the same technology of multi-element transducers devices able to work both in Transmit and Receive modes.

\section{Contributed Papers}

2:00

1pBBa4. Using a toroid transducer for thermal ablation by high-intensity-focused ultrasound increases the coagulated volume. David Melodelima (INSERM, U556, 151 Cours Albert Thomas, 69003 Lyon, France, melodelima@lyon.inserm.fr), William N'Djin (INSERM, U556, 151 Cours Albert Thomas, 69003 Lyon, France, ndjin@lyon.inserm.fr), Hubert Parmentier (INSERM, U556, 151 Cours Albert Thomas, 69003 Lyon, France, hubert.parmentier@chu-lyon.fr), Michel Rivoire (Institut de Chirurgie Experimentale - Centre Léon Berard, 28 rue Laennec, 69008 Lyon, France, rivoire@lyon.fnclcc.fr), Jean Yves Chapelon (INSERM, U556, 151 Cours Albert Thomas, 69003 Lyon, France, chapelon@1yon.inserm.fr)

Here, we report that a new design of High Intensity Focused Ultrasound transducer can significantly enlarge the coagulated volume over short periods of time, and that treatment in the liver can be guided in real-time using an integrated ultrasound imaging probe. Eight ultrasound emitters, divided into 256 elements, were created by sectioning a single toroid piezocomposite transducer. The focal zone was conical in shape and located $70 \mathrm{~mm}$ from the transducer; enabling the treatment of deep-seated tumors. A single thermal lesion was created when the eight emitters performed alternative and consecutive $5 \mathrm{~s}$ ultrasound exposures. This paper presents in vivo evidence that the coagulated volume obtained from a $40 \mathrm{~s}$ total exposure in the liver was $7.0 \pm 2.5 \mathrm{~cm}^{3}\left(\min 1.5-\max 20.0 \mathrm{~cm}^{3}\right)$. All lesions were visible with high contrast on sonograms. The correlation between the diameter of lesions observed on sonograms and during gross examination was $92 \%$. This method also allowed the user to easily enlarge the coagulated volume by juxtaposing single lesions. This approach may have a role in treating unresectable colorectal liver metastases and may also be used in conjunction with resection to extend its limits.

\section{2:20}

1pBBa5. Temperature measurements and determination of cavitation thresholds during High Intensity Focused Ultrasound (HIFU) Exposures in ex-vivo porcine muscle. Subha Maruvada (U.S. Food and Drug Administration, 10903 New Hampshire Ave., Silver Spring, MD 20993, USA, subha.maruvada@fda.hhs.gov), Yunbo Liu (U.S. Food and Drug Administration, 10903 New Hampshire Ave., Silver Spring, MD 20993, USA, yunbo.liu@fda.hhs.gov), Bruce A. Herman (U.S. Food and Drug Administration, 10903 New Hampshire Ave., Silver Spring, MD 20993, USA, bruce.herman@fda.hhs.gov), William F. Pritchard (U.S. Food and Drug Administration, 10903 New Hampshire Ave., Silver Spring, MD 20993, USA, william.pritchard@fda.hhs.gov), Gerald R. Harris (U.S. Food and Drug Administration, 10903 New Hampshire Ave., Silver Spring, MD 20993, USA, gerald.harris@fda.hhs.gov)

Cavitation in HIFU procedures can yield unpredictable results, particularly when the same location is targeted for more than several seconds. To study this effect, temperature rise was measured in fresh ex-vivo porcine tissue during HIFU exposures. Immediately following euthanasia, a section of back muscle (latissimus dorsi) was resected and a $50 \mu \mathrm{m}$ diameter fine bare wire thermocouple was placed via needle through the tissue. $825 \mathrm{kHz}$ HIFU was then applied to the tissue focused at the thermocouple junction. Thirty second HIFU exposures of increasing pressure from 1-7.5 MPa were applied and the temperature rise and decay during and after sonication were recorded. B-mode imaging was used to monitor any cavitation activity during sonication. If cavitation was noted during the sonication, the sonication was repeated at the same pressure level two more times at 20 minute intervals in order to characterize the repeatability given that cavitation has occurred. The cavitation threshold of porcine muscle was determined to be between 4 and $7 \mathrm{MPa}$. Temperature traces obtained at various pressure 
levels demonstrated a wide range of heating profiles in fresh ex-vivo tissue due to both the occurrence of cavitation and viscous heating artifacts. (This research was supported by DARPA IAG \# 224-05-6016).
lac@apl.washington.edu),Steven Kargl(Applied Physics Laboratory, University of Washington, 1013 NE 40th Street, Seattle, WA 98105, USA, kargl@troutmask.apl.washington.edu), Gavriel Speyer (Center for Industrial and Medical Ultrasound, Applied Physics Lab., University of Washington, 1013 NE 40th St., Seattle, WA 98105, USA, gavriel@u.washington .edu)

High-power, short-exposure time, High Intensity Focused Ultrasound (HIFU) treatment protocols are under development that offer the potential to increase procedure throughput and optimize individual therapies. Histological examination and optical image analysis of tissues following dynamic HIFU exposure in ex vivo bovine liver have revealed that cells undergo a fundamentally different form of cell death. The rapid temperature rise due to the HIFU exposure leaves the cells structurally intact but no longer viable, similar to the cell "fixation" induced by snap-freezing. These results suggest that careful choice of both staining technique and metric for determining cell death are important in quantifying type and morphology of cell ablation, and more broadly, safety and efficacy of treatment. This finding is similar to those obtained and under discussion in the laser and RF ablation communities. Specifically, the NADH staining technique is superior to H\&E for assessing cell viability, and an alternative measure of cell death may be preferable to the binary thermal dose threshold currently the standard for HIFU treatment. [Work supported by NIH.]

and Medical Ultrasound, Applied Physics Lab., University of Washington 1013 NE 40th St., Seattle, WA 98105, USA,

\section{Invited Papers}

\section{3:00}

1pBBa7. Ultrasound interstitial applicators for thermal ablation in liver. Cyril Lafon (INSERM, U556, 151 Cours Albert Thomas, 69003 Lyon, France, lafon@lyon.inserm.fr), Rares Salomir (INSERM, U556, 151 Cours Albert Thomas, 69003 Lyon, France, salomir@lyon.inserm.fr), Guillaume Bouchoux (INSERM, U556, 151 Cours Albert Thomas, 69003 Lyon, France, bouchoux@lyon.inserm.fr), Alain Birer (INSERM, U556, 151 Cours Albert Thomas, 69003 Lyon, France, birer@lyon.inserm.fr), Neil Owen (INSERM, U556, 151 Cours Albert Thomas, 69003 Lyon, France, Neil.Owen@lyon.inserm.fr), Eric Delabrousse (INSERM, U556, 151 Cours Albert Thomas, 69003 Lyon, France, delabrousse@lyon.inserm.fr), François Mithieux (INSERM, U556, 151 Cours Albert Thomas, 69003 Lyon, France, mithieux@lyon.fnclcc.fr), Jean Yves Chapelon (INSERM, U556, 151 Cours Albert Thomas, 69003 Lyon, France, chapelon@lyon.inserm.fr)

Aggressive treatment of localized hepatic metastases, by surgery or other means, was proven to be a viable strategy for improving the prognoses of many patients. In that context, thermal ablation by high intensity ultrasound was proposed and used in clinics. However, for treating deep-seated tumors and in most cases, radiofrequency and cryotherapy probes are applied interstitially. Interstitial ultrasound applicators were proposed as an intermediate solution. The treatment can be focused, deeper than with other physical agents, and the transducer can eventually both treat and image tissues. In our experience, two approaches were investigated: percutaneous and intratissular, or endo vascular. The active element was a miniature flat transducer operating at a frequency of $5 \mathrm{MHz}$, for a satisfactory tradeoff between beam penetration and energy absorption. In vivo trials on a porcine model demonstrated that both procedures are minimally invasive and that large thermal lesions, up to $20 \mathrm{~mm}$ deep, can be obtained. Technological improvements such as the use of dual mode transducers (for imaging and therapy) or the performance under MRI guidance allowed monitoring the treatment in real-time.

\section{3:20}

1pBBa8. Histotripsy and the developing role of microbubbles in ultrasound therapy. Jeffrey B. Fowlkes (University of Michigan, Department of Radiology, Kresge III, R3320, Ann Arbor, MI 48109-0553, USA, fowlkes@umich.edu)

Microbubbles in therapeutic ultrasound has seen increasing interest in recent years. Once thought to be problematic, bubble production and interactions with ultrasound fields are yielding promising new methods and this presentation will review two areas of collaborative research at the University of Michigan investigating therapeutic applications utilizing microbubbles. Histotripsy uses short pulses of high intensity ultrasound produce cavitation in vivo and subdivide tissue at a subcellular level. The highly localized bubble activity homogenizes tissue to the point of reducing acoustic backscatter enabling lesion detection. Lesion margins are remarkably fine, even yielding fractional disruption of cells. The homogenized material is readily absorbed thus debulking tissue with little residual scar. At the other end of the acoustic intensity scale, microbubbles can be produced by triggering vaporization of superheated liquid droplets, termed acoustic droplet vaporization (ADV). ADV requires diagnostic levels of ultrasound to yield localized microbubbles production. The method is being investigated for direct occlusion therapy and perfusion control to augment other therapeutic methods including drug delivery among other potential applications. These methods highlight the growing field of microbubble-based therapies, which will expand with our increasing understanding of microbubbles and their interactions with surrounding environments. Supported in part by NIH EB00281, HL077629 and EB006476.

\section{3:40-4:00 Break}




\section{Contributed Papers}

\section{4:00}

1pBBa9. Localization and enhancement of cavitation and heating during HIFU exposure using microparticles of high surface roughness. Manish Arora (University of Oxford, Medical Engineering Unit, 43 Banbury Road, OX2 6PE Oxford, UK, manish.arora@eng.ox.ac.uk), Costas Arvanitis (University of Oxford, Medical Engineering Unit, 43 Banbury Road, OX2 6PE Oxford, UK, costas.arvanitis@eng.ox.ac.uk), Emma Cox (University of Oxford, Medical Engineering Unit, 43 Banbury Road, OX2 6PE Oxford, UK, eflcox@gmail.com), Constantin C. Coussios (University of Oxford, Medical Engineering Unit, 43 Banbury Road, OX2 6PE Oxford, UK, constantin.coussios@eng.ox.ac.uk)

The occurrence of cavitation during HIFU exposure is associated with higher heating rates than in the absence of bubble activity. However, the peak rarefaction pressures required to initiate cavitation in vivo are high (7-10 MPa), making it difficult to localize, sustain, and confine cavitation activity and its associated bioeffects. In this work, we explore the use of rough microparticles to promote and localize cavitation nucleation during HIFU exposures. Polystyrene microparticles of mean diameter $\sim 2 \mu \mathrm{m}$ are embedded in a polyacrylamide tissue mimicking phantom, which has a high cavitation threshold similar to tissue. The temperature rise during HIFU exposure is measured using an embedded needle thermocouple, whilst cavitation activity is simultaneously monitored using a passive cavitation detector (PCD). The presence of particles is shown to lower the cavitation threshold significantly and, above this threshold, to result in higher heating rates than in the absence of particles for the same focal intensity. Furthermore, it is found that particles make it possible to repeatedly initiate cavitation at the same location and lead to increased broadband noise emissions. Finally, a strong correlation is found between enhanced heating and the broadband emissions arising from inertial cavitation.

\section{4:20}

1pBBa10. Local heating by a bubble excited by high intensity focused ultrasound. Wayne Kreider (Center for Industrial and Medical Ultrasound, Applied Physics Lab., University of Washington, 1013 NE 40th St., Seattle, WA 98105, USA, wkreider@u.washington.edu), Michael S. Canney (Center for Industrial and Medical Ultrasound, Applied Physics Lab., University of Washington, 1013 NE 40th St., Seattle, WA 98105, USA, mcanney@u.washington.edu), Michael R. Bailey (Center for Industrial and Medical Ultrasound, Applied Physics Lab., University of Washington, 1013 NE 40th St., Seattle, WA 98105, USA, bailey@apl.washington.edu), Vera A. Khokhlova (Center for Industrial and Medical Ultrasound, Applied Physics Lab., University of Washington, 1013 NE 40th St., Seattle, WA 98105, USA, vera@acs366.phys.msu.ru), Lawrence A. Crum (Center for Industrial and Medical Ultrasound, Applied Physics Lab., University of Washington, 1013 NE 40th St., Seattle, WA 98105, USA, lac@ap .washington.edu)

A current topic of interest for high intensity focused ultrasound (HIFU) treatments involves the relative roles of bubbles and nonlinear acoustic propagation as heating mechanisms. At high amplitudes, nonlinear propagation leads to the generation of boiling bubbles within milliseconds; at lower amplitudes, cavitation bubbles can enhance heating through viscous dissipation, acoustic radiation, and heat conduction. In this context, understanding the physics attendant to HIFU bubbles requires consideration of gas-vapor bubble dynamics, including thermal effects in the nearby liquid. To this end, recent experimental observations with high-speed photography suggest that bubbles undergo a brief period of growth after application of HIFU has stopped. To explain this observation, a model is implemented that couples the thermodynamic state of a strongly driven bubble with thermal conditions in the surrounding liquid. From model simulations, liquid heating in the vicinity of a HIFU bubble is estimated. Calculations suggest that thermal conduction and viscous dissipation can lead to the evolution of a nontrivial thermal boundary layer. Development of a boundary layer that reaches superheated temperatures would explain the aforementioned experimental observation. As such, cavitation bubbles and boiling bubbles share important characteristics during HIFU. [Work supported by NIH DK43881 and NSBRI SMS00402.]

\section{4:40}

1pBBa11. Temperature and size-dependence of the vaporization threshold of phase-shift emulsions. Tyrone Porter (Boston University, 110 Cummington Street, Boston, MA 02215, USA, tmp@bu.edu), Peng Zhang (Boston University, 110 Cummington Street, Boston, MA 02215, USA, pzhang@bu.edu)

Acoustic cavitation has proven to be important for several therapeutic applications of ultrasound. However, acoustic cavitation is difficult to initiate and sustain in the absence of cavitation nuclei, particularly in tissue. Phase shift emulsions are ideal candidates for cavitation nuclei for in vivo applications. These emulsions, which consist of superheated liquid perfluorocarbon droplets enclosed by albumin shells, may be vaporized with acoustic pulses, a process known as acoustic droplet vaporization (ADV). In this study, we determined the ADV threshold at $2 \mathrm{MHz}$ as a function of temperature and droplet size. Studies were conducted with micro- and nanosized emulsions in a closed-flow system immersed in a temperature controlled water bath. The emulsions were injected into a flow system and exposed to high intensity focused ultrasound (HIFU; acoustic parameters: 2 $\mathrm{MHz}, 10$ cycles, $100 \mathrm{~Hz}$ pulse repetition frequency). A portable diagnostic ultrasound scanner was used to monitor for vaporization. Upon vaporization, the peak rarefactional pressure of the acoustic pulse and the water bath temperature were recorded. It was determined that the vaporization threshold was independent of droplet size and inversely proportional to temperature. The utility of these emulsions in cancer therapy, particularly for bubbleenhanced heating during HIFU exposure, will be discussed.

\section{5:00}

1pBBa12. In vitro evaluation of an oscillating 5-element dual-mode transducer. Neil Owen (INSERM, U556, 151 Cours Albert Thomas, 69003 Lyon, France, Neil.Owen@lyon.inserm.fr), Guillaume Bouchoux (INSERM, U556, 151 Cours Albert Thomas, 69003 Lyon, France, bouchoux@1yon.inserm.fr), Alain Birer (INSERM, U556, 151 Cours Albert Thomas, 69003 Lyon, France, birer@lyon.inserm.fr), Rémi Berriet (Imasonic, Z.A. rue des Savourots, 70190 Voray sur l'Ognon, France, Remi.Berriet@imasonic.com), Jean Yves Chapelon (INSERM, U556, 151 Cours Albert Thomas, 69003 Lyon, France, chapelon@1yon.inserm.fr), Gérard Fleury (Imasonic, 15 rue Alain Savary, 25000 Besançon, France, Gerard.Fleury@imasonic.com), Cyril Lafon (INSERM, U556, 151 Cours Albert Thomas, 69003 Lyon, France, lafon@lyon.inserm.fr)

Miniature dual-mode transducers can be used for minimally invasive treatment of deep-seated tumors. While in contact with the tissue, the transducer guides and monitors localized necrosis. Here, an oscillating 5-element piezo-composite transducer was characterized, and then evaluated in vitro using porcine liver. Each element was $3.0 \times 3.8 \mathrm{~mm}^{2}$ with a geometric cylindrical focus of $14 \mathrm{~mm}$. The transmit frequency was determined by the maximal electro-acoustic efficiency, $65 \%$, which was found at $5.6 \mathrm{MHz}$. The transmit-receive impulse response was $400 \mathrm{~ns}$ long at $-6 \mathrm{~dB}$, and the $-6 \mathrm{~dB}$ fractional bandwidth, centered at $5.6 \mathrm{MHz}$, was $30 \%$. Axial and lateral resolution measured with a $0.1 \mathrm{~mm}$ diameter wire was $0.5 \mathrm{~mm}$ and $2.0 \mathrm{~mm}$, respectively. For therapy, all elements radiated simultaneously, and for imaging, independently. Treatment was performed at increments of $20^{\circ}$ to form a composite volume of necrosis. At each angle, ultrasound was applied for $60 \mathrm{~s}$ at a transducer surface intensity of $15 \mathrm{~W} / \mathrm{cm}^{2}$. Pulse-echo data were recorded while the transducer oscillated over a $180^{\circ}$ sector to form images before and after treatment at each angle. Gross examination of lesion size agreed well with echogenic region size in the images. [Supported by ANR and Inserm Post-doctoral Fellowship.] 


\title{
Session 1pBBb
}

\section{Biomedical Ultrasound/Bioresponse to Vibration: Quantitative Ultrasound Methods for Diagnosis and Therapy I}

\author{
Jonathan Mamou, Cochair \\ Riverside Research Institute, 156 William St., 9th Floor, New York, NY 10038, USA
}

Geneviève Berger, Cochair

Université Paris 6, Laboratoire d'Imagerie Paramétrique, 15 rue de l'Ecole de médecine, Paris, France

\section{Invited Papers}

\author{
$1: 00$
}

1pBBb1. Techniques and modalities for high resolution imaging and assessment of skin with high-frequency ultrasound (20 to 100 MHz). Michael Vogt (Institute of High-Frequency Engineering, Ruhr-University Bochum, IC 6/133, 44780 Bochum, Germany, Michael.Vogt@rub.de), Helmut Ermert (Institute of High-Frequency Engineering, Ruhr-University Bochum, IC 6/133, 44780 Bochum, Germany, Helmut.Ermert@rub.de)

High-frequency ultrasound (HFUS) in the $20 \mathrm{MHz}$ range is routinely used in dermatological diagnosis for morphological skin imaging in order to support the identification of suspicious skin lesions and to evaluate the success of therapies. In this presentation, technical developments for the utilization of higher ultrasound frequencies in the range up to $100 \mathrm{MHz}$ are presented, which allow for high-resolution imaging and assessment of skin structures with an axial resolution down to $10 \mu$ m. Furthermore, a new HFUS system for the assessment of elastic skin properties and quantitative strain imaging with $20 \mathrm{MHz}$ ultrasound is presented as a new imaging modality. Also, a reflection-tomography skin imaging concept was implemented with a $20 \mathrm{MHz}$ ultrasound limited-angle spatial compound system, which allows for multi-directional analysis of backscattered and reflected ultrasound waves from different insonation angles. The proposed techniques and modalities have been evaluated by measurements on phantoms and in vivo. Results show that high-resolution qualitative and quantitative skin assessment methods have been successfully implemented. HFUS based strain imaging enables the non-invasive assessment of skin and lesion properties, and HFUS limited-angle spatial compounding allows for improved skin analysis compared to the conventional linear-scan imaging approach.

\section{$1: 20$}

1pBBb2. Quantitative intravascular ultrasound elasticity imaging as an imaging biomarker in clinical trials. Ton Van Der Steen (Thorax centre ErasmusMC, Ee23.02, pobox 2040, 3000 CA Rotterdam, Netherlands, a.vandersteen@erasmusmc.nl), Johannes A. Schaar (Biomedical Engineering, Erasmus MC, P.O. Box 2040, 3000 CA Rotterdam, Netherlands, j.schaar@erasmusmc.nl), Frits Mastik (Biomedical Engineering, Erasmus MC, P.O. Box 2040, 3000 CA Rotterdam, Netherlands, f.mastik@erasmusmc.nl), Hector Garcia (Biomedical Engineering, Erasmus MC, P.O. Box 2040, 3000 CA Rotterdam, Netherlands, HGarcia@cardialysis.nl), Mike Danilouchkine (Biomedical Engineering, Erasmus MC, P.O. Box 2040, 3000 CA Rotterdam, Netherlands, m.danilouchkine@erasmusmc.nl), Radj Baldewsing (Biomedical Engineering, Erasmus MC, P.O. Box 2040, 3000 CA Rotterdam, Netherlands, radj.baldewsing@hewitt.com), Patrick W. Serruys (Biomedical Engineering, Erasmus MC, P.O. Box 2040, 3000 CA Rotterdam, Netherlands, p.w.j.c.Serruys@erasmusmc.nl)

The composition and morphology of an atherosclerotic lesion are currently considered more important determinants of acute coronary ischemic syndromes that the degree of stenosis. When a lesion is unstable, it can rupture and cause an acute thrombotic reaction. An unstable plaque can be characterized by a lipid core that is covered by a thin fibrous cap, which has been locally weakened by inflammatory cells. Intravascular Ultrasound Palpography is an intravascular ultrasound based technique that is capable to measure the local strain in coronaries and atherosclerotic plaque. This strain is induced by varying intraluminal pressure. This lecture will show principles of the technology and how this technology is used in clinical trials. Results from a trial with traditional lipid lowering treatment (IBIS1) and from a trial on the efficacy of a new medication (IBIS2) will be presented. Furthermore the potential of Intravascular Ultrasound Modulography will be discussed. This work is financially supported by the Dutch Technology Foundation, The Dutch Heart Foundation and a research grants from Volcano Corporation and Glaxo Smith Kline

\section{$1: 40$}

1pBBb3. Recent progress of acoustic microscopy for medicine and biology. Yoshifumi Saijo (Tohoku University, 4-1 Seiryomachi, Aoba-ku, 980-8575 Sendai, Japan, saijo@idac.tohoku.ac.jp), Kazuto Kobayashi (Honda Electronics Co. Ltd., 20 Oyamazuka, Oiwa-cho, 441-3193 Toyohashi, Japan, kazuto@honda-el.co.jp), Takahiro Iwamoto (Tohoku University, 4-1 Seiryomachi, Aoba-ku, 980-8575 Sendai, Japan, iwamoto@yoshizawa.ecei.tohoku.ac.jp), Nagaya Okada (Honda Electronics Co. Ltd., 20 Oyamazuka, Oiwacho, 441-3193 Toyohashi, Japan, nagaya@honda-el.co.jp), Akira Tanaka (Fukushima University, 1 Kanayagawa, 960-1296 Fukushima, Japan, a-tanaka@sss.fukushima-u.ac.jp), Naohiro Hozumi (Aichi Institute of Technology, 1247 Yachigusa, Yakusa-cho, 4700392 Toyota, Japan, hozumi@aitech.ac.jp)

Dramatic shortening of the calculation time provided by the recent progress of the computer technology has made biomedical researchers possible to assess nonlinear acoustic phenomena in soft materials which had been assumed as acting linearly. Besides, the spread of Windows-based personal computer and peripheral devices enabled easier and cheaper configuration of the whole acoustic microscope system such as pulse generation, analogue/digital conversion, mechanical scanning and image processing. According to 
these progresses, conventional acoustic microscopy with only C-mode imaging has widened its data acquisition mode to B-mode, C-mode, surface acoustic impedance mode, and three-dimensional (3D) imaging. The base of our acoustic microscope system was consisted of (1) PVDF transducer with the central frequency of $100 \mathrm{MHz}$, (2) ultrasonic pulser made of high speed semiconductor switching, (3) mechanical scanner using two linear servo motors, (4) high speed PCI card digitizer with the sampling frequency of 2 $\mathrm{GHz}$, and (5) personal computer controlling the whole system. For skin imaging, 3D imaging of the fingerprint was reconstructed by consecutive B-mode imaging. Surface acoustic impedance imaging of the fingerprint can be obtained by just putting a finger on a thin plastic plate of the transducer. Conventional C-mode imaging of thinly sliced skin sample presented quantitative values of thickness, attenuation and sound speed of the tissue.

1pBBb4. Improved cancer detection and classification using multi-parameter quantitative ultrasound. Michael L. Oelze (University of Illinois at Urbana-Champaign, Beckman Institute, 405 N Mathews, Urbana, IL 61801, USA, oelze@uiuc.edu), William D. O’Brien, Jr. (University of Illinois at Urbana-Champaign, Beckman Institute, 405 N Mathews, Urbana, IL 61801, USA, wdo@uiuc .edu)

Quantitative ultrasound (QUS) has been used successfully to differentiate benign from malignant solid tumors in animal models of breast cancer. In these studies, QUS made use of estimates of average scatterer diameter (ASD) and average acoustic concentration (AAC) from the ultrasonic backscatter. However, initial attempts to classify different kinds of malignant tumors based on ASD and AAC estimates were not successful. New models for ultrasonic backscatter were created by considering the cytoskeletal structure of cells. In addition, the homodyned $\mathrm{K}$ distribution was used to model the amplitude of the envelope from regions-of-interest in the malignant tumors. The homodyned $\mathrm{K}$ distribution yielded two parameters: the $\mathrm{S}$ parameter, which quantified the randomness of scatterer spacings, and the $\beta$ parameter, which quantified the amount of clustering of scatterers in the interrogated tissue. Statistically significant differences $(\mathrm{P}<0.05)$ were observed between average $\mathrm{S}$ and $\beta$ parameters from the malignant tumors. Furthermore, statistically significant differences $(\mathrm{P}<0.05)$ were observed between ASD and AAC estimates from the malignant tumors using the new scattering models at ultrasonic frequencies above $16 \mathrm{MHz}$. The use of four parameters, as opposed to two, improved the ability to uniquely classify different kinds of malignant tumors. [Supported by NIH R01CA111289.]

1pBBb5. Modeling scattering from cells and biological structures. Michael C. Kolios (Ryerson University, Department of Physics, 350 Victoria Street, Toronto, ON M5B2K3, Canada, mkolios@ryerson.ca), Gregory J. Czarnota (Sunnybrook Health Sciences Centre, Department of Radiation Oncology and Imaging Research, 2075 Bayview Ave, T2-Wing, Toronto, AB M4N 3M5, Canada, Gregory.Czarnota@sunnybrook.ca)

The goal of ultrasonic tissue characterization is to extract information over and above the information conventionally displayed on imaging instruments which is typically the backscatter strength (B-mode image). The driving hypothesis of our work is that as the ultrasound wavelength approaches the size of a cell, the backscatter characteristics become more sensitive to the cells' physical attributes (size, structure, and composition) and changes in the cell structure due to therapeutic intervention. A better understanding of the interaction of acoustic waves with cells is required to interpret the backscatter data. An overview of our efforts to model scattering from individual cells, cell ensembles, and in-vivo cancer will be presented. Theoretical results will be presented comparing scattering from individual cells with experimental data from a variety of cells in suspension, cell ensembles with pellets (compact aggregates of cells) and in-vivo tumors. It will be shown that for the tumors we have studied there is a very good correlation of scattering characteristics between cell pellets and in-vivo tumors, indicating that in the tumors studied cell structure was the factor that dominated the scattering response. The goal of this work is to use quantitative ultrasound methods for diagnosis and monitoring the response to therapy.

\section{2:40}

1pBBb6. Estimation of heterogeneity of scatterer densities for diagnosis of liver fibrosis. Tadashi Yamaguchi (Research C. for Frontier Med. Eng., Chiba University, 1-33 Yayoicho, Inageku, 263-8522 Chiba, Japan, yamaguchi@faculty.chiba-u.jp), Hiroyuki Hachiya (Dept. of Mech. and Cntr. Eng., Tokyo Inst. of Tech., 2-12-1 Ookayama, Meguroku, 152-8550 Tokyo, Japan, hachiya@ctrl .titech.ac.jp)

In the quantitative diagnosis using ultrasound, existence of the high or low echo which was indistinguishable from speckle serves as an index of diagnosis. In this study, we tried to clarify the relation between the scattering condition in the tissue and the probability density function of echo amplitude. Parameters to estimate the scattering condition were derived by Q-Q probability plot at computer simulation models and the clinical data of liver fibrosis. In the simulation model of heterogeneous medium, the result of Q-Q plot became a curve and the curvature was dependent on the difference of the scatterer density of two intermingled media. In the clinical data of liver fibrosis, curvature was large when many fibers were contained. On the other hand, curvature was small when cysts or minute blood vessels were intermingled in the speckle and the whole distribution function was able to be approximated by k-distribution. Moreover, the crooked point of the Q-Q plot was changed depending on the scatterer density of a mixture part. These results show that the mixture rate and scatterer distributions of two different distribution functions can be recognized parametrically in the liver in which minute diseased tissue was intermingled. 
1pBBb7. Noninvasive temperature estimation using diagnostic ultrasound: In vivo results. Dalong Liu (University of Minnesota, 200 Union St SE, Rm 4-174 EECS Bldg, Minneapolis, MN 55455, USA, liuxx293@umn.edu), Ajay Shrestha (University of Minnesota, 200 Union St SE, Rm 4-174 EECS Bldg, Minneapolis, MN 55455, USA, ajay@ece.umn.edu), Rachana Visaria (University of Minnesota, 200 Union St SE, Rm 4-174 EECS Bldg, Minneapolis, MN 55455, USA, visar001@umn.edu), John Bischof (University of Minnesota, 200 Union St SE, Rm 4-174 EECS Bldg, Minneapolis, MN 55455, USA, bischof@umn.edu), Emad S. Ebbini (University of Minnesota, 200 Union St SE, Rm 4-174 EECS Bldg, Minneapolis, MN 55455, USA, emad@umn.edu)

In this paper, we present temperature imaging data obtained before and after HIFU-induced lesions in implanted tumors in the hind limb of nude mice in vivo. The RF data was acquired using a $9 \mathrm{MHz}$ 192-element linear probe on a diagnostic scanner at 90 frames per second. Approximately $2 \mathrm{~s}$ of frame data was collected before, during and after sub-therapeutic exposures of $0.2 \mathrm{~s}$ duration. The frame rates were sufficiently high to capture the effects of local tissue deformation due to breathing and pulsation near blood vessel. Using a physics-based spatial-temporal filtering of tissue displacement, we were able to produce accurate spatial-temporal maps of the temperature change with fine spatial and temporal resolution. These results are in good agreement with directly measured temperatures using fine temperature sensors near the HIFU focal spot. The results show that robust temperature estimation in the presence of tissue motion/deformation is feasible. Applications of this method in measuring the local thermal properties of tissue will be addressed. In particular, tissue absorption can be estimated and it appears to increase by a factor of 2 - 4 after lesion formation.

\section{$3: 20$}

1pBBb8. Transient elastography: changing clinical practice in hepatology. Laurent Sandrin (Echosens, R\&D department, 153 avenue d'Italie, 75013 Paris, France, laurent.sandrin@echosens.com), Sylvain Yon (Echosens, R\&D department, 153 avenue d'Italie, 75013 Paris, France, sylvain.yon@echosens.com), Céline Fournier (Echosens, R\&D department, 153 avenue d'Italie, 75013 Paris, France, celine.fournier@echosens.com), Veronique Miette (Echosens, R\&D department, 153 avenue d'Italie, 75013 Paris, France, veronique.miette@echosens.com)

Transient elastography is one of several new approaches that have recently been proposed to manage liver diseases. This quantitative method is used in clinical practice to assess liver fibrosis noninvasively and rapidly. The technique consists in generating low-frequency elastic shear waves through the liver and measuring their velocity using ultrafast pulse-echo ultrasound acquisition. Liver stiffness is directly related to shear waves velocity. Nowadays more than 400 devices (Fibroscan ${ }^{\circledR}$, Echosens, Paris, France) are being used worldwide in clinical practice. Studies reported a strong correlation between liver stiffness and the fibrosis stage obtained by liver biopsy in patients with chronic liver diseases: hepatitis B and/or C, HCV co-infection, HIV, alcoholism, etc. Stiffness measurements obtained using transient elastography are in good agreement with measurements performed using other elastography techniques (magnetic resonance elastography and radiation force). Main limitations of transient elastography are related to the morphology of the patients. Examinations may be difficult or impossible in overweighted to obese patients and in children. New probes have been developed for children and obese patients. Using these probes and dedicated measurement procedures the performance of transient elastography is significantly improved. The development of transient elastography currently focuses on heterogeneous stiffness measurement.

3:40-4:00 Break

\section{Contributed Papers}

\section{4:00}

1pBBb9. Transient elastography in heterogeneous tissues. Cécile Bastard (Echosens, R\&D department, 153 avenue d'Italie, 75013 Paris, France, cecile.bastard@echosens.com), Jennifer Oudry (Echosens, R\&D department, 153 avenue d'Italie, 75013 Paris, France, jennifer.oudry@echosens.com), Yassine Mofid (Echosens, R\&D department, 153 avenue d'Italie, 75013 Paris, France, mofid@echosens.com), Laurent Sandrin (Echosens, R\&D department, 153 avenue d'Italie, 75013 Paris, France, laurent.sandrin@echosens.com)

The Fibroscan ${ }^{\circledR}$ (Echosens, Paris, France) is a transient elastography based device used to quantify liver fibrosis by following the propagation of a low frequency shear wave and measuring the mean Young's modulus of the liver. This device has been successfully applied to homogeneous tissues such as liver in patients with chronic hepatitis C. Current developments in transient elastography are now headed toward the characterization of heterogeneous tissues. The estimation of the shear wave velocity can be achieved by solving the elastic wave equation taking into account either the 1D, the $2 \mathrm{D}$, or the $3 \mathrm{D}$ components of the displacement spatial derivatives. The objective of this study is to characterize focal nodules in human liver and to quantify heterogeneous fibrosis. We present the methods used to estimate the local shear wave velocity and the results of experiments conducted on heterogeneous phantoms and in the liver in vivo.

$4: 20$

1pBBb10. Improvements in liver diseases evaluation using transient elastography for obese patients. Magali Sasso (Echosens, R\&D department, 153 avenue d'Italie, 75013 Paris, France, magali.sasso
@ echosens.com),Veronique Miette(Echosens, R\&D department, 153 avenue d'Italie, 75013 Paris, France, veronique.miette@echosens.com), Laurent Sandrin (Echosens, R\&D department, 153 avenue d'Italie, 75013 Paris, France, laurent.sandrin@echosens.com)

Transient elastography (Fibroscan ${ }^{\circledR}$, Echosens, Paris, France) is a non invasive and rapid technique used to assess liver fibrosis by measuring liver stiffness. Clinical interest of liver stiffness measurement using Fibroscan ${ }^{\circledR}$ has been demonstrated for chronic liver diseases in adult patients. Clinically, the liver stiffness evaluation can be tricky in obese patients due to their large subcutaneous fat thickness. Obesity is a growing public health concern which requires dedicated liver evaluation procedures due to the patients' morphology and/or the specific diseases associated with obesity. Our study aims to show how Fibroscan ${ }^{\circledR}$ can adapt for liver diseases evaluation in obese patients. Clinical adaptations dedicated to over-weighted patients are shown: ultrasound guidance procedure, development of a dedicated probe for patients with a large subcutaneous fat thickness, development of new algorithms based on attenuation of both ultrasonic and shear waves. Performance is assessed and clinical interest of each adaptation is discussed in over-weighted, obese and morbid obese patients (body mass index greater than 25). These new procedures dedicated to obese patients improve liver diseases assessment using Fibroscan ${ }^{\circledR}$.

\section{$4: 40$}

1pBBb11. Towards the modeling of high-frequency ultrasound scattering from cells. Omar Falou (Ryerson University, Department of Physics, 350 Victoria Street, Toronto, ON M5B2K3, Canada, ofalou 
@ ryerson.ca),J. Carl Kumaradas(Ryerson University, Dept. of Physics, 350 Victoria Street, Toronto, ON M5B 2K3, Canada, ckumarad@ryerson.ca), Michael C. Kolios (Ryerson University, Department of Physics, 350 Victoria Street, Toronto, ON M5B2K3, Canada, mkolios@ryerson.ca)

High-frequency ultrasound is a novel method to detect cell death based on changes in cell morphology that cause alterations in the acoustic properties of cell ensembles in tissues. In previous work, our group has suggested that for cells with a nucleus to cell volume ratio of 0.33 , the backscatter response from single cells was best modeled as a fluid sphere. However, for cells with a larger nucleus, neither the fluid nor the elastic sphere models showed a good agreement. We hypothesize that cells with a large nucleus ratio ( $>0.33)$ may be modeled as an elastic sphere surrounded by a fluid shell. In this work, the backscatter response from non-nucleated cells (Strongylocentrotus purpuratus oocytes) was measured Experimental RF lines were acquired using the VisualSonics VS40B ultrasound biomicroscope using 20 and $40 \mathrm{MHz}$ transducers. A finite-element model of wave propagation was developed to solve high-frequency ultrasound scattering from spheres. A very good agreement was found suggesting that the cytoplasm of the oocytes is of fluid nature. Finally, a finite-element model treating the cells as having an elastic nucleus surrounded by a fluid shell is compared to experimental measurements. The implications of these findings on the prediction of ultrasound backscatter from cells are discussed.

\section{5:00}

1pBBb12. High-frequency quantitative ultrasound imaging methods for human lymph nodes characterization ex vivo. Jonathan Mamou (Riverside Research Institute, 156 William St., 9th Floor, New York, NY 10038, USA, mamou@ @rinyc.org), Alain Coron (Université Paris 6, Laboratoire d'Imagerie Paramétrique, 15, rue de l'Ecole de Médecine, 75006 Paris, France, alain.coron@lip.bhdc.jussieu.fr), Masaki Hata (J. A. Burns School of Medicine, 405 N. Kuakini Street, Honolulu, HI 96817, USA, masakihata4999@yahoo.co.jp), Junji Machi (J. A. Burns School of Medicine, 405 N. Kuakini Street, Honolulu, HI 96817, USA, junji@hawaii.edu), Eugene Yanagihara (J. A. Burns School of Medicine, 405 N. Kuakini Street, Honolulu, HI 96817, USA, kplety@earthlink.net), Pascal Laugier (Université Paris 6, Laboratoire d'Imagerie Paramétrique, 15, rue de l'Ecole de Médecine, 75006 Paris, France, laugier@lip.bhdc.jussieu.fr), Ernest J. Feleppa (Riverside Research Institute, 156 William St., 9th Floor, New York, NY 10038, USA, feleppa @ rrinyc.org)

High-frequency ( $>15 \mathrm{MHz}$, i.e., wavelength $<100 \mu \mathrm{m}$ ) ultrasound has the potential to characterize biological tissues quantitatively at the microscopic level. In this study, quantitative ultrasound (QUS) methods were developed to evaluate freshly excised human lymph nodes from patients with possible metastatic cancer. The objectives were to improve detection of small but clinically significant lymph-node metastases that often are missed during routine histological evaluation. Three-dimensional (3D) ultrasound data acquisition was conducted on freshly excised nodes using a sphericallyfocused transducer with a center frequency of $21 \mathrm{MHz}$. Average scatterer sizes and acoustic concentrations were estimated using QUS methods with a Gaussian scattering model over a bandwidth extending from 11 to $27 \mathrm{MHz}$. Strategies were developed to recover lymph-node orientation and size after histological sectioning to allow for spatially matching QUS estimates to histology. The signal- and image-processing aspects of QUS estimation are presented and the preliminary results are shown that demonstrate our ability to acquire 3D ultrasound and histological data, to co-register them spatially, and to obtain scatterer-size and acoustic-concentration estimates for specific tissue types (cancer versus non-cancer) within the nodes. In future studies, these QUS estimates will be used to identify histologically difficult-to-detect micrometastases in lymph-nodes. [Supported by NIH R01CA100183.]

\section{$5: 20$}

1pBBb13. Extraction of the multiple scattering contribution in weakly scattering media: Application to human soft tissues. Alexandre Aubry (Laboratoire Ondes et Acoustique, ESPCI, Université Paris 7, CNRS, 10 rue Vauquelin, 75005 Paris, France, alexandre.aubry@espci.fr), Arnaud Derode (Laboratoire Ondes et Acoustique, ESPCI, Université Paris 7, CNRS, 10 rue Vauquelin, 75005 Paris, France, arnaud.derode@ujf-grenoble.fr), Mickael Tanter (Laboratoire Ondes et Acoustique, ESPCI, Université Paris 7, CNRS, 10 rue Vauquelin, 75005 Paris, France, michael.tanter@espci.fr)

Waves scattered by a weakly scattering random medium contain a predominant single scattering contribution as well as a multiple scattering contribution which is usually neglected. But its investigation can be fruitful for characterization purposes because it provides measurements of statistical parameters such as the scattering mean free path. Our aim is to extract the multiple scattering contribution in a weakly scattering random medium. The experimental set up consists in an array of programmable transducers placed in front of the sample. The impulse responses between each couple of transducers are measured and form the interelement matrix. Our technique allows the extraction of multiple scattering signals by taking advantage of their randomness, contrary to single scattering contributions which are shown to exhibit a deterministic coherence along the antidiagonals of the array response matrix, whatever the distribution of scatterers. To illustrate the interest of this technique, we applied it to a synthetic medium (Agar gel) and to breast tissues. Surprisingly, the multiple scattering contribution is far from negligible in the breast around 4.3 $\mathrm{MHz}$. The temporal evolution of its intensity can provide a new tool to complete information provided by standard echography; it also constitutes an experimental test of the Born approximation

\section{$5: 40$}

1pBBb14. Ultrasonic vibrometer for tissue characterization. James $S$. Martin (Georgia Institute of Technology, Mechanical Engineering, 771 Ferst Drive, Atlanta, GA 30332-0405, USA, james.martin@me.gatech.edu), Peter H. Rogers (Georgia Institute of Technology, Mechanical Engineering, 771 Ferst Drive, Atlanta, GA 30332-0405, USA, peter.rogers@me.gatech.edu), Michael D. Gray (Georgia Institute of Technology, Mechanical Engineering, 771 Ferst Drive, Atlanta, GA 303320405, USA, michael.gray@me.gatech.edu)

Ultrasonic vibrometers are well suited to a variety of tissuecharacterization tasks because they exploit the lateral resolution and depth of field available with diagnostic ultrasound systems for measurements at lower frequencies. In the past, ultrasonic vibrometers have been used to measure the motion of surfaces with high impedance contrast to their surroundings (otoliths, swim bladders, and lung tissue). New ultrasonic vibrometry techniques have been developed and tested that permit calibrated realtime (amplitude and phase) transduction of sub-nanometer-amplitude vibrations at audio frequencies. These can be configured using either analog or digital demodulation for carrier signals up to $10 \mathrm{MHz}$. In the fully digital configuration, the vibrometer offers better displacement resolution than has been previously reported for analog systems. It also has the capability to simultaneously distinguish between the displacements of multiple discrete vibrating scatterers or regions within a continuum of scatterers by exploiting a multi-sine carrier signal. Individual vibrating targets are separated by pulse compression and windowing. This reduces the problem of carrier drop-out or speckle noise that occurs when multiple reflections destructively interfere at the receiver of a narrow-band signal. The technique should permit the vibrometer a depth resolution comparable a typical pulse-echo ultrasound system. [Work supported by ONR.]

\section{6:00}

1pBBb15. An acoustic technique for mapping and sizing particles following needle-free transdermal drug and vaccine delivery. Jamie Condliffe (University of Oxford, Medical Engineering Unit, 43 Banbury 
Road, OX2 6PE Oxford, UK, jamie.condliffe@magd.ox.ac.uk),Heiko Schiffter (University of Oxford, Medical Engineering Unit, 43 Banbury Road, OX2 6PE Oxford, UK, heiko.schiffter@eng.ox.ac.uk), Constantin C. Coussios (University of Oxford, Medical Engineering Unit, 43 Banbury Road, OX2 6PE Oxford, UK, constantin.coussios@eng.ox.ac.uk)

Needle-free ballistic particle injection enables painless transdermal delivery of pharmaceuticals. To improve the efficacy of this technique, a noninvasive method of assessing particle penetration depth, distribution and changes in particle size following injection is required. Polydisperse distributions of polystyrene particles, mean diameter $25 \mu \mathrm{m}$, and insulin particles, mean diameter $44 \mu \mathrm{m}$, were injected into both tissue-mimicking phantoms and porcine skin, which they penetrate to a mean depth of $300 \mu \mathrm{m}$. Following injection, the surface of the targets was scanned using a $50 \mathrm{MHz}$ focused ultrasound transducer driven in pulse-echo mode. The received waveforms were post-processed to estimate particle penetration depth and distribution in the plane perpendicular to injection. The targets were then sectioned and optical microscopy was used to validate the acoustic results. Furthermore, computational implementation of an exact solution for sound scattering by an elastic sphere allows prediction of the frequency response of the insonified particles. Direct comparison of the theoretical model with the frequency content of experimental data is shown to provide an accurate means of estimating particle size, and is being extended to correlating particle size with penetration depth. The acoustic technique shows great promise as a noninvasive means of mapping, sizing, and assessing stability of drug particles following needle-free injection.

\section{6:20}

1pBBb16. Noninvasive vascular ultrasound elastography of carotid arteries for stroke prevention. Guy Cloutier (Laboratory of Biorheology and Medical Ultrasonics, University of Montreal Hospital Research Center, 2099 Alexandre de Sève (room Y-1619), Montreal, QC H2L 2W5, Canada, guy.cloutier@umontreal.ca), Roch Maurice (Laboratory of Biorheology and Medical Ultrasonics, University of Montreal Hospital Research Center, 2099 Alexandre de Sève (room Y-1619), Montreal, QC H2L 2W5, Canada, maurice.roch.chum@ssss.gouv.qc.ca), Elizabeth Mercure (Laboratory of Biorheology and Medical Ultrasonics, University of Montreal Hospital Research Center, 2099 Alexandre de Sève (room Y-1619), Montreal, QC H2L 2W5, Canada, elizabeth.mercure@UMontreal.CA), Marie-France Giroux (Department of Radiology, University of Montreal Hospital, 1560 Sherbrooke Est, Montreal, QC H2L 4M1, Canada, m_fgiroux@videotron.ca), Gilles Soulez (Department of Radiology, University of Montreal Hospital, 1560 Sherbrooke Est, Montreal, QC H2L 4M1, Canada, gilles.soulez.chum@ssss.gouv.qc.ca)

Carotid plaque rupture leading to stroke involves alteration of arterial wall mechanical properties. This paper addresses reproducibility and potential clinical impact of non-invasive vascular elastography. Fifteen symptomatic and 15 asymptomatic patients with greater than $50 \%$ stenoses of their internal carotids, and 15 control subjects were scanned independently by two radiologists. Radio-frequency cine-loops were acquired on left and right common and proximal internal carotids. For control subjects, homogeneous strains with successive compression and dilatation of the vascular wall from systole to diastole and few shear patterns were observed. The axial strain did not differ between recording sites, sides and radiologists (ANOVA, p > 0.19). For patients, heterogeneous axial strain patterns with both compression and dilatation of tissues within plaques were noted along with shear concentrations at interfaces of tissue structures. Despite similar blood pressures, larger mean instantaneous axial strains were measured in symptomatic $(1.5 \pm 0.6 \%)$ versus asymptomatic $(1.2 \pm 0.5 \%)$ patients in systole for com- mon carotids (t-test, $\mathrm{p}<0.02$ ). To conclude, reproducible results were obtained for control subjects and in patients, larger axial strains in common carotids may be associated with plaque rupture. This new imaging method may become a unique approach to characterize vulnerable plaques for stroke prevention.

1pBBb17. ANAIS: An ultrasound mammograph. Serge
ANA Mensah (Laboratory for Mechanics and Acoustics CNRS, 31 chemin Joseph Aiguier, 13009 Marseille, France, mensah@1ma.cnrs-mrs.fr), Philippe Lasaygues (Laboratory for Mechanics and Acoustics CNRS, 31 chemin Joseph Aiguier, 13009 Marseille, France, lasaygues@1ma.cnrs-mrs.fr), Eric Debieu (Laboratory for Mechanics and Acoustics CNRS, 31 chemin Joseph Aiguier, 13009 Marseille, France, debieu@1ma.cnrs-mrs.fr), Emilie Franceschini (CNRS - LMA, 31 Chemin Joseph Aiguier, 13009 Marseille, France, franceschini@1ma.cnrs-mrs.fr)

It is well established that ultrasonography is a cost-effective, patientfriendly imaging modality available for breast imaging. The main aim of this paper is concerned with a tomographic ultrasound mammograph allowing 3D-reconstruction of the uncompressed breast immersed in a water tank. The scanner, based on a 1024 semi-circular motorized antenna, provides more efficient way to view anatomical ductal structures of the breast. Quantitative volume parameter cartography is the unique means to make objective, reproducible, and operator-independent diagnoses (Computer Aided Diagnosis). The recovery of quantitative acoustical parameters requires solving a nonlinear inverse scattering problem from large amount of measurements. This is achieved using advanced computing resources; a front-end acquisition system based on $32 * 1024$ multiplexed channels and specific reconstruction procedures. The objective is not only to offer a multiparameter tissue characterization using reflection and transmission measurements but also to provide a systemic inspection of the ductal tree.

\section{7:00}

1pBBb18. A reflex transmission method for ultrasound thermometry. Caleb H. Farny (Harvard Medical School, Focused Ultrasound Lab - BWH Radiology, 221 Longwood Ave RM 521, Boston, MA 02115, USA, cfarny@bwh.harvard.edu), Gregory Clement (Harvard Medical School, Focused Ultrasound Lab - BWH Radiology, 221 Longwood Ave RM 521, Boston, MA 02115, USA, gclement@hms.harvard.edu)

It is of great interest to develop ultrasound-based thermal imaging for monitoring thermal ablation procedures and as a tissue characterization technique. A phase contrast analysis for temperature mapping has been investigated, based on the temperature dependence of sound speed of water and tissue. The method employs reflex transmission imaging principles, planar projection and tomographic reconstruction techniques, for interrogating a region featuring a temperature contrast. Through-transmission and pulseecho configurations were both used to image phase contrasts caused from a change in sound speed, in experimental measurements and numerical simulations. Tissue phantoms were used as a medium in which to create a steady-state sound speed contrast, and a raster scan was performed to acquire the sound field with a hydrophone. Two scans were acquired, a reference sound field and a scan featuring a phase contrast which corresponded to a $7-10^{\circ} \mathrm{C}$ peak temperature rise. The through-transmission configuration employed a 0.2 -mm diameter needle hydrophone, while the pulse-echo measurement employed a custom-built thin-wire hydrophone to acquire the sound field between the interrogation transducer and tissue phantom. The method successfully located the location and amplitude of the phase contrast, and future steps necessary for tracking dynamic temperature changes will be discussed. 


\title{
Session 1pBBc
}

\section{Biomedical Ultrasound/Bioresponse to Vibration and Engineering Acoustics: High-Intensity Focused Ultrasound Metrology and Standards I}

\author{
Peter Kaczkowski, Cochair \\ Center for Industrial and Medical Ultrasound, Applied Physics Lab., University of Washington, 1013 NE 40th St., \\ Seattle, WA 98105, USA \\ Gail Ter Haar, Cochair \\ Institute of Cancer Research, 15 Cotswold Road, Belmont, Sutton, Surrey, SM2 5NG, UK
}

\section{Invited Papers}

\section{5:40}

1pBBc1. On the need for basic theoretical, numerical, and experimental research in the development of useful standards for focused ultrasound therapy. Peter Kaczkowski (Center for Industrial and Medical Ultrasound, Applied Physics Lab., University of Washington, 1013 NE 40th St., Seattle, WA 98105, USA, peter@apl.washington.edu), Michael R. Bailey (Center for Industrial and Medical Ultrasound, Applied Physics Lab., University of Washington, 1013 NE 40th St., Seattle, WA 98105, USA, bailey@apl.washington.edu), Vera A. Khokhlova (Center for Industrial and Medical Ultrasound, Applied Physics Lab., University of Washington, 1013 NE 40th St., Seattle, WA 98105, USA, vera@ acs366.phys.msu.ru), Oleg A. Sapozhnikov (Center for Industrial and Medical Ultrasound, Applied Physics Lab., University of Washington, 1013 NE 40th St., Seattle, WA 98105, USA, oleg@acs366.phys .msu.ru)

High intensity focused ultrasound (HIFU) is rapidly gaining widespread clinical use in China, and is undergoing regulatory evaluation in Europe and the US for many target diseases. There is rising concern within the HIFU community that no standards exist for measuring or reporting of HIFU fields, inhibiting broad clinical adoption of HIFU. This paper addresses current technical gaps for the development of such standards. High power and strong focusing combine to make accurate measurements of focal fields difficult. No simple way exists to extrapolate in-water field measurements directly to patients, and thus to calculate temperature rise and therapeutic dose in tissue. To arrive at a set of characteristic parameters for HIFU systems that are both clinically relevant and are practical to measure reliably, we believe several avenues of research are needed. Numerical models that include nonlinear acoustic propagation must be carefully validated with experiments, and both numerics and experimental methods must be standardized for broad dissemination. Mechanisms of enhanced tissue heating due to nonlinear effects must be experimentally quantified, and robust accurate models developed. These advances in understanding must be transitioned into practical recommendations for standard metrics and methodologies to facilitate industrial development and regulatory oversight.

\section{6:00}

1pBBc2. A method of HIFU field characterization in water and deration to tissue. Vera A. Khokhlova (Center for Industrial and Medical Ultrasound, Applied Physics Lab., University of Washington, 1013 NE 40th St., Seattle, WA 98105, USA, vera@acs366.phys.msu.ru), Michael S. Canney (Center for Industrial and Medical Ultrasound, Applied Physics Lab., University of Washington, 1013 NE 40th St., Seattle, WA 98105, USA, mcanney@u.washington.edu), Michael R. Bailey (Center for Industrial and Medical Ultrasound, Applied Physics Lab., University of Washington, 1013 NE 40th St., Seattle, WA 98105, USA, bailey@apl.washington.edu), Olga V. Bessonova (Center for Industrial and Medical Ultrasound, Applied Physics Lab., University of Washington, 1013 NE 40th St., Seattle, WA 98105, USA, olga@ acs366.phys.msu.ru), Oleg A. Sapozhnikov (Center for Industrial and Medical Ultrasound, Applied Physics Lab., University of Washington, 1013 NE 40th St., Seattle, WA 98105, USA, oleg@acs366.phys.msu.ru), Lawrence A. Crum (Center for Industrial and Medical Ultrasound, Applied Physics Lab., University of Washington, 1013 NE 40th St., Seattle, WA 98105, USA, lac@apl.washington.edu)

Acoustic characterization of nonlinear HIFU fields is important for both the accurate prediction of ultrasound induced bioeffects and the development of regulatory standards for clinical HIFU devices. In this work a new characterization method is proposed and tested in water, tissue phantoms, and ex-vivo tissues. The method is based on the combined use of measurements and modeling. Experiments were performed with a $2 \mathrm{MHz}$ transducer of $4.2 \mathrm{~cm}$ aperture and $4.5 \mathrm{~cm}$ focal length. Low amplitude measurements in water were used to establish boundary conditions for modeling based on the KZK-type equation. High amplitude focal waveforms then were simulated and measured with a fiber optic probe hydrophone in water, within tissue phantom, or adjacent to excised tissue. It was shown that at high amplitudes, the simulations of shock waveforms were more accurate than the measurements. The focal waveforms obtained in water were found to be in a good agreement with those produced in tissue with higher source pressure scaled to compensate for the linear attenuation on the way to focus. This result establishes a method to derate the focal HIFU pressures determined in water to tissue. [Work supported by NIH DK43881, NSBRI SMS00402 and RFBR.] 
1pBBc3. Uncertainty of power and intensity measurement in focused fields. Adam Shaw (National Physical Laboratory, Hampton Road, Teddington, TW11 0LW Middlesex, UK, adam.shaw@npl.co.uk), Pierre Gelat (National Physical Laboratory, Hampton Road, Teddington, TW11 0LW Middlesex, UK, pierre.gelat@npl.co.uk)

Currently, the methodology employed to measure acoustic intensity and acoustic power relies on the plane wave assumption, resulting in a simple relation between acoustic power and the normal component of the radiation force vector, and acoustic intensity and pressure magnitude. For a range of ultrasonic transducers, and for positions in the field beyond the last axial maximum, this assumption is a valid one. Nevertheless, there is a range of situations where the plane wave assumption may introduce significant uncertainty in the measurement, such as in near field measurements and in fields resulting from highly focused devices. This investigation looks at the acoustic fields generated by axisymmetric rigidly vibrating curved pistons over a range of diameters and radii of curvature at megahertz frequencies. Finite element and boundary element methods are used to evaluate the acoustic pressure field and the components of the particle velocity vector at specified field positions. The time-averaged acoustic intensity vector, the acoustic power, as well as the normal component of the radiation force vector on a perfectly absorbing target may then be derived. Experimental results are will also be presented comparing theory with radiation force measurements and with the new and more accurate buoyancy change method.

\section{6:40}

1pBBc4. Towards a standardized approach for quantifying inertial cavitation activity. Jamie R. Collin (University of Oxford, Medical Engineering Unit, 43 Banbury Road, OX2 6PE Oxford, UK, jamie.collin@magd.ox.ac.uk), Ian Webb (University of Oxford, Medical Engineering Unit, 43 Banbury Road, OX2 6PE Oxford, UK, ian.webb@eng.ox.ac.uk), Ronald A. Roy (Boston University, Dept. of Aerosp. and Mech. Eng., 110 Cummington St., Boston, MA 02215, USA, ronroy@bu.edu), Constantin C. Coussios (University of Oxford, Medical Engineering Unit, 43 Banbury Road, OX2 6PE Oxford, UK, constantin.coussios@eng.ox.ac.uk)

Relative measures of the energy radiated as broadband noise emissions by inertially cavitating bubbles, such as the mean square voltage received using a passive cavitation detector (PCD), have been recently shown to be directly relatable to cavitation-mediated bioeffects. Even though numerous techniques exist to detect inertial cavitation qualitatively, there is presently a lack of a unified approach that makes it possible to quantify and compare levels of cavitation activity across different experimental setups and conditions. A technique has been developed that uses a calibrated sound source, a hydrophone, and a point spherical scatterer of known frequency response embedded in a tissue-mimicking material to achieve an absolute calibration of a PCD on receive. This makes it possible to relate the PCD signal during inertial cavitation activity to the acoustic power radiated as broadband noise in the proximity of the bubble cloud. The technique is validated against PCD measurements of single-bubble inertial cavitation activity in a tissue-mimicking material and compared to theoretical predictions using the Keller-Miksis model. Calibrated measurements of acoustic power radiated as broadband noise emissions could thus provide a quantitative measure of cavitation dose that can be directly related to resulting bioeffects, such as increased sonoporation or enhanced heating.

1pBBc5. Standardisation and metrology for High Intensity focused ultrasound: a clinical perspective. Gail Ter Haar (Institute of Cancer Research, 15 Cotswold Road, Belmont, Sutton, SM2 5NG Surrey, UK, gail.terhaar@icr.ac.uk)

The clinical application of HIFU is a young and rapidly expanding field, and it is essential that validated methods for measurement and testing are made available as soon as possible. These issues have not been addressed to date in any systematic fashion. There is, therefore, an urgent need to produce standard registration, testing equipment and methodology to allow users to characterize clinical HIFU systems for checking safety and reproducibility of a machine's output, comparing different devices or commissioning new systems. HIFU is delivered to the target volume via a number of routes. Extra-corporeal and trans-rectal probes are the most commonly used, although preliminary testing of intra-cavitary, catheter based devices is also underway 19 . These different devices present a wide range of different transducer geometries, f-numbers $(\sim 0.8-1.8)$, operating frequencies $(\sim 0.8-5 \mathrm{MHz})$, and focal peak intensities $(\sim 7.5$ $10^{2}-3104 \mathrm{~W} \mathrm{~cm}^{-2}$ ). Clinical HIFU systems are currently assessed on an ad hoc basis by individual clinical departments and manufacturers, using methods, many of which are unpublished. The main requirements from a clinical perspective are for the quantification of parameters which relate directly to individual HIFU treatments, allow reproducible treatments between different patients, and allow clinical trial data to be compared. 


\title{
Session 1pEAa
}

\section{Engineering Acoustics, Underwater Acoustics, Signal Processing in Acoustics, and ECUA: Sensor Technologies for Autonomous Acoustic Sensing Systems I}

\author{
Henrik Schmidt, Cochair \\ MIT, 77 Massachusetts Ave, 5-204, Cambridge, MA 02139, USA \\ Andrea Caiti, Cochair \\ University of Genova, Via Opera Pia 13, 15614 Genova, Italy
}

\section{Contributed Paper}

1:00

1pEAa1. SLITA: A new slim towed array for AUV applications. Alain Maguer (NURC Nato Undersea Research Centre, Viale San Bartolomeo 400, 19026 La Spezia, Italy, maguer@nurc.nato.int), Rodney Dymond (NURC Nato Undersea Research Centre, Viale San Bartolomeo 400, 19026 La Spezia, Italy, dymond@nurc.nato.int), Marco Mazzi (NURC Nato Undersea Research Centre, Viale San Bartolomeo 400, 19026 La Spezia, Italy, mazzi@nurc.nato.int), Stefano Biagini (NURC Nato Undersea Research Centre, Viale San Bartolomeo 400, 19026 La Spezia, Italy, biagini@nurc.nato.int), Stefano Fioravanti (NURC Nato Undersea Research Centre, Viale San Bartolomeo 400, 19026 La Spezia, Italy, fioravanti@nurc.nato.int), Piero Guerrini (NURC Nato Undersea Research Centre, Viale San Bartolomeo 400, 19026 La Spezia, Italy, guerrini@nurc .nato.int)

The typical $70 \mathrm{~mm}$ diameter towed array was developed for blue-water detection at long range and low frequencies in the 1960s. Since then, there has been a need for towed arrays that are lighter and less expensive, espe- cially since the maturing field of autonomous vehicles has expanded the potential of such arrays. The marriage of AUVs and lightweight towed arrays is a natural progression in the development of littoral autonomous sensing networks for applications such Anti-Submarine Warfare, marine mammals, or ambient noise measurements. In August 2007, NURC began to design and build a new thin diameter $(31 \mathrm{~mm}$ ) high-frequency (up to $20 \mathrm{kHz}$ ) nested towed array for ASW purposes. An engineering at-sea trial of the array towed by OEX AUV was performed beginning of November 2007. The flow noise level of the array while towed and the potential influence of the AUV self-noise on the acoustic array were also measured. This paper will first describe the array design, its acquisition system and its integration on the OEX AUV. Then, the results obtained from the data analysis are presented. It is shown that the SLITA array has performance that will make it easily fit requirements of the applications previously mentioned.

\section{Invited Paper}

\section{$1: 20$}

1pEAa2. An autonomous moored ocean profiler. Benjamin Cray (Naval Undersea Warfare Center, Code 821,1176 Howell Street, Newport, RI 02841-1708, USA, crayba@npt.nuwc.navy.mil)

An unconventional set of environmental and acoustic data, measured from an autonomous moored ocean profiler, is presented. The profiler is a programmable robotic underwater winch and data acquisition system that cycles vertically through the water column. The system profiles at pre-determined time intervals, surfaces to transmit data via RF links, and can be deployed for up to 180 days. Built by WETLabs Incorporated (Western Environmental Technology Laboratories) for the Naval Underwater Systems Center (NUWC), the system is outfitted with three environmental sensors and two acoustic vector sensors. The environmental sensors include; a Nortek Vector velocimeter which samples current components and characterizes surface wave velocities, a Seabird FastCAT Conductivity, Temperature, Depth (CTD) sensor; and a Wetlabs Fluorometer-Turbidity optical sensor that measures both chlorophyll-a and turbidity. The Wilcoxon acoustic vector sensors measure acoustic pressure and the three components of acoustic particle acceleration. Wilcoxon Research (a subsidiary of Meggitt Corporation) and Applied Physical Sciences (APS) have developed underwater acoustic vector sensors for many applications. The quality and sensitivity of these vector sensors has steadily improved. A review of early (pre-2002) vector sensor development efforts is discussed.

\section{Contributed Papers}

\section{1:40}

1pEAa3. Experimental validation of a chirp-based underwater acoustic communication method. Maria Palmese (DIBE - Dept. of Biophysical and Electronic Engineering - University of Genoa, Via Opera Pia 11a, 16145 Genova, Italy, palmese@dibe.unige.it), Giacomo Bertolotto (Fincantiere Cantieri Navali Italiani S.p.A., Via Cipro 11, 16126 Genova, Italy, Giacomo.bertolotto@fincantieri.it), Alessandro Pescetto (Vulnerability \& Signature Department, CETENA S.p.A., Via Ippolito d'Aste 5, 16121 Genova, Italy, alessandro.pescetto@cetena.it), Andrea Trucco (DIBE Dept. of Biophysical and Electronic Engineering - University of Genoa, Via Opera Pia 11a, 16145 Genova, Italy, trucco@ieee.org)
A wireless underwater acoustic communication algorithm based on the combination of chirp modulation and direct-sequence spread-spectrum signaling is presented. In this paper, the processing chain design is proposed, discussed, and demonstrated using real data. The communication algorithm is made of a transmission block encoding the bits with adequate linear chirps multiplied by pseudo noise (PN) sequences, and a rake receiver that allows one to positively exploit the energy present in the most significant propagation paths. The use of chirp signals takes advantage of the low Doppler sensitivity in the matched filter operation whereas the choice of PN sequences allows one to reduce narrowband interference arising from other users and self-interference due to multipath propagation. Moreover a tracking 
procedure that allows an adaptation to the instantaneous Doppler shift has been devised and tested. Some experiments have been carried out changing the distance and the speed between transmitter and receiver. Results show that the developed communication method is able to handle the multipath phenomenon and the Doppler effect, allowing one to achieve a bit error rate less than $10(-3)$ for long ranges for bit rates of about 15 and $230 \mathrm{bit} / \mathrm{s}$.

\section{2:00}

1pEAa4. Broadband transducers for underwater communications David Brown (BTech Acoustics, LLC and Universtity of Massachusetts Dartmouth, ATMC, Electro-Acoustics Research Laboratory and ECE Dept., 151 Martine St, Fall River, MA 02723, USA, dbAcoustics@cox.net), Corey Bachand (BTech Acoustics, LLC and Universtity of Massachusetts Dartmouth, ATMC, Electro-Acoustics Research Laboratory and ECE Dept., 151
Martine St, Fall River, MA 02723, USA, corey.bachand@cox.net),Boris Aronov (BTech Acoustics, LLC and Universtity of Massachusetts Dartmouth, ATMC, Electro-Acoustics Research Laboratory and ECE Dept., 151 Martine St, Fall River, MA 02723, USA, baronov@comcast.net)

Properties of underwater acoustic cylindrical piezoelectric (PZT) transducers currently used for broadband underwater communications with unmanned underwater vehicles (UUV) and new transducers under development utilizing single crystal relaxor ferroelectrics (PMN-PT) with significantly higher material coupling coefficients and lower sound speed are presented. Characteristics including resonance frequency, coupling coefficient, transmit (TVR) and receive (OCVS) frequency response, maximum drive and achievable source levels, tuned and untuned power factor, and directivity factors are discussed. Results for experimental calibrations and field tests will be compared with detailed equivalent electrical circuit models of the transducer and transducer channel. Work supported in part by BTech Acoustics and Office of Naval Research.

\section{Invited Papers}

1pEAa5. High resolution angular measurements with single vector sensors and arrays. Joseph A. Clark (NSWCCD, Code 7340, 9500 MacArthur Blvd., West Bethesda, MD 20817-5000, USA, joseph.a.clark1@navy.mil), Dehua Huang (Naval Undersea Warfare Center, 1176 Howell St., Newport, RI 02841, USA, HuangD@Npt.NUWC.Navy.Mil)

The direction finding capability of acoustic sensors that directly measure acoustic particle velocity alone or in combination with pressure measurements (vector sensors) is well known. The angular resolution of the measurements can be improved by using arrays of vector sensors. It is also possible to improve angular resolution by additional processing of data from even a single vector sensor. This approach could be especially advantageous for sonar systems designed to operate from small platforms such as autonomous underwater vehicles. In this talk linear and non-linear methods for processing data from one or more vector sensors to achieve high angular resolution will be reviewed. Experimental investigations comparing the methods will be reported and factors found to limit the localization and detection performance of sonar systems employing the methods will be discussed.

\section{2:40}

1pEAa6. Hydroflown: MEMS-based Underwater Acoustical Particle Velocity Sensor. Tuncay Akal (SUASIS: Underwater Systems Technology Development, Tubitak-Marmara Research Center, Tech. D. Free Zone, Block A - L4, Gebze, 41470 Kocaeli, Turkey, tuakal@yahoo.com), Hans-Elias De Bree (Microflown Technologies Inc., PO BOX 300, 6900 AH Zevenaar, Netherlands, debree@microflown.com), Piero Guerrini (NURC Nato Undersea Research Centre, Viale San Bartolomeo 400, 19026 La Spezia, Italy, guerrini@nurc.nato.int), Alain Maguer (NURC Nato Undersea Research Centre, Viale San Bartolomeo 400, 19026 La Spezia, Italy, maguer@nurc.nato.int)

The increasing problems related to homeland security and harbour/infrastructure protection have increased the level of interest on vector sensors. Market surveys carried out during the last three years gave the conclusion that there is a need for a new generation, small size, and low-cost underwater sensors capable of measuring particle velocity in three dimensional plain within a broad frequency band $(2 \mathrm{~Hz}-50 \mathrm{kHz})$ and with high angular resolution. The small size MEMS-based sensors developed by Microflown Technologies BV Inc are the world's only commercially available transducers that are capable of measuring the particle velocity, instead of pressure, in air. The development of a new generation, innovative and low-cost underwater sensors and technologies based on that in-air nano technology is therefore considered. This technology has a great potential to become a revolutionary underwater acoustic sensor using nanotechnology, capable of finding many applications like sensors for Autonomous Underwater Vehicles, sensors for directional receivers for underwater acoustic systems, Floating autonomous systems, Sensors for seismic towed arrays for underwater oil and mineral prospecting and harbour and water-side infrastructure protection. This paper describes how Microflown technology can be adapted to underwater applications.

\section{Contributed Papers}

3:00

1pEAa7. Concurrent detection, classification and localization of seabed targets using virtual time reversal. Alexis J. Dumortier (MIT, 77 Mass Ave, 5-204, Cambridge, MA 02139, USA, adumorti@mit.edu), Henrik Schmidt (MIT, 77 Mass Ave, 5-204, Cambridge, MA 02139, USA, henrik@mit.edu), Karim G. Sabra (Georgia Institute of Technology, School of Mechanical Engineering, 771 Ferst Drive, NW, Atlanta, GA 30332-0405, USA, karim.sabra@me.gatech.edu)

During the last decade, the use of unmanned vehicles to detect seabed objects has been revolutionized by the rapid development of AUV technology. The reduced power budget imposed by autonomous operations limits the amount of processing allowable onboard and calls for simple and efficient detection algorithms. Time reversal techniques have proved to be a robust way of focusing sound on reflective objects in complex environments and only require minimal computation. The method proposed here uses a virtual time reversal mirror in monostatic configuration to localize the origin of the field scattered by objects located on the seafloor. The waveguide is insonified with a low frequency source $(\sim \mathrm{kHz})$ mounted on the AUV and the resulting scattered field is sampled by a receiving array towed behind the vehicle. The recorded signals are then used to simulate the time reversed transmissions onboard, generating a map of reflectors present on the seabed. The rejection of clutter using singular value decomposition and the sensitivity of this method to environmental misknowledge are addressed along with the presentation of experimental results. [Work supported by ONR.] 


\section{3:40}

1pEAa8. Computational fluid dynamic analysis of optimized windscreens for UAV based acoustic arrays. Wayne E. Prather (Miltec Research \& Technology, 9 Industrial Park Dr., Oxford, MS 38655, USA, wprather@mil-tec.com), David H. Bridges (Mississippi State University, Dept. of Aerospace Engineering, Mississippi State, MS 39762, USA, dbridges@AE.MsState.EDU), Tom Edwards (Raspet Flight Research Laboratory, 114 Airport Road, Starkville, MS 39759, USA, edwards@raspet.msstate.edu), David S. Thompson (Mississippi State University, Dept. of Aerospace Engineering, Mississippi State, MS 39762, USA, dst@ simcenter.msstate.edu)

As part of an overall effort to place acoustic sensors on airborne platforms for surveillance applications, analysis studies were performed of acoustic probe windscreens that could minimize wind noise and be made relatively insensitive to small angle of attack and sideslip excursions. Through a collaborative effort between Miltec Research \& Technology, Mississippi State University Department of Aerospace Engineering, Raspet Flight Research Laboratory, and the Engineering Research Center several probe designs were developed and analyzed using inviscid and viscous simulations with the objective of characterizing their flow fields and identifying potential modifications that could improve suitability for use as acoustic measurement probes. Several of the recommended probe shapes were fabricated and tested under real world conditions on an acoustically optimized 1/3 scale Schleicher ASH26 glider test bed.
1pEAa9. Acoustical methods for azimuth, range and heading estimation in underwater swarms. Uwe R. Zimmer (The Australian National University, $0200 \quad$ Canberra, Australia, uwe.zimmer@ieee.org), Navinda Kottege (The Australian National University, 0200 Canberra, Australia, navinda.kottege@anu.edu.au)

Acoustical Methods for Azimuth, Range and Heading Estimation in Underwater Swarms Enhanced, embodied autonomy in small submersibles enables the design and deployment of practical swarms of autonomous underwater vehicles (AUVs). The swarming paradigm requires for each vehicle location awareness of at least its near neighbors. The Serafina AUV swarming project additionally requires a localisation system which could cope with the dynamic and fast changing vehicle configurations while being small, reliable, robust, and energy efficient and not dependent on pre-deployed acoustic beacons. The short-range acoustical relative localisation system proposed here, uses hyperbolic and spherical localization concepts and provides each vehicles with the azimuth, range and heading of its near neighbours. The implementation utilises an acoustically transmitted MLS signal which provides extremely high robustness against interference by stochastic and systematic disturbances which are typical for underwater environments. The azimuth is obtained via hyperbolic positioning with improved resolution and accuracy with respect to conventional methods. Range and heading estimation is achieved by two independent methods for increased robustness; one uses the implicit synchronisation provided by the underlying inter-vehicle communication scheduling system to measure the difference of TOAs of an acoustic and a long-wave radio signals; the second relies on TDOAs and a reverse hyperbolic localisation scheme.

\section{Invited Paper}

\section{4:20}

1pEAa10. Integrated sensing, modeling, and control in undersea sensor networks. Arjuna Balasuriya (MIT, 77 Mass Ave, 5-204, Cambridge, MA 02139, USA, arjunab@mit.edu), Henrik Schmidt (MIT, 77 Mass Ave, 5-204, Cambridge, MA 02139, USA, henrik@mit.edu), Michael B. Benjamin (MIT, 77 Mass Ave, 5-204, Cambridge, MA 02139, USA, mikerb@csail.mit.edu)

The way forward in adaptively sampling the vast ocean environment is by deploying a network of sensors with different capabilities. Due to the complex nature of the environment, covering such an area cannot be achieved by simply deploying underwater sensor nodes at predefined locations. The undersea network topology should dynamically adapt to the events and changes in the environment. However, the main challenge for undersea network autonomy is due to the limitations in underwater acoustic communication. In order to overcome such limitations, Nested Autonomy architecture is being developed and demonstrated in programs such as PLUSNet and Ocean Observatories. This paper looks into the implementation of such autonomy architecture in different sensor nodes with different capabilities. This is achieved by separating the "low-level" tasks of a sensor node with its "high-level" tasks. Low-level tasks will be sensor specific while the high-level tasks looks into the autonomy required for the adaptive sampling applications, which here refers to as the "Backseat-driver paradigm". The "backseat" carries the on-board intelligence required for the mission at hand. Behavior-based autonomy architecture which is used in the back-seat significantly improves the sensor performance by selecting the best behavior for the given state of the vehicle, mission at hand and the observations being made.

\section{Contributed Paper}

4:40

1pEAa11. Artificial characteristic landmarks for SLAM navigation. Yan Pailhas (Ocean Systems Laboratory, School of Eng. \& Phys. Sciences, Heriot-Watt University, EH14 4AS Edinburgh, UK, Y.Pailhas@hw.ac.uk), Chris Capus (Ocean Systems Laboratory, School of Eng. \& Phys. Sciences, Heriot-Watt University, EH14 4AS Edinburgh, UK, C.Capus@hw.ac.uk), Keith E. Brown (Ocean Systems Laboratory, School of Eng. \& Phys. Sciences, Heriot-Watt University, EH14 4AS Edinburgh, UK, K.E.Brown@hw.ac.uk)

The use of autonomous underwater vehicles (AUVs) for a variety of purposes is set to increase in the future. A key issue in the navigation processing, especially for survey applications, is the lack of accuracy or cumulative error introduced by the various position sensors: accelerometer; DVL; compass. Algorithms such as SLAM (simultaneous localisation and mapping) rely on accurate landmark recognition in order to correct the vehicle position. This paper proposes a solution based on broadband sonar and artificial coded landmarks to improve the navigation. Through resolution of the wave equation for acoustic propagation in a multilayer concentric sphere, we will show that there is a great diversity in the echo spectrum with small changes in internal structure. This enables the design of a set of passive landmarks which can be identified unambiguously, since each has a characteristic signature or 'spectral code' when insonified with a broadband sonar.

\section{Invited Paper}

\section{5:00}

1pEAa12. Acoustic sensor systems on a flying wing underwater glider and two prop-driven autonomous underwater vehicles. Gerald D’Spain (Marine Physical Lab, Scripps Institution of Oceanography, 291 Rosecrans St., San Diego, CA 92106, USA, gld@mpl.ucsd.edu), Richard Zimmerman (Marine Physical Lab, Scripps Institution of Oceanography, 291 Rosecrans St., San Diego, 
CA 92106, USA, richard@mpl.ucsd.edu),Scott A. Jenkins (Marine Physical Lab, Scripps Institution of Oceanography, 291 Rosecrans St., San Diego, CA 92106, USA, sjenkins@ucsd.edu), Dennis B. Rimington (Marine Physical Lab, Scripps Institution of Oceanography, 291 Rosecrans St., San Diego, CA 92106, USA, drimington@ucsd.edu), James C. Luby (Applied Physics Lab, Univ. of Washington, 1013 NE 40th St., Seattle, WA 98105, USA, jcl@apl.washington.edu), Peter Brodsky (Applied Physics Lab, Univ. of Washington, 1013 NE 40th St., Seattle, WA 98105, USA, brodsky@apl.washington.edu)

The Marine Physical Laboratory, Scripps Institution of Oceanography operates several underwater vehicles including an autonomous underwater glider based on a flying wing design and two prop-driven autonomous underwater vehicles (AUV) manufactured by Bluefin Robotics. The objective of this presentation is to describe the acoustic sensor systems on these platforms and provide sample results from the at-sea data. The glider, with a 6.1-m wing span, was developed jointly by the Marine Physical Lab and the Applied Physics Laboratory, University of Washington. It is designed to maximize the horizontal distance traveled between changes in buoyancy (i.e., maximize its "finesse") while quietly listening to sounds in the ocean. A 27 -element hydrophone array with $5 \mathrm{kHz}$ per channel bandwidth is located in the sonar dome all along the wing's leading edge. In addition, it has a four-component acoustic vector sensor in its nose. The two prop-driven AUVs have been equipped with hull-mounted hydrophone arrays with $10 \mathrm{kHz}$ bandwidth for passive synthetic aperture studies, an acoustic vector sensor, and active acoustic imaging systems for ocean bottom/subbottom mapping. Results from the data processing illustrate the tight coupling between acoustic sensor systems, signal/array processing methods, and vehicle behavior. [Work supported by the Office of Naval Research.]

MONDAY AFTERNOON, 30 JUNE 2008

ROOM 353, 1:00 TO 4:00 P.M.

\title{
Session 1pEAb
}

\section{Engineering Acoustics and Signal Processing in Acoustics: Microphone Array Signal Processing I}

\author{
Gary Elko, Cochair \\ mh acoustics LLC, 25-A Summit Ave., Summit, NJ 07901, USA \\ Walter Kellermann, Cochair \\ Univ. of Erlangen-Nuremberg, 90158 Erlangen, Germany
}

\section{Contributed Papers}

1:00

1pEAb1. Spherical harmonic analysis applied to the reconstruction of the sound field radiated by a loudspeaker. Filippo M. Fazi (ISVR, University of Southampton, Highfield, SO171BJ Southampton, UK, ff1@isvr.soton.ac.uk), Vincent Brunel (ISVR, University of Southampton, Highfield, SO171BJ Southampton, UK, Vincent.Brunel@eurocopter.com), Philip A. Nelson (ISVR, University of Southampton, Highfield, SO171BJ Southampton, UK, P.A.Nelson@ soton.ac .uk)

The three dimensional acoustic radiation patterns generated by different models of loudspeaker have been measured in an anechoic environment using an array of 40 omnidirectional microphones, arranged on a hemispherical surface. The acquired data have been numerically processed in order to obtain the complete three dimensional reconstruction of the analysed sound field. The theory of the applied method is based on the spherical harmonic decomposition of the sound field defined over the surface of a sphere containing the sound source and sampled at a finite number of positions. The reconstruction of the sound field in the region of the space inside and outside the measurement surface has been obtained by multiplying each coefficient of the spherical harmonic series by the corresponding spherical propagator, defined as the ratio of two spherical Hankel functions. The conditioning of the inverse problem related to the reconstruction of the sound field in the interior of the measurement surface has been analysed and put in relation to the behaviour of the spherical propagators of different orders.

\section{$1: 20$}

1pEAb2. Surround sound echo cancellation in the spherical harmonic domain. Joshua Atkins (Johns Hopkins University, 853 West 35th Street, Baltimore, MD 21211, USA, joshatkins@jhu.edu), James West (Johns
Hopkins University, Department of Electrical Engineering, 3400 North Charles Street, Baltimore, MD 21218, USA, jimwest@jhu.edu)

The problem of creating a multiuser hands-free immersive telecommunications environment poses many challenges for acoustic signal processing. The most pressing is the creation of a fast multichannel acoustic echo canceller (MCAEC) to eliminate acoustic feedback created in the speakermicrophone loop. Traditional multichannel adaptive algorithms for echo cancellation are not fast enough to work in systems with highly correlated multichannel signals like those found in the traditional telecommunications setup. This work presents analysis of a proposed acoustic echo cancellation system that operates in the spherical harmonic domain. Transforming the microphone and speaker signals into the spherical harmonic domain reduces both the number of channels and the correlation between channels for faster convergence of the adaptive MCAEC filters. This method also has the added benefit of being independent of the microphone and speaker array configuration, resulting in a hybrid mono/stereo/multichannel acoustic echo canceller. This work is funded by NSF Grant IIS-0534221.

\section{$1: 40$}

1pEAb3. Improving the directivity of a Soundfield microphone using least-squares estimation. Craig Jin (University of Sydney, School of Electrical and Information Engineering, Bldg. J03, 2006 Sydney NSW, Australia, craig@ee.usyd.edu.au), Alan Kan (University of Sydney, School of Electrical and Information Engineering, Bldg. J03, 2006 Sydney NSW, Australia, alan@carlab.ee.usyd.edu.au), Andre Van Schaik (University of Sydney, School of Electrical and Information Engineering, Bldg. J03, 2006 Sydney NSW, Australia, andre@ee.usyd.edu.au)

Faller [1] has recently shown that highly directive microphone systems can be obtained using coincident microphones and appropriate signal processing to separate direct and ambient sound. We apply this approach to a Soundfield microphone in which coincident virtual first-order microphone patterns pointing in any direction are available. The variation with our ap- 
proach is that we apply a specific gain pattern across a set of coincident cardiod or hypercardiod microphones to determine the pick-up direction. A least-squares estimate is made of the spectrum of the on-axis sound. We empirically investigate the performance of this directive microphone technique. [1] C. Faller, "A highly directive 2-capsule based microphone system," in Proc. of 123rd Audio Engineering Convention, New York, U.S.A., October $5-8,2007$.

\section{2:00}

1pEAb4. A comparison of measured and theoretical performance of a co-centred rigid and open spherical microphone array. Craig Jin (University of Sydney, School of Electrical and Information Engineering, Bldg. J03, 2006 Sydney NSW, Australia, craig@ee.usyd.edu.au), Abhaya Parthy (University of Sydney, School of Electrical and Information Engineering, Bldg. J03, 2006 Sydney NSW, Australia, aparthy@it.usyd.edu.au), Andre Van Schaik (University of Sydney, School of Electrical and Information Engineering, Bldg. J03, 2006 Sydney NSW, Australia, andre@ee.usyd.edu.au)

We present a comparison of the measured and theoretical performance of a dual co-centred spherical microphone array that consists of an open spherical microphone array with a smaller, rigid spherical microphone array at its centre. The dual co-centred spherical microphone array has 64 microphones, with 32 microphones on the open spherical microphone array of radius 6.30 $\mathrm{cm}$ and 32 microphones on the rigid spherical microphone array of radius $1.63 \mathrm{~cm}$. We have previously shown [1] that this even distribution of microphones, between the two spherical microphone arrays, provides a greater frequency range of operation for a third-order, 64-channel spherical microphone array compared to a single rigid 64-channel spherical array. The performance of the dual co-centred spherical microphone array is measured in an anechoic chamber using a speaker mounted on a robotic arm. A comparison is made between the theoretical and measured directivity pattern for various frequencies. [1] A. Parthy, C. Jin, and A. van Schaik "Optimisation of Co-centred Rigid and Open Spherical Microphone Arrays," in Proc. of 120th Audio Engineering Convention, Paris, France, May 20-23, 2006.

\section{2:20}

1pEAb5. Towards 'not so spherical' microphone arrays. Nicolas Epain (France Telecom R\&D, 2 avenue Pierre Marzin, 22300 Lannion, France, nicolas.epain@orange-ftgroup.com), Jérôme Daniel (France Telecom R\&D, 2 avenue Pierre Marzin, 22300 Lannion, France, jerome.daniel @ orange-ftgroup.com)

An increasing number of applications require that the sound field spatial properties are accurately described. Using arrays consisting of omnidirectional microphones distributed at the surface of a rigid sphere has been proven to be an efficient yet practical strategy to obtain such three dimensional sound field descriptions. However these sensor arrays are known to suffer from frequency limitations, mainly due to the sphere directivity properties. In this paper, it is proposed to optimize the shape of the object upon which the microphones are located, in order to make them more directive. Results of measurements and simulations show that such "not so spherical" shapes could help at widening the microphone array frequency range.

\section{2:40}

1pEAb6. Global synthesis of superdirective frequency-invariant beam patterns. Marco Crocco (DIBE - Dept. of Biophysical and Electronic Engineering - University of Genoa, Via Opera Pia 11a, 16145 Genova, Italy, crocco@dibe.unige.it), Stefania Repetto (DIBE - Dept. of Biophysical and Electronic Engineering - University of Genoa, Via Opera Pia 11a, 16145 Genova, Italy, stefania@ dibe.unige.it), Andrea Trucco (DIBE - Dept. of Biophysical and Electronic Engineering - University of Genoa, Via Opera Pia 11a, 16145 Genova, Italy, trucco@ieee.org)

Frequency-invariant beam patterns are often required by systems using an array of sensors to process broadband signals. If the spatial aperture is shorter than the involved wavelengths, the use of a superdirective beam pattern is essential to attain an efficient system. In this context, robustness to array imperfections is a crucial feature. In the literature, only a few approaches have been proposed to design a robust, superdirective, frequency- invariant beamformer, based on a filter and sum architecture: in all of them, the frequency invariance is achieved imposing an a priori desired beam pattern. However the choice of a suitable desired beam pattern is not trivial and depends on the specific design case: an improper selection of the desired beam pattern can produce unsatisfactory performances. We propose a new method of global synthesis, computationally inexpensive, allowing to design a robust broadband beam pattern with an optimal trade-off between the frequency invariance and the directivity, without the need of imposing a priori a desired beam pattern. The results show that the synthesized beam patterns have a directivity, a frequency-invariance, and a robustness that are very similar to or better than those of the beam patterns obtained by the literature methods

\section{3:00}

1pEAb7. The generation of binaural signals from a regularly sampled soundfield using a beamforming approach. Mark Poletti (Industrial Research Ltd., PO Box 31-310, 310 Lower Hutt, New Zealand, M.Poletti@irl.cri.nz), Peter Svensson (Norwegian Univ. of Science and Technology, O.S. Bragstads plass 2B, Dept. of Electronics and Telecommunications, NO-7491 Trondheim, Norway, svensson@iet.ntnu.no)

Auditorium designs can be evaluated prior to construction by numerica modeling of the design. Subjective assessment of the design requires auralization of the computed soundfield at a desired listener position and the binaural format is dominant for representation of the soundfield. For high accuracy the modeling is based on solving the wave equation numerically, which typically produces the sound pressure on a rectangular grid. Such a sampled soundfield is not straightforward to convert to a binaural format. This paper investigates binaural conversion from the sound pressure at a selected number of grid points using a least squares beamforming approach Low-frequency axisymmetric emulations are derived assuming a solid sphere model of the head, and an open spherical array of 640 microphones is used to emulate ten measured HRTF data sets from the CIPIC database for half the audio bandwidth. The microphone positions might be displaced from the exact spherical surface to fit the mesh sample points. The spherica array can produce high-accuracy band-limited emulation of any human subject's measured HRTFs for a fixed listener position by using individual sets of beamforming impulse responses.

\section{3:20}

1pEAb8. New measurements of head-related transfer functions with an optimized ear-canal microphone. Christopher Haut (Oldenburg University, Institute for Physics, 26111 Oldenburg, Germany, christopher@aku.physik.uni-oldenburg.de), Jacqueline Rausch (Oldenburg University, Institute of Physics - Acoustics, Carl-von-Ossietzky Str. 9-11, $26111 \quad$ Oldenburg, Germany, jacqueline@aku.physik.uni-oldenburg.de), Volker Mellert (Oldenburg University, Institute for Physics, 26111 Oldenburg, Germany, volker.mellert @uni-oldenburg.de)

Affecting the ear-canal impedance by inserting a microphone produces considerable changes in the directional characteristics of the ear with increasing frequency. Head-related transfer functions (HRTFs) are measured in the horizontal and median plane under systematic change of the ear-canal microphone more or less blocking the ear-canal entrance. A microphone arrangement is developed which minimizes the influence on the given impedance of the ear canal entrance. New measurements of HRTFs are reported and compared with well-known literature results. The "undisturbed" HRTFs serve as objective functions for a microphone array which substitutes the dummy head in head-related (stereophonic) recordings. The (complex) objective functions are modified for the microphone array according to the relevance in directional hearing. 


\section{3:40}

1pEAb9. Study of a concert harp's radiation using acoustic imaging methods. Quentin Leclere (Laboratoire Vibrations Acoustique - INSA Lyon, 25 bis avenue Jean Capelle, Bâtiment Saint-Exupéry, F-69621 Villeurbanne cedex, France, quentin.leclere@insa-lyon.fr), Jean-Loic Le Carrou (Laboratoire d'Acoustique de l'Université du Maine, Avenue Olivier Messiaen, 72085 Le Mans, France, jean-loic.le_carrou@univ-lemans.fr), François Gautier (Laboratoire d'Acoustique de l'Université du Maine, Avenue Olivier Messiaen, 72085 Le Mans, France, francois.gautier@univlemans.fr)

Recent studies have been conducted to understand the low frequency radiation of a concert harp. Experiments have been carried out in a semianechoic room: the harp's soundboard is excited with a shaker and the pres- sure is measured at more than 600 positions on a nearly hemispherical surface at a distance of about $2 \mathrm{~m}$ from the instrument. Simple source models with a set of monopoles have been optimized to fit the measured acoustic radiation. These models are very satisfactory in the low frequency domain but cannot properly reproduce the measured field above $350 \mathrm{~Hz}$. The aim of this paper is to present the application of acoustic imaging tools to this academic case: volumetric velocity and acoustic power maps in the harp plane are computed with an optimized beamforming and with a regularized inverse FRF method up to $1 \mathrm{kHz}$. The optimization of the beamforming is realized using a variable windowing factor, and the regularization of the inverse FRF method with a Tikhonov approach. The presented results permit the evaluation of the importance of the bass reflex effect in the harp, of the contribution of the first bending modes and of the contribution of the soundbox vibrations in the radiated sound field.

\title{
Session 1pEAc
}

\section{Engineering Acoustics and Psychological and Physiological Acoustics: Hearing Aid Engineering I}

\author{
Daniel M. Warren, Cochair \\ Knowles Electronics, 1151 Maplewood Dr, Itasca, IL 60143, USA \\ Stefan Launer, Cochair \\ Phonak AG, Laubisrütistr. 28, CH-8712 Stäfa, Switzerland
}

\section{Invited Paper}

$4: 20$

1pEAc1. Model-based signal processing for hearing aids. Volker Hohmann (Carl von Ossietzky Universität Oldenburg, Ammerländer Heerstraße 114-118, 26111 Oldenburg, Germany, volker.hohmann@uni-oldenburg.de)

The aim of the work presented in this talk is to investigate potential applications of models of human auditory processing to signal processing in hearing aids. In particular, it is shown that auditory models may be used as objective indicators of the perceptual quality of single- and multichannel noise reduction strategies. Furthermore, a general framework for computational auditory scene analysis (CASA) is introduced and applied to the problem of estimating spatial direction and spectral envelope of super-imposed sound sources from a binaural input signal. Finally, implications of using nonlinear peripheral models for the design of compression systems in hearing aids are discussed. In particular, results from a multiband instantaneous compression scheme are presented, which is based on a novel nonlinear auditory filterbank controlled by the instantaneous frequency calculated in frequency sub-bands.

\section{Contributed Paper}

\section{4:40}

1pEAc2. The sound field in life-size replicas of human ear canals occluded by a hearing aid. Mike R. Stinson (Inst. for Microstructural Sciences, National Research Council of Canada, Bldg. M-36, Ottawa, ON K1A 0R6, Canada, mike.stinson@nrc-cnrc.gc.ca), Gilles A. Daigle (Inst. for Microstructural Sciences, National Research Council of Canada, Bldg. M-36, Ottawa, ON K1A 0R6, Canada, gilles.daigle@nrc-cnrc.gc.ca)

In addition to the longitudinal sound pressure distributions that form in the human ear canal, large transverse variations can arise in the vicinity of an occluding hearing aid. These effects are being studied, numerically and experimentally, making use of life-size ear canal replicas. Using digital representations of real ear canal geometries [Stinson and Lawton, J. Acoust.
Soc. Am. 85, 2492-2503 (1989)], a polyjet fabrication process forms replicas with a spatial accuracy of better than $0.1 \mathrm{~mm}$. A hearing aid test fixture, with vent, receiver, and an inner microphone, occludes the replica canal and provides the acoustical input. The sound field inside the canal is measured using a $0.2 \mathrm{~mm}$ o.d. probe microphone. In parallel, the interior sound field is calculated using a boundary element method, using the same ear canal geometry as the replicas and accounting for the acoustical boundary conditions presented by the eardrum and the hearing aid. In the current series of ear canal replicas, the eardrum is rigid. Measurements and calculations are in good agreement. Large transverse variations of sound pressure level, as much as $20 \mathrm{~dB}$ at $8 \mathrm{kHz}$, are observed across the inner face of the hearing aid, particularly near the receiver and vent ports. 


\section{Invited Papers}

\section{5:00}

1pEAc3. Acoustics of open fittings. Matthias Blau (Institut für Hörtechik + Audiologie, FH OOW, Ofener Str. 16, D-26121 Oldenburg, Germany, matthias.blau @ fh-oldenburg.de), Tobias Sankowsky (Institut für Hörtechik + Audiologie, FH OOW, Ofener Str. 16, D-26121 Oldenburg, Germany, tobias.sankowsky@ @ fh-oldenburg.de), Alfred Stirnemann (Phonak AG, Laubisrütistr. 28, CH-8712 Stäfa, Switzerland, alfred.stirnemann@phonak.ch), Hannes Oberdanner (Phonak AG, Laubisrütistr. 28, CH-8712 Stäfa, Switzerland, hannes.oberdanner@phonak.ch), Nicola Schmitt (Phonak AG, Laubisrütistr. 28, CH-8712 Stäfa, Switzerland, nicola.schmitt@phonak .ch)

The use of so-called "open fittings" instead of individual ear shells has become very popular in hearing aid fitting, in particular because the open fittings avoid the occlusion effect, but also because they are more comfortable, easier to manufacture, and cosmetically preferred. On the other hand however, there are acoustical issues with open fittings, including the mixture of direct and amplified sound, a poor low-frequency performance, an increased risk of feedback and a supposedly less reproducible position of the sound delivering device (tubing or speaker) in the ear canal, which in turn may result in a greater variability of acoustic parameters such as RECD and REOG. The two latter issues are addressed here in a study with 20 subjects, for individual shell and a number of open fittings, comprising closed and open domes of different diameters with tubings as well as with ear canal receivers. It was observed that in comparison to individual shell fittings, the open fittings did not exhibit higher interindividual differences of RECD and REOG, but up to 15 $\mathrm{dB}$ (closed domes) to $25 \mathrm{~dB}$ (open domes) lower feedback thresholds.

\section{$5: 20$}

1pEAc4. Estimation of maximum stable gain in a hearing aid. Ivo L. Merks (Starkey Laboratories, 6600 Washington Ave S, Eden Prairie, MN 55344, USA, ivo_merks@ starkey.com), Karrie Recker (Starkey Laboratories, 6600 Washington Ave S, Eden Prairie, MN 55344, USA, karrie_recker@ @starkey.com), Harikrishna P. Natarajan (Starkey Laboratories, 6600 Washington Ave S, Eden Prairie, MN 55344, USA, harikrishna_natarajan@starkey.com), Lalin S. Theverapperuma (Starkey Laboratories, 6600 Washington Ave S, Eden Prairie, MN 55344, USA, lalin_theverapperuma@starkey.com)

Feedback cancellation (FBC) algorithms have become an important part of hearing aids, allowing the Maximum Stable Gain (MSG) to be increased by up to $25 \mathrm{~dB}$ beyond what is possible without an FBC. Although FBC algorithms have improved the usability of hearing aids, it is difficult to predict the MSG for a given individual with a particular hearing aid. Knowledge of this information would enable the audiologist to make more informed decisions regarding the appropriateness of a hearing aid/earmold, and it could be used to counsel the patient regarding feedback. A method of estimating the MSG of a hearing aid with and without an FBC will be presented. The method uses a finite impulse response filter to approximate the acoustic path. The filter coefficients are estimated during the initialization of the FBC. From these filter coefficients, the MSG for the hearing aid with and without FBC are calculated. This method has been implemented in the firmware and fitting software. Using a variety of device styles, microphone modes and feedback paths, it has been verified that the estimated MSG matches the actual MSG within $6 \mathrm{~dB}$ for $90 \%$ of hearing aids.

\section{$5: 40$}

1pEAc5. "Auditory scene analysis" in hearing instruments. Matthias Froehlich (Siemens Medical Solutions SAT, 91058 Erlangen, Germany, Matthias.froehlich@siemens.com)

The term "auditory scene analysis" generally refers to a categorization of a given acoustic situation based on the acoustic signal only, where the results determine the subsequent processing of the acoustic signal within some auditory context. According to this definition, several approaches can be differentiated in the field of hearing instrument development. They differ in the computational complexity of the particular analysis methods applied, as well as in the subsequent action. Some of these approaches have been realized in commercially available hearing instruments, others lie still ahead. A simple example of the former category is noise reduction algorithms that address different classes of noises, examples of the latter are MPEG4-like virtual arrangements of media objects. In the presentation, different approaches will be discussed in terms of potential benefit and technical realization, as well as their limitations. For approaches already realized in commercially available hearing instruments, the expected benefit will be aligned with results from clinical studies.

\section{Contributed Paper}

\section{6:00}

1pEAc6. Prediction of perceived sound quality of hearing aids (algorithms) using perceptual models. Rainer Huber (Kompetenzzentrum HörTech, Marie-Curie-Str. 2, 26129 Oldenburg, Germany, Rainer.Huber@HoerTech.de)

This contribution presents an overview of basic approaches for predicting the perceived sound quality of hearing aids and hearing aid algorithms using auditory processing models for hearing impaired. Comparison-based concepts will be considered in particular. The main characteristic of these concepts is the comparison of internal representations (the outputs of the auditory models) of a test and a reference signal. While this approach is straight-forward and has proven to be successful for the prediction of sound quality of lossy speech and audio processing systems perceived by normalhearing listeners, the requirement of a reference representing the optimal quality can pose a problem in the case of hearing aids and hearing-impaired listeners. The potential and limitations of comparison-based approaches will be illustrated by example results from different studies obtained with an extended version of the perceptual audio quality model PEMO-Q. 


\section{Invited Papers}

6:20

1pEAc7. Real-world effectiveness of directional microphone hearing aids. Ruth Bentler (University of Iowa, 250 Hawkins Drive, Wendell Johnson Clinic, Iowa City, IA 52242, USA, ruth-bentler@uiowa.edu)

Single, dual, triple, and array microphone designs that are static, automatic and/or adaptive are now available in marketed hearing aids. The cost of designing and producing these more sophisticated systems is significant; yet, studies of user benefit are often equivocal, particular those involving self-report or field-obtained measures. In a series of studies we have attempted to characterize (model) the function of a number of these designs in simple and complex environments in an effort to explain the results obtain in both laboratory and field studies. Rather than directivity limitations, factors such as internal noise, loudness preference and visual cues have all been implicated in the limited user benefit, and will be discussed in this presentation.

\section{6:40}

1pEAc8. Whispering in your ear: a recent history of subminiature transducers. Daniel M. Warren (Knowles Electronics, 1151 Maplewood Dr, Itasca, IL 60143, USA, daniel.warren@knowles.com)

For 50 years, the history of hearing aid transducer development could be read in a simple photograph and timeline, where the size of the transducer was inversely correlated to the year it was developed. This size curve corresponds to hearing aid development over the same time period, progressing from body-worn aids in the 1940s to large behind-the-ear (BTE), smaller BTE's, in-the-ear (ITE), and successively deeper in- the-canal (ITC) devices through the 1990s. Although size still matters, in the last decade there has been less effort in reducing the size of hearing aids and more on increasing features, usability, and comfort. Development of hearing aid transducers has become similarly multidimensional. This paper charts the progress from the linear smaller-is-better design model to lower vibration, higher acoustic output, lower current draw, reduced radio frequency interference, wider bandwidth, and other multifaceted challenges of modern hearing aid transducer design.

\section{Contributed Papers}

\section{7:00}

1pEAc9. High sound quality and concha headphones: where are the limitations? Lola Blanchard (Bang\&Olufsen ICEpower/DTU, Gl. Lundtoftevej 1b, st., 2800 Lyngby, Denmark, lob@bang-olufsen.dk)

Concha headphones (the small type of headphone that rests at the entrance of the ear canal) are the most popular type of headphones for everyday use. However, the sound quality produced by even "high quality" concha headphones is rather poor. This paper gives an overview of the different factors that affect the quality of this type of headphones, describes the measurement difficulties, and presents a model. The leakage between the headphone and the ear changes every time the headphone is inserted in the ear. This alters the perceived sound quality, especially at low frequencies, and makes measurements very difficult. Moreover, because of energy efficiency considerations, a typical transducer design is prone to nonlinear behaviour, and a high distortion rate can be measured for most concha headphones. The measured transducer parameters and leakage have been implemented in a model realised in Simulink. The simulation model, which makes it possible to study the influence of the various parameters including the uncontrolled leakage, is the first step towards developing a compensation technique.

$$
7: 20
$$

1pEAc10. A two-stage binaural speech enhancement approach for hearing aids with preserving binaural benefits in noisy environments. Junfeng Li (Japan Advanced Institute of Science and Technology, 1-1,
Asahidai, Nomi, 923-1292 Ishikawa, Japan, junfeng@jaist.ac.jp),Shuichi Sakamoto (R.I.E.C., Tohoku University, 2-1, Katahira, Aoba-ku, 980-8577 Sendai, Japan, saka@ais.riec.tohoku.ac.jp), Satoshi Hongo (Faculty of Design and Computer Applications, Miyagi National College of Technology, 48, Nodayama, Medeshima Shiote, 981-1239 Natori, Japan, hongo@miyagi-ct.ac.jp), Masato Akagi (Japan Advanced Institute of Science and Technology, 1-1, Asahidai, Nomi, 923-1292 Sendai, Japan, akagi@jaist.ac.jp), Yôiti Suzuki (R.I.E.C., Tohoku University, 2-1, Katahira, Aoba-ku, 980-8577 Sendai, Japan, yoh@ais.riec.tohoku.ac.jp)

Speech enhancement is one of the most crucial functions, if not the most, in hearing aids, as hearing impaired people have great difficulty in understanding speech in noisy environments. In this paper, we propose a two-stage binaural speech enhancement approach for hearing aids, which consists of interference estimation by pre-trained adaptive filters and speech enhancement using the Wiener filters. Main attention is then paid to the theoretical analysis of this system and the experimental comparisons with the traditional binaural speech enhancement approaches. The comparisons are conducted with the following two considerations: interference suppression performance and the ability in preserving binaural cues which give birth to listener's own "binaural gain." We finally give the general discussion on this proposed binaural speech enhancement algorithm from the viewpoints of theory, through implementation, to evaluation. 


\title{
Session 1pEAd
}

\section{Engineering Acoustics, Underwater Acoustics, and ECUA: Sonar Transducer Design and Modeling I}

\author{
John B. Blottman, Chair \\ Naval Undersea Warfare Center, Division Newport, 1176 Howell Street, Newport, RI 02841-1708, USA
}

Invited Papers

$5: 40$

1pEAd1. Advanced application-motivated design techniques for high performance piezocomposite underwater sonar transducers and arrays. Sandy Cochran (Institute for Medical Science and Technology, University of Dundee, Wilson House, 1 Wurzburg Loan, DD2 1FD Dundee, UK, s.cochran@dundee.ac.uk), Christine E. Demore (Institute for Medical Science and Technology, University of Dundee, Wilson House, 1 Wurzburg Loan, DD2 1FD Dundee, UK, c.demore@dundee.ac.uk), Pablo Marin (Piezo Composite Transducers Ltd, Aberdeen Science Park, Balgownie Drive, Bridge of Don, AB22 8GU Aberdeen, UK, pablo.marin@pct-ltd.co.uk), Richard Marsh (Tritech International Ltd, Peregrine Road, Westhill Business Park, Westhill, AB32 6LJ Aberdeen, UK, richard-marsh@tritech.co.uk), Keith Mayne (Piezo Composite Transducers Ltd, Aberdeen Science Park, Balgownie Drive, Bridge of Don, AB22 8GU Aberdeen, UK, keith.mayne@pct-ltd.co.uk), Graeme McRobbie (University of the West of Scotland, High Street, PA1 2BE Paisley, UK, graeme.mcrobbie@uws.ac.uk), Michelle Wallace (Doosan Babcock Energy, Porterfield Road, PA4 8DJ Renfrew, UK, mwallace@ doosanbabcock.com), Mark P. Walsh (Piezo Composite Transducers Ltd, Aberdeen Science Park, Balgownie Drive, Bridge of Don, AB22 8GU Aberdeen, UK, mark.walsh@pct-ltd.co.uk), Richard Wright (Tritech International Ltd, Peregrine Road, Westhill Business Park, Westhill, AB32 6LJ Aberdeen, UK, dick-wright @ tritech.co.uk)

Developments in areas as diverse as advanced piezoelectric materials and low cost digital electronics continue to offer new possibilities to the designer of high performance underwater sonar transducers and arrays. Many of these are likely to be realised with complex composite materials comprising a polymer matrix with rods of piezoelectric ceramic such as PZT 5H or crystal such as PMN-PT which require advanced design techniques. This paper first briefly reviews relevant capabilities of conventional equivalent circuit and wave equation transducer models, and the composite homogenisation technique of Smith and Auld. The increasing possibilities offered by finite element analysis (FEA) running on relatively low-cost computing hardware are also considered. Whichever design technique is adopted, accurate material properties are crucial and this is reported through reference to a range of piezoelectric and passive materials. The potential variation of such properties across the operating temperature range of underwater transducers is also considered, illustrated with experimental measurements. As understanding of high performance transducer structures grows, future possibilities also become evident, such as the 3-1 connectivity multilayer composite. The paper is therefore completed with an outline of these complex structures and a stochastic mathematical technique that can be used to optimise their designs automatically.

\section{6:00}

1pEAd2. Leveraged motion underwater sound piston transducers. John Butler (Image Acoustics, Inc., 97 Elm Street, Cohasset, MA 02025, USA, jbutler@imageacoustics.com), Alexander L. Butler (Image Acoustics, Inc., 97 Elm Street, Cohasset, MA 02025, USA, abutler@imageacoustics.com)

Flextensional transducers use leveraged shell motion to produce enhanced acoustic radiation from compact sources, which are often used in low frequency underwater sound applications. The most commonly used flextensional is the Class IV where an oval shell is driven along its major axis by a stack of piezoelectric ceramic elements. We present here a review of a new class of leveraged transducers in which the shell motion drives pistons attached to the shell at the location of maximum magnified motion. Because of the uniform motion of the pistons, greater source strength and radiation load is attained compared to the tapered motion of flextensional shells. The leveraged motion typically yields a motion magnification of approximately three and an impedance load magnification of approximately nine, resulting in reasonably low $\mathrm{Q}$ for a compact low frequency transducer. Various designs are presented and illustrated including one in which the piezoelectric drive simultaneously acts as a tail mass and one which operates in a high impedance velocity control mode under array conditions. Lumped models as well as FEA models and measured results are presented to illustrate the concept and performance of PZT and PMN-PT driven designs. [Work supported in part by ONR.]

6:20

1pEAd3. Electro-active polymer transduction for distributed netted sensing. Dennis F. Jones (Defence R\&D Canada - Atlantic, P.O. Box 1012, Dartmouth, NS B2Y 3Z7, Canada, dennis.jones@drdc-rddc.gc.ca), John B. Blottman (Naval Undersea Warfare Center, Division Newport, 1176 Howell Street, Newport, RI 02841-1708, USA, blottmanjb@npt.nuwc.navy.mil), Roger T. Richards (Naval Undersea Warfare Center, Division Newport, 1176 Howell Street, Newport, RI 02841-1708, USA, richardsrt@npt.nuwc.navy.mil)

The Naval Undersea Warfare Center in Newport, RI is developing an electro-active polymer-based sensing node for use in a persistent distributed underwater surveillance system. Persistence demands extreme energy conservation measures. The node will resemble a jellyfish in form, complete with tentacles housing a volumetric array of light-weight piezoelectric polyvinylidene fluoride trifluoroethylene (PVDF-TrFE) copolymer cylindrical hydrophones designed for an ultra-low power acoustic receiver. Each cylinder has con- 
ductive silver electrodes on the inside and outside surfaces, a length of $2.5 \mathrm{~cm}$, an outside diameter of $11 \mathrm{~mm}$, a $1 \mathrm{~mm}$ wall thickness, and a mass less than $2 \mathrm{~g}$. Operating in the hydrostatic mode, the hydrophone sensitivity is typically $-195 \mathrm{~dB} / / 1 \mathrm{~V} / \mu \mathrm{Pa}$ and is stable with both hydrostatic pressure (50 to $1000 \mathrm{psi})$ and temperature $\left(-1\right.$ to $\left.35^{\circ} \mathrm{C}\right)$. [Work supported by the Office of Naval Research.]

\title{
$6: 40$
}

1pEAd4. Magnetostrictive compacted sonar transducer design. Stephen C. Butler (Naval Undersea Warfare Center, 1176 Howell St, Newport, RI 02841, USA, butlersc@npt.nuwc.navy.mil), Julie Slaughter (Etrema Products, Inc., 2500 N. Loop Drive, Ames, IA 50010, USA, julie.slaughter@etrema.com)

A compact modular high power magnetostrictive sound source that is capable of producing scanning Cardioid beams every 45 degrees in the horizontal plane and operates over one octave has been fabricated. The device consists of 8 transducer elements in the form of a ring that radiates acoustic energy radially outward. This design uses the operation principles of a peizoceramic transducer of similar design [J. L. Butler and A. L. Butler, J. Acoust. Soc. Am. 119, 3409 (2006)], which operated in a higher frequency band. The sound source can be driven to form omni-directional, dipole, and quadrupole beam patterns. By combining the measured electrical drive and acoustic pressure amplitude and phase coefficients of these beam patterns a narrower type Cardioid beam pattern is generated. The transducer elements are of a tonpilz type vibrator consisting of magnetostrictive Terfenol-D drive rods sandwiched between radiating head mass and a tail mass that is common to all elements with tie bolts consolidating the parts together. The Terfenol-D rods are interlaced with rare earth magnets to provide the DC magnetic bias field for the AC drive field provided by a coil. FEA models using ATILA and COMSOL are used for structural and magnetic analysis predictions of the device. [Work supported by ONR.]

$$
\text { 7:00 }
$$

1pEAd5. Broadband tonpilz transducers based on single crystal relaxor ferroelectrics: Design and modeling. Richard J. Meyer (The Applied Research Laboratory/ The Pennsylvania State University, P.O. Box 30, State College, PA 16801, USA, rmeyer@psu.edu), Douglas C. Markley (The Applied Research Laboratory/ The Pennsylvania State University, P.O. Box 30, State College, PA 16801, USA, dcm13@psu.edu)

Very broad bandwidth transduction capability has been made possible by high coupling piezoelectric single crystal materials. Recent work has shown that PMN-PT based tonpilz elements with a single resonance have a device electromechanical coupling coefficient greater than 0.86 and can easily achieve more than two octaves of source level bandwidth. Implementing single crystal based tonpilz elements reduces array packaging volume and weight while maintaining source level. In addition, high coupling projectors reduce transmit system complexity when compared to traditional materials. This paper will describe approaches to creating broadband SONAR projectors using single crystal materials. Crystal anisotropy plays a key role in transducer design. This anisotropy requires final designs to be modeled in three dimensions. ATILA finite element code was used to capture all of the modes of vibration and performance predictions. Motor section geometries and material compositions will be compared. The model predictions will also be compared to measured data.

AMPHI MAILLOT, 1:00 TO 4:00 P.M.

\section{Session 1pMUa}

\section{Musical Acoustics: Acoustic Measurements on Wind Instruments I}

\author{
Noam Amir, Cochair \\ Tel Aviv University, Dept. of Communications Disorders, Sheba Medical Center, Tel Hashomer, 52621, Israel \\ David B. Sharp, Cochair \\ Acoustics Research Group, DDEM, MCT Faculty, Open University, Walton Hall, Milton Keynes, MK7 6AA, UK
}

\section{Invited Papers}

\section{1:00}

1pMUa1. A new impedance sensor for wind instruments. Jean-Pierre Dalmont (Laboratoire d'Acoustique de l'Université du Maine, Avenue Olivier Messiaen, 72085 Le Mans, France, Jean-Pierre.Dalmont@univ-lemans.fr), Jean Christophe Le Roux (CTTM, 20, rue Thales de Milet, 72000 Le Mans, France, jcleroux@cttm-lemans.com)

Our aim was to build a low cost but accurate portable impedance sensor. The adopted technique is the one using a source with a back cavity. A first elctret microphone on the front of the source measures the pressure at the input of the instrument weather a second one measures the pressure in the cavity, which is proportional to the volume flow supplied by the source. By choosing a sufficiently small sealed cavity and a small piezoelectric source the system do not exhibit any cut-off frequency in the measurement range. The calibration is then simplified and only few parameters are needed to model the three complex calibration functions. This calibration is performed with three nonresonant calibration loads. Moreover, it is shown that the geometry of the sensor being known, the measurement with a rigid wall is sufficient to calibrate the sensor. Results show that it is possible to achieve a measurement with an accuracy lower than $1 \mathrm{~dB}$ in the range $50-4000 \mathrm{~Hz}$ 
1pMUa2. Input impedance measurements of conical acoustic systems using the two-microphone technique. Antoine Lefebvre (Schulich School of Music, McGill University, 555 Sherbrooke Street West, Montreal, QC H3A 1E3, Canada, antoine.lefebvre2@mail.mcgill.ca), Gary Scavone (Schulich School of Music, McGill University, 555 Sherbrooke Street West, Montreal, QC H3A 1E3, Canada, gary@music.mcgill.ca)

The two-microphone technique with a broadband excitation has been used for the measurement of various objects including straight conical waveguides and alto saxophones. An improved procedure has been developed whereby the noise excitation signal is pre-filtered by the inverse frequency response of the system under consideration. This approach helps reduce distortion and improves the SNR of the measurement. Results with and without pre-filtering are compared for different fingerings of an alto saxophone. The input impedance of a straight conical waveguide is compared with theory and discrepancies are analyzed. The input impedance of saxophones are evaluated with the transmission matrix approach and compared with measurements. A software environment for efficient comparison and analysis of measurement data with theoretical calculations is presented. We also propose a framework for sharing raw measurement data among researchers to allow comparison of results obtained with different apparatus on similar objects to better quantify their accuracy.

\section{Contributed Paper}

\section{$1: 40$}

1pMUa3. Improved precision in acoustic impedance measurements by using calibration loads without resonances. Paul Dickens (University of New South Wales, Music Acoustics, School of Physics, NSW 2052 Sydney, Australia, PDickens@resmed.com.au), John Smith (University of New South Wales, Music Acoustics, School of Physics, NSW 2052 Sydney, Australia, john.smith@unsw.edu.au), Joe Wolfe (University of New South Wales, Music Acoustics, School of Physics, NSW 2052 Sydney, Australia, J.Wolfe@unsw.edu.au)

Although the input impedance of a one-dimensional waveguide is simply defined by the ratio of pressure to volume flow measured at its input, the deficiencies of available transducers require a more complicated approach for precise measurements. These can involve multiple transducers at points within an impedance head connected to the measured load. However, reso- nances and/or singularities during calibration and/or measurement often limit the precision of acoustic impedance spectra. This paper reviews and compares several established techniques, and describes a technique that incorporates three features that can considerably improve precision. The first feature involves minimising the problems due to resonances by calibrating the instrument using up to three different acoustic reference impedances that do not themselves exhibit resonances: an acoustically infinite waveguide, an acoustic open circuit and an acoustically infinite flange. The second feature involves using multiple pressure transducers to reduce the effects of measurement singularities. The third involves iteratively tailoring the spectrum of the stimulus signal to control the distribution of errors across the particular measured impedance spectrum. Examples are given of the performance of the technique on simple cylindrical waveguides and some wind instruments, including the saxophone and clarinet.

\section{Invited Paper}

\section{2:00}

1pMUa4. A practical way to measure intonation quality of woodwind instruments using standard equipment without custom made adapters. Wilfried Kausel (Inst. f. Wiener Klangstil, Univ. f. Music, Anton von Webernplatz 1, A-1030 Vienna, Austria, kausel@mdw.ac.at), Helmut Kuehnelt (Inst. f. Wiener Klangstil, Univ. f. Music, Anton von Webernplatz 1, A-1030 Vienna, Austria, Helmut.Kuehnelt@arsenal.ac.at)

Woodwinds are not much different from brass instruments in how intonation is determined by air column resonances. Nevertheless, it is easier to measure input impedance of brasses because the input cross-section at the mouthpiece rim is circular, plane and big enough to be easily coupled to standard measuring heads. In reed instruments the input cross-section is neither flat nor circular nor well defined. Flute instruments not even have any definable input cross-section as this is controlled by the player's lower lip. On top of that, flutes are played at an open end where some coupled impedance considerably influenced by the nearby environment like lips, mouth, and face of the player has to be taken into account. Existing approaches to measure input impedance of woodwind instruments usually require custom made adaptors optimised to yield nearly accurate intonation results for a specific instrument and playing range only. The proposed new approach is to separate instrument head or mouthpiece from the body and measure both parts from their cylindrical ends. During the measurement a natural playing condition can be simulated at the now available end. Both measurements can be assembled computationally yielding useful intonation results as will be demonstrated for flutes and saxophones.

\section{Contributed Paper}

\section{2:20}

1pMUa5. Measured and calculated sounding frequencies of pipes coupled with free reeds. James Cottingham (Coe College, 1220 First Avenue NE, Cedar Rapids, IA 52402, USA, jcotting@coe.edu), Eric A. Dieckman (Truman State Univ., Physics Dept., Kirksville, MO 63501, USA, ead603@truman.edu)

The Asian free-reed mouth organs incorporate approximately symmetric free reeds coupled to pipe resonators. Previous research has shown that the reeds in these instruments behave as "blown-open" reeds in which the playing frequency is above both the natural frequency of the reed and the first peak of the measured impedance curve. Measurements of the input impedance have been made for a variety of these instruments. Detailed calculations of input impedance have also been made using transmission matrices, taking into account the position of the reed along the pipe, tuning slots, finger holes, and nonuniform cross sections. These calculations are in good agreement with the measured impedances of the same instruments. If the reed is treated as a damped, driven harmonic oscillator, the sounding frequencies of these reed-pipes can be predicted using a phase relation between the reed vibration and the phase of the complex impedance. [Research supported by National Science Foundation REU Grant PHY-0649007.] 


\section{Invited Papers}

\section{2:40}

1pMUa6. Calibration of apparatus for wave separation in wind instruments. Giovanni De Sanctis (Queen's University Belfast, University Road, BT7 1NN Belfast, UK, gdesanctis01@qub.ac.uk), Maarten Van Walstijn (Sonic Arts Research Centre, Queen's University Belfast, BT7 1NN Belfast, UK, m.vanwalstijn@qub.ac.uk)

Knowledge of the forward and backward traveling waves inside the bore of a wind instrument is a useful starting point for the estimation of its playing parameters. These are mainly parameters describing the mouthpiece embouchure and the states of the tone holes. Due to the highly nonlinear behaviour of the excitation mechanism, a precise estimation is needed. The separation method under investigation relies on a model inversion, starting from the pressure measured by three microphones. For this reason the estimation is very sensitive to the relative positions of the microphones, and the latter must therefore be known very precisely. A geometrical measurement would not be reliable because of construction tolerances, including uncertainties about the acoustic centers of the microphones; therefore the distances are gathered through audio measurements. A number of approaches to such a calibration of the measurement system have been investigated, using a purposely constructed apparatus which will also be described.

\section{3:00}

1pMUa7. Acoustic pulse reflectometry for the measurement of horn crooks. Jonathan Kemp (Edinburgh University, 4201 JCMB, Kings Buildings, Mayfield Road, EH9 3JZ Edinburgh, UK, jonathan@ph.ed.ac.uk), John Chick (Edinburgh University, 4201 JCMB, Kings Buildings, Mayfield Road, EH9 3JZ Edinburgh, UK, john.chick@ed.ac.uk), Murray Campbell (Edinburgh University, 4201 JCMB, Kings Buildings, Mayfield Road, EH9 3JZ Edinburgh, UK, dmc@ph.ed.ac.uk), Darren Hendrie (Edinburgh University, 4201 JCMB, Kings Buildings, Mayfield Road, EH9 3JZ Edinburgh, UK, darrenhendrie@ hotmail.com)

The echo-based technique of acoustic pulse reflectometry can be used to measure tubular objects to determine the bore profile. In this paper, measurements of historic orchestral horn crooks are presented showing how the technique can help to determine the method of construction of historic crooks and can provide valuable information to manufacturers of reproduction period instruments. Comparison of the bore profile measured by pulse reflectometry and the known exit radius of the crook can be used to determine the presence of leaks. The technique is shown to be sensitive enough to find a leak in a horn crook that behaves reasonably under playing conditions and was not suspected of having a leak prior to testing.

\section{Contributed Papers}

3:20

1pMUa8. Industrial applications of acoustic pulse reflectometry. Noam Amir (Tel Aviv University, Dept. of Communications Disorders, Sheba Medical Center, 52621 Tel Hashomer, Israel, noama@post.tau.ac.il), Oded Barzelay (AcousticEye LTD., 4 Harechev st., 67771 Tel Aviv, Israel, oded@acousticeye.com), Tal Pechter (AcousticEye LTD., 4 Harechev st., 67771 Tel Aviv, Israel, tal@acousticeye.com)

Acoustic pulse reflectometry (APR) has been used extensively in the study of wind instruments, for measuring input impedance, bore reconstruction, and fault detection. Industrial applications have often been mentioned in the literature, though they have barely been exploited. Academic APR systems are extremely bulky, which limits their industrial use severely. Two requirements from such an industrial system are that the source tubes be as short as possible, and that they be able to measure long objects, often much longer than most musical instruments, e.g., condenser tubes which are at least $8 \mathrm{~m}$ long. This creates difficult calibration problems and difficulties in obtaining sufficient SNR levels, especially at low frequencies. In this paper we describe a system with the necessary adaptations for industrial use. We demonstrate the problems that are encountered, and introduce solutions which we have implemented. Modifications to the deconvolution phase improve low frequency SNR, which is further improved using nonlinear filtering. Adaptive filters are then used to discriminate between a faulty and intact system. When faults are detected, they are identified using several identification algorithms based on matched filtering and local inverse scattering.

\section{$3: 40$}

1pMUa9. Influence of the bocal on the sound of the bassoon. Johannes Baumgart (Technische Universität Dresden, Institute for Aerospace Engineering, $01062 \quad$ Dresden, Germany, johannes.baumgart@tu-dresden.de), Timo Grothe (Technische Universität Dresden, Institute for Aerospace Engineering, 01062 Dresden, Germany, timo.grothe@tu-dresden.de), Roger Grundmann (Technische Universität Dresden, Institute for Aerospace Engineering, 01062 Dresden, Germany, roger.grundmann@tu-dresden.de)

The bocal of a bassoon is characterized by geometrical parameters such as the bore, the outer contour and the wall-thickness as well as material parameters and the coating. Professional bassoonists select bocals extremely carefully and report influences on the playability and sound colour. In order to understand the underlying effects, several experiments were performed with bocals of the same bore bent in two different shapes and with two different materials. Images of a high-speed camera show a periodic motion of the bocal tip while the instrument is artificially blown. A modal analysis of the bocals was performed experimentally and numerically to investigate the dynamic behaviour of the structure. The first bending mode of the bocals is around $70 \mathrm{~Hz}$. While the material properties shift the modal frequencies less than $2 \%$, the shift due to the geometry is more than $10 \%$. The experimental program included playing experiments with professional bassoonists. The setup was fixed and the players used the same reed. For each player, slight shifts in the sound spectra could be found due to the use of different bocals. The harmonic structure showed changes in the frequency bands where the bending modes differ. 


\title{
Session 1pMUb
}

\author{
Musical Acoustics: Edge Tone and Flue Pipes I \\ Shigeru Yoshikawa, Cochair \\ Dept. of Acoustic Design, Kyushu University, Fukuoka 815-8540, Japan \\ Judit Angster, Cochair \\ Fraunhofer Institute for Building Physics, Nobelstrasse 12, Stuttgart, 70569, Germany
}

Invited Papers

$4: 20$

1pMUb1. Influence of the geometrical parameters in flue instruments on the vorticity modulation near the separation points of the jet. François Blanc (Institut Jean Le Rond d'Alembert/LAM (UPMC/CNRS/Ministère Culture), 11, rue de Lourmel, 75015 Paris, France, blanc@lam.jussieu.fr), Pierre-Yves Lagrée (Institut Jean le Rond d'Alembert - FCIH team, 4 place Jussieu, 75006 Paris, France, pyl@ ccr.jussieu.fr), Patricio De La Cuadra (Centro de Investigación en Tecnologias de Audio (CITA), Universidad Católica de Chile, Alameda 340, Oficina 13, Casilla 114-D Santiago, Chile, pcuadra@uc.cl), Benoit Fabre (Institut Jean Le Rond d'Alembert/LAM (UPMC/CNRS/Ministère Culture), 11, rue de Lourmel, 75015 Paris, France, fabreb@ccr.jussieu.fr)

In the making of the recorder, great care is given to the shape of the chamfers at the exit of the channel. Makers insist on the need of sharp chamfers, for a good tone quality. Recorders made with rounded chamfers present a noisy sound. On the other hand, in the flute, the channel is made by the lips of the musician, defining a rounder geometry. Previous work show that variations of the geometry of the exit of the channel has little influence on the velocity profile of the jet and on the position of the separation points. Thus, variations of the geometry are expected to affect mainly the interaction between the air jet and the acoustic field. The paper will present measurements together with direct Navier Stokes calculations for different geometrical configurations of a jet emerging in a transverse oscillating velocity field. We investigate the modulation of the vorticity of the jet shear layers by the acoustical field, in the vicinity of the separation points, in an attempt to develop a model that allows to predict the influence of the geometry.

\section{4:40}

1pMUb2. Mode transition of a flue organ pipe. Seiji Adachi (Fraunhofer Institute for Building Physics, Nobelstrasse 12, 70569 Stuttgart, Germany, seiji.adachi@ibp.fraunhofer.de), Judit Angster (Fraunhofer Institute for Building Physics, Nobelstrasse 12, 70569 Stuttgart, Germany, an@ibp.fhg.de), Andras Miklos (Fraunhofer Institute for Building Physics, Nobelstrasse 12, 70569 Stuttgart, Germany, an@ibp.fhg.de)

A flue organ pipe can be excited in various acoustic modes by changing the air pressure supplied to it. This research aims to reconstruct this behavior from the result of numerical flow simulation of a jet deflected by sound and from physical modeling simulation of the total sound production system. In the numerical flow simulation, motion of the jet in the pipe mouth was replicated: The jet emerges from a flue and travels in a space where the air oscillates laterally to the jet direction. As a result, the jet oscillates with the same frequency as the oscillation of the air i.e., sound. From the flow simulation, a model of the jet deflection was developed. This model was then used as a model of the sound source in the physical modeling simulation where not only the sound source but the resonance of the pipe is also modeled in a set of differential equations with delayed feedback. The mode transition observed in the physical modeling simulation was discussed by comparing with that experimentally observed.

\section{5:00}

1pMUb3. Frequency and phase characteristics of the edge tone. György Paál (Budapest University of Technology and Economics, P.O. Box 91, 1521 Budapest, Hungary, paal@hds.bme.hu), István Vaik (Budapest University of Technology and Economics, P.O. Box 91, 1521 Budapest, Hungary, vaik@hds.bme.hu)

Extensive experimental and computational work was performed to characterize the edge tone phenomenon. The dependence of the oscillating frequency on some of the main parameters of the configuration including the nozzle-wedge distance and the profile and the mean velocity of the jet was measured. The frequency was obtained from the FFT of a pressure sensor signal. The measured and computed frequencies are compared and show a very good agreement. For both the CFD simulations and the experiments the phase of the oscillation and the pressure at specified points on the wedge are correlated. For the experiments the phase of the flow oscillation is determined with the help of flow visualization pictures obtained by a high speed digital camera. From the CFD results spatial distribution of acoustical sources are also observed and correlated with pressure distribution on the wedge and with the phase of the oscillating flow. Some aspects of the initial transients are also presented. 
1pMUb4. Influence of the edge tone (mouth tone) on the sound of flue organ pipes as a function of pipe scaling. Judit Angster (Fraunhofer Institute for Building Physics, Nobelstrasse 12, 70569 Stuttgart, Germany, an@ibp.fhg.de), Andras Miklos (Steinbeis Transfer Center Applied Acoustics, Landauer Str. 24, D-70499 Stuttgart, Germany, Andreas.Miklos@urz.uni-heidelberg.de)

The sound quality of an organ pipe is mainly influenced by the attack transients. This onset is first dominated by the edge tone, while later the pipe resonator will play a more important role. To understand the physics of a flue organ pipe, it is necessary to analyse the edge tone, the acoustic properties of the pipe resonator, the attack transient and the stationary sound of the pipe. Several pipe ranks have been investigated in the anechoic room of the Fraunhofer IBP: Nachthorn, Diapason, Gamba, Octave, Flute, Geigenprinzipal, Salizional. By the evaluation all physical effects contributing to the production of sound were taken into account. In the present paper about the physical effect of the edge tone on the production of the pipe sound will be reported.

\title{
$5: 40$
}

1pMUb5. Development of an adjustable pipe-foot model of a labial organ pipe. Hubert Außerlechner (Fraunhofer Institute for Building Physics, Nobelstrasse 12, 70569 Stuttgart, Germany, hubert.ausserlechner@ibp.fraunhofer.de), Judit Angster (Fraunhofer Institute for Building Physics, Nobelstrasse 12, 70569 Stuttgart, Germany, an@ibp.fhg.de), Andras Miklos (Steinbeis Transfer Center Applied Acoustics, Landauer Str. 24, D-70499 Stuttgart, Germany, Andreas.Miklos@urz.uni-heidelberg.de)

In order to understand the physical processes involved in the build-up of the sound signal in a pipe (in our case a labial organ pipe) a pipe-foot model has been developed, with which the main important parameters, such as positions of the lower- and upper-labium, the wind pressure in the foot and the width of the flue can be adjusted. Moreover, different types of languids and pipe bodies (resonators) can be attached to the model. For the reason of corresponding to a real metal organ pipe these parts of the model are made of "organ metal." The reproducibility of measurements is provided by the micrometer screws applied for the adjustments. Flow and edge-tone measurements were carried out with the help of this model. A comparison with real organ pipes gives some indications for the range of the parameters. Because of the flexible adjustments and the large range of values of every parameter, it is possible to find different ranges with varying parameter settings for the occurrence of the edge-tone. The analysis of these measurement results shows the dependency of the edge-tone of the adjusted parameters.

\section{Session 1pMUc}

\section{Musical Acoustics and Psychological and Physiological Acoustics: Acoustics and Psychoacoustics of Pipe Organs}

\author{
Jonas Braasch, Cochair \\ Rensselaer Polytechnic Institute, Greene Bldg., 110 8th St., Troy, NY 12180, USA \\ David M. Howard, Cochair \\ University of York, Department of Electronics, Heslington, York, YO10 5DD, UK
}

\section{Invited Papers}

6:00

1pMUc1. Voicing documentation of a pipe organ. Christophe D'Alessandro (LIMSI-CNRS, B.P. 133, 91403 Orsay, France, cda @limsi.fr)

The paper begins with a brief presentation on the Parisian organ landscape, and then focuses on sound documentation of the historical organ of Sainte Elisabeth (Suret 1853, 3 keyboards and pedal, 40 stops, about 2500 pipes). Calibrated recordings of all the notes have been performed inside and outside the organ case, using several microphone positions. The methodology proposed by Pollard ( $\mathrm{J}$. Acoust. Soc. Am. 106(1), July 1999, 360-370) is extended and applied to sound analysis for all the notes of the instrument. The analogies between the proposed sound description (or "voicing quality," described in terms of sharpness, loudness, tristimulus coordinates, transient building, and spectral centre of gravity) and human voice quality description are discussed. Both voicing of individual pipes and voicing of the instrument are analysed. "Voicing of the instrument" means voicing variations of the pipes for different notes or registers of a same stop, for different stops of a same division, and for different divisions of the instrument. This methodology may be applied to documentation and characterisation of the "style" of the instrument in terms of its acoustic signature, provided additional information on recording conditions and room acoustics are available. 
1pMUc2. Documenting the acoustics of the Silbermann pipe organ at the Catholic Cathedral in Dresden before and after the restoration of 2002. Christian Ahrens (Musikwissenschaftliches Institut, Ruhr-Universität Bochum, Universitätsstr. 150, 44780 Bochum, Germany, christian.ahrens@rub.de), Jonas Braasch (Rensselaer Polytechnic Institute, Greene Bldg., 110 8th St., Troy, NY 12180, USA, braasj@rpi.edu), Sebastian Schmidt (Institute of Communication Acoustics, Ruhr-Universität Bochum, IC 1/142, Universitätsstr. 150, 44780 Bochum, Germany, sebastian.schmidt@ rub.de)

Gottfried Silbermann's pipe organ at the Catholic Cathedral of the Saxonian Court in Dresden was, with 47 stops, the largest instrument he had ever built. The instrument was evacuated during World War II and luckily survived intact, while the church itself was badly damaged. The instrument received a more romantic sound ideal after its resurrection in 1971. The biggest deviation from the original concept of Silbermann was the change from church pitch $\left(\mathrm{A}_{4}=415 \mathrm{~Hz}\right)$ to chamber pitch $\left(\mathrm{A}_{4}=440 \mathrm{~Hz}\right)$. In 2002 , the instrument was renovated again with the goal to restore the instrument to the original design of 1755 . The work was commissioned to two local organ builders, Kristian Wegscheider and Jehmlich Orgelbau, and the authors received the unique opportunity to measure the instrument before and after the restoration. During the measurements, the pipes of each stop were recorded in the near field in steps of a third. The acoustical comparison between each pipe before and after the restoration shows significant differences that match the informal observations of expert listeners. In general, the pipes received more energy in the higher partials and had a better attack response. Another interesting finding is the improvement of the pipes' formants after the restoration.

\section{Contributed Papers}

6:40

1pMUc3. The physical characteristics of mechanical pipe organs and how much they allow the organist to influence the initial transient. Jose Grossinho (R. Francisco da Silva Marques, n ${ }^{\circ}$, $2^{\circ}$ Dto, 2825-125 Caparica, Portugal, zefino@netcabo.pt)

It has been accepted by organists that one can change the timbre of the organ simply by varying the velocity of the key. Previous research (Nolle, 1941, 1992) shows clearly that there is a change in the initial transient of the sound with different pressure rise times (PRT) on the foot of the pipe. More recent research (Woolley, 2006) shows that in mechanical action organs there is no major difference in the velocity of the key when the organist thought there was. The velocity of opening of the pallet is one of the main aspects that influence the PRT. Calculations of the flexibility of mechanical parts from organs in Mafra, Portugal, were taken, as well as motion of the key, pressure on the pipe's foot and sound measurements on a model organ, using human touch on the key. Due to the pressure differences in the wind chest and the groove, it is difficult to control the pallet movement after it starts to open. The results show that there is a more efficient control of the velocity of opening of the pallet on more rigid actions, giving a better control to the organist over the timbre of the instrument.

\section{7:00}

1pMUc4. Very-low frequency range influence for free reed instruments physical modeling. Laurent P. Millot (IDEAT (University Paris 1), ENS Louis-Lumière, 7 allée du Promontoire, 93161 Noisy-le-grand, France, 1.millot@ens-louis-lumiere.fr)

Measurements of the inner and outer over-pressure for a diatonic harmonica were performed during blown, drawn, and bend notes notably. Using an energy analysis based on a nonregular spectral decomposition of the energy with only 10 frequency subbands, the IDS analysis, we have compared the relative weights of each subbands for both inner and outer pressure signals. This comparison underlines the key role played by the very low frequency range $(0-50 \mathrm{~Hz})$ and justifies a physical modeling without any waves occurrences but taking into account only acoustical flows descriptions. Within the presentation the audience will listen these phenomena and will access an explanation of the details of the physical modeling which has been used and which permits to refind chromatical playing on a diatonic harmonica, including the vocal tract player. We will also propose some clues to include a pipe in the modeling in order to study instruments like the sheng or the harmonium for instance. 


\title{
Session 1pNSa
}

\section{Noise, Physical Acoustics, and EURONOISE: Aeroacoustics I}

\author{
Philip J. Morris, Cochair \\ Penn State University, 233C Hammond Building, University Park, PA 16802, USA \\ Christophe Bailly, Cochair \\ Ecole Centrale de Lyon, 36, avenue Guy de Collongue, LMFA, Ecully, 69134, France
}

\section{Invited Papers}

\author{
1:00
}

1pNSa1. Jet noise from large-scale turbulent structures. Philip J. Morris (Penn State University, 233C Hammond Building, University Park, PA 16802, USA, pjm@psu.edu)

The generation sound by large-scale turbulent structures in jets, where the structures are convecting supersonically with respect to the ambient speed of sound, is well understood. The pressure pattern generated by the large-scale structures couples directly with the radiated sound field. An instability wave model for the development of the large-scale structures gives excellent predictions of both the near and far acoustic fields. However, when the structures are convecting subsonically, there is no general agreement as to how or even whether they generate any noise directly. Recent experimental evidence has shown a remarkable similarity between the jet noise spectra in the vicinity of the peak noise radiation direction for both subsonic and supersonic jets. This suggests that the large-scale structures do contribute directly to jet noise radiation at all jet operating conditions. In the present paper a model is developed for the noise generation mechanism. Using experimental measurements of the far field noise, the near field pressure pattern required to generate the noise can then be calculated. The question of whether this pattern is consistent with a reasonable physical model for the evolution of the largescale turbulent structures is then discussed.

\section{$1: 20$}

1pNSa2. Effect of jet temperature on jet noise: data and requirements for modeling. Krishnamurthy Viswanathan (The Boeing Company, M/S 67-ML, P.O. Box 3707, Seattle, WA 98124, USA, k.viswanathan@ boeing.com)

The modifications to the jet spectra caused by the heating of the jet have been the subject of experimental and theoretical research since the early 1970s. Early experimental data produced ambiguous and contradictory trends, which complicated the modeling efforts. Disparate theories have been proposed; however, no fundamental explanation or theory exists to date. First of all, a comprehensive experimental program has been carried out to establish the effect of temperature on jet noise. Detailed analyses indicate that in addition to the jet velocity $(\mathrm{Vj} / \mathrm{a})$, the jet temperature ratio, either stagnation or static, is an independent controlling parameter. The spectral shape at the lower radiation angles (measured from the jet inlet) is universal and independent of the jet Mach number and temperature. In the aft directions, the spectral shape is controlled by the jet temperature ratio, regardless of the jet velocity. These recently established trends have not been incorporated in any existing theory. The modeling of the effects of jet temperature and the suitability of the proposed formulations in existing theories are examined; the need for fresh thinking and the requirements for a good model are highlighted.

\section{Contributed Paper}

\section{$1: 40$}

1pNSa3. Correlations between the turbulent and the acoustic fields of a hot co-axial jet simulated by large-eddy simulation. Christophe Bogey (Ecole Centrale de Lyon, 36, avenue Guy de Collongue, LMFA, $69134 \quad$ Ecully, France, christophe.bogey@ec-lyon.fr), Francois Coiffet (Ecole Centrale de Lyon, 36, avenue Guy de Collongue, LMFA, 69134 Ecully, France, francois.coiffet@ec-lyon.fr)

Correlations between the turbulent and the acoustic fields of a highspeed hot co-axial jet are calculated, in order to study noise generation mechanisms by a causality method. The jet flow was computed by a largeeddy simulation using specific aeroacoustic schemes. The simulation also provided directly the near-presure field, which was then extrapolated in the far field by solving the linear acoustic equations. The causality method is applied using flow quantities such as the velocity components, density, and temperature in the jet and the far field pressure, in the temporal as well as in the spectral domains. To exhibit and characterize the regions of noise generation, we are focusing on the correlation peaks, and in particular their location and corresponding frequencies. Moreover, there is an attempt to link the noise generation with the jet turbulence properties including intermittency and convection velocity. For instance, the turbulence at the end of the primary potential core was found to show high correlations with the far field pressure and significant intermittency, in a similar way as in single-stream jets. 


\section{Invited Papers}

\section{2:00}

1pNSa4. Simulation of jets exhausting from chevron nozzles and their noise. Ali Uzun (Florida State University, School of Computational Science, 400 Dirac Science Library, Tallahassee, FL 32306, USA, uzun@scs.fsu.edu)

Environmental concerns and strict noise regulations around major airports have made jet noise a crucial problem in present day aeroacoustics research, as it is the jet engine exhaust that is responsible for much of the noise generation during aircraft takeoff. Chevron nozzles have drawn a lot of attention recently due to their noise reduction benefits. Chevrons typically reduce low frequency noise at aft angles while providing an increase in high frequency noise at broadside angles relative to the jet. The streamwise vorticity generated by the chevrons affects the jet shear layer mixing and thus is responsible for the decrease or increase in noise over certain frequency ranges. The ultimate goal in chevron design is to decrease low frequency noise as much as possible while preventing the increase in high frequency noise. In our research, we are performing high-fidelity numerical simulations of jets exhausting from chevron nozzles to gain a better understanding of chevron jet shear layer mixing. These simulations employ a large eddy simulation tool utilizing state-of-the-art numerical techniques. In this talk, representative results from our research will be presented and an assessment of our current prediction capability of chevron jet noise will be provided.

1pNSa5. Reduced-order models for jet noise. Ann P. Dowling (University of Cambridge, Department of Engineering, Trumpington Street, CB2 1PZ Cambridge, UK, apd1@cam.ac.uk), Mohammed Afsar (University of Cambridge, Department of Engineering, Trumpington Street, CB2 1PZ Cambridge, UK, ma357@cam.ac.uk), Sergey Karabasov (University of Cambridge, Whittle Laboratory, Department of Engineering, Madingley Road, CB3 0DY Cambridge, UK, sak36@eng.cam.ac.uk), Tom P. Hynes (University of Cambridge, Whittle Laboratory, Department of Engineering, Madingley Road, CB3 ODY Cambridge, UK, tph@eng.cam.ac.uk)

The research reported here leads to a simple prediction methodology based on a reduced-order model for jet noise. The approach is a hybrid one made up of three components. Each component uses modeling and numerical techniques optimised to suit a particular purpose. The propagation of noise to the far field is captured via a new method for solution of the adjoint linearised Euler equations, describing how sound emitted by the jet is modified by propagation through the time-averaged but spatially varying jet flowfield. The directivity of the quadrupole sources is more general than is usually assumed and their statistical properties are modeled based on a RANS solution for the jet: the cross-correlation of the turbulent quadrupoles is modelled as Gaussian with length and time parameters proportional to the local length and timescales from the RANS solution, The constants of proportionality are determined through comparison with correlations from LES and from experimental data. Hence the source model is determined entirely from near field data and the far-field sound is then predicted with no empirical constants. Comparison between this predicted noise and experimental data is very good, across a wide spectral range and even at angles close to the jet axis.

\section{Contributed Paper}

\section{2:40}

1pNSa6. Numerical study of the influence of temperature and microjets on the radiated noise for a subsonic jet. Maxime Huet (ONERA, BP 72-29 avenue de la Division Leclerc, 92322 Châtillon, France, Maxime.Huet@onera.fr), Gilles Rahier (ONERA, BP 72 - 29 avenue de la Division Leclerc, $92322 \quad$ Châtillon, France, Gilles.Rahier@onera.fr), François Vuillot (ONERA, BP 72 - 29 avenue de la Division Leclerc, 92322 Châtillon, France, francois.vuillot@onera.fr)

Jet noise remains the principal nuisance source for an aircraft at take-off conditions. A previous work [1] showed that using micro-jets could be effective to modify the turbulence development and thus decrease the radiated pressure. This micro-jets effect is numerically studied for two jets. LES simulations of a cold $(\mathrm{M}=0.9)$ and a hot $(\mathrm{M}=0.636)$ jets, with and with- out micro-jets, are computed and the acoustic post-processing is performed using the Ffowcs-Williams and Hawkings surface formulation. The temperature effect on the noise is analyzed for the reference computations and, with micro-jets, the noise reduction is compared to measurements for a similar configuration [1]. Aerodynamic comparisons with experiments give a good comparison, excepted a too short potential core and overestimated turbulent kinetic energy. The noise decrease with the higher temperature and the modifications of the spectral densities are well captured by the simulations. The micro-jets provide a reduction up to more than $2 \mathrm{~dB}$ for the best angles. Turbulence modification is still under investigation and results will be presented. [1] Castelain, T., Béra, J., Sunyach, M., and Juvé, D., "Effect of microjets on a high-subsonic jet. Parametric study of far-field noise reduction," 12th AIAA/CEAS Aeroacoustics Conference (2006).

\section{Invited Papers}

1pNSa7. On the separation of hydrodynamic and acoustic waves in linear free-shear flows. Anurag Agarwal (Institute of Sound and Vibration Research, University of Southampton, Highfield, SO17 1BJ Southampton, UK, aa2@isvr.soton.ac.uk), Gwenael Gabard (Institute of Sound and Vibration Research, University of Southampton, Highfield, SO17 1BJ Southampton, UK, gabard@soton.ac.uk)

The governing equations for sound propagation through free-shear flows, like jets and mixing layers, are the linearized Euler equations. These equations support both hydrodynamic and acoustic waves. For an aeroacoustician wishing to study the refraction effects of a sound source by shear flows, it is important to distinguish the acoustic solution from the hydrodynamic waves. Agarwal et al. (AIAA J., Vol. 42, No. 1, 2004) presented a technique to achieve this in the frequency domain. In this talk, we present a time-domain technique to separate the hydrodynamic and acoustic waves. The idea is to implement a filter that filters out only the acoustic wave solution from the linearized Euler equations. Some sample solutions are presented for two-dimensional free-shear flows and comparisons are made against known analytical solution for parallel flows and from solution obtained by approximate methods, which have a limited range of applicability. The advantage for the present technique is that it is applicable to arbitrary frequencies and flow profiles. 
1pNSa8. Turbulent flow noise around a zero-incidence airfoil. Olivier Marsden (Ecole Centrale de Lyon, 36, avenue Guy de Collongue, LMFA, 69134 Ecully, France, olivier.marsden@ec-lyon.fr), Christophe Bogey (Ecole Centrale de Lyon, 36, avenue Guy de Collongue, LMFA, 69134 Ecully, France, christophe.bogey@ec-lyon.fr), Christophe Bailly (Ecole Centrale de Lyon, 36, avenue Guy de Collongue, LMFA, 69134 Ecully, France, christophe.bailly@ec-lyon.fr)

A large eddy simulation (LES) of the flow around a NACA 0012 airfoil at zero incidence, at a chord-based Reynolds number of 500,000 and a Mach number of 0.22 , is presented. The aim is to show that high-order numerical schemes can successfully be used to perform direct acoustic computations of compressible transitional flow on curvilinear grids. At a Reynolds number of 500,000, the boundary layers around the airfoil transition from an initially laminar state to a turbulent state before reaching the trailing edge. Results obtained in the LES show a well-placed transition zone, and turbulence levels in the boundary layers in agreement with analytical developments as well as experimental data. Furthermore, the radiated acoustic field is determined directly by the LES, without the use of an acoustic analogy. Third-octave acoustic spectra are compared to experimental data, with deviations of around $3 \mathrm{~dB}$ per band exhibited.

\section{Contributed Paper}

3:40

1pNSa9. Preliminary screening of flow behavior around airfoils using a microphone phased array. Marcel C. Remillieux (Virginia Tech, Mechanical Engineering, 153 Durham 0238, Blacksburg, VA 24061, USA, mremilli@vt.edu), Ricardo A. Burdisso (Virginia Tech, Mechanical Engineering, 153 Durham 0238, Blacksburg, VA 24061, USA, rburdiss@vt.edu), William J. Devenport (Virginia Tech, Aerospace and Ocean Engineering, 224E Randolph Hall, Blacksburg, VA 24061, USA, devenport@vt.edu)

Recently, as a part of research projects for the National Renewable Energy Laboratory (NREL) and Sandia National Laboratories, several fullscale, wind-turbine airfoils were tested at various speeds (high Reynolds numbers) and angles of attack in the Virginia Tech Stability Wind Tunnel. This unique facility is aerodynamically closed and acoustically open, which allows better aerodynamic performance than a free jet facility, while maintaining comparable acoustic performance. Flow measurements consisted of monitoring the surface pressure around the airfoils with a distribution of pressure taps on the airfoil surface. Acoustic measurements were carried out with a microphone phased array. The phased-array data was post-processed to generate acoustic maps of the noise generated by the airfoils exposed to the flow as well as their noise spectra. In addition to the tests, twodimensional CFD computations of the flow field around the airfoils were carried out using a $\mathrm{k}-\epsilon$ turbulent model. The acoustic maps clearly show the noise sources generated by potential turbulent flow around the airfoils. The very good correlation between the acoustic maps and the flow characteristics of the airfoils, both numerical and experimental, allows for phased-array measurements to be used as a tool for preliminary screening of the flow behavior around a given airfoil.

4:00-4:20 Break

\section{Invited Paper}

1pNSa10. Experimental analysis of the wall aerodynamics and acoustic radiation of the trailing edge of an airfoil in subsonic flow. Cyrille Bonamy (Laboratoire d'Etudes Aérodynamiques (LEA), Université de Poitiers - ENSMA - CNRS, Bâtiment K, 40 Avenue du Recteur Pineau, 86022 Poitiers, France, cyrille.bonamy@lea.univ-poitiers.fr), Yves Gervais (Laboratoire d’Etudes Aérodynamiques (LEA), Université de Poitiers - ENSMA - CNRS, Bâtiment K, 40 Avenue du Recteur Pineau, 86022 Poitiers, France, yves.gervais@lea.univ-poitiers.fr)

This experimental study treats of the trailing edge noise mechanisms, especially broadband noise, resulting from the hydrodynamic wave diffraction on the sharp edge of a foil in flow, with the aim of improving its modeling. A detailed review of the dynamic behaviour of the phenomenon characteristic aerodynamic quantities (wall pressure and velocity fluctuations) has been made on a Naca 0012 airfoil and particular statistical data analysis have been performed (POD, temporal and spectral visualizations, spatial filtering by transducer array). Some models from the literature related to the wall pressure statistics (Corcos, Chase) have been adjusted and validated, and finally used for the modeling of the noise from the trailing edge of the airfoil. To conclude, measurements of the radiated acoustic pressure have been made in an anechoic wind tunnel, showing as a result the validity and limits of the implemented aeroacoustic model. The approach proposed by Chase in terms of wall pressure statistic modeling appears to be able to correctly represent the influence of the problem main parameters (Reynolds number, airfoil incidence).

\section{Contributed Papers}

\section{4:40}

1pNSa11. A numerical insight into the effect of confinement on trailing edge noise. Thomas Le Garrec (Arts et Métiers Paris Tech - Sinumef Lab, 151 bd de l'Hopital, 75013 Paris, France, thomas.le-garrec@paris.ensam.fr), Xavier Gloerfelt (Arts et Métiers Paris Tech - Sinumef Lab, 151 bd de l'Hopital, 75013 Paris, France, xavier.gloerfelt@paris.ensam.fr), Christophe Corre (LEGI Lab, BP 53, 38041 Grenoble Cedex 9, France, christophe.corre@hmg.inpg.fr)

The flow and the acoustic field around a 3D NACA 0018 airfoil at Reynolds 160000 with an angle of attack of $6^{\circ}$ are investigated numerically by direct noise computation to make comparisons with the experimental results of Nakano et al. (Experiments in Fluids, 2005) and the numerical simulations of Kim et al. (Heat and Fluid Flow, 2006). The direct noise computation of the flow around a 3D airfoil reaches the limits of current computational capacities. Indeed the main difficulty of such simulations comes from the large disparities between the fine scales of turbulence and the large wavelengthes of acoustic radiation which impose severe constraints on the meshes. In order to limit the number of points and to reduce the calculation cost, a multisize-mesh multitime-step strategy is adopted. The main purpose of the paper is to study numerically the influence of the confinement due to the top and bottom surfaces of the wind tunnel used in the experiments of Nakano et al. on the flow around the airfoil. The numerical results for a con- 
fined airfoil will be compared to the results obtained when the top and bottom walls are removed. So the noise can be directly linked to the vortices which develop in the wake of the airfoil.

\section{5:00}

1pNSa12. Coupled oscillations in the aeroacoustics of a Katana blade. Michel Roger (Ecole Centrale de Lyon, 36 Avenue Guy de Collongue, Centre Acoustique, 69134 Ecully, France, michel.roger@ec-lyon.fr)

The paper investigates experimentally the tonal characteristics of the aerodynamic sound of a Katana blade handled at high speed. Katana is the name of the Japanese sword used in martial arts. It is designed with a sharp cutting edge, a thick black-edge, and sometimes so-called blood-grooves. When handled through the air, the blade radiates sound at different frequencies. First a low-frequency, dominant sound is heard due to the von Karman vortex shedding in the wake of the blade. Second high-frequency tones are produced in the case of a Katana with grooves. The tones result from the self-sustained oscillations of the detached shear layers over the grooves which behave like cavities under a grazing flow. In the case of a Katana with no groove, other high-frequency tones are heard due to acoustic back-reaction on the Tollmien-Schlichting waves growing in the boundary layers. The variations of the acoustic signature with varying angle of attack and relative flow speed are analyzed in the paper, with emphasis on the coupling between the different mechanisms. The main trends are compared to simple self-sustained oscillation formulae. Finally the possible use of the sound as a criterion of inaccuracies in the handling of the Katana is discussed

\section{$5: 20$}

1pNSa13. Large-eddy simulation of roughness-generated boundary-layer noise. Meng Wang (University of Notre Dame, Departmernt of Aerospace and Mechanical Engineering, Notre Dame, MD 46556, USA, m.wang@nd.edu), Qin Yang (University of Notre Dame, Departmernt of Aerospace and Mechanical Engineering, Notre Dame, MD 46556, USA, qyang@nd.edu)

A computational study is carried out to predict rough-wall boundary layer noise and elucidate noise generation mechanisms. As a first step, the sound radiation from a single hemispherical roughness element in a turbulent boundary layer at $R e_{\theta}=7500$ is investigated. The roughness height is $3.6 \%$ of the boundary layer thickness, or 95 wall units. The flow field is computed by large-eddy simulation. The velocity statistics show reasonable agreement with the experimental data measured at Virginia Tech. Acoustic calculations are performed based on the Curle-Powell integral solution to the Lighthill equation. The sound radiation is dominated by unsteady drag dipoles and their images in the wall, with the spanwise dipole of similar magnitude or stronger compared to the streamwise dipole. The viscous contribution to the drag dipole is negligible relative to the pressure contribution. Numerical experiments are performed to isolate the roles of vortex shedding and diffraction of convected hydrodynamic pressure by the roughness element; both are shown to be important noise source mechanisms. The effects of roughness height, upstream wake, and multiple roughness elements are discussed as well.

\section{$5: 40$}

1pNSa14. The mechanisms of roughness noise. William J. Devenport (Virginia Tech, Aerospace and Ocean Engineering, 224E Randolph Hall, Blacksburg, VA 24061, USA, devenport@vt.edu), Stewart A. Glegg (Florida Atlantic University, Department of Ocean Engineering, Boca Raton, VA 33431, USA, glegg@oe.fau.edu), Nathan Alexander (Virginia Tech, Aerospace and Ocean Engineering, 224E Randolph Hall, Blacksburg, VA 24061, USA, alexande@vt.edu), Ben Smith (Virginia Tech, Aerospace and Ocean Engineering, 224E Randolph Hall, Blacksburg, VA 24061, USA, besmith5@vt.edu)

The interaction of turbulence and a rough surface results in the radiation of sound that is not produced in smooth wall flows. The mechanisms by which this sound is generated are not well understood. Scattering of the convected turbulent pressure field by the surface irregularities seems to be a probable source when the roughness size is small compared to the boundary layer. The bluff body mechanisms of vortex shedding and unsteady drag from the roughness elements appear more likely to be dominant sources when those elements are large compared to the boundary layer. Demonstrating the existence of these mechanisms, their range of dominance, the extent to which they are distinct and can be characterized in terms of flow parameters that can be realistically modeled (such as the surface pressure spectrum), are all pre-requisites to the development of a robust prediction method. New insight into these issues has been obtained through measurements made with using a purpose built roughness noise facility in combination with fundamental theoretical analysis of the problem. The measurements include roughness noise spectra, wall pressure spectra and aerodynamics for a large variety of rough surfaces. This work is supported by the Office of Naval Research.

\section{Invited Paper}

\section{6:00}

1pNSa15. Modeling and prediction of fan noise. Edmane Envia (NASA Glenn Research Center, Acoustics Branch, M.S. 54-3, 21000 Brookpark Road, Cleveland, OH 44135, USA, edmane.envia-1@nasa.gov)

Fan noise is a significant contributor to the total noise signature of a modern high bypass ratio aircraft engine and, with the advent of ultrahigh bypass ratio engines like the geared turbofan, it is likely to remain so in the future. As such, accurate modeling and prediction of the basic characteristics of fan noise are necessary ingredients in designing quieter aircraft engines in order to ensure compliance with ever more stringent aviation noise regulations. In this paper, results from a comprehensive study aimed at establishing the utility of current tools for modeling and predicting fan noise will be summarized. It should be emphasized that these tools exemplify present state of the practice and embody what is currently used at NASA and industry for predicting fan noise. The ability of these tools to model and predict fan noise is assessed against a set of benchmark fan noise databases obtained for a range of representative fan cycles and operating conditions. Detailed comparisons between the predicted and measured narrowband spectral and directivity characteristics of fan noise will be presented in the full paper. General conclusions regarding the utility of current tools and recommendations for future improvements will also be given. 


\section{Contributed Papers}

6:20

1pNSa16. Contra-rotating fans noise prediction for jet engine performance optimization. Dax Au (ISAE, 10, av Edouard Belin, 31055 Toulouse, France, dax.au@isae.fr), Alexandre Leblanc (ISAE, 10, av Edouard Belin, 31055 Toulouse, France, alexandre.leblanc@isae.fr)

This work fits within the framework of the European research project VITAL, which is dedicated to the development of technologies aimed at reducing the environmental impact of jet engines. In this respect, a new concept of ducted contra-rotating fans is investigated. The present contribution to this project is to elaborate a noise prediction tool for performances optimization. On the basis of the helicoidal surface theory, Hanson previously developed a harmonic formulation for the propfan rotor/rotor interaction. This method is here extended in order to take into account the duct effects and the noise radiated by the rear struts. A computer program has been written to predict the pressure distribution in the near field and to estimate the noise spectra in the far field. The results are validated with computational fluid dynamics simulations and compared with available public data. Those predictions are then included in a global noise computation with the other engine acoustic sources (coaxial jet, combustor and so on), and a multidisciplinary optimizer is used for the engine noise minimization.

\section{6:40}

1pNSa17. Design of a low noise radial fan with computational aeroacoustics. Esra Sorgüven (Yeditepe University, Kayisdagi, 34755
Istanbul, Turkey, sorguven@yeditepe.edu.tr),Yilmaz Dogan(Arcelik A.S., Tuzla, 34950 Istanbul, Turkey, yilmaz.dogan@arcelik.com), Ergin Arslan (Arcelik A.S., Tuzla, 34950 Istanbul, Turkey, ergin.arslan@arcelik .com)

Aim of this study is to employ computational aeroacoustics methods in order to design a fan system. Design objectives of this study are to decrease the flow-induced noise, to provide the necessary flow rate and pressure increase. During the design, modern computational methods will be employed in addition to traditional turbomachinery design methods. The design procedure begins with a baseline fan system design, which is based on traditional semi-empirical correlations. Flow through this fan system is analyzed via computational fluid dynamics. Pressure fluctuations on rotating and stationary solid surfaces are determined to calculate sound sources of the system. The baseline design is improved by eliminating the areas where powerful sound sources occur. Flow analysis and sound prediction is repeated to improve fan system further. This step is repeated until the desired sound power level is achieved. This design procedure employs numerical analysis instead of experiments. Therefore it will be more effective and less time consuming than the experimental trial and error design procedures. The number of prototypes and necessary measurements are decreased dramatically.

\section{Invited Paper}

1pNSa18. Computational AeroAcoustics of Realistic Co-Axial Engines. Stephane Redonnet (ONERA (French Aerospace Center), CFD \& Aeroacoustics Department, BP 72, 29 avenue de la division Leclerc, 92322 Chatillon Cedex, France, stephane.redonnet@onera.fr), Ciprian D. Mincu (ONERA (French Aerospace Center), CFD \& Aeroacoustics Department, BP 72, 29 avenue de la division Leclerc, 92322 Chatillon Cedex, France, dmincu@onera.fr), Eric Manoha (ONERA (French Aerospace Center), CFD \& Aeroacoustics Department, BP 72, 29 avenue de la division Leclerc, 92322 Chatillon Cedex, France, eric.manoha@onera.fr), Yann Druon (Airbus S.A.S, Department of Acoustic \& Environment, 316 route de Bayonne, 31000 Toulouse, France, yann.druon@airbus.com), Bastien Caruelle (Airbus S.A.S, Department of Acoustic \& Environment, 316 route de Bayonne, 31000 Toulouse, France, bastien.caruelle@airbus.com)

This study, that is relevant from the turbofan engines noise prediction/reduction, aims at CAA-computing the aft fan noise propagation/radiation of a realistic full-3D exhaust (with pylon and internal bifurcations), the latter being affected of (i) typical in-flight (take-off) thermodynamic conditions and of (ii) a representative fan noise modal content. As for previous studies conducted over baseline geometries, this CAA computation is conducted following the usual hybrid process, where a preliminary aerodynamic calculation provides a heterogeneous steady mean flow on which an acoustic calculation is then conducted A RANS computation is first performed, delivering the stationary jet mean flow characterizing the 3D exhaust in its typical 'take-off flight' $(\mathrm{M} \infty=0.25)$. A CAA grid (22 blocks, 28 millions cells) is then derived from the CFD one, before the RANS steady jet mean-flow is interpolated on it. After what the CAA computation is computed, a fan noise mode $(26,1)$ being emitted at a reduced frequency of $\mathrm{kR}=30(1 \mathrm{BPF})$ in the upstream of the engine's secondary exhaust, and numerically propagated along and outside the latter. Finally, a Kirchhoff post-treatment provides the far-field radiation characterizing these engine geometry, modal content and thermodynamic conditions. 


\title{
Session 1pNSb
}

\section{Noise and EURONOISE: Action Planning and Global Solutions for Urban Noise I}

\author{
Klaus Genuit, Cochair \\ HEAD acoustics GmbH, Ebertstrasse 30a, Herzogenrath, 52134, Germany \\ Itziar Aspuru Soloaga, Cochair \\ Labein-Tecnalia, C/Geldo-Parque Tecnologico de Bizkaia, Derio, 48160, Spain
}

\section{Invited Papers}

1:00

1pNSb1. Strategies for noise action plans. Paul De Vos (DHV BV, Postbus 1132, NL 3800 BC Amersfoort, Netherlands, paul .devos@dhv.com)

Following the requirements of the Environmental Noise Directive, Noise Action Plans should have been produced. The plans are supposed to build on two important pillars: the strategic noise maps and the consultation with the local residents. In some cases, the plan is considered as a mere statement of the long term policy of the authority under concern. In other cases, it is set up as an implementation plan with clear and measurable targets. Also the focus varies: only the highest noise levels could be attacked or the large numbers of exposed citizens, or even the overall annoyance. Target noise levels may lead to confusion both for local decision makers and citizens. Also, the preservation and possible creation of quiet areas can be envisaged. The use of an equal annoyance indicator helps to set the targets in the right perspective. Translation into different levels of quality of life is recommended. In terms of cost and benefits, the options for city councils are very limited. Due to a lack of European legislative power, cities tend to set their own rules, e.g. for road vehicles. Several initiatives to propose toolkits for action plans, comparable to the best practice guide for noise mapping, are welcomed but require harmonization and dissemination.

\section{$1: 20$}

1pNSb2. From strategic noise maps to action plan: Perspective of Spanish main roads. Maria Dolores Jimenez (Dirección General de Carreteras del Ministerio de Fomento, Paseo de la Castellana 67, 28071 Madrid, Spain, mdjimenez@fomento.es), Jesus Rubio (Dirección General de Carreteras del Ministerio de Fomento, Paseo de la Castellana 67, 28071 Madrid, Spain, jjrubio@fomento.es), Fernando Segues (CEDEX Centro de Estudios de Técnicas Aplicadas, Alfonso XII, 3-5, 28014 Madrid, Spain, fsegues@cedex.es), Pilar Fernandez (Labein Tecnalia, Parque Tecnologico Bizkaia, 48160 Derio, Spain, pfernandez@labein.es)

The Direccion General de Carreteras of the Spanish Ministry of Transport is finishing the process of Strategic Noise Mapping of Spanish major roads (EGRA). The whole National road network that has been noise mapped for the first END has a length of $6.400 \mathrm{~km}$ round. The present paper contains - Some conclusions from the process of noise mapping; - A general view of the process of making decisions about Action Plans; - Definition of the first steps to decide the scope of Action Plans: design of a pilot study to establish the elements needed technical studies, coordination with other administration bodies and public involvement.

$$
1: 40
$$

1pNSb3. Decision support system for Action Planning in the framework of the European Noise Directive. Jeroen Borst (TNO Science and Industry, Stieljesweg 1, 2628CK Delft, Netherlands, jeroen.borst@tno.nl), Walter J. Lohman (TNO, P.O. Box 49, NL2600 AA Delft, Netherlands, walter.lohman@tno.nl), Arno R. Eisses (TNO, Stieltjesweg 1, P.O. Box 155, 2600 AD Delft, Netherlands, Arno.Eisses@tno.nl), Henk M. Miedema (TNO, P.O. Box 49, NL-2600 AA Delft, Netherlands, henk.miedema@tno.nl)

The European Noise Directive (END) requires assessment of noise exposures as well as the formulation of Action Plans for the reduction of the number of people harmfully affected by environmental noise. TNO is developing a decision support system for noise mitigating measures. The proof of concept of such a system for road noise is presented here. On the basis of a detailed noise map, for each road segment an indication is given for the amount of negative effect (e.g., number of people being highly annoyed) per meter it is causing. On the basis of the characteristics of the road segment, the system suggests possible noise mitigation measures. The effect of the measure chosen by the user, such as the application of silent road surface types or lowering speed limits, can be interactively explored with the system. It directly shows the updated detailed noise contour maps as well as indicators describing the impact after a measure has been applied though the interactive interface. 
1pNSb4. Urban Noise Action Planning - an integrated approach towards noise abatement in the frame of urban development: Tools and recommendations developed within the SILENCE project. Melanie Kloth (Polis, rue du Trône 98, 1050 Brussels, Belgium, mkloth@polis-online.org)

Noise action planning following the European Directive on Environmental Noise is for many cities a new, additional task. It will probably be not very successful when considered as mere duty separated from other urban policy areas. Today, noise does not rank high on the local political agenda. Human and financial resources provided for noise abatement tend to be low. Furthermore, looking at concrete noise abatement measures it becomes clear that most effective measures also impact on other policy areas (air quality, mobility, etc.). Therefore it is advisable to link noise action planning closely with other urban plans like development plans, mobility plans, clean air programmes, etc. This requires a strong involvement of the relevant stakeholders as well as knowledge about the potential impact of noise abatement measures on other policy areas. To support local authorities in this complex task the SILENCE project developed a guidebook which comprises recommendations on organisational settings, on the involvement of stakeholders and the public, the action planning process as well as on a range of noise abatement measures. The description of these measures refers not only to their noise abatement potential but takes also into account their - positive or negative - impact on other policy areas.

\section{$2: 20$}

1pNSb5. Noise Action Plan in the Ile-de-France region: a complex process. Fanny Mietlicki (Bruitparif, 25 rue Coquillière, 75001 Paris, France, fanny.mietlicki@bruitparif.fr)

The transposition of the European Noise Directive (END) into French law was achieved in April 2006. Noise maps have to be established before June 30, 2007 and action plans have to be set up before July 18, 2008 for agglomerations with more than 250,000 inhabitants and for major infrastructures. The State representatives at the departmental scale are in charge of the infrastructure maps, while the local authorities are in charge of the agglomeration maps. The Paris agglomeration which counts 11 million inhabitants is made of 396 towns. Since some of them are grouped into cooperation structures for noise issues, there are 238 entities in charge of the application of the END in the Paris agglomeration. To face this administrative complex situation, Bruitparif has developed a program to help local authorities in setting up the END. Different actions are lead: - providing information and technical assistance on how to establish noise maps and action plans, - conducting noise measurements in order to validate maps and to provide complementary information, - coordinating actions and initiatives, - consolidating the map of the Paris agglomeration from the local maps in order to estimate the noise exposure, - preparing guidelines for the action plans.

\section{2:40}

1pNSb6. Action planning procedures and realized action plans of municipalities and cities - results from the implementation of END. Markus Petz (ACCON GmbH, Ingenieurbüro für Schall- und Schwingungstechnik, Gewerbering 5, 86926 Greifenberg, Germany, markus.petz@accon.de)

For many federal states of Germany the Strategic Noise Maps were calculated and published in 2007. For some cities it will be shown, how a standard procedure for preparation of a noise action plan was defined together with responsible authorities from the municipality. The chosen procedure also considered the deadline for finalisation and the commencement of an action plan at July, 2008. Based on Strategic Noise Maps and hot spot analyses a catalogue of measures for noise reduction within a community was investigated and evaluated (cost/effectiveness). Together with representatives of traffic-, town- and environmental planning departures of the municipalities efficient measures were discussed and selected considering other local planning interests, financial possibilities and public acceptance. The draft action plan were published and public demurs have been weighted. In July, 2008 the action plans came into force.

\section{Contributed Papers}

\section{3:00}

1pNSb7. Action plans (PPBE) : experience of municipal noise fighting plans. Eric Gaucher (Acoustique \& Conseil, 17-19 rue des Grandes Terres, 92508 Rueil-Malmaison, France, eg@acoustique-conseil.com)

The European Noise Directive (END) relating to the assessment and management of environmental noise requires the elaboration of action plans (Plans de Prévention du Bruit dans l'Environnement PPBE). However, these plans are limited to their concerned sources: trains, roads, planes and industrial plants. Municipal noise fighting plans (Plans Municipaux de Lutte contre le Bruit PMLB) taking equally into account any annoying sound situation, regardless of its nature, have been developed and implemented in around forty towns in France, with increasing success before the END was published. Acoustique \& Conseil have already implemented thirty PLMBs. This experience has lead us to think that the strict application of PPBEs will in most cases not answer all the expectations of the concerned populations, in terms of solutions to their daily noise problems. It therefore seemed relevant for us to present a synthesis of the situations encountered during our works in order to enrich the approach of towns willing to enlarge their actions beyond those of the END. Now that it is time to elaborate the PPBE, it seems relevant and appropriate to implement PLMBs equally for the towns concerned.

\section{3:20}

1pNSb8. Urban and building acoustics management in the next decades: a matter of prevention, simplification and education. João G. Baring (Univ. of São Paulo - Facty. of Archt. and Urban., Rua Rafael Clark 85, 05526 - 010 São Paulo, Brazil, jbaring@uol.com.br)

In Brazil, in cities like São Paulo and Rio de Janeiro, noise pollution control has been carried out mainly in the form of repression. In the seventies and eighties, people joined together to fight against noisy government projects, and government fought noisy industries and commercial establishments. Since then, people have become aware of their rights, but have learned little about their own obligations on the matter. The author researched this process in order to find a better way for the future and concluded that prevention must be enforced through noise codes, standards must include easier survey methods, and low cost devices for noise control must be available. Most important, education must be improved in all levels, to make every citizen a partner of noise control at home, at work and elsewhere in the cities. This paper points out some ways of dealing with this process.

\section{$3: 40$}

1pNSb9. NERS-analysis extended to include the existence of neighbouring quiet areas. Åsa Stenman (Acoustic Control AB, Tumstocksvägen 1, SE-187 66 Täby, Sweden, stenman@acoustic.se), Peter 
Malm(Acoustic Control AB, Tumstocksvägen 1, SE-187 66 Täby, Sweden, peter.malm@acoustic.se), Magnus Lindqvist (Stockholm Environmental \& Health Administation, Box 38024, SE-100 64 Stockholm, Sweden, magnus .lindqvist@miljo.stockholm.se

One of the goals for the EC financed Integrated Project Quiet City Transport (QCITY), is to provide European city administrations with validated analysis tools and technical noise control solutions for the efficient production of noise action plans. A part of the QCITY project work is to produce complete noise maps and hot-spot analysis for a part of Stockholm. This paper will focus on the Noise Environmental Rating System (NERS), a tool for performing hot-spot analysis on noise maps, developed within the QCITY project. The input parameters that have been used in previous studies [Internoise-07_411, Å. Stenman, P.-Malm, 2007] are outdoor noise on al floors, number of inhabitants per building and the facade sound insulation index. Previous studies, such as the "Stockholm score rating model (Trafikbuller och planering II, Stockholm, 2004) show a strong correlation between noise annoyance and the existence of quiet nearby areas. The NERS-analysis in this work has therefore been extended to include quie areas.

\section{4:00-4:20 Break}

4:20

1pNSb10. NERS-analysis extended to include noise levels measured on city courtyards. Peter Malm (Acoustic Control AB, Tumstocksvägen 1, SE-187 66 Täby, Sweden, peter.malm@acoustic.se), Åsa Stenman (Acoustic Control AB, Tumstocksvägen 1, SE-187 66 Täby, Sweden, stenman@acoustic.se), Thomas Hammarlund (Environment Administration, Karl Johansgatan 23, SE-414 59 Göteborg, Sweden, thomas .hammarlund@miljo.goteborg.se)

One of the goals for the EC financed Integrated Project Quiet City Transport (QCITY), is to provide European city administrations with validated analysis tools and technical noise control solutions for the efficient production of noise action plans. A part of the QCITY project work is to produce complete noise maps and hot-spot analysis for the entire Gothenburg area. Earlier studies have shown that calculated noise levels from traffic at inner city courtyard are lower compared to actual measurements Gothenburg city have performed over 700 measurements on city courtyards. The measurements are included in the NERS-analysis to show the influence of the actual courtyard noise levels. The study covers a smaller area in the central part of Gothenburg where the measurements have been made.

\section{Invited Papers}

\section{4:40}

1pNSb11. Auralisation and psychoacoustic evaluation of traffic noise scenarios. Sandro Guidati (HEAD acoustics GmbH, Ebertstrasse 30a, 52134 Herzogenrath, Germany, claudia.erens@head-acousitcs.de)

The evaluation of vehicle noise is mostly done using the A-weighted sound pressure level, e.g., the Lmax for pass-by noise or the Leq for community noise. Unfortunately, the $\mathrm{dB}(\mathrm{A})$-value does not reflect the annoyance effect of vehicle noise correctly. During the first phase of the European Research Project QCity a metric for psychoacoustic evaluation of single pass-by events has been developed. In the next step this metric is adapted for the evaluation of complex traffic scenarios calculated by traffic flow simulations. Psychoacoustic evaluation requires time signals. Single values or third octave spectra are not sufficient to investigate all relevant effects (e.g., annoying time patterns, masking). The time signals are calculated combining advanced resynthesis with exterior noise simulation techniques. Hereby, it is possible to investigate the effect of e.g., low noise surfaces or various traffic control measures (traffic light vs. roundabout). Since a standard traffic scenario includes a very large number of single pass-by events special focus lies on fast processing techniques. This paper gives an overview of the developed algorithms and presents first results of the psychoacoustic evaluation of traffic scenarios.

1pNSb12. Lions Bay noise mitigation program. Duane E. Marriner (Wakefield Acoustics Ltd., 301-2250 Oak Bay Avenue, Victoria, BC V8R 1G5, Canada, duane@wakefieldacoustics.com)

The Ministry of Transportation (MoT) of the Province of B.C. noise abatement policy requires that environmental community noise impacts of highways projects involving new or substantially upgraded highways be assessed and mitigation implemented where warranted. This paper presents an extraordinary noise model developed to mitigate residential areas of the Village of Lions Bay on the rugged B.C. coastline from the Sea-to-Sky Highway connecting Vancouver, B.C. to Whistler, B.C. home of the Winter Olympic and Para-Olympic Games in 2010. The Cadna/A Version 3.9.15 software was used to develop a new 4 lane split grade alignment model incorporating two mini-interchanges. With the objective of achieving a $10 \mathrm{dBA}$ noise reduction benefit, mitigation in the form of quiet pavement (OGA), traffic calming and 5 meter high absorptive sound walls or noise barriers were introduced into the model which included 150 mountain side residential receptors along the $2 \mathrm{~km}$ long corridor at elevations up to 35 meters above the project. Noise reduction benefits for dwellings were further analyzed to determine contributions during each stage of project development by creating a super user, modular, multi-layer noise model of Lions Bay. This work was carried out under the sponsorship of BCMoT.

1pNSb13. Tire/road noise reduction. Alexander Ossipov (Goodyear, Ave G. Smith, L-7750 Colmar - Berg, Luxembourg, alexander_ossipov@goodyear.com), Marc Engel (Goodyear, Ave G. Smith, L-7750 Colmar - Berg, Luxembourg, marc_engel @ goodyear.com)

Traffic running on high speed roads is a considerable source of noise annoyance and is frequently a source of complaint by the general public. One of the components of this noise is generated by the interaction between motor vehicles tires and the road surface. The amplitude and frequency content of this noise is a function of many parameters, including the road surface texture, tire dimensions, tire materials, and construction and the tread pattern design. The paper provides detailed insight concerning the factors influencing exterior (environmental) and interior (vehicle) tire/road noise reduction and includes recommendations with regard to optimize tire design. 


\section{Contributed Papers}

\section{$5: 40$}

1pNSb14. Calculations of low height noise barriers efficiency by using Boundary Element Method and optimisation algorithms. Marine Baulac (CSTB, 24 rue Joseph Fourier, 38400 Saint-Martin-d'Hères, France, marine.baulac@cstb.fr), Arnaud Guillou (Institut National de Métrologie (LNE-INM/Cnam), 61 rue du Landy, 93210 La Plaine Saint Denis, France, guillou.arnaud@gmail.com), Jérôme Defrance (CSTB, 24 rue Joseph Fourier, $38400 \quad$ Saint-Martin-d'Hères, France, jerome.defrance@cstb.fr), Philippe A. Jean (CSTB, 24 rue Joseph Fourier, 38400 Saint-Martin-d'Hères, France, philippe.jean@cstb.fr)

Nowadays, roads and railways are often quoted as the most annoying source of noise for the living environment. This problem of ground transportation noise is present in extra-urban environments as well as in town centres. Traditional noise barriers are in many cases not suited for acoustic protection in urban areas mainly because of their height. Previous studies have shown a significant efficiency of low height noise protections (kind of urban furniture) for the decreasing of noise levels in semi-opened areas such as city parks, places, and large avenues. However, the simulations of low height noise protections requires specific numerical codes since ray tracing methods are not suited for barriers lower than around $2 \mathrm{~m}$. This paper proposes a way to implement an approach with allow to simulate low height noise protections in engineering calculation methods. This can be done by creating a database using a Boundary Element Method numerical code combined with optimisation algorithms. Work is still in progress since only $2 \mathrm{D}$ configurations are considered at the moment.

\section{6:00}

1pNSb15. Status of noise abatement measures for roads in Switzerland. Laurent Cosandey (Office Fédéral de l'Environnement, OFEV, 3003 Berne, Switzerland, laurent.cosandey@bafu.admin.ch), Kirk Ingold (Office Fédéral de l'Environnement, OFEV, 3003 Berne, Switzerland, kirk.ingold@bafu.admin.ch), Tommaso Meloni (Office Fédéral de l'Environnement, OFEV, 3003 Berne, Switzerland, tommaso.meloni@bafu.admin.ch), Nicole Luethi (Grolimund und Partner AG, Entfelderstr. 41, 5000 Aarau, Switzerland, Nicole.Luethi@gundp.ch), Hansueli Pestalozzi (Grolimund und Partner AG, Entfelderstr. 41, 5000 Aarau, Switzerland, hansueli.pestalozzi@gundp .ch)

In 2006, a comprehensive survey on the status of noise abatement measures for all roads was carried out in Switzerland for the first time. This survey recorded the costs of remedial work and noise protection measures completed to date (approx. CHF 1 billion) or still outstanding (approx. CHF 3 billion). An account is also given of the distribution among different types of measures, and of the protective effects on the public. As well as indicating the financial resources required for remediation of the Swiss road network on schedule, the survey highlights measures for controlling noise emissions at source which need to be implemented urgently.

\section{6:20}

1pNSb16. Influence of noise source representation on the estimation of specific descriptors close to traffic signals. Arnaud Can (LICIT, ENTPE/INRETS - Université de Lyon, rue Maurice Audin, 69518 Vaulx-enVelin Cedex, France, can@entpe.fr), Ludovic Leclercq (LICIT, ENTPE/INRETS - Université de Lyon, rue Maurice Audin, 69518 Vaulx-enVelin Cedex, France, leclercq@entpe.fr), Joel Lelong (INRETS, 25 av. F. Mitterrand, case 24, 69675 Bron, France, lelong@inrets.fr)

Considering traffic dynamics greatly improves noise estimation in urban area. This can be achieved by coupling a dynamic traffic model with both emission laws and sound propagation calculation. This paper focuses on the influence of noise source representations in the combination with the traffic model. Several representations are tested: point sources and homogeneous line sources of different sizes. We aim at evaluating how these representations correctly estimate classical descriptors (LAeq and statistical descriptors) and specific descriptors able to capture noise dynamics at a traffic signal scale. Three typical urban situations are studied: in front of, upstream and downstream a traffic signal. Noise source representation can be coarse for classical descriptors calculation if traffic dynamics is precisely described. On the contrary, noise source representation should be refined to precisely assess noise dynamics.

\section{$6: 40$}

1pNSb17. Evaluation of Directive 2000/14/EC on outdoor machinery noise. Michael G. Dittrich (TNO Science and Industry, PO Box 155, 2600 AD Delft, Netherlands, michael.dittrich@tno.nl)

The European Directive 2000/14/EC sets a requirement for noise labeling for the sound power level of 57 types of outdoor equipment, and sound power limits for 22 of these. In the NOMEVAL project the Directive and its amendment 2005/88/EC were evaluated, resulting in recommendations for an update of the equipment list, the noise limits and the test codes. A European database of noise emission data was assessed, and environmental bodies and industry were consulted. The recommendations were also based on environmental, technical and economic impact assessments. A new environmental indicator was applied to rank the impact of the different equipment types. It was found that many types currently without noise limits have a higher impact than those with noise limits. Some new types of equipment have been identified including snowmobiles, mobile waste breakers and screens, motorised brooms, handheld cut-off saws, and power pruners. The technical impact assessment was based on current technology and trends and the feasibility of new or stricter limits. The economic impact assessment was based on estimated societal benefits versus the estimated additional cost to the consumer or purchaser. Finally, a number of instruments for further reduction of outdoor equipment noise were proposed.

\section{7:00}

1pNSb18. Some algebra and statistics on isolated noise events. Michel Maurin (INRETS-LTE, case 24, 69675 Bron cedex, 69500 Bron, France, maurin@inrets.fr)

Noise indices related to isolated emerging noise events are calculated on variable finite durations $\Delta \mathrm{T}_{\mathrm{ev}}$, as opposed to instantaneous levels or indices on constant conventional periods $\Delta \mathrm{T}(1 \mathrm{~h}, 24 \mathrm{~h}, \ldots)$. Here they are defined by TEL (transit exposure levels) or Leq ${ }_{\Delta \mathrm{Tev}}$ on every $\Delta \mathrm{T}_{\mathrm{ev}}$. There are classical formulas to pass from many TEL to the Leq on a longer period including many events. When $\mathrm{TEL}_{\mathrm{i}}$ are supposed equal to TEL one gets classicaly $\mathrm{Leq}_{\Delta \mathrm{T}}=\mathrm{TEL}+10 \log \mathrm{n}+\mathrm{K},(\mathrm{CNR}$, NEF for instance). In algebra, the approach develops some algebraic relations and conditions between total duration $\Delta \mathrm{T}$, the global part of all $\Delta \mathrm{T}_{\mathrm{ev}, \mathrm{i}}$ and their number $\mathrm{n}$, in a way that TEL is clearly emerging from noise level without the isolated events. It is a manner to find a general result like "events kill events," say that too many events render not emerging any new one. One finds the same with a statistical approach and other appropriate technics (quantiles for instance). And coming on with probabilistic tools, the Bêta distribution renders possible some characterisation of the graphical look of the time evolution of noise level during $\Delta \mathrm{T}_{\mathrm{ev}}$. This work has partly been done with $\mathrm{V}$. Catto of Lyon 1 University. 


\title{
Session 1pNSc
}

\section{Noise and EURONOISE: Noise Mapping Techniques and Uncertainties I}

\author{
Richard Peppin, Cochair \\ Scantek, Inc., 7060 Oakland Mills Rd., Columbia, MD 20146, USA \\ Wolfgang Probst, Cochair \\ DataKustik GmbH, Gewerbering 5, Greifenberg, 86926, Germany
}

\section{Contributed Paper}

1:00

1pNSc1. Optimizing localization of noise monitoring stations for the purpose of inverse engineering applications. Malgorzata Reiter (Gdansk University of Technology, Multimedia Systems Department, 11/12 Gabriela Narutowicza Street, 80-952 Gdansk, Poland, reiter.m@sound.eti.pg.gda.pl), Jozef Kotus (Gdansk University of Technology, Multimedia Systems Department, 11/12 Gabriela Narutowicza Street, 80-952 Gdansk, Poland, joseph@sound.eti.pg.gda.pl), Andrzej Czyzewski (Gdansk University of Technology, Multimedia Systems Department, 11/12 Gabriela Narutowicza Street, 80-952 Gdansk, Poland, andcz@sound.eti.pg.gda.pl)

Long-term environmental monitoring of noise levels can be done using autonomous measurement stations. Because of the high cost of monitoring systems and management of these stations, it is essential to identify how many of measuring localization points are really required. In cases related to complex noise generation schemes, when there are various noise sources, the differences between calculations and measurements can be difficult to estimate. Therefore, it is vital to find some most appropriate locations for measurement stations which would ensure obtaining an adequate number of measurement results to be employed in the reverse engineering. These measurements can be then utilized to update dynamic noise maps. Furthermore, predictive noise models may be developed accordingly to certain local requirements. This could result in a better accuracy of dynamic noise maps. The paper focuses on defining the proper choice of the measurement points localizations. The experiments described include a comparison between real-life measurement results performed with the Multimedia Noise Monitoring System developed at the Multimedia Systems Department of the Gdansk University of Technology and the noise level prediction results. The optimization of the number and location of noise monitoring points with regard to the measurement accuracy is also discussed.

\section{Invited Paper}

1pNSc2. Acoustical design margins: Uncertainty in prediction and measurement of community noise. Robert Putnam (Siemens Power Generation, 4400 Alafaya Trail, Orlando, FL 32826, USA, bob.putnam@ siemens.com), Roland Hetzel (Siemens Power Generation, Beethovenring 8a, 91090 Effeltrich, Germany, roland.hetzel@siemens.com)

Compliance with regulatory requirements for sound levels in communities adjacent to industrial or power generating facilities is typically a contractual commitment, with the potential for significant financial penalties in the event of noncompliance. Uncertainties at any stage of the design, specification or prediction of plant sound level may be accounted for as part of the overall plant acoustical design margins. There are also additional uncertainties in terms of compliance sound measurement surveys, that are commonly referred to as either "test tolerance," or "instrumentation tolerance and measurement uncertainty." From the viewpoint of the plant equipment supplier all of the uncertainties associated with equipment design and specification are simply additive to all of the uncertainties of compliance measurements, since they all contribute to, or affect the selection of, the overall plant acoustical design margin. The discussion will address the types of uncertainties in source sound power levels and measurement error, tolerances, and confidence limits of field sound surveys, highlighting some seldom-treated aspects of uncertainty. Measurement uncertainties and the applicable combinatorial rules will be treated.

\section{Contributed Papers}

\section{$1: 40$}

1pNSc3. Possibilities of supporting municipal authorities in processes of acoustical environment management. Jan Ka Mierczak (Silesian University of Technology, Faculty of Organization and Management, ul. Roosevelta 26/28, 41-800 Zabrze, Poland, Jan.Kazmierczak@polsl.pl)

EU directives and state law acts oblige municipal authorities of European towns to create and exploit strategic acoustic maps. The further decisions of these authorities, considering both sustainable local development strategies and results of analysis of environmental effects (such as noise), ought to form suitable living conditions in the towns. The people, responsible for such decisions, ought to dispose suitable knowledge and skills. Author of this paper leaded in recent years the consortium of R\&D units involved in the research project, focussed on preparing technology of creating acoustic maps of urban areas. The project resulted in the method of acoustic mapping, based on GIS technology. Apart of "purely acoustical" problems, the forming of competencies of these members of municipal staff, responsible on preparation and then exploitation of acoustic maps, has been mentioned as a problem of essential importance in this research. In result of discussions with potential users of strategic acoustic maps, the need was formulated to give municipal units some effective tools of supporting them by means of offering necessary knowledge and expertises in the field of management of acoustical environment in urban areas. The concept of network-based system focussed on such a target is presented in the paper.

\section{2:00}

1pNSc4. Evaluation models for the noise diminution due to the phonique barrier walls. Mariana Arghir (Technical University of ClujNapoca, Faculty of Machines Design; Department of Mechanics and Computer Programming, B-dul Muncii; Nr. 103-105, 400641 Cluj-Napoca, 
Romania, marianaarghir@yahoo.com),Dan Borza(National Institute of Applied Sciences of Rouen, INSA Rouen, LMR, Ave l'Universite, BP8 76800 Rouen, France, dan.borza@insa-rouen.fr), Florin Blaga (University of Oradea, Str. Universitatii nr.1, 400641 Cluj-Napoca, Romania, florin_blaga2000@yahoo.com), Tiberiu Vesseleny (University of Oradea, Str. Universitatii nr.1, 400641 Cluj-Napoca, Romania, vasitata@yahoo.com), Geamilia Solea (Technical University of ClujNapoca, Faculty of Machines Design; Department of Mechanics and Computer Programming, B-dul Muncii; Nr. 103-105, 400641 Cluj-Napoca, Romania, marylandprod@gmail.com), Mariana Runcan (National Institute of Applied Sciences of Rouen, INSA Rouen, LMR, Ave 1'Universite, BP8 76800 Rouen, France, mariruncan@yahoo.com)

The present paper contains our study regarding the diminution of the urban noise using the phonique barrier walls conformed to the EMPARA procedure, which is based on the simplified of the standard method for the measurements and computes of the surface traffic inside the urban agglomeration, as they are given in SRM2 laws (Netherlands standards). There are made the studies taking into account all the parameters that characterize the the noise and they are graphical representations having the "n-1" constant parameters and only one having the time variation law. In this way we are obtained the influence of each parameter in the diminution of the urban noise using the phonique barrier walls. In addition we realized a measurement for a real barrier wall inside the Oradea town. The comparison between them was enough good, that means our theoretical study is correct and characterizes the noise pollution and its diminution on the urban agglomeration. The measurement device was a CENTER 322 Sound Lever
Meter. This study contains the two steps for the "noise map" asked for the Romania integration in the European Union, and was made by the researchers of the Oradea University under the direction of the Prof. Dr. Ing. Mariana ARGHIR from the Technical University of Cluj-Napoca.

\section{2:20}

1pNSc5. Environmental impact of noise from mobile outdoor equipment. Foort De Roo (TNO Science and Industry, Stieljesweg 1, 2628CK Delft, Netherlands, foort.deroo@tno.nl), Eddy Gerretsen (TNO Science and Industry, Stieljesweg 1, 2628CK Delft, Netherlands, eddy.gerretsen@tno.nl), Jeroen Borst (TNO Science and Industry, Stieljesweg 1, 2628CK Delft, Netherlands, jeroen.borst@tno.nl)

As part of an evaluation study of European Directive 2000/14/EC relating to the noise emission by equipment for use outdoors, an environmental impact assessment was required to investigate the need for new or tighter noise limits. In contrast to other environmental noise sources, the operating location of outdoor machines is not fixed. As conventional environmental noise mapping could not be used for this purpose, an environmental indicator was developed. This indicator takes the following factors into account: numbers of equipment in use in the EU, typical average sound power level, tonal/impulsive/intermittent sound content, annual duration of operation, day/night operation and typical areas of use. The numbers of people affected were assessed by means of a series of noise maps, resulting in distribution curves characteristic for different types of environment (urban, suburban, rural, and others). The newly developed environmental indicator is a relatively simple quantity taking all relevant factors into account including annoyance.

\section{Invited Papers}

\section{2:40}

1pNSc6. Procedures of large scale noise mapping - examples based on German projects. Markus Petz (ACCON GmbH, Ingenieurbüro für Schall- und Schwingungstechnik, Gewerbering 5, 86926 Greifenberg, Germany, markus.petz@accon.de)

Noise mapping projects for the German Federal States Baden-Wuerttemberg, Hesse, and Schleswig-Holstein and for the cities Munich, Frankfurt, and Wiesbaden are presented. These large projects have been performed using newest software technology like fully automated parallel computing applying the PCSP technique (Program Controlled Segmented Processing) and by using all CPU cores of the noise mapping computer cluster (multithreading). With the noise mapping for all main roads and railways in Austria the 64 Bit technology has been applied - this allows to handle even files of more than $10 \mathrm{~GB}$ without the need of manual intervention. The influence of these techniques on uncertainties is discussed using the strategy of DIN 45687 and it is shown that such complete 3D models are the optimal basis to develop noise reduction measures in the frame of Action Planning.

\section{3:00}

1pNSc7. Noise mapping for large urban areas - the city of Vienna. Werner Talasch (Magistrat der Stadt Wien - Magistratsabteilung 22, Dresdner Straße 45, A-1200 Wien, Austria, office@talasch.at)

The Environmental Noise Directive 2002/49/EG requires noise maps for the main noise sources railway, road and aircraft and as well for agglomerations. For agglomerations there are also industrial sites (restricted to IPPC plants) a relevant noise source. The noise maps for the agglomeration of Vienna includes the City of Vienna and 5 further municipalities. These are Brunn am Gebirge, Maria Entersdorf, Mödling, Perchtoldsdorf, and Wiener Neudorf. The area of the agglomeration is about $460 \mathrm{~km}^{2}$. The most difficult part in the task of creating the noise maps was the 3D model of the agglomeration. Most of the relevant objects were available as 3D objects. Only some objects are only available as $2 \mathrm{D}$ objects and caused therefore a lot of work.

\section{3:20-3:40 Break}

\section{$3: 40$}

1pNSc8. The United Kingdom noise mapping experience. Nick Hawkins (ACCON UK Limited, Unit B, Fronds Park, Frouds Lane, Aldermaston, RG7 4LH Reading, UK, nick.hawkins@accon-uk.com), Graham Parry (ACCON UK Limited, Unit B, Fronds Park, Frouds Lane, Aldermaston, RG7 4LH Reading, UK, graham.parry@accon-uk.com)

The paper describes the noise mapping of the Bristol and Bournemouth agglomerations for the United Kingdom Noise Mapping Initiative in compliance with the European Directive on Environmental Noise and the challenges of delivering both compliant noise mapping, noise mapping outputs and shape files, and the associated methodology reports. The paper examines the noise model utilised for the mapping, the various efficiencies built into the system to provide error checking, the external data acquisition and the effects that any uncertainties may have in strategic noise mapping. Additionally, the use of a GIS based toolkit for identifying and amending the location of noise barrier objects was utilised. 


\section{Contributed Paper}

4:00

1pNSc9. A comparison between predicted and measured noise levels at the Rijeka - Zagreb motorway. Sanja Grubesa (Faculty of EE and Computing, Unska 3, Department of Electroacoustics, HR-10000 Zagreb, Croatia, sanja.grubesa@fer.hr), Marko Horvat (Faculty of EE and Computing, Unska 3, Department of Electroacoustics, HR-10000 Zagreb, Croatia, marko.horvat@fer.hr), Hrvoje Domitrovic (Faculty of EE and Computing, Unska 3, Department of Electroacoustics, HR-10000 Zagreb, Croatia, hrvoje .domitrovic@fer.hr)

The current calculations used for predicting the levels of road traffic noise can and usually do give results that can differ significantly from the results of measurements of noise levels performed in the field. In order to examine these discrepancies, levels of road traffic noise have been measured on two locations on the Rijeka - Zagreb Motorway. After that, the geographical layout of these locations has been prepared and then input into a computer simulation program with the goal of obtaining simulated road traffic noise levels. Following the assumption that these results will be different, the goal is to determine which of the simulation models used today will provide results that are in the best agreement with the results obtained from the actual field measurements, with the emphasis on this particular geographic region, namely, the Republic of Croatia.

\section{Invited Paper}

\section{$4: 20$}

1pNSc10. City noise mapping without traffic data. Laurent Droin (Acouphen-Environnement, Campus de La Doua, 66 BD Niels Bohr, BP 52132, 69603 Villeurbanne, France, laurent.droin@acouphen-environnement.com), Pierre Odent (MVAConsultancy, 12-14 rue Jules Cesar, 75012 Paris, France, podent@mvaconsultancy.com)

The authers have a large experience on transport and noise planning and mapping with projects on many communities of different size in France. Within the time scale of a mapping project, it is very common to have a large variation of the accuracy in traffic data, especially for roads (largest source data base). In many cases, no traffic data is available on less exposed roads. This paper illustrates how variation of input data accuracy and lack of data were tackled in both large and small territories (population between 50000 and $1000000)$ in order to provide maps with the required accuracy and information for political action and communication.

\section{Contributed Papers}

\section{4:40}

1pNSc11. Definition of traffic scennaries, application on a practise case of the criteria followed by the guide of good practises for elaboration of strategic traffic noise maps in urban routes. Igone García Pérez (Labein-Tecnalia, C/Geldo-Parque Tecnologico de Bizkaia, 48160 Derio, Spain, igarcia@labein.es), Itziar Aspuru Soloaga (Labein-Tecnalia, C/Geldo-Parque Tecnologico de Bizkaia, 48160 Derio, Spain, iaspuru @1abein.es)

Configuration of noisy environment in towns mostly depends upon the characterization of traffic noise in urban routes. Frequently there is a lack of information when it is a question of having data from the whole urban area. To solve this lack of information the above-mentioned guide (WG-AEN 002 .2006) proposes some suppositions for this characterization which can turn out to be very general in practical applications. Through the presentation of these criteria applied to a practical case it can be seen the tools necessary to adjust real traffic characterization to the elaboration of noise maps and definition of an Action Plan. The analyzed variables are: AVT, \% of heavy vehicles, time distribution and speed considering, for this last factor, the lacks shown by the interim method and the applicable supposes based upon the state of the art.

\section{5:00}

1pNSc12. Urban noise mapping - an approach to the establishment of standard making procedure. Predrag Vukadin (Brodarski institut d.o.o., Avenija V.Holjevca 20, 10020 Zagreb, Croatia, prevuk@hrbi.hr), Ivan Bublic (Brodarski institut d.o.o., Avenija V.Holjevca 20, 10020 Zagreb, Croatia, ivan.bublic@hrbi.hr), Ivan Tudor (Brodarski institut d.o.o., Avenija V.Holjevca 20, 10020 Zagreb, Croatia, ivan.tudor@hrbi.hr)

The Directive 2002/49 relating to the assessment and management of environmental noise and the Croatian legislative derived from it defines the basic rules to carry out strategic noise mapping. However, in real life situations, each mapping project implies a different approach, depending on various case related distinctions e.g. availability and quality of input data, extent of noise annoyance, the form and the volume of output data to be presented, etc. Based on the experience from various urban noise mapping projects, this work is an approach to defining key steps in urban noise map production and establishing standard step-by step procedure, and an attempt to define the procedure for each step. Examples for each step are given based on the case studies from our previous urban noise mapping projects. 


\title{
Session 1pNSd
}

\section{Noise and EURONOISE: Environmental Noise Mapping I}

\author{
Kenneth Cunefare, Cochair \\ Woodruff School of Mechanical Engineering, The Georgia Inst. of Technol., Atlanta, GA 30332 USA \\ Gaetano Licitra, Cochair \\ ARPAT - Dept. Firenze, Via Porpora, 22, Firenze, 50144, Italy
}

\section{Invited Papers}

\section{5:40}

1pNSd1. Differences among European noise mapping methods. Stylianos Kephalopoulos (European Commission, via e. fermi, 1, 21020 Ispra, Italy, Stylianos.Kephalopoulos@jrc.it), Marco Paviotti (European Commission, via e. fermi, 1, 21020 Ispra, Italy, marco.paviotti@jrc.it)

This article will present the differences among noise maps of Lden and Lnight levels produced following the EU Interim methods and those produced following other national methods used in the EU MS. Lden and Lnight are the two indicators required by the European Environmental Noise Directive (2002/49/EC) defined at the position of $4 \mathrm{~m}$ height and $2 \mathrm{~m}$ away from the facade of a building. Four different comparisons will be presented for road, railway, industrial, and aircraft noise. This article will also focus on the methodology used to assess the equivalence among the national methods against the interim ones as well as the statistical approach used to consider uncertainties. Finally, the input values used, the parameters and the lay-outs of the four protocols corresponding to the four environmental noise sources (i.e., road traffic, railway, industrial, and aircraft noise) adopted for the equivalence exercise will be also presented.

\section{6:00}

1pNSd2. Challenges and opportunities for noise mapping in the United States. Kenneth Kaliski (Resource Systems Group (RSG), 55 Railroad Row, White River Junction, VT 05001, USA, kkaliski@rsginc.com)

Primarily through the impetus of European Union Directive 2002/49/EC, EU cities have been on the forefront of noise mapping and the community planning that results from it. The United States has no similar legislation at federal or state levels. As a result, noise mapping for towns and cities in the U.S., and indeed, awareness of noise issues by planners, appears to lag behind their European counterparts. However, metropolitan areas in the U.S. have invested a great deal in transportation modeling. As a result, the data foundation for noise mapping, that is, road and rail geometry and traffic volumes, is in place in many metropolitan areas. In addition, digital terrain elevation data, aerial photography, and GIS data are generally available on a state or national basis at no charge over the internet. The Chittenden County, Vermont noise map is one example of how publically available data can be used to create a useful community noise map over a large area. Other examples will be discussed.

\section{6:20}

1pNSd3. Presenting Noise Mapping Information to the Public. John F. Hinton (Birmingham City Council, Regulatory Services, 581 Tyburn Road, B24 9RF Birmingham, UK, bham.acoustics@dsl.pipex.com), Soren Rasmussen (Cowi-Odense, Odensevej 95, Postboks 444, Hjallese, DK-5260 Odense, Denmark, SRS@ @ cowi.dk)

Directive 2002/49/EC of the European Parliament and of the Council of 25 June 2002 relating to the assessment and management of environmental noise requires Member States to undertake strategic noise mapping. It also refers to legislation regarding access to the information from strategic noise maps, since it requires "that the strategic noise maps ... are made available and disseminated to the public in accordance with relevant Community legislation ... and in conformity with Annex IV ... to this Directive." Under its terms of reference for 2007 the European Environment Agency's (EEA) Working Group on the Assessment of Exposure (WG-AEN) was asked to produce a Position Paper containing the Group's ideas and suggestions on good practice and associated strategies for presenting noise mapping information to the public at local and national levels. Many of these ideas and suggestion were developed from the outcomes of a workshop held in London in October, 2006. The final draft of the Position Paper was completed in December, 2007. This conference paper and associated verbal presentation will provide an overview of the contents of the Position Paper and report on developments since December, 2007. 
1pNSd4. Limits and advantages of Good Practice Guide to Noise Mapping. Gaetano Licitra (ARPAT - Dept. Firenze, Via Porpora, 22, 50144 Firenze, Italy, g.licitra@arpat.toscana.it), Gianluca Memoli (Imperial College London, Department of Chemical Engineering, Exhibition road, SW7 2AZ London, UK, g.memoli@imperial.ac.uk)

The Pisa Noise Mapping Project has recently presented to the public what turned out to be the first noise map for road traffic in Italy, developed taking into account the Good Practice Guide version 2 (GPG2) of WG-AEN and the main results of the IMAGINE project. This paper will discuss the results of this noise map, relative to road traffic, in terms of Lden and Lnight and their uncertainties, obtained by comparing the calculated values with a set of noise measurements taken across the territory. The uncertainties so defined were compared with the ones predicted by GPG2 considering, in particular, two different ways to model the source. To do this, input traffic flow were assigned first by taking direct measurements and performing a road classification and then, at a second stage, using a static traffic model (the latter method should give less uncertainty, according to GPG2). The expected change in uncertainty will be discussed, together with advantages and disvantages of the two different choices. A comparison of exposed population with other EU realities will be also presented.

\title{
Contributed Paper
}

7:00

1pNSd5. A noise mapping study of a large metropolitan city with a heterogeneous traffic. Ramachandra Alur (Indian Institute of Technology, Acoustics Laboratory, Dept of Civil Engg, IIT- M, 600036 Chennai, India, ram_alur@yahoo.com), Kalaiselvi Ramasamy (Indian Institute of Technology, Acoustics Laboratory, Dept of Civil Engg, IIT- M, 600036 Chennai, India, kalai_archi@yahoo.com)

Traffic noise characteristics in some of the cities in a developing country like India are slightly varied by virtue of the fact that the composition of the traffic is heterogeneous accompanied by variance in road geometry and varying density of the buildings on the either side of the road. To study the feasibility of noise reduction in some of the areas a noise mapping study has been attempted along with field measurements of L10, L50, L90 and Leq. In the noise mapping parameters such as Ld, LN, Lden have been arrived at by taking into consideration the geometrical features of the roads and varying heights of the buildings. In this study noise mapping through computer simulation model (soundplan) is used by considering several noise sources and propagation of noise to the receiver point. This paper describes how the local characteristics of the city affect the propagation of noise and describes an integrated approach to control noise pollution in these areas. A three dimensional acoustical model has been developed for two locations of the city. Methodologies of noise control through noise contours around traffic are illustrated.

MONDAY AFTERNOON, 30 JUNE 2008

AMPHI HAVANE, 1:00 TO 7:40 P.M.

\section{Session 1pPAa}

\section{Physical Acoustics: Acoustics of Porous Media I}

\author{
Walter Lauriks, Cochair \\ Lab. ATF, Katholieke Universiteit Leuven, Celestijnenlaan 200D, Leuven, B-3001, Belgium
}

Keith Attenborough, Cochair

Open University, Department of Design, Development, Materials and Environment, Walton Hall, Milton Keynes, MK7 6AA, UK

\section{Invited Papers}

\section{1:00}

1pPAa1. Nonlinear acoustic acceleration waves in porous media flow. Pedro M. Jordan (U.S. Naval Research Lab., Code 7181, Stennis Space Center, MS 39529-5004, USA, pjordan@nrlssc.navy.mil)

Acoustic acceleration waves are defined as jumps in the first derivatives of the velocity, pressure, or density across a propagating singular surface (or wavefront). In this talk, the temporal evolution of the amplitude and the propagation speed of such waves are investigated in the context of finite-amplitude acoustic propagation in Darcy-type porous media. It is shown that there exists a critical value, $\alpha^{*}(>0)$, of the initial jump amplitude such that the acceleration wave magnitude either goes to zero, as $t \leftarrow \infty$, or blows up, in finite time, depending on whether the given initial jump amplitude is less than or greater than $\alpha^{*}$. In addition, a connection to traveling wave solutions is noted and the linearized case is examined. Finally, the numerical solution of a (1D) nonlinear IBVP involving sinusoidal signaling in a fluid-saturated porous slab is used to illustrate the finite-time transition from acceleration wave to shock wave, which occurs when the initial jump amplitude is greater than $\alpha^{*}$. [Supported by ONR/NRL funding (PE 061153N).] 
1pPAa2. New equipment for the measurements of flow resistivity and porosity of open cell ceramic and metal foams. Benjamin Jados (Illinois Institute of Technology, Civil, Architectural and Environmental Engineering, 3201 S. Dearborn St., Room 228, Chicago, IL 60616, USA, jadoben@iit.edu), Ralph T. Muehleisen (Illinois Institute of Technology, Civil, Architectural and Environmental Engineering, 3201 S. Dearborn St., Room 228, Chicago, IL 60616, USA, muehleisen@iit.edu)

Two of the most important measurements in the acoustic analysis of porous media are the flow resistivity and porosity. To reduce the errors in the measurements of these parameters, new instruments have been developed, which incorporate recent technological advances. The new equipment helps to streamline the user experience and improve the confidence of the results. The current design includes the use of LabVIEW and COMSOL Multiphysics software, the use of thermal press-fit sample holders, and the use of low differential pressure transducers. The new instruments were used to study the flow resistivity and porosity of various pore sizes of open cell ceramic and metal foams. Data are presented with accompanying errors, as well as a discussion of the shortcomings and possible improvements of the measurement system.

\section{$1: 40$}

1pPAa3. Semi-empirical time domain model of sound attenuation in porous materials. Olga Umnova (University of Salford, Acoustics Research Centre, Newton Building, M5 4WT Salford, UK, o.umnova@salford.ac.uk), Diego Turo (University of Salford, Acoustics Research Centre, Newton Building, M5 4WT Salford, UK, d.turo@pgr.salford.ac.uk)

A semi-empirical model for complex tortuosity function, which satisfies physically correct low and high frequency limits and allows analytical transformation into the time domain has been developed. It is based on the assumption, that a network of pores with two characteristic sizes can approximate the internal structure of the material, and thus requires the knowledge of two relaxation times. It is proven, however, that the model can predict sufficiently well the acoustical properties of rigid porous materials with various microstructures when is complete with the tortuosity as an additional parameter. It is shown that relaxation times can be easily related to the "equivalent fluid" model parameters. Numerical time domain calculations confirm that the contribution of both viscous and inertial effects on the evolution of acoustical pulses as they propagate through a porous material can be accounted for simultaneously within the framework of the model. It is shown that the extended version of the model, which accounts for the thermal effects can also be explicitely used for time-domain computations.

1pPAa4. Acoustical and micro-structural properties of recycled grains and fibres. Kirill V. Horoshenkov (University of Bradford, School of Engineering, Design and Technology, BD7 1DP Bradford, UK, k.horoshenkov@Bradford.ac.uk), Amir Khan (University of Bradford, School of Engineering, Design and Technology, BD7 1DP Bradford, UK, a.khan72@bradford.ac.uk), Hadj Benkreira (University of Bradford, School of Engineering, Design and Technology, BD7 1DP Bradford, UK, h.benkreira@bradford.ac.uk), Giulio Pispola (Umbra Group, Umbra Cuscinetti S.p.A., Zona Industriale, Localita Paciana, 06034 Foligno, Italy, gpispola@umbracus.com)

This paper presents a systematic study into the production, characterisation and modeling of the acoustic behaviour of highly heterogeneous, low density porous layers having a complex pore size distribution. A new cold extrusion production method was developed at the University of Bradford to process recycled polymeric fibres and grains so that accurate control of the pore size distribution and the porosity of the resultant porous product could be attained. In this way high values of the acoustic absorption coefficient could be obtained in a relatively thin porous layer throughout the design frequency range. Two approaches were used to model the acoustic performance of the manufactured porous media. The first approach requires the direct numerical integration of the Biot viscosity correction function which depends on the probability density function of the pore size. The other approach assumes a low permeability contrast between the two porous scales so that the acoustic properties could be estimated using the semi-phenomenological models of Johnson and Lafarge for the viscous and thermal dynamic permeabilities. Numerical results predicted by the two models were then compared with impedance tube experimental data showing good accuracy of the selected prediction methods.

1pPAa5. Deduction of porous material properties using a point source. Jean François Allard (Laboratoire d'Acoustique de l’Université du Maine, Avenue Olivier Messiaen, 72085 Le Mans, France, jean-francois.allard@univ-lemans.fr), Olivier Dazel (Laboratoire d'Acoustique de l'Université du Maine, Avenue Olivier Messiaen, 72085 Le Mans, France, olivier.dazel@univ-lemans.fr), Laurens Boeckx (Lab. ATF, Katholieke Universiteit Leuven, Celestijnenlaan 200D, B-3001 Leuven, Belgium, laurens.boeckx@fys.kuleuven.be), Nathalie Geebelen (Lab. ATF, Katholieke Universiteit Leuven, Celestijnenlaan 200D, B-3001 Leuven, Belgium, Nathalie.Geebelen@bwk.kuleuven.be), Walter Lauriks (Lab. ATF, Katholieke Universiteit Leuven, Celestijnenlaan 200D, B-3001 Leuven, Belgium, Walter.Lauriks@ fys.kuleuven.be)

The acoustic field created by a point source above the plane boundary between two semi-infinite fluid layers has been previously studied by Brekhovskikh. A straightforward generalization can be performed by replacing the fluid under the source by the fluid equivalent to a rigid-framed porous medium. This leads to a method of evaluating, from pressure measurements, the surface impedance close to grazing incidence and the Brewster angle of total refraction of the porous medium. The model by Brekhovskikh can also be generalized when a thin porous layer is set under the point source. A pole of the reflection coefficient exists for an angle of incidence close to grazing incidence. This pole is related to an acoustic field similar to a surface wave above the layer. The measurement of this angle provides an evaluation of the surface impedance close to grazing incidence. The point source induces frame vibrations that can be predicted with the Biot theory. The rigidity coefficients of the frame at audible frequencies can be evaluated from measurements of the frame velocity with a laser velocimeter. 
1pPAa6. Acoustic waves propagation along mechanically free surface of unconsolidated granular porous media. Xavier Jacob (LPEC/UMR 6087/CNRS/Université du Maine, Avenue Olivier Messiaen, 72085 Le Mans Cedex 09, France, xavier.jacob@univ-lemans.fr), Vladislav Aleshin (LPEC/UMR 6087/CNRS/Université du Maine, Avenue Olivier Messiaen, 72085 Le Mans Cedex 09, France, aleshinv@mail.ru), Vincent Tournat (LPEC/UMR 6087/CNRS/Université du Maine, Avenue Olivier Messiaen, 72085 Le Mans Cedex 09, France, vincent.tournat@univ-lemans.fr), Philippe Leclaire (Lab. de Recherche en Mécanique et Acoustique, I.S.A.T - Univ. de Bourgogne, 49, rue Mademoiselle Bourgeois, 58000 Nevers, France, philippe.leclaire@gmail.com), Walter Lauriks (Lab. ATF, Katholieke Universiteit Leuven, Celestijnenlaan 200D, B-3001 Leuven, Belgium, Walter.Lauriks@fys.kuleuven.be), Vitali Gusev (LPEC/UMR 6087/CNRS/Université du Maine, Avenue Olivier Messiaen, 72085 Le Mans Cedex 09, France, vitali.goussev@univ-lemans.fr)

Unconsolidated granular materials exhibit strong dependence of elastic properties on pressure due to high sensitivity of the intergrain contacts to magnitude of loading. As a consequence the gravity field makes these granular assemblages highly elastically inhomogeneous particularly near mechanically free surface. Theoretical and experimental investigations conducted recently have demonstrated that multiple waveguide surface acoustic modes propagate along the free surface of the solid-state skeleton in the disordered air-saturated granular packings. These modes are localized near the surface. In ordered granular packing the acoustic waves can travel along the surface inside the horizontal channels, which are localized beneath the surface. The waves of different frequencies are travelling at different depths. The anisotropy of these inhomogeneous granular phononic crystals has an important influence of the existence of the waveguide modes. The experiments with acoustic waves traveling in the vicinity of a free surface provide fundamental information on the mechanical behaviour of unconsolidated granular media at very low pressures in the vicinity of the jamming transition. The perspectives of this research could be related to the analysis of the role which could be played in the considered phenomena by the acoustic waves predominantly travelling in these granular porous assemblages through the fluid-saturated pores.

1pPAa7. The Biot Type II wave in the fluid and matrix of soils. James M. Sabatier (University of Mississippi, NCPA, 1 Coliseum Drive, University, MS 38677, USA, sabatier@olemiss.edu), Chris McNeill (University of Mississippi, NCPA, 1 Coliseum Drive, University, MS 38677, USA, cmcneil1@olemiss.edu)

When an acoustic wave is incident on the surface of the porous ground energy is coupled into the soil matrix and fluid. To investigate the depth dependence of the Type I and II waves from the ground surface, the ground is modeled as a semi-infinite air-filled poroelastic medium. Using a modified form of Biot-Stoll theory the magnitude and phase of the matrix velocity and fluid pressure caused by the two possible dilatational waves are determined. Traditionally speaking the type I (fast) wave is the nondispersive wave traveling in the solid, and the type II (slow) wave is dispersive and travels primarily in the fluid (air). Previous experiments have shown the possibility that the type II wave travels in the solid near the surface and influences the measured matrix velocity and fluid pressure. Calculations have been done showing that near the surface of the ground the type II wave actually does have a large effect on the particle velocity of the matrix. After the first few centimeters the type II wave is completely attenuated and the type I wave is responsible for the displacement and pressure in the medium

\section{3:20}

1pPAa8. Anisotropy effects on the acoustical properties of porous materials. Olivier Dazel (Laboratoire d'Acoustique de l’Université du Maine, Avenue Olivier Messiaen, 72085 Le Mans, France, olivier.dazel@univ-lemans.fr), Jean François Allard (Laboratoire d'Acoustique de l'Université du Maine, Avenue Olivier Messiaen, 72085 Le Mans, France, jean-francois.allard@univ-lemans.fr), Laurens Boeckx (Lab. ATF, Katholieke Universiteit Leuven, Celestijnenlaan 200D, B-3001 Leuven, Belgium, laurens.boeckx@fys.kuleuven.be), Nathalie Geebelen (Lab. ATF, Katholieke Universiteit Leuven, Celestijnenlaan 200D, B-3001 Leuven, Belgium, Nathalie.Geebelen@bwk.kuleuven.be), Poonam Khurana (Lab. ATF, Katholieke Universiteit Leuven, Celestijnenlaan 200D, B-3001 Leuven, Belgium, Poonam.Khurana@fys.kuleuven.be), Walter Lauriks (Lab. ATF, Katholieke Universiteit Leuven, Celestijnenlaan 200D, B-3001 Leuven, Belgium, Walter.Lauriks@fys.kuleuven.be)

Porous materials are now widely used in noise control for their acoustic properties in sound absorption and transmission. These properties are function of the internal porous medium structure. Generally, most of the models assume that the porous medium is isotropic. Mineral wools (as well as some foams) clearly present an anisotropic structure. This communication is concerned with the adaptation of recent works on isotropic materials to the case of anisotropic porous materials. The general theory will be exposed for sound absorbing materials with anisotropic acoustical and mechanical parameters. The case of transverse isotropic materials (with fibers organized in planes running parallels one to each other) will then be detailed in particular in the case when the plane of fibers is not the same than the normal plane of the sample. The influence on the acoustical properties anisotropic porous materials will then be presented. Illustrations are then provided with measurements performed on wools and it will be shown that the rigidity coefficients of a sample can be evaluated at audible frequencies from measurements in particular from the speed of the Rayleigh wave in different directions. 


\section{Contributed Papers}

3:40

1pPAa9. Low frequency implementation of the full Kramers-Kronig relationships for the description of dispersion and attenuation of acoustical waves in porous media. Bernard R. Castagnede (Laboratoire d'Acoustique de l'Université du Maine, Avenue Olivier Messiaen, 72085 Le Mans, France, bernard.castagnede@univ-lemans.fr), Denis Lafarge (Laboratoire d'Acoustique de l'Université du Maine, Avenue Olivier Messiaen, 72085 Le Mans, France, denis.lafarge@univ-lemans.fr), Claude_Depollier (Laboratoire d'Acoustique de l'Université du Maine, Avenue Olivier Messiaen, 72085 Le Mans, France, claude.depollier@univ-lemans.fr), Naima Sebaa (Laboratoire d'Acoustique de l'Université du Maine, Avenue Olivier Messiaen, 72085 Le Mans, France, naima.sebaa@univ-lemans.fr), Michel Henry (Laboratoire d'Acoustique de l'Université du Maine, Avenue Olivier Messiaen, 72085 Le Mans, France, michel.henry@univ-lemans.fr)

It is well known that the causality principle which applies during the acoustic propagation of a wave packet inside any material should be described by the universal Kramers-Kronig (K-K) relationships, enabling to formally link dispersion to attenuation curves. The application of the K-K formalism to porous networks has been studied lately, but unfortunately, the metrology of dispersion features at very low frequency is intricate. The use of parametric arrays of nonlinear acoustics enables to get at the same time, some precise information on dispersion and absorption on various porous media, for instance in the frame of the "equivalent fluid" model. In the present work, we describe some very recent findings obtained along these ideas for a truly relevant application of the full $\mathrm{KK}$ formalism in various fibrous materials. We have checked numerical predictions of the absorption versus frequency computed from the dispersion curves with the K-K modeling, as well as confrontation of these numerical predictions with experimental data on absorption obtained with various set-ups, on different fibrous materials.

\section{4:00-4:20 Break}

\section{4:20}

1pPAa10. On the diffusion of sound energy in porous materials. Nicola Prodi (Engineering Dept. - Univ. of Ferrara, Via Saragat, 1, 44100 Ferrara, Italy, nicola.prodi@unife.it)

The sound intensity was used in the past to describe the surface properties of porous materials. Actually no extensive study used the same concepts to describe the properties of the energy flow inside the material. As known, the sound intensity has a twofold nature, that is an active part transferring the energy out of a point and a reactive one localizing the energy. These features are crucial in the development of the new model for the propagation of the energy inside porous materials developed in this work. In fact, by means of the intensimetric approach, it is shown that the one-dimensional transfer of sound energy can be fully described as a diffusion process. The role of the active and reactive sound intensities is outlined and specific parameters are defined to account for the material behaviour with respect to the transfer of the sound energy. Moreover the sinks of energy and the sources of reactivity in the material are derived and discussed.

\section{4:40}

1pPAa11. Optimal sound absorbing and manufacturable two-dimensional, hexagonal-like porous structure. Camille Perrot (Groupe d'Acoustique de l'Université de Sherbrooke, 2500, Boul. de 1'Université, Département de génie mécanique, Sherbrooke, QC J1K-2R1, Canada, camille.perrot@usherbrooke.ca), Fabien Chevillotte (Groupe d'Acoustique de l'Université de Sherbrooke, 2500, Boul. de l'Université, Département de génie mécanique, Sherbrooke, QC J1K-2R1, Canada, fabien.chevillotte@usherbrooke.ca), Raymond Panneton (Groupe d'Acoustique de l'Université de Sherbrooke, 2500, Boul. de l'Université, Département de génie mécanique, Sherbrooke, QC J1K-2R1, Canada, raymond.panneton@usherbrooke.ca)

Results from a numerical study examining micro-macro relations linking local geometry parameters to sound absorption properties are presented. For an hexagonal structure of solid fibers, the porosity $\phi$, the thermal characteristic length $\Lambda^{\prime}$, the static viscous permeability ko, the tortuosity $\alpha_{\infty}$, the viscous characteristic length $\Lambda$, and the sound absorption coefficient are computed. Numerical solutions of the steady Stokes and electrical equations are employed to provide ko, $\alpha_{\infty}$, and $\Lambda$. Hybrid estimates based on direct numerical evaluation of porosity $\phi, \Lambda^{\prime}$, ko, $\alpha_{\infty}, \Lambda$ and the analytical model derived by Johnson, Champoux, and Allard are used to relate varying (i) throat size, (ii) pore size, and (iii) fibers cross-section shapes to the sound absorption spectrum. The result of this paper tends to demonstrate the important effect of throat size in the sound absorption level, cell size in the sound absorption frequency selectivity, fibers cross-section shape in the porous material weight reduction. In a hexagonal porous structure with solid fibers, the sound absorption level will tend to be maximized with a $48 \pm 10$ $\mu \mathrm{m}$ throat size corresponding to an intermediate resistivity, a $13 \pm 8 \mu \mathrm{m}$ fiber radius associated with small pores, and convex triangular cross-section shape fibers allowing weight reduction.

\section{5:00}

1pPAa12. Analytical microstructural model for acoustical porous materials with single or double porosity. Emmanuel Gourdon (Université de Lyon, Ecole Nationale des Travaux Publics de l'Etat, CNRS, URA 1652, Département Génie Civil, 3, rue Maurice Audin, F-69120 Vaulx-en-Velin, France, emmanuel.gourdon@entpe.fr), Luc Jaouen (Matelys - Acoustique \& Vibrations, 20/24 rue Robert Desnos, 69120 Vaulx-en-Velin, France, luc.jaouen@matelys.com)

An analytical model of sound propagation for porous materials with single or double scale of porosity is described. For each scale, pores and interconnections between them are modeled by a serie of two cylinders; a cylindrical periodical cell is thus considered. Scales are supposed to be separated, the porous medium is supposed to be periodic and to have a motionless skeleton. The geometrical parameters needed to quantify visco-thermal effects are directly related to the microstructure of the material. These parameters: lengths and radii of pores and interconnections can be extracted from image analysis for example. From additional conditions on cell morphology, independent parameters per porosity scale can be reduced to a number of three. Good comparisons between theoretical calculations of the sound absorption coefficient at normal incidence and impedance tube measurements are obtained for single and double porosity (meso-perforated) materials.

\section{$5: 20$}

1pPAa13. Adsorption in activated carbon and its effects on the low frequency performance of hearing defenders. Fouad Bechwati (University of Salford, Acoustics Research Centre, Newton Building, M5 4WT Salford, UK, f.bechwati@pgr.salford.ac.uk), Trevor J. Cox (University of Salford, Acoustics Research Centre, Newton Building, M5 4WT Salford, UK, t.j.cox@salford.ac.uk), Mark R. Avis (University of Salford, Acoustics Research Centre, Newton Building, M5 4WT Salford, UK, m.r.avis@salford.ac.uk), Olga Umnova (University of Salford, Acoustics Research Centre, Newton Building, M5 4WT Salford, UK, o.umnova @ salford.ac.uk)

Activated carbon displays interesting behaviours at low frequencies due to its large internal surface area and complex network of pores of various sizes and shapes. The material can produce larger than expected absorption and change the compliance of acoustic enclosures. This paper investigates the performance of hearing defenders which utilize activated carbon as the lining material of the cup. Compared to a standard foam liner, the introduction of activated carbon increases the insertion loss by up to 15 to $20 \mathrm{~dB}$ at frequencies between 31.5 and $250 \mathrm{~Hz}$. The enhanced insertion loss is due to the increase in stiffness and apparent volume of the cup cavity as the activated carbon is introduced. This is probably due to the substantial change in local density as air molecules adsorb onto, and desorb from, the activated carbon pores during sound propagation. There is a change in entropy and energy loss from the sound wave during the adsorption/ desorption process due to the existence of a hysteresis loop. This additional absorption enhances performance. 
$5: 40$

1pPAa14. Ultrasonic behaviour of high pressure air filled porous media. Stephane Griffiths (LAUM, CNRS, Université du Maine, Av. O. Messiaen, 72085 Le Mans, France, stephane.griffiths@live.fr), Christophe Ayrault (LAUM, CNRS, Université du Maine, Av. O. Messiaen, 72085 Le Mans, France, christophe.ayrault@univ-lemans.fr)

This paper deals with the acoustic behaviour of porous media when the saturating fluid is high pressured. These observations are performed by ultrasonic transmission through a porous sample with variations of the static pressure of the saturating fluid. In order to characterize high damping materials, measurements are performed for high static pressure (up to 18 bars) It is shown that the behaviour of transmission coefficient and speed with pressure follow the Biot's theory. Moreover, measurements are strongly dependant on temperature, which is not visible in modelizations with Biot's model, although this parameter is taken into account in thermodynamical parameters. It is therefore assumed that mechanical characteristics vary with temperature and pressure. An estimation of mechanical parameters is then performed by minimization between the Biot's model and experimental data. First results, obtained in suitable cases for which measurements quality is good and minimization process converges correctly, show that mechanical parameters follow the evolution with frequency described by Pritz at low frequencies. Further researches are still necessary to determine dependance to temperature.

\section{6:00}

1pPAa15. Automatic characterisation of ground surfaces from in situ measurements. Guillaume Dutilleux (Lab. Régional des Ponts et Chaussées, 11, rue Jean Mentelin, BP 9, 67035 Strasbourg Cedex 2, France, Guillaume.Dutilleux@equipement.gouv.fr), David Ecotiere (Lab. Régional des Ponts et Chaussées, 11, rue Jean Mentelin, BP 9, 67035 Strasbourg Cedex 2, France, david.ecotiere@equipement.gouv.fr)

The study of long term acoustic performance of road pavements requires to access the evolution of the intrinsic parameters of these surfaces, such as resistivity or tortuosity. In long range sound propagation as well, it is well known that ground absorption is time varying. The understanding of this variation is an important issue. In both application cases, nondestructive measurement methods are required. The present contribution focuses on the 2-parameter model by Delany and Bazley for outdoor surfaces and the 4-parameter one by Hamet-Berengier for porous road pavements. It is shown that for both models the absorption coefficient spectrum obtained by an ISO 13472-1 compliant measurement contains enough data for the automatic identification. For the 2-parameter model the identification is based on a suitable cost function. The complexity of the function is low enough for a resolution by exhaustive search. It turns out that, for a large set of soft ground samples, the cost function is convex. Therefore the resolution can be done quite efficiently by classical gradient descent methods. Regarding the 4-parameter model, although the cost function is no longer convex, the resolution can be performed using a global optimization tool, like an evolutionary algorithm.

\section{6:20}

1pPAa16. A new setup for measuring the mechanical properties of porous materials. Nicolas Dauchez (Laboratoire d'Acoustique de l'Université du Maine, Avenue Olivier Messiaen, 72085 Le Mans, France, nicolas.dauchez@univ-lemans.fr), Olivier_ Doutres (Laboratoire d'Acoustique de l'Université du Maine, Avenue Olivier Messiaen, 72085 Le Mans, France, olivier.doutres.etu@univ-lemans.fr), Jean Michel Genevaux (Laboratoire d'Acoustique de l'Université du Maine, Avenue Olivier Messiaen, $72085 \quad$ Le Mans, France, jean-michel.genevaux@univ-lemans.fr), Guy Lemarquand (Laboratoire d'Acoustique de l'Université du Maine, Avenue Olivier Messiaen, 72085 Le Mans, France, guy.lemarquand@univ-lemans.fr)

A new device for determining complex Young's modulus of porous materials in a extended frequency range is proposed. Classical methods are based on quasitatic or dynamic response of porous material. These methods generaly neglect the coupling between the surrounding fluid and the porous frame so that they are restricted to low frequency range $(<100 \mathrm{~Hz})$ or specific sample shape. Dynamic methods provide relevant information only at the resonance frequencies of the frame. The proposed method extends the quasistatic method towards high frequencies: 1. the porous sample is setup in a cavity in order to avoid the coupling with the external fluid, 2. a specific electrodynamic transducer has been developped to get the mechanical impedance of the sample from the measurement of the electrical impedance, 3 . mechanical properties of the frame are derived by inverse method using Biot theory so that the frequency range is not restricted to the quasistatic domain First results obtained with a prototype validate the method in comparison with two classical methods.

\section{6:40}

1pPAa17. Quasistatic evaluation of mechanical properties of poroelastic materials: static and dynamic strain dependence and in vacuum tests. Paolo Bonfiglio (Dipartimento di Ingegneria - University of Ferrara, Via Saragat 1, 44100 Ferrara, Italy, paolo.bonfiglio@unife.it), Francesco Pompoli (Dipartimento di Ingegneria - University of Ferrara, Via Saragat 1, 44100 Ferrara, Italy, francesco.pompoli@unife.it), Paresh Shravage (Dipartimento di Ingegneria - University of Ferrara, Via Saragat 1, 44100 Ferrara, Italy, paresh.shravage @unife.it)

A complete description of the vibro-acoustical behavior of a poroelastic material requires the knowledge of both geometrical quantities, related to the structure of the fluid-filled pores to account the sound propagation within them, and mechanical parameters (i.e. Young Modulus, Poisson's ratio, and loss factor) in order to model the wave propagation through the elastic structure constituting its skeleton. Because the nonlinear nature of poroelastic media, those mechanical properties are shown depending on static preload and dynamic strain applied to them. In the present work a well established quasi-static method, based on the measurement of mechanical impedance and the use of adequate polynomial relations, has been used to determine the dependence of the mechanical properties on the applied deformations. Furthermore, tests have been also carried out in a vacuum chamber in order to evaluate the real contribution of the filling fluid on the total vibro-acoustical response of the material.

\section{7:00}

1pPAa18. Sound absorbing properties of materials made of rubber crumbs. Francesco Asdrubali (Università degli studi di Perugia, Via G. Duranti 67, 06125 Perugia, Italy, fasdruba@unipg.it), Francesco D’Alessandro (Università degli studi di Perugia, Via G. Duranti 67, 06125 Perugia, Italy, dalessandro.unipg@ciriaf.it), Samuele Schiavoni (Università degli studi di Perugia, Via G. Duranti 67, 06125 Perugia, Italy, schiavo81 @ libero.it)

Recycled tyre granules can be used for manufacturing acoustic insulating and absorbing materials, with applications in buildings and road barriers. Therefore, the production of these materials is a valid alternative to the disposal into landfill or incineration of used tyres. This paper presents the results of sound absorbing coefficient measurements of several samples manufactured at the Acoustics Laboratory of the University of Perugia. The sound absorbing panels were produced by mixing rubber crumbs and an adequate binder in a proper proportion and then by compacting the obtained mix. The methodology used to evaluate coefficient of absorption coefficient is indicated in ISO 10534-2 standard, thanks to an impedance tube. The influence on the absorption performance of granules size, binder concentration, thickness and compaction ratio of the samples was investigated and an optimization process was carried out, in order to produce a sample with satisfying acoustical performances

$$
7: 20
$$

1pPAa19. Measurement of acoustic and mechanical parameters of poroelastic materials by mean of active control and wave-based methods. Cedric Batifol (Centre Acoustique du LMFA, Ecole Centrale de Lyon, 36 avenue Guy de Collongue, 69134 Ecully cedex, France, cedric.batifol@ec-lyon.fr), Marie-Annick Galland (Centre Acoustique du LMFA, Ecole Centrale de Lyon, 36 avenue Guy de Collongue, 69134 Ecully 
cedex, France, marie-annick.galland@ec-lyon.fr),Mohamed Ichchou(Centre Acoustique du LMFA, Ecole Centrale de Lyon, 36 avenue Guy de Collongue, 69134 Ecully cedex, France, mohamed.ichchou@ec-lyon.fr)

Poroelastic materials are used in sound barriers for sound insulation and absorption. At low frequencies, the medium is modeled using the Biot theory. This homogenised model needs to be feed by at least eight parameters using the Johnson-Allard approach. The presented characterisation method relies on two specific test facilities. First, acoustic parameters are obtained thanks to a modified Kundt tube. The porosity and resistivity are obtained by direct measurements of the surface impedance with hard wall and zero pressure boundary conditions on the rear face of the sample, respectively. This last boundary conditions is achieved thanks to active control procedure. Finally, the remaining acoustic parameters are obtained in a least mean square sense. Mechanical parameters are then measured on a second test bench. A beam made of the poroelastic sample is subjected to mechanical broadband forcing. The transverse displacement is measured along the beam thanks to a laser vibrometre. Dispersion curves are then obtained by calculating the Inhomogeneous Wave Correlation (IWC) ratio. The Young modulus and loss factor of the sample are estimated thanks to an optimisation procedure applied to the finite element model of the test. Results are compared to those obtained using more classical quasi-static methods.

MONDAY AFTERNOON, 30 JUNE 2008

ROOM 351, 1:00 TO 7:00 P.M.

\title{
Session 1pPAb
}

\section{Physical Acoustics: Phononic Crystals I}

\author{
Pierre Deymier, Cochair \\ University of Arizona, Materials Science and Engineering Department, Mines Bldg., P.O. Box 210012, Tucson, AZ 85721, USA \\ Jérôme Vasseur, Cochair \\ IEMN, UMR CNRS 8520, avenue Poincaré, BP 60069, Villeneuve d'Ascq, 59652, France
}

\section{Contributed Papers}

\section{1:00}

1pPAb1. Rubber/air acoustic band gap materials: Elastic and viscoelastic effects. Bassam Merheb (University of Arizona, Materials Science and Engineering Department, Mines Bldg., P.O. Box 210012, Tucson, AZ 85721, USA, bassam@merheb.net), Pierre Deymier (University of Arizona, Materials Science and Engineering Department, Mines Bldg., P.O. Box 210012, Tucson, AZ 85721, USA, deymier@u.arizona.edu), Manish Jain (3M Corporation, Corporate Research Materials Laboratory, Bldg. 201-3N-04, 3M Center, St. Paul, MN 55144-1000, USA, mjain@mmm.com), Marie Aloshyna-Lesuffleur (3M Corporation, Corporate Research Materials Laboratory, Bldg. 201-3N-04, 3M Center, St. Paul, MN 55144-1000, USA, mlesuffleur@mmm.com), Richard Greger (3M Corporation, Corporate Research Materials Laboratory, Bldg. 201-3N-04, 3M Center, St. Paul, MN 55144-1000, USA, rgreger@mmm.com), Sanat Mohanty (3M Corporation, Corporate Research Materials Laboratory, Bldg. 201-3N-04, 3M Center, St. Paul, MN 55144-1000, USA, smohanty@mmm.com), Ali Berker (3M Corporation, Corporate Research Materials Laboratory, Bldg. 201-3N-04, 3M Center, St. Paul, MN 551441000, USA, aberker@mmm.com)

Two-dimensional elastic and viscoelastic phononic crystals structures are investigated theoretically and experimentally. These are two-dimensional arrays of cylinders of air inclusions in a solid silicone rubber matrix as well as arrays of silicone rubber cylinders in an air matrix. In this study, viscoelasticity is modeled with a compressible general linear viscoelastic fluid model. Numerical calculations of transmission spectra and band structures are conducted by extending the finite difference time domain (FDTD) method to account for linear viscoelastic materials which exhibit timedependent moduli. These systems demonstrate very wide band gaps in their transmission spectra that extend to frequencies in the audible range of the spectrum. Experimental and calculated transmission spectra for a rubber matrix/air inclusions phononic crystal show that the system behaves as a fluid/fluid composite with respect to the longitudinal polarization of acoustic waves due to the large contrast between the transverse and longitudinal speeds of sound. We also demonstrate that viscoelasticity can attenuate transmission over very wide ranges of frequency leaving only passing bands at very low frequency. These phononic crystals demonstrate the practical design of elastic or viscoelastic solid rubber/air acoustic band gap sound barrier with small dimensions.

\section{$1: 20$}

1pPAb2. Development of a suitable PML for an harmonic study of a finite 1D phononic crystal. Maxime Bavencoffe (LOMC FRE-3102 CNRS, Groupe Ondes Acoustiques, University of Le Havre, Place Robert Schuman, BP 4006, 76610 Le Havre, France, maxime.bavencoffe@univ-lehavre.fr), Bruno Morvan (LOMC FRE-3102 CNRS, Groupe Ondes Acoustiques, University of Le Havre, Place Robert Schuman, BP 4006, 76610 Le Havre, France, bruno.morvan@univ-lehavre.fr), Anne-Christine Hladky (IEMN, UMR CNRS 8520, avenue Poincaré, BP 60069, 59652 Villeneuve d'Ascq, France, anne-christine.hladky@isen.fr), Olivier Bou Matar (IEMN, UMR CNRS 8520, avenue Poincaré, BP 60069, 59652 Villeneuve d'Ascq, France, olivier.boumatar@iemn.univ-lille1.fr), Jean-Louis Izbicki (LOMC FRE3102 CNRS, Groupe Ondes Acoustiques, University of Le Havre, Place Robert Schuman, BP 4006, 76610 Le Havre, France, jean-louis.izbicki @ univ-lehavre.fr)

This paper deals with the interaction of ultrasonic Lamb waves with a 1D phononic crystal. The studied structure is a finite plate with a periodic corrugated surface. Two types of forbidden bands arise in the Lamb wave dispersion curves. The first one is located at the limit of the first Brillouin zone, the second one exists at the crossing of dispersion curves of two different Lamb modes. These forbidden bands lead to conversion phenomena. In order to study accuratly the conversion phenomena in the band gaps, harmonic finite element analysis are performed. Perfect matching layers (PML) on both sides of the plate are then necessary to avoid stationnary waves. PML, adapted for Lamb modes involved at a given frequency, must be designed. By using these PML, the attenuation of Lamb waves propagating in the phononic crystal is clearly shown and is related to the existence of the forbidden band.

\section{1:40}

1pPAb3. Plate waves in phononic crystals slabs. Jiu-Jiu Chen (CNRS and Paris VI University, INSP - 140 rue de Lourmel, 75015 Paris, France, chen99nju@gmail.com), Bernard Bonello (CNRS and Paris VI University, INSP - 140 rue de Lourmel, 75015 Paris, France, bernard.bonello@insp .jussieu.fr)

We have computed the dispersion curves of plate waves propagating in periodic composite structures composed of isotropic tungsten cylinders 
embedded in a isotropic silicon background. The phononic crystal has a square symmetry and the calculation is based on the plane wave expansion method. The direction of propagation is defined by the angle $\varphi$ between $\Gamma \mathrm{X}$ in the reduced Brillouin zone and the wave vector k. If $\varphi=0\{0\}$ or $\varphi=90$ $\{0\}$, SH modes are simple hyperbolas and do not couple to the Lamb wave modes polarized in the sagittal plane. If is different from $0\{0\}$ or $90\{0\}, \mathrm{SH}$ modes convert to Lamb wave modes and couple with the flexural and dilatational modes. The symmetric modes splitting in the band structures along this direction and all three components of the particle displacements demonstrate this phenomenon which is different from the pure isotropic plate case where $\mathrm{SH}$ waves decouple to Lamb waves whatever the propagating direction.

\section{2:00}

1pPAb4. Analysis of bandpass spectra of phononic defect-mode waveguides based on mode coupling between point defects. Toyokatsu Miyashita (Dept. Electronics \& Informatics, Ryukoku University, Seta Oecho Yokotani 1-5, 520-2194 Otsu, Japan, miya@ rins.ryukoku.ac.jp)

Pass bands of defect-mode waveguides fabricated in sonic/phononic crystals have desirable characteristics for their practical applications to acoustic bandpass filters. Their mechanism is considered to originate in mode-coupling characteristics of point defects, a chain of which composes a defect-mode waveguide. Especially, mode confinement in each point defect or their mode coupling is found to decide the flatness of the pass band. For example, with a relatively strong mode confinement, the transmission of the pass band swings between 0 and $-16 \mathrm{~dB}$ with frequency, although the transmission outside the pass band remains still typically at $-50 \mathrm{~dB}$. On the contrary with a moderate mode confinement, the transmission remains fairly flat only with a swing of $2 \mathrm{~dB}$. We have obtained these characteristics not only by an elastic FDTD method but also by acoustic experiments. These have been investigated theoretically and experimentally based on analyses of the mode coupling between point defects. Two sonic/phononic crystals are considered; one is composed of acrylic-resin cylinders in air, and the other steel cylinders in water.

\section{2:20}

1pPAb5. Design and characterization of stop-band filters using PZT layer on silicon substrate phononic crystals. Jérôme Vasseur (IEMN, UMR CNRS 8520, avenue Poincaré, BP 60069, 59652 Villeneuve d'Ascq, France, jerome.vasseur@univ-lille1.fr), Anne-Christine Hladky-Hennion (IEMN, UMR CNRS 8520, avenue Poincaré, BP 60069 , $59652 \quad$ Villeneuve d'Ascq, France, Anne-Christine.Hladky@isen.fr), Bertrand Dubus (IEMN, UMR CNRS 8520, avenue Poincaré, BP 60069, 59652 Villeneuve d'Ascq, France, Bertrand.Dubus@isen.fr), Bahram Djafari-Rouhani (IEMN, UMR CNRS 8520, avenue Poincaré, BP 60069, 59652 Villeneuve d'Ascq, France, Bahram.Djafari-Rouhani@univ-lille1.fr), Bruno Morvan (LOMC FRE3102 CNRS, Groupe Ondes Acoustiques, University of Le Havre, Place Robert Schuman, BP 4006, 76610 Le Havre, France, bruno.morvan@univlehavre.fr)

Phononic crystals are periodic structures exhibiting absolute band gaps i.e. frequency bands in which the propagation of elastic waves is forbidden in all directions. Filtering is then a possible application of phononic crystals. Recently, the existence of absolute band gaps has also been theoretically demonstrated for guided elastic waves in a piezoelectric plate on a substrate [J. Vasseur et al, J. Appl. Phys, 101, 114904 (2007)], which is a geometry of interest for possible co-integration on silicon chip. The $2 \mathrm{D}$ phononic crystal was constituted by a square arrangement of cylindrical holes in a PZT layer deposited on a silicon substrate. In this communication, the realization of a stop-band filter constituted by a periodically patterned PZT layer, polarized along thickness, on silicon substrate and interdigitated electrodes (IDE) for emission/reception of guided elastic waves, is investigated. The filter characteristics are theoretically evaluated by using finite element simulations: dispersion curves of patterned PZT layer are computed for various pattern geometries to obtain the absolute band gap. Complete structure is then modeled, with appropriate IDE to propagate a guided mode in the piezoelectric layer. Finally, filtering capability of the structure is evaluated. Work supported by STMicroelectronics (Nano2008 program of French ministry of industry).

\section{2:40}

1pPAb6. Acoustic response of a soniclike crystal within a rigid frame porous plate. Jean-Philippe Groby (DRE/L2S - UMR8506 CNRS/Supelec/Univ. Paris Sud 11, Département de Recherche en Electromagnétisme/Laboratoire des signaux et systèmes, 3 rue Joliot-Curie, 91192 Gif-sur-Yvette cedex, France, groby@1ss.supelec.fr), Armand Wirgin (CNRS-Laboratoire de Mécanique et d'Acoustique, 31 Chemin Joseph Aiguier, 13402 Marseille, France, wirgin@lma.cnrs-mrs.fr), Erick Ogam (Laboratoire de Mécanique et d'Acoustique CNRS UPR-7051, 31, Chemin Joseph Aiguier, 13402 Marseille Cedex 20, France, ogam@1ma.cnrs-mrs.fr), Laurent De Ryck (Lab. ATF, Katholieke Universiteit Leuven, Celestijnenlaan 200D, B-3001 Leuven, Belgium, Laurent.DeRyck@fys.kuleuven.be), Walter Lauriks (Lab. ATF, Katholieke Universiteit Leuven, Celestijnenlaan 200D, B-3001 Leuven, Belgium, Walter.Lauriks@fys.kuleuven.be), Claude_Depollier (Laboratoire d'Acoustique de l'Université du Maine, Avenue Olivier Messiaen, 72085 Le Mans, France, claude.depollier@univ-lemans.fr)

The acoustic response (in particular, the transmission) of a periodic distribution of macroscopic inclusions within a rigid frame, porous plate (similar to a sonic crystal) is studied by the multipole method. Numerical results show that the addition of grating stacks leads to band gaps within the audible frequency range for a small number of stacks, this being associated with a large decrease of the transmission coefficient of the initial plate. The first band gap is of practical interest for noise suppression. The second band gap enables total acoustic absorption within a narrow frequency range due to the fact that a modified mode of the plate lies within this band gap.

\section{3:00}

1pPAb7. K-dependent polarization of sagittal acoustic waves in phononic crystals. Felipe Ramos-Mendieta (Universidad de Sonora, Rosales y Boulevard Luis Encinas, Colonia Centro, 83000 Hermosillo, Mexico, framos@cajeme.cifus.uson.mx), Betsabe Manzanares-Martinez (Division de Ciencias e Ingenieria, Unidad Sur Universidad de Sonora, Boulevard Lazaro Cardenas 100, 85390 Navojoa, Mexico, mbmm@cajeme.cifus.uson $. \mathrm{mx})$

By calculating the longitudinal and transverse strain energy averages we studied the polarization states of sagittal acoustic waves in one- and twodimensional phononic crystals. Our theoretical results show the continuous variation of the field displacement components when the Bloch wave vector sweeps the Brillouin zone; thus, the vibrational modes of a same dispersion curve can have different polarization. First we present the polarization map of sagittal waves for an epoxy/Sn superlattice. Then we discuss the band polarization of an array of cylindrical holes in epoxy. As we shall see, the mixed modes can be either predominantly transverse of predominantly longitudinal. For calculations we have used an energy balance criterion.

\section{3:20}

1pPAb8. Proof of the existence of large complete band gaps in high frequency silicon phononic crystal plates. Saeed Mohammadi (Georgia Institute of Technology, 338083 Georgia Tech Station, Atlanta, GA 30332, USA, saeedm@gatech.edu), Ali Asghar Eftekhar (Georgia Institute of Technology, 338083 Georgia Tech Station, Atlanta, GA 30332, USA, eftekhar@ece.gatech.edu), Abdelkrim Khelif (Institut FEMTO-ST/CNRS, 32 avenue de l'Observatoire, 25044 Besançon cedex, France, abdelkrim.khelif@femto-st.fr), William D. Hunt (Georgia Institute of Technology, 338083 Georgia Tech Station, Atlanta, GA 30332, USA, bill.hunt@ee.gatech.edu), Ali Adibi (Georgia Institute of Technology, 338083 Georgia Tech Station, Atlanta, GA 30332, USA, adibi@ece.gatech .edu)

We show, for the first time, the evidence of the existence of large phononic band gaps (PBGs) in two-dimensional phononic crystal (PC) plates formed by etching a hexagonal (honeycomb) array of air holes through a free standing plate of silicon ( $\mathrm{Si}$ ). A CMOS compatible fabrication process is used on a $\mathrm{Si}$ on insulator (SOI) substrate to realize the PC devices. More than $30 \mathrm{~dB}$ attenuation is observed for eight periods of the hexagonal lattice $\mathrm{PC}$ at high frequency region, i.e. $133 \mathrm{MHz}$ with a band gap to mid gap ratio of $25 \%$. We show that the experimental results agree very well 
with the theoretical predictions for the PBG. This result opens a new direction in the implementation of high frequency practical PC structures for a variety of applications especially wireless communication, and sensing.

\section{3:40}

1pPAb9. Acoustic defect mode in a finite 1-dimension lattice Kanghyun Chu (Center for Noise and Vibration Control, Korea Advanced Institute of Science and Technology, 4114, Department of Mechanical Engineering, Guseong-dong, Yuseong-gu, 305-701 Daejon, Republic of Korea, kanghyunchu@kaist.ac.kr), Yang-Hann Kim (Center for Noise and Vibration Control, Korea Advanced Institute of Science and Technology, 4114, Department of Mechanical Engineering, Guseong-dong, Yuseong-gu, 305701 Daejon, Republic of Korea, yanghannkim@kaist.ac.kr)

If there is a defect in a periodic lattice, then the defect modes are expected. The wave localization can be one of representative examples of defect modes applications. However, it is not likely possible to deal with an infinite-periods lattice in practice, and this implies that it is impossible to obtain a perfect wave localization. This motivates us to study a lattice with a finite period which allows the wave tunneling through it. Therefore, the behavior of the acoustic wave in a 1-dimension periodic lattice containing a defect with a finite period has been studied. For simplicity, we considered the lattice composed of two kinds of media with different characteristic impedance in the repeating order with finite periods and the defect with arbitrary size and characteristic impedance Finally, the limitation of wave localization in the real world application is discussed.

\section{4:00-4:20 Break}

\section{4:20}

1pPAb10. Review of phononic crystals, nonlinear processes, devices and prospects. Sergey Nikitov (Institute of Radioengineering and Electronics, Russian Academy of Sciences, 11, bldg. 7, Mokhovaya St., 125009 Moscow, Russian Federation, nikitov@cplire.ru), Yurii Gulyaev (Institute of Radioengineering and Electronics, Russian Academy of Sciences, 11, bldg. 7, Mokhovaya St., 125009 Moscow, Russian Federation, nikitov@cplire.ru), Valery Grigorevsky (Institute of Radioengineering and Electronics, Russian Academy of Sciences, 11, bldg. 7, Mokhovaya St., 125009 Moscow, Russian Federation, vig324@ms.ire.rssi.ru), Alexander Grigorevsky (Institute of Radioengineering and Electronics, Russian Academy of Sciences, 11, bldg. 7, Mokhovaya St., 125009 Moscow, Russian Federation, vig324@ms.ire.rssi.ru), Ivan Lisenkov (Institute of Radioengineering and Electronics, Russian Academy of Sciences, 11, bldg. 7, Mokhovaya St., 125009 Moscow, Russian Federation, lisenkov@cplire.ru), Roman Popov (Institute of Radioengineering and Electronics, Russian Academy of Sciences, 11, bldg. 7, Mokhovaya St., 125009 Moscow, Russian Federation, rspopov@gmail.com)

The review of current state of the art of bulk and surface linear and nonlinear acoustic wave propagation in phononic crystals $(\mathrm{PhC})$ is given. First, theoretical analysis of bulk acoustic waves propagation in $2 \mathrm{D}$ phononic crystals composed of elastic medium with periodic systems of air holes with different symmetry is considered. The properties of hypersonic bulk and surface acoustic waves (Love, Lamb) in PhC are considered theoretically and expermentally. We studied bulk waves propagation in microstructured optical fiber preforms made of quartz. The study of simultaneous propagation of acoustic waves and light in structures being both photonic and phononic crystals was done. We study also properties of guided waves (Lamb modes) propagating in layered structures containing the magnetic films with twodimensional periodic structures. In these structures we discovered a strong coupling between elastic and magnetic properties leading to effective waves transformation between magnetic and elastic systems. Such structures are so called magnonic-phononic crystals. The recommendations of use such unusual BAW and SAW properties in PhC for development of solid state devices are given. This work is supported by RFBR grant 08-02-00785.

\section{4:40}

1pPAb11. Omnidirectional phononic reflection and selective transmission in solid/fluid superlattices. El Houssaine El Boudouti (Faculté des Sciences, Département de Physique, Université Mohamed I,
60000 Oujda, Morocco, elboudouti@yahoo.fr),Youssef El Hassouani (Faculté des Sciences, Département de Physique, Université Mohamed I, 60000 Oujda, Morocco, hassouani@yahoo.fr), Hassan Aynaou (Faculté des Sciences, Département de Physique, Université Mohamed I, 60000 Oujda, Morocco, haynaou_2004@yahoo.fr), Bahram Djafari-Rouhani (IEMN, UMR CNRS 8520, avenue Poincaré, BP 60069, 59652 Villeneuve d'Ascq, France, Bahram.Djafari-Rouhani@univ-lille1.fr)

We present a theoretical analysis of the occurrence of omnidirectional reflection in one-dimensional phononic crystal. We discuss the conditions for a one-dimensional layered structure, made of alternating solid and fluid layers, to exhibit total reflection of acoustic incident waves in a given frequency range for all incident angles. In general, this property cannot be fulfilled with a simple finite superlattice if the incident wave is launched from an arbitrary fluid. Therefore, we propose two solutions to obtain such an omnidirectional band gap, namely: (i) cladding of the superlattice with a layer of high acoustic velocities that acts like a barrier for the propagation of phonons, or (ii) the association in tandem of two different superlattices in such a way that the superposition of their band structures exhibits an absolute acoustic band gap. We discuss the appropriate choices of the material and geometrical parameters to realize such structures. The behavior of the transmission coefficients is discussed in relation with the dispersion curves of the finite structure embedded between two fluids. By inserting a defect layer in the structure, we show that selective transmission may occur in the forbidden bands.

\section{5:00}

1pPAb12. Bubble phononic crystals. Alice Bretagne (Laboratoire Ondes et Acoustique, ESPCI, Université Paris 7, CNRS, 10 rue Vauquelin, 75005 Paris, France, alice.bretagne@espci.fr), Arnaud Tourin (Laboratoire Ondes et Acoustique, ESPCI, Université Paris 7, CNRS, 10 rue Vauquelin, 75005 Paris, France, arnaud.tourin@espci.fr), Patrick Tabeling (MMN ESPCI, 10 rue vauquelin, 75005 Paris, France, patrick.tabeling@espci.fr), Valentin Leroy (Laboratoire Ondes et Acoustique, ESPCI, Université Paris 7, CNRS, 10 rue Vauquelin, 75005 Paris, France, valeroy77@yahoo.fr), Nicolas Pannacci (MMN ESPCI, 10 rue vauquelin, 75005 Paris, France, nicolas.pannacci@espci.fr), Hervé Willaime (MMN ESPCI, 10 rue vauquelin, 75005 Paris, France, herve.willaime@espci.fr), Mathias Fink (Laboratoire Ondes et Acoustique, ESPCI, Université Paris 7, CNRS, 10 rue Vauquelin, 75005 Paris, France, mathias.fink@espci.fr)

Our aim is to investigate phononic crystals consisting of periodic arrangements of micron size gas bubbles. Such crystals are of great potential interest. Because of the high compressibility and high acoustic impedance contrasts between gas and water, a gas filled bubble in water is indeed a strong acoustic scatterer with a large value of its resonant wavelength compared with its size and a scattering cross section several orders of magnitude larger than its geometrical cross section. In that sense a microbubble can be viewed as the equivalent of an acoustic atom and even a small size sample made with such bubbles is expected to have a dramatic impact on acoustic propagation. Using microfluidics techniques we were able to produce perfectly monodisperse gas bubbles in a liquid. When these bubbles (radius $=50$ $\mu \mathrm{m})$ are then injected into a liquid with surfactant, they are found to organize themselves to form an hexagonal close packed crystal. Its stability has still to be optimised before testing it successfully with acoustic waves. The calculation of the band structure of that crystal with the plane wave expansion (PWE) method confirms its interest for controlling propagation of $\mathrm{MHz}-$ ultrasounds: a $800-\mathrm{kHz}$ wide band gap around $1.85 \mathrm{MHz}$ is predicted.

\section{$5: 20$}

1pPAb13. Floquet Lamb modes in periodic plates. Vincent Pagneux (Laboratoire d'Acoustique de l'Université du Maine, UMR CNRS 6613, AV. O. Messiaen, 72085 Le Mans, France, vincent.pagneux@univ-lemans.fr), Agnes Maurel (LOA/ESPCI, 10 rue Vauquelin, 75005 Paris, France, agnes.maurel@espci.fr)

An elastic waveguide with periodic perturbation of its thickness or of its elastic properties can support Floquet modes that are the extension of the classical Lamb modes. In this work, we obtain the spectra of the Floquet modes by using a new numerical coupled mode method which is based on a 
pseudospectral discretization in the transverse direction and a Magnus exponential integration in the longitudinal direction. The Floquet mode spectra are analyzed and compared to the Lamb mode spectra for different configuration of the periodic perturbation of the plate. A particular emphasis is given to the existence of backward wave propagation and to the number of propagating modes as the frequency is increased.

\section{$5: 40$}

1pPAb14. Acoustic transparency by switching of phononic band gaps José Sanchez-Dehesa (Polytechnic University of Valencia, Cami de Vera s/n, $46022 \quad$ Valencia, Spain, jsdehesa@upvnet.upv.es), Helios Sanchis-Alepez (Polytechnic University of Valencia, Cami de Vera s/n, 46022 Valencia, Spain, hesana@alumni.uv), Daniel Torrent (Politechnic University of Valencia, Wave Phenomena Group. Department of Electronic Engineering, C/Camino Vera s.n, ES-46022 Valencia, Spain, datorma1@upvnet.upv.es), Liang-Wu Cai (Kansas State University, Mechanical and Nuclear Engineering, Manhattan, KS 66506, USA, cai@ksu .edu)

Acoustic transparency is here studied in two-dimensional phononic crystals made of hexagonal arrangements of layered cylinders. These layered cylinders, which have their acoustical properties radially dependent, can be achieved by using the recently proposed acoustical metamaterials [D. Torrent and J. Sanchez-Dehesa, New J. Phys., 9, 323 (2007)]. The transparency condition is here achieved by switching of the phononic band gaps, which is governed by the scattering form factor of the acoustic parameters (bulk modulus and sound speed). Various dependence have been studied. The feasibility of this proposal is demonstrated by multiple scattering simulations of the proposed metamaterials [Work supported by NSF of USA and MEC of Spain.]

\section{6:00}

1pPAb15. Three-dimensional phononic crystals made by brazing aluminum beads to form an opal structure. John H. Page (Dept. of Physics and Astronomy, Univ. of Manitoba, Winnipeg, MB R3T 2N2, Canada,jhpage@cc.umanitoba.ca), Hefei Hu (Dept. of Physics and Astronomy, Univ. of Manitoba, Winnipeg, MB R3T 2N2, Canada, hefeihu2@uiuc.edu), Yukihiro Tanaka (Division of Applied Physics, Graduate School of Engineering, Hokkaido University, 0608628 Sapporo, Japan, yuki@eng.hokudai.ac.jp), Takuro Okada (Department of Applied Physics, Hokkaido University, 060-8628 Sapporo, Japan, broaden_my_outlook@yahoo.co.jp), Shin Tamura (Department of Applied Physics, Hokkaido University, 060-8628 Sapporo, Japan, s-tamura@eng .hokudai.ac.jp)

Most phononic crystals studied so far are constructed from periodically arranged objects (e.g., spheres or rods) immersed in a continuous solid or fluid medium. In this presentation, we investigate the properties of a different type of phononic crystal made by weakly brazing 4-mm-diameter aluminum beads in a face-centred cubic array, producing a large-scale opal. In this crystal, wave propagation proceeds through the network of coupled beads rather than through the surrounding medium (air in our case), and the main band gaps that we observe result from coupled resonances of the beads rather than from Bragg scattering. Using ultrasonic techniques, we measure the transmission coefficient and the dispersion relation along the $\Gamma \mathrm{L}$ direction, and show that the lowest-frequency gap occurs between about 500 and
$600 \mathrm{kHz}$ - a frequency range that is just below the lowest resonance of an isolated aluminum sphere. We have also measured the group velocity, which we find to be negative in the lowest frequency band gap. Further insight into the properties of these phononic crystals is obtained by performing finite difference time domain (FDTD) calculations, in which the coupling between spheres is taken into account by allowing the spheres to overlap.

\section{6:20}

1pPAb16. Band gaps in a phononic crystal constituted by cylindrical dots on a homogeneous plate. Bahram Djafari-Rouhani (IEMN, UMR CNRS 8520, avenue Poincaré, BP 60069, 59652 Villeneuve d'Ascq, France, Bahram.Djafari-Rouhani@univ-lille1.fr), Yan Pennec (IEMN, UMR CNRS 8520, avenue Poincaré, BP 60069, 59652 Villeneuve d'Ascq, France, yan.pennec@univ-lille1.fr), Hocine Larabi (IEMN, UMR CNRS 8520, avenue Poincaré, BP 60069, 59652 Villeneuve d'Ascq, France, hocine.larabi@wanadoo.fr), Jérôme Vasseur (IEMN, UMR CNRS 8520, avenue Poincaré, BP 60069, 59652 Villeneuve d'Ascq, France, jerome.vasseur@univ-lille1.fr), Anne-Christine Hladky (IEMN, UMR CNRS 8520, avenue Poincaré, BP 60069, 59652 Villeneuve d'Ascq, France, anne-christine.hladky@isen.fr)

Using the finite difference time domain (FDTD) and the finite element (FE) methods, we investigate theoretically the band structure of a phononic crystal constituted by a periodic array of cylindrical dots deposited on a thin homogeneous plate. We demonstrate that such a structure can exhibit a very low frequency absolute band gap as compared to the wavelength in the constituent materials. The occurrence of this gap requires an appropriate choice of the geometrical parameters and, in particular, of the thickness of the plate as compared to the period or to the height of the cylinders. However, the same behavior can be obtained for various combinations of the materials constituting the homogeneous plate and the dots. We study in detail the width of this band gap as a function of the geometrical and physical parameters of the structure. On the other hand, the structure can also exhibit one or more higher gaps whose numbers can be increased by increasing the height of the cylinders.

\section{6:40}

1pPAb17. Laser-generated surface acoustic waves in copper line arrays on silicon. Alexei A. Maznev (Department of Applied Physics, Hokkaido University, Kita 13 Nishi 8, Kita-ku, 060-8628 Sapporo, Japan, alexei .maznev@gmail.com)

Laser-generated acoustics waves are widely used to control the fabrication on metal interconnect structures in the semiconductor industry. On the other hand, acoustic properties of such structures, typically comprised of periodic arrays of metal lines embedded in a dielectric film on a silicon substrate, pose some interesting wave propagation problems. We will report measurements of surface acoustic waves (SAWs) performed using laserinduced transient grating technique on periodic arrays of micron-wide copper lines. As expected, SAW dispersion curves yield small band gaps at the Brillouin zone boundary. However, much larger band gaps are observed within the 1st Brillouin zone. We believe that these giant band gaps are formed due to avoided crossing of the 1st and 2nd order surface acoustic modes of the film/substrate structure when the 1 st mode dispersion curve becomes folded due to periodicity. Another observed phenomenon is a spectacularly sharp increase in the acoustic attenuation occurring when the folded surface mode becomes coupled to a bulk wave. 


\title{
Session 1pPAc
}

\section{Physical Acoustics and Signal Processing in Acoustics: Acoustic Landmine Detection I}

\author{
James M. Sabatier, Cochair \\ University of Mississippi, NCPA, 1 Coliseum Drive, University, MS 38677, USA
}

Keith Attenborough, Cochair

Open University, Department of Design, Development, Materials and Environment, Walton Hall, Milton Keynes, MK7 6AA, UK

\section{Invited Papers}

\section{1:00}

1pPAc1. Combined seismic, radar, and induction sensor for landmine detection. Waymond R. Scott (Georgia Institute of Technology, School of Electrical and Computer Engineering, 777 Atlantic Dr., Atlanta, GA 30332-0250, USA, waymond.scott@ece.gatech.edu), Kangwook Kim (Dept. Machatronics, GIST, 1 Oryong-dong, Buk-gu, 500-712 Gwangju, Republic of Korea, mkkim@gist.ac.kr), Gregg D. Larson (Georgia Institute of Technology, G. W. Woodruff School of Mechanical Engineering, 777 Atlantic Dr., Atlanta, GA 30332-0250, USA, gregg.larson@me.gatech.edu), Ali C. Gurbuz (Georgia Institute of Technology, School of Electrical and Computer Engineering, 777 Atlantic Dr., Atlanta, GA 30332-0250, USA, gtg173p@mail.gatech.edu), James H. McClellan (Georgia Institute of Technology, School of Electrical and Computer Engineering, 777 Atlantic Dr., Atlanta, GA 303320250, USA, jim.mcclellan@ece.gatech.edu)

An experimental system to collect co-located ground penetrating radar (GPR), electromagnetic induction (EMI), and seismic data was developed to investigate possibilities of using the sensors in a cooperative manner and to investigate the benefits of the fusion of the sensors. These sensors were chosen because they can sense a wide range of physical properties. The seismic sensor is sensitive to the differences between the mechanical properties of a landmine and the soil while the GPR is sensitive to the dielectric properties, and the EMI sensor is sensitive to the conductivity. In the experiments, a range of mines and clutter objects were buried at various depths in a sandbox. Multiple burial scenarios were investigated with a variety of antipersonnel and antitank mines and typical clutter objects. The GPR makes use of modified resistive-vee antennas. The EMI sensor collects broadband data so that the relaxation spectra of the buried targets can be used to aid discrimination. The system uses electrodynamic shakers to generate seismic waves, and a radar is used to measure the displacement of the surface caused by the seismic waves. The responses of each of these sensors to the buried targets will be shown. [Work supported by ARO.]

\section{$1: 20$}

1pPAc2. Information-based sensor management for landmine detection using electromagnetic induction, ground-penetrating radar, and seismic sensors. Mark P. Kolba (Duke University, Department of Electrical and Computer Engineering, Box 90291, Durham, NC 27708, USA, mpk6@ee.duke.edu), Peter A. Torrione (Duke University, Department of Electrical and Computer Engineering, Box 90291, Durham, NC 27708, USA, pt@ee.duke.edu), Waymond R. Scott (Georgia Institute of Technology, School of Electrical and Computer Engineering, 777 Atlantic Dr., Atlanta, GA 30332-0250, USA, waymond.scott@ece.gatech.edu), Leslie M. Collins (Duke University, Department of Electrical and Computer Engineering, Box 90291, Durham, NC 27708, USA, 1collins@ee duke.edu)

An information-based sensor management framework is presented that enables the automated tasking of a suite of sensors when detecting static targets. The sensor manager chooses the sensors to use and the grid-based locations to observe in order to maximize the expected information gain that will be obtained with each new sensor observation. Initially, sensor probabilities of detection and false alarm, Pd and Pf, are assumed to be known by the sensor manager. In a field setting, however, Pd and Pf cannot be known exactly, and so uncertainty modeling for Pd and Pf is also presented. The sensor manager is tested on real landmine data using electromagnetic induction (EMI), ground-penetrating radar (GPR), and seismic sensors. A matched subspace detector is used to process the EMI data, an adaptive pre-screening algorithm based on the least mean squares (LMS) adaptive filter is used to process the GPR data, and whitening followed by an energy detector is used to process the seismic data. The sensor manager is able to detect the landmines more quickly and more effectively than an unmanaged, blind-search approach. Using all three sensor modalities also results in superior detection performance to that achieved by only a single modality.

\section{$1: 40$}

1pPAc3. Nonlinear seismo-acoustic landmine detection. Dimitri Donskoy (Stevens Institute of Technology, Dabvidson Lab., 711 Hudson Street, Hoboken, NJ 07030, USA, ddonskoy@stevens.edu)

The seismo-acoustic methods are among the most promising emerging techniques for the detection of landmines. Numerous field tests have demonstrated that buried landmines manifest themselves at the surface through resonance and nonlinear responses (or signatures) to acoustic/seismic excitation at the frequencies below $1000 \mathrm{~Hz}$. The resonance signatures are primarily due to mine's housing structural resonances. The nonlinear signatures explained by high contact nonlinearity at the mine-soil interface. These phenomena are utilized for landmine detection demonstrating high provability of detection and low false alarms. The paper provides overview of theoretical and experimental investigations conducted by the author and his colleagues at Stevens Institute of Technology. Among major 
accomplishments are discovery and explanation of mine's resonance behavior; soil/depth effect on buried mine's resonances; discovery and analysis of nonlinear acoustic interactions at the soil-mine interface; development of nonlinear quadratic and intermodulation detection algorithms based on dual-frequency excitation. Work supported by the U.S.Army NVESD, ARO and ONR.

1pPAc4. Hysteresis effects in nonlinear acoustic landmine detection. Murray S. Korman (Physics Dept., United States Naval Academy, Chauvenet Hall Room 295, 572 C Holloway Road, Annapolis, MD 21402, USA, korman@usna.edu)

In nonlinear acoustic landmine detection the vibration interaction of the top-plate of the buried plastic landmine with the adjacent soil is a subject of interest due to the strong nonlinear coupling. Using airborne sound excitation (in buried plastic anti-tank inert VS 1.6 and VS 2.2 landmine experiments) tuning curve behavior and generation of combination frequencies from two-tone tests were similar to the mesoscopic/nanoscale nonlinear elastic behavior observed in geomaterials like sandstone [J. Acoust. Soc. Am. 116, 3354-3369 (2004)]. Tuning curve measurements near resonance for increased acoustic amplitude (exhibiting softening) can be explained if hysteresis effects (characteristic of mesoscopic nonlinearity) are considered. The backbone curve (peak amplitude vs. corresponding resonant frequency from a family of tuning curves) exhibits mostly linear behavior for "off target" soil surface vibration measurements of a soil layer resonating over a rigid boundary. Backbone curves for "on target" measurements exhibit significantly more bending and curvature when a soil layer resonates over the compliant top-plate of the landmine. An oscillator with hysteresis modeled by a distribution of parallel spring elements each with a different threshold slip condition seems to describe the "off target" behavior, while a single bilinear hysteresis element describes the "on target" results. [Support by USNA.]

\section{2:20}

1pPAc5. Accelerometer measurements of acoustic-to-seismic coupling above buried objects. Keith Attenborough (Open University, Department of Design, Development, Materials and Environment, Walton Hall, MK7 6AA Milton Keynes, UK, Keith .Attenborough@ioa.org.uk)

The surface velocity of sand inside a large PVC container, induced by the sound pressure from either a large loudspeaker radiating into an inverted cone and pipe or a B\&K point source loudspeaker mounted with its axis vertical, has been measured using accelerometers. Results of white noise and stepped frequency excitation are presented. Without any buried object the mass loading of an accelerometer creates resonances in the spectral ratio of sand surface velocity to incident acoustic pressure i.e. the acoustic-to-seismic (A/S) admittance spectra. The A/S responses above a buried compliant object are larger and distinctive. The linear A/S admittance spectra in the presence of a buried electronic components box have been studied as a function of burial depth and sand state. The nonlinear responses above the buried box have been studied as a function of depth, sand state and amplitude. Predictions of a modified 1D lumped parameter model have been found to be consistent with the observed nonlinear responses. Also the modified model has been used to explain features of the A/S responses observed when using an accelerometer without any buried object [Work supported by DSTL, UK.]

\section{2:40}

1pPAc6. Acoustic-laser land mine detection: Operationally enabling technologies. Robert Haupt (MIT Lincoln Laboratory, 244 Wood St., Lexington, MA 02421, USA, haupt@LL.mit.edu)

Reliable and cost effective methods are critically needed to locate the vast number of land mines left behind from military conflicts. Many mine location systems being developed fall short of being operationally practical since they may detect only metal or a specific mine feature, have limited standoff ranges, or have slow search speeds. An approach that may overcome operational issues is the acoustic-laser method. Acoustic waves generated by a noncontact/standoff source and transmitted through air can couple to the ground and induce resonances from a large variety of mines. The vibration field above these mines can be measured remotely using laser Doppler vibrometry. In this paper, enabling technologies that can significantly increase search area speed, improve standoff range, and reduce size in an acoustic-laser system are discussed. We take a novel approach to excite mines by using a high powered parametric acoustic array source developed at MIT Lincoln Laboratory that transmits a highly directive sound beam from a safe distance. The avalanche photo diode (APD) array developed at MIT Lincoln Laboratory will also be discussed as the enabling technology of a proposed 4096 multipixel laser system that can sense a wide vibration field out in front of a moving vehicle.

\section{3:00}

1pPAc7. Fast acoustic landmine detection using multiple beam laser Doppler vibrometry. Vyacheslav Aranchuk (University of Mississippi, NCPA, 1 Coliseum Drive, University, MS 38677, USA, aranchuk@olemiss.edu), James M. Sabatier (University of Mississippi, NCPA, 1 Coliseum Drive, University, MS 38677, USA, sabatier@olemiss.edu), Richard D. Burgett (Planning Systems, Inc., NCPA, 1 Coliseum Drive, University, MS 38677, USA, rburgett@olemiss.edu)

An acoustic method of buried landmine detection consisting of excitation of the ground using airborne sound or seismic waves in the frequency range from about $50 \mathrm{~Hz}$ to $1000 \mathrm{~Hz}$, and obtaining a velocity image of the ground surface with a laser Doppler vibrometer (LDV) has been developed and successfully tested in the laboratory and field experiments. The presence of a buried landmine can be detected by an abnormality in the velocity image. Initially, the scanning single-beam LDV created a velocity image of the ground through point-by-point measurements, which resulted in a long measurement time. To reduce this measurement time, a multiple beam LDV having 16 beams configured in a linear array was developed and successfully used in field experiments. To further reduce the time of measurement, a full-field LDV capable of making 256 vibration measurements in parallel on the target, configured as $16 x 16$ square array of points equally separated in $\mathrm{x}$ - and $\mathrm{y}$-directions has been recently developed. The system is capable of creating a twodimensional vibrational velocity image of the ground surface in the time defined by the required frequency resolution. The parallel measurement makes the system a unique tool to measure transient vibrations. 
1pPAc8. Mechanical resonances in the low-frequency vibration spectrum of a cylindrically symmetric, anti-tank landmine. William C. Alberts (U.S. Army Research Laboratory, 2800 Powder Mill Rd, Adelhi, MD 21044, USA, kirk.alberts@arl.army.mil), James M. Sabatier (University of Mississippi, NCPA, 1 Coliseum Drive, University, MS 38677, USA, sabatier@olemiss.edu), Roger Waxler (University of Mississippi, NCPA, 1 Coliseum Drive, University, MS 38677, USA, rwax @ olemiss.edu)

Acoustic-based landmine detection methods have enjoyed success, in part, because of structural resonances in many landmines. The unburied VS 1.6, a member of a family of plastic, cylindrically symmetric anti-tank landmines, exhibits seven modes below $1.6 \mathrm{kHz}$ and a large frequency shift of the first symmetric mode such that its frequency is greater than that of the first asymmetric mode, a phenomenon observed in timpani of reduced kettle volume [Christian et al., J. Acoust. Soc. Am., 76(5), 1336-1345 (1984)]. An elastically supported, thin, elastic plate acceptably models the unperturbed modes of the pressure plate. Coupling of the acoustic analog of the first symmetric mode to the cavities beneath the plate shows those cavities to be the cause of the perturbation. Shallow burial in sand effectively removes the frequency shift of the first symmetric mode. Comparisons to burial simulation experiments in water will be made, thus allowing a qualitative explanation of the effect sand has on the first symmetric mode of the plate. [This work was sponsored by US Army Research, Development, and Engineering Command, Night Vision and Electronic Sensors Directorate under Contract DAAB15-02-C-0024.]

\title{
$3: 40$
}

1pPAc9. Review of a model for the response of a buried landmine to an airborne acoustic signal. Doru Velea (QinetiQ North America, Technology Solutions Group, 12030 Sunrise Valley Dr., Suite 400, Reston, VA 20191, USA, dvelea@plansys.com), Roger Waxler (University of Mississippi, NCPA, 1 Coliseum Drive, University, MS 38677, USA, rwax@ olemiss.edu), James M. Sabatier (University of Mississippi, NCPA, 1 Coliseum Drive, University, MS 38677, USA, sabatier@olemiss.edu)

Buried landmines can be found acoustically by insonifying the ground and detecting a contrast between the vibratory motion of the ground surface directly above the mine and a short distance away from the mine. Experiments have revealed a short-ranged spatial response and a frequency response that peaks around 100-200 Hz for anti-tank mines. The peak is believed to be a response to mechanical resonances of the mine itself. A model was developed to explain these experimental observations and to predict how the mine's resonance is influenced by the surrounding soil. In the model the soil was assumed to be an effective fluid while the mine was modeled as a right cylinder with a compliant top. The response of the top of the mine was modeled by an impedance condition. Predictions of the model are presented and compared with the phenomena observed in the field.

\section{Session 1pPAd}

\section{Physical Acoustics: Sonic, Ultrasonic, and Megasonic Cleaning I}

\author{
Ralph T. Muehleisen, Cochair \\ Illinois Institute of Technology, Civil, Architectural and Environmental Engineering, 3201 S. Dearborn St., Room 228, \\ Chicago, IL 60616, USA \\ Claus-Dieter Ohl, Cochair \\ University of Twente, P.O. Box 217, Department of Science and Technology, Enschede, 7500 AE, Netherlands
}

\section{Invited Papers}

4:00

1pPAd1. Global Trend of Precision Cleaning Technology and the Application in Hard Disk Drive Industry. Jun Zhang (Data Storage Institute, DSI bldg, 5 Eng Dr 1 (Off Kent Ridge Crescent, NUS), 117608 Singapore, Singapore, zhang_jun@dsi.a-star.edu.sg) , Yun Fook Liew (Data Storage Institute, DSI bldg, 5 Eng Dr 1 (Off Kent Ridge Crescent, NUS), 117608 Singapore, Singapore, zhang_jun@dsi.a-star.edu.sg)

As industry trends for hard disk drive and related electronic devices are moving toward high capacity and miniaturization. A big challenge is to find increasing the efficient ways to clean the parts which are regarded as the most stringent in cleanliness. The critical issues include the methodology for identifying the contaminants and how to choose a correct cleaning chemical and cleaning technology. Today's hard disk media, particularly the perpendicular recording media, is capable of storing up to $300 \mathrm{~GB}$ per disk under which the distance between the recording head/disk have to be reduced to a few nanometers. It will bring more difficulties for increasing the reliability and disk capacities. On the other hand, all components within the hard disk drive are required to avoid submicron particulate contamination $(<0.1 \mu \mathrm{m})$. Megasonic and ultrasonic cleaning are the most effective and promising technologies to remove such contaminants. This presentation will discuss the technical trend and possible solution of precision cleaning and the strategy for selecting the cleaning chemicals and technologies to meet the cleanliness requirement through a case study. 
1pPAd2. Accoustic cleaning in nanoelectronics. Paul Mertens (Imec, Kapeldreef 75, B-3001 Leuven, Belgium, paul.mertens @imec.be)

The fabrication of state-of-the-art electronic integrated circuits involves multiple cleaning steps in which residues of the order of ten or a few tens of $\mathrm{nm}$ need to be removed with very high efficiency. Historically cleaning was obtained by chemical undercutting (order of a few $\mathrm{nm}$ ). The dimensions of nanoelectronic devices become so small that the amount of substrate etching involved in a cleaning step should be kept below $0.05 \mathrm{~nm}$. Therefore other cleaning mechanism, involving a mechanical force, have to be considered such as acoustic agitation of the cleaning liquid or high-velocity aerosol bombardment. These forces, however, can damage fine structures already fabricated on the substrate, particularly the "up-features." This implies that a trade-off needs to be made between avoiding damage and obtaining an acceptable cleaning performance. This presentation includes typical hardware configurations and possible mechanisms for particle removal and use of megasonic agitation in nonaqueous liquid solutions.

\section{4:40}

1pPAd3. Cleaning of semiconductor substrates by controlled cavitation. Frank Holsteyns (SEZ AG, SEZ-Strasse 1, 9500 Villach, Austria, F.Holsteyns@at.sez.com), Alexander Lippert (SEZ AG, SEZ-Strasse 1, 9500 Villach, Austria, A.Lippert@at.sez.com), Florian Lechner (SEZ AG, SEZ-Strasse 1, 9500 Villach, Austria, F.Lechner@at.sez.com), Andrea Otto (Göttingen University, Friedrich-Hund-Platz 1, 37077 Göttingen, Germany, aotto@physik3.gwdg.de), Till Nowak (Göttingen University, Friedrich-Hund-Platz 1, 37077 Göttingen, Germany, tnowak@physik3.gwdg.de), Robert Mettin (Göttingen University, Drittes Physikalisches Institut, Friedrich-Hund-Platz 1, 37077 Göttingen, Germany, R.Mettin@physik3.gwdg.de), Alexander A. Doinikov (Belarus State University, 11 Bobruiskaya Street, 220030 Minsk, Belarus, doinikov@ bsu.by), Harald Okorn-Schmidt (SEZ AG, SEZStrasse 1, 9500 Villach, Austria, H.Okorn-schmidt@at.sez.com)

The continuing downscaling of device geometries in the semiconductor industry is driving the requirements for both process and contamination control. Historically, the physical and the chemical processes required for contamination control were evolutionarily scaled with device geometry. However, today's tailored wet-chemical cleaning approaches must strive to meet stringent requirements to assure a minimal material loss and no damage to extremely fragile structures. While chemical solutions exist for the control of molecular-organic and metallic ion contamination, the physico-chemical solutions for the removal of nanosized particulate contamination to critical diameters below $20 \mathrm{~nm}$ are still undetermined. Therefore, the potential and the limitations of megasonic cleaning, which is mainly based on cavitation, are carefully balanced and a detailed understanding of the ongoing physical mechanisms is necessary to maintain a stable window of operation. The relevant active mechanisms present in such a cavitation driven cleaning process, will depend in great part on the characteristics of the applied sound field; that is to say on frequency, amplitude and uniformity and also on the properties of the cleaning liquid, i.e., the level of gasification, temperature, surface tension, and many others. Different techniques in conjunction with simulations and modeling are under development to improve the design of a novel cleaning tool that can address the challenges.

\section{5:00}

1pPAd4. Using megasonic agitation to extend chemical cleaning for nanotechnology device production. Cole Franklin (1241 East Dyer Road, Santa Ana, CA 92672, USA, colesfranklin@yahoo.com)

Aqueous and solvent chemicals are widely used in the semiconductor industry and as the technology nodes shrink device makers are working to extend these cleaning fluids to the next technology node. Internal data shows megasonic agitation can enhance polymer removal without complete dissolution for both aqueous and solvent chemicals. Data has shown solvents and semi-aqueous solutions show better selectivity with advanced material stacks over aqueous solution. It has been established that megasonic energy can enhance particle removal from semiconductor devices during cleaning processes. However, applied megasonic energy can also damage sensitive semiconductor devices during cleaning, especially in aqueous solutions. Aqueous fluids appear to promote much more damage for the same applied megasonic power than do most solvents. We show that some solvents have a higher threshold for cavitation than deionized water mixes. Since device manufacturers are working to extend their current cleaning chemicals to the 65 and $45 \mathrm{~nm}$ pilot production and research nodes, we studied several ways to accomplish this goal for aqueous or solvent chemicals. Described here we apply a methods to predict the damage of nanostructures by various liquids using signal analysis to better understand the mechanical that promotes improved control of cleaning without damage.

\section{$5: 20$}

1pPAd5. On frequency scaling of ultrasonic cavitation and cleaning. Robert Mettin (Göttingen University, Drittes Physikalisches Institut, Friedrich-Hund-Platz 1, 37077 Göttingen, Germany, R.Mettin@physik3.gwdg.de)

In virtually all applications of acoustic cavitation, variations of the effects with sound frequency are observed. However, a simple comparison of analogical setups at different frequencies can be misleading, as other parameters, like wavelength or emitted transducer power, change jointly with the driving frequency. Indeed, the similarity of acoustically cavitating systems under scaling of frequency is not well defined yet, in particular because it is not clear what observable should stay the same. A possible way of characterization of "similar" systems could be the observation of similar cavitation bubble structures, "such as" for hydrodynamic flow patterns or hydrodynamic cavitation structures. This idea is debated on experimental and theoretical basis, and the consequences for cleaning applications in the higher frequency range are discussed.

1pPAd6. Surface cavitation on micro- and nanometer scales. Detlef Lohse (Physics of Fluids, University of Twente, P.O. Box 217, 7500 AE Enschede, Netherlands, d.lohse@utwente.nl), Nicolas Bremond (University of Twente, P.O. Box 217, Department of Science and Technology, 7500 AE Enschede, Netherlands, Nicolas.Bremond@espci.fr), Bram Borkent (University of Twente, P.O. 
Box 217, Department of Science and Technology, 7500 AE Enschede, Netherlands, b.m.borkent@tnw.utwente.nl),Claus-Dieter Ohl (University of Twente, P.O. Box 217, Department of Science and Technology, 7500 AE Enschede, Netherlands, c.d.ohl@utwente.nl), Manish Arora (University of Twente, P.O. Box 217, Department of Science and Technology, 7500 AE Enschede, Netherlands, m.arora@tnw.utwente.nl), Julius Vancso (University of Twente, P.O. Box 217, Department of Science and Technology, 7500 AE Enschede, Netherlands, g.j.vancso@tnw.utwente.nl), Holger Schoenherr (University of Twente, P.O. Box 217, Department of Science and Technology, 7500 AE Enschede, Netherlands, h.schonherr@utwente.nl)

Heterogeneous bubble nucleation at surfaces has been notorious because of its irreproducibility. Here controlled multibubble surface cavitation is achieved by heterogenous nucleation of bubbles on a hydrophobic surface patterned with microcavities. The expansion of the nuclei in the microcavities is triggered by an impulsive lowering of the liquid pressure. The procedure allows to control and fix the bubble-distance within the bubble cluster. We observe a perfect quantitative reproducibility of the cavitation events where the inner bubbles in the two-dimensional cluster are shielded by the outer ones, reflected by their later expansion and their delayed collapse. Apart from the final bubble collapse phase (when jetting flows directed towards the cluster's center develop), the bubble dynamics can be quantitatively described by an extended Rayleigh-Plesset equation, taking pressure modification through the surrounding bubbles into account. When repeating the same experiments with flat polyamide and hydrophobized silicon surfaces populated with surface nanobubbles (as can be seen through atomic force microscopy), these nanobubbles do not act as nucleation sites for cavitation bubbles, in contrast to the expectation. This implies that surface nanobubbles are not just stable under ambient conditions but also under enormous reduction of the liquid pressure down to $-6 \mathrm{MPa}$. We denote this feature as superstability.

\section{6:00}

1pPAd7. In-situ monitoring of megasonic cleaning. Aaldert G. Zijlstra (Physics of Fluids, University of Twente, P.O. Box 217, 7500 AE Enschede, Netherlands, a.g.zijlstra@tnw.utwente.nl), Tom Janssens (IMECvzw, Kapeldreef 75, 3001 Heverlee, Belgium, janssent@imec.be), Paul Mertens (Imec, Kapeldreef 75, B-3001 Leuven, Belgium, paul.mertens@imec.be), Michel Versluis (Physics of Fluids, University of Twente, P.O. Box 217, 7500 AE Enschede, Netherlands, m.versluis@utwente.nl)

Acoustic agitation is used in ultrasonic cleaning to induce a physical force to remove nanoparticles in semiconductor processing. The performance of cleaning tools is commonly quantified ex-situ by the particle removal efficiency $\eta$ (i.e. the ratio of the remaining to the original particle concentration). The resulting $\eta$-wafer maps often reveal spatial nonuniformities related to tool design. The local study of the cleaning dynamics, quantified by the removal frequency $\left(f_{R}\left[s^{-1}\right]\right)$ also indicates that the density of cleaning events over a wafer can be strongly non homogeneous. Because the sound frequencies used in industrial tools are in the MHz range, visualization of the cavitating bubbles (resonant size $3 \mu \mathrm{m}$ ) is highly challenging, both in time and in spatial resolution. Considering that the cleaning effect of a cavitating bubble is permanent a method is presented here to determine the acoustic cleaning event size $\left(A_{\text {event }}\left[\mu \mathrm{m}^{2}\right]\right)$ and the event flux $\left(\phi\left[\mu \mathrm{m}^{-2} \mathrm{~s}^{-1}\right]\right)$ through visualization techniques. The method consists of time-resolved imaging of the removal of a nanoparticle layer deposited on a transparent substrate. The microscopic cleaning activity can then be correlated to the actual removal frequencies $f_{R}$ through the relation $f_{R}=\phi A_{\text {event }}$ to further clarify the cleaning mechanisms in industrial tools.

\section{6:20}

1pPAd8. Potential uses of ultrasound in the dairy ultrafiltration processes. Shobha Muthukumaran (School of Engineering and Information Technology, Deakin University, Pigdons Road, Waurn Ponds, Victoria, 3217 Geelong, Australia, shobha.muthukumaran@deakin.edu.au), Sandra Kentish (Department of Chemical and Biomolecular Engineering, The University of Melbourne, Parkville, Victoria, 3010 Melbourne, Australia, sandraek@unimelb.edu.au), Muthupandian Ashokkumar (Department of Chemical and Biomolecular Engineering, The University of Melbourne, Parkville, Victoria, 3010 Melbourne, Australia, masho@unimelb.edu.au), Geoff Stevens (Department of Chemical and Biomolecular Engineering, The University of Melbourne, Parkville, Victoria, 3010 Melbourne, Australia, gstevens@unimelb.edu.au)

There has been a growing interest in the industrial application of ultrasound, especially in the food industry. Power ultrasound can have a number of physical effects; it can increase turbulence through both the introduction of vibrational energy and through acoustic streaming, it can cause both particle dispersion and particle agglomeration and clean surfaces with a scouring action. Our work in this area has focused on the use of ultrasound to enhance membrane processing. Low frequency ultrasound has been used to facilitate cross flow ultrafiltration of dairy whey solutions for both during the ultrafiltration production cycle and the cleaning cycle. During the production cycle, the use of ultrasound reduces both pore blockage and the specific resistance of the fouling cake layer. This leads to higher flux rates and the potential for longer production cycles. During the cleaning cycle, ultrasound systematically increases cleaning efficiency, thus has the potential to reduce both total chemical consumption and system downtime. There was no deterioration in cleaning effectiveness or membrane condition, which implies that sonication, has not damaged the membrane itself. Similarly, there was no change in the chemical nature of soluble proteins following sonication.

\section{$6: 40$}

1pPAd9. Ultrasonic cleaning of particle and natural organic matter fouled cross-flow membranes. Linda K. Weavers (The Ohio State University, 470 Hitchcock Hall, 2070 Neil Ave, Columbus, OH 43210, USA, weavers.1@ osu.edu), Harold W. Walker (The Ohio State University, 470 Hitchcock Hall, 2070 Neil Ave, Columbus, OH 43210, USA, walker.455@osu.edu), Dong Chen (Lamar University, Department of Civil Engineering, Beaumont, TX 77710, USA, dchen@my.lamar.edu), Mikko O. Lamminen (The Ohio State University, 470 Hitchcock Hall, 2070 Neil Ave, Columbus, OH 43210, USA, m.o.lamminen@gmail.com)

Membrane filtration has arguably been the most significant development in the area of water purification in the past 50 years. It has enabled many processes to become more reliable and to achieve purities that have not been achieved previously. One of the drawbacks of membrane use is fouling. In this research the ultrasonic cleaning of particle-fouled membranes was investigated. Specifically, we have explored the mechanisms responsible for ultrasonic cleaning and how solution conditions, particle conditions, cake layer effects, 
and ultrasonic conditions affect cleaning using scanning electron microscopy (SEM) and permeate flux measurements. We have investigated continual cleaning during membrane filtration in addition to cleaning after fouling. Results of this work will be discussed for cross-flow system configurations.

\section{Contributed Papers}

7:00

1pPAd10. Measurements and simulations on the wall shear stress from single cavitation bubbles. Rory Dijkink (University of Twente, Physics of Fluids, Building Meander, Postbus 217, 7500 AE Enschede, Netherlands, r.j.dijkink@tnw.utwente.nl), Evert Klaseboer (Institute of High Performance Computing, 1 Science Park Road, \#01-01 The Capricorn, 117528 Singapore, Singapore, evert@ihpc.a-star.edu.sg), Boo Cheong Khoo (National University of Singapore, Dept of Mechanical Engineering, 10 Kent Ridge Crescent, 119260 Singapore, Singapore, mpekbc@nus.edu.sg), Claus-Dieter Ohl (University of Twente, P.O. Box 217, Department of Science and Technology, 7500 AE Enschede, Netherlands, c.d.ohl@utwente.nl)

Cavitation bubbles collapsing near a solid boundary manifest a jetting effect towards the closest rigid boundary. After impacting on the boundary the jet spreads out radially thereby shearing the surface. It is speculated that cavitation bubbles clean through the resulting shearing forces, e.g. they drag adherent contaminants through the radial spreading jet. Although the cleaning through bubbles is used in a wide set of technologies, for example for pre-cleaning surgical equipment, for bio-film removal in medical and drinking water applications, and in ultraclean processing of semiconductor wafers, very limited experimental studies on the wall shear stress exist. In an effort to shed light on the shear forces caused by no spherical oscillating cavitation bubbles close to boundaries we implement a constant temperature anemometer together with high-speed imaging to elucidate the fluid dynamics near the boundary. Additionally, we perform potential flow simulations with a boundary element method. The boundary layer is resolved with a convolution integral to the solution of Stokes' first problem. In general we find the generation of wall shear stress both in experiment and simulations during the growth and collapse of the bubble. Yet, very high shear stress is generated once the jet impacts and spreads on the boundary.

$$
7: 20
$$

1pPAd11. Cinephotographic observations of particle removal from a surface by acoustic cavitation. Lawrence A. Crum (Center for Industrial and Medical Ultrasound, Applied Physics Lab., University of Washington, 1013 NE 40th St., Seattle, WA 98105, USA, lac@apl.washington.edu), Michael R. Bailey (Center for Industrial and Medical Ultrasound, Applied Physics Lab., University of Washington, 1013 NE 40th St., Seattle, WA 98105, USA, bailey@apl.washington.edu), Michael S. Canney (Center for Industrial and Medical Ultrasound, Applied Physics Lab., University of Washington, 1013 NE 40th St., Seattle, WA 98105, USA, mcanney@u.washington.edu), Hong Chen (Center for Industrial and Medical Ultrasound, Applied Physics Lab., University of Washington, 1013 NE 40th St., Seattle, WA 98105, USA,
hopechen@u.washington.edu),Thomas Matula(Center for Industrial and Medical Ultrasound, Applied Physics Lab., University of Washington, 1013 NE 40th St., Seattle, WA 98105, USA, matula@apl.washington .edu), Christopher McInnes (Ultreo, Inc., 9461 Willows Road NE, Redmond, WA 98052, USA, chris@ultreo.com)

It is commonly believed that ultrasonic cleaners remove particles from a surface through acoustic cavitation, presumably in which a pulsating bubble interacts directly with the particle. We are unaware of any direct evidence of this interaction. Recently, however, we have used a high-speed movie camera to observe the removal of biofilm attached to a solid surface during exposure to a cavitation field. The biofilm consisted of Streptococcus mutans, a common oral bacterium, grown on a glass slide and observed under magnification to be both thinly coating the glass surface and clustered in larger colonies. The cavitation field was created by an Ultreo ${ }^{\mathrm{TM}}$ toothbrush which combines both vibrating bristles and an ultrasound transducer with waveguide, operating at a frequency of $324 \mathrm{kHz}$. When the waveguide was immersed in water containing bubbles from an ultrasound contrast agent $\left(\right.$ Optison $\left.{ }^{\mathrm{TM}}\right)$, visual observations could be made with the naked eye of biofilm removal. With high-speed cinephotography, it was possible to observe bacteria removal by the direct interaction of a cavitation bubble (cloud) and the colony. These observations will be presented along with our interpretations of the data. [Work supported in part by Ultreo ${ }^{\mathrm{TM}}$, Inc.]

\section{$7: 40$}

1pPAd12. Pressure fields and their effects in membrane cleaning applications. Fabian Reuter (Göttingen University, Drittes Physikalisches Institut, Friedrich-Hund-Platz 1, 37077 Göttingen, Germany, freuter@physik3.gwdg.de), Robert Mettin (Göttingen University, Drittes Physikalisches Institut, Friedrich-Hund-Platz 1, 37077 Göttingen, Germany, R.Mettin@physik3.gwdg.de), Werner Lauterborn (Göttingen University, Friedrich-Hund-Platz 1, 37077 Göttingen, Germany, W.Lauterborn @ physik3.gwdg.de)

It is known that the use of ultrasonic cavitation can support the cleaning of filtration membranes. We study the final purification step in drinking water production by ultrafiltration through a submerged filter module. The module consists of parallel polymer membrane sheets. Its filtration efficiency is rapidly decreased by fouling. Here, the removal of membrane attached fouling layers by backflushing can successfully be enhanced using ultrasound without membrane damage. To better understand the cleaning mechanism and cavitation properties for further optimization of efficiency, the sound pressure field is measured and the bubble distribution is characterized. Different sound field arrangements are investigated with respect to cleaning performance, while membrane integrity is controlled by turbidity and particle monitoring of the permeate. Results show that a strong influence due to the geometrical configuration can be present, for instance due to impedance effects of the membrane layers. 


\title{
Session 1pPAe
}

\section{Physical Acoustics: Mathematical and Numerical Methods I}

\author{
Andrew Piacsek, Cochair \\ Central Washington University, Department of Physics, 400 E. University Way, Ellensburg, WA 98926, USA
}

B. D. Zaitsev, Cochair

Institute of Radio Engineering and Electronics of RAS, Saratov Branch, Zelyonaya str., 38, 410019 Saratov, Russian Federation

\section{Contributed Papers}

\section{7:00}

1pPAe1. The peculiarities of energy characteristics of acoustic waves. Boris D. Zaitsev (Institute of Radio Engineering and Electronics of RAS, Saratov Branch, Zelyonaya str., 38, 410019 Saratov, Russian Federation, zai-boris@yandex.ru), Andrey A. Teplykh (Institute of Radio Engineering and Electronics of RAS, Saratov Branch, Zelyonaya str., 38, 410019 Saratov, Russian Federation, teplykhaa@mail.ru), Iren E. Kuznetsova (Institute of Radio Engineering and Electronics of RAS, Saratov Branch, Zelyonaya str., 38, 410019 Saratov, Russian Federation, kuziren@yandex.ru)

Acoustic waves propagating in piezocrystals transport kinetic and potential energies. Potential energy consists of mechanical, electrical, and mutual contributions. At that the change in potential energy of the voluentary unit of medium is identified with the work of mechanical stress for infinitesimal displacement. Versus method of integrating this equation there are two different expressions for density of potential energy. In case 1 (traditional approach) the density of energy is equal to the half-product of mechanical stress and strain. At that for any type of plane acoustic wave the instantaneous density of total energy in given point of medium depends on time and there is no mutual transformation of potential and kinetic energies. For this case there exists well-known traditional expression for power flow. In case 2 suggested by authors the density of potential energy is equal to half-product of mechanical stress gradient and displacement. At that the density of total energy does not depend on time. It means the mutual transformation of potential and kinetic energies according to motion equation. The new expression for power flow is derived for this case. The peculiarities of energy characteristics for piezoactive waves are discussed. The work is supported by RFBR grant $\mathrm{N}^{\circ}$ 08-08-00793.

\section{$7: 20$}

1pPAe2. Audio acoustic modeling using full-wave methods. Timo Lahivaara (University of Kuopio, P.O. Box 1627, 70211 Kuopio, Finland, timo.lahivaara@uku.fi), Tomi Huttunen (University of Kuopio, P.O. Box
1627, 70211 Kuopio, Finland, tomi.huttunen@uku.fi),Simo-Pekka Simonaho (University of Kuopio, P.O.Box 1627, 70211 Kuopio, Finland, simo-pekka.simonaho@uku.fi)

The numerical simulation of wave propagation poses a significant challenge in scientific computation. Historically, several approaches are explored in order to get a stable method that can be efficiently used for approximating wave propagation without excessive numerical dissipation or dispersion. Unfortunately, the traditional approaches, such as the finite element and the finite difference, require many discretization points per wavelength to obtain reliable solutions. In this study, two alternative full-wave methods for reducing the computational complexity are consider. The methods are the time-domain discontinuous Galerkin method and the ultraweak variational formulation in the frequency domain. Using these techniques, the directivity and the frequency response of a loudspeaker are studied. Moreover, the simulated results are compared to experimental measurements.

\section{$7: 40$}

1pPAe3. Propagation in a dispersive medium: a phase-space approach. Leon Cohen (City University of New York, Hunter-Phyiscs, 695 Park Ave., New York, NY 10021, USA, leon.cohen@hunter.cuny.edu)

We derive exact moments for pulse propagation in a dispersive medium. These moments are not only inherently interesting but clarify the validity of a recently proposed approximation scheme for wave propagation. The approximation method for pulse propagation is based on the Wigner positionwave/number representation and is very accurate, easy to apply, and moreover is physically illuminating. In particular one obtains the evolved approximate Wigner distribution from the initial Wigner distribution by a simple linear translation in phase space. Propagation with damping is also taken into account. We will show that the reason for the high accuracy of the approximation is that the important low order moments are exactly given by the approximation and that these low order moments preserve very well the basic shape of the pulse. Moreover, now that we understand why the approximation method works well, the approximation can be systematically improved. We give a number of specific examples of exactly calculable moments to illustrate the method and we compare exact and approximate moments. Research supported by the AFOSR. 


\title{
Session 1pPPa
}

\section{Psychological and Physiological Acoustics: Integrated Approaches to Auditory Scene Analysis I}

\author{
Mounya Elhilali, Cochair \\ Johns Hopkins University, Dept of Electrical and Computer Engineering, Barton Hall 105, 3400 N. Charles Street, \\ Baltimore, MD 21218, USA \\ Daniel Pressnitzer, Cochair \\ CNRS UMR 8158 ; Univ. Paris Descartes; Ecole Normale Supérieure, 29 rue d'Ulm, Paris, 75005, France
}

\section{Invited Papers}

\section{1:00}

1pPPa1. Sequential integration in the perception of tone sequences. Leon Van Noorden (Rassonstraat 39, B-1030 Brussels, Belgium, leonvannoorden@mac.com)

The seminal book of Albert Bregman on Auditory Scene Analysis had not yet appeared at the time of my dissertation research (1970-1975). One of the major difficulties that I had was to come at grips with the concept of a sequence of tones that form a 'gestalt' of tones 'belonging' together. This Sequential Integration, as coined by Bregman, has not received as much attention as the 'Differentiation' aspect. A number of experiments show that this sequential integration process is characterised by a resonance process with a broad peak near $2 \mathrm{~Hz}$. This resonance process can explain several phenomena such as subjective rhyhmisation, the data on tapping along polyrhythms and the histogram of musical tempi. Confronting these findings with the measurements of the Temporal Cohence Boundary may indicate where pitch movement detectors may play a role. Recent publications have linked this resonance with a movement control process.

\section{$1: 20$}

1pPPa2. On the binding of successive tones: Implicit versus explicit pitch comparisons. Laurent Demany (CNRS UMR 5227; Univ. Victor Segalen, 146 rue Léo Saignat, 33076 Bordeaux, France, laurent.demany@psyac.u-bordeaux2.fr), Daniel Pressnitzer (CNRS UMR 8158; Univ. Paris Descartes; Ecole Normale Supérieure, 29 rue d'Ulm, 75005 Paris, France, Daniel.Pressnitzer@ens.fr), Catherine Semal (CNRS UMR 5227; Univ. Victor Segalen, 146 rue Léo Saignat, 33076 Bordeaux, France, catherine.semal@u-bordeaux2.fr)

Listeners were presented with sound sequences in which one pure tone (T) was followed by a set (S) of five synchronous or asynchronous pure tones 550 cents apart. In a "present/absent" condition, $\mathrm{T}$ was either identical to a randomly selected component of S or halfway in frequency between two components, and listeners had to indicate if T was present in S or not. In an "up/down" condition, $\mathrm{T}$ was 100 cents below or above a randomly selected component of S, and listeners had to identify the direction of the frequency shift. When the components of $\mathrm{S}$ were asynchronous, the present/absent task was easier than the up/down task. When the components of $\mathrm{S}$ were synchronous, the opposite trend was observed. In case of asynchrony, the components of S could be heard out individually, so listeners presumably compared explicit (conscious) pitch percepts to make their judgments. In case of synchrony, the components of S were difficult to hear out individually; apparently, pitch comparisons were then made implicitly by "frequency-shift detectors" (Demany and Ramos, 2005) which, we argue, participate in auditory scene analysis. It seems that such detectors relate automatically consecutive tones, and are generally less efficient for comparisons between nonconsecutive tones.

\section{$1: 40$}

1pPPa3. Concurrent sound segregation and peripheral frequency resolution. Christophe Micheyl (University of Minnesota, Department of Psychology, 75 E. River Road, Elliott Hall N218, Minneapolis, MN 55455, USA, cmicheyl@umn.edu), Michael V. Keebler (University of Minnesota, Department of Psychology, 75 E. River Road, Elliott Hall N218, Minneapolis, MN 55455, USA, keeb0002@umn.edu), Andrew J. Oxenham (University of Minnesota, Department of Psychology, 75 E. River Road, Elliott Hall N218, Minneapolis, MN 55455, USA, oxenham@umn.edu)

In everyday environments where multiple sound sources coexist, listeners often have to follow a harmonic source of interest (e.g., someone's voice) among other such sources (e.g., other talkers). Here, we review psychoacoustical findings suggesting that this form of auditory scene analysis is related to the degree of peripheral "resolvability" of harmonics in the auditory system. We present experimental results, which indicate a systematic relationship between harmonic resolvability and the ability of normal-hearing listeners to "track" (or discriminate) changes in the fundamental frequency (F0) of a target harmonic complex mixed with another (interferer). We show that poor harmonic resolvability is associated not only with poor performance, but also with an inability to take advantage of normally potent cues for concurrent sound segregation, including F0 differences, laterality differences, and onset-offset asynchronies. While it remains unclear whether this relationship is causal, these findings may have important implications for the design of artificial auditory-scene-analysis systems, and for our understanding of the listening difficulties experienced by hearing-impaired listeners in whom peripheral frequency resolution is reduced. [Work supported by NIDCD R01DC05216.] 
1pPPa4. Temporal fine structure cues in auditory stream segregation of complex tones. Nicolas Grimault (Laboratoire Neurosciences Sensorielles, Comportement, Cognition, UMR 5020, Université Lyon 1 - CNRS, 50 av. Tony Garnier, 69366 Lyon Cedex 07, France, ngrimault@olfac.univ-lyon1.fr), Etienne Gaudrain (Laboratoire Neurosciences Sensorielles, Comportement, Cognition, UMR 5020, Université Lyon 1 - CNRS, 50 av. Tony Garnier, 69366 Lyon Cedex 07, France, egaudrain@ gmail.com)

The ability to understand speech-in-speech is generally described to be related to the capacity to segregate one auditory stream among others sound sources. The channeling theory suggests that sequential stream segregation is basically sustained by frequency selectivity. However, it has been evidenced that sequences of stimuli that evoked the same excitation pattern can be streamed apart based on temporal cues only. Other results involving hearing impaired listeners with enlarged auditory filters hardly fit in with this theory. More recently, several reports in the literature and new attempts of modelization underline the potential interest of temporal fine structure cues for stream segregation. The current study is dedicated to test further the potential interest of temporal fine structure cues to stream apart sequences of unresolved complex tones with alternating fundamental frequencies. Several conditions of envelope saliency (positive and negative Schroeder phase complex tones) and several conditions of fine structure will be used in a streaming test leading to an objective measure related to segregation.

\section{$2: 20$}

1pPPa5. Auditory streaming and coherence. Shihab Shamma (ECE Department, AV Williams Bldg., 2203, University of Maryland, College Park, MD 20742, USA, sas@eng.umd.edu), Mounya Elhilali (Johns Hopkins University, Dept. of Electrical and Computer Engineering, Barton Hall 105, 3400 N. Charles Street, Baltimore, MD 21218, USA, mounyaelhilali@gmail.com)

Auditory streaming is a phenomenon that manifests itself in the everyday ability of humans and animals to parse complex acoustic information arising from multiple sound sources into meaningful auditory "streams." For instance, the ability to follow a conversation at a noisy cocktail party or hear the violin in the orchestra both rely on the formation of auditory streams. While seemingly effortless, the neural mechanisms underlying auditory streaming remain a mystery. In this talk, we shall discuss physiological experiments that address the role of coherence in mediating this percept. The experiments contrast the perception and neural responses evoked by the classic two-tone stimuli when presented in alternating or synchronous modes. Briefly, while the percepts in these two conditions are very different, being much more "streamed" in the alternating case, physiological responses to the tones did not differ significantly enough to explain this change in percept. This result casts doubt on the simple spatial segregation hypothesis, and instead argues for a substantial role of coherence of stimuli across different channels in mediating streaming.

\section{Contributed Paper}

\section{2:40}

1pPPa6. Auditory temporal edge detection in human auditory cortex Maria Chait (UCL Ear Institute, 332 Gray's Inn Road, WC1X 8EE London, UK, m.chait@ucl.ac.uk)

Auditory objects are detected if they differ acoustically from the ongoing background. In simple cases, the appearance or disappearance of an object involves a transition in power, or frequency content, of the ongoing sound. However, it is more realistic that the background and object possess substantial nonstationary statistics, and the task is then to detect a transition in the pattern of ongoing statistics. How does the system detect and process such transitions? We use magnetoencephalography (MEG) to measure early auditory cortical responses to transitions between constant tones, regularly alternating, and randomly alternating tone-pip sequences, where the dimension of change is either frequency, loudness, or spatial location. Such transitions embody key characteristics of natural auditory temporal edges. Our data demonstrate that the temporal dynamics and response polarity of the neural temporal-edge-detection processes depend in specific ways on the generalized nature of the edge (the context preceding and following the transition) and suggest that distinct neural substrates in core and noncore auditory cortex are recruited depending on the kind of computation (discovery of a violation of regularity, vs the detection of a new regularity) required to extract the edge from the ongoing fluctuating input entering a listener's ears.

\section{Invited Papers}

1pPPa7. Perception of simultaneous sentences with frequency-shifted voiced excitation. Brian Roberts (Aston University, Psychology, Life and Health Sciences, B4 7ET Birmingham, UK, b.roberts@aston.ac.uk), Stephen D. Holmes (Aston University, Psychology, Life and Health Sciences, B4 7ET Birmingham, UK, S.D.Holmes@aston.ac.uk), Chris J. Darwin (University of Sussex, Psychology, School of Life Sciences, BN1 9QG Brighton, UK, cjd@sussex.ac.uk)

Identification of key words in one of two simultaneous sentences is substantially improved when the sentences differ in fundamental frequency (F0). When the sentences are constructed to be almost continually voiced, identification can improve from $20 \%$ at 0 semitones difference to $80 \%$ at 8 semitones difference (J. Bird \& C.J. Darwin 1998 in Psychophysical and Physiological Advances in Hearing, Ed A.R. Palmer et al., Whurr, London, pp. 263-269). We recorded the same monotone sentences as Bird and Darwin. These were first resynthesised using PSOLA to give a range of harmonic F0 differences $(0,1,3$, and 10 semitones) and then additionally resynthesised by LPC with the LPC residual frequency shifted by $25 \%$ of F0, to give excitation with inharmonic but regularly spaced components. Experiment 1 showed that frequency-shifted sentences gave the same large improvement with nominal F0 difference as did the harmonic sentences, although overall performance was about 10\% poorer. Experiment 2 replicated the finding by Bird and Darwin that swapping the F0 difference across spectral regions only reduced the improvement in recognition for large F0 differences, and extended this finding to frequency-shifted voiced excitation. The results extend the range of perceptual phenomena originally attributed to harmonic processing. 
1pPPa8. Signal duration modulates age differences in neuromagnetic brain activity associated with concurrent sound segregation. Claude Alain (Rotman Research Institute, Baycrest Centre, 3560 Bathurst Street, Toronto, ON M6A 2E1, Canada, calain@rotman-baycrest.on.ca), Kelly McDonald (Rotman Research Institute, Baycrest Centre, 3560 Bathurst Street, Toronto, ON M6A 2E1, Canada, kmcdonald@rotman-baycrest.on.ca)

Listeners' ability to parse concurrent sounds is a prerequisite in solving the cocktail party problem. Here, we examine whether deficits in periodicity coding can account for older adults' difficulties in understanding speech in noise. In two experiments, we measured auditory evoked fields (AEFs) while young and older adults listened to complex sounds that either had all of their harmonics in tune or had the third harmonic mistuned by 4 or $16 \%$ of its original value. For each participant, the AEFs were modeled with a pair of dipoles in auditory cortex and the effects of age on the resulting source waveforms were examined. Older adults were less likely to report hearing the mistuned harmonic as a separate sound than young adults, but only for short duration signals (50 ms). This age difference was paralleled by reduced neuromagnetic activity indexing the processing of the mistuned harmonic. For longer duration signals (e.g., $200 \mathrm{~ms}$ ), older adults show comparable amplitude, but a delay in latency. These findings show an age-related decline in concurrent sound perception based on harmonicity, which could partly be alleviated by increasing signal duration. The implications of these findings for speech perception in noise will be discussed.

\section{Contributed Paper}

4:00

1pPPa9. Influence of noise type, degree of comodulation and interaural phase difference on the combined monaural and binaural masking release. Bastian Epp (Universität Oldenburg, Medizinische Physik, Carlvon-Ossietzky Str. 9-11, 26111 Oldenburg, Germany, Bastian.Epp@uni-oldenburg.de), Jesko Verhey (Universität Oldenburg, Medizinische Physik, Carl-von-Ossietzky Str. 9-11, 26111 Oldenburg, Germany, jesko.verhey@uni-oldenburg.de)

Several masking experiments have shown that the auditory system is able to use coherent envelope fluctuations of the masker across frequency within one ear as well as differences in interaural disparity between signal and masker to enhance signal detection. The two effects associated with these abilities are comodulation masking release (CMR) and binaural mask- ing level difference (BMLD). The aim of the present study was to investigate the combination of CMR and BMLD. Thresholds of a sinusoidal signal were measured in a flanking band paradigm, i.e., in the presence of several narrowband noise maskers, (i) for two noise types (Gaussian and multiplied noise), (ii) various degrees of comodulation, and (iii) various interaural phase differences (IPD) of the signal. Thresholds decreased as the signal IPD increased and decreased as the masker comodulation increased. For both noise types, the maximum CMR was about $10 \mathrm{~dB}$ and the maximum BMLD was about $14 \mathrm{~dB}$. Thresholds where monaural and binaural cues were present showed an addition of the single effects, i.e., a maximum masking release of $24 \mathrm{~dB}$. A simplified model of the auditory system assuming a serial alignment of the across-frequency and the binaural processing stages is able to reproduce the dataset.

\section{Invited Papers}

1pPPa10. Speech in noise and the medial olivo-cochlear efferent system. Raymond Meddis (University of Essex, Department of Psychology, Wivenhoe Park, CO7 9SW Colchester, UK, rmeddis@essex.ac.uk), Robert Ferry (University of Essex, Department of Psychology, Wivenhoe Park, CO7 9SW Colchester, UK, rferry @essex.ac.uk), Guy J. Brown (University of Sheffield, Dept. of Computer Science, Regent Court, 211 Portobello Street, S1 4DP Sheffield, UK, g.brown@dcs.shef.ac.uk)

A central question in auditory scene analysis is how we are able to follow speech against a background of interfering noise. The question is particularly acute for artificial speech recognition algorithms and the hearing impaired. The medial efferent system has been suggested as one contributor to our ability to hear speech in noise. We have recently added efferent suppression to our model of the auditory periphery and evaluated it against physiological observations at the level of the basilar membrane and auditory nerve. We have also replicated a study using compound action potentials where a tone in a noise background became more salient when the efferent system was artificially activated. Visual representations of the computed auditory nerve response to speech in noise show considerable improvement when the efferent system is activated. Tests using the auditory model as a front end to a connected-word recognition algorithm also showed improved performance in the presence of noise when efferent effects were included. The benefits of efferent suppression include reduced compression and extension of the dynamic range of individual auditory nerve fibers.

1pPPa11. Contributions of the brainstem and below to auditory scene analysis. Ian M. Winter (Centre for the Neural Basis of Hearing, The Physiological Laboratory, Downing Street, CB2 3EG Cambridge, UK, imw1001@cam.ac.uk)

We are increasingly aware of the cues the auditory system uses in the segregation and fusion of auditory objects, however, we have very little evidence for how, or where, these cues are extracted in the auditory pathway. Here I will show how the inner ear and cochlear nucleus contribute to three classical auditory scene analysis phenomena. In the first, grouping by common onset; adaptation, lateral suppression, and recovery from suppression all play a role. In the second phemonenon, a sequential streaming task, commonly referred to as $\mathrm{ABA}$, the contributing low-level mechanisms are frequency tuning and neural adaptation. Finally, in the third phenomenon, comodulation masking release, which describes the seemingly paradoxical situation whereby the detection of a masked signal is improved by the addition of coherently modulated energy remote from the signal frequency, lateral suppression is an important mediator. Taken together these studies suggest that neurophysiological processes at, or before, the level of the cochlear nucleus make an important contribution to auditory scene analysis. 
1pPPa12. The European starling as a model for understanding mechanisms underlying auditory scene analysis. Georg Klump (Oldenburg University, Zoophysiology \& Behavior Group, IBU, Fak 5, Carl von Ossietzky Str. 9-11, 26129 Oldenburg, Germany, georg .klump@uni-oldenburg.de)

The European starling is a songbird that has evolved mechanisms for the analysis of acoustic scenes exhibiting a number of similarities with human perception. Segregation of signals and background noise from different sources is enhanced due to the processing of differences in modulation patterns as is evident in experiments on the comodulation detection difference and and comodulation masking release. Similarly to auditory streaming in human subjects, integration of sequential signals into streams occurs in the European starling and it is affected by spectral and temporal properties of the sounds. The presentation reviews results from psychophysical experiments and compares these to observed neural response patterns of auditory forebrain neurons in starlings.

\section{$5: 20$}

1pPPa13. Identifying auditory events at a cocktail party: Principles of temporal integration and stream segregation. Elyse Sussman (Albert Einstein College of Medicine, Dept. of Neuroscience, 1410 Pelham Parkway S, Bronx, NY 10461, USA, esussman @ aecom.yu.edu)

The question of how sequential sound elements are grouped into meaningful percepts has been within the focus of researchers for decades. This talk takes a new perspective by addressing how within-stream temporal integration occurs in the context of a cocktail party. In a noisy environment, the ability to identify specific events depends upon multiple processes: the overall segregation of sounds into streams as well as the within-stream integration of sequential sounds into meaningful units. This talk presents two studies that address the interaction of these two complex processes. Event-related potentials were used to determine effects of timing (Experiment 1) and of attention (Experiment 2) on the perception of within-stream sound events in multistream environments. Experiment 1 shows that within-stream temporal integration of sequential elements uses previously segregated streams as input. Experiment 2 shows that attention can override stimulus-driven processing biases to facilitate task demands. These results demonstrate different time courses for segregation of sounds into separate streams and for integration of sound elements into within-stream units. Thus, the findings suggest that different neural mechanisms interact in the perceptual organization of sequential sound elements.

\section{Contributed Papers}

\section{$5: 40$}

1pPPa14. Object formation versus object selection. Barbara Shinn-Cunningham (Boston University Hearing Research Center, 677 Beacon Street, Boston, MA 02215, USA, shinn@cns.bu.edu)

Auditory scene analysis is intricately linked to our ability to understand one acoustic source amidst competing sources. However, studies of selective attention often fail to distinguish between the roles of object formation (estimating the spectro-temporal content of a particular auditory object) and object selection (determining which object will be the focus of attention) in performance. Part of the difficulty in trying to disentangle these effects is that they are intimately related. However, listeners in a selective attention task often report sound elements that are similar in a sound dimension they know is task irrelevant when trying to selectively attend to a different sound attribute (e.g., location, pitch, intensity, timbre). Such results suggest a model of selective attention in which the focus of attention is an auditory object that is formed through interactions between automatic grouping processes and top-down selection. A conceptual model of these interactions will be presented along with data in support of this framework. [Work supported by AFOSR and ONR.]

\section{6:00}

1pPPa15. Auditory scene analysis emerges from a distributed yet integrated network. Rhodri Cusack (MRC CBU, 15 Chaucer Road,
Cambridge, CB2 7EF Cambridge, UK, rhodri.cusack@mrc-cbu .cam.ac.uk), Daniel Pressnitzer (CNRS UMR 8158; Univ. Paris Descartes; Ecole Normale Supérieure, 29 rue d'Ulm, 75005 Paris, France, Daniel .Pressnitzer@ens.fr)

Three architectures that have been explicitly or implicitly proposed for auditory scene analysis were evaluated: (1) that some single module is responsible for scene analysis after feature extraction; (2) that scene structure is elaborated through feed-forward stages of auditory processing; and (3) that scene analysis is integrated yet distributed. It is argued that the available evidence points towards the third model. Against (1), scene analysis is influenced by features that are best represented across a broad range nodes in the auditory network, and transmitting this information to a single module would not be parsimonious. Furthermore, neurophysiology has shown a range of nodes reflect scene analysis, ranging from the cochlear nucleus, through auditory cortex to the parietal cortex. Against both (1) and (2) is the high degree of reciprocal interaction between the behavioural derivation of features and scene analysis, and the integration evident from the exclusivity of scene organisations. In contrast, model (3) naturally predicts these observations and is compatible with auditory anatomy, which is not "feed forward" or hublike. Finally, we highlight outstanding issues important to a fuller understanding of scene analysis, such as the neural code for perceptual groups and the mechanism for extraction of statistical regularities.

\section{Invited Papers}

\section{6:20}

1pPPa16. Perceptual dynamics of auditory streaming and its neural correlates. Makio Kashino (NTT Communication Science Laboratories, NTT Corporation, 3-1, Morinosato Wakamiya, 243-0198 Atsugi, Japan, kashino@avg.brl.ntt.co.jp), Hirohito M. Kondo (NTT Communication Science Laboratories, NTT Corporation, 3-1, Morinosato Wakamiya, 243-0198 Atsugi, Japan,hkondo@brl.ntt.co.jp), Minae Okada (ERATO Shimojo Implicit Brain Function Project, JST, 3-1, Morinosato Wakamiya, 2430198 Atsugi, Japan, mokada@ @ shimojo.jst.go.jp)

Prolonged listening to a repeated sequence consisting of low and high tones produces spontaneous transitions in the perceptual state between a single coherent stream and two segregated streams. The dissociation between constant physical stimulation and fluctuating perceptual experience in auditory streaming provides a compelling means for studying how auditory percepts are formed in the brain. First, we psychophysically examined the nature of such perceptual transitions in various frequency differences $(\Delta \mathrm{f})$ between low and 
high tones. After the initial buildup of streaming, perceptual transitions occurred frequently even at the $\Delta \mathrm{fs}$ that were previously thought to produce a stable percept, and the dominant perceptual state changed depending on $\Delta \mathrm{f}$. Next, we explored brain activities correlated with the perceptual transitions using functional magnetic resonance imaging (fMRI). An event- related analysis revealed that the auditory cortex and thalamus were activated at the timing of perceptual transitions. The response onset of the activity in the auditory cortex was earlier than that in the thalamus for the transition from dominant to non-dominant percepts, and later for the transition from nondominant to dominant percepts. These results imply that the interaction of the auditory cortex and thalamus plays a crucial role for perceptual transitions in auditory streaming.

\section{6:40}

1pPPa17. What can binocular rivalry tell us about auditory streaming? Susan Denham (University of Plymouth, Drake Circus, PL4 8AA Plymouth, UK, sdenham@plymouth.ac.uk), István Winkler (Department of General Psychology, Institute for Psychology, Hungarian Academy of Sciences, P.O. Box 398, 1394 Budapest, Hungary, iwinkler@cogpsyphy.hu), Kinga Gyimesi (Department of General Psychology, Institute for Psychology, Hungarian Academy of Sciences, P.O. Box 398, 1394 Budapest, Hungary, kgyimesi@ cogpsyphy.hu), Gábor Stefanics (Department of General Psychology, Institute for Psychology, Hungarian Academy of Sciences, P.O. Box 398, 1394 Budapest, Hungary, gstefanics@ cogpsyphy.hu)

Although sensory systems share the common goal of building accurate representations of the environment, differences in the physical nature of stimuli from different modalities seem to argue against similar processing strategies. Nevertheless, our experiments have revealed surprisingly deep parallels between the dynamics of perceptual organisation in auditory streaming and binocular rivalry. These parallels provide evidence for an interpretation of auditory streaming in terms of rivalry between competing temporal associations, which helps to explain the distribution of perceptual switching with respect to stimulus features, strong differences between first and steady-state phases in ongoing switching, the nonmonotonic dependence of switching rate on stimulus "strength," the range of relationships between phase duration and stimulus features, and the existence and distribution of "transition" phases during which subjects simultaneously experience what are usually thought to be mutually exclusive perceptual states.

1pPPa18. Multistability in audiovisual speech scene analysis: behavioral and neurophysiological data on multimodal verbal transformations. Jean-Luc Schwartz (ICP-GIPSA, INPG, 46 Av. Félix Viallet, 38031 Grenoble, France, schwartz@icp.inpg.fr), Anahita Basirat (ICP-GIPSA, INPG, 46 Av. Félix Viallet, 38031 Grenoble, France, basirat@icp.inpg.fr), Marc Sato (ICP-GIPSA, INPG, 46 Av. Félix Viallet, 38031 Grenoble, France, sato@icp.inpg.fr)

The multistable perception of speech refers to the perceptual changes experienced while listening to a speech form cycled in rapid and continuous repetition, the so-called Verbal Transformation Effect. Because distinct interpretations of the same repeated stimulus alternate spontaneously, this effect provides an invaluable tool to examine how speech percepts are formed in the listener's mind. In a series of behavioural studies, we demonstrated that (1) articulatory-based representations play a key part in the endogenously driven emergence of auditory speech percepts; (2) vision penetrates into the transformation process, intervening both in the stability of a given pattern, and in the temporal dynamics of perceptual switches. Furthermore, we determined the cortical circuit in charge for the emergence of a shift of percept associated to a given audio input. This circuit involves a cortical "dorsal route" for speech perception, linking temporal, parietal, and frontal regions. Current experiments involve further tests about the nature of both perceptuo-motor and audiovisual interactions in verbal transformations. Altogether, these results shed some new lights on the cognitive and neurobiological bases of speech perception, in which multimodal interactions between sensory and motor representations play a crucial role. 


\title{
Session 1pPPb
}

\section{Psychological and Physiological Acoustics: Cochlear Implants: Going Beyond the Envelope I}

\author{
Bernhard U. Seeber, Cochair \\ MRC Institute of Hearing Research, Science Rd / University Park, Nottingham, NG7 2RD, UK \\ H. Steven Colburn, Cochair \\ Boston University, 44 Cummington St., Boston, MA 02215, USA
}

\section{Contributed Paper}

1:00

1pPPb1. A new approach to electric-acoustic stimulation. Christopher A. Brown (Arizona State University, Dept. of Speech \& Hearing Science, P.O. Box 870102, Tempe, AZ 85287-0102, USA, c-b@asu.edu), Sid P. Bacon (Arizona State University, Dept. of Speech \& Hearing Science, P.O. Box 870102, Tempe, AZ 85287-0102, USA, spb@asu.edu)

When low-frequency acoustic stimulation is combined with either real or simulated electric stimulation from a cochlear implant (electric-acoustic stimulation, or EAS), speech intelligibility in noise can improve dramatically. This improvement has been shown in simulation to be due in part to the presence of fundamental frequency (F0) and amplitude envelope information in the low-frequency region. The current experiment extends those findings to implant patients. Six patients who had residual lowfrequency hearing in either their implanted or unimplanted ear participated. A target talker was combined with multitalker babble and presented to the implant. In the low-frequency region, patients heard either no stimulus, target speech, or a tone that was modulated in frequency to track the dynamic changes in F0, and in amplitude with the amplitude envelope of the low-pass target speech. Results showed that the tone provided, on average, about 58 percentage points of improvement over electric-only stimulation. Both the tone and target speech provided a statistically significant benefit over electric stimulation only $(\mathrm{p}<0.0001)$, and were statistically equivalent to each other $(\mathrm{p}>0.05)$. These results demonstrate that a tone that conveys F0 and amplitude envelope information can provide significant benefit in EAS.

\section{Invited Papers}

\section{$1: 20$}

1pPPb2. Temporal pitch processing by cochlear implant users. Robert P. Carlyon (MRC CBU, 15 Chaucer Rd., CB2 7EF Cambridge, UK, bob.carlyon@mrc-cbu.cam.ac.uk), Ying-Yee Kong (Notheastern University, 106A Forsyth, Boston, MA Ma 02115, USA, yingyeekong@googlemail.com), Cathy Lynch (Addenbrooke's NHS Trust, Hills Rd, CB2 2QQ Cambridge, UK, catherine.lynch@addenbrookes.nhs.uk), John Deeks (MRC CBU, 15 Chaucer Rd., CB2 7EF Cambridge, UK, john.deeks@mrc-cbu .cam.ac.uk)

Modern cochlear implants (CIs) convey fundamental frequency (F0) information using a purely temporal code. However, temporal pitch processing - as measured by the discrimination of the changes in the rate of a pulse train applied to a single electrode - is often worse than in normal hearing $(\mathrm{NH})$ at low pulse rates, and usually deteriorates dramatically at rates above about 300 pps. We will describe evidence that at low pulse rates, the value of the pitch perceived can be affected by refractory effects at the level of the auditory nerve $(\mathrm{AN})$, and can also be influenced by small $(<0.5 \mathrm{~dB})$ changes in stimulus level. In contrast, the deterioration in rate discrimination at high rates appears to be impervious to a wide range of manipulations that would be expected to strongly affect the representation of the stimulus at the level of the AN. One example of this is the finding that the variation in rate discrimination performance with baseline rate for single-pulse-per-period pulse trains correlates, across listeners, with discrimination of different rates of sinusoidal amplitude modulation imposed on a 5000-pps carrier. The implications of these findings for attempts to improve pitch coding in CIs will be discussed.

\section{$1: 40$}

1pPPb3. Investigating the influence of pulse rate and duration on pitch perception in cochlear implants. Joshua S. Stohl (Duke University, Department of Electrical and Computer Engineering, Box 90291, Durham, NC 27708, USA, jss@ee.duke.edu), Chandra S. Throckmorton (Duke University, Department of Electrical and Computer Engineering, Box 90291, Durham, NC 27708, USA, cst@ee.duke.edu), Leslie M. Collins (Duke University, Department of Electrical and Computer Engineering, Box 90291, Durham, NC 27708, USA, 1collins@ee.duke.edu)

Multirate sound processing strategies may potentially provide improved speech recognition and music perception in cochlear implants (Nie et al., 2005; Throckmorton et al., 2006), although it may be necessary to tune these strategies using psychophysical data to provide maximum benefit to the user (Throckmorton et al., 2006). To investigate the need for tuning, cochlear implant users participated in several rate-based psychophysical experiments. Subjects performed single- and two-rate pitch ranking tasks to investigate the influence of pulse rate on the overall pitch structure. The introduction of a second rate resulted in frequent pitch reversals between closely spaced electrodes. In addition to the multi-rate pitch structure, the duration necessary to perceive a rate change must also be considered. Experiments were performed using ABA stimuli, where rate B was higher than rate A. Subjects completed an embedded rate discrimination task in which the duration of B was fixed and its rate varied adaptively for durations ranging from 20-200 ms. The rate of segment B was then fixed, and its duration varied adaptively. Results imply that embedded rate difference limens may be a function of duration, and that the minimum duration required for detecting a change in pulse rate is subject specific. 
1pPPb4. Use of simultaneous stimulation to represent fine structure in cochlear implant processors. Leonid Litvak (Advanced Bionics, 12740 San Fernando Rd., Sylmar, CA 91342, USA, LeonidL@AdvancedBionics.Com), Aniket Saoji (Advanced Bionics, 12740 San Fernando Rd., Sylmar, CA 91342, USA, Aniket.Saoji@ AdvancedBionics.Com), Anthony Spahr (Arizona State University, Lattie F. Coor Hall, Room 3462, Tempe, AZ 85287, USA, Tony.Spahr@Asu.Edu), Abhijit Kulkarni (Advanced Bionics, 12740 San Fernando Rd., Sylmar, CA 91342, USA, Abhijit.Kulkarni@ AdvancedBionics.Com), Michael Marzalek (Advanced Bionics, 12740 San Fernando Rd., Sylmar, CA 91342, USA, mikemarz@sonic.net)

In cochlear implants, simultaneous stimulation of adjacent electrodes, can be used to shape the electric fields generated within the cochlea. For example, simultaneous in-phase stimulation of nearby electrodes can be used to create activation patterns which may have maxima at locations that are intermediate to the locations of the stimulated electrodes. Psychophysically, such "virtual channels" give rise to intermediate pitch sensations. Simultaneous out-of-phase stimulation of two flanking electrodes in addition to the center electrode can be used to presumably produce more focused activation at the cost of larger overall stimulation currents. Forward masking patterns indicate that for sufficiently large compensation currents, such "focused" stimulation can lead to a more spatially contained excitation. In some cases, current focusing also could lead to sensations that are more tonal relative to monopolar. This presentation will review recent results obtained in our laboratories with both "virtual channel" and "focused" configurations. We will also address how both techniques can be used to better represent the within-channel fine structure in a wearable sound processing strategy.

\section{$2: 20$}

1pPPb5. Effect of channel interactions on sensitivity to binaural timing cues in electrical hearing. Gary Jones (University of Wisconsin, 1500 Highland Ave, Waisman Center, Madison, WI 53705, USA, gjones@ waisman.wisc.edu), Ruth Litovsky (University of Wisconsin, 1500 Highland Ave, Waisman Center, Madison, WI 53705, USA, litovsky@waisman.wisc.edu), Richard Van Hoesel (CRC HEAR, 384-388 Albert St, VIC 3002 East Melbourne, Australia, rvanh@bionicear.org)

With bilateral cochlear implant (BICI) users it is possible to test sensitivity to interaural timing differences (ITDs) while bypassing peripheral auditory processing. Ongoing work in our lab suggests that at low pulse rates ITD JNDs in the tens of microseconds can be achieved by BICI users with postlingual onset of deafness at single pairs of electrodes at the base, middle or apex of the electrode array. The current project is concerned with channel interactions and, specifically, performance when there is activation of more than one electrode pair and when more than one ITD is presented to the auditory system simultaneously. We examined ITD sensitivity in BICI users with bilateral pairs of pitch-matched electrodes on which unmodulated pulse trains were presented at 100 pulses per second. A probe pulse train and an added pulse train were temporally interleaved. ITD JNDs for the probe train were measured while varying the added train's: a) frequency place, b) level, and c) ITD (matched to probe or fixed at $0 \mu \mathrm{s}$ ). Results suggest that stimulation of multiple electrodes can result in a range of interaction effects that can have either facilitatory or interfering effects on binaural sensitivity. Work supported by NIH-NIDCD (R01 DC003083-09, F31 DC009361).

\section{2:40}

1pPPb6. Binaural jitter improves sensitivity to interaural time differences in electric and acoustic hearing. Bernhard Laback (Austrian Academy of Science/Acoustics Research Institute, Wohllebengasse 12-14, 1040 Vienna, Austria, Bernhard.Laback@oeaw.ac.at), Piotr Majdak (Austrian Academy of Science/Acoustics Research Institute, Wohllebengasse 12-14, 1040 Vienna, Austria, piotr@majdak.com), Matthew J. Goupell (Austrian Academy of Science/Acoustics Research Institute, Wohllebengasse 12-14, 1040 Vienna, Austria, matt.goupell@gmail.com)

Cochlear implant (CI) listeners are often sensitive to fine structure ITD at low pulse rates, but their sensitivity declines at higher pulse rates which are required for speech coding. We hypothesized that this limitation is related to the binaural adaptation phenomenon [Hafter and Dye, J. Acoust. Soc. Am. 73 644-651 (1983)], associated with periodic stimulation. With five CI listeners, we tested the effect of introducing binaurally-synchronized jitter (binaural jitter) in the stimulation timing, assuming that this reduces the periodicity in the neural response and thus avoids binaural adaptation. Pulse rates from 400 to 1515 pulses per second (pps) were tested. In addition, we performed a similar experiment with normal-hearing $(\mathrm{NH})$ listeners using bandpass-filtered acoustic pulse trains and testing the pulse rates 600 and 1200 pps. The CI listeners showed large improvements in ITD sensitivity from binaural jitter at high pulse rates (800 1515 pps). The NH listeners also showed large improvements from binaural jitter. The results are consistent with the hypothesis that random temporal variation reactivates the adapted binaural auditory system. Thus, binaurally-jittered stimulation improves the access of bilateral CI listeners to ITD information. Partly supported by the Austrian Science Fund, FWF, project number P18401-B15.

\section{3:00}

1pPPb7. Parameters affecting the precedence-effect with cochlear implants. Bernhard U. Seeber (MRC Institute of Hearing Research, Science Rd., / University Park, NG7 2RD Nottingham, UK, seeber@ihr.mrc.ac.uk), Ervin R. Hafter (University of California, Department of Psychology, 3210 Tolman Hall, Berkeley, CA, CA 94720-1650, USA, hafter@ berkeley.edu)

Monaural spectral and temporal cues as well as binaural cues provide the information to analyze the auditory scene. Subjects using bilateral cochlear implants (CIs) have limited access to those cues, particularly to interaural time differences (ITDs) and to spectral information. Nevertheless, many subjects can localize sounds in quiet based on the evaluation of interaural level differences (ILDs) but this might be impaired by the presence of reflections. We studied the precedence effect with patients wearing bilateral CIs and found that localization was abolished in most subjects when the echo was present, but some subjects showed dominance of the leading sound on localization. The reasons for the different outcomes were studied with simulations of CIs. Using a noise-band vocoder simulation, subjects could not fuse lead and lag into a single object and they localized both separately. With a sinusoidal vocoder, lead-lag fusion and the precedence effect could be obtained provided that frequencies of the carrier sinusoids were matched across ears. Interaural phase of the carrier played only a limited role. We conclude that precedence of ongoing sounds can solely be based on ILDs and envelopeITDs and that proper place matching of CI-electrodes would help the analysis of concurrent sounds. 
1pPPb8. Neural cross-correlation metrics to quantify envelope and fine-structure coding in auditory-nerve responses. Michael Heinz (Purdue University, 500 Oval Drive, West Lafayette, IN 47907, USA, mheinz@purdue.edu), Jayaganesh Swaminathan (Purdue University, 500 Oval Drive, West Lafayette, IN 47907, USA, jswamy@purdue.edu)

Fundamental questions about the relative perceptual importance of envelope and fine structure are often addressed using specialized acoustic stimuli, such as temporal-fine-structure speech or auditory chimaeras. Interpretation of these perceptual studies assumes envelope and fine structure can be isolated at the output of the cochlea. Narrowband cochlear filtering constrains the ability to isolate fine structure from envelope; however, envelope recovery from fine structure has been difficult to evaluate physiologically. Separate neural cross-correlation coefficients (CCCs) for envelope and fine-structure were developed based on shuffled auto- and cross-correlograms. Neural CCCs have a wide dynamic range for both within-fiber, cross-stimulus and cross-fiber, within-stimulus correlations based on both model and recorded spike-train data from auditory-nerve fibers. Results provide physiological evidence consistent with perceptual findings that envelope recovery is reduced as the number of analysis bands increases, but is not completely eliminated for 8- and 16band conditions. Neural CCCs were also used to evaluate across-fiber temporal coding, which has been implicated in the difficulties that hearing-impaired listeners have in understanding speech in complex acoustic backgrounds. Results demonstrate the potential of these neural CCC metrics to quantitatively evaluate a wide range of perceptually significant temporal coding issues relevant to normal and impaired hearing. Supported by NIH-NIDCD.

\section{Contributed Paper}

\section{4:00}

1pPPb9. Transmission of temporal fine structure by a penetrating auditory nerve implant. John C. Middlebrooks (University of Michican, KHRI, 1301 E. Ann St., Ann Arbor, MI 48109-5506, USA, jmidd@umich.edu), Russell L. Snyder (University of California, Epstein Laboratory, Room U-490, P.O. Box 0526, San Francisco, CA 94143 0526, USA, rsnyder@ohns.ucsf.edu)

We are quantifying the transmission of temporal fine structure from a penetrating auditory nerve electrode array to the inferior colliculus (IC) in anesthetized cats. We stimulate with biphasic electrical pulse trains and determine the maximum pulse rate eliciting phase-locked activity. Many IC neurons showed significant phase locking to rates as high as 600 pulses per second (pps) when the nerve was stimulated with the penetrating array, whereas phase locking reached only 300 pps when stimulated with a conventional intrascalar cochlear implant. Phase locking to rates $>300$ pps was found primarily among IC units with characteristic frequencies (CFs) $<2$ $\mathrm{kHz}$. Such low frequency fibers are not stimulated selectively by conventional intrascalar cochlear implant. We selectively stimulated apical fibers by placing ball electrodes on the apical spiral lamina. Activation of these balls selectively stimulated low-CF IC neurons. These neurons phase locked to 600 pps. Based on these results, we conclude that transmission of temporal information from a penetrating intraneural electrode array is superior to that obtained with a conventional cochlear implant but only insofar as the intraneural electrodes provide more selective access to low-CF pathways. Supported by NIDCD NO1-DC-5-0005.

\section{Invited Papers}

1pPPb10. Why is sensitivity to interaural time differences (ITD) poorer than normal with bilateral cochlear implants? Neurophysiological considerations. Bertrand Delgutte (Massachusetts Eye \& Ear Infirmary, Eaton-Peabody Lab., 243 Charles St., Boston, MA 02114, USA, Bertrand_Delgutte@meei.harvard.edu), Kenneth E. Hancock (Massachusetts Eye \& Ear Infirmary, EatonPeabody Lab., 243 Charles St., Boston, MA 02114, USA, ken_hancock@MEEI.HARVARD.EDU)

Bilateral cochlear implantation improves sound localization and speech reception in noise. Yet ITD sensitivity is clearly poorer than normal with bilateral implants, even when the stimulation bypasses the processors (which discard the temporal fine structure of the stimulus). This poor behavioral ITD sensitivity is surprising from a physiological perspective because (1) electric stimulation produces precise temporal patterns of activity in the auditory nerve, and (2) most neurons in the inferior colliculus of acutely-deafened, bilaterally-implanted cats show precise tuning to ITD of pulse trains at the appropriate intensity (Smith and Delgutte, J. Neurosci. 27:6740). We will present physiological and modeling results bearing upon two hypotheses for reconciling neural and psychophysical observations. One hypothesis is that plastic changes to the neural circuitry resulting from binaural deprivation may impair the binaural processing in long-term deafened animals (and humans), particularly if deafening occurs during the neonatal period. Alternatively, the abnormal spatio-temporal pattern of activity across the population of binaural neurons (due in part to limited dynamic range with electric stimulation) may impair the ability of the central decoding stages to extract the ITD information available in individual binaural neurons.

1pPPb11. Modeling responses of brainstem neurons to electrical stimuli. H Steven Colburn (44 Cummington St., Boston, MA 02215, USA, colburn@bu.edu), Yoojin Chung (44 Cummington St., Boston, MA 02215, USA, yjchung@bu.edu), Andrew Brughera (44 Cummington St., Boston, MA 02215, USA, brughera@bu.edu), Yi Zhou (Johns Hopkins University, Dept. of Biomedical Eng.; School of Medicine, Ross 424 Wang, Baltimore, MD 21205, USA, zhouyi@jhu.edu)

This talk describes modeling efforts to understand brainstem neural responses to electrical cochlear stimulation. Our approach is to combine brainstem models developed for acoustic stimulation with descriptions of auditory-nerve (AN) responses to electric stimulation. Predictions for the behavior of neurons at several levels of the brainstem are compared to available physiological data. Specifically, the AN model predicts differences in discharge probability, degree of phase-locking, and adaptation of discharge rate of the AN response to stimuli, inlcuding both electrical and acoustical cases. Predicted neural responses are generated for several brainstem 
nuclei, including cell populations in the cochlear nucleus $(\mathrm{CN})$, the superior olive (SOC) and the inferior colliculus (IC). Specific attention is given to two special topics: 1) responses to the simultaneous stimulation of multiple electrodes, and 2) the effects of amplitude modulation on the tuning of binaural neurons to interaural time delay (ITD). [Work supported by US National Institutes of Health grants DC04663, DC00100, and DC05775 (Delgutte, PI).]

\section{Contributed Papers}

\section{5:00}

1pPPb12. Higher sensitivity of human auditory nerve fibers to positive electrical currents. Olivier Macherey (MRC CBU, 15 Chaucer Rd., CB2 7EF Cambridge, UK, olivier.macherey@mrc-cbu.cam.ac.uk), Robert P. Carlyon (MRC CBU, 15 Chaucer Rd., CB2 7EF Cambridge, UK, bob.carlyon@mrc-cbu.cam.ac.uk), Astrid Van Wieringen (ExpORL, Dept. Neurosciences, K.U. Leuven, Herestraat 49 bus 721, O. \& N2, B-3000 Leuven, Belgium, astrid.vanwieringen@med.kuleuven.be), John Deeks (MRC CBU, 15 Chaucer Rd., CB2 7EF Cambridge, UK, john.deeks@mrc-cbu.cam.ac.uk), Jan Wouters (ExpORL, Dept. Neurosciences, K.U. Leuven, Herestraat 49 bus 721, O. \& N2, B-3000 Leuven, Belgium, jan.wouters@med.kuleuven.be)

Most contemporary cochlear implants (CIs) stimulate the auditory nerve with trains of amplitude-modulated, symmetric biphasic pulses. Although both polarities of a pulse can depolarize the nerve fibers and generate action potentials, it remains unknown which of the two (positive or negative) phases has the stronger effect. Animal experiments have shown that cathodic (negative) current flows are more effective than anodic (positive) ones in eliciting neural responses, and this finding has motivated the development of novel speech-processing algorithms. Here we show electrophysiologically and psychophysically that the human auditory system exhibits the opposite pattern, being more sensitive to anodic stimulation. We measured electrically evoked compound action potentials in CI listeners for phase-separated pulses, allowing us to tease out the responses to each of the two oppositepolarity phases. At an equal stimulus level, the anodic phase yielded the larger response. This finding was corroborated by a measure of psychophysical masking patterns and may relate to a particular orientation of the nerve fibers relative to the electrode or to a substantial degeneration/demyelination of the peripheral processes. Potential applications to improve $\mathrm{CI}$ speech-processing strategies are discussed.

\section{$5: 20$}

1pPPb13. 3D-localization of virtual sound sources in normal-hearing and cochlear-implant listeners. Piotr Majdak (Austrian Academy of Science/Acoustics Research Institute, Wohllebengasse 12-14, 1040 Vienna, Austria, piotr@majdak.com), Bernhard Laback (Austrian Academy of Science/Acoustics Research Institute, Wohllebengasse 12-14, 1040 Vienna, Austria, Bernhard.Laback@oeaw.ac.at), Matthew J. Goupell (Austrian Academy of Science/Acoustics Research Institute, Wohllebengasse 12-14, 1040 Vienna, Austria, matt.goupell@gmail.com)

The ability to localize sound sources in 3D-space was tested in humans. Five normal-hearing $(\mathrm{NH})$ subjects listened via headphones to noises filtered with subject-specific head-related transfer functions. Four bilateral cochlear implant (CI) subjects listened via their clinical speech processors to noises filtered with subject-specific behind-the-ear head-related transfer functions. A virtual structured environment was presented via head mounted display. Two conditions were used: a condition where the subjects were naive and had no response feedback and a learning condition where the subjects were trained by providing extensive feedback during the test. Response feedback was provided via the visual virtual environment. The results show that the $\mathrm{CI}$ listeners performed generally worse than $\mathrm{NH}$ listeners, both in the horizontal and vertical dimensions. Both subject groups were able to learn to better localize sound sources, which is supported by lower localization errors in the learning condition. However, in the learning condition, the $\mathrm{CI}$ listeners showed a front/back confusion rate comparable to naive $\mathrm{NH}$ listeners, which was two times higher than for the trained NH listeners. These results indicate the necessity of new CI processing strategies which include spectral localization cues. Funding by FWF (P18401-B15).

\section{$5: 40$}

1pPPb14. Emergence of sound localization acuity in children who are fitted with bilateral cochlear implants. Ruth Litovsky (University of Wisconsin, 1500 Highland Ave, Waisman Center, Madison, WI 53705, USA, litovsky@waisman.wisc.edu), Tina Grieco-Calub (University of Wisconsin, 1500 Highland Ave, Waisman Center, Madison, WI 53705, USA, grieco@waisman.wisc.edu), Shelly Godar (University of Wisconsin, 1500 Highland Ave, Waisman Center, Madison, WI 53705, USA, godar @waisman.wisc.edu)

Sound localization was investigated in children who are born deaf and hear with either monaural or bilateral cochlear implants (CIs). Using a 2AFC discrimination task with stimuli to the right/left, in a sound proof booth, loudspeaker locations varied along the horizontal plane between +/-70 deg. Minimum audible angle (MAA) thresholds were estimated adaptively. In study 1, 4-14 year-old children who received two CIs in sequential procedures participated. They were first tested while functioning monaurally, then at 3-and 12-months following activation of bilateral hearing. Following bilateral activation, MAA thresholds were significantly lower, especially after 12 months. In study 2 children were 2.5 years old, with 6-20 months of bilateral experience. MAAs were typically better when children had $\geqslant 12$ months of exposure to bilateral stimulation. Overall, these findings suggest that binaural hearing abilities can be established in an auditory system that is strictly stimulated with electrically pulsed signals, but that unlike a normal auditory system, here a year of exposure may be required before localization acuity is better under bilateral than monaural conditions. These findings are especially relevant to considerations of localization in absence of fine-structure, a cue that is discarded by the speech processors utilized in CIs. 


\title{
Session 1pSAa
}

\section{Structural Acoustics and Vibration and EURONOISE: Vibration and Radiation from Complex Structural Systems I}

\author{
David Feit, Cochair \\ Applied Physical Sciences Corp., Ste. 300, 2 State St., New London, CT 06320, USA \\ Jean-Louis Guyader, Cochair \\ INSA de Lyon - LVA, Bâtiment St. Exupéry, 25 bis avenue Jean Capelle, Villeurbanne Cedex, F-69621, France
}

Invited Papers

1:00

1pSAa1. Development of a general SEA subsystem formulation using FE periodic structure theory. Vincent Cotoni (ESI Group, 12555 High Bluff Dr., Suite 250, San Diego, CA 92130, USA, vincent.cotoni@esi-group-na.com), Phil S. Shorter (ESI Group, 12555 High Bluff Dr., Suite 250, San Diego, CA 92130, USA, pjs@esi-group-na.com), Robin S. Langley (University of Cambridge, Trumpington Street, CB5 8HU Cambridge, UK, rs121@hermes.cam.ac.uk)

Statistical Energy Analysis (SEA) represents a field of study in which statistical descriptions of a system are employed in order to simplify the analysis of complicated vibro-acoustic problems. In SEA, a vibro-acoustic system is represented by a collection of subsystems that can receive, store, dissipate and transmit vibro-acoustic energy. Traditionally, the SEA parameters for a given subsystem are formulated analytically based on consideration of wave propagation through the subsystem. While such analytical algorithms can be readily applied to the majority of systems encountered in practical problems, there are certain types of sections that are difficult to describe using existing analytical formulations. Examples include: isogrid in launch vehicle fairings, extruded aluminum sections in train floors and modern corrugated aircraft fuselage constructions. This paper describes the development of a generic SEA subsystem formulation based on the use of finite element (FE) periodic structure theory. A small unit cell of the section is created and computationally efficient algorithms are developed to calculate wave propagation through a large array of such cells. The resulting algorithms are used to calculate the SEA parameters for the section. The approach is described and a number of numerical validation examples are presented.

\section{$1: 20$}

1pSAa2. Structure/cavity coupling using Statistical Energy Analysis: Coupling loss factors and energy maps into subsystems. Nicolas Totaro (INSA de Lyon - LVA, Bâtiment St. Exupéry, 25 bis avenue Jean Capelle, F-69621 Villeurbanne Cedex, France, nicolas.totaro@insa-lyon.fr), Jean-Louis Guyader (INSA de Lyon - LVA, Bâtiment St. Exupéry, 25 bis avenue Jean Capelle, F-69621 Villeurbanne Cedex, France, jean-louis.guyader@insa-lyon.fr)

Prediction of interior noise is one of the most concerning issues of vehicle industry. Statistical Energy Analysis theoretically allows to determine energy spread over a structure divided into subsystems when one subsystem is submitted to a rain-on-the-roof excitation. Subsystems can be either a part of the structure or a cavity. Recently, a method (SmEdA) based on modal bases of uncoupled subsystems have been derived. This method permits to compute CLF using standard FEM software. This method has been successfully applied on structure/structure coupling and is extended to estimation of CLF between a structure and a cavity in the present article. In addition, in the case of interior noise, SEA can only provide a global energy into the cavity on frequency bands. No information on energy repartition into the subsystem is given. In the present article, an extension of SmEdA method is proposed to quickly estimate energy repartition into subsystems (structure or cavity).

\section{$1: 40$}

1pSAa3. Theory and application of pseudo-damping in structures. Adnan Akay (Carnegie Mellon University, Mechanical Engineering Department, Pittsburgh, PA 15213, USA, akay@cmu.edu), Antonio Carcaterra (Università di Roma, 'La Sapienza,' Dipartimento di Meccanica ed Aeronautica, Via Eudossiana, 18, 00184 Roma, Italy, a.carcaterra@dma.ing.uniroma1.it)

This presentation describes the underlying physics and theory of pseudo damping to reduce vibration of conservative structures. The theoretical approach is based on particular properties of harmonic functions that lead to construction of a complex system that exhibits nearly irreversible energy transfer. This approach also provides the ability to tailor energy absorption from a particular structure in the absence of damping. The authors will also discuss extension of the concept to continuous systems and its application to waveguides. The presentation will illustrate test results including application to reduce vibrations in a satellite. [Research carried out while AA served at NSF.] 
1pSAa4. Localization and mesoscopics in structures and rooms I. Richard Weaver (Dept. of Physics, University of Illinois, Urbana, IL 61801, USA, r-weaver@uiuc.edu)

Diffuse field methods such as reverberation room acoustics and statistical energy analysis predict wave energy flow in complex structures. They are predicated on an incorrect assumption of incoherence. The consequent failures of otherwise successful theories are occasionally striking. The most noteworthy among these are related to Anderson - and other kinds of - localization. Residual coherence manifests also in time-reversal invariance and Green's function retrieval in field-field correlations. It is sometimes possible in special cases to make ad hoc corrections to the diffuse field theories. It would be preferable, however, to augment them with more a fundamental quantitative theory applicable to a wide variety of systems. This talk reviews mesoscopic phenomena such as weak Anderson localization, modal echoes, Anderson localization, Thouless localization, and Greens function retrieval. It sets out the phenomena and concepts behind the new theory to be presented in "Localization and Mesoscopics in Structures and Rooms II" at a later session.

\section{2:20}

1pSAa5. Spherical harmonic analysis of the field radiated by a source embedded in a two-layer coating of a spherical shell. Jerry H. Ginsberg (Georgia Insitute of Technology, G. W. Woodruff School of Mechanical Engineering, Atlanta, GA 30332-0405, USA, jerry.ginsberg@me.gatech.edu)

The acoustic field generated by a small source embedded in a viscoelastic layer encapsulating a spherical shell was modeled previously by treating each layer as a dissipative fluid [J. H. Ginsberg, JASA, 122, 3067 (2007)]. Using spherical harmonic series for each layer was shown to lead to profoundly ill-conditioned equations because of the enormous differences of scale of the spherical Bessel functions having complex argument and high order. Mie series is not directly applicable because the present source is embedded in a layer. Scaling of the spherical Bessel functions and the alternatives of using Bessel and Neumann or two Hankel functions are examined for their efficacy in addressing ill-conditioning. The specfic system that is analyzed consists of a soft inner layer that coats the shell, covered by an outer layer that encapsulates the source. The impedance of the outer layer nominally matches the surrounding water, so reflection of the source from the soft inner layer might seem to have the effect of creating a dipole. However, depending on the wavelength in the inner layer, it might seem that the source "sees" the shell, in which case one would expect the behavior to be like a simple source whose strength is doubled.

\section{Contributed Papers}

\section{2:40}

1pSAa6. Periodic assembly of multicoupled beams: Wave propagation and natural modes. Guillaume Gosse (Laboratoire Vibrations Acoustique - INSA Lyon, 25 bis avenue Jean Capelle, Bâtiment Saint-Exupéry, F-69621 Villeurbanne cedex, France, guillaume.gosse@insa-lyon.fr), Charles Pezerat (Laboratoire Vibrations Acoustique - INSA Lyon, 25 bis avenue Jean Capelle, Bâtiment Saint-Exupéry, F-69621 Villeurbanne cedex, France, charles.pezerat@insa-lyon.fr), François Bessac (CETIAT, Domaine Scientifique de la Doua, 25 avenue des Arts, BP 2042, F-69603 Villeurbanne cedex, France, francois.bessac@cetiat.fr)

The present work is concerned with the vibrations of a discrete multicoupled periodic system. It lies within a larger study on the vibroacoustic behaviour of a heat exchanger. These structures are usually made of a succession of huge number of identical parallel fins (around 600 per meter) connected by tubes conveying the coolant fluid. By now their behaviour can not be calculated using FE model. As a first step, the periodicity principles are applied on a simpler structure, i.e.. an assembly of identical beams linked by several damped springs. The basic unit is symmetric and composed of one flexural beam with several springs on each side. Using the Floquet-Bloch's theorem and the works of Denys Mead (receptance matrix), it is possible to completely describe the whole structure behaviour (natural modes, response) only from the vibroacoustic knowledge of the basic unit. This has be done analytically and the results were confirmed by a (very time consuming) FE model calculation. The study of the basic unit can also give valuable information on the physical phenomena governing the transmission from one unit to the next, and then the propagation in the whole structure.

\section{3:00}

1pSAa7. The response of a complex dynamical system to a complex external forcing. John McCoy (Catholic University, 620 Michigan Ave, Washington, DC 20064, USA, mccoy@cua.edu)

The response of a dominant grounded spring/mass to which are attached a large number of significantly less massive sprung masses, when subject to an external forcing acting on the dominant mass, is revisited. Distinguishing the new study is a focus on the net force that the attached systems cause to act on the dominant mass and the derivation of a semi-analytic, time domain representation of the system operator that transforms the history of the ex- ternal force acting on the adorned dominant spring/mass to the history of an effective force that acts on the unadorned dominant spring/mass. The mathematical framework provided by the concept of an "effective force" acting on the unadorned simple system gives additional insight to a commonly accepted concept of an "effective dissipation" modifying the unadorned simple system, when subject to simple external force histories; i.e., force histories with limited time extents or with limited bands of spectral content. Of greater practical interest is the mathematical framework allows investigation of external force histories that are not simple.

\section{3:20}

1pSAa8. General absorbing boundary for acoustic and elastic waves Denis Duhamel (ENPC, UR Navier, 6 et 8 Avenue Blaise Pascal, Cité Descartes, Champs sur Marne, 77455 Marne la Vallée, France, duhamel@lami .enpc.fr)

In the finite element computation of unbounded acoustic problems, the domains must be of finite size and artificial absorbing conditions have to be introduced in order to avoid reflections at the truncated boundary. This communication proposes a new method in the frequency domain to generate efficient absorbing boundary conditions without the need to consider high order derivatives on the boundary. Moreover the approach is quite general and can consider media with mean flow or be applied to structural vibrations. It needs the knowledge of the dynamic stiffness matrix of only one element which can be obtained from any finite element software. The final result is an impedance condition linking the forces and displacements on the boundary which is determined numerically from a wave analysis of one element of the media. Using this impedance condition a finite element problem can be solved by standard methods. This is then applied to study some examples for 2D standard acoustic, acoustic problems with mean flow and vibrations of plates.

\section{3:40}

1pSA9. Dynamic response of an insonified sonar window. Andrew J. Hull (Naval Undersea Warfare Center, Code 8212, 1176 Howell St., Newport, RI 02841, USA, hullaj@npt.nuwc.navy.mil)

This talk derives and evaluates an analytical model of an insonified sonar window in contact with an array of Tonpilz transducers operating in 
receive mode. The window is fully elastic so that all wave components are present in the analysis. The output of the model is a transfer function of a transducer element output voltage divided by input pressure versus arrival angle and frequency. This model is intended for analysis of sonar systems that are to be built or modified for broadband processing. The model is validated at low frequency with a comparison to a previously derived thin plate model. Once this is done, an example problem is studied so that the effects of higher order wave interaction with acoustic reception can be understood. It was found that these higher order waves cause multiple nulls in the region where the array detects acoustic energy and that their locations in the arrival angle-frequency plane can be determined. The effects of these nulls in the beam patterns of the array are demonstrated.

\section{4:00-4:20 Break}

\section{$4: 20$}

1pSAa10. Circumferential admittance approach for vibro-acoustic prediction of a submerged cylindrical shell with rib aperiodicity and transversal bulkheads. Laurent Maxit (DGA (Defence Agency), CTSN/SDP/CMPF, B.P. 28, 83800 Toulon, France, laurent.maxit@dga .defense.gouv.fr)

The circumferential admittance approach is presented to predict the vibro-acoustic behaviour of an externally fluid loaded shell with aperiodic stiffeners and transversal bulkheads. It consists to consider the circumferential spectral displacements of the shell and to use a partitioning of the structure such that the fluid loaded shell constitutes one subsystem and the stiffeners/bulkheads constitute others subsystems. Each subsystem is characterised independently by the circumferential admittances. For the stiffeners/bulkheads, a standard FE code is used to calculate these admittances with a shell description. The admittances of the fluid load shell are estimated by a spectral approach. Developments are proposed in this paper to improve the numerical convergence of the spectral approach. The final assembly is obtained using the continuity relations. Excitations like mechanical forces, acoustic diffuse fields or plane waves can be easily taken into account. For submarine applications, this model permits to study the noise radiated by the pressure hull and to analyse the acoustic target strength of the shell with its internal frames. The whole submarine pressure hull can be described with the assumption that it is extended by a cylindrical rigid baffle. Numerical validations are presented and results at several $\mathrm{kHz}$ are shown for a submarine application.

\section{4:40}

1pSAa11. Enhanced coupled mechanical-acoustic field computations on nonmatching grids. Simon Triebenbacher (Univ. ErlangenNuremberg, Dept. of Sensor Technology, Paul-Gordan-Str. 3/5, 91052 Erlangen, Germany, simon.triebenbacher@1se.eei.uni-erlangen.de), Manfred Kaltenbacher (Univ. Erlangen-Nuremberg, Dept. of Sensor Technology, Paul-Gordan-Str. 3/5, $91052 \quad$ Erlangen, Germany, manfred.kaltenbacher@1se.eei.uni-erlangen.de), Martin Meiler (Simetris $\mathrm{GmbH}$, Am Weichselgarten 7, 91058 Erlangen, Germany, martin.meiler@simetris.de), Hermann Landes (Simetris GmbH, Am Weichselgarten 7, 91058 Erlangen, Germany, hermann.landes@simetris.de)

In this work we extend our research on nonmatching finite element grids, as used in the analysis of coupled mechanical-acoustic systems, from standard (bi-)linear grids to higher order Lagrangian grids. In practical applications, such as the simulation of thin membranes, the use of higher order finite elements is often advantageous to prevent locking effects. For the comparison of the radiated sound from a loudspeaker with measurements an acoustic propagation domain is required which is very large in relation to the size of the speaker. To keep the number of unknowns in this domain low the discretization should be chosen as coarse as possible without becoming susceptible to numerical dispersion. Due to the same reason it is also desirable to use a uniform discretization and to be able to adjust the approximation order. To gain more flexibility, we introduce a nonmatching interface between the subdomains and discretize them independently, both in space and approximation order. Our enhanced scheme applies the mortar finite element method to coupled mechanical-acoustic field problems. We demonstrate the applicability of our approach to practical applications. We analyze the system response of an electrodynamic loudspeaker and the sound radiation of an electric transformer.

\section{5:00}

1pSAa12. Comparison of numerical techniques for the vibro-acoustic behaviour of a flexible structure. Yvon Goth (CETIM, 52 av Felix Louat, BP 80067, F-60304 Senlis Cedex, France, yvon.goth@cetim.fr)

The tractors bonnets are large flexible parts that radiate noise under the excitation of the forces exerted on the contact points and the sound pressure fluctuations coming from the engine. The vibro-acoustic behaviour of such a bonnet, excited by local forces or a diffuse pressure field, has been computed using various techniques: - In the low frequency domain, the finite element method has been used to compute the structural behaviour, while the acoustic radiation has been computed by using the boundary element method and the finite element method with approximated boundary conditions to simulate the free field conditions. - In the high frequency domain, the computation has been made using the SEA method, with an approximation of the radiation coefficients. -In the medium frequency domain, both techniques have been used and compared. Software used where VA-One, from ESI, and Code-Aster, from EDF. The paper presents the numerical results obtained, associated to the computing time. Approximated methods can be largely faster than 'exact' ones (finite elements associated with boundary elements) and, for engineering use, represent a good compromise between quality and efficiency.

\section{$5: 20$}

1pSAa13. Structure-borne modeling of a vehicle in the mid-frequency range using Virtual SEA: experimental validation. Denis Thenail (PSA Peugeot Citroën, Route de Gisy, 78943 Vélizy-Villacoublay Cedex, France, denis.thenail@mpsa.com), Julien Baratier (Faurecia AST, Center of Acoustic Technology, Dämmstoffwerk 100, 38524 Sassenburg, Germany, julien.baratier@faurecia.com), Arnaud Duval (Faurecia AST, Center of Acoustic Technology, Dämmstoffwerk 100, 38524 Sassenburg, Germany, arnaud.duval@faurecia.com), Gérard Borello (InterAC, 10 impasse BordeBasse, ZA. La Violette, 31240 L'Union, France, gerard.borello@interac.fr), Laurent Gagliardini (PSA Peugeot Citroën, Route de Gisy, 78943 Vélizy-Villacoublay Cedex, France, laurent .gagliardini@mpsa.com)

Virtual SEA is a modeling process using FE computations to build an SEA model including equivalent masses, modal densities, and CLF, but excluding DLF since damping modeling in the mid-high frequency range is still an open issue. This technique, previously proposed by some of the authors, is applied to a production vehicle in the range 200-1000 Hz. The actual vehicle is simultaneously measured at a subset of the FE nodes. The automated sub-structuring provided by Virtual SEA (20 subsystems at $630 \mathrm{~Hz}$ ) is used to favorably position 64 sensors on the body. Next, an experimental SEA procedure is performed: a full transfer matrix is measured between more than 1000 excitation (hammer) locations and the sensors. In order to compensate for structural heterogeneity, input mobilities are measured at every point and used to normalize the transfer matrix As all measurement points are associated to FE nodes, computed input mobilities can be compared to measurements. Finally, the SEA model identification is carried out for both experimental and virtual SEA. As far as damping (DLF) can only be known experimentally, comparisons of the numerical and experimental approach only concern the orher SEA parameters (CLFs, modal densities ...) and transfer functions.

\section{$5: 40$}

1pSAa14. Measurement of SEA damping loss factor for complex structures. Maxime Bolduc (Univ. de Sherbrooke, Mechanical Engineering Depart., 2500 Boulevard de l'Université, Sherbrooke, QC J1K 2R1, Canada, maxime.bolduc@usherbrooke.ca), Noureddine Atalla (Univ. de Sherbrooke, Mechanical Engineering Depart., 2500 Boulevard de l'Université, Sherbrooke, QC J1K 2R1, Canada, Nouredinne.Atalla @Usherbrooke.ca)

Statistical Energy Analysis has become extremely popular in the transportation industry. As a prediction tool, it offers appealing advantages such as its wide frequency range and short computational time, which conventional methods do not offer. Critical parameter in every SEA model, the 
damping characteristics of the subsystems must be determined by way of experimentations. A variety of different techniques of measuring the damping loss factor were developed. These techniques can be divided into three main groups: (i) method based on the identification of modal damping by curve-fitting frequency response function, (ii) decay techniques based ondetermination of the reverberation time and, (iii) steady-state techniques involving measurements of power input method much closely related to the definition of SEA since its starting point is the power balance. This work presents an experimental study of these techniques for various structures such as flat metallic panels, aircraft side walls (ribbed curved panels) and sandwich-composite panels in both low and high damping configurations (additions of damping materials, sound packages in both single wall and double wall configurations, mounting effects, ...). In particular, a critical discussion is made on the implementation, advantages and drawbacks of each method regarding its use in SEA modeling of complex structures.

\section{6:00}

1pSAa15. Transmission loss of a panel with tuned vibration absorbers. Carl Q. Howard (The University of Adelaide, School of Mechanical Engineering, North Terrace, 5005 Adelaide, Australia, carl.howard@adelaide.edu .au)

The transmission loss of a rectangular panel with numerous discrete tuned vibration absorbers is investigated both theoretically and experimentally. The 49 tuned vibration absorbers have a linear distribution of resonance frequencies and were arranged in grid pattern on the rectangular plate. The transmission loss of the plate was measured between two reverberation chambers and was compared with theoretical predictions. In addition, theoretical predictions and experimental measurements were conducted on a similar system where each vibration absorber was replaced with the equivalent mass of the absorber. The numerical computations were conducted on a distributed computing network comprising 200 desktop computers. The results indicate the improvement in sound transmission loss that can be achieved, greater than merely increasing the thickness of the panel (mass law), and also provides a data set for comparison with predictions involving the use of fuzzy-structure theory.

\section{$6: 20$}

1pSAa16. A 'concatenation' study of wave energy transport among three coupled plates. Nick Wolff (Dept. of Mechanical Science and Engineering, University of Illinois, 1206 W Green St. MC 244, Urbana, IL 61801, USA, nlwolff@uiuc.edu), Richard Weaver (Dept. of Physics, University of Illinois, Urbana, IL 61801, USA, r-weaver@uiuc.edu)

We address the problem of constructing predictions for the diffuse transport and distribution of mean spectral acoustic energy density in an undamped complex linear structure, without appeal to SEA and by taking information gleaned from short time cost-effective, direct numerical simulations (DNS). A numerical model is constructed consisting of three finite plates coupled by sets of springs. Coupling is chosen to be sufficiently strong to avoid localization and sufficiently weak that the notion of substructures remains valid. Numerical simulations of transient responses to each of several sources are carried out; responses are recorded at each of several receivers. The resulting time-domain diffuse waveforms are analyzed for the work done at each source, and the transient spectral energy density at each receiver at times shortly after the action of the sources. This information is used in a 'concatenation' ansatz to predict the evolution and distribution of spectral energy density at these receivers at later times. The resulting prediction is compared favorably with the actual energy densities observed in the DNS at late times. It is described how the results also apply directly to the more conventional but closely related problem of damped systems and/or steady state input. Work supported by NSF CMS-0528096.

\section{6:40}

1pSAa17. Modification of an interface parameter between sub-system and vehicle: case of a fan system attached to the front end of a car. Saul Mapagha (CEVAA, Technopole du Madrillet, 2 Rue Joseph Fourier, 76800 Saint Etienne du Rouvray, France, s.mapagha@cevaa.com), Vincent
Martin(Institut Jean Le Rond d'Alembert, UMR CNRS 7190, UPMC, 2 Place de la Gare de Ceinture, 78210 Saint-Cyr 1'Ecole, France, vmartin@ccr .jussieu.fr)

The car industry, as well as many others, is constantly undergoing modifications to comply security and comfort regulations. Among the major causes for concern is the integration of sub-systems on vehicles. In fact, when a sub-system runs on a vehicle, it can lead to vibrations on the body of the car and thus acoustical radiations, causing trouble for the passengers, and also outside the vehicle. The frequency response functions (FRF)-based substructuring technique and impedances coupling methods are used to predict the forces entering the vehicle from those measured on a test bed. When a given constraint on the forces entering the vehicle is not satisfied, the car supplier may choose to modify the interface parameters between the substructures. In this paper an analytical approach to filter the entering forces by elastic suspension is proposed. An application is given by way of numerical experiments on a fan system attached to the front end of a car, both subsystems being identified through measurements.

\section{7:00}

1pSAa18. Sound radiation induced force vibration of submerged and fluid filled multilayered spherical shell. Sadeq Malakooti (Acoustics Research Laboratory, Dept. of Mechanical Engineering, Iran University of Science and Technology, Narmak, 16844 Tehran, Iran, s_malakooti@mecheng .iust.ac.ir), Seyyed M. Hasheminejad (Acoustics Research Laboratory, Dept. of Mechanical Engineering, Iran University of Science and Technology, Narmak, 16844 Tehran, Iran, hashemi@iust.ac.ir), Nader Mohammadi (Univ. of Tehran, School of Mechanical Engineering, 1439957131 Tehran, Iran, nmohamady@ut.ac.ir)

Nowadays sound radiation from spherical shells is attracted for aviation industry. In this paper, the general approach is presented for predicting a coupled fluid solid interaction problem. Therefore, a fully elasticity solution is applied in conjunction with laminated shell model for considering of the sound field over the shell. For each plies the elasticity coefficients are assumed to be constant. The main objectives of this work are investigating the influence of internal and external acoustic mediums and also radially inhomogeneity on acoustic performance of the spherical shell. Numerical results are presented for a three layered spherical shell. For each excitation frequency, sound pressure over zenith angle in polar coordinate is plotted. Also pressure levels against excitation frequency for resonance spectroscopy are investigated.

$$
7: 20
$$

1pSAa19. Particular analysis of vibration energy transmission in connections of thin plates. Jacek Cieslik (AGH-UST University of Science and Technology, Al. Mickiewicza 30, 30-059 Krakow, Poland, cieslik @ agh.edu.pl)

Analyses of energy balance in the mechanical systems are done by the quantitative and qualitative assessment. Qualitative assessment is possibly by the use of energy flow descriptors and observation of energy density distribution in the mechanical structure. The work presents the results of investigation of welded connections of rectangular steel plates. The aim of the analysis was the quantitative estimation of vibrational energy transmitted though the welded connection depending on the inclination angle of plates. The structural intensity was used as the parameter for the analysis. Method of structural intensity evaluation enabled the elaboration of intensity vector field. Unification of shape and size of finite elements made possible the summation of only the magnitude of intensity. Summation was performed in the plate's cross sections far from the places of excitation and the damping. The detailed information on vibration energy flow was achieved. The obtained results of calculation give the quantitative information on amount of energy transmitted, reflected and the damped in welded joints of plates. The analyzed case was intentional to show the utility of intensity method in diagnostics of joints in mechanical constructions. The calculation results were verified experimentally with application of thermograph measurements of stress and vibration velocity. 
Session 1pSAb

\title{
Structural Acoustics and Vibration and EURONOISE: General Topics in Structural Acoustics and Vibration I
}

\author{
Wolfgang Kropp, Cochair \\ Chalmers University of Technology, Division of Applied Acoustics, Gothenburg, SE-41296, Sweden
}

Sean Wu, Cochair

Wayne State Univ., 5050 Anthony Wayne Dr., Detroit, MI 48202, USA

\section{Contributed Papers}

1:00

1pSAb1. Efficient computation of the radiated sound power of vibrating structures using a modal approach. Nicolas Roy (Top Modal, 5, rue de la ZA de Ribaute, 31130 Quint-Fonsegrives, France, nicolas.roy@topmodal.fr), Marc Lapi (DGA/DCE/CTSN, Site du Mourillon, BP 28, 83800 Toulon Armées, France, marc.lapi@dga.defense.gouv.fr)

The optimal design of structures in terms of noise control is of great interest in many fields such as automotive, aerospace, and naval. Since design strategies require simple design parameters, the sound power is often used to characterize the sound radiated from a structure. The CTSN in collaboration with TOP MODAL has recently developed a software tool to efficiently compute the radiated sound power of a vibrating structure using modes obtained by finite element analysis. The modal analysis has been enhanced to take into account the presence of fluid cavities and damping elements via the introduction of residual modes. The radiated sound power is computed from the volume velocities of the vibrating surface using a lumped parameter method requiring no explicit modeling of the acoustic medium. The modal contributions to the total sound power may also be calculated. The underlying methods are described including the improved coupled fluid-structure modal analysis and the computation of the radiated sound power. Next an overview of the tool's architecture is presented. Finally, several industrial applications are presented to illustrate the features and interest of the tool.

\section{1:20}

1pSAb2. High resolution modal analysis. Kerem Ege (Laboratoire de Mécanique des Solides, Ecole Polytechnique, 91128 Palaiseau Cedex, France, kerem.ege@1ms.polytechnique.fr), Xavier Boutillon (Laboratoire de Mécanique des Solides, Ecole Polytechnique, 91128 Palaiseau Cedex, France, boutillon@lms.polytechnique.fr), Bertrand David (Télécom Paristech (ENST) / TSI - CNRS LTCI, 46, rue Barrault, 75634 Paris Cedex 13, France, bertrand.david@enst.fr)

The Fourier-transform-based traditional modal analysis techniques perform poorly when modal overlap $\eta$ exceeds $30 \%$, due to the $\Delta \mathrm{T} . \Delta \mathrm{f}$ limitation. In view of filling the gap between the low- and the highfrequency domains where modal analysis and statistical methods respectively apply, a technique based on the high resolution analysis algorithm ESPRIT (Roy \& Kailath, IEEE Transactions on Acoustics Speech and Signal Processing, 37(7), 984-995, 1989) has been developed. A pulselike force is repeatedly applied to the structure and the response is measured in a number of points. In each point, the impulse response of the structure is retrieved by deconvolving the pulselike force and filtering the response with the result. In a second step, the number of modes in the reconstructed impulse response is evaluated by the novel procedure ESTER (Badeau et al., IEEE Transactions on Signal Processing, 54(2), 450-458, 2006) and various preconditioning techniques are applied. The ESPRIT algorithm finally extracts the modal frequencies, damping factors, and complex amplitudes at each point. Two applications are presented: the separation of twin modes of a square plate $(\eta$ $=200 \%$ ) and the partial modal analysis of a $1620 \times 1000 \times 5 \mathrm{~mm}$ aluminum plate up to a modal overlap $\eta=70 \%$.
$1: 40$

1pSAb3. Vibration of a submarine hull under harmonic propeller-shaft excitation. Mauro Caresta (University of New South Wales, 2052 Sydney, Australia, maurorestaca@yahoo.it), Nicole J. Kessissoglou (University of New South Wales, 2052 Sydney, Australia, n.kessissoglou@unsw.edu.au)

A model to describe the dynamic response of submarine hull to a harmonic propeller shaft excitation is presented. The submarine is modeled as a ring stiffened cylindrical shell, with bulkheads and end caps. The stiffeners are introduced using a smeared approach. The bulkheads are modeled as circular plates with in plane and bending motion, and the end closures are modeled as truncated conical shells. External fluid loading is introduced to take into account the interaction of the structure with the acoustic medium. The propeller introduces a harmonic varying force in both the axial and transverse directions. The force is transmitted to the structure through the shaft that is connected to the end plate of the cylindrical hull and supported by the conical end cap. The axial component excites the axisymmetric modes of the structure. The transverse force component excites the hull through the conical shell and excites the higher circumferential modes. Since these modes are mainly flexure in nature, they can result in a high noise signature level. Results are presented in terms of FRFs calculated analytically and compare the axisymmetric vibration caused by the axial excitation and the asymmetric response due to the transverse component of the force.

\section{2:00}

1pSAb4. Application of guided flexural waves in immersed plates to aquatic propulsion of mono-hull marine vessels. Victor V. Krylov (Loughborough University, Department of Aeronautical and Automotive Engineering, Ashby Road, LE11 3TU Loughborough, UK, V.V.Krylov@lboro.ac.uk), Ewan Porteous (Loughborough University, Department of Aeronautical and Automotive Engineering, Ashby Road, LE11 3TU Loughborough, UK, wan2001@hotmail.com)

The present paper describes the results of the experimental investigation of a small-scale mono-hull model boat propelled by a localised flexural wave propagating along the plate of finite width forming the boat's keel. Forward propulsion of the boat was achieved through flexural wave propagation in the opposite direction, which is similar to the aquatic propulsion used in nature by stingrays. The model boat under consideration underwent a series of tests both in a Perspex water tank and in the experimental pool. In particular, the forward velocity of the boat has been measured for different frequencies and amplitudes of the flexural wave. The highest velocity achieved was $32 \mathrm{~cm} / \mathrm{s}$. The thrust and propulsive efficiency have been measured as well. The obtained value of the propulsive efficiency in the optimum regime was $51 \%$. This indicates that efficiency of this type of aquatic propulsion is comparable to that of dolphins and sharks (around 75\%) and to that of a traditional propeller (around 70\%). In contrast to a propeller though, the wavelike aquatic propulsion has the following advantages: it does not generate underwater noise and it is safe for people and marine animals. 
1pSAb5. The structural acoustic properties of stiffened shells. Yu Luan (DTU, Building 352, Oersted. DTU, 2800 Lyngby, Denmark, yl @ oersted.dtu.dk)

Plates stiffened with ribs can be modeled as homogeneous isotropic or orthotropic plates, and modeling such an equivalent plate numerically with, say, the finite element method is, of course, far more economical in terms of computer resources than modelling the complete, stiffened plate. This is important when a number of stiffened plates are combined in a complicated structure composed of many plates. However, whereas the equivalent plate theory is well established there is no similar established theory for stiffened shells. This paper investigates the mechanical and structural acoustic properties of curved shells with stiffening ribs. Finite element simulations and experimental data will be compared and discussed.

\section{2:40}

1pSAb6. Multi-bay double panel system with heterogeneous blanket treatment: A comparison between theory and experiment. Kamal Idrisi (Virginia Tech, Mechanical Engineering, 143 Durham 0238, Blacksburg, VA 24061, USA, idrisi@vt.edu), Marty Johnson (Virginia Tech, Mechanical Engineering, 143 Durham 0238, Blacksburg, VA 24061, USA, martyj@vt.edu), Alessandro Toso (Virginia Tech, Mechanical Engineering, 143 Durham 0238, Blacksburg, VA 24061, USA, aletoso@vt.edu)

This study is part of an effort to improve the low frequency performance of acoustic blankets used to reduce the noise inside aircraft cabins. This is achieved by embedding small masses inside the poro-elastic layer such that they act like distributed mass spring damper systems. These mass-springdamper systems can then be designed to reduce the sound transmission through the double panel system at low frequencies where traditional poroelastic materials have little effect. A mathematical model of a multi-bay double panel system with frames, stringers, an acoustic cavity and porous/mass layer was developed using impedance and mobility methods (IMM). The multi-bay double panel system includes four skin pockets with four HG blankets of different dimensions such that the interaction between the panels can be analyzed. The predicted responses of the source and receiver panel due to a point force are validated with experimental measurements. The results indicate that proper tuning of the mass insertions can improve the broadband noise reduction below $500 \mathrm{~Hz}$ with less than $10 \%$ added mass without losing the performance of the acoustic foam at high frequencies.

\section{3:00}

1pSAb7. Acoustic radiation from vibrating panels subject to high-frequency broadband excitation. Donald B. Bliss (Duke University, Mechanical Engineering and Materials Science, 148B Hudson Hall, Durham, NC 27708, USA, dbb@duke.edu), Linda P. Franzoni (Duke University, Mechanical Engineering and Materials Science, 148B Hudson Hall, Durham, NC 27708, USA, franzoni@duke.edu)

Radiation from a baffled panel subject to high-frequency broadband excitation is studied. If the panel flexural waves are subsonic, the radiation comes from edges. For supersonic waves the entire surface is important. Characterization of the radiation is surprisingly straightforward in the highfrequency broadband limit, with simple analytical expressions for directivity patterns. For subsonic waves, a series expansion of the surface velocity wave number transform, convergent in the radiating region, can be reinterpreted physically as singularity functions along the panel perimeter, namely the delta function and its derivatives. This interpretation leads to monopole, dipole, and higher-order edge radiators with relative strengths that depend on surface-wave Mach number. Both propagating and evanescent structural waves contribute to the radiation. A proper physical explanation of the radiation is provided both in physical space and transform space. The common interpretation of edge radiation in terms of uncancelled volumetric sources is not correct; the higher order edge singularities are very significant. For low structural damping, this approach leads to a very simple way to calculate the radiated field. The relationship between radiation and structural power flow is potentially useful in energy-intensity based prediction methods with application to vehicle interior noise. (Sponsor NSF)
1pSAb8. Active noise control at a moving location in a modally dense three-dimensional sound field using virtual sensing. Danielle J. Moreau (The University of Adelaide, School of Mechanical Engineering, North $\quad$ Terrace, 5005 Adelaide, Australia, danielle.moreau@adelaide.edu.au), Ben S. Cazzolato (The University of Adelaide, School of Mechanical Engineering, North Terrace, 5005 Adelaide, Australia, benjamin.cazzolato@adelaide.edu.au), Anthony C. Zander (The University of Adelaide, School of Mechanical Engineering, North Terrace, 5005 Adelaide, Australia, anthony.zander@adelaide.edu.au)

Local active noise control systems generate a zone of quiet at the physical error sensor using secondary sources to cancel the acoustic pressure at the sensor location. The resulting zone of quiet is generally limited in size and as such, placement of the physical error sensor at the location of desired attenuation is required, which is often inconvenient. Virtual acoustic sensors overcome this by projecting the zone of quiet away from the physical error sensor to a remote location. While virtual acoustic sensors have shown potential to improve the performance of local active noise control systems, it is, however, likely that the desired location of maximum attenuation is not spatially fixed. The work described here presents a virtual sensing method capable of tracking a desired location in a modally dense three-dimensional sound field. The developed algorithm has been experimentally verified in a three-dimensional enclosure and the experimental results are presented.

\section{3:40}

1pSAb9. Active modal control in rooms - practical approach Pierre-Jean Rene (Acoustical Engineering \& Research, Ch. de la Vuachère, 20D, 1012 Lausanne, Switzerland, pjrene@a-e-r.ch), Hervé Lissek (Ecole Polytechnique Fédérale de Lausanne, EPFL STI LEMA, Station 11, CH 1015 Lausanne, Switzerland, herve.lissek@epfl.ch)

Engineers dealing with noise reduction in habitations close to transportation traffic or industrial facilities encounter several problems to decrease noise level in rooms at low frequencies. Passive materials and current building construction knowledge enable to avoid noise transmission in habitations at medium and high frequencies and the regulations based on the $\mathrm{dBA}$ scale can often be respected. But these regulations do not take into account the real unpleasantness of noise for the inhabitants who are still disturbed by low frequency noise. Because of the modal behavior of rooms, air-borne and structure-borne noise generate high sound pressure level at the first modal frequencies, even with small amount of energy. The aim of this work is to decrease noise level in rooms at these specific and most annoying frequencies. Usual techniques of room acoustics and acoustic insulation reach their limits and cannot be improved due to cost and volume constraints. To reach our goal, active modal control seems to be the best way. The principle of the AMC is to decrease only the 2 or 3 first modal frequencies with a unique microphone-controller-loudspeaker system. Several results are presented for different excitations and room configurations. The controller setup has to be the more autonomous and flexible system as possible.

\section{4:00-4:20 Break}

\section{$4: 20$}

1pSAb10. Resonant frequencies of a cantilevered timoshenko beam with an attached general point-connected vibratory system. Robert $M$. Koch (NUWC, 1176 Howell Street, Code 8232, Bldg. 1302, Newport, RI 02841-1708, USA, kochrm@npt.nuwc.navy.mil)

The literature is full of technical papers that have examined the free vibration of cantilevered Bernoulli-Euler beams with both rigidly and elastically-attached point masses at the free end. Additionally, much work has been published in the last ten years on cantilevered Timoshenko beams (which includes the effects of shear deformation and rotatory inertia) with a similar, yet more restricted, set of free end boundary conditions. Rossit and Laura published in the Journal of the Acoustical Society of America in 2001 a formulation for a Timoshenko beam with a simple single DOF spring-mass system attached to the free end. The present paper extends Rossit and Laura's mathematical formulation to account for a general, damped, multi- 
DOF system point-connected to a cantilevered Timoshenko beam's free end. To demonstrate the formulation, mathematical relations and numerical results for a single DOF damped system and both damped/undamped 2DOF vibration absorbers are presented.

\section{$4: 40$}

1pSAb11. Damage detection in composite laminates using coin-tap method. Sung Joon Kim (Korea Aerospace Research Institute, 45 EoeunDong, Youseong-Gu, 305-333 Daejeon, Republic of Korea, yaelin@kari.re $. \mathrm{kr})$

The "coin-tap" test has the ability to indicate damage in a composite structural element due to a localized change of stiffness. The change in vibration signature may be detected by ear or more precisely by measurement of the dynamic contact force. It has been shown that the characteristics of radiated sound from a structure during a tap are changed by the presence of defect beneath the surface of the composite structure. For structurally radiated noise, the sound field is directly coupled to the structural motion. Therefore, impact response analysis should be computed. In this study, the radiated sound induced by impact is obtained by solving the Rayleigh integral equation. Experimental results for the delaminated composite structure are presented and correlations between analytical predictions and measured data are shown. It is shown that the sound based tap test is useful and practical diagnostic tool for detecting localized delamination in composite laminates

\section{5:00}

1pSAb12. Identification of noise sources by means of inverse finite element method using measured data. Matthias Weber (Helmut-Schmidt-Universität - Universität der Bundeswehr Hamburg, Holstenhofweg 85, 22043 Hamburg, Germany, mweber@hsuhh.de), Thomas Kletschkowski (Helmut-Schmidt-Universität - Universität der Bundeswehr Hamburg, Holstenhofweg 85, 22043 Hamburg, Germany, thomas.kletschkowski@hsuhh.de), Delf Sachau (Helmut-Schmidt-Universität - Universität der Bundeswehr Hamburg, Holstenhofweg 85, 22043 Hamburg, Germany, sachau@hsuhh.de)

Identification of noise sources in airplane cabins proves to be difficult particularly at low frequencies. A new approach reconstructs the spatial distribution of sound pressure and particle velocity based on the inverse finite element (FE) method. This procedure requires measurements in the cavity of the cabin. If all sound sources are located on the boundary of the cabin, the equation system resulting from a matching FE model can be resorted in such way that computation of the unknown boundary data is possible, even with distorted measurement data. The method is explained using a simplified 2D laboratory experiment consisting of a flat sound-hard bounded rectangle with a loudspeaker included in the boundary. A corresponding FE model with given boundary conditions is verified by mapping the sound pressure in the test bed. The inner part of the measurement data is used to compute the boundary values. Regularization methods are applied to find an optimal solution. To prepare a verification of this technique in real world conditions, the sound field in the cavity of an airplane mock-up (Airbus A340) exited by both an interior and exterior noise source is mapped with a custom-built microphone array. Taking advantage of the results of the $2 \mathrm{D}$ examination, the current status of this verification process is shown.

\section{$5: 20$}

1pSAb13. Reconstruction of Rayleigh-Lamb dispersion spectrum based on noise obtained from an air-jet forcing. Eric Larose (LGIT CNRS - Université Joseph Fourier, Maison des Géosciences, 1381 rue de la Piscine, BP 53, 38041 Grenoble, France, Eric.Larose@obs.ujf-grenoble.fr), Philippe Roux (LGIT - CNRS - Université Joseph Fourier, Maison des Géosciences, 1381 rue de la Piscine, BP 53, 38041 Grenoble, France, philippe.roux@obs.ujf-grenoble.fr), Michel Campillo (LGIT - CNRS - Université Joseph Fourier, Maison des Géosciences, 1381 rue de la Piscine, BP 53, 38041 Grenoble, France, Michel .Campillo@obs.ujf-grenoble.fr)

The time-domain cross-correlation of incoherent and random noise recorded by a series of passive sensors contains the impulse response of the medium between these sensors. By using noise generated by a can of com- pressed air sprayed on the surface of a plexiglass plate, we are able to reconstruct not only the time of flight but the whole waveforms between the sensors. From the reconstruction of the direct $A_{0}$ and $S_{0}$ waves, we derive the dispersion curves of the flexural waves, thus estimating the mechanical properties of the material without a conventional electromechanical source. The dense array of receivers employed here allow a precise frequency-wave number study of flexural waves, along with a thorough evaluation of the rate of convergence of the correlation with respect to the record length, the frequency, and the distance between the receivers. The reconstruction of the actual amplitude and attenuation of the impulse response is also addressed in this paper [Larose et al, J. Acoust. Soc. Am 122 (2007)].

\section{$5: 40$}

1pSAb14. Interaction of a pair of complex dynamical systems under impact conditions. Joseph F. Vignola (Catholic University, 620 Michigan Ave., Washington, DC 20064, USA, vignola@cua.edu), John A. Judge (Catholic University, 620 Michigan Ave., Washington, DC 20064, USA, judge@cua.edu), John McCoy (Catholic University, 620 Michigan Ave., Washington, DC 20064, USA, mccoy@cua.edu)

An important class of complex dynamical systems has members that are comprised of a dominant simple system, made complex by attaching a large number of significantly less massive subsystems. The response of such systems to a simple forcing that acts on the dominant system has received considerable attention. A simple forcing is understood here to be represented by a time series that has very limited support when represented in either temporal or spectral space. Little attention has be paid to the response of such systems to a complex forcing, understood to be one represented by a time series that has extended support when represented in both temporal and spectral spaces. In the reported study, the interaction of two systems drawn from the described class, under impact conditions are investigated. Significantly, the action of each system on the other during the time interval of their contact is represented by an interaction force that is complex in the sense described. The investigation is accomplished via numerical simulation, physical experiments and analysis.

\section{6:00}

1pSAb15. Reduction of radiated sound from a continuum using low-damping vibration absorbers. Zenzo Yamaguchi (Kobe Steel, Ltd., 1-5-5, Takatsukadai, Nishi-ku, 651-2271 Kobe, Hyogo, Japan, zyamaguc@purdue.edu), Akio Sugimoto (Kobe Steel, Ltd., 1-5-5, Takatsukadai, Nishi-ku, 651-2271 Kobe, Hyogo, Japan, sugimoto.akio@kobelco.com), Yoshio Yano (Kobe Steel, Ltd., 1-5-5, Takatsukadai, Nishi-ku, 651-2271 Kobe, Hyogo, Japan, yano.yoshio @kobelco.com), J. Stuart Bolton (Ray W. Herrick Labs., School of Mech. Eng., Purdue University, 140 S. Martin Jischke Drive, West Lafayette, IN 47907-2031, USA, bolton@purdue.edu)

When reducing the sound radiated from a resonant structure, it is necessary to ensure that the driving frequency does not correspond to any of the system resonance frequencies. In addition, it may be desirable to use dynamic vibration absorbers to reduce the response at the driving frequency. In this paper, a low-damping vibration absorber is studied for solving noise radiation problems from a resonant, light-gauge structure driven by a force whose frequency and amplitude are constant and whose frequency does not match any of the system natural frequencies. When the driving frequency and the structure's natural frequency are different, the structure cannot be represented by as a SDOF system. Therefore, the conventional design method, based on equivalent mass, is inapplicable. In this paper, a design method based on the mobility of the structure is proposed. By means of the proposed method, the sound power radiated from a simply supported beam with an attached absorber is calculated and it leads to the following results: 1) When the absorber is installed at the driving point, it reduces the sound power as predicted; 2) An increase in radiated noise may occur when the absorber is not installed at the driving point. 
6:20

1pSAb16. A fluid level sensor using A0 Lamb wave mode. Fouad Boubenider (University of Science and Technology Houari Boumediene (USTHB), Laboratoire de Physique des Matériaux BP32, El Alia 16111, DZ Alger, Algeria, fboubenider@yahoo.fr)

The aim of this study was to examine the feasibility of creating a fluid level sensor operating in A0 Lamb wave mode. The experimental sensor is composed of a plate of stainless steel 1.3 meters high, in which, an A0 Lamb wave mode is generated. The choices of the material, the plate thickness and the wave vibration mode are all important. We therefore plotted the phase and group velocity curves, and the transversal and longitudinal displacements versus the product frequency x thickness. These curves enabled us to visualize the best target point for the excitation energy on a suitably guided wave mode at an appropriate frequency; this point is referred to as the operating point. When the chosen wave mode is generated, the echo from the reflection at the guide-water interface is easily detected by the transmitterreceiver transducer and can be worked efficiently.

MONDAY AFTERNOON, 30 JUNE 2008

ROOM 240, 1:00 TO 4:00 P.M.

\title{
Session 1pSCa
}

\section{Speech Communication: Speech Technology I}

\author{
Deliang Wang, Chair \\ Ohio State University, Department of Computer Science \& Engineering, 2015 Neil Ave., Columbus, OH 43210, USA
}

\section{Contributed Papers}

\section{1:00}

1pSCa1. Automatic testing of speech understanding. Tom Francart (ExpORL, Dept. Neurosciences, K.U. Leuven, Herestraat 49 bus 721, O. \& N2, B-3000 Leuven, Belgium, tom.francart@med kuleuven.be), Marc Moonen (ESAT/SISTA, K.U. Leuven, Kasteelpark Arenberg 10, B-3001 Leuven, Belgium, marc.moonen@esat .kuleuven.be), Jan Wouters (ExpORL, Dept. Neurosciences, K.U. Leuven, Herestraat 49 bus 721, O. \& N2, B-3000 Leuven, Belgium, jan.wouters @ med.kuleuven.be)

Speech reception tests are commonly administered by manually scoring the oral response of the test subject. This requires a test supervisor to be continuously present, which can be avoided by having the subject type the response on a computer keyboard and automatically scoring the response. However, spelling errors may then be counted as recognition errors, and hence influence the test results. We demonstrate an autocorrection approach based on two scoring algorithms to cope with spelling errors. The first algorithm deals with sentences and is based on word score. The second algorithm deals with single words and is based on phoneme score. Both algorithms are evaluated with a corpus of typed answers based on three different Dutch speech materials. The percentage of differences between the autocorrection algorithm and the manual correction was calculated, in addition to the mean difference in speech recognition threshold between automatic and manual scoring. The sentence correction algorithm performed at higher accuracy than commonly obtained with these speech materials. The word correction algorithm performed better than the human operator. Both algorithms can be used in practice and allow speech reception tests with open set speech materials over the internet.

\section{$1: 20$}

1pSCa2. A comparison between two methods for speech transmission quality assessment with noise simulation at receiver's side. Marcel Wältermann (Deutsche Telekom Laboratories, Berlin Institute of Technology, Ernst-Reuter-Platz 7, 10587 Berlin, Germany, marcel.waeltermann @ telekom.de), Nicolas Côté (France Télécom, 2 avenue Pierre Marzin, 22300 Lannion, France, nicolas.cote@orange-ftgroup.com), Alexander Raake (Deutsche Telekom Laboratories, Berlin Institute of Technology, Ernst-Reuter-Platz 7, 10587 Berlin, Germany, alexander.raake @ telekom.de), Sebastian Möller (Deutsche Telekom Laboratories, Berlin Institute of Technology, Ernst-Reuter-Platz 7, 10587 Berlin, Germany, sebastian.moeller@telekom.de)

Two auditory experiments are presented that were designed in order to compare two different methods for the assessment of speech quality when noise at listener's side is present: (1) Headphone presentation with samples pre-recorded over a head and torso simulator, and (2) test participants located in a noisy environment, rendered by a 4.1 loudspeaker system. Realworld types of background noise were employed as well as a variety of user terminals. The correlation between corresponding overall quality scores amounts to $r=0.9$. Hence, a headphone presentation can replace a loudspeaker noise simulation in principle, leading to the consequence that a simpler experimental set-up is sufficient for assessing the speech quality when noise is present at the receiver's side. However, there exist certain differences between both quality scores. For example, in most cases clean wideband $(50-7000 \mathrm{~Hz})$ and standard narrow-band $(300-3400 \mathrm{~Hz})$ speech are rated slightly lower if the samples are presented over a headphone and noise is simultaneously present. Since the separation between speech and noise is potentially easier when the noise is played back over loudspeakers, participants may mainly judge the speech rather than the overall quality, leading to higher scores for the clean channel conditions.

\section{$1: 40$}

1pSCa3. Predicting speech quality under noise in wideband speech transmission. Juan-Pablo Ramirez (Deutsche Telekom Laboratories, Berlin Institute of Technology, Ernst-Reuter-Platz 7, 10587 Berlin, Germany, juan-pablo.ramirez@telekom.de), Alexander Raake (Deutsche Telekom Laboratories, Berlin Institute of Technology, Ernst-Reuter-Platz 7, 10587 Berlin, Germany, alexander.raake@telekom.de), Marcel Wältermann (Deutsche Telekom Laboratories, Berlin Institute of Technology, Ernst-Reuter-Platz 7, 10587 Berlin, Germany, marcel.waeltermann@telekom.de), Sebastian Möller (Deutsche Telekom Laboratories, Berlin Institute of Technology, Ernst-Reuter-Platz 7, 10587 Berlin, Germany, sebastian.moeller@telekom.de)

Prediction of users' opinion of speech quality in telecommunication is of a major issue in speech transmission planning. For this purpose, reliable models, such as the E-model (ITU-T Rec. G.107, 2005) were developed for narrow-band $(\mathrm{NB})$ transmission. Meanwhile, telephony using the Internet Protocol (VoIP) has enabled an enlargement of the transmission band, introducing wideband (WB; $50-7000 \mathrm{~Hz}$ and beyond) to every day communication. The quality improvement thanks to the bandwidth extension was found to be $29 \%$, as compared with NB [3]. Consequently, an updated planning model is required to assess quality in WB speech transmission. We performed two listening only tests to study the quality impairment due to signal attenuation, different noise types and level and various codecs. The tests showed that user's quality perception in case of WB was similar to NB for the noisy conditions. However, considerably higher judgments were given to $\mathrm{WB}$ than to $\mathrm{NB}$ for the low-noised conditions. With increasing noise level, the quality of NB and WB become more and 
more alike, and quality differences between codecs decrease. Following the lines of thoughts of the E-model, we propose a model assessing the quality impairment in WB speech transmission resulting from noise and/or signal attenuation.

\section{2:00}

1pSCa4. Effect of degradations' distribution in a corpus test on auditory ratings. Nicolas Côté (France Télécom, 2 avenue Pierre Marzin, 22300 Lannion, France, nicolas.cote@orange-ftgroup.com), Virginie Durin (France Télécom, 2 avenue Pierre Marzin, 22300 Lannion, France, virginie.durin@orange-ftgroup.com)

Speech quality of telecommunications systems is usually evaluated thanks to auditory tests, which must be carried out in accordance with ITU-T Recommendations. In these tests, subjects are asked to assess the quality of speech sample by giving a score on a five-level scale. The averaging of subjects' scores yields the Mean Opinion Score (MOS) which represents the speech quality for a given condition. However, MOS values can be strongly influenced by many factors. In this paper, we focus on a specific bias: the distribution of the impairments in the stimuli set. In effect the rating of a narrow-band stimulus $(300-3400 \mathrm{~Hz})$ may have a lower quality score in a mixed-band set (mixed of narrow-band and wideband (50-7000 $\mathrm{Hz}$ ) conditions) than in a purely narrow band set. This effect is detailed through results of auditory tests recently carried out. Consequently, the validity of MOS value is theoretically limited within a test which prevents MOS comparison between different tests. Finally, two solutions are proposed to avoid this effect: first, an improvement of auditory tests methodology and then, a new approach to assess speech quality, based on the subject's behaviour.

\section{2:20}

1pSCa5. Influence of informational content of background noise on speech quality evaluation for VoIP application. Adrien Leman (France Telecom, 2, Avenue Pierre Marzin, 22300 Lannion, France, adrien.leman@orange-ftgroup.com), Julien Faure (France Telecom, 2, Avenue Pierre Marzin, 22300 Lannion, France, julien.faure@orange-ftgroup.com), Etienne Parizet (Laboratoire Vibrations Acoustique, Insa Lyon, 25 bis, av. J. Capelle, 69621 Villeurbanne Cedex, France, etienne.parizet@insa-lyon.fr)

With the rise of mobile communication systems, the background noise in the speaker's environment and its interaction with VoIP network impairment affects speech quality perception. This effect should be taken into account in nonintrusive models in order to improve accuracy of end user perception measurement. The aim of this study is to determine the impact of information contained in background noise (background speech, environmental sources) on speech quality perception. A subjective test on the speech quality perception in real network conditions has been done comparing the effect of stationary background noise mainly due to transmission equipment (electrical humming or blowing noises) with the effect of nonstationary environmental noise (public place, traffic noise, background conversation). Interactions between these different background noise condition and realistic network impairments (coders and packets loss) were also studied. The speech quality was evaluated through the Mean Opinion Score determined from an Absolute Category Rating method.

\section{2:40}

1pSCa6. Speech perception in noise with binary gains. Deliang Wang (Ohio State University, Department of Computer Science and Engineering, 2015 Neil Ave., Columbus, OH 43210, USA, dwang@cse.ohio-state.edu), Ulrik Kjems (Oticon A/S, Kongebakken 9, DK-2765 Smørum, Denmark, uk@Oticon.dk), Michael S. Pedersen (Oticon A/S, Kongebakken 9, DK-2765 Smørum, Denmark, msp@Oticon.dk), Jesper B. Boldt (Oticon A/S, Kongebakken 9, DK-2765 Smørum, Denmark, jeb@Oticon.dk), Thomas Lunner (Oticon Research Centre Eriksholm, Kongevejen 243, DK-3070 Snekkersten, Denmark, TLU @Oticon.dk)

For a given mixture of speech and noise, an ideal binary time-frequency mask is constructed by whether SNR within individual time-frequency units exceeds a local SNR criterion (LC). With linear filters, co-reducing mixture
SNR and LC does not alter the ideal binary mask. Taking this manipulation to the limit by setting both mixture SNR and LC to minus infinity produces an output that contains only noise with no target speech at all. This particular output corresponds to turning on or off the filtered noise according to a pattern prescribed by the ideal binary mask. Our study was designed to test on speech intelligibility of noise gated by the ideal binary mask obtained this way. It is observed that listeners achieve nearly perfect speech recognition from gated noise. Only sixteen filter channels and a frame rate of one hundred Hertz are sufficient for high intelligibility. The results show that, despite a dramatic reduction of speech information, a pattern of binary gains provides an adequate basis for speech perception in noise.

\section{3:00}

1pSCa7. Low-dimensional, auditory feature vectors that improve vocal-tract-length normalization in automatic speech recognition. Jessica J. Monaghan (Centre for the Neural Basis of Hearing, Department of Physiology, Development and Neuroscience, University of Cambridge, Downing Site, CB23EG Cambridge, UK, jjmm2@cam.ac.uk), Christian Feldbauer (Centre for the Neural Basis of Hearing, Department of Physiology, Development and Neuroscience, University of Cambridge, Downing Site, CB23EG Cambridge, UK, feldbauer@tugraz.at), Tom C. Walters (Centre for the Neural Basis of Hearing, Department of Physiology, Development and Neuroscience, University of Cambridge, Downing Site, CB23EG Cambridge, UK, tcw24@cam.ac.uk), Roy D. Patterson (Centre for the Neural Basis of Hearing, Department of Physiology, Development and Neuroscience, University of Cambridge, Downing Site, CB23EG Cambridge, UK, rdp1@cam.ac.uk)

Human speech recognition is robust to large changes in vocal tract length (VTL) but automatic speech recognition is not. In an effort to improve VTL normalization, an auditory model was used to derive formantlike features from syllables. The robustness supported by these auditory features was compared to the robustness provided by traditional MFCCs (MelFrequency Cepstral Coefficients), using a standard HMM recognizer (Hidden-Markov-Model). The speech database consisted of 180 syllables, each scaled with the vocoder STRAIGHT to have a wide range VTLs and glottal pulse rates. Training took place with syllables from a small, central range of scale values. When tested on the full range of scaled syllables, average performance for MFCC-based recognition was $73.5 \%$, with performance falling close to $0 \%$ for syllables with extreme VTL values. The feature vectors constructed with the auditory model led to much better performance; the average for the full range of scaled syllables was $91 \%$, and performance never fell below $65 \%$ even for extreme combinations of VTL and GPR. Moreover the auditory feature vectors contain just 12 features whereas the standard MFCC vectors contain 39 features. Research supported by the UK-MRC (G0500221) and EOARD (FA8655-05-1-3043).

\section{3:20}

1pSCa8. A Portable robot audition software system for multiple simultaneous speech signals. Hiroshi G. Okuno (Kyoto University, Graduate School of Informatics, Yoshida-Honmachi, Sakyo, 606-8501 Kyoto, Japan, okuno@i.kyoto-u.ac.jp), Shunichi Yamamoto (Kyoto University, Graduate School of Informatics, Yoshida-Honmachi, Sakyo, 606$8501 \quad$ Kyoto, Japan, shunichi@kuis.kyoto-u.ac.jp), Kazuhiro Nakadai (Honda Research Institute Japan Co., Ltd., 8-1 Honcho, Wako, 351-0114 Saitama, Japan, nakadai@jp.honda-ri.com), Jean-Marc Valin (CSIRO ICT Center, Cnr Vimiera \& Pembroke Rds, NSW 2122 Marsfield, Australia, jean-marc.valin@csiro.au), Tetsuya Ogata (Kyoto University, Graduate School of Informatics, Yoshida-Honmachi, Sakyo, 606-8501 Kyoto, Japan, ogata@kuis.kyoto-u.ac.jp), Kazunori Komatani (Kyoto University, Graduate School of Informatics, YoshidaHonmachi, Sakyo, 606-8501 Kyoto, Japan, komatani@kuis.kyoto-u.ac.jp)

Since a robot is deployed in various kinds of environments, the robot audition system should work with minimum prior information on environments to localize, separate and recognize utterances by multiple simultaneous talkers. For example, it should not assume either the number of speakers, the location of speakers for sound source separation (SSS), or specially 
tuned acoustic model for automatic speech recognition (ASR). We developed a portable robot audition that uses eight microphones installed on the surface of robot's body such as Honda ASIMO, and SIG-2 and Robovie-R2 at Kyoto University with different microphone configurations. The system integrates SSS and ASR by using the Missing-Feature Theory. For SSS, we use Geometric Source Separation (GSS) [Parra 2002] and multichannel post-filter [Cohen 2002] to separate each utterance. Since separated speech signals are distorted due to interfering talkers and sound source separation, multichannel post-filter enhanced speech signals. At this process, we create a missing feature mask that specifies which acoustic features are reliable in time-frequency domain. Multiband Julius [Nishimura 2004], a missingfeature-theory based ASR, uses this mask to avoid the influence of unreliable features in recognizing such distorted speech signals. The system demonstrated a waitress that accepts meal orders placed by three actual human talkers and a voice "Rock-Scissors-Paper" game referee that decides who wins the game.

\section{$3: 40$}

1pSCa9. Ambiguity in the recognition of phonetic vowels when using a bone conduction microphone. Véronique Zimpfer (ISL, 5 rue du Général Cassagnou BP 70034, 68301 Saint Louis, France, veronique
.zimpfer@isl.eu),Karl Buck(French German Institut of Saint Louis (ISL), 5 rue du Général Cassagnou, 68301 Saint-Louis, France, karl.buck@isl.eu)

When speaking, not only air conducted noise is generated, but also vibrations can be recorded at different places on the head using accelerometers. The bone conduction microphones are less sensitive to noise than regular acoustical microphones, they are usable in harsh environments and they are compatible with head equipment such as NBC protection devices. This paper reports the first results of a study designed to evaluate the differences in perception between speech recorded via a acoustic microphone and speech recorded using bone conduction. These differences may cause of a bad intelligibility of bone conduction communication, even if presented in an undisturbed environment. We study the recognition of ten French phonetic vowels recorded by a bone conduction microphone. A listening test is designed to show the confusions of phonetic vowels when listening to speech picked up by an air or bone conduction microphone. The tests show confusion between the vowels [i] [y] [u]. All of these have the frequency of their first formant in common. The sonograms are distinctly different when recorded with an aero-acoustic microphone, become almost identical if the bone conducted speech is analyzed. Moreover, the confusions of the phonetic vowels depend on the speaker.

MONDAY AFTERNOON, 30 JUNE 2008

ROOM 250B, 1:00 TO 4:00 P.M.

\title{
Session 1pSCb
}

\section{Speech Communication: Measurement of Sociophonetic Variation in Speech}

\author{
Ewa Jacewicz, Cochair \\ The Ohio State University, 110 Pressey Hall, 1070 Carmack Road, Columbus, OH 43210, USA \\ Jonathan Harrington, Cochair \\ Institute of Phonetics and Speech Processing, University of Munich, Munich, 80799, Germany
}

\section{Invited Papers}

$1: 00$

1pSCb1. Inter- and intra-speaker variation: some methodological consequences for sociophonetic research. Gerard J. Docherty (Newcastle University, School of ECLS, KG VI Building, NE3 1YB Newcastle upon Tyne, UK, g.j.docherty@ncl.ac.uk), Paul Foulkes (University of York, Department of Language \& Linguistic Science, Heslington, YO10 5DD York, UK, pf11@york.ac.uk)

In recent years there has been a rapidly growing awareness of the importance of sociophonetic variation for advancing models of speech production, perception and learning. Meanwhile within sociolinguistic research there has been an equally rapidly evolving recognition that full accounts of the social marking functions of speech communication require greater focus on the manner in which individuals deploy phonetic resources variably as a reflection of the fluid identities which they project across the diverse interactions which they participate in. The aim of this talk is to explore some of the methodological consequences of these developments. We explore the ways in which an approach to sociophonetic variation which rightly focuses on how it plays out in the performance of individual speakers can be reconciled with the development of models of speech production and perception which to date have typically been driven by what is found to be common across individual speakers/listeners and much less by those factors which differentiate them.

1pSCb2. Sociophonetic methods for studying substratal effects and new dialect formation. Erik R. Thomas (North Carolina State University, Department of English, Box 8105, Raleigh, NC 27695-8105, USA, ethomas@ social.chass.ncsu.edu)

Studies of substratal effects in the formation of new language varieties have seldom combined both acoustic sophistication and the examination of a broad suite of pronunciation features. This study provides an example of how such work can be conducted. The study focuses on Pearsall, Texas, a community in southern Texas in which Mexican Americans have formed an established community for four generations and exhibit a shift from Spanish dominance to English dominance. A variety of segmental and prosodic variables are examined, both for different generations of Mexican Americans and for Anglo speakers of the contact dialect, and the variables are compared statistically. Results show that some Spanish interference features are discarded, while others take on a life of their own as markers of Mexican American identity. 
1pSCb3. Modeling dialect-related variability in American English plosives. Thomas Purnell (University of Wisconsin, 1168 Van Hise, 1220 Linden Drive, Madison, WI 53706, USA, tcpurnell@wisc.edu)

Socially motivated variation in speech often lies in the differential use of phenomena described in identical or near-identical terms. This paper investigates the hypothesis that dialect differences may result from the relative distribution of an acoustic characteristic with respect to trading relations with other co-varying characteristics, or by variations in the characteristic within regions of linguistic stability. Plosive variation among European American and African American English speakers in the upper Midwest is examined. Speakers from both dialect groups may produce plosives instead of interdental fricatives in syllable initial position, and devoice or glottalize apical plosives in syllable final position. Additionally, speakers of both dialects have been observed to hypervoice voiced plosives word-initially. The current experiment explores the boundary characteristics of a trading relations model within the plosive system of both dialect groups. The specific question addressed by the paper is whether the range of plosive variation suggests a shared property of nonstandard dialects, or whether the relation among acoustic measures is dialect specific. Results are discussed in light of acoustic, perceptual and categorical stability.

\section{2:00}

1pSCb4. The acoustic and perceptual bases of diachronic /u:/-fronting in Standard Southern British. Jonathan Harrington (Institute of Phonetics and Speech Processing, University of Munich, 80799 Munich, Germany, jmh@phonetik.uni-muenchen.de), Felicitas Kleber (Institute of Phonetics and Speech Processing, University of Munich, 80799 Munich, Germany, kleber@phonetik.uni-muenchen.de), Ulrich Reubold (Institute of Phonetics and Speech Processing, University of Munich, 80799 Munich, Germany, reubold@phonetik.uni-muenchen.de)

In a recent experiment concerned with diachronic/u:/-fronting in Standard Southern British, it was shown both that the/u:/ category boundary was perceptually retracted for older compared with younger listeners of this variety, and that they compensated perceptually to a greater extent for the coarticulatory fronting effects of the preceding consonant on /u:/ in words like used. In the present study, these perceptual differences were related to the same speakers' productions of words containing a /u:/ nucleus in a fronting (e.g., 'used') and non-fronting (e.g., 'swoop') contexts. The relative acoustic distance of /u:/ between front and back vowels was measured in a threedimensional space formed by applying the discrete-cosine-transformation to the entire F2-trajectory shape separately for each speaker. Compatibly with their perceptual responses, older listeners had a more retracted /u:/ on this measure and exhibited a greater coarticulatory influence of the preceding consonant. The mechanism of this sound change is considered both from the point of view of a shift in the perceptual compensation for coarticulation and recent developments in episodic models of speech perception

$$
\text { 2:20 }
$$

1pSCb5. Speech processing and dialect variation in the American Midwest. Cynthia G. Clopper (Ohio State University, 1712 Neil Ave, Oxley Hall 222, Columbus, OH 43210, USA, clopper.1@osu.edu)

The Midwestern United States is divided into two dialect regions: North and Midland. The Northern dialect is characterized by the clockwise rotation of the low and low-mid vowels in the acoustic-phonetic vowel space. The Midland dialect is characterized by back vowel fronting and the merger of the low back vowels. A series of experiments exploring the effects of dialect variation on spoken word recognition has revealed systematic linguistic processing costs associated with these dialect-based vowel system differences for Midwestern listeners. While word recognition performance in noise is equally accurate for both dialects, the patterns of errors that listeners produce reveal systematic confusions between phonologically different vowels that overlap in the acoustic-phonetic space across the two dialects. In addition, speeded lexical classification performance is highly accurate for both dialects, but performance is slower for Northern vowels than Midland vowels of the same phonological class. The results from these studies suggest that even in the absence of striking intelligibility differences between the two dialects, processing costs associated with the perception of dialect variation can be observed using traditional speech perception and spoken word recognition tasks.

$$
\text { 2:40 }
$$

1pSCb6. Analysis of total vowel space areas in three regional dialects of American English. Robert A. Fox (The Ohio State University, 110 Pressey Hall, 1070 Carmack Road, Columbus, OH 43210, USA, fox.2@osu.edu), Ewa Jacewicz (The Ohio State University, 110 Pressey Hall, 1070 Carmack Road, Columbus, OH 43210, USA, jacewicz.1@osu.edu)

The acoustic characteristics of vowel systems in different varieties of American English are greatly affected by regional variation. Given the significant positional differences of vowels within the acoustic space across regional dialects of English, one should expect that the size and extent of the vowel space is also affected by this type of variation. Traditionally, the size of the acoustic vowel space has been measured as the triangular area defined by the three corner vowels. An obvious weakness of this approach is that it underestimates the actual "working space" of vowel system in that the onsets and/or offsets of other vowels are often found outside this triangular area. This paper proposes a procedure to estimate the area of a complete vowel space, taking into account dynamic formant pattern of all vowels and diphthongs. Complete vowel space areas are calculated for individual speakers and compared across three distinct regional varieties (representing Inland North, Southern Appalachian, and a Midland variety). The comparison also examines possible changes to vowel space area as a function of speaker generation (younger and older adults).

\section{3:00}

1pSCb7. Regional variation in vowels and vowel systems: normalization and optimization. Roeland Van Hout (Department of Linguistics - Radboud University, Post Box 9103, 6500 HD Nijmegen, Netherlands, hout@let.ru.nl)

We made an acoustical description of regional variation patterns in the vowel system of Dutch spoken in the Netherlands and Flanders. The speech material consisted of read monosyllabic utterances in a neutral consonantal context, representing the vowels of 
Dutch. A discriminant analysis applied on the raw measurements to classify each speaker into one of the eight regions involved (sociogeographic variation) gave a correct regional classification for $72.0 \%$ of the 160 speakers. When normalization procedures were applied, the percentages of proper classification increase to $82.5 \%$. Several questions have to be answered though. Which normalization procedure is the best one and why, and how can we be sure that specific parts of sociophonetic variation are not distorted by the normalization procedure? We will present additional materials collected on the same set of speakers in reading aloud a strictly controlled list of words containing all Dutch vowels in different consonantal contexts. Another question is how to reduce the amount of information used in a normalization procedure. The $\mathrm{z}$ transformation (Lobanov) performs well, but is rather expensive in the amount of information needed. Can we minimize the information needed and yet uncover the patterns of sociophonetic variation?

1pSCb8. A new speaker-intrinsic vowel formant frequency normalization algorithm for sociophonetics. Anne Fabricius (Roskilde University, Department of Culture and Identity, Postboks 260, DK4000 Roskilde, Denmark, fabri@ruc.dk), Dominic Watt (University of York, Department of Language \& Linguistic Science, Heslington, YO10 5DD York, UK, dw539@york.ac.uk)

This paper evaluates the strengths and weaknesses of a speaker-intrinsic vowel formant frequency normalization algorithm initially proposed in Watt and Fabricius (2002) and modeled by Thomas and Kendall (2007) for direct comparison with other normalization algorithms. We evaluate the merits of the new routine as a sociophonetic research tool relative to those of two well-known speakerintrinsic methods documented in Lobanov (1971) and Nearey (1977) through comparisons of the values of two parameters: degree of overlap of vowel spaces and vowel space area ratios. Measurements of angles and Euclidean distances between pairs of points in the vowel space (a method presented in Fabricius 2007) also provide a comparative parameter revealing how different algorithms model the vowel space. The study uses two existing datasets: 1) a corpus of RP vowels compiled from Hawkins and Midgley (2005) and Moreiras (2006) and 2) previously unpublished data from Aberdeen, northeast Scotland.

1pSCb9. Stability and variation in the pronunciation of French: A corpus-based approach. Jacques Durand (Laboratoire Cognition, Langues, Langage, Ergonomie (CLLE), Université Toulouse - Le Mirail \& CNRS, 5, Allées Antonio Machado, 31058 Toulouse, France, jacques.durand@univ-tlse2.fr), Noel Nguyen (LPL, Aix-Marseille Université \& CNRS, 29, avenue Robert Schuman, 13100 Aix-en-Provence, France, Noel.Nguyen@1pl-aix.fr)

This contribution relates to the project "La Phonologie du Français Contemporain" (PFC), the largest and most ambitious survey of modern French ever conceived. The PFC project involves over thirty researchers from a variety of countries and aims at the recording, partial transcription and phonetic analysis of over 600 speakers from the francophone world. It aims at a broad coverage of varieties of contemporary French by selecting groups of speakers from approximately 60 different locations in the francophone world. So far around 600 speakers have already been recorded in various parts of the francophone world beside France, including Belgium, Burkina Fasso, Canada, Côte d'Ivoire, Louisianne, and Switzerland. The recordings involve formal and informal conversation, the reading aloud of a word-list and a passage. Current work conducted on the database ranges from auditory exploitation of the data to automatic speech recognition via classical experimental phonetics. We address theoretical and empirical issues related to liaison as well as vowel systems, and examine sociogeographical variation and changes in progress in these respects within French, using a variety of quantitative methods for the empirical assessment of phonological hypotheses. 


\title{
Session 1pSCc
}

\section{Speech Communication: General Topics in Speech Communication I (Poster Session)}

\author{
Erika Levy, Cochair \\ Biobehavioral Sciences, Teachers College, Columbia University, New York, NY 10027, USA
}

Susanne Fuchs, Cochair

ZAS, Schuetzenstr. 18, 10117 Berlin, Germany

Patti Adank, Cochair

F.C. Donders Centre for Cognitive Neuroimaging, University College London, London WC1N 3AR, UK

\begin{abstract}
All posters will be on display from 4:20 p.m. to 6:00 p.m. To allow contributors an opportunity to see other posters, contributors of odd-numbered papers will be at their posters from 4:20 p.m. to 5:10 p.m. and contributors of even-numbered papers will be at their posters from 5:10 p.m. to 6:00 p.m.
\end{abstract}

\section{Contributed Papers}

1pSCc1. Acoustic validation of the analysis of diadochokinesis in normal speech using the motor speech profile program. Yu-Tsai Wang (National Yang-Ming University, School of Dentistry, Rm. 411, No. 155, Sec. 2, Li-Nong St. Beitou District, 11221 Taipei, Taiwan, yutsaiwang@ym.edu.tw), Yuh-Mei Chung (Dept. of Physical Medicine and Rehabilitation, Taipei Veterans General Hospital, No. 201, Sec. 2, Shih-Pai Rd., 11217 Taipei, Taiwan, ymchung@vghtpe.gov.tw), Ray D. Kent (Waisman Center, Rm. 435, University of Wisconsin-Madison, 1500 Highland Avenue, Madison, WI 53705-2280, USA, kentray@waisman.wisc.edu), Hsiu-Jung Lu (National Yang-Ming University, School of Dentistry, Rm. 411, No. 155, Sec. 2, Li-Nong St. Beitou District, 11221 Taipei, Taiwan, hsiujung_lu@ hotmail.com)

The purpose of this study is to evaluate the suitability, reliability, and concurrent validity of the analysis of diadochochinesis (DDK) in normal speech using a computer program, Diadochokinetic Rate Analysis (DRA) in the KayPENTAX Motor Speech Profile. Fifteen healthy participants were recorded at UW-Madison as they repeated various syllables as quickly and steadily as possible. The DDK samples were executed by the DRA protocol to generate immediate quantitative information at different thresholds and were also hand-measured. When the lowest peak intensity during consonantvowel syllables is lower than the highest peak intensity during intersyllable pauses, the DRA output is incorrect and the DDK sample is defined as nonexecutable. Analyses were based on the percentage of nonexecutable DDK samples and the comparisons of the results between repeated analyses at different thresholds and between automatic and manual measuring methods. Results: (1) One-ninth of the DDK samples was nonexecutable; (2) when the protocol was executable, the reliability at different thresholds and validity between different measuring methods were both satisfactory; and (3) the temporal variation between different measuring methods were larger than the intensity variation. Implications of the findings will be discussed in terms of the application of the DRA to general clinical purposes.

1pSCc2. Analysing cockpit and laboratory recordings to determine fatigue levels in pilots' voices. Claude Legros (Université de Toulouse-Le Mirail, Laboratoire d'Acoustique LAUTM, 5, allées Antonio Machado, 31058 Toulouse Cedex 1, France, legros@univ-tlse2.fr), Robert Ruiz (Université Toulouse II, LARA, 5 allée Antonio Machado, 31058 Toulouse Cedex, France, robert.ruiz@univ-tlse2.fr), Philippe Plantin De
Hugues(Ministère des Transports, Bureau Enquêtes Analyses, 200 rue de Paris, Bât 153, Aéroport du Bourget, 93352 Le Bourget Cedex, France, plantindehugues@bea-fr.org)

Analysis of pilots' voices was undertaken under controlled laboratory conditions, using aeronautical terminology, standard professional equipment, and a standardized recording environment. The aim of the experiment was to determine the way the acoustic characteristics of the voice are modified after a phase of sleep. The results, which are presented in this paper, show very significant variations in the dispersion parameters, like jitters, associated with the fundamental frequency. In an aircraft cockpit, the electroacoustic and environmental conditions are not so good. Therefore, it is essential to improve and adapt voice analysis methods to achieve reliable results. Cockpit Voice Recorder recordings have poor acoustic characteristics due to microphone quality and the signal to noise ratio, in addition to the non-predetermined vocabulary range. This paper will outline the modifications made to the laboratory methodology and the comparative results obtained, to improve the analysis of CVR recordings. Comparisons of the test recordings meant that it was feasible to determine, via voice analysis, the state of drowsiness of a pilot and, made possible the study of CVR recordings from accidents using new analytical techniques.

1pSCc3. The temporal location of rms peak in coarticulated vowels. Ewa Jacewicz (The Ohio State University, 110 Pressey Hall, 1070 Carmack Road, Columbus, OH 43210, USA, jacewicz.1@osu.edu), Robert A. Fox (The Ohio State University, 110 Pressey Hall, 1070 Carmack Road, Columbus, OH 43210, USA, fox.2@osu.edu)

The acoustic energy peak of the vowel, here defined as the rms peak, occurs typically before the temporal vowel midpoint. This study examines whether and how the location of the rms peak varies as a function of consonantal context and vowel duration. Four measures of rms peak location were explored: its distance (in $\mathrm{ms}$ ) from vowel onset and from $\mathrm{CVC}$-word onset (absolute measures) and its relative position (ranging from 0 to $100 \%$ ) within the vowel and within the CVC-word (relative measures). Analysis of eight American English vowels produced in ten consonantal contexts by twenty speakers shows that the two relative measures yielded the most comparable and consistent patterns. The relative location of rms peak varied significantly as a function of consonantal context. It occurred earlier (i.e., closer to vowel or word onset) in the context of fricatives (and not stops), voiced consonants (as opposed to voiceless), and labials (as opposed to 
alveolars). The temporal location of rms peak was also closer to the vowel onset (or word onset) for long vowels as opposed to short. Explanation of these patterns will be provided.

1pSCc4. Analysis of short-time speech transmission index algorithms. Karen Payton (ECE Dept., UMass Dartmouth, 285 Old Westport Rd., Dartmouth, MA 02747, USA, kpayton@umassd.edu), Mona Shrestha (ECE Dept., UMass Dartmouth, 285 Old Westport Rd., Dartmouth, MA 02747, USA,g_mshrestha@umassd.edu)

Various methods have been shown to compute the Speech Transmission Index (STI) using speech as a probe stimulus (Goldsworthy \& Greenberg, J. Acoust. Soc. Am., 116, 3679-3689, 2004). Frequency-domain methods, while accurate at predicting the long-term STI, cannot predict short-term changes due to fluctuating backgrounds. Time-domain methods also work well on long speech segments and have the added potential to be used for short-time analysis. This study investigates the accuracy of two time-domain STI methods: envelope regression (ER) and normalized correlation (NC), as functions of window length, in various acoustically degraded environments with multiple talkers and speaking styles. Short-time STIs are compared with a short-time Theoretical STI, derived from octave-band signal-to-noise ratios and reverberation times. For windows as short as $0.3 \mathrm{~s}$, the ER and NC Methods track the short-time Theoretical STI and both the Theoretical and ER Methods converge to the long-term result for windows greater than $4 \mathrm{~s}$. Short-time STIs are also compared to intelligibility measurements on clear/conversational speech. Correlations between STI and intelligibility scores are high at the sentence and word levels and, consistent with the scores, short-time methods predict a higher average value of STI for clear than for conversational speech.

1pSCc5. An acoustic investigation into coarticulation and speech motor control: high vs. low frequency syllables. Frank Herrmann (University of Sheffield, Human Communication Sciences, 31 Claremont Crescent, S10 2TA Sheffield, UK, F.Herrmann@Sheffield.ac.uk), Sandra P. Whiteside (University of Sheffield, Human Communication Sciences, 31 Claremont Crescent, S10 2TA Sheffield, UK, s.whiteside@sheffield.ac.uk), Stuart Cunningham (University of Sheffield, Human Communication Sciences, 31 Claremont Crescent, S10 2TA Sheffield, UK, s.cunningham@sheffield.ac.uk)

Levelt \& Wheeldon's concept of the mental syllabary (1994) suggests that articulatory routines for syllables are stored in the form of gestural scores in a library. Cholin et al. (2006) found a syllable frequency effect on naming latency of nonsense words and interpret this as supporting evidence for the existence of such a syllabary. This paper presents a data set from a project investigating speech motor learning and coarticulation. Acousticbased coarticulation measures, such as F2 locus equations, are used to indirectly determine the degree of gestural overlap in articulatory movements. Greater degrees of coarticulation can be indicators of cohesive articulatory gestures and therefore, motor speech skill. It is hypothesised that high frequency syllables, as well trained articulatory gestures, should exhibit a greater degree of coarticulation, less variation, and shorter duration times than low frequency syllables. Fifteen native speakers of English listened to, and repeated 16 mono-syllabic stimuli which belonged to either of two categories: high and low frequency (CELEX). Acoustic measurements included the transitions of the first three formants (C-to-V coarticulation), transition times and syllable duration. Patterns of coarticulation were quantified using F2 locus equations and the absolute changes in formant frequency. The results are presented and discussed.

1pSCc6. Segmental and supra-segmental contributions to cross-language speech intelligibility. Melanie Preece-Pinet (University College London, Department of Phonetics and Linguistics, 4, Stephenson Way, NW1 2HE London, UK, m.pinet@ucl.ac.uk), Paul Iverson (University College London, Department of Phonetics and Linguistics, 4, Stephenson Way, NW12HE London, UK, p.iverson@ucl.ac.uk)

Non-native accents affect speech recognition in noise, and previous work has shown that intelligibility is modulated by listener-talker interaction; a matched linguistic background between the two seems to maximise intelligibility. The present study examined the contribution of segmental and supra-segmental cues, as well as how the degree of L2 experience affects non-native speech perception. A group of monolingual native English listeners and two groups of French native listeners with different amounts of L2 experience were presented with English sentences produced by native and non-native (French) speakers in noise. The stimuli were digitally processed to swap the pitch and segment durations between recordings of the same sentences produced by different speakers (e.g., imposing a French-accented prosody onto recordings made from English speakers). The results revealed that English listeners were more accurate at recognizing speech with English segments but were little affected by prosody; low proficiency French listeners were more accurate when the speech had both French-accented segments and prosody; and high proficiency French listeners varied in the cues that they used depending on the noise level. The use of segmental and supra-segmental cues thus appears to vary with language experience and listening context.

1pSCc7. A multidimensional acoustic analysis of vowels in two Polish dialects. Wieslawa Cholewa (UMR 7018, CNRS/Univ. Paris III, 19, rue des Bernardins, 75005 Paris, France, Wieslawa.Cholewa@univ-paris3.fr), Charalampos Karypidis (UMR 7018, CNRS/Univ. Paris III, 19, rue des Bernardins, 75005 Paris, France, ch _karypidis@yahoo.com)

In this paper, we attempt to examine the acoustic (oral) vowel spaces of two main Polish dialects: the Polish spoken in Cracow and that spoken in the wider region of Warsaw. A semi-spontaneous corpus consisting of four speakers (two male, two female) of each dialect was recorded with approximately 2000 vowel occurrences for each speaker. The segmentation was performed manually and was subsequently corrected with an intensity-based algorithm. The tokens were submitted to acoustic analyses, and two multidimensional representations were then prepared, one based on formants (accompanied with bandwidths and amplitudes) and the other on critical bands. Both representations included measurements for duration, F0, spectral center of gravity, skewness, and kurtosis. These values served as input for discriminant and principal component analysis which allowed us: (a) to compare the relative weight of each cue, (b) to isolate those parameters that allow a differentiation of the members of a vowel system (in a language such as Polish, F1 and F2 are traditionally the sole cues regarded as relevant), and (c) to detect any (dis)similarities between the two dialects in the three aforementioned aspects.

1pSCc8. Automatic data enhancement for language identification using voice generation. Aaron Lawson (RADC, Inc., 525 Brooks Road, Rome, NY 13441, USA, aaron.lawson.ctr@rl.af.mil), Matthew Linderman (Air Force Research Laboratory, 525 Brooks Road, Rome, NY 13441, USA, matthew.linderman@gmail.com), Michael Carlin (Air Force Research Laboratory, 525 Brooks Road, Rome, NY 13441, USA, Michael.Carlin@rl.af.mil), Allen Stauffer (RADC, Inc., 525 Brooks Road, Rome, NY 13441, USA, stauffar@clarkson.edu)

Approaches to LID require very large sets of training (often greater than six hours per language) for accurate results. This study looks at ways of automatically reducing the amount of training data required to train a LID model, while maintaining or increasing accuracy. Initial experiments found that speaker density, i.e., the number of speakers per time unit, had a very dramatic influence on the accuracy of models (absolute increase of 15\%). In order to accomplish the goal of increasing the number of speakers available for LID training without having to collect additional audio, the STRAIGHT algorithm was used to synthesize "novel" speakers for use in training language models for a LID system. The mean pitch and vocal tract length of the speaker in each LID training file was scaled to generate four additional voices per original speaker to artifically augment the training data. The resulting models yielded an improvement of $10 \%$ over the baseline system (from $80 \%$ to $90 \%$ ). This study shows that automatically generated speakers 
have a great impact on the accuracy of LID models, in this case an initial training set of 15 minutes per language was augmented and performed comparably to a set trained with six hours.

1pSCc9. Channel mitigation approach for automatic language identification. Allen Stauffer (RADC, Inc., 525 Brooks Road, Rome, NY 13441, USA, stauffar@clarkson.edu), Aaron Lawson (RADC, Inc., 525 Brooks Road, Rome, NY 13441, USA, aaron.lawson.ctr@rl.af.mil)

A major obstacle to overcome in language identification (LID) performance is the impact of varying channel conditions. In house experiments show that LID performance drops between $10-12 \%$ across channels. The focus of this project is to mitigate the impact of channel conditions and artifacts on LID, and to provide an understanding of how channel robust models may be created by combining data from across corpora. Our approach involved creating composite cross-channel language models from multiple corpora that were tested with data from three corpora whose results were compared to results obtained from same-channel and pure cross-channel experiments. Our hypotheses were that 1) same-channel models would be the most accurate, 2) purely cross-channel models would be considerably less accurate, and 3) composite model accuracy would fall in between that of the same-channel and cross-channel models. Results were surprising: while pure cross-channel tests performed the worst, with an average of $11 \%$ loss in accuracy, composite models outperformed matched condition models in tests by $0.5 \%$ to $15 \%$ for all four combinations tested. These findings provide evidence that cross-channel augmentation does not just make for more robust LID models across channel conditions, but improves model robustness within channel conditions as well.

1pSCc10. Newborn pain cry analysis based on pitch frequency tracking. Delphine Bard (Lund University, Division of Engineering Acoustics, John Ericsson väg 1, 22100 Lund, Sweden, delphine.bard@acoustics.lth.se), Per Runefors (Department of Paediatrics, Växjö Hospital, $351 \quad 85 \quad$ Växjö, per.runefors@vaxjo.mail.telia.com), Einar Arnbjörnsson (Department of Paediatric Surgery, University Hospital Lund, 22100 Lund, Sweden, einar .arnbjornsson@telia.com)

The aim of the newborn pain cry analysis is to test the hypothesis that cry can be used as a tool to detect signs of nocioceptive pain. Previous studies applying signal processing techniques to analyze the sound of these cries have been done. The subject of this paper is to adapt and improve the original method with the help of new signal processing methods. The pitch frequency is extracted from the waveform of the recorded babies' cries using time domain methods. The fluctuations of this parameter are analyzed in terms of jitter. In particular, a sliding buffer approach is presented, as well as an improvement of the Average Mean Difference Function (AMDF). Comparison between original and news results has been done.

1pSCc11. Age effects of pitch-shifted auditory feedback on reflexive and volitional voice Fo control. Kyoko Nagao (Center for Pediatric Auditory and Speech Sciences, Nemours Biomedical Reserach, A.I. du Pont Hospital for Children, 1600 Rockland Road, Wilmington, DE 19803, USA, nagao@asel.udel.edu), Katie E. McCurdy (Indiana University, Dept. of Speech and Hearing Sciences, 200 S Jordan Avenue, Bloomington, IN 47405, USA, kemccurd@indiana.edu), Theresa A. Burnett (Indiana University, Dept. of Speech and Hearing Sciences, 200 S Jordan Avenue, Bloomington, IN 47405, USA, burnett@indiana.edu)

Research on voice fundamental frequency (F0) control suggests that when making a voluntary vocal response to a pitch change in voice auditory feedback, the pitch-shift reflex typically occurs before the volitional response is initiated. The current study examined the effects of aging on reflexive and volitional voice F0 responses to pitch-shifted auditory feedback. Thirty participants (age range 19 to 78 years) repeatedly sustained an /u/ vocalization at a steady pitch and loudness while listening to their voice fed backed over headphones. Once per vocalization, feedback pitch was randomly shifted upward or downward for 100 milliseconds and 20 or 100 cents. In the Sustain condition, participants attempted to ignore the pitch change in auditory feedback voice. In the Follow condition, participants immediately changed their pitch in the same direction as the auditory pitch shift they heard. Preliminary results from thirteen participants indicate that there appears to be no effect of aging on the first F0 responses for both magnitude and latency. Voluntary voice F0 changes were delayed in older participants compared to younger participants as expected due to age-related general slowing. Results broaden our understanding of typical reflexive and volitional voice control capabilities across the age span.

1pSCc12. Do Japanese speakers perceive nonexistent vowels in non-native consonant clusters? Seiya Funatsu (The Prefectural Univ. of Hiroshima, 1-1-71 Ujinahigashi Minami-ku, 734-8558 Hiroshima, Japan, funatsu@pu-hiroshima.ac.jp), Satoshi Imaizumi (The Prefectural Univ. of Hiroshima, 1-1 Gakuen-machi, 723-0053 Mihara, Japan, imaizumi@pu-hiroshima.ac.jp), Masako Fujimoto (The National Inst. for Japanese Language, 10-2 Midori-machi, 190-8561 Tachikawa, Japan, mfuji@ viola.ocn.ne.jp), Akira Hashizume (Hiroshima Univ., 1-2-3 Kasumi Minami-ku, 734-8553 Hiroshima, Japan, wj8uc6@bma.biglobe.ne.jp), Kaoru Kurisu (Hiroshima Univ., 1-2-3 Kasumi Minami-ku, 734-8553 Hiroshima, Japan, kuka422@hiroshima-u.ac.jp)

Vowel epenthesis is a well known phenomenon that non-native speakers insert epenthetic vowels inside non-native consonant clusters. Vowel epenthesis is assumed as perceptual "illusory vowels" (Dupoux et al. 1999). We analyzed vowel epenthesis shown by native Japanese speakers during reading and repetition tasks for non-native consonant clusters, and analyzed their brain responses using magnetoencephalographic methods. Under the reading task, in which subjects read English words and nonsense words, native Japanese speakers (eight females) inserted vowel /o/ after $/ \mathrm{t} /$ and $/ \mathrm{d} / \mathrm{in}$ consonant clusters, and vowel /u/ after other consonants. Under the repetition task, in which subjects repeated utterances of a native English speaker, native Japanese speakers did not produce epenthetic vowels with few exceptions. The length of exceptional epenthetic vowels found under the repetition task was shorter than those under the reading task. The magnetoencephalographic mismatch responses were elicited by epenthetic vowels, suggesting that the native Japanese detected epenthetic vowels as real segments. Based on these results, we conclude that vowel epenthesis by native Japanese speakers did not arise from "illusory vowels", but rather from the difficulty of articulating consonant clusters which are not found in Japanese and/or Japanese phonological rules.

1pSCc13. Fine-phonetic variation in a computational model of word recognition. Odette Scharenborg (Centre for Language and Speech Technology, Radboud University Nijmegen, Erasmusplein 1, 6525 HT Nijmegen, Netherlands, O.Scharenborg@1et.ru.nl)

There is now considerable evidence from psycholinguistic and phonetic research that fine-phonetic variation in the speech signal modulates human speech processing (HSP), and helps the listener segment a speech signal into syllables and words [e.g., Salverda et al., Cognition 90, 51-89 (2003)]. This kind of information also appears to help the human perceptual system distinguish short words (like 'ham') from the longer words in which they are embedded (like 'hamster'). Salverda et al. showed that the lexical interpretation of an embedded sequence is related to its duration; a longer sequence tends to be interpreted as a monosyllabic word more often than a shorter one. Until recently, no computational models of HSP existed that are able to model this fine-phonetic variation [Hawkins, J. of Phonetics 31, 373-405 (2003)]. In this paper, we present Fine-Tracker, a novel computational model of human word recognition that it is able to capture and use this finegrained acoustic-phonetic variation during speech recognition. Simulations using the recordings from the listener experiments by Salverda et al. show that Fine-Tracker is able to capture and use duration variation in the speech signal to distinguish short words from the longer words in which they are embedded. [Research supported by NWO.]

1pSCc14. Lombard speech: effects of task and noise type. Youyi Lu (Sheffield University, Computer Science Department, Regent Court, 211 Portobello St., S1 4DP Sheffield, UK, y.lu@dcs.shef.ac.uk), Martin 
Cooke(Sheffield University, Computer Science Department, Regent Court, 211 Portobello St., S1 4DP Sheffield, UK, m.cooke@dcs.shef.ac.uk)

An analysis of the effect of noise on speech production requires material recorded while undertaking realistic tasks in the presence of realistic noise backgrounds. This study examined speech produced in a number of types of "noise" in tasks with and without a communication factor. In one task, individual speakers were asked to speak aloud while solving "sudoku" puzzles, while in another task pairs of speakers solved these puzzles cooperatively. In both cases the background was quiet or contained one of three types of noise: competing talker, babble-modulated noise and speech-shaped noise. Individual digit words "one" to "nine" and speech/nonspeech sections were manually segmented for acoustic analysis. Both tasks produced Lombard effects observed in previous studies: increases in duration, energy, F0 and spectral centre of gravity. Relative to the non-communicative task, the presence of a communication factor led to words with shorter duration, more overall energy and higher spectral centre of gravity. Speakers actively avoided overlapping with the competing speech masker, with a stronger effect in the communicative task. These results suggest that speakers can, to some extent, compensate for both energetic and informational masking at the ears of the interlocutor.

1pSCc15. Differences in the time-course of accent adaptation: a comparison of adaptation to foreign-accented and unfamiliar regionally-accented speech. Bronwen G. Evans (Department of Phonetics and Linguistics, University College London, 4 Stephenson Way, NW12HE London, UK, bronwen.evans@ucl.ac.uk), Patti Adank (F.C. Donders Centre for Cognitive Neuroimaging, Kapittelweg 29, 6525EN Nijmegen, Netherlands, patti.adank@fcdonders.ru.nl)

Recent work in speech perception has demonstrated that listeners are able to rapidly adapt to foreign- accented speech (Clarke and Garrett, 2004). However, it is not clear whether adaptation to an unfamiliar regional accent operates in the same way. Although some studies have shown that listeners are able to adapt to different accents within the same language after only a short amount of exposure (e.g., Maye et al., in press), others have shown that listeners do not always alter their perceptual representations when listening to a non-native regional accent even if they are highly familiar with that accent (Evans and Iverson, 2004, 2007). In this study, we further explored perceptual adaptation to different accents by comparing the time course of adaptation to an unfamiliar regional accent with adaptation to foreign- accented speech. Listeners identified sentences in noise produced in either an unfamiliar regional accent (Glaswegian) or foreign-accented speech (Spanish-accented English). Preliminary results suggest a different pattern of adaptation for each accent: although listeners show greater adaptation to foreign- accented speech, they perform more poorly with foreignaccented speech overall and the rate of adaptation is slower.

1pSCc16. Production of Parisian French front rounded vowels by second-language learners. Erika S. Levy (Teachers College, Columbia University, Biobehavioral Sciences, Box 180, 525 W120th St., New York, NY 10027, USA, elevy@tc.columbia.edu), Franzo Law (CUNY Graduate Center, 365 5th Avenue, Program in Speech-Language-Hearing Sciences, New York, NY 10016, USA, flaw@gc.cuny.edu)

This study examined the effects of language experience and consonantal context on the production of Parisian French (PF) vowels by American English (AE) learners of French. A repetition task was performed, involving French vowels /y-œ-i-a-u/ uttered by a native speaker of Parisian French (PF) in bilabial /bVp/ and alveolar /dVt/ contexts embedded in the nonsense word/phrase / raCVCa/. Three groups of native AE speakers participated: speakers without French experience (NoExp), speakers with formal French experience (ModExp), and speakers with formal and extensive immersion experience (HiExp). Production accuracy was assessed by native PF listeners' judgments and by acoustic analysis. Native PF listeners identified second-language (L2) learners' productions more accurately as a function of speakers' increased language experience, although /u/, /y/ and /œ/, even produced by the HiExp group, were frequently misidentified. A consonantal context effect was revealed, including / $\mathrm{u} /$ being misidentified more often in alveolar context than in bilabial context by all three groups. Overall, all groups distinguished front rounded $/ \mathrm{y} /$ from $/ \mathrm{u} /$ in production, but often in a non-native manner, e.g., producing /y/ as /ju/. Individual differences, interactions between consonantal context and vowels, as well as implications for the perception-production link in L2 learning, are discussed.

1pSCc17. Development of assisting system for learning Chinese aspirated sounds. Akemi Hoshino (Toyama College of Maritime Technology, Ebie, Neria, 933-0293 Imizu, Toyama Prefecture, Japan, hoshino@toyama-cmt.ac.jp), Jianwu Dang (Japan Advanced Institute of Science and Technology, 1-1 Asahidai, Nomi, 923-1292 Ishikawa, Japan, jdang@jaist.ac.jp), Akio Yasuda (Tokyo University of Marine Science and Technology, 2-1-6 Etchujima, 135-8533 Tokyo, Japan, yasuda@kaiyodai.ac .jp)

Chinese aspirates are generally difficult for Japanese to pronounce and perceive. In order to assist Japanese students to learn Chinese, the authors attempt to build a system to automatically evaluate the pronunciation of Chinese aspirates by Japanese students. To do so, this study analyzed 21 singlevowel syllables of six different Chinese aspirates, bilabial, alveolar, velar, palatal, retroflex, and dental using two evaluation parameters: the voice onset time (VOT) and mean breathing power during VOT. The speech materials were uttered by nine Japanese students and nine native Chinese speakers. It is found that for a give aspirate, the longer the VOT is, the better the score is, as is generally said. But we found that an aspirate with a larger breathing power acquires good mark even with a shorter VOT in a certain range of VOT. The testing results showed that a combination of these two parameters can distinguish aspirated and unaspirated sounds quite well. To achieve an assisting system, we developed a method to automatically detect the VOT. To detect the burst of an aspirate, a filter bank was used to measure signal energy in frequency domain, and the burst timing was determined.

1pSCc18. Articulatory perturbation due to ultrasound imaging: an acoustic analysis. Rachael-Anne Knight (City University, Department of Language and Communication Science, Northampton Square, EC1V 0HB London, UK, knight@city.ac.uk), Christina Villafana Dalcher (City University, Department of Language and Communication Science, Northampton Square, EC1V OHB London, UK, cvdalcher@mac.com), Mark J. Jones (University of Cambridge, Phonetics Lab, Department of Linguistics, Raised Faculty Building, Sidgwick Avenue, CB3 9DA Cambridge, UK, markjjones@hotmail.com)

A safe, non-invasive imaging technique which captures articulatory movements in real time is the Holy Grail of articulatory analysis. Ultrasound imaging of the tongue seems suitable, albeit for a single articulator. However, ultrasound requires positioning a probe under the chin which may inhibit jaw movement. It is well known that speakers compensate rapidly for similar articulatory perturbations such as bite-blocks. Consequently, the images obtained by ultrasound may show compensatory articulations due to jaw inhibition, rather than natural articulations. This study assesses the effects of articulatory compensation during ultrasound imaging using acoustic analysis. The experiment compares acoustic data from three speech conditions: probe-free, probe held manually, probe fixed non-manually. It is hypothesised that sounds requiring a low jaw position, such as low vowels, velars, and velarised laterals, will be worst affected. High jaw sounds such as /s/ and /i/ are also assessed. Any differences between conditions are assumed to be due to compensation for jaw immobility rather than learned segment-specific register effects and are therefore unlikely to diminish as the subjects relax. Variability may diminish, however, as compensatory strategies become more practised. We expect to find some effects on certain segments, but that manual probe holding allows for more natural speech.

1pSCc19. Cortical mechanisms of speech perception in noise in elderly listeners. Edward R. Lee (Northwestern University, 860 Hinman Ave., Apartment 715, Evanston, IL 60202, USA, EdwardLee86@gmail .com), Patrick C. Wong (Northwestern University, 2240 Campus Drive, Evanston, IL 60208, USA, pwong@northwestern.edu), Sumitrajit Dhar (Northwestern University, 2240 Campus Drive, Evanston, IL 60208, USA,s-dhar@northwestern.edu), Geshri M. Gunesekera (Northwestern 
University, 2240 Campus Drive, Evanston, IL 60208, USA, geshri-g@northwestern.edu), Rebekah A. Abel (Northwestern University, 2240 Campus Drive, Evanston, IL 60208, USA, r-abel@northwestern.edu)

The present study examines the cerebral hemodynamics (measured by fMRI) associated with listening to speech in noise in elderly listeners with relatively normal peripheral hearing. We asked subjects to identify single words in quiet and in two multi-talker babble noise conditions $(+20$ and -5 dB SNRs). The behavioral results indicated that subjects performed similarly between the Quiet and +20 SNR conditions, both in accuracy and reaction time, but were less accurate and responded slower in the -5 SNR condition. There were performance-independent and SNR-dependent effects in the brain. A contrast of the +20 SNR and Quiet conditions revealed bilateral (especially left) auditory and prefrontal activation in noise despite equal behavioral performance (performance-independent but noisedependent activations). As noise increased and behavioral performance decreased (as in the -5 SNR condition), activation in these regions increased. These results reveal a cortical network involving acoustic analysis, working memory, and auditory attention associated with speech perception in noise by the elderly. [Work supported by NIH.]

1pSCc20. Computer simulations of intraoral pressure patterns during voiced stop consonant production. Jorge C. Lucero (Univ. Brasilia, Dept. Mathematics, 70910-900 Brasilia - DF, Brazil, lucero@unb.br), Laura L. Koenig (Haskins Labs. and Long Island Univ., Brooklyn, New York, NY 11201-8423, USA, koenig@ haskins.yale.edu)

Several past studies have used laryngeal and/or upper vocal tract models to explore aerodynamic control during production of obstruent consonants, yet many aspects of such control remain unclear. Of particular interest in this work is how varying laryngeal and vocal tract dimensions such as occur among men, women, and children influence phonatory behavior and the time course of intraoral pressure (Pio) increase during voiced stop consonant production. To maintain phonation during a voiced stop closure, Pio must remain below the subglottal pressure. Physiological and modeling studies indicate that adult speakers actively increase supraglottal volumes during voiced stops, which slows the rate of Pio increase. Speakers with smaller vocal tracts may either be less successful in slowing the rate of Pio buildup, or else may perform more extreme volumetric changes to achieve similar end results. The current study uses data recently collected on voicing and Pio during stop production in men, women, and children. We use a modified two-mass model of the vocal folds coupled to a model of the upper vocal tract to reproduce the observed Pio patterns, and explore how the behavior of the system reflects differences in anatomical and physiological dimensions. [Work supported by MCT/CNPq-Brazil.]

1pSCc21. The production of Mandarin and American English vowels by Mandarin-speakers. Jia-Shiou Liao (Department of Applied Foreign Languages, Chung Shan Medical University, No.100, Sec. 2, Dah-Ching St., 402 Taichung, Taiwan, jsliao@csmu.edu.tw)

This study examines whether there is any correspondence between how Mandarin-speakers pronounce Mandarin vowels and how they pronounce those vowels' English equivalents and non-equivalents. Twenty Mandarin graduate students, who spoke English as a second language and had stayed continuously in America for at least two years, produced a series of American English vowels in $/ \mathrm{hVd} /$ contexts from a randomly ordered list. To approximate English syllable structure, they also read a list of $/ \mathrm{hVdV} /$ syllables containing Mandarin vowels in the first syllable. The vowel in the second syllable is always a schwa. First- (F1) and second-formant (F2) values of each Mandarin and English vowel were analyzed. The statistical results suggest that the distances between the Mandarin corner vowels in the vowel space are similar to those between the English ones. The acoustic characteristics of the English vowels with Mandarin equivalents do not differ significantly from those equivalents. For the other English vowels, the formant values most often differ from those of their closest Mandarin equivalents in only one formant or in neither. For instance, Mandarin sub- jects do not distinguish English /u/ from the near-close near-back vowel in either formant - attributable to the absence of the near-close near-back vowel in Mandarin.

1pSCc22. The influence of the feature (sonorant) in lexical access in Spanish. Danny R. Moates (Ohio University, Department of Psychology, Porter Hall, Athens, OH 45701, USA, moates@ohio.edu), Emilia A. Marks (Ohio University, Department of Modern Languages, Athens, OH 45701, USA, markse@ohio.edu), Dario Barrera (Universidad de Sevilla, Facultad de Filologia, Departmento de Filologia Inglesa, Palos de la Frontera, s/n, 41004 Sevilla, Spain, dario@us.es)

Does the feature (sonorant) influence access to lexical entries? In current feature geometry, the feature (sonorant) is a root node feature, distinguishing resonants from obstruents. Marks, et al. (2002) demonstrated its influence in lexical access using the word reconstruction task in Spanish. Participants heard a nonword, e.g., dirmar, and were asked to change it into a real word (firmar) by changing just one consonant. Errors in recovering the correct word (firmar) were much higher when the word contained an obstruent that had been replaced with a resonant (nirmar) than when the word contained an obstruent that had been replaced by another obstruent (dirmar). Obstruent target words were easier to recover when the target segment and the replacing segment matched on the feature [sonorant]. Why do obstruents show this matching effect? The present study subdivided obstruents into stops and fricatives. Two experiments compared them separately to resonants in the word reconstruction task. Are fricatives alone or stops alone responsible for the observed obstruent effect? Results showed greater errors in the mismatch condition for both fricatives and stops, but significantly greater for fricatives than for stops, suggesting that the mismatch effect observed for obstruents is largely based on fricatives.

1pSCc23. Syllable production rate in conversational speech. Lawrence J. Raphael (Adelphi University, 1 South Avenue, Garden City, NY 11530, USA,raphael@adelphi.edu)

Most acoustic analyses of speech are based on samples obtained from carefully constructed corpora that are read or recited by subjects in a laboratory setting. Recently, speech scientists have attempted to obtain speech samples that are representative of a more casual, conversational style than might be obtained in the laboratory. One available and plentiful source of such speech samples can be found in "talkradio" shows in which listeners engage in phone conversations with radio hosts. Phone-in programs receive calls from thousands of callers each day across the continental United States. Moreover, the listeners who call generally speak in an essentially natural, unself-conscious manner, exhibiting a broad range of rates and emotional states. This paper reports the results of a study of syllable production rate from samples of speech obtained from a variety of types of phone-in programs, including those focusing on sports, politics and the arts. The data reveal that syllable production rates (in syllables per second) range from 3.05 to 7.50 . Subjective judgements of speech rate by naive subjects were found to correlate only moderately with actual rates of syllable production.

1pSCc24. A temporally stable representation of power spectra of periodic signals and its application to $\mathrm{F} 0$ and periodicity estimation. Hideki Kawahara (Faculty of Systems Engineering, Wakayama University, $930 \quad$ Sakaedani, 640-8510 Wakayama, Japan, kawahara@sys.wakayama-u.ac.jp), Masanori Morise (Faculty of Systems Engineering, Wakayama University, 930 Sakaedani, 640-8510 Wakayama, Japan, s055068@sys.wakayama-u.ac.jp), Toru Takahashi (Faculty of Systems Engineering, Wakayama University, 930 Sakaedani, 640-8510 Wakayama, Japan, tall@sys.wakayama-u.ac.jp), Ryuichi Nisimura (Faculty of Systems Engineering, Wakayama University, 930 Sakaedani, 640-8510 Wakayama, Japan, nisimura@sys.wakayama-u.ac.jp), Hideki Banno (Meijyo University, 501-1 Shiogamaguchi, Tenpakuku, 468-8502 Nagoya, Japan, banno@ccmfs.meijo-u.ac.jp), Toshio Irino (Faculty of Systems Engineering, Wakayama University, 930 Sakaedani, 640-8510 Wakayama, Japan, irino@sys.wakayama-u.ac.jp)

A unified framework for extracting F0 and periodicity is proposed based on a new formulation of STRAIGHT. Averaging power spectra, they are calculated using two time windows a half pitch period apart, yields a tempo- 
rally stable power spectrum for periodic signals (TANDEM spectrum). Applying consistent sampling theory also yields computationally efficient implementaiton of spectral envelope estimation used in STRAIGHT (STRAIGHT spectrum). Normalized TANDEM spectrum by STRAIGHT spectrum only represents periodicity information and can be used to detect periodicity both in the base-band (for F0 extraction) and other frequency bands (for mixed mode excitation). This unified framework provides a basis to represent the complex modes of voice excitation and enables greatly realistic remaking of extreme vocal expressions. [Supported by Grant in aids for scientific research and CrestMuse project.]

1pSCc25. Construction of MRI-based three-dimensional atlas of the human tongue for tongue modeling. Chao-Min Wu (Dept. Electrical Engineering, National Central University, \#300, Chung-Da Rd., 320 Chung-Li, Taiwan,wucm@ee.ncu.edu.tw), Sung-Yi Wang (Dept. Electrical Engineering, National Central University, \#300, Chung-Da Rd., 320 Chung-Li, Taiwan,945201073@cc.ncu.edu.tw)

The main purpose of this study is to build a MRI-based 3D tongue atlas for research on tongue morphometrics and physiological modeling of normal and disordered speech production. In this study, MRI data of 8 subjects ( 4 males and 4 females) were chosen from an orally-based MRI database of 20 male and 20 female college students without speech disorders. Sixteen landmarks were defined and selected from the $3 \mathrm{D}$ reconstructed MRI tongue images based on the subjects. Thin-plate spline analysis (TPS) was used to build a 3D tongue atlas for male and female subjects, respectively. Sagittal sections of the original MRI data were used to evaluate the accuracy of image registration and reconstruction. The resulted $3 \mathrm{D}$ tongue atlas was used to study subject-to subject, subject-to-atlas, and male-to-female morphometric variation. Preliminary results show the major difference among female subjects before and after the TPS analysis is in the area of tongue dorsum that is close to the velum and epiglottis, respectively. However, the major difference among male subjects is in the areas of tongue tip and body regardless of TPS analysis. In summary, our preliminary results imply that the 3D tongue atlas of female subjects show less subject-to-subject difference.

1pSCc26. Neural correlates on Chinese speech production: an fMRI study. Chao-Min Wu (Dept. Electrical Engineering, National Central University, \#300, Chung-Da Rd., 320 Chung-Li, Taiwan, wucm@ee.ncu.edu.tw), Yi-Syuan Lin (Dept. Electrical Engineering, National Central University, \#300, Chung-Da Rd., 320 Chung-Li, Taiwan, kenshin5718@yahoo.com.tw)

The aim of this study is to use functional magnetic resonance imaging (fMRI) method to find neural correlates on Chinese speech production. Three young normal adults (mean age $24 \mathrm{yrs}$ ) produced four tone Chinese characters "ma1, ma2, ma3, and ma4" in four ways: (A) normal speech, (B) mouthing speech, (C) unarticulated speech, (D) internal speech, and "ma1, ma1, ma1, and ma1" for the condition (E) normal speech. The fMRI results showed that the areas associated with the control of breathing for speech, vocalization, and hearing were the sensorimotor cortex, supplementary motor area, the superior temporal gyrus, the cerebellum, parietal association area, and the lingual gyrus. The areas associated with articulation were the sensorimotor cortex, the cerebellum, the inferior frontal gyrus, anterior and posterior cingulate gyrus, and the precuneus. The areas associated with tone variation were found in bilateral basal ganglia, hippocampal formation, and parietal association area, and the left pre-motor area, middle occipital gyrus, and the right cerebellum, superior temporal gyrus, and midbrain. Neural correlates of speech production and Chinese tone were all related to the cerebellum. In summary, our preliminary results showed the cerebellum plays an important role on Chinese speech production.

1pSCc27. Differences in the development of speech segmentation abilities in two French dialects. Thierry Nazzi (CNRS - Université Paris Descartes, 45 rue des Saints-Pères, 75006 Paris, France, thierry.nazzi @ univ-paris5.fr), Karima Mersad (CNRS - Université Paris Descartes, 45 rue des Saints-Pères, 75006 Paris, France, crocnotekary@yahoo.fr),Galina Iakimova (CNRS - Université Paris Descartes, 45 rue des Saints-Pères, 75006 Paris, France, galina.iakimova@free.fr), Megha Sundara (UCLA Department of Linguistics, 3125 Campbell Hall, Los Angeles, CA 90095 1543, USA, megha.sundara@humnet.ucla.edu), Linda Polka (McGill University, School of Communication Sciences and Disorders, Beatty Hall, 1266 Pine Avenue West, Montreal, QC H3G 1A8, Canada, linda.polka @mcgill.ca)

Speech segmentation skills develop in infancy and are influenced by many phonological properties of the native language and, in particular, the prosodic structure of the infant's native language. Studies using the HPP task show that American English infants appear to favor a stress-based procedure (Jusczyk et al., 1999) whereas Parisian French infants favor a syllable-based procedure (Nazzi et al., 2006), in line with a prosodic-based bootstrapping account of segmentation abilities (Nazzi et al., 1998). However, in a study using different stimuli, Polka and Sundara (2003) found results that might suggest a developmental trajectory for Canadian French infants that does not rely on syllable-based segmentation. Given that the stimuli used in both studies on French were different, both research teams tested their infant populations (at 8, 12, and 16 months of age) with the stimuli originally used by the other team The results suggest a complex interaction between specific stimuli and infants' native dialect, thus providing for the first time data suggesting that word segmentation is dialect specific. We will discuss how these findings, together with acoustic analyses, show that early word segmentation is influenced by linguistic (rhythmic/syllabic, dialectal) prosodic cues, extra-linguistic (speech style) prosodic cues, and distributional cues.

1pSCc28. Effect of consonant gemination on vowel coarticulation Maria-Gabriella Di Benedetto (University of Rome, Infocom Dept. Via Eudossiana 18, 00184 Rome, Italy, gaby@acts.ing.uniroma1.it), Daniele Domenicali (University of Rome, Infocom Dept. Via Eudossiana 18, 00184 Rome, Italy, dome@newyork.ing.uniroma1.it)

As well known V1CV2 structures are typically characterized by two different phenomena: the first vowel V1 spectral properties are affected by the presence and nature of the second vowel V2 (anticipatory effect), and V2 is influenced by the presence of the first one (cross-over effect). The intensity of anticipatory and the cross-over effects are language dependent and can vary significantly. Both effects have been observed in the past to be present in the Italian language through the analysis of VCV nonsense words. The Italian language has however a unique property, that is distinctive consonant gemination, i.e. consonant length is phonemic; two different words may thus differ by the sole presence or absence of gemination in one of this consonants. The Italian language offers therefore the ideal framework for analyzing the effect of gemination on across-consonant coarticulation. The object of this work is to analyze whether and how the presence of gemination may affect the acoustic properties of vowels in V1CCV2 words. First results of acoustic analysis of V1CV2 as well as their V1CCV2 counterparts, spoken by Italian native speakers, tend to show that gemination may have an effect on across-consonant coarticulation, with specific peculiarities for anticipatory vs. cross-over effects.

1pSCc29. Enhancement of voiced and voiceless stop categories in infant-directed speech to 11-month-old infants. Gina C. Cardillo (University of Washington, Dept. of Speech \& Hearing Sciences, and Institute for Learning \& Brain Sciences, Box 357988, Seattle, WA 98195, USA, ginacc@u.washington.edu), Jennifer R. Perry (University of Washington, Dept. of Speech \& Hearing Sciences, and Institute for Learning \& Brain Sciences, Box 357988, Seattle, WA 98195, USA jperry3@u.washington.edu), Patricia K. Kuhl (University of Washington, Dept. of Speech \& Hearing Sciences, and Institute for Learning \& Brain Sciences, Box 357988, Seattle, WA 98195, USA, pkkuhl@u.washington .edu)

It has been proposed that infant-directed speech (IDS) increases the discriminability of phonetic categories by exaggerating the acoustic differences between phonetic units (Kuhl et al., 1997, Science). However, reports show conflicting results on whether this principle holds for consonants. The current study measured English /p/ /b/ /t/ /d/ in both IDS and adult-directed 
speech (ADS) from connected speech in 27 mothers addressing their 11month-old infants. Findings supported the exaggeration hypothesis. The mean VOT of $/ \mathrm{p} /$ was longer in IDS than ADS, and approached significance for $/ \mathrm{t} /$. The mean VOT difference between $/ \mathrm{p} /$ and $/ \mathrm{b} /$ was greater in IDS than in ADS. Similarly, when phonemes were grouped into voiceless and voiced categories, the difference between categories was significantly greater in IDS than ADS. We will discuss ways to measure AD-ID differences. [Work supported by NIH HD37954.]

1pSCc30. Speaker-specific patterns of token-to-token variability Christine Mooshammer (Haskins lab and MIT Research Lab of Electronics, 300 George Street, Suite 900, New Haven, CT 06511, USA, tine@haskins.yale.edu), Pascal Perrier (ICP/GIPSA-lab, INPG, 46 Avenue Félix Viallet, 38031 Grenoble Cedex 01, France, Pascal.Perrier@gipsa-lab.inpg.fr), Susanne Fuchs (ZAS, Schuetzenstr. 18, 10117 Berlin, Germany, fuchs@zas.gwz-berlin.de)

One explanation for token-to-token variability is neural noise corrupting the motor commands. In a previous study using a two-dimensional tongue model, adding neural noise to motor commands was able to account for shapes and orientations of the dispersion ellipses of tongue positions for non-high vowels only. It was concluded that additional sources shaping lingual variability patterns of vowels should be examined: (a) stabilization of the tongue by lateral palate contact, (b) palate shape and (c) perceptual constraints due to the crowdedness of the vowel space. Variability patterns of three speakers of German were analyzed by means of EMMA, EPG, and formant frequencies. Only the most variable speaker used lingual-palatal contact to reduce lingual variability for high front vowels. The other two speakers achieved articulatory vowel targets in a very precise manner. A flat palate shape can explain the limited lingual variability of one of these two. Only for this speaker did the acoustic distance between vowel targets significantly limit acoustical variability with smaller Bark ellipsoid volumes in regions with closer neighbors. For the other precise speaker, neither palate shape nor perceptual demands were relevant. We conclude that the relative importance of the factors analyzed varies across speakers.

1pSCc31. Neural mechanism for understanding speakers' intentions: developmental analyses of children with and without communication disorders. Satoshi Imaizumi (The Prefectural Univ. of Hiroshima, 1-1 Gakuen-machi, 723-0053 Mihara, Japan, imaizumi@pu-hiroshima.ac.jp)

Ability to understand speakers' intentions through linguistic contents and affective prosody is examined for children between 5 and 12 years-old with and without communication disorders. Four types of spoken short phrases, expressing admiration, sarcasm, blame, and humor or joke, were presented. For each stimulus, the subjects were asked to choose between two cards, one with a written word "praise" and another with "no praise" accompanied by corresponding drawings. For children without any communication disorders, the percentage of the correct judgment of speaker intentions was high and stable for admiration and blame phrases which have congruent linguistic and affective valences. It was significantly low for 6-year-old children and increased with age for the sarcastic or joking phrases which have incongruent linguistic and affective valences. The percent correct was significantly lower for autistic children than normally developing children particularly for the phrases with incongruent valences. Although a significant difference was found between children with severe- and mild-hearing impairment, no significant difference was found in the percent correct between the congruent and incongruent phrases for them. Based on these results together with brain activation analyses using fMRI (Imaizumi et al., 2004, 2006), a model of neural mechanism for understanding speakers' intentions is discussed.

1pSCc32. Perception of two English front vowels by L1 Dutch speakers with different regional accents. Paola Escudero (University of Amsterdam, Institute of Phonetic Sciences, Spuistraat 210, 1012 VT Amsterdam,
Netherlands, P.R.EscuderoNeyra@uva.nl),Ellen Simon(Ghent University, English Department, Rozier 44, 9000 Ghent, Belgium, Ellen.Simon@UGent .be)

This paper reports the results of a categorization task of the English vowels "epsilon" and "ash" by native speakers of Dutch. Dutch has only one member of the contrast ("epsilon"), the acoustic realization of which is subject to considerable regional variation (Adank et al., 2007). Previous research has shown that that there is asymmetrical lexical competition between English words containing "epsilon" and "ash" for native speakers of Dutch (Weber \& Cutler, 2004; Escudero et al., in press). Weber \& Cutler (2004) argue that "epsilon" is the dominant category for native speakers of Dutch, because it is phonetically closer to the L1 Dutch sound. The present study investigates whether the dominant category may vary depending on the speakers' L1 dialect by examining how two groups of Dutch-speaking participants from different dialect regions (the Randstad region in the Netherlands and East-Flanders in Belgium) perceive the English vowels "epsilon" and "ash." Preliminary results indicate that participants from these two area's do indeed categorize English "epsilon" and "ash" differently as the result of the acoustic properties of their L1 vowel "epsilon" (F1, F2 and duration).

1pSCc33. Motor equivalent strategies in the production of $/ u /$ in perturbed speech. Jana Brunner (Humboldt-Universität Berlin/Gipsa-lab, INP Grenoble, ZAS, Schützenstr. 18, 10117 Berlin, Germany, brunner@zas.gwz-berlin.de), Phil Hoole (Institut für Phonetik und Sprachverarbeitung, Schellingstr. 3, 80799 München, Germany, hoole@phonetik.uni-muenchen.de), Pascal Perrier (ICP/GIPSA-lab, INPG, 46 Avenue Félix Viallet, 38031 Grenoble Cedex 01, France, Pascal.Perrier @ gipsa-lab.inpg.fr)

Several articulatory strategies are available during the production of /u/, all resulting in a similar acoustic output. For example, speakers can protrude the lips and compensate for that by widening the velar constriction. This study investigates whether speakers use this relation under perturbation. Five speakers were provided with palatal prostheses which were worn for two weeks. Speakers were instructed to make a serious attempt to produce normal speech. Their speech was recorded via EMA and acoustics several times over the adaptation period. Formant values of / $\mathrm{u} /$-productions were measured. Velar constriction width and lip protrusion were estimated. For four speakers a correlation between constriction width and lip protrusion was found. A correlation between lip protrusion and F1 or F2 was rare and no correlation occurred between constriction size and either of the formants. The acoustic output was thus constant. The results show that under perturbation speakers use motor equivalent strategies. The correlation between constriction size and lip protrusion is stronger than in studies investigating unperturbed speech (e.g., Perkell et al., 1993). This could be because under perturbation speakers are inclined to try out several strategies in order to reach the acoustic target and the co-variability might thus be greater.

1pSCc34. Using sets of combs to control pitch estimation errors. Jean-Sylvain Lienard (LIMSI-CNRS, BP133, 91403 Orsay Cedex, France, jean-sylvain.lienard@limsi.fr), Claude Barras (LIMSI-CNRS, BP133, 91403 Orsay Cedex, France, claude.barras@limsi.fr), Francois Signol (LIMSI-CNRS, BP133, 91403 Orsay Cedex, France, francois.signol @limsi.fr)

We analyze the errors of a Pitch Estimation Algorithm using the Pitch Function (response of the periodicity estimator as a function of the frequency parameter $F_{c}$ ). The estimator's maximum response for a single signal of fundamental frequency $F_{0}$ is expected to occur for $F_{c}=F_{0}$. Actually the pitch function exhibits many secondary peaks which occasionally cause the errors. When several signals are mixed, secondary peaks of a signal may take over the main peak of the other. By taking as periodicity estimator the correlation of the spectrum module with a uniform infinite spectral comb of fundamental frequency $F_{c}$ we show that each peak corresponds to a particular value of the ratio $F_{c} / F_{0}=p / q$ ( $p$ and $q$ integers $\geqslant 1$ ). It follows that some secondary peaks can be canceled either by augmenting the comb with intermediary negative teeth, or by setting to zero some of its teeth. These modified combs can be viewed as linear combinations of uniform combs of different $F_{c}$ s. The present study aims at precisely defining and combining the modified combs so that the main peaks of the new Pitch Function reliably 
indicate the $F_{0} \mathrm{~s}$ of the components. Examples are given on mixtures of voiced segments extracted from natural speech.

1pSCc35. Articulatory exaggerations during infant directed speech Jordan R. Green (University of Nebraska, 3180 Barkley Center, Lincoln, NE 68583-0738, USA, jgreen4@unl.edu), Ignatius Nip (San Diego State University, 5500 Campanile Dr., San Diego, CA 92182-1518, USA, inip@mail.sdsu.edu), Erin Wilson (Waisman Center, 1500 Highland Avenue, Madison, WI 53705-2280, USA, emhillman@wisc.edu), Antje Mefferd (University of Nebraska, 3180 Barkley Center, Lincoln, NE 68583-0738, USA, amefferd@unlserve.unl.edu)

Although a growing body of literature suggests a principal role for observational learning in the acquisition of speech, the facial movements of infant directed speech (IDS) have rarely been studied. Identifying both the auditory and visual features of IDS is an essential step toward understanding the contribution of environmental stimulation to the development of speech and language. The current project uses three-dimensional motion capture technology to describe how parents modify their articulatory movements when communicating with their infants. The following three experimental questions will be addressed: (1) Do parents exaggerate articulatory gestures during IDS?; (2) If so, are there individual differences among parents in the degree of IDS?; and (3) Is there a strong association between acoustic and movement characteristics of IDS? Mouth movements were recorded from twenty-four mothers while speaking to their infants and to an unfamiliar adult. Mouth shapes during four target vowels were measured for differences in maximum vertical separation, maximum horizontal spread, and duration across speaking conditions. The result indicated that the majority of mothers exaggerated their lip movements when speaking to their infants. Speculation is made regarding the potential role of articulatory exaggerations in early speech learning.

1pSCc36. Evaluation of the Pitch Estimation Algorithms in the monopitch and multipitch cases. Francois Signol (LIMSI-CNRS, BP133, 91403 Orsay Cedex, France, francois.signol@limsi.fr), Claude Barras (LIMSI-CNRS, BP133, 91403 Orsay Cedex, France, claude.barras@limsi.fr), Jean-Sylvain Lienard (LIMSI-CNRS, BP133, 91403 Orsay Cedex, France, jean-sylvain.lienard@limsi.fr)

Reliably tracking the fundamental frequency $F_{0}$ of the components is an important step in the separation of superimposed speech signals. Several Pitch Estimation Algorithms are potentially usable and a rigorous evaluation method is needed in order to compare them. However, even in the monopitch case, many variations between them render such a comparison difficult . The extent of the $F_{0} \min -F_{0} \max$ interval, the use of a priori information on the whole sequence or database, and above all the arbitrary setting of the voicing threshold, yield large differences in the results. These biases can be removed by setting the $F_{0}$ bounds to fixed values acceptable for many voices, by proceeding with the evaluation on a strictly frame-to-frame basis, and by fixing the voicing threshold in order to get an equal error rate for overvoiced and undervoiced frames. In the multipitch case any frame may exhibit 0,1 , or 2 valid voicings according to the coincidence between the voiced and unvoiced parts of both signals. This problem is treated by defining a similarity measure linking the PEA's hypotheses to the pitch values of the isolated signals. The proposed methodology is applied to several PEAs, using the Keele database in monopitch and multipich setups.

1pSCc37. An acoustic investigation of the Swedish child's acquisition of obstruent place of articulation. Jan Van Doorn (Umeå University, Department of Clinical Sciences, SE90187 Umeå, Sweden, jan.vandoorn@logopedi.umu.se), Fredrik Karlsson (Umeå University, Department of Language Studies, SE90187 Umeå, Sweden, fredrik.karlsson@ling.umu.se), Kirk P. Sullivan (Umeå University, Department of Language Studies, SE90187 Umeå, Sweden, kirk.sullivan@ling .umu.se)

Speech produced by children in the initial stages of development does generally not uphold as many phonetic distinctions as speech sounds produced by adults. A child's productions of different target words may therefore have similar acoustic properties and result in homonyms being perceived by the adult observer. This study presents a longitudional investigation into the development of place of articulation from nondistinctive to distinctive productions in word-initial obstruents produced by 22 Swedish children (aged 18 - 48 months). The data was collected through monthly recordings, approximatelly one year per child. The acoustic correlates analysed were spectral diffuseness, spectral skewness and spectral tilt for plosives and spectral skewness, spectral kurtosis, spectral variance and F2 onset frequency for fricatives. The results show a developmental trend in spectral skewness that is indicative of a increasing number of acquired phonetic contrasts. Spectral tilt change, F2 onset frequency, spectral mean and spectral variance provide evicence of within-category refinement wich is argued to be caused primarilly by advancements in motor control.

1pSCc38. Nonlinear dynamics of speech categorization: critical slowing down and critical fluctuations. Leonardo Lancia (LPL, Aix-Marseille Université \& CNRS, 29, avenue Robert Schuman, 13100 Aix-en-Provence, France, leonardo.lancia@lpl-aix.fr), Noel Nguyen (LPL, Aix-Marseille Université \& CNRS, 29, avenue Robert Schuman, 13100 Aix-en-Provence, France, Noel.Nguyen@lpl-aix.fr), Betty Tuller (National Science Foundation, 4201, Wilson Boulevard, Arlington, VA 22230, USA, tuller@ccs.fau .edu)

The dynamical system view of speech perception assumes that speech categorization depends on both the acoustic properties of the incoming sound and the state of the perceptual system (indexed by the subject's responses to preceding sounds). Tuller and colleagues [1] systematically manipulated the order of presentation of stimuli from a "say"--"stay" continuum in a binary-choice word identification task. Listeners' responses were modeled using a nonlinear dynamical system whose point attractors were associated with perceptual categories. In the present study, we asked French speakers to categorize stimuli on a continuum from "cèpe" / sep/ to "steppe" /step/. Our results provide support for two yet untested predictions from the model: the occurrence of a) critical slowing down and b) critical fluctuations

Critical slowing down relates to the increase in relaxation time that occurs as the system approaches points of instability. Critical fluctuations refer to the system's increased sensitivity to random fluctuations near the points of instability. [Work supported by the CNRS \& French Ministry of Research, and by NSF.] [1] P. Case, B. Tuller, D. Mingzhou, and J.A.S. Kelso. Evaluation of a dynamical model of speech categorization. Perception and Psychophysics, 57:977-988 (1995).

1pSCc39. The effects of vowel identification training on sentence-level speech recognition. Carolyn Richie (Communication Disorders, Butler University, 4600 Sunset Ave., Indianapolis, IN 46208, USA, crichie@butler .edu)

Recent work examined the effects of a novel computer-based approach to speech recognition training involving vowel identification [C. Richie, J. Acoust. Soc. Am. 121, 3045 (2007)]. When vowel identification training was done under auditory-visual conditions with noise participants demonstrated modest improvements in sentence recognition, and when training was done under visual-only conditions participants failed to show improvements in sentence recognition. However, sentence recognition was assessed for the CID Everyday Sentences in terms of words correctly recognized. Improvements in speech recognition may have occurred following training on a level lower than the word, and gone unrecognized given this scoring method. The purpose of the present study is to determine whether vowel identification training leads to improvements in speech recognition on a test of sentence recognition, as measured by correct identification of consonants and vowels. These findings have implications for sentence recognition test scoring methods. The role of acoustics in improving speech recognition will also be discussed. (Supported by Butler University HAC grant \#027096).

1pSCc40. An acoustic study on voiceless retroflex and dental sibilants of young native Min speakers in Taiwan. Yu-Ying Chuang (Graduate Institute of Linguistics, Grad. Inst. of Linguistics, National Taiwan Univ., 1, 
Roosevelt Rd. Sec. 4, 106 Taipei, Taiwan, r95142001@ntu.edu.tw),Janice Fon (Grad. Inst. of Linguistics, National Taiwan Univ., 1, Roosevelt Rd. Sec. 4, 106 Taipei, Taiwan, jfon@ntu.edu.tw)

The Mandarin spoken in Taiwan is influenced by Min, a Southern Chinese dialect, in many perspectives. Due to the absence of retroflex sibilants in Min, previous studies claimed that retroflex sibilants are replaced by their dental counterparts. Recent studies, however, showed that retroflex and dental sibilants are actually distinguished. Such contradictory result was due to the fact that different speaker groups were targeted at - one on older generation, acquiring Min as L1, whereas the other on younger generation, acquiring Min as L2. Since age and the acquisition order of Min were involved, it was unknown which factor affected the realization of retroflex and dental sibilants more. To clarify this point, we targeted at the younger generation that acquired Min as L1. Acoustic measurements were taken on all sibilants. Results showed that retroflex and dental sibilants had distinctive realization, suggesting age factor to be more determent. Gender effect was found significant, in which females showed a greater distinction than males. Moreover, retroflex sibilants pronounced in syllables were significantly different from those in sentences. Future studies could be done on the other group - older generation, acquiring Min as L2. Also, whether the frequency of using Min plays a role awaits further exploration.

1pSCc41. A comparison of native speaker and American adult learner Vietnamese lexical tones. Allison Blodgett (University of Maryland College Park, Center for Advanced Study of Language, 7005 52nd Ave, College Park, MD 20742, USA, ablodgett@casl.umd.edu), Jessica Bauman (University of Maryland College Park, Center for Advanced Study of Language, 7005 52nd Ave, College Park, MD 20742, USA, jbauman@hesp.umd.edu), Anita Bowles (University of Maryland College Park, Center for Advanced Study of Language, 7005 52nd Ave, College Park, MD 20742, USA, abowles@ casl.umd.edu), Lykara Charters (University of Maryland College Park, Center for Advanced Study of Language, 7005 52nd Ave, College Park, MD 20742, USA, lykarac@mac.com), Anton Rytting (University of Maryland College Park, Center for Advanced Study of Language, 7005 52nd Ave, College Park, MD 20742, USA, crytting@casl.umd.edu), Jessica Shamoo (University of Maryland College Park, Center for Advanced Study of Language, 7005 52nd Ave, College Park, MD 20742, USA, jshamoo@casl.umd.edu), Matthew Winn (University of Maryland College Park, Department of Hearing \& Speech Sciences, 0100 Lefrak Hall, College Park, MD 20742, USA, mwinn@hesp.umd.edu)

This study introduces a modification of the traditional carrier sentence elicitation task, provides new native speaker data regarding aspects of pitch and voice quality in Vietnamese lexical tones, and provides an empirical description of non-native speaker errors in tone production. Participants produce a series of three-word utterances in response to target words that appear individually on a computer screen in one of four colors. In this way, participants actively describe a changing event, while critical components remain constant: the identity of the word preceding the target, the target position (utterance medial), and the target's focus structure. Native speaker results are consistent with existing descriptions of fundamental frequency contours and creakiness. In addition, they provide evidence supporting claims that breathiness can occur in two different tones: the falling tone (huyen) and curve tone (hoi). Non-native speaker results suggest that adult learners struggle with voice quality distinctions and pitch shape.

1pSCc42. Lip protrusion and constriction in anticipation in English and French. Aude Noiray (Haskins Laboratories, 300 George St, New Haven, CT 06511, USA, aude.noiray@haskins.yale.edu), Lucie Ménard (Département de linguistique et de didactique des langues, Université du Québec à Montréal, Case postale 8888, succursale Centre-ville, Montréal, QC H3C 3P8, Canada, menard.lucie@uqam.ca), Marie-Agnès Cathiard (Centre de Recherche sur l'Imaginaire (CRI), Université Stendhal, Grenoble 3, BP 25, 38040 Grenoble cedex 9, France, Marie-Agnes
.Cathiard@u-grenoble3.fr),Christian Abry(Centre de Recherche sur l'Imaginaire (CRI), Université Stendhal, Grenoble 3, BP 25, 38040 Grenoble cedex 9, France, christian.abru@u-grenoble3.fr)

Though lip constriction is acoustically the most robust parameter for rounding, most studies on anticipatory coarticulation relied on the investigation of protrusion via EMG, optoelectrical systems or audiovisual processing. These various methods produced inconsistent anticipatory profiles across studies or even speakers. The Movement Expansion Model (MEM) of Abry and Lallouache has explained vocalic anticipatory coarticulation in four French adults and eight children via lip constriction, showing idiosyncratic behaviours, i.e. personal regression slopes but with a lawful variability validated by high correlation coefficients. In order to describe global anticipatory movement, the current study included measures of both protrusion and constriction over time. Four American English and four Canadian French adults were audio visually recorded, uttering $[\mathrm{iC}[\mathrm{n}] \mathrm{u}] \mathrm{se}-$ quences, in which $\mathrm{C}[\mathrm{n}]$ corresponded to a varying number of intervocalic consonants. Lip protrusion was measured via an Optotrak system, and constriction via a Lip-Shape Tracker (from ICP Grenoble). We found that when tested on lip area control, English speakers joined French speakers in a regular MEM temporal pattern, displaying their own anticipatory behaviour, with personal regression slope or expansion coefficient.

1pSCc43. The contribution of vowel production to the intelligibility and accentedness of nonnative speech. Jessica E. Alexander (Dept. of Psychology, Emory University, 532 Kilgo Cir, Atlanta, GA 30322, USA, jessica.alexander@emory.edu), Sabrina K. Sidaras (Dept. of Psychology, Emory University, 532 Kilgo Cir, Atlanta, GA 30322, USA, ssidara@emory.edu), Lynne C. Nygaard (Dept. of Psychology, Emory University, 532 Kilgo Cir, Atlanta, GA 30322, USA, 1nygaar@emory.edu)

The present study investigated the extent to which the perception of accentedness and intelligibility in nonnative speech depends on the specific acoustic realization of vowels produced by nonnative speakers. Twelve native speakers of Korean were recorded producing both word- and sentencelength English utterances. Acoustic analyses, including measures of vowel duration and formant frequencies, were conducted on tokens of individual English vowels identified from the word-length utterances. Vowel tokens were selected from a variety of phonetic contexts and were compared to productions of the same vowels produced in the same contexts by native English speakers. A separate set of native English-speaking listeners judged accentedness and intelligibility from the sentence-length utterances. The results showed that both the duration and spectral characteristics of Koreanaccented vowels were related to overall sentence intelligibility and ratings of perceived accentedness. These findings suggest that the detailed acoustic realization of the temporal and spectral structure of non-native vowels contributes to global measures of perceived accentedness and overall intelligibility.

1pSCc44. Word-initial voiceless geminate stops: production and perception. Rachid Ridouane (Laboratoire de Phonétique et PhonologieUMR7018 CNRS/Sorbonne Nouvelle, 19 rue des Bernardins, 75005 Paris, France, rachid.ridouane@univ-paris3.fr), Pierre Halle (Laboratoire de Phonétique et Phonologie-UMR7018 CNRS/Sorbonne Nouvelle, 19 rue des Bernardins, 75005 Paris, France, pierre.halle@univ-paris5.fr)

Quantity contrasts (i.e., singleton vs geminate consonants, short vs long vowels) are common in the languages of the world. Yet, most singletongeminate contrasts occur in word-medial position. Languages that allow gemination in other than word-medial position (word-initially or wordfinally) are quite few. Even less frequent is the occurrence in the languages of the world of word-initial voiceless geminate stops. Pattani Malay (Abramson, 1986), Turgovian Swiss German (Kraehenmann, 2003), Tashlhiyt Berber (Ridouane, 2007), are the only documented languages that allow singleton-geminate contrasts word-initially for all native consonants, including both voiced and voiceless plosives. In this work we deal with wordinitial voiceless geminate stops in Tashlhiyt Berber and present results from 3 experiments: 1) a production study including acoustic and electropalatographic measurements 2) a native-listener experiment with labelling and discrimination, and 3) a cross-language perceptual experiment, with listeners of 
two languages differing in germination: Italian (in which gemination only occurs word-medially) and French (in which gemination only occurs between words in continuous speech).

1pSCc45. Inter-speaker variability and the articulatory-acoustic relations in German and English /sh/. Susanne Fuchs (ZAS, Schuetzenstr. 18, 10117 Berlin, Germany, fuchs@zas .gwz-berlin.de), Martine Toda (CNRS \& ENST, 46, rue Barrault, 75634 Paris, France, martinetoda@yahoo.co.jp)

The aim of this study is to investigate speaker-specific articulatoryacoustic relations and their potential causes in the realisation of /sh/. By means of electropalatographic and acoustic data for 12 German and 12 English native speakers (6 males and 6 females for each language), we obtained the following results: 1) relatively invariant COG values of the frication noise among the subjects, 2) huge inter-speaker variability in the articulatory place of articulation, especially for the males. We suggest that in the realisation of $/ \mathrm{sh} /$ motor equivalence principles can be used speakerdependently. In particular, the length of palatal channel, the size of the front cavity (including its sublingual portion) as well as lip protrusion are the relevant articulatory parameters involved in the adjustment of the frication noise. Speaker-specific articulatory strategies will be discussed in the light of the formant transition characteristics, palatal morphology, and the prototypical articulatory patterns will be illustrated by means of acoustic modelling.

1pSCc46. Pitfalls of spectrogram readings of flaps. Minjung Son (Yale University and Haskins Labs, 300 George St. Suite 900, New Haven, CT 06511, USA, son@haskins.yale.edu)

Several acoustic studies have revealed stable characteristics of the speech signal for rhotics, whereas articulatory studies have found different articulatory strategies across speakers for producing these segments (Ladefoged and Maddison, 1996; Tiede et al., 2004). However, no study has showed that the phonologically reduced flap gesture, which is argued to be a lateral in the underlying representation, can sometimes fail to be reflected in the acoustic correlates of an articulatory event. Using electromagnetic midsagittal articulometry (Perkell et al., 1992), a case study has been conducted for Korean. Productions of two Seoul-Korean speakers were collected using real words in the within-word condition. The subjects read target words within a single accentual phrase, as in Jun (1993), and repeated eight times. Each target word (/ili/ and /ala/) appeared in its own distinct natural sentence at two speech rates (fast and comfortable). A total of 32 tokens were available for analysis. Tongue tip gestures were examined simultaneously with spectrograms. Results from one speaker indicated that sometimes no acoustic correlates of the tongue tip gesture for a flap were present (6.3\% of productions) although articulation was obviously produced [Work supported by NIH DC00403.]

1pSCc47. Clicks with noisy accompaniment in West !Xoon. Sven Grawunder (Max Planck Institute for Evolutionary Anthropology, Deutscher Platz 6, 04103 Leipzig, Germany, grawunder@eva .mpg.de), Christfried Naumann (Max Planck Institute for Evolutionary Anthropology, Deutscher Platz 6, 04103 Leipzig, Germany, naumann@eva .mpg.de)

The current study is concerned with ingressive stops (clicks) with a noisy accompaniment exemplified by data from West !Xoon, a Taa variety ("!Xóó", Tuu/Southern Khoisan) spoken in Namibia. Within the obstruent system of West !Xoon, we describe (1) aspirated clicks, (2) clicks followed by a laryngeal fricative, (3) clicks with an aspirated uvular accompaniment, and (4) clicks followed by a uvular fricative. In previous analysis for the variety of East !Xoon (Traill and Ladefoged, 1994), (2) were analysed as series of "delayed aspiration", while the contrast between (1) and (3) was ignored. A novelty discovery are prenasalized "voiced" clicks followed by $[\mathrm{h}]$, the voiced counterparts of clicks with "delayed aspiration", which are probably absent in East !Xoon. We present an acoustic analysis of alveolar and palatal clicks based on field data of 6 speakers ( 3 male $/ 3$ female), where for each click type plus noise accompaniment three tokens in \#CV context had been recorded repeatedly. As descriptive parameters served durational measures of burst length, cluster length, length of aspiration delay, and spectral measures of burst and friction parts. In opposite to the previous distinction our findings suggest a clear 3-way distinction of clicks (1-3) with noisy accompaniment.

1pSCc48. Blind source separation and directional audio synthesis for binaural auralization of multiple sound sources using microphone array recordings. Banu Gunel (I-Lab Multimedia and DSP Research Group, Centre for Communication Systems Research, University of Surrey, GU2 7XH Guildford, UK, B.Gunel@surrey.ac.uk), Huseyin Hacihabiboglu (I-Lab Multimedia and DSP Research Group, Centre for Communication Systems Research, University of Surrey, GU2 7XH Guildford, UK, h.hacihabiboglu@surrey.ac.uk), Ahmet Kondoz (I-Lab Multimedia and DSP Research Group, Centre for Communication Systems Research, University of Surrey, GU2 7XH Guildford, UK, A.Kondoz@surrey.ac.uk)

Microphone array signal processing techniques are extensively used for sound source localisation, acoustical characterisation and sound source separation, which are related to audio analysis. However, the use of microphone arrays for auralisation, which is generally related to synthesis, has been limited so far. This paper proposes a method for binaural auralisation of multiple sound sources based on blind source separation (BSS) and binaural audio synthesis. A BSS algorithm is introduced that exploits the intensity vector directions in order to generate directional signals. The directional signals are then used in the synthesis of binaural recordings using head related transfer functions. The synthesised recordings subsume the indirect information about the auditory environment conveying the source positions and the acoustics similar to dummy head recordings. Test recordings were made with a compact microphone array and a dummy head in different indoor environments. Subjective listening tests were performed to compare the synthesized recordings with the actual ones for their performance in providing presence, spaciousness and envelopment

1pSCc49. Bilinguals' and monolinguals' reaction-time in two language contexts: evidence for the double phonetic representation in bilinguals Adrian Garcia-Sierra (University of Washington, Institute for Learning and Brain Sciences, Box 357988, Seattle, WA WA 98195-7988, USA gasa@u.washington.edu), Craig Champlin (University of Texas, 1 University Station A1100, Austin, TX 78712-0114, USA, champlin@austin.utexas .edu)

The time between the presentation of a stimulus and the response given to it is occupied by a series of mental operations reflecting the decision perse. Reaction-time (RT) can be thought as the duration of the mental operations behind making a decision. Small RT values involve less mental operations than larger RT values. In speech categorization tasks, sounds with clear phonetic membership produce small RT values, and ambiguous speech sounds produce large RT values. In the present investigation a Go-No-Go task was implemented to assess RT from Spanish-English bilinguals $(\mathrm{N}=27)$ and English monolinguals $(\mathrm{N}=27)$ in two language contexts. Participants were asked to identify the speech sound "ta" from a 10 token speech continuum varying from -20 to +25 ms of VOT. It was expected that bilinguals, but not monolinguals, would perceive +VOT stimuli as clear representations of ' $t$ ' (faster RT) in the Spanish language context, but as ambiguous representations of ' $t$ ' in the English language context. The results confirmed the expectations. That is, language contexts affected the speed by which mental operations were produced in bilinguals. This outcome is in agreement with the idea that bilinguals possess a double phonemic representation. Challenges and limitations of the present study are discussed.

1pSCc50. Is there a sensitive period for representation of phoneme sequences? Mary Anne Welton (University of Prince Edward Island, 550 University Ave, Charlottetown, PE C1A 4P3, Canada, mwelton@upei.ca), Annabel J. Cohen (University of Prince Edward Island, 550 University Ave, Charlottetown, PE C1A 4P3, Canada, acohen@upei.ca)

A key issue in development of speech perception and production concerns the possible existence of an early sensitive period that facilitates language acquisition. Spoken language acquisition entails representation of grammatical sequences of phonemes. In a two-phase experiment, 120 participants in three age groups (pre-adolescents, adolescents, young adults) were presented with 64 words, 16 each of English, English pronounceable 
non-words, Spanish, and Mandarin. The task was to determine if the word was English. All age groups performed well, indicating that the words had been attended. A subsequent surprise recognition phase presented 64 words again, one half of which were old and one half new. Participants judged whether each word had previously been presented. Here performance overall was poorest for Spanish and Mandarin, and children performed relatively more poorly on English non-words as compared to the older groups, but showed no age-relative disadvantage for Chinese. Arguing that evidence for a sensitive period arises if children perform relatively better than older participants on foreign words as compared to English words or English nonwords, the recognition results are consistent with the proposal of a sensitive period for phoneme sequence representation. The paradigm provides a new way of examining the sensitive period hypothesis. (supported by NSERC)

1pSCc51. A detailed biomechanical finite element tongue model Reiner F. Wilhelms-Tricarico (Rewtnode, 27 Old Bay Road, Belchertown, MA 01007, USA, rewtnode@gmail.com), Maureen Stone (Vocal Tract Visualization Lab, Depts. of Biomedical Sciences and Orthodontics, University of Maryland Dental School, 650 W. Baltimore St., Baltimore, MD 21201, USA, mstone@umaryland.edu), Mark Carlson (MSC.Software Professional Services, 4332 Brookside Avenue, Minneapolis, MN 55436, USA, mark.carlson@mscsoftware.com), Paul Buscemi (Restore Medical, Inc, 2800 Patton Rd, Roseville, MN 55113, USA, pbuscemi@ restoremedical .com)

For research on surgical modifications of the tongue, e.g., implants for treatment of sleep apnea, we built a detailed finite element model of the human tongue, to experimentally estimate and investigate muscle activations corresponding to observed movement data, and to study possible effects of tissue modifications. Most of the extrinsic and all intrinsic muscles of the tongue are represented as parametrized volumes within the tongue, and muscle fiber directions are specified by a tangent in curvilinear coordinate systems of parametric solids that overlay each muscle region. Fiber regions of different muscles may overlap, for example, for the transverse and vertical intrinsic muscles. Mixed Ogden type hyper-elastic models of strain energy density are used to represent passive tissue properties, and active con- traction in muscle fibers is emulated by changing externally specified activation parameters in an anisotropic component of the strain energy density. The activation can vary over time and in its spatial distribution over a muscle, which is accomplished by specifying center point and spread of a Gaussian distribution in the muscle region. The tongue model is realized as a finite element implementation with large displacement/large strain calculations using incompressible 8-node elements in a commercial FE code (Marc by MSC-Software).

1pSCc52. Stable patterns of articulatory movements across inter-subject variability. Patrizia Bonaventura (Case Western Reserve University, 11206 Euclid Ave, Room 405, Cleveland, OH 44106, USA, pxb72@case.edu), Paul Taylor (Case Western Reserve University, 11206 Euclid Ave, Room 405, Cleveland, OH 44106, USA, paul.taylor@case.edu)

The goal of the present study was to test whether models of portions of curves, representing movements of the crucial articulator for production of place in syllables containing labiodental and alveolar consonants ('iceberg' portions of demisyllables), that had previously been found to be stable across different prosodic conditions (Bonaventura, 2003,2005, 2006; Bonaventura and Fujimura, 2007), a) remained stable across different subjects pronunciations, for each consonantal class b) were significantly different for the two different consonantal movements. Curves were previously extracted from microbeam articulatory data. Curve fitting models were obtained, by using a best fit fourth order polynomial, from 592 curves representing lower lip displacement for production of [f] in 'five' (3 subjects) and from 299 curves representing tongue tip displacement for production of [n] in 'nine' (3 subjects). Coefficients were statistically compared; results showed a) significant difference for all coefficients between subjects pronouncing same consonant, except for the 3rd coefficient, both in initial and final demisyllable, representing stability of slope around a position threshold. These results might indicate stable patterns across inter-subject variability; b) coefficients were significantly different for [f] vs [n] models across speakers, within initial vs. final demisyllable, possibly indicating properties of an identifiable articulatory gesture.

MONDAY AFTERNOON, 30 JUNE 2008

ROOM 343, 1:00 TO 2:20 P.M.

Session 1pSPa

Signal Processing in Acoustics: Sound Reproduction and Source Separation

\author{
William L. Martens, Cochair \\ McGill University, Schulich School of Music, 555 Sherbrooke Street West, Montreal, QC H3A 1E3, Canada
}

Jamie A. Angus, Cochair

University of Salford, 12 Lister Way, York, YO30 6NL, UK

\title{
Contributed Papers
}

\section{1:00}

1pSPa1. HRTF modeling in due consideration variable torso reflections. Markus Guldenschuh (Institute of Electronic Music and Acoustics, Inffeldgasse 10/3, 8010 Graz, Austria, markusgu@sbox.tugraz.at), Alois Sontacchi (Institute of Electronic Music and Acoustics, Inffeldgasse 10/3, 8010 Graz, Austria, sontacchi@iem.at), Franz Zotter (Institute of Electronic Music and Acoustics, Inffeldgasse 10/3, 8010 Graz, Austria, zotter@iem.at), Robert Höldrich (Institute of Electronic Music and Acoustics, Inffeldgasse 10/3, 8010 Graz, Austria, hoeldrich@iem.at)

Head related transfer functions (HRTFs) describe the physical path from an acoustical source to the ears. It can be gained within the relation of two measurements. The first will give the reference sound pressure in the virtual middle of the head the second has to be done in both ears. In literature exhaustive investigations concerning the idealized location of the second position can be found. Beyond that measurements with various artificial heads and human-beings considering various source directions (azimuth and elevation) with different angular resolutions have been undertaken. The obtained HRTFs exhibit, with subject to the examined source position, frequency dependent magnitude and phase. These complex transfer functions are caused by various reflections, diffractions, pressure superelevation, and shadowing, respectively. Reflections are caused form pinna, head and torso, too. Heretofore during determination of the transfer functions the influence of various head-torso arrangements have not be considered. Within this paper the differences which will be caused due to different head-torso angles will be highlighted. Beyond that a numerical model based on interpolation using spherical harmonics will be presented. By the aid of that model the 
integration within an existing binaural resynthesis model can be realized and perceptual differences within a dynamic system can be investigated.

\section{$1: 20$}

1pSPa2. Soundscape source extraction using wavelet-based sparse representations. Oliver Bunting (University of York, Department of Electronics, Heslington, YO10 5DD York, UK, ob103@ohm.york.ac.uk), David Chesmore (University of York, Department of Electronics, Heslington, YO10 5DD York, UK, edc1@ohm.york.ac.uk)

As part of the EPSRC Instrument for Soundscape Recognition, Identification and Evaluation (ISRIE) project, the work described here aims to extract sources from a soundscape, with the aim of improving the signal to interference ratio to aid automatic identification and classification. The paper will describe the project's background and aim. Results using directional audio coding (dirAC) to separate sparse signals will be presented. This method has been shown to have successfully separated two speakers at known positions from a virtual B-format soundscape. An extension of this method using the dual-tree complex wavelet transform will also be presented, which improves the sparsity of the time-frequency representation. Initial results for spatially known sources will be presented. It is expected that some results from current work on localising and tracking sources in a B-format soundscape will be presented.

\section{$1: 40$}

1pSPa3. Instrument for Soundscape Recognition, Identification and Evaluation (ISRIE): Signal Classification. Jon Stammers (University of York, Department of Electronics, Heslington, YO10 5DD York, UK, js185@york.ac.uk), David Chesmore (University of York, Department of Electronics, Heslington, YO10 5DD York, UK, edc1@ohm.york.ac.uk)

ISRIE is a collaborative project between the universities of York and Newcastle and ISVR in Southampton. The work being undertaken at York is in its second year and focuses on signal separation and classification. Developing novel methods for classifying urban and other sounds into distinct categories (such as transportation, industrial, human, animal, etc.) is the focus of this project. The classification system will consist of two main parts: a feature extractor and a classifier. Both time and time-frequency domain techniques have been considered for feature extraction and it is anticipated that a combination of domains will be required for this complex task. To date only simple classifiers have been implemented and development is now focussed on syntactic pattern recognition. The project background and an overview of the techniques involved will be presented along with preliminary results for the classification of urban and other sounds using syntactic methods.

\section{2:00}

1pSPa4. Real-time acoustic blind signal separation system based on the spatio-temporal gradient analysis. Kenbu Teramoto (Saga University, 1-Honjo, 8408502 Saga, Japan, tera@me.saga-u.ac.jp), Md. Tawhidul Islam Khan (Saga University, 1-Honjo, 8408502 Saga, Japan, khan@me.saga-u.ac .jp)

This paper presents an autonomous directivity microphone system for the blind source separation based on the newly proposed spatio-temporal gradient algorithm. The blind source separation technique principally uses no a priori knowledge about parameters of convolution, filtering, nor mixing. In the simplest case of the blind source separation problems, the observed mixing signals are the linear combinations of unknown source signals which are mutually and statistically independent and contain zero mean. The proposed blind signal separation algorithm utilizes the linearity among the four signals: (1) the sound pressure, (2) x, (3) y, and (4) z-directional particle velocities, all of which are governed by the wave equation and the equation of motion. The proposed method, therefore, has an ability to simplify the convoluted blind source separation problems into the instantaneous blind source separation problems over the spatio-temporal gradient space. Several acoustical experiments have been performed with acceptable performance of the proposed method of blind source separation. A sensor for measuring the particle velocity of acoustic signal has been used in the experiment.

MONDAY AFTERNOON, 30 JUNE 2008

ROOM 343, 4:00 TO 7:00 P.M.

\title{
Session 1pSPb
}

\section{Signal Processing in Acoustics: Acoustical Nondestructive Evaluation, Ultrasonics, and Imaging I}

\author{
Grace A. Clark, Cochair \\ Lawrence Livermore National Laboratory, 7000 East Ave., L-130, Livermore, CA 94550, USA \\ Ning Xiang, Cochair \\ Rensselaer Polytechnic Institute, Greene Building, School of Architecture, 110 8th Street, Troy, NY 12180, USA
}

\section{Contributed Papers}

\begin{abstract}
4:00
1pSPb1. Signal analysis in acoustic microscopy for nondestructive inspection of varnish layers on metal substrates. Sebastian Brand (University of Halle, Magdeburger St. 22, 06112 Halle, Germany, Sebastian.Brand@medizin.uni-halle.de), Peter Czuratis (SAM TEC GmbH, Gartenstrasse 133, 0553 Aalen, Germany, p.czurratis@stec-germany.com), Kay Raum (Martin Luther University of Halle-Wittenberg, Dept. of Orthopedics, Q-BAM Group, Magdeburger Str. 22, 06097 Halle, Germany, kay.raum@medizin.uni-halle.de)

For preventing corrosion and for surface protection metallic objects are commonly finished with layers of varnish. The integrity of the varnish and potential defect propagation influence the durability of the metal and hence are a measure for the quality of the finishing. Scanning acoustic microscopy provides high axial and lateral resolution, a sufficient penetration depth and
\end{abstract}

is nondestructive. The goal of this work was the development of a method for detection and evaluation of delaminations of varnish layers on metallic surfaces. Investigated were samples containing one and two layers of varnish. One group contained priming only whereas the second group contained varnish on top of the undercoat. The surface integrity of the finishing was destroyed by a scratch through all finishing layers. Defect aging was then performed by exposing the samples to a corrosion-friendly atmosphere. Scanning acoustic microscopy combined with signal analysis was performed for investigating the connectivity between the finishing layers and the substrate. A robust numerical deconvolution technique has been adapted and optimized to enable the separation of strongly overlapping pulses. Echoes originated at the substrate and the finishing layers have been localized and layer thicknesses/distances were estimated. Delaminated spots of the finishing were successfully evaluated using the method developed during this study. 
4:20

1pSPb2. Structural noise characterization and flaw detection in austenitic stainless steels using ultrasonic signals, wavelet analysis and significance testing. Mohamed Khelil (ENSIM - LAUM, Université du Maine, rue Aristote, 72085 Le Mans, France, mohamed.khelil@univ-lemans.fr), Jean-Hugh Thomas (ENSIM - LAUM, Université du Maine, rue Aristote, 72085 Le Mans, France, jean-hugh.thomas@univ-lemans.fr), Rachid El Guerjouma (ENSIM LAUM, Université du Maine, rue Aristote, 72085 Le Mans, France, Rachid.El_Guerjouma@univ-lemans.fr), Laurent Simon (LAUM, CNRS, Université du Maine, Lab. d'Acoustique Université du Maine, UMR CNRS 6613, 72085 Le Mans Cedex 9, France, Laurent.Simon@univ-lemans.fr), Malika Boudraa (USTHB Faculté d'électronique et d'informatique, BP 32, El-Alia, 16031 Alger, Algeria, mk .boudraa@yahoo.fr)

The aim of this study is to characterize the structural noise in order to better detect flaws in several heterogeneous materials (steels, welding, composites œ) using ultrasonic waves. For this purpose, a continuous wavelet transform is applied to ultrasonic Ascan signals acquired using an ultrasonic Non Destructive Testing (NDT) device. The time-scale representation provided, which highlights the temporal evolution of the spectral content of the Ascan signals, is relevant but can lead to misinterpretation. The problem is to identify if each pattern from the wavelet representation is due to the structural noise or a flaw. To solve it, a detection technique based on statistical significance testing in the time-scale plane is used. Typical structural noise signals are then described using an autoregressive model which seems relevant according to the spectral content of the signals. The approach is tested on experimental signals, obtained by ultrasonic NDT of metallic materials (austenitic stainless steel) then of a welding in this steel and indeed enables to separate various components from the signal that is two kinds of structural noises and flaw echoes.

\section{$4: 40$}

1pSPb3. Flaw detection and thin materials thickness measurement using time frequency and high resolution algorithms. Redouane Drai (Image and signal processing laboratory. Welding and NDT Centre, Route de Dely-Ibrahim, BP 64, Chéraga, 16035 Alger, Algeria, drai_r@yahoo.fr), Mohamed Khelil (ENSIM - LAUM, Université du Maine, rue Aristote, 72085 Le Mans, France, mohamed.khelil@univ-lemans.fr), Fouzia Megherbi (Welding and Control Research Center, Route de Dely-Ibrahim, B.P 64, Chéraga, 16035 Algiers, Algeria, fmegherbi@yahoo.fr), Abdelsaalam Benammar (Image and signal processing laboratory. Welding and NDT Centre, Route de Dely-Ibrahim, BP 64, Chéraga, 16035 Alger, Algeria, abs_benammar@yahoo.fr)

Several techniques of signal processing were introduced in ultrasonics NDT field. In thin samples the reflected signals are overlapping thus making detection of defects in these samples and accurate measurements impossible. It is thus necessary to enhance the visibility of the defect echo by signal processing techniques. In this context, we develop signal processing tools allowing detecting and locating the imperfections present in these materials. In this paper, we contribute by the development of some signal processing techniques based on time frequency and high resolution algorithms in order to enhance the resolution of flaw detection and to measure thin materials thickness. 1- We propose to implement temporal versions of methods known as high resolution like MUSIC, Root MUSIC and Eigen vectors method. These methods allow frequencies extraction in the case of the complex signals drowned in noise. 2- We apply time-frequency algorithms based on STFT, Wigner-Ville, Gabor transform on thin materials thickness measurement. A comparative study is carried out between all of these algorithms and is applied in separation of closer flaw echoes and thin materials thickness measurement. Satisfactory results are obtained with Gabor transform in measurement of few tenth $(0.1) \mathrm{mm}$.

\section{5:00}

1pSPb4. Investigation of damage mechanisms of composite materials: multivariable analysis based on temporal and wavelet features extracted from acoustic emission signals. Anne Marec (ENSIM - LAUM, Université du Maine, rue Aristote, 72085 Le Mans, France, anne.marec.etu@univ-lemans.fr), Jean-Hugh Thomas (ENSIM - LAUM, Université du Maine, rue Aristote, 72085 Le Mans, France, jean-hugh.thomas@univ-lemans.fr), Rachid El Guerjouma (ENSIM LAUM, Université du Maine, rue Aristote, 72085 Le Mans, France, Rachid .El_Guerjouma@univ-lemans.fr)

The aim of this work is the analysis of damage development and timeto-failure mechanisms within fibre-matrix composite materials in order to assess their remaining lifetime using in situ ultrasonic sensors. Thus Acoustic Emission (AE), which represents the generation of transient ultrasonic waves in a material under load, is used to discriminate the different damage mechanisms in composite materials. In this work, unsupervised pattern recognition analyses (fuzzy c-means) associated with a principal component analysis are used for classifying the AE events. A cluster analysis of AE data is achieved and the resulting clusters are correlated to the damage mechanisms of the material under investigation. This method gives good results on complex composite materials such as glass fibre/polyester cross-ply composites, sheet molding compound (SMC), concretes. Furthermore, AE signals of heterogeneous materials are not stationary. Thus, time-scale or timefrequency methods (continuous and discrete wavelet transforms) are used to determine new relevant descriptors from AE signals. These new descriptors are introduced in the clustering process to provide a better characterization and discrimination of the different damage mechanisms.

\section{$5: 20$}

1pSPb5. Ultrasonic structural health monitoring by multiple-input-single-output minimum variance processing. Jeffrey Krolik (Duke University, ECE Dept. Hudson Hall 130, P.O. Box 90291, Durham, NC 27708, USA, jk@ee.duke.edu), Granger Hickman (Duke University, ECE Dept. Hudson Hall 130, P.O. Box 90291, Durham, NC 27708, USA, gwh@ee.duke.edu), Lawrence Carin (Duke University, ECE Dept. Hudson Hall 130, P.O. Box 90291, Durham, NC 27708, USA, lcarin@ee.duke.edu), Jennifer E. Michaels (Georgia Institute of Technology, Van Leer Electrical Engineering Building, Atlanta, GA 30332, USA, jennifer.michaels@ece.gatech.edu)

This work concerns ultrasonic detection and localization of defects in platelike metal structures such as airfoils. In previous work, MIMO delayand-sum beamforming has been used to image these defects using changedetected ultrasonic signals between a sparse array of transmitters and receivers. However, mismatch of medium dispersion and unknown scattering properties of the defects can lead to significant imaging artifacts. To reduce imaging artifacts and sidelobes, a multiple-input-single-output (MISO) minimum variance (MV) approach is proposed here. MV-MISO imaging involves electronically scanning a transmit beam over the area of interest while, at each point being imaged, virutal transmit nulls are adaptively steered in the directions of interfering scatterers. For each imaging cell, this is achieved by adjusting transmit weights to minimize the variance of signals received at a single receiver subject to the constraint of achieving constant insonification. Using a model of dispersive Lamb wave propagation, the proposed algorithm has been successfully applied to change-detected data gathered with a six-sensor transmit-receive array coupled to a thin aluminum plate with artificial defects (e.g., drilled holes).

\section{$5: 40$}

1pSPb6. An accurate distance measurement by calibration of doppler-shift for ultrasonic sonar sensing. Hirata Shinnosuke (Tokyo Institute of Technology, 4259 Nagatutamati, Midori-ku, 226-8502 Yokohama, Japan, hirata.s.ab@m.titech.ac.jp), Minoru K. Kurosawa (Tokyo Institute of Technology, 4259 Nagatutamati, Midori-ku, 226-8502 Yokohama, Japan, mkur@ip.titech.ac.jp), Takasi Katagiri (Sutekina Inc., 20014 Akaho, 3994117 Komagane, Japan, takashi.katagiri@primemotion.com)

Ultrasonic distance measurement with the pulse-echo method is based upon the determination of the time of flight of ultrasonic waves. The pulsecompression technique, which involves the calculation of the cross- 
correlation function between the transmitted and the reflected waves, is the conventional method for improving the resolution of the measurement. The calculation of a cross-correlation operation requires high-cost digital signal processing, however. This paper presents a new sensor signal processing method of the pulse-compression technique using a delta-sigma modulated single-bit digital signal. The proposed method consists of three parts: an analyzing operation of the doppler-shifted frequency, a cross-correlation operation by single-bit signal processing, and a smoothing operation accomplished by a moving average filter. For the pulse-compression technique, this method reduces the calculation cost of digital signal processing. Since the frequency of the linear chirp signal is doppler-shifted, the distance from the moving transmitter to the moving object cannot be accurately measured in the pulse-compression technique. To solve this problem, the analyzing operation of the doppler-shifted frequency is used to calibrate the transmitted ultrasonic wave, thus effectively improving the accuracy of the measurement.

\section{6:00}

1pSPb7. Image processing and wavelets transform for sizing of weld defects using ultrasonic TOFD images. Ahmed Kechida (Image and Signal Processing Laboratory. Welding and NDT Centre, Route de DelyIbrahim, BP 64, Chéraga, 16035 Alger, Algeria, Abs_benammar@yahoo.fr), Redouane Drai (Image and Signal Processing Laboratory. Welding and NDT Centre, Route de Dely-Ibrahim, BP 64, Chéraga, 16035 Alger, Algeria, drai_r@yahoo.fr), Abdelsaalam Benammar (Image and Signal Processing Laboratory. Welding and NDT Centre, Route de Dely-Ibrahim, BP 64, Chéraga, 16035 Alger, Algeria, abs _benammar@yahoo.fr)

Ultrasonic time-of-flight diffraction (TOFD) is rapidly gaining prominence as a reliable nondestructive testing technique for weld inspection in steel structures, providing highly accurate positioning and sizing of flaws. A number of signal and image processing tools have been specifically developed for use with TOFD data and adapted to function autonomously without the need for continuous intervention, configuring automatically according to the nature of the data and the data acquisition settings. This paper presents the results of several innovative procedures based on Multiresolution analysis such as wavelet transforms and texture analysis for detecting edges of planar defects. The approach is based on the decomposition by packets of wavelets of the image while taking into account of the underimage content textural after each level of decomposition. The reconstruction of the image is done by eliminating the underimages of poor textural information and at the end the segmented image is got by a binarisation operation done on the image rebuilt histogram. The automation of sizing and positioning of weld flaws in TOFD data as an essential stage of a comprehensive TOFD inspection and interpretation aid is developed and implemented. Key words: U1trasonic Time-Of-Flight Diffraction., Texture Analysis, Wavelet Transform.

\section{6:20}

1pSPb8. Temporal approach of the synthetic aperture imaging using Hadamard matrix. Frédéric Mosca (Ixsea, 46 Quai François Mitterrand, 13600 La Ciotat, France, fmo@ixsea.com), Jean-Marie Nicolas (Ecole Nationale Supérieure des Télécommunication de Paris, Telecom ParisTech, Département TSI, 46 rue Barrault, 75634 Paris Cedex 13, France, nicolas@enst.fr), Laurent Kopp (IXwaves, 220 rue Albert Caquot, 06560 Sophia Antipolis, France, laurent.kopp@ixwaves.com), Mathieu Couade (Supersonic Imagine, Les Jardins de la Duranne, bât E, 510, rue René Descartes, 13857 Aix-en-Provence, France, mathieu.couade @ supersonicimagine.fr)

The synthetic aperture imaging is a very promising solution in the wellknown compromise between contrast and frame rate. Indeed this method leads to the measurement of each transmitter/receiver impulse response of the system. From this fact, synthetic aperture imaging reach the transmit/receive focus imaging quality for the cost in frame rate of the number of antenna's elements. The main inconvenient of this method is the very low signal to noise ratio provided. Indeed, using only one transmitter per sequence leads to a very poor penetration. To correct this, a method using spatial Hadamard sequences has been introduced. For each of this Hadamard sequence, a Hadamard beam is generated in the medium. By a temporal approach, some interesting properties of those beams are highlighted and a method using those properties is proposed. Some experiments have been done using those properties and the results show an important improvement of the frame rate for a very small cost in contrast.

\section{6:40}

1pSPb9. Elastic-wave reverse-time migration imaging with a new vector-imaging condition. Huseyin Denli (Los Alamos National Laboratory, Mail Stop D408, Los ALamos, NM 87544, USA, denli@lanl.gov), Lianjie Huang (EES-11 (Geophysics) - Los Alamos National Laboratory, MS D443, Los Alamos, NM 87545, USA, ljh@lanl.gov)

With increasing computing capability, the use of elastic waves in threedimensional subsurface imaging is becoming more feasible. However, the research in this area is still in the embryonic stage. In conventional seismic imaging, multicomponent data are decomposed into compressional and shear components, and solutions of the acoustic-wave equation are used to process each component independently. Such an imaging technique, however, cannot correctly handle elastic-wave conversions/couplings in complex regions, which is critically important for high-resolution and reliable imaging. In this paper, we develop elastic reverse-time migration imaging, applying a finite-difference solution to the pure elastic-wave equation in heterogeneous media. We implement numerical reverse-time migration imaging in a scheme similar to time-reversal acoustics in the laboratory. To correctly handle polarizations of compressional- and shear-waves during imaging, we also develop a novel vector-imaging condition for elastic-wave reverse-time migration. We use synthetic reflection data to demonstrate that, compared with the conventional imaging condition, our new vector-imaging condition increases image resolution and reduces image artifacts. The new imaging algorithm can significantly improve our capability to image complex subsurface structures. 


\title{
Session 1pUW
}

\section{Underwater Acoustics, Acoustical Oceanography, and ECUA: Seabed and Sea Surface Interaction Measurements and Modeling}

\author{
Peter H. Dahl, Cochair \\ Applied Physics Laboratory, University of Washington, 1013 NE 40th St, Seattle, WA 98105, USA \\ Michael A. Ainslie, Cochair \\ TNO Defence, Security and Safety, Oude Waalsdorperweg 63, The Hague, 2597 AK, Netherlands
}

\section{Invited Paper}

$1: 00$

1pUW1. Fine-grained sediment layers: an acoustic lens for sub-bottom sediment processes and structures. Charles W. Holland (Penn State University/Applied Research Lab, Applied Science Bldg., State College, PA 16804, USA, cwh10@psu.edu)

In many coastal and deep water regions, there is a layer of fine-grained sediment (e.g., clay and silty-clay) over coarser grained sediments or rock. This layer may range from scales of centimeters to hundreds of meters thick. Fine-grained sediments typically have a sound speed less than that of the interstitial fluid (i.e., overlying water column) with modest attenuation and thus act as a refracting lens for the acoustic field as it interacts with sub-bottom structures. The presence of fine-grained sediments frequently opens an effective window for acoustically probing very small-scale processes within the sediment. The presence of fine-grained sediments also has a profound impact on seabed reflection and scattering, hence propagation, reverberation, and clutter. The effect of fine-grained sediment layers (along with concomitant sub-bottom processes) on reflection and scattering is discussed using both theory and measurements. [Research supported by ONR OA321.]

\section{Contributed Paper}

$1: 20$

1pUW2. A parametric study of interactions between acoustic signals reflected by the seafloor. Samuel Pinson (Ecole Navale/Irenav, BP 600, 29240 Brest Armées, France, samuel.pinson@ecole-navale.fr), Laurent Guillon (Ecole Navale/Irenav, BP 600, 29240 Brest Armées, France, guillon @ecole-navale.fr)

This paper deals with the study of broadband acoustic signals reflected by the seafloor and recorded on a vertical array in shallow water areas. Only the first bottom reflected path is considered here. In previous studies (see e.g., Guillon and Holland, JASA, 122, 2974), two different aspects of the "coherence" of these signals were examined (the maximum of the cross- correlation coefficients and the phase of the cross-spectra) and it was shown that these parameters are sensitive to the geoacoustic nature of the seafloor. These results show that the interactions between signals can be used efficiently for improving geoacoustic inversion schemes or for transmission loss predictions. Before going to such applications, a detailed parametric study is needed to investigate the sensitivity of the interactions measurement to various parameters (range, geoacoustic structure,...). This is done using a numerical model, based on the spherical wave decomposition of plane waves, that simulates the signals reflected by the seafloor. The parametric study lead shows the relative importance of the various parameters and gives indications for the possibility of inverting these parameters.

\section{Invited Paper}

1pUW3. Mid-frequency bottom interaction measurements off the New Jersey continental shelf. Jee Woong Choi (Dept. of Environmental Marine Sciences, Hanyang Univ., 1271, Sa-3-dong, Sangrok-gu, 426-791 Ansan, Republic of Korea, choijw@hanyang.ac.kr), Peter H. Dahl (Applied Physics Laboratory, University of Washington, 1013 NE 40th St, Seattle, WA 98105, USA, dahl@apl.washington.edu)

Two kinds of bottom interaction measurements conducted in waters $80 \mathrm{~m}$ deep off the North American continental shelf as part of Shallow Water 06 (August, 2006) are discussed. In each, acoustic signals were recorded on two, colocated vertical line arrays of length $1.4 \mathrm{~m}$, one at depth $25 \mathrm{~m}$, and other at depth $50 \mathrm{~m}$. The source was deployed at depth $40 \mathrm{~m}$ from the R/V Knorr that could either be positioned or towed at rate $0.1 \mathrm{~m} / \mathrm{s}$. The first, "bottom reflection" is interpreted as a measure of the modulus of the plane wave reflection coefficient as functions of frequency (1-20 kHz) and grazing angle associated with the discrete set of ranges (50-300 m). The second represents a "move-out" type measurement with source towed away from the receiver, and reflection at continuous angles associated with the 50-300 m range. Frequency range was 1-2 kHz, and as the source was $5 \mathrm{~m}$ off the bottom, spherical wave effects are 
investigated. Both measurements were carried out over the same four directions originating from the receiver, each separated by 90 degrees. Physical processes responsible for the observations in each case are discussed and modeled. [Supported by ONR, with partial support from Hanyang University.]

\section{Contributed Paper}

2:00

1pUW4. Analysis of acoustic backscattering from the ocean bottom using radiative transfer theory. Jorge E. Quijano (NEAR Lab-Portland State University, 1900 SW 4th Avenue, Suite 160, Portland, OR 97201, USA, jorgeq@pdx.edu), Lisa M. Zurk (NEAR Lab-Portland State University, 1900 SW 4th Avenue, Suite 160, Portland, OR 97201, USA, zurkl @ cecs.pdx.edu)

Radiative Transfer (RT) theory is a heuristic formulation for analysis of wave propagation in random media based on the principle of conservation of energy rather than on the solution of the wave equation. The theory has been applied to electromagnetics and more recently to ultrasound and seismics, and it has the advantage of being computationally tractable for complex ran- dom environments. In this paper the RT formulation is proposed for the problem of layered ocean bottom sediment with random scatterers. The volume backscattering level from tenuous media is obtained by solving the RT equation at each individual layer and by applying the corresponding reflection and transmission coefficients at the rough boundaries. Simulations of the backscattered acoustic intensity from a finite layer with elastic spherical scatterers are presented. The results obtained from the RT equation are compared to those based on the wave equation. Single frequency steady state solutions are considered for different cases of sediment attenuation and layer thickness. The flexibility of the RT method is demonstrated by showing the individual effect of propagating longitudinal and shear waves in the elastic sediment. It is proved that the contribution of shear waves in consolidated sediments is considerable.

\section{Invited Paper}

\section{2:20}

1pUW5. Determination of seabed scattering law from long range reverberation. Christopher H. Harrison (NATO Undersea Research Centre, Viale San Bartolomeo 400, 19138 La Spezia, Italy, harrison@ nurc.nato.int)

Reverberation degrades sonar performance, and often the dominant cause in shallow water is scattering from the seabed. Usually scattering is weaker at low angles than at high whereas, in contrast, sound propagates better at low angles than at high. This, in turn, affects the range-dependence of signal-to-reverberation which influences the choice of source level. A better understanding of this scattering angle dependence (or scattering law) offers the potential of improved discrimination of targets against this reverberation background. This paper will discuss an experimental technique for deducing the scattering angle dependence and backscattering strength from long range reverberation (out to $25 \mathrm{~km}$ ) measured on a vertical line array (VLA). Well-sampled near simultaneous angle-dependent propagation measurements out to comparable ranges are used to separate the propagation effects without the need for any modeling. Applying the technique to the Malta Plateau (during BOUNDARY2003 and BOUNDARY2004), first the method appears to work, and second it suggests that the scattering law is close to Lambert when the seabed is flat.

\section{Contributed Paper}

\section{2:40}

1pUW6. Underwater stereo-photographic measurement of small scale roughness: limits to spatial correlation accuracy. Gaetano Canepa (NATO Undersea Research Centre, Viale San Bartolomeo 400, 19126 La Spezia, Italy, canepa@nurc.nato.int), Eric Pouliquen (NATO ACT, 7857 Blandy Road, Suite 100, Norfolk, 23551-2490, USA, pouliquen@act.nato.int), Anthony P. Lyons (Applied Research Laboratory, The Pennsylvania State University, P.O. Box 30, State College, 16804, USA, ap12@psu.edu)

In recent years stereo-photogrammetry measurements of seafloor small scale roughness have been reported in the literature. Stereo-photogrammetry algorithm consists of a comparison between areas (windows) belonging to corresponding regions of a pair of photos. This comparison may be per- formed numerically or by human eyes using a photogrammetric stereocomparator. The results of this process are then converted in an array of seafloor heights. Until now underwater stereo-photographic data are given with no quantitative measurement of the positions and position correlation lengths errors: this work addresses this problem applying the results to the SAPHO system (Seafloor Automatic PHOtogrammetry) developed at NURC. It is shown that positional error estimates for stereo-photographic systems must take into account not only line/matrix resolution and system geometry but also the effects of comparison window size and image quality. A method by which this can be achieved is described. It is also shown that the stereo-photographic systematic error has an important effect when the purpose of the measurement is to determine seafloor correlation length or power spectral density for acoustic scattering estimation.

\section{Invited Paper}

1pUW7. Bubbles at the top and bottom of the water column: the acoustical effects, and the use of acoustics to measure them. Timothy G. Leighton (Institute of Sound and Vibration, Univ. of Southampton, University Road, Highfield, SO17 1BJ Southampton, UK, T.G.Leighton@soton.ac.uk), David Coles (National Physical Laboratory, Hampton Road, TW11 OLW Teddington, UK, dc@isvr.soton.ac.uk), Michael A. Ainslie (TNO Defence, Security and Safety, Oude Waalsdorperweg 63, 2597 AK The Hague, Netherlands, michael.ainslie@tno.nl), Paul R. White (Institute of Sound and Vibration, Univ. of Southampton, University Road, Highfield, SO17 1BJ Southampton, UK, prw@isvr.soton.ac.uk)

Bubbles of atmospheric gas can be entrained by breaking waves at the top of the water column: this bubble population is dynamic, and will evolve through the effects of buoyancy, gas exsolution and dissolution, and the fragmentation and coalescence of bubbles. These processes are important to ambient noise, sonar operation, and the overall gas budget of the planet. At the base of the water column, methane bubbles can occur in marine sediments, a phenomenon important to the global methane budget, to the petrochemical industry, and to the stability of the sediment (e.g., for civil engineering purposes). This paper examines the acoustical effects of both of 
these populations, and the ways in which acoustics can be used to measure them. Data will be presented from field trials, including measurements of gassy marine sediments in UK waters, and of wave-generated bubble clouds measured by an $11 \mathrm{~m}$ spar buoy deployed from 16th June to 18th July 2007 at a distance of 400 miles off the west coast of Portugal.

\section{3:20-3:40 Break}

\section{Contributed Paper}

3:40

1pUW8. The effect of wind-generated bubbles on sea-surface backscattering at $940 \mathrm{~Hz}$. Robbert Van Vossen (TNO Defence, Security and Safety, Oude Waalsdorperweg 63, 2597 AK The Hague, Netherlands, robbert.vanvossen@tno.nl), Michael A. Ainslie (TNO Defence, Security and Safety, Oude Waalsdorperweg 63, 2597 AK The Hague, Netherlands, michael.ainslie@tno.nl)

Predictions of sea-surface backscattering strength are needed for sonar performance modelling. Such predictions are hampered by two problems. First, measurements on surface backscattering are not available at small grazing angles. These are of special interest to low-frequency active sonar since these mainly contribute to long range propagation. Second, existing theoretical models underestimate the surface backscattering strength at larger grazing angles. We investigate whether wind-generated bubbles can explain this deficit. Thus, we develop a theoretical description which includes the effect of refraction and scattering of sound by wind-generated bubbles. We compare the theoretical surface backscattering predictions to Critical Sea Test measurements. These are a set of backscattering measurements for different wind speeds, and grazing angles are in the range between 5 and 30 degrees. The comparison shows that a good fit can be obtained between the theoretical predictions and the surface backscattering measurements, depending on the population density of resonant bubbles. This indicates that wind-generated bubbles significantly contribute to rough-surface back-scattering. The bubble backscattering contribution is dominant at large wind speeds and also at small grazing angles.

\section{Invited Paper}

1pUW9. Quasi-deterministic forward scattering from the sea surface. Grant Deane (Scripps Inst. Oceanography, Univ. Califiornia, San Diego, La Jolla, CA 92093, USA, gdeane@ucsd.edu), James Preisig (Woods Hole Oceanographic Institution, Bigelow 404, MS\#9, Woods Hole, MA 02543, USA, jpreisig@whoi.edu), Chris T. Tindle (Physics Department, University of Auckland, Bag 92019, 1010 Auckland, New Zealand, c.tindle@auckland.ac.nz)

Gravity waves create focal regions and caustics in sound forward-scattered from the sea surface. Focal regions are associated with rapid variations in amplitude and Doppler shift, which can impact the performance of underwater communications systems and sonars operated near the shore. Single-bounce focal regions consist of a superposition of up to 3 micro-paths, each of which is associated with its own amplitude, time delay, and Doppler shift. Focal regions move systematically through the water column as the wave propagates. Studies of these effects in near-shore ocean experiments and a laboratory flume have shown that a quasi-deterministic analysis of wave scattering can lead to valuable insights about the underlying scattering process. [Work supported by ONR.]

\section{Contributed Papers}

$$
\text { 4:20 }
$$

1pUW10. Multipath reflection from surface waves. Chris $T$. Tindle (Physics Department, University of Auckland, Bag 92019, 1010 Auckland, New Zealand, c.tindle@auckland.ac.nz), Grant Deane (Scripps Inst. Oceanography, Univ. Califiornia, San Diego, La Jolla, CA 92093, USA, gdeane@ucsd.edu), James Preisig (Woods Hole Oceanographic Institution, Bigelow 404, MS\#9, Woods Hole, MA 02543, USA, jpreisig@whoi.edu)

A tank experiment was conducted at Scripps Institution of Oceanography to measure reflection of underwater sound from surface waves. Short pulses at a nominal $200 \mathrm{kHz}$ were transmitted beneath surface waves of wavelength $0.7 \mathrm{~m}$ to a receiver at $1.2 \mathrm{~m}$ range. The surface wave crests act as curved mirrors for underwater sound and lead to focussing and caustics in the surface reflected pulses. The locations of the foci and caustics move steadily as the wave progresses and lead to rapid variation of amplitude, phase and arrival time of the received pulses. Wavefront modeling has been used to calculate theoretical waveforms for the measured surface wave shape. The theory shows there are typically three distinct reflected eigenrays beneath a wave crest and they interfere to give rapid variation of the received signal. The theory gives good agreement with the details of the time dependent interference of the surface reflected pulses. [Work supported by ONR.]

$$
\text { 4:40 }
$$

1pUW11. Observations and modeling of angular compression and vertical spatial coherence in sea surface forward scattering. Peter $\mathrm{H}$. Dahl (Applied Physics Laboratory, University of Washington, 1013 NE 40th St., Seattle, WA 98105, USA, dahl@apl.washington.edu)

Measurements and modeling of vertical spatial coherence and related angular spreading associated with forward scattering from the sea surface are presented. The van Cittert-Zernike theorem is used in the modeling. The measurements were conducted in waters $80 \mathrm{~m}$ deep off the North American continental shelf (Shallow Water 06, Aug. 2006). Acoustic signals were recorded on a moored array system (MORAY) consisting of three arrays (each $1.4 \mathrm{~m}$ ): two vertical line arrays at depth $25 \mathrm{~m}$ and $50 \mathrm{~m}$, and one horizontal array at depth $27 \mathrm{~m}$. The source $(1-20 \mathrm{kHz})$ was deployed at depth $40 \mathrm{~m}$ from the R/V Knorr, at ranges 50-500 $\mathrm{m}$ from the MORAY. Conditions were characterized by a downward-refracting profile, e.g., sound speed going from $1530 \mathrm{~m} / \mathrm{s}$ at the surface to $1490 \mathrm{~m} / \mathrm{s}$ at depth $25 \mathrm{~m}$. Refraction modifies the vertical angular vertical spread due to scattering, which can understood from Snell's law. The Snell mapping is smooth, and a Taylor expansion around the mean grazing angle provides a functional relation between the angular variance near the surface and that at the receiver associated with scattering. The latter is measurably compressed owing to refraction, an effect mirrored in the spatial coherence data. [Research support from ONR.]

\section{5:00}

1pUW12. Unperturbed normal mode method for forward surface scattering. Frank S. Henyey (Applied Physics Laboratory, University of Washington, 1013 NE 40th St, Seattle, WA 98105, USA, frank@apl.washington.edu), Eric Thorsos (Applied Physics Laboratory, University of Washington, 1013 NE 40th St, Seattle, WA 98105, USA, eit @apl.washington.edu)

A technique is presented that uses an expansion in unperturbed modes to calculate acoustic scattering from ocean surface waves in a shallow water waveguide. The basic formalism as well as a useful extension to account for the difference between the water column and the domain in which the modes 
are calculated. The coupling between the modes due to the waves is local at the ocean surface, unlike the coupling of local modes. Numerical examples of the calculation are given for both a sinusoid surface wave and a random surface wave with a typical wind driven spectrum.

\section{$5: 20$}

1pUW13. Sonic-boom noise under a wavy air-water interface. Adam M. Fincham (University of Southern, Aerospace \& Mechanical Engineering, 854 W Downey Way, Los Angeles, CA 90089-1191, USA, afincham@usc.edu), Tony Maxworthy (University of Southern, Aerospa \& Mechanical Engineering, 854 W Downey Way, Los Angeles, CA 90089 1191, USA, maxworth@usc.edu), Hsien K. Cheng (University of Southern, Aerospace \& Mechanical Engineering, 854 W Downey Way, Los Angeles, CA 90089-1191, USA, afincham@usc.edu), Charles P. Griffice (The Aerospace Corporation, System Planning and Engineering, El Segundo, CA 90245-4691, USA, Charles.P.Griffice@aero.org), Johnson C. Wang (The Aerospace Corporation, System Planning and Engineering, El Segundo, CA 90245-4691, USA, Johnson.C.Wang@aero.org), John R. Edwards (U. S. Air Force, Air Force Space Command, 483 N. Aviation Blvd., El Segundo, CA 90245-2808, USA, John.Edwards@LOSANGELES.AF.MIL), Adel A.
Hashad(U. S. Air Force, Air Force Space Command, 483 N. Aviation Blvd., El Segundo, CA 90245-2808, USA, Adel.Hashad@LOSANGELES.AF .MIL)

Recent investigations of the underwater sound field produced by sonic booms show that the latter's interaction with surface waves can strongly alter the sound level, frequency range, and signal waveform reaching the deeper part of the ocean. Unlike conclusions from studies based on the flatocean model of Sawyers [1] which indicate little effects at depth beyond one signature length, the interaction of sonic-boom waves with a surface-wave train can generate nonevanescent, downward propagating waves which, owing to their lower spatial-attenuation rate, overwhelm the otherwise primary (flat-ocean) wave field. Theoretical results [2] substantiate and elucidate the significant differences between flat and wavy surfaces in waveform characteristics and their audibility in deep, and moderately deep water. Here we report on an extensive series of laboratory experiments performed specifically to validate this theoretical model and to ascertain the distinct differences in wave-field characteristics between a wavy and flat interface. The experimental measurements confirm the theoretical findings, showing the predicted differences between the sound fields measured under wavy and flat air-water interfaces stimulated by a passing sonic boom. [1] Sawyers, K. H. J. Acoust. Soc. Am., 44(2), 523 - 524, (1968) [2] Cheng, H. K. and Lee, C. J., J. Fluid Mech. 514, 281-312 (2004). 
Session 2aAAa

\title{
Architectural Acoustics: Acoustics of Concert Halls II
}

\author{
Takayuki Hidaka, Cochair \\ Takenaka R\&D Institute, 1-5-1, Otsuka, 270-1395 Inzai, Chiba, Japan \\ Daniel E. Commins, Cochair \\ Commins Acoustics Workshop, 15 rue Laurence Savart, Paris, 75020, France
}

\section{Contributed Papers}

\section{8:00}

2aAAa1. Acoustical design consideration for the new IFEZ concert hall. Jin Yong Jeon (Hanyang University, Department of Architectural Engineering, 133-791 Seoul, Republic of Korea, jyjeon@ @anyang.ac.kr), Yong Hee Kim (Hanyang University, Department of Architectural Engineering, 133-791 Seoul, Republic of Korea, kimyonghee@gmail.com), Shin-Ichi Sato (Hanyang University, Department of Architectural Engineering, 133791 Seoul, Republic of Korea, s_sato@mac.com)

The design of a new concert hall for the Incheon Free Economic Zone (IFEZ) Arts Center in Korea is in progress. The hall is supposed to be the home of the Asia Philharmonic Orchestra. It has over 1,700 seats, including 150 choirs. A vineyard seating arrangement will be applied, but the seats around the stage area will be minimized in order to form a solid stage enclosure. Lateral walls are designed for each seating block to increase acoustical intimacy. Average width between lateral walls has been designed to be less than $15 \mathrm{~m}$, and every seat is arranged within $7.5 \mathrm{~m}$ to the closest lateral wall. All lateral walls are designed to be inclined to lead first reflections and to improve spatial impression at the audience area. Diffusers are functionally installed to the effective reflecting surfaces from the source on stage. The design considerations are investigated through both computer simulation and scale modelling.

\section{8:20}

2aAAa2. Objective and subjective analysis of acoustical response in newly renovated Palais Moncalm, Quebec City, Canada. Jean-Philippe Migneron (Lab. d'acoustique, École d'architecture, 1, Côte de la Fabrique, Vieux Séminaire, Université Laval, Québec, QC G1K 7P4, Canada, jean-philippe.migneron.1@ulaval.ca), Jean-Gabriel Migneron (Lab. d'acoustique, École d'architecture, 1, Côte de la Fabrique, Vieux Séminaire, Université Laval, Québec, QC G1K 7P4, Canada, jgmigneron@hotmail.com), Jean-François Hardy (Lab. d'acoustique, École d'architecture, 1, Côte de la Fabrique, Vieux Séminaire, Université Laval, Québec, QC G1K 7P4, Canada, jean-francois.hardy.1@ulaval.ca)

To celebrate the 75th anniversary of Palais Montcalm, the building has been almost entirely rebuilt to convert it into the House of Music. The main room, named Salle Raoul-Jobin, has been designed specifically for the resident chamber orchestra: Les Violons du Roy. Collaboration between acoustical consultant, Larry S. King, and architect Jacques Plante of MUSE consortium results in a 979 seats concert hall with variable acoustic to accommodate different kinds of musical events. Since reopening in March 2007, the general acoustic has been enthusiastically acclaimed by professionals, by world known musicians, and by the public. The principal objective of this study is to characterize acoustical response of the room with various adjustments of wall curtains and motorized canopy. This important reflective area is software-controlled in a range of 3 meters depending on musical needs. Acoustical objective parameters, such as reverberation time, early decay time, impulse response or $\mathrm{C} 80$ factor will be analyzed and compared to musical subjective perception of a few instruments played at multiple positions on stage and for different listening locations.

\section{8:40}

2aAAa3. New design tendencies in modern concert hall design. Alban A. Bassuet (Arup Acoustics, 155 avenue of the americas, New York, NY 10013, USA, alban.bassuet@arup.com)

New concert hall design trends are emerging as seen from recently completed halls around the world and recent international architectural competitions. Analyzing the current situation, the paper starts by differentiating the acoustical characters of major traditional concert hall forms such as the large shoe-box, the Vineyard and the surround hall. It discusses in particular the balance of acoustical energy that characterize these different basic hall shapes, the sensation of intimacy in relation to the listener's distance to the performance area and the different early reflection patterns inherent from these hall shapes. As an example of modern concert halls design tendencies, the paper then describes a design option chosen by Arup Acoustics for the Paris Concert Hall Architectural design competition with Zaha Hadid architect. The paper describes the development of the form and shape of the hall in response to the brief, the concept chosen for the design of sending/receiving surfaces to improve the early reflection "efficiency" and "stability" and the use of an overhead reflector to improve the balance between soloist and orchestra and the balance between the orchestra and the reverberation in the hall.

\section{9:00}

2aAAa4. Acoustic design and evaluation of a multi-purpose hall of a new conference centre. Attila Balázs Nagy (Kotschy Bt., Álmos vezér u. 4, 2045 Törökbálint, Hungary, nagyab@hit.bme.hu), Ferenc Tamás (Kotschy Bt., Álmos vezér u. 4, 2045 Törökbálint, Hungary, tamas.ferenc@kotschy.hu), András Kotschy (Kotschy Bt., Álmos vezér u. 4, 2045 Törökbálint, Hungary, bandi@kotschy.hu)

At Forum Acusticum 2005 the building and room acoustic design of a new Conference Centre was presented. Since then the construction work has been finished and the Centre is now open. The Conference Centre itself is a multi-functional building, having three wings of different functions. The Main Hall of the Centre is a room for 750 people which can be extended with adjacent section rooms for 1100 persons, giving a total volume of $14000 \mathrm{~m}^{3}$. It was designed to host conferences, lectures, and - most of the time - to be used as a concert hall. The extremely different acoustical demands of being a concert hall and a lecture room had been fulfilled with appropriate room acoustic design and with variable acoustics by employing a DCR (Digital Control of Reverberation) system. In this paper we give a report on the achieved acoustical performance of the Main Hall of the Conference Centre. We have performed extensive room acoustic measurements in the Main Hall, the results of which are compared to the designed values and are presented in this paper. The design of the DCR system is discussed in a different paper. 
9:20

2aAAa5. Renovation of the concert hall The Doelen: A case study on the impact of a stage canopy on stage and room acoustics. Margriet $R$. Lautenbach (Peutz BV, PO Box 696, 2700 AR Zoetermeer, Netherlands, m.lautenbach@zoetermeer.peutz.nl), Martijn Vercammen (Peutz, De Grippen 1124, 6605 TA Wijchen, Netherlands, m.vercammen@mook .peutz.nl), Klaus-Hendrik Lorenz-Kierakiewitz (Peutz BV, Kolberger Strasse 19, D-40599 Duesseldorf, Germany, khl@peutz.de)

When opened in 1966, the main concert hall in De Doelen, Rotterdam, the Netherlands, was provided with six canopies above the stage platform. Their function was twofold: to provide a large part of the audience with early reflections; to create good ensemble conditions for the musicians on stage. Despite good reviews after the opening, a few years later the canopies were removed to get rid of unwanted reflections at the recording microphones positions just below the canopy. Since then, a significant percentage of the orchestra is unhappy about the acoustic conditions on stage. During the design process of the renovation, possibilities to re-introduce a stage canopy are investigated. Objective acoustic parameters obtained by carrying out measurements in the hall as well as in a 1:10 scale model and by calculations with a ray-tracing computer model are examined. Parallel, three questionnaire rounds gave an impression about the musicians' subjective judgement about the stage acoustics. In this paper the acoustic differences of three situations (without canopy, with original canopies and with new canopy design) and the search for an optimal balance between improving the stage acoustics without altering room acoustical conditions in the audience are discussed.

\title{
Invited Paper
}

2aAAa6. Evaluation of virtual acoustic stage support for musical performance. Wieslaw Woszczyk (McGill University, Schulich School of Music, 555 Sherbrooke Street West, Montreal, QC H3A 1E3, Canada, wieslaw@music.mcgill.ca), William L. Martens (McGill University, Schulich School of Music, 555 Sherbrooke Street West, Montreal, QC H3A 1E3, Canada, wlm@music.mcgill.ca)

The effects of performance space acoustics on musical performance can be evaluated most effectively by eliminating the influence of powerful non-auditory factors, such as the visual appearance of the performance space. To allow for such relatively unbiased evaluations, a virtual acoustic stage support system was set up for a live musical performance, and the performer was asked to make blind comparisons between a variety of architectural acoustic simulations. While results support the broad generalization that the preferred reverberation time for acoustical stage support depends upon the piece of music to be performed, it was also clear that preferences strongly depended upon performers' aural familiarity with architectural acoustic spaces in which they had considerable experience in previous performances. After some exposure to descriptive analysis techniques, performers could explain their preferences in terms of the perceptual characteristics that differed between presented aural architectures.

TUESDAY MORNING, 1 JULY 2008

ROOM 253, 8:00 TO 9:00 A.M.

\author{
Session 2aAAb
}

\section{Architectural Acoustics and Noise: Low Frequency Absorption: Mechanisms, Measurement Methods and Application II}

\author{
Peter D'Antonio, Cochair \\ RPG Diffusor Systems, Inc., 651-C Commerce Drive, Upper Marlboro, MD 20774, USA
}

Christian Nocke, Cochair

Akustikbüro Oldenburg, Katharinenstr. 10, Oldenburg, 26121, Germany

\section{Contributed Papers}

\section{8:00}

2aAAb1. Effects of Low-frequency Absorption on Perceived Tightness of Bass Imagery in Music Reproduction. William L. Martens (McGill University, Schulich School of Music, 555 Sherbrooke Street West, Montreal, QC H3A 1E3, Canada, wlm@music.mcgill.ca)

In order to enable unbiased observation of the effects of low-frequency absorption on auditory imagery associated with multichannel loudspeaker reproduction, binaural recordings were made of surround sound program material that was reproduced over full-range loudspeakers located in a room that was specially constructed to allow for variation in low-frequency acoustical treatment. These recordings were then presented via headphones to allow for double-blind comparison of the variation in auditory imagery associated with selected changes in room acoustics while holding listener and loudspeaker locations constant. Several perceptual attributes were examined, but the listeners were able to make the most clear distinctions between auditory spatial images in terms of the attribute identified as the "perceived tightness of bass imagery." Analysis of the signals presented to the listeners' ears in these binaurally-reproduced multichannel music samples showed that the tightest bass imagery was associated with high values of interaural coherence, with lower values producing more "muddy" bass imagery. [Work supported by Canada Foundation for Innovation.]

\section{8:20}

2aAAb2. Acoustical tests of custom-made, low-cost bass absorptive treatment for small rooms. Ioana Pieleanu (Acentech Incorporated, 33 Moulton Street, Cambridge, MA 02138, USA, ipieleanu@acentech .com), Jeffrey Fullerton (Acentech Incorporated, 33 Moulton Street, Cambridge, MA 02138, USA, jfullerton@acentech.com), Marc Choiniere (University of Nebraska, 101A Peter Kiewit Institute, Omaha, NE 68182, USA, mchoiniere@gmail.com)

Small music classrooms, practice rooms or small budget recording studios and control rooms are a few of the numerous applications where low frequency absorption is sought and necessary for a well-balanced acoustical spectrum. Often, low frequency absorption is achieved by using preengineered products, such as tuned panel resonators. Unfortunately, the cost of these pre-engineered products can be excessive for small budget projects, 
or projects that involve a large number of such spaces. For these applications, more affordable, custom-made low frequency absorbers are desired. Several types of custom-made treatments were physically tested, to determine if the degree of low frequency absorption they provide would make them an effective replacement for the pre-engineered counterparts. The test samples included glass fiber panels mounted as corner traps, custom-made plywood panel resonators and others. The testing was conducted in a space comparable in size to a small studio room/control room, or a medium practice room. This presentation describes the test method and measurement results.

\section{8:40}

2aAAb3. The importance of bass clarity in pop and rock venues. Niels W. Adelman-Larsen (Flex Acoustics, Diplomvej 377, 2800 Lyngby, Denmark, nwl@flexac.com), Eric R. Thompson (Centre for applied hearing research, Technical University of Denmark, DTU, Bygn. 352, 2800 Lyngby, Denmark, et@oersted.dtu.dk)

High levels of bass sound have been shown to stimulate the part of the brain that controls such basic instincts as sexual desire and hunger [Todd, 2000]. In rock and pop music, the bass frequencies from $40-125 \mathrm{~Hz}$ get amplified to very loud levels. Easily half of the electrical power of the PA and monitor system goes to these 1.5 octaves. A recent survey [Adelman-Larsen et al., 2007] showed that the most important subjective parameter for a rock and pop music hall to score a high rating was 'bass clarity' which correlated with a coefficient of 0.74 to 'overall impression'. Informal discussions with audio engineers and bass players give the perspective that artificial reverberation is rarely, if ever, added to bass-frequencies. In fact the ideal hall should be as dry as possible at low-frequencies. In the mid-treble frequency range, sound absorption, and thereby 'clarity', is easily obtained through the presence of the audience that absorbs 4-6 times more mid/high frequency sound energy than bass sound energy. In the low-frequency range 'clarity' is not so easily obtained. This paper discusses the challenge in depth and proposes design solutions.

\title{
Session 2aAAc
}

\section{Architectural Acoustics and Engineering Acoustics: Acoustics and Electroacoustics of Small Rooms}

\author{
Jiri Tichy, Cochair \\ Pennsylvania State University, 5552 N. Citation Road, Toledo, OH 43615, USA
}

Mendel Kleiner, Cochair

Chalmers Rm. Acoust. Group, Dept. of Appl. Acoust., Chalmers Univ. of Technology, Gothenburg, 41296, Sweden

\section{Invited Papers}

2aAAc1. Investigation of Bonello criteria as a practical tool in small room acoustics. Todd Welti (Harman International Industries, Inc., R\&D Group, 8500 Balboa Blvd, Northridge, CA 91329, USA, twelti@harman.com)

The Bonello criteria are often used as an objective metric for assessing room dimensions with respect to modal response. In reality, the modal response is not simply a property of the room dimensions, but is quite dependent on source/receiver locations as well. The legitimacy of Bonello's criteria when used in rooms with typical subwoofer and seating configurations has not been well investigated. In this study, a room model is used to calculate room responses for a large number of combinations of room dimensions, subwoofer positions, and seating locations. The Bonello criteria are evaluated for each combination and compared to perceptually relevant criteria such as flatness and seat to seat consistency of the calculated acoustical responses.

\section{8:40}

2aAAc2. Region of control for low frequency modal equalisation in small listening rooms. Aki Mäkivirta (Genelec Oy, Olvitie 5, FIN-74100 Iisalmi, Finland, aki.makivirta@genelec.com)

Small-size listening rooms are characterized by sparse modal density with pronounced spectral colouration when the decay times at low frequencies are large. Various active approaches for reducing the modal decay time have been suggested in the literature. A review of the salient principles of these approaches is given. Active control of the modal decay time at the primary listening position by using the primary radiator for control is demonstrated and evaluated for performance. The locality and size of the region of control, and the amount of control achieved, are studied in light of the experiment. Effect of the modal equalizer filter to the perceived response flatness, audibility of low frequency resonances, and the required amount of modal equalization are discussed in light of the experiment. 
2aAAc3. Improving room acoustics at low frequencies with multiple loudspeakers and time based room correction. Sofus Birkedal Nielsen (Aalborg University, Fredrik Bajers Vej 7 B, 9220 Aalborg Ø, Denmark, sbn@es.aau.dk), Adrian Celestinos (Oticon A/S, Kongebakken 9, 2765 Smørum, Denmark, adc@oticon.dk)

Small and medium size rectangular rooms are often used for sound reproduction. These rooms have substantial acoustical problems at low frequencies primarily caused by the reflections from the room boundaries. The spatial variation in sound pressure level (SPL) can be up to $30 \mathrm{~dB}$ in a room at low frequencies, and appear not only at modal frequencies. The problem is an acoustical issue in time, and should therefore be analyzed in the time-domain, instead of the traditional steady state frequency domain. The construction of a finitedifference time-domain approximation program (FDTD) has lead to a simple and untraditional solution called CABS (Controlled Acoustical Bass System) that makes use of multiple loudspeakers. With the proper placement of low frequency loudspeakers, CABS can create a plane wave from the front wall which will be absorbed by additional low frequency loudspeakers at the back wall. With the back wall reflection removed a homogeneous sound field will be created in the whole room at low frequencies. Simulations and measurements of normal size listening rooms show that 4 loudspeakers are enough to even the sound field in a room. The CABS system is controlled by a developed DSP system.

2aAAc4. Applications of a plane wave based room correction system for low frequencies using multiple loudspeakers. Adrian Celestinos (Oticon A/S, Kongebakken 9, 2765 Smørum, Denmark, adc@oticon.dk), Sofus Birkedal Nielsen (Aalborg University, Fredrik Bajers Vej 7 B, 9220 Aalborg Ø, Denmark, sbn@es.aau.dk)

When low frequency sound is radiated inside small listening spaces by loudspeakers, large uniformities occur over the sound field. This is due to the multiple reflection and diffraction of sound on the walls and different objects in the room. A developed system named Controlled Acoustically Bass System (CABS) produces uniform sound field at low frequencies. This is performed by utilizing loudspeakers at the front wall and extra loudspeakers at the opposite wall, processed to remove the rear-wall reflection of a rectangular room. Effectiveness of CABS on different room scenarios has been evaluated by using a computer simulation program based on the Finite Difference Time Domain Method (FDTD). CABS has been simulated in a small car cabin and in an irregular room. Non-ideal placement of loudspeakers in CABS have been evaluated. The influence of utilizing CABS with different types of loudspeakers has been evaluated by simulations and measurements.

\section{9:40}

2aAAc5. Active Sound Field Equalization. Akira Omoto (Kyushu University, 4-9-1, Shiobaru, Minamiku, 815-8540 Fukuoka, Japan, omoto@design.kyushu-u.ac.jp), Hisaharu Suzuki (Kyushu University, 4-9-1, Shiobaru, Minamiku, 815-8540 Fukuoka, Japan, hisaha@souldsp.jp), Akihiro Kakiuchi (Kyushu University, 4-9-1, Shiobaru, Minamiku, 815-8540 Fukuoka, Japan, kakirikakki @ hotmail.com)

Dominant acoustic modes often result in the inevitable non-uniform distribution of the acoustic quantities such as sound pressure, especially in the small sized enclosure. In our study, active control technique is thus introduced to overcome the adverse effects of such modes. Instead of the squared sound pressure which is normally adopted in active noise control, the quantity to be controlled is selected as the weighted values of acoustic intensities or the acoustic impedances in rectangular directions such as $\mathrm{x}, \mathrm{y}$ and $\mathrm{z}$ measured at plural points. The reasonably selected combinations of weights yield the control outputs which can alter the directions of propagating waves and result in reducing the complicated standing waves. Further, results of numerical simulations suggest that the well balanced arrangement of the primary and the secondary sources can 'rectify' the propagating direction and result in the uniform distributions of sound pressure. The strategies used in this study have possibilities of realizing useful controller for active sound field equalization.

\section{0:00}

2aAAc6. Different approaches for efficient finite element modelling of absorbers in small rooms. Marc Aretz (RWTH Aachen University, Institute for Technical Acoustics, Neustraße 50, 52066 Aachen, Germany, Marc.Aretz@akustik.rwth-aachen.de)

The FEM is a powerful tool for the numerical simulation of sound fields in enclosures. It accounts for the modal characteristics of the sound field, which are dominant at frequencies below the Schroeder frequency and it is also possible to model the mutual coupling between airborne and structure borne sound fields, when an appropriate structure model is implemented. When applying the FEM to complex room acoustics applications, like e.g. a control room in a recording studio, it is a challenging task to specify realistic boundary conditions. Different kinds of acoustical absorbers like Helmholtz resonators, plate absorbers and complex layered porous absorbers are found in these environments. While it is possible in principle to use complex and exhaustive models for these acoustical absorbers, it is often computationally much more efficient to use acoustic impedances or two-port network FEM elements to represent the fluid structure interactions. In the course of this study we compare different approaches for the modelling of Helmholtz resonators (without and without flow resistance), since it has proven very costly in terms of computation time to explicitly model every single hole in the resonator boxes. The simulation results are compared with results measured in a model room. 
2aAAc7. Direct and modal frequency response analysis of sound fields in small rooms by finite element method. Reiji Tomiku (Faculty of Engineering, Oita University, Dannoharu 700, 870-1192 Oita, Japan, tomiku-reiji@cc.oita-u.ac.jp), Toru Otsuru (Faculty of Engineering, Oita University, Dannoharu 700, 870-1192 Oita, Japan, otsuru@cc.oita-u.ac.jp), Noriko Okamoto (Faculty of Engineering, Oita University, Dannoharu 700, 870-1192 Oita, Japan, nokamoto@cc.oita-u.ac.jp), Yuka Kurogi (Faculty of Engineering, Oita University, Dannoharu 700, 870-1192 Oita, Japan, kurogi@cc.oita-u.ac.jp)

In this paper, sound pressures are computed by two techniques using finite element method. One is a technique by solving the system of linear equations directly (direct analysis) and the other is a technique by modal superposition (modal analysis). To confirm the accuracy of the direct analysis, sound pressures obtained by the technique are compared with those obtained by the modal analysis in a room with the volume of $10 \mathrm{~m}^{3}$. Then, as in the modal analysis, two methods are employed: one is a simplified method based on a real eigenvalue problem assuming that the damping matrix, [C], has orthogonality; and another is the method based on complex eigenvalue problem. Those obtained by the direct analysis are in good agreement with those obtained by the two kinds of modal analyses regardless of absorption conditions, even if the analysis is carried out at the frequency close to an eigen frequency. Next, diffuseness of sound field below $315 \mathrm{~Hz}$ in a room, which is used in the measurement of ISO140-3, is investigated by the direct analysis from the viewpoint of mean sound pressure level measurements.

\section{1:00}

2aAAc8. The effect of diffusers on low frequency modes. Jamie A. Angus (University of Salford, 12 Lister Way, YO30 6NL York, UK, j.a.s.angus@salford.ac.uk)

Small listening rooms are becoming prevalent, due to the availability of production facilities on personal computers, and the financial pressures faced by the industry. In these rooms, modal behaviour at low frequencies significantly affects the quality of the reverberant decay, due to their non-diffuse nature compared with other frequencies. Diffusion and absorption can help and may be a useful way of improving the low frequency performance of such rooms. This paper discusses the properties of these rooms at low frequencies, in particular the effect of diffusing boundaries on the modal behaviour of such rooms. The paper will first discuss what is meant by a mode and modal decay. It will then go on to examine the effect of diffusing boundaries on the frequency and density of modes. In particular, it will examine the effect of the scale of the diffuser on its efficacy in this task. For ease of visualisation this will be done using a two dimensional model and, for accuracy a finite element, element simulation. The effect of going to three dimensions on the results will also be discussed. Finally, the effect of absorption based diffusing boundaries will be examined.

\section{$11: 20$}

2aAAc9. Power output regularization in the active reproduction of sound fields in rooms. Nick Stefanakis (National Technical University of Athens, School of Electrical and Computer Engineering, Heroon Polytechniou 9, 15773 Athens, Greece, nstefan@mobile.ntua.gr), Sotiris Dalianis (National Technical University of Athens, School of Electrical and Computer Engineering, Heroon Polytechniou 9, 15773 Athens, Greece, dalias@central.ntua.gr), Tilemachos Karatzas (National Technical University of Athens, School of Electrical and Computer Engineering, Heroon Polytechniou 9, 15773 Athens, Greece, tilkar6@ hotmail.com), George Cambourakis (National Technical University of Athens, School of Electrical and Computer Engineering, Heroon Polytechniou 9, 157 73 Athens, Greece, gcamb@cs.ntua.gr)

In this paper we address the problem of using a multi-channel active control system in order to reproduce a harmonic sound field in a large part of the volume of a reverberant room. The problems associated with the calculation of the inverse system matrix are confronted by introducing a term that is proportional to the sound power-output of the system in the cost function that is obtained by the multiple point method. Simulation results show that this technique results to a better conditioning of the system matrix at low frequencies, comparing to other traditional regularization techniques. Moreover, it is shown that this method can be employed to increase the spatial robustness of the control sensor array inside the listening room.

\section{1:40}

2aAAc10. Low-frequency response in active acoustic practice rooms. Ronald Freiheit (Wenger Corporation, 555 Park Drive, Owatonna, MN 55060, USA, ron.freiheit@wengercorp.com)

Integrating active (virtual) acoustics into relatively small practice rooms to create a sense of envelopment is critical to the satisfaction of the musicians using these spaces for learning. The number of speakers and their locations play an important role in minimizing the ability to localize to the sources supporting this sense of envelopment. Equally important is the frequency response required for the speakers used in these applications, to more accurately simulate the acoustics of a performance environment. Excessive high frequencies increase the ability to localize and decrease the sensation of a larger space, since the air in larger spaces naturally absorbs many high frequencies. Another challenge is creating the sense of envelopment for instruments in the bass region ( $125 \mathrm{~Hz}$ octave band and below) by providing enough low-frequency energy response. Work will be presented on low-frequency response desired to satisfy a sampling 
of solo cello players using active acoustic practice rooms. The optimal sources for these low frequencies - single or multiple - will be discussed. Updated information will also be provided on the sound field coverage in active rooms with extended low-frequency response.

\section{Contributed Papers}

\section{2:00}

2aAAc11. Active playback of acoustic quadraphonic sound events. Domenico Stanzial (Italian National Research Council, FSSG-CNR Lab Acustica, c/o Fondazione Cini, Isola di San Giorgio Maggiore, 30124 Venezia, Italy, domenico.stanzial@cini.ve.cnr.it), Giorgio Sacchi (Physics Department - University of Ferrara, V. Saragat, 44100 Ferrara, Italy, giorgio.sacchi@student.unife.it), Giuliano Schiffrer (Physics Department University of Ferrara, V. Saragat, 44100 Ferrara, Italy, schgln@unife.it)

The reproduction in a given confined space - such as a cinema hall or a smaller room - of a sound event previously recorded in a completely different acoustical environment is an interesting and still open acoustical problem. A new method for hi-fi audio playback based on the general solution of the acoustic inverse problem is here pourposed. A feed-forward control based on overdetermination of conditions at active contours - i.e. loudspeakers - in order to obtain an optimal stable solution via least square approach is here proposed. This is easily possible even for complex configurations thanks to acoustic quadraphony, the application of sound intensimetry to audio technology developed in the last years within the IST-2511316-IP European project denominated IP-RACINE. After a short explanation of the model theory, the experimental application to the simplest case of 1-D confined field is presented and some obtained results are shown.

\section{2:20}

2aAAc12. Acoustics in a small control room. Sergio Beristain (Mexican Institute of Acoustics, P.O. Box 12-1022, Narvarte, 03001 Mexico, D.F., Mexico, sberista@hotmail.com)

Acoustics in small rooms is allways a problem, particularly at the low frequency range, because of their size and the fact that there is very limited space to install all the acoustic materials needed in order to obtain the desired acoustic response, but when an electroacoustic system has to be employed as it is the case in a small Control Room for a small recording studio, matters become even worse. First of all, the space is further reduced in order to accomodate the required sound system, and with the presence of hard to control low resonant frecuencies, which can be excited at any moment during any recording session, it can be produced highly different sound presure levels at those frequencies, changing the timbre of sounds. Some measurement results are presented.

TUESDAY MORNING, 1 JULY 2008

ROOM 253, 9:20 A.M. TO 1:00 P.M.

\section{Session 2aAAd}

\section{Architectural Acoustics and Noise: Acoustics and Privacy in Healthcare Facilities I: Emerging Policy Around the World}

David M. Sykes, Cochair

ANSI S12 Workgroup 44 and the Joint ASA/INCE/NCAC Subcommittee on Healthcare Acoustics \& Speech Privacy, 23 Buckingham Street, Cambridge, MA 02138, USA

Kerstin Persson Waye, Cochair

Dept. of Environ. Medicine, The Sahlgrenska Acad. of Gothenburg Univ., Box 414, Gothenburg, 405 30, Sweden

\section{Invited Papers}

\section{9:20}

2aAAd1. Waves of change: global policies \& their impacts on the acoustics profession. David M. Sykes (ANSI S12 Workgroup 44 and the Joint ASA/INCE/NCAC Subcommittee on Healthcare Acoustics \& Speech Privacy, 23 Buckingham Street, Cambridge, MA 02138, USA, david.sykes@remington-partners.com)

In a decade, five waves of change swept across the globe stimulating new interest in acoustics. This session examines their effects on the EU, North America and Japan. The Internet caused the first wave-the EU's privacy Directive 9546EC drove nations everywhere to develop privacy laws, many covering "Speech Privacy." A second wave, in 2000 when the "tech bubble" burst, produced laws to improve financial accountability and forcing organizations to find ways to shield their leaders. A third wave arose in 2001 with the increase in terrorism, producing a surge in security laws that challenge the right to privacy enshrined in the 1948 Declaration of Human Rights. The fourth wave is demographic: racing to build healthcare facilities for "boomers," this giant industry is now wrestling with a "noise epidemic" resulting from decades of neglecting the health effects of noise. The fifth wave came from the "green" movement-the concept of "indoor environmental quality" has renewed interest in noise as a pollutant. How countries balance such concerns as state security, citizen privacy and human health will be decided by courts but these five waves have catalyzed demand for understanding, insight, expertise, standards, codes, manpower and solutions from the acoustical profession. 
2aAAd2. Developing acoustical policies around in EU countries. Katrin Bergmark (Saint-Gobain Ecophon AB, Box 500, 26061 Hyllinge, Sweden, katrin.bergmark@ecophon.se), Marc R. Janssen (Saint-Gobain Ecophon AB, Box 500, 26061 Hyllinge, Sweden, marc.janssen@ecophon.se)

According to research, noise levels in hospitals worldwide have increased. At the same time, the awareness of the negative effects of noise on patients and healthcare staff has grown. To manage or eliminate noise in hospitals, standards and policies are a great help. An overview of international standards will be presented, showing various parameters and differences in values and angles of approach, e.g based on activity or room type. To further optimize the content, and stimulate the use of the standards, some countries have even defined healthcare specific standards. These can support the planning and the evaluation of the sound environment in those premises. New insights from research and case studies, as well as emerging laws, provide opportunities or even force us to extend the existing content of standards to meet future demands. Suggestions for that include extension of the amount of parameters, such as decrease of sound pressure level $(\triangle \mathrm{SPL})$ and parameters addressing privacy (PI, AC). Finally, revision of standards provides opportunities for end users to increase their understanding and interest in acoustic and to supply possibilities to define the desired quality of the sound environment.

\section{0:00}

2aAAd3. Effects of healthcare acoustics on medical outcomes. Roger Ulrich (Texas A\&M University, Department of Architecture, 3137 TAMU, College Station, TX 77843, USA, rulrich@archmail.tamu.edu)

Although considerable research has examined detrimental effects of noise on patient sleep quality in healthcare buildings, few studies have investigated the extent to which noise may worsen other types of patient clinical outcomes. Studies are also scarce concerning the effects of noise and poor acoustics on healthcare staff. The presentation describes a prospective controlled study conducted with colleagues in Sweden that examined the impact of higher versus lower noise levels, and longer in contrast to shorter reverberation times, on several patient and staff outcomes in a hospital coronary critical care unit (CCU). Acoustics were altered during the study period by changing the ceiling tiles throughout the CCU from sound-reflecting tiles to sound-absorbing tiles of similar appearance. Regarding patients, an improved acoustics environment significantly reduced physiological stress, increased satisfaction with quality of care, improved sleep quality, and lessened incidence of costly re-hospitalizations following discharge. Better acoustics also improved speech intelligibility, and healthcare staff experienced reduced work demands and less pressure and strain. The discussion concludes by outlining research directions, including the need for more outcomes studies to enable development of a strong business case for better healthcare acoustics.

\section{Contributed Papers}

10:20

2aAAd4. Influence of intenesive coronary care acoustics on the quality of care and phyiological state of patients. Inger Hagerman (Dept of Cardiology, Karolinska University Hospital, M52, Huddinge, 14186 Stockholm, Sweden, inger.hagerman@karolinska.se), Gundars Rasmanis (Dept of Cardiology, Karolinska University Hospital, M52, Huddinge, 14186 Stockholm, Sweden, gundars.rasmanis@karolinska.se), Vanja Blomkvist (Dept of Cardiology, Karolinska University Hospital, M52, Huddinge, 14186 Stockholm, Sweden, vanja.blomkvist@pubcare.uu.se), Roger Ulrich (Texas A\&M University, Department of Architecture, 3137 TAMU, College Station, TX 77843, USA, rulrich@archmail.tamu.edu), Töres Theorell (Dept of Cardiology, Karolinska University Hospital, M52, Huddinge, 14186 Stockholm, Sweden, tores.theorell@stressforskning.su.se)

Background unstable coronary disease is a stressful situation and environmental influences may increase stress mechanisms important for the cardiovascular status. Aim to evaluate acoustic influence on patients with coronary artery disease and to test if the effects of poor sound absorption on work environment affects quality of care and medical status of patients. Methods 94 patients admitted to the intensive coronary heart unit for chest pain evaluation participated. Patient groups were recruited during bad and good acoustic conditions respectively. Blood pressure, pulse amplitude, heart rate and heart rate variability were monitored. Patients were asked to fill in a questionnaire about the quality of the care and a follow up of rehospitalisation and mortality was made at 1 and 3 months. Results good and bad acoustics differed significantly with respect to pulse amplitude in acute myocardial infarction and unstable angina pectoris groups, with lower values at night during the good acoustics period. Re-hospitalisations were higher for the bad acoustics group. During the good acoustics period patients considered the staff attitude much better than during the bad acoustics period. Conclusion, bad acoustics environment during acute illness may have important physiological effects of importance for rehabilitation.

\section{0:40}

2aAAd5. The future of UK hospital design. Adrian Popplewell (Arup Acoustics, St Giles Hall, Pound Hill, CB3 OAE Cambridge, UK, adrian .popplewell@arup.com)

The importance of acoustic conditions within hospitals is highlighted by recent research indicating that patient recovery times are significantly influenced by the noise levels within wards. Added to this are concerns about speech privacy and confidentiality, patient and staff comfort, communication between patient and doctor, and the effects of vibration on sensitive medical equipment, all of which makes the acoustic design a key parameter in any hospital development. This paper will discuss the practical issues associated with the implementation of the current national guidance on hospital acoustics (Health Technical Memorandum 2045) and the new, currently unpublished replacement, Health Technical Memorandum 08-01. The experiences of the author with several large Private Finance Initiative projects, designed to the current standard, have illustrated the practical difficulties of fully complying with all its requirements and the design solutions which can be adopted. Speech privacy and confidentiality has been a critical issue in developing the new design standard. With Arup Acoustics as key contributors to this document, and acoustic designers of one of the first major projects in the UK to comply with this standard, the author is in a prime position to be able to assess the practical and technical implications of the future guidance.

11:00-11:20 Break 


\section{Invited Papers}

\section{1:20}

2aAAd6. Development of 'sound and vibration design guidelines for health care facilities'. Kurt Rockstroh (Steffian Bradley Architects, 100 Summer Street, Boston, MA 02110, USA, kurtr@steffian.com)

"The Guidelines for Design \& Construction of Healthcare Facilities" is utilized by the majority of the United States and its federal agencies involved in funding health care projects. As part of the development of the 2010 Edition of "The Guidelines" a joint subcommittee of ASA, INCE, NCAC and the Health Guidelines Revision Committee was formed to develop sound and vibration guidelines. This presentation will explain the collaborative process used to develop, publicize, review and formally vote acceptance of the sound and vibration guidelines. The presentation will also outline the major components of the new guidelines.

\section{1:40}

2aAAd7. Acoustics in green buildings: refining the concept of environmentally quality while improving occupant health and productivity synergistically. Sholem Prasow (Teknion Furniture Systems, 1150 Flint Rd., Thornhill, ON L3T 4 M9, Canada, sholem .prasow@teknion.com)

Environmental Quality has always been a keystone of the green building movement. The concept has evolved from "Indoor Air Quality", for example, in the first version of LEED seven years ago to a much broader and enriched concept - a concept that fully embraces Acoustics - today. This paper explores the evolution of Environmental Quality in both general and health care environments, and demonstrates the emerging role of Acoustics as an equal partner with Air Quality and Lighting as determinants of both health and productivity in the built environment. Specific references will be made to LEED rating systems and to emerging acoustical design requirements within LEED.

\section{2:00}

2aAAd8. Getting noise and speech privacy issues heard in design of healthcare facilities. Anjali Joseph (The Center for Health Design, 1850 Gateway Blvd., Suite 1083, Concord, CA 94520, USA, ajoseph@healthdesign.org)

Hospitals are extremely noisy, and noise levels in most hospitals far exceed recommended guidelines. The high ambient noise levels, as well as peak noise levels in hospitals, have serious impacts on patient and staff outcomes ranging from sleep loss and elevated blood pressure among patients to emotional exhaustion and burnout among staff. Poorly designed acoustical environments can pose a serious threat to patient confidentiality if private conversations between patients and staff or between staff members can be overheard by unintended listeners. At the same time, a poor acoustical environment impedes effective communication between patients and staff and between staff members by rendering speech and auditory signals less intelligible or detectable. This has serious implications for patient safety. A well-designed acoustical environment is critical in addressing these problems related to noise and communication of information. The purpose of this presentation is to examine how different aspects of sound - noise, speech privacy, speech intelligibility, and music - impact patient and staff outcomes in healthcare settings and the specific environmental design strategies that can be used to improve the acoustical environment of healthcare settings.

2aAAd9. Noise confounds in functional MRI research and potential solutions. Marc J. Kaufman (McLean Hospital, 115 Mill St., Brain Imaging Center, Belmont, MA 02478, USA, kaufman@mclean.harvard.edu), Blaise D. Frederick (McLean Hospital, 115 Mill St., Brain Imaging Center, Belmont, MA 02478, USA, BBFrederick@mclean.harvard.edu), Eric E. Ungar (Acentech Inc, 33 Moulton St., Cambridge, MA 02138, USA, eungar@ACENTECH.com), Jonathan D. Kemp (Acentech Inc, 33 Moulton St., Cambridge, MA 02138, USA, jdkemp@acentech.com), David M. Sykes (ANSI S12 Workgroup 44 and the Joint ASA/INCE/NCAC Subcommittee on Healthcare Acoustics \& Speech Privacy, 23 Buckingham Street, Cambridge, MA 02138, USA, david.sykes@ remingtonpartners.com)

High field functional MRI (fMRI) is becoming a neuroscience research technique of choice because it is noninvasive and can reveal brain circuitry regulating sensory, motor, and cognitive functions. Unfortunately, rapid scan fMRI results in high noise levels (100-140 dB) that can alter auditory, visual, and pain system function, and also can induce stress, which itself modulates brain responses to various stimuli. These effects can confound fMRI data interpretation. A number of solutions for this problem have been proposed including modifying MRI scanner hardware to reduce noise output, an expensive proposition limited to willing manufacturers, and modifying fMRI pulse sequences to reduce noise output, which is effective within certain limitations. Another approach is to develop acoustic noise isolating equipment that separates subjects from noise. This passive approach confers maximum flexibility because it is both hardware- and fMRI scan sequence-independent. We present initial data documenting efficacy of first generation acoustic noise isolating equipment for animal fMRI studies. As nearly 25\% of patients referred for clinical MRIs refuse scans because they are stressful, acoustic noise isolating equipment scaled for clinical MRI scanner use may decrease stress and increase patient compliance, thereby decreasing morbidity and mortality, and improve MRI center workflow. 
2aAAd10. Experimental study on applicability of sound masking system in medical examination room. Kanako Ueno (Institute of Industrial Science, University of Tokyo, Komaba 4-6-1, Meguro-ku, 153-8505 Tokyo, Japan, ueno@iis.u-tokyo.ac.jp), Hyojin Lee (Institute of Industrial Science, University of Tokyo, Komaba 4-6-1, Meguro-ku, 153-8505 Tokyo, Japan, leehj@iis.u-tokyo.ac.jp), Shinichi Sakamoto (Institute of Industrial Science, University of Tokyo, Komaba 4-6-1, Meguroku, 153-8505 Tokyo, Japan, sakamo@iis.u-tokyo.ac.jp), Atsuko Ito (Center for Advanced Sound Technologies, Yamaha, 203 Matsunokijima, 4380192 Iwata, Shizuoka, Japan, atsuko1_ito@gmx.yamaha.com), Mai Fujiwara (Center for Advanced Sound Technologies, Yamaha, 203 Matsunokijima, 4380192 Iwata, Shizuoka, Japan, mai_fujiwara@gmx.yamaha.com), Yasushi Shimizu (Center for Advanced Sound Technologies, Yamaha, 203 Matsunokijima, 4380192 Iwata, Shizuoka, Japan, yasushi_shimizu@gmx .yamaha.com)

Recently, speech privacy to avoid oral information leakage in healthcare facilities has become an important issue. This study investigated effectiveness of sound masking system in regard to masking efficiency, annoyance and its influence on speech conversation for medical examination rooms in an experimental approach. Considering actual application, two adjacent medical examination rooms partitioned by a low sound insulation wall in a typical healthcare facility were selected as an experimental field and sound masking system was temporally installed. In the rooms, acoustic environment was measured and reproduced in an anechoic room with a 3-D sound field simulation system using a 6-ch sound recording/reproduction technique. In the simulated acoustic condition, subjective tests were designed to quantify the masking efficiency and annoyance caused by the masking sound. The annoyance test was conducted in listening condition (with high attention to the sound) and in talking condition (with low attention). As a result, mixed maskers composed by water stream, synthesized speech signals, and steady state noise showed high performance in both aspect of masking efficiency and annoyance.

\title{
Session 2aAAe
}

\section{Architectural Acoustics, Musical Acoustics, Physical Acoustics, and Noise: Acoustics of Opera Houses I}

\author{
Robin Glosemeyer Petrone, Cochair \\ 918 16th St., Apt 3, Santa Monica, CA 90403, USA \\ Roberto Pompoli, Cochair \\ Engineering Dept. - Univ. of Ferrara, Via Saragat, 1, Ferrara, 44100, Italy
}

\section{Invited Papers}

$10: 20$

2aAAe1. Generous opera house acoustics for lyric and symphonic performances. Daniel E. Commins (Commins Acoustics Workshop, 15 rue Laurence Savart, 75020 Paris, France, d.commins@comminsacoustics.com)

It is believed that a short acoustic response is needed in an opera house to guarantee good intelligibility of lyrics. Some of the best opera houses have a relatively long reverberation time associated to good clarity. They are more suitable to symphonic concerts than "damped" rooms. The recently opened 1350-seat Grand Théâtre de Provence in Aix-en-Provence, France, is an example of an opera house with a long response and good clarity. An adequate orchestra shell and proper permanent acoustical treatment of the stage tower lead to suitable acoustical parameters in the symphony configuration. The measurements show that in an opera house of this capacity, it is possible to create conditions compatible with opera, symphony, chamber music and recitals. The rich opera configuration sound generates mostly positive reactions. Most concert goers consider that this room is now the reference for the region. One must note however that this approach is risky since the values that are considered ideal for opera must be stretched far. It may lead to excessive reverberation and even to unwanted reflections. This target requires genuine investigations, from early concept to final design, using the most advanced prediction and modeling techniques. The design of larger rooms under the same principle requires even more care.

\section{0:40}

2aAAe2. Opera House Reverberation Times - Design Considerations. Eckhard Kahle (Kahle Acoustics, 188 avenue Molière, 1050 Brussels, Belgium, kahle@kahle.be), Thomas Wulfrank (Kahle Acoustics, 188 avenue Molière, 1050 Brussels, Belgium, twulfrank@kahle.be), Yann Jurkiewicz (Kahle Acoustics, 188 avenue Molière, 1050 Brussels, Belgium, yjurkiewicz@kahle.be), Brian F. Katz (LIMSI-CNRS, B.P. 133, 91403 Orsay, France, brian.katz@limsi.fr)

The intimacy of many historic European Opera Houses, especially of the traditional Italian style, is highly cherished and many of these halls are considered to be among the best halls acoustically. From an acoustical point of view the generally small dimensions often combined with a moderate seat count - provide excellent source presence and clarity. On the other hand, the corresponding small volume leads to short reverberation times, and in recent decades higher reverberation times have been preferred and asked for by clients and audiences in many countries. Ideas will be presented on how this apparent dilemma between the preference for small dimensions (for intimacy, source presence and definition) and increased volume (in order to create longer reverberation times) can be addressed. 
2aAAe3. The "Teatro di San Carlo" in Naples and its smaller clone "Teatro Verdi" in Salerno. Raffaele Dragonetti (DETEC, University of Naples Federico II, Piazzale Tecchio 80, 80125 Naples, Italy, dragonet@ unina.it), Carmine Ianniello (DETEC, University of Naples Federico II, Piazzale Tecchio 80, 80125 Naples, Italy, ianniell@ unina.it), Francesco Mercogliano (DETEC, University of Naples Federico II, Piazzale Tecchio 80, 80125 Naples, Italy, francesco.mercogliano@unina.it), Rosario A. Romano (DETEC, University of Naples Federico II, Piazzale Tecchio 80, 80125 Naples, Italy, rosroman@unina.it)

The Teatro di San Carlo in Naples (Italy) is a well preserved baroque-type theatre. It was opened in 1737 and flourished up to a destructive fire. Nine months after the disastrous event the San Carlo was rebuilt completely as it was and reopened in 1817. It is still praised for its sound quality for opera performance. The Teatro Verdi was conceived by the Municipality of Salerno (Italy) in 1843. However, the "querelles" of local parties delayed much its construction so that it could be opened only in 1872 . The architects who designed the Teatro Verdi were influenced very much by the successful Teatro di San Carlo and tried to copy the older and not-far-one in a smaller scale. The Verdi has undergone few minor changes during its life and is used also for opera shows. The main halls of the two are in a scale about 1:5. This paper reports a comparison of their acoustical features in terms of objective room-acoustics parameters accepted for opera-house sound quality. Acoustic measurements were performed with the same instrumentation set. Similarities and differences are discussed.

\section{$11: 20$}

2aAAe4. Teatro 'La Fenice', Venice --- The secrets of the acoustical reconstruction of the destroyed theater according to historical and modern requirements. Jürgen Reinhold (Müller BBM GmbH, Robert Koch Strasse 11, D 82152 Planegg/München, Germany, Juergen.Reinhold@MuellerBBM.de)

The reconstruction of this artistically and architecturally very important opera house that was completely destroyed by a fire and which was famous throughout the world not least for its excellent acoustical qualities, was a brilliant challenge also for an acoustician. The historical planning requirements - complete reconstruction "as it was; where it was" - the latest findings in room acoustics as well as a huge number of "modern" requirements are to be brought in line. The theatre hall as heart of the Teatro "La Fenice" was reconstructed in its classical horseshoe shape with five tiers in pure wooden construction. Modern standard specifications had to be met for ventilation and air conditioning, the installation of a modern stage machinery as well as an improved safety and fire prevention concept. Furthermore the space allocation plan had to be extended by the integration of new rehearsal rooms and technical equipment rooms. From the point of view of building and room acoustics this was a very complex task - if in addition the cramped conditions of the Teatro "La Fenice" in Venice are taken into consideration. The lecture provides an insight into the building and room acoustical planning, its realization and the achieved acoustical results.

\section{1:40}

2aAAe5. The acoustics of the Beijing National Grand Theatre of China. Isabelle Schmich (CSTB, 24, Rue Joseph Fourier, 38400 Saint Martin D’Hères, France, isabelle.schmich@cstb.fr), Paul Chervin (CSTB, 24, Rue Joseph Fourier, 38400 Saint Martin D’Hères, France, paul.chervin@ cstb.fr), Zhu Xiangdong (School of Architecture, Tsinghua University, Room 104, Centre Main Building, 100084 Beijing, China, zxd@abcd.edu.cn), Yan Xiang (School of Architecture, Tsinghua University, Room 104, Centre Main Building, 100084 Beijing, China, yx@abcd.edu.cn), L Guo-Qi (The National Grand Theatre, 2, Chang an sast street, 100031 Beijing, China, liguoqi@fujisound.com)

The National Grand Theatre of China opened in December 2007 in Beijing. It is a complex of three performance halls: the Opera (2400 seats), the Concert Hall (2000 seats) and the Theatre (1100 seats). These three halls are covered by a super-ellipsoidal shell made out of titanium and glass. The history of the acoustic design evolution from the winning of the competition in 1999 by the Architect Paul Andreu to the final construction will be retraced including the preliminary design, the acoustic simulations and auralisations. The acoustic design of each hall will be presented with its specifications and detailed particularities. Final acoustic measurements have been done in the three empty halls and in the occupied concert hall. The results and acoustic criteria will be discussed. In addition, a subjective evaluation has been done with psychoacoustic questionnaires and the results will be presented and analysed.

\section{Contributed Papers}

\section{2:00}

2aAAe6. Acoustical design and scale model test for the opera house of Zhongshan city. Shuo Xian Wu (State Key Laboratory of Subtropical Building Science, South China University of Technology, 381 Wushan Street, 510640 Guangzhou, China, arshxwu@scut.edu.cn), Yue Zhe Zhao (State Key Laboratory of Subtropical Building Science, South China University of Technology, 381 Wushan Street, 510640 Guangzhou, China, arzhyzh@scut.edu.cn)

The opera house of Zhongshan City in Guangdong province of China is the performing art center of the city. It was completed in Oct. 2005. It has 1400 seats including 833 stall seats and another 484 seats on two levels of balcony. The volume of the auditorium is $12000 \mathrm{~m} 3$ and the $\mathrm{V} / \mathrm{N}$ is $9.1 \mathrm{~m} 3$ per seat. The main purpose of the building is for the performances of opera and ballet. Some music and conference events are also to be held there. Therefore, the occupied reverberation time at medium frequencies is set to be 1.6s and its background noise level has to meet NR-20. During the acoustical design, a 3D computer simulation model established with ODEON software and a 1:20 scale model was made to analyze the sound fields of the auditorium with and without a music shell on the stage. After the completion of the building, an acoustics test was taken. Several performances show that its acoustics reaches a quite high level and has won high praises.

$$
\text { 12:20 }
$$

2aAAe7. Subjective Assessments of Acoustical Environments for Un-assisted Traditional Peking Opera Performances. Wei-Hwa Chiang (National Taiwan University of Science and Technology, 43, sec. 4 Keelung Rd, Taiwan, 106 Taipei, Taiwan, edchiang1224 @ hotmail.com), Wei Lin (National Taiwan University of Science and Technology, 43, sec. 4 Keelung Rd, Taiwan, 106 Taipei, Taiwan, D9313001@mail.ntust.edu.tw), Ya-Jhen Yu (National Taiwan University of Science and Technology, 43, sec. 4 Keelung Rd, Taiwan, 106 Taipei, Taiwan, ianmelody@hotmail.com)

The prevalence of Western performing arts has impacted on how traditional Peking opera is performed. It is, however, necessary to preserve and pass on traditional Peking opera for it integrates the essence of Chinese 
opera. The research has been conducted regarding subjective assessment of the acoustical environment for traditional Peking opera. Recording of dry sound sources was also performed. Sound strength and listening direction were found to be the principle factors that determined evaluation for overall impression while reverberation time is less important. The $6000 \mathrm{~m} 3$ can be used as the upper limit for room volume when a thrust stage is used.

12:40

2aAAe8. Considerations about the acoustical properties of Teatro Nuovo in Spoleto after the restauration works. Alessandro Cocchi (University, DIENCA Dept. Facoltà di Ingegneria, Viale Risorgimento 2, 40136 Bologna, Italy, alessandro.cocchi@mail.ing .unibo.it), Marco Cesare Consumi (University, DIENCA Dept. Facoltà di Ingegneria, Viale Risorgimento 2, 40136 Bologna, Italy,
marcocesare.consumi@mail.ing.unibo.it),Ryota Shimokura(AIST, 1-8-31 Midorigaoka, Ikeda, 563-8577 Osaka, Japan, ryota.shimokura@ aist.go.jp)

In 2003 the municipality of Spoleto decided to stop the performances within the Teatro Nuovo, among the others also of "Two World Annual Festival", as it was necessary to modify some material, repair the floor, refurbish pictures and so on: the first author was charged with all the acoustical aspects and he decided to perform extensive acoustical measurements. During this measurement campaign, a flutter echo was detected in the stalls, so it became necessary to detect the origin of this problem, then to find some modification able to remove this problem: we have already presented our studies on this subject, from which a new design of the orchestra pit was derived. For fire safety reasons it was necessary also to remove some elastic panels from the boxes, and an acoustically equivalent technical solution was adopted. Even the stalls floor was removed and a new solution was adopted incorporating the heating plant and an acoustical solution. In this paper we will present the results of measurements performed after the opening of the theatre (a typical Italia Opera House of the end of the Eighteen Century) and some consideration about the acoustical results so obtained.

TUESDAY MORNING, 1 JULY 2008

ROOM 342B, 8:00 A.M. TO 1:20 P.M.

\title{
Session $2 \mathrm{aAB}$
}

\section{Animal Bioacoustics and ECUA: Animal Bioacoustic Censusing I}

\author{
Marie A. Roch, Cochair \\ San Diego State University, Dept. of Computer Science, San Diego, CA 92182-7720, USA \\ Cédric Gervaise, Cochair \\ E3I2 - EA3876, 2 rue François Verny, Brest Cedex, 29806, France
}

\section{Invited Papers}

\section{8:00}

2aAB1. Passive acoustic detection of grouper sound production. David Mann (University of South Florida, College of Marine Science, 140 7th Ave. S., St. Petersburg, FL 33701, USA, dmann@marine.usf.edu), James Locascio (University of South Florida, College of Marine Science, 140 7th Ave. S., St. Petersburg, FL 33701, USA, locascio@marine.usf.edu), Michelle Schärer (University of Puerto Rico-Mayaguez, Department of Marine Sciences, Isla Magueyes, La Parguera, 00667 Lajas, Puerto Rico, m_scharer@hotmail.com), Chris Koenig (Florida State University, Coastal and Marine Laboratory, St. Teresa Beach, FL 32358, USA, koenig@bio.fsu.edu), Michael Nemeth (University of Puerto Rico-Mayaguez, Department of Marine Sciences, Isla Magueyes, La Parguera, 00667 Lajas, Puerto Rico, michaelnemeth@hotmail.com), Misty Nelson (University of South Florida, College of Marine Science, 140 7th Ave. S., St. Petersburg, FL 33701, USA, mnelson@marine.usf.edu), Felicia Coleman (Florida State University, Coastal and Marine Laboratory, St. Teresa Beach, FL 32358, USA, coleman@ bio.fsu.edu), Richard Appeldoorn (University of Puerto Rico-Mayaguez, Department of Marine Sciences, Isla Magueyes, La Parguera, 00667 Lajas, Puerto Rico, rappeldo@uprm.edu)

Passive acoustic recordings were used to study the behavior of red hind (Epinephelus guttatus) at spawning aggregation sites off of Puerto Rico and Mona Island, and goliath grouper (Epinephelus itajara) and red grouper (Epinephelus morio) on the West Florida Shelf. The sounds produced by each species were unique, low-frequency pulsed sounds and associated with reproductive-related behavior. Male red hind produced sounds composed of a series of pulses that graded into a tonal-like sound, mostly during territorial patrolling. Long-term acoustic recorder (LARS) data from the west coast of Puerto Rico and Mona Island showed similar diel periodicities of sound production of red hind, but had different monthly peaks in sound production. Goliath grouper sounds consisted of low-frequency individual pulses $(50-100 \mathrm{~Hz})$ that are consistent with sounds produced by a large fish, and showed lunar periodicity in sound production. Peaks in sound production occurred nightly after midnight, but sounds were also produced throughout the day. Sound production and spawning by red grouper was recorded using a remotely operated vehicle (ROV) at depths of 80-100 m. Passive acoustic techniques can provide synoptic, long-term time series of sound production associated with reproductive activities of soniferous species at widely spaced sites.

$$
\text { 8:20 }
$$

2aAB2. The value of acoustic technologies for monitoring bird migration. Andrew Farnsworth (Cornell Laboratory of Ornithology, 420 E 54th St, 38J, New York, NY 10021, USA, af27@cornell.edu)

Many North American species of birds give distinctive flight calls during nocturnal migration. Monitoring these calls can be a powerful method for studying the timing and magnitude of migration, as well as for confirming the presence of individual species and 
potentially for quantifying passage rates. Recent technological advances in acoustic monitoring, such as increased processor speeds of computers, automated detection software, increased data storage capacities, and a comprehensive identification guide, permit recording of the vocalizations of passing migrants over entire nights for entire migration seasons, thus yielding data on species composition, migration timing and routing, and the magnitude of migration traffic. I will discuss several recent studies that have used acoustic technologies to monitor nocturnal migration, presenting data on species composition and relative abundance. Additionally, I will present some recent approaches to addressing quantification of passing migrants by their calls. I will also discuss wider applications of this technology beyond the realm of North American migrant species.

\section{Contributed Papers}

\section{8:40}

2aAB3. Automatic detection of short time periodic bird calls in realistic monitoring scenarios. Daniel Wolff (Department of Computer Science III, University of Bonn, Roemerstr. 164, 53117 Bonn, Germany, wolffd@bonn.edu), Klaus H. Tauchert (Humboldt-University Berlin, Institute of Biology, Invalidenstr. 43, 10115 Berlin, Germany, klaus.tauchert@gmx.de), Karl H. Frommolt (Humboldt-University Berlin, Museum für Naturkunde, Tierstimmenarchiv, Invalidenstr. 43, 10115 Berlin, Germany, karl-heinz.frommolt@rz.hu-berlin.de), Rolf Bardeli (Department of Computer Science III, University of Bonn, Roemerstr. 164, 53117 Bonn, Germany, bardeli@iai.uni-bonn.de), Frank Kurth (FGAN-FKIE, Abteilung KOM, Neuenahrer Str. 20, 53343 Wachtberg-Werthhoven, Germany, kurth@fgan.de), Michael Clausen (Department of Computer Science III, University of Bonn, Roemerstr. 164, 53117 Bonn, Germany, clausen@iai .uni-bonn.de)

In this contribution, we propose a method for the automatic detection and localisation of bird calls featuring simple as well as highly repetitive structures. Reporting from a research project focused at computer aided acoustical monitoring, a detector for Locustella luscinoides (Savi's Warbler) is presented, performing reliably even on highly distorted recordings. In cooperation with the Animal Sound Archive at the Humboldt University, Berlin, this detector was incorporated into a multimodal censusing method combining GPS-data and automatically annotated audio recordings to perform line mapping. An evaluation of the proposed techniques for unsupervised monitoring purposes was also performed on 20 hours of multichannel recordings from Lake Parstein, a cane brake area in Germany. Finally, exem- plifying the discriminative potential of the underlying features, we will point out the application to other members of the Warbler family as well as the detection of cricket and toad sounds.

\section{9:00}

2aAB4. Animal censusing using seismic cues: techniques used for African elephants. Jason D. Wood (Beam Reach Marine Science and Sustainability School, 7044 17th Ave NE, Seattle, WA 98115, USA, jason@beamreach.org), Caitlin E. O’Connell-Rodwell (Stanford University, Department of Otolaryngology, Head and Neck Surgery, Stanford, 94305, USA, ceoconnell@stanford.edu), Simon L. Klemperer (Stanford University, Department of Geophysics, Stanford, 94305, USA, sklemp @ stanford.edu)

Counting populations of animals has proven to be difficult and inexact for species that are difficult to detect visually. A growing number of researchers have successfully turned to detecting animals by their acoustic signals in order to count their numbers. To predict the number of animals present a regression line is generated from the relationship between such measures as calling rate and the number of animals documented visually during that recording by the researcher. Depending on the communication system of the species in question, a significant amount of the variation in calling rate can be attributed to other factors such as group behavior rather than the number of individuals present. For species where these other sources of variation are high it may be more appropriate to use acoustic cues to detect and count the number of animals. This paper will present techniques used to detect and estimate elephant numbers from geophone recordings of their footfalls; cues that are a byproduct of their locomotion and are thus not as dependent on group behavior.

\section{Invited Papers}

\section{9:20}

2aAB5. Species identification and measurement of activity in odontocete species of Palmyra Atoll by acoustic monitoring. Simone Baumann (Scripps Institution of Oceanography- UCSD, 9500 Gilman Dr. \#0205, La Jolla, CA 92093, USA, sbaumann@ucsd.edu), John A. Hildebrand (Scripps Institution of Oceanography- UCSD, 9500 Gilman Dr. \#0205, La Jolla, CA 92093, USA, jhildebrand@ucsd.edu), Sean M. Wiggins (Scripps Institution of Oceanography- UCSD, 9500 Gilman Dr. \#0205, La Jolla, CA 92093, USA, swiggins@ucsd.edu), Hans-Ulrich Schnitzler (Eberhard-Karls-Universität Tübingen, Zool. Institut, Abt. Tierphysiologie, Auf der Morgenstelle 28, 72076 Tübingen, Germany, hans-ulrich.schnitzler@uni-tuebingen.de)

Acoustic monitoring has been used to study odontocete presence at Palmyra Atoll, a remote island in the Northern Line Islands chain. Long-term recordings of high-frequency, broadband acoustic data have become possible with recent technological advances. A High-frequency Autonomous Recording Package (HARP) has been developed which samples at $200 \mathrm{kHz}$ with a duty cycle of $1 / 4$ for up to seven months. This instrument has recorded since October 2006 at Palmyra Atoll. Visual and acoustic surveys were conducted around Palmyra Atoll using a four-element towed hydrophone array sampling real-time at $200 \mathrm{kHz}$ to obtain species-specific acoustic data. These data are used as reference for automatic detection algorithms applied on the long-term recordings. To date, acoustically and visually detected odontocetes include bottlenose dolphins (Tursiops truncatus), spinner dolphins (Stenella longirostris), melon-headed whales (Peponocephala electra) and beaked whales of the genus Mesoplodon. The long-term HARP data reveal acoustic activity primarily at night time and predominantely odontocete clicks. Both the beaked as well as the melon-headed whales are present year round and show a distinct daily acoustic activity cycle.

2aAB6. Distribution patterns of delphinids in the California Current Ecosystem observed through acoustic monitoring of species-specific echolocation clicks. Erin M. Oleson (Scripps Institution of Oceanography- UCSD, 9500 Gilman Dr. \#0205, La Jolla, CA 92093, USA, eoleson@ucsd.edu), Melissa S. Soldevilla (Scripps Institution of Oceanography- UCSD, 9500 Gilman Dr. \#0205, La Jolla, CA 92093, USA, msoldevilla@ucsd.edu), John Calambokidis (Cascadia Research Collective, 218 1/2 W. 4th Ave., Olympia, WA 98501, USA, calambokidis@cascadiaresearch.org), Curtis Collins (Naval Postgraduate School, 833 Dyer Road, Rm 328, 
Monterey, CA 93943, USA, Collins@ nps.edu),Sean M. Wiggins (Scripps Institution of Oceanography- UCSD, 9500 Gilman Dr. \#0205, La Jolla, CA 92093, USA, swiggins@ucsd.edu), John A. Hildebrand (Scripps Institution of Oceanography- UCSD, 9500 Gilman Dr. \#0205, La Jolla, CA 92093, USA, jhildebrand@ucsd.edu)

Visual surveys along the U.S. west coast conducted in the 1980s-90s suggested that Pacific white-sided and Risso's dolphins likely undergo annual movement between California during winter-spring, and Oregon-Washington in the summer-fall. Using high-frequency autonomous acoustic recordings within the southern California, central California, and Washington regions of the California Current System, we evaluated the seasonal occurrence of these dolphin species through detection of their echolocation clicks. The clicks of Pacific white-sided and Risso's dolphins are characterized by a unique combination of local frequency peaks for each species, providing a statistically robust means of identification from autonomous acoustic records. Based on these unique spectral characters, we extracted periods of clicking by each species from concurrently sampled coastal and shelf/slope locations off southern California and Washington, and a single offshore site off central California. The number of hours that each species was heard per day was quantified and compared among seasons and regions. Both species were heard year-round at shelf/slope sites off southern California and Washington; however their patterns of occurrence at coastal sites differed somewhat. Our results indicate year-round presence of Pacific white-sided and Risso's dolphins in some regions, suggesting that the seasonal distribution of these species may have recently changed.

10:00-10:20 Break

\section{Contributed Papers}

10:20

2aAB7. Radial distance sampling with passive acoustics: The prospect of estimating absolute densities of cetaceans from static acoustic datalogger data. Jakob Tougaard (National Environmental Research Institute, University of Aarhus, Frederiksborgvej399, DK-4000 Roskilde, Denmark, jat@dmu.dk)

Recording animal vocalisations with a static acoustic datalogger can be viewed as a form of point transect sampling and results analysed within the framework of distance sampling theory. The key element is the radial detection function, which specifies the probability of detecting a vocalisation as a function of distance from the datalogger. This function can be modelled theoretically or preferably determined experimentally and from it the efficient radial detection distance can be determined. Radial detection functions were determined for two different harbour porpoise dataloggers (T-PODs, version 1 and version 3 ) by means of concurrent visual tracking of porpoises. $52 \%$ and $82 \%$ of the porpoises within $100 \mathrm{~m}$ from the T-PODs were detected by the V1 and V3 T-POD, respectively and $11 \%$ and $30 \%$, respectively were detected between 100 and $200 \mathrm{~m}$ from the T-PODs. Effective detection radius (EDR) was $97 \mathrm{~m}$ and $150 \mathrm{~m}$ for the two T-PODs, respectively. Porpoises echolocate almost continuously. If it is assumed that silent periods rarely exceeds 1 minute in duration an average detection rate on the version 3 T-POD for the study site of 2.7 detection positive minutes per hour can be converted into a density estimate of 0.69 porpoises $/ \mathrm{km}^{2}$.

\section{0:40}

2aAB8. Computer-aided detection of non-stereotyped bowhead whale calls in the presence of seismic airgun signals. Aaron Thode (Marine
Physical Laboratory, Scripps Institution of Oceanography, 9500 Gilman Dr, MC 0238, La Jolla, CA 92093-0238, USA, athode@ucsd.edu), Delphine Mathias (Marine Physical Laboratory, Scripps Institution of Oceanography, 9500 Gilman Dr, MC 0238, La Jolla, CA 92093-0238, USA, delphine.mathias@gmail.com), Miles McLennan (Greeneridge Sciences, Inc., 4512 Via Huerto, Santa Barbara, CA 93110, USA, billm @greeneridge.com), Charles R. Greene (Greeneridge Sciences, Inc., 4512 Via Huerto, Santa Barbara, CA 93110, USA, cgreene@greeneridge.com)

In 2007 thirty-five autonomous recording packages were deployed over a 150 mile swath in the Beaufort Sea to monitor the annual migration of the bowhead whale (Balaena mysticetus) population during seismic exploration activities. Over 1350 days worth of acoustic data were gathered, generating a need for computer-aided assistance in detecting calls. Bowhead whales produce over ten types of frequency modulated calls, and the frequency range, duration, and fine structure of individual calls vary considerably even within each call type, creating difficulties when using simple matchedfiltering or spectrogram correlation. Numerous airgun signals also display frequency-modulated structure, complicating the challenge. In this presentation a three-stage process is presented for detecting arbitrary bowhead whale calls in the presence of seismic airgun signals, implemented in JAVA and MATLAB, with components extracted from the industry-supported PAMGUARD software package. The first stage runs several "energy-based" detectors simultaneously across multiple frequency bands to capture events, and a second stage analyzes the timing of these detections to remove regular periodic sequences, such as those expected from airguns. The final stage attempts to trace contours on spectrograms. Both genetic algorithms and direct optimization are used to optimize the program's 21 input parameters. [Work supported by Shell Exploration and Production Company].

\section{Invited Papers}

\section{1:00}

2aAB9. Comparison of feature extraction methods for the identification of odontocete species based upon echolocation clicks. Marie A. Roch (San Diego State University, 5500 Campanile Dr, Dept of Computer Science, San Diego, CA 92182-7720, USA, marie.roch@sdsu.edu), Holger Klinck (Alfred Wegener Institute, P.O. Box 120161, 27515 Bremerhaven, Germany, holger.klinck@awi.de), David K. Mellinger (Oregon State Univ. and NOAA, 2030 SE Marine Science Dr., Newport, OR 97365, USA, David.Mellinger@oregonstate.edu), Melissa S. Soldevilla (Scripps Institution of Oceanography- UCSD, 9500 Gilman Dr. \#0205, La Jolla, CA 92093, USA, msoldevilla@ucsd.edu), John A. Hildebrand (Scripps Institution of Oceanography- UCSD, 9500 Gilman Dr. \#0205, La Jolla, CA 92093, USA, jhildebrand@ucsd.edu)

Recent work by several groups has shown that odontocete echolocation clicks contain information that can be used to detect or identify specific species. In this study, we compare the relative performance of cepstral and wavelet features on various Pacific Ocean species of odontocetes. Comparison of features within different systems is often complicated by the large number of variables unrelated to feature extraction that change between systems. By experimenting within a proven state of the art classification framework, it is possible to make meaningful comparisons of feature extraction performance with respect to common machine learning algorithms such as neural networks, support vector machines, and Gaussian mixture models. 
2aAB10. Long-term Passive Acoustic Monitoring of Delphinids in the Southern California Bight. Melissa S. Soldevilla (Scripps Institution of Oceanography- UCSD, 9500 Gilman Dr. \#0205, La Jolla, CA 92093, USA, msoldevilla@ucsd.edu), John A. Hildebrand (Scripps Institution of Oceanography- UCSD, 9500 Gilman Dr. \#0205, La Jolla, CA 92093, USA, jhildebrand@ucsd.edu), Sean M. Wiggins (Scripps Institution of Oceanography- UCSD, 9500 Gilman Dr. \#0205, La Jolla, CA 92093, USA, swiggins@ucsd.edu), Marie A. Roch (San Diego State University, 5500 Campanile Dr, Dept of Computer Science, San Diego, CA 92182-7720, USA, marie.roch@sdsu.edu)

Spectral characteristics of clicks are described for five species of delphinids in the Southern California Bight (long-beaked common dolphins, Delphinus capensis, short-beaked common dolphins, Delphinus delphis, Risso's dolphins, Grampus griseus, Pacific whitesided dolphins, Lagenorhynchus obliquidens, and bottlenose dolphins, Tursiops truncatus). Recent technological advancements allow long-term, broadband (100 kHz bandwidth), passive acoustic monitoring from autonomous sea-floor mounted instruments. The ability to record higher frequencies allows study of a broader range of odontocete vocalizations including echolocation clicks. To determine whether delphinid species could be identified by their clicks, concurrent ship-based visual and acoustic surveys were conducted. We find that clicks from Pacific white-sided and Risso's dolphins contain spectral peaks and notches that are unique and consistent for each species. These spectral patterns are also apparent in long-term autonomous acoustic recordings throughout the Southern California Bight. Utilizing this spectral classification method, we examine of diel, seasonal, and habitat use patterns of acoustically active Risso's and Pacific white-sided dolphins. The ability to monitor animals through the night provides insight into distinct diel patterns of acoustic activity for both species while the high temporal resolution acoustic data can be used to relate oceanographic time series to dolphin activity.

\section{Contributed Papers}

\section{1:40}

2aAB11. Sound production and spawning by black brum (Pogonias cromis) in southwest Florida. James Locascio (University of South Florida, College of Marine Science, 140 7th Ave. S., St. Petersburg, FL 33701, USA, locascio@marine.usf.edu), Ernst Peebles (University of South Florida, College of Marine Science, 140 7th Ave. S., St. Petersburg, FL 33701, USA, epeebles@marine.usf.edu), David Mann (University of South Florida, College of Marine Science, 140 7th Ave. S., St. Petersburg, FL 33701, USA, dmann@marine.usf.edu)

We used the Long Term Acoustic Recording System (LARS) to investigate patterns of sound production in spawning aggregations of black drum (Pogonias cromis) during 2004 - 2006 in southwest Florida. Our purposes were to document trends in black drum sound production on daily and seasonal time scales and to investigate the temporal and quantitative relationships between egg production and sound production. Sound production was strongly diel, beginning near dusk and lasting for several hours. Sound production occurred from October through April and peaked in FebruaryMarch, consistent with prior descriptions of the spawning season for this species based on the gonado-somatic index (GSI). To investigate the relationship between sound production and egg production, surface plankton tows were conducted hourly between 1600 - 0400 on two consecutive nights while continuous underwater acoustic recordings were made. This was done five times between January and April, 2006. Neither the timing nor quantity of sound production was correlated with egg production on a nightly basis. These results indicate that patterns in sound production are not useful for predicting patterns in egg production by black drum on a daily scale but do provide accurate characterization of spawning behavior on a seasonal basis.

\section{2:00}

2aAB12. Nearest-neighbor techniques for automated monitoring of nocturnal flight calls. Harold Figueroa (Cornell University, Bioacoustics Research Program, 159 Sapsucker Woods Road, Ithaca, NY 14850, USA, harold.figueroa@gmail.com), Andrew Farnsworth (Cornell Laboratory of Ornithology, 420 E 54th St, 38J, New York, NY 10021, USA, af27@ cornell .edu)

Flight-calls are short vocalizations used primarily during nocturnal flight Their observation provides a means for studying the timing, location, and composition of nocturnal migrations. As part of a three-year study the Cornell Lab of Ornithology is using autonomous recorders to sample flight-calls of nocturnal migrants in the Northeastern US. The resulting tens of thousands of hours of recording, make software-assisted detection and classification essential. Automatic processing and human evaluation have yielded a considerable collection of flight-calls, 5-1000 examples for $\sim 100$ species. The many-class classification problem, along with the availability of many examples from most of the classes, and established (condensation and editing) and recent (metric-trees) techniques used in prototype-based classification nearest-neighbor techniques, have led us to develop nearestneighbor based techniques and software to assist in the analysis of this data. We will present classification results on two examples, a set of thrushes (genera Catharus and Hylocichla, family Turdidae) consisting of six species and wood-warblers (family Parulidae) consisting of 48 species. The thrush flight-calls are visually and aurally distinctive, usually $100-400 \mathrm{~ms}$ in duration and occupy and the $2-5 \mathrm{kHz}$ band. Wood-warbler flight-calls are typically between 20-100 ms in duration and occupy the $5-10 \mathrm{kHz}$, and are difficult for many experienced observers to distinguish.

\section{Invited Paper}

\section{2:20}

2aAB13. Sperm whale monitoring with a deep acoustic platform: Results from NEMO ONDE experiment and way ahead. Gianni Pavan (CIBRA - Università di Pavia, Via Taramelli 24, 27100 Pavia, Italy, gianni.pavan@unipv.it)

Within the INFN NEMO Project on the underwater detection of high energy neutrino, a deep hydrophone station, named ONDE (Ocean Noise Detection Experiment), has been deployed on the seafloor $21 \mathrm{~km}$ offshore Catania (Sicily, Italy), at $2000 \mathrm{~m}$ depth. Acoustic data recorded in 2005 and 2006 provided long term information on the underwater noise and a huge amount of sperm whales' detections that indicate a presence of the species higher than previously believed. Only few sightings are available for the previous years and scarce literature is available for the area. With ONDE, in year 2005 sperm whales were detected in 117 of the 231 recorded days and in 31 of the 83 days in year 2006. Clicks were the most common vocalizations recorded. Chirrups and codas (dominated by the $3+1$ pattern) were present frequently, but creaks, possibly indicating feeding actions, were seldom heard. The whales were often detected, solitary or in groups, for time segments of only a few hours. This, with the low rate of creaks, may mean they were just in transit. Based on these results, a new project named (Listening Into the Deep Ocean) has been set with INGV to create a Mediterranean wide network by upgrading existing underwater seismic detectors with broadband acoustic sensors. 


\section{Contributed Paper}

12:40

2aAB14. Detection and classification of call types in the vocalizations of north-east pacific blue whales. Jack McLaughlin (University of Washington, 1013 NE 40th St, Seattle, WA 98105-6698, USA, jackm@apl.washington.edu), Nicolas Josso (GIPSA-lab, dep. DIS, 961, rue de la Houille Blanche, 38402 St Martin d'Hères, France, nicolas.josso@gispa-lab.inpg.fr), Cornel Ioana (GIPSA-lab, dep. DIS, 961, rue de la Houille Blanche, 38402 St Martin d'Hères, France, cornel.ioana @ gipsa-lab.inpg.fr)

Characterization of marine mammal vocalizations is an essential part of any program of marine mammal monitoring as well as being of great help for furthering understanding of subjects such as underwater communication, sonar, etc. The vocalizations of the North-East Pacific (NEPAC) blue whales are known to be made of at least three different call types: the A call, the B call and the $\mathrm{C}$ call. This study aims at the development of a wholly automatic process of detection and classification for the two most common call types of the NEPAC population which are the A call and the B call. We created one template for the A call and one for the B call in order to extract features with matched filtering operations. We show that a simple Gaussian Mixture Model classifier can be used to accurately track and identify the call types in 24-hour long records. The proposed methodology is applied to real data sets recorded by seismic sensors.

\section{Invited Paper}

\section{1:00}

2aAB15. Experience with VoxNet: a rapidly-deployable acoustic monitoring system for bio-acoustic studies. Lewis Girod (MIT/CSAIL, 32G-918, 32 Vassar St, Cambridge, MA 02139, USA, girod@nms.csail.mit.edu), Michael Allen (Cogent Computing ARC, Coventry University, Coventry, UK, allenm@lecs.cs.ucla.edu), Travis Collier (UCLA Dept. of Biology, 3563 Boelter Hall, Los Angeles, CA 90095, USA, travc@taylor0.biology.ucla.edu), Daniel T. Blumstein (UCLA Dept. of Biology, 3563 Boelter Hall, Los Angeles, CA 90095, USA, marmots@ucla.edu), Deborah Estrin (UCLA Dept. of Biology, 3563 Boelter Hall, Los Angeles, CA 90095, USA, destrin@cs.ucla.edu), Charles Taylor (UCLA Dept. of Biology, 3563 Boelter Hall, Los Angeles, CA 90095, USA, taylor @biology.ucla.edu)

Terrestrial bioacoustic census is a difficult problem because of propagation characteristics, obstructions, the diversity of bioacoustic sources, and the impact of noise. To address this problem we have developed VoxNet, a complete hardware and software platform for distributed acoustic monitoring applications. Each VoxNet node is a portable, self-contained processor with a small four-channel acoustic array. Using a distributed set of VoxNet nodes, a forested habitat can be monitored and the behavior of animals can be recorded and analyzed acoustically. In this work we present our experiences applying VoxNet to bioacoustic census. This work is based on data collected using the system during a deployment in Chiapas, Mexico at the Chajul Biological Field Station. The Chajul station is located in a region of dense rain forest and is home to Mexico's most diverse ecosystem. Using VoxNet in this harsh environment, we obtained census estimates based on observation of bird calls. 


\title{
Session 2aAOa
}

\section{Acoustical Oceanography and ECUA: Marine Ecosystem Acoustics II}

\author{
Kenneth G. Foote, Cochair \\ Woods Hole Oceanographic Institution, Woods Hole, MA 02543, USA \\ Olav R. Godoe, Cochair \\ Institute of Marine Research, PO Box 1870, Bergen, 5817, Norway
}

Invited Papers

2aAOa1. Spawning Behaviour and Spatial Distribution of Atlantic Herring on Georges Bank Revealed by Ocean Acoustics Waveguide Remote Sensing. Purnima Ratilal (Northeastern University, 302 Stearns Center, Rm 311, 360 Huntington Ave, Boston, MA 02115, USA, purnima@ece.neu.edu), Zheng Gong (Northeastern University, 302 Stearns Center, Rm 311, 360 Huntington Ave, Boston, MA 02115, USA, zgong@ece.neu.edu), Daniel Cocuzzo (Northeastern University, 302 Stearns Center, Rm 311, 360 Huntington Ave, Boston, MA 02115, USA, dcocuzzo@ece.neu.edu), Mark Andrews (Northeastern University, 302 Stearns Center, Rm 311, 360 Huntington Ave, Boston, MA 02115, USA, Andrews.mar@neu.edu), Srinivasan Jagannathan (Massachusetts Institute of Technology, Room 5-435, 77 Massachusetts Avenue, Cambridge, MA 02139, USA, jsrini@mit.edu), Ioannis Bertsatos (Massachusetts Institute of Technology, Room 5-435, 77 Massachusetts Avenue, Cambridge, MA 02139, USA, ibertsat@mit.edu), Tianrun Chen (Massachusetts Institute of Technology, Room 5-212, 77 Massachusetts Avenue, Cambridge, MA 02139, USA, trchen@ mit.edu), Hector Pena (Institute of Marine Research, PO Box 1870, 5817 Bergen, Norway, hector.pena@imr.no), Ruben Patel (Institute of Marine Research, PO Box 1870, 5817 Bergen, Norway, ruben.patel@imr.no), Olav R. Godo (Institute of Marine Research, PO Box 1870, 5817 Bergen, Norway, olav.rune.godoe@imr.no), J. Michael Jech (NOAA/Northeast Fisheries Science Center, 166 Water Street, Woods Hole, MA 02543, USA, michael.jech@noaa.gov), Thomas C. Weber (University of New Hampshire, Ctr. for Coastal and Ocean Mapping, 24 Colovos Road, Durham, NH 03824, USA, weber@ccom.unh.edu), Nicholas Makris (Massachusetts Institute of Technology, Room 5-212, 77 Massachusetts Avenue, Cambridge, MA 02139, USA, makris@mit.edu)

An ocean acoustics waveguide remote sensing (OAWRS) system was deployed in the Gulf of Maine, near Georges Bank to image Atlantic herring and other fish populations from Sep-Oct 2006. OAWRS provides spatially unaliased imaging of herring over wide areas, spannning over $100 \mathrm{~km}$ diameter. Migration and spawning behaviour of Atlantic herring was observed using OAWRS over several diurnal periods, including massive movements on and off the bank to spawn. Measurements made simultaneously with a conventional fish-finding echosounder (CFFS) and a multibeam sonar provide the depth distribution and local 3D morphology respectively of the herring schools in the water column. Concurrent trawl surveys provide identification of the fish species. Measurements mades by OAWRS and CFFS systems are highly correlated. Examples will be provided of the co-registration between the two systems over a one week period. Calibration of the OAWRS system using CFFS estimates of fish population densities along with a full-field scattering model that takes into account both coherent and incoherent scattering from a fish group is discussed. The fish swimbladder is modelled as a spheroidal bubble. Resonance scattering behaviour of herring is observed in the OAWRS system with significant changes in scattering amplitude over the $300 \mathrm{~Hz}$ to $1.5 \mathrm{kHz}$ frequency range of the OAWRS system.

2aAOa2. Passive acoustic mapping of marine biological choruses. Gerald D'Spain (Marine Physical Lab, Scripps Institution of Oceanography, 291 Rosecrans St., San Diego, CA 92106, USA, gld@mpl.ucsd.edu), Heidi Batchelor (Marine Physical Lab, Scripps Institution of Oceanography, 291 Rosecrans St., San Diego, CA 92106, USA, heidi@mpl.ucsd.edu), Catherine Berchok (Marine Physical Lab, Scripps Institution of Oceanography, 291 Rosecrans St., San Diego, CA 92106, USA, cberchok@ucsd.edu)

Since underwater sound is a critical aspect of the ocean environment for marine animals, passive acoustic recordings provide important information on marine habitats. Conversely, because biological sounds can be a dominant component of the ocean sound field, knowledge of their characteristics is important for assessing sonar system performance. This presentation summarizes the properties of three biological choruses observed in experiments off the southern California coast. Measurements were made with large aperture, well-filled hydrophone arrays, yielding highly resolved estimates of the chorus directionality. The first chorus generated energy in the 200-800 Hz band, occurred predominantly at night in spring and summer, and displayed an unusual spatiotemporal pattern where the region of chorusing periodically propagated upcoast over $25 \mathrm{~km}$ of coastline at nearly $1.5 \mathrm{~km} / \mathrm{sec}$. The other two choruses occurred in the 1-10 kHz frequency band and come from slowly-moving, or fixed, sources at, or near, the ocean bottom. One chorus was present only on two consecutive nights during a summer experiment, whereas the second occurred at all times throughout the 10-day duration of a late winter experiment. Numerical models predict some of the characteristics of the choruses. [Work supported by the Office of Naval Research]. 
2aAOa3. Understanding potential effects of using active sonar to study marine ecosystems. Mardi C. Hastings (Penn State University, Applied Research Laboratory, 801 North Quincy Street, Suite 120, Arlington, VA 22203-1708, USA, mch26@psu.edu)

Biological organisms can suffer various kinds of effects when exposed to high intensity sound. Active sonar systems used to investigate marine ecosystems typically have source levels exceeding $220 \mathrm{~dB}$ re: $1 \mu \mathrm{Pa}$ at $1 \mathrm{~m}$, which are high enough to have an effect on organisms in relatively close proximity. Although these systems operate at frequencies from the low kilohertz to megahertz ranges that are not usually associated with potentially harmful effects in the marine environment, fundamental interactions with sound occur at various biological scales that strongly depend on the size of the organism with respect to acoustic wavelengths. These effects range from subtle changes in behavior to various types of trauma that can result in temporary or permanent hearing loss, or in hemorrhage or even mortality. Examples from the literature will be used to illustrate the basic relationships between biological effects of sound and the size and structure of marine organisms, as well as potential concerns associated with using active sonar to study the marine environment.

\section{9:00}

2aAOa4. How toothed whales echolocate to find and capture prey in the deep ocean. Peter Tyack (Woods Hole Oceanographic Institution, Applied Ocean Physics \& Engineering Dept., Woods Hole, MA 02543, USA, ptyack@whoi.edu), Mark Johnson (Woods Hole Oceanographic Institution, Applied Ocean Physics \& Engineering Dept., Woods Hole, MA 02543, USA, majohnson@whoi.edu), Peter T. Madsen (University of Aarhus, Biological Sciences, Zoophysiology, C. F. Møllers Allé, Building 1131, DK-8000 Aarhus, Denmark, peter.madsen@biology.au.dk), Walter M. Zimmer (NATO Undersea Research Centre, Viale San Bartolomeo 400, 19126 La Spezia, Italy, walter@nurc.nato.int), Natacha A. Soto (University of La Laguna, BIOECOMAC Department of Animal Biology, 38207 La Laguna, Spain, naguilar@ull.es)

Sperm and beaked whales dive to feed on squid and deepwater fish. We have attached sound-and-orientation recording tags to study how these whales use echolocation to forage at depth. Tagged whales are usually silent when starting a dive, but start producing echolocation clicks at a few hundred meters depth, shallower than the depth at which they feed, suggesting that descending whales scan the deep layers where they will feed. Once sperm or beaked whales encounter prey, they switch from regular search clicks to a buzz of rapid clicks. Tags on beaked whales not only record outgoing clicks, but also echoes from prey at ranges out to 10-20 m. Beaked whales produce clicks every 0.2-0.4 sec when searching. Echoes from several targets are often detected after each beaked whale click. Beaked whales will pass by many targets before selecting one. Whales may switch from the search clicks to a buzz as they close within a body length of the prey. Sperm and beaked whales have an angular acceleration at the end of the buzz, which probably indicates turning to catch the prey.

\section{9:20}

2aAOa5. Hypotheses regarding exploitation of bubble acoustics by cetaceans. Timothy G. Leighton (Institute of Sound and Vibration, Univ. of Southampton, University Road, Highfield, SO17 1BJ Southampton, UK, T.G.Leighton@soton.ac.uk), Paul R. White (Institute of Sound and Vibration, Univ. of Southampton, University Road, Highfield, SO17 1BJ Southampton, UK, prw@isvr.soton.ac.uk), Daniel C. Finfer (Institute of Sound and Vibration, Univ. of Southampton, University Road, Highfield, SO17 1BJ Southampton, UK, d.c.finfer@soton.ac.uk)

Bubbles are the most acoustically active naturally occurring entities in the ocean, and cetaceans are the most intelligent. Having evolved over tens of millions of years to cope with the underwater acoustic environment, cetaceans may have developed extraordinary techniques from which we could learn. This paper outlines some of the possible interactions, ranging from the exploitation of acoustics by humpback whales (Megaptera novaeangliae) in bubble nets to trap prey, to techniques by which coastal dolphins (e.g. of the genus Cephalorhynchus) could successfully echolocate in bubbly water (a hypothesis which has led to the development of a man-made sonar which can penetrate bubble clouds, and a range of possibilities for homeland security).

2aAOa6. Marine ecosystem acoustics: contributions of sonar technology. Kenneth G. Foote (Woods Hole Oceanographic Institution, Woods Hole, MA 02543, USA, kfoote@whoi.edu)

Sonar is considered in the widest sense as an acoustic system consisting of a transmitter and receiver of underwater sound. It may be monostatic, bistatic, or multi-static; narrowband or broadband; with one or more fixed, rotating, or electronically formed beams. Transducers may be mounted on hulls of vessels and autonomous underwater vehicles, fixed structures, or seafloor; towed; or suspended. Spatial scales may be sampled from millimeters to thousands of kilometers; corresponding temporal scales vary from a fraction of a second, as in target-tracking, to seasons, as in observing animal migration. Several sonar types are described. Contributions of sonar technology to ecosystem studies, both actual and potential, are noted. These include, for example, fine-scale surveying of pelagic and semi-demersal fish by scientific echo sounder; mapping of the three-dimensional structure of fish aggregations and bathymetry by multibeam sonar; quantification of benthic egg beds of the squid Loligo opalescens by sidescan sonar; and large-scale surveying of swimbladdered fish by parametric sonar. Each of these sonar systems has potential for detection, quantification, and classification of marine organisms, as well as for characterization of the habitat, hence yielding the kind of information that is required for ecosystem assessment and management. 
2aAOa7. Use of broadband active acoustics to study marine organisms. Timothy K. Stanton (Woods Hole Oceanographic Institution, Applied Ocean Physics \& Engineering Department, 98 Water Street, MS \#11, Woods Hole, MA 02543, USA, tstanton@whoi.edu), Dezhang Chu (NOAA/NMFS/NWFSC/FRAMD, Montlake Blvd., E. Seattle, WA 98112, USA, dchu@whoi .edu)

Broadband signals inherently have more information than narrowband signals. In essence, they have more "channels" of information due to the wider range of frequencies spanned. It is therefore advantageous to use broadband signals in active acoustic studies of marine organisms and to apply techniques that best exploit the broadband nature of the signals. In this presentation, a brief background on the use of narrowband systems for studying marine organisms by these authors and other investigators is given. Limitations to those studies are outlined, with arguments made for the use of broadband acoustics. The past twenty years of studies centered at the Woods Hole Oceanographic Institution are subsequently reviewed in which broadband signals are used both in the laboratory and field to study a variety of organisms-- swimbladder-bearing fish and three major anatomical categories of zooplankton. The analyses are divided broadly into two major categories-- time and frequency domain-- for various types of analyses, including pulse compression processing and spectral analysis. Results are first shown based on studies, one organism at a time, in the laboratory. Those laboratory approaches are then applied to ocean studies of fish and large zooplankton using a new towed instrument spanning the frequency range $1.7 \mathrm{kHz}-100$ $\mathrm{kHz}$.

TUESDAY MORNING, 1 JULY 2008

ROOM 342A, 10:40 A.M. TO 6:40 P.M.

\title{
Session 2aAOb
}

\section{Acoustical Oceanography, Underwater Acoustics, Signal Processing in Acoustics, and ECUA: Geoacoustic Characterization of the Ocean Bottom and Geoacoustic Inversion I}

\author{
Peter Gerstoft, Cochair \\ Marine Physical Laboratory, Scripps Institute of Oceanography, 8602 La Jolla Shores Drive, La Jolla, CA 92093-0238, USA \\ Dag Tollefsen, Cochair \\ Norwegian Defence Research Establishment (FFI), Box 115, Horten, 3191, Norway
}

Invited Papers

10:40

2aAOb1. Characterization of seabed geo-acoustic structure for a shallow water environment. David Knobles (Applied Research Laboratories, UT at Austin, P. O. Box 8029, Austin, TX 78713, USA, knobles@arlut.utexas.edu)

Full-field inversion methods over large frequency bands and spatial scales attempt to exploit the sensitivity of the frequency and spatial dependence of the acoustic field of the properties to the seabed. A comprehensive set of broadband impulsive and narrowband tonal acoustic recordings in the 5-3000 Hz band from two L-arrays separated by $20 \mathrm{~km}$ were made in a shallow water area on the New Jersey continental shelf during the Shallow Water 2006 (SW06) experiment. In addition, surface ship of opportunity passages on both arrays were recorded along with noise during the passage of tropical storm Ernesto. Reverberation and transmission loss data in the 25-9200 Hz band from explosive sources were also collected at a nearby site prior to the SW06 experiment. Numerous full-field geoacoustic inversion methods and analyses of all these acoustic data with supporting range and azimuth-dependent geo-physical measurements are employed to characterize the seabed properties. For example, the analyses of these data permit inferences about the frequency-dependence of the seabed sound speed and attenuation to be made. The overlapping data types act to reduce the intrinsic ambiguities associated with inversion, quantify the spatial variability, and serve as independent validation.

11:00

2aAOb2. Effect of ocean sound speed uncertainty on matched-field geoacoustic inversion. Chen-Fen Huang (Department of Marine Environmental Informatics/National Taiwan Ocean University, 2 Pei-Ning Road, 202 Keelung, Taiwan, chenfen@ mail.ntou.edu .tw)

The effect of ocean sound speed uncertainty on matched field geoacoustic inversion is investigated using data from the SW06 experiment along a nearly range-independent bathymetric track. Significant sound speed differences were observed at the source and receiving array and several environmental parameterizations were investigated for the inversion including representing the ocean sound speed at both source and receivers with empirical orthogonal function (EOF) coefficients. A GA-based global optimization method was applied to the candidate environmental models. Then, a Bayesian inversion technique was used to quantify uncertainty in the environmental parameters for the best environmental model, which included an EOF description of the ocean sound speed. 


\section{Contributed Papers}

11:20

2aAOb3. Quantifying the uncertainty of geoacoustic parameter estimates in a dynamic environment using oceanographic data. Ross Chapman (University of Victoria, 3800 Finnerty Rd, Vcitoria, BC V8W 3P6, Canada, chapman@uvic.ca), Yong-Min Jiang (University of Victoria, 3800 Finnerty Rd, Vcitoria, BC V8W 3P6, Canada, minj@uvic.ca)

This paper presents results of Bayesian matched field geoacoustic inversion in a temporally and spatially varying shallow water environment. The acoustic data were collected by a vertical line array at ranges of 1,3 and 5 km in the SW06 (Shallow Water 06) experiment carried out in August 2006. The sound speed profiles measured at the array and at the source positions during the experiment showed significant variation in the thermocline in the middle part of the water column. The resulting uncertainty in the sound speed profile in the water impacts the performance of matched field inversion methods in estimation of geoacoustic model parameters. In previous work, an effective sound speed profile was estimated in the inversion, based on empirical orthogonal functions (EOFs) derived from a limited number of sound speed profiles measured at the time of the experiment. This paper explores the effect of using a larger number of profiles measured over several days in the SW06 experiment to include a greater diversity of ocean conditions in generating the EOFs. The inversion results for the two approaches are compared in terms of marginal probability distributions of the estimated geoacoustic parameters. (Work supported by Office of Naval Research).

\section{1:40}

2aAOb4. Short range geoacoustic inversion with a vertical line array. Yong-Min Jiang (University of Victoria, 3800 Finnerty Rd, Vcitoria, BC V8W 3P6, Canada, minj@uvic.ca), Ross Chapman (University of Victoria, 3800 Finnerty Rd, Vcitoria, BC V8W 3P6, Canada, chapman@uvic.ca), Peter Gerstoft (Marine Physical Laboratory, Scripps Institute of Oceanography, 8602 La Jolla Shores Drive, La Jolla, CA 920930238, USA, gerstoft@ucsd.edu)

During the SW06 (Shallow Water 06) experiments, short range geoacoustic inversion experiments were carried out at a source-receiver range of $230 \mathrm{~m}$ to determine the sea bed properties at fine spatial scales. Acoustic signals were collected at 16 hydrophones on a vertical line array from source depths of 15-65 m in $10 \mathrm{~m}$ intervals. For this experimental geometry, the angular coverage for reflectivity versus grazing angle is $7-25^{\circ}$. This paper combines the information extracted from low-frequency $(100-900 \mathrm{~Hz})$ and mid-frequency $(1500-4500 \mathrm{~Hz})$ LFM signals transmitted from the same source position to estimate multi-layered geoacoustic models. The model parameterization was based on the number of resolved sub-bottom reflections in the data. An inversion approach using adaptive simplex differential evolution was applied to the data to invert for the sub-bottom sound speeds and layer thicknesses. The temporal variation of the water column sound speed profile was included in the inversion. The estimated sound speeds are consistent with the range-averaged profile that was estimated previously from longer range data for the same radial path from the array. (Work supported by Office of Naval Research).

\section{2:00}

2aAOb5. Does a depth variable sound-speed profile matter for SW06 geoacoustic inversion? Alexandra Tolstoy (ATolstoy Sciences, 1538 Hampton Hill Circle, McLean, VA 22101, USA, atolstoy@ieee.org)

This work will discuss the effects of depth variability for a sound-speed profile with regard to goeacoustic inversion on some simulated SW06 "data". First, broadband $(400-800 \mathrm{~Hz})$ simulated "data" are generated using
RAM_PE (by Collins) in the time domain for some short range (less than 1 km) SW06 scenarios. Next, the first 4 boundary reflection arrivals (from surface, bottom, surface-bottom, bottom-surface) are studied as a function of source range and depth, phone depth, and sound-speed variability. Finally, we examine the effects of sound-speed depth variability on geoacoustic inversion via MFP. We note that knowledge of array phone locations has a large impact on inversion capabilities.

\section{2:20}

2aAOb6. Three dimensional geoacoustic inversion on the New Jersey shelf. Megan S. Ballard (Penn. State University, P.O. Box 30, State College, PA 16804, USA, msd200@psu.edu), K.m. Becker (Penn. State University, P.O. Box 30, State College, PA 16804, USA, kmbecker@psu.edu)

Perturbative inversion, based on a linearized relationship between sound speed in the sediment and modal eigenvalues, is applied to data from the Shallow Water Experiment 2006. Data were collected by towing a lowfrequency sound source out and back along radials, spanning a 90 degree angular sector, from a common receiver location. Range-dependent estimates of horizontal wave numbers are obtained along each of the radials using high-resolution signal processing techniques capable of detecting and localizing changes in sub-bottom properties, and that are particularly sensitive to changes in layer structure. Wave number estimates at each range are used in a linearized inversion algorithm to estimate local sediment properties. Locations of the R-reflector and other layering information are used as a priori information in the inversion algorithm. The additional information both constrains the solution of an otherwise ill-posed problem and emphasizes the layered structure of the sediment. These methods have been shown to yield accurate estimates of the sound speed profile deep into the sediment using very few perturbations to the forward model. Combining the local inversion results, a three-dimensional map of sediment sound speed structure is obtained for a $25 \mathrm{~km} 2$ region of the seafloor. [Supported by NDSEG and ONR]

\section{2:40}

2aAOb7. Experimental verification of range-dependent inverse method for geoacoustic parameters from modal dispersion data. Subramaniam Rajan (Scientific Solutions, Inc, 99 Perimeter Road, Nashua, NH 03063, USA, srajan@scisol.com), K.m. Becker (Penn. State University, P.O. Box 30, State College, PA 16804, USA, kmbecker@psu.edu)

The procedure for determining the sediment compressional wave speed profile from modal dispersion data in a range-independent case is well documented in the literature. In this paper, the procedure developed for the range-independent case is extended to cover the range-dependent case. In this procedure the region under investigation is divided into discrete range intervals and the sediment characteristics at each range interval estimated assuming that the sediment properties of each range interval are range independent. The approach has been demonstrated to yield range-dependent sediment properties when applied to synthetic data generated for an experiment consisting of multiple source/receiver combinations. During the Shallow Water Experiment 2006, broadband data transmitted by a single source were measured on multiple receivers. These data are used here to estimate range-dependent properties of the sediment. The results obtained in this manner are compared with inversion results obtained for the same region by other investigators who employed different approaches for estimating the geoacoustic properties. [Work supported by ONR, Ocean Acoustics].

\section{1:00-2:00 Lunch Break}




\section{Invited Paper}

2:00

2aAOb8. Modal inversion using the acoustic field emitted by a moving source and measured on a vertical line array of hydrophones. K.m. Becker (Penn. State University, P.O. Box 30, State College, PA 16804, USA, kmbecker@psu.edu), Megan S. Ballard (Penn. State University, P.O. Box 30, State College, PA 16804, USA, msd200@psu.edu)

As part of the Shallow Water Experiment 2006 (SW06), a low-frequency continuous wave acoustic source was towed out and back along radials from a fixed vertical line array of hydrophones. The resulting modal pressure field, measured on synthetic range apertures created by the relative source motion, is used to derive input data to inversion algorithms for estimating bottom properties. The inversion algorithms considered are based on well established relationship between modal eigenvalues and geoacoustic properties of the sediment. In this talk, linear inversion methods based on modal input data will be reviewed for their application to the SW06 data. The first step in these methods is the accurate estimation of modal eigenvalues from the pressure field data. For a moving source, theory predicts a Doppler shift along with frequency spreading proportional to both the transmitted frequency and tow speed. Propagating modes and their corresponding modal eigenvalues are predicted to correspond with the Doppler shifted frequencies. An emphasis of this work is on Doppler shift as an observable in the data and properly accounting for it in inversion. Sediment sound speeds estimated from data are consistent with sandy sediments found in the experimental area. [Work supported by ONR and NDSEG]

\section{Contributed Paper}

2:20

2aAOb9. Geoacoustic inversion using combustive sound source signals. Gopu R. Potty (University of Rhode Island, Department of Ocean Engineering, Narragansett Bay Campus, Narragansett, RI 02882, USA, gopu@uri.edu), James H. Miller (University of Rhode Island, Department of Ocean Engineering, Narragansett Bay Campus, Narragansett, RI 02882, USA, miller@egr.uri.edu), Preston S. Wilson (The University of Texas, Mechanical Engineering Department, Austin, TX 78712, USA, pswilson@mail.utexas.edu), James F. Lynch (Woods Hole Oceanographic Institution, 98 Water Street, Bigelow 203A, MS-11, Woods Hole, MA 02543, USA, jlynch@whoi.edu), Arthur Newhall (Woods Hole Oceanographic Institution, 98 Water Street, Bigelow 203A, MS-11, Woods Hole, MA 02543, USA, anewhall@whoi.edu)
In the summer of 2006, Combustive Sound Sources (CSS) were deployed in shallow waters off New Jersey during the Shallow Water experiment (SW 06). The depth of these sources were $26 \mathrm{~m}$ in water depths of the order of $100 \mathrm{~m}$. CSS are low frequency broadband sound sources and suitable for modal dispersion based inversion. CSS data collected on various receivers (Single Hydrophone Receiving Units (SHRU), Vertical Line Array, etc.) will be used for the inversions. The inversions are based on an iterative scheme using a new time-frequency analysis technique (dispersion based short-time Fourier transform) in which each time-frequency tiling is adaptively rotated in the time-frequency plane, depending on the local wave dispersion. Data from various receivers will be used to investigate the spatial variation in compressional wave speed. Compressional wave attenuation will also be estimated using modal amplitude ratios. Inversion results will be validated by comparing the data with model predictions. [Work supported by the Office of Naval Research]

\section{Invited Paper}

\section{2:40}

2aAOb10. Tomographic and bottom geoacoustic inversions using Genetic Algorithms and a statistical characterization of the acoustic signal. Michael Taroudakis (University of Crete \& FORTH/IACM, Vassilika Vouton, P.O.Box 1385, 71110 Heraklion, Greece, taroud@iacm.forth.gr), Costas Smaragdakis (University of Crete, Dept of Mathematics, Knossou Ave, 71409 Heraklion, Greece, kesmarag@gmail.com)

The paper deals with the problem of estimation of the parameters of the water column and/or the sea bed, using measurements of the acoustic field due to a known source at a certain point in the water column. Following the work by Taroudakis et al. (JASA 119, 1396-1405 (2006)) the acoustic signal is characterized using the statistics of the wavelet sub-band coefficients, which obey a certain statistical law, described by an Alpha-Stable distribution. Thus, the signal observables are the set of the parameters of the appropriate distributions at the various levels of the signal decomposition. In this work an inversion procedure based on a Genetic Algorithm and the statistical characterization of the acoustic signal, is described. The quality of a certain population of candidate model parameters (properties of the water columns and/or the sea-bed) is evaluated using the Kullback-Leibler divergence (KLD) of the wavelet sub-band coefficient distributions, between the measured and simulated acoustic signals. Following an appropriate population regeneration procedure, the final population is described by an a-posteriori statistical distribution of the model parameters, indicating among the others their sensitivity in the inversion procedure. Very good inversion results have been observed in simulated shallow water environments.

\section{Contributed Paper}

3:00

2aAOb11. Dispersion curve estimation with partcile filters for geoacoustic inversion. Ivan Zorych (Department of Mathematical Sciences, New Jersey Institute of Technology, 323 M L King Blvd, Newark, NJ 07102, USA, zorych@gmail.com), Zoi-Heleni Michalopoulou (Department of Mathematical Sciences, New Jersey Institute of Technology, 323 M L King Blvd, Newark, NJ 07102, USA, michalop@njit.edu)

In this work we extend our particle filtering method for dispersion curve extraction from spectrograms of acoustic signals that have propagated in underwater environments, the goal being to obtain accurate representation of modal dispersion for geoacoustic inversion. The approach combines particle filtering with modeling of sound propagation in ocean environments to track dispersion curves of multimodal signals in noisy environments. In addition to providing connected modal "trajectories" that facilitate the computation of maximum a posteriori estimates of modal arrival times, the method provides posterior probability distributions for arrival times, quantifying errors that are then propagated into the geoacoustic inversion process. The method, thus, allows the calculation of posterior probability distributions of geoacoustic parameters. We present results from both synthetic and real data from the Gulf of Mexico experiment. [Work supported by ONR.] 


\section{Invited Paper}

$3: 20$

2aAOb12. Geoacoustic characterization of the seafloor from a subbottom profiler applied to the BASE'07 experiment. Gwladys Theuillon (SHOM, 13 rue du Chatellier, CS 92803, 29228 Brest cedex 2, France, gwladys.theuillon@shom.fr), Yann Stephan (SHOM, 13 rue du Chatellier, CS 92803, 29228 Brest cedex 2, France, yann.stephan @shom.fr)

The most recent subbottom profilers present good performances in term of signal to noise ratio, resolution and penetration. These devices can thus be used to infer quantitatively the geoacoustic parameters of the seafloor. We have previously developed inversion methods which aim to estimate absorption, reflection, penetration, impedance contrast and micro-roughness in sediments from the SBP 120 subbottom profiler, manufactured by Kongsberg. These methods have been tested against real data and geoacoustic parameters derived from the SBP 120 are fully coherent with in situ measurements, which tends to confirm the possibility of seafloor characterization with subbottom profilers. In this work, the inversion methods are applied to a set of data acquired on the Malta Plateau during BASE'07 experiment. Geoacoustic parameters results are presented and discussed. They are in good agreement with the a priori knowledge of sediment properties in the area.

3:40-5:20 Posters

Lecture sessions will recess for presentation of poster papers on various topics in acoustics. See poster sessions for topics and abstracts.

\section{Invited Paper}

\section{$5: 20$}

2aAOb13. Bayesian model parameterization selection for seabed reflection-coefficient inversion. Jan Dettmer (University of Victoria, School of Earth and Ocean Sciences, Victoria, BC V8W 3P6, Canada, jand@uvic.ca), Stan E. Dosso (University of Victoria, School of Earth and Ocean Sciences, Victoria, BC V8W 3P6, Canada, sdosso@uvic.ca), Charles W. Holland (Penn State University / Applied Research Lab, Applied Science Bldg., State College, PA 16804, USA, cwh10@psu.edu)

This paper considers Bayesian inversion of seabed reflection-coefficient data for multi-layer geoacoustic models; in particular, the important issue of determining appropriate model parameterizations (e.g., number of layers, sound speed and density variations within layers). A poor model parameterization can lead to unreasonable inversion results. In particular, under-parameterization can introduce theory error in the inversion, causing biased results. However, more complex models always fit the data better. Therefore, parameterization quality cannot be quantified only in terms of data misfit, and Occam's razor is commonly applied to prefer simple parameterizations. In a Bayesian framework, Occam's razor is inherently included through Bayesian evidence. Bayesian inversion can be associated with two levels of inference. The first level assumes a specific model parameterization, and quantifies the data information content. This paper focuses on the second level: using Bayesian evidence to compare different model parameterizations. To this end, Gibbs sampling is applied, including full error covariance estimation, to sample the posterior probability density (PPD) for various parameterizations. Bayesian evidence is then computed from the PPD samples by numerical integration (reverse importance sampling).

\section{Contributed Papers}

\section{5:40}

2aAOb14. Combined inversion of mid-frequency propagation and reverberation sonar data. Peter Louring Nielsen (NURC, Viale S. Bartolomeo 400, 19126 La Spezia, Italy, nielsen@ nurc.nato.int), Christopher H. Harrison (NATO Undersea Research Centre, Viale San Bartolomeo 400, 19138 La Spezia, Italy, harrison@nurc.nato.int)

Sonar performance predictions in shallow-water regions are strongly dependent on a good knowledge of the geoacoustic and scattering properties of the seabed. The bottom properties are probably the most difficult parameters to determine, but inversion of measured acoustic data to infer the geoacoustic and scattering properties is a feasible technique. One of these techniques relies on inverting: (1) propagation data to estimate local bottom properties and (2) long-range reverberation data providing effective bottom properties over larger areas. A matched-field inversion approach is applied to propagation and reverberation data received on a towed horizontal array during the BASE'04 experiment conducted on the Malta Plateau, Mediterranean Sea. A total of 30 transmissions along a 10-km track were used to capture eventual range-dependent bottom properties. The inversion algorithm is composed of efficient prediction tools which can provide environmental parameter estimates within tactical time frames for in-situ sonar performance predictions. The experimental set-up mimics a mid-frequency active sonar system using only the suite of sensors available on the vessel towing the sonar system; also known as the through-the-sensor technique. The impact on sonar performance utilizing the environmental characterization approach is shown for various experimental scenarios and seasons. [Research sponsored by NURC and the BOUNDARY Partners]. 
2aAOb15. Reverberation inversion for seabed properties. Stan E. Dosso (University of Victoria, School of Earth and Ocean Sciences, Victoria, BC V8W 3P6, Canada, sdosso@uvic.ca), Peter Louring Nielsen (NURC, Viale S. Bartolomeo 400, 19126 La Spezia, Italy, nielsen@nurc.nato.int), Christopher H. Harrison (NATO Undersea Research Centre, Viale San Bartolomeo 400, 19138 La Spezia, Italy, harrison @ nurc.nato.int)

This paper applies nonlinear inversion to reverberation and/or propagation data recorded on a towed horizontal array for geoacoustic and scattering properties of the seabed. A Bayesian inversion approach is applied to estimate properties of the posterior probability density, such as the maximum a posteriori (most-probable) model, 1-D and 2-D marginal probability distributions, and covariance/correlation matrices, providing optimal parameter estimates and quantifying parameter uncertainties and inter-relationships. Of particular interest is quantifying the information content of different combinations of data (e.g., reverberation or propagation data; joint inversion of reverberation and propagation data) to resolve seabed parameters, and understanding strong parameter inter-relationships (correlations) which, in some cases, act as the limiting factor in resolving geoacoustic/scattering parameters. The inversion is also applied to shallow-water reverberation and propagation data recorded in Mediterranean Sea.
2aAOb16. Local bottom characterization using an autonomous underwater vehicle. Peter Louring Nielsen (NURC, Viale S. Bartolomeo 400, 19126 La Spezia, Italy, nielsen@nurc.nato.int), Charles W. Holland (Penn State University/Applied Research Lab, Applied Science Bldg., State College, PA 16804, USA, cwh10@psu.edu)

In the past decade the usage of autonomous underwater vehicles (AUV) to sample properties of the underwater environment has increased. The advantages of using platforms are their autonomy and that operations can be performed covertly. In 2007, the CLUTTER'07 experiment was conducted on the Malta Plateau with the main aim of characterizing the underwater environment. An AUV was deployed at a particular site on the Plateau to demonstrate the feasibility to infer bottom properties using such an autonomous platform. The AUV was equipped with 2 sound sources covering a frequency band $800-3500 \mathrm{~Hz}$, and the transmitted signals were acquired on a bottom moored vertical array. The mission of the AUV was to perform a linear track of $\sim 1000 \mathrm{~m}$ passing the vertical array as close as possible while transmitting every second from the sound sources. This experimental configuration is similar to the move-out or wide-angle reflection measurements The received signals are inverted for geoacoustic properties using both matched-field techniques and processed for direct bottom reflection properties. The results obtained are compared to independent findings from different experiments using various types of equipment at different seasons in the same area. [Research sponsored by NURC, ONR OA321 and the CLUTTER Partners]

\title{
Session 2aBB
}

\section{Biomedical Ultrasound/Bioresponse to Vibration and Physical Acoustics: Ultrasound Contrast Agents for Imaging I}

\author{
Charles C. Church, Cochair \\ University of Mississippi, NCPA, 1 Coliseum Drive, University, MS 38677, USA
}

Ayache Bouakaz, Cochair

INSERM U930, 2, Bvd Tonnelle, Tours Cedex 9, 37044, France

\section{Invited Paper}

\begin{abstract}
8:00
2aBB1. Ultrasound and microbubble interaction. Nico De Jong (Erasmus MC, Dr Molewaterplein 50 room Ee2302, 3015GE Rotterdam, Netherlands, n.dejong@erasmusmc.nl)

Ultrasound contrast agent (UCA) bubble response to ultrasound (US) is still the subject of several studies, since it determines the power of medical diagnostic imaging modalities. Generally, bubbles are studied using optical or acoustical methods, both having their specific advantages, in an environment that mimic the clinical practice as good as possible (small-diameter capillaries, red blood cells, blood viscosity). Acoustical observations could provide a better insight in the acoustical scattering of bubbles, which determines the final efficacy of contrast-enhanced diagnostic US imaging, and are better suitable to determine the properties of populations of bubbles. Optical observations allow for easier characterization of fewer bubbles and a more detailed characterisation in case where bubbles vibrate non spherically, which is expected for bubbles nearby a wall or red blood cells. In this presentation, we show optical recordings of the various bubble responses with the ultrahigh speed Brandaris-128 camera, and discuss the clinical implications of our findings.
\end{abstract}




\section{Contributed Papers}

8:20

2aBB2. Nonlinear pulsing schemes for the detection of ultrasound contrast agents. Michalakis A. Averkiou (University of Cyprus/Dept. of Mech. Engineering, 75 Kallipoleos Str., 1678 Nicosia, Cyprus, maverk@ucy.ac.cy), Christophoros Mannaris (University of Cyprus/Dept. of Mech. Engineering, 75 Kallipoleos Str., 1678 Nicosia, Cyprus, mannaris@ucy.ac.cy), Matthew Bruce (SuperSonic Imagine, Les Jardins de la Duranne - Bât. E, 510, rue René Descartes, F - 13857 Aix-enProvence, France, matt.f.bruce@gmail.com), Jeffry Powers (Philips Medical Systems, 22100 Bothell Everett Hwy, Bothell, WA 98021, USA, jeff .powers@philips.com)

Ultrasound contrast agents are used in cardiology for the assessment of myocardial perfusion and in radiology for the detection and characterization of tumors. One widely used approach of imaging contrast agents is to use a low Mechanical Index $(M I)$ nonlinear imaging technique to avoid bubble destruction and image both the macro- and micro-circulation in real-time. Various pulsing schemes are employed for the detection of nonlinear echoes from contrast microbubbles. The objective of this paper is to evaluate the various pulsing schemes for low MI imaging of contrast microbubbles and better understand their similarities and differences. The pulsing schemes considered are pulse inversion, power modulation, and their combination. Emphasis is placed on identifying whether nonlinearity due to propagation in tissue may be discriminated from nonlinearity due to scattering from bubbles. Bubble destruction (use of high $M I$ ) and tissue motion were not considered in this work. The evaluation of the different pulsing schemes was performed with numerical simulations from well established theoretical models and experimental data from microbubbles in tissue phantoms and human tissues.

\section{8:40}

2aBB3. Contrast agent response to chirp reversal. Anthony Novell (INSERM U930, CHRU Bretonneau, 37044 Tours Cedex 9, France, anthony.novell@etu.univ-tours.fr), Sander Van Der Meer (Physics of Fluids, University of Twente, P.O. Box 217, 7500 AE Enschede, Netherlands, s.m.vandermeer@utwente.nl), Michel Versluis (Physics of Fluids, University of Twente, P.O. Box 217, 7500 AE Enschede, Netherlands, m.versluis@utwente.nl), Nico De Jong (Erasmus MC, Dr Molewaterplein 50 room Ee2302, 3015GE Rotterdam, Netherlands, n.dejong@erasmusmc.nl), Ayache Bouakaz (INSERM U930, 2, Bvd Tonnelle, 37044 Tours Cedex 9, France, bouakaz@med.univ-tours.fr)

We investigate an excitation approach for contrast agents based on chirps. This technique, named chirp reversal, consists in transmitting an up sweep frequency chirp (UPF) followed by a down sweep frequency chirp (DNF). Simulations using a modified Rayleigh-Plesset equation were carried out. Chirps with center frequencies from 1.4 MHz to $2 \mathrm{MHz}$, pressures from $50 \mathrm{kPa}$ to $200 \mathrm{kPa}$ and frequency bandwidths from $30 \%$ to $65 \%$ were considered. High speed optical observations and acoustical measurements were performed using individual contrast bubbles of radii from $1 \mu \mathrm{m}$ to 5 $\mu \mathrm{m}$ and a diluted solution of contrast agent respectively. Simulations showed differences between bubbles' oscillations following UPF and DNF chirps in terms of amplitude and duration. Maximal differences occurred for bubbles that were around $80 \%$ and $140 \%$ of the resonance size. Bubbles at resonance or far away from resonance provided identical responses to UPF and DNF chirps. Larger bandwidths and higher acoustic pressures accentuate further the difference between the UPF and DNF responses. These findings were confirmed through optical data and acoustical measurements. The results reveal the potential of chirp reversal for contrast agent detection.

\section{9:00}

2aBB4. Dual-frequency insonation of single microbubbles. Marcia Emmer (Erasmus MC, Ee2302, P.O. Box 2040, 3000 CA Rotterdam, Netherlands, m.emmer@erasmusmc.nl), Hendrik J. Vos (Biomedical Engineering, Erasmus MC, P.O. Box 2040, 3000 CA Rotterdam, Netherlands,
H.J.Vos@ErasmusMC.nl),Nico De Jong(Erasmus MC, Dr Molewaterplein 50 room Ee2302, 3015GE Rotterdam, Netherlands, n.dejong@erasmusmc .nl)

Radial modulation imaging is a new medical imaging technique based on dual-frequency insonation of ultrasound contrast agents. The difference in echo between a high frequency 'imaging' pulse transmitted at either the compression or rarefaction phase of a low frequency 'modulating' pulse is detected by regular correlation techniques. Little is however known about the contrast agent microbubble dynamics in a dual-frequency ultrasound field, which were investigated in this study. Using a high-speed camera system, the radial excursions of single phospholipid-coated microbubbles were recorded. The microbubbles were simultaneously insonified with 2.5 cycles pulse at $0.5 \mathrm{MHz}$ and $30 \mathrm{kPa}$ and a 32 cycles pulse at $3.75 \mathrm{MHz}$ and $80 \mathrm{kPa}$. The microbubbles studied had diameters ranging from $1.1-5.2 \mu \mathrm{m}$. Microbubbles smaller than $1.4 \mu \mathrm{m}$ frequently showed shrinkage. Microbubbles larger than $2.6 \mu \mathrm{m}$ showed low $(<8 \mathrm{~dB})$ or no amplitude modulation of the high frequency radial excursion. Microbubbles with diameters between 1.4 and $2.6 \mu \mathrm{m}$ showed high amplitude modulation (up to $25 \mathrm{~dB}$ ) and strong compression-only oscillation, which both may be explained by nonlinear shell properties. The observed behaviour is beneficial for the detection of contrast agents.

\section{9:20}

2aBB5. Sub-harmonic response from polymer-shelled contrast agents with a 40-MHz excitation. Jeffrey A. Ketterling (Frederic L. Lizzi Center for Biomedical Engineering, Riverside Research Institute, 156 William St., New York, NY 10038, USA, ketterling@ rrinyc.org), Jonathan Mamou (Riverside Research Institute, 156 William St., 9th Floor, New York, NY 10038, USA, mamou@ rrinyc.org)

There is a growing interest in using acoustic contrast agents with highfrequency ultrasound $(>15 \mathrm{MHz})$ in order to better visualize microcirculation. Experiments were performed with polycaprolactoneshelled agents (POINT Biomedical, San Carlos, CA) having mean diameters of $0.56,1.1$, and $3.4 \mu \mathrm{m}$. The agents were heavily diluted in filtered water and injected through a $200 \mu \mathrm{m}$ channel into the focal zone of a $40-\mathrm{MHz}$ transducer that had a focal length of $12 \mathrm{~mm}$ and an outer diameter of $6 \mathrm{~mm}$. Backscatter signals from single agents were digitized using tone bursts of 5 to 20 cycles at peak-negative pressures of 0.6 to $6.3 \mathrm{MPa}$. 1000 valid singlebubble backscatter events at each exposure condition were digitized and then analyzed for 20-MHz subharmonic content. The data showed that the subharmonic response was initiated between 5 and 10 cycles and the likelihood of a subharmonic event increased as the number of cycles increased. A subharmonic backscatter response was most likely at $3.9 \mathrm{MPa}$ for the $3.4 \mu \mathrm{m}$ agent and $1.7 \mathrm{MPa}$ for the 0.56 and $1.1 \mu \mathrm{m}$ agents. The increased pressure for subharmonic activity for larger agent was consistent with its larger size.

\section{9:40}

2aBB6. Combined optical and acoustical characterization of single ultrasound contrast agent microbubbles. Jeroen Sijl (Physics of Fluids, University of Twente, P.O. Box 217, 7500 AE Enschede, Netherlands, j.sij1@utwente.nl), Timo Rozendal (Physics of Fluids, University of Twente, P.O. Box 217, 7500 AE Enschede, Netherlands, t.rozendal@student.utwente.nl), Hendrik J. Vos (Biomedical Engineering, Erasmus MC, P.O. Box 2040, 3000 CA Rotterdam, Netherlands, H.J.Vos@ErasmusMC.nl), Benjamin Dollet (Physics of Fluids, University of Twente, P.O. Box 217, 7500 AE Enschede, Netherlands, benjamin.dollet@univ-rennes1.fr), Nico De Jong (Erasmus MC, Dr Molewaterplein 50 room Ee2302, 3015GE Rotterdam, Netherlands, n.dejong@erasmusmc.nl), Detlef Lohse (Physics of Fluids, University of Twente, P.O. Box 217, 7500 AE Enschede, Netherlands, d.lohse@utwente.nl), Michel Versluis (Physics of Fluids, University of Twente, P.O. Box 217, 7500 AE Enschede, Netherlands, m.versluis @utwente.nl)

Contrast enhancement in medical ultrasound imaging is provided by the non-linear characteristics of coated microbubbles used as Ultrasound Contrast Agents (UCA). Optical time-resolved observations of the UCA mi- 
crobubble dynamics have revealed new non-linear bubble behavior such as "compression only" and "thresholding" behavior. Up to now, the contributions of such behavior to the non-linear acoustic response of UCA microbubbles is not known. Theoretically, the sound emission of an oscillating microbubble is derived from the unsteady Bernoulli equation and mass conservation assuming incompressible and irrotational flow. An experimental validation of this relation between the radial dynamics and the sound emission of a microbubble is not straightforward. We present a combined optical and acoustical setup to characterize individual BR-14 (Bracco Research S.A., Geneva, Switzerland) UCA microbubbles. The bubbles were isolated in a capillary fiber by an active flow control. During insonation the radial response of the single microbubble was recorded with the Brandaris ultra high-speed camera while the resulting acoustic response was measured with an accurately calibrated sensitive transducer. The sound emission calculated from the measured radius-time curves gives excellent quantitative agreement with the directly measured sound emission for both the linearly oscillating microbubbles and bubbles displaying "compression-only" and "thresholding" behavior, which indeed resulted in a strong non-linear sound emission.

\section{Invited Paper}

\section{0:00}

2aBB7. Secondary Bjerknes forces between ultrasound contrast agent microbubbles. Michel Versluis (Physics of Fluids, University of Twente, P.O. Box 217, 7500 AE Enschede, Netherlands, m.versluis@utwente.nl), Valeria Garbin (Physics of Fluids, University of Twente, P.O. Box 217, 7500 AE Enschede, Netherlands, v.garbin@tnw.utwente.nl), Benjamin Dollet (Physics of Fluids, University of Twente, P.O. Box 217, 7500 AE Enschede, Netherlands, benjamin.dollet@univ-rennes1.fr), Leen Van Wijngaarden (Physics of Fluids, University of Twente, P.O. Box 217, 7500 AE Enschede, Netherlands, 1.vanwijngaarden@tnw.utwente.nl), Nico De Jong (Erasmus MC, Dr Molewaterplein 50 room Ee2302, 3015GE Rotterdam, Netherlands, n.dejong@erasmusmc.nl), Detlef Lohse (Physics of Fluids, University of Twente, P.O. Box 217, 7500 AE Enschede, Netherlands, d.lohse@utwente.nl)

Acoustic radiation forces arise on UCA microbubbles from pressure gradients in the ultrasound pressure field. The ultrasound wave emitted by an oscillating UCA microbubble and experienced by a neighboring bubble results in the so-called secondary Bjerknes force. Typically, only the (time) averaged value of the secondary Bjerknes force is estimated from experiments. Here, the ability to resolve in time the radial dynamics of UCAs and the evolution of their relative distances, allows us to obtain a quantification of the instantaneous secondary Bjerknes force. Two bubbles were selected and confined within two separate optical traps and positioned away from the sample chamber wall prior to insonation. The radius-time curves and distance-time curves were then extracted from high-speed optical recordings. The camera fully resolved the alternating attractive-repulsive features of the secondary Bjerknes force in time. The effect of the time averaged secondary Bjerknes force results in a net attraction of the two bubbles, with a typical observed displacement of 1-2 $\mu \mathrm{m}$, with an instantaneous peak value of the secondary Bjerknes force of up to $10^{-6} \mathrm{~N}$. We predict the mutual interaction of the two coated bubbles in their translation with an accuracy better than $10 \%$.

\section{0:20-10:40 Break}

\section{Contributed Paper}

\section{0:40}

2aBB8. Mathematical models for contrast bubble dynamics. Charles C. Church (University of Mississippi, NCPA, 1 Coliseum Drive, University, MS 38677, USA, cchurch@olemiss.edu), John S. Allen (University of Hawaii-Manoa, Department of Mechanical Engineering, Holmes Hall Room 302, Honolulu, HI 96822, USA, alleniii@hawaii.edu)

Theoretical investigation of the acoustic responses of albuminencapsulated microspheres began over fifteen years ago when Albunex, the first agent approved for clinical use in the US, was still in development. Since that time, the number of potential ultrasound contrast agents has grown considerably. Depending on the agent, the shell may comprise a layer of proteins, synthetic polymers, surfactants, or lipids with a thickness from a few $\mathrm{nm}$ to $500 \mathrm{~mm}$ and surrounding air, sulfur hexafluoride, perfluorocarbon, or other gas. A thorough understanding of the interaction between ultrasound pulses and contrast microbubbles is essential for the successful clinical application of a particular agent. In this talk, the behavior of various contrast agents will be discussed, and appropriate models for each will be described. The basis for each of these theories is a free bubble model "supplemented" by the effect of the encapsulating shell. The differences among these models lie primarily in their treatment of the encapsulating layer and, to some extent, the surrounding medium. Comparisons among models will include predictions of radial responses, thresholds for bubble destruction, and clinically significant acoustic properties (resonance frequency, scattering strength, nonlinearity, etc.). [Supported by NIH 2 RO1 EB000350-04A2 (CCC) and by NIH 2 G12RR003061-21 (JSA).]

\section{Invited Papers}

\section{1:00}

2aBB9. Ultrasound contrast agents: from concept to clinical use. François Tranquart (INSERM U930, 2, Bvd Tonnelle, 37044 Tours Cedex 9, France, tranquart@med.univ-tours.fr), Ayache Bouakaz (INSERM U930, 2, Bvd Tonnelle, 37044 Tours Cedex 9, France, bouakaz@med.univ-tours.fr), Aurore Bleuzen (INSERM U930, 2, Bvd Tonnelle, 37044 Tours Cedex 9, France, bleuzen@chu-tours.fr), Peggy Palanchon (INSERM U930, 2, Bvd Tonnelle, 37044 Tours Cedex 9, France, palanchon@med.univ-tours.fr), Jean-Michel Correas (Hopital Necker, 75015 Paris, France, jean-michel.correas@nck.ap-hop-paris.fr)

Contrast ultrasound imaging techniques are of interest for almost every clinical application in all organs including not only liver and kidney but also superficial organs and brain or lungs. This technique is mainly powerful in terms of lesion detection and characterization especially for liver with diagnostic value greater than $90 \%$. This was the case for all categories of lesions (with values nearly $100 \%$ for liver metastases, FNH) but the accuracy was slightly lower for HCC. Specific recommendations were established by EFSUMB for the use of contrast agents in liver, pancreas, kidney, trauma and brain imaging for a proper and safe use to improve diagnosis accuracy. On the other hand, contrast-enhanced ultrasound may be used in evaluating response to therapy. The follow-up of vascularization under specific targeted treatments offers the capacity to early diagnose positive or negative local response for an adaptation of therapeutic way 
before or in absence of tumour size changes. This non invasive method could be repeated without adverse events early after starting this therapy in order to depict local response or recurrence for a better adaptation of dose with a strong impact on cost and limitation of adverse events.

\section{1:20}

2aBB10. Dynamics of microbubbles targeted to surfaces: numerical and experimental modelling. Richard Manasseh (CSIRO, Fluid Dynamics Group, PO Box 56, Highett, VIC 3190 Melbourne, Australia, Richard.Manasseh@csiro.au), Edward Payne (University of Melbourne, Department of Mechanical \& Manufacturing Engineering, Parkville, VIC 3010 Melbourne, Australia, Edward.Payne@csiro.au), Andrew Ooi (University of Melbourne, Department of Mechanical \& Manufacturing Engineering, Parkville, VIC 3010 Melbourne, Australia, a.ooi@unimelb.edu.au)

Numerical calculations and illustrative experiments are presented on the volumetric oscillations of microbubbles on and near surfaces. There is a considerable theoretical and experimental literature on the acoustic interactions of bubbles. In the present study, the surface was represented by a mirror-image bubble and the nonlinear frequency response calculated by integrating acoustically coupled sets of Rayleigh-Plesset-like equations. A significant shift was found in the peak nonlinear response frequency of a bubble targeted onto a surface. This effect is increased when other bubbles are nearby on the surface. Owing to the asymmetric influence of the surface, experimental images were dominated by shape-mode instabilities, making optical determination of the peak nonlinear response frequency difficult. Moreover, it was found that even if bubbles are separated by only a small fraction of the sound wavelength, time delays owing to the finite speed of sound have a surprisingly significant influence. Calculations on the symmetric mode of mutual oscillation showed that the introduction of time delays significantly modified harmonics of the spectrum.

\section{1:40}

2aBB11. Echogenic liposomes for image-guided drug delivery. Christy K. Holland (University of Cincinnati, Biomedical Engineering, MSB, 231 Albert Sabin Way, Cincinnati, OH 45267-0586, USA, Christy.Holland@uc.edu), David D. McPherson (University of Texas Health Science Center, 6431 Fannin St., MSB 1.252, Houston, TX 77030, USA, David.D.McPherson@ uth.tmc.edu)

Echogenic liposomes (ELIP) are under development to enable ultrasound-controlled drug delivery. Ultrasound-triggered release of hydrophilic and lipophilic agents was assessed from circulating ELIP in vitro using color Doppler ultrasound (6 MHz). Calcein, or recombinant tissue plasminogen activator (rt-PA), both hydrophilic agents, or papaverine, a lipophilic agent, were each loaded into ELIP and diluted in $0.5 \%$ bovine serum albumin. Ultrasound-triggered release of calcein, rt-PA or papaverine from ELIP was determined relative to detergent and untreated controls. Calcein concentration was measured by fluorimetry and release of rt-PA was assayed with a chromogenic substrate and a spectrophotometric method. Papaverine concentration was quantified by absorbance spectrophotometry and the amount of papaverine associated with P-ELIP was determined using a spin column filtration technique. Dynamic changes in echogenicity were assessed with low output B-mode ultrasound (0.04 MI) as mean digital intensity. Treatment with color Doppler ultrasound resulted in a statistically significant amount of calcein and rt-PA release from liposomes $(\mathrm{p}<0.01)$, but did not induce papaverine release $(\mathrm{p}>0.05)$. The differential efficiency of ultrasound-mediated pharmaceutical release from ELIP for water-soluble and lipid-soluble compounds suggests that water-soluble drugs are better candidates for the design and development of ultrasoundcontrolled drug delivery systems.

\section{Contributed Papers}

12:00

2aBB12. Ultrasound contrast agents in an in vivo murine melanoma model. François Tranquart (INSERM U930, 2, Bvd Tonnelle, 37044 Tours Cedex 9, France, tranquart@med.univ-tours.fr), Ayache Bouakaz (INSERM U930, 2, Bvd Tonnelle, 37044 Tours Cedex 9, France, bouakaz@med.univ-tours.fr), Sophie Serriere (INSERM U930, 2, Bvd Tonnelle, 37044 Tours Cedex 9, France, serrie_s@med.univ-tours.fr)

The purpose of the study was to test different types of microbubble contrast agents (SonoVue ${ }^{\circledR}$, Definity ${ }^{\circledR}$ and targeted microbubbles against vascular endothelial growth factor receptors (KDR, antiCD31)) to quantify angiogenesis. After SonoVue ${ }^{\circledR}$ and Definity ${ }^{\circledR}$ injections, a strong, rapid and heterogeneous signal enhancement was detected in all tumors. According to the tumor size, the quantification of the perfused area revealed major interindividual variations. Three groups of animals bearing tumors, following SonoVue ${ }^{\circledR}$ administration, were arbitrarily constituted to compare various sonographic parameters such as AUC, mTT, etcœ. The tumoral size increase seemed negatively correlated to a decrease in all quantified parameters. Non linear acoustic signal from microbubbles targeted to the molecular site was determined by an ultrasound-based destruction-reperfusion scheme. In tumor-bearing mice, an increase of the retention time ( $>10$ minutes) was revealed following KDR and antiCD31 targeted microbubbles administrations when compared to the retention time of non targeted microbubbles. The amount of remaining targeted bubbles remains always lower than non specific agents. Results showed that quantitative contrast-enhanced ultrasound imaging could be an effective method for monitoring angiogenesis process in mice and so could be used for the follow-up of tumours under specific treatment.

\section{$12: 20$}

2aBB13. Ultrasound contrast imaging of angiogenisis in a murine tumor model. Olivier Lucidarme (CHU Pitié-Salpétrière, 47-83 Boulevard de l'Hôpital, 75651 Paris, France, olivier.lucidarme@psl.aphp.fr), Laurent Taillade (Laboratoire d'Imagerie Paramétrique, 15 rue de l'Ecole de Médecine, 75006 Paris, France, laurenttaillade2@yahoo.fr), Aymeric Guibal (Laboratoire d'Imagerie Paramétrique, 15 rue de l'Ecole de Médecine, 75006 Paris, France, guibala@hotmail.com), Sebastien Mule (Lab d'Imagerie Fonctionnelle, 91 Boulevard de l'Hôpital, 75634 Paris, France, Sebastien.Mule@imed.jussieu.fr), Eva Comperat (CHU Pitié-Salpétrière, 47-83 Boulevard de l'Hôpital, 75651 Paris, France, eva.comperat@psl.aphp.fr), Yasmina Badachi (Laboratoire d'Imagerie Paramétrique, 15 rue de l'Ecole de Médecine, 75006 Paris, France, yasmina.badachi@gmail.com), Erwan Jouannot (Laboratoire d'Imagerie Paramétrique, 15 rue de l'Ecole de Médecine, 75006 Paris, France, Erwan.Jouannot@sanofi-aventis.com), Olivier Rixe (CHU PitiéSalpétrière, 47-83 Boulevard de l'Hôpital, 75651 Paris, France, olivier.rixe@psl.aphp.fr), Lori_Bridal (Laboratoire d'Imagerie Paramétrique, 15 rue de l'Ecole de Médecine, 75006 Paris, France, bridal @lip.bhdc.jussieu.fr)

Microvascularization modifications should precede tumor size-changes during anti-angiogenic therapy. We applied contrast functional ultrasound imaging (fUSI) to detect changes in Wilms tumors with anti-angiogenic treatment (Bevacizumab). Human Wilms tumor cells was grafted in left kidney of 32 mice. Once tumors had $>5 \mathrm{~mm}$ diameter, mice received : placebo, $\mathrm{N}=14$; Bevacizumab for 21days, $\mathrm{N}=11$; and Bevacizumab for 10days followed by placebo for 11 days, $\mathrm{N}=7$. On days $-1,+1,+9,+14$ and +21 
with respect to treatment start, fUSI was performed (CPS mode, SonoVue). Linear time intensity curves were obtained from regions in kidney cortex and matched-depth of tumor for first bolus passage and 50s following acoustic destruction of contrast. Excised tumor weight decreased with increased treatment duration: $3.7+/-1.8 \mathrm{~g}$ (placebo), 2.3+/-1.9 g (Bevacizumab10days, placebo-11days), 1.4+/-0.7 g (Bevacizumab-21 days) $[\mathrm{p}<0.05]$. Area under the bolus-passage curve (AUC) and the plateau intensity of the destruction-reperfusion were greater from $\mathrm{D}+9$ to $\mathrm{D}+21[\mathrm{p}<0.04]$ in the placebo than Bevacizumab-21day. For the group treated during the first 10 days, fUSI values were comparable to those of the treated group until $\mathrm{D}+14$, then increased to become slightly superior to those of the placebo group by $\mathrm{D}+21$. Noninvasive fUSI demonstrated revascularization after suspension of anti-VEGF therapy.

\section{2:40-1:40 Lunch Break}

\section{Invited Paper}

\section{$1: 40$}

2aBB14. Violent cavitation from optically configured microbubble pairs. Paul A. Campbell (University of Dundee, Ewing 1-6, Carnegie Physics Laboratory, Main Campus, DD14HN Dundee, UK, p.a.campbell@dundee.ac.uk)

The mutual interaction between two cavitating microbubbles was investigated using a novel optical trapping arrangement. This approach facilitated development of arbitrary, stable, and initial spatial configurations for two-bubble systems. Critically, exercising optical control over such a binary system can effectively isolate it from resident bubble populations during insonation. This ensures that any early stage dynamical evolution of the system is dominated by the mutual interaction of the two bubbles in view, rather than any extraneous influence arising from 'cross-talk'. In circumstances where the bubbles are located far from a rigid boundary, we observed, using high speed microphotography at framing rates of over $1 \mathrm{MHz}$, that the action of secondary radiation forces leads to mutual bubble attraction often followed by the development of violent microjetting. Microbubble coalescence, with subsequent fragmentation could be observed, and the generation of short-lived anti-bubbles can often occur also. Finally, when cells are present as confluent monolayers on rigid substrates, the statistics for microbubble outcome and dynamics are modified somewhat. I will relate statistics to the parameter space that we interrogated, which included: inter-bubble spacing; relative bubble radii; and individual bubble stand-off parameters.

\section{Contributed Papers}

\section{2:00}

2aBB15. Nonlinear propagation in microbubble cloud, does it make the distal myocardium appear brighter or darker? Meng-Xing Tang (Imperial College London, Dept. of Bioengineering Engineering, South Kensington, SW7 2AZ London, UK, mengxing.tang@ic.ac.uk), Robert Eckersley (Imperial College London, Imaging Sciences Department, Hammersmith Campus, Du Cane Road, W12 OHS London, UK, r.eckersley@ic.ac.uk)

A number of questions remain in contrast ultrasound imaging regarding the complex interaction between bubbles, US and tissue. E.g. in contrast echocardiography for perfusion imaging, it is sometimes difficult to interpret the images for the myocardium distal to the probe. Due to US nonlinear propagation through the bubble-filled chamber, the distal part can appear artefactually brighter or darker in e.g. Pulse Inversion images. Although tissue at target is likely to increase in brightness in contrast specific images when nonlinear propagation happens, it is less clear for microbubbles. This work tries to gain better understanding of this by investigating how nonlinear propagation of ultrasound pulses can change the appearance of microbubbles and tissues in Pulse Inversion images by altering their acoustic response. A series of specifically designed simulations and experiments were performed. The results show that nonlinear propagation can have a significant impact on the appearance of tissue containing bubbles, and this varies with insonating frequency and pressure. These results suggest the mechanism involves a balance between a reduction in nonlinear bubble scattering after propagation against an increase in the nonlinear tissue scattering. Consequently the deeper tissues containing bubbles may appear brighter or darker depending on the relative contribution of these two effects.
2:20

2aBB16. Frequency dependence of kidney injury induced by contrast-aided diagnostic ultrasound. Douglas Miller (University of Michigan, Department of Radiology, Ann Arbor, MI 48109-0553, USA, douglm@umich.edu), Chunyan Dou (University of Michigan, Department of Radiology, Ann Arbor, MI 48109-0553, USA, chunyand@umich.edu), Roger C. Wiggins (University of Michigan, Department of Internal Medicine (Nephrology), Ann Arbor, MI 48109, USA, rwiggins@med.umich.edu)

Glomerular capillary hemorrhage $(\mathrm{GCH})$ induced in rat kidneys by contrast aided diagnostic ultrasound was examined as a function of ultrasonic frequency. The right kidneys of anesthetized rats mounted in a water bath were exposed to image pulse sequences at $1 \mathrm{~s}$ intervals during intravenous infusion of diluted ultrasound contrast agent. Diagnostic ultrasound (DUS) scanners were utilized for exposure at $1.5,2.5,3.2,5.0$ and $7.4 \mathrm{MHz}$. A laboratory exposure system (LES) was used to simulate DUS exposure at 1.0, $1.5,2.25,3.5,5.0$ and $7.5 \mathrm{MHz}$ at relatively high peak rarefactional pressure amplitudes (PRPAs). The exposed kidneys were removed and fixed for histology. GCH was measured by counting glomeruli with blood in the urinary (Bowman's) space on histological sections. The percentage of GCH at a fixed exposure frequency showed a rapid rise with PRPA above an apparent threshold. The threshold values were approximately proportional to the ultrasound frequency, with proportionality constants of $0.5 \mathrm{MPa} / \mathrm{MHz}$ for DUS and $0.6 \mathrm{MPa} / \mathrm{MHz}$ for LES exposures. The increasing thresholds with increasing frequency limited the GCH effect for contrast aided DUS, and no GCH was seen for DUS at 5.0 or $7.4 \mathrm{MHz}$ for the highest available PRPAs. 


\title{
Session 2aEAa
}

\section{Engineering Acoustics, Underwater Acoustics, and ECUA: Sonar Transducer Design and Modeling II}

\author{
John B. Blottman, Cochair \\ Naval Undersea Warfare Center, Division Newport, 1176 Howell Street, Newport, RI 02841-1708, USA \\ Pascal Mosbah, Cochair \\ IEMN dpt ISEN, UMR CNRS 8520, Lille, France
}

\section{Invited Papers}

2aEAa1. Acoustic array interactions in the time domain. George W. Benthien (511 Savoy Street, San Diego, CA 92106, USA, benthien@cox.net)

In this paper I discuss the computation of acoustic array interactions in the time domain. Acoustic array interactions in the time domain are expressed in terms of mutual impulse response functions. The mutual impulse response between a pair of array elements is the acoustic force on the first element of the pair due to an acceleration impulse of the second with all other elements held rigid. The mutual response functions are calculated in the frequency domain using the boundary element program CHIEF and then Fourier transformed to the time domain. As the convergence in the frequency domain can be very slow, I introduce modified impulse response functions that converge much faster in the frequency domain and are simply related to the desired impulse response functions. This approach reduces the range of frequencies required in the CHIEF computations and gives better accuracy in the initial time period. Transient results are presented for an array of simple transducer elements. The mutual impulse response functions are combined with the transducer element equations to yield a system of differential-integral equations of delay type. A solution procedure for equations of this type is presented.

2aEAa2. Exact model of Langevin transducers with internal losses. D. D. Ebenezer (Naval Physical and Oceanographic Laboratory, Thrikkakara, 682021 Kochi, India, tsonpol@vsnl.com), P. A. Nishamol (Naval Physical and Oceanographic Laboratory, Thrikkakara, 682021 Kochi, India, dd_ebenezer@yahoo.com)

An exact method is presented to analyze classical Langevin transducers with internal losses. The transducers consist of an axially polarized piezoelectric cylinder sandwiched between two elastic cylinders. All three cylinders are of the same diameter. Exact solutions to the exact equations of motion of the piezoelectric and elastic cylinders and the Gauss electrostatic condition are used. Complex piezoelectric and elastic coefficients are used to model internal losses. For each cylinder, the first set of solutions contains Bessel functions that form a complete set in the radial direction. The second and third sets contain trigonometric functions that form complete sets in the axial direction. They are used to represent fields that are symmetric and anti-symmetric with respect to the plane midway between the ends of the cylinder, respectively. The interface and boundary conditions are satisfied by using the orthogonal properties of the functions. Transducers with identical elastic cylinders at the ends as well as those with a light head mass and a heavy tail mass are analyzed. Numerical results are presented to illustrate the input electrical admittances of transducers. They are compared with those obtained using ATILA - a finite element package for the analysis of sonar transducers.

2aEAa3. Numerical acoustic modeling code applied to sonar transducers and arrays: review and perspectives. Gerard Vanderborck (Thales Underwater Systems SAS, Acoustic Department, 525 route des Dolines, BP 157, 06903 Sophia Antipolis Cedex, France, gerard.vanderborck@fr.thalesgroup.com)

In this paper a review based on several examples of Thales Underwater Systems (TUS) design and modeling of transducers will be presented: several examples like wide band free flooded ring flextensional and bender transducers, high frequency transducers with thermal analysis modeling included, will be discussed. An approach of the problem of the mutual impedance computation appearing in array modeling will be also presented. Tus used since several decades FEM - BEM codes to design acoustic transducers and arrays. The perspectives in transducer and array modeling will be indicated like the possibility to take into account the non-linearities in the material, the time dependent problem and the use of new kind of piezoelectric material will be also discussed. Specific aspect of single crystal transducers modeling will be presented. The increase of the computing power will also permit to take into account the complex problem of the interaction between transducers in an array (mutual impedances). We will conclude on complex ultra wide band antenna (based on strong transducers interactions) optimization and the associated modeling architecture issues. 
2aEAa4. Finite element analysis simulations of a piezoelectric cymbal actuator using atila software. Thomas Tremper (Micromechatronics Inc., 200 Innovation Blvd, Suite 155, State College, PA 16803, USA, ttremper@mmech.com), Alfredo Vazquez Carazo (Micromechatronics Inc., 200 Innovation Blvd, Suite 155, State College, PA 16803, USA, avc@mmech.com), Kenji Uchino (Micromechatronics Inc., 200 Innovation Blvd, Suite 155, State College, PA 16803, USA, kenjiuchino@mmech.com)

The piezoelectric ultrasonic cymbal actuator is a well known flextensional type actuator. Because of the actuator's geometry, the small movements of the piezoelectric ceramic are amplified by the flextensional structure. Simulation of this device was performed using Atila finite element software, which is specially suited for piezoelectric devices. This study will focus on some of the necessary parameters to accurately simulate cymbal actuators and other piezoelectric actuators. The simulations discussed will be compared to actual experimental data. Based on the experimental results the simulations will be reexamined and modifications will be made to increase the models accuracy. Employing this iterative process will promote increased accuracy in future simulations, which would therefore decrease development time and increase productivity.

\section{Contributed Papers}

\section{9:20}

2aEAa5. Underwater tensor sensors based on optical fiber bragg gratings. Francois M. Guillot (Georgia Institute of Technology, Mechanical Engineering, 771 Ferst Drive, Atlanta, GA 30332-0405, USA, francois.guillot@me.gatech.edu), David H. Trivett (Georgia Institute of Technology, Mechanical Engineering, 771 Ferst Drive, Atlanta, GA 303320405, USA, david.trivett@me.gatech.edu), Peter H. Rogers (Georgia Institute of Technology, Mechanical Engineering, 771 Ferst Drive, Atlanta, GA 30332-0405, USA, peter.rogers@me.gatech.edu)

This paper deals with the development a new type of low-noise underwater tensor sensor aimed at improving the performance and the design of directional arrays. The transducer can be configured either as a particle velocity sensor (dipole) or as fluid shear sensor (quadrupole). The sensing principle of the device relies on the interference signal from two Bragg gratings written on the same fiber, and illuminated by a tunable, narrowband light source. The gratings are a few centimeters apart, and they each reflect a portion of the incident light. The fiber is epoxied to two spacers separated by a small gap situated between the gratings. This assembly is then adhered to two plates connected by a hinge, which is located below the gap. One plate is held rigidly and the tip of the other (free) plate experiences transverse vibrations, when ensonified. These vibrations produce periodic gap length changes, which modulate the interference signal from the two gratings. The modulation is related to the amplitude of the sound wave and is monitored with a photodetector. A noise analysis will be presented and the performance of prototype sensors will be discussed.

\section{9:40}

2aEAa6. Beampattern optimization for a conformal projecting array of transducers with baffle effect and mutual coupling among elements. Yuanliang Ma (Institute of Acoustic Engineering, Northwestern Polytechnical University, 710072 Xi'an, China, ylma@nwpu.edu.cn), Zhengyao $\mathrm{He}$ (Institute of Acoustic Engineering, Northwestern Polytechnical University, 710072 Xi'an, China, hezhengyao@163.com)

Beampattern optimization for projecting arrays is much more challenging in comparison with receiving arrays, particularly for conformal projecting arrays. It is because the array elements have to work in resonance state for efficient projecting and the variation of radiation impedance has strong effect on the array performance. The radiation impedance for each element is determined by a lot of factors, i.e, the element position, baffle property, mutual coupling and velocity weighting vector of the array. Thus beampattern optimization for a projecting conformal array must take into account all these factors. One thing makes the topic more difficult that the mutual impedance for each element is changeable along with the weighting vector change. In this paper a global solution approach is formulated and a boundary element model combined with transducer equivalent circuits is developed. The driving voltage weighting vector is deduced through an optimization algorithm. Computer simulation together with experiments are conducted for a 14-element conformal array. The results agree well. The multiple beampatterns formed within a wide observation sector exhibit uniform beamwidth and low sidelobes. The source level for each beam is maximized in constraint of the maximum driving voltage of array elements being constant.

\section{0:00}

2aEAa7. Advanced Single Crystal Piezoelectric Transducers for Naval Sonar and Medical Ultrasound Applications. Wesley Hackenberger (TRS Technologies, Inc., 2820 East College Avenue, State College, PA 16801, USA, wes@ trstechnologies.com), Jun Luo (TRS Technologies, Inc., 2820 East College Avenue, State College, PA 16801, USA, jun@trstechnologies.com), Kevin Snook (TRS Technologies, Inc., 2820 East College Avenue, State College, PA 16801, USA, kevin@trstechnologies .com)

Single crystal piezoelectrics have received much attention due to their very high piezoelectric and electromechanical coupling coefficients. Naval sonar and medical ultrasound transducers, fabricated from this material, exhibit unprecedented increases in bandwidth, source level, and sensitivity compared to ceramic based devices. In this paper we report many new developments in the growth and manufacture of single crystals. These include increased diameter $\left(>3^{\prime \prime}\right)$ crystal boules, improved compositional uniformity, increased thermal stability, and minimized surface damage. Manufacturing improvements are being applied to a broad range of crystal transducer applications. For navy sonar systems, crystals are resulting in very high bandwidths $(>100 \%)$ for transducer sizes that are $1 / 2$ the resonating length and $1 / 4$ the weight of ceramic counterparts with equivalent (or often lower) source level. Manufacturing improvements such as high tensile strength, achieved by precision polishing crystal surfaces, are enabling new transducer operational regimes not possible with ceramic. In particular, single crystal 1-3 composite projectors show promise for achieving high source level in a compact, integrated system designs. Single crystals are also enabling new applications in medical ultrasound including very broadband and extremely high frequency transducers. Further improvements are expected in the near future.

\section{0:20-10:40 Break}

\section{$10: 40$}

2aEAa8. Design of a phase array ultrasonic sensor using vibration decoupled concept. Chia-Yu Lin (National Taiwan University, Rm.433, Institute of Applied Mechanics, No.1, Sec.4, Roosevelt Rd., 10617 Taipei, Taiwan, cylinx@mems.iam.ntu.edu.tw), Chih-Chiang Cheng (National Taiwan University, Rm.433, Institute of Applied Mechanics, No.1, Sec.4, Roosevelt Rd., 10617 Taipei, Taiwan, cccheng@nbm.ntu .edu.tw), Wen-Jong Wu (Department of Engineering Science and Ocean Engineering, National Taiwan University, Rm.433, Institute of Applied Mechanics, No.1, Sec.4, Roosevelt Rd., 10617 Taipei, Taiwan, wjwu@ntu.edu.tw), Chuin-Shan Chen (Department of Civil Engineering, National Taiwan University, Rm.433, Institute of Applied Mechanics, No.1, Sec.4, Roosevelt Rd., 10617 Taipei, Taiwan, dchen@ntu.edu.tw), Jay Shieh (Department of Material Science and Engineering, National Taiwan University, Rm.433, Institute of Applied Mechanics, No.1, Sec.4, Roosevelt Rd., 10617 Taipei, Taiwan, jayshieh@ntu.edu.tw), Chih-Kung Lee (National Taiwan University, Rm.433, Institute of Applied Mechanics, No.1, Sec.4, Roosevelt Rd., 10617 Taipei, Taiwan, cklee@mems.iam.ntu .edu.tw)

Phase array ultrasonic sensors have been used widely to generate higher directional radiating patterns in which piezoelectric units are distributed 
sparsely in space. In this study, we present a novel design of a phase array ultrasonic sensor based on the concept of vibration decoupling. Decoupling is achieved by careful design of source aperture, and such design allows piezoelectric units tightly located in the same structure. The phase array sensor is designed herein as a cylinder with a dumbbell shape groove to decouple vibration, and finite element analysis is used to optimize the design. Two piezoelectric discs are adhered on the bottom plate of the sensor whereby desirable wave generation and detection are controlled adaptively. By electrical steering, the sensor thus operates as a dipole mode. Finally, prototypes of the sensor are made and experiments are conducted to verify simulation results.

\section{1:00}

2aEAa9. Nonlinear multifrequency transmitter for seafloor characterization. Lucilla Di Marcoberardino (Institut Jean le Rond D'Alembert, UMR 7190, 2 Place de la Gare de Ceinture, 78210 Saint Cyr L'Ecole, France, lucilladm@hotmail.com), Jacques Marchal (Institut Jean le Rond D'Alembert, UMR 7190, 2 Place de la Gare de Ceinture, 78210 Saint Cyr L'Ecole, France, jmarchal@ccr.jussieu.fr), Pierre Cervenka (Institut Jean le Rond D'Alembert, UMR 7190, 2 Place de la Gare de Ceinture, 78210 Saint Cyr L’Ecole, France, cvk@ccr.jussieu.fr)

In the underwater context, it is known that the frequency diversity provides essential information to derive the nature of the seafloor. This presentation deals with a new concept based on a transmitter that generates simultaneously several harmonic frequencies. Our final objective is to assert the feasibility of a multi-frequency tool whose desirable characteristics could be specified for applications such as detection of sunken oil slicks, sediment characterization, or surveys before cable or pipe laying. The acoustic beams are generated through the harmonic components of a shock wave radiated by an antenna driven at a high level. The source is unique in time and space so that the multi-frequency responses are inherently perfectly matched. A numerical model based on a generalized KZK equation has been developed to estimate the saturated fields. Measurements of the first harmonic fields obtained in our outdoor tank facility are compared with simulations.

\section{1:20}

2aEAa10. Modeling the acoustic radiation force in piezoelectrically driven micro fluidic chambers using ATILA. Karl Fisher (Lawrence Livermore National Laboratory, 7000 E Avenue, Brentwood, CA 94513, USA, fisher34@1lnl.gov)

A procedure is demonstrated to quantitatively evaluate the acoustic radiation forces in micro fluidic particle manipulation chambers. Typical estimates of the acoustic pressure and the acoustic radiation force are based on an analytical solution for a simple 1-dimensional standing wave pattern. The complexities of a typical micro fluidic channel limit the usefulness of this approach. By leveraging finite element approaches, and a generalized equation for the acoustic radiation force, channel designs can be investigated in two- and three-dimensions. Calculations and experimental observations are in good agreement. This work was performed under the auspices of the U.S. Department of Energy by University of California, Lawrence Livermore National Laboratory under Contract W-7405-Eng-48.

\section{1:40}

2aEAa11. Equivalent circuit models derived from finite element models using structural dynamics techniques. Julien Bernard (Thales Underwater Systems, 525 route des Dolines BP 157 Valbonne Parc d'Activités de Sophia Antipolis, 06903 Sophia Antipolis Cedex, France, julien.bernard@fr .thalesgroup.com)

Electroacoustic transducers can be divided into an active part, the driver, and a passive part, the structure. The driver ensures electromechanical transduction, while the structure performs various mechanical and acoustical functions, such as support, shock and pressure protection, and impedance transformation. For design purposes, one often needs an equivalent circuit model which gives a relationship between the acoustic characteristics of the overall device and that of its components. Transducer equivalent circuits are usually either physical or modal. Physical equivalent circuits lend themselves to the treatment of a transducer as an assembly, but in general yield frequency dependant parameters. Modal equivalent circuits are more adequate for resonant transducers, but describe transducers as a whole. This works shows how these two types of equivalent circuits can be obtained from a full finite element model by using common structural dynamics techniques: substructuring and modal expansion. It also shows that a third, hybrid type of equivalent circuit can be obtained by using a component mode synthesis technique derived from the Craig-Bampton method. This hybrid equivalent circuit combines the advantages of physical and modal equivalent circuits, enabling to express transducer modal parameters in terms of driver and structure modal parameters.

\section{2:00}

2aEAa12. Acoustic field calculation for a compact barrel-stave flextensional transducer array. Zhengyao He (Institute of Acoustic Engineering, Northwestern Polytechnical University, 710072 Xi'an, China, hezhengyao@163.com), Chao Sun (Institute of Acoustic Engineering, Northwestern Polytechnical University, 710072 Xi'an, China, csun@nwpu .edu.cn)

The boundary element method together with the finite element method is used to calculate the radiated acoustic field of a compact array of barrelstave flextensional transducers. At first, the surface vibration displacement distribution of one barrel-stave transducer is obtained by the finite element method using the commercial software ANSYS. The calculation results are then imported into the boundary element calculation software SYSNOISE. At the frequency of $1400 \mathrm{~Hz}$, the radiated acoustic field and radiation impedance are calculated by the boundary element method for a planar array which is composed of three identical barrel-stave flextensional transducers uniformly distributed on a circle with spacing much less than half wavelength. The calculation results show that the mutual interactions among elements are significant for the compact array. The mutual radiation resistance between two transducers is close to the self-radiation resistance of the transducers. And the transmitting source level of the 3-element array is $8.7 \mathrm{~dB}$ higher than that of one transducer if the surface vibration velocities of the transducers in the array are the same as that of one transducer. The proposed technique can be used to predict the performance of a transmitting transducer array at the stage of preliminary design.

\section{2:20}

2aEAa13. Finite Element Modeling of 2-D Transducer Arrays. Hind Mestouri (ISEN Brest (Institut Supérieur de l'Electronique et du Numérique), 20, rue Cuirassé Bretagne, C.S. 42807, 29228 Brest, France, hind.mestouri@isen.fr), Alain Loussert (ISEN Brest (Institut Supérieur de l'Electronique et du Numérique), 20, rue Cuirassé Bretagne, C.S. 42807, 29228 Brest, France, alain.loussert@isen.fr), Gilles Keryer (ISEN Brest (Institut Supérieur de l'Electronique et du Numérique), 20, rue Cuirassé Bretagne, C.S. 42807, 29228 Brest, France, gilles.keryer@isen.fr)

Active sonar detects objects underwater by sending out sound waves in pulses, scientists could measure the time it takes these pulses to travel through the water, reflect off of an object, and return to the ship. There are a number of factors limiting the performance of low frequency transducer arrays for active sonar systems including the crosstalk and housing interactions which affects the directivity pattern and the sensitivity. Many important issues in transducer arrays design, such as crosstalk, cannot be accurately studied using analytic method due to the complexity of the partial differential equations involved. Finite element method is the only appropriate way to gain more detailed information. In this paper, a 2-D finite element model is constructed to analyze the crosstalk and structure interactions, using ATILA code and GiD graphical interface, of transducer arrays, consists of six active piezoceramic bars, and developed for shallow water use. The directivity pattern strongly depends on the transducer size versus wavelength, each transducer elements interacts directly with its neighbour and indirectly with the other elements, and the radiated acoustic power may considerably vary from one element to another. It is shown that crosstalk and housing interactions can be reduced through the use of different interelement materials and housing characteristics. 


\title{
Session 2aEAb
}

\section{Engineering Acoustics: Acoustic Evaluation I}

\author{
David Brown, Chair \\ BTech Acoustics, LLC and Universtity of Massachusetts Dartmouth, ATMC, Electro-Acoustics Research Laboratory and ECE \\ Dept., 151 Martine St, Fall River, MA 02723, USA
}

\section{Contributed Papers}

\section{8:00}

2aEAb1. Measurement of Sound Velocity in Water Using Optical Probe and Acoustical Holography. Takeshi Ohbuchi (University of Tsukuba, 1-1-1 Tennoudai, 305-8573 Tsukuba, Japan, ohbuchi@aclab.esys.tsukuba.ac.jp), Koichi Mizutani (Tsukuba Univ., Tsukuba Science City, 305-8573 Ibaraki, Japan, mizutani@esys.tsukuba.ac.jp), Naoto Wakatsuki (Tsukuba Univ., Tsukuba Science City, 305-8573 Ibaraki, Japan, wakatuki@iit.tsukuba.ac.jp), Hiroyuki Masuyama (Toba National College of Maritime Technology, 1-1 Ikegami-cho, 517-8501 Toba, Japan, masuyama@toba-cmt.ac.jp)

We propose a method for a determination of a three-dimensional sound velocity using optical computerized tomography (O-CT) and near-field acoustical holography (NAH) for measuring a phase object. Ultrasonic waves affect a phase of the light passing through radiated sound fields. The zeroth order diffraction light including sound pressure information is acquired by an avalanche photodiode. Eighteen projections are acquired by rotational scanning, and each projection along the optical axis is obtained by single linear scanning and electronically quadrature-detected as the complex amplitude. The complex sound fields are reconstructed by O-CT in a region of $40 \times 40 \mathrm{~mm}^{2}$. Then the sound field on another plane is propagated using $\mathrm{NAH}$ from the acquired sound fields, and the sound field on the same plane is reconstructed by O-CT. Comparing the phase of propagated and reconstructed sound fields in wavenumber domain, we can obtain the sound velocity in three dimensional space in a region of $30 \times 30 \times 10 \mathrm{~mm}^{3}$. The experimental results are in agreement with the reference value measured by another mean.

\section{8:20}

2aEAb2. Influence of a load on the nonlinear behavior of a piezoelectric rod under high sinusoidal voltages. Denis Parenthoine (Lussi, Université F Rabelais de Tours, 40, rue de la Chocolaterie, 41000 Blois, France, parenthoine_denis@yahoo.fr), Lionel Haumesser (Lussi, Université F Rabelais de Tours, 40, rue de la Chocolaterie, 41000 Blois, France, lionel.haumesser@univ-tours.fr), François Vander Meulen (Lussi, Université F Rabelais de Tours, 40, rue de la Chocolaterie, 41000 Blois, France, vandermeulen@univ-tours.fr), Louis-Pascal Tran-Hhuu-Hue (Lussi, Université F Rabelais de Tours, 40, rue de la Chocolaterie, 41000 Blois, France, tran@univ-tours.fr)

At high levels of excitation, appear, in piezoelectric devices, nonlinear phenomena which can adversely affect the quality of the applications to which they are devoted. It is, in particular, true in non-destructive evaluation, where the generation of harmonics in the ultrasonic tranducer can make inacurrate measurements in materials. In a previous work, third-order constants in a piezoceramic rod of PZT-21 under high sinusoidal electric fields have been evaluted from the analysis of second harmonic generation in the mechanical displacement. All these measurements have been performed under condition of complete free stress. In order to allow a better understanding of the nonlinear behavior of the transducer, various loads, are, now, applied at one end of the piezoceramic rod. Velocity masurements performed by a laser probe at the free end of the active element provides then informations about the nonlinear behavior of the system. Influence of load on the linear and nonlinear behavior of the piezoelectric rod is then studied in the case of an aluminium bar, and in the case of more nonlinear materials. Results are compared both experimentally and theoritically in loaded and unloaded configurations.

\section{8:40}

2aEAb3. Uncertainty of Acoustical Material Characteristic Measurements. Samir N. Gerges (Federal University of Santa Catarina (UFSC), Campus Universitario - Trindade, 88040-900 Florianopolis, Brazil, samir@emc.ufsc.br), Peter K. Giesbrecht (Federal University of Santa Catarina (UFSC), Campus Universitario - Trindade, 88040-900 Florianopolis, Brazil, samir@emc.ufsc.br)

The experimental determination of the acoustic properties of sound absorption materials depends on numerous factors. The accuracy level in the determination of each property depends, for example, on the measurement apparatus and the type of material. The objective of this work is to present a study about the main sources of uncertainty in the measurement of properties such as sound absorption coefficient, acoustic impedance, flow resistivity and porosity. The uncertainties of the measurement apparatus of each property are quantified. The uncertainty of the materials heterogeneity is also assessed and its individual contribution highlighted. Important aspects of the Standards ASTM C 522 and ISO 10534-2, which present, respectively, recommendations and a procedure for flow resistivity and sound absorption measurements, are discussed. The methodology to calculate the uncertainty of each property is discussed with emphasis on the relevancy of each uncertainty source.

\section{9:00}

2aEAb4. Design and implementation of a three-dimensional seven microphone vector intensity probe with low and high frequency compensations. Khalid Miah (University of Texas, P.O. Box 7155, Austin, TX 78713, USA, miah@mail.utexas.edu), Elmer Hixson (Univ. of Texas, ECE Dept., 1 University Station 0803, Austin, TX 78712-0240, USA, ehixson@mail.utexas.edu)

A seven microphone vector intensity probe has been designed and implemented in this report with low and high frequency compensations. Measurements from plane wave tube and anechoic chamber are used for overall system calibration with corrected gain and phase mismatch errors. Measured and calculated intensity level from both plane wave tube and anechoic room settings showed close agreement with each other in comparison to separate low and high frequency components of the array. Particle velocity vectors and pressures determined for calculating intensity were also used to calculate sound power and total energy density components of various sources. This microphone array vector intensity probe is then used to explore various sound source identification and localization problems.

\section{9:20}

2aEAb5. Application of contrast optimisation autofocus to flexible ultrasonic arrays for non-destructive testing. Alan J. Hunter (University of Bristol, Queen's Building, University Walk, BS8 1SY Bristol, UK, a.hunter@bristol.ac.uk), Bruce W. Drinkwater (University of Bristol, Queen's Building, University Walk, BS8 1SY Bristol, UK, 
b.drinkwater@bristol.ac.uk),Paul D. Wilcox(University of Bristol, Queen's Building, University Walk, BS8 1SY Bristol, UK, p.wilcox@bristol.ac.uk)

Flexible ultrasonic arrays are used for imaging within objects with complicated geometries for non-destructive testing and evaluation (NDT/NDE), e.g., thick-walled pipes, weld caps, etc. We consider the application of autofocus techniques (routinely used in synthetic aperture sonar (SAS)) to this engineering problem. A flexible ultrasonic array is functionally similar to a wide-beam, stripmap SAS with a single transmitter and receiver. Unfortunately, there are few autofocus algorithms available for this configuration. Popular algorithms, such as echo/image correlation, PGA, DPCA, etc., are better suited for use with a narrow-beam or multiple-receiver SAS. However, contrast optimisation is a more general technique that is well suited to the single transmitter/receiver geometry. In this presentation, we describe our implementation of contrast optimisation autofocus and show experimental results using a flexible array prototype.

\section{9:40}

2aEAb6. Inspection of complex components with flexible phased array transducer. Olivier Casula (CEA/LIST, bat 611, 91191 Gif sur Yvette, France, olivier.casula@cea.fr), Gwenael Toullelan (CEA/LIST, bat 611, 91191 Gif sur Yvette, France, gwenael.toullelan@cea.fr), Philippe Dumas (ZA, rue des Savourots, 70190 Voray-sur-l'Ognon, France, philippe .dumas@imasonic.com)

Non destructive testing techniques using ultrasonic methods are often carried out in contact. But, the inspection performances are limited to regular surfaces. Thus, surface irregularities lead to thickness variations of the coupling layer that result in beam distortions and losses of sensitivity. In the context, CEA/LIST has designed flexible phased-array techniques for compensating the surface irregularities and fitting the surface. The independent piezoelectric elements composing the radiating surface are mechanically assembled to build an articulated structure. An embedded profilometer measures the local surface distortion allowing to compute the optimized delay laws and to master the characteristics of the focus beam. Those delay laws computed by the UT-acquisition system are applied in real-time to the piezoelectric elements. To evaluate inspection method capabilities, CEA/LIST develops a simulation software for non destructive testing, CIVA, able to simulate realistic configurations in particular with complex $2 \mathrm{D}$ and $3 \mathrm{D}$ applications. Matrix flexible phased-array probes have been designed and manufactured in collaboration with IMASONIC. This paper sums up examples of inspections in complex geometries where these flexible probes have been successfully used. Moreover, the data are reconstructed thanks to CIVA tools and allow to locate and size the flaw in the part.

\section{0:00-10:20 Break}

\section{0:20}

2aEAb7. Characterization of laminated glasses by means of an inversion method using Finite Elements. Jorge Frances Monllor (DFISTS. Univ. de Alicante, Carretera de Sant Vicent del Raspeig s/n, 03690 San Vicente del Raspeig, Spain, jfmonllor@ua.es), Jaime Ramis Soriano (DFISTS. Univ. de Alicante, Carretera de Sant Vicent del Raspeig s/n, 03690 San Vicente del Raspeig, Spain, jramis@ua.es), Jesús Alba Fernandez (Escola Politècnica Superior de Gandia, Universitat Politècnica de València, Crtra Natzaret-Oliva s/n, 46730 Gandia, Spain, jesalba@ fis.upv .es), Enrique E. Segovia Eulogio (Depto Ing. Construcción. Univ. de Alicante, Carretera de Sant Vicent del Raspeig s/n, 03690 San Vicente del Raspeig, Spain, Enrique.Gonzalo@ua.es), Jenaro Vera Guarinos (DFISTS. Univ. de Alicante, Carretera de Sant Vicent del Raspeig s/n, 03690 San Vicente del Raspeig, Spain, jenaro@dfists.ua.es)

The experimental determination of elastic modules and loss factor in laminated glass components is of great interest in building acoustics since at present there are a lot of building systems using these types of devices. This makes it necessary to predict the transmission losses in a partition. Even though described in regulation, the process is not exempt from serious difficulties. In this work we present a method in order to obtain the parameters mentioned above for a laminated glass composed of a sequence of isotropic layers by solving the model-based inverse problem for frequency admittance experimentally obtained. The parameter that best describes the mechanical constants of material of the layers is obtained by minimizing the discrepancy between the real numerically and numerically predicted waveform. This is done using an iterative optimization. A sensitivity study of the parameters uncertainty is performed in order to establish the feasibility of this technique.

\section{0:40}

2aEAb8. Detection of Shallow Underground Buried Object Using Air Vibration Probe. Yuji Sato (Tsukuba Univ., Tsukuba Science City, 3058573 Ibaraki, Japan, yuji@aclab.esys.tsukuba.ac.jp), Tomohiro Okamura (Univ. of Tsukuba, 1-1-1 Tennodai, 305-8573 Tsukuba, Japan, mizutani@esys.tsukuba.ac.jp), Koichi Mizutani (Tsukuba Univ., Tsukuba Science City, 305-8573 Ibaraki, Japan, mizutani@esys.tsukuba.ac.jp), Naoto Wakatsuki (Tsukuba Univ., Tsukuba Science City, 305-8573 Ibaraki, Japan, wakatuki@iit.tsukuba.ac.jp)

An air vibration probe is a device to measure an acoustic impedance using acoustic delay line oscillation. The frequency is changed when the acoustic impedance is changed. If some objects are varied, the frequency is expected to be changed in the case of the shallow underground detection. The advantage of this device is that it can detect the underground object easily without any contacts or destructions. Two experiments were practiced to study the relation among the frequency, the space between the probe and the ground, and the depth of object. The space was changed with the buried objects in various depths. The frequency became higher when the space became wider. In addition, the frequency was changed more obviously when the space was narrower. The probe scanned the buried objects in the fixed space. The objects were buried in various depths. The frequency became high when the object was buried shallower. In conclusion, the air vibration probe can detect the buried object because the frequency became high when something was buried.

\section{1:00}

2aEAb9. Ultrasonic Airflow Meter in Greenhouse Using Acoustic Reflection against Wall. Ikumi Saito (Tsukuba Univ., Tsukuba Science City, 305-8573 Ibaraki, Japan, saitou@aclab.esys.tsukuba.ac.jp), Naoto Wakatsuki (Tsukuba Univ., Tsukuba Science City, 305-8573 Ibaraki, Japan, wakatuki@iit.tsukuba.ac.jp), Koichi Mizutani (Tsukuba Univ., Tsukuba Science City, 305-8573 Ibaraki, Japan, mizutani@esys.tsukuba.ac.jp), Limi Okushima (National Institute for Rural Engineering, 2-1-6 Kannondai, 3058609 Tsukuba, Japan, limi@affrc.go.jp)

In this paper, we described an ultrasonic airflow meter in a greenhouse using an acoustic reflection against a wall. The ultrasonic airflow meter is available for measuring the spatial mean wind velocity and direction, which consists of two sound probes and the wall of the greenhouse. Use of sound probes has advantage that the airflow accumulated along sound paths from a loudspeaker to a microphone is obtained in contrast to point measurements. The wind velocity and direction are calculated from time of flights (TOFs) of direct and reflected signals. The wind velocity and direction were measured in a greenhouse of $7.2 \mathrm{~m} \times 29.0 \mathrm{~m}$ under factitious winds generated from two large electric fans located at the one side of the greenhouse. In addition, we detected air convection generated from two pairs of fans. These were measured every 20 seconds for 120 minutes. Regarding the measurement; by the proposed airflow meter and a reference; by a conventional one, their mean wind velocities were 0.12 and $0.15(\mathrm{~m} / \mathrm{s})$, respectively. The presented airflow meter using the acoustic reflection against the wall is convenient to measure the wind velocity and direction in large-size facilities such as the greenhouse.

\section{1:20}

2aEAb10. An alternative and industrial method using low frequency ultrasound enabling to measure quickly tortuosity and viscous characteristic length. François Fohr (Centre de Transfert de Technologie du Mans, 20, rue Thalès de Milet, 72000 Le Mans, France, ffohr@cttm-lemans.com), Damien Parmentier (Centre de Transfert de Technologie du Mans, 20, rue Thalès de Milet, 72000 Le Mans, France, 
dparmentier@cttm-lemans.com),Bernard R. Castagnede(Laboratoire d'Acoustique de l'Université du Maine, Avenue Olivier Messiaen, 72085 Le Mans, France, bernard.castagnede@univ-lemans.fr), Michel Henry (Laboratoire d'Acoustique de l'Université du Maine, Avenue Olivier Messiaen, 72085 Le Mans, France, michel.henry@univ-lemans.fr)

In the past, several different methods have been proposed in order to determine tortuosity and characteristic lengths, using various physical approaches. For instance, the BET (from S. Brunauer, P. Emmett and E. Teller) method used the adsorption of argon molecules within the surface of the pores to measure thermal characteristic length (or the so-called specific surface). Ultrasonic methods, during the two last decades, were efficiently implemented to measure these parameters, using large frequency bandwidth, different gazes including helium, and varying static pressures. The most standard and well-known method relies on phase spectrum measurements performed over short bursts, by plotting the inverse of the squared velocity as a function of the inverse of the square root of the angular frequency. Unfortunately, this method is quite sensitive on noise during the unwrapping procedure. We here propose a much simplier and efficient method, which is very robust with noisy signals, working at one single frequency, in most cases around $40 \mathrm{kHz}$. Measurements have been done onto a specific bench developped at CTTM, with some calibrated glass beads, enabling to recover the expected values of the physicalparameters. This method is particularly well suited for industrial and "on line" applications.

\section{1:40}

2aEAb11. A new impedance tube for large frequency band measurement of absorbing materials. Jean Christophe Le Roux (CTTM, 20, rue Thales de Milet, 72000 Le Mans, France, jcleroux@cttm-lemans.com), Jean-Pierre Dalmont (Laboratoire d'Acoustique de l'Université du Maine, Avenue Olivier Messiaen, 72085 Le Mans, France, Jean-Pierre.Dalmont@univ-lemans.fr), Bruno Gazengel (Laboratoire d'Acoustique de l'Université du Maine, Avenue Olivier Messiaen, 72085 Le Mans, France, bruno.gazengel@univ-lemans.fr)

The standard two microphones technique does not allow the measurement of absorbing materials characteristics at low frequency. Moreover, to cover a range from 100 to $6000 \mathrm{~Hz}$ two experiments have to be done with two different sample diameters. By using a sensor with a known volume velocity source developed by the LAUM together with the CTTM, it is demonstrated that the impedance can be obtained from 10 to $6000 \mathrm{~Hz}$ by performing only one measurement with a single material sample. Results showing the behaviour of some materials at low frequency are presented. On the other hand a comparison is done with classical Kundt tube results.

12:00

2aEAb12. An experimental study of Sound Transmission Loss (STL) measurement techniques using an impedance tube. Behrooz Yousefzadeh (Univ. of Tehran, School of Mechanical Engineering, 1439957131 Tehran, Iran, behrooz.j.y@gmail.com), Mohammad Mahjoob (Univ. of Tehran, School of Mechanical Engineering, 1439957131 Tehran, Iran, mmahjoob@ut.ac.ir), Nader Mohammadi (Univ. of Tehran, School of Mechanical Engineering, 1439957131 Tehran, Iran, nmohamady@ut.ac.ir), Ali Shahsavari (Univ. of Tehran, School of Mechanical Engineering, 1439957131 Tehran, Iran, shervin.shahsavari@gmail .com)

A comparison between the two sound transmission loss (STL) measurement techniques using an impedance tube (i.e. two-load method and anechoic termination method) is presented. A modified B\&K type $4206 \mathrm{im}-$ pedance tube has been designed and built. STL tests have been carried out for three homogeneous and isotropic materials with disk-type test samples of identical diameters and different thicknesses. In addition, the results have been compared with those of the classical and more reliable method of tworoom. For both methods, the effect of downstream (tube termination) boundary conditions have been completely studied. The two-load method yields results which matches with two-room measurements, especially when the two boundary conditions are considerably different. The anechoic termination method, on the other hand, is significantly dependant on the termination boundary conditions.

TUESDAY MORNING, 1 JULY 2008

AMPHI MAILLOT, 8:00 TO 10:40 A.M.

\title{
Session 2aMUa
}

\section{Musical Acoustics and Physical Acoustics: Brass Instrument Acoustics I}

\author{
Thomas Moore, Cochair \\ Rollins College, Department of Physics, Winter Park, FL 32789, USA
}

Joël Gilbert, Cochair

Laboratoire d'Acoustique de l'Université du Maine, Avenue Olivier Messiaen, Le Mans, 72085, France

\section{Invited Paper}

\section{8:00}

2aMUa1. Does mass matter? Examining a concrete didjeridu. Noam Amir (Tel Aviv University, Dept. of Communications Disorders, Sheba Medical Center, 52621 Tel Hashomer, Israel, noama@ post.tau.ac.il)

The influence of wall material on the acoustics of musical wind instruments has been debated widely. While this has been examined for some western instruments, it has been barely touched upon regarding the Australian didjeridu. This is very interesting, considering that didjeridus vary enormously in materials and dimensions. Indeed, musicians and manufacturers alike often have very definite opinions concerning the influence of material type and thickness on instrument quality. As a first step towards examining this issue, we conducted a blind test involving three cylindrical didjeridus of identical internal dimensions: all three shared a basic structure of identical plastic tubing. However, a cement casing was cast on the exterior of one of these, bringing its weight to 17 kilograms. The three 
instruments were fixed to a stationary wooden frame, then covered so that only the mouthpieces were visible. 32 players of varying expertise were allowed to play each instrument for as long as they wished, and then asked to judge which instrument was different from the other two. Results indicate that the responses were random, regardless of player level. Further research will be necessary to show whether this holds for noncylindrical bores and different internal finishings.

\section{Contributed Paper}

\section{8:20}

2aMUa2. Relationships among subjective assessments and measured physical properties in the didjeridu. John Smith (University of New South Wales, Music Acoustics, School of Physics, NSW 2052 Sydney, Australia, john.smith@unsw.edu.au), Guillaume Rey (University of New South Wales, Music Acoustics, School of Physics, NSW 2052 Sydney, Australia, guimms@free.fr), Joe Wolfe (University of New South Wales, Music Acoustics, School of Physics, NSW 2052 Sydney, Australia, J.Wolfe@unsw .edu.au)

Traditional didjeridus are unusual and ancient lip-valve instruments with an irregular bore that is largely constructed by termites eating the interiors of small eucalypt trees. This produces instruments with a broad range of bore geometries with many details not immediately apparent to a player. They are therefore well-suited for examining relationships between subjective assessments by players and their measured physical properties. In this study, seven experienced players assessed 38 didjeridus that spanned a wide range of quality, pitch and geometry. A control group of 11 plastic cylindrical pipes were also studied. Eight subjective parameters (backpressure, clarity, resonance, loudness, overtones, vocals, speed, and overall quality) were assessed and the ranking of each instrument correlated with measurements of their geometry and acoustic input impedance spectrum. A strong result of these experiments was the finding that the ranked quality of a didjeridu correlated negatively with the magnitude of its acoustic input impedance, particularly in the frequency range from 1 to $2 \mathrm{kHz}$. Maxima in the impedance of the player's vocal tract would have a greater effect on instruments with a low impedance, thus favouring the production of the varying spectral peaks or formants in the sound envelope that characterise this instrument.

\section{Invited Papers}

\section{8:40}

2aMUa3. A psychoacoustical investigation into the effect of wall material on the sound produced by lip-reed instruments. James W. Whitehouse (Acoustics Research Group, DDEM, MCT Faculty, Open University, Walton Hall, MK7 6AA Milton Keynes, UK, j.w.whitehouse@open.ac.uk), David B. Sharp (Acoustics Research Group, DDEM, MCT Faculty, Open University, Walton Hall, MK7 6AA Milton Keynes, UK, d.sharp@open.ac.uk)

In order to investigate whether a lip-reed instrument's material of manufacture plays a significant role in determining the timbre of the notes it produces, tests have been conducted on five post horns. These post horns have identical geometry but are manufactured from different copper alloys. Using a laser Doppler vibrometer, the structural resonances of each instrument have been established and the wall vibrations induced in each instrument when artificially blown have been measured. In this paper, these measurements are compared with findings from a series of blindfold playing tests carried out using professional musicians and with listening tests comprising notes produced by the different instruments.

\section{9:00}

2aMUa4. More experimental evidence favouring the hypothesis of significant wall vibration influence on radiated horn sound Wilfried Kausel (Inst. f. Wiener Klangstil, Univ. f. Music, Anton von Webernplatz 1, A-1030 Vienna, Austria, kausel@mdw.ac.at), Alexander Mayer (Inst. f. Wiener Klangstil, Univ. f. Music, Anton von Webernplatz 1, A-1030 Vienna, Austria, mayer@mdw.ac.at)

The question whether wall vibrations of wind instruments do or can affect the radiated sound has not been finally answered yet. Instrument makers and musicians make a strong claim that wall thickness, material and conditioning are crucial factors for sound quality and response of wind instruments, while acousticians rather tend to question that claim. Recent experiments on horns are presented favoring the hypothesis that wall vibrations do matter. Although horn sound in general appears to change quite significantly when wall vibrations, particularly those of the bell, are dampened during artificial playing, it becomes more and more evident that more than one single mechanism has to be considered to explain the whole phenomenon. Radiation of the bell as multi-pole will add directivity and strengthen higher harmonics while absorption by the wall will rather weaken them. Dynamic interaction between air column and oscillating bore profile theoretically depends on broken symmetry. Feedback of structural resonances to the oscillating lips can be avoided by decoupling the mouthpiece from the instrument. Experiments and statistical evaluation have been laid out in order to exclude or identify certain mechanisms as well as possible artifacts like changes of mouthpiece position, bore or acoustical environment caused by damping forces.

2aMUa5. Design and manufacturing of an artificial marine conch by bore optimisation. Jef Petiot (Ecole Centrale Nantes IRCCyN, 1 rue de la Noe, BP 92101, 44321 Nantes, France, jean-francois.petiot@irccyn.ec-nantes.fr), Francois Tavard (Ecole Centrale Nantes - IRCCyN, 1 rue de la Noe, BP 92101, 44321 Nantes, France, francois.tavard@eleves.ec-nantes.fr)

The marine conch is a traditional instrument of the brass family. The resonator is made of the inner shape of the shell, with which the lips of the player interact by the way of a hole pierced in the extremity of the shell. Several notes can be played with this instrument. Unfortunately, the marine conch has become very rare and expensive. In order to build an artificial conch (by injection moulding), we studied the acoustics property of a natural conch. This paper is dedicated to the description of the measurements we made on the conch, and to the presentation of the method used to design the bore. Firstly, the input impedance of the natural conch was measured. Secondly, the size of the bore of the natural conch was assessed by the way of pictures and image processing tools. An initial bore of the artificial 
conch was designed with a CAD software, by taking into account manufacturing constraints. We developed next an optimisation procedure to improve the harmonicity of the artificial conch. Finally, an artificial conch was next manufactured by rapid prototyping. As a result, we noticed that the artificial conch manufactured can clearly be used as an interesting musical instrument.

\section{Contributed Paper}

9:40

2aMUa6. A design strategy for brass instruments. Robert W. Pyle (S. E. Shires Co., 11 Holworthy Place, Cambridge, MA 02138, USA, rpyle @ post.harvard.edu)

This paper describes the processes being used to design a $\mathrm{C}$ trumpet. Since "one size fits all" is not a good approach for high-quality brass instruments, one of the goals is to create a basic design that, with minor variations, can be tailored to meet the needs of a variety of players. There are other goals, secondary to playing quality but nonetheless desirable. These include appearance and mechanical reliability ("fit and finish"), ease of manufacture (consistent quality at minimal possible cost), and ease of repair (accidents will happen, alas!). Existing instruments provide input data to the design process by way of physical dimensions, acoustic input impedance, and players' judgements. Computer modeling helps to predict the effect on intonation of changes of bore contour. Feedback from top professional players is essential to refine the design, especially the "black magic" aspects like brace placement and even the type of solder used to assemble the instrument.

\section{Invited Paper}

\section{0:00}

2aMUa7. Characterisation of brass musical instrument designs using the brassiness parameter. Arnold Myers (University of Edinburgh, Reid Concert Hall, Bristo Square, EH8 9AG Edinburgh, UK, am@ed.ac.uk)

Recent work has established a "brassiness" parameter as a measure of the support given over the sounding length of a brass instrument to non-linear propagation in sound production. This parameter is a function of the geometry (bore profile) of an instrument, and provides a useful means of relating the timbral characters to the designs of the various kinds of brass instrument. The geometries of over 1000 brass instruments belonging to museums worldwide and to individual musicians have been measured to determine the values of their brassiness parameter. Comparisons of these enable not only a more precise taxonomy but also provide a tool for studying the evolution of instrument design. This paper explores both the taxonomic analysis of the whole brasswind field and also gives examples of the use of brassiness in historical research.

\section{Contributed Paper}

10:20

2aMUa8. Influence of acoustic waveguides lengths on self-sustained oscillations: Theoretical prediction and experimental validation. Nicolas Ruty (Département Parole \& Cognition, GIPSA-lab, 46, avenue Félix Viallet, 38031 Grenoble Cedex, France, nicolas.ruty@gipsa-lab.inpg.fr), Xavier Pelorson (Département Parole \& Cognition, GIPSA-lab, 46, avenue Félix Viallet, 38031 Grenoble Cedex, France, pelorson@icp.inpg.fr), Annemie Van Hirtum (Département Parole \& Cognition, GIPSA-lab, 46, avenue Félix Viallet, 38031 Grenoble Cedex, France, annemie.vanhirtum@gipsa-lab.inpg.fr)

Human vocal folds and lips of brass instruments players produce selfsustained oscillations due to the interaction between airflow, acoustic waveguides and deformable tissues. This interaction is commonly modelled as a distributed one or two mass-spring system coupled with a simple airflow and acoustic description. This study focuses on the influence of the acoustic waveguide length on the resulting self-sustained oscillation characteristics, i.e. the minimum pressure required to sustain oscillations, the oscillation frequency. Both fixed and varying waveguide lengths are considered. Theoretical predictions with the simplified interaction model are compared to experimental data obtained with a deformable in-vitro replica suitable to produce self-sustained oscillations in presence of an upstream $(12,24$ or $32 \mathrm{~cm}$ ) and downstream (varying from 0 up to $235 \mathrm{~cm}$ ) acoustic waveguide. The current study shows the strong influence on the minimum pressure regardless the waveguide length. In addition the waveguide length is shown to impose the resonance frequency for waveguide length superior to $40 \mathrm{~cm}$. A rapid change in waveguide length introduces bifurcations between different oscillation regimes. 


\title{
Session 2aMUb
}

\section{Musical Acoustics: Interaction Between Instrument and Instrumentalist I}

\author{
Gary Scavone, Cochair \\ Schulich School of Music, McGill University, 555 Sherbrooke Street West, Montreal, QC H3A 1E3, Canada \\ Xavier Boutillon, Cochair \\ Laboratoire de Mécanique des Solides, Ecole Polytechnique, Palaiseau Cedex, 91128, France
}

Invited Papers

\section{1:00}

2aMUb1. Motor control in drumming: Influence of movement pattern on contact force and sound characteristics. Sofia Dahl (Institute of Music Physiology and Musicians' Medicine, Hanover University of Music and Drama, Hohenzollernstr. 47, 30161 Hannover, Germany, dr.sofia.dahl@gmail.com), Eckart Altenmüller (Institute of Music Physiology and Musicians’ Medicine, Hanover University of Music and Drama, Hohenzollernstr. 47, 30161 Hannover, Germany, altenmueller@hmt-hannover.de)

Whereas wind instrumentalists and string players have a continuous control of the acoustic sound parameters during playing, a percussionists' direct contact with the instrument is limited to a few milliseconds. The player has no possibilities to adjust grip or dampening during the actual contact. Whatever timbre and sound level the player is aiming for therefore has to be integrated in the entire striking gesture. How can the player control the complex interaction between drumstick and drumhead? In order to investigate how the players' grip and striking gestures influence the sound characteristics of drum strokes we recorded movements, audio, contact time and contact force during drumming. Different instructions were given with the intention to influence how the player's grip controlling the drumstick. "Normal" strokes were allowed to freely rebound from the drumhead. For "controlled" strokes the player was asked to control the ending position of the drumstick, stopping it as close as possible to the drumhead after the stroke. Preliminary analysis showed that the instructions influenced contact force, contact time, and perceptual ratings of the strokes. Further results and implications will be discussed.

\section{$11: 20$}

2aMUb2. Two dimensional finger-string interaction in the concert harp. Jean-Loic Le Carrou (Laboratoire d'Acoustique de l’Université du Maine, Avenue Olivier Messiaen, 72085 Le Mans, France, jean-loic.le_carrou@ univ-lemans.fr), Elio Wahlen (LAUM, CNRS, Université du Maine, Lab. d'Acoustique Université du Maine, UMR CNRS 6613, 72085 Le Mans Cedex 9, France, elio@elio.de), Emmanuel Brasseur (LAUM, CNRS, Université du Maine, Lab. d'Acoustique Université du Maine, UMR CNRS 6613, 72085 Le Mans Cedex 9, France, emmanuel.brasseur@univ-lemans.fr), Joël Gilbert (Laboratoire d'Acoustique de l'Université du Maine, Avenue Olivier Messiaen, 72085 Le Mans, France, joel.gilbert@univ-lemans.fr)

The sound of the concert harp partly depends on the way the string is plucked. The vibrating string is brought into a state of initial conditions by the finger-string interaction and then oscillates according to two transverse planes. In order to understand the plucking action of the concert harp, a one-dimensional model of this interaction has been developed in a previous paper [Le Carrou et al, Proc. ISMA (2007)]. The parameters of this model were deduced from measurements of the string's and finger's trajectories. The aim of the present paper is to extend this model to a more realistic one, including a two-transverse trajectory for each one of the interaction's elements. To do so, a special experiment with a high-speed camera, which films the interaction, is set up. Specific image processing, based on edges detection, helps to automatically track both objects' positions. The results show that the finger-string interaction takes place in two planes and permits us to obtain the parameters of the two-dimensional model for the finger-string interaction.

\section{1:40}

2aMUb3. The modern violin bow in off-string action. Knut Guettler (Norwegian Academy of Music, P.O.Box 5190 Majorstuen, 0302 Oslo, Norway, knut.guettler@nmh.no)

The convex camber of the modern Tourte-model bow permits off-string stroke techniques to be performed with resulting clean and crisp attacks. This paper discusses how different bow-, string-, and bowing parameters must combine in order for this to be achieved. Of particular interest are the geometrical changes imposed on the bow stick during bouncing strokes. A good spiccato bow is characterized by a stick that feels "lively", even when the bouncing is low or the hairs are not leaving the string at all. With lesser-quality bows the player has to work harder (on the lower-pitched double bass even sometimes synchronously "shake" the bow stick in the stringlength plane) in order to induce sufficient stick oscillation. Included in this study are measurements of finger action on bow sticks of different quality. 


\section{Contributed Paper}

12:00

2aMUb4. Observations on bow changes in violin performance. Matthias Demoucron (IRCAM, 1 Place Igor Stravinsky, 75004 Paris, France, demoucron@ircam.fr), Anders Askenfelt (Dept. of Speech, Music and Hearing, Royal Institute of Technology (KTH), Lindstedtsvägen 24, SE10044 Stockholm, Sweden, andersa@speech.kth.se), René E. Causse (IRCAM, 1 Place Igor Stravinsky, 75004 Paris, France, causse @ircam.fr)

Players of bowed instruments commonly separate notes by changing the direction of the bow motion ("bow changes"). The separation can be made more or less pronounced (detaché - portato - martellato). In contrast, long notes, requiring more than a full bow stroke, are played by making the bow changes as inaudible as possible. Acceptable bow changes require accurate control and coordination of a set of bowing parameters, in particular bow speed, bow force and bow-bridge distance. Long practice is required before optimal control is achieved. Detailed descriptions of basic bowing gestures such as bow changes are of great interest for various fields of violin-related studies, including realistic control of synthesis algorithms. We present recent measurements that provide an accurate description of the evolution of bow speed, acceleration and bow force during bow changes at the tip and the frog, respectively. Using these data for controlling a synthesis algorithm, we will discuss how modifications of the parameters influence the bow-string interaction and resulting string vibrations. The simulations are used to model efficient implementations of bow changes.

\section{Invited Paper}

2aMUb5. Extraction of lumped clarinet reed model parameters from numerically synthesised sound. Vasileios Chatziioannou (Sonic Arts Research Centre, Queen's University Belfast, BT7 1NN Belfast, UK, vchatziioannou01@qub.ac.uk), Maarten Van Walstijn (Sonic Arts Research Centre, Queen's University Belfast, BT7 1NN Belfast, UK, m.vanwalstijn@qub.ac.uk)

Fluid dynamical analysis and time-domain modelling of a single reed-mouthpiece-lip system can be used to inform the formulation of a lumped model of the woodwind excitation mechanism. Coupling this lumped model to a model of the instrument bore enables computationally efficient generation of sustained oscillations, using a small number of physical parameters that define the instrument and the way the player controls them. As such, the embouchure of the player as well as the geometry of the system is taken into account. In this paper, an attempt is carried out to use the numerically generated sound as an input to an inversion algorithm for the reedmouthpiece-lip system. Assuming that the reed motion is proportional to the pressure difference across it, a relationship can be established between the pressure and the total flow inside the mouthpiece that allows a first estimation of the physical parameters using standard optimisation techniques. Currently we are undertaking efforts to apply the inversion to data measured under real playing conditions, i.e. effectively capturing player gesture information in the form of physical control parameters.

\section{2:40-1:40 Lunch Break}

\section{Contributed Paper}

$1: 40$

2aMUb6. How to play the first bar of Rhapsody in Blue. Jer-Ming Chen (University of New South Wales, Music Acoustics, School of Physics, NSW 2052 Sydney, Australia, jerming@phys.unsw.edu.au), John Smith (University of New South Wales, Music Acoustics, School of Physics, NSW 2052 Sydney, Australia, john.smith@unsw.edu.au), Joe Wolfe (University of New South Wales, Music Acoustics, School of Physics, NSW 2052 Sydney, Australia, J.Wolfe@unsw.edu.au)

The two-and-a-half octave glissando opening Gershwin's Rhapsody in Blue is one of the great icons of 20th century music and one of the best known bars in music. Expert clarinettists combine unusual fingerings with even more unusual configurations of their vocal tract to achieve a nearly continuous rise in pitch. Using a novel method [1], we incorporated an acoustic impedance measurement head within a clarinet mouthpiece, allowing us to study the player's vocal tract at various stages in the glissando. We measured and compared vocal tract impedance spectra with the corresponding clarinet impedance spectra for the fingering used at that pitch. Partially uncovering an open finger-hole raises the frequency of clarinet impedance peaks in the lower register, thereby allowing smooth increases in the playing pitch. In the upper register, however, resonances in the clarinettist's vocal tract are manipulated to be comparable with those in the clarinet for frequencies in this range. Thus the pitch in the higher section of the glissando is largely controlled by smoothly varying a resonance of the player's vocal tract. [1] Chen, JM. Smith, J. and Wolfe, J., (2008) ' 'Experienced saxophonists learn to tune their vocal tracts'. Science, (in press).

\section{Invited Papers}

2aMUb7. Evaluating Vocal-Tract Influence in the Production of Saxophone Multiphonics. Gary Scavone (Schulich School of Music, McGill University, 555 Sherbrooke Street West, Montreal, QC H3A 1E3, Canada, gary@music.mcgill.ca), Antoine Lefebvre (Schulich School of Music, McGill University, 555 Sherbrooke Street West, Montreal, QC H3A 1E3, Canada, antoine.lefebvre2@mail.mcgill.ca), Andrey R. Da Silva (Schulich School of Music, McGill University, 555 Sherbrooke Street West, Montreal, QC H3A 1E3, Canada, andrey.dasilva@mail.mcgill.ca)

A new approach for the analysis of vocal-tract influence in single-reed woodwind instruments during performance was recently reported (Scavone et. al., 2008). Two types of vocal-tract influence were observed. When the downstream air column provides only weak support of a given note, players can use a strong and narrow-bandwidth upstream resonance to override the reed vibrations, such as when pitch bending or playing extended register notes. Performers can also use a more wide-bandwidth upstream resonance to affect subtle timbre variations when playing notes over the full range of the instrument. The research reported here addresses the performance of multiphonic tones, for which the results of the previously mentioned study were less conclusive. While it is clear that upstream influence is involved in the production of multiphonics, we are interested in determining whether performers must support a specific intermodulation component or a wider bandwidth range of components for proper production. The latest results of this research will be reported. 
2aMUb8. A study of flute control parameters. Benoit Fabre (Institut Jean Le Rond d'Alembert / LAM (UPMC / CNRS / Ministère Culture), 11, rue de Lourmel, 75015 Paris, France, fabreb@ccr.jussieu.fr), Nicolas Montgermont (Institut Jean Le Rond d'Alembert / LAM (UPMC / CNRS / Ministère Culture), 11, rue de Lourmel, 75015 Paris, France, montgermont@lam .jussieu.fr), Patricio De La Cuadra (Centro de Investigación en Tecnologias de Audio (CITA), Universidad Católica de Chile, Alameda 340, Oficina 13, Casilla 114-D Santiago, Chile, pcuadra@uc.cl)

The sound of musical instruments played in self-sustained oscillations can be interpreted as a sounding transposition of the player's gesture. The playing of wind instruments requires expert control of the blowing that may be difficult to grasp and to measure because it induces only very little motion of the player, as opposed to string or keyboard instruments. In the case of instruments of the flute family, the player seems to control mainly the air jet velocity for mode selection. Flutes in which the air jet is formed between the lips also allow for a control of the total jet flow by the player, through lip adjustments. We present measurements carried on several players, in different playing conditions. The score includes technical exercises such as scales and short musical excerpts from the flute repertoire. Time evolutions of the control parameters are analysed, based on pressure and geometrical measurement, in the framework of the current knowledge on the sound production in flutes, such as jet instability. The control over different parameters will be discussed, both for basic technical exercises and in a musical melodical context.

\section{Contributed Paper}

2:40

2aMUb9. Acoustical analysis of timbral modulations on the flute as controlled by phonetic gestures. Maryse Lavoie (Laboratoire informatique, acoustique et musique, Faculté de musique, Université de Montréal, C.P. 6128, succursale Centre-Ville, Montréal, QC H3C 3J7, Canada, maryse.lavoie@umontreal.ca), Caroline Traube (Laboratoire informatique, acoustique et musique, Faculté de musique, Université de Montréal, C.P. 6128, succursale Centre-Ville, Montréal, QC H3C 3J7, Canada, caroline.traube@umontreal.ca), Marie-Hélène Breault (Observatoire international de la création et des cultures musicales (OICCM), Faculté de musique, Université de Montréal, C.P. 6128, succursale Centre-Ville, Montréal, QC H3C 3J7, Canada, mhbreault@yahoo.ca)
The purpose of this project is to study the control of timbre on the flute by varying articulatory parameters (i.e. embouchure) which correspond to distinct phonetic gestures. The main goal is to compare the production of a specific timbral modulation on the flute and its vocal reproduction (diphthong) by means of acoustical analyses of these sounds. The recordings of timbral modulations performed on the flute (e.g. by increasing the mouth opening) and reproduced vocally as diphthongs (e.g. [u] -> [a] transition) have been analyzed acoustically for intensity as well as for the first two formant trajectories. The results of the formant analyses as presented in an F1-F2 plane reveal the cardinal vowel triangle and confirm a correlation between the flute timbre modulations and the vocal diphthongs corresponding to the underlying phonetic gestures. In addition, a listening test has shown that flutists are able to perceive and recognize these timbral modulations from the sound alone.

\section{Invited Papers}

2aMUb10. Lip control of brass instruments. Donald M. Campbell (Edinburgh University, 4201 JCMB, Kings Buildings, Mayfield Road, EH9 3JZ Edinburgh, UK, d.m.campbell@ed.ac.uk)

Brass instruments are frequently described in the acoustics literature as "lip reed" instruments, emphasising the fact that the sounding mechanism of this instrumental class is flow modulation by the vibration of the player's lips. As well as providing the source of sound, the lips act as the major interface through which the performer controls the intonation, dynamics, articulation and timbre of the performance. Indeed, on instruments such as the natural trumpet and the alphorn, the player's lips form the only control interface. This paper reviews recent studies which have examined the vibrational properties of brass players' lips, the types of motion which they undergo when playing different instruments, and the nature of the interaction between the lips and the instrument. It also considers the extent to which current physical models of the lips are capable of encompassing these control processes.

2aMUb11. Vocal tract interactions in saxophone performance. Jer-Ming Chen (University of New South Wales, Music Acoustics, School of Physics, NSW 2052 Sydney, Australia, jerming@phys.unsw.edu.au), John Smith (University of New South Wales, Music Acoustics, School of Physics, NSW 2052 Sydney, Australia, john.smith@unsw.edu.au), Joe Wolfe (University of New South Wales, Music Acoustics, School of Physics, NSW 2052 Sydney, Australia, J.Wolfe@unsw.edu.au)

Although acousticians have debated the importance of the vocal tract in reed instrument performance, expert saxophonists report adjusting their vocal tract for advanced techniques including altissimo playing, subtone playing, bugling and multiphonics. Using a novel method [1], we incorporated an acoustic impedance head within a saxophone mouthpiece to study the vocal tract directly during playing. For fingerings above the first register, the operating peak in the saxophone's input impedance decreases with increasing pitch, falling to below $20 \mathrm{MPa} . \mathrm{s}^{-3}$ after 2.7 octaves, thus ending the standard range that is readily available to amateurs. Above this, in the altissimo, professional saxophonists produce peaks in the impedance of their tracts of about 20 to $40 \mathrm{MPa}^{\mathrm{s} . \mathrm{m}^{-3}}$, which they tune to select the desired note. The crossover of the relative magnitudes of saxophone and tract impedance peaks coincides with the transition from standard to altissimo register. While professionals use the vocal tract thus for other extended effects, inexperienced players do not tune their tract resonances and are unable to produce advanced effects. [1] Chen, JM. Smith, J. and Wolfe, J., (2008) ',Experienced saxophonists learn to tune their vocal tracts". Science, (in press). 


\title{
Contributed Paper
}

2aMUb12. Reproducibility of piano playing. Nicolas Principeaud (Laboratoire d'Acoustique Musicale, 11 rue de Lourmel, 75015 Paris, France, nicolas.principeaud@free.fr), Xavier Boutillon (Laboratoire de Mécanique des Solides, Ecole Polytechnique, 91128 Palaiseau Cedex, France, boutillon@1ms.polytechnique.fr)

Mechanically, piano playing consists in giving to the hammer a certain escapement velocity at a certain time - $t_{e}, v_{e}$ - by means of a traditional keyboard and piano action. Numerous replacement systems for the keyboard, action or even for the pianist's finger have been proposed. They implicitly raise the question of the accuracy with which the $\left(t_{e}, v_{e}\right)$ information must be coded or reconstructed. The precision with which pianists are able to reproduce their playing sheds some light on this question. At this end we have asked several concert pianists to play the same passage several times with the highest reproducibility level they could achieve. The experiment was done on a traditional grand piano equipped with the Yamaha Disklavier system, used as a measurement tool for the escapement velocity of the hammers. The beginnings of a fugue by Bach and a study by Debussy were recorded. Results show that the reproducibility varies highly from note to note and can be as good as approximately $1 \%$. Additional tests on auditors show that deviations on note amplitudes are not perceived unless they reach a level which turns out to be significantly higher than the reproducibility level achieved by the pianists.

TUESDAY MORNING, 1 JULY 2008

ROOM 250A, 8:00 A.M. TO 8:00 P.M.

\section{Session 2aNSa}

\section{Noise, Physical Acoustics, and EURONOISE: Aeroacoustics II}

\author{
Philip J. Morris, Cochair \\ Penn State University, 233C Hammond Building, University Park, PA 16802, USA \\ Christophe Bailly, Cochair \\ Ecole Centrale de Lyon, 36, avenue Guy de Collongue, LMFA, Ecully, 69134, France
}

\section{Contributed Papers}

\section{8:00}

2aNSa1. Source location prediction of subsonic isothermal jet flows. Juan Battaner-Moro (Institute of Sound and Vibration Research, University of Southampton, University Road, SO17 1BJ Southampton, UK, jpb@isvr.soton.ac.uk), Mahdi Azarpeyvand (Institute of Sound and Vibration Research, University of Southampton, University Road, SO17 1BJ Southampton, UK, ma@isvr.soton.ac.uk), Rod Self (Institute of Sound and Vibration Research, University of Southampton, University Road, SO17 1BJ Southampton, UK, rhs@isvr.soton.ac.uk)

The location of jet noise sources is a far from trivial problem that is of great importance for both understanding the noise production and radiation mechanisms and also for finding new jet noise reduction strategies. This paper presents comparisons of theoretical results with data for a number of jets. The theory used is based on the MGBK method but including a novel time scale based on the rate of energy transfer through the turbulent cascade. This new technique has been shown to give a number of advantages over existing models. The experimental results were obtained using the Polar Correlation Technique and were made at QinetiQ's Jet Noise Facility in the UK as part of the EU FP6 programme CoJeN. The high resolution jet noise images resulted from using a 64 microphone polar arc array set at two reference angles, namely 60 and 90 degrees to the jet axis. Comparisons with experimental data are made for coplanar and short cowl nozzles at different working conditions for predictions from different theoretical models. It is shown that the best agreement is obtained for the prediction methodology using the energy transfer rate timescale

$$
\text { 8:20 }
$$

2aNSa2. A hybrid method for jet noise predictions based on Large Eddy Simulation and Reynolds-Averaged Navier-Stokes simulations. Guillaume Bodard (Snecma Villaroche, Rond point René Ravaud, 77550 Moissy-Cramayel, France, guillaume.bodard@snecma.fr), Christophe Bailly (Ecole Centrale de Lyon, 36, avenue Guy de Collongue, LMFA, 69134 Ecully, France, christophe.bailly@ec-lyon.fr)

A hybrid jet noise prediction method combining steady and unsteady flow calculations is discussed. The main objective is to merge advantages of each technique to obtain a robust acoustic prediction tool, which will be able to correctly evaluate design and installation effects such as chevron nozzles or jet-pylon interaction for instance. The low-frequency component of acoustic spectra is computed using large-eddy simulations and the integral formulation derived by Ffowcs-Williams \& Hawkings (1969, Phil. Trans. Roy. Soc. Lond., vol. 264). The high-frequency component associated with fine scale turbulence is obtained thanks to Tam \& Auriault's mixing noise theory (1999, AIAA Journal, vol. 37) from Reynolds-Averaged NavierStokes simulations. The oral presentation will detail the two methods, the LES simulations for subsonic round single and coaxial jets, and the matching between the two approaches to get a complete picture of the acoustic spectra.

\section{$8: 40$}

2aNSa3. Efficiency of optimized microjets on realistic nozzles Alexandre Vuillemin (Snecma, Etablissement de Villaroche Sud, Rondpoint René Ravaud - Réau, 77550 Moissy-Cramayel, France, alexandre .vuillemin@snecma.fr)

Even if chevrons nozzles are an efficient way to reduce jet noise during take off, they also decrease performances during cruise. An innovative way to avoid this decrease is the use of active devices that could be switch off after take off, such as microjets nozzles. Snecma led test campaigns at Martel Facility (LEA/CEAT) to optimize microjets settings (geometric and thermodynamic parameters) and to characterize their efficiency on both $2 \mathrm{D}$ axisymmetric and chevrons nozzles. Acoustic and PIV measurements were done on a single stream hot jet with and without external flow, and on a double stream hot jet with pylon.

\section{9:00}

2aNSa4. Numerical investigation of the effect of nonlinear propagation distortion on helicopter noise. Penelope Menounou (University of Patras, Department of Mechanical and Aeronautical Engineering, Rion, 26504 Patras, Greece, menounou@mech.upatras.gr), Panagiotis 
Vitsas(University of Patras, Department of Mechanical and Aeronautical Engineering, Rion, 26504 Patras, Greece, pvitsas@upnet.gr)

Nonlinear propagation distortion causes energy to be shifted to the high frequency end of the spectrum. This leads to underestimation of the noise levels at high frequencies. The effect has been demonstrated in the case of aircraft noise, but less attention has been given to helicopters. In the present work, the effect of nonlinear propagation distortion on helicopter noise is demonstrated based on measured data for low-speed descent and numerical calculations that predict the noise level away from the helicopter with and without nonlinear effects. It is shown that (i) for some frequency bands the difference between linear and nonlinear calculations can be as high as $8 \mathrm{~dB}$, (ii) frequencies between 1000 and $3000 \mathrm{~Hz}$ are more affected, and (iii) the effect is highly directional depending on the receiver location around the helicopter. It is further shown that nonlinear effects manifest themselves differently than in the case of aircraft noise and that they depend on the specific helicopter noise mechanism. More specifically, the following helicopterspecific noise source types are investigated with regards to nonlinear effects: advancing Blade Vortex Interaction (BVI) noise vs retreating BVI noise, High Speed Impulsive noise, and BVI vs Very Impulsive noise.

\section{Invited Papers}

2aNSa5. Recent developments in helicopter rotor noise prediction. Kenneth S. Brentner (Penn State University, 233C Hammond Building, University Park, PA 16802, USA, ksb16@psu.edu)

Prediction of discrete frequency noise for rotorcraft in steady flight has reached a high level of sophistication and understanding. The primary challenge in such rotor noise predictions is the accurate determination of the loading on and flow field around the blades. Although current rotor noise prediction tools have been demonstrated for steady flight conditions, the utility of rotorcraft comes from their unique ability to hover and maneuver. Such maneuvers often occur near the ground in close proximity to people. This paper describes an initial study to characterize maneuver noise. A maneuver noise prediction system has been developed, which consists of a flight simulation code, free-vortex wake code, and a maneuver noise prediction code, loosely coupled together. One of the key aspects of this system is the ability to investigate the additional noise caused during the transition from one flight state to another. Several maneuvers including turns, accelerations, and pop-up/popdown maneuvers were considered. Significant increases in the low-frequency noise can occur, depending on how aggressive the maneuver is performed. The status of advanced research on predicting acoustic scattering of the rotor noise by the aircraft and the first-principles prediction of rotor broadband noise will also be presented.

\section{9:40}

2aNSa6. Aeroacoustic research complex for aircraft source noise characterization. Micah Downing (Blue Ridge Research and Consulting, 13 1/2 W. Walnut St., Asheville, NC 28801, USA, micah.downing@blueridgeresearch.com), Robert McKinley (Air Force Research Laboratory, AFRL/RHCB, 2610 Seventh St., Bldg 441, Wright-Patterson AFB, OH 45433, USA, Robert.McKinley@wpafb.af.mil), John Hall (Air Force Research Laboratory, AFRL/RHCB, 2610 Seventh St., Bldg 441, WrightPatterson AFB, OH 45433, USA, John.Hall@wpafb.af.mil), Frank Mobley (Air Force Research Laboratory, AFRL/RHCB, 2610 Seventh St., Bldg 441, Wright-Patterson AFB, OH 45433, USA, Frank.Mobley@wpafb.af.mil), Michael James (Blue Ridge Research and Consulting, 13 1/2 W. Walnut St., Asheville, NC 28801, USA, michael.james@blueridgeresearch.com)

Aircraft noise has been traditionally measured with either a few ground-based microphones or a linear ground-plane array of microphones. These techniques capture one-dimensional and/or two-dimensional characteristics of aircraft flight noise. The US Air Force Research Laboratory has started the construction of a 3-dimensional measurement facility at White Sands Missile Range in New Mexico. This facility, the Aeroacoustic Research Complex (ARC), will allow aircraft to fly through the array, collecting fully 3D acoustic data. ARC is initially being developed in two phases The first phase includes two $91.4 \mathrm{~m}$ tall towers separated by $244 \mathrm{~m}$ and will focus on noise from rotary wing and UAV aircraft. The second phase will add two $366 \mathrm{~m}$ tall towers separated by $610 \mathrm{~m}$ and will focus on large and high performance fixed wing aircraft. This facility will allow more accurate characterization of in-flight noise directivity by providing synchronized 3-dimensional magnitude \& spectral acoustical signatures from $50+$ microphones. ARC responds to a critical need for validation of existing predictive acoustic models. Such models are used for aircraft design, survivability, nonlinear acoustic propagation research and assessing noise exposure to residents living adjacent to airfields.

\section{0:00-10:20 Break}

\section{Contributed Papers}

\section{0:20}

2aNSa7. Lateral directivity of aircraft noise. Walter Krebs (Empa, Ueberlandstrasse 128, 8600 Duebendorf, Switzerland, walter.krebs @empa.ch), Georg Thomann (Empa, Ueberlandstrasse 128, 8600 Duebendorf, Switzerland, georg.thomann@empa.ch)

A three-dimensional model was developed to characterise the directional sound emission of different aircraft. The model is based on spherical harmonics and defines the directional spectral sound pressure level at a reference distance. The parameters of the model are derived from acoustic measurements on real aircraft traffic. With the help of this model different physical effects on sound propagation and sound impact are analysed. Variations with respect to a rotational symmetric sound emission are outlined and compared to the engine installation corrections proposed in the revised Doc 29 3rd edition. In addition the influence of the ground effect on A-weighted sound levels is analysed for different receiver heights and ground impedances.

\section{0:40}

2aNSa8. Sonic booms, spectral analysis, and diffraction by buildings. Victor W. Sparrow (Penn State, Graduate Program in Acoustics, 201 Applied Science Bldg., University Park, PA 16802, USA, vws1@psu.edu)

An analysis has been performed upon conventional and low-amplitude $\mathrm{N}$-wave sonic boom data taken by NASA outside two houses in June 2006 and July 2007. The buildings were one-floor residences which were carefully instrumented with strategically-placed, multiple microphones. The incident sonic booms had a substantial variation in rise times, most likely due to atmospheric effects. Previous work using the June 2006 data [Sparrow, Klos, and Buehrle, J. Acoust. Soc. Am. 122 (5, Pt. 2) 3084] revealed maximum pressure loads near the ground and wall facing the incident boom. A new spectral analysis of the individual booms now confirms that the diffraction of sound over the house tops is substantially affected by the spectral content of each boom. These results indicate that the pressure loading of homes due to conventional or low-amplitude sonic boom will be affected by 
both the spectral content of each boom's rise phase and each house's exterior geometry. [Work supported by NASA.]

\section{1:00}

2aNSa9. Identification of aerodynamic sound sources: the key problem in noise control. Alexander Fedorchenko (Independent Researcher/Consultant, Dm. Ul'yanov Str. 27/12-1-50, 117449 Moscow, Russian Federation, fedorchenko@mail.com)

Despite the recent advances in the noise control technology, the key mechanisms of aerodynamic sound emission remained poorly studied. The "well-recognized" methods of TCAA did not lead to any breakthrough in this topical problem, and what seems most disappointing, the family of "acoustic analogies" is still used by many for definition of aerodynamic noise sources, though the sufficient set of mathematical proofs has been given by the author that this model is wrong. Experimental approaches aimed at localization of aerodynamic noise sources, including the method of acoustic imaging via using a microphone array, are considered, and their inherent limitations are pointed out. Unfortunately, no current experimental technique enables one to measure instantly all sound sources and sound disturbances inside the zone of generation, and so the latter is often regarded as a kind of "black box". The two-medium nonlinear theory of aerodynamic sound, based on the original decomposition of each flow variable into two components, for unsteady background flow and for acoustic field, has been created, that promotes better comprehension of the noise generation phenomena and opens new ways in flow/noise control. The main properties of this theory are now indicated in comparison with the most detrimental delusions originated from "acoustic analogies".

\section{Invited Paper}

2aNSa10. Direct aeroacoustic simulations based on domain decompositions. Jens Utzmann (University of Stuttgart, Institute for Aerodynamics and Gasdynamics, Pfaffenwaldring 21, 70569 Stuttgart, Germany, utzmann@iag.uni-stuttgart.de), Claus-Dieter Munz (University of Stuttgart, Institute for Aerodynamics and Gasdynamics, Pfaffenwaldring 21, 70569 Stuttgart, Germany, munz@iag.unistuttgart.de)

For CAA, an accurate and feasible direct simulation that considers both the generation of sound within the flow and its propagation into the far-field is hard to realize with one numerical method in a single computational domain. On the other hand, a direct approach contains automatically the interaction of the acoustic perturbations with the flow-field, a property which lacks the popular acoustic analogy models. The proposed method is basically a direct simulation, but it simplifies the problem that has to be solved for individual regions in the computational domain. The idea is to use a non-overlapping domain decomposition method where the equations, methods, grids and even the time steps are adapted to meet the local requirements. Inside the coupling framework, high-order solvers from different classes of methods are available: On unstructured grids, a reconstructed ADER finite volume method (ADER-FV) is used for linear and nonlinear problems, as well as a discontinuous Galerkin method. On structured grids, the ADER-FV and the ADER-FD method are efficiently implemented for nonlinear (FV) and linear (FV, FD) problems. These high-order accuracy methods ensure excellent wave propagation capabilities throughout the entire computational domain. In the subdomains, the Navier-Stokes, Euler and the linearized Euler equations are solved.

\section{Contributed Papers}

\section{$11: 40$}

2aNSa11. Direct Noise Computation in subsonic and transonic flows Frédéric Daude (LaMSID UMR EDF/CNRS, 1 avenue du Général de Gaulle, 92141 Clamart, France, frederic-externe.daude@edf.fr), Thomas Emmert (LaMSID UMR EDF/CNRS, 1 avenue du Général de Gaulle, 92141 Clamart, France, tommem@gmail.com), Philippe Lafon (EDF, 1, avenue du Général de Gaulle, 92141 Clamart, France, Philippe.lafon@edf.fr), Fabien Crouzet (EDF, 1, avenue du Général de Gaulle, 92141 Clamart, France, fabien.crouzet@edf.fr), Christophe Bailly (Ecole Centrale de Lyon, 36, avenue Guy de Collongue, LMFA, 69134 Ecully, France, christophe.bailly@ec-lyon.fr)

In order to model flow phenomena involving interactions between aerodynamics and acoustics, it is necessary to use Direct Noise Computation (DNC) instead of hybrid methods that are not suitable to take into accoun the feedback of acoustics on the flow. The methods that are now available in the field of Computational AeroAcoustics (CAA) allows us to deal with DNC in realistic configurations. The numerical code SAFARI (Simulation of Aeroacoustics in Fluids And with Resonance and Interactions) has been developed for this goal. The set of equations are the compressible 3-D NavierStokes equations. High order finite difference schemes are used. Multidomain capabilities are implemented in order to deal with complex geometries. Block decomposition is used in order to take advantage of parallel processing on large clusters Validation cases are presented: diffraction by a cylinder, shock tube. Results on realistic configurations are also shown: ducted cavity, transonic sudden enlargment, airfoil interactions.

\section{2:00}

2aNSa12. Asymptotic expressions for the directivity of round jets. Ricardo E. Musafir (UFRJ / COPPE, Universidade Federal do Rio de Janeiro, 21941-972 Rio de Janeiro, Brazil, rem@serv.com.ufrj.br)

Expressions for the directivity of round jets, in the high and low frequency limits, are derived, based on solutions of Lilley's equations. Two different forms of the equation in what concerns source terms representation are considered, along with general point sources of the appropriate type (i.e., equivalent stress and force sources and also, when necessary, volume sources), which are assumed to be statistically axisymmetric. Effects of mean shear and temperature gradients are accounted for, as well as of source movement. The expressions are compared with earlier ones, based on a plug flow model. The differences in the resulting expressions due to the choice of source description, which are relevant for hot jets, are discussed and used to derive a form consistent with both representations. Comparison with experimental data is also presented.

\section{$12: 20$}

2aNSa13. Investigations of roughness-generated TBL sound using coupled physical-computational experiments in conjunction with theoretical development. William Blake (Naval Surface Warfare Center (Ret.), 6905 Hillmead Road, Bethesda, MD 20817, USA, hydroacoustics@aol.com), Ki-Han Kim (Office of Naval Research, Randolph St., Arlington, VA 22202, USA, kihan.kim@navy.mil), Michael Goody (Naval Surface Warfare Center (Ret.), 6905 Hillmead Road, Bethesda, MD 20817, USA, michael.goody@navy.mil), Meng Wang (University of Notre Dame, Departmernt of Aerospace and Mechanical Engineering, Notre Dame, MD 46556, USA, m.wang@nd.edu), William J. Devenport (Virginia Tech, Aerospace and Ocean Engineering, 224E Randolph Hall, Blacksburg, VA 24061, USA, devenport@vt.edu), Stewart A Glegg (Florida Atlantic University, Department of Ocean Engineering, Boca Raton, VA 33431, USA, glegg@oe.fau.edu)

Sound produced by turbulent-boundary layers (TBL) over rough walls is being studied in a series of physical-computational experiments. At issue is the development of a knowledge of how the wall elements generate flow dipoles which directly determines how the sound is described in terms of dependent variables. The considered mechanisms include dipoles at the 
roughness elements due to their shed wakes, distributed surface dipoles due to convecting turbulence impinging onto elements, Rayleigh-like scattering into sound of aerodynamic pressures of hydrodynamic wave numbers of flow above the roughness. The LES of rough-wall TBL consists of "numeri$\mathrm{cal}^{\prime \prime}$ experiments being used to isolate the separate mechanisms. These simulations are benchmarked with analysis and with matching physical experiments on rough wall patches in which identical geometries of wall roughness and identical Reynolds numbers are used. In the physical measurements, array-based measurements of the radiated sound are being used to characterize the directivity and magnitude of the sound and to relate the sound to aerodynamic wall pressure and to classical characteristics of the turbulent boundary layer. The LES produces comparison for both radiated sound and detailed flow structure around the roughness elements. This project is funded by the Office of Naval Research, Washington, D.C., USA.

\section{2:40-2:00 Lunch Break}

\section{Contributed Paper}

\section{2:00}

2aNSa14. Experimental investigation of sound from flow over a rough surface. Michael Goody (Naval Surface Warfare Center (Ret.), 6905 Hillmead Road, Bethesda, MD 20817, USA, michael.goody@navy.mil), Jason Anderson (Naval Surface Warfare Center (Ret.), 6905 Hillmead Road, Bethesda, MD 20817, USA, Jason.M.Anderson@navy.mil), Devin Stewart (Naval Surface Warfare Center (Ret.), 6905 Hillmead Road, Bethesda, MD 20817, USA, Devin.Stewart@navy.mil), William Blake (Naval Surface Warfare Center (Ret.), 6905 Hillmead Road, Bethesda, MD 20817, USA, hydroacoustics@aol.com)

Measurements of radiated noise and unsteady surface pressures have been carried out in order to better understand the mechanism for sound production from flow over a rough surface. In order to investigate scaling re- lationships, the flow speed, roughness height, geometry and element distribution were varied systematically. When considered in total, previous investigations do not present a consistent picture of the scaling behavior of roughness noise, or the underlying physical mechanism. They have reported roughness noise levels that scale on flow velocity, roughness height, and fetch area and have indicated that the sound production may be dipole or quadrupole in nature. Prevailing analytical models assume that both dipole and quadrupole sources are present. The scaling of roughness noise for large roughness height has not been investigated previously and is part of the current study. A recent developed scattering model (dipole) developed by Glegg et al is interrogated using detailed measurements of the roughness element height distribution, turbulent boundary layer properties, and array-based radiated sound levels.

\section{Invited Paper}

2aNSa15. On the sound generated by boundary-layer vorticity. Umberto Iemma (University Roma Tre, via vasca navale 79, 00146 Rome, Italy, u.iemma@uniroma3.it), Luigi Morino (via vasca navale 79, 00146 Roma, Italy, 1.morino@uniroma3.it), Roberto Camussi (University Roma Tre, via vasca navale 79, 00146 Rome, Italy, camussi@uniroma3.it), Giovanni Caputi Gennaro (University Roma Tre, via vasca navale 79, 00146 Rome, Italy, gcaputi@uniroma3.it)

Turbulent boundary layers generate broadband noise as the effect of vortical-disturbances scattering into acoustic waves. The paper presents a formulation for evaluating of acoustic pressure in the field in terms of the transpiration velocity, here defined in terms of vorticity and closely related to Lighthill equivalent source. Specifically, the formulation used allows one to obtain, in the frequency domain, a matrix relationship between the transpiration velocity at a number of points on the body surface (those arising from boundaryelement discretization) and the pressure at given points in the irrotational region. From this, the relationship between the corresponding PDF is easily obtained using the Wiener-Khintchine theorem. The paper will include the general formulation, validating numerical results, and comparison with experimental data. The inverse problem, that is, determining the source intensity on the body surface from field noise (in particular, the invertibility of the operator), will be also addressed.

\section{Contributed Papers}

\section{2:40}

2aNSa16. A numerical study on multimode sound propagation in lined ducts and radiation to the far field. Rie Sugimoto (ISVR, University of Southampton, Highfield, SO17 1BJ Southampton, UK, rs@isvr.soton.ac.uk) , R Jeremy Astley (ISVR, University of Southampton, Highfield, SO17 1BJ Southampton, UK, rja@isvr.soton.ac.uk), Claire R. McAleer (ISVR, University of Southampton, Highfield, SO17 1BJ Southampton, UK, crm@soton.ac.uk), Iansteel Achunche (ISVR, University of Southampton, Highfield, SO17 1BJ Southampton, UK, ia1@isvr.soton.ac.uk)

In previous articles, the authors developed a hybrid scheme for analysing bypass duct noise, in which a numerical analysis using finite element method for in-duct propagation and an analytic radiation code with fully represented effects of bypass shear layer are coupled. Such procedure permits detailed study on the interaction between duct configurations, such as geometry and acoustic liner impedances, and modal propagation and attenuation, and also the effects on the radiation pattern, within practical timescale and at modest computational cost. The scheme has been applied to realistic aero-engine bypass ducts and has been integrated with an optimisation programme. The numerical results obtained so far have revealed that for ducts with acoustic liners highly attenuated modes are not necessarily those with high mode angles, which is contrary to general anticipation. The aim of the study in the current paper is to understand the physics behind this phe- nomenon and its effect on the radiation to the far field. Detailed investigation on modal attenuation is performed by using the hybrid scheme. The effect of modal power distribution at the duct exit on the directivity pattern of the radiated noise to the far field is also discussed.

\section{3:00}

2aNSa17. Modeling of sound propagation in nonuniform waveguides. Wenping Bi (Laboratoire d'Acoustique de l'Université du Maine, UMR CNRS 6613, AV. O. Messiaen, 72085 Le Mans, France, wenping.bi@univ-lemans.fr), Vincent Pagneux (Laboratoire d'Acoustique de l'Université du Maine, UMR CNRS 6613, AV. O. Messiaen, 72085 Le Mans, France, vincent.pagneux@univ-lemans.fr), Denis Lafarge (Laboratoire d'Acoustique de l'Université du Maine, Avenue Olivier Messiaen, $72085 \quad$ Le Mans, France, denis.lafarge@univ-lemans.fr), Yves Aurégan (Laboratoire d'Acoustique de l'Université du Maine, Avenue Olivier Messiaen, 72085 Le Mans, France, yves.auregan@univ-lemans.fr)

Sound propagation in waveguides is modeled by a Multimodal Method. The waveguides geometries may involve bends, variable cross-sections, or their combinations. The waveguide boundaries may involve axially or circumferentially nonuniform impedance conditions or acoustically rigid 
conditions. Uniform flow may also be included for a simple uniform geometry. The pressure (displacement potential for uniform flow) is expanded in terms of the modes of acoustically rigid waveguides and an additional function that carries the information about the impedance boundary. The rigid waveguide modes and the additional function are known a priori so that calculations of the true modes of waveguides with impedance boundary, which are difficult, are avoided. By matching the pressure and axial velocity (displacement potential and axial derivative for uniform flow) at the interface between different axially uniform segments, scattering matrices are obtained for each individual segment; these are then combined to construct a global scattering matrix for multiple segments. After calculating the scattering matrix, the transmitted and reflected sound fields or intensities may be obtained for any kind of modal sources. The method allows modeling sound propagation in waveguides with axially and circumferentially nonuniform impedance boundaries up to dimensionless frequency $\mathrm{K}=70$ in just hours on a personal computer, which advantageously compares with other techniques.

\section{3:20}

2aNSa18. An Active Network Representation to Predict the Flow Noise Characteristics of Corrugated Ducts. Wim De Roeck (K.U.Leuven Dept. of Mechanical Engineering, Celestijnenlaan 300B - bus 2420, 3001
Heverlee, Belgium, wim.deroeck@mech.kuleuven.be),Vasilisa Solntseva (Andreev Acoustics Institute, Shvernik, 4, Moscow, 117036 Moscow, Russian Federation, mironov@akin.ru), Wim Desmet (K.U.Leuven Dept. of Mechanical Engineering, Celestijnenlaan 300B - bus 2420, 3001 Heverlee, Belgium, Wim.Desmet@mech.kuleuven.be)

In this paper, an active network representation is used to numerically predict and gain more insight in the internally generated flow noise sources in corrugated ducts (the active bi-port component) as well as to describe the acoustic transmission characteristics of this type of application (the passive bi-port components) in the presence of a non-uniform mean flow. For this purpose a numerical approach is chosen, using compressible Large Eddy Simulations (LES) to predict the noise generation mechanisms and Linearized Euler Equations (LEE) with a plane pulse excitation to obtain the transmission characteristics for these components. The accuracy of the active network component determination is increased by separating the aerodynamic and the acoustic fluctuating field of the LES using both an aerodynamic/acoustic splitting technique and multiple plane mode-matching strategies. In this way, a numerical method is proposed to analyze the flow-acoustic behavior of corrugated tubes, which can be generally used for all types of duct system applications.

Lecture sessions will recess for presentation of posters on various topics in acoustics. See poster sessions for topics and abstracts.

\section{Contributed Papers}

\section{$5: 20$}

2aNSa19. Validation of a hybrid method of aeroacoustic noise computation applied to internal flows. Mélanie Piellard (Delphi Thermal, Avenue de Luxembourg, 4940 Bascharage, Luxembourg, melanie.piellard@delphi.com), Christophe Bailly (Ecole Centrale de Lyon, 36, avenue Guy de Collongue, LMFA, 69134 Ecully, France, christophe .bailly@ec-lyon.fr)

A hybrid method of aeroacoustic noise computation based on Lighthill's acoustic analogy is first validated, and then applied to investigate the noise radiated by a low Mach number flow through a diaphragm in a duct. The simulation method is a two-step hybrid approach relying on Lighthill's acoustic analogy, assuming the decoupling of noise generation and propagation. The first step consists in an incompressible Large Eddy Simulation of the turbulent flow field, during which the Lighthill's source term is recorded. In the second step, a variational formulation of Lighthill's Acoustic Analogy using a finite element discretization is solved in the Fourier space. The validation of this method is briefly presented: a general validation is performed on the case of two corotating vortices in a medium at rest: the exit of turbulent structures from the computational domain is accounted for by a spatial filtering; and a study of spatial interpolation from the CFD mesh to the acoustic mesh shows an acceptable level of error. This method is applied to a three-dimensional diaphragm with low Mach number flow, showing good agreement with both experimental results and Direct Noise Computation performed by Gloerfelt \& Lafon (Computers \& Fluids, 2007).

\section{$5: 40$}

2aNSa20. Numerical strategies for investigation of gust-airfoil interaction. Florent Margnat (Arts et Métiers Paris Tech - Sinumef Lab, 151 bd de 1'Hopital, 75013 Paris, France, florent.margnat@paris .ensam.fr), Thomas Le Garrec (Arts et Métiers Paris Tech - Sinumef Lab, 151 bd de 1'Hopital, 75013 Paris, France, thomas.le-garrec@paris .ensam.fr), Djaafer Fedala (Lab. d'Energétique et de Mécanique des Fluides Interne, Arts et Métiers ParisTech, 151 boulevard de l'Hôpital, 75013
Paris, France, djaafer.fedala@paris.ensam.fr),Xavier Gloerfelt(Arts et Métiers Paris Tech - Sinumef Lab, 151 bd de l'Hopital, 75013 Paris, France, xavier.gloerfelt@paris.ensam.fr), Smaine Kouidri (LIMSI-CNRS, BP 133, 91403 Orsay Cedex, France, smaine.kouidri@limsi.fr)

The noise generated by the interaction between a gust and an airfoil in a uniform flow is investigated. This problematic is of major industrial interest, regarding fans, turbomachinery, or wind turbine applications. A twodimensional symmetric Joukowski-type airfoil is immersed without incidence in a flow at Mach number 0.5 , disturbed by a harmonic gust at $45^{\circ}$ of incidence (4th CAA Workshop on Benchmark Problems, 2004). Our methodology is first to perform a high-order direct resolution of Euler's equations of the disturbed flow over the airfoil and the associated acoustic emission, which is taken as a reference simulation. Second, the near aerodynamic field is simulated with Fluent 6.3 solver based on finite volume method with second-order schemes. The aerodynamic data thus obtained are used for far field acoustic prediction, based on Ffowcs Williams and Hawkings analogy. Finally, following another hybrid approach, the noise is predicted by using integral formulations with source field from the DNS. The aim of the study is to provide insight into the efficiency and validity of these numerical strategies commonly used. Comparisons with results of the CAA workshop are given, covering various wavenumbers values.

\section{6:00}

2aNSa21. A stochastic source model for turbulent noise prediction including sweeping time dynamics. Malte Siefert (German Aerospace Center, Lilienthalplatz 7, 38108 Braunschweig, Germany, malte.siefert@dlr.de), Roland Ewert (DLR/Institute of Aerodynamics and Flow Technology, Lilienthalplatz 7, 38108 Braunschweig, Germany, Roland .Ewert@dlr.de)

We extend a low-cost computational aeroacoustic approach by taking into account temporal effects of the modeled turbulent flow. As the noise production is determined by the turbulent frequency spectrum rather than the wavenumber spectrum, temporal properties of the turbulent flow play a role in many cases. We focus on the most dominant temporal effect, on sweeping, i. e. the advection of inertial range structures by the energy containing large scales. By introducing a feedback-mechanism of the turbulent field onto itself, we are able to incorporate this effect in the fast random particle method, which was successfully applied to different cases such as 
slat noise, jet noise and others. It is shown that sweeping reproduces important properties of spatio-temporal correlations of the turbulent flow, which are not grasp by most turbulence-models. The influence on the sound generation will be discussed for aeroacoustic simulations of jet and trailing edge noise.

\section{6:20}

2aNSa22. Aeroacoustic simulation based on linearized Euler equations and stochastic sound source modelling. Hervé Dechipre (Volkswagen AG, Brieffach 1777, $38436 \quad$ Wolfsburg, Germany, herve.dechipre@volkswagen.de), Michael Hartmann (Volkswagen AG, Brieffach 1777, 38436 Wolfsburg, Germany, michael.Hartmann2 @ volkswagen.de), Jan W. Delfs (DLR/Institute of Aerodynamics and Flow Technology, Lilienthalplatz 7, 38108 Braunschweig, Germany, jan.delfs@dlr.de), Roland Ewert (DLR/Institute of Aerodynamics and Flow Technology, Lilienthalplatz 7, 38108 Braunschweig, Germany, Roland .Ewert@dlr.de)

In order to continually improve passenger car acoustic comfort, effective methods are needed to simulate the noise generated by external flows as well as the noise produced by duct flows in HVAC systems. The present paper illustrates the use of two methods based on the Linearized Euler equations and derivatives thereof to compute the acoustic field. The two methods implemented in the DLR's aeroacoustic code PIANO are the introduction of a perturbation (vortex) in the flow and the stochastic sound source modelling by Ewert. The application considered by this study is the noise produced by a flat plate in a two-dimensional duct at a thickness related Reynolds number of 1300. Different sizes and edge shapes of the flat plate have been tested. The simulations show a good agreement of the results obtained by the two methods as well as the existence of a vortex street behind the plate corresponding to a Strouhal number of 0.18 . It could be shown computationally, that even in the absence of the classical Aeolian tone generation (pure broadband turbulence related trailing edge noise generation) resonance type phenomena occur in the duct. The computed modes for the different configurations show encouraging correlations with the Parker-type modes or resonance phenomena described by Koch.

\section{6:40}

2aNSa23. Active Perturbation on Vortex-Induced Acoustic Resonance. Li Cheng (Hong Kong Polytechnic University, Hung Hom, Hong Kong, Hong Kong, mmlcheng@polyu.edu.hk), Y Zhou (Hong Kong Polytechnic University, Hung Hom, Hong Kong, Hong Kong, mmyzhou@ polyu.edu.hk) , Ming Ming Zhang (Hong Kong Polytechnic University, Hung Hom, Hong Kong, Hong Kong, mingming@jhu.edu)

Flow-induced acoustic resonance results from strong interactions between unsteady separated flows and the acoustic modes of a cavity. This work explores the feasibility of using piezoelectric-actuator-based perturbation technique in the control of vortex-induced noise through systematic experimental studies. A thick rectangular plate was used as the vortex generator and placed upstream of a cavity. Curved piezo-ceramic actuators were embedded in a slot on the top side of the plate to provide a perturbation to the flow. Uncontrolled flow-acoustic interaction was first examined to provide a baseline for comparison. Results show that noises induced by flow separation from the thick rectangular plate and the wall ahead of the cavity have very different critical flow velocities so that their effects can be well separated. Open-loop control tests indicate that vortex-induced acoustic resonance can be successfully controlled using the proposed technique. Analyses suggest that the convection of votices separated from the leading edge along the plate surface was accelerated by the surface perturbation, which interacted vigorously with the formation of the dominant trailing edge vortex, thus weakening the vortex strength in the wake of the plate. This weakened vortex strength substantially alters the flow-acoustic interaction, resulting in a significant impairment of vortex-induced acoustic resonance. (Supported by Research Grants Council of HKSAR. PolyU 5132/07E)

\section{7:00}

2aNSa24. Sound amplification in a lined duct with flow: PIV measurements. David Marx (Laboratoire d'Etudes Aerodynamiques CNRS, Bat K, 40 avenue du recteur Pineau, 86022 Poitiers, France, david.marx@lea.univ-poitiers.fr), Yves_ Aurégan (Laboratoire d'Acoustique de l'Université du Maine, Avenue Olivier Messiaen, 72085 Le Mans, France, yves.auregan@univ-lemans.fr), Helène Bailliet (Laboratoire d'Etudes Aerodynamiques - CNRS, Bat K, 40 avenue du recteur Pineau, $86022 \quad$ Poitiers, France, helene.bailliet@lea .univ-poitiers.fr), Jean-Christophe Valière (Laboratoire d'Etudes Aérodynamiques (LEA), Université de Poitiers, ENSMA, CNRS, Bat K, 40 avenue du recteur Pineau, 86022 Poitiers, France, jean-christophe.valiere@lea.univpoitiers.fr)

An experimental investigation of the acoustic behavior of a liner in a rectangular channel with grazing flow has been conducted. The liner is a locally reacting structure. When increasing the velocity of the grazing flow the transmission coefficient increases at resonance frequency. The transmission coefficient can even become larger than 1 meaning that acoustical energy is produced by the liner. This amplification of the sound wave is accompanied by an increase in the stationary pressure drop induced by the liner (up to $300 \%$ ). This effect is attributed to a modification of the flow induced by the acoustic wave. Thus, the flow is measured using Particle Image Velocimetry (PIV) imaging technique and a comparison of velocity maps with and without sound amplification is performed. Some differences occur and will be presented.

$$
7: 20
$$

2aNSa25. Acoustic PIV: Measurement of the acoustic particle velocity using synchronized PIV-technique. André Fischer (German Aerospace Center (DLR), Mueller-Breslau-Str. 8, 10623 Berlin, Germany, andre.fischer@dlr.de), Emilie Sauvage (Ecole Polytechnique de 1'Univerité d'Orléans, 8 rue Léonard de Vinci, 45072 Orléans, France, milie.sauvage@gmx.net), Ingo Roehle (German Aerospace Center (DLR), Mueller-Breslau-Str. 8, 10623 Berlin, Germany, ingo.roehle@dlr.de)

This paper outlines a technique for measuring the acoustic particle velocity and the flow field simultaneously by applying synchronized particle image velocimetry (PIV). As test set-up a squared acrylic glass chamber was chosen. One side of the test section is connected to a loudspeaker, which allows a sinusoidal excitation of the chamber. To point out constrains of this method the investigation includes an analysis of excitation amplitude and frequency as well as the effect of the mean flow magnitude. Therefore a small PC fan can be mounted inside the test section to produce an adjustable mean flow. It can be shown that for a low number of averaged images (80) reasonable results can be achieved up to a certain level of fan rotation speed. Beyond this level the turbulence sensitivity increases and more images are necessary for the calculations. However, the acoustic particle velocity can be computed in the presence of turbulent flow. The presented method called acoustic PIV is a non intrusive technique, applied successfully in measuring acoustic particle velocity fields over a wide range of conditions.

\section{$7: 40$}

2aNSa26. A finite element method for time harmonic acoustics in arbitrary flows. Anne-Sophie Bonnet- Ben Dhia (CNRS, ENSTA - 32 Boulevard Victor, 75015 Paris, France, bonnet@ensta.fr), Jean-Francois Mercier (CNRS, ENSTA - 32 Boulevard Victor, 75015 Paris, France, jmercier@ensta.fr), Florence Millot (CERFACS, 42 avenue Gaspard Coriolis, 31057 Toulouse, France, millot@cerfacs.fr), Sebastien Pernet (CERFACS, 42 avenue Gaspard Coriolis, 31057 Toulouse, France, pernet@cerfacs.fr)

The reduction of noise in aeronautics motivates an intensive research in aeroacoustics. In particular, there is a need for efficient tools to simulate acoustic propagation in a mean flow. We are interested here by solving the linearized problem in the frequency domain, by a finite element method able to take into account general geometries and flows. To our knowledge, only 
the potential case has been completely handled. Recently, a new approach has been developed and validated in the case of a parallel shear flow: it relies on a regularized formulation of Galbrun's equation, well-suited for a discretization by Lagrange finite elements, combined with Perfectly Matched Layers. A drawback of the method comes from the additional term of regularization, which requires the evaluation of an oscillating integral, coupling all degrees of freedom located on the same streamline. This difficulty can be avoided by replacing this non-local term by its Low-Mach approximation We show here how to extend this Low-Mach approach to the case of a non parallel flow. Numerical experiments are done. In the case of a potential mean flow, a good agreement with the exact approach is observed, even for quite large Mach numbers.

\title{
Session 2aNSb
}

\section{Noise, Biomedical Ultrasound/Bioresponse to Vibration, ASA Committee on Standards, and EURONOISE: Session in Honor of Henning von Gierke}

\author{
Paul Schomer, Cochair \\ Schomer and Associates, Inc., 2117 Robert Drive, Champaign, IL 61821, USA
}

Brigitte Schulte-Fortkamp, Cochair

TU Berlin, Institute of Fluid Mechanics and Engineering Acoustics, Einsteinufer 25, Sekr. TA 7, Berlin, D-10587, Germany

\section{Invited Papers}

\section{8:00}

2aNSb1. Where do we stand on standards for noise? William Lang (Noise Control Foundation, 29 Hornbeck Ridge, Poughkeepsie, NY 12603, USA, lang1ww@gmail.com)

Almost 40 years ago, Henning von Gierke organized and chaired a symposium at the ASA Cleveland meeting with the above title. The chairs of nine writing groups of the ANSI-predecessor working on methods for the measurement and rating of noise presented summaries of their tasks and their progress in 1968. If a symposium on the same theme were to be held today, there would be reports from fifty-four working groups of ANSI Accredited Standards Committees on Acoustics (S1), Bioacoustics (S3), and Noise (S12). These committees are cornerstones of the ASA standards program. As the first ASA Standards Director, Henning revitalized the Society's standards program to become the productive organization it is today. The progress in standardization in America during the past four decades is a tribute to his foresight and participation. He also contributed to international standardization as the leader of the U.S. delegation to ISO TC/43 (Acoustics) and its subcommittee on noise (SC1), and for three decades he chaired ISO TC/108/SC4 on human exposure to mechanical shock and vibration. Trained as an engineer, Henning was a leader of national and international standardization efforts to relate the mechanical energy in sound waves to the responses of the human organism.

8:20

2aNSb2. Henning E. von Gierke and human biodynamics. Anthony J. Brammer (Ergonomic Technology Center, Univ. of Connecticut Health Center, 263, Farmington Ave., Farmington, CT 06030, USA, brammer@uchc.edu)

In addition to his well-known involvement in protecting humans from exposure to excessive noise, Dr. Henning E. von Gierke was deeply involved in protecting humans from exposure to excessive mechanical shocks, impacts and vibration. From exploring the limits of survivability during the landing phase of space and aircraft capsules to the more everyday issues of occupational exposure to vibration, he provided scientific insight into problems and leadership in developing solutions. While most of Henning's contributions are buried in past literature, the spirit of his efforts lives on in a review chapter published in Harris' Shock and Vibration Handbook that I have had the privilege to co-author with him through two editions, now extending back more than a decade. The challenge of encompassing the original content of his contribution while introducing the results of more recent research has, so far, yielded a composition that he has endorsed. Henning's involvement and interests in human biodynamics will be illustrated by examples taken from this work.

\section{$8: 40$}

2aNSb3. Service to his fellow men. Richard McKinley (AFRL, Wright-Patterson Air Force Base, Dayton, OH 45433-7901, USA, richard.mckinley@wpafb.af.mil)

Henning Von Gierke's life of service followed in the footsteps of his family legacy. This presentation will focus on Henning's 50 years of research at the Air Force Research Laboratory at Wright-Patterson Air Force Base, Dayton, Ohio and the recollections of Henning gathered from $20+$ hours of video interviews. Henning's life and his research were dedicated to helping mankind. Beginning with his dissertation on noise produced by jet flow and continuing with the accomplishments of his division at the Air Force Research Laboratory his contributions to acoustics were outstanding. His contributions include the Air Force hearing conservation program, the EPA levels document, the BENOX report, the development of Bionics in the 1960's, car airbag research in the 60's, testing of the 
original 7 US astronauts, human limits of survival for impact, human limits of performance in vibration, pioneering work in hearing protector modeling, bone conduction, and microwave hearing, speech communication, as well as his extensive work in American National Standards and international standards. His contributions have in one way or another affected nearly everyone. He was a great researcher and a great mentor. He will be missed by his colleagues at the Air Force Research Laboratory and around the world.

\section{Contributed Paper}

\section{9:00}

2aNSb4. Henning von Gierke, leader, consensus builder, mentor, and friend. Alice H. Suter (Alice Suter \& Associates, 575 Dogwood Way, Ashland, OR 97520, USA, ahsuter@charter.net)

Over his long and illustrious career, Henning von Gierke has had an enormous effect on public policy in noise and vibration, not only because he was active in the field for more than half a century, but because of his intense dedication, his persistence, and his unique, almost legendary ability to build consensus. EPA's Office of Noise Abatement and Control could not possibly have produced its Criteria and Levels documents in response to the timeframe imposed by Congress without Henning's leadership. Those of us who were lucky enough to be mentored by him were able to accomplish more than we ever could have without his guidance. In addition to his invaluable assistance in my doctoral program, I knew him as ASA's Standards Director, then President of ASA, chairman of interagency task forces, chairman of several standards committees, and participant in numerous international activities. He was always willing to share the burden of work and to encourage the rest of us to forge ahead in an effort to make the world a quieter and healthier place.

\section{Invited Papers}

2aNSb5. Dr. Henning von Gierke-My mentor, everyone's mentor. Paul Schomer (Schomer and Associates, Inc., 2117 Robert Drive, Champaign, IL 61821, USA, schomer@ SchomerAndAssociates.com)

Dr. Henning von Gierke was a leading force in acoustics for a majority of the 20th century. He led in human response to noise AND vibration and its assessment. He was a leading force in the US government and in the National Academy of Science (NAS) Committee on Hearing and Bioacoustics (CHABA). He was a leader in hearing assessment and conservation, and in Standards. And to many working in these various areas, he was a mentor, a confidant, and a friend. Mentoring did not stop at the doors of his laboratory, but rather, his mentoring extended to the world. He was a mentor to me in my first years after graduation when we developed the $\mathrm{C}$-weighted procedure for the assessment of large-amplitude impulse noise, and in the development of related Standards and NAS studies. He thrust me into ISO work and CHABA studies, and, later, he chose me to succeed him as ISO delegation chair in acoustics and noise. Not enough can be said about Henning's impact on noise and vibration research and policy in the USA and in the world, and on the expanded reach of Henning's influence through the many like me that he mentored.

\section{9:40}

2aNSb6. Henning von Gierke's continuing contribution: Underwater whole-body vibration. Sarah Gourlie (Applied Research Laboratories, The University of Texas, P.O. Box 8029, Austin, TX 78713-8029, USA, sarah.gourlie@ gmail.com), Mark F. Hamilton (Applied Research Laboratories, The University of Texas, P.O. Box 8029, Austin, TX 78713-8029, USA, hamilton@mail.utexas.edu)

Henning von Gierke was a leader in the characterization of human response to sound and vibration and in the development of international standards in this area. One focus of his research was using a multidisciplinary approach to create biodynamic models for whole-body vibration with the aim of predicting and preventing injury in vehicles. The focus of this presentation is whole-body vibration underwater with the aim of predicting discomfort or possibly injury to divers from low-frequency sonar. The frequency range of interest is 40-80 Hz, which encompasses the resonance of human lung. For this purpose, a biodynamic model developed by von Gierke to simulate thoracic, abdominal, and spinal responses to different vibrational excitations in air [H. E. von Gierke, J. Acoust. Soc. Am. $50,1397(1971)]$ is adapted for underwater conditions. It is assumed that the diver is neutrally buoyant and will therefore experience whole-body acceleration equal to the particle acceleration produced by the sound field in the absence of the diver. Emphasis is placed on determining the appropriate source distribution on the body as well as adjusting von Gierke's model to accommodate the decrease in lung compressibility with diver depth and mass loading on the chest. [Work supported by ONR.] 


\title{
Session 2aNSc
}

\section{Noise and EURONOISE: Physical and Psychophysical Evaluation of Vehicle Exterior Noise I}

\author{
Paul Donavan, Cochair \\ Illingworth \& Rodkin, Inc., 505 Petaluma Blvd. South, Petaluma, CA 94952, USA \\ Hugo Fastl, Cochair \\ AG Technische Akustik, MMK, TU München, Arcisstr. 21, München, 80333, Germany
}

Invited Papers

8:00

2aNSc1. Low speed exterior vehicle voise and the effect of pavement type. Paul Donavan (Illingworth \& Rodkin, Inc., 505 Petaluma Blvd. South, Petaluma, CA 94952, USA, pdonavan@illingworthrodkin.com)

For operating conditions of cruise and moderate acceleration, the exterior noise emission of light vehicles is typically dominated by tire/pavement noise at speeds of $50 \mathrm{~km} / \mathrm{h}$ or greater. At a test speed of $56 \mathrm{~km} / \mathrm{h}$, it has been found that pavement type can create a 10 $\mathrm{dB}$ or more variation in tire/pavement noise. This has significant implications for both community noise and vehicle noise emission testing. In this paper, the results tire/pavement noise measurements for over 40 different pavements in Europe and the United States are reported. These pavements include research surfaces, existing roadways, and ISO 10844 passby test surfaces. Measurements were conducted using an on-board sound intensity methodology that has been correlated to cruise-by noise levels. These results are discussed in terms of the revisions being considered for the newly revised ISO 362 passby test procedure and the ISO 10844 test surface specification. Additionally, a case history of community traffic noise reduction achieved by use of a quieter pavement is reviewed to demonstrate the importance of the pavement in low speed vehicle noise emissions.

8:20

2aNSc2. Noise source mapping for trucks, part 1: development and design. Kenneth J. Plotkin (Wyle Laboratories Inc., 241 18th Street S., Suite 701, Arlington, VA 22202, USA, kenneth.plotkin@wylelabs.com), Yuriy Gurovich (University of Mississippi, NCPA, 1 Coliseum Drive, University, MS 38677, USA, yuriy.gurovich@wylelabs.com), William Blake (Naval Surface Warfare Center (Ret.), 6905 Hillmead Road, Bethesda, MD 20817, USA, hydroacoustics@aol.com), Paul Donavan (Illingworth \& Rodkin, Inc., 505 Petaluma Blvd. South, Petaluma, CA 94952, USA, pdonavan@illingworthrodkin.com)

Mapping and quantifying noise sources on trucks under actual operating conditions on the road are important for traffic noise modeling and mitigation. The purpose of this study is to develop a practical truck noise source localization technique using acoustic beamforming. An experimental $70+$ microphone elliptical array was designed and fabricated for truck testing. Beam-forming software was developed and implemented using a computerized data acquisition system. Proof-of-concept tests were performed at low-speed and high-speed truck testing facilities for a representative sample of trucks with widely different characteristics to validate the measurement system performance. The measurement system design parameters were verified experimentally, and certain improvements to the system were recommended for future implementation based on the field experience. The developed beam-forming measurement system provided adequate noise mapping and localization for various noise sources on trucks, stationary and moving with the speed up to 50 mph. The results of the proof-of-concept testing presented in an accompanying paper (Part 2) confirm that the developed microphone array, data acquisition system and beam-forming software performed generally as expected and required no major adjustments. This ongoing project is funded by the National Cooperative Highway Research Program of the Transportation Research Board of the National Academies, USA.

\section{8:40}

2aNSc3. Noise source mapping for trucks, Part 2: Experimental results. William Blake (Naval Surface Warfare Center (Ret.), 6905 Hillmead Road, Bethesda, MD 20817, USA, hydroacoustics@aol.com), Kenneth J. Plotkin (Wyle Laboratories Inc., 241 18th Street S., Suite 701, Arlington, VA 22202, USA, kenneth.plotkin@wylelabs.com), Yuriy Gurovich (University of Mississippi, NCPA, 1 Coliseum Drive, University, MS 38677, USA, yuriy.gurovich@wylelabs.com), Paul Donovan (505 Petaluma Blvd, South, Petaluma, VA 94952, USA, pdonovan@illingworthrodkin.com)

The elliptical array described in the previous paper (Part 1) was deployed road-side at a test track for measuring the sound emitted from trucks during passby. Measurements were made on various truck models in various operating states with and without trailers. Extensive spherical-source calibrations confirmed the array's beamforming at both on-normal and steering angles (to $45^{\circ}$ ); they also disclosed the magnitudes of ground reflection paths for localized sources at different elevations. In all cases, the array output was computationally inverted to produce two-dimensional spatial maps of source levels in the side profile of the truck. In the cases of stationary trucks, the source maps were correlated with simultaneously-obtained intensity maps. Comparisons verified that the arraybased source maps for the trucks ranked sound sources of disparate levels in the same order as did the sound power levels deduced from the sound intensity data. Acoustic source maps obtained during truck passbys were then used to provide time-histories and spatial distributions of sources and source paths from the engine, muffler, tires, and certain body components. This project is funded by the National Cooperative Highway Research Program of the Transportation Research Board of the National Academies, USA. 
2aNSc4. Vehicle exterior noise from the view point of new experts. Brigitte Schulte-Fortkamp (TU Berlin, Institute of Fluid Mechanics and Engineering Acoustics, Einsteinufer 25, Sekr. TA 7, D-10587 Berlin, Germany, brigitte.schulte-fortkamp@tu-berlin.de)

The combination of physical and psycho- acoustical measurements with scientific evaluation of perceptual responses to environmental noise, known as Soundscaping, is an essential method for assessing and actualizing environments. Empirically, the perception of sounds and their evaluation will be explored under the premise of combining human judgment and physical factors. In a recent Soundscape Project, environmental noise perception and evaluation in a defined urban area were investigated in detail. Classical measurements and open, narrative, but issue-centred interviews with residents were carried out, and those interviews allowed the interviewed persons to set their own focus on certain aspects concerning the development of a public space in Berlin. Method and procedure will be discussed in detail to introduce to the New Expert Concept. The Soundscape Project is a module of the Project "Nauener Platz - Remodelling for Young and Old" in the framework of the research program "Experimental Housing and Urban Development (ExWoSt)" of the "Federal Ministry of Transport, Building, and Urban Affairs (BMVBS)" by the "Federal Office for Building and Regional Planning $(\mathrm{BBR})$ ". It is related to the fields of research (ExWoSt) concerned with "Innovation of Urban Neighbourhoods for Families and the Elderly". The project executing organization is the Regional Office Berlin-Mitte.

2aNSc5. Significance of Psychoacoustic Aspects for the Evaluation of Vehicle Exterior Noise. Klaus Genuit (HEAD acoustics GmbH, Ebertstrasse 30a, 52134 Herzogenrath, Germany, klaus.genuit@ head-acoustics.de)

For 25 years acoustic engineers have been using specific measurement technologies and analyses to determine sound quality of vehicle interior noise. However, with regard to exterior vehicle noise only standard measurements, e.g. the A-weighted sound pressure level (ISO 362), have been used and interpreted so far. Extensive knowledge and experiences concerning the evaluation of interior noise are still unutilized for an advanced assessment of vehicle exterior noise. Within EU research projects, such as SVEN or QCity, exterior noise was investigated, methods established and psychoacoustic analyses carried out. The relevance of vehicle exterior noise with respect to perceived product quality was underestimated for a long time. Today, manufacturers recognize the importance of exterior noise beyond the annoyance perspective. Vehicle exterior noise conveys an impression of product quality. By optimizing the product sound quality manufacturers can compete against others and meet increased requirements. Customers prefer vehicle sounds which match their expectations, which also applies to the exterior sound of a vehicle. The acoustical fingerprint of a vehicle - inside and outside - provides enormous marketing opportunities. This paper shows how the knowledge of sound quality analyses with regard to interior noise can be transferred to the evaluation of exterior vehicle noise.

9:40

2aNSc6. Rating the Dieselness of engine-sounds. Hugo Fastl (AG Technische Akustik, MMK, TU München, Arcisstr. 21, 80333 München, Germany, fastl@mmk.ei.tum.de), Bernhard Priewasser (AG Technische Akustik, MMK, TU München, Arcisstr. 21, 80333 München, Germany, bernhard@prie.de), Markus Fruhmann (AG Technische Akustik, MMK, TU München, Arcisstr. 21, 80333 München, Germany, Markus.Fruhmann@gmx.de), Herbert Finsterhölzl (BMW Group, Knorrstr. 147, 80788 München, Germany, herbert.finsterhoelzl@bmw.de)

Sounds of idling Diesel engines were rated according to their Dieselness with two different psychoacoustic methods. On the one hand, a yes/no procedure was used. Subjects were presented sounds of two seconds duration and had to answer by yes or no to the following question: Does this sound stem from a Diesel engine? Histograms were calculated and a hypothesis was put forward as follows: The more positive answers are given to a specific sound, the larger is the Dieselness of this sound. On the other hand, the method of paired comparison was used, applying a kind of Bradley-Terry-Luce (BTL) procedure. Results are discussed with respect to the psychophysical method used and possible differences in ratings by expert versus naive listeners. Finally, psychoacoustic data are compared to predictions of Dieselness calculated by four different algorithms.

10:00

2aNSc7. Perceptive temporal features of train passbys. Jonathan Terroir (Laboratoire MRTE - UCP, 5, mail Gay-Lussac, Neuville-sur-Oise, 95031 Cergy-Pontoise Cedex, France, jterroir@u-cergy.fr), Catherine Lavandier (Laboratoire MRTE - UCP, 5, mail Gay-Lussac, Neuville-sur-Oise, 95031 Cergy-Pontoise Cedex, France, catherine.lavandier@u-cergy.fr)

This work deals with characterization of temporal features linked to train passages which may act upon annoyance. Two varying parameters in the recordings have been selected: distance from the railway ( 7.5 meters, 50 and 100 meters) and train category (four types of French trains). A first series of psychoacoustic tests are carried out in order to mainly extract temporal aspects which could be important for sound perception. To avoid the level saliency, a preliminary equalization has been made on $\mathrm{L}_{\mathrm{A} 5}$. The selection of perceptive factors is carried out through a multidimensional analysis (INDSCAL). Dissimilarity tests highlight several dimensions: train category, slope of the level increase linked to train arrival and passage length. In addition to the dissimilarity between two stimuli estimation, subjects have to choose the one they prefer and explain their answers. The verbalization task helps illustrate multidimensional analysis axis. A second series of tests, designed with recordings varying on two independent parameters (the level and the slope of the temporal evolution) makes it possible to compare perceptive variations on temporal effects to loudness. 
2aNSc8. Synthesis of pass-by railway noise. Shafiquzzaman Khan (Kungliga Tekniska Hogsklan, Department of Aeronautical and Vehicle Engineering, SE - 10044 Stockholm, Sweden, Shafik@kth.se), Mohite Ulhas (Indian Institute of Technology Roorkee, Graduta Student, Dept. of Mech. \& Ind. Engg, 247667 Roorkee, India, goelvfme@iitr.ernet.in), Virendra Goel (Indian Institute of Technology Roorkee, Graduta Student, Dept. of Mech. \& Ind. Engg, 247667 Roorkee, India, goelvfme@iitr.ernet.in)

Pass-by railway noise is one of the main problems in the community. Typically pass-by railway noise is estimated using Leq or Lden in $\mathrm{dB}(\mathrm{A})$ and often these methods do not reflect the listener perceptual overview. It is therefore necessary to reduce the pass-by railway noise by synthesizing the noise characters into various segments. The segments could be based on the physical properties of rail vehicles like rolling, traction, pantograph, cooling system noise, etc. There are few other segments which are also based on the perceptual view of the pass-by railway noise like squeaking, rattling or beating (tadak, tadak). In this study only a few of the physical characteristics of the railway noises are examined. The idea of synthesization is to determine what segments in the noise characters are annoying. And thereby make a virtual pleasant pass-by railway sound. Several pass-by noises from Swedish rail vehicles were recorded using binaural technology according to ISO 3095. Recorded noise signals were then analyzed with the help of spectrogram analysis using Matlab. The results of the studies indicate that the rolling noise and broadband noise are most annoying for the long distance passenger rail vehicles.

TUESDAY MORNING, 1 JULY 2008

ROOM 251, 10:20 A.M. TO 4:00 P.M.

\title{
Session 2aNSd
}

\section{Noise and EURONOISE: Environmental Noise Mapping II}

\author{
Kenneth Cunefare, Cochair \\ Woodruff School of Mechanical Engineering, The Georgia Inst. of Technol., Atlanta, GA 30332-0405, USA \\ Gaetano Licitra, Cochair \\ ARPAT - Dept. Firenze, Via Porpora, 22, Firenze, 50144, Italy
}

\section{Invited Papers}

\section{$10: 20$}

2aNSd1. Managing Risk by Utilising an Integrated Approach to Quality Assurance During Strategic Noise Mapping. Simon J. Shilton (Acustica Ltd, Trident One, Styal Road, M22 5XB Manchester, UK, simon.shilton@acustica.co.uk), Alan Štimac (DARH2 Acoustics \& Civil Eng. Ltd., Ljubicin prolaz 3, HR-10430 Samobor, Croatia, alan@darh2.hr), James Trow (Hepworth Acoustics Ltd, 5 Bankside, Crosfield Street, WA1 1UP Warrington, UK, james.trow@ hepworth-acoustics.co.uk), Nathan Archer (Hepworth Acoustics Ltd, 5 Bankside, Crosfield Street, WA1 1UP Warrington, UK, nathan.archer@hepworth-acoustics.co.uk), Vincent Hii Jiu Ta (Hepworth Acoustics Ltd, 5 Bankside, Crosfield Street, WA1 1UP Warrington, UK, vincent.hii@hepworth-acoustics.co.uk), Nigel Jones (Extrium Ltd, Calverley House, 55 Calverley Road, TN1 2TU Tunbridge Wells, UK, nigel.jones@extrium.co.uk)

With the proclamation of the Directive on Environmental Noise (2002/49), the process of noise mapping and action planning has begun around Europe. As the development of strategic noise maps is arguably a new experience for many end-users, the first round of noise mapping could potentially lead to uncertainties within many aspects of the process. Noise maps represent a baseline for the implementation of noise management systems and any systematic errors within the noise maps could reduce the effective implementation of the whole management system. The extensive range of data inputs required in strategic noise mapping are usually obtained from different data sources or base datasets and as such, cataloguing with the use of metadata is key. The implementation of a quality assurance system is imperative to maintain consistency between technicians and within multi-disciplinary teams. It is also essential to ensure the control of processes and the ability to review inputs, intermediaries and deliverables. A similar approach may also be applied to calculation and post processing of noise levels. This paper presents collective experience of the implementation of quality assurance procedures used in several EU countries during the successful completion of projects within the first round of mapping.

\section{0:40}

2aNSd2. A methodology for mapping neighbourhood impacts. Ronny Klaeboe (Institute of Transport Economics, Gaustadalleen 21,0349 Oslo, Norway, rk@toi.no), Erik Engelien (Statistics Norway, Oterveien 23, N-2225, 2225 Kongsvinger, Norway, erik.engelien@ssb.no), Margrete Steinnes (Statistics Norway, Oterveien 23, N-2225, 2225 Kongsvinger, Norway, margrete.steinnes @ssb.no)

A methodology for the spatial analysis of environmental impacts in urban areas and social gradients is presented. The methodology is computational intensive and requires access to geographically located data at the dwelling level. Neighbourhood information is harvested to provide contextual information, and a spatial smoothing technique used to provide powerful visualisations of residential and neighbourhood impacts. By focussing on impacts rather than exposures, the construction of accumulative non-monetarised and monetarised indicators of impacts such as disability adjusted life years (DALY) and health costs is facilitated. Since the residential dwelling location is, or can be made, the focal point for social, dwelling, environment, neighbourhood, and transportation related indicators, a rich network of information in a uniform format is available for construction of novel indicators. By focussing on neighbourhoods rather 
than individual dwellings, privacy is enhanced while maintaining useful level of detail for involving the public and politicians in local planning. By combining information on social status and environmental exposures, questions on whether transportation related impacts are distributed fairly and whether social inequity is increased or decreases as a result of a particular measure can be addressed. The methodology is not applicable to rural areas and low probability events.

\section{1:00}

2aNSd3. Noise emissions at intersections: comparing microscopic and macroscopic traffic simulation approaches. Bert De Coensel (Ghent University - Department of Information Technology, Sint-Pietersnieuwstraat 41, 9000 Ghent, Belgium, bert.decoensel@intec.ugent.be), Dick Botteldooren (University Ghent - Department Information Technology, Sint-Pietersnieuwstraat 41, 9000 Gent, Belgium, dick.botteldooren@intec.ugent.be), Luc Dekoninck (Ghent University - Department of Information Technology, Sint-Pietersnieuwstraat 41, 9000 Ghent, Belgium, luc.dekoninck@intec.ugent.be), Dominique Gillis (Ghent University - Department of Civil Engineering, Krijgslaan 281 S8, 9000 Ghent, Belgium, dominique.gillis@ ugent.be), Dirk Lauwers (Ghent University - Department of Civil Engineering, Krijgslaan 281 S8, 9000 Ghent, Belgium, dirk.lauwers@ugent.be)

Environmental noise mapping often involves the use of traffic simulation software. In most cases, traffic flows are simulated in a macroscopic way, considering only traffic flow parameters averaged over road segments. This approach does not allow to correctly account for the typical deceleration and acceleration patterns of traffic at intersections, which influence local noise emissions. Microscopic traffic simulation models, which model each vehicle individually, incorporate these dynamic effects. However, they require much larger amounts of input data for calibration, and are therefore less suited to map large areas. In this paper, we investigate the possible influence of the choice of simulation detail on simulated traffic flow parameters and, as a consequence, on noise emissions near intersections. As a case study, several types of road intersections under various traffic demand and traffic composition conditions are considered. Simulation results are obtained using a microscopic (Paramics) as well as a mesoscopic (MaDAM) and a macroscopic (OmniTRANS) traffic simulation model, and predicted traffic flow parameters and noise emission values are compared between these approaches.

\section{Contributed Papers}

11:20

2aNSd4. Environmental Noise Directive - results of noise mapping in Germany. Matthias Hintzsche (Federal Environment Agency, Woerlitzer Platz 1, 06844 Dessau-Rosslau, Germany, matthias.hintzsche@uba.de)

The Environmental Noise Directive intends the development of a concept to avoid, prevent or reduce on a prioritised basis the harmful effects, including annoyance, due to exposure to environmental noise and to inform the public on environmental noise and its effects. In a first step noise maps were made for all agglomerations and for all major roads, major railways and major airports. This noise maps are the basics for noise action plans. These plans have the target to prevent and to reduce environmental noise where necessary to preserve environmental noise quality where it is good. The results of the first round for noise mapping in Germany will be presented.

\section{1:40}

2aNSd5. Noise mapping of public roads in Norway: New calculation tool based on the Nord2000 Road Engineering Method. Ingunn Milford (Norwegian Public Roads Administration, Brynsengfaret 6A, P.O .Box 8142 Dep., N-0033 Oslo, Norway, ingunn.milford@ vegvesen.no)

To comply with requirements in the Directive 2002/49/EC on environmental noise (END) and to national guidelines on land use and transport noise, the Norwegian Public Roads Administration has developed the calculation tool called NorStoy, which is composed of two main modules: 1. A calculation module with the Nord2000 Road Engineering Method algorithms. 2. An ArcGIS module to arrange the input data, delimit the calculation area, locate the calculation points and report the results. Nord2000 Road Engineering Model was completed in 2006, in cooperation with the Nordic countries. The model has very much in common with the coming joint European Harmonoise model. The challenge in using these models is to obtain an acceptable calculation time. To prevent NorStoy from stopping every time insufficient data occurs, we use default values based on either a typical mean value or the nearest registered value. At this time preparing input data is quite time consuming. We are able to produce the noise maps for the EC directive, but NorStoy is not yet an efficient tool for noise mapping in general. The development of NorStoy is still in progress.

\section{2:00}

2aNSd6. The national noise map of the Netherlands $A$ trend in exposure since 1990. Rik Van Haaren (DHV, P.O. box 1132, 3800BC Utrecht, Netherlands, rik.vanhaaren@dhv.com)

Noise maps according to the environmental noise directive have been provided by several actors in the Netherlands. The results of the noise maps show the significance of each of the source types, as well as the distribution within and outside agglomerations. In the Netherlands 2.7 million people are exposed to noise levels above $55 \mathrm{~dB}(\mathrm{~A})$. In some cases up to 4000 people are exposed to level higher than $75 \mathrm{~dB}(\mathrm{~A})$. In the night period 2.3 million people are exposed to noise levels higher than $50 \mathrm{~dB}(\mathrm{~A})$. Road noise is the dominant source for annoyance and sleep disturbance. Within agglomerations road noise is responsible for $88 \%$ of the highly annoyed population. Major roads, outside agglomerations, contribute less than $10 \%$ to the total annoyance due to road traffic. Railways are a second source in producing annoyance and sleep disturbance. $57 \%$ of the high annoyance due to railways is produced outside agglomerations. The results of the noise maps for road traffic noise have been compared with results for a selection of cities in 1990. The results show that the total number of exposed people has increased. However, the exposition to the higher bands has decreased.

\section{2:20}

2aNSd7. Noise Mapping and Noise Scoring - Software Techniques and Result Presentation. Wolfgang Probst (DataKustik GmbH, Gewerbering 5, 86926 Greifenberg, Germany, wolfgang.probst@datakustik.com)

Noise scoring is based on noise level maps and on exposure distribution. Different techniques can be used to develop the areal distribution of these values and to present them as coloured maps. Especially more complex ratings that are not only based on an exposure level and on population densities need relatively complex procedures to develop them from noise maps. It must be taken into account that there are two important steps in this procedure: One is the calculation of the noise score itself and the other is the presentation as coloured map to detect and focus on Hot Spots. Different techniques have been developed, were integrated in the software and have been applied in the frame of the QCity project. The consequences of different parameter settings are presented and discussed. The second step is the assignment of colours to a given scale of result values and even this simple process must be handled carefully to transport the needed information. 


\section{Contributed Papers}

2:00

2aNSd8. The Milan agglomeration Strategic Noise Map. Giovanni Zambon (Department of Environmental Sciences of the University of Milano - Bicocca, Piazza della Scienza, 1, 20126 Milan, Italy, giovanni.zambon@unimib.it), Simone Radaelli (Department of Environmental Sciences of the University of Milano - Bicocca, Piazza della Scienza, 1, 20126 Milan, Italy, s.radaelli@unimib.it)

The aim of this paper is to describe the procedure used for the Milan agglomeration Strategic Noise Mapping, as defined by Environmental Noise Directive (2002/49/EC). City of Milan is a complex case study because of the presence of a plurality of sources: road traffic, railway traffic (also tram traffic) and aircraft noise (Linate airport). The assessment method is based on the guidelines contained in the "Good Practice Guide for Strategic Noise Mapping and the Production of Associated Data on Noise Exposure" (WGAEN). The study of noise generated from several sources was carried out with the integrated use of both numerical models and Geographic Information Systems (GIS). Numerical models allow us to estimate of noise levels in large areas near to a specific noise source and GIS enable the efficient acquisition, management and elaboration of geo-referenced data representative of territory, sources and buildings. By means of the study of specific GIS application it was possible to predict the global noise exposure for overall sources, by overlapping the noise maps of the single noise sources.

\section{2:20}

2aNSd9. Budapest Noise Mapping Project I. - Experiences. András Muntag (Enviroplus Kft., Telepy u. 3., 1096 Budapest, Hungary, muntaga@zajan.hu), Mihály Berndt (Enviroplus Kft., Telepy u. 3., 1096 Budapest, Hungary, berndt@mgx.hu)

The Hungarian capital Budapest, and their agglomeration have recently finished their first strategic noise map. According to the Hungarian noise regulations and to the European Directive 2002/49/EC, this is a legal obligation. The main part of the costs was supported by the EU. The preparation of the strategic noise maps based on a computer model and describing the present noise situation of the municipalities, should be seen as the first step to build a true noise information and management system, based on the computer model initially developed. The main parameters of the project were the planned area $1100 \mathrm{qkm}$, number of dwellings 440 thousand, length of the mapped roads ca. $2000 \mathrm{~km}$ This paper based on the experience of 23 municipal noise maps - Budapest and other 22 settlements in the agglomeration. We successfully finished the project on time - the results (all maps) can be found on the internet-page: http://terkep.budapest .hu/website/zajterkep The first paper deals with the technical experiences of the project.

\section{2:40}

2aNSd10. Budapest Noise Mapping Project II. - Results. Mihály Berndt (Enviroplus Kft., Telepy u. 3., 1096 Budapest, Hungary, berndt@mgx.hu), András Muntag (Enviroplus Kft., Telepy u. 3., 1096 Budapest, Hungary, muntaga@zajan.hu)

The Hungarian capital Budapest, and their agglomeration have recently finished their first strategic noise map. According to the Hungarian noise regulations and to the European Directive 2002/49/EC, this is a legal obligation. The main part of the costs was supported by the EU. The preparation of the strategic noise maps based on a computer model and describing the present noise situation of the municipalities, should be seen as the first step to build a true noise information and management system, based on the computer model initially developed. The main parameters of the project were the planned area $1100 \mathrm{qkm}$, number of dwellings 440 thousand, length of the mapped roads ca. $2000 \mathrm{~km}$ This paper is based on the experience of 23 municipal noise maps - Budapest and other 22 settlements in the agglomeration. We successfully finished the project on time - the results (all maps) can be found on the internet-page: http://terkep.budapest .hu/website/zajterkep The second paper shows the results of the project.

\section{3:00}

2aNSd11. The Port of Livorno noise mapping experience. Mario Morretta (Sintesis - Enginering and Consulting S.r.l., via Martin Luther King, 15, 57128 Livorno, Italy, mario.morretta@sintesis.toscana.it), Andrea Iacoponi (ARPAT - Dipartimento di Livorno, via Marradi, 114, 57128 Livorno, Italy, a.iacoponi@arpat.toscana.it), Fabrizio Dolinich (Sintesis Enginering and Consulting S.r.l., via Martin Luther King, 15, 57128 Livorno, Italy, fabrizio.dolinich@gmail.com)

Livorno Municipality and its seaport are requested to produce the strategic noise map according to the European Directive only in 2012. However Livorno Port Authority, following the principle of environmental sustainability and in coherence with its EMAS status, started the investigation about the interaction between the noise generated by port activities and the nearby city. This analysis has been carried out within the Life funded project NoMEPorts, whose goal is to develop and provide tools and expertise to European ports for implementing their strategic noise maps, by means of a collaborative mapping of 8 Port Authority technical representatives from all around Europe. The work presented outlines in detail the process followed in performing the noise mapping of the Livorno port area and the port-city interface area, focusing in the problems encountered in the data collection, in port noise model building steps and in the consequent solutions adopted. The paper also talks about the result obtained by showing the noise maps in six detail levels and reports about the action plan proposed. As a result this experience emphasises the importance of noise maps as a powerful tool for decision-makers in port planning.

\section{3:20}

2aNSd12. Strategic Noise Mapping of the City of Ljubljana, Slovenia Alan Štimac (DARH2 Acoustics \& Civil Eng. Ltd., Ljubicin prolaz 3, HR10430 Samobor, Croatia, alan@darh2.hr), Aleš Globevnik (A-PROJEKT Natasa Kepe-Globevnik s.p., Vinarje 110b, SI-2000 Maribor, Slovenia, aprojekt@siol.net), Dinko Stipanicev (DARH2 Acoustics \& Civil Eng. Ltd., Ljubicin prolaz 3, HR-10430 Samobor, Croatia, dinko@darh2.hr), Lilijana Kuhelj (Environmental Agency of the Republic of Slovenia, Vojkova 1B, SI-1000 Ljubljana, Slovenia, lilijana.kuhelj@gov .si)

Currently Ljubljana is the only agglomeration according to the definition of the directive 2002/49/EC in Slovenia. For this agglomeration, separate noise maps not only for road and rail traffic but also for industrial sources were made. As for the first phase of preparation there is no precise methodology stated for representing industrial noise sources in strategic noise maps regarding END and following the vision of other EU countries, only industrial plants classified as IPPC installations were taken into consideration of noise mapping. Short term noise measurements around industrial plants conducted according to ISO 1996 during previous three years were used as reverse engineering techniques for the determination of sound power levels of industrial plants. Elaboration of road and rail traffic noise maps required development of perfect acoustical model with 3D terrain modelling including all possible obstacles (barriers, walls, bridges, viaducts, etc.). For calibration of noise maps more then 30 measurements were performed following the standard DIN 45642. During all stages of elaborating noise maps, quality assurance procedures were performed with a view to estimate accuracy of the results. Strategic noise maps of the agglomeration performed at municipal level of Ljubljana will be used mainly for drawing up action plans and spatial planning of areas with different noise levels allowed. 
3:40

2aNSd13. Mixed Industry and Traffic Noise Mapping. Céline Boutin (Acouphen Environnement, Campus de la DOUA, 66, BD Niels Bohr, BP 52132, 69603 Villeurbanne, France, celine.boutin @ acouphen-environnement.com), Alexis Bigot (Acouphen Environnement, Campus de la DOUA, 66, BD Niels Bohr, BP 52132, 69603 Villeurbanne, France, alexis.bigot@acouphen-environnement.com)

The authors have a large experience on Noise Mapping of both transportation sources and industrial plants. This paper provides examples of
Noise Maps for Communities of different sizes with road, railway and industrial sources. The difficulties to estimate Noise Emission from industrial sites are discussed. Recommandations are provided. A Methodology to create the Industry data base for a Noise Map with a large number of industrial sites in the territory is presented. It is shown how Noise Maps can be an attractive tool to be used in the Environmental Management System of the Plant Manager to communicate on the Plant environmental integration and Noise Abatement Plan.

\section{Session 2aNSe}

\section{Noise and EURONOISE: General Topics in Noise I}

\section{Contributed Papers}

\section{1:00}

2aNSe1. Comparison of International Standards for Measuring Sound Power in Tool-Machines. Jose A. Ballesteros (Universidad de Castilla-La Mancha, Campus Universitario, 16071 Cuenca, Spain, Josea.Ballesteros@uclm.es), Marcos D. Fernandez (Universidad de Castilla-La Mancha, Campus Universitario, 16071 Cuenca, Spain, Marcos.Fernandez@uclm.es), Samuel Quintana (Universidad de Castilla-La Mancha, Campus Universitario, 16071 Cuenca, Spain, Samuel.Quintana@uclm.es), Ivan Suarez (Universidad de Castilla-La Mancha, Campus Universitario, 16071 Cuenca, Spain, ivan.suarezcasal@gmail.com), Laura_Rodriguez (Universidad de Castilla-La Mancha, Campus Universitario, 16071 Cuenca, Spain, Laura .Rodriguez@uclm.es)

Industrial noise is one of the most important contaminant agents in the industrialised countries due to its effects on health. Sound power is used in order to characterize the noise emitted by the machines because it does not depend on aspects like place or distance of the measurement. These measurements are handled by international standards like UNE-EN ISO 374x and UNE-EN ISO 9614-x. The advantages and disadvantages of each one of these standards have been evaluated after making a representative number of measurements in several types of tool-machines and then, a set of recommendations have been derived to choose the best standard depending on the machine that is going to be measured.

\section{1:20}

2aNSe2. Activated Resonance Systems as Silencers and Sound Absorbers. Philip Leistner (Fraunhofer IBP, Nobelstrasse 12, 70569 Stuttgart, Germany, philip.leistner@ibp.fraunhofer.de)

A great variety of classical resonance systems is used for sound absorption and attenuation in technical systems. Apart from other requirements such as minimum size, high reliability and low costs the acoustic efficiency can be fulfilled in a wide range. However, the performance is limited and focused on a certain frequency band. These restrictions can significantly be overcome by activation. A very clear system comprises a membrane (mass) and a rear air volume (spring) which can be assembled by using a conventional loudspeaker in front of a cassette. Even the 'passive' system is causing a respectable sound attenuation also at low frequencies. The activation is based on a microphone close to the membrane the signal (according to the exciting sound pressure) of which is linearly amplified and fed back to the loudspeaker. A lot of details have to be considered prior to the real applicability of such an activated resonator. But the remarkable benefits can be documented for numerous practical scenarios. Theoretical and experimental results are based on a sophisticated model of the resonant structure and its acoustic environment. Additionally, several practical challenges, e.g. the presences of turbulent air flow or high temperatures, can be solved by using specific modifications.

\section{1:40}

2aNSe3. Sound radiation of the end of cylindrical duct application on industrial stacks. Thierry Simoneau (Acoustique \& Conseil, 17-19 rue des Grandes Terres, 92508 Rueil-Malmaison, France, ts@ acoustique-conseil .com)

In order to determine the sound field emitted by the top end of an industrial stack, a study of the acoustical radiation of unbaffled semi-infinite cylindrical duct ends was conducted. Firstly, the main publications on the subject were studied. A calculation model of the radiation directivity of the end of a chimney, based on H. Levine and J. Schwinger's results for the fundamental mode and on the Geometrical Theory of Diffraction for the modes of superior order, was established. In order to validate the model, measurements on scaled model were made in an anechoic chamber. Tallying between theoretical and experimental results is very good, especially at low frequencies, where the number of radiating modes is low. Moreover, measurements on a real size industrial site have been made. They also show satisfying tallying between the theory and experimentation.

\section{2:00}

2aNSe4. Austrian investigation on the influence of sound leakage in noise reducing devices. Marco Conter (Arsenal Research, Giefinggasse 2, 1210 Vienna, Austria, marco.conter@arsenal.ac.at), Manfred Haider (Arsenal Research, Giefinggasse 2, 1210 Vienna, Austria, manfred .haider@arsenal.ac.at)

Noise barriers are the most widely used means for road traffic noise abatement. Sound absorption and sound insulation are the key properties for noise barrier elements. The standard method of determining these properties by reverberation room measurements has recently been complemented by an in-situ method following CEN/TS 1793-5, also known as Adrienne method. This method allows flexible assessment of the acoustic performance of noise barriers in almost arbitrary places by means of mobile measurement equipment. Using the possibility to perform in-situ measurements the authors have investigated the presence of sound leakage due to structural imperfections which limit the sound insulation performance of noise barriers. This paper summarizes the results of Austrian research on different kinds of barriers regarding the difference in sound insulation between measurements carried out in front of the supporting post (where leakage is likely to occur) and in the middle of the barrier. A statistical correlation of the data was also performed. 
12:20

2aNSe5. A study of twenty-one cases of low-frequency noise complaints. Christian Sejer Pedersen (Acoustics, Aalborg University, Fredrik Bajers Vej 7 B5, 9220 Aalborg $\varnothing$, Denmark, cp@acoustics .aau.dk), Henrik Møller (Acoustics, Aalborg University, Fredrik Bajers Vej 7 B5, 9220 Aalborg Ø, Denmark, hm@acoustics.aau.dk), Kerstin Persson Waye (Dept. of Environ. Medicine, The Sahlgrenska Acad. of Gothenburg Univ., Box 414, 40530 Gothenburg, Sweden, kerstin.persson-waye@amm .gu.se)

From 203 cases of low-frequency complaints a random selection of twenty-one previously unsolved cases were investigated. The main aim of the investigation was to answer the question whether the annoyance is caused by an external physical sound or by a physically non-existing sound, i.e. low-frequency tinnitus. Noise recordings were made in the homes of the complainants, and the complainants were exposed to these in blind test listening experiments. Furthermore, the low-frequency hearing function of the complainants was investigated, and characteristics of the annoying sound was matched. The results showed that some of the complainants are annoyed by a physical sound $(20-180 \mathrm{~Hz})$, while others suffer from low-frequency tinnitus (perceived frequency 40-100 Hz). Physical sound at frequencies below $20 \mathrm{~Hz}$ (infrasound) is not responsible for the annoyance - or at all audible - in any of the investigated cases, and none of the complainants has extraordinary hearing sensitivity at low frequencies. For comparable cases of low-frequency noise complaints in general, it is anticipated that physical sound is responsible in a substantial part of the cases, while low-frequency tinnitus is responsible in another substantial part of the cases.

\section{2:40-2:00 Lunch Break}

\section{Contributed Papers}

\section{2:00}

2aNSe6. Measuring low-frequency noise indoors. Steffen Pedersen (Acoustics, Aalborg University, Fredrik Bajers Vej 7 B5, 9220 Aalborg Ø, Denmark, stp@acoustics.aau.dk), Henrik Møller (Acoustics, Aalborg University, Fredrik Bajers Vej 7 B5, 9220 Aalborg $\varnothing$, Denmark, hm@acoustics.aau.dk), Kerstin Persson Waye (Dept. of Environ. Medicine, The Sahlgrenska Acad. of Gothenburg Univ., Box 414, 40530 Gothenburg, Sweden, kerstin.persson-waye@amm.gu.se)

At low frequencies, the sound pressure level may vary 20-30 dB in a room due to standing waves. For assessment of annoyance, mainly areas with the highest occurring levels are relevant, since persons present in such areas are not helped by the existence of lower levels in other areas. The level that is exceeded in $10 \%$ of the volume of a room $L_{10}$ is proposed as a rational and objective target for a measurement method. In Sweden and Denmark rules exist for measuring low-frequency noise indoors. The performance of these procedures was investigated in three rooms. The results from the Swedish method were close to the $L_{10}$ target, but, due to a doubtful use of C-weighting in the scanning, it may give too low results in case of complex sounds. The Danish method was found to have a high risk of giving results substantially below the target, unless complainants can precisely appoint measurement positions, where the sound is loudest/most annoying which they often cannot. An alternative method using measurements in four three-dimensional corners of the room is proposed. This easy and straightforward method seems to give reliable results close to the proposed target.

\section{2:20}

2aNSe7. The use of communication device in background noise. Esko Toppila (Finnish Institute of Occupational Health, P.O:Box 486, 33101 Tampere, Finland, esko.toppila@ttl.fi), Pekka Airre (FIOH, P.O.Box 486, 33101 Tampere, Finland, erkko.airo@ttl.fi), Pekka Olkinuora (FIOH, Topeliuksenkatu 41,00250 Helsinki, Finland, pekka.olkinuora@ttl.fi)

Communication devices are used more and more often in industrial premises. We have evaluated how the users experience these device in a call center $(\mathrm{N}=41)$, in a low noise warehouse $(\mathrm{N}=67)$ and in a noisy $(\mathrm{L}>80 \mathrm{~dB})$ warehouse $(\mathrm{N}=25)$. For all subjects the same questionnaire was sent. The questionnaire contained questions were about the quality of communications device and self-evaluated hearing symptoms. The self-evaluated speech intelligibility was lowest in the high background environments. Still in the call center five persons and five in the silent warehouse had great difficulties with speech intelligibility. All these persons evaluated that they have always difficulties when communicating with people. In the noisy environments this relationship was not found. The need to rise the voice was highest in the warehouses ad did not depend on the background noise. Tinnitus was more frequent among process industry workers. The results suggests that lowered hearing function cause problems in low background noise. The selfevaluated nuisance depends on the complexity of the communication. The quality of the communication channel may also play a vital role in the communication problems especially for workers with hearing problems.

\section{2:40}

2aNSe8. Phase spectral processing for improved time-domain soft microphone based noise estimation. Ioannis Paraskevas (T.E.I. Piraeus / Department of Electronics, 250, Thivon str., GR-12244 Athens-Aigaleo, Greece, paraskevas@env.aegean.gr), Maria Rangoussi (T.E.I. Piraeus / Department of Electronics, 250, Thivon str., GR-12244 Athens-Aigaleo, Greece, mariar@teipir.gr), Stylianos M. Potirakis (T.E.I. Piraeus / Department of Electronics, 250, Thivon str., GR-12244 Athens-Aigaleo, Greece, spoti@teipir.gr), Stylianos Savvaidis (T.E.I. Piraeus / Department of Electronics, 250, Thivon str., GR-12244 Athens-Aigaleo, Greece, ssavaid@ teipir .gr)

'Soft Microphones' (SM) constitute a cost-effective, yet quality alternative to the multiple microphones measurement, in applications related to noise mapping. SM offer a solution of great interest to real field applications, e.g., industrial plants. The SM approach, proposed in previous work of the authors, is based on the estimation of the noise signal and the calculation of noise levels over a set of points within the space of interest. To this end, a novel, frequency domain method was introduced and verified in a real field, textile plant experiment, with satisfactory results. However, in order to expand the use of SM from accurate noise mapping to a full Active Noise Control application, it is necessary to obtain accurate noise signal estimates in the time rather than the frequency domain. Further research into the deconvolution step of the proposed method reveals that discontinuities, appearing across the phase spectrum of the estimated signals, cause ambiguities that affect the deconvolution process. We propose here the use of the Hartley transform phase spectrum, which conveys fewer discontinuities as compared to its Fourier Transform counterpart, while it allows for a discontinuities compensation scheme. Experimental results verify that phase spectrum preprocessing provides accurate time domain signal estimates.

\section{3:00}

2aNSe9. Research into the improvement of the management of helicopter noise in the UK. David C. Waddington (Acoustics Research Centre, School of Computing, Science \& Engineering, University of Salford, M5 4WT Salford, UK, d.c.waddington@salford.ac.uk), Paul Kendrick (Acoustics Research Centre, School of Computing, Science \& Engineering, University of Salford, M5 4WT Salford, UK, p.kendrick@salford.ac.uk), Geoff Kerry (Acoustics Research Centre, School of Computing, Science \& Engineering, University of Salford, M5 4WT Salford, UK, g.kerry@salford.ac.uk), Matthew Muirhead (QinetiQ Ltd, Cody Technology Park, Ively Road, GU14 OLX Farnborough, UK, mmuirhead@qinetiq.com), Ray Browne (QinetiQ Ltd, Cody Technology Park, Ively Road, GU14 0LX Farnborough, UK, rwbrowne@qinetiq.com)

Helicopter noise has a negative impact on the quality of life for many people. Effected populations are not just those living close to heliports, but include those exposed to noise from helicopters used by emergency services, the military, and commercial companies. One problem identified in the UK is that it is often difficult to complain about helicopter noise, since it is un- 
clear which organisation is responsible for dealing with the complaint. Consequently, the Department for Environment, Food and Rural Affairs (Defra, UK) has commissioned research to summarise the following: i. the nature and extent of the concern about helicopter noise in the UK. ii. rules and regulations governing operations. iii. existing procedures for handling complaints. This stage of the project will produce a detailed report into the improvement of the management of helicopter noise. Also to be produced is a short non-technical guide including the means of redress for perceived disturbance. This paper will report on the findings of this project. [Work funded by Defra, UK]

TUESDAY MORNING, 1 JULY 2008

AMPHI HAVANE, 8:00 A.M. TO 12:40 P.M.

\title{
Session 2aPAa
}

\section{Physical Acoustics: Acoustics of Porous Media II}

\author{
Walter Lauriks, Cochair \\ Lab. ATF, Katholieke Universiteit Leuven, Celestijnenlaan 200D, Leuven, B-3001, Belgium
}

\author{
Keith Attenborough, Cochair \\ Open University, Department of Design, Development, Materials and Environment, Walton Hall, Milton Keynes, MK7 6AA, UK
}

\section{Contributed Papers}

\begin{abstract}
8:00
2aPAa1. Sensitivity analysis and non linear parameters estimation of porous materials from normal sound coefficient absorption measurements. Mohammed Garoum (Ecole Supérieure de Technologie de Salé, Laboratoire Energétique Matériaux et Environnement, Avenue du Prince Héritier, BP 227, 10000 Salé, Morocco, garoum1@yahoo.fr), Rachida Idchabani (Ecole Supérieure de Technologie de Salé, Avenue du Prince Héritier, BP 227, Salé, Morocco, idchabani@mcinet.gov.ma), Mohammed Tajayouti (Ecole Supérieure de Technologie de Salé, Avenue du Prince Héritier, BP 227, Salé, Morocco, mtajayouti@gmail.com), Mohammed Rhachi (Ecole Supérieure de Technologie de Salé, Laboratoire Energétique Matériaux et Environnement, Avenue du Prince Héritier, BP 227, 10000 Salé, Morocco, mrhachi@hotmail.com), Antonio Moreno (Instituto de Acústica, CSIC, 144 Callé Serrano, 28006 Madrid, Spain, amoreno@ia.cetef.csic.es)
\end{abstract}

In literature, various models have been proposed in order to predict characteristic impedance $\mathrm{Zc}$ and wave number kc of porous materials. To use these models their involved nonacoustical parameters (i. e. flow resistivity, tortuosity porosityœ) are usually measured. Unfortunately measurements require specialised equipments and are often difficult on loose porous materials leading most often to erroneous values. Moreover, the inverse estimation of theses parameters from experimental data is not an easy task as believed. This is due to the non linearity of models and the presence of some parameters with weak and/or linearly dependent influences. In this work an approach based on the tradeoff between magnitude and linear independence of the sensitivity matrix components is presented in order to achieve an efficient ranking of parameters according to their inherent ease of estimation. Next this approach is associated to Genetic Algorithms to minimize the least squares norm between measured and modelled normal sound absorption coefficient. For five widely used models in literatures, the application of the proposed inverse estimation procedure on loose granular cork and vegetable fibres, shows that, unlike classical gradient approaches methods, better estimation has been obtained.

$$
\text { 8:20 }
$$

2aPAa2. Characterization of an acoustic ceramic liner in a subsonic flow by mean of Laser Doppler Velocimetry. Romain Pethieu (ONERA, 2, av. Edouard Belin - BP 4025 - Cedex 4, 31055 Toulouse, France, romain .pethieu@onecert.fr)

As aircraft traffic constantly increases, serious efforts are made to reduce engine noise. Among them, the design of high performance absorbing materials (liners) placed on the nacelle's internal walls is an exciting challenge. Optimizing these media requires to know the mechanisms of acoustical propagation near them in the conditions encountered in jet engines: high speed hot shear flows. Particular interest is being granted to the influence of the grazing flow in contact with the liners. This is due to its known effects on the liners characteristics: efficiency of absorption and frequency resonance. This influence is remarkable for those configurations based on perforated plates. Therefore, a testing method based on non-intrusive measurements has been developed at ONERA. This technique consists in measuring acoustic velocities by laser Doppler velocimetry to determine acoustic quantities under grazing turbulent high-speed flow, thanks to an EulerianLagrangian description of the perturbations. Experimental activity is performed on a honeycomb material, via the achievement of acoustical pressure and intensity fields, for different Mach numbers. Moreover, these measurements bring into evidence a coupling between acoustical modes and hydrodynamic modes (from Kelvin-Helmholtz instability) which modify the material acoustical behaviour.

\section{8:40}

2aPAa3. The effect of mechanical elasticity on the surface impedance of a organic/inorganic composite aerogel. Winny Dong (California State Polytechnic University, 3801 West Temple Avenue, Chemical and Materials Engineering, Pomona, CA 91768, USA, winnydong@csupomona.edu), Wayland Dong (Veneklasen Associates, 1711 Sixteenth Street, Santa Monica, CA 90404, USA, wdong@veneklasen.com), Tanya Faltens (California State Polytechnic University, 3801 West Temple Avenue, Chemical and Materials Engineering, Pomona, CA 91768, USA, tafaltens@ csupomona.edu), Elizabeth Scott (California State Polytechnic University, 3801 West Temple Avenue, Chemical and Materials Engineering, Pomona, CA 91768, USA, escott@csupomona.edu), Travis Thompson (California State Polytechnic University, 3801 West Temple Avenue, Chemical and Materials Engineering, Pomona, CA 91768, USA, trthompson@csupomona.edu)

Previous reports on the acoustical properties of monolithic silica aerogels have indicated that although the material's high porosity, pore tortuosity, and surface area contribute to a low sound velocity in the material, the rigid matrix of the aerogel exhibit a high reflection coefficient. Work by Forest et al. and others have shown that using granules instead of silica aerogel monoliths, the acoustical properties can be significantly enhanced. We report the acoustic properties of a polydimethylsilicate (PDMS)/silica composite aerogel that has both high surface area and a mechanically elastic matrix. Both monoliths and granules are studied. The surface impedance (at normal incidence) as a function of open porosity, pore size distribution, surface area, elasticity, and granule size will be reported. 
9:00

2aPAa4. Numerical modeling of transient poroelastic waves in the low frequency range. Guillaume Chiavassa (Ecole Centrale Marseille MSNM-GP, Technopole de Chateau-Gombert, 13013 Marseille, France, guillaume.chiavassa@ec-marseille.fr), Bruno Lombard (Laboratoire de Mécanique et d'Acoustique LMA, 31 chemin Joseph Aiguier, 13402 Marseille, France, lombard@1ma.cnrs-mrs.fr), Joel Piraux (Laboratoire de Mécanique et d'Acoustique LMA, 31 chemin Joseph Aiguier, 13402 Marseille, France, piraux@1ma.cnrs-mrs.fr)

A numerical method is proposed to simulate the propagation of transient poroelastic waves across heterogeneous media, in the low frequency range. A velocity-stress formulation of Biot's equations is followed, leading to a first-order differential system. The latter is splitted in two parts: a propagative one discretized by a fourth-order ADER scheme, and a diffusive one solved analytically. Near sources and interfaces, a space-time mesh refinement is implemented to capture the small scales of evolution of the diffusive slow compressional wave. Lastly, an immersed interface method is implemented to accurately model the jump conditions at interfaces between the different media. Numerical experiments in one and two dimensions are shown, with porous/porous or fluid/porous interfaces. Comparisons with analytical solutions confirm the efficiency of the approach. [1] G. Chiavassa, B. Lombard, J. Piraux, Numerical modeling of 1-D transient poroelastic waves in the low-frequency range, soumis au J. Comput. Appl. Math., (2007), disponible sur http://hal.archives-ouvertes.fr/hal-00193103/fr/

\section{9:20}

2aPAa5. On the scattering of a plane wave by porous sound-absorbing strip. Alex De Bruijn (Independent Acoustical Consultant, Schonenvaardersstraat 18014, 7418 CC Deventer, Netherlands, alex.bruijn@ planet.nl)

The analysis concerning the scattering of a plane wave by a porous sound-absorbing strip embedded in an infinite sound-hard surface is most relevant for the evaluation of measurements with respect to absorbing materials tested in a reverberation room. This diffraction phenomenon around the edges leads to an additional sound absorption, the so-called edge effect. This extra absorption can be analyzed and computed - among other wellknown methods - by using a spatial Fourier field expansion just on the absorbing strip. The field in the space above the reflecting surface can be put into a contour integral of an angular spectrum in the complex plane or -in other words- a superposition of plane waves with complex angles of incidence. Matching both field representations leads to the solution of the unknown coefficients of the Fourier expansion and hence to the amplitudes of the total diffracted field. It would be interesting to compare these results with diffraction data obtained via an alternative technique: the FiniteDifference Time-Domain method using the direct Euler equations, also within the porous sound-absorbing material. The paper will show the data for the field amplitudes obtained by both methods and will discuss the agreement and differences.

\section{9:40}

2aPAa6. Analysis of porous plate/water layered structures by means of the transition terms method. Ferroudja Belhocine (LOMC FRE CNRS 3102, Université du Havre, Place Robert Schuman, 76610 le Havre, France, ferroudja.belhocine@univ-lehavre.fr), Serge Derible (LOMC FRE CNRS 3102, Université du Havre, Place Robert Schuman, 76610 le Havre, France, serge.derible@univ-lehavre.fr), Cole Franklin (1241 East dyer road, Santa Ana, CA 92672, USA, colesfranklin@yahoo.com)

This paper is devoted to the study of water-saturated porous plate/water layered structures by means of the transition terms defined from the reflection and transmission coefficients $R$ and $T$ as follows: $\mathrm{TT}_{\text {sym }}=(1-(\mathrm{R}+\mathrm{T})) /(2 \mathrm{i}), \mathrm{TT}_{\mathrm{asym}}=(1-(\mathrm{R}-\mathrm{T})) /(2 \mathrm{i})$. They are the eigenvalues of the scattering matrix of the water-immersed structure and are directly connected with its symmetric or antisymmetric vibrations. The $\mathrm{N}$ porous plates associated in our structures obey Biot's theory which is first used to calculate the reflection and transmission coefficients of a unique water-saturated plate. An induction on $\mathrm{N}$ process allows to find the reflection and transmission coefficients of a given $\mathrm{N}$ plate/water-layer structure. The plates used in the experiments at normal incidence are $5 \mathrm{~mm}$ thick. The reflection and transmission coefficients of sets of $1,2,3$, and 4 water immersed plates, separated from each other by a $1 \mathrm{~cm}$ water gap, are measured thanks to two wideband transducers with central frequency $0.5 \mathrm{MHz}$. There are good agreements between the calculated and experimental transition terms which exhibit the symmetric and antisymmetric resonances of the structures. They obey the Breit-Wigner resonant form which characteristics can be obtained

\section{0:00}

2aPAa7. On the adsorption-desorption relaxation time of carbon in very narrow ducts. Timothy J. Mellow (Nokia, Nokia House, Summit Avenue, GU14 0NG Farnborough, UK, tim.mellow@nokia.com), Olga Umnova (University of Salford, Acoustics Research Centre, Newton Building, M5 4WT Salford, UK, o.umnova@salford.ac.uk), Konstantinos Drossos (University of Southampton: Institute of Sound and Vibration Research, University Road, Highfield, SO17 1BJ Southampton, UK, kd806@soton.ac.uk), Keith Holland (University of Southampton: Institute of Sound and Vibration Research, University Road, Highfield, SO17 1BJ Southampton, UK, krh@isvr.soton.ac.uk), Andrew Flewitt (University of Cambridge: Centre for Advanced Photonics and Electronics, 9, JJ Thomson Ave., CB3 0FA Cambridge, UK, ajf@eng.cam.ac.uk), Leo Kärkkäinen (Nokia Research Center, Itämerenkatu 11 - 13, 00180 Helsinki, Finland, leo.m.karkkainen@nokia.com)

Loudspeakers generally have boxes to prevent rear wave cancellation at low frequencies. However, the stiffness of the air in a small box reduces the diaphragm's excursion at low frequencies. Hence the size of the box is generally a compromise between low frequency performance and practicality. Activated carbon has been found to increase the apparent size of a given box through adsorption of the air molecules when the pressure increases and likewise desorption when it decreases. However, the exact viscous effects in the granular structure are difficult to model. Thus it is impossible determine the high frequency limit due to the natural adsorption/desorption relaxation time in the absence of viscous losses. In this study, a tube model is presented which takes into viscous and thermal losses with boundary slip together with adsorption. Impedance measurements are performed on an array of 12 million holes, each 2 micrometers in diameter, etched in a $0.5 \mathrm{~mm}$ thick silicon wafer so that the viscous and thermal losses can be verified against the model without adsorption. Impedance measurements are then performed on an array of holes coated with graphite in order to create an activated carbonlike structure, thus enabling the adsorption/desorption relaxation time to be evaluated.

\section{$10: 20$}

2aPAa8. Acoustic pulse attenuation and transmission in rigid porous media: Experimental investigation and numerical simulations. Diego Turo (University of Salford, Acoustics Research Centre, Newton Building, M5 4WT Salford, UK, d.turo@pgr.salford.ac.uk), Olga Umnova (University of Salford, Acoustics Research Centre, Newton Building, M5 4WT Salford, UK, o.umnova@salford.ac.uk)

This paper presents an investigation on acoustic pulse attenuation and transmission in rigid porous media. Transmission measurements of acoustic pulses through porous material with a rigid frame were performed using an appropriate standing wave tube. A vertical tube was built to make measurements on granular materials. The influence of pulse duration, layer thickness and material microstructure on the transmission has been investigated. $\mathrm{Nu}$ merical time domain simulations based on two different semi-empirical models with one and two viscous relaxation times, respectively, were performed to compare with existing data. First, material microstructure and pulse durations were adapted to investigate the influence of viscous and inertial effects on pulse propagation separately. The simultaneous contribution of both viscous and thermal effects was then investigated. In the finite difference time domain (FDTD) model, different approaches are used to correctly simulate the pulse transmission through a porous layer. Accuracy and computational time required for the numerical methodologies have been compared and their advantages and drawbacks applied to this particular case shown. 


\section{1:00}

2aPAa9. On the influence of the micro-geometry on sound propagation through periodic array of cylinders. Rodolfo Venegas (University of Salford, Acoustics Research Centre, Newton Building, M5 4WT Salford, UK, R.G.VenegasCastillo@pgr.salford.ac.uk), Olga Umnova (University of Salford, Acoustics Research Centre, Newton Building, M5 4WT Salford, UK, o.umnova@salford.ac.uk)

Sound propagation in rigid porous media has been widely studied by using macroscopical models. These models make use of averaged quantities in which the microscopic details of the porous media geometry are represented by macroscopical parameters and, in a certain way, the influence of the microscopic geometry is not directly identified. In this paper, homogenization theory and finite element method are used for solving the full microscopic dynamic flow and dynamic heat problems for a porous medium modelled as an idealized geometry consisting of a periodic array of cylinders. Different cross-section shapes of the cylinders (circular, ellipsoidal and square crosssection shapes) and a wide range of porosity values are considered. The influence of the microscopic features of the porous media on dynamic permeability and dynamic compressibility is also studied.

\section{1:20}

2aPAa10. Acoustic emission before avalanches in granular media. Vincent Gibiat (Université Paul Sabatier, PHASE, 118, route de Narbonne, 31062 Toulouse cedex 9, France, gibiat@cict.fr), Eric Plazza (Université Paul Sabatier, PHASE, 118, route de Narbonne, 31062 Toulouse cedex 9, France, ericvpp@hotmail.com), Pierre De Guibert (Université Paul Sabatier, PHASE, 118, route de Narbonne, 31062 Toulouse cedex 9, France dguibert@cict.fr)

Avalanches of granular media are mainly characterized by the observation and the measurement of the main angles of avalanche corresponding first to the movement of isolated beads and to the whole movement of a great part of the grains. These characterisations do not give any information about the rearrangements of the grains inside the layer of granular beads. As any movement of a grain produces a deformation of the structure it is quite normal to expect for a sound that will propagate inside the granular medium. We present an experimental study of the precursors of avalanches on spherical granular glass beads and silica aerogels in powder (size of grains less than 80 micrometers). Acoustic emission has been recorded with two piezoelectric transducers placed on the lower part of the material layer. Our results show clearly that before any movement on the upper part of the beads layer, so for an angle less than the first angle of avalanche, movements inside the material produce pulsed sounds that can be recorded. Theses vibrating events are occurring more and more when the angle is increasing until the first angle of avalanche where acoustic emission becomes intense.

\section{1:40}

2aPAa11. On heterogeneous blankets: Analytical solution for the interaction between masses and poro-elastic layers. Kamal Idrisi (Virginia Tech, Mechanical Engineering, 143 Durham 0238, Blacksburg, VA 24061, USA, idrisi@vt.edu), Andreas Wagner (Virginia Tech, Mechanical Engineering, 143 Durham 0238, Blacksburg, VA 24061, USA, andreasw@vt.edu), Marty Johnson (Virginia Tech, Mechanical Engineering, 143 Durham 0238, Blacksburg, VA 24061, USA, martyj@vt.edu), David Bartylla (Virginia Tech, Mechanical Engineering, 143 Durham 0238, Blacksburg, VA 24061, USA, bartylla@vt.edu)

There has been substantial research over the last five decades on control of aircraft cabin noise. One new passive approach is the heterogeneous (HG) blanket where a traditional acoustic blanket treatment is altered by adding mass inhomogeneities into the poro-elastic/viscoelastic layers. These masses act like distributed vibration absorbers and can be used to reduce vibration and sound transmission by targeting modes of the fuselage. The natural frequency of a mass inhomogeneity is determined by the mass itself and by the effective stiffness of the porous layer due to the mass/poro interaction. An experimental 1st order approach to predict the effective stiffness based on the shape of the mass inhomogeneities is reviewed. An analytical model for poro-elastic media proposed by Allard et al. was simplified for low frequencies and is used to validate and extend the 1st order approach. It is shown that the effective stiffness depends on mass shape, the foam thickness and material constants such as the modulus of elasticity and Poisson's ratio. Furthermore the model can be used to calculate the stress and displacements fields in the blanket in order to give further insight into the behaviour of the HG blankets.

12:00

2aPAa12. Influence of boundary slip on the acoustical properties of microfibrous absorbents. Olga Umnova (University of Salford, Acoustics Research Centre, Newton Building, M5 4WT Salford, UK, o.umnova@salford.ac.uk), David Tsiklauri (University of Salford, Acoustics Research Centre, Newton Building, M5 4WT Salford, UK, d.tsiklauri@salford.ac.uk), Rodolfo Venegas (University of Salford, Acoustics Research Centre, Newton Building, M5 4WT Salford, UK, R.G .VenegasCastillo@pgr.salford.ac.uk)

In the past decades a variety of new highly porous materials with unusually small pores have been manufactured. In aerogels, for instance, pores can be less than $20 \mathrm{~nm}$ in diameter. The conventional models have to be modified when applied to describe acoustical properties of those materials. The non-slip condition on a pore surface is no longer valid and needs to be replaced by the Knudsen boundary condition. In attempt to provide an insight into the behaviour of microfibrous materials, an analytical model has been developed, which accounts for the boundary slip in a medium consisting of rigid parallel fibres assuming different directions of sound propagation with respect to fibres. It has been shown that the presence of the boundary slip leads to a significant change in model predictions. For instance, in a material with fibre radius $80 \mathrm{~nm}$ and $95 \%$ porosity the sound speed decreases and attenuation increases by more than $20 \%$ compared to the values obtained assuming no boundary slip. The effect is stronger for smaller size fibres, lower porosity values and for sound propagating parallel to fibres. Numerical computations have been performed to simulate oscillatory flow around the cylindrical fibres assuming Knudsen boundary conditions and the results have been compared with the analytical model predictions

\section{2:20}

2aPAa13. Time-Resolved Spectroscopy of Water confined in Vycor. Riccardo Cucini (European Lab. for Non-Linear Spectroscopy (LENS), Univ. di Firenze, via Nello Carrara 1, 50019 Sesto Fiorentino (Fi), Italy, cucini@lens.unifi.it), Andrea Taschin (European Lab. for Non-Linear Spectroscopy (LENS), Univ. di Firenze, via Nello Carrara 1, 50019 Sesto Fiorentino (Fi), Italy, taschin@lens.unifi.it), Paolo Bartolini (European Lab. for Non-Linear Spectroscopy (LENS), Univ. di Firenze, via Nello Carrara 1, 50019 Sesto Fiorentino (Fi), Italy, bart@lens.unifi.it), Renato Torre (European Lab. for Non-Linear Spectroscopy (LENS), Univ. di Firenze, via Nello Carrara 1, 50019 Sesto Fiorentino (Fi), Italy, torre@lens .unifi.it)

We present measurements on water confined in Vycor 7930 by heterodyne-detected transient grating experiments (HD-TG) [1]. We studied the acoustic wave propagation, the thermal diffusion and the liquid viscous flow through the pores as a function of temperature $\left(-15-90{ }^{\circ} \mathrm{C}\right)$ and of the exchanged q-vector $\left(0.63-2.5 \mu \mathrm{m}^{-1}\right)$. The data show interesting aspects due partly to the confinement effects and partly to the peculiarities of bulk water. Acoustic results are compared with the predictions of the Biot theory finding a good agreement only for the sound velocity data, while the predictions for the acoustic wave attenuation is poor as just reported in [2]. Our technique is able to induce a thermal grating inside the sample. The analysis of the data requires an extension of the Biot theory, that takes into account the temperature variations. Surprisingly, our data show that the acoustic oscillation are not affected by the temperature. The induced thermal grating is also used to analyze the viscous flow of water inside the pores and the thermal diffusion. [1] Time-resolved spectroscopy of complex liquids, edited by Torre R. (Springer, New York) 2008 [2] A. Taschin, R. Cucini, P. Bartolini, R. Torre, Europhys. Lett., in press. 


\section{Session 2aPAb}

\section{Physical Acoustics: Diffraction of Waves on Periodical Structures: Acoustic, Ultrasonic, and Acousto-Optical Diffraction Phenomena I}

Nico F. Declercq, Cochair

Georgia Tech Lorraine - G.W. Woodruff School of ME, UMI Georgia Tech - CNRS 2958, 2 rue Marconi, Metz, 57070, France

Nataliya Polikarpova, Cochair

M.V. Lomonosov Moscow State University, Department of Physics, Moscow, 119991, Russian Federation

\section{Invited Papers}

\section{8:00}

2aPAb1. Spectral instruments based on acousto-optical tunable filters: advantages and prospects. Vladislav Pustovoit (Scientific Technological Center of Unique Instrumentation of RAS, ul.Butlerova, 15, 117342 Moscow, Russian Federation, np@ckbup.dol.ru), Vitold Pozhar (Scientific Technological Center of Unique Instrumentation of RAS, ul.Butlerova, 15, 117342 Moscow, Russian Federation, aoslab@ckbup.dol.ru)

A review of modern spectral techniques and instruments using acousto-optical tunable filters (AOTF) is presented. New approaches for spectrometers and spectral systems development are considered. The following instruments are described: a series of compact spectrometers and spectrophotometers of ultra-violet, visible, and infra-red ranges for Raman, fluorescence, and absorption spectroscopy for various applications, including out-of-door measurements; spectroradiometers for environment monitoring (open air, Earth surface, subsurface and depth seawater), specialised spectral equipment for industrial monitoring (microelectronics, alcoholometry), imaging spectral devices, time-resolve fluorescent spectrometers, and instruments for differential spectroscopy. Also some fundamental problems of AOTF-based spectroscopy are considered: spectrogram correction of instrument function distortions, ultimate spectral resolution of different methods, modulation techniques for instrument function synthesizing, optimization of measurement procedures, including adaptive algorithms. Basic trends of the past 30 years of AOTF-based spectroscopy development are formulated. The most promising ideas are discussed.

\section{8:20}

2aPAb2. Advances in acousto-optic devices based on frequency intermodulations suppression. Valeriy V. Proklov (IRE RAS, 11 Mokhovaya str., build.7, 125009 Moscow, Russian Federation, proklov@mail.cplire.ru), Sergey Antonov (IRE RAS, 11 Mokhovaya str., build.7, 125009 Moscow, Russian Federation, olga-ant@yandex.ru), Yury Rezvov (IRE RAS, 11 Mokhovaya str., build.7, 125009 Moscow, Russian Federation, rezvov@newmsk.tula.net), Alexander Vainer (IRE RAS, 11 Mokhovaya str., build.7, 125009 Moscow, Russian Federation, sawashi@yandex.ru)

The limitative influence of the frequency intermodulation effect on the most important parameters in a big family of acousto-optic (AO) devices is well known. The proposed presentation deals with new principles and related technical tools to perform significant suppression of the inherent intermodulation effects within broadband AO devices. The different AO interaction mechanisms in solids offering the intermodulations decrease have been considered. On this basis there were theoretically established and experimentally verified facilities to design a few types of advanced AO devices for a wideband signal processing with the extended spurious free dynamic range, as well as for a flexible laser beam forming with extremely high efficiency and etc.

\section{Contributed Paper}

\section{$8: 40$}

2aPAb3. Periodically non-homogeneous acoustic beams and their application in acousto-optics. Vladimir I. Balakshy (Dept. of Physics, M.V.Lomonosov Moscow State Univ., Vorobyevy Gory, Bldg. 1, 119991 Moscow, Russian Federation, balakshy@phys.msu.ru), Bogumil B. Linde (Inst. of Exper. Physics, Univ. of Gdańsk, Ul. Wita Stwosza, Bldg. 57, 80-952 Gdańsk, Poland, fizbl@univ.gda.pl)

Light diffraction by ultrasonic waves is of great interest in the view of both fundamental physics and diverse applications. Acousto-optic devices are used for modulation of optical wave parameters, for optical information processing in real time, etc. In the given research, basic attention has been focused on the analysis of characteristics of anisotropic acousto-optic cells in which acoustic waves are excited by means of a phased array of transducers. Current technology makes it possible to create easily such transducers with antiphase excitation of adjacent elements of the array. The directional diagram of this composite transducer consists of several lobes whose spatial orientation varies with frequency. This peculiarity results in changing the Bragg condition. In the case of the anisotropic acousto-optic diffraction, every branch of the Bragg angle frequency dependence is split into several curves. This opens up new possibilities for optimization of acousto-optic devices. In this work, we have analyzed amplitude, frequency and angular characteristics of the phased transducer cells depending on crystal cut and transducer parameters. In particular, it has been shown that, in spite of a noticeable phase mismatch between interacting waves, the diffraction efficiency can approach $100 \%$. 


\section{Invited Paper}

\section{9:00}

2aPAb4. Differential properties of acousto-optical tunable filters in phase-modulation mode. Vitold Pozhar (Scientific Technological Center of Unique Instrumentation of RAS, ul.Butlerova, 15, 117342 Moscow, Russian Federation, aoslab@ckbup.dol.ru), Vladislav Pustovoit (Scientific Technological Center of Unique Instrumentation of RAS, ul.Butlerova, 15, 117342 Moscow, Russian Federation, np@ckbup.dol.ru), Sergey Beryoza (Scientific Technological Center of Unique Instrumentation of RAS, ul.Butlerova, 15, 117342 Moscow, Russian Federation, bruce_lee_22@mail.ru)

Tailoring the transmission function of acousto-optical tunable filters (AOTF) via acoustic waves control is one of the most challenging tasks, which is promising for optical spectroscopy applications. In our early work it was theoretically predicted and experimentally demonstrated that collinear AOTF with fast periodic phase manipulation exhibits differential properties, in particular capability of detection spectrum derivatives. In the report, the form of the instrument function of such AOTF was measured with use of Ne-lamp linear spectrum. Comparison to theoretically calculated function shows rather good agreement. Also the form of window of equivalent differential filter was determined and proved to be quite similar to AOTF classical window. Advantages of using this differential detection technique are considered. Its applicability to fluorescence spectroscopy is discussed.

\section{Contributed Papers}

9:20

2aPAb5. Quasicollinear acoustooptic tunable filters based on KDP single crystal. Vladimir Molchanov (Technological State Univ., Moscow Steel and Alloys Institute, Leninsky prospect, 4, 119049 Moscow, Russian Federation, v_molchanov@smtp.ru), Sergei Chizhikov (Technological State Univ., Moscow Steel and Alloys Institute, Leninsky prospect, 4, 119049 Moscow, Russian Federation, aocenter@mail.ru), Oleg Makarov (Molecular Technology GmbH, Rudower Chaussee 29-31, 12489 Berlin, Germany, moltech@mt-berlin.com)

The paper is devoted to the theoretical and experimental investigation of acoustooptical tunable filters, based on quasicollinear geometry of lightsound interaction in KDP single crystal. This geometry uses the effect of acoustic anisotropy in KDP, as well as peculiarities of acoustic wave reflections from the free boundary of KDP crystal. The mathematical approach for determination of optical and consructional characteristics of the filters is elaborated. Different types of quasicollinear filters are considered. The filters are intended for UV-VIS regions of optical radiation. The experimental acoustooptical filter was designed and investigated. Typical tunable spectral band is 220-500 nm, the spectral resolution doesn't exceed $0.1 \mathrm{~nm}$ in UV region of spectra. The experimental data are in a good agreement with the predicted one. This research is supported by RFBR grants \# 07-02-12238 and \# 07-02-01195.

\section{9:40-10:00 Break}

\section{0:00}

2aPAb6. Surface profile investigations by means of acousto-optic technique. Boris S. Gurevich (Scientific Instruments, Rizhsky prospekt 26, $190103 \quad$ St. Petersburg, Russian Federation, bgurevich@mail.ru), Valentine V. Shapovalov (Intelligent Software Systems, Nevsky pr. 190, 193317 St. Petersburg, Russian Federation, Shapovalov@incomsys.ru), Sergey V. Andreyev (Scientific Instruments, Rizhsky prospekt 26, 190103 St. Petersburg, Russian Federation, svan51@mail.ru), Andrey V. Belyaev (Institute for analytical instrumentation RAS, Rizhsky pr. 26, 190103 St. Petersburg, Russian Federation, bgurevich@mail.ru), Ilya A. Kolesov (Intelligent Software Systems, Nevsky pr. 190, 193317 St. Petersburg, Russian Federation, ilkak@mail.ru)

Acousto-optic tunable filters (AOTF) represent a very strong tool in different kinds of investigations where high rate light wavelength variations are required. The rough surfaces profile investigation is among the problems which can be solved partially by this way. Our investigations have been connected with use of AOTF and lens components with strong chromatic aberration. One of the problems was to choose the $\mathrm{z}$-distance resolving power criterion. The proposed criterion is defined by the admissible probability to miss information unit regarding the object characterization in z-distance. In our experiments we used the specially elaborated AOTF based on tellurium dioxide crystal with transmission bandwidth of several $\mathrm{nm}$, depending on the central wavelength position. Also we have used the lenses fabricated with tellurium dioxide because this material provides high chromatic aberrations. The results of experiments are discussed from the point of view of the AOTF possibilities to increase the $\mathrm{z}$-distance resolving power in comparison with existing systems. The $90 \%$-probability distinguishing of defocusing while electric frequency variation of $200 \mathrm{kHz}$, can be considered as serious advantage. The device improvement can be attained by means of the noise level decreasing to the level taking place for electric frequency of $94 \mathrm{MHz}$.

10:20

2aPAb7. Transformation of Acoustic Modes in Case of Arbitrary Reflection in Acousto-Optic Crystals. Nataliya Polikarpova (M.V. Lomonosov Moscow State University, Department of Physics, Leninskie gory 1 bldg. 2, Division of Oscillations, 119991 Moscow, Russian Federation, polikarp@phys.msu.ru), Vitaly Voloshinov (M.V. Lomonosov Moscow State University, Faculty of Physics, Vorob'evy Gory, MSU, 119991 Moscow, Russian Federation, volosh@phys.msu.ru), Nico F. Declercq (Georgia Tech Lorraine - G.W. Woodruff School of ME, UMI Georgia Tech - CNRS 2958, 2 rue Marconi, 57070 Metz, France, nico .declercq@me.gatech.edu)

The phenomenon of unusual reflection of plane elastic waves is examined in the crystalline compounds of tellurium and mercury. It is predicted and registered in experiments that energy flow of one of the reflected waves may propagate practically in a back direction with respect to an incident energy flow. This phenomenon is observed in the materials with a strong anisotropy of elastic properties. In the case of inclined incidence, the reflection may take place strictly in the back direction with respect to the intrinsic energy flow. Moreover, all incident elastic energy may be reflected in a form of a back elastic wave. The analysis was carried out for all exisiting cases of inclined incidence in the crystals. It was proved that relative intensity of the unusually reflected wave might be close to a unit in a wide variety of incidence and cut angles in the crystals. A comparative analysis of the effect in new anisotropic materials and in the well-known crystals has been carried out. Since the crystals under investigation possess relatively high magnitudes of acousto-optic figure of merit, e.g. paratellurite and calomel, principal results of the research are of significance in development of new generations of acousto-optic devices.

\section{0:40}

2aPAb8. Optimization of KDP-based acousto-optic imaging filters. Konstantin B. Yushkov (Moscow State University, Phisical Faculty, Leninskie Gory, 119991 Moscow, Russian Federation, protonoa@mail.ru)

Acousto-optic devices can perform spatial and spectral tunable filtration of light with the use of anisotropic Bragg diffraction. In the near UV range 
of spectrum, KDP single crystals provide the highest acousto-optic figure of merit among uniaxial crystals. The report is devoted to the problem of optimization of the filter parameters in order to maximize angle aperture of the instruments. It was found that the maximum angle aperture of wide angle tunable acousto-optic filters is equal to 3 degrees in air at output of the KDP crystals. This magnitude of the angle aperture is limited by a narrow deflection angle of light in KDP that is due to a relatively low birefringence of the material. The investigation showed that the diffraction efficiency of light depends on the dimensions of the acousto-optic cell, i.e. linear aperture and length of a piezoelectric transducer. A discussion on trade-off between transmission coefficient and the spatial resolution in the filter is presented in the report.

\section{1:00}

2aPAb9. Acousto-optic collinear diffraction of arbitrary polarized light. Sergey Mantsevich (Dept. of Physics, M.V.Lomonosov Moscow State Univ., Vorobyevy Gory, Bldg. 1, 119991 Moscow, Russian Federation, manboxx@mail.ru), Vladimir I. Balakshy (Dept. of Physics, M.V
.Lomonosov Moscow State Univ., Vorobyevy Gory, Bldg. 1, 119991 Moscow, Russian Federation, balakshy@phys.msu.ru)

Acousto-optic collinear diffraction of light is a type of light scattering interesting as a physical phenomenon and important from practical point of view. This type of diffraction is used in tunable acousto-optic filters. In this case, the incident light has to be linearly polarized along one of the acoustooptic crystal anisotropy axes. If the incident light is not primordially polarized, a polarizer should be disposed at the input of the acousto-optic cell. Unfortunately, this leads to loss of half the light power. The present work is devoted to theoretical and experimental investigation of collinear acoustooptic interaction peculiarities that arise when the incident optical beam is not polarized. It is shown that the output diffraction spectrum in the general case contains four components which have different polarization and frequency. Beating of these components leads to modulation of light intensity passed through the output analyzer. In this work, dependences of modulation components amplitudes on frequency and power of the acoustic wave are examined for different polarizer and analyzer orientations.

\section{Invited Paper}

\section{$11: 20$}

2aPAb10. Ultrasonic diffraction grating spectroscopy: particle size measurements and investigation of the inertial model for attenuation. Margaret Greenwood (Pacific Northwest National Laboratory, P. O. Box 999, Mailstop K5-26, Richland, Wa, WA 99352, USA, margaret.greenwood@pnl.gov)

The experimental setup consists of a unit with a grating machined on the surface of the unit. Send and receive transducers are placed at equal angles to the grating surface that is in contact with a slurry. The transmitted beam of spectral order $\mathrm{m}=1 \mathrm{reaches}$ an angle of $90^{\circ}$ at the so-called critical frequency, where it becomes an evanescent wave that interacts with particles in the slurry. As a result of this interaction, the signal in the receive transducer exhibits a dependence upon particle size of the slurry. Data will be presented for slurries of polystyrene spheres, ranging in size from $45 \mu \mathrm{m}$ to $467 \mu \mathrm{m}$, as a function of the volume fraction. The velocity of sound is also determined from the critical frequency. The distributions will be compared with the viscous and inertial models. The results show that larger particle sizes can be understood in terms of the inertial model, while the smaller sizes can be explained as a result of scattering. Also, arguments will be presented to show that this technique can probe the inertial model, without the simultaneous observation of scattering effects. Such studies can improve the theoretical interpretation of standard attenuation measurements.

\section{Contributed Paper}

\section{1:40}

2aPAb11. Non-reciprocity of acousto-optic interaction at high acoustic frequencies. Yury Dobrolenskiy (M.V. Lomonosov Moscow State University, Faculty of Physics, Vorob'evy Gory, MSU, 119991 Moscow, Russian Federation, dobrolenskiy@phys.msu.ru), Vitaly Voloshinov (M.V. Lomonosov Moscow State University, Faculty of Physics, Vorob'evy Gory, MSU, 119991 Moscow, Russian Federation, volosh@phys.msu.ru), Yury Zyuryukin (Saratov State Technical University, Politechnicheskaja str., 77, 410054 Saratov, Russian Federation, phys@sstu.ru)

Modern acousto-optic (AO) tunable filters have reached the values of spectral resolution exceeding 104 and operate with acoustic frequencies up to a few gigahertzes. At such high frequencies and such narrow bandwidths, there appear effects so far neglected. Among them there is non-reciprocity of
AO interaction. It consists in the fact that acoustic frequency of light diffraction by ultrasound is different when optic beams propagate in directions opposite to each other. In the present research, the new effect has been studied theoretically and experimentally for the example of collinear AO diffraction. Theoretical analysis and calculations related to particular AO materials have shown that the effect is essential at acoustic frequencies about $1 \mathrm{GHz}$ and higher. The value of the non-reciprocal shift of acoustic frequency can be as high as the frequency bandwidth of the filter. The effect has also been registered and investigated experimentally in a sample of lithium niobate crystal. The experimental data has totally confirmed the theoretical analysis. Therefore, it has been proved that the non-reciprocal effect influences the parameters of AO diffraction and, consequently, operation of AO filters at high frequencies. On the other hand, one can design devices, e.g. directional couples, exactly based on the non-reciprocity.

\section{Invited Paper}

\section{2:00}

2aPAb12. Critical cone channelling in directly bonded wafers. Evgeny Twerdowski (Institute of Experimental Physics II, University of Leipzig, Linnéstr. 5, 04103 Leipzig, Germany, twerdowski@physik.uni-leipzig.de), Moritz Von Buttlar (Institute of Experimental Physics II, University of Leipzig, Linnéstr. 5, 04103 Leipzig, Germany, vbuttlar@physik.uni-leipzig.de), Reinhold Wannemacher (Institute of Experimental Physics II, University of Leipzig, Linnéstr. 5, 04103 Leipzig, Germany, wannemacher@physik.uni-leipzig.de),Wolfgang Grill (Institute of Experimental Physics II, University of Leipzig, Linnéstr. 5, 04103 Leipzig, Germany, grill@physik.uni-leipzig.de)

Directly bonded semiconductor wafers have been investigated using ultrasonic transmission tomography and imaging of the phonon focusing patterns at ultrasonic frequencies. Beside of total disbonds, several bonded wafers contained defects that are fully transparent to normally incident waves of longitudinal polarization, and are fully opaque to those of transverse polarization. These defects, which are due to slip boundary conditions at the wafer-wafer interface, generate an additional acoustic mode by mode conversion at the interface. The additional mode is clearly observable in the experimental phonon focusing patterns and is indicative of the critical cone 
channelling phenomenon, which is caused by the generation of pseudo-surface and head waves at weakly bonded solid-solid interfaces. The effect is also expected to be present in the case of weakly-bonded isotropic materials. Since the strong pseudo-surface wave resonance exists only in the case of boundary conditions that allow for the relative displacement of the two adjoining media along the interface, the critical cone channelling effect can serve as a measure of the type and quality of the bond.

\section{Contributed Papers}

12:20

2aPAb13. Sound waves in 3D periodic granular materials. Orion Mouraille (UTwente, Multi Scale Mechanics, P.O.Box 217, 7500 AE Enschede, Netherlands, o.j.p.mouraille@utwente.nl), Stefan Luding (UTwente, Multi Scale Mechanics, P.O.Box 217, 7500 AE Enschede, Netherlands, s.luding@utwente.nl)

The sound propagation mechanisms inside dense granular matter, even for periodical structures, are challenging the attempts to describe it because of the discrete nature of the material. Phenomena like dissipation, scattering, and dispersion are hard to predict based on the material state and/or properties and vice-versa. We propose here a simulation method using dynamic discrete elements in order to get more insight in this problem. The small perturbation created on one side of a dense, static regular packing of grains is examined during its propagation and when it arrives at the opposite side. Both longitudinal and shear perturbation are studied and an interesting wave-acceleration is observed [1]. Moreover the rotational degree of freedom permits to observe the role of rotations in the wave propagation. The control of the inter-particle forces like, contact potential, cohesion and friction make possible to observe the effect of these micro-parameters on the macro-behavior (at the wave scale). Long term goal is to predict the largescale macroscopic material behavior from the microscopic structure and material parameters. [1] O. Mouraille, W A Mulder and S. Luding: Sound wave acceleration in granular materials, J. Stat. Mech. (2006) P07023.

\section{2:40}

2aPAb14. Bragg light diffraction in nontransparent crystals. Farkhad Akhmedzhanov (Navoi State Mine Institute, 27a Yuzhnaya Street, 210100 Navoi, Uzbekistan, farkhad2@yahoo.com)

As is well known, in absolutely transparent crystals an acoustooptical interaction is lacking. In this connection's acoustooptical investigations are presented greatest interest in optical range, in which the crystal is not transparent. In order to carry out similar experiments, it is necessary to use the crystal with a small light absorption coefficient at applied wavelength. The main problem is selection of an appropriate buffer crystal that the light beam can penetrate into sample by Bragg angle at required frequency. At present work the acoustooptical properties of Si crystal were investigated at light wavelength $632.8 \mathrm{~nm}$. Dy $\mathrm{S}_{3}$ crystals were used as a buffer sample. It was detected the diffracted light intensity is much more powerful in comparison with that for $\mathrm{LiNbO}_{3}$ crystals. Acoustooptical efficiency M2 (it is defined the acoustic-optical quality of material) has been calculated from the values of optic coefficients and elastic constants for appropriate directions of sound and light. The results of executed investigations have shown the possibility of determination of acoustooptical properties of nontransparent crystals by Bragg diffraction method. At that rate, the very high intensity of diffracted light can be obtained in comparison with that in transparent crystals.

1:00-2:00 Lunch Break

\section{Invited Paper}

2:00

2aPAb15. Theory of resonant acoustic transmission through subwavelength apertures. Johan Christensen (Universidad Autónoma de Madrid, Departamento de Física Teórica de la Materia Condensada, Facultad de Ciencias, C-V, 28049 Madrid, Spain, johan.christensen@uam.es), Luis Martín-Moreno (Universidad de Zaragoza, Departamento de Física de la Materia Condensada, Instituto de Ciencia de Materiales de Aragón, C/ Pedro Cerbuna 12, 50009 Zaragoza, Spain, lmm@unizar.es), Francisco Jose García-Vidal (Universidad Autónoma de Madrid, Departamento de Física Teórica de la Materia Condensada, Facultad de Ciencias, C-V, 28049 Madrid, Spain, fj.garcia@uam.es)

The discovery of the phenomenon of extraordinary optical transmission through a two-dimensional array of subwavelength holes in a metallic film has opened a new line of research within optics. The key role played by surface plasmons in transferring light efficiently from the input side of the metal film to the output region was soon realized. This fundamental knowledge enabled extension of this surface-plasmon ability to achieve extraordinary optical transmission and strong collimation of light in a single hole surrounded by a finite periodic array of indentations. Here, we show how these ideas developed for electromagnetic radiation can be transferred to other classical waves such as acoustic waves.

\section{Contributed Papers}

\section{2:20}

2aPAb16. Theoretical and experimental ultrasonic investigation of a thin plate with regions of different periodic double-corrugations. Sarah W. Herbison (Georgia Tech Lorraine - G.W. Woodruff School of ME, UMI Georgia Tech - CNRS 2958, 2 rue Marconi, 57070 Metz, France, sherbison@gatech.edu), Nico F. Declercq (Georgia Tech Lorraine - G.W. Woodruff School of ME, UMI Georgia Tech - CNRS 2958, 2 rue Marconi, 57070 Metz, France, nico.declercq@me.gatech.edu)

In order to provide a qualitative measurement of the depth of periodic double-corrugations existing on both sides of a thin plate, theoretical and experimental ultrasonic techniques have been applied. The aesthetic galvanized steel sheets under investigation contain many regions of periodic double-corrugations, each region with a different corrugation depth. An ultrasonic technique is investigated as a means to improve existing optical testing techniques in quality and speed. Theoretical simulations using the theory of diffraction from periodically corrugated surfaces have been employed to obtain reflection spectra to facilitate qualitative observations regarding the depth of the corrugation in the different regions. Experiments to obtain reflection spectra correlate well with the theoretical simulations, and show the application of ultrasound to the qualitative measurement of the corrugation depth. Because the wavelengths necessary to make the investigations produce Lamb waves, some difficulty lies in the generation of diffracted bulk waves and Lamb waves. The research shows that correlations between theory and experiments exist, but they can only be obtained after thorough simulations that do not produce easy to apply rules of thumb. 
2:40

2aPAb17. BEM analysis of plane waves scattered from periodic surfaces. Mahesh Bansal (Institute of Technical Acoustics, Tech. Univ. Berlin, Einsteinufer 25, 10587 Berlin, Germany, mbansal.iitk @ gmail.com), Wolfgang Ahnert (Ahnert Feistel Media Group, Arkonastr. 45-49, 13189 Berlin, Germany, wahnert@ada-acousticdesign.de), Stefan Feistel (Ahnert Feistel Media Group, Arkonastr. 45-49, 13189 Berlin, Germany, sfeistel@afmg.eu)

Periodic surfaces like stairs, seats and repetitive designs on walls are very common and integral part of room acoustics. Effective simulation of the sound field inside enclosures requires the investigation of scattering from such periodic surfaces. In this work, we perform BEM analysis to calculate the scattering coefficients of different samples. For comparison purposes we also introduce a simple point-source based model to calculate the scattered wave fronts. Both approaches are implemented in a computational tool called EASE Scatterer. The incident plane waves are considered at various angles and scattered waves computed in both models are then compared with the measured data. It is found that while the point-source model can give reasonable asymptotic results, the BEM model matches with the measurement data significantly better in quantity and quality. Moreover, a study by varying the number of periods for the given sample is also performed.

\section{3:00}

2aPAb18. Diffraction phenomena associated with a composite plate containing an interior periodically corrugated interface. Sarah W. Herbison (Georgia Tech Lorraine - G.W. Woodruff School of ME, UMI Georgia Tech - CNRS 2958, 2 rue Marconi, 57070 Metz, France, sherbison@gatech.edu), Nico F. Declercq (Georgia Tech Lorraine - G.W. Woodruff School of ME, UMI Georgia Tech - CNRS 2958, 2 rue Marconi, 57070 Metz, France, nico.declercq@me.gatech.edu)

The interest in the study and applications of phononic crystals has naturally lead to the investigation of other novel periodic structures. The present work examines the case of a plate constructed of two solid layers of differing elastic properties separated by a periodically corrugated interface. It is shown how the dispersion curves are influenced by the internal corrugated interface and how they evolve as a function of the magnitude of this corrugation. Internal diffraction effects alter the dispersion properties and thus have an important effect on the composite when it is used as an acoustic filter. These effects are also important for the transmission and reflection of sound when the composite is used as a panel or when it is the intention to generate Lamb waves to investigate the composite plate nondestructively.

$3: 20$

2aPAb19. Multiple scattering of acoustic waves from two transversely isotropic cylinders. Sina Sodagar (Faculty of Mechanical Engineering, K. N. Toosi University of Technology, Pardis St., Molla Sadra Ave., Vanak Sq., Postal code 1999143344, 16579 Tehran, Iran, ssodagar@alborz.kntu.ac.ir), Farhang Honarvar (Faculty of Mechanical Engineering, K. N. Toosi University of Technology, Pardis St., Molla Sadra Ave., Vanak Sq., Postal code 1999143344, 16579 Tehran, Iran, honarvar @ mie.utoronto.ca), Anthony N. Sinclair (Department of Mechanical Engineering, University of Toronto, 5 King's College Road, Toronto, ON M5S 1A4, Canada, sinclair@mie.utoronto.ca)

The study of the interaction of acoustic waves with cylindrical structures has numerous applications including the ultrasonic nondestructive testing of materials. The scattered pressure field from a submerged cylinder contains valuable information about its physical properties. Scattering of acoustic waves from single cylindrical components including solid cylinders, shells, and multilayered cylinders has been of interest during the past two decades. These studies include theoretical modeling, numerical calculations, and experimental measurements of the scattered field of these objects. The more complex problem of multiple scattering from a grating of cylindrical components has also been considered during the past few years. These studies usually deal with either rigid or isotropic cylindrical objects. In this paper, the mathematical modeling for the scattering of plane acoustic waves from two adjacent infinite anisotropic solid cylinders will be presented. The type of anisotropy considered is transverse isotropy (hexagonal symmetry). The mathematical model accounts for the effects of the scattered filed of each cylinder on the total resultant pressure field. Numerical calculations are used to verify the validity of the developed mathematical model.

3:40-5:20 Posters

Lecture sessions will recess for presentation of poster papers on various topics in acoustics. See poster sessions for topics and abstracts.

\section{Contributed Papers}

$5: 20$

2aPAb20. Phononic crystals in the diffraction regime. Vincent Laude (Institut FEMTO-ST/CNRS, 32 avenue de l'Observatoire, $25044 \mathrm{Be}-$ sançon cedex, France, vincent.laude@femto-st.fr), Sarah Benchabane (Institut FEMTO-ST/CNRS, 32 avenue de l'Observatoire, 25044 Besançon cedex, France, sarah.benchabane@femto-st.fr), Abdelkrim Khelif (Institut FEMTO-ST/CNRS, 32 avenue de l'Observatoire, 25044 Besançon cedex, France, abdelkrim.khelif@femto-st.fr)

Phononic crystals are periodic composite materials exhibiting amazing wave propagation properties. In many works, complete band gaps are being looked for, i.e. the materials constituting the phononic crystal and its lattice arrangement are chosen such that propagation for all waves within a prescribed frequency range is forbidden. In other studies, the phononic crystal is considered a metamaterial, the anisotropic spatial dispersion of which can be tuned, and in which negative refraction can even be observed under certain circumstances. Such effects are usually considered in the sub-diffraction regime, i.e. below some critical onset frequency. In this work, we specifically examine phononic crystals in the diffraction regime. Indeed, the boundaries of a finite size phononic crystal embedded in a host propagation medium can be viewed as diffraction gratings, as we show. We will specifically consider two cases: two-dimensional phononic crystals composed of steel rods in water, and two-dimensional phononic crystals for surface acoustic waves achieved by etching cylindrical holes in a solid substrate.

\section{$5: 40$}

2aPAb21. Methods to investigate the possibilities of using a three element periodic structure to suppress the transmission of energy in an elastic tube. Ole Holst-Jensen (Minus10dB, Stokrosevej 29, 8330 Beder Denmark, ole.holst@minus10db.dk), Sergey Sorokin (Univ. of Aalborg, Institute for mechanical engineering, Pontoppidanstraede 101, 9220 Aalborg East, Denmark, svs@ime.aau.dk)

In industrial applications, such as pump and compressor systems, pipe vibrations can exceed an acceptable level. Periodically spaced masses added to the piping are an appropriate method of attenuation, and investigations to develop valid prediction models and measurement techniques has been initiated in the Danish Makunet network. The present investigation concern the effect of three masses attached periodically to a pipe of small diameter. The pipe is small enough that it can be treated as a beam. The masses are eccentric to the center of the beam, to achieve a large change in the moment of inertia by the added elements. The theoretical model is formulated as a system of boundary equations, which describe propagation of flexural, axial and torsion waves within each segment of a tube between periodic elements An exact solution of this system is obtained and the power transfer is dramatically reduced in some frequency 'stop bands' regardless the excitation conditions. Transfer impedance measurements between the force input and the acceleration on selected positions are used to find the insertion loss due to the application of the periodic elements, showing good agreement with theory. 
6:00

2aPAb22. Diffraction influence on the SAW tag characteristics. Boris V. Sveshnikov (Lebedev Research Center in Physics, 23, Lenin Av., 119991 Moscow, Russian Federation, bvs@ieee.org)

The diffraction of acoustic beams may influence notably on the responses of the SAW based RFID systems. In order to prevent such an influence one cannot increase the tag acoustic aperture too much because of ohmic loss in a transponder. On the other hand, usually the tag length should be large enough. There is a very specific and unique feature of the acoustoelectric scattering of SAW beam on the coding sequence of the reflective metal electrodes placed on a piezoelectric substrate. The point is that the SAW itself is formed by two coupled sub-systems, namely: elastic displacements and electric field. Both of them are scattered on every discontinuity because of two kinds of the boundary condition perturbation: electric shorting beneath electrodes and mass-loading effect. A uniform metal electrode scatters differently from each other the "elastic" and "electric" subsystems with non-uniform wave-front caused by diffraction. The closed form expressions are found, allowing us to describe consistently the mentioned phenomena. Their numerical analysis has been added by the analytical one in case of parabolic approximation of the crystal slowness curve. The synthesis algorithm of SAW tags with high capacity is proposed taking into account the diffraction effect in a natural way.

\section{6:20}

2aPAb23. Frequency-domain diffraction for edges of arbitrary length with efficient numerical integration. Peter Svensson (Norwegian Univ. of Science and Technology, O.S. Bragstads plass 2B, Dept. of Electronics and Telecommunications, NO-7491 Trondheim, Norway, svensson@iet.ntnu.no), Paul Calamia (Rensselaer Polytechnic Institute, Greene Bldg., 110 8th St., Troy, NY 12180, USA, calamp@rpi.edu)

Frequency-domain edge diffraction from an infinite wedge insonified by a point source has been widely studied, with various analytical solutions dating back nearly a century. In this talk we present an alternative frequency- domain solution which can be used for finite as well as infinite edges. The expression, given as a line integral along the diffracting edge, is derived from an analytical time-domain expression presented in [U. P. Svensson et al., J. Acoust. Soc. Am. 106, 2331-2344 (1999)]. The new formulation is shown to be equivalent to an exact, analytical, contour-integral formulation for the infinite wedge via a variable transformation. Results for various finite and infinite edge cases will be presented, and numerical integration using an efficient quadrature method for highly oscillatory integrals will also be discussed.

\section{$6: 40$}

2aPAb24. Acoustic cloaking by two-dimensional sonic crystals. Daniel Torrent (Politechnic University of Valencia, Wave Phenomena Group. Department of Electronic Engineering, C/ Camino Vera s.n, ES-46022 Valencia, Spain, datorma1@upvnet.upv.es), José Sanchez-Dehesa (Polytechnic University of Valencia, Cami de Vera s/n, 46022 Valencia, Spain, jsdehesa @upvnet.upv.es)

It has been previously shown that a two dimensional sonic crystal made of a periodic arrangement of solid cylinders in air behaves in the low frequency limit (homogenization) like an acoustic metamaterials with anisotropic effective density and isotropic effective bulk modulus [D. Torrent and J. Sanchez-Dehesa, New J. Phys. February 2008]. Here, the homogenization method is extended to the case of sonic crystals with two types of cylinders in the unit cell, and analytical expressions for the anisotropic effective density are then obtained. Moreover, it will be shown how these new acoustic metamaterials can be used to physically realize the solution for the acoustic cloaking proposed by Cummer and Schurig [New. J. Phys., vol. 9, 45, 2007] [Work supported by MEC of Spain.]

TUESDAY MORNING, 1 JULY 2008

ROOM 352A, 8:00 A.M. TO 7:00 P.M.

\title{
Session 2aPAc
}

\section{Physical Acoustics: Outdoor Sound Propagation and Uncertainties I}

\author{
Keith Wilson, Cochair \\ U.S. Army Cold Regions Research and Engineering Laboratory, 72 Lyme Road, Hanover, NH 03755-1290, USA
}

Michel C. Berengier, Cochair

Laboratoire Central des Ponts et Chaussées, Centre de Nantes - Route de Bouaye, BP 4129, Bouguenais cedex, 44341, France

\section{Invited Papers}

8:00

2aPAc1. Expected mean in an environmental noise measurement and its related uncertainty. Marco Paviotti (European Commission, via e. fermi, 1, 21020 Ispra, Italy, marco.paviotti@jrc.it), Stylianos Kephalopoulos (European Commission, via e. fermi, 1, 21020 Ispra, Italy, Stylianos.Kephalopoulos@jrc.it)

In the context of the implementation of the Environmental Noise Directive 2002/49/EC a study on noise measurement uncertainty was developed. In performing any noise measurement average, there might be assumptions over the distribution and independency of the samples. In a concrete example dealing with environmental noise, this might be the case of a series of measurements of a constant noise source like an industrial plant or a fluctuating noise source like road traffic. Using a series of 1 day Leq in the first case, or a series of 15 minutes samples in the second case, the average of these values is usually considered as the expected mean, however, the error caused by the specific selection of the samples is not evaluated. Statistically speaking, before establishing an average value, at least the lognormal distribution of the samples and the effect of adding-up several uncertainties should be evaluated. This article will focus on the formulas to be used and will discuss differences in assessing the expected mean for normally distributed values, or for lognormally distributed, and will suggest an approach to properly add-up all uncertainties related to an environmental noise measurement campaign. 
2aPAc2. Criterion to select meteorological factors to evaluate uncertainties in sound propagation. Panu Maijala (VTT, Tekniikankatu 1, 33101 Tampere, Finland, Panu.Maijala@vtt.fi)

Most of the environmental factors have some effect on sound propagation outdoors. Many of these factors can be properly implemented to a sound propagation model. However, it is not easy to handle sound scattering due to the turbulence, and at the same time, the turbulence is the most important source of uncertainties. Concurrently with the studies of turbulence models we have developed a concept to get an estimate of the excess attenuation using a state-of-the-art physical model and to evaluate the uncertainties using a statistical model. This statistical model is based on two years continuous measurements using extensive acoustical and meteorological measurement facilities and producing over 100 factors hourly. Many meteorological factors had a strong and significant correlation with the excess attenuation, but between each other too. To avoid instable model due to the collinearity many factors were abandoned. In this paper the criterion and methods to select best explaining factors and to form this statistical model are considered.

\section{8:40}

2aPAc3. Methodological and metrological benchmarks for determining experimental values of meteorological parameters. Benoit Gauvreau (Laboratoire Central des Ponts et Chaussées, Centre de Nantes - Route de Bouaye, BP 4129, 44341 Bouguenais cedex, France, benoit.gauvreau@lcpc.fr)

Outdoor sound propagation is largely influenced by meteorological conditions: mean refraction, intermittency and atmospheric turbulence. Thus, acoustic engineers and scientists have to rigorously characterize those propagation conditions through several parameters, both for numerical predictions (input data) and for operational reports (impact studies). One of these influent parameters is the well-known (and widely used) effective vertical sound speed gradient, which depends on spatial and temporal mean values of wind and temperature between source and receiver. However, the experimental characterization of wind and temperature (and next sound speed) vertical profiles remains delicate: it requires accurate meteorological devices and adapted measuring methods. At this purpose, an experimental campaign has been carried in 2007 on a perfectly flat and open ground, in order to quantify the confidence, uncertainty and spreading of such parameter values for different time scales. This campaign involved different meteorological devices: instrumented towers, classical sensors, 3D sonic anemometers, etc. The benchmark results will be presented and next analysed in terms of metrological and methodological requirements for acousticians in charge of environmental studies for standard organizations and/or scientific institutes.

2aPAc4. Sources and potential influences of uncertainty in ground impedance measurements and estimations. Shahram Taherzadeh (The Open University, Faculty of Mathematics, Computing and Technology, Walton Hall, MK7 6AA Milton Keynes, UK, s.taherzadeh@open.ac.uk), Keith Attenborough (Open University, Department of Design, Development, Materials and Environment, Walton Hall, MK7 6AA Milton Keynes, UK, Keith.Attenborough@ioa.org.uk)

Knowledge of acoustic impedance of ground surface is essential for determining noise levels outdoors. At distances up to a few hundred metres from the source ground effect may be the dominant factor. The short-range measurement of sound level spectra from an omni-directional source has been used as a standard method to deduce the acoustic impedance spectrum of ground surfaces (e.g. ANSI S1.18, 1999). However, there remain a number of uncertainties in such measurement methods. For example, at low frequencies the difficulties in fitting theoretical or phenomenological models to short range data may result is a large uncertainty in predictions of sound levels at larger distances. Another source of uncertainty is the variability of the acoustic impedance of apparently uniform ground. Another potential influence on predictions of long term equivalent noise levels is seasonal variation. This paper discusses the extent and potential influences of such uncertainties in measured and deduced ground impedance spectra.

2aPAc5. Facing the challenge of calculating outdoor sound propagation using a 3D multi domain approach based on linear euler equations. Fabrice Junker (EDF, 1, avenue du Général de Gaulle, 92141 Clamart, France, fabrice.junker@edf.fr), Fabien Crouzet (EDF, 1, avenue du Général de Gaulle, 92141 Clamart, France, fabien.crouzet@edf.fr), Philippe Lafon (EDF, 1, avenue du Général de Gaulle, 92141 Clamart, France, Philippe.lafon@edf.fr)

Solving the Linear Euler Equations (LEE) is the reference method that allows to take into account all the phenomena involved in Outdoor Sound Propagation. However, the huge size of the problems to be treated is still a great limitation to the practical application of this method. The concept of multi domain computations associated with the use of massively parallel computers now pushes the limits away. The code SAFARI, recently developed by EDF, solves LEE with high order numerical schemes on structured grids. To deal with complex and large geometries, a multi domain approach is used. The computational domain is composed of several partially overlapping grids (overset grids). Computations are parallelized by domain decomposition to be runned on cluster facilities. The presentation means to show the capability of SAFARI to deal with propagation over realistic 3D domains. The strategy used to carry out the calculations is detailed. The new perspectives of this kind of method are finally given. 
2aPAc6. Computing the parameter sensitivities of outdoor sound propagation in a random environment. Chris L. Pettit (U.S. Naval Academy, Aerospace Engineering Dept., 590 Holloway Rd., MS 11-B, Annapolis, MD 21402, USA, petitcl@usna.edu), Keith Wilson (U.S. Army Cold Regions Research and Engineering Laboratory, 72 Lyme Road, Hanover, 03755-1290, USA, D.Keith.Wilson @erdc.usace.army.mil)

Computational forecasts of near-ground sound levels are compromised by uncertainty (e.g., randomness) and error (e.g., grid resolution) in the atmosphere and terrain representations, and by simplified or incorrect physics. For an incompletely known environment, a model's predictive power cannot be assessed without first quantifying the sensitivity of its forecasts to the full range of every parameter. Knowledge of these sensitivities throughout the spatial domain also is essential for effectively investing data-gathering resources to support sound propagation forecasts. Sensitivity analysis therefore is central to raising the relevance of computational acoustics in practical applications. These considerations should motivate practitioners of computational acoustics to adopt a consistent framework for sensitivity and uncertainty analyses. Topics to be discussed include: (1) standard uncertainty taxonomies in computational mechanics, (2) why uncertainty about a parameter should be distinguished from sensitivity of a model to that parameter, (3) sources of uncertainty in the near-ground acoustics, (4) a sampling-based sensitivity analysis framework that facilitates estimating typical and extreme values of sensitivities at each point in the spatial domain (i.e., full-field sensitivities), (5) factors to be aware of when applying sensitivity analysis to forecasts of near-ground sound propagation, and (6) ways of representing sensitivity estimates to facilitate insight.

\section{0:00-10:20 Break}

\section{0:20}

2aPAc7. Field experiments on the influences of wind speed and direction on outdoor sound propagation over flat ground. Takatoshi Yokota (Kobayasi Institute of Physical Research, 3-20-41 Higashi-Motomachi Kokubunji, 185-0022 Tokyo, Japan, t-yokota@kobayasi-riken.or.jp), Koichi Makino (Kobayasi Institute of Physical Research, 3-20-41 Higashi-Motomachi Kokubunji, 185-0022 Tokyo, Japan, makino@kobayasi-riken.or.jp), Kohei Yamamoto (Kobayasi Institute of Physical Research, 3-20-41 HigashiMotomachi Kokubunji, 185-0022 Tokyo, Japan, yamamoto@kobayasi-riken.or.jp), Yasuaki Okada (Faculty of Science and Technology, Meijo University, 1-501 Shiogamaguchi Tempaku-ku, 468-8502 Nagoya, Japan, okada@ccmfs.meijo-u.ac.jp), Koichi Yoshihisa (Faculty of Science and Technology, Meijo University, 1-501 Shiogamaguchi Tempaku-ku, 468-8502 Nagoya, Japan, yosihisa@ccmfs .meijo-u.ac.jp)

The influences of wind on outdoor sound propagation are investigated both by field experiments and numerical simulations. At first, measurements of sound propagation at a distance of $160 \mathrm{~m}$ were carried out under various wind speed and direction. The relationship between vector wind speed and the variation of sound pressure level has been examined not only for the up/down wind conditions but also for the cross wind conditions. Secondly, the short term sound fluctuations were investigated by the use of a high energy impulsive sound generator and a loud speaker as sound sources. Sound propagation up to $300 \mathrm{~m}$ was examined with frequency range from $16 \mathrm{~Hz}$ to $4 \mathrm{kHz}$ octave band. Finally, the sound speed profiles in the measurement field were estimated by two methods; one was based on the time interval during the sound propagation from the source to the receivers, the other was based on the wind speed at height of $0.6 \mathrm{~m}$, $1.2 \mathrm{~m}, 2.4 \mathrm{~m}$ and $4.8 \mathrm{~m}$, and the temperature. By using those sound speed profiles, the excess attenuation was calculated by the PE method. The measured and the calculated values have been compared and the methods to estimate the influences due to wind condition have been discussed.

\section{0:40}

2aPAc8. Variation in measured sound level as a function of propagation environment and distance. Michelle E. Swearingen (Norwegian Defense Research Establishment/US Army, Postboks 25, 2027 Kjeller, Norway, michelle.swearingen@ffi.no), Morten Huseby (Norwegian Defense Research Establishment/US Army, Postboks 25, 2027 Kjeller, Norway, Morten.Huseby@ffi.no), Michael J. White (US Army Engineer Research and Development Center, 2902 Newmark Drive, Champaign, IL 61826, USA, Michael.J.White @ usace.army.mil)

The propagation environment exerts a large influence on the range of received levels of impulsive events. This talk focuses on the variation in excess attenuation over durations of less than approximately 15 minutes. Data are presented for greatly different measurement distances $(25 \mathrm{~m}$ to $7 \mathrm{~km}$ ) and propagation environments (sparse vegetation to forested), illustrating the effects of distance and terrain cover on sounds from a propane cannon and an artillery source. Over sparse vegetation $7 \mathrm{~km}$ from an artillery source, the received CSEL varied $11 \mathrm{~dB}$ within a 12-minute duration. In measurements up to approximately $300 \mathrm{~m}$ from the source, variation in received level (both peak and SEL) was less than $1 \mathrm{~dB}$ within the forest, and much more in the open. The control of the forest canopy on the micrometeorology seems to explain the effect.

\section{1:00}

2aPAc9. The revision of the French method for road traffic noise prediction. Guillaume Dutilleux (Lab. Régional des Ponts et Chaussées, 11, rue Jean Mentelin, BP 9, 67035 Strasbourg Cedex 2, France, Guillaume.Dutilleux @equipement.gouv.fr), Jérôme Defrance (CSTB, 24 rue Joseph Fourier, 38400 Saint-Martin-d'Hères, France, jerome.defrance@ cstb.fr), Benoit Gauvreau (Laboratoire Central des Ponts et Chaussées, Centre de Nantes - Route de Bouaye, BP 4129, 44341 Bouguenais cedex, France, benoit.gauvreau@lcpc.fr), Francis Besnard (Lab. Reg. Régional des Ponts et Chaussées de l'Est Parisien, Rue de l'égalité prolongée, BP 34, 93352 Le Bourget, France, francis.besnard@equipement.gouv.fr)

A revision of the French method for road traffic noise prediction (NMPB-Routes-2008) has been released. The major principles of NMPB-Routes-2008 are outlined. The most important modifications regarding the source are the reduction of its height and the introduction of two different spectra. In this complete revision, the main change is the replacement of the ISO 9613-2 - based ground attenuation formula in downward conditions by the formula for ground attenuation in homogeneous conditions of NMPB-Routes-1996 
with corrected heights in order to take into account the mean curvature of rays (refraction) and its spreading (turbulence). The revised NMPB adds an attenuation term for an occasional cutting embankment. Regarding diffraction the $\Delta$ dif formula is now suitable for low height barriers. The validation of the revised NMPB with respect to experiment is presented. It is based on measurement campaigns on 6 sites with complex geometries and shows that the predicted noise levels obtained from the revised NMPB are significantly closer to experimental results than in the case of the original method.

2aPAc10. Long range propagation of high speed train noise: Sound level variations before and after the pass-bys. Benjamin Cotte (Ecole Centrale de Lyon, LMFA, UMR CNRS 5509, Université de Lyon, Bât. KCA, 36 Avenue Guy de Collongue, 69134 Lyon, France, benjamin.cotte@ec-lyon.fr), Philippe Blanc-Benon (Ecole Centrale de Lyon, LMFA, UMR CNRS 5509, Ecully, 69134 Lyon, France, Philippe.Blanc-Benon@ec-lyon.fr), Franck Poisson (SNCF DIR, 45, rue de Londres, 75379 Paris, France, franck.poisson@sncf.fr), Cora Cremezi-Charlet (UIC, 16,rue J. Rey, F-75015 Paris, France, cremezi@uic.asso.fr)

It has been observed relatively frequently that high speed train (TGV) noise could be heard tens of seconds before or after the actual train pass-bys. This study is aimed at characterizing this phenomenon and the conditions in which it occurs, both experimentally and numerically. Acoustic measurements of TGV pass-bys have been performed under controlled conditions. A relatively strong wind was blowing from the South during the experiment. The measurements show that the TGV noise can be heard before the pass-bys when the TGV was coming from the South (same wind and train directions), and after the pass-bys when the TGV was coming from the North (opposite wind and train directions). This noise is relatively low in frequency (around $400 \mathrm{~Hz}$ ), and corresponds to propagation distances that can exceed $1 \mathrm{~km}$. Levels associated with this phenomenon can vary significantly over short time intervals (5-10 minutes), which raises the issue of the representativeness of TGV measurements at long ranges. It will be shown using numerical prediction methods (parabolic equation in the frequency domain, linearized Euler equations in the time domain) that these acoustic variations are mostly due to variations in the meteorological conditions between the pass-bys.

\section{1:40}

2aPAc11. A model based monitoring system for aircraft noise. Frank Van Den Berg (TNO - Science and Industry, Stieltjesweg 1, 2628 CK Delft, Netherlands, Frank.vandenBerg@tno.nl), Arno R. Eisses (TNO, Stieltjesweg 1, P.O.Box 155, 2600 AD Delft, Netherlands, Arno.Eisses@tno.nl), Pieter J. Van Beek (TNO, Stieltjesweg 1, P.O.Box 155, 2600 AD Delft, Netherlands, Pieter.vanBeek @ tno.nl)

A new approach for an airport noise monitoring system is presented that comprises not only a number of measuring stations, but also a dedicated sound propagation model and an aircraft noise emission model. This approach enables estimation of noise levels in the whole area around the airport and not only at the location of the measuring stations. An additional advantage is that the locations of the measuring stations can be chosen more freely. Traditionally the measuring stations must be located near residential areas where the influence of other noise sources (such as cars or wind) cannot be eliminated. Better locations can be found that yield more reliable data and therefore more reliable noise levels. To update the sound propagation model frequently, the measuring stations not only measure noise levels, but also other parameters, like temperature and wind profile. The sound emission of the aircraft is derived from a directional aircraft noise emission model. A tool was developed to visualize the power of combining data and models in this model-based monitoring system. The use of models opens possibilities for interesting applications such as i) short term noise forecasts, ii) scenario studies with various aircraft distributions and iii) enforcement of noise limits.

\section{Contributed Papers}

12:00

2aPAc12. The variability of acoustical turbulence in the atmospheric boundary layer. Sylvain Cheinet (ISL, 5 Rue du General Cassagnou, BP 70034, 68301 Saint-Louis, France, sylvain.cheinet@isl.eu)

Outside sound propagation is affected by small-scale turbulence in the atmospheric boundary layer through refractive processes. These effects modulate the performance of acoustical sensors, and are at the basis of the atmospheric remote sensing by sodar. Previous studies have documented the impact of a mean turbulence profile or a statistical distribution of turbulence. In this study, we use a high-resolution atmospheric model called LES to reveal the spatial and temporal variability of the small-scale turbulence. First, we introduce some standard results on the role of various eddy sizes on acoustical propagation. Second, we discuss the methodology to derive the acoustical refractive index fluctuations from LES data. Third, we analyze the LES prediction in the case of a fair-weather boundary layer, emphasizing the impact of large-scale convective organization. Finally, we discuss some challenges in attempting to predict the acoustical turbulence from presentday weather forecasts.
$12: 20$

2aPAc13. Sound propagation in areas with a complex meteorology: a meteorological-acoustical model. Frits Van Der Eerden (TNO - Science and Industry, Stieltjesweg 1, 2628 CK Delft, Netherlands, frits.vandereerden@tno.nl), Frank Van Den Berg (TNO - Science and Industry, Stieltjesweg 1, 2628 CK Delft, Netherlands, Frank.vandenBerg@tno .nl)

Long range sound propagation is largely affected by the vertical wind and temperature gradients. These gradients are more complicated in areas where the meteorology can be complex, such as: coastal areas, islands, and lake districts. Furthermore, the gradients usually vary as a function of the horizontal distance, for instance at water-land crossings near a coast or a lake. As a result the sound propagation in these areas cannot be calculated with "standard" acoustic models, such as the ISO 9613 or Harmonoise engineering model. By using an advanced meso-scale meteorological model, which is using large-scale weather forecast data, and an acoustical model, that incorporates horizontal and vertical meteorological variations, long range sound propagation in such complex areas is calculated. In this paper 
we present two applications of this hybrid meteorological-acoustical calculation scheme: i) propagation of impulse noise in a coastal area, and ii) propagation of industrial noise in a rural area with a lake. For the coastal area sound level contours have been calculated as a function of the meteorology for a complete year. For the lake area the effect of the lake on the sound propagation is demonstrated.

\section{2:40-2:00 Lunch Break}

\section{Contributed Papers}

2:00

2aPAc14. Horizontal wave number spectra of atmospheric acoustic fields in range-dependent environments. Kenneth E Gilbert (National Center for Physical Acoustics, University of Mississippi, University, MS 38677, USA, kgilbert@olemiss.edu), Xiao Di (National Center for Physical Acoustics, University of Mississippi, University, MS 38677, USA, xiaodi@olemiss.edu), Santosh Parakkal (National Center for Physical Acoustics, University of Mississippi, University, MS 38677, USA, sparakka @ olemiss.edu)

The parabolic equation (PE) is a powerful method for computing sound propagation in range-dependent environments. However, the PE gives only the total field, with no information on the horizontal wave number spectrum of the acoustic field. In many situations, e.g., for propagation over irregular terrain, the horizontal wave number spectrum is valuable for analyzing the modal content of the acoustic field. For example, one might want to know the strength of the surface wave component at different ranges. A spectral decomposition method developed by Gilbert and Evans for ocean acoustics is applied here to atmospheric propagation over irregular terrain. This paper shows that for a PE field $p(z)$ at range $r$, the horizontal wave number spectrum $\phi(\kappa, z)$ can be obtained directly and efficiently by solving a onedimensional elliptic equation that uses the field $p(z)$ as a source term. The derivation of the spectral decomposition equation is given along with a numerical method for solving it. Examples are presented and discussed for propagation over irregular terrain. [Research supported by the U. S. Army TACOM-ARDEC at Picatinny Arsenal, New Jersey]

\section{2:20}

2aPAc15. Numerical modeling of sonic boom propagation from hypersonic aircraft. Alexandra Loubeau (Institut Jean Le Rond d'Alembert, Université Pierre et Marie Curie, Boites 161 et 162, 4 place Jussieu, 75252 Paris Cedex 05, France, loubeau@1mm.jussieu.fr), François Coulouvrat (Institut Jean Le Rond d'Alembert, Université Pierre et Marie Curie, Boites 161 et 162, 4 place Jussieu, 75252 Paris Cedex 05, France, coulouvr@ccr.jussieu.fr)

A numerical study of sonic boom propagation from hypersonic aircraft is performed including the effects of nonlinearity, atmospheric absorption and dispersion, and atmospheric stratification. A second-order split-step algorithm, which alternates application of nonlinearity in the time domain and complex absorption in the frequency domain, allows for a faster convergence of results with fewer range steps than with conventional first-order algorithms. Nonlinearity is calculated using the potential, the integral of the pressure, as proposed by Burgers and later applied to sonic booms by Hayes et al. This method, an alternative to Landau's law of equal areas, efficiently locates the shock position by selecting the maximum potential in multivalued regions. Definition of atmospheric absorption at high altitudes is important for modeling the propagation of sonic booms from hypersonic aircraft. Some aspects of an extended absorption model by Sutherland and Bass are adopted, therefore, which extend absorption predictions above the $20 \mathrm{~km}$ limit of the current ISO and ANSI standards. The study is completed using the meteorological conditions at two locations, Le Havre, France and Edwards Air Force Base, CA, USA, over the course of a year. [Work supported by European Union through ATLLAS AST5-CT-2006-030729, meteorological data provided by ECMWF.]

\section{2:40}

2aPAc16. Road noise: characterization and estimation of uncertainty due to meteorological effects. David Ecotiere (Lab. Régional des Ponts et Chaussées, 11, rue Jean Mentelin, BP 9, 67035 Strasbourg Cedex 2, France, david.ecotiere@equipement.gouv.fr)

Meteorological effects can lead to important temporal fluctuations of the sound level in outdoor sound propagation. These fluctuations lead to sound level uncertainties that are rarely estimated. A method based on the coupling of a model of sound propagation and a temporal micrometeorological model is used to quantify sound level fluctuations only due to meteorological fluctuations, over a very long period (typ. 10-30 years). Some statistical analysis are presented: diary or seasonal fluctuations, influence of the duration of observation on the accuracy of the estimation of a LAeq. A semi-analytical method is also proposed for characterizing the uncertainty of the sound level of a distribution of punctual sources. An application to a road noise source is presented.

\section{3:00}

2aPAc17. A numerical study of sound propagation over urban canyons. Martin Schiff (Division of Applied Acoustics, Chalmers University of Technology, 41296 Göteborg, Sweden, schiff@student.chalmers.se), Maarten Hornikx (Applied Acoustics, Chalmers University of Technology, Sven Hultins Gata 8a, SE-41296 Gothenburg, Sweden, maarten.hornikx@chalmers.se), Jens Forssén (Division of Applied Acoustics, Chalmers University of Technology, 41296 Göteborg, Sweden, jens .forssen@chalmers.se)

Because quiet areas in dense urban environments are important, there is high interest in propagation to areas shielded from direct road traffic noise. Sound levels in shielded areas are strongly influenced by distant sources, so intermediate propagation factors such as metrology, screening, and intermediate canyons must therefore be addressed in a realistic propagation model. A numerical investigation of sound propagation across the open tops of intermediate urban canyons has been performed, using the Parabolic Equation and Equivalent Sources methods. Results have been collected for various canyon geometries, and the influence of multiple canyons, canyon/rooftop absorption, variable rooftop height, and correlated versus uncorrelated source models has been investigated. By characterizing the "insertion loss" of canyons intermediate to the source and receiver, the influence of these intermediate canyons could be addressed simply, without the overhead of a detailed numerical calculation.

\section{$3: 20$}

2aPAc18. A validation test for the Acoustic Footprint Mission Planning System. Doru Velea (QinetiQ North America, Technology Solutions Group, 12030 Sunrise Valley Dr., Suite 400, Reston, VA 20191, USA, dvelea@plansys.com), Troy D. Schultz (Wyle Laboratories Inc., 241 18th Street S., Suite 701, Arlington, VA 22202, USA, troy.schultz@wylelabs.com), Kenneth J. Plotkin (Wyle Laboratories Inc., 241 18th Street S., Suite 701, Arlington, VA 22202, USA, kenneth.plotkin@wylelabs.com), Andy Rogers (QinetiQ North America, Technology Solutions Group, 12030 Sunrise Valley Dr., Suite 400, Reston, VA 20191, USA, arogers@plansys.com)

QinetiQ North America, Technology Solutions Group (QNA) and Wyle Laboratories have integrated two mature, proprietary technologies into a prototype Acoustic Footprint Mission Planning System. Wyle's Noise Model Simulation (NMSim) uses validated linear noise propagation routines in combination with ray-tracing algorithms based on weather data provided by QNA's Precision Airdrop System (WindPADS) to predict the time his- 
tory of spectral noise levels from a flying aircraft at any number of userdefined receptor locations. Through interpolation over a ground-based receptor grid, the impact of noise propagated through a stratified atmosphere may be quantified in terms of audibility at any point inside the acoustic footprint. Weather data for the NMSim ray-tracing routines is extracted from the WindPADS three-dimensional forecast of wind, pressure, temperature and humidity in the atmosphere as a function of time. The three-dimensiona field is the result of physical and dynamic assimilation of forecast and measured atmospheric data, with wind-forcing by the underlying terrain. This paper describes the results of a validation test performed at Yuma Proving Ground, AZ. It is shown that the noise propagation model predictions agree satisfactorily with the measurements of a C-130 cargo plane. [Work supported by Natick Soldier Center.]

3:40-5:20 Posters

Lecture sessions will recess for presentation of poster papers on various topics in acoustics. See poster sessions for topics and abstracts.

\section{Contributed Papers}

\begin{abstract}
2aPAc19. Modal probabilistic analysis. Christophe Heinkele (ENTPE/DGCB, Rue Maurice Audin, 69518 Vaulx-en-Velin, France, heinkele@entpe.fr), Claude-Henri Lamarque (rue Maurice Audin, 69100 Vaulx-en-Velin, France, lamarque@entpe.fr)

In this paper, we first recall a method for estimating the numerical Probability Density Function (PDF) with Parzen-Rosenblatt estimators of experimental data (like the sound absorption coefficient with the Kundt's tube). Then we focus on the model of Mikki which is used as a predictive tool. We are interested then in inversing the model and in identifying the 3 parameters $(\mathrm{q}, \sigma, \phi)$ of Mikki's model. But we want to go further and to identify from the estimated PDF directly the PDF of the 3 parameters. We explain first when it is possible, then we give some examples to illustrate the method. This work allows for evaluating the robustness of a model from experimental data.
\end{abstract}

\section{$5: 40$}

2aPAc20. An experimental evaluation of a new approach to aircraft noise modelling. Foort De Roo (TNO Science and Industry, Stieljesweg 1, 2628CK Delft, Netherlands, foort.deroo@tno.nl), Erik Salomons (TNO Science and Industry, Stieljesweg 1, 2628CK Delft, Netherlands, erik .salomons@tno.nl)

Common engineering models for aircraft noise, such as INM, yield noise levels by interpolation of Noise Power Distance (NPD) tables. In the European project Imagine (2004 2006), a different approach was proposed: the source is characterized by an emission spectrum and the received noise spectrum is calculated by subtracting the propagation attenuation spectrum from this emission spectrum. This is the usual approach for noise mapping of most noise sources. The aircraft emission spectrum is a function of (downward) emission direction, so each aircraft is represented by a hemisphere of emission spectra. This has been described by Butikofer in Acta Acustica 93 (2007). As hemisphere emission data are not yet available for all aircraft types, a 'reverse engineering' scheme was developed within Imagine to derive first order estimates of hemispheres from NPD tables. To gain experience with this approach, we have performed an experiment near Amsterdam airport. Various types of data were collected for a set of aircraft departures, including noise data at eleven positions and flight data. The Imagine approach was used to calculate noise contours, and noise spectra at the eleven positions. The differences between measured and calculated spectra may be used as a basis for improving the first order estimates of the hemispheres.

\section{6:00}

2aPAc21. Sound propagation in a street canyon: A study by modal decomposition. Adrien Pelat (Laboratoire d'Acoustique de l'Université du Maine, Avenue Olivier Messiaen, 72085 Le Mans, France, adrien.pelat.etu@univ-lemans.fr), Simon Félix (Laboratoire d'Acoustique de l'Université du Maine, Avenue Olivier Messiaen, 72085 Le Mans, France, simon.felix@univ-lemans.fr), Vincent Pagneux (Laboratoire d'Acoustique de l'Université du Maine, UMR CNRS 6613, AV. O. Messiaen, 72085 Le Mans, France, vincent.pagneux@univ-lemans.fr), Christophe
Ayrault(LAUM, CNRS, Université du Maine, Av. O. Messiaen, 72085 Le Mans, France, christophe.ayrault@univ-lemans.fr), Olivier Richoux (Laboratoire d'Acoustique de l'Université du Maine, Avenue Olivier Messiaen, 72085 Le Mans, France, olivier.richoux@univ-lemans.fr)

An urban, U-shaped, street canyon being considered as an open waveguide in which the sound may propagate, one is interested in a multimodal approach to describe the sound propagation within. The key point in such a multimodal formulation is the choice of the basis of local transversal modes on which the acoustic field is projected. For a classical waveguide, with a simple and bounded cross-section, a complete orthogonal basis can be analytically obtained. The case of an open waveguide is more difficult, since no such a basis can be exhibited. However, an open resonator, as displays for example the U-shaped cross-section of a street, presents resonant modes with complex eigen frequencies, owing to radiative losses. This work first presents how to numerically obtain these modes and, then, how they can be used as a basis for the modal decomposition of the sound field in a street canyon. Results are compared with experimental measurements on a scale model.

\section{$6: 20$}

2aPAc22. Acoustical waves propagation in rough walls street. Bertrand Lihoreau (Laboratoire d'Acoustique de l'Université du Maine, Avenue Olivier Messiaen, 72085 Le Mans, France, bertrand.lihoreau@univ-lemans.fr), Simon_ Félix (Laboratoire d'Acoustique de l'Université du Maine, Avenue Olivier Messiaen, 72085 Le Mans, France, simon.felix@univ-lemans.fr), Claude Depollier (Laboratoire d'Acoustique de l'Université du Maine, Avenue Olivier Messiaen, 72085 Le Mans, France, claude.depollier@univ-lemans.fr)

We present a method for the numerical calculation of a sound wave propagating in a two dimensional rough-sided street. Is this situation, the wave is subject to multiple scattering at the two boundaries. The propagation is governed by a parabolic equation. We show that the amplitude of a pulse may be expressed as the fractional Fourier transform of the incident pulse, and that the order of the transformation is related to the distance between the source and the point of observation. Numerical simulations are proposed as illustration of this model and are compared to experimental results.

\section{6:40}

2aPAc23. A high-density infrasound array of particle velocity sensors in the Netherlands. Arnout Tim Van Zon (Royal Netherlands Meteorological Institute (KNMI), PO Box 201, 3730 AE De Bilt, Netherlands, zon@knmi.nl), Laeslo G. Evers (Royal Netherlands Meteorological Institute (KNMI), PO Box 201, 3730 AE De Bilt, Netherlands, evers@knmi.nl)

A small aperture, High-Density Infrasound Array (HDIA) is being operated by the Royal Netherlands Meteorological Institute (KNMI). This 80 sensor array is part of the geophysical application within LOFAR, i.e. an astronomical low-frequency array in the Netherlands. HDIA occupies an area of 100 by 100 meter, so about the size of a noise reducer used in infrasound arrays for verification purposes. HDIA will be able to estimate the wind-noise correlation length, which has implications for noise reducer design. Of the 80 instruments, 74 are Microflowns. These measure particle velocity and have a directional sensitivity. If two of them are closely spaced then the azimuth of the incoming sound wave can be calculated from the 
amplitude ratio. The field setup has 37 elements, each containing a pair of Microflowns. Six elements also have a pressure microphone. We will present the first results of using the vector properties of the particle velocity to estimate the direction of arrival (DOA) of events. These will be compared with DOA-estimates from beamforming of 1) the pressure data, 2) the particle velocity data and 3) pressure data of a nearby 6-element microbarometer array. Furthermore, the observed correlation length of wind will be discussed.

TUESDAY MORNING, 1 JULY 2008

ROOM 362/363, 8:00 A.M. TO 1:20 P.M.

\title{
Session 2aPAd
}

\section{Physical Acoustics: Photoacoustics I}

\author{
Gerald Diebold, Cochair \\ Department of Chemistry, Brown University, Providence, Rhode Island 02912, USA \\ Christ Glorieux, Cochair \\ Lab. ATF, Katholieke Universiteit Leuven, Celestijnenlaan 200D, Leuven, B-3001, Belgium
}

Invited Papers

\begin{abstract}
8:00
2aPAd1. On the optical generation and detection of high frequency ultrasounds: thermal and non-thermal processes. Pascal Ruello (LPEC/UMR 6087/CNRS/Université du Maine, Avenue Olivier Messiaen, 72085 Le Mans Cedex 09, France, pascal.ruello@univ-lemans.fr), Philippe Babilotte (LPEC/UMR 6087/CNRS/Université du Maine, Avenue Olivier Messiaen, 72085 Le Mans Cedex 09, France, philippe.babilotte.etu@univ-lemans.fr), Shuo Zhang (INSP - UMR 7588 CNRS \& Université Pierre et Marie Curie, 140 Rue de Lourmel, 75015 Paris, France, Shuo.Zhang @insp.jussieu.fr), Denis Mounier (LPEC/UMR 6087/CNRS/Université du Maine, Avenue Olivier Messiaen, 72085 Le Mans Cedex 09, France, denis.mounier@univ-lemans.fr), Jean-Marc Breteau (LPEC/UMR 6087/CNRS/Université du Maine, Avenue Olivier Messiaen, 72085 Le Mans Cedex 09, France, jean-marc.breteau@univ-lemans.fr), Mathieu Edely (LPEC/UMR 6087/CNRS/Université du Maine, Avenue Olivier Messiaen, 72085 Le Mans Cedex 09, France, mathieu.edely@univ-lemans.fr), Patrick Laffez (Université François Rabelais, Tours, IUT Blois, 6, place Jean Jaures, 41029 Blois, France, patrick.laffez@univ-tours.fr), Alain Bulou (LPEC/UMR 6087/CNRS/Université du Maine, Avenue Olivier Messiaen, 72085 Le Mans Cedex 09, France, alain.bulou@ univ-lemans.fr), Bernard Perrin (INSP - UMR 7588 CNRS \& Université Pierre et Marie Curie, 140 Rue de Lourmel, 75015 Paris, France, bernard.perrin@insp.jussieu.fr), Vitali Gusev (LPEC/UMR 6087/CNRS/Université du Maine, Avenue Olivier Messiaen, 72085 Le Mans Cedex 09, France, vitali.goussev@univ-lemans.fr)

We report on the results of the generation and detection by femtosecond laser pulses of the acoustic waves at frequencies of tens to hundreds $\mathrm{GHz}$ in semiconductors and in oxides compounds exhibiting phase transition. We focus first on the generation mechanisms involved to achieve opto-acoustic transformations. Particular attention will be paid to the cases where classical thermal effects (thermoelastic coupling) drive the mechanism of generation of acoustic phonons and those where non-thermal effects become significant and sometimes dominant sources of acoustic phonons field. In the latter cases, we will especially focus on the phonons generation based on photo-induced modifications of microscopic internal electric fields (potential deformation) and also on the use of photoexcited carriers dynamics (carriers recombination) as a tuning parameter of the photo-generated ultrasounds spectrum. Secondly, we give the examples demonstrating that the choice of optical frequency for ultrasound detection influences not only the amplitude of the detected signal but provides an opportunity to detect separately high or low frequencies in the spectrum of ultrasounds. This study was supported by CPER and ANR project No. BLAN06-3-136284.
\end{abstract}

$8: 20$

2aPAd2. Optical detection of longitudinal and shear acoustic waves with laser picosecond acoustics. Osamu Matsuda (Division of Applied Physics, Graduate School of Engineering, Hokkaido University, 0608628 Sapporo, Japan, omatsuda@eng.hokudai.ac.jp), Oliver B. Wright (Division of Applied Physics, Graduate School of Engineering, Hokkaido University, 0608628 Sapporo, Japan, olly@eng.hokudai.ac.jp), David H. Hurley (Materials Characterization Department, Idaho National Laboratory, P.O.Box 1625, Idaho Falls, ID 83415-2209, USA, David.Hurley@inl.gov), Vitali Gusev (LPEC/UMR 6087/CNRS/Université du Maine, Avenue Olivier Messiaen, 72085 Le Mans Cedex 09, France, vitali.goussev@univ-lemans.fr), Ken’Ichi Shimizu (University Chemical Laboratory, Keio University, 4-1-1 Hiyoshi, Kohoku-ku, 223-8251 Kanagawa, Japan, shimizuk@econ.keio.ac.jp)

The absorption of picosecond light pulses in a medium can generate sub-THz acoustic waves. These cause a transient optical reflectance change that can be monitored by delayed probe light pulses. This technique, termed laser picosecond acoustics, can be used for the nondestructive evaluation of the physical properties of thin films and substrates. This paper describes a general method for quantitative analysis of such reflectance changes. It is applicable to multiple anisotropic layers that may be opaque or transparent. Longitudinal or shear acoustic waves propagating along the stacking direction of the multilayers modulate the dielectric permittivity anisotropically and inhomogeneously through the photoelastic effect, through local rotation, or through the surface and interface displacements. We describe how the optical reflectance for obliquely incident probe light can be calculated for the modulated medium. 
We then demonstrate the method with reference to experimental results for a sample consisting of a silica film on a zinc substrate in which both longitudinal and shear acoustic waves are generated and detected. The analysis yields the film thickness, sound velocity, and photoelastic tensor components, for example. The method is also applicable to various light scattering problems involving the inhomogeneous modulation of optical properties such as in photothermal experiments.

\section{Contributed Papers}

8:40

2aPAd3. Experimental studies of generation and propagation of high frequency acoustic waves in various solid materials using ultraviolet picosecond laser pulses. Eirini Tzianaki (Department of Electronics, Technological Educational Institute of Crete, Romanou 3, 73133 Chania, Greece, i.tzianaki@mail.chania.teicrete.gr), Michael Tatarakis (Department of Electronics, Technological Educational Institute of Crete, Romanou 3 , 73133 Chania, Greece, m.tatarakis@chania.teicrete.gr), Makis Bakarezos (Department of Music Technology and Acoustics, Technological Educational Institute of Crete, 1 E. Daskalaki Str., 74100 Rethymnon, Greece, bakarezos@stef.teicrete.gr), Maria Elefteriou (Department of Music Technology and Acoustics, Technological Educational Institute of Crete, 1 E. Daskalaki Str., 74100 Rethymnon, Greece, marel@physics.uoc.gr), Nektarios Papadogiannis (Department of Music Technology and Acoustics, Technological Educational Institute of Crete, 1 E. Daskalaki Str., 74100 Rethymnon, Greece, npapadogiannis@stef.teicrete.gr), Spyros Kazianis (Department of Physics, University of Ioannina, 45110 Ioannina, Greece, skaziannis@in.gr), Costas Kosmidis (Department of Physics, University of Ioannina, 45110 Ioannina, Greece, kkosmidis@uoi.gr), Andreas Lyras (Department of Physics, University of Ioannina, 45110 Ioannina, Greece, alyras@uoi.gr)

The generation of high frequency acoustic waves by picosecond laser pulses in the ultraviolet region and their detection by optical interferometric schemes, is presented. The two main acoustical modes, longitudinal and shear are clearly apparent in the time resolved spectra of solid materials, for various absorbing energies, extending from the thermoelastic to the ablative regime. The ultraviolet light is strongly absorbed by insulator materials like Pyrex and thus strong elastic waves are produced. From the time separation of the longitudinal waves we have deduced values for the speed of sound in various materials and of different thickness, that are in very good agreement with those reported in the literature. Also the time bandwidth of the sound waves is measured and significant differences, originating from different sample thickness, are apparent.

\section{9:00}

2aPAd4. Effect of photoacoustic generation mechanisms on transient grating measurements of thin films on silicon. Alexei A Maznev (Department of Applied Physics, Hokkaido University, Kita 13 Nishi 8, Kita-ku, 060-8628 Sapporo, Japan, alexei.maznev@gmail.com)

Laser induced transient gratings technique is widely used for noncontact measurements of surface acoustic waves (SAWs). One major application of such measurements is characterization of thin films used in microelectronics. A peculiar phenomenon has been observed in transient gratings measurements of thin films on a silicon substrate: if the film is transparent, there is a systematic discrepancy between the measured and expected SAW velocity values that cannot be accounted for by instrumental errors. However, as soon as the sample is coated by an opaque metal film, the discrepancy disappears. In this presentation, we will show that the origin of the effect is in the competition of thermal and electronic mechanisms of the optical generation of sound in silicon. We will also describe another effect of the same origin observed on very thin semi-transparent metal films: at a certain film thickness, the SAW signal vanishes as the thermal expansion of the film and the contraction of silicon caused by photoexcited carriers cancel each other.

\section{9:20}

2aPAd5. Thickness measurement of submicron metal coatings on transparent substrate by laser optoacoustic method. Ivan Pelivanov (International Laser Center of Moscow State University, Vorob’yovy Gory, 1, 119992 Moscow, Russian Federation, pelivanov@ilc.edu.ru), Daria Kopylova (International Laser Center of Moscow State University, Vorob'yovy Gory, 1, 119992 Moscow, Russian Federation, dskopylova@mail.ru), Nataliya Podimova (Moscow State University, MSU, 1, building 2, GSP-2, Leninskiye Gory, 119992 Moscow, Russian Federation, npodymova@mail.ru), Alexander Karabutov (Moscow State University, MSU, 1, building 2, GSP-2, Leninskiye Gory, 119992 Moscow, Russian Federation, akarabutov@gmail.com)

New nondestructive method for measurement of the thickness of submicron metal coatings on a transparent dielectric substrate is developed. Theoretical background includes the consecutive solution of the thermal and acoustic problems. The experimental part is based on the measurement of frequency dependence of the laser-ultrasound excitation efficiency on the metal coating thickness for the system where the coating is covered by a transparent liquid. Three chrome coatings of various thicknesses $(0.2,0.3$ and 0.6 micrometers) deposited on a quartz substrate were tested experimentally. A rough estimate of their thickness was obtained from the sputtering time. Two different experimental modes were used: forward mode (laser pulses irradiate the metal film through the substrate, excited acoustical transients are detected in the liquid) and backward mode (both laser irradiation of the film and detection of excited acoustical transients are performed in the liquid). Spectral dependencies of the thermo-optical transformation efficiency were calculated analytically and obtained experimentally for both signal detection modes. The values of the film thicknesses were determined by the least squares fitting of the theoretical curves to experimental data. It is demonstrated, that the developed optoacoustic method can be used for metal coatings thickness measurement in the range of $50 \mathrm{~nm}-5$ microns with inaccuracy of $50 \mathrm{~nm}$.

\section{9:40-10:00 Break}

\section{Invited Papers}

10:00

2aPAd6. Acoustic phenomena in porous media studied by transient grating spectroscopy: a critical test of the Biot theory. Riccardo Cucini (European Lab. for Non-Linear Spectroscopy (LENS), Univ. di Firenze, via Nello Carrara 1, 50019 Sesto Fiorentino (Fi), Italy, cucini@lens.unifi.it), Andrea Taschin (European Lab. for Non-Linear Spectroscopy (LENS), Univ. di Firenze, via Nello Carrara 1, 50019 Sesto Fiorentino (Fi), Italy, taschin@lens.unifi.it), Paolo Bartolini (European Lab. for Non-Linear Spectroscopy (LENS), Univ. di Firenze, via Nello Carrara 1, 50019 Sesto Fiorentino (Fi), Italy, bart@lens.unifi.it), Renato Torre (European Lab. for Non-Linear Spectroscopy (LENS), Univ. di Firenze, via Nello Carrara 1, 50019 Sesto Fiorentino (Fi), Italy, torre@lens.unifi.it)

The propagation of sound in a porous solid filled by a liquid can be described by a phenomenological model introduced by M. A. Biot, that is still extensively used to predict the essential acoustic properties of a wide variety of porous media. Nevertheless testing of the Biot theory at ultra/hypersonic frequencies and in porous media with nanometric scale heterogeneities remains an open issue. We 
studied the propagation of acoustic waves in two liquid-filled porous glasses by heterodyne detected transient grating experiments [1]. A test of the Biot theory is presented under two new regimes, namely, for a new frequency range up to $1.3 \mathrm{GHz}$ and for porous media with nanometric scale heterogeneities [2]. We show that the Biot theory describes the sound velocity data correctly, but does not account for the acoustic attenuation. We suggest that the acoustic damping is mainly due to the dissipation mechanisms intrinsic of the matrix and the liquid which are not accounted for in the theory. [1] Time-resolved spectroscopy of complex liquids, edited by Torre R. (Springer, New York) 2008. [2] A. Taschin, R. Cucini, P. Bartolini, R. Torre, Europhys. Lett. In press.

\section{0:20}

2aPAd7. Laser ultrasonic study of Lamb wave propagation in different film loading configurations. Xiaodong Xu (Modern Acoustics, Institute of Acoustics, Nanjing University, 210093 Nanjing, China, xdxu@nju.edu.cn), G. Shkerdin (Laboratorium voor Akoestiek en Thermische Fysica - Departement Natuurkunde en Sterrenkunde - Katholieke Universiteit Leuven, Celestijnenlaan 200D, B-3001 Leuven, Belgium, shkerdin@yahoo.com), Christ Glorieux (Lab. ATF, Katholieke Universiteit Leuven, Celestijnenlaan 200D, B-3001 Leuven, Belgium, christ.glorieux@fys.kuleuven.be)

A study is performed concerning the propagation Lamb waves in technologically and scientifically interesting configurations. In a first configuration a thin film was loaded with different liquids on its two sides. Three experimental laser ultrasonic configurations, line excitation with scanning detection, grating excitation with single point detection, and grating excitation with scanning probe beam, were implemented. All experimental results were found to be consistent between experimental methods and with theoretical predictions. The configuration can be implemented in monitoring applications where elastic properties of liquids that are kept in containers can be determined via the outside container wall. In a second configuration laser excited and detected Lamb waves in a rubber-metal bi-layer were used to determine the highly damped elastic properties of rubber.

\section{Contributed Papers}

\section{0:40}

2aPAd8. Zero-group velocity modes and local vibrations of an elastic plate. Claire Prada (Laboratoire Ondes et Acoustique, ESPCI, Université Paris 7, CNRS, 10 rue Vauquelin, 75005 Paris, France, claire.prada-julia@espci.fr), Dominique Clorennec (Laboratoire Ondes et Acoustique, ESPCI, Université Paris 7, CNRS, 10 rue Vauquelin, 75005 Paris, France, dominique.clorennec@espci.fr), Daniel Royer (Laboratoire Ondes et Acoustique, ESPCI, Université Paris 7, CNRS, 10 rue Vauquelin, 75005 Paris, France, daniel.royer@espci.fr)

Elastic plates or cylinders can support guided modes with zero group velocity $(\mathrm{ZGV})$ at a nonzero value of the wave number. The conditions required for the existence of $\mathrm{ZGV}$ Lamb modes in isotropic plates are discussed. It is shown that these modes appear in a range of Poisson's ratio over the value for which the cut-off frequency curves of modes belonging to the same family intercept, i.e for a bulk wave velocity ratio equal to a rational number. An interpretation of this phenomenon in terms of a strong repulsion between a pair of modes having a different parity in the vicinity of the cutoff frequencies is given. Using laser-based ultrasonic techniques, we experimentally investigate some properties of these ZGV Lamb modes: resonance, backward wave propagation, interference between backward and forward waves. Experiments performed with materials of various Poisson's ratio demonstrate that the resonance spectrum of an unloaded elastic plate, locally excited by a laser pulse, is dominated by the ZGV Lamb modes. From these local resonance frequencies, thickness variations can be measured accurately and material properties like the Poisson's ratio, bulk acoustic wave velocities or material attenuation can be determined without any mechanical contact.

\section{1:00}

2aPAd9. Mechanical contacts probed with picosecond ultrasonics. Thomas Dehoux (Division of Applied Physics, Graduate School of Engineering, Hokkaido University, 060-8628 Sapporo, Japan, dehoux@eng.hokudai.ac.jp), Motonobu Tomoda (Division of Applied Physics, Graduate School of Engineering, Hokkaido University, 060-8628 Sapporo, Japan, mtomoda@eng.hokudai.ac.jp), Oliver B. Wright (Division of Applied Physics, Graduate School of Engineering, Hokkaido University, 0608628 Sapporo, Japan, olly@eng.hokudai.ac.jp)

We investigate the contact between a thin metal film and a ball bearing indented to nanometre depths using picosecond ultrasonics. The area of contact is acoustically imaged to micron spatial resolution using $\mathrm{GHz}$ acoustic pulses produced by ultrashort pulsed optical excitation of the film through a transparent substrate. In particular, acoustic echoes are detected through transient optical reflectance changes that are monitored by probe optical pulses. In this way we image the acoustic reflection coefficient at the interface between the film and the indenter. In addition, by imaging the arrival time of the acoustic echoes we determine the penetration profile of the indenter to nanometre precision. Furthermore, imaging the transient thermoreflectance gives a different means for measuring the contact area through spatial variations in thermal diffusion. We thus demonstrate that picosecond ultrasonics and thermoreflectance provide powerful tools for the non-contact evaluation of mechanical contacts. These techniques could be applied to the in situ characterization of contact interfaces between machine elements.

\section{Invited Paper}

\section{1:20}

2aPAd10. Photoacoustic imaging and laser-ultrasonics using Fourier domain reconstruction methods. Peter Burgholzer (Upper Austrian Research, Hafenstr. 47, 4020 Linz, Austria, peter.burgholzer@uar.at), Thomas Berer (Upper Austrian Research, Hafenstr. 47, 4020 Linz, Austria, thomas.berer@uar.at), Bernhard Reitinger (Upper Austrian Research, Hafenstr. 47, 4020 Linz, Austria, bernhard.reitinger@uar.at), Robert Nuster (Karl-Franzens-Universität Graz, Universitätsplatz 5, 8010 Graz, Austria, ro.nuster@uni-graz.at), Günther Paltauf (Karl-Franzens-Universität Graz, Universitätsplatz 5, 8010 Graz, Austria, guenther.paltauf @uni-graz.at)

Laser-ultrasonics as well as photoacoustic imaging use optically generated acoustic waves detected at the sample surface to image its interior. In laser-ultrasonics a laser pulse is absorbed at the sample surface generating an ultrasound pulse that propagates into the sample, is subsequently reflected at internal structures, and finally detected at the surface by an interferometer. In photoacoustic imaging ultrasound is generated by heating of light-absorbing structures inside of an optical semitransparent sample. The goal in photoacoustic imaging is to recover the spatial distribution of the absorbed energy density inside the sample from the acoustic pressure signals measured outside the sample (photoacoustic inverse problem). Fourier reconstruction is based on the decomposition into plane waves and is a fast and efficient method used in photoacoustic imaging. Interpolation is needed when signal Fourier components are mapped to 
source Fourier components. We have shown that the synthetic aperture focusing technique (SAFT) in frequency domain, which needs no interpolation, and the Fourier reconstruction method are mathematically equivalent if the step size of the spatial discretization goes to zero. Both imaging methods are compared using simulated data and measurement data acquired with our interferometer set-up. This work has been supported by the Austrian Science Fund (FWF), project P18172-N02 and project L418-N20.

\section{Contributed Papers}

\section{1:40}

2aPAd11. Stroboscopic interferometric full-field imaging of laser-induced surface acoustic waves. Bart Sarens (Laboratorium voor Akoestiek en Thermische Fysica - Departement Natuurkunde en Sterrenkunde - Katholieke Universiteit Leuven, Celestijnenlaan 200D, B-3001 Leuven, Belgium, bart.sarens@fys.kuleuven.be), Osamu Matsuda (Division of Applied Physics, Graduate School of Engineering, Hokkaido University, 0608628 Sapporo, Japan, omatsuda@eng.hokudai.ac.jp), Xiaodong Xu (Modern Acoustics, Institute of Acoustics, Nanjing University, 210093 Nanjing, China, $\quad x d x u @ n j u . e d u . c n)$, Georgios Kalogiannakis (Laboratorium voor Akoestiek en Thermische Fysica - Departement Natuurkunde en Sterrenkunde - Katholieke Universiteit Leuven, Celestijnenlaan 200D, B-3001 Leuven, Belgium, Georgios.Kalogiannakis@vub.ac.be), Robbe Salenbien (Lab. ATF, Katholieke Universiteit Leuven, Celestijnenlaan 200D, B-3001 Leuven, Belgium, robbe.salenbien@fys.kuleuven.be), Renaud Côte (Lab. ATF, Katholieke Universiteit Leuven, Celestijnenlaan 200D, B-3001 Leuven, Belgium, renaud.cote@ fys.kuleuven.be), Christ Glorieux (Lab. ATF, Katholieke Universiteit Leuven, Celestijnenlaan 200D, B-3001 Leuven, Belgium, christ .glorieux@fys.kuleuven.be)

Surface acoustic waves (SAW) have the interesting property that they specifically interact with surface and sub-surface regions, rendering them suitable for non-contact investigation of sub-surface properties and heterogeneities. Transmission, reflection and diffraction effects of SAW propagation are analysed in order to reveal information on the region over which they propagate. Laser excitation allows to efficiently excite SAW with short wavelengths, enhancing both the lateral and the depth resolution. Typically information on the waves is collected by scanning a part of the surface of interest with a fast laser probe, using the surface displacement or slope as a real time witness of the wave field. Here we present results obtained by a full field imaging technique [1], in which the sample is repetitively excited by a pulsed pump laser, and the wave displacement field is stroboscopically gathered by illuminating the sample surface with an electronically delayed pulsed probe laser, whose displacement induced optical path variations are visualized by interferometrically analyzing it. In this work, the technique is applied on different samples, varying the geometry of the pump laser beam pattern, the interferometric configuration, and the heterogeneities of the sample. [1] "Phase mask-based interferometer: operation principle, performance, and application to thermo-elastic phenomena", C. Glorieux, J.D. Beers, E.H. Bentefour, K. Van de Rostyne and K.A. Nelson, Rev. Sci. Instrum. 75(9), 2906-2920 (2004).

\section{2:00}

2aPAd12. Laser-ultrasonic defectoscope with focused transducer. Alexander Karabutov (Moscow State University, MSU, 1, building 2, GSP-2, Leninskiye Gory, 119992 Moscow, Russian Federation, akarabutov@gmail.com), Nataliya Podimova (Moscow State University, MSU, 1, building 2, GSP-2, Leninskiye Gory, 119992 Moscow, Russian Federation, npodymova@mail.ru)

The novel focused laser-ultrasonic transducer for ultrasonic microscopy of constructive materials is presented. Short and sharp ultrasonic pulse is produced by optoacoustic effect - excitation of ultrasound by absorption of nanosecond laser pulse in special layer. Absorbing layer is integrated with the wide-band ultrasonic receiving system into common unit - laserultrasonic transducer. Ultrasound beam is focused by acoustical lens with relatively low numerical aperture (convergence angle $-15^{\circ}$, focal distance $20 \mathrm{~mm}$ ). An acoustic impedance of the lens matches that of the laserultrasonic transducer. The duration of the irradiated ultrasonic pulse was $\sim 0.1 \mu \mathrm{s}$. The focused laser-ultrasonic transducer was mounted on 3-D linear translation system. The object under control and the transducer was placed in a tank with distilled water. The transducer was moved across the object with $2.5 \mu \mathrm{m}$ step. The caustic of the probe ultrasonic beam was analyses by sliding the transducer across sharp beard of a knife. The reflected ultrasonic signal was recorded by 12-bit ADC. The diameter of the focal area was 0.53 $\mathrm{mm}$. The length of caustic of ultrasonic was $28 \mathrm{~mm}$. The amplitude of the probe ultrasonic pulse was $\sim 20 \mathrm{kPa}$ at laser energy $0.1 \mathrm{~mJ}$. The dynamic range of ultrasonic trace measurement exceeded $90 \mathrm{~dB}$.

\section{$12: 20$}

2aPAd13. Acoustic waves generated by a laser point pulse in a micrometric fiber. Damien Segur (LMP, UMR CNRS 5469, Université Bordeaux I, 351, cours de la Libération, 33405 Talence, France, d.segur@1mp.u-bordeaux1.fr), Alexander Shuvalov (LMP, UMR CNRS 5469, Université Bordeaux I, 351, cours de la Libération, 33405 Talence, France, a.shuvalov@1mp.u-bordeaux1.fr), Yong Dong Pan (Institute of Acoustics, Tongji University, 200092 Shangai, China, ypan@mail.tongji.edu.cn), Nikolay Chigarev (LPEC/UMR 6087/CNRS/Université du Maine, Avenue Olivier Messiaen, 72085 Le Mans Cedex 09, France, Nikolay.Chigarev@univ-lemans.fr), Clément Rossignol (LMP, UMR CNRS 5469, Université Bordeaux I, 351, cours de la Libération, 33405 Talence, France, c.rossignol@lmp.u-bordeaux1.fr), Bertrand Audoin (LMP, UMR CNRS 5469, Université Bordeaux I, 351, cours de la Libération, 33405 Talence, France, b.audoin@Imp.u-bordeaux1.fr)

Having emerged in the 1980s, the laser ultrasonics technique with its non-contact generation and detection process overpasses the difficulties of coupling piezoelectric transducers with curved surfaces. To date, the authors [1] have been interested in acoustic generation for cylinders opaque at a given laser wavelength and for the acoustic source located at the cylinder surface. In this presentation, assuming point focusing of the laser pulses, we propose a three-dimensional (3D) semi-analytical model for acoustic waves generation and propagation in a partly transparent isotropic cylinder. First, the radial displacement at any position on the free surface is derived, in a 3D Fourier domain, for an inner point source. The response to a volume-source distribution along a radius is obtained as a convolution of the above Green function with the corresponding source distribution caused by optical absorption. Three inverse transforms are then applied to obtain the radial displacement at the cylinder surface. Picosecond ultrasonics experiments are performed on different micrometric fibers and compared with calculated waveforms for different optical absorptive properties. References [1] Y.D. Pan, C. Rossignol and B. Audoin, Appl. Phys. Lett. 82, 4379 (2003).

\section{$12: 40$}

2aPAd14. Simulations of Thermally Induced Photoacoustic Wave Propagation Using a Pseudospectral Time-Domain Method. Yae-Lin Sheu (Dept. Electrical Engineering, National Taiwan University, No.1, Sec. 4, Roosevelt Road, 106 Taipei, Taiwan, b88901147@ntu.edu.tw), Pai-Chi Li (Institute of Biomedical Electronics and Bioinformatics, National Taiwan University, No.1, Sec. 4, Roosevelt Road, 106 Taipei, Taiwan, paichi@cc.ee .ntu.edu.tw)

Physical models used to evaluate thermally induced photoacoustic waves in biomedical applications are mostly approximations based on certain hypotheses, such as the thermal and stress confinements, for the sake of obtaining analytical results. On the other hand, using numerical methods to solve the general photoacoustic wave equations gives detailed information of wave phenomena without making as many assumptions. The photoacoustic wave generated by thermal expansion involves the heat conduction theorem and the state, continuity, and Navier-Stokes equations. In this study a numerical approach was developed in $2.5 \mathrm{D}$ axis-symmetric cylindrical coordinates using a pseudospectral time-domain (PSTD) scheme. The method is efficient for large scale simulations in that only two grids for the smallest 
wavelength are required, where in conventional methods $10 \sim 20$ grids are typically needed. The numerical techniques include Berenger's perfectly matched layers (PMLs) for free wave simulations, and linear-perturbation analytical solutions are used to validate the simulation results. The numeri- cal results using 2 grids for the minimum wavelength in simulation domain agree with theory to within an error of $7 \times 10^{-3}$ in the absolute differences. On the other hand, conventional methods such as finite-difference timedomain method requiring 10 grids result in an error of $1.3 \times 10^{-3}$.

\title{
Invited Paper
}

\section{1:00}

2aPAd15. Laser ultrasonic study of Lamb wave propagation in different film loading configurations. Xiaodong Xu (Modern Acoustics, Institute of Acoustics, Nanjing University, 210093 Nanjing, China, xdxu@nju.edu.cn), G. Shkerdin (Laboratorium voor Akoestiek en Thermische Fysica - Departement Natuurkunde en Sterrenkunde - Katholieke Universiteit Leuven, Celestijnenlaan 200D, B-3001 Leuven, Belgium, shkerdin@yahoo.com), Christ Glorieux (Lab. ATF, Katholieke Universiteit Leuven, Celestijnenlaan 200D, B-3001 Leuven, Belgium, christ.glorieux@fys.kuleuven.be)

A study is performed concerning the propagation Lamb waves in technologically and scientifically interesting configurations. In a first configuration a thin film was loaded with different liquids on its two sides. Three experimental laser ultrasonic configurations, line excitation with scanning detection, grating excitation with single point detection, and grating excitation with scanning probe beam, were implemented. All experimental results were found to be consistent between experimental methods and with theoretical predictions. The configuration can be implemented in monitoring applications where elastic properties of liquids that are kept in containers can be determined via the outside container wall. In a second configuration laser excited and detected Lamb waves in a rubber-metal bi-layer were used to determine the highly damped elastic properties of rubber.

\section{Session 2aPPa}

\section{Psychological and Physiological Acoustics and ASA Committee on Standards: Applications of Psychoacoustics I}

\author{
Patricia Davies, Cochair
}

Ray W. Herrick Lab., School of Mechanical Engineering, Purdue Univ., 140 S. Martin Jischke Drive, West Lafayette, IN 47907-2031, USA

Hugo Fastl, Cochair

AG Technische Akustik, MMK, TU München, Arcisstr. 21, München, 80333, Germany

\section{Invited Papers}

\section{8:00}

2aPPa1. Determination of filtering parameters for dichotic-listening binaural hearing aids. Yôiti Suzuki (R.I.E.C., Tohoku University, 2-1, Katahira, Aoba-ku, 980-8577 Sendai, Japan, yoh@ais.riec.tohoku.ac.jp), Atsunobu Murase (Panasonic Shikoku Electronics Co., Ltd, 600, Saedo-cho, Tsuzuki-ku, 224-8539 Yokohama, Japan, murase.atsunobu@jp.panasonic.com), Motokuni Itoh (Matsushita Electric Industrial Co., Ltd, 600, Saedo-cho, Tsuzuki-ku, 224-8539 Yokohama, Japan, ito.gempo@jp.panasonic.com), Shuichi Sakamoto (R.I.E.C., Tohoku University, 2-1, Katahira, Aoba-ku, 980-8577 Sendai, Japan, saka @ ais.riec.tohoku.ac.jp)

Sensorineural hearing-impaired people have difficulty in hearing sounds not only because of the increased hearing threshold, but also because of their reduced dynamic range of hearing (loudness recruitment), as well as the large and extensive masking resulting from the reduced frequency selectivity, especially masking by which middle-frequency and high-frequency components are masked by the intense low-frequency component, the so-called upward spread of masking. Dichotic listening, listening to complementary filtered speech signals given to the two ears, has been proposed to cope with these problems. We introduce a dichotic listening technique for binaural hearing aids and the relationship between the effect of this technique and auditory characteristics of hearing-impaired people. The results of listening tests imply that some relationship exists between the width of the auditory filter and the effect of this technique. Moreover, intelligibility test results obtained using the low-frequency-boosted sounds suggest that this dichotic listening reduced the upward spread of masking. We are applying this technique to hearing aid systems and have begun evaluating the performance of this hearing aid. 
2aPPa2. Binaural auralization based on spherical-harmonics beamforming. Wookeun Song (Brïel \& Kjær Sound \& Vibration Measurement A/S, Skodsborgvej 307, DK-2850 Nærum, Denmark, wksong@bksv.com), Wolfgang Ellermeier (Institut für Psychologie, Technische Universität Darmstadt, Alexanderstraße 10, D-64283 Darmstadt, Germany, ellermeier@psychologie .tu-darmstadt.de), Jørgen Hald (Brüel \& Kjær Sound \& Vibration Measurement A/S, Skodsborgvej 307, DK-2850 Nærum, Denmark, JHALD@bksv.com)

The binaural auralization of a 3D sound field using spherical-harmonics beamforming (SHB) techniques was investigated and compared with the traditional method using a dummy head. The new procedure was verified by comparing simulated room impulse responses with directly measured ones both monaurally and binaurally. The objective comparisons show that there is good agreement in the frequency range between 0.1 to $6.4 \mathrm{kHz}$. Psychoacoustic attributes of multi-channel reproduced sounds were measured in a listening experiment to validate the method subjectively. The results show that subjective ratings of the width, spaciousness and preference of different audio reproduction modes auralized based on SHB were not significantly different from those obtained for dummy head measurements. Thus binaural synthesis using SHB may be a useful tool to reproduce a 3D sound field binaurally while saving considerably on measurement time because head rotation can be simulated based on a single recording.

\section{8:40}

2aPPa3. Simulation of wave field synthesis. Florian Völk (AG Technische Akustik, MMK, TU München, Arcisstr. 21, 80333 München, Germany, florian.voelk@mytum.de), Josef Konradl (AG Technische Akustik, MMK, TU München, Arcisstr. 21, 80333 München, Germany, koj@mmk.ei.tum.de), Hugo Fastl (AG Technische Akustik, MMK, TU München, Arcisstr. 21, 80333 München, Germany, fast1@mmk.ei.tum.de)

Wave field synthesis utilizes a large number of loudspeakers to generate a desired wave field. It therefore is necessary to drive each speaker with an independent signal, which requires as many amplifier and soundcard channels as there are loudspeakers. These enormeous hardware costs make research and development very expensive and time consuming. Additionally, different rooms influence the wave field synthesis arrays in different ways. For this reason a simulation technique is of advantage which permits the evaluation of the perceived properties of arbitrary wave field synthesis configurations without the need to physically construct them. This paper proposes a simulation system capable to simulate wave field synthesis systems in different rooms based on physical measurements of one loudspeaker in each room. The techniques used are presented, and possiblities as well as limits of the system are discussed.

\section{9:00}

2aPPa4. The next generation of artificial heads. Janina Fels (Institute of Technical Acoustics, RWTH Aachen University, Neustr. 50, 52056 Aachen, Germany, Janina.Fels@akustik.rwth-aachen.de), Michael Vorlaender (Institute of Technical Acoustics, RWTH Aachen University, Neustr. 50, 52056 Aachen, Germany, mvo@akustik.rwth-aachen.de)

Standardised artificial heads are vital means when it comes to describing the binaural transmission from the sound field into the ear canal or rather the eardrum. In recent years numerous fields of application were created ranging from room acoustics, to product sound design or telecommunications, all based on the well-known KEMAR standard IEC TR 959. In the meantime, however, it has become a well-known fact, that a) specific artificial heads with natural heads or replicas in hearing experiments are superior to standard artificial heads as far as the quality of spatial hearing is concerned and that $b$ ) the standardised heads do not comply with the dimensions of an average population (the standard heads are too small). In this contribution first of all today's situation will be assessed and then a possible way will be outlined that could lead to a new future artificial head standard. This includes an adequate match with adult population for various continents, and also new approaches such as children-size artificial heads to measure and fit hearing aids or for new measurement techniques for classroom acoustics.

2aPPa5. Auditory memory and evaluation of environmental sounds. Sonoko Kuwano (Graduate School of Human Sciences, Osaka University, 1-2 Yamadaoka, 565-0871 Suita, Osaka, Japan, kuwano@see.eng.osaka-u.ac.jp), Seiichiro Namba (2-7-5-604 Obana, 666-0015 Kawanishi, Hyogo, Japan, QZW00041@nifty.com), Tohru Kato (Otemon Gakuin University, 2-1-15 Nishiai, 5678502 Ibaraki, Osaka, Japan, tkatou@res.otemon.ac.jp)

A series of experiments were conducted concerning the memory of environmental sounds. Twelve kinds of sound were presented with soft background noise of about $6 \mathrm{~min}$ to participants. They were asked to recall or recognize the sound sources and to judge the loudness of the recalled or recognized sounds some period after they listened to the sounds. The recalled or recognized loudness was examined in relation to the length of the period between the presentation of sounds and the judgment of loudness, the method to measure the memory, LAeq of each sound source, etc. The results suggest that it is possible to judge the loudness of the memorized sounds and that the judgments seems reliable. 
2aPPa6. Comparison of subjective impression of copy machine noise between Japanese, American and German participants. Tatsuya Furukawa (Ricoh Co., Ltd., 16-1 Shinei-cho, Tsuzuki-ku, 224-0035 Yokohama, Japan, furukawa@rdc.ricoh.co.jp), Osamu Takehira (Ricoh Co., Ltd., 16-1 Shinei-cho, Tsuzuki-ku, 224-0035 Yokohama, Japan, osamu.takehira@nts.ricoh.co.jp), Masaki Nagamiya (Ricoh Co., Ltd., 16-1 Shinei-cho, Tsuzuki-ku, 224-0035 Yokohama, Japan, masaki.nagamiya@nts.ricoh.co.jp), Sonoko Kuwano (Graduate School of Human Sciences, Osaka University, 1-2 Yamadaoka, 565-0871 Suita, Osaka, Japan, kuwano@see.eng.osaka-u.ac.jp), Seiichoro Namba (Graduate School of Human Sciences, Osaka University, 1-2 Yamadaoka, 5650871 Suita, Osaka, Japan, namba.seiichiro@ nifty.com), Hugo Fastl（AG Technische Akustik, MMK, TU München, Arcisstr. 21, 80333 München, Germany, fastl@mmk.ei.tum.de)

Psychological evaluations of copy machine noise are in progress to lower acoustical noise and to avoid disturbing the office environment. Psychological experiments were made for Japanese, American and German participants to examine cultural differences in sensitivity to copy machine noise using the Semantic Differential. Seventeen copy machine and laser printer sounds were used for the experiments. The results were analyzed statistically. The experiments revealed that, just as with Japanese, Americans and Germans were mostly sensitive to "sound pressure levels." However, the second influential factor for Japanese participants was "impulsiveness" but "sharpness" for Americans and Germans. Evaluation formulas for "pleasing" noise using physical values were decided for each country. This suggests that copy machine noise will be evaluated without psychological experiments. The information gathered will possibly be used to adapt copy machine noise to levels that are comfortable to users in different countries.

\section{0:00}

2aPPa7. Applications of psychoacoustics to information technology products. Willem M. Beltman (Intel Corporation, $2111 \mathrm{NE}$ 25th Avenue, M/S JF2-86, Hillsboro, OR 97124, USA, willem.m.beltman@intel.com), Rina A. Doherty (Intel Corporation, 5200 NE Elam Young Parkway, Hillsboro, OR 97124, USA, rina.a.doherty@intel.com), Eric Salskov (University of Twente, Dept. Mechanical Engineering, P.O. Box 217, 7500 AE Enschede, Netherlands, eric.salskov@intel.com), Philip J. Corriveau (University of Twente, Dept. Mechanical Engineering, P.O. Box 217, 7500 AE Enschede, Netherlands, philip.j.corriveau@intel.com), Doug Gabel (Intel Corporation, 2111 NE 25th Avenue, M/S JF2-86, Hillsboro, OR 97124, USA, doug.gabel@intel.com), Eric Baugh (Intel Corporation, 15400 NW Greenbrier Parkway, Beaverton, OR 97006, USA, eric.baugh@intel.com)

Emerging usage models for computing devices require low acoustic noise, for example in home entertainment systems. Studies have shown that not only the overall level, but also the psychoacoustic aspects matter. This paper provides an overview of testing techniques that are used in the information technology industry and outlines two specific case studies. First, an extensive subjective psychoacoustic study was designed and conducted in multiple geographies to determine the aspects of sound that best describe the annoyance to sound from information technology products in a home type environment. Over 200 participants in four countries participated in this carefully controlled experiment and rated typical steady state sounds on a 5 point annoyance scale. The relevant sound quality metrics were extracted and geographical variations quantified. Second, in a paired comparison study the influence of modulation on annoyance was investigated by superimposing different frequency and amplitude modulated sounds onto a baseline sound. The results indicate that modulation can have a significant effect on subjective perception.

\section{0:20-10:40 Break}

\section{0:40}

2aPPa8. Identification of transient events from a hard disk drive using non-stationary loudness. Dave Ali (Western Digital, 5863 Rue Ferrari, San Jose, CA 95127, USA, dave.ali@wdc.com)

The Hard Disk Drive (HDD) industry has been pushed so much to lower its limits of acoustic emissions by OEMs and customers that the levels of most HDDs flirt with the threshold of hearing and in some cases go below. With these much reduced levels come different problems and complaints from customers, namely transient events; latching, de-latching, spin up, spin down, etc. The difference in amplitude and duration of these events typically stand out to the human ear above the steady state nature of the HDD during idle and sometimes when it's active. This paper shows a method of characterizing these events with confidence to help in designing a better product for the industry.

\section{1:00}

2aPPa9. Automotive tire/road sound quality. Gabriella Cerrato Jay (Sound Answers Inc, 4856 Alton Drive, Suite 100, Troy, MI 48085, USA, gabriella.cerratojay@soundanswers.net), Todd Freeman (Sound Answers Inc, 4856 Alton Drive, Suite 100, Troy, MI 48085, USA, todd.freeman@soundanswers.net), Chris Raglin (Cooper Tire \& Rubber Company, 701 Lima Avenue, Findlay, OH 45840, USA, caraglin@coopertire.com), Timothy Carson (IAC North America, 47785 W Anchor CT, Plymouth, MI 48170, USA, tcarson@iacna.com)

Tire/road noise and sound quality are increasingly important factors for customer satisfaction. As vehicle interior sound levels decrease, the noise from tires and their interaction with the road become more noticeable. Both tire and vehicle manufacturers need to assess as early as possible the impact of any tire-vehicle combination on perceived interior sound quality. In this paper, we describe two projects in which psychoacoustics concepts were applied to help, on one hand, tire manufacturers to screen for designs likely to generate poor sound quality in vehicle, and, on the other hand, vehicle manufacturers to measure the impact of road noise and interior acoustic treatment on speech transmission in the cabin. In both projects, the activities were driven by the understanding of the psychoacoustic features of tire/road noise and by the need to improve customer satisfaction. 


\section{Contributed Paper}

11:20

2aPPa10. Acoustical aspects of travel comfort in the aircraft cabin. Ingo Baumann (Oldenburg University, Institute of Physics - Acoustics, Carl-von-Ossietzky Str. 9-11, 26111 Oldenburg, Germany, ingo @ aku.physik.uni-oldenburg.de), Sandra Buss (Oldenburg University, Institute of Physics - Acoustics, Carl-von-Ossietzky Str. 9-11, 26111 Oldenburg, Germany, sandra@aku.physik.uni-oldenburg.de), Nils Freese (Oldenburg University, Institute of Physics - Acoustics, Carl-von-Ossietzky Str. 9-11, 26111 Oldenburg, Germany, nils_f@aku.physik.uni-oldenburg.de), Volker Mellert (Oldenburg University, Institute for Physics, 26111 Oldenburg, Germany, volker.mellert@uni-oldenburg.de), Reinhard Weber (Oldenburg University, Institute of Physics - Acoustics, Carl-von-Ossietzky Str. 9-11, 26111 Oldenburg, Germany, Reinhard.Weber@uni-oldenburg.de)
In several European projects tests were conducted during real flights and in aircraft simulators in order to identify important parameters of travel comfort. Physical environmental, physiological and questionnaire data were measured and investigated with respect to human perception. Besides numerous intrinsic quantities affecting health, well-being and awareness of ambience defined measurable environmental parameters influence flight and cabin crews' as well as passengers' perception, psychology and physiology. Sound and vibration are relevant environmental parameters with impact on passenger and crew. Other important influence is caused by e.g. air quality, pressure, local climate. Statistical analysis of the collected data reveals significant correlations between environment and human response for selected groups of test persons. A specific acoustic comfort is not well defined but part of common well-being and comfort. The demand for a general human response model is discussed, which relates ambience and perception.

\section{Invited Papers}

\section{$11: 40$}

2aPPa11. The influence of speed bumps on perceived annoyance. Anna Preis (Institute of Acoustics, Adam Mickiewicz University, Unultowska 85, 61-614 Poznan, Poland, apraton@amu.edu.pl), Tomasz Kaczmarek (Institute of Acoustics, Adam Mickiewicz University, Unultowska 85, 61-614 Poznan, Poland, tomek@spl.ia.amu.edu.pl), Barbara Griefahn (Institute for Occupational Physiology, Ardeystr. 67, 44139 Dortmund, Germany, griefahn@ifado.de), Truls Gjestland (O.S. Bragstads plass 2, N-7464 Trondheim, Norway, Truls.Gjestland@sintef.no)

Recently, several attempts to use speed bumps as a noise reduction method have been made. Objective analyses of the effect of speed bumps on noise have been shown to result in a rather small reduction of noise. In the present paper the influence of speed bumps on perceived annoyance is investigated. The annoyance rating of a situation in which a passenger car approaches with constant velocity, then decelerates, crosses the bump, accelerates, and then recedes at a constant speed was compared with a car pass-by at a constant velocity without a bump. Three different velocities were analyzed: 40,50 and $60 \mathrm{~km} / \mathrm{h}$, and two types of driving conditions: normal, and aggressive. Listeners judged their annoyance for all the investigated scenarios using the ICBEN scale (0-10) for annoyance assessment. Objective analyses showed a significant reduction of LAeqT in the bump situation for all tested velocities, and for both driving conditions. The results of this psychoacoustic experiment show no effect of the bump on annoyance rating for normal driving conditions. However, in aggressive driving conditions the bump resulted in a significant increase in annoyance. In the light of these results, speed bumps cannot be considered as a noise reduction method.

\section{2:00}

2aPPa12. The Adequate Sound Levels for Acoustic Signs for Visually Impaired in the Sound Environment with Ambient Musics from shops. Katsuya Yamauchi (Faculty of Engineering, Nagasaki University, 1-14 Bunkyo-machi, 852-8521 Nagasaki, Japan, yamauchi@cis.nagasaki-u.ac.jp), Koji Nagahata (Faculty of Symbiotic Systems Science, Fukushima University, 1 Kanayagawa, 9601296 Fukushima, Japan, nagahata@sss.fukushima-u.ac.jp), Mari Ueda (Faculty of Human-Environment, Kyushu University, 6-19-1 Hakozaki, Higashik-ku, 812-8581 Fukuoka, Japan, mari-u@gsd.design.kyushu-u.ac.jp), Shin-Ichiro Iwamiya (Kyushu University, 4-9-1 Shiobaru, Minami-ku, 815-8540 Fukuoka, Japan, iwamiya@design.kyushu-u.ac.jp)

Providing auditory signs for the visually impaired is one of the most effective ways to support their orientation and mobility. Although the use of such sounds by the visually impaired has been revealed qualitatively, the acoustical properties of ideal sound-designs for them have not known sufficiently, even a basic property such as the sound level of these sounds. On the other hand, the advertising sounds from shops such as ambient musics are the one of the typical sounds in the Japanese downtown. These sounds also disturb their sound information listening and make their mobility difficult. Our previous studies revealed the adequate sound levels of acoustic signs under the road traffic noise environment. This study discusses the the effect of the ambient musics from shops on the adequate sound levels of acoustic signs through the psychoacoustical experiment. The results showed that the difference between the adequate sound level of acoustic signs and the environmental noise levels are larger than that under the road traffic noise environment. This trend is thought to be attributed to the frequency characteristics of the acoustical signs and the environmental noise.

$$
12: 20
$$

2aPPa13. An algorithm modelling the Irrelevant Sound Effect (ISE). Sabine J. Schlittmeier (Work, Environmental and Health Psychology, Catholic University of Eichstaett-Ingolstadt, Ostenstr. 26-28, 85072 Eichstaett, Germany, sabine.schlittmeier@ku-eichstaett.de), Tobias Weissgerber (AG Technische Akustik, MMK, TU München, Arcisstr. 21, 80333 München, Germany, tobias@p-weissgerber.de), Stefan Kerber (AG Technische Akustik, MMK, TU München, Arcisstr. 21, 80333 München, Germany, stefan@ihr.mrc.ac.uk), Hugo Fastl (AG Technische Akustik, MMK, TU München, Arcisstr. 21, 80333 München, Germany, fastl@mmk.ei.tum.de), Juergen Hellbrueck (Work, Environmental and Health Psychology, Catholic University of Eichstaett-Ingolstadt, Ostenstr. 26-28, 85072 Eichstaett, Germany, juergen.hellbrueck@ku-eichstaett.de)

Verbal short-term memory capacity is reduced significantly during certain background sounds. Remembering a series of digits is significantly impaired by speech or music with prominent staccato passages whereas, for example, music with prominent legato passages does not disturb performance in comparison to silence. This so-called Irrelevant Sound Effect (ISE) occurs although the background sounds are irrelevant with respect to the digit sequences to be remembered. Until now, a multitude of cognitive psychological experiments explored the ISE and collected behavioral performance data during different sound conditions. The talk presents an algo- 
rithm which models performance data in ISE experiments, i.e. the detrimental impact of background sounds on memory performance. The data base of this algorithm is about 50 background sounds and corresponding performance data, which have been collected in cognitive psychological experiments at the KU Eichstaett-Ingolstadt. The algorithm is based on the instrumental measuring of the hearing sensation fluctuation strength and is able to reconstruct the performance results in about $90 \%$ of cases within the interquartile ranges. The algorithm will be discussed within the scope of cognitive short-term memory models, which claim to explain the ISE and with respect to practical implications.

\section{2:40-2:00 Lunch Break}

\section{Invited Papers}

2aPPa14. Fusion and masking threshold of a tone in narrowband noise. Hans Hansen (IRCAM - Sound Perception \& Design, 1 place Igor Strawinsky, 75004 Paris, France, Hans_Hansen@gmx.de), Reinhard Weber (Oldenburg University, Institute of Physics Acoustics, Carl-von-Ossietzky Str. 9-11, 26111 Oldenburg, Germany, Reinhard.Weber@uni-oldenburg.de)

When comparing different pitch phenomena, it is not clear whether the expression pitch strength points towards a unique perceptive phenomenon or object. As many studies concentrate on one special phenomenon, the question, what is actually judged, seems only implicitly answered. Kubovy and Van Valkenburg (2001) define a perceptual object as "that what is susceptible to figure-ground segregation". Pitch plays a major role in these grouping processes. In this context the judgment of pitch strength refers to two perceptual cases. The first one is the pitch strength of a tone-in-noise, i.e. the case where the pitch is linked to a separate contour within the narrowband noise $(\mathrm{NBN})$, while the second is the pitch strength of tonal noise. Here, the noise evokes a pitch sensation that is not related to a separate object. In order to explore the transition from case $/ 1$ tone-in-noise to case $/ 2$ tonal noise, the identification threshold hearing a tone in NBN centered on the tone is determined in a lab experiment. This segregation or fusion threshold is compared to the measured masking threshold for center frequencies $250-4000 \mathrm{~Hz}$ octave-wise at $60 \mathrm{~dB}$ SPL noise level. The bandwith of the NBN is varied from $50-250 \mathrm{~Hz}$ accordingly.

2aPPa15. Are absolute thresholds and loudness judgements influenced by different colours? Daniel Menzel (AG Technische Akustik, MMK, TU München, Arcisstr. 21, 80333 München, Germany, menzel@tum.de), Elias Faccinelli (AG Technische Akustik, MMK, TU München, Arcisstr. 21, 80333 München, Germany, fac@mmk.ei.tum.de), Hugo Fastl (AG Technische Akustik, MMK, TU München, Arcisstr. 21, 80333 München, Germany, fastl@mmk.ei.tum.de)

Previous experiments showed that images of differently colored trains and sports cars can influence the loudness ratings given by subjects via free magnitude estimation. Red vehicles caused subjects to rate the loudness of simultaneously presented train or car sounds higher relative to green vehicles. To investigate whether these loudness differences correspond to shifts in absolute threshold, subject's threshold in quiet was measured via Békésy-tracking while viewing red, green, and neutral color patches. Also, the influence of color on the loudness of broadband noise was measured using a method of adjustment, in which subjects had to adjust the level of a test sound until it was perceived as loud as a reference sound. In both cases, no influence of color on either absolute threshold or loudness perception measured via adjustment could be found. These results support the hypothesis that, compared to previously used methods like magnitude estimation, methods that require subjects to concentrate on the auditory stimulus do not seem to be as applicable for measurements of audio-visual interactions.

\section{2:40}

2aPPa16. An efficient masking model for audio coding exploiting spectro-temporal masking. Steven Van De Par (Philips Research Europe, Digital Signal Processing (MS WO02), High Tech Campus 36, 5656 AE Eindhoven, Netherlands, steven.van.de.par@philips.com), Jeroen Koppens (Philips Research Europe, Digital Signal Processing (MS WO02), High Tech Campus 36, 5656 AE Eindhoven, Netherlands, jeroen.koppens@philips.com), Armin Kohlrausch (Philips Research Europe, Digital Signal Processing (MS WO02), High Tech Campus 36, 5656 AE Eindhoven, Netherlands, armin.kohlrausch@philips.com), Werner Oomen (Philips Research Europe, Digital Signal Processing (MS WO02), High Tech Campus 36, 5656 AE Eindhoven, Netherlands, werner .oomen@philips.com)

Perceptual audio coding achieves part of its coding efficiency by spectrally shaping the quantization noise such that it is masked by the audio signal to be encoded. In order to determine how much quantization noise is allowed within each frequency band and time interval a masking model is used to predict a masking curve specifying the maximally allowed quantization noise level within each frequency band. In most audio coders only spectral masking properties of the audio signal are used. The model by Dau et al. [J. Acoust. Soc. Am. 99, Vol. 3615, 1996] provides an interesting approach to also model temporal masking. Since this model operates as an artificial observer it only predicts whether the quantization noise is audible or not in the presence of the audio signal. In order to determine the most efficient quantization noise shape, the encoder needs to iteratively adapt the noise shape and evaluate each option with the model. This implies a highly computational complex encoding algorithm. In this contribution we will present an efficient masking model based on the Dau et al. model that only requires a single evaluation of the input signal to determine the maximally allowed noise level within each frequency band. 
2aPPa17. Factor analyses of critical-band-filtered speech of British English and Japanese. Kazuo Ueda (Perceptual Psychology Unit, Kyushu University, 4-9-1 Shiobaru, Minami-ku, 815-8540 Fukuoka, Japan, ueda@design.kyushu-u.ac.jp), Yoshitaka Nakajima (Perceptual Psychology Unit, Kyushu University, 4-9-1 Shiobaru, Minami-ku, 815-8540 Fukuoka, Japan, nakajima@ design.kyushu-u .ac.jp)

Two-hundred sentences of British English and Japanese, each uttered by 10 native speakers ( 5 females and 5 males) in each language, were analyzed through 20 bands of critical band filters. Smoothed power fluctuations derived from the filters were submitted to principal component analyses followed by varimax rotation. The first three factors explained $34-37 \%$ of variance. One of the factors exhibited two peaks along frequency axis in the standardized scores and two of the factors showed one peak for each. These three factors divided the whole frequency range of speech sound into four bands. The structure of the factors and frequency bands was essentially the same across the two languages. These frequency bands can be used for speech perception in general, because intelligible noise-vocoded speech sounds can be synthesized with the frequency bands.

\section{Contributed Paper}

\section{3:20}

2aPPa18. A Structuralistic Approach to Acoustic-Auditory Functions of Meaning. Ute Jekosch (Chair of Communication Acoustics, TU Dresden, Helmholtzstr. 10, 01069 Dresden, Germany, ute.jekosch@tu-dresden.de), Ercan Altinsoy (Chair of Communication Acoustics, TU Dresden, Helmholtzstr. 10, 01069 Dresden, Germany, ercan.altinsoy@ias.et.tu-dresden.de), Sebastian Merchel (Chair of Communication Acoustics, TU Dresden, Helmholtzstr. 10, 01069 Dresden, Germany, sebastian.merchel@tu-dresden.de)

In this paper we introduce a methodology of semio-acoustics to get information on how human listeners associate meaning to acoustic-auditory events. We concentrate on identifying cues in the auditory stream listeners base the association of meaning on as well on modelling major characteristics of the reference system of meaning. The methodology we use is closely related to structuralism, an approach that has its origins in semiotics. In principle, structuralism differentiates between creating functions, carrying functions and changing functions of systems of meaning. We concentrate on carrying and changing functions here using the following procedure: a signcarrier (in our case an acoustic-auditory event the association of meaning is based on) is decomposed into sub-units. By a minimal pair analysis we investigate carrying and changing functions of acoustic-auditory features with regard to the associated meaning. We will introduce the methodology used and discuss first results of a pilot study.

3:40-5:20 Posters

Lecture sessions will recess for presentation of poster papers on various topics in acoustics. See poster sessions for topics and abstracts.

\section{Contributed Papers}

\section{$5: 20$}

2aPPa19. Optimization of a dual recognition tasks for speech quality assessment. Virginie Durin (France Télécom, 2 avenue Pierre Marzin, 22300 Lannion, France, virginie.durin@orange-ftgroup.com), Laetitia Gros (France Télécom, 2 avenue Pierre Marzin, 22300 Lannion, France, laetitia.gros@orange-ftgroup.com)

This paper deals with perceptive test methodologies to assess speech quality of telecommunication systems. Faced with drawbacks of typical methodologies recommended by ITU-T, a new way to assess speech quality is investigated. The new approach requires collecting reaction times and performances when subjects are achieving tasks involving degraded speech signals; it is shown that reactions times lengthen and performances decrease in a specific task when quality is impaired. The proposed task is a dual task with a digit recognition memory task and a letter recognition task. Three different quality levels are applied to audio signals describing digits and letters. Different experimental designs are examined to reinforce the effect of speech quality on performances and reaction times. The results show significant differences of performances and reaction times between the three quality levels, depending on the experimental design.

\section{5:40}

2aPPa20. Electronic pass-through hearing protection and directional hearing restoration integrated in a helmet. Wouter K. Vos (TNO, Kampweg 5, 3769ZG Soesterberg, Netherlands, wouter.vos@tno.nl), Adelbert W. Bronkhorst (TNO, Kampweg 5, 3769ZG Soesterberg, Netherlands, adelbert.bronkhorst@tno.nl), Jan A. Verhave (TNO, Kampweg 5, 3769ZG Soesterberg, Netherlands, jan.verhave@tno.nl)

Compared to standard earplugs, electronic pass-through earplugs provide better sound localisation. Provided that the bandwidth is sufficiently wide and the earplugs do not change the shape of the pinnae. However, when a helmet is worn that partially or completely covers the ears, the directional hearing capability is diminished. We attempt to restore directional hearing when wearing a helmet by attaching a microphone array to the helmet. The signals from the microphone array are filtered with Finite Impulse Response (FIR) filters to recreate an individual or generic open-ear Head Related Transfer Function (HRTF). The filters are designed by minimisation of an error measure in the frequency domain. The error measure incorporates both the log magnitude and the phase differences between the original and the recreated HRTF. The global minimum is found using modern optimisation techniques like Particle Swarm Optimisation (PSO) or Differential Evolution (DE). The total system is evaluated with subject experiments. Participants have to localise sounds and rate the quality of sounds. Independent variables are the number of microphones that should be used and their positions on the helmet.

\section{6:00}

2aPPa21. How many psycho-acoustic attributes are needed? Torben Holm Pedersen (Delta Acoustics \& SenseLab, Venlighedsvej 4, 2970 Hørsholm, Denmark, thp@delta.dk), Nick Zacharov (Delta Acoustics \& SenseLab, Venlighedsvej 4, 2970 Hørsholm, Denmark, nvz@delta.dk)

Sounds may be characterized by objective perceptive attributes (for which there may exist physical metrics) or by subjective (affective or connotative) attributes. This paper will deal with the perceptive attributes. Within product sound quality the metrics for classical the psycho-acoustic attributes (loudness, sharpness, roughness and fluctuation strength) -maybe supplemented with tone and impulse prominence- are often used as the only attributes to characterize the sounds. But are these 4-6 attributes or dimensions sufficient to characterize a sound? Within room acoustics and reproduced sound many other attributes are used and in the language around 100 direct sound describing words may be found. This paper will give an overview over attributes used within different acoustic areas. The latter part of the paper will discuss the role of sensory evaluation methods as a means to systematically developing attributes for the objective qualification and quantification of sound characteristics. 
6:20

2aPPa22. Noise perception of wall-hung gas boilers. Patrick Chevret (01dB-Metravib, 200 Chemin des Ormeaux, 69578 Limonest Cedex, France, patrick.chevret@01db-metravib.com), Anne Coulon (CETIAT, Domaine Scientifique de la Doua, 25 avenue des Arts, BP 2042, F-69603 Villeurbanne cedex, France, anne.coulon@cetiat.fr), François Bessac (CETIAT, Domaine Scientifique de la Doua, 25 avenue des Arts, BP 2042, F-69603 Villeurbanne cedex, France, francois.bessac@cetiat.fr), Etienne Parizet (Laboratoire Vibrations Acoustique, Insa Lyon, 25 bis, av. J. Capelle, 69621 Villeurbanne Cedex, France, etienne.parizet@insa-lyon.fr)
The aim of this study is to assess the quality image for domestic wallhung gas-fired boilers, based on their noise. Nine boilers were recorded using an acoustic manikin in a hemi-anechoic room, for different operating conditions. Two of these operating conditions (maximum heat input, hot water tapping) were first studied. Five-second sequences were presented (through headphones) to sixty listeners using the mixed assessment method allowing for the comparison between signals. Analyses showed several assessment strategies: according to listeners, the relevant noise parameters could be the loudness, the sharpness or the presence of tonal components. A second experimental phase focused on tonal components by artificially modifying some sounds to offer a relevant perceptive indicator. The results of this second experiment will also be presented.

\section{Invited Papers}

\section{6:40}

2aPPa23. Methodological aspects in the determination of the auditory filters and critical band at low and mid-frequencies. Carlos A. Jurado (Acoustics, Aalborg University, Fredrik Bajers Vej 7 B5, 9220 Aalborg Ø, Denmark, cjo@es.aau.dk), Henrik Møller (Acoustics, Aalborg University, Fredrik Bajers Vej 7 B5, 9220 Aalborg Ø, Denmark, hm@acoustics.aau.dk), Christian Sejer Pedersen (Acoustics, Aalborg University, Fredrik Bajers Vej 7 B5, 9220 Aalborg Ø, Denmark, cp@acoustics.aau.dk)

In order to evaluate loudness or audibility of complex sounds, knowledge of the auditory filter characteristics is necessary. At low frequencies, where both the threshold of hearing and dynamic range become considerably frequency dependent, care must be taken to account for this both in the psycho-acoustical model and the methodological approach. To account for variation in hearing sensitivity at low frequencies, equal loudness contours have been used to weight the stimuli accordingly. At mid and high frequencies, threshold of hearing curves have been used. These stimuli weightings can be applied before or after the experiment, normally being applied afterwards. Due to the non-linear characteristics of the cochlear amplifier, it is arguable whether post-experimental weighting is a proper approach, or whether at low frequencies there will be any difference between pre or post stimuli weighting. Listening experiments are then to be performed to test possible differences in pre or post filtering the stimuli. The most appropriate approach will then be discussed. Measurements will be done at low and mid frequencies. To obtain accurate auditory filter estimates, individual ELC or threshold curves will be determined. Methods such as the notched-noise method and the classical band-widening approach will be tested with these conditions

7:00

2aPPa24. Effect of recording/playback technique and experimental method on assessments of noise. Emine Çelik-Christensen (Aalborg University / Rockwool International A/S, Rockwool International, Building Knowledge Center, Hovedgaden 584, Entrance C, 2640 Hedehusene, Denmark, emine.christensen@rockwool.com), Kerstin Persson Waye (Dept. of Environ. Medicine, The Sahlgrenska Acad. of Gothenburg Univ., Box 414, 40530 Gothenburg, Sweden, kerstin.persson-waye@amm.gu.se), Henrik Møller (Acoustics, Aalborg University, Fredrik Bajers Vej 7 B5, 9220 Aalborg Ø, Denmark, hm@acoustics.aau.dk)

The study investigated possible effects of recording/playback technique and experimental method on assessments of annoyance, loudness and unpleasantness. A possible effect of exposure duration was also studied. Sounds were recorded with two different techniques: monophonic and binaural (dummy-head technique). In addition, they were reproduced with three different techniques: monophonic recordings presented through a loudspeaker system, binaural recordings presented through closed (circum-aural) and completely open (free-of-the-ear headphones. The study adopted three psychometric methods for collecting responses from test-subjects. Fifty-four subjects participated, and three types of sounds were used: everyday restaurant sound, road traffic sound and ventilation sound dominated by low frequencies. Each sound was played back at three different levels. The results show that there is no significant main effect of recording and playback technique for any of the three perceptual attributes; however, significant interactions between techniques and sounds were found. Since the effect of recording and playback technique differs depending on sound, this finding is of importance for future design of experiments and interpretation of results. The results also show that long-term annoyance and unpleasantness are poorly predicted by short-duration methods.

\section{Contributed Papers}

\section{$7: 20$}

2aPPa25. Relation between the overall unpleasantness of a long duration sound and the one of its event6: application to a delivery truck. Emilie Geissner (Laboratoire Vibrations Acoustique - INSA Lyon, 25 bis avenue Jean Capelle, Bâtiment Saint-Exupéry, F-69621 Villeurbanne cedex, France, emilie.geissner@insa-lyon.fr), Etienne Parizet (Laboratoire Vibrations Acoustique, Insa Lyon, 25 bis, av. J. Capelle, 69621 Villeurbanne Cedex, France, etienne.parizet@insa-lyon.fr)

The goal of this study was to investigate the link between the unpleasantness assessment of an unstationary long duration sound composed of several distinct sound events and the corresponding judgments of each of those events. For that purpose, a sound sequence of a delivery truck was evaluated by 16 listeners during a test in laboratory: first, subjects had to continuously quantify the perceived unpleasantness of the sequence by moving a sliding cursor along a five levels graduated scale and then give a global rating by using the same scale. In a second step, listeners had to express their overall judgment of unpleasantness for eight samples of the delivery sequence. As previously shown for loudness by Kuwano and Namba (1985), the global rating of the unpleasantness of long sound could not be estimated by the arithmetic mean of the continuous assessment. It also appeared that the overall judgment corresponds to the arithmetic mean on the local values of unpleasantness of each main sound event. This last result was similar to the conclusions of Hellbrück et al. (2001) for the loudness scaling of traffic noise.

\section{$7: 40$}

2aPPa26. Just noticeable differences of loudness and sharpness for earth moving machines. Francesca Pedrielli (CNR-Imamoter, via Canal Bianco 28, 44100 Ferrara, Italy, f.pedrielli@imamoter.cnr.it), Eleonora 
Carletti(CNR-Imamoter, via Canal Bianco 28, 44100 Ferrara, Italy, e.carletti@imamoter.cnr.it), Camilla Casazza (CNR-Imamoter, via Canal Bianco 28, 44100 Ferrara, Italy, c.casazza@imamoter.cnr.it)

This paper describes some results of a research concerning the improvement of the noise climate at the operator station of construction machines during real working conditions. Binaural noise signals were previously recorded and then used in subjective listening tests aimed at identifying the set of acoustic and psychoacoustic parameters which affect the auditory perception of these signals with respect to the annoyance sensation. Results showed that loudness and sharpness are the parameters best correlated to the annoyance. In order to verify the efficacy of some noise control solutions in improving the operator comfort conditions, the next necessary step is to build a specific metrics able to show the minimum differences in these parameters which are subjectively perceived. This paper describes the results of specific listening tests carried out in order to evaluate the differential thresholds of loudness and sharpness by the Method of Limits. The test was repeated at two different signal presentation levels. The loudness value of each original stimulus was varied by interval steps of 0,3 sone, while the Sharpness by interval steps of 0,02 acum. At this moment in time, the data analysis is still in progress and the ultimate results will be presented in the manuscript.

\title{
Session 2aPPb
}

\section{Psychological and Physiological Acoustics: Auditory Perception and Signal Processing by Prostheses I}

\author{
Huanping Dai, Cochair \\ Univ. of Arizona, Speech, Language and Hearing Science, Tucson, AZ 85721, USA \\ Birger Kollmeier, Cochair \\ Universität Oldenburg, Medizinische Physik, Carl-von-Ossietzky Str. 9-11, Oldenburg, 26111, Germany
}

\section{Invited Papers}

2aPPb1. Advantages and disadvantages of fast and slow compression in hearing aids. Brian Moore (University of Cambridge, Department of Experimental Psychology, Downing Street, CB2 3EB Cambridge, UK, bcjm@cam.ac.uk)

Compression is used in hearing aids to compensate for the effects of loudness recruitment. However, there is no consensus about the "best" compression speed. The theoretical advantages and disadvantages of slow and fast compression will be discussed. Studies comparing the relative merits of slow and fast compression have led to a great variety of outcomes. It is argued, following the work of Gatehouse and colleagues, that this is partly the result of a failure to consider individual differences and the auditory ecology of each individual. It is argued that listening in the dips of a fluctuating background sound, such as a competing talker, depends on the ability to process the temporal fine structure (TFS) of sounds, as represented in patterns of phase locking in auditory neurons. For people with a good ability to process TFS, fast compression can amplify sounds in the dips, increasing the effectiveness of dip listening. However, for people with a poor ability to process TFS, envelope cues may be critical for speech intelligibility and fast compression may disrupt such cues. It is proposed that a test of the ability to process TFS might be useful for selecting compression speed for an individual.

\section{$8: 20$}

2aPPb2. Dynamic compression in hearing aids based on an auditory model. Volker Hohmann (Carl von Ossietzky Universität Oldenburg, Ammerländer Heerstraße 114-118, 26111 Oldenburg, Germany, volker.hohmann@uni-oldenburg.de)

A multichannel dynamic compression algorithm is proposed that uses a novel nonlinear auditory filterbank which aims at effectively describing the basilar membrane (BM) response to arbitrary signals. It is based on a linear Gammatone filterbank, a subsequent instantaneous compression stage and a frequency-synthesis stage. In order to model the linear response to off-frequency tones and suppression effects from signals below the characteristic frequency (low-side suppression) the compression characteristics is controlled in each filter band by the deviation of the current sub-band instantaneous frequency from the band's center frequency. If the deviation is small, on-frequency components are prominent, and full gain and compression is applied. If the deviation is large, off-frequency components are prominent, and gain and compression is reduced. Simulations of responses to sinusoids as a function of frequency and level at a fixed BM place (i.e., within a fixed filter band) and for all filter bands (i.e., BM excitation patterns) show good correspondence with psychoacoustical excitation pattern models. Responses to two-tone stimuli quantitatively simulate psychoacoustical two-tone suppression. Likewise, nonlinear growth of simultaneous masking is quantitatively modeled. First results show that speech reception in modulated noise may be improved by the system for a subgroup of hearing-impaired subjects.

\section{8:40}

2aPPb3. Speech perception in fluctuating noise with signals compensated for hearing loss. Joost M. Festen (VU University Medical Center, de Boelelaan 1117, 1081 HV Amsterdam, Netherlands, jm.festen@vumc.nl)

For speech reception in noise normal-hearing listeners gain from masker modulations up to about $12 \mathrm{~dB}$ depending on rate, duty cycle, and depth of the modulations. Listeners with sensorineural hearing loss need a better signal-to-noise ratio to improve signal quality as a compensation for their auditory deficits. Generally, a larger compensation is needed for fluctuating interferences leading to 
reduction or even absence of release from masking for modulated maskers. With the Speech Intelligibility Index adapted for modulated maskers, SIImod [Rhebergen and Versfeld, J. Acoust. Soc. Am. 117, 2181-92 (2005)] it is shown that these elevated thresholds are needed to compensate not only reduced hearing sensitivity but also impaired auditory and non-auditory processing. After frequencydependent compensation for hearing loss as offered by a hearing aid, more of the speech is presented at impaired frequency regions. As a consequence the effect of a hearing aid on speech intelligibility will be less than predicted by SIImod. Speech reception thresholds in noise are affected by peripheral spectro-temporal processing (bottom-up) and by cognitive processing capabilities, like working memory (top-down). Effects of cognitive processing on speech perception are found especially in fluctuating noise as this masker presents a more complex and demanding environment than steady noise.

\section{9:00}

2aPPb4. Signal processing algorithms for speech in fluctuating noise. Peggy Nelson (University of Minnesota, 164 Pillsbury Drive SE, Minneapolis, MN 55455, USA, peggynelson@umn.edu), Janet Rutledge (University of Maryland Baltimore County, 1000 Hilltop Circle, Baltimore, MD 21250, USA, jrutledge@umbc.edu), Juan Carlos Tejero-Calado (University of Malaga, Boulevar Louis Pasteur 35, Campus de Teatinos - Complejo Tecnologico, 29071 Malaga, Spain, jctejero@uma.es)

Fluctuating background noise is a significant problem for listeners with sensorineural hearing loss (SNHL). Data indicate that fluctuating noise significantly affects both speech understanding and satisfaction with hearing aids. Listeners with SNHL do not take advantage of momentary dips in the noise and thus to not experience release from masking in fluctuating noise, as normal-hearing listeners do. Our results have shown that listeners with SNHL obtain about half of the masking release of their normal-hearing counterparts, and that this masking release is generally related to the audibility of the speech in the noise dips. Current slow-acting amplitude compression is based on the level of the background noise and does not improve the audibility of speech in the dips of fluctuating noise. Fast-acting multiband compression can improve audibility but may produce unwanted artifacts. Fast-acting algorithms based on spectral peaks shows potential for maximizing the audibility of speech in fluctuating noise to improve speech intelligibility, with limited artifact. Portions of this work are supported by the University of Minnesota and Starkey Laboratories.

\section{9:20}

2aPPb5. Signal processing in hearing aids: results of the HEARCOM project. Jan Wouters (ExpORL, Dept. Neurosciences, K.U. Leuven, Herestraat 49 bus 721, O. \& N2, B-3000 Leuven, Belgium, jan.wouters@ med.kuleuven.be), Heleen Luts (ExpORL, Dept. Neurosciences, K.U. Leuven, Herestraat 49 bus 721, O. \& N2, B-3000 Leuven, Belgium, heleen.luts@med.kuleuven.be), Koen Eneman (ExpORL, Dept. Neurosciences, K.U. Leuven, Herestraat 49 bus 721, O. \& N2, B-3000 Leuven, Belgium, koen.eneman@med.kuleuven.be), Ann Spriet (ESAT/SISTA, K.U. Leuven, Kasteelpark Arenberg 10, B-3001 Leuven, Belgium, ann.spriet@esat.kuleuven.be), Marc Moonen (ESAT/SISTA, K.U. Leuven, Kasteelpark Arenberg 10, B-3001 Leuven, Belgium, marc.moonen@esat.kuleuven.be), Michael_Büchler_ (University Hospital, 8091 Zürich, Switzerland, michael.buechler@usz.ch), Norbert Dillier (University Hospital, 8091 Zürich, Switzerland, norbert.dillier@usz.ch), Wouter A. Dreschler (AMC, Clinical and Experimental Audiology, 1105 Amsterdam, Netherlands, w.a.dreschler@amc.uva.nl), Matthias Froehlich (Siemens Medical Solutions SAT, 91058 Erlangen, Germany, Matthias.froehlich@ siemens.com), Giso Grimm (Carl von Ossietzky Universität Oldenburg, Ammerländer Heerstraße 114-118, 26111 Oldenburg, Germany, giso.grimm@vegri.net), Volker Hohmann (Carl von Ossietzky Universität Oldenburg, Ammerländer Heerstraße 114-118, 26111 Oldenburg, Germany, volker.hohmann@uni-oldenburg.de), Rolph Houben (AMC, KNO-Audiologie, 1105 Amsterdam, Netherlands, a.c.houben@amc.uva.nl), Arne Leijon (KTH, Royal Institute of Technology, 10044 Stockholm, Sweden, arne.leijon@ee.kth.se), Anthony Lombard (University of Erlangen, 91058 Erlangen, Germany, lombard@nt.e-technik.uni-erlangen.de), Dirk Mauler (Ruhr University of Bochum, 44780 Bochum, Germany, dirk.mauler@ruhr-uni-bochum.de), Henning Puder (Siemens Medical Solutions SAT, 91058 Erlangen, Germany, henning.puder@siemens.com), Michael Schulte (Hoerzentrum Oldenburg, 26129 Oldenburg, Germany, M.Schulte@ hoerzentrum-oldenburg.de), M Vormann (Hoerzentrum Oldenburg, Hoerzentrum Oldenburg, 26129 Oldenburg, Germany, matthias.vormann@web.de)

Digital hearing aids of today allow the application of advanced signal processing strategies. In recent years a number of promising signal processing approaches have been designed and developed. However, most of these different evolutions have been evaluated only in a limited way. Within the framework of the HEARCOM EU-research project a number of signal enhancement techniques have been further developed and evaluated based on a representative set of real-life recordings and physical performance measures. Different auditory profiles, representing common categories of hearing aid users, have been taken into account. A selection of 5 of these signal enhancement techniques (single-channel noise suppression, blind source separation, dereverberation, multi-microphone adaptive processing, feedback reduction) has been implemented on a single common hard- and software test platform, the Master Hearing Aid (MHA). These signal processing strategies have been evaluated perceptually based on speech reception thresholds, listening effort and preference rating, at 5 different test-sites for a number of speech-and-noise listening scenarios. Fifty normal hearing subjects and 100 hearing aid users according to 2 auditory profiles, took part in this study.

\section{9:40}

2aPPb6. Model-based objective assessment of noise reduction systems for hearing aids. Birger Kollmeier (Universität Oldenburg, Medizinische Physik, Carl-von-Ossietzky Str. 9-11, 26111 Oldenburg, Germany, birger.kollmeier@uni-oldenburg.de), Rainer Huber (Kompetenzzentrum HörTech, Marie-Curie-Str. 2, 26129 Oldenburg, Germany, Rainer.Huber@HoerTech.de), Thomas Rohdenburg (Universität Oldenburg, Medizinische Physik, Carl-von-Ossietzky Str. 9-11, 26111 Oldenburg, Germany, thomas.rohdenburg@uni-oldenburg.de), Rainer Beutelmann (Universität Oldenburg, Medizinische Physik, Carl-von-Ossietzky Str. 9-11, 26111 Oldenburg, Germany, rainer.beutelmann@uni-oldenburg.de), Volker Hohmann (Carl von Ossietzky Universität Oldenburg, Ammerländer Heerstraße 114-118, 26111 Oldenburg, Germany, volker.hohmann@uni-oldenburg.de)

Since the ultimative goal of hearing-aid development is the (subjective) judgment of the individual hearing-impaired listener, timeconsuming tests with the end user are indispensable. However, time- and effort-saving objective methods to assess the potential benefit of different versions and parameter sets of hearing aid algorithms are gaining importance. This contribution reviews perception-modelbased approaches to predict the hearing-impaired judgement and speech reception performance achieved with various noise reduction 
schemes. The perceptual similarity measure PSM evaluates the similarity between a tested condition and an "ideal" reference condition not on the physical level, but rather on the perceptual level at the output of a perception model for the individual hearing-impaired listener. The binaural extention of the SII approach uses a binaural preprocessing stage followed by a speech intelligibility index (SII)based prediction scheme capable of predicting the relative benefit of binaural signal presentation and signal enhancement in complex spatial signal and noise source configurations. Both model-based schemes can be combined to assess the effect of noise reduction algorithms (such as adaptive beamformers) and to optimize their respective performance for different acoustical situations.

\section{Contributed Papers}

10:00

2aPPb7. Quantifying and modeling the acoustic effects of compression on speech in noise. Koenraad S. Rhebergen (AMC - Dept. of Clinical and Experimental Audiology, AMC, Clinical and Experimental Audiology, 1105 Amsterdam, Netherlands, k.s.rhebergen@amc.uva.nl), Niek J. Versfeld (AMC - Dept. of Clinical and Experimental Audiology, Meibergdreef 9, 1105AZ Amsterdam, Netherlands, n.j.versveld@amc .uva.nl), Wouter A. Dreschler (AMC, Clinical and Experimental Audiology, 1105 Amsterdam, Netherlands, w.a.dreschler@amc.uva.nl)

In this presentation a method is proposed that is able to separate a speech signal out of a noise signal after processing of the signal through widedynamic-range compression (WDRC). This technique reconstructs the speech signal and noise signal sample by sample separately using the gain factor of the WDRC, and can be used to quantify the acoustic effects of WDRC in noise. It will be shown that this technique is more accurate than a frequently used inversion technique, because the method is not affected by phase shifts that introduce distortion products in the reconstructed speech signal. As a result, the acoustic effects of WDRC can be measured more accurately. In addition, this reconstruction method allows modeling the speech intelligibility after non-linear signal processing in the Speech Intelligibility Index. With the aid of Speech Reception Threshold data it will be shown that this approach can give a good account for most existing data.

10:20

2aPPb8. Acoustical frequency discrimination and pitch matching in bimodal and hybrid hearing. Uwe Baumann (Univ. of. Frankfurt -
ZHNO - Audiologische Akustik, Theodor-Stern-Kai 7, Haus 8D, 60590 Frankfurt a.M., Germany, uwe.baumann@kgu.de), Tobias Rader (Univ. of. Frankfurt - ZHNO - Audiologische Akustik, Theodor-Stern-Kai 7, Haus 8D, 60590 Frankfurt a.M., Germany, tobias.rader@kgu.de), Silke Helbig (Univ. of. Frankfurt - ZHNO - Audiologische Akustik, Theodor-Stern-Kai 7, Haus 8D, 60590 Frankfurt a.M., Germany, silke.helbig@kgu.de), Wolfgang Gstöttner (Univ. of. Frankfurt - ZHNO - Audiologische Akustik, TheodorStern-Kai 7, Haus 8D, 60590 Frankfurt a.M., Germany, a.jan@em.unifrankfurt.de)

Frequency discrimination and pitch matching of implantees using combined electric and acoustic stimulation in either the same ear (EAS) or the opposite ear (bimodal condition) was assessed by means of adaptive procedures. EAS patients received either the MED-EL standard electrode or the recently introduced FLEX design with reduced diameter. Acoustic JNDF in EAS patients ranged from close to normal to grossly abnormal compared to a group of matched SNHL listeners. The median JNDF was 7.1\% in the SNHL and $7.5 \%$ in the EAS group. There was no statistically significant difference in terms of JNDF between both groups of listeners. Frequency mapping was studied by means of an adjustment method where subjects were instructed to control the pitch of an acoustically presented sinusoid in reference to electrical stimulation. The findings demonstrate that the insertion of an intra-cochlear electrode does not significantly hamper the average frequency discrimination ability in EAS patients.

\section{Invited Paper}

10:40

2aPPb9. Combining hearing aids and cochlear implants to solve the cocktail party problem. Fan-Gang Zeng (University of California Irvine, 364 Med Surge II, Irvine, CA 92697, USA, fzeng@uci.edu)

The cocktail party problem refers to the difficulty in speech recognition in noise that a hearing-impaired listener must face in daily life. Combining a hearing aid with a cochlear implant can provide complementary information that may have a great potential to solve this problem. On one hand, a hearing aid may provide low-frequency temporal fine structure cues that are not conveyed by a cochlear implant. On the other hand, a cochlear implant can provide high-frequency temporal envelope cues that are not effectively delivered by a hearing aid. This talk will provide psychophysical and speech recognition evidence for combining hearing aids and cochlear implants to solve the cocktail party problem. One interesting finding along this line of research is that in many important functional tasks, the hearing aid and cochlear implant combination provides a more effective solution than bilateral cochlear implants. Another interesting finding is that the fundamental frequency cue alone can significantly improve speech perception in noise, especially when the noise is a competing voice. The latter finding suggests that combining a tactile aid and a cochlear implant can potentially achieve the same benefit as combining a hearing aid and a cochlear implant in patients with no residual acoustic hearing. 


\title{
Session 2aPPc
}

\section{Psychological and Physiological Acoustics: Binaural Perception by Hearing-Aid Wearers}

\author{
Sridhar Kalluri, Cochair \\ Starkey Hearing Research Center, 2150 Shattuck Ave, Suite 408, Berkeley, CA 94704, USA \\ Michael A. Akeroyd, Cochair \\ MRC Institute of Hearing Research, Glasgow Royal Infirmary, 16 Alexandra Parade, Glasgow, G31 2ER, UK
}

\section{Invited Paper}

\section{1:20}

2aPPc1. Binaural function and its benefits for wearers of hearing aids. Sridhar Kalluri (Starkey Hearing Research Center, 2150 Shattuck Ave, Suite 408, Berkeley, CA 94704, USA, sridhar_kalluri@starkey.com), Brent Edwards (Starkey Hearing Research Center, 2150 Shattuck Ave, Suite 408, Berkeley, CA 94704, USA, brent_edwards@starkey.com)

Hearing-aid design has been predicated traditionally on improving the reception of speech in background noise, a goal that has, at least in part, been successfully attained. Despite the advances, hearing-aid wearers continue to have difficulty in complex acoustic environments and in auditory tasks where normal-hearing listeners benefit greatly from binaural hearing. In order to improve hearing-aid wearers' performance in such challenging conditions, recent research has begun examining the effects of hearing aids on binaural perception. Interest in the topic is heightened by the rapidly approaching prospect of bilateral hearing aids that communicate wirelessly and thereby allow implementation of more complex signal-processing algorithms than currently possible. This talk will review binaural function and its benefits, with particular emphasis on the aspects that hearing aids can affect. The talk will also speculate on which hearing-aid algorithms can affect binaural perception.

\section{Contributed Paper}

\section{1:40}

2aPPc2. Auditory localization with linear and compression hearing aids. Helen J. Simon (Smith-Kettlewell Eye Research Institute, 2318 Fillmore St., San Francisco, CA, CA 94115, USA, helen@ski.org), E. William Yund (VAMC, 150 Muir Road, Martinez, CA 94553, USA, yund@ebire.org), Harry Levitt (Advanced Hearing Concepts, PO Box 610, Bodega Bay, CA 94923, USA, harrylevitt@earthlink.net)

The question of how well hearing-impaired individuals can localize sound (with or without amplification) is still not fully resolved. This study was designed to compare sound localization with two types of hearing-aid (HA) processing, wide dynamic range multichannel compression (WDRMCC) and linear amplification (LA) with compression limiting, during the first 32 weeks of HA use. HAs from two different manufacturers were included to compare different digital signal processing implementations, (1) fast Fourier transform (FFT), necessitating a $10 \mathrm{~ms}$ delay, and (2) non-FFT signal processing with a shorter time delay ( $\sim \mathrm{ms})$. We found an initial degradation of sound localization, relative to original unaided performance, for both WDRMCC and LA in both FTT and non-FTT platforms. We found no difference between WDRMCC and LA processing. However, sound localization with non-FFT platform improved consistently throughout 32 weeks of HA use and was better than the original unaided measurements at 16 and 32 weeks. In contrast, localization with the FFT platform showed no consistent change throughout the 32 -week test period and remained inferior to original unaided performance. The continuing localization problems present for the FFT, but not the non-FFT, implementations of LA and WDRMCC may be due to its $10-\mathrm{ms}$ processing delay.

\section{Invited Papers}

12:00

2aPPc3. Effects of noise type and location on binaural benefit in asymmetric directional fittings. Benjamin Hornsby (Vanderbilt University, Room 8310 Medical Center East, South Tower, 1215 21st Ave. South, Nashville, TN 37232-8242, USA, ben.hornsby @ vanderbilt.edu)

The benefits of bilateral directional processing for improving speech understanding in noise are well documented. However, these fittings are not universally accepted by hearing aid wearers. Research suggests that an asymmetric fitting (omnidirectional in one ear/directional in the other) may provide benefit in noise comparable to symmetric directional fittings (directional in both ears). This study evaluated factors that may affect the relative benefit provided by an asymmetric directional fitting. Specific factors evaluated included noise configuration, reverberation and noise type. Twenty individuals with mild-moderate SNHL participated. Aided speech understanding in cafeteria babble was assessed in bilateral omnidirectional and directional modes and in an asymmetric mode in four (4) different noise configurations. Measures were made in both an anechoic and reverberant (RT $\sim 20 \mathrm{~ms}$ ). In a second experiment the effects of noise type were evaluated by comparing performance in symmetric and asymmetric modes in both steady state noise and cafeteria babble. Results suggest that noise configuration has a significant effect on the relative benefit provided by asymmetric fittings. No significant differences due to noise type were observed. Individual differences in bilateral directional benefit also appear to have a significant effect on the reduction in benefit resulting from an asymmetric fitting. 
2aPPc4. Speech-in-noise enhancement and sound localization with improved binaural hearing instruments. Jan Wouters (ExpORL, Dept. Neurosciences, K.U. Leuven, Herestraat 49 bus 721, O. \& N2, B-3000 Leuven, Belgium, jan.wouters @ med.kuleuven.be), Simon Doclo (ESAT/SISTA, K.U. Leuven, Kasteelpark Arenberg 10, B-3001 Leuven, Belgium, simon.doclo@esat.kuleuven.be), Marc Moonen (ESAT/SISTA, K.U. Leuven, Kasteelpark Arenberg 10, B-3001 Leuven, Belgium, marc.moonen@esat.kuleuven.be), Tim Van Den Bogaert (ExpORL, Dept. Neurosciences, K.U. Leuven, Herestraat 49 bus 721, O. \& N2, B-3000 Leuven, Belgium, tim.vandenbogaert@med.kuleuven.be)

Multi-microphone noise reduction schemes have become standard in commercial hearing aids and cochlear implants. Recent studies with bilateral hearing aids have shown that common adaptive directional microphone systems tend to distort localization cues, leading to inappropriate and reduced spatial awareness for bilateral hearing aid users. Here we show that binaural multi-microphone signal processing based on multi-channel Wiener filter (MWF) are capable of combining noise reduction with the preservation of directional hearing. Physical simulations and perceptual results from 10 listeners have been studied for different noise source scenarios, in different reverberant conditions, and for a number of signal processing schemes using up to 4 microphone inputs ( 2 each side). An overview is given of the localization performance and the speech reception benefits in these different listening conditions for the different noise reduction strategies. An adaptive directional microphone system (ADM) is used as a reference system. Signal processing based on MWF does, unlike ADM, provides a combination of noise reduction and preservation of spatial awareness. Moreover, in some conditions it even offers an improved spatial release from masking. The MWF outperforms the ADM in terms of localization and noise reduction if signals are not arriving from the most forward field of view.

12:40-2:00 Lunch Break

\section{Invited Papers}

2:00

2aPPc5. Interaction between stimulus and compression type in precedence situations with hearing aids. Bernhard U. Seeber (MRC Institute of Hearing Research, Science Rd / University Park, NG7 2RD Nottingham, UK, seeber@ihr.mrc.ac.uk), Cheryl Eiler (Starkey Hearing Research Center, 2150 Shattuck Ave, Suite 408, Berkeley, CA 94704, USA, cheryl_eiler@starkey.com), Sridhar Kalluri (Starkey Hearing Research Center, 2150 Shattuck Ave, Suite 408, Berkeley, CA 94704, USA, sridhar_kalluri@starkey.com), Ervin R. Hafter (University of California, Department of Psychology, 3210 Tolman Hall, Berkeley, CA, CA 94720-1650, USA, hafter@berkeley.edu), Brent Edwards (Starkey Hearing Research Center, 2150 Shattuck Ave, Suite 408, Berkeley, CA 94704, USA, brent_edwards@ @starkey.com)

Multiband compression in hearing aids has the potential to interfere with binaural perception by altering binaural cues. We compared binaural precedence in patients fitted with compression hearing aids or linear hearing aids and tested one month later with a variety of stimuli. In an open field environment simulated in an anechoic chamber, subjects localized the direction of a leading sound accompanied by a lagging copy played from a different direction. Precedence, defined by a strong influence of the first sound, was found in $6 / 7$ of the subjects when the stimulus was a sentence, but in only $4 / 7$ when it was a high-pass or a wideband burst of noise. The fact that increasing the noise-bandwidth to include low frequencies did not produce precedence in the two patients who had shown it with a sentence may indicate a need for them to accumulate information through the successive pseudo onsets in the speech-envelope. The choice of amplification, compressive or linear, did not significantly affect precedence for any sound, except for one subject who showed weak influence of compression with the high-pass noise. We conclude that although compression might alter interaural level cues, for most, especially with long sounds, localization dominance appears unimpaired.

2aPPc6. Effect of hearing aids on distance perception. Michael A. Akeroyd (MRC Institute of Hearing Research, Glasgow Royal Infirmary, 16 Alexandra Parade, G31 2ER Glasgow, UK, maa@ihr.gla.ac.uk)

The two primary auditory cues to distance in rooms are the overall level of the sounds received by the listener and the ratio of the level of the direct sound to the reverberant sounds. In previous work we have demonstrated that hearing-impaired listeners showed no overall deficits in the ability to use the overall-level cue, but they did have deficits in the ability to use the direct-to-reverberant cue [M. Akeroyd, S. Gatehouse, and J. Blaschke, J. Acoust. Soc. Am., 121, 1077-1089 (2007)]. These deficits would be expected to contribute to the auditory disability suffered by the listeners. But both of these auditory cues are level cues, as they require some measurement of intensity, and so they would be expected to be affected adversely by the amplitude compression found in most modern hearing aids. Initial results from an experiment measuring the just-noticeable difference (JND) for changes in distance (at $2 \mathrm{~m}$ and $5 \mathrm{~m}$ ) with experienced hearing-aid users suggest, however, that their JNDs are no different to those of unaided but impaired listeners. If confirmed by the final results, this surprising result may be interpretable if listeners have acclimatized to the effects of their aids on level.

$$
\text { 2:40 }
$$

2aPPc7. Binaural hearing abilities of bilaterally fitted hearing aid users assessed using objective and subjective outcome measures. Thomas Behrens (Eriksholm Research Centre, Oticon A/S, 243 Kongevejen, 3070 Snekkersten, Denmark, tbs@oticon.dk), Tobias Neher (Eriksholm Research Centre, Oticon A/S, 243 Kongevejen, 3070 Snekkersten, Denmark, ton@oticon $. \mathrm{dk})$

Aided spatial hearing in the hearing impaired remains a rather sparsely explored topic. Therefore we do not know enough about the ability of the hearing impaired to exploit auditory cues for spatial hearing. In an attempt to advance our knowledge the following 
experiment was set up. A group of 21 experienced hearing aid users took part in a test using modern completely in the canal hearing aids in the field for at least 7 weeks. After acclimatization to the devices they were tested on a number of outcome measures. These included spatial unmasking, an interview administered selection of questions from the Speech Spatial and Qualities of hearing scale, and a baseline speech in noise measure. Spatial unmasking was assessed using three concurrent female talkers, with the target talker always presented directly ahead and the maskers presented either at $+/-50$ degrees or both at 180 degrees. This presentation will provide results from the study described above along with possible relations to auditory and non-auditory predictors of spatial hearing performance.

\title{
Contributed Paper
}

\section{3:00}

2aPPc8. The effect of binaural processing techniques on speech quality ratings of assistive listening devices in different room acoustics conditions. Johan Odelius (Luleå University of Technology, Dept Human Work Sciences, Div of Sound and Vibration, SE-97187 Luleå, Sweden, johan.odelius@1tu.se), Örjan Johansson (Luleå University of Technology, Dept Human Work Sciences, Div of Sound and Vibration, SE-97187 Luleå, Sweden, orjan.johansson@1tu.se)

External microphone systems, referred to as assistive listening devices (ALD), are used in classrooms for hearing impaired students. The objective is to investigate the effect of binaural processing techniques in different room acoustic conditions. A listening experiment was conducted with 10 normal hearing adults. Response variables were judgements of clarity, pleas- antness, listening effort and overall speech quality. Design variables were binaural processing, room acoustics and ALD bandwidth. Stimuli were generated using the room acoustic modelling software CATT Acoustic. Three speech sources, two male voices and one female voice, were placed at a table in the centre of a room and one Brown noise source was placed in one corner of the room. Microphones were placed $0.5 \mathrm{~m}$ in front of each speech source. Target source was a random choice of one of the two male voices. The binaural processing was utilized by a simple HRTF filtering. Depending on the angle to the source from a fictitious listening position at the table, corresponding interaural time difference (ITD) and the interaural level difference (ILD) was applied to the signal. Stimuli were presented by loudspeakers using cross-talk cancellation. The hypothesis is that binaural processing will give a significant improvement in speech quality.

TUESDAY MORNING, 1 JULY 2008

ROOM 242B, 8:00 TO 10:40 A.M.

\author{
Session 2aSAa
}

\section{Structural Acoustics and Vibration and EURONOISE: Vibration and Radiation from Complex Structural Systems II}

\author{
David Feit, Cochair \\ Applied Physical Sciences Corp., Ste. 300, 2 State St., New London, CT 06320, USA \\ Jean-Louis Guyader, Cochair \\ INSA de Lyon - LVA, Bâtiment St. Exupéry, 25 bis avenue Jean Capelle, Villeurbanne Cedex, F-69621, France
}

\section{Contributed Papers}

\begin{abstract}
8:00
2aSAa1. On the comparison of symmetric and unsymmetric formulations for experimental vibro-acoustic modal analysis. Morvan Ouisse (FEMTO-ST UMR CNRS, 23 chemin de l'Epitaphe, 25000 Besançon, France, morvan.ouisse@univ-fcomte.fr), Emmanuel Foltete (FEMTO-ST Applied Mechanics, 24 chemin de l'épitaphe, 25000 Besançon, France, emmanuel.foltete@univ-fcomte.fr)

The classical u-p formulation for vibro-acoustic problems is very convenient for experimental vibro-acoustic modal analysis since the physical variables are directly those which are measured by operators. In this particular context, the objective is to identify from experimental measurements a reduced model which has the same behaviour as the measured one. The complex mode shapes which are identified using this technique must satisfy a properness condition. When they do not verify it, they should be modified to be able to represent the behaviour of a physical system. Some techniques have been proposed in order to develop a strategy to obtain the modified eigenshapes, but this is a quite difficult point because of the unsymmetric topology of the equations. In this paper, a symmetric formulation is used in order to be able to directly apply the classical methodology which has been developed for structural modal analysis to obtain the physical reduced system. The methodology is described and compared with the u-p formulation, in terms of efficiency and precision, in particular when some absorbing
\end{abstract}

devices are considered. All results are first presented on an ideal numerical test-case, and applications on experimental data are finally shown.

\section{8:20}

2aSAa2. Numerical modeling of Panphonics's G1 flat loudspeaker. Krisztián Gulyás (Budapest University of Technology and Economics, BME Dept. of Telecommunications, Magyar tudósok körútja 2, H-1117 Budapest, Hungary, gulyas@ hit.bme.hu), Fülöp Augusztinovicz (Budapest University of Technology and Economics, BME Dept. of Telecommunications, Magyar tudósok körútja 2, H-1117 Budapest, Hungary, fulop@ hit.bme .hu)

The basic idea behind AABC (Active Acoustic Barrier Control) is to reduce the sound radiation of a structure acoustically without influencing the vibration behavior of the structure. The large surface acoustic polymer material actuator/sensor systems are primarily meant to form the actuator/sensor system for the AABC concept. The main component of this package is a special active device: the rEMA - revised Elastic Mass Actuator based on the Panphonics' G1 panel loudspeaker element. This paper deals with the modeling and the low-frequency performance issues of the G1 flat loudspeaker. The aim was to create an accurate mathematical model to understand the operational principles of the loudspeaker, and then this model was used to optimize its low-frequency performance. Due to the special 
structure and the optimization tasks, a new numerical method was developed to model special multi-layer coupled vibro-acoustics systems based on the Finite Difference and Boundary Element Method. The investigation focuses on the mechanical behavior of the panel and describes the sound radiation properties also. The performed work was a part of the research of the project InMAR (Intelligent Materials for Active Noise Reduction) which was funded by the European Union.

\section{8:40}

2aSAa3. Metrology and prediction for integrating a subsystem on a vehicle: Application to a fan system attached to the front end of a car. Marie-Hélène Moulet (CEVAA, Technopole du Madrillet, 2 Rue Joseph Fourier, 76800 Saint Etienne du Rouvray, France, mh.moulet@cevaa.com), Saul Mapagha (CEVAA, Technopole du Madrillet, 2 Rue Joseph Fourier, 76800 Saint Etienne du Rouvray, France, s.mapagha@cevaa.com), Vincent Martin (Institut Jean Le Rond d'Alembert, UMR CNRS 7190, UPMC, 2 Place de la Gare de Ceinture, 78210 Saint-Cyr 1’Ecole, France, vmartin@ccr.jussieu.fr)

The car industry must satisfy physical acoustic and vibratory objectives in order to comply with safety and comfort norms. Among others, efforts entering the chassis should be limited. When a host structure is excited by a vibratory system, called subsystem, it also vibrates and may radiate an acoustic field. Usually, the subsystem is first tested on a bench and the question is then to deduce the efforts entering the chassis from those entering the test bench. Globalising notions of impedance already made evident via deformable structure configurations are used presently through measurements in a technological configuration, the complexity of which does not allow modelling. The case of a fan system attached to the front end of a car is under study. Some metrological conclusions, as well as more dedicated ones concerning the fan system itself, will be given. Finally, on the calculation and prediction sides, expected properties that are not satisfied (such as symmetry in measured matrices) are seen to be of no great consequence in the present case.

\section{9:00}

2aSAa4. Numerical study of the transition to chaos in nonlinear forced vibrations of plates. Cédric Camier (ENSTA, Chemin de la Hunière, 91761 Palaiseau cedex, France, cedric.camier@ensta.fr), Cyril Touze (ENSTA, Chemin de la Hunière, 91761 Palaiseau cedex, France, cyril.touze@ensta.fr), Olivier Thomas (CNAM, 2, rue Conté, 75003 Paris, France, olivier.thomas@cnam.fr), Stefan Bilbao (University of Edinburgh, Room 7306B, JCMB, King's Bldgs., Mayfield Rd., EH9 3JZ Edinburgh, UK, sbilbao@staffmail.ed.ac.uk)

Geometrically nonlinear vibrations of free edge circular plates subjected to a harmonic excitation are discussed. Particularly, transition from periodic to chaotic motion is observed when increasing the amplitude of the forcing. The present work is devoted to reproduce numerically these highly nonlinear behaviours. The temporal integration of such dynamics, including instabilities and chaotic regimes, is not straight forward because a stiff problem with a very large number of dofs is at hand. Consequently, numerical instabilities are observed when typical Runge-Kutta schemes are applied. To settle the matter, two methods have been tested and compared. They both rely on a modal approach applied to the von Kármán's model for large amplitude vibrations of plate. For the first one, the energies of the plates are expressed at the continuous level. The Hamiltonian of the system is then derived and discretized using the eigenmodes. The Hamiltonian formulation ensures the conservation of energy. An implicit time discrete scheme is then chosen to approximate the equations of motion. For the second one, the Gear's BDF method, implemented in the IMSL Fortran library, is used to integrate the nonlinear oscillator equations.

\section{9:20}

2aSAa5. Numerical investigations into the squeal propensity of a railway disc brake. Olivier Chiello (INRETS, Transport and Environment Lab., 25 avenue François Mitterrand, 69675 Bron cedex, France, olivier.chiello@inrets.fr), Xavier Lorang (SNCF, Innovative \& Research Dept., 45 rue de Londres, 75379 Paris, France, xavier.lorang@sncf.fr)

This paper comes within the scope of a research program concerning with the reduction of the squeal noise generated by high power railway disc brakes. It focuses on the numerical results provided by a finite element model of the brake including unilateral contact and Coulomb friction at the disc/pad interfaces. In particular, the dynamic stability of the sliding equilibrium is investigated by performing a complex eigenvalue analysis of the linearized equations verified by the structural displacement fields. Complex eigenvalues and complex modes are used to estimate the squeal propensity of the brake in a given frequency range. The effect of various mechanical and geometrical parameters is studied in order to better understand the mechanism leading to the system instability.

\section{$9 \cdot 40$}

2aSAa6. Three dimensional orthogonality of the Lamb modes in layered plates of elastic and viscoelastic materials and their implementation to the far field evaluation. Dmitry Zakharov (LMP, UMR CNRS 5469, Université Bordeaux I, 351, cours de la Libération, 33405 Talence, France, dmitrii.zakharov@gmail.com)

The 3D guided waves in the linearly viscoelastic laminates are considered. On the plate surfaces any of the homogeneous boundary conditions are allowed, e.g., the Lamb waves, waves in clamped plates, etc. are taken into account. The fundamental property of these waves is their generalized orthogonality, which is deduced and discussed. The applications of the orthogonality relations for solving some particular boundary value problems are suggested. A method for the exact calculation of the far field caused by an acoustic source of a finite size is suggested. The only restriction is that the distance required must exceed the longitudinal radius of the source. The obtained results can be used for evaluating the fields radiated by ultrasonic transducers of arbitrary aperture and by other realistic sources.

\section{0:00}

2aSAa7. Sound Radiation of a Large Truck Oil Pan: Estimation and Experimental Investigation. Olaf Heintze (German Aerospace Center (DLR), Institute of Composite Structures and Adaptive Systems, Lilienthalplatz 7, 38108 Braunschweig, Germany, olaf.heintze@dlr.de), Volker Wittstock (Physikalisch-Technische Bundesanstalt, Bundesallee 100, 38116 Braunschweig, Germany, volker.wittstock@ptb.de), Carl Fredrik Hartung (Volvo Technology Corporation, Götaverksgatan 10, 40508 Göteborg, Sweden, carl.fredrik.hartung@volvo.com)

The oil pan of large diesel engine trucks has been identified as a significant contributor to their external noise radiation. This undesired broadban noise is caused by the oil pan's structural vibration and can not be treated effectively by passive measures especially in the low frequency regime up to $500 \mathrm{~Hz}$. In order to address this challenge, an active structural acoustic control system consisting of structural sensors and actuators is suitable to alter the oil pans vibrations in a sound reducing manner. A first step is however to classify the broadband sound radiation such that it allows for a proper and efficient sound power estimation resulting from structural measurements Therefore, an acoustical model was set up based on a geometrical surface scan of a serial production large truck oil pan mounted in a laboratory tes stand. This model served for the numerical computation of a set of principle velocity patterns contributing independently to the active sound power, where its hybrid estimation has been performed employing additionally the measured structural response of the oil pan assembly due to a shake excitation. Finally, the sound power radiation of the test stand has been measured in a reverberation room to validate this hybrid estimation. 
10:20

2aSAa8. Sound propagation on a high pressure gas pipe. Rolf Schirmacher (Müller-BBM, Robert-Koch-Straße 11, 82110 Planegg, Germany, Rolf.Schirmacher@MuellerBBM.de), Robert Baars (M+P raadgevende ingenieurs B.V., Visserstraat 50, 1431 GJ Aalsmeer, Netherlands, robertbaars@mp.nl)

At a two stage high pressure gas compressor with intermediate cooler, the cooler radiate a tone at the compressor rotational frequency of approx.
$160 \mathrm{~Hz}$. For the design of noise reduction devices, the mechanism of sound propagation in between the compressor an the cooler on a DN 400 steel pipe with $24 \mathrm{~mm}$ wall thickness and 160 Bar internal gas pressure was to be determined. By non-invasive vibration measurements on the pipe, the dominance of the fluid borne sound (natural gas) over the structure borne sound was found. Later, pressure measurements in the pipe clearly approved this result. The concepts upon which the measurements and data evaluation were based (propagating modes of coupled system, wave separation, etc) as well as the results are presentend.

TUESDAY MORNING, 1 JULY 2008

ROOM 243, 8:00 A.M. TO 12:20 P.M.

Session 2aSAb

\title{
Structural Acoustics and Vibration and EURONOISE: General Topics in Structural Acoustics and Vibration II
}

\author{
Wolfgang Kropp, Cochair \\ Chalmers University of Technology, Division of Applied Acoustics, Gothenburg, SE-41296, Sweden \\ Sean Wu, Cochair \\ Wayne State University, 5050 Anthony Wayne Dr., Detroit, MI 48202, USA
}

\section{Contributed Papers}

8:00

2aSAb1. Wavelet-based data processing for comparative study of noncontact measurement techniques for vibroacoustics. Dan Borza (National Institute of Applied Sciences of Rouen, INSA Rouen, LMR, Ave l'Universite, BP8 76800 Rouen, France, dan.borza @insa-rouen.fr), Ioana Nistea (Institut National des Sciences Appliquées de Rouen, BP8 avenue de l'Université, 76801 Saint-Etienne du Rouvray, France, ioana.nistea@insa-rouen.fr)

In vibration analysis of structures complex measurement information is required in order to perform modal analysis, therefore the choice of the measurement technique to be applied is essential. Non contact measurements are preferred to classical transducer based methods, mainly due to the absence of influence upon the structure under test. The various techniques available today in vibroacoustics produce results which are quite different in terms of spatial and temporal resolution or measured quantities and therefore a choice has to be made of the experimental tool best adapted for different fields of research (acoustics, mechanical structures, dynamics). In this paper, we present a comparative study of several optical, acoustical and numerical techniques for vibration measurement or simulation, namely Digital Speckle Interferometry, Laser Doppler Vibrometry and a FE model. The tests were made for the free and the forced vibrations of a highly dumped, non-metallic plate. In the data processing stage, discrete wavelet decomposition has been applied on the experimental data in order to match up the spatial maps of vibration amplitudes.

\section{8:20}

2aSAb2. Analysis of the acoustic signals backscattered by a tube using the time-frequency representations. Mustapha Laaboubi (Ibn Zohr University, FS Agadir, 80000 Agadir, Morocco, laaboubi @ gmail.com), Elhoucien Aassif (Ibn Zohr University, FS Agadir, 80000 Agadir, Morocco, aassif@hotmail.com), Rachid Latif (ESSI - ENSA, BP 1136, Ibn Zohr University, 80000 Agadir, Morocco, latif@ensa-agadir.ac.ma), Gerard Maze (LAUE, Université du Havre, Place Robert Schuman, F-76610 Le Havre, France, gerard.maze
$@$ univ-lehavre.fr),Dominique Decultot(LOMC FRE 3102 CNRS Groupes Ondes Acoustiques, Université du Havre (IUT), Place Robert Schuman, 76610 Le Havre, France, dominique.decultot@univ-lehavre.fr), Ali Moudden (Ibn Zohr University, FS Agadir, 80000 Agadir, Morocco, ali_moudden@yahoo.fr), Abdelilah Dariouchy (LMTI, univirsté ibn zohr Faculté des Sciences LMTI bp 8106, 80000 Agadir, Morocco, abdelilah _dariouchy@yahoo.fr)

The normal excitation of a tube immersed in water by the acoustic plane wave, circumferential waves are generated inside the shell. These circumferential waves, standing form stationary waves on the circumference of the tube for some frequencies. These stationary waves, constituting resonances of the tube which are perfectly visible on the backscattered spectrum. Moreover, the studies carried out on the diffusion of a plane acoustic wave by target were based primarily on the use of the monodimensional methods (Temporal domain and/or frequencial domain). To exceed the disadvantages of these methods, in this work, we used the time-frequency representations such as the Short-Term Fourier Transform (STFT), Wigner-Ville Distribution (WVD) and Wavelet Transform method. These representations are applied to a theoretical signal backscattered by a tube of aluminium, copper and steel with radii ratio $\mathrm{b} / \mathrm{a}=0.95$ ( $\mathrm{a}$ is the external radius, and $\mathrm{b}$ the internal radius). From the time-frequency images obtained we have visualized the dispersion of circumferential waves (S0, A1, S1,œ) and identified these different waves. This analysis permits to compare between these timefrequency representations. And also we have compared between the cut-off frequencies of circumferential waves obtained from these representations and those computed by the proper modes theory of the vibration.

\section{8:40}

2aSAb3. Weak radiator design using dimples. D. Chih-Chun Cheng (National Chung Cheng University, Dept. of Mechanical Engineering, 160, San-Hsing, Ming-Hsiung, 621 Chia-Yi, Taiwan, imecce@ccu.edu.tw), Wen-Nan Cheng (National Chung Cheng University, Dept. of Mechanical Engineering, 160, San-Hsing, Ming-Hsiung, 621 ChiaYi, Taiwan, zzs1003@hotmail.com), Cary H. Koopmann (Pennsylvania 
State University, Dept. of Mechanical and Nuclear Engineering, 157 Hammond Building, University Park, PA 16802, USA, ghk@engr.psu.edu)

A design method for achieving minimum radiation of sound from a beam by creating cylindrical dimples on its surface is presented. Two strategies of determining the dimple size and its location on the beam are presented. The first is based on the optimization method, in which the dimple size and its location are the design variables. The design variables that minimize the sound power are obtained directly using an optimization subroutine. The second is to synthesize the beam's weak radiator mode using a set of dimples, in which the mode shape of the dimpled beam is a close fit to the beam's weak radiator mode. As a comparison in sound power reduction between these strategies, numerical results for a simply supported beam in a rigid baffle excited by a harmonic force are presented.

\section{9:00}

2aSAb4. Estimation for vibration mode of membrane by NAH method. Nabilah Ibrahim (Shibaura Institute of Technology, 3-7-5, Toyosu, Koto-ku, 135-8548 Tokyo, Japan, m107068@sic.shibaura-it.ac.jp), Hideo Shibayama (Shibaura Institute of Technology, 3-7-5, Toyosu, Koto-ku, 135$8548 \quad$ Tokyo, Japan, sibayama@sic.shibaura-it.ac.jp), Toru Itakura (Shibaura Institute of Technology, 3-7-5, Toyosu, Koto-ku, 1358548 Tokyo, Japan, m106009@sic.shibaura-it.ac.jp)

Near Field Acoustic Holography (NAH) is the method of measuring sound information on the radiant surface in the near field as a hologram. By use the technique, we can visualize the vibration of the radiant surface. Thus, we can easily understand the object of the acoustic conditions in acoustic image processing. We made the measurement on one side of the drum using array microphone while the driven source is attached to the other side. In addition, the measurement is not touched on the vibrating membrane surface. This paper describes the estimated results of the vibration mode on the vibration membrane of a drum. And this is possible by apply it to the vibration controlling field for detecting the sound structure on the membrane.

\section{9:20}

2aSAb5. Computing high frequency vibrations of polygonal plates by the Image Source Method. Jacques Cuenca (LAUM, CNRS, Université du Maine, Lab. d'Acoustique Université du Maine, UMR CNRS 6613, 72085 Le Mans Cedex 9, France, Jacques.Cuenca.etu@univ-lemans.fr), François_Gautier (Laboratoire d'Acoustique de l'Université du Maine, Avenue Olivier Messiaen, 72085 Le Mans, France, francois.gautier@univ-lemans.fr), Laurent Simon (LAUM, CNRS, Université du Maine, Lab. d'Acoustique Université du Maine, UMR CNRS 6613, 72085 Le Mans Cedex 9, France, Laurent.Simon@univ-lemans fr)

The aim of this paper is to show that the Image Source Method (ISM) can be used for predicting medium and high frequency vibrations of arbitrarily shaped polygonal plates with controllable precision. Modeling the vibrations of polygonal plates by ISM consists in superposing the contributions of elementary image sources, which allows the plate boundary conditions to be satisfied. For particular plate shapes leading to a spatially periodic pattern of image sources, it is shown that ISM allows the exact computation of the Green's function by means of a Poisson summation formula. For arbitrarily polygonal plates, a criterion for truncating the image source generation process is examined and used as a control parameter for the precision of the estimations. The given examples show that the results are in good agreement with exact analytical solutions for simple plate shapes and with results obtained by the finite element method for more complex shapes. The convergence towards reference solutions as the number of image sources is increased is investigated. The main advantage of the method is that the accuracy is improved with structural damping and with frequency for a given number of image sources.

\section{9:40}

2aSAb6. Estimation of received acoustic levels in the near-field of a ship. Christopher Barber (Penn State University, PO Box 30, State College, PA 16804-0030, USA, cbarber@psu.edu)

Measured acoustic levels at the face of a receiver in close proximity to a ship in shallow water can differ substantially from the levels predicted by applying standard propagation models to a source level determined from a far-field radiated noise measurement. At distances typical of mine-ship engagements, the dimensions of the source (ship) can be large with respect to range, ocean depth and acoustic wavelength, and the ship cannot be approximated as a single point source, nor is the receiver necessarily located outside the acoustic near field. Direct free-field measurements of ship radiated noise at short range are problematic, so that the received acoustic level in the nearfield of a ship must be estimated from other available information. Options for generating such estimates are presented, including a hybrid approach combining empirically-derived transfer functions, a virtual distributedsource representation of the ship, and in-situ hull vibration measurements. The potential for computational methods such as BEM - FEM and measurements such as Nearfield Acoustic Holography to provide an improved understanding of the vibration sources, structural responses, radiation mechanisms and propagation paths associated with the radiated noise field of realistic ship structures is also explored. (Work sponsored by ONR Code $331)$.

\section{0:00}

2aSAb7. Some notes on the sound reduction index of pax cabins panels on cruise ships. Marco Biot (DINMA, University of Trieste, Via Valerio, 10, 34100 Trieste, Italy, biot@units.it), Francesco De Lorenzo (Fincantieri SpA, Passeggio S. Andrea, 6, 34100 Trieste, Italy, Francesco.Delorenzo @ fincantieri.it)

The issue of comfort of passenger ships has became in the last years of paramount importance; in this context the noise and vibration control plays a leading role. The concept of comfort on board is subjective and it is impossible to define it with simple formula. On the other hand, a few number of significant parameters is commonly used to characterize the level of comfort on board of ships, and the sound insulation index in one of the most important. As known, it has a paramount importance in defining the contractual comfort on board of ships. The report is related with the problem of setting up the sound insulation index for pax cabins, specifically in relation to the nature and characteristics of the panels between cabins.

\section{0:20-10:40 Break}

\section{0:40}

2aSAb8. Acoustic and vibration measurements of NASA spacesuits Durand R. Begault (NASA Ames Research Center, Mail Stop 262-2, NASA ARC, Moffett Field, CA 94035, USA, Durand.R.Begault@nasa.gov), James L. Hieronymus (NASA Ames Research Center, Mail Stop 262-2, NASA ARC, Moffett Field, CA 94035, USA, JimH@riacs.edu), Bernard D. Adelstein (NASA Ames Research Center, Mail Stop 262-2, NASA ARC, Moffett Field, CA 94035, USA, Bernard.D.Adelstein@nasa.gov)

Research was conducted to examine interactions between acoustic signals and mechanical factors relevant to the design of improved auditory displays and noise mitigation for future NASA Spacesuits. Data were collected at NASA Johnson Space Center for the current Mark III advanced prototype surface suit during walking, walking with arm motion, and while seated. Measurements were made using multiple microphones (both head mounted and helmet mounted) and a tri-axial accelerometer to evaluate the effects of suit-borne vibration on overall background noise. Footfall impacts were found to create temporary depolarization of condenser microphone signals, and microphone positioning had a strong effect on signal-noise ratio, partially due to the effect of the helmet enclosure. The results indicate noise mitigation and signal conditioning strategies for improving lunar suit audio voice communications under extra-vehicular (EVA) conditions. Funded by a directed research program of NASA's Space Human Factors Engineering project and by NASA-JSC's Constellation EVA system audio group. 
11:00

2aSAb9. Influence of material properties on sound radiation for flat loudspeakers. Jaime Ramis Soriano (DFISTS. Univ. de Alicante, Carretera de Sant Vicent del Raspeig s/n, 03690 San Vicente del Raspeig, Spain, jramis@ua.es), Jesús Alba Fernandez (Escola Politècnica Superior de Gandia, Universitat Politècnica de València, Crtra Natzaret-Oliva s/n, 46730 Gandia, Spain, jesalba@fis.upv.es), Jorge Frances Monllor (DFISTS. Univ. de Alicante, Carretera de Sant Vicent del Raspeig s/n, 03690 San Vicente del Raspeig, Spain, jfmonllor@ua.es), Jorge P. Arenas Bermudez (Universidad Austral de Chile, Institute of Acoustics, 567 Valdivia, Chile, jorgep.arenas@gmail.com)

This work aims to analyze numerically, analytically and experimentally, the influence on sound power radiated, frequency response, and directionality of different materials in flat loudspeakers. Concerned experimental setup, the excitation force consists in a shaker which his mechanical characteristics are completely known. Finite Element Method has been used to simulate this configuration. The analytical model used couples the electrical, mechanical and acoustical system of the panel. The parameters of the panel material which we are focused are elastic modulus and loss factor. The analytical formalism serves as the basis to study the structural and vibrational behaviour of the system that also is analyzed with experimental results. The results obtained from different materials and different excitation position serves as basis for optimum configuration in this type of speakers.

11:20

2aSAb10. Acoustical Effectiveness of Damping Coating. Raymond Fischer (Noise Control Engineering Inc, 799 Middlesex Tnpk, Billerica, MA 01821, USA, rayf@noise-control.com), Leonid Boroditsky (Noise Control Engineering Inc, 799 Middlesex Tnpk, Billerica, MA 01821, USA, leob@noise-control.com)

Damping coatings have again become popular in the shipbuilding industry to reduce onboard and underwater noise. Understanding of the actual effectiveness of damping tiles applied to ship structures is important for noise prediction during the design stage and for optimizing noise control. The commonly held opinion that energy loss is proportional to loss factor value is not confirmed by tests conducted on actual structures. This paper discusses differences between loss factors measured on the Oberst beam and on two-dimensional real structures. The paper shows how loss factor change may influence transmission loss, radiation efficiency and acceptance. The results of loss factor measurements in different conditions including waterloaded media are demonstrated. influences of resonant and non- resonant modes on damping tile effectiveness is discussed. Corrections for SEA noise prediction algorithms, taking into account results of this research, are proposed.

\section{1:40}

2aSAb11. On the prediction of absorption coefficient of porous materials with Finite Elements. Sabine Langer (TU Braunschweig, Institute of Applied Mechanics, Spielmannstr. 11, 38106 Braunschweig, Germany, s.langer@tu-bs.de), Meike Wulkau (TU Braunschweig, Institute of Applied Mechanics, Spielmannstr. 11, 38106 Braunschweig, Germany, m.wulkau@tu-bs.de)

The absorption coefficient gives the relation between incited and reflected sound power on a surface and is a measure for the damping properties of materials. Experimentally it can be investigated by using the impedance tube for perpendicular incitation and the echo chamber in the case of a diffuse sound field as is known. To optimize the sound absorption properties of materials in the pre-prototype stage, numerical simulation can support the design of materials. A detailed finite element simulation based on Biots Theory for poroelastic-media is used to predict the absorption coefficient of materials with open-pored surfaces. Prospects and limits of this strategy are discussed.

\section{2:00}

2aSAb12. Ground-borne vibration case studies in residential constructions. James Perry (Cerami \& Associates, 404 Fifth Avenue, New York, NY 10018, USA, jperry@ ceramiassociates.com)

Case studies are examined for several new high-rise residential and hotel buildings proposed for construction over or near underground and surface transit systems. Baseline vibration measurements were taken on grade and existing building structures and projected against standard functional vibration criteria (ANSI S3.29 / ISO 2631 2). Through post-construction testing and client feedback, the vibration and structure-borne noise impacts were field verified. Study results offer insight into the correlation between these vibration criteria and the subjective human perceptions of various sensitivities. Further items of discussion include the relative effects of various architectural constructions, building types, and applications as well as mitigating sound and vibration controls. 


\section{Session 2aSAc}

\section{Structural Acoustics and Vibration and EURONOISE: Source Characterization in Structure Borne Noise Problems I}

Evan Davis, Cochair

The Boeing Company, P.O. Box 3707, Seattle, WA 98124, USA

Charles Pezerat, Cochair

Laboratoire Vibrations Acoustique - INSA Lyon, 25 bis avenue Jean Capelle, Bâtiment Saint-Exupéry, Villeurbanne cedex, F-69621, France

Invited Papers

11:00

2aSAc1. Structure-borne sound transmission from machines into ribbed structures. Barry M. Gibbs (University of Liverpool, School of Architecture, Abercromby Square, L693BX Liverpool, UK, bmg@liv.ac.uk), Andreas R. Mayr (Stuttgart University of Applied Sciences, Schellingstrasse 24, 70174 Stuttgart, Germany, andreas.mayr@hft-stuttgart.de)

The total structure-borne sound power of an installed machine is a function of the source activity and mobility, and the receiver mobility, at each contact and for each component of excitation. The data and computational requirements for prediction therefore are large. Manufacturers view their products as single entities and desire corresponding single values of source strength. A laboratory reception plate measurement procedure has been proposed which yields single equivalent values of source strength and source mobility. The source data, in combination with an estimate of the single equivalent value of receiver mobility, yields the approximate total installed power. The accuracy of the estimate is dependent on the spatial variation in contact conditions over the connections. In addition, phase information has been lost in the simplification. Case studies are described for multiple contact sources on a non-homogeneous plate (a timber-joist floor) where the approximate estimates of structure-borne power are compared with exact values obtained from full mobility formulations.

11:20

2aSAc2. Time domain identification of loads on plate-like structures using an array of acoustic velocity sensors. Quentin Leclere (Laboratoire Vibrations Acoustique - INSA Lyon, 25 bis avenue Jean Capelle, Bâtiment Saint-Exupéry, F-69621 Villeurbanne cedex, France, quentin.leclere@insa-lyon.fr), Charles Pezerat (Laboratoire Vibrations Acoustique - INSA Lyon, 25 bis avenue Jean Capelle, Bâtiment Saint-Exupéry, F-69621 Villeurbanne cedex, France, charles.pezerat@insa-lyon.fr)

The FAT (Force Analysis Technique) method has been developped to identify loads on beams or plates from the knowledge of their flexural displacements. The method is based on a local discretisation of the differential operator of the studied structure : all derivatives of the displacement field are assessed at a given point from a finite difference scheme. The estimation of the operator gives as an output the value of the force distribution. Up to now, applications of the FAT method have been made in the frequency domain, scanning the studied structure with accelerometers or with a laser vibrometer, and using phase references to get the phase relation between different points. The aim of the present study is to show that the FAT method allows to identify loads in the time domain. This operation requires the simultaneous measurement of at least 13 points on the plate, that can be realized without contact using an array of acoustic velocity sensors in the very near field of the plate. The method has been applied on a plate excited by an acoustic diffuse field. The identified force distribution is compared to the parietal acoustic pressure measured in the reverberant room.

\section{1:40}

2aSAc3. Measurements of the bending moment at boundaries of a structure. Simon Chesne (LaMCoS - INSA-Lyon - CNRS UMR5259, 18-20, rue des Sciences, Bâtiment Jean d'Alembert, F-69621 Villeurbanne, France, simon.chesne@ insa-lyon.fr), Baptiste Chomette (LaMCoS - INSA-lyon - CNRS UMR5259, 18-20, rue des Sciences, Bâtiment Jean d'Alembert, F-69621 Villeurbanne, France, baptiste.chomette@insa-lyon.fr), Charles Pezerat (Laboratoire Vibrations Acoustique - INSA Lyon, 25 bis avenue Jean Capelle, Bâtiment Saint-Exupéry, F-69621 Villeurbanne cedex, France, charles.pezerat@insa-lyon.fr)

In the vibration transmission process, the part due to the moment excitation is often neglected, because of the difficulty to measure them, even if these terms are sometimes important. Indeed, several studies show that the influence of the moment in structure borne power transmission can be higher than simple force. Moment measurement or identification is an old problem especially at boundaries. It has been mainly investigated in mobility methods. In fact, bending moment expression can be seen as spatial derivatives of displacements. These derivatives can be approximated from measured displacements but two major difficulties appear: derivatives are highly sensitive to measurement errors and the usual methods used to obtain them (finite differences, modal approach, etc.) are not well adapted at boundary points. In this paper, three different moment identification methods are investigated where the considered structure is a beam. Both of these approaches are already developed by authors, the third method is based on the use of particular mechanical and electric setups of piezoelectric patches. These 3 methods are numerically and experimentally implemented allowing us to compare and discuss on results. 
2aSAc4. Characterisation of structure borne sound sources from measurement in-situ. Andrew Elliott (University of Salford, Acoustic Research Centre, Newton Building, M5 4WT Salford, UK, a.s.elliott@pgr.salford.ac.uk), Andy T. Moorhouse (University of Salford, Acoustic Research Centre, Newton Building, M5 4WT Salford, UK, a.t.moorhouse@salford.ac.uk)

In-situ source characterisation methods are those which involve measurements made whilst source and receiver are coupled as they would be in a real installation. Potentially in-situ source characterisation may account for the physical reality lost in the "black box" approach. There are other potential benefits such as ease of measurement. In this work a structure borne sound source is characterised using in-situ measurements of blocked force and coupled mobility. Promising results from the method have been presented previously. Further to this, an extension of the method allowing the use remote measurement positions has been developed. Using reciprocity, the extended method will further ease measurement for situations where access poses a problem. The extended method is outlined and some preliminary validation results are presented.

12:20

2aSAc5. General model of a structure-borne sound source and its application to shock vibration. Yuri Bobrovnitskii (Mechanical Engineering Research Institute, 4, M. Kharitonievky Str., 101990 Moscow, Russian Federation, yuri@imash.ac.ru), Tatiana Tomilina (Mechanical Engineering Research Institute, 4, M. Kharitonievky Str., 101990 Moscow, Russian Federation, tatiana@imash .ac.ru)

One of the well-known models of structure-borne sound sources is described by the quantities defined at the source-receiving structure interface: by the matrix of the source impedances or mobilities and by the vector of the blocked forces or free velocities. Together with the receiver impedances or mobilities it allows one to predict the vibration field in the receiver and to develop and examine candidates for source characterization. In this paper, it is shown analytically that this model is valid when the sound is generated inside the source by a kinematic excitation as well as by force excitation or their combination and, thus, it represents a rather general model with wide range of applications. Most attention is paid to its peculiarities and limitations when applied to shock sources. Results are illustrated by the data measured in the shock tests of some scientific space instruments.

TUESDAY MORNING, 1 JULY 2008

ROOM 240, 8:20 TO 10:40 A.M.

\title{
Session 2aSCa
}

\section{Speech Communication: Speech Technology II}

\author{
Indranil Dutta, Chair \\ Rice University, Center for the Study of Languages, MS \#36, 6100 Main St., Houston, TX 77005, USA
}

\section{Contributed Papers}

$8: 20$

2aSCa1. Evaluation of acoustic environments using deteriorated speech sound. Yoshiki Nagatani (Nara Medical University, 840 Shijo-cho, 634-8522 Kashihara, Japan, naramed-u@nagatani.ne.jp), Takefumi Sakaguchi (Nara Medical University, 840 Shijo-cho, 634-8522 Kashihara, Japan, t-saka@naramed-u.ac.jp), Hiroshi Hosoi (Nara Medical University, 840 Shijo-cho, 634-8522 Kashihara, Japan, hosoi@naramed-u.ac.jp)

Aged or hearing-impaired people require better acoustical environments for higher QOL. Many methods of evaluating the quality of speech in acoustical environments have been developed. However, since they mainly focus on quality of speech in bad conditions (e.g. environments with huge noise or long reverberation), they are not suited for evaluations in generic environments such as normal houses or public facilities for aged people. For instance, the scores of D value (deutlichkeit) or speech transmission index (STI) are too high and not sensitive enough to refer to such environments. The intelligibility test using normal speech sounds cannot clarify the differences between different room environments because the intelligibility scores reach almost 100 percent in ordinary room environments. Therefore, we proposed a new evaluation method using deteriorated speech sounds. In this method, signal-processed speech sounds are presented to trial subjects under target sound environments. In this study, Japanese monosyllabic speech sounds convoluted by the impulse responses of room reverberations were presented through a headphone in order to simulate the architectural acous- tic environments. As a result, it was shown that this new method could detect the small difference of sound environments, which the conventional methods could hardly evaluate.

\section{$8: 40$}

2aSCa2. Measurement of Speech Intelligibility Using Low Level Output - Threshold Efficient S/N Ratios. Christos Nestoras (London South Bank University, FESBE, Borough Road, SE1 0AA London, UK, nestorasc@gmail.com), Stephen Dance (London South Bank University, FESBE, Borough Road, SE1 0AA London, UK, dances@1sbu.ac.uk)

Excessive background noise levels or perhaps annoyance due to a high test signal level are the main reasons for having a low signal to noise ratio $(\mathrm{S} / \mathrm{N})$ or low level output during a measurement session. A significant error, in this sense, can be introduced in the resulting data since the measurement technique requires, among others, a minimum $\mathrm{S} / \mathrm{N}$ ratio for an accurate result. A validation is presented of low level output intelligibility measurements in an attempt to establish a point of reference for the verification of data accuracy for a given space. An indication of the functions taking place in this respect could be obtained through practical experimentation. Results are reported here. 
9:00

2aSCa3. Vowels recognition using mellin transform and plp-based feature extraction. Mahdi Jamaati (Technical University of Shahrood, 12345 Shahrood, Iran, mahdi.jamaati@gmail.com), Hossein Marvi (Technical University of Shahrood, 12345 Shahrood, Iran, h_marvi@shahroodut.ac.ir), Milad Lankarany (Technical University of Shahrood, 12345 Shahrood, Iran, milad.lankarany@gmail.com)

Feature extraction for speech recognition is a subject of majer interest today. Different feature have been investigated in speech recognition systems. The scale transform is a particular restriction of the Mellin transform. The key property of the scale transform is the scale invariance .The mel frequency(MFCC) and perceptual linear predicate (PLP) have usually reported to have yielded good performance. In this paper, a new method is presented which combines feature extracted from mellin transform with the plp features. To evaluate the performance of the proposed features the comparative tests with the lpc features, MFCC and PLP were performed on the vowels based speech recognition system. Preliminary experiment show that this approach posses promissing result.

9:20

2aSCa4. A system for automatic detection and correction of detuned singing. Michał Lech (Gdansk University of Technology, Multimedia Systems Department, 11/12 Gabriela Narutowicza Street, 80-952 Gdansk, Poland, mlech@sound.eti.pg.gda.pl), Bozena Kostek (Gdansk University of Technology, Multimedia Systems Department, 11/12 Gabriela Narutowicza Street, 80-952 Gdansk, Poland, bozenka@sound.eti.pg.gda.pl)

The aim of the paper is to show a system engineered for automatic detection and correction of detuned singing. For this purpose, existing methods of fundamental frequency detection and pitch correction are reviewed. In addition, main characteristics of some existing detuning systems are presented. As algorithms for fundamental frequencies detection and pitch correction, the fast autocorrelation and HPS (Harmonic Product Spectrum), and the modified phase vocoder and PSOLA (Pitch-Synchronous Overlap-Add) are chosen and examined. Four possible combinations of the algorithms are reviewed not only in the context of fundamental frequency detection and pitch shifting correctness but also with regard to the quality of the resulting singing signal. Experiments are performed on both male and female singing samples consisting of a variety of tones and various articulations. Basing on the obtained results, it is concluded that the HPS and PSOLA algorithms are the optimum choice as means to correct detuned singing. In addition, listening tests are performed in order to confirm objective measurements of pitch detection and correction. The system is implemented in JAVA. Conclusions are drawn and proposals of improvements are provided.

\section{9:40}

2aSCa5. Spectrotemporal and f0 evidence for a theory of enhancement. Indranil Dutta (Rice University, Center for the Study of Languages, MS \#36, 6100 Main St., Houston, TX 77005, USA, indranil.dutta@rice.edu)

According to Keyser \& Stevens, 2006, apart from universal features that are utilized to calculate motoric instructions, there is a second parallel and language-specific process called enhancement, where additional motoric instructions are processed for the enhancement of the saliency of features that are in danger of being obliterated. The "Standard View" on the distinction between voiced stops (VS) and voiced aspirated stops (VAS) in Hindi proposes that the breathy-murmured release following VAS is sufficient to make the contrast between the VS and VAS (Ladefoged \& Maddieson, 1996). Hence, audible distinctions between VAS and VS during closure (CD/VLT) are not relevant in maintaining the contrast. Results from our studies show that $\mathrm{CD}$ is a relevant cue in making a distinction between aspirated and unaspirated stops. Spectral intensity measures show that speakers employ different glottal configurations to obtain the breathy/modal voicing contrast. VLT durations are inversely correlated with f0, such that longer
VLT for VAS leads to further lowering of $f_{0}$. This evidence suggests that $f_{0}$ perturbations, differences in the durations of closure, and nature and extent of aspiration, all contribute in making the four-way stop contrast possible in Hindi, in support of a theory of enhancement.

\section{$10: 00$}

2aSCa6. A Statistical Prosodic Model for Voice Conversion. Jan Schwarz (Institute for Circuit and System Theory, Christian-AlbrechtsUniversity of Kiel, Kaiserstrasse 2, 24143 Kiel, Germany, js@tf.uni-kiel.de), Ulrich Heute (Institute for Circuit and System Theory, Christian-Albrechts-University of Kiel, Kaiserstrasse 2, 24143 Kiel, Germany, uh@tf.uni-kiel.de)

Nowadays Text-to-Speech (TTS) systems adapt the output voice to the user and the corresponding application. The aim is a personalisation. Thereby, the user is set into familiar surroundings, increasing the TTS acceptance. For example, an e-mail client that may read the incoming messages with the synthesised voice sounding like that of the sender. Such a personalised TTS system is costly; so, voice-conversion (VC) techniques are used to save resources. VC transforms the voice of a "source speaker" in such a way that the converted voice sounds like that of another "'target speaker'. This voice sounds only natural, if it includes all features relevant for the true target voice. Here, a main problem is the mapping of the prosody which is one of the essential features. This contribution introduces a statistical prosodic model for voice conversion. It is based on Gaussian-Mixture Models (GMM), trained for the pitch and the duration of diphones. To ensure sufficient data for the GMM training, seven diphone classes are separated as related to the international phonetic alphabet. The suitability for VC as well as limitations, necessary extensions (stress) and problems are pointed out

\section{0:20}

2aSCa7. Modelling acoustic parameters of prosody for read and acted-speech synthesis. Milan Rusko (Institute of Informatics of the Slovak Academy of Sciences, Dubravska cesta 9, 84507 Bratislava, Slovakia, milan.rusko@savba.sk), Marián Trnka (Institute of Informatics of the Slovak Academy of Sciences, Dubravska cesta 9, 84507 Bratislava, Slovakia, trnka@savba.sk), Sakhia Darjaa (Institute of Informatics of the Slovak Academy of Sciences, Dubravska cesta 9, 84507 Bratislava, Slovakia, utrrsach@savba.sk), Richard Kováč (Institute of Informatics of the Slovak Academy of Sciences, Dubravska cesta 9, 84507 Bratislava, Slovakia, richard.kovac@savba.sk), Juraj Hamar (Philosophical Faculty, Comenius University, Gondova 2, 81801 Bratislava, Slovakia, juraj.hamar@chello.sk)

The prosody model is one of the most important parts of every speech synthesizer, influencing mainly its naturalness. The intonation contour and phoneme lengths (together with speech quality) bear a great deal of extralinguistic and paralinguistic information contained in the synthesized speech. The features reflecting personality, mood and emotions of the speaker are in strong interaction with those reflecting speech styles. Anyway the appropriate choose of prosody model and training material can make it possible to create special model for every speaking style. The paper presents our approach to modelling of acoustic parameters of prosody in two different speech styles in Slovak. Our model is based on Classification and regression trees (CARTs). It uses independent CART for phoneme lengths and three CARTs for fundamental frequency (F0) at the beginning, centre, and end of every syllable. Two hours of read speech were used for training a model of read speech. The recordings of a puppet player were used to train a model of acted speech. The models were implemented in the Kempelen 2.2 unit selection Slovak speech synthesizer. The listening tests have shown that the models are capable of modelling significant amount of the differences of the two speaking styles. 


\title{
Session $2 \mathrm{aSCb}$
}

\section{Speech Communication: How do Physical and Motor Knowledge Matter to Speech Perception?}

\author{
Carol A. Fowler, Cochair \\ Haskins Laboratories, 300 George St, Suite 900, New Haven, CT 06511, USA
}

Mikko Sams, Cochair

Helsinki University of Technology, Lab. of Computational Engineering, Cognitive Science and Technology, Otakaari 5A, 02150 Espoo ('Magnet house'), Finland

Invited Papers

2aSCb1. Action-based multisensory integration in striking events. Bruno L. Giordano (Centre for Interdisciplinary Research in Music Media \& Technology (CIRMMT) - Schulich School of Music - McGill Univ., 555 Sherbrooke Street West, Montreal, QC H3A1E3, Canada, bruno.giordano@music.mcgill.ca), Stephen McAdams (Centre for Interdisciplinary Research in Music Media \& Technology (CIRMMT) - Schulich School of Music - McGill Univ., 555 Sherbrooke Street West, Montreal, QC H3A1E3, Canada, smc@music.mcgill.ca), Paolo Crosato (Dept. of Information Engineering, Univ. of Padova, Via G. Gradenigo 6/A, 35131 Padova, Italy, jimbobaus@virgilio.it), Federico Avanzini (Dept. of Information Engineering, Univ. of Padova, Via G. Gradenigo 6/A, 35131 Padova, Italy, avanzini@dei.unipd.it), Carmine Casciato (Centre for Interdisciplinary Research in Music Media \& Technology (CIRMMT) - Schulich School of Music - McGill Univ., 555 Sherbrooke Street West, Montreal, QC H3A1E3, Canada, casciato@music.mcgill.ca), Stephen Sinclair (Centre for Interdisciplinary Research in Music Media \& Technology (CIRMMT) Schulich School of Music - McGill Univ., 555 Sherbrooke Street West, Montreal, QC H3A1E3, Canada, sinclair@music.mcgill.ca), Marcelo M. Wanderley (Centre for Interdisciplinary Research in Music Media \& Technology (CIRMMT) - Schulich School of Music - McGill Univ., 555 Sherbrooke Street West, Montreal, QC H3A1E3, Canada, marcelo.wanderley@mcgill .ca)

Many theories of multisensory integration focus on action-independent perception. This approach has limited ecological validity, because under everyday conditions we frequently act upon multisensory events through motor behavior. We present a novel methodology for the study of multisensory integration in action-directed perception, focusing on striking events. Observers repeatedly hit a virtual object with a target striking velocity, and are presented with various types of sensory information about the striking event: auditory and/or haptic and/or visual. For each of the experimental trials, they initially receive feedback on the tracking of the target striking velocity. In a second phase, feedback is eliminated. In a third phase, the sensory properties of the striking event are changed. We quantify the extent to which a variation in each of the sensory modalities disrupts performance in the tracking of the target striking velocity. Multisensory dominance hierarchies are inferred from the comparison of the patterns of performance disruption in unimodal and multisensory contexts.

\section{$8: 40$}

2aSCb2. Amodal specification of talker-specific motor behavior. Lawrence D. Rosenblum (University of California, Riverside, Department of Psychology, 900 University Ave., Riverside, CA 92521, USA, rosenblu @ citrus.ucr.edu)

There is substantial evidence that the speech perception function incorporates information for articulatory motor behavior. Research over the last 15 years has shown that speech perception also uses information for talker-specific motor behavior. Interestingly, this research reveals that talker information can facilitate speech perception whether it is apprehended through auditory or visual speech (lipread) means. The multimodal nature of these effects could mean that the talker-specific properties used by the system are amodal and motoric-or gestural-in nature. Evidence for this proposition-that talker-specific facilitation of speech is based on amodal specification of gestural style-will be discussed. This evidence includes findings that isolated talker-specific phonetic information, available both optically and acoustically, can be informative about talker identity to the degree that it supports cross-modal talker matching. Other evidence shows that the talker familiarity gained through one modality can facilitate perception of speech in the other. Finally, recent evidence reveals that speech perceivers will align to (inadvertently imitate) talker-specific properties of utterances they are asked to shadow, whether that speech is presented auditorily or visually. These results suggest that some of the 'motor knowledge' bearing on speech perception takes the form of talker-specific gestural properties, amodally specified. 
2aSCb3. Cross-modal synchrony perception reveals aspects of categorical perception. Armin Kohlrausch (Philips Research Europe, Digital Signal Processing (MS WO02), High Tech Campus 36, 5656 AE Eindhoven, Netherlands, armin.kohlrausch@philips.com), Rob L. Van Eijk (Technische Universiteit Eindhoven, Human-Technology Interaction, P.O. Box 513, 5600 MB Eindhoven, Netherlands, r.1.j.v.eijk@tue.nl), Steven Van De Par (Philips Research Europe, Digital Signal Processing (MS WO02), High Tech Campus 36, 5656 AE Eindhoven, Netherlands, steven.van.de.par@philips.com), James F. Juola (University of Kansas, Department of Psychology, 1415 Jayhawk Blvd., Lawrence, KS KS 66045, USA, juolas@ku.edu), Michael Vitevich (University of Kansas, Department of Psychology, 1415 Jayhawk Blvd., Lawrence, KS KS 66045, USA, mvitevit@ku.edu)

The phenomenon of "categorical perception" has played an important role in speech research. When a specific (combination of) feature(s) of a speech stimulus is varied along a physical dimension, categorical perception is reflected by two observations: (1) the percept of the sound changes abruptly from one category to another, e.g., from "ba" to "da" to "ga" for changes in formant transition frequencies, and (2) physical changes of a given amount lead to more easily perceivable differences for stimuli close to a category boundary, compared to stimuli in the center of a category. In this talk, I present data about audio-visual synchrony perception, which indicate that perceived synchrony also reveals properties of categorical perception. Depending on the physical delay between the auditory and visual component, the percept changes from "audio first," to "synchronous" to "video first." When measuring sensitivity to changes in audio-visual delays, we observe that the thresholds are small for stimuli at the transitions between the earlier determined categories, while they are larger for stimuli within a category.

2aSCb4. Do we perceive articulatory gestures when we listen to speech? Pierre Divenyi (VA Northern Calif. Health Care Syst. and East Bay Inst. for Research and Education, Bldg. R4, 150 Muir Rd., Martinez, CA 94553, USA, pdivenyi@ebire.org), Adam Lammert (VA Northern Calif. Health Care Syst. and East Bay Inst. for Research and Education, Bldg. R4, 150 Muir Rd., Martinez, CA 94553, USA, alammert@ebire.org)

Articulatory synthesis methods, classic and contemporary, have demonstrated that it is possible to generate speech from an ensemble of functions derived from articulatory gestures. Such gesture-to-waveform transforms suggest that, inversely, the speech signal should be also decomposable into the same set of gesture, or gesture-like, functions. These functions vary slowly in time and their association with the speech waveform (words as well as sentences) can be established by machine learning algorithms. In a recent study at our laboratory, listeners were asked to type the word or the sentence they heard, with speech (degraded in diverse ways) as the stimulus. The subjects' responses were synthesized, time-aligned with the stimulus, and decomposed into a set of eight gestures, as specified by the Haskins Laboratories TADA system (http://www.haskins.yale.edu/tada_download/index.html). When the running distance between input and response gesture functions is calculated, results indicate a significant degree of gesture information transmitted even during severely degraded speech segments, suggesting that the perceptual system may track speech via underlying functions similar to gestures. Epochs at which this running distance estimate fails, i.e., exceeds a certain threshold, may be considered to signal periods during which insufficient bottom-up information had to be supplemented using higher-order linguistic knowledge.

\section{9:40}

2aSCb5. Hearing the tongue and lips of vowel gestures: A new differential paradigm. Jean-Luc Schwartz (ICP-GIPSA, INPG, 46 Av. Félix Viallet, 38031 Grenoble, France, schwartz@icp.inpg.fr), Nathalie Vallée (ICP-GIPSA, INPG, 46 Av. Félix Viallet, 38031 Grenoble, France, vallee@icp.inpg.fr), Sonia Kandel (LPNC, Université Pierre Mendès-France - BP 47, 38040 Grenoble, France, Sonia.Kandel@upmf-grenoble.fr)

It is an old question to know to what extent a listener can recover the articulatory dimensions of a speaker's gesture. In the case of vocalic configurations, a number of experiments have been done on expert phoneticians, showing that vowel height can be reasonably well estimated from the sound, but the front-back and lip rounding dimensions are much less well recovered. However, almost nothing has been done on naive listeners, due to the difficulty to perform absolute estimations in the lack of explicit phonetic knowledge. In the past years, we have developed an original paradigm, exploiting differential rather than absolute estimations. We show that French listeners, even naïve, are able to discriminate to a certain extent which vowel in a given pair has a higher vs. lower or more front vs. more back tongue position, or more or less rounded lips. From these data, we have elaborated an algorithm enabling to estimate what are the internal representations of vowel height, frontness and rounding, and correlated these representations with acoustic parameters, F1, F2-F1 and F'2 in Barks appearing to play a key role in the auditory recovery of these three motor dimensions.

\section{0:00}

2aSCb6. Mathematical Evidence For Motor Theories of Speech Perception. Gordon Ramsay (Haskins Laboratories, 300 George Street, New Haven, CT 06511, USA, ramsay@ haskins.yale.edu)

Theories of speech perception have often proposed, controversially, that recovery of phonological information from the acoustic signal necessarily requires implicit knowledge of the physical processes underlying speech production. Most of the evidence supporting this hypothesis has been derived empirically from experiments in speech perception. In this paper, we show that many of the basic ideas underlying motor theories of speech perception can also be derived mathematically from first principles, using classic results in stochastic nonlinear filtering theory. Two key results, the Duncan-Mortenson-Zakai and Fujisaki-Kallianpur-Kunita theorems, show that the optimal state estimator for any partially-observed nonlinear stochastic dynamical system always takes the form of a "matched filter", which is itself a nonlinear stochastic dynamical system, the structure of which mimics, and resonates with, the structure of the original. Interpreting this in the context of speech perception, recovery of phonological information from sound produced by a human vocal tract necessarily involves construction of an internal model of the processes implicated in speech production. A key prediction, which we explore, is that any such model need not reproduce all the details of these processes, but is only required to predict the lawful conditional correlation between gesture and sound. 


\title{
Session 2aSCc
}

\section{Speech Communication: Speech Recognition in Noisy Environments}

\author{
Olivier Siohan, Cochair \\ Advanced Large Vocabulary Speech Recognition, IBM Watson Research Center, Yorktown, NY 10598, USA
}

\author{
Georges Linares, Cochair \\ Université d'Avignon et des Pays de Vaucluse, Laboratoire Informatique d'Avignon, 339, chemin des Meinajaries, Agroparc BP \\ 1228, 84911 Avignon Cedex 9, France
}

\section{Contributed Paper}

\section{0:40}

2aSCc1. Speech recognition with body-conducted speech using differential acceleration. Masashi Nakayama (Hiroshima City University / National Institute of Advanced Industrial Science and Technology, 3-4-1 Ozuka-Higashi, Asa-Minami-Ku, 731-3194 Hiroshima, Japan, m.nakayama @ aist.go.jp), Shunsuke Ishimitsu (Hiroshima City University / National Institute of Advanced Industrial Science and Technology, 3-4-1 OzukaHigashi, Asa-Minami-Ku, 731-3194 Hiroshima, Japan, ishimitu @hiroshima-cu.ac.jp), Seiji Nakagawa (National Institute of Advanced Industrial Science and Technology (AIST), 1-8-31 Midorigaoka, 563-8577 Ikeda, Osaka, Japan, s-nakagawa@aist.go.jp)

Speech-recognition rates decrease in noisy environments. The bodyconducted speech, conducted in solids such as body and skins, has a noise- robust characteristics and can be served for recognition systems even in 98 dBSPL-noise (-20 dBSNR) environments. However, the body-conduction could not capture high frequency sounds. Conventional methods for the improvement in sound quality of body-conducted speeches needs both speeches and body-conducted speeches. In this paper, a new body-conducted speech retrieval technique in sound quality without a speech signal itself is proposed. First, high-frequency components in the body-conducted speech were emphasized using differential acceleration. Second, conventional noise reduction method was adopted to make a clear body-conducted speech from a retrieval speech which contains constant noise. The recognition experiments using the proposed method showed that it improved recognition rate in all speakers.

\section{Invited Paper}

\section{1:00}

2aSCc2. Exploiting confidence measures for missing data speech recognition. Christophe Cerisara (LORIA UMR 7503, Campus Scientifique, 54506 Vandoeuvre-lès-Nancy, France, Christophe.Cerisara@loria.fr)

Automatic speech recognition in highly non-stationary noise, for instance with a competing speaker or background music, is an extremely challenging and still unsolved problem. Missing data recognition is a robust approach that is well adapted to this kind of noise. A standard missing data technique consists in marginalizing out, from the observation likelihoods computed during decoding, the contribution of the spectro-temporal fragments that are dominated by noise. However, such an approach can hardly be applied to advanced parameterization domains that do not separate speech from noise frequencies, such as the cepstrum or ETSI AFE. We propose in this work to extend this technique to such parameterization domains, and not only to spectrographic-like front-ends as it was the case before. This is realized by masking the observations that favor erroneous decoding paths, instead of masking the features that are dominated by noise. These new missing data "masks" are now estimated based on speech recognition confidence measures, which can be considered as indicators of the reliability of decoding paths. A first version of this robust algorithm is evaluated on the French broadcast news ESTER corpus.

\section{Contributed Paper}

\section{1:20}

2aSCc3. An MTF-based blind restoration of temporal power envelopes as a front-end processor for automatic speech recognition systems in reverberant environments. Xugang Lu (Japan Advanced Institute of Science and Technology, 1-1, Asahidai, Nomi, 923-1292 Sendai, Japan, xugang@jaist.ac.jp), Masashi Unoki (JAIST, 1-1 Asahidai, 923-1292 Nomi, Japan, unoki@jaist.ac.jp), Masato Akagi (Japan Advanced Institute of Science and Technology, 1-1, Asahidai, Nomi, 923-1292 Sendai, Japan, akagi@jaist.ac.jp)

To reduce speech degradation in reverberant environments, we previously proposed a modulation transfer function (MTF) based method for speech dereverberation. It is based on the MTF relation that the sub-band temporal power envelope of reverberant speech can be represented as the convolution between temporal power envelopes of clean speech and the room impulse response. Therefore, the sub-band power envelope of clean speech can be estimated using inverse MTF filtering without measuring the room impulse response. We tested the effectiveness of this method as a front-end for automatic speech recognition (ASR) in both artificial and real reverberant environments. Reverberant speech signals were created by simple convolution of clean speech (AURORA-2J) and artificially-produced or real room impulse responses. The relative spectral filtering of the auditory-power-spectrum based method was used as a baseline. Compared with the baseline, our proposed method had $36.64 \%$ and $21.68 \%$ improvements in error reduction rate for artificial reverberant environments (reverberation times from 0.2 to $2.0 \mathrm{~s}$ ) and real reverberant environments (43 reverberant impulse responses), respectively. These results indicate that our proposed method can be used as a robust front-end for ASR. [Work supported by a Grant-in-Aid for Science Research from the Japanese Ministry of Education (No. 18680017).] 


\section{Invited Paper}

\section{1:40}

2aSCc4. Front-end processing of a distant-talking speech interface for control of an interactive TV system. Maurizio Omologo (Fondazione Bruno Kessler - IRST, Via Sommarive, 18, Povo, 38050 Trento, Italy, omologo@fbk.eu)

This work addresses a research activity being conducted for the development of a user-friendly interface for the access to a virtual smart assistant enabling the interaction with TV-related digital devices and infotainment services. In the given scenario, the users can speak in a natural and comfortable way, not encumbered by any hand-held or head-mounted microphone. The environment is typically a living room, equipped with digital TV, Hi-Fi audio devices, etc., and populated by a group of people (e.g., family members). Among the most challenging issues involved in this scenario are a multi-microphone front-end for an effective processing of the given acoustic scene, an Acoustic Echo Cancellation (AEC) component to compensate the sound produced by loudspeakers, and a multi-modal distanttalking spoken dialogue system. As far as the front-end is concerned, multiple speaker localization, speech activity detection, speaker identification, and speech recognition will have to perform accurately even when AEC is applied to the given microphone array. The paper aims to present preliminary results of this research, which is being conducted under the European Project DICIT.

\section{Contributed Paper}

\section{2:00}

2aSCc5. Speaker adaptation combined with missing data reconstruction. Ulpu Remes (Adaptive Informatics Research Centre, Helsinki University of Technology, P.O. Box 5400, 02015 Espoo, Finland, ulpu.remes@tkk.fi), Kalle J. Palomäki (Adaptive Informatics Research Centre, Helsinki University of Technology, P.O. Box 5400, 02015 Espoo, Finland, kalle.palomaki@tkk.fi), Mikko Kurimo (Adaptive Informatics Research Centre, Helsinki University of Technology, P.O. Box 5400, 02015 Espoo, Finland, mikko.kurimo@tkk.fi)

Missing data methods offer an attractive framework for automatic speech recognition (ASR) under time-varying and unpredictable noise conditions. The motivation for the methods comes from the human speech perception and auditory scene analysis, studies on which have suggested that the more intense sound components in an auditory scene dominate the neu- ral response to the scene. The missing data methods developed for ASR divide the noisy speech signal to speech and noise dominated regions. Speech components in the noise dominated regions are viewed as missing but may be reconstructed based on the observed components. Missing data methods have performed well in previous studies with noise corrupted speech but have not become a standard in ASR. Especially considering ASR on large vocabularies, the methods have a disadvantage: it is not straightforward to combine them with common speaker adaptation methods such as constrained maximum likelihood linear regression (CMLLR). In this work, we investigate efficient ways to combine missing data reconstruction with speaker adaptation in large vocabulary ASR task with speech data recorded in noisy real-world environments. The experiments show that speaker adaptation combined with the missing data reconstruction can improve the missing data approach in noisy large vocabulary ASR.

\section{Invited Paper}

2aSCc6. An efficient frame selection approach to variable frame rate analysis for noise robust speech recognition. Zheng-Hua Tan (Department of Electronic Systems, Aalborg University, Niels Jernes Vej 12, 9220 Aalborg, Denmark, zt@es.aau.dk), Børge Lindberg (Department of Electronic Systems, Aalborg University, Niels Jernes Vej 12, 9220 Aalborg, Denmark, bli@es.aau.dk)

This paper presents a low-complexity, effective variable frame rate (VFR) analysis method that conducts frame selection on the basis of a posteriori signal-to-noise ratio (SNR) weighted energy distance. It has two characteristics. First, energy distance (instead of cepstral distance) is used to make it computationally efficient and thus enable a finer granularity in search as compared with cepstral distance criterion. Secondly, SNR weighting is used to emphasize the reliable regions in noisy speech signals. In terms of frame selection, it is experimentally found that the method is able to assign a higher frame rate to fast changing events such as consonants, a lower frame rate to steady regions like vowels and no frames to silence, even for very low SNR signals. The VFR method is applied to speech recognition in noisy environments to improve noise robustness. Being a method that takes effect in the time-domain, it is moreover combined with spectral- and cepstral-domain techniques to gain further improvement. Experiments are conducted on the Aurora 2 database, which is the TI digits database artificially distorted by adding different noises, and very encouraging results are obtained. 


\title{
Session 2aSCd
}

\section{Speech Communication: Speech Perception I}

\author{
Mirjam Broersma, Chair \\ Radboud University Nijmegen, PO Box 9104, Nijmegen, 6500 HE, Netherlands
}

\section{Contributed Papers}

11:00

2aSCd1. Phonological specificity of vowels and consonants in 20-month-olds' word representations. Anders Højen (Univ. of Southern Denmark, Dept. Lang. Communication, Campus Vej 55, 5230 Odense, Denmark, anders@andershojen.dk), Thomas O. Madsen (Univ. of Southern Denmark, Dept. Lang. Communication, Campus Vej 55, 5230 Odense, Denmark, tom@language.sdu.dk), Werner Vach (Univ. of Southern Denmark, Dept. Lang. Communication, Campus Vej 55, 5230 Odense, Denmark, werner.v@stat.sdu.dk), Torkil Østerbye (Univ. of Southern Denmark, Dept. Lang. Communication, Campus Vej 55, 5230 Odense, Denmark, oesterbye@sdu.dk), Karina F. Christensen (Univ. of Southern Denmark, Dept. Lang. Communication, Campus Vej 55, 5230 Odense, Denmark, kf@language.sdu.dk), Hans Basbøll (Univ. of Southern Denmark, Dept. Lang. Communication, Campus Vej 55, 5230 Odense, Denmark, hba@language.sdu.dk), Sueli Caporali (Univ. of Southern Denmark, Dept. Lang. Communication, Campus Vej 55, 5230 Odense, Denmark, s.caporali@widex.com), Dorthe Bleses (Univ. of Southern Denmark, Dept. Lang. Communication, Campus Vej 55, 5230 Odense, Denmark, bleses @ sdu.dk)

At the onset of word learning around the age of 12 months, infants are highly capable of perceiving native phonological distinctions. However, required to make word-object associations, infants sometimes fail to perceive certain phonological distinctions. The present study used an inter-modal preferential looking technique to examine Danish 20-month-olds' sensitivity to mispronunciations involving either a vowel or a consonant in familiar words. Each mispronounced word could be "reconstructed" to either of two familiar Danish words. By way of example, the English nonword "bock" can be turned into either "rock" or "book" depending on whether the mispronunciation is perceived to be in the first consonant or in the vowel. The results so far indicate that the infants were not differentially sensitive to vowel vs. consonant mispronunciations. This result, in turn, suggests that vowels and consonants do not differ in constraining lexical access to familiar words in Danish 20-month-olds.

\section{1:20}

2aSCd2. Perceptual asymmetries induced by category learning Laurent Bonnasse-Gahot (CAMS-EHESS, 54 bd Raspail, 75270 Paris Cedex 06, France, lbg@ehess.fr)

This paper takes an information-theoretic approach to study the perceptual consequences of the neural encoding of categories (e.g. vowels). We focus on two well-known psychophysical phenomena: categorical perception, characterized by greater cross-category than within-category discrimination, and perceptual magnet effect, stating that perceptual space is wrapped around prototypical instances of a given category, leading to a better ability to discriminate stimuli near non-prototypical exemplars of a category than near prototypical ones. Introducing a perceptual distance based on the Kullback-Leibler distance between the patterns of activity evoked by two stimuli, we show that both categorical perception and prototypical effects emerge from maximizing information contained in the neural representation about a set of categories. We discuss the relations between these two psychophysical phenomena and show that they go in hand with another kind of asymmetric effect. The discriminability between a category prototype and a non-prototype is not symmetric, depending on which stimuli serves as a referent. Non-prototypical stimuli are judged closer to prototypical exem- plars than the reverse. Quantitative and qualitative comparisons with experimental data and previous theoretical work are presented and discussed.

\section{1:40}

2aSCd3. Order effects and peripherality: a cross-linguistic perceptual study using an [i]-[e] articulatory continuum. Charalampos Karypidis (UMR 7018, CNRS / Univ. Paris III, 19, rue des Bernardins, $75005 \quad$ Paris, France, ch_karypidis@yahoo.com), Antonia Colazo-Simon (UMR 7018, CNRS / Univ. Paris III, 19, rue des Bernardins, 75005 Paris, France, simonantonia@hotmail.com), Angelica V. Costagliola (UMR 7018, CNRS / Univ. Paris III, 19, rue des Bernardins, 75005 Paris, France, angelicacostagliola@yahoo.it), Cirineu Cecote Stein (UMR 7018, CNRS / Univ. Paris III, 19, rue des Bernardins, 75005 Paris, France, cirineustein@uol.com.br), Gilles Guglielmi (ARP / UFRL Univ. Paris VII - Denis Diderot, 30, rue du Chateau des Rentiers, 75013 Paris, France, gillesgug@yahoo.fr)

This paper attempted to provide further evidence on whether peripherality is a universal bias triggering stimulus order effects. Participants from four linguistic systems - Brazilian Portuguese, Salentinian Italian, French and Spanish - participated in the study. In Experiment 1, listeners identified as /i/ or /e/ the ten stimuli of a continuum prepared with an articulatory model. Experiment 2 consisted in an AX 'same-different' discrimination task including pairs of stimuli differing in one or two steps along the continuum. Discrimination was better when the more peripheral stimulus was presented second in a pair. However, these order effects were found mainly within the /i/ category and only for Italian and French. Experiment 3 was a replication of Experiment 2, except that two modifications were introduced: a. identical pairs were added, and b. one- and two-step pairs were separately examined. Order effects were again found but, this time, they covered a wider area of the continuum. Peripherality alone was not able to account for order effects. The role of focalization was later discussed.

\section{2:00}

2aSCd4. Perception of an infrequent assimilation: Labial-to-alveolar assimilation in German. Holger Mitterer (Max Planck Institute for Psycholinguistics, Wundtlaan 1, 6525 XD Nijmegen, Netherlands, holger .mitterer@mpi.nl)

In German (and many languages) the alveolar nasal /n/ can assimilate to $[\mathrm{m}]$ if followed by a labial (e.g. 'in Berlin' $\leftarrow$ "i $[\mathrm{m}]$ Berlin"). In a German spontaneous speech corpus (KielCorpus), one also finds a few cases in which an $/ \mathrm{m} /$ followed by an alveolar consonant surfaces as [n] (e.g., 'samstag' $\leftarrow$ "sa[n]stag", Engl. Saturday). Four experiments investigated whether there is a similar pre-lexical compensation process for these labial-toalveolar assimilations as previous research uncovered for alveolar-to-labial assimilations. This turns out to be the case: German and Dutch listeners-the latter potentially unfamiliar with this type of assimilation-tend to perceive the assimilated [n] in "sanstag" as /n/ if presented in isolation ("an"), but as $/ \mathrm{m} /$ if presented with minimal context ("ansta"). For German listeners, this context effect is larger if they hear the complete word "sanstag", introducing an additional lexical bias to perceive the $[\mathrm{n}]$ as $/ \mathrm{m} /$. Finally, phonetic detail such as transitions of the [s] fricative pole are also important: If the assimilated "an" is spliced into another /s/-initial syllable by the same speaker, the context effect is reduced. Perceiving infrequent assimilations seems therefore similar to perceiving frequent assimilations. 
12:20

2aSCd5. Nonnative listeners prefer perceptual cues they know from their L1: Dutch listeners use vowel duration less than English listeners for English final /v/-/f/. Mirjam Broersma (Radboud University Nijmegen, PO Box 9104, 6500 HE Nijmegen, Netherlands, mirjam .broersma@mpi.nl)

Two 2AFC experiments investigated Dutch and English listeners' use of preceding vowel duration for the English nonword-final /v/-/f/ contrast. Like English, Dutch has a /v/-/f/ contrast, but unlike English, Dutch has no final /v/. Dutch listeners therefore have no native language experience with the use of preceding vowel duration as a final voicing cue. Previous research showed that Dutch listeners used preceding vowel duration less than English listeners when it was invariable in the experiment (Broersma, 2005, JASA, 117, 3890-3901). The present results show that they also used it less when it was varied. An 11-step fricative voicing continuum from a natural /v/ to a natural /f/ and a 7-step vowel duration continuum from a long (originally /v/-preceding) to a short (originally /f/-preceding) vowel were created. In Experiment 1, all steps of the fricative voicing continuum were combined with one long and one short vowel. In Experiment 2, all steps of the vowel duration continuum were combined with the /v/ and /f/ endpoints and with one ambiguous fricative. In both experiments, Dutch listeners used fricative voicing more and vowel duration less than English listeners did. Thus, the nonnative listeners especially used the perceptual cues they knew from their native language.

\title{
Session 2aSPa
}

\section{Signal Processing in Acoustics, Physical Acoustics, Biomedical Ultrasound/Bioresponse to Vibration, and Underwater Acoustics: Overview of Time Reversal in Acoustics I}

\author{
David Chambers, Cochair \\ Lawrence Livermore Natl. Lab., L-333, 7000 East Ave., Livermore, CA 94550, USA
}

Claire Prada, Cochair

Laboratoire Ondes et Acoustique, ESPCI, Université Paris 7, CNRS, 10 rue Vauquelin, Paris, 75005, France

\section{Invited Papers}

\section{8:00}

2aSPa1. An overview of time-reversal acoustics. Mathias Fink (Laboratoire Ondes et Acoustique, ESPCI, Université Paris 7 , CNRS, 10 rue Vauquelin, 75005 Paris, France, mathias.fink@espci.fr)

Time-reversal invariance is a very powerful concept in physics. In the field of acoustics where time reversal invariance occurs, time-reversal experiments may be achieved simply with arrays of transmit-receive transducers, allowing an incident wave field to be sampled, recorded, time-reversed and re-emitted. Time reversal mirrors (TRMs) may be used to study random media and chaotic reverberating structures. Common to these complex media is a remarkable robustness exemplified by observations that the more complex the medium between the probe source and the TRM, the sharper the focus. TRMs open the way to new signal processings that interest imaging, detection, telecommunications and therapy. Time reversal mirrors have plenty of applications including ultrasonic therapy and medical imaging, non destructive testing, telecommunications, underwater acoustics, seismology, sound control, home automation. An overview of these fields will be presented.

2aSPa2. Ocean acoustic time reversal. William A. Kuperman (MPL, Scripps Institution of Oceanography, University of California, San Diego, La Jolla, CA 92093-0238, USA, wkuperman@ucsd.edu)

A series of ocean acoustic time experiments have been jointly conducted in the last ten years by the NATO Undersea Research Centre and the Marine Physical Laboratory. The experiments demonstrated a robustness of the ocean acoustic time reversal mirror process similar to that found in more controlled, ultrasonic laboratory experiments. Results from these experiments have pointed to potential applications to SONAR signal processing methods and to acoustics communications. In addition, the experimental procedures themselves have yielded data that provide further understanding of the ocean acoustic environment and the increased stability of time reversal process over one way propagation in a fluctuating medium. 


\section{Contributed Paper}

8:40

2aSPa3. 2004 M6.0 Parkfield earthquake characterization using Time Reversal. Carene Larmat (EES-11 (Geophysics) - Los Alamos National Laboratory, MS D443, Los Alamos, NM 87545, USA, carene@lanl.gov), Paul A. Johnson (EES-11 (Geophysics) - Los Alamos National Laboratory, MS D443, Los Alamos, NM 87545, USA, paj@lanl.gov), Lianjie Huang (EES-11 (Geophysics) - Los Alamos National Laboratory, MS D443, Los Alamos, NM 87545, USA, ljh@lanl.gov)

Time reversal has proved to be a robust source location method in acoustics and is now being developed for a number of seismic applications. One problem of particular interest is locating sources where the signal-to-noise ratio is small. These include small earthquakes $(<$ M5.5) or atypical seismic sources with a small seismic energy radiation (e.g., tremor, slow earthquakes). Time reversal has been shown to be very robust and work in the presence of poor data, low signal to noise ratio, etc. We present a prototype study showing the power of time reversal, using seismic data from the 2004 M6.0 Parkfield earthquake, which is the world's best recorded event to date and thus one of the most studied. The back-propagation of recorded seismic data in a 3D Earth velocity model is numerically carried out. We show that the reconstructed reverse wave-field exhibits clear focusing at the source point but also displays a four-lobe radiation pattern for each type of rebroadcast waves (body, surface), which is consistent with the known source mechanism: a right-lateral strike slip along the almost-vertical San Andrea fault.

\section{Invited Paper}

\section{9:00}

2aSPa4. Time reversal and subwalength focal spot. Julien De Rosny (Laboratoire Ondes et Acoustique, ESPCI, Université Paris 7, CNRS, 10 rue Vauquelin, 75005 Paris, France, julien.derosny@espci.fr), Arnaud Tourin (Laboratoire Ondes et Acoustique, ESPCI, Université Paris 7, CNRS, 10 rue Vauquelin, 75005 Paris, France, arnaud.tourin@espci.fr), Geoffroy Lerosey (Laboratoire Ondes et Acoustique, ESPCI, Université Paris 7, CNRS, 10 rue Vauquelin, 75005 Paris, France, geoffroy.lerosey@espci.fr), Mathias Fink (Laboratoire Ondes et Acoustique, ESPCI, Université Paris 7, CNRS, 10 rue Vauquelin, 75005 Paris, France, mathias.fink@espci.fr)

When a wave is time-reversed inside a homogeneous medium, the focal spot width at best equals half a wavelength. This limit comes from the loss of evanescent wave during propagation. An analysis of the time reversed field in terms of the Green's function formalism shows that in order to get a finer spot, not only the field has to be time reversed but also the initial source. In such a case, an acoustic sink is obtained. Experimental results are presented. Then we show two methods to obtain subwavelength focal spot without time-reversed source. The first method consists of setting the time reversal mirror in the near field of the initial source. Despite the evanescent wave transmission, we will see that subwavelength focusing is only observed for a special time-reversal mirror. The second method consists of surrounding the initial source by many scatterers. In such a case, the evanescent waves emitted by the initial source are converted into propagating ones. During the time reversal step, back-conversion occurs that leads to a sub wavelength focal spot. Thanks to this principle, a focal spot of a thirtieth of a wavelength has been reported in the case an electromagnetic experiment.

\section{Contributed Papers}

9:20

2aSPa5. Model-based time reversal method for photoacoustic imaging of heterogeneous media. Peter Burgholzer (Upper Austrian Research, Hafenstr. 47, 4020 Linz, Austria, peter.burgholzer@uar.at), Hubert Gruen (Upper Austrian Research, Hafenstr. 47, 4020 Linz, Austria, huber.gruen@uar.at), Robert Nuster (Karl-Franzens-Universität Graz, Universitätsplatz 5, 8010 Graz, Austria, ro.nuster@uni-graz.at), Günther Paltauf (Karl-Franzens-Universität Graz, Universitätsplatz 5, 8010 Graz, Austria, guenther.paltauf@uni-graz.at), Markus Haltmeier (University of Innsbruck, Technikerst. 21a/2, 6020 Innsbruck, Austria, markus.haltmeier @uibk.ac.at)

In photoacoustic (also called optoacoustic or thermoacoustic) tomography acoustic pressure waves are generated by illumination of a semitransparent sample with pulsed electromagnetic radiation. Subsequently the waves propagate towards the detection surface enclosing the sample. The inverse problem consists of reconstructing the initial pressure sources from those measurements. In certain applications of photoacoustic imaging one has to deal with media with spatially varying sound velocity, e.g. bones in soft tissue. Image reconstruction without any compensation of this effect leads to a poor image quality. It is therefore essential to develop reconstruction algorithms that take spatially varying sound velocity into account and are able to reveal small structures in acoustically heterogeneous media. A model-based time reversal reconstruction method is presented that is capable of reconstructing the initial pressure distribution despite variations of sound speed. This reconstruction method calculates the time reversed field directly with a second order embedded boundary method by retransmitting the measured pressure on the detector positions in reversed temporal order. Numerical simulations and experiments with phantoms consisting of areas with spatially varying sound velocity are presented.

\section{9:40}

2aSPa6. The effects of transducers on the time reversal process in solids. Brian E. Anderson (EES-11 (Geophysics) - Los Alamos National Laboratory, MS D443, Los Alamos, NM 87545, USA, bea@lanl.gov), Michele Griffa (EES-11 (Geophysics) - Los Alamos National Laboratory, MS D443, Los Alamos, NM 87545, USA, mgriffa@lanl.gov), Paul A. Johnson (EES-11 (Geophysics) - Los Alamos National Laboratory, MS D443, Los Alamos, NM 87545, USA, paj@lanl .gov)

Every experimental implementation of Time Reversal (TR) involves the use of transducers to convert wave motion, whether mechanical or acoustical, into electrical signals, and vice versa. Practical considerations of transducers are not included in the basic theory of time reversal, which is based on idealized point-like sources. These considerations include temporal ring down at a narrowband transducer resonance, the finite size of the transducer giving rise to directivity, and the impedance contrast between the transducer and the medium. The effects of these considerations on the TR process will be characterized by presenting data from various TR experiments.

\section{0:00}

2aSPa7. Estimation of guided waves from cross-correlations of diffuse Wavefields for passive Structural Health Monitoring. Adelaide Duroux (Georgia Institute of Technology, School of Mechanical Engineering, 771 Ferst Drive, NW, Atlanta, GA 30332-0405, USA, adelaide.duroux@gatech.edu), Karim G. Sabra (Georgia Institute of Technology, School of Mechanical Engineering, 771 Ferst Drive, NW, Atlanta, GA 30332-0405, USA, karim.sabra@me.gatech.edu), Massimo 
Ruzzene(Georgia Institute of Technology, School of Aerospace Engineering, 270 Ferst Drive, Atlanta, GA 30332-0150, USA, massimo .ruzzene@ae.gatech.edu), Vin Sharma (Georgia Institute of Technology, School of Aerospace Engineering, 270 Ferst Drive, Atlanta, GA 30332-0150, USA, vin.sharma@millenniumdynamics.com), James Ayers (Georgia Institute of Technology, School of Aerospace Engineering, 270 Ferst Drive, Atlanta, GA 30332-0150, USA, jayers3@gatech.edu)

Recent theoretical and experimental studies in a wide range of applications (ultrasonics, underwater acoustics, seismicœ) have demonstrated that Green's functions (impulse responses) can be extracted from crosscorrelation of diffuse fields using only passive sensors. The technique, whose validity is supported by a physical argument based on time-reversal invariance, effectively uses a correlation process between the point source and points located in the focal zone. Indeed, the coherent noise source distributions can be considered as a time-reversal mirror and the crosscorrelation operations gives the field measured at one receiver after refocusing on the other receiver. Passive-only reconstruction of coherent Lamb waves $(80-200 \mathrm{kHz})$ in an aluminum plate and thickness comparable to aircraft fuselage and wing panels will be presented. In particular, the influence of the noise source characteristics (location, frequency spectrum) on the signal-to-noise ratio the emerging coherent waveform will be investigated using a scanning laser Doppler velocimeter. This study suggests the potential for a structural health monitoring method for aircraft panels based on passive ultrasound imaging reconstructed from diffuse fields.

\title{
Session 2aSPb
}

\section{Signal Processing in Acoustics, Biomedical Ultrasound/Bioresponse to Vibration, and Underwater Acoustics: Time Reversal Methods for Array Imaging and Signal Processing I}

\author{
David Chambers, Cochair \\ Lawrence Livermore Natl. Lab., L-333, 7000 East Ave., Livermore, CA 94550, USA
}

Claire Prada, Cochair

Laboratoire Ondes et Acoustique, ESPCI, Université Paris 7, CNRS, 10 rue Vauquelin, Paris, 75005, France

\section{Invited Papers}

\section{$10: 40$}

2aSPb1. The detection and characterization of buried resonant targets by iterative, single-channel time reversal. Ronald A. Roy (Boston University, Dept. of Aerosp. and Mech. Eng., 110 Cummington St., Boston, MA 02215, USA, ronroy@bu.edu), Zachary J. Waters (Boston University, Dept. of Aerosp. and Mech. Eng., 110 Cummington St., Boston, MA 02215, USA, zjwaters@bu.edu), Benjamin R. Dzikowicz (Naval Surface Warfare Center, Panama City Division, Code HS-11, 100 Vernon Ave., Panama City, FL 32407, USA, benjamin.dzikowicz@navy.mil), R. Glynn Holt (Boston University, Dept. of Aerosp. and Mech. Eng., 110 Cummington St., Boston, MA 02215, USA, rgholt@bu.edu)

A technique for detecting buried resonant targets is described in which enhanced signal-to-noise ratio and convergence to a narrowband signal is achieved using the iterative time reversal of backscattered echo returns. [Waters et al., J. Acoust. Soc. Am. 122, 3023 (2007).] The center frequency of the converged-upon signal is typically a resonance frequency of the target and thus could be used for classification and identification purposes. This technique could offer a straightforward means for enhancing target return levels in a noisy or cluttered environment using existing SONAR systems. The procedure consists of exciting the transducer with a broadband pulse, digitizing the echo return windowed about the target, reversing the data stream in time, and using this signal as the source waveform for the next interrogation pulse. We report results derived from a multi-pronged investigation that includes numerical modeling, high-frequency $(100 \mathrm{kHz}-2 \mathrm{MHz})$ scaled tank experiments employing both free-field and buried targets, and mid-frequency (20 kHz $-200 \mathrm{kHz}$ ) buried target experiments run in a shallow pond. [Work supported by The Office of Naval Research and the Center for Subsurface Sensing and Imaging Systems (NSF ERC Award No. EEC-9986821).]

2aSPb2. Green's function estimation in speckle using the FDORT method. Jean-Luc Robert (Philips Research, 345 Scarborough Road, Briarcliff Manor, NY 10510, USA, jean-luc.robert@philips.com), Mathias Fink (Laboratoire Ondes et Acoustique, ESPCI, Université Paris 7, CNRS, 10 rue Vauquelin, 75005 Paris, France, mathias.fink@espci.fr)

The FDORT method (French acronym for Decomposition Of the Time Reversal Operator using Focused beams) is a Time Reversal based method that can detect point-scatterers in a heterogeneous medium and extract their Green's function. It is particularly useful when focusing in a heterogeneous medium. In this presentation, the theory of the FDORT method is generalized to random media (speckle), and it is shown that it is possible to extract Green's functions from speckle signal using this method. Therefore it is possible to achieve a good focusing even if no point scatterers are present. Moreover, a link is made between FDORT and the Van Cittert Zernike theorem. We deduce from this interpretation that the normalized first eigenvalue of the Focused Time Reversal Operator is a well-known 
focusing criterion. The concept of an equivalent virtual object is introduced, that allows the random problem to be replaced by an equivalent deterministic problem and leads to an intuitive understanding of FDORT in speckle. Applications to aberration correction are presented. The reduction of the variance of the Green's function estimate is discussed.

\section{Contributed Papers}

11:20

2aSPb3. Separation of single and multiple scatterring: Application to the ultrasonic detection of a target embedded in a diffusive medium. Alexandre Aubry (Laboratoire Ondes et Acoustique, ESPCI, Université Paris 7, CNRS, 10 rue Vauquelin, 75005 Paris, France, alexandre.aubry@espci.fr), Arnaud Derode (Laboratoire Ondes et Acoustique, ESPCI, Université Paris 7, CNRS, 10 rue Vauquelin, 75005 Paris, France, arnaud.derode@ujf-grenoble.fr)

We present a new imaging technique dedicated to the detection of a target embedded in a strongly scattering medium. Classical imaging techniques such as echography fail in this kind of configuration because of multiply scattered echoes and aberration distorsions. The experimental set up consists in an array of programmable transducers placed in front of a collection of steel rods randomly distributed. The slab thickness is three times the mean free path. Behind this strongly diffusive slab, we set an echogene steel cylinder that we want to detect and localize. The impulse responses between each couple of transducers are measured and form the interelement matrix. Our technique separates the single-scattered echoes from the multiple scattering background. This is possible because of a deterministic coherence of single-scattering signals along the antidiagonals of the array response matrix, whatever the distribution of scatterers. Once this operation is performed, the detection of the target is achieved by applying the DORT method (French acronym for decomposition of the time reversal operator). The quality of detection is assessed theoretically with Random Matrix Theory and shown to be, by far, better than what is obtained with echography and the classical DORT method.

\section{1:40}

2aSPb4. Time reversal processing to forward scattering waves of underwater targets. Yoshiaki Tsurugaya (NEC @Corp., 1-10 @Nissincho, Fuchu, 183-8501 Tokyo, Japan, y-tsurugaya@bp.jp.nec.com), Toshiaki Kikuchi (National Defence Academy, 39-21 Uhoyabe 4-chome, Yokosuka, 238-0024 Kanagawa, Japan, ADS01881@nifty.com), Koichi Mizutani (Tsukuba Univ., Tsukuba Science City, 305-8573 Ibaraki, Japan, mizutani@esys.tsukuba.ac.jp)

This paper presents the detection of underwater targets using a time reversal. When a target exists between a sound source and a time reversal array in shallow water, the time reversal array receive the sound waves from the sound source and the waves scattering by the target. If the time reversal processing to them is carried out and they are re-transmitted from the time reversal array, it will be thought that they are converged at the position of the sound source and the target. However, since the waves converging at the sound source have a high level, the waves converging at the target position are usually masked by the high level sounds. Then, we cannot observe the waves converged at the target. We eliminate only the high level sounds from the sound fields. In each array element, the signals in case of non target are subtracted from the signals including the target. As a result of subtraction, the components of the scattering wave by the target are left on the array elements. The time reversal fields of the scattering wave are constructed by radiating the components of the scattered waves from each element again.

\section{2:00}

2aSPb5. Super-resolution imaging of active sound and vibrational sources using a time-reversal sink. Eric Bavu (Univ. de Sherbrooke, Mechanical Engineering Depart., 2500 Boulevard de l'Université, Sherbrooke, QC J1K 2R1, Canada, eric.bavu@usherbrooke.ca), Alain Berry (Univ. de Sherbrooke, Mechanical Engineering Depart., 2500 Boulevard de l'Université, Sherbrooke, QC J1K 2R1, Canada, alain.berry @usherbrooke.ca), Jean-Dominique Polack (Institut Jean le Rond
d'Alembert, Laboratoire d'Acoustique Musicale, 11, rue de Lourmel, 75015 Paris, France, polack@ccr.jussieu.fr), Vincent Gibiat (Université Paul Sabatier, PHASE, 118, route de Narbonne, 31062 Toulouse cedex 9, France, gibiat@cict.fr), Charles Besnainou (Institut Jean le Rond d'Alembert, Laboratoire d'Acoustique Musicale, 11, rue de Lourmel, 75015 Paris, France, chbesnai@ccr.jussieu.fr)

Theory and experiments of super-resolution focusing using a timereversal sink have been investigated in high-frequency regime [Rosny and Fink, Phys. Rev. Lett. 89] and in audible range [Bavu, Besnainou, Gibiat, Rosny and Fink, Act. Acoust., 93]. This technique, generalized to the case acoustic and vibrational imaging of active sources, allows super-resolution imaging and provides a new method of characterization of active sources in a known background medium. This imaging technique involves a measurement in the background medium using an array, and the simulation of the backpropagating-field in a fictive medium. An ideal numerical time-reversal sink (NumTRAS) is then used to refine results and obtain high-contrast, high-resolution imaging of initial sources. The algorithm has been validated in parallel supercomputer simulations, in both vibrational and acoustics fields and has been used to detect active vibrational sources in a clamped Mindlin plate and active sound sources in an anechoic room. All results show high-resolution imaging capabilities when compared with classical time-reversal backpropagation. NumTRAS provides an alternative to other imaging and source detection techniques, such as acoustic holography and beamforming. Beyond the applications of acoustic and vibrational nondestructive evaluation of industrial structures, NumTRAS has applications in evaluation of musical structures and is being tested to detect and characterize moving sources.

\section{2:20}

2aSPb6. Invariants of the time reversal operator for an elastic target in a water waveguide. Franck D. Philippe (Laboratoire Ondes et Acoustique, ESPCI, Université Paris 7, CNRS, 10 rue Vauquelin, 75005 Paris, France, franck.philippe@espci.fr), Claire Prada (Laboratoire Ondes et Acoustique, ESPCI, Université Paris 7, CNRS, 10 rue Vauquelin, 75005 Paris, France, claire.prada-julia@espci.fr), Julien De Rosny (Laboratoire Ondes et Acoustique, ESPCI, Université Paris 7, CNRS, 10 rue Vauquelin, $75005 \quad$ Paris, France, julien.derosny@espci.fr), Jean-Gabriel Minonzio (Laboratoire Ondes et Acoustique, ESPCI, Université Paris 7, CNRS, 10 rue Vauquelin, 75005 Paris, France, jean-gabriel.minonzio@espci.fr), Mathias Fink (Laboratoire Ondes et Acoustique, ESPCI, Université Paris 7, CNRS, 10 rue Vauquelin, 75005 Paris, France, mathias.fink@espci.fr)

Detection and characterization of a target in shallow water is an active field of research. In general, in a waveguide, the dispersion is such that the frequency signature of a target cannot be extracted from a single backscatterd signal unless the waveguide properties as well as the target's position are known (Mignerey et al., JASA 1992 and Yang et al., JASA 1994). We propose to apply the Decomposition of the Time-Reversal Operator method to recover the target's signature in an unknown waveguide. Using a modal theory, we show that provided the target is far from the boundaries of the guide the first singular value of the time reversal operator is proportional to its signature. Using the same approach, the second singular value is shown to be proportional to the second derivative of the angular dependant form function which is a relevant parameter for target identification. Ultrasonic laboratory experiments are presented that confirm these theoretical results. 


\title{
Session 2aUW
}

\section{Underwater Acoustics and ECUA: Fifty Years of Progress in Sonar Acoustic Research: The Role of NURC/SACLANTCEN}

\author{
Henrik Schmidt, Cochair \\ MIT, 77 Massachusetts Ave, 5-204, Cambridge, MA 02139, USA \\ Finn B. Jensen, Cochair \\ NATO Undersea Research Centre, Viale San Bartolomeo 400, La Spezia, 19126, Italy
}

8:40-9:00 Director's Welcome

Invited Papers

9:00

2aUW1. Saclant ASW Research Centre contributions to underwater acoustics during the first sixteen years of its existence: Personal records. Robert Laval (1 rue Emile Duclaux, 75015 Paris, France, robert-laval@wanadoo.fr)

In less than one year the NATO Undersea Research Centre, previously called Saclant ASW Research Centre, will celebrate its 50th anniversary. I had the great privilege to be one of the first scientists joining the Centre in 1959 and to work there until 1975 . The main purpose of the research conducted in underwater acoustics was to reach a physical understanding of the different processes of sound propagation, including multipath effects, transformations introduced by a multilayered bottom and random scattering by the volume inhomogeneities and by the surface and bottom roughness. Experiments at sea first conducted from the research ships Aragonese and then Maria Paolina used explosives charges and active sonars FM pulses as sound sources. The digital analysis equipment, which had been designed to record, process and facilitate the interpretation of the received signals, had no equivalent at the time. The Centre has been a very active platform of intercommunications for most of the civilian and military organizations, which were involved in this discipline. The result of this continuous exchange of ideas has facilitated the harmonization of the research programs in the different NATO countries and has greatly contributed to the creation of a large international research community in underwater acoustics.

9:20

2aUW2. Shallow water environmental acoustics at NURC/SACLANTCEN. William A. Kuperman (MPL, Scripps Institution of Oceanography, University of California, San Diego, La Jolla, CA 92093-0238, USA, wkuperman@ucsd.edu)

The Centre has a long tradition of studying shallow water acoustics. The research has been experimental and theoretical, the latter effort often involving either using or developing models. Examples include innovations such as the concept of the optimum frequency of an acoustic channel, first measured and then precisely explained with modeling, to, for a recent example, the utilization of ocean ambient noise based on its special properties. Consequently, the Centre has continued to remain at the leading-edge for a half century. The common goals at the Centre that provided the impetus for the progression of research are presented. Then a brief historical review of a selection of the contributions the Centre has made in shallow water acoustics is given.

9:40

2aUW3. Seafloor studies at NURC/SACLANTCEN: The first 50 years (1959-2008). Michael Richardson (Naval Research Laboratory, Marine Geosciences Division, Stennis Space Center, MS 39529-5004, USA, Mike.Richardson@nrlssc.navy.mil)

Characterization of the seafloor has always been one of the central research themes at NURC/SACLANTCEN. These studies, reviewed in this presentation, generally supported acoustic measurement and modeling programs. Motivation for bottom-interacting acoustic research began with the deep-water ASW programs of the 1960s and 1970s (long-range propagation, reflection, and bottom loss measurements) and slowly evolved into support for high-frequency, shallow-water MCM programs of the 1990s and 2000s (acoustic propagation within sediments, penetration into and scattering from the sediment surface). Seafloor characterization has included scales appropriate for plate tectonics to sub-mm scale sediment microstructure used to statistically characterize sediment heterogeneity for high-frequency acoustic studies. Although collection and analysis of sediment cores has been the dominate methods of seafloor characterization, direct in situ measurements and remote acoustic characterization has often been used. Seafloor studies were first rate, cutting edge research as demonstrated by quality and quantity of peer-reviewed publications by NURC/SACLANTCEN scientists and their post-SACLANTCEN research careers. The excellent engineering department made possible the development of unique acoustic and seafloor sampling equipment. Ship support for seafloor studies has always been a NURC/SACLANTCEN advantage that has attracted many scientists to multi-national, multi-institutional experiments and symposia. 
2aUW4. My personal perspective of sonar research at the NATO Undersea Research Centre. Peter Wille (Kitzeberger Strasse 31, 24226 Heikendorf, Germany, p.c.wille@t-online.de)

As the first German Director of the NATO Undersea Research Centre I was contemporary to the fall of the Berlin Wall in November 1989 and the unification of Germany in October 1990, the breathtaking termination of the Cold War without bloodshed. NATO's Undersea Research Centre, thirty years of age when the Berlin Wall fell, has been a key player and focal point of a most important and demanding field of defense research. It has been exemplary in combining customer oriented applied research on sonar concepts up to system demonstrators with basic research to understand, to model and to predict the variability of the ocean environment, the most difficult challenge of successful surveillance and reconnaissance. Some unique capabilities enable the Centre to serve the nations and the NATO commands: Above all, the ability to attract first class scientists to work together at the Centre for a few years and then return to their home countries, forming an ever growing international network of intense scientific cooperation. The advanced sea trial related technology and data treatment realized by a capable and flexible technical staff is no less decisive. The many high-ranking visitors, scientific, military and political underline the international standing of the Centre.

\section{0:20-10:40 Break}

\section{0:40}

2aUW5. 19 years in the SCNR for NURC: A recollection. Leif Bjørnø (UltraTech Holding, Stendiget 19, DK-2630 Taastrup, Denmark, prof.lb@mail.dk)

I joined the SCNR for NURC by January 1st 1980 and left it again by the end of 1998. This period, on which I will concentrate my talk, includes the toughest period of the "Cold War" up to 1989 and the period after, when money to NURC became sparse and new ways of funding had to be found. This presentation will emphasize some of the highlights in NURC's contributions to Underwater Acoustics, ASW, MCM and to engineering aspects of underwater research. These highlights include for instance towed arrays, underwater acoustic modelling, MILOC, the MCM and the REA programmes, time-reverse underwater acoustics, operations research and engineering of underwater instruments. These important contributions over 19 years were supervised by 10 chairmen of the SCNR and by 6 directors, all putting their special fingerprint, closely related to their personality, on the course of NURC's development. A few humoristic features and experiences from the 19 years will briefly be referred to.

\section{1:00}

2aUW6. The Centre: It's Impact on Ocean Science---Past, Present and Future. David Bradley (Pennsylvania State University, Post Office Box 30, State College, PA 16804-0030, USA, dlb25@psu.edu)

The Centre (NATO Undersea Research Centre) was formed in 1959, in response to the (then) clear Soviet Union expansion of submarine operations and consequent threat to the NATO Alliance. It began as an experiment to provide a "water leveling" of ASW capability to the Nations of the Alliance. The combination of multi-national staff, on site for 3-5 years for the most part; together with the resident staff, has made this "experiment" an incredible success. The contributions to this success come in two parts: Specific work and scientific progress on site at the Centre and equally importantly, continued international collaboration upon return to one's Nation. The primary purpose of this presentation is to provide some "data" to illustrate both the impact of the Centre on Ocean Science and it's role within the Alliance. Given the rather specific circumstances that lead to the creation of the Centre, the question should be (and is) asked: "Should the experiment end?" The simple answer is: "NO!" The Centre is poised to have an even more critical international role in the future: The knowledge and stewardship of vital marine resources.

$$
\text { 11:20 }
$$

2aUW7. NURC/SACLANTCEN contributions to sonar signal processing and ASW systems research. Douglas Abraham (CausaSci LLC, PO Box 5892, Arlington, VA 22205, USA, abrahad@ieee.org)

The NATO Undersea Research Centre (NURC) was established in 1959 as the SACLANT ASW Research Centre and tasked with providing scientific research and technical analysis in the area of antisubmarine warfare (ASW) to NATO nations. This presentation will review NURC's contributions to sonar acoustic research in the areas of sonar signal processing and ASW systems. These contributions span at-sea testing and experimentation of novel sonar system concepts to algorithm development and theoretical advances in sonar signal processing. The diversity of expertise across fields such as oceanography, acoustics, signal processing, systems, and operations research brought by international scientists into the cauldron of a small, focused research centre with world-class technicians, engineers, and at-sea capabilities produced innovative, interdisciplinary research. [Supported by the Office of Naval Research Code 321US under contract number N0001407C0092]

\section{$11: 40$}

2aUW8. NURC/SACLANTCEN milestone experiments toward solving inverse problems in ocean acoustics. Jean-Pierre Hermand (Université libre de Bruxelles (U.L.B.) - Environmental hydroacoustics lab, av. Franklin D. Roosevelt 50, CP 194/5, 1050 Bruxelles, Belgium, jhermand@ulb.ac.be)

This paper reviews milestone experiments conducted by Saclant Undersea Research Centre to support the development and validation of techniques for obtaining and taking into account environmental information in sonar. The experiments brought together underwater acousticians, geophysicists and oceanographers with the aim to collect comprehensive acoustic and environmental groundtruth data. Environmental-adaptive signal processing was first demonstrated in a deep water area (WESTSARDINIA'89\&90). From ducted propagation measurements, a model-based matched filter (MBMF) receiver that fully incorporates the physics of wave propagation determined a source range, depth and Doppler. For environmental inversion the broadband extension of matched-field processing 
was investigated in a shallow water area south of Elba island (YELLOWSHARK'94\&95). Geoacoustic properties of the sea bottom were determined by finding the best fit between predicted and observed sound fields at multiple frequencies using genetic search algorithms. The same inversion results were obtained by MBMF processing of broadband linearly-frequency-modulated signals enabling the use of an array of a few hydrophones instead of a dense and large vertical array. This motivated the development of drifting acoustic buoys which were tested successfully over the southern continental shelf off Marettimo island, Sicily (ENVERSE'97\&98). A recent experiment demonstrated an integrated concept of Rapid Environmental Assessment (MREA/BP'07) using sparse arrays of hydrophones and pressure/temperature sensors, hand-deployed from small vessels.

\title{
Session 2pAAa
}

\section{Architectural Acoustics and ASA Committee on Standards: Comparison of US and European Standards in Building/Room Acoustics I}

\author{
Kenneth P. Roy, Cochair \\ Armstrong Building Products, 2500 Columbia Ave, Lancaster, PA 17603, USA \\ Eddy Gerretsen, Cochair \\ TNO Science and Industry, Stieljesweg 1, Delft, 2628CK, Netherlands
}

\section{Invited Papers}

2pAAa1. Building acoustics standards in the USA - ASTM and ISO. Sean D. Browne (Armstrong World Industries, 2500 Columbia Avenue, Lancaster, PA 17604, USA, sdbrowne@armstrong.com), Kenneth P. Roy (Armstrong Building Products, 2500 Columbia Ave, Lancaster, PA 17603, USA, kproy@armstrong.com)

In the US most architectural design and acoustical performance specifications are based on the ASTM International standards for Construction/Building \& Environmental Acoustics. Those standards are developed as a responsibility of ASTM International committee E 33 on Building \& Environmental Acoustics. The US is of course a participating member in the development of ISO standards through the American National Standards Institute (ANSI), and this participation relative to standards in architectural acoustics is within ISO TC $43 \mathrm{SC} 2$ which deals with building acoustics. The actual ISO standard development and voting responsibilities in the US rests with the ANSI Technical Advisory Group (TAG) to ISO TC43 SC2 which is delegated to ASTM International committee E 33.06 International Standards. This paper will present the process whereby international participation is involved in the development of both ASTM and ISO standards from the US perspective.

2pAAa2. Prediction models for building performance - European need and world wide use. Eddy Gerretsen (TNO Science and Industry, Stieljesweg 1, 2628CK Delft, Netherlands, eddy.gerretsen@tno.nl)

The development of the unified European market made it necessary to create CE-marking to indicate a certain performance of products. For building products the performance had to enable the fulfilment of essential requirements by buildings, constructed with those products. This made it essential for acoustic requirements to standardize predictions models: the link between acoustic product performance and building performance. In the mean time all six parts of that standard (EN 12354) have been published and are used. Some parts have also been published as ISO standard (ISO 15712) indicating the wider interest in the subject. To be of use in Europe and elsewhere it is important that all types of building structures are covered and indeed work is going on to extend the models to lighter building elements, even more common outside Europe than within. Furthermore, a very important aspect of prediction models is the input data, hence an increased need of standards to determine product performance in an appropriate way. This is even more an item of world wide interest. So the existing standards, the current developments for improvement and the identified need for product standards will be addressed.

$$
\text { 2:40 }
$$

2pAAa3. Comparison of ASTM and ISO sound absorption test methods. Marsha S. Bischel (Armstrong Building Products, 2500 Columbia Ave, Lancaster, PA 17603, USA, msbischel@armstrong.com), Kenneth P. Roy (Armstrong Building Products, 2500 Columbia Ave, Lancaster, PA 17603, USA, kproy@armstrong.com), Joan V. Greenslade (Armstrong Building Products, 2500 Columbia Ave, Lancaster, PA 17603, USA, jvgreenslade@armstrong.com)

A series of planned experiments has been conducted in which the differences between ASTM C423 and ISO 354 have been compared. These experiments focused on the differences in the two test methods, with the goal of identifying and understanding differences in the ultimate test results. A variety of acoustical ceiling tiles were tested in the same NVLAP-certified facility, generating data that cover a broad range of acoustical performance. The factors examined were: the effect of sample size on absorption; the effect of 
plenum depth on measured absorption; and the differences between the SAA and $\alpha_{\mathrm{w}}$ curves resulting from the two test methods. The results of these experiments will then be used to identify areas in which additional research is needed if harmonization of the two standards is to occur.

\section{Contributed Papers}

3:00

2pAAa4. Evaluation of standards for transmission loss tests. Matthew Cassidy (Queen's University Belfast, School of Mechanical and Aerospace Engineering, Ashby Building, Stranmillis Road, BT9 5AH Belfast, UK, mcassidy06@qub.ac.uk), Richard K. Cooper (Queen's University Belfast, School of Mechanical and Aerospace Engineering, Ashby Building, Stranmillis Road, BT9 5AH Belfast, UK, R.Cooper@qub.ac.uk), Richard Gault (Queen's University Belfast, School of Mechanical and Aerospace Engineering, Ashby Building, Stranmillis Road, BT9 5AH Belfast, UK, r.gault@qub.ac.uk), Jian Wang (Queen's University Belfast, School of Mechanical and Aerospace Engineering, Ashby Building, Stranmillis Road, BT9 5AH Belfast, UK, j.wang@qub.ac.uk)

FG Wilson, a generator set manufacturer in Larne, UK, (part of the CAT Electric Power Division), has an acoustics facility for obtaining noise emission of current products, future developments and research. A wall between a hemi-anechoic chamber and a reverberation room contains an aperture which is used for testing canopy panels to determine their sound transmission loss. Two different standard procedures for transmission loss testing are compared; the J1400, and ISO 15186. Tests were carried out on both to determine which is most feasible for the company to use. The J1400 is a variation of the ASTM E90 which uses the same method but is specifically for a reverberation room to hemi-anechoic chamber. A known 'limp' material, chosen to be lead, is used to obtain a transmission loss correction. ISO 15186 is based on a sound intensity method. A microphone on the source side recorded the sound pressure levels, and an intensity mapping was done on the receiving side, from which transmission loss was calculated. Two tests were completed; a lead sheet, and a steel plate. Both standards showed an acceptable accuracy in relation to the mass law.
3:20

2pAAa5. Evaluation of Metrics for Field Airborne Sound Isolation. Wayland Dong (Veneklasen Associates, 1711 Sixteenth Street, Santa Monica, CA 90404, USA, wdong@veneklasen.com), John Loverde (Veneklasen Associates, 1711 Sixteenth Street, Santa Monica, CA 90404, USA, jloverde@veneklasen.com)

ASTM E336 and ISO 140 define several metrics describing airborne sound isolation for field measurements of wall and floor/ceiling assemblies. The current standards include provisions for the measurements of an apparent sound power reduction (ASTC and $\mathrm{R}^{\prime}{ }_{\mathrm{w}}$ ), a non-normalized noise reduction (NIC and $\mathrm{D}_{\mathrm{n}, \mathrm{w}}$ ), and a normalized noise reduction (NNIC and $\mathrm{D}_{\mathrm{nT}, \mathrm{w}}$ ). In a previous paper [LoVerde and Dong, J. Acoust. Soc. Am. 119, 3219 (2006)], the relationships between these metrics were reviewed. The desired metric for isolation in residential uses is discussed, specifically whether it should be based on sound power and related to the performance of the partition system (ASTC and $\mathrm{R}_{\mathrm{w}}$ ), or based on sound pressure and related to the acoustic isolation between spaces (NNIC or NIC or ISO equivalents). The analysis indicates that the sound pressure-based metric is preferred. Since power and pressure-based airborne sound metrics have small differences in field tested assemblies in most cases, it appears engineers have assumed that ASTC (and R' ${ }_{w}$ ) correlates with isolation. Field tests where this assumption does not hold are presented. In these cases, the power metric provides an inaccurate assessment of the acoustic isolation and can lead to engineering evaluations inconsistent with actual performance.

3:40-5:20 Posters

Lecture sessions will recess for presentation of poster papers on various topics in acoustics. See poster sessions for topics and abstracts.

\section{Invited Papers}

2pAAa6. The adaptation of the façade sound insulation requirements in the Belgian standard. Bart Ingelaere (BBRI, rue du Lombard, 42, B-1000 Brussel, Belgium, bart.ingelaere@bbri.be), Lieven De Geetere (Belgian Building Research Institute, Lombardstraat 42, B-1000 Brussel, Belgium, lieven.de.geetere@bbri.be), Gerrit Vermeir (Lab. ATF, Katholieke Universiteit Leuven, Celestijnenlaan 200D, B-3001 Leuven, Belgium, Gerrit.Vermeir@bwk.kuleuven.be), Manuel Vandamme (BBRI, rue du Lombard, 42, B-1000 Brussel, Belgium, manuel.vandamme@bbri.be)

In a previous paper, a draft of the new Belgian requirements for the sound insulation in dwellings in the prNBN S01-400-1 was presented. During the public enquiry, there was protest of the architectural associations against the façade sound requirements and this part of the draft had to be revised. Architects complained that it would become almost impossible to build even smaller projects without the help of acoustical engineers, what would increase building costs. The building market for dwellings is mainly a liberal market of an individual architect working for a single family, and only the intervention of a structural engineer is usual. The façade requirements are expressed as a function of the outdoor noise and concern the finished building. Architects found the relation of these requirements with the performance of the façade elements as can be calculated by EN 12 354-3:2000 too complicated. A simplified approach was developed and got the approval of the building sector and will be detailed in the presentation. Due to federal law on "the obligatory use of good craftsmanship in building", requirements in standards in Belgium are obligatory. The new standard will be of application from the 15 th of February 2008.

\section{5:40}

2pAAa7. Latinoamerican perspective of acoustical standards - the Chilean approach. Kenneth P. Roy (Armstrong Building Products, 2500 Columbia Ave, Lancaster, PA 17603, USA, kproy@armstrong.com), Micaelina Campos (Armstrong Building Products, 2500 Columbia Ave, Lancaster, PA 17603, USA, mcampos@armstrong.com)

A review will be made of current acoustical standards in several countries of Latinoamérica: Brasil, Mexico, Costa Rica, Argentina, Chile, and Perú. Federal standards will be discussed relative to typical end user approaches to meeting requirements. Additionally, press articles warning of health concerns related to acoustics will be presented. Most Latinoamerican acoustical standards include measure- 
ments of exterior noise, but do not mention interior noise requirements as mandatory. Brazilian standards establish overall maximum noise levels for different areas, but do not mention acoustical testing of materials. However, this is changing as a multi-professional team in Mexico is working to definition the acoustical coefficients required for ceilings and walls. And, this team is also working to establish levels of acoustical comfort in different office spaces. Most countries in Latinoamerica have federal institutions that deliver technical standards for the building industry. In Chile, we have the "Instituto Nacional de Normalizacion"(National Standards Institute), and they base their standards on the ISO standards. In 2006 they have delivered many acoustical standards and regulations, including those on transmission loss for private housing including social housing parameters. They are planning to work on standards for Healthcare and School segments.

6:00

2pAAa8. A special procedure to guarantee sound isolation in dwellings. Wilhelmus Beentjes (Lichtveld Buis \& Partners, Postbus 1475, 3430 BL Nieuwegein, Netherlands, beentjes@lbp.nl)

Brochures of projects for newly build dwellings and apartments create many expectations about the various qualities of the new buildings. This also concerns the quality of the sound isolation. But what happens if the quality is not as one expected and one cannot come to an agreement with the contractor? Where can one get one's rights. In the Netherlands we therefore have the Guarantee Institute Housing ( In Dutch GIW). This organisation guarantees that the building fulfills the requirements of the Dutch Building Decree. In this paper the procedure will be explained in a few cases. The topics can vary from airborne- and impact sound insulation between buildings, the sound insulation of facades to the sound levels produced by domestic installations. What are the possibilities to improve the sound isolation if the measured values do not fulfill the guaranteed values?

\title{
Contributed Paper
}

6:20

2pAAa9. Analysis and comparison of acoustic insulation in dwellings and buildings, recently build. Leonardo Meza (Pontificia Universidad Católica de Chile, Av. Vicuña Mackena 4860, Macul, 7820436 Santiago, Chile,1mezam@uc.cl)

At this work the acoustic quality of new dwellings is analyzed from acoustic insulating measurements taken in both single-family dwellings and buildings. Through this work is intended to conduct a case study in order to know the acoustic quality of dwellings built after the year 2001, which fulfill the in force regulations called Basic Standards for Building. Therefore, acoustic insulation tests where realized in-situ in sitting rooms and bedrooms of the dwellings. We performed a total of 30 trials according to the ISO standard measure 140-5. This work is part of an investigation that will continue with the implementation of the Basic Document HR Code Technical Construction in Spain, which recently entered into force, so that this future will be how to compare the real benefits obtained with the implementation of this new regulation, to make a new campaign measures in dwellings built fulfilling this new regulation.

\section{Session 2pAAb}

\section{Architectural Acoustics, Musical Acoustics, Physical Acoustics, and Noise: Acoustics of Opera Houses II}

\author{
Robin Glosemeyer Petrone, Cochair \\ 918 16th St., Apt 3, Santa Monica, CA 90403, USA \\ Roberto Pompoli, Cochair \\ Engineering Dept., Univ. of Ferrara, Via Saragat, 1, Ferrara, 44100, Italy
}

\section{Contributed Papers}

\section{2:00}

2pAAb1. Sound-focusing effects in the plan of horse-shoe shaped opera theatres. Gino Iannace (Built Environment Control Laboratory Ri.A.S., Second University of Naples, Abazia di S. Lorenzo, 81031 Aversa, Italy, gino.iannace@unina2.it), Elvira Ianniello (DIMEC - University of Salerno, via Ponte don Melillo, 84084 Fisciano, Italy, gino.iannace@unina2.it)

An acoustical feature reported specifically for Italian-style opera houses is the non uniformity of early sound in the stalls. The curvature of the lower part of the lateral walls produces a sort of partial wave-guiding effect, like the well known "whispering gallery" effect, that is responsible of the concentration of early sound energy at seats located back in the stalls when the sound source radiates from the foreground of the stage floor. As much of the global room impression depends on the early part of the impulse response it can be supposed that the perceived sound quality of singers' voice and instrumentalists' music performing on the stage will be much different if the listener seats in the last rows with respect to different locations in the stalls. This paper reports an objective analysis on this subject based on measurements carried out in the stalls of a baroque-type theatre. Relevant computer simulations were also implemented.

$$
\text { 2:20 }
$$

2pAAb2. Opera Performance in a Broadway Roadhouse. Dawn Schuette (Threshold Acoustics, 53 W. Jackson Blvd, Suite 1734, Chicago, IL 60604, USA, dschuette@thresholdacoustics.com)

The John A. Williams Theatre at The Cobb Energy Centre for the Performing Arts is a facility that was designed to accommodate touring, large- 
scale musical theatre productions, such as presentations of off-Broadway shows, as its primary revenue source. The 2750 -seat space has recently received rave reviews, not just for its amplified music capabilities but for nonamplified opera presented by the Atlanta Opera, the first resident company in the facility. This paper discusses how the room form as well as fixed and adjustable acoustic elements in the space have allowed it to be successful for its various uses being conscious of its construction budget limitations. [Work completed while author was employed as a Principal Consultant at Kirkegaard Associates.]

$$
\text { 2:40 }
$$

2pAAb3. Acoustics of a selection of famous 18th century opera houses: Versailles, Markgräfliches, Drottningholm, Schweitzingen. Alban A. Bassuet (Arup Acoustics, 155 avenue of the americas, New York, NY 10013, USA, alban.bassuet@arup.com)

This paper presents the analysis of the acoustical measurements conducted in 8 renowned baroque Opera Houses located in France and Germany. Still in authentic condition to their time of usage, and incorporating the historical changing scenery system (shutter and groove), these Houses have undergone little renovations. The acoustical analysis investigates the performances of these great Houses using energy parameters, balance between stage and orchestra parameters, 3D intensity plots, new spatial distribution parameters and timbre frequency analysis. The objective of the study is to deduce (for the Constellation Project) design targets for a medium size Opera House against these benchmarks and also to revisit the use of the lateral changing scenery system as used in the Baroque era, as an original scenery design concept for a new House. The paper presents the unique qualities of the rooms, illustrates some of acoustical benefits of the 18th century Opera House design characteristics, discusses some differences in the design approach between the Italian and the French traditions and proposes new perspectives for modern Opera Houses design.
3:00

2pAAb4. The Ellie Caulkins Opera House: A study in simplified predictive modeling. Robert Mahoney (Robert F Mahoney \& Associates, 310 Balsam Avenue, Boulder, CO 80304, USA, rfm@rfma.com)

Recent advances in predictive modeling and experience in understanding and interpreting the significance of the data provided by such models have given rise to a spate of wonderful new performance spaces. However the models - whether computer models or physical scale models - demand an investment in time, money and organizational resources that are not always available due to the funding and political climate surrounding some projects. In order to secure public funding for the Ellie Caulkins Opera House in Denver, CO USA, the architectural design team had to combine elements of modeling from both the recent past and decades ago in order to present to public authorities a definitive and detailed design and an equally firm estimate of construction costs quickly and with little investment. This paper discusses how the predictive techniques of the last half-century were synthesized to provide a confident estimate of the proposed design's acoustical character as well as definitive construction information.

\section{$3: 20$}

2pAAb5. Opera House Acoustics - Case Studies of Renovations. Mark Holden (JaffeHolden, 114a Washington St, Norwalk, CT 06854, USA, mholden@jaffeholden.com)

In the Unites States, there has been a rapid increase in the number and type of perfomance halls built and renovated in the last 10 years. New Opera Houses are few, however three significant Opera Houses have been renovated by our firm, including the Seattle Opera House, The Houston Grand Opera House, and the Kennedy Center Opera House in Washington DC, home of the National Opera. This paper will discuss, compare and contrast approaches to pit design, wall shaping and ceiling shaping, materials for surfaces and orientation and distribution of acoustic volume. Design calculations and completed measurement data, where applicable, will be presented.

\title{
Session 2pAAc
}

\section{Architectural Acoustics and Noise: Acoustics and Privacy in Healthcare Facilities II: Emerging Research Around the World I}

\author{
William J. Cavanaugh, Cochair \\ Cavanaugh Tocci Associates, Inc., 327F Boston Post Road, Sudbury, MA 01776, USA
}

Kerstin Persson Waye, Cochair

Dept. of Environmental Medicine, The Sahlgrenska Academy of Gothenburg Univ., Box 414, Gothenburg, 405 30, Sweden

\section{Invited Papers}

2pAAc1. AIA/FGI interim acoustical guidelines for health care facilities. Gregory C. Tocci (Cavanaugh Tocci Associates, Inc., 327F Boston Post Road, Sudbury, MA 01776, USA, gtocci@cavtocci.com), William J. Cavanaugh (Cavanaugh Tocci Associates, Inc., 327F Boston Post Road, Sudbury, MA 01776, USA, wcavanaugh@cavtocci.com)

Currently, noise is one of, if not the most prominent complaint of hospital care by patients and staff. In response to a growing concern for sound in hospitals and other types of healthcare facilities, the Facility Guidelines Institute (FGI) of the American Institute of Architects (AIA) has asked for assistance in incorporating acoustical guidelines into their Guidelines for the Design and Construction of Health Care Facilities. A group of 40 acoustical consultants and persons from the architectural and medical professions were assembled to develop the resulting 32-page document entitled "Interim Sound and Vibration Design Guidelines for Hospital and Healthcare Facilities." The document divides the variety of health care facility acoustical design issues into 6 sections as follows 1) site exterior noise, 2) interior finishes, 3) room noise levels, 4) sound isolation and speech privacy, 5) electroacoustics and alarms, and 6) building vibration. The presentation will provide an overview of the Interim Guides as they are expected to be adopted into the 2010 issue of the AIA/FGI Guidelines for the Design and Construction of Health Care Facilities. 
2pAAc2. Relating the hospital sound environment to occupant psychological and physiological response. Kerstin Persson Waye (Dept. of Environ. Medicine, The Sahlgrenska Acad. of Gothenburg Univ., Box 414, 405 30 Gothenburg, Sweden, kerstin.persson-waye@amm.gu.se), Erica Ryherd (Woodruff School of Mechanical Engineering, Georgia Institute of Technology, Atlanta, GA 30332-0405, USA, erica.ryherd@me.gatech.edu), Berit Lindahl (School of Health and Sciences, Borås University College, 50190 Borås, Sweden, berit.lindahl@hb.se), Ingegerd Bergbom (Institute of Health and Caring Sciences, The Sahlgrenska Academy at Göteborg University, PO Box 457, 40530 Göteborg, Sweden, ingegerd.bergbom@fhs.gu.se)

Examining the relationship between specific acoustical characteristics of noise and occupant response is a fundamental step in determining the current hospital soundscape. Hospitals should be conducive to patient recovery and safety as well as employee health and productivity. Therefore, the sound environment that occupants are exposed to should be attended to from a health standpoint. Previous research on hospital patients has indicated negative effects of hospital sounds such as sleep disturbance, cardiovascular response, increased incidence of rehospitalization, extended hospital stay, and increased dosages of pain medication. There is some evidence that overall levels of hospital noise may impact staff mental efficiency, short-term memory and stress. A series of studies are currently being conducted by the authors to evaluate the modern hospital soundscape and the associated psycho-physiological responses of occupants, incorporating both quantitative and qualitative approaches. One phase in which detailed sound measurements, staff evaluations, and patient physiological measurements were conducted in a general intensive care unit will be highlighted, focusing also on methodological aspects related to physiological measurements. This phase also includes evaluation of patient ICU delirium, a condition that may negatively impact patient health and length of stay in the hospital. [Work supported by ASA and Swedish FAS].

2pAAc3. Quantifying the noise environment: effects of the wearer's voice on body-mounted noise dosimeter measurements. Steve Ryherd (Chalmers Rm. Acoust. Group, Dept. of Appl. Acoust., Chalmers Univ. of Technology, 41296 Gothenburg, Sweden, srryherd@arpeggioacoustics.com), Kerstin Persson Waye (Dept. of Environ. Medicine, The Sahlgrenska Acad. of Gothenburg Univ., Box 414, 40530 Gothenburg, Sweden, kerstin.persson-waye@amm.gu.se), Mendel Kleiner (Chalmers Rm. Acoust. Group, Dept. of Appl. Acoust., Chalmers Univ. of Technology, 41296 Gothenburg, Sweden, mendel.kleiner@chalmers.se), Erica Ryherd (Woodruff School of Mechanical Engineering, Georgia Institute of Technology, Atlanta, GA 30332-0405, USA, erica.ryherd@me.gatech.edu)

The purpose of this project was to investigate the effects of the wearer's voice as a dominant sound source on body-mounted noise dosimeters in medium-level acoustic environments. Noise dosimeters provide a convenient method of quantifying an occupant's acoustical exposure. In recent years, interest in personal noise exposure has expanded beyond a necessary safety measure used in industrial settings to be used as a means of documenting a person's exposure to sound pressure levels in lower level environments like hospitals and schools. This study quantifies the contribution of the wearer's voice to the dosimeter measurement in sixteen different office conditions. Statistical analysis was used to determine the effects of the following experimental variables on the measured levels: 1) the type of room in which the measurement is taken, 2) the type of background noise present, 3) the level of background noise. Preliminary statistical results suggest significant main effects of the experimental variables with no significant interaction effects. The statistical effect of the subject's hearing level was also considered. The results of this study can improve the interpretation of dosimeter measurements in medium-level environments and may provide further motivation for alternate methods of quantifying sound pressure levels associated with body-mounted measurements.

3:00

2pAAc4. Acoustics in the Environment of Care: An Interdisciplinary Harvard Medical School Research Project. Joanne Solet (Harvard Medical School, 25 Shattuck Street, Boston, MA 02115, USA, joanne_solet@hms.harvard.edu), Andrew Carballeira (Cavanaugh Tocci Associates, Inc., 327F Boston Post Road, Sudbury, MA 02421, USA, acarballeira@cavtocci.com), Orfeu Buxton (Harvard Medical School, 25 Shattuck Street, Boston, MA 02115, USA, orfeu@hms.harvard.edu), Grace Wyshak (Harvard Medical School, 25 Shattuck Street, Boston, MA 02115, USA, unknown@unknown.com)

Noise in hospitals and healthcare facilities is a growing concern. Anticipating the wave of aging baby-boomers, the US healthcare industry is committed to significant construction and renovation over the next decade. New construction guidelines from the American Institute of Architects/Facility Guidelines Institute and the LEED-based "Green Guide for Healthcare" recognize improved acoustical conditions as vital to quality of care quality. To set these specific standards for acoustical performance in healthcare facilities, clinical evidence is needed on the impact of the acoustical environment on patients. An interdisciplinary team of Harvard Medical School faculty and acoustical engineers, in concert with public policy professionals, have designed and implemented a simulation methodology to test acoustic disruption of sleep in human subjects. This research collaboration uses soundscapes derived from recordings made in real healthcare environments, and then quantifies the human response to them. The methodology also enables virtual design changes with corresponding subject exposure to acoustically improved environments. Studies of the human sleep response using this methodology are providing objective evidence for establishing and validating design and construction standards to integrate improved acoustics with healthcare facility design.

3:20

2pAAc5. Speech privacy in healthcare buildings:review of early studies and current procedures for analysis. William J. Cavanaugh (Cavanaugh Tocci Associates, Inc., 327F Boston Post Road, Sudbury, MA 01776, USA, wcavanaugh@cavtocci.com), Gregory C. Tocci (Cavanaugh Tocci Associates, Inc., 327F Boston Post Road, Sudbury, MA 01776, USA, gtocci@cavtocci.com)

Studies on speech privacy problems in buildings, including healthcare facilities, since the mid-1950s, have convincingly shown that a person's sense of acoustical privacy is directly related to the intelligability of the intruding speech in adjacent spaces [Cavanaugh et al, "Speech Privacy in Buildings" J. Acoust. Soc. Am. 34, 475-492 (1962)]. Both in laboratory and in real-world settings people feel they have adequate "confidential" privacy when the articulation index (AI) of the intruding speech is 0.05 or less. Higher values of AI, up to 
about 0.15 , are acceptable for less demanding tasks, i.e. "normal" or "everyday" privacy. The analysis procedure quantifies each of the significant variables involved and compares summations of these metrics with response data from prior case histories both to predict and, in the case of existing situations, to evaluate speech privacy conditions. In the mid-1960s independent review of the analysis method showed that sufficient accuracy is preserved if A-scale metrics are used in place ofmore cumbersome frequency-based metrics. later in the 1960s the method was successfully applied to open plan spaces although achieving confidential levels of speech privacy in such spaces proved difficult. Lessons leared in applying this relatively simple analysis method over the years are discussed.

3:40-5:20 Posters

Lecture sessions will recess for presentation of poster papers on various topics in acoustics. See poster sessions for topics and abstracts.

\section{Invited Papers}

2pAAc6. New Measurement Procedure and Rating Categories for Speech Privacy of Closed Rooms. John S. Bradley (National Research Council, 1200 Montreal Rd, Ottawa, ON K1A 0R6, Canada, john.bradley@nrc-cnrc.gc.ca), Brad N. Gover (National Research Council, 1200 Montreal Rd, Ottawa, ON K1A 0R6, Canada, Brad.Gover@nrc-cnrc.gc.ca)

A procedure for assessing the speech privacy of enclosed rooms is described based on measured level differences between roomaverage levels in the room and levels at spot receivers $0.25 \mathrm{~m}$ from the outside boundaries of the room. The procedure is not sensitive to the acoustics of the receiving space, assesses conditions at more sensitive listening positions, and can describe variations in speech privacy due to weaker elements such as doors. The arithmetic average of the level differences over speech frequencies from 160 to $5 \mathrm{k}$ $\mathrm{Hz}$ is used to determine uniform-weighted signal-to-noise ratios found to indicate the audibility and intelligibility of transmitted speech (J. Acoust. Soc. Am. 116 (6) 3480-3490 (2004)). The degree of speech privacy is related to the speech levels in the room, the measured transmission characteristics and ambient noise levels outside the room. The probability of transmitted speech being audible or intelligible to eavesdroppers is related to the likelihood of louder speech in the meeting room. The measured statistics of speech levels in a large number of meeting rooms have been used to describe categories of speech privacy of rooms ranging from minimal privacy to situations where transmitted speech would be very rarely audible.

\section{$5: 40$}

2pAAc7. Evaluation of Speech Privacy Index and Noise Isolation Class rating of medical examination rooms and comparison with predictions. Steve Pettyjohn (The Acoustics \& Vibration Group, Inc., 5700 Broadway, Sacramento, CA 95820-1852, USA, spettyjohn@acousticsandvibration.com)

The Health Insurance Portability and Accountability Act of 1996 (HIPAA) set speech privacy requirements for medical related information without defining speech privacy for all situations. Areas of special concern for medical organizations are examination rooms because of the need to exchange information between patient and care giver. These rooms are usually constructed in groups with up to three walls common to other examination rooms and a fourth wall next to a corridor. Each adjacency can pose special problems because of construction and position of the equipment in the room. Medical groups are attempting to design and build examination rooms to achieve what they perceive as acceptable levels of privacy while staying within budget and waiting for definitions and requirements. This study provides results of NIC and SPI tests of examination rooms for two medical groups using several different designs. A comparison is given between measured and predicted values based on wall heights and construction details. The wall designs ranged from standard products and construction to employing specialized products, but with only partial height walls. All rooms employed acoustical tile ceilings rather than hard lids. Construction details were found to be the major factor affecting SPI.

\section{Contributed Papers}

6:00

2pAAc8. Prediction of speech privacy between rooms. Jukka S. Keränen (Finnish Institute of Occupational Health, Lemminkäisenkatu 14-18 B, 20520 Turku, Finland, jukka.keranen@ttl.fi), Petra Virjonen (Finnish Institute of Occupational Health, Lemminkäisenkatu 14-18 B, 20520 Turku, Finland, petra.virjonen@gmail.com), Valtteri O. Hongisto (Finnish Institute of Occupational Health, Lemminkäisenkatu 14-18 B, 20520 Turku, Finland, valtteri.hongisto@ttl.fi)

The main purpose of sound insulation is usually to provide an appropriate level of speech privacy between rooms. However, the desired privacy is not always reached if, e.g. the background noise level of receiving room has not been considered or rooms are very small. This is often the case in dwellings and office rooms. Thus, sound reduction index does not correlate well with speech privacy. The aim of this study is to present a simple and validated model to predict the speech privacy between two arbitrary rooms when basic room and wall parameters are known. Speech privacy was described using Speech Transmission Index, STI. STI can be determined when the speech effort, room dimensions, early decay times of the rooms and background noise level of the reception room are known. The model was validated in several cases. The model is useful when economic optimization is appreciated. It can lead to major reductions or increments of conventional recommendations for $\mathrm{R}_{\mathrm{w}}$ value, especially, when room dimensions are unusual or the rooms have special purposes. The optimization is very easy using a web-based software.

\section{6:20}

2pAAc9. A systematic review on the influence of noise on patients' sleep in the Intensive Care Units. Hui Xie (School of Architecture, University of Sheffield, Western Bank, S10 2TN Sheffield, UK, h.xie@sheffield.ac.uk), Jian Kang (School of Architecture, University of Sheffield, Western Bank, S10 2TN Sheffield, UK, j.kang@sheffield.ac.uk)

Noise level in the Intensive Care Units (ICU) often ranges far above the recommended standards. Patients in the ICUs are usually critically ill, making them more susceptible to the unfavorable environment in the hospital. Several objective and subjective methods have been used for the sleep measurement in previous intensive care studies, which showed that the sleep of ICU patients is remarkably and extraordinarily disrupted. While a number of 
factors may affect sleep in the ICUs, many earlier studies reported that noise is one of the most common causes for disturbed nocturnal sleep among ICU patients, although some other studies also suggested that noise in the hospital environment is not the most frequent cause of patients' awakenings.
Moreover, patients would become accustomed to noise accompanied with their hospital stay. In this paper, a systematic review is given to the influence of noise on patients' sleep in the ICUs, and some strategies to improve the sleep quality are also discussed.

\title{
Invited Paper
}

\section{6:40}

2pAAc10. Vibration effects in healthcare facilities. Anthony Nash (Charles M. Salter Associates, 130 Sutter, 5th Floor, San Francisco, CA 94104, USA, anthony.nash@cmsalter.com)

In the USA, the principal rating system for "green" buildings is named, "Leadership in Energy and Environmental Design" (LEED). There is now a proposed LEED rating system for healthcare facilities as described in a "pilot draft" document that was released for public comment in November 2007. This document, in turn, cites the 2006 AIA/AHA Draft Interim Sound and Vibration Design Guidelines. These AIA/AHA Guidelines focus on both the human perceptual effects of vibration as well as the control of structure-borne noise. This paper addresses another aspect of vibration in healthcare facilities - the effects of vibration upon sensitive diagnostic equipment installed on floors above grade. Such equipment may include magnetic resonance imagers, surgical microscopes, and computed tomography (CT) scanners, some of which do not have specific vibration limits provided by the manufacturer. The obvious need for attaining low-level building vibration may conflict with other design constraints imposed on modern healthcare facilities as architects and engineers strive for lightweight floor bays having widely-spaced support columns. This paper reviews the generic vibration design criteria found in the AIA/AHA Guidelines and discusses them in light of several case studies.

Session 2pAAd

\section{Architectural Acoustics and Noise: Acoustics of Open-Plan Spaces II (Poster Session)}

\author{
Kenneth P. Roy, Cochair \\ Armstrong Building Products \\ Valtteri Hongisto, Cochair \\ Finnish Institute of Occupational Health
}

\begin{abstract}
All posters will be on display from 3:40 p.m. to 5:20 p.m. To allow contributors an opportunity to see other posters, contributors of odd-numbered papers will be at their posters from 3:40 p.m. to 4:30 p.m. and contributors of even-numbered papers will be at their posters from 4:30 p.m. to 5:20 p.m.
\end{abstract}

\section{Contributed Papers}

2pAAd1. Acoustic Design for Open Plan Spaces. Björn Hellström (ÅF-Ingemansson AB, Box 47321, SE-100 74 Stockholm, Sweden, Bjorn.Hellstrom@afconsult.com), Erling Nilsson (Saint-Gobain Ecophon AB, Box 500, SE-260 61 Hyllinge, Sweden, erling.nilsson @ecophon.se), Björn Berthelsen (Saint-Gobain Ecophon AB, Box 500, SE26061 Hyllinge, Sweden, bjorn.berthelsen@ecophon.se)

In an ongoing Nordic cooperation project (Nordic Innovation Center Sound Design of Open-Plan Offices) the acoustical conditions in open-plan spaces are investigated (see also the adjacent paper Room Acoustical Measures for Open Plan Spaces). The project's starting point is that acoustic qualities in terms of communication, orientation, comfort and privacy are crucial for open-plan spaces. Therefore, beside acoustic parameters, the aim is to develop methodologies in order to measure and specify such qualities. Beside site analyses, one major methodological tool concerns the development of a questionnaire, intended to be utilized as a compliment to acoustic measurements, and thereby function as a tool for design measures of large open-plan offices. Its purpose is to aid acousticians as well as architects and designers to identify different types of acoustic design criteria. The questionnaire covers not only the perception of the sound environment, but also psychosocial criteria, architectural design and the furnishing of the workplace. These qualitative criteria will be discussed and suitable parameters for design and evaluation of open-plan spaces will be suggested.

2pAAd2. Adaptive sound masking. Robert Chanaud (Secure Sound, 3044 Chaco Circle, Prescott, AZ 86305, USA, 3044sound@cableone .net), Ronald Zuydervliet (STCSound, Drususlaan 20, 6932NS Westervoort, Netherlands, ronald@STIsound.com)

Because activity sound levels vary widely during the day in open offices, fixed level sound masking spectra do not keep employees free of distractions. Programmed level control, time scheduling that permits hourly changes in level, is considerably better, but requires pre-knowledge of the activity. An adaptive masking system detects and responds to activity sound, both human and nonhuman, to provide constancy of privacy as opposed to constancy of masking level. These systems use several sound detectors in an area, average the sound level from them, and develop a running table of percentile levels with a given sensitivity. Two percentile levels (L10 and L99) are used to separate the masking from the activity sound. The system acts to continually keep the difference between them constant. The advantage of adaptive masking will be shown by comparison with other methods. The distraction potential will be discussed in terms of $\mathrm{dB}$-minutes. The overall design of these systems will be discussed and examples of experience with existing systems will be given. 


\title{
Session 2pAAf
}

\section{Architectural Acoustics, Musical Acoustics, Physical Acoustics, and Noise: Acoustics of Opera Houses III (Poster Session)}

\author{
Robin Glosemeyer Petrone, Cochair \\ Roberto Pompoli, Cochair \\ Engineering Dept. - Univ. of Ferrara
}

\begin{abstract}
All posters will be on display from 3:40 p.m. to 5:20 p.m. To allow contributors an opportunity to see other posters, contributors of odd-numbered papers will be at their posters from 3:40 p.m. to 4:30 p.m. and contributors of even-numbered papers will be at their posters from 4:30 p.m. to 5:20 p.m.
\end{abstract}

\section{Contributed Papers}

2pAAf1. Diagnosis of an historical performance hall: case study. Maud Serra (Peutz \& Associés, 10 rue des Messageries, F75010 Paris, France, m.serra@peutz.fr)

The Opera of Vichy is an historical Italian style performance hall. Initially solely devoted to opera performance, the economical trends have pushed towards concert and theatre. A first major refurbishment was performed in the 1990s and prior to that a diagnosis of that hall was performed in order to characterize the acoustics of the facility prior to actual work being carried out. While the historical preservation constraints did not allow for much acoustical work to be performed, the hall proved to be quite decent. However, in order to improve the intelligibility for theatre purposes, a new diagnosis was recently carried out so as to support the relevant computer simulations. After a brief reminder of the historical background of the project, this paper submits the diagnosis procedure that was followed, as well as its main conclusions regarding the fitness of the hall for the various performances. It turns out that such a diagnosis can be a convincing tool in order to help both the design team and the end user understand the implications - and the limitations too!- of the existing facility and its possible developments.

2pAAf2. Acoustics of new and renovated Russian opera houses: Decade 1998-2007. Michael Lannie (MNIIP, Acoustic Lab., 2-nd Brestskaja Str, 29a, 123056 Moscow, Russian Federation, mlannie@mail.ru)

Four new opera houses have been built in Russia and two existing opera houses have been fully renovated during the last 10 years. New chairs were installed and new materials were used on the inner surfaces of the renovated theatres. All the opera houses are not very large and the biggest one has 1200 seats. The plan of the two theatres has a horse-shoe form that is typical for old classic opera houses. The other three theatres have plans which are close to rectangular and one renovated theatre has a semicircular form of the back walls behind the stalls and the large balcony. The subject of the presentation is the acoustic design of these theatres. The results of the acoustic measurements which have been done in the halls are going to be discussed as well. Acoustics of the recently opened fully renovated Moscow academic musical theatre for 1100 seats is going to be presented in a more substantial way.

2pAAf3. The influence of the stage layout on the acoustics of the auditorium of the Grand Theatre in Poznan. Anna Sygulska (Poznan University of Technology, ul. Nieszawska 13C, 60-965 Poznan, Poland, annasygulska@wp.pl)

The paper concerns the influence of the stage layout on the acoustic properties of the auditorium of the Grand Theatre in Poznan. An experimental investigation and a numerical calculation were carried out. During the experimental investigation three cases were analyzed. Firstly, the stage was prepared for a concert (acoustical shell), secondly in had sets for the "Wedding of Figaro", and thirdly it was empty. The reverberation time and the acoustic pressure distribution were analyzed. The research was done by the interrupted noise method and by the true impulsive sources (pistol shots). The modified ray method was used in numerical calculations of acoustic field parameters. It turned out that the light weight structures (materials used in construction of stage sets and acoustical shell) had only decorative function and their influence on the acoustics of the auditorium opera theatre is small. Moreover, the investigation proved that the distribution of the sound pressure level in the auditorium is asymmetrical. It is because of the geometrical asymmetry of the stage (only one side wing). An acoustical correction of the stage was suggested i.e. applying of slided acoustics walls to separate the stage from the background and the side wing.

2pAAf4. Resonant cavities and acoustics vases in Italian Opera Houses; the "Teatro Principal" of Valencia and the eighteenth century treatises about theatres. Arturo Barba Sevillano (Grup d'Acustica Arquitectònica, Ambiental i Industrial, E.T.S.I.I, Univ. Politécnica de Valencia, Camino de Vera, s/n, 46022 Valencia, Spain, arbarse@ doctor.upv.es), Alicia Giménez (Grup d'Acustica Arquitectònica, Ambiental i Industrial, E.T.S.I.I, Univ. Politécnica de Valencia, Camino de Vera, s/n, 46022 Valencia, Spain, agimenez@ fis.upv.es), Radu Lacatis (Grup d'Acustica Arquitectònica, Ambiental i Industrial, E.T.S.I.I, Univ. Politécnica de Valencia, Camino de Vera, s/n, 46022 Valencia, Spain, rala1@doctor.upv.es), Rosa Cibrián (Facultad de Medicina, Univ. de Valencia, Av. Blasco Ibáñez, 15, 46010 Valencia, Spain,rosa.m.cibrian@uv.es)

This paper describes the research job carried out at Polytechnic University of Valencia -Spain- about resonant cavities placed under the orchestra pits of Italian Opera Houses, and others acoustics mechanisms like this one. The Teatro Principal of Valencia was built following the project developed by the Italian architect Felipe Fontana in 1774 and it opened its doors in 1832. Fifteen years ago, in the last architectonic intervention in this theatre, almost a hundred of acoustic vases were found in one of those resonant cavities. What acoustics role they accomplish? How they "functioned"? This was the starting point to study this kind of artifices, throughout the eighteenth century European treatises about theatres and in our Teatro Principal.

2pAAf5. CARMEN $\circledast$ in the Norwich Theatre Royal, UK. Christophe Rougier (CSTB, 24, Rue Joseph Fourier, 38400 Saint Martin D'Hères, France, christophe.rougier@cstb.fr), Isabelle Schmich (CSTB, 24, Rue Joseph Fourier, 38400 Saint Martin D'Hères, France, 
isabelle.schmich@cstb.fr),Paul Chervin(CSTB, 24, Rue Joseph Fourier, 38400 Saint Martin D’Hères, France, paul.chervin@cstb.fr), Paul Gillieron (Paul Gillieron Acoustic Design, 130 Brixton Hill, SW2 1RS London,UK,paul@pgacoustics.org)

Since 1999 the CARMEN® system has been installed in several halls in Europe. The last installation took place in November 2007 at the Norwich Theatre Royal. This classical stage venue has been completely refurbished to become a multipurpose hall with 1280 seats and a single very deep balcony. To fulfil the different acoustic requirements for each performance type (from speech drama to symphonic music) it was decided to install an electroacoustic Reverberation Enhancement System. CARMEN® is a regenerative one which is based upon the virtual wall principle. It can increase the Reverberation Time (RT) of a hall up to $100 \%$ and adapt the acoustics to each performance. The installed system has been tuned with 6 configurations: Theatre, Chamber Music, Opera, Concerto, Symphony, and Chorus. The RT of the empty hall now ranges from $1.1 \mathrm{~s}$ without CARMEN® to $2.2 \mathrm{~s}$ in the Chorus preset. A brief description of the CARMEN® principle will be presented first. Then the tuning of the different configurations in the hall will be described. Empty hall measurements have been done and the measured acoustic criteria will be analysed. The deep balcony of the Norwich Theatre Royal separates it into two slightly coupled volumes. This particularity will be discussed.
2pAAf6. Italian opera house Mantua's Teatro Scientifico acoustical parameters measurements' comparison between Neumann KU 100 and Schoeps KFM 6 binaural recording systems. Franco Policardi (University, DIENCA Dept. Facoltà di Ingegneria, Viale Risorgimento 2, 40136 Bologna, Italy, franz@nemail.it), Ryota Shimokura (AIST, 1-8-31 Midorigaoka, Ikeda, 563-8577 Osaka, Japan, ryota.shimokura@aist.go.jp), Marco C. Consumi (University, DIENCA Dept. Facoltà di Ingegneria, Viale Risorgimento 2, 40136 Bologna, Italy, marco.cesare@mail.ing.unibo.it), Alessandro Cocchi (University, DIENCA Dept. Facoltà di Ingegneria, Viale Risorgimento 2, 40136 Bologna, Italy, alessandro.cocchi@mail.ing.unibo.it)

From the dawning of modern acoustics, researchers tryed to develop useful measurement techniques to describe acoustical field. Sound field measurement improvement's first endeavours implementing microphone capsule miniaturization technology date back to the thirtyes of the past century. Long human perception reproduction path through recording tecnique came to the attempt to position transducers similarly to the human natural hearing system, frequently obtaining as alluring results as difficult comparison among them and reapply. This paper describes processes, first results and comparison between as much as possible identical acoustical measurement sessions in Mantua's Teatro Scientifico opera house using Neumann KU 100 and Schoeps KFM 6 binaural recording systems at the same time.

TUESDAY AFTERNOON, 1 JULY 2008

P2-B, LEVEL 2, 3:40 TO 5:20 P.M.

\title{
Session 2pAAg
}

\section{Architectural Acoustics: Acoustics of Concert Halls II (Poster Session)}

\author{
Takayuki Hidaka, Cochair \\ Takenaka $R \& D$ Institute \\ Daniel Commins, Cochair \\ Commins Acoustics Workshop
}

\begin{abstract}
All posters will be on display from 3:40 p.m. to 5:20 p.m. To allow contributors an opportunity to see other posters, contributors of odd-numbered papers will be at their posters from 3:40 p.m. to 4:30 p.m. and contributors of even-numbered papers will be at their posters from 4:30 p.m. to 5:20 p.m.
\end{abstract}

\section{Contributed Papers}

2pAAg1. Measuring absorption coefficients of open ceilings in multi-purpose halls. Yong Hee Kim (Hanyang University, Department of Architectural Engineering, 133-791 Seoul, Republic of Korea, kimyonghee @ gmail.com), Hye Mi Lee (Hanyang University, 133-791 Seoul, Republic of Korea, ymihoo@daum.net), Jin Yong Jeon (Hanyang University, Department of Architectural Engineering, 133-791 Seoul, Republic of Korea, jyjeon@hanyang.ac.kr)

This paper investigates the acoustical characteristics of open ceilings in multi-purpose halls using a 1:25 scale model. A steel truss, duct system, catwalk and opening were selected for the major components of open ceilings from a field survey of 17 existing halls. Absorption characteristics of the equipped ceilings with the 1:25 scale model were measured in a reverberation chamber according to ISO 354. It was found that different volume and materials of the ceiling spaces cause the composite decay characteristics to be similar to those of coupled room effects. Results showed that the absorption coefficients of an empty ceiling with $50 \%$ opening and $6 \mathrm{~m}$ of depth are 0.2-0.3 above $500 \mathrm{~Hz}$. Adding steel trusses to the ceiling increases the absorption coefficient by 0.1 at $125-2,000 \mathrm{~Hz}$; ducts and catwalks with steel trusses yield an increase in the absorption coefficient at high frequencies The average absorption coefficients of the various composite ceiling structures were 0.19 to 0.61 .

2pAAg2. Singers' preferences for acoustical characteristics of concert halls. Kathleen Stetson (Rensselaer Polytechnic Institute, Greene Bldg., 110 8th St., Troy, NY 12180, USA, kstetson@post.harvard.edu), Jonas Braasch (Rensselaer Polytechnic Institute, Greene Bldg., 110 8th St., Troy, NY 12180, USA, braasj@rpi.edu)

It is the goal of acousticians aiding in concert hall design to create an ideal acoustical environment for experiencing music. To this end, parameters have been developed to quantify the listener's experience in a hall, and while many contribute positively toward listener satisfaction, they only tell part of the story. In reality, the experience of a listener is dependent on the quality of the performance occurring in the hall in addition to the acoustics; even great performers can only perform their best when in comfortable environments conducive to their art. Yet only one widely used parameter, 
Stage Support, considers a performer's acoustical needs, and this metric only applies to instrumentalists playing in ensemble. This study examines the unexplored case of a solo singer in a concert hall, augmenting the little existing research on this subject as a first step toward a new parameter. Subjective responses from a survey given to a number of experienced singers and the preferences of singers for acoustical aspects of various virtual concert halls, auralized real-time as the singers sang, are examined. The limitations in using typical acoustical measurement techniques to capture data for voice self-perception are discussed.

2pAAg3. The Temporal and Spectral characteristics of Gamelan Sunda Music. I Gde Nyoman Merthayasa (Institute of Technology Bandung, Dept. of Engineering Physics - ITB, Jln. Ganesha no. 10, 40132 Bandung, Indonesia, ignmerth@tf.itb.ac.id), Bayu Pratomo (Institute of Technology Bandung, Dept. of Engineering Physics - ITB, Jln. Ganesha no. 10, 40132 Bandung, Indonesia, rah_220@yahoo.com)

In order to design a dedicated Concert hall for Gamelan Sunda, first of all, it is important to determine the temporal and spectral parameters of Gamelan Sunda Music (Degung Musics). Gamelan Sunda's music compositions are strongly affected by the sound envelope and timbre. The fundamental frequencies of instrument keys also affect the musical scale, notes placings and the communication between the musical ensembles. The acoustical parameters of the anechoic studio recorded sound waves has been analyzed to determine the acoustical parameters. The sound envelopement factors have been compared with the note configuration in the musical composition, in order to analyze the influence of the sound envelopement to the music composition. In addition, the effective duration of the autocorrelation has also been analyzed to determine the preffered reverberation time in the proposed Concert hall for Gamelan Sunda.

2pAAg4. Diffuser design for both auditorium and stage acoustics in concert halls. Jae Ho Kim (Hanyang University, 133-791 Seoul, Republic of Korea, nosaer4@gmail.com), Yong Hee Kim (Hanyang University, Department of Architectural Engineering, 133-791 Seoul, Republic of Korea, kimyonghee@gmail.com), Jin Yong Jeon (Hanyang University, Department of Architectural Engineering, 133-791 Seoul, Republic of Korea, jyjeon@hanyang.ac.kr)

This study investigates the effect of the shapes and the locations of diffusers on acoustical characteristics, both in the audience area and on the stage, using a 1:50 scale model. Random-incidence scattering coefficients of the diffusers were measured in a reverberation chamber together with the RT, EDT, C80, G and ST1 of the model hall. The results show that an appropriate design of diffusers in halls affects the sound field of both the auditorium and the stage of the hall. It was also found that the effective number of surface reflections at a receiver position actually quantifies the sound diffusion. Through instillation of omni-directional diffusers close to stage walls, the number of reflected rays increases not only in the audience area but also in the stage area.

2pAAg5. New (stage) parameter for conductor's acoustics? Elisabeth W. Van Den Braak (DHV Building and Industry, Larixplein 1, 5616 VB Eindhoven, Netherlands, bertie.vandenbraak@dhv.com), Laurentius C. Van Luxemburg (LeVeL Acoustics BV, De Rondom 10, 5612 AP Eindhoven, Netherlands, 1.c.j.v.luxemburg@tue.nl)

During fine-tuning of the stage acoustics of the main auditorium of Casa da Musica, designed by the Office for Metropolitan Architecture, in Porto (Portugal) we were confronted with conflicting comments with respect to the acoustic effect of different canopy positions. The well known stage parameters, ST and CS, did not point out a difference in results for the different canopy positions, while the conductor's experience did. So we carried out several measurements and tried to find a parameter that could clarify the contradictory experiences of the musicians and the conductor. We needed a parameter that correlated with the 'transversely support over the stage' instead of or additional to the known parameters. It was also not clear to what degree the commonly used parameters describe the acoustics at the conductor's position. This study aims at proposing a new (stage) parameter as well as trying to find out more about the 'conductor's acoustics'. In the main auditorium $(\mathrm{CdM})$ the newly defined parameter seems to describe the musi- cians' and conductor's experiences on the hearing 'ensemble' on stage even better than the known stage parameters. The same measurements have been carried out in other halls in order to check the validity of this new parameter.

2pAAg6. On the interaction between double basses and the stage floor. Knut Guettler (Norwegian Academy of Music, P.O.Box 5190 Majorstuen, 0302 Oslo, Norway, knut.guettler@nmh.no), Anders Askenfelt (Dept. of Speech, Music and Hearing, Royal Institute of Technology (KTH), Lindstedtsvägen 24, SE-100 44 Stockholm, Sweden, andersa@speech.kth.se), Anders Buen (Brekke \& Strand akustikk as, Hovfaret 17,0275 Oslo, Norway, Anders.Buen@bs-akustikk.no)

Double bassists unanimously claim the importance of a compliant stage floor for producing a warm and nuanced orchestra sound. However, in the limited number of reports studying the stage floor's contribution to radiated sound no clear conclusion has been reached. The present study, based on measurements of three concert halls and three double basses, points at some measurable features that should be considered when trying to settle the question: (1) With a compliant floor the velocity transfer between the bass bridge and floor is often higher than $0 \mathrm{~dB}$ in the low-frequency range. In these cases the bass largely acts as a mass (viewed from the end pin) while the floor acts like a spring. (2) The floor properties affect the bridge mobility in the low-frequency range. (3) Below the Helmholtz resonance, around 60 $\mathrm{Hz}$, the radiation of the bass corpus falls about $40 \mathrm{~dB}$ within one octave while the ratio between the input power at the bridge and the power transferred to the floor via the end pin has been observed to boost from 3 to $40 \%$ in the same range. (4) The effect of a compliant floor may be more pronounced for the player than for the audience.

2pAAg7. Historical and chronological evolution of the concert hall acoustics parameters. Radu Lacatis (Grup d'Acustica Arquitectònica, Ambiental i Industrial, E.T.S.I.I, Univ. Politécnica de Valencia, Camino de Vera, s/n, 46022 Valencia, Spain, rala1@doctor.upv.es), Alicia Giménez (Grup d'Acustica Arquitectònica, Ambiental i Industrial, E.T.S.I.I, Univ. Politécnica de Valencia, Camino de Vera, s/n, 46022 Valencia, Spain, agimenez@fis.upv.es), Arturo Barba Sevillano (Grup d'Acustica Arquitectònica, Ambiental i Industrial, E.T.S.I.I, Univ. Politécnica de Valencia, Camino de Vera, s/n, 46022 Valencia, Spain, arbarse@doctor.upv.es), Salvador Cerdá (Grup d'Acustica Arquitectònica, Ambiental i Industrial, E.T.S.I.I, Univ. Politécnica de Valencia, Camino de Vera, s/n, 46022 Valencia, Spain, salcerjo@mat.upv.es), José Romero (Grup d'Acustica Arquitectònica, Ambiental i Industrial, E.T.S.I.I, Univ. Politécnica de Valencia, Camino de Vera, s/n, 46022 Valencia, Spain, romerof@fis.upv.es), Rosa Cibrián (Facultad de Medicina, Univ. de Valencia, Av. Blasco Ibáñez, 15, 46010 Valencia, Spain, rosa.m.cibrian@uv.es)

The present paper examines the historical evolution of the concert halls acoustic parameters, in the international field. From the beginnings of twentieth century, the acoustic parameters have had a continuous evolution, covering more and more aspects of the concert hall acoustics qualification. Since the bibliography about these is fragmented, we performed in this article, a global and updated presentation on the chronology and the evolution of the studied parameters. Considering the great variety of the same, we presents the appearance of each parameter, located in decades, who has proposed them and, where is possible, indicates articles or publications where they have been presented for the first time. Thus, the historical presentation of the majority of the acoustic parameters of concert halls, it optimizes and can be useful for future research as a central platform in a bibliographical study. The presentation of the same is also grouped according to quality criteria.

2pAAg8. Designing a desirable medium-sized multipurpose hall using ODEON. Jason Duty (Charles M Salter Associates, Inc., 130 Sutter St, Suite 500, San Francisco, CA 94104, USA, jason.duty@cmsalter.com), Hari V. Savitala (Charles M Salter Associates, Inc., 130 Sutter St, Suite 500, San Francisco, CA 94104, USA, hari.savitala@cmsalter.com)

This presentation focuses on using the room acoustics program ODEON for modeling a desirable medium-sized multipurpose hall (400-600 seats). A sampling of medium-sized halls was taken and impulse response measurements were conducted with drapes deployed and retracted. In each hall, the 
same configurations were modeled in ODEON. Acoustical parameters, such as T20, T30, C50, C80, were used to check the agreement of the model to the measurements. A systematic approach was then used to adjust model parameters to match the real-world measurements. These results and modifications are presented to better understand how to accurately model and design medium-sized multipurpose halls.

2pAAg9. The acoustical design of rehearsal rooms for orchestra and choir in the Teatro Lirico in Cagliari. Andrea Farnetani (Engineering Dept. - Univ. of Ferrara, Via Saragat, 1, 44100 Ferrara, Italy, andrea.farnetani@unife.it), Nicola Prodi (Engineering Dept. - Univ. of Ferrara, Via Saragat, 1, 44100 Ferrara, Italy, nicola.prodi@unife.it), Roberto Pompoli (Engineering Dept. - Univ. of Ferrara, Via Saragat, 1, 44100 Ferrara, Italy, roberto.pompoli@unife.it)

The acoustical conditions of rehearsal rooms are of primal importance during the training process of an orchestra or a choir. For this reason these spaces should be specifically designed to allow the musicians to clearly ear themselves and each other. At the same time an appropriate sound level should be maintained to avoid extensive exposure to high levels, which is a risk seldom lamented among musicians. Notwithstanding the peculiar role of these rooms in the musical production process, their acoustical requirements are still not sufficiently clarified. In particular the most effective parameters to employ and the best ranges to adopt need further investigations. This work reports the final acoustical optimization of the orchestra and choir rehearsal rooms in the Teatro Lirico in Cagliari (Italy). The comparison between the acoustical parameters before and after the refurbishment will be shown and discussed, with a focus on the difference between the sound level generated by the performer himself and by the others.

2pAAg10. The influence of the source directivity on the measurement uncertainty. Renzo Vitale (Institute of Technical Acoustics, Templergraben 55, 52056 Aachen, Germany, renzo.vitale@akustik .rwth-aachen.de), Ingo Witew (Institute of Technical Acoustics, Templergraben 55, 52056 Aachen, Germany, Ingo.Witew@akustik.rwth-aachen.de)

Modelling can play a key role in assessing the measurement uncertainties, as specified in the "Guide to the expression of Uncertainty in Measurements" (GUM). A model is presented that is used to quantify the influence of the source directivity on the measurement uncertainty. On the grounds of image sources and radiosity methods the propagation of sound in rooms is simulated. In this model the characteristics of dodecahedron sound sources are implemented with their statistical properties and characteristics. In Monte Carlo Simulations these two core concepts are combined and the results are compared with measurement results. This comparison allows a quality assessment of the model. In a conclusion the input quantities that affect the measurement uncertainty that is introduced by the source directivity are identified.
2pAAg11. Establishment a methodology for an objective-subjective acoustic analysis in a multifunctional hall: Paraninfo of the Polytechnic University of Valencia. Radu Lacatis (Grup d'Acustica Arquitectònica, Ambiental i Industrial, E.T.S.I.I, Univ. Politécnica de Valencia, Camino de Vera, s/n, 46022 Valencia, Spain, rala1@doctor.upv.es), Alicia Giménez (Grup d'Acustica Arquitectònica, Ambiental i Industrial, E.T.S.I.I, Univ. Politécnica de Valencia, Camino de Vera, s/n, 46022 Valencia, Spain, agimenez@fis.upv.es), Salvador Cerdá (Grup d'Acustica Arquitectònica, Ambiental i Industrial, E.T.S.I.I, Univ. Politécnica de Valencia, Camino de Vera, s/n, 46022 Valencia, Spain, salcerjo@mat.upv.es), Rosa Cibrián (Facultad de Medicina, Univ. de Valencia, Av. Blasco Ibáñez, 15 46010 Valencia, Spain, rosa.m.cibrian@uv.es), José Romero (Grup d'Acustica Arquitectònica, Ambiental i Industrial, E.T.S.I.I, Univ. Politécnica de Valencia, Camino de Vera, s/n, 46022 Valencia, Spain, romerof@fis.upv.es), Arturo Barba Sevillano (Grup d'Acustica Arquitectònica, Ambiental i Industrial, E.T.S.I.I, Univ. Politécnica de Valencia, Camino de Vera, s/n, 46022 Valencia, Spain, arbarse@doctor.upv.es)

This study presents a methodology for the acoustic analysis, from a comprehensive study of a multifunctional hall, of the Polytechnic University of Valencia, as a pilot hall in a Spanish Coordinated Project. The acoustic parameters studied were those who the most acoustics experts consider of interest. In the objective measures were use the equipment and methods conforming to ISO 3382. The questionnaire used to analyze the subjective response has been patented in Spain, by the research group that developed the project. The correlation between objective and subjective results, along with the accurate simulation of the hall provides a valid tool for design, restoration and improvements the enclosures where the sound quality is preferred.

2pAAg12. Relationship between room shape and acoustics of rectangular concert halls. Andrzej K. Klosak (Institute of Building Materials and Structures, Faculty of Civil Eng., Cracow University of Technology, Warszawska 24, 31-155 Cracow, Poland, andrzej.klosak @pk.edu.pl), Anders C. Gade (Dept. of Acoustic Technology, Technical University of Denmark, Building 352, DK 2800 Lyngby, Denmark, acg @ oersted.dtu.dk)

Extensive acoustics computer simulations have been made using Odeon computer simulation software. In 24 rectangular rooms representing "shoebox" type concert halls with volumes of $8000 \mathrm{~m}^{3}, 12000 \mathrm{~m}^{3}$ and $16000 \mathrm{~m}^{3}$ from 300 to 850 measurements positions have been analysed. Only room averaged objective measures are considered here, in particular Clarity $\left(\mathrm{C}_{80}\right)$, Strength $(\mathrm{G})$ and Early Lateral Energy Fraction $\left(\mathrm{LF}_{80}\right)$. Results from simulations have been compared with regression models created based on real hall measurements. In general, simulated results of $\mathrm{C}_{80}$ and $\mathrm{G}$ are found to be in good agreement with regression models. Divergences are found in $\mathrm{LF}_{80}$ behaviour; these have been associated with influence of proportions of rectangular halls. Updated formula for predicting of $\mathrm{LF}_{80}$ in rectangular halls has been proposed, which takes into the account both width and length of hall. 


\title{
Session 2pAAh
}

\section{Architectural Acoustics and Noise: Low Frequency Absorption: Mechanisms, Measurement Methods and Application III (Poster Session)}

\author{
Peter D'Antonio, Cochair \\ RPG Diffusor Systems, Inc. \\ Christian Nocke, Cochair \\ Akustikbüro Oldenburg
}

\begin{abstract}
All posters will be on display from 3:40 p.m. to 5:20 p.m. To allow contributors an opportunity to see other posters, contributors of odd-numbered papers will be at their posters from 3:40 p.m. to 4:30 p.m. and contributors of even-numbered papers will be at their posters from 4:30 p.m. to 5:20 p.m.
\end{abstract}

\section{Contributed Papers}

2pAAh1. Sound absorption control at low frequencies in the new symphonic concert Hall of VillaPrado Valladolid. Victoria Chavez Thielemann (University of Chile \& Xu Acoustique, 17, rue Danton, 94270 Le Kremlin Bicêtre, France, v.chavez@yahoo.fr)

In large concert halls for symphonic orchestras the sound absorption control at low frequencies is a compromise between acoustics and architecture. In general the architectural approach is imposed over acoustical wills. In this way the acoustician must adapt his work to the architectural choice of finishing materials. Commonly is the case of the wood material's choice (usage of thin wood panels in medium density), whose architectural expression is related to orchestra instruments and to the visual warmth. Today the acoustical trend is to obtain in concert halls the appropriate acoustical "warmth". As convention, this acoustical quality in a concert hall is related to the bass sound spotlessness, and an appropriate strength of the bass tones arriving to the audience. In this context we will present the solutions and constructive compromises realised in the auditorium of Villa Prado in the way to obtain the desired warmness. This concert hall has a "shoe box" shape, an audience capacity of 1720 seats and it was made completely - walls, ceiling - in wood panels (architect: R. Bofiil, acoustician: XuAcoustique, inaugurated in April 2007, Spain).

2pAAh2. Signal analysis in the sound absorption measurement procedure: The importance of time subtraction and reference surface corrections. Paolo Guidorzi (DIENCA - Univ. of Bologna, Viale Risorgimento 2, 40136 Bologna, Italy, paolo.guidorzi@ mail.ing.unibo.it), Massimo Garai (DIENCA - Univ. of Bologna, Viale Risorgimento 2, 40136 Bologna, Italy, massimo.garai@mail.ing.unibo.it)

The sound absorption coefficient measurement (following ISO 13472-1 normative) of highly absorbing materials, which are tipically employed in room acoustic correction, presents some inherent difficulties. These materials present highly sound absorption coefficient indeed, but usually low sound insulation index and are installed over highly reflective surfaces. This leads to some measurement problems, partially solved by means of the reference surface method. In this work some measurement examples on the same materials but with different contour conditions are analyzed and the improvements on the results, due to reference surface normalization and time domain subtraction of free field response, are discussed. 


\title{
Session 2pAAi
}

\section{Architectural Acoustics and ASA Committee on Standards: Comparison of US and European Standards in Building/Room Acoustics II (Poster Session)}

\author{
Kenneth P. Roy, Cochair \\ Armstrong Building Products \\ Eddy Gerretsen, Cochair \\ TNO Science and Industry
}

\begin{abstract}
All posters will be on display from 3:40 p.m. to 5:20 p.m. To allow contributors an opportunity to see other posters, contributors of odd-numbered papers will be at their posters from 3:40 p.m. to 4:30 p.m. and contributors of even-numbered papers will be at their posters from 4:30 p.m. to 5:20 p.m.
\end{abstract}

\section{Contributed Paper}

2pAAi1. Sound Insulation Requirements in Hospitals: Comparisons and case studies. Simone Secchi (Univ. of Florence, Via San Niccolò 89/a, 50125 Florence, Italy, simone.secchi@taed.unifi.it), Gianfranco Cellai (Univ. of Florence, Via San Niccolò 89/a, 50125 Florence, Italy, gianfranco.cellai@unifi.it), Elisa Nannipieri (Univ. of Florence, Via San Niccolò 89/a, 50125 Florence, Italy, elisa.nannipieri@taed.unifi.it), Giorgio Raffellini (Univ. of Florence, Via San Niccolò 89/a, 50125 Florence, Italy, giorgio@studioraff.it)

Italian and EU regulations for sound insulation of hospitals are very hard to be satisfied. Especially with reference to sound insulation from outside noise, the simultaneous presence of important sound sources (refrigerating units, fans and others) and of sensitive receivers (the patients) imposes high requirements for façade sound insulation. Moreover, the necessity to assure high values of natural lighting in interiors imposes the use of large windows on façades. By using the tabular estimation model defined by annex B of the European Norm 14351 for the calculation of the performance of windows and the model of EN 12354-3 for the performance of façades, it is easy to show that the limiting values defined by the Italian Law can be achieved only with a perfect realisation of the building construction. In the paper the comparison between EU regulations for hospitals is shown. Moreover, different case studies of hospital buildings are analysed either with a theoretical approach or with measures carried out in site. 


\title{
Session $2 \mathbf{p A A j}$
}

\section{Architectural Acoustics and Musical Acoustics: Surround Sound Acoustics II (Poster Session)}

\author{
Anthony Hoover, Cochair \\ McKay Conant Hoover Inc. \\ Alexander Case, Cochair \\ Fermata Audio and Acoustics \\ Etienne Corteel, Cochair \\ Sonic Emotion
}

\begin{abstract}
All posters will be on display from 3:40 p.m. to 5:20 p.m. To allow contributors an opportunity to see other posters, contributors of odd-numbered papers will be at their posters from 3:40 p.m. to 4:30 p.m. and contributors of even-numbered papers will be at their posters from 4:30 p.m. to 5:20 p.m.
\end{abstract}

\section{Contributed Papers}

2pAAj1. Directivity control with Wave Field Synthesis: implications for live music performances. Terence Caulkins (Arup, 155 Ave of the Americas, New York, NY 10013, USA, terence.caulkins@arup.com), Etienne Corteel (Sonic Emotion, Eichweg, 6, CH-8154 Oberglatt, Switzerland, etienne.corteel@ sonicemotion.com), Joseph Sanson (IRCAM, 1 Place Igor Stravinsky, 75004 Paris, France, joseph.sanson@ircam.fr), Olivier Warusfel (IRCAM, 1 Place Igor Stravinsky, 75004 Paris, France, Olivier .Warusfel@ircam.fr)

Loudspeaker room interaction has a large impact on the perception of surround sound, especially in the context of "mixed concert", in which a loudspeaker system is used to synthesize virtual instruments that share the acoustic space of the stage with real musicians. Loudspeakers excite the listening environment in a way that differs from real instruments since, by design, they tend to exhibit constant directivity characteristics as a function of frequency. Moreover, real instruments exhibit radiation patterns that vary according to pitch, fingering and expressive movements. We present an approach based on Wave Field Synthesis which enables a control of radiation properties of the loudspeaker system which can be dynamically monitored in real time. Applications and potential perceptual impacts are discussed, focusing on the possibility of using directivity control to vary the excitation of the concert hall in order to monitor early reflections level and direct/reverberant energy ratio associated to virtual sources.

2pAAj2. Acoustic Radiation Properties of Dynamic Loudspeaker Arrays versus Multiactuator Panels for Wave Field Synthesis. Basilio Pueo (University of Alicante, Signals, Systems and Telecommunications, Cta San Vicente del Raspeig s/n, E-03690 Alicante, Spain, basilio@ua.es) , José J. Lopez (Tech. Univ. of Valencia, Camino de Vera S/N, 46021 Valencia, Spain, jjlopez@dcom.upv.es)

Wave Field Synthesis (WFS) is a spatial sound rendering technique that generates a true sound field using loudspeaker arrays. Dynamic loudspeaker arrays, that were used since the beginning of WFS, is the technology employed in most prototypes. Alternatively, Distributed Mode Loudspeakers can be used to build arrays for WFS, also known as Multiactuator Panels (MAPs). For that purpose, multiple vibrating exciters are attached to a single flat panel of a light and stiff material, creating bending waves that are then radiated as sound fields. An analysis of the radiation properties and spatial performance of such loudspeaker arrays for WFS reproduction is presented in this paper. Wave fields have been interpreted in the wavenumber domain, where the source radiation is decomposed into plane waves for arbitrary angles of incidence. Then, measurement and analysis of the radiation per- formance, evaluation of the spatial aliasing frequency and associated sampling artefacts for both linear loudspeaker arrays are addressed. In addition, parameters that can modify spatial aliasing artifacts, such as truncation effects caused by the array geometry or array directivity are also discussed. Simulations and experiment results are discussed through several case studies, comparing dynamic loudspeaker arrays and MAPs in WFS operation.

2pAAj3. Acoustical design of multichannel audio listening environments. Timothy Gulsrud (Kirkegaard Associates, 954 Pearl Street, Boulder, CO 80302, USA, tgulsrud@kirkegaard.com)

The acoustical design of spaces for surround sound music production raises issues concerning room size and shape, loudspeaker placement, and placement of sound-absorbing and sound-diffusing finishes that require different solutions than for 2-channel stereo listening environments. Design and construction of a new room for multichannel audio, rather than renovating a room originally conceived for a 2-channel stereo loudspeaker system, presents an opportunity to address the issues from first principles. This paper presents a case study of the design, construction, and commissioning of a new audio control room for multichannel classical music recording, and discusses unique solutions to the critical issues for these listening environments. The use of a computer model as a design tool and in situ measurements in the completed room are also discussed.

2pAAj4. Effects of practical loudspeaker characteristics on virtual acoustic imaging systems. Xiaojun Qiu (Key Laboratory of Modern Acoustics and Institute of Acoustics, Nanjing University, 210093 Nanjing, China, xjqiu@nju.edu.cn), Michael Vorlaender (Institute of Technical Acoustics, RWTH Aachen University, Neustr. 50, 52056 Aachen, Germany, mvo@akustik.rwth-aachen.de)

Loudspeakers used in virtual acoustic imaging systems are usually considered as monopoles in the models; however, it is only an approximation at low frequency range, and practical loudspeaker boxes behave complex directivity in mid to high frequency range and almost only radiate in the front direction above a certain frequency. Some studies have taken into account of the frequency response characteristics of the loudspeakers in virtual acoustic imaging systems, and others have considered their directivity characteristics separately. This paper presents a complete model of a virtual acoustics imaging system which takes account of both the temporal and spatial characteristics of the sound sources, and the effects of practical loudspeaker characteristics on virtual acoustic imaging systems are studied. 
Session 2pAAk

\title{
Architectural Acoustics: Case Studies and Design Approaches
}

\author{
Bryon Harrison, Cochair \\ 124 South Boulevard, Oak Park, IL, 60302 USA \\ Witew Jugo, Cochair \\ Institut für Technische Akustik, RWTH Aachen University Neustrasse 50, 52066 Aachen, Germany
}

\section{Contributed Papers}

$5: 20$

2pAAk1. Towards a classification of the "sonic character" of the architectural space. Fausto E. Rodriguez-Manzo (Departamento de Procesos y Tecnicas, CyAD, Universidad Autonoma MetropolitanaAzcapotzalco, Av. San Pablo 180, Edificio H-PB, Col. Reynosa Tamaulipas, Delegacion Azcapotzalco, 02200 Mexico, D.F., Mexico, rfme@correo.azc .uam.mx)

Today it is well-known that an architectural space can be compared with a musical instrument. One of the main characteristics of a musical instrument is its timbre, which makes unique the sound produced by it. We can understand this timbre as the sound quality of the instrument that is produced due to the specific physical characteristics of the instrument. In an architectural space these concepts of timbre and sound quality could be applied through the concept of "sonic character" of the space. The "sonic character" of the architectural space is the sound personality of the space due to the specific physical or architectural characteristics of it. Understanding this concept in architecture could be of great importance to architectural design purposes since this could be the way by which sound can be embodied in an integral way of designing architectural spaces. This paper presents a proposal for the classification of the "sonic character" of the architectural space as an important step of a research towards a better understanding of the need of taking into account the sound as an essential component of the architectural space. The presentation includes also some architectural space examples to improve the understanding of this important concept.

\section{$5: 40$}

2pAAk2. High Environmental Quality (HQE), the GIAc/ADEME approach. Eric Gaucher (Acoustique \& Conseil, 17-19 rue des Grandes Terres, 92508 Rueil-Malmaison, France, eg@acoustique-conseil.com)

The "Haute Qualité Environnementale" HQE concept is increasingly important for French building. The French acoustics consultants association, GIAc, has been working, with the ADEME's support in order to define acoustically a building that aims to be HQE. The approach is to come back to the analysis of a building acoustics situation: the activity developed in a room generates a sound which can disturb activities held in surrounding rooms. In return, this very activity requires quietness to be held in adequate conditions. If we define rules allowing quantification of this aggressivity and sensitivity, we can deduce the insulation criteria corresponding to the quality we are looking for. The main advantage of this method is the homogeneity it induces on the different criteria, which were usually defined independently one from the other. Many present situations show that the equilibrium between acoustic criteria is at least as important as their absolute values. Even though this approach has not been included in the QEB reference book, it can be used to validate the absolute values, to extend the qualification to other kinds of rooms, and also to adapt objective criteria to different situations.

\section{6:00}

2pAAk3. Auditory sketching as a tool in architectural design education. Tim Frank (Georgia Institute of Technology, College of Architecture, Atlanta, GA 30332-0155, USA timothy.frank@coa.gatech.edu), Selen Okcu (Georgia Institute of Technology, College of Architecture, Atlanta, GA 30332-0155, USA, okcuse @yahoo.com)

Architectural spaces are perceived through embodied experiences that are made up of both visual and non-visual stimuli. However, the conventional means that a designer uses to depict non -visual stimuli such as aural phenomena contain a visual bias. We have introduced a new aural drawing system called, "Auditory Sketching" as a way for design students to visualize the auditory fields that structure one's perception of architectural space. The Auditory Sketching procedure requires that the design student first mediate aural perceptions linguistically through meticulous observation during which they are encouraged to define aural qualities within the space such as notes, tones, clusters, bands, and pitches that would be difficult to capture using a graphic system of notation. Using this written language as a guide for graphic expression, they explore different ways of expressing these time and space notations with ink pens; creating an auditory system of 'tones' by building up marks in different ways: hatching, layering up, scribbling, etc. The eventual translation of aural perception into graphic patterns is crucial because designers need to visualize the sound in order to consider it a significant medium of architecture that they can define and shape.

\section{Invited Paper}

2pAAk4. Concepts \& Methods in Architectural Acoustics from the Classical Period to the Enlightenment. Adam Di Angelo (Rensselaer Polytechnic Institute, Greene Bldg., 110 8th St., Troy, NY 12180, USA, diangelo@secondstation.com), Paul Calamia (Rensselaer Polytechnic Institute, Greene Bldg., 110 8th St., Troy, NY 12180, USA, calamp@rpi.edu)

Herein is presented a critical examination of the early concepts in architectural acoustics in the western world. This study suggests that the historical extent of architectural acoustics is much more sophisticated than is generally considered. The presentation focuses on specific contributors and their discoveries in the nascent art of architectural acoustics. Among those studied are Marcus Vitruvius Pollio, the ancient Roman architect responsible for the tome De Architectura; German polymath Athanasius Kircher who delicately balanced empirical science with magic to explain acoustical phenomena in his two books, the Phornugia Nova and the encyclopedic Musurgia 
Universalis; and English architects-cum-scientists Sir Samuel Morland and Sir Robert Hooke whose wide and varied research often entered into acoustics. In the works of these gentlemen are elegant, precocious, and sometimes absurd revelations on the science of sound and surface.

\title{
Contributed Paper
}

6:40

2pAAk5. Acoustic characterization of rehabilitated cloisters. Antonio P. Carvalho (FEUP - Laboratory of Acoustics, R. Dr. Roberto Frias, P-4200465 Porto, Portugal, carvalho@fe.up.pt), Silvia R. Vilela (FEUP - Laboratory of Acoustics, R. Dr. Roberto Frias, P-4200-465 Porto, Portugal, ec01199@fe.up.pt)

This paper presents the results of field measurements in eight rehabilitated cloisters of old monasteries in Portugal (length: 20 to $35 \mathrm{~m}$ and height:
3.3 to $6.3 \mathrm{~m}$ ) regarding their acoustic behavior to two objective parameters: RT and RASTI. The goal is to characterize the acoustic effect of the rehabilitation done on theses spaces to adapt them to new uses. All these cloisters had recently their galleries' openings to the central yard closed with glass panels. Simple formulas were obtained that relate among the objective acoustic parameters and between the acoustic and architectural parameters. This paper also presents the variation on the RASTI and RT average values resulting of the rehabilitation work done on these buildings.

\section{Session 2pABa}

\section{Animal Bioacoustics, Underwater Acoustics, Acoustical Oceanography, Signal Processing in Acoustics, and ECUA: Animal Sonar Systems I}

\author{
James A. Simmons, Cochair \\ Brown University, Department of Neuroscience, Box G-LN, Providence, RI 02912, USA
}

Michel Andre, Cochair

Laboratori d'Aplicacions Bioacústiques, Universitat Politècnica de Catalunya, avda. Rambla Exposició s/n, Vilanova i la Geltrú, 08800, Spain

\section{Invited Paper}

2pABa1. The echolocating bat controls the direction and distance of its acoustic gaze. Cynthia Moss (University of Maryland, Department of Psychology, Biology-Psychology 2123M, College Park, MD 20742, USA, cmoss@psyc.umd.edu), Kaushik Ghose (University of Maryland, Department of Psychology, Biology-Psychology 2123M, College Park, MD 20742, USA, kghose@umd.edu), Annemarie Surlykke (University of Southern Denmark, Institute of Biology, Campusvej 55, -DK-5230 Odense, Denmark, ams@biology.sdu.dk)

Echolocation is an active sensing system that depends upon the dynamic interplay between auditory information processing and adaptive motor control. The echolocating bat produces ultrasonic vocalizations and uses information contained in the returning echoes to build a three-dimensional auditory representation of its surroundings. The timing, bandwidth, and duration of echolocation signals used to probe the environment directly impact the information available to the bat's acoustic imaging system. In turn, the bat's auditory representation of space guides its actions--ear movements, head aim, flight path, and the features of subsequent sonar vocalizations. This talk will summarize the bat's adaptive vocal behavior as it engaged in complex spatial tasks. The bat's 3D flight path was recorded with high-speed stereo IR video; its sonar signals were recorded with a microphone array that permitted reconstruction of the emission pattern. The sonar emission pattern of the big brown bat, Eptesicus fuscus, is directional, but broad enough to collect echo information from objects within a 60-90 deg cone, which would enable simultaneous inspection of objects in the frontal plane. Here, we report that bats encountering a complex environment shifted the direction and distance of their sonar gaze to inspect closely spaced obstacles and targets sequentially. 


\section{Contributed Papers}

2:40

2pABa2. Simulating bat sonar using vector sound intensity. Robert Hickling (Sonometrics Inc., 8306 Huntington Road, Huntington Woods, MI 48070-1643, USA, sonometrics@comcast.net)

In general bats use ultrasound to determine their surroundings and to detect flying insects. Generally the beam width of ultrasonic signals emitted by bats is too wide to distinguish closely-spaced objects and the receiving pattern of bats' ears is similar (J. A. Simmons, "Directionality of biosonar broadcasts and reception by the ears", Tutorial Lecture, ASA Spring Meeting, 2002). To locate an object requires knowing both distance and direction. Distance can be readily determined using time-of-flight. How a bat determines direction is less clear. In this presentation, an ultrasonic device is demonstrated that measures both distance and direction (R. Hickling, "Method and Apparatus for Echolocation" US Patent No. 7,054,226, May 2006). This uses a combination of a pulsed ultrasonic source and a vector sound-intensity probe. The probe has four ultrasonic receivers at the vertices of a regular tetrahedron and determines direction from the sound-intensity vector, using finite-difference approximations and the cross-spectral formulation. The frequencies of the echoes received by the probe have to be heterodyned down to lower frequencies for accuracy in the finite-difference approximations. Echolocation data using the device are presented.

\section{3:00}

2pABa3. An autocorrelation model of bat sonar. Lutz Wiegrebe (Biocenter, University of Munich, Großhaderner Str. 2, 82152 Munich, Germany, lutzw@lmu.de)

Their sonar system allows echolocating bats to navigate with high skill through a complex, three-dimensional environment at high speed and low light. The auditory analysis of the echoes of their ultrasonic sounds requires a detailed comparison of the emission and echoes. Here an auditory model of bat sonar is introduced and evaluated against a set of psychophysical phantom-target, echo-acoustic experiments. The model consists of a relatively detailed simulation of auditory peripheral processing in the bat, Phyl- lostomus discolor, followed by a functional module consisting of a strobed, normalised, autocorrelation in each frequency channel. The model output is accumulated in a sonar image buffer. The model evaluation is based on the comparison of the image-buffer contents generated in individually simulated psychophysical trials. The model provides reasonably good predictions for both temporal and spectral behavioural sonar processing in terms of sonar delay, roughness, and phase sensitivity and in terms of sensitivity to the temporal separations in two-front targets and the classification of spectrally divergent phantom targets.

\section{3:20}

2pABa4. Object-oriented echo perception and cortical representation in the bat Phyllostomus discolor. Uwe Firzlaff (Biocenter of the LudwigMaximilians University of Munich, Großhaderner Str. 2, 82152 PlaneggMartinsried, Germany, firzlaff@zi.biologie.uni-muenchen.de)

Echolocating bats can identify three-dimensional objects exclusively through the analysis of acoustic echoes of their ultrasonic emissions. However, objects of the same structure can differ in size and the auditory system must achieve a size-invariant, normalized object representation for reliable object recognition. In this talk both the neural representation in the auditory cortex and the behavioral classification of echoes of complex virtual objects that vary in object size are described. Electrophysiological experiments revealed a population of units in the auditory cortex of the bat Phyllostomus discolor which showed an object-size invariant response (14/109 units, $13 \%)$. These units respond preferentially to echoes from objects in which echo duration (encoding object depth) and echo amplitude (encoding object surface area) co-varies in a meaningful manner. The electrophysiological results are corroborated by the results of a phantom-target playback experiment, in which it is shown that P. discolor spontaneously classifies most scaled versions of objects according to trained standards. These results indicate that at the level of the bat's auditory cortex, an object-oriented rather than a stimulus-parameter oriented representation of echoes is achieved. This work was supported by the Volkswagen Foundation (I/79782)

All sessions will recess for presentation of poster papers on various topics in acoustics. See poster sessions for topics and abstracts.

\section{Invited Papers}

\section{5:00}

2pABa5. Absolute target range and target range profile in separate computational compartments: Synthetic aperture echolocation. James A. Simmons (Brown University, Department of Neuroscience, Box G-LN, Providence, RI 02912, USA, james _simmons@brown.edu)

Echolocating big brown bats emit wideband (20-100 kHz) FM sounds and perceive target range from echo delay with an accuracy determined by echo relative bandwidth $(\mathrm{Q})$. They distinguish among target shapes by echo spectra, but they perceive shape in terms of distances to different parts (glints), or the target's range profile. Shape resolution based on glint delay differences extracted from target interference spectra is in fractions of millimeters. The bat's flight velocity is about $5 \mathrm{~m} / \mathrm{s}$, so that echo delay changes continuously during production of broadcasts and then again during reception of echoes, smearing and displacing delay estimates for target ranging by several hundred microseconds. Previous workers have identified a particular distance at which smearing is minimal, but it is not known whether this "distance of focus" has significance for the bat. However, perception of target shape is based on a spectral representation that is only slightly distorted by the bat's motion, and it is kept in separate computational path from absolute delay until after absolute delay is determined, whereupon it is attached to absolute delay to place the target's image in an approximate spatial position. The bat's system achieves synthetic aperture imaging of shape while avoiding motion-related blurring.

\section{$5: 20$}

2pABa6. Vocal motor control in horseshoe bats - a bottom-up approach. Kota Kobayasi (UCLA, Dept. Physiol. Sci., Box 951606, Los Angeles, CA 90095, USA, kobayash@ucla.edu), Steffen Hage (UCLA, Dept. Physiol. Sci., Box 951606, Los Angeles, CA 90095, USA, hage@ucla.edu), Jie Ma (UCLA, Dept. Physiol. Sci., Box 951606, Los Angeles, CA 90095, USA, majie@ucla.edu), Walter Metzner (UCLA, Dept. Physiol. Sci., Box 951606, Los Angeles, CA 90095, USA, metzner@ucla.edu)

Most animals constantly adjust the spectrotemporal composition of their vocalizations depending on the information content intended to be conveyed by these signals. Whereas most studies focus on the sensory processing of spectrotemporal features of vocalizations in various vertebrates, it is still widely unknown how vocal motor structures in the brain adjust these call parameters. We used 
echolocation and acoustic communication behaviors in horseshoe bats to analyze the neurobiological and biomechanical basis for the motor control of their vocalizations. Results from pharmacological studies of the vocal motor nucleus, the nucleus ambiguus, lead us to developed a novel model for call frequency control by vocal motor neurons. Neurophysiological recordings combined with pharmacological manipulations at the single cell level in spontaneously vocalizing bats substantiated this model. Biomechanical properties of the larynx were also found to aid in call frequency control: Altering tracheal air flow in an isolated larynx preparation resulted in two distinct frequency bands that were non-harmonically related and reflected the main frequency components of echolocation pulses and communication signals, respectively. In addition, changes in air pressure revealed transitions from periodic to chaotic that occurred within a single signal cycle, giving rise to spectral features observed in horseshoe bat communication signals.

\section{$5: 40$}

2pABa7. Echolocation-like pulse emission for communication by Japanese horseshoe bats, Rhinolophus ferrumequinum nippon. Hiroshi Riquimaroux (Faculty of Engineering, Doshisha Univ., 1-3 Miyakodani Tatara, 610-0321 Kyotanabe, Japan, hrikimar@mail.doshisha.ac.jp), Ryota Shimazawa (Faculty of Engineering, Doshisha Univ., 1-3 Miyakodani Tatara, 610-0321 Kyotanabe, Japan, bte0060@mail4.doshisha.ac.jp), Shizuko Hiryu (Faculty of Engineering, Doshisha Univ., 1-3 Miyakodani Tatara, 6100321 Kyotanabe, Japan, shiryu@mail.doshisha.ac.jp)

It has been reported that the bats communicate with conspecifics by various types of vocalizations, which are different from echolocation pulses. However, we have found pulses used for communication, which were emitted when a bat walked to another individual, appeared to be very similar to those for echolocation. Data have shown two evidences. First, the bats never approached another one without emitting pulses. Second, the bats emitted echolocation-like pulses whose CF2 frequency sequentially changed during the approaching behavior. Such pulses were never emitted by an isolated bat. The changes in CF2 frequency were clearly different from Doppler-shift compensation. If this frequency shift was caused by Doppler-shift compensation, the bat should be moving as fast as 1-5 $\mathrm{m} / \mathrm{s}$ in the cage. Thus, we suggest that these CF2 frequency shifts may play an important role for communication during the approaching behavior to a conspecific animal. [The research supported by a grant to RCAST at Doshisha Univ. from MEXT and by the Innovative Cluster Creation Project promoted by MEXT.]

\section{Contributed Paper}

\section{6:00}

2pABa8. A model of range discrimination of multiple objects by using the linear period modulation signal. Ikuo Matsuo (Tohoku Gakuin University, Tenjinzawa 2-1-1, Izumi-ku, 9813193 Sendai, Japan, matsuo@cs .tohoku-gakuin.ac.jp)

Using the echolocation, bats can capture moving objects in 3D space. The big brown bats, Eptesicus fuscus, emit the linear period modulation sound and can identify objects with an accuracy of less than a millimeter. The delay separation of three or more closely spaced objects can be determined through analysis of the echo spectrum. However, delay times cannot be properly correlated with objects using only the echo spectrum because the sequence of delay separations cannot be determined without information on temporal changes in the interference pattern of the echoes. We previously proposed the model to determine delays of multiple objects from the echoes by using the linear frequency modulation sound. In order to extract the temporal changes, Gaussian chirplets with a carrier frequency compatible with bat emission sweep rates were used. In this paper, we examined the effect of the window's shape which was changed to extract the temporal changes dependent on delays of multiple objects by using the linear period modulation sound.

\section{Invited Paper}

2pABa9. Acoustic focussing: how flying bats control spatial distribution of Doppler-ranging errors by signal sweep rate. Marc W. Holderied (School of Biological Sciences, University of Bristol, Woodland Road, BS8 1UG Bristol, UK, marc.holderied@bristol .ac.uk)

Echolocating bats obtain three-dimensional images of their surroundings in complete darkness by emitting sonar signals and evaluating returning echoes. When flying close to objects, bats risk collision and therefore depend on the accuracy of images - particularly in the perceived distance of obstacles, which is coded by the time delay between call and echo. Yet, during flight, such accuracy is perturbed first because bats call and receive echoes at different positions and second because echoes are modified by Doppler shifts. The spatial distribution of such ranging errors is range dependent - objects at one particular distance from the bat have zero ranging errors, while ranging-errors increase for closer or more distant objects. Interestingly, this distance of zero ranging error depends on signal design, in particular sweep rate. By adjusting signal design flying bats could shift this distance adaptively to their target of interested. Because this has similarities with focusing (i.e. accommodation) in vision, this distance is called distance of focus (DOF). We will present examples for actual distances of focus of different bat species in different behavioural contexts, such as search flight, obstacle avoidance and target approach. DOF gives a novel perspective to the adaptive relevance of frequency modulated sonar signals. 


\title{
Contributed Paper
}

6:40

2pABa10. Passive acoustic localization techniques of Eastern Pacific grey whales. Jennifer L. Wladichuk (University of Bath, Dept. Mechanical Engineering, Claverton Down, BA2 7AY Bath, UK, jlw23 @ bath.ac.uk), William M. Megill (University of Bath, Dept. Mechanical Engineering, Claverton Down, BA2 7AY Bath, UK, enswmm@bath.ac.uk), Philippe Blondel (University of Bath, Department of Physics, Claverton Down, BA2 7AY Bath, UK, pyspb@bath.ac.uk)

Eastern Pacific grey whales (Eschrichtius robustus) apparently do not actively echolocate, yet still they thrive in shallow water environments where visibility is much reduced. Along their migration route and in their feeding grounds these whales are exposed to high levels of ambient noise, highlyturbid waters and many underwater obstacles. To test possible passive acoustic localization mechanisms (e.g. Acoustic Daylight Imaging and Passive Synthetic Aperture), we made extensive acoustic measurements during comprehensive field studies of these whales on their summer feeding grounds in British Columbia (Canada). In combination with visual observations of the whales and their behaviours, we investigated the acoustical sources available to the whales when navigating within a feeding bay. First, we measured ambient noise levels to construct the acoustic landscape around the whales. Second, we investigated how sound is altered when objects such as kelp beds and rocks are present. We also measured acoustic changes induced by direct, controlled modifications of the near-shore environment. The aim of this research is to understand how grey whales might be finding their way around, and what impact, if any, increased levels of ambient noise might have on the whales' ability to find food and navigate within the feeding grounds.

TUESDAY AFTERNOON, 1 JULY 2008

P3-C, LEVEL 3, 3:40 TO 5:20 P.M.

Session 2pABb

\section{Animal Bioacoustics and ECUA: Animal Bioacoustic Censusing II (Poster Session)}

\author{
Marie Roch, Cochair \\ San Diego State University \\ Cédric Gervaise, Cochair \\ E3I2 - EA3876
}

\begin{abstract}
All posters will be on display from 3:40 p.m. to 5:20 p.m. To allow contributors an opportunity to see other posters, contributors of odd-numbered papers will be at their posters from 3:40 p.m. to 4:30 p.m. and contributors of even-numbered papers will be at their posters from 4:30 p.m. to 5:20 p.m.
\end{abstract}

\section{Contributed Papers}

2pABb1. Acoustic detection of beaked whales from autonomous recording buoys. Antón Arias (Departamento de Zoología y Antropología Física, Facultad de Biología, Universidad Complutense de Madrid, 28040 Madrid, Spain, amarias@estumail.ucm.es), Mark Johnson (Woods Hole Oceanographic Institution, Applied Ocean Physics \& Engineering Dept., Woods Hole, MA 02543, USA, majohnson@whoi.edu), Natacha Aguilar Soto (Univ. de La Laguna, Department of Animal Biology, 38256 La Laguna (Tenerife), Spain, naguilar@ull.es), Peter T. Madsen (University of Aarhus, Biological Sciences, Zoophysiology, C. F. Møllers Allé, Building 1131, DK-8000 Aarhus, Denmark, peter.madsen@biology.au.dk), Peter Tyack (Woods Hole Oceanographic Institution, Applied Ocean Physics \& Engineering Dept., Woods Hole, MA 02543, USA, ptyack@whoi.edu), Bertel Møhl (University of Aarhus, Biological Sciences, Zoophysiology, C. F. Møllers Allé, Building 1131, DK-8000 Aarhus, Denmark, bertel.moehl@biology.au.dk)

Beaked whales are among the least known cetaceans but may be especially vulnerable to mid-frequency sonars. Effective implementation of passive acoustic monitoring (PAM) of beaked whale vocalizations may help both to study the biology of these elusive species and to mitigate human impacts. Here we report results of a feasibility study of PAM performed off El Hierro (Canary Islands) where there are coastal resident populations of two species of beaked whales. An acoustic recording system, comprising a GPS-equipped buoy with an archival acoustic tag (Dtag) suspended $200 \mathrm{~m}$ below, was deployed in an area with visual coverage from land. The DTAG recorded broadband acoustic data sampled at $96 \mathrm{kHz}$ along with timing and position information from the GPS. The buoy was deployed on 6 days and clicks were clearly detectable in all recordings. A majority of these were confirmed to be beaked whale clicks by comparison against sounds previously recorded from the same species. Visual sightings were then compared against acoustic detections to develop a methodology for estimating the probability of detection as a function of distance and group size. Results demonstrate the effectiveness of sonobuoy-type PAM systems for beaked whales and the possibility for low-cost tests of acoustic detection models.

2pABb2. Automated real-time detection of cetaceans at the Station ALOHA Cabled Observatory. Julie N. Oswald (Univ. of Hawaii, P.O. Box 1106, Kailua, HI 96734, USA, oswald.jn@gmail.com), Whitlow W. Au (Univ. of Hawaii, P.O. Box 1106, Kailua, HI 96734, USA, wau@ hawaii.edu), Fred K. Duennebier (Univ. of Hawaii, Dept. of Geology and Geophysics, 2525 Correa Rd., Honolulu, 96822, USA, fred@ soest .hawaii.edu)

Hawaiian waters support a wide variety of cetacean species, however, little is known about their occurrence in offshore waters. A cabled ocean bottom observatory located at Station ALOHA (Station ALOHA Cabled Observatory - ACO) provides a unique opportunity to study the occurrence of cetaceans in real-time and over long time scales at a deep ocean research site located $100 \mathrm{~km}$ north of Oahu. A retired electro-optical telecommunications cable provides power and broadband Ethernet communications capability to 
the ACO, allowing real-time continuous acoustic monitoring. The ACO has been operational since February 2007 and includes a hydrophone with a bandwidth of $0.01 \mathrm{~Hz}$ to $40 \mathrm{kHz}$. Automated methods for detection and identification of cetacean calls have been developed and data are processed in nearly real-time. Several cetacean species have been detected, including: dolphins and humpback, minke, fin, and sperm whales. The relationship between the occurrence of different species and changes in the environment has been explored using near-monthly shipboard oceanographic observations collected at Station ALOHA. In addition, seasonal trends in the occurrence of minke whales, a species that has been especially difficult to study using visual methods due to their cryptic behavior, have been examined.

2pABb3. Validating passive acoustic monitoring data loggers by visual observations. Line A. Kyhn (National Environmental Research Institute, University of Aarhus, Frederiksborgvej399, DK-4000 Roskilde, Denmark, lky@dmu.dk), Jakob Tougaard (National Environmental Research Institute, University of Aarhus, Frederiksborgvej399, DK-4000 Roskilde, Denmark, jat@dmu.dk), Mats Amundin (Kolmardens Djurpark \& Biology Dep., Inst. of Physics, Chemistry and Biology, Linkoping University, Kolmardens Djurpark, SE-61892 Kolmarden, Sweden, MATS.AMUNDIN @ KOLMARDEN.COM), Joanna Stenback (Kolmardens Djurpark \& Biology Dep., Inst. of Physics, Chemistry and Biology, Linkoping University, Kolmardens Djurpark, SE-61892 Kolmarden, Sweden, JOANNA .STENBACK@GMAIL.COM), Jonas Teilmann (National Environmental Research Institute, University of Aarhus, Frederiksborgvej399, DK-4000 Roskilde, Denmark, jte@dmu.dk), Daniel Wennerberg (Kolmardens Djurpark \& Biology Dep., Inst. of Physics, Chemistry and Biology, Linkoping University, Kolmardens Djurpark, SE-61892 Kolmarden, Sweden, daniel .wennerberg@gmail.com)

The use of passive acoustic monitoring (PAM) is increasingly used as a monitoring tool in e.g environmental impact assessments. However, very few studies have focused on validating PAM data against independent observations which is critical in order to compare data from different studies and areas, and over time. In August 2007 we tested two types of odontocete PAM equipment: version 5 T-PODs (Chelonia, U.K.) and PCL's (Aquatec, Lochborough, UK) by comparing acoustic detections to visual theodolite tracings of wild harbour porpoises. Eight T-PODs with known detection thresholds and four PCLs were mounted in four clusters. Detection thresholds of the T-PODs varied from 117 to $125 \mathrm{~dB}$ re $1 \mu$ Papp. Based on visual tracks of 28 porpoises it is shown that detection ranges correlated inversely with detection threshold. However, PAM-detection also strongly depended on the angle between the swim track of porpoises and the line to the data logger. Some porpoises were not detected despite swimming within $20 \mathrm{~m}$ of the nearest data logger. It was not possible to distinguish between recordings from one and more than one animal on the PAM-recordings, despite that the number of animals was known from the corresponding tracks. All in all, the results showed good correspondence between PAM recordings and visual tracings, as well as among PAM recordings.

2pABb4. Data Handling of a Perennial Acoustic Observatory in the Antarctic Ocean. Lars Kindermann (Alfred Wegener Institute, P.O. Box 120161, 27515 Bremerhaven, Germany, lars.kindermann@awi.de), Holger
Klinck(Alfred Wegener Institute, P.O. Box 120161, 27515 Bremerhaven, Germany, holger.klinck@awi.de), Ilse Catharina Van Opzeeland (Alfred Wegener Institute, P.O. Box 120161, 27515 Bremerhaven, Germany, Ilse .Van.Opzeeland@awi.de)

In December 2005, 4 broadband hydrophones were deployed through boreholes under the $100 \mathrm{~m}$ thick floating ice shelf near Atka Bay, Antarctica. Since then, more than 10,000 hours of underwater sounds have been recorded, containing marine mammal vocalizations and the unique background sounds generated by ice and the occasional ship. These records from this pristine region are analyzed in terms of the local noise budget, the behaviour of marine mammals and anthropogenic impacts. The observatory is energetically self-sufficient and connected by a $15 \mathrm{~km}$ WLAN link to the year-round manned German Neumayer Base, where a leased satellite line allows live streaming of highly compressed audio to http://icecast.awi.de. While the recording device is capable of four channels / $192 \mathrm{kHz} / 24 \mathrm{Bit}$, this cannot be transferred continuously through the WLAN and satellite link, so a multi-stage automatic buffering and selection scheme is implemented to allow for both, continuous coverage and high quality recordings of selected events. About two terabytes per year are automatically streamed into the petabyte data storage of the World Data Centre for Marine Environmental Data, hosted by AWI, where they are analysed and prepared for publishing under an open access license.

2pABb5. Development of a prototype electronic tag for studying the migratory behaviour of marine species. Prajas John (Department of Electronics, Cochin University of Science \& Technology, Thrikkakara, 682 022 Ernakulam, India, prajas@cusat.ac.in), Jinto George (Department of Electronics, Cochin University of Science \& Technology, Thrikkakara, 682 022 Ernakulam, India, jintokg@cusat.ac.in), Supriya M. Hariharan (Department of Electronics, Cochin University of Science \& Technology, Thrikkakara, 682022 Ernakulam, India, supriya @cusat.ac.in), Saseendran Pillai (Department of Electronics, Cochin University of Science \& Technology, Thrikkakara, 682022 Ernakulam, India, prspillai@cusat.ac.in)

Archival electronic tags can be used as standalone data loggers for sampling the ocean for gathering the environmental parameters and studying the migratory patterns of marine species, identifying their feeding and spawning grounds, etc. A prototype archival electronic tag for monitoring the ocean parameters like temperature, pressure and light intensity has been developed. A digital temperature sensor is used to sample the temperature from the tag's surroundings, while a micro machined piezoresistive silicon digital pressure sensor, which is capable of measuring absolute pressure levels upto 14 bars, provides the depth information. One of the important parameters to be measured is the geolocation of the species, which is computed from the ambient light intensities recorded by the digital light sensor in the tag. These parameters can be sampled and recorded in the memory at preset time intervals, as set at the time of deployment of the tag. This minuaturised tag provides the temperature data with 13 bit resolution, while the pressure and light intensity values have 15 bit resolutions. When used in fisheries studies, the size of the device has to be miniaturised, so that by way of attaching such devices, the normal behaviour of the species remain unaffected. 


\title{
Session 2pABc
}

\section{Animal Bioacoustics, Noise, and ECUA: Anthropogenic Noise Effects on Animals II (Poster Session)}

\author{
Michael Stocker, Cochair \\ Ocean Conservation Research \\ Jonathan Gordon, Cochair \\ Sea Mammal Research Unit
}

\begin{abstract}
All posters will be on display from 3:40 p.m. to 5:20 p.m. To allow contributors an opportunity to see other posters, contributors of odd-numbered papers will be at their posters from 3:40 p.m. to 4:30 p.m. and contributors of even-numbered papers will be at their posters from 4:30 p.m. to 5:20 p.m.
\end{abstract}

\section{Contributed Papers}

2pABc1. Discovery of Sound in the Sea website: Educational and media resources. Kathleen Vigness Raposa (Marine Acoustics, Inc., 809 Aquidneck Ave., Middletown, RI 02842, USA, kathleen.vigness@marineacoustics.com), Gail Scowcroft (University of Rhode Island, Graduate School of Oceanography, Narragansett, RI 02882, USA, gailscow@gso.uri.edu)

The scientific community and the public have become increasingly aware of, and concerned about, underwater sound. There is growing interest in learning about sources and uses of underwater sound, and potential effects of sound on the marine environment. Underlying this interest, however, is a need to provide scientific information at a level appropriate for the general public and for educational and media professionals that communicate with the public. The "Discovery of Sound in the Sea" website (http://www.dosits .org) provides scientific content introducing the physical science of underwater sound and how people and animals use sound to accomplish various tasks. In addition, it has recently been expanded to include three major resource sections: (1) Media Resources, (2) Teacher Resources, and (3) Student Resources. The Media Resources include a Facts \& Myths quiz, Frequently Asked Questions, and PDF reprints of a tri-fold pamphlet and a 12page educational brochure highlighting the content of the website. The Teacher and Student Resources include structured tutorials, educational games, and PowerPoint presentations. These resource sections provide easy, efficient access to timely information on the science of underwater sound and the current state of knowledge of the effects of underwater sound on marine mammals and fishes.

2pABc2. The effects of noise emanating from an adjoining architectural space on beluga whale audition in an aquarium exhibit. Katie Starke (University of Connecticut, 70 Pinney Hill Rd., \#95, Willington, CT 06179, USA, kathryn.starke@uconn.edu), Peter Scheifele (University of Cincinnati, French-East 345A, Cincinnati, OH 45221, USA, scheifpr@UCMAIL.UC.EDU)

Aquaria must have regulations regarding the intensity levels of anthropogenic noise that make their way into their captive belugas' tank, thus affecting the animals' ability to hear and vocalize naturally. My research focuses on the sound transmission across the acrylic and cement boundary that separates the beluga tank from the ballroom in the Georgia Aquarium. This ballroom hosts many social events which are often accompanied by high levels of noise that cross the barrier into the animal enclosure. I focus on the intensity range of 90-100dBA and experiment with a variety of frequencies, specifically those that are included in the range of beluga hearing. Though the data will be specific to the Georgia Aquarium, the results will apply to all aquaria that keep beluga whales, as high intensity sound will always travel through the exhibit boundaries and affect the animals living on the other side. 


\title{
Session 2pABd
}

\section{Animal Bioacoustics, Underwater Acoustics, Acoustical Oceanography, Signal Processing in Acoustics, and ECUA: Animal Sonar Systems II (Poster Session)}

\author{
James Simmons, Cochair \\ Brown University \\ Michel Andre, Cochair \\ Laboratori d'Aplicacions Bioacústiques, Universitat Politècnica de Catalunya
}

\begin{abstract}
All posters will be on display from 3:40 p.m. to 5:20 p.m. To allow contributors an opportunity to see other posters, contributors of odd-numbered papers will be at their posters from 3:40 p.m. to 4:30 p.m. and contributors of even-numbered papers will be at their posters from 4:30 p.m. to 5:20 p.m.
\end{abstract}

\section{Contributed Paper}

2pABd1. An area sensitive to apparent acoustic motion in the auditory cortex of the bat Phyllostomus discolor. Susanne Hoffmann (Biocenter of the Ludwig-Maximilians University of Munich, Großhaderner Str. 2, $82152 \quad$ Planegg-Martinsried, Germany, hoffmann@zi.biologie .uni-muenchen.de), Uwe Firzlaff (Biocenter of the Ludwig-Maximilians University of Munich, Großhaderner Str. 2, 82152 Planegg-Martinsried, Germany, firzlaff@zi.biologie.uni-muenchen.de), Gerd Schuller (Biocenter of the Ludwig-Maximilians University of Munich, Großhaderner Str. 2, 82152 Planegg-Martinsried, Germany, gschuller@1mu.de)

The processing of apparent acoustic motion was investigated in neurons in the auditory cortex of anaesthetized bats (Phyllostomus discolor). Apparent motion in the horizontal plane was generated by presenting pairs of pure tones with different interaural intensity differences (IIDs) and temporal separations, i.e. inter-pulse intervals (IPIs). Thus the spatial extent, motion direction and velocity of motion changed for each stimulus pair. A complete stimulus matrix consisted of 81 IID-combinations in the range between -40 to $+40 \mathrm{~dB}$ IID and was randomly presented via earphones with ten repetitions for up to five different IPIs $(6.25$ to $150 \mathrm{~ms})$. Thirty percent $(71)$ of the 236 extracellularly recorded cortical single cells or small neuronal clusters showed facilitatory responses to acoustic motion compared to static stimulation and were classified as motion sensitive. With decreasing IPI, all motion sensitive neurons changed their azimuthal receptive field in size or spatial position. Twenty two percent (15) of them preferred small movements in the frontal area at very short IPIs. Most interestingly, the motion sensitive neurons were almost exclusively $(97 \%)$ found in the dorsal area of the caudal part of the auditory cortex indicating that this cortical area is specifically involved in the processing of acoustic motion. 
Session 2pAOa

\title{
Acoustical Oceanography and ECUA: Marine Ecosystem Acoustics III (Poster Session)
}

\author{
Kenneth Foote, Cochair \\ Woods Hole Oceanographic Institution \\ Olav Godoe, Cochair \\ Institute of Marine Research
}

\begin{abstract}
All posters will be on display from 3:40 p.m. to 5:20 p.m. To allow contributors an opportunity to see other posters, contributors of odd-numbered papers will be at their posters from 3:40 p.m. to 4:30 p.m. and contributors of even-numbered papers will be at their posters from 4:30 p.m. to 5:20 p.m.
\end{abstract}

\section{Contributed Papers}

2pAOa1. An acoustic finite-element model to study sonar interactions with marine mammals. Gonzalo R. Feijoo (Woods Hole Oceanographic Institution, Woods Hole, MA 02543, USA, gfeijoo@whoi.edu), Kenneth G. Foote (Woods Hole Oceanographic Institution, Woods Hole, MA 02543, USA, kfoote@whoi.edu)

A computer model based on the finite-element method (FEM) is being developed to study the interaction of sonar signals with marine mammals. This model solves the Helmholtz equation in a computational box that includes the animal and the surrounding medium, water. The FEM code has been validated with analytical solutions for scattering of a plane wave by a fluid sphere over a range of parameters and frequencies of interest. The same FEM code has been applied to a 142-cm-long specimen of the common dolphin (Delphinus delphis); internal pressure and displacement fields have been computed. The animal is represented in the computer model by a set of tissue groups whose acoustic properties, density and sound speed, are taken from the literature. The geometry of each tissue group was constructed from segmented computerized tomography images. Results are presented for harmonic signals in the $1-10 \mathrm{kHz}$ frequency range. [Work supported by NOPP through ONR award N000140710992.]

2pAOa2. Mid-frequency pelagic imaging multi-beam sonar. Christopher D. Jones (Applied Physics Laboratory, University of Washington, 1013 NE 40th Street, Seattle, WA 98105, USA, cjones@ apl.washington .edu)

A unique circular array multi-beam sonar is used to investigate volumetric imaging of pelagic marine environments. Imaging of a radial volume of the ocean up to a kilometer in range and at $8-12 \mathrm{kHz}$ will be discussed, focusing mainly on the imaging of large aggregations of fish in shallow water. Several imaging geometries are presented including backscatter imaging in a shallow water waveguide used to resolve the two-dimensional horizontal structure of large fish schools, and the autonomous deployment of the sonar in a fixed location to create a time series of images and observations of variability over a 24 hour period. The potential for new imaging geometries will be explored including vertical profiling to create volumetric images of different pelagic zones in the ocean. Operational issues related to specific scientific question, and signal processing issues related to circular arrays will be discussed. Preliminary field data will be presented.

2pAOa3. Using multi-angle reflections to enhance ecosystem characterization. Jules S. Jaffe (Scripps Institution of Oceanography, Marine Physical Laboratory, 8820 Shellback Way, La Jolla, CA 92093, USA,
jules@mpl.ucsd.edu),Olav R. Godo(Institute of Marine Research, PO Box 1870, 5817 Bergen, Norway, olav.rune.godoe@imr.no), Hector Pena (Institute of Marine Research, PO Box 1870, 5817 Bergen, Norway, hector.pena@imr.no), Ben Maurer (Scripps Institution of Oceanography, Marine Physical Laboratory, 8820 Shellback Way, La Jolla, CA 92093 , USA, bmaurer@ucsd.edu)

Improved knowledge about target tilt can be used to improve acoustic estimates of abundance and to gain insights into the behavioural patterns of exploited marine species. This is because tilt angle strongly affects backscatter, especially at high frequencies. In order to estimate tilt, a dualtransducer system for synchronous observation of marine organisms at two angles (0o and 9o) at $38 \mathrm{kHz}$ was tested. Data was characterized via the "angle response"; the area backscatter from one transducer divided by the sum of both. This metric was computed for layers of small planktonic organisms as well as fish. While the angle response is almost negligible for small organisms at night and slightly in favour of the tilted transducer during day, there is a significant angle response from fish in layers. A theoretical model supports the idea that this effect is due swimbladder tilt. Backscatter from 13 schools of horse mackerel was also analyzed. Although no statistically significant difference was observed, the variability indicate that the angle response is highly dynamic and dependent on the actual behaviour of the school at time of observation. Further studies are planned to improve upon data collection as well as the theoretical aspects of interpretation.

2pAOa4. Application of multiple angle acoustic scatter to remote fish classification. Paul L. Roberts (University of California San Diego, Electrical and Computer Engineering Department, 9500 Gilman Dr., La Jolla, CA 92093, USA, plrobert@ucsd.edu), Jules S. Jaffe (Scripps Institution of Oceanography, Marine Physical Laboratory, 8820 Shellback Way, La Jolla, CA 92093, USA, jules@mpl.ucsd.edu)

Observation of broadband acoustic scatter from fish at multiple angles has the potential to offer advantages for remote classification in cases where the species exhibit differences in size and or body shape, and animal orientation is unknown or difficult to estimate. However, the total angular coverage of the observations, available bandwidth, and the inter- and intra-species variability in size can significantly influence classification accuracy. These parameters are investigated using laboratory data collected from ten different fish species using a linear array of receivers and a single transmitter with an effective angular coverage of up to 25 degrees with a 3 degree sampling increment. Results indicate that when the species exhibit significant differences in length, even two observations covering only 10 degrees are sufficient to obtain an 80 percent improvement in classification accuracy over a single observation angle. This holds true for a wide range of animal 
orientations. As inter-species size similarity increases, and intra-species size variability increases, more observations covering a larger range of angles are required to maintain a similar improvement in classification accuracy.

2pAOa5. Simultaneous acoustic observations of turbulence and zooplankton. Tetjana Ross (Dalhousie University, Department of Oceanography, 1355 Oxford Street, Halifax, NS B3H 4JI, Canada, tetjana.ross@dal.ca), Isabelle Gaboury (Jasco Research, Suite 432 - 1496 Lower Water Street, Halifax, NS B3J 1R9, Canada, isabelle.gaboury@gmail.com), Rolf Lueck (Rockland Scientific International Inc., 520 Dupplin Road, Victoria, BC V8Z 1C1, Canada, rolf @ rocklandscientific.com)

Models and laboratory experiments show that zooplankton may locate food more easily in turbulent waters, but whether plankton seek or avoid turbulence in the ocean is an open question. It is difficult to measure turbulence and plankton simultaneously and with the necessary spatial resolution using traditional methods (nets and airfoil shear sensors). Acoustics is commonly used to survey zooplankton abundance and recent studies have shown that stratified turbulence can also be a significant source of sound scatter. This may seem like more of a complication than a boon for those aiming to use acoustics to observe plankton in turbulence. We present acoustic data, however, that show that at least under certain circumstances, zooplankton and turbulence can be observed simultaneously with a single $307 \mathrm{kHz}$ sounder. The different natures of the two targets (discrete targets versus a volume effect) allow them to be distinguished. The key is sampling the same targets at multiple ranges and having low enough concentrations that there is no more than one animal within the beam at the closest ranges.

2pAOa6. Potential influence of shells on multibeam backscatter imagery within the Te Matuku Marine Reserve, New Zealand Alexandre C. Schimel (Univ. of Waikato - Coastal Marine Group, Ruakura Satellite Campus, PB3105 Hamilton, New Zealand, alex.schimel@gmail.com), David Johnson (MetOcean Solutions Ltd, 3/17 Nobs Line, PO Box 441 New Plymouth, New Zealand, d.johnson@metocean.co.nz), Terry Healy (Univ. of Waikato - Coastal Marine Group, Ruakura Satellite Campus, PB3105 Hamilton, New Zealand, t.healy@waikato.ac.nz), Peter J. McComb (MetOcean Solutions Ltd, 3/17 Nobs Line, PO Box 441 New Plymouth, New Zealand, p.mccomb@metocean.co.nz), Brett Beamsley (MetOcean Solutions Ltd, 3/17 Nobs Line, PO Box 441 New Plymouth, New Zealand, b.beamsley@metocean.co.nz), Dirk Immenga (Univ. of Waikato - Coastal Marine Group, Ruakura Satellite Campus, PB3105 Hamilton, New Zealand, d.immenga@waikato.ac.nz)

Backscatter imagery from a KONGSBERG EM3000 multibeam echosounder $(300 \mathrm{kHz})$ has been analyzed to investigate its potential for benthic habitat mapping. A MATLAB code has been developed to process both bathymetry and coregistered echo-strength, which includes a simple correction to remove the typical multibeam echo strength swathe contrast introduced by the backscatter incident-angle dependence. Although the correction algorithm does completely remove the effect, it is sufficient to generate maps of suitable quality for further analysis. The processing code was applied to a dataset from a survey performed on two sites in the Tamaki Strait, New Zealand, in August 2007, including a section of the Te Matuku marine reserve. Within the marine reserve, a $400 \mathrm{~m} 2$ area displays a strong reflectivity that contrasts with its surroundings while a past ground-truth data collection shows the full area is dominated by mud with only some differences in shell fragments distribution. Hypotheses to explain the origin of this reflectivity contrast are formulated and verified against past sediment and benthic survey results with emphasis on the potential influence of shells on multibeam backscatter. The potential of multibeam surveying for detecting the coverage of shells patches in benthic habitat mapping is discussed.

2pAOa7. Acoustic methods for water mass delineation in coastal marine ecosystems. John Proni (AOML/NOAA, 4301 Rickenbacker Cswy, Miami, FL 33149, USA, john.proni@noaa.gov), Jack Stamates (AOML/NOAA, 4301 Rickenbacker Cswy, Miami, FL 33149, USA, Jack.Stamates@noaa.gov), Thomas Carsey (AOML/NOAA, 4301 Rickenbacker Cswy, Miami, FL 33149, USA, thomas.p.carsey@noaa.gov), Jia-Zhong Zhang (AOML/NOAA, 4301 Rickenbacker Cswy, Miami, FL 33149, USA, Jia-Zhong.Zhang@noaa.gov), Chris Sinigalliano(AOML/NOAA, 4301 Rickenbacker Cswy, Miami, FL 33149, USA, Christopher.Sinigalliano@noaa.gov), Kevin Sullivan (AOML/NOAA, 4301 Rickenbacker Cswy, Miami, FL 33149, USA, Kevin.Sullivan@noaa .gov)

Acoustical methods play an important role in the in the study of nutrient sources for coral reef ecosystems in the south Florida coastal ocean. Many nutrients released into the coastal ocean are released in distinct water masses such as inlet discharge plumes, wastewater outfall discharge plumes, and hurricane-induced re-suspensions. Using water column acoustic backscatter profiles, obtained via either ship-borne instrumentation or in-situ instrumentation, multidimensional images of nutrient bearing water masses are derived. Said images are of assistance in the design of chemical and biological sampling efforts. Examples of water mass imaging will be presented as well as the use of such images in the design of water quality sampling programs. Using a combination of acoustic backscatter, Doppler current profilers, nutrient (nitrate, nitrite, silica, phosphate, ammonia) samples and microbiological (fecal, protozoa, viruses) samples, flux estimates of said quantities may be made for inlet discharges. Results will be presented for a south Florida inlet having an average net seaward discharge of approximately 200 million gallons per day. Selected acoustic images of treated waste water effluent plumes will be presented and compared with plume spatial distributions inferred from conservative plume tracer studies.

2pAOa8. Acoustic data from platforms of opportunity as fuel for ecosystem models. Nils Olav Handegard (Institute of Marine Research, C. Sundtsgt 64, Nordnes, 5817 Bergen, Norway, nilsolav@imr.no), Olav Rune Godoe (Institute of Marine Research, C. Sundtsgt 64, Nordnes, 5817 Bergen, Norway, olavrune@imr.no), Patrick Lehodey (Collecte Localisation Satellites, 8-10, rue Hermès, Parc Technologique du Canal, 31520 Ramonville Saint-Agne, France, plehodey@cls.fr)

Echo sounders are a widely used tool for observing marine ecosystems. Traditionally, rigid designed surveys are used to integrate biomass to a global estimate, which is coupled to traditional stock assessment models. More recently, the focus has shifted from single species assessment to an ecosystem approach, taking into account ecosystem function and dynamics. As a response, several ecosystem models have been developed. The data requirements for these models are different than for the traditional models, and new sensors and platforms have been developed as a response to this need. However, examples on how to integrate these observations with these new models are scarce. We propose a state-space observation-space approach, and compare the results from a vertically structured eco-system model with observations from an echo sounder positioned at the Mid Atlantic ridge. We argue that this approach is less vulnerable to assumptions in the data analysis process, and discuss adequate observations regimes for these models. 
Session 2pAOb

\title{
Acoustical Oceanography and ECUA: Acoustical Oceanography of Polar Environments II (Poster Session)
}

\author{
Peter N. Mikhalevsky, Cochair \\ Science Applications International Corporation \\ Hanne Sagen, Cochair \\ Nansen Environmental and Remote Sensing Center
}

\begin{abstract}
All posters will be on display from 3:40 p.m. to 5:20 p.m. To allow contributors an opportunity to see other posters, contributors of odd-numbered papers will be at their posters from 3:40 p.m. to 4:30 p.m. and contributors of even-numbered papers will be at their posters from 4:30 p.m. to 5:20 p.m.
\end{abstract}

\section{Contributed Papers}

2pAOb1. The factors that influence the design of an underwater acoustic modem for Arctic missions. Andrew M. Smerdon (Aquatec Group Ltd, High Street, RG27 8NY Hartley Wintney, UK, asmerdon@aquatecgroup .com)

As summer Arctic sea ice cover continues to reduce, there is a growing need to monitor the ice-ocean interface to aid in understanding and quantifying Arctic climate change. As part of the DAMOCLES EU 6th Framework programme, several subsea elements of a new Arctic Ocean observing system will be deployed in the Arctic Ocean during 2008. To ensure that the observing system is effective, it is essential that measured data from the ocean environment be delivered in a timely fashion for assimilation with data sets from the ice surface and atmosphere. As several of the observing platforms are drifting freely in the water below the ice, there is a need to communicate data to the surface using underwater acoustic techniques. We discuss the factors that influence the specification of an acoustic modem to achieve this data transfer. These include development of standards for data transfer and storage processes, standby power considerations, logistics of establishing a communication link, and under-ice acoustics.

2pAOb2. High-frequency broadband acoustic scattering for investigating double- diffusive convection. Tetjana Ross (Dalhousie University, Department of Oceanography, 1355 Oxford Street, Halifax, NS B3H 4JI, Canada, tetjana.ross@dal.ca), Andone Lavery (Woods Hole Oceanographic Institution, Applied Ocean Physics \& Engineering Department, 98 Water Street, MS \#11, Woods Hole, MA 02543, USA, alavery@whoi.edu)

Polar regions, with their supercooled and relatively fresh surface water, are highly susceptible to the diffusive regime of double-diffusive convection (DDDC). The fluxes associated with DDDC can play a significant role in their heat/buoyancy budgets. The use of high-frequency acoustics as a tool to map the extent and evolution of DDDC in the ocean is explored through a series of laboratory $200-300 \mathrm{kHz}$ broadband acoustic backscattering measurements. Pulse compression signal processing allows centimeter-scale interface thicknesses to be rapidly and remotely measured, and the evolution, and ultimate merging, of multiple centimeter-scale interfaces to be observed. Combining the acoustically measured interface thicknesses with knowledge of the relatively-constant temperatures within the surrounding layers allows the estimation of DDDC fluxes. Thus, broadband acoustics offers a rapid and remote method to infer fluxes, without the need for timeconsuming microstructure measurements, suggesting that this technique could benefit field studies of DDDC. Using simple models to extrapolate to the thermohaline steps typically found associated with DDDC in polar regions, diffusive-convection interfaces are predicted to scatter at measurable levels in many areas. Narrowband (120 and $200 \mathrm{kHz}$ ) acoustic backscattering observations of two scattering layers in the Western Antarctic Peninsula support this prediction.
2pAOb3. Acoustic contributions to marine ecosystem studies. Andrzej J. Orlowski (Sea Fisheries Institute in Gdynia, Kollataja 1, 81-332 Gdynia, Poland, orlov@mir.gdynia.pl)

Since the early 1970s, acoustic data collected in a form of calibrated measurements of integrated echo energy have been applied by the Sea Fishery Institute in Gdynia to observe the relationships among fish distribution and marine environment. Measurements were collected primarily with scientific echo sounders operating at $38 \mathrm{kHz}, 24 \mathrm{~h}$ a day, and stored in the form of standardized intervals of sailed distance and depth for comparison with values of selected environmental parameters, measured concurrently. Acoustic, biological, and hydrological data were transferred to the complex data base, enabling spatial correlation and four-dimensional analyses of relationships characterising a wide range of the marine organism behaviour. Selected methods and standards of comparison are described to explain how to improve the recognition of relationships between three-dimensional spatial environmental gradients and fish distribution. Results of several case studies, including the influence of hydrologic and seabed characteristics, illustrate the practical application and validity of the methods. Particular attention is given to indicators of the dependence of local fish biomass density on temperature structure in the sea and diel cycles of fish behaviour. Animations of time-dependent processes, modelled on the collected data, will be included as a new tool for marine ecosystem analysis.

2pAOb4. Estimation of macrophytes using single-beam and multibeam echosounding for environmental monitoring of arctic fjords (Kongsfjord, West Svalbard Island). Aleksandra Kruss (Institute of Oceanology Polish Academy of Sciences, Powstancow Warszawy 55, 81712 Sopot, Poland, kruss@iopan.gda.pl), Philippe Blondel (University of Bath, Department of Physics, Claverton Down, BA2 7AY Bath, UK, pyspb@bath.ac.uk), Jaroslaw Tegowski (Institute of Oceanology Polish Academy of Sciences, Powstancow Warszawy 55, 81-712 Sopot, Poland, tegowski@iopan.gda.pl), Jozef Wiktor (Institute of Oceanology Polish Academy of Sciences, Powstancow Warszawy 55, 81-712 Sopot, Poland, wiktor@iopan.gda.pl), Agnieszka Tatarek (Institute of Oceanology Polish Academy of Sciences, Powstancow Warszawy 55, 81-712 Sopot, Poland, derianna@iopan.gda.pl)

This paper presents results of a study on the spatial distribution and biomass of macrophytobentos in a fjord of Arctic Svalbard. Kongsfjord represents a periglacial environment with intense morphodynamic processes and rapidly progressing changes in the biotic environment, making it one of the most promising areas to research climate impact on ecosystems. The main objective was to provide an acoustic tool for the evaluation of benthic habitats. The 2007 field survey included systematic, co-registered, singlebeam and multibeam echosounder measurements. Acoustic observations were verified by biological samplings and observations for the classification 
algorithm development and verification. Analyses of acoustic signals scattered on bottoms covered by algae indicate the good quality of the data recorded, providing a map of phytobenthos distribution and biomass estimation in Kongsfjord. The algorithms designed and tested for processing single- and multibeam data allow extracting the morphological forms of the bottom and determine the areas covered by algae. This survey was the first instance where a multibeam imager was used to map macrophytes in an Arctic environment, in a wide variety of depths and ranges. Comparing the two types of echosounders was a great opportunity to understand the visualisation of macrophytes by multibeam systems and their identification.

\title{
Session 2pAOc
}

\section{Acoustical Oceanography, Underwater Acoustics, Signal Processing in Acoustics, and ECUA: Geoacoustic Characterization of the Ocean Bottom and Geoacoustic Inversion II (Poster Session)}

\author{
Peter Gerstoft, Cochair \\ Marine Physical Laboratory, Scripps Institute of Oceanography \\ Dag Tollefsen, Cochair \\ Norwegian Defence Research Establishment (FFI)
}

\begin{abstract}
All posters will be on display from 3:40 p.m. to 5:20 p.m. To allow contributors an opportunity to see other posters, contributors of odd-numbered papers will be at their posters from 3:40 p.m. to 4:30 p.m. and contributors of even-numbered papers will be at their posters from 4:30 p.m. to 5:20 p.m.
\end{abstract}

\section{Contributed Papers}

2pAOc1. Extracting Green's functions from noise correlation of SW06 data. Laura Brooks (Marine Physical Laboratory, Scripps Institute of Oceanography, 8602 La Jolla Shores Drive, La Jolla, CA 92093-0238, USA, lbrook02@gmail.com), Peter Gerstoft (Marine Physical Laboratory, Scripps Institute of Oceanography, 8602 La Jolla Shores Drive, La Jolla, CA 92093-0238, USA, gerstoft@ucsd.edu)

Ambient noise was recorded on an L-shaped array during the SW06 (Shallow Water 2006) sea trials. The data were cross-correlated in order to approximate the Green's function, and subsequently the acoustic travel time, between hydrophone pairs. Examination of the individual noise spectra and their mutual coherence revealed that the coherently propagating noise is dominated by frequencies less than $100 \mathrm{~Hz}$. Both time and frequency domain preprocessing techniques, and their effect upon the resulting correlation, were investigated. Times corresponding to the envelope peak of the noise cross-correlation time-derivative are in agreement with both the expected direct, and surface reflected, inter-hydrophone travel times.

2pAOc2. Single parameter description of seafloors for shallow oceans Adrian D. Jones (Defence Science and Technology Organisation, P.O Box 1500, 5111 Edinburgh, Australia, bearjones@chariot.net.au), Graham J. Day (Defence Science and Technology Organisation, P.O Box 1500, 5111 Edinburgh, Australia, Graham.Day@dsto.defence.gov.au), Paul A. Clarke (Defence Science and Technology Organisation, P.O Box 1500, 5111 Edinburgh, Australia, Paul.Clarke@dsto.defence.gov.au)

For a shallow ocean, sound transmission beyond short range is dominated by seafloor interactions at small grazing angles, for which the loss in $\mathrm{dB}$ on each bottom reflection may be approximated by a function $\mathrm{F}$ dB/radian which is linear with grazing angle. Acoustic inversion techniques have been shown to be able to obtain the value of $\mathrm{F}$ for a particular frequency. The suitability of this single parameter $\mathrm{F}$ as a seafloor descriptor has now been studied for an extensive range of seafloor types. It is shown that the phase-incoherent transmission loss (TL) obtained using the parameter F is not greatly different to TL predicted using complete knowledge of a particular seafloor, for a shallow ocean. Further, if the phase angle for a seafloor reflection is linked to the parameter F via a simple approximation, the phase- coherent properties of the shallow water interference field may be estimated to an accuracy which is surprising. This paper reviews the relevant theory and presents comparisons between TL predicted using full geoacoustic parameters versus TL based on the single parameter, for both uniform halfspace and layered seafloor types.

2pAOc3. On some possibilities and properties of matched-field geoacoustic inversion in shallow water. Alexander Zharikov (M.V. Lomonosov Moscow State University, Faculty of Physics, Alexander Zharikov, ul. Simonovskiy val 14, kv. 58, 115088 Moscow, Russian Federation, alexandr-j@yandex.ru), Pavel Kravchun (Moscow State University, Faculty of Physics, Department of Acoustics, Box 15, 125130 Moscow, Russian Federation, gedackt@mail.ru)

The aim of this paper is to estimate possibilities of matched-field geoacoustic inversion (MFI) in shallow water, and to recommend optimal arrangements of signal source and hydrophone array in variety of particular environments. We assumed ocean to be range independent with bottom consisting of homogeneous liquid layers. Sound fields were calculated as superposition of normal modes and continuous spectrum for tonal point source and vertical line array. MFI based on Bartlett processor was used. Possibilities of MFI were characterized by MFI penetration depth, sensitivity to various bottom parameters and non-uniqueness of inverted data. These characteristics were analysed as functions of frequency and the source depth and range for different values of sound attenuation in sediments and for various sound-speed profiles. To estimate possibilities of MFI in real ocean conditions, influence of array tilt and inadequacy of geoacoustic model were analysed. The influence of continuous spectrum was also discussed. Among the major results, optimal source ranges corresponding to the maximum penetration depth of MFI were calculated, and high influence of attenuation in sediments on possibilities of MFI was revealed.

2pAOc4. A mixin algorithm for geoacoustic inversion. Hefeng Dong (Dept. of Electron. \& Teleco., Norwegian Univ. of Science and Technology, NO-7491 Trondheim, Norway, dong@iet.ntnu.no), Arne Johannes 
Jenssen(Norwegian Defense Research Establishment, NO-3191 Horten, Norway, Arne-Johannes.Jenssen@ffi.no)

Shear wave velocities in the sediment can be inverted by using the dispersion curves of interface wave. It can be done by use of an algorithm based on singular value decomposition (SVD). This method can only invert the shear wave velocities and requires knowledge of the thicknesses and densities of the sediment layers. This paper presents a new algorithm for inversion of geoacoustic parameters based on dispersion curves that we have coined "mixin inversion". Mixin inversion combines SVD and global search using genetic algorithm (GA). The GA searches the depths and densities by doing a shear wave velocity inversion with SVD for each of its parameter values. This algorithm can be applied on cases where the depths, densities and shear wave velocities of the sediments are unknown. The advantage is that it is faster than pure GA since the search space is much smaller and it can be applied on cases where pure SVD inversion fails because necessary information about depths and densities is not known.

\title{
Session 2pBBa
}

\section{Biomedical Ultrasound/Bioresponse to Vibration: Ultrasound Contrast Agents for Therapy I}

\author{
Tyrone Porter, Cochair \\ Boston University, 110 Cummington Street, Boston, MA 02215, USA
}

Eleanor Stride, Cochair

University College London, Department of Mechanical Engineering, Torrington Place, London, WC1E 7JE, UK

\section{Invited Papers}

2pBBa1. Acceleration of ultrasonic tissue heating by microbubble agent. Shin-Ichiro Umemura (Tohoku University, Aoba 6-605, Aramaki, Aoba-ku, 980-8579 Sendai, Japan, sumemura@ecei.tohoku.ac.jp), Shin Yoshizawa (Tohoku University, Aoba 6-6-05, Aramaki, Aoba-ku, 980-8579 Sendai, Japan, syoshi@ecei.tohoku.ac.jp), Kazuaki Sasaki (Tokyo University of Agriculture and Technology, 3-5-8 Saiwai-cho, Fuchu, 183-0054 Tokyo, Japan, skazuaki@cc.tuat.ac.jp), Ken-Ichi Kawabata (Hitachi Central Research Laboratory, 1-280, Higashi-Koigakubo, Kokubunji, 185-8601 Tokyo, Japan, kenichi.kawabata.ap@ hitachi.com)

Ultrasound in the existence of microbubbles, whether they have been generated by acoustic cavitation or administered into the body, is known to induce bioeffects normally through mechanical or sonochemical mechanisms. Recently, it is reported that ultrasonic tissue heating can be accelerated several times by an administered microbubble agent. A microbubble, subjected to ultrasonic pressurein the frequency range of its resonanance converts the acoustic energy to heat through its volume oscillation. The acoustic power converted to heat by a microbubble through viscous heating was numerically calculated, and it was predicted that tissue ultrasonic absorption would be doubled if a microbubble agent is delivered to the tissue at a concentration in the order of 100 microbubbles $/ \mathrm{mg}$. Exteriorized murine kidneys were exposed to focused ultrasound at $3.2 \mathrm{MHz}$ in degassed saline and the tissue temperature change was measured. Optison at a dose of $0.2 \mathrm{ml} / \mathrm{kg}$ multiplied the temperature elevation induced by ultrasonic exposure by several times. In order to use this effect to treat a deep-seated tumor, microbubbles should be somehow delivered to the tumor selectively. A novel method of selective delivery, in which nano liquid particles are administered and converted into microbubbles by ultrasonic stimulation, will also be discussed.

\section{3:00}

2pBBa2. Ultrasound contrast agents pushing drug delivery: high speed optical observations. Annemieke Van Wamel (Erasmus MC, Dr Molewaterplein 50 room Ee2302, 3015GE Rotterdam, Netherlands, Annemieke@ieee.org), Klazina Kooiman (Erasmus MC, Dr Molewaterplein 50 room Ee2302, 3015GE Rotterdam, Netherlands, k.kooiman@erasmusmc.nl), Nico De Jong (Erasmus MC, Dr Molewaterplein 50 room Ee2302,3015GE Rotterdam, Netherlands, n.dejong@erasmusmc.nl)

Ultrasound-activated-microbubbles can cause transient non-lethal sonoporation of cells. Studies of influx of cell-membrane impermeable-dye, genetic materials, and nanoparticles have confirmed that the action of ultrasound-microbubbles on the cell-membrane is to alter both the cell-permeability. The sonoporation action of ultrasound-contrast-agent on cells lies in the fact that microbubbles oscillate while irradiated with ultrasound resulting in a mechanical-deformation of the cells. Oscillation microbubbles nearby cells have been optically recorded using a microscope and a high-speed camera. This camera is able to record the MHz oscillation microbubbles and therefore the interaction between oscillation microbubbles and cells. Micro-mechanical forces generated by the oscillating microbubble may also be used to locally 'push' the drugs into the cells. Ultrasound-activated-microbubbles are able to transiently increase the vascular-endothelial-layer permeability. These features may be used for future ultrasound-guided drug-delivery-systems. Next to increasing the drugs internalization, microbubbles can also be used as drug/particle carrier. Drug-loaded contrast-agents can be intentionally ruptured by diagnostic ultrasound. Using microbubbles to carry drugs to targets sites and rupturing the microbubbles by localized ultrasound energy, will result in a high local concentration of drugs. High-speed-optical-recordings of ultrasound-contrast-agent induced cell and vascular-endothelial-layer permeability will be demonstrated. Furthermore, recordings of ultrasound-controlled drugdelivery using pay-loaded ultrasound-contrast-agents will be shown. 
2pBBa3. Characterization and Optimization of Ultrasound-induced Molecular Delivery In Vivo. Elisa Konofagou (Dept. of Biomedical Engineering, Columbia Univ., 351 Engineering Terrace MC 8904, 1210 Amsterdam Ave., New York, NY 10027, USA, ek2191@columbia.edu), James Choi (Dept. of Biomedical Engineering, Columbia Univ., 351 Engineering Terrace MC 8904, 1210 Amsterdam Ave., New York, NY 10027, USA, jjc2132@ columbia.edu), Shougang Wang (Dept. of Biomedical Engineering, Columbia Univ., 351 Engineering Terrace MC 8904, 1210 Amsterdam Ave., New York, NY 10027, USA, sw2401@columbia.edu), Barclay Morrison (Dept. of Biomedical Engineering, Columbia Univ., 351 Engineering Terrace MC 8904, 1210 Amsterdam Ave., New York, NY 10027, USA, bm2119@columbia.edu), Mark Borden (Dept. of Biomedical Engineering, Columbia Univ., 351 Engineering Terrace MC 8904, 1210 Amsterdam Ave., New York, NY 10027, USA, mb2910@ columbia.edu)

Current treatments of neurological and neurodegenerative diseases are limited due to the lack of a truly noninvasive, transient, and regionally selective brain drug delivery method. The brain is particularly difficult to deliver drugs to because of the blood-brain barrier (BBB). Over the past few years, we have been developing methods that combine Focused Ultrasound (FUS) and microbubbles in order to noninvasively, locally and transiently open the BBB so as to treat neurodegenerative diseases. In this paper, we will focus on the characterization of the type of molecular delivery that can be induced through the opened BBB. More specifically, we willl characterize important properties of the BBB opening such as its size and permeability using fluorescence and MR imaging techniques, respectively. The role of the microbubble type, size and concentration on the BBB diffusion properties, its reversibility and the pressure threshold for the opening will also be described in vivo. Finally, results will be shown in both non-transgenic (normal) and transgenic (Alzheimer's) mice in order to determine the variability of the properties of the opened BBB in the presence and absence of disease.

3:40-5:20 Posters

Lecture sessions will recess for presentation of poster papers on various topics in acoustics. See poster sessions for topics and abstracts.

\section{Invited Papers}

\section{$5: 20$}

2pBBa4. Echogenic liposomes for vasoactive gas delivery and inhibition of intima hyperplasia in atheroproliferative disease. Huang Shaoling Huang (MSB 1.246, 6431 Fannin St., Houston, TX 77030, USA, Shaoling.Huang@uth.tmc.edu), David D. McPherson (University of Texas Health Science Center, 6431 Fannin St., MSB 1.252, Houston, TX 77030, USA, David.D.McPherson@uth.tmc.edu), Christy K. Holland (University of Cincinnati, Biomedical Engineering, MSB, 231 Albert Sabin Way, Cincinnati, OH 45267-0586, USA, Christy.Holland@uc.edu)

Our group has developed targeted echogenic liposomes (ELIP) for targeted ultrasound enhancement of atheroma as well as targeted therapeutic delivery. Nitric oxide (NO) has potent biological activities but is too labile for in-vivo vascular delivery. We have developed a methodology to entrap NO, cause local prolonged release and retain NO effect. NO-loaded ELIP were injected into the common carotid artery after balloon injury. Fourteen days later, the carotids were removed. Administration of NO-ELIP resulted in $51 \pm 6 \%$ inhibition of intimal thickening when compared with controls. Using a $6.0 \mathrm{MHz}$ clinical Doppler diagnostic ultrasound system, we (Smith et al) have identified the optimal pressure threshold (MI 0.08) for NO diffusion from ELIP. Using these parameters we have enhanced vascular permeability of drugs and stem cells to help stabilize atherosclerotic plaques. We have developed a novel method for encapsulating NO into ELIP demonstrating their capacity for NO delivery, inhibition of intimal hyperplasia and controlled gas diffusion with ultrasound. This methodology provides a new approach for delivering a variety of bioactive gases to target tissues, which otherwise would have negligible effects when administered systemically.

2pBBa5. Echogenic liposomes for molecular targeted therapeutic delivery. David D. McPherson (University of Texas Health Science Center, 6431 Fannin St., MSB 1.252, Houston, TX 77030, USA, David.D.McPherson@uth.tmc.edu), Shaoling Huang (University of Texas Health Science Center, 6431 Fannin St., MSB 1.252, Houston, TX 77030, USA, Shaoling.Huang@uth.tmc.edu), Christy K. Holland (University of Cincinnati, Biomedical Engineering, MSB, 231 Albert Sabin Way, Cincinnati, OH 45267-0586, USA, Christy.Holland@uc.edu)

Our group has developed targeted echogenic liposomes (ELIP) that can entrap therapeutics and release them at the target site. A key therapeutic is t-PA (tissue plasminogen activator). We have demonstrated entrapment and release following ultrasound application with t-PA effect on clot dissolution. We have demonstrated that t-PA can act as a targeting agent. Thus this drug can be both a targeting agent and a lytic agent minimizing the development of complicated formulations required for targeting and lysis. We have entrapped glitazones and demonstrated release following ultrasound application with drug effect by suppressing vascular hyperplasia. We have demonstrated that ultrasound with our ELIP can enhance delivery of agents into all areas of the atheroma, including the intima, media, the loose and dense adventitia. Heretofore these regions close to the arterial media have been impenetrable using standard intravenous drug therapy. These projects are helping to develop our echogenic immunoliposomes to; optimize targeted contrast delivery into vascular beds that are poorly penetrable by standard imaging agents; and optimize local release and concentration of therapeutics at the site of interest. This will ultimately help to direct therapeutic delivery, increase local effect, while minimizing the systemic effects of drugs and genes. 


\section{Contributed Papers}

6:00

2pBBa6. Enhancement of microbubble mediated gene delivery by simultaneous exposure to ultrasonic and magnetic fields. Eleanor Stride (University College London, Department of Mechanical Engineering, Torrington Place, WC1E 7JE London, UK, e_stride@meng.ucl.ac.uk), Colin Porter (The Institute of Cancer Research, Gene Therapy Group, Section of Cell and Molecular Biology, 237 Fulham Road, SW3 6JB London, UK, colin.porter@icr.ac.uk), Ana-Garcia Prieto (University College London, London Centre for Nanotechnology, 17-19 Gordon Street, WC1H 0AH London, UK, a.prieto@ucl.ac.uk), Quentin Pankhurst (University College London, London Centre for Nanotechnology, 17-19 Gordon Street, WC1H 0AH London, UK, q.pankhurst@ucl.ac.uk)

It has been shown in previous studies that ultrasound mediated gene delivery can be greatly enhanced by the presence of contrast agent microbubbles and that transfection efficiency is highly dependent upon both bubble/cell separation and the ultrasound field parameters. It has also been shown that gene delivery can be promoted by exposing cells to a magnetic field in the presence of DNA conjugated to magnetic nanoparticles. The aim of this work was to investigate whether it was possible to combine the advantages of both these techniques. It was found that transfection of Chinese hamster ovary cells by naked plasmid DNA was enhanced by simultaneous exposure to ultrasound (40-cycle sinusoidal pulses, centre frequency 0.5 $3 \mathrm{MHz}$, peak negative pressure $0.25-1.5 \mathrm{MPa}$, repetition frequency $1 \mathrm{kHz}$ for $10 \mathrm{~s})$ and a uniform magnetic field (3-5Nm-3) in the presence of two different microbubble/nanoparticle preparations. The first consisted of phospholipid-coated microbubbles mixed with micelles containing magnetic nanoparticles; the second of microbubbles which were themselves magnetically active. These preparations were found to be much more effective than either magnetic micelles or phospholipid-coated microbubbles alone. The mechanisms underlying these observations in terms of microbubble dynamics, the sensitivity to the ultrasound parameters and their significance for potential therapeutic applications will be discussed.

\section{6:20}

2pBBa7. Acoustic properties of multifunctional nano/microbubbles used in ultrasonography and ultrasound-mediated chemotherapy. Natalia Rapoport (University of Utah, 72 S. Central Campus Dr. room 2646, Department of Bioengineering, Salt Lake City, UT 84112, USA, natasha.rapoport@utah.edu), Kwon-Ho Nam (University of Utah, 72 S. Central Campus Dr. room 2646, Department of Bioengineering, Salt Lake City, UT 84112, USA, kwonho@gmail.com), Zhonggao Gao (University of Utah, 72 S. Central Campus Dr. room 2646, Department of Bioengineering, Salt Lake City, UT 84112, USA, zggao@yahoo.com), Douglas A. Cristensen (University of Utah, 72 S. Central Campus Dr. room 2646, Department of Bioengineering, Salt Lake City, UT 84112, USA, christen@ee.utah.edu), Anne M. Kennedy (University of Utah, 72 S. Central Campus Dr. room 2646, Department of Bioengineering, Salt Lake City, UT 84112, USA, Anne.Kennedy@hsc.utah.edu)

Multifunctional nano/microbubbles were developed that combine properties of drug carriers, ultrasound imaging contrast agents, and enhancers of ultrasound-mediated drug delivery. At room temperature, the formulations comprised perfluoropentane (PFP) nanodroplets stabilized by biodegradable diblock copolymers. The nanodroplets converted into nano/microbubbles upon heating or ultrasound irradiation. The acoustic properties of the droplets and corresponding bubbles depended on the type of the bubble stabilizing copolymer. Two different copolymers were tested, poly(ethylene oxide)co-poly(L-lactide) (PEG-PLLA) and poly(ethylene oxide)-copolycaprolactone (PEG-PCL). For the same quantitative composition, the PEG-PLLA-stabilized bubbles demonstrated higher inertial cavitation threshold and coalescence propensity than PEG-PCL-stabilized bubbles. Nanobubble tendency to coalescence is advantageous for using PEG-PLLA bubbles as tumor-targeted contrast agents.On the other hand, a lower cavitation threshold for PEG-PCL-stabilized bubbles is useful for ultrasoundmediated drug delivery. The drug doxorubicin (DOX) was localized in the bubble walls; it was released from the bubbles in response to sonication by therapeutic ultrasound, which resulted in a significant degree of drug tumortargeting and effective tumor chemotherapy.

\section{6:40}

2pBBa8. Trans-blood-brain barrier delivery of compounds at pharmacologically relevant molecular weights in the hippocampus of mice using Focused Ultrasound. James Choi (Dept. of Biomedical Engineering, Columbia Univ., 351 Engineering Terrace MC 8904, 1210 Amsterdam Ave., New York, NY 10027, USA, jjc2132@columbia.edu), Shougang Wang (Dept. of Biomedical Engineering, Columbia Univ., 351 Engineering Terrace MC 8904, 1210 Amsterdam Ave., New York, NY 10027, USA, sw2401@columbia.edu), Yao-Sheng Tung (Dept. of Biomedical Engineering, Columbia Univ., 351 Engineering Terrace MC 8904, 1210 Amsterdam Ave., New York, NY 10027, USA, yt2235@columbia.edu), Barclay Morrison (Dept. of Biomedical Engineering, Columbia Univ., 351 Engineering Terrace MC 8904, 1210 Amsterdam Ave., New York, NY 10027, USA, bm2119@columbia.edu), Elisa Konofagou (Dept. of Biomedical Engineering, Columbia Univ., 351 Engineering Terrace MC 8904, 1210 Amsterdam Ave., New York, NY 10027, USA, ek2191@ columbia.edu)

Molecular engineering has recently contributed immensely to the development of neuropharmaceuticals. However, most of these compounds are greater than $400 \mathrm{Da}$, which are too large to traverse the brain's natural defense, the blood-brain barrier (BBB). In this study, molecular delivery at pharmacologically relevant molecular weights through a Focused Ultrasound (FUS) induced BBB opening was investigated. The left hippocampus of mice $(n=13)$ was sonicated (frequency: $1.525 \mathrm{MHz}$, pressure: $0.64 \mathrm{MPa}$, duty cycle: $20 \%$, duration: 1 -min) in vivo through the intact skin and skull following intravenous injection of microbubbles (SonoVue®; 25 $\mu 1$ ). After sonication, otherwise BBB-impermeable fluorescent-tagged dextrans at various molecular weights were administered intravenously. Ex vivo fluorescent microscopy determined BBB opening by a significant increase in fluorescence in the left over the right hippocampus. The threshold for the molecular weights allowed through the hippocampal BBB was between $70 \mathrm{k}$ and 2,000k Da. Smaller compounds (i.e., 3k Da) were more spatially uniformly deposited throughout the hippocampus than larger (i.e., 70k Da) compounds. In conclusion, dextrans at neuropharmaceutically-relevant sizes were deposited in the left hippocampus of mice. This study thus demonstrates that FUSinduced $\mathrm{BBB}$ opening could allow neuropharmaceuticals previously impermeable to the $\mathrm{BBB}$, such as inhibitors and antibodies, to target tissue they were designed to treat. 


\title{
Session 2pBBb
}

\section{Biomedical Ultrasound/Bioresponse to Vibration: Ultrasound Contrast Agents for Therapy II (Poster Session)}

\author{
Tyrone Porter, Cochair \\ Boston University
}

\author{
Eleanor Stride, Cochair \\ University College London, Department of Mechanical Engineering, Torrington Place, London WC/E 7JE, UK
}

\begin{abstract}
All posters will be on display from 3:40 p.m. to 5:20 p.m. To allow contributors an opportunity to see other posters, contributors of odd-numbered papers will be at their posters from 3:40 p.m. to 4:30 p.m. and contributors of even-numbered papers will be at their posters from 4:30 p.m. to 5:20 p.m.
\end{abstract}

\section{Contributed Papers}

2pBBb1. Comparison of Spectral and Temporal Criteria for Inertial Cavitation Collapse. Mathieu Santin (Laboratoire d'Imagerie Paramétrique, 15 rue de l'Ecole de Médecine, 75006 Paris, France, santin@lip.bhdc.jussieu.fr), Alexander Haak (University of Illinois at Urbana-Champaign, Beckman Institute, 405 N Mathews, Urbana, IL 61801, USA, ahaak@uiuc.edu), Lori Bridal (Laboratoire d'Imagerie Paramétrique, 15 rue de l'Ecole de Médecine, 75006 Paris, France, bridal@lip.bhdc.jussieu.fr), William D. O’Brien, Jr. (University of Illinois at Urbana-Champaign, Beckman Institute, $405 \mathrm{~N}$ Mathews, Urbana, IL 61801, USA,wdo@uiuc.edu)

Dilute solutions of Optison and Definity were studied using a passive cavitation detector (PCD) with a $2.8-\mathrm{MHz}$ transmitter and $13-\mathrm{MHz}$ receiver. The dilution was such that each received signal should, on average, arise from a single microbubble. Several hundred microbubble responses were acquired at each of three rarefactional pressures $(1.6+/-0.2,2.0+/ 0.2$ and $2.4+/-0.2 \mathrm{MPa}$ ). Each microbubble response was grouped with signals presenting post-excitation emissions (100\% Inertial Cavitation-Collapse) or those with no evidence of post-excitation emission (0\% IC-Collapse). For each incident pressure, we compared discrimination of $100 \%$ and $0 \%$ ICCollapse groups with peak-voltage, broadband noise (12-17.6 MHz) and power at the fundamental, $2^{\text {nd }}, 3^{\text {rd }}$, and $4^{\text {th }}$ harmonic peaks. In addition to increased peak-voltage and broadband noise, spectra from 100\% ICCollapse groups consistently presented increased $2^{\text {nd }}, 3^{\text {rd }}$ and $4^{\text {th }}$ harmonics compared to the $0 \%$ IC-Collapse group. Throughout the studied pressure range, best separation between $0 \%$ and $100 \%$ IC-Collapse groups was obtained with peak-voltage $(4.7+/-1.8 \mathrm{~dB})$, broadband noise $(4.4+/-1.8 \mathrm{~dB})$ and $4^{\text {th }}$ harmonic $(5.6+/-2.2 \mathrm{~dB})$ for Optison. For Definity, all harmonics $\left(2^{\text {nd }}\right.$ to $4^{\text {th }}$ ) increased strongly for the $100 \%$ IC-Collapse group (on the order of 6 $\mathrm{dB})$ as well as peak-voltage $(5.3+/-1.2 \mathrm{~dB})$ and broadband noise $(5.8+/$ $2 \mathrm{~dB})$. Results should contribute to relating PCD criteria and IC activity.

2pBBb2. Extraction of green fluorescent proteins with sonoporation Kadija Kaddur (INSERM U930, 2, Bvd Tonnelle, 37044 Tours Cedex 9, France, $\quad$ kadija.kaddur@etu.univ-tours.fr), Anthony Delalande (CNRS-UPR4301, Centre de Biophysique Moléculaire, 45071 Orléans Cedex 2, France, anthony.delalande@cnrs-orleans.fr), François Tranquart (INSERM U930, 2, Bvd Tonnelle, 37044 Tours Cedex 9, France, tranquart@med.univ-tours.fr), Patrick Midoux (CNRS-UPR4301, Centre de Biophysique Moléculaire, 45071 Orléans Cedex 2, France, patrick.midoux@cnrs-orleans.fr), Chantal Pichon (CNRS-UPR4301, Centre de Biophysique Moléculaire, 45071 Orléans Cedex 2, France,
pichon@cnrs-orleans.fr),Ayache Bouakaz(INSERM U930, 2, Bvd Tonnelle, 37044 Tours Cedex 9, France, bouakaz@med.univ-tours.fr)

The mechanism of permeabilization with sonoporation is not understood but the recurrent hypothesis assumes the formation of pores in the cell membrane. The aim of our study is to comfort this assumption by investigating whether ultrasound (US) and microbubbles could also facilitate outward transport of molecules across the plasma membrane through these probable pore formations. Stably transfected Hela-GFP cells were used and insonified with a $1 \mathrm{MHz}$ unfocused transducer in presence of BR14 microbubbles (Bracco Research, Geneva) at different acoustic parameters. The percentage of Hela-GFP cells and the mean cell fluorescence intensity (FI) were measured by flow cytometry to evaluate the GFP release. While US alone did not affect the cells, the addition of BR14 microbubbles induced a significant decrease of FI and the percentage of Hela-GFP cells. A reduction of more than 50\% of FI and GFP + cells was achieved between 400-600 $\mathrm{kPa}$ and $40-75 \%$ of duty cycle during 2 minutes insonation. However a progressive recovery of Hela-GFP cells percentage and FI post-insonation has been observed which indicates a viable situation of Hela-GFP permeabilized cells allowing a restoration of GFP cell content. These results comfort pore formation hypothesis allowing transmembrane transport following molecular concentration gradient.

2pBBb3. Blood-brain barrier disruption using a diagnostic scanner and Definity in mice. Kristin Frinkley (Biomedical Engineering, Duke Univ., 136 Hudson Hall, Durham, NC 27708, USA, kdf2@duke.edu), Gabriel Howles-Banerji (Center for In Vivo Microscopy, Duke Univ., 141D Bryan Res Bldg., Durham, NC 27710, USA, gabriel.howles@duke.edu), Yi Qi (Center for In Vivo Microscopy, Duke Univ., 141D Bryan Res Bldg., Durham, NC 27710, USA, yi.qi@duke.edu) , G. Allan Johnson (Center for In Vivo Microscopy, Duke Univ., 141D Bryan Res Bldg., Durham, NC 27710, USA, gaj@orion.duhs .duke.edu), Kathryn Nightingale (Biomedical Engineering, Duke Univ., 136 Hudson Hall, Durham, NC 27708, USA, kathy.nightingale@duke.edu)

Objective: To quantify localized disruption of the blood-brain barrier $(\mathrm{BBB})$ in a murine model using focused, diagnostic ultrasound (at higher frequencies than previously studied) and contrast agent and to explore the optimal parameters for opening without causing irreversible damage. Methods: Definity (US contrast) and Magnevist (MR contrast) were injected before a custom ultrasound transmission sequence. The volume of tissue over which the BBB disruption allowed Magnevist to enter the brain was quantified by the contrast in T1-weighted MR images. Results: Preliminary results suggest increased BBB opening with decreased time between Definity injection and insonification. A range of typical diagnostic frequencies (e.g. 5-8 MHz) has shown BBB disruption, with maximum contrast occur- 
ring at 5.7 $\mathrm{MHz}$. Increasing pressure had an apparent threshold for visible opening, while increasing $\mathrm{F} / \#$ led to a larger region of $\mathrm{BBB}$ opening and increasing duty cycle produced more contrast. Histology showed blood cell extravasation from $\mathrm{B}$-mode imaging $(\mathrm{MI}=1.5$, duty cycle $=0.4 \%)$, but no damage was noted after the low-pressure, custom sequences. Conclusions: This study has shown the ability of a diagnostic ultrasound system and contrast agent to open the BBB for nanometer-scale particles (possibly drugs) and examined the effects of various parameters on this opening.

\title{
Session 2pBBc
}

\section{Biomedical Ultrasound/Bioresponse to Vibration and Physical Acoustics: Ultrasound Contrast Agents for Imaging II (Poster Session)}

\author{
Charles C. Church, Cochair \\ University of Mississippi \\ Ayache Bouakaz, Cochair \\ INSERM U930
}

\begin{abstract}
All posters will be on display from 3:40 p.m. to 5:20 p.m. To allow contributors an opportunity to see other posters, contributors of odd-numbered papers will be at their posters from 3:40 p.m. to 4:30 p.m. and contributors of even-numbered papers will be at their posters from 4:30 p.m. to 5:20 p.m.
\end{abstract}

\section{Contributed Papers}

2pBBc1. Influence of a rigid boundary on the resonance of an ultrasound contrast agent. Marie-Christine Pauzin (CNRS - LMA, 31 Chemin Joseph Aiguier, 13009 Marseille, France, pauzin@1ma.cnrs-mrs.fr), Serge Mensah (Laboratory for Mechanics and Acoustics CNRS, 31 chemin Joseph Aiguier, 13009 Marseille, France, mensah@1ma.cnrs-mrs.fr), Jean-Pierre Lefebvre (Laboratory for Mechanics and Acoustics CNRS, 31 chemin Joseph Aiguier, 13009 Marseille, France, lefebvre@1ma.cnrs-mrs.fr)

Recent experimental studies showed that the behavior of an Ultrasound Contrast Agent (UCA) is strongly modified when located close or targeted to a vessel wall. Asymmetrical oscillations can occur and the frequency response differs from those of free UCA. To design new techniques for diagnostic or therapeutic applications with UCA, it is important to have a better knowledge of the acoustic behavior and the dynamics of UCA in vessels. We used a finite element model (FEM) to focus on how the resonance frequency is affected by a boundary. A FEM had been first developed with COMSOL Multiphysics to calculate the pressure field scattered by two types of spherical objects (air microbubble or UCA with a viscoelastic shell) immersed in an unbounded liquid. The results obtained with the FEM being coherent with analytical models, a rigid boundary was then added to study its influence on the frequency responses of both objects. We observed a decrease of the resonance frequency of both objects when they are close to the boundary. This behavior is in good agreement with experimental results found in literature. The model has been used to perform parametrical studies (distance to the wall, UCA radius, shell parameter...).

2pBBc2. Simulation of the oscillation dynamics and translational motion of ultrasound contrast agents. Alexander Teterev (Belarus State University, 4, Nezavisimosti Ave., 220030 Minsk, Belarus, Teterev@bsu.by), Larisa Rudak (Belarus State University, 4, Nezavisimosti Ave., $220030 \quad$ Minsk, Belarus, rudak@bsu.by), Natalia Misuchenko (Belarus State University, 4, Nezavisimosti Ave., 220030 Minsk, Belarus, misuchenko@bsu.by)

A numerical model has been developed to simulate the oscillatory dynamics and the translational motion of both free and encapsulated bubbles in an ultrasound field. The model allows for the compressibility of the sur- rounding liquid and the bubble shell, which makes it possible to model the bubble dynamics at high acoustic pressures. Simulations can be carried out either by solving Rayleigh-Plesset-type equations, or in the hydrodynamic approximation taking into account the rheological properties of the liquid and the shell, or by a combined method. In the latter case, the gas pressure at the bubble surface, which is found from the gas-dynamic problem, is used as an input parameter for Rayleigh-Plesset-type equations at every time layer. The resulting radius of the bubble is then used as the boundary condition for the gas-dynamic equations. The model is a handy and flexible tool to investigate the effect of different factors on the oscillatory dynamics of the bubble, such as the thickness of the bubble shell, the rheological properties of the shell, and the translational displacement of the bubble. Using the developed model, the resonance frequency and translational displacement of contrast agents were numerically estimated.

2pBBc3. Improvement of the GMM-AR classification of multiframe contrast ultrasound images using gaussian filter. Bilal Ghazal (Univ. of Balamand Lebanon and Univ. of Claude Bernard France, Main Road Abdel Halim Ghazal bldg Kab-Elias Bekaa, 00961 Kab-elias, Lebanon, bilalghazal00@hotmail.com), Maha Khachab (Univ. of Balamand, Biomedical Dept., El Koura, 00961 Tripoli, Lebanon, maha.khachab@balamand.edu.lb), Denis Friboulet (Université de Lyon, 43 boulevard du 11 Novembre 1918, 69622 Villeurbanne, France, denis.friboulet@creatis.insa-lyon.fr), Chafic Mokbel (Univ. of Balamand, Biomedical Dept., El Koura, 00961 Tripoli, Lebanon, chafic.mokbel@balamand.edu.lb), Christian Cachard (Université de Lyon, 43 boulevard du 11 Novembre 1918, 69622 Villeurbanne, France, christian .cachard@creatis.insa-lyon.fr)

Despite the use of contrast agents that enhance the visualization of vascular zones, the backscattered signals from the contrast agent and tissue are still close which prevents the direct wide ultrasonic use in diagnosis. Thus, it was necessary to implement image-processing techniques that enhance the contrast echo and have the capability of classification. We have applied a new approach based on the autoregressive model where an image of prediction errors is calculated in the first phase. Then, a Gaussian filter is applied in order to model well afterward both agent and tissue behaviors by a Gaussian mixture model. The Agent to Tissue Ratio (ATR)factor and Fisher cri- 
terion are adopted to compare the performance of this method with existing techniques as the harmonic and B mode techniques. The experiments conducted have shown the advantages of our proposed approach where an increasing of ATR and Fisher are recorded. In fact, our ATR attains $19.20 \mathrm{~dB}$ which represents a good improvement in comparison with B mode $(9.50 \mathrm{~dB})$ and Harmonic technique $(12.13 \mathrm{~dB})$. Whereas Fisher, the parameter of classification feasibility, it reaches 2.01 which matches an excellent amelioration with respect the mentioning techniques with 0.97 and 1.00 respectively.

2pBBc4. Finite difference time domain numerical simulation of ultrasonic propagation in coated contrast agents. Galaz Belfor (Université Paris 6, Laboratoire d'Imagerie Paramétrique, 15, rue de l'Ecole de Médecine, 75006 Paris, France, bgalaz@fisica.usach.cl), Guillaume Haiat (CNRS, Laboratoire de Recherches Orthopédiques, 10, Avenue de Verdun, 75010 Paris, France, haiat@univ-paris12.fr), Taulier Nicolas (Université Paris 6, Laboratoire d'Imagerie Paramétrique, 15, rue de l'Ecole de Médecine, 75006 Paris, France, Nicolas.Taulier@lip.bhdc.jussieu.fr), Urbach Wladimir (Université Paris 6, Laboratoire d'Imagerie Paramétrique, 15, rue de l'Ecole de Médecine, 75006 Paris, France, urbach@1ps.ens.fr)

Acoustic wave propagation in solutions of ultrasound contrast agents (UCA) designed for in situ drug delivery remains unclear due to the complex interaction between ultrasound and the coated particles (mode conversion, scattering, multiple reflections). To address this problem, twodimensional Finite Difference Time Domain numerical simulations of ultrasound transmission and reflection were performed in solutions of an aqueous suspension of spheres made of fluid surrounded by a polymeric capsule. Simulations were made for particle concentrations equivalent ranging from 2 to $87 \mathrm{mg} / \mathrm{ml}$. For each concentration 15 aqueous solutions with randomly located particles were considered. The speed of sound $\mathrm{c}$, the attenuation coefficient and the integrated backscattered coefficient were computed from the transmitted and backscattered signals. The values of $\mathrm{c}$ decrease from $1498 \mathrm{~m} / \mathrm{s}$ to $1432 \mathrm{~m} / \mathrm{s}$ when the concentration increases, in good agreement with an effective medium model. The attenuation coefficient at $5 \mathrm{MHz}$ is an increasing function of the concentration, from 0.05 to $1.2 \mathrm{~dB} / \mathrm{cm}$. The integrated backscattered coefficient increases with the concentration for low concentration values but decreases for high concentration (with a maximum value for a concentration of about $50 \mathrm{mg} / \mathrm{ml}$ ). Our numerical simulations successfully mimic experimental results of ultrasound propagation in solutions of UCA.

2pBBc5. Photoacoustical evaluation of thermal response of microbubbles. Angelo Biagioni (Sapienza Università di Roma, Via A. Scarpa, 14, 00161 Rome, Italy, angelo.biagioni@uniroma1.it), Adriano Alippi (Sapienza Università di Roma, Via A. Scarpa, 14, 00161 Rome, Italy, adriano.alippi@uniroma1.it), Andrea Bettucci (Sapienza Università di Roma, Via A. Scarpa, 14, 00161 Rome, Italy, andrea.bettucci @uniroma1.it), Annunziata D’Orazio (Sapienza Università di Roma, Via A. Scarpa, 14, 00161 Rome, Italy, annunziata.dorazio @uniroma1.it), Massimo Germano (Sapienza Università di Roma, Via A. Scarpa, 14, 00161 Rome, Italy, massimo.germano@uniroma1.it), Daniele Passeri (Sapienza, Università di Roma, Via A. Scarpa, 14, 00161 Rome, Italy, daniele.passeri@uniroma1.it)

Photoacoustic technique has emerged as a tool for the study of liquid, gel, suspension behaviour and has been recently employed in a number of new biomedical applications. In the present paper, a new photoacoustic sensor is presented which was designed and realized to analysing photoacoustic signals from solution filled with microbubbles, usually used as contrast agents in echotomography imaging. The device is a closed cell where photothermal expansion in acqueous solution samples causes the deflection of a thin membrane closing a short pipe $0.7 \mathrm{~mm}$ by radius. The overall set up acts as a Helmoltz resonator, where the solution in the pipe is the mass oscillating under the driving force produced by the alternating expansion of the solution in the cell body. Displacement of the membrane was measured using a laser probe interferometer, whereas photoacoustic signal was generated by chopping a laser light beam impinging into the solution through a glass window in the cell. The response of the cell filled with shelled microbubble solution was investigated with respect to water behaviour, at the cell resonance frequency and for different temperatures.

2pBBc6. Frequency response of bubble pulsations in tubes with arbitrary wall impedance. Todd A. Hay (Applied Research Laboratories, The University of Texas, P.O. Box 8029, Austin, TX 78713-8029, USA, haymaker@mail.utexas.edu), Theresa Y. Cheung (Applied Research Laboratories, The University of Texas, P.O. Box 8029, Austin, TX 78713-8029, USA, cheung.ty@gmail.com), Mark F. Hamilton (Applied Research Laboratories, The University of Texas, P.O. Box 8029, Austin, TX 78713-8029, USA, hamilton@mail.utexas.edu), Yurii A. Ilinskii (Applied Research Laboratories, The University of Texas, P.O. Box 8029, Austin, TX 787138029, USA, yura@arlut.utexas.edu)

A model is presented for the linear pulsation of a small bubble in a tube with locally reactive walls but with otherwise arbitrary wall impedance. The model is based on the normal mode expansion of the Green's function presented by Morse and Ingard [Theoretical Acoustics (McGraw-Hill, 1968), Eq. (9.2.10)]. The specific case of a cylindrical tube is considered. For a bubble that is located in the center of the tube and that is small compared with both tube diameter and wavelength, the radiation impedance on the bubble is given by a summation of the normal modes evaluated in the center of the tube. From the radiation impedance, the frequency response of the bubble to an applied sound field is obtained. For tube walls that are either rigid of pressure release, the solution agrees with the frequency response calculated using the method of images for a square tube having the same cross-sectional area. In tubes with hard walls the resonance frequency decreases as tube radius decreases because of radiation damping. In tubes with very soft walls the radiation damping is negligible below the cutoff frequency of the lowest mode, and the resonance frequency increases slightly as tube radius is decreased.

2pBBc7. New theoretical models for lipid-shelled ultrasound contrast agents. Alexander A. Doinikov (Belarus State University, 11 Bobruiskaya Street, 220030 Minsk, Belarus, doinikov@bsu.by)

Analysis of existing theoretical models for lipid-coated contrast agents is presented. A general theoretical approach to the development of zerothickness encapsulation models, allowing the testing of different rheological laws for encapsulation, is proposed. Based on available experimental data, analysis of the rheological behavior of encapsulating lipid shells is made. The problems of the existing models, such as the dependence of shell parameters on the initial bubble radius and the "compression-only" behavior, are discussed. Physical explanations for the observed effects and new theoretical models for their simulation are offered. In particular, it is shown that the inclusion of nonlinear shell viscosity allows one to model the "compression-only" behavior. It is also very important to select an appropriate rheological law describing the dependence of the shell viscosity on the shear rate. A correct choice reduces considerably the spread of the experimentally estimated values of the shell parameters and makes them look more ordered. Whereas with an inappropriate rheological law, the experimental data may look rather chaotic.

2pBBc8. Non-linear extensions to shell models of ultrasound contrast agents: theory and experiment. Nikos A. Pelekasis (Dept. Mechanical Engineering, University of Thessaly, Pedion Areos, 38334 Volos, Greece, pel@uth.gr), Kostas Tsiglifis (Dept. Mechanical Engineering, University of Thessaly, Pedion Areos, 38334 Volos, Greece, kotsig1@uth.gr), Benjamin Dollet (Physics of Fluids, University of Twente, P.O. Box 217, 7500 AE Enschede, Netherlands, benjamin.dollet@univ-rennes1.fr), Nico De Jong (Erasmus MC, Dr Molewaterplein 50 room Ee2302, 3015GE Rotterdam, Netherlands, n.dejong@erasmusmc.nl), Detlef Lohse (Physics of Fluids, University of Twente, P.O. Box 217, 7500 AE Enschede, Netherlands, d.lohse@utwente.nl), Michel Versluis (Physics of Fluids, University of Twente, P.O. Box 217, 7500 AE Enschede, Netherlands, m.versluis @utwente.nl)

The standard UCA models, characterized by the area dilatation modulus and viscosity of the encapsulating membrane, are extended in order to account for deviations from linear material behaviour and for non-spherical 
pulsations. The former effect is captured by introducing a parameter that measures the degree of membrane softness, whereas the latter is modelled by introducing a bending elasticity. Lipid shells exhibit the phenomenon of 'thresholding behavior' where excursions in the relative area dilatation are measured only beyond a critical sound amplitude. Treating the lipid shell as strain softening reproduces this non-linear behaviour which can be further optimized by controlling the shell softness parameter. Such a tuning can be used to estimate the degree of softness for the contrast agent BR-14. Stabil- ity analysis allowing for shape distortions provides an estimate for the bending elasticity that reproduces the experimentally observed threshold for growth of surface modes. Phase diagrams are constructed for BR-14, based on high-speed optical observations and stability analysis. Both experiment and numerical analysis exhibit a smaller variation between the stability threshold of subsequent shape modes in the case of UCA's, as compared to free bubbles. Numerical simulations reproduce the experimentally observed saturation of growing shape modes for pre-stressed UCA's.

\title{
Session 2pBBd
}

\section{Biomedical Ultrasound/Bioresponse to Vibration and Engineering Acoustics: High-Intensity Focused Ultrasound Metrology and Standards II (Poster Session)}

\author{
Peter Kaczkowski, Cochair \\ Center for Industrial and Medical Ultrasound, Applied Physics Lab. \\ Gail Ter Haar, Cochair \\ Institute of Cancer Research
}

\begin{abstract}
All posters will be on display from 3:40 p.m. to 5:20 p.m. To allow contributors an opportunity to see other posters, contributors of odd-numbered papers will be at their posters from 3:40 p.m. to 4:30 p.m. and contributors of even-numbered papers will be at their posters from 4:30 p.m. to 5:20 p.m.
\end{abstract}

\section{Contributed Papers}

2pBBd1. Simultaneous measurement of pressure and temperature in a focused ultrasound field with a fiber optic hydrophone. Michael S. Canney (Center for Industrial and Medical Ultrasound, Applied Physics Lab., University of Washington, 1013 NE 40th St., Seattle, WA 98105, USA, mcanney@u.washington.edu), Michael R. Bailey (Center for Industrial and Medical Ultrasound, Applied Physics Lab., University of Washington, 1013 NE 40th St., Seattle, WA 98105, USA, bailey@apl.washington.edu), Vera A. Khokhlova (Center for Industrial and Medical Ultrasound, Applied Physics Lab., University of Washington, 1013 NE 40th St., Seattle, WA 98105, USA, vera@acs366.phys.msu.ru), Oleg A. Sapozhnikov (Center for Industrial and Medical Ultrasound, Applied Physics Lab., University of Washington, 1013 NE 40th St., Seattle, WA 98105, USA, oleg@acs366.phys.msu.ru), Lawrence A. Crum (Center for Industrial and Medical Ultrasound, Applied Physics Lab., University of Washington, 1013 NE 40th St., Seattle, WA 98105, USA, lac@apl.washington.edu)

The characterization of high intensity focused ultrasound (HIFU) fields is important for both clinical treatment planning as well as for regulation of HIFU medical devices. In previous work, we have used a $100-\mu \mathrm{m}$ fiber optic probe hydrophone (FOPH) to measure pressure waveforms from a $2-\mathrm{MHz}$ HIFU source with 42-mm aperture and 44-mm focal length. The formation of shock waves with peak positive pressure of up to $80-\mathrm{MPa}$ were measured and modeled in transparent tissue-mimicking gel phantoms and boiling was achieved in milliseconds [Canney MS, et al., J. Acoust. Soc. Am., 120:3110 (2006)]. In this work, the FOPH was also used to measure temperature changes in tissue phantoms from HIFU at peak focal intensities of 5000$20,000 \mathrm{~W} / \mathrm{cm}^{2}$. Temperature measurements were obtained by first low-pass filtering the voltage signal measured from the FOPH to remove the acoustic part of the measurement. Then, calibration of voltage to temperature was performed using results from a separate calibration experiment. Experimental measurements were compared with numerical modeling using a KZKtype model for acoustic propagation coupled with a heat transfer model. In summary, temperatures of $100^{\circ} \mathrm{C}$ were measured at the HIFU focus in milliseconds, in agreement with modeling [Work supported by NIH DK43881, NSBRI SMS00402, and RFBR.]

2pBBd2. Visualization of transducer fields using a two-dimensional acousto-optic sensor. Charles Pergantis (US Army Research Laboratory, Aberdeen Proving Ground, Abredeen, MD 21005-5069, USA, cpergan@arl.army.mil), Jaswinder Sandhu (Santec Systems, Inc., 716 South Milwaukee Ave., Wheeling, IL 60090, USA, j-sandhu@santecsystems.com), Witold Popek (Santec Systems, Inc., 716 South Milwaukee Ave., Wheeling, IL 60090, USA, w-popek@ santecsystems .com)

In this paper we will report on the use of a novel two-dimensional acousto-optic (2D AO) sensor as a simple, fast and cost-effective method of mapping transducer fields, which could be useful for quality control and quality assurance (QC/QA) of diagnostic and therapeutic transducers. This capability could be particularly useful for monitoring power output of therapeutic transducers prior to administering ultrasound based thermo-therapy treatment. It may also provide a quick tool for determining beam position and other transducer field characteristics such as propagation angle, beam diameter, divergence, and cross-sectional uniformity. Current standard transducer field mapping practices requires point-by-point scanning over a ball reflector or a hydrophone to map the transducer field. This approach is tedious, requiring hours of scanning time to generate the full cross-sectional and axial field distributions. The 2D AO system could overcome some of the drawbacks of current conventional scanning methodologies.

2pBBd3. Uncertainty Evaluation of Effective Radiation Area of Ultrasound Transducers: Preliminary Results of Inmetro's Laboratory of Ultrasound. Andre V. Alvarenga (Laboratory of Ultrasound - Inmetro, Av. N. Sra. das Gracas, 50, predio 1 - Xerem, 25250-020 Duque de Caxias - Rio de Janeiro, Brazil, avalvarenga@inmetro.gov.br), Rodrigo Costa-Felix (Laboratory of Ultrasound - Inmetro, Av. N. Sra. das Gracas, 
50, predio 1 - Xerem, 25250-020 Duque de Caxias - Rio de Janeiro, Brazil, rpfelix@inmetro.gov.br)

According to the "Guide to the Expression of Uncertainty of Measurement" (GUM), on calculation of the uncertainty of a given measurement it is necessary to take into account two types of uncertainty: Type A and Type B. Type A uncertainty is obtained from the statistical analysis of a series of observations, while Type B comes from sources that can not be evaluated considering statistical analysis, but can be obtained from previous measurements, knowledge on the behaviour of the measuring equipment, manufacturer's specifications, and data from certificates or handbooks.
Herein, the Effective Radiation Area (ERA) of ultrasound transducers has been estimated at Inmetro's Laboratory of Ultrasound using an acoustic pressure field mapping system. ERA calculation protocol developed was based on Standard IEC 61689. Besides, the Type A uncertainty was estimated from 4 repetitions of the full procedure for the determination of ERA, and Type B one was estimated from the mathematical model for ERA calculation, obtained based on IEC 61689 and the GUM. Initial tests using US transducer of $1.0 \mathrm{MHz}$ and $2.25 \mathrm{MHz}$ indicated an expanded uncertainty inferior to $7.0 \%$. This preliminary result encourages further research and development in order to increases frequency range to be assessed.

\title{
Session 2pBBe
}

\section{Biomedical Ultrasound/Bioresponse to Vibration: High-Intensity Focused Ultrasound II (Poster Session)}

\author{
Lawrence Crum, Cochair \\ Center for Industrial and Medical Ultrasound, Applied Physics Lab. \\ Jean Yves Chapelon, Cochair \\ INSERM, U556
}

\begin{abstract}
All posters will be on display from 3:40 p.m. to 5:20 p.m. To allow contributors an opportunity to see other posters, contributors of odd-numbered papers will be at their posters from 3:40 p.m. to 4:30 p.m. and contributors of even-numbered papers will be at their posters from 4:30 p.m. to 5:20 p.m.
\end{abstract}

\section{Contributed Papers}

2pBBe1. High intensity ultrasound activation of coagulation factors Stuart B. Mitchell (Applied Physics Laboratory, University of Washington, 1013 NE 40th Street, Seattle, WA 98105, USA, sbmitch@u .washington.edu), Noemie C. Juaire (Center for Industrial and Medical Ultrasound, Applied Physics Lab., University of Washington, 1013 NE 40th St., Seattle, WA 98105, USA, noemiej@apl.washington.edu), Yak-Nam Wang (Applied Physics Laboratory, University of Washington, 1013 NE 40th Street, Seattle, WA 98105, USA, ynwang @apl.washington.edu)

Although it has been demonstrated that High Intensity Focused Ultrasound (HIFU) can induce vascular cauterization, acoustic hemostasis technology has not been successful in making the transition from proof-ofconcept to clinical settings. One reason for this lack of acceptance is the limited understanding of the fundamental mechanisms involved in ultrasound-vessel and ultrasound-blood interactions. The aim of this research was to investigate the hematological and biochemical mechanisms which are influenced by HIFU induced coagulation. HIFU was used to induce coagulation in an in vitro hematological flow system and in animal models. The flow circuit and in vivo arteries were instrumented with flow probes and thermocouples in the treatment region. Physiological parameters were recorded throughout the in vivo experiments. Blood samples were drawn for analysis prior to, during, and immediately following each HIFU treatment. Clotting time, complete blood count, and biochemical analysis were performed immediately and citrated samples were immediately centrifuged and frozen for future analysis. Results indicate that HIFU can change local and systemic levels of thombin/anti-thrombin complex (TAT) and tissue plasminogen activator (tPA), as well as induce changes in activated clotting time (ACT). These results indicate that HIFU can induce coagulation via the coagulation cascades (TAT) and that normal hematological response to thrombus formation is unaffected.
2pBBe2. Dynamical focusing method for high-intensity ultrasound applications. Andrey Rybianets (Ultrashape Ltd., New Industrial Park, POB 80, 20692 Yoqneam, Israel, arybyanets@gmail.com)

New method for direct synthesis of dynamically focused acoustic field suitable for HIFU applications is proposed. The method is based on the continuous cyclic generation of different focal patterns at optimal repetition frequency by simultaneous applying of " $\mathrm{M}$ " different frequency signals to " $\mathrm{N}$ " sectors of spherically shaped sectored transducer array. The HIFU transducer arrays comprising a spherical piezoceramic cap with back electrode divided circumferentially into " $\mathrm{N}$ " regular or specially shaped sectors were designed and tested. The sectors were simultaneously powered by "M" (M $\leqslant$ N) sinus or burst drive signals with different frequencies from $6 \mathrm{~dB}$ bandwidth of the transducer. Calculation and modeling of acoustic field patterns for different array configurations and frequencies sets were performed. Acoustic pressure in focal planes was measured in water using calibrated hydrophone and 3D acoustic scanning system. In vitro experiments on different tissues confirming the advantages of dynamical focusing method were performed. The benefits of the method are creating bigger treated tissue volumes and enhancement of the cavitational, mechanical or thermal influences due to coincidence of the repetition frequency with a specific resonance/ relaxation times for tissue and/or for cavitation "cloud". Applications of the method for ultrasonic therapy, hyperthermia, and body aesthetics were considered.

2pBBe3. Delivery of antibacterial-nanoparticles into dentinal tubules with high intensity focused ultrasound. Siew Wan Fong (Institute of High Performance Computing, 1 Science Park Road, \#01-01 The Capricorn, 117528 Singapore, Singapore, fongsw@ihpc.a-star.edu.sg), Annie Shrestha (Department of Restorative Dentistry, Faculty of Dentistry, 
National University of Singapore, 5 Lower Kent Ridge road, 119074 Singapore, Singapore, g0600129@nus.edu.sg), Zhilong Shi (Department of Chemical and Biomolecular Engineering, National University of Singapore, 10 Kent Ridge Crescent, 119260 Singapore, Singapore, chesz@nus.edu.sg) , Boo Cheong Khoo (National University of Singapore, Dept of Mechanical Engineering, 10 Kent Ridge Crescent, 119260 Singapore, Singapore, mpekbc@nus.edu.sg), Anil Kishen (Department of Restorative Dentistry, Faculty of Dentistry, National University of Singapore, 5 Lower Kent Ridge road, 119074 Singapore, Singapore, rsdak@nus.edu.sg)

High-Intensity-Focused-Ultrasound (HIFU) when applied clinically often result in the formation of cavitation bubbles in the nearby fluid or tissue which collapse with high-speed jets and can be utilized for particle delivery. Bacteria residing in anatomical complexities and dentinal tubules resist conventional disinfection procedures during root canal treatment. This study aims is to evaluate the efficacy of jet flow produced by collapsing cavitations to push antibacterial nanoparticles into the areas inaccessible to conventional treatment. Eight dentin blocks of $8 \times 3 \times 1.5 \mathrm{~mm}^{3}$ were prepared from the root region of freshly extracted single rooted tooth samples and divided into two groups: (1) Control and (2) Nanoparticles. The samples were placed in sterile deionized water and nanoparticles suspension $(1 \mathrm{mg} / \mathrm{ml})$ for groups 1 and 2 respectively. HIFU at $27 \mathrm{kHz}$ for two minutes was employed. The samples were then sectioned to expose the dentinal tubules and viewed under field emission scanning electron microscopy and Energy Dispersive $\mathrm{X}$-ray analysis to ascertain the depth of penetration of the nanoparticles. The findings from this study suggested that HIFU treatment resulted in the significant penetration and coating of the dentinal tubules with nanoparticles. HIFU can be used as a potential tool for the delivery of antibacterial nanoparticles to disinfect the root canals.

2pBBe4. The effect of high intensity focussed ultrasound (HIFU) on pH responsive PEGylated micelles. Moira J. Smith (Chemical Engineering Department, Cambridge University, New Museums Site, CB2 3RA Cambridge, UK, mjs224@cam.ac.uk), Mark E. Eccleston (Chemical Engineering Department, Cambridge University, New Museums Site, CB2 3RA Cambridge, UK, mark.eccleston@ vivamer.com), Nigel K. Slater (Chemical Engineering Department, Cambridge University, New Museums Site, CB2 3RA Cambridge, UK, nigel_slater@cheng.cam.ac.uk)

The effect of HIFU on $\mathrm{pH}$ responsive PEGylated micelles was investigated. Micelles can be used as drug carrier vehicles reducing unwanted drug toxicity. HIFU is able to release drugs from the circulating micelles, as well as improving intracellular uptake of both micelleencapsulated and free drugs non-invasively. Large molecules generally enter cells by endocytosis. Endosomes gradually become acidic and fuse with enzyme containing lysosomes degrading the endosomal contents and preventing them from reaching their intracellular target. Using $\mathrm{pH}$ responsive polymers enables endosomes to be distrupted, releasing their contents into the cytoplasm before degradation occurs. Addition of poly(ethylene glycol), referred to as PEGylation, prolongs circulatory half-life and reduces degradation within the bloodstream. HIFU did enable release of encapsulated molecules from the modified micelles, and the micelles were taken up by $\mathrm{H} 69$ human carcinoma cells in vitro. Further work will investigate optimization of the micelles to maximize encapsulated drug release. The combined approach of using both $\mathrm{pH}$ responsive PEGylated micelles and HIFU to deliver drugs would provide more accurate targeting of therapies allowing higher therapeutic doses to be administered, reduce unwanted side effects and give patients a higher quality of life.

2pBBe5. The interaction of microbubbles with high intensity pulsed ultrasound. Siew Wan Fong (Institute of High Performance Computing, 1 Science Park Road, \#01-01 The Capricorn, 117528 Singapore, Singapore, fongsw@ihpc.a-star.edu.sg), Evert Klaseboer (Institute of High Performance Computing, 1 Science Park Road, \#01-01 The Capricorn, 117528 Singapore, Singapore, evert@ihpc.a-star.edu.sg), Boo Cheong
Khoo(National University of Singapore, Dept of Mechanical Engineering, 10 Kent Ridge Crescent, 119260 Singapore, Singapore, mpekbc@nus.edu $. s g)$

High intensity pulsed ultrasound when interacting with microbubble contrast agents is potentially useful for biomedical applications such as drug delivery, cancer treatment and tissue ablation. To establish a fundamental understanding of the interaction between a microbubble with a sound wave, numerical simulations are performed using the Boundary Element Method (BEM). Bubble dynamics in terms of shape changes in time, maximum bubble radius obtained, jet velocity and translational movement of the bubble center is studied. The effect of varying ultrasound intensity and initial bubble size is examined as well. One important result is the determination of the conditions under which a clear high speed jet will be formed in the microbubble when it is hit by a specific sound wave. The jet is crucial to the success of the biomedical applications mentioned. Also, it is shown that one cycle of ultrasound consisting of a single negative part followed by a single compressive part would be the optimized wave form because collateral damage by re-expanding remnants of a collapsed microbubble by the subsequent negative pressure wave is prevented. The BEM model has greater computational efficiency in terms of speed and storage space over other full domain methods because only surface meshing is needed.

2pBBe6. A passive array technique for cavitation mapping during HIFU treatment. Miklós Gyöngy (University of Oxford, Medical Engineering Unit, 43 Banbury Road, OX2 6PE Oxford, UK, miklos.gyongy@jesus.ox.ac.uk), Manish Arora (University of Oxford, Medical Engineering Unit, 43 Banbury Road, OX2 6PE Oxford, UK, manish.arora@eng.ox.ac.uk), Alison Noble (University of Oxford, Wolfson Medical Vision Laboratory, Department of Engineering, OX13PJ Oxford, UK, noble@ robots.ox.ac.uk), Constantin C. Coussios (University of Oxford, Medical Engineering Unit, 43 Banbury Road, OX2 6PE Oxford, UK, constantin.coussios@eng.ox.ac.uk)

During exposure of tissue to HIFU, cavitation has been shown to enhance the rate of heat deposition in tissue and could provide an effective means of treatment monitoring. However, cavitation activity occurring prefocally or growing unstable around the focal region is known to impede safe and effective treatment delivery. A combined computational and experimental approach is being applied to develop a passive array-based technique for mapping cavitation activity in real-time, using a novel cross-correlation algorithm to combine single-channel information received by an imaging array co-axial to the HIFU transducer. The cavitation maps produced in this manner were validated using two single-element $15-\mathrm{MHz}$ focused PCDs during continuous-wave insonation of a polyacrylamide-based tissue mimicking material by $1.1 \mathrm{MHz}$ HIFU. Initial experiments have shown bubble cloud movement towards the HIFU transducer, in agreement with previously reported observations. Future work will involve applications of advanced imaging techniques to achieve a high spatiotemporal resolution, which is essential because of the unstable nature of cavitation activity and the requirement that treatment be well localized.

2pBBe7. Quantitative assessment of thermal dose using photographic measurements of tissue discoloration. Gavriel Speyer (Center for Industrial and Medical Ultrasound, Applied Physics Lab., University of Washington, 1013 NE 40th St., Seattle, WA 98105, USA, gavriel@u .washington.edu), Peter Kaczkowski (Center for Industrial and Medical Ultrasound, Applied Physics Lab., University of Washington, 1013 NE 40th St., Seattle, WA 98105, USA, peter@apl.washington.edu), Andrew Brayman (Center for Industrial and Medical Ultrasound, Applied Physics Lab., University of Washington, 1013 NE 40th St., Seattle, WA 98105, USA, brayman@apl.washington.edu), Marilee Andrew (Center for Industrial and Medical Ultrasound, Applied Physics Lab., University of Washington, 1013 NE 40th St., Seattle, WA 98105, USA, marilee@apl.washington .edu), Steven Kargl (Applied Physics Laboratory, University of Washington, 1013 NE 40th Street, Seattle, WA 98105, USA, kargl@troutmask 
.apl.washington.edu),Lawrence A. Crum(Center for Industrial and Medical Ultrasound, Applied Physics Lab., University of Washington, 1013 NE 40th St., Seattle, WA 98105, USA, lac@apl.washington.edu)

High Intensity Focused Ultrasound (HIFU) is rapidly gaining widespread clinical use in China, and is undergoing regulatory evaluation in Europe and the US for many target diseases. Nevertheless, tools for therapy planning, monitoring, and assessment remain at a rudimentary level. In particular, measurement of thermal dose in tissues exposed with HIFU has not been sufficiently quantitative to make detailed comparisons with numerical simulations, required for validation of therapy planning models. Indeed, model validation is complicated by high sensitivity of the results to small changes in parameter values and by the general difficulty of performing geometrical registration with sufficient precision to meaningfully compare millimeter scale features typical of HIFU lesions. Our work uses photographic measurement of visible tissue discoloration so that it can be used to accurately and rapidly quantify HIFU-induced bioeffects at scales of several centimeters for comparison with the prior therapy plan. Precise comparison between nonlinear acoustic simulation and macroscopic lesion data indicates that a newly defined "blanching index" is nearly linearly proportional to the logarithm of predicted thermal dose over a very wide range of exposure, including well below the 240 minute (at 43 degrees) necrotic threshold up to about 10,000 minutes.

TUESDAY AFTERNOON, 1 JULY 2008

P3-B, LEVEL 3, 3:40 TO 5:20 P.M.

Session 2pBBf

\title{
Biomedical Ultrasound/Bioresponse to Vibration: Quantitative Ultrasound Methods for Diagnosis and Therapy II (Poster Session)
}

\author{
Jonathan Mamou, Cochair \\ Riverside Research Institute \\ Geneviève Berger, Cochair \\ LIP, CNRS, Paris, France
}

\begin{abstract}
All posters will be on display from 3:40 p.m. to 5:20 p.m. To allow contributors an opportunity to see other posters, contributors of odd-numbered papers will be at their posters from 3:40 p.m. to 4:30 p.m. and contributors of even-numbered papers will be at their posters from 4:30 p.m. to 5:20 p.m.
\end{abstract}

\section{Contributed Papers}

2pBBf1. Liver fibrosis identification by spectral slope of the backscattering curve. Mahmoud Meziri (Univ Badji Mokhtar Annaba, Bp 12 Sidi- Amar Wilaya Annaba, 23000 Annaba, Algeria, mmahmouddz@yahoo.fr), Wagner C. Pereira (Biomedical Engineering Program - COPPE, Federal University of Rio de Janeiro, CEP. 21.941-972 Rio de Janeiro, Brazil, wagner@peb.ufrj.br), Naceur Tiah (Univ Badji Mokhtar Annaba, Bp 12 Sidi- Amar Wilaya Annaba, 23000 Annaba, Algeria, nac_tiah@yahoo.fr), Naamane Remita (Univ 20 Aout 55 Skikda, BP 24, 21000 Skikda, Algeria, n.remita@yahoo.fr), Bouzid Boudjema (Univ 20 Aout 55 Skikda, BP 24, 21000 Skikda, Algeria, boudjema_b@yahoo.fr), Pascal Laugier (Université Paris 6, Laboratoire d'Imagerie Paramétrique, 15, rue de l'Ecole de Médecine, 75006 Paris, France, laugier@lip.bhdc.jussieu.fr)

Ultrasonic tissue characterization is primarily based on radio-frequency (RF) signals. Different studies have demonstrated that the RF signals are closely linked to tissue structures. The processing of these signals using spectral methods has shown the possibility of deriving quantitative parameters (attenuation and backscattered coefficients) and also of providing a means to estimate the elementary properties of tissue (scatterer size, concentration, periodicity) by evaluating spectral parameters (slope, intercept etc). We have estimated the spectral slope of the average backscattering curve to test its potential in the discrimination of fibrosis stages (F0, F1, F2, F3, and F4, METAVIR scale) from 20 in-vitro human liver samples, insonified at $20 \mathrm{MHz}$. The slope estimations were $(\mathrm{dB} / \mathrm{MHz})$ : 0.95,0.24 (F0), $1.15,0.43$ (F1), 1.20,0.26 (F3) and 1.07,0.33 (F4). The KolmogorovSmirnov test $(\mathrm{p}>0.05)$ indicated that the slope alone cannot discriminate all fibrosis groups (as well as the other mentioned parameters). When associated to integrated backscattered coefficient, discriminant analysis has correctly classified $80 \%$ of liver samples $(\mathrm{p}<0.0000)$. The misclassification resulted from some F0 samples grouped as F4 or vice-versa, which agrees with our previous results that suggested some parameter overlapping for normal and cirrhotic hepatic tissue. Nevertheless, combination of these parameters can help the diagnosis of liver fibrosis.

2pBBf2. Ultrasonic method to define human serum blood total protein and protein fractions. Anna Klemina (Dept. of Acoustics, Radiophysical Faculty, Nizhny Novgorod State University, 23 Gagarin Ave., 603950 Nizhny Novgorod, Russian Federation, klemina@rf.unn.ru)

Research of ultrasonic characteristics of biological liquids for purposes of medical diagnostics is the important task. Ultrasonic method to define total protein and protein fractions - albumin, $\alpha 1-, \alpha 2-, \beta-, \gamma$-globulin's of human serum blood is discussed. This method is based on the precise measurements of velocity and absorption of ultrasonic waves in serum blood and its modified solutions under different temperatures from range (15-40)0C. The measurements of ultrasonic velocity and absorption in samples serum blood fulfils by means of method ultrasonic interferometer constant length or resonator method. The precision of relative measurements of velocity by means of this method is $10-5$ and absorption is $10-3$, temperature stability is the order of 10-3 0C. Protein fractions define by means of decision of linear system of equations where in the character of unknown components consider these protein fractions. Comparison of data of different patient obtained by traditional electrophoreses method with data of ultrasonic method shows very high correlation of all protein fractions and total protein. This work was supported by RFBR and Leading Scientific Schools grants.

2pBBf3. Evaluation of performance in some ultrasonic procedures for non-invasive thermal estimation into hyperthermia phantoms. Ivonne Bazan (CINVESTAV, Dpto. Ingen. Electrica, Mexico DF, Mexico, 
ibazan@cinvestav.mx),Antonio Ramos(Instituto de Acustica CSIC, Serrano 144, 28006 Madrid, Spain, aramos@ia.cetef.csic.es), Monica Vazquez (UNAM, IIMAS, Mexico DF, Mexico, moni260577 @ yahoo.com.mx), Arturo Vera (CINVESTAV, Dpto. Ingen. Electrica, Mexico DF, Mexico, arvera@cinvestav.mx), Lorenzo Leija (CINVESTAV, Dpto. Ingen. Electrica, Mexico DF, Mexico, lleija@cinvestav.mx)

To dispose of a precise and non-invasive temperature measurement over the treated area becomes very important during hyperthermia treatments, in order to make possible an optimization of their healing effects. A performance analysis of some previously reported ultrasonic techniques, which were proposed for non-invasive temperature estimation, is made in this work over phantoms mimicking human tissues. A first technique is based on discrete scattering modeling for tissue characterization and spectral analysis of frequency distributions related to average scatters spacing [R. Seip \& E.S. Ebbini]. Other technique uses certain relations between tissue temperature changes and time-shifts in echoes due to thermally induced sound velocity changes and expansions [R. Maass-Moreno \& C.A. Damianou]. And finally, the third procedure analyzed here, is a recently proposed alternative, based on phase demodulation processing, to estimate the indirect effects of echoes time-shifts in the phase domain [M. Vázquez, A. Ramos, et al.]. The three options are analyzed for the same simulated \& measured multi-pulse echo patterns, looking for detecting possible advantages and inconveniences in each case. Finally, some improvement paths over the basic estimation methods are also explored.

2pBBf4. Sensitivity study of the acoustic nonlinearity parameter for measuring temperatures during High Intensity Focused Ultrasound treatment. Koen W. Van Dongen (Lab. of Acoustical Imaging and Sound Control, Faculty of Applied Sciences, Delft Univ. of Technology, PO Box 5046, 2600 GA Delft, Netherlands, K.W.A.vanDongen@tudelft.nl), Martin D. Verweij (Lab. of Electromagnetic Research, Faculty of Electrical Engineering, Mathematics and Computer Science, Delft Univ. of Technology, Mekelweg 4, 2628 CD Delft, Netherlands, m.d.verweij@tudelft.nl)

The aim of High Intensity Focused Ultrasound (HIFU) is to locally increase the temperature in a body. For an adequate application of the treatment it is important to measure non-invasively the temperature profiles in the heated region. Most efficiently, this is done with the same modality as being used for heating. Consequently, the preferred measuring method should rely on the temperature dependence of an acoustic medium parameter. The goal of this study is to determine which parameter is most sensitive to temperature changes. To find the most suitable parameter, the temperature dependence of the speed of sound (SOS), the density of mass and the acoustic nonlinearity parameter B/A are compared for water. The temperature dependence of the SOS and the mass density are obtained by interpolating values found in literature. Since measured values of the B/A parameter are only known for a few coarsely distributed temperature values, it has been synthesised from a two-dimensional function describing the SOS versus temperature and pressure. It turns out that the B/A parameter is far more sensitive for temperature changes than the other parameters. Comparison with measured B/A values confirms the idea that nonlinear acoustics is a favourite candidate to measure temperature profiles.

2pBBf5. The scattering of sound by a moving sphere in a stratified liquid. Jason Bach (Georgia Tech Lorraine - G.W. Woodruff School of ME, UMI Georgia Tech - CNRS 2958, 2 rue Marconi, 57070 Metz, France, jsbach82@yahoo.com), David Ku (Georgia Tech Lorraine - G.W. Woodruff School of ME, UMI Georgia Tech - CNRS 2958, 2 rue Marconi, 57070 Metz, France, david.ku@me.gatech.edu), Nico F. Declercq (Georgia Tech Lorraine - G.W. Woodruff School of ME, UMI Georgia Tech - CNRS 2958, 2 rue Marconi, 57070 Metz, France, nico.declercq@me.gatech.edu)

The interaction of sound with a sphere and the Doppler shift caused by scatterers in motion are well described in the literature. A numerical study is performed on the interaction of sound with a moving sphere and with multiple moving spheres. The study shows how the reflected sound fields are influenced by the relative motion and relative position of the spheres. The aim of the research is to discover efficient acoustic methods to detect and characterize moving spheres at different levels of complexity determined by the number of spheres, their relative velocities and the velocity of the sur- rounding liquid. At a first stage the interaction of sound with a single sphere in a stratified fluid flow is investigated. The research is performed in the framework of the use of ultrasound for biomedical applications.

2pBBf6. Two coupling media method for ultrasonic velocity measurement in biological tissues. Jérôme Mathieu (IES - Univ. Montpellier 2, place Eugène Bataillon, cc 082, 34095 Montpellier Cedex 5, France, mathieu@lain.univ-montp2.fr), Audrey Leydier (IES - Univ. Montpellier 2, place Eugène Bataillon, cc 082, 34095 Montpellier Cedex 5, France, leydier@lain.univ-montp2.fr)

Measuring the ultrasonic velocity in soft materials, especially in biological tissues, is never easy. One of the main problems is that the thickness of the sample in test is badly defined. From time-of-flight measurement method uncertainties in the thickness induce important uncertainties in experimental results. This causes that the ultrasonic velocity is often a poor criteria whereas it could be a good one. This paper brings a new method to measure the ultrasonic velocity in soft materials without knowing the sample thickness The main idea of this method is to use two different coupling media for making two relative time-of-flight measurements. The comparison of these two measurements gives the ultrasonic velocity in the sample in test. Furthermore, this comparison can give the local sample thickness of the sample. The uncertainty calculi show up that the effect on the thickness uncertainties is drastically reduced by using this new ultrasound method. This method is validated on reference samples, in silicon. Before concluding, some experimental results obtained with mouse skin samples are presented.

2pBBf7. Ultrasound elasticity assessment of in vivo human achilles tendons during a quick-release exercise. Marielle Defontaine (LUSSI, 10 Bd Tonellé, 37032 Tours, France, defontai@med.univ-tours.fr), Mourad Bentahar (ENSIM - LAUM, Université du Maine, rue Aristote, 72085 Le Mans, France, Mourad.Bentahar@univ-lemans.fr), Francis Canon (CNRS UMR6600, Centre de Recherche de Royallieu - UTC, 60200 Compiègne, France, francis.canon@utc.fr)

An ultrasound (US) axial transmission technique has been developed to assess in vivo real time tendon biomechanical properties during calibrated exercises. The US propagation velocity changes are indeed related to the tendon visco-elasticity variations. We propose to monitor the propagation velocity changes during a very short period of time corresponding to the tendon/muscle complex release just after a sub-Maximal Voluntary isometric Contraction (MVC). This measurement is repeated for several sub-MVC amplitudes. The propagation velocity variations, linearly related to the torque measurements, allow to estimate a stiffness index, essentially due to the Series Elastic Component of the Achilles tendon. This study has been performed in collaboration with the UMR 6600 (UTC, France), and we have used the Ergometer they developed a few years ago. The US device is composed of a $1.8 \mathrm{MHz}$ US probe (1 emitter, 20 receivers), and an electronic module (Althaïs Technologies, France) allowing real time and synchronous acquisitions of US RF signals and auxiliary inputs (Torque, angular position). Four healthy subjects were asked to develop several submaximal voluntary isometric contractions, and the sole was suddenly released (sub MVC to relaxed state, $2 \mathrm{~s}, 2 \mathrm{kHz}$ ). This protocol was repeated two times with foot and probe repositioning.

2pBBf8. Carotid Inter-adventitial Distance based Vascular Age and Heart Disease Risk Estimation from B mode Ultrasound. Jayaraj Joseph (Indian Institute of Technology, ESB 313 ; Dept. of Electrical Engineering, IIT Madras, 600036 Chennai, India, jayaraj85@yahoo .com), Kishore Kumar (Indian Institute of Technology, ESB 313 ; Dept. of Electrical Engineering, IIT Madras, 600036 Chennai, India kishorekumar015@yahoo.co.in), V Jayashankar (Indian Institute of Technology, ESB 313 ; Dept. of Electrical Engineering, IIT Madras, 600036 Chennai, India, jshankar@ee.iitm.ac.in)

A method to estimate the risk of heart disease in a person, based on diagnostic ultrasound is proposed here. The "vascular age" obtained from carotid ultrasound is very commonly used as a measure of cardiac health. Existing methods of vascular age estimation make use of the Carotid Intima Media Thickness (CIMT) measurements to arrive at an estimate of the vascular age. Recent work has demonstrated that the carotid inter adventitial 
distance $(\mathrm{CAD})$ has a direct relation with the risk of coronary heart disease in persons. It has also been demonstrated that CAD correlates better to a number of risk factors accounting for heart disease than the CIMT. Hence, we propose a method to estimate vascular age based on CAD. B mode images of the carotid artery are analyzed to find out the carotid inter adventitial distance. The vascular age is estimated from carotid diameter using a statistical model developed based on the Atherosclerosis Risk In Communities Limited access database. This vascular age is then used to modify standard risk assessment procedures like the Framingham risk equation to give a better estimate of coronary heart disease risk. A comparison of the method with the CIMT based method is also performed.

2pBBf9. Effect of inclination of articular surface on ultrasonic surface reflection and surface roughness in diagnosing cartilage degeneration. Erna Kaleva (University of Kuopio, Department of Physics, POB 1627, FI70211 Kuopio, Finland, erna.kaleva@uku.fi), Simo Saarakkala (University of Kuopio, Department of Physics, POB 1627, FI-70211 Kuopio, Finland, simo.saarakkala@uku.fi), Juha Töyräs (Kuopio University Hospital, POB 1777, 70211 Kuopio, Finland, Juha.Toyras@kuh.fi)

High-frequency Quantitative Ultrasound Imaging is a potential method for detecting early osteoarthrotic changes in articular cartilage. However, uncontrolled inclination of the ultrasound transducer or the curvature of the cartilage surface can jeopardize the reliability of the method. Visually intact and mechanically degraded osteochondral bovine patellae samples were imaged using a scanning acoustic microscope equipped with a $50 \mathrm{MHz}$ ultrasound transducer. The surfaces of the sample and transducer were adjusted to a known relative inclination in three sequential scans (0,2 and 5 degrees). Surface reflection was evaluated in time and frequency domains and surface roughness in time domain as a function of the inclination. Inclination of the surface of the sample had a greater effect on the reflection than on the roughness value. Despite the inclination-induced error, the degraded sample could be distinguished from the intact one at all inclinations with both the reflection and roughness parameters. However, as the inclination affected the reflection parameters significantly more, their reliability at greater inclinations is questionable. Furthermore, the proportion of scattering vs. reflection may change as a function of inclination. Provided that the amount of scattering varies against cartilage surface fibrillation, this can affect the reliability of ultrasound diagnostics significantly.

2pBBf10. Acoustothermometrical control during hyperthemia of biological objects. Andrej Anosov (Institute for Radio-Engineering and Electronics of the RAS, Starosadskij per, 8, 101000 Moscow, Russian Federation, aanosov@atom.ru), Aleksandr Kazanskij (Institute for RadioEngineering and Electronics of the RAS, Starosadskij per, 8, 101000 Moscow, Russian Federation, kazansky@ihome.ru), Anton Sharakshane (Institute for Radio-Engineering and Electronics of the RAS, Starosadskij per, 8, 101000 Moscow, Russian Federation, iva@hotmail.ru)

A number of acoustothermometrical experiments modelling hyperthermia of biological objects was carried out. After that the first preliminary acoustothermometrical measurement of laser hyperthermia of human tissues was conducted. In all experiments the changes of the thermal acoustic radiation intensity in 1.4-2.2 $\mathrm{MHz}$ region were controlled during both the heating and the cooling of the object (about 8-10 minutes) with the help of set of acoustothermometers. As model objects we used the beef liver and glycerin water solution in which plasticine was placed. We reconstructed the 2-D inner temperature distribution by using acoustothermometrical experimental data, i.e. we calculated position, size and temperature of thermal source. The object surface temperature was controlled with the help of IRthermograph. Acoustothermometrical measurements were carried out during laser hyperthemia of mammary and thyroid glands for seven patients. Obtained data allowed us to estimate inner temperature changes which were a result of laser hyperthemia.

2pBBf11. Measurement of dynamic shear modulus in soft solids using laser vibrometry. Maria Y. Izosimova (Center for Industrial and Medical Ultrasound, Applied Physics Lab., University of Washington, 1013 NE 40th
St., Seattle, WA 98105, USA, maria.izossimova@mail.ru),Alexandr I. Korobov (Dept. of Acoustics, Physics Faculty, M.V. Lomonosov Moscow State University, Leninskie gory 1, 119991 Moscow, Russian Federation, akor@acs465a-1.phys.msu.ru), Ekaterina V. Prokhorova (Center for Industrial and Medical Ultrasound, Applied Physics Lab., University of Washington, 1013 NE 40th St., Seattle, WA 98105, USA, e-v-p@ rambler.ru), Oleg A. Sapozhnikov (Center for Industrial and Medical Ultrasound, Applied Physics Lab., University of Washington, 1013 NE 40th St., Seattle, WA 98105, USA, oleg@acs366.phys.msu.ru)

Shear modulus is an important property of biological tissue and can be imaged for diagnostic purposes. A related application involves the acquisition of precise shear modulus measurements in excised soft tissue. Because the mechanical properties of tissue are frequency-dependent, it is important to develop methods that characterize the elasticity of soft solids at various frequencies. Here, two methods of measuring shear modulus are presented that employ laser vibrometry. At low frequencies $(1-10 \mathrm{~Hz})$, the tissue sample is fixed between plates so that the displacement of one plate induces a shear strain. Vibrometer measurements of the plate displacement define the induced strain so that shear modulus can be deduced if the applied shear force is known. At higher frequencies $(0.1-10 \mathrm{kHz})$, the sample lays flat on a surface and an impulsive force is applied to the exposed surface. Using the vibrometer to measure displacements along the exposed surface enables estimation of the surface wave speed and the implied shear modulus. To demonstrate the capabilities of these techniques, experiments were performed using plastisol tissue phantom samples. These experiments indicated a shear modulus that was $50 \%$ greater at high frequencies $(300 \mathrm{~Hz})$ than at low frequencies $(10 \mathrm{~Hz})$. Work supported by RFBR.

2pBBf12. Applying Genetic Algorithms to Optimise Breast Ultrasound Images Segmentation Procedure Based on Morphological Operators. Andre V. Alvarenga (Laboratory of Ultrasound - Inmetro, Av. N. Sra. das Gracas, 50, predio 1 - Xerem, 25250-020 Duque de Caxias - Rio de Janeiro, Brazil, avalvarenga@inmetro.gov.br), Wagner C. Pereira (Biomedical Engineering Program - COPPE, Federal University of Rio de Janeiro, CEP. 21 .941-972 Rio de Janeiro, Brazil, wagner@peb.ufrj.br), Antonio F. Infantosi (Biomedical Engineering Program - COPPE, Federal University of Rio de Janeiro, CEP. 21.941-972 Rio de Janeiro, Brazil, afci@peb.ufrj.br), Carolina M. Azevedo (Brazilian National Cancer Institute - INCa, Praça da Cruz Vermelha, 23, 20230-130 Rio de Janeiro, Brazil, azevedocma@bol.com.br)

Ultrasound (US) image segmentation is a complex problem due its textural nature. To address this difficulty, different segmentation procedures have been described to support radiologists in recognising abnormal USimage areas. This work proposes a procedure, based on Genetic Algorithms (GA) and Morphological Operators (MO), to segment breast tumours on ultrasound images. Each chromosome represents a complete image processing sequence, composed of MOs and structuring elements (SE). The GA generates 500 chromosomes of 93 genes, where the three first genes summed indicate the number of performed operations, the following 45 genes represent the operations' sequence, and the last 45, the SE-disk diameter (in pixel). The best segmentation sequence (best chromosome) is selected using an objective function, based on the weighted sum of the number of false-positive and false-negative pixels. The gold standards are the tumour contours depicted by an experienced radiologist. Twenty images are used to train the GA procedure, and the best chromosome tested with 40 different images. The performance of the procedure is assessed using the overlap ratio among the obtained segmentation and the gold standard. All tested images present an area overlap ratio superior to $70 \%$. This initial result encourages us to go further by increasing the number of images.

2pBBf13. Measuring physiological properties of lymphoedemous tissues by ultrasound: theoretical foundations. Paul E. Barbone (Boston University, 110 Cummington St, Boston, MA 02215, USA, 
barbone@bu.edu),Ricardo Leiderman(Federal University of Rio de Janeiro, Program of Mechanical Engineering, Ilha do Fundo, 21945-970, P.B. 68509 Rio de Janeiro, Brazil, leider@mecanica.coppe.ufrj.br), Jeff Bamber (Institute of Cancer Research, 15 Cotswold Road, Belmont, Sutton, SM2 5NG Surrey, UK, jeff.bamber@icr.ac.uk), Gearoid Berry (Institute of Cancer Research, 15 Cotswold Road, Belmont, Sutton, SM2 5NG Surrey, UK, gearoid.berry@icr.ac.uk), Assad A. Oberai (Rensselaer Polytechnic Institute, Mechanical, Aerospace and Nuclear Engineering, 5048 JEC, 110 8th Street, Troy, NY 12180, USA, oberaa@rpi.edu), Yixiao Zhang (Rensselaer Polytechnic Institute, Mechanical, Aerospace and Nuclear Engineering, 5048 JEC, 110 8th Street, Troy, NY 12180, USA, zhangy12@rpi.edu)

Roughly one in four breast cancer survivors report some degree of arm oedema. Lymphoedema is a build-up of excess lymph fluids in the tissues. Persistent lymphoedema leads to pain, diminished limb function, increased risk of infection, soft tissue fibrosis, and severe cases can be grossly disfiguring. From a mechanics perspective, the lymphoedemous tissue may be thought of as a two phase composite, consisting of both fluid and solid phases. Here we discuss the use of composites mixture theory to model the mechanics of lymphoedemous tissues. By treating the tissue as a fluid-solid composite, rules-of-mixtures may be used to estimate the effective moduli in terms of the properties of the individual components and their respective volume fractions in these two states. The mechanical properties of the tissue may be measured in vivo using a generalization of the methods of ultrasound elasticity imaging. We discuss how the measured "effective stiffness" depends upon whether the tissue is drained or undrained, and how ultrasound may be used to measure these properties. Thus we explore the possibility of evaluating volume fractions and component properties of the individual tissue phases from ultrasound elasticity imaging.
2pBBf14. Multilayered non-invasive temperature estimation from backscattered ultrasound. Cesar A. Teixeira (University of Algarve, Faculty of Sciences and Technology, Campus de Gambelas, 8005-139 Faro, Portugal, cteixei@gmail.com), Maria Graça Ruano (University of Algarve, Faculty of Sciences and Technology, Campus de Gambelas, 8005-139 Faro, Portugal, mruano@ualg.pt), António Ruano (University of Algarve, Faculty of Sciences and Technology, Campus de Gambelas, 8005-139 Faro, Portugal, aruano@ualg.pt), Wagner C. Pereira (Biomedical Engineering Program - COPPE, Federal University of Rio de Janeiro, CEP. 21.941-972 Rio de Janeiro, Brazil, wagner@peb.ufrj.br)

A major drawback on the application of thermal therapies is the lack of reliable knowledge about temperature in the region under treatment. The most attractive approach is non-invasive temperature estimation (NITE) strategies and, among them, techniques based on backscattered ultrasound (BSU) are a convenient choice for its portability and simplicity. Using BSU some methods have been proposed for temperature estimation basically for homogeneous media, and simple operating situations. More complex scenarios can be explored by non-linear approaches. In this work, a methodology based on neural networks is presented for temperature estimation in a multilayer phantom. A three-layer gel-based phantom was heated at eight intensities, between 0.30 and $2.0 \mathrm{~W} / \mathrm{cm}^{2}$ (at $1 \mathrm{MHz}$ ) for 15 minutes. Temperature and BSU signals were acquired from five spatial-points at each 10 seconds. Temporal echo-shifts, induced by temperature change, were computed from the BSU signals, and used as model's input information. Neural models were developed to estimate temperature at four intensities. Then models were validated in data including all the intensities. The best model presents a maximum absolute error of $0.43{ }^{\circ} \mathrm{C}$ for untrained situations. To the best of our knowledge this is the first NITE approach in multilayered media.

TUESDAY AFTERNOON, 1 JULY 2008

ROOM 353, 2:00 TO 7:00 P.M.

Session 2pEAa

\section{Engineering Acoustics: Silicon Microphones}

James West, Cochair

Johns Hopkins University, Department of Electrical Engineering, 3400 North Charles Street, Baltimore, MD 21218, USA

Gerhard Sessler, Cochair

Darmstadt University of Technology, Merckstrasse 25, 64283 Darmstadt, Germany

\section{Invited Paper}

2pEAa1. Overcoming Scaling Problems in Miniaturized Silicon Microphones. Sung B. Lee (Knowles Acoustics, 1151 Maplewood Dr, Itasca, IL 60143, USA, sung.lee@knowles.com), David E. Schafer (Knowles Acoustics, 1151 Maplewood Dr, Itasca, IL 60143, USA, dave.schafer@knowles.com), Peter V. Loeppert (Knowles Acoustics, 1151 Maplewood Dr, Itasca, IL 60143, USA, pete .loeppert@knowles.com)

In 2008 it is projected that global demand for mobile devices will reach 1.2 billion units. Compared to electronic gadgets from 2002 when 430 million cell phones were sold world wide, these devices are smaller, have more features, and will generally be priced lower. In order to keep up with designers and manufacturers who relentlessly pursue efficient use of space at lower cost, it is required that component manufacturers pursue the same goal. For a microphone manufacturer like Knowles, this led to the introduction of world's 
first commercialized surface-mountable microphone, the SiSonic ${ }^{\mathrm{TM}}$ microphone, in 2002. MEMS technology is used to manufacture the complex miniaturized components used in SiSonic ${ }^{\mathrm{TM}}$ microphone. Straightforward miniaturization of microphone diaphragms made of silicon, a common material in MEMS technology, instead of traditional diaphragm materials leads to poor performing microphones. To take advantage of MEMS technology without sacrificing microphone performance we developed the free plate technology. In this presentation we discuss the use of free plate technology to overcome difficulties related to miniaturization in MEMS.

\section{Contributed Paper}

2:20

2pEAa2. Silicon microphones: a commercial success? and what comes next? Stephen C. Thompson (Applied Reearch Laboratory, The Pensylvania State University, P.O. Box 30, State College, PA 16804, USA, sct12 @psu.edu)

The first silicon microphone commercial product was introduced to the market in late 2005. As of early 2008 the cumulative production is well over a half billion units and the production rates continue to rise. To date the acoustical performance of these microphones is only as good as that of the of the lowest priced miniature electret microphones. Yet acoustical systems manufacturers often prefer silicon microphones because they are compatible with automated insertion and wave soldering assembly methods. Silicon microphones are also more environmentally stable in sensitivity than are electrets which may be important in matched microphone applications. Current research activities are investigating methods to reduce the internal noise improve matching tolerances, and further reduce manufacturing costs. This paper will review the current state of silicon microphones in commercial manufacture, and will survey some of the areas of current research in an attempt to suggest some possible directions of future market development.

\section{Invited Paper}

2pEAa3. Optimization of miniaturized silicon microphones using a two-wafer approach. Jianmin Miao (Nanyang Technological University, School of Mechanical and Aerospace Engineering, 50 Nanyang Avenue, 639798 Singapore, Singapore, mjmmiao@ntu .edu.sg), Chee Wee Tan (Nanyang Technological University, School of Mechanical and Aerospace Engineering, 50 Nanyang Avenue, 639798 Singapore, Singapore, cheewee@ntu.edu.sg), Zhihong Wang (Nanyang Technological University, School of Mechanical and Aerospace Engineering, 50 Nanyang Avenue, 639798 Singapore, Singapore, ezhwang@ntu.edu.sg)

A two-wafer concept is proposed for the fabrication of silicon microphones with emphasis on deep reactive ion etching and wafer bonding techniques. For miniaturized sensor structures with an air gap of 1-2 microns, the viscous damping effect dominates the dissipation mechanism, which can have an adverse influence on the microphone performance, namely frequency response characteristic and mechanical-thermal noise. Therefore, an optimum microphone performance has its origin in a well-designed backplate structure. A silicon backplate with carefully placed acoustic slots and holes is attached to a silicon nitride/metal-based diaphragm. An impediment to achieve high sensitivity is the residual stress that is presented in the diaphragms. Besides the process optimization of less stress silicon nitride layer and the introduction of corrugated diaphragm, an investigation is carried out to determine the effects of sputtering parameters of $\mathrm{Cr} / \mathrm{Au}$ metal electrode film (thickness, sputtering process pressure and process power) on the residual stress of silicon nitride/metal diaphragm. Details of modeling, fabrication and experimental results will be presented.

\section{Contributed Papers}

\section{3:00}

2pEAa4. Piezoelectric Cantilevers for Low-Noise Silicon Microphones. Robert Littrell (University of Michigan, 2250 G G Brown Bldg, 2350 Hayward St., Ann Arbor, MI 48109, USA, rlittrel@umich.edu), Karl Grosh (University of Michigan, 2250 G G Brown Bldg, 2350 Hayward St., Ann Arbor, MI 48109, USA, grosh@umich.edu)

Microphones fabricated using microelectromechanical systems (MEMS) technology are one of the fastest growing applications of MEMS. Capacitive sensing has been the dominant detection principle used in MEMS microphones. Piezoelectric sensing, however, offers advantages including simpler accompanying circuitry and the possibility for simpler fabrication. Piezoelectric microphones have been limited primarily by a high noise floor, typically at least an order of magnitude higher than, otherwise similar, capacitive microphones. We present a low noise piezoelectric cantilever microphone to overcome the main limitation of previously constructed piezoelectric microphones. Aluminum Nitride (AlN) has been selected as the piezoelectric material because its piezoelectric coupling coefficient, in combination with its electric permittivity, and its piezoelectric loss coefficient enable low-noise devices. Through both mechanical and electrical optimization, models indicate that by combining several short, thin cantilevers made exclusively of Molybdenum and AlN, microphones with a die size of $1 \mathrm{~mm}$ $\mathrm{x} 1 \mathrm{~mm}, 10 \mathrm{kHz}$ bandwidth, $2 \mathrm{mV} / \mathrm{Pa}$ sensitivity, and noise floor below 40 $\mathrm{dBA}$ can be constructed using a simple 4 mask process. Analytical and numerical models and experimental results will be presented.

\section{3:20}

2pEAa5. A low-noise biomimetic differential microphone. Ronald N. Miles (State University of New York, PO 6000, Vestal Parkway East, Binghamton, NY 13902-6000, USA, miles@binghamton.edu), Quang T.
$\mathrm{Su}$ (State University of New York, PO 6000, Vestal Parkway East, Binghamton, NY 13902-6000, USA, be83190@binghamton.edu), Weili Cui (State University of New York, PO 6000, Vestal Parkway East, Binghamton, NY 13902-6000, USA, weilicui@yahoo.com), Stephen A. Jones (State University of New York, PO 6000, Vestal Parkway East, Binghamton, NY 139026000, USA,saj0716@gmail.com), F. Levent Degertekin (Georgia Institute of Technology, G. W. Woodruff School of Mechanical Engineering, 801 Ferst Dr. NW, Atlanta, GA 30332-0405, USA, levent.degertekin@me.gatech.edu), Baris Bicen (Georgia Institute of Technology, G. W. Woodruff School of Mechanical Engineering, 801 Ferst Dr. NW, Atlanta, GA 30332-0405, USA, baris@gatech.edu), Caesar Garcia (Georgia Institute of Technology, G. W. Woodruff School of Mechanical Engineering, 801 Ferst Dr. NW, Atlanta, GA 30332-0405, USA, caesar@gatech.edu), Neal A. Hall (Georgia Institute of Technology, G. W. Woodruff School of Mechanical Engineering, 801 Ferst Dr. NW, Atlanta, GA 30332-0405, USA, nahall@alumni.utexas.net)

A miniature differential microphone is described that has a noise floor that is substantially lower than that of existing devices of comparable size. The sensitivity of a differential microphone suffers as the distance between the two pressure sensing locations decreases, resulting in an increase in the input sound pressure-referred noise floor. In the microphone described here, the two sources of microphone internal noise, the diaphragm thermal noise and the electronic noise, are minimized by a combination of novel diaphragm design and the use of low-noise optical sensing. The differential microphone diaphragm measures $1 \mathrm{~mm}$ by $2 \mathrm{~mm}$ and is fabricated out of polycrystalline silicon. The diaphragm design is based on the coupled ears of the fly Ormia ochracea. The sound pressure input-referred noise floor of this miniature differential microphone has been measured to be less than 36 dBA. 


\section{Contributed Paper}

$5: 20$

2pEAa6. Thin film thermo-viscous damping in miniature condenser microphones. Thierry Le Van Suu (Laboratoire d'Acoustique de l’Université du Maine, Avenue Olivier Messiaen, 72085 Le Mans, France, thierry.levansuu@univ-lemans.fr), Petr Honzik (CVUT v Praze, Fakulta Elektrotechnická, K13137 Katedra Radioelektroniky - Technická 2, 16627 Praha 6, Czech Republic, Petr.Honzik@lf1.cuni.cz), Stephane Durand (Laboratoire d'Acoustique de l'Université du Maine, Avenue Olivier Messiaen, 72085 Le Mans, France, stephane.durand@univ-lemans.fr), Nicolas Joly (Laboratoire d'Acoustique de l'Université du Maine, Avenue Olivier Messiaen, 72085 Le Mans, France, nicolas.joly@univ-lemans.fr), Zdeněk Škvor (CVUT v Praze, Fakulta Elektrotechnická, K13137 Katedra Radioelektroniky - Technická 2, 16627 Praha 6, Czech Republic, skvorzdn@feld.cvut.cz), Michel Bruneau (Laboratoire d'Acoustique de l'Université du Maine, Avenue Olivier Messiaen, 72085 Le Mans, France, michel.bruneau@univ-lemans.fr)
The thermo-viscous damping due to the thin fluid film between the membrane and the backing electrode strongly influences both the sensitivity of the condenser microphones in the lower frequency range and the upper limit of the frequency bandwidth. Nowadays, most of the MEMs microphones use a perforated backing electrode while some authors suggest that a continuously curved backing electrode could enhance their performances (among avantages in the design when etching). The present paper provides two kinds of modeling for such microphones with a tapered fluid film: the first one lies on Kirchhoff-network analysis (neglecting cross-coupling between elements) whereas the second one is based upon the direct resolution of the set of basic equations (including heat transfer phenomena). The results are presented and discussed for both models in the cases of flat, parabolic, and stepped shapes backing electrode. Finally, the pressure field in the fluid film, computed (for an axisymmetrical configuration) using the abovementioned models, is compared to the one computed with a new FEM formulation taking into account both viscous and thermal phenomena in the boundary layers.

\section{Invited Paper}

\section{$5: 40$}

2pEAa7. Miniature diffraction-based optical MEMS microphones with integrated optoelectronics. Levent Degertekin (Georgia Institute of Technology, G. W. Woodruff School of Mechanical Engineering, 801 Ferst Dr. NW, Atlanta, GA 30332-0405, USA, levent@gatech.edu), Kamran Jeelani (Georgia Institute of Technology, G. W. Woodruff School of Mechanical Engineering, 801 Ferst Dr. NW, Atlanta, GA 30332-0405, USA, gtg804t@mail.gatech.edu), Shakeel Qureshi (Georgia Institute of Technology, G. W. Woodruff School of Mechanical Engineering, 801 Ferst Dr. NW, Atlanta, GA 30332-0405, USA, shakeel@ece.gatech.edu), Paul Hasler (Georgia Institute of Technology, School of Electrical and Cumputer Engineering, 777 Atlantic Drive NW, Atlanta, GA 30332-0250, USA, phasler@ece.gatech.edu), Baris Bicen (Georgia Institute of Technology, G. W. Woodruff School of Mechanical Engineering, 801 Ferst Dr. NW, Atlanta, GA 30332-0405, USA, baris@gatech.edu), Weili Cui (State University of New York, PO 6000, Vestal Parkway East, Binghamton, NY 13902-6000, USA, weilicui@yahoo.com), Quang T. Su (State University of New York, PO 6000, Vestal Parkway East, Binghamton, NY 13902-6000, USA, be83190@binghamton.edu), Ronald N. Miles (State University of New York, PO 6000, Vestal Parkway East, Binghamton, NY 13902-6000, USA, miles@binghamton.edu)

Diffraction-based optical interferometric sensing has been shown to be a low-noise displacement detection method for MEMS microphones. For this method to be useful in many important applications such as hearing aids, it needs to be packaged in a small volume and should have power consumption levels suitable for operation with a battery. In this talk, we describe miniature, packaged optical microphones that use solid state vertical cavity surface emitting lasers (VCSELs) as light sources and custom designed photodetectors. The package carries silicon chips with two micromachined biomimetic differential microphones as well as an omnidirectional microphone. It is made by 3-D laser stereo lithography process and has dimensions suitable for behind-the-ear hearing aids. With this configuration, the input referred noise floor for the differential microphone is measured as $42.6 \mathrm{dBA}$, limited by the VCSEL intensity noise in this particular case. In addition to miniature packaging, an optoelectronic chip including VCSEL pulser, photodetectors, transimpedance amplifiers and 1 bit Sigma-Delta ADC has been implemented in 1.5V, 0.35u CMOS technology. The second order ADC structure providing 14-bits of theoretical resolution with 64 over sampling ratio and $20 \mathrm{kHz}$ input signal is described and initial characterization results are presented.

\section{Contributed Papers}

6:00

2pEAa8. On determination of microphone response and other parameters by a hybrid experimental and numerical method. Salvador Barrera-Figueroa (Danish Fundamental Metrology, Matematiktorvet 307, 2800 Kgs. Lyngby, Denmark, sbf@dfm.dtu.dk), Finn Jacobsen (Acoustic Technology Department, Technical University of Denmark, Ørsted Plads, B352, DK-2800 Lyngby, Denmark, fja@oersted.dtu.dk), Knud Rasmussen (Danish Fundamental Metrology, Matematiktorvet 307, 2800 Kgs. Lyngby, Denmark, kra@dfm.dtu.dk)

Typically, numerical calculations of the pressure, free-field and randomincidence response of a condenser microphone are carried out on the basis of an assumed displacement distribution of the diaphragm of the microphone; the conventional assumption is that the displacement follows a Bessel function. This assumption is probably valid at frequencies below the resonance frequency. However, at higher frequencies the movement of the membrane is heavily coupled with the damping of the air film between membrane and back plate, and with resonances in the back chamber of the microphone. A solution to this problem is to measure the velocity distribution of the membrane by means of a non-contact method, such as laser vibrometry. The measured velocity distributions can be used together with a numerical formulation such as the Boundary Element Method for estimating the microphone response and other parameters such as the acoustic centres. In this work, a hybrid method is presented. The velocity distributions of condenser Laboratory Standard microphones were measured using a laser vibrometer. This measured velocity distribution was used for estimating the microphone responses and parameters. The agreement with experimental data is good. This method can be used as an alternative for validating the parameters of the microphones determined by classical calibration techniques. 
6:20

2pEAa9. Diffraction based optical MEMS microphones and accelerometers with active electrostatic force feedback. Baris Bicen (Georgia Institute of Technology, G. W. Woodruff School of Mechanical Engineering, 801 Ferst Dr. NW, Atlanta, GA 30332-0405, USA, baris@gatech.edu), Caesar Garcia (Georgia Institute of Technology, G. W. Woodruff School of Mechanical Engineering, 801 Ferst Dr. NW, Atlanta, GA 30332-0405, USA, caesar@gatech.edu), Neal A. Hall (Georgia Institute of Technology, G. W. Woodruff School of Mechanical Engineering, 801 Ferst Dr. NW, Atlanta, GA 30332-0405, USA, nahall@alumni .utexas.net), Murat Okandan (Sandia National Laboratories, New Mexico, PO Box 5800, Albuquerque, NM 87185-1080, USA, mokanda@sandia .gov), Weili Cui (State University of New York, PO 6000, Vestal Parkway East, Binghamton, NY 13902-6000, USA, weilicui@yahoo.com), Quang T. Su (State University of New York, PO 6000, Vestal Parkway East, Binghamton, NY 13902-6000, USA, be83190@binghamton.edu), Ronald N. Miles (State University of New York, PO 6000, Vestal Parkway East, Binghamton, NY 13902-6000, USA, miles@binghamton.edu), Levent Degertekin (Georgia Institute of Technology, G. W. Woodruff School of
Mechanical Engineering, 801 Ferst Dr. NW, Atlanta, GA 30332-0405, USA, levent@gatech.edu)

Diffraction-based optical displacement detection method and its use in low noise micromachined microphones have been shown earlier. [Hall et al., J. Acoust. Soc. Am. 118, 3000-3009 (2005), Garcia et al., J. Acoust. Soc. Am. 121, 3155 (2007)]. In these devices, the integrated electrostatic port of the sensor is uncoupled from the integrated optical sensing. This structure enables one to use this port for sensitivity tuning, self characterization, and active control to adjust the device dynamics. Given that the displacement noise of integrated optical sensor is below the thermal-mechanical noise of the mechanical structure, one can implement force feedback methods such as active Q-control, or adjust device stiffness without adding substantial noise to the system. We implemented micromachined optical microphones and accelerometers with integrated optoelectronics integrated in a $1.5 \mathrm{~mm} 3$ volume. We present experimental results on force feedback Q-control of low noise omnidirectional, and biomimetic directional optical microphones, as well as adjusting the stiffness of accelerometers to improve their frequency response. [Work supported by NIH Grant 5R01DC005762-03 and the Catalyst Foundation.]

\section{Invited Paper}

\section{$6: 40$}

2pEAa10. Piezoelectric silicon microphones for aeroacoustics applications. Mark Sheplak (University of Florida, $231 \mathrm{MAE}-\mathrm{A}$ Building, PO Box 116250, Gainesville, FL 32611-6250, USA, sheplak@ufl.edu)

This talk presents the development of several microelectromechanical systems (MEMS)-based piezoelectric measurement microphone technologies for aeroacoustic applications. Piezoelectric MEMS microphones offer the promise of reducing cost, improving performance, and increasing mounting flexibility over existing conventional microphone technologies. Specifically, a microphone with no external power requirement has a key advantage for a large-channel count, widespread deployment. The modeling and design aspects of these devices are reviewed. First, the electroacoustic transduction is predicted via piezoelectric composite plate theory. Lumped element models are then synthesized to describe the dynamic characteristics of the microphone diaphragm and the cavity/vent structure. Constrained nonlinear design optimization using a sequential quadratic programming scheme is then performed to determine the microphone design parameters. From a fabrication perspective, the main drawback to silicon-micromachined piezoelectric devices is material integration and compatibility with standard micromachining. Several different device structures and fabrication approaches will be discussed in this talk. Representative results from a lead zirconate-titanate-based device will then be presented. Finally, unresolved technical issues are summarized for future sensor development. 


\title{
Session 2pEAb
}

\section{Engineering Acoustics and Signal Processing in Acoustics: Microphone Array Signal Processing II (Poster Session)}

\author{
Gary Elko, Cochair \\ mh acoustics LLC \\ Walter Kellermann, Cochair \\ Univ. of Erlangen-Nuremburg
}

\begin{abstract}
All posters will be on display from 3:40 p.m. to 5:20 p.m. To allow contributors an opportunity to see other posters, contributors of odd-numbered papers will be at their posters from 3:40 p.m. to 4:30 p.m. and contributors of even-numbered papers will be at their posters from 4:30 p.m. to 5:20 p.m.
\end{abstract}

\section{Contributed Papers}

2pEAb1. Advanced Processing of Microphone Array Data for Engineering Applications. Sandro Guidati (HEAD acoustics $\mathrm{GmbH}$, Ebertstrasse 30a, 52134 Herzogenrath, Germany, claudia.erens@headacousitcs.de)

Microphone arrays are tools for the localisation and quantification of sound sources. The use of the technology is strongly related to the progresses in computer technology. For a long time the application was limited to military or scientific use. The focus of the systems designed for industrial applications lies on fast setup and basic evaluation (e.g. delay-andsum beamforming). The resulting boundaries are mainly a limited dynamic range and the limitation to free field environments. Although a wide range of algorithms for advanced evaluation has been developed, only few of them are integrated in industrial systems. This is mainly due to the fact that these algorithms require considerably longer computation time, expert knowledge and the integration of additional hardware. This paper presents techniques for almost real-time processing of microphone array data including multiband beamforming, coherence/incoherence filtering and the integration of measured source characteristics for the application in strongly reverberant environments. By combining the signals of multiple cameras it is possible to detect the distance between the array and a three dimensional source distribution increasing the accuracy of the localisation and quantification.

2pEAb2. Instrumentation Synchonization Techniques for Large Microphone Arrays. Kurt Veggeberg (National Instruments, $11500 \mathrm{~N}$. Mopac C, Austin, TX 78759, USA, kurt.veggeberg@ni.com)

In many acoustic measurement applications, there is a need to correlate data acquired from different systems or synchronize systems together with precise timing. Signal Based and Time Based are the two basic methods of synchronizing instrumentation. In Signal Based synchronization, clocks and triggers are physically connected between systems. Typically this provides the highest precision synchronization. In many acoustic applications size and distance constrains physically connecting the systems needed for making measurements though the inter-channel phase information of simultaneously sampled signals is crucial. In Time Based synchronization, system components have a common reference of what time it is. Events, triggers and clocks can be generated based on this time. This is an overview of how you can use a variety of time references including GPS, IEEE-1588, and IRIG-B to correlate and synchronize measurements anywhere in the world with absolute timing with and without a direct connection between the measurement systems. The level of precision of the variety of methods that can be used for timestamping, generating a trigger at a user specified time as well as synchronizing multiple instrumentation types is covered. Specific applications where this has been applied to large microphone arrays will be described.

2pEAb3. Differential microphone array for speech recognition. Philippe Martin (Ecole Polytechnique Fédérale de Lausanne, EPFL STI LEMA, Station 11, CH 1015 Lausanne, Switzerland, philippe.martin@epfl.ch), Hervé Lissek (Ecole Polytechnique Fédérale de Lausanne, EPFL STI LEMA, Station 11, CH 1015 Lausanne, Switzerland, herve.lissek@epfl.ch), John Dines (IDIAP, Rue du Simplon 4, 1920 Martigny, Switzerland, john.dines@idiap.ch)

Speech recognition applications embedded on a PDA are already available on the market. The usual hardware for this kind of system is a single microphone mounted on the PDA, giving good results within quiet environments. Though, the recognition rate falls drastically as the signal to noise ratio decreases. Arrays of microphones are then particularly interesting, allowing the discrimination of useful sounds sources within parasitic ones, thanks to an improved directivity pattern. In speech recognition applications on PDA, a trade-off is to be found on an array small enough to guarantee the ergonomics of the whole system, while operating on a bandwidth covering the frequency range of human voice, from $300 \mathrm{~Hz}$ up to $6 \mathrm{kHz}$ and low distortion ratio. Differential arrays are well adapted to fulfill these specifications, since they are known to be robust and allow small dimensions for a high directivity index. The performances could be improved by the addition of adaptive post-filtering and noise reduction algorithms. This work describes the design and the implementation of a recording device dedicated to speech recognition applications on PDA, based on overlapping differential arrays. The assessment of its performances in a noisy environment is carried out and show the system efficiencies.

2pEAb4. Speech separation based on law of causality. Kensaku Fujii (University of Hyogo, 2167 Shosha, 671-2280 Himeji, Japan, fujiken@eng.u-hyogo.ac.jp), Hirofumi Nakano (University of Hyogo, 2167 Shosha, 671-2280 Himeji, Japan, er06j025@steng.u-hyogo.ac.jp), Mitsuji Muneyasu (Kansai University, 3-3-35 Yamate-cho, 564-8680 Suita, Japan, muneyasu@ipcku.kansai-u.ac.jp)

This paper proposes a microphone array system separating speech signals, based on a different principle from independent component analysis (ICA). This system applies linear prediction error filters to microphone outputs, and using the prediction errors, adjusts the coefficients of adaptive filters. In this case, only the prediction errors satisfying the low of causality become available for the adjustment; consequently, this system can steer a null toward a direction satisfying it. The permutation problem discussed in 
ICA can be thereby avoided. This system also can compensate the separated speech signals by using adaptive filters, and finally, can provide high quality speech signals. This paper moreover verifies the performance of the proposed system by using speech signal data measured in an ordinary room. This result shows that the proposed system works well even in reverberation environment.

2pEAb5. Time-Shift Sensor Noise Suppression Algorithm. Alain De Cheveigne (CNRS, Universite Paris 5, Ecole Normale Superieure, 29 rue d’Ulm, 75230 Paris, France, alain.de.cheveigne@ens.fr)

We propose a new method to remove sensor noise from multichannel data recordings. Each channel is projected on the span of the time-shifted neighboring channels, and replaced by its projection. Noise specific to any sensor is attenuated, whereas any signal component that loads more than one sensor is preserved. The inclusion of a range of time shifts allows the method to compensate for any delay or convolutional mismatch in the signal paths between sensors. The method is expected to be of use for microphone arrays to reduce sensor-specific noise, e.g. thermal noise, wind noise, or overloading. The new method extends a previous method for sensor noise suppression in physiological recordings (EEG, MEG), that performed the same operation without the delays.
2pEAb6. Acoustic payload on an aerostat detects and locates transient sources. Michael V. Scanlon (US Army Research Laboratory, AMSRDARL-SE-SA, 2800 Powder Mill Road, Adelphi, MD 20783-1197, USA, mscanlon@arl.army.mil), Christian Reiff (US Army Research Laboratory, AMSRD-ARL-SE-SA, 2800 Powder Mill Road, Adelphi, MD 20783-1197, USA, creiff@arl.army.mil), Latasha Solomon (US Army Research Laboratory, AMSRD-ARL-SE-SA, 2800 Powder Mill Road, Adelphi, MD 207831197, USA, 1solomon@arl.army.mil)

The US Army Research Laboratory (ARL) has conducted experiments using acoustic sensor arrays suspended below tethered aerostats to detect and localize transient signals from mortars, artillery, and small arms fire. The airborne acoustic sensor array calculates an azimuth and elevation to the originating transient, and immediately cues a collocated imager to capture the remaining activity at the site of the acoustic transient. This single array's vector solution defines a ground-intersect region or grid coordinate for threat reporting. Unattended ground sensor (UGS) systems can augment aerostat arrays by providing additional solution vectors from several ground-based acoustic arrays to perform a 3D triangulation on a source location. The aerostat array's advantage over ground systems is that it is not as affected by diffraction and reflection from man-made structures, trees, or terrain, and has direct line-of-sight to most events. Helicopter detection and tracking algorithms can also be implemented.

\title{
Session 2pEAc
}

\section{Engineering Acoustics: Acoustic Evaluation II (Poster Session)}

\begin{abstract}
All posters will be on display from 3:40 p.m. to 5:20 p.m. To allow contributors an opportunity to see other posters, contributors of odd-numbered papers will be at their posters from 3:40 p.m. to 4:30 p.m. and contributors of even-numbered papers will be at their posters from 4:30 p.m. to 5:20 p.m.
\end{abstract}

\section{Contributed Papers}

2pEAc1. Mechanism detection of stress corrosion cracking by acoustic emission and effect of manufacturing process on AE signals. Hossein Heidary (Polytechnic of Tehran, AmirKabir University of Technology, Department of Mechanical Engineering, NDT Lab., 424 Hafez Ave, 158754413 Tehran, Iran, hosseinheidary@gmail.com), Amir Refahi Oskouei (Polytechnic of Tehran, AmirKabir University of Technology, Department of Mechanical Engineering, NDT Lab., 424 Hafez Ave, 15875-4413 Tehran, Iran, amir.refahi@cic.aut.ac.ir), Mehdi Ahmadi (Polytechnic of Tehran, AmirKabir University of Technology, Department of Mechanical Engineering, NDT Lab., 424 Hafez Ave, 15875-4413 Tehran, Iran, ahmadin@cic.aut .ac.ir)

Stress corrosion cracking could occur wherever a specific corrodent and sufficient tensile stresses coexist. In the objective to monitor online the SCC on real structures, it seems reasonable to characterize and recognize acoustic emission during static U-bend tests. The present study is concerned with static tests on 304 stainless steels in two different media $(5 \% \mathrm{HCl}$ and $5 \%$ $\mathrm{H} 2 \mathrm{SO} 4)$ in order to find a criterion to distinguish, the two different mechanism of SCC (anodic dissolution and hydrogen embrittlement) by characteristic parameters and waveform analysis of $\mathrm{AE}$ signals. In the next stage, effect of residual stress on stress corrosion cracking in chloride solution has been studied. Three samples have been prepared: one of them was stress relieved; the second one was made sensitive to SCC by heat treatment and the last one was cold worked by rolling. Cumulative count, amplitude distribution and waveform analysis were selected as AE-parameters. AE with amplitudes ranging from 39 to $65 \mathrm{~dB}$ with different counts and energy occurred during SCC of SS-304 at room temperature. According to the results, acoustic emission is able to be used as robust technique for mechanism detection of SCC. In addition it can be used to measure the effect of residual stress in manufacturing process on SCC.

2pEAc2. Underground Sonar Using Shear Waves -Resolution improvement Using Pulse Compression and Dynamic Focusing-. Hiraku Kawasaki (Toin University of Yokohama, 1614 Kurogane-cho, Aoba-ku, 225-8502 Yokohama, Japan, hot.hu2.feel.good@gmail .com), Tsuneyoshi Sugimoto (Toin University of Yokohama, 1614 Kurogane-cho, Aoba-ku, 225-8502 Yokohama, Japan, tsugimot@cc.toin.ac .jp)

In order to detect the buried relics and ruins at shallow depth, a method by using shear waves has proposed. The effect of the pulse compression processing is examined for improving the underground imaging resolution. Using a super-magnetostriction vibrator which can change waveforms and use high frequency waves, we generate both chirp waves and high frequency waves. To confirm the resolution of underground images, pulse compression is simulated. And, to confirm the lateral resolution, dynamic focusing is simulated in our laboratory. Then, its exploration experiment is carried out where the buried position has already known. The axial resolutions are smaller than $0.2 \mathrm{~m}$ when the range of start and stop frequencies are more 
than $700 \mathrm{~Hz}$ in the simulation. And the underground images obtained from exploration experiment confirm that the images by the pulse compression have almost the same axial resolution as by the result of the simulation.

2pEAc3. PVB mechanical constants characterization in laminated glasses using low frequency ultrasound. Jaime Ramis Soriano (DFISTS. Univ. de Alicante, Carretera de Sant Vicent del Raspeig s/n, 03690 San Vicente del Raspeig, Spain, jramis@ua.es), Jorge Frances Monllor (DFISTS. Univ. de Alicante, Carretera de Sant Vicent del Raspeig s/n, 03690 San Vicente del Raspeig, Spain, jfmonllor@ua.es), Jesús Alba Fernandez (Escola Politècnica Superior de Gandia, Universitat Politècnica de València, Crtra Natzaret-Oliva s/n, 46730 Gandia, Spain, jesalba@fis.upv.es), Enrique E. Segovia Eulogio (Depto Ing. Construcción. Univ. de Alicante, Carretera de Sant Vicent del Raspeig s/n, 03690 San Vicente del Raspeig, Spain, Enrique.Gonzalo@ua.es), Jenaro Vera Guarinos (DFISTS. Univ. de Alicante, Carretera de Sant Vicent del Raspeig s/n, 03690 San Vicente del Raspeig, Spain, jenaro@dfists.ua.es)

To predict the behaviour of a partition made of laminated glasses, it's necessary to know the parameters of the layers composing it. These types of devices are composed by monolithic glass and an intermediate muffling layer usually made of PVB. In this study, we present a method to find the mechanical constants of PVB in laminated glasses. Mechanical constants characterization in these materials is studied using a model-based inverse problem and the data obtained from an ultrasonic transmission setup: a numerical simulation of the system is proposed using a lineal finite elements model of the ultrasonic propagation on the multilayered solid. Parameters are obtained by minimizing divergences between experimental and numerically predicted waveform

2pEAc4. Ultrasonic Technique for the Quality Control of Water Containing Clay. Adil Hamine (Ibn Zohr University, FS Agadir, 80000 Agadir, Morocco, adil.hamine@Gmail.com), Bouazza Faiz (Ibn Zohr University, FS Agadir, 80000 Agadir, Morocco, faizbou@ hotmail.com), Driss Izbaim (Ibn Zohr University, FS Agadir, 80000 Agadir, Morocco,
driss_izbaim@yahoo.fr),Ali Moudden(Ibn Zohr University, FS Agadir, 80000 Agadir, Morocco, ali_moudden@yahoo.fr)

In this work a new method is developed to control the stopping water using an ultrasonic technique. This technique allows controlling the presence of clay grains by measuring the attenuation of the ultrasonic waves. The dimension of clay grains used in this study is about $10 \mu \mathrm{m}$. For slurries with different weight percent of clay $1 \%$ or less, high sensitivity is gained by analyzing attenuation measurements. At high clay concentrations, sufficient sensitivity is obtained by analyzing data from a simple transmission. The experimental results show that the sound attenuation due to particles varies linearly with mass fraction.

2pEAc5. Acoustic Computerized Tomography for Temperature Distribution Measurement in Rectangular Space. Ayumu Minamide (Grad. Sch. of Sys. Inf. Eng., Univ. of Tsukuba, 1-1-1, Tennodai, 305-8573 Tsukuba, Japan, minamide@aclab.esys.tsukuba.ac.jp), Naoto Wakatsuki (Tsukuba Univ., Tsukuba Science City, 305-8573 Ibaraki, Japan, wakatuki@iit.tsukuba.ac.jp), Koichi Mizutani (Tsukuba Univ., Tsukuba Science City, 305-8573 Ibaraki, Japan, mizutani@esys.tsukuba.ac.jp)

In this research, we propose an improved algorithm of Acoustic Computerized Tomography to measure temperature distribution in rectangular space. Temperature distribution measurement is used in various fields, such as, air-conditioning control in offices, temperature control in greenhouse. If acoustic transducers are fixed, they are ordinarily arranged at equal intervals on a circle. However, it is difficult to take circular arrangements of transducers in the rectangular spaces. Thus, we devise to arrange the transducers on a rectangle. Therefore, a problem is occurs that projection data could not be obtained at equal angular intervals. To solve this problem, we propose non-uniform angular interval Back-Projection to weight the projection data with respect to its angular intervals. We confirm usefulness of the proposed method by numerical simulations. In the simulations, 24-acoustic transducers are arranged at equal intervals on a rectangle whose aspect ratio is two. By using the proposed method, the shape approaches to the given temperature distribution, and RMS error of the reconstructed distribution to the given distribution decreases about $25 \%$ compared to the error of conventional CT. 


\title{
Session 2pEAe
}

\section{Engineering Acoustics, Underwater Acoustics, Signal Processing in Acoustics, and ECUA: Sensor Technologies for Autonomous Acoustic Sensing Systems II (Poster Session)}

\author{
Henrik Schmidt, Cochair \\ MIT \\ Andrea Caiti, Cochair \\ University of Genova
}

\begin{abstract}
All posters will be on display from 3:40 p.m. to 5:20 p.m. To allow contributors an opportunity to see other posters, contributors of odd-numbered papers will be at their posters from 3:40 p.m. to 4:30 p.m. and contributors of even-numbered papers will be at their posters from 4:30 p.m. to 5:20 p.m.
\end{abstract}

\section{Contributed Papers}

2pEAe1. High Accuracy Multisensor Hydroacoustic System for Models of Trawl Geometry Measurements. Jacek Marszal (Gdansk University of Technology, Narutowicza 11/12, 80-952 Gdansk, Poland, marszal@eti.pg .gda.pl)

The new high accuracy multisensor hydroacoustic system for models of trawl geometry measurements was designed for developing the construction of cod trawl net for the Baltic fishery. The system consist of 20 miniature hydrophones attached by the light-gauge cables to the measuring microprocessor device and notebook computer. The pulse excitation at high frequency and correlation digital signal processing make it possible to obtain high resolution of measurements. Additionally each of hydrophones is equipped with the thermistor which is used to calculate the local sound velocity and enable precision calibration of obtained measururing data. This method of calibration is particularly important for measurements investigated in the surface water layer with the high temperature gradient. The construction details, the measuring signal processing algorithms as well as examples of obtained data and the accuracy verification will be presented in the paper.

2pEAe2. Ultra-deep low-frequency sub-bottom profiler for AUV and ROV. Yves Le Gall (IFREMER, BP 70, 29280 Plouzané, France, Yves.Le.Gall@ifremer.fr), Anne Pacault (IFREMER, BP 70, 29280
Plouzané, France, Anne.Pacault@ifremer.fr),Frédéric Mosca(Ixsea, 46 Quai François Mitterrand, 13600 La Ciotat, France, fmo@ixsea.com), Gilles Greninguey (Ixsea, 46 Quai François Mitterrand, 13600 La Ciotat, France, ggr@ixsea.com), Marcel Vial (Ixsea, 46 Quai François Mitterrand, 13600 La Ciotat, France, mvi@ixsea.com), Jean-Claude Aissa (Ixsea, 46 Quai François Mitterrand, 13600 La Ciotat, France, jca@ixsea.com)

Acoustical techniques for ultra-deep sub-bottom exploration are still little investigated. IXSEA and IFREMER have launched in 2006 the development of a new deep-sea low-frequency sub-bottom profiler, working up to 6000 meters depth. Based on the Janus-Helmholtz broadband technology, two acoustic sources have been modelled, achieved and in-tank and at-sea measured : a single transducer working in the $[1.8,6.2 \mathrm{kHz}]$ frequency band, and a three-transducer array operating in the $[2,8 \mathrm{kHz}]$ frequency band. In both cases, vertical resolution is better that $20 \mathrm{~cm}$. Comparison between finite element modelling and in-tank measurements is presented, together with the specific constraints linked to very-deep water and AUV mounting. Power electronics and impedance matching unit have been specifically optimised to deliver a sound level of $190 \mathrm{~dB}$ (ref. $1 \mu \mathrm{Pa} @ 1 \mathrm{~m}$ ), with 48 VDC supply voltage and $250 \mathrm{~W}$ available electric power. The receiving part is made up of a three-hydrophone array with a loss of sensitivity lower than 1 $\mathrm{dB}$ between 0 and 600 bar. This ultra-deep light system has been mounted on an IFREMER's AUV and the first results at sea are presented and discussed. 


\title{
Session 2pEAf
}

\section{Engineering Acoustics and Psychological and Physiological Acoustics: Hearing Aid Engineering II (Poster Session)}

\author{
Daniel Warren, Cochair \\ Knowles Electronics \\ Stefan Launer, Cochair \\ Phonak $A G$
}

\begin{abstract}
All posters will be on display from 3:40 p.m. to 5:20 p.m. To allow contributors an opportunity to see other posters, contributors of odd-numbered papers will be at their posters from 3:40 p.m. to 4:30 p.m. and contributors of even-numbered papers will be at their posters from 4:30 p.m. to 5:20 p.m.
\end{abstract}

\section{Contributed Papers}

2pEAf1. Two-input two-output speech enhancement with binaural spatial information using a soft decision mask filter. Satoshi Hongo (Faculty of Design and Computer Applications, Miyagi National College of Technology, 48, Nodayama, Medeshima Shiote, 981-1239 Natori, Japan, hongo@miyagi-ct.ac.jp), Ai Sasaki (R.I.E.C., Tohoku University, 2-1, Katahira, Aoba-ku, 980-8577 Sendai, Japan, asasaki@ais.riec.tohoku.ac.jp), Shuichi Sakamoto (R.I.E.C., Tohoku University, 2-1, Katahira, Aoba-ku, 980-8577 Sendai, Japan, saka@ais.riec.tohoku.ac.jp), Junfeng Li (Japan Advanced Institute of Science and Technology, 1-1, Asahidai, Nomi, 923-1292 Ishikawa, Japan, junfeng@jaist.ac.jp), Yôiti Suzuki (R.I.E.C., Tohoku University, 2-1, Katahira, Aoba-ku, 980-8577 Sendai, Japan, yoh@ais.riec.tohoku.ac.jp)

A two-input two-output speech enhancement method that preserves binaural spatial information in the output is effective for realizing a comfortable auditory communication system. Such a system benefits from the noise reduction capability provided by the signal processing technology, in addition to the binaural processing of the human auditory system. We investigated a two-input two-output speech enhancement method that calculates soft decision mask filters to attenuate a noisy time-frequency bin. The soft decision mask filter is estimated for each direction of arrival (DOA) based on a target noise ratio calculated using an adaptive filter that cancels the target signal. Results of computer simulations show that the proposed method has superior capabilities for maintaining spatial information in the two-output signals and for segregating the target signal in arbitrary azimuth and elevation DOA.

2pEAf2. Improvement of speech intelligibility by audio hearing systems. Hannes Seidler (Clinic of ORL, Dept. of Medicine, TU Dresden, Fetscherstrasse 74, 01307 Dresden, Germany, Hannes.Seidler@tu-dresden .de)

All hearing aids and cochlea implants have algorithm to improve the speech intelligibility. The idea is to share human speech and noise to find different ratings. The result should be easier to understand by impaired people. On the market there are big efforts to recognize speech and to separate it from noise. In this field we can notice a remarkable progress in the last years. But the reverberant sound in rooms or noise from same direction like the signal need a lot of work to suppress them effective. The simplest way seems to be to gets the original speech signal direct from the source and to processes it individual in hearing devices. Induction loop systems as well as wireless infrared or radio frequency systems are used in churches, cinemas, theatres and conference rooms. But the available systems are very different in costs and benefit for management and clients. If these systems are used there is a considerable improvement of speech intelligibility. The presentation will show the level of most used external audio hearing systems, their possibilities to improve the signal to noise ratio, the speech transmission index (STI) and the benefit for hearing aid or cochlear implant users. 


\title{
Session 2pEAg
}

\section{Engineering Acoustics, Underwater Acoustics, and ECUA: Sonar Transducer Design and Modeling III (Poster Session)}

\author{
John Blottman, Cochair \\ Naval Undersea Warfare Center, Division Newport \\ Pascal Mosbah, Cochair \\ IEMN dpt ISEN
}

\begin{abstract}
All posters will be on display from 3:40 p.m. to 5:20 p.m. To allow contributors an opportunity to see other posters, contributors of odd-numbered papers will be at their posters from 3:40 p.m. to 4:30 p.m. and contributors of even-numbered papers will be at their posters from 4:30 p.m. to 5:20 p.m.
\end{abstract}

\section{Contributed Papers}

2pEAg1. Finite element and boundary element modeling of multimodal arrays. Julien Bernard (Thales Underwater Systems, 525 route des Dolines BP 157 Valbonne Parc d'Activités de Sophia Antipolis, 06903 Sophia Antipolis Cedex, France, julien.bernard@fr.thalesgroup.com)

Modern active sonar arrays exhibit the following characteristics: low operating frequency, large number of transducers, small transducer spacing compared to wavelength, and in some cases, multimodal transducers. This results in strong, complex acoustic interaction phenomena which can involve both a large number of array elements and multiple transducer modes. To predict the performance of such arrays, this paper presents a modeling approach which is both accurate and computationally economical. First, the generic equivalent circuit for an array of multimodal transducers is described. The field of application of such a model, the definition of the parameters, and the characteristics which can be calculated from it are provided. Second, the computation of the equivalent circuit parameters is performed using commercial finite element and boundary element codes. Specifically, a method is described to compute the mechanical, electrical, self and mutual radiation parameters of the various transducer modes. Finally, the methodology is applied to a 12 element volumetric array of flextensional transducers, with both $\lambda / 2$ and $\lambda / 4$ spacings.

2pEAg2. The design of a wideband and widebeam piston transducer in a finite closed circular baffle. Zekeriyya Sahin (Aselsan A.S., Mehmet Akif Ersoy Mah. 16.Cad. no:16, Macunkoy, 06370 Ankara, Turkey, zsahin@aselsan.com.tr), Hayrettin Koymen (Bilkent Univ., Dept. of Electrical and Electronics Engineering, 06800 Ankara, Turkey, koymen@ee .bilkent.edu.tr)

The design of a high power piezoelectric underwater transducer operating at frequency range $45 \mathrm{kHz}-85 \mathrm{kHz}$ with acoustic power capability in excess of $150 \mathrm{~W}$ is described. The transducer consists of two back-to-back elements. Each element is formed by stacked PZT-4 ceramic rings, a matching and a steel backing layer, and placed in a finite rigid circular baffle. We investigate the dependence of bandwidth and beamwidth to the combination of piston and baffle radii, a and b, respectively. With a ka of 2.45 (k:wave number) and b/a ratio of 2, the transducer resonates at $65 \mathrm{kHz}$ with $70 \%$ bandwidth and has a beamwidth of $60^{\circ}$ at each half space. We show that when two transducers are placed at right angles spatially and driven in parallel, we can obtain an omnidirectional beam pattern horizontally in the lower frequency band. The beam pattern exhibits two dips in each quadrant at the higher end of the frequency band, which are within $7 \mathrm{~dB}$. We also investigated power handling capability of the transducer from thermal point of view using finite element analysis. The input impedance measurements agree well with the numerical results within the pass band.

2pEAg3. A lossy, one-dimensional, linear systems model for piezoelectric transducers containing opposing zones of polarization. Sivaram Nishal Ramadas (Institute of Sound and Vibration Research, University of Southampton, University Road, Highfield, S017 1BJ Southampton, UK, nishal@ieee.org)

Inversion layer transducers (ILT), consisting of two or more active, piezoelectrically opposed regions along their thickness direction, have attracted considerable interest in the recent past, due to the potential for wide bandwidth and even harmonic sensitivity. However, design and manufacture of such ILT devices are not straightforward due to the large number of degree of freedom involved. This paper presents the development of a lossy, computationally efficient analytical model to design ILT configuration. The mathematical formulation for a generalized linear systems model, applicable to arbitrary ILT configurations is presented. The model describes multiple active and passive layers, including zones of variable piezoelectric polarization, matching layers and bondlines. The model is extended further to include mechanical wave absorption in both the active transducer element and the propagating channel, and the effect of such frequency dependant loss on the operational characteristics of an ILT device is reported. Several simulation results are shown to demonstrate the feasibility of the approach together with a comparisonal analysis to conventional finite modeling techniques and experimental evaluations. The results indicate clearly that such approximate analytical techniques can be used effectively to design optimal ILT configurations.

2pEAg4. An analytical model for piezoelectric/elastic axisymmetric bender disks radiating in water. Raphaël Lardat (Thales Underwater Systems, 525 Route des Dolines, BP 157, 06930 Sophia Antipolis cedex, France, raphael.lardat@fr.thalesgroup.com)

Flextensional transducers have been widely studied for low frequency SONAR application due to the low celerity of the flexion wave. In this work, we will describe in detail a complete analytical model for an axisymmetric disk made of one elastic plate on which two piezoelectric disks of smaller radius have been reported on each side. The model, starting from flexural axisymmetric theory and from the piezoelectric equations, finally comprises local radiation impedance and mutual coupling with neighboring disks. In particular, we will explain the continuity conditions that are to be imposed at the external radius of the piezoelectric disk. We also show how to include a radiation force opposed and proportional to the normal velocity 
inside the analytical solution given by a fourth order differential equation. The described model is then able to compute a large number of parameters such as Eigen modes, harmonic admittance, radiated pressure, coupling coefficient and static and dynamic stresses. The validity of the model is as- sessed by comparison to solutions of the coupled problem in water using classical FEM/BEM. We will show an extremely good agreement enabling us to use this simple model for geometry optimization and design improvement.

TUESDAY AFTERNOON, 1 JULY 2008

P2-D, LEVEL 2, 3:40 TO 5:20 P.M.

\title{
Session 2pMUa
}

\section{Musical Acoustics and Physical Acoustics: Brass Instrument Acoustics II (Poster Session)}

\author{
Thomas Moore, Cochair \\ Rollins College \\ Joël Gilbert, Cochair \\ Laboratoire d'Acoustique de l'Université du Maine
}

\begin{abstract}
All posters will be on display from 3:40 p.m. to 5:20 p.m. To allow contributors an opportunity to see other posters, contributors of odd-numbered papers will be at their posters from 3:40 p.m. to 4:30 p.m. and contributors of even-numbered papers will be at their posters from 4:30 p.m. to 5:20 p.m.
\end{abstract}

\section{Contributed Papers}

2pMUa1. Pedal notes of brass instruments, a mysterious regime of oscillation. Joël Gilbert (Laboratoire d'Acoustique de l'Université du Maine, Avenue Olivier Messiaen, 72085 Le Mans, France, joel.gilbert@univ-lemans.fr), Pierre Aumond (Lab. d'Acoustique Université du Maine, UMR CNRS 6613, 72085 Le Mans Cedex 9, France, pierre .aumond.etu@univ-lemans.fr)

In reed and brass instruments, sound is produced by self-sustained mechanical oscillations driven by an airflow coming from a pressure supply such as the player's lungs. Most of the time, the fundamental frequency of a periodic oscillation is close to, and mainly controlled by one given acoustic mode. Even though, these oscillations are the result of a complex non-linear coupling between all the acoustic modes and the mechanical valve. The pedal note, which is the lowest note that can be sounded on a brass instrument, is well-known as a counter-example. Eighty years ago, Bouasse did an experiment by replacing the brass mouthpiece by a reed mouthpiece on a brass instrument, and obtained a pedal note a fifth below! An elementary model dedicated to both cane-reed and lip-reed instruments can be used. In order to investigate the sound production of pedal notes, a simulation method based on modal decomposition of bore and reed dynamics has been carried out. Then, the periodic solutions of this model are obtained, and the mysterious oscillation regimes of Bouasse experiment are discussed.

2pMUa2. Nonlinear effects in the propagation of outgoing and reflected pulses inside a trombone. Pablo L Rendón (CCADET, Universidad Nacional Autónoma de México, Ciudad Universitaria, 04510 Mexico, Mexico, pablo.rendon@ccadet.unam.mx), Felipe Orduña-Bustamante (CCADET, Universidad Nacional Autónoma de México, Ciudad Universitaria, 04510 Mexico, Mexico, felipe.orduna@ccadet.unam.mx), Jacques Sorrentini (Institut Fresnel, UMR 6133 CNRS, Campus de Saint Jérôme, Av. Escadrille Normandie 13397, Cedex 20 Marseille, France, jacques.sorrentini@ec-marseille.fr), Antonio Pérez-López (CCADET, Universidad Nacional Autónoma de México, Ciudad Universitaria, 04510 Mexico, Mexico, antonio.perez@ccadet.unam.mx)

While sound propagation in a trombone can be generally quite properly described by linear models, it is commonly acknowledged that the "brassiness" of sound produced at high amplitudes is associated to nonlinear effects. Whether these effects are due principally to propagation inside a long, narrow pipe, or to the production of sound at the mouthpiece is still unclear. We present experimental measurements taken both from a long, narrow tube, and an actual slide trombone from which it is observed that the scale of nonlinear effects seems to be greater for outgoing pulses than for the reflected pulses at the open end of the trombone or tube. A theoretical model is also proposed, based on weakly nonlinear perturbations of linear theory, and is validated both by experimental results and by numerical simulations. The results indicate that both the amplitude and the shape of the initial perturbation produced at the trombone mouthpiece have an effect on the scale of propagation nonlinearity present inside the trombone resonator, and thus also on the "brassy" quality of sound.

2pMUa3. External sound radiation of vibrating trombone bells Guillaume Nief (Laboratoire d'Acoustique de l'Université du Maine, Avenue Olivier Messiaen, 72085 Le Mans, France, guillaume .nief.etu@univ-lemans.fr), François Gautier (Laboratoire d'Acoustique de l'Université du Maine, Avenue Olivier Messiaen, 72085 Le Mans, France, francois.gautier@univ-lemans.fr), Jean-Pierre_Dalmont (Laboratoire d'Acoustique de l'Université du Maine, Avenue Olivier Messiaen, 72085 Le Mans, France, Jean-Pierre.Dalmont@univ-lemans.fr), Joël Gilbert (Laboratoire d'Acoustique de l'Université du Maine, Avenue Olivier Messiaen, 72085 Le Mans, France, joel.gilbert@univ-lemans.fr)

The issue of the influence of bell vibrations on the sound of brass instruments is still debated. For such instruments, external sound field is the superposition of the sound field resulting from acoustic oscillations of the air column and the sound field resulting from direct radiation of the bell and walls. The aim of this paper is to quantify the bell contribution with respect to the air column one, and to examinate the conditions for which the former may become audible. For this purpose, the structural modes of a trombone bell are identified using an experimental modal analysis. For each mode shape, the radiation efficiency is computed using a model of the radiated sound based on a distribution of equivalent monopoles. A critical frequency is defined for the bell and allows us to determine at which condition a given structural mode is radiating. Measurements of the sound power radiated by a trombone excited by an harmonic acoustic source are carried out when the bell is free to vibrate and when bell vibrations are damped. Comparison between the two configurations is analyzed using the developed model and is used to quantify the sound power due to bell vibrations. 
2pMUa4. Acoustic radiation of wind instruments resonators. Bastien Mallaroni (Laboratoire de Mécanique et d'Acoustique - CNRS, 31 chemin Joseph Aiguier, 13402 Marseille, France, mallaroni@lma.cnrs-mrs .fr), Pierre-Olivier Mattei (Laboratoire de Mécanique et d'Acoustique CNRS, 31 chemin Joseph Aiguier, 13402 Marseille, France, mattei@lma.cnrs-mrs.fr), Philippe Herzog (Laboratoire de Mécanique et d'Acoustique - CNRS, 31 chemin Joseph Aiguier, 13402 Marseille, France, herzog@1ma.cnrs-mrs.fr)

Optimization of the acoustic radiation of wind instruments resonators may be based on the study of finite length circular ducts radiating in unbounded medium, as this is a model realistic enough, but which allows advanced analytics developments. Our approach is based on the calculation of resonance frequencies of such a simplified wind instrument. These are the complex frequency singularities of the operator describing the global resonator/external fluid system. A Newton's method is used to search for the singularities of the multimodal Green's function of the duct. This is obtained by the product of impedance matrices which relate pressure and velocity between two abscissae inside the duct. A variable coupling factor is introduced with external acoustic radiation, described by a multimodal radiation impedance based on the Zorumski formulation. Examples of variation of the resonance frequencies with this coupling factor will be given. Surprisingly, regular variations of the coupling lead to non regular evolution of the resonances. Physical interpretation of this behaviour will be discussed.

2pMUa5. Transient behaviour of human and artificial brass players' lips. Samuel Stevenson (Edinburgh University, 4201 JCMB, Kings Buildings, Mayfield Road, EH9 3JZ Edinburgh, UK, samuel.stevenson @ed.ac.uk), Donald M. Campbell (Edinburgh University, 4201 JCMB, Kings Buildings, Mayfield Road, EH9 3JZ Edinburgh, UK, d.m.campbell@ed.ac.uk), John Chick (Edinburgh University, 4201 JCMB, Kings Buildings, Mayfield Road, EH9 3JZ Edinburgh, UK, john.chick@ed.ac.uk), Joël Gilbert (Laboratoire d'Acoustique de l'Université du Maine, Avenue Olivier Messiaen, 72085 Le Mans, France, joel.gilbert@univ-lemans.fr), Stefan Bilbao (University of Edinburgh, Room 7306B, JCMB, King's Bldgs., Mayfield Rd., EH9 3JZ Edinburgh, UK, sbilbao@staffmail.ed.ac.uk)

It is widely accepted that the starting transient is an extremely significant perceptual feature of musical sound. For players, the ease with which a note can be started on a particular instrument is also of great importance. However, until recently research has concentrated on the analysis of steady state sounds rather than the study of the starting behaviour of the instrument. The work reported here uses a high speed camera to capture the motion of lip reeds during the starting transient. Simultaneous recordings are made of the pressure signals generated in the mouthpiece of the instrument and radiated from the bell. Preliminary results on human players, already presented, have been extended and supplemented by measurements using an artificial mouth in order to separate effects due to the instrument from those dependent on the expertise of a particular human player. Computational simulations have been compared to these experimental findings.

2pMUa6. Tristan trumpet or English horn? Robert W. Pyle (S. E. Shires Co., 11 Holworthy Place, Cambridge, MA 02138, USA, rpyle@post.harvard.edu), Sabine K. Klaus (National Music Museum, The
University of South Dakota, PO Box 190, Landrum, SC 29356, USA, sabineklaus@alltel.net)

In Act III of his opera "Tristan und Isolde", Richard Wagner wrote an instrumental tune to be (apparently) played by a shepherd. This was originally scored for, and is customarily played by, an off-stage cor anglais. Wagner indicated that he would have preferred some form of wooden trumpet, similar to a small-scale alphorn. A few such instruments have been built. These are pitched in 4-foot $\mathrm{C}$, with a pear-shaped bell similar to that of the cor anglais, and a single metal whole-tone valve. The Utley Collection at the National Music Museum, The University of South Dakota, has two such instruments: one made by Gebrüder Alexander (Mainz), formerly used in the National Opera in Prague (Volkstheater), the other a copy of a trumpet by Martin Lehner, Munich, in the possession of the Staatsoper Dresden, made by Andreas Schöni and Rainer Egger (Bern and Basel). We will compare sound spectra of the Tristan trumpet with that of the cor anglais. We will also compare the acoustic input impedance of the Tristan trumpet with that of an ordinary trumpet of the same pitch.

2pMUa7. Harmonicity of trumpet modes. Peter L. Hoekje (Baldwin-Wallace College, 275 Eastland Rd., Berea, OH 44017, USA, phoekje@bw.edu), Michael Attaway (Richardson ISD, 400 S. Greenville Ave., Richardson, TX 75081, USA, michael_attaway@hotmail .com)

The mouthpiece on a brass instrument is generally removable but constitutes an important part of the whole instrument. In the frequency domain, it can be considered to provide a frequency dependent equivalent length that increases with frequency up to the Helmholtz resonance frequency $f_{\text {pop }}$ of the mouthpiece itself. Geometrical changes that affect the total volume or the $f_{\text {pop }}$ of the mouthpiece will affect the harmonicity of the nearly harmonic normal modes of the instrument. Using heterodyne filter analysis, the development of the frequency components and their amplitudes were measured for low trumpet notes, both for repeated attacks of short notes and for sustained note crescendos from pianissimo to fortissimo. The measurements were repeated after the mouthpiece was modified to improve the harmonicity of the normal modes. During both attack and crescendo tests, the modified mouthpiece was associated generally with more stable frequencies of the partials and more even development of the amplitudes of the partials.

2pMUa8. The influence of braces on the playability of trumpets. Alexander Mayer (Inst. f. Wiener Klangstil, Univ. f. Music, Anton von Webernplatz 1, A-1030 Vienna, Austria, mayer@mdw.ac.at), Rainer Egger (Blechblas-Instrumentenbau Egger, Turnerstrasse 32, 4058 Basel, Switzerland, info@eggerinstruments.ch), Gregor Widholm (Inst. f. Wiener Klangstil, Univ. f. Music, Anton von Webernplatz 1, A-1030 Vienna, Austria, Widholm@mdw.ac.at)

The influence of braces on the playability, response and sound properties of the trumpet is an intense discussed subject for players and instrument makers. In addition to the problem of finding the proper position of the braces, inserted braces can produce stress between the instruments tubes. In this paper the influence of the tube stress is investigated. To ensure an adjustable and reproducible stress between the trumpet tubes special adjustable braces in conjunction with a force monitor are used. The results of blind playing tests are compared with spectral analysis of the played sounds. In addition, the acoustic input impedance, the wall vibrations and the transfer function of the instrument are investigated. 


\title{
Session 2pMUb
}

\section{Musical Acoustics: Edge Tone and Flue Pipes II (Poster Session)}

\author{
Shigeru Yoshikawa, Cochair \\ Kyushu University \\ Judit Angster, Cochair \\ Fraunhofer Institute for Building Physics
}

\begin{abstract}
All posters will be on display from 3:40 p.m. to 5:20 p.m. To allow contributors an opportunity to see other posters, contributors of odd-numbered papers will be at their posters from 3:40 p.m. to 4:30 p.m. and contributors of even-numbered papers will be at their posters from 4:30 p.m. to 5:20 p.m.
\end{abstract}

\section{Contributed Papers}

2pMUb1. Vortex sound of the flute and its interpretation. Andreas Bamberger (Physics Institute University Freiburg, Hermann-Herder-Str. 3, 79104 Freiburg, Germany, bamberger@physik.uni-freiburg.de)

The flute as an example of flue instruments is investigated for its properties of the jet-labium interaction at $1 \mathrm{kHz}$. The jet and the externally excited acoustic flow in the embouchure especially near the labium is recorded with endoscopic Particle-Image-Velocimetry. Extracting the vorticity the data allows us to calculate the vortex sound according to M. How (1975) The far field acoustic power of the flute is measured as well and compared with the vortex sound power, which matches in sign and magnitude up to a factor 2 for different sound levels. The non-trivial interpretation of the sound production is based on the near cancellation of the two vortex layers of the jet along its path except near the labium. In order to gain insight of the interplay of the different ingredients of the vortex sound power the coriolis force, the acoustic field and their phase relation are determined. Scaling of laws for these quantities as a function of the jet velocity/pressure of the standing wave are deduced and compared with the acoustic powe dependence. A comparison with recently available numerical simulations (ISMA 2007) is discussed.

2pMUb2. Experimental investigation of the aeroacoustic coupling of self-sustained tones to non-planar modes of a resonator. Martin Glesser (CNRS - LMA, 31 Chemin Joseph Aiguier, 13009 Marseille, France, glesser@1ma.cnrs-mrs.fr), Vincent Valeau (Laboratoire d'Etudes Aérodynamiques (LEA), Université de Poitiers - ENSMA - CNRS, Bâtiment K, 40 Avenue du Recteur Pineau, F-86022 Poitiers, France, vincent.valeau@lea.univ-poitiers.fr), Anas Sakout (LEPTIAB Université de La Rochelle, Avenue Michel Crépeau, 17042 La Rochelle Cedex 01, France, asakout@univ-lr.fr)

Recent studies have shown that a jet-slot oscillator (ie, an aeroacoustic self-sustained source consisting of the impingement of the vortical structures of a plane jet on a slotted plate) can be coupled to non-planar modes of the flow-supply duct. This phenomenon occurs in the particular case where the jet-exit and the obstacle are misaligned in the spanwise direction. This paper presents an experimental investigation of the excited modes as a function of the plate inclination angle, and of the distance between the jet-exit and the slot. It is shown that: (i) the vortex-tubes spanwise morphology is governed by the nature of the transverse excited modes, and (ii) the vortices tend to impinge on the inclined obstacle almost in phase in the spanwise direction, in order to enhance the coherence of the interaction along the slot. Moreover, an elementary analytical model of the resonant acoustic field shows that the ratio between the transverse and planar modes amplitudes is tuned by the system in order to keep this coherence maximum. The existence of a so-called "opposed transverse mode" is also shown, generating vortices with an inclination angle opposed to the plate inclination angle.

2pMUb3. Open and closed loop characterization of an air-jet reflection amplifier. John C. Price (University of Colorado, Department of Physics, 390 UCB, Boulder, CO 80309-0390, USA, john.price@ colorado.edu)

Our laboratory has embarked on an effort to understand the steady-state behavior of a Yamaha YRT-304B tenor recorder. The instrument is split at the head-joint, and both sides are studied with a multi-microphone reflectometer using sinusoidal excitation. For the head joint, data is obtained versus frequency, blowing pressure, and excitation amplitude. We have two goals: First, to use our open-loop reflectometry data to understand the internal acoustic spectrum of the assembled instrument, as a function of blowing pressure and fingering; and second, to provide a well-defined set of openloop observables for comparison with hydrodynamic calculations of the airjet dynamics. This particular instrument is attractive for several reasons: it disassembles at a straight cylindrical section of the bore, and so is easily coupled to straight waveguide; it is large enough for accurate reflection coefficient and internal pressure measurements; it can be studied closed-loop as a complete instrument; and it represents a fiducial geometry that is readily available to any laboratory. 


\title{
Session 2pMUc
}

\section{Musical Acoustics: Acoustic Measurements on Wind Instruments II (Poster Session)}

\author{
Noam Amir, Cochair \\ Tel Aviv University \\ David B. Sharp, Cochair \\ Open Univeristy
}

\begin{abstract}
All posters will be on display from 3:40 p.m. to 5:20 p.m. To allow contributors an opportunity to see other posters, contributors of odd-numbered papers will be at their posters from 3:40 p.m. to 4:30 p.m. and contributors of even-numbered papers will be at their posters from 4:30 p.m. to 5:20 p.m.
\end{abstract}

\section{Contributed Papers}

2pMUc1. Sound power level measurement of Sheng, a Chinese wind instrument. Yue Zhe Zhao (State Key Laboratory of Subtropical Building Science, South China University of Technology, 381 Wushan Street, 510640 Guangzhou, China, arzhyzh@scut.edu.cn), Shuo Xian Wu (State Key Laboratory of Subtropical Building Science, South China University of Technology, 381 Wushan Street, 510640 Guangzhou, China, arshxwu@ @cut .edu.cn), Jian Zhen Qiu (State Key Laboratory of Subtropical Building Science, South China University of Technology, 381 Wushan Street, 510640 Guangzhou, China, qjianzhen@gmail.com), Li Ling Wu (Dept. of Musicology, Xinghai Conservatoire of Music, 510500 Guangzhou, China, wushijun@scut.edu.cn), Hong Huang (Dept. of Musicology, Xinghai Conservatoire of Music, 510500 Guangzhou, China, yyxyhuanghong@163.com)

Sheng is one of the Chinese traditional wind instruments. But its sound power level has never been carefully measured. In this paper, the sound power measurements of Sheng were performed for the first time in a reverberation chamber according to ISO standard and Chinese national standard. Two qualified musicians performed on their own instruments in the center of the reverberation chamber. The radiated sound energy and the dynamic ranges of the Sheng were investigated by four channel acoustic measuring equipments. Typical sound power values were obtained through averaging and the results were reported in this paper. It was showed that the mean forte sound power level can reach up to $98 \mathrm{~dB}$ with a dynamic range of $22.5 \mathrm{~dB}$ when music scale was performed. The method discussed here is valuable for the sound power measurements of other musical instruments. The measurement of the sound power radiated by national musical instruments lays foundations for the investigation into the acoustics of national music halls.

2pMUc2. Measurements of woodwind tone-hole parameters using a double impedance head method. Paul Dickens (University of New South Wales, Music Acoustics, School of Physics, NSW 2052 Sydney, Australia, PDickens@resmed.com.au), Yakov Kulik (University of New South Wales, Music Acoustics, School of Physics, NSW 2052 Sydney, Australia, ykulik@phys.unsw.edu.au), Joe Wolfe (University of New South Wales, Music Acoustics, School of Physics, NSW 2052 Sydney, Australia, J.Wolfe @unsw.edu.au)

A woodwind tone hole is often represented as a T-junction with a shunt and series impedance. We measured the frequency dependence of the series and shunt impedances of open and closed tone holes using a pair of impedance heads, one on either side of a symmetric section of short bore pipe with a finger hole, and each calibrated on resonance-free loads. The shunt impedance is most accurately measured when the hole is located at a pressure antinode (speakers in phase) and the series impedance at a pressure node (speakers in anti-phase). We use both conditions, in this way, to measure series and shunt impedances for all frequencies studied. Pipes with the same length and diameter, but having wall thicknesses 1.5-5.0 $\mathrm{mm}$ and tone hole diameters 1.5-15.0 mm were used. For open holes, results are compared with calculations and results measured using other methods. The results for holes closed with fingers are also used to calculate the effective length of finger intrusion. Examples of the inclusion of the results into woodwind models are given.

2pMUc3. Investigation of clarinet reed auto-oscillations with digital Fresnel holography. Denis Mounier (LPEC/UMR 6087/CNRS/Université du Maine, Avenue Olivier Messiaen, 72085 Le Mans Cedex 09, France, denis.mounier@univ-lemans.fr), Pascal Picart (Laboratoire d'Acoustique de l'Université du Maine, Avenue Olivier Messiaen, 72085 Le Mans, France, pascal.picart@univ-lemans.fr), Julien Leval (Laboratoire d'Acoustique de l'Université du Maine, Avenue Olivier Messiaen, 72085 Le Mans, France, julien.leval.etu@univ-lemans.fr), Francis Piquet (Laboratoire d'Acoustique de l'Université du Maine, Avenue Olivier Messiaen, 72085 Le Mans, France, francis.piquet@univ-lemans .fr), Jean-Pierre Boileau (Laboratoire d'Acoustique de l'Université du Maine, Avenue Olivier Messiaen, 72085 Le Mans, France, jean-pierre.boileau@univ-lemans.fr), Thomas Guimezanes (Laboratoire d'Acoustique de l'Université du Maine, Avenue Olivier Messiaen, 72085 Le Mans, France, thomas.guimezanes@univ-lemans.fr), Jean-Pierre Dalmont (Laboratoire d'Acoustique de l'Université du Maine, Avenue Olivier Messiaen, 72085 Le Mans, France, Jean-Pierre.Dalmont@univlemans.fr)

This paper describes a full field digital holographic method for studying the cinematic of the vibration of the clarinet reed in playing conditions in an artificial mouth. Since many years, LAUM is interested in studying musical instruments and particularly the clarinet. The behaviour of such an instrument is particularly complex because it depends on aerodynamics, contact between reed and beak, and interaction with the lip of the musician. Thus, visualisation of the full movement of the reed in playing conditions is necessary to better understand physics of the clarinet. However, free oscillations have high amplitude of several hundreds of micrometers, thus corresponding to several thousand times the laser wavelength. The analysis of the movement is performed by a laser tracking of the vibration. It is demonstrated that it is possible to reconstruct a synthetic high amplitude deformation of auto-oscillations encoded with digital Fresnel holograms. The setup is applied to the auto-oscillation of the clarinet reed in a synthetic mouth. Tracking of the vibration is performed by using the pressure signal delivered by the mouth. Experimental results show the four steps of the reed movement and especially emphasize the shocks of the reed on the mouthpiece. 


\title{
Session 2pMUd
}

\section{Musical Acoustics: Interaction Between Instrument and Instrumentalist II (Poster Session)}

\author{
Gary Scavone, Cochair \\ Schulich School of Music, McGill University \\ Xavier Boutillon, Cochair \\ Laboratoire de Mécanique des Solides
}

\begin{abstract}
All posters will be on display from 3:40 p.m. to 5:20 p.m. To allow contributors an opportunity to see other posters, contributors of odd-numbered papers will be at their posters from 3:40 p.m. to 4:30 p.m. and contributors of even-numbered papers will be at their posters from 4:30 p.m. to 5:20 p.m.
\end{abstract}

\section{Contributed Papers}

2pMUd1. Chest wall dynamics and respiratory muscle recruitment during flute playing. Isabelle Cossette (McGill University, 555, Sherbrooke St, Montréal, QC H3A 1E3, Canada, isabelle.cossette1@mcgill.ca), Pierpaolo Monaco (McGill University, 555, Sherbrooke St, Montréal, QC H3A 1E3, Canada, pierpal@yahoo.it), Andrea Aliverti (Politecnico di Milano, Dipartimento di Bioingegneria, P.zza Leonardo da Vinci, 32, 20133 Milano, Italy, andrea.aliverti@polimi.it), Peter Macklem (PO Box 250, Lansdowne, ON K0E 1L0, Canada, peter.macklem @ gmail.com)

Respiratory parameters and sound were recorded during professional flute playing in order to assess what physiological processes were associated with the control of sound production that results in 'breath support' which in turn is associated with high quality playing. Four standing young professional flautists played flute excerpts with different technical requirements with and without breath support. Recordings included optoelectronic plethysmographic measurements of chest wall volume (Vcw) and its compartments, surface electromyography of the scalene, lateral abdominal, rectus abdominus, parasternal and sternocleidomastoid muscles, mouth pressure, and sound. Flow was estimated from differentiating Vcw during playing. Comparison in between various types of playing were performed. Results showed that flute support entails antagonistic contraction of nondiaphragmatic inspiratory muscles that tends to hold the rib cage at higher lung volume during long legato phrase playing. This relieves the expiratory muscles from the task of producing the right mouth pressure, especially a the end of the phrases, so they can contribute more to the finer control of mouth pressure modulations required for high quality playing.

2pMUd2. A concise overview of present knowledge of bowed-string gestures and their acoustical consequences. Knut Guettler (Norwegian Academy of Music, P.O.Box 5190 Majorstuen, 0302 Oslo, Norway, knut .guettler@nmh.no)

During the few years of our young millennium the picture of what happens acoustically when the bow is drawn across the string has become considerably more complete. A few surprises emerged along the way: the bow's position on the string does not seem to influence the spectral envelope-and different from what Schelleng predicted-there seems to be no clear correlation between minimum bow force and the bow speed. The intention of this presentation is to give a brief survey of related studies and their results, as well as pointing out some of the blank spots still remaining on the canvas. 


\title{
Session 2pMUe
}

\section{Musical Acoustics and Speech Communication: Singing Voice and Source-Filter Interaction}

\author{
Brad Story, Cochair \\ University of Arizona, Dept. of Speech and Hearing Sciences, 1131 E. 2nd Street, Tucson, AZ 85721-0071, USA \\ Nathalie Henrich, Cochair \\ Département Parole \& Cognition, GIPSA-lab, 46, avenue Félix Viallet, Grenoble Cedex, 38031, France
}

\section{Contributed Paper}

\section{$5: 20$}

2pMUe1. How whisper and croak phonation affect vocal tract resonances. Yoni Swerdlin (University of New South Wales, Music Acoustics, School of Physics, NSW 2052 Sydney, Australia, yoniswerdlin@hotmail.com), John Smith (University of New South Wales, Music Acoustics, School of Physics, NSW 2052 Sydney, Australia, john.smith@unsw.edu.au), Joe Wolfe (University of New South Wales, Music Acoustics, School of Physics, NSW 2052 Sydney, Australia, J.Wolfe @ unsw.edu.au)

The whisper and croak modes of phonation provide convenient broad band excitation of the vocal tract and thus give relatively precise information about tract resonances. How closely do the measured resonances for these mechanisms approximate those of normal speech? We measured the frequencies of the first four resonances (R1-R4) in normal, whisper and croak phonation. Subjects produced pairs of these phonations in the same vocal gesture. Formants were used to measure the frequencies (R1-R4) for the non-periodic phonations and broad band excitation at the mouth was used to measure them with similar precision in normal speech. For R1 to R4 respectively, whispering raised the resonant frequencies by $255 \pm 90 \mathrm{~Hz}$ $(92)^{*}, 115 \pm 105 \mathrm{~Hz}(119)^{*}, 125 \pm 125 \mathrm{~Hz}(109)^{*}$ and $75 \pm 120 \mathrm{~Hz}(118)^{*}$, (mean \pm standard deviation $(\mathrm{n})$, asterisks show significance at the $5 \%$ level). These values, and their decrease with increasing frequency, are consistent with the effect of the increased glottal opening in whispering only if the increased opening is large. A supra-glottal narrowing in the tract could also help raise the frequencies of resonance. Croak phonation raises the resonant frequencies respectively by $45 \pm 50 \mathrm{~Hz}(121)^{*}, 10 \pm 60 \mathrm{~Hz}(124), 65 \pm 120$ $\mathrm{Hz}(109)^{*}$ and $15 \pm 110 \mathrm{~Hz}(108)$.

\section{Invited Papers}

2pMUe2. Vocal folds and ventricular bands in interaction: comparison between 'in vivo' measurements and theoretical predictions. Lucie Bailly (Département Parole \& Cognition, GIPSA-lab, 46, avenue Félix Viallet, 38031 Grenoble Cedex, France, Lucie.Bailly@gipsa-lab.inpg.fr), Nathalie Henrich (Département Parole \& Cognition, GIPSA-lab, 46, avenue Félix Viallet, 38031 Grenoble Cedex, France, Nathalie.Henrich@gipsa-lab.inpg.fr), Xavier Pelorson (Département Parole \& Cognition, GIPSA-lab, 46, avenue Félix Viallet, 38031 Grenoble Cedex, France, pelorson@icp.ingg.fr), Joël Gilbert (Laboratoire d'Acoustique de l'Université du Maine, Avenue Olivier Messiaen, 72085 Le Mans, France, joel.gilbert@univ-lemans.fr)

Period-doubling occurrences have been found during singing phonations such as Mongolian Kargyraa throat singing or Sardinian A Tenore Bassu singing. The combined vibrations of vocal folds and ventricular bands have been observed during the production of such low-pitch bass-type sounds. The present study aims at better understanding the physical interaction between the ventricular-bands vibration and the vocal-folds self-sustained oscillations. In this paper, the vibratory properties of both vocal folds and ventricular bands in interaction are analysed on a professional singer by means of acoustical, electroglottographic signals and synchronized glottal images obtained by high-speed cinematography. Using the detected glottal and ventricular areas, the aerodynamic behavior of the laryngeal system is simulated using a simplified aerodynamic modelling previously validated "in vitro" on vocal-folds and ventricular-bands replica. An estimate of the subglottal pressure along with the ventricular aperture extracted from the "in vivo" data allows a theoretical prediction of the glottal aperture. The "in vivo" measurements of the glottal aperture are then compared to the simulated estimations. The influence of the subglottal pressure is also discussed.

\section{6:00}

2pMUe3. Longitudinal voice source and acoustic analysis of English girl cathedral choristers. David M. Howard (University of York, Department of Electronics, Heslington, YO10 5DD York, UK, dh@ohm.york.ac.uk)

Since 1998, the authors have been recording girl choristers who sing at Wells Cathedral in England to enable a longitudinal quantitative exploration of voice development for girls aged between 8 and 16 who are involved in professional choral singing on a daily basis. The recordings are made of their acoustic output and the output from an electrolaryngograph for a series of vocal tasks including speaking and singing. Changes have been observed on a longitudinal basis for larynx closed quotient (the percentage of each cycle for which the vocal folds remain closed), long-term average spectra as well as in the overall spectral coverage in terms of frequency and dynamic ranges. Evidence of observations will be presented and their implications will be discussed with reference to source-filter interaction as appropriate. 


\section{Contributed Papers}

6:20

2pMUe4. Phonetograms of laryngeal source parameters for different vowels and laryngeal mechanisms. Sylvain Lamesch (IJLRA-LAM, 11, rue de Lourmel, 75015 Paris, France, lamesch@lam.jussieu.fr), Boris Doval (LIMSI-CNRS, B.P. 133, 91403 Orsay Cedex, France, boris.doval@limsi.fr), Michèle Castellengo (IJLRA-LAM, 11, rue de Lourmel, 75015 Paris, France, castel@ccr.jussieu.fr)

This paper explores how the laryngeal source is adjusted by the singer when the sung vowel changes. As the source parameter adjustments may depend on intensity and pitch, comparisons are done through phonetograms computed for each vowel. Distinction is made in the phonetograms between the two main laryngeal mechanisms (M1 and M2). Male and female subjects were recorded producing crescendos and decrescendos from $\mathrm{C} 3$ to $\mathrm{C} 5$ on /a/, /i/ and /o/ in order to obtain a reduced phonetogram for each vowel and each laryngeal mechanism. Sound, electroglottographic signals and vertical larynx position (VLP) were recorded. Investigations were done on the open quotient and the VLP. The results show a smaller vocal dynamic on /i/ (and in a smaller extent on /o/) in each laryngeal mechanism, and differences on the intensity low limit of the highest part of the M1-phonetogram. Clear tendencies about the larynx position were measured. They are similar for both laryngeal mechanisms, but differ among singers. The Oq seems to be lower on /i/ than on /a/ and /o/ for M1 productions.

\section{6:40}

2pMUe5. Resonance strategies and glottal behaviour in the two main laryngeal mechanisms for professional operatic singers. Nathalie
Henrich(Département Parole \& Cognition, GIPSA-lab, 46, avenue Félix Viallet, 38031 Grenoble Cedex, France, Nathalie.Henrich@gipsa-lab .inpg.fr), John Smith (University of New South Wales, Music Acoustics, School of Physics, NSW 2052 Sydney, Australia, john.smith@unsw .edu.au), Joe Wolfe (University of New South Wales, Music Acoustics, School of Physics, NSW 2052 Sydney, Australia, J.Wolfe@unsw.edu.au)

In lyrical singing, two principal laryngeal mechanisms are used to produce all the notes across the singer's tessitura. Laryngeal mechanisms M1 and M2 are characterised by a greater and lesser vibrating mass respectively. Different timbres between the two are at least partly explained by their different glottal behaviours. However, different adjustments of the vocal-tract resonances, which can also contribute to different timbres, may also be involved, but have been neglected. We studied seven professional operatic singers, who produced sustained vowels at the same pitch using both laryngeal mechanisms. Vocal-tract resonances were directly measured during phonation, by broadband acoustic excitation at the mouth. Glottal behaviour and laryngeal movement were measured indirectly with an electroglottograph. As expected, we measured lower values of open quotient and higher sound pressure levels in M1 than in M2. In most cases, the first two vocal-tract resonances were characterised by lower frequencies in M2 than in M1. In the singer's formant region, similar resonance-frequency values were found for both laryngeal mechanisms. The effect on the sound spectrum of an open-quotient increase together with a first-formant decrease may be modelled by source-filter theory. The theoretical and measured amplitudes of the first two harmonics will be compared.

\section{Invited Paper}

\section{7:00}

2pMUe6. Chest, head and whistle registers in an untrained female singer analyzed by videokymography, strobolaryngoscopy and sound spectrography. Jan G. Svec (Palacky University Olomouc, Faculty of Science, Dept. Experimental Physics, Laboratory of Biophysics, Tr. Svobody 26, CZ-771 46 Olomouc, Czech Republic, svecjan@vol.cz), Johan Sundberg (KTH, Department of Speech, Music and Hearing, Lindstedtsvägen 24, SE-100 44 Stockholm, Sweden, jsu@csc.kth.se), Stellan Hertegard (Karolinska University Hospital Huddinge, Dept. Logopedics and Phoniatrics, SE-141 86 Stockholm, Sweden, Stellan.Hertegard@ki.se)

There has been a lack of objective data on the singing voice registers, particularly on the so called "whistle" register, occurring in the top part of the female pitch range, which is accessible only to some singers. This study offers unique strobolaryngoscopic and high-speed (7812.5 images/s) videokymographic data on the vocal fold behavior of an untrained female singer capable of producing three distinct voice qualities, i.e., the chest, head and whistle registers. The sound was documented spectrographically. The transition from chest to head register, accompanied by pitch jumps, occurred around tones B4-C\#5 (500-550 Hz) and was found to be associated with a slight decrease in arytenoids adduction, resulting in decrease of the closed quotient. The register shifts from head to whistle, also accompanied by pitch jumps, occurred around tones E5-B5 (670-1000 Hz) without any noticeable changes in arytenoids adduction. Some evidence was found for the vocal tract influence on this transition. The mechanism of the vocal fold vibration in whistle register was found principally similar to that at lower registers: vibrations along the whole glottal length and vertical phase differences (indicated by sharp lateral peaks in videokymography) were seen on the vocal folds up to the highest tone G6 (1590 Hz).

\section{Contributed Paper}

\section{$7: 20$}

2pMUe7. Sopranos' secondo passagio: a resonance adjustment and/or a laryngeal transition to the whistle voice? Maeva Garnier (University of New South Wales, Music Acoustics, School of Physics, NSW 2052 Sydney, Australia, maeva.garnier@gmail.com), Nathalie Henrich (Département Parole \& Cognition, GIPSA-lab, 46, avenue Félix Viallet, 38031 Grenoble Cedex, France, Nathalie.Henrich @ gipsa-lab.inpg.fr), John Smith (University of New South Wales, Music Acoustics, School of Physics, NSW 2052 Sydney, Australia, john.smith@unsw.edu.au), Joe Wolfe (University of New South Wales, Music Acoustics, School of Physics, NSW 2052 Sydney, Australia, J.Wolfe @unsw.edu.au)

How are glottal source and vocal tract involved in the sopranos' passagio around E5? This pilot study investigates a legit soprano singing C5 to C6 with a lyrical technique and C5 to D7 in a "light" voice. On (de)crescendi and glissandi over the passagio, we observed clear bifurcations from "light" to lyrical mode, with discontinuous changes in f0, larynx vertical position, amplitude and shape of the electroglottographic signal. No such discontinuities occurred from C5 to D7 within the "light" mode. We established phonetograms of both modes and characterised differences in voice quality (Spectrum, Harmonicity, Open quotient). For [oe] in both modes, this singer tunes the first vocal tract resonance (R1) to fo over the passagio. For a free vowel, a single and strong resonance is observed in the "light" mode, which may correspond to the merging of R1 and R2 and which is tuned to 2 fo below C6, then to f0 until F6. Above F\#6, two resonances are observed again and R2 is tuned to f0. All these observations suggest that, for this singer, the secondo passagio may correspond to both a resonance adjustment and a transition from the second laryngeal mechanism to the third, also called whistle voice. 


\title{
Session 2pNSa
}

\section{Noise, ASA Committee on Standards and EURONOISE: Sound Quality Tools and Applications I}

\author{
Wade R. Bray, Cochair \\ HEAD acoustics, Inc., 6964 Kensington Road, Brighton, MI 48116, USA \\ Klaus Genuit, Cochair \\ HEAD acoustics GmbH, Ebertstrasse 30a, Herzogenrath, 52134, Germany
}

Etienne Parizet, Cochair

Laboratoire Vibrations Acoustique, Insa Lyon, 25 bis, av. J. Capelle, Villeurbanne Cedex, 69621, France

\section{Invited Papers}

\section{2:00}

2pNSa1. How loud is low frequency noise? Norm Broner (Sincliar Knight Merz, 590 Orrong Road, Armadale, 3143 Melbourne, Australia, nbroner@skm.com.au)

When it comes to assessing noise, the conventional wisdom is that the loudness of a sound will correlate well with its perceived annoyance. On this basis, the A-weighted sound pressure level is also assumed to correlate with the potential percieved annoyance of a sound. This might well be a reasonable assumption for mid range frequency sounds. But for low frequency sounds, particularly below $63 \mathrm{~Hz}$, the assumption that loudness is correlated highly with perceived annoyance breaks down. Indeed, while loudness adaptation may occur for low frequency sound, the annoyance can increase with time. Thus, with time, the annoyance may keep increasing while the apparent loudness decreases!

2pNSa2. Metric assessment of subjective annoyance due to low frequency aircraft noise. Kathleen Hodgdon (Applied Research Laboratory, The Pennsylvania State University, P.O. Box 30, State College, PA 16804, USA, kkh2@ psu.edu), Matthew L. Nickerson (IBM, 3039 Corwallis Road, Durham, NC 27709, USA, mlnicker@us.ibm.com), Anthony A. Atchley (The Graduate Program in Acoustics, The Pennsylvania State University, PO Box 30, State College, PA 16804, USA, atchley@engr.psu.edu), Thomas Gabrielson (The Graduate Program in Acoustics, The Pennsylvania State University, PO Box 30, State College, PA 16804, USA, tbg3@psu.edu)

A low frequency noise study was conducted at the Pennsylvania State University to investigate human response to the low frequency content of aviation noise. Metric assessment included level based and loudness metrics, including time-varying loudness. Current predictive noise models and metrics may underestimate the impact of low-frequency noise produced by aviation noise. Assessing the role of low frequency noise on human response will facilitate understanding how this type of noise can impact communities. Indoor recordings of aircraft arrivals and departures at Washington Dulles International Airport made in 2004 were used to assess subjective noise annoyance. These recordings and low-frequency variants of these recordings were reproduced for subjects through the Gulfstream Supersonic Acoustic Signature Simulator II (SASS II); the signatures were then rated for annoyance. Subjective judgments were statistically analyzed and compared against objective metrics that were calculated for each signature. Results are shown that all things being equal higher levels of low-frequency content in aircraft noise can result in increased annoyance in subjects. The C-weighted sound exposure level was found to correlate well with the subjective annoyance response.

\section{2:40}

2pNSa3. Sound character ratings by experienced and naive listeners. Reinhard Weber (Oldenburg University, Institute of Physics - Acoustics, Carl-von-Ossietzky Str. 9-11, 26111 Oldenburg, Germany, Reinhard.Weber@uni-oldenburg.de), Hans Hansen (IRCAM - Sound Perception \& Design, 1 place Igor Strawinsky, 75004 Paris, France, Hans_Hansen@gmx.de)

In the frame of a cross-cultural study on pitch perception of tones in noise, two different groups of participants took part in the listening tests in France. Trained in psycho-acoustic evaluation tasks, members of the laboratory belonged to the first group, whereas the second one consisted of naïve students. 25 stimuli with different tone-to-noise ratios were presented via headphones and, using a semantic differential of 14 adjective pairs, the participants made their judgements on common seven step scales. For both listening groups a PCA of the results led to the same three orthogonal dimensions of the perceptual spaces. They were identified in accordance to Namba's findings as 'pleasant', 'metallic' and 'powerful' factors with an interesting difference: For the trained, the 'metallic' factor, preferably describing the sound character, explained the largest amount of total variance as first factor. The 'pleasant' factor as 'evaluation' dimension took the second place and the 'powerful' factor ranked behind. However, the order of the first two factors was reversed for the untrained listeners. For them the variance of 'pleasant' factor was a bit higher compared to the 'metallic' factor. So the variance in sound character judgements was higher for the trained compared to the untrained listeners. 


\section{Contributed Papers}

\section{3:00}

2pNSa4. Optimising the value of sound quality evaluations by observing assessors' driving strategies. Sebastiano S. Giudice (WMG, University of Warwick, Gibbet Hill Road, CV47AL Coventry, UK, S.D.Giudice@warwick.ac.uk), Paul Jennings (WMG, The University of Warwick, International Manufacturing Centre, CV4 7AL Coventry, UK, paul.jennings@warwick.ac.uk), Rebecca Cain (WMG, The University of Warwick, International Manufacturing Centre, CV4 7AL Coventry, UK, R.Cain.1@warwick.ac.uk), Garry Dunne (Jaguar \& LandRover, Jaguar Engineering Center Abbey Road, Whitley, CV3 4LF Coventry, UK, gdunne@jaguar.com), Mark Allman-Ward (Sound Evaluations Ltd, 18 Walsworth Road, Hitchin, Hertfordshire, SG49SP Hitchin, UK mallman@soundevaluations.com), Roger Williams (Sound Evaluations Ltd, 18 Walsworth Road, Hitchin, Hertfordshire, SG49SP Hitchin, UK, rwilliams@soundevaluations.com)

Traditionally vehicle sound evaluations have been conducted either whilst driving a car or by auditioning fixed test conditions in a listening room. On-road testing provides the right context but the results are often inconsistent and unrepeatable. Furthermore it is not possible to evaluate prototype sounds or carry out back-to-back comparisons. In-room evaluations improve the statistical confidence, but the context of the assessments is unrepresentative. Interactive NVH vehicle simulators enable assessments to be performed in a setting representative of real appraisals. Accurate sounds can now be generated in real-time, and assessors can adopt a driving strategy that allows their own interpretation of the attributes. The benefits include the opportunity to understand how preferences are formed by assessors, albeit with added complexities. Different assessors may be associating their preferences on different operating conditions which have different acoustical properties. Therefore for engineers to identify the key features that influence perception they need to be able to relate the driving strategies with the subjective preferences. This paper reports on new observational methods which capture assessors' decision making strategies. It demonstrates how these help in relating subjective preferences to vehicle operating conditions and how to design a structured evaluation to reduce sources of experimental error

\section{3:20}

2pNSa5. Perception of simple sounds from an emotional point of view. Anders Sköld (Chalmers University of Technology, Division of Applied Acoustics, Sven Hultinsgata 8a, 41296 Gothenburg, Sweden, anders.skold@chalmers.se), Penny Bergman (Chalmers University of Technology, Division of Applied Acoustics - Chalmers Room Acoustics Group, Sven Hultins gata 8a, 41296 Gothenburg, Sweden, penny@chalmers.se), Daniel Vastfjall (Chalmers University of Technology, Division of Applied Acoustics - Chalmers Room Acoustics Group, Sven Hultins gata 8a, 41296 Gothenburg, Sweden, daniel.vastfjall@psy.gu.se), Andreas Colebring (Chalmers University of Technology, Division of Applied Acoustics, Sven Hultinsgata 8a, 41296 Gothenburg, Sweden, andreas.colebring@gmail.com)

In sound perception the focus often lies in the cognitive aspect of the sound. We argue that the emotional aspect has to be added to get a fuller picture of sound perception. By using emotions as parameter in design of auditory alerts, one can reach a more accurate reaction to the alert. In this paper we studied the emotional connection to some attributes, common in music psychology, that are possible to describe by simple parameters. Short stimuli were created from these parameters in a factorial test design. The sounds were presented over headphones, with same signal fed to both ears, to 30 participants. The participants were asked to rate level of valence and activation, using a pictorial scale (SAM). Statistical differences was mostly found in ratings of activation, but differences were also shown in valence ratings. Results will be discussed in relation to theories of sound perception as well as music psychology.

Lecture sessions will recess for presentation of poster papers on various topics in acoustics. See poster sessions for topics and abstracts.

\section{Contributed Paper}

\section{5:00}

2pNSa6. Emotional bias for the perception of rising tones. Ana Tajadura-Jiménez (Chalmers University of Technology, Division of Applied Acoustics, Sven Hultinsgata 8a, 41296 Gothenburg, Sweden, ana.tajadura@chalmers.se), Aleksander Väljamäe (Laboratory for Synthetic Perceptive, Emotive and Cognitive Systems (SPECS) - Universitat Pompeu Fabra, Ocata 1, 08003 Barcelona, Spain, aleksander.valjamae@iua.upf.edu), Daniel Vastfjall (Chalmers University of Technology, Division of Applied Acoustics - Chalmers Room Acoustics Group, Sven Hultins gata 8a, 41296 Gothenburg, Sweden, daniel.vastfjall @psy.gu.se)

Sounds with rising and falling intensity are often perceived, respectively, as approaching or receding sound sources. Research has shown the existence of biases both at perceptual and neural levels towards approaching versus receding sounds. It has been suggested that these effects might be accounted to a greater biological salience of approaching sounds. In the present study we investigated whether this asymmetry could be also explained by emotional theories. Approaching and receding tones, followed by neutral, negative or positive photographs, were presented. Participants were required to make a speeded three-alternative forced choice (3AFC) task regarding their feelings towards the photographs. Reaction times (RTs) to this task, together with self-reported valence (pleasantness) and arousal (activation) ratings for the photographs were collected. In addition, participants' electrodermal activity (EDA) and facial electromyography (EMG) when listening to the sounds were measured. Participants responded faster to photographs preceded by approaching tones, especially for photographs with a negative emotional content. Both the intensity range and the period of intensity change of the sounds had a significant effect in RTs. Taken together, these results suggest that approaching sounds induce a greater emotional response on listeners, which might modulate subsequent attentional and perceptual processes.

\section{Invited Papers}

2pNSa7. Survey of sound quality research and applications in the information technology industry. Terry Baird (HewlettPackard Co., 11311 Chinden Blvd, Boise, ID 83714, USA, terry.baird@hp.com)

Traditionally, emission sound power level has been the key attribute measured and reported for describing IT equipment acoustic performance. More recently, designers and manufacturers of IT equipment have come to realize that customer satisfaction or annoyance is not dictated by sound power alone, but is dependent on many sound quality attributes as well. Additionally, sound power level tends 
to be a poor indicator of equipment condition or component degradation where other tools are required for diagnosis and characterization. This paper reviews key research and trends over the last several years using psychoacoustic principles and analyses, as well as case studies of specific applications in the IT sector.

\section{5:40}

2pNSa8. Identification and Classification of Noise Patterns. Roland Sottek (HEAD acoustics GmbH, Ebertstrasse 30 a, 52134 Herzogenrath, Germany, claudia.erens@head-acoustics.de)

Sound quality evaluation is a challenge due to spectral and temporal structures of noise. Tonal components, howling sounds and modulated signals are often the cause of customer complaints. Thus, besides frequency-weighted level like $\mathrm{dB}(\mathrm{A})$ or loudness, additional parameters are required. Besides time-varying loudness, other psychoacoustic parameters like sharpness and roughness can be used for sound quality evaluation. Sharpness considers the amount of high frequency components of a noise, and roughness evaluates modulation characteristics. In addition, a metric combining modulation spectral analysis with loudness calculation has been introduced. Some years ago, a "Hearing Model" was developed with the intention of explaining and describing psychoacoustic effects. Applying the Hearing Model to sound quality tasks allows evaluating the spectral and temporal patterns of a sound ("Relative Approach" analysis) where absolute level or loudness is often without significance. The Relative Approach analysis emphasizes all relevant signal components concerning human auditory perception: tonal and transient signals. For extracting and evaluating individual patterns further signalprocessing steps are necessary. The paper presents different methods for effective sound quality evaluation of noise and their application to several examples.

\section{6:00}

2pNSa9. The Calibration and Validation of a Binaural Room Scanning System Used for Subjective Evaluation of Automotive Audio Systems. Sean E. Olive (Harman International Industries, Inc., R\&D Group, 8500 Balboa Blvd, Northridge, CA 91329, USA, solive@harman.com), Todd Welti (Harman International Industries, Inc., R\&D Group, 8500 Balboa Blvd, Northridge, CA 91329, USA, twelti@harman.com)

Binaural room scanning (BRS) is a method of capturing the acoustical response of audio systems in rooms and automotive cabins as a set of binaural impulse responses that are stored and later reproduced through head-tracking headphones. All BRS systems have errors that without careful calibration limit their usefulness for subjective measurements of sound quality. This paper discusses a method for calibrating and testing a proprietary BRS system based on how well it reproduces listeners' preference ratings of different automotive audio systems made in situ. A group of trained listeners gave preference ratings for 5 different equalizations of a high quality automotive audio system made both in situ and through the BRS capture/playback system. The tests were repeated in mono and stereo using 6 individualized listener calibrations. The results can be summarized as follows: there were no significant differences in preferences between the in situ and BRS-based evaluations for either the mono or stereo tests. A significant interaction was found between the different equalizations and individualized calibrations that were largely confined to 1 particular listener calibration. The results suggest that a generalized BRS calibration may provide sufficiently accurate preference measurements of audio system sound quality.

\section{Contributed Papers}

\section{6:20}

2pNSa10. Head and torso simulators for acoustic measurements. Gunnar Rasmussen (G.R.A.S. Sound \& Vibration A/S, Skovlytoften 33, 2840 Holte, Denmark, gr@gras.dk)

Head and torso simulators which reproduce the acoustical effect of a median human adult, including diffraction and direction-dependent transformation from free field to the ear drum as well as allow for objective testing of sound systems involving hearing aids, headphones, earphones, telephones, mobile phones, headsets and sound quality type of measurements may be realized based on different philosophies. It may be based on geometrically simplified shape or mean values of individuals. The geometrically simplified shape will appear like no human being. The mean values may result in non human like features, while average anthropometric dimensions of an adult human may offer data closer to a subjective test. Especially the shape of the pinna may often be critical to test of earphones and headphones as well as mobile phones. Subjective tests on groups of people may be supplemented with objective tests on a manikin for reference and quality control purposes. Variations in pinna size may have significant influence on the measurement of earphones as well as leakage in telephone testing. The influence of the dress worn may also be of significance especially for the head related transfer function and on the directional response in the middle frequency range.

\section{6:40}

2pNSa11. Capturing blocked-entrance binaural signals from open-entrance recordings. Dorte Hammershøi (Acoustics, Aalborg University, Fredrik Bajers Vej 7 B5, 9220 Aalborg Ø, Denmark, dh@es.aau.dk), Pablo F. Hoffmann (Acoustics, Aalborg University, Fredrik Bajers Vej 7 B5, 9220 Aalborg Ø, Denmark, pfh@es.aau.dk), Søren K. Olesen (Acoustics, Aalborg University, Fredrik Bajers Vej 7 B5, 9220 Aalborg Ø, Denmark, sko@es.aau.dk), Per Rubak (Acoustics, Aalborg University, Fredrik Bajers Vej 7 B5, 9220 Aalborg Ø, Denmark, pr@es.aau.dk)

Binaural recordings enable us to capture all sound attributes including spatial information, room effect, and source characteristics in a given environment. It has been shown that blocked-entrance binaural recordings provide advantages over open-entrance recordings, primarily because the blocked-entrance recordings is not influenced by the ear canal acoustics of the individual for which it is recorded. However, blocking the ear canal for recoding imposes an obvious disruption to normal hearing conditions, which may be unacceptable for applications in which binaural audio capturing is desired but without interfering the individual's hearing and doing. In this work we propose a strategy for the recording of binaural audio with minimal hearing interference, and for transforming these recordings to blockedentrance versions that are more suitable for analysis and reproduction of binaural audio in a more general context. To this purpose, equalization filters are derived from the ratio between blocked and open ear canal transfer functions. Different transfer-function measuring techniques and inverse filtering methods are evaluated. 


\section{Invited Paper}

\section{7:00}

2pNSa12. PsySound3: a program for the analysis of sound recordings. Densil Cabrera (University of Sydney, Faculty of Architecture, Design and Planning, NSW 2006 Sydney, Australia, densil@usyd.edu.au), Sam Ferguson (University of Sydney, Faculty of Architecture, Design and Planning, NSW 2006 Sydney, Australia, samferguson@ihug.com.au), Farhan Rizwi (Empirical Musicology Group, University of New South Wales, NSW 2052 Sydney, Australia, farhan.rizwi@unswalumni.com), Emery Schubert (Empirical Musicology Group, University of New South Wales, NSW 2052 Sydney, Australia, E.Schubert@unsw.edu.au)

This paper demonstrates the sound analysis software PsySound3, which was written by the authors. The software currently includes a range of DSP-based analysis techniques (e.g., spectrum, cepstrum, autocorrelation, Hilbert transform, sound level meter emulator), as well as implementations of psychoacoustical algorithms often associated with sound quality (e.g., loudness, sharpness, loudness fluctuation, roughness, pitch, binaural attributes). In some cases, PsySound3 makes available multiple models of the one auditory attribute - for example it implements dynamic and static loudness models using Erb- and Bark-based auditory filters. The program is extensible, and so has the potential to allow researchers to share their analysis models using a common interface. PsySound3 is written in Matlab, and is also available as a stand-alone program. The software is freely available from www.psysound.org.

\section{Contributed Papers}

$7: 20$

2pNSa13. Hartis, a re-synthesis tool for vehicles sound design. Gaylord Desoeuvre (GIST, 62 bis avenue André Morizet, 92100 Boulogne Billancourt, France, gaylord.desoeuvre@ext.mpsa.com), Florent Richard (PSA Peugeot Citroën, Centre Technique de Vélizy, Route de Gisy, 78943 VélizyVillacoublay, France, florent.richard@mpsa.com), Vincent Roussarie (PSA Peugeot Citroën, Centre Technique de Vélizy, Route de Gisy, 78943 VélizyVillacoublay, France, vincent.roussarie@mpsa.com), Marie Céline Bezat (PSA Peugeot Citroën, Centre Technique de Vélizy, Route de Gisy, 78943 Vélizy-Villacoublay, France, marieceline.bezat@mpsa.com)

For more than thirty years, the main goal for the automotive acoustic engineer was the car's noise level reduction. Within new customers' behaviours, the car manufacturers have now to put products sound quality at the same level as its aesthetic qualities or ergonomics. To improve customer's satisfaction, one solution is to increase sensory coherence and perceived quality by controlling the sound identity and character of a vehicle. To reach this new objective, the Research Department of PSA Peugeot Citroën is developing his own sound design software HARTIS [1] (HArmonics Real TIme Synthesis). HARTIS is a complete re-synthesis tool useful in many stages of vehicle sound design process. In this paper we first present the last version of HARTIS fully interfaced for an easy use in project teams. The second part of the paper presents new integrated module that deals with impulsional noise for diesel application and other sources models. Each type of source can be controlled and filtered to recreate different driving situation.
References [1] Sound design in car passenger compartment Process and tool for the control of engine sound character. V. Roussarie, F. Richard, Journées du Design Sonore 2004

\section{$7: 40$}

2pNSa14. Ride quality and noise in high speed elevators. Mauro Pierucci (San Diego State University, 5500 Campanile drive, San Diego, CA 92182-3020, USA, mpierucci@mail.sdsu.edu), Michael Frederick (San Diego State University, 5500 Campanile drive, San Diego, CA 92182-3020, USA, mike.frederick-1@nasa.gov)

Ride quality in an elevator is the latest "hot topic" in elevator design and construction. In skyscrapers being built in Asia, Dubai and the US, the new elevators travel close to and beyond $10 \mathrm{~m} / \mathrm{s}$. The rides to the highest floors will last well over one minute. From a comfort point of view, it is important that the ride be as smooth and quiet as possible. There are many factors that influence the vibration of the elevator and the concomitant noise. In this paper we will examine the sudden forces that the elevator experiences as it passes each floor. The side forces on the elevator are caused by the asymmetric flow field about the elevator cab. The pressure and streamline flow field was evaluated by using the CFD code FLUENT. Comparison between the numerical and the measured results are given and the pressure disturbances caused by the passage of the elevator and its appendages are discussed together with some solutions to alleviate the pressure disturbances. 
Session 2pNSb

\title{
Noise, Physical Acoustics, and EURONOISE: Aeroacoustics III (Poster Session)
}

\author{
Philip Morris, Cochair \\ Penn State University \\ Christophe Bailly, Cochair \\ Ecole Centrale de Lyon
}

\begin{abstract}
All posters will be on display from 3:40 p.m. to 5:20 p.m. To allow contributors an opportunity to see other posters, contributors of odd-numbered papers will be at their posters from 3:40 p.m. to 4:30 p.m. and contributors of even-numbered papers will be at their posters from 4:30 p.m. to 5:20 p.m.
\end{abstract}

\section{Contributed Papers}

2pNSb1. Nonlinear propagation of spark-generated $\mathrm{N}$-waves in atmosphere: theoretical and experimental assessment of the shock front structure. Petr V. Yuldashev (Center for Industrial and Medical Ultrasound, Applied Physics Lab., University of Washington, 1013 NE 40th St., Seattle, WA 98105, USA, petr@acs366.phys.msu.ru), Mikhail V. Averiyanov (Center for Industrial and Medical Ultrasound, Applied Physics Lab., University of Washington, 1013 NE 40th St., Seattle, WA 98105, USA, misha@acs366.phys.msu.ru), Vera A. Khokhlova (Center for Industrial and Medical Ultrasound, Applied Physics Lab., University of Washington, 1013 NE 40th St., Seattle, WA 98105, USA, vera@acs366.phys.msu.ru), Oleg A. Sapozhnikov (Center for Industrial and Medical Ultrasound, Applied Physics Lab., University of Washington, 1013 NE 40th St., Seattle, WA 98105, USA, oleg@acs366.phys.msu.ru), Ollivier Sebastien (Ecole Centrale de Lyon, LMFA, UMR CNRS 5509, Ecully, 69134 Lyon, France, Sebastien.Ollivier@ec-lyon.fr), Philippe Blanc-Benon (Ecole Centrale de Lyon, LMFA, UMR CNRS 5509, Ecully, 69134 Lyon, France, Philippe .Blanc-Benon@ec-lyon.fr)

Extensive outdoor and laboratory-scale experiments on sonic boom propagation in turbulent atmosphere have shown that shock wave amplitude and rise time are important parameters responsible for sonic boom annoyance. However, accurate measurement of the shock front structure with standard microphone remains a challenge due to the broadband spectrum of the $\mathrm{N}$-wave shock front. In this work the experimental setup utilizing a spark source has been designed and built to investigate nonlinear $\mathrm{N}$-wave propagation in homogeneous medium. Short duration $(30 \mu \mathrm{s})$ and high amplitude $(1 \mathrm{kPa})$ spherically divergent $\mathrm{N}$-waves were generated. In addition to acoustic measurements with $1 / 8^{\prime \prime} \mathrm{B} \& \mathrm{~K}$ microphones, the shadowgraphy method using short duration flash lamp (20 ns) and CCD camera was employed to assess the shock front structure at different distances from the spark. It is shown that the shock rise time measured by the shadowgraphy method was in a good agreement with the theoretical predictions and it was 10 times shorter than in microphone measurements. The widening of the shock in acoustic measurements was therefore due to the limited bandwidth of the microphone. The combination of modeling, acoustic and optical measurements provided an accurate calibration of the shock wave measuring system. [Work supported by RFBR and INTAS.]

2pNSb2. Doppler effect in aeroacoustics. Jean Varnier (ONERA, 29 avenue de la Division Leclerc, 92320 Châtillon, France, jean.varnier@onera .fr)

Doppler effect in aeroacoustics The prediction of acoustic fallout caused at the ground level by aircraft in flight or by launchers at take-off sets the problem of the distortion of the emitted sound spectrum by the Doppler effect. Former studies applied to this problem use a geometrical formalism based on simplifying hypotheses such as homogeneous atmosphere, rectilin- ear trajectory and constant velocity of the sound source. Other studies consider the supersonic case using a model of spherical waves inside the Mach cone. These approaches are in fact not well adapted to the real cases, and the literature does not give many practical examples concerning this topic. In this study, we propose a new time approach of the Doppler effect that allows to avoid the complexity of the geometrical models and to take into account real atmosphere, curvilinear trajectories or accelerations of moving sound sources. This model allows to easily calculate the frequency shift and the change of acoustic level of the signal recorded at a given hearing point, in both cases of subsonic and supersonic sound sources. We present a first validation of this method applied to signals recorded in Kourou during the atmospheric phase of Flight 521 of Ariane 5.

2pNSb3. Noise computation of an axisymmetric free jet using general purpose CFD code. Péter Tóth (Dep. of Fluid Mechanics, Budapest Univ. of Technology and Economics, Bertalan Lajos street 4-6, 1111 Budapest, Hungary, toth@ara.bme.hu), Máté Márton Lohász (Dep. of Fluid Mechanics, Budapest Univ. of Technology and Economics, Bertalan Lajos street 4-6, 1111 Budapest, Hungary, lohasz@ara.bme.hu)

The increasing interest in the noise prediction of the turbulent flows is leading the general purpose Computational Fluid Dynamics (CFD) software developers to integrate noise predicting methods into their code. However the aeroacoustic simulation of free shear flows, where the mean source of noise is the turbulent fluctuations in the free space is challenging with a general purpose CFD application. The aim of this work is to investigate the capabilities of commercial CFD software in the field of noise prediction of free shear flows. The important acoustical properties of a low Reynolds number, low Mach number axisymmetric (2D) jet is computed by means of hybrid Computational Aeroacoustic method using Fluent. The noise source is computed without modeling instantaneously using the compressible solver of the code, and the far field acoustical data is evaluated by the Ffowcs-Williams-Hawkings acoustical analogy. The results is compared to the Direct Numerical Simulation of [Jiang2004 J. Sound Vib.] in terms of frequency spectra in the far field and directivity pattern of the jet.

2pNSb4. Application of RANS modelling and LES to the prediction of noise from a simple cold propane jet. Tanya Stanko (The University of Leeds, School of Process, Environmental and Materials Engineering, LS2 9JT Leeds, UK, pmtss@leeds.ac.uk), Michael Fairweather (The University of Leeds, School of Process, Environmental and Materials Engineering, LS2 9JT Leeds, UK, M.Fairweather@leeds.ac.uk), Mohamed Pourkashanian (The University of Leeds, School of Process, Environmental 
and Materials Engineering, LS2 9JT Leeds, UK, m.pourkashanian @ leeds.ac.uk), Derek Ingham (The University of Leeds, School of Process, Environmental and Materials Engineering, LS2 9JT Leeds, UK, D.B.Ingham @ leeds.ac.uk)

Numerical simulation, combining computational fluid dynamic and acoustic models, is useful as an aeroacoustic tool that is effective in identifying components or surfaces that generate significant amounts of noise, and can thereby provide opportunities for early design changes. In this work, numerical solutions of a cold turbulent jet of propane issuing from a circular nozzle are used to provide velocity information at a Reynolds number of 68,000 . Predictions provided by large eddy simulation are compared with Reynolds-averaged Navier-Stokes solutions derived using a second-moment turbulence closure, as well as with experimental data available in the literature. Reasonable agreement with data is found, and these solutions are used subsequently as the basis for noise predictions. The first acoustic model employed is based on Lighthill's theory using the Goldstein broadband noise source formalisation that postulates axisymmetric turbulence superposed on the mean flow. The second approach is based on Ffowcs-Williams and Hawkings theory which implies that the far field sound is computed from an integral formulation with all quantities evaluated on a control surface, with the control surface itself assumed to enclose all nonlinear flow effects and noise sources. The simulation results reported in this paper confirm previous experimental works presented in the literature demonstrating that jet noise is modified by inlet conditions.

2pNSb5. Uncertainties of Measured and Calculated Aircraft Noise and Consequences in Relation to Noise Limits. Georg Thomann (Empa, Ueberlandstrasse 128, 8600 Duebendorf, Switzerland, georg.thomann@empa .ch)

The uncertainties of measuring and calculating aircraft noise have been analysed in a thesis at the ETH Zurich. The thesis provides information and methods for estimating uncertainties. It shows possibilities for handling these uncertainties and provides guidance to legal and administrative bodies on how to deal with such uncertainties when applying the legal noise limits. To estimate the uncertainties of calculations and measurements the Swiss aircraft noise calculation program FLULA2 and a great deal of measured data have been analysed. Using radar data it is possible to achieve a standard uncertainty of the calculated Leq between $0.5 \mathrm{~dB}$ for day-time and $1.0 \mathrm{~dB}$ for night-time. The analyses also show that the uncertainty of measurements at automated stations is in the same order of magnitude as the calculations. Therefore the annual calculations and measurements of aircraft noise show no significant deviations. Furthermore with FLULA2 it is possible to present the uncertainty at a confidence level of $90 \%$. It is now up to administrative and legal bodies to set up rules on how to account for uncertainties of calculations in evaluating noise situations close to the legal noise limits.

2pNSb6. Acoustic test facility for aero engine fans. Yuri Khaletskiy (Central Institute of Aviation Motors, 2, Aviamotornaya Str., 111116 Moscow, Russian Federation, noise@ciam.ru), Victor Mileshin (Central Institute of Aviation Motors, 2, Aviamotornaya Str., 111116 Moscow, Russian Federation, mileshin@ciam.ru), Rollan Shipov (Central Institute of Aviation Motors, 2, Aviamotornaya Str., 111116 Moscow, Russian Federation, noise@ciam.ru)

The facility for acoustic tests of aero engine fans in conditions of anechoic chamber is created in Central Institute of Aviation Motors (Moscow). The facility is designed for testing models of single rotating fans and counter rotating fans. The measurements of the sound are carried out in forward and rearward semi-spheres simultaneously. Design features of the anechoic chamber and its acoustic performance are presented. Also a fan drive system and systems of acoustic and aerodynamic measurements are described. In particular fan rotor max rotation frequency makes up 13,000 rpm at each shaft max power 2.5 MW. The rig acquisition system allows checking in real time results of complete three-octave analysis of machine noise on all 24 channels in a measurement frequency range of $0 œ 80 \mathrm{kHz}$.
2pNSb7. Nonlinear Propagation of Screech Noise. Jothi Punekar(School of Engineering and Materials, Queen Mary, University of London, 327 Mile End Road, E1 4NS London, UK, j.punekar@qmul.ac.uk), Eldad Avital (School of Engineering and Materials, Queen Mary, University of London, 327 Mile End Road, E1 4NS London, UK, e.avital@qmul.ac.uk)

This study investigates the non-linear propagation of screech noise emitted by supersonic jets. Supersonic jet noise consists of three components: mixing, broadband and screech noise. In order to generate broadband and screech noise the jet has to expand imperfectly, meaning shock waves are formed inside the potential core and interact with the surrounding shear layer. The feedback between the generated noise and the nozzle cause the screech noise pattern. It is associated with high frequency (annoying to the human ear). Near field screech noise obtained from Large Eddy Simulation (LES) of circular supersonics jets is investigated using the Burgers equation, taking into account thermo-viscous effects. The Burgers equation is commonly used to investigate non-linear propagation effects. The initial screech signal is found to consist of two main frequency modes. Significant nonlinear effects appear during propagation although the pressure amplitude is no more than a few percentages of the ambient pressure. Saw tooth waves are generated and the amplitude is found to decrease as $1 / \operatorname{sqrt}(\mathrm{t})$ for low viscous medium according to Lighthill' 'bunching' theory. Further high frequencies are also generated. Finally, geometrical attenuation is discussed by transforming the plane wave propagation to cylindrical form.

2pNSb8. Instability wave control in a subsonic round jet. Georgy Faranosov (TsAGI, Acoustic Division, 17, Radio str, 105005 Moscow, Russian Federation, georgefalt@ rambler.ru)

It is generally accepted that one of the main sources of acoustic radiation from a turbulent jet is due to spatial instability wave packets propagating downstream within the jet. This approach has enabled to explain and predict the principal features of sound radiated by a supersonic jet. Therefore the problem of noise control for the jet could be considered as a problem of instability wave control. Instability wave developed in the tone-excited jet issued from axisymmetric, semi-infinite duct is considered. The flow field in the vicinity of the nozzle edge is investigated for the purpose of identifying instability wave parameters. Instability wave turned out to be damped completely on the condition that the amplitude and the phase of the external forcing are chosen in accordance with those of the instability wave. The robust strategy of instability wave experimental suppression based on the near field measurements is formulated. Similar problem in two-dimensional case was recently analyzed in Kopiev \& Faranosov (Acoust. Phys. 2008, V.54, N.2).

2pNSb9. Decomposition of the Lighthill source term and analysis of acoustic radiation from mixing layers. Florent Margnat (Arts et Métiers Paris Tech - Sinumef Lab, 151 bd de l'Hopital, 75013 Paris, France, florent.margnat@paris.ensam.fr), Véronique Fortuné (Univ. Poitiers, CNRS Laboratoire d'Etudes Aérodynamiques, Bat. K - 22 avenue du recteur Pineau, $86022 \quad$ Poitiers, France, veronique.fortune@lea.univ-poitiers.fr), Peter Jordan (Univ. Poitiers, CNRS Laboratoire d'Etudes Aérodynamiques, 43, rue de l'Aérodrome, $86036 \quad$ Poitiers, France, peter.jordan@lea.univ-poitiers.fr), Yves Gervais (Laboratoire d'Etudes Aérodynamiques (LEA), Université de Poitiers - ENSMA - CNRS, Bâtiment K, 40 Avenue du Recteur Pineau, 86022 Poitiers, France, yves.gervais@lea.univ-poitiers.fr)

Acoustic radiation from jet flow has been studied extensively by means of theoretical, experimental and numerical approaches over the past decades. Unfortunately, the mechanisms responsible for the production of sound by unbounded turbulence in subsonic flows remain unclear. For advancing our fundamental understanding of these mechanisms, the development of specific analysis tools is needed. Our study is based on a decomposition of the Lighthill source term, which is known to contain all the existing links between the fluid flow and the acoustic field. Ten subterms are written with the help of physically meaningful quantities such as velocity, density, dilatation and vorticity. The methodology is tested through the two-dimensional compressible mixing layer flow in spatial development, at a Reynolds number of 400 and a Mach number of 0.25 . A direct numerical simulation of the flow and its acoustic radiation is performed and used as a reference solution. 
Acoustic field generated by each source terms is predicted computing the integral solution of Lighthill's equation. Effect of the compressibility on the balance between the contribution of major subterms is of key importance. Directivity and convective effects are identified as well.

2pNSb10. Acoustic and aerodynamic dissipations induced by a sound wave-impacted Helmholtz resonator. Jean-Michel Roche (ONERA, BP 72 - 29 avenue de la Division Leclerc, 92322 Châtillon, France, jean-michel.roche@onera.fr), Laurent Leylekian (ONERA, BP 72 - 29 avenue de la Division Leclerc, 92322 Châtillon, France, laurent.leylekian@onera.fr), François Vuillot (ONERA, BP 72 - 29 avenue de la Division Leclerc, 92322 Châtillon, France, francois.vuillot@onera.fr)

The purpose of the work introduced herein is to study the dissipation of the acoustic energy carried by sound waves, by a Helmholtz resonator. Twodimensional direct numerical simulations (DNS) are performed with the CEDRE code, a Navier-Stokes equations solver developed by Onera, for a resonator with a $0.8 \times 0.8 \mathrm{~mm}$ square opening. The incident sound pressure level (SPL) varies between $100 \mathrm{~dB}$ and $150 \mathrm{~dB}$ : it is checked that when the SPL is low, the acoustic energy is dissipated in the resonator opening by viscous wall friction, whereas when the SPL is high, the acoustic energy is converted in kinetic energy carried by micro-vortices induced at the opening mouths. However, when the SPL takes intermediate values, both mechanisms coexist. Thus, for each case, we aim at splitting the aeraoacoustic field into respective acoustic and aerodynamic contributions, and at evaluating the ratio between these two distinct mechanisms in order to quantify their relative weight on the global dissipation. Our results are compared with those obtained by Tam et al. for the same geometry. A three-dimensional study for a Helmholtz resonator with a cubic opening is eventually considered.

2pNSb11. The multiple side-branch system as a model for a corrugated pipe. Devis Tonon (Technische Universiteit Eindhoven, CC.2.25, N-LAAG, Postbus 513, 5600 MB Eindhoven, Netherlands, d.tonon@tue.nl), Stefan Belfroid (TNO Science and Industry, Stieltjesweg 1 PO Box 155, 2600 AD Delft, Netherlands, stefan.belfroid@tno.nl), Jan F. Willems (Technische Universiteit Eindhoven, CC.2.25, N-LAAG, Postbus 513, 5600 MB Eindhoven, Netherlands, J.F.H.Willems@tue.nl), Avraham Hirschberg (Technische Universiteit Eindhoven, CC.2.25, N-LAAG, Postbus 513, 5600 MB Eindhoven, Netherlands, a.hirschberg@tue.nl)

Corrugated pipes are used as flexible risers in offshore natural gas production and for vacuum cleaners. Such pipes can display whistling. As theoretical models are available to predict the aero-acoustical behaviour of closed side branches [1], we consider a row of equally spaced closed side branches along a pipe, as a model for a corrugated pipe. We consider side branches with a diameter $\mathrm{D}$ and depth $\mathrm{L}$ equal to the main pipe diameter, placed at a distance of three diameters from each other. For systems of 11 or 12 side branches the lowest resonance modes are reasonably well predicted by assuming $n(\lambda / 2)$ standing wave modes with an effective speed of sound $c_{\text {eff }}[2]$. Whistling is observed for the $\mathrm{n}=2$ and $\mathrm{n}=3$ modes with a pressure fluctuation amplitude $p^{\prime} /\left(\rho_{0} \mathrm{c}_{0} \mathrm{U}_{0}\right)=\mathrm{O}\left(2 \times 10^{-2}\right)$ similar to that observed for sharp edges corrugations in a corrugated pipe (with $\rho_{0}$ the fluid density, $c_{0}$ the speed of sound and $U_{0}$ the main flow velocity). The Strouhal number of these oscillations is $S r_{D}=(f \cdot D) / U_{0} \simeq 0,70$. This is higher than typical values observed for corrugated tubes [3]. References: [1] J.C. Bruggeman et al., J. Sound and Vibration 1991 150(4) 371-393. [2] J.W. Elliot, in "Lecture Notes on the Mathematics of Acoustics" M.C.M. Wrigth, Imperial College Press, London (2005). [3] S. Belfroid et al., ASME paper PVP2007-26503.

2pNSb12. The sound wave reflection from the corrugated plane streamlined by the low Mach number shear flow (generalized Miles mechanism). Mikhail Mironov (Andreev Acoustics Institute, Shvernik, 4, Moscow, 117036 Moscow, Russian Federation, mironov@akin.ru), Ivan Belyaev (Central Aerohydrodynamics Institute (TsAGI), Acoustic Division, 17 Radio str., 105005 Moscow, Russian Federation, belyaev_iv@bk.ru), Vasilisa Solntseva (Andreev Acoustics Institute, Shvernik, 4, Moscow, 117036 Moscow, Russian Federation, mironov@akin.ru)

The plane sound wave reflection from the periodically corrugated plane streamed by the parallel shear flow is studied. The incident wave scatters on corrugations and gives both the mirror reflected sound wave and two inhomogeneous nonpropagating in the normal to the plane direction waves. One of the inhomogeneous waves goes in the same direction along the plane of the flow and the second one in the opposite direction. The first inhomogeneous wave interacts with critical layer of flow according to Miles mechanism (which was originally applied to the generation of surface waves by the wind (JFM: 1957, 1959)) and reflects from this layer back to the plane. After that this inhomogeneous wave scatters from the corrugated plane and gives some additional part to the mirror reflected sound wave. The total reflection coefficient of sound wave appeared to be more than 1 . The angle dependence of the reflection coefficient is analyzed. The equivalent impedance is calculated. The obtained amplification is compared with thermal and viscous surface losses

2pNSb13. On a stability of the shear flow with a tangential discontinuity in the presence of a small scatterer. Nikolay Kanev (Andreev Acoustics Institute, Shvernik, 4, Moscow, 117036 Moscow, Russian_Federation, nikolay.kanev@mail.ru), Mikhail Mironov (Andreev Acoustics Institute, Shvernik, 4, Moscow, 117036 Moscow, Russian Federation, mironov@akin.ru)

It is well known a tangential discontinuity of velocity in the shear flow is unstable. That instability is convective i.e. all disturbances of the tangential discontinuity grow exponentially but they are carried away by the flow downstream so that after all the amplitude of sound field generated by the tangential discontinuity will decrease at any fixed point. In the presented paper we consider the problem when a small scatterer is placed near the tangential discontinuity. It appears this system can be absolutely unstable under the definite position of the scatterer. It means that the appearance of any disturbance of the tangential discontinuity will result in rising of the amplitude of sound field at every point of space. The cause of absolute instability is a feedback provided by the scatterer and the type of instability does not depend on the size of the scatterer. An absolutely unstable system behaves as an autogenerator and radiates tone or multitone sound. So the obtained result explains the possible mechanism of noise generation in systems with tangential discontinuities and gives some hints about noise control in such systems.

2pNSb14. Spectral attenuation of sound in dilute suspensions with nonlinear particle relaxation. Max Kandula (ASRC, Mailstop ASRC5211, NASA Kennedy Space Center, Kennedy Space Center, FL 32899, USA, max.kandula-1@ksc.nasa.gov), Michael Lonergan (NASA, Kennedy Space Center, Mailstop NE-M1, Kennedy Space Center, FL 32899, USA, michael.lonergan-1@ksc.nasa.gov)

Theoretical studies on the dissipation and dispersion of sound in twophase suspensions have been briefly reviewed. Previous studies (theory of Temkin \& Dobbins, JASA, Vol. 40, No. 2, pp. 314-324, 1966,) on the sound attenuation in particle-laden flows under Stokesian drag and conductioncontrolled heat transfer have been extended to accommodate the nonlinear drag and heat transfer. It has been shown that for large particle-to-fluid density ratio, the particle Reynolds number bears a cubic relationship with $\{$ lomega $\{\backslash$ tau $\}$, where $\{$ lomega $\}$ is the circular frequency and $\{\mid t a u\}$ the Stokesian particle relaxation time. This dependence leads to the existence of a peak value in the linear absorption coefficient occurring at a finite value of \{lomega\} $\{$ tau\}. Comparison of the predictions with the test data of Norum (AIAA-2004-2976) for the spectral attenuation of sound with water injection in a perfectly expanded supersonic jet (Mach number of 1.45) shows a satisfactory trend of the proposed theory. It is found that the nonlinear particle relaxation processes (particle drag and heat transfer) are primarily responsible for reduction in the linear absorption coefficient of sound at high frequencies.

2pNSb15. Aeroacoustic simulation of automotive ventilation outlets. Jean-Luc Adam (Renault SAS, 1 avenue du golf, 78288 Guyancourt, France, jean-luc.adam@ renault.com), Denis Ricot (Renault SAS, 1 avenue 
du golf, 78288 Guyancourt, France, denis.ricot@ renault.com),Flavien Dubief (Renault SAS, 1 avenue du golf, 78288 Guyancourt, France, flavien.dubief@renault.com), Christine Guy (Ligeron, Les Algorithmes Bâtiment Euclide, 91194 Saint Aubin, France, christine.guy@ligeron.com)

In this work we have numerically studied aeroacoustics of automotive ventilation outlets. Simulations are performed with the CFD software PowerFLOW based on Lattice Boltzmann method (LBM). Low dissipative LBM scheme enables to compute aeroacoustic sources generated by turbulence fluctuations and to propagate them in the same simulation. In a first step we validate the ability of LBM for propagating acoustic waves in ducts and radiating them at open end terminations. In a second step, aeroacoustic simulations on automotive vents will be presented and compared with experimental data obtained from a DoE (Design of Experiment). This DoE is based on an idealized outlet with variing parameters (number and lenght of grid blades, grids spacing ...) which gives 18 distinct geometrical configurations. All these configurations have been simulated with PowerFLOW and measured with a new test facility (built in the Renault Research Department). The large number of tested geometries and the statistical analysis of the DoE give an accurate idea of the capability of PowerFLOW, and more generally LBM, to correctly simulate the generation and propagation of aeroacoustic sources for a complex geometry. Results will be presented and discussed.

2pNSb16. Increase of sound proofing of air duct. Igor Shubin (Research Institute of Building Physics (NIISF RAABS), 21, Lokomotivny pr., 127238 Moscow, Russian Federation, marina_niisf@mail.ru), Vladimir Gusev (Research Institute of Building Physics (NIISF RAABS), 21, Lokomotivny pr., 127238 Moscow, Russian Federation, marina_niisf@mail.ru), Marina Pestereva (Research Institute of Building Physics (NIISF RAABS), 21, Lokomotivny pr., 127238 Moscow, Russian Federation, marina_niisf@mail.ru)

One of ways of noise protection from transit air duct is increase of sound insulation of air duct by different acoustical materials. The main interest of this work is in comparative analysis of acoustic efficiency different thermal insulation materials for use as sound insulation coverings. The report con- tains analysis of the distribution of noise through round and rectangular air ducts. There was discovered different tipes of thermal insulation materials such as easy made foam materials and fibrous materials. Tests are executed in the reverberation chamber. The received results have shown advantage of a fibrous material.

2pNSb17. On the correlation of the acoustic signal of microphones mounted on a flat plate to the turbulence of an impinging jet. Christoph Reichl (Arsenal Research - Austrian Research Centers, Giefinggasse 2, 1210 Wien, Austria, Christoph.Reichl@arsenal.ac.at), Michelle Boeck (Arsenal Research - Austrian Research Centers, Giefinggasse 2, 1210 Wien, Austria, michelle.boeck@arsenal.ac.at), Wolfgang Tilser (Arsenal Research - Austrian Research Centers, Giefinggasse 2, 1210 Wien, Austria, wolfgang.tilser@arsenal.ac.at), Hermann Lang (Arsenal Research - Austrian Research Centers, Giefinggasse 2, 1210 Wien, Austria, hermann.lang@arsenal.ac.at), Klaus Haindl (AKG Acoustics GmbH, Lemboeckgasse 21-25, 1230 Wien, Austria, HaindlK@akg.com), Friedrich Reining (AKG Acoustics GmbH, Lemboeckgasse 21-25, 1230 Wien, Austria, ReiningF@akg.com), Martin Opitz (AKG Acoustics GmbH, Lemboeckgasse 21-25, 1230 Wien, Austria, OpitzM@akg.com)

An axial symmetric fan driven by a brushless actuator with variable frequency is used to generate a turbulent circular jet leading to typical corevelocities of around $10 \mathrm{~m} / \mathrm{s}(\operatorname{Re}=99000)$. The jet impinges on a flat plate. Turbulence is generated using different sets of turbulence generators in the pipe consisting of meshes, rods, cubes and spikes. The turbulent flow field downstream of the pipe outlet is measured using two-axis hot wire anemometry with a temporal resolution of a minimum of $48 \mathrm{kHz}$. The CTA probe is positioned using an automated three-axis traversing system. 12 Microphones are mounted on a flat plate and are used to capture the wall pressure fluctuations. CFD calculations are performed for the different turbulence generators and are compared to the acoustic and CTA signals. An important aspect of the work is the simultaneous recording of both the CTA- and the acoustic signals in the experimental and numerical approach. This allows for the calculation of correlation patterns between turbulence and the acoustic signals of the plate mounted microphones. 


\title{
Session 2pNSc
}

\section{Noise, ASA Committee on Standards, and EURONOISE: Comparing Noise Regulations and Codes in USA and Europe (Poster Session)}

\author{
Robert Bruce, Cochair \\ CSTI, 15835 Park Ten Place, Suite 105, Houston, TX 77084-5131, USA \\ Wolfgang Probst, Cochair \\ DataKustik GmbH, Gewerbering 5, 86926 Greifenberg, Germany
}

\begin{abstract}
All posters will be on display from 3:40 p.m. to 5:20 p.m. To allow contributors an opportunity to see other posters, contributors of odd-numbered papers will be at their posters from 3:40 p.m. to 4:30 p.m. and contributors of even-numbered papers will be at their posters from 4:30 p.m. to 5:20 p.m.
\end{abstract}

\section{Contributed Papers}

2pNSc1. Noise limits and noise levels along motorways in Europe. Wiebe Alberts (Rijkswaterstaat: Centre for Transport and Navigation, Postbus 5044, 2600 GA Delft, Netherlands, wiebe.alberts@rws.nl)

Environmental noise caused by traffic is one of the main local environmental problems in Europe. The National Road Authorities (NRA's), combined in the Conference of European Directors of Roads (CEDR), are aware of the environmental noise problem along their motorways. One of the main objectives of CEDR is to facilitate knowledge sharing on noise abatement issues among European NRA's. The CEDR noise group has made a comprehensive questionnaire survey on how the CEDR member states handle noise issues. The paper focuses on the part of the survey on noise limits and noise levels along motorways throughout Europe. It will deal with the: - status of noise limits; - noise indicators to express noise limits; - noise limits (outdoor and indoor) for motorways; - noise models to calculate noise levels. Due to the use of different noise indicators and different noise calculation models, the comparison of noise levels and noise limits along motorways in Europe is complicated to say the least. The only way to solve this problem is to have and to use a European noise model for road traffic based on the outcome of the HARMONOISE/IMAGINE projects.

2pNSc2. Origins of the modern noise ordinance in the County of San Diego, California. John C. Bennett (6110 Loukelton Circle, San Diego, CA 92120, USA, jc.bennett@cox.net)

An unofficial history of the origins of San Diego County's noise regulations has been compiled as an investigative tool to review current and future zoning schemes in this jurisdiction. These regulations date back to the 1970's and overlap with the development of city regulations. The focus of the history is the acoustical aspect of their development. A general discussion of performance standards is included in a comparison with similar regu- lations in two other California jurisdictions developed within the last five years. Some practical similarities and differences with European regulations in local jurisdictions are identified to encourage an open discussion in this session.

2pNSc3. Implementation of the noise directive in music and entertainment sector in Finland. Esko Toppila (Finnish Institute of Occupational Health, P.O:Box 486, 33101 Tampere, Finland, esko.toppila@ttl.fi), Pekka Olkinuora (FIOH, Topeliuksenkatu 41, 00250 Helsinki, Finland, pekka.olkinuora@ttl.fi), Pekka Airre (FIOH, P.O.Box 486, 33101 Tampere, Finland, erkko.airo@ttl.fi)

In the noise directive $(2003 / 10 / \mathrm{EC})$ the member countries were obliged to develop codes of conduct for the music and entertainment sector. In Finland the code of conduct was adopted into use in February 2007. According to this code of conduct the workers in the music and entertainment sector consist of performers (musicians, singers, conductors and actors), staff exposed to music (waiters,...) and teacher who teach music in schools. Guidelines were designed based on the worker group and the type of employment. In this approach the performers, who actually produce the "noise", must be able to monitor the sound levels in real time. Restaurants and music halls design the protective actions for other staff based on the assumption that predefined sound level is not exceeded the premises. The same principle is applied to schools. For temporary staff there must be hearing protectors available. Also the need for damping and use of quieter instruments is recognised. The code of conduct give the principles for the hearing conservation, but for the music and entertainment sector direct application of the principles is too complicated. Implementation examples are needed for all exposure patterns. This work is going on now in Finland. 


\title{
Session 2pNSd
}

\section{Noise, ASA Committee on Standards, and EURONOISE: Sound Quality Tools and Applications II (Poster Session)}

\author{
Wade Bray, Cochair \\ HEAD acoustics, Inc. \\ Klaus Genuit, Cochair \\ HEAD acoustics $\mathrm{GmbH}$ \\ Etienne Parizet, Cochair
}

Laboratoire Vibrations Acoustique, Insa Lyon

\begin{abstract}
All posters will be on display from 3:40 p.m. to 5:20 p.m. To allow contributors an opportunity to see other posters, contributors of odd-numbered papers will be at their posters from 3:40 p.m. to 4:30 p.m. and contributors of even-numbered papers will be at their posters from 4:30 p.m. to 5:20 p.m.
\end{abstract}

\section{Contributed Papers}

2pNSd1. Two-level description of environmental sounds: comparison and generalization of $\mathbf{4}$ timbre studies. Antoine Minard (IRCAM - UMR CNRS 9912, Equipe Perception et Design Sonores, 1, place Igor Stravinsky, 75004 Paris, France, antoine.minard@ircam.fr), Patrick Susini (IRCAM UMR CNRS 9912, Equipe Perception et Design Sonores, 1, place Igor Stravinsky, $75004 \quad$ Paris, France, susini@ircam.fr), Nicolas Misdariis (IRCAM - UMR CNRS 9912, Equipe Perception et Design Sonores, 1, place Igor Stravinsky, 75004 Paris, France, misdarii@ircam .fr), Guillaume Lemaitre (IRCAM - UMR CNRS 9912, Equipe Perception et Design Sonores, 1, place Igor Stravinsky, 75004 Paris, France, lemaitre@ircam.fr), Stephen McAdams (Centre for Interdisciplinary Research in Music Media \& Technology (CIRMMT) - Schulich School of Music - McGill Univ., 555 Sherbrooke Street West, Montreal, QC H3A1E3, Canada, smc@music.mcgill.ca), Etienne Parizet (Laboratoire Vibrations Acoustique, Insa Lyon, 25 bis, av. J. Capelle, 69621 Villeurbanne Cedex, France, etienne.parizet@insa-lyon.fr)

The purpose of this study is to propose a scheme of environmental sound description based on the comparison and generalization of 4 timbre studies of diverse environmental sounds. In the medium term, the aim is to make the indexing and classification processes of this kind of sound automatic, which is essential for sound content-based searching and browsing methods using perceptual models of environmental sounds. First, we inventoried the stimuli, experiments and perceptual results of these 4 studies. Then, we experimentally identified 3 main environmental sound categories that constitute this overall sound dataset: impact sounds, motor sounds and instrumentlike sounds. We developed an efficient tool that predicts these categories according to only two acoustic features calculated on the signal. However, because of the specificities of the sound production of these three categories, each of them has its own timbre space. Therefore, we finally used perceptually relevant acoustic features to explain these timbre spaces, according to the main acoustic characteristics that define each sound class. We found that timbral brightness is a common feature used to discriminate sounds in all classes, while other particular features are specifically used within each class. [Work partially supported by the SamplerOrchestrator project of the ANR program]

2pNSd2. Relations between acoustic parameters and perceptual properties: an approach by regressions tree applied to car door closure sounds. Marie Céline Bezat (PSA Peugeot Citroën, Centre Technique de
Vélizy, Route de Gisy, 78943 Vélizy-Villacoublay, France, marieceline.bezat@mpsa.com), Vincent Roussarie (PSA Peugeot Citroën, Centre Technique de Vélizy, Route de Gisy, 78943 VélizyVillacoublay, France, vincent.roussarie@mpsa.com), Richard Kronland-Martinet (CNRS-LMA, 31, chemin Joseph Aiguier, 13402 Marseille, France, kronland@1ma.cnrs-mrs.fr), Solvi Ystad (CNRS-LMA 31, chemin Joseph Aiguier, 13402 Marseille, France, ystad@1ma.cnrs-mrs .fr)

The aim of this study is to identify perceptually pertinent parameters for the evaluation of car door closure sounds. For this purpose, door closing sounds are finely decomposed into perceptual properties: analytical properties (which are obtained thanks to sensory analysis), natural properties (linked to perception of sources and events) and evocations. The acoustic characterisation of the sound is then processed by means of an analysissynthesis model which aims, not at reproducing the exact replica of the door closing sounds, but to synthesize sounds that preserve perceptual properties with a reduced number of signal parameters. The model consists in decomposing the sound in several independent impact sources, each impact being modelled by a set of gains and damping factors in frequency bands. Listening tests on controlled sounds are then carried out to observe the effects of acoustic parameters on the perceptual properties. The complex relations between acoustic parameters and perceptual properties are finally established via regressions tree analyses.

2pNSd3. Acoustic feedback of control elements in cars. Alexander S. Treiber (Heilbronn University, Max-Planck-Str. 39, 74081 Heilbronn, Germany, treiber@hs-heilbronn.de), Gerhard Gruhler (Heilbronn University, Max-Planck-Str. 39, 74081 Heilbronn, Germany, gruhler@hs-heilbronn.de)

Due to the complexity of modern car's on-board systems, car manufacturers tend to use menu based user interfaces. These interfaces offer a relatively low number of control elements for a large number of functions. Optimization of both haptical and acoustical feedback of the remaining control elements can improve both acceptance and security of operation. Since cars nowadays are no longer sold simply as technical but as lifestyle products it is crucial for the success that the potential customer perceives every single aspect of the car to be valuable. Obvious examples are exterior design and material quality. Since the user interface of the car can be judged even before a test drive the feeling of buttons and switches is a key aspect. This work presents the results of a jury test which is part of an ongoing research project focused on acoustical feedback. Subjects were asked to judge both 
recordings of real control elements as well as synthesized signals which were presented on purpose-built hardware. Based on the subjective results the recorded signals were analyzed in order to identify spectral features which correlate with the subject's responses.

2pNSd4. Influence of time-varying booming noise on automotive comfort. Florent Richard (PSA Peugeot Citroën, Centre Technique de Vélizy, Route de Gisy, 78943 Vélizy-Villacoublay, France, florent.richard@mpsa.com), Francois Costes (PSA Peugeot Citroën, Centre Technique de Vélizy, Route de Gisy, 78943 Vélizy-Villacoublay, France, francois.costes@free.fr), Vincent Roussarie (PSA Peugeot Citroën, Centre Technique de Vélizy, Route de Gisy, 78943 Vélizy-Villacoublay, France, vincent.roussarie@mpsa.com)
Noise level reduction is the main preoccupation for acoustic engineers in the automotive industry. Huge progresses were made during the past decades thanks to the use of acoustic materials and specific intake or exhaust silencers. In the future, vehicles will have to be lighter and engines optimized to ensure very low energy consumption. This sets new compromises between mass, performances and acoustics, in particular for booming noise that can be very affected by mass reduction or exhaust modifications. Acoustic specifications must therefore be optimized to guarantee customer's comfort in spite of these new constraints. In this paper, we study the impact of time-varying booming noise on customer's comfort on acceleration sound samples. We performed several customer tests to evaluate the impact of different time-varying booming profiles on comfort and to find out an acceptability threshold for booming.

\title{
Session 2pNSe
}

\section{Noise and EURONOISE: General Topics in Noise II (Poster Session)}

\begin{abstract}
All posters will be on display from 3:40 p.m. to 5:20 p.m. To allow contributors an opportunity to see other posters, contributors of odd-numbered papers will be at their posters from 3:40 p.m. to 4:30 p.m. and contributors of even-numbered papers will be at their posters from 4:30 p.m. to 5:20 p.m.
\end{abstract}

\section{Contributed Papers}

2pNSe1. Measurement of the acoustic pressure distribution occurring around an aerial substation adjacent to apartment buildings. Sebastian Borucki (Technical University of Opole, ul. Miko ajczyka 5, 45-271 Opole, Poland, s.borucki@po.opole.pl), Tomasz Boczar (Technical University of Opole, ul. Miko ${ }^{3}$ ajczyka 5, 45-271 Opole, Poland, t.boczar@po.opole.pl), Andrzej Cichon (Technical University of Opole, ul. Miko ${ }^{3}$ ajczyka 5, 45-271 Opole, Poland, a.cichon@po.opole.pl)

The subject matter of the research work presented in this paper refers to the measurements of the values of the acoustic pressure levels (noise) occurring around a main feeding-point aerial substation adjacent to apartment buildings. The paper presents the values of the noise accompanying the particular power devices, mainly transformers, during their regular operation. The main aim of this research work was the comparison and assessment of the acoustic pressure values measured with the permissible values defined by environmental standards binding in Poland. The research analysis carried out proved that during a long-term operation of power appliances installed in substations the acoustic pressure level that they emit into the environment is not constant but is subject to changes. Thus the increase of the noise level above the permissible values can be the cause of violation of environmental standards. Due to a significant increase of people's awareness and readiness to claim their rights, the main consequence of violating the standards is a growing number of claims. Therefore it is imperative that the level of the acoustic pressure be monitored during routine tests, especially around highpower transformers.

2pNSe2. Investigation and application of theoretical acoustic field model evaluating the change of environmental conditions. Robertas Mikalauskas (Kaunas University of Technology, K.Donelaicio str. 73, LT44029 Kaunas, Lithuania, robertas.mikalauskas@ktu.lt), Vitalijus Volkovas (Kaunas University of Technology, K.Donelaicio str. 73, LT44029 Kaunas, Lithuania, tsdi@ktu.lt)

The effectiveness of the means used to reduce the noise depends on many factors. One of the main factors is the sources which generates acoustic field. In industrial premises there are many sources, their impact to acoustic field differs, and the source may also change due to environmental conditions. Therefore the task of the creation of the mobile noise reduction means, their rational arrangement and control becomes more and more relevant. In this case theoretical modeling of interaction of acoustic noise control systems with environment has significant importance for creating such systems. This paper provides acoustic field model using FEM, which imitates changing conditions of real industrial premise, evaluates the effectiveness and application possibilities of the mobile noise reduction system. It also provides the comparison of the results of theoretical experiment with experimental results. The results showed that with the help of the model mobile noise reduction system can be modeled, their effectives might be evaluated considering the change of sources and reflection planes, and the structural model of the investigative room can be supplemented with acoustic noise reduction means - noise reduction screens, selecting their geometric dimensions, arrangement in the space and selecting the materials and predicting the values of acoustic field parameters in the real object point under consideration.

2pNSe3. Effects of personal stereo use: pilot results from 20 university students. Eric De Santis (Acoustics, Aalborg University, Fredrik Bajers Vej 7 B5, 9220 Aalborg $\varnothing$, Denmark, ems@es.aau.dk), Rodrigo Ordoñez (Acoustics, Aalborg University, Fredrik Bajers Vej 7 B5, 9220 Aalborg Ø, Denmark, rop@es.aau.dk), Karen Reuter (Acoustics, Aalborg University, Fredrik Bajers Vej 7 B5, 9220 Aalborg $\varnothing$, Denmark, kr@es.aau.dk), Dorte Hammersh øi (Acoustics, Aalborg University, Fredrik Bajers Vej 7 B5, 9220 Aalborg Ø, Denmark, dh@es.aau.dk)

Modern personal stereo systems have the ability to store thousands of music files in small, relatively low-cost, compact devices. The popularity and widespread presence of the MP3 player cannot go unnoticed in contemporary society. Given a sufficient noise level and listening duration, consumers are at risk of developing a noise-induced hearing loss. A study into the effects of personal stereo use will be carried out with the intention of investigating common noise exposure levels in realistic settings, user listening habits and the hearing status in a group of listeners. Noise exposure levels are to be obtained by using the manikin technique described in the ISO 
11904-2 standard. The questionnaire to be presented to interested participants is designed to report personal stereo listening habits and to screen subjects for the hearing status evaluation. The hearing status of selected subjects will be evaluated through the use of conventional and possibly extended high-frequency audiometry, transient-evoked otoacoustic emissions and distortion product otoacoustic emission fine structures.

2pNSe4. Classroom noise in schools containing children learning English as a second language. Gina Ramirez (5924 N. Campbell Avenue, Chicago, IL 60659, USA, gina.ramirez@loop.colum.edu)

This study analyzes the acoustics of two elementary schools containing both native English speaking children and large English learning populations, to determine if facilities are best being utilized acoustically. As stated by the ASA, children learning English require a more favorable signal-to- noise ratio and should thus be instructed in quieter learning environments The study examined at least 20 classrooms in each facility by measuring ambient noise level and reverberation time by methodologies outlined in the ANSI S12.60 (2002) standard. Creating a noise mapping allowed for the evaluation of each school based on utilization of pre-existing facilities Based on preliminary results all classrooms tested failed to meet the maximum A-weighted steady background noise level specified in the standard furthermore schools placed English learning students in environments that were up to $10 \mathrm{~dB}$ louder than classrooms used for native English speaking students, demonstrating that more attention to ambient noise level and its effect on speech intelligibility is needed when planning the layout of learning facilities containing large English learning populations. The ongoing analysis of the data aims at assessing the impact of the measured acoustical environments in terms of perceived intelligibility by using a survey tool among the student population.

TUESDAY AFTERNOON, 1 JULY 2008

P2-A, LEVEL 2, 3:40 TO 5:20 P.M.

\title{
Session 2pNSf
}

\section{Noise, Computational Acoustics, and EURONOISE: Time-Domain Modeling Methods in Acoustics I (Poster Session)}

\author{
Paul Calamia, Cochair \\ Rensselaer Polytechnic Institute
}

\author{
Maarten Hornikx, Cochair \\ Applied Acoustics, Chalmers University of Technology
}

\begin{abstract}
All posters will be on display from 3:40 p.m. to 5:20 p.m. To allow contributors an opportunity to see other posters, contributors of odd-numbered papers will be at their posters from 3:40 p.m. to 4:30 p.m. and contributors of even-numbered papers will be at their posters from 4:30 p.m. to 5:20 p.m.
\end{abstract}

\section{Contributed Papers}

2pNSf1. Optimization of compact finite difference method for wave acoustic simulation. Hideo Tsuru (Nittobo Acoustic Engineering, 1-21-10 Midori Sumida-ku, 130-0021 Tokyo, Japan, tsuru@noe.co.jp), Reima Iwatsu (Tokyo Denki University, Kanda-Nishikicho 2-2, Chiyoda-ku, 1018457 Tokyo, Japan, iwatsu@cck.dendai.ac.jp)

Recently, the finite difference method, a tool for wave acoustic simulations, can be applied to practical analysis. However, the numerical dispersion which makes propagation speed in simulations change according to the wave length, is a severe problem to maintain high accuracy in the analysis. The numerical error accompanied by the time integration hinders to carry out an accurate simulation. An examination of a compact finite difference scheme which can approximate differentiation precisely on a comparatively large grid spacing was made. It was shown that the adjusting the coefficients in the compact finite difference scheme can simulate a wave propagation accurately with a small amount of computation. The improvement method of the time integration was also discussed. The possibility of improvement of accuracy through a multi-step integration is shown.

2pNSf2. Time-domain modeling of porous media acoustics. David K. Wilson (U.S. Army Engineer Research and Development Center, 72 Lyme Rd., Hanover, NH 03755-1290, USA, D.Keith.Wilson@usace .army.mil), Sandra L. Collier (U.S. Army Research Lab, Attn: AMSRDARL-CI-EE, 2800 Powder Mill Rd., Adelphi, MD 20783, USA, sandra.collier@us.army.mil), Vladimir E. Ostashev (NOAA/Earth System Research Laboratory, 325 Broadway, Boulder, CO 80305, USA,
vladimir.ostashev@noaa.gov),David H. Marlin(U.S. Army Research Lab, Attn: AMSRD-ARL-CI-EE, 2800 Powder Mill Rd., Adelphi, MD 20783, USA, dmarlin@arl.army.mil), David F. Aldridge (Sandia National Laboratories, PO 5800, MS 0750, Albuquerque, NM 87185-0750, USA dfaldri@sandia.gov), Neill P. Symons (Sandia National Laboratories, PO 5800, MS 0750, Albuquerque, NM 87185-0750, USA, npsymon@sandia .gov)

Sound waves propagating in porous media are subject to strong dissipation and dispersion. This paper elaborates upon several recent publications by the authors regarding the time-domain description of these effects. The foundation is a relaxational description of the viscous and thermal dissipation in a rigid porous medium, which is shown to possess an exact, analytical conversion from the frequency to the time domain. The complex density and bulk modulus operators transform to temporal convolutions between a causal response function and the acoustic field variables. When the convolutions are neglected, the equations reduce to the well known ZwikkerKosten phenomenological model. The relaxation function can be inverted to provide an equivalent time-domain formulation from the complex volume and compressibility operators. Although a direct time-domain transformation of the specific impedance from the relaxation model has not been found, an accurate broadband approximation thereof can be transformed. The resulting time-domain boundary condition (TDBC) describes the absorptive and reactive response of the material. The response of the boundary decays slowly, as the inverse square root of time, but efficient numerical procedures are formulated that allow the TDBC to be approximated with a small number of recursive filters. 


\title{
Session 2pNSg
}

\section{Noise and EURONOISE: EU Projects for Aircraft Noise Reduction I (Poster Session)}

\author{
Dominique Collin, Chair \\ Snecma - Safran Group
}

\begin{abstract}
All posters will be on display from 3:40 p.m. to 5:20 p.m. To allow contributors an opportunity to see other posters, contributors of odd-numbered papers will be at their posters from 3:40 p.m. to 4:30 p.m. and contributors of even-numbered papers will be at their posters from 4:30 p.m. to 5:20 p.m.
\end{abstract}

\section{Contributed Papers}

2pNSg1. Effect of curvature on the scattering coefficients of Herschel-Quincke tubes. Benjamin Poirier (Université de Technologie de Compiègne, Centre de Recherche Royallieu, BP20529, 60205 Compiègne, France, bpoirier@utc.fr), Jean-Michel Ville (Université de Technologie de Compiègne, Centre de Recherche Royallieu, BP20529, 60205 Compiègne, France, jean-michel.ville@utc.fr), Cédric Maury (Université de Technologie de Compiègne, Centre de Recherche Royallieu, BP20529, 60205 Compiègne, France, cedric.maury@utc.fr), Félix Foucart (Université de Technologie de Compiègne, Centre de Recherche Royallieu, BP20529, 60205 Compiègne, France, felix.foucart@utc.fr)

The concept of Herschel-Quincke (HQ) tubes has been known since the beginning of the 20th century and was shown to be of interest to reduce tonal and broadband noise from turbofan engine. To help to understand the physical phenomena underlying the HQ concept, Hallez \& Burdisso have developed a 3D theoretical model. The 3D modelling technique considers the tubes-inlet interfaces as finite piston sources that couple the acoustic field inside a hard-walled duct with the acoustic field within the HQ tubes. This model makes a geometrical approximation by considering the tube as a straight duct whereas in reality it corresponds to a curved duct. In this paper, a model is presented which takes into account the curvature of the bend by integrating two differential equations for the pressure and velocity in the bend, projected on the local transverse modes. Results of some typical coefficients of the scattering matrix [S] deduced from the curved HQ model are compared with the 3D straight tube model, the experimental results and the numerical results. The curvature is shown to modify the HQ resonances and then have to be taken into account in the analytical formulation.

2pNSg2. Assessment of noise impacts of the air traffic. Katrin Ohlau (Universität Stuttgart / IER, Heßbrühlstraße 49a, 70565 Stuttgart, Germany, katrin.ohlau@ier.uni-stuttgart.de), Peter Bickel (Universität Stuttgart/IER, Heßbrühlstraße 49a, 70565 Stuttgart, Germany, peter.bickel@ier .uni-stuttgart.de), Rainer Friedrich (Universität Stuttgart/IER, Heßbrühlstraße 49a, 70565 Stuttgart, Germany, rainer.friedrich@ier.uni-stuttgart.de)
Against the background of the generally increasing traffic and especially the expansion plans in doubling the air traffic until 2020, this paper presents an assessment of noise effects of air traffic and underlines its future meaning. In several projects including the EC funded projects HEATCO (Developing Harmonised European Approaches for Transport COsting and Project Assessment), GRACE (Generalisation of Research on Accounts and Cost Estimation) and ASSET (ASsessing SEnsitiveness to Transport), methodologies for assessing noise including aircraft noise have been developed, improved and applied. The methodologies focus on the impact pathway approach, i.e. first noise levels are estimated, and then level- response functions are applied to calculate health impact and annoyance level distributions. Afterwards it is evaluated by transforming the impacts into monetary levels. Our studies for assessing annoyance have been carried out with the contingent valuation method. Results of the monetary valuation will be shown for the airports Frankfurt, Hamburg, Berlin-Tegel, Düsseldorf, Köln/Bonn, München and Stuttgart. The total air traffic noise costs for Germany range from EUR 150 million to EUR 1200 million per year.

2pNSg3. Efficiency of shielding effect on installed double stream nozzles. Sébastien Aeberli (SNECMA - groupe SAFRAN, Villaroche Rond-point R. Ravaud, 77550 Moissy-Cramayel, France, sebastien.aeberli @ snecma.fr)

In spite of acoustic improvements on jet noise brought by innovative technologies such as chevrons, a way to ensure important acoustic gains is to optimize the power plant integration on aircrafts in order to benefit of the shielding effect due to wing, empennage and fuselage surfaces. In this presentation, these effects are illustrated thanks to a jet noise test campaign at 1/10th scale in an anechoic chamber as part of a European project. This campaign gives a better understanding of shielding effect by a wing and also of the acoustic impact of pylon azimutal direction on two representative BPR 9 nozzles (Baseline - smooth - and Low Noise - with chevrons). The efficiency of shielding effect on jet noise sources in these configurations is assessed. 


\title{
Session 2pNSh
}

\section{Noise and EURONOISE: Action Planning and Global Solutions for Urban Noise II (Poster Session)}

\author{
Klaus Genuit, Cochair \\ HEAD acoustics $\mathrm{GmbH}$
}

\author{
Itziar Aspuru Soloaga, Cochair \\ Labein-Tecnalia
}

\begin{abstract}
All posters will be on display from 3:40 p.m. to 5:20 p.m. To allow contributors an opportunity to see other posters, contributors of odd-numbered papers will be at their posters from 3:40 p.m. to 4:30 p.m. and contributors of even-numbered papers will be at their posters from 4:30 p.m. to 5:20 p.m.
\end{abstract}

\section{Contributed Papers}

2pNSh1. A proposal for doing a touch of anova with noise levels. Michel Maurin (INRETS-LTE, case 24, 69675 Bron cedex, 69500 Bron, France, maurin@inrets.fr)

Because noise levels are logarithm of additive variables, the usual numerical processings are not suitable for them (mind $60+60 \neq 120$ ). Then one has to deal with them differently, following some other syntaxic rules which may be called in a general way the "logic of levels". Of course this question is correctly resolved with the "energetic mean" but it fails as soon as one has to deal with variances of levels or covariances with other variables (data analysis, linear regressions, œ). Some (necessarily) approximate compromises may be developped between the logic of levels and the arithmetical logic, (transforming variables in statistics for instance). Here we introduce a new statistic, the h-dispersion, which is not a variance but has many properties of variance, and very fortunatly takes account of the logarithmic status of data. This new compromise may be employed for levels in some cases of design of experiments, such as one-way cases and comparisons "beforeafter" in relation to acoustics operations for instance, and also more or less for two-ways cases. It remains approximate (a syntaxic compromise), but in any time this is an improvement in comparison with a crude ANOVA on levels.

2pNSh2. Study of the surface traffic inside the Cluj-Napoca urban agglomeration. Geamilia Solea (Technical University of Cluj-Napoca, Faculty of Machines Design; Department of Mechanics and Computer Programming, B-dul Muncii; Nr. 103-105, 400641 Cluj-Napoca, Romania,
marylandprod@gmail.com),Mariana Arghir(Technical University of ClujNapoca, Faculty of Machines Design; Department of Mechanics and Computer Programming, B-dul Muncii; Nr. 103-105, 400641 Cluj-Napoca, Romania, marianaarghir@yahoo.com), Dan Borza (National Institute of Applied Sciences of Rouen, INSA Rouen, LMR, Ave l'Universite, BP8 76800 Rouen, France, dan.borza@insa-rouen.fr), Mariana Runcan (National Institute of Applied Sciences of Rouen, INSA Rouen, LMR, Ave l'Universite, BP8 76800 Rouen, France, mariruncan@yahoo .com)

The environment's protection is one of the most actual problems, because of the fact that pollution is a major danger for planet life. The environment's pollution is caused because some pollution agents are in too large a quantity for a long time and they become dangerous for human health, for the flora, for the fauna and for the constructions too. The pollution sources can be classified as natural and artificial sources. The quality of the environment from a specific area at a certain time can be determined by: a) the air quality; b) the water quality; c) the soil quality; d) the health level of the population; e) the decrease of animals and plants species that are accounted for. In the paper there are given the urban traffic on the principal routes inside the Cluj-Napoca municipality. The traffic is the constituent part of the research activity of the national project having the destination the monitoring of the noise and vibrations into an urban agglomeration, applied to the Cluj - Napoca town. This study is the first step for the "noise map" asked for by the Romania integration in the European Union for the team researchers of TUCN under the the direction of Dr. Mariana Arghir. 


\title{
Session 2pNSi
}

\section{Noise and EURONOISE: Railway Noise and Vibration I (Poster Session)}

\author{
Richard Carman, Cochair \\ Wilson, Ihrig \& Associates \\ Franck Poisson, Cochair \\ SNCF DIR \\ David Thompson, Cochair \\ Inst. of Sound and Vibration
}

\begin{abstract}
All posters will be on display from 3:40 p.m. to 5:20 p.m. To allow contributors an opportunity to see other posters, contributors of odd-numbered papers will be at their posters from 3:40 p.m. to 4:30 p.m. and contributors of even-numbered papers will be at their posters from 4:30 p.m. to 5:20 p.m.
\end{abstract}

\section{Contributed Papers}

2pNSi1. On effectiveness of vibration isolation using super-elastic rail support combined with booted sleeper or floating slab. Tianxing X. Wu (Shanghai Jiao Tong University, School of Mechanical Engineering, 800 Dong Chuan Road, 200240 Shanghai, China, txwu@sjtu.edu.cn)

Ground vibration or structure vibration of viaduct due to railway traffic can be reduced by use of super-elastic rail support, booted sleeper and floating slab to isolate vibration transmission from the track to the infrastructure. In this study modeling in the frequency domain of track structure dynamics and wheel/rail interaction is carried out. Wheel/rail interaction and transmission to the infrastructure of the interaction force due to a relative displacement excitation are simulated. The effectiveness of vibration isolation is analyzed for booted sleeper or floating slab combined with super-elastic rail fastener. It is found that the combination of super-elastic rail fastener with booted sleeper or floating slab can effectively reduce the wheel/rail interaction force in the medium frequencies, compared with the booted sleeper or floating slab track using usual rail pad. The combination also shows better ability to block vibration transmission from the track to the infrastructure in the medium and high frequencies.

2pNSi2. Overview of Railway Noise Control in Europe. Jakob Oertli (Swiss Federal Railways, Schanzenstrasse 5, 3000 Bern, Switzerland, jakob.oertli@sbb.ch)

European and national legislation, increasing freight traffic, political pressure and many more factors contribute to make noise an issue of increasing importance for the railways. This paper provides an overview of railway noise abatement efforts throughout Europe. Different countries in Europe have adopted varying noise abatement strategies, ranging from an emphasis on infrastructure based measures to a combination of rolling stock measures with noise barriers. Important in the current European discussion is the development and retrofitting of freight wagons with K- and LL-brake blocks. To support this retrofitting a wide variety of incentives, such as differential track access charges, operational restrictions or direct subsidies are being discussed. The current efforts by the European Commission, the International Union of Railways (UIC), the Community of European Railways (CER) and other international organisation to promote silent railways are presented.

2pNSi3. A guideline for prediction and control of noise in railroads maintenance activities. Sergio Luzzi (Vie En.Ro.Se. Ingegneria, Via Stibbert, 1, 50134 Florence, Italy, sergio.luzzi@vienrose.it)

In railroads routine maintenance as well as emergency maintenance can be represented by the acoustic point of view as a system of classified yard activities, including catalogued single and grouped noise sources, that is moved along the tracks areas. RFI (Rete Ferroviaria Italiana), the Italian National Railway Company, has provided a guideline for the correct evaluation of noise impact on receivers surrounding the yards, which can be adapted to many situations fitting the specific national law and local regulations disposals about noise assessment, prediction and reduction in temporary activities. Starting from their long term experience, the authors have developed a method for the production of local reports on maintenance railroad yard activities based on the modelling of standard scenarios. Each machinery employed in yards (for superstructure laying or repairing, electric traction, etc, has been measured and characterized as noise source or as part of a noise sources system (emission box). The noise propagation scenarios are then build using an MS Excel based adaptation software. This methodology represents the technical and procedural architecture of the guideline which provides materials for the management of all the different typologies of maintenance yards operating on the RFI railroad network.

2pNSi4. Railway vibration mitigation - An innovative concept. Frederic Le Corre (Alstom Transport, TGS Engineering - 33, rue des Bateliers, 93400 Saint-Ouen, France, frederic.le-corre@transport.alstom .com), Christine Charles (Alstom Transport, TGS Engineering - 33, rue des Bateliers, 93400 Saint-Ouen, France, christine.charles@transport.alstom .com), Marcel Girardi (Alstom Transport, TGS Engineering - 33, rue des Bateliers, 93400 Saint-Ouen, France, marcel.girardi@transport.alstom.com)

These last decades, rail transportation has known an amazing development. Hence, the resulting pollution on people living alongside lines or above underground lines has become a crucial subject of consideration. The disturbance has two origins: the direct noise or vibration from the railway traffic and the noise produced by vibration of the building walls, the so-called re-radiated noise. Noise and vibration excitation is essentially due to wheel and rail roughness at the contact point. From this excitation point, the track acts as a filter of vibrations. Hence, spectrum of emitted vibrations can be controlled with the design of railway track. Providing vibration mitigation with the track is now commonly encountered, especially on urban projects (LRT MRT). This is usually achieved by introducing an adequate resilient layer to provide the required attenuation. Alstom Transport and Sateba are developing a system to mitigate vibrations. This system called AFST is based on the booted sleeper system. After a complete analysis of critical parameters, a special design has been drawn. The resulting antivibratile solution is a high-performance system suiting areas where vibration mitigation and/or attenuation of re-radiated noise are required. 
2pNSi5. Noise and vibrations at tram track intersection. Stjepan Lakusic (University of Zagreb, Faculty of Civil Engineering, Kaciceva 26, 10000 Zagreb, Croatia, laki@grad.hr), Marijan Bogut (KONCAR - Electrical Engineering Institute, Fallerovo setaliste 22, 10000 Zagreb, Croatia, mbogut@koncar-institut.hr), Visnja Tkalcevic Lakusic (University of Zagreb, Faculty of Civil Engineering, Kaciceva 26, 10000 Zagreb, Croatia, visnjat@grad.hr

In urban areas noise and vibration due to public traffic diminish the quality of life. In Zagreb, tram traffic is the most important form of public transport. Tram network has $120 \mathrm{~km}$ of the tracks and 190 trams are used for passengers transport every day. In the movement of the vehicle over track, the interaction between the wheel and track plays the most important role in the field of noise and vibrations. The increasing of noise and vibrations are particularly emphasized at track intersection where a given number of track switches and crossings are installed. This paper presents an experimental study of noise and ground vibrations through the passage of three types of tram vehicles. The measurements were done on the tram intersection with the highest traffic volume in Zagreb's tram network. Two types of track construction were analyzed, discrete and continuous rail fastening system. During the track reconstruction at this intersection, the existing discrete rail fastening system was replaced with a continuous. Because of that, the measurements were carried before and after reconstruction at the same tes points. Test results have showed significant reductions of noise and vibrations at track intersection in the case of continuous rail installation.

2pNSi6. Numerical and analytical models for high speed train pantograph radiated noise prediction. Massimo Viscardi (Dept. of
Aerospace Engineering - University of Naples, Via Claudio, 21, 80125 Naples, Italy, massimo.viscardi@unina.it), Michele Iadevaia (Dept. of Aerospace Engineering - University of Naples, Via Claudio, 21, 80125 Naples, Italy, miadevaia@inwind.it), Salvatore Melchionna (Dept. of Aerospace Engineering - University of Naples, Via Claudio, 21, 80125 Naples, Italy, s.melchionna@active-group.it), Leonardo Lecce (Dept. of Aerospace Engineering - University of Naples, Via Claudio, 21, 80125 Naples, Italy, leonardo@unina.it)

The present work deals with the development and comparison of an analytical and numerical models for the evaluation of the noise radiated from a pantograph of an high speed train. Under the numerical point of view, a simplified model has been developed for the pantograph; the model that substructures the problem approaching a complex structure as a combination of simple components, has been derived from a similar aeronautical problem (landing gear noise) but has been self-modified to adapt the formulation to the specific problem. The output of the model is the radiated noise level and spectra as a function of the pantograph speed. The CFD numerical model has been developed for the pantograph based upon a commercial code; only the upper part of the system has been herein simulated because it was addressed as the main noise source during previous laboratory acoustic tests As an output of the model, the radiated noise has been derived with special reference to two main speed to which experimental data could be referred. Analytical and numerical results will be within the paper discussed and compared to highlight the single approach's advantage and drawbacks. At the and of the paper, some line experimental results will be also introduced and discussed.

\title{
Session 2pNSj
}

\section{Noise and EURONOISE: Noise, Structure Borne Noise from Building Technical Equipment, and Ground Borne Noise from Railways I (Poster Session)}

\author{
James Nelson, Cochair \\ Wilson, Ihrig and Associates, Inc. \\ Michel Villot, Cochair \\ CSTB
}

\begin{abstract}
All posters will be on display from 3:40 p.m. to 5:20 p.m. To allow contributors an opportunity to see other posters, contributors of odd-numbered papers will be at their posters from 3:40 p.m. to 4:30 p.m. and contributors of even-numbered papers will be at their posters from 4:30 p.m. to 5:20 p.m.
\end{abstract}

\section{Contributed Papers}

2pNSj1. Low frequency noise reduction from building technical equipment: A case study. Kathleen Kondylas (NEVA Associates Noise Control, 15 Beck Street, Newburyport, MA 01950, USA, kondylas@earthlink.net), Chris R. Fuller (Virginia Tech, 131 Durham Hall, Blacksburg, VA 24061, USA, christopher.r.fuller@nasa.gov), Joseph A. King (DuPont, 5401 Jefferson Davis Highway, Richmond, VA 23234, USA, joseph.a.king@usa.dupont.com), Natalia Levit (DuPont, 5401 Jefferson Davis Highway, Richmond, VA 23234, USA, natalia.v.levit@usa.dupont .com)

Mechanical equipment (fans, chillers, motors) produces airborne and structure-borne noise with a significant low frequency component. However, in many applications, mitigation of the low frequency noise requires imple- mentation of significant mass barrier in combination with an air space. This work presents case studies on the use of a novel lightweight and thin acoustical material to mitigate low frequency noise from mechanical equipment. This advanced material, based on patented distributed absorber technology, was used to treat the metal housing of the mechanical equipment. The nove material effectively increased transmission loss of the metal housing below $300 \mathrm{~Hz}$. The total noise reduction was 10-14 dB (A) with $5 \mathrm{~dB}$ improvement over typical flexible acoustical blankets below $300 \mathrm{~Hz}$.

2pNSj2. Experimental and numerical study of the ground transmission of structure-borne sound generated by trams. Guillaume Coquel (RATP - CSTB, 13, Rue Jules Vallès, 75547 Paris Cedex 11, France. 
guillaume.coquel@cstb.fr),Philippe A.Jean(CSTB, 24 rue JosephFourier, 38400 Saint-Martin-d'Hères, France, philippe.jean@cstb.fr), Michel Villot (CSTB, 24 rue Joseph Fourier, 38400 Saint-Martin-d'Hères, France, michel.villot@cstb.fr)

The increase of railway traffic in urban areas leads to developments in modelling and understanding the propagation of structure borne sound through soil, foundation and structure. Vibration propagation through the ground, is still not well known. The present paper focuses on the propagation of trams vibrations $(20-250 \mathrm{~Hz})$ in the ground and the energy transmission to the structure. In the first part, calculations performed using a $2 \mathrm{D}$ code based on a FEM/BEM approach are compared to measurements performed on a test site. The railway excitation is produced with a vibrator in order to recreate a line of uncorrelated forces. Soil properties are evaluated by an analysis of surface waves. Both bending and in-plane waves are measured on the structure. The ratio of the soil velocity over the structure velocity is computed in order to evaluate the accuracy of the computation. A 2,5D modelisation is also performed in order to evaluate the effect of a point source on the velocity fields on the soil and on the structure. In the second part, a parametric study using the $2 \mathrm{D}$ code is performed on typical cases in order to evaluate the modification of vibration transmission from the ground to the foundation.

2pNSj3. A parametric study on the isolation of ground-borne noise and vibrations in a building using a coupled numerical model. Péter Fiala (Budapest University of Technology and Economics, BME Dept. of
Telecommunications, Magyar tudósok körútja 2, H-1117 Budapest, Hungary, fiala@hit.bme.hu), Shashank Gupta (KU Leuven, Department of Civil Engineering, Kasteelpark Arenberg 40, B-3001 Leuven, Belgium, shashank.gupta@bwk.kuleuven.be), Geert Degrande (KU Leuven, Department of Civil Engineering, Kasteelpark Arenberg 40, B-3001 Leuven, Belgium, geert.degrande@bwk.kuleuven.be), Fülöp Augusztinovicz (Budapest University of Technology and Economics, BME Dept. of Telecommunications, Magyar tudósok körútja 2, H-1117 Budapest, Hungary, fulop@ hit.bme .hu)

Underground traffic induced vibrations and noise in buildings are a major environmental concern in urban areas. To quantify these vibrations a numerical prediction model has been developed and validated. A coupled FE-BE model is used to compute the incident ground vibrations due to the passage of a train in the tunnel. A dynamic soil-structure interaction model is used to determine the vibration levels of the building. The soil-structure interaction problem is solved by means of a 3D boundary element method for the soil coupled to a 3D finite element method for the structural part. An acoustic 3D spectral finite element method is used to predict the acoustic response. The coupled numerical model is used to quantify the efficiency of vibration and noise mitigation measures at different stages of the vibration propagation chain. Vibration isolation with a floating slab track is modeled on the source side, base isolation is incorporated in the structure model, and a box-within-box arrangement is considered for the isolation of re-radiated noise in the building's rooms. The insertion gain of the three methods is compared using the model of a multi-story portal frame office building subjected to ground-borne vibrations from an underground railway line.

TUESDAY AFTERNOON, 1 JULY 2008

P2-A, LEVEL 2, 3:40 TO 5:20 P.M.

\title{
Session 2pNSk
}

\section{Noise and EURONOISE: Physical and Psychophysical Evaluation of Vehicle Exterior Noise II (Poster Session)}

\author{
Paul Donavan, Cochair \\ Illingworth \& Rodkin, Inc.
}

Hugo Fastl, Cochair

AG Technische Akustik, MMK, TU München

\begin{abstract}
All posters will be on display from 3:40 p.m. to 5:20 p.m. To allow contributors an opportunity to see other posters, contributors of odd-numbered papers will be at their posters from 3:40 p.m. to 4:30 p.m. and contributors of even-numbered papers will be at their posters from 4:30 p.m. to 5:20 p.m.
\end{abstract}

\section{Contributed Papers}

2pNSk1. Questionnaire survey on vehicle horn use. Masahiko Takada (Omihachiman Community Medical Center, 1379 Tsuchida-cho, 523*0082 Omihachiman, Japan, takada@design.kyushu-u.ac.jp), Yoko Fukuda (Foster Electric Company, Limited, 512 Miyazawa-cho, Akishima City, 196-8550 Tokyo, Japan, yoko_19831228@yahoo.co.jp), Shin-Ichiro Iwamiya (Kyushu University, 4-9-1 Shiobaru, Minami-ku, 815-8540 Fukuoka, Japan, iwamiya@ design.kyushu-u.ac.jp)

The Japanese Road Traffic Law provides that a vehicle horn should be utilized in the dangerous situation or the place where the horn sign was installed. However, drivers are frequently seen to use their vehicle's horn as a signal in the situations when they pass each other. Given such situations, the mentioned above law is likely to become a law in name only. Therefore, a questionnaire survey was carried out to ascertain the current circumstance of a vehicle horn use. The respondents were asked the latest or last remem- bered case of horn use in various situations of both their driving or as pedestrians. With regards to an experience of being tooted at by another driver, the questionnaire included questions concerning place, traffic volume, the aim of the horn use, time pattern of horn tooting, respondent's mental reaction when tooted at by another driver, etc. As a result, drivers mostly had negative mental reactions (i.e., startled, noisy and irritating) by long toots. Pedestrians' negative mental reactions were mainly aroused by horn use calling another's attention to something or to inform of a danger. Furthermore, nearly 40 percent of the respondents were discontented with the volume and the sound quality of horn.

2pNSk2. Sound Quality Evaluation for the Axle Gear Noise in the vehicle. Hyun Ho Lee (Inha University, Mechanical Engineering, 253 Yonghyun Dong, 402-751 Inchon, Republic of Korea, hyunho0424@naver 
.com),Ho Wuk Kim(Inha University, Mechanical Engineering, 253 Yonghyun Dong, 402-751 Inchon, Republic of Korea, mowugi@gmail.com) , Sang Kwon Lee (Inha University, Mechanical Engineering, 253 Yonghyun Dong, 402-751 Inchon, Republic of Korea, sangkwon@inha.ac.kr)

A gear whine sound due to the axle system is one of the most important sound qualities in a sport utility vehicle (SUV). In the previous works about the gear whine sound, it was known that it is difficult to evaluate the gear whine sound objectively by using the only A-weighted sound pressure level because of the masking effect. In this paper, for the objective evaluation of the axle-gear whine sound, the characteristics of the axle-gear whine sound is at the first investigated based on the synthetic sound technology and the new objective evaluation method for the axle-gear whine sound is developed by using the sound metrics, which is the psychoacoustic parameters, and the artificial neural network (ANN) used for the modeling of the correlation between objective evaluation and subjective evaluation. This model is successfully applied the objective evaluation of the axle-gear whine sound for real sport utility vehicles and the output of the model are compared with subjective evaluation. The results have a good correlation over 90 per cent.

2pNSk3. Effect of coolant temperature on combustion and combustion noise fluctuations in a Diesel engine at idle conditions. Matthieu Gazon (Coria CNRS UMR6614, Site Universitaire du Madrillet, Avenue de l’Université BP12, 76801 St Etienne du Rouvray, France, gazon@coria.fr) , Jean Bernard Blaisot (Coria CNRS UMR6614, Site Universitaire du Madrillet, Avenue de l'Université BP12, 76801 St Etienne du Rouvray, France, Jean-Bernard.Blaisot@coria.fr), Nicolas Duclaux-De-L'Estoille (Renault SAS, 1 Allée Cornuel, 91510 Lardy, France, nicolas.duclaux-de-lestoile @ renault.com), Shanjin Wang (Renault SAS, 67, rue des Bons Raisins, 92508 Rueil Malmaison, France, shanjin.wang@renault.com)

At idle conditions, the cycle-to-cycle fluctuations in Diesel engines lead to combustion noise variations that could be annoying for people, even if the mean level is low, and the cold start condition is one of the worst cases. This study focuses on the effect of injection parameters (duration and timings) and the effect of coolant temperature on combustion characteristics and on the combustion noise. A Direct Injection Diesel engine (four cylinders, 16 valves) equipped with a common-rail injection system and piezoelectric injectors was used. The coolant temperature is changed at idle from the ambient temperature to the temperature at normal working conditions. The combustion is analysed in terms of rate of heat release and combustion noise through the measurement of the cylinder pressure. The results show the dependency of the combustion behavior on the increase of coolant temperature at constant engine adjustment parameters. It is also shown that acting on adjustment parameters can modify the combustion behavior and, consequently, the cycle-to-cycle fluctuations. A trade-off between combustion noise and engine emissions can thus be reached.

2pNSk4. Sound perception of urban delivery trucks. Florence Manca-Demirdjian (Laboratoire Vibrations Acoustique - INSA Lyon, 25 bis avenue Jean Capelle, Bâtiment Saint-Exupéry, F-69621 Villeurbanne cedex, France, florence.manca@gmail.com), Etienne Parizet (Laboratoire Vibrations Acoustique, Insa Lyon, 25 bis, av. J. Capelle, 69621 Villeurbanne Cedex, France, etienne.parizet@insa-lyon.fr)

The study deals with the annoyance of urban delivery trucks noises Sound sequences of two lorries were recorded during a typical delivery : a commercial one and a prototype specially designed to reduce sound emissions. Various sources could be heard during these sequences (engine, doors, hydraulic rear doors). These recordings (the duration of which being more than 5 minutes) were evaluated by subjects in a sound proofed room by two methods. The first one was a continuous assessment of sequences : the listeners had to continuously assess their annoyance by moving a cursor sliding along a five levels graduated scale (from "not at all annoying" to "extremely annoying"). The second one was an overall evaluation (using the same scale) of each event of the sequences (for example : the arrival of the truck, or the closing of the driver's door, etc...). The comparison of the results obtained from these two methods for both lorries will be presented, as well as the reduction of the annoyance could be realized on the prototype. 


\title{
Session 2pNSI
}

\section{Noise and EURONOISE: Environmental Noise Mapping III (Poster Session)}

\author{
Kenneth Cunefare, Cochair \\ The Georgia Inst. of Technol. \\ Gaetano Licitra, Cochair \\ ARPAT - Dept. Firenze
}

\begin{abstract}
All posters will be on display from 3:40 p.m. to 5:20 p.m. To allow contributors an opportunity to see other posters, contributors of odd-numbered papers will be at their posters from 3:40 p.m. to 4:30 p.m. and contributors of even-numbered papers will be at their posters from 4:30 p.m. to 5:20 p.m.
\end{abstract}

\section{Contributed Papers}

2pNSI1. Low frequency noise impact of road traffic in the Netherlands J. Jabben (RIVM, A. van Leeuwenhoeklaan 9, 3720 BA Bilthoven, Netherlands, jan.jabben@rivm.nl), Eric Schreurs (RIVM, A. van Leeuwenhoeklaan 9, 3720 BA Bilthoven, Netherlands, eric.schreurs@ @rivm.nl)

Current traffic noise impact assessments are usually based on broadband A-weighted noise indicators. A more complete picture and better correlation with annoyance and health effects may result from indicators that include temporal aspects and frequency character. These aspects are currently under investigation at RIVM. This paper gives an evaluation of low frequency noise impact from road traffic in the Netherlands. The results are based on low frequency noise maps covering the entire road network. In particular the effectiveness of various types of noise control measures, such as noise barriers or walls, porous asphalt layers and reduction of noise emission, with regards to low frequency impact were studied. The results indicate for low frequency noise levels, powertrain noise and freight traffic in general, are of major importance for environmental quality.

2pNSI2. In Situ Characterization and Noise Mapping of Ships Moored in the Port of Venice. Antonino Di Bella (Dept. Technical Physics - University of Padova, Via Venezia 1, 35131 Padova, Italy, antonino.dibella@unipd.it), Andrea Tombolato (Studio di Acustica, Via Tripoli 16, 35141 Padova, Italy, tombolato@acusticapd.191.it), Stefano Cordeddu (Studio di Acustica, Via Tripoli 16, 35141 Padova, Italy, cordeddu@acusticapd.191.it), Emanuele Zanotto (Autorità Portuale di Venezia, Porto Commerciale - Molo A - Fabbricato 448, 30175 Marghera, Italy, emanuele.zanotto@port.venice.it), Marco Barbieri (Autorità Portuale di Venezia, Porto Commerciale - Molo A - Fabbricato 448, 30175 Marghera, Italy, marco.barbieri@port.venice.it)

The acoustical characterization and the mapping of noise emitted from ships were carried out on behalf of the Venice Port Authorithy in the frame of the European Project NoMEPorts (Noise Management in European Ports). According to the Directive 2002/49/CE (END), different noise sources (like roads, railways, aircraft, industrial sites, ports) have to be considered and mapped separately; so, in the first step, the acoustical characterization of ships is required. Annex IV of the Directive, in particular, establishes that the strategic noise maps for agglomerations shall put a special emphasis on the noise emitted by, inter alia, industrial activities, including ports. Three kinds of ships were investigated, using the Italian technical standards UNI 10855 and UNI 11143: 1. inland navigation ships (along wharves); 2. ferries (along wharves and when maneuvering); 3. cruises (along wharves and when maneuvering). As for the maneuvering, it could be said that a supplementary noise indicator is needed, as the END, Annex I, forsees in the case the average number of noise events in one or more of the day periods is very low (for example, less than one noise event an hour).

2pNS13. Mapping urban noise quota generated by a transport service. Sergio Luzzi (Vie En.Ro.Se. Ingegneria, Via Stibbert, 1, 50134 Florence, Italy, sergio.luzzi@vienrose.it)

The paper refers to a recent noise mapping experience, regarding the determination of quotas generated by urban transport services in complex areas. The first results of this study have been presented at Euronoise 2006 in Tampere and at Transport Noise 2006 congress in Saint Petersburg. Now the study is completed. Italian Law established that noise levels produced by buses, coaches and trams, must respect noise limits of area and road buffers classification. Public and Private transport companies are directly requested to point out their specific contributions levels and action plans for the reduction of those levels. At the same time, data for 2002/49/EC Directive have to be collected by them and given to authorities in agreement with formats and standards. The original methodology of source analysis, noise mapping and action planning studied and experienced by the authors in the Florentine area, applies a multi-variable couple-checking algorithm for the optimal choice of critical sites, to the whole road network of each company, reducing the number of calculations and conducting measurement campaigns only for the chosen critical "pivot" scenarios. Noise contribution quotas, generated by bus and coach traffic on linear sources and road systems, have been investigated and considered in models.

2pNSI4. Uncertainty of noise mapping software. Jacek Wierzbicki (AGH University of Science and Technology, Al. Mickiewicza 30, 30-059 Kraków, Poland, wierzbic@agh.edu.pl), Wojciech Batko (AGH University of Science and Technology, Al. Mickiewicza 30, 30-059 Kraków, Poland, batko@agh.edu.pl)

Noise map is a tool to come to noise policy and especially to an action plan. The most important question connected with noise mapping realized by computational methods is about reliability of results. It is closely connected with quality of input data and calculation algorithms and their parameters. An influence of elements location, buildings height and number of vehicles accuracy on calculated results was considered in first stage. Before implementation of Harmonoise/Imagine P2P algorithms there are differences between various software. The paper presents first results from investigations with two commercial prediction programs. The absolute accuracy (uncertainty) of predicted by software sound levels is very difficult to quantify because an uncertainty in the measurements. The main result of 
investigations is a table of differences in sound level calculations related to variation of input data (geometry of objects and acoustic parameters) and calculation algorithm.

2pNSI5. Noise mapping of major roads in Milan District. Alessandro Bisceglie (Department of Environmental Sciences of the University of Milano - Bicocca, Piazza della Scienza, 1, 20126 Milan, Italy, alessandro.bisceglie@unimib.it), Giovanni Zambon (Department of Environmental Sciences of the University of Milano - Bicocca, Piazza della Scienza, 1, 20126 Milan, Italy, giovanni.zambon@unimib.it), Fabrizio Artom (Phoneco S.r.1., Via San Cristoforo 84, 20090 Trezzano Sul Naviglio, Italy, artom@phoneco.it), Mattia Viganò (Phoneco S.r.l., Via San Cristoforo 84, 20090 Trezzano Sul Naviglio, Italy, vigano@phoneco.it), Franco Bertellino (Microbel s.r.l., via Livorno 60, 10144 Torino, Italy, franco.bertellino@microbel.it), Marco Longoni (Provincia di Milano, Corso di Porta Vittoria 27, 20122 Milano, Italy, m_longoni@provincia .milano.it)

The paper deals with noise map calculation, according to the first deadline of the END (Environmental Noise Directive, 2002/49/EC), for Milan district major roads. The roads with more than six million vehicle passages per year have been estimated to cover $405 \mathrm{~km}$ out of $1090 \mathrm{~km}$ total. Noise emission has been calculated from peak hour flow data for each segment of the whole road network and from some local traffic direct measurements. A statistical method to assign mean flow values to each road segment has been used, according to Italian reference periods. These data enable to use an acoustic model on a wide area with several line sources in order to obtain noise propagation maps of Lden and Lnight indicators. A procedure to estimate people exposed to different noise levels, as required by the END, has been also studied. Through a geographic information system it has been possible to intersect population input data (demographic density for different areas, buildings use type and buildings volumetric data) and noise mapping data. Every step of the procedure has been carried out complying with WGAEN Good Practice Guide recommendations, on the basis of the best available data in Milan district.

2pNSI6. 3D noise map of an hospital and noise sources evaluation. Paulo J. Sequeira Goncalves (EST / Instituto Politecnico de Castelo Branco, Av Empresario, 6000-767 Castelo Branco, Portugal, pgoncalves@est.ipcb .pt)

This paper presents the tri-dimensional acoustical characterization of Castelo Branco Hospital. Traffic and air conditioning machines produce noise that influences the Hospital and the buildings nearby. The results are presented in two dimensions at a height of four meters above the ground and in the vertical direction to access the noise levels in the building floors. For the construction of the model were used and compared to types of measurements of vehicles counting, for characterization of the traffic noise. The first approach was performed at every hour during the 24 hours of the day, by counting periods of 10 minutes. In the second approach counting was performed for 30 minutes on six time intervals, identified by inquiries to the nearby population and by a 24 hour noise measurement. The air conditioning machines were modelled using the Portuguese norm, NP EN ISO 3744. Correlation results taken from the two previous approaches and the noise measurements used to validate the Cadna/A model showed values of 0.87 and 0.85 . Since they are very similar, one can conclude that both approaches can be used in practice. Taking the economical factor, the second approach is preferable because it takes less time to be performed.

2pNS17. Management of the Tourist \& Entertainment Noise Using Noise Mapping Technique. Alan Štimac (DARH2 Acoustics \& Civil Eng. Ltd., Ljubicin prolaz 3, HR-10430 Samobor, Croatia, alan@darh2.hr), Aleš Globevnik (A-PROJEKT Natasa Kepe-Globevnik s.p., Vinarje 110b, SI-2000 Maribor, Slovenia, aprojekt@siol.net), Lilijana Kuhelj (Environmental Agency of the Republic of Slovenia, Vojkova 1B, SI-1000 Ljubljana, Slovenia, lilijana.kuhelj@gov.si)

In countries with strong orientation in tourism and entertainment, especially during summer months, noise problems are always present. This paper represents some applications of noise mapping techniques for the management of tourist \& entertainment noise that were conducted in Croatia and
Slovenia. The noise mapping techniques were applied for the prediction of noise levels on several places inside the cities of Varaždin and Pola (Croatia) including famous Roman amphitheatre, and in the city of Nova Gorica (Slovenia) especially during entertainment live-shows (concerts, movie festival etc). Such approach enables the city authorities to prepare the base for the implementation of noise policy with introducing such a system that would represent background documents for providing criteria of setting out areas on one side intended to entertainment and quiet areas on the other. For the daily activities in areas that are the most attractive for tourists, noisemodelling techniques have been used for predicting noise emitted from pubs and bars including terraces and loudspeakers. Such approaches have been used by city authorities to establish technique for "fixed quotas" that will be able to designate maximum area of terraces concerning number of seating places in relation with the type of bar/pub and noise emission.

2pNS18. Managing Input Data Uncertainty within Strategic Noise Mapping by Utilising Data Schema Specifications. Simon J. Shilton (Acustica Ltd, Trident One, Styal Road, M22 5XB Manchester, UK, simon.shilton@acustica.co.uk), Nigel Jones (Extrium Ltd, Calverley House, 55 Calverley Road, TN1 2TU Tunbridge Wells, UK, nigel.jones@extrium.co.uk), James Trow (Hepworth Acoustics Ltd, 5 Bankside, Crosfield Street, WA1 1UP Warrington, UK, james.trow @hepworth-acoustics.co.uk)

With the proclamation of the Directive on Environmental Noise (2002/49), the process of noise mapping and action planning has begun around Europe. Large scale wide area noise modelling requires an extensive range of data inputs. The requirements for these inputs are driven by several factors, including the noise calculation methodologies, noise mapping software system, GIS and the deliverables set out within the Directive and recommended Reporting Mechanism. Utilising a staged approach to project design led to the development of detailed noise mapping data schema specifications prior to the collation of input datasets for the calculation process. The data schema may be used throughout the mapping project as a framework to manage dataset concatenation, data capture or the use of defaults and GPGv2 Toolkits to infill data gaps. They also enable data preprocessing guidelines to be passed to non-acoustic GIS specialists, and help to guide and catalogue data processing steps. This paper presents experience gained whilst developing a number of noise mapping data schema specifications and their implementation within successful projects during the first round of mapping. Railway noise mapping is used as an example of how a data schema may be used to develop solutions to technical problems as well as coordinate data capture.

2pNS19. On railway noise modelling - an approach to the european interim method. J. Luis Bento Coelho (CAPS, Instituto Superior Técnico, TU Lisbon, Av. Rovisco Pais, P-1049-001 Lisbon, Portugal, bcoelho@ist.utl.pt), Diogo Alarcão (CAPS, Instituto Superior Técnico, TU Lisbon, Av. Rovisco Pais, P-1049-001 Lisbon, Portugal, diogo.alarcao@ist .utl.pt)

Railway noise results from a number of sources, with airborne and structure borne transmission mechanisms being responsible for the noise radiation and propagation. The European Directive 2002/49/EC requires the drawing of noise maps for areas near major transport infrastructures. The Portuguese Noise Act of 2007 further requires the drawing of noise maps for all transport infrastructures. This paper reports on our experience on noise mapping of the major Portuguese railway lines. The interim calculation model RMR96/SRMII, recommended in Directive 2002/49/EC, was adopted for the prediction of the railway noise, where a 1/1 octave band noise spectrum curve fitting approach was followed. Details on the implementation, adaptation and validation of the calculation procedure are reported. Emphasis on the required data such as train categories, superstructure, and speed profiles will be given. Examples and results for a number of major railway lines will be presented. 
2pNSI10. Is DNL appropriate for airport noise zoning? Jules G. Slama (UFRJ, Av. Horacio Macedo, 2030, Centro de Tecnologia, B1. G, Sala 204, Cidade Universitária, 21941-914 Rio de Janeiro, Brazil, julesslama@yahoo.com.br), Félix A. Mora-Camino (Ecole Nationale de l'Aviation Civile, 7 avenue Edouard-Belin BP 54005, 31055 Toulouse, France, felix.mora@enac.fr), Teo C. Revoredo (UFRJ, Av. Horacio Macedo, 2030, Centro de Tecnologia, B1. G, Sala 204, Cidade Universitária, 21941-914 Rio de Janeiro, Brazil, teorevoredo@yahoo.com.br)

The use of the DNL metric in airport noise zoning is based in the works of Schultz, that were later improved by Fidell. The basic idea is to use the percentage of highly annoyed people as a parameter to organize the area around airports. In urban zoning it is recommended to use the equivalent sound level LAEQ, which uses different criteria to day and night periods to define the land use. With regards to airport noise one may use LAD and LAN metrics which are the equivalent sound levels determined for day and night period, respectively. In this case, the maximum levels chosen to the establishment of the zoning are based on the land use. It is possible, through a simple equation, to determine the DNL level associated to a LAD, LAN pair. The inverse problem, however, has not a unique solution, once this relation depends on the number of daily and night flights of the airport. Thus, the use of DNL in airport noise zoning may lead to completely different results for different airports. Exemples are presented and discussed for two main brazilian airports: Recife and Congonhas.

\title{
Session 2pNSm
}

\section{Noise and EURONOISE: Noise Mapping Techniques and Uncertainties II (Poster Session)}

\author{
Wolfgang Probst, Chair \\ DataKustik GmbH, Gewerbering 5, Griefenberg, 86926, Germany
}

\begin{abstract}
All posters will be on display from 3:40 p.m. to 5:20 p.m. To allow contributors an opportunity to see other posters, contributors of odd-numbered papers will be at their posters from 3:40 p.m. to 4:30 p.m. and contributors of even-numbered papers will be at their posters from 4:30 p.m. to 5:20 p.m.
\end{abstract}

\section{Contributed Papers}

2pNSm1. Strategic noise mapping in slovak republic. Milan Kamenicky (Norsonic Slovensko s.r.o., Letisko M.R. Stefanika 63, 82001 Bratislava, Slovakia, kamenicky@norsonic.sk), Peter Petrak (AkusTech s.r .o., Lubovnianska 14, 85107 Bratislava, Slovakia, akustech@akustech.sk)

In the EU there is an ongoing process of strategic noise mapping and action planning. The goal is to determine the noise produced by the main noise sources - road, rail and air transport and industrial facilities and the exposure caused by them in densely populated agglomerations. Subsequently it is necessary to consider possibilities, how to reduce additional increase of noise levels in the environment. Authors participated in the strategic noise map development process and this paper comprises the information about the measurement and calculation results of traffic and industrial noise in Bratislava and in the slovak republic. Presentation of strategic noise maps and actions plans in Slovakia.

2pNSm2. Mapping of noise caused by military airplane operation. Milan Kamenicky (Norsonic Slovensko s.r.o., Letisko M.R. Stefanika 63, $82001 \quad$ Bratislava, Slovakia, kamenicky@norsonic.sk), Peter Petrak (AkusTech s.r.o., Lubovnianska 14, 85107 Bratislava, Slovakia, akustech@akustech.sk)

Noise from military airplane operations is a serious problem in affected surroundings of military airports. Usually, military airplane noise emission data are not available in databases of common programs, which causes problems in noise load modeling. In such cases it is necessary to obtain emission data by measurement. Measurement must be performed in modes which are not used in ordinary airplane operation. Based on these measurements, acoustic emission data will be processed in the required form according to a used mathematical model. This paper will include examples of acoustic data acquisition and their utilization in mapping of noise load caused by military airplane operations in the surroundings of military airports. Also, verification of data acquired by prediction and measurement in the airport surrounding ordinary airplane operations will be present.

2pNSm3. Effect of limitations of ray-tracing software on predicting community from petrochemical plants. Frank Brittain (Bechtel, Corp., 2255 Peavine Valley Road, Reno, NV 89523, USA, fhbritta@bechtel.com), Marlund Hale (Advanced Engineering Acoustics, 663 Bristol Ave., Simi Valley, CA 93065, USA, noisedoc@aol.com)

Ray-tracing software has proven to be a valuable and powerful tool to predict community noise from outdoor petrochemical and power plants. Accurate predictions are necessary to determine noise reductions needed to meet regulations and/or project noise limits, and then to determine individual equipment noise limits, select add-on noise controls, and confirm the plant will comply with its noise limits. Modeling using ray-tracing software is much more powerful and versatile than a spreadsheet model, which had often been used. Ray tracing and similar image source software have many limitations, particularly for outdoor petrochemical and power plants. To effectively use ray-tracing software, the user needs to understand those limitations. For example, ray tracing does not adequately predict for configurations where distances are significant compared to the wave length of sound, reflections from dense pipe racks, or screening by pipelines and equipment. This paper identifies and discusses some of the limitations of ray-tracing software for predicting community noise. Examples are given. This paper emphasizes limitations of ray-tracing methodology, and not those arising from atmospheric effects. 


\title{
Session 2pNSn
}

\section{Noise and EURONOISE: Railway Noise and Vibration II}

\author{
Richard A. Carman, Cochair \\ Wilson, Ihrig \& Associates, 5776 Broadway, Oakland, CA 94618, USA \\ Franck Poisson, Cochair \\ SNCF DIR, 45, rue de Londres, Paris, 75379, France \\ David Thompson, Cochair \\ Institute of Sound and Vibration Research, University Road, Highfield, Southampton S017 1BJ, UK
}

\section{Invited Papers}

2pNSn1. The railway noise reductions achieved in the Silence project. Franck Poisson (SNCF DIR, 45, rue de Londres, 75379 Paris, France, franck.poisson@sncf.fr), Pierre Etienne Gautier (SNCF DIR, 45, rue de Londres, 75379 Paris, France, pierre-etienne .gautier@sncf.fr)

The European project Silence is dedicated to the reduction of railway and roadway noise in urban areas. Within the framework of the subproject $\mathrm{E}$ and $\mathrm{G}$, the reduction of the railway noise is addressed. Source ranking of state of the art rolling stocks has been carried out. Then, the Diesel engine, the exhaust, the cooling unit, the electrical engine, the wheels and the track have been optimised by the manufacturers and prototypes have been constructed. The most promising have been selected through laboratory measurement to be installed on the trains and trams. Last measurement campaigns have been carried out to characterise the combination of optimised rolling stocks running on optimised track. Then, relevant reductions of the pass-by noise have been achieved for electrical and Diesel multiple unit trains, freight trains and trams. In the same time, a software called VAMPPASS has been developed in subproject B to simulate the time signal of a train pass-by. The paper focuses on the results of the last measurement campaigns which validate the noise reduction achieved on the global pass-by noise of several rolling stocks and tracks. A comparison with the reduction predicted by VAMPPASS will be also presented. Some improvement of the solutions will be also addressed.

$$
5: 40
$$

2pNSn2. Principles for selection of rail fastening systems for control of railway noise and vibration. Stephen J. Cox (Pandrol Rail Fastenings Ltd, 63 Station Road, KT15 2AR Addlestone, UK, s.cox@pandrol.com)

Railways generate noise and vibration as trains pass along the track. It is usually ultimately noise that causes annoyance to those above or alongside the track, but this noise is often generated as a result of vibrations transmitted along and away from the track. Examples of ground-borne vibration at the surface above railway tunnels; airborne noise from trains passing at grade; and noise from trains passing over structures such as viaducts and bridges are each considered in turn. The selection of suitable rail fastening systems, and in particular their stiffness, to control each of these problems is discussed. This often requires compromise - for example, reduction of the noise emitted from the rail may suggest a high stiffness, while reduction of noise from the supporting structure may require a low stiffness. These considerations lead to different solutions to different problems. Some general conclusions are drawn as to how, and to what extent, each of the different types of railway noise and vibration problem may be addressed by modifications to the track fastening system.

\section{6:00}

2pNSn3. Cost-effectiveness of railway noise measures in practice. Chiel Roovers (ProRail, P.O. Box 2038, Department VMJB, Railway Noise Knowledge Centre, NL-3500 GA Utrecht, Netherlands, Chiel.Roovers@prorail.nl)

The Dutch noise nuisance law enforces railinframanager ProRail to take noise measures when railway transport is growing, new lines are built or changes are made to the existing lines. Also, measures are taken by the Dutch government at existing "hotspots". At the moment the toolbox of measures includes noise barriers, sleeper renewal, raildampers, acoustic grinding and façade insulation. The life-cycle costs of these measures have been derived from recent projects. This paper focuses on the evaluation of the cost-effectiveness of these measures in typical situations where the choice of the application of noise mitigation measures was made recently. In what circumstances is it considered to be appropriate to take measures, and how much money will be available? Are source measures like raildampers more cost-effective compared to noise barriers? The introduction of a track-access-charge system which differentiates on the noise emission of trains concludes this paper. 


\section{Contributed Papers}

\section{6:20}

2pNSn4. Comparing railway noise prediction results for passenger trains using various models. Malgorzata Reiter (Gdansk University of Technology, Multimedia Systems Department, 11/12 Gabriela Narutowicza Street, 80-952 Gdansk, Poland, reiter.m@sound.eti.pg.gda.pl), Bozena Kostek (Gdansk University of Technology, Multimedia Systems Department, 11/12 Gabriela Narutowicza Street, 80-952 Gdansk, Poland, bozenka @ sound.eti.pg.gda.pl)

Railway noise modeling is a requirement for all European countries. Under the European Directive on the Assessment and Management of Environmental Noise, 2002/49/EC all member states were obliged to create strategic noise maps of the major railways by June 30th, 2007. Some of the participating states have their own national schemes dedicated to noise prediction. For those not having their own noise prediction model, the recommendation is to use the Dutch SRM II Model. The most important assessment criterion is an inaccuracy value, which is defined as a difference between the result calculated based on a model and the actual measurements under the same atmospheric conditions simulated. Therefore, it is essential to check out which of the main calculation schemes produces most accurate results. Therefore, all above models were implemented numerically and calculations of the noise maps were performed with the use of: Schall 03 developed in Germany, Dutch SRM II and the Nordic model engineered in Norway. Those models were tested employing sample passenger trains data exploited in Gdansk, Poland. Noise maps were drawn in CadnaA software and then were compared to the field noise measurement test results.

\section{6:40}

2pNSn5. Dynamic wheel/rail forces induced by trams at low frequencies. Olivier Chiello (INRETS, Transport and Environment Lab., 25 avenue François Mitterrand, 69675 Bron cedex, France, olivier.chiello@inrets.fr), Marc Maldonado (Research Inst. of Civil Eng. and Mech., UMR CNRS 6183, 1 rue de la Noë, 44321 Nantes Cedex 3, France,marc.maldonado@ec-nantes.fr)

This work is part of various experimental and theoretical investigations into the noise and the vibrations caused by trams in Nantes, France. It focuses on the problem of the low frequency vibrations induced in the track and the ground and particularly on the excitation mechanism at the wheel/rail interface. In order to estimate the corresponding dynamic wheel/rail forces, axle-box vibrations have been measured on a carrying bogie in various vehicle and track configurations. Additionally, a specific instrument has been used to measure the rail unevenness at large wavelengths likely to excite the vehicle/track system in the low frequency range. Finally, the vertical receptance of the different tracks has been measured by using an impact hammer. In the paper, all these experimental results are presented and the validity of a simple vehicle/track interaction model is discussed.

\section{7:00}

2pNSn6. A fast time-domain model for wheel/rail interaction demonstrated for the case of impact forces caused by wheel flats. Astrid Pieringer (Chalmers University of Technology, Division of Applied Acoustics, SE-41296 Gothenburg, Sweden, astrid.pieringer@chalmers .se), Wolfgang Kropp (Chalmers University of Technology, Division of Applied Acoustics, SE-41296 Gothenburg, Sweden, wolfgang.kropp @ chalmers.se)

The prediction of impact forces caused by wheel flats requires the application of time-domain models that are generally more computationally demanding than are frequency-domain models. In this paper, a fast timedomain model is presented to simulate the dynamic interaction between wheel and rail, taking into account the non-linear processes in the contact zone. Track and wheel are described as linear systems using impulse- response functions that can be precalculated. The contact zone is modelled by non-linear contact springs, allowing for loss of contact. This general model enables the calculation of the vertical contact forces generated by any kind of roughness excitation between wheel and rail. Here, the model is adapted to the excitation caused by wheel flats by introducing the irregular wheel shape as a form of extreme roughness. A brief parameter study is presented to demonstrate the functioning of the model. The results from the model are discussed and compared with results from literature.

\section{$7: 20$}

2pNSn7. Testing a new rail roughness measurement standard. Chris J. Jones (Institute of Sound and Vibration Research, Univeristy of Southampton, Highfield, SO17 1BJ Southampton, UK, cjcj@isvr.soton.ac.uk), Fabien Létourneaux (SNCF - Agence d'Essai Ferroviaire, 21 avenue Salvador Allende, F-94407 Vitry-Sur-Seine, France, fabien.letourneaux@sncf .fr), Pascal Fodiman (SNCF - Direction Générale Déléguée Infrastructure, 34 rue du Commandant Mouchotte, 75699 Paris Cedex 14, France, pascal .fodiman@sncf.fr)

Railway rolling noise arises from the combined roughness of the wheel and rail surfaces. The rail roughness is therefore an important parameter in the assessment of train pass-by noise. The Technical Specifications for Interoperability (TSI's) in Europe require noise to be measured on a 'reference track' the conditions of which are controlled. A spectral limit for the rail roughness is a major part of that control. The TSIs needed a new standard for rail roughness measurement so the CEN commissioned TC 256 Working Group 3 to draft one. The final draft of the new standard is now complete and will soon be published. This paper describes a 'road test' that was part of the standard development process. This test involved asking eight teams from all over Europe with seven different instruments to measure roughness at the same site, independently making their own interpretation of the standard. The paper outlines the aims of the new standard and describes the road test. It presents results and conclusions of the test that have been used already to improve the standard. Consideration had to be made of how railhead defects are treated and how different measurement technologies are used.

\section{7:40}

2pNSn8. Brake noise measurements on mixed freight trains with composite brake blocks. Erwin H. Jansen (TNO Science and Industry, PO Box 155, 2600 AD Delft, Netherlands, erwin.jansen@tno.nl), Michael G. Dittrich (TNO Science and Industry, PO Box 155, 2600 AD Delft, Netherlands, michael.dittrich@tno.nl), Erik L. Sikma (Lloyd's Register Rail Europe B.V., PO Box 2016, 3500 GA Utrecht, Netherlands, erik.sikma @1rrail.com)

In the Netherlands, the noise emission of shunting yards, which are often located in urban areas, are a source of disturbance. The noise emission of shunting yards is legislated in terms of maximum allowable equivalent sound pressure levels and peak noise levels of the façade of residences. Brake noise is known to be a major contributor to the total sound emission of shunting yards, in particular from trains with cast-iron block brakes. Within the scope of the Dutch Innovation Programme on Noise Reduction, the rolling noise reducing effect of alternative composite block brakes, such as LL and $\mathrm{K}$ blocks, was successfully demonstrated. Additionally, the effect of composite brake blocks on braking noise was studied. For this purpose, noise measurements were conducted on mixed freight trains according to the applicable protocols. Measurements were conducted on trains slowing down from a speed of $30 \mathrm{~km} / \mathrm{h}$ to a standstill and trains passing-by with brake systems applied. Some composite brake blocks show a considerable reduction of both braking pass-by levels and peak noise levels. Results are presented. If squeal noise is absent, braking noise of most considered brake blocks show a speed dependency similar to that for rolling noise. 


\title{
Session 2pNSo
}

\section{Noise and EURONOISE: Noise, Structure Borne Noise from Building Technical Equipment, and Ground Borne Noise from Railways II}

\author{
James Nelson, Cochair \\ Wilson, Ihrig \& Associates, Inc., 5776 Broadway, Oakland, CA 94618, USA \\ Michel Villot, Cochair \\ CSTB, 24 rue Joseph Fourier, Saint-Martin-d'Hères, 38400, France
}

\section{Invited Papers}

2pNSo1. Prediction method for structure borne noise generated in buildings by tools such as drills. Michel Villot (CSTB, 24 rue Joseph Fourier, 38400 Saint-Martin-d'Hères, France, michel.villot@cstb.fr), Philippe A. Jean (CSTB, 24 rue Joseph Fourier, 38400 Saint-Martin-d'Hères, France, philippe.jean@cstb.fr)

Tools such as drills generate structure borne noise which propagate in the building structure and radiate loud noise even in rooms far from the source. This paper presents a method for predicting this type of noise, frequent in buildings under construction or renovation. First the structural power injected by the tool to the receiving structure (floors or walls) is estimated from field measurements of the vibrational (bending) energy stored in the receiving plate. Then the vibration propagation through the building structures and the noise radiated in the receiving room is estimated using Statistical Energy Analysis (SEA), where both bending and in plane waves are taken into account. Comparisons between predictions and results measured in an apartment building under construction show that SEA gives quite acceptable results down to $1 / 3$ octave $50 \mathrm{~Hz}$ for structure borne sound sources such as drills.

\section{$5: 40$}

2pNSo2. Structure borne sound source data of building service equipments for EN 12354 part 5 - Round Robin measurements. Christian Simmons (Simmons Akustik \& Utveckling, Krokslätts Fabriker 1, 43137 Mölndal, Sweden, info@simmons.se)

At the conference, results will be presented of a round robin test where sound pressure levels and vibration data have been measured in 7 sites using two laundry machines and one heating compressor as sources. The vibrations are determined as transversal vibration levels of the concrete slabs (with the structural loss factors) according to a draft standard prEN 15657-1, as well as relative levels compared to the standardized tapping machine (ISO 140-8). The purpose is to study whether these data may be applied to predict structure borne sound in nearby spaces, according to the draft standard prEN 12354 parts 5 and 2, with a reasonable accuracy as compared to the measured sound pressure levels. The variations in space and time will be monitored carefully to observe any modal coincidence and whether the time and spatial average is the best descriptor of these types of source.

\section{6:00}

2pNSo3. A combined approach for base isolation design. Albano Neves E Sousa (IST, Department of Civil Engineering and Architecture, Av. Rovisco Pais, 1, 1049-001 Lisbon, Portugal, albano.nsousa@civil.ist.utl.pt), Luís Guerreiro (IST, Department of Civil Engineering and Architecture, Av. Rovisco Pais, 1, 1049-001 Lisbon, Portugal, luisg@civil.ist.utl.pt), Rui Gomes (IST, Department of Civil Engineering and Architecture, Av. Rovisco Pais, 1, 1049-001 Lisbon, Portugal, ruigomes@civil.ist.utl.pt)

New buildings in the vicinity of underground railway lines should be protected against noise and vibrations induced by railway traffic. In the present paper a case study is presented. A 2D FEM model of the tunnel, ground and building was built in order to identify the transfer function of vibration from the tunnel to building foundations. A 3D model of the building was built, including not only structural elements, but also other non structural walls, in order to identify the transfer function of vibration from foundations to walls and floors. As the impedance of heavy walls and floors is much higher than the acoustic impedance of air, the numerically assessed vibration fields of walls and floors were used to calculate sound fields in rooms by means of natural mode analysis. The vibration and sound fields were then compared with comfort criteria in order to design a base isolation system. With this combined approach, the analysis was performed up to $170 \mathrm{~Hz}$, which is more than one can usually do with integrated 3D models.

\section{6:20}

2pNSo4. Variance in predicted structure borne sound power due to simplified characterisation of the source. Tomos A. Evans (University of Salford, Acoustic Research Centre, Newton Building, M5 4WT Salford, UK, t.a.evans@pgr.salford.ac.uk), Andy T. Moorhouse (University of Salford, Acoustic Research Centre, Newton Building, M5 4WT Salford, UK, a.t.moorhouse@ salford.ac.uk)

Theoretically the structure borne sound power transmission from a vibration source to a receiving structure can be predicted using the mobility method. In order for all the power transmission mechanisms to be accounted for the source and receiver mobility matrices must contain the mobilities for all degrees of freedom (three translational and three rotational) for each contact point and the transfer mobilities between; each contact point, each degree of freedom and each degree of freedom at each contact point. In practice reliable 
measured data for some degrees of freedom is difficult to obtain, so simplified source characterisation methods are required, for example using only certain degrees of freedom. The novel contribution of this paper is to investigate the variance in the predicted structure borne sound power transmission calculated when some degrees of freedom are missing, and when the source is approximated as a single equivalent excitation. The investigation was carried out using numerical simulations of simplified source and receiver structures.

\section{6:40}

2pNSo5. Uncertainties and repeatability of the Reception Plate Method. Lieven De Geetere (Belgian Building Research Institute, Lombardstraat 42, B-1000 Brussel, Belgium, lieven.de.geetere@bbri.be), Bart Ingelaere (BBRI, rue du Lombard, 42, B-1000 Brussel, Belgium, bart.ingelaere@bbri.be), Pieter Schevenels (Lab. ATF, Katholieke Universiteit Leuven, Celestijnenlaan 200D, B-3001 Leuven, Belgium, pieter.schevenels@bwk.kuleuven.be)

The Reception Plate Method (RPM) as proposed by CEN/TC126/WG7 in prEN 15657-1 estimates the structure-borne sound power injected from a (high mobility) vibrating source into a (low mobility) building structure. In the near future, a round-robin test is planned to investigate repeatability and reproducibility issues of the RPM. As a preparation of this round-robin, uncertainties of the RPM are studied using the standard ISO tapping machine, placed on a 4 feet table on a reception plate. The injected power into the reception plate is calculated using different methods. The influence of the source position, accelerometer positions and airborne excitation are investigated, together with repeatability tests. Also, differences in injected power between two different brands of the ISO tapping machine will be studied. Finally, in-situ installed structure-borne sound power level will be predicted using prEN 12354-5 and compared to measurements on a simple building structure.

\section{7:00}

2pNSo6. Investigation of a vibro-acoustic reciprocal method to derive the contact forces of building equipment. Pieter Schevenels (Lab. ATF, Katholieke Universiteit Leuven, Celestijnenlaan 200D, B-3001 Leuven, Belgium, pieter.schevenels@bwk.kuleuven.be), Arne Dijckmans (Lab. ATF, Katholieke Universiteit Leuven, Celestijnenlaan 200D, B-3001 Leuven, Belgium, arne.dijckmans@bwk.kuleuven.be), Peter J. Van Der Linden (Qsources BVBA, Ketelwinning 38, 3293 Diest, Belgium, info@qsources.be), Gerrit Vermeir (Lab. ATF, Katholieke Universiteit Leuven, Celestijnenlaan 200D, B-3001 Leuven, Belgium, Gerrit.Vermeir@bwk.kuleuven.be)

Structure-borne sound caused by installations can be derived from the injected structure-borne sound power into the connected building element. Theoretically, this is as simple as summing the products of force and velocity at each contact point of the installation. The velocity can be well approximated by the velocity measured close to the contact point. But to measure the force, installation and building element would have to be separated to insert a force sensor. This is generally impractical or even impossible. In this paper, a method is analyzed to derive the force between an installation and a building element in a vibro-acoustic reciprocal way. First, a dedicated volume sound source is placed in an adjacent room and the transfer function of its volume acceleration with the acceleration of the building element is determined. Next, the sound pressure is measured at the source's position while the installation is operating. Since most installations have an important lower frequency contribution, below $50 \mathrm{~Hz}$, the sound source must cover this frequency range with sufficient level. Moreover, an accurate measure of the in-situ volume acceleration of the used source is required. Finally, the method is compared to other methods that approximate the injected structure-borne sound power.

\section{Contributed Papers}

\section{$7: 20$}

2pNSo7. Building Isolation Design for Noise Critical Applications George P. Wilson (Wilson, Ihrig \& Associates, Inc., 5776 Broadway, Oakland, CA 94618, USA, gwilson@wiai.com)

Because it is a complex, multi-degree-of-freedom system, the success of a building isolation installation depends on careful selection of a number of design factors. The new Toronto Opera House, the Four Seasons Centre for the Performing Arts, opened in June 2006, is located directly adjacent to a surface light rail trackway and to a subway rail transit system. The results characterized as "the quietest Opera House in the world" demonstrate that groundborne noise and vibration is not a factor precluding the use of a noisy site for a noise-sensitive facility. Measurement of the existing groundborne noise provided the basis to determine the required noise reduction. Very stiff and massive foundation elements with stiff and massive structure directly above the isolation bearings allows the isolation design to be simplified to a single-degree-of-freedom system for deriving the performance of the isolation at low frequencies. Natural rubber bearings with thickness adjusted to achieve a specific amount of insertion loss for the structure-borne noise can be used to determine the expected performance at frequencies above 50-80 Hz. Isolation system design parameters for the new 2000-seat Opera House Performance Hall and graphical results from vibration and noise projections and tests are presented.

\section{$7: 40$}

2pNSo8. Ground vibration requirements in vehicle projects. Janne Färm (Bombardier Transportation Mainline and Metros Division, Östra
Ringvägen 2 MLN/TSSV, 72173 Västerås, Sweden, janne.farm@se .transport.bombardier.com), Siv Leth (Bombardier Transportation Centre

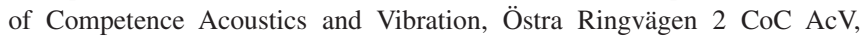
72173 Västerås, Sweden, siv.leth@se.transport.bombardier.com), Philipp Rüst (Bombardier Transportation Bogie Division, Zürcherstrasse 39 Postfach 414, 8401 Winterthur, Switzerland, philipp.ruest@ch.transport .bombardier.com)

The climate is right for trains and the environmental targets regarding noise and vibration have to be met to enhance the competitiveness of rail over other forms of transport. Ground vibration is one part that needs to be further addressed. Customer requirements on ground vibration are often included in vehicle contracts even if the vehicle is only one part of the system determining the ground vibration at the receiver point. For the design of new lines there is a need for a better optimization of overall generation of noise and vibration where both the infrastructure and vehicle parameters are included. An overview of the diversity of ground vibration requirements in different vehicle projects is given and the relation to a number of vehicle and track parameters. The potential conflict between ground vibration reduction and increased airborne noise generation is analyzed for particular examples. The background analysis clearly points out the need for a standardization regarding both setting and evaluation of requirements. There is also a need for developing one commonly used, widely accepted, validated prediction tool for ground vibration. It should facilitate engineering level decision making and allow assessment of mitigation measures. 


\title{
Session 2pPAa
}

\section{Physical Acoustics: Nonlinear Acoustics in Earthquake Processes and Other Earth Processes I}

\author{
Paul A. Johnson, Cochair \\ EES-11 (Geophysics) - Los Alamos National Laboratory, MS D443, Los Alamos, NM 87545, USA \\ Fabrice Cotton, Cochair \\ Université Joseph Fourier, Laboratoire de Géophysique Interne et Tectonophysique, CNRS, BP53, France
}

\section{Invited Papers}

2pPAa1. Stick-slip dynamics of a granular medium. Alberto Petri (Institute of Complex Systems - CNR, via del Fosso del Cavaliere 100, 00133 Rome, Italy, alberto.petri@isc.cnr.it), Andrea Baldassarri (Dipartimento di Fisica -Università di Roma La Sapienza, P.le Aldo Moro 5, 00186 Rome, Italy, andrea.baldassarri@roma1.infn.it), Fergal Dalton (Institute of Complex Systems - CNR, via del Fosso del Cavaliere 100, 00133 Rome, Italy, fergal.dalton@isc.cnr.it), Giorgio Pontuale (Institute of Complex Systems - CNR, via del Fosso del Cavaliere 100, 00133 Rome, Italy, giorgio.pontuale@isc.cnr.it), Stefano Zapperi (CNR-INFM, S3, Dipartimento di Fisica Universita’ di Modena e Reggio Emilia, via Campi 213/A, 41100 Modena, Italy, stefano.zapperi@roma1.infn.it)

Under certain limits, granular materials share many features with fluids and solids. Most of the time, however, they exhibit peculiar features which originate in the non-equilibrium and dissipative character of the grain dynamics. We have investigated the dynamics of a granular medium in a channel, when subject to continuous shear stress from a plate connected to a motor by an elastic spring. We have shown [1] that the statistical features of granular stick-slip dynamics are not simply Gaussian and that [2] can be described in a quantitative way by a simple stochastic equation. Extant work, such as the statistical properties of friction in solid-on-solid systems, further suggests that a large class of driven instabilities can be described in terms of similar general mechanisms. The intensity of the acoustic signals emitted by the granular medium during the slip events displays a non trivial correlation with the plate velocity. [1] F. Dalton et al., Phys. Rev. Lett. 95, 138001 (2005) [2] A. Baldassarri et al., Phys. Rev. Lett. 96, 118002 (2006)

\section{2:20}

2pPAa2. Monitoring changes in crustal properties with seismic noise. Michel Campillo (LGIT - CNRS - Université Joseph Fourier, Maison des Géosciences, 1381 rue de la Piscine, BP 53, 38041 Grenoble, France, Michel.Campillo@obs.ujf-grenoble.fr), Florent Brenguier (Laboratoire de Sismologie IPGP, 4 place Jussieu, 75252 Paris, France, fbrengui@ipgp.jussieu.fr), Celine Hadziioannou (LGIT Université Joseph Fourier/CNRS, Maison des Géosciences Domaine Universitaire, 38041 Grenoble, France, eline.hadziioannou@ujf-grenoble.fr), Nikolai Shapiro (Laboratoire de Sismologie IPGP, 4 place Jussieu, 75252 Paris, France, nshapiro@ipgp.jussieu.fr), Eric Larose (LGIT - CNRS - Université Joseph Fourier, Maison des Géosciences, 1381 rue de la Piscine, BP 53, 38041 Grenoble, France, Eric.Larose@obs.ujf-grenoble.fr)

The seismic noise is a continuous source of waves due to the interaction between the solid Earth and its fluid envelopes. Theoretically, the time correlation of the field measured at two distant points allows retrieving the Green function between the points for a random distribution of sources or in presence of a diffuse field. We use this property and study the stability of the correlation functions. We show that the velocity measurements made with correlations are very stable. Using doublet analysis, we found that that we can monitor relative velocity changes smaller than $10^{-4}$. We present applications to the forecast of volcanic eruptions and to the monitoring of an active fault.

\section{Contributed Paper}

\section{2:40}

2pPAa3. Force fluctuations in granular disks near the jamming threshold. Robert E. Ecke (Los Alamos National Laboratory, Center for Nonlinear Studies, MS-B258, Los Alamos, NM 87545, USA, ecke@lanl.gov), Mahesh Bandi (Los Alamos National Laboratory, Condensed Matter and Thermal Physics, MS-K764, Los Alamos, NM 87545, USA, mbandi@lanl.gov), Michael K. Rivera (Los Alamos National Laboratory, Condensed Matter and Thermal Physics, MS-K764, Los Alamos, NM 87545, USA, mkrivera@lanl.gov)

We study experimentally the force fluctuations felt by a probe disk as it is dragged at a nominally constant rate through a two-dimensional bi- disperse system of randomly packed photo-elastic disks. As the packing fraction increases from loose packing to a value where the disks are jammed, lines of connected force - stress chains - develop that couple the entire domain. We consider the transition from an unjammed state with no long range force distribution to a jammed state with stress chains spanning the system size. We characterize this transition using the measured force fluctuations and the determination of stress chains as visualized by the photo-elastic disks. Length and time scales obtained from these measurements are used to describe both the transition to jamming and the nature of the jammed state. The reorganization of disks and the force fluctuations have a stick-slip character in the jammed state. 


\section{Invited Paper}

\section{3:00}

2pPAa4. Rheology and mobility in a sono-fluidized granular packing. Eric Clement (Laboratoire PMMH, ESPCI, 10 rue Vauquelin, 75231 Paris Cedex 5, France, erc@ccr.jussieu.fr), Gabriel Caballero (Laboratoire PMMH, ESPCI, 10 rue Vauquelin, 75231 Paris Cedex 5, France, g.a.caballerorobledo@tnw.utwente.nl), Chay Goldenberg (Laboratoire PMMH, ESPCI, 10 rue Vauquelin, 75231 Paris Cedex 5, France, chayg@pmmh.espci.fr)

Dynamics near jamming (glassy phase, aging, memory effects and intermittency) shows amazing analogies among a variety of very different systems (colloids, dense suspensions, foams, granular materials). Recently, several proposals have emerged with the aim of describing in a general and unified way this behavior. With the purpose of testing experimentally some of these ideas, we performed several experimental studies on dry granular materials under vibration. We found that even with a weak level of vibration, such as sound injection, the behavior of granular matter is changed dramatically. We directly observe that the grains undergo a sub diffusive collective dynamics on a very long time scale which results on global compaction effects. In this regime, we have studied the rheology of an intruder moving in the bulk as a function of the level of energy injected, the driving velocity and the shape of the moving object. We also present a simple model to account for the observed behavior.

\section{Contributed Paper}

$$
\text { 3:20 }
$$

2pPAa5. Irreversible interaction of sound waves with dense granular matter. Jérôme Laurent (Université Paris-Est Marne la Vallée, 5 Bd. Descartes, 77454 Marne la Vallée, France, jerome.laurent @univ-mlv.fr), Thomas Brunet (Université Paris-Est Marne la Vallée, 5 Bd. Descartes, 77454 Marne la Vallée, France, Thomas.Brunet @insp.jussieu.fr), Xiaoping Jia (Université Paris-Est Marne la Vallée, 5 Bd. Descartes, 77454 Marne la Vallée, France, jia@univ-mlv.fr)

In this talk, we will describe quantitatively the irreversible interaction between sound and the granular medium under low confining pressure $(<$ $0.1 \mathrm{MPa}$ ). Both resonance measurement and pulsed wave propagation show that as the acoustic pressure goes up to a few percent of the confining pressure, the dynamic bulk modulus and shear one can be reduced to $10 \%$ and
$20 \%$, respectively. This observation of modulus softening is qualitatively in agreement with the prediction by a model based on the Mindlin hysteretic nonlinearity at the grain contact level. Beyond certain thresholds of acoustic amplitude, the irreversible sound-granular matter interaction is accompanied by continue or intermittent sample compactions. No visible grain motion is observed at this stage; however the multiply scatted shear waves [1] allow probing a significant evolution of the contact networks, which coincide with slow recovery of elastic moduli. This work may have the implication for better understanding the physical origin of dynamic triggering of the fault core [2]. [1] X. Jia, "Coda-like multiple scattering of elastic waves in dense granular media," Phys. Rev. Lett. 93, 154303 (2004) [2] P.A. Johnson \& X. Jia, "Nonlinear dynamics, granular media and dynamic earthquake triggering," Nature 437, 871-874 (2005)

3:40-5:20 Posters

Lecture sessions will recess for presentation of poster papers on various topics in acoustics. See poster sessions for topics and abstracts.

\section{Contributed Papers}

$$
5: 20
$$

2pPAa6. Acoustic emissions for the prediction of avalanches. Pierre De Guibert (Université Paul Sabatier, PHASE, 118, route de Narbonne, 31062 Toulouse cedex 9, France, dguibert@cict.fr), Vincent Gibiat (Université Paul Sabatier, PHASE, 118, route de Narbonne, 31062 Toulouse cedex 9, France, gibiat@cict.fr), Eric Plazza (Université Paul Sabatier, PHASE, 118, route de Narbonne, 31062 Toulouse cedex 9, France, ericvpp@ hotmail .com)

Although we know well enough the main zones where occur avalanches, the main difficulty results in forecasting avalanches because of the constant evolution of the snow coat. The coat, composed of various slopes have different properties according to the weather conditions not only during falls but also after. Weather modifications after snow falls generate instability of slopes which create stress, fissure and failure states. These different states generate acoustic emissions whose parameters can lead to forecast avalanches. The aim is to detect in situ these precursors, that initiate a low frequency signal. We have settled an experiment to monitor and register this signal. It includes two geophones to detect the acoustic signals, an acquisition system, one aerial of temperature sensor and a wireless connection to a deported PC. The system is controlled by ADSL in our offices. It will be shown on examples that any movement of the snow cap provides a signal. The rate of repetition of these elementary signals is the indication of an increasing instability of the snow precursor to an avalanche. By comparing the acoustic signals to temperature variation evolution of the snow pack we could define an alarm degree allowing to secure the area.

\section{$5: 40$}

2pPAa7. The effect of acoustic waves on stick-slip behaviour in sheared granular media, with implications to earthquake processes. Paul A.
Johnson(EES-11 (Geophysics) - Los Alamos National Laboratory, MS D443, Los Alamos, NM 87545, USA, paj@lanl.gov), Heather Savage (University of California, Department of Earth and Planetary Science, Santa Cruz, CA 95064, USA, hsavage@ucsc.edu), Matt Knuth (University of Wisconsin, Department of Geology and Geophysics, Madison, WI 53707, USA, mwknuth@wisc.edu), Joan Gomberg (US Geological Survey, University of Washington, Department of Earth and Space Sciences, Box 351310, Seattle, WA 98195-1310, USA, gomberg@usgs.gov), Robert Behringer (Duke University, Department of Physics, Durham, NC 27708, USA, bob@phy.duke.edu), Chris Marone (Pennsylvania State University, Department of Earth and Planetary Science, University Park, PA 16802, USA, cjm@geosc.psu.edu)

We are studying the effects of acoustic waves on sheared granular material, with two goals in mind: one is to understand the intriguing physics that arises in this experimental system, and the other is to see if such experiments offer insight into earthquake processes, in particular the phenomenon where one earthquake triggers another nearby, or distant, earthquake ('dynamic earthquake triggering'). We conducted laboratory experiments of stick-slip in granular media using a double-direct, shear apparatus, while applying low amplitude vibration as well as pulsed waves. We find that vibration and pulses significantly perturb the shearing behaviour of the granular material, and that the manifestation of vibration is extremely complex, including strong material memory of the acoustic perturbation, that persists. We note that the wave disturbance must take place near the critical point, where the granular material is near failure, otherwise no effect is observed. Also, horizontal loads on the system can eliminate the effect if they arelarge $(\geqslant 4-5 \mathrm{MPa})$. 


\title{
Invited Paper
}

6:00

2pPAa8. Observations and models of dynamic earthquake triggering. Joan Gomberg (US Geological Survey, University of Washington, Department of Earth and Space Sciences, Box 351310, Seattle, WA 98195-1310, USA, gomberg@usgs.gov), Paul A. Johnson (EES-11 (Geophysics) - Los Alamos National Laboratory, MS D443, Los Alamos, NM 87545, USA, paj@lanl.gov)

Seismologists have long accepted the idea that step-function perturbations to the deformation field acting on a fault can change its likelihood of, or 'trigger', failure as fault slip. We review the observations that have lead to the very recent recognition that transient perturbations (e.g., associated with seismic waves) also affect failure probabilities, and more broadly, observations of the spatial and temporal variations in both triggering deformations and triggered responses. Many of these cannot be explained by conventional models of earthquake nucleation, requiring consideration of ideas developed in other disciplines, such as those describing and explaining nonlinear dynamic elasticity from rock-mechanics. In addition to the scientific challenges, these observations and models significantly impact earthquake forecasts and hazard assessments. We focus on observations of natural earthquakes and from the rock-mechanics laboratory, and some of the explanatory models that we and others have proposed. While many of these observations and models are just being vetted now, even newer ones related to slow aseismic fault slip and non-volcanic tremor (seismic radiation that scales very differently from that from earthquakes) may lead to substantive modifications and advances. We conclude with a few tantalizing examples as a prelude to a companion presentation.

\section{Contributed Paper}

6:20

2pPAa9. Dynamic asperity failure triggering during the rupture process? Carene Larmat (EES-11 (Geophysics) - Los Alamos National Laboratory, MS D443, Los Alamos, NM 87545, USA, carene@lanl.gov)

Dynamic triggering by the transient deformations associated with seismic waves has been proposed as an alternative mechanism for inducing aftershocks in contrast to the Coulomb stress. This idea is supported by observations of triggered seismicity like for the $\mathrm{Mw}=7.3$ Landers earthquake (1992), the $\mathrm{Mw}=7.1$ Hector Mine (1999), and after the Mw=7.4 Izmit earthquake for which small events began in Greece immediately after the passage of the mainshock surface waves (Brodsky et al., 2000). Gomberg et al. (2003) conducted a rigorous analysis of aftershock distribution for 15 mainshocks and showed that most of the triggered events are located in the rupture direction which corresponds to a maximum of the radiated seismic energy. Another argument is the fact that the correctly scaled amplitude of the dynamic deformations are consistent with laboratory observations of failure processes (Gomberg \& Johnson, 2005). Finally, work by Felzer and Brodsky (2006) indicates that aftershocks are due to dynamic triggering rather than quasistatic stress changes. Thus instabilities can be triggered as aftershocks as well as at remote distances by seismic waves, suggesting asperities located on the mainshock fault may fail due to transient strains. In this study, we propose to explore that hypothesis.

\section{Session 2pPAb}

\section{Physical Acoustics: Nonlinear Acoustics of Unconsolidated Granular Media I}

\author{
Paul A. Johnson, Cochair \\ EES-11 (Geophysics) - Los Alamos National Laboratory, MS D443, Los Alamos, NM 87545, USA \\ Vitali Gusev, Cochair \\ LPEC/UMR 6087/CNRS/Université du Maine, Avenue Olivier Messiaen, Le Mans Cedex 09, 72085, France
}

\section{Invited Papers}

\section{2:20}

2pPAb1. Strongly nonlinear behavior of granular chains and granular composites. Vitali F. Nesterenko (University of California, San Diego, 9500 Gilman Drive, Department of Mechanical and Aerospace Engineering, La Jolla, CA 92093-0411, USA, vnesterenko@ucsd.edu), Eric B. Herbold (University of California, San Diego, 9500 Gilman Drive, Department of Mechanical and Aerospace Engineering, La Jolla, CA 92093-0411, USA, eherbold@gmail.com), David J. Benson (University of California, San Diego, 9500 Gilman Drive, Department of Mechanical and Aerospace Engineering, La Jolla, CA 92093-0411, USA, dbenson@ucsd.edu), Jeonghoon Kim (University of California, San Diego, 9500 Gilman Drive, Materials Science and Engineering Program, La Jolla, CA 92093, USA, jek008@ucsd.edu), Chiara Daraio (California Institute of Technology, Aeronautics (GALCIT) and Applied Physics, Pasadena, CA 91125, USA, daraio@caltech.edu)

Strongly nonlinear behavior of granular phononic crystals composed of stainless steel cylinders and PTFE spheres is considered experimentally and numerically. The tenability of phononic band gap in this two-mass prestressed chain was investigated. The results show that the phononic band gap exists within audible frequency regime $(20-20,000 \mathrm{~Hz})$ and can be tuned by changing the value of prestress. Influence of the weak and strong nonlinearity on the propagation of signals with frequencies inside band gap was investigated. The mechanical properties of reactive materials (e.g., high density mixtures of polytetraflouroethylene (PTFE), aluminum (Al) and 
tungsten (W) powders) where unconsolidated granular packing of metal particles plays significant role in the dynamic behavior was investigated. It was found that dynamic strength can be tailored by changing the morphology of the particles and porosity. Cold isostatically pressed PTFE-Al-W powder composites with fine metallic particles and a higher porosity exhibited higher ultimate compressive strength than less porous composites having equivalent mass ratios with coarse W particles. Based on the numerical analysis it was concluded that a mesoscale force chains between the fine metallic particles are responsible for this unusual phenomenon. This work was supported by the NSF DCMS03013220 and by MURI ONR N00014-07-1-0740.

\section{2:40}

2pPAb2. Elastic waves in one-dimensional unconsolidated medium with Hertz nonlinearity. Alexandr I. Korobov (Dept. of Acoustics, Physics Faculty, M.V. Lomonosov Moscow State University, Leninskie gory 1, 119991 Moscow, Russian Federation, akor@acs465a-1.phys.msu.ru), Maria Y. Izosimova (Center for Industrial and Medical Ultrasound, Applied Physics Lab., University of Washington, 1013 NE 40th St., Seattle, WA 98105, USA, maria.izossimova@mail.ru), Yurii A. Brazhkin (Dept. of Acoustics, Physics Faculty, M.V. Lomonosov Moscow State University, Leninskie gory 1, 119991 Moscow, Russian Federation, akor@acs465a.phys.msu.su), Elizaveta Sovetskaya (Dept. of Acoustics, Physics Faculty, M.V. Lomonosov Moscow State University, Leninskie gory 1, 119991 Moscow, Russian Federation, akor@acs465a.phys.msu.su)

Results of experimental study of elastic longitudinal harmonic wave's propagation in one-dimensional unconsolidated medium are presented. A 1-D chain of identical pre-pressed beads is used as an experimental model. To describe elastic properties of such medium the Hertz model is used. Presence of periodical structure causes elastic waves to be dispersive in such medium and to have a cutoff frequency. A cutoff frequency depends not only on material's elastic properties and beads' geometrical sizes but also on pre-press value. Velocity dependence on frequency of longitudinal harmonic wave of small magnitude is measured experimentally, and dispersion curves are built under different pre-press value. Pre-press value is regulated with applied external static force along chain axis. Measured dispersion dependences are in good agreement with theoretically calculated ones. Dispersion impedes observation of higher harmonics generation in such chain. Therefore to study nonlinear properties of 1-D structure, velocity dependence on longitudinal wave amplitude were investigated. The wave velocity depends not only on its magnitude but also on pre-press value on beads. It was experimentally shown the one-dimensional chain of elastic beads is a medium with dispersion, linear and nonlinear properties to be controlled with the applied external static force. Work supported by RFBR.

\section{Contributed Papers}

\section{3:00}

2pPAb3. Breathing in Granular Chains and Conversion of Wave Energy to Useful Forms. Surajit Sen (State University of New York at Buffalo, Dept of Physics, SUNY-Buffalo, Buffalo, NY, Buffalo, NY 142601500, USA, sen@nsm.buffalo.edu)

Estimates suggest that a meter of a wave front carries $\sim 100 \mathrm{~kW}$ of power. A significant amount of renewable energy is hence dissipated across the time scale of minutes as surface gravity waves dissipate on any beach. Given that the majority of the world population lives near the ocean, it makes sense to explore the conversion of wave energy into electrical energy. Here we consider a new concept - that of a potentially scalable system that converts wave energy into energy in mechanical pulses and then to voltage drops. The envisioned system would exploit the well studied physics of nonlinear repulsion between elastic grains encased in cylinders to continuously convert the incident wave energy into dispersionless energy bundles, which can be subsequently converted to stress pulses in a piezo-electric slab. The key points involved in the concept will be presented.

\section{3:20}

2pPAb4. Wave propagation in wetted Hertzian granular chains: analysis of a single contact dynamics. Stéphane Job (Supméca Paris, 3 rue Fernand Hainaut, 93407 Saint-Ouen Cedex, France, stephane.job @ supmeca.fr), Francisco Santibañez (Univ. de Santiago de Chile - Dept. de Fisica, Avenida Ecuador 3493, Casilla 307, Correo 2, Santiago de Chile, Chile, fsantibanez@fisica.usach.cl), Francisco Melo (Univ. de Santiago de Chile - Dept. de Fisica, Avenida Ecuador 3493, Casilla 307, Correo 2, Santiago de Chile, Chile, fmelo@usach.cl)

A one-dimensional dry granular medium, a chain of beads which interact via the nonlinear Hertz potential, exhibits strongly nonlinear behaviors. When such an alignment contains some viscous fluid between grains, it exhibits new interesting features. We report some recent experiments, analysis and numerical simulations concerning nonlinear wave propagation in dry and wet chains of spheres. We consider first a monodisperse chain as a reference case. We then analyze how the pulse characteristics are modified in the presence of an interstitial viscous fluid. The fluid not only induces dissipation but also strongly affects the intergrain stiffness: in a wet chain, wave speed is enhanced and characteristic pulse duration is shorter. Our observations are in a satisfactory agreement with ultrasound propagation measurements in 3D wetted granular beds reported in the literature. Recent experiments performed with a single sphere colliding a wall covered by a thin film of fluid allowed us to determine the rheology of the contact dynamics, and confirmed that a complex elastohydrodynamic interaction takes place close to solids contact. 


\section{Invited Paper}

5:00

2pPAb5. Dynamic effective mass of granular media. David L. Johnson (Schlumberger-Doll Research, One Hampshire St., Cambridge, MA 02139, USA, johnson10@slb.com)

We report an experimental and theoretical investigation of the frequency-dependent effective mass, $\mathrm{M}(\omega)$, of loose granular particles which occupy a rigid cavity to a filling fraction of $48 \%$. We demonstrate that this is a sensitive and direct way to measure those properties of the granular medium that are the cause of the changes in acoustic properties of structures containing grain-filled cavities. The dominant features of $\mathrm{M}(\omega)$ are a sharp resonance and a broad background, which we analyze within the context of simple models as well as with molecular dynamic simulations. We find that: a) These systems may be understood in terms of a height-dependent and diameter-dependent effective sound speed $(\sim 130 \mathrm{~m} / \mathrm{s})$ and an effective viscosity $\left(\sim 2 \times 10^{4}\right.$ Poise $)$. b) There is a dynamic Janssen effect in the sense that, at any frequency, and depending on the method of sample preparation, approximately one-half of the effective mass is borne by the side walls of the cavity and one-half by the bottom. c) On a fundamental level, dissipation is dominated by adsorbed films of water at grain-grain contacts in our experiments, not by global viscous damping.

\section{Contributed Paper}

$5: 20$

2pPAb6. Mechanisms of acoustic absorption in weakly wet granular media. Thomas Brunet (Université Paris-Est Marne la Vallée, 5 Bd. Descartes, 77454 Marne la Vallée, France, Thomas.Brunet@insp.jussieu .fr), Xiaoping Jia (Université Paris-Est Marne la Vallée, 5 Bd. Descartes, 77454 Marne la Vallée, France, jia@univ-mlv.fr), Pierre Mills (Université Paris-Est Marne la Vallée, 5 Bd. Descartes, 77454 Marne la Vallée, France, mills@univ-mlv.fr)

Multiply scattered elastic waves provide a sensitive probe for studying the viscoelastic properties of a granular medium at the length scale of grains.
Based on a diffusion model, we can infer the structural and viscoelastic properties of the material from the fitting parameters such as the diffusion constant D and quality factor Q. In this work, we characterize quantitatively the acoustic dissipation occurring at the grain contacts by measuring the $\mathrm{Q}$ factor in different granular samples. We focus a particular attention on the effects of on the internal loss by adding a small amount of liquid in the granular medium $(\sim 0.05 \%)$. Using the Hertz-Mindlin contact theory we have identified two different mechanisms of acoustic absorption: frictional and viscous dissipations.

\section{Invited Papers}

2pPAb7. Static and dynamic nonlinear behaviors of unconsolidated granular media. Zhiqu Lu (University of Mississippi, NCPA, 1 Coliseum Drive, University, MS 38677, USA, zhiqulu@olemiss.edu), James M. Sabatier (University of Mississippi, NCPA, 1 Coliseum Drive, University, MS 38677, USA, sabatier@olemiss.edu)

Unconsolidated granular media such as sands, soils, and glass beads, exhibit astonishing static and dynamic nonlinear behaviors that are quite different from those of elastic materials such as liquids and crystalline solids. They can generally classified as mesoscopic hysteretic nonlinear materials since hysteresis is a universal phenomenon for these materials. In order to understand the mechanism and to quantify the nonlinearity of unconsolidated granular materials, a series of studies have been conducted on re-molded unconsolidated soils. The systematic research consists of three parts: (1) a triaxial cell test with pre-defined stress-paths and sound speed measurement, focusing on the exploration of the role of hysteresis in both linear and nonlinear acoustics; (2) the phase shift method, using nonclassical nonlinear acoustic theory to measure the hysteretic nonlinearity parameter; (3) the study of fluid effects on the hysteretic nonlinearity parameter. The study reveals many static and dynamic nonlinear behaviors of soils, including: nonlinear stress/strain relationship, hysteresis loop, end-point memory, much greater dynamic elastic modulus than static elastic modulus, strain-amplitude dependence of elastic modulus, linear resonant frequency shift and phase shift relationships against strain, extremely high values of the hysteretic nonlinearity parameter, harmonics generation, and moisture effects.

2pPAb8. Acoustic waves in granular materials. Stefan Luding (UTwente, Multi Scale Mechanics, P.O.Box 217, 7500 AE Enschede, Netherlands, s.luding@utwente.nl), Orion Mouraille (UTwente, Multi Scale Mechanics, P.O.Box 217, 7500 AE Enschede, Netherlands, o.j.p.mouraille@utwente.nl)

The numerous sound propagation mechanisms in granular materials are rather complex and strongly depend on the material microscale parameters, such as inter-particle friction or damping. The use of DEM (Discrete Element Simulations) allows us to tune these parameters and hence to understand their influence. In both regular (crystal-like) and polydisperse (sand-like) systems of spheres, a small perturbation is created on one side of the simulation box and examined during its propagation and on arrival at the opposite side. Starting from a regular packing, a tiny size variation comparable to the typical contact deformation already changes sound propagation considerably [1]. The transmission spectrum becomes discontinuous, i.e., a lower frequency band is transmitted well, while higher frequencies are not, mainly due to scattering effects. Furthermore, the wave propagation and transmission properties are studied on fully polydisperse, frictional and cohesive packings under increasing load until breakage of the material [2]. [1] O. Mouraille and S. Luding, Sound wave propagation in weakly polydisperse granular materials, Ultrasonics, submitted. [2] O. Mouraille, O. Herbst and S. Luding, Testing cohesive and frictional granular materials with the discrete element model, in preparation. 
2pPAb9. Acoustic wave propagation in granular systems. Ellak Somfai (University of Warwick, Department of Physics and Centre for Complexity Science, CV4 7AL Coventry, UK, E.Somfai@warwick.ac.uk), Jean-Noel Roux (Laboratoire des Materiaux et des Structures du Genie Civil, Institut Navier, 2 allee Kepler, Cite Descartes, 77420 Champs-sur-Marne, France, jean-noel.roux@lcpc.fr), Jacco H. Snoeijer (University of Bristol, Department of Mathematics, University Walk, BS8 1TW Bristol, UK, jacco.snoeijer@bristol.ac.uk), Martin Van Hecke (Universiteit Leiden, Kamerlingh Onnes Laboratory, P.O. Box 9504, 2300RA Leiden, Netherlands, mvhecke@lorentz.leidenuniv.nl), Wim Van Saarloos (Universiteit Leiden, Kamerlingh Onnes Laboratory, P.O. Box 9504, 2300RA Leiden, Netherlands, saarloos@lorentz.leidenuniv.nl)

We present numerical simulations of acoustic wave propagation in granular systems consisting of particles interacting with the three-dimensional Hertz-Mindlin force law. The response to a short mechanical excitation on one side of the system is found to be a propagating coherent wavefront followed by random oscillations made of multiply scattered waves. We find that the coherent wavefront is insensitive to details of the packing: force chains do not play an important role in determining this wavefront. The coherent wave has a well defined velocity, which is roughly compatible with the predictions of macroscopic elasticity, and its amplitude and width depend as a power law on distance. As there is at present no theory for the broadening and decay of the coherent wave, we numerically and analytically study pulse-propagation in a one-dimensional chain of identical elastic balls. The results for the broadening and decay exponents of this system differ significantly from those of the disordered packings. We briefly discuss the eigenmodes of the system and effects of damping are investigated as well.

\section{Contributed Papers}

\section{6:40}

2pPAb10. Localization of sound in a three-dimensional elastic network John H. Page (Dept. of Physics and Astronomy, Univ. of Manitoba, Winnipeg, MB R3T 2N2, Canada, jhpage@cc.umanitoba.ca), Hefei Hu (Dept. of Physics and Astronomy, Univ. of Manitoba, Winnipeg, MB R3T 2N2, Canada, hefeihu2@uiuc.edu), Anatoliy Strybulevych (Dept. of Physics and Astronomy, Univ. of Manitoba, Winnipeg, MB R3T 2N2, Canada, anatoliy@physics.umanitoba.ca), Sergey Skipetrov (Université Joseph Fourier, Laboratoire de Physique et Modélisation des Milieux Condensées/CNRS, Maison des Magistères, 38042 Grenoble, France, Sergey.Skipetrov@grenoble.cnrs.fr), Bart Van Tiggelen (Université Joseph Fourier, Department of Applied Physics, Hokkaido University, 060-8628 Sapporo, Japan, Bart.Van-Tiggelen@grenoble.cnrs.fr)

A long standing question in physical acoustics has been whether or not the Anderson localization of sound can be demonstrated unambiguously in three dimensions. Here we address this question by reporting evidence for the localization of ultrasonic waves in a three-dimensional granular network of weakly sintered aluminum beads. In the upper part of the intermediate frequency regime, where the ultrasonic wavelength is comparable with the sizes of the pores and beads, the intensity time-of-flight profile of the multiply scattered waves exhibits non-exponential decay, which may be construed as a slowing down of the diffusion coefficient with propagation time and is consistent with predictions for localized waves. We use a quasi point source and detector to demonstrate how localization cuts off the transverse spreading of the multiply scattered waves, an effect, which we call 3D transverse localization, that has not been observed previously for any type of wave. These results are interpreted using recent theoretical predictions based on the self-consistent theory of the dynamics of localization, allowing the localization length to be determined. Further evidence is obtained from intensity statistics, which reveal a Thouless conductance g less than unity. These results unambiguously demonstrate the localization of sound in this system.

\section{7:00}

2pPAb11. Surface elastic waves in granular media under gravity and their relation to booming avalanches. Lénaïc Bonneau (Laboratoire PMMH, ESPCI, 10 rue Vauquelin, 75231 Paris Cedex 5, France, bonneau@pmmh.espci.fr), Bruno Andreotti (Laboratoire PMMH, ESPCI, 10 rue Vauquelin, 75231 Paris Cedex 5, France, Andreotti@pmmh.espci.fr), Eric Clement (Laboratoire PMMH, ESPCI, 10 rue Vauquelin, 75231 Paris Cedex 5, France, erc@ccr.jussieu.fr)

Due to the nonlinearity of Hertzian contacts, the speed of sound $\mathrm{c}$ in granular matter is expected to increase with pressure as P1/6. A static layer of grains under gravity is thus stratified so that the bulk waves are refracted toward the surface. The reflection at the surface being total, there is a discrete number of modes (both sagittal plane and transverse ones) localized close to the free surface. The shape of these modes and the corresponding dispersion relation are investigated in the framework of an elastic description taking into account the main features of granular matter: the nonlinearity between stress and strain and the existence of a yield transition. We show in this context that the surface modes localized at the free surface exhibit a waveguide effect related to the nonlinear Hertz contact. Sound propagation measurements in a laboratory scale channel as well as in the field (slip face of a singing dune), will be presented and compared to the theoretical predictions. Finally, these results will be used to constrain the possible dynamical mechanisms at the origin of booming avalanches. 


\title{
Session 2pPAc
}

\section{Physical Acoustics: Diffraction of Waves on Periodical Structures: Acoustic, Ultrasonic and Acousto-Optical Diffraction Phenomena II (Poster Session)}

\author{
Nico Declercq, Cochair \\ Georgia Tech Lorraine - G.W. Woodruff School of ME \\ Nataliya Polikarpova, Cochair \\ M.V. Lomonosov Moscow State University, Department of Physics
}

\begin{abstract}
All posters will be on display from 3:40 p.m. to 5:20 p.m. To allow contributors an opportunity to see other posters, contributors of odd-numbered papers will be at their posters from 3:40 p.m. to 4:30 p.m. and contributors of even-numbered papers will be at their posters from 4:30 p.m. to 5:20 p.m.
\end{abstract}

\section{Contributed Papers}

2pPAc1. Information losses minimization in spectral devices based on acousto-optic Bragg cells. Boris S. Gurevich (Scientific Instruments, Rizhsky prospekt 26, 190103 St. Petersburg, Russian Federation, bgurevich@mail.ru), Simon B. Gurevich (A.F. Ioffe Physical-Technilcal Institute RAS, Politekhnicheskaya, 26, 194021 St. Petersburg, Russian Federation, sbgurevich@mail.ru), Sergey V. Andreyev (Scientific Instruments, Rizhsky prospekt 26, 190103 St. Petersburg, Russian Federation, svan51@mail.ru), Andrey V. Belyaev (Institute for analytical instrumentation RAS, Rizhsky pr. 26, 190103 St. Petersburg, Russian Federation, bgurevich@mail.ru)

Spectral devices such as spectrophotometers are intended, as many other devices, for information transmission and processing. Hence, they can be characterized by specific information characteristics such as information transmission capability and information capacity, which can be connected with some physical and technical parameters of the devices. The use of acousto-optic components in these devices introduce some peculiarities in information transmission and processing in spectrophotometers. We have considered the information transmission limitations based on signal and noise in acousto-optic Bragg cells. The spectral resolving power is also considered as one of decisive characteristics influencing on acousto-optic spectral device transmission capability.

2pPAc2. Theoretical study of the diffraction fields generated by a fluid-loaded plate with two-surface corrugation. Sarah W. Herbison (Georgia Tech Lorraine - G.W. Woodruff School of ME, UMI Georgia Tech - CNRS 2958, 2 rue Marconi, 57070 Metz, France, sherbison@gatech.edu), Nico F. Declercq (Georgia Tech Lorraine - G.W. Woodruff School of ME, UMI Georgia Tech - CNRS 2958, 2 rue Marconi, 57070 Metz, France, nico.declercq@me.gatech.edu)

A renewed interest has recently emerged in the study of the interaction of elastic waves with periodic structures, including periodically corrugated surfaces. From prior theoretical and experimental studies of the diffracted fields generated by such surfaces, it is well known that periodically corrugated surfaces can be used to generate ultrasonic surface waves, which are especially important in the field of ultrasonic non-destructive evaluation. In general, anomalies appear in the reflection and transmission spectra due to the presence of the corrugation, and these anomalies can be correlated with the surface waves generated. The present work has extended the theory of diffraction on periodically corrugated surfaces to the case of a fluid-loaded plate with both sides corrugated. The unique filtering effects and diffraction phenomena that can be achieved with such a structure are examined and related to the creation of surface and plate waves.

2pPAc3. 2D-ultrasonic tomography using first-order Born and canonical approximations. Philippe Lasaygues (Laboratory for Mechanics and Acoustics CNRS, 31 chemin Joseph Aiguier, 13009 Marseille,
France, lasaygues@1ma.cnrs-mrs.fr),Loic Le Marrec(Institute of Mathematical Research of Rennes IRMAR, Campus de Beaulieu, 35042 Rennes, France, loic.lemarrec@univ-rennes1.fr), Thierry Scotti (CNRS - LMA, 31 Chemin Joseph Aiguier, 13009 Marseille, France, scotti@1ma.cnrs-mrs.fr)

This paper deals with the two-dimensional image reconstruction of an elastic tubes using ultrasonic tomographic method based on first-Born approximation and a canonical approximations. The latter improvement makes it possible to extend the scope of tomography from lower impedance contrast media to higher impedance contrast situations, even when the ultrasonic propagation is greatly perturbed by the difference in acoustic impedance between the scatterer and the surrounding medium. The strategy used to solve this problem was based on comparisons between the experimental diffracted field and the canonical solution approached as a forward problem. The algorithm adopted, using the analytical solution to the local forward problem and an iterative process to recover the unknowns, is fast enough to yield real-time information about the shape. A single frequency does not suffice to determine the real solution as the global minimum of the cost function, and the inversion is improved by using all the frequencies present in the broadband of the transducers. The method presented is robust and is not perturbed by the experimental measurements or small errors in the material properties. The results are most promising. This method gives an image with an error, which is lower than the wavelength.

2pPAc4. Light interaction with leaky acoustic wave radiation in YX-LiTaO3. Paulius Kazdailis (Vilnius Univ., Dept. of Radiophysics, Saulëtekio al. 9, LT-10222 Vilnius, Lithuania, paulius.kazdailis@ff .vu.lt), Romualdas Rimeika (Vilnius Univ., Dept. of Radiophysics, Saulëtekio al. 9, LT-10222 Vilnius, Lithuania, romualdas.rimeika@ff .vu.lt), Daumantas Ciplys (Vilnius Univ., Dept. of Radiophysics, Saulëtekio al. 9, LT-10222 Vilnius, Lithuania, daumantas.ciplys@ff.vu.lt)

The theoretical model and experimental studies of $633 \mathrm{~nm}$ laser light interaction with radiation from leaky surface acoustic waves (LSAW) at various frequencies in YX-lithium tantalate (LiTaO3) are presented. The acousto-optic diffraction with and without polarization rotation has been observed. The values of BAW propagation angle and of the corresponding velocity have been calculated and compared to the ones extracted from the experiment. The differences of the light diffraction process in YX-lithium tantalate from the one in ZX-lithium niobate have been pointed. The potential of the described acousto-optic interaction for applications in light control devices and acoustic wave probing has been demonstrated.

2pPAc5. Scattering of ultrasonic waves in randomly layered materials Michal Pakula (Université Paris 6, Laboratoire d'Imagerie Paramétrique, 15, rue de l'Ecole de Médecine, 75006 Paris, France, michalp@ukw.edu.pl), Mieczyslaw Cieszko (Institute of Environmental 
Mechanics and Applied Computer Science, Kazimierz Wielki University in Bydgoszcz, ul. Chodkiewicza 30, 85-064 Bydgoszcz, Poland, cieszko@ukw.edu.pl), Jozef Kubik (Institute of Environmental Mechanics and Applied Computer Science, Kazimierz Wielki University in Bydgoszcz, ul. Chodkiewicza 30, 85-064 Bydgoszcz, Poland, kubik@ukw.edu.pl)

The goal of the paper is the proposal of a new macroscopic description of scattering of elastic waves at internal inhomogeneities of the material. Within the studies the microscopic inhomogeneity of the medium is modeled as the alternately arranged two kinds of elastic layers of random thicknesses and different mechanical properties. Calculations of the acoustical characteristics of such medium (reflection and transmission coefficients) are performed in two stages: (i) first the problem of interaction of plane harmonic wave with the half space of the randomly layered medium for the case of normal wave incidence is analyzed; (ii) then the interaction of the harmonic wave with the slice composed of randomly layered structure is considered. Such approach allowed to derive analytical relations for the phase velocity of wave propagation and attenuation as the explicit functions of frequency, stochastic structural parameters of the medium and material properties of layers. The obtained formulas are helpful for interpretation of experimental ultrasonic data and may serve for identification of the characteristic size of material inhomogeneity based on the measured wave parameters like frequency dependent phase velocity, attenuation as well as backscattering coefficient.

2pPAc6. Design of III-Nitride multi-layer structures for optical and surface acoustic wave interaction. Samuel Dupont (IEMN CNRS 8520 DOAE, Université de Valenciennes et du Hainaut Cambrésis Le Mont Houy, 59313 Valenciennes, France, samuel.dupont@univ-valenciennes .fr), Jean-Claude Kastelik (IEMN CNRS 8520 DOAE, Université de Valenciennes et du Hainaut Cambrésis Le Mont Houy, 59313 Valenciennes, France, jean-claude.kastelik@univ-valenciennes.fr), Victor Y. Zhang (IEMN-CNRS, Av. Poincare, Cite Scientifique, B.P. 60069, 59652 Villeneuve d'Ascq Cedex, France, victor.zhang@iemn.univ-lille1 .fr), Federica Causa (Dept. of Electronic and Electrical Engineering, Univ. of Bath, BA2 7AY Bath, UK, f.causa@bath.ac.uk), Qingbin Meng (Dept. of Electronic and Electrical Engineering, Univ. of Bath, BA2 7AY Bath, UK, Q.Meng @ bath.ac.uk), Jayanta Sarma (Dept. of Electronic and Electrical Engineering, Univ. of Bath, BA2 7AY Bath, UK, J.Sarma@bath.ac.uk)

Research efforts into Surface Acoustic Waves (SAW) devices built on III-Nitrides has recently significantly intensified because of the increasing availability of good-quality epitaxial material. Owing to their strong piezoelectricity and high acoustic velocity, high-Al-content materials are of particular interest in high-speed applications (GHz electronics). For optoelectronics applications III-Nitrides cover a wide range of wavelengths from ultra-violet $(\mathrm{AlN})$ to blue $(\mathrm{GaN})$ to infra-red $(\mathrm{InN})$; thus providing great flexibility in the choice of operating wavelength. III-Nitrides, therefore, become particularly attractive for the development of acousto-optic modulators where the SAW is used to create a controllable (moving) optical grating, causing the diffraction of the Optical Guided Wave (OGW). The interaction is governed by the momentum and energy conservation and importantly by the field overlap of the two waves. We present here design guidelines and modelling results for the development of III-Nitride-based multi-layer structures to support both OGW and SAW. The purpose of this study is to attempt to optimise the device efficiency compared to single GaN-layer structures typically used in the literature where only the SAW characteristics are optimised.

2pPAc7. Short access time acousto-optic deflector based on two cascaded Paratellurite devices. Nabil Ouail (IEMN CNRS 8520 DOAE, Université de Valenciennes et du Hainaut Cambrésis Le Mont Houy, 59313 Valenciennes, France, nabil.ouail@univ-valenciennes.fr), Jean-Claude Kastelik (IEMN CNRS 8520 DOAE, Université de Valenciennes et du Hainaut Cambrésis Le Mont Houy, 59313 Valenciennes, France,
jean-claude.kastelik@univ-valenciennes.fr),Michel_Pommeray(IEMN CNRS 8520 DOAE, Université de Valenciennes et du Hainaut Cambrésis Le Mont Houy, 59313 Valenciennes, France, michel.pommeray @ univ-valenciennes.fr), Samuel Dupont (IEMN CNRS 8520 DOAE, Université de Valenciennes et du Hainaut Cambrésis Le Mont Houy, 59313 Valenciennes, France, samuel.dupont@univ-valenciennes.fr)

Acousto-optic deflectors (AODs) are used in many applications requiring optical scanning devices with various specifications regarding access time, resolution and wavelength. High time-bandwidth products can be achieved with AODs made in a tellurium dioxide (TeO2) crystal. As paratellurite crystals are available with relatively large dimensions, the timebandwidth product can be increased by enlarging the optical aperture. However, the access time is increased which is not suitable for random access scanning mode. A new laser scanning system is then presented based on two wide-band paratellurite acousto-optic deflectors. Anisotropic interactions take place under two different tangential phase matching (TPM) configurations in such a way that the acousto-optic bandwidths add up. The optical arrangement of the two cascaded AODs is detailed. The feasibility of such a cascade deflection system has been demonstrated for the green wavelength $\lambda=514 \mathrm{~nm}$ of an argon laser. The total frequency bandwidth is $\Delta \mathrm{ft}=100$ $\mathrm{MHz}$, equally distributed between the two acousto-optic deflectors. The total angular scan at the output is $\Delta \theta t=4.5^{\circ}$ leading to more than 120 resolvable spots for a $1 \mathrm{~mm}$ truncated Gaussian beam and a short access time (as low as $1.5 \mu \mathrm{s})$.

2pPAc8. Peculierities of acoousto-optic interaction in nanodimensional laser heterostructures. Liudmila Kulakova (Ioffe Physico-Technical Institute RAS, Polytekhnicheskaya 26, 194021 St. Petersburg, Russian Federation, L.Kulakova@mail.ioffe.ru)

In the report, we present results of study of acousto-optic and acoustoelectron interactions in active and optical resonator regions of nanodimensional laser heterostructures. It was established that an ultrasonic wave introduced into a laser heterostructure produces modulation of the laser radiation frequency with a period equal to that of the acoustic wave. The static and dynamic analysis of the spectral parameters change under the ultrasonic strain has been fulfilled. The appreciable contribution of the acousto-optic interaction (comparable with the acousto-electron), resulting in the modulation in time of the positions of the heterolaser optical resonator lines was found out. The assumption about basic influence of quasi-two dimensional configuration of the laser optical resonator on the photoelastic properties causing unexpectedly large acousto-optic efficiency is stated.

2pPAc9. 3D Simulation of periodic surface wave inter-digital transducer using finite element/boundary element analysis. Sylvain Ballandras (CNRS FEMTO-ST, 32 Avenue de l'Observatoire, 25044 Besançon, France, sylvain.ballandras@femto-st.fr), William Daniau (CNRS FEMTO-ST, 32 Avenue de l'Observatoire, 25044 Besançon, France, william.daniau@femto-st.fr), Mikaël Wilm (Imasonic, ZA rue des Savourots, $\quad 70190 \quad$ Voray/Ognon, France, mikael.wilm@imasonic.com), Alexandre Reinhardt (CEA-LETI, 17 rue des Martyrs, 38054 Grenoble, France, alexandre.reinhardt@cea.fr), Raphaël Lardat (Thales Underwater Systems, 525 Route des Dolines, BP 157, 06930 Sophia Antipolis cedex, France, raphael.lardat@fr.thalesgroup.com), William Steichen (THALES Safare, 525 Route des Dolines, 06560 Sophia Antipolis, France, william.steichen @ fr.thalesgroup.com)

Spectacular advances have been made during the past years in the development of surface acoustic wave thanks to technology improvements but also to a strong effort in the modelling and design of such devices, taking into account their actual structure to benefit from second order effects (for instance the influence of the metal strip shape used in inter-digital transducers on the wave propagating under periodic gratings). In this paper, we present a 2D-periodic 3D-simulation approach to compute the spectral response of one period of a surface wave transducer, accounting for the actual shape of the electrodes as well as realistic guiding conditions and acoustic properties of the propagation surface. The idea consists in meshing the inhomogeneous part of the transducer, i.e. the electrodes and the bus bar, as well as a thin layer of the substrate to match the finite element section with 
surface boundary elements simulating the acoustic contribution of the substrate (propagation and radiation). Periodic boundary conditions are applied in both surface direction, allowing for the exploitation of spectral Green's function-based boundary elements, but additional absorbing conditions are added in the transverse direction (from both part of the bus bars) to suppress any unwanted modes generated by the mesh.

2pPAc10. Acoustic wave scattering from infinite cylinders made from functionally graded materials. Jalil Jamali (Faculty of Mechanical Engineering, K. N. Toosi University of Technology, Pardis St., Molla Sadra Ave., Vanak Sq., Postal code 1999143344, 16579 Tehran, Iran, ja_ja032@yahoo.com), Farhang Honarvar (Faculty of Mechanical Engineering, K. N. Toosi University of Technology, Pardis St., Molla Sadra Ave., Vanak Sq., Postal code 1999143344, 16579 Tehran, Iran, honarvar@mie .utoronto.ca), Mohammad Hasan Naei (Faculty of Mechanical Engineering, K. N. Toosi University of Technology, Pardis St., Molla Sadra Ave. Vanak Sq., Postal code 1999143344, 16579 Tehran, Iran, mhnaei@ut.ac.ir)

Functionally graded materials (FGM's) are superheat-resistive materials which show attractive properties in many applications including furnace liners. FGM's consist of two distinct materials, for example a ceramic and a metal alloy. These two materials are mixed such that the composition of each material changes continuously along a specific direction resulting in a continuous change of microstructure along that direction. The change in microstructure induces chemical, material, and microstructural gradients, and makes functionally graded materials different in behavior from homogeneous materials and traditional composites. In this paper, the scattering of an incident plane acoustic wave from an infinite solid cylinder made from functionally graded materials is studied. Expressions are derived for the far-field scattered pressure generated by illumination of the infinite FGM cylinder by an infinite plane acoustic wave. The propagation direction of the incident wave can make an arbitrary angle with the normal to the cylinder axis. The mathematical equations are derived and numerical results for cylinders made from specific functionally graded materials are presented at different incidence angles.
2pPAc11. Pendellösung phenomenon in two-dimensional sonic crystals Mitko Angelov (Politechnic University of Valencia, Wave Phenomena Group. Department of Electronic Engineering, C/ Camino Vera s.n, ES 46022 Valencia, Spain, mitan@doctor.upv.es), Daniel Torrent (Politechnic University of Valencia, Wave Phenomena Group. Department of Electronic Engineering, C/ Camino Vera s.n, ES-46022 Valencia, Spain, datorma1@upvnet.upv.es), Francisco Cervera (Politechnic University of Valencia, Wave Phenomena Group. Department of Electronic Engineering, C/ Camino Vera s.n, ES-46022 Valencia, Spain, fcervera@fis.upv.es), José Sanchez-Dehesa (Polytechnic University of Valencia, Cami de Vera s/n, 46022 Valencia, Spain, jsdehesa@upvnet.upv.es)

The phenomenon of "Pendelösung" oscillations, which is well known in X-ray diffraction [P.P. Ewald, Phys. Z. 14, 465 (1913); Ann. Phys. 54, 519 (1917); Rev. Mod. Phys. 37, 189 (1965)] is predicted here and experimentally verified for the case of two-dimensional sonic crystals made of hexagonal arrays of fluidlike cylindrical bars embedded in water. The dependence of diffraction intensity is studied as a function of layer thickness; i.e. of the layer number N. Nearly periodic oscillations have been observed at specific frequencies and the oscillation period is also a function of the frequency. The periodic behaviour is evidence of the exchange of energy between two modes in the sonic crystal. [Work supported by MEC of Spain.]

2pPAc12. Sound diffraction in periodic surfaces in ancient architectural structures. Jorge Antonio Cruz Calleja (Instituto Pólitecnico Nacional, Av Santa Ana No. 1000 col. San Francisco Culhuacan, Del Coyoacan, 04430 México, Mexico,jorgeacruzc@hotmail.com)

In this work is analyzed the sound diffraction in architectural structures caused by periodic surfaces. Is studied the sound interaction with architectural structures using different types of sources, materials and sizes. The apparently scattering effect of the staircases in ancient constructions (like the staircase of the Kukulcan pyramid in Chichen Itza), that allows the speech communication to large distances and the spatial filter effect using the glide repetition pitch theory are also analyzed (Is the Chirp echo of the ancient prehispanic pyramids an intentional effect?). Computational models of the phenomenon and real measurements were used to establish the phenomena physics principles. 


\title{
Session 2pPAd
}

\section{Physical Acoustics: Outdoor Sound Propagation and Uncertainties II (Poster Session)}

\author{
Keith Wilson, Cochair \\ U.S. Army Cold Regions Research and Engineering Laboratory \\ Michel Berengier, Cochair \\ Laboratoire Central des Ponts et Chaussées
}

\begin{abstract}
All posters will be on display from 3:40 p.m. to 5:20 p.m. To allow contributors an opportunity to see other posters, contributors of odd-numbered papers will be at their posters from 3:40 p.m. to 4:30 p.m. and contributors of even-numbered papers will be at their posters from 4:30 p.m. to 5:20 p.m.
\end{abstract}

\section{Contributed Papers}

2pPAd1. Lossy propagation of the Gulfstream Quiet Spike. Joe Salamone (Gulfstream Aerospace, 500 Gulfstream Rd., M/S R-01, Savannah, GA 31402, USA, joe.salamone@gulfstream.com)

The Gulfstream Quiet Spike is a telescoping nose extension with multiple segments of decreasing diameter intended to reduce the outdoor loudness of a sonic boom. This work examines the influence of the atmosphere on a propagated sonic boom signature for this device. First, atmospheric data, averaged by season, has been collected as a function of latitude to reasonably bound the range of meteorological conditions. These atmospheric profiles are subsequently input to nonlinear, lossy propagation code that computes the primary sonic boom carpet for a supersonic aircraft in steady, level flight. This code is an augmented implementation of the KZK model equation for one-dimensional propagation. The confluence of shocks generated by the Quiet Spike remains distinct and do not coalesce, as intended per the design. The amplitudes, rise times and spacing of the shocks from the individual spike segments are strongly governed by dispersion and attenuation. Loudness levels calculated from the resulting ground signatures show an observable change of over $4 \mathrm{~dB}$ at certain azimuths due to the various atmospheric conditions. Based on these results, minor design changes are recommended to improve the acoustic performance of the Gulfstream Quiet Spike.
2pPAd2. Practical analysis of the influence of basic meteorological phenomena on the long-term noise level. Barbara Joanna Lebiedowska (Warsaw University of Technology, ul. Lukasiewicza 17, 09400 Plock, Poland, barbara_lebiedowska@yahoo.fr)

Current regulations on the prognosis of the acoustic climate outdoors require that meteorological conditions should be examined as part of longterm noise level calculations. The impact of meteorological conditions on the noise level was analysed using simulation calculations performed with NMPB-96 method and Mithra software. Their aim was to determine the relationship between the value of these influences and the distance between the receiver point and the noise source and its height above the ground.The distance between a source and the built-up area varied. A number of its locations in relation to the built-up area ranging between $200 \mathrm{~m}$ and $4000 \mathrm{~m}$ were considered. The built-up area consisted of a row of 11-storey buildings ca. $600 \mathrm{~m}$ long. The ground floor height and the floor height were accepted to be $3.5 \mathrm{~m}$ and $3.0 \mathrm{~m}$, respectively. Calculations were performed for various meteorological conditions: from $\mathrm{P}=0$ (homogenous conditions) to $\mathrm{P}=1.0$, increasing by every 0.1 . The receiver point was located $2 \mathrm{~m}$ away from the façade at varying heights: from the lowest point positioned at $5 \mathrm{~m}$ to the highest point at $27.5 \mathrm{~m}$, increasing by $2.5 \mathrm{~m}$, which corresponds to the median position of each successive storey, from first floor to tenth floor. 


\title{
Session 2pPAe
}

\section{Physical Acoustics: Phononic Crystals II (Poster Session)}

\author{
Pierre Deymier, Cochair \\ University of Arizona \\ Jérôme Vasseur, Cochair \\ IEMN, UMR CNRS 8520
}

\begin{abstract}
All posters will be on display from 3:40 p.m. to 5:20 p.m. To allow contributors an opportunity to see other posters, contributors of odd-numbered papers will be at their posters from 3:40 p.m. to 4:30 p.m. and contributors of even-numbered papers will be at their posters from 4:30 p.m. to 5:20 p.m.
\end{abstract}

\section{Contributed Papers}

2pPAe1. Advances in acoustics of liquid crystals. Olga Kapustina (Institute of Acoustics, 4 Shvernik Street, 117036 Moscow, Russian Federation, oakapustina@yandex.ru)

Advances in the fundamental problem of the orientation transition in liquid crystals produced by acoustical action are considered. The theoretica models to describe a variety of liquid crystals orientation transitions in acoustic fields for the homeotropically and planar oriented layers which were developed in the framework of the Leslie-Ericksen hydrodynamics and the new methodology based on the ideas of non-equilibrium thermodynamics and nonlinear hydrodynamics are discussed and are substantiated experimentally. The orientation phenomena in liquid crystals were classified and the physical factors defining a kind of transition (threshold or nonthreshold type) and a choice of approach correct to describe adequately the experimental data on a new molecular arrangement induced by acoustica action were established. Among them a macrostructure type of liquid crystals layer, a layer thickness-viscous wavelength in liquid crystal ratio, an angle between the medium optical axe (the liquid crystal director) and the line of acoustical wave propagation, a sound intensity, a type of acoustical boundary conditions at the layer edges. The modern ideas on the distortion peculiarities of liquid crystals above a certain threshold of the structure instability under non-stationary dynamic action on the liquid crystal layer of sound-induced oscillating flows for a wide frequency band were considered.

2pPAe2. SH-wave propagation and localization anomalies in semi-infinite 1D magnetic phononic crystal. Tetyana V. Laptyeva (Donetsk Institute for Physics and Technology, 72, R. Luxemburg str., 83114 Donetsk, Ukraine, lapteva@mail.fti.ac.donetsk.ua), Sergey V. Tarasenko (Donetsk Institute for Physics and Technology, 72, R. Luxemburg str., 83114 Donetsk, Ukraine, tarasen@mail.fti.ac.donetsk.ua), Olga S. Tarasenko (Donetsk Institute for Physics and Technology, 72, R. Luxemburg str., 83114 Donetsk, Ukraine, tarasen@mail.fti.ac.donetsk .ua), Vladimir M. Yurchenko (Donetsk Institute for Physics and Technology, 72, R. Luxemburg str., 83114 Donetsk, Ukraine, yurch@mail.fti.ac .donetsk.ua)

At the present, the composite magnetic materials with a superstructure (magnetic photonic crystals) are actively investigated in view of conditions of electromagnetic wave transmission. Some of these materials are acoustically continuous media and, then, can be considered as magnetic phononic crystals (MPC). However, the dynamic acoustic peculiarities of such structures have been disregarded. Theoretical investigation of $\mathrm{SH}$-wave propagation in the gyrotropic and nongyrotropic semi-infinite one dimensional magnetic phononic crystals (1D MPC) is presented here. We have taken into account magnetoelastic interaction and the commensurability a wavelength and 1D MPC period. The transfer matrix method allowed us to determine the specific features of volume $\mathrm{SH}$-wave localization and propagation near the rigid interface of 1D MPC and nonmagnetic medium. In particular, the necessary conditions of existence and dispersion relations for three types of shear surface acoustic waves localized near the external surface of 1D MPC have been found. This type of elastic excitation are exists also for a case when elastic properties of MPC components are identical. The conditions of reflectionless transmission of volume $\mathrm{SH}$-waves through a finite $1 \mathrm{D}$ MPC have been determined.

2pPAe3. Zone center phonons in thalium fluoroborate. Hem Chandra Gupta (Indian Institute of Technology, Physics Department, 110016 New Delhi, India, hcgupta@physics.iitd.ac.in), Jaya Singh (Indian Institute of Technology, Physics Department, 110016 New Delhi, India, hcgupta @ physics.iitd.ac.in)

The study of phonons in thalium fluoroborate is very important in order to understand the phenomenon of phase transition in perchlorates and fluoroborates.Few studies of structural phase transition have been made of perchlorates and fluoroborates with a higher thalium ion, but their zone center phonons have not been investigated thoroughly. In order to understand the above, the Raman and the infrared frequencies of thalium fluoroborate have been investigated for the first time theoretically in its orthorhombic phase of space group Pnma. A short range force constant model (SRFCM) as well as rigid ion model (RIM) with stretching and bending force constants has been applied to obtain the zone center Raman and infrared phonons. The calculated values of Raman and infrared phonons are in very good agreement with the available observed values. The effect of these calculated interatomic force constants on the phase transition of thalium fluoroborate is discussed.

2pPAe4. Study of full band gaps and propagation of acoustic waves in two-dimensional piezoelectric phononic plates. Jin-Chen Hsu (Institute of Applied Mechanics, National Taiwan University, No. 1, Sec. 4, Roosevelt Road, 106 Taipei, Taiwan, hsu@ndt.iam.ntu.edu.tw), Tsung-Tsong Wu (Institute of Applied Mechanics, National Taiwan University, No. 1, Sec. 4, Roosevelt Road, 106 Taipei, Taiwan,wutt@ndt.iam.ntu.edu.tw)

Acoustic wave propagation in periodic structures composed of multiconstituents, namely the phononic crystals (PCs), has received much attention in the past decade. Recently, PCs constructed in the form of plate structures with two-dimensional lattices have been investigated, and some important phenomena, such as the full band-gap and waveguiding effects, induced by the periodicities of the phononic plates were reported. One of the virtues using Lamb waves is to perfectly confine the acoustic energy within the plate thickness and guided in structures with only 2D lattices rather than 3D lattices; however, the additional free surfaces derive much more complicated characteristics which are still awaiting study further. In this study, we 
investigate the band-gap and propagation properties of waves in twodimensional phononic plates that consist of either elastic or piezoelectric materials by employing a revised full 3D plane wave expansion (PWE) method and so on. To apply the PWE method efficiently, Fourier expansions are performed in the thickness direction for an imaginary 3D periodic structure by stacking the phononic plates and vacuum layers alternatively. Moreover, effects of piezoelectricity, lattices, and filling ratios on band gaps are discussed.

2pPAe5. Shannon Entropy as a Characterization Tool in Acoustics. Helios Sanchis-Alepuz (Polytechnic University of Valencia, Cami de Vera s/n, 46022 Valencia, Spain, hesana@alumni.uv.es), José SanchezDehesa (Polytechnic University of Valencia, Cami de Vera s/n, 46022 Valencia, Spain, jsdehesa@upvnet.upv.es)

Shannon entropy [1] was introduced as a measure of the uncertainty associated with a random variable and initially it was used to quantify the information contained in a binary message. In this work, a definition of Shannon's information entropy is introduced in acoustics, defined in terms of the displacement field, as a measure of the localization of resonant modes. As an example, it is applied to study the avoided crossing appearing in the resonant Zener-like phenomenon in ultrasonic superlattices made of two different fluidlike metamaterials. It is shown that acoustic Shannon entropy gives a correct physical insight of the localization effects taking place and manifest the informational exchange of the involved acoustic states in the narrow region of parameters where the avoided crossing occurs. Results for ultrasonic structures consisting of alternating layers of polymethylmethacrylate (Plexiglas) and water cavities, in which the acoustic Zener effect were recently demonstrated [2], are also reported. It is concluded that Shannon entropy is a useful tool to characterize localization effects in acoustical systems. [1] C.E. Shannon, Bell Sys. Tech. J. 27, 623 (1948). [2] H. Sanchis-Alepuz, Yu.A. Kosevich, and J. Sanchez-Dehesa, Phys. Rev. Lett. 98134301 (2007).

2pPAe6. Acoustic wave band gaps in triangular and honeycomb two-dimensional phononic crystals. Fu-Li Hsiao (Institute of Optical Sciences, National Central University, 320 Jung-Li, Taiwan, flhsiao@ios.ncu.edu.tw), Abdelkrim Khelif (Institut FEMTO-ST/CNRS, 32 avenue de l'Observatoire, 25044 Besançon cedex, France, abdelkrim.khelif@femto-st.fr), Boujemaa Aoubiza (Institut FEMTOST/CNRS, 32 avenue de l'Observatoire, 25044 Besançon cedex, France, boujemaa.aoubiza@univ-fcomte.fr), Abdelkrim Choujaa (Institut FEMTOST/CNRS, 32 avenue de l'Observatoire, 25044 Besançon cedex, France, abdelkrim.choujaa@femto-st.fr), Hanane Moubchir (Institut FEMTOST/CNRS, 32 avenue de l'Observatoire, 25044 Besançon cedex, France, hanane.moubchir@femto-st.fr), Chii-Chang Chen (Institute of Optical Sciences, National Central University, 320 Jung-Li, Taiwan, trich@ios.ncu.edu.tw), Vincent Laude (Institut FEMTO-ST/CNRS, 32 avenue de l'Observatoire, 25044 Besançon cedex, France, vincent.laude @ femto-st.fr)

The ultrasonic band gap properties of phononic crystals composed of arrays of steel cylinders immersed in water and arranged according to square, triangular or honeycomb lattices are compared theoretically and experimentally. In all three cases, complete band gaps are obtained, and the conditions of existence are identified by observing the complete band gap width as a function of the size of the inclusions. However, the measured transmission spectra reveal in the hexagonal symmetry cases (triangular and honeycomb lattices) the existence of deaf bands that cause strong attenuation in the transmission, with no band gap being involved. Band gaps and deaf bands are identified by comparing band structure computations for the infinite phononic crystal, obtained by a periodic-boundary finite element method (FEM), with transmission simulations for the finite phononic crystal, obtained using the finite difference time domain (FDTD) method. The possibility of managing phononic wave guides within a defect line channel inside an otherwise perfect triangular lattice phononic crystal is further demonstrated experimentally.
2pPAe7. Shock waves in nanomechanical resonators. Robert $H$. Blick (University of Wisconsin-Madison, Electrical and Computer Engineering, 1415 Engineering Drive, Madison, WI 53705, USA, blick@engr.wisc.edu), Florian W. Beil (University of Munich, Center for NanoScience, Geschwister Scholl Platz 1, 80539 Munich, Germany, beil_florian@bah.com), Achim Wixforth (University of Augsburg, Universitaetstrasse 1, 86135 Augsburg, Germany, achim.wixforth@physik .uni-augsburg.de), Werner Wegscheider (University of Regensburg, Fakultaet fuer Physik, 93040 Regensburg, Germany, werner.wegscheider @ physik.uni-regensburg.de), Dieter Schuh (University of Regensburg, Fakultaet fuer Physik, 93040 Regensburg, Germany, dieter.schuh @ physik.uni-regensburge.de), Max Bichler (Technical University of Munich, Coulombweg 1, 86748 Garching, Germany, max.bichler@wsi.tum.de)

The dream of every surfer is an extremely steep wave propagating at the highest speed possible. The best waves for this are would be shock waves, but are very hard to surf. In the nanoscopic world the same is true: the surfers in this case are electrons riding through nanomechanical devices on acoustic waves. Naturally, this has a broad range of applications in sensor technology and for communication electronics for which the combination of an electronic and a mechanical degree of freedom is essential. But this is also of interest for fundamental aspects of nano-electromechanical systems (NEMS), when it comes to quantum limited displacement detection and the control of phonon number states. Here, we study the formation of shock waves in a NEMS resonator with an embedded two-dimensional electron gas using surface acoustic waves. The mechanical displacement of the nanoresonator is read out via the induced acoustoelectric current. Applying acoustical standing waves we are able to determine the anomalous acoustocurrent. This current is only found in the regime of shock wave formation. We ontain very good agreement with model calculations.

2pPAe8. Experimental measurements of the band structure of Lamb waves in phononic lattices. Bernard Bonello (CNRS and Paris VI University, INSP - 140 rue de Lourmel, 75015 Paris, France, bernard.bonello@insp.jussieu.fr), Thomas Brunet (CNRS and Paris VI University, INSP - 140 rue de Lourmel, 75015 Paris, France, thomas.brunet@insp.jussieu.fr), Jiu-Jiu Chen (CNRS and Paris VI University, INSP - 140 rue de Lourmel, 75015 Paris, France, chen99nju@gmail .com)

We have measured the band structure of Lamb waves propagating in $2 \mathrm{D}$ phononic crystals. Different cases were investigated. First we have studied the phononic film deposited on a homogeneous substrate. The phononic film is made of cylindrical iron scatterers embedded into a copper background; the substrate is a $700 \mu \mathrm{m}$ silicon plate. The propagation is along the crystallographic direction [100] of Si. At low filling fraction, a frequency band gap for the antisymmetric mode arises at reduced Brillouin edge. At high filling fraction, a band gap opens also on the symmetric branch. We have also studied the case of phononic slabs with very high contrast between the scatterers and the matrix. The samples are made of $200 \mu \mathrm{m}$ silicon plates with air holes lattices drilled throughout. Whatever the symmetry of the lattice, square of centered rectangular, broad band gaps open at first and second reduced Brillouin zone edges. Experimental data are then compared to theoretical predictions obtained using a plane wave expansion method.

2pPAe9. Order/disorder transition for ultrasonic propagation in 2D scatterer arrays. Alice Bretagne (Laboratoire Ondes et Acoustique, ESPCI, Université Paris 7, CNRS, 10 rue Vauquelin, 75005 Paris, France, alice.bretagne@espci.fr), Arnaud Tourin (Laboratoire Ondes et Acoustique, ESPCI, Université Paris 7, CNRS, 10 rue Vauquelin, 75005 Paris, France, arnaud.tourin@espci.fr), Mathias Fink (Laboratoire Ondes et Acoustique, ESPCI, Université Paris 7, CNRS, 10 rue Vauquelin, 75005 Paris, France, mathias.fink@espci.fr)

Recently, intriguing phenomena in quantum mechanics, such as the Aharonov-Bohm effect [P. Roux et al., Phys. Rev. Lett. 79, 3170 (1997)], weak localization in disordered media [A. Tourin et al., Phys. Rev. Lett. 79, 3637 (1997)] and maybe one of the most striking, tunnelling [S. Yang et al., Phys. Rev. Lett. 88, 104301 (2002)], have been revisited using acoustic waves. The advantages of using ultrasound for such studies lie in the macroscopic character of samples and the possibility of directly measuring the 
phase of the wavefield. Here we explore propagation of $\mathrm{MHz}$-ultrasound in a medium which is either ordered or disordered in the two transverse dimensions $(\mathrm{x}, \mathrm{y})$ but invariant in the propagation direction $(\mathrm{z})$. An equivalent scheme has been recently used in optics to demonstrate transverse localization of light [T. Schwartz et al., Nature 446, 52 (2007)]. Our samples are made of a parallel arrangement of cylindrical scatterers $(0.8 \mathrm{~mm}$ in diameter) embedded in a PVA matrix. In the ordered case, the probe beam undergoes ballistic transport. In the disordered case transport becomes diffusive and as the sample length increases the wave tends to be laterally confined.

2pPAe10. Experimental and theoretical investigations of focusing of ultrasonic waves by two-dimensional flat phononic crystals. Alexey Sukhovich (Universidad de Murcia, Dept. of Physics and Astronomy, Univ. of Manitoba, Winnipeg, MB R3T 2N2, Canada, alexei@physics.umanitoba.ca), John H. Page (Dept. of Physics and Astronomy, Univ. of Manitoba, Winnipeg, MB R3T 2N2, Canada, jhpage@cc.umanitoba.ca), Bassam Merheb (University of Arizona, Materials Science and Engineering Department, Mines Bldg., P.O. Box 210012 , Tucson, AZ 85721, USA, bassam@merheb.net), Jérôme Vasseur (IEMN, UMR CNRS 8520, avenue Poincaré, BP 60069, 59652 Villeneuve d'Ascq, France, jerome.vasseur@univ-lille1.fr), Pierre Deymier (University of Arizona, Materials Science and Engineering Department, Mines Bldg., P.O. Box 210012, Tucson, AZ 85721, USA, deymier@u.arizona.edu)

We compare experimental and theoretical results demonstrating focusing of sound fields emitted by subwavelength sources using two-dimensional flat phononic crystals. Our phononic crystals are made of stainless steel rods assembled in a triangular crystal lattice and immersed in a liquid. Focusing is achieved due to negative refraction of ultrasonic waves propagating through the crystal, which is realized at the frequencies corresponding to the 2nd band, where the group velocity and wave vector are antiparallel. High quality images are obtained experimentally with a methanol-filled phononic crystal surrounded by water, in which the source and detecting transducers are located. Excellent resolution approaching the diffraction limit is achieved for the frequency at which the equifrequency contours inside the crystal match those in water. Our experimental results are compared with theoretical predictions by the Finite Difference Time Domain (FDTD) method. Good agreement is observed for the width of the focal spot in the direction parallel to the crystal surface, while along the perpendicular direction, we find experimentally that the focal spot is narrower and closer to the crystal surface than in the theoretical predictions.

2pPAe11. Complete band gap in the phonon spectra of compact ceramics. Evgenii I. Salamatov (Physical-Technical Institute UrB RAS, 132 Kirov str., 426000 Izhevsk, Russian Federation, salam@otf.pti.udm.ru)

The processes of sound propagation in inhomogeneous systems have long the focus of researchers attention. The new interest in this problem $i$ last years has been inspired by the appearance of artificial elastic periodic structures - phononic crystals. Since the lattice parameter artificial phononic crystals is about several millimetres the complete bandgap is assumed to lie in the megahertz range. It is of fundamental importance for modern radio equipment to obtain phononic crystals with forbidden bandgap in the gigahertz range, which requires generation of phononic lattices with the period of the order of tens and hundreds nanometers. The author believes nanoceramics compacted from a superfine powder in a special way to be very promising systems for the generatio such phononic lattices. In this work the phonon spectra of the model two-dimensional ceramics numerically calculated using the finite-difference time-domain (FDTD) method. To interpret the results, the analytical expressions obtained for an elastic limit are used, which enables formalization of the conditions necessary for the appearance of a gap in a phonon spectrum. The possibility of creation of bulk acoustic waves resonators is discussed.

2pPAe12. A Transient Grating approach to elastic wave and thermal propagation in a 2D free-standing micrometric phononic crystal Iacopo Malfanti (European Lab. for Non-Linear Spectroscopy (LENS), Univ. di Firenze, via Nello Carrara 1, 50019 Sesto Fiorentino (Fi), Italy,
malfanti@lens.unifi.it),Renato Torre(European Lab. for Non-Linear Spectroscopy (LENS), Univ. di Firenze, via Nello Carrara 1, 50019 Sesto Fiorentino (Fi), Italy, torre@lens.unifi.it), Paolo Bartolini (European Lab. for Non-Linear Spectroscopy (LENS), Univ. di Firenze, via Nello Carrara 1, 50019 Sesto Fiorentino (Fi), Italy, bart@lens.unifi.it), Andrea Taschin (European Lab. for Non-Linear Spectroscopy (LENS), Univ. di Firenze, via Nello Carrara 1, 50019 Sesto Fiorentino (Fi), Italy, taschin@lens.unifi.it), Francesco Vita (via Brecce Bianche, 1, 60131 Ancona, Italy, f.vita@univpm.it), Francesco Simoni (via Brecce Bianche, 1, 60131 Ancona, Italy, f.simoni@univpm.it)

We investigate the phonon propagation in a $2 \mathrm{D}$ phononic crystal (with typical dimensions on the micrometric scale) by means of a transient grating (TG) heterodyne detected experiment. In a TG experiment both a temperature and a density grating are induced by means of optical techniques. The relaxation dynamics of the induced grating are then monitored live-time over 6 temporal decades with a probe beam. Our sample is a freestanding 100 micrometer thick polymer matrix with empty rods (filled with gas) arranged in a triangular lattice. Evidence of the presence of two different bulk wave acoustic modes are experimentally found. The excited acoustic modes show a correlation with the orientation of the sample with respect to the induced grating wave vector, while the thermal properties show a significant dependence on the magnitude of the induced grating wave vector.

2pPAe13. Thermal shifting of phononic bandgaps in barium strontium titanate-based structures. Kwok Lung Jim (Department of Applied Physics and Materials Research Centre, The Hong Kong Polytechnic University, Hunghom, Kowloon Hong Kong, China, jim.kl@polyu.edu.hk), Chi Wah Leung (Department of Applied Physics and Materials Research Centre, The Hong Kong Polytechnic University, Hunghom, Kowloon Hong Kong, China, apleung@inet.polyu.edu.hk), Chung Loong Choy (Department of Applied Physics and Materials Research Centre, The Hong Kong Polytechnic University, Hunghom, Kowloon Hong Kong, China, scclchoy@ine .polyu.edu.hk), Helen L. W. Chan (Department of Applied Physics and Materials Research Centre, The Hong Kong Polytechnic University, Hunghom, Kowloon Hong Kong, China, apahlcha@inet.polyu.edu.hk)

Phononic crystals, which are structures with periodic variations of density and/or sound velocities, can exhibit phononic bandgaps where propagation of acoustic waves is forbidden. The ability of phononic crystals to manipulate sound, in a similar manner which photonic crystal control light, makes them particularly useful for applications such as acoustic filters or very efficient waveguides. Since the positions of the phononic bandgaps depend solely on the densities and the sound velocities of the constituent materials, it is expected that a modulation in the densities and/or sound velocities of the constituent materials would result in a phononic bandgap shift. It is known that ferroelectric ceramic, such as barium strontium titanate (BST) will undergo a ferroelectric-to-paraelectric phase transition across the Curie temperature. During the phase transition, there is a large variation in the sound velocities of BST which result in a shift of the phononic bandgaps. In this work, we calculated the phononic bandstructures of BST-based structures by the plane-wave expansion method and showed that the phononic bandgaps can be shifted thermally.

2pPAe14. Nondiffractive propagation of sound in sonic crystals. Isabel Perez-Arjona (IGIC - Universitat Politècnica de València, Cra. NazaretOliva S/N, E-46730 Gandia, Spain, iparjona@upvnet.upv.es), Victor Sánchez-Morcillo (IGIC - Universitat Politècnica de València, Cra Nazaret-Oliva S/N, E-46730 Gandia, Spain, victorsm@fis.upv.es), Víctor Espinosa (IGIC - Universitat Politècnica de València, Cra. Nazaret-Oliva S/N, E-46730 Gandia, Spain, vespinos@fis.upv.es), Kestutis Staliunas (Departament de Fisica i Enginyeria Nuclear, Colom 11, 08222 Terrassa (Barcelona), Spain, Kestutis.Staliunas@icrea.es), Javier Redondo (IGIC - Universitat Politècnica de València, Cra. Nazaret-Oliva S/N, E-46730 Gandia, Spain, fredondo@fis.upv.es)

In this work we present the study of the nondiffractive propagation regime of ultrasonic waves in a two-dimensional periodic acoustic media (sonic crystal) both from the theoretical and the experimental point of view. An analytical treatment of this regime sonic wave propagation is presented which allows evaluating the parameters of the nondiffractive regime, just as 
the minimum size of the nondiffractively propagating acoustic beam. Experimentally, we report on the first experimental demonstration of the subdiffractive propagation (self-collimation) of an ultrasound beam in a sonic crystal formed by steel cylinders in water. The experimental measurements show a good agreement with the theoretical predictions, where the effect of the finite size of the crystal was also considered. The preliminary results on self-collimation of sound in three-dimensional sonic crystal, in progress at the present moment, will be also presented.

TUESDAY AFTERNOON, 1 JULY 2008

P3-A, LEVEL 3, 3:40 TO 5:20 P.M.

\title{
Session 2pPAf
}

\section{Physical Acoustics: Acoustics of Porous Media III (Poster Session)}

\author{
Walter Lauriks, Cochair \\ Lab. ATF, Katholieke Universiteit Leuven \\ Keith Attenborough, Cochair \\ Open University
}

\begin{abstract}
All posters will be on display from 3:40 p.m. to 5:20 p.m. To allow contributors an opportunity to see other posters, contributors of odd-numbered papers will be at their posters from 3:40 p.m. to 4:30 p.m. and contributors of even-numbered papers will be at their posters from 4:30 p.m. to 5:20 p.m.
\end{abstract}

\section{Contributed Papers}

2pPAf1. In-situ measurement of interface permeability. Lin Lin (Universisty Of Maine, 5711 Boardman Hall, Orono, ME 04469, USA, lin.lin@umit.maine.edu), Michael Peterson (Universisty Of Maine, 5711 Boardman Hall, Orono, ME 04469, USA, michael.peterson @ maine.edu), Alan Greenberg (Univesity of Colorado, 427 UCB Dept. of Mechanical engineering, Boulder, CO 80309, USA, Alan.Greenberg @ colorado.edu), Benjamin McCool (ExxonMobil Research and Engineering Company, 1545 Route 22 East, Annandale, NJ 08801, USA, Benjamin .a.mccool@exxonmobil.com)

Permeability of a porous interface is important for many areas of research and technology ranging from transport of nutrients in marine sediments to produce separations in bio-pharmaceuticals. Biot theory has shown that porous materials are able to support the propagation of two longitudinal waves: fast and slow wave. By measuring the propagation threshold of the slow longitudinal wave when the wave number is higher than the critical wave number $\mathrm{kcr}$, we can obtain the intrinsic permeability of a porous interface. The ability to sense permeability change due to differences in pore size and fluid viscosity is demonstrated. The measurement is compared to an exact solution for kcr as well as previously published analytical solutions. This technique for measuring permeability has been shown in a model material and can now be extended to a wide range of materials of scientific and technical importance.

2pPAf2. Sound absorption of advanced textile materials. Ivan Bolkovac (Faculty of EE and Computing, Unska 3, Department of Electroacoustics, HR-10000 Zagreb, Croatia, ibolkovac@gmail.com), Hrvoje Domitrovic (Faculty of EE and Computing, Unska 3, Department of Electroacoustics, HR-10000 Zagreb, Croatia, hrvoje.domitrovic@fer .hr), Martina Bozic (Faculty of textile technology, University of Zagreb, Ljubijska 51a, 10000 Zagreb, Croatia, martina.bozic.ttf@gmail.com)

Today's acoustic applications demand an ever greater array of materials used for acoustic treatment of spaces for both professional or nonprofessional use. Elements like absorbers, diffusers and silencers are met with increasingly strict demands, both aesthetic and functional. That is the reason why we were interested in acoustic qualities (absorption coef.) of different kinds of textile materials that could be used in building or finishing such elements. Textile materials range from conventional to high- performance textiles. The latter, with their advanced qualities (both acoustic and mechanical) promise a much wider area of application and further specialized use, both as stand-alone elements or their parts.

2pPAf3. Influence of sound absorption on close proximity noise of porous pavement. S. E. Paje (Laboratory of Acoustics Applied to Civil Engineering (LA2IC), Universidad de Castilla-La Mancha, Department of Applied Physics, 13071 Ciudad Real, Spain, santiago.exposito@uclm .es), Moises Bueno (Laboratory of Acoustics Applied to Civil Engineering (LA2IC), Universidad de Castilla-La Mancha, Department of Applied Physics, 13071 Ciudad Real, Spain, moises.bueno@uclm.es), Urbano Viñuela (Laboratory of Acoustics Applied to Civil Engineering (LA2IC), Universidad de Castilla-La Mancha, Department of Applied Physics, 13071 Ciudad Real, Spain, urbano.vinuela@uclm.es), Fernando Terán (Laboratory of Acoustics Applied to Civil Engineering (LA2IC), Universidad de Castilla-La Mancha, Department of Applied Physics, 13071 Ciudad Real, Spain, fernando.teran@uclm.es)

The main objective of this research is to correlate sound absorption of a new bituminous porous pavement (air void content 21\%) with the close proximity noise. Different core samples were taken from a porous road surface. The normal incidence sound absorption spectra were measured for these samples using the two-microphone impedance tube. Noise levels close to the tire/pavement contact patch were measured continuously in a georeferenced way, when the test vehicle was rolling. The emission properties of the porous surface as a function of vehicles speed are analyzed in the acoustic frequency range showing different behaviors for the relationships between noise levels and speed. An abrupt variation in the relationship between the coefficient $\mathrm{B}$ and frequencies below $1 \mathrm{kHz}$ was observed $(\mathrm{Lcp}=\mathrm{A}+\mathrm{B} \cdot \log (\mathrm{V}))$. This feature could be highly influenced by sound absorption mechanism, particularly noticeable for a non-clogged porous surface with a high content of air voids.

2pPAf4. Physico-chemical properties and ultrasonic characterization of calcium phosphate ceramics. Daniela Predoi (National Institute of Materials Physics, Atomistilor 105 bis, 77123 Magurele, Romania, 
dpredoi_68@yahoo.com),Serge Derible(LOMC FRE CNRS 3102, Université du Havre, Place Robert Schuman, 76610 le Havre, France, serge.derible@univ-lehavre.fr), Hugues Duflo (LOMC FRE CNRS 3102, Université du Havre, Place Robert Schuman, 76610 le Havre, France, hugues.duflo@univ-lehavre.fr), Mihai Valentin M. Predoi (University Politechnica of Bucharest, Department of Mechanics, 060032 Bucharest, Romania, predoi@cat.mec.pub.ro), Cristian Catalin Petre (University Politechnica of Bucharest, Department of Mechanics, 060032 Bucharest, Romania, cristian.petre@promteh.ro)

Calcium phosphate compounds have been studied for biomedical applications due to their chemical and structural similarity to the mineral phase of bone and tooth. The composition, physical and chemical properties, crystal size and morphology of synthetic apatite are extremely sensitive to preparation conditions and sometimes it resulted into non- stoichiometric calcium deficient hydroxyapathite (Hap) powders. The present paper refers to calcinations of calcium phosphate ceramics at 800 and $1000^{\circ} \mathrm{C}$. The effect of heat treatment were investigated by X-ray diffraction (XRD), Fourier transform infrared (FTIR), differential thermal analysis (DTA) and thermal gravimetric analysis (TGA). FTIR spectra showed the presence of various $\mathrm{PO}_{4} 3$ - and $\mathrm{OH}$-groups present in the powders. Powders compacted and sintered at 800 and $1000^{\circ} \mathrm{C}$ showed an increase in density. The main objective of the paper is a comparison of the results obtained by the previous methods to those obtained using the ultrasonic air-coupling technique. Modulated ultrasonic signals of $500 \mathrm{kHz}$ central frequency have been transmitted through the calcium phosphate ceramics specimens. Correlation between signals allowed some conclusions concerning density, porosity and preparation temperature influence on these specimens. These comparisons and correlation of methods, allow a better characterisation of such important materials

2pPAf5. Ultrasonic monitoring of hardening concrete. Julien Bue (Institut Jean le Rond D'Alembert / UMR 7190, 2 Place de la Gare de Ceinture, 78210 Saint Cyr L'Ecole, France, julien.bue@wanadoo .fr), Frederic Cohen Tenoudji (Institut Jean le Rond D'Alembert / UMR 7190, 2 Place de la Gare de Ceinture, 78210 Saint Cyr L'Ecole, France, fcohentenoudji@yahoo.fr)

Monitoring results of velocities and attenuations of compressional and transverse ultrasonic waves in hardening concrete using short pulse throughtransmission are presented. A fluid concrete is a highly attenuating heterogeneous medium, composed of several phases and granular classes. Different attenuation mechanisms are considered in order to account for experimental results: high impedance mismatch between air layers and solid-fluid clusters at early ages, Energy loss by viscous dissipation and wave scattering by heterogeneities (Rayleigh scattering at low frequencies, single or multi diffusion scatterings at intermediate and high frequencies) during the beginning of hardening. At early ages, the medium may be described as composed of a succession of large slabs of fresh concrete separated by very thin slabs of air. Attenuation is then computed from the effective transmission coefficient of this medium. This model is in good agreement with experiments. During the beginning of hardening, the medium may be seen as composed of big particles immersed in an effective medium formed by the ensemble of the particles of lesser granular sizes and water. Then, the Rayleigh scattering model at low frequencies and the multiple diffusion model predictions at intermediate frequencies are in good agreement with experiments.

2pPAf6. Flow resistivity profile inversion for a porous medium. Claude Depollier (Laboratoire d'Acoustique de l'Université du Maine, Avenue Olivier Messiaen, 72085 Le Mans, France, claude.depollier@univ-lemans .fr), Naima Sebaa (Laboratoire d'Acoustique de l'Université du Maine, Avenue Olivier Messiaen, 72085 Le Mans, France, naima.sebaa@univ-lemans .fr), Mouna Naas (Laboratoire d'Acoustique de l'Université du Maine, Avenue Olivier Messiaen, 72085 Le Mans, France, mouna.naas.etu @ univ-lemans.fr), Bernard R. Castagnede (Laboratoire d'Acoustique de
l'Université du Maine, Avenue Olivier Messiaen, 72085 Le Mans, France, bernard.castagnede@univ-lemans.fr), Zine Fellah (CNRS-Laboratoire de Mécanique et d'Acoustique, 31 Chemin Joseph Aiguier, 13402 Marseille, France, fellah@lma.cnrs-mrs.fr), Walter Lauriks (Lab. ATF, Katholieke Universiteit Leuven, Celestijnenlaan 200D, B-3001 Leuven, Belgium, Walter.Lauriks@fys.kuleuven.be)

Predictions for the low frequency sound waves propagation in a porous medium needs the knowledge of the flow specific resistance of the medium. We present a scheme for the flow resistivity profile inversion for a layered medium. In the framework of the fluid equivalent model a closed-form relation of the resistivity profile with the Fourier transform of the reflection is derived. It provide a straightforward way to perform the reconstruction of the profile. The result show a strong correlation between the flow resistivity profile and the reflection coefficient in this model. Some numerical simulations are given as examples of the applicability of this scheme.

2pPAf7. Wave equation in non-integer-dimensional porous media. Claude Depollier (Laboratoire d'Acoustique de l'Université du Maine, Avenue Olivier Messiaen, 72085 Le Mans, France, claude.depollier @ univ-lemans.fr), Jean-Baptiste Legland (Laboratoire d'Acoustique de l’Université du Maine, Avenue Olivier Messiaen, 72085 Le Mans, France, jean-baptiste.legland.etu@univ-lemans.fr)

Over some range of scales, porous media display properties of fractals. It particular both pore sizes and pore-interfaces exhibit the fractal characteristics. One of the fascinating interests of fractals is their capability to model objects with complicate structure. This is due to an important property of fractal objects that their structure is characterized by a small number of parameters. One of them is the fractal dimension which tells how the fractal fills the space in which it lies. Very early, fractal concept has been incorporated in the study of porous media to investigate various phenomena as flow in porous media, pores and fractures of geological media. We present generalized Biot's wave equations for a fractional dimensional system of 3-spatial coordinates used as an effective description of porous media. Some specific examples are given as an application of this model.

2pPAf8. Investigation of the limp model validity for porous materials Olivier Doutres (Laboratoire d'Acoustique de l'Université du Maine, Avenue Olivier Messiaen, 72085 Le Mans, France, olivier.doutres.etu @ univ-lemans.fr), Nicolas Dauchez (Laboratoire d'Acoustique de l'Université du Maine, Avenue Olivier Messiaen, 72085 Le Mans, France, nicolas.dauchez@univ-lemans.fr), Jean Michel Genevaux (Laboratoire d'Acoustique de l'Université du Maine, Avenue Olivier Messiaen, 72085 Le Mans, France, jean-michel.genevaux@univ-lemans.fr), Olivier Dazel (Laboratoire d'Acoustique de l'Université du Maine, Avenue Olivier Messiaen, 72085 Le Mans, France, olivier.dazel@univ-lemans.fr)

A new criterion to test the validity of using the limp model for porous materials is proposed. The limp model is derived from the poroelastic Biot model assuming that the frame has no bulk stiffness. Being an equivalent fluid model accounting for the motion of the frame, it has fewer limitations than the usual equivalent fluid model assuming a rigid frame. A criterion is derived to identify the porous materials for which the limp model can be used. This criterion relies on a new parameter, the Frame Stiffness Influence (FSI) based on porous material properties. The critical values of FSI under which the limp model can be used, are determined using a 1D analytical modeling for three boundary sets: absorption of a porous layer backed by a rigid wall, radiation of a vibrating plate covered by a porous layer and transmission loss of a double leaf panel filled in by a porous layer. Compared with other criteria, the criterion associated with FSI provides information in a wider frequency range and can be used for configurations which include vibrating plates.

2pPAf9. An index to quantify the through-thickness symmetry of sound absorbing materials. Yacoubou Salissou (Groupe d'Acoustique de l'Université de Sherbrooke, 2500, Boul. de l'Université, Département de génie mécanique, Sherbrooke, QC J1K-2R1, Canada, Yacoubou.Salissou 
@USherbrooke.ca),Raymond Panneton(Groupe d'Acoustique de l'Université de Sherbrooke, 2500, Boul. de l'Université, Département de génie mécanique, Sherbrooke, QC J1K-2R1, Canada, raymond.panneton @ usherbrooke.ca)

In this work, an extension of the theoretical formulation of the transfer matrix to non symmetrical sound absorbing porous materials is carried out. From this extension, an index of asymmetry is proposed and discussed. This index allows one to quantify the through-thickness asymmetry of a sound absorbing porous material. This index may be used for quality control or to assess the symmetry of the material in terms of its acoustic properties (absorption, reflection, impedance, propagation constant). To validate the application of the index of asymmetry, samples made up from two layers of porous materials are studied. Each so-constructed two-layered sample is seen as an equivalent asymmetrical single porous layer with a sudden change in its physical properties. The acoustic properties of each sample are then measured in the direct and inverted configurations (i.e., when both sides of the sample are facing successively the incident wave). Their values are compared in terms of the asymmetry index of the equivalent single layer. From these tests, an index value is suggested. Above this value, the acoustic properties of a sound absorbing material should be considered as being not symmetrical.

2pPAf10. Ultrasonic critical angle reflectometry applied to porous nuclear fuel mechanical characterization. Vivian Cereser Camara (Université Montpellier II, Place Eugène Bataillon, 34095 Montpellier, France, cereser@lain.univ-montp2.fr), Didier Laux (Université Montpellier II, Place Eugène Bataillon, 34095 Montpellier, France, laux@lain .univ-montp2.fr), Gilles Despaux (Université Montpellier II, Place Eugène Bataillon, 34095 Montpellier, France, despaux@lain.univ-montp2 .fr), Daniel Baron (EDF, CEA Cadarache, 13108 St Paul Lez Durance, France, daniel.baron@edf.fr)

An ultrasonic reflectometer working from 2 to $10 \mathrm{MHz}$ on zones around $4 \mathrm{~mm}$ has been built in order to assess in a non destructive way the reflection coefficient $\mathrm{R}(\theta, \mathrm{f})$ as a function of incident angle and frequency. Many experiments were performed on various materials (glass, aluminium,...) showing that with this goniometer and the related signal processing it is possible to assess very accurately $\mathrm{R}(\theta, \mathrm{f})$ for bulk or thin samples (Lamb modes). Longitudinal and shear velocities (VL, Vs) deduced from $\mathrm{R}(\theta, \mathrm{f})$ have been used to determine the elastic moduli $\mathrm{E}$ and $\mathrm{G}$ which values match very favourably with literature. Hence the Ultrasonic Critical angle Reflectometry is a powerful tool for materials characterization. In a second step, this device has been used on civil power plants fuel (UO2) with various volume fraction of porosity. The elastic moduli obtained are in good agreement with the literature data. The relations between VL, VS and the porosity (p) on the one hand, and $\mathrm{E}$ and $\mathrm{G}$ with $\mathrm{p}$ on the other hand will be presented and discussed. In particular, a link between these relations and the pores morphology (deduced with numerical simulations) will be pointed out.

2pPAf11. Hybrid inversion technique for predicting geometrical parameters of porous materials. Paresh Shravage (Dipartimento di Ingegneria - University of Ferrara, Via Saragat 1, 44100 Ferrara, Italy, paresh.shravage@unife.it), Paolo Bonfiglio (Dipartimento di Ingegneria University of Ferrara, Via Saragat 1, 44100 Ferrara, Italy, paolo.bonfiglio @unife.it), Francesco Pompoli (Dipartimento di Ingegneria - University of Ferrara, Via Saragat 1, 44100 Ferrara, Italy, francesco.pompoli@unife.it)

In prediction of acoustical behavior of porous materials, five geometrical parameters play a very important role, but some of these geometrical properties are very difficult to measure directly. So many authors have suggested different inversion strategies for getting these properties from directly measured both characteristic and surface properties of the material using standing wave tube. These approaches can be divided in two different categories: analytical (based on the limit behaviour of the bulk properties) and minimization based methods (which make use of searching algorithms to determine the best solution that minimizes a cost function calculated by means of a prediction model). Recent studies have shown the reliability of the analytical methods for the determination of the airflow resistivity and the minimization based approach by using genetic algorithms for getting the other physical parameters. Consequently, a hybrid inversion technique can be proposed for the complete calculation of the geometrical quantities and here it is presented in detail. Moreover the paper compares the results from the hybrid approach with the experimentally measured parameters and the values of the five parameters obtained by using genetic algorithms. Finally, the paper presents the effect of both inversion techniques on acoustical properties using Johnson-Allard-Champoux model.

2pPAf12. Absorption and dispersion of acoustical waves in synthetic and natural compressed fibrous materials. Bernard $R$. Castagnede (Laboratoire d'Acoustique de l'Université du Maine, Avenue Olivier Messiaen, 72085 Le Mans, France, bernard.castagnede @univ-lemans.fr), Bruno Brouard (Laboratoire d'Acoustique de l'Université du Maine, Avenue Olivier Messiaen, 72085 Le Mans, France, bruno.brouard@univ-lemans.fr), Claude_ Depollier (Laboratoire d'Acoustique de l'Université du Maine, Avenue Olivier Messiaen, 72085 Le Mans, France, claude.depollier@univ-lemans.fr), Olivier Dazel (Laboratoire d'Acoustique de l'Université du Maine, Avenue Olivier Messiaen, 72085 Le Mans, France, olivier.dazel@univ-lemans.fr), Denis Lafarge (Laboratoire d'Acoustique de l'Université du Maine, Avenue Olivier Messiaen, 72085 Le Mans, France, denis.lafarge@univ-lemans.fr)

When a fibrous slab is compressed along its thickness, there are some changes in the basic physical parameters which describe the propagation of sound waves. For instance during compression, the acoustical resistivity and the tortuosity increase, while at the same time the porosity as well as the viscous and thermal characteristic lengths diminish. For a 1-D compression along the thickness of the porous layer, these changes are decribed by straightforward linear equations, as long as the compression ratio is kept small (e.g. between 1 and 5). Such simple expressions are derived theoretically on the base of conservation principles relying on some fundamental metrology indicators. Next, the influence of these changes on the 5 fundamental physical properties of any porous networks are studied numerically on the dispersion and attenuation curves versus frequency, in the frame of the "equivalent-fluid" model [4]. Finally, experiments are performed in order to check some of these predictions on various felt fibrous materials having different compression ratio.

2pPAf13. Acoustic properties of partly saturated porous soils. Kirill V. Horoshenkov (University of Bradford, School of Engineering, Design and Technology, BD7 1DP Bradford, UK, k.horoshenkov@Bradford .ac.uk), Mostafa H. Mohamed (University of Bradford, School of Engineering, Design and Technology, BD7 1DP Bradford, UK, m.h.a.mohamed@Bradford.ac.uk), Siow N. Ting (University of Bradford, School of Engineering, Design and Technology, BD7 1DP Bradford, UK, s.n.ting@bradford.ac.uk)

Controlled experiments are performed on soil samples saturated with different types of liquid such as water and oil to determine the relations between the degree of saturation, type of saturated liquid and the acoustic surface admittance. These experiments are conducted using a Buchner funnel attached directly to an acoustic impedance tube. The results show a very sensitive dependence of the acoustic admittance upon the degree of saturation irrespective of the liquid present. It is found that the relationship between the volumetric water content and the real part of the surface admittance in the frequency range of $500-1000 \mathrm{~Hz}$ can be expressed using a logarithmic equation. The coefficients of the proposed equation can be determined using a soil property called "uniformity coefficient" and the acoustic admittance of dry soil. Results of volumetric water content obtained using the proposed equation are validated using those obtained from an independent test. The results of the validation exercise demonstrate that the proposed relations can be used to determine very accurately the volumetric water content within soil from the acoustical data. The accuracy of the acoustically measured degree saturation is found to be within $2.0 \%$. 


\title{
Session 2pPAg
}

\section{Physical Acoustics: Photoacoustics II (Poster Session)}

\author{
Gerald Diebold, Cochair \\ Brown Univ. \\ Christ Glorieux, Cochair \\ Lab. ATF, Katholieke Universiteit Leuven
}

\begin{abstract}
All posters will be on display from 3:40 p.m. to 5:20 p.m. To allow contributors an opportunity to see other posters, contributors of odd-numbered papers will be at their posters from 3:40 p.m. to 4:30 p.m. and contributors of even-numbered papers will be at their posters from 4:30 p.m. to 5:20 p.m.
\end{abstract}

\section{Contributed Papers}

2pPAg1. Fiber Bragg grating applied pulsed photoacoustic detection technique for online monitoring concentration of liquid. Atsushi Yarai (Osaka Sangyo University, 3-1-1 Nakagaito, 574-8530 Daito, Osaka, Japan, yarai@osaka-sandai.ac.jp), Takuji Nakanishi (Osaka Sangyo University, 3-1-1 Nakagaito, 574-8530 Daito, Osaka, Japan, a.yarai@ieee.org)

The need for real-time monitoring of the concentration of raw liquids and wastewater flowing in ducts is one of the significant process controls in chemical plants. A concentration sensor applicable to acidic fluids, however, has not been practically developed because of the maintenance required to sustain performance due to the metal erosion of the electrode from the acidity. To overcome this problem, we propose the fiber Bragg grating applied pulsed photoacoustic (PA) detection technique for online monitoring concentration of liquid. In our technique, optical fiber made of quartz is used for the PA signal generation and detection, for which a 0.6-mm-diameter step index fiber and Bragg grating composed of single mode fiber are used as an object, respectively, so that our metal-free sensor head is not only of small size but is also maintenance-free. The concentration of dye (Rhodamine 6G) dissolved into distilled water was measured to estimate the capability. A Q-switched frequency doubled Nd:YAG laser was used as the PA exiting beam, which is fed to the PA sensor through the fiber. It was experimentally confirmed that the detection linearity was significantly obtained in a concentration range from a few ppm to approximately $200 \mathrm{ppm}$.

2pPAg2. Film thickness determination by laser ultrasonics. Michaël Lemaire (Institut d'Electronique de Microélectronique et de Nanotechnologie, Département Opto-Acousto-Electronique (UMR CNRS 8520), Université de Valenciennes et du Hainaut-Cambrésis, Le Mont-Houy, 59313 Valenciennes cedex 9, France, Michael.Lemaire@meletu.univ-valenciennes .fr), Frédéric Jenot (Institut d'Electronique de Microélectronique et de Nanotechnologie, Département Opto-Acousto-Electronique (UMR CNRS 8520), Université de Valenciennes et du Hainaut-Cambrésis, Le Mont-Houy, 59313 Valenciennes cedex 9, France, frederic.jenot@univ-valenciennes .fr), Mohammadi Ouaftouh (Institut d'Electronique de Microélectronique et de Nanotechnologie, Département Opto-Acousto-Electronique (UMR CNRS 8520), Université de Valenciennes et du Hainaut-Cambrésis, Le Mont-Houy, 59313 Valenciennes cedex 9, France, mohammadi.ouaftouh @univ-valenciennes.fr), Wei Jiang Xu (Institut d'Electronique de Microélectronique et de Nanotechnologie, Département Opto-AcoustoElectronique (UMR CNRS 8520), Université de Valenciennes et du Hainaut-Cambrésis, Le Mont-Houy, 59313 Valenciennes cedex 9, France, weijiang.xu@univ-valenciennes.fr), Marc Duquennoy (Institut d'Electronique de Microélectronique et de Nanotechnologie, Département
Opto-Acousto-Electronique (UMR CNRS 8520), Université de Valenciennes et du Hainaut-Cambrésis, Le Mont-Houy, 59313 Valenciennes cedex 9, France, Marc.Duquennoy@univ-valenciennes.fr), Mohamed Ourak (Institut d'Electronique de Microélectronique et de Nanotechnologie, Département Opto-Acousto-Electronique (UMR CNRS 8520), Université de Valenciennes et du Hainaut-Cambrésis, Le Mont-Houy, 59313 Valenciennes cedex 9, France, Mohamed.Ourak@univ-valenciennes.fr), Renaud Côte (Lab. ATF, Katholieke Universiteit Leuven, Celestijnenlaan 200D, B-3001 Leuven, Belgium, renaud.cote@fys.kuleuven.be), Robbe Salenbien (Lab. ATF, Katholieke Universiteit Leuven, Celestijnenlaan 200D, B-3001 Leuven, Belgium, robbe.salenbien@ @ys.kuleuven.be), Bart Sarens (Laboratorium voor Akoestiek en Thermische Fysica - Departement Natuurkunde en Sterrenkunde - Katholieke Universiteit Leuven, Celestijnenlaan 200D, B-3001 Leuven, Belgium, bart.sarens@fys .kuleuven.be), Walter Lauriks (Lab. ATF, Katholieke Universiteit Leuven, Celestijnenlaan 200D, B-3001 Leuven, Belgium, Walter.Lauriks@fys .kuleuven.be), Christ Glorieux (Lab. ATF, Katholieke Universiteit Leuven, Celestijnenlaan 200D, B-3001 Leuven, Belgium, christ.glorieux@fys .kuleuven.be)

The thickness of films deposited on substrates is crucial for their thermal, electrical, optical behaviour. These properties are essential in thin film applications, especially in the field of microelectronics. In this study, we are interested in thickness determination of silver and gold films deposited by evaporation on a silicon substrate by using an ultrasonic non-destructive technique. In particular, the well-known laser ultrasonic technique is used to generate and detect the surface acoustic waves. Results obtained by two complementary methods allowing a non-contact measurement in a large bandwidth (from $5 \mathrm{MHz}$ to $200 \mathrm{MHz}$ ) are presented, and the dispersion of the Rayleigh wave propagation velocity is analyzed to determine the film thickness.

2pPAg3. Photoacoustic metrology of nanoimprint polymers. Timothy Kehoe (Tyndall National Institute, Lee Maltings, University College Cork, Prospect Row, Cork, Ireland, tim.kehoe@tyndall.ie), Juerg Bryner (ETH Zurich, Institute of Mechanical Systems, Dept. of Mechanical and Process Engineering, CH 8092 Zurich, Switzerland, bryner@imes.mavt .ethz.ch), Jacqueline Vollmann (ETH Zurich, Institute of Mechanical Systems, Dept. of Mechanical and Process Engineering, CH 8092 Zurich, Switzerland, vollmann@imes.mavt.ethz.ch), Clivia Sotomayor Torres (Tyndall National Institute, Lee Maltings, University College Cork, Prospect Row, Cork, Ireland, clivia.sotomayor@tyndall.ie), Laurent Aebi (ETH Zurich, Institute of Mechanical Systems, Dept. of Mechanical and Process Engineering, CH 8092 Zurich, Switzerland, aebi@imes.mavt.ethz.ch), Juerg 
Dual(ETH Zurich, Institute of Mechanical Systems, Dept. of Mechanical and Process Engineering, CH 8092 Zurich, Switzerland, juerg.dual@imes .mavt.ethz.ch)

Nanoimprint lithography (NIL) is an alternative lithography method for patterning of thin polymer films using a rigid stamp, which is being developed as desired minimum feature sizes reduce to the scale of tens of nanometres. To characterise nanoimprinted structures, there is a need for more convenient and non-destructive wafer-scale metrologies to complement scanning electron microscopy and atomic force microscopy. The photoacoustic method, with a resolution in the range of $10 \mathrm{~nm}$, and normally used to measure metal and dielectric layer thicknesses and physical properties, has been used for the first time to study nanoimprinting polymer layers. A good signal was obtained from the top and the bottom interfaces of two polymers, mr-I PMMA 75k300 and mr-NIL 6000.3, with thicknesses ranging from 100 to $500 \mathrm{~nm}$. From the measured time of flight of the acoustic wave, and modelling physical parameters of the polymers, thicknesses calculated agree well with those measured by profilometry. The measurements are performed on a short pulse laser pump-probe setup, where bulk wave packets are excited and detected using near infrared laser pulses of less than $100 \mathrm{fs}$ duration. The entire experimental setup is also simulated numerically.

2pPAg4. Line-focus beam photoacoustic imaging of surface and undersurface defect simulated on a planar specimen. Tsutomu Hoshimiya (Tohoku Gakuin University, 13-1, Chuo 1, 985-8537 Tagajyo, Japan, tpth@tjcc.tohoku-gakuin.ac.jp), Mika Hatake-Yama (Tohoku Gakuin University, 13-1, Chuo 1, 985-8537 Tagajyo, Japan, hatakemail @yahoo.co.jp)

A theoretical formulation of photoacoustic (PA) imaging with a linefocus beam (LFB) for surface and undersurface simulated defect was performed. Equivalence between 2D surface defect photoacoustic tomography (PAT) and X-ray CT was derived. PAT imaging experiment was carried out A second harmonics of a LD-pumped YAG laser was used as a LFB. The laser power was $45 \mathrm{~mW}$. The length and width of a laser beam on a specimen was $25 \mathrm{~mm}$ times $0.65 \mathrm{~mm}$ The measured area was $27 \mathrm{~mm} \times 27 \mathrm{~mm}$, while the reconstructed area was $18 \mathrm{~mm} \times 18 \mathrm{~mm}$. 64 times 64 resolution image was reconstructed from the rotation and translation scanning. Reconstructed PA image agreed with the PA image obtained with a point-focus PA imaging. The frequency dependence of thermally diffused image agreed well with the theoretical prediction (thermal diffusion length is inversely proportional to the square root of modulation frequency) Under surface PAT image was obtained by a thermal wave diffraction formula, and the simulated image agreed well with the experimental data.

2pPAg5. Generation of Acoustic Wavelength Shorter Than 10nm By Means Of Nonlinear Acoustic. Shuo Zhang (INSP - UMR 7588 CNRS \& Université Pierre et Marie Curie, 140 Rue de Lourmel, 75015 Paris, France, Shuo.Zhang@insp.jussieu.fr), Emmanuel Péronne (INSP - UMR 7588 CNRS \& Université Pierre et Marie Curie, 140 Rue de Lourmel, 75015 Paris, France, emmanuel.peronne@insp.jussieu.fr), Laurent Belliard (INSP - UMR 7588 CNRS \& Université Pierre et Marie Curie, 140 Rue de Lourmel, 75015 Paris, France, lbelliar@ccr.jussieu.fr), Bernard Perrin (INSP UMR 7588 CNRS \& Université Pierre et Marie Curie, 140 Rue de Lourmel, 75015 Paris, France, bernard.perrin@insp.jussieu.fr)

As the size of the structure decrease toward the nanoscale, their acoustic properties get closer to the $\mathrm{THz}$ range. Traditional techniques based on piezoelectric transducer or the Brillouin diffusion can hardly reach the adequate frequency range. However, if we tightly focus short optical pulses, we can expect to generate high frequencies due to nonlinear effect during the propagation of the acoustic pulses. Moreover, such small source could be used to image buried structures. We have developed an pump-probe experiment based on a Ti:Sa oscillator using microscope objectives and 2 axis displacement stage to study nonlinear propagation and diffraction of acoustic wave in 3 dimension. We have studied 356- $\mu \mathrm{m}$ thick GaAs substrate and have demonstrated the generation of acoustic longitudinal wavelength as short as $10 \mathrm{~nm}$. Due to the acoustic dispersion during the propagation, acoustic solitons are observed for high excitation power. Thanks to the use of interferometric detector, the acoustic pulses are characterized in great details. Such experimental development can help us to study the properties of transmission and reflection of buried nanosystem such as single quantum well or phonon nanocavities.

2pPAg6. The theoretical analyse of the surface acoustic waves propagated on the surface of the bulk materials with subsurface defect. Xiaodong Xu (Modern Acoustics, Institute of Acoustics, Nanjing University, 210093 Nanjing, China, xdxu@nju.edu.cn), Ran Ding (Nanjing University, P.O.Box44\#, Pukou Campus, 210089 Nanjing, China, snail_dr@hotmail.com), Christ Glorieux (Lab. ATF, Katholieke Universiteit Leuven, Celestijnenlaan 200D, B-3001 Leuven, Belgium, christ.glorieux@fys.kuleuven.be), Xiaojun Liu (Modern Acoustics, Institute of Acoustics, Nanjing University, 210093 Nanjing, China, xjliu@nju .edu.cn)

A theoretical model based on finite element method is used to analyse the properties of surface acoustic wave (SAW) propagated on the surface of the bulk materials with subsurface defect. The simulation results reveal the modes conversion between SAW and Lamb wave at the border of the defect and explain the diffusion properties of SAW propagated on the bulk material with subsurface defect. The modes conversion and wave reflection at the border of the defect are theoretically explained why there are a lot of riffles after the SAW passed through Al sample with circle subsurface defect, which detected by laser ultrasonic method. The relationships between the wavelength of wave and the physical state of the defect in the bulk material are also fully described in theoretical simulation.

2pPAg7. LED-based Stroboscopic Schlieren System. Kalle Hanhijärvi (Electronics Research Unit, University of Helsinki, P.O.Box 64 (Gustaf Hällströmin katu 2), FIN-00014 Helsinki, Finland, kalle.hanhijarvi@helsinki.fi), Ivan Kassamakov (Electronics Research Unit, University of Helsinki, P.O.Box 64 (Gustaf Hällströmin katu 2), FIN00014 Helsinki, Finland, ivan.kassamakov@helsinki.fi), Juha Aaltonen (Electronics Research Unit, University of Helsinki, P.O.Box 64 (Gustaf Hällströmin katu 2), FIN-00014 Helsinki, Finland, juha.aaltonen@helsinki.fi), Edward Hæggström (Electronics Research Unit, University of Helsinki, P.O.Box 64 (Gustaf Hällströmin katu 2), FIN00014 Helsinki, Finland, edward.haeggstrom@ helsinki.fi)

A stroboscopic Schlieren system is proposed to characterize acoustic fields in transparent media. Schlieren imaging has been used to visualize beam profiles of ultrasonic transducers mostly in liquids. The method is sensitive to gradients in refractive index, and can thus be used with solids and fluids. A stroboscopic illumination synchronized with the ultrasonic vibration (controllable phase delay between the illumination and the ultrasonic drive signal), allows obtaining an image of a propagating tone burst. An LED source provides relatively fast response time for high measurement bandwidth. Incoherent light doesn't suffer from unwanted interference, as with laser sources. Our setup employs two $90^{\circ}$ off-axis parabolic mirrors. Non-reflected light is blocked with spatial filtering which is achieved with a knife-edge, placed at the focal point of the focusing mirror. We first present a time-averaged Schlieren image of the acoustic field of a $7.5 \mathrm{MHz}$ focusing transducer, as a proof-of-principle result. Then a custom-built LED-based stroboscopic illumination system is used to visualize wave propagation in water emitted by a high power $20 \mathrm{kHz}$ transducer. Schlieren imaging is useful in applications, where non-contact characterization of acoustic fields is necessary.

2pPAg8. Stroboscopic White Light Interferometry for Dynamic Characterization of Capacitive Pressure Sensors. Ivan Kassamakov (Electronics Research Unit, University of Helsinki, P.O.Box 64 (Gustaf Hällströmin katu 2), FIN-00014 Helsinki, Finland, ivan.kassamakov@helsinki.fi), Kalle Hanhijärvi (Electronics Research Unit, University of Helsinki, P.O.Box 64 (Gustaf Hälströmin katu 2), FIN$00014 \quad H e l s i n k i, \quad$ Finland, kallehanhijarvi@helsinki.fi), Juha Aaltonen (Electronics Research Unit, University of Helsinki, P.O.Box 64 
(Gustaf Hällströmin katu 2), FIN-00014 Helsinki, Finland, juha.aaltonen@helsinki.fi), Lauri Sainiemi (Micro and Nanosciences Laboratory, Helsinki University of Technology, P.O.Box 3500, FI-02015 TKK, Finland, lauri.sainiemi@tkk.fi), Kestutis Grigoras (Micro and Nanosciences Laboratory, Helsinki University of Technology, P.O.Box 3500, FI02015 TKK, Finland, kestas.grigoras@tkk.fi), Sami Franssila (Micro and Nanosciences Laboratory, Helsinki University of Technology, P.O.Box 3500, FI-02015 TKK, Finland, sami.franssila@tkk.fi), Anu Kärkkäinen (VTT Technical Research Centre of Finland, P.O.Box 1000, FIN-02044 VTT, Finland, anu.karkkainen@vtt.fi), Edward Hæggström (Electronics Research Unit, University of Helsinki, P.O.Box 64 (Gustaf Hällströmin katu 2), FIN-00014 Helsinki, Finland, edward .haeggstrom@helsinki.fi)

Scanning white light interferometry (SWLI) is a well-established method for accurate static out-of-plane 3-D profiling of micromechanical devices. Periodic displacement can be measured using stroboscopic illumination synchronized to an arbitrary phase angle of the sample oscillation. We modified an existing SWLI setup for dynamic MEMS characterization. A two-channel function generator drives the sample and the stroboscopic illumination, and controls the inter-channel phase. A phosphorous white light LED or single-color LED is used as light source. Currently our shortest stroboscopic pulse is $50 \mathrm{~ns}$. We measured the out-of-plane displacement of a thermal microbridge fabricated on SOI wafer with $20 \mathrm{~nm}$ accuracy. The microbridge was driven with sinusoidal and square wave signals $(1 \mathrm{~Hz}-960$ $\mathrm{Hz})$. The stroboscopic duty cycles were $0.1 \%-5 \%$. We also characterize the vibration modes of the membrane of a capacitive pressure sensor. Stroboscopic SWLI is useful for nanoscale profile measurements of periodic oscillations.

2pPAg9. Laser-generated narrow-band ultrasonic wave for detection of subsurface defect. Ran Ding (Nanjing University, P.O.Box44\#, Pukou Campus, 210089 Nanjing, China, snail_dr@hotmail.com), Xiaodong Xu (Modern Acoustics, Institute of Acoustics, Nanjing University, 210093 Nanjing, China, xdxu@nju.edu.cn)

Laser ultrasonics employs laser beams to excite ultrasound. Spatial an$\mathrm{d} /$ or temporal modulated intensity of laser on the surface of the specimen (aluminum) can serve to narrow the band of the generated ultrasound around the desired central frequency and meanwhile obtains higher signal-noise ratio. In the present work, we use Computer Generated Holograms (CGHs) to spatial-modulate the laser beam. CGHs provide flexibility of adjustment of the intensity profile of the laser on the specimen and the online reconstruction of CGHs by digital and electronic devices helps to reduce the complexity of the experimental system and is promising in detection of subsurface defect and depth profiling as the surface acoustic waves penetrate into the solids a depth proportional to the wavelength. Discussion was carried out between experimental results and a theoretical model which based on finite element analysis.

2pPAg10. Laser-Ultrasonic Measurement of Stress in Metal. Aleksei Podolsky (Moscow State University, MSU, 1, building 2, GSP-2, Leninskiye Gory, 119992 Moscow, Russian Federation, uzh@yandex .ru), Vladimir Koshkin (Moscow State University, MSU, 1, building 2, GSP-2, Leninskiye Gory, 119992 Moscow, Russian Federation, uzh@ya.ru), Viktor Bel'Ko (Moscow State University, MSU, 1, building 2, GSP-2, Leninskiye Gory, 119992 Moscow, Russian Federation, uzh@ya.ru), Andrei Fetisov (Moscow State University, MSU, 1, building 2, GSP-2, Leninskiye Gory, 119992 Moscow, Russian Federation, uzh@ya.ru), Alexander Karabutov (Moscow State University, MSU, 1, building 2, GSP-2, Leninskiye Gory, 119992 Moscow, Russian Federation, akarabutov@gmail.com)

Stress in metal was investigated by acousto-elastic effect. The dependence of longitudinal ultrasonic wave velocity on transverse stress was considered. A laser-ultrasonic defectoscope was used for precise ultrasonic velocity measurement. Optoacoustic transducer provides laser excitation and piezoelectric wide-band detection of ultrasonic pulse. The accuracy of the measurement exceeded $0.1 \%$ for $2 \mathrm{~mm}$ thick metal sample. Samples in the form of plates with the thickness of 2 to $4 \mathrm{~mm}$ made of aluminum and titanium alloys and stainless steel were tested. The samples were loaded quasistatically, the load was changing from zero to yield point stress. Load, deformation and velocity of ultrasound were measured. It is shown that the velocity changes significantly with the load. Some features of the velocity change under tensile stress are presented. The possibility of the local residual stress measurement with the laser-ultrasonic technology is discussed.

2pPAg11. Application of nonlinear laser photoacoustic technique to crack detection. Jacek Zakrzewski (LPEC/UMR 6087/CNRS/Université du Maine, Avenue Olivier Messiaen, 72085 Le Mans Cedex 09, France, jzakrzew@fizyka.umk.pl), Nikolay Chigarev (LPEC/UMR 6087/CNRS/Université du Maine, Avenue Olivier Messiaen, 72085 Le Mans Cedex 09, France, Nikolay.Chigarev@univ-lemans.fr), Vincent Tournat (LPEC/UMR 6087/CNRS/Université du Maine, Avenue Olivier Messiaen, 72085 Le Mans Cedex 09, France, vincent.tournat@univ-lemans .fr), Denis Mounier (LPEC/UMR 6087/CNRS/Université du Maine, Avenue Olivier Messiaen, 72085 Le Mans Cedex 09, France, denis.mounier@univ-lemans.fr), Vitali Gusev (LPEC/UMR 6087/CNRS/Université du Maine, Avenue Olivier Messiaen, 72085 Le Mans Cedex 09, France, vitali.goussev@univ-lemans.fr)

Nonlinear acoustics is a promising method for non-destructive testing (NDT) as it allows to improve the sensitivity and contrast of defects detection. The application of contactless laser photoacoustic technique for the tasks of nonlinear acoustics looks very promising. Meanwhile, real examples of industrial systems using nonlinear photoacoustics are still absent. In this work nonlinear photoacoustic response of artificially prepared cracks has been studied. Acoustic vibrations have been excited through the loASAHGRcal heating caused by the absorption of a focused beam of visible or near-infrared laser radiation. Several methods have been applied for the detection of surface displacement including piezo-transducers, aircoupled transducers and optical laser interferometry. It has been shown, that nonlinear acoustic response increases drastically when both pump and probe points are localized near the crack. For this reason, the last two methods allowing local probing look very promising. Two dimensional scans of generation and detection points on the sample surface could be used to obtain the images of crack at new spectral components induced by crack nonlinearity. The goal of this work is to find optimal methods of the excitation and detection of nonlinear photoacoustic response of the crack. It should make the technique attractive for the industrial applications.

2pPAg12. Looking for shear waves in glass forming liquids. Thomas Pezeril (Massachusetts Institute of Technology, 77 Mass. Avenue, room 6-026, Cambridge, MA 02215, USA, pezeril@mit.edu), Christoph Klieber (Massachusetts Institute of Technology, 77 Mass. Avenue, room 6-026, Cambridge, MA 02215, USA, cklieber@mit.edu), Stephane Andrieu (Laboratoire de Physique des Matériaux UMR7556, Université H. Poincaré/Nancy I, 54506 Vandoeuvre, France, stephane.andrieu@1pm .u-nancy.fr), Keith Nelson (Massachusetts Institute of Technology, 77 Mass. Avenue, room 6-026, Cambridge, MA 02215, USA, kanelson@mit .edu)

The ability of the generation of picosecond shear acoustic pulses in solids using ultrashort optical pulses have been employed to look for high frequency shear waves in glass forming liquids. As a transducer we used a canted iron thin film deposited on a glass substrate. The strong shear efficiency of generation provided by this kind of samples allowed the study of several glass forming liquids. As a first try, we have detected shear brillouin scattering in m-toludine at $100 \mathrm{~K}$, that is below the glass transition temperature, at a frequency of $9 \mathrm{GHz}$. Then, we observed shear waves propagating at room temperature in glycerol at frequencies below $50 \mathrm{GHz}$. 


\title{
Session 2pPAh
}

\section{Physical Acoustics and Signal Processing in Acoustics: Acoustic Landmine Detection II (Poster Session)}

\author{
James Sabatier, Cochair \\ University of Mississippi \\ Keith Attenborough, Cochair \\ Open University
}

\begin{abstract}
All posters will be on display from 3:40 p.m. to 5:20 p.m. To allow contributors an opportunity to see other posters, contributors of odd-numbered papers will be at their posters from 3:40 p.m. to 4:30 p.m. and contributors of even-numbered papers will be at their posters from 4:30 p.m. to 5:20 p.m.
\end{abstract}

\section{Contributed Papers}

2pPAh1. Vector surface velocity measurement using contour scanning laser vibrometers for the detection of landmines. Patrick $F$. O'Malley (Catholic University, 620 Michigan Ave, Washington, DC 20064, USA, 68omalley@cua.edu), Woods J. Teresa (Catholic University, 620 Michigan Ave, Washington, DC 20064, USA, 59woods@cua.edu), Joseph F. Vignola (Catholic University, 620 Michigan Ave, Washington, DC 20064, USA, vignola@cua.edu), John A. Judge (Catholic University, 620 Michigan Ave, Washington, DC 20064, USA, judge@cua.edu), Jacek Jarzynski (Catholic University, 620 Michigan Ave, Washington, DC 20064, USA, jjarzynski@comcast.net)

Much work has been done studying the detection of buried landmines using acoustic excitation and measuring the vibration response of the ground using Laser Doppler Vibrometry (LDV). We examine the benefits of measuring the full three-dimensional velocity response of the ground surface when a landmine is present. Three-dimensional velocity measurements provide a richer data set for identification of buried targets and avoid potential errors associated with projecting the surface velocity vector onto a single laser line of sight direction. We present results of a study conducted using landmines buried in sand in a laboratory environment. The data were obtained using a novel five-axis laser vibrometry system, which uses a single point LDV system to measure the surface of the sand from multiple angles at each measurement location. Data will be presented showing the consequences of a buried landmine on the three-dimensional velocity response of a contoured ground surface.

2pPAh2. Scattering of a buried circular membrane imbedded in a rigid substrate in a layered fluid waveguide. Michelle B. Mattingly (Physics Dept., United States Naval Academy, Chauvenet Hall Room 295, 572 C Holloway Road, Annapolis, MD 21402, USA, mini_matisick@ hotmail.com) , James L. Buchanan (Mathematics Dept., United States Naval Academy, 572 C Holloway Road, Annapolis, MD 21402, USA, jlb@usna.edu), Reza Malek-Madani (Mathematics Dept., United States Naval Academy, 572 C Holloway Road, Annapolis, MD 21402, USA, mm@usna.edu), Murray S. Korman (Physics Dept., United States Naval Academy, Chauvenet Hall Room 295, 572 C Holloway Road, Annapolis, MD 21402, USA, korman @usna.edu)

A study of mathematical modeling of the buried landmine detection problem involves wave propagation in a layered waveguide in the presence of a flush mount buried circular target. In this study, emphasis is placed on acoustic to seismic coupling of an airborne $\mathrm{CW}$ point source located over an iso-velocity fluid layer. The top plate of the buried landmine is modeled as a circular elastic membrane stretched flush over a cylindrical cavity in a rigid substrate beneath the fluid layer. The finite fluid layer affords a manageable study of the modal resonances in the waveguide system with and without the target. The Helmholtz equation is solved in the atmospheric layer using cylindrical coordinates and a point source. The homogeneous Helmholtz equation is used in the fluid layer. Green's function techniques involving vibrations of the membrane are incorporated into the boundary conditions. Results of the closed form normal mode solution will be presented in a MATLAB ${ }^{\mathrm{TM}}$ user interface; describing the effects of frequency, depth, density, sound, absorption, including radius and elastic parameters of the membrane. Comparison of the results (involving the fluid surface particle velocity profiles across the target) will be made with experiments reported in the literature to evaluate its usefulness.

2pPAh3. Nonlinear scattering of a surface wave by an object buried in soil. Evgenia A. Zabolotskaya (Applied Research Laboratories, The University of Texas, P.O. Box 8029, Austin, TX 78713-8029, USA, zhenia@arlut.utexas.edu), Yurii A. Ilinskii (Applied Research Laboratories, The University of Texas, P.O. Box 8029, Austin, TX 78713-8029, USA, yura@arlut.utexas.edu), Mark F. Hamilton (Applied Research Laboratories, The University of Texas, P.O. Box 8029, Austin, TX 78713-8029, USA, hamilton@mail.utexas.edu)

Nonlinear scattering of a surface wave by an object buried in soil is investigated theoretically. The object is supposed to be excited by a monochromatic seismic wave and its pulsations are considered to be nonlinear. Specific features of soil are taken into account, namely, shear modulus less than compressibility modulus and anomalously high nonlinearity. Two analytical approaches are applied. One is based on theory of elasticity and the other is based on fluid mechanics. The two approaches yield identical results for the second harmonic in the scattered field. The second harmonic is evaluated in the near field where the medium can be considered as incompressible. The analysis is performed with perturbation theory for small displacement amplitudes in the soil. The second harmonic is also investigated with an asymptotic expression obtained from the general equation for the second harmonic generated by a pulsating object in a compressible medium. The equation is expanded for a small object at distances that are small compared with a wavelength. The influence of a stress-free boundary on the scattered field is evaluated using Green's functions. [Work supported by ONR.]

2pPAh4. Numerical simulations of resonant granular layers. Joseph A. Turner (University of Nebraska, Dept. of Engineering Mechanics, W317.4 NH, Lincoln, NE 68588, USA, jaturner@unl.edu), Florin Bobaru (University of Nebraska, Dept. of Engineering Mechanics, W317.4 
NH, Lincoln, NE 68588, USA, fbobaru2@unl.edu),Kitti Rattanadit (University of Nebraska, Dept. of Engineering Mechanics, W317.4 NH, Lincoln, NE 68588, USA, kittir@bigred.unl.edu)

A coupled Discrete Element Method-Finite Element Method (DEMFEM) model is developed and implemented for simulating the dynamic response of a dry granular layer on top of an elastic beam. The implementation is first validated against the quasi-static results for spherical particles which are compared with well-bonded effective-medium models. In the dynamic regime however, the effective-medium analytical result differs from experi- mental values in terms of the dependence of particle size. The simulations developed are used to understand the discrepancy between the theory and the experiments. The first resonance of the system is examined with respect to particle size using a variety of particle sizes and a frequency sweep input. The particle size dependence observed experimentally is reproduced in the simulations only through inclusion of cohesive forces between the particles. Rolling resistance and friction between particle is shown to be of minor importance. This new modeling tool offers promise for understanding the dynamic interactions of granular materials. [Work supported by ARL]

TUESDAY AFTERNOON, 1 JULY 2008

P3-A, LEVEL 3, 3:40 TO 5:20 P.M.

\title{
Session 2pPAi
}

\section{Physical Acoustics: Nonlinear Acoustics of Unconsolidated Granular Media II (Poster Session)}

\author{
Paul Johnson, Cochair \\ EES-11 (Geophysics) - Los Alamos National Laboratory \\ Vitali Gusev, Cochair \\ LPEC/UMR 6087/CNRS/Université du Maine
}

\begin{abstract}
All posters will be on display from 3:40 p.m. to 5:20 p.m. To allow contributors an opportunity to see other posters, contributors of odd-numbered papers will be at their posters from 3:40 p.m. to 4:30 p.m. and contributors of even-numbered papers will be at their posters from 4:30 p.m. to 5:20 p.m.
\end{abstract}

\section{Contributed Papers}

2pPAi1. Low-frequency acoustoelasticity in glass beads saturated with water using a nonlinear wave-coupling technique. Guillaume Renaud (LUSSI, 10 Bd Tonellé, 37032 Tours, France, guillaume.renaud28 @etu.univ-tours.fr), Samuel Callé (LUSSI, 10 Bd Tonellé, 37032 Tours, France, calle_s@med.univ-tours.fr), Jean-Pierre Remenieras (LUSSI, 10 Bd Tonellé, 37032 Tours, France, remenier@med.univ-tours.fr), Marielle Defontaine (LUSSI, 10 Bd Tonellé, 37032 Tours, France, defontai@med .univ-tours.fr)

To study "non-classical" acoustical nonlinearities in cracked materials, we developed a nonlinear (NL) wave coupling technique. Propagation velocity and amplitude of short high-frequency (HF, $600 \mathrm{kHz})$ bursts are modulated as result of nonlinear interaction with a low-frequency (LF, 3 $\mathrm{kHz}$ ) wave. Time Of Flight Modulation (TOFM) is indeed related to both elasticity and density variations. Because high acoustic nonlinearities in glass beads have been reported, we applied our technique to glass beads with different diameters (hundreds micrometers). A small container is filled up with glass beads saturated with water and placed below the LF source. The large HF to LF ratio (200) and the small sample size $(6 \mathrm{~cm})$ compared to the LF wavelength in water $(50 \mathrm{~cm})$ allow: 1$)$ to measure instantaneous Time Of Flight Modulation (TOFM) and attenuation as functions of the instantaneous LF pressure, 2) to consider the LF pressure field a quasi-static hydrostatic variation of the ambient pressure in the medium. The LF pressure amplitude in water is approximately $10 \mathrm{kPa}$. Different TOFM and NL attenuation behaviors in tension and in compression, as well as different hysteresis patterns, are observed. The influence of the LF pressure amplitude is discussed.

2pPAi2. Sound in a linear array of magnetic spheres. J. Carlos Ruiz-Suarez (CINVESTAV-IPN, Antigua Carretera a Progreso $\mathrm{Km} 6$, $97310 \quad$ Merida, Mexico, cruiz@mda.cinvestav.mx), Florent Malloggi (PMMH-ESPCI Paris, 10 Rue Vaquelin, 75005 Paris, France,
F.G.J.Malloggi@tnw.utwente.nl),Osvaldo Carvente(CINVESTAV-IPN, Antigua Carretera a Progreso $\mathrm{Km}$ 6, 97310 Merida, Mexico, ocarven@yahoo.com.mx), Jose Cruz-Damas (CINVESTAV-IPN, Antigua Carretera a Progreso Km 6, 97310 Merida, Mexico, josia.damas @ gmail.com), Felipe Pacheco (CINVESTAV-IPN, Antigua Carretera a Progreso Km 6, 97310 Merida, Mexico, fpacheco@mda.cinvestav.mx)

We study the propagation of sound in a chain of strongly magnetized spheres. The strong dipole-dipole magnetic force allows us to hold the chain in different positions to evaluate the effect gravity produces on sound transmission. This strong cohesion permits us also to insert in the chain a periodical array of impurities and study the effect this array has in the transmission. A well defined gap of frequencies, where no sound propagation exists, is observed. We investigate as well square lattices, and look into the effects dipole anisotropy causes to sound propagation. Finally, we study the effects produced by vacancies.

2pPAi3. Linear and non linear acoustic waves in macroscopically inhomogeneous unconsolidated granular crystals. Aurélien Merkel (LPEC/UMR 6087/CNRS/Université du Maine, Avenue Olivier Messiaen, 72085 Le Mans Cedex 09, France, aurelien.merkel.etu @univ-lemans.fr), Xavier Jacob (LPEC/UMR 6087/CNRS/Université du Maine, Avenue Olivier Messiaen, 72085 Le Mans Cedex 09, France, xavier.jacob@univ-lemans.fr), Vincent Tournat (LPEC/UMR 6087/CNRS/Université du Maine, Avenue Olivier Messiaen, 72085 Le Mans Cedex 09, France, vincent.tournat@univ-lemans.fr), Vitali Gusev (LPEC/UMR 6087/CNRS/Université du Maine, Avenue Olivier Messiaen, 72085 Le Mans Cedex 09, France, vitali.goussev@univ-lemans.fr)

Ordered unconsolidated structure of spherical beads in the absence of external loading except the gravity field constitutes a macroscopically inhomogeneous and strongly nonlinear phononic crystal. We report the experimental and theoretical investigation of linear and nonlinear acoustic phenomena in these granular crystals of finite thickness along the gravity 
direction. In particular the dependence of the resonance frequencies on the thickness of the crystal (the number of layers) is evaluated. The linear transmission of acoustic waves from the bottom to the free surface of the granular crystal exhibits complicated features. It is compared to the linear transmission through the same structure but with a macroscopically homogeneous static stress. Some frequency regions of the acoustic response function are shown to be insensitive to the transition from an inhomogeneous static stress (gravity induced) to a homogeneous one (additional ex- ternal load). The nonlinear acoustic phenomena of resonance frequency shift and resonance curve broadening with increasing amplitude of acoustic oscillations are observed. It allows to measure the nonlinear parameters of the crystal for different amounts of layers, accounting for the absolute particle velocity amplitude detected at the free surface with a laser vibrometer. Corresponding theoretical models describing the acoustic eigenmodes in macroscopically homogeneous and inhomogeneous granular crystals are developed.

\title{
Session 2pPAj
}

\section{Physical Acoustics: Nonlinear Acoustics in Earthquake Processes and Other Earth Processes II (Poster Session)}

\author{
Paul Johnson, Cochair \\ Fabrice Cotton, Cochair \\ Université Joseph Fourier
}

EES-11 (Geophysics) - Los Alamos National Laboratory

\begin{abstract}
All posters will be on display from 3:40 p.m. to 5:20 p.m. To allow contributors an opportunity to see other posters, contributors of odd-numbered papers will be at their posters from 3:40 p.m. to 4:30 p.m. and contributors of even-numbered papers will be at their posters from 4:30 p.m. to 5:20 p.m.
\end{abstract}

\section{Contributed Papers}

2pPAj1. Elastic-anisotropic properties of rocks along the Finnish Drill Hole (OKU) section in the depth range down to $1 \mathrm{~km}$. Mikhail Kovalevskiy (Geological Institute of Kola Science Centre of Russian Academy of Sciences, 14, Fersman St., 184209 Apatity, Russian Federation, koval@geoksc.apatity.ru)

The Finnish drill hole OKU was drilled in the south-eastern Baltic Shield (Finland). The drill hole limiting depth is $2516 \mathrm{~m}$. The goal of investigations was determination of elastic and non-elastic characteristics of 14 rock samples taken within $1.0 \mathrm{~km}$ depth. The determinations were done on the basis of the latest improvement of the acoustopolarization method with devices for determining elastic properties [1]. The study of the rock sample properties from the drill hole section showed that they are all elastic anisotropic and pertain to the transverse-isotropic and orthorhombic symmetry types. A slight change in the compression and shear wave velocities with depth can be observed. The effect of linear acoustic anisotropic absorption has been registered in the samples. The nature of this effect manifestation is related to the presence of microcracks of the natural character. The effect of depolarization of shear waves was registered in some samples which suggests the presence of the angular unconformity between the directions of the LAAA elements and elastic symmetry elements. The pattern of change in the anisotropy factors for compression and shear waves with depth is manifested in a similar way. REFERENCES 1. Gorbatsevich F.F. Acoustopolariscopy of rocks. Apatity, Acad. Sciences, 1995, 204 p.

2pPAj2. Generation and Evolution of Cavitation in Magma under Decompression Waves. Valeriy K. Kedrinskiy (Lavrentyev Institute of Hydrodynamics, Siberian Division of the Russian Academy of Sciences, Lavrentyev prospect 15, 630090 Novosibirsk, Russian Federation, kedr@hydro.nsc.ru), Maxim N. Davydov (Lavrentyev Institute of Hydrodynamics, Siberian Division of the Russian Academy of Sciences,
Lavrentyev prospect 15, 630090 Novosibirsk, Russian Federation, davydov @hydro.nsc.ru)

It's considered that presence of the dissolved water (with concentration up to $5-7 \%$ (wt.)) and viscosity of magma play one of the key roles in volcanic processes. According to the geophysical data, both a character and a structure of explosive volcano eruptions are defined by dynamics of cavitation development and the viscosity increasing orders during the diffusion of the dissolved gas from magma in bubbles. This paper represents a full system of the equations, including the kinetics of phase transitions, which allows us to describe dynamics of a magma melt state in a gravity field behind the front of a decompression wave. The problem is solved numerically, using a known expression for the nucleation frequency as a function of current concentration of gas dissolved in magmatic melt. The notion of diffusion zones which arise around cavitation nuclei is introduced. The latter allow us to define real density of cavitation kernels generated in an unit of magmatic melt volume as a result of phase transitions behind a rarefaction-wave front. The dynamics of the size distribution of cavitation bubbles along a magmamelt column ( $1 \mathrm{~km}$ height) and also of the magma viscosity changing dynamically vs. a concentration of dissolved water are investigated. (RFBR 06-01-00317a financial support).

2pPAj3. Features of acoustic emission at various influences on rock samples. Alexander S. Voznesenskiy (Moscow State Mining University, Leninskiy Prospect 6, 119991 Moscow, Russian Federation, al48@mail.ru), Sergey V. Viljamov (Moscow State Mining University, Leninskiy Prospect 6, 119991 Moscow, Russian Federation, serjo@mail.ru)

In the report special features of acoustic emission (AE) in the rock samples during its deforming, loading, dissolving and heating are discussed. The AE-activity and spectral characteristics of AE at the frequency band from on $30 \mathrm{kHz}$ up to $500 \mathrm{kHz}$ versus time at various influence stages and forms are analyzed. For example the amplitudes at the frequencies 30-90 $\mathrm{kHz}$ during deforming of carnallite are higher than these ones during its 
dissolving. It means the cracks at the deforming are about $4-10 \mathrm{~mm}$ and these ones at the dissolution are about 1-3 mm. These results can be used for the recognition of influence forms and stages.

2pPAj4. Formation of ripples on sand surface as result of nonlinear interaction of sound waves and wind drift particles. Nora A. Vilchinska (LAA-Latvian Acoustics Association, 3 Kurzemes pr, LV-1064 Riga, Latvia, vilcinska@hotmail.com)

The phenomenon speaks within the limits of nonlinear acoustics, considering it as amplification of surface acoustic waves (SAWs) by drift of carriers. Interaction and amplification of SAWs, borne from falling sand particles on dry sand surface and drift sand particles under wind action, is responsible for the first ripple forming on a dry sand flat surface. SAWs interact with drift sand particles in the moment, when the velocity of drift is equal to velocity of a sound propagation. The sound wave acts on drifting particles so that sand grains, a little bit advancing wave, will be braked, and lagging behind be accelerated. Particles will gather in a phase of a wave take place grouping of particles and ripple forming. The distance between groups is a ripples wave length $\lambda$. Experimental in-situ are measured weak sound field on frequencies $25 \mathrm{~Hz}$ in just forming sand massif, and near surface velocity of sand drug under wind action. Measured ripples wave lengths $\lambda$ are in good agreement with measured diapason of wind velocitys Measurements were carried out on the sand beach of the gulf of Riga, Latvia. Theoretically the phenomenon speaks within the limits of nonlinear acoustics or within the limits of nonlinear mechanic.

2pPAj5. A numerical study of the onset of granular avalanches. Lydie Staron (Institut Jean le Rond d'Alembert, 4 place Jussieu, case 161-162, 75252 Paris Cedex 05, France, staron@1mm.jussieu.fr)

Granular media can either exist in a jammed state, in which their behaviour can be seen as solid-like, or they can exist in a dilute state and flow as a fluid would. In this contribution, we are interested in the mechanisms prevailing in the transition between solid-like and fluid-like behaviour, specifically in the case of the onset of granular avalanches. Therefore, discrete numerical simulations of granular flows are carried out. The focus is set on the analysis of the stick-slip dynamics preceeding the trigger of the avalanche; the existence of precursors to the flow, and their distribution in space and time will be investigated.

\title{
Session 2pPAk
}

\section{Physical Acoustics: Sonic, Ultrasonic, and Megasonic Cleaning II (Poster Session)}

\author{
Ralph Muehleisen, Cochair \\ Illinois Institute of Technology \\ Claus-Dieter Ohl, Cochair \\ University of Twente
}

\begin{abstract}
All posters will be on display from 3:40 p.m. to 5:20 p.m. To allow contributors an opportunity to see other posters, contributors of odd-numbered papers will be at their posters from 3:40 p.m. to 4:30 p.m. and contributors of even-numbered papers will be at their posters from 4:30 p.m. to 5:20 p.m
\end{abstract}

\section{Contributed Paper}

2pPAk1. Ultrasonic cleaning of submerged membranes for drinking water applications. Sonja Lauterborn (TU Darmstadt, Institut WAR, Petersenstraße 13, 64287 Darmstadt, Germany, s.lauterborn@iwar .tu-darmstadt.de), Wilhelm Urban (TU Darmstadt, Institut WAR, Petersenstraße 13, 64287 Darmstadt, Germany, w.urban@iwar.tu-darmstadt.de)

Ultrasonic cleaning of membranes used in water purification and waste water treatment is under investigation for avoiding fouling and scaling on the membranes. So far chemicals are used, but their use is under scrutinity for safety, waste removal and health issues. Chemicals often even do not solve the cleaning problem durably. For applications in the part of drinking water treatment it is necessary to ensure the filtered water is really clean.
Therefore in the presented experiment the outflow is constantly controlled by turbidity measurements and by using a particle counter in an online system. A pilot plant for sonication of submerged membranes to produce drinking water from surface water was constructed and placed at the Rhine water works in Biebesheim. It could be shown that sonication with $130 \mathrm{kHz}$ when backflushing the membranes only works with following air overflow. Then the permeability keeps high. No damage of the membranes occurs like often has been found in former studies. Only 30 seconds of sonication after $30 \mathrm{~min}$ of filtration are enough to keep high performance of the membranes. Thus energy demand is low, which is a pre-condition for an economical use in technical applications. 


\title{
Session 2pPAl
}

\section{Physical Acoustics: Mathematical and Numerical Methods II (Poster Session)}

\begin{abstract}
All posters will be on display from 3:40 p.m. to 5:20 p.m. To allow contributors an opportunity to see other posters, contributors of odd-numbered papers will be at their posters from 3:40 p.m. to 4:30 p.m. and contributors of even-numbered papers will be at their posters from 4:30 p.m. to 5:20 p.m.
\end{abstract}

\section{Contributed Papers}

2pPAl1. Finite element modelling of thermoviscous acoustics in closed cavities. Nicolas Joly (Laboratoire d'Acoustique de l'Université du Maine, Avenue Olivier Messiaen, 72085 Le Mans, France, nicolas.joly @univ-lemans.fr)

A numerical methodology is presented to compute the acoustic field in a closed domain filled by a thermoviscous fluid, using the finite element method. The formulation based upon temperature variation and particle velocity is well suited for both (i) modelling the acoustic, thermal and viscous effects in the fluid bulk and (ii) accounting for the non-slip and thermal boundary conditions on the solid. The set of coupled equations presents usual scalar- and vector- operators for finite element modelling. Due to the development of thin thermal- and viscous- boundary layers compared to the acoustic wavelength, very different scales are present in the computed fields: the finite-element mesh needs to be dramatically refined along the direction normal to the boundary, while a coarse mesh is often sufficient along the directions parallel to the boundary, and far from the boundaries. The optimised mesh is obtained iteratively, using a loop procedure computing (a) the thermoviscous solution, (b) the metric map suited for this field, and (c) the unstructured anisotropic adapted mesh based on this metric, until convergence. Results are presented for two-dimensional and axisymmetric three-dimensional applications, illustrating thermoviscous effects for various geometries of cavities.

2pPA12. Wave propagation in stratified fluid flows: Application of the Stroh formalism to Lagrangian acoustic perturbations. Olivier Poncelet (LMP, UMR CNRS 5469, Université Bordeaux I, 351, cours de la Libération, 33405 Talence, France, o.poncelet@1mp.u-bordeaux1 .fr), Mélanie Ottenio (INRETS - Univ. Claude Bernard Lyon 1, UMR-T 9406, Laboratoire de Biomecanique et Mecanique des Chocs, 25, Avenue Francois Mitterand, 69675 Bron, France, melanie.ottenio@inrets.fr)

We consider the problem of harmonic waves propagating in non uniform fluid flows in presence of either solid interfaces (elastic walls), or jumps of mechanical properties or jumps of fluid convection-speed. The acoustic field is described by taking into account Lagrangian perturbations referred to an Eulerian frame related to the moving fluid. In contrast to the case of eulerian perturbations, the lagrangian description enables us to introduce the acoustical displacement in the problem and therefore to write the continuity conditions in a simple and unambiguous way: normal acoustical displacement is always continuous through an interface between either solids or (moving) fluids; the normal acoustical stress is also continuous in most of the practical cases (boundary layers, vibrating walls). We express the wave equation as an ordinary differential system by making use of the Stroh formalism from which analytical and semi-analytical results can be obtained for any arbitrary profile of the flow properties. This formalism, coupled with PeanoNeumann series expansion and Frobenius power series, enable us to compute modes dispersion in elastic ducts, and reflective properties of a mixing layer in between two fluids in relative motion. Some analytics on those problems is provided.

2pPAI3. Treatment of the asymptotic behaviour of the piezolectric Green's function for finite element/boundary element analysis of surface waveguides. Sylvain Ballandras (CNRS FEMTO-ST, 32 Avenue de
l'Observatoire, 25044 Besançon, France, sylvain.ballandras@femto-st .fr), Michel Lenczner (CNRS FEMTO-ST, 32 Avenue de l'Observatoire, 25044 Besançon, France, michel.lenczner@femto-st.fr), Thierry Larroche (CNRS FEMTO-ST, 32 Avenue de l'Observatoire, 25044 Besançon, France, thierry.larroche@femto-st.fr)

The simulation of surface waveguides has been dramatically improved by the combination of analytic description of piezoelectric materials using surface Green's function and numerical approaches sur as plane wave expansion, finite diffrence, finite element, etc. A lot of work has been dedicated to treat the singularities of such Green's function generally derived in the spectral domain. An interesting approach consists in using the Green's function which relates the surface stresses to the displacements which is particularly well-suited for mixed finite element/boundary element formulations. This Green's function does not exhibit any pole but presents an asymptotic behavior which tends to infinity along increasing wavenumber values, which prevents the computation of its Fourier transform. In this work, we show how this difficulty can be overcome and we propose a formulation in which the Green's function is factorized in order to change its asymptotic behaviour to a form allowing for Fourier transform computation for non periodic problems and an analytic treatment of its asymptotic behaviour for the simulation of periodic structures. Examples are provided to show the interest of the proposed approach in terms of computation delays and precision.

2pPA14. 'Maxwellian' macroscopic acoustics and acoustic metamaterials. Denis Lafarge (Laboratoire d'Acoustique de l'Université du Maine, Avenue Olivier Messiaen, 72085 Le Mans, France, denis.lafarge@univ-lemans.fr), Aroune Duclos (Laboratoire d'Acoustique de l'Université du Maine, Avenue Olivier Messiaen, 72085 Le Mans, France, aroune.duclos@univ-lemans.fr)

For electromagnetic wave propagation it was found that there exist composite media exhibiting strong spatial dispersion. This has raised the question of the relevance of the spatial dispersion in the characterization of the new metamaterials. The present communication intends to show that exactly the same problematic occurs in acoustics when considering long-wavelength acoustic wave propagation in a fluid in presence of solid obstacles of any arbitrary shape. When these obstacles resemble "Helmholtz" resonators the macroscopic equivalent medium may exhibit strong spatial dispersion. It is demonstrated that the corresponding Macroscopic Acoustics, which may be worked out in detail, is very similar to the general nonlocal Maxwellian Macroscopic Electrodynamics. This raises the question of the relevance of the spatial dispersion in the characterisation of porous materials, and specifically the question of the possible design of acoustic metamaterials exhibiting enhanced absorption due to spatial dispersion.

2pPA15. Theoretical proof of acoustic source property single definition in liquid and solid. Leo Zagorskiy (Moscow Mining Institute, Lenin Street, 6, 16-14, Settl. Vugi, 140004 Luybertsy, Russian Federation, ZAGORSKIY_LEV@MAIL.RU)

The problem of acoustic waves property sourse single definition consists in unknown density and elastic modules of the medium. The Green's function definition is difficult as well as the real sourse isn't point and has the 
time dependence. In the early author's papers Green's function had been defined by using Levitan's polynomials. In the liquid or solid medium it is necessary for that to know only the first eigen values and shear or P-wave velocity at the first step from the free surface. It provides to calculate amplitudes of first mode of SH waves, $\alpha$ and $\beta$ - the limits of lacunas in the spectrum. The time function of not moving sourse $F$ is defined from the equation $\mathrm{F}^{*} \mathrm{G} \equiv \mathrm{U}$, where $\mathrm{U}$ is displacement, G- Green's function, * denominates rolling up. That equation is solved by direct and inverse fast Fourie transform. The further definition of source's location from the direct task for P or S body waves with defined velocity isn't hard by using modern finite element method. The rounding of the fronts gives the location of the sourse.

TUESDAY AFTERNOON, 1 JULY 2008

P2-C, LEVEL 2, 3:40 TO 5:20 P.M.

\title{
Session 2pPPa
}

\section{Psychological and Physiological Acoustics: General Topics in Psychological and Physiological Acoustics I (Poster Session)}

\author{
Elizabeth Strickland, Cochair \\ Purdue University
}

Armin Kohlrausch, Cochair

Philips Research Europe

Alain De Cheveigne, Cochair

CNRS, Universite Paris 5, Ecole Normale Superieure

\begin{abstract}
All posters will be on display from 3:40 p.m. to 5:20 p.m. To allow contributors an opportunity to see other posters, contributors of odd-numbered papers will be at their posters from 3:40 p.m. to 4:30 p.m. and contributors of even-numbered papers will be at their posters from 4:30 p.m. to 5:20 p.m.
\end{abstract}

\section{Contributed Papers}

2pPPa1. Detection and Cortical Representations of the Break in Interaural Correlation of Narrowband Noises Are Affected by Center Frequency and Interaural Delay. Ying Huang (Dept. of Psychology, Peking Univ., 5 Yeheyuan Road, Haidian District, 100871 Beijing, China, innhuang@gmail.com), Lingzhi Z. Kong (Dept. of Psychology, Peking Univ., 5 Yeheyuan Road, Haidian District, 100871 Beijing, China, konglingzhi@gmail.com), Silu Fan (Graduate School of the Chinese Academy of Sciences, 100049 Beijing, China, slfan@gucas.ac.cn), Xihong H. Wu (Dept. of Machine Intelligence, Speech and Hearing Research Center, 2 Science Building, Peking Univ., 5 Yeheyuan Road, Haidian District, 100871 Beijing, China,wxh@cis.pku.edu.cn), Liang Li (Dept. of Psychology, Peking Univ., 5 Yeheyuan Road, Haidian District, 100871 Beijing, China, liangli@pku.edu.cn)

When either broadband or narrowband arbitrary noises presented at the two ears are correlated, a fused noise image is perceived inside the head if the interaural interval (interaural time difference, ITD) is sufficiently short, indicating that acoustic-waveform information can be binaurally integrated At both the perceptual level and neurophysiological level, this study investigated whether the binaural integration of correlated noises is affected by center frequency (for narrowband noise) and ITD. Results of the psychophysical experiment show that the duration threshold for detecting a break in correlation (BIC) in the correlated noises at the two ears was higher for high-frequency noises than for low-frequency noises, and dramatically elevated with the increase of the ITD from 0 to $4 \mathrm{~ms}$. Moreover, the ITDinduced threshold elevation was much larger for high-frequency narrowband noises than for low-frequency narrowband noises. Results of the neurophysiological experiments show that the cortical components of scalp eventrelated potentials to the BIC were markedly modulated by both the center frequency (for narrowband noise) and ITD. Thus, temporal integration of acoustic waveform details of correlated noises at the two ears is both frequency and ITD dependent. Supported by the National Natural Science Foundation of China.

2pPPa2. Object-related negativity indexes perception of sounds beyond the echo threshold. Lisa D. Sanders (Univ. of Massachusetts, Department of Psychology, Tobin Hall, Amherst, MA 01003, USA 1sanders@psych.umass.edu), Benjamin Zobel (Univ. of Massachusetts, Department of Psychology, Tobin Hall, Amherst, MA 01003, USA, benjaminzobel@yahoo.com), Rachel Keen (University of Virginia, Department of Psychology, P. O. Box 400400, Charlottesville, VA 22904-4400, USA, rachelkeen@ virginia.edu), Richard L. Freyman (Univ. of Massachusetts, Dept. of Communication Disorders, 358 N. Pleasant St., Amherst, MA 01003, USA, rlf@comdis.umass.edu)

The ability to isolate a single sound source among concurrent sources and reverberant energy is crucial for understanding the auditory world. The precedence effect describes an experimental finding that when listeners are presented with identical sounds from two locations with a short onset asynchrony, they report hearing a single source with a location dominated by the lead sound. We recently showed that event-related potentials (ERPs) elicited by click pairs differ for trials on which listeners do and do not report hearing the lag sound as a separate source. Specifically, when participants report hearing two sounds, we observe a negativity between 100 and $250 \mathrm{~ms}$, previously termed the object-related negativity (ORN). These results indicate that the precedence effect reflects top-down cognitive influence over early neurosensory processing. Additionally, the study provides support for the claim that the ORN, initially reported for sounds perceived as two concurrent pitches, indexes auditory object perception. The effects on the ORN of repeating identical click pairs, a condition that has previously been shown to 
increase echo thresholds, will also be discussed in the context of listeners forming complex models of room acoustics based on what they hear in a specific setting.

2pPPa3. Improving source localisation in multi-source, reverberant conditions: exploiting local spectro-temporal location cues. Heidi Christensen (University of Sheffield, Regent Court, Dept. of Computer Science, 211 Portobello Road, s1 4dp Sheffield, UK, h.christensen@dcs .shef.ac.uk), Ning Ma (University of Sheffield, Dept. of Computer Science, Regent Court, 211 Portobello Street, S1 4DP Sheffield, UK, n.ma@dcs.shef.ac.uk), Stuart N. Wrigley (University of Sheffield, Regent Court, Dept. of Computer Science, 211 Portobello Road, s1 4dp Sheffield, UK, s.wrigley@dcs.shef.ac.uk), Jon Barker (University of Sheffield, Dept. of Computer Science, Regent Court, 211 Portobello Street, S1 4DP Sheffield, UK, j.barker@dcs.shef.ac.uk)

This paper examines the use of binaural cues for estimating the location of individual sources in multi-source acoustic mixtures. In most environments, location cues such as interaural time difference (ITD) and interaural intensity difference (IID) are rendered unreliable by noise due to competing sources and reverberation. We propose a method that examines spectrotemporal regions where such effects are minimised. Source location is estimated by combining two processing stages. First, following [Christensen et al, Interspeech (2007)], as pitch cues are generally less affected by noise, a multi-pitch estimation and tracking algorithm is used to identify local spectro-temporal 'fragments' in which the SNR is high. Second, the confidence of ITD and IID estimates arising from each time-frequency 'pixel' is estimated based on interaural coherence [Faller and Merimaa, J. Acoust. Soc. Am. 116, 3075--3089 (2004)] and reverberation estimation [Heckmann et al, Int. Conf. Int. Robots and Systems (2006)]. A location estimate is then constructed by integrating suitably weighted cues from each pixel within the fragment. Experiments are carried out on a large corpus of multi-speaker data, mixed from binaural recordings in a real, reverberant environment. The fragment based processing is shown to provide significant improvements when compared to competitive baseline approaches.

2pPPa4. The effect of spectral overlap on sensitivity to interaural time differences. Christopher A. Brown (Arizona State University, Dept. of Speech \& Hearing Science, P.O. Box 870102, Tempe, AZ 85287-0102, USA, c-b@asu.edu), William A. Yost (Arizona State University, Dept. of Speech \& Hearing Science, P.O. Box 870102, Tempe, AZ 85287-0102, USA, William.Yost@asu.edu)

Cochlear implant users who have some residual low-frequency hearing in both their implanted and unimplanted ears may have access to interaural time differences (ITDs), which could provide a cue for sound source segregation. Speech intelligibility has been shown in simulation to improve under these conditions. However, implant patients who stand to benefit from ITDs may have a significant asymmetry in the configuration of loss in the low-frequency region. It is unknown how a difference in corner frequency of loss across ears will affect ITD sensitivity. As a first step toward characterizing this relationship, the present study measured ITD just-noticeable differences (JNDs) of bands of noise that varied in the amount of spectral overlap across ears in five normal-hearing listeners. In one ear, the center frequency was $250 \mathrm{~Hz}$, and the bandwidth was either $1 / 3$ or $2 / 3$ octaves. In the other ear, the center frequency was $250,281,315,354,397$, or $794 \mathrm{~Hz}$, and the bandwidth was $1 / 3$ or $2 / 3$ octaves. Results showed that although ITD JNDs increased as spectral overlap decreased, ITDs were only detectable so long as some spectral overlap was present.

2pPPa5. Perception of speaker's facing angle. Hiroaki Kato (ATR Cognitive Information Science Laboratories, 2-2-2 Hikaridai, Seika-cho Soraku-gun, 619-0288 Kyoto, Japan, kato@atr.jp), Hironori Takemoto (ATR Cognitive Information Science Laboratories, 2-2-2 Hikaridai, Seika-cho Soraku-gun, 619-0288 Kyoto, Japan, takemoto@atr.jp), Ryouichi Nishimura (National Institute of Information and Communications Technology/ATR Cognitive Information Science Laboratories, 2-2-2 Hikaridai, Seika-cho, 619-0288 Kyoto, Japan, ryou@atr .jp)

In pursuit of an ultimately realistic human-to-human telecommunication technology, the ability to auditorily perceive the facing direction of a human speaker was explored. A male speaker sat on a pivot chair in an anechoic chamber and spoke a short sentence (about 5 seconds) while facing either of eight azimuth angles $(0=$ listener's direction, 45, 90, 135, 180, 225, 270, or 315 degrees) and either of three elevation angles $(0=$ horizontal direction, -45 , or 45 degrees). The azimuth angles were set solely by turning the pivot chair. Twelve blindfolded listeners heard the spoken sentence at a distance of either 1.2 or 2.4 meters from the speaker and were asked to indicate the speaker's facing angle. In separate sessions, the speaker changed facing angles while speaking and the listeners indicated the perceived direction of horizontal movement (clockwise or counter-clockwise) or vertical movement (up or down). Overall results showed that the listeners were more accurate in indicating the movement of the speaker than in simply indicating the facing angles. Effective acoustic cues were then discussed on the basis of the transfer characteristics from the speaker's mouth to the listener's ears measured by the cross-spectral method using the speaker's own voice.

2pPPa6. Auditory capture in a spatial cueing task. Marieke Van Der Hoeven (TNO, Kampweg 5, 3769ZG Soesterberg, Netherlands, marieke.vanderhoeven@tno.nl), Adelbert W. Bronkhorst (TNO, Kampweg 5, 3769ZG Soesterberg, Netherlands, adelbert.bronkhorst@tno.nl)

This study examined how an auditory non-informative spatial cue influences detection and localization of auditory targets. In a go-no go task subjects attended a centrally placed loudspeaker surrounded by 6 other loudspeakers and were asked to respond only to centre targets. The cue came from one of the loudspeakers and preceded the target tone with an SOA of 100 or $400 \mathrm{~ms}$. A baseline condition with a spatially diffuse cue was included, using out-of-phase presentation through two loudspeakers. Results show that, compared to the baseline, cues shortened reaction times at small cue-target angles (up to $7^{\circ}$ ) and increased them at larger angles. At the shortest SOA, the reaction time difference was up to $80 \mathrm{~ms}$. Furthermore, cueing seems to have no effect on sound localization: false alarms were normally distributed around the hits. These findings demonstrate that strong auditory capture effects occur that depend on cue-target distance.

2pPPa7. Spatiotemporal interactions between audition and vision. Adelbert W. Bronkhorst (TNO, Kampweg 5, 3769ZG Soesterberg, Netherlands, adelbert.bronkhorst@tno.nl), Thomas Koelewijn (Vrije Universiteit, van der Boechorststraat 1, 1081 BT Amsterdam, Netherlands, T.Koelewijn $@$ psy.vu.nl), Erik Van Der Burg (Vrije Universiteit, van der Boechorststraat 1, 1081 BT Amsterdam, Netherlands, E.van.der.Burg@psy.vu.nl)

It is well known that spatial auditory cues can affect performance on spatial visual tasks. One example is crossmodal cueing: response times for a visual spatial discrimination task are shortened when the location of the visual stimuli is cued auditorily, and prolonged when an invalid location is cued. Another example is auditory support of visual search: we normally find lower search times for visual items when the location of the target item is indicated by an auditory cue. In our research we have explored two questions: one related to crossmodal cueing, and the other to audiovisual search. In our crossmodal cueing paradigm we have looked at the interaction between (exogenous) cueing and endogenous information given before each trial. In this way we wanted to determine whether auditory cues are able to capture visual attention even when this attention has already been focused endogenously. In our audiovisual search paradigm we have studied whether nonspatial auditory cues, presented simultaneously with the visual target item, are able to improve visual search. Because of the temporal synchrony, the auditory stimuli do not act as actual cues but they are probably fused with the visual stimuli into an integrated percept.

2pPPa8. The acoustical bright spot and the erroneous localization of tones by human listeners. Brad Rakerd (Michigan State University, Dept. of Communicative Sciences and Disorders, East Lansing, MI 48824, USA, 
rakerd@msu.edu),Zach Ryan(Michigan State University, Dept. of Physics and Astronomy, East Lansing, MI 48824, USA, ryanzach@msu.edu), Eric Macaulay (Michigan State University, Dept. of Physics and Astronomy, East Lansing, MI 48824, USA, macaula5@msu.edu), William M. Hartmann (Michigan State University, Dept. of Physics and Astronomy, East Lansing, MI 48824, USA, hartmann@ pa.msu.edu)

In free-field sound localization experiments, human listeners estimated the locations of sine tones originating at 13 loudspeakers that spanned a range of azimuths from 0 degrees (straight ahead) to 90 degrees (extreme right). During the experiments the tone amplitudes and phases were measured in the listener's ear canals. Because of the acoustical bright spot, measured interaural level differences (ILD) were found to be a nonmonotonic function of azimuth at frequencies above about $1000 \mathrm{~Hz}$. In source identification experiments at $1500 \mathrm{~Hz}$, there was no correlation between estimated location and the interaural phase difference. There was high correlation with ILD, causing listeners to make large localization errors for azimuths greater than about 55 degrees. In a second experiment, listeners judged the direction of movement of sounds from one source to another. Errors in the second experiment could be well predicted from the localization responses in the source identification experiment. The ear canal measurements showed that, in principle, listeners could localize successfully by using the levels in the two ears independently. However, localization performance remained poor even after extensive training. Work supported by the NIDCD, grant DC00181.

2pPPa9. Human cortical responses to change in sound lateralization suggest non-topographical coding of interaural time differences. David A. Magezi (MRC Institute of Hearing Research, University Park, NG7 2RD Nottingham, UK, david.magezi@ihr.mrc.ac.uk), Katrin Krumbholz (MRC Institute of Hearing Research, University Park, NG7 2RD Nottingham, UK, katrin@ihr.mrc.ac.uk)

It is generally assumed that interaural time differences (ITDs) are coded by a parametric array of finely tuned ITD channels (topographic code). An alternative theory is that ITD is coded by a rate code, which involves only one channel in each hemisphere, broadly tuned to the contralateral hemispace. The aim of the current study was to investigate ITD coding in humans by using electroencephalography (EEG) with a continuous stimulation paradigm (CSP). In this paradigm, a test sound, with a given ITD, is immediately preceded by an appropriately matched control sound with a different ITD. At the transition from the test to the control sound, only neural elements that respond to the test but not the control ITD would be expected to be activated. Thus, the size of the transition response would be assumed to be determined by the degree of overlap between the neural responses to the control and test ITDs. The pattern of results was found to be inconsistent with the topographic model, and thus lend support to the hemispheric channel model.

2pPPa10. Both prime length and prime position affect the spatial release of nonsense speech from informational masking. Zhigang Yang (Dept. of Psychology, Peking Univ., 5 Yeheyuan Road, Haidian District, 100871 Beijing, China, synrhine@163.com), Jing Chen (Dept. of Machine Intelligence, Speech and Hearing Research Center, 2 Science Building, Peking Univ., 5 Yeheyuan Road, Haidian District, 100871 Beijing, China, chenj@cis.pku.edu.cn), Qiang Huang (Dept. of Machine Intelligence, Speech and Hearing Research Center, 2 Science Building, Peking Univ., 5 Yeheyuan Road, Haidian District, 100871 Beijing, China, QIANG.HUANG@SPREADTRUM.COM), Ying Huang (Dept. of Psychology, Peking Univ., 5 Yeheyuan Road, Haidian District, 100871 Beijing, China, innhuang@gmail.com), Xihong H. Wu (Dept. of Machine Intelligence, Speech and Hearing Research Center, 2 Science Building, Peking Univ., 5 Yeheyuan Road, Haidian District, 100871 Beijing, China, wxh@cis.pku.edu.cn), Yanhong Wu (Dept. of Psychology, Peking Univ., 5 Yeheyuan Road, Haidian District, 100871 Beijing, China, wuyh@pku.edu.cn), Liang Li (Dept. of Psychology, Peking Univ., 5 Yeheyuan Road, Haidian District, 100871 Beijing, China, liangli@pku.edu.cn)

When masking speech is present, pre-presentation of early part of nonsense target speech improves recognition of the rest of target speech, indicating a content and/or voice priming effect (Freyman et al., 2004; Yang et al., 2007). Here, we examined both the prime-length effect and the primeposition effect on recognition of nonsense target speech with twelve syllables and three keywords. Target speech started 1 sec. after the onset of two-talker masking speech. The results show that a longer prime with 10 syllables (including the 1st and 2nd keywords) significantly improved recognition of the last (3rd) keyword in target speech. However, when the $1 \mathrm{st}$ four syllables (including the 1st keyword) were pre-presented, recognition of either the 2 nd or 3rd keyword was not improved. Interestingly, when the 2nd four syllables (including the 2nd keyword) were pre-presented, recognition of the 1 st but not the 3rd keyword was significantly improved. Thus under speech-on-speech masking conditions, both the prime length and the prime position in the sentence influence the priming effect on recognition of target speech, and listeners contribute more attentional resource to the initial part of target speech. Supported by the National Natural Science Foundation of China.

2pPPa11. Masking release based on fine-grain temporal pitch cues in speech-like stimuli - mechanisms and implications for the cocktail-party phenomenon. Nicholas R. Clark (MRC Institute of Hearing Research, University Park, NG7 2RD Nottingham, UK, n.clark@ihr.mrc .ac.uk), Katrin Krumbholz (MRC Institute of Hearing Research, University Park, NG7 2RD Nottingham, UK, katrin@ihr.mrc.ac.uk)

One of the most important cues for segregating wanted from unwanted sounds when listening in noisy environments is temporal regularity (or harmonicity), which gives rise to the perception of pitch. This study measured masked signal detection thresholds when both the signal and the masker are complex tonal stimuli similar to speech. Detection was considerably easier when the pitch of the masker differed from that of the signal. A control experiment involving non-simultaneous masking revealed that this pitch-based release from masking was not due to spectral differences between the signal and the masker. A second experiment investigated the effect of a pitch difference between the signal and the masker on the audibility of certain perceptual features of the signal when the signal is presented at a supra-threshold level. Two features were used that are known to be particularly important for speech perception: amplitude modulation and spectral profile related to formant frequencies. The supra-threshold tasks enabled to quantify the unmasking effect of pitch on the processing of these features over and above the effect on the signal detection threshold. The results provide new insights into the low-level processes involved in the cocktail-party phenomenon. Acknowledgement: Work supported by the Medical Research Council.

2pPPa12. Perceptual sensitivity to high-frequency interaural time differences created by rustling sounds. Lutz Wiegrebe (Biocenter, University of Munich, Großhaderner Str. 2, 82152 Munich, Germany, lutzw @1mu.de)

ITDs are recruited to localize sounds in azimuth. ITDs can be extracted from low-frequency sounds or from the envelopes of high-frequency, complex sounds. Studies of the latter have included amplitude-modulated or transposed tones. A recent study showed that not the degree of envelope fluctuation, as quantified by the envelope 4 th moment, determines sensitivity to envelope ITDs but the envelope spectrum and with it the interaural crosscorrelation function of the auditory envelopes provide a good description of envelope ITD sensitivity. Here we use a different class of high-frequency stimuli, namely noise stimuli generated with different degrees of roughness which resemble natural rustling sounds. Stimuli are generated with a Gaussian-noise carrier and an aperiodic, rectangular modulator of statistically different duty cycle. The results show that ITD sensitivity increases both with increasing roughness and increasing bandwidth of the rustling sounds. While the effect of bandwidth on ITD sensitivity is in line with previous conclusions, the effect of roughness is not. ITD sensitivity for rustling sounds elicited ITD thresholds as low as $32 \mu$ s, i.e., considerably lower than reported in previous studies with transposed stimuli. The data show that high-frequency rustling sounds provide strong temporal localization cues which the auditory system can effectively exploit. 
2pPPa13. Lateralization of binaural stimuli with independent fine-structure and envelope-based temporal disparities. Mathias Dietz (Carl von Ossietzky Universität Oldenburg, Ammerländer Heerstraße 114-118, 26111 Oldenburg, Germany, mathias.dietz@uni-oldenburg .de), Stephan D. Ewert (Carl von Ossietzky Universität Oldenburg, Ammerländer Heerstraße 114-118, 26111 Oldenburg, Germany, stephan.ewert@uni-oldenburg.de), Volker Hohmann (Carl von Ossietzky Universität Oldenburg, Ammerländer Heerstraße 114-118, 26111 Oldenburg, Germany, volker.hohmann@uni-oldenburg.de)

A computational model for the lateralization of binaural stimuli, motivated by recent physiological findings in the literature and psychoacoustic data is presented. The model is based on the evaluation of the interaural phase difference (IPD). In the model, IPDs are separately assessed for the stimulus' fine-structure and envelope. Psychoacoustic measurements were conducted and compared to model predictions. Sinusoidally amplitude modulated $1-\mathrm{kHz}$ tones with a modulation frequency of 25,50 , and $100 \mathrm{~Hz}$ were employed. The IPD of the fine-structure and the envelope IPD were independently matched with an interaural level difference or were traded against each other. Lateralization increased for increasing IPDs up to $135^{\circ}$ of either the fine-structure or envelope independent of the modulation frequency. However, trading a fine-structure IPD with an opposing envelope IPD revealed a most persistent fine-structure IPD at $45^{\circ}$. The data could be modeled assuming a physiological distribution of the best IPDs of binaural neurons clustered around $45^{\circ}$. The model was also utilized to correctly predict the perceived lateralization of critical stimuli from literature. Individual differences in the perceptual salience of envelope and fine-structure cues, also known from the literature, could be modeled by a personal weighting coefficient for the fine-structure cue.

2pPPa14. A binaural advantage in the subjective modulation transfer function with simple impulse responses. Eric R. Thompson (Centre for applied hearing research, Technical University of Denmark, DTU, Bygn. 352, 2800 Lyngby, Denmark, et@oersted.dtu.dk), Torsten Dau (Centre for applied hearing research, Technical University of Denmark, DTU, Bygn. 352, 2800 Lyngby, Denmark, tda@oersted.dtu.dk)

The speech transmission index (STI) has been a popular method for predicting speech intelligibility in rooms. It is based on the magnitude of the modulation transfer function, which can be derived from the impulse response of the room and the background noise levels. However, it does not take into account that humans listen with two ears. There can be large interaural phase differences in the modulation transfer functions, which can create detectable interaural level difference fluctuations. Measurements were made to determine whether these interaural modulation phase differences can be used to enhance the detectability of sinusoidal intensity modulations imposed on a broadband noise carrier and then convolved with simple, dichotic impulse responses. The results show that there can be a significant advantage to listening with two ears over listening with just one. Some further investigations were made to determine whether this advantage in signal detection can also be used as an advantage for speech intelligibility.

2pPPa15. Temporal weighting of interaural level differences in high-rate click trains. Christopher Stecker (Dept. of Speech and Hearing Sciences, University of Washington, 1417 NE 42nd St, Seattle, WA 98105, USA, cstecker@u.washington.edu)

When high-carrier-frequency stimuli are amplitude modulated at rates above $\sim 200 \mathrm{~Hz}$, lateralization based on envelope interaural time differences (ITD) becomes dominated by onset ITD. The role of onset cues in interaural-level-difference (ILD) processing, however, is less well understood. Although theoretical predictions suggest that onset dominance should have similar effects upon ILD as upon ITD, some experimental data suggest that ILD processing retains greater sensitivity during post-onset segments of a high-rate stimulus. In this study, subjects lateralized ILDs carried by trains of 16 Gaussian-filtered clicks (carrier frequency $4000 \mathrm{~Hz}$ ) that varied in interclick interval (ICI) from 2 to $10 \mathrm{~ms}$ (modulation rate 100-500 $\mathrm{Hz}$ ). ILDs of individual clicks in each train were randomly perturbed. Multiple linear regression of listener responses onto the perturbed ILD values was used to generate temporal weighting functions (TWFs) that describe how a listener's sensitivity to ILD changes over the stimulus duration. The resulting TWFs were compared to predictions from models of onset dominance as well as to TWFs measured for ITD and in free-field conditions. The results have implications for binaural processing of high-rate pulsatile stimuli experience by users of bilateral cochlear implants.

2pPPa16. Modeling the precedence effect in inferior colliculus neurons using converging excitatory and inhibitory inputs. Jing Xia (Boston University Hearing Research Center, 677 Beacon Street, Boston, MA 02215, USA, xiajing0928@ hotmail.com), Andrew Brughera (44 Cummington St., Boston, MA 02215, USA, brughera@bu.edu), H Steven Colburn (44 Cummington St., Boston, MA 02215, USA, colburn@bu.edu), Barbara Shinn-Cunningham (Boston University Hearing Research Center, 677 Beacon Street, Boston, MA 02215, USA, shinn@cns.bu.edu)

Responses of a population model of low-frequency inferior colliculus (IC) neurons to a pair of broadband clicks were evaluated. IC inputs came from Hodgkin-Huxley models of medial superior olive (MSO) and cochlear nucleus bushy cells driven by a stochastic auditory nerve model. Each IC cell received converging inputs that were excitatory from ipsilateral MSO and inhibitory from contralateral MSO. Convergence of multiple excitatory, ipsilateral MSO inputs with similar interaural time difference (ITD) tuning improved ITD sensitivity and reduced the influence of MSO onset responses caused by monaural, rather than binaural, coincidences. Inhibition that lasted over several milliseconds suppressed IC responses to the lagging click. The effect of the inhibition depended on the ITD tuning of the excitation and inhibition converging on a given IC neuron. With appropriate convergence, the IC population showed no response to the lagging click at short delays, and responded to the lagging click but did not encode its ITD at intermediate delays. Only at longer inter-click delays did IC responses show sensitivity to the lagging click ITD, consistent with the perceptual phenomenon of the precedence effect. [Work supported by NSF and NIH].

2pPPa17. Relationship between reading and language ability and auditory temporal processing measured with the precedence effect. Cynthia M. Zettler (University of Wisconsin, Waisman Center, 1500 Highland Ave, Madison, WI 53705, USA, zettler@waisman.wisc.edu), Rose A. Sevcik (Georgia State University, Dept of Psychology, University Plaza, Atlanta, GA 30303, USA, psyras@langate.gsu.edu), Robin D. Morris (Georgia State University, Dept of Psychology, University Plaza, Atlanta, GA 30303, USA, psyrdm@langate.gsu.edu), Mary Ann Romski (Georgia State University, Dept of Psychology, University Plaza, Atlanta, GA 30303, USA, mromski@gsu.edu), Colleen O'Rourke (Georgia State University, Dept of Psychology, University Plaza, Atlanta, GA 30303, USA, corourke@gsu.edu), Marsha G. Clarkson (Georgia State University, Dept of Psychology, University Plaza, Atlanta, GA 30303, USA, mclarkson @gsu.edu)

Processing of timing information by the auditory system contributes to numerous abilities, including spatial hearing and higher-order language and reading skills. However, relationships among these abilities are poorly understood. The present study was aimed at investigating potential common mechanisms that involve timing. Auditory timing was measured by click stimuli presented via two loudspeakers at a rate of $1.5 / \mathrm{sec}$. Fourteen-ms clicks were presented from one (single-source condition) or two (dualsource condition) loudspeakers. In the latter condition, the onset of one loudspeaker was delayed relative to the other by $5,10,20,50,100,200$, or $400 \mathrm{~ms}$. Seventy-five naïve adults (mean age 21.23 years) listened to the auditory stimuli and were asked to identify the source of the leading sound. Additionally, all participants completed a battery of language and reading measures. Results on the auditory task revealed that performance was significantly above chance on single-source trials, dropped at short delays, and improved with increasing delay. The mean threshold for correctly identifying the source of the sound was $43.48 \mathrm{~ms}$. A significant correlation $(\mathrm{p}<.05)$ found between this threshold and a measure of word identification suggests a relationship between timing required for auditory localization and timing required for reading ability.

2pPPa18. Lateralization of unidirectional frequency sweeps at high frequencies. I-Hui Hsieh (University of California, Department of Cognitive Sciences, Irvine, CA 92697, USA, ihsieh@uci.edu), Kourosh 
Saberi(University of California, Department of Cognitive Sciences, Irvine, CA 92697, USA, saberi@uci.edu)

The ability to lateralize FM sweeps at high frequencies was investigated in a 2IFC 2-down 1-up adaptive design. Four conditions were examined in which the unidirectional FM stimulus swept either up or down in frequency, and either linearly or logarithmically between 3 and $8 \mathrm{kHz}$ with the onset frequency roved by $15 \%$ on each presentation. The signal was an interaural delay in the waveform's fine-structure and hence its instantaneous frequency. The waveforms to the left and right ears had simultaneous envelopes and were filtered with the inverse of the headphone transfer functions to eliminate frequency-dependent interaural level differences. Results showed that sweep durations exceeding $25 \mathrm{~ms}$ produce near-chance performance. Thresholds monotonically improved with increasing sweep rate to a lower asymptote of approximately 100 microseconds. Counterintuitive reversals of lateral position percepts were observed for down-sweeps which were inconsistent with lateralization based on envelope outputs of frequency-matched filters.

2pPPa19. The localization of multiple simultaneous sounds is mediated by attention. Brian D. Simpson (AFRL, 2610 Seventh Street, WPAFB, OH 45433, USA, Brian.Simpson@wpafb.af.mil), Douglas S. Brungart (AFRL, 2610 Seventh Street, WPAFB, OH 45433, USA, douglas.brungart@wpafb.af.mil), Robert H. Gilkey (Wright State University, Department of Psychology, 335 Fawcett Hall, Dayton, 45435, USA, gilk@wright.edu), Nandini Iyer (AFRL, 2610 Seventh Street, WPAFB, OH 45433, USA, nandini.iyer@wpafb.af.mil), James T. Hamil (Ohio State University, Department of Electrical Engineering, Columbus, 43210, USA, hamil.3@osu.edu)

Real-world listening experiences suggest that listeners possess a much greater ability to recover spatial information from multiple simultaneous sounds than would be predicted from the results of most multisource listening experiments. Recent data from our laboratory are consistent with these real-world experiences, and indicate that listeners can reliably report the location of a sound that has been deleted from a multisource scene. However, performance degrades as the number of competing sounds increases, and it is unclear if this 'set size effect' arises from changes in signal-to-noise ratio (SNR) related to the number of competing sources, or from attentional constraints that limit the number of simultaneous sources that can be monitored. In this study, SNR was held roughly constant by fixing the number of competing sources at 8 , and the 'relevant set size' was varied by displaying on a visual monitor, prior to an observation interval, a list of $1,2,3,4,6$, or 8 environmental sounds, one of which was the target sound (the sound that would be deleted). The ability to localize this target sound decreased as the relevant set size increased, suggesting that the ability to monitor complex auditory scenes is mediated by attentional constraints.

2pPPa20. Better-ear listening strategies for normal-hearing and hearing-impaired individuals. William M. Whitmer (GN Auditory Research Laboratory, 2601 Patriot Blvd, Chicago, IL 60026, USA, whitmerb@beltone.com), Maureen Coughlin (GN Auditory Research Laboratory, 2601 Patriot Blvd, Chicago, IL 60026, USA, mcoughlin@gnresound.com), Jeff Bondy (GN Auditory Research Laboratory, 2601 Patriot Blvd, Chicago, IL 60026, USA, jbondy@gnresound.com), Andrew Dittberner (GN Auditory Research Laboratory, 2601 Patriot Blvd, Chicago, IL 60026, USA, adittberner @ gnresound.com)

The current study was designed to examine how hearing-impaired (HI) listeners use better-ear listening. Better-ear performance was initially assessed for normal-hearing (NH) and HI participants using a connectedspeech recognition test at two signal-to-noise-ratio (SNR) levels presented over insert earphones. Test conditions consisted of monaural, symmetric and asymmetric combinations of better and worse SNR conditions. SNRs were different for NH and HI groups. Stimuli were presented at each participant's most comfortable level; to account for audibility with HI participants, stimuli were spectrally shaped based on audiometric data. A second experiment investigated the role of spatial information using recordings of the same stimuli presented in a sound-dampened chamber at the same SNR differences, with the speech signal at $0^{\circ}$ azimuth/elevation, and uncorrelated babble presented from eight speakers at the corners of the chamber. Listening strategies between $\mathrm{NH}$ and $\mathrm{HI}$ groups were similar across experiments; neither group consistently used an optimal listening strategy. Results will be discussed in terms of hearing-impaired pathologies, rehabilitation and amplification strategies.

2pPPa21. Numerical Analysis of the Effects of Pinna Shape and Position on the Characteristics of Head-Related Transfer Functions. Yukio Iwaya (R.I.E.C., Tohoku University, 2-1, Katahira, Aoba-ku, 9808577 Sendai, Japan, iwaya @riec.tohoku.ac.jp), Yôiti Suzuki (R.I.E.C., Tohoku University, 2-1, Katahira, Aoba-ku, 980-8577 Sendai, Japan, yoh@ais .riec.tohoku.ac.jp)

There are distinctive notches and peaks in Head-Related Transfer Functions (HRTFs). Some of them are considered as important cues in the perception of the elevation angle and thus the roles of these peaks and notches should be clarified. It is known that the characteristics of HRTFs are deeply related to listener's anthropometry. It is thus naturally expected that frequencies of the peaks and notches also change according to the individuality of listeners' anthropometry. Therefore, in this study, effects of ear shapes and positions on frequency positions of the peaks and notches are examined by numerical analyses. The analysis was performed with boundary element method (BEM). A three-dimensional model of a dummy-head was constructed with a three-dimensional laser scanner and HRTFs of the model were numerically computed with a BEM solver. The model was modified on some features as follows: 1) pinna position, 2) pinna size, 3) angle of pinna toward listener's head, and 4) existence of wrinkles of pinna. HRTFs of the modified models were also computed. From the comparison among the results of calculation, several systematic changes were found. For example, the frequency position of notches around $8 \mathrm{kHz}$ is shifted toward lower frequency as the size of pinna increases.

2pPPa22. Are interaural time and level cues processed by separate or integrated networks in the human auditory cortex? Barrie A. Edmonds (MRC Institute of Hearing Research, University Park, NG7 2RD Nottingham, UK, barrie@ihr.mrc.ac.uk), Katrin Krumbholz (MRC Institute of Hearing Research, University Park, NG7 2RD Nottingham, UK, katrin @ihr.mrc.ac.uk)

Listeners use two cues to localise the position of a sound source in the horizontal plane: interaural time differences (ITDs) and interaural level differences (ILDs). It is thought that these two cues are encoded separately at the level of the brainstem, but are likely to be combined at higher levels in order to produce a representation of sound azimuth. In the current study, we used electro-encephalography (EEG) to investigate whether the cortical representation of a stimulus containing both ITDs and ILDs reflects separate processing of ITDs and ILDs or a combined code for perceived sound location. Stimuli were presented over headphones using a continuous stimulation paradigm; a diotic control sound was presented for 1.5 seconds followed immediately by a dichotic test sound for 250 milliseconds. Evoked responses were recorded for test stimuli containing an ITD only, an ILD only, or both an ITD and an ILD indicating either the same or opposite source azimuths. When the two cues were in opposition, the stimuli were perceived as central and were indistinguishable from the diotic control sound. However, they still elicited a strong cortical response. This suggests that ITDs and ILDs are encoded separately in the auditory cortex. 


\title{
Session $2 \mathrm{pPPb}$
}

\section{Psychological and Physiological Acoustics and ASA Committee on Standards: Applications of Psychoacoustics II (Poster Session)}

\author{
Patricia Davies, Cochair \\ Ray W. Herrick Lab., School of Mechanical Engineering, Purdue Univ. \\ Hugo Fastl, Cochair \\ AG Technische Akustik, MMK, TU München
}

\begin{abstract}
All posters will be on display from 3:40 p.m. to 5:20 p.m. To allow contributors an opportunity to see other posters, contributors of odd-numbered papers will be at their posters from 3:40 p.m. to 4:30 p.m. and contributors of even-numbered papers will be at their posters from 4:30 p.m. to 5:20 p.m.
\end{abstract}

\section{Contributed Papers}

2pPPb1. Loudspeaker sound quality: comparison of assessment procedures. Vincent Koehl (LISyC EA 3883, 6 avenue Victor Le Gorgeu, CS 93837, 29238 Brest Cedex 3, France, vincent.koehl @univ-brest.fr), Mathieu Paquier (LISyC EA 3883, 6 avenue Victor Le Gorgeu, CS 93837, 29238 Brest Cedex 3, France, mathieu.paquier@univbrest.fr)

In listening tests involving different loudspeakers and aimed at assessing the sound quality of these sound-reproducing systems, the level is generally adjusted to compensate for differences in sensitivity. The loudness sensation must be alike for each system under test. Because of the non-stationary nature of the musical signals used as test material in loudspeaker ratings, loudness assessment by using the current models (Zwicker, Mooreœ) remains slightly inaccurate. In practice, loudness is often equalized by ear by the experimenter. This study deals with the comparison of various test procedures. The first experiment was a paired comparison of loudspeakers where short-duration stimuli were presented to listeners for preference ratings. In the second experiment, the same listeners were allowed to switch, at any time, from one loudspeaker to another one so that the proposed stimuli were longer. In both experiments the loudness was equalized by the experimenter. However, under normal listening conditions, the listener is usually free to adjust by himself the reproduction level. At last, in a third experiment, the listeners had the opportunity, at any time, to not only to switch from one system to another one, but also to adjust the loudness of the stimuli.

2pPPb2. Study of the perceptive space linked to dashboard tapping sounds. Francois Montignies (Renault Technocentre, FR TCR LAB 252, 1 avenue du Golf, 78288 Guyancourt Cedex, France, francois.montignies @insa-lyon.fr), Etienne Parizet (Laboratoire Vibrations Acoustique, Insa Lyon, 25 bis, av. J. Capelle, 69621 Villeurbanne Cedex, France, etienne .parizet@insa-lyon.fr)

It was observed that dashboard tapping sounds can be produced by customers exploring static vehicles. To master the perceived quality of the dashboard, a global sound design process has thus been engaged. The specific aim of the present study was to identify the acoustical and technologica characteristics being major determinants in the discrimination of the sounds and in the evaluation of the sound sources. 47 sounds were used for a perceptive test in laboratory. They were recorded in 22 vehicles by hand tapping on different dashboard parts. In a same session, each subject performed 3 different tasks: categorisation (free sorting of sounds), description (free verbalisations) and evaluation (the score of a freely selected prototype was attributed to the whole group). Data analysis took advantage of the combination of various multidimensional analysis techniques, already used in psychoacoustics or sensory analysis. This low-cost methodology allowed us to describe a wide range of product samples with complementary psychological data. Results will help us to propose a sound metric which represent the evaluation of dashboard quality evoked by the sound. Furthermore, they give first hypotheses for the technological variables that could be decisive in the design process.

2pPPb3. Perceptual pitch compensation for low frequency bandwidth extension. Kim Seung (So. EECS/EE at Seoul National University, INMC, 132-302 Seoul National University, 599 Gwanangno, Gwanak-gu, 151-742 Seoul, Republic of Korea, bandwalk@acoustics.snu .ac.kr), Koeng-Mo Sung (Applied Acoustics Lab., INMC, 132-302, Seoul National University, San 56-1, Sillim-dong, Kwanak-gu, 151-742 Seoul, Republic of Korea, kmsung@snu.ac.kr)

As the size of speakers decreases with miniaturization of audio devices and thickness of TV, there is increased demand to ensure low-frequency sound quality. Psychoacoustics bandwidth extension for low-frequency has been applied to recent audio devices. A perceptual pitch does not correspond with fundamental frequency of missing fundamental, because the perceptual pitch is heard highly than desirable fundamental frequency. Consequently, the algorithm compensating perceptual pitch with difference is needed. This paper describes the algorithm compensating the difference using mel scale curve, and the listening test result of sound source applying the algorithm in comparison with sound source applying conventional algorithm.

2pPPb4. Towards an hearing threshold prediction model in car noise. Jean-François Sciabica (PSA Peugeot Citroën, Centre Technique de Vélizy, Route de Gisy, 78943 Vélizy-Villacoublay, France, jean-francois.sciabica @mpsa.com), Florent Richard (PSA Peugeot Citroën, Centre Technique de Vélizy, Route de Gisy, 78943 Vélizy-Villacoublay, France, florent.richard@mpsa.com), Vincent Roussarie (PSA Peugeot Citroën, Centre Technique de Vélizy, Route de Gisy, 78943 Vélizy-Villacoublay, France, vincent.roussarie@mpsa.com)

Sound design is an important challenge at PSA Research Center. Engine noise is rich in harmonics and interacts with aerodynamic and road noise. In this context, comprehension of masking phenomenon is indispensable to obtain a good description of sound attributes like roughness and booming. In this paper, we present a study about interaction between engine harmonics and road and aerodynamic noise. Thresholds for the detection of one harmonic in noise are firstly measured as a function of noise level. We propose also a prediction model for thresholds as a linear function of noise level in critical band. Results were compared to data available in the literature. Secondly, masking of harmonics by a harmonic and noise is measured. The 
masker frequency is $100 \mathrm{~Hz}$ and its level varies from 65 to $85 \mathrm{~dB}$ SPL in 5 $\mathrm{dB}$ steps. Noise level is $80 \mathrm{~dB}$ SPL. Masking patterns are traced for 8 frequencies (50 to $225 \mathrm{~Hz}$ ).

2pPPb5. The noise induced harmful effects assessment using psychoacoustical noise dosimeter. Jozef Kotus (Gdansk University of Technology, Multimedia Systems Department, 11/12 Gabriela Narutowicza Street, 80-952 Gdansk, Poland, joseph@sound.eti.pg.gda.pl), Bozena Kostek (Gdansk University of Technology, Multimedia Systems Department, 11/12 Gabriela Narutowicza Street, 80-952 Gdansk, Poland, bozenka@sound.eti.pg.gda.pl), Andrzej Czyzewski (Gdansk University of Technology, Multimedia Systems Department, 11/12 Gabriela Narutowicza Street, 80-952 Gdansk, Poland, andcz@sound.eti.pg.gda.pl), Krzysztof Kochanek (The Institute of Physiology and Pathology of Hearing, Pstrowskiego 1, 01-943 Warsaw, Poland, k.kochanek@ifps.org.pl), Henryk Skarzynski (The Institute of Physiology and Pathology of Hearing, Pstrowskiego 1, 01-943 Warsaw, Poland, h.skarzynski@ifps.org.pl)

A new way of assessment of noise-induced harmful effects on human hearing system was presented in the paper. Employing the developed psychoacoustical noise dosimeter the new indicators of noise harmfulness were verified on the basis of hearing examinations and noise measurement results. The indicators were based on some psychoacoustical properties of the human hearing system and, at the same time, on evaluation of the time and frequency characteristics of noise. Additionally, time properties of the Temporary Threshold Shift are calculated during the noise exposure. The evaluation of the proposed indicators were conducted on the basis of hearing examinations in the real noise exposure situations and also on the basis of simulation results employing standard test signals (such as: white, pink and brown noise). The standard noise dose analysis results were also presented for the purpose of comparison. The performed analysis and obtained results confirmed correctness and practical usefulness of the proposed indicators.

2pPPb6. Subjective and objective evaluation of localisation accuracy in wave field sysnthesis. Joseph Sanson (IRCAM, 1 Place Igor Stravinsky, 75004 Paris, France, joseph.sanson@ircam.fr), Etienne Corteel (Sonic Emotion, Eichweg, 6, CH-8154 Oberglatt, Switzerland, etienne.corteel@sonicemotion.com), Olivier Warusfel (IRCAM, 1 Place Igor Stravinsky, 75004 Paris, France, Olivier.Warusfel@ircam.fr)

Wave Field Synthesis (WFS) is a holophonic technique that relies on the reproduction of physical properties of sound fields in an extended listening area. Limitations of this technique are due to approximations regarding the mathematical basis and have been studied in [Ber88] [dB04]. The synthesis remains correct up to a corner frequency referred to as the spatial aliasing frequency and inside an area limited by the length of the WFS array and the position of the virtual sources. A Multichannel Equalization filter (MEQ) is applied to the driving limentation signals of the loudspeakers, as described in [Cor06]. The aim of the present study is to evaluate localisation in WFS depending on both listening and source positions. An objective analysis of the binaural signals is compared to the results of a localisation task for which spatialised high-pass filtered white noises served as stimuli. The results of the psycho-acoustic test are compared to the binaural localisation cues, namely ITD (interaural time differences) and ILD (interaural leve differences). Above the aliasing frequency, ITD and ILD are conflicting and ILD errors account in most cases for the perceptual errors.

2pPPb7. Analysis of everyday sounds which are extremely annoying for children with autism. Örjan Johansson (Luleå University of Technology, Dept Human Work Sciences, Div of Sound and Vibration, SE-97187 Luleå, Sweden, orjan.johansson@1tu.se), David Lindegren (Luleå University of Technology, Dept Human Work Sciences, Div of Sound and Vibration, SE-97187 Luleå, Sweden, david.lindegren@gmail.com)

Extreme sensitivity to noise is a problem that almost all autistic children suffer from. A sound that is extremely annoying does not need to be loud. However, the characteristics and temporal variations of these sounds are sparsely investigated. The aim of this study is to increase the knowledge about these extremely annoying sounds so they can be avoided by better design criteria for classrooms and venues like that. By interviewing teachers and parents a number of everyday sounds were identified and binaurally recorded. Examples are vacuum cleaners, ventilation noise, washing machines and pouring water. Detailed psychoacoustic analyses of this type of sounds were achieved by a listening test procedure in three parts. First 16 children composed different types of vacuum cleaner sounds trying to minimize annoyance in two different tests, a) keeping original sound pressure level. b) adjusting to acceptable loudness. In the second part, teachers working with autistic children performed a listening test to evaluate some of the composed sounds from part 1 and modified versions of them. The third part was performed by children to validate the results. The results showed that Roughness, Loudness and an index defined as High frequency tonality were the most important characteristics.

2pPPb8. Application of psychoacoustic tests for the evaluation of acoustical characteristics of Bartók Béla Concert Hall in Palace of Arts, Budapest. Zsuzsanna Alabardos (St Stephan King Technical School of Music Arts, Columbus u.11, H-1143 Budapest, Hungary, alabardos.zsuzsanna@aratokft.hu), Eva Arato-Borsi (Arato Acoustics Ltd, Mezo u.7, H-1038 Budapest, Hungary, arato.eva@aratokft.hu)

The objective characterisation of the subjective listening effect of single number quantities are well known in concert halls such: reverberation time, clarity, centre time, lateral energy fraction and so on. The Bartók Béla Concert Hall in the Palace of Arts in Budapest has been inaugurated at 15th of March 2005. The acoustic consultant office of the hall was ARTEC Ltd. A set of objective measurements has been carried out in the Concert Hall and a series of listening tests were accomplished. Results of the experiment show clear correlations in the relationship of architectural and room acoustical parameters. Apart from the important conclusions, there are some questions revealed.

2pPPb9. Parametric quality prediction for IP-based audio. Alexander Raake (Deutsche Telekom Laboratories, Berlin Institute of Technology, Ernst-Reuter-Platz 7, 10587 Berlin, Germany, alexander.raake @ telekom.de), Marieneige Garcia (Deutsche Telekom Laboratories, Berlin Institute of Technology, Ernst-Reuter-Platz 7, 10587 Berlin, Germany, marie-neige.garcia@telekom.de), Bernhard Feiten (T-Systems Enterprise Services GmbH, Goslarer Ufer 35, 13437 Berlin, Germany, bernhard.feiten@telekom.de), Sebastian Möller (Deutsche Telekom Laboratories, Berlin Institute of Technology, Ernst-Reuter-Platz 7, 10587 Berlin, Germany, sebastian.moeller@telekom.de)

Different multimedia services are more and more transmitted over a common network infrastructure, e.g. using the Internet Protocol (IP). Examples are the widespread voice over Internet Protocol (VoIP), and Internet Protocol Television (IPTV). The streaming of pure audio over IP even has a longer tradition, with applications such as internet radio. For an efficient development, planning and monitoring of such services, models can be used that predict user-perceived quality based on technical service characteristics. Speech quality models for telephony are among the most advanced ones in this context, with different model types like the signal-based PESQ (ITU-T Rec. P.862, 2001) or the parametric E-model (ITU-T Rec. G.107, 2005). In this paper, we describe a parametric approach for predicting the quality of IP-based audio. The main parameters are the audio codec, codec bitrate, packet loss characteristics and the audio content. We base our considerations on own listening tests conducted in the framework of IPTV quality assessment, on approaches and test data described in the literature and on complementary knowledge from the fields of speech and video quality models. In this context, we identify similarities and discrepancies between different types of services in the light of a common model framework. 


\title{
Session 2pPPc
}

\section{Psychological and Physiological Acoustics: Loudness, from Controlled Stimuli to Environmental Sounds I (Poster Session)}

\author{
Jeremy Marozeau, Cochair \\ Northeastern University \\ Sabine Meunier, Cochair \\ CNRS-LMA
}

\begin{abstract}
All posters will be on display from 3:40 p.m. to 5:20 p.m. To allow contributors an opportunity to see other posters, contributors of odd-numbered papers will be at their posters from 3:40 p.m. to 4:30 p.m. and contributors of even-numbered papers will be at their posters from 4:30 p.m. to 5:20 p.m.
\end{abstract}

\section{Contributed Paper}

2pPPc1. Effect of reverberation on loudness perception. Andrew Raimond (Reading University, Department of Psychology, RG6 6AL Reading, UK, a.raimond@ reading.ac.uk), Anthony J. Watkins (Reading University, Department of Psychology, RG6 6AL Reading, UK, syswatkn@rdg.ac .uk)

Stimuli with a slow-attack/fast-decay (S-F) envelope are judged to be louder than stimuli with a temporally-reversed, equal-energy version of the same envelope (fast-attack/slow-decay or F-S). It may be that the energy in the tails of F-S stimuli is discounted from loudness judgments because it is perceptually attributed to room reverberation. Here we examine the characteristics of real-room reverberation that affect loudness perception, particularly the way that reverberant tails tend to be de-correlated at the two ears. Our experiments used reverberation from real-room measurements of binaural room impulse responses (BRIRs). We ask if the perceived reduction in loudness of F-S stimuli relative to S-F stimuli is still apparent with dichotic sounds processed by the BRIRs. 


\title{
Session 2pPPd
}

\section{Psychological and Physiological Acoustics: Integrated Approaches to Auditory Scene Analysis II (Poster Session)}

\author{
Mounya Elhilali, Cochair \\ Johns Hopkins University \\ Daniel Pressnitzer, Cochair \\ CNRS UMR 8158; Univ. Paris Descartes; Ecole Normale Supérieure
}

\begin{abstract}
All posters will be on display from 3:40 p.m. to 5:20 p.m. To allow contributors an opportunity to see other posters, contributors of odd-numbered papers will be at their posters from 3:40 p.m. to 4:30 p.m. and contributors of even-numbered papers will be at their posters from 4:30 p.m. to 5:20 p.m.
\end{abstract}

\section{Contributed Papers}

2pPPd1. The ability of temporally integrating acoustic waveforms is associated with release of speech from informational masking under reverberant conditions. Liang Li (Dept. of Psychology, Peking Univ., 5 Yeheyuan Road, Haidian District, 100871 Beijing, China, liangli@pku.edu.cn), Ying Huang (Dept. of Psychology, Peking Univ., 5 Yeheyuan Road, Haidian District, 100871 Beijing, China, innhuang@gmail.com), Qiang Huang (Dept. of Machine Intelligence, Speech and Hearing Research Center, 2 Science Building, Peking Univ., 5 Yeheyuan Road, Haidian District, 100871 Beijing, China, QIANG.HUANG@SPREADTRUM.COM), Xun Chen (National Key Lab. on Machine Perception, Peking Univ., 5 Yiheyuan Road, 100871 Beijing, China, Xun.Chen@sonyericsson.com), Xihong H. Wu (Dept. of Machine Intelligence, Speech and Hearing Research Center, 2 Science Building, Peking Univ., 5 Yeheyuan Road, Haidian District, 100871 Beijing, China, wxh @ cis.pku.edu.cn)

Perceptual integration of the sound wave directly emanating from the source with reflections of the source needs both bridging temporal gaps and calculating correlations between sound waves. In this study, we examined whether the temporal integration of source/reflection signals is frequency dependent and associated with speech unmasking under simulated reverberant conditions. In Experiment 1, a break in correlation (BIC) between correlated wideband or narrowband noises at the two ears was detectable even when an interaural interval (IAI) was introduced. The longest IAI varied markedly across listeners. In wideband noise, it could be up to $21 \mathrm{~ms}$; in narrowband noise, it decreased as the center frequency was increased. In Experiment 2, when the interval between target speech and its single-reflection simulation (inter-target interval, ITI) was reduced from 64 to $0 \mathrm{~ms}$, intelligibility of target speech was markedly improved under the speech-masking condition but not the noise-masking condition. The longest effective ITI under the speech-masking condition significantly correlated with the longest IAI for detecting the BIC in low-frequency $(<$ or $=400 \mathrm{~Hz})$ narrowband noises. Thus the frequency-dependent temporal integration of soundwaveform signals is critical for releasing speech from informational masking in reverberant environments. Supported by the National Natural Science Foundation of China.

2pPPd2. A computational model for auditory scene analysis. Maria E. Niessen (University of Groningen, Dept. Artificial Intelligence, P.O. Box 407, 9700 AK Groningen, Netherlands, m.niessen@ai.rug.nl), Ronald A. Van Elburg (University of Groningen, Dept. Artificial Intelligence, P.O. Box 407, 9700 AK Groningen, Netherlands, r.van.elburg@ai.rug.nl), Dirkjan J. Krijnders (University of Groningen, Dept. Artificial Intelligence, P.O. Box
407, 9700 AK Groningen, Netherlands, j.d.krijnders@ai.rug.nl),Tjeerd C Andringa (University of Groningen, Dept. Artificial Intelligence, P.O. Box 407, 9700 AK Groningen, Netherlands, t.andringa@ai.rug.nl)

Primitive auditory scene analysis (ASA) is based on intrinsic properties of the auditory environment. Acoustic features such as continuity and proximity in time or frequency cause perceptual grouping of acoustic elements Various grouping attributes have been translated into successful signal processing techniques that may be used in source separation. A next step beyond primitive ASA is source identification through schema-based ASA. We present a computational model for ASA that is inspired by models from cognitive research. It dynamically builds a hierarchical network of hypotheses, which is based on (learned) knowledge of the sources. Each hypothesis in the network, initiated by bottom-up evidence, represents a possible sound event. The network is updated for each new input event, which may be any sound in an unconstrained environment. The analysis of new input events is guided by knowledge of the environment and previous events. As a result of this adaptive behavior, information about the environment increases and the set of possible hypotheses decreases. With this method of continuously improving sound event identification we make a promising advance in computational ASA of complex real-world environments.

2pPPd3. Pitch-based streaming of vowel sequences, speech-in-speech segregation, and frequency selectivity. Etienne Gaudrain (Laboratoire Neurosciences Sensorielles, Comportement, Cognition, UMR 5020, Université Lyon 1 - CNRS, 50 av. Tony Garnier, 69366 Lyon Cedex 07, France, egaudrain@gmail.com), Nicolas Grimault (Laboratoire Neurosciences Sensorielles, Comportement, Cognition, UMR 5020, Université Lyon 1 CNRS, 50 av. Tony Garnier, 69366 Lyon Cedex 07, France, ngrimault@olfac.univ-lyon1.fr), Eric W. Healy (University of South Carolina, Speech Psychoacoustics Laboratory, Department of Communication Sciences and Disorders, William Brice Bldg., 1621 Greene St., Columbia SC 29208, USA, ewh@sc.edu), Jean-Christophe Béra (INSERM U556, 151 cours Albert Thomas, 69424 Lyon Cedex 03, France, jean-christophe .bera@ec-lyon.fr)

Simultaneous and sequential segregation are described as the base mechanisms for auditory scene analysis and are likely to be involved in concurrent speech segregation. However, speech-in-noise perception has been found to be uncorrelated to simultaneous segregation, but related to puretone fusion threshold. This study aimed to clarify the relationship between pitch-based speech-in-speech segregation, pitch-based streaming and frequency selectivity. Twenty-five listeners with close to normal hearing were involved. Auditory filter widths were derived from a notched-noise method. Speech-in-speech perception was measured using words presented in a time reversed single talker background, with various pitch differences between 
target and masker. The streaming performance was measured using an objective order-naming task on vowel sequences. The results showed a correlation between frequency selectivity and performance in speech-in-speech perception suggesting that intelligibility relies on simultaneous masking. A correlation was also found between the effect of pitch on speech-in-speech perception and the effect of pitch on streaming performance. However, no correlation was found between streaming and frequency selectivity. These latter results suggest that pitch-based segregation probably relies on pitch discrimination which is only weakly correlated to frequency selectivity.

2pPPd4. Speech- synchronized visual cues release speech from informational masking. Mengyuan Y. Wang (Dept. of Psychology, Peking Univ., 5 Yeheyuan Road, Haidian District, 100871 Beijing, China, motoluto@163.com), Jingyu Y. Li (Dept. of Machine Intelligence, Speech and Hearing Research Center, 2 Science Building, Peking Univ., 5 Yeheyuan Road, Haidian District, 100871 Beijing, China, lijy@cis.pku.edu.cn), Ying Huang (Dept. of Psychology, Peking Univ., 5 Yeheyuan Road, Haidian District, 100871 Beijing, China, innhuang@gmail.com), Yanhong Wu (Dept. of Psychology, Peking Univ., 5 Yeheyuan Road, Haidian District, 100871 Beijing, China, wuyh@pku.edu.cn), Xihong H. Wu (Dept. of Machine Intelligence, Speech and Hearing Research Center, 2 Science Building, Peking Univ., 5 Yeheyuan Road, Haidian District, 100871 Beijing, China, wxh@cis.pku.edu.cn), Liang Li (Dept. of Psychology, Peking Univ., 5 Yeheyuan Road, Haidian District, 100871 Beijing, China, liangli@pku.edu.cn)

Visual speech information, such as lipreading cues, can assist listeners to segregate a target voice from competing voices (Helfer and Freyman, 2005). However, because signals contained in lipreading are multidimensional, it is not clear whether a simple visual cue, such as the light flash that is synchronous to the onset of each syllable in target speech, is sufficient to release target speech from noise or speech masking. In this study, when target speech was of a constant rate, the speech-synchronized light flash had no effects on speech recognition under either speech or noise masking condition. However, when the rate of target speech was artificially manipulated unstable or an intense noise burst occurred in the middle of the target sentence, the speech-synchronized light flash improved speech recognition when the two-talker speech masker but not the speech-spectrum noise masker was co-presented. These data suggest that only when the rate of target speech cannot be predicted and the masker is speech, speechsynchronized visual cues play a role in helping listeners attend to the target voice and follow the stream of target speech, leading to a release of target speech from informational masking. Supported by the National Natural Science Foundation of China.

2pPPd5. Phonemic restoration with hearing-impaired listeners. Deniz Baskent (Starkey Hearing Research Center, 2150 Shattuck Ave, Suite 408, Berkeley, CA 94704, USA, deniz_baskent@starkey.com), Cheryl Eiler (Starkey Hearing Research Center, 2150 Shattuck Ave, Suite 408, Berkeley, CA 94704, USA, cheryl_eiler@starkey.com), Brent Edwards (Starkey Hearing Research Center, 2150 Shattuck Ave, Suite 408, Berkeley, CA 94704, USA, brent_edwards@starkey.com)

Baskent et al. (ISAAR 2007) showed that simulated undershoot that may happen due to release from hearing-aid compression may reduce phonemic restoration by normal-hearing $(\mathrm{NH})$ listeners. Phonemic restoration was measured as the increase in recognition of interrupted speech when the silent intervals were filled with loud noise bursts. These results would be more crucial for hearing-impaired (HI) listeners who might encounter such problems in real life as hearing-aid users. However, it has not been previously shown if $\mathrm{HI}$ listeners benefit from phonemic restoration similar to $\mathrm{NH}$ listeners. Factors such as adverse effects of background noise on speech perception and increased forward masking observed with HI listeners might affect phonemic restoration. The present study explored phonemic restoration with mildly and moderately HI listeners with a method similar to the previous study. NH listeners participated as the control group. Perception of speech interrupted at rates of 1.5 and $2.2 \mathrm{~Hz}$ was measured with silent intervals and with noise bursts filling the silent intervals. The preliminary re- sults showed that many HI listeners, especially with mild hearing loss, were able to benefit from phonemic restoration. However, in each subject group, there were a small number of listeners who did not show any benefit.

2pPPd6. Effect of lip movement cues on auditory streaming of concurrent speech. Aymeric Devergie (Université Lyon 1, UMR 5020 CNRS, 50 av. T. Garnier, 69366 Lyon cedex 07, France, aymeric.devergie@olfac.univ-lyon1.fr), Nicolas Grimault (Laboratoire Neurosciences Sensorielles, Comportement, Cognition, UMR 5020, Université Lyon 1 - CNRS, 50 av. Tony Garnier, 69366 Lyon Cedex 07, France, ngrimault@olfac.univ-lyon1.fr), Frédéric Berthommier (Gipsa-lab UMR 5216, 46 avenue Félix Viallet - INPG, F - 38031 Grenoble cedex, France, frederic.berthommier@gipsa-lab.inpg.fr), Etienne Gaudrain (Laboratoire Neurosciences Sensorielles, Comportement, Cognition, UMR 5020, Université Lyon 1 - CNRS, 50 av. Tony Garnier, 69366 Lyon Cedex 07, France, egaudrain@gmail.com), Eric W. Healy (University of South Carolina, Speech Psychoacoustics Laboratory, Department of Communication Sciences and Disorders, William Brice Bldg., 1621 Greene St., Columbia, SC 29208, USA, ewh@sc.edu)

Lip reading is known to induce audio-visual integration processes that enhance speech-in-speech intelligibility. Recent work in auditory scene analysis [see Gaudrain et al., Acoustic'08] found correlations between sequential segregation performance and intelligibility of speech-in-speech. Our working hypothesis is that an audiovisual binding process is involved in speech understanding in multi-talker situations. However, the potential effect of audio-visual integration on auditory stream segregation remains unclear. A single previous study [Gaudrain et al., J. Acoust. Soc. Am. 122, 3065 (2007)] found that lip movements congruent with the presentation of vowels may enhance segregation. In this paradigm, audio-visual sequences of vowels with alternating low and high fundamental frequencies have been generated, in which the lip movements are paired with the second ones. The current experiment is built to test further the potential effect of visual cues on segregation. As in the previous study, lips movements were either steady (control condition), open-closed (rhythm condition) or congruent with each particular vowel. Moreover, a congruent but asynchronous audio-visual condition has been introduced as a new control. This is expected to disrupt the audiovisual binding process. Results are interpreted as an evaluation of the contribution of visual cues in Cocktail Party situations.

2pPPd7. Modelling the temporal response of the auditory nerve to the pitch of complex tones in reverberation. Lowel P. O'Mard (Centre for the Neural Basis of Hearing, The Physiological Laboratory, Downing Street, CB2 3EG Cambridge, UK, 1po21@cam.ac.uk), Mark Sayles (Centre for the Neural Basis of Hearing, The Physiological Laboratory, Downing Street, CB2 3EG Cambridge, UK, ms417@cam.ac.uk), Ian M. Winter (Centre for the Neural Basis of Hearing, The Physiological Laboratory, Downing Street, CB2 3EG Cambridge, UK, imw1001@cam.ac.uk)

A computational model of the auditory nerve has been used to investigate the effect of reverberation on the response to the fundamental frequency (F0) of frequency-swept harmonic complexes. The model used a computationally efficient dual resonance non-linear (DRNL) model of basilar membrane frequency selectivity. The DRNL architecture comprises two parallel filter paths providing linear and non-linear responses. The model successfully reproduced the results of a physiological investigation using the same stimuli in a population of single units from the ventral cochlear nucleus (VCN). In particular the effect of reverberation was dependent upon unit best frequency, F0 and source-to-receiver distance. The model results suggest that the effects of reverberation observed at the level of the VCN are already present in the auditory periphery. The use of the model has also enabled us to examine the effect of hearing impairment by the removal of the non-linear path response. This simulation of a sensorineural hearing loss accentuated the loss of the temporal representation of F0 in the presence of reverberation. 
2pPPd8. Blind source separation and sound source localization on time-frequency domain considering time lag information. Shogo Ueda (Department of Architecture, Tokyo University of Science, 1-3 Kagurazaka, Shinjuku-ku, 162-8601 Tokyo, Japan, shogo_0604@yahoo .co.jp), Fumio Sasaki (Department of Architecture, Tokyo University of Science, 1-3 Kagurazaka, Shinjuku-ku, 162-8601 Tokyo, Japan, fsasaki@rs.kagu.tus.ac.jp), Osamu Tanaka (Department of Architecture, Tokyo University of Science, 1-3 Kagurazaka, Shinjuku-ku, 162-8601 Tokyo, Japan, otanaka@rs.kagu.tus.ac.jp), Masahito Yasuoka (Department of Architecture, Tokyo University of Science, 1-3 Kagurazaka, Shinjuku-ku, 162-8601 Tokyo, Japan, shogoarc@ybb.ne.jp)

The blind source separation and sound source localization based on independent component analysis on time-frequency domain considering time lag information between source signals and observation signals are conducted. The formulation based on the independency of time-frequency domain and the linearity of source signal is presented. The method which can be conducted not only the separation of source signals but also the specification of location of source signals is proposed through the consideration of time lags. Using this method, it can be analyzed even if observation signals include an intermittent noise, under the assumption of some independency of source signals. First of all, the number of source signals is specified through the quotient of complex valued time-frequency information of two observation signals. Next, the locations of source signals are specified using the relationship of relative time lags between source signal and observation signal. Then, the source signals are obtained by use of the Fourier information. The numerical test is conducted to confirm our method, and then the locations of source signals and source signals are obtained by high accuracy.

TUESDAY AFTERNOON, 1 JULY 2008

P2-C, LEVEL 2, 3:40 TO 5:20 P.M.

\title{
Session 2pPPe
}

\section{Psychological and Physiological Acoustics: Cochlear Implants: Going Beyond the Envelope II (Poster Session)}

\author{
Bernhard Seeber, Cochair \\ $M R C$ Institute of Hearing Research \\ H Steven Colburn, Cochair \\ Boston University
}

\begin{abstract}
All posters will be on display from 3:40 p.m. to 5:20 p.m. To allow contributors an opportunity to see other posters, contributors of odd-numbered papers will be at their posters from 3:40 p.m. to 4:30 p.m. and contributors of even-numbered papers will be at their posters from 4:30 p.m. to 5:20 p.m.
\end{abstract}

\section{Contributed Papers}

2pPPe1. Perception of speech and environmental sounds in cochlear implant patients. Valeriy Shafiro (Rush University Medical Center, Dept. Communication Disorders, 600 S. Paulina Str., 1015 AAC, Chicago, IL 60612, USA, valeriy_shafiro@rush.edu), Brian Gygi (East Bay Institute for Research and Education, 150 Muir Road 151-I, Martinez, CA 94552, USA, bgygi@ebire.org), Min-Yu Cheng (Rush University Medical Center, Dept. Communication Disorders, 600 S. Paulina Str., 1015 AAC, Chicago, IL 60612, USA, min-yu_cheng@rush.edu), Megan Mulvey (Rush University Medical Center, Dept. Communication Disorders, 600 S. Paulina Str., 1015 AAC, Chicago, IL 60612, USA, megan_mulvey@rush.edu), Blythe Holmes (Rush University Medical Center, Dept. Communication Disorders, 600 S. Paulina Str., 1015 AAC, Chicago, IL 60612, USA, blythe_holmes @rush.edu)

Limited research has investigated cochlear implant (CI) patients' ability to perceive environmental sounds. In this study, environmental sound perception was assessed with a large-item test composed of 40 sound sources, each represented by four different tokens. The relationship between speech and environmental sound perception and the role of working memory and basic auditory abilities was examined based on patient performance on a battery of speech tests (HINT, CNC, and individual consonant and vowel tests), tests of basic auditory abilities (audiometric thresholds, gap detection, temporal pattern and temporal order tests), and a backward digit recall test. Twelve postlingually deaf adult CI patients, having 1-5 years of implant experience participated. The results indicate substantially reduced ability to identify common environmental sounds in CI patients. Speech test scores correlated strongly with the scores on the environmental sound test. Both speech and environmental sound tests moderately correlated with gap detection, temporal order test and backward digit recall test. However, the correlation between speech and environmental sounds changed little after partial- ling out the variance due to other variables. These results suggest that speech and environmental sounds may overlap considerably in their perceptual processing, being largely independent of peripheral limitations that may affect both sound classes.

2pPPe2. Intelligibility of interrupted speech in normal-hearing listeners and cochlear implantees. Dan Gnansia (Univ Paris Descartes, CNRS, Ecole Normale Superieure, DEC, 29 rue d'Ulm, 75005 Paris, France, dan.gnansia@ens.fr), Vincent Pean (MXM / Neurelec, 2729 Chemin St Bernard, 06224 Vallauris Cedex, France, vpean@neurelec .com), Christian Lorenzi (Univ Paris Descartes, CNRS, Ecole Normale Superieure, DEC, 29 rue d'Ulm, 75005 Paris, France, lorenzi@ens.fr)

The intelligibility of a target speech signal is substantially degraded in the presence of a competing talker in cochlear implantees. The current study aimed to test whether this degradation is caused by an inability to i) make use of the partial target speech information glimpsed into the competing talker "valleys", and ii) fuse perceptually those successive glimpses into coherent speech streams. This hypothesis was tested by assessing the intelligibility of periodically interrupted vowel-consonant-vowel (VCV) signals pronounced by a male $(\mathrm{F} 0=113 \mathrm{~Hz})$ and female $(\mathrm{F} 0=216 \mathrm{~Hz})$ speaker in normal-hearing listeners and implantees. A 4-Hz square-wave modulator with random phase was used to interrupt periodically each signal. The interrupted VCVs from each speaker were either presented alone (Experiment 1) or interleaved (Experiment 2: the two speakers were alternated). In experiment 1 , the mean identification score calculated for each voice was about $90 \%$ in normal-hearing listeners, and $40 \%$ in implantees. In experiment 2 , the mean identification score corresponding to correct identification of both voices was about $50 \%$ in normal-hearing listeners, but at chance 
level in implantees. Taken together, these data suggest that implantees can make use of partial speech information, but cannot organize this partial speech information into coherent streams.

2pPPe3. Electrode Discrimination and the effect on Speech Perception for Adults with Cochlear Implants. Debi A. Vickers (UCL Ear Institute, 332-336 Gray's Inn Road, WC1X 8EE London, UK, d.vickers@ucl.ac.uk), Leah Meerton (UCL Cochlear Implant Team, 332336 Gray's Inn Road, WC1X 8EE London, UK, leah.meerton@royalfree .nhs.uk)

Zwolan et al (1997) showed that electrode discrimination can have an effect on speech perception for adult cochlear implant users with the Nucleus Mini 22 multi-channel device. The purpose of this study was to evaluate electrode discrimination in subjects using different implant systems and speech processing strategies to observe the effect that switching off undiscriminable electrodes has on speech perception. 17 postlingually deafened adults were tested; they used either a Nucleus 24 device (either using SPEAK or ACE processing strategies) or an Advanced Bionics HiRes cochlear implant (either using HiRes or HiRes120 strategies). Only electrodes that were activated in the subjects' clinical maps were tested in the electrode discrimination task. Electrode discrimination was performed using standard clinical software at comfortable listening level and pairs of electrodes that scored less than $60 \%$ were deemed undiscriminable. One of the undiscriminable pair was switched off and the electrode selected was determined from the results of the other electrode pairs. Performance was evaluated prior to and immediately after switch off and at a follow up appointment 1 month later. Zwolan T, Collins L and Wakefield G (1997). J. Acoust. Soc. Am. 102 (6), 3673-3685

\title{
Session 2pPPf
}

\section{Psychological and Physiological Acoustics: Auditory Perception and Signal Processing by Prostheses II (Poster Session)}

\author{
Huanping Dai, Cochair \\ University of Arizona
}

\author{
Birger Kollmeier, Cochair \\ Universität Oldenburg, Medizinische Physik
}

\begin{abstract}
All posters will be on display from 3:40 p.m. to 5:20 p.m. To allow contributors an opportunity to see other posters, contributors of odd-numbered papers will be at their posters from 3:40 p.m. to 4:30 p.m. and contributors of even-numbered papers will be at their posters from 4:30 p.m. to 5:20 p.m.
\end{abstract}

\section{Contributed Papers}

2pPPf1. A model for predicting hearing aid benefit. Michael Nilsson (Sonic Innovations, 2795 East Cottonwood Parkway, Suite 660, Salt Lake City, UT 84121, USA, mnilsson@sonici.com), Victor Bray (Sonic Innovations, 2795 East Cottonwood Parkway, Suite 660, Salt Lake City, UT 84121, USA, vbray@sonici.com)

A 'large n' database was created incorporating demographic information as well as speech-recognition-in-noise measurements from several hundred subjects who participated in field trials with wearable DSP hearing aids. Performance measures were obtained across multiple hearing aid styles (CIC to BTE) with multiple signal processing technologies (multi-channel compression, digital noise reduction, directionality). The database was previously analyzed using various regression techniques (results presented at AAS2005, IHCON-2006, AAS-2007). The current analysis includes additional parameters that were not in the earlier models and uses a more robust statistical analysis. Aided speech-understanding-in-noise can be predicted based on factors of (a) unaided thresholds for tones (b) unaided thresholds for speech in quiet, (c) unaided thresholds for speech in noise, (d) hearing aid technology, and (e) subject age. We conclude that a model for hearing aid benefit can be used as a new tool for evidence-based management of hearing aid clients. Patient parameters may be entered into the model to serve as a basis for prediction of benefit in speech-understanding-in-noise using various forms of amplification treatment.
2pPPf2. Temporal manipulations in multi-channel compression effecting sound quality and performance. Michael Nilsson (Sonic Innovations, 2795 East Cottonwood Parkway, Suite 660, Salt Lake City, UT 84121, USA, mnilsson@sonici.com), Michelle Hicks (Sonic Innovations, 2795 East Cottonwood Parkway, Suite 660, Salt Lake City, UT 84121, USA, mhicks@sonici.com), Robert Ghent (Sonic Innovations, 2795 East Cottonwood Parkway, Suite 660, Salt Lake City, UT 84121, USA, bghent @ sonici.com), Victor Bray (Sonic Innovations, 2795 East Cottonwood Parkway, Suite 660, Salt Lake City, UT 84121, USA, vbray@sonici.com)

Multi-channel compression (in four or more compression channels) is expected in modern hearing aids, but compression temporal characteristics vary without an understanding of what is optimal and why. This study takes a 16 channel system using linearly spaced compressors with minimal overlap and evaluates sound quality ratings and speech recognition performance with the HINT in quiet and noise with various attack and release settings in a $2 \times 2 \times 2$ design. The first variable is the relationship between attack and release times, with asymmetric time constants (attack faster than release) or symmetric time constants (attack and release set the same). The second variable is the relationship of time constants across frequency channels, with uniform settings across frequencies, or frequency dependent time constants (faster settings for higher frequencies with shorter wavelengths). The third variable is absolute speed, with fast or slow time constants. No characteristics other than the temporal settings of the compressor were changed between conditions. Results will be presented showing optimal settings for 
sound quality as well as speech recognition. The interaction between compression characteristics and additional signal processing, such as singlemicrophone noise reduction, will be discussed.

2pPPf3. Speech Intelligibility in Virtual Environments Simulating an Asymmetric Directional Microphone Configuration. Lorenzo Picinali (De Montfort University, Music, Technology and Innovation Research Centre, LE1 9BH Leicester, UK, lorenzo@limsi.fr), Silvano Prosser (Dipartimento di Audiologia, Università degli Studi di Ferrara, Corso Giovecca 203, 44100 Ferrara, Italy, prssvn@unife.it), Antonio Mancuso (DICO-LIM, Università degli Studi di Milano, Via Comelico, 39, 20135 Milano, Italy, knmbm@tin.it), Giancarlo Vercellesi (DICO-LIM, Università degli Studi di Milano, Via Comelico, 39, 20135 Milano, Italy, giancarlo.vercellesi@dico.unimi.it)

The benefit of directional processing and binaural listening in terms of speech intelligibility for frontal sound sources has been well documented in recent and past studies. Nevertheless, only the $20 \%$ of the situations in real life present a speaker located exactly in the frontal position. Different attempt have been done so far in order to allow, manually or automatically, a change in the directional pattern of the hearing aid, but the results of these studies are far from being satisfying. The purpose of this study is to explore the advantages brought by the asymmetric directional microphone configuration of hearing aids in terms of speech intelligibility for frontal, lateral and rear sound sources in noisy environments, and in terms of the quality of the "spatial perception" of the surrounding sound-scape. Through a 3D Ambisonic virtual environment manipulation, the presence of two microphones (the two hearing aids) is simulated in a noisy environment with a speech sound source. The listeners are presented with the signal recorded from the two simulated microphones, calibrated with symmetrical and asymmetrical directional patterns, and played through a pair of headphones. The speech intelligibility is measured for all the directional microphones configurations and for all the speech sound source positions.

2pPPf4. Simulation of an electro-acoustic implant (EAS) with a hybrid vocoder. Fabien Seldran (Univ. Lyon 1 - Lab. Neurosciences, Service Pr Collet, Pavillon U, Hôpital Edouard Herriot, F-69003 Lyon, France, fseldran@yahoo.fr), Eric Truy (Hôpital Edouard Herriot, Service ORL, Place d'Arsonval, 69003 Lyon, France, eric.truy@chu-lyon.fr), Stéphane Gallégo (Univ. Lyon 1 - Lab. Neurosciences, Service Pr Collet, Pavillon U, Hôpital Edouard Herriot, F-69003 Lyon, France, iakhoun@olfac.univ-lyon1.fr), Christian Berger-Vachon (Univ. Lyon 1 Lab. Neurosciences, Service Pr Collet, Pavillon U, Hôpital Edouard Herriot, F-69003 Lyon, France, iakhoun@olfac.univ-lyon1.fr), Lionel Collet (Univ. Lyon 1 - Lab. Neurosciences, Service Pr Collet, Pavillon U, Hôpital Edouard Herriot, F-69003 Lyon, France, iakhoun@olfac.univ-lyon1.fr), Hung Thai-Van (Univ. Lyon 1 - Lab. Neurosciences, Service Pr Collet, Pavillon U, Hôpital Edouard Herriot, F-69003 Lyon, France, iakhoun@olfac.univlyon 1.fr)

Electroacoustic stimulation (EAS) is indicated for hearing impaired patients with enough residual hearing in low frequencies and severe hearing loss in high frequencies. We aimed at simulating the speech intelligibility provided by EAS with a hybrid vocoder model. The French Fournier word set was used in this study. We therefore tested several parameters on 24 normal hearing adults. First, the boundary between acoustic and electric stimulation frequency areas $(\mathrm{Fc})$ was taken at 500, 707, 1000 and $1414 \mathrm{~Hz}$. Second, we assessed the effect of electrical stimulation channel numbers (1 to 4). Third, we tested the effect of background noise with a cocktail party noise at $-6,0$ and $+6 \mathrm{~dB}$ SNR. It appeared that the 3 electrical channels \& $707 \mathrm{~Hz}$ Fc condition produced normal-hearing-like results (at least in quiet). In noisy auditory scene, 4 electrical channels \& $500 \mathrm{~Hz} F c$ could produce fair speech intelligibility. [Work supported by CNRS, Lyon1 University and Medel].

2pPPf5. Cartilage conduction hearing aid for the patient with atresia auris. Takefumi Sakaguchi (Nara Medical University, 840 Shijo-cho, 634$8522 \quad$ Kashihara, Japan, t-saka@naramed-u.ac.jp), Osamu Saito (Department of Otorhinolaryngology, Nara Medical University, 840 Shijo-cho, 634-8522 Kashihara, Japan, osam0502@yahoo.co.jp), Hiroshi Hosoi (Nara Medical University, 840 Shijo-cho, 634-8522 Kashihara, Japan, hosoi@naramed-u.ac.jp)

Although bone conduction hearing aid is considered to be better to compensate the hearing loss of the patients who have atresia auris, some of these patients tend to wear air conduction hearing aid because of the feeling of tightness when wearing bone conduction haring aid. We expected cartilage conduction would reduce feeling of tightness compared to bone conduction, and improve compensation compared to air conduction. In this study, we report the result of the basic studies performed to evaluate the usefulness of cartilage conduction hearing aid. Patient who has atresia auris participated in the hearing test. Stimuli were presented to the patient by means of two types of transmitter. One was piezoelectric transducer placed on antilobium, another one was insertion earphone. We found that the hearing threshold of the patient improved about $25 \mathrm{~dB}$ or greater when the transducer was located on antilobium compared to the result when insertion earphone was used. These results suggest that the cartilage conduction hearing aid can be an option of the hearing aid for the patient who has atresia auris which is better than air conduction hearing aid in compensating their hearing, and more comfortable to wear compared to the bone conduction hearing aid.

2pPPf6. Verifying the attenuation of earplugs in situ: variability of transfer functions among human subjects. Annelies Bockstael (Ghent University, De Pintelaan 185 2P1, 9000 Gent, Belgium, annelies.bockstael@ugent.be), Dick Botteldooren (University Ghent - Department Information Technology, Sint-Pietersnieuwstraat 41, 9000 Gent, Belgium, dick.botteldooren@intec.ugent.be), Bart Vinck (Ghent University, De Pintelaan 185 2P1, 9000 Gent, Belgium, bart.vinck@ugent.be)

The use of in situ measurements of hearing protectors' (HPD's) attenuation following the MIRE-protocol (Microphone In Real Ear) is increasing. The attenuation is hereby calculated from the difference in sound levels outside the ear and inside the ear canal behind the HPD. Custom-made earplugs have been designed with an inner bore that allows inserting a miniature microphone. A thorough understanding of the difference, henceforth called 'transfer function', between the sound pressure of interest at the eardrum and the one measured at the inner bore of the HPD is indispensable for optimizing this technique and extending its field of application. Of particular interest is the variation of these transfer functions among humans. This was checked experimentally on 19 subjects. Differences in sound pressure were measured at the HPD's inner bore, by the MIRE-microphone, and at the eardrum by inserting an extra tube microphone in the ear canal. All transfer functions showed a comparable shape, however variability was substantial for the exact frequency and amplitude of the resonance peaks. The link between this variability and the morphology of the individual's HPD and ear canal was addressed using FDTD-simulations (Finite-Difference TimeDomain) of the outer ear canal occluded by an earplug with inner bore. 


\title{
Session 2pPPg
}

\section{Psychological and Physiological Acoustics: Loudness, from Controlled Stimuli to Environmental Sounds II}

\author{
Jeremy Marozeau, Cochair \\ Northeastern University, 360 Huntington Ave - 106A Forsyth Building - SLPA, Institute for Hearing, Speech \& Language, \\ Boston, MA 02115, USA
}

Sabine Meunier, Cochair

CNRS-LMA, 31, chemin Joseph Aiguier, Marseille, 13402, France

\section{Invited Papers}

2pPPg1. Does induced loudness reduction explain contextual effects in loudness judgment? Lawrence E. Marks (John B. Pierce Laboratory, 290 Congress Avenue, New Haven, CT 06519, USA, MARKS@JBPIERCE.ORG)

Briefly presenting an inducing tone of $70-80 \mathrm{~dB}$ can substantially reduce the loudness of a subsequent test tone at or near the inducer's frequency, a phenomenon called Induced Loudness Reduction (ILR). The study of ILR emerged from earlier observations on differential contextual effects in loudness judgment: Tones of a given SPL and frequency were judged softer when presented as part of an ensemble of high rather than low SPL tones at the same frequency, relative to judgments of loudness of tones at a different sound frequency. At first, these effects of stimulus context on loudness judgment were assumed to reflect decisional processes, that is, to reflect biases in loudness judgment. On the other hand, ILR is often assumed to reflect a depression in the intensity response of the auditory system. While it is tempting to explain differential contextual effects in loudness judgment wholly in terms of ILR, the properties of ILR and the properties of contextual effects may not be identical, leaving open a possible role for decisional processes as well as sensory processes in contextual effects, and perhaps also in ILR, in both laboratory and 'real-world' settings.

\section{$5: 40$}

2pPPg2. The effects of induced loudness reduction on tone-burst otoacoustic emissions. Michael Epstein (Northeastern University, 360 Huntington Ave - 106A Forsyth Building - SLPA, Institute for Hearing, Speech \& Language, Boston, MA 02115, USA, m.epstein@neu.edu), Jeremy Marozeau (Northeastern University, 360 Huntington Ave - 106A Forsyth Building - SLPA, Institute for Hearing, Speech \& Language, Boston, MA 02115, USA, marozeau@gmail.com)

Induced loudness reduction (ILR) is a phenomenon by which the loudness of a sound is reduced when it is preceded by a higherlevel sound (inducer). Because the effects of ILR can last for at least several minutes after exposure to an inducer, many clinical and laboratory test procedures may be unintentionally confounded. However, little is known about the physiologic manifestation of ILR or how it might affect clinical tests. The present study examined whether ILR could be observed in otoacoustic emission measurements, which have been shown to correlate well with loudness. Otoacoustic emissions were measured monaurally in 12 normal-hearing listeners in response to a $1 \mathrm{kHz} 70 \mathrm{~dB}$ SPL tone-burst stimulus before and after a series of $1 \mathrm{kHz} 90 \mathrm{~dB}$ SPL tone-burst inducers. Additionally, otoacoustic emissions were measured in two control conditions in which ILR was not expected to occur. For most listeners, the level of otoacoustic emissions decreased after inducer exposure and did not decrease in the control conditions. The results indicate that at least some component of ILR is likely to arise as a cochlear process and that although the observed effects are small, ILR may affect measures beyond just loudness judgments.

\section{6:00}

2pPPg3. Modelling categorical loudness perception for arbitrary listeners and sounds. Birger Kollmeier (Universität Oldenburg, Medizinische Physik, Carl-von-Ossietzky Str. 9-11, 26111 Oldenburg, Germany, birger.kollmeier@uni-oldenburg.de), Jens E. Appell (Universität Oldenburg, Medizinische Physik, Carl-von-Ossietzky Str. 9-11, 26111 Oldenburg, Germany, jens.appell@offis.de), Jesko Verhey (Universität Oldenburg, Medizinische Physik, Carl-von-Ossietzky Str. 9-11, 26111 Oldenburg, Germany, jesko.verhey@uni-oldenburg.de), Volker Hohmann (Carl von Ossietzky Universität Oldenburg, Ammerländer Heerstraße 114-118, 26111 Oldenburg, Germany, volker.hohmann@uni-oldenburg.de)

While "classical" loudness models predict loudness in sone using the concepts of Stevens' compressive power law, (subdivided) categorical loudness perception after Heller follows the compressive logarithmic Weber-Fechner law. To bridge the gap between both approaches, this contribution reviews various steps towards a loudness model that predicts categorical loudness (in categorical units, $\mathrm{CU}$ ) for normal and hearing-impaired listeners for arbitrary sounds. It uses a (modified) classical loudness model for stationary signals to derive the loudness in sone and a nonlinear transformation from sone to CU. This transformation is approximated by a cubic polynomial equation wich is derived from categorical loudness data of 84 normal-hearing subjects. The model parameters are further set to predict the standard isophones that are in good agreement with the equal loudness level contours derived from categorical loudness data. Also, the model predicts the loudness functions near threshold both for normal and hearing-impaired listeners and can be extended to predict duration-dependent loudness perception. Since categorical loudness can be measured more easily and directly than loudness in sone, the current modelling approach can be experimentally tested and can be used in various applications, such as, e.g. hearing aid processing and fitting procedures. 
2pPPg4. Binaural loudness summation in and out of the laboratory. Mary Florentine (Northeastern University, 360 Huntington Ave - 106A Forsyth Building - SLPA, Institute for Hearing, Speech \& Language, Boston, MA 02115, USA, florentin@neu.edu), Michael Epstein (Northeastern University, 360 Huntington Ave - 106A Forsyth Building - SLPA, Institute for Hearing, Speech \& Language, Boston, MA 02115, USA, m.epstein@ neu.edu)

Textbooks state that a tone presented binaurally is louder than the same tone presented monaurally. This is called Binaural Loudness Summation, BLS. Recent data and classroom demonstrations of BLS using speech stimuli from a visually present talker yield much less BLS than previously reported. Are conclusions about BLS drawn from tones presented via earphones in the laboratory applicable to live- voice speech in a room? To answer this question, eight normal listeners were presented three types of stimuli [monitored live voice (MLV) spondees, recorded spondees, and tones] monaurally and binaurally across a wide range of levels. The same stimuli were presented via earphones and loudspeakers. Loudness was measured using magnitude estimation. Results show that the amount of BLS was significantly less for MLV spondees than for tones or recorded spondees. It was also significantly less for loudspeaker presentation than for earphone presentation. The amount of BLS was least for MLV spondees presented via loudspeakers. Results indicate that BLS in loudspeaker conditions is significantly less than BLS in typical laboratory test conditions using earphones. A new phenomena-called "Binaural Loudness Constancy"-will be described that may result from expectations about loudness of a visually present talker. [Work supported by NIH-NIDCD grant R01DC02241]

\section{6:40}

2pPPg5. Loudness asymmetry ratings between accelerating and decelerating car sounds. Patrick Susini (IRCAM - UMR CNRS 9912, Equipe Perception et Design Sonores, 1, place Igor Stravinsky, 75004 Paris, France, susini@ircam.fr), Stephen McAdams (Centre for Interdisciplinary Research in Music Media \& Technology (CIRMMT) - Schulich School of Music - McGill Univ., 555 Sherbrooke Street West, Montreal, QC H3A1E3, Canada, smc@music.mcgill.ca)

Loudness change has been studied for tones with linearly varying levels revealing an asymmetry depending on the direction of change (increasing vs. decreasing) and the range of levels (high vs. low). Different assumptions were proposed to explain this asymmetry in favour of linearly increasing sounds. Teghtsoonian et al. (2005) and, more recently, Susini et al. (2006) explain that loudness of an increasing sound is influenced by the end level. Neuhoff (1999) describes this result by a survival advantage for detecting an approaching sound source. Whatever the assumption is, the results show that loudness judgments for abstract sounds (1000-Hz tones) and synthetic vowel sounds were significantly higher for increasing ramps. Those results are compared here with continuous and global ratings obtained on everyday sounds such as accelerating and decelerating car sounds with a same duration (43 s), but with different temporal functions and range of levels. Global loudness judgments made after the end of the sound are significantly higher for accelerating than decelerating car sounds, but are judged on average similar using continuous judgments. In addition, an increase in the speed (acceleration) is evaluated primarily in terms of its level at the end. These results confirm the previous studies.

\section{Contributed Papers}

\section{7:00}

2pPPg6. Temporal weighting in loudness judgments of time-varying sounds containing a gradual change in level. Daniel Oberfeld (Dept. of Psychology, Johannes Gutenberg-Universitaet Mainz, Staudingerweg 9, 55128 Mainz, Germany, oberfeld@uni-mainz.de)

Two experiments studied how listeners weight the level information from different portions of a noise consisting of ten temporal segments, the levels of which were sampled independently from a normal distribution. If a gradual increase in level ("fade-in") is imposed on the first few segments in such a sound, temporal weights in global loudness judgments in a oneinterval paradigm show a delayed primacy effect [Oberfeld and Plank, Fortschritte der Akustik - DAGA2005, 227-228 (2005)]. The maximum weight is assigned to the first segment presented at the full level, while the weights assigned to the fade-in segments are close to zero. In Experiment 1, a similar pattern of weights was observed in a two-interval paradigm. Thus, listeners do not use intensity information from the fade-in segments even when making within-trial comparisons. In Experiment 2, the first three segments gradually decreased rather than increased in level (i.e., the first segment had the highest level). Listeners gave near exclusive weight to the first segment. The results are compatible with a tendency to assign greater weight to loud elements [Lutfi and Jesteadt, J. Acoust. Soc. Am. 120, 3853-3860 (2006)] rather than with segmentation of the noise into a "gradually changing" and a "stable" part.

\section{$7: 20$}

2pPPg7. Magnitude judgments of loudness change for discrete, dynamic, and hybrid stimuli. Jesse D. Flint (Binghamton University (SUNY), Department of Psychology, Binghamton, NY 13902-6000, USA, jdflint@binghamton.edu), Richard E. Pastore (Binghamton University (SUNY), Department of Psychology, Binghamton, NY 13902-6000, USA,
pastore@binghamton.edu),Jeremy R. Gaston(Binghamton University (SUNY), Department of Psychology, Binghamton, NY 13902-6000, USA jgaston1@ binghamton.edu)

Recent investigations of loudness change across dynamically varying stimuli have identified differences between increasing versus decreasing power that depends upon stimulus complexity and intensity range [e.g., Canévet, Acustica, 62, 2136-2142 (1986); Neuhoff, Nature, 395(6698), 123 124, (Sep 1998)]. Some explanations of these findings are based upon known psychophysical effects [Teghtsoonian, Teghtsoonian \& Canévet, Perception \& Psychophysics, 67(4), 699-712 (2005)], whereas others posit qualitative differences in the perception of discrete laboratory and dynamic natural sound structures (Neuhoff, 1998). For discrete, static stimuli, the relationship between loudness and power is dependant upon a number of factors, including frequency, intensity range, perceptual anchoring, temporal order, and integration of power across time. The current investigation examines listener perception of loudness for sound structures that vary systematically from discrete to dynamic changes in power, allowing evaluation of the contributions of static versus dynamic change, as well as other factors in the perception of loudness change within complex stimuli. Relevant methodology, as well as commonalities and differences with predictions of traditional psychophysics, are discussed.

$$
7: 40
$$

2pPPg8. Loudness of impulsive sounds as a function of duration: a parametric study of the effect of level and frequency. Sabine Meunier (CNRS-LMA, 31, chemin Joseph Aiguier, 13402 Marseille, France, meunier@lma.cnrs-mrs.fr), Guy Rabau (CNRS-LMA, 31, chemin Joseph Aiguier, 13402 Marseille, France, rabau@1ma.cnrs-mrs.fr)

Loudness of brief signals increases when signal duration increases up to about $100 \mathrm{~ms}$, this increase is called temporal summation. The question examined in this study is to determine how loudness change as a function of 
duration. Literature shows different results that lead to different conclusions: when duration increases (i) the loudness increases in direct proportion to energy (intensity x duration); (ii) the loudness increases faster than energy; (iii) the loudness increases slower than energy. We have shown, in previous experiments, that loudness change with duration can follow either (i) or (ii) depending on signal level. The present study provides a parametric exami- nation of this question. Loudness was measured for signals whose energy was kept constant while the duration was varied. The parameters of the experiment were the signal frequency and level. The results allow us to explain part of the discrepancies found in literature. Moreover, the results of this study are used into a model of impulsive sounds that calculate with a good precision the loudness of environnemental short-duration sounds.

\title{
Session 2pSAa
}

\section{Structural Acoustics and Vibration and EURONOISE: Acoustic Imaging in Confined Space I}

\author{
Earl G. Williams, Cochair \\ Naval Research Laboratory, 4555 Overlook Ave, Washington, DC 20375, USA
}

Alexandre Garcia, Cochair

CNAM, 292 rue Saint Martin, Paris, 75003, France

\section{Invited Papers}

\section{2:00}

2pSAa1. Analysis of enclosed sound fields using spherical microphone array processing. Boaz Rafaely (Ben-Gurion University of the Negev, Department of Electrical and Computer Engineering, 84105 Beer-Sheva, Israel, br@ee.bgu.ac.il)

Enclosed sound fields in rooms and auditoria are characterized by multiple reflections in addition to the direct sound. The spatial and temporal manner in which sound propagates from the sound source to a listener determines the acoustics of the enclosed space. Recent studies showed that spherical microphone arrays and associated array processing can be used effectively in the experimental study of the acoustics of auditoria. The main advantages presented are the true three-dimensional analysis and the effectiveness of array processing in the spherical harmonics domain. Spherical microphone array design issues will first be reviewed in this work, in particular concerning the design of open-sphere arrays useful in room acoustics analysis with high spatial resolution. Then, beam-forming methods will be presented which can facilitate identification of individual room reflections, and computation of directional room impulse responses. Finally, the use of optimal array processing methods will be presented which facilitates improved sound field analysis.

$$
\text { 2:20 }
$$

2pSAa2. Resolution improvement of beamformers using spherical microphone array. Jean-Claude Pascal (ENSIM - LAUM, Université du Maine, rue Aristote, 72085 Le Mans, France, Jean-Claude.Pascal@ univ-lemans.fr), Jing-Fang Li (Visual VibroAcoustics, 51 rue d'Alger, 72000 Le Mans, France, jingfang.li@visualvibroacoustics.com)

Spherical microphone array has been studied for various applications. In enclosed space, spherical array eliminates forwardbackward grating lobes occurring in two-dimensional arrays. Beamforming and spherical harmonic decomposition are both used to map the distribution of source strength. The two approaches show similar performance at frequencies where the upper spherical harmonic order equals the product of the wave number and sphere radius. However, at lower frequencies, processing using spherical harmonics maintains the same directivity while the spatial resolution for delay-and-sum beamformer deteriorates. It has been shown that the scattering of sound by a rigid sphere improves the directivity of the same open sphere microphone beamformer, by increasing the path length travelled by incident sound. This paper concerns focused beamformer using rigid and open sphere. The concept of statistically optimized array processing (SOAP) is applied to adapt to a particular interior geometry, considering the scattered field in case of rigid sphere. The regularization process makes it possible to use a model of partially coherent sound field in the enclosed space. A deconvolution post-processing based on the knowledge of the actual steering vector leads to an efficient and robust solution to improve considerably the resolution of the source strength distribution.

\section{2:40}

2pSAa3. Measurement of low-frequency sources in non-anechoic room using near-field acoustic holography. Manuel Melon (CNAM, 292 rue Saint Martin, 75003 Paris, France, melon@cnam.fr), Christophe Langrenne (CNAM, 292 rue Saint Martin, 75003 Paris, France, christophe.langrenne@cnam.fr), Alexandre Garcia (CNAM, 292 rue Saint Martin, 75003 Paris, France, garcia@cnam.fr), Philippe Herzog (Laboratoire de Mécanique et d'Acoustique - CNRS, 31 chemin Joseph Aiguier, 13402 Marseille, France, herzog@1ma.cnrs-mrs.fr)

Low frequency $(20 \mathrm{~Hz}-200 \mathrm{~Hz})$ measurements of sound sources are rather difficult to perform because free field conditions can not be easily achieved properly. Moreover, some industrial sources have to be measured in situ. In such a case, a Field Separation Method (FSM) can be used to subtract the pressure field reflected by walls of the testing room from the measured data. This approach required the knowledge of both acoustic pressure and velocity on a closed surface surrounding the source. In this paper, a spherical harmonic expansion of measured data is used to solve the problem. The proposed method is applied to the measurement of the frequency response 
of a closed box subwoofer tested under various conditions: in a room with variable reverberation time (6.4 s to $0.6 \mathrm{~s})$. Theoretical frequency response of the subwoofer is also calculated using the Thiele and Small model. Results show a good agreement between separated data and simulations. The influences of the measurement distance and of the measurement point number required on the separation process are discussed.

2pSAa4. Vector intensity reconstructions in a volume surrounding a rigid spherical measurement array. Earl G. Williams (Naval Research Laboratory, 4555 Overlook Ave, Washington, DC 20375, USA, earl.williams@nrl.navy.mil), Kazuhiro Takashima (Nittobo Acoustic Engineering Co., Ltd., 1-21-10, Midori, Sumida-Ku, 130-0021 Tokyo, Japan, taka@ noe.co.jp)

An approach is presented that provides a prediction of the vector intensity field throughout a volume exterior to a rigid spherical measurement array consisting of 31 flush mounted microphones. The theory is based on spherical harmonic expansions of the measured field with the radial variation of the near-field pressure obtained using the Greens function with vanishing normal derivative at the rigid sphere surface. Experimental results with rigid spherical arrays of differing radii are presented using multiple incoherent sources. Successful intensity reconstructions are obtained over a volume three times the sphere radius up to a frequency of $1.5 \mathrm{kHz}$ that clearly reveal the locations and levels of the two sources. This volumetric intensity probe is very similar mathematically to one described recently by the author (EGW) that used 50 microphones in an open array. The latter was used successfully inside an aircraft cabin in flight to uncover sources of noise. This work was supported by the US Office of Naval Research and Nittobo Acoustic Engineering Co. Ltd.

3:20

2pSAa5. A boundary element method for near-field acoustical holography in bounded noisy environment. Christophe Langrenne (CNAM, 292 rue Saint Martin, 75003 Paris, France, christophe.langrenne@cnam.fr), Manuel Melon (CNAM, 292 rue Saint Martin, 75003 Paris, France, melon@cnam.fr), Alexandre Garcia (CNAM, 292 rue Saint Martin, 75003 Paris, France, garcia @cnam.fr)

This paper presents a boundary element method to recover free field conditions from noisy bounded space situations. The proposed approach is based on the Helmholtz integral formulation and requires the knowledge of double layer pressure fields on two parallel closed surfaces surrounding the source. First, the outgoing and ingoing pressure fields are separated. Then, the incident field scattered by the tested source is subtracted from the outgoing field to estimate the pressure field which would have been radiated in free field. The method had been numerically tested and an experimental example is given here. The source is a rectangular box with seven loudspeakers mounted on it driven by bandwith limited white noise. The source is put at $0.4 \mathrm{~m}$ from the ground of a semi-anechoïc room. The ground plays a disturbant role because it produces secondary sources. The results show the effectiveness of the method particularly at frequencies where stationary waves between the ground and the underside of the box exist.

3:40-5:20 Posters

Lecture sessions will recess for presentation of poster papers on various topics in acoustics. See poster sessions for topics and abstracts.

\section{Invited Papers}

$5: 20$

2pSAa6. Acoustically invisible cylinder. Yuri Bobrovnitskii (Mechanical Engineering Research Institute, 4, M. Kharitonievky Str., 101990 Moscow, Russian Federation, yuri@imash.ac.ru)

Coatings of new type recently proposed by the author (Acoustical Physics, 2007, vol. 53, N5, pp. 535-545) are applied to bodies of cylindrical geometry to reduce reflection or scattering of sound and thus to make them undetectable by imaging systems. Such a coating, called as a coating with extended reaction, represents a periodic set of small elements with coupling between the neighboring elements. Appropriate choice of the coupling parameters makes its efficiency much higher than that of commonly used coatings. In the present paper, it is shown by computer simulation that a rather simple coating of this type can reduce the back-scattered pressure amplitude more than $40 \mathrm{~dB}$ (with respect to the rigid cylinder) practically at all frequencies. Considerable reduction of the scattered power can also be achieved in a low frequency range. The width of this range and the reduction index depend on the number of couplings introduced into the coating.

\section{$5: 40$}

2pSAa7. Acoustic source identification in an enclosed space using the inverse phased beam tracing at medium frequencies. Jeong-Guon Ih (KAIST, Dept. of Mechanical Engineering, Science Town, 305-701 Daejeon, Republic of Korea, J.G.Ih@kaist.ac.kr), Cheol-Ho Jeong (Acoustic Technology, DTU Elektro, Technical University of Denmark, Building 352, Ørsted plads, DK-2800 Kgs. Lyngby, Denmark, chj@oersted.dtu.dk)

When the source is enclosed by room surfaces, it is not easy, though not totally impossible, to apply conventional modal methods for source identification. This is because there are too many complicated wave interferences and effects of wall impedance, in particular at medium frequencies. The phased beam tracing method was suggested as a fast and efficient acoustic simulation tool at the medium frequencies in an enclosure, which overcomes the defects of geometrical acoustics techniques. In this study, the phased beam tracing method, implemented in its inverse form, was applied to the identification of the acoustic sources inside a chamber. In the simulation 
tests, spherical and rectangular shaped sources operating in a room were taken as examples. When the source pressure distribution was reconstructed by this numerical inverse technique, the sound power spectrum radiated from the source could be estimated by eliminating the contribution from surface reflections. Reconstruction error was investigated by the Monte Carlo simulation in terms of the signalto-noise ratio.

\section{Contributed Papers}

\section{6:00}

2pSAa8. Noise source characterisation using patch impedance technique. G Pavić (INSA de Lyon - LVA, Bâtiment St. Exupéry, 25 bis avenue Jean Capelle, F-69621 Villeurbanne Cedex, France, goran.pavic @insa-lyon.fr), Nicolas Totaro (INSA de Lyon - LVA, Bâtiment St. Exupéry, 25 bis avenue Jean Capelle, F-69621 Villeurbanne Cedex, France, nicolas.totaro@insa-lyon.fr)

A novel approach of an environment-independent sound source characterisation is discussed. The source is defined via a suitable enveloping interface surface by its blocked sound pressure and its surface impedance. Both the blocked pressure and the impedance are discretised using the averaging patch concept. Such a definition avoids singularity of point acoustic impedance and is suitable for numerical as well as experimental implementation. The characterisation of a source by the patch concept allows for the acoustical sub-structuring, which in turn enables the prediction of the sound field created by the source coupled to an arbitrary environment. Numerical simulations are presented which demonstrate the feasibility of the approach. It is hoped that the proposed approach can serve as a universal tool for noise synthesis of complex equipment incorporating noise sources.

\section{6:20}

2pSAa9. Local patch acoustic holography methods in enclosed spaces. Zdenek Havranek (Brno University of Technology, UAMT-FEEC, Kolejni
4, 61200 Brno, Czech Republic, havranek@feec.vutbr.cz),Ludvik Bejcek (Brno University of Technology, UAMT-FEEC, Kolejni 4, 61200 Brno, Czech Republic, bejcek@feec.vutbr.cz)

The paper compares reconstruction accuracy and computational cost of three patch acoustic holography algorithms applicable in the enclosed spaces for prediction of sound field near sources. Algorithms under investigation were DL-SONAH, IBEM and classical NAH with spatial transformations and hologram aperture enlargement. All of the selected algorithms take an advantage of using double layer microphone array for measurement of sound pressure field. The reconstruction accuracy of all algorithms is determined by using of simple 3D model of curved radiating surface on the basis of calculation of difference between true acoustic quantity (pressure, velocity or intensity) values very close to the surface and predicted sound field at same positions. All these methods use different calculation procedure to obtain predicted sound field near source surface, thus comparison of effectiveness of these algorithms including prediction accuracy-to-computational cost ratio is useful to determination their applicability in practice. Results of prediction error and calculation time including regularization with different parameter choice methods (L-curve, GCV, etc., are presented and compared. Initial comparison of the selected methods confirms shorter calculation time in non-element based methods, especially in NAH with transformations, while reconstruction error is in the same order.

\section{Invited Papers}

\section{6:40}

2pSAa10. Identification of source velocities with Inverse Patch Transfer Functions method. Mathieu Aucejo (INSA de Lyon LVA, Bâtiment St. Exupéry, 25 bis avenue Jean Capelle, F-69621 Villeurbanne Cedex, France, mathieu.aucejo@insa-lyon.fr), Nicolas Totaro (INSA de Lyon - LVA, Bâtiment St. Exupéry, 25 bis avenue Jean Capelle, F-69621 Villeurbanne Cedex, France, nicolas.totaro@insa-lyon.fr), Jean-Louis Guyader (INSA de Lyon - LVA, Bâtiment St. Exupéry, 25 bis avenue Jean Capelle, F-69621 Villeurbanne Cedex, France, jean-louis.guyader@insa-lyon.fr)

The identification of source velocities remains an important problem in noise control. For this purpose, several methods were developed such as Near-field Acoustic Holography (NAH) or inverse Boundary Elements Method (iBEM). An alternative method, based on the double measurement of pressure and particle velocity fields surrounding the source is presented. This method has been developed in the SILENCE European project framework. In this method, called inverse Patch Transfer Functions method (iPTF), measurement and identification surfaces are divided into elementary areas called patches. Then, source velocities are computed from acoustic field and inversion of impedances matrices obtained by FEM. Theoretically, this method presents two main advantages: it can be applied to sources with complex 3D geometries and measurements can be carried out in a non-anechoic environment, even in the presence of other sources. In the present paper, theoretical background of iPTF is exposed and results are presented on a source with simple geometry (an L-shaped plate) and discussed.

2pSAa11. Detection of acoustic radiating areas of a generic helicopter cabin by beamforming. Gérard Martin (ONERA, 2 avenue Edouard Belin, BP 4025, 31055 Toulouse, France, Gerard.Martin@ onecert.fr), Frank Simon (ONERA, 2 avenue Edouard Belin, BP 4025, 31055 Toulouse, France, Frank.Simon@onecert.fr), Daniel Biron (ONERA, 2 avenue Edouard Belin, BP 4025, 31055 Toulouse, France, Daniel.Biron@onecert.fr)

Improvement of the helicopter internal noise is essential to decrease the fatigue effects of passengers. This requires having a metrological tool, able to give information on acoustic radiating areas in cabin in order to target appropriate acoustic passive or active solutions. In this context, the authors have shown, in previous papers, capability of modified beamforming using a cross-shaped array of microphones, associated with an acoustic mask, to localize isolated sources in a generic composite helicopter cabin (VASCo), thus, in spite of free field's hypothesis. The present paper deals with the feasibility of this measurement method to identify main acoustic pressure areas radiated by an helicopter mechanical deck whose vibration is generated by gear box beams between 800 and $5000 \mathrm{~Hz}$. This configuration is performed, thanks to simulations and experiments on a sandwich composite panel of VASCo, excited by 4 cor- 
related shakers. It appears that beamforming can be used to identify main acoustic area coming from the radiating of many coupled vibration modes. Nevertheless, the dynamic range decreases with the number of pressure anti-nodes and it is necessary to introduce an inverse method to reject the ghost images and to reconstruct amplitude and phase of synthesized sources.

\title{
Contributed Papers
}

$$
7: 20
$$

2pSAa12. 3D localization of acoustic sources with a spherical array. Karim Haddad (Brüel \& Kjær Sound \& Vibration Measurement A/S, Skodsborgvej 307, DK-2850 Nærum, Denmark, khaddad@bksv .com), Jørgen Hald (Brüel \& Kjær Sound \& Vibration Measurement A/S, Skodsborgvej 307, DK-2850 Nærum, Denmark, JHALD@bksv.com)

This paper describes a technique dedicated for the localization of acoustic sources in all directions and in the far-field. Classical beamforming techniques based on planar arrays provides an acoustic map restricted to a limited aperture, but a spherical array does not have such a limitation since there is no preferential direction. In this processing called Spherical Harmonics Beamforming, the sound field on the sphere is decomposed with spherical harmonics functions, and then a corrected summation gives the acoustic contribution from a given direction. We have used a rigid spherical array, which has the advantage that cabling of microphones and integrated cameras can be hidden inside the sphere. A rigid surface also provides better numerical stability in connection with Spherical Harmonics Beamforming. In this study, this technique is evaluated in connection with the resolution and the dynamic range. Simulated and experimental results are presented.

$$
7: 40
$$

2pSAa13. Near-field Acoustic Holography for partial measurements inside complex structures. Nicolas Valdivia (Naval Research Laboratory,
4555 Overlook Ave, Washington, DC 20375, USA, valdivia@pa .nrl.navy.mil), Earl G. Williams (Naval Research Laboratory, 4555 Overlook Ave, Washington, DC 20375, USA, earl.williams@nrl.navy.mil)

Near-field acoustical holography (NAH) requires the measurement of the near-field pressure field over a closed surface in order to recover the acoustic field on a nearby conformal surface. Very often we encounter applications where pressure measurements are available only over a patch of the measurement surface. In these cases the strict NAH theory does not hold, but still there are techniques that have been developed to overcome this difficulty. The best-known technique for planar surfaces is patch NAH and, recently proposed, for arbitrarily shaped surfaces patch IBEM and patch ESM. It was found in a recent study by the authors that these techniques will be affected by the problem of back-source contamination for interior NAH, but it was showed that this problem could be overcome by the use of "Cauchy" measurements. In this work we will compare the reconstruction of the acoustic field from patch based techniques with the technique that uses Cauchy measurements, and discuss the problem of back-source contamination in more detail. We use a cylindrical surface excited by a point force as an example to validate our results. This work was supported by the US Office of Naval Research.

\section{Session 2pSAb}

\section{Structural Acoustics and Vibration and EURONOISE: Source Characterization in Structure Borne Noise Problems II (Poster Session)}

\author{
Evan Davis, Cochair \\ The Boeing Company \\ Charles Pezerat, Cochair \\ Laboratoire Vibrations Acoustique - INSA Lyon
}

\begin{abstract}
All posters will be on display from 3:40 p.m. to 5:20 p.m. To allow contributors an opportunity to see other posters, contributors of odd-numbered papers will be at their posters from 3:40 p.m. to 4:30 p.m. and contributors of even-numbered papers will be at their posters from 4:30 p.m. to 5:20 p.m.
\end{abstract}

\section{Contributed Papers}

2pSAb1. The significance of cross-order terms in interface mobilities for structure-borne sound source characterization. Hannes A. Bonhoff (Berlin Institute of Technology, Einsteinufer 25, TA7, 10587 Berlin, Germany, hannes.bonhoff@tu-berlin.de), Björn A. Petersson (Berlin Institute of Technology, Einsteinufer 25, TA7, 10587 Berlin, Germany, b.a.t .petersson@tu-berlin.de)

For the characterization of structure-borne sound sources and the description of the associated transmission process, the source descriptor and coupling function were introduced. The concept of source descriptor and coupling function can be reformulated by incorporating the interface mobilities. The applicability for source-receiver assemblies with a multipoint or continuous connection is thereby granted. The accuracy of the results and consequently the validity of this approach, however, depends on the significance of the so-called cross-order terms. Such cross-order terms consist of force orders and cross-order interface mobilities. In recent theoretical and experimental work, the influence of cross-order interface mobilities as well as the distribution of force-orders have been investigated. Based on this knowledge, the significance of the cross-order terms is assessed in the present contribution. 
2pSAb2. Measurement of force and moment mobilities using a finite difference technique. Andrew Elliott (University of Salford, Acoustic Research Centre, Newton Building, M5 4WT Salford, UK, a.s.elliott @ pgr.salford.ac.uk), G Pavić (INSA de Lyon - LVA, Bâtiment St. Exupéry, 25 bis avenue Jean Capelle, F-69621 Villeurbanne Cedex, France, goran.pavic@insa-lyon.fr), Andy T. Moorhouse (University of Salford, Acoustic Research Centre, Newton Building, M5 4WT Salford, UK, a.t .moorhouse@salford.ac.uk)

Measuring only force mobilities it is possible to derive a moment mobility without the need for an externally applied moment. The method uses finite differences of both forces and velocities about a central point. In this way pure forces and moments can be extracted as can pure velocities and angular velocities. There are, however, errors associated with finite difference techniques. In this case the purity of the extracted quantities depends on the area over which they are measured and its significance in terms of wavelength. Meanwhile experimental errors such as noise dictate that the area is sufficiently large to provide differences which are incorruptible. The paper aims to quantify the finite difference error in a general way free of situation specifics.

TUESDAY AFTERNOON, 1 JULY 2008

P2-B, LEVEL 2, 3:40 TO 5:20 P.M.

\title{
Session 2 pSAc
}

\section{Structural Acoustics and Vibration and EURONOISE: Acoustic Imaging in Confined Space II (Poster Session)}

\author{
Earl Williams, Cochair \\ Naval Research Laboratory \\ Alexandre Garcia, Cochair \\ CNAM
}

\begin{abstract}
All posters will be on display from 3:40 p.m. to 5:20 p.m. To allow contributors an opportunity to see other posters, contributors of odd-numbered papers will be at their posters from 3:40 p.m. to 4:30 p.m. and contributors of even-numbered papers will be at their posters from 4:30 p.m. to 5:20 p.m.
\end{abstract}

\section{Contributed Papers}

2pSAc1. Experimental feasibility of in-duct sound source reconstruction. Teresa Bravo (Université de Technologie de Compiègne, Centre de Recherche Royallieu, BP20529, 60205 Compiègne, France, teresa.bravo-maria@utc.fr), Cédric Maury (Université de Technologie de Compiègne, Centre de Recherche Royallieu, BP20529, 60205 Compiègne, France, cedric.maury@utc.fr)

Due to the expected air transport growth and stringent environmental regulations, there is a strong need to develop noise reduction techniques at acceptable cost in the aeronautical sector. A sound characterisation of the aero-acoustic sources in the nacelle acoustic duct problem plays a crucial role. In this study, it is shown how the liners optimized impedances strongly depend on the noise source characteristics under both tonal and broadband excitation conditions, and in the latter case, for varying degree of correlations between the random source strengths. The limitations for source identification methods, such as pointwise model-based inverse techniques, have been studied. These methods are able to provide reliable models of equivalent sound sources from a limited number of in-duct measurements, but require a priori knowledge of the source location. The performance of such techniques are compared with two different approaches, namely the use of focussed in-duct beamforming techniques to locate the unknown sources, and a decomposition of the assumed source strengths into angular Fourier series for both the location and the determination of the source amplitudes. Experimental results are presented for the location and the reconstruction of the particle velocity spectrum of wall-mounted compression drivers from induct measurements.

2pSAc2. Vector intensity measurement with a rigid spherical microphone array in a vehicle cabin. Kazuhiro Takashima (Nittobo Acoustic Engineering Co., Ltd., 1-21-10, Midori, Sumida-Ku, 130-0021 Tokyo, Japan, taka@noe.co.jp), Hiroshi Nakagawa (Nittobo Acoustic
Engineering Co., Ltd., 1-21-10, Midori, Sumida-Ku, 130-0021 Tokyo, Japan,nakagawa@noe.co.jp), Earl G. Williams (Naval Research Laboratory, 4555 Overlook Ave, Washington, DC 20375, USA, earl.williams@nrl.navy .mil)

Measurement results showing intensity vector reconstructions using a rigid spherical microphone array in a vehicle cabin are presented. The measurement equipment developed by Nittobo was used in previous demonstrations to visualize the acoustic field using a beamforming technique superimposed on a picture from an on-board camera. In the present study intensity reconstructions are shown and are successful in locating and quantifying sources, demonstrating the usefulness of this technique in an enclosed space like a vehicle cabin, aircraft cabin, small room, etc. This work was supported by the US Office of Naval Research and Nittobo Acoustic Engineering Co. Ltd.

2pSAc3. An improved method of visualizing the energy flow: the Rayleigh wave. Cleon E. Dean (Physics Department, Georgia Southern University, P. O. Box 8031, Statesboro, GA 30460-8031, USA, cdean @ GeorgiaSouthern.edu), James P. Braselton (Department of Mathematical Sciences, Georgia Southern University, P.O.B. 8093, Statesboro, GA 304608093, USA, jbraselton@GeorgiaSouthern.edu)

A variety of ways of visualizing the energy flux or elastodynamic Poynting vector field are demonstrated including gridded vector field, color coding, and other methods for the simple example of a Rayleigh wave. In particular an improved version of a technique shown at the 8th International Conference on Theoretical and Computational Acoustics, ICTCA 2007 is demonstrated [David M. F. Chapman, "Visualizing acoustic energy flow into the seabed using energy streamlines," Eighth International Conference on Theoretical and Computational Acoustics, ICTCA 2007, Heraklion, Crete, GREECE, 2-5 July 2007.]. The improved streamline method shows both the direction and the intensity of the energy flow. 


\title{
Session 2pSAd
}

\section{Structural Acoustics and Vibration and EURONOISE: Vibration and Radiation from Complex Structural Systems III (Poster Session)}

\author{
David Feit, Cochair \\ Applied Physical Sciences Corp. \\ Jean-Louis Guyader, Cochair \\ INSA de Lyon - LVA
}

\begin{abstract}
All posters will be on display from 3:40 p.m. to 5:20 p.m. To allow contributors an opportunity to see other posters, contributors of odd-numbered papers will be at their posters from 3:40 p.m. to 4:30 p.m. and contributors of even-numbered papers will be at their posters from 4:30 p.m. to 5:20 p.m.
\end{abstract}

\section{Contributed Papers}

2pSAd1. Implementation of the high order asymptotic models of linings and coatings to the SAW spectra evaluation. Dmitry Zakharov (LMP, UMR CNRS 5469, Université Bordeaux I, 351, cours de la Libération, 33405 Talence, France, dmitrii.zakharov@gmail.com), Alexander Shuvalov (LMP, UMR CNRS 5469, Université Bordeaux I, 351, cours de la Libération, 33405 Talence, France, a.shuvalov@1mp.u-bordeaux1 .fr), Olivier Poncelet (LMP, UMR CNRS 5469, Université Bordeaux I, 351, cours de la Libération, 33405 Talence, France, o.poncelet@1mp.ubordeaux1.fr)

High order asymptotic models of the relatively thin linings and coatings are used to reduce the problem dimension when dealing with a reasonable frequency range. The essential point consists in the use of asymptotic expansion for the internal stress and strain state of a laminate, subjected to the tight conditions at least on one of its faces, i.e., the conditions formulated for the displacements. The respective algorithm, based on it, is shorter than for the direct evaluation of the SAW spectra. It can be implemented to the calculation of Rayleigh waves or Lamb waves in layered media. Numerical examples are presented with both asymptotical and numerical evaluation of error.

2pSAd2. Plate Sound Radiation Prediction in Machines Using Multiple Input Techniques. Armand Nejade (Institut National de Recherche et de Sécurité (INRS), Ave. de Bourgogne, B.P. 27, F-54501 Vandoeuvre Cedex, France, armand.nejade@inrs.fr)

In systems with various types of noise sources, the characterization and ranking of each source may require a different approach. Also, one of the main challenges in such investigations, consists of isolating each source from the influence of the others. Often in the machines, the constituting plates are efficient radiators whose specific contributions need to be properly determined. In this presentation, we describe a method of plate radiation characterization using multiple input techniques. These techniques have frequently been used in case of discrete sources such as, for instance, vehicle engine cylinders where the pressure variations are modelled as the inputs. This application differs from the traditional ones by the fact that the inputs are now, the elements of a continuous medium. Here the surface of a plate is meshed, each element is taken as a sub-source and consequently, the plate is modelled as an assembly of sub-sources. The plate contributions at the operational modes, are then obtained through vibration measurements and therefore, are not affected by the noise from the other sources. Investigations on structures of various geometries and materials have so far, demonstrated the efficiency of this type of application.
2 pSAd3. Forced vibrations of a cylindric panel with regular orthogonal system of stiffeners. Leonid A. Lazarev (Central Aerohydrodynamic Institute (TsAGI), 17, Radio Str., 105005 Moscow, Russian Federation, leonid174@mail.ru), Boris M. Efimtsov (Central Aerohydrodynamic Institute (TsAGI), 17, Radio Str., 105005 Moscow, Russian Federation, efimtsov @ prob-lab.ru)

Forced vibrations of a cylindrical panel freely supported in contour, with regular orthogonal system of stiffeners (typical of aircraft fuselage structure) are investigated. The connection of all three components of displacement of the skin and of discrete stiffeners as well as of the respective elastic and inertial forces and moments are taken into account in the case of excitation by normal and tangential forces. This connection can be described correctly at joint application of the recreptance method and the method of spaceharmonics. The task of forced vibrations is solved directly except the procedure of solving the task in terms of eigen-values. Some illustrative examples of the aircraft fuselage panel by a concentric harmonic force are presented. They demonstrate a high efficiency of applying this combined method. Potential possibilities of using this combined method for solving the tasks related to vibrations of a framed skin modeling the aircraft fuselage section and to the sound field in the volume bounded by it are considered.

2pSAd4. Optimisation of building vibration isolation by means of numerical methods. Fülöp Augusztinovicz (Budapest University of Technology and Economics, BME Dept. of Telecommunications, Magyar tudósok körútja 2, H-1117 Budapest, Hungary, fulop@ hit.bme.hu)

Ground-borne vibrations pose a steadily increasing environmental problem in densely populated areas of large cities. Although not the cheapest, the application of resilient elements in the construction of new buildings is an effective and viable method. Obviously, experimentation with parameters and placement of the isolators is not possible in this case. Therefore, a careful and watchful optimization of the static and dynamic characteristics of the supporting elements is a must. The proposed paper gives an overview of the vibration isolation design and reports on the expected effect of some basic design versions of a typical multi-storey building. Standard structural finite element method is used as a basic design tool, but a number of vibroacoustic aspects are also considered and treated. Experimental results as well as comparisons of measurements and predictions will be reported too. 
2pSAd5. Refracto-vibrometry - a novel method for visualizing sound waves in transparent media. Lothar Zipser (HTW University of Applied Sciences, Friedrich-List-Pl. 1, D-01069 Dresden, Germany, zipser @et.htw-dresden.de), Heinz H. Franke (HTW University of Applied Sciences, Friedrich-List-Pl. 1, D-01069 Dresden, Germany, zipser@et.htwdresde.de)

For optimization of new sonic techniques, for development of acoustic sensors or for understanding of complex sonic phenomena it is often desirable to visualize the generation and distribution of the invisible sound waves. A novel method for measuring and visualizing the sound waves in transparent media was developed and tested by the authors. This non-contact method is based on a scanning laser-Doppler vibrometer. It can be used for measuring and visualizing sound fields in gases, fluids and even pellucid solid objects. The variation of the optical refractive index $n$ of transparent media, caused by the sound waves is used as measuring effect. This justifies the denomination "refracto-vibrometry". The paper describes the theoretical fundamentals and the technical equipment required for the novel method. As examples, the generation and radiation of sound in air, water and transparent solid objects are presented. The animation of the results as videos is very impressive and instructive. In research, education and system design refracto-vibrometry has a large potential to make acoustics clearer and more feasible for application.

2pSAd6. Influence of the pre-stress state on the wave propagation in a shearable Timoshenko beam. Nicolas Bideau (Institute of Mathematical Research of Rennes IRMAR, Campus de Beaulieu, 35042 Rennes, France, nicolas.bideau@univ-rennes1.fr), Polynice Eyi-Assoumou (Institut de Recherche Mathématique de Rennes, Université de Rennes 1, Campus de Beaulieu, 35042 Rennes, France, polynice.eyi-assoumou @univ-rennes1.fr), Loic Le Marrec (Institute of Mathematical Research of Rennes IRMAR, Campus de Beaulieu, 35042 Rennes, France, loic.lemarrec@univ-rennes1.fr), Lalaonirina Rakotomanana (Institut de Recherche Mathématique de Rennes, Université de Rennes 1, Campus de Beaulieu, 35042 Rennes, France, lalaonirina.rakotomanana-ravelonarivo @ univ-rennes1.fr)

It is very common to use pre-stressed beam models in the structure design. However in the nonlinear domain, the modal analysis remains difficult especially when shearability is taken into account. This work aims to study the natural vibrations of pre-stretched nonlinear shearable Timoshenko beam using Cosserat continuum mechanics. In this paper, a three dimensional nonlinear beam model is first developed. Then we analyse waves which are superimposed on a finite pre-stretch state. Namely, analytical dispersion relations are given for different values of pre-strech. The complete spectrum, including propagating and evanescent traction/compression, shear and bending modes, of a pre- stretched shearable beam is calculated through dispersion pattern. Spectrum strongly depends on initial pre-stress value. A significant qualitative and quantitative difference with the classical Timoshenko beam theory is pointed out even for small pre-stretch values.

2pSAd7. Temporal prediction of the acoustic radiation of vibrating structures: subjective evaluation of a simplified approach. Nacer Hamzaoui (LVA, INSA de Lyon, Bat. Saint-Exupéry, 25 bis avenue Jean Capelle, $69621 \quad$ Villeurbanne, France, nacer.hamzaoui @ insa-lyon.fr), Juliette Pierangelo (LVA, INSA de Lyon, Bat. SaintExupéry, 25 bis avenue Jean Capelle, 69621 Villeurbanne, France, juliette.pierangelo@insa-lyon.fr), Antoine Levy (LVA, INSA de Lyon, Bat. Saint-Exupéry, 25 bis avenue Jean Capelle, 69621 Villeurbanne, France, antoine.levy@insa-lyon.fr)

The goal of the approach presented in this paper is to predict the noise radiated by vibrating structures with acquired vibratory measurements, in the temporal field, on their surface. In the frequential field this prediction is based on the resolution of the Kirchhoff integral equation, which requires a lot of computing time and is thus limited to low and intermediate frequencies; the boundary element method (BEM) is used for the resolution. Simplifications were already brought to low $(<$ Flim1) and to high $(>$ Flim2) frequencies, leading to a simple calculation of a fast integral. The limiting frequencies (Flim1 and Flim2) depend on the structure dimensions and its position towards the observation point. In this paper we will present an approach developed in the temporal field in which a complete calculation (BEM) is avoided by choosing a minimal distance in order to have Flim1 = Flim2 and use only the simplified calculations. The vibratory data is measured in the temporal field and the studied structure is a parallelepipedic box excited by two mechanical excitation pots which excite one or two faces. The acoustic pressures calculated in the temporal field will be confronted with measurements, by using similarity subjective tests and integrating a variation of the principal parameters intervening in this approach.

2pSAd8. Radiation efficiency of natural modes of plates with beam stiffeners. Andreas Rousounelos (Loughborough University, Department of Aeronautical and Automotive Engineering, Ashby Road, LE11 3TU Loughborough, UK, a.rousounelos@lboro.ac.uk), Stephen J. Walsh (Loughborough University, Department of Aeronautical and Automotive Engineering, Ashby Road, LE11 3TU Loughborough, UK, S.J.Walsh@lboro.ac.uk), Victor V. Krylov (Loughborough University, Department of Aeronautical and Automotive Engineering, Ashby Road, LE11 3TU Loughborough, UK, V.V.Krylov@lboro.ac.uk)

In this paper, the radiation efficiency of the natural modes of finite plates with attached beams is considered. Firstly, the scattering of flexural waves, by the beams and the boundaries, in the plate is considered. The reflection and transmission coefficients along with the near-field coefficients of the beam are used to calculate the vibration field of the plate, by taking into account an infinite number of reflections from the boundaries. A wavenumber transform of the vibration velocity field is used to calculate the sound power and the radiation efficiency. Numerical results are presented, for a number of attached beams, and show that the beams introduce ripples in the radiation efficiency curve, below a certain frequency. The amplitude of the ripples is controlled by the mass and the torsional stiffness of the attached beams whereas the stiffness of the beam has little influence. The results are compared with measurements of the radiation efficiency of plates with attached beams.

2pSAd9. Virtual statistical energy analysis for vibroacoustic industrial prediction. Gérard Borello (InterAC, 10 impasse Borde-Basse, ZA. La Violette, 31240 L'Union, France, gerard.borello@interac.fr), Laurent Gagliardini (PSA Peugeot Citroën, Route de Gisy, 78943 VélizyVillacoublay Cedex, France, laurent.gagliardini@mpsa.com), Denis Thenail (PSA Peugeot Citroën, Route de Gisy, 78943 Vélizy-Villacoublay Cedex, France, denis.thenail@mpsa.com)

In the mid-frequency range $(200-2000 \mathrm{~Hz})$, difficulty is encountered when modeling car body vibroacoustic interactions, mainly due to the complexity of automotive design. Analytical Statistical Energy Analysis (ASEA) is efficient to bring to the fore regions of interest regarding NVH design but was proven to be accurate only above $2000 \mathrm{~Hz}$. To overcome ASEA limitations at lower frequencies, Virtual SEA (VSEA) technique was introduced to translate the dynamic information contained in a finite element (FE) model into an SEA model. Any FE model, whatever its complexity, can thus be processed thanks to an automatic sub-structuring algorithm and a built-in VSEA modal synthesis solver, fast leading to robust numerical SEA model. VSEA also addresses structure-borne noise problems by coupling structural VSEA subsystems to analytical acoustic subsystems through a virtual wave number. Investigation of damping and trim treatment effects on mixed acoustic and structural subsystems are thus possible. While reviewing VSEA theory, industrial application cases will be presented.

2pSAd10. Noise Prediction for a Pumping Loop using the Noise Synthesis Technology Approach. Henri Pepin (CETIM, 52 Avenue Felix Louat, 60300 Senlis, France, henri.pepin@ cetim.fr), Axel Hass (52, avenue Félix Louat, 60304 Senlis, France, Axel.Haas@cetim.fr)

A collective project involving the french manufacturers of industrial pumps and valves is dedicated to the noise prediction in pumping plants. This paper presents a methodology based on virtual acoustic prototyping, and specifically on the noise synthesis technology (NST). Prediction of radiated noise in particular locations, such as operator stations or contractual specifications, allows us to control from the conception stage if the industrial plant will meet the prescribed noise criteria (regulations or user specifications). In addition, related noise control recommendations can be 
achieved by comparison of data obtained from the individual response of the components and their interaction. This methodology does not work as an absolute predictive process, and thus requires experimental validation. Components are taken into account by establishing their detailed vibroacoustic characteristics (technical data or measurements); in the present work on a simplified pumping loop, selected components are a centrifugal pumping unit, an open pipe circuit and several type of valves. Vibroacoustic and hydroacoustic measurements have to be adjusted according to the NST requirements. Comparison of the NST results with experimental data show a wide validity domain, covering the $100-10 \mathrm{kHz}$ third-octave range.

2pSAd11. Design of air springs for improved frequency response characteristics using computational fluid dynamics. Grace $R$. Kessenich (IPTRADE, Inc, 1 Gateway Center Suite 601, Newton, MA 02458, USA, grace.kessenich@iptrade.com), Allan D. Pierce (College of Enginering, Boston University, 110 Cummington St, Boston, MA 02215 , USA, adp@bu.edu), Baruch Pletner (IPTRADE, Inc, 1 Gateway Center Suite 601, Newton, MA 02458, USA, baruch.pletner@iptrade.com)
High performance air springs can be limited as vibration isolation devices by aeroacoustic cavity resonances. Such systems require detailed knowledge of their frequency response over all frequencies of interest. Transient computational fluid dynamics (CFD) simulations can derive quantitative magnitude and phase data early in the design cycle, before manufacturing. CFD models are unaffected by ambient noise, resonances from other sources, and sensor sensitivity, major experimental concerns. Unlike analytical methods, CFD modeling is applicable to complex as well as simple geometries. The modeling methodology is illustrated in the present study with 2D and 3D simulations of three different prototypical chambers. A chamber wall section, representing a piston, is moved with a sinusoidal displacement at constant frequency. Measurement of the magnitude and phase from the resulting sinusoidal force on this surface provides one point of a frequency response. By running multiple simulations over a wide frequency range, the null and resonant frequencies are readily apparent. Comparison with analytical predictions for simple geometries validates this technique. Insertion of porous membranes in the air spring cavity is modeled and is shown to be an effective method for mitigating aeroacoustic effects on air spring performance. Simulations provide crucial information for membrane parameter and placement optimization.

TUESDAY AFTERNOON, 1 JULY 2008

P2-B, LEVEL 2, 3:40 TO 5:20 P.M.

\title{
Session 2pSAe
}

\section{Structural Acoustics and Vibration and EURONOISE: General Topics in Structural Acoustics and Vibration III (Poster Session)}

\author{
Wolfgang Kropp, Cochair \\ Chalmers University of Technology \\ Sean Wu, Cochair \\ Wayne State University
}

\begin{abstract}
All posters will be on display from 3:40 p.m. to 5:20 p.m. To allow contributors an opportunity to see other posters, contributors of odd-numbered papers will be at their posters from 3:40 p.m. to 4:30 p.m. and contributors of even-numbered papers will be at their posters from 4:30 p.m. to 5:20 p.m.
\end{abstract}

\section{Contributed Papers}

2pSAe1. The investigation on sound souce indentification in semi-space by NAH. Dejiang Shang (Harbin Engineering University, College of Underwater Acoustic Engineering, 150001 Harbin, China, chizhafengyun1979@126.com), Yongwei Liu (Harbin Engineering University, College of Underwater Acoustic Engineering, 150001 Harbin, China, chizhafengyun1979@126.com)

The near field acoustic holography (NAH) theory model in semi-space has been constructed on the foundation of NAH theory in full-space. The technique of semi-space acoustic field reconstruction by NAH has been developed on Fast Fourier Transform Algorithm. This method has considered the water surface as soft boundary condition. The equivalent free sound field can be acquired if the measured sound field has been handled dissymmetrically by the principle of the mirror. This method can overcome the disadvantages of the boundary element method which can be used to resolve the problems of sound source identification in semi-space, such as the using of the semi-space Green function, complex transformation matrix calculation, more time consummation and singularity solution existence. This method has been verified by numerical calculation and by experiment respectively. The experimental frequency is between 3 and $10 \mathrm{kHz}$. The distance between the hologram plane and source plane is 3 centimeter. The results show that it's an efficiency way to deal with the problem of sound source identification in semi-space.

2pSAe2. An analysis of the modes of wave propagation in cylindrical shells. Yu-Cheng Liu (Graduate Institute of the Mechanical \& Aeronautical Engineering, Feng Chia University, No. 100 Wenhwa Rd., Seatwen, $40724 \quad$ Taichung, Taiwan, p9245215@fcu.edu.tw), Yun-Fun Hwang (Electroacoustic Graduate Program, No. 100 Wenhwa Rd., Seatwen, $40724 \quad$ Taichung, Taiwan, yfhwang@fcu.edu.tw), Jin-Huang Huang (Department of Mechanical and Computer-Aided Engineering, No. 100 Wenhwa Rd., Seatwen, 40724 Taichung, Taiwan, jhhuang@fcu.edu.tw)

Similar to a recent paper by Karczub [J. Acoust. Soc. Am., Vol. 119(6), 3553-3557, 2006], this work utilizes a symbolic math solution to obtain analytical solutions for the dispersion relation in a thin circular cylindrical shell. Analyses on the propagating wave mode shapes are particularly emphasized. The analytical solutions provide a straightforward calculation for all four branches of the dispersion curves, and the cumbersome numerical root searching technique is avoided. The dispersion relations for the breathing and torsional modes $(n=0$ circular harmonic), which were neglected by 
Karczub, are included. Mode shapes that characterize the types of wave that are predominantly transversal, longitudinal, circumferential, or in any combination of the three are analyzed in considerable detail. Effects by thickness and Poisson ratio variations are considered and examined. The cutoff frequencies for the higher order propagating modes are compared among the various orders of circular harmonics, from $n=0$ to 10 . Finally, comparison among several shell theories in the dispersion relation prospective, which is directly related to their accuracy in predicting the vibratory resonant frequencies of a finite shell, is also included in the discussion.

2pSAe3. Influence of Constructional Parameters of Small Reciprocating Compressors on Sound Power Emissions. Peter J. Kral (Vienna University of Technology, Institute for Engineering Design and Logistics Engineering, Getreidemarkt 9/E307, 1230 Vienna, Austria, peter.kral@tuwien.ac.at)

Noise in the environment is an increasing problem in industrial countries and is often strongly restricted by governmental laws. Therefore reducing sound levels must be one target in every design process. This paper describes in detail the influence of constructional parameters on the total sound power emitted by a small reciprocating compressor $\left(V_{H}=159 \mathrm{~m}^{3}\right)$ with reed valves. Measurements (sound power levels, sound pressure levels, FFTanalysis, time-signal analysis) on a test bed situated in an anechoic room by varying the operating conditions - for example - rotational speed, maximum pressure, and suction terms, were done. Additionally sound power measurements with regard to reed valve parameters (thickness of the reed, area of the inlet and outlet port of the valve plate, material of the valve plate) were achieved. Theoretically researches with the aid of computational methods (FEM-Analysis with Ansys, Calculations with MatLab) are presented and show very good accordance compared to the sound level measurements. These results should lead to a better understanding of the causal connection between constructional (valve) parameters/operating conditions and the resulting sound power emissions. Finally the differentiation of airborne and structure born noise may be helpful to minimize the noise emissions of compressors already within the design process.

2pSAe4. Modelling of the hydro and aero acoustic sources in piping Véronique Villouvier (EDF/R\&D, 1 avenue du Général de Gaulle, 92141 Clamart, France, veronique.villouvier@edf.fr)

In the industrial piping networks, various components are crossed by flows, generally in air or in water. The pressure fluctuations generated locally close to these singularities are propagated then in all the network and can lead to important vibrations of the structures. A research program based on many laboratory tests made it possible to highlight a hydroacoustic modelling of the butterfly valves in the low frequencies domain. The model, which describes the acoustic sources, generated by the turbulence, has a general applicability. In this paper, one presents the application of this model to orifices (openings and slits) working in air and in water. These various orifices were studied from an acoustic point of view on different test loops and for several hydraulic conditions (flow rate and head loss). These experimental cases were then simulated using the source model of turbulence noise, associated to the transfer matrix of each singularity, which is determined by another way. The comparison between these calculations and the corresponding measurements makes it possible to consolidate the general character of the model of turbulence noise for singularities in piping.

2pSAe5. Hydroacoustic modeling of the butterfly valves - some industrial applications. Véronique Villouvier (EDF/R\&D, 1 avenue du Général de Gaulle, 92141 Clamart, France, veronique.villouvier@edf.fr)

The French PWR units include a large number of control valves which are sometimes at cavitation working for certain operating conditions. The very high excitations which are then created may lead to the cracking of some small lines located nearby. These devices have been tested on laboratory test loops. An hydroacoustic behaviour model has been set up on the basis of very precise identifications obtained from numerous experimental tests. The model obtained describes the acoustic transfers through the valve and the sources generated by turbulence and cavitation in the low frequency domain, according to the main operating parameters: aperture, flow rate, cavitation number, upstream pressure and diameter. Hydraulic and vibratory measurements were carried out on several Residual Heat Removal circuits for many control configurations. When this circuit is functioning under certain conditions, the two valves which regulate the hot and cold flow rates operate according to a cavitation system. The hydroacoustic valve model is used to simulate of the valves under the hydraulic conditions of measurements. The results are compared at the vibratory levels recorded on site. The model also makes it possible to determine the optimal operating conditions in order to limit the vibrations of the circuit.

2pSAe6. Use of noise and vibration signal for detection and monitoring of cavitation in kinetic pumps. Jan Černetič (University of Ljubljana, Faculty of Mechanical Engineering, Aškerčeva 6, 1000 Ljubljana, Slovenia, jan.cernetic@fs.uni-lj.si), Jurij Prezelj (University of Ljubljana, Faculty of Mechanical Engineering, Aškerčeva 6, 1000 Ljubljana, Slovenia, jurij.prezelj@fs.uni-lj.si), Mirko Čudina (University of Ljubljana, Faculty of Mechanical Engineering, Aškerčeva 6, 1000 Ljubljana, Slovenia, mirko .cudina@fs.uni-lj.si)

Cavitation in kinetic pumps causes lowering performance, mechanical damage and increase of vibrations and noise. Therefore, it is important to detect inception and development of cavitation phenomenon in the pump. This paper deals with signals of vibrations and noise, which will be used for detection and monitoring of cavitation in kinetic pumps, and also to prevent the effect of cavitation in the pump and pumping system. When the cavitation is increasing, the flowing conditions are changing, which leads to an increase of vibrations of the pump and emitted noise in the surroundings. Because vibrations and noise are transferred from the pump through its casing, the signal is non-uniformly distorted due to transfer losses and structure of the casing surfaces. Even so, it is possible to determine development of cavitation phenomena from the measured signal. Noise and vibrations are increasing steadily, but in some specific frequency ranges the signal is more pronounced than in other part of the spectrum. Experimental results have shown that when the cavitation is fully developed, the measured signals at a characteristic frequency or range of frequencies start to decrease. This characteristic frequency or band of frequency is discussed in this paper, and also comparison between theoretical expectations and measurement results was performed.

2pSAe7. The wavelet analysis the acoustic emission signals generated by multi-source partial discharges. Andrzej Cichon (Technical University of Opole, ul. Miko ${ }^{3}$ ajczyka 5, 45-271 Opole, Poland, a.cichon@po.opole.pl), Sebastian Borucki (Technical University of Opole, ul. Mikoªjczyka 5, 45-271 Opole, Poland, s.borucki@po.opole.pl), Tomasz Boczar (Technical University of Opole, ul. Miko ${ }^{3}$ ajczyka 5, 45-271 Opole, Poland, t.boczar@po.opole.pl)

Within the research work, the results of which are presented in this paper, a comparative analysis of the acoustic emission (AE) signals generated by multi-source partial discharges (PDs) was carried out. The research investigations were carried out in a model system in which PDs were generated with two spark-gaps. A spark-gap in the surface system and a pointplane spark-gap were used due to the fact that these are the most commonly occurring PD forms in power transformers. The AE signals were registered with a contact transducer placed on the external part of the tub. The analysis in the time-frequency domain using a short-time Fourier transform (STFT) was carried out for the AE signals generated by PDs. The results of the analysis are shown in the form of two- and three-dimensional spectrograms of the power spectral density and three-dimensional spectrograms of the amplitude spectrum.

2pSAe8. Using wavelet transform to locate acoustic emission source in composite plate with one sensor. Amir Refahi Oskouei (Polytechnic of Tehran, AmirKabir University of Technology, Department of Mechanical Engineering, NDT Lab., 424 Hafez Ave, 15875-4413 Tehran, Iran, amir.refahi@cic.aut.ac.ir), Mehdi Ahmadi (Polytechnic of Tehran, 
AmirKabir University of Technology, Department of Mechanical Engineering, NDT Lab., 424 Hafez Ave, 15875-4413 Tehran, Iran, ahmadin@cic.aut .ac.ir)

In this paper a new method for locating the AE source with one sensor is presented. When acoustic emission signals propagate in wave-guided, they have multi-mode and dispersion characteristics. The separation of the modes at the sensors could make it possible to extract the exact information about the source that produced the waves. Based on the modal nature of $\mathrm{AE}$, it can be understood that a good location would have two methods to determine the arrival times. One is determined on the same part of the waves (not only the same mode, but also the same frequency) at all sensors. The other is determined on the different modes at one sensor, which makes it possible to reduce the number of sensors needed. First, through modal analysis of the propagation of elastic waves in a thin plate, the dispersion characteristics of the modes are predicted. Second, the wavelet transform theory is briefly outlined and its application in elastic waves is explained. It is shown that by using the peak of the magnitude of the wavelet transform, the arrival times of the different modes can be determined. Additionally, experiments were undertaken using a lead break on the edge of the plate. These demonstrated the feasibility of the one sensor linear location scheme.

2pSAe9. Effect of boundary restraints on sound radiation. Wen Li (Wayne State University, Department of Mechanical Engineering, 5050 Anthony Wayne Dr., Detroit, MI 48202, USA, wli@wayne.edu)

This study is focused on the effect of boundary restraints on the sound radiation from a rectangular plate. The modal and acoustic characteristics of square plates are studied for different restraining stiffnesses and configurations. It is shown that the modes of the restrained plates can be considerably different from those for the familar simply supported boundary condition, so are the corresponding modal radiation efficiencies. The proposed method generally applies to the plates elastically restrained along any edge(s) and for any stiffness values. The acoustic calculations are also valid for any acoustic wavenumber, instead of only for some extreme acoustic/structural wavenumbers as in other investigations.

2pSAe10. Progress in Primary Calibration of Acoustic Emission Sensors. Jiri Keprt (Brno University of Technology, UAMT-FEEC, Kolejni 2906/4, 61200 Brno, Czech Republic, keprt@feec.vutbr.cz), Petr Benes (Brno University of Technology, UAMT-FEEC, Kolejni 2906/4, 61200 Brno, Czech Republic, benesp@ feec.vutbr.cz)

The paper reviews the background of the primary calibration of acoustic emission sensors and the determination of uncertainty by this calibration. There are discussed the aims and the purpose of the primary calibration and main sources of uncertainty in practical usage of calibration results. The comparison of the results of the both method is presented. The shape of calibration characteristics corresponds well up to $500 \mathrm{kHz}$. The calculation of the uncertainty in the reciprocity and step function calibration is closely described. The problem of propagation of uncertainty in the fast fourier algorithm was solved. Uncertainty of measurement by primary calibration is determined and presented in the paper. The uncertainty in reciprocity calibration of UT 1000 in range from $60 \mathrm{kHz}$ to $285 \mathrm{kHz}$ was $\pm 3 \mathrm{~dB}$ and from $285 \mathrm{kHz}$ to $1 \mathrm{MHz}$ up to $\pm 8 \mathrm{~dB}$ and the same results in the calibration by step function. The calculation of the uncertainty includes all the presumable sources. Such as variation of input coefficient of reciprocity parameter, impact of thickness of glass capillary Also the main problems, that were solved and have to be solved, are presented.

2pSAe11. High-speed electronic speckle pattern interferometry of a struck flat plate. Thomas Moore (Rollins College, Department of Physics, Winter Park, FL 32789, USA, tmoore@rollins.edu), Daniel Zietlow (Rollins College, Department of Physics, Winter Park, FL 32789, USA, dzietlow@rollins.edu), Christopher Gorman (Rollins College, Department of Physics, Winter Park, FL 32789, USA, cgorman@ rollins.edu)

An electronic speckle pattern interferometer capable of imaging 33,000 frames per second has been used to study the transient motion of a flat plate which was struck at a point near the edge. Multiple interferograms, each with an exposure time of 25 microseconds, were combined to construct a video of the plate motion that can be used for both qualitative and quantitative analysis. The interferograms reveal the expected evolution of the bending waves created when a thin circular plate is struck near the edge; however, anomalous displacement of the surface of the plate is also observed. This unusual displacement of the surface takes the form of a transverse wave that precedes the motion directly attributable to the normal propagation of the initial bending wave.

2pSAe12. The Investigation on Measuring the Reflection Coefficient of Materials in Semi-space by Spatial Fourier Transforms. Yongwei Liu (Harbin Engineering University, College of Underwater Acoustic Engineering, 150001 Harbin, China, chizhafengyun1979@126.com), Dejiang Shang (Harbin Engineering University, College of Underwater Acoustic Engineering, 150001 Harbin, China, chizhafengyun1979@126.com), Qi Li (Harbin Engineering University, College of Underwater Acoustic Engineering, 150001 Harbin, China, chizhafengyun1979@126.com)

A new method using SFT (Spatial Fourier Transforms) has been investigated to measure reflection coefficient at arbitrary angles of incidence by $\mathrm{NAH}($ Near-field Acoustical holography ) in semi-space. The paper gives an experimental verification of this method. The experimental frequency is from $3 \mathrm{kHz}$ to $10 \mathrm{kHz}$. The size of test steel plate is $1.6 \mathrm{~m} \times 1.5 \mathrm{~m}$, the size of hologram surfaces is $1.32 \mathrm{~m} \times 1.14 \mathrm{~m}$, the distance between two hologram surface and the test material is about $2 \mathrm{~cm}$, the distance between sound source and water surface is about $4 \mathrm{~cm}$. The measured results are generally in good agreement with the theoretical value; the mean-square error of data is below 0.06. The experimental results show that this method eliminates the effect of the scattered sound field of the water surface completely. Because the sound source and the test material are set near the water surface during the experiment, it is very easy to set the measuring system, and this is very useful in practice.

2pSAe13. Optimizing the Dynamic Behaviour of a Large Portal Robot. Christian R. Koenig (University of Bremen, Badgasteiner Str. 1, FZB Room 2140, 28359 Bremen, Germany, chkoenig@uni-bremen.de), Markus Kochmann (University of Bremen, Badgasteiner Str. 1, FZB - Room 2140, 28359 Bremen, Germany, kochmann@uni-bremen.de), Jan Ohlendorf (University of Bremen, Badgasteiner Str. 1, FZB - Room 2140, 28359 Bremen, Germany, johlendorf@uni-bremen.de), Dieter H. Müller (University of Bremen, Badgasteiner Str. 1, FZB - Room 2140, $28359 \quad$ Bremen, Germany, ml@biba.uni-bremen.de), K.-D Thoben (University of Bremen, Badgasteiner Str. 1, FZB - Room 2140, 28359 Bremen, Germany, tho@biba.uni-bremen.de)

The object under investigation in this work is a portal robot used for the production of large fibre reinforced structures. The achieved product quality and the production speed of the device are influenced by the vibration and the damping behaviour of the system. The required process time is determined by kinematic parameters, i.e. speed and acceleration of the system and the dead time required for the vibration level to decay to a threshold level given by the required precision of process. In the first step, a Design of Experiments is used in order to identify the ideal combination of acceleration and speed. At this optimum, the sum of the drive time and the dead time reaches a minimum for a given precision. In the second step, an experimental Modal Analysis is performed in order to identify the potential for an optimization of the structure.

2pSAe14. Evaluation of hand-transmitted vibration exposure on basis of a questionnaire. Rauno Pääkkönen (Finnish Institute of Occupational Health, P.O:Box 486, 33101 Tampere, Finland, rauno.paakkonen@ttl.fi), Riitta Sauni (Finnish Institute of Occupational Health, P.O:Box 486, 33101 Tampere, Finland, riittta.sauni@ttl.fi), Jukka Uitti (Finnish Institute of Occupational Health, P.O:Box 486, 33101 Tampere, Finland, jukka.uitti@ttl.fi), Esko Toppila (Finnish Institute of Occupational Health, P.O:Box 486, 33101 Tampere, Finland, esko.toppila@ttl.fi)

We made a survey to see if metal workers had symptoms caused by vibration. The vibration exposure over lifetime was also asked. The cumulative HAV dose was calculated as a HAV index determined on the basis of the questionnaire, in which jobs, tools and active work time (hours/day, days/week, moths/year) had been requested. An index, I, describing the total 
HAV exposure was determined by $\mathrm{I}=\mathrm{a} 8 \mathrm{~h} 2 \times \mathrm{x}$ y $\mathrm{x}$, where $\mathrm{I}=$ cumulative exposure index $(\mathrm{m} 2 \mathrm{ad} / \mathrm{s} 4), \mathrm{y}=$ exposure time in years $(\mathrm{a})$, and $\mathrm{d}=$ annual exposure time in days. The exposure of metal workers varied $1-5 \mathrm{~m} / \mathrm{s} 2$, and the average was $2.4 \mathrm{~m} / \mathrm{s} 2$. The limit value of European directive $5 \mathrm{~m} / \mathrm{s} 2$ is not usually exceeded. However, there is a wide unaccuracy area that needs to be cleared one way or another. We also connected symptoms and exposure on statistical level. The total exposure times did not differ significantly in these groups, but, when the daily HAV exposure was taken into account in the calculation of the cumulative index, the difference was statistically significant.

2pSAe15. Experimental investigation of the acoustic black hole effect for vibration damping in elliptical plates. François Gautier (Laboratoire d'Acoustique de l'Université du Maine, Avenue Olivier Messiaen, 72085 Le Mans, France, francois.gautier@univ-lemans.fr), Jacques Cuenca (LAUM, CNRS, Université du Maine, Lab. d'Acoustique Université du Maine, UMR CNRS 6613, 72085 Le Mans Cedex 9, France, Jacques.Cuenca.etu@univ -lemans.fr), Victor V. Krylov (Loughborough University, Department of Aeronautical and Automotive Engineering, Ashby Road, LE11 3TU Loughborough, UK, V.V.Krylov@lboro.ac.uk), Laurent Simon (LAUM, CNRS, Université du Maine, Lab. d'Acoustique Université du Maine, UMR CNRS 6613, 72085 Le Mans Cedex 9, France, Laurent.Simon@univ-lemans.fr)

It is well known that flexural waves in beams or plates slow down if their thickness decreases. A beam or a plate with a decreasing thickness in the vicinity of one of their boundaries can be designed so that the travel time needed for a wave to reach the edge becomes infinite. Thus, the reflection coefficient associated with the corresponding region is zero. This effect is referred to as the acoustic black hole effect. The use of 'acoustic black holes' in combination with thin damping layers for wedges of power-law profile has been theoretically and experimentally studied by V.V. Krylov and R.E.T.B. Winward (JSV 300 (2007) 43-49). The aim of this paper is to show experimentally the acoustic black hole effect in a two-dimensional configuration comprising an elliptical plate with a pit of power-law profile placed in one of its focuses. The elliptical shape of the plate induces a focalization of waves towards the black hole, which is observed by means of scanning laser vibrometry. Comparison of input mobility measurements for plates with and without black hole shows the reduction in vibrational level induced by the black hole effect.
2pSAe16. Characterising elastic layers as non-lightly damped SDOF systems for the reduction of impact transmission noise. Francisco Simon (Instituto de Acústica (CSIC), C/ Serrano 144, 28006 Madrid, Spain, psimon@ia.cetef.csic.es), David K. Anthony (Instituto de Acústica (CSIC), C/ Serrano 144, 28006 Madrid, Spain, iaca344@ia.cetef.csic.es)

There is a wealth of literature dealing with modal determination of lightly damped viscous systems, however there is relatively little reported for medium and heavily damped systems or with non-viscous damping. The most commonly reported and applied methods have significant errors in the determination of both modal frequency and damping ratio for systems with high damping. For the lightly damped case simplification can be often conveniently made to allow the performance of each method to be studied theoretically, however for the non-lightly damped case this is not possible and the analysis must proceed on a heuristic basis. For the application modal parameter determination of elastic floor layers designed to reduce building transmission noise by impact testing, traditional and new methods of modal parameter determination for non-lightly are presented and heuristically evaluated for both viscous and non-viscous SDOF systems. It is also shown the performance often depends on the type of damping. This either must be known a priori or can be gleaned by comparing results from different methods.

2pSAe17. Eigenvalue imaging method for subsurface defects detection. Kenbu Teramoto (Saga University, 1-Honjo, 8408502 Saga, Japan, tera @ me.saga-u.ac.jp)

This paper proposes the eigenvalue imaging method to be independent of frequency and phase velocity and to characterize the Lamb-wave field. Lamb-waves play important roles in NDE fields. Being placed on the surface of a plate, a sound source excites several kinds of waves: (a) pressure and shear waves, (b) a Rayleigh wave, and (c) symmetric and antisymmetric Lamb-waves traveling along the plate. The first two waves are utilized for traditional ultrasonic techniques such as pulse-echo methods. In contrast to these classical techniques, in which the wavelength is shorter than the plate thickness, the Lamb-wave has the advantage of propagating over large area. The variation of the Lamb-wave velocity, however, causes difficulties for interpretation of observed signals. It is important, therefore, to establish the crack detection criterion independent of local wave numbers. This paper proposes a novel imaging method which utilizes the 3rd and 4th eigenvalues of the covariance matrix, which is defined over the 4- dimensional vector space which is spanned by following components: (1) a vertical displacement, (2) its particle velocity, (3) the orthogonal pair of out-ofplane strains. This study shows that the 3rd eigenvalue detects a wave scattered by defects and the 4th one has an ability to localize defects. 


\title{
Session 2pSCa
}

\section{Speech Communication: Speech Perception II}

\author{
Souhila Messaoud-Galusi, Chair \\ University College London, Wolfson House, Department of Phonetics and Linguistics, 4 Stephenson Way, London, NW1 2HE, \\ $U K$
}

\section{Contributed Papers}

2:00

2pSCa1. Cue switching in the perception of approximants: Evidence from two English dialects. Rachael-Anne Knight (City University, Department of Language and Communication Science, Northampton Square, EC1V 0HB London, UK, knight@ city.ac.uk), Christina Villafana Dalcher (City University, Department of Language and Communication Science, Northampton Square, EC1V OHB London, UK, cvdalcher@mac.com), Mark J. Jones (University of Cambridge, Phonetics Lab, Department of Linguistics, Raised Faculty Building, Sidgwick Avenue, CB3 9DA Cambridge, UK, markjjones@ @otmail.com)

A surprising dissimilarity is attested in the perception of approximants by speakers of American and Standard Southern British English. Eighteen subjects (6 AE and $12 \mathrm{SSBE}$ ) performed an identification task in which they judged whether stimuli were more like $/ \mathrm{r} /$ or $/ \mathrm{w} /$. The stimuli comprised five sounds, copy-synthesised from a source / $\mathrm{r} /$, where the values of $\mathrm{F} 2$ and F3 we adjusted to fall between the frequencies typical for ["turned $\left.\mathrm{r}^{\prime \prime}\right]$ and $[w]$. The only significant difference between the two dialect groups' performance occurred with a token in which F3 was at a frequency typical for $/ \mathrm{r} /$ and F2 was lowered to that of $/ \mathrm{w} /$. AE and SSBE speakers identified this token as $/ \mathrm{r} / 90 \%$ and $59 \%$ of the time, respectively. This is unexpected as $/ \mathrm{r} /$ in both dialects is characterised by a low F3. However, the difference may be due to the existence of the 'labiodental' variant of $/ \mathrm{r} /$ in SSBE. As this variant does not have a low F3, SSBE speakers must tolerate a wider diversity of / $\mathrm{r}$ /-types than AE speakers. Therefore, the / $\mathrm{r} /$ category in SSBE may be becoming increasingly defined by F2, rather than F3, which also has implications for the future production of $/ \mathrm{r} /$ in this accent

2:20

2pSCa2. Dialectal effects in the perception of vowels produced by first and second language speakers: North Carolinian versus Southern Welsh listeners. Ricardo Augusto Hoffmann Bion (SISSA - Cognitive Neuroscience, VL Stock 2/2, 34135 Trieste, Italy, ricardobion@gmail.com), Paola Escudero (University of Amsterdam, Institute of Phonetic Sciences, Spuistraat 210, 1012 VT Amsterdam, Netherlands, P.R.EscuderoNeyra@uva.nl) , Geoffrey Stewart Morrison (Australian National University, School of Language Studies, Building 110, ACT 0200 Canberra, Australia, geoff .morrison@anu.edu.au)

This paper investigated the effect of listeners' regional origin on the perception of vowels. Listeners from North Carolina and South Wales were presented with natural tokens of the four English vowels /i/, /I/, /E/ and /\{/ which were produced by L1 and L2 speakers. Randomization tests revealed a significant difference between the listener groups' confusion matrices. Examination of the confusion matrixes suggested that the dialectal differences were primarily in the vowel contrasts /I/-/E/ and /E/-/\{/. In order to explore these findings, logistic regression models were fitted to each listener group's responses (predictor variables in the models were initial and final formant values and duration). Territorial maps based on the logistic regression models indicated that there was a substantial difference in the locations of the $/ \mathrm{E} /-/\{/$, and a smaller difference in the location of the /I/-/E/ boundary.
2pSCa3. Is there evidence of an allophonic mode of speech perception in dyslexic children. Souhila Messaoud-Galusi (University College London, Wolfson House, Department of Phonetics and Linguistics, 4 Stephenson Way, NW1 2HE London, UK, souhila@phon.ucl.ac.uk), Valerie Hazan (University College London, Wolfson House, Department of Phonetics and Linguistics, 4 Stephenson Way, NW1 2HE London, UK, val@phon.ucl.ac.uk), Stuart Rosen (UCL, Wolfson House, 4, Stephenson Way, NW1 2HE London, UK, stuart@phon.ucl.ac.uk)

Recent studies have suggested that dyslexics perceive phonemic categories in a less categorical fashion than individuals of average reading ability due to increased within-category discrimination ('allophonic' mode of perception). In the present study, 62 dyslexic and 51 typically-developing (TD) children aged between 6;6 and 13;7 years old were tested on the perception of a /pi-bi/ continuum using an adaptive identification task presented in clear and in background noise, and a fixed-level discrimination task presented in clear. They also undertook a battery of tests of reading, IQ, phonological awareness, phonological short-term memory and word perception in noise. The gradient of the identification function was significantly weaker for the dyslexic than TD group in clear, but the two groups did not differ in the noisy condition. In the discrimination test, there was no evidence of increased within-category discrimination in the dyslexic group but the TD group showed better cross-category discrimination. These results do not support the existence of an allophonic model of speech perception in dyslexic children. Also, they do not support the view that dyslexic children are more affected by noise as a result of more poorly specified phonemic categories. [Work supported by Wellcome Trust].

\section{3:00}

2pSCa4. fMRI evidence for central auditory processing of speech in deaf infants under sedation. Scott K. Holland (Cincinnati Children's Hospital Medical Center, Imaging Research Center, 3333 Burnet Ave, Cincinnati, OH 45229, USA, scott.holland@cchmc.org), Prasanna Karunanayaka (Cincinnati Children's Hospital Medical Center, Imaging Research Center, 3333 Burnet Ave, Cincinnati, OH 45229, USA, KAR4RP@cchmc.org), Akila Rajagopal (Cincinnati Children's Hospital Medical Center, Imaging Research Center, 3333 Burnet Ave, Cincinnati, OH 45229, USA, RAJA7Y @ cchmc.org), Kristen Smith (Cincinnati Children's Hospital Medical Center, Imaging Research Center, 3333 Burnet Ave, Cincinnati, OH 45229, USA, SMIJ6D@cchmc.org)

Functional MRI (fMRI) performed in infants with congenital hearing loss provides evidence that auditory language stimulation produces activation of the central auditory system in the brain. Babies are normally sedated for clinical MRI scans. With IRB approval we have added a 10 minute fMRI scanning procedure to clinically indicated MRI scans in infants with severe to profound hearing impairment $(\mathrm{n}=18)$ or normal hearing $(\mathrm{n}=22)$. Sedation is performed according to clinical guidelines at our institution using either Propofol $((200-250 \mathrm{mcg} / \mathrm{kg} / \mathrm{min}$ i.v. $)$ or pentobarbital $(5 \mathrm{mg} / \mathrm{kg}$ orally). fMRI scanning is performed using Echo-planar gradient echo acquisitions on a 3 Tesla clinical MRI system while a mothers voice reads stories to the babies at a sound level $10 \mathrm{~dB}$ above the measured hearing threshold. Brain activation is measured in primary auditory cortex and planum temporale speech recognition areas. Results in the normal hearing group of infants demonstrates that the central auditory system is stimulated by speech in in- 
fants, although sedation does attenuate brain activation in a dose dependent manner. Central auditory and language activation is also detected in hearing impaired infants with levels of activation correlating to measured hearing thresholds.

\section{$3: 20$}

2pSCa5. Left parietal activation during the production of pointing in several modalities: prosodic focus, syntactic extraction, digital- and ocular- pointing. Hélène Loevenbruck (Speech and Cognition Department, GIPSA-lab, 46 avenue Félix Viallet, 38031 Grenoble, France, Helene.Loevenbruck@gipsa-lab.inpg.fr), Coriandre Vilain (Speech and Cognition Department, GIPSA-lab, 46 avenue Félix Viallet, 38031 Grenoble, France, coriandre.vilain@gipsa-lab.inpg.fr), Francesca Carota (Centre de Neuroscience Cognitive, 67, Boulevard Pinel, 69675 Bron, France, Francesca.Carota@isc.cnrs.fr), Monica Baciu (Laboratoire de Psychologie et NeuroCognition, UFR Sciences de l'Homme et de la Société, BP47, 38040 Grenoble, France, monica.baciu@upmf-grenoble .fr), Christian Abry (Centre de Recherche sur l'Imaginaire (CRI), Université Stendhal, Grenoble 3, BP 25, 38040 Grenoble cedex 9, France, christian.abru@u-grenoble3.fr), Laurent Lamalle (Plateforme régionale
IRM 3Tesla, IFR n ${ }^{\circ}$ 1, RMN Biomédicale : de la cellule à l'homme, CHU de Grenoble, BP 217, 38043 Grenoble Cedex 9, France, Laurent.Lamalle @ujf-grenoble.fr), Cédric Pichat (Laboratoire de Psychologie et NeuroCognition, UFR Sciences de l'Homme et de la Société, BP47, 38040 Grenoble, France, cedric.pichat@upmf-grenoble.fr), Christoph Segebarth (Grenoble Institut de Neurosciences, CHU de Grenoble - B.P. 217, 38043 Grenoble, France, csegebar@ujf-grenoble.fr)

Deixis, or pointing, is the ability to draw the viewer/listener's attention to an object, a person, a direction or an event. Pointing is gradually acquired by children, first with the eyes, then with the finger, then with intonation and finally with syntax. The crucial role of digital pointing in language acquisition suggests that all modalities of pointing may share a common cerebral network. An fMRI study of the production of multimodal pointing was carried out on 15 subjects. Subjects were scanned during the execution of index finger pointing gestures, eye pointing gestures, prosodic pointing (focus) and syntactic pointing (extraction). The results of a random effect group analysis show that the left superior parietal lobule (BA 7) was activated in all three digital, ocular and prosodic pointing but not in syntactic pointing. These results indicate that pointing in different modalities may recruit the left superior parietal lobule, with ocular pointing more anterior than prosodic pointing, itself more anterior than digital pointing. A grammaticalisation process is suggested to explain the lack of parietal activation in syntactic pointing.

TUESDAY AFTERNOON, 1 JULY 2008

ROOM 250B, 2:00 TO 3:40 P.M.

\title{
Session $2 \mathrm{pSCb}$
}

\section{Speech Communication: Speech Articulation I}

\author{
Helen M. Hanson, Chair \\ Union College, 807 Union St., Schenectady, NY 12308, USA
}

\section{Contributed Papers}

\section{2:00}

2pSCb1. Acoustic cues to the voicing contrast in coda stops in the speech of 2-year-olds learning American English. Helen M. Hanson (Union College, 807 Union St., Schenectady, NY 12308, USA, hansonh@union.edu), Stefanie Shattuck-Hufnagel (Massachusetts Institute of Technology, Research Laboratory of Electronics, Speech Communication Group, 77 Massachusetts Ave., Cambridge, MA 02139, USA, stef@ speech .mit.edu)

Developing a model of how children acquire the phonological feature contrasts and phonetic cues of their adult speech community requires a detailed understanding of how production changes during development. Earlier studies based on listening provided an initial estimate of these patterns; recent instrumental studies of the acoustic and articulatory details of production during development have revealed some surprises, including covert contrasts between distinctive feature categories (e.g., Scobbie et al., 1996) and of incomplete acquisition of adult feature-cue patterns (Imbrie, 2005), which can be difficult for adults to hear. Few instrumental studies, however, have focused on the child's cue patterns for coda consonants (although see Song and Demuth, in press). Our study of cues to the feature [voice] for coda stops used quantitative acoustic analyses of tokens from the Imbrie corpus of 2-year-old speech to compare child productions with those of their adult caretakers. Results show that a) many children produce a noisy region at the end of the vowel for voiceless codas (in duck, cup) but a long strong voice bar during closure for voiced codas (in bug, tub), and b) these cues may be exaggerated versions of the feature cues of their adult caretakers.

\section{2:20}

2pSCb2. Are acoustic and articulatory changes of speech produced in noise only related to the increase in vocal intensity? Maeva Garnier (University of New South Wales, Music Acoustics, School of Physics, NSW 2052 Sydney, Australia, maeva.garnier@gmail.com), Nathalie Henrich (Département Parole \& Cognition, GIPSA-lab, 46, avenue Félix Viallet, 38031 Grenoble Cedex, France, Nathalie.Henrich@gipsa-lab .inpg.fr), Danièle S. Dubois (CNRS, LCPE/LAM 11 rue de Lourmel, 75015 Paris, France, ddubois@ccr.jussieu.fr)

Speech acoustics and articulation are modified in speech produced in noisy environments. Is this simply a result of the increase in vocal intensity or may these modifications be some communicative strategies to increase audiovisual intelligibility? We recorded a first acoustic database with ten speakers and a second acoustic and articulatory database with three speakers, for quiet and for two kinds of background noise. We first verified previous observations about speech modifications in noise and observed that some are not strongly correlated to the increase in vocal intensity and cannot be entirely explained by previous models of vocal effort. We also made additional observations. Pitch does not only rise in noise but also extends its dynamic. For female speakers, pitch not only rises but also fits spectrum "holes" of cocktail-party noise. Speech modifications are not only global 
over the whole utterance but also specific to some of its units. Articulatory modifications consist not only of an amplification of lip movements, but also correspond to an enhancement of the potentially visible contrast between vowels. Lastly, some articulatory changes may be compensation for formant modifications induced by the increase in vocal intensity, rather than a consequence of it.

\section{2:40}

2pSCb3. Muscle saturation effect in /i/ production: Counterevidence from a 3D biomechanical model of the tongue. Stéphanie Buchaillard (ICP/GIPSA-lab, INPG, 46 Avenue Félix Viallet, 38031 Grenoble Cedex 01, France, stephanie.buchaillard@gipsa-lab .inpg.fr), Pascal Perrier (ICP/GIPSA-lab, INPG, 46 Avenue Félix Viallet, 38031 Grenoble Cedex 01, France, Pascal.Perrier@gipsa-lab .inpg.fr), Yohan Payan (TIMC-IMAG, Pavillon Taillefer, Faculté de Médecine, 38706 La Tronche cedex, France, yohan.payan@imag.fr)

Numerous works suppose the existence of a saturation effect to facilitate the control of the constriction area when producing vowel lil. This study exploits a 3D biomechanical Finite Element model of the vocal tract to evaluate the effectiveness of this assumption. The model includes the tongue and its major muscles, represented as a hyperelastic body, inserted in the oral cavity including jaw, palate, pharyngeal walls and hyoid bone. Muscles activations are controlled with the lambda model (Equilibrium-Point Hypothesis). After determining a set of possible motor commands to generate vowel \i〉, the impact of local variations of these commands on tongue shape was studied. The main tongue muscles motor commands were independently modified (by $\pm 2 \%, 5 \%, 8 \%$ around their values at target) and the effect on the tongue shape and on the acoustic signal was studied. The simulations showed a global stabilization of the tongue body, thanks to the palatal contacts, but also variability in the alveolar groove due to the anterior genioglossus activation. Though extremely localized, this variation has an important impact on the constriction size and, then, on the acoustic signal. Consequently, a specific control of the articulatory variability could be necessary to explain experimental data for vowel lil.

\section{3:00}

2pSCb4. Compensatory tongue patterns in glossectomy patients. Emi Murano (University of Maryland Dental School, $650 \mathrm{~W}$ Baltimore St. \#8402, Department of Biomedical Sciences, Baltimore, MD 21201, USA, emurano@umaryland.edu), Maureen Stone (Vocal Tract Visualization Lab, Depts of Biomedical Sciences and Orthodontics, University of Maryland Dental School, 650 W. Baltimore St., Baltimore, MD 21201, USA, mstone@umaryland.edu), Xiaofeng Liu (The Johns Hopkins University, 3400 N Charles Street, 105 Barton Hall, Baltimore, MD 21218, USA, xiaofeng@jhu.edu), Jiachen Zhuo (University of Maryland Medical
System, 22 South Greene Street, Department of Diagnostic Radiology, Baltimore, MD 21201, USA, JZHUO@umm.edu), Andrew Salama (University of Maryland Dental School, $650 \mathrm{~W}$ Baltimore St. \#8402, Department of Biomedical Sciences, Baltimore, MD 21201, USA, asalama@umm.edu), Rao Gullapalli (University of Maryland Medical System, 22 South Greene Street, Department of Diagnostic Radiology, Baltimore, MD 21201, USA, RGULLAPALLI@umm.edu), Robert Ord (University of Maryland Dental School, 650 W Baltimore St. \#8402, Department of Biomedical Sciences, Baltimore, MD 21201, USA, rord@umm.edu), Jerry Prince (The Johns Hopkins University, 3400 N Charles Street, 105 Barton Hall, Baltimore, MD 21218, USA, prince@jhu .edu)

To examine the effects of glossectomy surgery on tongue deformation strategies, a unique combination of data was used, including internal tongue strain patterns from tagged-cine-MRI and detailed muscle architecture from high-resolution MR images. Two glossectomy patient groups were studied and compared to normal controls. They differed in the type of reconstruction after cancer excision: primary closure or reconstruction with forearm flap. All patients included in this study had the same tumor type (Squamous Cell Carcinoma), size (T1 and T2) and location (unilateral mid-third of ora tongue). The tongue developed distinct deformation strategies that move the remaining tongue tissue, the flap and the scar tissue. Compensatory patterns such as pivot points and areas of rigidity will be shown and discussed. These appear to be compensatory strategies to optimize speech output after surgery. This presentation is based on viewing the tongue as a muscular hydrostat, which provides the basis for hypotheses about the formation and release of areas of rigidity within the tongue. The rigidity might be a way to simplify sound-specific strategies for moving the tongue and controlling changes in its shape in normal and patient subjects. Supported by NIH K99DC009279, and R01DC01758 from the NIDCD.

\section{3:20}

2pSCb5. Statistical identification of critical, dependent and redundant articulators. Philip J. Jackson (University of Surrey, Centre for Vision, Speech and Signal Processing, GU2 7XH Guildford, UK, p.jackson@surrey.ac.uk), Veena D. Singampalli (University of Surrey, Centre for Vision, Speech and Signal Processing, GU2 7XH Guildford, UK v.singampalli@surrey.ac.uk)

An automatic method for identifying critical, dependent and redundant roles in speech articulation is presented. Critical articulators are identified using the Kullback-Leibler divergence between phone-specific and model pdfs, which are initialised to the grand pdfs for each articulator. Model pdfs of critical and dependent articulators, those significantly correlated with the critical ones, are updated accordingly for both 1D and 2D cases, as long as the divergence exceeds the threshold. Those unaffected are termed redundant. Algorithm performance is evaluated on the MOCHA-TIMIT database by comparison with phonetic features. Results are also given for an exhaustive search, and principal component analysis of articulatory fleshpoints. Implications of being able to extract phonetic constraints automatically from articulatory recordings are discussed. 


\title{
Session $2 \mathrm{pSCc}$
}

\section{Speech Communication: General Topics in Speech Communication II (Poster Session)}

\author{
Yukari Hirata, Cochair \\ Colgate University, Dept. of East Asian Lang. and Lit., 13 Oak Dr., Hamilton, NY 13346, USA
}

James Hillenbrand, Cochair

Western Michigan University, Dept. Speech Path. and Aud., 1903 W. Michigan Ave., Kalamazoo, MI 49008, USA

\author{
Natasha Warner, Cochair \\ University of Arizona, MPI NIjmegen, Dept. of Linguistics, Box 210028, Tucson, AZ 85721, USA
}

\begin{abstract}
All posters will be on display from 3:40 p.m. to 5:20 p.m. To allow contributors an opportunity to see other posters, contributors of odd-numbered papers will be at their posters from 3:40 p.m. to 4:30 p.m. and contributors of even-numbered papers will be at their posters from 4:30 p.m. to 5:20 p.m.
\end{abstract}

\section{Contributed Papers}

2pSCc1. A grouping approach to harmonic complexes. Dirkjan J. Krijnders (University of Groningen, Dept. Artificial Intelligence, P.O. Box 407, 9700 AK Groningen, Netherlands, j.d.krijnders@ai.rug.nl), Maria E. Niessen (University of Groningen, Dept. Artificial Intelligence, P.O. Box 407, 9700 AK Groningen, Netherlands, m.niessen@ai.rug.nl), Tjeerd C. Andringa (University of Groningen, Dept. Artificial Intelligence, P.O. Box 407, 9700 AK Groningen, Netherlands, t.andringa@ai.rug.nl)

Humans seem to perform sound-source separation for quasi-periodic sounds, such as speech, mostly on harmonicity cues. To model this function, most machine algorithms use a pitch-based approach to group the speech parts of the spectrum. In these methods the pitch is obtained either explicitly, in autocorrelation methods, or implicitly, as in harmonic sieves. If the estimation of pitch is wrong, the grouping will fail as well. In this paper we show a method that performs harmonic grouping without first calculating the pitch. Instead a pitch estimate is associated with each grouping hypothesis. Making the grouping independent of the pitch estimate makes it more robust in noisy settings. The algorithm obtains possible harmonics by tracking energy peaks in a cochleogram. Co-occuring harmonics are compared in terms of frequency difference. Grouping hypotheses are formed by combining harmonics with similar frequency differences. Consistency checks are performed on these hypotheses and hypotheses with compatible properties are combined into harmonic complexes. Every harmonic complex is evaluated on the number of the harmonics, the number of subsequent harmonics and the presence of a harmonic at the pitch position. By using the number of subsequent harmonics octave errors are prevented. Multiple concurrent harmonic complexes can be found as long as the spectral overlap is small.

2pSCc2. The role of generalization in learning perceptual contrasts Meghan Sumner (Stanford University, Department of Linguistics, Margaret Jacks Hall, Stanford, CA 94305-2150, USA, sumner@stanford.edu)

Understanding spoken language is a difficult task. Variation caused by accents make this task even more daunting. This project examines the generalization of novel phonetic cues by listeners of non-native speech. Native speakers of English were trained on Korean-English pronunciations of minimal pairs with word-final contrasts (e.g., bed - bet). While native English speakers rely on vowel duration to cue a final voicing contrast, native Korean speakers of English do not produce a vowel duration contrast, but use a different distinguishing phonetic cue (e.g., voicing into closure duration). Listeners were trained on a subset of minimal pairs ending in final stops (bet - bed). Following successful training, listeners participated in an immediate or long-term form priming task. Four conditions included: Trained Identity (bet - bet), Trained Non-identity (bed - bet), Novel Non-identity, Trained Rime (wet - wed), and Novel Non-identity, Novel Rime (beat - bead). Preliminary results show episodic treatment of words in the immediate priming task, but generalization to novel forms in the long-term priming task. These data suggest that early learning is mediated by episodic representations learning proceeds episodically until sufficient information is available to support a more abstract generalization.

2pSCc3. Vocalizations of deaf infants before and after cochlear implantation. Mary K. Fagan (Indiana University School of Medicine, Riley Research Wing 044, 699 West Drive, Indianapolis, IN 46202, USA, mkfagan@indiana.edu)

Critical aspects of spoken language depend on perceiving and understanding speech sounds. For deaf infants, however, neither sound perception nor awareness of the sound-making consequences of their actions is available. The goal of this study was to uncover and document early, measurable effects of hearing loss on infant vocalization and changes in these behaviors following cochlear implantation. Participants were 8 deaf infants, 7-11 months old, and 8 infants with cochlear-implants, 12-20 months old and 1-6 months post-implantation. Dependent variables include number of vocalizations, mean vocalization duration, and mean frequency before and after cochlear implantation and in relation to reduplicated babble onset and word production. Post-implant changes in vocal behaviors may have clinical implications for decisions regarding age of implantation and auditory habilitation as well as for understanding post-implant variability.

2pSCc4. Contrastive Focus in Taiwan Mandarin. Ho-Hsien Pan (Dept. of Foreign Languages and Literatures, National Chiao Tung University, 1001 TA Hsueh Road, 300 Hsinchu, Taiwan, hhpan@faculty.nctu .edu.tw), Hui-Ying Tsou (Dept. of Foreign Languages and Literatures, National Chiao Tung University, 1001 TA Hsueh Road, 300 Hsinchu, Taiwan, hytsou@ms11.hinet.net), Wan-Ting Huang (Dept. of Foreign Languages and Literatures, National Chiao Tung University, 1001 TA Hsueh Road, 300 Hsinchu, Taiwan, caissybelle@yahoo.com.tw)

This study investigated the production and perception of given, new, and contrastive information in Taiwan Mandarin. Spontaneous dialogues elicited through a game were analyzed to access the acoustical parameters marking different information structures. In perceptual studies, two perceptual experiments, (1) a dialogue history experiment, of which listeners identified the questions preceding the sentences, and (2) a pair comparison experi- 
ment, of which subject compared sentence with same wording but different information structures were used. The stimuli were either natural utterances form elicited spontaneous dialogues or resynthesized utterances with conflicting duration and fo cues swapped from utterances of different information structures. Preliminary results of production experiments showed that duration elongation is a more consistent cue for marking narrow and contrastive foci than fo range expansion. Listeners performed better in the pair comparison experiments than in the dialogue history experiment. For nature utterances, listeners were able to identify sentences with given information, given versus new information, and given versus contrastive information, but were not able to identify utterances with more than two contrastive foci. Listeners' performances declined when resynthesized utterances with conflicting duration and fo cues were presented. When presented with conflicting cues, listeners relied more on durational than f0 cues.

2pSCc5. Comparison of Japanese expressive speech perception by Japanese and Taiwanese listeners. Chun-Fang Huang (Japan Advanced Institute of Science and Technology, 1-1, Asahidai, Nomi, 923-1292 Sendai, Japan, chuang@jaist.ac.jp), Donna Erickson (Showa University of Music, 808 Sekiguchi, Atsugi, 243-8521 Kanagawa, Japan, EricksonDonna2000@gmail.com), Masato Akagi (Japan Advanced Institute of Science and Technology, 1-1, Asahidai, Nomi, 923-1292 Sendai, Japan, akagi@jaist.ac.jp)

Language is an important tool in speech communication. Even without the understanding of one language, we can still judge the expressive content of a voice, such as happiness or sadness. However, sometimes misunderstanding of emotional communication occurs. It is not clear what the common/different features are that help or hinder people with different culture/native-languages background in making judgments about the expressivity of speech. In order to explore this question, we focus on Japanese and Taiwanese listeners perception of Japanese expressive speech utterances. We used the perceptual model proposed by [Huang and Akagi, InterSpeech 2005; 2007], which involves a concept called "semantic primitives"-- adjectives for describing voice perception. This concept simplifies and clarifies the discussion of common/different features in terms of acoustic cues and expressive speech perception categories. Using this model, we found some common/different features in the perception of expressive speech. Specifically, our results suggest that there may be primary and secondary semantic primitives associated with acoustic speech characteristics which are involved in the perception of expressive speech, and that people from different cultures/native-language background tend to use the same primary semantic primitives in perceiving expressive speech but different secondary ones.

2pSCc6. Acoustic analysis of the vocal tract during vowel production by finite-difference time-domain method. Hironori Takemoto (ATR Cognitive Information Science Laboratories, 2-2-2 Hikaridai, Seika-cho Soraku-gun, 619-0288 Kyoto, Japan, takemoto@atr.jp), Parham Mokhtari (ATR Cognitive Information Science Laboratories, 2-2-2 Hikaridai, Seika-cho Soraku-gun, 619-0288 Kyoto, Japan, parham@atr.jp), Tatsuya Kitamura (Konan University, Okamoto 8-9-1, Higashinada, 658-8501 Kobe, Japan, t-kitamu@konan-u.ac.jp)

An acoustic simulator based on the finite-difference time-domain (FDTD) method was evaluated by acoustic measurements on solid models of the vocal tract. Three-dimensional vocal tract (3D VT) shapes for a male subject during production of the five Japanese vowels were measured by magnetic resonance imaging. Transfer functions of the 3D VT shapes were computed by the acoustic simulator. The accuracy of the finite-difference algorithm was second-order in time and fourth-order in space. From the same 3D VT shapes, solid models were made of epoxide resin by a stereolithographic technique, and their acoustic transfer functions were measured using a time-stretched pulse signal. The calculated and measured spectra were compared up to $8 \mathrm{kHz}$. Although locations of major poles and zeros were common between calculated and measured spectra, the calculated spectra showed a larger number of small zeros, possibly caused by underestimation of VT acoustic losses in the simulation. However, the lower four formants were simulated remarkably accurately, with a mean absolute error of only $2.2 \%$ compared with the acoustic measurements. These facts indicated that the acoustic simulator can reasonably account for acoustic phenomena within the VT.

2pSCc7. Speech intelligibility determined with various tests presented against noise. Edward Ozimek (Umultowska 85, 61-614 Poznan, Poland ozimaku@amu.edu.pl), Dariusz Kutzner (Umultowska 85, 61-614 Poznan, Poland, konsbol@wp.pl), Anna Warzybok (Umultowska 85, 61-614 Poznan, Poland, a_warzybok@poczta.onet.pl)

The paper describes three Polish tests for assessment of speech intelligibility under noisy conditions: the matrix test, the sentence test and the digit triplet test. The matrix test contains semantically unpredictable sentences of fixed syntactical structures. This test is very useful for long-term intelligibility measurements and is applicable for subjects with profound hearing loss or cochlear implants users. The sentence test comprises utterances taken from everyday speech and aims at clinical speech intelligibility measurements. The digit triplet test contains sequences of three digits that are spoken separately and is aimed at screening measurements, especially via telephone. The statistical and phonemic properties of the tests are analyzed and compared. The correlation between results obtained in the respective tests and the tests applicability are also discussed.

2pSCc8. Consonants, but not vowels, prime lexical decision following masked priming. Boris New (CNRS - Université Paris Descartes, 71 Avenue Edouard Vaillant, 92100 Boulogne-Billancourt, France, boris.new@univ-paris5.fr), Veronica Araujo (CNRS - Université Paris Descartes, 71 Avenue Edouard Vaillant, 92100 Boulogne-Billancourt, France, veronica.cruzaraujo@gmail.com), Nathalie Bour (CNRS - Université Paris Descartes, 71 Avenue Edouard Vaillant, 92100 BoulogneBillancourt, France, psynat93@hotmail.com), Thierry Nazzi (CNRS - Université Paris Descartes, 45 rue des Saints-Pères, 75006 Paris, France, thierry .nazzi@univ-paris5.fr)

Recently, Nespor et al (2003) have argued that consonants play a more important role at the lexical level than vowels. This proposal has received direct support from studies showing that 16/20/30-month-old infants are better at processing specific consonantal than vocalic information while learning new words (Nazzi, 2005; Nazzi \& New, 2007). This proposal is also supported, although indirectly, by studies on adults. Adults have been found to rely more on consonants than on vowels when asked to transform a nonword into a real word (Cutler et al., 2000). Moreover, adults presented with a continuous stream of syllables are able to segment it into "lexical units" by relying on consonantal but not vocalic information (Bonatti et al., 2005). In our study, we directly investigate this issue using a visual masked priming lexical decision task (an oral version being currently implemented). The test items are bisyllabic (CVCV or VCVC) and trisyllabic (CVCVCV or VCVCVC) words, which are presented following four different primes: identity (e.g., joli-joli), unrelated (vabu-joli), same consonants (jalu-joli) and same vowels (vobi-joli). Priming was found for the identity and same consonants conditions, not for the same vowels condition, supporting the privileged role of consonants at the lexical level.

2pSCc9. Frequency importance function of Mandarin Chinese speech. Jing Chen (Dept. of Machine Intelligence, Speech and Hearing Research Center, 2 Science Building, Peking Univ., 5 Yeheyuan Road, Haidian District, 100871 Beijing, China, chenj@cis.pku.edu.cn), Tianshu S. Qu (Dept. of Machine Intelligence, Speech and Hearing Research Center, 2 Science Building, Peking Univ., 5 Yeheyuan Road, Haidian District, 100871 Beijing, China, qutianshu@cis.pku.edu.cn), Xihong H. Wu (Dept. of Machine Intelligence, Speech and Hearing Research Center, 2 Science Building, Peking Univ., 5 Yeheyuan Road, Haidian District, 100871 Beijing, China, wxh@cis.pku.edu.cn), Qiang Huang (Dept. of Machine Intelligence, Speech and Hearing Research Center, 2 Science Building, Peking Univ., 5 Yeheyuan Road, Haidian District, 100871 Beijing, China, QIANG 
.HUANG@SPREADTRUM.COM),Ying Huang(Dept. of Psychology, Peking Univ., 5 Yeheyuan Road, Haidian District, 100871 Beijing, China, innhuang@gmail.com), Liang Li (Dept. of Psychology, Peking Univ., 5 Yeheyuan Road, Haidian District, 100871 Beijing, China, liangli@pku.edu.cn), Huisheng S. Chi (Dept. of Machine Intelligence, Speech and Hearing Research Center, 2 Science Building, Peking Univ., 5 Yeheyuan Road, Haidian District, 100871 Beijing, China, chi@pku.edu.cn)

The speech intelligibility index (SII) theory objectively assesses speech intelligibility, and the frequency-importance function (FIF), which reflects the relative importance of various frequency bands to speech intelligibility for various languages, occupies the central part of the theory. However, the FIF has not been examined for tonal Chinese Mandarin speech. In this study, considering the characteristics of Mandarin speech, 50 phonemicallybalanced one-syllable words were selected as the speech stimuli from the speech corpus "A Method for Calculating Articulation Index (GB/T 155081995)", which meets the National Standards of China, and the 1/3 octave FIF was measured and computed with the Fletcher's method. The results show that spectrum regions with frequencies $2000-4000 \mathrm{~Hz}$ are more important in Chinese speech than those in English speech. The results predicted by SII model with the new FIF fit the result of human psychophysical studies well, which indicate that the new FIF model is effective and appropriate for assessing the intelligibility of Mandarin speech even when a steady-state noise masker is co-presented. [Supported by the NSFC 60435010; 60535030; 60605016; 30670704]

2pSCc10. Arabic Diagnostic Rhyme Test using minimal pairs. Bachir Boudraa (USTHB Faculté d'électronique et d'informatique, BP 32, El-Alia, 16031 Alger, Algeria, b.boudraa@yahoo.fr), Malika Boudraa (USTHB Faculté d'électronique et d'informatique, BP 32, El-Alia, 16031 Alger, Algeria, mk.boudraa@yahoo.fr), Bernard Guerin (INPG, Avenue Félix Viallet, 38031 Grenoble, France, Bernard.Guerin@enserg.fr)

In this paper, we try to adapt to Arabic the diagnostic rhyme test (DRT) developed by Voiers for English and adapted to French by Peckels \& Rossi. DRT is specific to each language and is essential to subjective assessments of coders and synthesizers. Six Arabic pertinent dimensions are used which are acuteness, compactness, tenseness, stridence, nasality and flatness. 72 monosyllabic meaningful pairs of words called minimal pairs have been developed. The apprehensibility of every attribute is tested in each of six vowel contexts. Every feature has been repeated six times and has been paired twice with every vowel.

2pSCc11. A fascinating personage in the history of phonetics: The phonetics and the politics of Elise Richter. Natasha Warner (University of Arizona, MPI NIjmegen, Box 210028, Dept. of Linguistics, Tucson, AZ 85721-0028, USA, nwarner@u.arizona.edu)

Elise Richter (1865-1943) was the first woman lecturer or professor in Austria or Germany, and she was also an experimental phonetician and even an experimental phonologist. Richter's career was delayed by the roles allowed for women at the time, and the end of her career, and her life, came about through the Nazis' policies toward Jews. Richter began her scientific work in Romance philology, but decided to seek explanations for historical sound change in phonetics. This led her to a productive career in experimental phonetics, and she even applied phonetic methods to study questions of phonological structure. Richter was a leader in exploring new methods and bringing together questions across widely differing fields. Although her specific experimental findings were superseded with time, Richter's refusal to be constrained by the limits of a field led her to ideas that have become current in phonetics just recently. In addition to her scientific career, Richter was politically active, both in working to expand women's opportunities and in general politics. This poster presents a new perspective on Richter's contributions to phonetics and on her view of feminism. Furthermore, it makes new observations of a parallel between Richter's political activities and her scientific work.
$2 \mathrm{pSCc12}$. The role of tongue articulation for $/ \mathrm{s} /$ and $/ \mathrm{z} /$ production in whispered speech. Hirohide Yoshioka (University of Tsukuba, 1-4-5 Shinmei, Urawa, 336-0023 Saitama, Japan, hirohide-y@mtc.biglobe.ne.jp)

Although the timing of the initiation and cessation of vocal fold vibrations is crucial to characterize the voiced and voiceless cognates, other cues, such as the duration of preceding vowels, the patterns of the formant transitions in the following vowels, and the period of stop closure, may also play important roles in performing these distinctions. The present study is to further investigate the role of tongue articulatory movements during voiced and unvoiced consonant production, specifically when the vocal folds do not vibrate during the production of fricative consonants, /s/ and /z/ in whispered speech. A normal Japanese speaker served as the subject. The palato-lingual contact patterns during the intervocalic consonant $/ \mathrm{s} /$ and $/ \mathrm{z} /$ in whispered speech were recoded using dynamic electro-palatography. The results show that the area of palato-lingual contact is clearly wider during / $\mathrm{z} /$ production than $/ \mathrm{s} /$ production; the opening for fricative turbulent noise production is narrower and longer for $/ z /$ than for $/ \mathrm{s} /$. In addition, the contact pattern is unstable for $/ \mathrm{s} /$ production.

2pSCc13. Production and perception of V1V2 described in terms of formant transition rates. René Carré (Laboratoire Dynamique du Langage, UMR 5596, CNRS, Université Lyon 2, 14 Avenue Marcelin Berthelot, 69363 Lyon cedex 07, France, recarre@wanadoo.fr)

Vowels can be produced with static articulatory configurations leading to stable formant frequencies (targets). Here, an algorithm computes area functions according to the criterion of minimal deformation leading to maximal acoustic variations. Within this evolutionary dynamics, the deformations of the tube are not performed to reach targets, unknown during the process, but to move in the acoustic space in order to increase acoustic contrast. The corresponding formant trajectories in the acoustic space can be described in terms of formant transition rates. For example, following this dynamic approach, to produce [ae] from [a], the transition rates of F1 and F2 are necessary and sufficient to represent $[\mathrm{e}]$ and at the very beginning of the transition and throughout the transition there is sufficient information to detect [e]. This assertion means that the transition duration is more or less constant. Studies of V1V2 production and perception characterized by their formant transition rates are presented. Such a representation leads to new interpretations of vowel reduction, coarticulation, perceptual overshoot, hyper and hypo speech, normalization.

2pSCc14. The effect of facal dynamics on infant perception of adult-directed speech in noise. Linda Polka (McGill University, School of Communication Sciences and Disorders, Beatty Hall, 1266 Pine Avenue West, Montreal, QC H3G 1A8, Canada, linda.polka@mcgill.ca), Robin Panneton (Virginia Polytechnic Instit. \& State Univer., Williams Hall, Blacksburg, VA 24061-0436, USA, Panneton@vt.edu), Jessica Versele (Virginia Polytechnic Instit. \& State Univer., Williams Hall, Blacksburg, VA 24061-0436, USA, Versele@vt.edu)

Recently Polka and Rvachew (in press) found that 6- to 8-month-old infants discriminated a native contrast("bu" - "gu") when familiarized and tested with syllables presented in quiet, but not when familiarized with syllables mixed with a competing noise, even when testing was conducted in quiet. Because the competing noise (segments of cricket and bird song) did not create energetic masking, infant failure to encode the familiarization syllable was due to a disruption in attention to the speech stream. Importantly, in this study, infants watched a checkerboard while listening to syllables spoken in an adult-directed (AD) style. The current study investigated if the addition of a dynamic face facilitates infants' speech processing in the same task. Six-month-olds were habituated to repetitions of the native syllable "ba" mixed with the same noise but these infants saw and heard syllables produced in $\mathrm{AD}$ style. Following habituation, infants were presented familiar (repetitions of "ba") and novel test trials (repetitions of "ga"). Infants failed to show syllable discrimination as indexed by a reliable preference for 
the novel test trials. These findings show that a dynamic face producing AD speech does not engage infant attention to speech enough to support syllable discrimination in noise.

2pSCc15. Voicing offsets and onsets in relation to intraoral pressure values in lingual obstruents of German. Laura L. Koenig (Haskins Labs. and Long Island Univ., Brooklyn, New York, NY 11201-8423, USA, koenig@haskins.yale.edu), Susanne Fuchs (ZAS, Schuetzenstr. 18, 10117 Berlin, Germany, fuchs@zas.gwz-berlin.de)

Phonation requires that tracheal pressure remain higher than intraoral pressure (Pio). In obstruent consonants, a major constriction in the upper vocal tract yields an increase in Pio, inhibiting phonation. The degree of Pio increase in consonants varies as a function of laryngeal and supraglottal apertures. Voiceless stops involve a rapid buildup and discharge of Pio, whereas fricatives involve more gradual changes in Pio. This work quantifies phonation offsets and onsets in German obstruents in relation to the Pio at these times. Pio signals were recorded via a pressure transducer affixed to the posterior end of an EPG palate while 9 speakers of standard German produced intervocalic voiceless consonant sequences (stops, fricatives, affricates, and clusters). Past theoretical work suggests that phonation offsets and onsets will show a hysteresis effect, with onsets requiring higher driving pressures than offsets. Of particular interest here is whether the extent of hysteresis differs among stops, fricatives, and obstruent sequences. Data on intraoral pressure change will also be compared with EPG data to explore how supraglottal constrictions affect Pio, and thus, phonation.

2pSCc16. Magnetic Resonance investigation of palatalized stop consonants and spirants in Russian. Galina Y. Kedrova (Moscow State Lomonosov University, GSP-2, Leninskije Gory, MGU, 1st Humanities, R. 983, Centre for New Technologies in Humanities, 119992 Moscow, Russian Federation, kedr@philol.msu.ru), Nikolay V. Anisimov (Moscow State Lomonosov University, GSP-2, Leninskije Gory, MGU, 1st Humanities, R. 983, Centre for New Technologies in Humanities, 119992 Moscow, Russian Federation, kedr@philol.msu.ru), Leonid M. Zaharov (Moscow State Lomonosov University, GSP-2, Leninskije Gory, MGU, 1st Humanities, R. 983, Centre for New Technologies in Humanities, 119992 Moscow, Russian Federation, kedr@philol.msu.ru), Yurij A. Pirogov (Moscow State Lomonosov University, GSP-2, Leninskije Gory, MGU, 1st Humanities, R. 983, Centre for New Technologies in Humanities, 119992 Moscow, Russian Federation, kedr@philol.msu.ru)

The research in question is aimed at the experimental MRI-investigation of articulatory gestures corresponding to the palatalized Russian stop con-

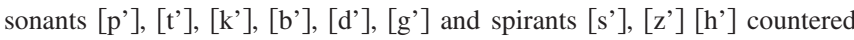
with their non-palatalized counterparts $[\mathrm{p}],[\mathrm{t}],[\mathrm{k}],[\mathrm{b}],[\mathrm{d}],[\mathrm{g}],[\mathrm{s}],[\mathrm{z}][\mathrm{h}]$ in Russian speech production. An experimental dataset was based upon 2D MR-images, audio- and video recordings taken from 4 native speakers of Russian (3 males and 1 female) producing VCCV sequencies (Russian pseudo-words with the second vowel stressed) containing Russian consonants under investigation in the vocalic contexts [a]_[a] and [a]_[i]. All speaking subjects had standard pronunciation and were without any perceptible articulation disease. MRI investigation of the Russian consonantal phonemes was based upon admitted procedures and techniques though expanded with several new original methods elaborated by the Russian team of experimentalists. Experimental data was collected through several MRI sessions (done in a month and in a year with participation of the same speaking subjects), ensuring thereby credibility and robustness of the experimental results. A detailed analysis of the whole dataset of MR-images of palatalised Russian consonants countered with their non-palatalized counterparts revealed special articulatory pattern of palatalisation in Russian.

2pSCc17. Duration of Japanese singleton and geminate obstruents in two- to four-mora words. Yukari Hirata (Colgate University, 13 Oak Drive, Department of East Asian Languages and Literatures, Hamilton, NY 13346, USA, yhirata@mail.colgate.edu), Hiroaki Kato (ATR Cognitive
Information Science Laboratories, 2-2-2 Hikaridai, Seika-cho Soraku-gun, 619-0288 Kyoto, Japan, kato@atr.jp), Keiichi Tajima (Hosei University, 2-17-1 Fujimi, Chiyoda-ku, 102-8160 Tokyo, Japan, tajima@hosei.ac.jp)

Hirata and Whiton [J. Acoust. Soc. Am. 118, 1647-1660 (2005)] found an invariant durational structure for Japanese stop quantity distinction in two- and three-mora words across different speaking rates. The present study examined whether their finding extends to include fricative and affricate quantity distinctions and three- and four-mora words. Stimuli were stop, fricative, and affricate contrasts in four types of words, including (1) a long vowel (e.g., ka.so.o vs. ka.s.so.o), (2) a moraic nasal (e.g., ji.se.n vs. ji.s.se .n), (3) CV sequence (e.g., ho.so.ku vs. ho.s.so.ku), and (4) shorter words (e.g., i.shi vs. i.s.shi), spoken in isolation at three speaking rates by four native Japanese speakers. Duration of contrasting obstruents, words, and the interval between the onsets of the first and the second vowels (VOI) was measured. Results indicated durational patterns similar to those found previously for stop contrasts. In addition, duration of words, regardless of their segmental composition, reflected well the number of moras they contained Finally, the ratio of the contrasting obstruent to the word and the ratio of the VOI to the mean mora duration were useful in classifying the singleton and geminate categories across rates. [Supported by Grant-in-Aid for Scientific Research, JSPS]

2pSCc18. Effects of hand gesture and lip movements on auditory learning of second language speech sounds. Spencer Kelly (Colgate University, 13 Oak Drive, Department of Psychology, Hamilton, NY 13346, USA, skelly@mail.colgate.edu), Yukari Hirata (Colgate University, 13 Oak Drive, Department of East Asian Languages and Literatures, Hamilton, NY 13346, USA, yhirata@mail.colgate.edu), Jen Simester (Colgate University, 13 Oak Drive, Department of Psychology, Hamilton, NY 13346, USA, jsimester@gmail.com), Jackie Burch (University of Rochester, 585 Elmwood Ave., Box 645, Rochester, NY 14642, USA, Jaclyn_Burch @ mail.urmc.rochester.edu), Emily Cullings (Colgate University, 13 Oak Drive, Department of East Asian Languages and Literatures, Hamilton, NY 13346, USA, ecullings@mail.colgate.edu), Jason Demakakos (Colgate University, 13 Oak Drive, Department of Psychology, Hamilton, NY 13346, USA, jdemakakos@mail.colgate.edu)

Previous research found that auditory training helps native English speakers to perceive phonemic vowel length distinction in Japanese, but that their performance has never reached native levels (Hirata et al., 2007) Given that multimodal information, such as hand gesture and lip movements, influences semantic aspects of language processing and development (Kelly et al., 2002), we examined whether this multimodal information helps to improve native English speakers' ability to perceive Japanese vowel length distinction. Forty-five native English speakers participated in one of three types of training: (1) audio alone; (2) audio with hand gestures; and (3) audio with lip movements and hand gestures. Before and after training, participants were given phoneme perception tests that measured their ability to distinguish between short and long vowels in Japanese, e.g., / kato/ versus /kato:/. Our original prediction was that more modalities in training would result in greater learning. Although all three groups improved from pre- to post-test, there were no significant differences among the three training groups. Unlike the original prediction, hand gestures and lip movements did not seem to augment learning of difficult sound distinctions. We will discuss possible benefits and limitations of using multimodal information in second language speech learning.

2pSCc19. MRI-based 3-D vocal tract acoustic analysis of an American English lateral sound. Xinhui Zhou (Speech communication lab, Institute of systems research and department of electrical and computer engineering, University of Maryland, College Park, MD 20742, USA, zxinhui @ umd.edu), Carol Y. Espy-Wilson (Speech communication lab, Institute of systems research and department of electrical and computer engineering, University of Maryland, College Park, MD 20742, USA, espy 
@ glue.umd.edu),Mark Tiede(Haskins lab and MIT Research lab of electronics, 300 George street suite 900, New Haven, CT 06511, USA, tiede@haskins.yale.edu), Suzanne Boyce (Department of communication sciences and disorders, University of Cincinnati, Mail location 0394, Cincinnati, OH 45267, USA, boycese@email.uc.edu)

The lateral sound /1/ in American English involves a complex articulatory configuration that includes one or two lateral channels along the sides of the tongue, and sometimes a lingual-alveolar contact. The acoustic characteristics of /1/ consist of a third formant (F3) that is close in frequency to the F3 of the adjacent vowel(s), but is often considerably weaker in amplitude. Generally, there is relatively weak energy in the F3-F5 region. The articulatory-acoustic relationship is not well understood. Previous studies did not consider the 3-D vocal tract model and the area function extraction was based on some assumed model. In this study, we constructed a 3-D vocal tract geometry based on magnetic resonance images from one subject during sustained production of syllabic dark /1/ (as in "pole"), and performed finite-element analysis. The harmonic analysis showed that the frequency response of the 3-D vocal tract is comparable in many ways to the spectrum of the acoustic signal, suggesting good agreement between the actual and reconstructed vocal tracts. A pole-zero pair is found in F3-F4 region, which may explain the weak energy level in this frequency region. Application of the 3-D wave propagation property to vocal tract area function extraction will also be discussed. [Research supported by NIH.]

2pSCc20. The acoustics of yer and non-yer vowels [e] and [o] in Slovak Stefan Benus (Constantine the Philosopher University, Stefanikova 67, 94901 Nitra, Slovakia, sbenus@ukf.sk)

Yers of Slavic languages are vowels that alternate with zero and historically developed from high short lax vowels. In Slovak, both front and back yers were preserved and surface as [e] and [o] respectively. For example, the second [o] in kotol 'cauldron-Nom-Sg' is a yer because it disappears with adding a suffix vowel: kotla 'cauldron-Gen-Sg'. Compare with a non-yer [o] in kostol 'church-Nom-Sg', kostola 'church-Gen-Sg'. Traditional phonological accounts of this difference (e.g. Rubach 1993) assume that yer vowels are underlyingly different from non-yer vowels and merge with [e] and [o] through a phonological process. Therefore, these accounts predict that yer and non-yer vowels should be phonetically identical since they enter the phonetic component already merged as [e] or [o]. The results of our acoustic experiments show that yer vowels are phonetically different from non-yer vowels. The most salient differences were observed in the first formant and duration: yers have lower F1 than non-yers, and for some subjects they are also shorter. This finding supports the view that the phonetic component has access to deep phonological alternations and that phonetics-phonology is a single cognitive system in which the components have different granularities and interact bidirectionally.

2pSCc21. Behavioral and physiological correlates of language preference. Megha Sundara (UCLA Department of Linguistics, 3125 Campbell Hall, Los Angeles, CA 90095-1543, USA megha.sundara@humnet.ucla.edu), Christine Kitamura (MARCS Auditory Laboratories, Bankstown Campus, Building 1, University of Western Sydney, Locked Bag 1797, 1797 Penrith South DC NSW, Australia, c.kitamura@uws.edu.au), Thierry Nazzi (CNRS - Université Paris Descartes, 45 rue des Saints-Pères, 75006 Paris, France, thierry.nazzi@univparis5.fr)

With increasing experience listening to language, infants' sensitivity to the patterns of their native language becomes more specialized. In monolingual, hearing infants, this tuning-in into the native language has been demonstrated in an elegant experiment. Hayashi et al. (2001) tested Japaneselearning infants between 4 and 14 months on their preference for listening to Japanese when compared to English. Not surprisingly, Japanese-learning infants preferred to listen to Japanese over English. Critically, the magnitude of this native language preference increased linearly from 4- to 14-months. In this study, we replicated and extended Hayashi et al.'s results. We tested 65 Australian-English learning infants aged 3 - 16 months on their preference for Australian-English compared to Japanese sentences. Our results confirm the findings of Hayashi et al.: Australian-English hearing infants' prefer their native language, and this preference increases linearly with age.
We also tested preference when the same infants heard Australian-English and German sentences. English and German are rhythmically similar, while differing in segmental characteristics. Results indicate that similar to early language discrimination, rhythmic distance affects the emergence of language preference. We are currently analysing concomitant heart rate measured from these infants when they were listening to native and non-native sentences.

2pSCc22. Improvement of speech recognition thresholds by spectral modulation enhancement. Chang Liu (University of Texas, Dept. of Comm. Sci. \& Dis., 1 University Station A1100, Austin, TX 78712, USA, changliu@mail.utexas.edu), David A. Eddins (University of Rochester, Department of Otolaryngology, Rochester, NY 14618, USA, David_Eddins @URMC.Rochester.edu)

Our previous studies have shown that vowel identification in noise was significantly improved by spectral enhancement resulting from modifications in the spectral modulation domain. The present study investigates whether spectral enhancement results in improved speech recognition in background competition. Speech recognition thresholds (SRT) were measured in a long-term speech shaped (LTSS) noise and in multi-talker babble using NU-6 words with and without spectral enhancement for young normal-hearing listeners. Results indicated that SRTs were significantly reduced, by $2.2 \mathrm{~dB}$, in LTSS noise and in babble when speech sounds were spectrally enhanced between 2.0 and 2.5 cycles/octave, while no significant changes in SRTs occurred for enhancement between 1.5 and 2 cycles/octave or between 1.5 and 2.5 cycles/octave. Spectral modulation transfer functions were also measured for each listener. Linear regression analyses showed that SRT improvement was moderately correlated with modulation detection thresholds at a spectral modulation frequency of 0.5 cycles/octave. Thus, it appears that better modulation detection at low spectral modulation frequencies is associated with greater improvement in word recognition in background competition.

2pSCc23. Perception of sinewave vowels. James $M$. Hillenbrand (Western Michigan University, Dept of Speech Path \& Aud., 1903 W. Michigan Ave., Kalamazoo, MI 49008, USA, james.hillenbrand@wmich.edu), Michael J. Clark (Western Michigan University, Dept of Speech Path \& Aud., 1903 W. Michigan Ave., Kalamazoo, MI 49008, USA, michael.clark@wmich.edu)

There is a significant body of research examining the intelligibility of sinusoidal replicas of naturally spoken utterances. Discussion has followed about what the sinewave speech phenomenon might imply about the auditory and perceptual mechanisms that are involved in phonetic recognition. However, this work has typically been conducted using meaningful and syntactically well formed sentences, making it unclear what the relative contributions are of higher level, top-down processes as opposed to lower level auditory and phonetic mechanisms. The simple purpose of this study was to measure vowel intelligibility using sinusoidal replicas of naturally spoken $/ \mathrm{hVd} /$ syllables. The sinusoidal signals were modeled after 300 utterances selected from a database of 1,668 syllables spoken by men, women, and children [Hillenbrand, Getty, Clark, and Wheeler, J. Acoust. Soc. Am., 97, 3099-3111 (1995)]. Listeners consisted of 74 students enrolled in an introductory phonetics course. Recognition rates for the sinusoidal vowels averaged $55 \%$, with considerable variability across listeners. This figure is substantially lower than the $\sim 95 \%$ intelligibility (measured in several earlier studies) of the naturally spoken signals upon which the sinusoidal replicas were modeled. Attempts to improve performance using three different training methods met with modest success, with post-training recognition rates rising by $\sim 5-12$ percentage points, depending on the training method.

2pSCc24. Complexity of acoustic-production-based models of speech perception. Geoffrey Stewart Morrison (Australian National University, School of Language Studies, Building 110, ACT 0200 Canberra, Australia, geoff.morrison@anu.edu.au)

Discriminant analysis models trained on acoustic vowel production data have been found to have significant correlation with listeners' perception. Two regularised discriminant analysis models were trained on monolingual speakers' vowels. One model was trained on North Central Peninsular Span- 
ish vowel tokens, and the other on Western Canadian English vowel tokens. For each language the model which resulted in the lowest cross-validated classification error rate was close to the least complex model possible, i.e., close to linear discriminant analysis using the variances of each acoustic variable but not the covariances between variables. In order to make crosslanguage vowel perception predictions the Spanish model was used to classify English vowel tokens and the English model was used to classify Spanish vowel tokens. Results suggest that monolingual North Central Peninsular Spanish listeners would assimilate most tokens of Western Canadian English /i/ and /I/ to Spanish /i/ and /e/ respectively, and thus for this combination of dialects, Spanish-speaking learners of English would not be expected to have difficulty with the English /i/-/I/ contrast.

2pSCc25. Letter sound and letter name recognition for automated literacy assessment of young children. Shrikanth Narayanan (University of Southern California, 3740 McClintock Ave, EEB430, Los Angeles, CA 90089-2561, USA, shri@sipi.usc.edu), A Kazemzadeh (University of Southern California, 3740 McClintock Ave, EEB430, Los Angeles, CA 90089-2561, USA, kazemzad@usc.edu), Matthew Black (University of Southern California, 3740 McClintock Ave, EEB430, Los Angeles, CA 90089-2561, USA, Matthew.Black@usc.edu), Joseph Tepperman (University of Southern California, 3740 McClintock Ave, EEB430, Los Angeles, CA 90089-2561, USA, tepperma@usc .edu), Sungbok Lee (University of Southern California, 3740 McClintock Ave, EEB430, Los Angeles, CA 90089-2561, USA, sungbokl@usc.edu), Abeer Alwan (University of California, 405 Hilgard Ave, Los Angeles, CA 90095, USA, alwan@ee.ucla.edu)

Evaluations of letter naming and letter sounding are commonly used to measure a young child's growing reading ability, since performance in them is well-correlated with future reading development. Assessing a child's oral reading skills requires teachers, as well as technologies that attempt to automate such assessment, to form an item-level accept/reject decision based on speech cues and prior knowledge of the child's literacy level and linguistic background. With data collected from 171 K-2 children, both learners and native speakers of American English, we designed and evaluated an automated letter naming assessment method using a simple word-loop HMM decoding for the word-level letter names. The automated accept/reject evaluation performance, $81.9 \%$, approached the agreement of human raters, $83.2 \%$ (0.62 kappa). However, the task where children must produce the sound that the letter represents was more difficult: English orthography allows one-to many letter-to-sound mapping, teachers showed less agreement in their assessment $(80.9 \%, 0.55 \mathrm{kappa})$, and the brief durations of some of the letter sounds made it difficult to distinguish them from each other and from background noises. Phone-level HMM based evaluation accuracy was $58.2 \%$. Preprocessing the recordings into speech, silence, and noise improved these results, especially for plosive sounds. [Supported by NSF]

2pSCc26. Neighbors as competitors: Phonological analysis of spoken word recognition errors. Adam Buchwald (NYU, 665 Broadway, Suite 910, New York, NY 10012, USA, buchwald@nyu.edu), Robert A Felty (Indiana University - Speech Research Laboratory, 1101 E 10th St., Dept. of Psychology, Bloomington, IN 47401, USA, robfelty@indiana.edu), David B. Pisoni (Indiana University - Speech Research Laboratory, 1101 E 10th St., Dept. of Psychology, Bloomington, IN 47401, USA, pisoni@indiana.edu)

Theories of speech perception and spoken word recognition widely assume that phonetically similar words compete for selection during lexical access. These competitors - called lexical neighbors - are classically defined as differing by a single segment, with little attention paid to other levels of phonological structure such as featural content or syllabic structure. This paper reports on analyses of over 15,000 word recognition errors made on a representative sample of the lexicon $(\mathrm{N}=1428)$ that includes the same variety of syllable structures, lengths, and lexical frequencies that exists in the English lexicon. The analyses reveal that competitors share segmental information as has been previously suggested, but that the classic definition of neighbor cannot capture the level at which featural and syllabic properties of the target constrain the error responses. We will discuss several analyses on these issues and discuss the implications for our understanding of the organization of the lexicon. [Work supported by NIH-NIDCD R01 00111 and T32 00012]

2pSCc27. The prosody of second position clitic placement and focus in Croatian. Kristine Yu (Dept. of Linguistics, University of California, 3125 Campbell Hall, Los Angeles, CA 90095, USA, krisyu@humnet.ucla .edu)

Since Browne 1974, the placement of second position clitics in Bosnian/Croatian/Serbian has inspired debate about interaction at the syntaxphonology interface. The placement of these clitics can alternate quite freely: either after the first phonological word or after the first syntactic constituent. While it's generally agreed that prosodic phonology, in addition to morphosyntax, plays a role in clitic placement in Bosnian/Croatian/Serbian, the prosodic patterns for the different placements have not been studied acoustically. In addition, it has been suggested that clitic placement and pragmatic focus may interact (Bošković 2001), but this has not been systematically studied. We recorded adult Zagreb Croatian speakers producing subject noun phrases with initially stressed trisyllabic adjectives and nouns in transitive sentences. We varied clitic placement (after the first word or first constituent) and focus domain (broad focus, and narrow focus on adjective, noun, both the adjective and the noun, or the entire noun phrase) and controlled for dialect and pitch accents. Preliminary results from three speakers indicate that gross differences in prosodic patterns occur only as a function of focus domain but not clitic placement. Further work will confirm if these patterns hold for more speakers and if clitic placement is correlated with finer-grained prosodic differences.

2pSCc28. English /r/-/l/ pronunciation training for Japanese speakers Kota Hattori (University College London, Department of Phonetics and Linguistics, 4, Stephenson Way, NW1 2HE London, UK, k.hattori@ucl.ac.uk), Paul Iverson (University College London, Department of Phonetics and Linguistics, 4, Stephenson Way, NW1 2HE London, UK, p.iverson@ucl.ac.uk)

Previous studies have demonstrated that perceptual training improves both perception and production by adult second-language (L2) learners. The present study examined whether production training likewise improves both perception and production. Japanese speakers underwent ten sessions of production training for English $/ \mathrm{r} /$ and $/ 1 /$. The training combined three methods: (1) subjects received audio-visual examples and pronunciation instructions for $/ \mathrm{r} /$ and $/ 1 /$; (2) they produced mono syllables (i.e., /ra/ and $/ \mathrm{la} /$ ) and minimal-pair words, with online feedback about their formant frequencies using a real-time spectrogram as well as pronunciation feedback from an instructor; and (3) they made recordings of minimal-pair words and compared them to "enhanced" versions of these recordings (i.e., signalprocessed versions with the correct F3 frequencies and transition durations). All participants completed a battery of pre/post training tests (i.e., English /r/-/1/ identification, discrimination, perceptual mapping of best exemplars, and production). The preliminary results indicate that this training approach improves production; the results will be further discussed in terms of its effect on perception and the underlying representation of these categories.

2pSCc29. Production of American English [eI] and [ $\varepsilon$ ] by Mandarin speakers: mono- vs. disyllabic words. Chung-Lin Yang (Dept. of Linguistics, Indiana Univ.-Bloomington, Memorial Hall 322, Bloomington, IN 47405, USA, cy1@indiana.edu)

Mandarin productions of English tense vs. lax vowels are difficult for English speakers to differentiate (Chen et al. 2006). In this study the production of American English $[\mathrm{eI}]$ and $[\varepsilon]$ by Mandarin and American participants was investigated. The target vowels were embedded in monosyllabic and disyllabic words with a stop-V-voiceless stop context in carrier sentences of variable length. There were two formant measurements for each vowel (20\% after the onset and before the end of the vowel). In the monosyllabic condition, Mandarin speakers' performance was similar to American speakers in duration and formant values except that [eI] started too low. However, in the disyllabic condition, Mandarin speakers' formants for $[\mathrm{eI}]$ and $[\varepsilon]$ were overlapped and there was no significant durational distinction between $[\mathrm{eI}]$ and $[\varepsilon]$. One possible account for this difference is that 
when producing a disyllabic word, Mandarin speakers tend to shorten the vowel duration of the first syllable to compensate for the production of the following syllable. The difference in syllable conditions may reflect a tendency that Chinese regularizes word durations regardless of the number of syllables. Reference: Chen, Y. et al. (2006). Vowel production by Mandarin speakers of English. Clinical linguistics and phonetics, 15, 427-440

$2 \mathrm{pSCc} 30$. A numerical analysis of fluctuations in pressure wave within the larynx using two-dimensional asymmetrical vocal folds model Hideyuki Nomura (Dept. of Electronic Eng., Univ. of ElectroCommunications, 1-5-1, Chofugaoka, 182-8585 Chofu-shi, Japan, nomu@ee.uec.ac.jp), Tomoo Kamakura (Dept. of Electronic Eng., Univ. of Electro-Communications, 1-5-1, Chofugaoka, 182-8585 Chofu-shi, Japan, kamakura@ee.uec.ac.jp), Tetsuo Funada (Div. of Electronic Eng. and Computer Sci., Kanazawa Univ., Kakuma-machi, 920-1192 Kanazawa-shi, Japan, funada@t.kanazawa-u.ac.jp)

Numerical simulations of pathological voice production and estimations of pressure wave fluctuations are performed based on a two-dimensional asymmetrical vocal folds (VFs) model. The asymmetrical VFs model takes into account of geometrical asymmetries (the thickness, effective depth of vibration region, and lateral rest position) and mechanical asymmetries (the Young's modulus, density, and viscosity of VF tissues). Simulation results based on the asymmetrical VFs model show that the left and right VFs vibrate with a phase difference. Obtained pressure waves within the larynx and vocal tract indicate fluctuations of fundamental frequency, amplitude, and waveform. In order to quantitatively evaluate the fluctuations, the coefficient of variation of the fundamental frequency, the coefficient of variation of the amplitude, and the harmonic-to-noise ratio are estimated. With increasing the VF asymmetries, especially on the effective depth and the density of VF elements, remarkable fluctuations are observed not only at the glottis but also in the regions downstream of the glottis and faraway from the glottis. This suggests that the estimation of fluctuation in pressure wave is useful for the diagnosis of pathological VFs.

2pSCc31. Differential effects of the phonemes on identification of previously unknown speakers. Kanae Amino (Dept. of Electrical and Electronics Engineering, Sophia University, 7-1 Kiyoi-cho, Chiyoda-ku, 102-8554 Tokyo, Japan, amino-k@sophia.ac.jp), Takayuki Arai (Dept. of Electrical and Electronics Engineering, Sophia University, 7-1 Kiyoi-cho, Chiyoda-ku, 102-8554 Tokyo, Japan, arai@sophia.ac.jp)

In perceptual speaker identification, it is known that the identification accuracy depends on the contents of the stimuli presented to the listeners. In our previous experiments, we found that the stimuli containing a nasal sound are effective for human speaker identification, and that coronal sounds are more effective than labial ones. This tendency was observed in the identifications of both familiar and previously unknown speakers. In this present study, we investigated the effects of the speech contents again, by focusing on the vowels in CV monosyllabic stimuli. Through the experiment we obtained several findings: 1) stimuli containing a nasal gained significantly higher accuracy compared to stimuli with only oral sounds; 2) coronal sounds were more effective than labial sounds; 3) palatalisation caused an improvement in performance; and 4) back vowels were more effective than front vowels significantly. These results can be explained by speakerspecific morphologies of the nasal cavity and the paranasal sinuses, and also of the pharyngeal cavity. We will also show analyses and discussions on the acoustical properties of the stimuli and the performance differences among the subjects. [Work supported by Sophia University Open Research Centre from MEXT.]

2pSCc32. Coarticulation in CV sequences: Locus Equation data Simone Graetzer (University of Melbourne, Royal Parade, Parkville Victoria, 3010 Melbourne, Australia, n.graetzer@pgrad.unimelb.edu.au)

Background: Speech sounds are known to be influenced by the context in which they occur, and to be produced in an overlapping way. These processes are referred to by the term "coarticulation". Objective: The aim of the study was to compare the magnitude of anticipatory coarticulation of oral voiceless plosives by following vowels in four Australian languages, Arrernte, Burarra, Gupapuyngu, and Warlpiri. Methods: The corpus consists of approximately six hundred isolated real words spoken by eight female adult speakers of the four languages. Locus equations are calculated for intervocalic consonants /p,c,t,tr,k/ (where "/tr/" represents a voiceless oral retroflex plosive) and all following vowels in consonant-vowel sequences. Measurement points are 0.1 and 0.5 into the vowel. The relationship between these two measurement points is linear and is expressed as a locus equation. The slope of the fitted line associated with the equation is a measure of the magnitude of coarticulation of the consonant by the following vowels. Results: There was a general tendency towards relatively highly coarticulated bilabial and velar plosives, weakly coarticulated retroflex plosives, and moderately coarticulated alveolar plosives. Results were variable for palatal plosives. These results are for the most part in accordance with findings for other Australian languages.

2pSCc33. Lexical analysis of spoken word recognition errors. Robert A. Felty (Indiana University - Speech Research Laboratory, 1101 E 10th St., Dept. of Psychology, Bloomington, IN 47401, USA, robfelty@indiana.edu), Adam Buchwald (NYU, 665 Broadway, Suite 910, New York, NY 10012, USA, buchwald@nyu.edu), David B. Pisoni (Indiana University - Speech Research Laboratory, 1101 E 10th St., Dept. of Psychology, Bloomington, IN 47401, USA, pisoni@indiana.edu)

In speech perception and spoken word recognition, a lexical "neighbor" of a target word is a phonetically similar word that competes with the target during lexical access. A commonly-used definition is that neighbors differ from one another by a single segment. While this basic definition accounts for many results of lexical decision and word recognition tasks among monosyllabic words, it also suggests that approximately half of the words in the English lexicon are hermits (words without neighbors). To generate a more inclusive definition of neighbor, we created a representative sample of the lexicon $(\mathrm{N}=1428)$ to examine spoken word recognition errors, and we used the incorrectly selected competitors as a means of indexing "neighbor" status. Analysis of over 15,000 errors reveals several patterns: (1) Errors tend to be of higher frequency than the target word; (2) Less than $1 / 3$ of the errors were neighbors according to the traditional definition of neighborhood density; and (3) Errors differed in phonetic similarity more as the $\mathrm{S} / \mathrm{N}$ ratio decreased. We use these data to test several alternative definitions of "neighbor" and their ability to predict actual word recognition errors. [Work supported by NIH-NIDCD R01 00111 and T32 00012]

2pSCc34. Articulatory constaints on coarticulation in fricatives as a function of tongue involvement. Carole E. Gelfer (William Paterson University, 300 Pompton Road, Wayne, NJ 07470, USA, gelferc@wpunj.edu), Fredericka Bell-Berti (St. John's University, 8000 Utopia Parkway, Queens, NY 11439, USA, bellf@stjohns.edu)

Numerous studies have shown coarticulatory effects of vowel rounding on the spectra of sibilants. However, the extent to which the tongue contributes to coarticulation is not clear because most studies have used only fricatives whose constriction is formed with the tongue. This study examines the effects of vowel environment on coarticulation in fricatives under two conditions: when the constriction is formed with the tongue (i.e., [s]) and when it is not (i.e., [f]), paired with the English vowels occurring at the extremes of the vowel quadrilateral. Preliminary results for adult native speakers of American English suggest that in the absence of tongue constraint ( $\mathrm{i}$ .e., [f]), there is a greater effect of vowel context on the fricative spectrum. This result reinforces the importance of considering the freedom of an articulator to anticipate an upcoming gesture when examining the extent of coarticulation.

2pSCc35. Acquisition of rhythm: evidence from spontaneous L2 speech. Irina Shport (University of Oregon, Department of Linguistics, 1290, Eugene, OR 97403, USA, ishport@uoregon.edu)

This study examined the acquisition of Japanese rhythm by comparing the rhythmic characteristics of elicited spontaneous speech of English learners in the setting of an oral proficiency test. Two metrics for the quantification of speech rhythm that have been shown to reliably discriminate rhythmic classes in both L1 and L2 speech (White and Mattys, 2007) were utilized for the analysis - 1) the proportion of vowel intervals within the sentence $(\% \mathrm{~V})$, and 2$)$ the standard deviation of vowel intervals divided by the 
mean vowel interval duration and multiplied by 100 (VarcoV). A difference in the rhythm scores of novice-mid and intermediate-high learners of Japanese was found. Furthermore, the learners' scores were significantly different from Japanese native speakers' scores, suggesting that speakers start acquiring speech rhythm relatively early, but they are still short of the target at the intermediate level of proficiency. By comparing the obtained scores with scores of stress-timed languages, such as English, the difference in the scores may be attributed to an L1 transfer into the rhythmic organization of the L2 speech.

2pSCc36. Effects of training, style, and rate of speaking on speech perception of young people in reverberation. Nao Hodoshima (Dept. of Electrical and Electronics Engineering, Sophia University, 7-1 Kiyoi-cho, Chiyoda-ku, 102-8554 Tokyo, Japan, n-hodosh@sophia.ac.jp), Takayuki Arai (Dept. of Electrical and Electronics Engineering, Sophia University, 7-1 Kiyoi-cho, Chiyoda-ku, 102-8554 Tokyo, Japan, arai@sophia.ac.jp), Kiyohiro Kurisu (TOA Corporation, 2-1 Takamatsucho, Takarazuka,665-0043 Hyogo, Japan, kurisu_kiyohiro@toa.co.jp)

Because of the difficulty of listening to speech in reverberation (e.g. train stations or airports), we need to find characteristics of intelligible speech sounds that are appropriate for announcements over loudspeakers in public spaces. This study investigated the effects of training (seven talkers who have received speech training or not), style (conversational/clear) and rate (normal/slow) of speaking on speech perception of young people in simulated reverberant environments. The talkers were instructed to speak nonsense words embedded within a carrier sentence clearly or normally in an anechoic room, and listening tests were carried out with young people in simulated reverberant environments. Results showed that correct rates significantly differed among the talkers, but no difference in correct rates was found between the two speaking rates, and conversational speech had significantly higher correct rates than clear speech. Casual inspections of the stimuli indicate that vowels are enhanced as well as consonants in clear speech so that clear speech had lower correct rates than conversational speech due to increased reverberant masking in clear speech compared to conversational speech. [Work supported by Sophia University Open Research Center from MEXT.]

2pSCc37. Prosody in French theatrical declamation traced backwards in time. George Brock-Nannestad (Patent Tactics, Resedavej 40, DK-2820 Gentofte, Denmark, pattac@image.dk)

Listening to early recordings of a French theatrical personality, such as Sarah Bernhardt, the emphatic emission of words is very noticeable. It is sometimes ascribed to Bernhardt's theatrical mannerisms, but possibly also caused by an erroneous speed of reproduction. But even at correct speed, huge variations in intenseness are very noticeable. Literature does not give a clear indication whether this manner of performance was considered deviating at the time (ca. 1900). New research into the original handwritten patent documents of Édouard-Léon Scott (filed 1857 and 1859) has analyzed his method of recording the performance of the human voice. Scott documented the performance of a complete stanza from a French play, and looking at his graphical output and interpretation it becomes clear that the prosody of Mme. Bernhardt was already in place in 1859. Having identified the French play, even earlier descriptions of the prosodic approach in French declamation were found. The paper also discusses the patents of ÉdouardLéon Scott and his desire for the use of his invention, which was different from that of his manufacturer, Rudolph Koenig. Furthermore, the prosodic documentation will be demonstrated, and a historically informed reproduction of a recording by Sarah Bernhardt will be presented.

2pSCc38. Articulatory coordination in German and English onset and coda clusters. Marianne Pouplier (Institute of Phonetics and Speech Processing, University of Munich, 80799 Munich, Germany, pouplier@phonetik.uni-muenchen.de), Stefania Marin (Institute of Phonetics and Speech Processing, University of Munich, 80799 Munich, Germany, stefania.marin@gmail.com)

Articulatorily, onset consonants differ from coda consonants in their spatial as well as their temporal characteristics in that onsets exhibit greater spatial displacement and are generally less variable than codas. As to their temporal organization, it has been claimed that onsets, but not codas exhibit a so-called c-center organization in which all consonant gestures of a cluster are organized as a single complex structure with respect to the vowel, while consonant clusters in codas have been hypothesized to be organized sequentially [Browman \& Goldstein 1988 Phonetica]. While some studies have confirmed the c-center hypothesis for onsets but not codas, others found a c-center organization for both onset and coda consonants. The current study uses German and English onset and coda clusters to test systematically the c-center hypothesis on the basis of EMA data. Results for both languages indicate that a c-center organization may be observed in both onset and coda position, yet it does not appear consistently for all clusters. Also cross wordboundary clusters may exhibit c-center organization, possibly indicating resyllabification.

2pSCc39. Acoustic consequences of gestural intrusion errors. Stefania Marin (Institute of Phonetics and Speech Processing, University of Munich, 80799 Munich, Germany, stefania.marin@gmail.com), Marianne Pouplier (Institute of Phonetics and Speech Processing, University of Munich, 80799 Munich, Germany, pouplier@phonetik.uni-muenchen de), Jonathan Harrington (Institute of Phonetics and Speech Processing, University of Munich, 80799 Munich, Germany, jmh@phonetik .uni-muenchen.de), Susanne Waltl (IPS, LMU, Schellingstr. 3/II, 80799 München, Germany, susanne@phonetik.uni-muenchen.de)

An increasing number of studies have elicited speech errors in articulatory experiments, and thus evaluated errors on the basis of their articulatory properties, yet there has been no study that relates the articulatory variability observed in errors to the acoustic consequences. The present study aims to evaluate the acoustic properties of errorful/error-free $[\mathrm{k}]$ and $[\mathrm{t}]$ stimuli with known articulatory configurations (and articulatorily categorized as errorful/error-free), and determine the extent to which articulatory errors have acoustically observable consequences. The most frequent error observed in the articulatory data is an intrusion of a second gesture, resulting in a simultaneous presence of both tongue tip and tongue body gestures during production of $[\mathrm{k}]$ or $[\mathrm{t}]$. Spectral analysis of these stimulis' bursts shows that $[\mathrm{k}]$ and $[\mathrm{t}]$ are differently affected by intrusion gestures: intrusion errors during intended $[\mathrm{k}]$ tokens are more robustly grouped with error-free $[\mathrm{k}]$ across speakers, while intrusion errors during intended $[\mathrm{t}]$ tokens are more variable, some being acoustically like $[\mathrm{t}]$, some like $[\mathrm{k}]$, and some like neither category, matching previous perceptual results (Pouplier \& Goldstein 2004, Journal of Phonetics). These results will be discussed in the context of different timing patterns between the two co-occurring gestures during intrusion errors.

2pSCc40. Embodiment, not imitation, leads to the replication of timing phenomena. Piers Messum (112 Warner Road, SE5 9HQ London, UK, p.messum@gmail.com)

In many languages, a spoken vowel is shorter before phonologically voiceless consonants than before voiced ones. In West Germanic languages, including English, tense and lax vowels have different lengths and there is a characteristic 'stress-timed' rhythm. For these phenomena and some others, it is generally assumed that time is the controlled variable for production, and that children replicate these speech behaviors through the reproduction of timing patterns abstracted from the adult input (i.e. by imitation). The developmental data does not support these assumptions, and an imitative mechanism would present a young speaker with a highly complex challenge. Instead, these behaviors are more plausibly the result of the embodiment of speech. Embodiment goes beyond laryngeal and articulatory function. A child's speech breathing is not a scaled-down version of the adult model but a distinctly different skill, and one that must be learnt during speech production. Similarly, the aerodynamic setting of child speech differs significantly from that of adults. The constraints that these factors impose become manifest in speech as changes in timing, but these changes are epiphenomenal, not modelled directly. Phenomena particular to West Germanic languages reflect the style of speech breathing these languages require of a child. 
2pSCc41. Investigating the perception of noise-vocoded speech - an individual differences approach. Carolyn McGettigan (University College London, Institute of Cognitive Neuroscience, 17 Queen Square, WC1N 3AR London, UK, c.mcgettigan@ucl.ac.uk), Stuart Rosen (UCL, Wolfson House, 4, Stephenson Way, NW1 2HE London, UK, stuart@phon.ucl.ac.uk), Sophie K. Scott (University College London, Institute of Cognitive Neuroscience, 17 Queen Square, WC1N 3AR London, UK, sophie.scott@ucl.ac.uk)

We used a cochlear implant simulation (noise-vocoded speech) to investigate speech recognition and perceptual learning in normal-hearing adult speakers of English. In two separate sessions (1-2 weeks apart), 28 listeners were tested on recognition of noise-vocoded Sentences, Words, and isolated segments (Consonants and Vowels). There was evidence of significant perceptual learning that survived until Session 2 for all tasks. An individual differences analysis of Session 1 data suggested two independently-varying 'levels' of processing at work in the initial perception of the distorted speech stimuli - a 'top-down' listening mode making use of contextual and lexical information, and a 'bottom-up' mode focussed on acoustic-phonetic discriminations. By Session 2, a more generalised listening mode emerged, reflecting listeners' consolidation of basic sound-to-representation mappings. Further exploration of Consonant and Vowel confusion data (using Information Transfer analyses) suggested that better speech recognition performance may be achieved through more efficient use of the preserved cues to duration and voicing in noise-vocoded stimuli, but that listeners failed to take full advantage of such information. We conclude that training regimes involving directed attention to specific features, such as vowel length, may help to improve performance with noise-vocoded speech.

2pSCc42. Speed-curvature relations in speech production: a multilingual experimental and modeling study. Pascal Perrier (ICP/GIPSA-lab, INPG, 46 Avenue Félix Viallet, 38031 Grenoble Cedex 01, France, Pascal.Perrier@gipsa-lab.inpg.fr), Susanne Fuchs (ZAS, Schuetzenstr. 18, 10117 Berlin, Germany, fuchs@zas.gwz-berlin.de)

Regularities in the relations between tangential velocity and trajectory curvature have been found in a number of human movements. They were formalized in the $1 / 3$ power law [Viviani and Terzuolo, Neuroscience 7: 431-437, 1982]. Viviani and colleagues also found that modifying the speedcurvature relations would influence the perception of synthetic movements in terms of naturalness and classification. Hence, these relations could be at the core of the perception-action link in human movements. In this paper, tongue movement collected from speakers of French, German and Mandarin are analyzed. They are compared with data from simulations using a biomechanical tongue model, which is controlled on a target-to-target basis. Our results for experimental data and simulations are very similar. It is found that the $1 / 3$ power law offers a fair description of the velocity-curvature relations when a large amount of movements are considered together. However, a refined analysis of individual movements reveals that the exceptions to this law are numerous. It is concluded that the velocity-curvature relation is not controlled in speech movements, and that the $1 / 3$ power law only accounts for general characteristics of movements, which could arise from dynamical properties of the articulators.

2pSCc43. Vowel spaces in Swedish children with cochlear implants Tina Ibertsson (Lund University, Dept. Logopedics, Phoniatrics \& Audiology, University Hospital, SE-221 85 Lund, Sweden, Tina.Ibertssont@med.lu.se), Birgitta Sahlén (Lund University, Dept. Logopedics, Phoniatrics \& Audiology, University Hospital, SE-221 85 Lund, Sweden, Birgitta.Sahlen@med.lu.se), Anders Lofqvist (Lund University, Dept. Logopedics, Phoniatrics \& Audiology, University Hospital, SE-221 85 Lund, Sweden, Anders.Lofqvist@med.lu.se)

This study analyzed vowel productions of Swedish children with cochlear implants, capitalizing on the rich vowel system of Swedish with nine vowels. Minimal word pairs were used as linguistic material. A group of 12 children with CI (mean age 184 months, range 144-229 months), and a group of children with normal hearing (mean age 105 months, range 103118 months) were recruited. The age at implantation of the CI was 79 months (range 31-155 months). The first two formants were measured for each vowel. To estimate the vowel space, the average Euclidean distance in the F1-F2 plane between each vowel and the mean first and second formant frequencies of all the vowels was calculated for each child. The results show a significantly smaller vowel space for the children with a cochlear implant. However, there was no overall significant correlations between the vowel space and measures of speech recognition and vowel production in the CI group. Although the present results show that speech recognition ability affects intelligibility and vowel production, the vowel space may not be a suitable measure for assessing speech production in this population. [Work supported by the Sunnderdahl Disability Foundation.]

2pSCc44. Detection of obstruent consonant landmark for knowledge based speech recgonition system. Jung-In Lee (Yonsei University, 134 Sinchon-dong, Seodaemun-gu, 120-749 Seoul, Republic of Korea, junginida@dsp.yonsei.ac.kr), Jeung-Yoon Choi (Yonsei University, 134 Sinchon-dong, Seodaemun-gu, 120-749 Seoul, Republic of Korea, jychoi @yonsei.ac.kr)

Obstruent consonant landmarks are detected using spectral energy difference profiles. This study expands upon previous work by Liu. A[J. Acoust. Soc. Am. 100, 3417-3430, 1996]. The proposed algorithm detects four types of landmarks : [stop closure], [stop release], [fricative closure] and [fricative release], where affricates are detected by combining [stop closure], [fricative closure] and [fricative release]. In addition to finding abrupt changes in energy differences, we use energy contours, relative energy and spectral center of gravity differences. This method results in improved performance particularly for CV obstruents. Overall detection rates for stop closure and release are $76.9 \%$ and $85.7 \%$ for obstruent landmarks in TIMIT, and fricatives yield $82.2 \%$ and $83.6 \%$ respectively. For strident fricatives, the figures are $94.7 \%$ and $93.6 \%$.

2pSCc45. Acoustic-phonetic features for stop consonant place detection in clean and telephone speech. Jung-Won Lee (Yonsei University, 134 Sinchon-dong, Seodaemun-gu, 120-749 Seoul, Republic of Korea, jaesuk2002@dsp.yonsei.ac.kr), Jeung-Yoon Choi (Yonsei University, 134 Sinchon-dong, Seodaemun-gu, 120-749 Seoul, Republic of Korea, jychoi @yonsei.ac.kr)

This work classifies voiceless stop consonant place in CV tokens of English using burst release cues for clean (TIMIT) and telephone speech (NTIMIT). We compared the performance of cepstral coefficients to acoustic phonetics-motivated features such as center of gravity, burst amplitude and relative difference of formant amplitudes. In clean speech, cepstral coefficients resulted in better classification. However, for test data from NTIMIT, acoustic phonetic-based features outperformed cepstral coefficients, particularly if models were trained on clean speech. In addition, augmenting cepstral coefficients with acoustic phonetic-based measurements resulted in the best performance. These findings suggest that cepstral coefficients are able to model speech in a given environment in finer detail, whereas acoustic phonetic-based features are more robust to changes in environment, so that combining both types of measurements leads to the best performance.

2pSCc46. Vowel place detection for a knowledge-based speech recognition system. Sukmyung Lee (Yonsei University, 134 Sinchondong, Seodaemun-gu, 120-749 Seoul, Republic of Korea, pooh390@dsp.yonsei.ac.kr), Jeung-Yoon Choi (Yonsei University, 134 Sinchon-dong, Seodaemun-gu, 120-749 Seoul, Republic of Korea, jychoi @yonsei.ac.kr)

This work aims to detect vowel place as part of a knowledge-based speech recognition system. Vowel place was classified into 6 groups based on tongue advancement [Front/Back] and height [High/Mid/Low]. Experiments were performed using $300 / \mathrm{hVd} /$ utterance data from Hillenbrand [J. Acoust. Soc. Am. 97, 3099-3111] and 6600 TIMIT vowels. Features used include fundamental frequency (F0) and formant value (F1 F3), where formant measurements were classified into separate groups using F0 measurements. The nearest class was found using a simple Mahalanobis distance measure, and yielded a $91.5 \%$ classification rate for the $/ \mathrm{hVd} /$ data. 
The results for the TIMIT data were $64.4 \%$, and error analysis with regard to adjacent segment manner and place was carried out to observe the effects of coarticulation, which was not observed in the $/ \mathrm{hVd} /$ data.

2pSCc47. Cross-modal perceptual learning of spectrally degraded speech: Behavioral and neuroimaging studies. Frank Eisner (University College London, Institute of Cognitive Neuroscience, 17 Queen Square, WC1N 3AR London, UK, f.eisner@ucl.ac.uk), Carolyn McGettigan (University College London, Institute of Cognitive Neuroscience, 17 Queen Square, WC1N 3AR London, UK, c.mcgettigan@ucl.ac.uk), Stuart Rosen (UCL, Wolfson House, 4, Stephenson Way, NW1 2HE London, UK, stuart@phon.ucl.ac.uk), Andrew Faulkner (UCL, Wolfson House, 4, Stephenson Way, NW1 2HE London, UK, andyf@phon.ucl.ac.uk), Sophie K. Scott (University College London, Institute of Cognitive Neuroscience, 17 Queen Square, WC1N 3AR London, UK, sophie.scott@ucl.ac.uk)

We investigated normal-hearing listeners' ability to adapt to a speech signal that simulates aspects of the stimulation received from a cochlear implant. The training materials were spoken sentences which were spectrally degraded by noiseband-vocoding and shifted upwards in frequency. A control condition consisted of spectrally inverted, unintelligible versions of these stimuli. Participants listened passively to these sentences, and on each trial received visual feedback which could be either a written version of the sentence or a video of the talker who originally produced it. Learning under both feedback conditions was relatively fast: Subjects improved on average by $25 \%$ on keyword recognition scores after 100 trials. No learning effects were observed in the control condition. We further used functional magnetic resonance imaging to investigate which cortical areas may be recruited during learning. A comparison of degraded and learnable sentences with the unlearnable control stimuli showed activity in the left superior temporal sulcus both during passive listening and receiving feedback. In contrast, the left inferior frontal gyrus was activated only when subjects were receiving feedback in the learnable condition. These results suggest that the inferior frontal gyrus plays an important role in integrating acoustic-phonetic processing with externally provided feedback.

2pSCc48. Robust harmonic grouping by octave error correction. Hedde Van De Vooren (University of Groningen, Dept. Artificial Intelligence, P.O. Box 407, 9700 AK Groningen, Netherlands, h.van.de.vooren@ai.rug.nl), Renante R. Violanda (University of Groningen, Dept. Artificial Intelligence, P.O. Box 407, 9700 AK Groningen, Netherlands, R.Violanda@ai.rug.nl), Tjeerd C. Andringa (University of Groningen, Dept. Artificial Intelligence, P.O. Box 407, 9700 AK Groningen, Netherlands, t.andringa@ai.rug.nl)

Harmonic grouping is a frequently applied technique in computational auditory scene analysis and automatic speech recognition systems. However, grouping is easily disrupted by noise and reverberation. For instance, a noise induced signal component positioned roughly between two harmonics, might undesirably be assigned to the harmonic complex (HC) as well. This results in an octave error: harmonics in an $\mathrm{HC}$ are assigned to harmonic numbers twice as high as the correct values. We propose a cost function based method to correct these octave errors. This function is designed to, on the one hand, improve the balance between odd and even harmonic numbers, and, on the other hand, minimize the amount of signal components to be rejected. As a preprocessing step we applied short-time Fourier analysis to derive an instantaneous frequency representation from which we obtained the signal components. We used these as input for our harmonic grouping algorithm to obtain the HCs. Then we selected the optimal solution from the cost function and modified the composition of the HCs accordingly. As long as enough harmonics are sufficiently above the local noise level, this octave error correction mechanism works well for various sorts of harmonic sounds including speech.

2pSCc49. A cross-language familiar talker advantage? Susannah Levi (University of Michigan, 440 Lorch Hall, 611 Tappan Street, Ann Arbor, MI 48109, USA, svlevi@umich.edu), Stephen Winters (University of
Calgary, Social Sciences Bldg. Room 820, Department of Linguistics, Calgary, AB T2N 1N4, Canada, swinters@basesproduced.com), David B. Pisoni (Indiana University - Speech Research Laboratory, 1101 E 10th St. Dept. of Psychology, Bloomington, IN 47401, USA, pisoni@indiana.edu)

Previous research has shown that familiar talkers are more intelligible than unfamiliar talkers. In the current study, we tested the source of this familiar talker advantage by manipulating the type of talker information available to listeners. Two groups of native English listeners were familiarized with the voices of five German-English bilingual talkers; one group learned the voices from German stimuli and the other from English stimuli. Thus, English-trained listeners had access to both language-independent and English-specific talker information, while German-trained listeners had access to language-independent and German-specific talker information. After three days of voice learning, all listeners performed a word recognition task in English. Consistent with previous findings, English-trained listeners found the speech of familiar talkers to be more intelligible than unfamiliar talkers, as measured by whole words and phonemes correct. In contrast, German-trained listeners showed no familiar talker advantage, suggesting that listeners must have knowledge of talker-specific, linguistically relevant information to elicit the familiar talker advantage and that knowledge of language-independent talker information - such as size and shape of the vocal tract - does not facilitate speech perception.

2pSCc50. Prosodic effects on articulatory coordination in initial consonant clusters in German. Lasse Bombien (Institut für Phonetik und Sprachverarbeitung, Schellingstr. 3, 80799 München, Germany, lasse@phonetik.uni-muenchen.de), Christine Mooshammer (Haskins lab and MIT Research lab of electronics, 300 George street suite 900, New Haven, CT 06511, USA, tine@ haskins.yale.edu), Phil Hoole (Institut für Phonetik und Sprachverarbeitung, Schellingstr. 3, 80799 München, Germany, hoole@phonetik.uni-muenchen.de), Barbara Kuehnert (Laboratoire de Phonétique et Phonologie - UMR 7018 CNRS - Paris 3, 19, rue des Bernadins, 75005 Paris, France, barbara.kuhnert@univ-paris3.fr)

This study examines how articulatory coordination in heterorganic initial consonant clusters is modulated by prosodic condition. Extending the paradigm of articulatory strengthening at domain edges to clusters, we provide EPG data of 7 speakers as well as EMA data of two speakers including domain-initial clusters $(/ \mathrm{kl} /, / \mathrm{kn} /$ and $/ \mathrm{sk} /)$ in different prosodic positions, varying the strength of the preceding boundary and the position of lexical stress. (e.g. 'Claudia vs. Klau'sur, i.e. stressed vs. unstressed target cluster). The results show that $\mathrm{C} 1$ was consistently lengthened at higher boundaries, but only if the cluster was preceded by a clear pause. C2 was only weakly and inconsistently affected. There were no effects on the spatial domain at all. No consistent effects of lexical stress were found for either $\mathrm{C} 1$ or $\mathrm{C} 2$. Regarding overlap of $\mathrm{C} 1$ and $\mathrm{C} 2$ there was a tendency, albeit quite weak, in the direction of more overlap at the lower boundary levels and in unstressed condition. However, differences in timing between segmentally different clusters were consistent and much larger than boundary and stress effects. Especially for $/ \mathrm{kl} / \mathrm{vs}$. $/ \mathrm{kn} /$ we find that the lateral's timing to $/ \mathrm{k} /$ is much closer than the nasal's.

2pSCc51. Hierarchical approach to voice activity detection in realistic environmental noise. Jörg-Hendrik Bach (Universität Oldenburg, Medizinische Physik, Fakultät V, 26111 Oldenburg, Germany, j.bach@uni-oldenburg.de), Jörn Anemüller (Universität Oldenburg, Medizinische Physik, Fakultät V, 26111 Oldenburg, Germany, joern.anemueller @uni-oldenburg.de)

The automatic identification of acoustic objects, particularly speech, in adverse backgrounds can pose a difficult problem. In this work, the problem of speech detection in different backgrounds is tackled using a hierarchical decision tree: The root node provides a coarse classification of the noise background, while the next nodes are optimised to detect speech in one particular background each. The results of this approach are compared to the ITU G729B standard VAD and to a binary classifier trained on speech vs a mixture of the backgrounds. The features employed are amplitude modulation spectra of the signals (Kollmeier \& Koch, 1993) that represent the modulation content of a signal as a two-dimensional (modulation-frequency vs centre frequency) spectral pattern. The best features for classification at 
each node are selected independently (node-specific) using a sequential floating forward search algorithm. The statistical learner is an open source support vector machine (Chang \& Lin, 2001).

2pSCc52. Visual Influences on Alignment to Voice Onset Time Kauyumari Sanchez (University of California, Riverside, Department of Psychology, 900 University Ave., Riverside, CA 92521, USA, ksanc004@student.ucr.edu), Rachel M. Miller (University of California, Riverside, Department of Psychology, 900 University Ave., Riverside, CA 92521, USA, rmill002@ucr.edu), Lawrence D. Rosenblum (University of California, Riverside, Department of Psychology, 900 University Ave., Riverside, CA 92521, USA, rosenblu@ citrus.ucr.edu)

In speech alignment, interlocutors inadvertently imitate aspects of each others utterances. Alignment also occurs when talkers are asked to shadow (identify out loud) recorded words heard over headphones. For example, the voice onset time (VOT) of consonants produced by subjects will be greater when shadowing heard words whose consonants have been modified to have extended VOTs [K. Shockley, et al., Percept. Psychophys. 66, 422 (2004)]. An experiment was conducted to test whether alignment to VOT has a purely acoustic basis. For this purpose, visual speech tokens composed of a face articulating /ba/ syllables at three different rates, were each dubbed onto a series of seven audio /ba/ syllables varying in VOT. Subjects were asked to simply shadow the audiovisual /ba/s. Results revealed that both the visible syllable rate and audio VOT of the stimuli influenced the VOTs of the shadowers' produced syllables. The implications of these results for theories of alignment, audiovisual integration, and the perceptual primitives of speech are discussed.

2pSCc53. The '[+English]' effect in acquisition of English as a second language: Novel- category biases in second language perception and production. Kenneth De Jong (Indiana University, 322 Memorial Hall, 1021 E. 3rd Street, Bloomington, IN 47405, USA, kdejong@indiana .edu), Yen-Chen Hao (Indiana University, 322 Memorial Hall, 1021 E. 3rd Street, Bloomington, IN 47405, USA, yehao@indiana.edu), Hanyong Park (Indiana University, 322 Memorial Hall, 1021 E. 3rd Street, Bloomington, IN 47405, USA, hanypark@indiana.edu), Noah H. Silbert (Indiana University, 322 Memorial Hall, 1021 E. 3rd Street, Bloomington, IN 47405, USA, nosilber@indiana.edu)

Silbert \& de Jong (JASA, 122: 3092) investigated correlations between response bias and segment frequency in a number of previously published segment identification data sets. In general, bias tends to be positively correlated with frequency. When applied to second language (L2) learning, this pattern predicts biases toward identifying novel L2 categories as L1 categories, since the number of instances of L1 categories far outweighs those of novel categories in learners' experience. Nagao, Lim, \& de Jong (2003, $\mathrm{ICPhS}$ ) confirm this: Japanese listeners tend to label ambiguous English coda consonants as onsets. However, younger and less experienced learners of English tend to label ambiguous tokens as codas; they tend toward using the novel category even though it is less frequent in their experience. This paper reports experiments in which Korean EFL learners identified English obstruents and in which they produced them. Cases of novel category response bias are observed in identification, and less so in production. Such novel category effects suggest a model in which novel categories serve, in part, as indicators of the L2 itself and stand out as response options in L2 identification tasks. These effects are likely counteracted by motor difficulties in L2 production tasks.

2pSCc54. Study on voice quality parameters for anger degree estimation. Yoshiko Arimoto (Tokyo University of Technology, Graduate School of Bionics, Information and Media Science, Faculty of MS, Iida labolatory, 1404-1 Katakura, Hachioji, 192-0982 Tokyo, Japan, ar@mf.teu.ac.jp), Sumio Ohno (Tokyo University of Technology, 1404-1 Katakura, 192-0982 Hachioji, Japan, ohno@cc.teu.ac.jp), Hitoshi Iida (Tokyo University of Technology, 1404-1 Katakura, 192-0982 Hachioji, Japan, iida@media.teu.ac.jp)

With great advance of automatic speech recognition (ASR) systems and a voice command system are demanded to be more sensitive to user's intention or emotion. These systems currently process linguistic information, but not process nonlinguistic information or paralinguistic information which users expressed during dialogs. For that reason, computers can obtain less information about a user through a dialog than human listeners can. If computers will recognize user's emotions conveyed by acoustic information, more appropriate response can be made toward users. For realization of emotion recognition, we have continued our study on anger degree estimation by both prosodic features and segmental features with anger utterances which were recorded during two kinds of pseudo-dialogs. This report focuses on only segmental features related to voice quality and examines them for capabilities to estimate anger degree. The first cepstral coefficient of anger utterances has been analyzed to obtain acoustic parameters related to spectral tilt in our previous works. In addition to the previous voice quality parameters, features related to amplitude of formants and harmonics were extracted from the same anger utterances as that in the previous works. Comparisons were made with the previous parameters to evaluate estimation accuracy of each voice quality parameters.

2pSCc55. Identification of place of articulation and voicing in white and babble noise. Magnus Alm (Norwegian University of Science and Technology, Psychology Dept, NO 7491 Trondheim, Norway, magnusal@stud.ntnu.no), Dawn Behne (Norwegian University of Science and Technology, Psychology Dept, NO 7491 Trondheim, Norway, dawn .behne@svt.ntnu.no)

Previous research shows that white noise influences the degree auditory and visual modalities are used in audio-visual (AV) speech perception. This study assesses identification of voicing and place of articulation (POA) in the infrequently studied natural babble noise. Incongruent monosyllabic AV stimuli were presented to 15 young adults in white and babble noise at 0 and $-12 \mathrm{~dB}$ SNR where voicing stimuli differed in voicing and voicing structure and POA stimuli differed in POA and POA structure. In white noise POA stimuli received fewer audio responses than in babble whereas voicing stimuli received more audio responses in white noise than in babble. Voiced syllables received more audio responses than voiceless. Findings suggest that differences in noise type for POA and voicing identification are attributable to discrepancies in acoustical attributes of noise and target stimuli. In voicing identification, the spectral transition between aspiration (stabile signal) and voicing (fluctuating signal) is less distinct in the fluctuating babble noise than in the flat power white noise. Voiceless consonants are more spectrally similar to white noise than voiced consonants, making the latter more auditorily accessible. Visual cues aid voicing identification, but only when POA is visually salient and auditorily susceptible to the noise type.

2pSCc56. A comparison of task and non-task related variability in speech production and acoustics, with implications to speech perception. Khalil Iskarous (Haskins Laboratories, 300 George St, New Haven, CT 06511, USA, iskarous@haskins.yale.edu)

Task Dynamics provides a way of testing whether production goals are acoustic or articulatory. According to this theory, during the production of a segment, the vocal tract can be divided into locations where a task is specified and locations where no task is specified. The goal is for articulators to synergetically achieve the constriction in the task location. Articulatory variability in the non-task locations is permitted due to overlap. However, articulatory activity in both task and non-task locations affects the area function and acoustic output. Therefore it is possible that an articulatory synergy stabilizes an articulatory task variable, without stabilizing the acoustics, evidence that the goal is articulatory. An experiment on the articulation and acoustics of /Vd/ in Ameican English by 26 subjects, using the Wisconsin Xray Microbeam database, shows that there is a synergy between jaw and tongue tip that explains $79 \%$ of the variability in articulator locations. This synergy stabilizes the position of the tongue tip in the alveolar region. But the formant transitions and bursts are highly variable, since the back of the tongue and jaw assume positions consistent with the preceding vowelevidence that the goal is articulatory (Funded by NIH DC-02717). 
2pSCc57. Evaluation of a simplified phonetic annotation scheme for disordered speech. Kathleen Murray (Head and Neck Surgery, Drexel University College of Medicine, Department of Otolaryngology - Head and Neck Surgery, 1721 Pine Street, Philadelphia, PA 19103, USA, kathleen.murray@drexel.edu), David Graff (Linguistic Data Consortium, 3600 Market St. Suite 810, Philadelphia, PA 19104-2653, USA, graff@ldc.upenn.edu), Yolanda D. Heman-Ackah (Head and Neck Surgery, Drexel University College of Medicine, Department of Otolaryngology Head and Neck Surgery, 1721 Pine Street, Philadelphia, PA 19103, USA, Phillyvoicemd@aol.com), Robert T. Sataloff (Head and Neck Surgery, Drexel University College of Medicine, Department of Otolaryngology Head and Neck Surgery, 1721 Pine Street, Philadelphia, PA 19103, USA, rtsataloff@phillyent.com)

Attempts to train a computer to mimic a vocal pathology expert's perception of perceivable voice problems have had limited success. A recent study successfully used a Cepstrum-based calculation (CPPs) to detect dysphonic speech, but it made significant false negative and false positive errors [1]. Appropriate training data could improve calculation accuracy, but logistical and legal issues from the medical domain render large amounts of detailed, consistent training data difficult to produce. A simple scheme has been developed based on labeling one point in time for each of a few vowels in a read statement. Calculations from [1] and [2], centered on neighborhoods of these vowels will augment the CPPs classifier. The annotation scheme will be evaluated with respect to its ability to assist a classifier to automatically predict perceptual labels [1] from data, and practicalities such as inter/intra-annotator agreement. 1. Heman-Ackah, Y.D., Heuer, R.J., Michael, D.D., Ostrowski, R., Horman, M., Baroody, M., Hillenbrand, J.M., and Sataloff, R.T. (2003). "Cepstral peak prominence: A more reliable measure of dysphonia," Annals of Otology, Rhinology, and Laryngology, 112, 324-333. 2. "Perceived Level of Noise by Mark VII and Decibels (E)" S. S Stevens, J. Acoustical Soc. Am. v. 51(2, part 2) 1972. pp. 575-602.

2pSCc58. Neural correlates of speech and non-speech processing: Role of language experience in brain activation. Alexis $\mathrm{N}$. Bosseler (University of Washington, Dept. of Speech \& Hearing Sciences, and Institute for Learning \& Brain Sciences, Box 357988, Seattle, WA 98195, USA, bosseler@u.washington.edu), Toshiaki Imada (University of Washington, Dept. of Speech \& Hearing Sciences, and Institute for Learning \& Brain Sciences, Box 357988, Seattle, WA 98195, USA imada@u.washington.edu), Elina Pihko (BioMag Laboratory, Helsinki University Central Hospital, 00029 Helsinki, Finland, pihko@biomag .hus.fi), Jyrki Mäkelä (BioMag Laboratory, Helsinki University Central Hospital, 00029 Helsinki, Finland, jyrki.makela@hus.fi), Samu Taulu (Elekta-Neuromag Oy, 22 Elimäenkatu, 00510 Helsinki, Finland,
samu@squid.neuromag.fi),Antti Ahonen(Elekta-Neuromag Oy, 22 Elimäenkatu, 00510 Helsinki, Finland, antti.ahonen@elekta.com), Patricia K Kuhl (University of Washington, Dept. of Speech \& Hearing Sciences, and Institute for Learning \& Brain Sciences, Box 357988, Seattle, WA 98195 , USA, pkkuhl@u.washington.edu)

In adults, neural responses to the acoustic properties of native and nonnative speech sounds differ. Recent magnetoencephalography (MEG) studies indicate that two regions of the brain, the superior temporal area and inferior parietal area (Broca's), in both the left and right hemispheres, may contribute to how the adult brain responds to the acoustic properties of speech. The aim of the present study was to investigate neural activation in adults when listening to native and non-native speech, and to non-speech analogs of speech. We used MEG to examine activation of the superior temporal and inferior parietal areas. Native speakers of Finnish were presented with Finnish (/pa/ and / ta/) and Chinese (/t "looped c" "superscript $\mathrm{h}^{\prime \prime} \mathrm{i} /$ and /"looped c" i/) syllables, and sine-wave replicas of /pa/ and / ta/, in a passive oddball paradigm. Our analysis examines the properties of localized neural activities to determine how activation of sensory and motor speech areas differs for speech and non-speech, and the role of language experience in brain activation.

2pSCc59. Why words do not have a psychological alphabet. Robert F. Port (Indiana University, 330 Memorial Hall, Bloomington, IN 47405, USA, port@indiana.edu)

Linguists and psychologists share a powerful intuition that the psychological form of language (in memory) employs letter-like units for spelling words and any other linguistic material. However evidence accumulating over the decades shows that memory for linguistic material actually consists of continuous-time representations containing much more information than we thought, plus there are episodic representations with much redundancy. So Liberman's /di/ and /du/ share nothing in their representation. There need not be an abstract /d/ in the memory representations of language. Our intuition that a letter-like representation is necessary for speech perception and production turns out to be shared only with literate people. Because we cultivate our reading and writing skills, we bias our brains (and consciousness) to model speech using something like letters (e.g, phones) either from an orthographic or a technical alphabet. So our conscious experience of language is shaped by our cultural tradition of training children to hear speech as letters and letters as speech - all to exploit the technology of literacy. When we can overcome this education-based bias toward phones and phonemes, we will discover the phonemic emperor has no supporting evidence. Linguistic material in memory is richly detailed - relative to what an alphabet represents. 


\title{
Session 2pSPa
}

\section{Signal Processing in Acoustics: Beamforming, Localization, and Tracking I}

\author{
Michael Roan, Cochair \\ Virginia Tech, 917 Village Way North, Blacksburg, VA 24060, USA \\ Natalia Sidorovskaia, Cochair \\ Department of Physics, University of Louisiana, UL BOX 44210, Lafayette, LA 70504-4210, USA
}

\section{Contributed Papers}

\section{2:00}

2pSPa1. Acoustic vector-sensor array beamforming based on fourth-order cumulants. Tingting Li (College of Underwater Acoustic Engineering, Harbin Engineering University, 150001 Harbin, China, liqiaocheng@hrbeu.edu.cn), Xiukun Li (College of Underwater Acoustic Engineering, Harbin Engineering University, 150001 Harbin, China, xiukun _li@yahoo.com.cn)

Abstract: Some high-resolution direction-of-arrival (DOA) estimation methods, such as the MUSIC method, have been developed based on vectorsensor array. However, these high-resolution methods suffer from serious drawbacks. Indeed, they are not able to estimate coherent signals and sensitive to calibration errors. Mainly to overcome these limitations and in particular to satisfy practical engineering application, vector-sensor array beamforming based on fourth-order cumulants is adopted in this paper. The main interest in using fourth-order cumulants instead of second-order ones in vector-sensor array processing application is the aperture extension property of higher-order cumulants, which makes it possible to increase both the resolution and the number of sources to be processed from a given array, and suppress the Gaussian noise. Based on this method, smaller size of array and higher accuracy of DOA estimation can be realized, besides, this method is less sensitive to the coherence of the signals and calibration errors, which makes it possible to work well in engineering application. The results of computer simulation and analysis of data from outfield trials show that this method excels traditional direct estimation and can improve estimation capability of vector-sensor arrays.

\section{2:20}

2pSPa2. Robust array pattern synthesis with uncertain manifold vector. Shefeng Yan (Department of Electronics and Telecommunications, Norwegian University of Science and Technology, O.S. Bragstads plass 2A, 7491 Trondheim, Norway, sfyan@ieee.org)

The knowledge of the array manifold vectors of an acoustic array can be imprecise, which is often the case in practice. This may cause undesirably high sidelobes for a nominal optimal beamformer where the array manifold vectors are assumed to be known exactly. Although the norm constraint on beamformer weights can be imposed to improve the robustness of the optimal beamformer, it is not clear how to choose the optimal constrained parameter based on the known level of uncertainty of the array manifold vectors. A pattern synthesis approach to arbitrary arrays with robustness against array manifold vectors errors is developed. Our technique optimizes the worst-case performance by minimizing the worst-case sidelobe level while maintaining a distortionless response to the worst-case signal steering vector. The parameters can be optimally chosen based on the uncertainty of the array manifold vector. The robust beamformer problem is shown to be convex, which can be efficiently solved using second-order cone programming. A simple lower bound on how much worse the robust optimal beamformer will be compared to the nominal one is also derived. Computer simulations show better performance of the proposed robust beamformer.
2:40

2pSPa3. Analytical prediction of sample eigenvector quality deterioration in large arrays due to SNR or sample size constraints. Raj Nadakuditi (MIT, Department of Mathematics, 77 Massachusetts Avenue, Cambridge, MA 02139, USA, raj@mit.edu), Arthur B. Baggeroer (MIT, Department of Mathematics, 77 Massachusetts Avenue, Cambridge, MA 02139, USA, abb@boreas.mit.edu)

It is well-known that subspace-based estimation methods in adaptive array processing suffer a rapid degradation in performance as either the signalto-noise ratio (SNR) or the number of available snapshots drops below a certain threshold value. In the large system, relative large sample size limit, one can use random matrix theory to analytically predict this threshold and the degradation in the "'quality" of the corresponding subspace estimates. In certain settings, one observes a "phase transition" phenonemon so that if the signals are too weak or there are insufficient number of snapshots or both, the subspace estimates are, statistically speaking, noise-like. We discuss the implication of these results for the subspace based detection of signals in white and colored noise using large arrays and illustrate the accuracy of the predictions with numerical simulations.

\section{3:00}

2pSPa4. Efficient Replica Generation for Space Time Adaptive Processing of Acoustic Signals. Neena Imam (Oak Ridge National Laboratory, 1 Bethel Valley Road, Oak Ridge, TN 37831-6015, USA, imamn@ornl.gov), Jacob Barhen (Oak Ridge National Laboratory, 1 Bethel Valley Road, Oak Ridge, TN 37831-6015, USA, barhenj@ornl.gov), Michael Traweek (Office of Naval Research, 875 North Randolph Street, Arlington, VA 22203, USA, Mike.Traweek@navy .mil)

This paper addresses an innovative method for real-time replica generation using generalized Green's function for Space Time Adaptive Processing (STAP) technology for sonar applications. In hypothesis processing for STAP algorithms, the first step is to generate replicas for various target hypothesis such as the infinite-range fixed-direction (IRFD) target hypotheses or the tracking (trajectory) hypotheses. Once generated, these replicas are used in multiple steps of the STAP algorithm for computing system parameters such as the signal to noise ratio. Replicas are traditionally precomputed or hard-wired due to computational load constraints. Such a precomputation precludes direct application of array shape corrections, and limits the ability to process arbitrary target trajectories. W propose to use a generalized Green's function for efficient and ultra-fast real-time generation of replicas on multi-core processors Specifically, for an arbitrary target trajectory $r_{T}\left(t_{i}\right)=\left[x_{T}\left(t_{i}\right), \quad y_{T}\left(t_{j}\right), \quad z_{T}\left(t_{i}\right)\right]$, arbitrary receive array trajectory $r_{R(k)}\left(t_{i}\right)=\left[x_{R(k)}\left(t_{i}\right), y_{R(k)}\left(t_{i}\right), z_{R(k)}\left(t_{i}\right)\right],\left(k=1, \ldots, N_{E L}\right)$, arbitrary vector of frequencies in homogeneous acoustic medium $f=\left[f_{1}, f_{2}, \ldots . f_{n}, \ldots\right]$, and sound speed $c_{0}$, we can write $G\left[r_{T}\left(t_{i}\right), r_{R(k)}\left(t_{i}\right), f_{n} \mid c_{0}\right]=\exp \left[j\left(2 \pi f_{n} / c_{0}\right)\left|r_{R(k)}\left(t_{i}\right)-r_{T}\left(t_{i}\right)\right|\right] / \mid$ $r_{R(k)}\left(t_{i}\right)-r_{T}\left(t_{i}\right) \mid$ In terms of rationale, this is the simplest possible replica set for any STAP algorithm. The efficient implementation of the generalized Green's function on a multi-core processor will allow real-time adjustment/modification of acoustical target tracking resulting in improved numerical accuracy and time performance. 
3:20

2pSPa5. Beam-forming with progressive focalisation. Gaetano Caronna (Università La Sapienza - Dept. Fisica Tecnica, Via Eudossiana 18, $00184 \quad$ Roma, Italy, gaetano.caronna@uniroma1.it), Pierluigi Testa (Università La Sapienza - Dept. Fisica Tecnica, Via Eudossiana 18, 00184 Roma, Italy, pierluigi.testa@uniroma1.it)

This investigation is aiming to detect and track a moving target using its acoustic emissions and applying a new beam-forming approach. An original algorithm of medium computational complexity is reported that is capable of locating, with good accuracy, the direction of an acoustic source with only a few microphones. This algorithm circumvents the known localisation ambiguities due to the unwanted "grating lobes", by using all spectral content of the acoustic source and progressively and narrowly focusing its beam in the direction of the target. The algorithm starts by locating the direction of the target with low angular resolution, using only low frequency components of the acoustic emissions; in this way, no grating lobes occur even with only a few microphones. Then, the angular resolution is increased by processing higher frequencies of the spectrum, but profiting of the information on the angular position achieved in the previous stages. Some algorithmic means are introduced to increase the performance of the algorithmic against environmental noise. This scheme is described in detail. Some experimental tests are included and discussed.

TUESDAY AFTERNOON, 1 JULY 2008

P3-C, LEVEL 3, 3:40 TO 5:20 P.M.

\title{
Session 2pSPb
}

\section{Signal Processing in Acoustics: Beamforming, Localization, and Tracking II (Poster Session)}

\author{
Michael Roan, Cochair \\ Virginia Tech \\ Natalia Sidorovskaia, Cochair \\ Department of Physics, University of Louisiana
}

\begin{abstract}
All posters will be on display from 3:40 p.m. to 5:20 p.m. To allow contributors an opportunity to see other posters, contributors of odd-numbered papers will be at their posters from 3:40 p.m. to 4:30 p.m. and contributors of even-numbered papers will be at their posters from 4:30 p.m. to 5:20 p.m.
\end{abstract}

\section{Contributed Papers}

2pSPb1. Particles filter applied in the real-time bearings-only tracking problem of a sonar target. Cleide V. Rodrigues (Ipiru 2 Ilha do Governador, 21931-095 Rio de Janeiro, Brazil, clevital@ hotmail.com), William Soares-Filho (Ipiru 2 Ilha do Governador, 21931-095 Rio de Janeiro, Brazil, wsoaresf@hotmail.com)

The development of a passive sonar tracking system in real-time needs algorithms each time more accurate and that can be able to follow rapid changes in the signal characteristics. In a bearings-only tracking problem, the bearing model is a nonlinear function of the target states. Nowadays, methods that deal with nonlinear and non-Gaussian estimation are receiving great attention. Particles filter is one of these methods. Particles filters are Sequential Monte Carlo methods that represent the required probability density function as a set of random samples. This present paper describes the particles filter application in the bearings-only tracking problem. Real data of targets are displayed in energy versus bearing graphics obtained in an operational naval environment. These data were used with the Sampling Importance Resampling algorithm (SIR). The particles filter is formulated in the Cartesian coordinates and then are transformed to modified polar coordinates. Results are compared with Kalman Extended Filter and the effectiveness and limitations of SIR algorithm for tracking target using a bearing/time record are examined.
2pSPb2. Tracking of underwater acoustic tone sources with linear antenna arrays. Denis Orlov (Institute of Applied Physics of the Russian Academy of Sciences, 46 Ulyanov Street, 603950 Nizhny Novgorod, Russian Federation, denis@hydro.appl.sci-nnov.ru), Alexander Rodionov (Institute of Applied Physics of the Russian Academy of Sciences, 46 Ulyanov Street, 603950 Nizhny Novgorod, Russian Federation, alexr@hydro.appl.sci-nnov.ru)

Track determination is a necessary task for a variety of underwater measurement applications, such as measurement of acoustic fields of ships. The use of linear antenna arrays provides great advantages for measurements and tracking. The investigated ship may be equipped by a special sound source of either pulse or tone signal utilized for tracking. The work considers two scenarios involving the case of a tone source. The scenarios are conditioned by parameters of the array and the source, which may vary depending on task. When a source is of relatively high frequency (several thousands $\mathrm{Hz}$ ), tracking can be based on the dependence of the Doppler frequency shift and bearing on time; in this case, the parameters to be estimated are the ones of the whole track (velocity, traverse instant, etc.). If the source frequency is relatively low (up to $300 \mathrm{~Hz}$ ), the array is large (greater than a typical distance from the source to the array aperture), the array focusing ability allows to perform the determination of "instant" locations of the source at various 
instances; estimating of parameters of the track as a whole is then based on the set of the obtained coordinates. The developed methods have been successfully tested using a large amount of experimental data.

2pSPb3. Robust beamforming and beamspace DOA estimation using measured array manifold of arbitrary geometry acoustic array. Yixin Yang (Institute of Acoustic Engineering, Northwestern Polytechnical University, 710072 Xi'an, China, yxyang@nwpu.edu.cn), Chao Sun (Institute of Acoustic Engineering, Northwestern Polytechnical University, 710072 Xi’an, China, csun@nwpu.edu.cn)

The acoustic diffraction of array supporting structure will result in the large difference of the amplitude and phase response of each hydrophone, which leads to the distortion of beampattern. A method for obtaining the array manifold of arbitrary geometry acoustic array is proposed in this paper.
We measured the array response vector in many directions with a fixed angular step in anechoic water tank. The array response vectors were smoothed to eliminate the measurement system error. Using these smoothed array response vectors, the robust beampattern of arbitrary geometry array is obtained via beam synthesis method. A 16 element volumetric acoustic array was constructed and tested in an anechoic water tank. Robust low sidelobe beampatterns based on the measured manifold were formed, and then the beamouputs were used to apply to beamspace high resolution DOA estimation methods. The experimental results show that our method can suppress noise and interferences outside the beam coverage regions, and get high resolution DOA estimation results. When the array geometry has some distortion, the weighting vectors of beamforming can still work well to form good beampatterns, and then get accurate DOA estimation results. This verifies the effectiveness of our proposed method.

TUESDAY AFTERNOON, 1 JULY 2008

P3-C, LEVEL 3, 3:40 TO 5:20 P.M.

\title{
Session 2pSPc
}

\section{Signal Processing in Acoustics: Acoustical Nondestructive Evaluation, Ultrasonics, and Imaging II (Poster Session)}

\author{
Grace Clark, Cochair \\ Lawrence Livermore National Laboratory \\ Ning Xiang, Cochair \\ Rensselaer Polytechnic Institute
}

\begin{abstract}
All posters will be on display from 3:40 p.m. to 5:20 p.m. To allow contributors an opportunity to see other posters, contributors of odd-numbered papers will be at their posters from 3:40 p.m. to 4:30 p.m. and contributors of even-numbered papers will be at their posters from 4:30 p.m. to 5:20 p.m.
\end{abstract}

\section{Contributed Papers}

2pSPc1. Identification of Noise Emission in a Gear Unit. Ales Belsak (University of Maribor, Faculty of Mechanical Engineering, Smetanova ulica 17, 2000 Maribor, Slovenia, ales.belsak@uni-mb.si), Jurij Prezelj (University of Ljubljana, Faculty of Mechanical Engineering, Aškerčeva 6, 1000 Ljubljana, Slovenia, jurij.prezelj@fs.uni-lj.si)

Today it is very important to ensure a stable production without unscheduled outages. To achieve this objective it is required to use advanced production technologies, to ensure adequate maintenance of mechanical systems and to monitor the condition of a device or machine. Reliable and accurate operation of machines and devices with as few outages as possible is desired. The significance of a life cycle design of machines and devices is growing. Possible damages in gear units can be defined by means of monitoring acoustic emission. A crack in the tooth root is usually indicated by significant changes in tooth stiffness. A difference in dynamic responses of an undamaged gear and of a damaged gear can be noted. The possibility of the use of an acoustic method in the field of condition diagostics is dealt with. The noises produced by a gear unit have been analysed, the noise sources within a gear unit have been determined and the corresponding time-frequency analysis of these sources have been performed, using an acoustic camera.
2pSPc2. Detection of Ultrasonic closer flaws using Nonlinear signal processing. Abdessalem Benammar (Image and signal processing laboratory. Welding and NDT Centre, Route de Dely-Ibrahim, BP 64, Chéraga, 16035 Alger, Algeria, Abs_benammar@yahoo.fr), Redouane Drai (Image and signal processing laboratory. Welding and NDT Centre, Route de Dely-Ibrahim, BP 64, Chéraga, 16035 Alger, Algeria, drai_r@yahoo.fr), Ahmed Kechida (Image and signal processing laboratory. Welding and NDT Centre, Route de Dely-Ibrahim, BP 64, Chéraga, 16035 Alger, Algeria, Abs_benammar@yahoo.fr), Abderrezak Guessoum (Image and signal processing laboratory. Welding and NDT Centre, Route de Dely-Ibrahim, BP 64, Chéraga, 16035 Alger, Algeria, Abs _benammar@yahoo.fr)

The ultrasonic flaw detection is an important problem in the nondestructive evaluation (NDE) of materials. In order to successfully detect and classify flaw echoes from high scattering grain echoes, an efficient and robust method is required. In this paper, a method using split-spectrum processing (SSP) combined with a neural network (NN) has been developed and applied on the ultrasonic signals to perform the detection of closer echoes. SSP can display signal diversity and is therefore able to provide the signal feature vectors for signal classification. The neural network (NN) performs 
highly complex nonlinear mapping by which signals can be classified according to their feature vectors. Therefore, the combination of SSP and NN (SSP-NN) presents a powerful technique for ultrasonic NDE. The SSP is achieved by using Gaussian bandpass filters. Then, an adaptive three layer neural network using a backpropagation learning process is applied to perform the classification processing of frequency diverse data. The SSP-NN method has been tested using both simulated and experimental ultrasonic signals, and the results show that SSP-NN has good sensitivity in the detection of ultrasonic closer flaws echoes drowned in the noise.

2pSPc3. Quantitative Ultrasonics for Inclusion and Pore Characterization of Steel Billets. Ville Kananen (Univ. of Helsinki / Dept. of Physical Sciences, POB 64 (Gustaf Hällströmin katu 2), 00014 Helsinki, Finland, ville.kananen@helsinki.fi), Joona Eskelinen (Univ. of Helsinki / Dept. of Physical Sciences, POB 64 (Gustaf Hällströmin katu 2), 00014 Helsinki, Finland, joona.eskelinen@helsinki.fi), Edward Hæggström (Electronics Research Unit, University of Helsinki, P.O.Box 64 (Gustaf Hällströmin katu 2), FIN-00014 Helsinki, Finland, edward .haeggstrom@helsinki.fi)

We present ultrasonic quantitative inclusion and pore characterization in bearing steel $100 \mathrm{Cr} 6$. A $9.5 \mathrm{MHz}$ focused transducer $(14 \mathrm{~cm}$ focal length, $6.3 \mathrm{MHz}$ bandwidth) scanned across the top surface of immersed $22 * 12 * 6 \mathrm{~cm}^{3}$ production samples (8pcs). Automatic pre-processing was employed to detect inclusions in samples. Recorded RF-data were analyzed. Continuous wavelet transform and cross-correlation were applied to measured and registered RF-signals from known (verified by SEM) inclusions and pores. This allowed characterizing the echoes by shape from wavelet coefficients (WC) and cross-correlation coefficients: the echo is a superposition of the reflection from the inclusion front and back surface, whereas pores exhibit no back surface echo due to their large acoustic impedance mismatch. Fourier transform allows characterizing echo RF-signals by frequency content. Dissimilarities -signal phase and WCs- in echo characteristics between different inclusion and pore classes allows quantitative inclusion and pore characterization. We discriminate three classes: oxideinclusions (aluminium-oxides, calcium-aluminates), sulphides (manganesesulphide), and pores. We also estimate inclusion size, position, and orientation. Rolled (reduction ratio 4 and 10) and unrolled production samples are characterized. The instrument functionality was validated by blind tests using SEM. Inclusion distributions in different classes of steel 100Cr6 and classification probabilities are presented. Our software saves sample preparation and image analysis work.

2pSPc4. Digital High Frequency Coded Imaging System. Marcin Lewandowski (Institute of Fundamental Technological Research, Swietokrzyska 21, 00-049 Warsaw, Poland, mlew@ippt.gov.pl), Andrzej
Nowicki(Institute of Fundamental Technological Research, Swietokrzyska 21, 00-049 Warsaw, Poland, anowicki@ippt.gov.pl)

Coded transmission is an approach to solve the inherent compromise between penetration and resolution required in ultrasound imaging. It is universally acknowledged that this technique gives major improvement in SNR. A novel high frequency (20-35 MHz) ultrasound real-time imaging system for research and evaluation was developed. The digital programmable coder-digitizer module based on the field programmable gate array (FPGA) supports arbitrary waveform coded transmission and RF echoes sampling up to $200 \mathrm{MSPS}$, as well as real-time streaming of digitized RF data via a high speed USB interface to the PC. All RF and image data processing were implemented in the software. A novel balanced software architecture using CPU and GPU processing supports real-time processing and display at rates up to 30 frames/sec. The system was used to acquire data for sine burst and 16-bit Golay code excitation using a single element scanning head with thick film focused spherical transducer $25 \mathrm{MHz}$ center frequency and $56 \%$ system bandwidth. SNR gain for the Golay codes (referenced to single burst) of $15 \mathrm{~dB}$ for $20 \mathrm{MHz}$ and $16 \mathrm{~dB}$ for $35 \mathrm{MHz}$ were obtained. In water the axial resolution for both single burst and the Golay codes was the same $\mathrm{FWMH}=20 \mathrm{~ns}$ at $20 \mathrm{MHz}$ and $\mathrm{FWMH}=15 \mathrm{~ns}$ at $35 \mathrm{MHz}$.

2pSPc5. Wavelet threshold enhancement by an energetic characterization of ultrasonic signal Ndt analysis. Fairouz Bettayeb (Research Center on Welding and Control, CSC, Route de Delly Brahim, Bp: 64. Chéraga, 16800 Algiers, Algeria, fairouz_bettayeb@email .com)

In ultrasonic NDE of industrial components, the visibility of flaw echoes is corrupted by noise due to multiple scattering. Grain boundaries can reach the size of the same order of magnitude than discontinuities to be detected, becoming scatters of ultrasonic noise and could be confused with defect indications. Many studies have been conducted on the use of the wavele theory for ultrasonic signal de-noising, but nothing has been done on the structural noise features and its analyzing wavelet function. In the framework of the automation of the ultrasonic signal analysis project, we have followed the exploration of the wavelet theory, from the continuous transforms to the discrete ones, and the experiments give us some ambivalent results. So for a best threshold control, our idea was directed to the investigation of the noise analyzing function. In this work the noise features were extracted by an energetic smoothing algorithm that allows the exploration of the noise analyzing function; by which the random nature of the noise in the spatial domain is bypassed. The energetic characterization of the noise and the defects allows an improved filtering process. The new smoothing algorithm performs an accurate signal reconstruction in an interesting computing time. 


\title{
Session 2 pSPd
}

\section{Signal Processing in Acoustics and Physical Acoustics: Biomedical Applications of Time-Reversal II (Poster Session)}

\author{
Oleg Sapozhnikov, Cochair \\ Center for Industrial and Medical Ultrasound, Applied Physics Lab. \\ Mickael Tanter, Cochair \\ Laboratoire Ondes et Acoustique
}

\begin{abstract}
All posters will be on display from 3:40 p.m. to 5:20 p.m. To allow contributors an opportunity to see other posters, contributors of odd-numbered papers will be at their posters from 3:40 p.m. to 4:30 p.m. and contributors of even-numbered papers will be at their posters from 4:30 p.m. to 5:20 p.m.
\end{abstract}

\section{Contributed Papers}

2pSPd1. Time reversal elastography in soft solids. Thomas Gallot(LGIT Université Joseph Fourier/CNRS, Maison des Géosciences Domaine Universitaire, 38041 Grenoble, France, thomas.gallot@obs.ujf-grenoble .fr), Stefan Catheline (LOA, Université Paris 7 Denis Diderot, ESPCI, 10 rue Vauquelin, 75005 Paris, France, stefan.catheline@espci.fr), Nicolas Benech (Science University of Montevideo, 11400 Montevideo, Uruguay, nbenech@fisica.edu.uy), Javier Brum (Science University of Montevideo, $11400 \quad$ Montevideo, Uruguay, jbrum@fisica.edu.uy), Carlos Negreira (Science University of Montevideo, 11400 Montevideo, Uruguay, carlosn@fisica.edu.uy), Philippe Roux (LGIT - CNRS - Université Joseph Fourier, Maison des Géosciences, 1381 rue de la Piscine, BP 53, 38041 Grenoble, France, philippe.roux @obs.ujf-grenoble.fr)

One channel time-reversal (TR) experiments allow focalization of waves in reverberant cavities. According to Rayleigh criteria, the focal spot width is directly related to wavelength and therefore depends on the mechanical properties of the medium. Thus the general idea of this work is to extract quantitative estimations of these mechanical properties from TR experiments. They were performed inside 3D soft solid cavities (gelatin based phantoms). An external source creates mechanical waves in the audible frequency range and one component of the vectorial field is measured as function of time by ultrasonic techniques developed in elastography. The wavelength information of shear waves, that dominates the vectorial field, is deduced from TR experiments. The advantage is that the technique works with sources of any shape and with any time dependence. This robustness as regard to shear wave source allows to think about many applications in the medical field, including deep or moving organs. Examples of elasticity image will be shown in inhomogeneous soft tissues.

2pSPd2. Time Reversal Acoustic focusing with random reverberator. Laurent Fillinger (Artann Laboratories/ Stevens Institute of Technology, 711 Hudson St, Hoboken, NJ 07030, USA, laurent.fillinger@artannlabs .com), Alexander Sutin (Stevens Institute of Technology, Castle Point on Hudson, Hoboken, NJ 07030, USA, asutin@stevens.edu), Armen Sarvazyan (Artann Laboratories, 1753 Linvale-Harbourton, Lambertville, NJ 08350, USA, armen@artannlabs.com)

Time Reversal Acoustic (TRA) focusing system based on an external reverberator can provide good focusing with minimum side lobes even with a few transducers. The focusing ability of such system can be increased using reverberator with rough boundaries or by adding internal random scatterers. The experiments were conducted with a reverberator made of a polyethylene bottle filled with water and a single piezoceramic disk attached externally to its wall. Experiments demonstrated that inserting in the bottle random hard scatterers or altering of the bottle surface significantly decreases the size of the focused spot and the level of side lobes. The new pseudo-impulse method of TRA focusing allowed suppressing piezotransducer resonances and provided formation of short wide band signals. The ability of such TRA focusing system based on random reverberator to form simultaneously multiple focuses and produce focal spots of the complex shape has been demonstrated. The application of binary radiation regime led to several fold increasing of the amplitude of the focal spot while the focal structure practically was not affected. Applications of such random focusing system for medical imaging and HIFU treatment is discussed. 


\title{
Session 2pSPe
}

\section{Signal Processing in Acoustics, Acoustical Oceanography, and ECUA: Bayesian Signal Processing I (Poster Session)}

\author{
Zoi-Heleni Michalopoulou, Cochair \\ Department of Mathematical Sciences, New Jersey Institute of Technology \\ Simon Godsill, Cochair \\ Dept. of Engineering, University of Cambridge, Trumpington St., CB2 1PZ Cambridge, UK
}

\begin{abstract}
All posters will be on display from 3:40 p.m. to 5:20 p.m. To allow contributors an opportunity to see other posters, contributors of odd-numbered papers will be at their posters from 3:40 p.m. to 4:30 p.m. and contributors of even-numbered papers will be at their posters from 4:30 p.m. to 5:20 p.m.
\end{abstract}

\section{Contributed Papers}

2pSPe1. Continuous estimation of VTL from vowels using a linearly VTL-covariant speech feature. Christian Feldbauer (Centre for the Neural Basis of Hearing, Department of Physiology, Development and Neuroscience, University of Cambridge, Downing Site, CB23EG Cambridge, UK, feldbauer@tugraz.at), Jessica J. Monaghan (Centre for the Neural Basis of Hearing, Department of Physiology, Development and Neuroscience, University of Cambridge, Downing Site, CB23EG Cambridge, UK, jjmm2 @ cam.ac.uk), Roy D. Patterson (Centre for the Neural Basis of Hearing, Department of Physiology, Development and Neuroscience, University of Cambridge, Downing Site, CB23EG Cambridge, UK, rdp1@cam.ac.uk)

Vowel sounds encode information about the speaker's vocal tract length (VTL) as well as vowel type. This paper shows how VTL can be estimated on a frame-by-frame basis from vowels to facilitate speaker tracking and make automatic speech recognition (ASR) more robust in multi-speaker environments. The algorithm is based on a new, VTL-covariant speech feature that encodes the size information linearly. The paper shows that this new speech feature is better suited for VTL estimation than the more traditional cepstral coefficients. The VTL estimation is based on a Gaussian mixture model that was trained on the speech material of speakers with known body height but unknown VTL. Research supported by the Austrian Science Fund (FWF) [J2541-N15], EOARD [FA8655-05-1-3043], and the UK-MRC [G0500221].

2pSPe2. A sequential Bayesian approach to vertical slice tomography of a shallow water environment. Olivier Carrière (Université libre de Bruxelles (U.L.B.) - Environmental hydroacoustics lab, av. Franklin D. Roosevelt 50, CP 194/5, 1050 Bruxelles, Belgium, ocarrier@ulb.ac .be), Jean-Pierre Hermand (Université libre de Bruxelles (U.L.B.) - Environmental hydroacoustics lab, av. Franklin D. Roosevelt 50, CP 194/5, 1050 Bruxelles, Belgium, jhermand@ulb.ac.be)

A major challenge of acoustic tomography in shallow water environments is to track physical features that are highly variable in time and space and to properly account for sound interaction with the subbottom. In a previous work an extended Kalman filter (EKF) scheme was proposed to track the sound-speed field variations in a vertical slice of the water column for known bottom geoacoustic parameters. Although the results were encouraging, it was shown that some biases were encountered when using the standard EKF, principally because of the high nonlinearity between the observations (the multi-frequency pressure field) and the environmental parameters (here the sound-speed field). In this paper, we show that Ensemble Kalman or sequential Monte Carlo filtering significantly enhance the estimation of the sound-speed field, for both range-independent and rangedependent cases. Reproducible results show that the temporal variations are well tracked, even in presence of measurement noise and model uncertainty. Furthermore the sampling approach allows to increase the time interval between successive measurements, which is an advantage from an operational viewpoint. The performances of the different nonlinear filters are also discussed.

2pSPe3. Source localization and tracking in a waveguide via sequential bayesian estimation. Kevin Cockrell (MIT, 77 Mass Ave, 5-204, Cambridge, MA 02139, USA, cockrell@mit.edu), Henrik Schmidt (MIT, 77 Mass Ave, 5-204, Cambridge, MA 02139, USA, henrik@mit.edu)

This talk addresses the problem of sequentially estimating the location of a moving acoustic source in a waveguide, given a series of acoustic field measurements from an array. The approach taken, in it's most general form, is known as sequential Bayesian estimation. A well known special case of sequential Baysian estimation is the Kalman filter. However, the Kalman filter is only optimal when the relationship between the measured parameters and the ones to be estimated is linear with Gaussian noise. Given the highly non-linear relationship between the acoustic source location and the measured acoustic field, other approaches must be taken. The performance and computational feasibility of various approaches, such as grid-based methods, will be explored using simulated data.

2pSPe4. The role of reproducing densities in a joint likelihood approach to multi-sensor detection and estimation. Michael Roan (Virginia Tech, 917 Village Way North, Blacksburg, VA 24060, USA, mroan@vt.edu), Elizabeth Hoppe (Virginia Polytechnic Institute and State University, 141 Durham Hall, Blacksburg, VA 24060, USA, ehoppe@vt .edu)

In this work, a Bayesian, joint estimation-detection approach is used for computation of sufficient statistics and development of a general multisensor information fusion architecture. An approach borrowed from sequential Bayesian processing is used to compute prior densities for joint Bayesian estimation-detection. In this approach, a posteriori densities calculated at one sensor become priors at the next sensor after a coordinate transformation that transforms the outputs of each sensor to a common reference frame for all sensors. Reproducing prior densities are used to simplify the Bayesian iteration scheme and reduce communications requirements. The framework that is developed is equally applicable to networks where all sensors communicate with a centralized detection and estimation processor as well as those networks where sensors relay information from point to point 
We anticipate using Bayesian iteration to convert posterior information into prior information on the next data gather cycle, iterate on estimates by exchanging information between sensors, i.e., "turbo likelihood fusion," and having the central decision maker decide when sensors should or should not communicate, depending on a cost criterion which combines information gain versus communication energy expenditure. Simulation are used to show detection and estimation performance under various conditions of sensor position and orientation uncertainty.

\title{
Session 2pSPf
}

\section{Signal Processing in Acoustics, Biomedical Ultrasound/Bioresponse to Vibration, and Underwater Acoustics: Time Reversal Methods for Array Imaging and Signal Processing II (Poster Session)}

\author{
David Chambers, Cochair \\ $L L N L$ \\ Claire Prada, Cochair \\ Laboratoire Ondes et Acoustique
}

\begin{abstract}
All posters will be on display from 3:40 p.m. to 5:20 p.m. To allow contributors an opportunity to see other posters, contributors of odd-numbered papers will be at their posters from 3:40 p.m. to 4:30 p.m. and contributors of even-numbered papers will be at their posters from 4:30 p.m. to 5:20 p.m.
\end{abstract}

\section{Contributed Papers}

2pSPf1. Isolation of backscattering resonances of a thin spherical shell using iterative time reversal. Zachary J. Waters (Boston University, Dept. of Aerosp. and Mech. Eng., 110 Cummington St., Boston, MA 02215, USA, zjwaters@bu.edu), Benjamin R. Dzikowicz (Naval Surface Warfare Center, Panama City Division, Code HS-11, 100 Vernon Ave., Panama City, FL 32407, USA, benjamin.dzikowicz@ navy.mil), R. Glynn Holt (Boston University, Dept. of Aerosp. and Mech. Eng., 110 Cummington St., Boston, MA 02215, USA, rgholt@bu.edu), Ronald A. Roy (Boston University, Dept. of Aerosp. and Mech. Eng., 110 Cummington St., Boston, MA 02215, USA, ronroy@bu.edu)

The backscattering spectrum of thin spherical shells show resonance peaks due to several different physical processes including high frequency Lamb wave excitation and low frequency modal ringing [Kaduchak et al., J. Acoust. Soc. Am. 97, 2699-2708 (1995)]. These different processes can be isolated in both time and frequency by using simulated iterative time reversal. This is accomplished by windowing in the time domain and/or filtering in the frequency domain. Iterative time reversal techniques developed for buried target detection [Waters et al., J. Acoust. Soc. Am. 122, 3023 (2007)] are applied to the partial wave series solution for the backscattering of plane acoustic waves by a thin-walled spherical shell. Tank experiments are performed to verify theoretical results. Targets loaded with both water and sediment are considered. Understanding the relationship between the time reversal window's size/position and the dominant target scattering mechanisms is key to the development of time reversal as a detection and identification technique. [Work supported by The Office of Naval Research.]

2pSPf2. The Decomposition of the Time Reversal Operator with virtual transducers. Jean-Luc Robert (Philips Research, 345 Scarborough Road, Briarcliff Manor, NY 10510, USA, jean-luc.robert@philips.com), Mathias Fink (Laboratoire Ondes et Acoustique, ESPCI, Université Paris 7, CNRS, 10 rue Vauquelin, 75005 Paris, France, mathias.fink@espci.fr)

The FDORT method (French acronym for Decomposition Of the Time Reversal Operator using Focused beams) is a variant of the DORT method using focused transmits instead of single element transmits. An intuitive interpretation of the method is presented here. It is shown that the FDORT method is equivalent to a DORT method with two different arrays: the actual array and a virtual array whose transducers are located at the foci of the transmits. By changing the focal depth, it is possible to change the position of the virtual array and thus obtain different information. An application to aberration correction in a far field phase screen model will be presented.

2pSPf3. Reconstruction of temporal invariants of the time-reversal operator for communication in shallow water. Franck D. Philippe (Laboratoire Ondes et Acoustique, ESPCI, Université Paris 7, CNRS, 10 rue Vauquelin, 75005 Paris, France, franck.philippe @espci.fr), Thomas Folégot (NATO Undersea Research Center, Viale San Bartolomeo 400, 19126 La Spezia, Italy, folegot@nurc.nato.int), Claire Prada (Laboratoire Ondes et Acoustique, ESPCI, Université Paris 7, CNRS, 10 rue Vauquelin, 75005 Paris, France, claire.prada-julia@espci.fr), Mathias Fink (Laboratoire Ondes et Acoustique, ESPCI, Université Paris 7, CNRS, 10 rue Vauquelin, 75005 Paris, France, mathias.fink@espci.fr)

Time-reversal techniques allow focusing in waveguides with application for detection or telecommunication (Kupperman et al., J. Acoust. Soc. Am. 103(1), 25-40, (1998)). It has been shown that the DORT method (French acronym for Decomposition of the Time Reversal Operator) can be used to achieve detection and selective focusing on pointlike scatterers in a waveguide (Mordant et al., J. Acoust. Soc. Am. 105, 2634-2642 (1999)). However, the decomposition is done in frequency domain providing eigenvectors $\mathrm{V}(\omega)$ of undetermined phase. In order to achieve temporal focusing, it is necessary to built 'temporal eigenvectors' $\mathrm{V}(\mathrm{t})$ as an appropriate combination of the eigenvectors $\mathrm{V}(\omega)$. Due to dispersion, the reconstruction of the temporal eigenvectors is difficult in waveguides. One solution was proposed by Mordant et al., using the symmetry of the array response matrix and assuming continuous frequency dependence of the invariants. However this method does not work if eigenvalues crossings occur. Furthermore, it cannot be applied to distinct arrays of transmitters and receivers. We propose a simple method to reconstruct the temporal invariants by using the back propagation of eigenvector in free space. Application to data from a laboratory experiment and from the DOREV 2005 shallow water experiment are presented. 
2pSPf4. Modeling time-reversal focusing in a multiple scattering medium. Kevin J. Haworth (University of Michigan, Department of Radiology, Kresge III, R3320, Ann Arbor, MI 48109-0553, USA, khaworth@umich.edu), Jeffrey B. Fowlkes (University of Michigan, Department of Radiology, Kresge III, R3320, Ann Arbor, MI 48109-0553, USA, fowlkes@umich.edu), Paul L. Carson (University of Michigan, Department of Radiology, Kresge III, R3320, Ann Arbor, MI 48109-0553, USA, pcarson@umich.edu), Oliver D. Kripfgans (University of Michigan, Department of Radiology, Kresge III, R3320, Ann Arbor, MI 48109-0553, USA, greentom@umich.edu)

Time-reversal acoustics has been shown to be effective at focusing sound through high-order multiple-scattering media. In addition to signals being refocused, large amplitudes via pulse compression have also been observed. Previously, a statistical model was developed to simulate a timereversal experiment where an impulse is sent into the scattering medium and the entire response is time-reversed [A. Derode et al., J. App. Phys. 85, 6343-6352 (1999)]. The high-order multiple-scattering process is modeled as a shot noise process. Again postulating a shot noise process the model has been extended to allow for arbitrary input functions and arbitrary windowing before time-reversal. The model has been shown to accurately predict the variance and expectation value of ensembles of simulated data. The analytical model is also tied directly to the governing physical processes, which is useful in attempting to optimize refocused signals. This extension is motivated by a desire to achieve high-amplitude long-pulse focusing, as would be useful in thermal therapies and radiation force experiments. However, the model can find application beyond this specific end-point. The model, it's physical implications, and several time-reversal simulations will be presented.

2pSPf5. Spectral properties of the backscattering matrix in random scattering media. Alexandre Aubry (Laboratoire Ondes et Acoustique, ESPCI, Université Paris 7, CNRS, 10 rue Vauquelin, 75005 Paris, France, alexandre.aubry@espci.fr), Arnaud Derode (Laboratoire Ondes et Acoustique, ESPCI, Université Paris 7, CNRS, 10 rue Vauquelin, 75005 Paris, France, arnaud.derode@ujf-grenoble.fr)

We investigate, both experimentally and theoretically, the spectral properties of the backscattering operator in random media. The experimental set up consists in an array of 64 programmable ultrasonic transducers placed in front of a random scattering medium. The impulse responses between each couple of transducers are measured and form the interelement matrix.The evolution of the singular values with time and frequency is computed by means of a short-time Fourier analysis. Our aim is to compare the mean distribution of the singular values with the predictions of Random Matrix Theory (RMT). Two kinds of random media have been investigated: a highly scattering medium in which multiple scattering predominates and a weakly scattering medium. In both cases, residual correlations that may exist between matrix elements are shown to be a key parameter. The mean distribution of singular values exhibits, surprisingly, very different behaviors in the single and multiple scattering regimes. The agreement between experimental results and RMT predictions is found to be very good in both cases. The results are applied to the detection of a target embedded in a random scattering medium with the DORT method.

\title{
Session 2pSPg
}

\section{Signal Processing in Acoustics, Physical Acoustics, Biomedical Ultrasound/Bioresponse to Vibration, and Underwater Acoutics: Overview of Time Reversal in Acoustics II (Poster Session)}

\author{
David Chambers, Cochair \\ $L L N L$ \\ Claire Prada, Cochair \\ Laboratoire Ondes et Acoustique
}

\begin{abstract}
All posters will be on display from 3:40 p.m. to 5:20 p.m. To allow contributors an opportunity to see other posters, contributors of odd-numbered papers will be at their posters from 3:40 p.m. to 4:30 p.m. and contributors of even-numbered papers will be at their posters from 4:30 p.m. to 5:20 p.m.
\end{abstract}

\section{Contributed Paper}

2pSPg1. Numerical modeling of the effects of finite size transducers for time reversal acoustics in solid media. Michele Griffa (EES-11 (Geophysics) - Los Alamos National Laboratory, MS D443, Los Alamos, NM 87545, USA, mgriffa@lanl.gov), Brian E. Anderson (EES-11 (Geophysics) - Los Alamos National Laboratory, MS D443, Los Alamos, NM 87545, USA, bea@lanl.gov), Paul A. Johnson (EES-11 (Geophysics) Los Alamos National Laboratory, MS D443, Los Alamos, NM 87545, USA paj@lanl.gov)

Time Reversal Acoustics (TRA) has been shown to be very robust not only in fluids but also in solid bounded media. The most relevant limitations to the Time Reversal Process (TRP) in solid specimens are the co-existence of several propagation modes, mode conversion at each interface (inhomogeneities or boundaries), attenuation mechanisms and the multidimensional nature of the propagating wave fields. Additional limitations arise in practical applications, for example for Non Destructive Evaluation purposes, when the Time Reversal Mirror (TRM) piezoelectric transducers are usually attached to the surface of the solid. We have investigated the role of the finite size of the TRM transducers and their sensitivity to only certain components of the incident wave fields in the TRP when they are attached to the surface of the sample under study. We have developed a theoretical analysis and performed numerical simulations and laboratory experiments in order to examine the robustness of TRA in solid media, including where the TRM is composed of finite size elements attached to the specimen surface. The results lead to useful information about the efficiency of the TRP as well as the optimization of the TRM setup in terms of transducer size. 
Session 2pSPh

\title{
Signal Processing in Acoustics: Advances in Acoustic Sensors and Networks for Defense Applications I
}

\author{
Brian G. Ferguson, Cochair \\ Defence Science and Technology Organisation, PO Box 44, Pyrmont NSW, Sydney, 2009, Australia \\ Pierre Naz, Cochair \\ ISL, 5 rue du Général Cassagnou, 68300 Saint Louis, France
}

\section{Contributed Papers}

\section{5:00}

2pSPh1. Acoustic signal processing methods for detecting intrusions and threats in the battlespace. Brian G. Ferguson (Defence Science and Technology Organisation, PO Box 44, Pyrmont NSW, 2009 Sydney, Australia, Brian.Ferguson@dsto.defence.gov.au), Kam W. Lo (Defence Science and Technology Organisation, PO Box 44, Pyrmont NSW, 2009 Sydney, Australia, Kam.Lo@dsto.defence.gov.au)

The processing of acoustic sensor data enables the detection, classification, localization and tracking of intrusions and threats in the battlespace. Initially, passive acoustic signal processing methods that are used in unattended ground sensor networks and onboard uninhabited aerial vehicles are reviewed. These methods process radiated noise (acoustic signature) data generated by sources of military interest and extract tactical information on air and ground vehicles as well as direct (rifle) and indirect (artillery/mortar) fire weapons. Examples of applying various passive acoustic signal processing methods to real sensor data collected during field experiments are presented. The tactical parameter estimates derived from these acoustic methods are then compared with ground truth data. Next, the application of active sonar signal processing to the automatic detection and tracking of a fast inshore watercraft in a cluttered harbor environment is demonstrated using real data. The air bubbles associated with wakes from high-speed surface craft are highly reflective of incident high frequency sonar signal transmissions. Finally, various high frequency active sonar methods that use both real and synthetic apertures to image sea mines are reviewed and demonstrated.

\section{$5: 20$}

2pSPh2. Networking and Fusion of Disparate Acoustic Sensors for Battlespace Applications. Tien Pham (US Army Research Laboratory, 2800 Powder Mill Road, Attn: AMSRD-ARL-SE-SA, Adelphi, MD 207831197, USA, tien.pham1@us.army.mil), Gene Whipps (US Army Research Laboratory, 2800 Powder Mill Road, Attn: AMSRD-ARL-SE-SA, Adelphi, MD 20783-1197, USA, gene.whipps@us.army.mil)

A network of distributed acoustic sensor systems on the ground and/or in the air can be used effectively for autonomous and remote intelligence, surveillance, and reconnaissance (ISR) applications. However interoperability of disparate sensor systems is a major challenge for current coalition force applications. Specifically, a network of acoustic sensor nodes can exhibit heterogeneity in a variety of dimensions. At the sensor-level, the acoustic sensors can vary in their types (e.g., cardioid vs. omni-direction) and in their responsiveness to transient and continuous sources. At the node-level, the acoustic systems can vary in their array configuration, platform mobility, node reactiveness (e.g., timeliness of response), and information processing and output. At the network-level, the acoustic systems can vary in their communication protocols and access mediums. Current R\&D efforts within the US-UK International Technology Alliance seek to develop a "sensor fabric" technology to seamlessly connect disparate systems for networked sensing applications. Results from a recent field experiment to detect and locate moving ground targets, weapon firings, and explosions via a network of disparate acoustic sensor systems and sensing platforms (e.g., PDA's, workstations, motes and unattended ground sensor systems) are presented.

\section{$5: 40$}

2pSPh3. Performace Bounds for a Multi-Array Network Approach to Gunfire Detection and Localization. Michael Roan (Virginia Tech, 917 Village Way North, Blacksburg, VA 24060, USA, mroan@vt.edu), Elizabeth Hoppe (Virginia Polytechnic Institute and State University, 141 Durham Hall, Blacksburg, VA 24060, USA, ehoppe@vt.edu), Marty Johnson (Virginia Tech, Mechanical Engineering, 143 Durham 0238, Blacksburg, VA 24061, USA, martyj@vt.edu)

Acoustics-based gunfire detection and sniper localization have become important in both military and civilian sectors. For the case of rifle fire, most current localization algorithms rely on estimating parameters using the projectile's cone-shaped shockwave and the muzzle blast. Previous studies have attempted to quantify shockwave detection performance as a function of range for various approaches such as wavelet matched filtering for the " $\mathrm{N}$ " shaped shockwave. Accurately detecting the shockwave is important because the shockwave shape can be used to determine bullet trajectory if the bullet velocity is known. A second layer of direction of arrival (DOA) estimation can be added by using the time difference of arrival between the shockwave and the muzzle blast. These DOA methods require a two step process of detection and estimation. In this work, a signal model is developed for a network of microphone arrays that receives the acoustic waveform generated by supersonic projectiles. The best-case localization performance of the multi-array network is quantified via the Cramer-Rao lower bound on the error in estimating shooter position. Results are illustrated using simulated data to generate CRLB ellipses on shooter position estimation error and via live fire experiments using several calibers and a multi-array network of microphones.

\section{6:00}

2pSPh4. Automated Identification of Vehicles using Acoustic Signal Processing. Naoko Evans (University of York, Department of Electronics, Heslington, YO10 5DD York, UK, ne502@ohm.york.ac.uk), David Chesmore (University of York, Department of Electronics, Heslington, YO10 5DD York, UK, edc1@ohm.york.ac.uk)

Automated identification of unauthorised intruding vehicles approaching protected infrastructure is becoming increasingly important for security purposes. This three-year project, which is now in its second year, aims to develop a real-time acoustic vehicle type recognition system that will be predominantly composed of three main parts; acoustic signal pre-processing, feature extraction, and decision making (or classification). The main study area covers various signal processing techniques in time, time-frequency and potentially frequency domains with signal classification implemented using a range of artificial intelligence techniques such as artificial neural networks. So far the focus has been on time domain signal processing and neural network classification. Whilst the work is at an early stage, the time domain methods such as Time Domain Signal Coding (TDSC) and CoOccurrence Matrix combined with neural networks have already shown some promise. The presentation will introduce the project, describe methodologies involved as well as the results to date for the novel acoustic identification of categories of vehicles. 
6:20

2pSPh5. Source detection and tracking in 3-D space using efficient subspace methods. Colin Barnhill (Johns Hopkins University, $3400 \mathrm{~N}$. Charles St, Baltimore, MD 21218, USA, shaolin_cb@yahoo.com)

This presentation will demonstrate the versatility and robustness of a MUSIC-based subspace algorithm for acoustic source detection and tracking. The new algorithm (MUSIC3D) is designed for use with spherical arrays and performs three dimensional source detection and tracking. MUSIC3D operates on a spherical decomposition of the received array signals which results in its high efficiency. Spherical decomposition of the array signals allows for data reduction, frequency selection, and direct subspace calculations. The appeal of the MUSIC3D algorithm is that it offers a large variety of implementations which can be tailored for specific environments, signal sources or noise sources. Source tracking is accomplished through a combination of a Kalman-based filter and the MUSIC3D algorithm
6:40

2pSPh6. Optimal estimation of aircraft noise with four microphones in a spatial configuration. Nico Roosnek (Roosnek Research \& Development, Vlaskamp 82, 2592 AC The Hague, Netherlands, nico@ roosnek.nl)

By using all time shifts between the arrivals of the acoustic signal at the four microphones - in a spatial configuration - the source direction is obtained. The used correlation technique reduces significantly noise from other sources and the error in the estimated level of noise from the tracked aircraft/source. By taking into account a motion model for the source, the history of that motion and the measured acoustic data a very robust sound monitor system is obtained. The sensitivity for other sources, wind and rain noise is greatly reduced - theoretically almost unlimited - in comparison with classical monitor systems. Four aircraft can be tracked simultaneously. Another system capability is the estimation of the true flightpath with a system error depending on the system configuration and the environmental conditions. Using this path information the soundscape can be calculated by inter- and extrapolation. The design goal of the system is truly met!

\title{
Session 2pUWa
}

\section{Underwater Acoustics and ECUA: Auralization of Sonar Signals}

\author{
Paul C. Hines, Cochair \\ Defence R\&D Canada - Atlantic, P.O. Box 1012, Dartmouth, NS B2Y3Z7, Canada
}

Paul R. White, Cochair

Institute of Sound and Vibration, Univ. of Southampton, University Road, Highfield, Southampton, SO17 1BJ, UK

\section{Invited Papers}

\section{2:00}

2pUWa1. Active sonar clutter and auralization. Douglas Abraham (CausaSci LLC, PO Box 5892, Arlington, VA 22205, USA, abrahad@ieee.org)

Target-like false alarms generically termed clutter are the primary hindrance to active sonar in shallow water operational areas. Clutter can arise from a myriad of sources with geologic, biologic and anthropogenic origin. The clutter-source echoes are often determined by automatic signal processing to be target-like and therefore potentially of interest. These numerous false alarms consume either the sonar operator's time or flood automated data and information processing algorithms. Aural analysis of clutter echoes (auralization) can increase active sonar performance through improved operator or algorithmic classification. An overview of active sonar clutter is presented with a focus on the role of auralization for clutter reduction. [Supported by the Office of Naval Research Code 321US under contract number N0001407C0092]

2pUWa2. Insights to dolphin sonar discrimination capabilities with human listening experiments. Whitlow W. Au (Univ. of Hawaii, P.O. Box 1106, Kailua, HI 96734, USA, wau@ hawaii.edu), Caroline M. Delong (Brown University, Box 1953, Providence, RI 02912, USA, Caroline_Delong@brown.edu)

Dolphins have a very keen sonar system that is able to make fine discriminations between complex targets such as proud and buried mines. To understand the cues that dolphins use for fine target discrimination, human listening experiments have been conducted with echoes from real targets using a simulated broadband dolphin echo-ranger. Echoes are stretched in time to translate the echoes into the human auditory range. The human performance is usually as accurate as the dolphin, with the additional benefit of being able to get feedback on cues used. Various types of discrimination and detection experiments have been conducted paralleling dolphin sonar experiments. Discrimination of material composition of cylinders and spheres, detection of target in clutter and cylinder wall thickness difference discrimination experiment have been performed. The human listeners performed as well or better than the dolphins at the task of discriminating between a standard target and comparison targets. Click pitch, echo duration, time-separation pitch and timbre are some of the cues used by human listeners to discriminate targets. Dolphins may use some of the same echo features as humans. Human listening studies can quickly identify salient combinations of echo features that permit object discrimination and also help refine dolphin experiments. 
2pUWa3. Similarity-Based Perceptual Feature Identification for Active Sonar Signal Classification. Scott M. Philips (MIT Lincoln Laboratory, 244 Wood Street, Lexington, MA 02420-9108, USA, scott.philips@11.mit.edu), James W. Pitton (Office of Naval Research Global, Blenheim Crescent, West Ruislip, Middlesex, HA4 7HL London, UK, jpitton@onrglobal.navy.mil)

In many acoustic signal processing applications human listeners are able to outperform automated processing techniques, particularly in the identification and classification of acoustic events. This paper develops a framework for employing perceptual information from human listening experiments to improve automatic classification of active sonar signals. We focus on the identification of new signal features that are able to predict the human performance observed in formal listening experiments. Using this framework, our newly identified features have the ability to elevate automatic classification performance closer to the level of human listeners. We develop several new methods for learning a perceptual feature transform from human similarity measures. In addition to providing a more fundamental basis for uncovering perceptual features than previous approaches, these methods also lead to a greater insight into how humans perceive sounds in a dataset. We also develop a new approach for learning a perceptual distance metric. This metric is shown to be applicable to modern kernel-based techniques used in machine learning and provides a connection between the fields of psychoacoustics and machine learning.

2pUWa4. Auditory-motivated techniques for detection and classification of passive sonar signals. Guy J. Brown (University of Sheffield, Dept. of Computer Science, Regent Court, 211 Portobello Street, S1 4DP Sheffield, UK, g.brown@ dcs.shef.ac.uk), Robert W. Mill (University of Sheffield, Dept. of Computer Science, Regent Court, 211 Portobello Street, S1 4DP Sheffield, UK, r.mill@dcs.shef.ac.uk), Simon Tucker (University of Sheffield, Dept. of Information Studies, Regent Court, 211 Portobello Street, S1 4DP Sheffield, UK, s.tucker@dcs.shef.ac.uk)

Hydrophone arrays used to make passive sonar recordings are becoming increasingly complex, thus placing great demands on sonar operators who are required to identify transient and tonal events. Machine hearing systems can mitigate this problem, by performing initial classification and detection of passive sonar signals based on perceptually relevant principles. We describe two such systems. In the first system, transient sounds are characterised using measures of timbre and acoustic pattern. Acoustic features relating to timbre were identified by a multidimensional scaling study, in which human listeners judged the similarity of transient sonar sounds. The perceptual features give improved classification performance compared to commonly-used statistical measures derived from the power spectrum. The second system is an auditory-motivated approach to narrowband detection, which is based on information in zerocrossing intervals rather than signal power. This is motivated by the observation that the fine time structure of an acoustic stimulus is preserved by the phase locking of auditory nerve fibres. We show that a power detector outperforms the interval detector when the signal is centred on the analysis filter. However, when the signal frequency deviates from the centre of the analysis band, interval-based detection improves and power-based detection worsens.

2pUWa5. Auditory models and nonlinear filterbanks in underwater auralization. Stefan Bleeck (National Physical Laboratory, Hampton Road, TW11 OLW Teddington, UK, bleeck@gmail.com), Paul D. Fox (National Physical Laboratory, Hampton Road, TW11 OLW Teddington, UK, pf@isvr.soton.ac.uk), Paul R. White (Institute of Sound and Vibration, Univ. of Southampton, University Road, Highfield, SO17 1BJ Southampton, UK, prw@isvr.soton.ac.uk), Niamh O’Meara (National Physical Laboratory, Hampton Road, TW11 OLW Teddington, UK, niamh17@gmail.com)

Mammals like dolphins and humans have the ability to distinguish between different objects by listening to their scattered signals. We explore this phenomenon by evaluating the "Auditory Image Model" (AIM), a biologically inspired model of the human auditory system from the outer ear up to central processing. AIM aims to simulate the spectral analysis of the basilar membrane, the neural encoding and the temporal integration performed by the auditory system. Simulated scattered signals from the same object objects of different sizes and different objects of same size subjected to an incident pulse were analysed numerically with AIM, simulating the response of the human auditory system. The resulting neuronal activity patterns were analysed in two independent dimensions using the filter centre frequency and the relative timing intervals of the responses. The results are interpreted globally as reflecting the size and the shape/properties of the object respectively. We show for a series of calibration stimuli that the model can distinguish between same objects of different size and different objects of the same size. We also discuss how a nonlinear model of the basilar membrane, performing a Gammachirp/Mellin transformation, may act as a possible feature extraction tool for classification methods

3:40-5:20 Posters

Lecture sessions will recess for presentation of poster papers on various topics in acoustics. See poster sessions for topics and abstracts. 


\section{Contributed Papers}

$5: 20$

2pUWa6. What it is like to be a bat: A sonar system for humans. Cameron Morland (Boston University, Dept. of Cognitive and Neural Systems, 677 Beacon St., Boston, MA 02215, USA, cjmorlan@bu.edu), David Mountain (Boston University, Biomedical Engineering Dept., 44 Cummington St., Boston, MA 02215, USA, dcm@bu.edu)

Echolocation is a method of perceiving the world by emitting noises then listening to the reflections of these noises off objects in the world. It is used by animals for hunting and navigation. Some visually impaired humans use echolocation as part of their orienting repertoire. This work describes a device designed to enhanced one's existing echolocation ability. The device repeatedly emits an inaudible, ultrasonic "'click", several times per second. Each click reflects off surfaces; this reflection is detected by head-mounted microphones. Computer processing converts the signals into audible signals, which are presented to the user over custom open-ear earphones. ILD, ITD, HRTF, and Doppler shifts in the reflections allow the user to distinguish the location and size/surface properties of objects. The spatial cues presented by our device match those used to localize ordinary audible objects. The auditory "image" generated in this way causes objects in the world to seem to emit sounds, and for objects with different shapes/textures to have subtly different sounds. A prototype has been constructed. Simple detection of objects and open spaces is readily possible, as well as Doppler-based gross surface texture. Further refinement in the areas of emission design, spectral spatialization, externalization, and miniaturization is required.

\section{$5: 40$}

2pUWa7. Modeling the mechanism and neural substrate for aural categorization of sonar echoes. Jason E. Summers (U.S. Naval Res. Lab., Acoust. Div., Code 7142, 4555 Overlook Ave. SW, Washington, DC 20375 , USA, jason.summers@nrl.navy.mil), Charles F. Gaumond (U.S. Naval Res. Lab., Acoust. Div., Code 7142, 4555 Overlook Ave. SW, Washington, DC 20375, USA, charlie.gaumond@nrl.navy.mil), Derek Brock (U.S. Naval Res. Lab., Acoust. Div., Code 7142, 4555 Overlook Ave. SW, Washington, DC 20375, USA, derek.brock@nrl.navy.mil), Ralph N. Baer (U.S. Naval Res. Lab., Acoust. Div., Code 7142, 4555 Overlook Ave. SW, Washington, DC 20375, USA, ralph.baer@nrl.navy.mil)

Previously, human perception of impulsive active-sonar echoes was investigated through paired-comparison ratings and multidimensional scaling (MDS). In the resulting MDS configuration, stimuli formed clusters representing aurally distinct categories. An interpretation is presented which suggests that dissimilarity judgments reflect separate processes for within- and between- category comparisons. The process of categorization is not based on decision boundaries in a low- dimensional space of ordinal-scale perceptual features, while within-category judgments do reflect such features. A class-specific paradigm seems appropriate as a model of human categorization: signals are compared to category archetypes, each described by a set of features that may be unique to that category. Regardless of the specific mechanism, listeners must carry out categorization on a particular signal representation: the neural substrate of the process. The suitability of various temporal, spectral, and time-frequency signal representations are investigated through observing categories formed by hierarchical clustering. A signal-processing model of the spectrotemporal receptive fields of the auditory cortex and the processing of the auditory periphery [T. Chi et al., J. Acoust. Soc. Am. 118, 887-906 (2005)] is shown to yield good agreement with human categorization. Techniques for feature selection from this representation are discussed. [Work supported by ONR.]

\section{6:00}

2pUWa8. Automatic classification of vocalizations with Gaussian Mixture Models and Hidden Markov Models. Judith C. Brown (MIT Media Lab, 37 Indian Ridge Rd, Natick, MA 01760, USA, brown@media.mit.edu), Paris Smaragdis (Adobe Systems Inc., 275 Grove St., Newton, MA 02466, USA, paris@media.mit.edu)

The automatic classification of marine mammal sounds is very attractive as a means of assessing massive quantities of recorded data, freeing humans and offering rigorous and consistent output. Calculations on a set of vocalizations of Northern Resident killer whales using Dynamic Time Warping have been reported recently. [Brown, J. C., and P.J.O. Miller, 'Automatic classification of killer whale vocalizations using dynamic time warping," J. Acoust. Soc. Am. 122, 1201-1207, (2007).] Since this method requires the time-consuming pre-processing measurement of the frequency contours, we have explored the use of Gaussian Mixture Models (GMM) and Hidden Markov Models (HMM). These methods can be applied directly to timefrequency decompositions of the recorded signals. Calculations have been made on a set of 75 calls previously classified perceptually into 7 call types. Preliminary results give an agreement of roughly $85 \%$ agreement with the perceptual classification for the GMM and over $90 \%$ for an HMM.

\section{6:20}

2pUWa9. Incorporation of acoustic level analysis with sonar operator workstation workflow using hierarchical hidden Markov models. Justin Nevitt (Naval Research Laboratory, 4555 Overlook AVE SW, Washington, DC 20375, USA, justin.nevitt@nrl.navy.mil), James Ballas (Naval Research Laboratory, 4555 Overlook AVE SW, Washington, DC 20375, USA, james.ballas@nrl.navy.mil)

Future Anti Submarine Warfare (ASW) systems will be highly automated, and utilize multiple sonar signal processing, non-acoustic information, off platform information, and environmental information to maximize the detection, tracking, and classification of undersea threats. Recently, a hidden Markov model (HMM) was developed to describe and characterize the workflow strategies that expert sonar operators used during the IUSW-21 (Integrated UnderSea Warfare for the 21st Century) Sea Test. Abandoning the normative workflow designed into the system, the sonar operators developed and utilized unanticipated strategies to gather evidence to confirm or disconfirm automated classifications or to manually classify contacts. The workflow included detailed visual inspection of and listening to the sonar return. This initial HMM did not include a characterization of the auditory processing. Hidden Markov models are well suited to this purpose. Adding this component to the operator workflow was achieved by a hierarchical HMM which represents the acoustic processing as an embedded HMM in the overall workflow HMM. The resulting model is not just an operator workflow but defines a system processing model that could be used as a framework to develop automated processing of selected components. 


\title{
Session 2pUWb
}

\section{Underwater Acoustics and ECUA: Sonar System and Transducer Calibration Methodology I}

\author{
Kenneth G. Foote, Cochair \\ Woods Hole Oceanographic Institution, Woods Hole, MA 02543, USA \\ Stephen P. Robinson, Cochair \\ National Physical Laboratory, Hampton Road, Teddington, TW11 OLW, UK
}

\section{Invited Papers}

$$
\text { 2:00 }
$$

2pUWb1. The sonar equations: definitions, dimensions and units of individual terms. Michael A. Ainslie (TNO Defence, Security and Safety, Oude Waalsdorperweg 63, 2597 AK The Hague, Netherlands, michael.ainslie@tno.nl)

The passive and active sonar equations are reviewed with regard to the definitions of individual terms and their units. It is shown on the one hand that ambiguities exist in conventional definitions that can lead to misinterpretation, and on the other that under special conditions correction terms are needed that are not included in the standard equations. Revised definitions are proposed that remove the need for some of the correction terms and reduce the likelihood of misunderstanding. Particular emphasis is placed on the terms 'propagation loss' and 'source level' for both active and passive sonar. For example, the effect on changes in impedance between the source and receiver of the sound is considered in the case of passive sonar, and between the source, receiver and scatterer of sound in the case of active sonar.

\section{2:20}

2pUWb2. Validation of primary hydrophone calibrations by inter-laboratory comparisons and by independent calibration methods. Stephen P. Robinson (National Physical Laboratory, Hampton Road, TW11 OLW Teddington, UK, Stephen.Robinson@npl.co.uk), Peter D. Theobald (National Physical Laboratory, Hampton Road, TW11 OLW Teddington, UK, pdt @ npl.co.uk)

A description is presented of two approaches which may be used to validate primary calibration methods for hydrophones and transducers. Firstly, a comparison may be made with another independent absolute calibration method, preferably one based on a different physical principle (and therefore with few common sources of uncertainty). Secondly, an inter-laboratory comparison of calibrations may be undertaken between different institutes operating at a similar level. This paper describes the results of such exercises for free-field calibration of hydrophones in the range from $1 \mathrm{kHz}$ to $500 \mathrm{kHz}$. Firstly, two independent calibration methods are compared: the three-transducer reciprocity method and a method based on optical interferometry. The differences observed in the results are typically less than $0.5 \mathrm{~dB}$, which is of the same order as the overall uncertainties of each of the methods. Secondly, the results are shown of a recent international comparison of hydrophone calibrations involving institutes from Canada, China, Germany, Russia, South Africa, UK, and USA. Here, the agreement was generally within quoted uncertainties, the results generally lying within a $\pm 0.5 \mathrm{~dB}$ band for frequencies up to $300 \mathrm{kHz}$. A discussion is given of the general sources of uncertainties in the calibrations.

\section{2:40}

2pUWb3. Phase calibration of hydrophone and its application in the reconstruction of underwater acoustic waveforms. Yuebing Wang (Hangzhou Applied Acoustics Research Institute, 96 Huaxing Road, 310012 Hangzhou, China, wang_yuebing@tom.com), Shui Li (Hangzhou Applied Acoustics Research Institute, 96 Huaxing Road, 310012 Hangzhou, China, lishui9861@163.com), Yongjun Huang (Hangzhou Applied Acoustics Research Institute, 96 Huaxing Road, 310012 Hangzhou, China, pink2701@126.com)

Abstract: In this paper, both the reciprocal method and the optical method for the calibration of plural sensitivities of hydrophones are reviewed firstly. Then an experimental system is introduced in which with a $1.5 \mathrm{MHz}$ focusing transducer acting as a position indicator, phase responses of hydrophones can be calibrated easily and accurately. Measurements are carried out for different types of hydrophones and results are given in the frequency range $20 \mathrm{kHz}$ to $400 \mathrm{kHz}$. Finally, with the plural responses of hydrophones included in the construction for the transfer function of the receiving system, acoustic waveforms produced by a projector are reconstructed in wide band from received signals, and a few conclusions are summarized from the experiment. 


\section{Contributed Papers}

3:00

2pUWb4. The hydrophone free-field calibration in the non-anechoic water tank using continuous radiation mode. Alexander Isaev (VNIIFTRI, Mendeleevo, 141570 Moscow, Russian Federation, isaev@vniiftri.ru), Anton Matveev (VNIIFTRI, Mendeleevo, 141570 Moscow, Russian Federation, matveev@ vniiftri.ru)

Anechoic chambers are used at free-field measurements in acoustic. Quality of the anechoic chamber estimate by comparing the dependence of sound pressure with distance between a projector and the receiver with the inversely proportional law. Creation of an anechoic water tank is a difficult technical problem. Therefore measurements in a water tank usually use tone burst radiation technique. In a non-anechoic water tank it is possible to provide free-field conditions at a continuous radiation mode. It is achieved by using a signal with the distributed spectrum and mathematical processing of results of measurements. The measurement procedure and results of free field calibration of this method are considered in the paper. Efficiency of measuring procedure was estimated by a check of the inversely proportional law in conditions of a non-anechoic water tank.

$$
\text { 3:20 }
$$

2pUWb5. Calibration of vector sensors. Joseph A. Clark (NSWCCD Code 7340, 9500 MacArthur Blvd., West Bethesda, MD 20817-5000, USA, joseph.a.clark1@navy.mil)

Vector sensors that combine a hydrophone and a triaxial arrangement of accelerometers in a neutrally buoyant package are currently being used for underwater radiated noise measurements and other applications. Precise amplitude and phase calibration is required to obtain high quality measurements. A basic procedure that has been used successfully to calibrate measured radiated noise spectra in one-third-octave and narrowband formats will be described. Results of validation experiments that compared vector sensor measurements with other reference data will be presented. An iterative calibration scheme for improving the performance of a vector sensor employed as part of an active sonar system will also be presented.

\section{3:40-5:20 Posters}

Lecture sessions will recess for the presentation of poster sessions on various topics in acoustics. See poster sessions for topics and abstracts.

\section{Invited Papers}

\section{$5: 20$}

2pUWb6. Array gain degradation due to nearby bubbles. Richard Lee Culver (ARL Penn State, PO Box 30, State College, PA 16804, USA, rlc5@psu.edu), Mario F. Trujillo (ARL Penn State, PO Box 30, State College, PA 16804, USA, mft10@psu.edu)

Signal-to-noise ratio (SNR) is signal power divided by noise power, usually expressed in $\mathrm{dB}$ ( $\mathrm{SNR}=20 * \log 10$ (Psig/Pnoise). Array gain (AG) is the increase in SNR at an array output relative to that at a single element. AG assumes spatially-compact signals embedded in spatially-diffuse noise or interference. When the array is steered in the direction of a signal, AG is maximum when the signal is fully coherent across the array (i.e. the signals add in phase for all elements) while the noise or interference is incoherent across the array (i.e. adds with random phase). For an acoustic array operating in the ocean, we would like to understand the degree to which nearby bubbles degrade AG. Scattering by bubbles represents interference at the array. Bubble attenuation can also degrades array performance by attenuating the signal of interest, but that is separate from AG degradation. Previously we applied the single scattering approximation of Ishimaru (1977, Chap. 6) and found that scattering from bubbles very close to the array can generate correlated interference, thereby decreasing AG. Here we validate the theory using in-water measurements of AG as the distance of from the array to the bubbles varies. Work sponsored by Office of Naval Research, Code 321 Undersea Signal Processing.

$$
5: 40
$$

2pUWb7. A method for calibrating hydrophones immersed in sandy sediment. Gary B. Robb (National Oceanography Centre, University of Southampton Waterfront Campus, European Way, SO14 3ZH Southampton, UK, gbor199@noc.soton.ac.uk), Gary Hayman (National Physical Laboratory, Hampton Road, TW11 OLW Teddington, UK, Gary.Hayman@npl.co.uk), Peter D. Theobald (National Physical Laboratory, Hampton Road, TW11 OLW Teddington, UK, pdt@npl.co.uk), Victor F. Humphrey (Institute of Sound and Vibration, Univ. of Southampton, University Road, Highfield, SO17 1BJ Southampton, UK, vh@isvr.soton.ac.uk), Stephen P. Robinson (National Physical Laboratory, Hampton Road, TW11 OLW Teddington, UK, Stephen.Robinson@npl.co.uk), Timothy G. Leighton (Institute of Sound and Vibration, Univ. of Southampton, University Road, Highfield, SO17 1BJ Southampton, UK, T.G.Leighton@soton.ac.uk), Justin K. Dix (National Oceanography Centre, University of Southampton Waterfront Campus, European Way, SO14 3ZH Southampton, UK, jkd@ noc.soton.ac.uk), Angus I. Best (National Oceanography Centre, University of Southampton Waterfront Campus, European Way, SO14 3ZH Southampton, UK, aib@noc.soton.ac.uk)

Hydrophones are frequently used as receivers for in situ sediment acoustic experiments. At present, processing techniques use receiver sensitivities measured from water-based calibrations. It is, however, accepted that the receive sensitivity will depend on the medium surrounding the hydrophone, particularly at frequencies close to the transducer's resonance frequency. To assess this affect, a series of calibrations were performed over the frequency range of 10 to $200 \mathrm{kHz}$ on two types of hydrophones (with cylindrical and spherical elements) inserted into degassed sandy sediment. Sensitivities were measured using a modified three-transducer reciprocity technique, which uses a co-linear arrangement to allow the sediment attenuation to be omitted from the sensitivity calculation. The insertion of the hydrophones into the sediment reduced the measured receive sensitivities by a maximum value of $3.8 \mathrm{~dB}$ with respect to the equivalent water-based calibrations. The co-linear arrangement adopted allowed the transmission between the outer devices to be recorded with and without the central hydrophone present. Repeat measurements indicated that the sediment disturbance associated with the removal of the central hydrophone caused sensitivity differences of less than $1.2 \mathrm{~dB}$, while the inclusion of the central hydrophone caused a shadowing effect which increased sensitivities by between 1.3 to $4.0 \mathrm{~dB}$. 
2pUWb8. Standard-target method of calibrating active sonars: principles, applications, benefits. Kenneth G. Foote (Woods Hole Oceanographic Institution, Woods Hole, MA 02543, USA, kfoote@whoi.edu)

The standard-target method of calibrating active sonar involves suspending a target at a known position in a sonar beam, insonifying it with a known signal, measuring the scattered pressure field, and relating this to the scattering properties of the target. These properties are known a priori for standard targets, rendering the calibration primary. Repetition of the procedure for different relative sonar-target positions can enable determination of directional and nearfield characteristics. Following a review of standard-target calibration principles, the method is illustrated for both narrowband and broadband scientific echo sounders and multibeam sonars, with operating frequencies in the range $18-3200 \mathrm{kHz}$. Applications to sidescan sonar and to low-frequency parametric sonars and other mid-frequency sonars in the nominal range 1-10 kHz have not yet been developed, but are evidently feasible given preliminary standard-target designs, which are described. In every case, the standard targets are of a proven manageable size, with immersion weight less than $200 \mathrm{~N}$. Benefits of standard-target calibration, including accuracy, operational convenience, and cost-effectiveness, among others, are emphasized. The method, which is useful for both quantitative and qualitative imaging applications, extends to borehole acoustic sensors and medical ultrasonic devices.

\title{
Contributed Paper
}

6:20

2pUWb9. Calibration of broadband active acoustic systems using a single standard spherical target. Timothy K. Stanton (Woods Hole Oceanographic Institution, Applied Ocean Physics \& Engineering Department, 98 Water Street, MS \#11, Woods Hole, MA 02543, USA, tstanton@whoi.edu), Dezhang Chu (NOAA/NMFS/NWFSC/FRAMD, Montlake Blvd., E. Seattle, WA 98112, USA, dchu@whoi.edu)

When calibrating a broadband active acoustic system with a single standard target such as a sphere, the inherent resonances associated with the scattering by the sphere pose a significant challenge. In this research, a method is developed which completely eliminates the source of resonances through isolating and exploiting the echo from the front interface of a sphere. This echo is relatively insensitive to frequency over a wide range of frequencies, lacking resonances, and relatively insensitive to small changes in material properties and, in the case of spherical shells, shell thickness. The research builds upon the concept of using this echo for calibration in the work of Dragonette et al. (J. Acoust. Soc. Am. 69, 1186-1189 (1981)). This current work generalizes that of Dragonette by 1) incorporating a pulse compression technique to significantly improve the ability to resolve the echo, and 2) rigorously accounting for the scattering physics of the echo so that the technique is applicable over a wide range of frequencies and material properties of the sphere. The utility of the new approach is illustrated through application to data collected at sea with an air-filled aluminum spherical shell and long broadband chirp signals $(30-105 \mathrm{kHz})$.

TUESDAY AFTERNOON, 1 JULY 2008

P3-C, LEVEL 3, 3:40 TO 5:20 P.M.

\section{Session 2pUWd}

\section{Underwater Acoustics and ECUA: Sonar System and Transducer Calibration Methodology II (Poster Session)}

\author{
Kenneth Foote, Cochair \\ Woods Hole Oceanographic Institution \\ Stephen Robinson, Cochair \\ National Physical Laboratory
}

\begin{abstract}
All posters will be on display from 3:40 p.m. to 5:20 p.m. To allow contributors an opportunity to see other posters, contributors of odd-numbered papers will be at their posters from 3:40 p.m. to 4:30 p.m. and contributors of even-numbered papers will be at their posters from 4:30 p.m. to 5:20 p.m.
\end{abstract}

\section{Contributed Paper}

2pUWd1. Low frequency hydrophone calibration with using tensometric pressure sensor. Alexander Isaev (VNIIFTRI, Mendeleevo, 141570 Moscow, Russian Federation, isaev@vniiftri.ru), Alexander Shchelkunov (VNIIFTRI, Mendeleevo, 141570 Moscow, Russian Federation, for_my_reg@mail.ru)

Usually when calibrating a hydrophone in an acoustic coupler the piezoelectric hydrophones use an auxiliary transducer. Widely applicable reciprocity calibration is high-ly accurate, but difficult in realization and laborious. The method of comparison with a reference transducer is more simple and quick, but less accurate. In the paper the method of low frequency hydrophone calibration using the auxiliary transducer with a flat frequency response up to $3 \mathrm{kHz}$ is considered. The auxiliary transducer is calibrated accurately by changing the water column in the acoustic coupler. It finally allows us to calibrate measuring hydrophones more accurately too. The measuring equipment on the basis of tensometric pressure sensor type MPX 5010 is considered. 


\title{
Session 2pUWe
}

\section{Underwater Acoustics and ECUA: Geoacoustic Sediment Modeling I (Poster Session)}

\author{
Nicholas Chotiros, Cochair \\ Applied Research Laboratories, University of Texas \\ Jean-Pierre Sessarego, Cochair \\ Laboratory for Mechanics and Acoustics CNRS
}

\begin{abstract}
All posters will be on display from 3:40 p.m. to 5:20 p.m. To allow contributors an opportunity to see other posters, contributors of odd-numbered papers will be at their posters from 3:40 p.m. to 4:30 p.m. and contributors of even-numbered papers will be at their posters from 4:30 p.m. to 5:20 p.m.
\end{abstract}

\section{Contributed Papers}

2pUWe1. Estimating the frequency dependent behavior of marine sediment sound speeds using low frequency aircraft sound. Alireza Amiri-Simkooei (Acoustic Remote Sensing Group, Delft Institute of Earth Observation and Space Systems, Delft University of Technology, Kluyverweg 1, 2629 HS Delft, Netherlands, a.amirisimkooei@tudelft.nl), Mirjam Snellen (Acoustic Remote Sensing Group, Delft Institute of Earth Observation and Space Systems, Delft University of Technology, Kluyverweg 1, 2629 HS Delft, Netherlands, m.snellen@tudelft.nl), Dick G. Simons (Delft University of Technology, P.O. Box Postbus 5048, 2600 GA Delft, Netherlands, d.g.simons@tudelft.nl), Michael Buckingham (Marine Physical Lab, Scripps Institution of Oceanography, UCSD, 9500 Gilman Dr. M/C 0238, La Jolla, CA 92093-0238, USA, mjb@mpl.ucsd.edu)

For investigating the dispersion behavior of sediment sound speeds, broadband sound speed measurements are required. Sediment sound speed dispersion models predict the largest sound speed variations typically at low frequencies. Aircraft fly-over noise acquired on receivers placed in sediment proved to be a promising approach towards broadband low-frequency measurements. Since the magnitude of the Doppler-shift depends on sound speed at the receiver position, the observed Doppler-shift can be used to derive the sound speed. We propose a new approach using the entire time series instead of employing only the two observed frequencies at approaching and departing. It is based on a model for received signals, accounting for the Doppler-shifted frequency. By maximizing the match between modeled and measured time series as a function of sound speed and frequency in a leastsquares framework, estimates of the frequency-dependent sound speed are obtained. The method is applied to data acquired at receivers placed in air, water and sediment. Statistical tests indicate that the marine sediment sound speed depends indeed on frequency, i.e. sound speed decreases significantly below $300 \mathrm{~Hz}$. A comparison is made with a modified viscous fluid model from which realistic values for the geotechnical parameters of the considered sediment were obtained.

2pUWe2. Imaging of Large-Scale Sediment Transport Dynamics with Multibeam Sonar. Stephen M. Simmons (University of Leeds, School of Earth and Environment, University of Leeds, LS29JT Leeds, UK, s.m.simmons@leeds.ac.uk), Dan R. Parsons (University of Leeds, School of Earth and Environment, University of Leeds, LS29JT Leeds, UK, parsons.daniel@btinternet.com), Jim L. Best (Departments of Geology and Geography and Ven Te Chow Hydrosystems Laboratory, 1301 W. Green St., University of Illinois, Urbana, IL 61801, USA, jimbest@uiuc.edu), Chris Malzone (Reson Inc., Goleta, Santa Barbara, CA 93117, USA, CMalzone @ reson.com), Gareth M. Keevil (University of Leeds, School of Earth and
Environment, University of Leeds, LS29JT Leeds, UK, g.keevil@see.leeds .ac.uk)

Multibeam Echo-Sounder (MBES) systems have developed rapidly over recent decades and are routinely deployed to provide high-resolution bathymetric imaging. Modern data handling and storage technologies have facilitated the logging of the back-scatter information previously discarded by these systems. This paper develops a novel methodology to exploit this logging capability to quantify the concentration and dynamics of suspended sediment within the water column. This development provides a multipurpose tool for the holistic surveying of sediment transport by imaging suspended sediment concentration, associated flow structures and providing concurrent high-resolution bathymetry. This paper presents the results obtained from the deployment a RESON 7125 MBES in the field, with the aim of examining the dynamics of suspended sediment transport over dune bedforms and in the region of flow mixing between large rivers of significantly different suspended sediment concentration. Results from controlled tank tests are also presented, obtained using different types and mixes of sediment. The results demonstrate the capability of MBES systems to successfully resolve the contrast in suspended sediment concentrations. The large sets of data recorded in the two-dimensional MBES swath enables the real-time monitoring of suspended sediment transport and related flow processes on a scale previously unrealisable with single-beam acoustic systems.

2pUWe3. The influence of topography on sidescan sonar images. Dietmar Bürk (GKSS Research Centre, Max-Planck-Str. 1, Institute for Coastal Research, 21502 Geesthacht, Germany, dietmar.buerk @ gkss.de), Ingo Klaucke (IFM-GEOMAR, Leibniz-Institute of Marine Sciences, Wischhofstraße 1-3, 24148 Kiel, Germany, iklaucke@ifm-geomar de)

The backscattering signal on sidescan sonar images is to a large degree dependent on the incident angle of the acoustic beam onto the seafloor. As sidescan sonar systems are often used for mapping seafloor lithologies, it is necessary to reduce or even remove the effect of different backscattering strengths caused by varying incidence angles. This study evaluates the influence of seafloor morphology on the acoustic backscattering signal of the deep-towed IFM-GEOMAR DTS-1 sidescan sonar system. Data used are from the Pacific continental slope offshore Nicaragua in a water depth between 800 and 2400 metres. There authigenic carbonate patches formed by cold fluid venting are imaged with a high backscattering level. The carbonates are often located on top or on the flanks of mound structures with a strong morphology. The specific DTS-1 backscattering function is determined on normal, uniform seafloor sediment, and then applied in a new processing algorithm to the raw data of two test areas. The change in amplitude strength when considering seafloor morphology is calculated. The topographic influence on the backscattering signal can be quite significant when 
imaging mound structures. Nevertheless the high backscatter on mounds is not completely removed, leading to the conclusion that it originates from a different seafloor lithology and roughness.

2pUWe4. Theoretical justification of a coherent forward model for subbottom profiler data inversion. Sandrine Rakotonarivo (Laboratoire E3I2, ENSIETA, 2, rue François Verny, 29806 Brest, France, rakotosa@ensieta.fr), Michel Legris (Laboratoire E3I2, ENSIETA, 2, rue François Verny, 29806 Brest, France, legrismi@ensieta.fr), Rozenn Desmare (Institut de Recherche de 1'Ecole Navale, IRENav, BP 600, 29240 Brest Armées, France, desmare@ecole-navale.fr), Frédéric Jean (IXSEA, 46, quai François Mitterrand, 13600 La Ciotat, France, frj@ixsea.com)

Subbottom profiling systems measure and identify sediment layers that exist below the sediment/water interface. Nowadays, very-high resolution chirp profilers provide calibrated wideband signals which may enable quantitative seabed characterisation. In literature, sediment classification is based on attenuation and reflectivity estimation and backscattering models used for the inversion consider plane wave propagation through layered homogeneous attenuated bottom. But this bottom modelling may be in contradiction with the emitted spherical front wave and core samples observations which point out a heterogeneous structure of marine subbottom. Therefore, impacts of profiler features, geometry measurement and subbottom structure (layering, attenuation, rugosity, and volumetric inhomogeneity) on backscattering signal are all reviewed in this paper. This analysis shows that classical model of wave coherent propagation through layered homogeneous attenuated media offers the best compromise between its accuracy and inversion possibility, if bottom heterogeneities are insignificant (Rayleigh scatterers). For bigger heterogeneities (Rayleigh parameter close to unity), coherent backscattering still prevails though wave propagation is affected by scattering. In this case, the same coherent forward model is used with modified reflectivity and transmission coefficients. Then, sensitiviy and inversion possibility of the coherent bottom backscattering model are discussed.

2pUWe5. Use of dual methods to infer methane bubble populations in gassy sediments: Inversion of combination-frequency data. Agni Mantouka (Institute of Sound and Vibration, Univ. of Southampton, University Road, Highfield, SO17 1BJ Southampton, UK, am2@isvr.soton .ac.uk), Timothy G. Leighton (Institute of Sound and Vibration, Univ. of Southampton, University Road, Highfield, SO17 1BJ Southampton, UK, T.G.Leighton@soton.ac.uk), Gary B. Robb (National Oceanography Centre, University of Southampton Waterfront Campus, European Way, SO14 3ZH Southampton, UK, gbor199@noc.soton.ac.uk), Angus I. Best (National Oceanography Centre, University of Southampton Waterfront Campus, European Way, SO14 3ZH Southampton, UK, aib@noc.soton.ac.uk), Justin K. Dix (National Oceanography Centre, University of Southampton Waterfront Campus, European Way, SO14 3ZH
Southampton, UK, jkd@noc.soton.ac.uk),Zygmunt Klusek(Institute of Oceanography, Polish Academy of Science, P.O. Box 148 Sopot, Poland, klusek@iopan.gda.pl), Paul R. White (Institute of Sound and Vibration, Univ. of Southampton, University Road, Highfield, SO17 1BJ Southampton, UK, prw@isvr.soton.ac.uk)

Bubbles can dramatically change the acoustic properties of their host medium even if they are present in very small amounts. This paper describes the combination-frequency component of tank and field measurements taken using a device which measures bubbles in marine sediments using multiple acoustic techniques (allowing the results of the various techniques to be compared). The combination-frequency method uses the nonlinear scattering property of bubbles when insonified by two primary frequencies. For low void fractions, there is a monotonic relationship between the scattered field and the population of bubbles resonant at either of the primary frequencies or combination of these and/or their subharmonics. This principle is used to infer the bubble size distribution. In contrast to the case of gas bubbles in water, in marine sediments the shear properties of the host medium must be incorporated into the model for the bubble dynamics and a new model for this is presented. This model is then inverted to obtain the bubble size distribution. The predictions of this method were compared with the method of inversion of propagation data (detailed in a companion paper), obtained from in situ experiments on the South coast of England.

2pUWe6. Comparisons of Biot and grain-shearing theories: sound speed, attenuation and acoustic impedance. Brian T. Hefner (Applied Physics Laboratory, University of Washington, 1013 NE 40th St, Seattle, WA 98105, USA, hefner@apl.washington.edu), Kevin L. Williams (Applied Physics Laboratory, University of Washington, 1013 NE 40th St, Seattle, WA 98105, USA, williams@apl.washington.edu)

The comparison of sediment sound speed and attenuation measurements to predictions has been the primary method used to test Biot theory and the grain-shearing model. Examples of data/model comparisons will be shown. Subsequent refinements made to these models result in similar predictions for sound speed and attenuation. However, the underlying physics is substantially different suggesting other, more indirect means for discriminating between sediment propagation theories. One technique that has received recent attention is the measurement of forward scattering from the sediment interface. Model predictions of these measurements depend not only the sound speed and attenuation, but also on the acoustic impedance of the medium. Examination of the physics incorporated into Biot Theory shows that the ${ }^{3}$ effective density ${ }^{2}$ seen by the acoustic wave is lower than the bulk density, thus lowering the acoustic impedance. This results in a difference in the predicted flat surface reflection coefficient for Biot-type models as compared to grain-shearing models. The flat surface reflection coefficients derived from experiment will be compared with predictions using the Biot model and the viscosity grain shearing (VGS) model for a sand sediment. The validity of obtaining reflection coefficients using forward scattering from rough surfaces will also be discussed. [Work supported by ONR.] 


\title{
Session 2pUWf
}

\section{Underwater Acoustics, Signal Processing in Acoustics, and ECUA: Acoustic Vector Fields and Sensor Processing I (Poster Session)}

\author{
Kevin Smith, Cochair \\ Naval Postgraduate School/Naval Undersea Warfare Center \\ Jean-Pierre Hermand, Cochair \\ Université libre de Bruxelles (U.L.B.) - Environmental hydroacoustics lab
}

\begin{abstract}
All posters will be on display from 3:40 p.m. to 5:20 p.m. To allow contributors an opportunity to see other posters, contributors of odd-numbered papers will be at their posters from 3:40 p.m. to 4:30 p.m. and contributors of even-numbered papers will be at their posters from 4:30 p.m. to 5:20 p.m.
\end{abstract}

\section{Contributed Papers}

2pUWf1. Vector-phase methods of bottom reflecting properties research in shallow shelf area. Elena Ermolaeva (Moscow Lomonosov State University, Leninskie Gory, Physics Faculty, 119992 Moscow, Russian Federation, ermolaeva@phys.msu.ru), Boris Goncharenko (Moscow Lomonosov State University, Leninskie Gory, Physics Faculty, 119992 Moscow, Russian Federation, goncharenko@phys.msu.ru), Valery Gordienko (Moscow Lomonosov State University, Leninskie Gory, Physics Faculty, 119992 Moscow, Russian Federation, gordienko@phys.msu.ru)

It is known, that parameters of bottom have essential influence on the signal propagation in the shallow water. Variation of the attenuation coefficient can amount to tens $\mathrm{dB}$. Therefore, research of bottom properties and its structure (first of all - thickness and acoustics parameters of alluvial soil) is actual problem, especially at low frequencies. Using of receivers, which record both sound pressure and its gradient make it easier to solve this problem in a number of cases. The following methods are considered: impedance method, which is based on direct measurement of relation between pressure and vertical component of oscillatory velocity in one point on the water/ bottom boundary; method, which is based on measuring phase difference between pressure and horizontal or vertical component of particle velocity; method in which additive combination of sound pressure and vertical component of oscillatory velocity are used. The essence of the last method consists in formation of minima of reception system directional characteristic in the direction at the signals extending from surface to bottom and reflected from bottom. The suggested methods of acoustic characteristics research of a ground repeatedly have been checked in shallow shelf area of the Baltic and the Black seas, and also in conditions of shallow inland water areas.

2pUWf2. Geoacoustic Inversion Based on a Vector Hydrophone Array. Fenghua Li (National Laboratory of Acoustics, Institute of Acoustics, Chinese Academy of Sciences, No 21, Beisihuanxilu, 100080 Beijing, China, 1fh@mail.ioa.ac.cn), Renhe Zhang (National Laboratory of Acoustics, Institute of Acoustics, Chinese Academy of Sciences, No 21, Beisihuanxilu, 100080 Beijing, China, zrh@mail.ioa.ac.cn)
Vector sensor has attracted much attention in recent years. However, there are few papers involving in geoacoustic inversion from vector sensor. In this research, a geoacoustic inversion scheme employing a vector hydrophone array has been developed based on the fact that vector hydrophone can provide more acoustic field information than traditional pressure hydrophones. The inversion scheme is the combination of the Matched Field Processing and the difference of transmission losses between pressure and velocity. The advantages of this method are that it can decrease the uncertainty of the inverted sediment sound speed and the inversion of the sediment attenuation is independent on the source level. [Work supported by the National Natural Science Foundation of China under Grand No. 10574136]

2pUWf3. Underwater low frequency sound field simulation with the digital waveguide mesh method. Shengchun Piao (College of Underwater Acoustic Engineering, Harbin Engineering University, 145 Nantong Street, 150001 Harbin, China, shengchunpiao@hrbeu.edu.cn), Lingai Tian (Hangzhou Applied Acoustics Research Institute, 96 Huaxing Road, 310012 Hangzhou, China, tianling_ai@hrbeu.edu.cn), Jing Yang (College of Underwater Acoustic Engineering, Harbin Engineering University, 145 Nantong Street, 150001 Harbin, China, bambooyang7@163.com)

Digital waveguide mesh method has been widely used to model musical instruments and simulation for room acoustics. In this paper, this method was applied to calculation the acoustic vector fields in the Pekeris waveguide. After introducing a boundary condition treatment method for the ocean bottom in the digital waveguide mesh, the acoustic pressure and particle velocity fields are calculated in spatial and temporal dimensions. Using these calculation results, the waveforms for the received signals and distribution of acoustic intensity in the underwater sound channel can also be obtained. Numerical simulation shows that the digital waveguide mesh method can be used to simulate the low frequency two-dimensional acoustic vector fields in shallow water and this method is easy to applied to threedimensional acoustic fields calculation. 


\title{
Session 2pUWg
}

\section{Underwater Acoustics and ECUA: High Frequency Variability I (Poster Session)}

\author{
Marcia Isakson, Cochair \\ Applied Research Laboratories, University of Texas \\ Thomas Folégot, Cochair \\ NATO Undersea Research Center
}

\begin{abstract}
All posters will be on display from 3:40 p.m. to 5:20 p.m. To allow contributors an opportunity to see other posters, contributors of odd-numbered papers will be at their posters from 3:40 p.m. to 4:30 p.m. and contributors of even-numbered papers will be at their posters from 4:30 p.m. to 5:20 p.m.
\end{abstract}

\section{Contributed Paper}

2pUWg1. Correcting bathymetry measurements for water sound speed effects using inversion theory. Jelle De Plaa (Delft Institute of Earth Observation and Space Systems, Delft University of Technology, Kluyverweg 1, 2629 HS Delft, Netherlands, j.deplaa@tudelft.nl), M. Snellen (Delft Institute of Earth Observation and Space Systems, Delft University of Technology, Kluyverweg 1, 2629 HS Delft, Netherlands, M.Snellen@tudelft.nl), Dick G. Simons (Delft University of Technology, P.O. Box Postbus 5048, 2600 GA Delft, Netherlands, d.g.simons@tudelft.nl)

We present a method to accurately estimate the bathymetry and water sound speed in shallow waters using overlapping swaths obtained from a Multi-Beam Echo Sounder (MBES). The method is designed to correct the errors in the bathymetry manifested mainly in the outer beams of the MBES.
The errors are caused by deviations in sound speed, which occur, for example, in estuaries where fresh river water mixes with seawater. Simulations show that we are able to simultaneously estimate the mean sound speed and the bathymetry. This is accomplished by minimizing the differences between the MBES measurements in the overlap region using a LevenbergMarquardt optimization routine. We also present an application of the method to real data obtained at the fairway to the harbour of Rotterdam, The Netherlands. Analysis shows that the inverted sound speed agrees well with the measured sound speed at the transducer. However, the uncertainties are larger when the track-to-track distance is $\sim 2$ times the depth. Therefore, we also discuss the optimal distance between sailed tracks. The method appears to be a promising tool for the accurate mapping of sea floors in shallowwater areas with a complex sound-speed profile. 
Session 3aAAa

\title{
Architectural Acoustics: Case Studies and Design Approaches I
}

\author{
Bryon Harrison, Cochair \\ 124 South Boulevard, Oak Park, IL, 60302 \\ Witew Jugo, Cochair \\ Institut für Technische Akustik, RWTH Aachen University, Neustrasse 50, 52066 Aachen, Germany
}

\section{Contributed Papers}

\section{8:00}

3aAAa1. Acoustic and audience response analyses of eleven Venetian churches. Davide Bonsi (The Acoustics Lab., Fondazione Scuola di San Giorgio, Isola di San Giorgio Maggiore, I-30124 Venezia, Italy, davide.bonsi@scuoladisangiorgio.it), Malcolm Longair (Cavendish Lab., University of Cambridge, JJ Thomson Avenue, CB3 OHE Cambridge, UK, pjg47@cam.ac.uk), Philip Garsed (Dept. of Engineering, University of Cambridge, Trumpington Street, CB2 1PZ Cambridge, UK, ms11000@hermes.cam.ac.uk), Raf Orlowski (Arup Acoustics, St Giles Hall Pound Hill, CB3 0AE Cambridge, UK, Raf.Orlowski@arup.com)

A research project has been carried out by the University of Cambridge (UK), Arup Acoustics (Cambridge, UK) and the Fondazione Scuola di San Giorgio-CNR (Venice, Italy) with the purpose of investigating the relationship between music and the architectural spaces within which it was performed in the context of Venetian vocal polyphony during the 16th century. The most representative churches (San Marco, Parish churches, hospitals and monasteries) were selected for this study and the music was performed within them by the Choir of St John's College of Cambridge University. Members of the audience completed questionnaires assessing the acoustic qualities of the spaces. The results of the audience and expert scores were then statistically analyzed and correlated with a set of room acoustic indices which had been measured in the same locations. In the present paper technical aspects of the experiments and the data analysis will be illustrated. The principal conclusions were as follows: 1 . There is a strong correlation between the subjective impression of reverberance with EDT and T30 and musical clarity with C80; 2 . A grouping of the churches by typology was found by analysing the reverberation times; 3 . The poor quality of clarity was assessed for churches with longest EDT for the performance of music involving polyphony.

\section{8:20}

3aAAa2. A Comparative Study of Four Concert Halls in Istanbul: Correlation of Subjective Evaluation with Objective Acoustic Parameters. Nurgun Bayazit Tamer (Istanbul Technical University, Taskisla Taksim Istanbul, 34437 Istanbul, Turkey, nurgun@itu.edu.tr), Anil Vural (Istanbul Technical University, Taskisla Taksim Istanbul, 34437 Istanbul, Turkey, vuralanist@yahoo.com)

In the studies of room acoustics, the parameters that are taken into consideration while evaluating the acoustic performance of a hall are examined in two sections: objective acoustic parameters which can be measured and calculated and, subjective acoustic parameters which define the impacts of a hall's acoustical qualities over the audiences. During the first half of the century, the reverberation time was considered to be the most important objective acoustic parameter which defined the acoustic quality of a hall. But, the later studies indicated that this parameter wasn't sufficient alone to define a subjective evaluation. Consequently, studies have been made to define the subjective influence on people from the acoustic performance of a hall. In this study, it has been attempted to define the statistical correlation between the subjective evaluation of the acoustic characteristics of the four concert halls, which was done by the members of orchestra together with the ordinary concert-goers, and their objective acoustic parameters are explained.
The detailed objective acoustic parameters are presented for measurements in four concert halls in Istanbul. The halls are used by many different performances, from concerts to ballet or opera. The measured octave band quantities are RT, EDT, G,C80 and D50.

\section{8:40}

3aAAa3. Computer modelling and simulation of sound field in 24 rectangular concert halls. Andrzej K. Klosak (Institute of Building Materials and Structures, Faculty of Civil Eng., Cracow University of Technology, Warszawska 24, 31-155 Cracow, Poland, andrzej.klosak@pk.edu.pl)

Extensive acoustics computer simulations has been made using Odeon computer simulation software. From 300 to 850 measurements positions have been analysed in 24 rectangular rooms representing "shoe-box" type concert halls with volumes of $8000 \mathrm{~m}^{3}, 12000 \mathrm{~m}^{3}$ and $16000 \mathrm{~m}$. In total 14 000 receiver positions have been analysed. For each receiver position eight objective measures have been calculated, in particular Reverberation Time $\left(\mathrm{T}_{30}\right)$, Early Decay Time $(\mathrm{EDT})$, Clarity $\left(\mathrm{C}_{80}\right)$, Strength $\left(\mathrm{G}, \mathrm{G}_{\text {early }}, \mathrm{G}_{\text {late }}\right)$, Early Lateral Energy Fraction $\left(\mathrm{LF}_{80}\right)$ and Late Lateral Strength $\left(\mathrm{G}_{\mathrm{LL}}\right)$. Most of measures have been calculated in six octave bands from $125 \mathrm{~Hz}$ to $4000 \mathrm{~Hz}$. Extensive amount of generated data required developing of new methods for processing, comparing and graphical presentation of results. Those methods together with description of modelling and simulation procedures are described in this paper.

\section{9:00}

3aAAa4. Improvement of acoustic conditions in a historical interior by means of a DSP-controlled sound-reinforcement system. Tadeusz Fidecki (F. Chopin Academy of Music, Okolnik 2, 00-368 Warszawa, Poland, fidecki@chopin.edu.pl), Andrzej Miskiewicz (F. Chopin Academy of Music, Okolnik 2, 00-368 Warszawa, Poland, misk@chopin.edu.pl), Jan Zera (Centr. Inst. for Labour Prot. - Natl. Res. Inst., Czerniakowska 16, 00701 Warsaw, Poland, jazer@ciop.pl)

Stringent building conservation requirements in historical interiors pose substantial limitations in acoustical treatment and sound reinforcement systems used to secure proper sound quality. This presentation discusses the means undertaken to improve the acoustic conditions in the historic Great Ballroom of the Royal Castle in Warsaw. Originally, the room was too reverberant $\left(\mathrm{T}_{30}=2.9 \mathrm{~s}\right.$ at $\left.500 \mathrm{~Hz}\right)$ what resulted in a lack of clarity of performed music and poor speech intelligibility (STI $<0.3)$. A precise laser scan of the interior was made to obtain input data for a computer acoustic model of the room. This model was used for selection of a DSP-controlled sound reinforcement system to improve listening conditions for speech and music. [Work supported by the Polish Ministry of Science and Higher Education, grant No. R17 004 02]. 
9:20

3aAAa5. On the acoustics of a specifically designed library discussion room with corrugated ceiling. Nico F. Declercq (Georgia Tech Lorraine G.W. Woodruff School of ME, UMI Georgia Tech - CNRS 2958, 2 rue Marconi, 57070 Metz, France, nico.declercq@me.gatech.edu), Katrien Dewijngaert (Georgia Tech Lorraine - G.W. Woodruff School of ME, UMI Georgia Tech - CNRS 2958, 2 rue Marconi, 57070 Metz, France, declercq@ieee.org), Katelijn Vanderhaeghe (Georgia Tech Lorraine - G.W. Woodruff School of ME, UMI Georgia Tech - CNRS 2958, 2 rue Marconi, 57070 Metz, France, declercq@ieee.org), Patricia Verleysen (Ghent University, Mechanics of Materials and Structures, Sint-Pietersnieuwstraat 41, 9000 Ghent, Belgium, Patricia.Verleysen@ugent.be)

There is a discussion room at the library of Viipuri in Finland where the ceiling has been especially designed to enhance the acoustics and make every position within the room acoustically equivalent. In other words no matter where a speaker is standing, he is supposed to be heard equally well all over the room. For that purpose a corrugated ceiling has been constructed that must distribute sound optimally, at least from the point of view of a ray theoretical analysis. The numerical study presented here shows that the corrugation is such that the ray approach is not exactly valid and a plane wave expansion technique shows that the acoustics is much worse than it is supposed to be. A detailed description of the sound distribution as a function of the position of the sound source and the receiver is presented. The acquired knowledge is important for future construction of similar rooms.

\section{9:40}

3aAAa6. Acoustic climate inside a canteen and mitigation solutions Giuseppe Marsico (APAT, Via Vitaliano Brancati 48, 00144 Rome, Italy, giuseppe.marsico@apat.it), Giovanni Brambilla (CNR Institute of Acoustics, Via del Fosso del Cavaliere 100, 00133 Rome, Italy, giovanni.brambilla@idac.rm.cnr.it), Salvatore Curcuruto (APAT, Via Vitaliano Brancati 48, 00144 Rome, Italy, salvatore.curcuruto @apat.it), Monica Clapiz (Via Edoardo Jenner 102, 00151 Rome, Italy, mony.c@libero.it), Rinaldo Betti (APAT, Via Vitaliano Brancati 48, 00144 Rome, Italy, rinaldo.betti@apat.it), Michele Riccardi (APAT, Via Vitaliano Brancati 48, 00144 Rome, Italy, michele.riccardi@apat.it)

The acoustic environment inside a canteen is an important feature to guarantee speech intelligibility and speech privacy, as well as to reduce the exposure levels. Unfortunately this aspect is not always properly taken into account as others, like cleanliness. This paper describes the case-study of the canteen at the National Agency for Environmental Protection (APAT), having a capacity of 220 seats. The acoustic environment has been characterized by measuring the main acoustic descriptors for speech intelligibility (Reverberation Time, STI, C80 and D50) and evaluating the noise exposure levels for the users. The comparison of the results with the recommended values showed a bad situation, especially in the speech frequency range, which requires acoustic corrections to improve the quality of the acoustic environment. To identify these corrections a numerical model of room acoustics, implemented on a commercial software, has been used to evaluate the effects produced by different corrections. The proposed correction improves the acoustic performances up to a good level, without modifying the structural parts and existing walls. Moreover, the solution allows to maintain the hall's usability without obstructing user mobility, screening natural lighting and reducing number of seats.

\section{0:00}

3aAAa7. Cost Benefit Analysis of Acoustic Treatments for Entertainment Venues. David M. Borgeaud (Environmental Resources Management Australia Pty Ltd, Level1, 60 Leichhardt Street, Spring Hill QLD, 4000 Brisbane, Australia, david.borgeaud@erm.com)

Fortitude Valley, in Brisbane City, Australia is an inner city area combining retail, commercial, residential, and entertainment uses. It has been the starting point for several major Australian bands. To address the increase in apartments in the Valley, the Brisbane City Council has developed a noise policy (called The Valley Music Harmony Plan) using entertainment precincts with specific noise criteria within the Valley, to allow informed decisions to be made regarding future development while minimising potential noise conflicts. An earlier paper by the Author has addressed the ambient noise mapping undertaken for the Valley, and this paper discusses the results of the cost benefit study undertaken into the acoustic treatment of selected venues. The purpose of this work was to provide Council with information on both the feasibility of treating the entertainment venues to reduce noise emissions and the order of cost for the treatments. The study of four venues found that attenuation of $3-22 \mathrm{~dB}(\mathrm{~A})$ or $1-17 \mathrm{~dB}(\mathrm{C})$ could be achieved with asociated costs ranging from $\$ 30,000$ to over $\$ 400,000$ (Australian dollars). The costing work provided invaluable knowledge in the development of the Valley Music Harmony Plan.

\section{0:20-10:40 Break}

\section{0:40}

3aAAa8. Open-air theatre in the centre of the city: Acoustic design and noise environment control. Nicolaos Barkas (Democritus University of Thrace/ Greece, Vas. Sofias street 1, Department of Architecture, 67100 Xanthi, Greece, nbarkas@arch.duth.gr)

The municipal open-air Garden Theatre of Thessaloniki has for decades been located in the biggest urban park of the city next to a busy crossroads, where five major traffic roads meet. The intensification of traffic noise over the years led to the degradation of its use. In 1997, the organisation of the Cultural Capital of Europe served as a springboard for a study conducted by the author on redesigning the theatre. The design comprised a variety of arrangements, such as the rotation of the auditorium according to the results of the noise environment study, the choice of a closed plan in the type of the Greek-Roman Odeion, the sinking of the theatre space and the construction of barriers and shades. Various problems (such as archaeological findings, existence of big trees inside the auditorium, high level of underground water) and budget cuts during the design and construction, led to restricted execution of the planned architectural interventions. This paper deals with the design parameters that optimise acoustic function and maximise sound protection in an urban, open-air theatre and includes an analysis of the theatre's initial state, acoustic proposals and an assessment of the final applications.

\section{1:00}

3aAAa9. New acoustic test chambers at the University of Palermo. Marco Gullo (DREAM, Univ. of Palermo, Viale delle Scienze, Edificio 9, 90128 Palermo, Italy, mgullo@dream.unipa.it), Armando La Pica (DREAM, Univ. of Palermo, Viale delle Scienze, Edificio 9, 90128 Palermo, Italy, lapica@unipa.it), Giuseppe Rodono' (DREAM, Univ. of Palermo, Viale delle Scienze, Edificio 9, 90128 Palermo, Italy, rodon@unipa.it), Vincenzo Vinci (DREAM, Univ. of Palermo, Viale delle Scienze, Edificio 9, 90128 Palermo, Italy, vincenzo_vinci @ hotmail.it), Vincenzo Conti (DREAM, Univ. of Palermo, Viale delle Scienze, Edificio 9, 90128 Palermo, Italy, conti@csai.unipa.it)

Acoustic test chambers at the University of Palermo have been completed after a design optimization of existent building structures. A unique building houses five reverberation chambers, differently coupled each other in order to achieve a large flexibility on testing procedures, and offices for the staff. Main measurements offered to national and international standards include determination of sound power, sound insulation and sound absorption. Reverberation chambers present peculiar geometries in order to achieve the desidered sound fields. Chambers structure and layout within the whole building, housing all the acoustic facilities, has been designed in order to achieve strong sound insulation requirements. The present work describes design solutions and reportes preliminary results from a large, still running, validation in order to characterize the chamber acoustic behaviour. Design targets and objective achievements are listed and commented.

\section{1:20}

3aAAa10. Design and construction of an acoustic lab facility. Douglas Winker (ETS-Lindgren, 1301 Arrow Point Drive, Cedar Park, TX 78763, USA, douglas.winker@ets-lindgren.com)

This paper will discuss the design and construction of a new acoustic lab facility. The lab consists of a hemi anechoic chamber and a reverberation chamber suite. The hemi anechoic chamber was designed to obtain a $0 \mathrm{dBA}$ 
noise floor in the frequency range of interest. It features a 200 cubic meter volume inside the wedges. To achieve the design goals, several aspects were considered including ambient levels, vibration isolation, HVAC noise, and free field performance. The design solutions for each of these areas will be presented. The reverb chamber suite consists of two reverberation chambers designed to perform transmission loss tests and sound absorption testing. The source chamber has an internal volume of 214 cubic meters and the receive room has an internal volume of 418 cubic meters. Design considerations will be discussed including vibration isolation, wall panel design, and diffuser placement. Additionally, the design and construction of the transmission loss coupling will be addressed.

\section{1:40}

3aAAa11. Measurements of existing modular construction classroom acoustics in the Midwestern United States. Norman Philipp (University of Nebraska - Lincoln, 1110 S. 67th St., Omaha, NE 68182-0681, USA, nphilipp@mail.unomaha.edu), Lily M. Wang (University of Nebraska Lincoln, 1110 S. 67th St., Omaha, NE 68182-0681, USA, LWang4@UNL .edu)

Modular construction classrooms in the United States currently total more than 300,000 units, with $37 \%$ nationally of all public schools utilizing portable classrooms, according to the Modular Building Institute and a 2007 survey of Public School Principals conducted by the National Center for Education Statistics. Given this ever growing number there is a desire to improve their acoustics to meet ANSI S12.60-2002 guidelines for classroom acoustics. This paper gives a survey of existing acoustic conditions of relocatable classrooms currently in use in the Midwestern United States. Data to be presented include reverberation times, interior/exterior noise reduction, and site noise measurements. The results of these measurements and additional qualitative surveys will aid in understanding the performance of modular classrooms and assist in drafting a standard for them to supplement the guidelines in ANSI S12.60-2002.

\section{$12: 00$}

3aAAa12. The influence of the scattering coefficient on the reverberation time. Franz Huber (Ziviltechnikerbüro Dipl. - Ing. Franz Huber, Neumühl 44, A-3250 Wieselburg, Austria, fh@zthuber.at), Thomas Bednar (University of Technology, Karlsplatz 13/206, A-1040 Vienna, Austria, thomas.bednar@tuwien.ac.at)

The reverberation time is still seen as an important measure of quality in architectural acoustic. The absorption coefficient is to be considered as the largest influencing factor on the reverberation time. The formulas after Sabine and Eyring give us the opportunity to calculate the reverberation time, when the absorption coefficients are known. In contrast to this, is the investigation of the influence of the scattering coefficient on the reverberation time a relatively new field for research. In this study the reverberation time for a "shoebox" was simulated, where the parameters were the values of the absorptions coefficient and the scattering coefficient and the even and uneven distribution of the absorption coefficient. The results of the simulation were compared with the results from the formulas by Sabine and Eyring. The simulations indicate a significant influence of the scattering coefficient on the reverberation time especially at low absorption coefficients. You can see by comparing the results of the simulation with the results of the formulas by Sabine and Eyring, that the theoretical formulas assume a minimum of scattering coefficient. It shows that the variation of the scattering coefficient is a possibly way to influence the reverberation time.

\section{2:20}

3aAAa13. Air Absorption Error in Room Acoustical Modeling. Remy H. Wenmaekers (LeVeL Acoustics BV, De Rondom 10, 5612 AP Eindhoven, Netherlands, r.h.c.wenmaekers@tue.nl), Constant C. Hak (Technische Universteit Eindhoven, De Rondom 10, 5612 AP Eindhoven, Netherlands, c.hak@inter.nl.net), Heiko J. Martin (Eindhoven University of Technology, Den Dolech 2, BWK/BPS, 5600 MB Eindhoven, Netherlands, h.j.martin@tue.nl), Laurentius C. Van Luxemburg (LeVeL Acoustics BV, De Rondom 10, 5612 AP Eindhoven, Netherlands, 1.c.j.v .luxemburg@tue.nl)

Most statistical and ray-tracing computer models take into account the absorption of sound by air when calculating the reverberation time. Extensive research by many scientist lead to the standardized calculation model for pure tone air absorption. The phenomenon was discovered from a room acoustical point of view by Sabine, while the further development of the calculation model took place in the fields of physics and environmental noise. As a result, several parameters and units are used for the same phenomenon. However, air absorption is calculated for pure tones, while room acoustics calculations are performed for frequency bands. Most computer models use the center frequency of the normalized frequency bands to calculate the air absorption by the pure tone method. Frequency band reverberation measurements under laboratory and practical conditions show that errors larger than the JND are made in calculating the air absorption by this 'center frequency method'. No literature is found that provides an accurate air absorption calculation for frequency bands. 


\title{
Session 3aAAb
}

\section{Architectural Acoustics: Prediction Methods in Building Acoustics I}

\author{
Berndt Zeitler, Cochair \\ NRC - Institute for Research in Construction, 1200 Montreal Road, Building M-27, Ottawa, ON K1A OR6, Canada \\ Catherine Guigou-Carter, Cochair \\ CSTB, 24, rue Joseph Fourier, Saint Martin D’Hères, 38400, France
}

\section{Contributed Papers}

\section{8:00}

3aAAb1. FEM based prediction model for the impact sound level of floors. Andreas Rabold (Technische Universität München, Fakultät für Bauingenieur- und Vermessungswesen, Arcisstraße 21, 80290 München, Germany, rabold@bv.tum.de), Alexander Düster (Technische Universität München, Fakultät für Bauingenieur- und Vermessungswesen, Arcisstraße 21, 80290 München, Germany, duester@bv.tum.de), Ernst Rank (Technische Universität München, Fakultät für Bauingenieur- und Vermessungswesen, Arcisstraße 21, 80290 München, Germany, rank@bv .tum.de)

Up to now the research and development in the field of building acoustics is based mainly on experiments. The consequence of this approach is that the development and optimization of a new building component is a very tedious and expensive task. A considerably reduction of these costs could be achieved, if the optimization relying on experiments would be replaced - at least to some extent - by a computational approach. Common prediction models of the impact sound level from lightweight floors are based on measured impact sound level data for the component parts under consideration. These models are very useful for the evidence of performance of known building components, but they are not suited for the development of new components. An alternative approach in this context is the application of the finite element method (FEM). Based on a FE model, a modaland spectral analyses provides the framework for an optimization of the building component. This contribution will present the overall approach consisting of the three-dimensional modeling of a lightweight-floor and the excitation source (tapping machine), the subsequent modal- and the spectralanalyses and the computation of the radiated sound from the floor.

\section{$8: 20$}

3aAAb2. Human footsteps induced floor vibration. Delphine Bard (Lund University, Division of Engineering Acoustics, John Ericsson väg 1, 22100 Lund, Sweden, delphine.bard@acoustics.lth.se), Julia Sonnerup (Lund University, Division of Structural Mechanics, John Ericsson väg 1, 22100 Lund, Sweden, julia.sonnerup@byggmek.lth.se), Göran Sandberg (Lund University, Division of Structural Mechanics, John Ericsson väg 1, 22100 Lund, Sweden, goran@byggmek.1th.se)

The generation of the impact sound by the act of the human walk involves two factors, the characters of the footfall and the shape of the induced floor deflection. The footfall noise is created by the impact excitation and the characters of the footfall depend on the foot-ware: the heels and the frequencies of the footfall. The shape of the floor deflection depends rather on the geometrical walking pattern and the construction of the floor structure. In this investigation, the vibration patter of the light-weight construction floor is created by the same walking object, a male with common height. The excitation from the person to the floor in the FE simulations is a function of the length of the foot and the weight of the walking object. The geometrical time history is of the foot step allows it to have different directions in the room. Since the excitation is assumed to be deterministic, differences between the excitation frequencies are estimated from the video records. The goal of this investigation is to determine the difference of the floor structure deflections between two different walking paths: one is perpendicular to the bearing beams and the other is the diagonal path.

\section{Invited Papers}

3aAAb3. Vibration of a rib-reinforced floor/ceiling structures with irregularities. Hyuck Chung (The University of Auckland, Department of Mathematics, Private Bag 92019, 1001 Auckland, New Zealand, hyuck@math.auckland.ac.nz), Colin Fox (Department of Physics, The University of Otago, 730 Cumberland Street, P O Box 56, 9010 Dunedin, New Zealand, fox@physics.otago.ac.nz)

This paper describes mathematical modelling procedure of the rib-reinforced floor/ceiling structures, which are made up of components with irregular shapes and physical parameters. Exact determination of the vibration of a composite structure becomes impossible beyond the low-frequency range because one cannot determine all the necessary parameters of the components. Even if every possible parameter of the structure is known, the results from such deterministic model would not represent the real behaviour of the structure. Therefore, the prediction model in the mid- to high-frequency range must include the effects of the irregularities. We show how the power spectra of the irregular features of each component can be included in the model. The model gives statistical estimates of the solutions, which can give appropriate mean and variance of the vibration of the structure for the given severity of the irregularities.

\section{9:00}

3aAAb4. Parameter survey of a rib stiffened wooden floor using sinus modes model. Lars-Göran Sjökvist (Acoustic Technology Department, Technical University of Denmark, Ørsted Plads, B352, DK-2800 Lyngby, Denmark, lars-goran.sjokvist@sp.se), Jonas Brunskog (Dept. of Acoustic Technology, Technical University of Denmark, Building 352, DK 2800 Lyngby, Denmark, jbr@oersted.dtu.dk), Finn Jacobsen (Acoustic Technology Department, Technical University of Denmark, Ørsted Plads, B352, DK2800 Lyngby, Denmark, fja@oersted.dtu.dk)

In buildings built with new techniques there exists a need for better understanding of their acoustical performance. The development of large wooden houses slows down by the uncertainty and costly testing that have to be performed many times before gaining good 
results. A greater understanding of the sound insulation for lightweight buildings have the possibility to speed up the development of new techniques and in the end give tenants better quality of life. This study uses Fourier sinus series to calculate the vibrations on a rib stiffened plate. The beams are modelled as line forces and moments that reacts onto the plate vibrations. A parameter study is performed with the aim to identify the most important parameters and their behaviour. The preliminary results show that the attenuation of the system is by far most evident in the direction across the beams. The influence from the basic input parameters on the attenuation is then studied. And it is preliminary shown that the placement of the excitation force within a bay actually is irrelevant for the calculated attenuation.

\section{9:20}

3aAAb5. The modelling of vibration transmission through plate/beam structures typical of lightweight buildings. Laurent Galbrun (Heriot-Watt University, School of the Built Environment, EH14 4AS Edinburgh, UK, 1.g.u.galbrun@hw.ac.uk)

Vibration transmission through plate/beam structures typical of lightweight buildings is examined in this paper. Modelling difficulties are analysed and related to the several modelling choices available, including connection type, beam modelling, modal properties and material properties. A number of key experiments carried out on structures made of one or more parallel plates attached to a single beam are discussed, and the applicability and limitations of fundamental theories are identified through the use of Statistical Energy Analysis (SEA) models. It is shown that the interrelation between the various modelling factors can lead to complex models. The systems for which existing models can be used are identified, as well as those for which more complex models need to be developed.

\section{Contributed Papers}

9:40

3aAAb6. Approximation of single equivalent mobilities of timber joist floors. Andreas R. Mayr (Stuttgart University of Applied Sciences, Schellingstrasse 24, 70174 Stuttgart, Germany, andreas.mayr@hft-stuttgart .de), Barry M. Gibbs (University of Liverpool, School of Architecture, Abercromby Square, L693BX Liverpool, UK, bmg@liv.ac.uk)

To estimate the structure-borne sound power of vibrational active machines or components two source quantities, corresponding to the source activity and mobility, and one receiver quantity, the mobility, are usually required. A practical, laboratory-based measurement procedure for the characterisation of mechanical installations has recently been proposed, which yields single equivalent values of source strength and source mobility. In order to predict the power in the installed condition, an estimate of the receiver mobility also is required, again, for practical reasons, in the form of a single equivalent value. This paper considers the single equivalent receiver mobilities for lightweight building structures - timber joist floors. The value obtained is in the form of the average magnitude of the effective mobilities over the contact points. A simple prediction method is proposed for pointand transfer mobilities (both are required to yield the effective mobility at each contact) based on infinite beam and infinite plate behaviour. Estimates for point-, transfer- and single equivalent receiver mobilities are discussed and compared with measured data.

\section{0:00}

3aAAb7. Towards a prediction of the sound transmission from lightweight stairs. Jochen Scheck (Stuttgart University of Applied Sciences, Schellingstrasse 24, 70174 Stuttgart, Germany, jochen.scheck @ hft-stuttgart.de), Barry M. Gibbs (University of Liverpool, School of Architecture, Abercromby Square, L693BX Liverpool, UK, bmg@liv. ac.uk), Andreas Drechsler (University of Applied Sciences, Schellingstr. 24, 70174 Stuttgart, Germany, andreas.drechsler@hft-stuttgart .de), Heinz-Martin Fischer (University of Applied Sciences, Schellingstr. 24, 70174 Stuttgart, Germany, heinz-martin.fischer@hft-stuttgart.de)

The sound transmission from lightweight stairs which are connected to separating walls often gives rise for complaints. One reason for this is that present there is no prediction method available. Treating the stair as an ac- tive component in a similar manner like vibrating machines stairs can be characterised as structure-borne sound sources. The source data then can be used to predict the sound transmission in buildings using parts of EN 12354. Following this approach investigations on a timber stair have been carried out in a staircase test facility. Based on a full characterisation by contact free velocity and mobility and in-situ measurement using an indirect method, more practical methods like the reception plate method and a characterisation based on a reference power calibration are investigated. The source data obtained was used to predict the sound transmission in buildings.

\section{0:20}

3aAAb8. Prediction of the impact sound transmission from lightweight stairs in buildings based on a simplified laboratory characterisation. Andreas Drechsler (University of Applied Sciences, Schellingstr. 24, 70174 Stuttgart, Germany, andreas.drechsler@hft-stuttgart.de), Jochen Scheck (Stuttgart University of Applied Sciences, Schellingstrasse 24, 70174 Stuttgart, Germany, jochen.scheck@hft-stuttgart.de), Heinz-Martin Fischer (University of Applied Sciences, Schellingstr. 24, 70174 Stuttgart, Germany, heinz-martin.fischer@hft-stuttgart.de)

Based on a simplified laboratory characterisation of lightweight stairs the normalized impact sound pressure level in buildings can be predicted using parts of EN 12354. For the characterisation an approach is followed where the stair is treated as an active component with respect to an external excitation (e.g. by the tapping machine). The laboratory characterisation was performed for a timber stair and the normalized impact sound pressure level was predicted for different building situations. The various transmission paths via the separating and flanking walls were investigated and compared to the prediction according to EN 12354. Within the presentation the proceeding will be outlined and the restrictions are discussed. By comparison of measured and calculated values some references for the accuracy of the method are obtained. 


\section{Invited Paper}

11:00

3aAAb9. Impedance of standard impact sources and their effect on impact sound pressure level of floors. Berndt Zeitler (NRC - Institute for Research in Construction, 1200 Montreal Road, Building M-27, Ottawa, ON K1A 0R6, Canada, berndt .zeitler@nrc-cnrc.gc.ca), Trevor Nightingale (NRC - Institute for Research in Construction, 1200 Montreal Road, Building M-27, Ottawa, ON K1A 0R6, Canada, Trevor.Nightingale@nrc-cnrc.gc.ca)

This paper reports on a systematic study of the Impact Sound Pressure Level (ISPL) due to different standard sources (ISO hammer box, Ball, and "Bang Machine") when applied to a wood-joist floor-ceiling construction with and without various floor toppings. Measurement data show that for each floor, the ISPL was different for each source. Not surprising since the blocked force for each source is different. But, after applying corrections for the applied force the ISPL's remain different, suggesting problems since the sources simulate the same human walking activity. Perhaps more importantly, the rank of topping effectiveness (which is simply the difference in ISPL with and without the topping) was different for the three sources. Measurements of the drive point impedance of sources and floors are presented to explain this behaviour and to recognise that the power injected is not just related to the applied force, but also a function the impedance match (or miss-match) between the source and floor. The paper shows using a simple model, it is possible to predict ISPL's for any source if the forces and impedances are known, and using this model floor treatment effectiveness can be correctly ranked by each of the three standardised sources.

\section{Contributed Papers}

11:20

3aAAb10. Conversion of floor impact sound levels in apartment buildings. Pyoung Jik Lee (Hanyang University, Department of Architectural Engineering, 133-791 Seoul, Republic of Korea, pyoungjik@daum .net), Shin-Ichi Sato (Hanyang University, Department of Architectural Engineering, 133-791 Seoul, Republic of Korea, s_sato@mac.com), Jin Yong Jeon (Hanyang University, Department of Architectural Engineering, 133791 Seoul, Republic of Korea, jyjeon@hanyang.ac.kr)

A procedure to convert the floor impact sound level of the bang machine as well as the tapping machine into that of the impact ball was suggested. Because the bang machine has been the only standard heavy-weight impact source for a long time in Japan and Korea, much of its data is available. The conversion factor of the sound pressure level between the impact ball and other standard impact sources was applied to the sound pressure level of a bang machine and a tapping machine. In addition, a conversion method using a correlation analysis between the sound pressure level of impact ball and that of other standard impact sources at each octave band was also investigated. Results indicate that bang machine and tapping machine data can be successfully converted into impact-ball data, in the case of boxframed type, reinforced concrete structures.

\section{1:40}

3aAAb11. A simplified approach to impact sound insulation rating without using a tapping machine. Ming Li (Acoustics Research Centre, School of Architecture, The University of Auckland, 1010 Auckland, New Zealand, mli072@ec.auckland.ac.nz), George Dodd (Acoustics Research Centre, School of Architecture, The University of Auckland, 1010 Auckland, New Zealand, mli072@ec.auckland.ac.nz)

The aim of this study is to investigate a simplified approach for obtaining ratings of impact insulation of floors which requires only airborne sound measurements and correction factors depending on the particular type of floor finish. One third octave frequency-dependent correction factors have been obtained by processing published results of both airborne and impact insulation on floors. The uncertainty of predicted $\mathrm{Ln}, \mathrm{w}$ values using correction factors shows a good agreement with ISO recommended limits. The proposed technique has value in reducing the cost and effort for field insulation measurements. Further work is underway to refine the method and to extend the range of constructions for which we have correction factor.
12:00

3aAAb12. Reciprocity and the Prediction of the Apparent Sound Reduction Index for Lightweight Structures According to EN12354. Jeffrey Mahn (University of Canterbury, Department of Mechanical Engineering, Private Bag 4800, 8140 Christchurch, New Zealand, jma251 @ student.canterbury.ac.nz), John Pearse (University of Canterbury, Depart-ment of Mechanical Engineering, Private Bag 4800, 8140 Christchurch, New Zealand, john.pearse@ canterbury.ac.nz)

EN12354 makes use of reciprocity to cancel the radiation efficiency terms from the equations used to predict the apparent sound reduction index. The use of reciprocity works well for the application of EN12354 to massive, concrete structures with diffuse wave fields and with critical frequencies below the frequency range of interest. However, lightweight constructions may have critical frequencies above the frequency range of interest and may not support diffuse wave fields. The use of reciprocity for application to lightweight structures may introduce errors into the predictions. This study investigates the uncertainty introduced into the predictions of the apparent sound reduction index of lightweight constructions due to the use of reciprocity.

\section{$12: 20$}

3aAAb13. Innovative building systems to improve the acoustical quality in lightweight masonry constructions: Application of resilient joints at junctions - PART 1: Analysis of the experimental results. Charlotte Crispin (BBRI, rue du Lombard, 42, B-1000 Brussel, Belgium, charlotte.crispin@bbri.be), Bart Ingelaere (BBRI, rue du Lombard, 42, B-1000 Brussel, Belgium, bart.ingelaere@bbri.be), Gerrit Vermeir (Lab. ATF, Katholieke Universiteit Leuven, Celestijnenlaan 200D, B-3001 Leuven, Belgium, Gerrit.Vermeir@bwk.kuleuven.be)

The more severe acoustic requirements imposed by the new Belgian standard for dwellings are a real challenge for the building professionals (architects, contractors, building elements manufacturers and suppliers, and more particularly for the market of lightweight materials. An important brick producer in partnership with the BBRI has succeeded to propose efficient acoustic solutions for these kinds of materials by treating in particular the flanking transmission. Indeed, to obtain high sound insulations, the structural transmission paths of noise through the flanking walls cannot be neglected any more. By the application of resilient rubber interlayers at the junctions, these transmission paths are nearly eliminated. A large number of measurements was carried out in order to study in detail the effect of these flexible joints on the sound transmission. These measurements were made in a specially designed laboratory where vibration reduction indexes can be measured for all types of connections and for different loads. We present, in this paper, the measurement campaign and the analysis of the results.

\section{2:40-1:40 Lunch Break}




\section{Contributed Paper}

$1: 40$

3aAAb14. Innovative building systems to improve the acoustical quality in lightweight masonry constructions: Application of resilient joints at junctions - PART 2: Study cases modelled according to the standard 12354-1 (2000). Charlotte Crispin (BBRI, rue du Lombard, 42, B-1000 Brussel, Belgium, charlotte.crispin@bbri.be), Bart Ingelaere (BBRI, rue du Lombard, 42, B-1000 Brussel, Belgium, bart.ingelaere@bbri.be), Debby Wuyts (BBRI, rue du Lombard, 42, B-1000 Brussel, Belgium, debby.wuyts@bbri.be)

An important brick manufacturer in partnership with the BBRI has suc- ceeded to propose efficient acoustic solutions for this kind of lightweight materials by treating the flanking transmission using flexible interlayers at junctions and foundations. A large measurement campaign has been carried out to study the efficiency of the resilient joint and to provide a prediction model on airborne sound insulation in brickwork constructions. The prediction model used is based on the standard 12354-1 but was adapted in order to take into account higher orders for the flanking transmission paths. This adapted model thus allowed to study different applications of the flexible joint and their contribution to the improvement of the global sound insulation. This paper presents a discussion on the calculation model, the input data and some early results.

\section{Invited Papers}

\section{2:00}

3aAAb15. Direct and flanking sound transmission of solid gypsum walls - practical experience and numerical prediction. Andreas Ruff (Stuttgart University of Applied Sciences, Schellingstrasse 24, 70174 Stuttgart, Germany, andreas.ruff @ hft-stuttgart.de), Heinz-Martin Fischer (University of Applied Sciences, Schellingstr. 24, 70174 Stuttgart, Germany, heinz-martin .fischer@hft-stuttgart.de)

In modern multi storey buildings gypsum blocks are often used for solid inner walls without static requirements. However, these relatively light walls with a mass per unit area of about $90 \mathrm{~kg} / \mathrm{m}^{2}$ are not connected rigidly to the adjacent building elements. They are decoupled with elastic interlayers made of bitumen, cork or polyethylene foam. These elastic interlayers have a significant influence on the direct and the flanking sound transmission of the gypsum walls. For this reason the decoupled walls have to be considered different to rigidly connected walls. Within a recent research project there was an extensive investigation into the direct and the flanking sound transmission of gypsum walls in different test facilities in the laboratory. For several kinds of elastic interlayers the sound reduction index of gypsum walls was measured in the test stand for direct sound transmission. The vibration reduction index was investigated in a test facility for flanking transmission as well as in different building situations. The measured values are used as input data for the prediction model according EN 12354-1. The overall sound reduction index calculated with this prediction model is compared with the results measured in the buildings.

$$
\text { 2:20 }
$$

3aAAb16. Some practical aspects of the prediction of structure-borne sound caused by house-hold equipment. Eddy Gerretsen (TNO Science and Industry, Stieljesweg 1, 2628CK Delft, Netherlands, eddy.gerretsen@tno.nl)

Sound levels in rooms due to service equipment in the building can be an important reason for disturbance and more so in light weight building structures. Though the recently published draft standard prEN 12354-5 gives a framework for the prediction of the structure-borne sound as caused by this type of sources, there is still a lot to be studied and developed. That is certainly the case for light weight building structures. Some possibilities to simplify the indicated models have been studied and experimental data has been gathered for house hold equipment, taking a washing machine as an example, applied both in a 'heavy' and in a 'light weight' building. Various possibilities to apply substitution methods to characterise such a source as structure-borne sound source have been tried and compared.

\section{Contributed Papers}

\section{2:40}

3aAAb17. An hybrid method for sound transmission through opened windows. Philippe A. Jean (CSTB, 24 rue Joseph Fourier, 38400 SaintMartin-d'Hères, France, philippe.jean@cstb.fr), Michel Villot (CSTB, 24 rue Joseph Fourier, 38400 Saint-Martin-d'Hères, France, michel.villot@cstb .fr)

An hybrid model for the calculation of sound transmission between apartments when windows are opened is presented. It is an extension of Kropp's model for balconies (integral/modal/wave approach). Two rectangular rooms are set in a baffle with rectangular openings. A point source is placed in one room and sound attentation between rooms is computed. The external field may include the ground and a nearby building by means of a combined image source approach to compute a Green function in the integral description at the openings. Results are validated against BEM and published measurements. SEA is employed to assess the importance of vibration transmission relative to airborne transmission through the external medium.

\section{3:00}

3aAAb18. Directional distribution of acoustic energy density incident to a surface under reverberant condition. Cheol-Ho Jeong (Acoustic Technology, DTU Elektro, Technical University of Denmark, Building 352, Ørsted plads, DK-2800 Kgs. Lyngby, Denmark, chj@oersted.dtu .dk), Jeong-Guon Ih (KAIST, Dept. of Mechanical Engineering, Science Town, 305-701 Daejeon, Republic of Korea, J.G.Ih@kaist.ac.kr)

Most of acoustic measurements presume ideal conditions: one ideal condition is a diffuse and reverberant field. In a practical situation, diffuse sound field in a reverberation chamber can not be achieved. In particular, the directional diffusion which means the uniform angular distribution of incident direction can not be satisfied. Angle dependency of incident energy density was simulated by the phased beam tracing simulation. Various results were found by changing room shape and source position, and number of sources. Acoustic energy generally decreases with the angle of incidence. This result can be used as a weighting function for the calculation of sound transmission index and random incidence absorption coefficient. 


\title{
Invited Paper
}

$3: 20$

3aAAb19. Exposure of building roofs to road traffic noise; consequences on the field performance of skylights. Michel Villot (CSTB, 24 rue Joseph Fourier, 38400 Saint-Martin-d'Hères, France, michel.villot@cstb.fr), Philippe A. Jean (CSTB, 24 rue Joseph Fourier, 38400 Saint-Martin-d'Hères, France, philippe.jean@cstb.fr)

In this paper, the exposure to road traffic noise of building roofs is studied both theoretically and experimentally. First, the incident field is estimated using a BEM approach, taking into account the sound diffraction on the roof edge. The theoretical results show significant differences in noise exposure between roofs and vertical façades, particularly with relatively tall buildings; these differences are then confirmed by field measurements. The grazing incidence of the sound fields on roofs raises the question of the acoustic performances of skylights in real situation, as opposed to their laboratory performances measured under diffuse sound field; this problem is experimentally investigated by laboratory measurements of glazing under different angles of incidence, using a big size plane wave generator.

\section{Session 3aABa}

\section{Animal Bioacoustics, Underwater Acoustics, Acoustical Oceanography, Signal Processing in Acoustics, and ECUA: Animal Sonar Systems III}

\author{
James Simmons, Cochair \\ Brown University, Dept. of Neuroscience, Box G-LN, Providnece, RI 02912, USA
}

\author{
Michel Andre, Cochair \\ Laboratori, d’Aplicacions Bioacútques, Universitat Politècnica de Catalunya, avda. Rambla Exposicío s/n, 08800 Vilanova, \\ la Geltrú, Spain
}

\section{Contributed Papers}

\section{8:00}

3aABa1. Localization of brief sounds by a bottlenose dolphin. Gennadi L. Zaslavski (University Authority for Applied Research, RAMOT, TelAviv University, str. Gordon 51 app 7, 42442 Netanya, Israel, gennadi .zaslavski@gmail.com)

Spatial hearing in dolphins is believed to be similar to that in humans. The same binaural phenomenon known for humans is used to explain sound localization in dolphins. However, the ability of a bottlenose dolphin to localize a sound in the vertical plane as accurate as in the horizontal plane appears to undermine binaural phenomenon concept in dolphin sonar. We found that directionality of the bottlenose dolphin transmit beam could account for most results on the bottlenose dolphin underwater object localization. In this paper passive localization and spatial discrimination of brief signals by a bottlenose dolphin is examined. The bottlenose dolphin was found capable of discriminating clicks with different intensity as well as a click and brief noise pulse simultaneously transmitted via transducers separated in azimuth by less than $1^{\circ}$. The dolphin was also able to discriminate a lead from a lag noise pulse at the transducer azimuth separation as small as $0.3^{\circ}-0.4^{\circ}$ and the lag delay as small as 20 microseconds. The results are discussed with regard to the bottlenose dolphin auditory time resolution and so called precedence effect phenomenon well known for the binaural system of humans.

\section{8:20}

3aABa2. The interaction of emitted pulses and echoes in a whale's biosonar: A partial contribution to biosonar automatic gain control. Alexander Y. Supin (Institute of Ecology and Evolution, 33 Leninsky Prospect, 119071 Moscow, Russian Federation, alex_supin@mail.ru), Paul E. Nachtigall (University of Hawaii, Hawaii Institute of Marine Biology, P.O. Box 1106, Kailua, HI 96734, USA, nachtiga@hawaii.edu), Marlee Breese (University of Hawaii, Hawaii Institute of Marine Biology, P.O. Box 1106, Kailua, HI 96734, USA, mbreese@hawaii.edu)
In a false killer whale Pseudorca crassidens, echo perception thresholds were measured using a go/no-go psychophysical paradigm and one-up-onedown staircase procedure. Electronically synthesized echoes were triggered by whale-emitted echolocation pulses, and the echo amplitudes were presented proportional to the triggering biosonar pulse amplitudes, thus imitating the basic feature of real target echoes. Echo thresholds were measured in terms of attenuation relative to the source level of the biosonar pulses. The echo threshold dependence on echo delay was investigated. With increasing echo delay, the threshold echo attenuation decreased from $-49.3 \mathrm{~dB}$ at $2 \mathrm{~ms}$ to $-79.5 \mathrm{~dB}$ at $16 \mathrm{~ms}$, with a regression slope of $-31.5 \mathrm{~dB}$ per delay decade. At delays longer than $16 \mathrm{~ms}$, the threshold remained nearly constant around $-80.4 \mathrm{~dB}$. This echo threshold dependence on delay is interpreted as a release from forward masking by the preceding emitted pulse. This release may well compensate for the echo level decrease with distance which is from 20 to $40 \mathrm{~dB}$ per distance decade, depending on target properties. This compensation may keep the echo sensation level independent of, or little dependent on, target distance. Supported by Office of Naval Research (USA), Ministry of Science and Education (Russian Federation).

\section{8:40}

3aABa3. Investigation into the possible role of dolphins' teeth in sound reception. Sabine Graf (University of Bath, Dept. Mechanical Engineering, Claverton Down, BA2 7AY Bath, UK, sg283@bath.ac.uk), William M. Megill (University of Bath, Dept. Mechanical Engineering, Claverton Down, BA2 7AY Bath, UK, enswmm@bath.ac.uk), Philippe Blondel (University of Bath, Department of Physics, Claverton Down, BA2 7AY Bath, UK, pyspb@bath.ac.uk), Sally E. Clift (University of Bath,Dept. Mechanical Engineering, Claverton Down, BA2 7AY Bath, UK, enssec@bath.ac.uk)

Odontocetes use active sonars for echolocation, navigation and socialisation. These sonars are characterised by narrow transmission and reception directivity patterns, over a variety of ranges. There is physiological 
and behavioural evidence to suggest that dolphins hear the echoes of their high-frequency clicks through their lower jaws. Current theory suggests that sound is transmitted through a thin region at the base of the jaw into a waveguide leading to the ear. The angular precision predicted by this theory is however much less than dolphins have been observed behaviourally to be able to do. A novel hypothesis is that the teeth of the dolphin's lower jaw act as an end-fire sonar array. This paper will start by putting these competing hypotheses into their context, presenting bioacoustics in the ocean, dolphin echolocation physiology and acoustic behaviour, and the mechanical properties of bones and teeth. This information is used to model the reception of different sounds by bottlenose dolphins (tursiops truncatus) with each possible mechanism, investigating the variations with nerve delay, the role of the jaw relative to the teeth, and the resulting changes in frequency sensitivity and directivity. The results can then be compared with observations of behavioural patterns.

\section{9:00}

3aABa4. Acoustic behavior of echolocating porpoises during prey capture. Stacy Deruiter (Woods Hole Oceanographic Institution, Applied Ocean Physics \& Engineering Dept., Woods Hole, MA 02543, USA, deruiter@mit.edu), Alexander Bahr (MIT, 32 Vassar St., Room 32-331, Cambridge, MA 02139, USA, abahr@mit.edu), Marie-Anne Blanchet (Fjord\&Bælt / University of Southern Denmark, Margrethes Plads 1, 5300 Kerteminde, Denmark, marie@fjord-baelt.dk), Sabina Hansen (Fjord\&Bælt / University of Southern Denmark, Margrethes Plads 1, 5300 Kerteminde, Denmark, sabina@fjord-baelt.dk), Jakob Kristensen (Fjord\&Bælt / University of Southern Denmark, Margrethes Plads 1, 5300 Kerteminde, Denmark, jakob@fjord-baelt.dk), Peter T. Madsen (University of Aarhus, Biological Sciences, Zoophysiology, C. F. Møllers Allé, Building 1131, DK-8000 Aarhus, Denmark, peter.madsen @ biology.au.dk), Peter Tyack (Woods Hole Oceanographic Institution, Applied Ocean Physics \& Engineering Dept., Woods Hole, MA 02543, USA, ptyack@whoi.edu), Magnus Wahlberg (Fjord\&Bælt / University of Southern Denmark, Margrethes Plads 1, 5300 Kerteminde, Denmark, magnus @ fjord-baelt.dk)

Porpoise echolocation has been studied in target detection experiments using stationed animals and steel spheres as targets, but little is known about the acoustic behavior of free swimming porpoises as they echolocate for prey. Here we used archival tags (modified Dtags) recording audio (400 $\mathrm{kHz}$, 16-bit stereo sampling rate) and animal movements (depth, heading, $3 \mathrm{D}$ pitch and roll, and acceleration) to study echolocation in trained porpoises catching fish. The prey capture experiments were conducted with and without suction cups covering the porpoises' eyes. Without the eye cups, the porpoises could use both echolocation and vision to locate and ingest their prey, but with eye cups they could use only echolocation. Our data show that time to prey capture is about $27 \%$ greater with eye cups (T-test, $\mathrm{p}<0$ $.01)$. They also allow us to describe and compare prey capture in the two experimental conditions, including analysis of the animals' detailed movements and echolocation behavior leading up to and following prey capture events.

\section{9:20}

3aABa5. Biosonar model for obtaining fine target structure in complex targets. John R. Buck (UMass Dartmouth, ECE Dept, 285 Old Westport Rd, N. Dartmouth, MA 02740-2300, USA, johnbuck@ieee.org)

Bats use echolocation to discriminate targets with multiple glints in which the glints are much closer together in time than the integration time of the bat auditory system. This indicates the bats cannot be using purely timedomain processing to image the target. It is likely that the bats exploit cues such as spectral peaks and notches due to the interference between the glints at specific frequencies. However, these interference patterns quickly grow complicated as the number of glints increases. The cepstrum is a signal processing algorithm designed to identify these interference intervals. We previously demonstrated that simple two-glint targets can be accurately resolved using a time-varying version of the cepstrum, or cepstrogram. This talk will present an algorithm for interpreting the evolution of cepstral peaks across a target with many glints to estimate the inter-glint intervals. This algorithm has the desirable quality of processing the data causally as it is received, and thus is possible to implement in real time. The performance of the algorithm will be evaluated using experimental data from an ultrasonic bat simulator. [Work supported by US Office of Naval Research]

\section{Invited Paper}

3aABa6. Acoustics of shape recognition by a dolphin in a cross-modal matching-to-sample paradigm. Matthias Hoffmann-Kuhnt (Acoustic Research Laboratory, National University of Singapore, 12A Kent Ridge Road, 119223 Singapore, Singapore, mahoku@nus.edu.sg), Mandar A. Chitre (Acoustic Research Laboratory, National University of Singapore, 12A Kent Ridge Road, 119223 Singapore, Singapore, mandar@arl.nus.edu.sg), Paul J. Seekings (Acoustic Research Laboratory, National University of Singapore, 12A Kent Ridge Road, 119223 Singapore, Singapore, paul@arl.nus.edu.sg), Grant Abel (Ocean Park Hong Kong, Aberdeen, 118336 Hong Kong, Hong Kong, grant.abel@oceanpark.com.hk)

A pacific bottlenose dolphin was trained in a two-alternative cross-modal matching-to-sample paradigm. The animal was able to inspect complex PVC-pipe shapes through echolocation or vision but never through both senses simultaneously. Acoustic data was collected through a 3-channel high-frequency recording system while the dolphin performed one of the following tasks: (1) match a complex shape with its sonar only (pure echoic matching), (2) match from vision to echolocation and (3) match from echolocation to vision. Simultaneously, synchronized in-air and underwater video was recorded documenting the approach path of the dolphin to either the sample object or to the alternative objects. The collected data was analyzed for type of click signals used, the frequency range of the emitted clicks, number of clicks emitted before a successive match and variations of click type with different objects. 


\title{
Session 3aABb
}

\section{Animal Bioacoustics and ECUA: Odontocete Acoustics I}

\author{
David K. Mellinger, Cochair \\ Oregon State Univ. and NOAA, 2030 SE Marine Science Dr., Newport, OR 97365, USA
}

\author{
Michel Andre, Cochair \\ Laboratori d'Aplicacions Bioacústiques (Universitat Politècnica de Catalunya), avda. Rambla Exposició s/n, Vilanova i la \\ Geltrú, 08800, Spain
}

\section{Invited Paper}

10:20

\begin{abstract}
3aABb1. Annotated odontocete recordings for developing automated detection and classification methods. David K. Mellinger (Oregon State Univ. and NOAA, 2030 SE Marine Science Dr., Newport, OR 97365, USA, David.Mellinger@oregonstate.edu), Holger Klinck (Oregon State Univ. and NOAA, 2030 SE Marine Science Dr., Newport, OR 97365, USA, Holger.Klinck@oregonstate .edu), Sara Heimlich (Oregon State Univ. and NOAA, 2030 SE Marine Science Dr., Newport, OR 97365, USA, Sara.Heimlich @ oregonstate.edu), Sharon Nieukirk (Oregon State Univ. and NOAA, 2030 SE Marine Science Dr., Newport, OR 97365, USA, Sharon.Nieukirk@oregonstate.edu)
\end{abstract}

A free archive has been developed for research in automatic detection/classification of cetacean sounds. The archive contains many datasets, with each dataset comprising recordings and metadata for a given species and geographic area. This archive differs from other sound archives in three important respects: (1) Recordings are annotated to indicate where in time and frequency the sounds of a given species occur. These annotations are done manually to remove the bias of any automatic detection system. (2) The archive deliberately includes poor-quality recordings, recordings encountered in any realistic detection/classification application. (3) Since performance of detection/classification methods depends heavily on the SNR of target sounds, the archive includes each vocalization's SNR so performance can be effectively represented. Until recently, most recordings in the archive were of baleen whales [Mellinger and Clark, Applied Acoustics 67(11), 2006]. However, datasets for toothed whales and dolphins are now being incorporated. Initial efforts have been focused on beaked whales because of high interest for management and because of the difficulty of visual detection. To date, recordings of Cuvier's, Blainville's, Baird's, and Arnoux's beaked whales have been entered into the archive, as have those of bottlenose whales, pilot whales, and some delphinid species. Further contributions are welcomed.

\section{Contributed Papers}

10:40

3aABb2. Dynamic tracking of free-swimming whale groups using digital acoustic recording tags. Charles White (University of Rhode Island, Department of Ocean Engineering, Narragansett Bay Campus, Narragansett, RI 02882, USA, cwhite@oce.uri.edu), James H. Miller (University of Rhode Island, Department of Ocean Engineering, Narragansett Bay Campus, Narragansett, RI 02882, USA, miller@egr.uri .edu), Gopu R. Potty (University of Rhode Island, Department of Ocean Engineering, Narragansett Bay Campus, Narragansett, RI 02882, USA, gopu@uri.edu), Mark Johnson (Woods Hole Oceanographic Institution, Applied Ocean Physics \& Engineering Dept., Woods Hole, MA 02543, USA, majohnson@whoi.edu)

Understanding the group behavior of free-swimming whales is critical to the management and conservation of many whale species. Moreover, understanding how whales coordinate their activities could serve as a model for improving the communication and navigation systems of underwater robots and autonomous underwater vehicles (AUVs). One method for studying the group behavior of free-swimming whales involves simultaneously attaching acoustic recording tags to multiple animals within a close-swimming conspecific group. Acoustic recording tags can sample an animal's acoustic environment simultaneously with other physical parameters such as diving depth and acceleration. In this paper, methods are presented for dynamically tracking whales that vocalize regularly such as odontocetes using the DTAG, a digital acoustic recording tag developed by the Woods Hole Oceanographic Institution. A trilateration model is reviewed for tracking groups of whales that include three or more tagged whales using time of flight data and results are given from applying this model to a novel data set of three simultaneously diving and cross-audible short-finned pilot whales
(Globicephala macrorhyncus). A new model is subsequently proposed for tracking whale groups that include only two tagged whales using a generalization of multilateration and data captured by stereo DTAGs.

\section{1:00}

3aABb3. Spatial confirmation of vocal communication between a killer whale calf and its natal family. Scott Veirs (Beam Reach Marine Science and Sustainability School, 7044 17th Ave NE, Seattle, WA 98115, USA, scott@beamreach.org), Val Veirs (Colorado College, Department of Physics, Colorado Springs, CO 80903, USA, vveirs@coloradocollege .edu), Jason D. Wood (Beam Reach Marine Science and Sustainability School, 7044 17th Ave NE, Seattle, WA 98115, USA, jason@beamreach .org)

We recorded an interchange of vocal signaling between a four-year-old Southern Resident killer whale and its natal family members (mother and brother). This information was recorded on a 30-m-long linear array of hydrophones that was towed behind our electric motor powered research vessel. Over the course of about $1 / 2$ hour we recorded signals from these isolated orcas about $500 \mathrm{~m}$ offshore of San Juan Island, WA. Surface locations of the orcas were documented with visual accounts, photographs, video, and - during the latter portion of the encounter - with a hand-bearing compass. During a six minute interval of the recording we have localized $\sim 70$ vocalizations that we can associate with the surface positions of either the juvenile or the mother-brother pair (they were close together). The central three minutes of this interval document an event in which the juvenile left the family group, swam toward our vessel, approached it within about $10 \mathrm{~m}$, turned abruptly away during a rapid call-response sequence with the 
mother and/or brother, and then rejoined the natal group. This rare event informs killer whale vocal development, repertoire, communication, and active space.

\section{$11: 20$}

3aABb4. Killer whales (Orcinus orca) and Bell Laboratories: What can information theory tell us about the communication system of this species? Kenna D. Lehmann (University of California, Dept. of Evolution and Ecology, Davis, CA 95616, USA, kdlehmann@ucdavis.edu), Jason D. Wood (Beam Reach Marine Science and Sustainability School, 7044 17th Ave NE, Seattle, WA 98115, USA, jason@beamreach.org)

In the 1950's Shannon and Weaver developed information theory as a statistical tool to determine the structure and organization within communication systems. More recently information theory has been applied to the study of animal communication. We gathered acoustic recordings of the Southern Resident killer whales in the inland waters of Washington State, USA, and British Columbia, Canada, from both land based and boat based acoustic arrays. Recorded calls were categorized using the accepted call catalog for this population. In this paper we will present our results from applying various Shannon entropies to the documented call sequences. One notable result is that the call repertoire is more redundant than is ideal to convey the maximum amount of information. This may be a result of their particular social system and behavioral ecology, or may be an indication of the amount of noise present in their acoustic environment. Comparisons will also be made with measures of information theory in other species.

\section{1:40}

3aABb5. Whistle repertoire of captive bottlenose dolphins, Tursiops truncatus. Rebeca M. Lopez-Rivas (UNAM, Facultad de Ciencias, Depto. Física, Lab Acústica, Circuito exterior s/n, Cd. Universitaria, 04510 México, D.F., Mexico,rmlopez.rivas@gmail.com), Volker B. Deecke (University of British Columbia, Marine Mammal Research Unit, Vancouver, BC V6T 1Z4, Canada, deecke@zoology.ubc.ca), Carmen Bazúa Durán (UNAM, Facultad de Ciencias, Depto. Física, Lab Acústica, Circuito exterior s/n, Cd. Universitaria, 04510 México, D.F., Mexico, bazua@servidor.unam.mx)

The whistle repertoire of bottlenose dolphins has been poorly described in the literature, thus little information is known to support the hypothesis that whistles are used by dolphins to communicate. In this study, whistles emitted by four captive bottlenose dolphins housed in two aquaria in Mexico City were recorded to analyze the whistle repertoire and whistle emission rate for each aquarium. The whistle repertoire was described using human and automated categorizations. Results show that whistle emission rate and the whistle repertoire are both larger for the two dolphins that are more active and which are housed in the same aquarium. As for whistles shared in the repertoire of both aquaria, these are very few, probably because dolphins of the two aquaria were captured in different oceans and are of different ages, besides of the different activities that dolphins can perform in each aquarium. Additionally, all four dolphins could use similar whistles for a specific activity, like "swimming", while different whistles were used for another specific activity, like "observing". These results show that dolphins use whistles selectively, supporting the hypothesis that whistles may be used to communicate information for particular situations. [Work supported by PAPIIT, UNAM \& CoNaCyT]

\section{2:00}

3aABb6. Unsupervised clustering of whistle-sounds of bottlenose dolphins (Tursiops truncatus). Sebastian V. Huebner (Pestalozzistrasse 5, 14482 Potsdam, Germany, sebastian@sejona.de)

An unsupervised symbolic clustering algorithm for whistles of bottlenose dolphins (Tursiops truncatus) was tuned on an artificial data set containing 12 ideal synthetic signature whistles. Afterwards approximately 25,000 representations of natural whistles were clustered with the tuned algorithm. Recordings were made from a group of twelve adult semi-free living animals in Eilat (Israel). Obtained clusters were evaluated with the help of their individual compression rates. The clustering algorithm perfectly converged on all twelve artificial whistle types. Also, automatic whistle extraction produced good representations of both artificial and natural whistles, but clustering of natural whistles provided low support for the signature whistle hypothesis: The six most frequent induced whistle types could be interpreted as slight variations of only two signature whistles. Also, a great variety of different but rare types in automatically generated clusters could be observed. Results suggest that certain aspects of the group behavior are manifest in the frequency whistle types occur.

\section{$12: 20$}

3aABb7. Acoustic studies of dolphins in their natural habitat: Challenges and successes. Katherine Kim (HLS Research, Inc., 3366 N. Torrey Pines Ct., Ste. 310, La Jolla, CA 92037, USA, Katherine.Kim @ HLSResearch.com), Paul Hursky (HLS Research, Inc., 3366 N. Torrey Pines Ct., Ste. 310, La Jolla, CA 92037, USA, paul.hursky@hlsresearch .com), Michael B. Porter (HLS Research, Inc., 3366 N. Torrey Pines Ct., Ste. 310, La Jolla, CA 92037, USA, michael.porter@hlsresearch.com), John A. Hildebrand (Scripps Institution of Oceanography- UCSD, 9500 Gilman Dr. \#0205, La Jolla, CA 92093, USA, jhildebrand@ucsd.edu), Elizabeth Henderson (Scripps Institution of Oceanography- UCSD, 9500 Gilman Dr. \#0205, La Jolla, CA 92093, USA, e0hender@ucsd.edu), John Calambokidis (Cascadia Research Collective, 218 1/2 W. 4th Ave., Olympia, WA 98501, USA, calambokidis@ cascadiaresearch.org), Erin Falcone (Cascadia Research Collective, 218 1/2 W. 4th Ave., Olympia, WA 98501, USA, EFalcone@ cascadiaresearch.org)

Acoustic methods for studying marine mammals is a research area of great potential, with applications which include population estimation, spatial distribution, behavioral studies, and the effects of anthropogenic noise on animals. Until recently, much of the acoustic research on free-ranging animals has focused on mysticetes and sperm whales; however, concern over highly-publicized dolphin strandings has raised interest in other odontocetes. From acoustic and signal processing perspectives, dolphins pose numerous new challenges: They typically aggregate in pods composed of tens or hundreds of individuals, often vocalizing simultaneously, and their calls are highly variable and high-frequency. Detection algorithms will be presented which are robust (or, conversely, can be fine-tuned) to these variable calls and have been automated to overcome the ordinarily laborintensive analysis requirements of such large data sets. Historically, acoustic studies of marine mammals used relatively simple sensor systems comprised of one or few hydrophones. In our studies, multiple sensor systems were deployed. Doing so enabled the utilization of array and co-array processing techniques for improved localization estimation which, like our detection algorithms, were fully automated. They also raised important deployment considerations due to the acoustic propagation physics as well as challenges in call association between different systems.

\section{$12: 40$}

3aABb8. Narrow band high frequency clicks from Hourglass dolphins (Lagenorhynchus cruciger). Line A. Kyhn (National Environmental Research Institute, University of Aarhus, Frederiksborgvej399, DK-4000 Roskilde, Denmark, lky@dmu.dk), Jakob Tougaard (National Environmental Research Institute, University of Aarhus, Frederiksborgvej399, DK-4000 Roskilde, Denmark, jat@dmu.dk), Magnus Wahlberg (Fjord\&Bælt / University of Southern Denmark, Margrethes Plads 1, 5300 Kerteminde, Denmark, magnus@fjord-baelt.dk), Kristian Beedholm (Institute of Biology, University of Southern Denmark, Campusvej 55, 5230 Odense M, Denmark, beedholm@mail.dk), Peter T. Madsen (University of Aarhus, Biological Sciences, Zoophysiology, C. F. Møllers Allé, Building 1131, DK-8000 Aarhus, Denmark, peter.madsen@biology.au.dk)

The source properties of toothed whale echolocation clicks are essential for evaluating the performance of biosonar systems. However, such data only exist for a small number of free ranging toothed whale species. Here we present a quantitative analysis of the first recordings of echolocation sounds from hour glass dolphins (Lagenorhynchus cruciger) made in the Drake Strait. Recordings were made with a vertical array of four broadband, calibrated hydrophones that allows for acoustic localization and source parameter derivation. Only apparent on-axis clicks were used for analysis. Hour glass dolphins produce clicks with a mean peak and centroid frequency around $128 \mathrm{kHz}$, a narrow, mean RMS bandwidth of $10 \mathrm{kHz}$ with no energy below $100 \mathrm{kHz}$ and a mean signal duration of $161 \mu \mathrm{s}$. Echolocation clicks of hour glass dolphins are thus of the high frequency narrow band- 
width type (NBHF type) found in porpoises, a few delphinids of the Cephalorhynchus genus and the pygmy sperm whale Kogia breviceps, but are strikingly different from other dolphins. Acoustic convergence from vastly different genera and ecotypes suggest that evolution of NBHF clicks are adaptations to special auditory processing or acoustic crypsis rather than to habitat.

\title{
Session 3aAO
}

\section{Acoustical Oceanography, Underwater Acoustics, Signal Processing in Acoustics, and ECUA: Geoacoustic Characterization of the Ocean Bottom and Geoacoustic Inversion III}

\author{
Peter Gerstoft, Cochair \\ Marine Physical Laboratory, Scripps Institute of Oceanography, 8602 La Jolla Shores Drive, La Jolla, CA 92093-0238, USA \\ Dag Tollefsen, Cochair \\ Norwegian Defence Research Establishment (FFI), Box 115, Horten, 3191, Norway
}

Invited Papers

8:00

3aAO1. Location of Targets and Scatterers with Ambient Noise. Christopher H. Harrison (NATO Undersea Research Centre, Viale San Bartolomeo 400, 19138 La Spezia, Italy, harrison@nurc.nato.int)

By placing a vertical array in an ambient noise field and forming an upward and downward beam one obtains two time series which can be cross-correlated to reveal a sub-bottom profile of the seabed [Siderius, et al., J. Acoust. Soc. Am., 120, 1315-1323, (2006)]. Here the cross-correlation approach is extended to the location in range and bearing of a point target. An experiment was designed using floats and weights mounted (and dismounted) on the same cable as the vertical array. Careful measurements were made of the location of all likely floats, ballast weights, array terminations, and so on. After suitable coherent averaging, peaks were seen at delays (correlation offsets) agreeing with the reflector positions and were shown to be absent when reflectors were removed. An implication for geoacoustic inversion is that, in priciple, the technique can be used to explore scattering as well as reflection properties of layers. A simple extension of the theory developed in [Harrison and Siderius, J. Acoust. Soc. Am., (2007), accepted] is used to explain the amplitudes of the reflections. The approach differs from "acoustic daylight" principally in determining a target range.

\section{8:20}

3aA02. Wide-area geoacoustic inversion using distant ship noise. Altan Turgut (Naval Research Laboratory, Acoustics Division, Code 7120, Washington, DC 20375, USA, altan.turgut@nrl.navy.mil)

Broadband $(50-500 \mathrm{~Hz})$ noise data from fast-traveling surface ships in the vicinity of a VLA and a bottomed HLA are used to estimate the geoacoustic properties on the New Jersey Shelf. Matched-field inversion of geometric and geoacoustic parameters are presented by using twenty days of ship noise data, collected during the RAGS03 winter experiment. Noise data from different ships at different ranges provided robust geoacoustic inversions for both VLA and bottomed HLA data. Acoustic propagation tracks are determined from the HLA beamforming results and a 3-D geoacoustic parameter map is constructed. The constructed geoacoustic parameter map shows good agreement with the results from chirp sonar inversion and acoustic probe measurements, indicating silty seafloor sediments on the outer-shelf area and sandy sediments on the inner-shelf area. Efficiency of the 3-D geoacoustic inversion and extension to range-dependent inversions at longer ranges are also discussed. [Work supported by ONR.]

$8: 40$

3aA03. Doppler Geo-Spectroscopy in the Makai Experiment. David R. Barclay (Marine Physical Lab, Scripps Institution of Oceanography, UCSD, 9500 Gilman Dr. M/C 0238, La Jolla, CA 92093-0238, USA, dbarclay@mpl.ucsd.edu), Michael Buckingham (Marine Physical Lab, Scripps Institution of Oceanography, UCSD, 9500 Gilman Dr. M/C 0238, La Jolla, CA 92093-0238, USA, mjb @mpl.ucsd.edu)

Using data collected during the 2005 Makai experiment, acoustic parameters of a coral-sand seabed were obtained using the Doppler spectroscopy inversion technique. A light aircraft was used as a low frequency $(80-400 \mathrm{~Hz})$ sound source over an isovelocity shallow water channel. A microphone near the ocean surface and a hydrophone near the seabed were used to record the Doppler spreading of each harmonic of the aircraft's engine and propeller. In the water column, spectral peaks with the greatest up and down Doppler shifts are associated with the shallow equivalent rays of normal modes. The least mean squares (LMS) adaptive filter was used to track and enhance the frequency of these propagating modes. The inversion relies on a full wave number spectral model of a moving source in 
a three-layer waveguide. The Doppler shifted modes in the water column are observed in the data and simulated in the model. The comparison of the two, in symphony with the aircraft's signal in the air, returns the geoacoustic parameters of the seabed. The inversion was performed on numerous over-flights and the results were averaged to show statistical confidence. Work supported by ONR.

\section{Contributed Papers}

9:00

3aA04. Geoacoustic inversion in the frequency range $0.8-1.6 \mathrm{kHz}$ with drifting sparse arrays during MREA/BP'07 experiment. Jean-Claude Le Gac (NATO Undersea Research Center, Viale San Bartolomeo 400, 19126 La Spezia, Italy, legac@nurc.nato.int), Jean-Pierre Hermand (Université libre de Bruxelles (U.L.B.) - Environmental hydroacoustics lab, av. Franklin D. Roosevelt 50, CP 194/5, 1050 Bruxelles, Belgium, jhermand@ulb.ac.be), Sergio Jesus (ISR, Universidade do Algarve, PT-8005-139 Faro, Portugal, sjesus@ualg.pt)

In order to evaluate properly the acoustic propagation characteristics in shallow water environments, it is well established that appropriate knowledge of the acoustic properties of the seabottom is required. In the last decade, full-field geoacoustic inversion techniques have been demonstrated to provide adequate methodologies to assess those properties. However, several of the developed techniques may suffer a lack of adequacy to the design of low-frequency active sonar systems (LFAS) for which the assessment of seabottom characteristics are drawn. For instance most matched-field inversion techniques demonstrated so far use acoustical signals at much lower frequencies than those of the sonar. Furthermore, some of the techniques may be difficult to be handled in an "operationally relevant context" since they are based on relatively complex designed systems such as highly instrumented vertical line arrays spanning the whole water column. In this paper, we investigate the potential of medium-frequency acoustical signals ( 0 .8-1.6 kHz) received at several ranges on a field of drifting sparse arrays, eventually reduced to a couple of hydrophones, for spatially-coherent geoacoustic inversion purposes. The experimental datasets of the Maritime Rapid Environmental Assessment MREA/BP'07 sea trial south of Elba Island in the Mediterranean Sea are used to support this study.

\section{9:20}

3aA05. Using offshore seismic surveys as acoustic sources of opportunity for geoacoustic inversion. Alec J. Duncan (Curtin University of Technology, Centre for Marine Sci \& Tech, GPO Box U1987, 6845 Perth, WA, Australia, A.J.Duncan@curtin.edu.au), Alexander Gavrilov (Curtin University of Technology, Centre for Marine Sci \& Tech, GPO Box U1987, 6845 Perth, WA, Australia, a.gavrilov@cmst.curtin .edu.au), Tao Hu (Institute of Acoustics, Chinese Academy of Science, PostBox 2712, NO.21, Bei-Si-huan-Xi Road, 100080 Beijing, China, hutao76@yahoo.com.cn)

Commercial offshore seismic surveys involve the use of powerful acoustic sources consisting of arrays of airguns. These sources produce frequent, loud, impulsive sounds at precisely timed intervals, at accurately known positions during surveys that may last for several months. By deploying a lowcost acoustic receiving system in the vicinity of such a survey scientists can acquire high quality data for geoacoustic inversion experiments in an extremely cost-effective manner. This paper discusses the various factors that must be considered when using such data for geoacoustic inversion, including the prediction of source spectra and array directionality, and provides some examples.

\section{9:40-10:00 Break}

10:00

3aAO6. Geo-acoustic inversion with adaptive beamformed data. Steven A. Stotts (Applied Research Laboratories/The University of Texas, 10000 Burnet Rd, Austin, TX 78758, USA, stotts@arlut.utexas.edu), Robert A. Koch (Applied Research Laboratories/The University of Texas, 10000 Burnet Rd, Austin, TX 78758, USA, koch@arlut.utexas.edu)

Geo-acoustic inversion with matched field replicas constructed by adaptive techniques lacks robustness, because localizations with such techniques are sensitive to model mismatch [Soares, Jesus, and Coelho, J. Acoust. Soc. Am. 122, 3391-3404 (2007)]. Instead of adapting the matched field weights, inversion with adaptive beam-formed $(\mathrm{ABF})$ plane wave sub-aperture data produces accurate source localizations for ships of opportunity [Stotts and Hawkins, J. Acoust. Soc. Am. 118, 1857 (2005)]. Model inputs to the matched-field cost function are evaluated at sub-aperture phase centers in this approach. The main purpose of this talk is to demonstrate the robustness and accuracy of solutions obtained from geo-acoustic inversion using plane wave $\mathrm{ABF}$ data. Improvements over either single element or conventional plane wave beam-formed $(\mathrm{CBF})$ sub-aperture data for geo-acoustic inversions and simultaneous geo-acoustic inversions/source localizations using a source of opportunity in real data containing interferers will be demonstrated.

\section{0:20}

3aA07. Geoacoustic inversion with recreational boat noise. A. Vincent Van Leijen (Netherlands Defence Academy, P.O. Box 10 000, 1780 CA Den Helder, Netherlands, av.v.leijen@nlda.nl)

During the MREA07 sea trials various experiments involved geoacoustic inversion with sound sources of opportunity. A particular experiment that focussed on exploiting the self noise of autonomous vehicles suffered from interfering noise due to increased boating activity in the weekend. In this work the same data is re-examined to study the potential of recreational boats as sound sources for geoacoustic inversion. Even though recreational boats are non cooperative sound sources, in the sense that weekend traffic does not sail predefined tracks and navigational logging is absent, various boats swarm out and cover large areas thus acting as a distributed set of sound sources. A typical small boat signature counts numerous harmonics of narrowband tones that cover the frequency spectre from $100 \mathrm{~Hz}$ up to $2 \mathrm{kHz}$. With these tones matched field inversion and traditional Doppler techniques are used to find an initial geometry of the experiments and these results are fine tuned during the final geoacoustic inversion of a locally rangeindependent model of a coastal environment. Results of inversions with recreational boating sounds are shown to match with previous analysis results with self noise of autonomous vehicles, bottom grab samples and seismic data.

\section{0:40}

3aA08. Geoacoustic uncertainties in matched-field inversions of ship-noise data. Dag Tollefsen (Norwegian Defence Research Establishment (FFI), Box 115, 3191 Horten, Norway, dag.tollefsen@ffi.no), Stan E. Dosso (University of Victoria, School of Earth and Ocean Sciences, Victoria, BC V8W 3P6, Canada, sdosso@uvic.ca)

This paper applies geoacoustic inversion to ship-noise data recorded on a horizontal array deployed on the seafloor in an experiment conducted by FFI in the Barents Sea. A Bayesian matched-field inversion method is employed which quantifies geoacoustic uncertainties and allows for quantitative comparisons of results from different data sets. Estimates of sediment parameters and their uncertainties were obtained by inversions of lowfrequency narrowband data from a quiet research ship at source-array ranges of 1-6 km and different orientations with respect to the array, as well as from a merchant ship at ranges of 6-8 $\mathrm{km}$. Multiple, independent data segments are included in the inversion and shown to significantly reduce geoacoustic parameter uncertainties. The effect of the state of prior information on the ship track (e.g., well known or unknown) on geoacoustic information content is also examined. Estimates of the sound-speed profile and density of sediment are compared with results from inversion of data from a towed controlled-source, and with reference values from other geophysical data collected in the area. 
11:00

3aA09. Optimal source tracking in an unknown ocean environment. Michael J. Wilmut (University of Victoria, School of Earth and Ocean Sciences, Victoria, BC V8W 3P6, Canada, mjwilmut@uvic.ca), Stan E. Dosso (University of Victoria, School of Earth and Ocean Sciences, Victoria, BC V8W 3P6, Canada, sdosso@uvic.ca)

This paper develops and compares two approaches to localizing and tracking a low-level acoustic source when ocean environmental properties are unknown. Both approaches are based on a Bayesian formulation in which source and environmental parameters are considered random variables constrained by noisy acoustic data and by prior information on parameter values (e.g., physical limits for water-column and seabed properties) and on inter-parameter relationships (e.g., limits on horizontal and vertical source speed). One approach is based on determining the source track and environmental parameters that maximize the posterior probability density (PPD) using adaptive hybrid optimization. A key to solving this challenging problem efficiently is that the Viterbi algorithm is applied to compute the highest-probability source track for each environmental realization considered in the optimization-this provides the optimal track while requiring the optimization is applied only to environmental parameters. The second approach involves integrating the PPD over unknown environmental parameters to represent source-track information as a series of joint marginal probability surfaces over range and depth. The Viterbi algorithm is applied to extract the optimal track from these surfaces. For realistic environmental models (e.g., more than a dozen unknown parameters), the integration is carried out using efficient Markov-chain Monte Carlo methods.

WEDNESDAY MORNING, 2 JULY 2008

ROOM 352B, 8:00 TO 10:40 A.M.

\title{
Session 3aBBa
}

\section{Biomedical Ultrasound/Bioresponse to Vibration and Physical Acoustics: Shock Waves in Medicine}

\author{
Robin O. Cleveland, Cochair
}

Boston University, Dept. of Aerosp. and Mech. Eng., 110 Cummington St., Boston, MA 02215, USA

Oleg A. Sapozhnikov, Cochair

Center for Industrial and Medical Ultrasound, Applied Physics Lab., University of Washington, 1013 NE 40th St., Seattle, WA 98105, USA

\section{Invited Paper}

3aBBa1. Short- and long-delay tandem shock waves for application in medicine. Achim M. Loske (Universidad Nacional Autónoma de México, Centro de Física Aplicada y Tecnología Avanzada, AP 1-1010, 76000 Querétaro, Mexico, loske@fata .unam.mx), Francisco Fernandez (Universidad Nacional Autónoma de México, Centro de Física Aplicada y Tecnología Avanzada, AP 1-1010, 76000 Querétaro, Mexico, francisco@fata.unam.mx), Miguel De Icaza (Universidad Nacional Autónoma de México, Centro de Física Aplicada y Tecnología Avanzada, AP 1-1010, 76000 Querétaro, Mexico, icaza@fata.unam.mx), Guillermo Canseco (Universidad Nacional Autónoma de México, Centro de Física Aplicada y Tecnología Avanzada, AP 1-1010, 76000 Querétaro, Mexico, gcanseco@fata.unam.mx)

Long-delay tandem shock waves with an inter-pulse delay between about 200 and 500 microseconds have shown to enhance fragmentation of artificial kidney stones during in vitro and in vivo extracorporeal shock wave lithotripsy (SWL) in the past. Cellpermeabilization and inactivation of bacteria using tandem shock waves have also been proposed. The purpose of this research was to study the influence of short-delay (less than 50 microseconds) tandem shock waves on cavitation induced damage to kidney stones during SWL. A multipurpose piezoelectric shock wave generator was designed to generate shock waves at variable delays. The effect of short- and long-delay dual-pulse shock waves on the surface of artificial kidney stones was evaluated by microscopy. An adequate selection of delays was possible by means of a numerical model and high speed photography. Stone fragmentation was compared to that of standard (single-pulse) shock waves. Our results indicate that specific combinations of short- and long-delay tandem shock waves could improve damage to kidney stones by acoustic cavitation. 


\section{Contributed Papers}

8:20

3aBBa2. Correlation of vasoconstriction and kidney protection during shock wave lithotripsy. Michael R. Bailey (Center for Industrial and Medical Ultrasound, Applied Physics Lab., University of Washington, 1013 NE 40th St., Seattle, WA 98105, USA, bailey@apl.washington.edu), Marla Paun (Center for Industrial and Medical Ultrasound, Applied Physics Lab., University of Washington, 1013 NE 40th St., Seattle, WA 98105, USA, paun@u.washington.edu), Rajash K. Handa (Department of Anatomy and Cell Biology, Indiana University School of Medicine, 635 Barnhill Dr. MS5055, Indianapolis, IN 46202, USA, rhanda@iupui.edu), Sujuan Gao (Department of Medicine, Division of Biostatistics, Indiana University School of Medicine, 410 West 10th Street, Suite 3000, Indianapolis, IN 46202, USA, sgao@iupui.edu), Lynn R. Willis (Department of Pharmacology, Indiana University School of Medicine, 635 Barnhill Dr. MS5055, Indianapolis, IN 46202, USA, willis1@iupui.edu), Bret A. Connors (Department of Anatomy and Cell Biology, Indiana University School of Medicine, 635 Barnhill Dr. MS5055, Indianapolis, IN 46202, USA, connors@anatomy.iupui.edu), James A. McAteer (Department of Anatomy and Cell Biology, Indiana University School of Medicine, 635 Barnhill Dr. MS5055, Indianapolis, IN 46202, USA, mcateer @ anatomy.iupui.edu), Andrew P. Evan (Department of Anatomy and Cell Biology, Indiana University School of Medicine, 635 Barnhill Dr. MS5055, Indianapolis, IN 46202, USA, aevan@iupui.edu)

Kidney injury in lithotripsy can lead to long-term adverse effects, so minimizing injury is beneficial. We have found that injury is significantly reduced when shock wave (SW) administration is halted briefly- 3-minutesearly in treatment. Previous studies have shown that SWs stimulate renal blood vessels to constrict. Therefore, we tested the idea that vasoconstriction mediates SW-induced protection of the kidney. Doppler ultrasound was used to measure resistive index (RI), a ratio of systolic and diastolic velocities, indicating vasoconstriction. RI was determined for single intralobar arteries in targeted porcine kidneys treated by standard versus protection protocols (2,000SW, uninterrupted vs 500SW-3-min pause-2,000SW) using a DornierHM3 lithotripter $(2 \mathrm{~Hz}, 24 \mathrm{kV})$. Significant differences in RI from baseline within a group and between groups at various time points were determined using mixed-effect models for repeated measures with Holm's step-down method for multiple comparison adjustment. All animals had similar baselines. Sham pigs (no-SWs) showed no significant change in RI. The protection protocol produced a significant rise $(\mathrm{p}<0.05, \mathrm{n}=8)$ in RI 15 minutes into treatment, while the standard protocol did not yield a significant rise $(\mathrm{p}<0.05, \mathrm{n}=7)$ until 45 minutes after treatment. Thus, the treatment protocol shown to protect against injury, induces early vasoconstriction. [NIH-DK43881, NSBRI-SMS00402]

\section{8:40}

3aBBa3. Fundamental study of shock induced acceleration of cell growth by plane shock waves. Masaaki Tamagawa (Kyushu Institute of Technology, Hibikino 2-4, Wakamatsu-ku, 808-0196 Kitakyushu, Japan, tama@life.kyutech.ac.jp)

This paper describes effects of shock waves on cells to certificate the angiogenesis by shock wave in the clinical application. Especially, the effects of plane shock waves without cavitation bubbles on the endothelial cells are investigated by experiments and computation. In the experiments, the plane shock waves using shock tube apparatus are applied to the endothelial cells. The peak pressure working on the cells in the test case is 0.4 $\mathrm{MPa}$, which is under disintegration level for endothelial cells. After working shock waves on suspended cell, the disintegration and growth rate are measured. It is found that the initial growth rate of the shock-worked cells are clearly higher than that of control ones. As for the effect of rise time of working shock waves, to change the rise time of shock wave, Air and He gas are used as driver gas in the shock tube. As a result, the shorter rise time brings higher growth rate. In the computation, to check the effects of rise time on the pressure and stress distribution on the cells, fluid and structural interaction model are analyzed. The result shows that shorter rise time makes high stress level distribution on the cell membrane.

\section{9:00}

3aBBa4. Beamwidth measurement of single lithotripter shock waves. Wayne Kreider (Center for Industrial and Medical Ultrasound, Applied Physics Lab., University of Washington, 1013 NE 40th St., Seattle, WA 98105, USA, wkreider@u.washington.edu), Michael R. Bailey (Center for Industrial and Medical Ultrasound, Applied Physics Lab., University of Washington, 1013 NE 40th St., Seattle, WA 98105, USA, bailey@apl .washington.edu), Jeffrey A. Ketterling (Frederic L. Lizzi Center for Biomedical Engineering, Riverside Research Institute, 156 William St., New York, NY 10038, USA, ketterling@rrinyc.org)

New lithotripters with narrower foci and higher peak pressures than the original Dornier HM3 electrohydraulic lithotripter have proven to be less effective and less safe. Hence, accurate measurements of the focal characteristics of lithotripter shock waves are important. The current technique for measuring beamwidth requires collection of single-point measurements over multiple shock waves, thereby introducing error as a result of any shock-toshock variability. This work reports on the construction of a hydrophone array sensor and on array measurements of individual lithotripter shock waves. Beamwidths for an electrohydraulic lithotripter with a broad-focus, HM3-style reflector and a narrow-focus, modified reflector were measured using both new and worn electrodes as well as two different electrical charging potentials. The array measured the waveform, beamwidth, and focal location of individual shock waves. The HM3-style reflector produced repeatable focal waveforms and beam profiles at an $18-\mathrm{kV}$ charging potential with both new and worn electrodes. Accordingly, these measurements agreed with averaged point measurements acquired under the same conditions. However, a lack of consistency in the measured focal locations and beam profiles at $23 \mathrm{kV}$ underscores the value in obtaining beam profile measurements from individual shock waves. Work supported by Riverside Research Institute, NIH DK43881, and NSBRI SMS00402.

\section{9:20}

3aBBa5. Numerical simulation of shock wave generation and focusing in shock wave lithotripsy. Jeffrey Krimmel (California Institute of Technology, 1200 E. California Blvd, Pasadena, CA 91125, USA, jkrimmel @ caltech.edu), Tim Colonius (California Institute of Technology, 1200 E. California Blvd, Pasadena, CA 91125, USA, colonius@ caltech.edu)

Shock wave lithotripsy is a procedure where focused shock waves are fired at kidney stones in order to pulverize them. Lithotripters with different source mechanisms and reflector shapes (or lenses) are in clinical use, but prediction of focal region pressure is made difficult by nonlinearity and cavitation. We report on development of a numerical simulation framework aimed at accurate prediction of focal region pressures and bubble dynamics. Shock wave generation and beam focusing are simulated via the Euler equations with MUSCL-type shock-capturing scheme and adaptive mesh refinement (AMR). Electrohydraulic, electromagnetic, and piezoelectric-array lithotripters are modeled with axisymmetric geometries. In the electrohydraulic case, an expanding bubble model simulates spark firing. In the piezoelectric case, a boundary condition prescribing the motion of individual elements is used. Calculated peak pressures and pulse widths agree well with experimental data for the electrohydraulic and electromagnetic lithotripters. For the piezoelectric case, peak pressures are increasingly overpredicted by the model when more elements are fired, and reasons for this discrepancy will be discussed. Finally, we compare the spatial and temporal characteristics of the focal pressure fields for several clinical lithotripters in the context of observed stone comminution efficacy and tissue damage. This work is supported by NIH grant PO1 DK043881 and ONR Grant N0001406-1-0730.

\section{9:40}

3aBBa6. Effect of elastic waves in the metal reflector on bubble dynamics at the focus of an electrohydraulic lithotripter. Oleg A Sapozhnikov (Center for Industrial and Medical Ultrasound, Applied Physics Lab., University of Washington, 1013 NE 40th St., Seattle, WA 98105 
USA, oleg@acs366.phys.msu.ru),Wayne Kreider(Center for Industrial and Medical Ultrasound, Applied Physics Lab., University of Washington, 1013 NE 40th St., Seattle, WA 98105, USA, wkreider@u.washington .edu), Michael R. Bailey (Center for Industrial and Medical Ultrasound, Applied Physics Lab., University of Washington, 1013 NE 40th St., Seattle, WA 98105, USA, bailey@ apl.washington.edu), Vera A. Khokhlova (Center for Industrial and Medical Ultrasound, Applied Physics Lab., University of Washington, 1013 NE 40th St., Seattle, WA 98105, USA, vera@acs366 .phys.msu.ru), Francesco Curra (Center for Industrial and Medical Ultrasound, Applied Physics Lab., University of Washington, 1013 NE 40th St., Seattle, WA 98105, USA, fcurra@apl.washington.edu)

In extracorporeal electrohydraulic lithotripters, a hemi-ellipsoidal metal reflector is used to focus a spherical wave generated by an electrical discharge. The spark source is positioned at one of the ellipsoid foci (F1); this makes the reflected wave focused at the other focus (F2). Despite the common assumption that the reflector behaves as a rigid mirror, the true reflection phenomenon includes the generation and reverberation of elastic waves in the reflector, which reradiate to the medium. Although these waves are much lower in amplitude than the specularly reflected wave, they may influence cavitation at F2. To explore such effects, waves in water and a brass reflector were modeled in finite differences based on the linearized equations of elasticity. The bubble response was simulated based on a Rayleigh-type equation for the bubble radius. In addition, the role of acoustic nonlinearity was estimated by numerical modeling. It is shown that the elastic waves in the reflector give rise to a long "ringing" tail, which results in nonmonotonic behavior of the bubble radius during its inertial growth after shock wave passage. This numerical result is qualitatively confirmed by experimental observations of bubble behavior using high-speed photography. Work supported by NIH-DK43881, NSBRI-SMS00402, NIHDK075090, and RFBR.

\section{0:00}

3aBBa7. Elastic waves in human kidney stones: Shear dominates spall in shock wave lithotripsy. Robin O. Cleveland (Boston University, Dept. of Aerosp. and Mech. Eng., 110 Cummington St., Boston, MA 02215, USA, robinc@bu.edu), Haibiao Luo (Boston University, Dept. of Aerosp. and Mech. Eng., 110 Cummington St., Boston, MA 02215, USA, hluo @ bu.edu), James C. Williams (Indiana Univ. School of Medicine, 635 Barnhill Dr, Indianapolis, IN 46202, USA, williams@ anatomy.iupui.edu)

We investigated the role of shear waves and spall in the fragmentation of human kidney stones. Seven human kidney stones, approximately $5 \mathrm{~mm}$ in diameter, were scanned with micro computed tomography to obtain a 3D representation that was imported into an elastic wave code. The code was initialized with a lithotripsy waveform and the evolution of stresses and strains was tracked through space and time. The evolution of the principal stress indicated that reflection from the distal surface of the stone (spallation) did not induce large tensile stresses $(<40 \mathrm{MPa})$ due to the curved geometry of the proximal and distal surfaces. Instead as the shock wave in the fluid passed the mid-section of the stone there was efficient coupling into shear waves which then propagated into the interior of the stone and generated the largest regions of high tensile stress (>60 MPa). The results indicate that for the human stones studied here shear waves generated at the stone periphery are more important than spall in generating high tensile stress. These data suggest that the focal width of a lithotripter needs to be larger than the stone in order to generate large stresses. [Work supported by NIH DK43881 and DK59933].

\section{0:20}

3aBBa8. Damage potential of single-bubble collapse in shockwave lithotripsy. Eric Johnsen (California Institute of Technology, $1200 \mathrm{E}$. California Blvd, Pasadena, CA 91125, USA, johnsen@stanford.edu), Tim Colonius (California Institute of Technology, 1200 E. California Blvd, Pasadena, CA 91125, USA, colonius@caltech.edu)

In shockwave lithotripsy, the combined effect of focused shockwaves and cavitation pulverizes kidney stones. Although cavitation is known to play an important role in stone comminution, the underlying mechanism is not fully understood. The goal of the present study is to quantify the potential damage caused by Rayleigh collapse (RC) and shock-induced collapse (SIC) of a single bubble near a stone. A high-order accurate, quasiconservative, shock- and interface-capturing scheme [E. Johnsen and T. Colonius, J. Comput. Phys. 2006] is employed to simulate both phenomena. A high-speed re-entrant jet forms during the collapse and hits the distal side of the bubble, thereby generating a water-hammer pressure wave. A high pressure is measured along the stone surface upon the impact of this wave, thus providing potential for erosion. In SIC, this pressure may reach $1 \mathrm{GPa}$ and bubbles within approximately 15 initial radii generate a pressure higher than the incoming pulse. In addition, by using the present results as timedependent boundary conditions for an elastic wave propagation code, it is shown that a tension large enough to lead to failure may be achieved within small stones or fragments. This work is supported by NIH grant PO1 DK043881 and ONR Grant N00014-06-1-0730. 


\title{
Session 3aBBb
}

\section{Biomedical Ultrasound/Bioresponse to Vibration and Physical Acoustics: Light and Sound for Medical Imaging and Therapy I}

\author{
Ronald A. Roy, Cochair \\ Boston University, Dept. of Aerosp. and Mech. Eng., 110 Cummington St., Boston, MA 02215, USA \\ Emmanuel Bossy, Cochair \\ Laboratoire Photons et Matière, ESPCI/CNRS, 10 rue Vauquelin, Paris Cedex 05, 75231, France
}

\section{Invited Papers}

11:00

3aBBb1. Photoacoustic imaging for characterising the structure and function of vascular networks. Paul C. Beard (University College London, Gower Street, London, WC1E 6BT London, UK, pbeard@medphys.ucl.ac.uk)

Photoacoustic imaging is a new non invasive soft tissue imaging method in which low energy nanosecond pulses of visible or near infrared (NIR) laser light are used to excite subsurface ultrasound waves. The distinguishing advantage of the technique is that provides the high contrast and spectroscopic based specificity of optical techniques along with the high spatial resolution of ultrasound. Haemoglobin represents the most important source of naturally occurring photoacoustic contrast due its relatively strong optical absorption in the visible and NIR making the technique particularly well suited to imaging blood vessels. In addition, functional information can be provided by obtaining images at multiple wavelengths and applying a spectroscopic analysis to quantify the concentrations of endogenous chromophores such as oxy and deoxyhaemoglobin. These attributes make the technique well suited to studying a wide range of tissue abnormalities such as tumours and other pathologies characterised by changes in the structure and oxygenation status of the vasculature. A novel optical ultrasound imaging system has been developed and used to obtain 3D images of the superficial vascular anatomy of the mouse brain, skin and implanted tumours and demonstrate the spectroscopic capability of the technique.

$11: 20$

3aBBb2. The role of ultrasound and photoacoustic imaging in laser therapy of cancer. Stanislav Y. Emelianov (Applied Research Laboratories, The University of Texas, P.O. Box 8029, Austin, TX 78713-8029, USA, emelian@mail.utexas.edu)

Laser therapy is an alternative cancer treatment approach when traditional surgery cannot be used to remove small, poorly defined lesions embedded within vital organs. For example, in photothermal laser therapy, a localized temperature increase to cause tumor necrosis is achieved by using a continuous wave laser and molecular targeted and optically tuned photoabsorbers. However, for laser therapy to be successful, it is necessary to identify the presence of photoabsorbers in the tumor prior to therapy, to monitor the spatiotemporal temperature changes during therapy, and, finally, to identify thermal/mechanical damage and tumor destruction after therapy. To guide laser therapy, we developed a combined ultrasound/photoacoustic imaging system and bioconjugated nanoabsorbers. In our approach, ultrasound imaging is utilized to identify the anatomy of the tumor while photoacoustic imaging is used to ensure the presence of targeted nanoparticles before therapy. Both ultrasound and photoacoustic imaging techniques are then used to monitor temperature during therapy, ensuring tumor necrosis and protection of the surrounding healthy tissue. Finally, ultrasound and elasticity imaging is used to confirm thermal damage and cancer destruction. The results of our studies indicate that photoacoustic and ultrasound imaging can be used to effectively guide laser therapy.

\section{$11: 40$}

3aBBb3. Photoacoustic cancer imaging. Wiendelt Steenbergen (University of Twente /Institute for Biomedical Technology, PO Box 217, NL-7500 AE Enschede, Netherlands, w.steenbergen@utwente.nl)

Photoacoustics, or optoacoustics, involves ultrasound imaging based on ultrasound generated by absorption of light by tissue chromophores such as blood and melanin. Ultrasound is generated when pulsed or intensity modulated light is used which locally deposits a sufficient amount of energy in a sufficiently short time to cause an efficient generation of internal stress in the tissue. When this stress relaxes, an ultrasound wave is emitted which can be detected at the surface of the tissue. On the basis of these ultrasound waves, a threedimensional image the internal distribution of absorption can be constructed. We will discuss various technical implementations of the technique, and will illustrate various medical applications. A particularly important application field is oncology: in cancer, the presence of blood vessels is essential for the tumor to grow and spread. We will show results of photoacoustic imaging performed on small animals with implanted tumors. It appears, that measurement speed is a key issue in obtaining images with sufficient quality. Furthermore, we will show results obtained with our photoacoustic mammoscope on patients with breast cancer. 
3aBBb4. Theory and applications of frequency domain photoacoustic microscopy. Todd W. Murray (Boston University, 110 Cummington Street, Aerospace and Mechanical Engineering, Boston, MA 02215, USA, twmurray@bu.edu), Suraj Bramhavar (Boston University, 110 Cummington Street, Aerospace and Mechanical Engineering, Boston, MA 02215, USA, suraj10@bu.edu), Ashwin Sampathkumar (Boston University, 110 Cummington Street, Aerospace and Mechanical Engineering, Boston, MA 02215, USA, suraj10@bu.edu), Bruno Pouet (Bossa Nova Technologies, 606 Venice Boulevard, Venice, CA 90291, USA, Bpouet@BossaNovaTech .com)

In frequency domain photoacoustic microscopy, the pulsed laser source used for ultrasound excitation in conventional photoacoustic imaging is replaced by a low power, amplitude modulated laser source. The acoustic signals are detected using an interferometer or contact transducer, coupled to a RF lock-in amplifier or vector network analyzer. The detection bandwidth reduction afforded by this technique allows for a significant improvement in signal-to-noise ratio (SNR) over systems using pulsed laser excitation. In this paper, the method of frequency domain photoacoustic microscopy is reviewed and compared to the pulsed-laser based approach. Methods for processing the frequency domain data to extract the information of interest are discussed, along with the effects of measurement bandwidth and frequency resolution. A new technique to optically downshift acoustic signals detected using an optical interferometer to a fixed intermediate frequency is presented, which allows for the detection of high frequency (100's of MHz- GHz) acoustic signals using low frequency, low cost detection electronics. Several applications of frequency domain photoacoustic microscopy are presented including the inspection of thin films and environmental barrier coatings and the photothermal operation of nano-electro-mechanical systems. Finally, potential applications of this technique for the characterization of biological media are discussed.

\section{Contributed Papers}

12:20

3aBBb5. Photoacoustic microscopy for high-resolution imaging Wolfgang Bost (Fraunhofer IBMT, Ensheimer Strasse 48, 66386 Sankt Ingbert, Germany, wolfgang.bost@ibmt.fhg.de), Robert M. Lemor (Fraunhofer IBMT, Ensheimer Strasse 48, 66386 Sankt Ingbert, Germany, robert.lemor@ibmt.fhg.de)

Photoacoustics is a hybrid technology based on the photoacoustic effect that detects absorbed photons ultrasonically. Photoacoustic imaging combines ultrasonic resolution with high contrast due to light absorption depending on the physiology of the examined biological tissue. The resolution of conventional systems is not sufficient for in-vitro measurements of small tissue samples or individual cells. In this work, we present a high-resolution photoacoustic microscopy platform based on the SASAM acoustic microscope that allows high resolution imaging on living cells. The system based on an inverted optical microscope consists of a laser source for optical multi wavelength excitation (diode- and solid-state-laser) which emits nanosecond laser pulses with a wavelength in the near infrared spectrum (optical window). It allows the usage of different ultrasound transducers in the frequency range up to $300 \mathrm{MHz}$. In addition to the photoacoustic imaging mode, all common optical modalities are implemented. Pure acoustic imaging mode is used for reference imaging. The developed photoacoustic microscopy platform is a system for high-quality volumetric imaging in different scanning modes. 3D offline reconstruction is possible based on the presented 2D imaging. The system was characterized by in-vitro measurements with a lateral resolution better than $100 \mu \mathrm{m}$ in the confocal mode.

\section{2:40}

3aBBb6. Acoustic signals from gold nanoparticles irradiated with pulsed lasers. Constantin Ungureanu (Faculty of Science and Technology
(TNW), Biophysical Engineering Group (BPE), Twente University, 7500AE Enschede, Netherlands, c.ungureanu@tnw.utwente.nl), Tamara Van Weperen (University Twente, Building Meander, 7500 AE Enschede, Netherlands, t.m.vanweperen@student.utwente.nl), Jeroen Sijl (Physics of Fluids, University of Twente, P.O. Box 217, 7500 AE Enschede, Netherlands, j.sijl@utwente.nl), Raja Rayavarapu (Faculty of Science and Technology (TNW), Biophysical Engineering Group (BPE), Twente University, 7500AE Enschede, Netherlands, R.Rayavarapu@tnw.utwente.nl), Srirang Manohar (University of Twente, Biophysical Engineering Group (BPE), Faculty of Science and Engineering (TNW), P.O. Box 217, 7500 AE Enschede, Netherlands, s.manohar@tnw.utwente.nl), Michel Versluis (Physics of Fluids, University of Twente, P.O. Box 217, 7500 AE Enschede, Netherlands, m.versluis@utwente.nl), Ton G. Van Leeuwen (Univ. of Twente, P.O. Box 217, 7500 AE Enschede, Netherlands, a.g.j.m .vanleeuwen@tnw.utwente.nl)

Photoacoustic imaging is a new imaging technique, which enables imaging of living tissue with high resolution. The technique analyses ultrasound pulses generated when absorbing structures in tissue are irradiated with pulsed light. Due to the phenomenon of plasmon resonance gold nanoparticles possess high optical absorption coefficients which makes them potential contrast agents in photoacoustics. Further, the heat generated around these particles when irradiated with pulsed light can lead to nonlinear effects including bubble formation. The acoustic signals produced by gold nanoparticles in the linear thermoelastic and non-linear regimes are expected to have specific acoustic signatures. In this study, we investigate the acoustic signals generated by two sets of gold spheres having $25 \mathrm{~nm}$ and $60 \mathrm{~nm}$ diameter irradiated by laser pulses with increasing incident fluence rates. We identify and discuss the differences in acoustic signals belonging to the thermoelastic expansion regime and the non-linear regime. Finally, the experimental results are compared with different theoretical models available in literature.

\section{1:00-1:40 Lunch Break}




\section{Invited Papers}

\section{$1: 40$}

3aBBb7. Imaging tissue and wavefront control: Application to acousto-optics and photoacoustics. François Ramaz (Laboratoire Photons et Matière, ESPCI/CNRS, 10 rue Vauquelin, 75231 Paris Cedex 05, France, francois.ramaz@espci.fr), Emmanuel Bossy (Laboratoire Photons et Matière, ESPCI/CNRS, 10 rue Vauquelin, 75231 Paris Cedex 05, France, emmanuel .bossy@espci.fr), Vincent Loriette (Laboratoire Photons et Matière, ESPCI/CNRS, 10 rue Vauquelin, 75231 Paris Cedex05, France, vincent.loriette@espci.fr), Sylvain Gigan (Laboratoire Photons et Matière, ESPCI/CNRS, 10 rue Vauquelin, 75231 Paris Cedex 05, France, sylvain.gigan@espci.fr), Albert-Claude Boccara (Laboratoire Photons et Matière, ESPCI/CNRS, 10 rue Vauquelin, 75231 Paris Cedex 05, France, boccara@optique.espci.fr)

Although optical waves do not exhibit the same ability to be manipulated as e.g. electromagnetic waves up to a few GHz or acoustic waves, for which field emitters-detectors are available, various tools are nevertheless available in the optical domain which open the path to tricks which can be used in the context of sound-light detection. In the purely optical domain, one knows the impact of deformable mirrors led to adaptive optics which is now coupled to OCT for retinal examination. Real time holography leads to complex wavefront synthesis and are useful in acousto-optics (AO) to monitor very complex speckle field distributions. Phase conjugation which is in certain conditions equivalent to time reversal, starts to be used for wavefront retrieval after propagation in turbid media, one can think to use it for AO signal monitoring; moreover matrices of spatial light modulators allow to control light not only spatially but also in the frequency domain and open the field to new astonishing experiments. We will discuss a few applications and perspectives offered by these new devices to the field of biomedical imaging.

3aBBb8. Optoacoustics for biomedical sensing, monitoring, and imaging. Rinat O. Esenaliev (University of Texas Medical Branch, 301 University Blvd., Galveston, TX 77555-0456, USA, riesenal@utmb.edu)

Optoacoustics can provide diagnostic information with high optical contrast and ultrasound resolution. It is based on detection of broadband ultrasound induced by short optical pulses and utilizes absorption contrast in tissues. We proposed and developed sensing, monitoring, and imaging optoacoustic systems for in a variety of biomedical applications including pulsed laser tissue ablation, accurate measurement of tissue optical properties, tumor detection, monitoring of tissue hyperthermia, coagulation, freezing, and important blood parameters such as oxygenation and hemoglobin concentration. Recently, we built a novel, portable, multi-wavelength optoacoustic system which includes optical parametric oscillator operating in the range from 680 to $2400 \mathrm{~nm}$ and sensitive, broadband optoacoustic probes developed in our laboratory. We tested the system performance in animal and clinical studies. We monitored continuously and in real time cerebral blood oxygenation and total hemoglobin concentration. Direct probing of blood vessels with high contrast and resolution allowed for monitoring of these parameters with accuracy approaching that of the standard invasive techniques. Results on other important clinical applications of the optoacoustic technique will be presented as well.

\section{Contributed Papers}

2:20

3aBBb9. Photoacoustic tomography with a single detector in a reverberant cavity. Ben Cox (University College London, Gower Street, London, WC1E 6BT London, UK, bencox@mpb.ucl.ac.uk), Paul C. Beard (University College London, Gower Street, London, WC1E 6BT London, UK, pbeard@medphys.ucl.ac.uk)

In biomedical photoacoustic tomography (PAT), acoustic pulses, generated by the absorption of pulses of near-infrared light, are recorded on an array of ultrasound detectors, and the measured pressure time series are subsequently transformed into an image of the absorbed optical energy density within the tissue. For high resolution imaging, large-area detector arrays with a high density of sensitive, small elements are required. Such arrays can be expensive, so reverberant-field PAT is proposed as a means of obtaining PAT images using smaller arrays or even a single detector. By recording the reflections from a reverberant cavity surrounding the detector(s), in addition to the primary acoustic waves, sufficient information can be captured to allow a PAT image to be reconstructed, without the requirement for a large-area array. Image reconstruction, time reversal, non-uniqueness, and related ideas in quantum chaos will be discussed.

\section{2:40}

3aBBb10. Detection of elastic and optical contrasts in tissue mimicking phantoms based on the interaction of coherent laser light and transient displacements caused by the acoustic radiation force. Khalid Daoudi (Laboratoire Photons et Matière, ESPCI/CNRS, 10 rue Vauquelin, 75231 Paris Cedex 05, France, khalid.daoudi@espci.fr), Emmanuel Bossy (Laboratoire Photons et Matière, ESPCI/CNRS, 10 rue Vauquelin, 75231 Paris Cedex 05, France, emmanuel.bossy@espci.fr), Mickael Tanter (Laboratoire Ondes et Acoustique, ESPCI, Université Paris 7, CNRS, 10 rue Vauquelin, 75005 Paris, France, michael.tanter@espci
.fr),Mathias Fink(Laboratoire Ondes et Acoustique, ESPCI, Université Paris 7, CNRS, 10 rue Vauquelin, 75005 Paris, France, mathias.fink@espci .fr), Albert-Claude Boccara (Laboratoire Photons et Matière, ESPCI/CNRS, 10 rue Vauquelin, 75231 Paris Cedex 05, France, boccara@optique .espci.fr)

We recently demonstrated experimentally the possibility to optically detect transient perturbations generated by the acoustic radiation force, applied several centimetres deep in a tissue-mimicking phantom [Bossy et al., APL 90(17), 2007]. We proposed a detection scheme based on the transient decorrelation (millisecond time scale) of optical speckle patterns formed by coherent laser light that propagates through the phantom during the application of the radiation force. We demonstrated that this approach allows to detect optical contrasts embedded several centimetres deep in the phantom, based on the time-evolution of the correlation coefficient during the transient motion of the medium. In the present work, we demonstrate that this approach also allows the detection of shear mechanical contrast. Moreover, the shape of the observed decorrelation curve provides a way to discriminate between an optical contrast and a shear mechanical contrast. We are now currently investigating the feasibility of detecting with spatial resolution the propagation of a shear wave, based on its effect on localized acousto-optic interaction.

\section{3:00}

3aBBb11. Hybrid photoacoustic - ultrasound transmission parameter imaging in a miniature photoacoustic imager. Srirang Manohar (University of Twente, Biophysical Engineering Group (BPE), Faculty of Science and Engineering (TNW), P.O. Box 217, 7500 AE Enschede, Netherlands, s.manohar@tnw.utwente.nl), Rene G. 
Willemink(University of Twente, Signals and Systems Group (SaS), Faculty of Electrical Engineering, Mathematics and Computer Sciences (EWI), P.O. Box 217, 7500AE Enschede, Netherlands, G.H.Willemink@utwente .nl), Johan C. Van Hespen (University of Twente, Biophysical Engineering Group (BPE), Faculty of Science and Engineering (TNW), P.O. Box 217, 7500 AE Enschede, Netherlands, J.C.G.vanhespen@utwente.nl), Ton G. Van Leeuwen (Univ. of Twente, P.O. Box 217, 7500 AE Enschede, Netherlands, a.g.j.m.vanleeuwen@tnw.utwente.nl)

Photoacoustic imaging is based on detecting laser pulse induced ultrasound transients from absorbing structures in tissue. The technique combines the advantages of a high optical absorption exhibited by tumors for example with the high resolution possible with ultrasound. A conventional photoacoustic imager operating in a CT geometry comprises a light source, an ultrasound detector array and a coupling medium usually water between the subject under investigation and detector. The imaging protocol usually consists of obtaining projections at angles around the subject. Recently we reported a method of measuring the local speed-of-sound variations by the addition of an absorber to the conventional imager, arranged between the light source and subject. This generates an ultrasound signal which interacts with the subject which can then be detected at the far-end by the detector. This allows the measurement of the integrated speed-of-sound (SOS) at projections around the subject leading to the formation of SOS images. In this article we discuss further analysis of the ultrasound obtained at projections to obtain images of the acoustic attenuation as well. In addition conventional photoacoustic images of the subject are also formed. The principle is demonstrated on well-characterized phantoms and we discuss the potential applications of this technique.

\title{
Session 3aEAa
}

\section{Engineering Acoustics and Signal Processing in Acoustics: Transducers and Signal Processing for the Oil and Gas Industry I}

\author{
Fernando Garcìa-Osuna, Cochair \\ Schlumberger, Sugar Land, TX, USA \\ Benoit Froelich, Cochair \\ Etudes et Productions Schlumberger, 1, rue Becquerel, BP 202, Clamart, 92142, France
}

\section{Contributed Paper}

\begin{abstract}
8:00
3aEAa1. Sonic gas analyzer for hydrogen and methane. Steven Garrett (Penn State, Applied Research Laboratory, PO Box 30, State College, PA 16804, USA, sxg185@psu.edu)

In the late-1800s, Germans used a "methane whistle", known as a Schlagwetterpfeife, to determine the presence of hydrogen or methane in the air that was circulated in mines. This paper describes a modern version of that concept that should be suitable for safety-related applications in mines, sewers, garages that shelter hydrogen or methane-powered vehicles, etc. A compact, self-resonant standing wave tube, $2 \mathrm{~cm}$ in diameter and $3 \mathrm{~cm}$ long, is capped at both ends by two small electrodynamic speakers. The cylindri-
\end{abstract}

cal copper resonator has two slits at the mid-plane to allow a $25 \mathrm{~mm}$ fan to continuously sample the air which is brought to the resonator's temperature after being drawn along the outside of the resonator body. Variation in the resonator's temperature is compensated electronically by an integratedcircuit temperature sensor that produces one microampere per Kelvin. The sensor can measure the concentration of hydrogen to an accuracy of better than $\pm 30 \mathrm{ppm}$ or methane to $\pm 0.02 \%$ in air using phase-locked loop frequency tracking electronics and a voltage-controlled oscillator. Concentration data will be presented for several different gas mixtures and will be compared to concentrations determined by other techniques (e.g., thermal conductivity gauges).

\section{Invited Papers}

3aEAa2. Cylindrical ultrasonic array for borehole applications. Kenneth Liang (Schlumberger-Doll Research, One Hampshire Street, Cambridge, MA 01775, USA, kliang@boston.oilfield.slb.com), Gérard Fleury (Imasonic, 15 rue Alain Savary, 25000 Besançon, France, Gerard.Fleury@imasonic.com), Benoit Froelich (Etudes et Productions Schlumberger, 1, rue Becquerel, BP 202, 92142 Clamart, France, bfroelich@ @ clamart.oilfield.slb.com), Jean-Luc Guey (Imasonic, 15 rue Alain Savary, 25000 Besançon, France, jean-luc.guey@imasonic.com), Pascal Schoeb (Statice Etudes \& Developpement, 9, rue Thomas Edison, 25000 Besançon, France, p.schoeb@statice.com)

A cylindrical ultrasonic array has been developed for operating environments that can reach extremes of $175 \mathrm{C}$ and 20,000 psi. The array is a key component of the PharUSIT* (Phased Array Ultrasonic Transducer for Inspection of Tubing), a research demonstrator developed for borehole applications. The full array consists of 800 elements (10 rings of 80 elements each) and can provide a whole range of beam-forming versatilities and capabilities in 3-D, such as variable focusing, beam steering, electronic scanning, etc, all accomplished without mechanical movements. Special piezo-composites have been developed for the transduction layer, and new polymeric composites have been formulated for the backing material. The center frequency was chosen to be about $500 \mathrm{kHz}$ to accommodate attenuation of the propagation media. A novel technique utilizing custom flexible circuit provides electrical connections between the array and the front-end electronics. Special fabrication processes have been developed to construct the array in a cylindrical geometry. A customized testing protocol has been implemented to demonstrate the survivability of the array technology and to evaluate 
the performance characteristics of individual elements under high-temperature/high-pressure conditions. The unique experimental setup will be presented. Data from electroacoustic measurements such as electrical impedance, bandwidth, sensitivity, angular directivity, and inter-element cross-talks will be shown. *EC GROW project PharUSIT (GIRD-CT-2002-000689)

\section{8:40}

3aEAa3. Acoustic modeling for designing sonic logging tools. Toshihiro Kinoshita (Schlumberger K.K., 2-2-1 Fuchinobe, 2290006 Sagamihara, Japan, tkinoshi@fuchinobe.oilfield.slb.com), Hiroshi Hori (Schlumberger K.K., 2-2-1 Fuchinobe, 229-0006 Sagamihara, Japan, hori@fuchinobe.oilfield.slb.com)

Formation acoustic properties such as velocity and attenuation provide useful information regarding rock type and formation fluid. Sonic logging is one of the principal measurements to evaluate the presence of hydrocarbons in the well and to enable an efficient and safe production of oil. Unfortunately the sonic measurement is affected by the tool itself. Suppressing or attenuating the tool wave, which propagates along the logging tool and is received by the sensors, is directly related to the accuracy of the answer products. In order to improve the measurement quality and to increase the efficiency of logging operations, the structure of sonic logging tools must be well understood. A sonic tool with known effects is decidedly more complex and acoustic modeling incorporating the tool structure details is becoming essential to better sonic tool design. The methodology of numerical modeling used to predict the response of a sonic tool is to be presented.

\section{9:00}

3aEAa4. Extracting dispersion curves of acoustic data with continuous wavelet transform. Henri-Pierre Valero (Schlumberger Doll Research, 1 Hampshire Street - MD- B 213, Cambridge, MA 02139, USA, hvalero@boston.oilfield.slb.com), Sandip Bose (Schlumberger Doll Research, 1 Hampshire Street - MD- B 213, Cambridge, MA 02139, USA, bose1@ boston.oilfield.slb.com), Suchin Aeron (Boston University College of Engineering, One Sherborn Street, Boston, MA 02215, USA, shuchin@bu.edu)

Group and phase velocity (i.e. dispersion) of acoustic data carry important information about the acoustic state of the rock and are important tools for interpretation and for quantitative inversion. In this paper we propose an approach to automatically extract the velocity dispersion (group and phase) of acoustic waves received by an array of receivers without the use of any physical model, user inputs or supervision. The first step consists in applying the continuous wavelet transform to the waveforms recorded by each receiver. Then, the peak on the modulus map is used to determine the time locus of the energy of each mode as a function of frequency. At each frequency, a method of data association is used to find the corresponding loci for all receivers. A straight line is fit to these loci and its slope is used as an estimate for the group velocity at that frequency. Moreover the rate of change of the phase of the complex wavelet coefficients at the locations corresponding to this best-fit straight line can be used to compute an estimate of the phase slowness at the same frequency. Repeating this for all frequencies and all separated modes of interest yields the desired dispersion curves for these modes.

\section{Contributed Paper}

9:20

3aEAa5. Small scale adaptation of the seismic full waveform inversion method - Application to civil engineering applications. Francois Bretaudeau (LCPC, Centre de Nantes BP4129, 44341 Bouguenais, France, francois.bretaudeau@1cpc.fr), Donatienne Leparoux (LCPC, Centre de Nantes BP4129, 44341 Bouguenais, France, donatienne.leparoux @lcpc.fr), Odile Abraham (LCPC, Centre de Nantes BP4129, 44341 Bouguenais, France, odile.abraham@1cpc.fr)

Full Waveform Inversion (FWI) is a very general multi-parameters quantitative imaging method originally developed to obtain high resolution images of velocities and attenuation in the natural underground media. FWI promises interesting performances for civil engineering applications like cavity detection. Performances of the FWI method in seismic exploration are difficult to quantify because in real experimentations, the properties of the media are not known. Furthermore, characteristics of the source and coupling of receivers are not controlled. In order to quantify the performances of FWI and its adaptability to near subsurface applications, small scale physicals models are realized and a non-contact laser ultrasonic laboratory is dedicated to simulate seismic reflection measurement configurations at ultrasonic scale. Seismograms well reproduce real scale data in terms of waveforms and repartition of energy. A FWI algorithm developed by Geosciences Azur and applied in geological investigation is used. The direct part of the algorithm uses a frequency domain finite difference modelisation. The inverted model is obtained by using a Gauss-Newton method of optimization. Results of inversion of multi-receivers and multi-sources data obtained from simple physical models are presented.

\section{Invited Paper}

\section{9:40}

3aEAa6. Simulating Sonic Scanner responses in an interactive Web-based High Performance Computing environment. Valery Polyakov (Schlumberger Doll Research, 1 Hampshire Street - MD- B 213, Cambridge, MA 02139, USA, valp@computer .org), Henri-Pierre Valero (Schlumberger Doll Research, 1 Hampshire Street - MD- B 213, Cambridge, MA 02139, USA, hvalero@boston.oilfield.slb.com), Dzevat Omeragic (Schlumberger Doll Research, 1 Hampshire Street - MD- B 213, Cambridge, MA 02139, USA, omeragic@boston.oilfield.slb.com), Raymond L. Kocian (Schlumberger Doll Research, 1 Hampshire Street - MD- B 213, Cambridge, MA 02139, USA, rkocian@boston.oilfield.slb.com), Tarek M. Habashy (Schlumberger Doll Research, 1 Hampshire Street - MD- B 213, Cambridge, MA 02139, USA, thabashy@boston.oilfield.slb.com), Jahir Pabon (Schlumberger Doll Research, 1 Hampshire Street - MD- B 213, Cambridge, MA 02139, USA, jahir@boston.oilfield.slb.com)

Sonic Scanner logging tool collects a wealth of data about the geological formation. The drawback of this is that we are now discovering new features on the acoustic logs that have never been observed before; only rigorous modeling can help properly interpret the data. Invariably, it is difficult to learn quickly how to run a modeling code, set the parameters properly, and be able to detect possible errors in the input. In addition, complex modeling requires high power computing resources, which are not always easily accessible the user. To address these problems we developed a multi-tier Web-based log modeling environment where the Sonic Scanner simulator is 
easily accessible from the common Web browser. The user builds the model in an intuitive AJAX-like interface and submits the simulation to a remote High Performance Cluster. The computed waveforms are played back in the browser using Scalable Vector Graphics in a variety of customizable displays. The Web application is easily available to any user with an Internet access. In addition, a programmatically accessible Web service is available to application developers who desire to build their own interpretation applications using the Sonic Scanner simulator engine.

\title{
Session 3aEAb
}

\section{Engineering Acoustics: Ultrasonic Acoustic MEMS I}

\author{
F. Levent Degertekin, Cochair \\ Georgia Institute of Technology, G. W. Woodruff School of Mechanical Engineering, 801 Ferst Dr. NW, Atlanta, GA \\ 30332-0405, USA \\ Dominique Certon, Cochair \\ Lab. LUSSI Inserm U930 CNRS FRE 2448 Univ. François Rabelais, 10, Boulevard Tonnellé, Tours, 37032, France
}

\section{Contributed Paper}

\section{0:20}

3aEAb1. Micro-viscometer using acoustic wave absorption in micro-chamber and channel. Sungjoon Choi (Postech, San 31, HyojaDong, Namgu, 790-784 Pohang, Republic of Korea, csjangel@postech .ac.kr), Wonkyu Moon (Postech, San 31, Hyoja-Dong, Namgu, 790-784 Pohang, Republic of Korea, wkmoon@postech.ac.kr), Geunbae Lim (Postech, San 31, Hyoja-Dong, Namgu, 790-784 Pohang, Republic of Korea, limmems@postech.ac.kr)

A micro-viscometer is designed for real-time measurement on the viscosity change of biomaterial including DNA or RNA, etc. It is composed of two chambers and several micro-channels connecting between them. Each chamber has a PZT unimorph membrane for generating and sensing the sound waves. Namely, one is regarded as an actuator, the other as a sensor. Micro-channels, which have the large attenuation of acoustic waves caused by wall effect, are applied to the micro-viscometer for increasing viscosity effect. When the actuator is driven at a resonance frequency under the condition of continuous flowing of liquid through an inlet/outlet hole, the sensor can detect the amplitude differences caused by viscosity change. Therefore, it is possible to measure the viscosity change in real time by detecting the signal change of sensor. The micro-viscometer can be also applied to the integration kit such as LOC (Lab-On a Chip) to detect whether DNA is amplified by micro-PCR (Polymerase Chain Reaction) and it will give a first opportunity for experimentally evaluating the viscosity effect on the propagation of acoustic waves in micro-channel. [Research supported by MRCnd of DAPA]

\section{Invited Paper}

\section{0:40}

3aEAb2. Use of rapid protyping techniques in transducer manufacture. David Hutchins (University of Warwick, School of Engineering, CV4 7AL Coventry, UK, D.A.Hutchins@warwick.ac.uk), Duncan R. Billson (University of Warwick, School of Engineering, CV4 7AL Coventry, UK, drb@eng.warwick.ac.uk), Robert J. Bradley (University of Warwick, School of Engineering, CV4 7AL Coventry, UK, R.J.Bradley@warwick.ac.uk), Kwok S. Ho (University of Warwick, School of Engineering, CV4 7AL Coventry, UK, K.S.Ho@warwick.ac.uk)

This paper will describe the use of a rapid protyping technique, known as micro-sterolithography, for the manufacture of ultrasonic transducers. There are two main types to be described. The first is a MEMS-type capacitive transducer, this being an example of a capacitive micromachined ultrasonic transducer (CMUT). This can be made with all the main structural elements fabricated from polymers, so that no silicon processing is required. Experiments have investigated the properties of these devices, operating in air, water, and in contact with a metal surface. The technique has also been used to make electromagnetic acoustic transducers (EMATs), by forming the main structure from polymers and including a metal coil. These are interesting developments, which may have application to the fabrication of many types of acoustic MEMS devices. 


\section{Contributed Papers}

11:00

3aEAb3. Design, fabrication and characterisation of piezoelectric micromachined ultrasonic transducers. Anne-Christine HladkyHennion (IEMN, UMR CNRS 8520, avenue Poincaré, BP 60069, 59652 Villeneuve d'Ascq, France, Anne-Christine.Hladky@isen.fr), Didace Ekeom (Microsonics, 39 rue des Granges Galand, 37550 Saint Avertin, France, didace.ekeom@microsonics.fr), Fabrice Duval (IEMN, UMR CNRS 8520, avenue Poincaré, BP 60069, 59652 Villeneuve d'Ascq, France, fa_duval@yahoo.fr), Eric Cattan (IEMN, UMR CNRS 8520, avenue Poincaré, BP 60069, 59652 Villeneuve d'Ascq, France, Eric.Cattan@univvalenciennes.fr)

Micro-machined ultrasonic transducers (MUT) are investigated for phased arrays in high frequency acoustic imaging to overcome resolution and frequency limits of bulk PZT transducers currently used. The advantage of structures on silicon substrate is that transducers could be integrated with the electronics of the system. From a design point of view, finite element (FEM) codes are commonly used to model MUT, but to take into account the fluid surrounding the device radiation, scattering and the inter-mutual acoustic influence of the MUT arrays cells - the boundary element method (BEM) coupled with the finite element method is advantageously used. This paper describes the design, the fabrication and the characterisation of bending mode circular piezoelectric membranes, with operating frequency around $10 \mathrm{MHz}$. The devices are based on a PZT (lead zirconate titanate) film $1 \mu \mathrm{m}$ thick supported by a membrane of polysilicon, $2 \mu \mathrm{m}$ thick and $\mathrm{SiO}$, $500 \mathrm{~nm}$ thick. The PZT thin film was deposited following a sol-gel route. Fully-supported membranes as well as suspended cells are considered with a view to increase the resonant frequency and decrease the cross coupling between cells.

\section{1:20}

3aEAb4. Capacitive micromachined ultrasonic transducers for generation of highly directional sound with a parametric array. Ira $\mathrm{O}$. Wygant (E. L. Ginzton Laboratory, Stanford University, Stanford, CA
94305, USA, iwygant@stanford.edu),Mario Kupnik(E. L. Ginzton Laboratory, Stanford University, Stanford, CA 94305, USA, kupnik@stanford .edu), Jeffry C. Windsor (Applied Research Laboratories, The University of Texas, P.O. Box 8029, Austin, TX 78713-8029, USA, jwindsor@arlut.utexas.edu), Wayne M. Wright (Applied Research Laboratories, The University of Texas, P.O. Box 8029, Austin, TX 78713-8029, USA, wwright@arlut.utexas.edu), Mark S. Wochner (Applied Research Laboratories, The University of Texas, P.O. Box 8029, Austin, TX 78713 8029, USA, mwochner@mail.utexas.edu), Goksen G. Yaralioglu (E. L. Ginzton Laboratory, Stanford University, Stanford, CA 94305, USA, goksenin@gmail.com), Mark F. Hamilton (Applied Research Laboratories, The University of Texas, P.O. Box 8029, Austin, TX 78713-8029, USA, hamilton@mail.utexas.edu), Butrus (pierre) T. Khuri-Yakub (E. L. Ginzton Laboratory, Stanford University, Stanford, CA 94305, USA, pierreky @ stanford.edu)

Capacitive micromachined ultrasonic transducers (CMUTs) with vacuum-sealed cavities have been fabricated and used to project directional sound using parametric arrays. The wafer bonding approach used to fabricate the CMUTs provides good control over device dimensions, a singlecrystal silicon membrane that has predictable mechanical properties, and the capability to fabricate CMUTs with large-diameter membranes and deep cavities. The fabricated CMUTs are about $8 \mathrm{~cm}$ in diameter and comprise 284 circular membranes, each $4 \mathrm{~mm}$ in diameter. Testing of CMUTs with $40-\mu \mathrm{m}$ and $60-\mu \mathrm{m}$-thick membranes shows they have center frequencies of $46 \mathrm{kHz}$ and $55 \mathrm{kHz}$ and $3-\mathrm{dB}$ bandwidths of $1.9 \mathrm{kHz}$ and $5.3 \mathrm{kHz}$ respectively. With application of DC bias voltages of $380 \mathrm{~V}$ and $350 \mathrm{~V}$ and an $\mathrm{AC}$ excitation of $200 \mathrm{~V}$ peak-to-peak, the devices generate effective source levels of $139 \mathrm{~dB}$ and $131 \mathrm{~dB}$ (re $20 \mu \mathrm{Pa}$ ), respectively. We used the CMUT design with a $60-\mu$ m-thick membrane to produce $5 \mathrm{kHz}$ sound at $3 \mathrm{~m}$ with a $6-\mathrm{dB}$ beamwidth of $8.7 \mathrm{deg}$ and a sound pressure level of $58 \mathrm{~dB}$. The results demonstrate that large-area CMUTs, which produce high pressure ultrasound, can be fabricated for transmitting directional sound with parametric arrays. [Work supported by DARPA.]

\section{Invited Papers}

\section{$11: 40$}

3aEAb5. A finite element tool for the analysis and the design of capacitive micromachined ultrasonic transducer (cMUT) arrays for medical imaging. Alessandro Caronti (Dept. of Electronic Engineering, University Roma Tre, via della Vasca Navale, 84,00146 Roma, Italy, caronti@uniroma3.it), Giosue' Caliano (Dept. of Electronic Engineering, University Roma Tre, via della Vasca Navale, 84, 00146 Roma, Italy, giosue.caliano@uniroma3.it), Philipp Gatta (Dept. of Electronic Engineering, University Roma Tre, via della Vasca Navale, 84, 00146 Roma, Italy, gatta@uniroma3.it), Cristina Longo (Dept. of Electronic Engineering, University Roma Tre, via della Vasca Navale, 84, 00146 Roma, Italy, clongo@uniroma3.it), Alessandro Savoia (Dept. of Electronic Engineering, University Roma Tre, via della Vasca Navale, 84, 00146 Roma, Italy, savoia@uniroma3.it), Massimo Pappalardo (Dept. of Electronic Engineering, University Roma Tre, via della Vasca Navale, 84, 00146 Roma, Italy, pappalar@uniroma3.it)

The fabrication technology of capacitive micromachined ultrasonic transducers (cMUTs) is now mature enough to exploit the potential of this new generation of electro-acoustic transducers in the field of diagnostic medical imaging and non-destructive evaluation. Converting a demonstrator into a commercial product often requires many design and process refinements, and sometimes significant modifications to the fabrication process. Because each design-fabrication-and-test cycle is time-consuming and costly, the development of a modeling tool for the computer simulation of cMUTs is an essential aid to reduce the time to market and costs. Much effort has been put by researchers to develop more and more accurate and smart simulation tools, that advanced from the standard equivalent circuit modeling with lumped parameters to more powerful techniques based on the finite element method (FEM). This paper aims to give an overview of the most ordinary modeling approaches used for the simulation of cMUTs. It is shown that the pulse-echo operation of cMUT arrays for medical imaging applications can be analysed by a set of FEM models developed using a commercial software. The important aspects of the cMUT operation, including electro-mechanical-acoustical coupling and nonlinear effects in transmission, are taken into account. 
3aEAb6. Wafer bonded, local oxidation defined, capacitor micromachined ultrasonic transducers for medical applications Butrus (pierre) T. Khuri-Yakub (E. L. Ginzton Laboratory, Stanford University, Stanford, CA 94305, USA, pierreky@stanford.edu) , Kwan Kyu Park (E. L. Ginzton Laboratory, Stanford University, Stanford, CA 94305, USA, kwankyup@ stanford.edu), Hyunjoo Jenny Lee (E. L. Ginzton Laboratory, Stanford University, Stanford, CA 94305, USA, hyunjoo@stanford.edu), Mario Kupnik (E. L. Ginzton Laboratory, Stanford University, Stanford, CA 94305, USA, kupnik@ stanford.edu), Omer Oralkan (E. L. Ginzton Laboratory, Stanford University, Stanford, CA 94305, USA, ooralkan@stanford.edu)

We present a technology for making capacitive micromachined ultrasonic transducers (CMUTs) that is based on direct wafer bonding of silicon on insulator wafer (SOI), and where the vertical dimensions and geometry of the CMUT are defined by local oxidation (LOCOS). The advantages of this approach for making CMUTs is the excellent control, no only over the membrane properties, but also over the shape and dimensions of the cavity. We demonstrated the ability to make devices with cavities in the 10 s of nanometer range which enables devices up to a frequency of $100 \mathrm{MHz}$. The devices exhibit reduced charging where we measure a voltage shift of 0.03 $\mathrm{V}$ for devices cycled in and out of collapse at $50 \mathrm{~V}$. The devices also have reduced parasitic capacitance as thicker oxides are grown in the regions of the SOI bond to the membrane. These are typically the regions that contribute most to the parasitic capacitance. Finally, because of the reduced charging, it is possible to operate these devices in and out of collapse which results in higher output pressure than devices operating in conventional mode. Hence, these CMUTs are much more predictable and reproducible in their operation and performance.

\section{Contributed Paper}

12:20

3aEAb7. Acoustic microelectromechanical viscometer. Arthur Ballato (US Army Communications-Electronics R\&D Center, AMSRDCER-CS, Fort Monmouth, NJ 07703-5201, USA, Arthur.Ballato@us.army .mil)

Current dynamic techniques for measuring fluid shear viscosities using quartz, or other piezoelectrics, rely on the resonator surface being exposed to a measurand bath whose extent greatly exceeds the penetration depth of the evanescent shear mode excited by the active element. This configuration allows the effect of the loading parameters to be expressed concisely. Perturbation of the electrical equivalent circuit parameters of the resonator by the fluid loading permits calculation of the mass density - shear viscosity product. In this paper, we explore the interesting, albeit more complicated situation where the separation between the resonator and a confining wall is less than the penetration depth of the fluid occupying the intervening region. It turns out that the resonator perturbation in this case is a sensitive function of the separation. This important fact permits extreme miniaturization, since for gases between $200 \mathrm{~K}$ and $400 \mathrm{~K}$, pressures between 0.01 to $100 \mathrm{~atm}$, and frequencies between $10 \mathrm{MHz}$ and $1 \mathrm{GHz}$, the penetration depth varies from micrometers to nanometers. Variations in the spacing is effected by using a second, nonresonant piezoelectric as the wall. Micro-electro-mechanics (MEMS) versions of viscometers and associated types of fluid sensors are thereby enabled.

\section{2:40-2:00 Lunch Break}

\section{Contributed Papers}

\section{2:00}

3aEAb8. Thin Film Piezoelectric MEMs Devices. Mareike Dr. Klee (Philips Research Laboratories, High Tech Campus 4, 5656 AA Eindhoven, Netherlands, mareike.klee@philips.com), Henk Boots (Philips Research Laboratories, High Tech Campus 4, 5656 AA Eindhoven, Netherlands, henk.boots@philips.com), Biju Kumar (Philips Research Laboratories, High Tech Campus 4, 5656 AA Eindhoven, Netherlands, biju.kumar @ philips.com), Wilco Keur (Philips Research Laboratories, High Tech Campus 4, 5656 AA Eindhoven, Netherlands, wilco.keur@philips .com), Marco De Wild (Philips Research Laboratories, High Tech Campus 4, 5656 AA Eindhoven, Netherlands, marco.de.wild@philips.com), Peter Dirksen (Philips Research Laboratories, High Tech Campus 4, 5656 AA Eindhoven, Netherlands, peter.dirksen@philips.com), Klaus Reimann (NXP Research, HighTech Campus 4, 5656 AA Eindhoven, Netherlands, klaus.reimann@nxp.com), Olaf Wunnicke (NXP Research, HighTech Campus 4, 5656 AA Eindhoven, Netherlands, olaf.wunnicke@nxp .com), Christel Renders (NXP Research, HighTech Campus 4, 5656 AA Eindhoven, Netherlands, christel.renders@nxp.com), Harry Van Esch (Philips Research Laboratories, High Tech Campus 4, 5656 AA Eindhoven, Netherlands, harry.van.esch@nxp.com), Chris Van Heesch (Philips Research Laboratories, High Tech Campus 4, 5656 AA Eindhoven, Netherlands, chris.van.Heesch@philips.com), Georg Schmitz (Medical Engineering, University Bochum, 44680 Bochum, Germany, georg.schmitz@rub .de), Martin Mienkina (Medical Engineering, University Bochum, 44680 Bochum, Germany, martin.mienkina@rub.de), Michal Mleczko (Medical Engineering, University Bochum, 44680 Bochum, Germany, michal .mleczko@rub.de)

Thin film piezoelectric devices, processed in Si-related processes, are attractive for ultrasound transducers and piezoelectric switches. Thin film ultrasound transducer enable large bandwidth $(>100 \%)$, high frequency operation. In piezoelectric micromachined ultrasonic transducers (PMUTs) the ultrasonic waves are generated by flexural motion of the membrane, which is coupled to strain in the piezoelectric film. We have investigated the piezoelectric properties of thin films for ultrasound transducers and piezoelectric switches. Thin film piezoelectric ultrasound transducers as well as piezoelectric switches have been designed, processed and characterized. Thin film piezoelectric devices with excellent quality and reliability have been realized.

\section{2:20}

3aEAb9. Increasing power-efficiency of a micro-machined pump transducer for a parametric transmitting array in air. Haksue Lee (Postech, San 31, Hyoja-Dong, Namgu, 790-784 Pohang, Republic of Korea, jolary@postech.ac.kr), Wonkyu Moon (Postech, San 31, HyojaDong, Namgu, 790-784 Pohang, Republic of Korea, wkmoon@postech.ac .kr)

Parametric array applications in air, such as high directional loudspeaker system, have been studied with a large radiator (generally $\mathrm{D} \geqslant 300 \mathrm{~mm}$ ) to generate high intensive sound beams required for nonlinear interactions. However, conventional transducer requires high electrical power because its electro-acoustic efficiency is too low due to an acoustic impedance mismatch. For mobile applications, a high efficient transducer is required. As an alternative pump transducer, a feasibility of a micro-machined ultrasonic transducer is studied. The efficiency can be improved by applying a thin-film membrane, overcoming the impedance mismatch. The efficiency of micro-machined transducer is simulated with a reduced dynamic model and compared with that of a conventional transducer. Two types of unimorph piezoelectric micro-machined ultrasonic transducers are designed and fabricated as small pump transducers for a ranging sensor and a loudspeaker. The 
electro-acoustic efficiency and dynamic characteristics related with primary wave generation are mainly examined in this work. The radiation characteristics, directional beam patterns and axial pressure distributions, of primary and secondary waves are also measured and compared with theoretical results. Throughout this work, feasibility of the proposed pump transducer is shown, but further work is also required for practical applications. [Research supported by MRCnd and ADD-UD070054AD of DAPA]

\section{2:40}

3aEAb10. Ultraviolet detector based on a surface acoustic wave oscillator system with ZnO-nanostructure sensing material. Tsung-Tsong Wu (Institute of Applied Mechanics, National Taiwan University, No. 1, Sec. 4, Roosevelt Road, 106 Taipei, Taiwan, wutt@ndt.iam.ntu.edu.tw), Wei-Shan Wang (Institute of Applied Mechanics, National Taiwan University, No. 1, Sec. 4, Roosevelt Road, 106 Taipei, Taiwan, dainty@ndt.iam.ntu.edu.tw), Tai-Hsu Chou (Institute of Applied Mechanics, National Taiwan University, No. 1, Sec. 4, Roosevelt Road, 106
Taipei, Taiwan, deshi@ndt.iam.ntu.edu.tw),Yung-Yu Chen(Department of Mechanical Engineering, Tatung University, No. 40, Sec. 3, Chungshan N. Rd., 104 Taipei, Taiwan, yychen@ttu.edu.tw)

Recently, ultraviolet (UV) detectors based on surface acoustic wave (SAW) devices utilizing the acoustoelectric effect have been demonstrated However, most of presented cases were based on semiconductor sensing films. Sensors with nanostructure sensing material not only possess high sensitivity but also accelerate response and recovery time due to their high surface-to-volume ratio and fast charge diffusion rate. In this paper, $\mathrm{ZnO}$ nanostructure and a dual delay line SAW oscillator system are combined to develop a high precision UV detector. The UV detector is made of $\mathrm{ZnO}$ nanorod on a $1280 \mathrm{YX}-\mathrm{LiNbO} 3$ based SAW device. The operating frequency is around $145 \mathrm{MHz}$. The system is illuminated by a UV source consists of a Xe lamp and a monochromator, resulting in frequency shifts. The frequency shifts related to different light wavelengths and power are measured and discussed. Results show the $\mathrm{ZnO}$-nanostructure based SAW oscillator is a promising candidate for a real-time and high precision UV detector.

\section{Invited Paper}

3aEAb11. Silicon Micromachined Energy Concentrating Acoustic Transducers for Biomedical Applications. Amit Lal (Cornell University, 118 Phillips Hall, School of Electrical and Computer Engineering, Ithaca, NY 14853, USA, lal@ece.cornell.edu), Abhishek Ramkumar (Cornell University, 118 Phillips Hall, School of Electrical and Computer Engineering, Ithaca, NY 14853, USA, ar339@cornell.edu), Muhamet Araz (Cornell University, 118 Phillips Hall, School of Electrical and Computer Engineering, Ithaca, NY 14853, USA, mka22@cornell.edu)

Silicon's high maximum ultrasonic particle velocity, high thermal conductivity, and low internal loss, make it an ideal material for for high-intensity ultrasonic actuators. We have designed and microfabricated silicon ultrasonic horns, which when actuated at their $\lambda / 2$ longitudinal resonance (using ceramic PZT transducers) concentrate ultrasonic energy and magnify displacements. By incorporating microprobes at the high energy sites we have successfully demonstrated reduction of penetration force on biological tissues. We have also reported cardiac action potential measurement from multiple sites simultaneously within the heart wall by incorporating platinum electrodes on the microprobes. We have reported on mechanical and spatial characterization of seminiferous tubules in the testis in order to extract sperm for artificial insemination. We are currently exploring silicon ultrasonic horns in detecting testicular and prostate cancer in the early stages. In addition, silicon ultrasonic horns can be used for low power ultrasonic pumping and also atomization. By using bulk micromachining techniques an enclosed conduit is opened along the horn. Due to the highly amplified acoustic field at the tip, fluid enclosed in the channel can be atomized from the tip. Reversibly when the tip is immersed in fluid, fluid can also be pumped into the channel due to changing boundary conditions.

\section{Contributed Paper}

3:20

3aEAb12. Reproducibity and life duration assessment of cMUT transducers. Cyril Meynier (Vermon SA, 180 rue Général Renault, 37000 Tours, France, c.meynier@vermon.com), Dominique Certon (Lab. LUSSI Inserm U930 CNRS FRE 2448 Univ. François Rabelais, 10, Boulevard Tonnellé, 37032 Tours, France, dominique.certon@univ-tours.fr), Franck Teston (Lab. LUSSI Inserm U930 CNRS FRE 2448 Univ. François Rabelais, 10, Boulevard Tonnellé, 37032 Tours, France, franck.teston @ univ-tours.fr), Guillaume Ferin (Vermon SA, 180 rue Général Renault, 37000 Tours, France, g.ferin@vermon.com)

Cmut-based transducers are often expected to be superior in terms of reproducibility to piezoelectric transducers due to the use of microelectronic technology instead of more traditional machining techniques. During the conventional (sacrificial-layer based) production process of cmut transduc- ers, various process steps can display inhomogenities and uncertainty in flow rate, temperature profile, stochiometry, or other aspects, resulting in variations in layers thicknesses, gap height, and material properties, each having an impact on transducer performances. In this study, we choose to use impedance measurement technique as evaluation tool for the stability of the properties of cmut transducers. A large number of cMUT transducers with the same design, were characterized, allowing to compare their properties from wafer to wafer, and according to their position of origin in the wafer. The resistance of cmut transducers to temperature cycling was also investigated, using impedance and optical measurements control before and after a 3-days $+60 /-20^{\circ} \mathrm{c}$ test. The reliability was tested under a longduration electroacoustic measurement, where a cMUT transducer was submitted to periodic large amplitude excitation, and its pulse-echo response recorded for several hours 


\title{
Session 3aMUa
}

\section{Musical Acoustics and Speech Communication: Interdisciplinary Research on the Science of Singing: A Tribute to Johan Sundberg}

\author{
Annabel J. Cohen, Cochair \\ University of Prince Edward Island, 550 University Ave, Charlottetown, PE C1A 4P3, Canada \\ Sten Ternstrom, Cochair \\ KTH, Department of Speech, Music and Hearing, Lindstedtsvägen 24, S-100 44 Stockholm, Sweden
}

Invited Papers

8:00

3aMUa1. Listener-oriented motor control: Comparing evidence from speech and singing. Björn Lindblom (Stockholm University, Universitetsvägen 10C, SE10691 Stockholm, Sweden, lindblom@ling.su.se)

Singing and speech make different use of the vocal tract but work in similar ways with respect to how sound production is controlled. A principle that they share is: output-oriented control. This mode of organization implies that the task of the motor system is defined in terms of the desired properties of the output sound, rather than in terms of motor processes. This claim is strongly supported by Johan Sundberg's experimental work on singing and by 'compensatory articulation' and 'hyper-speech', phenomena that remind us that, for speech, output-directed control is also readily acknowledged. Our comparative review of singin and speech will be placed in the context of the current debate on the 'invariance' and true nature of phonetic units: 'articulatory gestures' or 'auditory goals'?

3aMUa2. Methods for singing voice control and synthesis. Xavier Rodet (Ircam, 1, place I. Stravinsky, 75004 Paris, France, Xavier.Rodet@ircam.fr)

There are various methods to synthesize the singing voice. Whatever the method, in order to obtain high quality voice output, it is essential to respect the precise parameter values for the first three to five formants of the vowels. These parameters depend on many factors including the singer, note, vowel, vocal effort, etc. Articulation of the notes is achieved by variations of fundamental frequency, vibrato, vocal effort and eventually formant characteristics of the vowels. Finally, the succession of consonants and vowels also has to be taken into account and implemented. With the expertise of J. Sundberg, a method called Formant Waveform Synthesis and a program for the control of all the above mentioned variations, except consonants, were developed at Ircam in the 1980s. This program permitted high quality singing voice synthesis but without consonants. Another synthesis method is to is to concatenate units of recorded singing voice. This provides good consonant-to-vowel articulations. But it necessitates to transpose and change the duration of these units which often degrades the output quality and precise control over the formant characteristics is rather difficult. These various methods will be discussed and examples given.

\section{8:40}

3aMUa3. A developmental approach to voice science. Stefanie Stadler Elmer (University of Zürich, Dachsweg $8 \mathrm{~b}$, CH - 4410 Liestal, Switzerland, stefanie.stadler@access.uzh.ch)

Vocal development had been studied mostly with a focus on speaking, and only rarely, on singing. Traditional theories on singing development are often based on wrong premises, e.g. eurocentrism, and reliable analyses of singing are missing or selective. A new theory - inspired by the principles of Piaget's theory -, and a new methodology - based on acoustic measures - are proposed. The voice starts to organise at birth, and gradually adapts to the cultural surrounding and its conventions concerning language, music, and social rules. Vocal and musical behaviour are highly adaptive and constructive, and concern two symbolic systems: music and language. The child develops the voice by playing and imitating. The development proceeds from sensorimotor activities towards more and more conscious actions and thoughts. In order to study children's singing, computer aided programs were devised to analyse and represent pitch, timing, pitch qualities, and syllables. This method yields complex configurations of these parameters describing children's song singing. Detailed descriptions allow to reconstruct the strategies children apply to invent or learn new songs. The empirical results from children at various ages demonstrate that the focus on the analysis of the organisation of the vocal expression is a promising research strategy. 
3aMUa4. Observing a chameleon: How to bridge a gap between the voice training and its scientific description. Jaan Ross (Koidu 122-61, 10139 Tallinn, Estonia, jaan.ross@ut.ee), Allan Vurma (Estonian Academy of Music and Theatre, Rävala Puiestee 16, 10143 Tallinn, Estonia, vurma@ema.edu.ee)

An overview of the authors' research on perception and production of singing voice as well as on the methods of voice training is presented. In the first study, a correlation was found for a group of voice students between the duration of training and the strength of the singer's formant, while the tone quality estimates were not significantly higher for students with longer practice years. In the second study we investigated possible acoustical correlates of the 'forward'/'backward' placing of a voice. A 'forward' placed voice may have higher F1, F2 and/or the singer's formant frequencies as well as a higher level of the singer's formant. In the third study we investigated the intonation accuracy in a cappella performance. There are considerable inter-individual differences between performers as to their adjustment of pitch level. The stability of intonation varies significantly both within a single rendition of the vocal exercise as well as between its consecutive renditions. There is a positive correlation between the deviation of a melodic interval from its equally tempered standard value and the number of out-of-tune judgments by the listeners. The dispersion of out-of-tune judgments is considerable, which suggests that listeners might have adopted different criteria for intonation accuracy.

3aMUa5. Rate of change of Fo in performance singing. Ronald C. Scherer (Bowling Green State University, Department of Physics and Astronomy, Bowling Green, OH 43403, USA, ronalds@bgnet.bgsu.edu), Nandhu Radhakrishnan (University of Missouri, 316 Lewis Hall, Department of Communication Science and Disorders, Columbia, MO 65211, USA, radhakrishnann @ health.missouri.edu), Prakash Boominathan (Sri Ramachandra Medical College \& Research Institute (DU), Dept. of Speech Language \& Hearing Sciences, Porur, Chennai, 600116 TamilNadu, India, Praxb77@yahoo.com), Haidee Tan (Bowling Green State University, 200 Health Center, Department of Communication Disorders, Bowling Green, OH 43403, USA, haideetan@ hotmail.com)

Johan Sundberg has had a strong interest in the vocal behavior of singers of different styles and nationalities. This tribute talk emphasizes the voluntary change of rate of fundamental frequency in performance singing of ornaments and vibrato. The "taan" gestures from Northern Indian classical singing, the "pulse patterns" in Carnatic Southern Indian classical singing, and pitch change in classical western coloratura singing constitute the primary corpus for this study of Fo rate change. Rate of Fo change varied from approximately 20 to $120 \mathrm{ST} / \mathrm{s}$ for soprano pitch change, 8 to $20 \mathrm{ST} / \mathrm{s}$ for the Southern Indian pulse patterns, and 7 to $60 \mathrm{ST} / \mathrm{s}$ for the Northern Indian taan gestures. What these rates depend upon and rates from other ornaments will be discussed, as well as the relation to maximum Fo rates in the literature, performance needs, perceptual characteristics, and control.

\section{9:40}

3aMUa6. Singing out of tune: Disturbances of vocal performance in the general population. Simone Dalla Bella (Dept. of Cognitive Psychology, University of Finance and Management in Warsaw, Pawia Street 55, 01-030 Warsaw, Poland, sdallabella@ vizja .pl)

Acoustical methods can provide a reliable and objective estimate of singing proficiency in the general population, in terms of pitch and temporal accuracy (e.g., Dalla Bella, Giguère, \& Peretz, 2007). The majority, when asked to sing a well-known song at a slow tempo, are as proficient as professional singers. Nonetheless, some nonmusicians exhibit poor singing. This deficit is mostly limited to the pitch domain and sometimes is not accompanied by impaired perception. More recently singing proficiency was examined in nonmusicians with tasks extending beyond singing familiar melodies. Forty participants imitated single pitches, intervals, and short melodies; in addition, participants sung three well-known melodies at a spontaneous tempo and at a fixed slow tempo. Additional tasks (e.g., Montreal Battery of the Evaluation of Amusia) were carried out to assess participants' perceptual abilities. Acoustical analyses of vocal performance revealed that the majority of nonmusicians sung in tune and in time, thus confirming previous findings. Still, various patterns of poor singing emerged from the analyses of pitch and time accuracy (e.g., poor pitch singing with or without perceptual deficits). The relationship between perception and performance mechanisms in vocal production will be discussed.

\section{0:00}

3aMUa7. Hi-Fi voice: observations on the distribution of energy in the singing voice spectrum above $5 \mathrm{kHz}$. Sten O. Ternström (Kungliga Tekniska Högskolan, Dept. of Speech, Music \& Hearing, Lindstedtsvägen 24, SE-100 44 Stockholm, Sweden, stern@kth.se)

Current audio technology enables the weak spectrum of the voice above 4-5 kHz to be studied reliably. It is known that energy in the $5-20 \mathrm{kHz}$ range can be perceived even when it is $50 \mathrm{~dB}$ or more below the main voice spectrum peak. Also, these upper frequencies are conventionally emphasized in the production of popular vocal music; yet very few studies of the acoustic content of this range have been made. High fidelity recordings were made of singers sustaining vowels at varying levels of vocal effort. A general characterization of the two highest octaves $(5-20 \mathrm{kHz})$ was sought. The prevalence of high-frequency energy, the variation of harmonics to noise over frequency, and the covariation with overall SPL were all highly variable, but several landmark features were identified. In addition to the commonly observed zero at 4-5 kHz, zeroes were often seen also around 6 and $12 \mathrm{kHz}$, as were clusters of resonances in the regions 7-10 kHz and 13-16 kHz. Harmonic energy was observed to over $16 \mathrm{kHz}$ in strong female voices. The audibility of these features was assessed by listening tests with selective filtering. A feature-based nomenclature for these uppermost frequency bands is suggested. 
3aMUa8. Advancing Interdisciplinary Research in Singing through a Shared Digital Repository. Annabel J. Cohen (University of Prince Edward Island, 550 University Ave, Charlottetown, PE C1A 4P3, Canada, acohen@upei.ca)

The domain of research in singing encompasses numerous disciplines, countless styles, and many lifespan stages of skill development. A comprehensive understanding of this domain would benefit from a vast digital repository for storing, accessing, and annotating recordings of singing in all its manifestations. A cross-cultural strategy for acquiring and storing the essential audio, audiovisual, and symbolic data parallels the Child Language Data Exchange System (CHILDES) developed and maintained by Brian MacWhinney (http://childes.psy.cmu.edu/) and used by hundreds of psycholinguistics researchers worldwide. A prototype of a digital repository for singing (http://vre.upei.ca/chimes) is under development using infrastructure at UPEI associated with a Canada Foundation for Innovation project on the enhancement of education through media. The database will support and be supported by an international team aiming to Advance Interdisciplinary Research in Singing (AIRS) on the following themes: (1) acquisition of singing (2) comparison of singing and speech acquisition (3) singing pedagogy (4) singing and intercultural understanding (5) singing and intergenerational understanding (6) singing and well-being. The breadth of the work of Johan Sundberg, an honoured member of this team, encompasses these themes and exemplifies the international, interdisciplinary scope of the initiative. (Supported by Canada Foundation for Innovation CFI).

WEDNESDAY MORNING, 2 JULY 2008

AMPHI MAILLOT, 11:00 A.M. TO 12:40 P.M.

Session 3aMUb

\title{
Musical Acoustics: Plucked Stringed Instruments I
}

\author{
François Gautier, Cochair \\ Laboratoire d'Acoustique de l'Université du Maine, Avenue Olivier Messiaen, Le Mans, 72085, France \\ Chris Waltham, Cochair \\ University of British Columbia, Department of Physics \& Astronomy, Vancouver, BC V6T 1Z1, Canada
}

\section{Contributed Papers}

11:00

3aMUb1. A vibrating sitar string: Modeling the 3D dynamics of a plucked string impacting a spatial obstacle with friction. Chandrika $\mathrm{P}$. Vyasarayani (University of Waterloo, Department of Systems Design Engineering, Waterloo, ON N2L 3G1, Canada, cpvyasar@engmail.uwaterloo .ca), Stephen Birkett (University of Waterloo, Department of Systems Design Engineering, Waterloo, ON N2L 3G1, Canada, sbirkett@real .uwaterloo.ca), John McPhee (University of Waterloo, Department of Systems Design Engineering, Waterloo, ON N2L 3G1, Canada, mcphee@real .uwaterloo.ca)

In some musical instruments of Indian origin, such as sitar, veena, or tambura, the string termination is a shallow curved (parabolic) bridge. The string is usually plucked obliquely and interacts with the bridge, thus producing a buzzing or ringing tone which is significantly different from stringed instruments with knife edge supports like guitar. The string is also not restricted to planar motion and friction between bridge and string plays an important role in damping the non-planar motion. A mathematical model is presented for simulating a $3 \mathrm{D}$ vibrating string as it impacts a curved obstacle with friction. The bridge is represented as a foundation model with nonlinear visco-elastic springs; multiple distributed spatial impacts occur between the bridge and string, however the locations of these are not known in advance so must be determined during the simulation. Galerkin approximation is used to reduce the partial differential equations of motion to a set of nonlinear ordinary differential equations. These equations have been solved numerically for different contact and friction conditions. Parametric studies show the effect of bridge geometry, impact damping, and friction on the bidirectional vibration behavior of the string subject to an oblique plucking idealized as an initial condition.

\section{1:20}

3aMUb2. Acoustical measurement of Indian musical instruments (vina-s): Towards greater understanding for better conservation. Sandie Le Conte (Musée de la Musique, 221 avenue Jean Jaures, 75019
Paris, France, sleconte@ cite-musique.fr),Stéphane Vaiedelich(Musée de la Musique, 221 avenue Jean Jaures, 75019 Paris, France, svaiedelich @ cite-musique.fr), Philippe Bruguiere (Musée de la Musique, 221 avenue Jean Jaures, 75019 Paris, France, pbruguiere@ cite-musique.fr)

Among the Musée de la musique collection of non-Western musical instruments are some rare and delicate ones from India, which have been the subject of acoustical investigation for a greater understanding, and hence a better conservation. In the case of the vina-s, the vibration of the string (exciter) plucked by the musician is transmitted to the resonators made from gourd through a flat bridge thus giving the instrument a remarkably singular timber. Acoustical and vibrational measurements were applied in order to determine the acoustic properties of resonators. These techniques are based on gauging the radiated acoustical field triggered by a light impact or a frequency sweep. This analysis confirms the importance given by instrument makers to the painstaking choice of gourd resonators. Thanks to the estimation of resonant frequency - and from the vibrating length - it is possible to hypothesise on the type of strings used (the material, diameter and strain). In short, these analyses make it possible the diagnosis of the mechanical state of constituent elements. These descriptors could facilitate decisions to optimise the choice of gourds in the production of instruments (sitar, tampura) that have today taken on semi-industrial shades.

\section{1:40}

3aMUb3. The influence of plectrum thickness on the radiated sound of the guitar. Sandra Carral (University of Music and performing Arts, Anton-von-Webern-Platz 1, Gebäudeteil M, 2. Stock, A-1030 Vienna,Austria, carral@mdw.ac.at), Martin Paset (University of Music and performing Arts, Anton-von-Webern-Platz 1, Gebäudeteil M, 2. Stock, A-1030 Vienna, Austria, martin.paset@students.mdw.ac.at)

It is generally thought that the human ear is very sensitive to subtle changes in sounds. In the context of musical instruments, one important aspect to study is how much the physical attributes of any given instrument have to differ so that a human can perceive a difference in the produced 
sound. In the case of the guitar, it is unquestionable that when the string is plucked at a particular position, some of the differences on the produced sound of the instrument are introduced by changes in how the player plucks the string (playing technique). However, given the wide variety of plectrum types, materials and thicknesses, it is hypothesised that the player is not the only parameter that influences the sound, but that the plectrum itself plays a significant role in the sound production. This paper presents a study whereby a guitar is played with three plectra of different thicknesses with an artificial plucking machine. The radiated sound is recorded, and subsequently analysed using the program SNDAN. Physical and psychoacoustical attributes of the sound are calculated from the resulting analysis. A thorough comparison of these results obtained for the three different plectra is presented and discussed.

\section{2:00}

3aMUb4. Acoustics of the French Baroque Lute. Nicholas Makris (Massachusetts Institute of Technology, Room 5-212, 77 Massachusetts Avenue, Cambridge, MA 02139, USA, makris@mit.edu)

Design innovations of the French baroque lute characterize the final plateau in the lutes' millennia long evolution in Europe before its eventual extinction at the end of the baroque period. They came with a dramatic increase in number of strings, a new tuning, the first physical description of string vibration, by Marin Mersenne, and led to the "style brisee" which became ubiquitous in baroque music. Compared to the violin, the lute experienced relatively large variations in design and construction material over its lifetime. The aesthetic effect of these variations on musical acoustics were and are often described in compelling but qualitative terms, even by players such as Aristotle and Constantjin Huygens. Here we will use fundamental theoretical principles to quantitatively analyze the acoustic effects of typical design and construction variations found in historic instruments, ranging from the material and thickness of sound boards, bracing and ribs, to rosette design and stringing. We will show how quantitative analysis can be reconciled with qualitative descriptions to help match lute design with desired sound quality.

12:20

3aMUb5. Vibrational Characteristics of Harp Soundboards. Chris Waltham (University of British Columbia, Department of Physics \& Astronomy, Vancouver, BC V6T 1Z1, Canada, cew@phas.ubc.ca)

The musical quality of a harp depends on many factors, but key among these is the soundboard. This work sets out to define general vibrational qualities of a good harp soundboard. First, in order to understand the relationship between the vibrational behavior of a bare soundboard and that of the completed instrument, a 36-string harp was built from scratch. Measurements were made at each stage of construction and compared to finiteelement calculations. The results showed how the bare soundboard properties affect those of the finished harp. Second, the soundboards of several harps of different sizes were assessed by measuring the admittances along the string bar. These data, and the views of the musicians who play the harps, showed that the most crucial relationship is that between the modal shapes and frequencies of the soundboard, and the position and pitches of the strings attached to it. This allows a general statement to be made about the vibrational qualities of a good soundboard, and suggests a recipe for improving poor soundboards.

WEDNESDAY MORNING, 2 JULY 2008

ROOM 250A, 8:00 TO 9:40 A.M.

\title{
Session 3aNSa
}

\section{Noise, Physical Acoustics, and EURONOISE: Aeroacoustics IV}

\author{
Philip J. Morris, Cochair \\ Penn State University, 233C Hammond Building, University Park, PA 16802, USA \\ Christophe Bailly, Cochair \\ Ecole Centrale de Lyon, 36, avenue Guy de Collongue, LMFA, Ecully, 69134, France
}

\section{Contributed Papers}

\section{8:00}

3aNSa1. Finite Volume Solvers and Moving Least Square Approximations for the Linearized Euler Equations on Unstructured Grids. Sofiane Khelladi (Lab. d'Energétique et de Mécanique des Fluides Interne, Arts et Métiers ParisTech, 151 boulevard de l'Hôpital, 75013 Paris, France, sofiane.khelladi@paris.ensam.fr), Xesús Nogueira (Group of Numerical Methods in Engineering, GMNI Dept. of Applied Mathematics, School of Civil Engineering, University of A Coruña, Campus de Elviña, 15071 A Coruña, Spain, xnogueira@udc.es), Farid Bakir (Lab. d'Energétique et de Mécanique des Fluides Interne, Arts et Métiers ParisTech, 151 Boulevard de l'Hôpital, 75013 Paris, France, farid.bakir @ paris.ensam.fr), Luis Cueto-Felgueroso (Aerospace Computational Design Lab., Dept. of Aeronautics and Astronautics, Massachusetts Institute of Technology, 77 Massachusetts Avenue, Cambridge, MA 02139, USA, lcueto@mit.edu), Ignasi Colominas (Group of Numerical Methods in Engineering, GMNI Dept. of Applied Mathematics, School of Civil Engineering, University of A Coruña, Campus de Elviña, 15071 A Coruña, Spain, icolominas@udc.es)

This paper presents the application of a high-order finite volume scheme based on the Moving Least Squares approximations (FVMLS) to solve Linearized Euler Equations (EEL) on unstructured grids. The proposed method allows the direct reconstruction of the (convective) fluxes using compact stencils, and without introducing new degrees of freedom, which is a real advantage over the most popular existing high-order methods. This particularity confers to the proposed methodology a significant reduction in workload and memory storage capability. The proposed scheme is compared to a second-order accurate finite volume scheme with centered fluxes around a uniform flow, with absorbing and/or reflecting boundary conditions. Some test cases are used to illustrate the potential of the FVMLS approximations on acoustic wave propagation. The ultimate objective of our research action is to simulate acoustic wave propagation into confined geometries and complex flows provided by subsonic turbomachines.

\section{8:20}

3aNSa2. Weak Compressibility Method for Prediction of Fluid Noise Generation by Cavity Flow at Low Mach Number. Ming Zhu (ESI Group, 99 rue des Solets, BP 80112, 94513 Rungis, France, Ming.Zhu @esi-group.com), Kjell Herfjord (Statoil Hydro, P.O. Box 7190, 5020 Bergen, Norway, kjher@statoilhydro.com), Mustafa Megahed (ESI Group, Mergenthalerallee 15-21, 65760 Eschborn, Germany, mme@esi-group 
.com),Argiris Kamoulakos(ESI Group, 99 rue des Solets, BP 80112, 94513 Rungis, France, Argiris.Kamoulakos@esi-group.com)

This paper presents the implementation and validation of a numerical simulation method that takes into account the air-borne acoustic wavepropagation within the turbulent flow solution at low Mach number, which is so-called weak compressibility, in order to cope with fully coupled aeroacoustics problems in a nonlinear system. An academic single $3 \mathrm{D}$ cavity case is used to validate the method in comparison with the experiment. The simulated results showed excellent agreement with the experimental data in regard to the acoustic pressure and frequency for the dominant peak of the sound pressure level (SPL) spectra over a wide speed range up to 55 meter per second. An industrial case of so-called singer riser in the system of ocean natural gas rig is studied using this method. There is fluid sound generation due to the interaction of the gas flow field with the pipe grooves. This sound causes strong resonance and vibration over the equipments on the gas rig platform therefore forces the gas producer to control the gas production rate. By using numerical simulation, the aero-acoustic behaviour of a gas flowing in a lab-scale corrugated pipe has been captured which provides deep insights of fluid sound generation mechanism of this singer riser. Experimental results are used to validate the numerical models.

\section{8:40}

3aNSa3. Transparent boundary condition for acoustic propagation in lined guide with mean flow. Emmanuel Redon (Université de Bourgogne, LRMA, 9, avenue Alain Savary, 21000 Dijon, France, Emmanuel .Redon@u-bourgogne.fr), Boureima Ouedraogo (Université de Bourgogne, LRMA, 9, avenue Alain Savary, 21000 Dijon, France, Boureima .Ouedraogo@u-bourgogne.fr), Anne-Sophie Bonnet-Ben Dhia (CNRS, ENSTA - 32 Boulevard Victor, 75015 Paris, France, Anne-Sophie.Bonnet -Bendhia@ensta.fr), Jean-Francois Mercier (CNRS, ENSTA - 32 Boulevard Victor, 75015 Paris, France, jmercier@ensta.fr), Colin Chambeyron (CNRS, ENSTA - 32 Boulevard Victor, 75015 Paris, France, colin.chambeyron@ensta.fr)

A finite element analysis of acoustic radiation in an infinite lined guide with mean flow is studied. In order to bound the domain, transparent boundary conditions are introduced by means of a Dirichlet to Neumann (DtN) operator based on a modal decomposition. This decomposition is easy to carry out in a hard-walled guide. With absorbant lining, many difficulties occur even without mean flow. Since the eigenvalue problem is no longer selfadjoint, acoustic modes are not orthogonal with respect to the L2-scalar product. However, an orthogonality relation exists which permits writing the modal decomposition. For a lined guide with uniform mean flow, modes are no longer orthogonal but a new scalar product allows us to define the DtN operator. We consider first the case of an infinite rectangular two- dimensional lined guide with uniform mean flow in order to present the methodology. Then, some extensions will be presented: non-uniform twodimensional geometries by calculating potential mean flow, and cylindrical axisymmetric three-dimensional problems with uniform mean flow.

\section{9:00}

3aNSa4. Sound radiation from annular jet ducts with impedance walls. João M. Oliveira (Instituto Superior Tecnico, Av. Rovisco Pais, 1049-001 Lisboa, Portugal, joliveira@ist.utl.pt)

An analytical model for sound radiation from a semi-infinite annular duct with cylindrical infinite centre section and carrying a jet issuing into an uniform mean flow is presented. The interior of the outer wall of the duct is allowed to have an acoustic impedance. This is an extension to a recent model where only rigid walls were considered. The unstable cylindrical vortex layer attached to the edge of the duct, between the jet and the uniform mean flow, is considered. The geometry considered aims to model a turbofan exhaust, where sound generated at the inlet propagates along the annular duct and refracts through the bypass jet before radiating into the far field. In this paper the effect of the acoustic impedance of the duct outer wall on the radiated acoustic field is studied.

\section{9:20}

3aNSa5. Acoustics of Low Mach Number Nozzles with Area Expansions. Fernando J. Lau (Instituto Superior Tecnico, Av. Rovisco Pais, 1049-001 Lisboa, Portugal, lau@ist.utl.pt), João M. Oliveira (Instituto Superior Tecnico, Av. Rovisco Pais, 1049-001 Lisboa, Portugal, joliveira@ist.utl.pt)

The acoustic wave equation for quasi-one-dimensional propagation is obtained, along a cylindrical duct with a significant area expansion and containing a low Mach number mean flow. The motivation is the study of the reflection in cross-sectional expanding area. The ray approximation, which holds only for wavelengths which are short compared with the length scales of the variation of the cross-section and mean flow velocity, is used as a factor to reduce the wave equation to a Schrodinger form, in a nozzle with a hyperbolic tangent expansion. The exact solutions are obtained, without restriction, as power series solutions around the middle of the duct; since this solution fails to converge at the two ends of the duct it is matched to the other solutions there, by application of the causality principle. In this way it is possible to calculate everywhere the pressure and velocity perturbation profiles, for both the transmitted and reflected waves, as well as the reflection and transmission coefficients. These are plotted as a function of the longitudinal coordinates along the duct for several values of the three dimensionless parameters in the problem, viz. (i) the area expansion ratio, ii) the Mach number of the mean flow at the central section and iii) the wavenumber. 


\title{
Session 3aNSb
}

\section{Noise and EURONOISE: Railway Noise and Vibration III}

\author{
Richard A. Carman, Cochair \\ Wilson, Ihrig \& Associates, 5776 Broadway, Oakland, CA 94618, USA \\ Franck Poisson, Cochair \\ SNCF DIR, 45, rue de Londres, Paris, 75379, France \\ David Thompson, Cochair \\ ISVR, University of Southampton, Highfields, SO17 1BT, UK
}

\section{Contributed Papers}

\section{8:00}

3aNSb1. Noise reduction of freight wagons achieved by LL-blocks implementation: Last investigations and results from IPG and NICOBB projects. Fabien Létourneaux (SNCF - Agence d'Essai Ferroviaire, 21 avenue Salvador Allende, F-94407 Vitry-Sur-Seine, France, fabien .letourneaux@sncf.fr), Jasper Peen (Lloyd's Register Rail Europe B.V., Catherijnesingel 33, PO Box 2016, 3500 GA Utrecht, Netherlands, jasper.peen@1rrail.com), Nicolas Meunier (Deutsche Bahn AG, Messungen Akustik (TZV12.1), Völckerstraße 5, D-80939 München, Germany, Nicolas.Meunier@bahn.de), Erwin H. Jansen (TNO Science and Industry, PO Box 155, 2600 AD Delft, Netherlands, erwin.jansen@tno.nl), Cora Cremezi-Charlet (UIC, 16,rue J. Rey, F-75015 Paris, France, cremezi@uic .asso.fr)

The high noise emission levels of freight wagons during rolling are mainly due to uneven wheel treads produced during the braking sequences when the vehicle is equipped with cast iron blocks. This has lead to the development of two types of composite brake shoes (K- and LL-blocks), which are far less aggressive to the wheel.It has been demonstrated that K-blocks achieve a noise reduction of about $10 \mathrm{~dB}$ but they are mainly dedicated, at term, to the new vehicles as their friction coefficient is very different to the cast iron one. LL-blocks are on the contrary especially designed for retrofit vehicles and do not require major modifications of the braking equipment. But as they were only very recently made available by the manufacturers due to difficulties in their development, noise efficiency of LLblocks products is not well-established. This paper focuses on the noise reduction and noise emission values of freight vehicles equipped with LLblocks. It aims at presenting on-going studies on this topic and at providing reliable and accurate results from different measurement campaigns carried out on a TSI reference track. This work is the result of a collaboration between the UIC project NICOBB and the Dutch Noise Innovation Programme.

\section{8:20}

3aNSb2. Measurements of curve squeal from Oslo's subway. Sigmund Olafsen (Brekke \& Strand akustikk as, Hovfaret 17, N-0275 Oslo, Norway, so@bs-akustikk.no), Tore Killengreen (Brekke \& Strand akustikk as, Hovfaret 17, N-0275 Oslo, Norway, tkn@bs-akustikk.no)

Curve squeal from the subway line at Brattlikollen, Oslo, has led to severe noise complaints through the years. Two important steps have been taken to reduce the problem. An automatic rail lubrication system has been installed on the critical spot, and Oslo's old subway trains (1300 series) are being replaced. One of the new trains (MX) has also been fitted with wheel dampers. Continuous measurements during 16 days and nights with 2238 train passages have been analyzed to verify the effectiveness of these steps to reduce noise. The main results are as follows: 1300 series trains without rail lubrication give severe curve squeal during $18 \%$ of the train passages. 1300 series trains with rail lubrication give severe curve squeal during less than $1 \%$ of the train passages MX trains give substantially less curve squeal than 1300 series trains, with or without lubrication The MX trains with wheel dampers gives no curve squeal, even without lubrication

\section{8:40}

3aNSb3. Reduction of Noise from Composite Steel/Concrete Aerial Structures by Damping Steel Plates. George P. Wilson (Wilson, Ihrig \& Associates, Inc., 5776 Broadway, Oakland, CA 94618, USA, gwilson @ wiai.com), Francis Kirschner (FGK Consulting, Inc., East Hills, Long Island, NY 11576, USA, francis516@aol.com)

Noise radiated from steel girders at bridges and aerial structures has been a long standing problem for steel wheel and rail systems. During the initial design period for the San Francisco Bay Area Rapid Transit System, a full scale model girder and deck was constructed in 1964 and tested with and without constrained layer damping on the steel girder plates. The radiated noise from $20 \mathrm{~Hz}$ to $150 \mathrm{~Hz}$ was reduced by $9 \mathrm{~dB}$ with full plate area damping. A test track installation in 1965 also showed about $9 \mathrm{~dB}$ reduction in wayside noise with the overall result within about $1 \mathrm{~dB}$ of the noise radiated by an all-concrete structure. Because full plate area constrained damping is expensive, a scheme with small area damping pads was developed in the 1970's and applied to composite steel/concrete aerial structures at the Atlanta rail transit system. The result was 9 to $10 \mathrm{~dB}$ reduction of low frequency radiated noise, allowing sound barrier walls at the outer edges of the decks to be fully effective in reducing overall wayside noise levels from trains. Drawings of the aerial structures and graphical results from vibration and noise tests are presented.

\section{9:00}

3aNSb4. Noise reduction applied to an engine cooling system for a diesel railway vehicle. Anders Frid (Bombardier Transportation, Östra Ringvägen 1, SE-72173 Vasteras, Sweden, anders.r.frid@se.transport .bombardier.com), Karl-Richard Fehse (Bombardier Transportation, Am Rathenaupark, 16761 Hennigsdorf, Germany, karl-richard.fehse@de .transport.bombardier.com)

In recent years there there has been growing attention to reduce the noise from rail traffic in urban areas. In or around stations and depots, noise from cooling systems and traction and auxiliary equipment normally dominates. New legislation setting noise limits for stationary and accelerating trains puts further emphasis on increased noise reduction of such sources. An experimental investigation has been carried out in which several design changes on a roof-mounted diesel engine cooler from the AGC (Autorail Grande Capacité) train have been assessed in laboratory and on a train. Even though this train is considered as a state-of-the-art product having very low noise emission, the engine cooler is identified as the dominating noise source when operating at its maximum capacity. The wide range of minor and major modifications tested includes different ways to guide the air flow into the fans, reduced tip clearance, absorption material in various positions 
inside and outside the cooler. Also different fan types have been tried (an axial fan with swept blades and a radial fan). The work has been carried out within the 6th framework EU-project "Silence" (ref TIP4-CT-2005-516288)

\section{9:20}

3aNSb5. Upgraded BB64700 locomotives - Noise Management Plan during the redesign process. Sebastien Dalle (CETIM, 52, av. Felix Louat, 60300 Senlis, France, sebastien.dalle@cetim.fr)

To extend the working life of its BB 64700 locomotives, SNCF decided to upgrade a fleet of 19 tractive units. Priorities for the upgrading work include changing the motor and its cooling system, adding air conditioning to the cab while meeting new environmental obligations and improving cab acoustics. An acoustic model that combines calculations and experimental data is used as a platform to specify maximum allocated acoustic power to each system suppliers. The global approach to sub-structuring and acoustic synthesis is based on decomposition of the product in a main structure and several noise sources, and that their acoustic and vibration energy propagates via different identified transfer paths. The model, which is updated throughout the design process, serves as a decision tool to enable corrective action plans if a clause might be not respected. In the context of this project, measured noise levels on the upgraded locomotive meet SNCF specifications for interior and exterior acoustic according to the defined operating conditions (idling, max load traction).

\section{9:40}

3aNSb6. Noise reduction strategies for a light metro rail traction system. Nicola Rusciano (Dept. of Aerospace Engineering - University of Naples, Via Claudio, 21, 80125 Naples, Italy, nruscian@unina.it), Massimo Viscardi (Dept. of Aerospace Engineering - University of Naples, Via Claudio, 21, 80125 Naples, Italy, massimo.viscardi@unina.it), Stefano Ferraiuolo (Ansaldobreda, Via Argine, 80100 Naples, Italy, ferraiuolo .stefano@ansaldobreda.it)

Inside the paper the vibro-acoustic characterization of a light rail vehicle is presented, with special reference to the traction noise emission identification. As a first step, on board measurements and pass by measurements, were performed in order to separate traction noise from rolling noise; these test revealed the electrical engine, as the most relevant sources at low speed. The electrical engine has then been characterized during specific laboratory test and innovative noise control solution were proposed to be tested. The first one could be a modification of inverter algorithm; this trying to reduce the influence of most relevant acoustic tones. This approach is essentially based on a software modification of the PWM algorithm through which modify the engine's spectral response. The second possible tested approach involved the enclosure of the engine in a sort of semi-active skin panels co-located at short distance from the engine; it has been demonstrated that this approach, by the merging of passive and active peculiarities, potentially lead to a wide frequency range application area. Following laboratory tests, the PWM solution was also decided to be implemented during "on board" test; a wide set of experimental results will be presented and discussed.

\section{0:00}

3aNSb7. Tram noise emission: Spectral analysis of the noise source contributions. Marie-Agnès Pallas (INRETS, 25 av. F. Mitterrand, case 24, 69675 Bron, France, marie-agnes.pallas@inrets.fr), Joel Lelong (INRETS, 25 av. F. Mitterrand, case 24, 69675 Bron, France, lelong@inrets.fr), Roger Chatagnon (INRETS, 25 avenue F. Mitterrand, case 24, 69675 Bron, France, roger.chatagnon@inrets.fr)

In France we can assist to a wide development of tram networks for public transportation in main cities. At the same time arises questioning on the noise emission of trams and possible consequences for resident exposure. A French research project has been conducted for studying noise and vibration emission of trams : this paper concerns the description of the noise emission of passing-by trams. A measurement campaign was achieved on the city of Nantes network, involving two kinds of trams with distinct technologies and equipment, and tested on two types of tracks having different platform surfaces. Acoustic measurement included both a 2D-array for noise source identification and a microphone set for vertical directivity analysis. The dominant noise sources are mainly the bogie areas (powered bogies, unpowered bogies) and an extended noise source along the track and the lower part of the tram, all of them involving rolling noise. This paper focuses on the spectral description of the main sources. A parametric study is presented, pointing out the effect of speed, tram type and track type on the frequency distribution of the emitted noise. 


\title{
Session 3aNSc
}

\section{Noise, Computational Acoustics, and EURONOISE: Source Identification in Radiation and Scattering I}

\author{
J. Stuart Bolton, Cochair \\ Ray W. Herrick Labs., School of Mech. Eng., Purdue University, 140 S. Martin Jischke Drive, West Lafayette, IN 47907-2031, \\ USA \\ Peter Juhl, Cochair \\ Institute of Sensors, Signals and Electrotechnics, University of Southern Denmark, Niels Bohrs Allé 1, Odense S, 5230, \\ Denmark
}

Invited Paper

8:00

\begin{abstract}
3aNSc1. Spherical near field acoustic holography with microphones on a rigid sphere. Finn Jacobsen (Acoustic Technology Department, Technical University of Denmark, Ørsted Plads, B352, DK-2800 Lyngby, Denmark, fja@oersted.dtu.dk), Jørgen Hald (Brüel \& Kjær Sound \& Vibration Measurement A/S, Skodsborgvej 307, DK-2850 Nærum, Denmark, JHALD@bksv.com), Efrén Fernandez (Acoustic Technology Department, Technical University of Denmark, Ørsted Plads, B352, DK-2800 Lyngby, Denmark, efrenfer@ hotmail.com), Guillermo Moreno (Acoustic Technology Department, Technical University of Denmark, Ørsted Plads, B352, DK-2800 Lyngby, Denmark, s051216@ student.dtu.dk)

Spherical near field acoustic holography (SNAH) is a recently developed technique that makes it possible to reconstruct the sound field inside and just outside an acoustically transparent spherical surface on which the sound pressure is measured with an array of microphones with negligible scattering. Because of the versatile geometry of a sphere SNAH is potentially extremely useful for source identification. On the other hand a rigid sphere is somewhat more practical than an open sphere, and it is possible to modify the SNAH theory so that a similar sound field reconstruction can be made with an array of microphones flush-mounted on a rigid sphere. However, this approach is only valid if it can be assumed that the sphere has a negligible influence on the incident sound field, in other words if multiple scattering can be ignored, and this is not necessarily a good assumption when the sphere is close to a radiating surface. This paper describes the modified SNAH theory and examines the matter through simulations and experimentally.
\end{abstract}

\section{Contributed Paper}

8:20

3aNSc2. Applications of Near-field Acoustic Holography on Small Apertures. Rick Scholte (Eindhoven University of Technology, Dept. of Mechanical Engineering, Dynamics \& Control, P.O. Box 513, 5600MB Eindhoven, Netherlands, r.scholte@tue.nl), Ines Lopez (Eindhoven University of Technology, Dept. of Mechanical Engineering, Dynamics \& Control, P.O. Box 513,5600MB Eindhoven, Netherlands, i.lopez@tue.nl), N.b. Roozen (Eindhoven University of Technology, Dept. of Mechanical Engineering, Dynamics \& Control, P.O. Box 513, 5600MB Eindhoven, Netherlands, n.b.roozen@tue.nl), Henk Nijmeijer (Eindhoven University of Technology, Dept. of Mechanical Engineering, Dynamics \& Control, P.O. Box 513, 5600MB Eindhoven, Netherlands, h.nijmeijer@tue.nl)

Small, finite measurement apertures are a major impediment for accurate and fast application of near-field acoustic holography (NAH). In theory, FFT-based NAH methods are very powerful and acquire extremely fast in- verse calculations of the sound field. However, in practice the usually small amount of sensors in a measurement array limit the possibilities of classical implementations of NAH, especially near the edges of the array or measurement grid. An alternative method to, for example, spatial windowing, patch $\mathrm{NAH}, \mathrm{SONAH}$, etc. is the recently introduced border-padding technique. This method makes it possible to apply FFT-based NAH with a large increase of accuracy compared to the original methods, specifically at the measurement grid edges, while maintaining the high speed of the inverse process PNAH is known for. This work focuses on the application of borderpadding and the practical implementation in a newly developed PNAH system. It illustrates a large variety of extended possibilities for PNAH that border-padding enables. Apart from the earlier introduced standard borderpadding, experimental results are shown for a highly improved borderpadding method. Measurements and calculations on industrial products illustrate the wide applicability of the presented method.

\section{Invited Papers}

\section{$8: 40$}

3aNSc3. A study on regularization parameter choice in Near-field Acoustical Holography. Jesper Gomes (Brüel \& Kjær Sound and Vibration Measurement A/S, Skodsborgvej 307, DK-2850 Nærum, Denmark, jgomes@bksv.com)

Regularization plays an important role in Near-field Acoustical Holography (NAH), and choosing the right amount of regularization is crucial in order to get a meaningful solution. An automated method such as the L-curve or Generalized Cross-Validation (GCV) is often used in NAH to choose a regularization parameter. These parameter choice methods (PCMs) are attractive, since they require no a priori knowledge about the noise. However, there seems to be no clear understanding of when one PCM is better than the other. This paper presents comparisons of three PCMs: GCV, L-curve and Normalized Cumulative Periodogram (NCP). The latter method is new within NAH and it is based on the Fourier transform of the residual vector. The methods are used in connection with three NAH methods: Statistically Optimized Near-field Acoustical Holography (SONAH), the Inverse Boundary Element Method (IBEM), and the 
Equivalent Source Method (ESM). All combinations of the PCMs and the NAH methods are investigated using simulated measurements with different types of noise added to the input. Finally, the comparisons are carried out for a practical experiment. This aim of this work is to create a better understanding of which mechanisms that affect the performance of the different PCMs.

\section{9:00}

3aNSc4. Regularization method applied to deconvolution problem in Real-Time Acoustic Holography. Sebastien Paillasseur (ENSIM - LAUM, Université du Maine, rue Aristote, 72085 Le Mans, France, sebastien.paillasseur.etu@univ-lemans.fr), Jean-Hugh Thomas (ENSIM - LAUM, Université du Maine, rue Aristote, 72085 Le Mans, France, jean-hugh.thomas@univ-lemans .fr), Jean-Claude Pascal (ENSIM - LAUM, Université du Maine, rue Aristote, 72085 Le Mans, France, Jean-Claude.Pascal@univlemans.fr)

Near-field Acoustic Holography (NAH) [Maynard et Williams, 1985] is a measuring process for locating stationary sound sources from measurements made by an antenna of microphones positioned near the acoustic source plane. In order to characterize nonstationary sources, a new formulation has been introduced [Grulier, 2004] to propagate signals on a forward plane using a convolution product with an impulse response in the time-wavenumber domain. The purpose of this study is to solve the deconvolution problem in order to introduce the Real-Time Acoustic Holography and to test its accuracy. Taking the evanescent waves into account improves the spatial resolution of the solution but makes the deconvolution problem "ill-posed". Thus, the inverse of the impulse response is neither unique nor stable. Then, regularization methods that consist of giving an other constraint to the solution to solve this problem are studied. In particular, the standard Tikhonov regularization is used, which is based on the minimisation of the solution's energy, combined with generalized cross validation to estimate the regularization parameters. This method provides an image representation of the time dependent pressure of the source plane in order to localize and characterize the different sound sources.

\section{9:20}

3aNSc5. A comparison of SONAH and IBEM for near-field acoustic holography. Peter Juhl (Institute of Sensors, Signals and Electrotechnics, University of Southern Denmark, Niels Bohrs Allé 1, 5230 Odense S, Denmark, pmjuhl@ sense.sdu.dk), Jesper Gomes (Brüel \& Kjær Sound and Vibration Measurement A/S, Skodsborgvej 307, DK-2850 Nærum, Denmark, jgomes@bksv.com)

Among the popular techniques for acoustic source identification in complex environments are the Statistically Optimal Near Acoustic Holography (SONAH) and the Inverse Boundary Element Method (IBEM). These two methods are quite different regarding the underlying assumptions and the practical implementations: Whereas SONAH performs the back-propagation of the sound field to a plane surface; the IBEM has no restrictions on the radiating geometry. On the other hand, IBEM requires the generation of a surface mesh and a time consuming solution process. The present paper compares the performance of the two methods for a number of simulated test cases and studies the influence on the performance of the models when changing selected parameters. Finally, the performance of the methods is compared in a simple measurement setup.

\section{9:40}

3aNSc6. Precise localisation of noise source using statistically optimal beamforming and post -processing deconvolution. Jean-Claude Pascal (ENSIM - LAUM, Université du Maine, rue Aristote, 72085 Le Mans, France, Jean-Claude.Pascal@univ-lemans.fr), Jing-Fang Li (Visual VibroAcoustics, 51 rue d'Alger, 72000 Le Mans, France, jingfang.li@visualvibroacoustics.com), Xavier Carniel (Centre Technique des Industries Mécaniques (CETIM), 52, avenue Félix Louat, 60304 Senlis, France, Xavier.Carniel@cetim.fr), Axel Hass (52, avenue Félix Louat, 60304 Senlis, France, Axel.Haas@cetim.fr), Laurent Bollade (DYVA - DYnamique Vibration \& Acoustique, 10 Rue Maryse Bastié, 69008 Lyon, France, laurent.bollade@dyva.fr)

Conventional data-independent beamforming with microphone array is widely used to locate sound sources and build acoustic model of equipments, engines and vehicles. The particularities of this technique are the use of a relatively few number of microphones and a simple signal processing. So, this technique is fast and easy to use. Other approaches using experimental data for computing the steering vector have been proposed to increase the resolution, for example, minimum variance method, and high-resolution methods. However these methods are often delicate to put in use in acoustic engineering where they are often reproached for lack of robustness in perturbed acoustic fields. Whereas, the most used methods nowadays are those by which the determination of the steering vector is data-independent. Among those methods we consider a method using a Statistically Optimal Array Processing (SOAP) completed by techniques of post-processing deconvolution, by which the resolution of the conventional beamforming is considerably increased, particularly at low frequencies. Some relevant indicators evaluate the performance of the proposed method by using perturbed data and by comparing between conventional and high-resolution beamformers. Examples of industrial measurements in a multi-sources environment demonstrate the practical interest of this technique.

10:00-10:20 Break

10:20

3aNSc7. Directional Recording and Analysis of Sounds from Musical Instruments. Franz Zotter (Institute of Electronic Music and Acoustics, Inffeldgasse 10 / 3,8010 Graz, Austria, zotter@iem.at)

The sound of musical instruments doesn't radiate omni directionally into the sound field. Moreover, each component of the sound (e.g. a partial) can have its own directivity pattern which, in turn, may also vary over time. In general, the radiated sound in free space will have a different timbre at different locations around the sound source. In many cases the description of a particular radiation pattern contour may not be valid for other instruments, tones, playing techniques or even the same musical gesture on the same instrument. Consequently, this work focuses on the synchronous capture and analysis of musical sound sources with a spherical microphone array. 
In order to analyze the directivity in its very detail, a spatial model for radiated sound is suggested. Assuming coherent signals, a common signal decomposition is applicable. The relations between the signal components yield the desired directivity patterns. Spherical harmonics interpolation between the discrete measurement points allows for the estimation of the radiation in a continuous angular space.

\section{Contributed Paper}

10:40

3aNSc8. Improving the localisation of sources based on shaped arrays with a reduced number of microphones. Lucille L. Lamotte (MicrodB, 7b allée Claude Debussy, 69130 Ecully, France, 11@microdb.fr), Quentin Leclere (Laboratoire Vibrations Acoustique - INSA Lyon, 25 bis avenue Jean Capelle, Bâtiment Saint-Exupéry, F-69621 Villeurbanne cedex, France, quentin.leclere@insa-lyon.fr)

The capacity of hardware today allows carrying out noise sources locali- sation without moving the microphone array at a limited financial and time cost. The single shot measurement processed with beamforming is comfortable for the user. But the quality of the results limits the interest of such systems: bad resolution, low dynamics and no quantitative levels. This article proposes complementary methods to beamforming to improve the results based on the same single measurement. First a near field beamforming algorithm is developed improving the resolution, and then an inverse method is applied based on the transfer function between the sources and the acoustic pressure at microphone positions or on the hologram in order to "clean" the localisation map and give quantitative results.

\section{Invited Paper}

\section{1:00}

3aNSc9. Array based measurement of radiated and absorbed sound intensity components. Jørgen Hald (Brüel \& Kjær Sound \& Vibration Measurement A/S, Skodsborgvej 307, DK-2850 Nærum, Denmark, JHALD@ @ksv.com), Jakob Mørkholt (Brüel \& Kjær Sound \& Vibration Measurement A/S, Skodsborgvej 307, DK-2850 Nærum, Denmark, jmorkholt@bksv.com), Pierre Hardy (Dassault Aviation, 80 quai marcel Dassault - Cédex 300, 92552 Saint Cloud, Cédex, France, Pierre.Hardy@dassault-aviation.com), Dominique Trentin (Dassault Aviation, 80 quai marcel Dassault - Cédex 300, 92552 Saint Cloud, Cédex, France, Dominique.Trentin @dassault-aviation.com), Martin Bach-Andersen (Ødegaard \& Danneskiold-Samsøe A/S, Titangade 15, DK-2200 København Ø, Denmark, martin.bach-andersen@1r-ods.com), Graeme Keith (Ødegaard \& Danneskiold-Samsøe A/S, Titangade 15, DK-2200 København Ø, Denmark, Graeme.Keith@1r-ods.com)

Calibration of energy flow models for cabin environments requires tools able to measure the energy flow between the sub-systems of the models. Here we shall specifically consider the energy flow between acoustic energy in the cabin and vibration energy in the panels. Sound intensity measurement, for example with a two-microphone intensity probe, can provide the total sound intensity at some distance from panels. It cannot resolve, how much of the intensity is due to radiation from the surface and how much is due to absorption of energy from the sound field. And it cannot provide the information right on the panel surface, leading to a degraded resolution. The ability to distinguish between radiated and absorbed intensity is important for energy flow modeling, and for identification of the areas where noise energy enters the cabin. The present paper describes some work done to measure the radiated and absorbed intensity components by the use of a double layer microphone array. Two measurements are performed: A measurement of surface absorption coefficient and an operational measurement. Non-locally reacting surfaces represent a particular challenge, the handling of which will be discussed. Results from both loudspeaker measurements and in-flight measurements will be presented.

\section{Contributed Papers}

\section{1:20}

3aNSc10. Cyclic Sound Intensity and Source separation from NAH measurements on a Diesel engine. Brice Lafon (Renault, 67 rue des Bons Raisins, CTR A03 3 37, 92500 Rueil Malmaison, France, brice.lafon @ renault.com), Jerome Antoni (Université de Technologie de Compiègne, Centre de Recherche de Royallieu, rue Personne de Roberval, 60200 Compiègne, France, jerome.antoni@utc.fr)

Noise source separation and acoustic imaging are two research fields of interest in car industry. For this, RENAULT (French car manufacturer) and the University of Technology of Compiègne (UTC, France) are conducting an original study aiming to couple these fields to solve in an efficient way engine NVH problems. Previously, in the first part of this study, combustion noise separation on Diesel engine radiation was implemented from classical sound intensity measurements. The separation, based on Wiener filtering, was performed by taking advantage from the cyclostationary property of engine signals. The second part, presented here, aims to overcome the main shortcoming of the method: time-consuming measurements. Our method is then applied to Near-field Acoustic Holography that is faster and more adapted to an industrial context. Source separation is implemented to complete global radiation maps by specific-source radiation maps such as combustion and other sources radiating in low frequencies since NAH performs rather in low frequencies. Furthermore, the exploitation of cyclostationarity enables to define a cyclic sound intensity (an instantaneous intensity estimated during an average engine cycle). Then, radiation movies can be set to allow a precise location of a specific radiation emergence in space, in frequency and in crankshaft angle.

\section{1:40}

3aNSc11. Tire radiation in vehicle environment: A review of some source identification methods. Christophe Picard (PSA Peugeot Citroën, Route de Gisy, 78943 Vélizy-Villacoublay Cedex, France, christophe .picard3@mpsa.com), Matthieu Fiack (Segula Technologies for PCA, Route de Gisy, 78943 Vélizy-Villacoublay Cedex, France, matthieu .fiack1@ext.mpsa.com), Olivier Tanneau (Altran AIT for PCA, Route de Gisy, 78943 Vélizy-Villacoublay Cedex, France, tanneau77@hotmail .com), Olivier Sauvage (PSA Peugeot Citroën, Route de Gisy, 78943 Vélizy-Villacoublay Cedex, France, olivier.sauvage@mpsa.com), Laurent Gagliardini (PSA Peugeot Citroën, Route de Gisy, 78943 VélizyVillacoublay Cedex, France, laurent.gagliardini@mpsa.com)

The REBECA research project aims at defining new architectural concepts and reduction strategies of vehicle external noise emission regarding the international pass-by noise certification standard (ISO 362). The first task of the project is to qualify and quantify the major contributor of the external emission, the tires/road surface source. To this end, a 3D array composed of 164 microphones has been set up in the near and far field around vehicle. Measurements have been performed for several operational conditions with the use of a chassis dyno in a semi-anechoic chamber. A review and a comparison of some source identification methods has been led including experimental FRFs based on inverse techniques (Nelson), conventional and optimized beamforming approaches (Elias, Dougherty, Brooks and Humphreys, Ravetta) and inverse BEM method (Hamdi, Omrani). This 
study permits to identify the more appropriate microphone array technique with respect to source identification robustness, power level and directivity radiation reconstruction in the far field.

12:00

3aNSc12. Large array optimization using a genetic algorithm and application to wide band moving sources imaging. Sébastien Debert (PSA-Peugeot-Citroën, Route de Gizy, CC : VV013 - Bât. 14, F-78943 Vélizy-Villacoublay Cedex, France, sebastien.debert@mpsa .com), François Ollivier (UPMC Univ Paris 06, CNRS UMR 7190 Institut Jean Le Rond D'Alembert, 2 Place de la Gare de Ceinture, 78210 Saint Cyr l'Ecole, France, frol@ccr.jussieu.fr)

In order to design a microphone array able to follow moving wide band acoustic sources, and improve the quality of the images obtained with a classical beam forming technique, numerical simulations were carried out. The various steps of the study are presented here. Searching the best design for a large microphone array, a simple genetic algorithm was developed. Considering a constant outer dimension of the array and technological constraints in order to design an adaptive array of 60 microphones, the algorithm selects among a large set several combinations of their positions. The functional being optimized is the directivity pattern, in terms of side lobes level. The genetic algorithm always converges to solutions comparable to a randomized set of microphones. Besides, taking advantage of symmetry properties of the array, an improvement of the speed of beamforming calculation was introduced. The optimum array being chosen, wide band moving sources were simulated and images were processed. In order to provide beam formed images with a constant resolution over the band a homoteticbalanced array of 120 microphones was assembled. Finally the dynamic of the images was significantly improved by performing a non-coherent image synthesis technique.

12:20

3aNSc13. Non stationary tire-road noise analysis using large array beam forming in the time and frequency domains. François Ollivier (UPMC Univ Paris 06, CNRS UMR 7190 Institut Jean Le Rond D'Alembert, 2 Place de la Gare de Ceinture, 78210 Saint Cyr l'Ecole, France, frol@ccr.jussieu.fr)

In the framework of the REBECA Project founded by the ADEME, one seeks to provide a description of the tire-road noise source from the exterior of the vehicle perspective. Far field acoustic imaging techniques were proposed to identify the sources. Therefore an optimised array of 120 microphones, $4 \mathrm{~m}$ in diameter, was modelled and built. Measurements of the noise produced by a car mounted on a roll were performed in a semi anechoïc room. The front car axle was driven at various constant speeds and to rising speeds as well. Beam formed images and point signals have been postprocessed with a $125 \mathrm{kHz}$ sampling rate in order to derive space and time analysis of the pressure field in the vicinity of the tire-roll contact point. The high rate movies processed clearly exhibit periodic phenomena generated around the contact point, while the tire structure borne source appears to be insignificant. Results of frequency and order analysis are also presented in this attempt to provide an interpretation of the tire/road contact noise in terms of source models.

\title{
Session 3aNSd
}

\section{Noise and EURONOISE: Tire-Road Noise from the Road Perspective I}

\author{
Paul Donavan, Cochair \\ Illingworth \& Rodkin, Inc., 505 Petaluma Blvd. South, Petaluma, CA 94952, USA \\ Fabienne Anfosso-Ledee, Cochair \\ Laboratoire Central des Ponts et Chaussées, BP 4129, Bouguenais Cedex, 44341, France
}

\section{Invited Papers}

\section{8:00}

3aNSd1. Tyre/road noise prediction: A comparison between the SPERoN and HyRoNE models - Part 1. Thomas Beckenbauer (Müller-BBM GmbH, Robert-Koch-Straße 11, 82152 Planegg, Germany, T.Beckenbauer@MuellerBBM.de), Philippe Klein (INRETS, 25 av. F. Mitterrand, case 24, 69675 Bron, France, philippe.klein@inrets.fr), Jean-François Hamet (INRETS, 25 av. F. Mitterrand, case 24, 69675 Bron, France, hamet@inrets.fr), Wolfgang Kropp (Chalmers University of Technology, Division of Applied Acoustics, SE41296 Gothenburg, Sweden, wolfgang.kropp@chalmers.se)

The SPERoN and HyRoNE models predict the pass-by tyre/road noise of a passenger car from intrinsic characteristics of the road surface. Both models are hybrid: they combine statistical laws with physical models. With a computing time of a few minutes (very quick compared to full physical models), they provide operational tools for tyre/road noise prediction. Particular fields of interest are road surface optimisation with respect to noise at the laboratory scale, conformity of production of a new surface and acoustic monitoring of roads. They are now implemented as user-friendly stand-alone applications. The presentation will address the principles of the models, their performances and their respective main fields of application. Part 1 will address the principles of the models and their respective fields of application. 
3aNSd2. Tyre/road noise prediction: A comparison between the SPERoN and HyRoNE models - Part 2. Philippe Klein (INRETS, 25 av. F. Mitterrand, case 24, 69675 Bron, France, philippe.klein@inrets.fr), Thomas Beckenbauer (Müller-BBM GmbH, Robert-Koch-Straße 11, 82152 Planegg, Germany, T.Beckenbauer@MuellerBBM.de), Jean-François Hamet (INRETS, 25 av. F. Mitterrand, case 24, 69675 Bron, France, hamet@inrets.fr), Wolfgang Kropp (Chalmers University of Technology, Division of Applied Acoustics, SE-41296 Gothenburg, Sweden, wolfgang.kropp@chalmers.se)

The SPERoN and HyRoNE models predict the pass-by tyre/road noise of a passenger car from intrinsic characteristics of the road surface. Both models are hybrid: they combine statistical laws with physical models. With a computing time of a few minutes (very quick compared to full physical models), they provide operational tools for tyre/road noise prediction. Particular fields of interest are road surface optimisation with respect to noise at the laboratory scale, conformity of production of a new surface and acoustic monitoring of roads. They are now implemented as user-friendly stand-alone applications. The presentation will address the principles of the models, their performances and their respective main fields of application. Part 2 will address the performances of the models.

\section{8:40}

3aNSd3. Experimental study of dynamical contact forces for tyre/road noise application. Julien Cesbron (Université d'Evry Val d'Essonne, Laboratoire de Mécanique d'Evry, EA3332, 40, rue du Pelvoux, 91020 Evry Cedex, France, cesbron.julien@neuf.fr), Fabienne Anfosso-Ledee (Laboratoire Central des Ponts et Chaussées, BP 4129, 44341 Bouguenais Cedex, France, fabienne.anfosso@lcpc.fr), Denis Duhamel (ENPC, UR Navier, 6 et 8 Avenue Blaise Pascal, Cité Descartes, Champs sur Marne, 77455 Marne la Vallée, France, duhamel@lami.enpc.fr), Hai Ping Yin (ENPC, UR Navier, 6 et 8 Avenue Blaise Pascal, Cité Descartes, Champs sur Marne, 77455 Marne la Vallée, France, yin@lami.enpc.fr), Donatien Le Houedec (Ecole Centrale de Nantes, GeM, 1 rue de la Noe, BP 92101, 44321 Nantes Cedex 3, France, Donatien.Lehouedec@ec-nantes.fr)

This paper deals with the experimental study of tyre/road contact forces in rolling conditions for tyre/road noise investigation. In situ measurements of contact forces and CPX noise levels were carried out for a slick tyre rolling on six different road surfaces at rolling speeds between $30 \mathrm{~km} / \mathrm{h}$ and $50 \mathrm{~km} / \mathrm{h}$. Contact stresses were measured at a sampling frequency around $10000 \mathrm{~Hz}$ using a single array of digital pressure sensitive cells both in the transverse and in the longitudinal directions. The contact areas obtained during rolling were smaller than the one measured in statics. This could be due to an influence of the viscoelastic behavior of the rubber of the tyre tread during rolling. Additionally the global energy of the longitudinal contact forces at different speeds were in the same order for the six road surfaces, while their spectrums at different speeds were quite different. This may be explained by a spectral influence of the vibration of the tyre during rolling. Finally, the levels of longitudinal contact forces and CPX noise measured at $30 \mathrm{~km} / \mathrm{h}$ were correlated. A high positive correlation was found around $800 \mathrm{~Hz}$, which frequency could be directly linked to the road texture.

\section{9:00}

3aNSd4. Optimized thin layers for urban roads. Sigurd N. Thomsen (Danish Road Institute/Road Directorate, Guldalderen 12, 2640 Hedehusene, Denmark, snt@vd.dk), Hans Bendtsen (Danish Road Institute/Road Directorate, Guldalderen 12, 2640 Hedehusene, Denmark, hbe@vd.dk), Bent Andersen (Danish Road Institute/Road Directorate, Guldalderen 12, 2640 Hedehusene, Denmark, bea@vd.dk)

In the EU project SILENCE work has been carried out to optimize the noise reduction of different types of pavements for urban roads. The Danish Road Institute (DRI) participated in this work together with the Swedish National Road and Transport Research Institute (VTI) and BAST in Germany. Joint international development work has been carried out including laboratory experiments. The objective was to find pavements with promising noise reduction. It was decided to carry out a full scale field testing in some countries. DRI has in cooperation with the municipality of Copenhagen and the Colas road construction company tested series of SMA pavements and open graded pavements, optimized for noise reduction by using small aggregate size of 4 to $6 \mathrm{~mm}$ and by constructing a relatively high builtin air void. Eight different pavements were constructed in June 2007 on Kastrupvej in Copenhagen. DRI has conducted SPB and CPX noise measurements. An initial noise reduction for passenger cars of $4.3 \mathrm{~dB}$ in relation to a DAC 11 reference pavement has been achieved. It is planned to continue the measurements in the coming years in order to be able to analyse the long time acoustical and structural performance of these test pavements.

3aNSd5. Traffic noise levels after replacing the top of two-layer porous asphalt at Øster Søgade. Jørgen Kragh (Danish Road Institute/Road Directorate, Guldalderen 12, 2640 Hedehusene, Denmark, kragh@vd.dk), Hans Bendtsen (Danish Road Institute/Road Directorate, Guldalderen 12, 2640 Hedehusene, Denmark, hbe@vd.dk)

In 1999 three different types of two-layer porous asphalt pavement with $5 \mathrm{~mm}$ or $8 \mathrm{~mm}$ maximum aggregate in the top layer were built in a city street in Copenhagen. A section with dense asphalt concrete was built as a reference. The pavements have been monitored since then. The initial noise reduction of $6-7 \mathrm{~dB}$ compared with the noise level at the reference section has vanished over the years due to gradual clogging of the porosities in the pavement. After 8 years in service the drainage asphalt in the spring of 2007 was worn and ravelling had begun. The municipality of Copenhagen decided to have the top layer milled away and replaced by new porous asphalt with $8 \mathrm{~mm}$ maximum aggregate. This type had proven most durable, acoustically and structurally. Noise measurements were carried out in 2007 as part of the SILENCE project, both before and after replacing the top layer. The measurements showed that at two of the test sections most of the initial noise reduction was regained while this was not the case at the one test section that was thinnest (a total of $55 \mathrm{~mm}$ while the other were $70 \mathrm{~mm}$ and $90 \mathrm{~mm}$, respectively). 


\section{Contributed Papers}

\section{9:40}

3aNSd6. The effect of grinding and grooving on the noise generation of Portland Cement Concrete pavement. Tanya Wulf (Purdue Univ., 140 Martin Jischke Dr., Herrick Lab., West Lafayette, IN 47907, USA, twulf@purdue.edu), Tyler Dare (Purdue Univ., 140 Martin Jischke Dr., Herrick Lab., West Lafayette, IN 47907, USA, tdare@purdue.edu), Robert Bernhard (Purdue Univ., 140 Martin Jischke Dr., Herrick Lab., West Lafayette, IN 47907, USA, rbernhar@nd.edu)

In this investigation, studies have been done to understand the effects of various grinding and grooving parameters to investigate their effect on noise generation at the tire-pavement interface. Grinding uses diamond-infused blades that are closely-spaced such that the fins between the blade tracks break off exposing an entirely new surface. For grooving, the blades are more widely spaced such that the fins do not break off and the surface texture remains largely unchanged except for grooves that are used for moisture control. Both procedures, used independently or in combination, have an effect on the noise produced by the tire-pavement interaction. Variation of grinding parameters was shown to have as much as a $3 \mathrm{~dB}$ effect on noise generation. Variation in grooving parameters has a secondary effect, which allows grooves to be added to texture without overall effect on overall noise. This paper will illustrate the effects on noise of the different parameters, such as grinding depth, blade width, and blade spacing, for grinding and grooving.

\section{0:00}

3aNSd7. Monitoring 20 Silent Roads Over 10 Years In The Municipality Of Groningen. Wim Van Keulen (Vankeulen Advies BV, De Savornin Lohmanlaan 68, 5252 AJ Vlijmen, Netherlands, info @ vankeulenadvies.nl)

The municipality of Groningen in the northern part of the Netherlands has a wide experience with the application of silent roads. This experience covers a period of about 20 tracks with 2-layered porous asphalt and various types of thin layers. These tracks have been and are monitored regularly. The obtained data from 10 years monitoring offer a vast amount of valuable information on noise reduction, durability, maintenance, costs, contracts and policy. The municipality of Groningen in the northern part of the Netherlands has a wide experience with the application of silent roads. This experience covers a period of about 20 tracks with 2-layered porous asphalt and various types of thin layers. These tracks have been and are monitored regularly. The obtained data from 10 years monitoring offer a vast amount of valuable information on noise reduction, durability, maintenance, costs, contracts and policy.

\section{0:20-10:40 Break}

\section{0:40}

3aNSd8. A quiet poroelastic road surface manufactured in a normal asphalt mixing plant. Nils-Åke Nilsson (Acoustic Control AB, Tumstocksvägen 1, SE-187 66 Taeby, Sweden, na.nilsson@acoustic.se), Nils Ulmgren (NCC Roads Sweden AB, R\&D Center, Bryggervägen 13, SE194 36 Upplands Väsby, Sweden, nils.ulmgren@ncc.se), Ake Sandin (City of Goteborg, Traffic and Public Transport Authority, PO Box 2403, 40316 Goteborg, Sweden, ake.sandin@trafikkontoret.goteborg.se)

A poroelastic road surface for low tire/road noise has been developed. It is manufactured using conventional asphalt mixing plants and conventional paving machines. Crumb rubber was added to the mix for attaining the desired elastic characteristics. The crumb rubber was subject to a long term pre-treatment before feeding it to the plant mixer. This ensures both long term durability and excellent adhesion to the binder. The crumb rubber grain size and amount in the mix has been carefully tuned to the aggregate grain size. By the pre-treatment of the crumb rubber the crumb rubber amount can be freely selected since it is not locked to the amount of binder (as for the hot bitumen pre-mixer technique) Single layer poroelastic road surface sections has been paved in Gothenburg. The reduction of noise relative to a SMA16 was found to be 4-6 $\mathrm{dB}(\mathrm{A})$ depending on the vehicle speed and amount of crumb rubber. The surface is almost unaffected by wear after 6 months winter traffic with studded tyres. Tuning the recipe for further increased reduction of the tire/road noise continues. Fundings from European Commission (project Quiet City Transport (QCITY)) is acknowledged.

\section{Invited Paper}

\section{1:00}

3aNSd9. Evaluation of the ASTM Standard Reference Test Tire for purposes of standardized measurement of on-bound tire/pavement noise. Paul Donavan (Illingworth \& Rodkin, Inc., 505 Petaluma Blvd. South, Petaluma, CA 94952, USA, pdonavan @illingworthrodkin.com)

Currently in the US, efforts are underway to develop standard methods for on-board sound intensity (OBSI) measurement of tire/pavement noise. Up until recently, the default standard tire was the Goodyear Aquatred 3 tire originally selected due to its apparent similarity to Tire A specified in the ISO CPX procedure. Because of longer-term availability, the ASTM Standard Reference Test Tire (SRTT) is the primary candidate for replacement of the Aquatred 3. Issues of concern for the SRTT include tire-to-tire variation, the relation of the SRTT to previously used reference tire, and the "break-in" period required for stable test tires. To address tire-to-tire variability, six SRTT's were tested on variety of asphalt (AC) and Portland cement concrete (PCC) surfaces. These included four new tires and two that had been in use for some time. Two of the new tires were retested with increasing use to examine any break-in period effect. For comparison, the older Aquatred 3 was also tested on these same surfaces using both OBSI and controlled pass-by measurements. The results of these measurements are presented along with their implication to for reference tire selection.

\section{Contributed Papers}

\section{1:20}

3aNSd10. Evaluation of effects of pavement characteristics on the OBSI levels using Principal Components Regression. Aybike Ongel (Kultur University, Ataköy Kampüsü Bakırköy, 34156 Istanbul, Turkey, aybikeongel@gmail.com), Erwin Kohler (Dynatest, University of California, Davis, CA 95616, USA, ekohler@dynatest.com), John Harvey (University of California, Civil and Environmental Engineering, Davis, CA 95616, USA, jtharvey@ucdavis.edu)

Tire/pavement noise is a major contributor to traffic noise at highway speeds. Tire/pavement noise is affected by different pavement properties. A study conducted in California measured the noise levels of different mix types and the mix characteristics affecting noise levels. In this study, tire/pavement noise was measured using the on-board sound intensity (OBSI) method. Data was collected on four different types of pavement mixes: conventional open graded asphalt concrete (OGAC), rubberized asphalt concrete that are open graded (RAC-O), rubberized asphalt concrete that are gap graded (RAC-G), and dense graded asphalt concrete mixes (DGAC). A total of 72 field pavement sections were included in the study, all of which were less than 8 years old at the time of the measurements. This paper evaluates the pavement characteristics affecting noise levels using principal components regression. This technique was used due to the multicollinearity found among the variables. Two principal components were extracted from 
the measured parameters such as air void content, gradation properties, pavement roughness, age, and pavement surface condition.

\section{$11: 40$}

3aNSd11. Austrian experience with the backing board method for statistical pass-by measurements. Marco Conter (Arsenal Research, Giefinggasse 2, 1210 Vienna, Austria, marco.conter@arsenal.ac .at), Manfred Haider (Arsenal Research, Giefinggasse 2, 1210 Vienna, Austria, manfred.haider@arsenal.ac.at)

Traffic noise is one of the major environmental concerns within the countries of the European Union. 80 to $90 \%$ of the traffic noise pollution is generated by road traffic. The major part of the noise emitted by vehicles on roads in the mid- to high-speed range is nowadays due to tyre/road noise. It is generated by the interaction between tyre and road surface, and therefore the measurement of the typical noise emission is essential for the classification of pavements with regard to noise. The SPB method (Statistical PassBy, ISO 11819-1) is nowadays the most widely used method to characterise road surfaces, but it is difficult to apply especially in the presence of roadside noise barriers. The backing board method, further developed within the EU Project SILENCE in the past years, offers a possibility to measure and to characterise surfaces also close to reflective objects and in urban environments. This paper describes the first Austrian experimental experience with the application of this variant of the SPB method.

\section{2:00}

3aNSd12. Acoustical characterisation and life-cycle of porous road surfaces. Beate Altreuther (Mueller-BBM GmbH, Robert-Koch-Str. 11, 82152 Planegg, Germany, beate.altreuther@muellerbbm.de), Wolfram Bartolomaeus (Bundesanstalt fuer Strassenwesen, Bruederstr. 53, 51427 Bergisch Gladbach, Germany, bartolomaeus@bast.de)

The German Federal Highway Research Institute (Bundesanstalt für Straßenwesen, BASt) is planning to develop an assessment procedure to characterize the acoustical properties of porous road surfaces. Final objective is to specify the life-cycle of these pavements with respect to their acoustical performance based on civil engineering properties. In 2007 comprehensive measurements of the acoustically relevant parameters absorption coefficient, air flow resistance and surface texture have been performed on a multitude of bavarian motorways with porous asphalt pavements of differen types of construction and different ages. Additional measurements of the near-field rolling noise (CPX) as well as measurements of the statistical pass-by-noise (SPB) have been performed. From precedent projects for the sections under investigation there is an extensive database covering details on asphalt mixtures as well as the results of drill core investigations concerning void content, binder content etc. The results of the present investigation allow to draw new conclusions with respect to acoustical performance and durability of porous asphalt pavements. We gratefully acknowledge the project funding by the German Federal Ministry of Transport, Building and Urban Affairs (project No. FE 02.276/2006/LRB).

\section{$12: 20$}

3aNSd13. DEUFRABASE: A German-French acoustic database on road pavements. Michel C. Berengier (Laboratoire Central des Ponts et Chaussées, Centre de Nantes - Route de Bouaye, BP 4129, 44341 Bouguenais cedex, France, Michel.Berengier@lcpc.fr), Bettina
Droste(Bundesanstalt für Strassenwesen, Brüderstrasse 53, 51427 Bergisch Gladbach, Germany, droste@bast.de), Benoit Gauvreau (Laboratoire Central des Ponts et Chaussées, Centre de Nantes - Route de Bouaye, BP 4129, 44341 Bouguenais cedex, France, benoit.gauvreau@lcpc.fr), Denis Duhamel (ENPC, UR Navier, 6 et 8 Avenue Blaise Pascal, Cité Descartes, Champs sur Marne, 77455 Marne la Vallée, France, duhamel@lami .enpc.fr), Markus Auerbach (Bundesanstalt für Strassenwesen, Brüderstrasse 53, 51427 Bergisch Gladbach, Germany, m.auerbach@bast.de)

In the framework of a DEUFRAKO (German/French cooperation) project on the Prediction and Propagation of Rolling Noise (P2RN), it has been proposed to rank the different German and French road pavements with respect to noise for different long distance configurations representative of real road topographies, ground characteristics and meteorologica situations. After identification of those typical 28 configurations, the attenuations between a reference point in the near field and several receiving points in the far field have been computed according to the most relevant and adapted modelling methods currently available. The main goal being the accuracy of the prediction and not the computation time, analytical (ray tracing) and numerical (BEM, Parabolic Equation) approaches have been implemented. In the following, a technique permitting to estimate day and night LAeq and Lden has been used to classify the various pavements for the whole configurations. All those results have been gathered in a common database (DEUFRABASE) which will be directly available on the website of the authors' Institutes. The paper deals with the description of the ranking procedures, how the database is implemented on the web and how to use it for road traffic noise prediction.

\section{2:40}

3aNSd14. Road noise characterization by Harmonoise procedures reviewed for the Italian case. Luca Alfinito (University of Pisa - Dept. of Civil Engineering, Via Diotisalvi, 2, 56100 Pisa, Italy, 1.alfinito@arpat .toscana.it), Gaetano Licitra (ARPAT - Dept. Firenze, Via Porpora, 22, 50144 Firenze, Italy, g.licitra@arpat.toscana.it), Mauro Cerchiai (ARPAT Dept. Pisa, Via V. Veneto, 27, 56127 Pisa, Italy, m.cerchiai@arpat.toscana it), Lorenzo Magni (ARPAT - Dept. Pisa, Via V. Veneto, 27, 56127 Pisa, Italy, 1.magni@arpat.toscana.it), Massimo Losa (University of Pisa - Dept. of Civil Engineering, Via Diotisalvi, 2, 56100 Pisa, Italy, losa@ing.unipi.it)

Several methods have been introduced to describe acoustical properties of road pavements in situ, such as the extended surface technique (Adrienne), the Statistical Pass-By and the Close Proximity methods. In general the aim of these techniques, normed in ISO standards, is to evaluate some indexes that may be representative of noise generation and propagation with respect to tyre-road interaction and surface absorption. In this paper we use results obtained in the LEOPOLDO project, that involves measurements carried out on many sites all over Tuscany Region characterized by typical conditions of Italian roads, to review the state-of-the-art of acoustical parameters in the modelling of road vehicles showed in the HARMONOISE documents. This task has been accomplished by comparing the theoretical results obtained from the HARMONOISE prediction method with the energy levels measured at the microphone locations close to the road borders, in order to apply corrections to the parameters influencing sound generation and propagation. As a consequence, we introduce a new parameter, related to transfer function between source and receiver sound energy levels, which involves directivity and ground reflection effects, obtaining a more suitable characterization of road surface acoustical behaviour 


\title{
Session 3aNSe
}

\section{Noise, Structural Acoustics and Vibration, Signal Processing in Acoustics, and EURONOISE: Airframe Noise Measurement, Prediction, and Control I}

\author{
Joe W. Posey, Cochair \\ NASA, Langley Research Center, MS 461, Hampton, VA 23681, USA
}

Denis Gely, Cochair

Département Simulation Numérique des écoulements et Aéroacoustique (DSNA), Onera, BP72 - 29 avenue de la Division Leclerc, 92320 Chatillon, France

\section{Invited Paper}

\section{0:00}

\begin{abstract}
3aNSe1. Airframe noise prediction. Yueping Guo (Boeing, 5301 Bolsa Ave, Huntington Beach, CA 92647, USA, yueping.guo @boeing.com)

This presentation discusses the prediction of aircraft airframe noise, namely, the noise that is generated by non-propulsion components of aircraft, including the leaning edge slats, the side edges of flaps and the landing gears. An overview will be given on various methodologies, ranging from empirical prediction to more physics-based modeling to full-blown numerical simulation. The advantages of each method will be discussed and compared with each other to reveal the most suitable application domain of each method. Detailed discussions will be given to the physics-based modeling, such as the acoustic analogy, which is ranked in between empirical and numerical methods in various aspects, including accuracy, turn-around time, limitations on geometry and flow conditions, and applicability/ feasibility in practical design applications. Examples will be given to demonstrate the applications of acoustic analogy in both noise prediction and noise reduction treatment design. Critical elements of the method that can and need to be improved to further mature the method will also be identified and discussed, providing potential directions for future research and development.
\end{abstract}

\section{Contributed Paper}

\section{0:20}

3aNSe2. ONERA's activities on airframe noise numerical simulation. Eric Manoha (ONERA (French aerospace Center), CFD \& Aeroacoustics Department, BP 72, 29 avenue de la division Leclerc, 92322 Chatillon Cedex, France, eric.manoha@onera.fr)

Airframe noise, or noise generated by flow around the airframe (high lift devices, landing gears and cavities), is a major contributor to the overall noise of large commercial aircraft in the approach phase. For several years, ONERA has been involved in the development of methodologies for the numerical simulation of airframe noise. These hybrid methods generally combine (i) CFD computations for the near field flow, including unsteady CFD techniques such as LES, DES and NLDE, and (ii) acoustic (CAA) propagation techniques in the mid- and far-field. A large range of acoustical methods is available for the propagation of sound waves in uniform or heterogeneous mean flows, either from the family of integral methods (Lighthill, FWH, $\mathrm{BEM}$ ) or in the domain of (non-linear or linearized) Euler equations in perturbation. The key challenges of these hybrid methodologie are (i) the ad-hoc coupling between individual techniques and (ii) the availability of reliable and dedicated experimental database for the validation. The paper presents a survey of ONERA's activities in this field, with applications to (i) single symmetrical airfoil, (ii) high lift wing with deployed slat and flap, (iii) landing gears and (iv) cylindrical cavities.

\section{Invited Papers}

3aNSe3. Measurement techniques for airframe noise source identification. Ricardo A. Burdisso (Virginia Tech, Mechanical Engineering, 153 Durham 0238, Blacksburg, VA 24061, USA, rburdiss@vt.edu)

Despite the significant progress achieved over the last three decades, noise pollution generated by aircraft around airports is still an obstacle to the further growth of the aviation industry. The engines and airframe constitute the main sources of the overall aircraft noise. Airframe noise is as important as the engine noise on approach due to the engines operating at low thrust. High-lift devices and landing gears are the dominant airframe noise components. Many efforts have recently been devoted to accurately locate and control the specific components of the airframe noise. Experimental studies on airframe noise include full-scale flight tests, and tests conducted on isolated small-scale models in both hard-walled and anechoic wind tunnels. These experimental efforts on airframe noise have been greatly helped by the advent of the microphone phased array technology. This presentation will focus on microphone phased array measurement techniques, in particular for model scale testing in wind tunnels. Issues such as beamforming algorithms, data corrections, effect of flow and acoustic environment on the measurements (hard-wall vs anechoic tunnels), etc. will be discussed. Illustrative results from experimental measurements of landing gear and high lift devices performed at the Virginia Tech wind tunnel will also be presented. 
3aNSe4. Reduction technologies and prediction of high lift noise. Jan W. Delfs (DLR/Institute of Aerodynamics and Flow Technology, Lilienthalplatz 7, 38108 Braunschweig, Germany, jan.delfs@dlr.de)

In the final approach phase airframe noise represents the ultimate aircraft noise barrier for future aircraft when equipped with quiet UHBR engines. This paper presents some of the main dependencies of high-lift noise sources on flow conditions as derived from extensive noise testing at DLR. Some successful reduction technologies for high-lift noise are discussed in view of their influence on the noise generation mechanisms. The remainder of the paper focusses on the numerical prediction of high-lift noise. The prediction concept as implemented in DLR's PIANO code is discussed. It is based on a two step approach, a) the computation of the time averaged turbulent flow field as a result of a $\mathrm{CFD}(=$ Computational Fluid Dynamics $)$ simulation and b) the highly accurate CAA (=Computational Aeroacoustics) simulation of perturbations about this mean flow. The prediction relies on an advanced stochastic modelling of the turbulence related sources and thus avoids time consuming turbulence simulations. The method represents an accurate yet "low cost" prediction of high-lift noise. This is particularly important for real applications at flight (very high) Reynolds numbers and corresponding design trade-off studies, which typically require the computation of large numbers of configurations. Examples for such kind of applications are given.

\section{Contributed Paper}

\section{1:20}

3aNSe5. Acoustic characterization and noise reduction strategies for a general aviation aircraft. Nicola Rusciano (Dept. of Aerospace Engineering - University of Naples, Via Claudio, 21, 80125 Naples, Italy, nruscian@unina.it), Massimo Viscardi (Dept. of Aerospace Engineering University of Naples, Via Claudio, 21, 80125 Naples, Italy, massimo .viscardi@unina.it)

This work concerns with the vibro-acoustic characterization of a general aviation aircraft with the objective of designing solutions to reduce the inside noise level during the flight; first of all a new passive insulation, and then verifying the possibility of an active noise control approach. The acoustic characterization was performed into two different step; in-flight acoustic and vibration measurements during a typical mission of the vehicle were initially measured, to characterize the acoustic levels and spectra elated to the different flight operations. Ground measurement were also performed to characterize the sound insulation properties of the aircraft structure. The next item was the partial re-design of the sound insulation pack for the vehicle by the use of innovative material; the choice of the best technical solution has been performed by the use of numerical prediction models and through experimental tests on modified samples of the aircraft structure. The in-flight acoustic and vibration measurement showed also the presence of dominant low frequencies tonal components related to the BPF's and upper harmonics. This situation suggested the possibility to implement an active noise control approach. All these activities will be herein presented and discussion of the main results will be performed within the paper.

\section{Invited Paper}

11:40

3aNSe6. On the reduction of flap side edge noise. Florence Hutcheson (NASA Langley Research Center, Hampton, VA 23681, USA, Florence.V.Hutcheson@nasa.gov), Thomas F. Brooks (NASA Langley Research Center, Hampton, VA 23681, USA, thomas.f .brooks@nasa.gov)

Results from flap side edge noise reduction experiments are presented. The test model used was a NACA 632-215 main-element airfoil with a half-span Fowler flap. The flap side edge was flat for the baseline configuration. The effect that small geometric variations of the flap edge have on the noise spectra is shown, and noise measurements from a "continuous mold-line link" (CML) flap configuration are presented. These measurements are compared to those obtained from the baseline flap. The results indicate that a large level of noise reduction is achieved using the CML flap. Finally, Particle Image Velocimetry (PIV) measurements of the flow in the region of a flap side edge are presented for a blowing flap configuration. In this active control approach, air was blown from small slots located along the side edge of the flap to weaken the vortex system that is present in that region of the flap. These measurements are presented to show the effects that the flap tip jets have on the structure of the flap side edge flow, and the potential that this blowing flap configuration has in reducing flap side edge noise.

\section{Contributed Paper}

\section{2:00}

3aNSe7. Landing gear noise assessment on Airbus aircraft. Nicolas Molin (Airbus France, 316 route de Bayonne, Acoustics and Environment Dept EDEA, 31060 Toulouse, France, nicolas.molin@airbus.com)

During the last two decades, airframe noise sources (high lift devices and landing gears) have become noticeable in new aircraft technology, especially during approach. Aircraft manufacturers need prediction tools, in order to include noise modelling at the early stages of aircraft design. Such tools can be developed on the basis of simple formulations. As long as the dominant features are preserved in the simplified problems, the solution is believed to behave correctly in terms of EPNdB. Such a model of landing gear noise prediction was developed in the late 1990s. It is based on the decomposition of a landing gear into different noise sources: the primary structure (massive elements) and the secondary structure (wire, pipes, œ), whose noise is estimated with existing classical source noise model fed with empirical constants based on wind tunnel test results. Airbus implemented this model into its total aircraft noise program and validated it with dedicated flight tests. As the model takes into account all main components with their geometry, it is well adapted to predict the impact of add-on treatment such as fairings on the gear or the impact of an advanced low noise gear with simplified geometry. 


\title{
Invited Paper
}

12:20

3aNSe8. A design-oriented approach to landing gear noise prediction. Philip J. Morris (Penn State University, 233C Hammond Building, University Park, PA 16802, USA, pjm@psu.edu), Kenneth S. Brentner (Penn State University, 233C Hammond Building, University Park, PA 16802, USA, ksb16@psu.edu), Leonard V. Lopes (Penn State University, 233C Hammond Building, University Park, PA 16802, USA, 1vl105@psu.edu)

Landing gear noise is one of the most important sources of airframe noise. But it is the most difficult to predict. The difficulty is that the landing gear is made up of components of substantially different scales. The small-scale features such as tubes and hoses are known to contribute to the landing gear noise, especially in the mid to high frequency range. Existing semi-empirical models are only reliable for the range of landing gear geometries for which test data are available. The present paper describes a component-based landing gear noise prediction model. The method uses an application of simple, fast, and scalable models called acoustic elements that are used to represent the complex landing gear geometry with a high level of geometric detail. Extensions to an earlier model developed by the authors is described, including the replacement of the original time domain approach with a frequency domain method. The local mean flow is also taken into account through the use of low-fidelity computational fluid dynamics simulations. Estimates of the effects of acoustic shielding are also described. Comparisons are made with available experimental data to demonstrate the capabilities of the method. Future improvements are also described.

WEDNESDAY MORNING, 2 JULY 2008

ROOM 251, 10:40 A.M. TO 12:40 P.M.

\section{Session 3aNSf}

\section{Noise and EURONOISE: Soundscape in the Heritage of Urban and Natural Areas I}

\author{
Bennett Brooks, Cochair \\ Brooks Acoust. Corp., 27 Hartford Turnpike, Vernon, CT 06066, USA \\ Giovanni Brambilla, Cochair \\ CNR Institute of Acoustics, Via del Fosso del Cavaliere 100, Rome, 00133, Italy
}

Invited Papers

$10: 40$

3aNSf1. Modeling auditory attention focusing in multisource environments. Bert De Coensel (Ghent University - Department of Information Technology, Sint-Pietersnieuwstraat 41, 9000 Ghent, Belgium, bert.decoensel@intec.ugent.be), Dick Botteldooren (University Ghent - Department Information Technology, Sint-Pietersnieuwstraat 41, 9000 Gent, Belgium, dick.botteldooren@intec .ugent.be)

In this paper, a mathematical submodel for auditory attention focusing is discussed in the framework of our ongoing research towards a unified model for soundscape perception. The submodel implements a balance between top-down focusing, in which higher level cognition guides attention towards expected sources, and bottom-up focusing, in which attention is triggered by the noticing of sound events. Attention elasticity - the ability to switch attention between different environmental sounds - depends on the current context (natural, urban...) as well as on the current activity of the modeled individual. The soundscape perception model is applied to case studies of simulated sound exposure in open area and in environments with a mixture of natural and man-made sounds. A comparison of the simulation results with survey results shows that introducing a submodel for auditory attention focusing into our model for soundscape perception enhances its ability to predict the emergence of annoyance.

11:00

3aNSf2. SOUND-SCAPE: A framework for characterising positive urban soundscapes. Rebecca Cain (WMG, The University of Warwick, International Manufacturing Centre, CV4 7AL Coventry, UK, R.Cain.1@warwick.ac.uk), Paul Jennings (WMG, The University of Warwick, International Manufacturing Centre, CV4 7AL Coventry, UK, paul.jennings@warwick.ac.uk), Mags Adams (Acoustics Research Centre, University of Salford, Newton Building, Salford, M5 4WT Manchester, UK, M.D.Adams@salford.ac.uk), Neil Bruce (Acoustics Research Centre, University of Salford, Newton Building, Salford, M5 4WT Manchester, UK, N.S.Bruce@pgr.salford.ac.uk), Angus Carlyle (London College of Communication, University of the Arts, Elephant \& Castle, SE1 6SB London, UK, angus_carlyle@yahoo.co.uk), Peter Cusack (London College of Communication, University of the Arts, Elephant \& Castle, SE1 6SB London, UK, pcusack@btinternet.com), William Davies (Acoustics Research Centre, University of Salford, Newton Building, Salford, M5 4WT Manchester, UK, w.davies@ salford.ac.uk), Ken Hume (School of Chemistry, Biological and Health Science, Manchester Metropolitan University, Chester Street, M1 5GD Manchester, UK, K.I.Hume@mmu. ac.uk), Christopher J. Plack (Psychology Department, Lancaster University, LA1 4YF Lancaster, UK, c.plack@lancaster.ac.uk)

People's assessments of urban soundscapes are dependent upon many factors. For example, perceptions of a soundscape may depend on the activity that the listener is doing and their associated listening state at the time. Temporal variations (daily, weekly, seasonal) can also affect perceptions of the soundscape, as can the type and usage of urban space, architectural characteristics and the cultural and historical setting. The context of listening is also a factor in terms of the cognitions that people bring to the listening situation by way 
of memories, preferences, attitudes, values and meaning. In order to inform the planning process with regard to assessing and creating positive urban soundscapes, a framework is under development. The framework attempts to bring together all the influencing factors in urban soundscape assessment in a new and novel way, and considers how the identification of sources and the variety and mix of sources interact to produce a soundscape. The framework presented in this paper forms the basis of multi-disciplinary soundscape research in the EPSRC funded "Positive Soundscapes Project", which seeks to develop a rounded view of human perception of soundscapes by combining methods from several disciplines.

\section{1:20}

3aNSf3. Can urban squares be recognized by means of their Soundscape? Giovanni Brambilla (CNR Institute of Acoustics, Via del Fosso del Cavaliere 100, 00133 Rome, Italy, giovanni.brambilla@idac.rm.cnr.it), Maria Di Gabriele (Built Environment Control Laboratory Ri.A.S., Second University of Naples, Abazia di S. Lorenzo, 81031 Aversa, Italy, maria.digabriele@unina2.it), Luigi Maffei (Built Environment Control Laboratory Ri.A.S., Second University of Naples, Abazia di S. Lorenzo, 81031 Aversa, Italy, luigi.maffei@unina2.it), Patrizio Verardi (CNR Institute of Acoustics, Via del Fosso del Cavaliere 100, 00133 Rome, Italy, patrizio .verardi@idac.rm.cnr.it)

Squares have always played important functions in urban areas, being a reference place in the citizen's life. During last decades, road traffic noise has influenced the perception of the sonic environment in many squares. However, there are squares where historical and typical sound sources are still present. In both cases the sonic environment remains a feature of the square together with other factors, like the visual impact. The present paper deals with the above issue and describes an experiment aimed to study how the soundscape of a square can contribute to recognize the square itself. For this purpose some well known squares in Naples and Rome have been selected to cover a range of different characteristics. In each square binaural recordings and pictures have been taken simultaneously. Then the binaural recordings have been played back by headphone to a group of subjects in laboratory simultaneously to the display of the pictures of all squares on a screen. After listening each sound the subject was asked to choose among the pictures the one showing the square judged to have the sonic environment most appropriate to the sound just heard. The comparison between subjective responses and correct answers are in progress.

3aNSf4. Fountains as a natural component of urban soundscape. Catherine Semidor (GRECAU-ENSAPBx, Domaine de Raba, 33400 Talence, France, catherine.semidor@bordeaux.archi.fr), Flora Venot-Gbedji (GRECAU-ENSAPBx, Domaine de Raba, 33400 Talence, France, flora.venot@bordeaux.archi.fr)

Fountains play an important role in urban landscape as well as being a cultural mark in a city. They also have an environmental function. For example, their effect in the control of the microclimate is well known. The paper deals with the sound effects which big fountains located in large squares have on the urban soundscape of the area. In particular, we deal with how their natural character can change the global perception of the site. Indeed, the representation of water sounds is positively appreciated by most city-dwellers. Depending on their size and shape, they are able to give a quiet or a dynamic character to the site. But in the case of areas with a lot of traffic we ask ourselves what are the interactions with strong background noise level and others sound sources. GRECAU uses the soundwalk method to assess the acoustic environment. The following case studies, Plaza de Cataluna in Barcelona and Piazza Ferrari in Genoa, are two examples of such a situation. Some of the results we give here try to answer the previous questions.

\section{2:00}

3aNSf5. Contemporary soundscape of ancient theatres. Kalliopi Chourmouziadou (School of Architecture, University of Sheffield, Western Bank, S10 2TN Sheffield, UK, pepitahou@ hotmail.com), Jian Kang (School of Architecture, University of Sheffield, Western Bank, S10 2TN Sheffield, UK, j.kang@ sheffield.ac.uk)

Ancient drama is performed every summer in ancient theatres in the Mediterranean countries, especially in Greece. In many cases the performance conditions in terms of acoustics are poor, due to the effects of external noise sources. There is often a motorway constructed near an archaeological site, without careful consideration regarding the impact on the overall soundscape. Previous studies have demonstrated the effectiveness of purposely designed scenery on improving the internal acoustic conditions, and this study investigates the contribution of scenery to the improvement of the acoustic environment of ancient theatres in terms of reducing nearby traffic noise. The acoustic simulation for the theatre of Philippi, with scenery design in the form of a background wall in different sizes, suggests that there is a decrease in traffic noise by about 4dB. Further improvements have also been demonstrated with noise barriers of various heights and lengths. Different levels of traffic flow have been examined. It is expected that the results will be verified by on-site measurements and recordings of summer performances will be presented during the conference.

\section{Contributed Paper}

\section{$12: 20$}

3aNSf6. Soundscape and California planning guidelines. Richard B. Rodkin (Illingworth \& Rodkin, Inc., 505 Petaluma Boulevard South, Petaluma, 94952, USA, rrodkin@illingworthrodkin.com), Michael S. Thill (Illingworth \& Rodkin, Inc., 505 Petaluma Boulevard South, Petaluma, 94952, USA, mthill@illingworthrodkin.com)

Community noise has been recognized as a significant environmental concern in California for more than 30 years. All cities and counties are mandated by state law to include a Noise Element as a part of the community's General Plan to provide "a basis for comprehensive local programs to control and abate environmental noise and to protect citizens from excessive exposure." What is, and has been missing from this process, is how the soundscape in a particular setting may affect the compatibility of land uses there. Generalized noise and land use planning guidelines are no longer sufficient. Specific attention must be given to the development of guidelines and policies that address "smart growth" in and around urban and suburban transportation corridors, residential and agricultural interactions, mixed-use developments, industrial-to-residential conversions (e.g., the gentrification of traditionally industrial areas like ports). A new soundscape vocabulary must be introduced to describe the acoustical environments in these settings to facilitate other goals in land use planning. Examples are presented of specific communities where planning goals and traditional noise and land use compatibility guidelines were in conflict and how these were resolved. 


\title{
Session 3aPAa
}

\section{Physical Acoustics: Nonlinear Acoustics of Consolidated Materials and Non Destructive Testing I}

\author{
Jim A. Tencate, Cochair \\ EES-11 (Geophysics) - Los Alamos National Laboratory, MS D443, Los Alamos, NM 87545, USA \\ Koen Van Den Abeele, Cochair \\ K.U.Leuven Campus Kortrijk, E. Sabbelaan 53, Kortrijk, 8500, Belgium
}

\section{Contributed Papers}

\section{8:00}

3aPAa1. Three wave mixing test of hyperelasticity in sedimentary rocks. David L. Johnson (Schlumberger-Doll Research, One Hampshire St., Cambridge, MA 02139, USA, johnson10@slb.com), Kenneth Winkler (Schlumberger-Doll Research, One Hampshire St., Cambridge, MA 02139, USA, kwinkler@boston.oilfield.slb.com), Ralph D'Angelo (Schlumberger-Doll Research, One Hampshire St., Cambridge, MA 02139, USA, dangelo2943@boston.oilfield.slb.com)

We report measurements of 3-wave mixing amplitudes on solids whose 3rd order elastic constants have also been measured by means of the elastoacoustic effect. Because attenuation and diffraction are important aspects of our measurement technique we analyze our results using a frequency domain version of the KZK equation, modified to accommodate an arbitrary frequency dependence to the attenuation. We find that the value of $\beta$ so deduced for polymethylmethacrylate (PMMA) agrees quite well with that predicted from the stress-dependent sound speed measurements, establishing that PMMA may be considered a hyperelastic solid, in this context. The $\beta$ values of sedimentary rocks, though they are typically two orders of magnitude larger than e.g. PMMA's, are still a factor 3-10 less than those predicted from the elasto-acoustic effect. Moreover, these samples exhibit significant heterogeneity on a centimeter scale, which heterogeneity is not apparent from a measurement of the position dependent sound speed.

\section{$8: 20$}

3aPAa2. Fatigue Damage Monitoring and Remaning Life Assessment using Nonlinear Vibro-Modulation Technique. Dimitri Donskoy (Stevens Institute of Technology, Dabvidson Lab., 711 Hudson Street, Hoboken, NJ 07030, USA, ddonskoy@stevens.edu), Alexander Chudnovsky (Univ. of Illinois at Chicago, Fracture Mechanics \& Material Durability Lab., 842 W. Taylor Styreet, Room 2095 ERF, Chicago, IL 60607, USA, achudnov@uic.edu), Andrei Zagrai (New Mexico Institute of Mining and Technology, Department of Mechanical Engineering, 801 Leroy Pl., Weir Bld., Room 124, Socorro, NM 87801, USA, azagrai@nmt .edu), Edvard Golovin (Univ. of Illinois at Chicago, Fracture Mechanics \& Material Durability Lab., 842 W. Taylor Styreet, Room 2095 ERF, Chicago, IL 60607, USA, egolovin@cs.com), Vinod Agarwala (Naval Air Systems Command, 48110 Shaw Road, Bldg. 2187/Suite 2373, Patuxent River, MD 20670, USA, vinod.agarwala@navy.mil)

Assessment of structural deterioration due to in-service and environmental loads is an essential element in ensuring safety, operability and long life of various structures and structural components. We applied vibro-acoustic modulation technique to monitor material degradation at the micro/meso scale before the onset of the macro-scale fracture. The technique explores nonlinear acoustic interaction of high frequency ultrasound and low frequency structural vibration at the site of the incipient damage. It is shown that micro/meso scale degradation increases the material nonlinearity leading to modulation of the high frequency ultrasonic signal by low frequency vibration, quantified by the nonlinear acoustic Damage Index (DI). Numerous tests with hundreds of test coupons proved high sensitivity of the developed technique to micro/meso scale fatigue damage accumulation. The study further demonstrated that the remaining life of the fatigued material can be predicted using phenomenological damage accumulation rule expressed through measured Damage Index. Work supported by NAVAIR.

\section{8:40}

3aPAa3. Thermal ageing evaluation with nonlinear elastic wave spectroscopy method of carbon fiber reinforced plastic composite plates. Faouzi Guenab (LOMC FRE-3102 CNRS Groupe Ondes Acoustique, Place Robert Schuman BP 4006, 76610 le Havre, France, guenab_faouzi @yahoo.fr), Hugues Duflo (LOMC FRE CNRS 3102, Université du Havre, Place Robert Schuman, 76610 le Havre, France, hugues.duflo@univ-lehavre .fr)

The excellent and specific properties of composite materials in the various fields such as aeronautics, transport, energy, the nuclear power and civil engineering led to the need of knowing their behaviours in presence of defect (crack, ageing, delamination...). Therefore, the aim of this paper is to study the thermal ageing of the carbon fibre reinforced composite plates, using their nonlinear behaviours. The method used to achieve this work is the NEWS method (Non-linear Elastic Wave Spectroscopy), where two alternatives of this one are used. The first one is SIMONRUS which involves a study of the nonlinear response of a single resonant mode of the plate according to various amplitudes of excitation. In this case, the nonlinear phenomena analysed are resonance frequency shift and damping characteristic of different level ageing of plate. The second one consists in studying the frequential response and the harmonics generated at the multiple of a resonance frequency $f(a t f, 2 f, 3 f . .$.$) caused by the ageing. Evolution of non$ linear effetcts are then correled to ageing duration

\section{9:00}

3aPAa4. Mesoscopic study of the enhancement of the nonlinearity of an ultrasonic wave in inhomogeneous media. André Moura (University of Heidelberg, Institute of Physical Chemistry, AK Hess, Im Neuenheimer Feld 253,69120 Heidelberg, Germany, Moura@uni-heidelberg.de)

In quantitative nondestructive evaluation of materials it is not still clear why linear elastic waves often exhibit substantial nonlinearity when traveling through inhomogeneous medium, whereas this effect exists but remains minor in most homogeneous media. A current dominant thesis claims that when damaged, intact brittle materials become nonlinear mesoscopic elastic materials. Although this model is consistent with macroscopic observations in the framework of continuum mechanics, it eludes, however, a fundamental aspect, i.e., the detailed nature of the wave/microstructure interaction like scattering and transmission through microcrack. To construct a unified theory, from the mesoscopic to the macroscopic scale, that could take account altogether such so different mesoscopic elastodynamic effects is our objective. Toward this aim, many aspects of the wave/slit interaction are thoroughly analyzed among the clapping effect and nonlinear scattering, and experimental schemes are scheduled. At the current state of our knowledge, a first general postulate is claimed. 
3aPAa5. A phase-pegistration acoustic method for nondestructive testing of porous materials. Valery M. Tsaplev (North-West State Technical University, 5, Millionnaya Street, 191186 Saint-Petersburg, Russian Federation, valery@lek.ru)

The well-known method of complex vibrator is modified for the purpose of meas-urements of complex elastic moduli (Young's and shear moduli) and corresponding values of internal friction, that change under the influence of different external factors. These external factors are: mechanical uniaxial compressive or tension stresses, temperature, electric and/or magnetic fields, etc. Different variants of phase-control methods in the selfexcitation system of the complex vibrator are considered depending on the connection of the active piezoelec-tric bars: active external control or adaptive self-control at prescribed level of the in-put excitation level. The system contains the recording device to record quick changes of elastic and unelastic parameters of the test specimen. The system also contains the termostatic switch with the active control device to provide the specimen temperature control at prescribed algorithm. The method is used for the nondestructive testing of the acoustic properties of different materials under the influence of the stepwise applied mechanical stress.

\section{0:00}

3aPAa6. Nonlinear-nonequilibrium wave propagation in sandstones Donatella Pasqualini (Los Alamos National Laboratory, POBox 1663, Los Alamos, NM 87545, USA, dondy@lanl.gov), Jim A. Tencate (EES-11 (Geophysics) - Los Alamos National Laboratory, MS D443, Los Alamos, NM 87545, USA, tencate@lanl.gov), Salman Habib (Los Alamos National Laboratory, POBox 1663, Los Alamos, NM 87545, USA, habib@lanl.gov)

The transition from linear to nonlinear-nonequilibrium dynamical elasticity in rocks is of considerable interest in seismic wave propagation as well as in understanding the basic dynamical processes in consolidated granular materials. We have carried out a careful experimental investigation of this transition for Berea and Fontainebleau sandstones. These experiments have showed the existence of two strain regimes. At low strain (10-9 up to 10-7) the rocks behave elastically as classical (Landau-Lifshitz theory) nonlinear materials. At higher strains memory effects due to a driven nonequilibrium state complicate the characterization of the nonlinear behavior. The understanding of this second region is not trivial. The main focus of this work is to review the behavior of the low strain, nonlinear region and to report on new experiments meant to understand more about the behavior in the nonequilibrium regime.

\section{Invited Paper}

3aPAa7. Scattering of Rayleigh wave by microcrack with interacting faces. Alexey M. Lomonosov (General Physics Institute, Vavilova str. 38, 119991 Moscow, Russian Federation, lom@kapella.gpi.ru), Peter Hess (Physical Chemistry Institute, University of Heidelberg, Im Neuenheimer Feld 253, 69120 Heidelberg, Germany, peter.hess@urz.uni-heidelberg.de)

Scattering of short Rayleigh pulse by a partially closed surface-breaking microcrack is studied both experimentally and numerically. The microcrack was generated by a nonlinear surface wave with shock. In practice such type of cracks corresponds to fatigue or thermally generated ones. The crack was irradiated by a probe laser-generated Rayleigh. The scattered acoustic field at the surface, monitored by means of optical detector, consisted of the reflected and transmitted Rayleigh waves, longitudinal and shear acoustic waves excited by mode-conversions at the crack. Characteristic size of the crack was 50-100 $\mu \mathrm{m}$, experimental bandwidth was 5-200 MHz. Scattering was simulated numerically in finite differences. For modeling the partially closed crack we proposed a simple one-parameter model, which takes into account weak interaction between the crack faces. It was shown, that interaction changes the predicted frequency-dependent transmission and reflection coefficients significantly. On the basis of fitting the simulated acoustic field to the measured one, the evaluation of the crack size has been performed. In this work frequency dependence of the following three parameters were employed for that purpose: transmission and reflection coefficients, and the time delay of the transmitted pulse. Obtained estimates were compared to the direct microscopic measurement of the crack.

\section{Contributed Papers}

\section{0:40}

3aPAa8. Finite element simulation of the linear and nonlinear interaction of progressive acoustic waves with a standing acoustic wave field. Ebrahim Lamkanfi (Ghent University, Sint-Pietersnieuwstraat 41, 9000 Ghent, Belgium, Ebrahim.Lamkanfi@ugent.be), Nico F. Declercq (Georgia Tech Lorraine - G.W. Woodruff School of ME, UMI Georgia Tech - CNRS 2958, 2 rue Marconi, 57070 Metz, France, nico.declercq@me.gatech.edu), Wim Van Paepegem (Ghent University, Sint-Pietersnieuwstraat 41, 9000 Ghent, Belgium, wim .vanpaepegem@ugent.be), Joris Degrieck (Ghent University, SintPietersnieuwstraat 41, 9000 Ghent, Belgium, joris.degrieck@ugent.be)

Throughout the 20th century studies have been published on the scattering of sound by sound. It has been shown that a combination of nonlinear effects and diffraction effects exists. Approximate models have been developed and some have been verified by experiments. The report presents a finite element simulation of the effect and results in exact solutions for configuration under consideration. It is shown that, depending on the amplitude of the standing wave field, orthogonally incident sound waves diffract. A simplified model is developed for linear interactions, followed by a more realistic model incorporating nonlinear elastic effects. The study is performed in a liquid and also in a solid. Analysis is achieved by Fourier transformation and a comparison is made with diffraction laws governed by the grating equation. For simplicity a 2D model is presented that is extendible however to a $3 \mathrm{D}$ problem should that be necessary. Comparison with some of the earlier published approximate results is also performed. The study shows the detailed influence of the amplitude on the strength of the diffraction effect.

\section{$11: 00$}

3aPAa9. Use of synchronous nonlinear interaction of flexural Lamb waves in NDT-application. Maria Y. Izosimova (Center for Industrial and Medical Ultrasound, Applied Physics Lab., University of Washington, 1013 NE 40th St., Seattle, WA 98105, USA, maria.izossimova@mail .ru), Alexandr I. Korobov (Dept. of Acoustics, Physics Faculty, M.V Lomonosov Moscow State University, Leninskie gory 1, 119991 Moscow, Russian Federation, akor@acs465a-1.phys.msu.ru), Dmitri Mekhedov (Center for Industrial and Medical Ultrasound, Applied Physics Lab., University of Washington, 1013 NE 40th St., Seattle, WA 98105, USA akor@acs465a.phys.msu.ru)

Workability of synchronous nonlinear interaction of flexural Lamb waves in NDT-application is demonstrated. During experiments traveling and standing $\mathrm{A}_{0}$ waves were used. Lamb waves are dispersive, so to provide synchronous nonlinear mode for traveling waves, non-collinear interaction of two waves with frequency $f$ were realized in aluminum alloy plate with defects. In the area of waves' nonlinear interaction, traveling wave of $2 f$ frequency appeared. Standing Lamb waves were excited with amplitudemodulated signal in a cylindrical resonator of aluminum plate. Carrier 
frequency $f$ and modulating frequency $\mathrm{F} \ll f$ are equal to natural modes of the resonator. Spectrum of amplitude-modulated waves contained oscillations with frequencies $f, f+F$, and $f-F$. As a result of interaction, oscillation on heterodyne frequency $F$ appeared in the plate and excited a natural mode. Amplitudes of traveling and standing waves on heterodyne frequencies depend on presence of defects in investigated area (non-classic nonlinearity).
Spatial distribution of amplitudes of initial waves and ones on heterodyne frequency in samples were measured with laser vibrometer PolytecPSV300. In area containing heterogeneities, amplitude increase of waves on heterodyne frequencies was observed. The possibility to define the spatial distribution of defects using measurements of heterodyne wave amplitude distribution was shown experimentally. Work supported by RFBR.

\section{Invited Papers}

\section{$11: 20$}

3aPAa10. Microdamage detection and monitoring using nonlinear elastic wave spectroscopy over a wide frequency range. Bart Van Damme (K.U.Leuven Campus Kortrijk, E. Sabbelaan 53, 8500 Kortrijk, Belgium, Bart.vandamme@kuleuven-kortrijk .be), Pierre-Yves Le Bas (EES-11 (Geophysics) - Los Alamos National Laboratory, MS D443, Los Alamos, NM 87545, USA, pylb@lanl.gov), Koen Van Den Abeele (K.U.Leuven Campus Kortrijk, E. Sabbelaan 53, 8500 Kortrijk, Belgium, koen.vandenabeele @kuleuven-kortrijk.be)

Innovative diagnostic techniques based on Nonlinear Elastic Wave Spectroscopy (NEWS) have been implemented for global inspection of microdamage in intact and damaged aeronautical components using low and high frequency sound waves. For low frequency analysis, we focused on the amplitude dependent analysis of the resonance behavior, both in time and frequency domain. Alternatively, we have analyzed the time-windowed interaction of a hammer impact with a high frequency continuous wave. The results of both techniques agree extremely well, and prove their potential for quick and global microdamage detection. For local inspection, we have implemented a combination of the traditional elastic wave time reversal technique with a phase coded pulse sequence selective excitation. Alternatively, sparse array tomographic reconstruction with NEWS pre-treatment could be used. The nonlinearity based imaging techniques are illustrated on aeronautical components, and discussed in terms of the feasibility and usefulness of the methodologies as new tools for microdamage imaging.

\section{1:40}

3aPAa11. Simulations of nonlinear ultrasonic NDT of plate-like structures. Marieke Geeraert (K.U.Leuven Campus Kortrijk, E. Sabbelaan 53, 8500 Kortrijk, Belgium, Marieke.Geeraert@student.kuleuven.be), Els Janssen (K.U.Leuven Campus Kortrijk, E. Sabbelaan 53, 8500 Kortrijk, Belgium, Els.Janssen@kuleuven-kortrijk.be), Steven Delrue (K.U.Leuven Campus Kortrijk, E. Sabbelaan 53,8500 Kortrijk, Belgium, steven.delrue@kuleuven-kortrijk.be), Olivier Bou Matar (IEMN, UMR CNRS 8520, avenue Poincaré, BP 60069, 59652 Villeneuve d'Ascq, France, olivier.boumatar@iemn.univ-lille1.fr), Erik Blomme (Katholieke Hogeschool Zuid-WestVlaanderen dept. VHTI, Doorniksesteenweg 145, 8500 Kortrijk, Belgium, Erik.Blomme@katho.be), Herbert De Gersem (K.U.Leuven Campus Kortrijk, E. Sabbelaan 53, 8500 Kortrijk, Belgium, Herbert.DeGersem@kuleuven-kortrijk.be), Koen Van Den Abeele (K.U .Leuven Campus Kortrijk, E. Sabbelaan 53, 8500 Kortrijk, Belgium, koen.vandenabeele@kuleuven-kortrijk.be)

Inclusions in materials generally affect the linear wave propagation characteristics of sound waves, observed through scattering, and reduced reflection or transmission with respect to pure materials. The smaller the inclusions, for a fixed ultrasonic frequency, the harder it is to interpret these observations. Therefore, zones of microdamage initiated from fatigue or thermal loading might not induce any linear scattering at all. Nevertheless, the damage information is definitely carried along within the propagating sound wave. In this case, however, it is necessary to investigate the spectral changes of the propagating sound waves, in the form of harmonics and intermodulation frequencies. This contribution reports on simulations of the nonlinear signatures arising in Lamb, Rayleigh and bulk wave propagation in media with localised microdamaged zones. The simulations are performed with a Finite Difference Time Domain solver accounting for microdamage though the inclusion of hysteretic nonlinear stress-strain relations at the microscale. Depending on the configuration it is possible to obtain one or more harmonic images of the defect, their number and relative position containing information about the location and depth of the defect. The simulations allow simple prediction of the response images in complicated experimental conditions.

\section{Contributed Paper}

\section{2:00}

3aPAa12. Fast and Slow Dynamics in Damaged Materials: Correlation to Acoustic Emission. Mourad Bentahar (ENSIM - LAUM, Université du Maine, rue Aristote, 72085 Le Mans, France, Mourad.Bentahar @univ-lemans.fr), Anne Marec (ENSIM - LAUM, Université du Maine, rue Aristote, 72085 Le Mans, France, anne.marec.etu@univ-lemans .fr), Rachid El Guerjouma (ENSIM - LAUM, Université du Maine, rue Aristote, 72085 Le Mans, France, Rachid.El_Guerjouma@univ-lemans .fr), Jean-Hugh Thomas (ENSIM - LAUM, Université du Maine, rue Aristote, 72085 Le Mans, France, jean-hugh.thomas@univ-lemans.fr)

Results are reported of the study of anomalous nonlinear fast dynamics (FD) and slow dynamics (SD) in damaged solids. Experimental observations characterizing FD and SD may be used to detect and eventually characterize induced damage of structural components. Here, we analyze the evolution of the non-linear dynamic behaviour of two polymer-based composites of different reinforcing elements as Sheet Moulding Compound (SMC) and Polymer Concrete (PC) as a function of their gradual damage. More particularly, non-linear FD and SD parameters (time and frequency shift) have been found to be very sensitive to damage creation and evolution for both materials. Besides, acoustic emission monitoring was performed and allowed to calculate the elastic energy released by SMC and PC at every damage stage. Interesting logarithm-like evolution of the followed non-linear parameters as a function of the calculated energy is found. A classification of the collected acoustic emission signals is proposed to understand the contribution of the different damage mechanisms to the evolution of the non-linear behaviour.

\section{2:20-1:40 Lunch Break}




\section{Contributed Papers}

\section{$1: 40$}

3aPAa13. Chirp-coded excitation applied with advanced pulse inversion for nonlinear acoustics in complex steel samples. Serge Dos Santos (ENI Val de Loire, Université François Rabelais de Tours, LUSSI CNRS, rue de la Chocolaterie, 41034 Blois, France, serge.dossantos @univ-tours.fr), Thomas Goursolle (ENI Val de Loire, Université François Rabelais de Tours, LUSSI CNRS, rue de la Chocolaterie, 41034 Blois, France, thomas.goursolle@univ-tours.fr), Martin Drab (Czech Technical University in Prague, Trojanova 13, 12000 Praha 2, Czech Republic, drab@kepler.fjfi.cvut.cz), Tomas Slunecko (ZD Rpety - DAKEL, Ohrobecka 408, 14200 Praha 4, Czech Republic, drab@kepler.fjif.cvut.cz)

Nonlinear acoustics for nondestructive testing is still an emergent technique in complex materials with localized cracks because linear effects can be cancelled using specific symmetries for the pre- and post-signal processing. The local nonlinear signature is accessible even in complex aeronautic structure by using the symmetry properties: i. e. time reversal invariance and pulse inversion associate to chirp-coded excitation. Excitation Symmetry Analysis Method [Dos Santos et al., JNLM, 2007] which is an extension of the pulse inverted method for extraction of third order nonlinearity is applied experimentally for the measurement of relative level of nonlinearity on a airplane steel bracket, on which visible crack was created by fatigue tests. This pre-processing method has been coupled to a 100-250 $\mathrm{kHz}$ bandwidth chirp-coded ultrasonic excitation, realized at various amplitude $\left(A, A / 2,3^{1 / 2} A / 2\right.$, etc.), and performed with DAKEL IDK09 $200 \mathrm{kHz}$ PZT sensors. Signal processing based on correlation measurements, allow an extraction of the nonlinear signature versus fatigue level. Experiments and data processing have been conducted with a 12 bit DAKEL-DTR system in the bandwidth $80-800 \mathrm{kHz}$ and compared with a continuous 4 channel acoustic emission device.

2:00

3aPAa14. Determination of symmetry relations between higher order material constants for the study of nonlinear acoustic properties of piezoelectric crystals of any symmetry class. Nico F. Declercq (Georgia Tech Lorraine - G.W. Woodruff School of ME, UMI Georgia Tech - CNRS 2958, 2 rue Marconi, 57070 Metz, France, nico.declercq@me.gatech.edu)

Each crystal is invariant under a given point symmetry operation. This results in relations between the different material property constants, such as second order elastic constants, second order piezoelectric constants and second order dielectric constants. Those relations are well reported in text books for the linear behavior of crystals. When nonlinear effects are studied, higher order constants play a significant role and their symmetry relations have only been reported in the literature for a limited number of crystal classes. The problem with higher order constants is that it is rather complicated and very time consuming to study the symmetry relations. This paper presents a newly developed semi-automatic computer program that is able to handle any kind of symmetry relation. Results are reported for all the different crystal classes for the higher order constants, i.e. third order elastic constants, third order piezoelectric constants, third order dielectric constants and electrostrictive constants. The results are important for the study of nonlinear properties of crystals and composites and will ease the experimental quantitative determination of all linear and nonlinear material constants of crystals.

\section{2:20}

3aPAa15. Nonlinearity, hysteresis, end-point memory, and congruence in sandstones. Kevin E. Claytor (Los Alamos National Laboratory, POBox 1663, Los Alamos, NM 87545, USA, ke.claytor@gmail .com), Joseph R. Koby (Los Alamos National Laboratory, POBox 1663, Los Alamos, NM 87545, USA, jkoby@lanl.gov), Jim A. Tencate (EES-11 (Geophysics) - Los Alamos National Laboratory, MS D443, Los Alamos, NM 87545, USA, tencate@lanl.gov)

Very low frequency stress-strain measurements on many rocks show definite and repeatable hysteresis loops. Much of the modeling of this behavior uses Preisach or Ising-like models adapted from models developed for magnetic systems which exhibit hysteresis. To date, the experimental data on many rocks showing end-point memory are noisy and only qualitative. The congruence property, in fact, has never been examined for rocks. Just how well does the behavior of a sandstone fit a Preisach model? To find the answer to this question, we performed a new set of careful stressstrain experiments on several sandstones to examine end-point memory (in detail) and also to see if the congruence property does indeed hold for these rocks. In addition we explored the dependence of measurement rate on the relaxation time of these rocks (the analog to magnetic aftereffect). For very slow measurement rates (low frequencies), hysteresis disappears, leaving only a simple nonlinear stress-strain curve.

\section{2:40}

3aPAa16. Foundations of the ultrasonic non-destructive method of determination of stresses in near-the-surface layers of solids. Alexander Guz (Institute of Mechanics, Nesterov str. 3, Kiev, 03057 Kiev, Ukraine, guz@carrier.kiev.ua)

The ultrasonic non-destructive method under consideration is intended for measurements of uniaxial and two axial stresses in the near-the-surface layers of relatively rigid materials (metals, alloys and similar material). This method is intended for measurements of the actual, assemble, operating, preload prestress and other stresses. Above mentioned stresses must be considered as initial or residual stresses taking into account the theory [1] under consideration. In this case the disturbances (displacements and stresses of the 3-D linearized theory [1] of elastic waves in bodies with initial or residual stresses) arise due to ultrasonic vibrations. Description of the nondestructive method under consideration and information on instruments and devices for measurements are presented. Some examples of non-destructive determination of stresses in near-the-surface layers of materials are presented also as applied to the residual stresses arising at electric welding and to the operating stresses arising at loading. Additional information is presented in monograph [1]. References: 1.Guz A.N. Elastic waves in bodies with initial (residual) stresses. Kiev: "A.C.K." Publishers, 2004. - 672 p. (In Russian).

\section{3:00}

3aPAa17. Effect of Water Saturation on the Nonlinear Elastic Response of Concrete. Cédric Payan (LCND - Université de la Méditerranée, IUT Aix Provence, Avenue Gaston Berger, 13625 Aix en Provence Cedex, France, cedric.payan@univmed.fr), Vincent Garnier (LCND - Université de la Méditerranée, IUT Aix Provence, Avenue Gaston Berger, 13625 Aix en Provence Cedex, France, vincent.garnier@univmed.fr), Joseph Moysan (LCND - Université de la Méditerranée, IUT Aix Provence, Avenue Gaston Berger, 13625 Aix en Provence Cedex, France, joseph.moysan @ univmed.fr)

Nonlinear interaction of a monochromatic elastic wave with a low frequency should be a good tool for non-destructive evaluation of existing concrete structures. This technique has already proofed efficient in detecting global damage. However, it is necessary to understand the influence of some structural parameters as porosity, stress state, or water saturation on the nonlinear processes. In this way, a thermodynamically based model containing all of them is presented in this paper. It is sustained by nonlinear interaction measurements in order to quantify the evolution of the nonlinear behaviour of concrete with water saturation state.

\section{3:20}

3aPAa18. The Parametric Array in Berea Sandstone, Revisted Pierre-Yves Le Bas (EES-11 (Geophysics) - Los Alamos National Laboratory, MS D443, Los Alamos, NM 87545, USA, pylb@lanl.gov), Jim A Tencate (EES-11 (Geophysics) - Los Alamos National Laboratory, MS D443, Los Alamos, NM 87545, USA, tencate@lanl.gov)

Previous measurements of the characteristics of the parametric array in sandstone by Johnson and Shankland [J. Geophys. Res., 94, 17729-17734 (1989)] were difficult to perform and, given the apparatus of the day, only qualitative. Since then, memory effects have been found to play a strong role in the dynamic behavior of rocks. This experimental study was performed to 
find out how well the "classical" theory of nonlinear acoustics, i.e., all compressional waves with no memory effects, works at describing the parameteric array in a granular solid. An array of PZT disk transducers (every other one broadcasting one of two frequencies) was epoxied to a large block of Berea sandstone $(1.2 \times 0.4 \times 0.4 \mathrm{~m})$. The two primary frequencies were near $100 \mathrm{kHz}$ and chosen to produce a difference frequency around $15 \mathrm{kHz}$. Using a scanning laser vibrometer (Polytec), several scans of the beampatterns were made at the opposite end of the rock and the results compared with calculations based on those commonly used for parametric arrays in water. A narrow beam generated from the two primaries was measured and the beampattern compared with those of the primaries. The agreement is surprisingly good using a beta of around 200 and a $\mathrm{Q}$ of about 50 .

\title{
Session 3aPAb
}

\section{Physical Acoustics: Acoustic Probes of Planetary Environments I}

\author{
Andi Petculescu, Cochair \\ University of Louisiana, Department of Physics, PO Box 44210, Lafayette, LA 70504, USA \\ Martin C. Towner, Cochair \\ PSSRI, The Open University, Walton Hall, Milton Keynes, MK7 6AA, UK
}

\section{Invited Paper}

8:00

\begin{abstract}
3aPAb1. The atmospheres of Mars, Venus and Titan: observed and modelled structures. Sebastien Lebonnois (Laboratoire de Meteorologie Dynamique, IPSL/CNRS, 4 place Jussieu, box 99, 75252 Paris Cedex 05, France, sllmd@1md.jussieu.fr)

In our solar system, three bodies possess atmospheres presenting similar characteristics with our own environment: our neighbours Mars and Venus, and also Saturn's largest satellite Titan. The comparative study of these different atmospheric systems provides many insights on their respective behaviour, and helps understand the processes at work within these complex systems. In this review, the observed vertical and latitudinal structures of these atmospheres are presented, including their variability and seasonal evolution. Waves that have been observed are also shown, e.g., thermal tides, gravity waves. Global Climate Models that have been developed to study such atmospheric systems are also presented. Using these tools, production and role of the different waves present in each atmosphere may be studied.
\end{abstract}

\section{Contributed Paper}

\section{$8: 20$}

3aPAb2. Bach on other planets or why acoustic sensors make sense in planetary science. Andi Petculescu (University of Louisiana, Department of Physics, PO Box 44210, Lafayette, LA 70504, USA, andi @louisiana.edu), Timothy G. Leighton (Institute of Sound and Vibration, Univ. of Southampton, University Road, Highfield, SO17 1BJ Southampton, UK, T.G.Leighton@soton.ac.uk)

The European Huygens probe, which landed on Titan in 2005, is the only planetary probe so far to incorporate passive and active acoustic sensing. Acoustic wave motion is intimately coupled with an atmosphere and thus can give first-hand information on that environment. Motivation for acoustic sensing in planetary atmospheres abounds: anemometry, turbulence measurements, soil characterization, sound generation by lightning, acoustics of liquid hydrocarbon flows, cryovolcanoes, dust devils, quantitative fluid spectroscopy, thermometry, atmospheric boundary layers etc. In this paper it is shown quantitatively how the environments of Mars, Venus, and Titan affect the generation and propagation of audible sound differently. To exemplify, a musical fragment with a rich frequency content was chosen (Bach's Toccata and Fugue in D-minor played by one of the authors) and passed through the three planetary "'filters." Appreciation of the nature of these sounds will inform the design of acoustic sensors for future space probes. Furthermore, a demonstration that the physics is sufficiently understood to predict these sounds indicates that the same understanding can be applied quantitatively to interpret alien sounds to estimate the environmental conditions.

\section{Invited Papers}

3aPAb3. Use of microphones to record windspeed and turbulence in planetary environments. Ralph D. Lorenz (JHU Applied Physics Lab, 11100 Johns Hopkins Road, Laurel, MD 20723, USA, ralph.lorenz@jhuapl.edu)

A major feature of any planetary acoustic measurement is noise due to ambient winds, or to the motion of the measurement platform through the air. Venera 13 and 14 recorded sound on the surface of Venus: the amplitude of the microphone signal was interpreted (by dynamic pressure scaling) to recover a windspeed. The Huygens probe at Titan also recorded sound levels during descent and on the surface with a passive microphone and an acoustic sounder. The author has made passive microphone measurements made on a rotating aerospace vehicle (an instrumented Frisbee) which show spin modulation and a variation with flight speed and angle of attack. He has also used microphones as a crude wind sensor on an array of 20 meteorological stations used to study dust devils in Arizona. All of these observations will be reviewed. Lessons learned for future planetary measurements, and prospects and methods for microphonic anemometry (especially with limited telemetry bandwidth) will be discussed. 
3aPAb4. The Huygens Surface Science Package sound speed measurements and the methane content of Titan's atmosphere. A. Hagermann (PSSRI, Open University, Walton Hall, MK7 6AA Milton Keynes, UK, unknown@unknown.com), Martin C. Towner (PSSRI, The Open University, Walton Hall, MK7 6AA Milton Keynes, UK, m.c.towner@open.ac.uk), Philip D. Rosenberg (PSSRI, Open University, Walton Hall, MK7 6AA Milton Keynes, UK, p.d.rosenberg@open.ac.uk), James R. Garry (PSSRI, Open University, Walton Hall, MK7 6AA Milton Keynes, UK, jrcgarry@hotmail.com), Hakan Svedhem (European Space Agency, Keplerlaan 1, NL 2201 AZ Noordwijk ZH, Netherlands, hsvedhem@ rssd.esa.int), Mark R. Leese (PSSRI, Open University, Walton Hall, MK7 6AA Milton Keynes, UK, m.r.leese@open.ac.uk), Brijen Hathi (PSSRI, Open University, Walton Hall, MK7 6AA Milton Keynes, UK, b.hathi@open.ac.uk), Ralph D. Lorenz (JHU Applied Physics Lab, 11100 Johns Hopkins Road, Laurel, MD 20723, USA, ralph.lorenz@jhuapl.edu), John C. Zarnecki (PSSRI, Open University, Walton Hall, MK7 6AA Milton Keynes, UK, j.c.zarnecki @open.ac.uk)

The Huygens probe descended through Titan's atmosphere in January 2005. On board was the Surface Science Package (SSP), a set of nine sensors, which included a speed-of-sound sensor. We present a detailed description of the SSP speed of sound measurements and report constraints on the methane content in Titan's lower atmosphere based on these measurements. After extensive instrument calibration and subsequent Bayesian analysis of the data, the most likely result derived from our measurements in Titan's lower atmosphere is a methane fraction of approximately $2 \%$ at $10 \mathrm{~km}$, increasing to $3.5 \%$ at lower altitudes. These estimates are based on a binary composition. Our data show that any large scale variation of methane within the lower $11 \mathrm{~km}$ of Titan's atmosphere is unlikely. Within experimental and theoretical uncertainties, our estimates are lower than, but compatible with earlier estimates obtained from the mass spectrometry experiment.

\section{9:20}

3aPAb5. Waterfalls in space, and other problems of 'underwater' acoustics on a small planet. Timothy G. Leighton (Institute of Sound and Vibration, Univ. of Southampton, University Road, Highfield, SO17 1BJ Southampton, UK, T.G.Leighton @ soton.ac.uk), Paul R. White (Institute of Sound and Vibration, Univ. of Southampton, University Road, Highfield, SO17 1BJ Southampton, UK, prw@isvr.soton.ac.uk), Daniel C. Finfer (Institute of Sound and Vibration, Univ. of Southampton, University Road, Highfield, SO17 1BJ Southampton, UK, d.c.finfer@ soton.ac.uk)

Whilst extraterrestrial liquids do occur in the Solar System, today's acoustical oceanographers have fewer sites to which they can apply their experience of Earth's oceans than perhaps they would have had in the early Solar System, with its magma oceans. Possible sites are Saturn's moons Titan and Enceladus, and Jupiter's moon Europa. The ability to transfer our understanding of Earth's acoustical oceanography to other moons and planets is particularly valuable, given that current understanding is sufficient to undertake complex inversions to estimate Earth's ocean environmental parameters from relatively sparse, or even naturally-occurring, acoustical signals. However such transference should be done with care, as terms familiar in Earth's acoustical oceanography may not be correct on other worlds. For example, in a deep ocean on a small world (such as Europa), the hydrostatic pressure will not equal the product of the density, the depth, and the surface value of the acceleration due to gravity, since the latter can vary with depth, and because vertical lines are not parallel on a small planet. This paper explores two cases of transferring our terrestrial experience off world, to the ice seas of Europa, and to the methane lakes and waterfalls of Titan.

\section{9:40-10:00 Break}

\section{0:00}

3aPAb6. Acoustic Anemometry on Mars. Jean-Pierre Williams (California Institute of Technology, Division of Geological and Planetary Sciences, MC 150-21, Padadena, CA 91125, USA, jpierre@gps.caltech.edu), Ian McEwan (California Institute of Technology, Division of Geological and Planetary Sciences, MC 150-21, Padadena, CA 91125, USA, ijm@gps.caltech.edu)

Acoustic anemometers provide wind measurements with high sensitivity, temporal resolution, and accuracy, and are in many ways superior to the hot wire technique anemometers currently and previously flown to Mars. An anemometer is under development that employs an internal model of how sound is changed by a particular gas mixture with respect to wind speed and temperature to derive these values. This is done by measuring the transfer function (attenuation and phase versus frequency) of sound between two transducers with a very wide spread-spectrum signal and comparing this with its internal model. While sonic anemometry is the premier technique for research studies of winds on Earth, the acoustic properties differ substantially from atmosphere to atmosphere and it is not valid to assume that an existing terrestrial system can provide heritage for a planetary instrument. Further, current multi-gas acoustic models rely on constants derived from experiments performed in conditions far from the planetary application intended here. Since the parameters of the model must be well defined in our approach, the instrument's ability to accurately measure transfer functions will be used in experiments to improve our knowledge of the behavior of various calibrated gas mixtures over relevant temperatures and pressures.

\section{0:20}

3aPAb7. An ultrasonic anemometer for Mars. Colin Wilson (University of Oxford, Department of Atmospheric, Oceanic and Planetary Physics, OX1 3PU Oxford, UK, wilson@atmos.ox.ac.uk), Lee A. Davis (University of Warwick, School of Engineering, CV4 7AL Coventry, UK, lee.davis@warwick.ac.uk), David Hutchins (University of Warwick, School of Engineering, CV4 7AL Coventry, UK, D.A.Hutchins@warwick.ac.uk), Martin C. Towner (PSSRI, The Open University, Walton Hall, MK7 6AA Milton Keynes, UK, m.c.towner@open.ac.uk)

Ultrasonic anemometers are often used for studies of 3-D atmospheric turbulence on Earth, due to their robust calibration and fast operation $(>10 \mathrm{~Hz})$. The same qualities make ultrasonic anemometry attractive for use on Mars, where similar atmospheric turbulence is found. The low density of Martian atmosphere - a hundredth that of the Earth's - is problematic, because of the large acoustic impedance mismatch between the atmosphere and piezoelectric transducers. One solution to this problem is to use piezoelectric transduc- 
ers with a hornlike resonator bonded to their front surface. Another solution is to use a capacitive membrane transducer instead. We report on performance of various ultrasonic transducers in carbon dioxide Martian pressures, and present an instrument design capable of meeting the stringent mass, power, and environmental requirements of the European ExoMars lander.

10:40

3aPAb8. Solid-fluid coupling on planets: from seismology to acoustics and beyond. Raphael F. Garcia (DTP-OMP Univ. Toulouse, 14 Avenue E. Belin, 31400 Toulouse, France, garcia@dtp.obs-mip.fr), Philippe Lognonné (IPGP, 4 avenue de Neptune, 94107 Saint Maur des Fossés, France, lognonne@ipgp.jussieu.fr), Giovanni Occhipinti (IPGP, 4 avenue de Neptune, 94107 Saint Maur des Fossés, France, ninto@ipgp.jussieu.fr)

On Earth, solid-fluid coupling is responsible for acoustic signals created by quakes or volcanic eruptions which have been observed by infrasonic sensors or through ionospheric perturbations produced by infrasounds. Similarly volcanic explosions, ocean surface waves and ocean internal gravity waves are producing seismic signals. A specific normal mode coupling theory has been developed for the whole solid/ocean/atmosphere Earth system in order to model these phenomena. Recent developments for the observation and modeling of the infrasonic waves created by quakes and tsunamis have focused on the ionospheric perturbations produced by the exponential amplification of vertically propagating infrasonic and gravity waves. On Earth, these tools are particularly interesting for the observation of seismic surface waves and tsunamis in the open ocean where sensors are not present. However, the interest is even stronger to infer the internal structure of planets for which the deployment of seismometers is almost impossible. Theoretical modeling of solid/atmosphere coupling on Venus is presented, including open questions concerning the attenuation of infrasounds by Venus atmosphere. An attempt to observe quake signals with VIRTIS instrument on board Venus Express ESA mission is also described. Finally, a mission analysis tool and other ideas for future planetary investigations of atmospheric acoustic normal modes are discussed.

\title{
Session 3aPAc
}

\section{Physical Acoustics and Computational Acoustics: Combustion Noise and Thermo-Acoustics I}

\author{
Tim Lieuwen, Cochair \\ School of Aerospace Engineering, Georgia Institute of Technology, Atlanta, Georgia 30332-0150, USA
}

Maria A. Heckl, Cochair

Keele University, Staffordshire, Newcastle-under-Lyme, ST5 5BG, UK

Rafael Piscoya, Cochair

Technische Fachhochschule Berlin, Univ. of Applied Sciences, Luxemburger Str. 10, Berlin, 13353, Germany

\section{Invited Papers}

\section{8:00}

3aPAc1. Sound production by partially premixed flames in a stagnation flow. Thierry Schuller (Ecole Centrale Paris, Laboratoire EM2C - CNRS, Grande-Voie des Vignes, 92295 Chatenay-Malabry, France, thierry.schuller@em2c.ecp.fr), Patrick Duchaine (Ecole Centrale Paris, Laboratoire EM2C - CNRS, Grande-Voie des Vignes, 92295 Chatenay-Malabry, France, patrick.duchaine @em2c.ecp.fr), Laurent Zimmer (Ecole Centrale Paris, Laboratoire EM2C - CNRS, Grande-Voie des Vignes, 92295 ChatenayMalabry, France, laurent.zimmer@em2c.ecp.fr)

In the perspective of combustion noise reduction, understanding mechanisms of sound generation from combustion of non perfectly mixed reactants is a major challenge. Sound production is thus investigated for vortices impinging a flat laminar flame in a stagnation flow. Such a configuration helps to better understand sound production from turbulent flames. A study with different compositions of the main flow and surrounding gases - mixtures of nitrogen, air and methane - is carried out under the same mean flow and perturbations conditions. Time traces of radiated sound pressure and chemiluminescence emission from radical species present in the reaction zone are characterized together with detailed imaging of the flame and flow motions. Elements of classical combustion noise theory are used to identify the main noise sources when air or fuel is entrained by the vortex interacting with the flame. 
3aPAc2. Flow field and acoustic characteristics of an acoustically forced burner impinging jet. Yang Zhang (The University of Manchester, School of Mechanical, Aerospace and Civil Engineering, George Begg Building, M60 1QD Manchester, UK, yang.zhang@manchester.ac.uk), Saad Hajim (The University of Manchester, School of Mechanical, Aerospace and Civil Engineering, George Begg Building, M60 1QD Manchester, UK, saad.hajim@manchester.ac.uk), Jason Bassi (The University of Manchester, School of Mechanical, Aerospace and Civil Engineering, George Begg Building, M60 1QD Manchester, UK, jason.bassi @ manchester.ac.uk), S Farhat (The University of Manchester, School of Mechanical, Aerospace and Civil Engineering, George Begg Building, M60 1QD Manchester, UK, s.farhat@manchester.ac.uk)

The flow field and acoustic characteristics of a turbulent impinging jet from an impinging burner have been investigated experimentally. The effect of acoustically excited upstream flow with a range of driving frequencies from 0 to $3000 \mathrm{~Hz}$ and use of perforated plate near the nozzle have been studied to achieve modifications to the near-field and far-field of the jet. The acoustic response of the burner to upstream acoustically excited flow under different geometry configurations was investigated in detail. The burner components were found to have a significant effect on the burner acoustic response, with overall damping and enhancement achieved over the full driving frequency range. The velocity flow field of the impinging jet, including spectra and rms data, were mapped from the burner nozzle to the plate with CTA measurements with three acoustic excitation frequencies and no excitation; $0 \mathrm{~Hz}, 52.5 \mathrm{~Hz}, 75 \mathrm{~Hz}$ and $530 \mathrm{~Hz}$ (within acoustic mode bands of burner). The results show that excitation frequencies of $52.5 \mathrm{~Hz}$ and $75 \mathrm{~Hz}$ were found to propagate all the way to the plate, whilst $530 \mathrm{~Hz}$ dissipates near the nozzle exit. ( $\mathrm{Re}=16,200, \mathrm{H} / \mathrm{D}=3$, nozzle velocity profile).

\section{8:40}

3aPAc3. Prediction of Self Excitation Frequencies and Amplitudes for a Model Gas Turbine Burner. Catherine Gardner (Queen Mary University of London, School of Engineering and Material Science, Mile End Road, E1 4NS London, UK, catherine .gardner@qmul.ac.uk), S. M. Reza Hosseini (Queen Mary University of London, School of Engineering and Material Science, Mile End Road, E1 4NS London, UK, s.m.hosseini@qmul.ac.uk), Chris J. Lawn (Queen Mary University of London, School of Engineering and Material Science, Mile End Road, E1 4NS London, UK, c.j.lawn@qmul.ac.uk)

Forced excitation of a swirl stabilised methane/air flame by acoustic waves at atmospheric pressure has been characterised in order to show that the frequencies and amplitudes where self-excitation occurs, but the natural limit cycle takes effect, can be predicted from these data. Chemiluminescent emission was therefore recorded to measure the Flame Transfer Function (FTF), as the flame was acoustically excited by two loud speakers. The experiments covered a wide range of frequencies and amplitudes with particular emphasis on the FTF at high amplitudes of excitation, where a non-linear response is often reported. The system was modelled by the 1D thermoacoustic element method, in which each acoustic element, such as a duct or a contraction, is described by a four-pole matrix. The flame is incorporated as another four-pole matrix that describes the measured flame transfer function. The solution of this set of equations without forcing predicts self-excitation frequencies and amplitudes that can be compared with the experimental data. The minimum magnitudes of the FTF for excitation may also be calculated. When proven at this small scale, the procedure will be applied to predict the limit cycling of actual installations from rig tests.

\section{9:00}

3aPAc4. Low-order modelling of unsteady flame behaviour in a spray combustor. David M. Arthur (University of Cambridge, Department of Engineering, Trumpington Street, CB2 1PZ Cambridge, UK, david.arthur@ cantab.net), Ann P. Dowling (University of Cambridge, Department of Engineering, Trumpington Street, CB2 1PZ Cambridge, UK, apd1@ cam.ac.uk), Stewart Cant (University of Cambridge, Department of Engineering, Trumpington Street, CB2 1PZ Cambridge, UK, rsc10@eng.cam.ac.uk)

The effect of mass flow rate perturbations on the stability of a liquid-fulled annular aeroengine gas turbine is investigated using a three-dimensional uRANS code. The simulations are performed on a single sector of an idealised annular gas turbine geometry. A Lagrangian fuel spray description is used with a Monte Carlo solution of Williams' spray equation. Broadband mass flow fluctuations are imposed at the combustor inlet and dilution ports, and the effect on heat release is quantifed using flame transfer functions. The excitation of inlet and dilution flows leads to signifcant unsteady combustion and the implications for self-excited oscillations are considered. The numerical results are then compared to a low-order model which is developed to account for the different physical mechanisms responsible for unsteady heat release when the inlet and dilution port air mass flow rates are perturbed. The low-order model is used to predict the possibility of self-excited oscillations as well as to investigate the potential of altering the phase and relative magnitude of the inlet and dilution port excitation as a possible means of controlling thermoacoustic oscillations.

9:20

3aPAc5. Mode switching of combustion instabilities associated with flame transfer function non-linearities. Daniel Durox (Ecole Centrale Paris, Laboratoire EM2C - CNRS, Grande-Voie des Vignes, 92295 Chatenay-Malabry, France, daniel.durox@em2c.ecp.fr), Nicolas Noiray (Ecole Centrale Paris, Laboratoire EM2C - CNRS, Grande-Voie des Vignes, 92295 Chatenay-Malabry, France, nicolas.noiray@em2c.ecp.fr), Thierry Schuller (Ecole Centrale Paris, Laboratoire EM2C - CNRS, GrandeVoie des Vignes, 92295 Chatenay-Malabry, France, thierry.schuller@em2c.ecp.fr), Sebastien Candel (Ecole Centrale Paris, Laboratoire EM2C - CNRS, Grande-Voie des Vignes, 92295 Chatenay-Malabry, France, sebastien.candel@em2c.ecp.fr)

Studies of combustion instabilities indicate that the frequency of oscillation changes during the growth of oscillations before a limit cycle is reached. It is also found that a system can switch from one mode to another when the amplitude exceeds a certain threshold. It is shown that these features can be linked to the flame response non-linearity. The analysis is carried out by examining combustion instabilities appearing on a multipoint injection burner, placed a the end of a cylindrical manifold. This element acts like a resonator and its length can be modified easily. Experiments indicate that mode switching takes place between two resonant modes of the cavity and this is shown to be caused by the non-linearity of the flame response. The phenomenon can be analyzed by considering the non-linear 
evolution of the flame transfer function. It is possible to combine this information with a stability analysis of the burner, and define conditions which give rise to mode switching. It is found that this behavior is related to a change in phase difference between heat release rate fluctuations and velocity perturbations at the burner outlet. This phase shift takes place as the amplitude of oscillation increases.

9:40

3aPAc6. Impact of acoustic boundary conditions on confined combustion noise. Ammar Lamraoui (Ecole Centrale Paris, Laboratoire EM2C - CNRS, Grande-Voie des Vignes, 92295 Chatenay-Malabry, France, ammar.lamraoui@em2c.ecp.fr), Franck Richecoeur (Ecole Centrale Paris, Laboratoire EM2C - CNRS, Grande-Voie des Vignes, 92295 Chatenay-Malabry, France, franck.richecoeur@em2c.ecp.fr), Thierry Schuller (Ecole Centrale Paris, Laboratoire EM2C - CNRS, Grande-Voie des Vignes, 92295 Chatenay-Malabry, France, thierry.schuller@em2c.ecp.fr), Sébastien Ducruix (Ecole Centrale Paris, Laboratoire EM2C - CNRS, Grande-Voie des Vignes, 92295 Chatenay-Malabry, France, sebastien.ducruix@ecp.fr)

The noise produced by aircraft engines at take-off and landing can be very harmful for airport neighbors, an issue that became recently particularly crucial. While combustion instabilities, where a strong coupling between flames and acoustic modes of the combustion chamber cause very high levels of harmonic sound, the wide bandwidth noise emitted by confined flames in stable situations is less well-documented. It has been recently shown by Tran et al. (2007) that varying the burner upstream acoustic boundary condition was a very efficient method for damping combustion instabilities and creating ideal conditions for the study of combustion noise. In the present study, the noise level induced by stable combustion is investigated for various upstream conditions. The test bench consists in a swirl stabilized burner, connected to a rectangular combustion chamber, equipped with microphone ports for acoustic measurements. The $40 \mathrm{~kW}$ burner is composed of two identical stages fed with air and propane. A photomultiplier equipped with a narrow band filter measuring the $\mathrm{CH}^{*}$ spontaneous emission from the flame is used to study heat release fluctuations in the flame. Time-resolved measurements are carried out and spectral analysis of pressure measurements and light emissions are performed for various inlet impedances.

\section{0:00-10:20 Break}

10:20

3aPAc7. Green's function model for a Rijke tube with a distributed heat source. Maria A. Heckl (Keele University, Staffordshire, ST5 5BG Newcastle-under-Lyme, UK, m.a.heckl@keele.ac.uk)

A Rijke tube is an open-ended tube with a compact heat source (flame or hot gauze) inside. The heat source is commonly modelled by the "n $\tau$ law", which assumes a point source releasing heat at a rate that is proportional to the velocity (delayed by a time lag $\tau$ ) at the heat source. The present paper aims to extend this model to include distributed heat sources. A distributed source is simulated by a row of point sources with individual heat release rates and time lags. The acoustic processes in the tube will be modelled by Lighthill's acoustic analogy equation, combined with a Green's function approach. The occurrence of thermo-acoustic instabilities will be predicted for different properties of the heat source. Geometrical complications in the tube, such as a blockage and a jump in cross-section, as well as a jump in mean temperature, will be taken into account.

10:40

3aPAc8. Experimental and Numerical Investigation of the Acoustic Response of Multi-slit Bunsen Burners. Philip De Goey (Eindhoven Univesity of Technology, Den Dolech 2, 5600 MB Eindhoven, Netherlands, 1.p.h.d.goey@tue.nl), Victor Kornilov (Eindhoven Univesity of Technology, Den Dolech 2, 5600 MB Eindhoven, Netherlands, v.n.kornilov@tue.nl), Ronald Rook (Eindhoven Univesity of Technology, Den Dolech 2, 5600 MB Eindhoven, Netherlands, r.rook@tue.nl), Jan Ten Thije Boonkkamp (Eindhoven Univesity of Technology, Den Dolech 2, 5600 MB Eindhoven, Netherlands, tenthije@win.tue.nl)

Acoustic resonances in combustion systems like central heating boilers prohibit further technological advances in these systems. The design and construction is obstructed by acoustic problems because they are largely misunderstood, in spite of our increase in knowledge over the last decades. The flame often acts as an active element in the acoustic field, because the flame transfer function of acoustic waves has a large amplitude at low frequencies. Current models of the phase of the flame transfer function of Bunsen-type flames, based on kinematic behavior of the flame dynamics, completely miss the experimentally observed phase, unless the measured flow field is used in the model. In the current paper we analyze numerical results of the flame dynamics, flow field and flame transfer function found with a 2D detailed numerical model of the flow and structure of the flame on a multiple-slit burner. The model is validated with experiments of the flame dynamics (using chemiluminescence), flow dynamics (using PIV) and flame transfer function (using OH luminescence for the heat release fluctuations and heated wire probe for the acoustic distortions) on exactly the same configuration. A very good agreement is found which indicates the importance of predicting all the influences of the flow on the flame and vise-versa.

\section{1:00}

3aPAc9. Numerical prediction of interaction between combustion, acoustics and vibration in gas turbines. Artur Pozarlik (University of Twente, P.O. Box 217, 7500 AE Enschede, Netherlands, a.k.pozarlik@utwente.nl), Jim B. Kok (University of Twente, P.O. Box 217, 7500 AE Enschede, Netherlands, j.b.w.kok@utwente.nl)

The turbulent flame in the lean combustion regime in a gas turbine combustor generates significant thermo-acoustic instabilities. The flame can amplify fluctuations in the released heat, and thus in the acoustic field as well. The induced pressure oscillations will drive vibrations of the combustor walls and burner parts. Stronger fluctuating pressure results in stronger fluctuations in the wall structure. Due to fatigue the remaining life time of the hard ware will be reduced significantly. This paper investigates modeling of acoustic oscillations and mechanical vibrations induced by lean premixed natural gas combustion. The mutual interaction of the combustion processes, induced oscillating pressure field in the combustion chamber, and induced vibration of the liner walls are investigated with 
numerical techniques. A partitioned procedure is used here: CFX-10 for the CFD analysis and Ansys-10 for the CSD analysis are coupled to give insight into a correlation between acoustic pressure oscillations and liner vibrations. These results will be compared with the available experimental data. The data are gathered in a purpose built $500 \mathrm{~kW} / 5$ bar premixed natural gas test rig.

\section{$11: 20$}

3aPAc10. Influence of Temperature Gradients on the Sound radiated from Flames. Rafael Piscoya (Technische Fachhochschule Berlin, Univ. of Applied Sciences, Luxemburger Str. 10, 13353 Berlin, Germany, piscoya@tfh-berlin.de), Martin Ochmann (Technische Fachhochschule Berlin, Univ. of Applied Sciences, Luxemburger Str. 10, 13353 Berlin, Germany, ochmann@tfh-berlin.de)

The far field pressure of a turbulent flame can be determined using the standard boundary element method (BEM) if the sound pressure or its derivative is known at a closed surface (control surface) surrounding the flame, as long as the medium outside the control surface is homogeneous. If temperature gradients are present, the homogeneous Helmholtz equation is no more valid. In that case, the wave equation can be rewritten in form of an inhomogeneous Helmholtz equation with a source term that depends also on the unknown pressure. Using the "Dual Reciprocity BEM" the integral form of this wave equation can be solved involving only surface integrals, so that the sound field can still be computed from field values at the control surface. The cases under study consider a volume of hot gas with a temperature distribution that is prescribed or obtained from a CFD simulation. The influence of the temperature gradients on the sound field can be evaluated by comparison of characteristic quantities like sound power and radiation patterns, with and without temperature gradient.

\section{1:40}

3aPAc11. Localization of Sound Sources in Combustion Chambers. Christian Pfeifer (Institut für Strömungsmechanik und Technische Akustik, Technische Universität Berlin, Mueller-Breslau-Str. 8, 10623 Berlin, Germany, christian.pfeifer@pi.tu-berlin.de), Jonas P. Moeck (Institut für Strömungsmechanik und Technische Akustik, Technische Universität Berlin, Mueller-Breslau-Str. 8, 10623 Berlin, Germany, jonas.moeck@pi.tu-berlin.de), Lars Enghardt (DLR - German Aerospace Center, Mueller-Breslau-Str. 8, 10623 Berlin, Germany, lars.enghardt@dlr.de), Christian Oliver Paschereit (Institut für Strömungsmechanik und Technische Akustik, Technische Universität Berlin, Mueller-Breslau-Str. 8, 10623 Berlin, Germany, oliver.paschereit@tu-berlin.de)

To optimize the efficiency and to minimize the emissions of pollutants, modern combustion chambers for aeroengines or stationary gas turbines run in high temperature ranges with lean fuel-air mixtures. The disadvantage of this combustion mode is its susceptibility to the formation of thermoacoustic instabilities. These self-excited oscillations are an unacceptable noise source and, furthermore, they reduce the durability of the combustion chamber significantly. The focus of this paper is the localization of combustion noise sources in enclosed combustion chambers. This can be achieved using the acoustical nearfield information from pressure measurements with wall-flush mounted sensors. The spatially bounded region, where the sound sources are suspected, is discretized by a grid of monopole sources. A system of linear equations for the source strengths is built based on the relations between the sound sources and the sound pressure field, theoretically caused by the assumed sources of the grid. The source strengths are calculated by inverting the system of equations. A spatial variation of the source grid allows the scan of likely regions of sound sources to finally deduce their original distribution. Numerical simulation results will be shown to illustrate the potential and the limits of the proposed method.

\section{2:00}

3aPAc12. Computation of Indirect Combustion Noise by a CAA Method. Christoph Richter (Technische Universität Berlin, Sekr. MB1, Müller-Breslau-Str. 11, ISTA, 10623 Berlin, Germany, christoph.richter@tu-berlin.de), Frank H. Thiele (Technische Universität Berlin, Sekr. MB1, Müller-Breslau-Str. 11, ISTA, 10623 Berlin, Germany, Frank.Thiele@TU-Berlin.de)

Indirect combustion noise, which is generated by the acceleration of hot gases from a turbulent swirling flame, has become an important issue to understand turbo-machinery core noise. This indirect combustion noise it is dominated by a strong interaction of hydrodynamic and acoustic perturbations due to the transonic base flow in the turbine stages and combustion chamber exit nozzle. Current numerical simulation methods for the phenomenon based on compressible Large Eddy Simulation (LES) are very expansive. Thus LES applications are still limited to one design or few variations with immense computational costs. The most simple model fully covering indirect combustion noise, are the linearized non-isentropic Euler equations over an arbitrary mean flow. Therefore the gap between theory and LES is closed by a method adopted from Computational Aeroacoustics (CAA). It combines a high cost efficiency in terms of required points per wavelength with a high adaptability to realistic mean flow conditions. The CAA method is first validated with a simplified experiment and then applied to predict the acoustic properties of two model combustion chambers, one of them with transonic termination nozzle. By means of the acoustic intensity the sources of the noise are located.

\section{2:20}

3aPAc13. Study of oscillating regimes in air-staged diffusion flames. Javier Ballester (University of Zaragoza / LITEC, Centro Politecnico Superior, Maria de Luna, 3, 50018 Zaragoza, Spain, ballester@unizar.es), Ricardo Hernández (University of Zaragoza / LITEC, Centro Politecnico Superior, Maria de Luna, 3, 50018 Zaragoza, Spain, richard@ litec.csic.es), Ana Sanz (University of Zaragoza / LITEC, Centro Politecnico Superior, Maria de Luna, 3, 50018 Zaragoza, Spain, anasanz@litec.csic.es)

The occurrence of fluctuating behaviours in air-staged diffusion flames was studied experimentally in a gas-fired furnace. Burner settings (swirl levels, air distribution) were systematically varied, leading to a broad range of flame characteristics and pollutant emissions. Pressure fluctuations reached significant amplitudes and displayed characteristic frequencies for certain operating conditions. However, heat release fluctuations (as estimated from $\mathrm{OH}^{*}$ chemiluminescence) were correlated with pressure only in a part of those cases. Therefore, thermo-acoustic coupling is thought to exist only under certain conditions, whereas other oscillating regimes are not ascribed to acoustic feedback but to changes in the location of the flame stabilization region. This conclusion was supported by the values of the Rayleigh index calculated for the different regimes. An enhanced tendency to the onset of thermo-acoustic instabilities was observed for highly staged flames. 
3aPAc14. The acoustic wave propagation equation in a turbulent combusting flow. Jim B. Kok (University of Twente, P.O. Box 217, 7500 AE Enschede, Netherlands, j.b.w.kok@utwente.nl)

Sound generation by turbulent flames originates from the fluctuating heat release in the flame. The description of this fluctuating heat release and its effect on acoustics in turbulent flames is complicated due to the interaction of chemical reactions with turbulence, mixing and pressure fluctuations. In a turbulent flame the instantaneous density, velocity, pressure, temperature and species concentrations are determined by the transport equations for mass, momentum, enthalpy and species and by the equation of state. In the proposed paper an equation is formulated that describes the propagation of acoustic pressure fluctuations, and that determines the source terms. These source terms are compared with various terms derived in the literature. Significant differences are found between several approaches. An effort is made to come to a reliable and generally acceptable formulation. Subsequently events are ordered on basis of their typical time scale. That way source terms can be evaluated for the situation where the combustion is described with the use of time averaged chemical reaction progress variables and a mixture fraction variable. Subsequently the consequences of Reynolds and Favre averaging on these source terms and conservation of acoustical variables in a domain with turbulent flow are discussed.

WEDNESDAY MORNING, 2 JULY 2008

ROOM 362/363, 8:00 TO 9:20 A.M.

Session 3aPAd

Physical Acoustics: Ultrasonics: Material Characterization I

\author{
Bart Lipkens, Cochair \\ Western New England College, 1215 Wilbraham Road, Box S-5024, Springfield, MA 01119, USA
}

Nicolas Wilkie-Chancellier, Cochair

ECIME Université de Cergy-Pontoise, rue d'Eragny, Neuville-sur-Oise, 95031, France

\title{
Contributed Papers
}

\begin{abstract}
8:00
3aPAd1. Frequency sweeping and fluid flow effects on particle trajectories in ultrasonic standing waves. Bart Lipkens (Western New England College, 1215 Wilbraham Road, Box S-5024, Springfield, MA 01119, USA, blipkens@wnec.edu), Jason Dionne (Western New England College, 1215 Wilbraham Road, Box S-5024, Springfield, MA 01119, USA, jdionne@flodesign.org), Michael Costolo (Physical Sciences Inc., 20 New England Business Center, Andover, MA 01810, USA, mac@psicorp .com), Edward Rietman (Physical Sciences Inc., 20 New England Business Center, Andover, MA 01810, USA, rietman@psicorp.com)
\end{abstract}

Particle concentration in ultrasonic standing waves by the acoustic radiation force is discussed. The acoustic radiation force is a function of the density and compressibility of the fluid and the suspended particles. A twodimensional theoretical model is developed for particle trajectory calculations. An electro-acoustic model is used to predict the acoustic field in a resonator. The results of the linear acoustic model are used to calculate the acoustic radiation force acting on a particle suspended in the resonator. A particle trajectory model is developed that integrates the equation of motion of a particle subjected to a buoyancy force, a fluid drag force, and the acoustic radiation force. Computational fluid dynamics calculations are performed to calculate the velocity field. For a fixed frequency excitation, the particles are concentrated along the stable node locations of the acoustic radiation force. Through a periodic sweeping of the excitation frequency particle translation is achieved. Two types of frequency sweeps are considered, a ramp approach and a step-change method. Numerical results of particle trajectory calculations in a resonator with dimensions much larger than a typical wavelength are presented. A movie of experimental observations of particle trajectories is shown to illustrate the method.

\section{8:20}

3aPAd2. Elastic constants identification of anisotropic composite rectangular parallelepipeds. Thomas Delaunay (Université Montpellier II, Place Eugène Bataillon, 34095 Montpellier, France, delaunay@lain .univ-montp2.fr), Didier Laux (Université Montpellier II, Place Eugène Bataillon, 34095 Montpellier, France, laux@lain.univ-montp2
fr),Jean-Yves Ferrandis(Radio Application Division, NEC Corporation, Université Montpellier II, Place Eugène Bataillon, 34095 Montpellier, France, ferrandi@lain.univ-montp2.fr)

For many years, composite materials have been investigated due to their potential in many applications such as nuclear fuel. Nowadays, the improvement of structure conception and simulation requires many reliable properties, especially the entire elastic tensor. For these reasons, the implementation of simulation and characterization methods adapted to the geometry of samples and the high anisotropy degree of such materials are then crucial issues to developers and end users. The work presented here reports first macroscopic properties of two composites Duralumin/Air and Duralumin/Tungsten Carbide) calculated using periodical homogenization method from elastic constants of each phase measured by high frequency acoustic microscopy. Then a resonant ultrasonic spectroscopy method is used to identify the entire elastic tensor. First, computation predicting the resonant frequencies and the mode shapes of free vibrations of cubes are presented. Excitation is then ensured by ultrasonic transducers and detection is performed by means of a laser vibrometer. A fit process of the theoretical frequencies on the measured data is then described. This method allows us to conclude about the accuracy of the homogenization method.

\section{8:40}

3aPAd3. Viscoelastic material monitoring using an acoustical cylindrical spectrometer. Nicolas Wilkie-Chancellier (ECIME Université de Cergy-Pontoise, rue d'Eragny, 95031 Neuville-sur-Oise, France, Nicolas.Wilkie-Chancellier@u-cergy.fr), Loic Martinez (ECIME Université de Cergy-Pontoise, rue d'Eragny, 95031 Neuville-sur-Oise, France, loic.martinez@u-cergy.fr), Pascal Griesmar (ECIME Université de CergyPontoise, rue d'Eragny, 95031 Neuville-sur-Oise, France, pascal.griesmar @u-cergy.fr), Stéphane Serfaty (ECIME Université de Cergy-Pontoise, rue d'Eragny, 95031 Neuville-sur-Oise, France, stephane.serfaty@u-cergy.fr)

Acoustic sensors often have a plane geometry due to manufacturing constrains. In the present work, the cylindrical geometry is investigated with a normal incident beam in order to suppress the edge effects on mode conversion. The experimental setup uses an aluminium cylindrical shell. A 
classical immersion transducer ( $2 \mathrm{MHz}$ central frequency) sends a plane pulse wave on the shell at normal incidence. A second immersion transducer can turn around, enabling the observation of the waves scattered by the cylindrical shell. Previous works have shown that the acoustic field scattered by the shell as a rainbow structure enabling the acoustic spectrometry of the material poured inside the shell. From these results, the present study proposes an acoustical model linking the resonances parameters to the material ones, enabling the monitoring of time varying viscous materials. Several gelation monitoring experiments are performed and analysed using the proposed model. Results are in good agreement with quartz sensor measurements and offer a good alternative to wide frequency range measurements.

\section{9:00}

3aPAd4. Ultrasonic and photoacopustic investigations of water mixtures with dioxanes 1-3 \& 1-4. Bogumil B. Linde (Inst. of Exper. Physics, Univ. of Gdańsk, Ul. Wita Stwosza, Bldg. 57, 80-952 Gdańsk, Poland, fizbl@univ.gda.pl), Anna K. Kiszel (Inst. of Exper. Physics, Univ. of
Gdańsk, Ul. Wita Stwosza, Bldg. 57, 80-952 Gdańsk, Poland, ania_tczew84 @wp.pl), Anna Sikorska (Inst. of Exper. Physics, Univ. of Gdańsk, Ul. Wita Stwosza, Bldg. 57, 80-952 Gdańsk, Poland, fizams@univ.gda.pl)

Ultrasonic velocities, densities and thermal effusivity dependence on concentration were measured in the mixtures of water with dioxanes 1-3 and $1-4$ in the temperature range from 291.15 to $303.15 \mathrm{~K}$. Adiabatic compressibilities were calculated from Laplace's equation based on the experimental results obtained. Variations of these values with concentration and temperature were studied. Structural interactions and the formation of a compact pseudo-stable structure at very low concentrations of these liquids were observed. The plots of the adiabatic compressibility versus the mole fraction of these molecules display two characteristic points at low concentrations: the intersection of the isotherms and their minimum. Such relations between adiabatic compressibility, concentration and temperature are usually attributed to the formation of pseudo-stable molecular structures. To formulate a model of local structures present in the investigated molecular systems it is indispensable to get an insight into hydration of molecules and the formation of hydrogen bonds. Therefore, the attention was focused particularly on these problems.

WEDNESDAY MORNING, 2 JULY 2008

ROOM 362/363, 9:20 A.M. TO 12:40 P.M.

\title{
Session 3aPAe
}

\section{Physical Acoustics: Ultrasonics: NDT and Layered Systems I}

\author{
Vincent Pagneux, Cochair \\ Laboratoire d'Acoustique de l'Université du Maine, UMR CNRS 6613, AV. O. Messiaen, Le Mans, 72085, France \\ Claire Prada, Cochair \\ Laboratoire Ondes et Acoustique, ESPCI, Université Paris 7, CNRS, 10 rue Vauquelin, Paris, 75005, France
}

\section{Contributed Papers}

\section{9:20}

3aPAe1. Thickness determination of a multilayered system of different materials by natural frequencies. Changzhi Zhou (Institute of Acoustics, Chinese Academy of Sciences, NO.21, Bei-Si-huan-Xi Road, 100080 Beijing, China, zcz@mail.ioa.ac.cn), Mingxuan Li (Institute of Acoustics, Chinese Academy of Sciences, NO.21, Bei-Si-huan-Xi Road, 100080 Beijing, China, 1mx@mail.ioa.ac.cn), Jie Mao (Institute of Acoustics, Chinese Academy of Sciences, NO.21, Bei-Si-huan-Xi Road, 100080 Beijing, China, maojie@mail.ioa.ac.cn), Xiaomin Wang (Institute of Acoustics, Chinese Academy of Sciences, NO.21, Bei-Si-huan-Xi Road, 100080 Beijing, China, wangxiaomin@mail.ioa.ac.cn)

This paper is focusing on the relationship between the natural frequencies of a multilayered system of different isotropic elastic materials and the thickness of each layer. A relevant ultrasonic method for thickness determination of a multilayered system by natural frequencies has been developed. The natural frequencies are numerically calculated from the normally incident reflection coefficient of a "steel-epoxy resin-aluminum-thin polymer" structured lamination. Some natural frequencies are sensitive to one layer thickness in certain range while the others are invariable. This phenomenon is explained by the defined strain energy ratio, and used for our thickness inversion. The measured natural frequencies of a specimen show good agreement with the theoretical ones. All the natural frequencies are taken into account, and the simulated annealing method is employed for the thickness inverse calculation of the multilayered structure. The average relative errors of calculated thickness of the each layer are $-0.7 \%, 35 \%,-1.3 \%$ and $-6.1 \%$, corresponding to the real values: $1957 \mu \mathrm{m}, 16 \mu \mathrm{m}, 1913 \mu \mathrm{m}$ and $54 \mu \mathrm{m}$.

\section{9:40}

3aPAe2. Bonding quality evaluation of layered materials by guided wave dispersion. Young H. Kim (Korea Science Academy, 111 Backyangkwanmoonro, Busanjin-ku, 614-822 Busan, Republic of Korea, youngkim@paran.com), Minseok Bae (Korea Science Academy, 111 Backyangkwanmoonro, Busanjin-ku, 614-822 Busan, Republic of Korea, bms1991@empal.com), Sunhee Choi (Korea Science Academy, 111 Backyangkwanmoonro, Busanjin-ku, 614-822 Busan, Republic of Korea, cjtwo120@hanmail.net), Jisu Kim (Korea Science Academy, 111 Backyangkwanmoonro, Busanjin-ku, 614-822 Busan, Republic of Korea, boone90@hotmail.com), Seokchan Hong (Korea Science Academy, 111 Backyangkwanmoonro, Busanjin-ku, 614-822 Busan, Republic of Korea, charliehongn@hanmail.net)

Multi-layered materials have been widely used for high performance materials with advanced properties required for the critical parts of structures. The characteristics of bonding quality in multi-layered materials have been hot issue for a long time. The bonding quality was evaluated by using dispersion of guided wave. Broadband guided waves were generated at oblique incidence angle, and waves reflected at the edge of the specimen were detected by using a pulse-echo setup. The dispersion curves were obtained from edge-reflected leaky guided wave for various plates: Single layered plates of aluminum, copper and stainless steel, and double layered plates of aluminum-stainless steel cladding, epoxy-bonded aluminumstainless and epoxy-bonded aluminum-copper. The aluminum-stainless steel cladding specimen showed similar dispersion for both sides, whereas epoxybonded specimens showed different dispersions which are similar to that of upper layer only. These results imply that the cladding specimen used in the 
present work is well bonded, whereas epoxy-bonded specimens are acoustically isolated. The dispersion curve of guided wave has high potential to evaluate bonding quality of layered materials.

\section{0:00}

3aPAe3. Acoustical imaging with bending waves based on sparse inversion. Lars Hörchens (University of Technology Delft, Lorentzweg 1, 2628 CJ Delft, Netherlands, 1.horchens@tudelft.nl), Diemer De Vries (University of Technology Delft, Lorentzweg 1, 2628 CJ Delft, Netherlands,d.devries@tudelft.nl)

Bending waves can be employed in the context of acoustical imaging for the detection of material defects or localization of vibration sources. The obtainable spatial resolution is limited by the minimal wavelength contained in the excitation signal. The evanescent part of the wave field can usually not be used for the imaging of sources at distances exceeding a wavelength. Therefore only the propagating part of the field can be employed, which means that the minimum size of the region where energy can be focused is in the order of half a wavelength due to the diffraction limit. If the recovery of vibration sources and reflections is posed as an inverse problem, regularization techniques can be applied in order to force a sparse solution. Preliminary results indicate that the assumption of a sparse source distribution can be used to resolve features significantly smaller than half a wavelength. The approach is therefore applied as a high-resolution imaging technique for bending wave fields.

\section{0:20-10:40 Break}

\section{0:40}

3aPAe4. An experimental method of corrugated surface reconstruction investigated in combination with finite-element simulation. Sarah W. Herbison (Georgia Tech Lorraine - G.W. Woodruff School of ME, UMI Georgia Tech - CNRS 2958, 2 rue Marconi, 57070 Metz, France, sherbison@gatech.edu), Ebrahim Lamkanfi (Georgia Tech Lorraine - G.W. Woodruff School of ME, UMI Georgia Tech - CNRS 2958, 2 rue Marconi, 57070 Metz, France, Ebrahim.Lamkanfi@ugent.be), Nico F. Declercq (Georgia Tech Lorraine - G.W. Woodruff School of ME, UMI Georgia Tech - CNRS 2958, 2 rue Marconi, 57070 Metz, France, nico .declercq@me.gatech.edu)

Current theory applied to ultrasonic diffraction from periodically corrugated surfaces is valid only in the far-field; it cannot be applied to determine the diffracted field within the surface corrugations themselves. Therefore, information regarding the corrugation is difficult to extract from experiments that are necessarily conducted in the far-field. The present work aims to simulate a potential experimental method for investigating the corrugations themselves. Using near-field results obtained from a finite-element code, the far-field diffraction is constructed as a series of time-harmonic plane waves. By back-propagating this diffracted field, information about the corrugations is obtained. Comparing the results of the back-propagated field to the field generated by the finite-element code shows the limitations of determining details of periodically corrugated surfaces from far-field experiments.

\section{1:00}

3aPAe5. Determination of the width of an axisymmetric deposit on a metallic pipe by means of Lamb type guided modes. Mustapha El Moussaoui (LOMC FRE 3102 CNRS Groupes Ondes Acoustiques, Université du Havre (IUT), Place Robert Schuman, 76610 Le Havre, France, elmoussm@univ-lehavre.fr), Farid Chati (LOMC FRE 3102 CNRS Groupes Ondes Acoustiques, Université du Havre (IUT), Place Robert Schuman, 76610 Le Havre, France, farid.chati@univ-lehavre.fr), Fernand Leon (LOMC FRE 3102 CNRS Groupes Ondes Acoustiques, Université du Havre (IUT), Place Robert Schuman, 76610 Le Havre, France, fernand.leon@univ-lehavre.fr), Aleksander Klauson (Tallinn University of Technology, Dept. of Mechanics, Ehitajate tee 5, 19086 Tallinn, Estonia,
aklauson@staff.ttu.ee),Gerard Maze(LAUE, Université du Havre, Place Robert Schuman, F-76610 Le Havre, France, gerard.maze@univ-lehavre.fr)

The non-destructive evaluation of pipes using guided waves is extensively used for the detection and the size estimation of defects. The important number of investigations concerning with the interaction between a guided wave and a defect show the growing need to improve our knowledge of this phenomenon in order to detect and to characterize as well as possible the defect. This work is devoted to the evaluation of the width of an axisymmetric resin deposit coupled to the wall of a metallic tube by means of longitudinal modes S0, S1. To that end, we interest to damping of these modes provoked by the deposit. The study is carried out both from the numerical point of view and from the experimental point of view in considering a deposit of variable width. The finite element method is employed to model the propagation and the diffraction of Lamb-type waves. The experiment is based on the generation of axisymmetric waves on the section of a tube by a piezoelectric transducer. Measurements of temporal echoes reflected by the deposit and transmitted after the deposit are achieved. A mathematical law of the attenuation as function of the width is deduced for each wave.

\section{1:20}

3aPAe6. Scattering by a defect in an elastic waveguide: Coupling of finite elements and modal representations. Vahan Baronian (CNRS, ENSTA - 32 Boulevard Victor, 75015 Paris, France, vahan.baronian @ensta.fr), Anne-Sophie Bonnet- Ben Dhia (CNRS, ENSTA - 32 Boulevard Victor, 75015 Paris, France, bonnet@ensta.fr), Colin Chambeyron (CNRS, ENSTA - 32 Boulevard Victor, 75015 Paris, France, colin.chambeyron@ensta.fr), Eric Luneville (ENSTA - CEA, 32 Boulevard Victor, 75015 Paris, France, eric.luneville@ensta.fr)

The potentiality of guided ultrasonic waves in nondestructive testing is currently investigated. Indeed, guided waves can allow a rapid inspection of large areas and of non accessible parts of particular structures like plates or pipes, compared to conventional techniques. This work concerns the numerical finite element computation, in the frequency domain, of the diffracted wave produced by a defect (crack, heterogeneity, discontinuity, local bend etc.) located in an infinite elastic waveguide. The computational domain is chosen as a portion of the waveguide, containing the perturbation, and our purpose is to build transparent conditions on its artificial boundaries by using modal representations. This cannot be achieved in a classical way, due to nonstandard properties of elastic modes. However, a biorthogonality relation is established which allows to derive a transparent condition relating hybrids displacement/stress vectors. An original mixed formulation is then implemented, whose unknowns are the displacement field in the bounded domain and the normal component of the normal stresses on the artificial boundaries. Numerical validations are presented in the two-dimensional and three-dimensional cases.

\section{1:40}

3aPAe7. Interaction of elastic waves with dislocations. Agnes Maurel (LOA/ESPCI, 10 rue Vauquelin, 75005 Paris, France, agnes.maurel@espci.fr), Vincent Pagneux (Laboratoire d'Acoustique de l'Université du Maine, UMR CNRS 6613, AV. O. Messiaen, 72085 Le Mans, France, vincent.pagneux@univ-lemans.fr), Felipe Barra (Universidad de Chile, av. Blanco encalada, 0 Santiago, Chile, fbarra@uchile.cl), Fernando Lund (CIMAT, Universidad de Chile, 0 Santiago, Chile, flund@cimat.cl)

This work concerns the effective propagation of elastic waves in a medium filled with randomly placed dislocations. Multiple scattering theory is applied to derive the effective velocity and attenuation of the waves (both transverse and longitudinal) because of the effect of the dislocations. Our work is a generalization of Granato \& Lucke theory since it takes into account the polarization of the elastic waves. Two principal results have been obtained, in connexion with experiments: 1) we have explained the different attenuations experimentally measured between longitudinal and transverse waves. The difference between the two values is obtained in quantitative agreement with no adjustable parameter. 2) we have offered a scenario for the frequency law in the attenuation measured in polycrystals (a combina- 
tion of quadratic and quartic law). This has been done by taking into account the dislocations at the grain boundaries that can contribute to the attenuation process.

12:00

3aPAe8. Particle deposit formation during filtration characterisation using ultrasonic waves. Didier Laux (Université Montpellier II, Place Eugène Bataillon, 34095 Montpellier, France, laux@lain .univ-montp2.fr), Hugues Blasco (Université Montpellier II, Place Eugène Bataillon, 34095 Montpellier, France, blasco@lain.univ-montp2 .fr), Jean-Yves Ferrandis (Radio Application Division, NEC Corporation, Université Montpellier II, Place Eugène Bataillon, 34095 Montpellier, France, ferrandi@lain.univ-montp2.fr), Julie Mendret (INSA Toulouse, Av de Rangueil, 31077 Toulouse, France, julie.mendret@insa-toulouse .fr), Christelle Guigui (INSA Toulouse, Av de Rangueil, 31077 Toulouse, France, guigui@insa-toulouse.fr), Corinne Cabassud (INSA Toulouse, Av de Rangueil, 31077 Toulouse, France, cabassub@insa-toulouse.fr), Philippe Schmitz (INSA Toulouse, Av de Rangueil, 31077 Toulouse, France, philippe.schmitz@insa-toulouse.fr)

Membranes filtration processes are widely used because of their ability to remove particles, colloidal species and micro-organisms from different liquids feeds. However an inherent process limitation is the membrane fouling due to deposition of suspended matter during filtration. Therefore the understanding of formation and transport properties of particle deposit responsible for membrane fouling is a necessary step to optimize membrane processes. These deposits are non homogeneous, highly porous and very thin (less than $500 \mu \mathrm{m}$ ). Thus, it is necessary to obtain local information in order to analyze and model the basic mechanisms involved in deposit formation and then to further predict process operation. As local parameters such as cake thickness and porosity are hardly reachable with conventional techniques, we propose in this paper the use of an ultrasonic echographic method. In a first step this method is validated on deposits of small glass balls. We show that the porosity and thickness of the deposit is in good agreement with theory. Then, the ultrasonic technique has been adapted on a filtration cell in order to give in line results during filtration. First results are presented and discussed.

\section{2:20}

3aPAe9. Single-sided air-coupled ultrasonic NDT of thick plates Steven Delrue (K.U.Leuven Campus Kortrijk, E. Sabbelaan 53, 8500 Kortrijk, Belgium, steven.delrue@kuleuven-kortrijk.be), Pieter Lust (Katholieke Hogeschool Zuid-West-Vlaanderen dept. VHTI, Doorniksesteenweg 145, 8500 Kortrijk, Belgium, pieter.lust@katho .be), Jurgen Deveugele (Katholieke Hogeschool Zuid-West-Vlaanderen dept. VHTI, Doorniksesteenweg 145, 8500 Kortrijk, Belgium, jurgen.deveugele@katho.be), Erik Blomme (Katholieke Hogeschool ZuidWest-Vlaanderen dept. VHTI, Doorniksesteenweg 145, 8500 Kortrijk, Belgium, Erik.Blomme@katho.be), Olivier Bou Matar (IEMN, UMR CNRS 8520, avenue Poincaré, BP 60069, 59652 Villeneuve d'Ascq, France, olivier.boumatar@iemn.univ-lille1.fr), Koen Van Den Abeele (K.U.Leuven Campus Kortrijk, E. Sabbelaan 53, 8500 Kortrijk, Belgium, koen .vandenabeele@kuleuven-kortrijk.be)

Non-contact air-coupled ultrasonic inspection using single sided access to the material offers interesting possibilities for the development of ultrasonic in-line NDT-systems. This contribution reports observations and simulations obtained from a single-sided air-coupled pitch-catch configuration The feasibility of the technique is demonstrated with respect to artificial defects in aluminium samples. Depending on the configuration it is possible to establish one or more ultrasonic images of the defect, their number and relative position containing information about the location and depth of the defect. The experiments are simulated using a spectral solution obtained within Comsol, and compared with a full 2D Finite Difference Time Domain simulation and a ray tracing (shadow) approach. The simulations allow simple prediction of the response images in more complicated experimental conditions.

\title{
Session 3aPAf
}

\section{Physical Acoustics: Ultrasonics: Industrial NDT I}

\author{
Bertrand Nongaillard, Cochair \\ IEMN - DOAE, Université de Valenciennes, Le Mont - Houy, Valenciennes, 59313, France \\ Christophe Aristegui, Cochair \\ LMP, UMR CNRS 5469, Université Bordeaux I, 351, cours de la Libération, Talence, 33405, France
}

\section{Contributed Papers}

$11: 20$

3aPAf1. Simple and versatile non-contact technique for measuring the interfacial tension of a liquid-liquid interface using pulsed acoustic radiation pressure. Bruno Issenmann (Bordeaux University - $\mathrm{CPMOH}$, 351 cours de la Liberation, 33405 Talence Cedex, France, b.issenmann @ cpmoh.u-bordeaux1.fr), Regis Wunenburger (Bordeaux University - CPMOH, 351 cours de la Liberation, 33405 Talence Cedex, France, r.wunenburger@cpmoh.u-bordeaux1.fr), Jean-Pierre Delville (Bordeaux University - CPMOH, 351 cours de la Liberation, 33405 Talence Cedex, France,jp.delville@cpmoh.u-bordeaux1.fr)

We present a versatile non-contact technique for measuring the interfacial tension of a liquid-liquid interface using the acoustic radiation pressure. This technique is based on the analysis of the shape of the time-dependent deformation of the liquid interface induced by the radiation pressure of a focused, pulsed ultrasonic beam. It combines a simple optical detection step and a novel analytical model of interface deformation dynamics that accurately takes into account interfacial, gravitational, as well as viscous effects in both weakly and highly viscous limits. The accuracy of this technique is experimentally demonstrated on liquid-air and liquid-liquid interfaces.

\section{1:40}

3aPAf2. Diffuse ultrasonic backscatter at oblique incidence for heterogeneous solids. Joseph A. Turner (University of Nebraska, Dept. of Engineering Mechanics, W317.4 NH, Lincoln, NE 68588, USA, jaturner@unl.edu), Goutam Ghoshal (University of Nebraska, Dept. of Engineering Mechanics, W317.4 NH, Lincoln, NE 68588, USA, goutamghoshal@rediffmail.com)

Diffuse ultrasonic backscatter techniques are useful for probing heterogeneous materials. They can be used to extract microstructural parameters and to detect flaws which cannot be detected by conventional ultrasonic 
techniques. Such experiments, usually done using a modified pulse-echo technique, utilize the spatial variance of the signals as a primary measure of microstructure. Quantitative ultrasonic scattering models include components of both transducer beams as well as microstructural scattering information. Of particular interest for interpretation of many experiments is the propagation through a liquid-solid interface at normal and oblique incidence. Here, the Wigner distribution of the beam pattern of an ultrasonic transducer through a liquid-solid interface is used in conjunction with the stochastic wave equation to model this scattering problem within a single scattering formulism. The Wigner distribution represents a distribution in space and time of the spectral energy density as a function of wave vector and frequency. A Gaussian beam is used to model the transducer beam pattern. The scattered response in the time domain is then compared with experimental results for materials of common interest. These results are anticipated to impact ultrasonic nondestructive evaluation and characterization of heterogeneous media.

\section{Invited Paper}

\section{2:00}

3aPAf3. Model-based reconstruction methods of NDT ultrasonic array data. Pierre Calmon (CEA LIST, Centre de Saclay, F-91191 Gif sur Yvette, France, pierre.calmon@cea.fr), Ekaterina Iakovleva (CEA LIST, Centre de Saclay, F-91191 Gif sur Yvette, France, ekaterina.iakovleva@cea.fr), Alex Fidahoussen (CEA LIST, Centre de Saclay, F-91191 Gif sur Yvette, France, alex.fidahoussen@cea.fr), Guillemette Ribay (CEA LIST, Centre de Saclay, F-91191 Gif sur Yvette, France, guillemette.ribay@cea.fr)

Ultrasonic array techniques are more and more used in NDT applications because of their flexibility and of their adaptability to complex configurations. In addition they can provide richer data than conventional methods. An optimal exploitation of such data in the aim of localizing and characterizing the detected flaws requires the application of imaging and reconstruction techniques based on a direct modeling of the propagation of ultrasound from the transmitter to the receiver. The quantitative models for the propagation which are available in the CIVA software platform developed at CEA-LIST allow such direct modelling in parts of possibly complex geometries and materials. Different array data reconstruction methods based on the exploitation of these direct models have been studied and implemented in the plat-form. Thus, in this communication we present in particular algorithms derived of synthetic focusing approach. These algorithms exploit the time of flight identification and can be apply to any set of array data. We also present a reconstruction method based on the post-processing of the transfert matrix of the array and the application of MUSIC algorithm. We show and discuss results obtained with these methods both on simulated and experimental data.

\section{Contributed Paper}

\section{2:20}

3aPAf4. Low frequency composite acoustic sensor for highly absorbing media characterization. Alain Skaf (IEMN - DOAE, Université de Valenciennes, Le Mont - Houy, 59313 Valenciennes, France, alain.skaf@univ -valenciennes.fr), Georges Nassar (IEMN - DOAE, Université de Valenciennes, Le Mont - Houy, 59313 Valenciennes, France, gnassar@univvalenciennes.fr), Fabrice Lefebvre (IEMN - DOAE, Université de Valenciennes, Le Mont - Houy, 59313 Valenciennes, France, Fabrice.Lefebvre @ univ-valenciennes.fr), Bertrand Nongaillard (IEMN - DOAE, Université de Valenciennes, Le Mont - Houy, 59313 Valenciennes, France, Bertrand .Nongaillard@univ-valenciennes.fr)

Ultrasonic techniques are widely used in nondestructive testing and evaluation of media. But when media are highly absorbing, it becomes often impossible to operate at classical ultrasonic frequencies. In this work, we propose a low frequency acoustic device to characterize such highly absorbing media. This device is composed of a piezoelectric disc embedded in a metallic ring. The technique consists in bringing into resonance the entire structure. Firstly, we will study analytically and numerically the main resonance modes of the sensor, which are the flexion modes and the radial modes. The results of the modelization are compared to measurements obtained through an impedance analyzer. In a second part, we will use this model of sensor to characterize two different media. The first type consists of a series of polymer disks with different viscoelastic properties. Using radial modes of vibration, we will characterize these polymer disks through measurement of the acoustic velocity. After that, we will characterize using the flexion modes a very complex medium which present a highly absorbing phenomenon such as dough in the fermentation phase. The measurements show the aptitude of this technique to follow the evolution of the physical properties of complex media. 


\title{
Session 3aPPa
}

\section{Psychological and Physiological Acoustics and ASA Committee on Standards: Applications of Psychoacoustics III}

\author{
Patricia Davies, Cochair
}

Ray W. Herrick Lab., School of Mechanical Engineering, Purdue Univ., 140 S. Martin Jischke Drive, West Lafayette, IN 47907-2031, USA

\author{
Hugo Fastl, Cochair \\ AG Technische Akustik, MMK, TU München, Arcisstr. 21, München, 80333, Germany
}

\section{Contributed Papers}

8:00

3aPPa1. Psychoacoustic evaluation of compression drives. Joan Cruanes Catala (Escola Politècnica Superior de Gandia, Universitat Politècnica de València, Crtra Natzaret-Oliva s/n, 46730 Gandia, Spain, joacrca@upvnet.upv.es), Jorge Frances Monllor (DFISTS. Univ. de Alicante, Carretera de Sant Vicent del Raspeig s/n, 03690 San Vicente del Raspeig, Spain, jfmonllor@ua.es), Jaime Ramis Soriano (DFISTS. Univ. de Alicante, Carretera de Sant Vicent del Raspeig s/n, 03690 San Vicente del Raspeig, Spain, jramis@ua.es), Jesús Alba Fernandez (Escola Politècnica Superior de Gandia, Universitat Politècnica de València, Crtra NatzaretOliva s/n, 46730 Gandia, Spain, jesalba@ fis.upv.es)

The following work tries to be an approximation to the sound quality valuation of radiant systems, in concrete, the case of compression drives. This approach is realized from different perspectives: on one hand, an evaluation of the quality of these systems from the point of view of the psychoacoustic classic parameters described by Zwicker will be done. On the other hand, a perceptual model described by Moore and based on the human auditory system will be applied. Both models will be applied in signals registered on different commercial models of compression drives. The work also includes the accomplishment of a psychoacoustic experiment in which a hearing will realize a valuation of perceived quality. Finally, one will try to establish a correlation with the information obtained in the three mentioned approaches.

\section{8:20}

3aPPa2. A study of evaluating the button sounds. Shunsuke Ishimitsu (Hiroshima City University / National Institute of Advanced Industrial Science and Technology, 3-4-1 Ozuka-Higashi, Asa-Minami-Ku, 731-3194 Hiroshima, Japan, ishimitu@hiroshima-cu.ac.jp), Koji Sakamoto (Hiroshima City University, 3-4-1 Ozuka-Higashi, Asa-MinamiKu, 731-3194 Hiroshima, Japan, koji_s@tm.ten.fujitsu.com), Takayuki Arai (Univ. of Hyogo, 2167, Shosha, 671-2280 Himeji, Japan, es07g001 @ steng.u-hyogo.ac.jp), Toshikazu Yoshimi (Pioneer Corp., 25-1, Nisimachi, Yamada, 350-8555 Kawagoe, Japan, toshikazu_yoshimi@post .pioneer.co.jp), Yuichi Fujimoto (Pioneer Corp., 25-1, Nisimachi, Yamada, 350-8555 Kawagoe, Japan, yuichi_fujimoto@post.pioneer.co.jp), Kenichi Kawasaki (Pioneer Corp., 25-1, Nisimachi, Yamada, 350-8555 Kawagoe, Japan, kenichi_kawasaki@post.pioneer.co.jp)
In recent years, much attention has been directed at the sound design which designs various sound treated as noise, such as automobile acceleration sound and cleaner sound, because the point of view sound is a part of products, has permeated. This research considered the sound design and its evaluation about 11 kinds of the button sounds. First, the impression was extracted by the SD method and relevance with time frequency analysis was investigated. Moreover, we also confirmed that an impression changed, when the sound of a bad impression is processed into the sound of a good impression using adaptive control.

\section{8:40}

3aPPa3. Factoring end-user expectations into the sound design process of computer keyboards. Romain Boulandet (Ecole Polytechnique Fédérale de Lausanne, EPFL STI LEMA, Station 11, CH 1015 Lausanne, Switzerland, romain.boulandet@epfl.ch), Hervé Lissek (Ecole Polytechnique Fédérale de Lausanne, EPFL STI LEMA, Station 11, CH 1015 Lausanne, Switzerland, herve.lissek@epfl.ch), Sylvain Sauvage (Logitech Europe S.A., Z.I. Moulin du choc D, CH 1122 Romanel/Morges, Switzerland, sylvain_sauvage@eu.logitech.com), Jacques Robert (Logitech Europe S.A., Z.I. Moulin du choc D, CH 1122 Romanel/Morges, Switzerland, jacques_robert@eu.logitech.com)

The poor acoustic comfort of usual commercial products is often perceived as annoying. Today, more and more companies have realized that sound can be an important component of product attributes and image. They are interested in having acoustic guidelines for enhancing their products sound quality. The objective of this research is to quantify the objective and subjective noise production of computer keyboards, and hence to translate the pleasantness in an objective way. Our strategy consists in developing both benchmarks and listening tests to ensure that sound design process never looses sight of the end-users expectations. The presented work proposes an original methodology to define what keystroke sound matches the image of a pleasant keyboard. We also approach what acoustic characteristics have a significant influence on the global appreciation of keystroke sounds. Good results have been obtained showing a strong correlation between perceptive cues and metrics. 


\title{
Session 3aPPb
}

\section{Psychological and Physiological Acoustics: Auditory Perception of Sound Source Properties I}

\author{
John Neuhoff, Cochair \\ The College of Wooster, 930 College Mall, Wooster, OH 44691, USA \\ Anna Preis, Cochair \\ Institute of Acoustics, Adam Mickiewicz University, Unultowska 85, Poznan, 61-614, Poland
}

\section{Invited Paper}

\begin{abstract}
8:00
3aPPb1. Non-visual identification of walking grounds. Bruno L. Giordano (Centre for Interdisciplinary Research in Music Media \& Technology (CIRMMT) - Schulich School of Music - McGill Univ., 555 Sherbrooke Street West, Montreal, QC H3A1E3, Canada, bruno.giordano@music.mcgill.ca), Stephen McAdams (Centre for Interdisciplinary Research in Music Media \& Technology (CIRMMT) - Schulich School of Music - McGill Univ., 555 Sherbrooke Street West, Montreal, QC H3A1E3, Canada, smc@music.mcgill.ca), Yon Visell (Centre for Interdisciplinary Research in Music Media \& Technology (CIRMMT) - Schulich School of Music - McGill Univ., 555 Sherbrooke Street West, Montreal, QC H3A1E3, Canada, yon@cim.mcgill.ca), Jeremy Cooperstock (Centre for Intelligent Machines - CIRMMT - Dept. of Electrical and Computer Engineering - McGill Univ., 3480 University Street, Montreal, QC H3A 2A7, Canada, jer@cim.mcgill.ca), Hsin-Yun Yao (Centre for Intelligent Machines - McGill Univ., 3480 University Street, Montreal, QC H3A 2A7, Canada, hyyao@cim.mcgill.ca), Vincent Hayward (Centre for Intelligent Machines - McGill Univ., 3480 University Street, Montreal, QC H3A 2A7, Canada, hayward@cim.mcgill.ca)
\end{abstract}

We investigated the role of haptic, proprioceptive and auditory information in the non-visual identification of walking grounds. We selected four solid materials (e.g., marble) and four aggregate materials (e.g., fine gravel). Five observers identified the materials in each of four experimental conditions: multisensory, haptic, proprioceptive, and auditory. In the auditory condition, they were presented with walking sounds they produced. In the other conditions, observers walked blindfolded on the materials. In the haptic and proprioceptive conditions auditory information was masked. In the proprioceptive condition haptic information was masked. No masking took place in the multisensory condition. In all conditions, solids and aggregates were seldom confused, and aggregates were better identified than solids. Chance identification performance was observed only for solids in the presence of simultaneous haptic and auditory masking, suggesting a secondary role of proprioceptive information. In the proprioceptive condition, identification of aggregates was better than chance, suggesting a significant role of proprioception. Identification of aggregates was at its best in the haptic condition, and not in the multisensory condition, suggesting either an inconsistency of information between auditory and non-auditory channels, or conflicting cognitive strategies for the exploitation of these sources of information in multisensory contexts.

\section{Contributed Paper}

$8: 20$

3aPPb2. The effect of tone envelope on sensory integration: support for the 'unity assumption'. Michael Schutz (University of Virginia, 102 Gilmer Hall, Charlottesville, VA 22904, USA, schutz@virginia .edu), Michael Kubovy (University of Virginia, 102 Gilmer Hall, Charlottesville, VA 22904, USA, kubovy@virginia.edu)

Research on the integration of auditory and visual sensory information consistently confirms the optimal integration hypothesis, according to which information is weighted according to its relative quality. Thus, since the auditory system has greater temporal resolution, this hypothesis predicts that visual information will not affect auditory judgments of duration. In conflict with this hypothesis, Schutz \& Lipscomb (2007) report that percussionists use visual information to alter audience perception of note duration. To show that this discrepancy arises from the acoustic characteristics of percussive sounds, we paired visual information derived from a striking motion with pure tones exhibiting two types of envelopes: percussive (sharp onset followed by exponential decay) and flat (sharp onset to a fixed level, followed by sharp offset) envelopes. Visual information affected auditory duration judgments only for the tones with percussive envelopes (e.g. the sounds produced by a striking motion). As ratings of percussive tones were no more variable than ratings of flat tones, the difference cannot be explained by "ambiguity" of percussive-tone duration. In contrast to the notion of optimal integration, we conclude that envelope is an important acoustic cue for cross-modal integration and offers important information regarding event identification.

\section{Invited Papers}

3aPPb3. Subjective verification of simulation of a vehicle pass-by. Tomasz Kaczmarek (Institute of Acoustics, Adam Mickiewicz University, Unultowska 85, 61-614 Poznan, Poland, tomek@ spl.ia.amu.edu.pl)

The simulation of a moving vehicle can be a very powerful tool for investigating the perception of vehicle motion (velocity, acceleration, road traffic annoyance studies etc). Before applying a simulated vehicle in psychoacoustic noise annoyance studies, one should perform a subjective validation of this tool. The present paper presents the subjective validation of simulation method created by the author. Real pass-by recordings were used to calculate the one-third octave power spectra of a vehicle. Based on these spectra, the simulated pass-bys were created. The simulation was validated in terms of annoyance level and perception of velocity by comparing (in 
psychoacoustic experiment) the original pass-bys with their simulated replicas. Two types of engines - otto and diesel - were tested, for velocities ranging from 30-110 km/h. For velocities above $50 \mathrm{~km} / \mathrm{h}$, the annoyance of simulated pass-bys is very close to the original signal. For lower velocities, simulated pass-bys result in lower annoyance ratings. The solution to the problem would be a reconstruction of the tonal components from the exhaust system.

\section{9:00}

3aPPb4. Experimentation in physics-based sound modeling. Davide Rocchesso (IUAV University of Venice, Department of Art and Design, Dorsoduro 2206, 30123 Venezia, Italy, roc@iuav.it)

Since 2001, as part of the Sounding Object and CLOSED projects, we have been developing physics-based sound models for everyday events and processes. The procedure of describing a sound-producing phenomenon in physical terms, building a model, and accessing its parameters, easily gives the illusion of controlling sound production in the most effective and convenient way. For example, it is trivial to connect a force sensor to the rubbing pressure variable of a friction model. However, one soon realizes that it is often difficult to predict changes in perceived sound in response to physical changes, and that robust models are difficult to achieve. These issues can partially be addressed with experimental research in perception, psychophysics, and phenomenology. Although this approach is inherently time consuming, it will eventually lead to sound models whose parametric layout is more suitable to human manipulation and control. Conversely, in the practice of designers we find that problems are tackled by rapidly producing a multiplicity of attempts, or sketches, and by critically comparing them. In our practice of sound modelers, there are several examples where both approaches, the one based on experimental psychology and the one based on design, proved to be compelling and useful.

\section{9:20}

3aPPb5. Size from sound: Size estimates of balls dropped from various height. Massimo Grassi (Dipartimento di Psicologia Generale, via Venezia 8, 35131 Padova, Italy, massimo.grassi@unipd.it)

Three experiments investigated the perception of the size of a ball (i.e., a non sounding object) from the sound it produces when dropped upon a plate (i.e., a sounding object) from various heights. Experiment one: listeners estimated metrically the size of seven balls $(\varnothing 1,1.5,2,2.5,3,4,5 \mathrm{~cm})$ when dropped upon a baked clay plate from $6 \mathrm{~cm}$ of height. Experiment two: a new group of listeners estimate the size of the balls, however, balls could be dropped from either $6 \mathrm{~cm}$ or $12 \mathrm{~cm}$ of height. In the last experiment, balls could be dropped from either 3, 6 or $12 \mathrm{~cm}$ of height. In all experiments listeners received no foregoing information about the sound source event, such as the material of the balls, the material of the plate, etc. Overall, listeners' estimations matched veridically the actual size of the balls. However, in the second and third experiment, listeners' estimations were dependent on the height: balls were judged slightly larger when dropped from the highest heights.

\section{Contributed Paper}

\section{9:40}

3aPPb6. The texture of natural sounds. Tjeerd C. Andringa (University of Groningen, Dept. Artificial Intelligence, P.O. Box 407, 9700 AK Groningen, Netherlands, t.andringa@ai.rug.nl)

The texture, a spectro-temporal pattern, of many sound sources is a robust and characteristic perceptual property that listeners use for sound source recognition. The robustness of the texture ensures that we can recognize sound sources like a helicopter, flowing water, or a surf breaking on pebbles in a wide variety of acoustic environments. This robustness suggests that textures can be used for automatic source identification or environment classification. We introduce a method to determine the presence of sound textures associated with, for example, flat noise, pulsed noises (helicopter), sweep based textures (running water), and tonal noises (babble). The cumulative probability density of time-frequency fluctuations are matched with prototypical cumulative probability density functions (cpdf) with a running variant of the Kolmogorov-Smirnov test. Textures with a similar distribution as the target distribution, contribute approximately equally to all values of the cpdf. The flatness of this distribution is used as a distance measure. When the pdf's of the target textures do not overlap strongly, the method can determine the texture of time-frequency-regions as small as $100 \mathrm{~ms}$ by 6 semi-tones. This method can therefore also be used to determine the texture of the background.

\section{Invited Papers}

\section{0:00}

3aPPb7. 'Behavioral Relevance' as a guiding principle in investigating the perception of sound source properties. John Neuhoff (The College of Wooster, 930 College Mall, Wooster, OH 44691, USA, jneuhoff@wooster.edu)

Perceiving a sounding object is the result of a complex interaction between physical, physiological, and cognitive factors. However, early in the history of modern psychacoustics, the difficulty in generating complex, controlled, dynamic stimuli limited many investigations of auditory perception to monaural presentation of static sine wave tones or bursts of noise. Thus, very influential models of auditory processing were developed using sounds that are not frequently encountered in a natural listening environment. Although this approach was useful in developing models of the function of the peripheral auditory system, it was less so in developing models of auditory cognition and behavior. If one accepts the position that our perceptual abilities have evolved specifically to help us survive and deal with the stimuli that occur in a natural environment, perhaps it is not surprising that there are differences in processing naturally occurring stimuli and those that are more artificial. Recent paradigmatic shifts spurred by technical innovations have spawned more ecologically valid investigations of complex auditory perception and cognition that include the perception of sound source properties. The future for this approach is promising if "behavioral relevance" can serve a guiding principle. 
10:40

3aPPb8. Classification of everyday sounds: Influence of the degree of sound source identification. Olivier Houix (IRCAM UMR CNRS 9912, Equipe Perception et Design Sonores, 1, place Igor Stravinsky, 75004 Paris, France, olivier.houix @ircam.fr), Guillaume Lemaitre (IRCAM - UMR CNRS 9912, Equipe Perception et Design Sonores, 1, place Igor Stravinsky, 75004 Paris, France, lemaitre@ircam.fr), Nicolas Misdariis (IRCAM - UMR CNRS 9912, Equipe Perception et Design Sonores, 1, place Igor Stravinsky, 75004 Paris, France, misdarii@ircam.fr), Patrick Susini (IRCAM - UMR CNRS 9912, Equipe Perception et Design Sonores, 1, place Igor Stravinsky, 75004 Paris, France, susini@ircam.fr)

Based on a review of everyday sound classification studies, we define three types of similarity that might underlie the formation of categories: acoustical similarity, related to the perception of the sound signal properties; causal similarity, related to the perception of the cause of the sound; semantic similarity, related to the meaning of the sounds. This study focuses on the influence of the degree of sound source identification on the type of similarity used during a free sorting task. Our hypothesis is that well-identified sounds will lead to categories based on causal or semantic similarities, while non-identified sounds will lead to categories based on acoustical similarity. First we measure the causal uncertainty of each sound of a set of kitchen sounds. We ask to participants to describe the cause of the sounds in order to measure the degree of identification for each sound. The results of a free sorting task on this sound corpus indicate that participants groups together sounds according to our assumptions. The classification of the well-identified sounds shows a clear distinction between classes of sound events (gas, liquid, solids, motor) confirming a taxonomy proposed in literature. [Work supported by the CLOSED project of the NEST program]

\section{Contributed Paper}

11:00

3aPPb9. Verbal imitations of sound events enable recognition of the imitated sound events. Karine Aura (Université de Toulouse II Le Mirail, Laboratoire Octogone-Lordat URI 41 56, IFR96, Pavillon de la Recherche, 5 allée Antonio Machado, 31058 Toulouse, France, aura@univ -tlse2.fr), Guillaume Lemaitre (IRCAM - UMR CNRS 9912, Equipe Perception et Design Sonores, 1, place Igor Stravinsky, 75004 Paris, France, lemaitre@ircam.fr), Patrick Susini (IRCAM - UMR CNRS 9912, Equipe Perception et Design Sonores, 1, place Igor Stravinsky, 75004 Paris, France, susini@ircam.fr)

We report a study investigating how verbal imitations of sound events might convey information enabling the recognition of the imitated sounds. We sampled twenty-eight sounds across a taxonomy of kitchen sounds, obtained from a previous experiment focused on the perceived properties of the causes of the sounds. First, we recorded speakers while imitating the sounds. They were specifically required not to use words. Then we selected the imitations of six speakers (three men, three women) imitating twelve sounds. Finally a group of twenty listeners performed a sorting task (they had to group together imitations thought to imitate the same events), and were asked to describe how they built each category. The categories of imitations, obtained from a hierarchical tree representation, fit very well with the categories of the original sound events. This suggests that speakers have succeeded in capturing the information sufficient to enable listeners to recognize the sound events. This is further confirmed by the analyses of the verbalizations. Phonetic analyses show that imitations grouped together share some phonetic similarities, yet the main similarities seem rather to be based on prosodic indexes (duration, intonation, rhythmic patterns). [This work is founded by the FP6 NEST Pathfinder European project CLOSED]

\section{Invited Papers}

\section{$11: 20$}

3aPPb10. Auditory Perception of Silent Object Properties. Lawrence D. Rosenblum (University of California, Riverside, Department of Psychology, 900 University Ave., Riverside, CA 92521, USA, rosenblu@ citrus.ucr.edu)

While much of what we consciously hear is based on sound sources, we also hear properties of objects that are themselves silent, but act to reflect and obstruct sound. A research program has been initiated to examine the degree to which listeners can judge properties of silent, sound-structuring objects. Results of this research have revealed impressive sensitivity to many of these properties. Thus far, this work has shown that (sighted) listeners have some ability to: a) determine the location, dimensions, and general shape of soundobstructing panels; and b) identify various rooms based on how rooms structure emitted sounds; and c) determine their location in a room based on how the room structures sound at different positions. These results indicate that listeners are sensitive to ambient, as well as emitted acoustic properties, and call for a modified conception of human audition that takes into account these sensitivities.

\section{$11: 40$}

3aPPb11. Evaluation and modification of listener decision strategy in human gait perception. Richard E. Pastore (Binghamton University (SUNY), Department of Psychology, Binghamton, NY 13902-6000, USA, pastore@ binghamton.edu), Jesse D. Flint (Binghamton University (SUNY), Department of Psychology, Binghamton, NY 13902-6000, USA, jdflint@binghamton.edu), Jeremy R. Gaston (Binghamton University (SUNY), Department of Psychology, Binghamton, NY 13902-6000, USA, jgaston1@ binghamton.edu)

Beyond obvious differences, there are many similarities in the challenges faced in studies of speech and natural (nonspeech) sounds. The source events are dynamic, complex and highly variable. Source properties map only statistically in the sounds produced, resulting in many acoustic properties that are only sometimes accurate indicators of specific source properties. Finally, beyond the nonlinear mapping of acoustic to perceptual properties, listeners vary in decision weights assigned to the perceived attributes of the sounds. Beginning with this complex conceptualization of source-sound-perception relationships, our research on the perception of human gait from walking sounds investigated the perception of walker posture. The current research extends this project on human gait perception, evaluating both the decision strategy of listener individual listeners and alternative approaches to improving that decision strategy. Implications for studying of both natural nonspeech and speech will be briefly discussed. 


\title{
Contributed Papers
}

12:00

3aPPb12. Source Perception of Everyday and Self Produced Sounds: Factors in the Evolution of Human Auditory Cognitive Capability. James Ballas (Naval Research Laboratory, 4555 Overlook AVE SW, Washington, DC 20375, USA, james.ballas@nrl.navy.mil)

The everyday world was making meaningful sound well before language developed. Self-produced sound exhibits a tight binding between source event and interpretation. These assertions naturally prompt thoughts about the role of these types of sound in the evolution of human auditory capability, especially language. The recent resurgence in theorizing about the origin of language has included other human capabilities as potential generative factors (e.g., gestures), but there has been little discussion about the possible role of capability to process everyday and self-produced sound. This is surprizing given that recent neurocognitive research has supported common processing for speech and meaningful sound. Key attributes of language processing are the ability to interpret not just signs but symbols, and the ability to employ generative grammar processing to produce and interpret symbol strings. Association of sound events with causes and sequences, auditory representation of meaning, persistence of meaning under event and sequence variation, and persistence of event interpretations beyond what is currently present (temporal and spatial abstraction) are all fundamental requirements for these language attributes, and it is asserted that these capabilities might have developed to accurately perceive everyday and selfproduced sound.
12:20

3aPPb13. Detection of missing modal frequencies. Christophe $\mathrm{N}$. Stoelinga (University of Wisconsin-Madison, Department of Communicative Disorders, 1975 Willow drive, Madison, WI 53706, USA stoelinga@wisc.edu), Robert A. Lutfi (University of Wisconsin-Madison, Department of Communicative Disorders, 1975 Willow drive, Madison, WI 53706, USA,ralutfi@wisc.edu)

The amount of information about a source that can be recovered from sound is naturally limited by the ear's ability to resolve individual modal frequencies unique to that source. To measure these limits, listeners were asked to detect, in a standard two-interval, forced-choice task with feedback, which of two sounds on each trial contained a missing partial. The frequencies of the partials corresponded to the ideal modes of a simply-supported, rectangular plate. Plate surface areas and height-wide ratios were chosen to produce the same bandwidths $(125-1125,250-2250$ or $500-4500 \mathrm{~Hz})$ for different numbers of partials $(11,16$ or 24$)$. Overall level of the sounds was roved to discourage detection based on simple level differences. Detection of five highly-practiced listeners was largely independent of the frequency of the lowest partial, being best for partials 1-3, 1-4 or 1-6 for 11, 16 or 24 partials in total, respectively. Mutual masking among the higher-number partials is given as the likely cause. The results are discussed in terms of their implications for the identification of rudimentary source attributes from sound. [Work supported by NIDCD grant 5 R01 DC006875].

\section{Session 3aPPc}

\section{Psychological and Physiological Acoustics, Architectural Acoustics, Noise, and Signal Processing in Acoustics: Jens Blauert and His Contributions}

Ervin R. Hafter, Cochair

University of California, Department of Psychology, 3210 Tolman Hall, Berkeley, CA, CA 94720-1650, USA

Armin Kohlrausch, Cochair

Philips Research Europe, Digital Signal Processing (MS WO02), High Tech Campus 36, Eindhoven, 5656 AE, Netherlands

\section{Invited Papers}

\begin{abstract}
9:00
3aPPc1. A means for comparing normal and impaired listeners in spatial hearing. Ervin R. Hafter (University of California, Department of Psychology, 3210 Tolman Hall, Berkeley, CA, CA 94720-1650, USA, hafter@berkeley.edu)

In our computer controlled Simulated Open Field Environment (SOFE), a computer generates simulated sources and echoes in an anechoic chamber. The SOFE has evolved over many years through ideas and hard work from a superb group of graduate students and post docs including Francoise Briolle, Tom Buell, Poppy Crum, Eric Jensen, Kourosh Saberi, Chris Stecker, Bernhard Seeber, and Miriam Valenzuela, as well as advice from such luminaries as Georg von Bekesy, Arnold Leiman and, of course, Jens Blauert. The ready use of Head Related Transfer Functions (HRTFs) with headphones raises the question, "Why the SOFE?" The answer is that each subject enters the environment with his or her natural HRTF and hearing apparatus intact, thus allowing comparisons between patients with cochlear implants or hearing aids, as well as listeners with normal hearing. Since its inception, this environment has been used to examine precedence, the role of relative level in echo suppression, multiple factors in auditory motion perception, and ventriloquism. This presentation will review some of the more interesting results.
\end{abstract}


3aPPc2. The salience of interaural temporal disparities (ITDs) conveyed by high-frequency signals is determined by the temporal features of their ongoing envelopes. Constantine Trahiotis (University of Connecticut Health Center, Departments of Neuroscience and Surgery (Otolaryngology), 263 Farmington Avenue, Farmington, CT 06030-3401, USA, tino@ neuron .uchc.edu), Leslie R. Bernstein (University of Connecticut Health Center, Departments of Neuroscience and Surgery (Otolaryngology), 263 Farmington Avenue, Farmington, CT 06030-3401, USA, les@neuron.uchc.edu)

Many studies of binaural hearing reveal that envelopes of complex, high-frequency stimuli convey useful interaural time differences (ITDs). Envelope-based ITDs have, typically, been found to be less "potent" than those conveyed by low-frequency waveforms. When considering such findings, Blauert, in his classic textbook, noted that monaural, peripheral processing would result in 1) low-frequency inputs to the binaural processor that are essentially half-wave rectified versions of the input waveforms and 2) high-frequency inputs that are essentially full-wave rectified versions of only the envelopes of the input waveforms. Taking this into account, Blauert, echoing Colburn and Esquissaud (1976), surmised that low-frequency signals would ultimately convey "more distinct time cues" having greater "transient features" than would high-frequency signals and, therefore, be expected to provide more precise ITD information. By using high-frequency "transposed" stimuli having envelopes constructed to provide "more distinct time cues" we have shown in a variety of experimental contexts that the potency of envelope-based ITDs, can rival that measured with low-frequency stimuli. Our most recent experiments use "raised sine stimuli" in order to vary directly the temporal features of the envelope waveform including its "sharpness," "off-time," and interaural cross-correlation function. The new data will be discussed in terms of Blauert's insight.

3aPPc3. Cognitive influences on spatial hearing. Rachel Keen (University of Virginia, Department of Psychology, P. O. Box 400400, Charlottesville, VA 22904-4400, USA, rachelkeen@virginia.edu)

In reverberant rooms a listener perceives a sound coming from its source but simultaneously suppresses reflected sound or echoes. This phenomenon is known as the precedence effect because the preceding (original) sound is given heavier weight than its subsequent reflections. Our research has explored this perceptual process and found that listeners are sensitive to the acoustic information contained in the reflected sound even though it is "suppressed". Specifically, below echo-threshold information relevant to room acoustics (e.g., delay between lead and lag sounds, filtering of the echo's spectrum, presence or absence of an echo at a particular location) is processed by the listener and influences perception. Listeners' expectations about ongoing input can raise or lower echo threshold. Recent ERP data on listeners' responses to click pairs near echo threshold found that when listeners perceived lead and lag sounds as separate sources, a negativity between 100 and $250 \mathrm{~ms}$ was elicited, termed object-related negativity (ORN). When the same stimulus was heard as a single fused sound, no ORN was elicited. These findings suggest that higher-level cognitive processes exert top-down influences over early neurosensory processing.

10:00

3aPPc4. Modeling the Precedence Effect -- how we arrived where we are today. Jonas Braasch (Rensselaer Polytechnic Institute, Greene Bldg., 110 8th St., Troy, NY 12180, USA, braasj@rpi.edu)

Throughout his career, Jens Blauert was interested in further exploring the precedence effect, an auditory phenomenon that enables us to localize sounds in rooms and other enclosures. A series of models that were developed at Bochum's Institute of Communication Acoustics under his leadership have changed the way we think about the precedence effect. While this effect as a psychoacoustic phenomenon is known for its complexity -- for which Jens Blauert's talent to define precise terminology and listen critically came in handy -- the modeling aspects are equally challenging due to the non-linear nature of the underlying auditory processes. Blauert and Cobben's binaural model from 1978 was already able to simulate observations related to the precedence effect. A few years later, Blauert's doctoral student Werner Lindemann introduced a binaural model with specific stages to model the precedence effect. Since then, almost all approaches to simulate the precedence effect are based on inhibitory elements. In this talk, the evolution of this class of models over several student generations at Bochum will be described, and the fruitful scientific exchange with other institutions such as Steven Colburn's laboratory at Boston University will be highlighted as well.

3aPPc5. A History of Binaural Interaction Modeling - Walks with Jens Blauert. H Steven Colburn (44 Cummington St., Boston, MA 02215, USA, colburn@bu.edu), Suzanne Carr (44 Cummington St., Boston, MA 02215, USA, ruiwan@bu.edu), Rui Wan (44 Cummington St., Boston, MA 02215, USA, suzy@bu.edu)

Jens Blauert has been cut an impressively wide path through diverse areas of binaural and spatial hearing. He has consistently integrated viewpoints based on empirical results, intuitive interpretations, and mathematical and computational models of binaural interaction and spatial hearing. This talk focuses on the development of ideas about binaural interaction as represented in quantitative models and the strong influence that Blauert exerted on these models. These influences start with simple models of the extraction and use of interaural time and level differences, include mechanisms that incorporate complex interactions of these differences, and lead to models that are appropriate for the interpretation of complex acoustic scenes. Finally, some current modeling activities will be outlined and related to these historical developments. [Work supported by the US National Institutues of Health DC00100.] 
3aPPc6. Localization-based segregation of acoustic sources: Advantages and limitations. Pierre Divenyi (VA Northern Calif. Health Care Syst. and East Bay Inst. for Research and Education, Bldg. R4, 150 Muir Rd., Martinez, CA 94553, USA, pdivenyi@ebire .org)

It has been suggested [Divenyi, P., \& Oliver, S.K., (1989), JASA 85, 2042-2052] that segregation of simultaneous pairs of sounds in the horizontal plane requires both resolution of the azimuthal separation between the sources, and correct assignment of each signal to the correct source. In fact, contrary to our a priori expectations, localization did not emerge as the dominant dimension underlying segregation of source pairs: its importance often fell behind that of segregation based on differences of spectral or temporal envelope structure, except for some listeners. Nevertheless, the possibility remains that these limitations can be attributed to localization dominance by one of the sources or both. The dual requirement of localization and signal-to-source assignment, in addition to reverberation, may represent a limiting factor for the role of localization in the cocktail-party effect.

\section{Contributed Paper}

11:20

3aPPc7. From binaural hearing to auralization: A triumph of Blauert's 40 years in room acoustics. Ning Xiang (Rensselaer Polytechnic Institute, Greene Building, School of Architecture, 110 8th Street, Troy, NY 12180, USA, xiangn@rpi.edu), Wolfgang Hess (Harman/Becker Automotive Systems, EG - Advanced Signal Processing, 76307 Karlsbad, Germany, WHess@harmanbecker.com)

In the late 60's when Jens Blauert's work on sound localization in the median plane was recognized by the psychoacoustics community, he had already started on a long journey from binaural-hearing research to many aspects of what nowadays is called Communication Acoustics. In addition to initiating many new research activities such as binaural auditory modelling, binaural technology, sound-quality, and speech synthesis, his unremitting endeavour in room acoustics has led to innovative developments in the fields of analysis and synthesis of auditory scenes. For example, binaural roomacoustics modelling, real-time dynamic room-impulse response processing, and auditory virtual environments (nowadays known as "auralization") were particularly driven by his work and his supervision of over 50 doctoral students. In this regard the first head-tracking and fast-convolution patent application was filed in the early 70 's. Recently auditory models have been applied in auditory spatial evaluations of rooms and cars. Not only his excellent analytical skills and theoretical knowledge, but also his vision for transfer to practical applications in room acoustics and human-auditory spatial perception led to an outstanding life-work. This paper will highlight just a few of Jens Blauert's major contributions to room-acoustics.

\section{Invited Papers}

\section{1:40}

3aPPc8. Binaural technology for e.g. rendering auditory virtual environments. Dorte Hammershøi (Acoustics, Aalborg University, Fredrik Bajers Vej 7 B5, 9220 Aalborg Ø, Denmark, dh@es.aau.dk)

Jens Blauert's research up through the late 1960s and later, pioneered the field of binaural technology and auditory virtual environments. He mastered the measurement of head-related transfer functions (HRTFs) before the term was introduced, and his methods were standard for decades. While most acknowledge his efforts in understanding the binaural hearing and the significance of interaural differences, appreciation is also in place for his evaluation of localization with identical ear input signals. The relations between the hearing's "directional" bands and the transfer functions' "boosted" bands, helped mediate the understanding that if the transfer functions could be mastered, then important dimensions of the auditory percept could also be controlled. He early understood the potential of using the HRTFs and numerical sound transmission analysis programs for rendering auditory virtual environments. Jens Blauert participated in many European cooperation projects exploring this field (and others), among other the SCATIS project addressing the auditory-tactile dimensions in the absence of visual information.

\section{2:00}

3aPPc9. How can we model a quality event? Some considerations on describing quality judgment and prediction processes. Sebastian Möller (Deutsche Telekom Laboratories, Berlin Institute of Technology, Ernst-Reuter-Platz 7, 10587 Berlin, Germany, sebastian.moeller@telekom.de)

Jens Blauert has introduced a systemic view on a listener in an auditory experiment. This view helps to separate sound events from auditory events and from their descriptions, and to identify and describe the processes involved in such experiments. The notion has later been extended to listeners in a quality judgment situation by Jekosch and Raake, leading to the notion of a "quality event". On the one hand, knowledge of the involved processes is necessary to design appropriate measurement processes for, e.g., sound quality, transmission quality, auditory-scene quality, or product-sound quality. On the other hand, such knowledge enables us to define algorithms which estimate quality - or sub-aspects of it - in the system design process. The talk will mainly follow the second line and will identify components which are necessary for an algorithmic description of the processes involved in the formation of a quality event. Taking the example of telecommunication services, it will be shown which components of quality prediction models are already available, and which others are still out-of-reach and require further study.

\section{$12: 20$}

3aPPc10. Semio-acoustics: a domain of communication acoustics. Ute Jekosch (Chair of Communication Acoustics, TU Dresden, Helmholtzstr. 10, 01069 Dresden, Germany, ute.jekosch@tu-dresden.de)

For user-oriented design of modern information and communication systems we investiate the perception of acoustic signals as sign carriers: How do listeners process acoustic/auditory events when they treat them as information carriers and which meaning do they associate with them? Sign carriers (in our case the acoustic signals) transport information across space and time. When being perceived, they are decoded, analyzed and interpreted with regard to their cultural meaning and their relevance for the actual living and commu- 
nication situation. These processes are universal to a certain degree. It is the aim of our research in Dresden to investigate the fundamental structures and organisational principles underlying this assignment of meaning to acoustic and their accompanying auditory events. In this talk, some semio-acoustic paradigms will be introduced. As a Ph.D. student of Jens Blauerts' and as a post doc in his team, the semio-acoustic approach has developed as a logical continuation of his way of thinking, of his research approaches, and of his scientific goals, based on and fed by his basic statement: Communication acoustics is not conceivable without audition, and only if we are able to understand auditory events, and even cognitive processes, we are able to make use of acoustic communication and information technology in a user-friendly way.

\title{
Session 3aSAa
}

\section{Structural Acoustics and Vibration, Computational Acoustics, and EURONOISE: Efficient Boundary Element Methods I}

\author{
Ramani Duraiswami, Cochair \\ University of Maryland Institute for Advanced Computer Studies, 115 A.V. Williams Bldg, College Park, MD 20742, USA \\ Lothar Gaul, Cochair \\ Institute of Applied and Experimental Mechanics, University of Stuttgart, Pfaffenwaldring 9, Stuttgart, 70550, Germany
}

\section{Invited Papers}

\section{8:00}

3aSAa1. Vibroacoustic Simulation of Partly Immersed Bodies by a Coupled Fast BE-FE Approach. Dominik Brunner (Institute of Applied and Experimental Mechanics, University of Stuttgart, Pfaffenwaldring 9, 70550 Stuttgart, Germany, brunner@iam.uni-stuttgart.de), Michael Junge (Institute of Applied and Experimental Mechanics, University of Stuttgart, Pfaffenwaldring 9, 70550 Stuttgart, Germany, junge@iam.uni-stuttgart.de), Christian Cabos (Germanischer Lloyd, Vorsetzen 35, 20459 Hamburg, Germany, christian.cabos@gl-group.com), Lothar Gaul (Institute of Applied and Experimental Mechanics, University of Stuttgart, Pfaffenwaldring 9, 70550 Stuttgart, Germany, gaul@iam.uni-stuttgart.de)

Simulation of vibroacoustic behavior of submerged bodies necessitates dealing with fluid-structure coupled problems. In case of a high density of the fluid, the feedback of the acoustic pressure onto the structure cannot be neglected and a fully coupled system must be investigated. In this work, the finite element method (FEM) is used for the structural part. The commercial finite element package ANSYS is applied for setting up the mass and stiffness matrices. The boundary element method (BEM) is well suited for simulating the sound propagation in the unbounded exterior acoustic fluid domain. Here, the fast multipole method (FMM) is applied to overcome the known bottleneck of classical BE-methods. In the case of partly immersed bodies, Dirichlet boundary conditions on the fluid surface additionally have to be incorporated. This is done by applying a halfspace formulation. The extension of the FMM to this scenario is discussed. Iterative preconditioned solvers are employed and the numerical efficiency is discussed. The applicability of the coupling scheme is demonstrated using a realistic model problem.

\section{8:20}

3aSAa2. A half-space BEM for the simulation of sound propagation above an impedance plane. Haike Brick (TFH Berlin University of Applied Sciences, FB II, Mathematics - Physics - Chemistry, Luxemburger Str. 10, 13353 Berlin, Germany, brick@tfh-berlin.de), Martin Ochmann (Technische Fachhochschule Berlin, Univ. of Applied Sciences, Luxemburger Str. 10, 13353 Berlin, Germany, ochmann@tfh-berlin.de)

The Boundary-Element-Method is a powerful tool for the simulation of sound radiation and scattering. Classically, it was developed for the free 3D-space, but it can be modified easily for half-space solutions as long as the half-space is delimited by a perfectly rigid or soft plane. In this case, the Green's function, the core of the BEM, can be derived from a simple image source ansatz, which however cannot be used for a more general impedance boundary condition. The alternative, an additional discretisation of a finite but large part of the plane leads to an enormous increase of the size of the set of equations. In this presentation, an appropriate Green's function will be introduced, which is able to describe the sound propagation above an impedance plane and is suitable for an implementation into a BEM code. It bases on the superposition of sound sources with complex source points. The numerical evaluation of this Green's function will be presented along with several test cases including sound radiation from burning flames above ground and tire-road noise. The computational costs of the developed "Complex-Source-Point-BEM" (CBEM) in comparison with a classical BEM together with a discretisation of the impedance plane will be discussed. 


\section{Contributed Papers}

8:40

3aSAa3. Simulation of noise barrier insertion loss using the boundary element method. Holger Waubke (Austrian Academy of Sciences / Acoustics Research Institute, Wohllebengasse 12-14, 1040 Vienna, Austria, holger.waubke@oeaw.ac.at), Wolfgang Kreuzer (Austrian Academy of Sciences / Acoustics Research Institute, Wohllebengasse 12-14, 1040 Vienna, Austria, wolfgang.kreuzer@oeaw.ac.at), Zhensheng Chen (Austrian Academy of Sciences / Acoustics Research Institute, Wohllebengasse 12-14, 1040 Vienna, Austria, zhensheng.chen@oeaw.ac.at)

Within a project of the European Commission "Calm Tracks \& Routes" the performances of different styles of noise barriers were investigated. Additionally new methods for increasing the absorption were investigated. A central theme was to investigate the mitigation of curved walls with high absorption coefficients, because studies made before the project gave the hint that noise barriers curved away from the source should give satisfying insertion loss. Due to the curvature the view in the landscape is hindered to a lesser degree compared to straight noise barriers. The investigation of the noise barriers was done by boundary element methods. An absorptive model for grassland was added and implemented into Green's functions. Due to the existence of smeared sources the application of the fast multipole method was not possible and the simulations were limited to the two-dimensional case. It became obvious that the distance of the source is important for the mitigation of curved noise barriers with respect to straight ones. Therefore different wall types and source positions were investigated. The results of the numerical simulation were compared to measurements in situ and in a large anechoic chamber.

\section{9:00}

3aSAa4. A GWBEM method for high frequency acoustic scattering Emmanuel Perrey-Debain (Université de Technologie de Compiègne, Lab. Roberval UMR 6253, Dept. Acoustique, BP 60319, 60203 Compiègne, France, emmanuel.perrey-debain@utc.fr), Hadrien Bériot (Université de Technologie de Compiègne, Lab. Roberval UMR 6253, Dept. Acoustique, BP 60319, 60203 Compiègne, France, beriotha@gmail.com), Mabrouk Ben Tahar (Université de Technologie de Compiègne, Lab. Roberval UMR 6253, Dept. Acoustique, BP 60319, 60203 Compiègne, France, mabrouk.bentahar@utc.fr), Catherine Vayssade (Université de Technologie de Compiègne, Lab. Roberval UMR 6253, Dept. Acoustique, BP 60319, 60203 Compiègne, France, catherine.vayssade@utc.fr)

This paper considers the problem of scattering of a time-harmonic acoustic incident wave by a hard obstacle. The numerical solution to this problem is found using a Galerkin Wave Boundary Element Method (GWBEM) whereby the functional space is built as the product of conventional low order piecewise polynomials with a set of plane waves propagating in various directions. In this work we present strategies for finding the appropriate plane wave basis locally on each boundary element in order to deal efficiently with very irregularly meshed structures exhibiting both large smooth scattering surfaces as well as corners and small geometrical features. Numerical results clearly demonstrate that these improvements allow the handling of scatterers with complicated geometries while maintaining a low discretization level of 2.5 to 3 degrees of freedom per full wavelength.

\section{9:20}

3aSAa5. Fast multipole accelerated boundary element method (FMBEM) for solution of 3D scattering problems. Nail A. Gumerov (University of Maryland Institute for Advanced Computer Studies, 115 A.V. Williams Bldg, College Park, MD 20742, USA, gumerov @ umiacs.umd.edu), Ramani Duraiswami (University of Maryland Institute for Advanced Computer Studies, 115 A.V. Williams Bldg, College Park, MD 20742,USA,ramani@umiacs.umd.edu)

Wideband FMBEM codes are challenging to implement since there are problems at both very low and high frequencies. Substantially different schemes for function representation and translation are efficient for low and high frequency ranges. We present a method which is suitable for solution both high and low frequency problems since it implements a switch between different representations and uses fast translation methods appropriate to each representation. For a high frequency problem the switch between representations may occur at some intermediate levels of hierarchical space subdivision of the FMM. We also present an FMM-based preconditioner used in the flexible GMRES iterative solver for scattering problems and discuss example problems computed in range $0.001<\mathrm{kD}<200$, where $\mathrm{k}$ is the wave number and $\mathrm{D}$ is the size of the computational domain. The theoretical and experimental computational complexity of our algorithm is approximately $\mathrm{O}\left((\mathrm{kD})^{3}\right)$ at large $\mathrm{kD}$ with relatively small asymptotic constant and the algorithm allows to solve problems with up to million nodes used for surface discretization in the range tested on a conventional personal computer.

\section{9:40}

3aSAa6. An efficient distributed BEM solver for acoustic and vibroacoustic analyses on a standard PC network. Paolo Di Francescantonio (STS, Via Dalmazia 30, 21100 Varese, Italy, pdifra@stsweb.it)

An efficient BEM approach that permit to solve large acoustic and vibroacoustic problems distributing analyses on a standard PC network is presented. The high efficiency is based on a mix of elements including: 1) A proprietary and high efficient communication and synchronization library (not MPI) 2) A Multi Domain Approach for reducing memory requirements and solution time 3) Efficient iterative solvers The proprietary communication / synchronization library permits to take full advantage of recent multi core PC, enabling to set up clusters with a large number of computing units with a reduced number of PC. A "distributed memory" approach permit to have available the sum of all the PCs RAM, ensuring the possibility to solve large and huge problems that cannot be faced with standard approaches. The Multi Domain approach permit to further reduce the memory requirements, while preserving all the required flexibility in terms of boundary condition and coupling with structural modes. Examples will be showed of large an huge analyses in different application fields including purely acoustics, and fully coupled structural-acoustic analyses. 


\title{
Session 3aSAb
}

\section{Structural Acoustics and Vibration, Computational Acoustics, and EURONOISE: Fluid-Structure Interaction I}

\author{
Noureddine Atalla, Cochair \\ Univ. de Sherbrooke, Mechanical Engineering Depart., 2500 Boulevard de l'Université, Sherbrooke, QC J1K 2R1, Canada
}

Vicente Cutanda Henriquez, Cochair

Institute of Sensors, Signals and Electrotechnics, University of Southern Denmark, Niels Bohrs Allé 1, Odense S, 5230, Denmark

Stefan Schneider, Cochair

Laboratoire de Mécanique et d'Acoustique, UPR 7051 CNRS, 31,Chemin Joseph-Aiguier, Marseille, 13402, France

\section{Invited Papers}

\section{0:40}

3aSAb1. Practical modeling of acoustic losses in air due to heat conduction and viscosity. René Christensen (Oticon A/S, Kongebakken 9, 2765 Smoerum, Denmark, rch@oticon.dk), Peter Juhl (Institute of Sensors, Signals and Electrotechnics, University of Southern Denmark, Niels Bohrs Allé 1, 5230 Odense S, Denmark, pmjuhl@ sense.sdu.dk), Vicente Cutanda Henriquez (Institute of Sensors, Signals and Electrotechnics, University of Southern Denmark, Niels Bohrs Allé 1, 5230 Odense S, Denmark, vch@sense.sdu .dk)

Accurate acoustics models of small devices with cavities and narrow slits and ducts should include the so-called boundary layer attenuation caused by thermal conduction and viscosity. The purpose of this paper is to present and compare different methods for including these loss mechanisms in analytical and numerical models. A simple circular geometry with a narrow tube has been used as a reference and is investigated both through measurements and the different models. The simulation methods compared are: i) traditional analytical approaches such as lumped parameter modelling and transmission line modelling, ii) numerical methods implemented into commercial packages, such as the low reduced frequency models as proposed by W. M. Beltman and implemented in ACTRAN and the linearized Navier-Stokes equations used in COMSOL Multiphysics, and iii) an implementation specifically made for this purpose using BEM and the full linearized model by M. Bruneau.

\section{1:00}

3aSAb2. An overview of models for viscothermal wave propagation, including fluid structure interaction. Willem M. Beltman (Intel Corporation, 2111 NE 25th Avenue, M/S JF2-86, Hillsboro, OR 97124, USA, willem.m.beltman@intel.com), Ysbrand H. Wijnant (University of Twente, Dept. Mechanical Engineering, P.O. Box 217, 7500 AE Enschede, Netherlands, Y.H.Wijnant@ctw.utwente.nl), Marten J. Nijhof (University of Twente, Dept. Mechanical Engineering, P.O. Box 217, 7500 AE Enschede, Netherlands, m.j.j.nijhof@ctw.utwente.nl)

In acoustics, the standard wave propagation models neglect the effects of viscosity and thermal conductivity. When waves propagate in narrow tubes or thin layers these simplifications might not be accurate. This paper presents an overview of models that take the effects of inertia, viscosity, thermal conductivity and compressibility into account. Based on the use of dimensionless parameters, three classes of models are outlined. The most important dimensionless parameter is the shear wave number, an unsteady Reynolds number that indicates the ratio between inertial and viscous effects. These viscothermal wave propagation models can be coupled to structural models to capture the fluid structure interaction. Analytical solutions can be found for these coupled analysis cases only for simple geometries and boundary conditions. For more complex geometries, numerical models were developed. Examples of applications of these models are also presented.

11:20

3aSAb3. A 3 dimensional finite element modeling of "smart foam" and its experimental validation. Pierre M. Leroy (Laboratoire de Mécanique et d'Acoustique - CNRS, 31 chemin Joseph Aiguier, 13402 Marseille, France, leroy@1ma.cnrs-mrs.fr), Noureddine Atalla (Univ. de Sherbrooke, Mechanical Engineering Depart., 2500 Boulevard de 1'Université, Sherbrooke, QC J1K 2R1, Canada, Nouredinne.Atalla@Usherbrooke.ca), Alain Berry (Univ. de Sherbrooke, Mechanical Engineering Depart., 2500 Boulevard de l'Université, Sherbrooke, QC J1K 2R1, Canada, alain.berry@usherbrooke.ca), Philippe Herzog (Laboratoire de Mécanique et d'Acoustique - CNRS, 31 chemin Joseph Aiguier, 13402 Marseille, France, herzog@1ma.cnrs-mrs.fr)

The "smart foam" concept and design originate from the combination of passive dissipation of acoustic foams in the medium and high frequency range and the active absorption ability of piezoelectric actuators (generally PVDF) in the low frequency range. This results in a passive/active absorption control device that can efficiently operate over a broad range of frequencies. In this paper, a $3 \mathrm{D}$ finite element model of smart foam and its experimental validation are presented. The finite element model uses quadratic poroelastic elements with a (u,p) formulation, as well as elastic, fluid and piezoelectric elements. The weak integral formulation of the different 
involved domains and their coupling conditions are presented. In particular, an isotropic and a simplified orthotropic model of poroelastic media are presented and compared. The fabrication of a prototype smart foam based on melamine foam with a curved, bonded PVDF film is discussed. Passive absorption and acoustic radiation of this prototype are measured in an impedance tube. The measured data are in good agreement with the numerical results, thereby validating the finite element model. This modeling tool constitutes a powerful platform to simulate and optimize various configurations of smart foams.

\section{1:40}

3aSAb4. Prediction of the sound field in anechoic rooms : comparison of two different approaches. François-Xavier Bécot (Matelys - Acoustique \& Vibrations, 20/24 rue Robert Desnos, 69120 Vaulx-en-Velin, France, francois-xavier.becot @ matelys.com), Stefan Schneider (Laboratoire de Mécanique et d'Acoustique, UPR 7051 CNRS, 31,Chemin Joseph-Aiguier, 13402 Marseille, France, schneider@1ma.cnrs-mrs.fr), Luc Jaouen (Matelys - Acoustique \& Vibrations, 20/24 rue Robert Desnos, 69120 Vaulx-en-Velin, France, luc.jaouen@matelys.com)

Despite the numerical advances, predicting the sound field in anechoic rooms is still challenging because it requires a fine modeling of the wall surface, acoustic properties and geometry, and the computation of the room sound field. It is proposed here to compare two innovative approaches to predict the performance of anechoic wedges, in particular to quantify the influence of porous frame motion, and to propose a numerically efficient model for the prediction of the sound field in anechoic room sounds at low frequencies. The first approach relies on the theory for modeling the sound propagation in double porosity media first presented by Olny \& Boutin [J. Acoust. Soc. Am. 114 (1), 2003]. It has been shown that this approach could accurately predict the cut-off frequency of anechoic wedges. The interest is that this approach is numerically costless and that complex geometry could be considered. The second approach is based on the combination of FEM and BEM. It allows to predict the sound field in anechoic rooms. Compared to the previous approach, the latter one has the advantage of being able to identify structural effects inside the wedges and to propose an accurate prediction of the sound field in the room.

\section{2:00}

3aSAb5. Improvements on the directional characteristics of a calibration sound source using the Boundary Element Method. Vicente Cutanda Henriquez (Institute of Sensors, Signals and Electrotechnics, University of Southern Denmark, Niels Bohrs Allé 1, 5230 Odense S, Denmark, vch@sense.sdu.dk), Salvador Barrera-Figueroa (Danish Fundamental Metrology, Matematiktorvet 307, 2800 Kgs. Lyngby, Denmark, sbf@dfm.dtu.dk), Peter Juhl (Institute of Sensors, Signals and Electrotechnics, University of Southern Denmark, Niels Bohrs Allé 1, 5230 Odense S, Denmark, pmjuhl@sense.sdu.dk)

The project Euromet-792 aims to investigate and improve methods for secondary free-field calibration of microphones. In this framework, the comparison method is being studied at DFM in relation to the more usual substitution method of microphone calibration. The design of the sound source is of particular importance to achieve a sound field that reaches both microphones with the same level and that is sufficiently uniform at the microphone positions, in order to reduce the effect of misalignment. An existing sound source has been modeled using the Boundary Element Method, and the simulations have been used to modify the source and make it suitable for this kind of calibration. It has been found that a central plug, already present in the device, can be re-shaped in such a way that makes the sound field on the microphone positions more uniform, even at rather high frequencies. Measurements have been carried out in order to verify the goodness of this solution.

\section{$12: 20$}

3aSAb6. Analysis of the resonance frequency shift in cylindrical cavities containing a sphere and its prediction based on the Boltzmann-Ehrenfest principle. Arturo Santillan (Universidad Nacional Autonoma de Mexico, Centro de Ciencias Aplicadas y Desarrollo Tecnologico, CCADET-UNAM, Circuito Exterior s/n, Cd. Universitaria, A. P. 70-186, 02510 Mexico, D.F., Mexico, arturo.orozco@ccadet.unam.mx), Vicente Cutanda Henriquez (Institute of Sensors, Signals and Electrotechnics, University of Southern Denmark, Niels Bohrs Allé 1, 5230 Odense S, Denmark, vch@sense.sdu.dk)

It is known that forces generated by high-level acoustic waves can compensate for the weight of small samples, which can be suspended in a fluid. To achieve this, a standing wave is created in a resonant enclosure, which can be open or closed to the external medium. This phenomenon, called Acoustic levitation, has numerous applications in containerless study and processing of materials. Although it is possible to levitate a sample for long periods of time, instabilities can appear under certain conditions. One of the causes of oscillational instabilities is the change of the resonance frequency of the cavity due to the presence of the levitated object. The Boltzmann-Ehrenfest principle is used to find an analytical expression for the resonance frequency shift in a cylindrical cavity produced by a small sphere, with $k r<1$, where $k$ is the wavenumber and $r$ is the radius of the sphere. The validity of this expression has been investigated by means of the Boundary Element Method and experiments. In addition, the effect of the dispersion of the sound field by the sphere and the boundaries of the cavity on the resonance frequency shift has been analyzed using the BEM.

\section{2:40-2:00 Lunch Break}




\section{Invited Paper}

\section{2:00}

3aSAb7. A hybrid SEA/image sources approach for the prediction of the insertion loss of enclosures. Franck C. Sgard (IRSST, Service de la recherche, 505 Boulevard de Maisonneuve O, Montréal, QC H3A3C2, Canada, frasga @irsst.qc.ca), Hugues Nelisse (IRSST, Service de la recherche, 505 Boulevard de Maisonneuve O, Montréal, QC H3A3C2, Canada, hugnel@irsst.qc.ca), Noureddine Atalla (Univ. de Sherbrooke, Mechanical Engineering Depart., 2500 Boulevard de l'Université, Sherbrooke, QC J1K 2R1, Canada, Nouredinne.Atalla@Usherbrooke.ca), Celse Kafui Amedin (GAUS, Université de Sherbrooke - 2500 Bd de l'Université, Sherbrooke, QC J1K2R1, Canada, Celse-kafui.amedin@Usherbrooke.ca), Rémy Oddo (GAUS, Université de Sherbrooke - 2500 Bd de l’Université, Sherbrooke, QC J1K2R1, Canada, Remy.Oddo@USherbrooke.ca)

Enclosures are a classical solution to reduce the sound exposure of workers to the noise radiated by machinery. Their acoustic design can be achieved with the help of predictive tools based on simple analytical tools or sophisticated numerical deterministic models. However, there is no simple and fast tool allowing to account for the complexity of the enclosure configuration, capable of better simulating the non-diffuse nature of the field inside the enclosure and covering the typical frequency range $[100 \mathrm{~Hz} ; 5000 \mathrm{~Hz}]$. This paper presents the development of such a tool for the prediction of the acoustic performance of enclosures. It is based on a hybrid model: the statistical energy analysis (SEA) for the sound transmission across the various elements of the enclosure and the method of image sources for the sound field inside the enclosure. The approach is validated by comparing calculation and experimental results carried out in a semi-anechoic room on rectangular and L-shape enclosures for several inner source locations. The effect of an opening is also investigated. The comparisons between the models and the experimental results show a good agreement for most of the tested configurations.

\section{Contributed Papers}

2:20

3aSAb8. Vibroacoustic interface conditions between prestressed structures and moving fluids. Fabien Treyssède (Nantes Atlantique University - LCPC, Division for Metrology and Instrumentation, Route de Pornic - BP 4129, 44341 Bouguenais, France, fabien.treyssede@ @ cpc.fr)

Many applications involve coupling between prestressed solids and fluids (possibly flowing). Typical problems might be given by vibroacoustics of fluid-filled pressurized cavities, wave propagation, dynamics and stability of pipes conveying fluids. The goal of this work is to investigate jump conditions that hold for small linear perturbations at any impermeable interfaces, slip or bonded, plane or not, between fluids and structures in the presence of initial flow and prestress. First, the mixed Eulerian-Lagrangian description is briefly recalled. It yields an interesting unification between existing formulations for inviscid fluids (Galbrun's equation) and solids (updated Lagrangian formulation). Based on conservative equations obtained from this description, interface conditions are derived in an elegant manner thanks to the concept of generalized functions in distribution theory. These conditions are written in terms of a curvilinear coordinate system attached to the interface. They are shown to coincide with a direct linearization of standard Eulerian jump conditions. General interface conditions for displacement, stress, heat flux and temperature are given. As an example, these conditions are applied to the analysis of elastoacoustic wave propagation inside an inviscid fluid-filled pressurized duct. The combined effects of slip, prestress and initial flow are briefly discussed.

\section{2:40}

3aSAb9. Experimental and numerical investigation of the dynamics in spatial fluid-filled piping systems. Jan Herrmann (Institute of Applied and Experimental Mechanics, University of Stuttgart, Pfaffenwaldring 9, 70550 Stuttgart, Germany, herrmann@iam.uni-stuttgart.de), Thomas Haag (Institute of Applied and Experimental Mechanics, University of Stuttgart, Pfaffenwaldring 9, 70550 Stuttgart, Germany, thomasshaag@gmx .de), Lothar Gaul (Institute of Applied and Experimental Mechanics, University of Stuttgart, Pfaffenwaldring 9, 70550 Stuttgart, Germany, gaul@iam .uni-stuttgart.de)

Hydraulic piping systems, such as fluid-filled break and fuel pipes in automotive applications, undergo strong acoustic excitation due to pressure pulsations of pump and valve operation. By fluid-structure coupling the sound transmission within the pipe may lead to a structural excitation of other car components causing excessive noise levels or even structural failure. In order to obtain a complete and reliable understanding of the wave propagation and vibration phenomena in spatial piping systems, a test rig is presented, consisting of a pressure source and a fluid-filled break pipe with an attached target structure. With this experimental setup, it is possible to quantify the acoustic sound transmission and to examine the dynamic behavior by transfer functions. The experimental results are compared with harmonic and transient finite element simulations employing efficient model order reduction techniques for the fluid-structure coupled system. This research focuses on the identification of hydraulic resonances and the optimal mounting of the fluid-filled break pipe in order to minimize the structureborne sound induced on the target structure.

\section{3:00}

3aSAb10. Noise generation in an unstable boundary-layer flow over a flexible wall. Marie-Laure Gobert (IRENav, BP 600, 29240 Brest Armées, France, marie-laure.gobert@ecole-navale.fr), Uwe Ehrenstein (IRPHE, 49 rue Joliot-Curie, BP 146, 13384 Marseille Cedex 13, France, ehrenstein @ irphe.univ-mrs.fr), Jacques Andre Astolfi (IRENav, BP 600, 29240 Brest Armées, France, astolfi@ecole-navale.fr), Patrick Bot (IRENav, BP 600, 29240 Brest Armées, France, bot@ecole-navale.fr), Jean Audet (Thales Underwater Systems, 525 route des Dolines, BP 157, 06903 Sophia Antipolis, France, Jean.Audet@fr.thalesgroup.com), Guillaume Oudot (DCNS Divison Navires Armés, Le Mourillon, BP 1306, 83076 Toulon cedex, France, guillaume.oudot@densgroup.com)

The boundary-layer flow, for instance along a sonar dome, gives rise to hydrodynamic noise due to the pressure fluctuations. The prediction of the resulting self-noise received by the sonar antenna is based on models, which in general take only partially into account the flexibility of the dome wall. The present work readdresses the problem of hydrodynamic noise, considering the geometrically simplified model of a two-dimensional unstable boundary-layer flow along an elastic plate with clamped ends. The incompressible Navier-Stokes equations are fully coupled to the elastic plate model and the system is numerically solved for various plate materials. The unstable flow dynamics is analyzed with respect to the wall properties. The Fourier-transformed stress tensor is then used in the framework of Lighthill's analogy to determine the generated radiative sound, emphasizing the effect of wall-flexibility. This work is supported by Thales Underwater System and DCNS. 


\title{
Session 3aSCa
}

\section{Speech Communication: Speech Prosody and How it Relates to Segmental Aspects of Speech}

\author{
Yi Xu, Cochair \\ University College London, Wolfson House, 4 Stephenson Way, London, NW1 2HE, UK \\ Hansjörg Mixdorff, Cochair \\ TFH Berlin University of Applied Sciences, Luxemburger Str. 10, Berlin, 13353, Germany
}

\section{Invited Papers}

\section{8:00}

3aSCa1. Segmental variability related to speech prosody - An articulatory-functional perspective. Yi Xu (University College London, Wolfson House, 4 Stephenson Way, NW1 2HE London, UK, yi.xu@ucl.ac.uk)

Just like in the tonal aspect, variability abounds in the segmental aspect of speech. Here I argue that segmental variability, similar to supra-segmental variability, can be understood from an articulatory- functional perspective. That is, two independent sources of segmental variability co-exist: a) multiple layers of communicative functions, and b) articulatory mechanisms of their encoding. From an articulatory perspective, much of the segmental variability arises from the nature of the syllable as a product of generating time markers, namely, synchronized onsets of consonant, vowel, tone and phonation register at the start of each syllable as recurrent events that serve like clock ticks to enable time tracking by both speakers and listeners. Such time marking results in complete overlap of initial consonant and the following vowel, which is responsible for most of the variability known as coarticulation, and sequential offset of coda components in a syllable, making them susceptible to deletion and merger. From a functional perspective, segments as supralaryngeal articulatory movements encode not only lexical contrast, but also other functional contrasts such as focus, grouping, attitude, etc. Like the tonal aspect of speech, these extra-lexical information are probably encoded as modification of segmental targets that have been lexically assigned.

$$
8: 20
$$

3aSCa2. Prosodic Variation: Understanding Scope, Categoricity, and Recursion in Speech Production and Perception. Dani Byrd (Dept of Linguisitcs, University of Southern California, 3601 Watt Way, GFS 301, Los Angeles, CA 90089-1693, USA, dbyrd@usc.edu), Jelena Krivokapic (Department of Linguistics, Yale University, 370 Temple St, Room 204, PO Box 208366, New Haven, CT 06520-8366, USA, jelena.krivokapic@yale.edu)

Prosodic structure refers to the level of linguistic structure above the segmental level, namely prominence and phrasal organization. We will examine both articulatory and perceptual characteristics of phrasal organization, and their interrelation. An experiment using articulatory movement tracking examines how far in time remotely from a boundary the effect of the boundary extends. In a second experiment (Krivokapic 2007), we examine how structural properties of a range of prosodic boundaries are reflected in perception-specifically, whether boundary perception is categorical or gradient. A third study considers whether prosodic junctures of the same category type (e.g, an intonational phrase boundary) can, when embedded, give rise in articulation to boundary signatures of measurably different strengths. That is, we evaluate the possibility of recursive prosodic structures of the kind that Ladd (1996) postulates. Taken together, these experiments inform us about structurally induced variability. They provide the opportunity to evaluate standard assumptions about prosodic structure such as the question of boundaries as symbolic or temporal events, categoricity, and strict layering. Throughout, we will consider how the pi-gesture framework (Byrd \& Saltzman 2003) offers theoretical insight regarding issues of prosodic representation and realization. [Supported by $\mathrm{NIH}]$

\section{Contributed Papers}

\section{8:40}

3aSCa3. Temporal organization of prosodic and segmental features in spoken Japanese. Hiroya Fujisaki (University of Tokyo (Prof. emeritus), 3-31-12 Ebisu, Shibuya-ku, 150-0013 Tokyo, Japan, fujisaki@alum .mit.edu), Sumio Ohno (Tokyo University of Technology, 1404-1 Katakura, 192-0982 Hachioji, Japan, ohno@cc.teu.ac.jp)

It is apparent that prosodic and segmental features of speech must be temporally coordinated in order to produce a consistent and meaningful message. The precise mechanism for the coordination, however, has not been clear. The present study looks into this problem in the case of word accent in spoken Japanese. The speech material consisted of utterances of Japanese words that were identical in the word accent type, in the number of morae, in vowel constituents, but were different in consonantal constituents (including a 'null' consonant) at a certain intervocalic position. As for the prosodic features, the fundamental frequency contours were analyzed using the command-response model, and the onset and the offset of the extracted accent command were used as indices of prosodic timing. As for the segmental features, the formant frequency trajectories of the vowels were analyzed using another command-response model, which allowed extraction of the onset of the articulatory command for a vowel nucleus as an index of segmental timing. Comparison of the timings of these commands provides a means for quantitative analysis of the relationship between segmental and prosodic features of speech, and leads to an understanding of the underlying mechanism for their temporal coordination.

\section{9:00}

3aSCa4. Stress, focus, and the roots of segmental variation. Kenneth De Jong (Indiana University, 322 Memorial Hall, 1021 E. 3rd Street, Bloomington, IN 47405, USA, kdejong@indiana.edu)

de Jong (JASA, 1995) proposed a definition of linguistic stress as variation in the articulation of segmental material; stress is variation on the hypoto hyper-articulation dimension (Lindblom, 1990) localized to the syllable. 
This definition has suggested that the interaction of stress and segmental articulation will involve 1) linguistic conventionalization, since stress is a property of particular linguistic systems, 2) the structure of segmental contrasts, since hyper-articulation is driven by contrast enhancement, and 3) propensities in articulation, since hypo-articulation is driven by motor considerations. This paper reviews evidence for effects of linguistic conventionalization of stress. It further examines and compares the results of a number of experiments in segmental variation related to focus. Some dimen- sions of acoustic contrast, vowel quality, transition dynamics, and glottal timing, exhibit readily detectable and gradient variation as expected by a hyperarticulation model, and such variation is specific to the language's contrastive structure. Not all contrastive dimensions, however, are so affected, for example, noise associated with obstruent consonants. These patterns of results together suggest a functional interaction between the various speech gestures which may define a unit of speech at about the level of the syllable; the level which is the domain of expression of stress.

\section{Invited Papers}

3aSCa5. Close coupling or points of rendezvous? Connections between intonational events and the segmental grid. Hansjörg Mixdorff (TFH Berlin University of Applied Sciences, Luxemburger Str. 10, 13353 Berlin, Germany, mixdorff@tfh-berlin .de), Hartmut Pfitzinger (Christian-Albrechts-University, Leibnizstr. 10, 24118 Kiel, Germany, hpt@phonetik.uni-muenchen .de), Stefan Werner (University of Joensuu, PL111, 80101 Joensuu, Finland, stefan.werner@joensuu.fi)

The authors examine the connection between the fundamental frequency (f0) contour and the segmental grid, employing the Fujisaki model for decomposing an fo contour into phrase and accent components. As shown in earlier work by the first author, accent commands are closely connected with the accented syllables of the constituent words of an utterance. They reflect the temporal alignment of intonational gestures by their on- and offset times, as well as the magnitude of the gestures by their amplitudes. Their timing relates to phonemic function, and the amplitudes are strongly correlated with the prominence of an accented syllable. In this study the authors attempt to model the intensity contour of an utterance as a concomitant of the articulatory gesture, namely by low-pass filtering pulsewise "syllable commands", yielding onset and offset points as well as amplitude values of the syllabic gestures and relate these data to the accent command data from the fo tier. We examine the influence of phonemic distinctions on the alignment between the f0 contour and the segmental grid, as well as the influence of the intonational gesture on the realization of segments. The work is carried out on spontaneous American English speech data from the Buckeye corpus.

\section{9:40}

3aSCa6. Segmental effects on the prosody of voice quality. Hartmut Pfitzinger (Christian-Albrechts-University, Leibnizstr. 10, 24118 Kiel, Germany, hpt@phonetik.uni-muenchen.de)

Voice quality variability is due to supra-segmental influences but also to segmental factors like phoneme class, vowel quality, nasalization, airstream mechanism etc. These factors determine a rather unexplored micro-prosodic phenomenon: the phone-intrinsic voice quality which causes voice quality coarticulation and voice quality transitions in fluent speech. I subsume all these phenomena under the high-frequency components of prosody. Since high-frequency and low-frequency components (supra-segmentals) of voice quality prosody are superposed and thus encoded the main goal of this paper is to separate them and make both accessible to speech research. In 2003 a holistic voice quality parameter extractor was introduced by Mokhtari, Pfitzinger, and Ishi: It applies a principal components analysis to a database of glottal-flow waveforms for the purpose of later reconstructing and interpolating all underlying glottal-flow waveforms from just a few principal components. Recently, by applying this basic principle to a large corpus of manually segmented glottal-flow waveforms of 44 speakers I dramatically improved its applicability to any speech signals. The application of this version to a large speech database and subsequent high-pass and low-pass filtering of the resulting voice quality parameters yielded phone-intrinsic voice quality parameter sets as well as slowly varying meaningful voice quality parameter contours. 
Session 3aSCb

\title{
Speech Communication: Speech Perception III
}

\author{
Eva Reinisch, Chair \\ Max Planck Institute for Psycholinguistics, Postbus 310, Nijmegen, 6500AH, Netherlands
}

\section{Contributed Papers}

\section{8:40}

3aSCb1. Lexical stress information modulates the time-course of spoken-word recognition. Eva Reinisch (Max Planck Institute for Psycholinguistics, Postbus 310, 6500AH Nijmegen, Netherlands, eva.reinisch @mpi.nl), Alexandra Jesse (Max Planck Institute for Psycholinguistics, Postbus 310, 6500AH Nijmegen, Netherlands, alexandra.jesse @ mpi.nl), James M. McQueen (Max Planck Institute for Psycholinguistics, Postbus 310, 6500AH Nijmegen, Netherlands, james.mcqueen@mpi.nl)

Segmental as well as suprasegmental information is used by Dutch listeners to recognize words. The time-course of the effect of suprasegmental stress information on spoken-word recognition was investigated by tracking Dutch listeners' looks to arrays of four printed words on a computer screen as they listened to spoken sentences. Each target was displayed along with a competitor that did not differ segmentally in its first two syllables but differed in stress placement (e.g., 'CENtimeter' and 'sentiMENT'; capitals marking stressed syllables). The listeners' eye-movements showed that suprasegmental stress information is used to recognize the target before distinct segmental information is available. Furthermore, the presence of initial-syllable stress affected the degree to which words compete for recognition: Targets with non-initial stress ('sentiMENT') suffered from stronger competition than initially stressed targets ('CENtimeter'). Follow-up experiments showed that initial-syllable duration, as a cue to lexical stress, is not interpreted dependent on the speaking rate of the preceding carrier sentence. This still held when other stress cues like pitch and amplitude were removed. Rather, the speaking rate of the preceding carrier affected the speed of word recognition globally, even though the speaking rate of the target itself was not altered.

\section{9:00}

3aSCb2. Using pause durations to discriminate between lexically ambiguous words and dialog acts in spontaneous speeech. Rajdip Dhillon (Dept. of Linguistics, Yale Univ., 370 Temple St., Rm. 204, New Haven, CT 06520, USA, rajdip.dhillon@yale.edu)

Using multiparty conversations conducted in spontaneous Scottish English speech, this study examines whether pause durations can be used to discriminate between lexically ambiguous words pertaining to different dia$\log$ acts and, more generally, whether pause durations signal different types of dialog acts. Dialog acts describe the pragmatic functions of utterances and their detection requires attention to pragmatic function, syntax, prosody, surrounding context, and interaction between speakers. In this study, the dia$\log$ acts examined are 'floor grabbers', which are used by speakers to gain attention or to interrupt a current speaker who has the floor, and 'acceptance/agreements', which express agreement to or acceptance of a previous speaker's question, proposal, or statement. Experiments 1 and 2 examine pause durations following the lexical items 'right' and 'good' respectively when used as 'floor grabbers' or 'acceptance/agreements'. Experiment 3 examines pause durations following 'floor grabbers' and 'acceptance/agreements', regardless of the lexical item. Results of all three experiments indicate that pauses following 'floor grabbers' are significantly longer than those following 'acceptance/agreements'. This study shows that pause durations are in fact a reliable means of discriminating between lexically ambiguous words and distinguishing dialog acts. The study also sheds light on how stable pause usage is in spontaneous speech.

\section{9:20}

3aSCb3. Identification of talkers speaking emotional speech. Kyung Wha Kim (Forensic Science Div, Supreme Prosecutors' Office, 706 Banporo, Seocho-gu, 137-730 Seoul, Republic of Korea, savoix@ spo.go .kr), Patrick C. Wong (Northwestern University, 2240 Campus Drive, Evanston, IL 60208, USA, pwong@ northwestern.edu)

Identification of talkers speaking emotional speech is common in many communicative situations and forensic "voice line ups". However, emotional speech is auditorily and acoustically different from neutral speech, which may cause difficulty in talker identification. In this study, we examined how listeners identify different talkers speaking emotional or neutral voices, and how short-term training may benefit talker identification. Recordings were obtained from 10 male speakers speaking in angry and neutral voices. Within one session, listeners were first trained to identify the talkers, and their ability to generalize to untrained sentences spoken by the same talkers was tested. We found that while there was no significant difference in identifying neutral and angry voices, the ability to generalize to untrained sentences was more impaired with angry voices. The theoretical and forensic implications of these results are discussed. [Work supported by NIH and NSF]

\section{9:40}

3aSCb4. An fMRI-based structural equation model for natural language processing shows age-dependent changes in brain connectivity. Scott K. Holland (Cincinnati Children's Hospital Medical Center, Imaging Research Center, 3333 Burnet Ave, Cincinnati, OH 45229, USA, scott.holland@cchmc.org), Prasanna Karunanayaka (Cincinnati Children's Hospital Medical Center, Imaging Research Center, 3333 Burnet Ave, Cincinnati, OH 45229, USA, KAR4RP@cchmc.org), Elena J. Plante (Univ. Arizona, Dept. Speech and Hearing Sciences, P.O. Box 210071, Tucson, AZ 85721-0071, USA, eplante@U.Arizona.EDU), Vincent J. Schmithorst (Cincinnati Children's Hospital Medical Center, Imaging Research Center, 3333 Burnet Ave, Cincinnati, OH 45229, USA, Vince .Schmithorst@cchmc.org)

Structural Equation Modeling (SEM) or path analysis is a multivariate analytic tool that is used to test hypothesis about causal influences among measured or latent variables. When applied to functional neuroimaging (fMRI) data, SEM combines interregional covariance and neuroanatomy to investigate brain connectivity and the dynamic flow of information across neural networks. We have investigate Linear SEM or LSEM as a first step in estimating connection strengths from fMRI data in children during acoustic stimulation with stories in English. An average LSEM was constructed based on the concatenated fMRI data from $\mathrm{N}=313$ children, age 5 to 18 , listening to stories. Time courses from the fMRI data is used as input to the LSEM as computed in AMOS (v.5.1, SmallWaters, Corp. Chicago, IL) to yield an average model for the entire group. The LSEM path coefficients were examined between brain regions involved in auditory speech perception and language processing. Connectivities estimated by LSEM are significantly age dependent in some brain areas, while the connectivity coefficient between other brain regions is not a function of age 


\title{
Session 3aSCc
}

\section{Speech Communication: Speech Articulation II}

\author{
Yves Laprie, Chair \\ LORIA CNRS, 615 rue du Jardin Botanique, Bâtiment C, Villers-lès-Nancy, 54600, France
}

\section{Contributed Papers}

\section{0:40}

3aSCc1. Modeling formant pattern in fricative-vowel sequences. Martine Toda (CNRS \& ENST, 46, rue Barrault, 75634 Paris, France, martinetoda@yahoo.co.jp), Shinji Maeda (CNRS \& ENST, 46, rue Barrault, 75634 Paris, France, maeda@tsi.enst.fr)

MRI data indicate that there are two subject-dependent articulatory patterns in French post-alveolar sibilant fricative /'integral of'/. Each type of articulations can correspond to the Polish fricatives /'curled c'/ and /'underdotted s'/ on the basis of their articulatory similarity: palatalized for the former, and the presence of a sublingual cavity as well as lip protrusion for the latter. The F2 transitions, in part, reflect the acoustic contrast between these Polish fricatives, which exhibit a higher locus in the palatalized /'curled c'/ than in /'underdotted s'/. However, no systematic transitional characteristic could be found in French. We hypothesize that the subjectspecific formant-cavity affiliation in the adjacent vowel /a/ and/or the difference of scale in vocal tract lengths manifest as the acoustic variation in French, whereas those VT morphological differences are articulatorily compensated in Polish to maintain the distinctiveness of these two post-alveolar fricatives. In order to assess this hypothesis, the F-patterns along /a 'integral of' a/ sequences are simulated for various vocal tract configurations. In calculations, the two different types of the fricative articulations are combined with the vocal-tract area functions appropriate for /a/ but different in shape and in size to taken into account for female and male speakers. The results will be discussed.

\section{1:00}

3aSCc2. Diffraction effects on speech phonemes during phonation Vijayakumar V (Multimedia University, FOSEE, Jalan Air Keroh Lama, 75450 Melaka, Malaysia, vijaya@mmu.edu.my), Eswaran C (Multimedia University, Faculty of Information Technology, Cyberjaya Campus, 63100 Cyberjaya, Malaysia, eswaran@mmu.edu.my)

In this paper we will show how the ventricular fold modifies speech phonemes and also causes the shimmering of speech. Diffraction of sound waves at the ventricular fold which can be considered as a circular aperture, can modify the vocalized glottal sound spectrum reaching the pharyngx. Thus during phonation, speech phoneme spectra are essentially enveloped by Bessel functions. Since Bessel beams are known to be nondiffracting, this step ensures that the phonemes do not undergo further diffraction effects which can destroy the unique harmonic structure of the phonemes. Note that the wavelengths of sound waves and the dimensions of the human vocal tract appendages are of the same order of magnitude which mean that diffraction should be commonplace in the vocal tract if not for the initial packaging of the speech phoneme at the ventricular fold.

\section{1:20}

3aSCc3. Evaluation of phonetic constraints in acoustic-to-articulatory inversion. Yves Laprie (LORIA CNRS, 615 rue du Jardin Botanique, Bâtiment C, 54600 Villers-lès-Nancy, France, Yves.Laprie@ @loria.fr), Blaise Potard (LORIA CNRS, 615 rue du Jardin Botanique, Bâtiment C, 54600 Villers-lès-Nancy, France, Blaise.Potard@ loria.fr), Anne Bonneau (LORIA
CNRS, 615 rue du Jardin Botanique, Bâtiment C, 54600 Villers-lès-Nancy, France, Anne.Bonneau@loria.fr)

The objective is to evaluate phonetic constraints intended to guarantee the phonetic realism of vocal tract shapes generated by an articulatory model, i.e. here that of Maeda. The constraints are based on the relation between vocal tract shapes allowed for a vowel and formants frequencies of the vowel. They are speaker independent since they are derived from standard phonetic knowledge. We used them to reduce the number of solutions in acoustic-to-articulatory inversion. The impact of constraints has been investigated by comparing vocal tract shapes recovered by inverting five French vowels /i, e, a, u, y/ to those obtained by outlining articulator contours in X-ray images. To that purpose we used X-ray images (used by Maeda to elaborate his articulatory model) with the corresponding speech signal. The evaluation shows that unrealistic vocal tract shapes are penalized by constraints, that vocal tract shapes recovered through inversion are in very good agreement with $\mathrm{X}$-ray images and that relevant articulatory variability is recovered when considering all vocal tract shapes corresponding to one vowel. These constraints thus complement the articulatory model very efficiently. Additionally, the inversion framework complemented by phonetic constraints is an efficient way of investigating the acoustic properties of an articulatory model.

\section{1:40}

3aSCc4. Acoustic-to-articulatory inversion of fricatives. Farid Feïz (LORIA CNRS, 615 rue du Jardin Botanique, Bâtiment C, 54600 Villers-lès-Nancy, France, Farid.Feiz@loria.fr), Blaise Potard (LORIA CNRS, 615 rue du Jardin Botanique, Bâtiment C, 54600 Villers-lès-Nancy, France, Blaise.Potard@loria.fr), Yves Laprie (LORIA CNRS, 615 rue du Jardin Botanique, Bâtiment C, 54600 Villers-lès-Nancy, France, Yves.Laprie @loria.fr)

The objective is to recover the vocal tract shape dynamics from the speech signal of vowels and fricatives. The method relies on the analysisby-synthesis paradigm and is an extension of the method proposed by Ouni and Laprie which exploits a hypercubic articulatory table to represent the synthesis facet, i.e. Maeda's articulatory model. The first major modification is the use of parallelepiped instead of cubes. The new construction strategy only subdivides the articulatory space in the articulatory direction which gives rise to the strongest non-linearities. This enables a substantial reduction of the table size. The second major modification is the inversion of fricative sounds. In addition to the articulatory parameters the relative location of the noise source downstream the constriction is taken into account. This gives rise to three different articulatory codebooks, each corresponding to the relative position of the source with respect to the main constriction. This new inversion method has been evaluated on VCV sequences. It turns out that the correct vocal tract dynamics is recovered even if the constriction area is slightly underestimated. Input data are the first three formant frequencies but MFCC coefficients are now investigated since they render the high frequency region of fricative spectra better. 


\title{
Session 3aSCd
}

\section{Speech Communication: Prosody I}

\author{
Volker Dellwo, Chair \\ UCL, Wolfson House, 4, Stephenson Way, London, NW1 2HE, UK
}

\section{Contributed Papers}

\section{0:40}

3aSCd1. Quantifying rhythm in running speech. Tristie Ross (Dept. of Linguistics, UCSD, 9500 Gilman Drive \#0108, La Jolla, 92093-0108, USA, tross@ling.ucsd.edu), Naja Ferjan (Dept. of Linguistics, UCSD, 9500 Gilman Drive \#0108, La Jolla, 92093-0108, USA, naja@ling .ucsd.edu), Amalia Arvaniti (Dept. of Linguistics, UCSD, 9500 Gilman Drive \#0108, La Jolla, CA 92093-0108, USA, amalia@ling.ucsd.edu)

In the past decade several metrics that reflect the variability of vocalic and consonantal intervals in speech have been used to quantify the impressionistic division of languages into stress- and syllable-timed. Although all such metrics successfully separate prototypical languages, such as stresstimed English and syllable-timed Spanish, their results for other languages are less clear. The problem is related to the limited datasets used, which consist of either a small number of sentences per language elicited from several speakers, or longer stretches of speech elicited from one speaker per language. Here we elicited short sentences, story reading and spontaneous speech from several speakers of stress-timed English, syllable-timed Spanish, Korean, a hitherto unclassified language, and Greek, which has shown to resist classification. Our results show that different metrics yield different classifications for some languages, like Greek, while scores for the same language differ depending on speaking style. Taken all together these results cast doubt on the robustness and usefulness of the popular metrics and suggest that alternative ways of conceiving of speech rhythm that do not rely exclusively on timing but take relative prominence into account may ultimately be more successful in explaining speech rhythm.

\section{1:00}

3aSCd2. Temporal Organization of the Compounds in Standard Chinese. Eric Zee (Phonetics Lab, Dept. of Chinese, Translation and Linguistics, City University of Hong Kong, 83 Tat Chee Avenue, Kowloon Hong Kong, Hong Kong, eric.zee@cityu.edu.hk), Wai-Sum Lee (Phonetics Lab, Dept. of Chinese, Translation and Linguistics, City University of Hong Kong, 83 Tat Chee Avenue, Kowloon Hong Kong, Hong Kong, w.s.lee @ cityu.edu.hk)

The study investigates the temporal organization of the bisyllabic, trisyllabic and quadrisyllabic compounds in Standard Chinese. Results of the analysis of the speech data from six speakers show that (a) the rime durations, the durations of the syllable-initial voiceless fricatives and the VOT of the syllable-initial voiceless aspirated stops and affricates are conditioned by the position of the component syllable in the compounds; (b) the rime duration decreases as the component syllable moves from the final toward the initial position in the compounds; (c) the mean duration of the rimes is longer in the final position than in the initial and medial positions; (d) the voiceless fricatives and the voiceless aspirated stops and affricates are shorter in the medial position than in the initial and final positions; (e) the cross-speaker variation is less in the rime duration than in the duration of the voiceless fricatives and VOT of the voiceless aspirated stops and affricates; and (f) no temporal patterns have been observed in the voiceless unaspirated stops and affricates. The results will be discussed in terms of articulatory programming and timing (Kozhevnikov and Chistovich, 1965; Allen, 1975). A temporal model of the compound production in Standard Chinese will be proposed.

\section{1:20}

3aSCd3. Second language rhythm and rhythm metrics. Naja Ferjan (Dept. of Linguistics, UCSD, 9500 Gilman Drive \#0108, La Jolla,
92093-0108, USA, naja@ling.ucsd.edu),Tristie Ross(Dept. of Linguistics, UCSD, 9500 Gilman Drive \#0108, La Jolla, 92093-0108, USA, tross@ling .ucsd.edu), Amalia Arvaniti (Dept. of Linguistics, UCSD, 9500 Gilman Drive \#0108, La Jolla, CA 92093-0108, USA, amalia@ling.ucsd.edu)

Several metrics that reflect the variability of vocalic and consonantal intervals in speech have been shown to successfully discriminate between the rhythm of first and second language (L2) speakers producing short sentences of a given language. Here we elicited running read speech from native speakers of English, German and Italian speaking English. We hypothesized that the scores of German L2 speakers would be closer to the scores of native English speakers, since both English and German are stress-timed, and that the scores of Italian L2 speakers would exhibit greater differences from native English scores, since Italian is stress-timed. Our results show instead that the scores of some German speakers are less close to those of the native English speakers than the scores of some Italian speakers, a difference that cannot be attributed to fluency, proficiency level, or length of exposure to English. It is possible that these results reflect over-compensation of some sort by some L2 speakers, though a more likely explanation is that the popular metrics used to quantify speech rhythm may be unreliable or at least not sufficiently robust to be used with L2 data. Reasons why this may be so for L2 speech in particular are discussed.

\section{$11: 40$}

3aSCd4. Influences of language typical speech rate on the perception of speech rhythm. Volker Dellwo (UCL, Wolfson House, 4, Stephenson Way, NW12HE London, UK, v.dellwo@ucl.ac.uk)

Human listeners can distinguish languages of different rhythmic classes (e.g. stress- and syllable-timed languages). The present study investigated the role of speech rate in this process. In experiment I, speech rate measurements (syllables/second) of read speech were performed in two stress-timed (English, German) and two syllable-timed languages (French, Italian). The results revealed that speech rate distinguishes as reliable between rhythmic class as previously proposed correlates of speech rhythm $(\% \mathrm{~V}$, VarcoC and nPVI). For a perceptual task (experiment II) sentences from French and German were delexicalized by turning consonantal intervals into white noise and vocalic intervals into complex periodic tones. 18 listeners rated the regularity of the noise-tone sequences on a 13 point scale. The results revealed that listeners rated French stimuli as significantly more regular than English stimuli. It was further shown that the rate of noise-tone sequences was the best predictor for the regularity rating (faster sequences were perceived as more regular) compared to standard rhythm measures (\%V, VarcoC and nPVI). Both experiments together show that a) syllable-timed languages are produced faster than stress-timed languages and b) listeners' perception of speech rhythm is highly influenced by these rate differences.

\section{2:00}

3aSCd5. Computing the fundamental frequency variation spectrum in conversational spoken dialogue systems. Kornel Laskowski (Carnegie Mellon University, 407 South Craig Street, Pittsburgh, PA 15213, USA, kornel@cs.cmu.edu), Matthias Wölfel (Universität Karlsruhe, am Fasanengarten 5, 76131 Karlsruhe, Germany, wolfel@ira.uka.de), Mattias 
Heldner(KTH, Lindstedtsvägen 24, SE-100 44 Stockholm, Sweden, mattias@speech.kth.se), Jens Edlund (KTH, Lindstedtsvägen 24, SE-100 44 Stockholm, Sweden, edlund@ speech.kth.se)

Continuous modeling of intonation in natural speech has long been hampered by a focus on modeling pitch, of which several normative aspects are particularly problematic. The latter include, among others, the fact that pitch is undefined in unvoiced segments, that its absolute magnitude is speakerspecific, and that its robust estimation and modeling, at a particular point in time, rely on a patchwork of long-time stability heuristics. In the present work, we continue our analysis of the fundamental frequency variation (FFV) spectrum, a recently proposed instantaneous, continuous, vectorvalued representation of pitch variation, which is obtained by comparing the harmonic structure of the frequency magnitude spectra of the left and right half of an analysis frame. We analyze the sensitivity of a task-specific error rate in a conversational spoken dialogue system to the specific definition of the left and right halves of a frame, resulting in operational recommendations regarding the framing policy and window shape.

\section{$12: 20$}

3aSCd6. A longitudinal study on vocal aging - Changes in F0, jitter, shimmer and glottal noise. Hideki Kasuya (International Univ. of Health and Welfare, 2600-1 Kita-kanemaru, 324-8501 Otawara, Japan, kasuyah
@ snow.ucatv.ne.jp),Hajime Yoshida(Yoshida Clinic, West 3, South 6, 0968585 Nayoro, Japan, nort3381@north.hokkai.net), Hiroki Mori (Fac. of Engr., Utsunomiya Univ., 7-1-2 Yoto, 321-8585 Utsunomiya, Japan, hiroki @ speech-lab.org), Hiroshi Kido (Tohoku Inst. of Technology, 35-1 Yagiyama Kasumicho, Taihaku-ku, 982-8557 Sendai, Japan, kidoh@tohtech .ac.jp)

A longitudinal study on vocal aging was conducted for voicing source parameters: fundamental frequency $(\mathrm{F} 0)$, jitter (period perturbation quotient, PPQ), shimmer (amplitude perturbation quotient, APQ) and glottal noise (normalized noise energy in a high frequency band, NNEb). Acoustic analyses were made on the sustained phonations of the Japanese vowel /a/ of 20 males and 38 females with no laryngeal disease, which were recorded once every year over periods ranging from 11 to 19 years. The acoustic parameters measured were subjected to statistical regression analysis, where the longitudinal change of the parameters was evaluated by the t-test. Two males $(10 \%)$ and 12 females $(32 \%)$ showed significant falling in F0 $(\mathrm{P}<0$ $.05)$ but no significant rising was observed. Five males $(25 \%)$ and 12 females $(32 \%)$ displayed a significant increase in APQ $(\mathrm{P}<0.05)$, whereas only six females $(16 \%)$ and no male revealed significant changes in PPQ $(\mathrm{P}<0.05)$. Increase in $\mathrm{NNEb}$ was significant for seven males $(35 \%)$ and 14 females $(37 \%)(\mathrm{P}<0.05)$. We conclude that 1$) \mathrm{F} 0$ falling is a more emblematic tendency of female vocal aging than male, 2) shimmer is a more observable indication of vocal aging than jitter, and 3) glottal noise in a high frequency region tends to increase with age.

\title{
Session 3aSPa
}

\section{Signal Processing in Acoustics and Physical Acoustics: Biomedical Applications of Time-Reversal II}

\author{
Oleg A. Sapozhnikov, Cochair \\ Center for Industrial and Medical Ultrasound, Applied Physics Lab., University of Washington, 1013 NE 40th St., Seattle, WA \\ 98105, USA
}

Mickael Tanter, Cochair

Laboratoire Ondes et Acoustique, ESPCI, Université Paris 7, CNRS, 10 rue Vauquelin, Paris, 75005, France

\section{Invited Papers}

\section{8:00}

3aSPa1. Time-Reversal Acoustics in Biomedical Engineering. Mathias Fink (Laboratoire Ondes et Acoustique, ESPCI, Université Paris 7, CNRS, 10 rue Vauquelin, 75005 Paris, France, mathias.fink@espci.fr), Gabriel Montaldo (Laboratoire Ondes et Acoustique, ESPCI, Université Paris 7, CNRS, 10 rue Vauquelin, 75005 Paris, France, gabriel.montaldo@espci.fr), Mickael Tanter (Laboratoire Ondes et Acoustique, ESPCI, Université Paris 7, CNRS, 10 rue Vauquelin, 75005 Paris, France, michael.tanter@espci.fr)

Time-reversal is a very powerful method for focusing through complex and heterogeneous media and shows very promising results in biomedical applications. In this presentation, we review some of the main applications investigated during the past decade. An iterative implementation of the time-reversal process allows tracking gallstones in real time during lithotripsy treatments. In this application domain, a smart exploitation of the reverberations in solid waveguides permits the focusing of high amplitude ultrasonic shock waves with a small number of transducers. Because time reversal is able to correct the strong distortions induced by the skull bone on ultrasonic propagation, this adaptive focusing technique allows brain ultrasonic therapy through the skull bone. Finally, time-reversal focusing guided by speckle noise can be implemented to correct distortion in medical ultrasonic imaging and recent results will be presented.

$$
8: 20
$$

3aSPa2. A comparison of planar projection and time reversal techniques. Gregory Clement (Harvard Medical School, Focused Ultrasound Lab - BWH Radiology, 221 Longwood Ave RM 521, Boston, MA 02115, USA, gclement@hms.harvard.edu)

Planar projection methods, such as the angular spectrum approach, have been shown to rapidly relate fields between two planes in space/time, with only a single measurement plane needed to characterize an entire field. Variants of the method have been used for medical transducer characterization, as well as for HIFU treatment planning. Presently, a potential advantage of the approach is considered: Operating in the temporal frequency domain, backward planer projection (i.e. "space reversal") is invariant with respect to 
the spatial dimension, even in the presence of anomalous dispersion. This ability can be contrasted with time reversal, which is violated in the presence of the absorption term. Time reversal is compared with planar back-projection under a varying set of conditions, including the presence of dispersive and nonlinear homogeneous and heterogeneous media. The specific case of a simulated impulsive signal from a 30-cm diameter piston radiator is directed through a $50-\mathrm{cm}$ section of a tissue-like medium $(\mathrm{B} / \mathrm{A}=5$ to $7, \mathrm{c}=1480$ to $1600 \mathrm{~m} / \mathrm{s}, \rho=0.9$ to $1.1 \mathrm{gm} / \mathrm{cm} 3, \alpha=0.5$ to $2 \mathrm{db} / \mathrm{cm} / \mathrm{MHz}$ ) over a range of pressures. Ability of backward projection to restore initiating signals is verified, provided that significant signal levels of all relevant frequencies can be reached at the initial plane.

\section{8:40}

3aSPa3. Nonlinear imaging of isoechogenic phantoms using selective phase conjugation of acoustic harmonics. Vladimir L. Preobrazhensky (IEMN, UMR CNRS 8520, avenue Poincaré, BP 60069, 59652 Villeneuve d'Ascq, France, vladimir .preobrajenski@iemn.univ-lille1.fr), Philippe Pernod (IEMN, UMR CNRS 8520, avenue Poincaré, BP 60069, 59652 Villeneuve d'Ascq, France, philippe.pernod@iemn.univ-lille1.fr), Leonid M. Krutyansky (WRC-GPI-RAS LEMAC, Vavilova street, 38, 119991 Moscow, Russian Federation, krut@orc.ru)

Selective phase conjugation of acoustic harmonics generated by an intense focused probing wave is applied for imaging of isoechogenic phantoms non detectable by the linear echography. The principle of imaging is based on the difference between the nonlinear properties of a phantom and its surrounding medium. As shown by theoretical modelling the analysis of the phase conjugate second harmonic $2 \omega$ of the probing wave provides an image contrast comparable with the relative difference between the nonlinear parameters of the phantom and its background. Second harmonic generation by the phase conjugate wave $2 \omega$ during its back propagation to the source of the probing wave provides an image contrast at the frequency $4 \omega$ even stronger than the difference of nonlinear parameters. These theoretical conclusions are confirmed by the experimental measurements carried out using 10MHz supercritical parametric phase conjugator. The objects were prepared with methanol water solutions of concentrations $20 \%$ and $60 \%$. The solutions filling either a cavity in the agar gel or an acoustically transparent container immersed in water represented isoechogenic phantoms with weaker and stronger nonlinearities relatively to water. The contrast values of $2 \omega$-images were measured as $-14 \%$ and $+10 \%$ respectively while for $4 \omega$-images the contrast achieved $-45 \%$ and $+37 \%$.

3aSPa4. Ultrasonic transducers for imaging and therapy based on time-reversal principles. Armen Sarvazyan (Artann Laboratories, 1753 Linvale-Harbourton, Lambertville, NJ 08350, USA, armen@artannlabs.com)

Focusing of ultrasonic waves is a fundamental aspect of most of the medical applications of ultrasound. There are three approaches in focusing acoustic energy: geometrical, using concave transducers and acoustic lenses, electronic, using phased arrays, and timereversal (TR), which allows both spatial and temporal concentration of acoustic energy. The aim of this talk is to make comparative analysis of advantages and disadvantages of ultrasound focusing techniques, demonstrate unique capabilities of TR focusing in medical imaging, surgery and therapy. Examples of embodiments of ultrasonic focusing transducers utilizing TR method for various biomedical applications will be presented. TR ultrasonic transducers are comprised of a reverberation chamber, which could be either an aluminum block or a water filled volume of irregular shape, and one or several piezotransducers attached to or incorporated in the reverberator. Optimization of the TR transducers for a particular application is considered, including the geometry of the TR reverberator, shape, type and dimensions of the piezotransducers. Unique abilities of TR transducers to steer the focal spot in 3D, produce pulses with arbitrary waveforms in a wide frequency band and generate focal regions of arbitrary predefined shapes using simple means such as single channel system with one piezoelement will be demonstrated.

\section{9:20}

3aSPa5. Doppler ultrasonography using wave phase conjugation. Yuri Pyl'Nov (Moscow Institute of Radio Engineering, Electronics \& Automation, Vernadsky prosp., 78, 119454 Moscow, Russian Federation, pylnov@yandex.ru), Nikolay Smagin (Moscow Institute of Radio Engineering, Electronics \& Automation, Vernadsky prosp., 78, 119454 Moscow, Russian Federation, pylnov@yandex.ru), Philippe Pernod (IEMN, UMR CNRS 8520, avenue Poincaré, BP 60069, 59652 Villeneuve d'Ascq, France, philippe.pernod@iemn.univ-lille1.fr), Vladimir L. Preobrazhensky (IEMN, UMR CNRS 8520, avenue Poincaré, BP 60069, 59652 Villeneuve d'Ascq, France, vladimir.preobrajenski@iemn.univ-lille1.fr)

Ultrasound wave phase conjugation (WPC) is an efficient tool for velocimetry of flows due to its sensibility to the break of time reversal invariance of acoustic fields in moving media. The presence of flow leads to the non compensated Doppler phase shift of the backward phase conjugate wave relatively to the primary probing wave. This phase shift provides detection, diagnostics and velocimetry of flows and does not require the presence of any scatterers in a flow. Furthermore the phase shift of phase conjugate wave can be used in liquid flowmeters. As it was shown in previous works the sensitivity of liquid flowmeters using phase conjugate ultrasonic waves essentially increases by using nonlinear effects appearing during propagation of phase conjugate waves in a moving liquid. In the present paper we report the theoretical and experimental results on imaging of flow velocity distributions in tubes and phantoms of blood vessel. The scanning auto-confocal system based on parametric ultrasonic wave phase conjugator at $10 \mathrm{MHz}$ operation frequency is used in the experiments. The preliminary results of experimental measurements of velocity of vascular blood flow in soft tissues in vitro are discussed. 
3aSPa6. Guiding of HIFU beams towards optical contrast agents by time-reversal of photoacoustic waves. Emmanuel Bossy (Laboratoire Photons et Matière, ESPCI/CNRS, 10 rue Vauquelin, 75231 Paris Cedex 05, France, emmanuel .bossy@espci.fr), Jean-François Aubry (Laboratoire Ondes et Acoustique, ESPCI, Université Paris 7, CNRS, 10 rue Vauquelin, 75005 Paris, France, jean-francois.aubry@espci.fr), Arik R. Funke (Laboratoire Photons et Matière, ESPCI/CNRS, 10 rue Vauquelin, 75231 Paris Cedex 05, France, arik.funke@espci.fr), Mathias Fink (Laboratoire Ondes et Acoustique, ESPCI, Université Paris 7, CNRS, 10 rue Vauquelin, 75005 Paris, France, mathias.fink@espci.fr), Albert-Claude Boccara (Laboratoire Photons et Matière, ESPCI/CNRS, 10 rue Vauquelin, 75231 Paris Cedex 05, France, boccara@optique.espci.fr)

This presentation will discuss the opportunity to use photoacoustics to guide high-intensity focused ultrasound (HIFU) in biological tissue. The photoacoustic effect consists in the emission of sound by an illuminated optically absorbing region, based on the thermoelastic expansion following the absorption of a light pulse. In the near-infrared range (700 nm to $900 \mathrm{~nm}$ ), the absorption of light in biological tissue is relatively low, and multiply scattered photons can travel as deep as a few centimetres in tissue. Photoacoustic waves can therefore be generated deep in tissue, and have recently been used in vivo to perform passive acoustical imaging of optical absorbing features, such as blood vessels. We recently demonstrated on tissue-mimicking phantoms that photoacoustics could be coupled to timereversal to automatically focus ultrasound towards an optical absorber [Bossy et al., APL 89(18), 2006]. We propose to further develop this approach to automatically guide HIFU therapy beams towards a targeted region labelled with an optically absorbing contrast agent. This presentation will discuss various aspects of such an approach, based on modelling and experimental results.

\section{Contributed Papers}

\section{0:00}

3aSPa7. Time Reversal Acoustic focusing using layered membranes structure. Yegor Sinelnikov (ProRhythm, 105 Comac St., Ronkonkoma, NY 11779, USA, yegor@prorhythm.com), Andrey Vedernikov (ProRhythm, 105 Comac St., Ronkonkoma, NY 11779, USA, vedernikov@prorhythm.com), Alexander Sutin (Stevens Institute of Technology, Castle Point on Hudson, Hoboken, NJ 07030, USA, asutin@stevens.edu), Armen Sarvazyan (Artann Laboratories, 1753 Linvale-Harbourton, Lambertville, NJ 08350, USA, armen@artannlabs .com)

In the recent years numerous Time Reversal Acoustic Focusing Systems (TRA FS) employing various reverberation chambers ("acoustic cavities") accumulating acoustic energy in time were described in the literature. We propose an alternative reverberator composed of a liquid-filled volume comprising a series of spatially distributed membranes with varying thickness in the range from one quarter to half wavelength of ultrasound. Random spatial variation of ultrasound reflection from such membranes leads to randomization of acoustic field that highly improve Time Reversal focusing of the system. A theoretical model of reflections from nonuniform membranes is suggested to evaluate spatial and temporal characteristics of TRA focusing formed as a result of numerous reflections and reverberation of ultrasound propagating though the plurality of membranes. The dependence of focal structure and focused waveform was investigated for suggested structure and their focusing efficiency has been compared with that of the conventional reverberators. In the experiment, the membrane were made of Polyethylene Terephthalate or Nylon thermoformed films. Ultrasonic signals were radiated by transducers with frequency in the range from 3 to $10 \mathrm{MHz}$.
Experiment demonstrated effective TRA focusing by membrane arrangement that can be used for ultrasound focusing and steering in various minimally-invasive biomedical applications.

\section{0:20}

3aSPa8. Time Reversed Elastic Waves within Soft Solids. Stefan Catheline (LOA, Université Paris 7 Denis Diderot, ESPCI, 10 rue Vauquelin, $75005 \quad$ Paris, France, stefan.catheline@espci.fr), Carlos Negreira (Science University of Montevideo, 11400 Montevideo, Uruguay, carlosn@fisica.edu.uy), Nicolas Benech (Science University of Montevideo, 11400 Montevideo, Uruguay, nbenech@fisica.edu.uy), Javier Brum (Science University of Montevideo, 11400 Montevideo, Uruguay, jbrum@fisica.edu.uy)

When a scalar far-field wave is time reversed from the surface of a cavity, it starts to converge toward its initial point source location, then collapses and finally diverges. Without near field evanescent waves, the symmetric focus spot is limited by the Rayleigh criterion. We present an experimental observation of a time-reversal vectorial wave in the volume of a soft solid. The elastic field is measured using the transient elastography technique. It is observed that the time reversed far field wave collapses and gives birth to near field effects. Numerical computation based on elastodynamic Green's functions in a time-reversal cavity confirms and completes the experimental conclusions: the time symmetry with respect to collapse time is broken and the Rayleigh criterion is direction dependant; the spatial collapse is larger in the direction of the point body force than in the perpendicular direction. The authors anticipate that the method can be used for shear wave beam forming in soft tissues as well as to tissue elasticity assessment. 


\title{
Session 3aSPb
}

\section{Signal Processing in Acoustics: Filter Design, Detection, and Estimation I}

\author{
Michael Roan, Cochair \\ Virginia Tech, 917 Village Way North, Blacksburg, VA 24060, USA \\ Jorge E. Quijano, Cochair \\ NEAR Lab-Portland State University, 1900 SW 4th Avenue, Suite 160, Portland, OR 97201, USA
}

\section{Contributed Papers}

\section{1:00}

3aSPb1. Wavelet filter bank based wide-band audio coder. Jan Nováček (Czech Technical University, Faculty of Electrical Engineering, Technicka 2, 16627 Prague, Czech Republic, novacj1@fel.cvut.cz)

New system for wide-band audio signals compression is presented. The system is based on a two stage wavelet filter bank principle. Special psychoacoustic model was designed for the system. Data compression is nowadays very topical issue. Due to the development of information technologies more sophisticated compression algorithms may be designed. Most of the present compression systems, e.g. MPEG, ATRAC or Vorbis, use a simple filter bank or DCT transformation. Both methods suffer from the origin of undesirable artefacts which are disturbing for the sound perception. Wavelet filter bank is used to suppress these artefacts and to increase data compression. Frequency dependant windowing function is used for improve of frequency resolution at low frequencies along with faster response at high frequencies. Wavelet transformation based coding algorithm can improve temporal masking effects over conventional perceptual coding algorithms for data compression.

\section{1:20}

3aSPb2. Investigation of a novel nonlinear detector of weak acoustic signals in noise. Julian L. Deeks (Institute of Sound and Vibration, Univ. of Southampton, University Road, Highfield, SO17 1BJ Southampton, UK, jd1@isvr.soton.ac.uk), Paul R. White (Institute of Sound and Vibration, Univ. of Southampton, University Road, Highfield, SO17 1BJ Southampton, UK, prw@isvr.soton.ac.uk)

Recent work has identified and explored a method of detecting weak signals in Gaussian noise, based on the response of a nonlinear system to small input perturbations. The technique is applicable to the detection of weak underwater acoustic signals in unwanted ambient sea noise. Much of the research to date has been carried out using a forced Duffing oscillator as the nonlinear system, with parameters selected so as to place the system at the point of transition from a chaotic state to a stable periodic state. The additive input perturbation, most commonly comprising a simulated single frequency embedded in Gaussian noise with a very low input SNR, has been found to initiate the transition as indicated by the appearance of a stable trajectory in phase space. The attendant increase in amplitude at the output of the detector, at or near to the signal frequency, constitutes a detection of that weak signal. This paper presents a quantitative investigation of the technique as applied to underwater acoustic signals (real and simulated), using receiver operating characteristics (ROC) as a measure of its performance. Both the Duffing system, and a more novel version of the technique using a forced Lorenz system, are investigated.

\section{1:40}

3aSPb3. Comparison of non linear system identification methods : the example of non linear propagation of acoustic waves in ducts. Antonin Novak (Laboratoire d'Acoustique de l'Université du Maine, Avenue Olivier Messiaen, 72085 Le Mans, France, antonin.novak.etu@univ
-lemans.fr),Laurent Simon(LAUM, CNRS, Université du Maine, Lab. d'Acoustique Université du Maine, UMR CNRS 6613, 72085 Le Mans Cedex 9, France, Laurent.Simon@univ-lemans.fr), Joël Gilbert (Laboratoire d'Acoustique de l'Université du Maine, Avenue Olivier Messiaen, 72085 Le Mans, France, joel.gilbert@univ-lemans.fr), Pierrick Lotton (Laboratoire d'Acoustique de l'Université du Maine, Avenue Olivier Messiaen, 72085 Le Mans, France, Pierrick.Lotton@univ-lemans.fr), Frantisek Kadlec (CVUT, Department of Radioelectronic, Technická 2, 16627 Praha 6, Czech Republic,kadlec@fel.cvut.cz)

The weakly non linear propagation of travelling acoustic waves in ducts is a well known problem leading to approximated analytical solutions. From an experimental point of view, the classical way for estimating the non linear parameters of propagation is to generate sine waves and to analyse the higher order harmonics as a function of the amplitude and the frequency of the excitation. In this work, new methods for estimating the non linear parameters of propagation are developed and compared to the sine excitation based method. The excitation signals associated to these new methods can be stationary noise or logarithmic chirps. For these excitation signals, the data processing is based on Multiple Input Single Output (MISO) direct path method. The comparison is made in terms of signal to noise ratio robustness and computation time. Experimental and theoretical results are also compared. We particularly show that a measurement using only one logarithmic chirp allows estimating accurate results for a broad band of amplitudes and frequencies. Associated computational time is then drastically reduced.

\section{2:00}

3aSPb4. The Use of Mel Cepstral Coefficients and Markov Models for the Automatic Identification, Classification and Sequence Modelling of Salient Sound Events Occurring During Tennis Matches. Gordon J. Hunter (Kingston University, Faculty of Computing, Information Systems and Mathematics, Penrhyn Road, KT1 2EE Kingston-upon-Thames, UK, G.Hunter@kingston.ac.uk), Krzysztof Zienowicz (Kingston University, Faculty of Computing, Information Systems and Mathematics, Penrhyn Road, KT1 2EE Kingston-upon-Thames, UK, zienowicz@btinternet .com), Ahmed I. Shihab (Kingston University, Faculty of Computing, Information Systems and Mathematics, Penrhyn Road, KT1 2EE Kingstonupon-Thames, UK, ais@kingston.ac.uk)

Some significant events in sports matches occur too quickly to be detected by conventional video. Audio signals, normally sampled at a much higher rate, provide a way to detect such short events. Here, we employ methods used in automatic speech recognition - templates of Mel Frequency Cepstral Coefficients (MFCCs) over several adjacent time windows, with Principal Components Analysis (PCA) - to identify and classify sound events : different tennis strokes, ball bounces, echos, speech and applause, occurring during tennis matches. Excellent success rates were achieved for both event detection ( $97.74 \%$ ) and correct classification (on average 98.64 $\%$ across all classes) of 1504 sound events in the available recordings. The successful classification rate varied between classes but no class had a success rate below $94 \%$. This could be valuable to spectators, officials and coaches in tennis and other sports - including cricket, baseball and golf - or 
to make video games (such as the Nintendo Wii) more realistic. We also model sequences of these events in both discrete (event oriented) and continuous time domains, using Markov and other models, to give our system predictive as well as reactive capability, help identify "unusual" or "unexpected" salient sounds and hopefully improve the correct classification rate for the classes where the performance was weakest.

\section{2:20}

3aSPb5. 3D Gabor analysis of transient space-time waves. Loic Martinez (ECIME Université de Cergy-Pontoise, rue d'Eragny, 95031 Neuville-sur-Oise, France, loic.martinez@u-cergy.fr), Nicolas Wilkie-Chancellier (ECIME Université de Cergy-Pontoise, rue d'Eragny, 95031 Neuville-sur-Oise, France, Nicolas.Wilkie-Chancellier@u-cergy .fr), Christ Glorieux (Lab. ATF, Katholieke Universiteit Leuven, Celestijnenlaan 200D, B-3001 Leuven, Belgium, christ.glorieux@fys .kuleuven.be)

Laser generation/detection methods allow the investigation of ultrasonic transient phenomena in both space and time dimensions. Used for the experimental investigation of surface wave propagation along a two dimensional medium, laser ultrasonics leads to three dimensional (3D) space-time signal collections. In order to extract the wave propagation information, the classical high resolution signal processing methods or 3D Fourier Transforms are not suitable to identify the transient and local aspect of wave propagation and mode conversion. In order to quantify these transient aspects in the Space-Time-Wave number-frequency domains, the 3D Gabor transform and some other suitable methods are presented. Their potential for the identification of the local and transient complex wave numbers is illustrated with the propagation of Lamb waves on a plane limited plate. The experimental results are in good agreement with the numerical simulations.

\section{2:40}

3aSPb6. Computer learning method for scattering junction identification. Adam Kestian (New York University, 77 Park Ave., apt 1214, Hoboken, NJ 07030, USA, apk240@nyu.edu), Agnieszka Roginska (New York University, 35 West Fourth St., Room 777, New York, NY 10012, USA, roginska@nyu.edu)

Acoustic reflectometry is a non-invasive, time-domain method of identifying the geometry of an acoustical space. A sound pulse is injected into a space and the resulting impulse response details particular changes of impedance, which is a result of a cross-sectional area change or an elbow/Tintersection. Each cause of reflection, known as a scattering junction, has a distinct reflection contour. Previous works were able to identify these scattering junctions via algorithms that attempt to extract particular contours from the impulse response. In the present study, the prominent reflections of the space are observed, isolated, and then compared to a training database of all possible scattering junctions. This method eliminates the necessity to create a contour identification algorithm, as scattering junctions are defined based on its most similar neighbors in the training database. Preliminary results suggest that this computer-learning algorithm can successfully identify reflection contours of a space with varying cross-sectional areas from those that were stored in the training database, which suggests that this method could be a more efficient and versatile alternative to previous identification processes.

\title{
Session 3aUWa
}

\section{Underwater Acoustics, Acoustical Oceanography, and ECUA: Impact of Environmental Variability on Mid-Frequency Sonar Performance}

\author{
Dajun Tang, Cochair \\ Applied Physics Laboratory, University of Washington, 1013 NE 40th St, Seattle, WA 98105, USA \\ Paul C. Hines, Cochair \\ Defence R\&D Canada - Atlantic, P.O. Box 1012, Dartmouth, NS B2Y3Z7, Canada \\ Peter Louring Nielsen, Cochair \\ NURC, Viale S. Bartolomeo 400, La Spezia, 19126, Italy
}

Invited Papers

8:00

3aUWa1. Operational Impacts of the Environment on Mid-Frequency Navy Sonar Systems In Shallow Water. Marcus M. Speckhahn (Planning Systems Inc., 3990 Old Town Avenue, Cabrillo Plaza, Suite A101, San Diego, CA 92110-2930, USA, marcus.speckhahn.ctr@navy.mil), Stephen C. Lingsch (Commander Naval Meteorology and Oceanography Command, 1100 Balch Boulevard, Stennis Space Center, MS 39529, USA, stephen.lingsch@navy.mil), Josette P. Fabre (Naval Research Laboratory, Code 7180, Stennis Space Center, MS 39529, USA, josie.fabre@nrlssc.navy.mil), Michael T. Garr (Naval Undersea Warfare Center, Code 1521, 1176 Howell Street, Newport, RI 02841, USA, michael.garr@navy.mil), Sandra K. Wetzel-Smith (Naval Mine and AntiSubmarine Warfare Command, 32444 Echo Lane, Suite 300, San Diego, CA 92147, USA, skw@ spawar.navy.mil)

The coastal oceans represent a challenging domain for mid-frequency maritime Anti-Submarine Warfare (ASW) sonar systems to operate effectively. Information on the advantages and disadvantages of, as well as general tactical employment criteria for the prevalent types of active mid-frequency sonar systems found in the world's navies is provided. Operational performance of these systems, which include hull-mounted sonars, monostatic buoys and multistatic active systems, as well as dipping sonars, is always affected by shallow water environmental parameters. These acoustic and oceanographic factors include frequent bottom interactions of the transmitted acoustic energy, highly variable sound speed and noise fields, strong coastal currents and high levels of acoustic clutter. Accurate and timely four-dimensional sensor performance predictions and optimization recommendations can mitigate some of the negative impact of 
the environment on sonar detection ranges. We discuss the trade-offs and assumptions made by modern computer-based tactical decision aids to deliver the most accurate environmental information balanced by the need to deliver these data in tactical time frames useful to the ASW mission planner and sonar operator at sea. We will also present thoughts on the quantification of acoustic uncertainty in support of ASW operations and the need for high-fidelity active sonar training systems.

\section{$8: 20$}

3aUWa2. Quantifying ocean acoustic sensitivity to environmental uncertainty/variability. Stan E. Dosso (University of Victoria, School of Earth and Ocean Sciences, Victoria, BC V8W 3P6, Canada, sdosso@uvic.ca)

It is well known that acoustic data used in sonar applications are strongly influenced by physical parameters of the ocean environment. Environmental sensitivity studies are often based on a qualitative comparison of acoustic fields computed for a reference environmental model and for a perturbed model in which one or more parameters have been changed. The acoustic-field perturbation generally includes a component representing a spatial shift of the field (i.e., local field structure remains coherent, but shifts in range and/or depth) and a component representing a change to the shifted field. Hence, the field change at a fixed point can indicate high sensitivity in cases where the field structure changes little but is simply offset, which can conflict with an intuitive understanding of sensitivity. This paper quantifies sensitivity rigorously in terms of a statistical measure of the relative uncertainty of acoustic fields or transmission loss due to realistic uncertainty/variability in environmental parameters. Further, accounting for field shifts can provide a more meaningful sensitivity measure for many applications. The sensitivity of acoustic fields to the state of knowledge of the soundspeed profile, bathymetry, and seabed geoacoustic parameters is examined for both range-independent and range-dependent cases. [Funded by DRDC-Atlantic Rapid Environmental Assessment Project.]

\section{$8: 40$}

3aUWa3. Tools and techniques to measure environmental parameters that improve mid-frequency sonar performance predictions. John C. Osler (Defence R\&D Canada - Atlantic, P.O. Box 1012, Dartmouth, NS B2Y3Z7, Canada, john.osler@drdc-rddc.gc.ca), Sean Pecknold (Defence R\&D Canada - Atlantic, P.O. Box 1012, Dartmouth, NS B2Y3Z7, Canada, sean.pecknold@drdc-rddc.gc.ca), Paul C. Hines (Defence R\&D Canada - Atlantic, P.O. Box 1012, Dartmouth, NS B2Y3Z7, Canada, Paul.Hines@drdc-rddc.gc.ca)

The performance of mid-frequency active sonar systems in littoral waters depends upon several environmental factors and their variability in space and time. These factors include seabed scattering strength, bottom loss, and the water column sound speed profiles. DRDC Atlantic has developed experimental apparatus and techniques to measure these environmental parameters in order to enhance the accuracy of sonar performance estimates in the mid-frequency band, 1 to $10 \mathrm{kHz}$. Experiments have been conducted during sea-trials on the Scotian Shelf and Strataform area on the New Jersey Shelf. In some instances, these experiments have been in conjunction with international research partners to provide an inter-comparison between experimental techniques and/or a comparison of rapid environmental assessment techniques with more traditional, and time-consuming, methods. In this talk, we will demonstrate that predictions of sonar system performance improve when the environmental parameters obtained with these techniques are used, rather than parameters from archival sources.

\section{Contributed Papers}

\section{9:00}

3aUWa4. A comparison of methods for approximating acoustic uncertainty in underwater sound channels. Kevin R. James (Univ. of Michigan, 2010 Lay Auto Lab, Ann Arbor, MI 48109, USA, krjames @ umich.edu), David R. Dowling (Univ. of Michigan, 2212 G. G. Brown Laboratory, Ann Arbor, MI 48109, USA, drd@umich.edu)

The accuracy and reliability of acoustic field calculations are often determined by uncertainty in environmental parameters. There are currently several approaches for predicting calculated-acoustic-field uncertainty arising from environmental uncertainties. This presentation outlines the current development of a method for predicting acoustic uncertainty based on correlations between variations in an uncertain parameter and spatial shifts within a calculated acoustic field. The results of this technique are compared with several other modern methods for predicting acoustic uncertainty such as direct sampling of environmental parameters, linearization and higherorder finite difference approaches, the adjoint method for approximating derivatives of the acoustic field with respect to environmental parameters, and polynomial chaos methods. The advantages and limitations of each technique are presented for range-independent shallow-ocean sound channels at nominal ranges of 1 to $10 \mathrm{~km}$ and frequencies from $100 \mathrm{~Hz}$ to $1 \mathrm{kHz}$. Comparisons of accuracy, computational speed, and applicability to particular uncertain parameters and field prediction routines are provided. Emphasis is placed on identifying the types of sound channel properties and environmental uncertainties for which each technique performs most reliably, as well as identifying situations for which current techniques are not yet adequate. [Sponsored by the Office of Naval Research, Code 321OA].

\section{9:20}

3aUWa5. Fluctuation of mid-frequency propagation in shallow water Dajun Tang (Applied Physics Laboratory, University of Washington, 1013 NE 40th St, Seattle, WA 98105, USA, djtang@ apl.washington.edu), Frank S. Henyey (Applied Physics Laboratory, University of Washington, 1013 NE 40th St, Seattle, WA 98105, USA, frank@ apl.washington.edu), Kevin L. Williams (Applied Physics Laboratory, University of Washington, $1013 \mathrm{NE}$ 40th St, Seattle, WA 98105, USA, williams@apl.washington.edu), Daniel Rouseff (Applied Physics Laboratory, University of Washington, 1013 NE 40th St, Seattle, WA 98105, USA, rouseff@apl.washington.edu), Peter H. Dahl (Applied Physics Laboratory, University of Washington, 1013 NE 40th St, Seattle, WA 98105, USA, dahl@apl.washington.edu), Zhongkang Wang (Hangzhou Applied Acoustics Research Institute, PO Box 1249, 310012 Hangzhou, China, zkwang@apl.washington.edu), Jorge E. Quijano (NEAR Lab-Portland State University, 1900 SW 4th Avenue, Suite 160, Portland, OR 97201, USA, jorgeq@pdx.edu), Jee Woong Choi (Dept. of Environmental Marine Sciences, Hanyang Univ., 1271, Sa-3-dong, Sangrok-gu, 426-791 Ansan, Republic of Korea, choijw@hanyang.ac.kr)

As part of the ONR-sponsored SW06 experiment, mid-frequency sound propagation was measured at ranges 1-10 km in the frequency band of 2-10 $\mathrm{kHz}$ in August, 2006. The water depth is $80 \mathrm{~m}$ and the source depth is $30 \mathrm{~m}$, close to the minimum of a duct with a thermocline above and a warm salty water below. The receivers are clustered into two groups, one at $25 \mathrm{~m}$ depth, the other at $50 \mathrm{~m}$. The region has active internal wave activity during this time. Because the source is near the axis of the sound channel, it is observed that propagation is dominated by trapped modes and behaves similar to 
sound propagation in a deep water duct. Amplitude fluctuations and crossfrequency correlations are estimated. The scintillation index as a function of frequency and bandwidth is calculated.

\section{9:40}

3aUWa6. Active Tracking using the Invariance Extended Kalman Filter (IEKF). Lisa M. Zurk (NEAR Lab-Portland State University, 1900 SW 4th Avenue, Suite 160, Portland, OR 97201, USA, zurkl@cecs .pdx.edu), Chensong He (NEAR Lab-Portland State University, 1900 SW 4th Avenue, Suite 160, Portland, OR 97201, USA, zurk1@pdx.edu), Jorge E. Quijano (NEAR Lab-Portland State University, 1900 SW 4th Avenue, Suite 160, Portland, OR 97201, USA, jorgeq@ pdx.edu), Christian Hempel (1176 Howell Street, Newport, RI 02841, USA, HempelCG@Npt.NUWC.Navy .Mil)

The pressure received from a moving source in a shallow water channel has a frequency dependent structure that is imposed by the propagation characteristics of the channel. While an exact description of this structure requires fine knowledge of the channel properties (including bottom composition), an approximate relationship which utilizes the so-called waveguide invariant can be used to describe the intensity variations as a function of frequency versus range. This relationship has been extensively explored and utilized for application to passive sonar systems. Recent work has demonstrated that a similar structure, called the active invariance, may be present in active sonar. In this paper the active invariance relationship is incorporated into an extended Kalman filter (EKF) to create a new tracking formulation called the Invariance EKF (IEKF). The IEKF differs from the conventional formulation by incorporating the time-dependent frequency content into both the state space representation and the update equations, where the relationship between target position and frequency is given by the active invariance expression. Performance of the IEKF relative to conventional approaches is explored with normal mode simulations and bistatic data collected in the Maltese channel. Sponsored by ONR-321US

\section{0:00}

3aUWa7. Comparison of sonar transmission models at mid-frequency for synthetic and real environments. Adrian D. Jones (Defence Science and Technology Organisation, P.O Box 1500, 5111 Edinburgh, Australia, bearjones@chariot.net.au), Janice S. Sendt (Thales Australia, 274 Victoria Road, 2116 Rydalmere, Australia, Janice.Sendt@au.thalesgroup.com), Alec J. Duncan (Curtin University of Technology, Centre for Marine Sci \& Tech, GPO Box U1987, 6845 Perth, WA, Australia, A.J.Duncan@ curtin.edu .au), Paul A. Clarke (Defence Science and Technology Organisation, P.O Box 1500, 5111 Edinburgh, Australia, Paul.Clarke@dsto.defence.gov .au), Zhiyong Zhang (Defence Science and Technology Organisation, P.O Box 1500, 5111 Edinburgh, Australia, Yong.Zhang@dsto.defence.gov .au), Han Vu (Defence Science and Technology Organisation, P.O Box 1500, 5111 Edinburgh, Australia, Han.Vu@dsto.defence.gov.au)

The suitability of sonar transmission loss models for application to the mid-range of acoustic frequencies has been studied by comparing the outputs from a number of models. Emphasis has been placed upon the requirements for models for circumstances relevant to both continental shelf and deep ocean zones within the Australian region. In particular, the ability to describe transmission in surface ducted environments for a deep ocean, and in a shallow ocean with both ducted and downward refracting environments has been considered. For the deep ocean case, a synthetic environment was selected, and the ability to describe duct trapping was examined. For the shallow ocean case, scenarios selected for comparison correspond with ocean tracks for which environmental parameters had been collected and received sound pressure time series measured. The subject transmission loss models are from a variety of types. To enable the most valid comparison, input parameters have been matched across models to the fullest extent. This paper presents the state of progress of this work, including a comparison of models amongst themselves and, for continental shelf scenarios, against measured transmission loss.
10:20-10:40 Break

\section{0:40}

3aUWa8. In ocean evaluation of low frequency active sonar systems. Karl Thomas Hjelmervik (FFI, Postboks 115, 3191 Horten, Norway, kth @ffi.no), Geir Helge Sandsmark (FFI, Postboks 115, 3191 Horten, Norway, ges@ffi.no)

Sonar performance measurements in the sea are always affected by uncontrollable and/or uncertain environmental conditions as sound speed variations, bottom topography or the acoustic properties of the sea floor. This paper presents a method to determine a sonar - target geometry which minimizes the uncertainty in target signal excess due to environmental variability. An acoustic model is used to estimate the signal excess for a large number of sound speed profiles measured in the relevant area. The results are compared seeking a target range and depth where the estimated signal excess is robust with respect to the variation of sound speed profiles to be expected in the actual area. Results from sea trials will be presented as well as simulated examples used to demonstrate the robustness or sensitivity of the signal excess to environmental changes at different test geometries.

\section{1:00}

3aUWa9. Effects of volume and boundary variability on the statistics of received signal frequency. Jeremy Joseph (ARL Penn State, PO Box 30, State College, PA 16804, USA, jmj269@psu.edu), Richard Lee Culver (ARL Penn State, PO Box 30, State College, PA 16804, USA, rlc5@psu.edu), Colin W. Jemmott (ARL Penn State, PO Box 30, State College, PA 16804, USA, cwj112@psu.edu)

The overall goal of our research is to incorporate statistical knowledge of received passive signal variability and uncertainty into the signal processing. To accomplish this goal we need to better understand the sources of this variability, which are due in part to propagation through the ocean channel. Propagation introduces significant variation in received signal parameters through rough surface and bottom scattering as well as volume inhomogeneities. This talk focuses on the variability introduced by these sources and its effect on the statistics of the frequency spectrum of the received signal. We consider low frequencies, for which theoretical predictions are compared with PE-based simulation results. Work supported by ONR Undersea Signal Processing Code 321US.

\section{1:20}

3aUWa10. Modeling higher order statistics of shallow water reverberation. Kevin D. Lepage (Naval Research Laboratory, 4555 Overlook Ave SW, Washington, DC 20375, USA, kevin.lepage@nrl.navy.mil)

The modeling of the higher moments of shallow water reverberation is addressed for problems where non-Rayleigh reverberation is caused by scattering from rough boundaries with non-Gaussian height distributions. Expressions for the first three spatial moments of exponentially heightdistributed roughness are derived for both Gaussian and exponential correlation functions corresponding to Gaussian or von-Karman second order spectra. The bi and tri-spectra for these roughness distributions are also derived, and differences are highlighted between these results and the results that are obtained for Gaussian height-distributed higher order spectra. Finally results for the higher moments of reverberation pressure are derived for exponentially height-distributed bottom roughness with the Gaussian spatial correlation function in the presence of multipath as a function of time, bandwidth, and correlation length scale. [Work supported by ONR]

\section{$11: 40$}

3aUWa11. Computational Predictions of sonar performance based on full wave acoustic propagation modeling. Michael Wolfson (Univ. of Washington, Applied Physics Lab., 101340 St NE, Seattle, WA 98105, USA, wolfson@apl.washington.edu)

For fishery acoustics, sonars offer the advantage of inferring the biomass of a given ocean volume at a lower operational cost of traditional echosounders, but wave guide effects which induce multi-pathing yield unknown variances in bio-mass estimates. A sonar performance model has recently been developed to assist in understanding this variance based on the para- 
axial approximation to the acoustic wave equation. The model takes into account the geometry of the sonar deployment (source depth, transmit/receive beam patterns, pulse shape) and the acoustic environment (water column and geo-acoustic bottom) and creates a data base of two-way transfer functions for a chosen set of range-depth cells. Then, given a hypothetical aggregation or school of fish, each with its own target strength, as well as noise and reverberation levels, one can create intensity maps which can be used to perform sensitivity studies to ascertain the performance characteristics of a fishery sonar. This talk will discuss the theoretical development of the model, its constraints, and provide an example which highlights its potential for future work. The example will be in a shallow water $(100 \mathrm{~m})$ environment using acoustic frequencies of around 10 kilohertz for imaging aggregations of pelagic fish. The Naval Ocean Partnership Program is gratefully acknowledged for this work.

\section{2:00}

3aUWa12. Temporal and spatial coherence of low frequency acoustic modes in shallow water. Harry Deferrari (Univ. of Miami, 4600 Rickenbacker Causeway, Miami, FL 33149, USA, hdeferrari@rsmas .miami.edu), Hien B. Nguyen (Univ. of Miami, 4600 Rickenbacker Causeway, Miami, FL 33149, USA, hnguyen@rsmas.miami.edu), Jennifer Whylie (Univ. of Miami, 4600 Rickenbacker Causeway, Miami, FL 33149, USA, jwhylie@rsmas.miami.edu)

Three recently collected shallow water acoustic propagation data sets have common experimental parameters of fixed sources with multiple frequency m-sequence transmissions, HLA and VLA receptions, and detailed measurements of the ocean acoustic environments. In each case, broad band signals allow the separation of modes by arrival time and the computation of spatial and temporal coherency for individual modes of propagation thus eliminating the effects of multipath interference. Here, coherency estimates are compared for several ranges from 10 to $80 \mathrm{Km}$ and depths of 80 to 250 $\mathrm{m}$, frequencies from 100 to $1600 \mathrm{~Hz}$ and for a wide range of potential energy levels of the internal wave field. There are a number of consisten findings. Low order modes are more coherent than high order modes in both space and time. Low frequency signals respond deterministically and in pro- portion to internal wave fluctuations whereas higher frequencies are randomized and have saturated statistics. In between, phase wrapping occurs. The number of bottom interactions is shown to be a critical factor in coherence especially for frequencies of $800 \mathrm{~Hz}$ and above. At very long ranges out of plane arrivals introduce multipath interference even for single mode reception.

12:20

3aUWa13. Effects of internal waves on acoustic coherent communications during SW06. Aijun Song (University of Delaware, College of Marine and Earth Studies, S. College Street, Newark, DE 19716, USA, ajsong@udel.edu), Mohsen Badiey (University of Delaware, College of Marine and Earth Studies, S. College Street, Newark, DE 19716, USA badiey@udel.edu), Arthur Newhall (Woods Hole Oceanographic Institution, 98 Water Street, Bigelow 203A, MS-11, Woods Hole, MA 02543, USA, anewhall@whoi.edu), James F. Lynch (Woods Hole Oceanographic Institution, 98 Water Street, Bigelow 203A, MS-11, Woods Hole, MA 02543, USA, jlynch@whoi.edu), Harry Deferrari (Univ. of Miami, 4600 Rickenbacker Causeway, Miami, FL 33149, USA, hdeferrari@rsmas.miami .edu)

During the SW06 experiment conducted east off the New Jersey continental shelf from July to September of 2006, internal wave surface expression and water temperature profiles were recorded when phase shift keying signals (PSK) were transmitted to study internal wave effects on coherent underwater acoustic communications at low to mid frequency bands. The collected communications data are processed using time reversal multichannel combining followed by a decision feedback equalizer. Due to the presence of internal waves, the acoustic channels show a time-varying property. Continuous channel updates prior to time reversal combining are required to combat fast channel fluctuations. The communications performance in terms of output signal-to-noise ratio exhibits significant change due to the interna waves. Further, the internal wave effects on the performance of coherent acoustic communications exhibit a frequency- and depth- dependency. The choices of receiver parameters also can be affected by the presence of internal waves.

\title{
Session 3aUWb
}

\section{Underwater Acoustics and ECUA: Sonar System and Transducer Calibration Methodology III}

\author{
Kenneth G. Foote, Cochair \\ Woods Hole Oceanographic Institution, Woods Hole, MA 02543, USA \\ Stephen P. Robinson, Cochair \\ National Physical Laboratory, Hampton Road, Teddington, TW11 OLW, UK
}

\section{Invited Paper}

\section{8:00}

3aUWb1. Calibration of wideband systems, need for a standard. Manell E. Zakharia (French Naval Acadamey, BP 600, 29240 Brest-Armees, France, manell.zakharia@ecole-navale.fr)

Although wide band sonar system are becoming more and more popular, there is no unified standard method for their calibration. Most of the wideband systems are calibrated at multiple frequencies using single frequency methods. Although such calibrations give correct figures, these figures do not correspond to the real use of the systems and do not display properly the problems encountered while using such systems such as signal deformation due to the directivity pattern. For instance, the zeroes of directivity patterns that correspond to destructive interference at one frequency are spatially smeared even for a band of $10 \%$ and appear as spectral variations. We will show two examples of such calibration with increasing difficulty. The first case considers a wideband sounder covering two 
octaves $(20-80 \mathrm{kHz}$ ) and the second one a parametric array (primaries around $100 \mathrm{kHz}$ and parametric generation from 10 to $20 \mathrm{kHz}$ ). In the second case, the difficulty is increased due to the factor of about ten between the primary and the secondary frequencies that are also associated to important level difference (about $40 \mathrm{~dB}$ ). It requires a high linearity constraint of the whole calibration chain.

\section{Contributed Paper}

$$
\text { 8:20 }
$$

3aUWb2. Calibration of broadband sonar systems using multiple standard targets. Philip Atkins (University of Birmingham, Department of Electronic, Electrical and Computer Engineering, Edgbaston, B15 2TT Birmingham, UK, p.r.atkins@bham.ac.uk), David T. Francis (University of Birmingham, Department of Electronic, Electrical and Computer Engineering, Edgbaston, B15 2TT Birmingham, UK, francdti@adf.bham .ac.uk), Kenneth G. Foote (Woods Hole Oceanographic Institution, Woods Hole, MA 02543, USA, kfoote@whoi.edu)

A seven-octave active sonar system spanning the nominal frequency range $25-3200 \mathrm{kHz}$ was deployed in Norwegian waters for the purpose of measuring the acoustic scattering characteristics of a range of marine organisms. This system transmitted linear frequency-modulated (LFM) signals in order to achieve good range resolution and to obtain spectral information on resolved targets. Total system performance was variously measured in situ and ex situ, depending on the particular octave band, using standard-target spheres. This enabled the frequency response of the entire system to be determined and the sidelobe level of the matched-filter receiver to be reduced. The effects of the deep nulls encountered in the backscattered spectrum of target spheres were partially reduced by using a string of up to six spheres of different sizes and material properties. Typical results will be presented showing that such calibration procedures are sensitive the relative alignment of the sonar-target and to sound-speed profile changes over the length of the string. [This work originated with EU RTD support through contract MAS3-CT95-0031.]

\section{Invited Paper}

\section{$8: 40$}

3aUWb3. Measuring the equivalent beam angle of echo sounders with split-beam transducers. Lars Nonboe Andersen (Simrad, P.O. Box 111, 3191 Horten, Norway, lars.nonboe.andersen@simrad.com), Egil Ona (Institute of Marine Research, P.O.Box 1870 Nordnes, 5817 Bergen, Norway, egil.ona@imr.no), Geir Pedersen (Institute of Marine Research, P.O.Box 1870 Nordnes, 5817 Bergen, Norway, geir.pedersen@imr.no), Frank Tichy (Simrad, P.O. Box 111, 3191 Horten, Norway, frank.tichy@kongsberg.com) , Even Borten Lunde (Simrad, P.O. Box 111, 3191 Horten, Norway, even.borten.lunde@kongsberg.com)

When trying to measure the numerical density of marine animal populations with scientific echo sounders, several technical system parameters are determined through a standard-target calibration. The acoustic scattering properties of standard targets are known $a$ priori. In a typical standard-target calibration the system gain and the transducer beam pattern are estimated. The system gain parameter is used both when measuring volume backscattering strength and when measuring target strength. The calibrated beam pattern is currently used only for target strength estimation. However, measurements of mean volume backscattering strength also require knowledge of the effective sampling volume of the acoustic beam, namely the equivalent beam angle. This parameter is typically not determined during an ordinary echo sounder calibration. A method for measuring the equivalent beam angle and the transducer angle sensitivity of a specific split-beam transducer is described, and results from exemplary calibrations are presented. Changes in the calibration parameters as a function of environmental parameters, especially the sound speed in water, are discussed.

\section{Contributed Paper}

\section{9:00}

3aUWb4. Multibeam sonar calibration techniques. Christian De Moustier (UNH, Center for Coastal and Ocean Mapping, Chase Ocean Engineering Lab, 24 Colovos Road, Durham, NH 03824, USA, cpm@ieee .org), Barbara J. Kraft (UNH, Center for Coastal and Ocean Mapping, Chase Ocean Engineering Lab, 24 Colovos Road, Durham, NH 03824, USA, bjkraft@cisunix.unh.edu), Glenn McGillicuddy (UNH, Center for Coastal and Ocean Mapping, Chase Ocean Engineering Lab, 24 Colovos Road, Durham, NH 03824, USA, glennm@ccom.unh.edu)

The acoustic calibration facility at the University of New Hampshire's Chase Ocean Engineering Laboratory has been used to calibrate several transducer arrays from multibeam sonars that operate at $100 \mathrm{kHz}$ and above. In each case, we mount the transducer array on a rotating colum and mea- sure the array's transmit and receive characteristics with a reference transducers leased from the Naval Undersea Warfare Center, Newport RI. Our rotation angles are known to within $0.2^{\circ}$ and our acoustic calibration measurements are repeatable to within $0.5 \mathrm{~dB}$. After adjusting for measured impedance mismatches between the elements of the array, we determine the actual element separation as the ratio of physical spacing over acoustic wavelength, and we use this result to estimate the beam pointing accuracy of the sonar. The beamforming gain is then estimated for all beam directions from broadside to the full range of steering angles used by the sonar. This work is motivated by research efforts to obtain calibrated acoustic backscatter measurements from multibeam echo-sounder that are used for seafloor characterization applications or for detection and identification of targets in the water column.

\section{Invited Papers}

3aUWb5. A comparison of hydrophone near-field scans and optical techniques for characterising high frequency sonar transducers. Victor F. Humphrey (Institute of Sound and Vibration, Univ. of Southampton, University Road, Highfield, SO17 1BJ Southampton, UK, vh@isvr.soton.ac.uk), Stephen P. Robinson (National Physical Laboratory, Hampton Road, TW11 OLW Teddington, UK, Stephen.Robinson@npl.co.uk), Peter D. Theobald (National Physical Laboratory, Hampton Road, TW11 OLW Teddington, UK, pdt@npl.co.uk), Gary Hayman (National Physical Laboratory, Hampton Road, TW11 OLW Teddington, UK, Gary.Hayman@npl.co.uk), Martin P. Cooling (National Physical Laboratory, Hampton Road, TW11 OLW Teddington, UK, mc@isvr .soton.ac.uk) 
Two potential methods of fully characterising the response of high frequency sonar transducers and arrays operating in the frequency range $100 \mathrm{kHz}$ to $500 \mathrm{kHz}$ are compared. In the first approach two-dimensional planar scans, with a spatial resolution of better than half a wavelength, are performed in the acoustic near-field using a small probe hydrophone. The measured two-dimensional data are propagated numerically using a Fourier Transform method to predict the far-field response. Alternatively the data can be back-propagated to re-construct the pressure distribution at the source, a powerful diagnostic technique which can identify defects in transducers and array elements. The second approach uses a scanning laser vibrometer to measure the velocity of the transducer surface; with the resulting velocity data also being used to predict the far-field response by numerical propagation. The two approaches are compared for a number of devices. Comparison of the propagated hydrophone near-field scan data with direct measurements at these ranges shows very good agreement, indicating the usefulness of the method for deriving far-field transducer responses from near-field measurements in laboratory tanks. The potential limitations introduced to the optical approach by the acousto-optic effect are discussed.

\section{9:40}

3aUWb6. Acousto-optic tomography for mapping of high-frequency sonar fields. Peter D. Theobald (National Physical Laboratory, Hampton Road, TW11 OLW Teddington, UK, pdt@npl.co.uk), Stephen P. Robinson (National Physical Laboratory, Hampton Road, TW11 OLW Teddington, UK, Stephen.Robinson@npl.co.uk), Gary Hayman (National Physical Laboratory, Hampton Road, TW11 OLW Teddington, UK, Gary.Hayman@npl.co.uk), Triantafillos Koukoulas (National Physical Laboratory, Hampton Road, TW11 OLW Teddington, UK, triantafillos.koukoulas@npl.co.uk)

This paper presents an acousto-optic method for tomographic mapping of acoustic fields generated by high-frequency sonar array transducers. The method uses a laser interferometer to measure the integrated refractive index change across the propagating acoustic wave generated by the transducer. An interferometer being a displacement or velocity measuring device, interprets this rate of change of optical path length as a displacement or velocity. Obtaining a series of these projections by scanning the laser beam or the transducer for a number of rotation angles of the transducer allow a two-dimensional plane of the acoustic field to be reconstructed using the techniques commonly used in X-ray Computed Tomography. Acousto-optic tomographic measurement results are presented for a 95 by $95 \mathrm{~mm}, 400 \mathrm{kHz}$ 1-3 composite, 4 element sonar array transducer and are compared to conventional planar hydrophone scans obtained using a $1.5 \mathrm{~mm}$ probe hydrophone. The optical method allows measurement of the acoustic field without perturbing the field being measured, which can occur when using the planar hydrophone scanning method. Different tomographic geometries are considered, including parallel beam and fan-beam methods, with the advantages and disadvantages of each being discussed.

\section{Contributed Paper}

\section{0:00}

3aUWb7. Prediction for the acoustic field of a high frequency cylindrical transducer. Tiejian Xia (Hangzhou Applied Acoustics Research Institute, 401\# Fuyang, Zhejiang, 311400 Fuyang, China, xiatiejian @263.net), Yuebing Wang (Hangzhou Applied Acoustics Research Institute, 96 Huaxing Road, 310012 Hangzhou, China, wang_yuebing@tom .com), Lisheng Zhou (Rensselaer Polytechnic Institute, Greene Building, School of Architecture, 110 8th Street, Troy, NY 12180, USA, 1szhou@163.com), Qiang Liu (Hangzhou Applied Acoustics Research Institute, 401\# Fuyang, Zhejiang, 311400 Fuyang, China, 715liuqiang@163 .com)
The BEM software is implemented in the prediction of the acoustic field produced by a cylindrical transducer, the transducer consists of 1200 PZT pillars which are fixed on the surface of a 156-mm-diameter and 120-mmheight cylinder. The acoustic field around the transducer is measured at the frequency $120 \mathrm{kHz}$ using an optical vibrometer, the measured data are fed into the BEM software as the boundary condition, and the directivities of the transducer are forecasted in the vertical and horizontal planes. To verify the calculation, the acoustic field in the far field of the transducer is measured using a hydrophone, and good agreements between the prediction and the measurement convince that the BEM software can be used to predict acoustic fields of cylindrical transducers through distributions of acoustic fields measured in their near fields. 


\title{
Session 3aUWc
}

\section{Underwater Acoustics, Signal Processing in Acoustics, and ECUA: Acoustic Vector Fields and Sensor Processing II}

\author{
Kevin B. Smith, Cochair \\ Naval Postgraduate School/Naval Undersea Warfare Center, Code PH/Sk, Department of Physics, Monterey, CA 93943, USA \\ Jean-Pierre Hermand, Cochair \\ Université libre de Bruxelles (U.L.B.) - Environmental hydroacoustics lab, av. Franklin D. Roosevelt 50, CP 194/5, Bruxelles, \\ 1050, Belgium
}

Invited Papers

10:40

3aUWc1. Acoustic vector sensors and vector/tensor processing in ocean acoustics research. Gerald D'Spain (Marine Physical Lab, Scripps Institution of Oceanography, 291 Rosecrans St., San Diego, CA 92106, USA, gld@mpl.ucsd.edu)

Several passive sensor systems that simultaneously measure acoustic pressure and the three components of acoustic particle motion have been designed and deployed in seagoing experiments by the Marine Physical Lab over the past two decades. Descriptions of these sensor systems and results from applying various signal/array processing methods with these data are presented in this talk. Although vector sensors do not provide any additional information on the sound field than that obtained by a suitable number of properly placed hydrophones, they do provide estimates of the sound field's directional properties from a very compact package. Applications where available space is severely limited include autonomous underwater vehicles (AUV). Recent results from acoustic vector sensors installed in a flying wing underwater glider and inside a prop-driven AUV to quietly measure sounds in the ocean illustrate the benefits, and drawbacks, of the use of vector sensors on autonomous platforms. The extension of the signal/array processing approaches to tensor sensors indicates the promise of additional gain, if the sensor design challenges can be overcome. [Work supported by the Office of Naval Research].

\section{1:00}

3aUWc2. Robust Adaptive Vector Sensor Processing. Andrew J. Poulsen (Massachusetts Institute of Technology, 77 Massachusetts Avenue, Cambridge, MA 02139, USA, poulsen@mit.edu), Arthur B. Baggeroer (MIT, Department of Mathematics, 77 Massachusetts Avenue, Cambridge, MA 02139, USA, abb@boreas.mit.edu), Jonathan Paul Kitchens (MIT Lincoln Laboratory, 244 Wood Street, Lexington, MA 02420, USA, jonpaul@mit.edu), Jennifer A. Watson (MIT Lincoln Laboratory, 244 Wood Street, Lexington, MA 02420, USA, watson@11.mit.edu)

A sizable literature exists on the theory of processing signals for many vector sensor array applications. In practice, however, mismatch and several noise processes can pose significant problems. If adaptive beamforming is also used, the "snapshot" issue potentially increases by a factor of four since each element in a vector sensor array consists of a scalar hydrophone and up to three spatially orthogonal particle motion sensors. Both of these sensor types have very different response and noise characteristics. Particle motion sensors are more sensitive to non-acoustic, motion-induced noise than hydrophones. In towed line array configuration, those sensors orthogonal to the direction of motion are exposed to higher intensities of flow noise at low frequencies than those coincident to the array axis. Similarly, different dipole sensors may be exposed to varying degrees of rotational mismatch. Sensors may also rest on the seafloor, creating asymmetries. Physical constraints also relate pressure and particle velocity measurements via conservation of momentum and mass equations. We examine adaptive processing methods customized to the unique characteristics of vector sensors. Array gain and other performance metrics in the presence of system mismatch and uncertainties is presented using common ocean noise models, including directional jamming, isotropic and surface noise.

\section{1:20}

3aUWc3. Using acoustic streamlines to visualize energy flow across boundaries. David M.F. Chapman (Scientific Consultant, 8 Lakeview Avenue, Dartmouth, NS B3A 3S7, Canada, dave.chapman@ns.sympatico.ca)

For spherical waves that radiate from a point source in a homogeneous fluid and propagate across a plane boundary into a dissimilar homogeneous fluid, the acoustic field may differ significantly from the geometric acoustics approximation if either the source or receiver is near the interface (in terms of acoustic wavelengths) or if the minimum-phase path is near the critical angle. Cross-boundary energy flow from a continuous-wave point source is visualized by tracing acoustic streamlines: those curves whose tangent everywhere is the average acoustic intensity vector. It is seen that the acoustic energy flow is not always in line with the "Snell's law" or minimum-phase ray path. Also, acoustic energy streamlines do not display unusual behaviour in the vicinity of the critical angle. Finally, it is shown that there exists a law of refraction of acoustic energy streamlines across density discontinuities, analogous to Snell's law of refraction of ray paths across sound speed discontinuities. Examples include water-to-seabed transmission and water-to-air transmission. [Work supported in part by ONR Code 32] 
3aUWc4. Real time sound field visualization in the near field, far field and at absorbing surfaces. Hans-Elias De Bree (Microflown Technologies Inc., PO BOX 300, 6900 AH Zevenaar, Netherlands, debree@microflown.com), Emiel Tijs (Microflown Technologies Inc., PO BOX 300, 6900 AH Zevenaar, Netherlands, tijs@microflown.com), Tom Basten (TNO Science and Industry, Stieltjesweg 1, 2600 AD Delft, Netherlands, tom.basten@tno.nl)

The Microflown is a small sensor that is capable of measuring the acoustic particle velocity. In combination with a small pressure microphone it is possible to measure the complete sound field. If the sound field close to an acoustic material is measured it make sense to measure the particle velocity normal to the surface and the sound pressure. With this, the acoustic impedance and absorption can be measured in real time, and with a high spatial resolution. If the sound field of a sound radiating object is measured, the sound field can be obtained directly. Three applications include: direct acoustic camera, that is the real time display of the sound field (velocity, pressure, intensity). Holography that is the pressure, velocity reconstruction on the surface based on the measured sound field (and other places). And cabin interior noise mapping, that is the visualization of the interior noise perceived on a certain location. With three dimensional sound probes (sound pressure and particle velocity in three dimensions), sources in the free field can be found for both bearings as distance. Most recent developments are presented.

\section{2:00}

3aUWc5. Measurement of total sound energy density in enclosures at low frequencies. Finn Jacobsen (Acoustic Technology Department, Technical University of Denmark, Ørsted Plads, B352, DK-2800 Lyngby, Denmark, fja@oersted.dtu.dk)

Many acoustic measurements rely on determining the total sound energy in an enclosure; and this quantity is usually estimated by measuring the mean square pressure at a number of discrete positions. Almost 30 years ago it was shown theoretically that the normalised spatial variance of the total sound energy density (potential and kinetic) is one third of the normalised spatial variance of the potential energy density (the mean square pressure) in a reverberant sound field above the Schroeder frequency. About ten years later this prediction was confirmed experimentally. However, until recently measurement of the total sound energy density (in air) has required an elaborate arrangement based on finite different approximations using at least four matched pressure microphones; therefore the method has never come into use. However, with the advent of a three-dimensional particle velocity transducer it has become somewhat easier to measure total rather than only potential energy density in a sound field. This paper examines the spatial uniformity of potential, kinetic and total sound energy density in enclosures theoretically and experimentally with particular emphasis on the frequency range below the Schroeder frequency.

\section{Contributed Papers}

\section{2:20}

3aUWc6. On the usefulness of waterborne measurement of particle velocity in geoacoustic inversion. Jean-Pierre Hermand (Université libre de Bruxelles (U.L.B.) - Environmental hydroacoustics lab, av. Franklin D. Roosevelt 50, CP 194/5, 1050 Bruxelles, Belgium, jhermand@ulb ac.be), Kevin B. Smith (Naval Postgraduate School/Naval Undersea Warfare Center, Code PH/Sk, Department of Physics, Monterey, CA 93943, USA, kbsmith@nps.edu)

Recent advances in sensor design have led to the development of receiving systems whose elements are vector sensors, i.e., sensors that simultaneously measure the acoustic pressure and fluid motion due to the propagation of acoustic energy at the sensor location. As such, arrays of vector sensors can provide more information about the sound field than arrays made of traditional hydrophones, and thus are attractive for various applications including the inversion for environmental properties. The fundamental question addressed by this paper is: does the use of acoustic vector data versus pressure-only data improve the results of an inversion scheme based on waterborne observations and matched field processing? To our knowledge no experimental data are available yet to allow a detailed comparison of performance between standard (pressure-only) arrays and vector sensor arrays for environmental inversions. For a preliminary study we will simulate the inversion of vector sensor data in the South Elba environment for which pressure-only inversion results and ground truth data are available following the Yellow Shark'94 and Blue Planet'07 experiments. Broadband signals received on fully-populated and sparse arrays will be considered.

\section{$12: 40$}

3aUWc7. Characterization of the Near Scattered Acoustic Vector Field Robert Barton (Naval Undersea Warfare Center, Code 1522 bldg1320, 1176 Howell st, Newport, RI 02841, USA, bartonrj@npt.nuwc.navy.mil), Kevin B. Smith (Naval Postgraduate School/Naval Undersea Warfare Center, Code PH/Sk, Department of Physics, Monterey, CA 93943, USA, kbsmith $@$ nps.edu)

In this study, we investigate the properties of the scattered acoustic vector fields generated by simple geometric objects, including the infinite rigid plate, disk, and sphere. Analytical solutions are derived from acoustic target strength scattering models in the near field region. Of particular interest is the understanding of the characteristics of energy flow of the scattered acoustic vector field in the near to far-field transition region. We utilize the time and space separable instantaneous active and reactive acoustic intensity to investigate the relative phase properties of the scattered field. Numerical results are presented for the near region scattered acoustic vector field of simple objects in both two and three dimensions. 


\title{
Session 3aUWd
}

\section{Underwater Acoustics and ECUA: Geoacoustic Sediment Modeling II}

\author{
Nicholas P. Chotiros, Cochair \\ Applied Research Laboratories, University of Texas, PO Box 8029, Austin, TX 78713-8029, USA \\ Jean-Pierre Sessarego, Cochair \\ Laboratory for Mechanics and Acoustics CNRS, 31 chemin Joseph Aiguier, Marseille, 13009, France
}

\section{Invited Papers}

\section{1:20}

3aUWd1. The Viscous-Grain-Shearing (VGS) theory of wave propagation in marine sediments. Michael Buckingham (Marine Physical Lab, Scripps Institution of Oceanography, UCSD, 9500 Gilman Dr. M/C 0238, La Jolla, CA 92093-0238, USA, mjb@mpl .ucsd.edu)

The grain-shearing (GS) theory of wave propagation in a marine sediment is based upon the idea that, as grains slide against one another, the dissipation becomes progressively stronger, a phenomenon known as strain hardening. The GS theory predicts a sound speed showing weak, logarithmic dispersion and an attenuation that scales linearly with frequency. At frequencies above about $10 \mathrm{kHz}$, such behavior matches the sound speed and attenuation data obtained during the SAX99 experiment in the Gulf of Mexico. But at lower frequencies, the measured dispersion curves show a lower sound speed and higher attenuation than predicted by the GS theory. A generalized version of the GS theory, designated the VGS theory, takes account of the effective viscosity of the molecularly thin layer of pore fluid between contiguous grains, which limits the degree of strain hardening that can occur during sliding. The VGS dispersion expressions are the same as those of the GS theory, except for the appearance of a simple algebraic function that has an effect only at low frequencies, below $10 \mathrm{kHz}$. The resultant VGS curves match the sound speed and attenuation measurements from SAX99 over the measurement frequency band from 1 to $400 \mathrm{kHz}$. (Research supported by ONR.)

\section{1:40}

3aUWd2. Analysis of acoustical transmission and reflection from a sandy stratified rough sediment using the grain shearing model. Laurent Guillon (Ecole Navale/Irenav, BP 600, 29240 Brest Armées, France, guillon@ecole-navale.fr), Vladislav Aleshin (LPEC/UMR 6087/CNRS/Université du Maine, Avenue Olivier Messiaen, 72085 Le Mans Cedex 09, France, aleshinv@mail.ru)

A number of applications, such as geoacoustic inversion or object detection, require an appropriate theoretical consideration of the sound reflection and transmission problem. The analysis we present here includes three essential aspects: account for a constitutive behavior of a saturated unconsolidated granular material, presence of vertical gradients in material properties, and roughness of the sediment surface. Accepting the Buckingham grain shearing model (see e.g. JASA, vol. 117, pp. 137-152) for the stress-strain relationship in the sediment, the gravity-induced variations of the longitudinal and shear rigidity can be described. The corresponding numerical solution for the plane interface is presented and the influence of infinite rigidity gradients at the surface is discussed. Another important contribution is provided by the interface roughness that considerably improves the penetration conditions. This problem is solved using a statistical treatment of a number of roughness realizations with a given spatial spectrum. The developed numerical tool enables to study the influence of all these factors and estimate the penetrated energy for different acoustical frequencies, roughness parameters, and grain sizes (This work was founded by DGA and Thales Underwater Systems).

3aUWd3. Bistatic scattering in sediments: comparison of model and scaled tank experiments at $238 \mathrm{kHz}$. Russell Howey (University of Bath, Department of Physics, Claverton Down, BA2 7AY Bath, UK, rph23@bath.ac.uk), Philippe Blondel (University of Bath, Department of Physics, Claverton Down, BA2 7AY Bath, UK, pyspb@bath.ac.uk)

Multiple-aspect scattering is increasingly used to investigate seabeds and objects, buried or not. However, high-frequency scattering processes on/in sediments need to be better understood, particularly when the structure and/or composition of these sediments is not fully homogeneous. Scaled tank experiments were conducted using the University of Bath facilities. A narrow acoustic beam (10 ${ }^{\circ}$ beamwidth) was generated by a transducer resonant at $238 \mathrm{kHz}$, imaging a silt seabed at $45^{\circ}$. Scattering angles varied between $\sim 16^{\circ}$ and $\sim 70^{\circ}$ (50 distinct values); bistatic angles varied $40^{\circ}$ either side of in-plane with a $2.5^{\circ}$ step (33 distinct values). Bottom returns were picked through two methods (automatic and manual) and converted into scattering strengths, accounting for the size of the scattering patch. This large dataset has been compared with the model of Jackson and Williams for bistatic scattering, intended for 10-100 kHz but successfully tested at $240 \mathrm{kHz}$ by other workers. Recursive fitting of model parameters to the experimental values shows the influence of sediment variations (backed by microscopic measurements of actual grain size distributions) and the importance of even small tilts in the surface of the sediments. These results can be used to test the validity of extending this scattering model to higher frequencies. 
3aUWd4. Dispersion and attenuation due to scattering from heterogeneities in the frame bulk and shear moduli of sand sediments. Brian T. Hefner (Applied Physics Laboratory, University of Washington, 1013 NE 40th St, Seattle, WA 98105, USA, hefner@apl.washington.edu), Darrell R. Jackson (Applied Physics Laboratory, University of Washington, 1013 NE 40th Street, Seattle, WA 98105, USA, drj@apl.washington.edu), Joseph Calantoni (Naval Research Laboratory, Marine Geosciences Division, Code 7440.3, Building 1005, MS 22217, USA, joec@nrlssc.navy.mil)

Previously, we presented the results of applying perturbation theory to the problem of fast compressional wave propagation through a Biot medium with heterogeneities in the frame bulk modulus (B. T. Hefner et al., J. Acoust. Soc. Am. 119, 3447 (2006)). It was found that the heterogeneities scattered energy into both the slow and fast compressional waves, thus increasing the attenuation of the fast compressional wave. This theory has since been generalized to account for heterogeneities in both the frame bulk and shear moduli. For the fast compressional wave, energy is now scattered into the shear wave as well as the fast and slow compressional waves, further increasing the attenuation of the coherent field. While shear wave propagation is unaffected by variations in the frame bulk modulus, scattering does occur when there are heterogeneities in the shear modulus. Energy in the shear wave is scattered into both shear and compressional waves as well. The generalized theory depends on the autocorrelation functions of both the shear and bulk moduli variations as well as the cross-correlation function between the moduli. Efforts are underway to estimate these statistics in simple random packings of spherical grains using discrete-element modeling. [Work supported by ONR.]

\section{2:40-2:00 Lunch Break}

\section{Invited Papers}

\section{2:00}

3aUWd5. Data-model comparisons for broadband sound speed and attenuation in sandy seabed. Ji-Xun Zhou (School of Mech. Eng., Georgia Institute of Technology, 771 Ferst Drive, Atlanta, GA 30332-0405, USA, jixun.zhou@me.gatech.edu), Xue-Zhen Zhang (School of Mech. Eng., Georgia Institute of Technology, 771 Ferst Drive, Atlanta, GA 30332-0405, USA, xuezhen.zhang@me .gatech.edu)

Sound speed and attenuation in sandy seabed were analyzed and reviewed from low-frequency (LF) acoustic measurements, conducted at 18 locations in different coastal zones around the world. [Zhou and Zhang, JASA 117(4), 2494 (2005) and 119 (5), 3447 (2006)]. The resultant sound speed and attenuation in a frequency range of $50-2000 \mathrm{~Hz}$ in sandy bottoms can be described equally well by the Biot-Stoll poro-elastic model, the Chotiros BICSQS model [JASA 116(4), 2011-2022 (2004)] or the Buckingham VGS model [JASA 122(3), 1486-1501(2007)]. The geophysical parameters were inverted from the best match between the LF data and each of these three geo-acoustic models. The values of the inverted geophysical parameters were reasonable based on both theoretical considerations and published experimental measurements. A combination of the LF field-inverted data with data from the SAX99 experiment and other recent high-frequency measurements offers a broadband reference data set of sound velocity and attenuation for sandy bottoms in shallow water over a frequency range of $50-400,000 \mathrm{~Hz}$. The data-model comparisons show that, with one set of adjustable input parameters, all three geo-acoustic models may perfectly match either the broadband velocity dispersion or broadband attenuation, but not both. These models generally either underestimate dispersion or overestimate LF attenuation. [Work supported by ONR.]

3aUWd6. Role of heterogeneity for sound speed and attenuation in unconsolidated ocean sediments. Jim G. Berryman (Lawrence Berkeley National Laboratory, 1 Cyclotron Road MS90R1116, Berkeley, CA 94720, USA, JGBerryman@LBL.GOV), Steven R. Pride (Lawrence Berkeley National Laboratory, 1 Cyclotron Road MS90R1116, Berkeley, CA 94720, USA, SRPride@LBL.GOV)

Although ocean sediments may have many types and degrees of heterogeneity, it has been determined that two-component models are tractable for poroelastic, thermoelastic, and thermoporoelastic studies. We have found in particular that a substantial part of the increased sound wave attenuation observed in both ocean sediments and laboratory glass-bead experiments above $50 \mathrm{kHz}$ can be explained by such a two-component poroelastic model. Furthermore, the inclusion of thermal expansion and entropy effects into a generalized binary thermoporoelastic model provides an additional attenuation enhancement (though not as large --- by itself --- as the poroelastic enhancement) of a mathematically very similar type that is also relatively easy to model and understand. The paper will present this extended model as well as comparisons to available data.

\section{2:40}

3aUWd7. Frequency and grain size dependence of longitudinal wave velocity in granular marine sediments. Masao Kimura (Tokai Univ., 3-20-1 Orido, Shimizu-ku, 424-8610 Shizuoka, Japan, mkimura@scc.u-tokai.ac.jp)

Frequency dependence of longitudinal wave velocity in granular marine sediments is important in underwater acoustics. Also, the grain size dependence is significant for the identification of sediments. In this study, the measurements of the velocity of three kinds of sands with different grain sizes are carried out in the frequency ranges from approximately $5 \mathrm{kHz}$ to $500 \mathrm{kHz}$ in the laboratory. The velocity at a low frequency is measured by using an acoustic tube method. Time-of-flight method is used for the measurements of the velocity at higher frequencies. These measured results are compared with the calculated results by using the Biot-Stoll model and a modified gap stiffness model incorporated into the Biot model (BIMGS model). It is shown that the frequency and grain size dependence of velocity can be explained by using the BIMGS model. 
3aUWd8. Biot-Stoll with squirt flow and shear (BICSQS) model: high-frequency correction. Nicholas P. Chotiros (Applied Research Laboratories, University of Texas, PO Box 8029, Austin, TX 78713-8029, USA, chotiros@arlut.utexas.edu)

The modeling of the grain to grain shear stiffness within the larger Biot-Stoll plus contact squirt flow and shear (BICSQS) model [N. P. Chotiros and M. J. Isakson, J. Acoust. Soc. Am. 116(4), 2011-2022, 2004] assumes Poiseuille flow, which must become invalid at high-frequencies. Specifically, the viscous shear drag term will be unphysically large. A correction is introduced based on Biot's solution for oscillatory flow between two parallel plane boundaries [M. A. Biot, J. Acoust. Soc. Am. 28, 179-191, 1956]. Although the original solution was derived for oscillatory fluid flow relative to stationary parallel plane boundaries, it can be readily adapted for oscillatory motion of the boundaries in opposite directions relative to the fluid, as in shear drag at the grain to grain contact. This correction extends the frequency range of the BICSQS model. Theoretical results, with and without the correction, will be compared. Work supported by the Office of Naval Research, Ocean Acoustics.

\title{
Contributed Paper
}

$3: 20$

3aUWd9. Use of dual methods to infer methane bubble populations in gassy sediment: Inversion of propagation data. Gary B. Robb (National Oceanography Centre, University of Southampton Waterfront Campus, European Way, SO14 3ZH Southampton, UK, gbor199@noc.soton.ac .uk), Timothy G. Leighton (Institute of Sound and Vibration, Univ. of Southampton, University Road, Highfield, SO17 1BJ Southampton, UK, T.G.Leighton@soton.ac.uk), Agni Mantouka (Institute of Sound and Vibration, Univ. of Southampton, University Road, Highfield, SO17 1BJ Southampton, UK, am2@isvr.soton.ac.uk), Angus I. Best (National Oceanography Centre, University of Southampton Waterfront Campus, European Way, SO14 3ZH Southampton, UK, aib@noc.soton.ac.uk), Justin K. Dix (National Oceanography Centre, University of Southampton Waterfront Campus, European Way, SO14 3ZH Southampton, UK, jkd@noc .soton.ac.uk), Victor F. Humphrey (Institute ofSound and Vibration, Univ. of Southampton, University Road, Highfield, SO17 1BJ Southampton, UK, vh@isvr.soton.ac.uk), Zygmunt Klusek (Institute of Oceanography, Polish
Academy of Science, P.O. Box 148 Sopot, Poland, klusek@iopan .gda.pl), Paul R. White (Institute of Sound and Vibration, Univ. of Southampton, University Road, Highfield, SO17 1BJ Southampton, UK, prw@isvr.soton.ac.uk)

The inversion of the acoustic properties of gassy sediments presents the optimum manner of determining the in situ distribution of sediment-based methane bubbles. An in situ device that measures both compressional wave attenuations and combination-frequency components in gassy sediment lying within $2 \mathrm{~m}$ of the seabed has been developed at the University of Southampton. This device was deployed at an inter-tidal site along the South coast of England. Compressional wave attenuations were measured from 10 to $100 \mathrm{kHz}$ though the analysis of propagation signals transmitted from a variety of sources to a buried co-linear hydrophone array, with propagation distances spanning 0.5 to $2 \mathrm{~m}$. Measured attenuations were inverted to infer in situ bubble size distributions using both established and new acoustic models for gassy sediment. The analysis and results of the combinationfrequency component are described in a companion paper.

\section{Session 3pAA}

\section{Architectural Acoustics: Architectural Acoustics Potpourri I}

\author{
David T. Bradley, Cochair \\ 124 Raymond Avenue, Poughkeepsie, NY, 12604
}

Andrea Farnetani, Cochair

University of Ferrara, Via Saragat 1, 44100 Ferrara, Italy

\section{Contributed Papers}

\section{2:00}

3pAA1. Shape optimization of polygonal rooms for a correct modal distribution at low frequencies based on psychoacoustic criterion. Sergio Floody (Universidad Tecnologica de Chile INACAP, Brown Norte 290, Nunoa, 7790569 Santiago, Chile, sfloody@utc.cl), Rodolfo Venegas (University of Salford, Acoustics Research Centre, Newton Building, M5 4WT Salford, UK, R.G.VenegasCastillo@pgr.salford.ac.uk)

Resonances in small rooms may lead to inadequate frequency responses. In rooms, where the exigencies on the listening conditions are important, these resonances may cause non wanted coloration effects, which implies a non desirable sound quality. By choosing the right shape and dimensions it is possible to reduce the audible effects of these resonances. The presented methodology aims to determine the shape and size of small and medium polygonal-shaped rooms based on the finite element method for modeling the physical acoustic behavior of the room; a neural network for loudness estimation and genetic algorithm for estimating the optimal dimensions. A comparison with previous techniques used to choose the dimension of rectangular room is also presented.

$$
\text { 2:20 }
$$

3pAA2. The reflected sound field by curved surfaces. Martijn Vercammen (Peutz, De Grippen 1124, 6605 TA Wijchen, Netherlands, m.vercammen@mook.peutz.nl)

Many spaces have curved walls or ceilings. With improved building technology and new fashions in architecture (blobs) there is an increasing number of problems due to the acoustic reflections by these surfaces. Sound reflected by concave surfaces will concentrate in a narrow area. In practical applications of room acoustics these curved surfaces will be calculated with mirror imaging or ray tracing programs, in which the structure is modeled by flat segments. Alternative is a geometrical approach. Both methods do 
not correspond to reality. The only valid calculation method is the calculation from a wave extrapolation method. It is shown that a theoretical correct solution of the sound field by curved surfaces is possible. A fairly simple expression for the sound pressure in the focal point is found and a more complicated description of the reflected sound field by small curved surfaces is presented. With these results the sound field in field applications can be calculated.

\section{2:40}

3pAA3. Calculation of temporal evolution of sound pressure levels in rooms, based on diffuse reflection. René Gamba (Gamba Acoustique, 2 rue de la découverte, BP 163, 31676 Labege Cedex, France, rene.gamba@acoustique-gamba.fr), Guillaume Cazard (Gamba Acoustique, 2 rue de la découverte, BP 163, 31676 Labege Cedex, France, guillaume.cazard@acoustique-gamba.fr), Claude Senat (Gamba Acoustique, 2 rue de la découverte, BP 163, 31676 Labege Cedex, France, claude .senat@acoustique-gamba.fr)

This paper describes a model which enables the temporal evolution of sound pressure levels in rooms to be calculated. It is built on the assumption that sound waves are totally scattered when reflected by the walls. This model prescribes the sampling of all the surfaces of the room and defines a process of time discretization, process which enables the temporal evolution of energetic exchanges between each sample to be known. Ultimately, the model allows the sound pressure level to be calculated in every point of the room for each time sample $\Delta \mathrm{t}$ (echograms). Thanks to the echograms, some useful criterias for room acoustic studies can be evaluated : reverberation time, EDT, D50, C80,... Measurements and calculations have been carried out for different kinds of rooms. We will describe them in the second part of this paper.

\section{3:00}

3pAA4. Sound absorption with fibre-free sintered aluminium in combination with thermally activated concrete slabs. Wouter Rottiers (Sonogamma, P.O.Box 49, Heverlee Ambassade, 3001 Leuven, Belgium,info@sonogamma.com)

Due to increasing ecological awareness and growing requirements of human well-being in working environments, contemporary interior design requires sustainable solutions. Consequently, cooling ceilings are an efficient alternative to conventional air conditioning systems with high energy demands. In order to maintain its thermal efficiency however, the ceiling surface must remain uncovered as much as possible. Conventional perforated or grooved acoustical materials allow for sound absorption only in combination with mineral wool or foam, thus presenting a thermal isolating barrier: they obviously decrease the performance of a thermal ceiling. Porous panels made of pure sintered aluminium offer a conductivity up to 96 $\mathrm{kcl} / \mathrm{mhC}$ with a thermal radiation of $18.4 \mathrm{~W} / \mathrm{m}^{2} \mathrm{~K}$, combined with a broadband sound absorption up to $\alpha \mathrm{w}=0.7$ without the use of any additional thermally isolating material. The panels meet the environmental, hygienic and fire safety requirements: they are fibre-free, non-combustible (Euroclass A1), rust-, moisture- and chlorine-proof and can be recycled. The unvisible porosity does not give away the outstanding acoustical qualities. Available in several designs and any colour, the sheets are optically identical to a plain, matt aluminium panel. Several possible configurations are discussed.

\section{$3: 20$}

3pAA5. Sound reduction of open noise screens. Jan Hardlooper (Cauberg-Huygen Consulting Engineers, Postbus 9222, 3007 AE Rotterdam, Netherlands, j.hardlooper@chri.nl)

Open noise screens consist of vertical absorbing screens with a depth of 1 meter, placed perpendicular to a building with a mutual distance of 1 meter. The screens are placed at a distance of approximately 1,5 meter. Open noise screens combine a sound reduction of $16 \mathrm{~dB}$ with a very open character for ventilation and daylight admittance. Measurements are carried out on a scale model (1:40) in a laboratory and in situ on a real size mock-up built in a 40-ft container. The results of the mock-up are comparable to the laboratory results. Frequency analysis shows the acoustical principles of the screens and the influence of several parameters. A calculation model is developed tot predict the results for other configurations. The principle of open noise screens is successfully applied for 200 new dwellings in Amsterdam. Suggestions are given for applying open noise screens near highways and railroads, instead of traditional closed screens. 


\title{
Session 3pAB
}

\section{Animal Bioacoustics, Psychological and Physiological Acoustics, and ECUA: Auditory Brainstem Response and Behavior Correlation I}

\author{
Elizabeth Brittan-Powell, Cochair \\ Dept of Psychology, University of Maryland, College Park, MD 20742, USA
}

Alexander Y. Supin, Cochair

Institute of Ecology and Evolution, 33 Leninsky Prospect, Moscow, 119071, Russian Federation

\section{Invited Papers}

\section{2:00}

3pAB1. Auditory brainstem responses in birds: How well do they compare to behavioral techniques for assessing hearing? Elizabeth Brittan-Powell (Dept of Psychology, University of Maryland, College Park, MD 20742, USA, bbrittanpowell@psyc .umd.edu), Bernard Lohr (Department of Biological Sciences, Northern Kentucky University, SC 204D, Nunn Drive, Highland Heights, AK 41099, USA, lohrb1@nku.edu), Isabelle Noirot (Dpt of Science/Oceanography, University of Liege, Bat B6c, 4000 SartTilman, B-4000 Liege, Belgium, isa_noirot@hotmail.com), Robert Dooling (Dept of Psychology, University of Maryland, College Park, MD 20742, USA, dooling@psyc.umd.edu)

Behavioral methods are the gold standard for assessing hearing sensitivity in birds. In many cases, however, behavioral techniques are simply impractical. The non-invasive auditory brainstem response (ABR) represents perhaps the next best approach, and researchers are increasingly turning to the ABR to obtain estimates of hearing sensitivity, auditory system function and development. Our lab has evaluated hearing sensitivity in over 11 species of birds using the ABR, and for six of these species, we have also obtained auditory thresholds by behavioral conditioning methods. These data, along with other results from the literature on bird hearing, now allow general conclusions about the use of ABR as a tool for measuring auditory sensitivity in birds. Waveform morphology is highly conserved across orders of birds. Regardless of how threshold is defined, the ABR audiogram reliably reflects the frequency range of hearing and the shape of the behavioral audiogram. However, ABR thresholds are higher than behavioral thresholds by $20-30 \mathrm{~dB}$ but with some variation across species. Aside from audiograms based on simple detection thresholds for tone bursts, ABR methods in birds have also been useful in assessing auditory development, hearing impairment, recovery of function with hair cell regeneration, masked thresholds and aspects of spectral and temporal processing.

$$
2: 20
$$

3pAB2. The Auditory Brainstem Response (ABR) Across Mammalian Species. Robert Burkard (University at Buffalo, Department of Rehabilitation Science, 511 Kimball Tower, Buffalo, NY 14214, USA, rfb@buffalo.edu)

The auditory brainstem response $(\mathrm{ABR})$ has been reported in a variety of mammalian species. I have published ABR studies in mammalian species including humans, gerbils, cats, rats, bats, and mice. In many cases, the non-human work was designed to extend observations made in humans. I will discuss data that addresses the variability in the slope of the ABR latency/intensity function across animal species. I will compare ABRs in humans and in gerbils to stimulus manipulations such as click level, click rate, noiseburst risetime and level of masking noise. I will discuss the effects of high stimulus rates across age in the kitten/cat, and compare this to reports in humans from the literature. I will end by discussing my views of the advantages as well as the challenges of using animal models when interested in human normative or pathologic processes.

\section{Contributed Paper}

\section{2:40}

3pAB3. Arbitrary evoked potentials: using AEPs to measure hearing in fishes. David Mann (University of South Florida, College of Marine Science, 140 7th Ave. S., St. Petersburg, FL 33701, USA, dmann@marine .usf.edu), Randy Hill (University of South Florida, College of Marine Science, 140 7th Ave. S., St. Petersburg, FL 33701, USA, randyjhill@yahoo .com)

Auditory evoked potential (AEP) techniques have become commonly used to measure hearing of a wide variety of animals. However, there is no standard way of defining a threshold from auditory evoked potential data. There are two arbitrary factors affecting the precision of AEP data: the number of sweeps averaged together to detect the AEP, and the method of calculating a threshold from the AEP data. While these arbitrary factors do not invalidate comparative studies where these variables are standardized, different laboratories use different techniques. We describe sources of variation in estimating thresholds using evoked potential techniques using the goldfish as an example. There was little variation in AEP thresholds due to variation in electrode placement or in the method of arbitrarily assigning an AEP threshold. The largest variation was due to differences in the number of sweeps averaged. Under controlled conditions using the same goldfish in the same test tank with the same number of sweeps averaged, AEP's better predicted behavioral thresholds at high frequencies than low frequencies. Since averaging reduces uncorrelated background noise (neural and electrical), it should theoretically be possible to obtain AEP measurements below behavioral thresholds with enough sweeps averaged together. 


\title{
Invited Papers
}

\section{3:00}

3pAB4. False killer whale hearing adjustment during echolocation measured with evoked potentials. Paul E. Nachtigall (University of Hawaii, Hawaii Institute of Marine Biology, P.O. Box 1106, Kailua, HI 96734, USA, nachtiga@hawaii.edu), Alexander Y. Supin (Institute of Ecology and Evolution, 33 Leninsky Prospect, 119071 Moscow, Russian Federation, alex_supin@mail.ru)

While much has been previously learned about the echolocation performance and characteristics of the outgoing signals of echolocating dolphins and small whales, we have measured hearing using evoked potentials during echolocation. We have found that: (1) the whale may hear her loud outgoing clicks and much quieter returning echoes at comparable levels, (2) the whale has protective mechanisms and hears her outgoing signals at a level about $40 \mathrm{~dB}$ lower than similar signals presented directly in front of her, (3) when echo return levels are lowered either by making the targets smaller or by placing the targets farther away - without changing the levels of her outgoing signals, the hearing of those echoes remains at almost the same level, (4) if targets are made much smaller and harder to echolocate, the animal will increase what she hears of her outgoing signal - as if to heighten overall hearing sensitivity to keep the echo level hearable, and (5) the animal has an active 'automatic gain control' mechanism in her hearing based on both forward masking that balances outgoing pulse intensity and time between pulse and echo and active hearing control. Overall, hearing during echolocation appears to be an actively changing process.

$$
\text { 3:20 }
$$

3pAB5. Temporal resolution in the hearing system and auditory evoked potentials. Kristian Beedholm (Institute of Biology, University of Southern Denmark, Campusvej 55, 5230 Odense M, Denmark, beedholm@mail.dk), Lee A. Miller (Institute of Biology, University of Southern Denmark, Campusvej 55, 5230 Odense M, Denmark, lee@biology.sdu.dk)

A popular type of investigation with auditory evoked potentials (AEP) consists of mapping the dependency of the envelope following response to the AM frequency. This results in what is called the modulation rate transfer function (MRTF). The physiological interpretation of the MRTF is not straight forward, but is often used as a measure of the ability of the auditory system to encode temporal changes. It is, however, shown here that the MRTF must depend on the waveform of the click-evoked AEP (ceAEP), which does not relate directly to temporal resolution. The theoretical relationship between the spectrum of the ceAEP and MRTF is such that the MRTF should be identical to the ceAEP if a $1 / \mathrm{f}$ weighting is applied to the ceAEP. Deviations from this relationship indeed reflect temporal resolution capabilities. We measured this in a harbour porpoise. Our devised stimulus was a sequence of $0.5 \mathrm{~ms}$ Hann weighted $130 \mathrm{kHz}$ tone pips presented at an increasing rate (chirped) over a time span of $32 \mathrm{~ms}$. The results reveal that the system's responsiveness declines roughly exponentially as a function of click rate with a rate constant of about $-0.7 \mathrm{kHz}$ and appears more rate limited than implied by traditional MRTF.

WEDNESDAY AFTERNOON, 2 JULY 2008

AMPHI MAILLOT, 1:40 TO 3:40 P.M.

Session 3pMUa

\section{Musical Acoustics: Bowed and Keyboard Stringed Instruments I}

\author{
Knut Guettler, Cochair \\ Norwegian Academy of Music, P.O.Box 5190 Majorstuen, Oslo, 0302, Norway \\ Claudia Fritz, Cochair \\ University of Cambridge, Music Faculty, West Rd, Cambridge, CB3 9DP, UK
}

\section{Contributed Papers}

$1: 40$
3pMUa1. On the dynamics of the clavichord. Christophe D’Alessandro (LIMSI-CNRS, B.P. 133, 91403 Orsay, France, cda@limsi .fr)

The clavichord is generally considered as the most sensitive and subtle among keyboard instruments. The player/instrument interaction is very direct: the mechanism is reduced to as simple lever, allowing for a direct contact between the finger and string through the key. Key velocity, two string-tangent contact signals, radiated acoustic signal have been synchronously measured for about 10 dynamic nuances and all the notes of four instruments (a 51 notes unfretted instrument, after a German XVIIIth century model, a 51 notes fretted instrument after Hubert (1754), a 45 notes fretted instrument, after a German XVIIth century model, a 37 notes fretted instrument after a medieval model). The instruments can be portrayed in terms of dynamic range, tonal/spectral colour and sound decay time. As for the dynamics, there is some evidence for a linear relationship between sound pressure level and the velocity of the tangent; and an almost constant spectral richness independently of loudness (in contrast with e.g. the piano). A simple model of the tangent/string interaction is proposed. This model reproduces well the behaviour of experimental data, and it may explain why sound quality of the clavichord depends much on the player's ability.

\section{2:00}

3pMUa2. Couchet Harpsichord soundboard vibroacoustics behaviour: An application of the Impact Nearfield Acoustical Holography (IPNAH). Sylvie Le Moyne (UPMC Univ Paris 06, CNRS UMR 7190 Institut Jean Le Rond D'Alembert, 2 Place de la Gare de Ceinture, 78210 Saint Cyr l'Ecole, France, slemoyne@ccr.jussieu.fr), Sandie Le Conte (Musée de la Musique, 
221 avenue Jean Jaures, 75019 Paris, France, sleconte@cite -musique.fr), François Ollivier (UPMC Univ Paris 06, CNRS UMR 7190 Institut Jean Le Rond D'Alembert, 2 Place de la Gare de Ceinture, 78210 Saint Cyr 1'Ecole, France, frol@ccr.jussieu.fr)

The Music Museum in Paris recently acquired a harpsichord made by Ioannes Couchet in 1652 in Anvers. As a masterpiece this instrument is considered as a "National Treasure" and therefore protected. A challenging problematic has risen when its restoration was decided since the aim was to play this instrument again in concert. In the objectives of increasing our understanding of the harpsichord ageing, improve a numerical model currently in process and develop a diagnostic method for conservation, an experimental modal analysis of the soundboard was performed by processing its sound field. A non intrusive method, the Impact Planar Nearfield Acoustic Holography, was used. This technique, developed by the authors, implements the well known inverse method NAH on the basis of the acoustic impulse response field and is well adapted to modal analysis. NAH is performed here in unusual conditions compared to literature, as they are far from the ideal: unbaffled source, low sound pressure level, unusually large measurement distance, preponderance of evanescent waves. An additional challenge was to muffle strings, as they should not be removed nor slackened. However, a very satisfying modal decomposition for [30-1500Hz] bandwidth is obtained. Results are confronted with literature and an energetic analysis is proposed.

\section{2:20}

3pMUa3. What have they done to the Strads? John McLennan (University of New South Wales, Music Acoustics, School of Physics, NSW 2052 Sydney, Australia, jmc11597@bigpond.net.au), Joe Wolfe (University of New South Wales, Music Acoustics, School of Physics, NSW 2052 Sydney, Australia, J.Wolfe@unsw.edu.au)

Almost every baroque violin, including those of Stradivarius etc, was extensively modified during the conversion to the romantic or modern configuration. What were the acoustic and playing changes? To answer this question, independently of the confounding factors of wood and manufacture, a baroque violin was made and subjected to acoustic and playing tests, before and after (i) replacement of the neck and fingerboard with longer, heavier more inclined parts, (ii) replacing gut with modern nylon-cored strings. Other changes were also made, including bridge style and position, bass bar and soundpost sizes and bow used. Loudness (Saunders plot) was not greatly changed, except for the E string with a long string length. Some acoustic features survived the changes, and professional baroque and modern style players reported that the instrument preserved some of its "personality". Comparisons were made with modern gut strings used by professional baroque players. As well as the acoustic differences, player assessments of bright/dull, full/thin, open/closed, ease of response, evenness, dynamic range are reported. Averaged over all ratings, the players ranked the romantic set up slightly better than the baroque (78\% vs $71 \%$ ).

\section{2:40}

3pMUa4. Perceptual studies of violin vibrato. Claudia Fritz (University of Cambridge, Music Faculty, West Rd, CB3 9DP Cambridge, UK, cf291@cam.ac.uk), Jim Woodhouse (University of Cambridge, Dept of Engineering, Trumpington St, CB2 1PZ Cambridge, UK, jw12@cam.ac.uk), Brian Moore (University of Cambridge, Department of Experimental Psychology, Downing Street, CB2 3EB Cambridge, UK, bcjm@cam.ac.uk), Ian Cross (University of Cambridge, Music Faculty, West Rd, CB3 9DP Cambridge, UK, ic108@cam.ac.uk)

Although it seems obvious to violinists that vibrato has a large influence on the perception of violin tone quality, published studies of violin vibrato have mainly concerned its influence on the sound from a scientific point of view, i.e. the characterisation of its time-frequency properties. Work will be described to explore the link between the perception of vibrato notes, the extent of frequency modulation and the level of damping of the resonance modes of the violin. Damping influences the "peakiness" of the frequency response function. The test methodology is based on liveliness ratings and triadic comparisons, where subjects have to select the most similar and the most different pair in each triad (presentation of three sounds). The sounds of the corpus are synthesised using sawtooth waves with frequency modulation, whose amplitude is varied, filtered through a set of admittances corresponding to a reference violin and two modified violins, one with the damping of all modes increased, the other one with the damping decreased.

\section{3:00}

3pMUa5. Performance comparison of violins through experimental force analyses. Enrico Ravina (University of Genoa - Centre of Research on Choral and Instrumental Music (MUSICOS), Via Opera Pia 15 A, 16145 Genova, Italy, enrico.ravina@unige.it), Paolo Silvestri (Univ. of Genoa DIMEC, Via Opera Pia 15 A, 16145 Genova, Italy, p.silvestri@unige.it), Federico Lowenberger (Master Violinmaker, Via Marussig 13, 16100 Genova, Italy, info@baroqueviolins.com)

The paper describes the most recent experiments developed on violins with different mounting (baroque, classical, modern) and specifically oriented to the dynamic forces analysis in significant points of the instruments. The vibrational behaviour of the soundboards are strongly influenced by the dynamic forces transmitted from the bridge: they are related also to the tension of the strings. An original testbench conceived to forces measurement has been set up: tensions on strings, forces detected under the bridge and deformations on the fingerboard and on the tailpiece are, in particular, monitored by means not intrusive thin-film tactile pressure measurement devices, micro load cells and micro strain gauges. Mapping of forces and strains are acquired in real time playing the instrument; the paper reports comparisons between differently mounted violins, playing notes under different techniques: continuous ("tenuta"), ghost ("strappata"), "detachet". Experiments are developed using different kind of strings (bowels, metallic,...). The results are used as input for experimental dynamic analyses and tests of the soundboard: in addition, they can be correlated to the acoustic response of the instrument.

\section{$3: 20$}

3pMUa6. Shaping and understanding sound: Violin makers, musicians and scientists from Renaissance to Romantism. Anne Houssay (Cité de la musique, 221, avenue Jean Jaurès, 75019 Paris, France, ahoussay@ citemusique.fr)

The training of instrument-makers in Renaissance Italy is linked to a rediscovery of theoretical works on physics and sound as well as to some new tools and skills. Then, the development of physics in the seventeenth and eighteenth century lead the establishment of acoustic as a modern science, with the distinction of partials from harmonics. The "western world" has given explanations on vibrations, modes and then elasticity theories, some of which have been included into general knowledge. By times, links between scientists and makers have developed and have participated to the development of musical instruments. Traditional violin makers are craftspeople, and they construct instruments by choosing step by step between many possibilities from the drawing of the model and the choice of materials, to the cutting out of the shapes to create air volumes and vibrating facings. Their parameters are the dimensions, shapes, weight, elasticity, quality and duration of sounds and notes. The test for judging the results is the playing of the instrument by a very competent musician. We will explore, with a point of view of the historian of techniques how the meeting of different cultures and knowledge have transformed the instruments from the Renaissance to the begining of the 19 th century. 
Session 3pMUb

\title{
Musical Acoustics: Wind Instruments I
}

\author{
Seiji Adachi, Cochair \\ Fraunhofer Institute for Building Physics, Nobelstrasse 12, Stuttgart, 70569, Germany \\ Simon Félix, Cochair \\ Laboratoire d'Acoustique de l'Université du Maine, Avenue Olivier Messiaen, Le Mans, 72085, France
}

\section{Contributed Papers}

$1: 40$

3pMUb1. Individual reed characteristics due to changed damping using coupled flow-structure and time-dependent geometry changing Finite-Element calculation. Rolf Bader (University of Hamburg, Institute of Musicology, Neue Rabenstr. 13, 20354 Hamburg, Germany, R_Bader@tonline.de)

The vibration of a reed of a saxophone is calculated using a 2D model of the mouth cavity, the mouth piece and the reed. The time-dependent FiniteElement calculation takes the flow-structure interaction into account and changes the geometry of the flow according to the reed vibration in time. The model is used to study the flow behaviour, pressure distribution, reed vibration and reaction to disturbances of the system. A constant pressure is assumed at the mouth cavity to simulate the pressure of the player's lungs. During the time-dependent process, an impulse is modeled at the end of the mouth piece travelling back from the end of the tube. The damping of the reed was changed to study the reed behaviour. Hard damped reeds show a simple and stable impulse behaviour causing a clear pressure impulse. When the damping is decreased, the impulse coming back from the end of the tube causes the reed not only to open and close but also to show additional vibrations caused by the interplay of the reed's eigenfrequencies and the pressure acting on the reed. These are more or less independent of the travelling impulse and so are a sound characteristic of the reed itself independent of the played note and used articulation.

\section{2:00}

3pMUb2. Aspects of vibrato and micromodulation in double reed instrument sounds. Michael Oehler (Institute for applied Musicology and Psychology, Saarstrasse 1A, 50677 Koeln, Germany, michael.oehler @iamp.info), Christoph Reuter (University of Cologne - Musicological Institute, Beethovenstrasse 4, 50674 Koeln, Germany, info@chr-reuter.de)

The perceived naturalness of real and synthesized oboe and bassoon vibrato sounds has been investigated in several perception experiments. The stimuli were generated by means of a currently developed synthesis and analysis framework for wind instrument sounds (first presented at 152nd ASA meeting), based on the pulse forming theory. The framework allows the control of amplitude and frequency parameters at different sound production levels. The stimuli were rated by 60 subjects from "natural" to "unnatural". A conducted ANOVA showed $(\mathrm{p}<.01)$ that the different types of modulation significantly affect the perceived naturalness of vibrato sounds: The synthesized stimuli with combined pulse width and cycle duration modulation (source modulation) are perceived as natural as the real sounds. The subsequently modulated synthesized stimuli (AM and/or FM near the end of the signal path) are perceived significantly less natural $(\mathrm{p}<.01)$. The results support the hypothesis, that source-affected timbre modulation is an important factor for the perceived naturalness of oboe and bassoon vibrato sounds. The use of the developed framework for wind instrument sounds is an alternative method to analyze (micro-)modulation effects. Further investigations may be useful for exploring new sound synthesis algorithms as well as for other experiments in the field of timbre research.
$2: 20$

3pMUb3. The influence of the mean flow on the transmission properties of wind instruments. Andrey R. Da Silva (Schulich School of Music, McGill University, 555 Sherbrooke Street West, Montreal, QC H3A 1E3, Canada, andrey.dasilva@mail.mcgill.ca), Gary Scavone (Schulich School of Music, McGill University, 555 Sherbrooke Street West, Montreal, QC H3A 1E3, Canada, gary@music.mcgill.ca)

The influence of mean flow on the transmission properties of wind instruments has traditionally been neglected due to the range of Strouhal numbers in which this family of instruments normally operate. However, this topic has gained considerable interest among the musical acoustic community during the last decade due to the appearance of new outcomes in research on duct acoustics. Nevertheless, very few contributions have investigated the effect of the mean flow on the transmission properties by taking into account the physical characteristics and dynamic peculiarities of wind instruments. The goal of this work is to present a numerical investigation of the influence of the mean flow on the end correction and on the magnitude of the reflection coefficient for different geometries and Strouhal numbers normally found in wind instruments. The results suggest that, excepting for a few cases, the mean flow can indeed be neglected and the aforementioned transmission properties can be described by the quiescent flow theory.

\section{2:40}

3pMUb4. Effect of bending portions of the air column on the acoustical properties of a wind instrument. Simon Félix (Laboratoire d'Acoustique de l'Université du Maine, Avenue Olivier Messiaen, 72085 Le Mans, France, simon.felix@univ-lemans.fr), Cornelis J. Nederveen (Acacialaan 20, 2641 AC Pijnacker, Netherlands, cjnederv@xs4all.nl), Jean-Pierre Dalmont (Laboratoire d'Acoustique de l'Université du Maine, Avenue Olivier Messiaen, 72085 Le Mans, France, Jean-Pierre.Dalmont @univ-lemans.fr), Joël Gilbert (Laboratoire d'Acoustique de l'Université du Maine, Avenue Olivier Messiaen, 72085 Le Mans, France, joel.gilbert @univ-lemans.fr)

The need to keep long wind musical instruments compact imposes the bending of portions of the air column. Although manufacturers and players mention its effects as being significant, the curvature is generally not included in physical models and only a few studies, in only simplified cases, attempted to evaluate its influence. The aim of our study is to quantify the influence of the curvature by modelling the wave propagation in an air column with a multimodal formalism. In a duct with a circular cross-section and a finite curvature, two infinite sets of coupled first-order differential equations are constructed for the components of the pressure and axial velocity, projected on the local transverse modes. From these an impedance matrix is defined, which can be easily calculated, particularly when considering a duct with a piecewise constant curvature. Influence of the curvature on the input impedance, effective length, or playing frequencies is then quantified, displaying notably a dependence to frequency such that, compared to an equivalent straight tube, the shift in resonance frequencies in a tube with bent sections is not always positive, as generally stated. Results are independently corroborated by numerical - finite differences computations. 
3:00

3pMUb5. Numerical investigations of the bassoons aeroacoustic. Andreas Richter (Technische Universität Dresden, Institute for Aerospace Engineering, 01062 Dresden, Germany, andreas.richter4@tu-dresden.de), Roger Grundmann (Technische Universität Dresden, Institute for Aerospace Engineering, 01062 Dresden, Germany, roger.grundmann@tu-dresden.de)

Solving the compressible, unsteady Navier-Stokes equations allows us to track single traveling waves in conjunction with a nonuniform superposed mean flow. Significant physical phenomena like wave steepening, viscothermal losses and acoustic streaming can be investigated. This may be helpful to understand the acoustical characteristics of different geometries, especially in musical woodwind instruments. Since acoustic perturbations are usually small compared to the driving pressure, the solution demands highorder accuracy and appropriate boundary conditions. We present a collection of our numerical results based on two- and threedimensional simulations of the aeroacoustic behavior inside the bassoon. This includes both simulations of the whole instrument under real playing conditions and also numerical studies of subsections of the instrument like tone holes. Here especially viscothermal effects are analyzed. Nonlinear effects like acoustic streaming which is primarily present in the crook of the instrument are also investigated and discussed. Flow field measurements based on Particle Image Velocimetry and pressure measurements inside and outside the instrument are performed to validate the earned results and show a good agreement between measurements and numerical results.
3:20

3pMUb6. Analysis of the timbre of Slovak folk reed aerophones using source-filter model. Milan Rusko (Institute of Informatics of the Slovak Academy of Sciences, Dubravska cesta 9, 84507 Bratislava, Slovakia, milan.rusko@savba.sk)

Bagpipes are the only group of traditional folk reed instruments that have survived in Slovak folk culture until now. Unlike Scottish highland pipe, gaita, cornemuse etc., all the types of Slovak bagpipes use single reeds in both chanters and drones. Moreover the horns of the drones are partly closed with a plate having only a small opening for air output and sound radiation. This forms a Helmholz-like resonator - acoustic filter having a strong influence on the acoustic and aesthetic properties of the sound. The ideal of the sound timbre of these pipes is "less open" (compare to open and closed vowels in speech) and, in spite of its relatively high loudness, "less agressive" in comparison to pipes with open horns. This paper presents our approach to study the ideal of sound timbre of the Slovak bagpipe using a source-filter all-pole model. To identify resonance frequencies/formants, we applied inverse filtering technique using discrete linear prediction. Some properties of the source (reed) were studied using parameters like amplitude qutient and open quotient. The possible relations between timbre of speech sounds (vowels) and the timbre of the sound of musical instruments are discussed.

\title{
Session 3pNSa
}

\section{Noise, ASA Committee on Standards, and EURONOISE: Sleep Disturbances and Other Health Effects}

\author{
Lily M. Wang, Cochair \\ University of Nebraska - Lincoln, 1110 S. 67th St., Omaha, NE 68182-0681, USA \\ Barbara Griefahn, Cochair \\ Institute for Occupational Physiology, Ardeystr. 67, Dortmund, 44139, Germany \\ Jacques Lambert, Cochair \\ Laboratoire Transport et Environnement, INRETS, 25, Avenue François Mitterrand, F-69675 BRON cedex, France
}

\section{Invited Paper}

\section{$1: 40$}

3pNSa1. Associations between road traffic noise level, road traffic noise annoyance and high blood pressure in the HYENA study. Wolfgang Babisch (Federal Environmental Agency, Corrensplatz 1, 14195 Berlin, Germany, wolfgang.babisch @ uba.de), Danny Houthuijs (The National Institute For Public Health and Environmental Protection, PO Box 1, 3720 B A Bilthoven, Netherlands, danny.houthuijs@rivm.nl), Goran Pershagen (Institute of Environmental Medicine, Karolinska Institutet, Box 210, 17177 Stockholm, Sweden, goran.pershagen@ki.se), Klea Katsouyanni (Department of Hygiene and Epidemiology, Medical School, University of Athens, 75 Mikras Asias Str, 11527 Athens, Greece, kkatsouy@med.uoa.gr), Manolis Velonakis (Laboratory of Prevention, Nurses School, University of Athens, 123 Papadiamadopoulou St, 11527 Athens, Greece, evelonak@nurs.uoa.gr), Ennio Cadum (Piedmont Regional Environmental Protection Agency, Via Sabaudia 164, 10095 Grugliasco (TO), Italy, e.cadum @arpa.piemonte.it), Lars Jarup (Imperial College London, Norfolk Place, W2 1PG London, UK, 1.jarup@imperial.ac.uk)

The HYENA study is a multi-centred study regarding the effects of aircraft noise and road traffic noise on blood pressure (BP) which was funded by the European Community. Study subjects were 4,861 males and females aged between 45 and 70 years, who had lived for at least 5 years in the vicinity of any of six major European airports. Aircraft noise contours and road traffic noise levels were modelled using the Integrated Noise Model (INM) and national calculation methods. The noise levels were linked to each participant's home address (most exposed façade) using graphical information systems (GIS). Noise annoyance was assessed using the 11-point ICBEN scale. High blood pressure was determined by measurements of systolic and diastolic blood pressure, anti-hypertensive medication and self-reported doctor diagnosed hypertension. The focus here is on the effects of road traffic noise. The road traffic noise level 
(LAFm, 24 hours) and noise annoyance due to road traffic noise were both significantly associated with high blood pressure. The association between road traffic noise and high blood pressure was stronger for the road noise level than for the road noise annoyance (90th percentile vs. 10th percentile of the exposure distributions).

\section{Contributed Paper}

$$
\text { 2:00 }
$$

3pNSa2. Computing Number of People Awakened by Aircraft Operations Noise. Nicholas P. Miller (Harris Miller Miller \& Hanson Inc., 77 South Bedford Street, Burlington, MA 01803, USA, nmiller@hmmh .com)

The acoustics literature documents a number of field studies in which aircraft noise was measured in people's bedrooms while their awakening was simultaneously monitored. Nearly all the field studies produced a doseresponse relationship between noise and an awakening response. These dose-response relationships generally show good agreement with each other. Virtually without exception, however, these dose-response relationships per- tain to (1) the indoor noise dose produced by a single aircraft flyover and (2) the chances that the noise dose will awaken an average person. These doseresponse relationships are too limited for application to a full night of operations and to a realistic population of varying individual sensitivities to noise-induced awakenings. The dose-response relationships do not account for multiple aircraft exposures during the night or for person-to-person variation in how deeply different people sleep. This presentation first briefly reviews a method previously reported for applying the study data to a full night of operations, accounting for time of night and for individual sensitivity to awakening. It then compares percent of population awakened for realistic situations, and shows the effects of including or excluding time of night or individual sensitivities to awakening.

\section{Invited Paper}

3pNSa3. Determination of noise sensitivity within an internet survey using a reduced version of the Noise Sensitivity Questionnaire. Barbara Griefahn (Institute for Occupational Physiology, Ardeystr. 67, 44139 Dortmund, Germany, griefahn@ifado .de)

The Noise Sensitivity Questionnaire (NoiSeQ), that determines noise sensitivity as a moderator of annoyance globally and separately for different everyday activities is with 35 items too long for extended social surveys. This study aimed at the development of a shorter version. Using 429 questionnaires (266 women, 163 men, 16-74 yrs) three factor analyses were performed leading to the NoiSeQ-R that consists of the three subscales 'Sleep', 'Habitation', and 'Work' with 4 items each. The scores are normally distributed, internal consistency is $\alpha=0.87$ and test-retest reliability varies between rtt $=0.66$ and 0.74 depending on the time gaps that varied between 1 to 37 months. To test the validity the NoiSeQ-R was applied to a field study where 190 residents (102 males, 88 females, 17-80 years, median: 51 years) in the vicinity of a large airport rated their chronic annoyance (for the previous 12 months) and their actual annoyance hourly during four consecutive days. As expected, noise sensitivity did not correlate with the individual noise load but significantly with annoyance and with age. Thus the NoiSeQ-R, which is available in ten languages, is regarded as a reliable and valid instrument which can be easily applied in even extended surveys.

\section{Contributed Paper}

\section{2:40}

3pNSa4. A case study on the validation of models that predict the impact of aircraft noise on sleep. Sarah McGuire (Ray W. Herrick Lab., School of Mechanical Engineering, Purdue Univ., 140 S. Martin Jischke Drive, West Lafayette, IN 47907-2031, USA, mcguires@purdue .edu), Patricia Davies (Ray W. Herrick Lab., School of Mechanical Engineering, Purdue Univ., 140 S. Martin Jischke Drive, West Lafayette, IN 47907-2031, USA, daviesp@ecn.purdue.edu)

The impact of aircraft noise on sleep disturbance is not accurately assessed by cumulative noise metrics that are often used to predict community impact, such as $L_{\mathrm{dn}}$, because sleep disturbance is highly dependent on the noise level of individual events. Most existing sleep disturbance models are dose-response relationships that relate the noise level of individual events, as measured by $\operatorname{SEL}(A)$ or $L_{A_{\max }}$ to the percent awakened, and independence of responses to individual events is assumed. There are a few models that also incorporate additional parameters such as time of night and noise sensitivity, and some that predict the impact of noise on sleep structure. In order to determine whether a model should be used as a general aircraft noise sleep disturbance prediction tool, its performance in a variety of situations must be evaluated. Data was collected from a number of sleep disturbance studies and comparisons were made between responses and model predictions of awakenings and sleep stages. The sufficiency of the collected data for estimating the parameters of more complex models of sleep disturbance is also discussed.

\section{Invited Paper}

3pNSa5. Effects of a hearing aid noise management feature on user perception and performance under noise. Lauren M. Ronsse (University of Nebraska - Lincoln, 1110 S. 67th St., Omaha, NE 68182-0681, USA, lronsse@mail.unomaha.edu), Lily M. Wang (University of Nebraska - Lincoln, 1110 S. 67th St., Omaha, NE 68182-0681, USA, LWang4@UNL.edu)

This paper presents the effects of a hearing aid noise management feature on user perception and performance under noise. Hearingimpaired subjects were exposed twice to seven different noise signals which simulate common mechanical system noise, once with the noise management feature enabled and once disabled. The seven noise signals vary in terms of level and spectral quality, but are all within the range of background noise conditions found in commercial offices. Performance is gauged on three types of tests (math, verbal and typing), while subjective perception is measured via a subjective questionnaire. The results of this investigation will show if the hearing aid noise management feature reduces detrimental effects caused by background noise. Additionally, the data from hearing- 
impaired subjects are compared to those from normal-hearing persons to assess if significant differences are present between the two groups. If so, they may lead to the development of different standards for noise criteria levels in spaces designed for the hearingimpaired. [Work supported by a Univ. of Nebraska Layman Award and an ASHRAE Graduate Student Grant-in-Aid]

\title{
Contributed Paper
}

$3: 20$

3pNSa6. Noise and health in the Greater Rotterdam Area. Henk Wolfert (DCMR EPA, P.O. Box 843, sGravelandseweg 565, 3100AV Schiedam, Netherlands, henk.wolfert@dcmr.nl)

In the Greater Rotterdam Area a second study to Noise and Health has been carried out in 2007 and 2008. In 2003 a first study was carried out and that study reported that around 12 percent of the people living in the Greater Rotterdam Area were highly annoyed by noise mainly caused by traffic. The number of people sleep was disturbed and suffered high blood pressure were reported and amounted to 6 percent for sleep disturbance and 3 percent were suffering hypertension. The 2003 study took place within the regularly framework of the Rotterdam Regional Council of Governments Environmental Monitoring program, a program that reports yearly the environmental performance indicators in the Greater Rotterdam Area. Beside this yearly report a theme report is published. Last year's theme reports were published about Air Quality and Energy. In 2007 the board of this program decided that in 2008 the theme should be Noise and Health. The 2008 study is not only an update of the 2003 study but is more detailed and more comprehensive as well, and based on recent insights in health effects, caused by long lasting noise too.

\section{Session 3pNSb}

\section{Noise, ASA Committee on Standards, and EURONOISE: Prominent Discrete Tones I}

\author{
Robert Hellweg, Cochair \\ 13 Pine Tree Road, Wellesley, MA 02482, USA \\ Lothar Schmidt, Cochair \\ Currenta GmbH \& Co. OHG, Building F1, Dormagen, 41538, Germany
}

Invited Papers

2:00

3pNSb1. Comparison of various procedures for the assessment of prominent discrete tones using a large number of sound samples. Lothar Schmidt (Currenta GmbH \& Co. OHG, Building F1, 41538 Dormagen, Germany, lothar.schmidt@currenta.de), Dirk Sagemuehl (Kattenbachstr. 77a, 51467 Bergisch-Gladbach, Germany, DSagemuehl@aol.com)

The procedures from the standards ISO 1996-2 and DIN 45681 as well as the two procedures (TNR and PR) from ISO 7779 are compared. In the first section, characteristic features of the procedures (e.g. signal analysis parameters, degree of automation and characteristics of the prominent discrete tone analysis) are compared. In the second section, the procedures are applied to approximately 70 sound samples. Besides natural sounds (e.g. from machines), synthetic sounds (e.g. peak in a trough) are also used. The results are compared against assessments of the sounds by human subjects. The results of the comparisons are discussed, with special attention paid to cases where significant deviations occur. The third section looks at the results of the comparisons under the aspect of requirements for a standard (e.g. robustness, precision). Overall it was determined that none of the procedures investigated provides optimal results for all sound samples.

3pNSb2. Prominent tones in noise - Proficiency testing among 30 laboratories of the ISO 1996-2 Annex C method and its predecessors. Torben Holm Pedersen (Delta Acoustics \& SenseLab, Venlighedsvej 4, 2970 Hørsholm, Denmark, thp@delta.dk)

Since 1979 the Danish Environmental Protection Agency's Reference laboratory for noise measurements have arranged proficiency testing for the around 30 approved Danish laboratories that are measuring environmental noise. Many of these "comparison measurements" have included objective analysis of the prominence of audible tones according to the Joint Nordic Methods which are the predecessors and very similar to the method described in ISO 1996 part 2 Annex C. This paper gives examples on (computerized) analyses of the samples from these proficiency tests and states the uncertainties that can be expected when a number of laboratories with different experience and different types of equipment analyze the same samples. The samples will be available for future reference and can be downloaded from DELTA's homepage. 
3pNSb3. Updates on Prominent Discrete Tone Procedures in ISO 7779, ECMA 74, and ANSI S1.13. Robert Hellweg (13 Pine Tree Road, Wellesley, MA 02482, USA, Hellweg@HellwegAcoustics.com)

There are two alternative procedures for determining if a tone is prominent, i.e. a "prominent discrete tone" in ISO 7779, ECMA 74 and ANSI S1.13: the Tone to Noise Ratio (TNR) method and the Prominence Ratio (PR) method. Both of these procedures have been used by the computer industry for more than a decade. Recent revisions to these procedures have refined the calculation of the critical bandwidth and modified the both procedures for low level tones. This paper describes these changes and the rationale behind them. Examples are presented that demonstrate the features of each procedure. The paper presents an overview on the applicability and limitations of each procedure.

3pNSb4. Issues in the evaluation of the tonality of nonstationary sounds containing time- varying harmonic complexes. Patricia Davies (Ray W. Herrick Lab., School of Mechanical Engineering, Purdue Univ., 140 S. Martin Jischke Drive, West Lafayette, IN 47907-2031, USA, daviesp@ecn.purdue.edu), Shashikant R. More (Ray W. Herrick Lab., School of Mechanical Engineering, Purdue Univ., 140 S. Martin Jischke Drive, West Lafayette, IN 47907-2031, USA, shashi@purdue.edu)

Nonstationary sounds that contain broadband noise and tone complexes whose fundamental frequency varies with time are encountered frequently. Aircraft flyovers and machines shifting operating speeds are examples. The tonalness of such sounds is a factor in how annoying they are. Most metrics used to quantify the tonalness of sounds have been developed for stationary sounds and are based on an analysis of spectra. Contributions from individual sinusoids are estimated and either summed or the maximum is taken. When sounds vary, spectral estimation can be problematic due to the conflicting needs to reduce the variance of the estimate, maintain high frequency resolution, and generate spectra at a rate where the varying tonal behavior can be tracked sufficiently. Harmonic complexes are often perceived holistically, having one dominant pitch, thus treating sinusoidal components independently and summing may not be appropriate. Tests conducted to analyze the tonalness of harmonic complexes in noise are described, and an analysis of aircraft flyovers is used to illustrate the issues that need to be addressed when estimating the tonalness of complex time-varying sounds.

3pNSb5. Implementation of ISO 1996-2 (2007) pure tone assessment in a sound level meter. Charles Greene (Brüel \& Kjær, Skodsborgvej 307, 2850 Nærum, Denmark, cgreene@bksv.com), Douglas Manvell (Brüel \& Kjær, Skodsborgvej 307, 2850 Nærum, Denmark,dmanvell@bksv.com), Matthias Scholz (Brüel \& Kjær, Skodsborgvej 307, 2850 Nærum, Denmark, mscholz@bksv.com) , Anne Lin Enggaard (Nordsjællands Akustik, Skræntevej 13, Holløselund, 3210 Vejby, Denmark, ale @nordsjaellandsakustik.dk)

Noise can be said to be tonal if it contains a distinguishable, discrete, continuous note. This may include a whine, hiss, screech, hum, etc., and any such subjective finding is open to discussion when reported. This is important when it is considered that the likelihood of a noise-provoking complaint depends on its relative level to background, and whether or not it has certain audible characteristics. Fortunately, ISO 1996-2 (2007) provides objective FFT (engineering) and 1/3-octave band (survey) assessment procedures to be used to verify the presence of audible tones if their presence is in dispute. Brüel \& Kjær has implemented the methodology of ISO 1996-2 in relation to assessing the audibility of prominent discrete tones in environmental noise into its innovative 2250 and 2270 hand-held analysers. This paper describes the implementation in hand-held instrumentation. 


\title{
Session 3pNSc
}

\author{
Noise and EURONOISE: Car Acoustics I \\ Luc Mongeau, Cochair \\ McGill University, 817 Sherbrooke St. West, Montreal, QC H3A 2K6, Canada \\ Virginie Maillard, Cochair \\ RENAULT, Groupe Acoustique, Technocentre, 1 avenue du Golf, 78288 Guyancourt Cedex, France
}

\section{Contributed Paper}

\section{2:20}

3pNSc1. Effects of notches on span wise correlation of surface pressure fluctuations downstream of a wall-mounted spoiler. Paloma Mejia (Purdue University, 140 S. Intramural Dr., West Lafayette, 47906, USA, umaphuyu@gmail.com), Jong Beom Park (McGill University, 817 Sherbrooke St. West, Montreal, QC H3A 2K6, Canada, jong.b.park @mail.mcgill.ca), Luc Mongeau (McGill University, 817 Sherbrooke St. West, Montreal, QC H3A 2K6, Canada, luc.mongeau@mcgill.ca)

Leading edge spoilers are widely-used for suppressing flow-induced cavity resonance such as buffeting due to open sunroofs in moving cars. Spoilers deflect the grazing flow over the opening into a region of greater flow velocity, thereby increasing the critical velocity. Notched spoilers have been observed to enhance resonance suppression while moving the flow reattachment region upstream, resulting in a decreased drag. The mechanisms involved in the effectiveness of the notched spoiler were investigated experimentally and numerically. Static and dynamic pressures on the surface behind a wall mounted notched spoiler were measured, and the spatial correlations of the measured pressures were compared to those for a spoiler without notches. The span wise pressure correlation was decreased by the presence of the notches, suggesting a breakdown of the span wise leading vortices predominantly responsible for the cavity excitation. Numerical flow simulations were performed using the lattice Boltzmann method (LBM) with turbulence modeling. Surface pressure results were compared with experimental data to better highlight surface pressure trends.

\section{Invited Papers}

3pNSc2. Simulation of vehicle interior wind noise at low frequencies: a case study. Robert Powell (Ford Motor Company, EVB, MD X-19, Rm 1EB08, 20800 Oakwood Blvd, Dearborn, MI 48121, USA, rpowell7@ford.com), Bijan Khatib-Shahidi (Ford Motor Company, EVB, MD X-19, Rm 1EB08, 20800 Oakwood Blvd, Dearborn, MI 48121, USA, bshahidi@ford.com)

Quietness of passenger vehicle interiors has become a critical-to-quality metric in designing modern passenger vehicles. At the same time, the necessity of bringing fresh designs to market quickly has greatly compressed the development time available to achieve the desired refinement of interior noise and vibration. This has forced manufacturers to place increasing responsibility on analytical simulations in developing countermeasures for noise problems. One aspect of car acoustics that has not received very much attention from the simulation community is the wind noise created by external air flow at speed. Significant contributions to interior loudness can come from underbody air flow, while improvements in simulation efficiency of Computational Fluid Dynamics (CFD) flow models and Finite Element Analysis (FEA) vehicle vibration and acoustic models now make it feasible to analytically simulate interior noise caused by wind excitation. This lecture describes a case study where existing vehicle models were adapted to first build a wind load case from CFD, and then to apply it in estimating and reducing interior noise in FEA. Topics to be covered include: spatial discretization of continuous panel pressures, application of random loads to deterministic vehicle FEA models and diagnostic imagery for visualizing noise and vibration responses.

\section{3:00}

3pNSc3. On the use of linear aero-acoustic models. Mats Abom (KTH-The Marcus Wallenberg Laboratory, Teknikringen 8, 100 44 Stockholm, Sweden, matsabom@kth.se)

The classical theory of aero-acoustics is based on Lighthills acoustic analogy which essentially leads to a wave equation with a source term defined by the flow field. This source term is assumed unaffected by the acoustic field and the resulting model can be seen as a linear model which, e.g., for duct acoustic problems can be formulated as an acoustic multi-port. Such linear aero-acoustic models can be applied to most aero-acoustic problems occurring in engineering practice with the exception of whistling, i.e., situations where the source term is affected by the acoustic field. At KTH this methodology has been applied to a number of applications over the years ranging from cooling and ventilation fans to flow generated noise from ducted orifices (valves) and air terminal devices. In this paper the experience from these works is summarized and the experimental techniques developed at KTH to characterize linear aero-acoustic sources are described. Recent efforts to extract linear aero-acoustic models from numerical calculations are also addressed. 


\title{
Contributed Paper
}

3:20

3pNSc4. Modeling and characterization of rattle noise encountered in an automotive environment. Ludovic Desvard (Renault, Technocentre, 1 avenue du Golf, 78288 Guyancourt, France, ludovic.desvard@renault .com), Nacer Hamzaoui (LVA, INSA de Lyon, Bat. Saint-Exupéry, 25 bis avenue Jean Capelle, 69621 Villeurbanne, France, nacer.hamzaoui@insa -lyon.fr), Jean-Marc Duffal (Renault, Technocentre, 1 avenue du Golf, 78288 Guyancourt, France, jean-marc.duffal@renault.com)

In an automotive cockpit, rattle noises deal with all noise due to normal impacts which radiate as annoying noises for customers. The state of the art shows studies focused on one automotive subsystem, describing the specific associated physic. The method proposed, in this study, is to generate auto- motive rattle noises using simple geometries. The interest of this approach is to know all the parameters of the system (impact location, material, geometries, excitation...) and to precisely describe the physical phenomena related to the apparition of rattle noises. Firstly an experimental bench was proposed to generate rattle noises using simple geometries. These rattle noises are similar to those encountered in an automotive environment. This experimental approach allows to measure the vibrational behaviour and the radiated sound of the system under a random impact excitation. Then an analytical model, which describes the experimental approach, is developed to predict the radiated sound, knowing the displacement of the impactor. $\mathrm{Nu}$ merical and experimental results are compared. Finally, thanks to the experimental approach, a database of rattle noises is created and used to find metrics to quantify the annoyance.

WEDNESDAY AFTERNOON, 2 JULY 2008

ROOM 351, 1:40 TO 3:40 P.M.

Session 3pPAa

\section{Physical Acoustics: Quantum Acoustics I}

\author{
Michel De Billy, Cochair \\ Institut Jean le Rond d'Alembert / UMR 7190, 2, place de la Gare de Ceinture, Saint Cyr l'Ecole, 78210, France
}

\author{
Walter Lauriks, Cochair \\ Lab ATF, Katholicke Universiteit Leuven, Celestijnenlaan 2000, Leuven, B-3001, Belgium
}

\section{Contributed Papers}

\begin{abstract}
$1: 40$
3pPAa1. Phonon dispersion in graphene. Leonid A. Falkovsky (Landau Institute for Theoretical Physics, Kosygina 2, 119334 Moscow, Russian Federation, falk@itp.ac.ru)

Taking into account constraints imposed by the lattice symmetry, we calculate analytically [1] the phonon dispersion for graphene with interactions between first and second neighbors. We find that the out-of-plane (bending) modes are not coupled with in-plane modes and described only with two force constants, one of which is determined by the corresponding Raman frequency and another by the smallest elastic constant $\mathrm{C} 44$. In contrast to calculations by Saito et al, we find the linear dispersion of the bending (outof-plane) mode around the $\Gamma$ point with a small but finite sound velocity $=1.57 \mathrm{~km} / \mathrm{s}$. The sound velocity of this mode is very sensitive to small variations of the force constants. The sound velocities of in-plane modes are $=20.3 \mathrm{~km} / \mathrm{s}$ and $=13.1 \mathrm{~km} / \mathrm{s}$. Because of the lack of information for graphene, we compare the present theory with experiments on graphite. The low phonon frequencies in the critical points turn out less than their values in graphite, since the atoms in graphene are more free to move in the outof-plane direction in comparison with graphite. Accuracy of the comparison can be estimated using the value of the observed splitting of the ZA and ZO' modes in graphite which is around $1301 / \mathrm{cm}$. [1] L.A. Falkovsky, condmat/0702409.
\end{abstract}

\section{2:00}

3pPAa2. Mechanical (acoustic-like) wave propagation along a vortex array in the superconducting heterostructure. Bogdan $T$. Maruszewski (Poznan University of Technology, Institute of Applied Mechanics, ul. Piotrowo 3, 60-965 Poznan, Poland, bogdan.maruszewski @ put.poznan.pl), Andrzej Drzewiecki (Poznan University of Technology, Institute of Applied Mechanics, ul. Piotrowo 3, 60-965 Poznan, Poland,
andrzej.drzewiecki@put.poznan.pl),Roman Starosta(Poznan University of Technology, Institute of Applied Mechanics, ul. Piotrowo 3, 60-965 Poznan, Poland, roman.starosta@put.poznan.pl)

Magnetic flux can penetrate the type - II superconductor in the form of Abrikosov vortices (also called flux lines, flux tubes or fluxons) each carrying a quantum of magnetic flux. These tiny vortices of supercurrent tend to arrange themselves in a triangular or quadratic flux-line lattice. Since the vortices are formed by the applied magnetic field, around of each of them the supercurrent flows. Moreover, there also exist some Lorentz force interactions among them. Those interactions form an origin of an additional mechanical (stress) field occurring in the type-II superconductor. The paper deals with an analysis of elastic (acoustic-like) wave propagation solely along vortices in a heterostructure consisted of the superconducting layer put on the superconducting substrate. Dispersion and the amplitude distribution of those waves in the vortex field existing in that structure has been presented.

$$
2: 20
$$

3pPAa3. Amplitude distribution of magnetoelastic waves propagating in a vortex field in a superconducting layer. Bogdan $T$. Maruszewski (Poznan University of Technology, Institute of Applied Mechanics, ul. Piotrowo 3, 60-965 Poznan, Poland, bogdan.maruszewski @ put.poznan.pl), Andrzej Drzewiecki (Poznan University of Technology, Institute of Applied Mechanics, ul. Piotrowo 3, 60-965 Poznan, Poland, andrzej.drzewiecki@put.poznan.pl), Roman Starosta (Poznan University of Technology, Institute of Applied Mechanics, ul. Piotrowo 3, 60-965 Poznan, Poland, roman.starosta@put.poznan.pl)

Magnetic field enters the type - II superconducting body along a discrete arrangement of magnetic vortex lines. In the dynamic case when the magnetic field vary in time, around each such a line a supercurrent flows. So, the vortices interact one to another with the help of the Lorentz force forming this way a new mechanical field of elastic properties. Moreover, those lines arrange themselves in a triangular or quadratic lattice. Such a set is observed 
if the intensity of the applied to the material magnetic field is close to its lower limiting value. The paper aims at investigating amplitude distributions of magnetoelastic waves propagating solely in the vortex field of the superconducting layer. Our attention have been focused on the applied magnetic field intensity influence on those amplitudes for various wave frequencies.

\section{2:40}

3pPAa4. Acoustical dissipation due to phonon-phonon interaction, thermoelastic loss and dislocation damping in $\mathrm{MnO}$ and $\mathrm{CoO}$. Rishi Pal Singh (Banaras Hindu University, 221005 Varanasi, India, rishisingh80 @ rediffmail.com), Manish Pratap Singh (Banaras Hindu University, 221005 Varanasi, India, mps_bhu@yahoo.co.in), Rajendra Kumar Singh (Department of Physics, Banaras Hindu University, 221005 Varanasi, India, rksingh_17@rediffmail.com)

The temperature dependent acoustical dissipation due to phonon-phonon (p-p) interaction, thermo-elastic mechanism and dislocation damping due to screw and edge dislocations have been evaluated in $\mathrm{MnO}$ and $\mathrm{CoO}$ in fcc (NaCl-B1 type) phase, in a wide temperature range $50 \leqslant \mathrm{~T} \leqslant 500 \mathrm{~K}$ for longitudinal and shear modes of propagation along three crystallographic directions viz. $\langle 100\rangle,<110\rangle$ and $<111>$. Electrostatic and Born repulsive potentials were used to obtain second order and third order elastic constants (SOEC and TOEC), taking interactions up to next nearest neighbours. The SOEC and TOEC obtained at diffrent temperatures have been used to obtain gruneisen parameters and non-linearity or anisotropic parameters which in turn were used to evaluate $(\alpha / \mathrm{f} 2)$ for longitudinal and shear waves. Specific heat (as function of Debye temperature), hardness parameter, lattice parameter have been used as input data. It has been found that $(\alpha / \mathrm{f} 2)$ increases with temperature and the acoustical dissipation is mainly influenced by $p-p$ interaction. The possible implications of results have been discussed.

\section{3:00}

3pPAa5. Numerical and experimental results on sonic band gaps in 1-D phononic crystals with a symmetric stub. Michel De Billy (Institut Jean le Rond d'Alembert / UMR 7190, 2, place de la Gare de Ceinture, 78210 Saint Cyr 1'Ecole, France, mdebilly@univ-paris-diderot.fr), Anne-Christine Hladky-Hennion (IEMN, UMR CNRS 8520, avenue Poincaré, BP 60069, 59652 Villeneuve d'Ascq, France, Anne-Christine.Hladky@isen.fr), Jérôme Vasseur (IEMN, UMR CNRS 8520, avenue Poincaré, BP 60069, 59652 Villeneuve d'Ascq, France, jerome.vasseur@univ-lille1.fr), Bahram Djafari-Rouhani (IEMN, UMR CNRS 8520, avenue Poincaré, BP 60069, 59652 Villeneuve d’Ascq, France, Bahram.Djafari-Rouhani@univ-lille1.fr)

The wave propagation in periodic systems has received a great deal of attention during the last years. By analogy with the studies driven on photonic crystals, many works were conducted on phononic crystals. In this pa- per the propagation of elastic waves through a one dimensional chain of beads with grafted stubs is experimentally as well as numerically investigated. The results obtained by both approaches are well correlated and show that the stub introduces a dip in the spectral response of the chain. The numerical analysis shows that this dip is due to the excitation of a stub mode that cancels the transmission from one extremity of the chain to the other. The position and the shape of the dip in the response are related to the geometry and nature of the stub. The results show that it is possible to adjust the position of the dip and open potential applications of these structures for filtering or demultiplexing. Finally first results on periodically grafted stubs in the chain are presented.

$$
\text { 3:20 }
$$

3pPAa6. Evidence for vortex transport by surface acoustic waves in a high-Tc superconductor. Carsten Hucho (Paul-Drude-Institut, Hausvogteiplatz 5-7, 10117 Berlin, Germany, hucho@pdi-berlin.de), Munise Rakel (Paul-Drude-Institut, Hausvogteiplatz 5-7, 10117 Berlin, Germany, rakel@pdi-berlin.de), Arno Wirsig (Paul-Drude-Institut, Hausvogteiplatz 5-7, 10117 Berlin, Germany, wirsig@pdi-berlin.de), Fabian Jachmann (Paul-Drude-Institut, Hausvogteiplatz 5-7, 10117 Berlin, Germany, jachmann@pdi-berlin.de)

The interaction of surface acoustic waves (SAW) with the magnetic vortex system in $\mathrm{YBa} 2 \mathrm{Cu} 3 \mathrm{O} 7$ is investigated. A 100nm YBCO film is deposited on a piezoelectric substrate and structured for electrical 4-point measurements. Interdigital transducers are fabricated on the same substrate. When applying an external magnetic field perpendicular to the film surface a SAW-induced dc-voltage perpendicular to the acoustic sound-path is observed. This is interpreted as consequence of directed dragging of vortices by the SAW induced dynamic pinning structure [1]. The piezoacoustic wave acts as a conveyor for mobile flux quanta. Additional ac-dc-conversion as result of the nonlinear current-voltage characteristics close to the superconducting transition temperature can be resolved and separated. In order to observe the sound-induced vortex motion directly, the flux distribution is analyzed magnetooptically. Magnetooptic imaging allows for time resolved analysis of flux distribution. Quantitative analysis of changes in the magnetization distribution when acoustic driving fields are applied is carried out. The influence of piezoacoustic waves on the pinning properties and soundinduced depinning is discussed. [1] F. Jachmann and C. Hucho, Sol. State Comm., 142 (4) (2007), 212 


\title{
Session 3pPAb
}

\section{Physical Acoustics: General Topics in Nonlinear Acoustics I}

\author{
Thomas Matula, Cochair \\ Center for Industrial and Medical Ultrasound, Applied Physics Lab., University of Washington, 1013 NE 40th St., \\ Seattle, WA 98105, USA \\ Murray S. Korman, Cochair \\ Physics Dept., United States Naval Academy, Chauvenet Hall Room 295, 572 C Holloway Road, Annapolis, MD 21402, USA
}

\section{Contributed Papers}

\section{2:00}

3pPAb1. Atomization and deviation of cylindrical water jets in a transverse acoustic field. Jean-Baptiste Carpentier (Coria UMR 6614, avenue de l'Université, 76800 Saint-Etienne-du-Rouvray, France, jean-baptiste.carpentier@univ-rouen.fr), Françoise Baillot (Coria UMR 6614, avenue de l'Université, 76800 Saint-Etienne-du-Rouvray, France, baillot@coria.fr), Jean-Bernard Blaisot (Coria UMR 6614, avenue de l'Université, 76800 Saint-Etienne-du-Rouvray, France, blaisot@coria .fr), Christophe Dumouchel (Coria UMR 6614, avenue de l'Université, 76800 Saint-Etienne-du-Rouvray, France, dumouchel@coria.fr)

This work deals with a particular breakup mode experienced by cylindrical liquid jets when submitted to an intense transverse acoustic wave. Experiments on low speed water jets $(<1 \mathrm{~m} / \mathrm{s})$ of diameters $3 \mathrm{~mm}$ and $6 \mathrm{~mm}$ show that sound waves with a frequency ranging from $500 \mathrm{~Hz}$ to $1800 \mathrm{~Hz}$ can produce bulges along the jet. When the sound level is high enough, these bulges can trigger an effective atomization mechanism where the jet flattens as a liquid sheet before disintegrating. Sound field can also induce steady deviation of the jet. Both phenomena are theoretically studied. A first model, which treats bulges as outward marks of one particular mode of vibration of the liquid column, is proposed. This model leads to a criterion for the onset of atomization that satisfactorily agrees with experimental observations of the present work. A second analysis identifies deviations as radiation pressure effects. It predicts the direction of experimental deviations with success.

\section{2:20}

3pPAb2. Numerical and experimental observations of azimutal shock waves. Régis Marchiano (Institut Jean Le Rond d'Alembert, Université Pierre et Marie Curie, Boites 161 et 162, 4 place Jussieu, 75252 Paris Cedex 05, France, marchi@1mm.jussieu.fr), Jean-Louis Thomas (Centre National de la Recherche Scientifique, Institut des NanoSciences de Paris, Université Pierre et Marie Curie, 4 place Jussieu, 75252 Paris Cedex 05, France, thomasj1@ccr.jussieu.fr), Thomas Brunet (CNRS and Paris VI University, INSP - 140 rue de Lourmel, 75015 Paris, France, thomas.brunet@insp .jussieu.fr), François Coulouvrat (Institut Jean Le Rond d'Alembert, Université Pierre et Marie Curie, Boites 161 et 162, 4 place Jussieu, 75252 Paris Cedex 05, France, coulouvr@ccr.jussieu.fr)

Acoustical Vortices (AV) are acoustical beams with a phase singularity of screw type. They possess a phase with an helical structure which is winding around the axis of propagation. These twists of phase engender special properties such as the existence of an associated angular momentum and imply a coupling between the axial and transverse components of the beam. Propagation of $\mathrm{AV}$ of finite amplitude follows the classic rule of nonlinear acoustics. Beyond a shock formation distance, AV develop shocks, but the structure of the latters is noticeable. It will be shown by numerical simulations and experimental measurements, that the nonlinear propagation of $\mathrm{AV}$ gives birth to 3D shock waves: a classical shock in the direction of propagation plus an azimutal one in the transverse plane. The numerical simulation, based on an original algorithm solving the 3D Khokhlov-Zabolotskaya equation, is used to investigate the dynamic of the formation of the azimutal shock. Experimental observations made with ultrasonics in water confirm the existence of particular shock waves. Finally, a brief discussion of the potential applications will be proposed.

\section{2:40}

3pPAb3. Water flow generation owing to interaction between pulsing bubble and rigid wall. Alexey P. Drozhzhin (Lavrentyev Institute of Hydrodynamics SB RAS, Lavrentyev ave., 15, 630090 Novosibirsk, Russian Federation, ruslan@hydro.nsc.ru), Vyacheslav S. Teslenko (Lavrentyev Institute of Hydrodynamics SB RAS, Lavrentyev ave., 15, 630090 Novosibirsk, Russian Federation, vteslenko@academ.org)

Generating a directed water flow in periodic forming a non-symmetrical acoustic field was experimentally investigated in the work. Periodic acoustic wave field was formed with the help of a vapor-gas bubble pulsing in the hole of a thin diaphragm near a rigid wall. Alternate formation of two water flows moving in opposite directions was found. During the bubble growth stage the flow at velocity V1 was formed in the opposite direction of the plate, and during the bubble collapse stage the flow at velocity V2 was formed towards the plate. By changing the parameter $k=R / r$ where $R$ was a distance from the hole center to the rigid wall, and $\mathrm{r}$ was a hole radius, the possibility to control average values $\mathrm{V} 1$ and $\mathrm{V} 2$ was shown. It was found that at $\mathrm{k}=\mathrm{R} / \mathrm{r}=5.8 \mathrm{~V} 1=\mathrm{V} 2$, at $\mathrm{k}>5.8 \mathrm{~V} 1>\mathrm{V} 2$, and at $\mathrm{k}<5.8 \mathrm{~V} 1$ was less than V2. It was shown that due to variation of the distance between a pulsing bubble and a rigid wall it was possible to control velocity $\mathrm{V}$ and direction of the total flow of water through the hole. The maximum value of velocity $\mathrm{V}$ reached $40 \mathrm{~cm} / \mathrm{s}$ in the experiment.

\section{3:00}

3pPAb4. Nonlinear ultrasound fields simulation of harmonics from exponential and bessel beams sources. Hicham Jakjoud (Lab. Electrical Systems and Telecommunications, Faculté des Sciences et Techniques Gueliz B.P. 549, 40000 Marrakech, Morocco, hicham.jakjoud@gmail .com), Ahmed Chitnalah (Lab. Electrical Systems and Telecommunications, Faculté des Sciences et Techniques Gueliz B.P. 549, 40000 Marrakech, Morocco, chitnalah@fstg-marrakech.ac.ma), Noureddine Aouzale (Lab. Electrical Systems and Telecommunications, Faculté des Sciences et Techniques Gueliz B.P. 549, 40000 Marrakech, Morocco, aouzale.nour@gmail.com), Djilali Kourtiche (Lab. d'Instrumentation, Electronique de Nancy, UHP Nancy I, BP 239, 54506 Vandœuvre, 54506 Nancy, France, djilali.kourtiche@lien.uhp-nancy.fr)

It's well known that the harmonic imaging quality can be improved by using sources that radiate narrower and attenuated sidelobe beams. Hence we try to enhance the harmonics' cartography by studying different source's power distributions. We developed a numerical code, using the spectral method, in order to resolve the parabolic wave equation. The numerical results are compared to those given by other researchers in order to validate our algorithm. Two source's power distributions (exponential and Bessel beams) are studied and compared to the uniform case. The use of exponential source leads to harmonics diagrams without sidelobes neither nearfield oscillations. But the beam width is increasing with propagation. The Bessel 
source presents a limited diffraction beam. The beam width is almost constant throughout the nearfield and the transition zone. The sidelobes have a weak level in the fundamental curves and they don't appear in the second harmonic ones.

\section{3:20}

3pPAb5. Experimental confirmation of the theory of acoustic radiation pressure applying on transparent interfaces. Bruno Issenmann (Bordeaux University - CPMOH, 351 cours de la Liberation, 33405 Talence Cedex, France, b.issenmann@cpmoh.u-bordeaux1.fr), Alice Nicolas (Laboratoire de Physique Matiere Condensee - Univ. Nice, Parc Valrose - 28, avenue Valrose, 06108 Nice Cedex 02, France, Alice .NICOLAS@unice.fr), Regis Wunenburger (Bordeaux University - CPMOH, 351 cours de la Liberation, 33405 Talence Cedex, France, r.wunenburger@cpmoh.u-bordeaux1.fr), Sébastien_Manneville (Ecole
Normale Supérieure de Lyon, 46 allée d'Italie, 69364 Lyon Cedex 07, France, sebastien.manneville@ens-lyon.fr), Jean-Pierre Delville (Bordeaux University - CPMOH, 351 cours de la Liberation, 33405 Talence Cedex, France,jp.delville@cpmoh.u-bordeaux1.fr)

Since Rayleigh and Brilloin, the acoustic radiation pressure has been the subject of several theoretical works, but of few quantitative tests. Whereas the radiation pressure acting on perfectly reflecting or perfectly absorbing solid targets is commonly used for the calibration of high intensity focused ultrasonic beams, it has never been quantitatively studied on acoustically transparent interfaces. Using an acoustically transparent liquid-liquid interface deformed by the radiation pressure of a focused, continuous wave beam, we have tested the theory of the acoustic radiation pressure acting on transparent interfaces for the first time. At large intensity, depending on the direction of propagation of the beam, we observe surprising interface shapes such as drop emitting jets and "nipple-like" deformations.

\title{
Session 3pPPa
}

\section{Psychological and Physiological Acoustics: General Topics in Psychological and Physiological Acoustics II}

\author{
Elizabeth Strickland, Cochair \\ Purdue University, 500 Oval Drive, West Lafayette, IN 47907, USA
}

Armin Kohlrausch, Cochair

Philips Research Europe, Digital Signal Processing (MS WO02), High Tech Campus 36, Eindhoven, 5656 AE, Netherlands

Alain De Cheveigne, Cochair

CNRS, Universite Paris 5, Ecole Normale Superieure, 29 rue d'Ulm, Paris, 75230, France

\section{Contributed Papers}

\section{2:00}

3pPPa1. A pair of spectral notches which plays a role as a spectral cue in the vertical localization, and it's application to estimation of sound source elevation from binaural signals. Kazuhiro Iida (Chiba Institute of Technology, 2-17-1 Tsudanuma, 275-0016 Narashino, Japan, kazuhiro.iida @it-chiba.ac.jp)

The author [Applied Acoustics, 68, 835-850 (2007)] proposed a parametric HRTF model for vertical sound localization. The parametric HRTF is recomposed only of the spectral peaks and notches extracted from the measured HRTF. The results of the median plane localization tests, which were carried out using the parametric HRTFs with various combinations of spectral peaks and notches, show that the pair of first and second notches (N1 and N2) above $5 \mathrm{kHz}$ can be regarded as spectral cues. In this research, estimation of the elevation of sound source in the upper median plane by extracting $\mathrm{N} 1$ and $\mathrm{N} 2$ frequencies from binaural input signal was carried out. The kinds of sound sources were female voice, male voice, music, white noise, and pink noise. The results show that the estimation is accurate for almost of all the elevation and of all the kind of sound sources.

\section{2:20}

3pPPa2. Trading of intensity and interaural correlation cues in the perception of loudness. John F. Culling (Cardiff University, School of Psychology, Tower Building, Park Place, CF10 3AT Cardiff, UK, CullingJ@cardiff.ac.uk), Hannah G. Lewis (Cardiff University, School of
Psychology, Tower Building, Park Place, CF10 3AT Cardiff, UK, lewishg @ Cardiff.ac.uk)

When a signal is added to noise in the $\operatorname{NoS} \pi$ binaural configuration, a reduction in interaural cross-correlation (IACC) occurs at the signal frequency. Increases in tone intensity produce decreases in IACC. Consistent with this relationship, direct manipulations of IACC can result in the perception of an added signal and progressive reduction in IACC produces progressive increases in the loudness of this signal [Culling et al. $2001 \mathrm{~J}$. Acoust. Soc. Am. 110, 1020-1029]. In the present study, a narrow sub-band of noise (460-540 Hz) embedded within a broadband $(0-3 \mathrm{kHz})$ diotic noise was manipulated in both intensity and IACC in a 3-interval, odd-one-out task. In the reference intervals, IACC was zero and the spectrum was flat. In the target interval, both the IACC and the intensity of the target band were incremented. These increases have opposing effects on loudness. Correct identification of the target interval followed a U-shape as a function of the size of intensity increment. The minimum of the function was at chance performance, indicating that the opposing cues were fully traded.

\section{2:40}

3pPPa3. A comparison between interaural level difference and interaural correlation uncertainty on binaural signal detection thresholds. Nicolas Le Goff (Technical University Eindhoven, Den Dolech 2, 5600 MB Eindhoven, Netherlands, n.legoff@tm.tue.nl), Armin Kohlrausch (Philips Research Europe, Digital Signal Processing (MS WO02), High Tech Campus 36, 5656 AE Eindhoven, Netherlands, 
armin.kohlrausch@philips.com),Jeroen Breebaart(Philips Research Europe, Digital Signal Processing (MS WO02), High Tech Campus 36, 5656 AE Eindhoven, Netherlands, jeroen.breebaart@philips.com)

Experiments were performed to study the effect of the presence of interaural level differences (ILDs) on binaural detection abilities. The subjects had to detect an interaurally out-of-phase $500-\mathrm{Hz}$ tone in the presence of a diotic noise masker that had a bandwidth of either $1 \mathrm{kHz}, 100 \mathrm{~Hz}$ or $10 \mathrm{~Hz}$. Experiments were conducted for both frozen and running-noise conditions and ILDs up to $30 \mathrm{~dB}$ were applied to both signal and masker. The results indicate an ILD dependency that varies with the bandwidth of the masker. Furthermore, with increasing ILDs, the difference in detection thresholds between frozen and running-noise conditions was larger for narrow-band noise conditions. Similar observations were made by previous investigations on the influence of reduced masker correlation. Both data sets are compared in order to find possible similarities in the mechanisms involved in the detection tasks.

\section{3:00}

3pPPa4. The effect of a speech target's motion on its recognition in the presence of simultaneous competing sentences. Wesley Grantham (Vanderbilt Bill Wilkerson Center, Department of Hearing and Speech Sciences, 1215 21st Avenue South, Room 8310, Nashville, TN 37232-8242, USA, d.wesley.grantham@vanderbilt.edu), Todd Ricketts (Vanderbilt Bill Wilkerson Center, Department of Hearing and Speech Sciences, 1215 21st Avenue South, Room 8310, Nashville, TN 37232-8242, USA, todd.a.ricketts@vanderbilt.edu), Daniel Ashmead (Vanderbilt Bill Wilkerson Center, Department of Hearing and Speech Sciences, 1215 21st Avenue South, Room 8310, Nashville, TN 37232-8242, USA, daniel.h.ashmead@vanderbilt.edu)

Prior knowledge of where to listen significantly improves speech recognition of target sentences presented in the presence of distracter sentences coming from different locations [G. Kidd et al., J. Acoust. Soc. Am. 118, 3804-3815 (2005)]. The present study extended the work of Kidd et al. by measuring the effect of a target's motion on its recognition when competing messages are present. In an anechoic chamber normal-hearing subjects were presented with three simultaneous sentences from the CRM corpus and were instructed to indicate key words from the target sentence (identified by a call-sign previously known to the subject). In the stationary condition the three sentences came from $-60^{\circ}, 0^{\circ}$, and $+60^{\circ}$ azimuth. In the moving condition, the target source moved during its on-time (e.g., from $-60^{\circ}$ to $0^{\circ}$ ) while the two distracter sentences were stationary (e.g., at $\left.\pm 60^{\circ}\right)$. In both cases, subjects either knew in advance where the target would be (Certain Condition) or did not know (Uncertain Condition). It is hypothesized that motion of the target will result in a release from informational masking. That is, the detrimental effect of location uncertainty observed with the stationary targets will be reduced or eliminated when the target is moving.

\section{3:20}

3pPPa5. Frequency selectivity in diotic and dichotic masking conditions for normal-hearing and hearing-impaired listeners. Marc Nitschmann (Universität Oldenburg, Medizinische Physik, Carl-vonOssietzky Str. 9-11, 26111 Oldenburg, Germany, marc.nitschmann @ uni-oldenburg.de), Jesko Verhey (Universität Oldenburg, Medizinische Physik, Carl-von-Ossietzky Str. 9-11, 26111 Oldenburg, Germany, jesko.verhey@uni-oldenburg.de), Birger Kollmeier (Universität Oldenburg, Medizinische Physik, Carl-von-Ossietzky Str. 9-11, 26111 Oldenburg, Germany, birger.kollmeier@uni-oldenburg.de)

Previous studies argued that, for broadband maskers, the operational binaural critical bandwidth is similar to the monaural critical bandwidth. The aim of the present study was to test this hypothesis using the notched noise paradigm. Thresholds were measured for a diotic masker and a signal that was either in phase or had an interaural phase difference of 180 degrees. For comparison, thresholds were also measured for a broadband masker (without a notch) at various masker levels to account for possible level effects of the binaural masking level difference (BMLD). Normal-hearing and hard-of-hearing listeners with various degrees of sensorineural hearing impairment participated in the experiment. For all subjects, the thresholds decreased faster for the diotic than the dichotic signal as the notch width increased. The comparison with the data for the broadband masker indicated that this decrease of the BMLD is not due to the decrease of masker level within the auditory filter centered at the signal frequency. The thresholds can be predicted by an equalization-cancellation model assuming a slightly broader auditory filter in the dichotic masking condition. A possible realization of this different binaural auditory filter shape is adding to the onfrequency filter fractions of the adjacent filters. 
Session 3pPPb

\title{
Psychological and Physiological Acoustics: General Topics in Psychological and Physiological Acoustics III
}

\author{
Elizabeth Strickland, Cochair \\ Purdue University, 500 Oval Drive, West Lafayette, IN 47907, USA
}

Armin Kohlrausch, Cochair

Philips Research Europe, Digital Signal Processing (MS WO02), High Tech Campus 36, Eindhoven, 5656 AE, Netherlands

\author{
Alain De Cheveigne, Cochair \\ CNRS, Universite Paris 5, Ecole Normale Superieure, 29 rue d'Ulm, Paris, 75230, France
}

\section{Contributed Papers}

\section{2:00}

3pPPb1. Effects on cochlear frequency selectivity after hypobaric pressure exposure. Jonas Brännström (Department of Audiology, Malmö University Hospital and Lund University, 20505 Malmö, Sweden, jonas.brannstrom@skane.se), Jan Grenner (Department of Audiology, Lund University Hospital, 22185 Lund, Sweden, jan.grenner@skane.se)

The effects of hypobaric pressure chamber exposure was measured in noise in ten patients with monaural fluctuating low-frequency hearing loss (FLFHL) such as Ménière's disease using psychophysical tuning curves (PTC), transiently evoked otoacoustic emissions (TEOAE), binaural pitch matches and speech recognition scores (SRS) in noise. In the literature, reversible hearing losses have been observed in about $50 \%$ of the patients, but sometimes improved SRS can be observed in patients without hearing threshold improvement. This indicates possible effects of pressure treatment on cochlear frequency selectivity. The relative overpressure in the middle ear obtained after repeated exposures in hypobaric pressure chamber (total duration 18.5 to 28 minutes) was used to impose pressure gradients to the inner ear. The results indicated that the treatment effects were small, but slightly improved SRS in noise, TEOAEs emission strength and PTCs were observed after treatment. Pure tone hearing thresholds improved only for patients exposed to longer treatment durations. Subjective improvement at follow-up could not be predicted from the results. Although the effects were small, the data suggest that hypobaric pressure treatment may improve cochlear frequency selectivity in the affected ear in patients with monaural FLFHL.

\section{2:20}

3pPPb2. IPods listening levels on London Underground. Stephen Dance (London South Bank University, FESBE, Borough Road, SE1 0AA London, UK, dances@lsbu.ac.uk), Phil Wash (Bickerdike Allen Partners, 117-121 Salusbury Road, Queens Park, NW6 6RG London, UK, pwash @ bickerdikeallen.com)

In the last 5 years the prevalence of the iPod/MP3 players has grown exponentially. The use of such in-earphones under urban conditions has been reported widely in the press at the anecdotal level. This study compared listening levels under quiet conditions and that representative of a London Underground train journey. Calibrated recordings of underground trains running in tunnels were played through loudspeakers in an anechoic chamber, whilst pop music or speech podcasts were played through the iBuds. Thirty-three participates listened to the iPod whilst a Binaural Head and Torso measured the noise levels through the in-earphones. The participants had time to adjust the volume setting on each occasion. A small audiometric study was undertaken for those participates with particularly high and low volume settings a week after the tests were completed. Results show very high volume settings were used when speech was played with the train noise.

\section{2:40}

3pPPb3. Assessment of hearing damage when listening to music through a personal digital audio player. Adriano Farina (Liceo Classico G.D. Romagnosi, Viale Maria Luigia 1, 43100 Parma, Italy, adriano @adrianofarina.it)

This study evaluates the sound pressure at the ears of users of personal digital audio players, equipped with in-ear earbuds. More than 50 devices were measured, provided by students aged 15/18. EN standard 50332 specifies the techniques for measuring the sound pressure level at the ears employing an head and torso simulator equipped with binaural microphones, when the gain control of the device is set to maximum. In this study, however, two measurmenets were performed on each device, one at maximum level, and another at the level at which the device had been left by the user after last listening. This way, on a statistical basis, it is possible to relate the effective exposure in daily usage with the maximum loudness of which the device is capable. Furthermore the study did also analyze the spectrum of the test signal: whilst the EN standard mandates for the IEC programme simulation noise, the analysis of thousands songs taken from the players under test revealed a slightly different spectrum, characterized by more boost at low frequencies and larger crest factor. The results of the study did show a relevant hearing risk for users of these personal digital audio players, which exceed noise exposure limits even when used for just one hour per day.

\section{3:00}

3pPPb4. The Healthy Benefits of Isolating Earphones. Jeremie Voix (Sonomax Hearing Healthcare Inc, 8375 Mayrand, Montreal, QC H4P 2E2, Canada, jvoix@sonomax.com), Cecile Le Cocq (Ecole de Technologie Superieure, 1100 Notre-Dame Ouest, Montreal, QC H4P 2E2, Canada, clecocq@mec.etsmtl.ca), Lee D. Hager (Sonomax Hearing Healthcare Inc, 8375 Mayrand, Montreal, QC H4P 2E2, Canada, lhager@ sonomax.com)

With the ubiquitous presence of Personal Stereo Players (PSPs), namely ${ }_{\text {iPods }}{ }^{\mathrm{TM}}$ and the like, many hearing conservationists have raised concerns about the temporary and permanent hearing damages that could result from long exposure to loud music playback, especially among adolescents and teenagers. The crux of the problem can be identified as an overexposure of the auditory system. In order to reduce the dose received by the PSP listener, the playback level and/or the duration should be reduced. Assuming that the duration of the music playback experience is really up to the user, the remaining parameter is the music playback level. The purpose of this study is to first understand - from the available public and scientific literature - what factors are influencing the PSP playback level; and second, to investigate if the use of earphones featuring good attenuation of the ambient noise level would lead to a reduced playback level, hence a reduced dose and eventually less auditory damage. Other benefits on sound quality that are associated with isolating earphones will be presented in a third part. Finally the article will review other safety mechanisms that could be used in earphones and PSP to make them safe for the hearing. 
tional hearing loss still occurs despite such standards may reflect a variety of issues ranging from enforcement of standards, to adequacy of standards, and problems of noise assessment. An additional factor that influences susceptibility to noise-induced hearing loss is the presence of chemical contaminants in the workplace that render the auditory system especially vulnerable to noise. We report on the ability of aviation fuels to potentiate noise-induced hearing loss in laboratory rats. Subjects were exposed to JP-8 jet fuel, a synthetic fuel produced from coal using the Fischer-Tropsch method, or to clean air. Half of the subjects received a moderate noise exposure following fuel or air exposure. The noise exposure was designed to produce a temporary threshold shift. JP-8 + noise produced greater disruption of distortion product otoacoustic emissions as well as the auditory threshold than did noise alone. Supported by VA Rehabilitation Service grants and the American Petroleum Institute.

\title{
Session 3pSCa
}

\section{Speech Communication: Phonetics}

\author{
Joseph S. Perkell, Chair \\ Massachusetts Institute of Technology, Research Laboratory of Electronics, Room 36-511, 50 Vassar St., Cambridge, MA \\ 02139-4307, USA
}

\section{Contributed Papers}

2:00

3pSCa1. Auditory and somatosensory goals for sibilants. Joseph S. Perkell (Massachusetts Institute of Technology, Research Laboratory of Electronics, Room 36-511, 50 Vassar St., Cambridge, MA 02139-4307, USA, perkell@mit.edu), Melanie L. Matthies (Massachusetts Institute of Technology, Research Laboratory of Electronics, Room 36-511, 50 Vassar St., Cambridge, MA 02139-4307, USA, matthies@bu.edu), Satrajit S. Ghosh (Massachusetts Institute of Technology, Research Laboratory of Electronics, Room 36-511, 50 Vassar St., Cambridge, MA 02139-4307, USA, satra@speech.mit.edu), Edwin Maas (Massachusetts Institute of Technology, Research Laboratory of Electronics, Room 36-511, 50 Vassar St., Cambridge, MA 02139-4307, USA, maas@bu.edu), Alexandra Hanson (Massachusetts Institute of Technology, Research Laboratory of Electronics, Room 36-511, 50 Vassar St., Cambridge, MA 02139-4307, USA, alex.hanson@gmail.com), Frank H. Guenther (Boston University, 677 Beacon Street, Boston, MA 02215, USA, guenther@cns .bu.edu), Harlan Lane (Massachusetts Institute of Technology, Research Laboratory of Electronics, Room 36-511, 50 Vassar St., Cambridge, MA 02139-4307, USA, lane@ @speech.mit.edu), Lucie Ménard (Département de linguistique et de didactique des langues, Université du Québec à Montréal, Case postale 8888, succursale Centre-ville, Montréal, QC H3C 3P8, Canada, menard.lucie@uqam.ca), Mark Tiede (Massachusetts Institute of Technology, Research Laboratory of Electronics, Room 36-511, 50 Vassar St., Cambridge, MA 02139-4307, USA, tiede@ @speech.mit.edu)

In prior studies [JSLHR 47, 1259-69, 2004; JASA 116, 2338-44, 2004], we found that speakers' auditory acuity for synthetic sibilant $(/ \mathrm{s} /, / \mathrm{sh} /)$ and vowel stimuli was correlated with the degree of acoustic contrast they produced. This outcome is consistent with the view that the articulatory movements underlying phonemic contrasts have auditory goals. There was also a cross-speaker relation between sibilant contrast and use of contact between the tongue tip and lower alveolar ridge for $/ \mathrm{s} /$ (but not $/ \mathrm{sh}$ ), indicating that such contact is a somatosensory goal for /s/. In the current study, another, larger group of subjects also showed relations between measures of auditory acuity and acoustic contrast for sibilants and vowels. To determine whether the idea of a somatosensory goal for /s/ would also be supported by a relation between acuity and contrast, we made measurements of somatosensory discrimination. Small plastic (JVP) domes with grooves of differ- ent spacing were pressed against each subject's tongue tip and the subject was asked to identify one of four possible orientations of the grooves. A wide range of individual performance was observed with a bias-corrected sensitivity measure. Correlations with produced sibilant contrast and further analyses will be reported. [Research supported by NIDCD, NIH.]

\section{2:20}

3pSCa2. Perceptual explanations of articulatory variability in the realisation of the nasal feature for the consonants. Jacqueline Vaissière (Laboratory of Phonetics and Phonology, 19 rue des bernardins, 75005 Paris, France, jacqueline.vaissiere@univ-paris3.fr)

This paper illustrates how the perceptual constraints explain the observed articulatory and aerodynamic inter-speakers and inter-languages variability between initial nasal consonants $(/ \mathrm{n} /)$, and unreleased final consonants $(/ \mathrm{N} /)$. In the case of $/ \mathrm{n} /$, only a short, well-defined region around the consonant release needs to be nasalized for the consonant to be perceived as nasal. In the case of $/ \mathrm{N} /$, because of the lack of a nasal release, a longer span is needed for deciphering the presence of nasalisation; contextual nasalization of the preceding vowel can be avoided, but in that case, the nasal murmur has to be long to be perceived (since the first part of the murmur is perceptually masked by the vowel), and/or /N/ has to be released. Such observations argue for the division of the nasal consonant into three parts, onset, murmur and release: the realisation of the nasal feature is aligned around the $/ \mathrm{N} /$ onset, and around the $/ \mathrm{n} /$ offset. It also argues for a better integration of the perceptual requirements and masking phenomena in modelling the observed variability. Finally, it shows that a feature can be realized very differently depending on the position of the phoneme in the syllable.

\section{2:40}

3pSCa3. Final consonant voicing and vowel height contrasts in whispered speech. Yana D Gilichinskaya (City University of New York, Graduate Center, 365 Fifth Avenue, New York, NY 10016, USA, ygilichinskaya@gc.cuny.edu), Winifred Strange (CUNY Graduate Center, 
3655 th Avenue, Program in Speech-Language-Hearing Sciences, New York, NY 10016, USA, strangepin@aol.com)

Whispered speech is a naturally distorted speech signal. Whereas it preserves some characteristics of fully phonated speech, some important acoustic cues are removed, diminished or altered. The prominence of acoustic cues in whispered speech may change due to the physical properties of the whispered speech signal, i.e., decreased intensity, the absence of periodic vibration of the vocal folds, damping of F1, shift of the formants and flattening of the amplitude envelope. Such changes affect the acoustic cues both for vowels (e.g. vowel height) and consonants (e.g. voicing contrasts). The objective of the present project was to explore the acoustic cues for poststressed syllable-final consonant voicing contrasts and the vowels preceding them in continuous whispered speech of American English speakers and to compare the results with those in fully phonated speech. The stimuli were recorded in the carrier sentence "I'll utter /habVC/ off the list". The consonant pairs included voiced/voiceless bilabial stops /b-p/ and labiodental fricatives /f-v/, each combined with $11 \mathrm{AE}$ vowels /i, I, "epsilon", e, æ, a, "turned-v", o, "open-o", u, "horseshoe"/. Preliminary results showed that vowels had longer duration in whispered speech than in fully phonated speech. Spectral dispersion, temporal contrastivness of vowels; F1, and vowel duration cues will be reported in the presentation.

\section{3:00}

3pSCa4. Production and perception of vietnamese short vowels. Nguyen Viet Son (MICA center HUT - CNRS/UMI2954 INP Grenoble, C10 Hanoi university of Technology, No1 Dai Co Viet street - Hai Ba Trung, 0084 Hanoi, Viet Nam, viet-son.nguyen@mica.edu.vn), René Carré (Laboratoire Dynamique du Langage, UMR 5596, CNRS, Université Lyon 2, 14 Avenue Marcelin Berthelot, 69363 Lyon cedex 07, France, recarre@wanadoo.fr), Eric Castelli (MICA center HUT - CNRS/UMI2954 INP Grenoble, C10 Hanoi university of Technology, No1 Dai Co Viet street - Hai Ba Trung, 0084 Hanoi, Viet Nam, Eric.Castelli@mica.edu.vn)

It is well known that vowels can be produced in isolation, acoustically stable in such a way that they are represented as points in the F1-F2-F3 space. Vietnamese language presents 13 vowels, however, Vietnamese can only pronounce 9 vowels $[\mathrm{i}, \mathrm{u}, \mathrm{e}, \mathrm{o}, \omega, \mathrm{a}, \sigma, \varepsilon, \gamma]$ in isolated mode. A pre- vious study showed that the 4 remaining vowels $[\alpha, \delta, \nu, \epsilon]$ have the same acoustic characteristics (F1, F2, F3) as, respectively, the vowels [a, $\varepsilon, \sigma, \gamma]$, but their dynamic characteristics (the rate of $\mathrm{CV}$ transitions) are clearly distinct. Measurements show us that vowels durations of $[\alpha, \nu]$ are always shorter than the one of corresponding classical vowels $[a, \gamma]$ and are not acoustically stable. For test perceptions (with 10 Vietnamese people), synthesized syllables $[\mathrm{a}-\mathrm{t}]$ and $[\gamma-\mathrm{t}]$ with changing vowel duration are recognized as $[\alpha-\mathrm{t}, \nu$-t $]$ when then duration of initial vowel $[\mathrm{a}, \gamma]$ are $60 \%-70 \%$ shorter. It means that the vowel duration is an important parameter that allows Vietnamese distinguishing the long vowels and short vowels in Vietnamese language. This paper further analyses the production of Vietnamese $\mathrm{CV}$, including long vowels and short vowels in terms of duration, formant evolution and rate of C.V transitions.

\section{3:20}

3pSCa5. Acoustic properties of the interdental approximant in Kagayanen. Kenneth S. Olson (SIL International, 7500 W Camp Wisdom Rd, Dallas, TX 75236, USA, Ken_Olson@sil.org), Jeff Mielke (University of Ottawa, Arts Hall, 70 Laurier Ave East, Room 401, Ottawa, ON K1N 6N5, Canada,jmielke@uOttawa.ca)

This paper describes the acoustic properties of the interdental approximant, a rare speech sound reported to date only in a dozen languages in the Philippines. The measurements analyzed here are based on recordings of one female speaker of Kagayanen. The interdental approximant exhibits acoustic characteristics typical of semi-vowels: the formant pattern is similar to that of vowels, and the formant transitions with adjoining vowels have a long duration, usually $35 \mathrm{~ms}$ or more. The values of F2 and F3 are analogous to those of the Kagayanen [1], a prototypical voiced alveolar lateral; the only significant formant value difference between the two sounds involves F1: the interdental approximant has a mean of $508 \mathrm{~Hz}(\mathrm{n}=9)$, whereas the mean for [1] is $368 \mathrm{~Hz}(n=9)$. On the other hand, while these two segments sound impressionistically similar, the interdental approximant differs acoustically from [1] in several other respects as well: there is no abrupt change in the formant pattern at the junctures with adjoining vowels, the higher formants (F6 and above) are not reduced in intensity, and F3 is not enhanced in intensity compared to semi-vowels.

\title{
Session 3pSCb
}

\section{Speech Communication: Prosody II}

\author{
Marion Dohen, Chair \\ Speech and Cognition Department, GIPSA-lab, 46 avenue Félix Viallet, Grenoble, 38031, France
}

\section{Contributed Papers}

\section{2:00}

3pSCb1. Audiovisual perception of prosodic contrastive focus in whispered French. Marion Dohen (Speech and Cognition Department, GIPSA-lab, 46 avenue Félix Viallet, 38031 Grenoble, France, marion.dohen@gipsa-lab.inpg.fr), Hélène Loevenbruck (Speech and Cognition Department, GIPSA-lab, 46 avenue Félix Viallet, 38031 Grenoble, France, Helene.Loevenbruck@gipsa-lab.inpg.fr)

This study investigates the potential contribution of visual information in the audiovisual perception of prosodic contrastive focus in French. Contrastive focus is used to attract the listener's attention to a specific part of the utterance. Mostly conceived of as auditory/acoustic, it also has visible correlates which have been shown to be perceived. This study aimed at analyzing the interaction between audition and vision for the perception of prosodic focus by using a whispered speech paradigm. It was based on audiovisual recordings from 4 speakers wearing no facial markers. It com- bined performance assessment to reaction time measurements and confirmed and extended preliminary results obtained on 2 speakers wearing facial markers (needed for a parallel articulatory analysis). The results showed that adding vision to audition for the perception of prosodic focus can not only improve perception performances but can also reduce perceptual cognitive load. A further analysis suggested that the two modalities are integrated for the perception of prosodic focus. Visual only perception was facilitated for whispered speech suggesting an enhancement of visual cues. Moreover, the absence of facial markers did not impair perception. Therefore facial markers do not seem to enhance the relative importance/salience of visual cues.

\section{2:20}

3pSCb2. Effects of boundary tones on accent-related F0 peak alignment. Yen-Liang Shue (Univ. of California Los Angeles, Dept. of Electrical Engineering, 405 Hilgard Ave., Los Angeles, CA 90095, USA, 
yshue@ee.ucla.edu),Markus Iseli(Univ. of California Los Angeles, Dept. of Electrical Engineering, 405 Hilgard Ave., Los Angeles, CA 90095, USA, iseli@ee.ucla.edu), Stefanie Shattuck-Hufnagel (Massachusetts Institute of Technology, Research Laboratory of Electronics, Speech Communication Group, 77 Massachusetts Ave., Cambridge, MA 02139, USA, stef@speech.mit.edu), Nanette Veilleux (Simmons College, Dept. of Computer Science, 300 The Fenway, Boston, MA 02115, USA, nanette.veilleux @simmons.edu), Sun-Ah Jun (Univ. of California Los Angeles, Dept. of Linguistics, 405 Hilgard Ave., Los Angeles, CA 90095, USA, jun@ @umnet.ucla.edu), Abeer Alwan (University of California, 405 Hilgard Ave, Los Angeles, CA 90095, USA, alwan@ee.ucla.edu)

Speakers sometimes delay the F0 peak of a high accent beyond the accented syllable (Silverman and Pierrehumbert, Papers in Laboratory Phonology I, 1990; Xu, Phonetica, 2001). Previous studies of factors affecting peak alignment focused on the phrasal position of the accented word and the segments of the accented syllable (Jilka and Möbius, Interspeech 2007). Shue et al. (Interspeech 2007) hypothesized that if an accented word also contains boundary tones, that could influence the position and height of the F0 peak. A test corpus controlling for vowel type, syllable number and position of the focused word, based on the utterances "Dagada gave Anne a dada (daily)" and "A dada gave Anne dagadas (daily)", was produced using four prosodically different contours, involving high $\left(\mathrm{H}^{*}\right)$ and low $\left(\mathrm{L}^{*}\right)$ pitch accents on either "dagada" or "dada", in declarative and interrogative forms eliciting different boundary tones. Analysis of five repetitions spoken by four male and four female native American English speakers reveals that for most speakers, the presence of boundary tones on the target word shifts the accentrelated F0 peak earlier when other factors are held constant. Preliminary results suggest effects of boundary tone on $\mathrm{H}^{*} \mathrm{~F} 0$ peak height as well.

\section{2:40}

3pSCb3. Double focus in general American English. Fang Liu (The University of Chicago, Department of Linguistics, 1010 E. 59th Street, Chicago, IL 60637, USA, liufang@uchicago.edu)

This study investigates the acoustic realization of double focus in statements and declarative questions in English. Five speakers produced eight sets of utterances with alternating focus (medial, final, or double) and sentence type (statement or declarative question) conditions. F0 analyses indicate that the effect of double focus on the global pitch range and the local pitch target is somewhat moderate when compared to that of single focus: 1) Post-focus pitch range suppression shows a smaller magnitude in doublethan in medial-focused statements, 2) Post-focus pitch range raising is less dramatic in double- than in medial-focused questions, and it ends before the stressed syllable of the second focus in double-focused questions, in order to realize a rising pitch target for the on-focus stressed syllable, and 3) pitch targets of on-focus stressed syllables are more fully realized (i.e., showing steeper slopes or higher registers) in single- than in double-focused sentences. Duration analyses indicate that the length of the double-focused item is in between those of the corresponding single- and non-focused item, with the single-focused stressed syllable having the longest duration. These findings demonstrate a delicate balance among the realization of lexical stress, focus, and sentence type in English.

\section{3:00}

3pSCb4. Melodic prominences structures: exploring to what extent the speaker variability is spreading. Geneviève Caelen-Haumont (MICA Center, C10, Hanoi Universirty of Technology, 1 Dai Co Viet Str., Hai Ba Trung, Hanoi, Hanoi, Viet Nam, genevieve.caelen@mica.edu.vn)

Melodic prominences in speech have a deserved reputation of conveying a great part of variability, as they are greatly based on subjective impulse and feelings. We think that these specific contours are nevertheless relevant, and that their structure, while obeying to internal laws of regulation, allow also a range of speaker variability. Generally no specific tool was used to describe these FO contours with precision. An automatic analysis tool MELISM was then developed by Caelen-Haumont and Auran (2004) allowing accurate descriptions of F0 salience (melisms). This paper aims at $1^{\circ} \mathrm{de}-$ scribing the MELISM tool, the automatic segmentation, the annotation, the melodic labelling of the melism structure it provides in the prosody domain $2^{\circ}$ presenting the main results over 4 speaker exploring to what extent the speaker variability spreads. The melism structures are explored and analysed such as the whole variability is reduced through three major components (onset, nucleus, coda) and their respective subparts, where only the nucleus is the compulsory part. Statistics running on these structures put to light a set of interesting laws about the internal regulation of melisms but in the other side, the part devoted to the speaker variability.

\section{3:20}

3pSCb5. How prosody correlates with syntax: An observation on Sakizaya, an endangered Formosan language. Sally Chen (Grad. Inst. of Linguistics, National Taiwan Univ., 1, Roosevelt Rd. Sec. 4, 106 Taipei, Taiwan, d93142002@ntu.edu.tw), Wen-Chi Shen (Grad. Inst. of Linguistics, National Taiwan Univ., 1, Roosevelt Rd. Sec. 4, 106 Taipei, Taiwan, r94142007@ntu.edu.tw), Li-May Sung (Grad. Inst. of Linguistics, National Taiwan Univ., 1, Roosevelt Rd. Sec. 4, 106 Taipei, Taiwan, limay@ntu.edu.tw), Janice Fon (Grad. Inst. of Linguistics, National Taiwan Univ., 1, Roosevelt Rd. Sec. 4, 106 Taipei, Taiwan, jfon@ntu.edu.tw)

This pioneering study investigates the relationship between prosody and syntax of Sakizaya, an endangered Formosan language in eastern Taiwan Both elicitation data and spontaneous data were collected from five informants (aged 48 to 74), in order to provide a more thorough sketch on this VSO language with clear case marking and voice systems. Preliminary results showed that in descriptive sentences, prosodic units and syntactic units do correlate with each other, depending on which of the four voices is used. (The four voices in Sakizaya: agent-, patient-, instrumental, and locative voices) However, once specific functions are imposed onto a sentence, they will override this "default" setting, and adopt corresponding prosodic patterns for the ease of communication. For example, in this language, the default position to receive a nuclear accent should be the ultimate syllable of a sentence; however, we observed an accent shift onto the penultimate syllable for yes/no questions, exclamations, as well as continuously progressive sentences. In negation sentences, pitch peaks are distinctively higher in the accented syllable of the negator (which posited sentence-initially), instead of the ultimate syllable in sentence-final position. Lastly, case markers and voice markers were found as preferred positions for speech planning and repair. 


\title{
Session 3pSP
}

\section{Signal Processing in Acoustics, Acoustical Oceanography, and ECUA: Model-Based Signal Processing I}

\author{
Sean Lehman, Cochair \\ Lawrence Livermore Natl. Lab., Livermore, CA 94551, USA \\ Christian Pichot, Cochair \\ Antennas \& Telecommunications Laboratory, University of Nice-Sophia Antipolis, France
}

Invited Papers

2:00

3pSP1. Model-based algorithms for detecting damage in ultrasonic nondestructive evaluation measurements. Grace A. Clark (Lawrence Livermore National Laboratory, 7000 East Ave., L-130, Livermore, CA 94550, USA, clark9@1lnl.gov)

This work addresses the "As-Built" modeling problem in ultrasonic nondestructive evaluation (NDE), in which one is given measurements of a mechanical part before and after use. The condition of the "as-built" part (prior to use) is known, so that after the part has been used, it can be tested for damage. This enables a two-step model-based approach: (1) Given input-output measurements (AScans), estimate a dynamic prediction-error model of the "as-built" measurement(s) using system identification algorithms. The model is validated by testing the innovations (residuals) for statistical whiteness and then stored for future use. (2) Later, when testing the part for damage, the error between the measurement and the output of the stored model is tested against a short-term whiteness confidence interval test statistic. If the part passes the test, this implies that the model remains valid and the part is declared undamaged. If the part fails the test, this indicates a model mismatch, which means that the part's acoustic properties have changed, and the part is declared damaged. Performance of the algorithms is demonstrated using real measurements, receiver operating characteristic (ROC) curves and a confidence interval about the probability of correct classification.

3pSP2. Signal-based ray tracing modeling in complex tectonics. Paul Cristini (CNRS-UMR5212 Modélisation et Imagerie en Géosciences, UPPA BP115, 64013 Pau, France, paul.cristini@univ-pau.fr), Eric De Bazelaire (11, Route du Bourg, 64230 Beyrie-enBéarn, France, edebaz@wanadoo.fr), Charles Revaux (50, Rue Lagardere, 64000 Pau, France, charles.revaux@wanadoo.fr)

The objective of this talk is to present a novel method which can perform the fast computation of the times of arrival of seismic waves which propagate between a source and an array of receivers in a stratified medium. This method combines signal processing concepts for the approximation of interfaces and wavefronts, and ray theory for the propagation of wavefronts. The main idea is to put in adequation the computations with the the precision and the resolution of the source signal in order to avoid unnecessary computational effort. This new approach leads to the redefinition and simplification of the model through which waves propagate. The modifications are governed by the spectral characteristics of the source signal. All rays are computed without any omission at a much lower cost in computing time than classical methods. In addition, we will show how to include surface waves such as head waves within the proposed method.

\section{Contributed Papers}

2:40

3pSP3. Imaging the earth's subsurface via regularized inversion Robert Clapp (Stanford University, 397 Panama Mall, Geophysics Department, Stanford, CA 94305, USA, bob@sep.stanford.edu)

Modern seismic exploration geophysics attempts to construct a model of the earth's subsurface using measurements taken at the earth's surface. The recorded measurements comprise datasets with billions to trillions of samples. Despite the size of the datasets, models are often poorly sampled because of the complexity of the earth's subsurface. Therefore, the resultant model is poorly determined for many components. The massive size of our problems introduce additional limitations. We are limited to adjoint-based inversion methods, we can never afford to iterate to true convergence, and most importantly we make approximations to the physics in our operators. These limitations introduce spurious events when inverting. Significantly improved results can be obtained by incorporating regularization that incorporates a priori knowledge of the physics and geology into the inversion process.

\section{3:00}

3pSP4. Characterization of targets buried in disordered medium Kamal Belkebir (Faculté St Jérôme, 13397 cedex 20 Marseille, France, kamal.belkebir@fresnel.fr), Faheem Ashraf (Faculté St Jérôme, 13397 cedex 20 Marseille, France, faheem.ashraf@fresnel.fr), Marc Saillard (Faculté St Jérôme, 13397 cedex 20 Marseille, France, marc.saillard@1seet.univ-tln.fr), Patrick Chaumet (Faculté St Jérôme, 13397 cedex 20 Marseille, France, patrick.chaumet@fresnel.fr)

We consider in this paper the problem of the determination of the permittivity profile of an unknown buried object from measurements of the electromagnetic scattered field. The target under test is assumed to be buried in one of the two involved media while the sources and the receivers are located in the other medium (limited-aspect data configuration). This illposed and non-linear inverse scattering problem is reformulated as an optimization problem that is solved iteratively. This method consists in building up a sequence of the parameter of interest by minimizing, at each iteration step, a cost functional representing the discrepancy between the data and 
those that would be obtained with the best available estimation of the parameter. In addition, when clutter is present, the decomposition of the time reversal operator method is used to improve the signal-to-clutter ratio, since it allows us to synthesize a wave that focuses on the scatterer. The data associated with this incident field are included in the iterative minimization procedure.

\title{
Invited Paper
}

3:20

3pSP5. Underwater channel characterization using opportunity sources : a time-frequency-phase approach. Cornel Ioana (GIPSA-lab, dep. DIS, 961, rue de la Houille Blanche, 38402 St Martin d’Hères, France, cornel.ioana@gipsa-lab.inpg.fr), Arnaud Jarrot (Schlumberger Riboud Product Center, 1 rue Becquerel, 92140 Clamart, France, ajarrot@clamart.oilfield.slb.com), Cédric Gervaise (E3I2 - EA3876, 2 rue François Verny, 29806 Brest Cedex, France, cedric.gervaise@ensieta.fr), Andre Quinquis (Scientific Research and Innovation Division, DET/GESMA BP 42, 29240 Brest, France, andre.quinquis@dga.defense.gouv.fr), Jérôme I. Mars (GIPSA-lab, dep. DIS, 961, rue de la Houille Blanche, 38402 St Martin d'Hères, France, jerome.mars@ gipsa-lab.inpg.fr)

Analyzing natural signals constitutes the main tool for characterization of physical phenomena. Underwater channel is an example of a natural environment potentially characterized by signals generated by various sources : underwater mammals, human activity noise, etc. In order to efficiently exploit the information from these signals two major problems should be addressed. First, since the signals are unknown or disturbed by unpredictable factors, we are deal with a blind processing context. That is, the lack of a priori hypothesis has to be considered. Generally, the signal has a complex shape characterized by multi-component non-linear time-frequency structures. The proposed solution consists in focusing our processing on non-parametric time-frequency analysis considering also fundamental signal items such as time-frequency energy and local phase analysis. The second problem is related to the complex connection between physical parameters of a phenomenon and parameters of signals characterizing this phenomenon. The proposed approach consists of combining the physical model with the information provided by a parametric representation of the signal. This framework helps to the definition of the concept of underwater passive tomography which provides the characterization of the underwater channel of interest by taking advantage of environmental signals : mammals vocalizations, motion sources, etc.

\section{Session 3pUWa}

\section{Underwater Acoustics and ECUA: Fluctuations and Statistics}

\author{
Barry J. Uscinski, Cochair \\ Cambridge University, Centre for Mathematical Sciences, Wilberforce Rd., Cambridge, CB3 OWA, UK \\ Kevin D. Lepage, Cochair \\ Naval Research Laboratory, 4555 Overlook Ave SW, Washington, DC 20375, USA
}

\section{Contributed Papers}

2:00

3pUWa1. Horizontal structure of acoustic intensity fluctuations in the ocean. Barry J. Uscinski (Cambridge University, Centre for Mathematical Sciences, Wilberforce Rd., CB3 0WA Cambridge, UK, bju1@cam .ac.uk), Jim Nicholson (QinetiQ Ltd., Winfrith Technology Centre, Dorchester, Dorset, DT2 8XJ Dorchester, UK, jrnicholson@qinetiq.com)

In April 1989 an acoustic experiment was performed over the abyssal plain south of Msderia in which transmissions were made, for about an hour, at 482,680 and $740 \mathrm{~Hz}$ from a ship steaming at $5 \mathrm{kts}$ to a receiving array towed by another ship $65 \mathrm{~km}$ away travelling on a parallel course at the same speed. The signals arrived by two paths, an upper path trapped in the surface duct and a lower path via the main sound channel. This paper describes the experiment and analyses the intensity fluctuations in the signal received by the lower path. We investigate the horizontal structure of intensity fluctuations in the ocean when these are mainly due to internal waves. This aspect of such acoustic intensity fluctuations has received little attention until now. The experimental results are compared with theoretical predictions based on the parabolic moment equations for propagation and scattering in randomly irregular media, and on the stnadard Garrett-Munk model for ocean internal waves. The experimental results and theoretical predictions agree quite well but some new questions arise about the correlation of intensity fluctuations as the acoustis transmission frequency is varied.

\section{2:20}

3pUWa2. Evolution of the statistics of the unscattered component of low order acoustic modes as a function of range. Tarun $\mathrm{K}$. Chandrayadula (George Mason University, 4400, University Drive, Fairfax, VA 22030, USA, tchandra@gmu.edu), Kathleen E. Wage (George Mason University, 4400, University Drive, Fairfax, VA 22030, USA, kwage@gmu .edu)

Scattering due to internal waves in the ocean causes the acoustic modes to exchange energy as they propagate. At a specific range, the mode signal consists of two components: the unscattered component is the energy that has propagated only in the designated mode and the scattered component contains the contributions from other modes. The unscattered component dominates the signal at short ranges, but decays to zero at longer ranges. If the unscattered component can be isolated from the scattered energy, it could be used in tomographic inversions. Signal processing techniques are needed to detect the unscattered component. A statistical model for the unscattered component that would help design signal processing techniques is currently unavailable. This talk describes a new model for the unscattered component that was developed using coupled mode and parabolic equation simulations. The model characterizes the statistics of the unscattered component using parameters such as frequency and time coherence. The characteristics of the unscattered component are then compared with the scat- 
tered component. The implications of these results for the design of detectors is briefly discussed. [Work supported by ONR Ocean Acoustics Graduate Traineeship Award]

\section{2:40}

3pUWa3. Exact and approximate moments for dispersive pulse propagation. Leon Cohen (City University of New York, Hunter-Phyiscs, 695 Park Ave., New York, NY 10021, USA, leon.cohen @ hunter.cuny.edu), Patrick Loughlin (University of Pittsburgh, 348 Benedum Engineering Hall, Dept. of Electrical \& Computer Engineering, Pittsburgh, PA 15261, USA, loughlin@engr.pitt.edu)

We derive exact moments for pulse propagation in a dispersive medium. These moments are not only inherently interesting but clarify the validity of a recently proposed approximation scheme for wave propagation. The approximation method for pulse propagation is based on the Wigner positionwave/number representation and is very accurate, easy to apply, and moreover is physically illuminating. In particular one obtains the evolved approximate Wigner distribution from the initial Wigner distribution by a simple linear translation in phase space. Propagation with damping is also taken into account. We will show that the reason for the high accuracy of the approximation is that the important low order moments are exactly given by the approximation and that these low order moments preserve very well the basic shape of the pulse. Moreover, now that we understand why the approximation method works well, the approximation can be systematically improved. We give a number of specific examples of exactly calculable moments to illustrate the method and we compare exact and approximate moments. The work of PL is supported by The Research of LC was supported by AFOSR and the work of PL is supported by ONR (N00014-06$1-0009)$

\section{3:00}

3pUWa4. Absolute intensities of acoustic shadow zone arrivals. Lora Van Uffelen (MPL, Scripps Institution of Oceanography, University of California, San Diego, La Jolla, CA 92093-0238, USA, lvanuffe@ucsd .edu), Peter Worcester (MPL, Scripps Institution of Oceanography, University of California, San Diego, La Jolla, CA 92093-0238, USA, pworcester@ucsd.edu), Matthew Dzieciuch (MPL, Scripps Institution of Oceanography, University of California, San Diego, La Jolla, CA 920930238, USA,mad@ucsd.edu)

Qualitative observations from bottom-mounted US Navy SOSUS receiving stations in the North Pacific reveal anomalously deep acoustic arrivals at travel times directly corresponding with timefronts expected to have turned much higher in the water column. The vertical structure of these shadow zone arrivals was studied during SPICEX, a long-range propagation experiment conducted from June to November 2004 in the North Pacific, utilizing moored sources 500 and $1000 \mathrm{~km}$ distant from two vertical line array receivers, which together virtually spanned the full ocean depth. Comparison of the measured absolute intensities of shadow zone arrivals with Monte Carlo parabolic equation simulations suggest that the amount of internal wave scattering associated with the standard Garrett-Munk (GM) internal wave spectrum is not adequate to account for the extent of scattering into the acoustic shadow evident in the experimental data, suggesting either that the GM spectrum is not an appropriate representation of the internal wave field or that some other mechanism, such as oceanic spice, may be also be contributing to the scattering.

\title{
Session 3pUWb
}

\section{Underwater Acoustics and ECUA: Array Processing}

\author{
David L. Bradley, Cochair \\ Pennsylvania State University, Post Office Box 30, State College, PA 16804-0030, USA \\ Claire Debever, Cochair \\ MPL, Scripps Institution of Oceanography, University of California, San Diego, La Jolla, CA 92093-0238, USA
}

\section{Contributed Papers}

2:00

3pUWb1. Effect of array element location on coherent inter-array processing. Claire Debever (MPL, Scripps Institution of Oceanography, University of California, San Diego, La Jolla, CA 92093-0238, USA, cdebever@ucsd.edu), William A. Kuperman (MPL, Scripps Institution of Oceanography, University of California, San Diego, La Jolla, CA 92093-

\section{8, USA, wkuperman@ucsd.edu)}

In the SWellEx-96 experiment, two 25-elements horizontal arrays, each of length $250 \mathrm{~m}$, were moored $3.5 \mathrm{~km}$ apart [N.O. Booth et al, IEEE, JOE, 25 , no 3, July 2000]. A broadband source (50 to $400 \mathrm{~Hz}$ tones) was towed from $1 \mathrm{~km}$ to $10 \mathrm{~km}$ away from the arrays. The data is processed using the simple conventional plane-wave beamformer for all the cases covering incoherent frequency and incoherent inter-array processing to coherent frequency and coherent inter-array processing as a function of source position relative to the arrays. The effect of noise and array element location mismatch on source localization and coherent processing gain is investigated. An attempt to improve array element localization is made using a selffocusing technique. [Work supported by ONR.]
2:20

3pUWb2. Longitudinal Correlation Improvement with Model-based Technique. Renhe Zhang (National Laboratory of Acoustics, Institute of Acoustics, Chinese Academy of Sciences, No 21, Beisihuanxilu, 100080 Beijing, China, zrh@mail.ioa.ac.cn), Fenghua Li (National Laboratory of Acoustics, Institute of Acoustics, Chinese Academy of Sciences, No 21, Beisihuanxilu, 100080 Beijing, China, lfh@mail.ioa.ac.cn)

Longitudinal correlation coefficient is one of the most important parameters for signal processing in ocean acoustics. Multi-path interference is an important effect on the decrease of the longitudinal correlation for low frequency sound propagation in shallow water. In this research, the FrequencyPhase-Shift Relationship (FPSR) between the Frequency Response Function (FRF) of sound propagation at two horizontally separated locations based on waveguide invariant is derived. The validity of the FPSR is demonstrated by both numerical simulations and data from two experiments. Experimental data also show that the decrease of the longitudinal correlation coefficient of low frequency signals from explosive source due to multi-path interference can be conquered significantly with the FPSR. [Work supported by the National Natural Science Foundation of China under Grand No 10734100]. 
2:40

3pUWb3. Performance analysis of GARCH based DOA estimation in SONAR. Hadi Amiri (Engineering Research Institute, Ministry of J-Agriculture, 13445-754 Tehran, Iran, h.amiri@ieee.org), Hamidreza Amindavar (Amirkabir University of Technology, Department of Electrical Engineering, 15914 Tehran, Iran, hamidami@aut.ac.ir), Mahmoud Kamarei (University of Tehran, Department of Electrical and Computer Engineering, 14395-515 Tehran, Iran, kamarei@ut.ac.ir)

In this paper we propose a new source localization method using additive noise modeling based on Generalized Autoregressive Conditional Heteroscedasticity $(\mathrm{GARCH})$ time-series. In an actual application such as underwater acoustics, the measurement of additive noise in a natural environment shows that noise can sometimes be significantly non-Gaussian and nonstationary, and therefore, signal processing algorithms that are optimized for Gaussian noise, may degrade significantly in this environment. GARCH models are feasible for heavy tailed PDFs and time varying variances of stochastic process and also has flexible forms. We use a more realistic $\operatorname{GARCH}(1,1)$ based noise model in the Maximum Likelihood Approach for the estimation of Direction-Of-Arrivals (DOAs) of impinging sources. In the performance analysis of the method, we examine the suitability of the proposed method in a passive sonar using simulation methods with cramer-rao bound (CRB) and perturbaion appraoch such as gain, phase and sensors positions errors.

\section{3:00}

3pUWb4. Direct wave suppression using wavelet transform for bistatic sonar. Bo Lei (Institute of Acoustic Engineering, Northwestern Polytechnical University, 710072 Xi’an, China, lei.bo.thunder@gmail .com), Yuanliang Ma (Institute of Acoustic Engineering, Northwestern Polytechnical University, 710072 Xi'an, China, ylma@nwpu .edu.cn), Kunde Yang (Institute of Acoustic Engineering, Northwestern Polytechnical University, 710072 Xi’an, China, ykdzym@nwpu .edu.cn), Chao Sun (Institute of Acoustic Engineering, Northwestern Polytechnical University, 710072 Xi'an, China, csun@nwpu.edu.cn)

The bistatic sonar is often influenced by the direct wave, especially when the target approaches the baseline of the system. The direct wave is above $50 \mathrm{~dB}$ higher than the scattering signal level. The paper analyzes the effect of the direct wave, and proposes a new method based on wavelet transform for direct wave suppression. The strong direct wave is well eliminated while maintaining the weak scattering signal almost unchanged. The basic idea is that the direct wave is firstly removed form the received signal after wavelet transform. Then the signal is reconstructed in time domain, finally the cross-correlation is achieved to shown the doppler frequency shift of the weak scattering signal. Computer simulation is given. The crosscorrelations of the received signal for both before and after the direct wave suppression are compared. It shows that the influence of the direct wave is correctly removed, the time delay and Doppler frequency shift are shown in the figure and the target is detectable.

\section{3:20}

3pUWb5. Adaptive beamforming applied to underwater acoustic measurements. Denis Orlov (Institute of Applied Physics of the Russian Academy of Sciences, 46 Ulyanov Street, 603950 Nizhny Novgorod, Russian Federation, denis@hydro.appl.sci-nnov.ru), Iosif Fiks (Institute of Applied Physics of the Russian Academy of Sciences, 46 Ulyanov Street, 603950 Nizhny Novgorod, Russian Federation, fiks@ @ydro.appl .sci-nnov.ru), Galina Fiks (Institute of Applied Physics of the Russian Academy of Sciences, 46 Ulyanov Street, 603950 Nizhny Novgorod, Russian Federation, galya@hydro.appl.sci-nnov.ru), Pavel Korotin (Institute of Applied Physics of the Russian Academy of Sciences, 46 Ulyanov Street, 603950 Nizhny Novgorod, Russian Federation, monitor@hydro.appl .sci-nnov.ru), Victor Turchin (Institute of Applied Physics of the Russian Academy of Sciences, 46 Ulyanov Street, 603950 Nizhny Novgorod, Russian Federation, tvi@hydro.appl.sci-nnov.ru)

In many applications, such as measuring the underwater noise level of moving ships, underwater acoustic measurements face serious difficulties related to several factors, including low signal-to-noise ratio, effects of surface and bottom on signal, as well as complicated spatial structure of the sea interference. The increase of the measurement interference resistance, compared to the case of a single receiver (hydrophone), can be provided by the use of spatially distributed receiving systems (antenna arrays), which are able to suppress the interference due to their spatial selectivity. The present work is aimed at developing adaptive methods for underwater acoustic measurements with the use of vertical antenna arrays. The method must provide the maximum reduction of external interference keeping the given measurement accuracy, i.e., the result must coincide with the output of a single receiver in the absence of interference. From the point of view of synthesis of array systems, the originality of the presented approach is mainly in conjunction of measurement functionality of the antenna array and the maximum interference suppression. The results of numerical simulation and experimental testing under sea conditions show that the proposed adaptive methods provide high precision of measurements under strong and/or complex interference conditions. 
Session 3pUWc

\title{
Underwater Acoustics and ECUA: Objects Scattering and Detection
}

\author{
Sven M. Ivansson, Cochair \\ Swedish Defence Research Agency, FOI Kista, Stockholm, SE-16490, Sweden \\ Ahmad Abawi, Cochair \\ HLS Research, Inc., 3366 N. Torrey Pines Ct., Ste. 310, La Jolla, CA 92037, USA
}

\section{Contributed Papers}

2:00

3pUWc1. Numerical design of Alberich anechoic coatings with superellipsoidal cavities of mixed sizes. Sven M. Ivansson (Swedish Defence Research Agency, FOI Kista, SE-16490 Stockholm, Sweden, sveni @ foi.se)

Thin rubber coatings with cavities in a doubly periodic lattice are able to reduce reflections of underwater sound by redistributing normally incident energy such that absorption in the surrounding rubber is enhanced. For spherical scatterers, the anechoic effect can be studied numerically by the layer multiple-scattering method. In comparison to more flexible but also more computer intensitive methods, such as FEM modeling, there are two important advantages. An improved physical understanding of the anechoic effect can be achieved by simplified semianalytical analysis, and the high computational speed allows modern global optimization techniques to be applied for coating design. In this paper, the flexibility of the layer multiscattering method is improved by combination with an efficient algorithm for numerical computation of transition matrices for superellipsoidal scatterers. Extensions to mixtures of nonspherical scatterers of different types are also considered, in order to enhance the broad-band performance. Symmetry properties are used to reduce the size of the pertinent equation systems. Examples of numerical coating design for underwater acoustic applications are presented, using differential evolution algorithms for the optimization.

\section{2:20}

3pUWc2. Scattering from rotationally-symmetric objects using only free space Green's functions. Ahmad Abawi (HLS Research, Inc., 3366 N. Torrey Pines Ct., Ste. 310, La Jolla, CA 92037, USA, Abawi@HLSResearch.com), Michael B. Porter (HLS Research, Inc., 3366 N. Torrey Pines Ct., Ste. 310, La Jolla, CA 92037, USA, michael.porter @ hlsresearch.com)

To compute scattering from an object, one has to solve the wave equation and impose the appropriate boundary conditions on the surface of the object. For objects for which the wave equation can be separated, like cylinders, spheres and spheroids, this problem can be solved analytically. For more general objects, methods like the finite element or boundary element techniques can be employed. The use of free space Green's functions offers another method for computation of scattering from a general-shaped object. This method, which is known as the method of field superposition or the virtual source technique, can be used to impose the appropriate boundary conditions on the surface of the object by using free space Green's function with complex amplitudes. These amplitudes are determined from a matrix equation that results when boundary conditions are imposed. In this paper we apply this technique to compute scattering from rotationally symmetric objects. These objects can be homogeneous solids or shells, filled with a homogeneous fluid. The versatility and robustness of the method is demonstrated by applying it to various objects in free space and in a waveguide.

\section{2:40}

3pUWc3. Modeling of underwater sonar barriers. Andrzej Elminowicz (R\&D Marine Technology Centre, Ul. Dickmana 62, 81-109 Gdynia, Poland, andrzeje@ctm.gdynia.pl), Leonard Zajaczkowski (R\&D Marine Technology Centre, Ul. Dickmana 62, 81-109 Gdynia, Poland, leonard@ctm.gdynia.pl)

Commonly used Diver Detection Sonar (DDS) are characterized by detection and tracking as well as intruder warning in limited range $300 \mathrm{~m} \div$ $600 \mathrm{~m}$. A new approach to underwater protection is focused on multimonostatic, bistatic and multistatic active sonar barriers (ASB). This paper describes key features of a multi-monostatic (MM), bistatic (BS) and multistatic (MS) operation in the littoral beginning from system configuration, its performance and constraints. A new type of acoustic devices in a form of a transmitting/receiving module and its application in active sonar barriers (ASB) has also been presented. Coverages and ranges of barriers for various technical parameters, environmental conditions and wave propagation as well as modules location (including transducers' depth, azimuth and inclination) have been studied. The application of active sonar barriers (ASB), especially in protection systems, has been presented as barriers protecting docks, harbour basins, ships at piers or harbour entries. The new type of a monostatic/bistatic acoustic barrier in technology demonstrator form, has been designed and constructed. In order to display detection and localization of the small underwater objects as well as their tracking and classification the performance tests of the barrier will be conducted. The results of the tests will be attached to the paper.

\section{3:00}

3pUWc4. Wideband sonar system for autonomous surveys using REMUS. Chris Capus (Ocean Systems Laboratory, School of Eng. \& Phys. Sciences, Heriot-Watt University, EH14 4AS Edinburgh, UK, C.Capus@hw.ac.uk), Yan Pailhas (Ocean Systems Laboratory, School of Eng. \& Phys. Sciences, Heriot-Watt University, EH14 4AS Edinburgh, UK, Y.Pailhas@hw.ac.uk), Keith E. Brown (Ocean Systems Laboratory, School of Eng. \& Phys. Sciences, Heriot-Watt University, EH14 4AS Edinburgh, UK, K.E.Brown@hw.ac.uk)

Many new roles are being proposed for Autonomous Underwater Vehicles (AUVs) to carry out hazardous tasks in harsh or remote locations and to free up valuable resources required for manned missions. These roles include marine environmental survey, target detection and classification and tracking of underwater pipes and cables. Suitable sensing and processing packages must be provided and building on recent wideband sonar research, the Ocean Systems Laboratory (OSL) is putting together a wideband system for deployment on board a REMUS AUV. The prototype sensor package comprises paired projectors and receivers mounted in a side-looking arrangement to provide complementary information to the standard REMUS sidescan modules. The sensor bandwidths cover a range from $30-130 \mathrm{kHz}$ similar to those used by the bottlenose dolphin. Data is gathered autonomously with a dedicated AMD Geode based PC104+ PC controlling a 4-channel $800 \mathrm{kHz}$ simultaneous sampling data acquisition module. Acquisition is triggered to coincide with mission requirements from a separate mission control PC on board the vehicle. 
3:20

3pUWc5. A multistatic synthetic aperture sonar to detect a cylinder lying on a rough interface: experimental results. Caroline Herve (Laboratory for Mechanics and Acoustics CNRS, 31 chemin Joseph Aiguier, 13009 Marseille, France, herve@1ma.cnrs-mrs.fr), Jean-Pierre Sessarego (Laboratory for Mechanics and Acoustics CNRS, 31 chemin Joseph Aiguier, 13009 Marseille, France, sessarego@1ma.cnrs-mrs.fr), Régine Guillermin (Laboratory for Mechanics and Acoustics CNRS, 31 chemin Joseph Aiguier, 13009 Marseille, France, guillermin@1ma.cnrs-mrs .fr), Françoise Schmitt (Groupe d'Electromagnétisme Appliqué, Pôle Scientifique et Technologique de l'Université Paris X Nanterre Site de Ville d'Avray, 50, rue de Sèvres, 92410 Paris Ville-d'Avray, France, fschmitt@u-paris10.fr), Franck Daout (Groupe d'Electromagnétisme Appliqué, Pôle Scientifique et Technologique de 1'Université Paris X Nanterre Site de Ville d'Avray, 50, rue de Sèvres, 92410 Paris Ville-d'Avray, France, fdaout@u-paris10.fr
Monostatic Synthetic Aperture Sonar (SAS) are high resolution systems for target imaging which are now of common use in the underwater acoustics domain. The objective of this work was to analyse what kind of information should be obtained from a multistatic SAS system. This idea has been applied in radar but very few works exist in the underwater acoustics domain. The applications could be detection and identification of buried mines or mines lying on the seabed and divers detection for harbour protection. These applications deal with the problem of target detection and identification near a rough surface. To show what can be obtained with a multistatic SAS processing, we have performed an experiment with a circular cylinder of $1 \mathrm{~cm}$ diameter lying on a rough interface made of sand grains of $1 \mathrm{~mm}$ diameter. Measurements were performed in a tank with both a multistatic and a monostatic SAS systems. The signal used to insonified the target area was a short impulse with a $2 \mathrm{MHz}$ central frequency which corresponds to a ka about 40 . Images of the cylinder in presence of clutter have been reconstructed with the matched filtering algorithm from monostatic and multistatic acquisitions and have been compared. 


\title{
Awards Ceremony
}

\author{
Gilles A. Daigle \\ President, Acoustical Society of America \\ Luigi Maffei \\ President, European Acoustics Association \\ Jean Kergomard \\ President, Société Française d'Acoustique
}

\section{Acoustical Society of America}

\section{Presentation of ASA Fellowship Certificates}

\author{
Michael A. Akeroyd \\ Susanna B. Blackwell \\ Dick B. Botteldooren \\ Dani M. Byrd \\ Brian R. Glasberg \\ Larry E. Humes \\ Vera A. Khokhlova
}

\author{
Christophe D. Micheyl \\ Anthony P. Nash \\ John C. Osler \\ Subramaniam D. Rajan \\ Richard M. Stern \\ Brad H. Story \\ Lily M. Wang
}

2007 Research Grant in Speech Science of the American Speech-Language-Hearing Foundation to Mary K. Fagan

Medwin Prize in Acoustical Oceanography to Mark V. Trevorrow

Distinguished Service Citation to Charles E. Schmid

R. Bruce Lindsay Award to Tyrone M. Porter

Helmholtz-Rayleigh Interdisciplinary Silver Medal to James V. Candy

Gold Medal to Patricia K. Kuhl

\section{European Acoustics Association}

Award for Lifetime Achievements in Acoustics to Gunnar Rasmussen

Award for Contributions to the Promotion of Acoustics in Europe to the Federation of Acoustical Societies of Europe (FASE) 1971-1996

Award for Outstanding Recent Scientific Results Published in Acta Acustica united with Acustica to Catherine Guastavino, Brian F. G. Katz, Jean-Dominique Polack, Daniel J. Levitin, and Danièle Dubois

\section{Société Française d'Acoustique}

The French Medal to Vitalyi Gusev

The Foreign Medal to Walter Lauriks 


\title{
Session 3eID
}

\section{Plenary Session}

\author{
Introduced by Dick Botteldooren, Ghent University, Belgium
}

\section{$5: 40$}

3eID1. Binaural Hearing and Systems for SOund Reproduction. Philip A. Nelson (ISVR, University of Southampton, Highfield, SO171BJ Southampton, UK, P.A.Nelson@aoton.ac.uk), M. Park (ISVR, University of Sothamton, Highfield, SO171BJ Southamption, UK, unknown@unknown.com), Takashi Takeuchi (OPSODIS Limited, c/o ISVR, University of Southamption, Highfield, SO17 1BJ Southampton, UK, ff1@isvr.soton.ac.uk)

Recent developments in models of binaural hearing can be usefully adapted and extedned to provide design tools for engineers engaged in the design of systems for sound reproduction. The particular focus of the work described is upon the development of models that give good statistical predictions of human sound localisation, based upon knowledge of the fluctuating acoustic pressures at the ears. Such models can be applied successfully to the prediction of stereophonic image localisation and reveal a number of important features of localisation relevant to audio system design. Developments will also be described in loudspeaker based systems for binaural reproduction that are finding their way into practical use. Binaural hearing models can be used to provide a preliminary evaluation of the performance of alternative designs. Finally, a brief review will be presented of multi-channel loudspeaker-based systems aimed at "full field" sound reproduction. Again, models of localisation provide some useful guidance for the designers of such systems.

\section{Introduced by William M. Hartmann, Michigan State University, USA}

\section{6:10}

3eID2. Optoacoustics: Can ultrasound become the preferred modality for molecular imaging? Matthew O'Donnell (University of Washington, 371 Loew Hall, Box 352180, Seattle, WA 98195-2180, USA, odonnel@engr.washington.edu)

Optoacoustics can be used to image the distribution of optical absorption in tissue, combining the specificity and sensitivity of optical imaging with the high resolution and penetration of ultrasound imaging. It represents one of the most promising techniques for molecular imaging because the optical absorption of bioconjugated nanoparticles can greatly exceed that of tissue over a range of wavelengths in which light can penetrate multiple centimeters into the body. We have explored several types of nanoparticles conjugated to a range of antibodies targeted to several important biological systems. Here we discuss two potential applications of bioconjugated gold nanorods, one for cancer cell targeting and the other to identify inflamed endothelial cells signaling the early stages of atherosclerosis. Optoacoustic images of cell cultures and animal models demonstrate the sensitivity and specificity of these nanosystems for molecular imaging. To translate these experimental findings into a clinically acceptable molecular imaging modality, we have also explored integrated optical systems able to deliver the optical pulse for optoacoustic excitation and detect the resultant ultrasonic waves using an all-optical transducer. The basic operating principles of this device, and the prospects for ultrasound-based molecular imaging using it, will be discussed. 


\title{
ACOUSTICAL SOCIETY OF AMERICA GENERAL ASSEMBLY
}

\section{Acoustical Oceanography Prize Lecture}

\author{
James H. Miller, Chair \\ Univ. of Rhode Island, Dept. of Ocean Engineering, Narragansett, RI 02882
}

Chair's Introduction-5:40

$$
5: 45
$$

The use of moored inverted echosounders for monitoring near-surface processes. Mark Trevorrow (Defence R\&D Canada Atlantic, 9 Grove St., PO Box 1012, Dartmouth, NS B2Y 3Z7, Canada, mark.trevorrow@drdc-rddc.gc.ca)

The near-surface ocean is a difficult place to conduct measurements of physical and biological processes. High-frequency inverted echo-sounders (IES), moored a short distance below the surface, provide a simple means for sustained, high-resolution monitoring. In particular, long duration deployments are useful in capturing transient events, such as storms or solitary wave trains, and placing their occurrence within the context of longer term trends. Lessons learned from use of these moored IES systems can be applied to modern ocean observatories. A number of long duration deployments of a self-contained 200kHz IES were made in the late 1990's. Three specific examples will be discussed. Firstly, an investigation of calibrated volume scattering from near-surface bubbles in the NE Pacific Ocean showed the frequent occurrence of vertical, plume-like structures drawn downwards up to $25 \mathrm{~m}$. Within the plumes, the backscatter cross-section exhibited an exponential decay with depth, with e-folding scale in the range 0.5 to $2.5 \mathrm{~m}$. Secondly, imaging of near-surface internal solitary waves was performed from a mooring near the Oregon coast, supplementing measurements made with nearby temperature sensors. These measurements showed some suggestion of scattering by turbulence. Thirdly, through the use of both backscatter intensity and echo statistics, a moored IES demonstrated quantitative monitoring of migratory zooplankton and fish populations.

\section{Celebration of the $60^{\text {th }}$ Anniversary of the French Acoustical Society}

On June 25, 1948, the so-called "Groupement des Acousticiens de Langue Française" (GALF) was created, with its first President, Yves Rocard, and was transformed into the "Société Française d'Acoustique" (SFA) in 1986. SFA now has 830 members and is composed of 8 technical groups and 3 regional sections. It is one of the founding members of the European Acoustics Association (EAA).

The $60^{\text {th }}$ anniversary will be focused on the presentation of the "White Book" of Acoustics in France, and the publishing of a DVD including the main part of the French journals in Acoustics since 1934.

\section{Agenda}

Opening by the current President of SFA and a former President (René-Guy Busnel)

Acoustical French journals since 1934: Presentation of the DVD

The White Book of Acoustics in France in 2008

White Book: An example of the position of acoustics in an activity sector: The automotive industry

60 years of acoustics in France: An overview 


\section{ACOUSTICAL SOCIETY OF AMERICA DISTINGUISHED SERVICE CITATION}

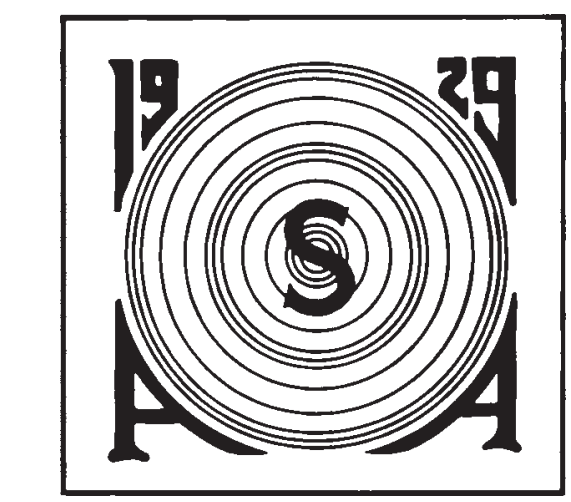

\section{Charles E. Schmid}

\section{8}

The Distinguished Service Citation is awarded to a present or former member of the Society in recognition of outstanding service to the Society.

\section{PREVIOUS RECIPIENTS}

Laurence Batchelder

Robert W. Young

Betty H. Goodfriend

Gerald J. Franz

Robert T. Beyer

Henning E. von Gierke

R. Bruce Lindsay

William S. Cramer

Stanley L. Ehrlich

Samuel F. Lybarger

1972
1973
1973
1974
1978
1978
1981
1984
1986
1986

1986
Frederick E. White

Daniel W. Martin

Murray Strasberg

William J. Cavanaugh

John C. Burgess

Alice H. Suter

Elaine Moran

John V. Bouyoucos

F. Avril Brenig

Thomas D. Rossing
1987

1989

1990

1994

1996

1997

1999

2000

2000

2006 


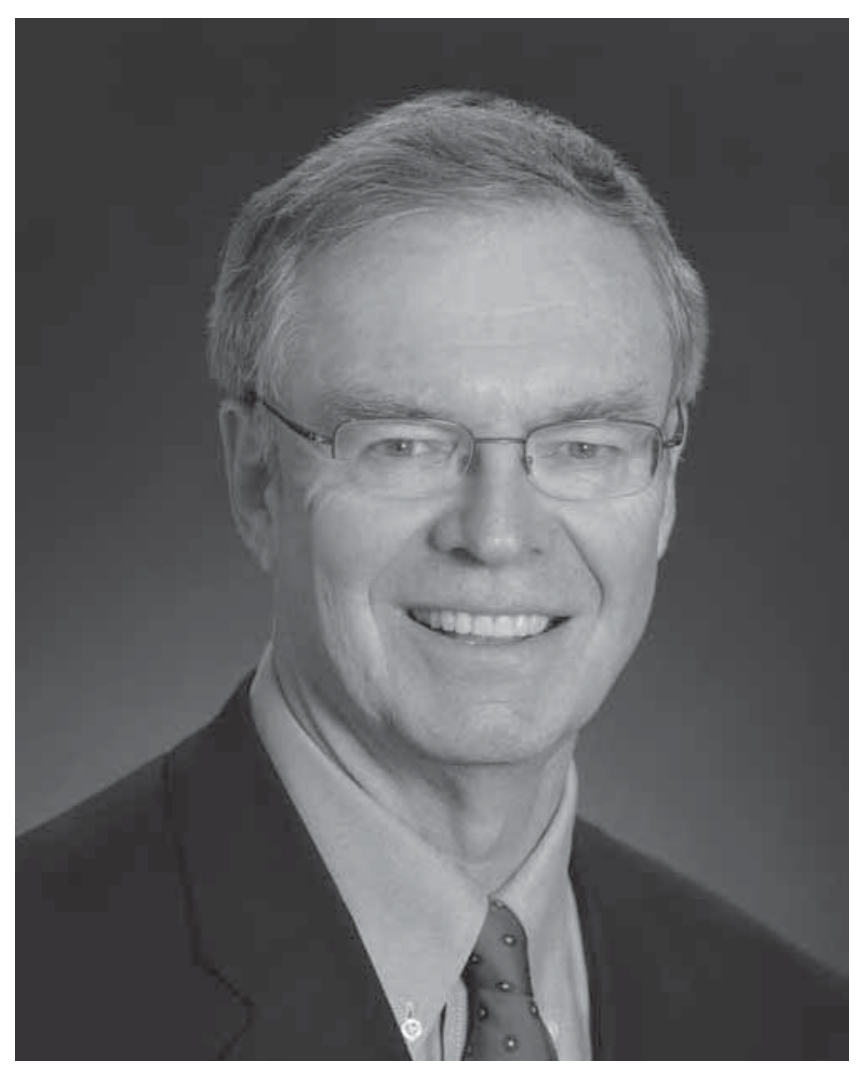




\section{CITATION FOR CHARLES E. SCHMID}

... for contributions as Executive Director of the Acoustical Society of America

\section{PARIS, FRANCE ・ 2 JULY 2008}

Charles Ernest Schmid grew up in East Williston, New York, about a 15-minute drive from the ASA's current headquarters in Melville. His father was an electrical engineer who ran the Shield Electric Company, which was founded by Charles's grandfather in 1909. They developed a process called thermo-welding for third rails for the Long Island Railroad and the New York City subway system. Charles's mother was a social worker who volunteered for the Red Cross. Long before the term "family values" became a cliché, Charles's parents were the genuine article, and they imparted those values to their children. His dad was a hard worker but tenderhearted and sometimes took in friends or relatives who were down on their luck. His mother was the practical one-very smart with a quick wit. Charles had one sibling, a younger sister Susan. There was much laughter in the Schmid household, and they had close and friendly relations with their neighbors and relatives. Summers were spent at their cottage on Fire Island, NY. There, Charles and Susan were free to roam-swimming, fishing, and clamming with their cousins and friends. It was an idyllic childhood for Charles, and that warmth and sense of optimism has carried over to his adult life.

At school Charles showed an early interest in mathematics, science, and history. Charles's great grandfather had emigrated from Ulm, Germany, "the city of artists and engineers," and that tradition apparently trickled down to Charles, who received a bachelor's degree in electrical engineering from Cornell University in 1963. Rowing was a big part of his undergraduate years. He still keeps in touch with fellow oarsmen, and can sometimes be found during lunch break taking a row in Eagle Harbor. Charles was also known on the Cornell campus for his 1929 Model-A Ford. It had a manifold heater and no defroster (you put your hand on the window to melt the ice) and a tendency to break down. It was truly an adventure to head off from Cornell in upstate New York to Long Island, especially during the winter.

After graduation from Cornell Charles moved to the seaside village of Stonington, Connecticut and went to work for Electric Boat designing sonar systems, where he participated in many submarine sea trials in the Atlantic and the Pacific. He also began working on his masters degree at the University of Connecticut and took courses in creative writing on the side. It was during this period that he met his wife Linda. Linda says that not only was he tall and nice looking, but by now he had ditched the old Model A and drove a red sports car!

Captivated by the natural beauty of the Pacific Northwest while on a sea trial there, Charles moved to Seattle in 1966 to work at Honeywell Marine Systems. He immediately joined the Seattle Mountaineers and learned glacier and rock climbing techniques, eventually leading seven climbs to the summit of Mt. Rainier, over 14,000 ft. This was one of the most productive times of Charles's life, working at Honeywell, helping to raise two children (Andrew and Jenny), and pursuing a PhD at the University of Washington. His dissertation was on computer recognition of musical instruments. When Charles finds any free time at an ASA meeting, he can often be found in a Musical Acoustics session.

Apparently the childhood memories of Fire Island remained with Charles because he and Linda found a place on Bainbridge Island, where they bought an old house that introduced Charles to the gentle arts of plumbing, wiring, and drywall hanging. On Bainbridge Island, Charles began community activities; he helped spearhead incorporation for Bainbridge Island and organized their new all-island government. (If you want to start a spirited conversation, just ask Charles what is happening in local politics on Bainbridge Island.)

In 1985, Charles took his interest in politics to Washington, DC as one of the first ASA Congressional Science and Engineering Fellows. The year in Washington, DC was a seminal one for Charles as he learned a new set of skills in his work to increase communications between scientists and Congress. A few years later, when the ASA announced an opening for its first Executive Director, Charles was an enthusiastic applicant. He assumed the position in 1990 and has held it ever since. Fortunately, Charles brought to the job of Executive Director qualities and skills he learned from his parents and 
developed over a lifetime: love of family, leadership, imagination, problem solving, abundant energy, persistence and patience, a good memory and sense of history, and a tolerance for diverse viewpoints. The ASA Executive Director must ensure that the Society not only survives, but flourishes. Directing an organization that depends so much on an ever-changing cadre of volunteers requires diplomacy of a high order. Charles has thrived in this environment as few others could, and the Society has thrived as well.

Charles has made many contributions to our Society, and to acoustics. He has been a member of the Governing Board of the American Institute of Physics (AIP) since 1990, and was elected to its Executive Committee for five separate terms. Anyone who has attended a meeting of the AIP Board will quickly recognize the high regard with which he is held by his AIP peers. He is also a member of the International Commission for Acoustics and regularly attends meetings of other national and regional acoustical societies. Charles played an indispensable role in leading the Society to reach out beyond its geographical borders. In 1998, he helped organize a joint meeting of the ASA and the International Congress on Acoustics, followed by a meeting with the European Acoustics Association in Berlin in 1999, a joint meeting with the Mexican Institute of Acoustics and Federation of Iberoamerican Acoustics held in Cancun, Mexico in 2002, and in 1996 and 2006 the third and fourth joint meetings with the Acoustical Society of Japan in Hawaii. It is appropriate that Charles should be recognized for his service to acoustics worldwide at another international conference, Acoustics'08 in Paris.

LAWRENCE A. CRUM

WILLIAM M. HARTMANN

PATRICIA K. KUHL 


\section{ACOUSTICAL SOCIETY OF AMERICA \\ R. BRUCE LINDSAY AWARD}

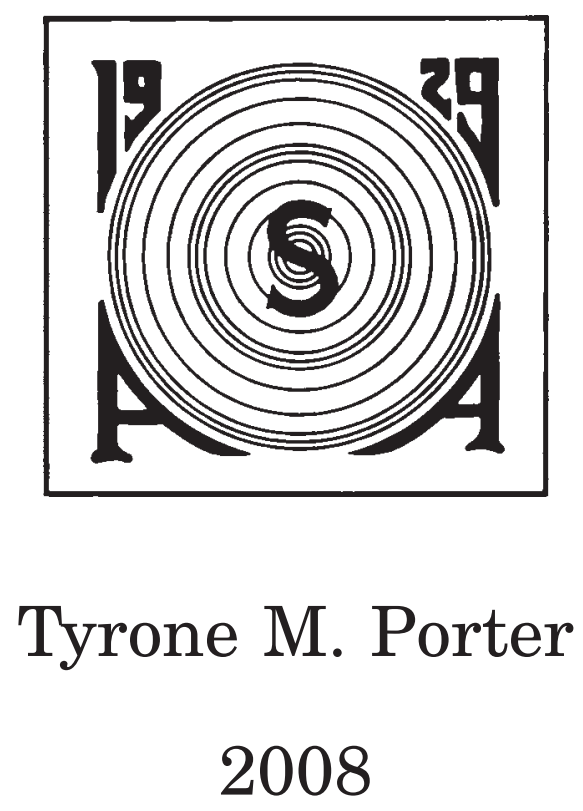

The R. Bruce Lindsay Award (formerly the Biennial Award) is presented in the Spring to a member of the Society who is under 35 years of age on 1 January of the year of the Award and who, during a period of two or more years immediately preceding the award, has been active in the affairs of the Society and has contributed substantially, through published papers, to the advancement of theoretical or applied acoustics, or both. The award was presented biennially until 1986. It is now an annual award.

\section{PREVIOUS RECIPIENTS}

$\begin{array}{ll}\text { Richard H. Bolt } & 1942 \\ \text { Leo L. Beranek } & 1944 \\ \text { Vincent Salmon } & 1946 \\ \text { Isadore Rudnick } & 1948 \\ \text { J. C. R. Licklider } & 1950 \\ \text { Osman K. Mawardi } & 1952 \\ \text { Uno Ingard } & 1954 \\ \text { Ernest Yeager } & 1956 \\ \text { Ira J. Hirsh } & 1956 \\ \text { Bruce P. Bogert } & 1958 \\ \text { Ira Dyer } & 1960 \\ \text { Alan Powell } & 1962 \\ \text { Tony F. W. Embleton } & 1964 \\ \text { David M. Green } & 1966 \\ \text { Emmanuel P. Papadakis } & 1968 \\ \text { Logan E. Hargrove } & 1970 \\ \text { Robert D. Finch } & 1972 \\ \text { Lawrence R. Rabiner } & 1974 \\ \text { Robert E. Apfel } & 1976 \\ \text { Henry E. Bass } & 1978 \\ \text { Peter H. Rogers } & 1980 \\ \text { Ralph N. Baer } & 1982 \\ \text { Peter N. Mikhalevsky } & 1984 \\ \text { William E. Cooper } & 1986\end{array}$

Ilene J. Busch-Vishniac 1987

Gilles A. Daigle 1988

Mark F. Hamilton 1989

Thomas J. Hofler 1990

Yves H. Berthelot 1991

Joseph M. Cuschieri 1991

Anthony A. Atchley 1992

Michael D. Collins 1993

Robert P. Carlyon 1994

Beverly A. Wright 1995

Victor W. Sparrow 1996

D. Keith Wilson 1997

Robert L. Clark 1998

Paul E. Barbone 1999

Robin O. Cleveland 2000

Andrew J. Oxenham 2001

James J. Finneran 2002

Thomas J. Royston 2002

Dani Byrd 2003

Michael R. Bailey 2004

Lily M. Wang 2005

Purnima Ratilal 2006

Dorian S. Houser 2007 


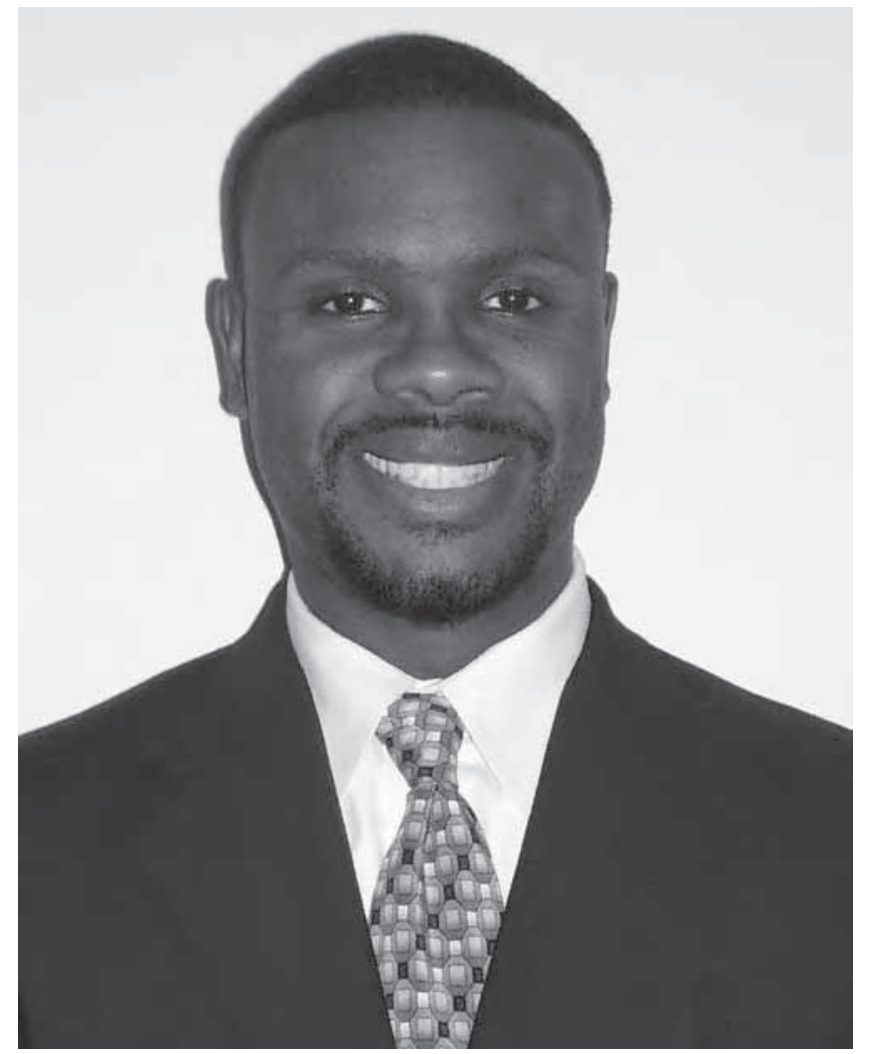




\section{CITATION FOR TYRONE M. PORTER}

. . . for contributions to ultrasound-guided drug delivery

PARIS, FRANCE ・ 2 JULY 2008

Tyrone Michael Porter was born in Detroit, Michigan on July 21, 1973. His parents, Dr. Elijah and Laverne Porter were teachers in the Detroit public school system. Elijah taught high school math early in his career and later served as Principal of Henry Ford High School and Laverne taught third through fifth grade at Vetal Elementary until their retirement a few years ago. Elijah and Laverne were strict disciplinarians and kept a close watch on Tyrone and his friends when they were teenagers. What Tyrone initially interpreted as an infringement of freedom was soon seen as a concern for his welfare, and he attributes much of his success in life to the important values he learned from his parents. Tyrone is a middle child with an older brother, Elijah Porter, II, and a younger sister, Dr. Dawne Jones. This birth order served as the quintessential training for Tyrone as a lively listener, a tolerant team-builder and a patient peacemaker. He continues to enjoy his role as proud uncle of five nephews and nieces. Tyrone is newly married to Dr. Monica Hall-Porter, and they reside in Roxbury, MA. Tyrone attended Renaissance High School in Detroit and graduated in 1991. While attending high school, Tyrone participated in the Detroit Area Pre-College Engineering Program, which served as a platform for pursuing a degree in engineering.

He left Michigan to attend Prairie View A\&M University, a historically Black university in southeast Texas. While earning a Bachelor of Science degree in Electrical Engineering (Magna Cum Laude), Tyrone participated in several organizations, including the National Society of Black Engineers (NSBE) and Alpha Phi Alpha Fraternity. He was later named 2002 Graduate Student of the Year by NSBE, and now directs their Bioengineering Special Interest Group. At the NSBE 2007 national convention in Columbus, Ohio, he organized a special session entitled, Sound Therapy: Innovation in Biomedical Acoustics. As an undergraduate, Tyrone also co-founded Peers Advising, Counseling, and Teaching (PACT) to motivate high school students to strive for academic excellence and attend college. In 1996 he was featured in USA Today as one of the nation's top undergraduate engineering students, and was awarded a position on the Collegiate All Academic Team.

Tyrone was encouraged to pursue a doctoral degree in engineering and a career in higher education. He attended graduate school at the University of Washington (UW), where he was awarded the Doctor of Philosophy in Bioengineering in 2003. Tyrone came cheap as a graduate student, arriving at UW with a National Science Foundation Fellowship, and when that ran out, a fellowship sponsored by the United Negro College Fund (UNCF) and Merck. Tyrone was so well known at the UW that the three recommendation letters for the UNCF/Merck award came from his thesis advisor, and the Provost and the President of the University of Washington. As a graduate student, Tyrone co-founded the Minority Think Tank, a social-activist group created to discuss and develop solutions to common problems among underrepresented groups. During his graduate days, Tyrone was always organizing things from trips to Whistler for skiing to faculty-student softball games. As an outspoken proponent of social justice, he soon gained the attention of local politicians, and was regularly on the evening news and in the local paper with an articulate defense of affirmative action or equal opportunity.

For his dissertation, Tyrone helped to develop a new class of "smart" polymeric carriers that could destabilize biological membranes to enhance DNA or protein drug delivery. Tyrone worked on the novel idea that the synergistic effects of ultrasound and these polymeric carriers might be used to enhance their biofunctionality by triggering membrane destabilization. In this way, drug delivery might be stimulated with an on/off type of switch by application of an acoustic stimulus. This project embodied the spirit of interdisciplinary study and collaborative research that is the cornerstone of biomedical engineering. To be successful, he had to learn the fundamental concepts of acoustics, polymer chemistry, biochemistry and biology. Tyrone was so well known as a graduate student at the University of Washington that he was asked to serve on the Search Committee for a new President...a committee that included the founder of Costco Wholesale Corporation, the CEO of Recreational Equipment, Inc. (REI), and the CEO of Delta 
Airlines. When he defended his dissertation, a lecture theater for over 200 people was reserved and it was nearly filled to capacity, including three Deans and a Vice Provost in attendance.

Tyrone was awarded the ASA's Frederick V. Hunt Postdoctoral Research Fellowship in Acoustics in 2003-04, which he chose to undertake at the University of Cincinnati. He worked with scientists in biomedical engineering, cardiology, biochemistry, and immunology to characterize the acoustic properties of echogenic liposomes, submicron-sized phospholipid vesicles that contain both gas and fluid. With antibody conjugation and drug incorporation, these liposomes can be used as novel targeted diagnostic and therapeutic ultrasound contrast agents. In addition to his scientific expertise, his sports acumen was legendary, and often led to rather heated and highly opinionated discussions on the merits of the Detroit Pistons versus anyone else. A detailed plan for the rehabilitation of the Cincinnati Bengals as a professional football team also resulted from these talks with his fellow researchers, as did some suggestions regarding recruitment ideas for the Reds.

At Boston University (BU), where he serves as a member of both the Department of Aerospace and Mechanical Engineering and the Department of Biomedical Engineering, Tyrone is crafting a world-class research program combining basic principles in chemistry, acoustics, and thermodynamics to develop novel drug delivery strategies for cancer therapy. He maintains research laboratories in both Departments and is forging true interdisciplinary collaborations involving team members with diverse interests and expertise. His research is currently focused on the development of targeted ultrasound contrast agents for image enhancement (molecular imaging), perfusion imaging, and image-guided drug delivery. Ultrasound-enhanced transport of drugs and genes across cell membranes and ultrasound-enhanced drug activity play an important role in this research.

At BU, Tyrone has been tasked with teaching the introductory dynamics course, which is a key college-wide curricular requirement populated by sizeable classes and students with diverse backgrounds. In this significant effort, Tyrone has acquitted himself extremely well, garnishing some of the finest teaching evaluations ever bestowed upon a freshman professor in the AME Department. Tyrone is destined to be a pedagogical star in a department populated with some of the best teachers in the BU College of Engineering. He is committed to the highest quality of education of students. His impeccable integrity and an infectious enthusiasm entice students to flock to him. Tyrone has the heart and heritage of an educator, and he will have a huge impact on younger people.

Tyrone has also been very active in the affairs of the ASA, having served on the Student Council, as a member of the organizing committee for the joint ASA/ICA meeting in Seattle in 1998, as one of the nine special presenters at ASA's 75th anniversary celebration, and as an organizer of several special sessions for the Technical Committee on Biomedical Ultrasound/Bioresponse to Vibration, of which he is also a member.

Tyrone Porter's parents, siblings, aunts and uncles, and a host of friends and colleagues are very proud of Tyrone's accomplishments and look forward to the exciting career that lies before him.

\author{
CHRISTY K. HOLLAND \\ RONALD A. ROY \\ LAWRENCE A. CRUM
}




\section{ACOUSTICAL SOCIETY OF AMERICA}

\section{Helmholtz-Rayleigh Interdisciplinary \\ Silver Medal in}

Signal Processing in Acoustics

and

Underwater Acoustics

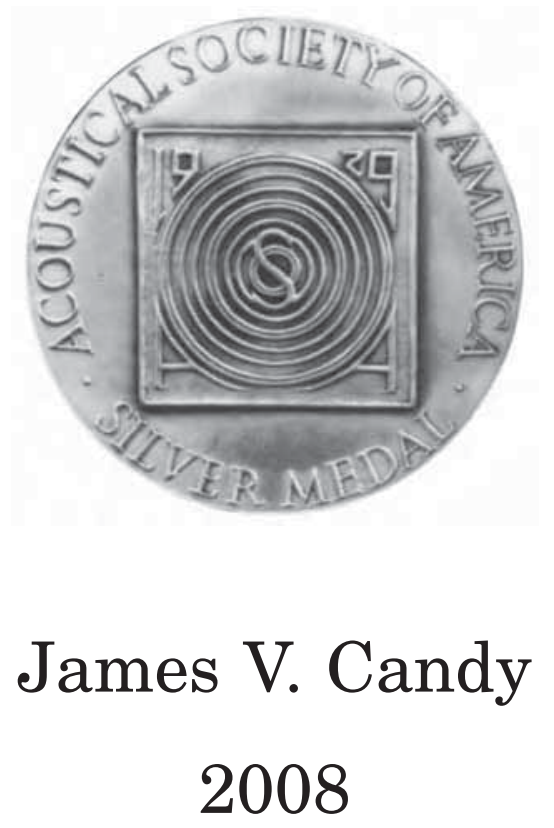

The Silver Medal is presented to individuals, without age limitation, for contributions to the advancement of science, engineering, or human welfare through the application of acoustic principles, or through research accomplishment in acoustics. 


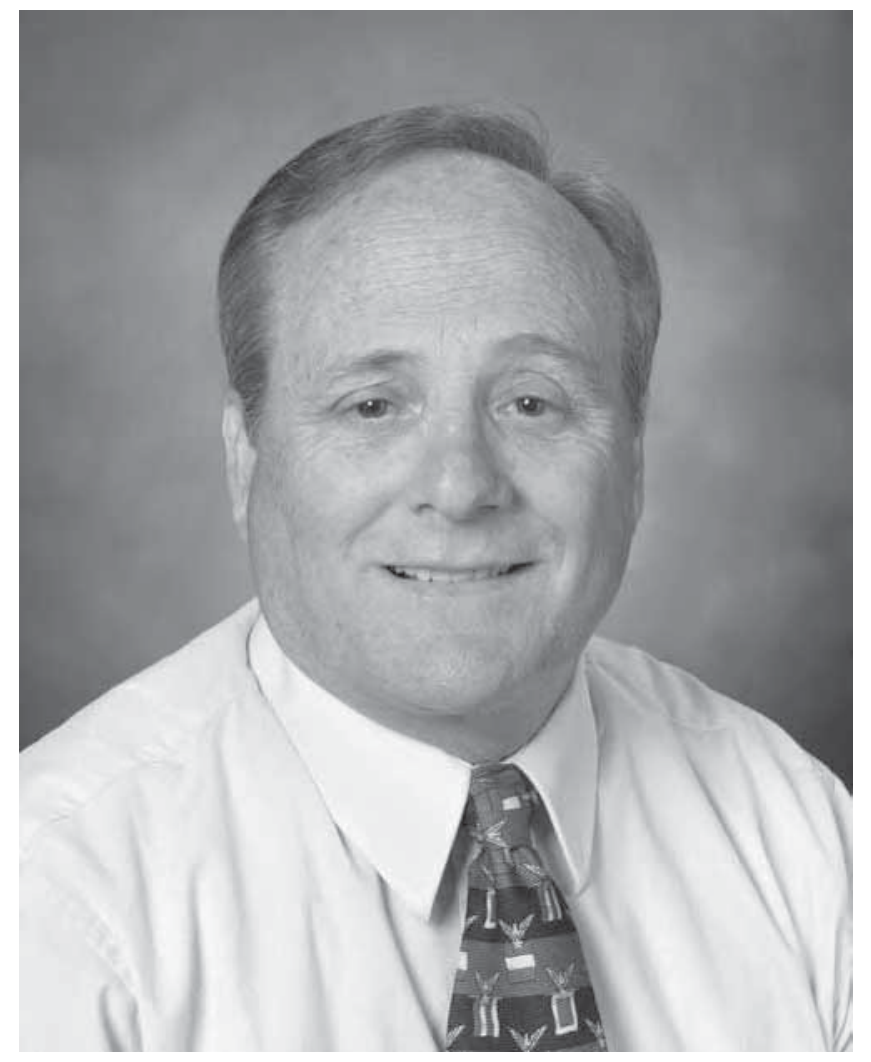




\section{CITATION FOR JAMES V. CANDY}

... for contributions to signal processing and underwater acoustics

PARIS, FRANCE ・ 2 JULY 2008

We have all heard the saying "Them that can, do; them that can't, teach." We also know that one exception disproves the theory. In the case of Jim Candy, we have that exception. He is a born teacher with a highly creative mind. When one first meets him, he comes across as a rather unassuming person. But once he becomes interested in a problem, he dives into it with a tenacious passion until he solves it. The teacher in him comes through by his ability to use simple analogies drawn from his wealth of knowledge and ideas. His intuitive creativity is displayed in clever innovative solutions to complex problems.

Many of his creative solutions are based on his background in control theory and recursive estimation theory. When he joined the staff of the Lawrence Livermore National Laboratory, Dr. Candy quickly found himself dealing with problems in electromagnetics, lasers, nuclear physics, internal waves, nuclear fusion, ultrasonics, array processing and inverse problems in general. It was not long before he gained the reputation as the "go to" guy when there was a need for a new approach in modeling, classification, estimation and detection, regardless of the subject. And anyone who deals with him on a collaborative basis walks away all the richer for it.

James Vincent Candy was born in Astoria, New York, on January 21, 1944. He received a B.S. in Electrical Engineering from the University of Cincinnati in 1966, an M.S.E. in Electrical Engineering from the University of Florida in 1972, and a Ph.D. in Electrical Engineering in 1976, also from the University of Florida. During the period, from 1967 to 1971, he served as a Captain in the United States Air Force and from 1966 to 1967, he worked as an Engineer for the General Electric Company. In 1976, Dr. Candy began his employment with the University of California, Lawrence Livermore National Laboratory (LLNL) as a scientist for the Engineering Directorate. At present, he is Chief Scientist for the Engineering Directorate and an Adjunct Professor at the University of California, Santa Barbara.

He began working in the field of oceanic signal processing during a short-term assignment at the NATO SACLANT ASW Research Center in 1987. Since that time, he has developed the "Model-Based" approach to oceanic signal processing. This has produced a wealth of applications in underwater acoustic signal processing to localization, tracking, signal enhancement, sound-speed inversion, noise cancellation, sequential detection and internal wave signal processing. His work led to the Bayesian solution for dispersive wave propagation, most notably as applied to internal waves in imaging and detection applications.

This model-based approach to signal processing evolved from Jim's intimate knowledge of Bayesian statistics and the Kalman filter. This type of processor has been used in the area of optimum control for many years, but it was not until he pointed out that in principle, there is really no limit to the sophistication of the physical models one can use in these processors, that it was recognized that they had great value in areas outside of control theory. This insight has laid the framework for a general scheme for including a priori information, in the form of physical models, into basically any processing scheme. This provides a self-consistent Bayesian structure, that avoids many of the difficulties associated with the use of physical models, including the so-called "mismatch problem," that arises when the physical parameters are not well known. Dr. Candy has closely followed, applied and contributed to advances made in recent years in this field, including the Unscented Kalman Filter and the particle filter, which avoid some of the limitations of the original Kalman filter, such as its inability to handle the cases of nonlinearity and non-Gaussian statistics.

Jim is a former Director of the LLNL Center for Advanced Signal and Image Sciences. He has been an Adjunct Professor at San Francisco State University, University of Santa Clara, and the University of California, Berkeley Extension, teaching graduate courses in signal and image processing. He is a Fellow of the Acoustical Society of America (ASA), the Institute of Electrical and Electronics Engineers (IEEE), and was recently elected as a Life Member (Fellow) of Clare Hall College at the University of Cambridge in the UK. 
He received the IEEE Distinguished Technical Achievement Award in 2002 for contributions of signal processing to oceanic engineering. Along with his many other publications, he has produced three texts: Signal Processing: The Model-Based Approach, Signal Processing: The Modern Approach, and Model-Based Signal Processing and Bayesian Signal Processing: Classical, Modern and Particle Filtering Methods (in press). He has conducted many short courses and tutorials on applied signal processing at meetings of the Acoustical Society of America and the Oceanic Engineering Society. He has also presented short courses in Applied Model-Based Signal Processing for the SPIE Optical Society. He is currently the Chair of the IEEE Technical Committee on Sonar Signal and Image Processing and was the Chair of the ASA Technical Committee on Signal Processing in Acoustics as well as an Associate Editor for Signal Processing for JASA Express Letters (JASA EL).

In addition to his technical accomplishments, Dr. Candy has been a mentor to many junior colleagues. His enthusiasm for signal processing in the ASA was contagious as he worked to bring the Signal Processing Committee into existence starting from an interdisciplinary technical group. He was its first Chair. He has also persuaded colleagues to become active members of the ASA and almost all of the active Society members from the Lawrence Livermore National Laboratory began their involvement through Jim's encouragement. His commitment and enthusiasm for acoustical signal processing is evidenced by the visibility of our Society at the Lawrence Livermore National Laboratory.

EDMUND J. SULLIVAN

WILLIAM M. CAREY 


\section{GOLD MEDAL \\ of the \\ Acoustical Society of America}

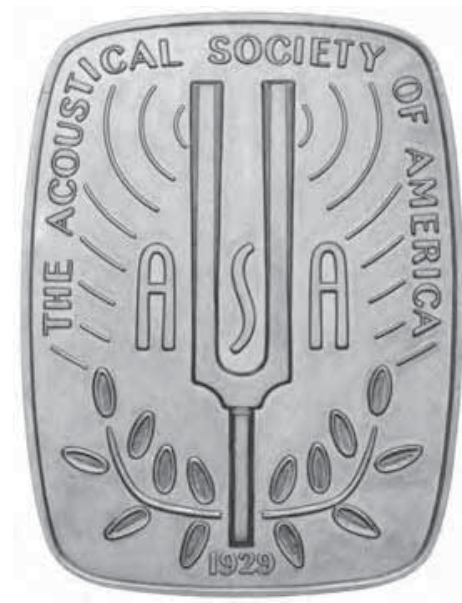

Patricia K. Kuhl

\section{8}

The Gold Medal is presented in the spring to a member of the Society, without age limitation, for contributions to acoustics. The first Gold Medal was presented in 1954 on the occasion of the Society's Twenty-Fifth Anniversary Celebration and biennially until 1981. It is now an annual award.

\section{PREVIOUS RECIPIENTS}

Wallace Waterfall

Floyd A. Firestone

Harvey Fletcher

Edward C. Wente

Georg von Békésy

R. Bruce Lindsay

Hallowell Davis

Vern O. Knudsen

Frederick V. Hunt

Warren P. Mason

Philip M. Morse

Leo L. Beranek

Raymond W. B. Stephens

1954

1955

1957

1959

1961

1963

1965

1967

1969

1971

1973

1975

1977

Richard H. Bolt

Harry F. Olson

Isadore Rudnick

Martin Greenspan

Robert T. Beyer

Laurence Batchelder

James L. Flanagan

Cyril M. Harris

Arthur H. Benade
1979

1981

1982

1983

1984

1985

1986

1987

1988
Richard K. Cook

1988

Lothar W. Cremer

1989

Eugen J. Skudrzyk

1990

Manfred R. Schroeder

1991

Ira J. Hirsh

1992

David T. Blackstock

1993

David M. Green

1994

Kenneth N. Stevens

1995

Ira Dyer

1996

K. Uno Ingard

1997

Floyd Dunn

1998

Henning E. von Gierke 1999

Murray Strasberg 2000

Herman Medwin 2001

Robert E. Apfel 2002

Tony F. W. Embleton 2002

Richard H. Lyon 2003

Chester M. McKinney 2004

Allan D. Pierce 2005

James E. West 2006

Katherine S. Harris 2007 


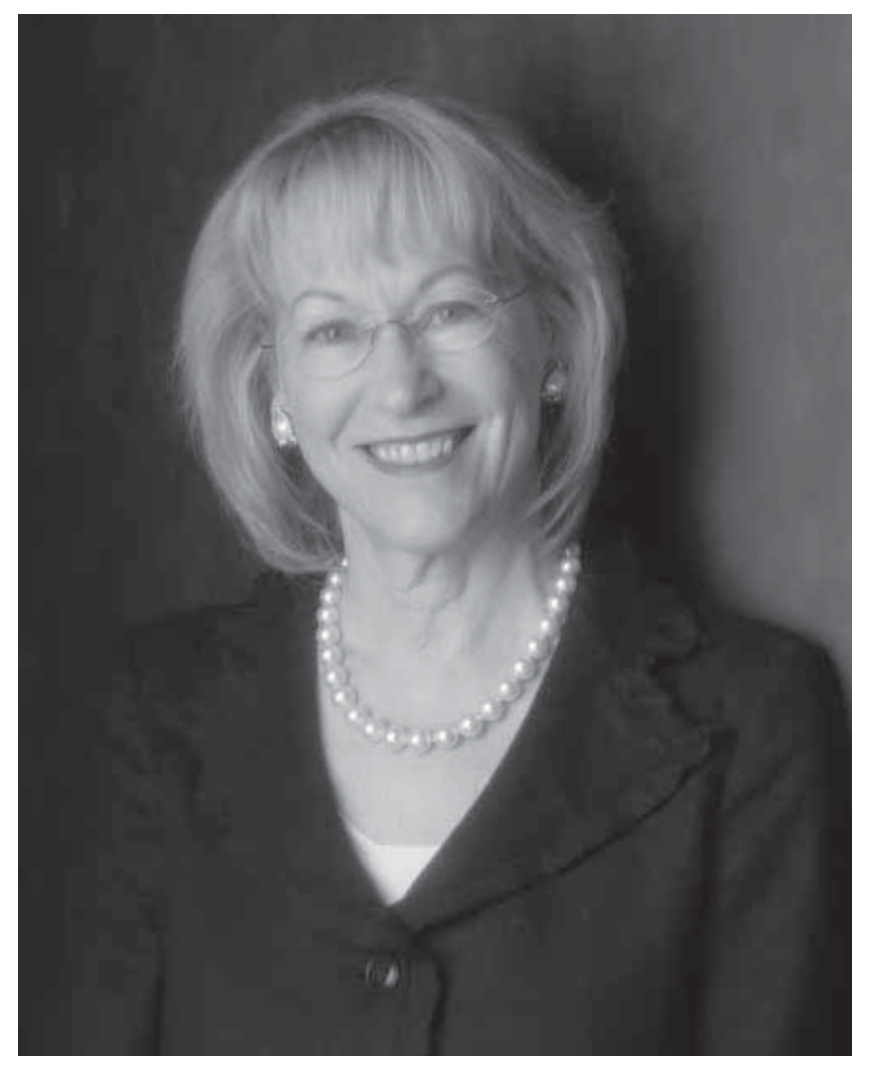




\section{CITATION FOR PATRICIA K. KUHL}

... for contributions to understanding how children acquire spoken language and for leadership in the Society.

\section{PARIS, FRANCE ・ 2 JULY 2008}

Patricia Katherine Kuhl was born and raised in St. Cloud, Minnesota, the second of five children of Joseph and Susan Kuhl. In high school, Pat was involved in many school activities, including the debate club, the honor society, and the basketball team. She was especially interested in music, showing great enthusiasm and talent for voice and piano. Luckily for the field of speech communication, Pat's parents stressed talking in addition to music. Every night before dinner in the Kuhl household, Pat's father unplugged the telephone so that the family could discuss the day's activities, the national political scene, and world affairs. Pat's father always read the newspaper before dinner, and expected the children to as well, so Pat read the papers before her father arrived home. Pat was always ready to debate her dad about anything and everything. For those of you who know Pat, she loves a lively discussion and is always prepared with facts as well as opinions.

Pat is married to Andrew Meltzoff, who along with Pat, is co-Director of one of the more visible research groups at the University of Washington-The Institute for Learning and Brain Sciences. Pat is also the Co-Director of another high-profile interdisciplinary research group on campus, an NSF Science of Learning Center-LIFE-which links neuroscience to education. Pat and Andy have enjoyed a long and productive collaboration in developmental cognitive science. According to Andy, the "best collaboration of all" was one that resulted in their daughter Katherine, who combines Andy's love of swimming and Pat's love of music. Katherine is one of the stars on the swim team at Trinity College in Connecticut and also had the singular distinction of participating in the first test of the acoustics in Seattle's Benaroya Hall by singing while former ASA President Cyril Harris, acoustical engineer for the Hall, dashed around taking measurements.

Pat completed her BA, MA, and Ph.D. degrees in Minnesota at St. Cloud State University and the University of Minnesota. Pat's interest in the development of speech perception in children began with her postdoctoral years at the Central Institute for the Deaf with James D. Miller. Those years led to a series of papers that examined the responses of mammals and children to speech. She established commonalities between mammals and human infants in their response to speech sounds, and, importantly, where their patterns of responses to speech stimuli diverged.

In the early 1980's, Pat's interest expanded beyond the auditory perception of speech to include studies of how infants integrate auditory and visual information about speech, and to the imitation of speech. Especially impressive has been Pat's insights into the future of speech research: She has consistently published important papers that inspire new lines of research, from her animal studies in the 70's and 80's, to her auditory-visual and imitation work in the 80 s, to her work on language experience and its effects on learning in the early 90's, and her most recent work which is forging new territory using the tools of modern neuroscience. She has most recently worked to develop baby Magnetoenchephalography (MEG). The technique is completely safe, non-invasive, and noiseless, and Pat and her team worked with engineers in Helsinki to develop ways to track an infant's head movements so the baby's brain activities can be precisely located in the infant brain as the child listens to language or music, and processes complex social information. Pat has a unique talent for spotting where the next interesting finding may lie, and an ability to put together the resources and interdisciplinary research teams needed to conduct work that few others would be able to accomplish. She has forged collaborations with speech scientists from Japan, Taiwan, Sweden, Russia, Finland, Spain, China, France, and Mexico to study how language experience affects speech processing in adults and young children. Pat's students also come from many different countries, and a tour through her Institute introduces a visitor to students from Taiwan, Japan, China, Mexico, and Finland. Once trained, many of these students return to their native countries to set up speech research laboratories of their own.

Pat's research has had theoretical as well as practical impact. If you have ever tried to learn a new language as an adult, you soon recognize that it is difficult to pronounce certain sounds, or even to hear the relevant differences, while your children seem to pick 
up foreign languages easily and speak without an accent. Pat has performed pioneering research in this field and learned from her studies with babies as young as 6 months that infants are born "citizens of the world" with regard to language. They can distinguish sounds from languages around the world, even if they have never heard them before. By the end of the first year of life, however, they become "language-specialists"- the ability to attend to native-language sounds increases substantially while the ability with foreign languages diminishes. Pat proposed the Native Language Neural Commitment Theory to account for this dramatic developmental change. The model shows that infants use computational abilities to "crack" the speech code and, interestingly, that infants' social skills may "gate" learning. She and her students showed that infants learn phonemes and words rapidly from a live foreign-language tutor at 9 months of age but that the same foreignlanguage material presented from a TV or audio-tape produces no learning. The finding has theoretical implications as well as implications for education and society.

Pat's research in acoustics has led to a number of singular honors: for example, she is a member of the American Academy of Arts and Sciences, the Rodin Academy, and the Norwegian Academy of Science and Letters. She was awarded the Silver Medal of the Acoustical Society of America in 1997, and the Kenneth Craik Research Award from Cambridge University in 2005. She received the University of Washington's Faculty Lectureship Award in 1998, and in 2007, she was awarded the University of Minnesota's Outstanding Achievement Award. Pat is a Fellow of the American Association for the Advancement of Science, the Acoustical Society of America, and the American Psychological Society.

Pat has also been an eloquent spokesperson for childhood learning and was one of six scientists invited to the White House in 1997 to make a presentation at President and Mrs. Clinton's Conference on "Early Learning and the Brain." In 2001 she was invited to make a presentation at President and Mrs. Bush's White House Summit on "Early Cognitive Development: Ready to Read, Ready to Learn." In 1999, she co-authored The Scientist in the Crib: Minds, Brains, and How Children Learn.

Pat has played an active role in the affairs of the Society for a number of years. She has served as an Associate Editor of the Journal, as a member of the Executive Council, and as a member or chair of several committees. Pat was cochair of the joint 135th meeting of the Acoustical Society of America and the 16th International Congress on Acoustics in 1998 as well as coeditor of its proceedings. She was cochair of the ASA's 75th Anniversary Celebration Committee with Leo Beranek in 2004. As part of the celebration, Pat organized a series of presentations by nine young investigators entitled "A Celebratory Look into the Future." She was elected the Society's Vice President in 1996, and in 1999 was the first woman elected President of the ASA. While President, Pat created the Student Council, one of the most successful presidential initiatives, and one that has generated great enthusiasm among our younger members.

The Gold Medal winners of the ASA are indeed a select group; they have been outstanding innovators and leaders in science and engineering and they have brought great recognition to our Society. Patricia Kuhl now joins this elite group and elevates its stature even more.

FREDERICKA BELL-BERTI

YANG ZHANG

PAUL IVERSON 


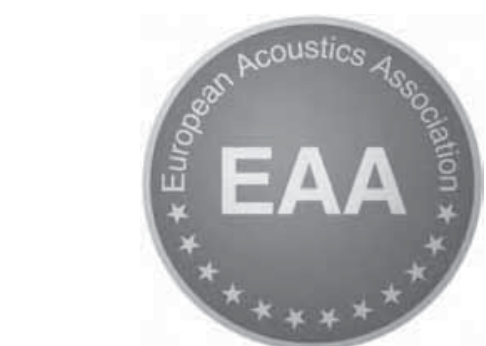

\section{EAA AWARD 2008}

\section{For lifetime achievements in acoustics}

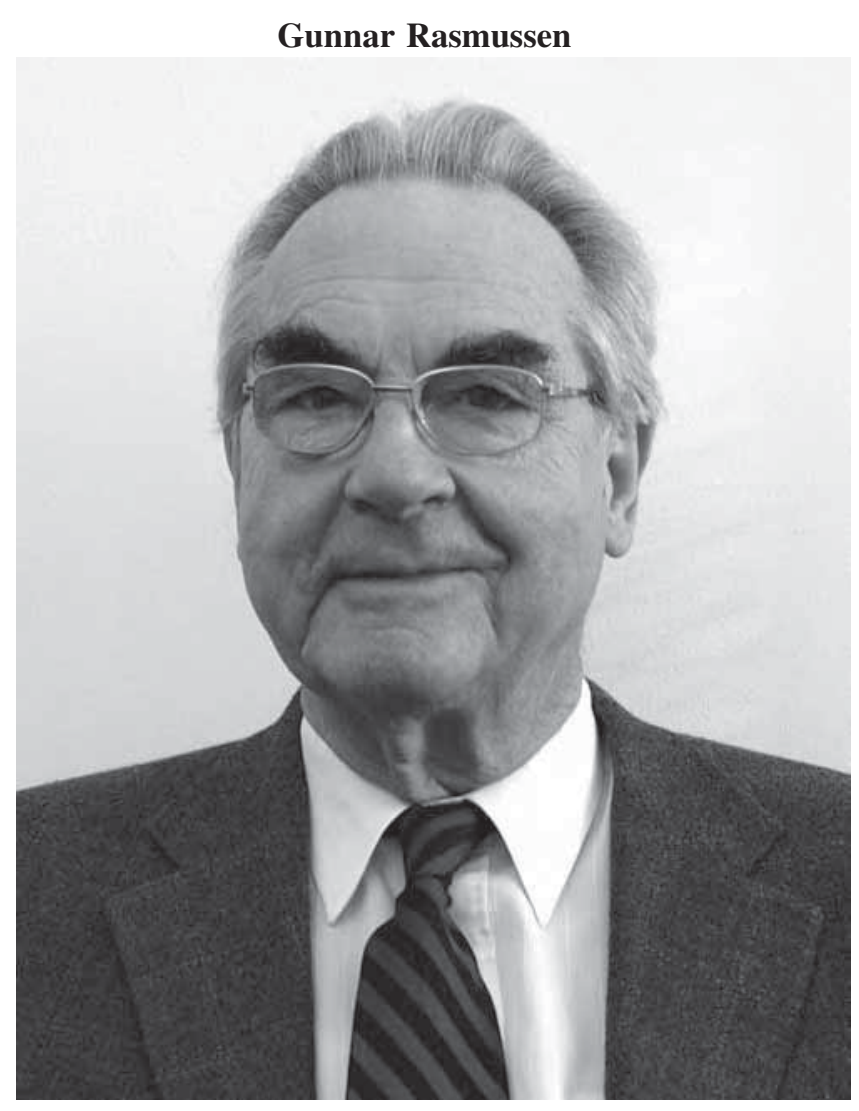

For his outstanding contribution in the field of acoustic measurements and instrumentation

Gunnar Rasmussen is a pioneer in the construction of acoustic instrumentation, particularly of pressure transducers and related devices. He was employed at Brüel \& Kjær Denmark as an electronics engineer immediately after his graduation in 1950. After holding various positions in development, testing and quality control, he spent one year in the United States working for Brüel \& Kjær in sales and service. After his return to Denmark in the mid-1950's he began the development of a new measurement microphone. This involved the invention of new methods for manufacturing microphone diaphragms in electro-galvanic deposited nickel, to replace the very corrosive aluminium foil diaphragms in use at that time. Also the mechanical construction was drastically simplified from more than 30 different parts to less than 10 parts. This resulted in superior mechanical stability, increased temperature and long term stability. The resulting one-inch pressure microphone soon became the de facto standard microphone for acoustical measurements.

The optimized mechanical design of the new generation of measurement microphones opened up the possibility for reducing the size of the microphones, first to a $1 / 2$ " microphone and then to $1 / 4$ " and $1 / 8$ " microphones with essentially the same superior mechanical, temperature and long term stability. Notably the $1 / 2$ " microphone is still the most widely used noise abatement tool today. Since the beginning of the 1960's, this microphone design has been preferred for all types of acoustic measurements and has formed the basis for the IEC 1094 series of international standards for measurement microphones.

Gunnar Rasmussen received the Danish Design Award in 1969 for his novel design of the microphones which were exhibited at the New York Museum of Modern Art. He also developed the first acoustically optimized sound level meter, where the shape of the body was designed to minimize the effect of reflections from the casing to the microphone. This type 2203 sound level meter was for many years seen as the archetype of sound level meters and its characteristic shape became the symbol of a sound level meter. It was also awarded the Danish Design Award in 1965. 
Other major inventions and designs include the Delta Shear accelerometer, the dual piston pistonphone calibrator for precision calibration, the face-to-face sound intensity probe and hydrophones, occluded ears, artificial mouth, etc. Gunnar Rasmussen is also the author of numerous papers on acoustics and vibration and has served as chairman and vice-chairman of various international organizations and standard committees. In 1990 he received the CETIM medal for his contributions to the field of intensity techniques. He is also a Fellow of the Acoustical Society of America.

In 1994 Gunnar Rasmussen started his own company, G.R.A.S. Sound and Vibration. Started as a company specializing in precision outdoor microphones for permanent noise monitoring around airports, it is now one of the world's leading companies in acoustic front-ends and transducers forming a wide range of general purpose and specialized microphones, electro-acoustic measurement devices such as ear couplers, precision calibration tools and multi-dimensional sound intensity probes. After many years of rich and fruitful work, Gunnar Rasmussen is still very active with the design of new transducers and solutions. At the same time he is an indispensable partner in discussions of measurement standards, where he provides valuable input and help for younger colleagues who admire his deep knowledge and experience and acknowledge his kindness and modest character.

EAA wishes to honour Gunnar Rasmussen for his tremendous impact on acoustics, for being an example for generations of scientists and engineers dealing with acoustic measurements, for the expertise he has given to us all. 


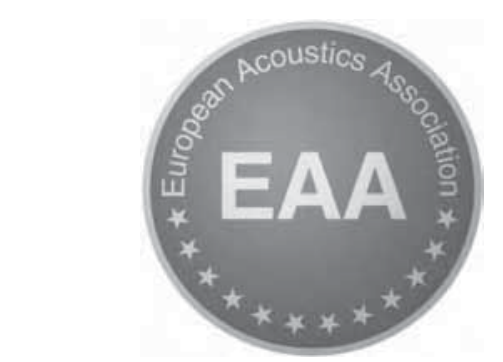

\title{
EAA AWARD 2008
}

\section{For contributions to the promotion of Acoustics in Europe}

\section{FASE Federation of Acoustical Societies of Europe 1971-1996}

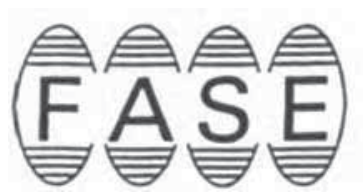

\author{
Federation of Acoustical Societies of Europe \\ Fédération Européenne des Sociétés d'Acoustique \\ Europäische Föderation der akustischen Gesellschaften \\ Фелерация Акустических Обществ Европы
}

\begin{abstract}
For the establishment, organization and support of new platforms of cooperation, partnership and friendship among European acousticians
\end{abstract}

Probably very few people in Europe know that on 9 May 1950 the first move was made toward establishing the European Union. On that day in Paris, against the background of the threat of a Third World War engulfing Europe as a whole, French Foreign Minister Robert Schuman read to the international press a declaration calling upon France, Germany and other European countries to pool together their coal and steel production as "the first concrete foundation of a European federation."

Probably very few acousticians in Europe know that on 26 August 1968 during the $6^{\text {th }}$ International Congress on Acoustics in Tokyo, the first move was made towards the creation of the European Acoustics Association (EAA). On that day in Tokyo, on the initiative of Prof. G. Kurtze and Prof. E. Zwicker, a semi-official meeting of the delegates of the European acoustical societies was organized in order to discuss the possibilities of founding the Federation of Acoustical Societies of Europe.

It took three more years to consult with different societies and to establish the main purposes of the new "learned society." Finally on 24 August 1971 in Budapest, representatives of 13 acoustical societies declared the foundation of FASE, the Federation of Acoustical Societies of Europe, and a provisional steering committee was elected.

On 5 May 1972, the first meeting of the FASE Council took place in Paris with the election of officers: Prof. J. Frenkiel (President), Prof. W. Furrer (Vice-President), Dr. F. Kolmer (Secretary), as well as the approval of a program of activities concerning the coordination of scientific acoustical events, information on possibilities of vacancies in acoustical institutions in Europe (universities, research institutes, firms, etc.) and a survey on education in acoustics in Europe.

During the next 25 years more European acoustical societies, academies of sciences and associations joined FASE, 7 Congresses on Acoustics and 12 Symposia on specific acoustic topics and in different European cities were organized, 25 Council meetings were held and more officers and members of the council were involved in these activities.

On a voluntary basis FASE has contributed to establish and sustain the contact between scientists in acoustics in the former Eastern Block countries and in Western Europe during a historical period in which travel and exchange of information was difficult. FASE has established fruitful collaboration, representing Europe, with international societies such as ICA, ASA, EEAA, ESCA, CEC, and ICSU. In 1986 FASE contributed to the publication of the volume Noise Pollution of the SCOPE (Scientific Committee on Problems of the Environment, ICSU) through Prof. A. Lara-Sáenz in collaboration with Prof. R.W.B. Stephens who called together 23 specialists in acoustics. FASE has promoted international journals and has organized surveys on education in acoustics.

During the development of the European Union it became necessary to build an association like an image of nations represented by their national societies rather than a group of societies, associations and academies of sciences. Accordingly, on 1 January 1997, with a natural process, FASE was integrated into the European Acoustics Association (EAA) which formally took the inheritance of FASE's purposes and activities.

The Federation of Acoustical Societies of Europe was not only the first and successful attempt of harmonization among acoustical societies in Europe but it also represented a generation of European acousticians, their knowledge and their efforts.

The award will be presented to Prof. Paul François, Prof. Felix Kolmer and Prof. Andrés Lara-Sáenz on behalf of FASE. 


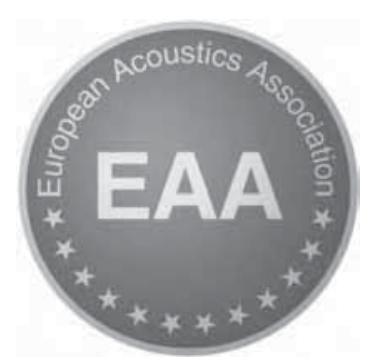

\section{EAA AWARD 2008}

\section{For outstanding recent scientific results published in ACTA ACUSTICA UNITED WITH ACUSTICA}

The award for outstanding recent research is given to a team of researchers for their contribution in the field of acoustics with the strict requirement that they published their results in the EAA scientific journal ACTA ACUSTICA UNITED WITH ACUSTICA within three years prior to the date that the award is given. In 2008 this EAA award goes to:

Catherine Guastavino, Brian F. G. Katz,

Jean-Dominique Polack, Daniel J. Levitin, Danièle Dubois

For their work on linguistic exploration of verbal data as a methodology for exploring the cognitive processing of environmental sounds, first published in:

\section{Ecological Validity of Soundscape Reproduction \\ ACTA ACUSTiCa United With ACUSTiCa Vol. 91 (2005) 333 - 341}

"A fundamental aim of psychoacoustics is to better understand how acoustic phenomena are perceived and represented at a cognitive level by individuals. Mental representations of sounds cannot be observed directly, but one way to study these representations empirically is through language, specifically, by analyzing how people talk about their sensory experiences." With these opening sentences the authors immediately point to the new line of thinking in psychoacoustics that they propose. Their methodology for digging into the cognitive level seems able to capture both the effect of source events and of the effect of background noise. Perception of environmental sound based on cognitive processes grounded in source recognition, have been well understood and can be studied using various methods. In environments with multiple sources, the soundscape is processed as a whole since source identification is hindered. The methodology proposed by the authors also allows the investigation of the perception of this background noise. In this case, mental representations related to both physical properties of the acoustic signal and semantic features and psychological effects can be identified.

In the awarded paper the authors apply the proposed methodology to investigate the ecological validity of reproduction of environmental soundscapes. They observed that ecological validity is easily obtained for sound events but that reproducing the urban background noise required immersive multichannel reproduction. This confirms existing knowledge on the cognitive processing of environmental sound. In later publications cited below the team applies their new methodology for exploring the meaning of urban soundscapes for its users and thus proves wider applicability of their methodology.

The awarded team goes beyond the classical paths of psychoacoustics and proposes a methodology for exploring mental representations of environmental sound. The novelty and potential impact of this approach were main motivations for the jury to select this work for the 2008 award for outstanding scientific results published in ACTA ACUSTICA UNITED WITH ACUSTICA.

The full paper can be downloaded from ACTA ACUSTICA UNITED WITH ACUSTICA ONLINE at www.ingentaconnect.com/ content/dav/aaua.

Further reading

Dubois, D., Guastavino, C., and Raimbault, M. (2006). “A cognitive approach to urban soundscapes: Using verbal data to access everyday life auditory categories," Acta Acustica United with Acustica 92, 865-874.

Guastavino, C., (2006) “The Ideal Urban Soundscape: Investigating the Sound Quality of French Cities," Acta Acustica United with Acustica, 92, 945-951. 


\section{FOREIGN MEDAL 2007 of the \\ French Acoustical Society}

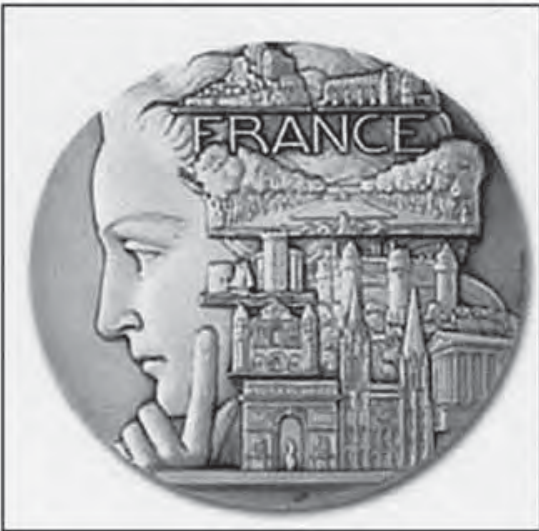

\section{Walter LAURIKS}

The Foreign Medal is annually presented to a renowned scientist having strong relations with French acousticians

PREVIOUS RECIPIENTS

\begin{tabular}{|c|c|}
\hline 1966 & BERANEK (USA) \\
\hline 1967 & L. PIMONOW (Poland) \\
\hline 1968 & SACERDOTE (Italy) \\
\hline 1969 & FRENKIEL (Belgium) \\
\hline 1970 & MALECKI (Poland) \\
\hline 1971 & $\begin{array}{l}\text { TARNOCZY (Hongrie) et KOBRYNSKI } \\
\text { (Poland) }\end{array}$ \\
\hline 1972 & FURRER (Switzerland) \\
\hline 1973 & STEPHENS (United Kingdom) \\
\hline 1974 & GRUTZMACHER (German) \\
\hline 1975 & RYFFERT (Poland) \\
\hline 1976 & LARA-SAENZ (Spain) \\
\hline 1977 & BOSQUET (Belgium) \\
\hline 1978 & A.STAN (Romania) \\
\hline 1979 & TARABA (Czechoslovakia) \\
\hline 1980 & WANG TE CHAO (China) \\
\hline 1981 & INGERSLEV (Denmark) \\
\hline 1982 & KURTOVIC (Yugoslavia) \\
\hline 1983 & CREMER (Germany) \\
\hline 1984 & LAUBER (Switzerland) \\
\hline 1985 & DOAK (United Kingdom) \\
\hline
\end{tabular}

\begin{tabular}{|c|c|}
\hline 1986 & MYNCKE (Belgium) \\
\hline 1987 & KUTRUFF (Germany) \\
\hline 1988 & W.G. MAYER (USA) \\
\hline 1989 & $\begin{array}{l}\text { J.E. FFOWCS WILLIAMS } \\
\text { (United Kingdom) }\end{array}$ \\
\hline 1990 & J. BLAUERT (Germany) \\
\hline 1991 & G. WEINREICH (USA) \\
\hline 1992 & O. B.WILSON (USA) \\
\hline 1993 & Z. SKVOR (Czech Republic) \\
\hline 1994 & F. FAHY (United Kingdom) \\
\hline 1995 & L. BJØRNØ (Denmark) \\
\hline 1996 & H. UBERALL (USA) \\
\hline 1997 & HIRSCHBERG (The Netherlands) \\
\hline 1998 & H. LEVINE (USA) \\
\hline 1999 & W. LAUTERBORN (Germany) \\
\hline 2000 & M. CAMPBELL (United Kingdom) \\
\hline 2001 & O. LEROY (Belgium) \\
\hline 2002 & G. DAIGLE (Canada) \\
\hline 2003 & M. ROSSI (Switzerland) \\
\hline 2004 & J. WOLFE (Australia) \\
\hline 2005 & V. PREOBRAJENSKI (Russia) \\
\hline 2006 & $\begin{array}{l}\text { M. LOWE and P. CAWLEY } \\
\text { (United Kingdom) }\end{array}$ \\
\hline
\end{tabular}




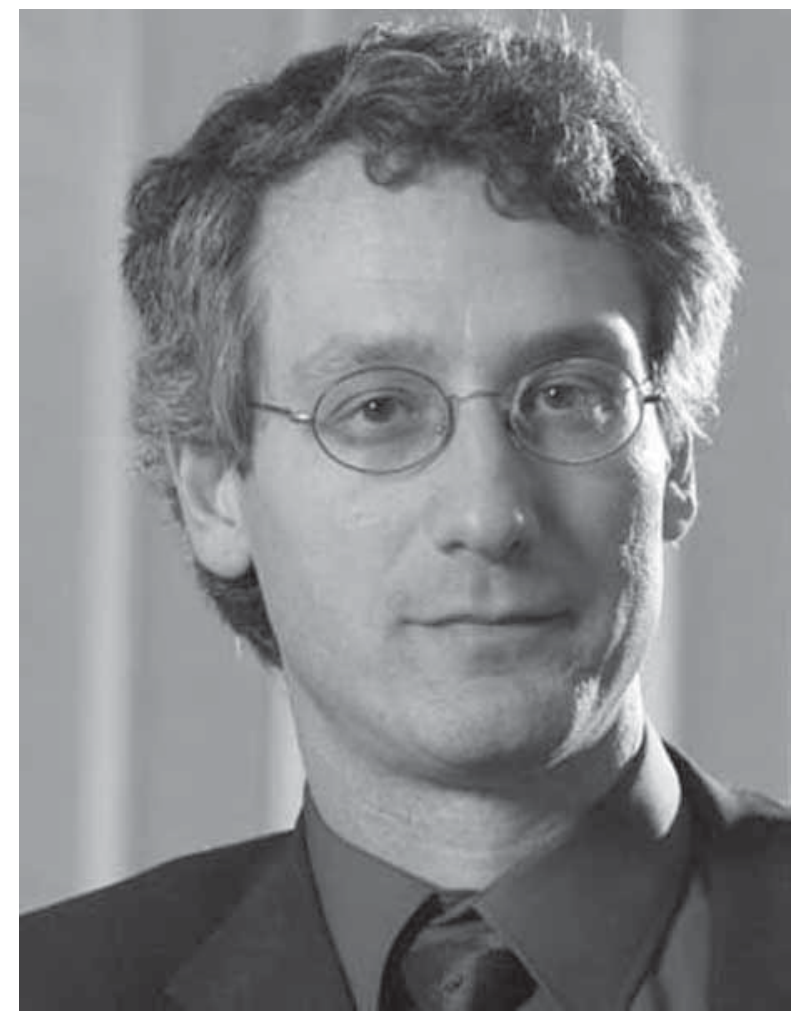


Walter Lauriks (born in 1961 in Reet - Belgium, married, 3 children) obtained a Master in Physics in 1983 and his Ph.D. in Physics in 1990, both at the University of Leuven (Belgium). Since then he worked as a research fellow at the Laboratory of Acoustics and Thermal Physics of the Department of Physics of the University of Leuven (K.U.Leuven), his main research area being sound propagation in elastic porous materials (also the subject of his Ph.D. thesis). He was responsible for multiple research projects in acoustics (among others for the Scientific Research Fund Flanders).

In 2005, Walter Lauriks became full professor and director of the Laboratory of Acoustics and Thermal Physics of the K.U.Leuven. At present, he is responsible for teaching different courses at the Faculty of Science and the Faculty of Engineering of the university: "Electricity and Magnetism", "Optics, Lasers and Acoustics" and "Physical Acoustics".

Until now, he supervised $13 \mathrm{PhD}$ theses (as advisor or promotor) and he was 37 times Jury member of Ph.D. committees, both national and international.

Walter Lauriks has developed a close and fruitful collaboration with the Laboratoire d'Acoustique de l'Université du Maine (UMR CNRS 6613, Le Mans, France) for more than twenty years and more recently with the LMA (Marseille, France) mainly in the domain of acoustics of air saturated porous media. He pioneered research in this field. The results of these works have many implications, ranging from fundamental studies to Applied Architectural Acoustics and the metrology of sound absorbing porous media.

Walter Lauriks is main author or co-author of more than 100 publications in international peer-reviewed journals and more than 110 conference proceedings for international conferences. At different occasions, he was session organiser and/or session chairman at these conferences. He also contributed to 7 book chapters.

Since 1997, Walter Lauriks is Associate Editor Physical Acoustics "Acustica united with Acta Acustica". He is member of several scientific organisations: Société Française d'Acoustique, Acoustical Society of America, Association Belge des Acousticiens (board member), Netherlands Akoestisch Genootschap (Dutch Acoustical Society NAG, board member), IEEE, The American Association of Physics Teachers. 


\section{FRENCH MEDAL 2007 of the}

\section{French Acoustical Society}

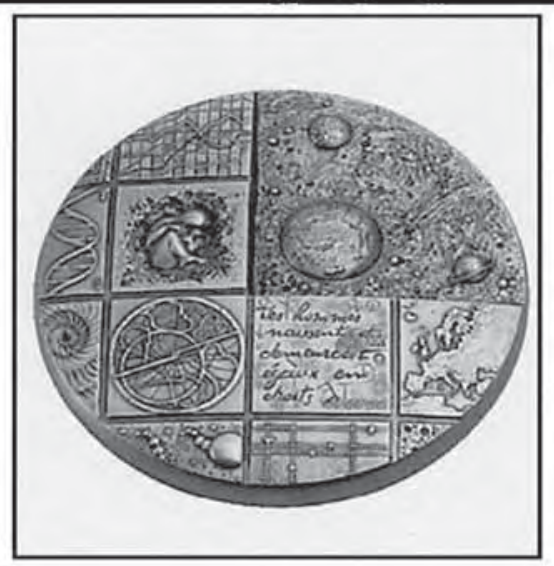

\section{Vitalyi GUSEV}

\section{7}

The French Medal is annually presented to a French scientist, who has contributed significantly to the reputation of French acoustics.

\section{PREVIOUS RECIPIENTS}

\begin{tabular}{|c|c|}
\hline 1966 & CANAC and P. CHAVASSE \\
\hline 1967 & A. BARON \\
\hline 1968 & R. LUCAS \\
\hline 1969 & J. BRILLOUIN \\
\hline 1970 & P. LIENARD \\
\hline 1972 & R-G. BUSNEL \\
\hline 1973 & T. VOGEL \\
\hline 1974 & J-J. MATRAS \\
\hline 1975 & R. CHOCHOLLE \\
\hline 1976 & J. PUJOLLE \\
\hline 1977 & R. SIESTRUNK \\
\hline 1978 & E. LEIPP \\
\hline 1979 & P. RAPIN \\
\hline 1980 & M. BURGEAT \\
\hline 1981 & A. DIDIER \\
\hline 1982 & A. BERGASSOLI \\
\hline 1983 & G. COMTE-BELLOT \\
\hline 1984 & J. MATTEI (refused) \\
\hline 1985 & R. LEHMANN \\
\hline 1986 & P. LORAND \\
\hline
\end{tabular}

\begin{tabular}{|c|c|}
\hline 1987 & P. FRANCOIS \\
\hline 1988 & E. DIEULESAINT \\
\hline 1989 & B. POIREE \\
\hline 1990 & R. CARRE \\
\hline 1991 & C. GAZANHES \\
\hline 1992 & JJF. ALLARD \\
\hline 1993 & M-C. BOTTE \\
\hline 1994 & J.P. HATON \\
\hline 1995 & J. RIPOCHE \\
\hline 1996 & A.M. BRUNEAU \\
\hline 1997 & J. POULIQUEN \\
\hline 1998 & D. ROYER \\
\hline 1999 & P. FILLIPI \\
\hline 2000 & A. DANCER \\
\hline 2001 & G. CANEVET \\
\hline 2002 & P. GATIGNOL \\
\hline 2003 & M. FINK \\
\hline 2004 & M. BRUNEAU \\
\hline 2005 & C. LESUEUR \\
\hline 2006 & D. CATHIGNOL \\
\hline
\end{tabular}




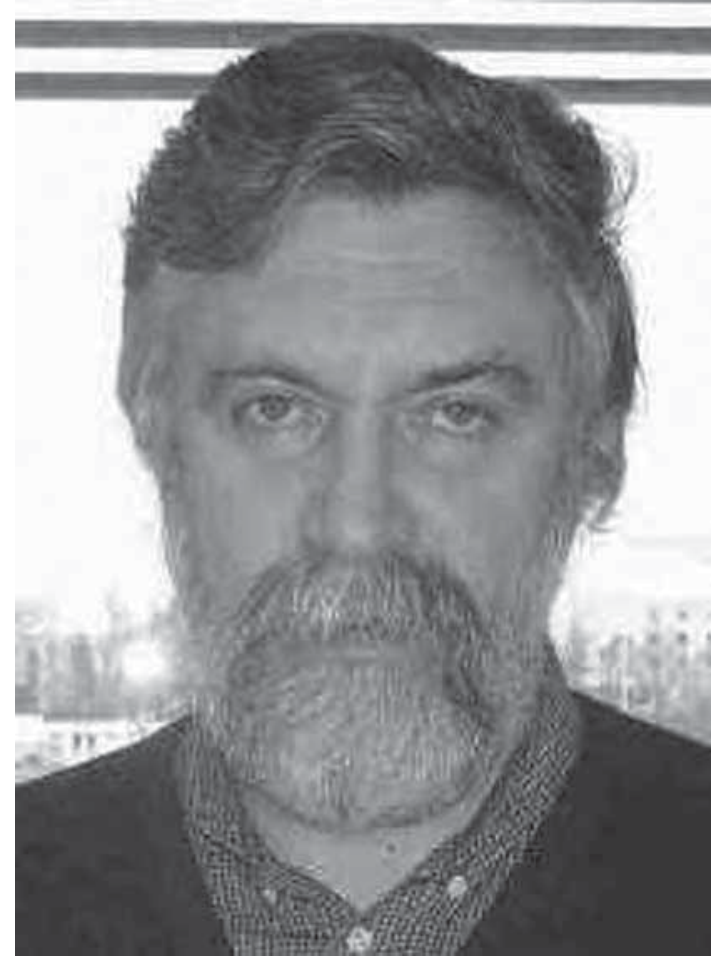


Professor Vitalyi GUSEV received degrees of PhD (1982) and Dr. Sc. (1991) in Physics and Mathematics from Moscow State University (Russia). After several years of being researcher and assistant professor at the same university, he joined in 1990 the International Laser Centre at Moscow State University where he was associated professor. In 1998, he joined University of Maine in Le Mans (France) where he became professor in a national engineering school (ENSIM). He is now full professor of University of Maine at the highest grade and he is since 2006 the Senior member of the "Institut Universitaire de France" (Institute of French Universities) because of his outstanding research achievements.

Pr. Gusev is the recipient of several awards, including the Lenin Comsomol Prize in Science and Technology (1987, Russia), the distinction of the International Science Foundation (1994), Senior Prize of International Photoacoustic and Photothermal Association" (2004).

He was invited in several universities (Sapporo, Japan - Heidelberg, Germany - Brown, USA - Toronto Canada - Leuven, Belgium - Paris, France) as fellow, visiting scientist, visiting professor, etc. He gave more than 50 lectures and seminars as an invited speaker. He is member of international scientific committees, among them: International Congress on Ultrasonics, International Conference on the Emerging Technologies in Quantitative Nondestructive Testing, International Conference on Photoacoustic and Photothermal Phenomena...

$\mathrm{He}$ is the author of more than 400 scientific publications, among them more than 180 are in international scientific journals or are book chapters. He also published a book "Laser Optoacoustics" with A. Karabutov (translated from Russian into English in 1993 by the American Institute of Physics, New York, USA).

His fields of research can be summarised mainly as: photothermal and photoacoustic phenomena, picosecond ultrasonics, nonlinear acoustics, acoustics of micro-inhomogeneous media, thermoacoustics. He has made deep and original contributions in all of his fields of interest and even he had a pioneering role in tackling theoretically several challenging 


\title{
Session 4aAAa
}

\section{Architectural Acoustics: Architectural Acoustics Potpourri}

\author{
David T. Bradley, Cochair \\ 124 Raymond Avenue, Poughkeepsie, NY, 12604
}

Andrea Farnetani, Cochair

Engineering Dept. - Univ. of Ferrara, Via Saragat, 1, Ferrara, 44100, Italy

\section{Contributed Papers}

8:00

4aAAa1. Irregular sound absorbers work better. Bernard Sapoval (Ecole Polytechnique, PMC-Polytechnique Route de Saclay, 91128 Palaiseau, France, bernard.sapoval@polytechnique.edu), Anna Rozanova-Pierrat (Ecole Polytechnique, PMC-Polytechnique Route de Saclay, 91128 Palaiseau, France, anna.rozanova-pierrat@polytechnique .edu), Simon Félix (Laboratoire d'Acoustique de l'Université du Maine, Avenue Olivier Messiaen, 72085 Le Mans, France, simon.felix@univlemans.fr), Marcel Filoche (Ecole Polytechnique, PMC-Polytechnique Route de Saclay, 91128 Palaiseau, France, marcel.filoche@polytechnique .edu)

The diffraction and absorption of waves by a system with both absorbing properties and irregular geometry is an open physical problem. In the same time, irregular absorbers have been shown to be extremely efficient (1). A more reachable and closely related goal is the understanding of wave oscillations in confined systems containing an absorbing material with an irregular shape. From the theoretical point of view, the difficulty lies in the fact that part of the propagation occurs in a lossy material for which the wave operator is non-hermitian. It is found here that, in resonators containing an irregular shaped absorbent material, there appears a new type of localization. This phenomemon, that we call "'astride" localization, describes the fact that these modes exist in both the lossless and the lossy regions. They are then both lossy and well coupled to sources in the air. A numerical computation of the time decay of acoustic energy shows that indeed sound absorbing devices work better when presenting a very irregular shape and that this is directly linked due to the existence of astride localisation. (1) Fractal Wall, product of Colas Inc., French patent N0203404; U.S. patent 10/508,119.

\section{8:20}

4aAAa2. A brief review on micro-perforated sound absorbers. Christian Nocke (Akustikbüro Oldenburg, Katharinenstr. 10, 26121 Oldenburg, Germany, nocke@akustikbuero-oldenburg.de), Catja Hilge (Akustikbüro Oldenburg, Katharinenstr. 10, 26121 Oldenburg, Germany, hilge@akustikbuero-oldenburg.de), Jean-Marc Scherrer (Normalu S.A.S, Route du Sipes, 68680 Kembs, France, jmscherrer@barrisol.com)

The theory and design of microperforated panel sound-absorbing constructions have been introduced by D.-Y. Maa in 1975. Since then many variations of micro-perforated sound absorbing devices and materials have been introduced. Materials that have been used to be micro-perforated have been metal, wood, plastics and many others. A survey of different applications of micro-perforation will be presented. Examples shown in more detail are the development of stretched foils and metal as micro-perforated panels. Results of sound absorption measurements of various assemblies for normal and statistical incidence of sound will be shown and compared with theoretical predictions. Finally the potential of the micro-perforated sound absorber will be discussed.

\section{8:40}

4aAAa3. The vibration sound absorption theory of soft materials. Xin An Zhang (Xi'an Polytechnic University, 134\#, Key Laboratary, 19 South Jin Hua Road, 710048 Xi'an, China, zxafafa@yahoo.com.cn)

During the past 2 years, a new theory has been established for soft materials that the vibration of materials brings the sound absorption, regardless whether they have pores in them or not. This theory is totally different from Classical theory, such as the Rayleigh model and the Zwikker and Kosten theory. Firstly, an empirical sound absorption coefficients formula of fibrous materials was found. Secondly, the theory sound absorption formula of thin fiber layers was obtained by the vibration sound absorption analysis and the applying of conservation law of energy. This formula agrees well with the empirical sound absorption formula of fibrous materials (mentioned above). Based on this achievment and applying classical laws of conservation of momentum and conservation of energy, the sound absorption theory formula of membrane (diaphragm) was also obtained, which have been well justified and also agree with the practice. This paper will review and discuss the main point about the vibration sound absorption theory and its establishment.

\section{9:00}

4aAAa4. The influence of absorption on statistical distribution of free path lengths in rooms. Dragana Sumarac Pavlovic (Faculty of Electrical Engineering, Bulevar Kralja Aleksandra 73, 11000 Belgrade, Serby, dsumarac@etf.bg.ac.yu), Miomir Mijic (Faculty of Electrical Engineering, Bulevar Kralja Aleksandra 73, 11000 Belgrade, Serby, emijic@etf.bg.ac.yu)

The ray tracing method in sound field simulation in a room allows the calculation of the free path lengths distribution (FPL) by superimposing all rays paths generated in the analysis. In room acoustics the statistical theory of sound field defined this distribution as an indicator of room geometrical properties which are independent of absorption. Accordingly, some commercial softwares for ray tracing simulation allow user to calculate that global distribution of FPL only. This paper is concerned with the analysis of the changes in the shape of FPL distribution as a consequence of nonuniform arrangement of absorption in room. Particularly is analysed the impact of absorptive auditorium in different global forms of the halls where all other interior surfaces are acoustically hard.

\section{9:20}

4aAAa5. The influence of geometrical features of rooms on their acoustic response - insight based on measurements in physical models. Miomir Mijic (Faculty of Electrical Engineering, Bulevar Kralja Aleksandra 73, 11000 Belgrade, Serby, emijic@etf.bg.ac.yu), Dragana Sumarac Pavlovic (Faculty of Electrical Engineering, Bulevar Kralja Aleksandra 73, 11000 Belgrade, Serby, dsumarac@etf.bg.ac.yu)

In a previous paper by the same authors, an analysis of the influence of geometrical features of rooms on their acoustic response was presented (Acta Acustica, Vol 93, 2007, 1012-1026). The analysis was based on ray tracing simulation. The results have shown that geometrical characteristics of rooms do influence reverberation time, and this influence is realised by the structure of sound energy paths through the room. It was concluded that 
room geometry influences reverberation time both at macro and micro levels, and this influence cannot be separated. To validate these conclusions in a real sound field, additional measurements have been conducted in specially prepared physical models of rooms. In several models of different shapes selected from previous results, scaled 1:10, the changes of scattering were introduced by appropriate modifications of interior surfaces. The results have confirmed the previous study conclusions.

\section{9:40}

4aAAa6. Room acoustics prediction based on multiple linear regressions and artificial neural networks. Maria Ribeiro (FEUP/DEC, Rua Dr. Roberto Frias, 4200-465 Porto, Portugal, mribeiro@fe.up .pt), Fernando Martins (FEUP/DEC, Rua Dr. Roberto Frias, 4200-465 Porto, Portugal, fgm@fe.up.pt)

Room acoustic quality is known to be dependent on several objective variables that are expected to be well correlated with subjective impressions of the room acoustics as judged by musical experts. Although subjects have different preferences and overall judgments are based in different criteria, it can be said that listeners in their subjective evaluation would give preference to reverberance, clarity, intimacy or spaciousness attributes. In this study, reverberance and clarity, expressed respectively by T30/EDT and C80 objective values, were predicted by multiple linear regression and artificial neural networks using normalized original data and principal components as dependent variables. The results obtained by these approaches were compared with predicted values using a computer simulation program based on the physics of ray-tracing and with measured data. Room acoustical quality was also evaluated based on preferred values as suggested by some researchers.

\section{0:00-10:20 Break}

\section{0:20}

4aAAa7. Minimum BRIR grid resolution for dynamic binaural synthesis. Alexander Lindau (Department of Audio Communication, Technical University of Berlin, Sekr. EN-08, Einsteinufer 17c, 10587 Berlin, Germany, alexander.lindau@tu-berlin.de), Hans-Joachim Maempel (Department of Audio Communication, Technical University of Berlin, Sekr. EN-08, Einsteinufer 17c, 10587 Berlin, Germany, hans-joachim.maempel@tu-berlin.de), Stefan Weinzierl (Department of Audio Communication, Technical University of Berlin, Sekr. EN-08, Einsteinufer 17c, 10587 Berlin, Germany, stefan.weinzierl@tu-berlin.de)

Binaural synthesis of acoustical environments is based on binaural room impulse responses (BRIRs) measured with a certain grid of spherical coordinates separated by angles of typically between $1^{\circ}$ and $15^{\circ}$. The resulting spatial resolution defines the size of the BRIR database as well as the duration of its measurement. Perceptual evaluations of datasets with a different spatial resolution using HRTFs (anechoic case) have been reported from. Most of these studies use the localization performance of listeners as a criterion; a more sensitive measurement for slight degradations in audio quality can be expected from a criterion-free comparison of datasets with different angular grids. Thus, to determine the minimum grid resolution required for dynamic binaural synthesis a listening test was performed. Following an adaptive $3 \mathrm{AFC}$ procedure, the spatial resolution of a recorded BRIR dataset was gradually lowered from a maximum of $1 \% 1^{\circ}$ resolution until audible artefacts were introduced. This was done for a sound source located at $0^{\circ} / 0^{\circ}$ presented with dynamical auralization in two degrees of freedom. To test for interaction effects the thresholds were derived independently for azimuth and elevation. The datasets used were acquired in an anechoic environment and in two rooms of different size and reverberation time.

\section{0:40}

4aAAa8. Optimum Room Acoustic Comfort ${ }^{\mathrm{TM}}$ (RAC $^{\mathrm{TM}}$ ) can be achieved by using a selection of appropriate acoustic descriptors. Carsten Svensson (Saint-Gobain Ecophon AB, Box 500, SE-260 61 Hyllinge, Sweden, carsten.svensson@ecophon.se), Erling Nilsson (SaintGobain Ecophon AB, Box 500, SE-260 61 Hyllinge, Sweden, erling.nilsson @ecophon.se)
In order to create an optimum Room Acoustic Comfort ${ }^{\mathrm{TM}}\left(\mathrm{RAC}^{\mathrm{TM}}\right)$ in rooms it is important to consider a variety of different acoustic descriptors. These descriptors must match and facilitate for wanted human qualities such as ability to concentrate, reduced stress, clear speech etc. In this process it is important to consider the people, what they do (the activity) and what room they will be in. Today, when designing ordinary rooms from an acoustic perspective, mainly reverberation time (T20) is utilised - both in practice but also in building regulation and standards. Reverberation time (T20) only describe the later part of the decay curve, and therefore only partly mirror the wanted acoustic reality. Thus, based upon a large number of acoustic measurements, we suggest a "cocktail" of acoustic descriptors for ordinary rooms in buildings like schools, offices, health care premises etc. These descriptors have to cover both early and late decay, sound levels and speech quality. Our suggestions are Speech Clarity (C50), Speech Transmission Index (STI), Early Decay Time (EDT), Reverberation Time (T20) and Strength $(\mathrm{G})$. Moreover, in open and long spaces we also suggest the acoustic descriptors Rate of Spatial Decay (DL2) and Excess of Sound Pressure Level (DLf).

\section{1:00}

4aAAa9. Distribution of Speech Intelligibility Metrics in Classrooms with Varied Signal to Noise (S/N) Ratios. Nurgun Bayazit Tamer (Istanbul Technical University, Taskisla Taksim Istanbul, 34437 Istanbul, Turkey, nurgun@itu.edu.tr)

In a classroom to provide adequate speech intelligibility is vital especially when young children are concerned. Room acoustical characteristics of classrooms like reverberation times and background noise mainly define the speech intelligibility in classrooms. Excessive background noise deteriorates the signal to noise ratio $(\mathrm{S} / \mathrm{N})$ and leads to reduction in learning efficiency. An extensive measurements study is being used in Istanbul Elementary Schools as part of a project. This paper explains the results of the investigation of the influence of varying signal to noise ratios on different speech intelligibility metrics. Background noise are includes external noises such as outdoor traffic noise, noise from playground or noise from adjacent classrooms. STI, RASTI, Alcon(\%) values of 20 different classrooms in 20 different schools are measured in 9 different positions. The measurements were repeated in each classroom while windows were open and closed respectively. The object of the work was to systematically study the influence of the $\mathrm{S} / \mathrm{N}$ ratio variations and reverberation time on the different speech intelligibility metrics. Finally to elucidate the effects of different absorption treatments achieving recommended reverberations on the measured speech metrics are also discussed.

\section{1:20}

4aAAa10. Speaker comfort and increase of voice level in lecture rooms. Jonas Brunskog (Dept. of Acoustic Technology, Technical University of Denmark, Building 352, DK 2800 Lyngby, Denmark, jbr@oersted .dtu.dk), Anders C. Gade (Dept. of Acoustic Technology, Technical University of Denmark, Building 352, DK 2800 Lyngby, Denmark, acg@oersted.dtu.dk), Gaspar Payà Bellester (C/ Sènia 1, 1er. C.P., 03640 Monòver Alacant, Spain, kansbarpb@gmail.com), Lilian Reig Calbo (Alacant, Spain,xusketa@hotmail.com)

Teachers often suffer health problems or tension related to their voice. These problems may be related to there working environment, including room acoustics of the lecture rooms which forces them to stress their voices. The present paper describes a first effort in finding relationships between the objectively measurable parameters of the rooms and the objective voice power produced by speakers. In rooms with different sizes, reverberation time and other physical attributes, the sound power levels produced by six speakers where measured while giving a short lecture. Relevant room acoustic parameters were also measured in the rooms and subjective impressions from about 20 persons who had experience talking in these rooms were collected as well. Analysis of the data revealed significant differences in the sound power produced by the speaker in the different rooms. It was also found that these changes were mainly related to the size of the room and to the gain or support produced by the room. To describe this quality, a new room acoustic quantity called 'room gain' is proposed. 
$11: 40$

4aAAa11. Measuring the mixing time in auditoria. Guillaume Defrance (Institut Jean le Rond d'Alembert, Laboratoire d'Acoustique Musicale, 11, rue de Lourmel, 75015 Paris, France, defrance@lam.jussieu.fr), Jean-Dominique Polack (Institut Jean le Rond d'Alembert, Laboratoire d'Acoustique Musicale, 11, rue de Lourmel, 75015 Paris, France,polack@ccr.jussieu.fr)

The goal of prediction, in Room Acoustics, is to synthesize the impulse responses (IRs) of a hall, in order to derive acoustic indices or to allow auralization. The process assumes the hall to be a time invariant linear system. Furthermore, the IR is known to behave stochastically when the sound field becomes diffuse, that is, after a certain time called mixing time. This study aims at characterizing the IR mixing time. Three methods are presented for visualizing and detecting the time evolution of the IR behaviour. The first one highlights the transition from early reflections to diffuse sound field by monitoring the phase evolution versus time. The two others exploit the gaussian distribution of pressure in a diffuse sound field, when the IR becomes statistical. These methods are applied to measurements, carried out in Salle Pleyel, and confirm the simple relationship found earlier between mixing time and volume.

\title{
Session $4 \mathrm{aAAb}$
}

\section{Architectural Acoustics: Prediction Methods in Building Acoustics II}

\author{
Berndt Zeitler, Cochair \\ NRC - Institute for Research in Construction, 1200 Montreal Road, Building M-27, Ottawa, ON K1A OR6, Canada \\ Catherine Guigou-Carter, Cochair \\ CSTB, 24, rue Joseph Fourier, Saint Martin D'Hères, 38400, France
}

\section{Invited Papers}

\section{8:00}

4aAAb1. Prediction method for the acoustic performance of permanent form systems. Jean-Baptiste Chene (CSTB, 84, Avenue Jean-Jaurès, 77447 Marne-la-Vallée Cedex 2, France, jean-baptiste.chene@cstb.fr), Catherine Guigou-Carter (CSTB, 24, rue Joseph Fourier, 38400 Saint Martin D’Hères, France, catherine.guigou@cstb.fr)

Permanent form systems considered in this paper combine a layer of porous or fibrous material onto which a concrete layer is poured. Such systems allow the fulfillment of French thermal regulations. However, their acoustic performance is quite limited. Indeed, the acoustic performance of the concrete layer is usually reduced by the presence of the porous or fibrous layer. The modeling of such multi-layered structures submitted to acoustic excitation is discussed in this work. The behavior of such a system is investigated by using a wave approach based prediction tool. The porous layer is modeled following Biot's theory. The effect of the metallic anchors that connect the fiber and the concrete layers is also investigated. The acoustic performance of such systems is studied both experimentally and analytically. A parametric study is performed to identify the most determinant parameters. The model is thus used to obtain insight into the behavior of such systems in order to develop solutions that result in improved acoustic performance.

4aAAb2. The directivity of the forced radiation of sound from panels and openings including the shadow zone. John L. Davy (RMIT University, Applied Physics, GPO Box 2476V, 3001 Melbourne, Victoria, Australia, john.davy@ rmit.edu.au)

This paper presents a method for calculating the directivity of the radiation of sound from a two dimensional panel or opening, whose vibration is forced by the incidence of sound from the other side. The directivity of the radiation depends on the angular distribution of the incident sound energy. For panels or openings in the wall of a room, the angular distribution of the incident sound energy is predicted using a physical model which depends on the sound absorption coefficient of the room surfaces. For an opening at the end of a duct, the sound absorption coefficient model is used in conjunction with a model for the directivity of the sound source in the duct. For angles of radiation approaching 90 degrees to the normal to the panel or opening, the effect of the diffraction by the panel or opening, or by the finite baffle in which the panel or opening is mounted, is included. A simple empirical model has been developed to predict the diffraction of sound into the shadow zone when the angle of radiation is greater than 90 degrees to the normal to the panel or opening. The method is compared with published experimental results. 


\title{
Contributed Papers
}

\section{8:40}

4aAAb3. Numerical study of sound transmission loss using an indirect boundary element method. Matthew Cassidy (Queen's University Belfast, School of Mechanical and Aerospace Engineering, Ashby Building, Stranmillis Road, BT9 5AH Belfast, UK, mcassidy06@qub.ac.uk), Richard K. Cooper (Queen's University Belfast, School of Mechanical and Aerospace Engineering, Ashby Building, Stranmillis Road, BT9 5AH Belfast, UK, R.Cooper@qub.ac.uk), Richard Gault (Queen's University Belfast, School of Mechanical and Aerospace Engineering, Ashby Building, Stranmillis Road, BT9 5AH Belfast, UK, r.gault@qub.ac.uk), Jian Wang (Queen's University Belfast, School of Mechanical and Aerospace Engineering, Ashby Building, Stranmillis Road, BT9 5AH Belfast, UK, j.wang@qub.ac.uk)

The purpose of this study was to simulate transmission loss tests at the acoustic facilities of FG Wilson, Larne, UK. A hemi-anechoic chamber adjoins a reverberation room via a transmission plug where canopy panel sections are mounted for testing. Boundary element methods in LMS Virtual .Lab are used in conjunction with a baffle model to simulate the test facilities for transmission loss. On one side of this wall the reverberation room is modelled as a diffuse field using a series of defined plane waves, and on the other the hemi-anechoic chamber is represented as a free field. Experiments were carried out on a steel plate and lead sheet following the ISO 15186 standard for measurement of sound insulation using sound intensity. Source room sound pressure levels were recorded with a microphone, and an intensity probe was used to map the sound intensity field on the receiving side. Transmission loss for a frequency range was calculated as stated in the standard and compared with the results for the computational analysis. Comparison of the computational simulation with the experimental yielded a sufficient agreement.

\section{Invited Paper}

\section{9:00}

4aAAb4. Prediction of the Sound Transmission Loss of Multi-layered Small Sized Elements. Stefan Schoenwald (Eindhoven University of Technology, Den Dolech 2, BWK/BPS, 5600 MB Eindhoven, Netherlands, s.schoenwald@tue.nl), Eddy Gerretsen (TNO Science and Industry, Stieljesweg 1, 2628CK Delft, Netherlands, eddy.gerretsen@tno.nl), Heiko J. Martin (Eindhoven University of Technology, Den Dolech 2, BWK/BPS, 5600 MB Eindhoven, Netherlands, h.j.martin@tue.nl)

In this paper an improved method for the prediction of the sound transmission loss of multilayered finite structures, like glazing will be presented. The sound transmission loss of an infinite structure is predicted with a common transfer matrix as a function of the angle of the incident sound wave. Then Villiot's spatial windowing method is applied to take into account the finiteness of the element. Usually an ideal diffuse distribution of the incident sound power is assumed and the prediction results are integrated over all angles of incidence. The obtained prediction results tend to underestimate sound transmission loss due to the dominance of the small values for gracing incidence. Often simple ad-hoc corrections are used for improvement, like Beranek's field incidence, that fail for multilayered structures. Kang suggests that the incident sound power on a surface of a room generally is Gaussian distributed on the angle of incidence and introduces a weighting function for the integration of the prediction results over the angles of incidence. New in this paper is that spatial windowing as well as a Gaussian distributed sound power is considered for the prediction of the transmission loss. The results of the prediction are validated by experiment.

THURSDAY MORNING, 3 JULY 2008

ROOM 243, 10:00 A.M. TO 7:00 P.M.

\section{Session 4aAAc}

\section{Architectural Acoustics: Measuring Methods and Uncertainty in Building Acoustics I}

\author{
Brandon Tinianow, Cochair \\ Quiet Solution, 1250 Elko Dr., Sunnyvale, CA 94089, USA \\ Werner Scholl, Cochair \\ Physikalisch-Technische Bundesanstalt, Bundesallee 100, Braunschweig, 38116, Germany
}

\section{Contributed Papers}

\section{0:00}

4aAAc1. A new technique for the measurement of the normal incidence absorption coefficient using an impedance tube and a single microphone with fixed position. Cedric Vuye (Hogeschool Antwerpen, Dept. of Industrial Sciences, Paardenmarkt 92, BE-2000 Antwerpen, Belgium, c.vuye@ha.be), Steve Vanlanduit (Vrije Universiteit Brussel, Acoustics and Vibration Research Group, Dept. of Mechanical Engineering, Pleinlaan 2, BE-1050 Brussels, Belgium, Steve.Vanlanduit@vub.ac.be), Karl Van Nieuwenhuyse (Vrije Universiteit Brussel, Acoustics and Vibration Research Group, Dept. of Mechanical Engineering, Pleinlaan 2, BE-1050 Brussels, Belgium, karl.van.nieuwenhuyse@skynet.be), Patrick Guillaume (Vrije Universiteit Brussel, Acoustics and Vibration Research
Group, Dept. of Mechanical Engineering, Pleinlaan 2, BE-1050 Brussels, Belgium, Patrick.Guillaume@vub.ac.be)

The normal incidence absorption coefficient of acoustic materials can be measured inside an impedance tube with different settings for the microphone(s). The two most widespread techniques are the standing wave method using a probe microphone and side-mounted two-microphone techniques. Errors that can occur here are related to phase mismatch between the two microphones and the knowledge of the exact locations of the (acoustic centre of the) microphones and test sample. These problems have (partially) been solved by, for example, calibrating the microphones by swapping them and calculating a calibration transfer function or by using one microphone techniques. In this article we will present a novel technique 
which will also avoid the need for the knowledge of the exact microphone location by continuously moving the sample under test. Different results will be presented and compared to the traditional techniques.

\section{0:20}

4aAAc2. New method for measuring sound absorption coefficients in an industrial hall. Joël Ducourneau (Faculté de Pharmacie de Nancy, Université Henri Poincaré, 5, rue Albert Lebrun, BP 80403, 54001 Nancy, France, Joel.Ducourneau@pharma.uhp-nancy.fr), Vincent Planeau (Institut National de Recherche et de Sécurité (INRS), Ave. de Bourgogne, B.P. 27, F-54501 Vandoeuvre Cedex, France, vincent.planeau@secav.fr), Jacques Chatillon (Institut National de Recherche et de Sécurité (INRS), Ave. de Bourgogne, B.P. 27, F-54501 Vandoeuvre Cedex, France, jacques.chatillon @inrs.fr), Armand Nejade (Institut National de Recherche et de Sécurité (INRS), Ave. de Bourgogne, B.P. 27, F-54501 Vandoeuvre Cedex, France, armand.nejade@inrs.fr)
Predicting the sound pressure level at a workplace requires in-situ characterization of the facings. This work describes a new method for the measurement of the sound absorption coefficient of flat panels present in industrial halls. In such room, it is necessary to separate the echo coming from the studied panel from the others due to the entire reverberation. This separation has been achieved in space by an acoustic array and in time by an impulse sound source. The array processing uses a multipolar weighting to achieve a directivity constant with frequency and with attenuated side lobes. This weighting requires a limited number of microphones. The impulse source has been designed using the inverse impulse response from the emission system (equalizer, amplifier, loudspeaker). This inverse filtering technique allows equalizing the response of the emission system in order to radiate very short pulses. Sound absorption coefficient of several flat facings have been evaluated by mean of this new device in a semi-anechoic chamber and in an industrial hall designed for testing. The results show a good agreement with others techniques except at low frequencies for which the array length is too small and the absorption coefficients too low.

\section{Invited Paper}

\section{0:40}

4aAAc3. Measurement uncertainty of the sound absorption coefficient. Anna Izewska (Building Research Institute, Filtrowa Str.1, 00-611 Warsaw, Poland, a.izewska@itb.pl)

The standard ISO/IEC 17025:2005 on the competence of testing and calibration laboratories requires that these laboratories shall apply procedures for estimating the uncertainty of their measurement results. One of the possibility is to evaluate the budget of uncertainty, taking into account all components that contribute significant uncertainty to the final result. In case of the sound absorption coefficient measurement, carried out according to the standard EN ISO 354:2003, the overall uncertainty is first of all influenced by the reverberation times T1, T2 and the power attenuation coefficients $\mathrm{m} 1$ and $\mathrm{m} 2$, calculated according to the ISO $9613-1$ standard and representing the climatic conditions in the reverberation room. In spite of very little difference between the values $\mathrm{m} 1$ and $\mathrm{m} 2 \mathrm{repre-}$ senting the change of climatic conditions (usually, it is the case in laboratory), exponential form of the coefficient's function causes that the uncertainty of measurement results increase with frequency very fast. Particularly for the high frequencies, the values of uncertainty are so important that the evaluation of the sound absorption coefficient is practically not possible.

\section{Contributed Papers}

11:00

4aAAc4. A comparison of impulse-like sources to be used in reverberation time measurements. Marko Horvat (Faculty of EE and Computing, Unska 3, Department of Electroacoustics, HR-10000 Zagreb, Croatia, marko.horvat@fer.hr), Kristian Jambrosic (Faculty of EE and Computing, Unska 3, Department of Electroacoustics, HR-10000 Zagreb, Croatia, kristian.jambrosic@fer.hr), Hrvoje Domitrovic (Faculty of EE and Computing, Unska 3, Department of Electroacoustics, HR-10000 Zagreb, Croatia, hrvoje.domitrovic@fer.hr)

As a part of an extensive ongoing research on reverberation time measurements, efforts have been made to find an impulse-like source that will comply with certain demands concerning its spectral content and sound pressure levels it can provide. Although the relevant standards state that such kind of sources should be avoided due to the lack of measurement repeatability, their use has proved to be necessary due to inability of conventional omni-directional loudspeakers to provide adequate sound pressure levels, especially at the low end of the frequency range of interest, thereby ensuring sufficient dynamic range. Therefore, the emphasis of this investigation has been made on peak sound pressure levels and the amount of low frequency content each source is able to provide. The investigated sources include $6 \mathrm{~mm}$ and $8 \mathrm{~mm}$ pistols, firecrackers with different amount of explosive compound and explosive mixtures of acetylene gas.

\section{1:20}

4aAAc5. An Application of Time-Reversal Acoustics to Sound Insulation Measurements in Buildings. Doheon Lee (University of Sydney, Faculty of Architecture, Design and Planning, NSW 2006 Sydney, Australia, dlee7117@mail.usyd.edu.au), Densil Cabrera (University of Sydney, Faculty of Architecture, Design and Planning, NSW 2006 Sydney, Australia, densil@usyd.edu.au)

This paper considers the possible application of time reversal acoustics (TRA) to airborne sound insulation measurements in buildings. In TRA, an array of transducers is set up to form a time-reversal 'mirror' or 'cavity' Using this array, the sound radiated from an initial source is collected and refocused spatially and temporally, thereby being reproduced at the initial source position with high signal-to-noise ratio $(\mathrm{S} / \mathrm{N})$. Most previous studies of TRA have been conducted underwater with ultrasonic sound sources, with only a few in the audible range in real buildings. The technique is best suited to non-dissipative systems, raising the question of whether any advantage could exist for transmission between rooms. This study applies TRA experimentally in the audible range using maximum length sequence signals for transmission between two rooms. Comparison is made between conventional measurements (with and without impulse response deconvolution) and TRA in terms of effective S/N and apparent level difference between the rooms. Substantially greater $\mathrm{S} / \mathrm{N}$ is achieved using TRA, but the interpretation of measurements is not straightforward, and the technique is much more demanding than conventional measurements.

\section{1:40}

4aAAc6. Measuring Sound Insulation using Deconvolution Techniques Constant C. Hak (Technische Universteit Eindhoven, De Rondom 10, 5612 AP Eindhoven, Netherlands, c.hak@inter.nl.net), Nicole H. Van Hout (LeVel Acoustics BV, De Rondom 10, 5612 AP Eindhoven, Netherlands, n.h.a.m.v.hout@tue.nl), Heiko J. Martin (Eindhoven University of Technology, Den Dolech 2, BWK/BPS, 5600 MB Eindhoven, Netherlands, h.j .martin@tue.nl)

To compare the acoustic performance of a building element with the given sound insulation requirements, measurements need to be done. Generally, a broadband noise source is used according to international standards. This method does not always work in practice due to high sound insulation values or high background noise levels. It is very inconvenient from a practical point of view or even impossible to perform an accurate sound insulation measurement for all frequency bands. A solution to this problem can be found in deconvolution techniques using mls or sweep signals. It is possible to increase the signal to noise ratio with these techniques by averaging mea- 
surements and spreading out the spectral sound energy in time. As a result an efficient use of available sound power is possible. In a laboratory is investigated how to use mls or sweep signals as a source signal and deconvolution as a measurement technique to obtain the sound insulation under noisy conditions.

\section{2:00}

4aAAc7. Characterising a washing machine as a structure borne sound source on a lightweight floor. Matthias Lievens (Institute of Technical Acoustics, Neustraße 50, 52056 Aachen, Germany, mli@akustik.rwthaachen.de)
The transfer of structure borne sound power depends on the mobility of the source and the receiver. If source and receiver are coupled through multiple points, the interaction between those points has to be accounted for. The force of a washing machine injected into a lightweight wooden floor is analysed to develop a simple measurement procedure for similar multiple point structure borne sound sources. A complete mobility model will be compared with a simplified model based on a reduced mobility matrix. The importance of different matrix components will be determined. Receiver structures used in real buildings will be investigated.

\section{Invited Paper}

\section{$12: 20$}

4aAAc8. On the use of scaled models in building acoustics. Volker Wittstock (Physikalisch-Technische Bundesanstalt, Bundesallee 100, 38116 Braunschweig, Germany, volker.wittstock@ptb.de), Martin Schmelzer (Physikalisch-Technische Bundesanstalt, PTB, Working group 1.71 'Building Acoustics', Bundesallee 100, 38118 Braunschweig, Germany, martin.schmelzer@ptb .de), Christoph Kling (Physikalisch-Technische Bundesanstalt, PTB, Working group 1.71 'Building Acoustics', Bundesallee 100, 38118 Braunschweig, Germany, christoph.kling@ptb.de)

Experimental studies of physical effects in building acoustics are usually time consuming and expensive. This is mainly caused by the building costs but also by the experimental effort. It is thus desirable to have another method for the investigation of basic effects in building acoustics. Building acoustic problems are characterized by the interaction between airborne and structure-borne sound fields. It is therefore possible to use scaled models when both sound fields are treated correctly. This means that the wavelengths in the airborne and in the structure-borne sound fields have to be scaled in the same way. With a scaling factor of typically $1: 8$, the costs can be reduced drastically and nearly all model parameters can be changed separately. Due to these advantages, this technique is used at PTB's building acoustics group. This contribution gives an overview on the physical background of scaled models, reports on validation experiments and on several applications e.g. investigations of the influence of temperature and static pressure, damping effects, geometry influence on the sound insulation of walls, the measurement of the flanking transmission of walls and the measurement of suspended ceilings.

\section{2:40-1:40 Lunch Break}

\section{Contributed Paper}

\section{$1: 40$}

4aAAc9. Influence of the source orientation on the measurement of acoustic parameters in a large reverberant cathedral. Miguel Arana (Public University of Navarre, Physics Department. Campus de Arrosadia, s/n, 31006 Pamplona, Spain, marana@unavarra.es), Ricardo San Martin (Public University of Navarre, Physics Department. Campus de Arrosadia, s/n, 31006 Pamplona, Spain, ricardo.sanmartin@unavarra.es) , Maria Luisa San Martin (Public University of Navarre, Physics Department. Campus de Arrosadia, s/n, 31006 Pamplona, Spain, sanmartin @unavarra.es)

ISO 3382 standard describes both definitions and measurement procedures of different acoustic parameters derived from the room impulse response. Regarding to sound sources, most of the commercial dodecahedron loudspeakers comply with the maximum allowed directional deviations of the source specified in the standard. However, the influence of its specific orientation may affect the results obtained on some parameters more than their subjective just noticeable difference-jnd- at least in rooms with no high reverberation times. An interesting aim is to study such influence in function of the liveliness of the room. A detailed measurement set is been carrying out in a reverberant place (Cathedral of Tudela, Spain) with the objective to analyze the influence of the source's orientation-apart from its acoustic characterization. In addition to dodecahedron loudspeakers, pseudoimpulsive sources are been used in order to compare results from a statistical point of view. Results obtained will be compared with those obtained in several concert and theater rooms.

\section{Invited Papers}

4aAAc10. Measurement of reverberation time with rotating microphone in test chamber and its problems. Hiroshi Sato (National Institute of Advanced Industrial Science and Technology, Central 6, 1-1-1 Higashi, $305-8566$ Tsukuba, Japan, sato.hiro@aist.go.jp), Junichi Yoshimura (Kobayasi Institute of Physical Research, 3-20-41 Higashi-Motomachi, Kokubunji, 185-0022 Tokyo, Japan, yoshi@kobayasi-riken.or.jp), Satoshi Sugie (Kobayasi Institute of Physical Research, 3-20-41 Higashi-Motomachi, Kokubunji, 185-0022 Tokyo, Japan, sugie@kobayasi-riken.or.jp), Takashi Koga (Kajima Technical Research Institute, 2-19-1 Tobitakyu, Chofu, 182-0036 Tokyo, Japan, tkoga@kajima.com), Emi Toyoda (Kobayasi Institute of Physical Research, 3-20-41 HigashiMotomachi, Kokubunji, 185-0022 Tokyo, Japan, toyoda@kobayasi-riken.or.jp), Jongkwan Ryu (National Institute of Advanced Industrial Science and Technology, Central 6, 1-1-1 Higashi, 305-8566 Tsukuba, Japan, jongkwan-ryu@aist.go.jp)

When measurement of reverberation time is done in a test chamber to evaluate acoustical property of materials, spatial averaging of reverberation time should be done. Using microphone rotator is recognized as one of the tool to do spatial averaging. This study compares between three methods of spatial averaging of reverberation times measured in small rectangular test chamber $(3 \mathrm{~m} x 4 \mathrm{~m} x 5 \mathrm{~m})$. The first method is averaging reverberation time measured at 5 of fixed position used as standard positions for testing, the second is at 72 fixed positions on the circle of microphone rotator, and the third is with microphone rotator (64 s/rotation). The result of comparison between three method revels that reverberation time measured by rotating microphone has more scatter than and presents different reverberation time from those measured by other method especially at lower frequency bands. Simulation of microphone rotation with 
the decay curves measured at 72 fixed positions suggests that spatial distribution of steady state sound pressure level, rotation speed of microphone, and reverberation time of test chamber are key factors of errors. As a conclusion, the strict guideline for measurement of reverberation time with microphone rotator should be presented to minimize errors.

4aAAc11. Uncertainty in building acoustics. Werner Scholl (Physikalisch-Technische Bundesanstalt, Bundesallee 100, 38116 Braunschweig, Germany, werner.scholl@ptb.de), Volker Wittstock (Physikalisch-Technische Bundesanstalt, Bundesallee 100, 38116 Braunschweig, Germany, volker.wittstock@ptb.de)

In many countries, legal requirements exist with respect to the acoustical performance of buildings and building elements. Therefore information about the uncertainty of measured or predicted building acoustic properties is urgendly needed. The complexity of the problem becomes obvious taking sound recuction index $\mathrm{R}$ as an example: $\mathrm{R}$ represents the ratio of incoming and transmitted sound power of a building element. For practical reasons, the direct measurement of $\mathrm{R}$ is replaced by a spatiotemporally averaged sound pressure level difference in two limited rooms, adjusted by the absorption of the receiving room. In doing so, unwanted influences occur like modal effects, flanking transmission, structural power exchange between laboratory and specimen, deviation from ideal diffuse sound fields with unknown consequences etc. For economic reasons, often only one sample is tested and declared 'typical' for the whole family of products without regarding their spread. As a consequence, the uncertainty most often is felt to be too large to decide about the compliance with regulations but cannot be quantified. PTB in Germany has investigated the problem for the German authorities by calculation, evaluation of Round-Robin-tests und large measurement series in model houses. The results are presented in the talk.

\section{Contributed Paper}

\section{2:40}

4aAAc12. Uncertainty evaluation in field measurements of airborne sound insulation. Ranny L.X. Michalski (Inmetro / CNPq, Av. N. S. das Graças, 50, Xerém, Duque de Caxias, 25250-020 RJ, Brazil, ranny_xavier@yahoo.com.br), Daiana Ferreira (Inmetro / CNPq, Av. N. S. das Graças, 50, Xerém, Duque de Caxias, 25250-020 RJ, Brazil, dpferreira@inmetro.gov.br), Marco Nabuco (Inmetro / CNPq, Av. N. S. das Graças, 50, Xerém, Duque de Caxias, 25250-020 RJ, Brazil, nabuco@inmetro.gov.br), Paulo Massarani (Inmetro / CNPq, Av. N. S. das Graças, 50, Xerém, Duque de Caxias, 25250-020 RJ, Brazil, pmmassarani @inmetro.gov.br)

The Brazilian Committee of Civil Engineering presented a set of standards concerning the evaluation of the performance of several topics for buildings up to five floors. The acoustic performance is one of them. The standards are in approval process and measurements in real buildings will be necessary. Different professionals using different equipment will emit certificates establishing which levels of insulation a certain flat provides and its uncertainties. The expanded measurement uncertainty can provide the basis to compare different measurement results for a same building. The international Guide to the Expression of Uncertainty in Measurement, the ISO GUM, is the document that specifies how to determine and evaluate the uncertainty of a measurement result. The standards concerning sound insulation measurements are ISO 140 and ISO 18233. Uncertainty estimates are available only for the classical technique described in ISO 140, based in repeatability and reproducibility tests performed in laboratories. Field measurements present some characteristics that can contaminate the results, as time variance. Several independent measurements were carried out in a one flat building using ISO 18233 specifications and the ISO GUM was applied to obtain the uncertainty for measurement results of airborne sound insulation between rooms in situ.

\section{Invited Paper}

4aAAc13. Uncertainty of Receiving Space Volume in Field Measurements of Transmission Loss Under ASTM E336-05. Jonah Sacks (Acentech, 33 Moulton Street, Cambridge, MA 02138, USA, jsacks@acentech.com)

As acoustical consultants, we are frequently asked by our clients to measure the sound isolating performance of constructions in the field. While it is often preferable to report "system-level" performance ratings such as Noise Isolation Class, there are compelling benefits both to us and to our clients to measuring and reporting "specimen-level" performance ratings such as Apparent Sound Transmission Class, defined by ASTM E336-05 (and E413-04). The accuracy of such ratings depends on accurate assessment of the amount of acoustical absorption present in receiving spaces at the time of testing, arrived at by means of reverberation time measurements and physical measurement of receiving spaces. When a receiving space is irregular in shape, one may feel pressed to use creative judgment to estimate its effective volume, and such judgments can have large impacts on the reported results. We will discuss the challenges of measuring apparent transmission loss in the field, and the compelling reasons to conduct these measurements despite the challenges.

\section{Contributed Paper}

\section{3:20}

4aAAc14. Field measurements of acoustic performance in buildings: a Round Robin Test. Fabio Scamoni (Construction Technologies Institute of Italian National Research Council, Viale Lombardia, 49, 20098 San Giuliano Milanese (MI), Italy, Fabio.Scamoni@itc.cnr.it), Maurizio Bassanino (ARPA Lombardy - Air and Physical Agents, 3/1, Viale F. Restelli, 20124 Milan, Italy, M.BASSANINO@arpalombardia.it), Giuseppe Bruno (Lombardy Region - Environmental Quality, Via Pola, 14, 20124 Milan, Italy, giuseppe_bruno@regione.lombardia.it), Giovanni Zambon (Department of Environmental Sciences of the University of
Milano - Bicocca, Piazza della Scienza, 1, 20126 Milan, Italy, giovanni .zambon@unimib.it)

This paper presents the experimental results of a round robin test performed on the same building by different teams working with three independent bodies: a research body, ITC-CNR, a university laboratory DISAT and the Regional Agency for Environment Protection of Lombardy, ARPA. A partition wall (the airborne sound insulation between rooms), a floor (the impact sound insulation between rooms) and a façade (the insulation of the façade against outdoor sound) were tested, using the measurement methods 
given in the relevant parts of the ISO 140 series. The accuracy of acoustic measurement methods in buildings depends on many factors. In this round robin test, the situation (varying from straightforward rectangular rooms to half open spaces with all kind of shapes) and the construction details were kept fix. The analysis was aimed at investigating the influence of the oper- ating condition of the equipment (repeatable settings) and the reproducibility, using different types of acoustic instrumentation and varying the method (choice of source and microphone positions). In particular the tolerance limits in the verification of the requirements of tender specifications were investigated.

Lecture sessions will recess for presentation of poster papers on various topics in acoustics. See poster sessions for topics and abstracts.

\section{Invited Papers}

\section{5:00}

4aAAc15. Handling of uncertainties for CE marking concerning Sound Transmission Loss of glazings. Marc Rehfeld (Saint Gobain Glass, CRDC, B.P. 40103, 60777 Thourotte Cedex, France, marc.rehfeld@saint-gobain.com)

A joint working group between CEN TC 126 (building acoustics) and CEN TC 129 (glass in building) has been created to handle the redaction of a test code, (give rules for CE marking) and particularly handle uncertainty problems. A round robin has been organised, at which 23 European labs have participated, and two configuration of double glazings have been tested: one with two monolithic glass components, the other with one monolithic and one highly damped laminate component. The paper will present the specifications and main results of this round robin, the questions that occurred concerning the possible ways to decrease uncertainty values, as well as the conclusions and decisions of the working group.

4aAAc16. Repeatability and reproducibility of field noise isolation testing. John Loverde (Veneklasen Associates, 1711 Sixteenth Street, Santa Monica, CA 90404, USA, jloverde@veneklasen.com), Wayland Dong (Veneklasen Associates, 1711 Sixteenth Street, Santa Monica, CA 90404, USA, wdong@veneklasen.com)

The reliability and precision of test measurements and methods are generally described in terms of repeatability and reproducibility. ASTM standards define and quantify these terms for noise isolation test methods in both laboratory and field conditions. Understanding these as reproducibility and repeatability of the measurement method, the authors extend the concept to the reproducibility and repeatability of a wall or floor/ceiling assembly design. Multiple instances of a floor/ceiling assembly on a multi-family residential project built by the same contractors is an example of design repeatability, while the same assembly design constructed on different projects is an example of design reproducibility. In a previous paper [LoVerde and Dong, J. Acoust. Soc. Am. 122, 2955 (2007)], definitions were suggested for field repeatability and reproducibility for Field Impact Insulation Class testing. Test data is presented to quantify the field repeatability and reproducibility of several assembly designs, which are compared to laboratory values. Field and laboratory repeatability and reproducibility of airborne noise isolation for a partition assembly are also examined.

\section{$5: 40$}

4aAAc17. Analysis of uncertainty in building acoustic predictions using Monte-Carlo methods. Ralph T. Muehleisen (Illinois Institute of Technology, Civil, Architectural and Environmental Engineering, 3201 S. Dearborn St., Room 228, Chicago, IL 60616, USA, muehleisen@iit.edu)

One of the most common tasks in architectural acoustics is the prediction of the acoustic performance of some aspect of a building, such as the background sound level of a room, the reverberation time of an enclosure, or the sound transmission of a wall construction. The accuracy of a prediction is not only dependent upon the computation model, but also upon the accuracy of the data of the model. Because of the complicated, non-linear interaction of various inputs, assessing the accuracy of a prediction can be difficult. One way to provide more accurate predictors and estimate the error in the prediction of complicated, multiple input systems is to utilize the MonteCarlo method. In this talk, the application of Monte-Carlo methods to building acoustic predictions is presented.

\section{Contributed Papers}

\section{6:00}

4aAAc18. Diffuseness and sound field distribution at room boundaries. Jean-Daniel Chazot (Université de Technologie de Compiègne, Lab. Roberval UMR 6253, Dept. Acoustique, BP 60319, 60203 Compiègne, France, jean-daniel.chazot@utc.fr), Jean-Louis Guyader (INSA de Lyon - LVA, Bâtiment St. Exupéry, 25 bis avenue Jean Capelle, F-69621 Villeurbanne Cedex, France, jean-louis.guyader@insa-lyon.fr)

In the present work, a classical modal analysis is used up to medium frequencies to study the sound field distribution, and its diffuseness, particularly at boundaries. Due to intensification zones at boundaries, the diffuse field distribution at room boundaries can not be assimilated to the distribution inside the room. Moreover, diffuseness at room boundaries, that is of interest for sound insulation measurement, is usually only related to an incidence angle while inside the room volume several descriptors such as a correlation function and the spatial uniformity are necessary to characterize a diffuse field. In this paper, we present a new descriptor adapted to characterize the sound field diffusivity at boundaries. This descriptor is called Boundary Diffuse Field Index. Its averaged value over a specific surface can be related to a limit incidence angle, and its standard deviation can be related to the spatial distribution over the surface. Finally, thanks to this descriptor, Sabine's assumptions of diffuse sound field are also evaluated in this study. 
4aAAc19. Previous results on the evaluation of the influence of sound level meter case in diffuse field. Alfonso R. Molares (University of Vigo,E.T.S.I de Telecomunicación, Rúa Maxwell s/n, 36310 Vigo, Spain, amolares@gts.tsc.uvigo.es), Manuel A. Sobreira-Seoane (University of Vigo, E.T.S.I de Telecomunicación, Rúa Maxwell s/n, 36310 Vigo, Spain, msobre@gts.tsc.uvigo.es)

In order to evaluate the influence of the sound level meter case on real measurements, two different case geometries are tested using Boundary Element Method. Solving the coupled structural-acoustic problem by means of an iterative procedure, their correction curves are obtained for various angles of incidence and for diffuse field. The deviation due to the case is obtained by comparison against the result of the reference microphone. The results reveal that the influence of the case can be relevant at the middle frequency range with deviations that can exceed $0.4 \mathrm{~dB}$ under free-field conditions for normal incidence. At the time of writing this abstract the available results show that the deviation for diffuse field at middle frequencies can reach $0.2 \mathrm{~dB}$, although further research is needed to evaluate the deviation at higher frequencies.
4aAAc20. Uncertainty analysis in acoustical modeling of room Dominik Mleczko (Romera 17, 33-300 Nowy Sacz, Poland, dmleczko@agh .edu.pl)

For a long time there is a need in industry of acoustical modeling of rooms. It is necessary for new production room design, machine exchange, renovation or enlargement of production rooms, change in a production profile or acoustical room adaptation for acoustical work conditions improvement. In such cases modeling quality is essential and thanks to uncertainty analysis it is possible to quantitatively estimate the effect that input parameters value variation has on model behavior. The article presents general rules for sound pressure level prediction uncertainty calculation in a room. By partial uncertainty calculation analysis of input parameters influence on uncertainty prediction an effort was taken to find parameters with biggest influence on the prediction process. As an example an industrial production room is presented which was modeled to predict noise level on a work stands after it was expanded.

\title{
Session $4 \mathrm{aABa}$
}

\section{Animal Bioacoustics, Psychological and Physiological Acoustics, and ECUA: Auditory Brainstem Response and Behavior Correlation II}

\author{
Elizabeth Brittan-Powell, Cochair \\ Dept of Psychology, University of Maryland, College Park, MD 20742, USA
}

Alexander Y. Supin, Cochair

Institute of Ecology and Evolution, 33 Leninsky Prospect, Moscow, 119071, Russian Federation

\section{Invited Papers}

8:00

4aABa1. Evoked-potential study of hearing directivity and sound-receiving apertures in dolphins. Vladimir V. Popov (Institute of Ecology and Evolution, 33 Leninsky Prospect, 119071 Moscow, Russian Federation, popov_vl@sevin.ru), Alexander Y. Supin (Institute of Ecology and Evolution, 33 Leninsky Prospect, 119071 Moscow, Russian Federation, alex_supin@mail.ru)

Positions of sound-receiving apertures were searched-for in bottlenose dolphins using the ABR technique. The receiving-area position was computed basing on ABR delays at various sound-source positions. Two acoustic apertures were revealed in such a way: for frequencies of $32 \mathrm{kHz}$ and higher, the receiving area was located near a proximal part of the lower jaw (the mandibular acoustic window); for lower frequencies, the receiving area was located near the tympanic bulla. In another experimental series, AEP thresholds to near-field stimuli were measured with transducer positioning next to various points of the dolphin's head. Again, at stimulus frequencies of $32 \mathrm{kHz}$ and higher, the lowest threshold area was next to the mandibilar acoustic window; at lower frequencies, the lowest threshold area was next to the bulla. The conclusion is that dolphins have at least two acoustic apertures differing in their frequency sensitivity. Directional sensitivity of these two apertures was investigated by measuring ABR thresholds at different frequencies and different sound source positions. At higher frequencies, the best-sensitivity direction estimated by ABR thresholds was near the head midline, at lower frequencies the best-sensitivity direction deviated laterally. These data were interpreted as indicating different axis directions of the two receiving apertures.

\section{$8: 20$}

4aABa2. Tuning curves derived from auditory brainstem responses point to a defect in outer hair cells of hypothyroid mice. Edward Walsh (Boys Town National Research Hospital, 555 North 30th Street, Omaha, NE 68132, USA, walsh@boystown.org), Megan Korte (Boys Town National Research Hospital, 555 North 30th Street, Omaha, NE 68132, USA, kortem@boystown.org), Joann McGee (Boys Town National Research Hospital, 555 North 30th Street, Omaha, NE 68132, USA, mcgee@boystown.org)

Based on an analysis of ABR derived tuning curves, recent reports suggest that the mechanics of passive transduction in hypothyroid mice, although delayed developmentally, eventually become indistinguishable from normal animals, whereas the mechanics of active transduction remain grossly abnormal throughout life, raising the possibility that the outer hair cell system is at least partially responsible for abnormalities observed in mutant animals. Moreover, results of in vitro studies have shown that although OHCs are electro- 
motile, they appear unable to withstand extensive voltage excursions in the whole cell voltage clamp environment; i.e., they appear fragile. These data led us to question the structural integrity of the OHC lateral wall in hypothyroid mice. Preliminary studies suggest that the cortical cytoskeleton is abnormal in at least some regions of the OHC lateral wall and preliminary confocal immunofluorescence images of the constituent proteins, f-actin and $\alpha$-II spectrin, suggest that spectrin is either absent or expressed in very low levels in the cytoskeleton of OHCs harvested from the hypothyroid progeny of Tshr mutant dams. We suggest that a cytoskeletal defect involving the f-actin cross-linking protein, spectrin, might compromise the efficient transfer of force along the long axis of OHCs, effectively diminishing the power of active amplification.

\section{Contributed Papers}

\section{8:40}

4aABa3. Electrophysiological and behavioral measures of temporary threshold shift in a bottlenose dolphin (Tursiops truncatus). James J. Finneran (US Navy Marine Mammal Program, Space and Naval Warfare Systems Center, 53560 Hull St., Code 71510, San Diego, CA 92152, USA, james.finneran@navy.mil), Carolyn E. Schlundt (EDO Professional Services, 3276 Rosecrans St., San Diego, CA 92110, USA, carolyn.melka @edocorp.com), Brian K. Branstetter (US Navy Marine Mammal Program, Space and Naval Warfare Systems Center, 53560 Hull St., Code 71510, San Diego, CA 92152, USA, branstet@hawaii.edu), Randall L. Dear (Science Applications International Corporation, 4065 Hancock St., San Diego, CA 92110, USA, RANDALL.L.DEAR@saic.com)

Auditory evoked potentials are being increasingly applied to more advanced studies of marine mammal hearing, such as frequency selectivity, temporal processing, and temporary threshold shift (TTS). In this study, both behavioral and electrophysiological techniques were used to measure TTS in a bottlenose dolphin exposed to $20-\mathrm{kHz}$ tones. Behavioral hearing thresholds were estimated using a modified staircase procedure and a whistle response. Electrophysiological thresholds were assessed using the multiple auditory steady-state response. Evoked potential stimuli consisted of seven frequency-modulated tones having carrier frequencies from $10-70 \mathrm{kHz}$ and unique modulation rates. Tones were simultaneously presented and the evoked response at each modulation rate independently tracked to test hearing at all seven frequencies simultaneously. The behavioral and evoked response data both showed frequency-dependent patterns of TTS, with the largest shifts at $30 \mathrm{kHz}$; however, TTS measured using evoked potentials (up to $40-45 \mathrm{~dB}$ ) was always larger than that observed behaviorally (19-33 dB). This discrepancy may be the result of the evoked response input-output function, which can be represented as the sum of two processes, a low threshold, saturating process and a higher threshold linear process, that react and recover to fatigue at different rates.

\section{9:00}

4aABa4. Directional sensitivity and hearing pathways in the beluga whale, Delphinapterus leucas. Aude Pacini (University of Hawaii, Hawaii Institute of Marine Biology, P.O. Box 1106, Kailua, HI 96734, USA, aude@hawaii.edu), Paul E. Nachtigall (University of Hawaii, Hawaii Institute of Marine Biology, P.O. Box 1106, Kailua, HI 96734, USA, nachtiga @ hawaii.edu), T. Aran Mooney (University of Hawaii, Hawaii Institute of Marine Biology, P.O. Box 1106, Kailua, HI 96734, USA, mooneyt @ hawaii.edu), Manuel Castellote (L'Oceanografic, C/. Junta de Murs i Valls, s/n, 46013 Valencia, Spain, mcastellote@oceanografic .org), Kristen A. Taylor (University of Hawaii, Hawaii Institute of Marine Biology, P.O. Box 1106, Kailua, HI 96734, USA, kristent@hawaii .edu), José-Antonio Esteban (Research Department, Parques Reunidos Valencia S. A. L'Oceanogràfic, Ciudad de las Artes y las Ciencias, 46013 Valencia, Spain, investigacion@oceanografic.org)

Although much variation exists in jaw morphology among species, odontocetes are believed to receive sound primarily through the pan bone region of the lower jaw. In order to further examine this jaw hearing hypothesis, we tested the head receiving sensitivity and directional hearing of a beluga whale, Delphinapterus leucas. Hearing measurements were conducted with a 9-yr-old female beluga using the auditory evoked potential technique. A preliminary audiogram indicated that the subject had very sensitive hearing $(45-55 \mathrm{~dB}$ from $32-80 \mathrm{kHz})$ and heard up to $128 \mathrm{kHz}$. The pathway investigation used a piezo-electric transducer to present the click stimuli, whereas the hearing directivity was measured in the far field, also using broadband clicks. Like the bottlenose dolphin, the subject had a region of high sensitivity around the pan bone region $(78 \mathrm{~dB})$, however, unlike the bottlenose dolphin, we found that the region of maximum sensitivity was at the tip of the lower jaw $(75 \mathrm{~dB})$. The subject also proved to have highly directional hearing. This study supports the shaded receiver hypothesis and also shows that hearing pathway variations appear to exist among odontocete species and are at least partially dependent on head morphology.

\section{9:20}

4aABa5. Evoked potential and behavioral hearing thresholds in nine bottlenose dolphins (Tursiops truncatus). Carolyn E. Schlundt (EDO Professional Services, 3276 Rosecrans St., San Diego, CA 92110, USA, carolyn.melka@edocorp.com), James J. Finneran (US Navy Marine Mammal Program, Space and Naval Warfare Systems Center, 53560 Hull St., Code 71510, San Diego, CA 92152, USA, james.finneran@navy.mil), Brian K. Branstetter (US Navy Marine Mammal Program, Space and Naval Warfare Systems Center, 53560 Hull St., Code 71510, San Diego, CA 92152, USA, branstet@hawaii.edu), Randall L. Dear (Science Applications International Corporation, 4065 Hancock St., San Diego, CA 92110, USA, RANDALL.L.DEAR@saic.com), Dorian S. Houser (Biomimetica, 7951 Shantung Dr., Santee, CA 92071, USA, dhouser@spawar.navy.mil), Erica Hernandez (University of Southern Mississippi, Department of Psychology, 118 College Dr., Box 5025, Hattiesburg, MS 39406, USA, erica.hernandez @yahoo.com)

Auditory evoked potentials are popular for assessing hearing in marine mammals because they do not require the same access to and training of animals as behavioral methods; however, within-subject comparisons of behavioral and electrophysiological thresholds are still necessary to benchmark evoked potential results against the more universally accepted behavioral data. In this study, auditory thresholds were measured in nine dolphins using both behavioral and electrophysiological methods. Subjects included eight males and one female, ages 21-43 yrs. Some had a full-range of hearing and others exhibited high-frequency hearing loss. Tests were conducted in-air, in San Diego Bay, and/or in quiet pools. Hearing test stimuli included frequency-modulated, amplitude-modulated, or pure tones projected in the direct field or via a "jawphone" contact transducer. Comparisons reveal good agreement between the evoked potential and behavioral methods, particularly when the testing environments, stimulus delivery methods, and stimulus waveforms are similar. The results show that evoked potential thresholds obtained in a variety of conditions provide reasonable approximations to underwater sensitivity, especially with respect to the shape of the audiogram and the upper limit of hearing.

\section{9:40}

4aABa6. Interactions of frequency components of multi-component envelope following response in a beluga. Alexander Y. Supin (Institute of Ecology and Evolution, 33 Leninsky Prospect, 119071 Moscow, Russian Federation, alex_supin@mail.ru), Vladimir V. Popov (Institute of Ecology and Evolution, 33 Leninsky Prospect, 119071 Moscow, Russian Federation, popov_vl@sevin.ru)

The envelope-following response (EFR) in odontocetes is composed of overlapping ABRs produced by each cycle of rhythmic sound stimulus at a rate of a few hundred cycles per sec. It has been shown recently (Finneran et al., JASA 2007, 121: 1775) that a complex stimulus consisting of a few carriers modulated by different rates produces a complex EFR composed of components reproducing all the modulation rates. Using this technique, interactions between different components of complex EFR were investigated in a beluga whale Delphinapterus leucas. When all carriers of the complex stimulus were equalized by SPL, the interaction depended on both the SL of carriers (their level relative the threshold) and inter-carrier frequency spacing. Addition of components of higher SL (at low-threshold 
frequencies) dramatically reduced the amplitudes of other EFR components, especially at short frequency spacing. The leading factor of this reduction was decreasing of the modulation depth of each of the carriers when the overall power of the stimulus increased by additional components. The threshold estimate of each carrier little depended on the number of components; however precision of threshold determination fell down with increasing the number of stimulus components due to the reduced amplitudes of EFR components.

\section{0:00}

4aABa7. Acoustic communication in Panthera tigris: A study of tiger vocalization and auditory receptivity revisited. Edward Walsh (Boys Town National Research Hospital, 555 North 30th Street, Omaha, NE 68132, USA, walsh@boystown.org), Douglas L. Armstrong (Henry Doorly Zoo, 3701 S. 10th, Omaha, NE 68107, USA, douga@omahazoo.com), Julie Napier (Henry Doorly Zoo, 3701 S. 10th, Omaha, NE 68107, USA julie.napier@omahazoo.com), Lee G. Simmons (Henry Doorly Zoo, 3701 S. 10th, Omaha, NE 68107, USA, 1simmons@omahazoo.com), Megan Korte (Boys Town National Research Hospital, 555 North 30th Street Omaha, NE 68132, USA, kortem@boystown.org), Joann McGee (Boys Town National Research Hospital, 555 North 30th Street, Omaha, NE 68132, USA, mcgee@boystown.org)
Preliminary findings reported at the 145 th meeting of the Society suggested that confrontational tiger roars contain energy in the infrasonic portion of the electromagnetic spectrum. This discovery generally supported the proposition that free ranging individuals may take advantage of this capability to communicate with widely dispersed conspecifics inhabiting large territories in the wild. Preliminary ABR findings indirectly supported this view suggesting that although tigers are most sensitive to acoustic events containing energy in the 0.3 to $0.5 \mathrm{kHz}$ band, they are most likely able to detect acoustic events in the near-infrasonic and infrasonic range based on the assumption that felid audiograms exhibit uniform shapes. In this study, the spectral content of territorial and confrontational roars was analyzed and relevant features of $\mathrm{ABR}$ based threshold-frequency curves were considered in relation to the acoustical properties of both roar types. Unlike the confrontational roar, infrasonic energy was not detected in the territorial roar; however, like the confrontational roar, peak acoustic power was detected in a frequency band centered on $\sim 0.3 \mathrm{kHz}$. In addition, ABR recordings acquired in a double walled sound attenuating chamber recently installed at the Henry Doorly Zoo suggest that acoustic sensitivity is significantly underestimated under "field" conditions.

THURSDAY MORNING, 3 JULY 2008

ROOM 342B, 10:40 A.M. TO 1:00 P.M.

\title{
Session $4 a A B b$
}

\section{Animal Bioacoustics: Sound Production and Reception in Amphibious Marine Mammals}

\author{
Jason Mulsow, Cochair \\ UCSC Institute of Marine Sciences, Long Marine Lab - University of California, 100 Shaffer Road, Santa Cruz, CA 95060, USA \\ Ronald J. Schusterman, Cochair \\ UCSC Institute of Marine Sciences, Long Marine Lab - University of California, 100 Shaffer Road, Santa Cruz, CA 95060, USA
}

Ian Boyd, Cochair

Sea Mammal Research Unit, Gatty Marine Laboratory, University of St Andrews, St Andrews, KY16 8LB, UK

\section{Invited Papers}

10:40

4aABb1. Vocal Learning in Pinnipeds: A Model System for Human Speech Evolution. William T. Fitch (Centre for Social Learning and Cognitive Evolution, University of St.Andrews, School of Psychology, KY16 9JP St.Andrews, Fife, UK, wtsf@st-andrews.ac.uk), Ronald J. Schusterman (UCSC Institute of Marine Sciences, Long Marine Lab - University of California, 100 Shaffer Road, Santa Cruz, CA 95060, USA, rjschust@ucsc.edu), Colleen Reichmuth (UCSC Institute of Marine Sciences, Long Marine Lab - University of California, 100 Shaffer Road, Santa Cruz, CA 95060, USA, coll@ ucsc.edu), Marija Spasikova (Centre for Social Learning and Cognitive Evolution, University of St.Andrews, School of Psychology, KY16 9JP St.Andrews, Fife, UK, marija.spasikova@gmail.com), Daniel Mietchen (Structural Brain Mapping Group, University of Jena, PF 07737 Jena, Germany, daniel.mietchen@googlemail.com)

Vocal learning is limited to a small subset of vertebrates: including birds (songbirds, parrots, hummingbirds), and mammals (humans, cetaceans, pinnipeds, and probably elephants and bats). Intriguingly, in most of these species, vocal production involves functionally or mechanistically novel systems: the avian syrinx, the nasal bursae in odontocetes, and ultrasonic echolocation in bats. The novel neural circuitry that evolved to control these systems may provide a "preadaptation" for vocal learning. Only two known vocal learners - humans and seals - definitely use the standard vertebrate vocal production system (larynx and vocal tract). Our studies of vocal production in harbour seals verify a surprisingly human-like vocal production system, and the critical question remaining is what neural mechanisms underlie the control of the Phoca vocal system, and whether such "human" specializations as direct cortico-ambiguual connections are also found in seals. Pinniped investigations also open the door to molecular exploration of the genetic bases for neural innovations. In addition to these mechanistic questions, we discuss evolutionary questions. What functional forces might select for vocal learning in aquatic mammals? One hypothesis is that selection for control of breathing may provide a preadaptation for enhanced cortical vocal control, explaining its prevalence in marine mammals. 
4aABb2. Sound production by pinnipeds can be modified by contingency learning. Ronald J. Schusterman (UCSC Institute of Marine Sciences, Long Marine Lab - University of California, 100 Shaffer Road, Santa Cruz, CA 95060, USA, rjschust@ucsc.edu), Colleen Reichmuth (UCSC Institute of Marine Sciences, Long Marine Lab - University of California, 100 Shaffer Road, Santa Cruz, CA 95060, USA, coll@ucsc.edu)

In contrast to terrestrial mammals, pinnipeds (seals, sea lions and walruses) have remarkable flexibility in the ways that they can learn to use and modify their amphibious sound emissions. The experiments that we will describe are drawn from captive studies which show that changes in sound production can occur as a result of contingency learning, using food as positive reinforcement. A range of specialized physiological and anatomical adaptations appear to play a critical role in controlling sound production in pinnipeds. These adaptations include breath-holding and buoyancy mechanisms, as well as fine muscular control of the mouth, lips and tongue that may be used primarily in feeding. The manipulation and modulation of air flow through these components of the vocal tract and associated super-laryngeal filters appears to be susceptible to some of the same reinforcing consequences that are routinely used to establish reliable control over motor behaviors, such as flipper waving, in operant conditioning contexts.

\section{$11: 20$}

4aABb3. Inertial and cochlear constraints for high-frequency hearing in phocid and otariid pinnipeds. Sirpa Nummela (University of Helsinki, Department of Biological and Environmental Sciences, PO Box 65 (Viikinkaari 1), FI-00014 Helsinki, Finland, snummela@fastmail.fm), Simo Hemilä (University of Helsinki, Department of Biological and Environmental Sciences, PO Box 65 (Viikinkaari 1), FI-00014 Helsinki, Finland, simo.hemila@welho.com), Annalisa Berta (San Diego State University, Biology Department, LS 250, San Diego, CA 92182-4614, USA, aberta@sciences.sdsu.edu), Tom Reuter (University of Helsinki, Department of Biological and Environmental Sciences, PO Box 65 (Viikinkaari 1), FI-00014 Helsinki, Finland, tom.reuter@helsinki.fi)

In air-borne hearing, mammals rely on sound transmission through the tympanic membrane and middle ear ossicles between the surrounding air and the cochlea. The high-frequency hearing limit (HFHL) is determined by the ossicular inertia, and also by the cochlear sensitivity. Due to coevolution, the sensitivity ranges of the middle and inner ear structures generally overlap, and the roles of inertial and cochlear constraints for the HFHL are difficult to discern. For studying this question we considered anatomical and experimental data for two phocid and two otariid pinnipeds. While any detailed mechanism for pinniped underwater hearing remains unclear, an underwater HFHL exceeding that in air is possible. Published in-air and underwater audiograms provide an opportunity for comparing the roles of ossicular mass inertia and cochlear sensitivity in HFHL. Phocid ossicles are very heavy, and their inertia explains the lower HFHLs in air - according to underwater audiograms the phocid cochlea is sensitive to higher frequencies. Otariids have normalsized mammalian ossicles, and their inertia should allow underwater hearing at higher frequencies than in air. However, the HFHL is approximately equal in air and water for otariids, hence their underwater HFHL is apparently set by the cochlea alone.

\section{1:40}

4aABb4. Evoked potential audiometry in sea lions. Jason Mulsow (UCSC Institute of Marine Sciences, Long Marine Lab - University of California, 100 Shaffer Road, Santa Cruz, CA 95060, USA, jmulsow@ucsc.edu), Colleen Reichmuth (UCSC Institute of Marine Sciences, Long Marine Lab - University of California, 100 Shaffer Road, Santa Cruz, CA 95060, USA, coll@ucsc.edu)

Auditory sensitivity in the otariid pinnipeds (sea lions and fur seals) has traditionally been studied using a relatively small number of trained subjects and psychophysical techniques. Recent refinement of auditory evoked potential techniques with odontocete cetaceans has elevated interest in adapting these methods for sea lion subjects, with the goal of increasing sample size and efficiency in audiometric studies. To date, several basic electrophysiological characteristics of the California sea lion (Zalophus californianus) auditory system have been described, and these findings have allowed for the development of more advanced techniques in investigations of sea lion hearing. Most notable is the recording of the envelope following response (EFR) evoked by narrow-band, sinusoidally amplitudemodulated tones. This method can provide significant advantages in the detection of low-amplitude electrophysiological signals in noise using Fourier analysis and objective statistical detection of responses. Currently, EFR audiometry in the California sea lion and Steller sea lion (Eumetopias jubatus) is proving to be a promising method for rapidly assessing the variation of hearing capabilities among individuals, including the detection of hearing loss.

\section{2:00}

4aABb5. Air and bone conduction evoked potential audiometry in the northern elephant seal. Dorian S. Houser (Biomimetica, 7951 Shantung Dr., Santee, CA 92071, USA, dhouser@spawar.navy.mil), Daniel Crocker (Sonoma State University, Department of Biology, 1801 East Cotati Avenue, Rohnert Park, CA 94928, USA, crocker@sonoma.edu), James J. Finneran (US Navy Marine Mammal Program, Space and Naval Warfare Systems Center, 53560 Hull St., Code 71510, San Diego, CA 92152, USA, james.finneran @ navy.mil)

Elephant seals (Mirounga angustirostris) are the largest and most aquatic of the pinnipeds, spending up to eight months of the year at sea diving to depths as great as $1600 \mathrm{~m}$. The pinna is absent in the elephant seal and the middle ear cavity and auditory canal are lined with a cavernous tissue, both of which are likely adaptations to deep diving. Elephant seals demonstrate a greater sensitivity to low frequency sounds than do other pinnipeds and an overall greater sensitivity to underwater sound than to airborne sound. The relative importance of sound conduction pathways in the elephant seal is undetermined, although it has been speculated that bone conduction pathways are important to underwater hearing in this species. To compare the sensitivity of the elephant seal to both air and bone conducted stimuli, auditory evoked responses were recorded in seals exposed to signals presented through headphones and via a bone vibrator. In comparison to airborne stimuli, bone conduction methods provide an opportunity to more effectively study sensitivity to low frequency sounds, but are challenged by a lack of reference equivalent threshold sound pressure levels. Future efforts should compare bone conduction and direct field audiometry results obtained within the same individual. 
4aABb6. Preliminary Results of a Behavioral Audiometric Study of the Polar Bear. Ann E. Bowles (Hubbs-Sea World Research Institute, 2595 Ingraham Street, San Diego, CA 92109, USA, abowles@ hswri.com), Megan A. Owen (Conservation and Research for Endangered Species, Zoological Society of San Diego, PO Box 120551, San Diego, CA 92112-0551, USA, mowen@sandiegozoo.org), Samuel L. Denes (Hubbs-Sea World Research Institute, 2595 Ingraham Street, San Diego, CA 92109, USA, sdenes@hswri.org), Stefanie K. Graves (Hubbs-Sea World Research Institute, 2595 Ingraham Street, San Diego, CA 92109, USA, sgraves@hswri.org), Jennifer L. Keating (Hubbs-Sea World Research Institute, 2595 Ingraham Street, San Diego, CA 92109, USA, jenniferlkeating@hotmail.com)

The hearing of polar bears is of great interest because little is known about hearing of large terrestrial carnivores, they are amphibious, their predatory habits differ from most bears, and there is an increasing need for data to manage anthropogenic noise in maternal denning habitat. Behavioral auditory thresholds were collected from two female polar bears at the San Diego Zoo (ZSSD) in 2006-2007, and are now underway with two females and a male at SeaWorld San Diego (SWSD). Thresholds were measured at 19 frequencies between $125 \mathrm{~Hz}$ and $31.5 \mathrm{kHz}$ using shaped $500 \mathrm{~ms}$ tones, a 'go/no-go' response protocol, and staircase presentation order with catch trials. Holding areas in both facilities were sound-isolated to the extent practicable. Threshold measurements were limited by background noise below $5 \mathrm{kHz}$, but sensitivity could be measured to below $0 \mathrm{~dB}$ at higher frequencies. To date, the bears have detected sounds down to the noise floor from $125 \mathrm{~Hz}$ to about $14 \mathrm{kHz}$. Their sensitivity declines rapidly above $20 \mathrm{kHz}$. The results suggest that their auditory threshold functions are narrower or shifted to lower frequencies than those of small carnivores. [Supported by Polar Bears International, ZSSD, SWSD and the author's organizations]

\section{$12: 40$}

4aABb7. Variation in pup vocalisations and mother-pup behaviour between harp seal whelping patches: effects of climate or geography? Ilse Catharina Van Opzeeland (Alfred Wegener Institute, P.O. Box 120161, 27515 Bremerhaven, Germany, Ilse.Van.Opzeeland@awi.de), Peter J. Corkeron (US NOAA, Northeast Fisheries Science Center, 166 Water Street, Woods Hole, MA 02543, USA, peter.corkeron@gmail.com), Denise Risch (US NOAA, Northeast Fisheries Science Center, 166 Water Street, Woods Hole, MA 02543, USA, drisch@whsun1.wh.whoi.edu), Gary B. Stenson (Dept of Fisheries and Oceans, P.O. Box 5667, St John's, NL A1C5X1, Canada, StensonG@DFO-MPO.GC.CA), Sofie Van Parijs (US NOAA, Northeast Fisheries Science Center, 166 Water Street, Woods Hole, MA 02543, USA, sofie.vanparijs@noaa.gov)

Harp seals breed in pack-ice, a substrate which can vary substantially between whelping patches depending on differing environmental and oceanographic conditions. This study demonstrates clear site differences in pup vocalizations and mother pup behaviour between Northeast (Greenland Sea) and Northwest (Canadian Front) Atlantic harp seal populations. Classification trees showed a distinctive split between Front and Greenland Sea pup vocalisations. No clear sex differentiation in vocalizations was present for pups at the Front; $42 \%(\mathrm{n}=12)$ of male and 38\% $(\mathrm{n}=13)$ of female calls could be attributed to a given individual. In the Greenland Sea, 55\% $(\mathrm{n}=42)$ of female vocalisations were attributed to individuals compared with only $8 \%$ for males $(\mathrm{n}=47)$. In addition behavioural observations of mother pup pairs were conducted (Front, $\mathrm{n}=58$; Greenland Sea, $\mathrm{n}=78$ ). Greenland Sea pups were found to nurse more, and were more alert than Front pups. Female attendance patterns also differed between sites: females at the Front were more likely to attend their pups than those in the Greenland Sea. This marked difference in female presence between sites could have several origins such as variability in ice conditions, predation pressure, or female condition. 


\title{
Session $4 \mathrm{aAO}$
}

\section{Acoustical Oceanography, Signal Processing in Acoustics, and ECUA: Adjoint Modeling for Geoacoustic Inversion}

\author{
Paul Hursky, Cochair \\ HLS Research, Inc., 3366 N. Torrey Pines Ct., Ste. 310, La Jolla, CA 92037, USA
}

Jean-Pierre Hermand, Cochair

Université libre de Bruxelles (U.L.B.) - Environmental hydroacoustics lab, av. Franklin D. Roosevelt 50, CP 194/5, Bruxelles, 1050, Belgium

\section{Invited Papers}

\section{1:00}

\begin{abstract}
4aAO1. Validation of adjoint-generated environmental gradients for the acoustic monitoring of a shallow water area. Matthias Meyer (Royal Netherlands Naval College (NLDA) - REA group, PO Box 10000, 1780 Den Helder, Netherlands, mmeyer@ulb.ac.be), Jean-Pierre Hermand (Université libre de Bruxelles (U.L.B.) - Environmental hydroacoustics lab, av. Franklin D. Roosevelt 50, CP 194/5, 1050 Bruxelles, Belgium, jhermand@ulb.ac.be), Mohamed Berrada (Laboratoire d'Océanographie et du Climat - Expérimentation et Approches Numériques, Université Pierre et Marie Curie, Tour 45-55 - 5ème étage - 4, place Jussieu, 75005 Paris, France, mohamed.berrada@locean-ipsl.upmc.fr), Mark Asch (Université de Picardie Jules Verne, LAMFA (CNRS UMR 6140), 33 Rue Saint Leu, 80039 Amiens, France, mark.asch@u-picardie.fr)
\end{abstract}

In the framework of the recent Maritime Rapid Environmental Assessment sea trial MREA07/BP'07 [Le Gac\&Hermand, 2007] that was conducted in the same area south of the island of Elba as the earlier Yellow Shark trial (YS94), this paper examines the original YS94 acoustic data and the recent MREA07 oceanographic data to demonstrate adjoint-based acoustic monitoring of environmental parameters in Mediterranean shallow waters. First, adjoint-generated environmental gradients are validated for the application in geoacoustic inversion where the bottom acoustic parameters of the YS94 layered seabed are determined from the long-range waterborne propagation of a multi-frequency signal. Then, for the application in ocean acoustic tomography, the temporal variability of the MREA07/BP'07 oceanographic data is analyzed in terms of empirical orthogonal functions and the adjoint-based inversion scheme is used to track the time-varying sound speed profile of the experimental transect.

\section{$11: 20$}

4aA02. Variational assimilation of simulated ocean acoustic tomography data in an ocean model. Elisabeth Remy (MercatorOcean, Parc Technologique du Canal, 8-10 rue Hermès, 31520 Ramonville Saint Agne, France, eremy @mercator-ocean.fr), Fabienne Gaillard (LPO-IFREMER, BP 70, 29280 Plouzane, France, fabienne.gaillard@ @ifremer.fr), Jacques Verron (Laboratoire des Ecoulements Géophysiques et Industriels (LEGI), BP 53, 38041 Grenoble Cedex 9, France, verron@hmg.inpg.fr)

In the concept of large scale observing system for the ocean, ocean acoustic tomography is an original tool to monitor the ocean interior. Analysis of tomographic travel time using inversion gives an estimate of the temporal evolution of the heat content along the observed sections, an important quantity to monitor the ocean climate evolution. At lower scales than the cell size defined by the observational array, it is not possible to estimate the ocean temperature field without using other sources of information. A possible approach is to combine the tomographic observations with a numerical dynamical ocean model to obtain a complete description consistent with the data on a given time interval. We propose to explore a variational method using the adjoint technic to assimilate those integral data. We studied the case of a basin scale observational array, as the one deployed in the Mediterranean sea for the Thetis 2 experiment. Only travel time anomalies due to the sea water properties are considered. The ability of tomographic data to constrain the ocean model circulation is evaluated using simulated observations with a model solution. This approach called twin experiments, allows to compare the result after assimilation with the "true" solution.

\section{Contributed Papers}

\section{1:40}

4aAO3. Probabilistic PCA and Ocean Acoustic Tomography Inversion with an Adjoint Method. Mohamed Berrada (Laboratoire d'Océanographie et du Climat - Expérimentation et Approches Numériques, Université Pierre et Marie Curie, Tour 45-55 - 5ème étage - 4, place Jussieu, 75005 Paris, France, mohamed.berrada@locean-ipsl.upmc.fr), Fouad Badran (Laboratoire CEDRIC, Conservatoire National des Arts et Métiers, 292, rue Saint Martin, 75003 Paris, France, badran@cnam.fr), Sylvie Thiria (Laboratoire d'Océanographie et du Climat - Expérimentation et Approches Numériques, Université Pierre et Marie Curie, Tour 45-55 - 5ème étage - 4, place Jussieu, 75005 Paris, France, Sylvie.Thiria@locean-ipsl .upmc.fr)
We present an Ocean Acoustic Tomography (OAT) inversion in a shallow water environment. The idea is to determine the celerity $\mathrm{c}(\mathrm{z}), \mathrm{z}$ is depth, knowing the acoustic pressures caused by a multiple frequencies source and collected by a sparse receiver array. The variational approach minimizes a cost function which measures the adequacy between the measurements and their forward model equivalent. This method introduces also a regularisation term in the form $\left(\mathrm{c}(\mathrm{z})-\mathrm{c}_{\mathrm{b}}\right)^{\mathrm{t}} \mathrm{B}^{-1}\left(\mathrm{c}(\mathrm{z})-\mathrm{c}_{\mathrm{b}}\right)$, which supposes that $\mathrm{c}(\mathrm{z})$ follows an a priori normal law. To circumvent the problem of estimating $\mathrm{B}^{-1}$, we propose to model the celerity vectors by a probabilistic PCA. In contrast to the methods which use PCA as a regularization method and filter the useful information, we take a sufficient number of axes which allow the modelization of useful information and filter only the noise. The probabilistic PCA intro- 
duces a reduced number of non correlated latent variables $\eta$ which act as new control parameters introduced in the cost function. This new regularization term, expressed as $\eta^{\mathrm{t}} \eta$, reduces the optimization computation time. In the following we apply the probabilistic PCA to an OAT problem, and present the results obtained when performing twin experiments.

12:00

4aA04. Geoacoustic Adjoint-Based Inversion via the Parabolic Equation. John S. Papadakis (Institute of Applied \& Computational Mathematics, Foundation for Research and Technology-Hellas (FORTH), Vassilika Vouton, P.O. Box 1385, GR-71110 Heraklion, Greece, johnsp@iacm.forth.gr), Jean-Pierre Hermand (Université libre de Bruxelles (U.L.B.) - Environmental hydroacoustics lab, av. Franklin D. Roosevelt 50 CP 194/5, 1050 Bruxelles, Belgium, jhermand@ulb.ac.be), Evangelia T. Flouri (Institute of Applied \& Computational Mathematics, Foundation for Research and Technology-Hellas (FORTH), Vassilika Vouton, P.O. Box 1385, GR-71110 Heraklion, Greece, flouri@iacm.forth.gr), Matthias Meyer (Royal Netherlands Naval College (NLDA) - REA group, PO Box 10000, 1780 Den Helder, Netherlands, mmeyer@ulb.ac.be)

In this paper an analytic method is exhibited for recovering the acoustic parameters of the sub-bottom region in the ocean. The acoustic propagation problem is modeled via the wide angle parabolic equation and the bottom boundary condition used is in the form of a Neumann to Dirichlet or Dirichlet to Neumann map. The sub-bottom region is assumed homogeneous or horizontally stratified with homogeneous layers. The inversion is modeled as an optimal control problem, and the solution is based on the adjoint method. Several cost functions are introduced which make use of the relative amplitude of the observed complex field. The method is applied to several test cases and satisfactory convergence of the inversion scheme is exhibited.

12:20

4aA05. Adjoint modeling for Acoustic Inversion based on an Adjoint Parabolic Equation. John S. Papadakis (Institute of Applied \& Computational Mathematics, Foundation for Research and Technology-Hellas (FORTH), Vassilika Vouton, P.O. Box 1385, GR-71110 Heraklion, Greece, johnsp@iacm.forth.gr), Evangelia T. Flouri (Institute of Applied \& Computational Mathematics, Foundation for Research and Technology-Hellas (FORTH), Vassilika Vouton, P.O. Box 1385, GR-71110 Heraklion, Greece, flouri@iacm.forth.gr)

In this paper the acoustic propagation problem is modeled by the wide angle parabolic equation and the bottom boundary condition is in the form of a Neumann to Dirichlet map. We formulate the inversion as an optimal control problem, the control parameters being the sound speed in the water and the sound speed, density and attenuation in the bottom. Using the adjoint operator to the wide angle parabolic equation, an inversion scheme is derived for the acoustic parameters of the water column and the bottom region. The optimization approach used is the method of steepest decent. Several test cases are exhibited.

\section{Session 4aBB}

\section{Biomedical Ultrasound/Bioresponse to Vibration: Theoretical and Computational Models of Ultrasonic Propagation in Bones I}

James G. Miller, Cochair

Washington University, Physics Box 1105, 1 BrookingsDrive, St. Louis, MO 63130, USA

Pascal Laugier, Cochair

Université Paris 6, Laboratoire d'Imagerie Paramétrique, 15, rue de l'Ecole de Médecine, Paris, 75006, France

\section{Invited Papers}

\section{8:00}

4aBB1. Recent developments in modeling and measuring scattering from trabecular bone. Keith A. Wear (U.S. Food and Drug Administration, Center for Devices and Radiological Health, 10903 New Hampshire Ave, Bldg 62, Rm 3108, Silver Spring, MD 20993, USA, keith.wear@fda.hhs.gov)

Laboratories at the US Food and Drug Administration, the University of Paris and elsewhere have investigated scattering from trabecular bone. Recent analysis of data from 23 human femur samples in vitro suggests that the Faran Cylinder Model and the Weak Scattering Model accurately predict frequency dependence of backscatter coefficient. Shear mode conversion of incident longitudinal waves may be a significant source of scattering loss. Other recent work involves the effect of errors of attenuation measurements on backscatter coefficient estimates. Backscatter measurements must be compensated for attenuation in order to estimate backscatter coefficient. However, attenuation is often overestimated because it is often measured using phase sensitive receivers that exhibit phase cancellation artifacts. Recent analysis of data from 16 human calcaneus samples in vitro suggests that backscatter coefficient estimates that are based on phase sensitive attenuation compensation tend to overestimate 1) average magnitude of backscatter coefficient at 500 $\mathrm{kHz}$ by a factor of about $1.6 \pm 0.3$ (mean \pm standard deviation) and 2 ) average exponent $(\mathrm{n})$ of frequency dependence by about 0.34 \pm 0.12 (where backscatter coefficient is assumed to be proportional to frequency to the nth power). 
4aBB2. The fast wave propagation in bovine cancellous bone-experiments and simulation. Mami Matsukawa (Doshisha University, 1-3, Tatara Miyakodani, 610-0321 Kyotanabe, Japan, mmatsuka@mail.doshisha.ac.jp), Katsunori Mizuno (Doshisha University, 1-3, Tatara Miyakodani, 610-0321 Kyotanabe, Japan, dtg0151@mail4.doshisha.ac.jp), Yoshiki Nagatani (Nara Medical University, 840 Shijo-cho, 634-8522 Kashihara, Japan, naramed-u@nagatani.ne.jp)

Cancellous bone is comprised of a complicated network of trabeculae and has strong anisotropy and inhomogeneity. In the cancellous bone, two types of longitudinal waves, fast and slow waves, are observed when the waves propagate parallel to the trabeculae direction. Paying attention to the wave front of observed waves, we have experimentally made clear the effect of anisotropy on the fast wave speeds and shown interesting relation between the mean trabeculae length and wave speeds (Mizuno et. al., IEEE Trans., UFFC, accepted), making use of the microstructural indices of the measured bone. We then compared the experimental results of fast waves with the simulation studies, using the three dimensional X-ray CT data and the Finite-Difference Time-Domain (FDTD) method. In spite of the lack of attenuation effects in the simulation, we can find interesting correlation between the fast wave speeds obtained by experiments and simulation. In addition, the characteristic attenuation behaviors of fast wave were found in both experiments and simulations. Attenuation of fast wave is always higher in the initial state of propagation, regardless of propagation direction and samples.

\section{Contributed Papers}

$8: 40$

4aBB3. Experimental confirmation of negative dispersion and Bayesian inversion of multimode propagation in a bone-mimicking phantom. Adam Q. Bauer (Washington University, Physics Box 1105, 1 Brookings Drive, St. Louis, MO 63130, USA, abauer@hbar.wustl .edu), Christian C. Anderson (Washington University, Physics Box 1105, 1 Brookings Drive, St. Louis, MO 63130, USA, canderson@wustl .edu), Karen R. Marutyan (Department of Radiology, Washington University, 1 Brookings Drive, St. Louis, MO 63130, USA, karenmarutyan@gmail .com), G Larry Bretthorst (Department of Radiology, Washington University, 1 Brookings Drive, St. Louis, MO 63130, USA, gbretthorst@wustl.edu), Keith A. Wear (U.S. Food and Drug Administration, Center for Devices and Radiological Health, 10903 New Hampshire Ave, Bldg 62, Rm 3108, Silver Spring, MD 20993, USA, keith.wear@fda.hhs.gov), Mark R. Holland (Washington University, Physics Box 1105, 1 Brookings Drive, St. Louis, MO 63130, USA, mrh@wuphys.wustl.edu), James G. Miller (Washington University, Physics Box 1105, 1 Brookings Drive, St. Louis, MO 63130, USA, james.g .miller@wustl.edu)

Previously we demonstrated using numerical simulations that negative dispersion observed in bone can result from the interference of two propagating modes, each of which exhibits positive dispersion, consistent with the Kramers-Kronig predictions. [J. Acoust. Soc. Am. 120, EL55-61 (2006)] One goal of the present study was to demonstrate this negative dispersion experimentally using the simplest example of a bone-mimicking phantom that is capable of producing two such interfering modes. An additional goal was to establish that, with the experimental data serving as input to a Bayesian approach to the inverse problem [J. Acoust. Soc. Am. 121, EL8-15 (2007)], reliable estimates of the underlying properties of the bonemimicking phantom could be obtained from the measured signals. The phantom consisted of a flat and parallel PlexiglasTM plate into which a step discontinuity was milled. The phase velocity and attenuation coefficient (3 to $7 \mathrm{MHz}$ ) of the phantom were measured with a 0.25 -inch piezoelectric receiver and calculated using both broadband and narrowband data. Negative dispersion was observed at specific spatial locations near the step where the attenuation coefficient rose approximately linearly with frequency. Results demonstrate that interference between modes can result in negative dispersion and that Bayesian inversion can yield underlying material properties.

\section{9:00}

4aBB4. Modeling of "anomalous" velocity dispersion in trabecular bone: effect of multiple scattering and of viscous absorption. Guillaume Haiat (CNRS, Laboratoire de Recherches Orthopédiques, 10, Avenue de Verdun, $75010 \quad$ Paris, France, haiat@univ-paris12.fr), Alain Lhémery (Commissariat à l'Energie Atomique, Bat 611, LIST, CEA Saclay, 91191 Gif-sur-Yvette, France, alain.lhemery@cea.fr), Frederic Padilla (Université Paris 6, Laboratoire d'Imagerie Paramétrique, 15, rue de
l'Ecole de Médecine, 75006 Paris, France, Frederic.Padilla@lip .bhdc.jussieu.fr), Pascal Laugier (Université Paris 6, Laboratoire d'Imagerie Paramétrique, 15, rue de l'Ecole de Médecine, 75006 Paris, France, laugier@lip.bhdc.jussieu.fr), Salah Naili (Université Paris 12, B2OA, 61, Avenue du Général de Gaulle, 94010 Créteil, France, naili @ univ-paris12.fr)

Speed of sound is now widely used in the clinic to assess bone strength and susceptibility to fracture. Therefore, much effort has been spent on the understanding of ultrasonic wave propagation in trabecular bone, which is an attenuating composite material in which negative values of velocity dispersion have been measured, but remain poorly explained physically. In this work, the physical determinants of velocity dispersion are described with a 2D homogenization model of the wave propagation in trabecular bone. The medium is assumed to be constituted of infinite viscoelastic cylinders (trabeculae) immersed in a saturating viscoelastic matrix (marrow). The coupling between multiple scattering and absorption phenomena allows the computation of phase velocity and of dispersion as a function of bone properties. Negative values of velocity dispersion are predicted, in good agreement with experimental results obtained in phantoms mimicking trabecular bone. In trabecular bone, mostly negative but also positive values of velocity dispersion are predicted, which spans within the range of values measured experimentally. Scattering effects are responsible for the negative values of dispersion whereas the frequency dependence of the attenuation coefficient in bone marrow and/or in the trabeculae is shown to induce an increase of dispersion.

\section{9:20}

4aBB5. Effects of elastic properties on the wave propagation in cancellous bones - a simulation study -. Yoshiki Nagatani (Nara Medical University, 840 Shijo-cho, 634-8522 Kashihara, Japan, naramed-u@nagatani.ne.jp), Takashi Saeki (Doshisha University, 1-3, Tatara Miyakodani, 610-0321 Kyotanabe, Japan, dth0915@mail4.doshisha.ac.jp), Mami Matsukawa (Doshisha University, 1-3, Tatara Miyakodani, 610-0321 Kyotanabe, Japan, mmatsuka@mail.doshisha.ac.jp), Takefumi Sakaguchi (Nara Medical University, 840 Shijo-cho, 634-8522 Kashihara, Japan, t-saka@naramed-u.ac.jp), Hiroshi Hosoi (Nara Medical University, 840 Shijo-cho, 634-8522 Kashihara, Japan, hosoi@naramed-u.ac.jp)

For diagnosing osteoporosis, ultrasonic systems are considered a powerful tool, because ultrasonic waves strongly depend on the elasticity and structure of cancellous bones. We have reported the separation of longitudinal waves into fast and slow waves, a phenomenon that is strongly connected to the alignment of bone trabeculae.In order to understand this complicated wave propagation, we have simulated the wave propagations with the finite-difference time-domain (FDTD) method using three-dimensional $\mathrm{X}$-ray CT images of actual cancellous bones. In this simulation, the effects of elastic properties in the solid portions are important. One idea is to adopt the experimentally observed ultrasonic properties of cortical bone. However, we should remind the possible problems like the elasticity difference between cancellous and cortical bones, individual differences, and anisotropy. 
In this study, then, we have investigated the influences of elastic properties, Poisson's ratio, and densities of solid portion (trabeculae). As a result, we confirmed that the small changes give strong influences on not only the wave speed but also the amplitudes of fast and slow waves. The influence on the amplitudes seems to come from the changes of acoustic impedance of trabeculae. The results show the importance of elastic properties in the simulation.

\section{9:40}

4aBB6. Wave propagation in cancellous bone in terms of Biot's theory Michal Pakula (Université Paris 6, Laboratoire d'Imagerie Paramétrique, 15, rue de l'Ecole de Médecine, 75006 Paris, France, michalp@ukw.edu.pl), Frederic Padilla (Université Paris 6, Laboratoire d'Imagerie Paramétrique, 15, rue de l'Ecole de Médecine, 75006 Paris, France, Frederic.Padilla@lip.bhdc.jussieu.fr), Mariusz Kaczmarek (Institute of Environmental Mechanics and Applied Computer Science, Kazimierz Wielki University, ul. Chodkiewicza 30, 85-064 Bydgoszcz, Poland, mkk@rose.man.poznan.pl), Pascal Laugier (Université Paris 6, Laboratoire d'Imagerie Paramétrique, 15, rue de l'Ecole de Médecine, 75006 Paris, France, laugier@lip.bhdc.jussieu.fr)

The paper is focused on modelling of wave propagation in cancellous bones using Biot's theory. Almost all required input mechanical and structural parameters for 31 pure femoral trabecular bone specimens were measured individually. Then frequency dependent wave parameters predicted by the model were compared with the results of ultrasonic tests performed on the same specimens. To compare the predictions to measurements, additional interactions of the plane harmonic wave with the slab of cancellous was considered. The most important finding is the significant contribution of the fluid/bone and bone/fluid boundaries on the global attenuation loss. The corrected values of attenuation coefficient are of the same order of magnitude compared to measured values. The theoretical results exhibit higher attenuation of fast wave compared to that of the slow wave in good agreement with experimental observations. Moreover the amplitude ratio of simulated time domain signals of both longitudinal waves (accordingly to the Biot's model with boundary corrections), are of the same order of magnitude compared to the amplitude ratio of experimental time records. However, an analysis in the frequency domain shows that the frequency content of the simulated pulses of the fast and slow wave differs from that observed in the experiments.

\section{0:00}

4aBB7. Experimental and numerical investigation of ultrasonic transmission through the skull bone and associated temperature rise. Mathieu Pernot (Laboratoire Ondes et Acoustique, ESPCI, Université Paris 7, CNRS, 10 rue Vauquelin, 75005 Paris, France, mathieu.pernot@espci .fr), Emmanuel Bossy (Laboratoire Photons et Matière, ESPCI/CNRS, 10 rue Vauquelin, 75231 Paris Cedex 05, France, emmanuel.bossy@espci .fr), Marie Muller (Laboratoire Ondes et Acoustique, ESPCI, Université Paris 7, CNRS, 10 rue Vauquelin, 75005 Paris, France, marie.muller@espci .fr), Christine Boué (Laboratoire Photons et Matière, ESPCI/CNRS, 10 rue Vauquelin, 75231 Paris Cedex 05, France, christine.boue@espci .fr), Jean-François Aubry (Laboratoire Ondes et Acoustique, ESPCI, Université Paris 7, CNRS, 10 rue Vauquelin, 75005 Paris, France, jean-francois.aubry@espci.fr), Mickael Tanter (Laboratoire Ondes et Acoustique, ESPCI, Université Paris 7, CNRS, 10 rue Vauquelin, 75005 Paris, France, michael.tanter@espci.fr), Mathias Fink (Laboratoire Ondes et Acoustique, ESPCI, Université Paris 7, CNRS, 10 rue Vauquelin, 75005 Paris, France, mathias.fink@espci.fr), Albert-Claude Boccara (Laboratoire Photons et Matière, ESPCI/CNRS, 10 rue Vauquelin, 75231 Paris Cedex 05, France, boccara@optique.espci.fr)

The feasibility of transcranial high-intensity focused-ultrasound (HIFU) therapy within the brain relies on the ability to transmit ultrasound through the skull bone at relatively high ultrasound power. Absorption of ultrasound through the skull bone may cause important temperature rises, and is therefore an important parameter to control. Ultrasonic measurements have shown that the ultrasound beam undergoes a significant attenuation when propagating through the skull, with values on the order of 10 to 20 $\mathrm{dB} / \mathrm{cm} / \mathrm{MHz}$. To predict temperature rise from such values, it is fundamental to weigh the relative role of absorption to the total ultrasonic attenuation (scattering + absorption + specular reflection). In this work, two types of numerical simulations and experiments are performed to investigate this relative role. Through-transmission of $1 \mathrm{MHz}$ ultrasound was performed numerically using a 3D Finite-Difference Time-Domain (FDTD) algorithm coupled to a 3D bone model obtained from high-resolution synchrotron microtomography, and compared to experimental measurements obtained with the same bone sample. Temperature rises were numerically simulated using the 3D bone model coupled to the heat equation, and compared to infrared thermography obtained experimentally while high-intensity ultrasound was propagating through the sample.

\section{0:20-10:40 Break}

\section{0:40}

4aBB8. Ultrasound simulation in the distal radius using clinical high-resolution CT images. Jonathan J. Kaufman (CyberLogic, Inc., 611 Broadway, Suite 707, New York, NY 10012, USA, jjkaufman@cyberlogic.org), Vincent Le Floch (Ecole Nationale Supérieure d'Arts et Métiers, Provence-Alpes-Cote-d'Azur, 13090 Aix-en-Provence, France, jjkaufman@cyberlogic.org), Donald J. McMahon (College of Physicians and Surgeons, Columbia University, 630 West 168th Street, New York, NY 10032, USA, jjkaufman@cyberlogic.org), Gangming Luo (CyberLogic, Inc., 611 Broadway, Suite 707, New York, NY 10012, USA, jjkaufman@cyberlogic.org), Adi Cohen (College of Physicians and Surgeons, Columbia University, 630 West 168th Street, New York, NY 10032, USA, jjkaufman@cyberlogic.org), Elizabeth Shane (College of Physicians and Surgeons, Columbia University, 630 West 168th Street, New York, NY 10032, USA, jjkaufman@cyberlogic.org), Robert S. Siffert (Mount Sinai School of Medicine, One Gustave L. Levy Place, New York, NY 10029, USA, jjkaufman@cyberlogic.org)

The overall objective of this research is to develop an ultrasonic method for non-invasive assessment of the distal radius. The specific objective of this study was to examine the propagation of ultrasound through the distal radius and determine the relationships between bone mass and architecture and ultrasound parameters. Twenty-six high-resolution peripheral-CT clinical images were obtained from a set of subjects that were part of a larger study on secondary osteoporosis. A single mid-section binary slice from each image was selected and used in the 2D simulation of an ultrasound wave propagating from the anterior to the posterior surfaces of each radius. Mass and architectural parameters associated with each radius, including total bone mass, volume fraction, trabecular number, and trabecular thickness were computed. Ultrasound parameters, including net time delay (NTD), broadband ultrasound attenuation (BUA), and ultrasound velocity (UV) were also evaluated. Significant correlations were found between NTD and total bone mass $(\mathrm{R} 2=0.92)$, BUA and trabecular number $(\mathrm{R} 2=0.78)$, and $\mathrm{UV}$ and trabecular bone volume fraction $(\mathrm{R} 2=0.82)$. The study shows that ultrasound measurements are correlated with bone mass and architecture at the distal radius, and thus ultrasound may prove useful as a method for noninvasive assessment of osteoporosis and fracture risk.

\section{1:00}

4aBB9. Guided ultrasound wave propagation in cortical bone with microstructure using the gradient elasticity theory. Maria G. Vavva (University of Ioannina, Unit of Medical Technology and Intelligent Information Systems, Department of Computer Science and Department of Material Science and Engineering, GR 45110 Ioannina, Greece, mvavva@cc.uoi.gr), Vasilios C. Protopappas (University of Ioannina, Unit of Medical Technology and Intelligent Information Systems, Department of Computer Science and Department of Material Science and Engineering, GR 45110 Ioannina, Greece, vprotop@cc.uoi.gr), Leonidas N. Gergidis (University of Ioannina, Department of Material Science and Engineering, GR 45110 Ioannina, Greece, lgergidi@uoi.gr), Antonios Charalambopoulos (University of Ioannina, Department of Material Science and Engineering, GR 45110 Ioannina, Greece, acharala@cc.uoi .gr), Dimitrios I. Fotiadis (University of Ioannina, Unit of Medical Technology and Intelligent Information Systems, Department of Computer Science and Department of Material Science and Engineering, GR 45110 
Ioannina, Greece, fotiadis@cc.uoi.gr),Demos Polyzos(University of Patras, Department of Mechanical Engineering and Aeronautics, GR 26500 Patras, Greece,polyzos@mech.upatras.gr)

Ultrasonic characterization of bone has been largely based on the linear theory of classical elasticity. However, the classical theory cannot adequately describe the mechanical behavior of materials with microstructure. In such materials, the stress state has to be defined in a nonlocal manner by employing theories, such as those proposed by Cosserat brothers, Mindlin (gradient elastic theory) and Eringen. In this study, we adopt the simplest form of gradient theory (Mindlin FormII) to model the cortical bone's microstructural effects in a macroscopic framework. The frequency characteristic equations are analytically derived for a bone plate $(4 \mathrm{~mm}$ thick, density $1.5 \mathrm{~g} / \mathrm{cm}^{3}$, bulk longitudinal velocity $4107 \mathrm{~m} / \mathrm{s}$ ). The plate is assumed free of stresses, as in the classical Lamb problem, and free of double stresses. The volumetric strain gradient energy coefficient, $g$ (a measure of internal length), is equal to $10^{-4}$ and $10^{-5} \mathrm{~m}$, i.e. of the order of the osteons size. The velocity dispersion curves of guided waves are numerically obtained using root-finding techniques and compared with those of the Lamb waves. It is shown that microstructure affects mode dispersion by inducing both material and geometrical dispersion. In conclusion, bone models with microstructure can contribute to the interpretation of in vivo measurements.

\section{1:20}

4aBB10. Investigation of the porous network as a determinant of the overall stiffness of cortical bone: Mori-Tanaka model vs. ultrasound propagation. Cécile Baron (Université Paris 6, Laboratoire d'Imagerie Paramétrique, 15, rue de l'Ecole de Médecine, 75006 Paris, France, norabelic@yahoo.fr), Quentin Grimal (Université Paris 6, Laboratoire d'Imagerie Paramétrique, 15, rue de l'Ecole de Médecine, 75006 Paris, France, quentin.grimal@lip.bhdc.jussieu.fr), Maryline Talmant (Université Paris 6, Laboratoire d'Imagerie Paramétrique, 15, rue de 1'Ecole de Médecine, 75006 Paris, France, talmant@lip.bhdc.jussieu.fr), Pascal Laugier (Université Paris 6, Laboratoire d'Imagerie Paramétrique, 15, rue de 1'Ecole de Médecine, 75006 Paris, France, laugier@lip.bhdc.jussieu.fr)

Assessing the effect of porosity on stiffness in cortical bone remains an important issue that has already been addressed with several models. The originality of the present work is to compare two models of cortical bone: one uses a realistic porous network (voxel 20 microns) reconstructed from synchrotron radiation tomography; the other considers cylindrical pores aligned in a single direction. In the first case, overall elastic properties are evaluated indirectly by means of finite difference time domain simulation of ultrasound bulk wave propagation at $1 \mathrm{MHz}$. In the second model, effective elasticity is calculated by means of a Mori-Tanaka scheme based on Eshelby solution for cylindrical inclusions with ellipsoidal cross section. Overall properties were evaluated with the two methods for 18 porosity values, each corresponding to a reconstructed bone volume. The diagonal stiffness coefficients of the overall bone material estimated with the two methods compared well. Results for the stiffness coefficient in the longitudinal bone direction are indistinguishable, which indicates that the detailed geometry and distribution of the pores have a negligible effect on the longitudinal stiffness. For the other stiffness coefficients, the Mori-Tanaka method slightly overestimates the stiffness compared to the wave propagation evaluation.

\section{$11: 40$}

4aBB11. Numerical modelling and in-vitro studies of ultrasound signal loss across fractures in cortical bone mimics. Victor $F$. Humphrey (Institute of Sound and Vibration, Univ. of Southampton, University Road, Highfield, SO17 1BJ Southampton, UK, vh@isvr.soton.ac.uk), Simon P. Dodd (University of Bath, School of Management, Claverton Down, BA2 7AY Bath, UK, S.Dodd@bath.ac.uk), Sabina Gheduzzi (University of Bath, Centre for Orthopaedic Biomechanics, Dept. of Mechanical Engineering, BA2 7AY Bath, UK, S.Gheduzzi@bath.ac.uk), James L. Cunningham (University of Bath, Centre for Orthopaedic Biomechanics, Dept. of Mechanical Engineering, BA2 7AY Bath, UK, J.L.Cunningham@bath.ac.uk), Anthony W. Miles (University of Bath, Centre for Orthopaedic Biomechanics, Dept. of Mechanical Engineering, BA2 7AY Bath, UK, A.W.Miles@bath.ac.uk)

The propagation of $200 \mathrm{kHz}$ ultrasonic waves along cortical bone mimics and across a simulated fracture has been investigated using a Finite Difference numerical model. The first arrival signal (FAS) transit time and peak amplitude have been calculated as a function of range at $200 \mathrm{kHz}$ in order to help understand the factors that determine the propagation across a fracture. The variation in the amplitude of the first peak of the reradiated wave is studied as a function of the gap width and shape. The results compare well with experimental measurements made in vitro using an axial transmission technique on cortical bone mimics and bovine cortical bone samples. The effects of various stages of the healing process have also been considered by introducing different fracture geometries into the plate model. Changing the geometry to an external callus with different mechanical properties causes the signal loss across the fracture to reduce significantly. The most significant changes are observed to occur from the initial inflammatory stage to the formation of a callus and in the remodelling stage after a significant reduction in the size of the callus has taken place.

\section{2:00}

4aBB12. The role of bone marrow on acoustic properties of cancellous bone - finite difference time domain modelling study. Antti S. Kallioniemi (University of Kuopio, POB 1627, 70211 Kuopio, Finland, antti.kallioniemi@uku.fi), Juha Töyräs (Kuopio University Hospital, POB 1777, $70211 \quad$ Kuopio, Finland, Juha.Toyras@kuh.fi), Mikko Hakulinen (University of Kuopio, POB 1627, 70211 Kuopio, Finland, Mikko.Hakulinen@uku.fi), Jukka Jurvelin (University of Kuopio, POB 1627, 70211 Kuopio, Finland, Jukka.Jurvelin@uku.fi)

Quantitative ultrasound (US) parameters are related to structure and properties of cancellous bone. The effect of bone marrow on US propagation, i.e. absorption and scattering, is still poorly understood. However, substitution of fatty marrow with water is known to significantly affect the US parameters. The present study investigates the role of marrow on US parameters, using microtomography based 3D-finite difference time domain (FDTD) modelling. Eleven human cancellous bone samples were analysed with a micro-CT system (SkyScan 1072) to determine microstructure and morphology. Wave 3000 Pro software (Cyberlogic Inc.) was used for simulations. Models were created to simulate experimental US measurement geometry with focused $1 \mathrm{MHz}$ transducers. Simulations were repeated before and after replacing the marrow with water. The voxel size of the simulation mesh significantly affected sample structure and simulations. US attenuation and speed decreased and increased, respectively, when marrow was replaced with water $(p<0.01)$. US reflection at sample surface and backscattering from internal structures increased $(p<0.01)$ when marrow was replaced with water. Contribution of bone marrow was stronger in samples with low bone volume fraction. This implicates that inter-individual differences in the composition of marrow may significantly affect measured ultrasound parameters, especially when investigating osteoporotic bone with low density. 


\title{
Session 4aEA
}

\section{Engineering Acoustics: Sound Fields I}

\author{
Raymond Kirby, Chair \\ Brunel University, School of Engineering and Design, Uxbridge, Middlesex, UB8 3PH, UK
}

\section{Contributed Papers}

\section{8:00}

4aEA1. A virtual headphone based on wave field synthesis. Klaus Laumann (Institut für Rundfunktechnik GmbH, Floriansmühlstraße 60, 80939 München, Germany, laumann@irt.de), Günther Theile (Institut für Rundfunktechnik GmbH, Floriansmühlstraße 60, 80939 München, Germany, theile@irt.de), Hugo Fastl (AG Technische Akustik, MMK, TU München, Arcisstr. 21, 80333 München, Germany, fastl@mmk.ei.tum.de)

The term "virtual headphone" refers to specially designed loudspeaker systems aiming for transmission characteristics equal to real headphones. Particularly of interest is the exact pre-filtering of the speaker signals to compensate the effect of head related transfer functions (HRTFs) between loudspeakers and ear canal. These so-called "HRTF inverse filters" are dependent on geometrical conditions and so they have to be updated with every head movement. In order to avoid problematic adaptive HRTF inverse filtering, the real loudspeakers are replaced by focussed sources generated according to the principles of Wave Field Synthesis (WFS). Head tracking controlled adjustment of driving functions allows easy source movement and thus fixed source positions in relation to the listener's ears, providing stable virtual headphone reproduction. A single static HRTF inverse filter network can be used. It is designed to ensure precise headphone equalization according to ITU-R BS.708 and offers accurate reproduction of e.g. binaural signals. A pilot study with a circular WFS array built in a panel above the listener's head has verified the functional capability of this concept.

\section{8:20}

4aEA2. Modelling sound propagation in a waveguide containing multiple obstacles. Raymond Kirby (Brunel University, School of Engineering and Design, Uxbridge, UB8 3PH Middlesex, UK, ray.kirby@brunel .ac.uk)

Acoustic waveguides often include relatively short area changes and/or complex non-uniform obstacles. Understanding the propagation of sound within such waveguides requires a detailed knowledge of the scattering of sound at each obstacle and how these obstacles interact with one another. Mathematically modelling sound propagation in waveguides containing multiple non-uniform obstacles is challenging, especially if one assumes that the waveguide is relatively large. Accordingly, a computationally efficient hybrid numerical method is presented here that uses the standard finite element method to model non-uniform obstacles, and maps this onto a wave-based modal solution that is used for uniform duct sections only. The hybrid method has the advantage, moreover, of removing the need to numerically enforce a non-reflecting boundary condition downstream of the obstacles, which is often encountered in studies that rely solely on the standard finite element method. In this way, transmission loss predictions for relatively large ducts and multiple obstacles may be generated efficiently, and predictions are presented here for two cylinders placed in a twodimensional waveguide.

\section{8:40}

4aEA3. Sound generation by airflow in a pipe having a small internal cavity. Ulf Kristiansen (Acoustics group, Norwegian University of Science and Technology, O.S. Bragstads plass 2B, N-7491 Trondheim, Norway, ulf.kristiansen@iet.ntnu.no), Børge Nygård (Acoustics group, Norwegian
University of Science and Technology, O.S. Bragstads plass 2B, N-7491 Trondheim, Norway, borge.b.nygaard@iet.ntnu.no)

Gas flow through corrugated pipes is known to excite strong acoustic resonances within the pipe. In an attempt to better understand the flow acoustic phenomena involved, we have investigated experimentally a short pipe $(0.6 \mathrm{~m}$ long and $0.04 \mathrm{~m}$ diameter) having a single small $(5 \mathrm{~mm}$ long, $2.5 \mathrm{~mm}$ deep) circumferential cavity. It was found that if placed close to the pipe's inflow end, strong acoustic resonances were generated. The experimental results were compared to a model based on describing-function theory. The model involves two transfer functions, one associated with the pipe resonator, and the other the shear layer above the cavity. These are combined to a feedback system. This model gives the frequencies generated and the acoustic pressure levels (to within a constant) for different flow velocities. Reasonable agreement was obtained between the experimental results and the model predictions.

\section{9:00}

4aEA4. Multi-modal acoustic propagation in pipes with arbitrary defects: theory and experiments. Raymond Kirby (Brunel University, School of Engineering and Design, Uxbridge, UB8 3PH Middlesex, UK, ray.kirby@brunel.ac.uk), Kirill V. Horoshenkov (University of Bradford, School of Engineering, Design and Technology, BD7 1DP Bradford, UK, k.horoshenkov@Bradford.ac.uk), Tareq Bin Ali (University of Bradford, School of Engineering, Design and Technology, BD7 1DP Bradford, UK, m.t.binali@Bradford.ac.uk)

Underground sewer systems are prone to flooding incidents caused by obstructions such as sediment deposits and wall deterioration. An efficient method for identifying and characterising these obstructions involves measuring the amplitudes of the reflected and transmitted acoustic normal modes excited by a point source in the sewer pipe. However, the behaviour of higher order modes in relatively large pipe work is often difficult to predict and interpret. In order to provide a greater physical insight into the measured data and to guide future experimental work, theoretical predictions have been developed and validated. The presented theoretical work is based on a finite element method and a mode matching technique. In this paper the predicted and measured sound fields are analysed for up to four acoustic modes reflected from two different obstacles (axisymmetric and nonaxisymmetric) deposited in a $150 \mathrm{~mm}$ diameter uPVC pipe.

\section{9:20}

4aEA5. Benchmarking for acoustic simulation software. Alfonso R Molares (University of Vigo, E.T.S.I de Telecomunicación, Rúa Maxwell s/n, 36310 Vigo, Spain, amolares@gts.tsc.uvigo.es), Manuel A Sobreira-Seoane (University of Vigo, E.T.S.I de Telecomunicación, Rúa Maxwell s/n, 36310 Vigo, Spain, msobre@gts.tsc.uvigo.es)

The validation of acoustic simulation software is still an obscure and imprecise matter. Validation studies of commercial implementations are rarely provided by vendors which are reluctant to show their product weakness. On the contrary in open academic implementations validation studies are one of the keys to make them used, rather than marketing. However, they demand from users a high level of knowledge and imply, generally, so long calculation times that make them impractical for common industry purposes. The aim of this study is to contribute to clarify this point setting a simple benchmark to measure the accuracy and performance of different software pack- 
ages for sound field calculations. The benchmark is presented by its application with two widely used commercial implementations of finite element method and with an open-source implementation of boundary element method developed at the Southern Denmark University. The validation is performed against analytical formulae and also against experimental results. In order to study the balance between accuracy and computational cost the results are finally presented in terms of relative error versus calculation time.

\section{9:40}

4aEA6. Spatialized additive synthesis. Charles Verron (Orange Labs, 2 avenue Pierre Marzin, 22307 Lannion, France, charles.verron @orange-ftgroup.com), Mitsuko Aramaki (CNRS - INCM and Université de Provence, 31, chemin Joseph Aiguier, 13402 Marseille, France, aramaki@1ma.cnrs-mrs.fr), Richard Kronland-Martinet (CNRS-LMA, 31, chemin Joseph Aiguier, 13402 Marseille, France, kronland@1ma .cnrs-mrs.fr), Grégory Pallone (Orange Labs, 2 avenue Pierre Marzin, 22307 Lannion, France, gregory.pallone@orange-ftgroup.com)

In virtual auditory environments, a spatialized sound source is typically simulated in two stages: first a "dry" monophonic signal is recorded or synthesized, and then spatial attributes (directivity, width and position) are applied by specific signal processing algorithms. In this paper, a unified analysis/spatialization/synthesis system is presented. It is based on the spectral modeling framework that analyses/synthesizes sounds as a combination of time-varying sinusoidal, noisy and transient contributions. The proposed system takes advantage of this representation to allow intrinsic parametric sound transformations, such as spatial distribution of sinusoids or diffusion of the noisy contribution around the listener. It integrates timbre and spatial parameters at the same level of sound generation, so as to enhance control capability and computational performance.

\section{0:00}

4aEA7. Notes on the reproduction of moving virtual sound sources Jens Ahrens (Deutsche Telekom Laboratories, Ernst-Reuter-Platz 7, 10587 Berlin, Germany, jens.ahrens@telekom.de), Sascha Spors (Deutsche Telekom Laboratories, Ernst-Reuter-Platz 7, 10587 Berlin, Germany, sascha .spors@telekom.de)

Wave field synthesis and higher-order Ambisonics are two representatives of sound reproduction systems that are based upon the concept of physical recreation of a desired sound field. Conventional implementations of such systems typically reproduce moving virtual sound sources as a concatenation of static source positions that change over time. This approach introduces various artifacts which are reported in the literature to be strongly audible. It was recently shown by the authors that the explicit consideration of the physical properties of the sound field of moving sources in the reproduction algorithm avoids these artifacts. It thus allows for the accurate reproduction of the Doppler Effect. In practical implementations of such sound field reproduction systems unavoidable artifacts arise. These are mainly a consequence of sampling and truncation of the loudspeaker distribution and appear both for static and moving virtual sound sources. For static sources, they are well documented in the literature. We revisit these investigations and point out the particularities of these artifacts with respect to the time-variant property of the reproduced sound field.

\section{0:20-10:40 Break}

\section{$10: 40$}

4aEA8. Loudspeakers simulation of sound environments for the car industry. Benoît Gauduin (Genesis S.A., Bâtiment Gérard Mégie, Domaine du Petit Arbois - BP 69, 13545 Aix-en-Provence Cedex 4, France, benoit.gauduin@genesis.fr), Sylvain Hourcade (Genesis S.A., Bâtiment Gérard Mégie, Domaine du Petit Arbois - BP 69, 13545 Aix-en-Provence
Cedex 4, France, sylvain.hourcade@genesis.fr),Nathalie Le Hir(Renault, TCR/AVA 163, Technocentre, 78288 Guyancourt Cedex, France, nathalie.le-hir@renault.com), Gaël Guyader (Renault, TCR/AVA 163, Technocentre, 78288 Guyancourt Cedex, France, gael.guyader@renault .com)

Car manufacturers are strongly interested in sound quality: they need to understand the expectations of their customers in terms of engine sound signature, door closure noise, etc. ... In order to allow engineering teams of research departments to perform psychoacoustics tests under controlled and realistic conditions, GENESIS supplies high fidelity listening 3D simulators on loudspeakers. Based on binaural recordings, these simulators, called transaural, are calibrated to obtain the best fidelity in terms of spatial and spectral components. In this presentation, we will discuss the requirements regarding sound system and room installation and present the precision reachable in terms of frequency response. Measurements and results achieved on such systems will be shown, with a particular focus on the two systems built for RENAULT and GENESIS. Finally, in order to perform equivalent psychoacoustics tests on both RENAULT and GENESIS sites, we will develop the procedure of comparison and validation of both transaural systems.

\section{1:00}

4aEA9. Development of a simple and accurate approximation method for the Gaussian beam expansion technique. Wei Liu (Institute of Acoustics, Chinese Academy of Sciences, Bei-Si-Huan-Xi Road, 100080 Beijing, China, liuwei9811@gmail.com), Peifeng Ji (Institute of Acoustics, Chinese Academy of Sciences, Bei-Si-Huan-Xi Road, 100080 Beijing, China, jipeifeng@mail.ioa.ac.cn), Jun Yang (Institute of Acoustics, Chinese Academy of Sciences, Bei-Si-Huan-Xi Road, 100080 Beijing, China, junyang.ioa@gmail.com)

The calculation of the sound field can be greatly simplified by using the Gaussian beam expansion technique. The source distribution function is expressed as the superposition of a small number of Gaussian functions, and the expansion coefficients could be obtained by minimizing an object function in the spatial or k-space domain. In this paper, a fast algorithm is developed to determine the Gaussian function coefficients for a more accurate approximation. Two-stage procedures are employed in the proposed method. Firstly, two real coefficients are estimated by a simple search approach, and then the least mean square (LMS) algorithm is adopted for determining the optimal expansion coefficients. Finally, the presented method is evaluated in the case of calculation of sound fields radiated from a piston and a rectangular planar source. Simulation results show that, compared with the previous approaches, the developed scheme is simple to implement with high accuracy.

\section{1:20}

4aEA10. Determination of Condition for Fastest Negative Group Velocities of Lamb-Type Waves under each Density Ratio of Solid and Liquid Layers. Kojiro Nishimiya (Tsukuba Univ., Tsukuba Science City, 305-8573 Ibaraki, Japan, nishimiya@aclab.esys.tsukuba.ac.jp), Koichi Mizutani (Tsukuba Univ., Tsukuba Science City, 305-8573 Ibaraki, Japan, mizutani@esys.tsukuba.ac.jp), Naoto Wakatsuki (Tsukuba Univ., Tsukuba Science City, 305-8573 Ibaraki, Japan, wakatuki@iit.tsukuba.ac.jp), Ken Yamamoto (Kansai Univ., 3-3-35 Yamate-cho, 564-8680 Suita, Japan, ken @ipcku.kansai-u.ac.jp)

Lamb-type waves are coupling modes of leaky Lamb waves on a layer structure. The Lamb-type waves have complicated propagation characteristics more than ordinary Lamb waves on a uniform elastic plate. In the characteristics, we examine the negative group velocities. Generally, the negative group velocities of Lamb waves are slower than positive group velocities under the same condition. If the negative group velocities are applied to fabricating some new application, it is desired that the speeds of negative group velocities are comparable to those of positive group velocities. Consequently, we aim to obtain the faster negative group velocities. Lamb-type waves show more discriminative characteristics in negative group velocities than ordinary Lamb waves. In this research, we consider the Lamb-type waves in a solid/liquid/solid structure. It is de- 
scribed the conditions for obtaining the fastest negative group velocities of Lamb-type waves. The conditions, which are the acoustical impedance ratio, are expressed as the function of the density ratio of solid and liquid layers. These results are verified by numerical calculations.

\section{$11: 40$}

4aEA11. Acoustic characterization of thin polymer layers for Love mode surface acoustic waveguide. Laurent Robert (CNRS FEMTO-ST, 32 Avenue de l'Observatoire, 25044 Besançon, France, laurent.robert @femto-st.fr), Lamia El Fissi (CNRS FEMTO-ST, 32 Avenue de l'Observatoire, 25044 Besançon, France, lamia.elfissi@femto-st .fr), Jean-Michel Friedt (CNRS FEMTO-ST, 32 Avenue de l'Observatoire, 25044 Besançon, France, jmfriedt@femto-st.fr), Frederic Cherioux (CNRS FEMTO-ST, 32 Avenue de l'Observatoire, 25044 Besançon, France, frederic.cherioux@femto-st.fr), Sylvain Ballandras (CNRS FEMTO-ST, 32 Avenue de l'Observatoire, 25044 Besançon, France, sylvain.ballandras @ femto-st.fr)

We investigate the use of thin (1-10 um) polymer films as guiding layer for Love mode surface acoustic wave sensors. Beyond the great gravimetric sensitivity provided by the polymer guiding layer resulting from the low acoustic velocity, the use of photoresists provide ecnonomical means of depositing guiding layers of optimal thicknesses compared to inorganic layer deposition processes (typically PECVD deposition of silicon dioxide lasting several hours). The limit of very thick ( $>100 \mathrm{um}$ ) layers provides means of propagating interface waves mostly insensitive to the environment (package-less sensors) whose properties only vary through modification of the bulk properties of the polymer. We here analyse the evolution of the properties of the guiding layer in terms of acoustic velocity and losses as a function of time (solvent evaporation following photoresist spin coating) and temperature (typical baking steps). The polymer films is deposited on AT-cut quartz patterned with interdigtated transducers for generating $40 \mathrm{um}$ wavelength shear waves converted to a guided Love mode in a delay line configuration. We complete the experimental results with data interpretation using a model of acoustic wave propagation yielding quantitative results including viscosity and density out of the velocity and insertion loss measurements, both for the guided Love mode and interface layers
12:00

4aEA12. From frequency to time domain: Signal features and physical characteristics for resonant acoustical systems. Samuel Rodriguez (Renault - Laboratoire PHASE, Centre Technique Renault - CTL L16 1 29, 1, allée Cornuel, 91510 Lardy, France, rodriguez.samuel@yahoo .fr), Vincent Gibiat (Université Paul Sabatier, PHASE, 118, route de Narbonne, 31062 Toulouse cedex 9, France, gibiat@cict.fr), Stephane Guilain (Renault - Laboratoire PHASE, Centre Technique Renault - CTL L16 1 29, 1, allée Cornuel, 91510 Lardy, France, stephane.guilain @ renault.com), Alain Lefebvre (Renault - Laboratoire PHASE, Centre Technique Renault - CTL L16 1 29, 1, allée Cornuel, 91510 Lardy, France, alain.a.lefebvre@ renault.com)

The determination of the impulse response or the reflection function of an acoustical system from data expressed in the frequency domain is not immediate. Signal processing from frequency domain to time domain should involve phenomena of large amplitude as oscillations known as "ripple" that does not correspond to any physical phenomenon. The "ripple phenomenon" will be analyzed from both a signal processing and a physical point of view with the help of simple duct acoustic examples. A map designed as a new time-frequency tool helps us to show that it cannot be removed in most cases without the use of processing techniques involving modifications in the computed signal. This work has been developed for the automotive research, but can be applied to musical acoustics or to any field connected with time domain exploration of acoustic cues.

4aEA13. Extension of Optimal Source Distribution principle. Takashi Takeuchi (OPSODIS Limited, c/o ISVR, University of Southampton, Highfield, SO17 1BJ Southampton, UK, tt@isvr.soton.ac.uk)

Binaural reproduction over loudspeaker requires system inversion which is often referred to as cross-talk cancellation. Such process is the major factor to degrade the quality of 3D sound reproduction but Optimal Source Distribution (OSD) provides simple and effective loudspeaker design principle and signal processing which enables lossless crosstalk cancellation process. OSD takes advantage of its physical property where in-phase and out-ofphase components of the binaural reproduction process are balanced, hence the bulk of the crosstalk cancellation is achieved by its loudspeaker design principle and natural interference in the sound field. It is also shown that the advantage of OSD is further enhanced by separating in-phase and out-ofphase components through its loudspeaker design. 


\title{
Session 4aEDa
}

\section{Education in Acoustics: Acoustics in the Public School Science Classrooms}

\author{
Uwe J. Hansen, Cochair \\ Indiana State University, 64 Heritage Dr, Terre Haute, IN 47803, USA
}

Malte Kob, Cochair

RWTH Aachen, Dept. of Phoniatrics, Pedaudiology, and Communication Disorders, Pauwelsstr. 30, Aachen, 52074, Germany

\section{Invited Papers}

\section{8:00}

4aEDa1. Acoustics in the public school classroom. Uwe J Hansen (Indiana State Univwersity, 64 Heritage Dr, Terre Haute, IN 47803, USA, u-hansen@indstate.edu), Corinne Darvennes (Tennessee Tech Univ., Dept. of Mechanical Engineering, PO Box 5014, Cookeville, TN 38501, USA, cdarvennes@tntech.edu)

As mentioned in a DAGA 07 paper 1, physical science preparation in American schools often leaves something to be desired. In recent years the ASA Committee on Education in Acoustics has made an effort to participate in finding relief for that problem. Three approaches have been of some influence. 1. Teacher workshops; 2. Hands-on student sessions. 3. Secondary school curriculum input. Teacher workshops have emphasized music as a vehicle to introduce science in the elementary classroom. Hands-on student sessions have included about 20 acoustics experiments of varying degrees of sophistication for students both in high school physics classes and in elementary general science classes. Secondary curriculum input has included both, development of laboratory experiments in acoustics, and exposure to relatively low cost educational versions of computational software. Examples of teacher workshop content, a number of hands-on experiments, and some finite element calculations will be discussed. 1Musik: Zugang zur Wissenschaft in der Grundschule. Uwe J. Hansen, DAGA 2007, Stutgart pp. 171-172.

\section{8:20}

4aEDa2. Math and science partnership program in the Upper Cumberland districts of Tennessee. Corinne Darvennes (Tennessee Tech Univ., Dept. of Mechanical Engineering, PO Box 5014, Cookeville, TN 38501, USA, cdarvennes@tntech.edu)

The Mathematics and Science Partnership (MSP) program by the US Department of Education "is intended to increase the academic achievement of students in mathematics and science by enhancing the content knowledge and teaching skills of classroom teachers. Partnerships between high-need school districts and the science, technology, engineering, and mathematics (STEM) faculty in institutions of higher education are at the core of these improvement efforts." This paper will present efforts in the Upper Cumberland districts of Tennessee to introduce engineering applications to Math and Science teachers in grades 8-12. The science teachers recruited represented the disciplines of Chemistry, Physics, and Physical Science. The 3-year program consisted of summer institutes, as well as bimonthly Saturday workshops. An overview of the program will be discussed. It will include background information about the Upper Cumberland region, Tennessee content standards related to acoustics, working with teachers, some of the hands-on activities that were used during summer workshops, and the equipment that was provided to the teachers.

\section{$8: 40$}

4aEDa3. Teaching Communication Acoustics and Physiology at the girl's day. Malte Kob (RWTH Aachen, Dept. of Phoniatrics, Pedaudiology, and Communication Disorders, Pauwelsstr. 30, 52074 Aachen, Germany, mkob@ukaachen.de)

The girl's day is an annual event for young female pupils who wish to have their own experiences during a day in a potential future work place. In our department a team of 19 phoniatricians, speech therapists, phoneticians, engineers, audiometrists and assistants works on diagnosis and therapy of disorders of voice, speech, hearing and swallowing. For several years, our department has been visited by a group of 16 to 20 pupils who are guided in small groups through a sequence of stations with hands-on exercises in the field of voice and hearing acoustics. The experiments include the measurement of a voice range profile, recording and visualisation of voice signals, subjective and objective assessment of voice disorders, and listening tests. The experiences with organisation and feedback of the girl's days are reported. 


\section{Contributed Paper}

9:00

4aEDa4. Investigating musical sound as a model for the scientific process. Andrew Piacsek (Central Washington University, Department of Physics, 400 E. University Way, Ellensburg, WA 98926, USA, piacsek@cwu .edu)

In recent years, science pedagogy at all levels has embraced active student learning, in which students are engaged in the process of discovery, rather than passively receiving information. One of the benefits of active learning in a science curriculum is that students have an opportunity to emulate scientists in their approach to producing knowledge. It is desirable that students at all levels, including those in primary and secondary schools, develop an understanding of (or at least an appreciation for) the scientific pro- cess, in addition to learning science content. Presented here is a description of a musical acoustics curriculum used at Central Washington University for non-science students, with emphasis on how the structure of the curriculum and the active-learning elements contribute to accomplishing the objectives of conceptual learning, problem-solving ability, and scientific thinking. An essential feature of this curriculum is the combination of guided and open scientific investigations by teams of students. Portions of this college curriculum have been successfully adapted to the high school classroom and to audiences of children as young as ten years of age. Because musical acoustics incorporates many fundamental topics in physics and engineering, and it is appealing and relevant to students of all ages, this topic can be a significant asset to any science curriculum.

\section{Invited Paper}

\section{9:20}

4aEDa5. Acoustics Modules Developed in the IIT Research Experience for Teachers Program. Ralph T. Muehleisen (Illinois Institute of Technology, Civil, Architectural and Environmental Engineering, 3201 S. Dearborn St., Room 228, Chicago, IL 60616, USA, muehleisen@iit.edu)

From 2004-2008, the Illinois Institute of Technology (IIT) received funding from the US National Science Foundation (NSF) to institute a program to bring K-12 (primary and secondary school) teachers into University research labs for a seven week summer program where teachers work with an IIT faculty Research Mentor and develop an educational module for their students based on their mentor's research. The module's goal is to introduce students to engineering design concepts, utilizing scientific inquiry techniques, and incorporating an ethics component and a design project. Additionally, the modules must be linked to the Illinois State Board of Education Learning Standards. In 2005, a teacher developed a module for teaching high school algebra, geometry, and physics using musical acoustics, culminating with a woodwind instrument design project. In 2006, a teacher developed a module for a 3rd grade science class that explores the basics of sound and hearing, culminating in a noise control design project. This paper will present and discuss these two acoustic teaching modules. This project was supported by NSF grant EEC-0502174.

\section{9:40-10:00 Break}

\section{Contributed Papers}

\section{0:00}

4aEDa6. Inquiring activities on the acoustic phenomena at the classroom using sound card in personal computer. Young $\mathrm{H}$. Kim (Korea Science Academy, 111 Backyangkwanmoonro, Busanjin-ku, 614-822 Busan, Republic of Korea, youngkim@paran.com)

Inquiring activities on acoustic phenomena have been carried out in the classroom of a high school for highly gifted children. Instead of expensive instruments such as function generators and oscilloscopes, sound cards, installed in a personal computer, were employed for the generation and detection of sound. The stereo function of the sound card offered two sound sources, so that phenomena of interference and beats can be realized in the classroom. The record function of the sound card offered detection of sound, permitting frequency spectrum analysis of sounds from two tuning forks or the sound from a moving fork. Using sound card, a lot of acoustic phenomena can be demonstrated in the classroom. In addition, sound from Rijke tube, which is a typical theromacoustic phenomena, was analyzed by using a sound card. Popup sound of a wine bottle and breaking of wine glasses, which are related to resonace and standing waves, were also investigated. Curiosity of students was greatly increased through a series of inquiring activities with sound cards, so that they were completely absorbed in research on acoustics.

\section{0:20}

4aEDa7. Ur[ban]sonate: Echoes of twentieth-century sound art in the urban elementary classroom. Kevin N. Summers (101 Woodbine Ave. Apt. 305, Syracuse, NY 13206, USA, knsummers@ @otmail.com), Jason E. Summers (ARiA Consulting LLC, 1222 4th St. SW, Washington, DC 20024, USA, jesummers@alumni.kenyon.edu)

The sonic arts provide a variety of cross-disciplinary entry points into the traditional lower-elementary curriculum. The exploration of sound poetry provides students with a workspace to deconstruct the connection between written and spoken language; phonemes; phonics; voiced, unvoiced, stressed and unstressed letter sounds and blends. Kurt Schwitters's "Ursonate," the music and scores of John Cage, various other sound art works and Deep Listening (TM) techniques provide a bridge to the study of acoustic sound and serve to validate/contextualize students' desires to creatively explore physical phonemes such as the relationship of pitch to frequency of vibration. After creating symbolic representations of sounds, writing sound poems and creating musical instruments, students synthesize learned material by scoring and directing the performance of original sound art pieces.

\section{0:40}

4aEDa8. Acoustics in the partial deaf student school music classrooms Filiz Bal Kocyigit (Karabuk University, 232. Str. No: 5/5 Ilkbahar Mah., Cankaya, 06550 Ankara, Turkey, filizbkocyigit@yahoo.com), Kubra Sevim X. Gulec (Karabuk University Fine Arts and Design Faculty, Safranbolu, 78600 Karabuk, Turkey, kubsev@gmail.com)

Music schools need some special acoustical design for good education. If this is also for children with disabilities, the effect is more important. Research is about musical education for partially hearing disabled children. At this point architectural acoustic design acquires importance. This is not only in the shape of the room but also in using covering materials and other solutions. The question is whether architectural acoustics solutions can affect the music education of partially hearing disabled children or not. Here the first discussion is whether we need some additional components of good classrom acoustics for partially hearing disabled children in the American National Standards Institute Standard S12.60 2002 for clasroom acoustics, and the cost impact of the Standard. Childhood hearing loss is a widespread problem with significant impact, an invisible condition resulting in communication problems that can ultimately interfere with learning and social development. Included are audio files that illustrate that even a mild hearing loss can have a significant impact on a child's ability to understand the teacher. Especially children with a partial hearing loss need a more lively room to amplify the instrument sound for improved hearing capacity. 
11:00

4aEDa9. The energy flow for a spherical acoustic lens: ray vs. wave methods. Cleon E. Dean (Physics Department, Georgia Southern University, P. O. Box 8031, Statesboro, GA 30460-8031, USA, cdean $@$ GeorgiaSouthern.edu), James P. Braselton (Department of Mathematical Sciences, Georgia Southern University, P.O.B. 8093, Statesboro, GA 304608093, USA, jbraselton@GeorgiaSouthern.edu)

A simple classroom demonstration consists of a weather balloon filled with carbon dioxide, a sound source, and a microphone. Since the speed of sound is slower in carbon dioxide than in air at room temperature and pressure, the balloon acts as a positive spherical acoustic lens. The accuracy of ray methods in locating the acoustic focus versus a full blown wave solution approach is probed. This problem presents particular difficulties if the sound source lies in the near field region. The sound emitter is treated as a dipole source equivalent to a rigid oscillating sphere of small size and amplitude of motion relative to the scatterer. The energy flux around the balloon is visualized by both ray methods and by the acoustic Poynting vector field. The geometrical ray results and the acoustic Poynting vector field resulting from the wave solution are compared.

THURSDAY MORNING, 3 JULY 2008

ROOM 202/203, 11:20 TO 12:20 P.M.

\title{
Session 4aEDb
}

\section{Education in Acoustics: Take 5's}

\author{
Uwe J Hansen, Cochair \\ Indiana State University, 64 Heritage Dr, Terre Haute, IN 47803, USA
}

Malte Kob, Cochair

RWTH Aachen, Dept. of Phoniatrics, Pedaudiology, and Communication Disorders, Pauwelsstr. 30, Aachen, 52074, Germany

Thomas D. Rossing, Cochair

Stanford University, CCRMA, Department of Music, Stanford, CA 94305, 26464 Taaffe Rd, Los Altos Hills, CA 94022, USA

For a Take-Five session no abstract is required. We invite you to bring your favorite acoustics teaching ideas. Choose from the following: short demonstrations, teaching devices, or videos. The intent is to share teaching ideas with your colleagues. If you can, bring a brief, descriptive handout with enough copies for distribution. Spontaneous inspirations are also welcome. You sign up at the door for a five-minute slot before the session starts. If you have more than one demo, sign up for non-consecutive slots. 


\title{
Session 4aMU
}

\section{Musical Acoustics: Virtual Musical Instruments I}

\author{
Julius O. Smith, Cochair \\ Stanford Univ., Center for Computer Research in Music and Acoustics (CCRMA), Dept. of Music, Stanford, CA 94305-8180, \\ USA \\ Antoine Chaigne, Cochair \\ ENSTA, Chemin de la Hunière, Palaiseau, 91761, France
}

\section{Invited Papers}

\section{8:00}

4aMU1. Interacting with virtual musical instruments at the junction nodes. Cumhur Erkut (Helsinki University of Technology (TKK), Lab. Acoustics and Audio Signal Processing, P.O. Box 3000, FI-02015 TKK Espoo, Finland, Cumhur.Erkut@tkk.fi), Antti Jylhä (Helsinki University of Technology (TKK), Lab. Acoustics and Audio Signal Processing, P.O. Box 3000, FI-02015 TKK Espoo, Finland, antti.jylha@tkk.fi), Matti Karjalainen (Helsinki University of Technology (TKK), Lab. Acoustics and Audio Signal Processing, P.O. Box 3000, FI-02015 TKK Espoo, Finland, matti.karjalainen@tkk.fi)

Sound synthesis by block-based physical modeling of musical instruments separates the tasks of component modeling and managing their interactions. The components are the exciters or the resonators, and their interactions are managed by explicit interaction blocks, which are obtained from the physical continuity and energy conservation rules. Well-known examples of the interactors include the wave- digital adaptors and the digital waveguide scattering junctions. When the virtual instruments need to be interfaced to the outside environment with sensors and actuators for bidirectional interaction, it is advantageous to reformulate the interactors to accept and provide signal inputs and outputs, respectively. In this contribution, we refer to these elements as nodes, introduce different types of nodes, and discuss their interconnection.

\section{$8: 20$}

4aMU2. Block based physical modeling for virtual musical instruments. Rudolf Rabenstein (University Erlangen-Nürnberg, Cauerstr.7, D-91058 Erlangen, Germany, rabe@LNT.de)

A variety of methods for physical modeling sound synthesis has been developed so far, mostly for single sound objects like strings, plates, etc. However, complex virtual musical instruments require not only advanced models but also methods for combining one or more sound objects with excitation mechanisms and resonating structures. This presentation shows how to derive modeling blocks from basic physical laws and how to connect them in a physically meaningful way. The first step is to establish a physical model of a dynamical structure in terms of potential and flow variables, like force and velocity. It is important to observe also the boundary conditions because firstly they shape the spectrum of the vibrating structure and thus the timbre of the sound and secondly they determine the exchange of energy with the environment. The second step is the discretization of the physical model. This procedure is shown for the functional transformation method, which delivers discrete-time models with direct access to the parameters of the physical model and which reproduces the original sound spectrum. In the last step, the resulting modeling blocks are connected by scattering elements which reflect energy back into the model or transmit it to neighboring blocks.

\section{$8: 40$}

4aMU3. Cymbal Synthesis. Stefan Bilbao (University of Edinburgh, Room 7306B, JCMB, King's Bldgs., Mayfield Rd., EH9 3JZ Edinburgh, UK, sbilbao@staffmail.ed.ac.uk)

Time domain sound synthesis based on a physical model of the cymbal presnts special problems, due to the need for a strongly nonlinear model of shell vibration. When standard numerical methods such as finite difference schemes are employed, various computational issues arise; among these are numerical stability, a proper treatment of numerical boundary conditions, which are nontrivial at the free edge and center of the the cymbal, and the extra concern of working in polar coordinates. Coupling with mallet and bow models, possibilities for increased computational efficiency using spectral methods, and general strengths and weaknesses of difference methods in this context will be discussed.

\section{9:00}

4aMU4. Passive admittance synthesis for sound synthesis applications. Balázs Bank (University of Verona, Ca' Vignal 2, strada le Grazie 15, 37134 Verona, Italy, bank@mit.bme.hu), Matti Karjalainen (Helsinki University of Technology (TKK), Lab. Acoustics and Audio Signal Processing, P.O. Box 3000, FI-02015 TKK Espoo, Finland, matti.karjalainen@tkk.fi)

In physics-based sound synthesis, it is in general possible to incorporate a mechanical or acoustical admittance/impedance in the form of a digital filter. Examples include modeling of the termination of a string or a tube. However, when digital filters are fitted to measured admittance or impedance data, care has to be taken that the resulting filter corresponds to a passive mechanical or acoustical 
system, otherwise the stability of the instrument model is at risk. This paper presents a simple method for designing inherently passive admittance or impedance filters. The admittance/impedance is composed as a linear combination of positive real (PR) functions, and the weights are determined by a constrained least squares optimization. The resulting filter is a parallel set of second-order sections. For wave-based modeling, such as digital waveguides (DWGs) or wave digital filters (WDFs), the admittance/impedance is converted to a reflectance filter. The parallel filter structure is retained during conversion. As an example, a guitar model based on DWG approach is presented, using mechanical admittance measurements of a guitar bridge behavior. The model is implemented as an efficient real-time sound synthesis algorithm.

\section{9:20}

4aMU5. Modal parameter estimation for shape-changing geometric objects. Cynthia Maxwell (Center for New Media and Audio Technologies, 1750 Arch Street, Berkeley, CA 94720, USA, cynthia@code404.com)

As a novel advancement in interactive sound synthesis, we would like to change the shape of a finite element model of an instrument and hear how the sound changes in real-time. Traditional modal synthesis methods require computing a new eigendecomposition for each geometric change -- a costly computation using today's hardware. However, by using the modes computed for one geometry to estimate modal frequencies for other nearby geometries, we can instantly hear the effects of changing the instrument shape on the sound produced. In this talk, we describe the process of estimating resonant frequencies of an instrument by combining information about the modes of similar instruments. We also propose a method for transferring the modal information from one finite element mesh to another. This method is used in situations where severe modifications to the object's geometry distort the finite element mesh and require an entirely new mesh to be created. We also describe the balance between computational speed and numerical accuracy of the computed resonances.

\section{9:40}

4aMU6. Applications of passivity theory to the active control of acoustic musical instruments. Edgar Berdahl (Stanford Univ., Center for Computer Research in Music and Acoustics (CCRMA), Dept. of Music, Stanford, CA 94305-8180, USA, eberdahl@ccrma.stanford.edu), Guenter Niemeyer (Stanford Univ., Mech. Eng., Bldg. 530, Stanford, CA 94305, USA, gunter.niemeyer@stanford.edu), Julius O. Smith (Stanford Univ., Center for Computer Research in Music and Acoustics (CCRMA), Dept. of Music, Stanford, CA 94305-8180, USA, jos@ccrma.stanford.edu)

The dynamic behavior of any acoustic musical instrument can be modified by closing a feedback loop around even a single sensor and actuator. The ultimate goal is to make the acoustics of the instrument programmable by way of a digital feedback controller, while the instrument retains its tangible form. In this talk, we describe a class of controllers that are applicable to passive acoustic musical instruments, and we present sound examples from laboratory experiments on a vibrating string. First, we briefly introduce positive real functions. Next, we design positive real controllers allowing the quality factor and resonant frequency of instrument modes to be individually controlled. Because positive real controllers are passive, they are stable if the instrument is passive. This means that neither a full instrument model nor complete state measurements are required. Finally, we describe a class of simple passive nonlinear controllers that can emulate various kinds of friction, stiffening and softening springs, etc. Passivity of these controllers follows from the local passivity of the controller components. Controller parameters may often be tweaked so that the controllers are no longer passive but still perform useful functions, such as bowing emulation.

10:00-10:20 Break

\section{Contributed Papers}

10:20

4aMU7. Vibrating-String Coupling Estimation from Recorded Tones Nelson Lee (Stanford Univ., Center for Computer Research in Music and Acoustics (CCRMA), Dept. of Music, Stanford, CA 94305-8180, USA, nalee@stanford.edu), Julius O. Smith (Stanford Univ., Center for Computer Research in Music and Acoustics (CCRMA), Dept. of Music, Stanford, CA 94305-8180, USA, jos@ccrma.stanford.edu)

Coupling of vibrational polarizations in a single string, for an instrument such as the acoustic guitar, produces psychoacoustically significant effects such as beating and two-stage decay (Weinreich, JASA v62 n6). Previous considerations of string coupling phenomena appear not to have addressed the practical problem of calibrating computational models based on recorded tones. In this work, we take a data-driven approach using measured data from a vibrating string from an acoustic guitar, the motion of the string in two orthogonal planes, and formulate a regularized least-squares problem for computing the coupling between the measurements. Such a formulation ensures that the resulting coupling is physically admissible, in that the resulting coupling factors do not generate energy, and are easily found as the problem is convex. Well-studied algorithms for solving convex problems, such as interior-point and gradient descent methods can be used and are widely available in the form of open-source libraries.

\section{0:40}

4aMU8. Sound synthesis of circular plates by finite differences. Kevin Arcas (ENSTA, Chemin de la Hunière, 91761 Palaiseau, France, arcas@ensta.fr), Antoine Chaigne (ENSTA, Chemin de la Hunière, 91761 Palaiseau, France, antoine.chaigne@ensta.fr), Stefan Bilbao (University of Edinburgh, Room 7306B, JCMB, King's Bldgs., Mayfield Rd., EH9 3JZ Edinburgh, UK, sbilbao@staffmail.ed.ac.uk)

This paper shows a method for simulating linear flexural vibrations of circular plates by finite differences (FD) for the purpose of sound synthesis. The vibrations are assumed to follow the Kirchhoff-Love model. In order to solve the continuous problem numerically, the equations are approximated in space and time by FD methods. Two schemes are presented and compared; depending on the coordinate system used for the grid, rectangular and polar, respectively. Cartesian FD are not easily adaptable to circular boundary conditions and generic conservative boundary conditions cannot be found. On the contrary, polar FD allow to find well-adapted conservative boundary conditions. With a polar grid, the distance between consecutive gridpoints decreases from the edge to the center. As a consequence the stability of the algorithm depends on the minimum radius of the grid, where this distance is the smallest. Because of this highly restrictive stability condition, numerical dispersion is high and the high-frequency content of the spectrum is badly reproduced. To avoid this problem an implicit polar FD 
scheme has been developed which yields simulations with acceptable numerical dispersion. The accuracy of the algorithm is estimated by computing the ratio between numerical and analytic eigenfrequencies in a simple case.

\section{1:00}

4aMU9. Digital algoritm for sound synthesis: Realism and complexity for creativity. Joël Bensoam (IRCAM, 1, place Igor Stravinsky, 75004 Paris, France, bensoam@ircam.fr)

Modalys, a sound synthesis software developed at Ircam for research and musical applications, make it possible to build virtual instruments based on physical models in order to obtain the broadest range of expressive variations in the instrument in response to intuitive controls. An instrument, as a complex structure, is described by the mechanical/acoustical interactions of its components (strings, tubes, soundboard, 3D FEM objects...). New research has recently been carried out in two directions: On one hand, a generic digital algorithm able to treat a large class of non linear interaction models (lip-reed, contact and friction, aero-acoustics jets, etc) was built. Based on a Gauss-Siedel strategy, this algorithm is used indifferently regardless of the modeled structure's complexity and guaranties convergence and robustness of the whole sound synthesis. On the other hand, efforts are made to infer from measurements the physical parameters needed for the sound synthesis (geometric or gesture parameters for example). Due to the lack of a complete analytical formulation (digital algorithm), derivative based methods to solve inverse problem (Newton, gradient, etc) are not allowed. Evolution Strategy (ES), especially for multiobjective optimization, are then investigated.

\section{$11: 20$}

4aMU10. Application of Volterra series to simulate dynamics of a Reissner beam. David Roze (IRCAM, 1, place Igor Stravinsky, 75004 Paris, France, david.roze@ircam.fr), Thomas Hélie (IRCAM, 1, place Igor Stravinsky, 75004 Paris, France, thomas.helie@ircam.fr), Joël Bensoam (IRCAM, 1, place Igor Stravinsky, 75004 Paris, France, bensoam @ircam.fr)

Sound synthesis of strings needs the use of nonlinear models to provide realistic sounds according to the amplitude and the method of excitation (pluck, bow...). In order to include coupling between the different degrees of freedom, the Reissner beam model will be used to simulate nonlinear dynamics of a string. Expression of equilibrium of Reissner beams using Lie groups and algebra allows us to write an exact, simple formulation including nonlinearities due to large strains. This work aims to apply Volterra series method to this formulation in order to perform simulation of a Reissner beam at a given order of nonlinearity. Volterra series requires a rewriting of the model with well-defined boundary and initial conditions and interconnection laws of the series adapted to the variables (vector and matrix). Once the linear part isolated and studied (calculation of the Green function, and therefore of the first kernel), nonlinear terms must be organized to define a recurent relation and solve the kernel equation. Finally, it can be possible to identify a structure of simulation using instantaneous sums and products of outputs of linear filters.

\section{1:40}

4aMU11. Singing Integrals or wind instruments modeling using Boundary Integral Equations. Umberto Iemma (University Roma Tre, via vasca navale 79, 00146 Rome, Italy, u.iemma@uniroma3.it)

The paper deals with the modeling of woodwind musical instruments using a Boundary Integral Equation (BIE) formulation. Specifically, the BIE is used to model the acoustic response of the instrument bore, and is numerically solved by means of a Boundary Element Method (BEM). The latter takes advantage of an analytical solution for the calculation of the BEM coefficients, thus allowing for the representation of the problem as an opendomain problem. This peculiarity avoids the use of approximated boundary conditions at the open end of the pipe. The formulation is used to: evaluate the input impedance of the resonating air column; identify the frequency dependent transfer function relating the inflow of the instrument with the signal measured at a specified location (the Reed-To-Microphone transfer function); extend the analysis to a realistic performing environment to obtain the "Reed-To-Listener" transfer function. Standard techniques are used to take into account the interaction of the bore with the nonlinear exciting device. Numerical results are obtained for single-reed instruments in terms of tuning properties, convergence of solution, directivity patterns, and simple synthesized sounds. Issues related to the possibility of real-time simulations are briefly addressed. Specifically, the identification of digital filters from the calculated transfer functions is investigated, and some preliminary numerical result included.

\section{2:00}

4aMU12. An approach and technique for acoustic modelling of contact Matthias Rath (Technische Universität Berlin, Deutsche Telekom Laboratories, Quality and Usability Lab, Ernst-Reuter-Platz 7, 10587 Berlin, Germany, matthias.rath@telekom.de)

Vibration of solid objects can often be modelled by modal description, which exists when certain operators in a differential equation are linear Discrete-time algorithms can represent the behaviour of "modal objects" without artefacts, exact (with precision of finite computer architecture), if based on analytical solution, not numeric approximation. In particular, the energy associated with the state of the system is then preserved and the algorithm stable. A scenario of two objects interacting only during contact, however, is non-linear: a non-zero linear function cannot be zero in a halfspace of the system's state-space. Existing computational models are based on approximate, numeric solution and cannot guaranty stability in situations with longer contact phases, such as rolling or sliding. General principle of our approach is: although any possible term for the interaction force must be non-linear, it may be piecewise-linear, and during each linear phase the whole system (of interacting solids) may be simulated by modal description. The question of "switching" between the different phases is here critical but may be solved in a way that assures control over the system's energy. Our implemented new contact model is stable in any condition and overcomes artefacts found with previous techniques. 


\title{
Session 4aNSa
}

\section{Noise and EURONOISE: EU Projects for Aircraft Noise Reduction II}

\author{
Dominique Collin, Chair \\ Snecma - Safran Group, Centre de Villaroche, Direction R\&T / UC, Moissy Cramayel, 77550, France
}

\section{Invited Papers}

\section{8:00}

4aNSa1. European Aircraft Noise Research Network (X3-Noise). Dominique Collin (Snecma - Safran Group, Centre de Villaroche, Direction R\&T / UC, 77550 Moissy Cramayel, France, dominique.collin@snecma.fr)

The X3-NOISE Coordination Action, through its network structure and comprehensive workplan involving expert groups, scientific workshops, stakeholder seminars and a common information system, addresses the aircraft noise challenges set by the ACARE 2020 Vision. To this end, X3-Noise undertakes the elaboration and coordination of research strategies, the dissemination of results and the integration of European research activities in the field of air transport related to noise. Over 4 years, the project involves 32 partners from 20 countries (FR, UK, ES, NL, BE, DE, SE, IT, PL, HE, HU, CZ, LT, IE, PT, RO, CH, UA, EG, BR), combining the complementary skills and expertise of industry partners, SMEs, university and research establishments to cover the whole field of interest. The international co-operation aspects of the research agenda to be developed through the project activity are further reinforced by the participation of 3 partners from Ukraine, Egypt and Brazil acting as Focal Points at Regional level.

\section{$8: 20$}

4aNSa2. The EU FP6 research project PROBAND - Objectives and first results. Lars Enghardt (DLR - German Aerospace Center, Mueller-Breslau-Str. 8, 10623 Berlin, Germany, lars.enghardt@dlr.de)

Fan broadband noise is a major aircraft noise challenge now and in the future will be even more important. Novel low-noise engine architectures, such as ultra-high-bypass-ratio engines and lower-speed fans, can help address jet noise and fan tone noise, but previous EC-funded programmes have shown they are unlikely to reduce significantly fan broadband noise without improved understanding of the source mechanisms. PROBAND will accomplish a major technical leap in providing industry with an improved understanding of the broadband noise source mechanisms, with validated broadband noise prediction methods, and with low fan broadband noise concepts. PROBAND will exploit the noise technology and methodology acquired in EC-funded projects and national programmes, to develop methods to allow the design of a fan system that will generate sufficiently low broadband noise to meet the EU noise level targets. This will be achieved by: 1. Developing a better understanding of broadband noise generation mechanisms using advanced experimental and computational techniques. 2. Developing and validating improved prediction methods using conventional computational fluid dynamics, and integrating them into industrial codes. 3. Exploring new prediction strategies using advanced computational techniques 4 . Developing low broadband fan noise concepts.

4aNSa3. SILENCE(R): A major step towards aircraft noise reduction. Eugène J. Kors (Snecma, Etablissement de Villaroche Sud - UE, Rond-point René Ravaud - Réau, 77550 Moissy-Cramayel cedex, France, eugene.kors@snecma.fr)

From 2001 up to 2007, SILENCE(R) has focused on the development of aircraft noise reduction technologies. As an European Union project coordinated by Snecma, SILENCE(R) brought together some 50 companies (including Airbus, Rolls-Royce, MTU Aero Engines and Snecma), research centers and universities. The overall budget was 112 million euros. Combined with innovative low-noise operational procedures studied at the same time, $\operatorname{SILENCE}(\mathrm{R})$ has achieved an impressive $5 \mathrm{~dB}$ noise reduction. This meets the medium-term objective of the European Commission's R\&D Framework Programs, and marks a significant advance towards ACARE's research goal of a $10 \mathrm{~dB}$ reduction in aircraft noise by 2020. SILENCE(R) carried out successful tests of more than 35 prototypes to check 10 noise reduction technology concepts. These included several advanced low noise fan rotors, as well as components for a complete low-noise nacelle (negatively scarfed intake, "squid" nozzle fitted with high frequency liner), flight tested on an Airbus A320. Flight tests were also carried out on an Airbus A340 with landing gear fitted with aerodynamic fairings. 


\section{Contributed Papers}

9:00

4aNSa4. TIMPAN - Technologies to IMProve Aiframe Noise. Stephane Perrin Decroux (Airbus France S.A.S, 316, route de Bayonne BP M0112/4, 31060 Toulouse, France, stephane.perrin-decroux@airbus.com)

TIMPAN is a 3-year Strategic Targeted Research Project of the 6th European Framework Programme, Priority "Aeronautics and Space", launched in 2006. It addresses the community noise reduction objective for commercial aircraft by focusing on main airframe noise sources: landing gear and high-lift devices - responsible, on recent aircraft, for about half of the total noise in approach situation. TIMPAN addresses both sources, within 3 main tasks: 1) Landing gear activity: investigation of both innovative low noise technologies on bluff body structures and the improvement of advanced low noise main gear design, as outcome from previous EC Technology Platform SILENCE(R). 2) High lift device activity: study of both innovative concepts based on flow control technologies and mid-term noise reduction solutions as absorptive wing leading edge treatments and high-lift settings optimization through computational aero-acoustic methods. 3) Technology evaluation: aims to prepare the exploitation phase by evaluating the noise reduction technologies under consideration in terms of noise benefit, integration, cost and performance. TIMPAN brings together 14 actors from the European aeronautics industry including aircraft manufacturers (Airbus and Dassault Aviation), landing gear manufacturers (Messier-Dowty and Messier Bugatti), key research institutes (DLR, ONERA, NLR, EADS-IW), universities (University of Southampton, Technical University of Braunschweig) and SMEs (ATECA, Free Field Technologies).
9:20

4aNSa5. Passive and active designs for noise and vibrations reduction in aircraft cabins. Vincent Marant (Acusttel - Acústica y Telecomunicaciones SL, Pol. Ind. Benieto, C/ Transport $n^{\circ}$ 12, 46702 Gandía (Valencia), Spain, vmarant@acusttel.com), Antonio Reig Fabado (Universidad Politécnica de Valencia, Camino de Vera s/n, 46022 Valencia, Spain, areig @ fis.upv.es), Juan Luís Aguilera De Maya (Acusttel - Acústica y Telecomunicaciones SL, Pol. Ind. Benieto, C/ Transport n ${ }^{\circ}$ 12, 46702 Gandía (Valencia), Spain, jlaguilera@acusttel.com), José Christian Donayre Ramírez (Acusttel - Acústica y Telecomunicaciones SL, Pol. Ind. Benieto, C/ Transport $n^{\circ}$ 12, 46702 Gandía (Valencia), Spain, cdonayre@acusttel .com)

Within the sixth European Commission framework programme, the main objective of the SEAT project initiated in September, 2006, consists of the development of a radically new concept, where the aircraft passenger comfort is considered at the highest level. Smart reactive seats and an interior environment able to detect on real time physiological and psychological changes in the passenger conditions will be developed. These data will be analysed and the appropriate parameters, like noise and vibration levels, temperature or air ventilation, will be adapted. Moreover, each passenger will be able to create his own configuration, with his personal entertainment and work characteristics. The project is focussed on the questions previous to the integration of the system, that is above all the creation of a more healthy and comfortable travel environment by means of noise and vibration reduction, as well as specific climatic controls. In this paper, the first passive and active designs under development are presented.

\section{Invited Paper}

\section{9:40}

4aNSa6. Reducing aircraft noise during approach and departure by efficient operations. Robert J. De Muynck (National Aerospace Laboratory NLR, Anthony Fokkerweg 2, 1059CM Amsterdam, Netherlands, demuynck@nlr.nl)

Reducing the noise from aircraft around airports is a serious challenge. Apart from making the noise source, the aircraft, more silent, by advanced engines and aerodynamics, additional noise reduction can be achieved by moving the noise source away, as well as by a more silent operation of the aircraft. One of these innovative approach procedures is the Continuous Descent Approach. The principle is that aircraft approaching an airport follow a continuous descent profile at low thrust setting instead of making gradual altitude steps. The higher altitude and the lower thrust of the aircraft allow decreasing significantly noise exposure around the airport. First results show considerable noise reduction from $3 \mathrm{~dB}$ to $8 \mathrm{~dB}$ compared to conventional practices. Additionally, this procedure allows reducing fuel consumption and emissions. To make CDA procedures operationally feasible however, efforts are needed to develop $* \mathrm{CDA}$ operating procedures so that they can be flown in busy traffic *improved onboard systems to fly the CDA *accurate planning and sequencing tools for air traffic controllers *better interaction and interoperability between aircraft and air traffic control systems The presentation will discuss progress made in EU projects such as Sourdine II, OPTIMAL and ERAT. 


\title{
Session 4aNSb
}

\section{Noise, ASA Committee on Standards, and EURONOISE: Measurement of Occupational Noise Exposure I}

\author{
William J. Murphy, Cochair \\ National Institute for Occupational Safety and Health, 4676 Columbia Parkway, Mailstop C-27, Cincinnati, OH 45226-1998, \\ USA
}

Beat W. Hohmann, Cochair

Suva, Physics Section, Roesslimattstrasse 39, P.O. Box 4358, Luzern, CH-6002, Switzerland

\section{Invited Paper}

\section{8:00}

\begin{abstract}
4aNSb1. Measurement strategies according to the new ISO/DIS 9612. Jürgen Maue (BGIA - Institut for Occupational Safety and Health, Alte Heerstraße 111, D-53757 Sankt Augustin, Germany, juergen.maue@dguv.de)

The European Directive 2003/10/EC on noise protection at the workplace has given the impact for the revision of the ISO 9612 describing the measurement and assessment of occupational noise. The ISO/TC 43 "Acoustics" decided that the revision should only contain requirements for the determination of the noise exposure for the purpose of assessing potential hearing damage. Meanwhile a new Draft ISO/DIS 9612 has been presented, which gives a detailed description of three alternative measurement strategies for the determination of the noise exposure level. Moreover the standard provides a method for estimating the uncertainty of the results. The measuring strategies, called "task based measurements", "job based measurements" and "full day measurements" are illustrated and compared to each other. The choice of the appropriate strategy is explained, depending on the complexity of the work situation and the movements of the worker doing his job. Based on practical experience some comments are given, which may help to reduce time and effort of measurements. The evaluation of measurement uncertainties is explained in a few words.
\end{abstract}

\section{Contributed Paper}

\section{8:20}

4aNSb2. Effective protection of the sense of hearing is prevented by ISO1999 noise standard. Gerald Fleischer (Liebig University School of Medicine, Aulweg 123, 35392 Giessen, Germany, gerald.fleischer@gmx .net)

To determine auditory performance in a simple way, pure-tone audiometry is being used. While health-related diagnostics is generally becoming more and more refined, ISO1999 is demanding rather rudimentary procedures. Both, frequency range and number of test frequencies are re- duced, making it impossible to use modern and effective analytical tools such as pattern recognition. There is a clear relation between pressure-timehistory of impulses and the details of the resulting auditory damage. But this relation can only be recognized if the restrictions of this standard are being ignored. Typical examples of audiograms from a large data base will be presented, showing simultaneously the data according to ISO1999 and in more modern ways. It will also be demonstrated how useful the tool of pattern recognition can be, for analysis of damages, as well as for preventive measures.

\section{Invited Paper}

4aNSb3. Comparison of impulse noise damage risk criteria using the Albuquerque blast overpressure walkup study data. William J. Murphy (National Institute for Occupational Safety and Health, 4676 Columbia Parkway, Mailstop C-27, Cincinnati, OH 45226-1998, USA, wjm4@cdc.gov), Amir Khan (National Institute for Occupational Safety and Health, 4676 Columbia Parkway, Mailstop C-27, Cincinnati, OH 45226-1998, USA, amk1@cdc.gov), Peter B. Shaw (National Institute for Occupational Safety and Health, 4676 Columbia Parkway, Mailstop C-27, Cincinnati, OH 45226-1998, USA, pbs3@cdc.gov)

The 1968 CHABA recommendations to limit impulsive noise exposure to levels below $140 \mathrm{~dB}$ SPL form the basis of current United States occupational and military standards. The U.S. military standard, MIL-STD-1474D, estimates the number of allowable shots to which a person may be exposed using peak level, B-duration, for varying levels of hearing protection usage. The European Union upper limit peak exposure action level is $137 \mathrm{~dB}$ C-weighted for the unprotected ear and $140 \mathrm{~dB}$ C-weighted for the protected ear. The U.S. Army blast overpressure studies in the early 1990's investigated the effects on the hearing of soldier subjects of simulated weapon blasts with varied levels and A-durations. The hearing thresholds of the subjects were tested before and after exposure to blasts. Exposures ranged from 6 shots to 100 shots per day and levels from 173 to $195 \mathrm{~dB}$ peak SPL. As judged by information criteria (AIC, BIC), the $L_{A e q 8}$ index with unprotected data yielded the best fit to logistic models; all the indices produced a better fit with unprotected data than with protected data. Other metrics including the MIL-STD-1474D and the Auditory Hazard Assessment Algorithm for Human were evaluated and will be presented in this paper. 


\section{Contributed Papers}

9:00

4aNSb4. Reconstruction of acoustic field horizontal layer in diffusive ambience. Arunas Pozera (Kaunas University of Technology/Technological Systems Diagnostics Institute, Kestucio st. 27, 3004-LT Kaunas, Lithuania, arunas.pozera@stud.ktu.1t), Vitalijus Volkovas (Kaunas University of Technology, K.Donelaicio str. 73, LT-44029 Kaunas, Lithuania, tsdi@ktu.lt)

This study introduces a method how to minimize the number of acoustic measurements in the diffusive ambience in order to reconstruct the horizontal layer of primary acoustic field. This reconstruction means determination of the level of the sound pressure at any point of surface, e.g. worker's occupational place, where there are a lot of sound sources. A mathematical modeling, based on regularity of sound spread, is used in the study. The elements of active experiment are applied in the research, the adequacy of mathematical model is evaluated by Fisher criterion, and the mathematical model of horizontal layer of the acoustic field is based on the polynomial of the regressive equation. The results are compared to the measurements received in the real experiment. This study also pays great attention to local and the EU legal regulations about the noise standards at the worker's occupational place and the measures to be taken to lower the noise level. The device of sound measurement INVESTIGATOR type 2260 and MAPLE 11 mathematics software tool have been used in the research.

9:20

4aNSb5. An experimental investigation of the measurement uncertainties in the assessment of exposure to noise in a working environment according to ISO 9612. Marco Nabuco(Inmetro / CNPq, Av N. S. das Graças, 50, Xerém, Duque de Caxias, 25250-020 RJ, Brazil, nabuco@inmetro.gov.br), Ana Claudia Fiorini (Catholic University of São Paulo - Audiology Dept., Rua Ministro Godoy, 969 - Perdizes, 05014-001 São Paulo, Brazil, acfiorini@pucsp.br), Gilberto Fuchs (GROM Acustica e Automação, Rua Indiana, 343 - Apt $^{\circ} 11$ - Broklin, 04562-000 São Paulo, Brazil, gilberto@grom.com.br)

The Brazilian regulation for workplace noise evaluation requires a comparison between the measured noise levels and the noise limits listed on a table. The table establishes the maximum work hours per day for each measured sound level. As in some other countries, the Brazilian table shows limit values with a resolution of $1 \mathrm{~dB}(\mathrm{~A})$ and considers a $5 \mathrm{~dB}(\mathrm{~A})$ exchange rate. The measured uncertainty is unknown. The use of type 2 equipment for instance can mean, theoretically, a uncertainty of $3 \mathrm{~dB}(\mathrm{~A})$. In Brazil it is not possible to estimate the impact of the measurement uncertainty on the costs of compensations paid due to noise exposure. It is also very difficult to assess the number of workers that are receiving unfair compensations or are not receiving any due to inaccurate noise exposition measurements. However it is possible to assure that the amount of money can reach incredible numbers in our country. This paper presents an experimental study of the uncertainty calculated on the ISO 9612 basis for different sound signals, in different workplaces, emitted by different noise sources. The main purpose is to show how important it can be to estimate the uncertainty for each measurement.

\section{Invited Paper}

\section{9:40}

4aNSb6. Simple evaluation of occupational noise exposure without measurements. Beat W. Hohmann (Suva, Physics Section, Roesslimattstrasse 39, P.O. Box 4358, CH-6002 Luzern, Switzerland, beat.hohmann@suva.ch)

In almost any country, employers must assess the noise exposure of the employees. But for small and medium-sized enterprises (SMEs), individual noise measurements are hardly feasible. Therefore, since almost 30 years, Suva publishes noise level tables, which list typical noise levels of many tools and machines. These 66 noise level tables cover almost any branch of the industry. Moreover, they include shooting noise, music, and so on. However, most SMEs were not able to calculate their workers' long-term noise exposure. Therefore, based on Suva's extensive database, typical noise exposure levels for occupations and activities were calculated. The new type of noise level tables includes now the typical noise exposures for the different occupations. Moreover, since 2007, the noise level tables also indicate the measures to be taken. Therefore, SMEs do not have to put their efforts into the determination of noise exposure but can start immediately to protect the workers against noise-induced hearing loss. The list of the noise level tables available (in French, German and Italian language) and the noise level tables themselves may be found at: www.suva.ch/waswo/86005

\section{Contributed Papers}

\section{0:00}

4aNSb7. A comparison of two active-speaker-detection methods suitable for usage in noise dosimeter measurements. Fredric Lindstrom (Dept. of Environ. Medicine, The Sahlgrenska Acad. of Gothenburg Univ., Box 414, 40530 Gothenburg, Sweden, fredric.lindstrom @amm.gu.se), Keni Ren (Umea University, Dept. of Applied Physics and Electronics, Teknikhuset, Box 414, 90187 Umea, Sweden, renkeni@gmail.com), Kerstin Persson Waye (Dept. of Environ. Medicine, The Sahlgrenska Acad. of Gothenburg Univ., Box 414, 40530 Gothenburg, Sweden, kerstin.persson-waye@amm.gu.se), Haibo Li (Umea University, Dept. of Applied Physics and Electronics, Teknikhuset, Box 414, 90187 Umea, Sweden, haibo.li@tfe.umu.se)

Measuring noise exposure in a working environment is often done by using standard noise dosimeters. This method is suitable for the evaluation of many working environments. However, in some situations the worker uses his/her voice a large amount during the day, e.g. teachers in a preschool environment. Thus, in these situations regular dosimeter measurements will not correspond to the actual noise exposure. In order to provide correct measurements, methods that can detect when the workers own voice is active are required. This paper presents a study of two such methods originating from voice research; the binaural and the throat microphone methods. The meth- ods are compared using a receiver operating characteristics based method, where the performance is assessed by the Probability-of-failure measure, i.e. the percentage of own voice that the method failures to detect correctly. The evaluation is performed in a lab environment as well as in real field conditions in a preschool. The results of the study show that both methods can be successful in a controlled low noise $(<45 \mathrm{dBA})$ environment (Probabilityof-failure $<0.1 \%$ for both methods), while in the preschool environment, the throat microphone method (Probability-of-failure $<0.1 \%$ ) is more suitable than the binaural method (Probability-of-failure $>6 \%$ ).

\section{0:20-10:40 Break}

\section{0:40}

4aNSb8. Recovery of distortion product otoacoustic emissions (DPOAE) after impulse vs. continuous equal-energy exposures. Miguel Angel Aranda De Toro (Acoustics, Aalborg University, Fredrik Bajers Vej 7 B5, 9220 Aalborg Ø, Denmark, maat@es.aau.dk), Rodrigo Ordoñez (Acoustics, Aalborg University, Fredrik Bajers Vej 7 B5, 9220 Aalborg Ø, Denmark, rop@es.aau.dk), Karen Reuter (Acoustics, Aalborg University, Fredrik Bajers Vej 7 B5, 9220 Aalborg $\varnothing$, Denmark, 
kr@es.aau.dk),Dorte Hammershøi(Acoustics, Aalborg University, Fredrik Bajers Vej 7 B5, 9220 Aalborg Ø, Denmark, dh@es.aau.dk)

The correct assessment of impulse noise from occupational environments for hearing-conservation purposes is still a controversial issue. Currently, no universally accepted standard defines impulse noise accurately nor does a standard method exist to measure impulses. Moreover, current impulse-damage risk-criteria suffer from lack of empirical data needed to quantify impulse noise exposures and assess potential damage. In this experiment human subjects are exposed to binaural recordings of noises from industrial environments. Stimuli consist of impulse noise, continuous noise, and combinations of impulse and continuous noise. Noise exposures are normalized to have the same energy $\left(\mathrm{L}_{\text {Aeq,8h }}=80 \mathrm{~dB}\right)$. The effects in the hearing of the subjects are monitored by measuring the recovery of the distortion product otoacoustic emissions (DPOAE) with high-time resolution. The results can be used to investigate the validity of current assessment methods and descriptors of the temporal characteristics of sound exposures and their relation to the temporal effects produced on the human hearing as well as investigating selected issues that may lead to possible improvements or alternative measuring methods. [Work supported by the Danish Research Council for Technology and Production.]

\section{Invited Paper}

\section{1:00}

4aNSb9. "Real world" noise exposure beneath hearing protectors : a scattered international practise. Pierre Canetto (INRS Institut National de Recherche et de Sécurité, Avenue de Bourgogne BP No 27, 54501 Vandoeuvre, France, pierre.canetto@inrs.fr), Nicolas Trompette (INRS - Institut National de Recherche et de Sécurité, Avenue de Bourgogne BP No 27, 54501 Vandoeuvre, France, nicolas.trompette@inrs.fr)

Assessing occupational noise exposure "beneath" Hearing Protection Devices (HPD) is a topical subject. Standardized methods allow to calculate the exposure by using the HPD attenuation. The HPD attenuations declared by manufacturers are much higher from the "real-world" ones. To reduce this difference, some "compensation" methods are proposed. The methods and their rules vary a lot from one country to another. The "derating" rmethod reduces the declared values from a certain amount, according to the HPD kind. "Doublelabelling" method uses attenuation values measured in laboratory with untrained subjects. "Statistical range enlargement" widens the statistical confidence of the laboratory-test results. All these methods propose a global solution: the reasons due to human behaviour (mainly bad HPD wearing), products quality, and the difference between laboratory and industrial situations are mixed. This "blind" approach could be considered as endorsing the lack of workers' training, and could impede the progress in HPD developments. But a "short-term" answer to the problem is needed. It should allow to build a more relevant medium-term answer, which could be worked out possibly on an international scale.

\section{Contributed Papers}

$11: 20$

4aNSb10. A comparison of earmuff protection measured in real-world and laboratory conditions. Emil Kozlowski (Centr. Inst. for Labour Prot. - Natl. Res. Inst., Czerniakowska 16, 00-701 Warsaw, Poland, emkoz@ciop.pl), Ewa Kotarbinska (Warsaw Univ. of Technology Inst. of Radioelectronics, Nowowiejska 15/19, 00-665 Warsaw, Poland, Ewa .Kotarbinska@ire.pw.edu.pl)

It has been well known and that noise protection provided by earmuffs in real-world conditions is lower than measured by a laboratory standardized REAT test. In this study, earmuff protection was tested by simultaneous measurement of the $\mathrm{L}_{\text {Aeq }}$ under and outside the earmuffs in 91 industrial workplaces and in the laboratory. In all cases, the $\mathrm{L}_{\text {Aeq }}$ measured under the earmuffs was compared with the level predicted according to the EN 458 standard, by an octave-band method for calculating the A-weighted SPL under the hearing protector. The $\mathrm{L}_{\text {Aeq }}$ levels measured under the earmuffs in real-world conditions were by more than $3 \mathrm{~dB}(\mathrm{~A})$ and $15 \mathrm{~dB}(\mathrm{~A})$ higher than predicted by the octave-band method, respectively in $65 \%$ and $17 \%$ of cases. The main causes of worse protection at workplaces were worn-out earmuffs due to prolonged usage (33\%), improper way of wearing earmuffs (15\%), or the use of eye-glasses $(8 \%)$. The data show that attenuation values measured by the REAT method overestimate by $1-5 \mathrm{~dB}$ the earmuff protection obtained in real-world conditions. [Work supported by the Polish Ministry of Labour and Social Policy, grants 4.S.03 and 3.S.02].
11:40

4aNSb11. Empirical evaluation using impulse noise of the level-dependency of various passive earplug designs. Elliott $\mathrm{H}$. Berger (E-A-R / Aearo Technologies, 7911 Zionsville Rd., Indianapolis, IN 46268-1657, USA, eberger@compuserve.com), Pascal Hamery (French German Institut of Saint Louis (ISL), 5 rue du Général Cassagnou, 68301 Saint-Louis, France, hamery@isl.tm.fr)

An objective in the development of hearing protection devices (HPDs) has been the design of a passive earplug that provides modest or no attenuation at low sound levels, with greater protection at high sound levels. This raises the issue of not only how to construct such a device, but also how to evaluate it. There is the related question of whether conventional HPDs are actually level independent. Passive level dependency is typically accomplished via an orifice that causes sound transmission to decrease as input level increases. We utilized an impulsive noise source (explosives) with peak levels from 110 to $190 \mathrm{~dB}$ SPL to measure the insertion loss of a variety of commercially available and developmental earplugs. The tests were conducted at frontal incidence in a reflection-free outdoor environment using the Institute of Saint-Louis acoustical test fixture specifically constructed for HPD attenuation measurements. Conventional foam and premolded earplugs exhibited attenuation that was essentially constant with level, whereas the best of the level-dependent designs provided attenuation that increased by about $25 \mathrm{~dB}$ over the $80-\mathrm{dB}$ range of test impulse levels. This latter design has been successfully utilized since 2000 in the Combat Arms ${ }^{\mathrm{TM}}$ Plug widely fielded in the U. S. Military. 


\section{Invited Paper}

\section{2:00}

4aNSb12. Impulse and continuous noise reduction of tactical hearing protection systems. Richard McKinley (AFRL, WrightPatterson Air Force Base, Dayton, OH 45433-7901, USA, richard.mckinley@wpafb.af.mil), Brian Hobbs (AFRL, Wright-Patterson Air Force Base, Dayton, OH 45433-7901, USA, brian.hobbs@wpafb.af.mil), Karl Buck (French German Institut of Saint Louis (ISL), 5 rue du Général Cassagnou, 68301 Saint-Louis, France, karl.buck@isl.eu), Dean Hudson (AFRL, Wright-Patterson Air Force Base, Dayton, OH 45433-7901, USA, harvey.hudson@wpafb.af.mil)

Tactical hearing protectors are devices designed to protect the wearer from high levels of impulse noise while providing some ambient listening and communication capability. Many of these devices also provide some attenuation of continuous noise. The efficacy of these types of devices depends on the amount of impulse noise protection and continuous noise protection, the quality of the ambient listening capability, and the intelligibility of the speech communication capability. This paper will describe the peak noise reduction of approximately $1 \mathrm{~ms}$ duration impulses with peak levels at $165 \mathrm{~dB}$ and $195 \mathrm{~dB}$, for several earplugs and earmuffs. The frequency dependent reduction of impulse noise measured using an acoustic test fixture (the French German Research Institute at Saint Louis Head). Generally the data show higher levels of impulse noise reduction with earplugs than with earmuffs. Additionally, continuous noise attenuation was measured using human subjects performing the ANSI S12.6 Real Ear At Threshold (REAT) test. High speed (20k frames/sec) video will show the dynamic motion of typical tactical hearing protection earplugs and earmuffs when stimulated with a 195 dB impulse.

\section{Contributed Papers}

\section{2:20}

4aNSb13. Sound path(s) to the ear protected by double hearing protection. Karl Buck (French German Institut of Saint Louis (ISL), 5 rue du Général Cassagnou, 68301 Saint-Louis, France, karl.buck@isl.eu), Pascal Hamery (French German Institut of Saint Louis (ISL), 5 rue du Général Cassagnou, 68301 Saint-Louis, France, hamery@isl.tm.fr), Richard McKinley (AFRL, Wright-Patterson Air Force Base, Dayton, OH 45433-7901, USA, richard.mckinley@wpafb.af.mil)

Studies have shown that the attenuation performance of double hearing protection is limited by bone or tissue conduction. We present the first results of a program to determine the different paths of the sound to the ear when a person is protected by double hearing protection. In order to determine the contribution of the noise signal passing by secondary sound path (bone or tissue conduction) into the volume of the ear canal behind the earplug, identical tests have been performed on humans and artificial heads. Time delay and attenuation through the hearing protection (NR) were measured on the ipsi- and contra-lateral side of the head. The measurements show differences in time delay and attenuation between artificial heads and humans which indicate that the main transmission mechanism is different. They also give a strong indication that the path of the acoustic signal depends on the angle of arrival when using double HP. This may partly explain the poor sound localization performance when using double HP that was reported by Brungart (2002). The single HP, and the double HP were then used in a sound localization experiment. The localization performance of these conditions will be presented along with the related parameters.

\section{2:40}

4aNSb14. Prediction of impulsive noise waveform under an earmuff worn by a real user. Rafal Mlynski (Centr. Inst. for Labour Prot. - Natl. Res. Inst., Czerniakowska 16, 00-701 Warsaw, Poland, rmlynski@ciop.pl), Jan Zera (Centr. Inst. for Labour Prot. - Natl. Res. Inst., Czerniakowska 16, 00-701 Warsaw, Poland, jazer@ciop.pl)

For the assessment of hearing damage risk caused by impulsive noise, it is important to know the impulse waveform a user is exposed to under the hearing protector. In this study, a complex transmittance of an earmuff was used to predict the waveform under a hearing protector. Earmuff's transmittance was calculated from impulses recorded outside and under the hearing protector, for a real user, as transmittance of the equivalent FIR filter. The transmittance determined in that way was then used to predict the impulse waveforms under the earmuffs produced in response to various outside impulses. Accuracy of predictions was assessed by a comparison of peak SPL, $\mathrm{L}_{\text {Aeq } 8}, \mathrm{~A}, \mathrm{C}$, or D duration of the impulse waveforms calculated and measured under the earmuffs. Results obtained for a real user were compared with the measurements made with the use of an artificial test fixture (ATF, ISO 4869-3). [Work supported by Polish grants R18004304 (MNiSzW) and 3.S.03 (MPiPS)]. 


\title{
Session 4aNSc
}

\section{Noise, Computational Acoustics, and EURONOISE: Time-Domain Modeling Methods in Acoustics II}

\author{
Paul Calamia, Cochair \\ Rensselaer Polytechnic Institute, Greene Bldg., 110 8th St., Troy, NY 12180, USA \\ Maarten Hornikx, Cochair \\ Applied Acoustics, Chalmers University of Technology, Sven Hultins Gata 8a, Gothenburg, SE-41296, Sweden
}

\section{Invited Papers}

\section{8:00}

4aNSc1. Time domain modeling of acoustic propagation with acoustic wave propagator and absorbing boundary conditions. Jan H. Ehrlich (FWG Underwater Acoustics and Marine Geophysics Research Institute, Klausdorfer Weg 2-24, 24148 Kiel, Germany, janehrlich@bwb.org)

The acoustic wave propagator (AWP) is the application of the time evolution operator on the acoustic wave equation for stationary systems in a polynomial expansion of Chebychev polynomials. It allows to increase the time step by more than one order of magnitude compared to finite difference time domain (FDTD) codes. In contrast to other implementations of the AWP the spatial differentiation is carried out with finite difference techniques because this allows the use of the perfectly matched layer formulation as absorbing boundary conditions. The formulation includes the direct implementation of acoustic sources with sinusoidal time evolution. Other sources can be synthesized by their Fourier components. For the calculation of large areas the explicit formulation of a large system matrix can be avoided by calculating the propagation equations for each time step at row and column level repeatedly which reduces memory requirements notably. This procedure and the suitability of the finite difference approach for parallelization makes the extension to fully three dimensional calculations possible. Examples for benchmark problems with sound propagation in air and water are given.

4aNSc2. A time domain boundary element method for compliant surfaces. Jonathan A. Hargreaves (University of Salford, M5 4WT Manchester, UK, j.a.hargreaves@salford.ac.uk), Trevor J. Cox (University of Salford, Acoustics Research Centre, Newton Building, M5 4WT Salford, UK, t.j.cox@salford.ac.uk)

The best way of representing compliant surfaces in time domain prediction models, such as the transient Boundary Element Method (BEM), is currently unresolved. This is not true of frequency-domain, time-invariant models, where the common practice is to represent the characteristics of a material by its surface impedance. A BEM may be used to predict the scattering of sound, and reduces the problem of modelling a volume of air to one involving surfaces conformal to the obstacles. Surface impedance is a convenient concept for inclusion in the frequency domain BEM as it abstracts the obstacle's characteristics into a property of the conformal surface. The time domain BEM predicts transient scattering of sound, and is usually solved in an iterative manner by marching on in time from known initial conditions. For surface impedance data to be utilised it must be Fourier transformed from a frequency dependent multiplication into a temporal convolution. This approach typically yields convolution kernels which involve future sound, hence is not compatible with time-marching solvers. In this paper an alternative time domain representation of compliant locally reacting materials is proposed to overcome this problem, and its implementation and limitations discussed.

\section{$8: 40$}

4aNSc3. Stability behaviour and results of a transient boundary element method for exterior radiation problems. Michael Stütz (Technische Fachhochschule Berlin, Univ. of Applied Sciences, Luxemburger Str. 10, 13353 Berlin, Germany, stuetz@tfh-berlin.de), Martin Ochmann (Technische Fachhochschule Berlin, Univ. of Applied Sciences, Luxemburger Str. 10, 13353 Berlin, Germany, ochmann@tfh-berlin.de)

Based on the Helmholtz integral equation, a boundary element method in time domain (TD-BEM) can be formulated. Because of instability issues, this method is rarely used in numerical acoustics. The stability and accuracy of the method for exterior radiation problems is investigated using some simple examples. A connection between the internal resonances of the closed structure and the instable behaviour of the method is assumed, but it is mathematically unproven. Numerical evidence of this connection is presented. Because of the sparse structure of the resulting system matrix, the use of iterative solvers is advantageous. The performances of different solvers are compared with respect to stability and numerical costs. For testing purposes, the acoustic radiation from an open turbulent flame is calculated and compared with results of a frequency domain BEM calculation. 
4aNSc4. Analytical validation of time-step interpolation in transient insular nodal analysis. Tom De Rybel (The University of British Columbia, Department of Electrical and Computer Engineering, 2332 Main Mall, Vancouver, BC V6T 1Z4, Canada, tomr@ece.ubc.ca), José Marti (The University of British Columbia, Department of Electrical and Computer Engineering, 2332 Main Mall, Vancouver, BC V6T 1Z4, Canada, jrms@ece.ubc.ca), Murray Hodgson (The University of British Columbia, Department of Electrical and Computer Engineering, 2332 Main Mall, Vancouver, BC V6T 1Z4, Canada, mhodgson@interchange.ubc.ca)

The transient insular nodal analysis method (TINA) combines elements from the finite differences (FD) and transmission-matrix methods (TLM) in one unified approach. In contrast to existing TLM-FD methods, TINA uses time-decoupled cells, avoiding the need for solving large system matrices. The time-decoupled cells allow for easy parallelisation, and the solution of large systems in detail. Due to the use of an exact transmission-line model in the cells, wave propagation can be computed without the need for discretisation of the equations, nor the use of prediction, yielding an unconditionally stable method. Boundary conditions are implicit, and are solely defined by the wave speed and characteristic impedance of the medium. One key difference with the TLM method is how cells whose transmission time is not an integer multiple of the simulation time step are integrated in the simulation. These mismatches occur due to the the varying wave speeds of the different media in the cells. In TINA, the match is obtained through interpolation, as opposed to the stub-matching methodology employed in TLM. In this paper, we will demonstrate the validity of the interpolation approach analytically, as well as compare the TINA method to a theoretical case.

4aNSc5. An eigenfunction expansion method to efficiently evaluate spatial derivatives for media with discontinuous properties. Maarten Hornikx (Applied Acoustics, Chalmers University of Technology, Sven Hultins Gata 8a, SE-41296 Gothenburg, Sweden, maarten.hornikx@chalmers.se), Roger Waxler (University of Mississippi, NCPA, 1 Coliseum Drive, University, MS 38677, USA, rwax@olemiss.edu)

Pseudo-Spectral methods are often used as an alternative to the Finite Difference Time Domain (FDTD) method to model wave propagation in heterogeneous moving media. The FDTD method is robust and accurate but is numerically expensive. Pseudo-Spectral methods make use of the wavelike nature of the solution to obtain more efficient time-domain algorithms. The most straightforward of the Pseudo-Spectral methods is the Fourier method in which a spatial Fourier transform is used to evaluate the spatial derivatives in the wave equation. Whereas this method is accurate for a weakly heterogeneous moving medium, it degenerates for media with discontinuous properties. The eigenfunction expansion method presented here is a way to accurately and efficiently evaluate spatial derivatives in media with interfaces. As in the Fourier method, transforms may be calculated using FFT's and spatial sampling is limited only by the Nyquist condition. The performance of the method is shown in a time-domain implementation for media with discontinuous density and sound speed.

4aNSc6. Optimal tree canopy shape for improving downwind noise barrier efficiency. Timothy Van Renterghem (University Ghent - Department Information Technology, Sint-Pietersnieuwstraat 41, 9000 Gent, Belgium, Timothy.Van.Renterghem@intec.Ugent .be), Dick Botteldooren (University Ghent - Department Information Technology, Sint-Pietersnieuwstraat 41, 9000 Gent, Belgium, dick.botteldooren@intec.ugent.be)

The presence of a row of trees behind a highway noise barrier significantly reduces the screen-induced refraction of sound by wind. In this paper, the influence of quantitative tree properties, such as the pressure resistance coefficient of the canopy and the distribution of biomass over height, was studied numerically. Three computational models were involved. First, computational fluid dynamics (CFD) software is used to accurately predict the wind fields. The finite-difference time-domain (FDTD) method is then used to simulate sound propagation in the direct vicinity of the noise barrier in combination with trees. In a last step, the Parabolic Equation (PE) method is used to predict sound fields at larger distances. As a general conclusion, it was found that coniferous trees are superior to deciduous trees to improve downwind noise barrier efficiency.

\section{0:00}

4aNSc7. Green roofs to enhance quiet sides. Timothy Van Renterghem (University Ghent - Department Information Technology, Sint-Pietersnieuwstraat 41, 9000 Gent, Belgium, Timothy.Van.Renterghem@intec.Ugent.be), Dick Botteldooren (University Ghent Department Information Technology, Sint-Pietersnieuwstraat 41, 9000 Gent, Belgium, dick.botteldooren@intec.ugent.be)

In this paper, the finite-difference time-domain method is used to study sound propagation over a green roof in an urban situation. Sound propagation between adjacent city canyons is considered, and the focus is on the reduction of the sound pressure level in the non-exposed canyon due to the presence of a vegetated (green) roof. Numerical calculations have been conducted for both intensive and extensive green roofs, showing that an important reduction of the sound pressure level in the shielded canyon can be achieved, compared to a rigid roof. In case of an extensive green roof, there is a strong dependence on the substrate layer thickness; a maximum reduction of $10 \mathrm{~dB}$ at the octave band of $1000 \mathrm{~Hz}$ was found. For intensive green roofs, the influence of the substrate layer thickness is limited. 


\title{
Session 4aNSd
}

\section{Noise and EURONOISE: Car Acoustics II}

\author{
Luc Mongeau, Cochair \\ McGill University, 817 Sherbrooke St. West, Montreal, QC H3A 2K6, Canada \\ Virginie Maillard, Cochair \\ RENAULT, Groupe Acoustique, Technocentre, 1 avenue du Golf, 78288 Guyancourt Cedex, France
}

\section{Invited Paper}

\begin{abstract}
8:00
4aNSd1. Trim FEM simulation of a dash and floor insulator cut out modules with structureborne and airborne excitations. Arnaud Duval (Faurecia AST, Center of Acoustic Technology, Dämmstoffwerk 100, 38524 Sassenburg, Germany, arnaud.duval@faurecia.com), Julien Baratier (Faurecia AST, Center of Acoustic Technology, Dämmstoffwerk 100, 38524 Sassenburg, Germany, julien.baratier@faurecia.com), Christian Morgenstern (Faurecia AST, Center of Acoustic Technology, Dämmstoffwerk 100, 38524 Sassenburg, Germany, christian.morgenstern@faurecia.com), Ludovic Dejaeger (Faurecia AST, Center of Acoustic Technology, Dämmstoffwerk 100, 38524 Sassenburg, Germany, ludovic.dejaeger@faurecia.com), Norimasa Kobayashi (Toyota Motor Corporation, 1, Toyota-cho, 471-8572 Toyota, Aichi, Japan, koba@nori.tec.toyota.co.jp), Hiroo Yamaoka (Toyota Motor Corporation, 1, Toyota-cho, 471-8572 Toyota, Aichi, Japan, yamaoka@giga.tec.toyota.co.jp)

During a vehicle development, measurements on cut out modules in large coupled reverberant rooms are often carried out in the middle and high frequency range in order to optimize the insulation performance of the trims (Transmission Loss). Using optimal controlled mounting conditions, we have been able to extend the frequency range to the low frequencies in order to validate trim FEM models of a dash and floor insulator modules with structureborne and airborne excitations. Both coupled response with movable concrete cavities (structureborne excitation) and Transmission Loss with the coupled reverberant rooms (airborne excitation) have been measured and simulated for various types of insulators on the same setup, without any change on the mounting conditions. An additional movable absorbing environment in the large reception room has been deployed in order to carry out laser vibrometer (skeleton velocity) and $\mathrm{p}-\mathrm{u}$ probes (particle velocity and intensity) measurements on the surface of the trims. By incorporating the maximal treatment mock-ups of the cut out modules as additional trims in the models, we have obtained good correlation results between measurements and simulations for both bare and trimmed configurations for a dash and floor insulators with structureborne and airborne excitations.
\end{abstract}

\section{Contributed Papers}

$8: 20$

4aNSd2. Practical aspects of implementing car interior active noise control systems. Rolf Schirmacher (Müller-BBM, Robert-Koch-Straße 11, 82110 Planegg, Germany, Rolf.Schirmacher@MuellerBBM.de), Roland Lippold (Müller-BBM, Robert-Koch-Straße 11, 82110 Planegg, Germany, Roland.Lippold@MuellerBBM.de), Frank Steinbach (Müller-BBM, Robert-Koch-Straße 11, 82110 Planegg, Germany, Frank.Steinbach @ MuellerBBM.de), Florian Walter (Müller-BBM, Robert-Koch-Straße 11, 82110 Planegg, Germany, Florian.Walter@MuellerBBM.de)

When implementing real-world, close-to-production active noise control (ANC) systems for car interiors, many aspects far beyond textbook theory have to be taken into consideration -- many of which might also be left out for first demonstrator systems. Due to the predominant role of robustness and reliability, any kind of uncertainty in the system has to be considered carefully. Among others, the uncertainties and changes of the acoustical environment, e.g. due to temperature changes, number of passengers, open windows and also of system components, e.g. loudspeakers and microphones have to be measured and/or modeled. This also gives some new insight into the acoustical environment inside cars at low frequencies. In addition, the quality of input data (e.g., the update rate for rpm information) is of major importance for the acoustical performance in terms of noise reduction. Finally, stability analyses and robustness calculations must be extended to incorporate uncertainties as well as time domain effects even for more or less freqency domain problems like engine noise. This requires to re-formulate feed-forward systems as feed-back systems and calculate system responses. Measurement results on the interior acoustics (and its uncertainty) as well as additional developments on ANC system robustness will be presented.

\section{8:40}

4aNSd3. Fluctuations under turbulent flows: Enhanced methods for separation of propagative wavenumbers from wall pressure dataset. Sébastien Debert (PSA-Peugeot-Citroën, Route de Gizy, CC : VV013 - Bât. 14, F-78943 Vélizy-Villacoublay Cedex, France, sebastien.debert @mpsa.com), Marc Pachebat (PSA-Peugeot-Citroën, Route de Gizy, CC : VV013 - Bât. 14, F-78943 Vélizy-Villacoublay Cedex, France, marc .pachebat@mpsa.com), Vincent Valeau (Laboratoire d'Etudes Aérodynamiques (LEA), Université de Poitiers - ENSMA - CNRS, Bâtiment K, 40 Avenue du Recteur Pineau, F-86022 Poitiers, France, vincent.valeau @lea.univ-poitiers.fr), Yves Gervais (Laboratoire d'Etudes Aérodynamiques (LEA), Université de Poitiers - ENSMA - CNRS, Bâtiment K, 40 Avenue du Recteur Pineau, 86022 Poitiers, France, yves.gervais@lea.univpoitiers.fr)

This work is a part of a more general study on automobile interior noise due to acoustic and aerodynamic wall-pressure fluctuations. Using experimental data of wall-pressure fluctuations measured with a microphone array beneath several kinds of flows, a wave-number analysis based on recently developed signal processing methods -- the spatial Empirical Mode Decomposition (sEMD) and the Ensemble Empirical Mode Decomposition (EEMD) -- is carried out, in order to separate acoustic and aerodynamic pressure fluctuations. In opposition with an existing classical method previously used, based on the spatial correlogram, these methods do not require a stationary uniform flow. A turbulent boundary layer on a flat plate and a detached/reattached flow downstream three different forward-facing steps are tested, with flow velocities from 0 to $40 \mathrm{~m} . \mathrm{s}^{-1}$, with or without a wellcontrolled artificial acoustic source. The sEMD method is first used as a wavenumber filter, and is shown to improve the detection of acoustic fluc- 
tuations of about $5 \mathrm{~dB}$ on classical wavenumber $(k, f)$ representations. The E-EMD method is developed in order to decompose the $(x, t)$ representation to make possible the separation of the acoustic and aerodynamic components, regardless of the stationarity or the uniformity of the flow.

\section{9:00}

4aNSd4. Stochastic modeling of the vibro-acoustic behavior of production cars. Laurent Gagliardini (PSA Peugeot Citroën, Route de Gisy, 78943 Vélizy-Villacoublay Cedex, France, laurent.gagliardini@mpsa .com), Jean-Francois Durand (Université Paris-Est, Laboratoire de Mécanique, 5 bd Descartes, 77454 Marne-la-Vallée, France, jean-francois .durand@astrium.eads.net), Christian Soize (Université de Marne la Vallée, 5, Boulevard Descartes, 77454 Marne la Vallée, France, soize@univ-mlv.fr)

Production cars -as any industrial product- are subject to various causes of variability including process uncertainties or product diversity. Many authors have shown that vibroacoustic problems sensitivity to small uncertainties increases dramatically with frequency until only statistical approaches remain relevant in the high frequency range. Moreover, modeling uncertainties due to numerous model simplifications induce similar dispersion effects on the computed responses. Both kind of uncertainties may be addressed when using a non-parametric stochastic modeling, based on the random matrices theory. Such a modeling, appears to be very practicable for industrial vibroacoustic problems while relying on a strong mathematical background. In a first part, the application of the non-parametric modeling of uncertainties to vibro-acoustics problems will be addressed. Stochastic aspects are controlled by only 7 dispersion parameters that provide most of the dynamic behaviors that can be observed experimentally. A Monte-Carlo simulation is performed to provide converged statistics of the stochastic problem solution. In a second part, the dispersion parameters are identified so that the stochastic model fits experimental data. Frequency Response Functions of 25 production vehicles were measured for this purpose and compared to the computed results in the low frequency range $(<200 \mathrm{~Hz})$.

\section{9:20}

4aNSd5. Improved spectrofilter applied to diesel engine noise : combustion and mechanical noise separation. Laurent Pruvost (Laboratoire Vibrations Acoustique, Insa Lyon, 25 bis, av. J. Capelle, 69621 Villeurbanne Cedex, France, laurent.pruvost@insa-lyon .fr), Quentin Leclere (Laboratoire Vibrations Acoustique - INSA Lyon, 25 bis avenue Jean Capelle, Bâtiment Saint-Exupéry, F-69621 Villeurbanne cedex, France, quentin.leclere@insa-lyon.fr), Etienne Parizet (Laboratoire Vibrations Acoustique, Insa Lyon, 25 bis, av. J. Capelle, 69621 Villeurbanne Cedex, France, etienne.parizet@insa-lyon.fr)

For engineers working on alternative fuels and diesel engine sound quality, being able to separate combustion noise and mechanical noise would be of prime interest. This separation can be attempted using a spectrofilter (also called Wiener filter) when in-cylinder pressure signals can be recorded. The major drawback of the spectrofilter is its unability to separate correlated sources like combustion and piston slap. An upgraded version of the spectrofilter can be computed. Its computation just requires to consider only the random parts of the engine signals. Actually, considering these random parts artificially uncorrelates the noise sources. Highly correlated signals have been synthesised and successfully separated by the upgraded spectrofilter. When dealing with real-life engine noise, the quality of the separation cannot be judged directly since the signals to separate are unknown. The spectrofilter's causality and stability have been used as criteria to judge its quality. These two criteria both confirmed the superiority of the spectrofilter computed using the random parts of the signals. A synchronous averaging step is required to estimate the signals random parts. This estimation has been found to depend on the phase-locking strategy.

\section{9:40}

4aNSd6. Prediction of the Excitation Force Based on the Dynamic Analysis for Flexible Model of a Powertrain. Yoon Sug Kim (Inha University, Mechanical Engineering, 253 Yonghyun Dong, 402-751 Inchon, Republic of Korea, black_boxter@hanmail.net), Sung Jong Kim (Inha University, Mechanical Engineering, 253 Yonghyun Dong, 402-751 Inchon,
Republic of Korea, kimsungjong@gmail.com),Min Geun Song(Inha University, Mechanical Engineering, 253 Yonghyun Dong, 402-751 Inchon, Republic of Korea, somiku@hanmail.net), Sang Kwon Lee (Inha University, Mechanical Engineering, 253 Yonghyun Dong, 402-751 Inchon, Republic of Korea, sangkwon@inha.ac.kr)

The powertrain is one of the import sources for the interior noise. In order to predict the interior noise due to a powertrain, the experimental method is has been used based on the TPA (transfer path analysis). Although this experimental method is a useful tool for the identification of the noise source and the transfer path due to the powertrain, it is difficult to modify the structure of a powertrain by using the experimental method for the reduction of vibration and noise. In order to solve this problem, the paper presents the noble approach for the prediction of interior noise caused by the vibration of the powertrain based on the hybrid TPA (transfer path analysis) technology. Therefore, the vibration of the powertrain in a vehicle is numerically analyzed by using FEM (finite element method). The vibration of the other part in a vehicle is investigated by using the experimental method based on VATF (vibrato-acoustic transfer function) analysis. These two methods are combined for the prediction of interior noise caused by powertrain. This paper present the prediction of the excitation force of the powertrain to the vehicle body based on numerical simulation.

\section{0:00-10:20 Break}

\section{0:20}

4aNSd7. Sound quality evaluation of air-conditioning sounds in a vehicle using psychoacoustic parameters. Ryota Nakasaki (Utsunomiya Univ., Calsonickansei Corp., 7-3 Sakae-cho, Tochigi-ken, 327-0816 Sanoshi, Japan, ryota_nakasaki@ck-mail.com), Takaharu Ogata (Utsunomiya Univ., 7-1-2 Yoto, Tochigi-ken, 321-8585 Utsunomiya-shi, Japan, mt076508@ced.is.utsunomiya-u.ac.jp), Hiroshi Hasegawa (Utsunomiya Univ., 7-1-2 Yoto, Tochigi-ken, 321-8585 Utsunomiya-shi, Japan, hasegawa@is.utsunomiya-u.ac.jp), Yukio Ozeki (Utsunomiya Univ., Calsonickansei Corp., 7-3 Sakae-cho, Tochigi-ken, 327-0816 Sano-shi, Japan, ryota_nakasaki@ck-mail.com), Masaharu Onda (Utsunomiya Univ., Calsonickansei Corp., 7-3 Sakae-cho, Tochigi-ken, 327-0816 Sano-shi, Japan, ryota_nakasaki@ck-mail.com), Masao Kasuga (Utsunomiya Univ., 7-1-2 Yoto, Tochigi-ken, 321-8585 Utsunomiya-shi, Japan, kasuga@ is .utsunomiya-u.ac.jp)

With recent developments of noise reduction technologies from mechanical components of a vehicle, air-conditioning systems become a major noise source in the compartment of a vehicle. To improve quietness in the compartment further, it is important not only to reduce the sound pressure level (SPL), but also to improve the sound quality. In this study, we tried to evaluate the air-conditioning sounds from a viewpoint of sound quality. First, we carried out a subjective evaluation experiment using some evaluation words that represent characteristics of the air-conditioning sounds. As a result of a factor analysis, noting that the air-conditioning sounds can be explained by three factors of "rough," "space" and "refreshing." "Rough factor" has strong correlation with the SPL, however, "space factor" and "refreshing factor" have little correlation with SPL. Next, to investigate parameters that correlate with these factors, we carried out an experiment to evaluate the air-conditioning sounds using the psychoacoustic parameters of loudness and sharpness. As a result, noting that "rough" factor strongly correlates with loudness, "space" and "refreshing" factors correlate with sharpness. This result shows that it is possible to evaluate the airconditioning sounds a viewpoint of sound quality by using the sharpness that is different from SPL.

\section{0:40}

4aNSd8. Low noise truck study for distribution in urban areas Nicolas Blairon (Volvo 3P, 99 route de Lyon, 69802 Saint - Priest, France, nicolas.blairon@volvo.com), Bruno Carton (Volvo 3P, 99 route de Lyon, 69802 Saint - Priest, France, bruno.carton@volvo.com)

Today, vehicle noise is identified in opinion pools as one of the main annoyance source in urban areas. To face this issue, truck manufacturers are today developing low noise vehicles in order to allow their customer to deliver goods in urban areas during night. An acoustic research program has 
been launched by Volvo 3P (Volvo Trucks, Renault Trucks and Mack Trucks): the LUT project as Low Noise Urban Truck. The partners of the project are Michelin, the Institut National des Sciences Appliquées de Lyon (INSA) - a French university - and Marmonier - a noise shield manufacturer. This project has been founded by ADEME The target in terms of noise reduction is ambitious because the low noise truck will emit an acoustic power four times lower than today's vehicles in specific rolling conditions! Methods and tools have been developed to analyze the vehicle noise sources in urban conditions and define acoustic solutions to reduce the main vehicle noise sources.

\section{1:00}

4aNSd9. Operational transfer path analysis: Comparison with conventional methods. Martin Lohrmann (Müller-BBM Vibroakustik Systeme, Robert Koch Strasse 13, 82152 Planegg / München, Germany, MLohrmann@MuellerBBM-vas.de)

Transfer Path Analysis describes how sound and vibration propagates through complex structures. The correct determination of transfer coefficients between sources and receivers is essential for a high quality analysis. Conventional methods use artificial excitations (forces or volume velocities) to evaluate transfer functions. Therefore, they do not consider the influence of different load conditions on the transfer function behaviour of complex structures. In a second step, operational data (vibrations or sound pressures) are applied to the transfer functions to calculate the different path contributions. In order to overcome these restrictions, transfer coefficients can be evaluated directly from operational data. Thus, the actual load conditions are taken into account and the quality of transfer characteristics is improved. The relation between simultaneously measured data of sources and receivers can be derived by statistical methods. Principal Component Analysis is used to separate the total signal into individual path contributions, while operating on airborne and structure-borne contributions simultaneously. Operating in the time-domain gives direct access to auralisation and in-depth analysis of dominant path contributions. This new single-step approach has been proven to generate more precise analysis results within a much shorter testing time.

\section{1:20}

4aNSd10. A transversal substructuring modal method for the acoustic analysis of dissipative mufflers with mean flow. José Albelda (Dept. Ingeniería Mecánica y de Materiales. Univ. Politécnica de Valencia, Camino de Vera, s/n, 46022 Valencia, Spain, jalbelda@mcm.upv.es), Francisco D. Denia (Dept. Ingeniería Mecánica y de Materiales. Univ. Politécnica de Valencia, Camino de Vera, s/n, 46022 Valencia, Spain, fradegu1@mcm.upv.es) , F. Javier Fuenmayor (Dept. Ingeniería Mecánica y de Materiales. Univ. Politécnica de Valencia, Camino de Vera, s/n, 46022 Valencia, Spain, ffuenmay@mcm.upv.es), Manuel J. Martínez (Dept. Ingeniería Mecánica y de Materiales. Univ. Politécnica de Valencia, Camino de Vera, s/n, 46022 Valencia, Spain, mamarbor@doctor.upv.es)

This work presents a modal approach to evaluate the transversal modes and wavenumbers for dissipative mufflers with mean flow. The method is based on the division of the transversal section of the muffler into subdomains, for which two simple sets of modes are considered. The first set of modes satisfies the condition of zero pressure at the common boundary between subdomains, while the second fulfils the condition of zero derivative in the direction normal to this boundary. From these sets, a substructuring procedure is applied that provides the final modes of the complete cross section, considering the presence of absorbent material, a perforate and mean flow. The technique avoids iterative schemes associated with the nonlinear characteristic equation found, for instance, in the analytical modelling of perforated dissipative circular mufflers. Once the final transversal modes have been calculated, the mode matching technique is applied at the geometrical discontinuities to completely define the acoustic field inside the muffler. The acoustic attenuation is then predicted by means of the transmission loss. Comparison with finite element calculations and results available in the literature show good agreement. The attenuation of some selected mufflers is analysed, including the effect of the perforate and the mean flow.

\section{1:40}

4aNSd11. Prediction of sound transmission through automotive door seal systems. Bertrand Andro (Renault, Technocentre, 1 avenue du Golf, 78288 Guyancourt, France, bertrand.andro@renault.com), Sébastien Chaigne (Renault, Technocentre, 1 avenue du Golf, 78288 Guyancourt, France, sebastien.chaigne@ renault.com), Alpha Diallo (Renault, Technocentre, 1 avenue du Golf, 78288 Guyancourt, France, alpha.diallo@renault.com), Matthieu Mermet (CEMEF, 1, rue Claude Daunesse, 06904 Sophia Antipolis, France, matthieu.mermet-renexter@renault .com)

In automotive industry the door seal systems is an important contributor to vehicle interior noise in the middle and high frequency range. The aim of the study was to develop a numerical model in order to predict the sound transmission loss through elastomeric seals. At the early stage of the development process, this type of numerical tool is very interesting to investigate the influence of the design parameters of the seal. Two steps were necessary: a static analysis to calculate the seal shape after compression (door closure event), an acoustic analysis based on dynamic parameters to determine the sound transmission. Finite element methods were used for both steps (commercial softwares). These two steps were validated experimentally for two types of geometry, different compression ratios and loading cases. One original contribution of the paper concerns the determination of a valid nonlinear model for the static part and of a good approximation of the dynamic behavior of the elastomer's Young modulus. Finally, a sensibility analysis was performed in order to evaluate the influence of the design parameters of the door seal system such as the compression ratio or the dynamic parameters.

\section{2:00}

4aNSd12. Quantification of structural damping of a multi-layered windshield at low and medium frequencies. Manuel Etchessahar (PSA Peugeot Citroën, Route de Gisy, 78943 Vélizy-Villacoublay Cedex, France, manuel.etchessahar@mpsa.com), Laurent Gagliardini (PSA Peugeot Citroën, Route de Gisy, 78943 Vélizy-Villacoublay Cedex, France, laurent .gagliardini@mpsa.com)

Structural damping is known as one of the most efficient design variable in order to reduce structure-borne noise. At low and medium frequencies (100-500 Hz), the vibro-acoustic behavior of the car is essentially governed by large panels as windshield, roof or front panel for example. In order to improve the car body design regarding cost, weight, performances and reliability, one must have a good understanding of the damping performances of these large panels submitted to various operating conditions. In a previous paper, the authors have proposed an improved method for structural damping numerical assessment of structural elements with non uniform damping using a stochastic distribution of input forces. In the present paper, this method is applied to quantify the structural damping of a multi-layered windshield at low and medium frequencies. Effects of the geometry, of the viscoelastic properties of the PVB layer and of the windshield glue bead on the total damping properties will be studied. 


\title{
Session 4aNSe
}

\section{Noise and EURONOISE: Noise from Wind Power Projects I}

\author{
Eddie Duncan, Cochair \\ Resource Systems Group (RSG), 55 Railroad Row, White River Junction, VT 05001, USA
}

Kerstin Persson Waye, Cochair

Dept. of Environ. Medicine, The Sahlgrenska Acad. of Gothenburg Univ., Box 414, Gothenburg, 405 30, Sweden

\section{Invited Paper}

\section{0:20}

\begin{abstract}
4aNSe1. Improving sound propagation modeling for wind power projects. Eddie Duncan (Resource Systems Group (RSG), 55 Railroad Row, White River Junction, VT 05001, USA, eduncan@rsginc.com), Kenneth Kaliski (Resource Systems Group (RSG), 55 Railroad Row, White River Junction, VT 05001, USA, kkaliski@ rsginc.com)

Sound propagation from wind power projects can be modeled in the same manner as other more common outdoor noise sources, but are these models suited to wind turbines' uniquely high source heights, operating under high wind conditions, and various degrees of terrain ruggedness. In "Propagation Modeling Parameters for Wind Turbines" (K. Kaliski and E. Duncan, Proceedings of Institute of Noise Control Engineers NOISECON 2007), the effects of ground attenuation and various adjustments for wind conditions on sound propagation modeling were discussed. This paper continues the discussion and explores the accuracy of existing sound propagation modeling methods for wind power projects including ISO 9613 and other standards. Model data for wind power projects and the implications of various terrain and ground coverage will be discussed.
\end{abstract}

\section{Contributed Papers}

10:40

4aNSe2. A review of the use of different noise prediction models for windfarms and the effects of meteorology. Graham Parry (ACCON UK Limited, Unit B, Fronds Park, Frouds Lane, Aldermaston, RG7 4LH Reading, UK, graham.parry@accon-uk.com)

As a result of involvement in a specific wind farm development at Guestwick, Norfolk and a requirement to determine the efficacy of competing noise prediction models a review of the potential impacts of a sixturbine wind farm was carried out. The paper considers the results of comparing three specific noise prediction methods and algorithms and determines the extent to which adherence to either one of the methodologies could result in relatively large differences in predicted noise levels under varying wind conditions and accordingly the potential for differing conclusions being reached as to the acceptability of the wind farm with respect to the ETSU-R-97 assessment methodology. The paper also examines other noise modelling research carried out on behalf of ETSU.
11:00

4aNSe3. Noise Impact of Wind Farms: Uncertainties due to wind data reference at 10m. David Slaviero (Acouphen Environnement, Campus de la DOUA, 66, BD Niels Bohr, BP 52132, 69603 Villeurbanne, France, david.slaviero@acouphen-environnement.com), Alexis Bigot (Acouphen Environnement, Campus de la DOUA, 66, BD Niels Bohr, BP 52132, 69603 Villeurbanne, France, alexis.bigot@acouphen-environnement.com)

Noise impact prediction or measurement of Wind Farms requires wind reference data at 10 meters. From data collected on more than 200 Farms in France, over the last 5 years, this paper shows that, in the sampling of noise and meteolological data for noise prediction and assessment, differences in the wind profil from one site to another, in the period of the day (day, night) can lead to different estimations of emission data selection and impact assessment. Different parameter studies are presented both with regard to the effect on impact studies and farms impact monitoring and control. The work presented is in accordance with the project of acoustic standard for the assessment of noise impact of Wind Farms under study in France.

\section{Invited Papers}

4aNSe4. Models of natural background noise and masking of wind turbine noise. Karl Bolin (KTH/MWL, Teknikringen 8, SE100 44 Stockholm, Sweden, kbolin@kth.se)

Wind turbine (WT) noise limits adjusted to background noise levels are used in several countries among others Britain and France. To determine the background noise level extensive measurements at locations near the proposed WT site are performed. This paper present methods to avoid these measurements in woodland and coastal areas, it also include a pre-study concerning the audibility of WT noise when mixed with background noise. A prediction model for noise from vegetation is described. This has been coupled to wind field simulations and fluctuations of vegetation noise can therefore be predicted. Measurements and a model for sea wave noise are also presented. Furthermore the paper present results from psycho acoustic tests with 8 subjects. These involve hearing thresholds and partial loudness when WT noise is mixed with background noise. These are compared to two loudness models. Two different WT sounds have been used as stimuli. The first sound is from a single WT and the second sound is from a WT park. Results show how natural background noises influence the audibility of WT noise and could be used as a tool to optimize the power generated from WTs without causing disturbance among nearby residents. 
4aNSe5. Criteria for wind farm noise: Lmax and Lden. Frits Van Den Berg (University of Groningen - Science \& Society Group, Nijenborgh 4, 9747AG Groningen, Netherlands, fvdberg@ggd.amsterdam.nl)

Wind turbine noise limits are based on either the highest sound immission level (Lmax) or several sound immission levels for a series of wind speed classes (Lmax,v). As yet no procedure has been proposed to determine the day-evening-night sound level (Lden) that is now commonly used in the European Union for all noise sources. Wind speed dependent rating wind turbine noise levels Lr,v can be predicted based on climatological data. This has been verified by measurements over a nine month period for a wind farm at a coastal location in the Netherlands. From these measurements also the long term average sound level Lden can be determined. Lden can also be determined from previously published wind speed measurements at an inland location over one year. The procedure shows that for a wind turbine or wind farm the Lden can be derived from Lmax by taking into account the regional climatology.

\section{2:00}

4aNSe6. Response to wind turbine noise in the Netherlands. Eja Pedersen (Occupational and Environmental Medicine, Göteborg University, PO Box 100, SE-405 30 Göteborg, Sweden, eja.pedersen@set.hh.se), Jelte Bouma (Northern Centre for Healthcare Research, University Medical Centre Groningen, PO Box 30001, 9700 RB Groningen, Netherlands, j.bouma@med.umcg.nl), Roel Bakker (Northern Centre for Healthcare Research, University Medical Centre Groningen, PO Box 30001, 9700 RB Groningen, Netherlands, R.H.Bakker@med.umcg.nl), Frits Van Den Berg (University of Groningen - Science \& Society Group, Nijenborgh 4, 9747AG Groningen, Netherlands, fvdberg@ggd.amsterdam.nl)

A cross-sectional study with the objective to explore the impact of wind turbine noise on people living in the vicinity of wind farms was carried out in the Netherlands in 2006. A postal questionnaire assessing response to environmental exposures in the living area, including wind turbine noise, was answered by 725 respondents (response rate: 37\%). Immission levels of wind turbine noise outside the dwelling of each respondent were calculated in accordance with ISO-9613. The risk for being annoyed by wind turbine noise outdoors increased with increasing sound levels $(\mathrm{rs}=0.501, \mathrm{n}=708, \mathrm{p}<0.001)$. The risk for annoyance was decreased for respondents who could not see wind turbines from their dwelling and for respondents who benefited economically from the turbines. No statistically significant correlations between immission levels of wind turbine noise and health or well-being were found. However, noise annoyance due to wind turbine noise was associated with stress symptoms, psychological distress and lowered sleep quality.

4aNSe7. Laboratory assessment of noise annoyance from large wind turbines. Steffen Pedersen (Acoustics, Aalborg University, Fredrik Bajers Vej 7 B5, 9220 Aalborg Ø, Denmark, stp@acoustics.aau.dk), Henrik Møller (Acoustics, Aalborg University, Fredrik Bajers Vej 7 B5, 9220 Aalborg Ø, Denmark, hm@acoustics.aau.dk)

An investigation of the annoyance from the wind turbine noise, to which neighbors may be exposed, is carried out. The aim is to obtain dose-response relationships and to uncover if specific noise components (e.g. low-frequencies) are primary contributors to the annoyance. In the experiments, sounds recorded close to large wind turbines are filtered (and levels adjusted accordingly) to represent indoor and outdoor positions at the neighbors' dwellings and played back in the laboratory. Challenges relating to the recording and transformation of sounds are discussed. The exposure technique is a combination of an advanced low-frequency chamber that can reproduce the frequency range $2-250 \mathrm{~Hz}$ (with uniform distribution in the room) and additional loudspeakers for the higher frequencies. The listening test is a randomized design. The stimuli, of 10 minute duration, are presented at three levels and in combinations of filtered versions (low- and mid-frequency) such that the influence of low-frequency tonal components and level fluctuations is investigated. 25 subjects are exposed to the stimuli while reading a novel and afterwards they rate annoyance on a visual analog scale. 


\title{
Session 4aNSf
}

\section{Noise and EURONOISE: Fan Noise and Low-Mach Number Rotating Blade Noise I}

\author{
Scott C. Morris, Cochair \\ Notre Dame, 109 Hessert Laboratory, University of Notre Dame, Notre Dame, IN 46556, USA \\ Michel Roger, Cochair \\ Ecole Centrale de Lyon, 36 Avenue Guy de Collongue, Centre Acoustique, Ecully, 69134, France
}

\section{Contributed Papers}

10:40

4aNSf1. Overview of turbofans noise prediction methods based on CFD computations. Cédric Morel (Snecma, Site de Villaroche, RondPoint René Ravaud, 77550 Moissy-Cramayel, France, cedric.morel @ snecma.fr), Benoit Farvacque (Snecma, Site de Villaroche, Rond-Point René Ravaud, 77550 Moissy-Cramayel, France, benoit.farvacque@snecma .fr)

Overview of turbofans noise prediction methods based on CFD computations The constant trend to increase bypass ratio in turbofan aircraft engines has led to an increase of the relative contribution of rotors to the overall engine noise. There is therefore a need for efficient and accurate prediction tools to achieve a silent design of the rotor components. Here is given an overview of the different aeroacoustic methods investigated by Snecma and used for prediction of tone interaction noise, broadband interaction noise and broadband fan self noise. Different levels of methods refinement, from various analytical models to steady and unsteady CFD, will be compared in terms of accuracy of prediction and computational cost. Examples of implementation of these methods on conventional turbofan and counter-rotating fan configurations will be presented.

\section{1:00}

4aNSf2. Benchmark of fan noise propagation tools. Jacky Mardjono (SNECMA Villaroche, 77550 Moissy Cramayel, France, jacky .mardjono@snecma.fr)

Various propagating codes based on different formulations have been developed over the last years to simulate the fan noise propagation into the nacelle of a turbofan engine and its radiation in the Far field. The abilities of the ACTRAN softwares (Potential formulation / FEM), SPACE (Linearised EULER equations / DGM) and ICARE (Rays-tracing method) to simulate typical industrial configurations in the frequential domain have been evaluated based on measurements carried out at the RACE acoustic test facility on a fan model at $1 / 2$ scale. The results of the computations carried out with each of these codes on intake and exhaust configurations (axisymmetric assumption) in hard wall and lined duct will be compared to the measured far field directivities. These comparisons will be discussed depending on the establishment of patterns (sources types, mean flows types, boundaries conditions) considered in the simulations of each of these codes. The impacts of the considered convected mean flow types (Uniform, Euler, NS,...) and the capacities of these codes to predict noise attenuation of the treatments will be investigated in particular.

\section{1:20}

4aNSf3. Comparative helicopter noise analysis in static and in-flight conditions. Doris Novak (University of Zagreb, Vukeliceva 4, 10000 Zagreb, Croatia, doris.novak@fpz.hr)

Rotary wing aircraft, i.e. helicopter, is a source of intense noise, external and internal alike, in conclusion becoming serious environmental and health issue. The generated noise is in some aspects similar to propeller noise in fixed wing aircraft (airplane), while differing in main noise source alignment in respect to the relative airflow: in helicopters, both rotors, i.e. main and tail, that produce forces necessary for flight, are inline with the direction of flight, while in airplanes rotor(s) are perpendicular to it. Another distinctive noise in helicopters, well known as "slapping", comes from the rotor cutting its own wake/vortex air inflow, especially while descending. In this article main helicopter noise sources will be discussed and most significant results of various static and in-flight noise measurements on two different types of helicopters will be presented and analyzed.

\section{1:40}

4aNSf4. Validation of Thickness and Loading Noise Codes by Isom's Formula Applied to Subsonic Axial and Centrifugal Fans. Sofiane Khelladi (Lab. d'Energétique et de Mécanique des Fluides Interne, Arts et Métiers ParisTech, 151 boulevard de l'Hôpital, 75013 Paris, France, sofiane.khelladi@paris.ensam.fr), Djaafer Fedala (Lab. d'Energétique et de Mécanique des Fluides Interne, Arts et Métiers ParisTech, 151 boulevard de l'Hôpital, 75013 Paris, France, djaafer.fedala@paris.ensam.fr), Farid Bakir (Lab. d'Energétique et de Mécanique des Fluides Interne, Arts et Métiers ParisTech, 151 Boulevard de l'Hôpital, 75013 Paris, France, farid.bakir@paris.ensam.fr), Robert Rey (Lab. d'Energétique et de Mécanique des Fluides Interne, Arts et Métiers ParisTech, 151 Boulevard de l’Hôpital, 75013 Paris, France, robert.rey@paris.ensam.fr)

The thickness noise predicted by the Ffowcs Williams and Hawkings $(F W \& H)$ equation depends on the normal velocity $v_{n}$ which is very sensitive to the meshing size. Isom showed that in far field a monopolar source is equivalent to a dipolar source induced by a uniform distribution of the load on the entire moving surface. Consequently, the calculation of the thickness noise becomes completely independent of the normal velocity $\mathrm{v}_{\mathrm{n}}$. Its expression, as suggested by Farassat, is for any moving surfaces. The main objective of this paper is to determine a specific expression of Isom's thickness noise in time and frequency domains for axial and centrifugal subsonic fans. The scope of the proposed expression of Isom's thickness noise is threefold: (1) highlight the effect of each geometrical parameter of the fan on the overall thickness noise, (2) a fast computational mean and low memory storage capability since the acoustic pressure in frequency domain is calculated for only one blade, and (3) a benchmark test of consistency for thickness and loading noise codes in both time and frequency domains when using the free field solution of FW\&H's equation.

\section{2:00}

4aNSf5. Prediction of the tonal noise radiation of an axial flow fan located downstream of a Bluff Body. Richard Gault (Queen's University Belfast, School of Mechanical and Aerospace Engineering, Ashby Building, Stranmillis Road, BT9 5AH Belfast, UK, r.gault@qub.ac.uk), Richard K. Cooper (Queen's University Belfast, School of Mechanical and Aerospace Engineering, Ashby Building, Stranmillis Road, BT9 5AH Belfast, UK, 
R.Cooper@qub.ac.uk),Jian Wang(Queen's University Belfast, School of Mechanical and Aerospace Engineering, Ashby Building, Stranmillis Road, BT9 5AH Belfast, UK, j.wang@qub.ac.uk), Christophe Schram (LMS International, Researchpark Z1, Interleuvenlaan 68, 3001 Leuven, Belgium, Christophe.Schram@1msintl.com)

This paper investigates the tonal noise radiated by a subsonic axial flow fan when installed downstream of a bluff body. Typically industrial axial flow fans operate in cluttered environments, and are usually driven through direct coupling from an engine. For this investigation, the fan is located in a knife-edged shroud and placed downstream of a bluff body having representative dimensions of a typical engine. Axial flow fans in general radiate broadband and discrete frequency noise, the latter of which modelling efforts are maturing. The numerical simulation is based on the aero-acoustic analogy where the unsteady flow is first computed using CFD and then passed to a BEM solver to compute the acoustic radiation. URANS and DES methods are examined for the turbulence modelling. The time-varying force on a single blade in the CFD solver over one complete rotation is used to construct the equivalent fan source in the BEM model. Experimental measurements of Sound Pressure Level are performed in a hemi-anechoic chamber, and a comparison between numerical predictions made. This numerical procedure can be used to further aid designers in understanding the effects of fan tonal noise in cluttered environments.

\section{Invited Papers}

\section{2:20}

4aNSf6. Near-field scattering of ducted fan noise using a boundary element method. Christophe Schram (LMS International, Researchpark Z1, Interleuvenlaan 68, 3001 Leuven, Belgium, Christophe.Schram @1msintl.com)

The noise emitted by rotating machinery is a concern in many applications, such as aeroengines, wind turbines, and cooling devices for IC engines or electronic appliances. A specific derivation of the Ffowcs Williams and Hawkings analogy for the tonal noise emitted by a fan was presented by Goldstein (1976), under the assumption that the listener is placed in the acoustical and geometrical far field. That formulation accounts for the modulation of the Doppler frequency shift during the fan revolution, but neglects near field effects. This work presents the application of an alternative derivation, introduced by Roger (2007), which preserves the near-field features of the sound field. This analytical method is compared with a second method in which the fan is modelled by a fixed azimuthal distribution of dipoles. A validation is performed for the case of a generic fan located in an infinite circular duct. The sound field within the duct is obtained by two means: i) calculating the sound field emitted by the fan, modelled by the above mentionned approaches, and scattered by the duct through the application of a Boundary Element Method, ii) computing the sound field by projecting the source in the duct modes.

\section{2:40}

4aNSf7. LES prediction of wall-pressure fluctuations and noise of a low-speed airfoil. Meng Wang (University of Notre Dame, Departmernt of Aerospace and Mechanical Engineering, Notre Dame, MD 46556, USA, m.wang@nd.edu), Stéphane Moreau (Valeo Thermal Systems, rue Louis Normand, 8, 78321 La Verrière, France, stephane.moreau@valeo.com), Gianluca Iaccarino (Stanford University, Dept. of Mechanical Engineering, Stanford, CA 94305, USA, jops@ctr.stanford.edu), Michel Roger (Ecole Centrale de Lyon, 36 Avenue Guy de Collongue, Centre Acoustique, 69134 Ecully, France, michel.roger@ec-lyon.fr)

The wall-pressure fluctuations and noise of a low-speed airfoil are computed using large-eddy simulation (LES). The results are compared with experimental measurements made in an open-jet anechoic wind-tunnel at Ecole Centrale de Lyon. To account for the effect of the jet on airfoil loading, a RANS calculation is conducted in the full wind-tunnel configuration, which provides velocity boundary conditions for the LES in a smaller domain within the potential core of the jet. The flow field is characterized by an attached laminar boundary layer on the pressure side and a transitional and turbulent boundary layer on the suction side. The predicted unsteady surface pressure field shows reasonable agreement with the experimental data in terms of frequency spectra and coherence in the trailing-edge region. In the nose region, characterized by unsteady separation and transition to turbulence, the wall-pressure fluctuations are highly sensitive to small perturbations and difficult to predict or measure with certainty. The LES, in combination with the Ffowcs Williams and Hall solution to the Lighthill equation, also predicts well the radiated trailing-edge noise. A finite-chord correction is derived and applied to the noise prediction, which is shown to improve the overall agreement with the experimental sound spectra.

\section{1:00-2:00 Lunch Break}

\section{Contributed Papers}

\section{2:00}

4aNSf8. The influence of the design parameters of centrifugal fans on the difference between outlet and inlet noise levels. Mikhail Y. Liberman (MOVEN Co., 17 Plekhanova str., 24/30 Zemlyanoi val str., apt. 27, 111141 Moscow, Russian Federation, mikhail.liberman@yahoo.com)

The operation of the industrial centrifugal fans is accompanied by the air-borne noise generation thanks to such phenomenon as: vortex formation within blade channels of impeller (because of flow separation in channel) and interactions between vortex wakes (at outlet of impeller) and cut-off of fan casing. Sound waves, which are formed at outlet of blade channels and within fan casing, propagate as through outlet side of casing, as in opposite direction: through rotating blade channels of impeller and inlet cone of casing. As it follows from theoretical analysis and experimental research the noise levels at outlet side of centrifugal fan are higher than noise levels at inlet of fan (as for broadband as for tonal noise), because of noise reduction, which is caused by sound wave propagation through inhomogeneous channel, consisted of impeller and inlet cone of casing. The efficiency of the noise reduction depends on the design parameters of impeller and casing. In particular, according to results of research the difference between levels of noise, radiated from outlet and inlet sides of fan, depend on: shape of blade channels, relation between width of casing and impeller.

\section{2:20}

4aNSf9. Prediction of flow induced noise in rotating devices using nonmatching grids. Jens Grabinger (Univ. Erlangen-Nuremberg, Dept. of Sensor Technology, Paul-Gordan-Str. 3/5, 91052 Erlangen, Germany, jens.grabinger@1se.eei.uni-erlangen.de), Branimir Karic (University Erlangen-Nuremberg, Institute of Fluid Mechanics, Cauerst. 4, 91058 Erlangen, Germany, branimir.karic@1stm.uni-erlangen.de), Simon Triebenbacher (Univ. Erlangen-Nuremberg, Dept. of Sensor Technology, Paul-Gordan-Str. 3/5, 91052 Erlangen, Germany, simon.triebenbacher @1se.eei.uni-erlangen.de), Manfred Kaltenbacher (Univ. ErlangenNuremberg, Dept. of Sensor Technology, Paul-Gordan-Str. 3/5, 91052 Erlangen, Germany, manfred.kaltenbacher@1se.eei.uni-erlangen.de), Stefan 
Becker(University Erlangen-Nuremberg, Institute of Fluid Mechanics, Cauerst. 4, 91058 Erlangen, Germany, stefan.becker@1stm.uni-erlangen .de), Reinhard Lerch (Univ. Erlangen-Nuremberg, Dept. of Sensor Technology, Paul-Gordan-Str. 3/5, 91052 Erlangen, Germany, reinhard.lerch@1se .eei.uni-erlangen.de)

With increasing number of electrical devices, e.g. air conditioning systems, used in homes and offices, noise pollution is becoming a more and more relevant topic. A large amount of this noise is generated by turbulent flows and laminar flows at leading and trailing edges, where mainly tonal noise is generated. The objective of our contribution is to investigate shape optimizations of rotating devices in order to reduce their noise levels. For this purpose, we conduct a simulation of the turbulent flow in a ventilator. The acoustic source terms are obtained from the fluid dynamics solution by using Lighthill's acoustic analogy. The acoustic domain is decomposed into a rotating part and a fixed part. The coupling between these two parts is enforced at their interface by a mortar finite element method, which uses Lagrange multipliers in order to "glue" the geometrically independent parts together. The mortar method takes into account the movement of the rotating part through a moving nonmatching grid, that is recomputed at each time step.

\section{2:40}

4aNSf10. Trailing edge noise computation of a fan blade profile. Julien Christophe (von Karman Institute, Chausee de Waterloo, 72, 1640 RhodeSaint-Genese, Belgium, christju@vki.ac.be), Jerome Anthoine (von Karman Institute, Chausee de Waterloo, 72, 1640 Rhode-Saint-Genese, Belgium, anthoine@vki.ac.be), Stéphane Moreau (Valeo Thermal Systems, rue Louis Normand, 8, 78321 La Verrière, France, stephane.moreau@valeo .com)

In problem involving noise generated by fans or high-lift devices in uniform stationary flow, trailing edge noise has a primary interest. This paper proposes to study the trailing edge noise produced by a Controlled-Diffusion (CD) airfoil specially developed for automotive engine cooling by Valeo. A LES flow computation is realised through the Fluent solver 6.3 for a Reynolds number based on the chord of $1.5 \times 10^{5}$ and an angle of attack of 8 degrees. This computation is compared to pressure and velocity measurements performed at ECL (France) and obtained by measurement techniques involing pressure probes and hot-wire anemometry. Amiet's theory using surface pressure spectra around the airfoil trailing-edge region is used to obtain far field acoustic predictions that are compared to microphone measurements.
3:00

4aNSf11. Optimization problem for the automatic positioning of flow obstructions to control tonal fan noise. Anthony Gerard (Univ. de Sherbrooke, Mechanical Engineering Depart., 2500 Boulevard de l'Université, Sherbrooke, QC J1K 2R1, Canada, Anthony.Gerard@USherbrooke .ca), Alain Berry (Univ. de Sherbrooke, Mechanical Engineering Depart., 2500 Boulevard de l'Université, Sherbrooke, QC J1K 2R1, Canada, alain.berry@usherbrooke.ca), Patrice Masson (Univ. de Sherbrooke, Mechanical Engineering Depart., 2500 Boulevard de l'Université, Sherbrooke, QC J1K 2R1, Canada, Patrice.Masson@USherbrooke.ca)

Tonal noise from subsonic axial fans can be controlled by adding obstructions in the downstream or upstream flow field of the rotor. These obstructions must be located with care to create secondary circumferential modes of the blade unsteady lift of equal magnitude but opposite in phase with the primary unsteady lift modes responsible for the tonal noise. The general optimization problem of controlling $\mathrm{N}$ circumferential modes using $\mathrm{N}$ obstructions with 2 degrees of freedom or $2 \mathrm{~N}$ obstructions with 1 degree of freedom is first posed. This optimization problem can be greatly simplified using $\mathrm{nB}$-perdiodic obstructions (where $\mathrm{B}$ is the number of blade) designed to control the most radiating mode of the blade unsteady lift at frequencies $n \times B P F$. In this case, the control of each frequency is uncoupled and a single error microphone in the axis of the fan can be used to globally control the selected frequencies.

\section{$3: 20$}

4aNSf12. Axial fan noise: Towards sound prediction based on numerical unsteady flow data - a case study. Hauke Reese (Ansys, Birkenweg 14a, 64295 Darmstadt, Germany, hauke.reese@ansys .com), Thomas Carolus (Institute of Fluid- and Thermodynamic, University of Siegen, Paul-Bonatz-Str. 9-11, 57068 Siegen, Germany, thomas .carolus@uni-siegen.de)

Objective of this work is to evaluate modern numerical methods for predicting flow induced fan noise. A generic fan assembly is investigated consisting of a low pressure axial impeller (diameter $0.3 \mathrm{~m}$, hub to tip ratio of 0.45 ) including an optional turbulence generator. The flow field is simulated with different state of the art unsteady computational fluid dynamic methods. All results are compared with each other and with hot wire flow velocity and surface pressure measurements. From the numerical data, the relevant dipole sound sources, i.e. the unsteady forces on the fan blades are derived. Eventually both, a free field formulation in the time domain (acoustical analogy by FFOWCS WILLIAMS and HAWKINGS), and a boundary element formulation in the frequency domain (SYSNOISE®) are employed to predict the radiated sound field based on the numerical source data. The acoustical results are compared and contrasted with measurements.

3:40-5:20 Posters

Lecture sessions will recess for presentation of poster papers on various topics in acoustics. See poster sessions for topics and abstracts.

\section{Contributed Papers}

5:20

4aNSf13. Aeroacoustic Computation of Ducted-Fan Broadband Noise Using LES Data. Gabriel Reboul (ONERA, 29 avenue Division Leclerc, $92320 \quad$ Châtillon, France, gabriel.reboul@onera.fr), Cyril Polacsek (ONERA, 29 avenue Division Leclerc, 92320 Châtillon, France, cyril.polacsek@onera.fr), Serge Lewy (ONERA, 29 avenue Division Leclerc, 92320 Châtillon, France, serge.lewy@onera.fr), Sebastien Heib (ONERA, 29 avenue Division Leclerc, 92320 Châtillon, France, sebastien.heib@onera.fr)

Following large efforts to reduce tone noise during the last decades in modern high-bypass ratio turbofans, fan broadband noise reduction has become now an industrial priority. A hybrid computational method providing source-to-far-field predictions of broadband noise due to rotor-stator interaction is presented. The acoustic model is based on the loading term of the
FWH (Ffowcs Williams and Hawkings) equation with a modal Green's function valid for an infinite annular duct, and a Kirchhoff approximation for the free-field radiation. The aerodynamic sources on the airfoils required by the model are expected to be directly issued from a LES (Large Eddy Simulation) computation. The method is applied to a simplified configuration tested in a laboratory rig. The first part of the study is concerned with the assessment of in- duct acoustic field. Usual assumptions about coherence and energy distribution between the acoustic modes are analyzed. PSD (Power Spectrum Density) are calculated through several ways. The second part is focused on the ability to generate an equivalent PSD by means of equivalent source distributions. The purpose is to validate a practical way for coupling LES with Computational Aero-Acoustics Euler solver, in order to include realistic geometry and mean flow effects. 
$5: 40$

4aNSf14. Improvements of a parametric model for fan broadband and tonal noise. Antoine Moreau (DLR - German Aerospace Center, MuellerBreslau-Str. 8, 10623 Berlin, Germany, antoine.moreau@dlr.de), Lars Enghardt (DLR - German Aerospace Center, Mueller-Breslau-Str. 8, 10623 Berlin, Germany, lars.enghardt@dlr.de)

The fan of an aero-engine is one of the most significant noise sources of civil aircraft. The purpose of the present work is to provide a tool for fan noise prediction, which can be integrated into the design process of innovative fans. Fan noise is predicted by means of an analytic model. A single noise source is considered: the rotor-stator interaction noise due to rotor blade wakes impinging onto the stator vanes. This source is known to be the major source of fan broadband and tonal noise. The formula for the sound power is based on the single airfoil theory in far field developed by Amiet. It accounts for the effect of subsonic compressible flow and source noncompactness. The model accounts for fan parameters such as geometry (blade and vane count, chord length, rotor-stator gap) and flow parameters (mean velocities, wake and turbulence characteristics). Future work will investigate a more detailed description of the sound field based on an acoustic modal approach, in which each frequency component of the sound spectrum is decomposed into a distribution of duct acoustic modes.

\section{6:00}

4aNSf15. Turbulence ingestion fan noise predictions using an advanced turbulence correlation model. Scott C. Morris (Notre Dame, 109 Hessert Laboratory, University of Notre Dame, Notre Dame, IN 46556, USA, s.morris@nd.edu)

The generation of sound by an isolated rotating blade row results from the interaction of the blades with turbulence in the approach flow. The relationship between the unsteady velocity field and the sound produced can be expressed directly in terms of the two point velocity correlation function of the approach flow field. It is often assumed that the turbulent flow can be approximated by a number of simplifying assumptions, such as homogeneous, isotropic flow. However, in the frequency range of interest for a number of applications these assumptions are not valid, and their use can lead to significant errors in the prediction of generated sound. The present work will describe a method for using advanced models of two-point velocity correlations and outline new experimental results that validate this approach.
6:20

4aNSf16. Investigation into the effect of altering incoming gust shape to far field noise radiation in Amiet's theory. Michael Bilka (von Karman Institute, Chausee de Waterloo, 72, 1640 Rhode-Saint-Genese, Belgium, bilka@vki.ac.be), Jerome Anthoine (von Karman Institute, Chausee de Waterloo, 72, 1640 Rhode-Saint-Genese, Belgium, anthoine@vki.ac.be)

Noise prediction for turbulent interactions with fan-blades or high-lift devices has been continuously developed for the last thirty years. A benchmark solution to this problem is Amiet's theory for acoustic radiation from an airfoil in a turbulent stream. In most aerodynamic theories the incoming gust is assumed to be sinusoidal, which is also the case for the theory of Amiet. This assumption affects the derivation of the unsteady lift function which is used to propagate the noise to the far-field. This paper proposes to show the effect of altering the shape of the incoming gust by assuming a Gaussian function. This analysis will then be compared to the case of the sinusoidal gust as well as to acoustic measurements of an airfoil placed in a jet taken in the VKI semi-anechoic chamber.

\section{6:40}

4aNSf17. Aeroacoustics of a low Mach number tip-gap flow. Julien Grilliat (Ecole Centrale de Lyon, 36, av. Guy de Collongue, 69134 Lyon, France, julien.grilliat@ec-lyon.fr), Marc C. Jacob (Ecole Centrale de Lyon, 36, av. Guy de Collongue, 69134 Lyon, France, marc.jacob@ec-lyon.fr)

A thorough experimental study was performed in several campaigns in the anechoic wind tunnel of the Ecole Centrale de Lyon on a single airfoil at a low Mach number to investigate the tip leakage flow and the associated broadband noise. The influence of the inflow velocity, the airfoil angle of attack and the gap size were characterized and hence an extensive data set was obtained. Both near field aerodynamic and far field acoustic features of this configuration were recorded. Statistical post-processing of the data highlighted some of the governing parameters of this jet-like flow configuration and two different noise generation mechanisms. Some scaling laws were derived from the experimental data which gave also inputs for semianalytical far field noise prediction models relying on the theory for linearised unsteady aerodynamics around a slender airfoil. The underlying sound generation mechanism is the scattering of the tip clearance flow perturbations by the airfoil trailing edge. The aerodynamic perturbation is described as a gust with a spanwise distribution that is concentrated near the gap region. The models are presented and compared to experimental results and CFD calculations. This work has been funded by the European Community as part of the 6th Framework Project PROBAND n AST4-CT-2005012222 . 


\title{
Session 4aPAa
}

\section{Physical Acoustics: Thermoacoustics I}

\author{
Steven Garrett, Cochair \\ Penn State, Applied Research Laboratory, PO Box 30, State College, PA 16804, USA \\ Philippe Blanc-Benon, Cochair \\ Ecole Centrale de Lyon, LMFA, UMR CNRS 5509, Ecully, Lyon, 69134, France
}

\section{Invited Papers}

\begin{abstract}
8:00
4aPAa1. Recent progress on thermoacoustic heat engines and refrigerators. Ercang Luo (Technical Institute of Physics and Chemistry, CAS, Beiyitiao Rd., Zhongguancun St., P.O.Box 2711, 100080 Beijing, China, Ecluo@cl.cryo.ac.cn)

In this talk, recent progress on thermoacoustic heat engines and refrigerators developed at the Technical Institute of Physics and Chemistry (TIPC), Chinese Academy of Sciences (CAS), is covered, which mainly includes three aspects: (i) Energy-focused thermoacoustic-Stirling heat engines (EF-TASHE) by using tapered resonators are highlighted for both low-frequency $(\sim 50 \mathrm{~Hz})$ and medium-frequency $(-300 \mathrm{~Hz})$ operation; (ii) A thermoacoustically driven two-stage pulse tube cryocoolers capable of achieving liquid hydrogen temperature $(\sim 20 \mathrm{~K})$ is described. In addition, a $300 \mathrm{~Hz}$ compact thermoacoustically driven pulse tube cryocooler operating below $80 \mathrm{~K}$ is also covered. In this part, an acoustical pressure amplifier is highlighted; (iii) A heat-driven thermoacoustic refrigeration system with double thermoacoustic-Stirling configuration for room temperature cooling is reported. This system is able to provide a cooling power of more than $300 \mathrm{~W}$ at $-20^{\circ} \mathrm{C}$, showing good prospect as an alternative of CFC refrigeration. Finally, consideration and prospect for future development are forecasted.
\end{abstract}

\section{$8: 20$}

4aPAa2. Study of a thermoacoustic-Stirling engine. Hassan Tijani (Energy research Centre of the Netherlands (ECN), Westerduinweg 3, 1755 LE Petten, Netherlands, tijani@ecn.nl), Simon Spoelstra (Energy research Centre of the Netherlands (ECN), Westerduinweg 3, 1755 LE Petten, Netherlands, spoelstra@ecn.nl), Gaelle Poignand (Energy research Centre of the Netherlands (ECN), Westerduinweg 3, 1755 LE Petten, Netherlands, poingnand@ecn.nl)

During the last decade most efforts in thermoacoustics have been focused on the development and understanding of the travelingwave thermoacoustic systems. These systems get much attention because they employ the inherently efficient Stirling cycle. This makes them much more efficient that the standing-wave counter parts which are intrinsically irreversible. A $1 \mathrm{~kW}$ thermal power thermoacoustic-Stirling engine is designed and performance measurements are performed. The engine incorporates a compact acoustic network to create the traveling-wave phasing necessary to operate in a Stirling cycle. The acoustic network consists of a regenerator unit, an acoustic compliance and a feedback inertance. The design, construction and performance measurements of the traveling-wave thermoacoustic engine will be presented and discussed.

\section{8:40}

4aPAa3. Low operating temperature integral thermo acoustic devices for solar cooling and waste heat recovery. Kees De Blok (Aster Thermoakoestische Systemen, Smeestraat 11, NL 8194 LG Veessen, Netherlands, c.m.deblok@aster-thermoacoustics.com)

Utilizing low temperature differences from solar vacuum tube collectors or waste heat in the range $70-200{ }^{\circ} \mathrm{C}$ seems to be the most promising field of applications for thermoacoustic systems. At these reduced temperatures overall system performance is increasingly affected by the ratio between amplified (useful) power and acoustic power stored in the resonance circuitry. Well known is that this ratio can be improved by deploying multiple regenerator units (hex-reg-hex). However, in commonly used torus or coaxial bypass configurations the correct timing (real and high acoustic impedance) is hardly realized inside more than two regenerator units (soft spot). Acoustic losses in the standing wave resonator account for another fundamental limitation because of the relatively low transferred power at a given pressure amplitude. Therefore a novel acoustic geometry will be presented in which a high and near real impedance can be maintained in even more than two regenerator units and in which acoustic feedback is performed by a true traveling wave. This approach improves the overall performance of integral thermoacoustic systems. Details and experimental results of a solar driven thermoacoustic cooler and ongoing work on thermoacoustic tri-generation utilizing exhaust gas of a standard gas-engine will be presented. 
4aPAa4. Commercial thermoacoustic products and next-step developments in acoustic cooling. John Corey (CFIC-Qdrive, 302 Tenth St., Troy, NY 12180, USA, jcorey@cficinc.com), Philip Spoor (CFIC-Qdrive, 302 Tenth St., Troy, NY 12180, USA, pspoor @ cficinc.com)

CFIC-Qdrive committed to development and commercialization of thermoacoustic energy conversion devices in 1999, by combining the newly developed acoustic perspective and tools developed at Los Alamos with a deep well of Stirling-cycle experience to achieve a total physics model with both inertial and viscous behavior of the working fluid. Such acoustic-Stirling devices combine the mechanical simplicity, robustness, and efficiency. Focusing first on refrigeration for cryogenics; this work has led to a family of standard products that have found uses worldwide, from air-quality sampling and oil refinery support to military aviation oxygen liquefaction. This paper details the basic operation of these acoustic- Stirling products and the key technological elements that make them viable and attractive in cryogenics; then examines the implications for less-cold uses like food-storage and air-conditioning; with a discussion of achievable performance in accessible applications. We review the work now underway to develop devices to meet those opportunities with environmentally benign cooling of superior performance.

9:20

4aPAa5. Generating electricity from burning wood using Thermo-acoustics for use in developing countries. Paul H. Riley (University of Nottingham, Room 1211 Tower Block, School of Electrical and Electronic Engineering, NG7 2RD Nottingham, UK, paul.riley@nottingham.ac.uk), Mark Johnson (University of Nottingham, Room 1211 Tower Block, School of Electrical and Electronic Engineering, NG7 2RD Nottingham, UK, mark.johnson@nottingham.ac.uk)

SCORE is a wood burning stove that will cook food, generate electricity and cooling for use in developing countries by means of thermoacoustics. The consortium of Nottingham (Lead), Manchester, Queen Mary, Imperial and City universities with the charity Practical Action, believe that the very demanding cost targets can be achieved by using thermo-acoustic technology due to the no-movingpart design. Standing wave and travelling wave designs are being evaluated with support from Los Alamos Laboratories in the US. The presentation will concentrate on the stove requirements and needs of the developing world and how this affects the thermo-acoustic and mechanical design. Results from mathematical modelling and measurements from an early demonstrator will be presented. More information can be found at Http://www.score.uk.com. The £2M Score research project is funded by EPSRC, a UK government agency.

\section{9:40}

4aPAa6. Recent developments on heat to electricity thermoacoustic conversion. Guadalupe Huelsz (UNAM, Privada Xochicalco Temixco Centro, Temixco Morelos, 62580 Temixco, Mexico, ghl@cie.unam.mx), Miguel Piñeirua (UNAM, Privada Xochicalco Temixco Centro, Temixco Morelos, 62580 Temixco, Mexico, mipim@cie.unam.mx), Alfonso A. Castrejon-Pita (UNAM, Privada Xochicalco Temixco Centro, Temixco Morelos, 62580 Temixco, Mexico, aacp@atm.ox.ac.uk), Fabrisio Gomez (UNAM, Privada Xochicalco Temixco Centro, Temixco Morelos, 62580 Temixco, Mexico, flgog@cie.unam.mx)

In this work we present recent developments for the conversion of heat into electricity based on the combined effects of a thermoacoustic prime mover coupled to a magnetohydrodynamic generator where different working fluids can be optimally chosen for each process. We consider the acoustically produced oscillatory motion of a liquid drop confined into a horizontal squared cross section capillary tube as a possible flow configuration for the system. We investigated the energy losses of the system and concluded that this system would be a convenient configuration for small systems. [Work supported by CONACYT 25116 project]

\section{0:00-10:20 Break}

\section{Contributed Paper}

\section{0:20}

4aPAa7. Fundamental study of a loop-tube-type thermoacoustic cooling system using heat energy from condensed sunlight. Shin-Ichi Sakamoto (Faculty of Engineering, Doshisha Univ., 1-3 Miyakodani Tatara, 610-0321 Kyotanabe, Japan, ssakamot@mail.doshisha.ac.jp), Shintaro Komiya (Faculty of Engineering, Doshisha Univ., 1-3 Miyakodani Tatara, 610-0321 Kyotanabe, Japan, bte1031@mail4.doshisha.ac.jp), Naoki Miya (Faculty of Engineering, Doshisha Univ., 1-3 Miyakodani Tatara, 610-0321 Kyotanabe, Japan, dtg0144@mail4.doshisha.ac.jp), Jiro Senda (Faculty of Engineering, Doshisha Univ., 1-3 Miyakodani Tatara, 610-0321 Kyotanabe, Japan, jsenda@mail.doshisha.ac.jp), Yoshiaki Watanabe (Faculty of Engineering, Doshisha Univ., 1-3 Miyakodani Tatara, 610-0321 Kyotanabe, Japan, kwatanab@mail.doshisha.ac.jp)
The feasibility of implementation of a sunlight-based loop-tube-type thermoacoustic cooling system is investigated. Sunlight is condensed using a $0.60 \mathrm{~m}$ diameter Fresnel lens and irradiated to the high-temperature heat exchanger. Then, the temperature of heat exchanger is risen. This heat energy from condensed sunlight is applied to the driving hear energy of the loop-tube-type thermoacoustic cooling system. The total length of the loop tube is $3300 \mathrm{~mm}$, and a gaseous mixture of $\mathrm{He}$ and $\operatorname{Ar}(50 \% / 50 \%)$ is used as the working fluid. A 50-mm-long ceramic honeycomb is used for the stack. The channel radius of the prime mover stack is $0.45 \mathrm{~mm}$; that of the heat pump is $0.35 \mathrm{~mm}$. The cooling point temperature falls from $29^{\circ} \mathrm{C}$ to $-4.3^{\circ} \mathrm{C}$ before sunlight is irradiated to the high-temperature heat exchanger. Consequently, a temperature drop of $33.3^{\circ} \mathrm{C}$ is achieved using sunlight. Result obtained in this experiment underscores the feasibility of implementation of a sunlight-based loop-tube-type thermoacoustic cooling system. 


\section{Invited Paper}

10:40

4aPAa8. Geometry effects and scaling in thermoacoustics. Jos Zeegers (Eindhoven University of Technology, Den Dolech 2, 5612 AZ Eindhoven, Netherlands, j.c.h.zeegers@tue.nl)

Current work at TU Eindhoven on thermoacoustics will be discussed. The end effects of the geometry of a stack on the performance of thermoacoustic machines will be shown. End effects and the formation of vortices is an issue that contributes to enhanced convective losses at the stack ends. Influence of Reynolds and Strouhal numbers on the oscillatory flow field in the stack are studied. It is possible to plot $\mathrm{Sr}$ and $\mathrm{Re}$ number diagrams in which various zones can be identified that display characteristic flow patterns. Furthermore the influence of the type of regenerator material is studied in traveling wave engines. Performance of honeycomb material of high-density pores is compared with wire screen regenerators in a thermoacoustic motor. As a last point the effects of downscaling to miniature systems is discussed. Limits of how far the size of a thermoacoustic cooler can be downscaled before conduction effects limit the performance are relevant

\section{Contributed Papers}

\section{1:00}

4aPAa9. Suppression of harmonics in a high frequency standing-wave thermoacoustic engine. Wei Dai (Technical Institute of Physics and Chemistry, CAS, Beiyitiao Rd., Zhongguancun St., P.O.Box 2711, 100080 Beijing, China, dwpeng@yahoo.com), Bo Yu (Technical Institute of Physics and Chemistry, Chinese Academy of Sciences, Zhongguancun BeiYiTiao 2, Hai Dian, 100080 Beijing, China, yubo@mail.ipc.ac.cn), Guoyao Yu (Technical Institute of Physics and Chemistry, Chinese Academy of Sciences, Zhongguancun BeiYiTiao 2, Hai Dian, 100080 Beijing, China, gyyao@cl.cryo.ac.cn), Ercang Luo (Technical Institute of Physics and Chemistry, CAS, Beiyitiao Rd., Zhongguancun St., P.O.Box 2711, 100080 Beijing, China, Ecluo@cl.cryo.ac.cn)

A thermoacoustic engine converts heat into acoustic power and could be used to driven a cooler for refrigeration purposes or to drive a piston for electric power generation. Due to non-linear effects inside the system, higher order harmonics could be generated which may deteriorate the thermal performance of the whole system. In this report, a $500 \mathrm{~Hz}$ standing wave thermoacoustic engine has been built. The occurrence of higher order acoustic oscillations has been closely observed. A series of experiments has been done to investigate the influence of resonance tube configuration on this phenomenon. The influence on the related thermal performance is also reported.

\section{$11: 20$}

4aPAa10. Energy conversion efficiency improvement of a thermoacoustic cooling system - The influence of a lamination mesh on cooling effect-. Atsushi Sakaguchi (Faculty of Engineering, Doshisha Univ., 1-3 Miyakodani Tatara, 610-0321 Kyotanabe, Japan, dth0165@mail4.doshisha.ac.jp), Shin-Ichi Sakamoto (Faculty of Engineering, Doshisha Univ., 1-3 Miyakodani Tatara, 610-0321 Kyotanabe, Japan, ssakamot@mail.doshisha.ac.jp), Yoshiyuki Tsuji (Faculty of Engineering, Doshisha Univ., 1-3 Miyakodani Tatara, 610-0321 Kyotanabe, Japan, dtg0925@mail4.doshisha.ac.jp), Yoshiaki Watanabe (Faculty of Engineering, Doshisha Univ., 1-3 Miyakodani Tatara, 610-0321 Kyotanabe, Japan, kwatanab@mail.doshisha.ac.jp)

It was generally considered that energy conversion efficiency of a thermoacoustic cooling system was improved by increasing proportion of a thermal boundary layer in the stack. Thinning down a channel radius of the stack is required to increase the thermal boundary layer per unit area. A ceramic stack is difficult to satisfy this requirement. To satisfy this requirement, we propose to use a lamination mesh which is formed by piled up a stainless mesh. Since the lamination mesh has complex channel, the effect of changing the mesh number on the cooling effect is obscure. The experiments were carried out by changing the mesh number and the insertion position of the stack using the straight acoustic tube to measure the cooling effect. From the experimental results, changing mesh number causes the distribution shift of the phase difference between pressure and particle velocity. The insertion position of the stack for which the maximum temperature decrease come close to center of the acoustic tube. This is corresponded to a result using the ceramic stack. It suggests that a same design method, which is used in case of applying the ceramic stack, can be adapted to the lamination mesh.

\section{1:40}

4aPAa11. Scalability of Thermoacoustic Refrigerator. Ehab Abdel-Rahman (The American University, 211 Science Building, 113 Kasr El Aini St., P.O. Box 2511, 11511 Cairo, Egypt, ehab_ab@aucegypt.edu)

The uses of thermoacoustic effect are the conversion of heat onto acoustic wave by thermoacoustic prime mover and pumping heat by acoustic wave using a thermoacoustic refrigerator. Thermoacoustic refrigerator is a good alternative to conventional vapor compression device. It can be very compact, using a minimum of uncomplicated, economical components. It can also provide variable cooling capacity and scalability to different sizes in ways that conventional vapor compression technology is not capable of We are investigating the minimum size of a thermoacoustic refrigerator that can meet the cooling requirement for different applications. The coefficien of performance (COP) of small thermoacoustic refrigerators is in the range of $20 \%$ of the ideal (Carnot cycle) COP, which is actually better than similarly scaled vapor compression coolers which is at about $10 \%$. The efficiency of thermoacoustic refrigerator can be improved by better designing of its components. In this paper the effect of components design on the performance of thermoacoustic refrigerator is discussed. The scalability of such devices is also investigated.

\section{2:00}

4aPAa12. Advanced flow measurements in thermoacoustic systems. Arganthaël Berson (Ecole Centrale de Lyon, LMFA, UMR CNRS 5509, Ecully, 69134 Lyon, France, arganthael.berson@ec-lyon.fr), Philippe Blanc-Benon (Ecole Centrale de Lyon, LMFA, UMR CNRS 5509, Ecully, 69134 Lyon, France, Philippe.Blanc-Benon@ec-lyon.fr)

One of the bottlenecks for the conception of highly efficient thermoacoustic systems is the poor performances of heat exchangers. A better understanding of both aerodynamical and thermal phenomena that occur at the interface between the stack and the heat exchangers is necessary for the improvement of heat transport between these components, especially at high acoustic amplitudes that are required for industrial applications. To this end, a specific Particle Image Velocimetry (PIV) method has been developed and measurements are performed within a standing-wave thermoacoustic refrigerator model driven at high drive ratios (up to 5\%). Vortex streets are observed behind the plates of a single stack at high acoustic level. The flow is characterized using advanced vortex analysis tools and dimensionless numbers. Vortices also appear within the gap between the stack and the hea exchangers. They will influence heat transport as was previously shown in numerical simulations from the literature. Moreover, as a first step toward enthalpy flux measurements, cold and hot wire anemometry methods are developed specifically for temperature and velocity measurements in oscillating flows. First results with these techniques will be presented and compared to available numerical and analytical models. This work is supported by ANR (project MicroThermAc NT051_42101). 


\section{Invited Paper}

4aPAa13. Powerful, efficient, robust, electro-acoustic transducers. John Corey (CFIC-Qdrive, 302 Tenth St., Troy, NY 12180, USA, jcorey@cficinc.com)

The STAR ${ }^{\mathrm{TM}}$ resonant, reciprocating transducer began as a lightweight linear alternator design for a space-power free-piston Stirling engine in the early 1990's. It has since been developed into a range of commercially available motors and alternators with rated powers from 100 to over 10,000 watts (acoustic). As motors, these are acoustic pressure drivers with unlimited operating life and typical transduction efficiencies of 80-90 percent. This paper explains the electrodynamics and operation of these moving- magnet Lorentz-force devices and the unique geometric configuration that has allowed scaling over such a wide range. We discuss the design and function of the unique single-degree-of-freedom flexure suspension that enables both the compact geometry and unlimited service life without wear. Data is presented from a large sample of units placed in service during the last decade, demonstrating the durability and performance of these remarkable devices.

\section{2:40-1:40 Lunch Break}

\section{Invited Paper}

\section{$1: 40$}

4aPAa14. Recent developments in miniaturization of thermoacoustic devices. Pierrick Lotton (Laboratoire d'Acoustique de l'Université du Maine, Avenue Olivier Messiaen, 72085 Le Mans, France, Pierrick.Lotton@univ-lemans.fr), Guillaume Penelet (Laboratoire d'Acoustique de l'Université du Maine, Avenue Olivier Messiaen, 72085 Le Mans, France, guillaume.penelet@univ-lemans.fr), Etienne Gaviot (Laboratoire d'Acoustique de l'Université du Maine, Avenue Olivier Messiaen, 72085 Le Mans, France, etienne.gaviot@univ-lemans.fr), Stephane Durand (Laboratoire d'Acoustique de l'Université du Maine, Avenue Olivier Messiaen, 72085 Le Mans, France, stephane.durand@univ-lemans.fr), Lionel Camberlein (Laboratoire d'Acoustique de l’Université du Maine, Avenue Olivier Messiaen, 72085 Le Mans, France, lionel.camberlein@univ-lemans.fr), Philippe Blanc-Benon (Ecole Centrale de Lyon, LMFA, UMR CNRS 5509, Ecully, 69134 Lyon, France, Philippe.Blanc-Benon@ec-lyon.fr), Michel Bruneau (Laboratoire d'Acoustique de l'Université du Maine, Avenue Olivier Messiaen, 72085 Le Mans, France, michel.bruneau@univ-lemans .fr)

This talk will present a review of recent works on thermoacoustic devices miniaturization conducted at Laboratoire d'Acoustique de l'Universite du Maine (LAUM), in collaboration with Laboratoire de Mecanique de Fluides et d'Acoustique (LMFA). A part of these works deals with new designs allowing higher compactness of devices. As an example, a compact non-resonant thermoacoustic refrigerator will be described, and experimental results obtained on a prototype will be presented. Another part of these works deals with the miniaturization of each element of a thermoacoustic device, especially the stack and the acoustic source. The acoustical and thermal sensors used to control these small devices have also to be miniaturized. Some specific actuators and sensors designed for miniaturized thermoacoustic refrigerators will be presented. Finally, potential applications of these miniaturized devices will be discussed.

\section{Contributed Papers}

\section{2:00}

4aPAa15. Miniaturation of thermoacoustic refrigerators. Yan Li (Eindhoven Univ. of Tech, Applied Physics, Low Temperature Physics, Cascade 3.13, PO Box 513, 5600MB Eindhoven, Netherlands, yan.li@tue .nl)

The possibility to miniature thermoacoustic refrigerators is theoretically investigated. Both standing-wave and traveling-wave systems are considered. In the consideration of standing-wave refrigerators, a system consisting of a resonator tube $(50 \mathrm{~cm})$ with a closed end and a PVC stack (length $5 \mathrm{~cm}$ ) is taken as a reference. Helium is used at a mean pressure of 10 bars and an amplitude of 1 bar. The operating frequency is $1 \mathrm{kHz}$. The variation of the performance of the refrigerator when scaled down in size is computed under the prerequisites that the temperature difference over the stack or the energy flux or energy flux density are fixed. The analytical results show, as expected, that there is a limitation for scaling-down of a standing-wave thermoacoustic refrigerator due to heat conduction. Similar scaling trends are also shown in traveling-wave refrigerators. The travelingwave reference system consists of a feedback inertance tube of $0.567 \mathrm{~m}$ long, inside diameter $78 \mathrm{~mm}$, a compliance volume of $2830 \mathrm{~cm}^{3}$ and a 24 $\mathrm{cm}$ thermal buffer tube. The regenerator is sandwiched between two heat exchangers. The system is operated at $125 \mathrm{~Hz}$ and filled with 30 bar helium gas. Again, the thermal conductance forms a practical limitation in downscaling.

$$
\text { 2:20 }
$$

4aPAa16. Nonlinear effects in standing-wave types of thermoacoustic devices. Paul Aben (Eindhoven University of Technology, Den Dolech 2, 5612 AZ Eindhoven, Netherlands, p.c.h.aben@tue.nl), Jos Zeegers (Eindhoven University of Technology, Den Dolech 2, 5612 AZ Eindhoven, Netherlands, j.c.h.zeegers@tue.nl)

In order to create high amplitudes $\left(p^{\prime} / p_{0}>10 \%\right)$ for a relatively low frequency range (between 5 and $125 \mathrm{~Hz}$ ), a large subwoofer is connected to a resonator tube by an exponential horn. A parallel-plate stack, with various plate thicknesses and separations, can be placed at different positions in the resonator tube. The position of the subwoofer membrane, the voltage and current of the subwoofer, as well as the pressure at six different positions in the resonator is measured. The measurements are in good agreement with simulations. Using a multi-microphone method the transfer matrix of a stack is determined experimentally. Using a PIV method a 2-D velocity field between and around the stack plates is measured. The vortex shedding at the end of stack plates is studied in particular. The amplitude, frequency, plate thickness, plate separation and plate-ending shape are varied. Also the streaming velocity field is studied. Small asymmetries in the geometry have a huge influence on the streaming velocity. 


\section{Invited Papers}

\section{2:40}

4aPAa17. Intensity measurement of a periodic acoustic shock wave in a resonator. Tetsushi Biwa (Dept. of Mechanical Systems and Design, Tohoku Univ., Aramaki 6-6-01, Aoba-ku, 980-8579 Sendai, Japan, biwa@ amsd.mech.tohoku.ac.jp), Taichi Yazaki (Aichi Univ. Education, Igaya, 448-8542 Kariya, Japan, tyazaki@auecc.aichi-edu.ac.jp)

A periodic shock wave of a gas column is formed in a duct, when the gas column is sinusoidally driven near the resonance frequency. This phenomenon has been one of the fundamental problems in nonlinear acoustics and has been studied extensively both theoretically and experimentally. In this work, we study the nonlinear effect leading to the shock formation through measurements of the acoustic intensity. A gas column of atmospheric air is filled in a resonator with a length of $1.15 \mathrm{~m}$ and internal radius of $10.5 \mathrm{~mm}$, and driven by an oscillating piston at $144.4 \mathrm{~Hz}$ near the fundamental resonance frequency. Pressure and axial acoustic particle velocity of the gas column are measured as a function of the resonator axis using small pressure transducers and a laser Doppler velocimeter. We show the spatial distribution of the acoustic intensity associated with the fundamental and the second modes, from which we show the nonlinear interaction between these oscillating modes.

4aPAa18. Transition to turbulence and acoustic Rayleigh streaming in thermoacoustic devices. Helene Baillet (Laboratoire d'Etudes Aérodynamiques (LEA), Université de Poitiers, ENSMA, CNRS, Bat K, 40 avenue du recteur Pineau, 86022 Poitiers, France, helene.baillet@lea.univ-poitiers.fr), Jean-Christophe Valière (Laboratoire d’Etudes Aérodynamiques (LEA), Université de Poitiers, ENSMA, CNRS, Bat K, 40 avenue du recteur Pineau, 86022 Poitiers, France, jean-christophe.valiere@lea.univ-poitiers.fr), Solenn Moreau (Laboratoire d'Etudes Aérodynamiques (LEA), Université de Poitiers, ENSMA, CNRS, Bat K, 40 avenue du recteur Pineau, 86022 Poitiers, France, solene.moreau@lea.univ-poitiers.fr), David Marx (Laboratoire d'Etudes Aerodynamiques - CNRS, Bat K, 40 avenue du recteur Pineau, 86022 Poitiers, France, david.marx@lea.univ-poitiers.fr)

Thermoacoustic engines and refrigerators with practical levels of heating or pumping power must generally operate at high pressure amplitudes. When used to describe the behavior of such high-amplitude thermoacoustic devices, the well-established foundations of thermoacoustics, based on the acoustic approximation, reach their limits. It is necessary to gain a deeper understanding of the highamplitude phenomena in order to improve the performances of thermoacoustic devices, and efforts of several research groups have been directed towards this goal over the last decade. In this presentation, we will consider recent advances in the understanding of some of the gas-dynamics phenomena leading to limitation of devices performances, namely transition to turbulence and acoustic Rayleigh streaming. The common point for these phenomena is that they owe their origin in the dynamic of oscillating flows in very near wall regions, so that their quantification implies measurements of acoustic particle velocity in adverse conditions. Recent progresses in Laser techniques used to perform such measurements will therefore also be reviewed.

4aPAa19. PIV contribution for measuring acoustic and streaming flow in thermoacoustic systems, using phase average dynamics. Diana Baltean Carlès (LIMSI-CNRS, BP 133, F-91403 Orsay, France, baltean @limsi.fr), Philippe Debesse (LIMSICNRS, BP 133, F-91403 Orsay, France, debesse@limsi.fr), François Lusseyran (LIMSI-CNRS, BP 133, F-91403 Orsay, France, francois.lusseyran@limsi.fr), Maurice-Xavier François (LIMSI-CNRS, BP 133, F-91403 Orsay, France, mxf@limsi.fr)

The present study deals with the experimental challenge of the measurement of the velocity field generated by a thermoacoustic wave. The system consists in a cylindrical standing-wave resonator, filled with gas confined at high mean pressure, driven by a thermoacoustic prime-mover. The axial and radial components of fluid velocity are measured using Particle Image Velocimetry (PIV) with an optical flow technique. The average cycle of acoustic oscillation of the velocity field is reconstructed from a temporally undersampled set of PIV snapshots, using an embedding method for building out a suitable phase space based on Singular Value Decomposition (SVD). This reconstruction allows us to extract both oscillation component of the velocity field (with the harmonic content) and time-averaged component of velocity (streaming flow). The measurements are confirmed using a second experimental procedure, based on a classical phase-averaged method: velocity measurements are synchronized with the pressure signal, the fundamental time period being decomposed in 16 phases. The measurements are repeated for different values of the drive ratio (acoustic pressure/mean pressure). The results are compared with available theory. Different experimental methods used in measuring the velocity field in thermoacoustic systems are analysed and compared with the present method. 


\section{Invited Papers}

4aPAa20. Interactive analysis, design, and teaching for thermoacoustics using DeltaEC. William C. Ward (Los Alamos National Laboratory, MS C914, Los Alamos, NM 87545, USA, ww@lanl.gov), Greg W. Swift (Los Alamos National Laboratory, MS C914, Los Alamos, NM 87545, USA, swift@lanl.gov), John P. Clark (Los Alamos National Laboratory, MS C914, Los Alamos, NM 87545, USA, jpclark@stanford.edu)

The 2008 release of the Los Alamos thermoacoustics code, DeltaEC, is distinctly different from the text-based program that was first made available in 1993. The physics captured by "Design Environment for Low-Amplitude Thermoacoustic Energy Conversion" has been steadily extended over the years. Toroids and other acoustic network topologies are now possible, along with superimposed steady flow, time-averaged pressure gradients, gas diodes, Gedeon streaming, thermoacoustic mixture separation, and resonator vibration solutions. Eight different stack pore geometries are supported, and a powerful algebraic user language allows complex, custom results to be derived without source code revisions at Los Alamos. Over the last year, the numerical methods of DeltaEC were condensed into a FORTRAN computational core and wrapped with a Python-based graphical user interface to provide modern interactive features: a multi-model tabbed interface, colorizing editors, scaled schematics, and 2D plotter windows. An intricate model can now be divided into interlinked sub-models that can be solved independently (and consistently). In addition to providing usability and new capabilities, the Python front end makes the legacy code more maintainable, extensible, and verifiable. The latest download of DeltaEC is available to all researchers at www.lanl.gov/thermoacoustics/.

\section{$5: 20$}

4aPAa21. Design road-map for thermoacoustic refrigerators. Kaveh Ghorbanian (Sharif Univ. of Technology, Dept. of Aerospace Engineering, Azadi Street, 14588-89694 Tehran, Iran, ghorbanian@sharif.edu), Hemed Hosseini (Sharif Univ. of Technology, Dept. of Aerospace Engineering, Azadi Street, 14588-89694 Tehran, Iran, hamed_hosseiny20042000@yahoo.com), Mahmoud Jafargholi (Sharif Univ. of Technology, Dept. of Aerospace Engineering, Azadi Street, 14588-89694 Tehran, Iran, mahmoud.jafargholi @ gmail.com)

The main purpose of this work is to develop a road-map to enhance the design procedure and performance analysis of thermoacoustic refrigerators (TAR). The basic mechanism of TAR is very simple and is based on the wave interaction processes of gas particles with their surrounding environment. As known, the performance of a TAR system is highly dependent to the number of gas particles involved in the process. The essential components of TAR include a sound generating device, a resonance tube, a stack of plates, and heat exchangers. In this paper, the parameters influencing the performance of a TAR system are grouped into four blocks: (I) Operation (drive ratio, operating frequency), (II) Fluid (Prandtl number), (III) Geometry (blockage ratio, tube diameter, stack length, stack positioning), and (IV) Material. A parametric study is executed to determine the optimized design of a TAR system for fixed Block II and IV scenario. First, an analysis is made on obtaining the optimum number of gas particles to be involved in the process. Then, based on this, an optimization approach is carried out to identify the best drive ratio, blockage ratio, stack positioning, stack length, and resonance tube diameter. Finally, results are compared with experimental data.

\section{Contributed Paper}

\section{$5: 40$}

4aPAa22. Thermoacoustics in random fibrous materials. Carl $R$. Jensen (The University of Mississippi - NCPA, 1 Coliseum Drive, University, MS 38677, USA, crjensen@olemiss.edu), Richard Raspet (National Center for Physical Acoustics, University of Mississippi, University, MS 38677, USA, raspet@olemiss.edu), Henry E. Bass (The University of Mississippi - NCPA, 1 Coliseum Drive, University, MS 38677, USA, pabass @olemiss.edu)

Current approaches to acoustics in fibrous and porous materials use fitting parameters to match theoretical models to measured values for the material's complex compressibility and wavenumber. In effect, these models treat the material as though it were composed of an array of rigid capillary tubes; they have proven accurate in fitting the model to data for various different porous materials such as wools and foams. However, these models do not address thermoacoustic heat transfer when the material is put under a static temperature gradient. A direct simulation has been performed using a three-dimensional thermal fluid solver to calculate both the acoustic properties and the thermoacoustic properties of a random fibrous material. The results of the simulation will be compared to experimental results for complex compressibility and wavenumber [Tarnow, H., J. Acoust. Soc. Am., 97(4) ,2272-81] as well as a proposed extension to porous theory that incorporates thermoacoustics [Roh et al., J. Acoust. Soc. Am., 121(3), 1413-22]. [Work supported by U.S. Army Space \& Missile Defense Command.]

\section{Invited Paper}

\section{6:00}

4aPAa23. Numerical study of the performance of thermally isolated thermoacoustic-stacks in the linear regime. Antonio Piccolo (Dept. of Civil Engineering - Univ. of Messina, Contrada di Dio, Villaggio S.Agata, 98100 Messina, Italy, apiccolo@unime.it), Giuseppe Pistone (Dept. of Matter Physics and Advanced Physical Technologies - Univ. of Messina, Contrada di Dio, Villaggio S.Agata, 98100 Messina, Italy, pistone@unime.it)

A simplified calculus model to investigate on the transverse heat transport near the edges of a thermally isolated thermoacoustic stack in the low acoustic Mach number regime is presented. The proposed methodology relies on the well known results of the classical linear thermoacoustic theory which are implemented into an energy balance calculus-scheme through a finite difference technique. Details of the time-averaged temperature and heat flux density distributions along a pore cross-section of the stack are given. It is shown that a net heat exchange between the fluid and the solid walls takes place only near the edges of the stack plates, at distances from the ends not exceeding the peak-to-peak particle displacement amplitude. The structure of the mean temperature field within a stack plate is also investigated; this last results not uniform near its terminations giving rise to a smaller temperature difference between the plate 
extremities than that predicted by the standard linear theory. This result, when compared with experimental measurements available in literature, suggests that thermal effects localized at the stack edges may play an important role as sources of the deviations found between linear theory predictions and experiments at low and moderate Mach numbers.

\title{
Contributed Paper
}

6:20

4aPAa24. Time-domain modelling of thermoacoustic devices: Reflections from the stack. Stig Kleiven (Chalmers University of Technology, Division of Applied Acoustics, SE-41296 Gothenburg, Sweden, stig.kleiven@chalmers.se), Krister Larsson (Chalmers University of Technology, Division of Applied Acoustics, SE-41296 Gothenburg, Sweden, k1@ta.chalmers.se), Wolfgang Kropp (Chalmers University of Technology, Division of Applied Acoustics, SE-41296 Gothenburg, Sweden, wolfgang .kropp@chalmers.se)

Thermoacoustic devices are today mainly simulated using frequencydomain models. Contrary to frequency-domain models, time-domain models may include time-varying boundary conditions and time varying effects. Furthermore, effects like reflections from the stack can be visualised which, in turn, can improve the understanding. The primary aims were: (1) to study the initial behaviour of the acoustic field in a thermoacoustic device using a time-domain method; and (2) to compare the results from the simulations with experiments. Since time-domain techniques are computational expensive, the detailed Finite-Difference Time-Domain (FDTD) method are combined with the quicker Equivalent Source Method (ESM). The acoustic field in the stack, only including viscous effects, was modelled using the FDTD method, and the ESM was used outside the stack. The experimental setup consisted of a loudspeaker connected to a circular tube containing a ceramic stack with rectangular pores. From the results of both the simulations and the experiments, clear reflections from the stack were seen. Since these reflections influence the total acoustic field in thermoacoustics devices, timedomain methods provide useful tools for further development of thermoacoustic devices.

\section{Invited Paper}

\section{6:40}

4aPAa25. Cooling load and coefficient of performance of thermoacoustic refrigerators: the role of the working fluid. Cila Herman (Johns Hopkins University, 3400 N. Charles Street, Baltimore, MD 21218, USA, cherman.jhu@gmail.com)

\begin{abstract}
Thermoacoustic refrigeration is a technology that uses mechanical energy in the form of sound waves to drive a heat pumping process that offers an environmentally safe, relatively low maintenance alternative to vapor compression refrigeration. Improving the design of the thermoacoustic core, composed of the stack plates and heat exchangers, may have the potential to bring thermoacoustic technology closer to commercial use. The stack plates have been analyzed to increase efficiency (expressed in terms of the coefficient of performance, COP) but in some applications a very high efficiency can lead to a smaller cooling load. The thermoacoustic stack was analytically optimized for maximum heat transfer (cooling load) and coefficient of performance (COP) for a range of working fluids of interest. Different noble gas mixtures were analyzed as the working fluid and helium was found to produce the highest cooling load because of its low molecular weight. The thermoacoustic stack plate center location, length, thickness and spacing were analyzed and optimum values to maximize cooling load and COP were found to exist for the specific input parameters considered. These optimization techniques may be used to design devices where maximum cooling load is more desirable than high efficiency.
\end{abstract}

\section{Contributed Papers}

7:00

4aPAa26. van der Waal gaz and direct simulation for thermoacoustics. Alain Fontaine (Université Paul Sabatier, PHASE, 118, route de Narbonne, 31062 Toulouse cedex 9, France, fontaine@cict.fr), Marie-Catherine Mojtabi (Univ. Paul Sabatier, PHASE, 118 route de Narbonne, 31062 Toulouse Cedex 9, France, cmojtabi@cict.fr), Abdelkader Mojtabi (IMFT, Allée du Professeur Camille Soula, 31400 Toulouse, France, mojtabi@cict.fr), Jean-Louis Breton (Univ. Paul Sabatier, PHASE, 118 route de Narbonne, 31062 Toulouse Cedex 9, France, breton@cict.fr), Vincent Gibiat (Université Paul Sabatier, PHASE, 118, route de Narbonne, 31062 Toulouse cedex 9, France, gibiat@cict.fr)

Thermoacoustic refrigerators work with high amplitube acoustic waves and lead to high thermal local gradient near the stack. In order to understand nonlinear thermoacoustic effects, an acoustic plane wave is propagated in a model with a specific geometry. It is a two-dimensional channel with adiabatic walls, including two conductive plates whose thickness is not regarded as null.The fluid is supposed to be a real gaz with thermodynamic properties described by a van der Waals law. A two-dimensional direct numerical model for compressible flow is used to investigate unsteady dynamic and energetic behaviours in the channel. This model relies on a finite volume formulation of the mass, momentum and energy equations for compressible flow. Thermal equilibrium between gaz and plates is assured by a Dirichlet boundary condition for the interface temperature and a thermal conduction equation in the plate. Adiabatic walls are described by a Neumann boundary condition for the temperature on the wall. The acoustic wave is generated at the input. Perfectly Matched Layer (PML) is used on input and output as a boundary condition to deal with wave reflections. Numerical investigation will be presented and compared with linear classical model.

$$
7: 20
$$

4aPAa27. Modeling and simulation of Taconis oscillations in a framework of the boundary-layer theory. Nobumasa Sugimoto (Osaka University, Department of Mechanical Science, Graduate School of Engineering Science, Toyonaka, 560-8531 Osaka, Japan, sugimoto@me .es.osaka-u.ac.jp), Dai Shimizu (Osaka University, Department of Mechanical Science, Graduate School of Engineering Science, Toyonaka, 5608531 Osaka, Japan, shimizu@mars.me.es.osaka-u.ac.jp)

This paper develops a simplified one-dimensional model to simulate numerically the onset of Taconis oscillation in a helium-filled, quarterwavelength tube in cryogenics. Introducing a boundary layer on the tube wall, nonlinear fluid-dynamical equations are averaged over the crosssection of the acoustic main-flow region outside of the boundary layer. The boundary layer gives rise to memory effects, which are taken into account in the form of half-order derivatives. For a smooth temperature distribution of the tube wall, an initial and boundary-value problem of the equations derived is solved numerically for evolution of a small disturbance. The boundary condition at the open end neglects radiation and requires the excess pressure to vanish, while the condition at the closed end takes account of the boundary layer. When the ratio of the temperature at the closed end to the one at the open end exceeds a critical value, the initial helium column becomes unstable to grow in amplitude and stationary self-excited Taconis os- 
cillations of finite amplitude emerge. It is shown that even the first-order boundary-layer theory can describe such an evolution of a small unstable disturbance into self-excited oscillations.

\section{$7: 40$}

4aPAa28. Numerical model of boundary layer effects in Rayleigh streaming. Brian Tuttle (Penn State, 412 W Foster Ave, State College, PA 16801, USA, btuttle@psu.edu), Victor W. Sparrow (Penn State, Graduate Program in Acoustics, 201 Applied Science Bldg., University Park, PA 16802,USA,vws1@psu.edu)

In thermoacoustic devices such as the pulse-tube refrigerator, efficiency is diminished by the formation of a second-order mean velocity known as
Rayleigh streaming. This flow emerges from the interaction of the working gas with the wall of the tube in a thin boundary layer. This research develops a numerical model to investigate Rayleigh streaming in straight and tapered tubes. Since the accuracy of the model depends on the correct representation of boundary layer effects, special consideration is given to the computation of thermal and viscous boundary layers including finite difference methods, computational grid refinement, and exaggeration of physical parameters for testing of boundary layers at low grid resolution. The model also allows for the inclusion or exclusion of temperature-dependent viscosity and thermal conductivity terms, the effects of which will be examined. [Work supported in part by the Office of Naval Research.]

THURSDAY MORNING, 3 JULY 2008

ROOM 351, 8:00 A.M. TO 7:40 P.M.

\title{
Session $4 \mathrm{aPAb}$
}

\section{Physical Acoustics: Ultrafast Acoustics I}

\author{
David H. Hurley, Cochair \\ Materials Characterization Department, Idaho National Laboratory, P.O.Box 1625, Idaho Falls, ID 83415-2209, USA
}

Clément Rossignol, Cochair

LMP, UMR CNRS 5469, Université Bordeaux I, 351, cours de la Libération, Talence, 33405, France

\section{Invited Paper}

\begin{abstract}
8:00
4aPAb1. Coherent phonons in semiconductor superlattice under DC electrical bias. Anthony Kent (University of Nottingham, School of Physics and Astronomy, University Park, NG9 3JE Nottingham, UK, anthony.kent@nottingham.ac.uk), Ryan Beardsley (University of Nottingham, School of Physics and Astronomy, University Park, NG9 3JE Nottingham, UK, ppxrb1@nottingham.ac.uk), Andrey Akimov (University of Nottingham, School of Physics and Astronomy, University Park, NG9 3JE Nottingham, UK, andrey.akimov@ nottingham.ac.uk), Mohamed Henini (University of Nottingham, School of Physics and Astronomy, University Park, NG9 3JE Nottingham, UK, mohamed.henini@nottingham.ac.uk)

Resonant pumping of superlattices SLs by femtosecond laser pulses results in the generation of coherent phonons with frequency centred approximately on $s / d_{\mathrm{SL}}$, where $s$ is the speed of longitudinal sound and $d_{\mathrm{SL}}$ is the SL period. The phonons can be detected by measuring the changes in reflectance of time-delayed probe pulses. To date, measurements have been made on nominally undoped SLs, and phonon frequencies are typically in the range $100 \mathrm{GHz}-1 \mathrm{THz}$. The motivation for these studies is that such hypersound could be used for acoustic probing of nanostructures. Here we describe the generation and detection of coherent phonons in a doped and electrically-biased SL. The studied SL consisted of $50 \times(6 \mathrm{~nm} \mathrm{GaAs}$ and $4 \mathrm{~nm} \mathrm{AlAs})$, uniformly doped with Silicon to density $2 \times 10^{22}$ $\mathrm{m}^{-3}$. Pump-probe measurements were made at $\lambda \sim 770 \mathrm{~nm}$ on an optical MESA at $T=12 \mathrm{~K}$. At zero bias, we observed a similar phonon spectrum as previously observed in comparable but undoped SLs, with a mode at $\sim 450 \mathrm{GHz}$. Under applied bias this mode increased in amplitude, and the decay time was also increased. We discuss these observations in terms of the effects of the bias on the coherent phonon generation and detection process and also the possibility of coherent phonon amplification occurring in the structure.
\end{abstract}

\section{Contributed Papers}

\section{8:20}

4aPAb2. Acoustic Bloch oscillations, Wannier-Stark ladders and negative refraction in ultra- and hypersonic superlattices. Yuriy A. Kosevich (Semenov Institute of Chemical Physics Russian Academy of Sciences, ul. Kosygina 4, 119991 Moscow, Russian Federation, yukosevich @yahoo.com)

A new and very efficient ultrasonic superlattice is realized for the study of such fundamental effects of quantum transport in a perturbed periodic potential as acoustic Bloch oscillations, Wannier-Stark ladders and resonant Landau-Zener tunneling. The acoustic equivalent of the Wannier-Stark ladders is employed in a set of water cavities, with a gradient of the thick- nesses, in a simple water-solid multilayer system. Bloch oscillations in different acoustic minibands are observed as time-resolved oscillations of the transmisssion of ultasonic pulses with corresponding spectral positions and widths. Acoustic Bloch oscillations with different temporal periods for the pulses centered in two neighboring acoustic minibands are observed. Experimental observations are in very good agreement with the transfer-matrix simulations [1]. The propagation of acoustic pulse in an even acoustic miniband in orthogonal or oblique with respect to the superlattice axis direction will result in negative refraction and Bloch oscillations of acoustic pulse with "negative effective mass", which can be visualized by mapping out of acoustic pressure field. Hypersonic phononic structures, which can be fabricated with the use of interference lithography, render possible the obser- 
vation of all the abovementioned acoustic phenomena in the hypersound frequency range. 1. H. Sanchis-Alepuz, Yu. A. Kosevich, and J. SanchezDehesa, 2007, Phys. Rev. Lett. v. 98, 134301.

\section{$8: 40$}

4aPAb3. Strain wave induced electron transport in superlattices Anthony Kent (University of Nottingham, School of Physics and Astronomy, University Park, NG9 3JE Nottingham, UK, anthony.kent@nottingham.ac.uk), Daivid Fowler (University of Nottingham, School of Physics and Astronomy, University Park, NG9 3JE Nottingham, UK, daivid.fowler@nottingham.ac.uk), Mohamed Henini (University of Nottingham, School of Physics and Astronomy, University Park, NG9 3JE Nottingham, UK, mohamed.henini@nottingham.ac.uk), Mark Greenaway (University of Nottingham, School of Physics and Astronomy, University Park, NG9 3JE Nottingham, UK, ppxmg@nottingham ac.uk), Alexander Belanov (University of Nottingham, School of Physics and Astronomy, University Park, NG9 3JE Nottingham, UK, alexander.belanov@nottingham.ac.uk), Mark Fromhold (University of Nottingham, School of Physics and Astronomy, University Park, NG9 3JE Nottingham, UK, mark.fromhold@nottingham.ac.uk)

We show that propagating high-amplitude coherent strain pulses, generated by ultrafast optical excitation of a metal film can induce a charge current in a GaAs/AlAs superlattice (SL). The studied SL had a period of of $12.5 \mathrm{~nm}$ and a miniband width of $12 \mathrm{meV}$. It was grown by MBE on a 0.35 mm-thick semi-insulating GaAs substrate, and a 100 micron device MESA fabricated. On the other side of the substrate a $100 \mathrm{~nm}$-thick Al film was deposited. A coherent picosecond strain pulse was generated opposite the device by exciting the Al film with $40 \mathrm{fs}, 800 \mathrm{~nm}$ pulses from a $5 \mathrm{KHz}, 2.5$ mJ Ti:Sapphire amplifier. A strong current pulse from the device was observed about $80 \mathrm{~ns}$ after the laser pulse was incident on the Al film, this time delay being equal to the time of flight of longitudinal polarized strain pulses across the GaAs substrate. We attribute the current pulse to electrons that are confined and dragged along by the potential generated by the strain wave Theoretical calculations show that this "wave dragging" effect in the presence of the SL potential can give rise to the generation of ultra-high $(\mathrm{THz})$ frequency electron dynamics.

\section{9:00}

4aPAb4. Thickness dependence of the acoustical response of ultra-thin metallic films studied by Colored Picosecond Ultrasonics. Arnaud Le Louarn (IEMN-CNRS, Cité Scientifique - Avenue Poincaré, BP 60069, 59652 Villeneuve d'Ascq Cedex, France, arnaud.lelouarn@isen.fr), Arnaud Devos (IEMN-CNRS, Cité Scientifique - Avenue Poincaré, BP 60069, 59652 Villeneuve d'Ascq Cedex, France, arnaud.devos@isen.fr), Clément Rossignol (LMP, UMR CNRS 5469, Université Bordeaux I, 351, cours de la Libération, 33405 Talence, France, c.rossignol@1mp.u-bordeaux1.fr)

We have previously demonstrated $[1,2]$ that a connection exists between Colored Picosecond Ultrasonic (CPU) experiments and electronic structure of metallic thin films. Indeed, a strong change of the detected acoustic echoes is observed when the laser is tuned around an interband transition wavelength. This connection suggests that CPU can be an useful tool for measuring interband transitions in thin metallic films. Surprisingly, by doing such a measurement on a series of ultra-thin Aluminium films, we observed a significant shift of the transition (from 880 to $970 \mathrm{~nm}$ ) as the film thickness is reduced (from 400 to $120 \mathrm{~A}$ ). We will discuss the origin of the phenomenon and propose some applications to the characterization of ultra-thin metallic films. [1] A. Devos and C. Lerouge, Phys. Rev. Lett. 86, 2669 (2001) [2] A. Devos and A. Le Louarn, Phys. Rev. B 68, 045405 (2003)

\section{9:20}

4aPAb5. Monochromatic high frequency coherent phonons propagation with superlattice transducers. Agnès Huynh (INSP - UMR 7588 CNRS \& Université Pierre et Marie Curie, 140 Rue de Lourmel, 75015 Paris, France, agnes.huynh@insp.jussieu.fr), Maria Florencia Pascual Winter (INSP - UMR 7588 CNRS \& Université Pierre et Marie Curie, 140 Rue de Lourmel, 75015 Paris, France, Florencia.PascualWinter@insp .jussieu.fr), Bernard Perrin (INSP - UMR 7588 CNRS \& Université Pierre et Marie Curie, 140 Rue de Lourmel, 75015 Paris, France, bernard.perrin@insp.jussieu.fr), Bernard Jusserand (INSP - UMR 7588 CNRS \& Université Pierre et Marie Curie, 140 Rue de Lourmel, 75015 Paris, France, Bernard.Jusserand@insp.jussieu.fr), Aristide Lemaitre (LPN, CNRS route de Nozay, 91460 Marcoussis, France, Aristide.Lemaitre @1pn.cnrs.fr), Alejandro Fainstein (Centro Atomico Bariloche \& Instituto Balseiro, C. N. E. A., 8400 S. C. de Bariloche, Argentina, afains@cab.cnea gov.ar)

The availability of efficient and compact phonons transducers in the $\mathrm{THz}$ range would be very interesting for phonons spectroscopy, acoustic microscopy and study of vibrational and electronic properties of nanostructures. Thanks to epitaxial growth of semiconductors multilayers, high quality phononic nanostructures with standard semiconductors, such as superlattices (SL) and nanocavities can be obtained for the $\mathrm{GHz}$ and $\mathrm{THz}$ transduction. Picosecond ultrasonics experiments have been performed in transmission geometry with pump and probe incident on opposite sides of the substrate, allowing discoupling acoustic generation and detection processes. By these means, we have shown independently that SL are very efficient high frequency monochromatic phonon generators and detectors. We report on experiments where two superlattices have been grown on the opposite sides of a substrate: a first SL with uniform layer thickness over the whole surface sample is used as a generator; the other one, used as the detector, presents a thickness gradient and the location of the detection is chosen in order to have the best matching with the emitted frequency. This setup is used to study the propagation of monochromatic high frequency coherent phonons as a function of temperature.

\section{9:40}

4aPAb6. Progress on coherent generation of terahertz acoustic phonons by resonant absorption of nanosecond-pulsed far-infrared laser radiation in silicon doping superlattices. Thomas E. Wilson (Marshall University, Department of Physics, One John Marshall Drive, Huntington, WV 25755-2570, USA, wilsont@marshall.edu)

We report progress on experiments to generate and detect coherent terahertz acoustic phonons. Coherent phonons are generated ${ }^{1}$ in a silicon-doping superlattice by the absorption of grating-coupled high peak-power cavitydumped far-infared laser radiation ${ }^{2}$. The superlattice period is chosen to match the phonon wavelength at the excitation frequency of the laser radiation. The phonons propagate across the $\mathrm{Si}: \mathrm{B}$ substrate and are detected by a novel superconducting granular aluminium/palladium bilayer microbolometer $^{3}$ with sub-nanosecond resolution. The phonon spectrum is obtained by piezo-phonon spectroscopy ${ }^{4}$ via the boron impurities in the silicon substrate. (1) P. Ruden and G.H. Dohler, "Anistropy Effects and Optical Excitation of Acoustic Phonons in n-i-p-i Doping Superlattices", Solid State Commun. 45 (1), 23 (1983). (2) T.E. Wilson, "A High-Power NH3 Laser Pumped in a Three-Mirror CO2 Laser Cavity with Optically-Switched Cavity-Dumping", International Journal of Infrared and Millimeter Waves 14 (2), 303 (1993). (3) T E Wilson, "Fabrication and characterization of granular aluminum/palladium bilayer microbolometer", Meas. Sci. Technol. 18 N53-N59 (2007). (4) S. Roshko and W. Dietsche, "Phonon Spectroscopy in High Magnetic Fields: The B+ Center in Si", Solid State Comm. 98(5), 453 (1996).

\section{0:00-10:20 Break}

\section{$10: 20$}

4aPAb7. Escape time of an acoustic nanocavity mode. Maria Florencia Pascual Winter (INSP - UMR 7588 CNRS \& Université Pierre et Marie Curie, 140 Rue de Lourmel, 75015 Paris, France, Florencia.PascualWinter @insp.jussieu.fr), Guillermo Rozas (Centro Atomico Bariloche \& Instituto Balseiro, C. N. E. A., 8400 S. C. de Bariloche, Argentina, rozasg@ib .cnea.gov.ar), Alejandro Fainstein (Centro Atomico Bariloche \& Instituto Balseiro, C. N. E. A., 8400 S. C. de Bariloche, Argentina, afains@cab .cnea.gov.ar), Bernard Jusserand (INSP - UMR 7588 CNRS \& Université Pierre et Marie Curie, 140 Rue de Lourmel, 75015 Paris, France, Bernard.Jusserand@insp.jussieu.fr), Bernard Perrin (INSP - UMR 7588 CNRS \& Université Pierre et Marie Curie, 140 Rue de Lourmel, 75015 
Paris, France, bernard.perrin@insp.jussieu.fr),Aristide Lemaitre(LPN, CNRS route de Nozay, 91460 Marcoussis, France, Aristide.Lemaitre@1pn .cnrs.fr)

We present a study of the escape time of a $1 \mathrm{THz}$ nanocavity phonon mode as a function of the Q-factor of the cavity. We compare results from picosecond acoustics and high resolution Raman scattering experiments, the latter obtained by means of a Fabry-Perot/triple-additive spectrometer tandem. A nanocavity consists of a GaAs spacer enclosed by two acoustic GaAs/AlAs Bragg mirrors. The number of periods of the inner mirror varies from sample to sample, spanning a range $64<\mathrm{Q}<2470$. This means that the cavity mode tunnels through the inner mirror to the substrate at theoretical time intervals that vary from 64 to 2470 ps for the different samples. An optic AlGaAs/AlAs Bragg mirror was grown between the substrate and the inner acoustic mirror in order to allow for the observation of the cavity mode in a backscattering configuration of the Raman experiments, otherwise forbidden by symmetry. At room temperature we observe escape times that vary from 65 to $278 \mathrm{ps}$. The theoretical values match the experimental results if a 3.0-GHz-wide lorentzian convolution is included to account for broadening effects. Possible explanations for this broadening will be discussed, as well as low temperature results.

\section{Invited Papers}

\section{0:40}

4aPAb8. Measurement of the velocity dispersion and attenuation in a liquid metal at GHz frequencies. Oliver B. Wright (Division of Applied Physics, Graduate School of Engineering, Hokkaido University, 0608628 Sapporo, Japan, olly@eng.hokudai.ac.jp), Bernard Perrin (INSP - UMR 7588 CNRS \& Université Pierre et Marie Curie, 140 Rue de Lourmel, 75015 Paris, France, bernard.perrin@insp.jussieu.fr), Osamu Matsuda (Division of Applied Physics, Graduate School of Engineering, Hokkaido University, 0608628 Sapporo, Japan, omatsuda@eng.hokudai.ac.jp), Vitali Gusev (LPEC/UMR 6087/CNRS/Université du Maine, Avenue Olivier Messiaen, 72085 Le Mans Cedex 09, France, vitali.goussev@univ-lemans.fr)

Ultrashort optical pulses are used to excite and interferometrically detect picosecond longitudinal acoustic pulses in thin films of liquid mercury sandwiched between sapphire plates. By analysing consecutive acoustic echoes we derive the dispersion of the ultrasonic attenuation and sound velocity for this liquid at frequencies up to $10 \mathrm{GHz}$. Two types of optical detection, from the same side of the film as the excitation light and from the opposite side to the excitation light, are presented. Significant effects of structural relaxation are observed and are compared to a simple model that indicates the presence of picosecond relaxation times in mercury.

11:00

4aPAb9. Pulse laser induced wave propagation in graded media and focusing devices. Jacqueline Vollmann (ETH Zurich, Institute of Mechanical Systems, Dept. of Mechanical and Process Engineering, CH 8092 Zurich, Switzerland, vollmann@imes.mavt.ethz.ch), Juerg Bryner (ETH Zurich, Institute of Mechanical Systems, Dept. of Mechanical and Process Engineering, CH 8092 Zurich, Switzerland, bryner@imes.mavt.ethz.ch), Laurent Aebi (ETH Zurich, Institute of Mechanical Systems, Dept. of Mechanical and Process Engineering, CH 8092 Zurich, Switzerland, aebi@imes.mavt.ethz.ch), Jurg Dual (ETH Zurich, Institute of Mechanical Systems, Dept. of Mechanical and Process Engineering, CH 8092 Zurich, Switzerland, dual@imes.mavt.ethz.ch)

Near-infrared-laser pulses having durations of $100 \mathrm{fs}$ are used to excite elastic waves thermoelastically propagating in a sub-THz frequency range. The elastic waves interact with inhomogeneities and carry information to the surface. The arrivals of the elastic pulses at the surface lead to transient changes of the optical reflectance which are monitored with short laser pulses which have a defined and controlled time delay relative to the initial pulses. Two activities of the research group are presented: The reflection and transmission behavior of acoustic waves propagating in graded media shows a frequency dependent nature and can therefore be used for filtering purpose. Time-boundary value problems are solved for various gradients with a finite-difference method. Results of the numerical simulation are presented and compared with laser-acoustic measurements. A 'classical' photoacoustic set-up provides an in-depth resolution of about $5 \mathrm{~nm}$ whereas the lateral resolution is in the order of 5 to 10 microns. To enhance the lateral resolution of the pump-probe technique, the elastic wave propagation along structures with arbitrary tip-like geometries consisting of orthotropic material is analyzed. With such structures representing ultrasonic lenses, the elastic energy is focused to a spot size given by the sharpness of the tip thereby leading to a higher lateral resolution.

\section{Contributed Papers}

11:20

4aPAb10. Acoustic dynamics in glasses in the mesoscopic range Giulio Monaco (ESRF, 6, rue Horowitz, 38043 Grenoble, France, gmonaco @esrf.fr)

The investigation of the high-frequency acoustic excitations in glasses and of their connection to the universal anomalies in the thermal properties remains a largely debated topic. For instance, one interpretation is based on the observation that the high-frequency acoustic dynamics in simulated harmonic glasses shares the same main features as those found in experiments on real glasses [1]. Another interpretation is based on the observation that both acoustic dispersion and attenuation measured in glasses and in the corresponding poly-crystals are indistinguishable [2]. A further interpretation is based on the observation that in some glasses the high-frequency acoustic attenuation increases as a power of $\mathrm{q}$ with an exponent of four or larger up to frequencies corresponding to the Boson peak [3]. Here, I will discuss the above approaches on the basis of recent inelastic x-ray scattering results on the high-frequency acoustic dynamics of glasses. [1] G. Ruocco et al., Phys. Rev. Lett. 84, 5788 (2000). [2] A. Matic et al., Phys. Rev. Lett. 93, 145502 (2004). [3] B. Rufflè et al., Phys. Rev. Lett. 96, 045502 (2006).
11:40

4aPAb11. Perspectives on spatial dispersion in cubic crystals provided by neutron scattering, phonon imaging, picosecond laser ultrasound and lattice dynamics models. Arthur G. Every (School of Physics, University of the Witwatersrand, PO Wits 2050 Johannesburg, South Africa, arthur.every@wits.ac.za), Kudakwashe Jakata (School of Physics, University of the Witwatersrand, PO Wits 2050 Johannesburg, South Africa, Kudakwashe.Jakata@students.wits.ac.za)

Spatial dispersion is the variation of acoustic wave speed with wavelength, and sets in when the wavelength approaches the natural scale of length of a medium, or lattice spacing in the case of a crystal. The first onset of dispersion can be treated within the context of continuum mechanics by the incorporation of third and fourth order spatial derivatives of the displacement field in the elastic wave equation. These additional terms yield corrections to the phase velocity which in general are quadratic in the spatial frequency $\mathrm{k}$. This paper will survey the experimental techniques that give one access to the coefficients in these expansions, in particular inelastic neutron scattering, ballistic phonon imaging and picosecond laser ultrasound. The main emphasis of the paper will be on deriving the numerical values of the 
dispersion coefficients for four cubic crystals, $\mathrm{Si}, \mathrm{Ge}, \mathrm{GaAs}$ and $\mathrm{InSb}$, from published neutron scattering data and demonstrating how modified continuum elastodynamics with these values of the coefficients is able to account well for the available dispersive phonon images of these crystals. Comparison will be made with values for the dispersion coefficients that have been obtained from laser ultrasound measurements and from lattice dynamics models.

\section{2:00}

4aPAb12. High frequency ultrasonic waves in metals and dielectrics Maria Eleftheriou (Department of Music Technology and Acoustics, Technological Educational Institute of Crete, 1 E. Daskalaki Str., 74100 Rethymnon, Greece, marel@physics.uoc.gr), Makis Bakarezos (Department of Music Technology and Acoustics, Technological Educational Institute of Crete, 1 E. Daskalaki Str., 74100 Rethymnon, Greece, bakarezos@stef.teicrete.gr), Andreas Lyras (Department of Physics, University of Ioannina, 45110 Ioannina, Greece, alyras@uoi.gr), Costas Kosmidis (Department of Physics, University of Ioannina, 45110 Ioannina, Greece, kkosmidis@uoi.gr), Michael Tatarakis (Department of Electronics, Technological Educational Institute of Crete, Romanou 3, 73133 Chania, Greece, m.tatarakis@chania.teicrete.gr), Nektarios Papadogiannis (Department of Music Technology and Acoustics, Technological Educational Institute of Crete, 1 E. Daskalaki Str., 74100 Rethymnon, Greece, npapadogiannis@stef.teicrete.gr)

We theoretically study the generation of high frequency ultrasonic waves by short laser pulses, as well as their propagation, in metals and dielectrics. For this purpose, we employ a theoretical model that applies to both cases of materials. In the case of the dielectric the theoretical model is reduced properly. We compute key physical quantities of the lattice deformation such as the temperature, the strain and the displacement of the bulk while we compare the obtained results for the two different abovementioned types of materials. The dependence of these quantities on the generating laser intensity and pulse duration is investigated, revealing interesting differences in their behavior.
12:20

4aPAb13. Imaging of 3D acoustic wave-fronts by means of picosecond laser ultrasonics in GaAs substrate. Emmanuel Péronne (INSP - UMR 7588 CNRS \& Université Pierre et Marie Curie, 140 Rue de Lourmel, 75015 Paris, France, emmanuel.peronne@insp.jussieu.fr), Laurent Belliard (INSP - UMR 7588 CNRS \& Université Pierre et Marie Curie, 140 Rue de Lourmel, 75015 Paris, France, lbelliar@ccr.jussieu.fr), Shuo Zhang (INSP - UMR 7588 CNRS \& Université Pierre et Marie Curie, 140 Rue de Lourmel, 75015 Paris, France, Shuo.Zhang@insp.jussieu .fr), Clément Rossignol (LMP, UMR CNRS 5469, Université Bordeaux I, 351, cours de la Libération, 33405 Talence, France, c.rossignol@1mp.u-bordeaux1.fr), Bernard Perrin (INSP - UMR 7588 CNRS \& Université Pierre et Marie Curie, 140 Rue de Lourmel, 75015 Paris, France, bernard.perrin@insp.jussieu.fr)

Picosecond ultrasonic experiments are widely used as pump-probe technique using longitudinal acoustic pulses and measuring time dependence of the signal. We present an experiment which combines an interferometer with the control of the pump-probe spatial overlap. Such set-up can perform a new kind of bulk phonon imaging based on ultrafast optical generation and detection scheme. When ultrafast acoustic longitudinal wave propagate in crystals they generate coherent transverse acoustic wave by diffraction. Transverse waves are known to give rise to phonon focusing patterns. The imaging of such patterns in time and space allowed us to partially reconstruct the wave front surface of bulk acoustic wave in crystals. The data may be viewed as $2 \mathrm{D}$ slices of the $3 \mathrm{D}$ acoustic wavefront revealing the basic anisotropy of the solid with unprecedented micronic lateral and in-depth resolution. Measurements are carried out in a $356 \mu \mathrm{m}$-thick GaAs sample as an example, but many different experimental conditions are compatible with the present set-up. This technique provides a new way to perform phonon energy and phonon polarisation dependent experiment with high frequency phonons.

12:40-1:40 Lunch Break

\section{Contributed Paper}

\section{$1: 40$}

4aPAb14. Surface acoustic waves propagating on microstructured phononic crystals. Dieter M. Profunser (Division of Applied Physics, Graduate School of Engineering, Hokkaido University, 0608628 Sapporo, Japan, dieter@profunser.net), Oliver B. Wright (Division of Applied Physics, Graduate School of Engineering, Hokkaido University, 0608628 Sapporo, Japan, olly@eng.hokudai.ac.jp), Osamu Matsuda (Division of Applied Physics, Graduate School of Engineering, Hokkaido University, 060 8628 Sapporo, Japan, omatsuda@eng.hokudai.ac.jp), Yukihiro Tanaka (Division of Applied Physics, Graduate School of Engineering, Hokkaido University, 0608628 Sapporo, Japan, yuki@eng.hokuda .ac.jp), Abdelkrim Khelif (Institut FEMTO-ST/CNRS, 32 avenue de l'Observatoire, 25044 Besançon cedex, France, abdelkrim .khelif@femto-st.fr), Vincent Laude (Institut FEMTO-ST/CNRS, 32 avenue de l'Observatoire, 25044 Besançon cedex, France, vincent .laude@femto-st.fr), Sarah Benchabane (Institut FEMTO-ST/CNRS, 32 avenue de l'Observatoire, 25044 Besançon cedex, France, sarah.benchabane @ femto-st.fr)

We investigate the interaction between high frequency surface acoustic waves and periodic microstructured patterns that form phononic crystals The experimental method combines an optical pump-probe setup with interferometric detection and provides picosecond temporal and micron spatial resolutions. Surface acoustic waves with frequency components up to 1.3 $\mathrm{GHz}$ are imaged in real-time propagating over the periodic metamaterial. We used a DRIE (deep reactive ion etching) process to fabricate $2 \mathrm{D}$ air-silicon phononic crystals in the form of a square lattice. We present real-time animations of surface acoustic waves scattered by the phononic crystals. In particular we describe the frequency and angular dependence of the surface acoustic wave reflection from a $2 \mathrm{D}$ phononic crystal boundary. Fourier analysis allows us to reveal details of the acoustic band structure including gaps. The presence of such phononic band gaps enables us to visualize surface acoustic waves in waveguides, cavities and other phononic circuits at $\mathrm{GHz}$ frequencies. 


\section{Invited Papers}

\section{2:00}

4aPAb15. Nanoacoustic waves in nanomaterials. Jaap I. Dijkhuis (Utrecht University, Ornsteinlaboratorium, Princetonplein 1, 3508TA Utrecht, Netherlands, j.i.dijkhuis@phys.uu.nl), Peter J. Van Capel (Utrecht University, Ornsteinlaboratorium, Princetonplein 1, 3508TA Utrecht, Netherlands, p.j.s.vancapel@phys.uu.nl)

We discuss the formation and detection of acoustic soliton trains in crystal slabs. High-amplitude amplitude strain pulses are generated by impact of intense femtosecond optical pulses on a metallic film and injected into the crystal slab. Nonlinear acoustic propagation leads to the formation of shock fronts (N-waves), that, in absence of viscous damping and by virtue of dispersion, may develop into soliton trains at the leading edge and high frequency tails at the trailing edge. Interferometric pump-probe optical experiments are discussed to directly detect the ultrafast surface displacements when a soliton is reflected at the surface of the crystal slab. Finally, we experimentally prove that these acoustic solitons are capable of impulsively exciting $\mathrm{THz}$ transitions in electronic centers in solids. The coherent terahertz acoustic pulse trains are applied to manipulate the optical response of two-dimensional excitons in a III-V quantum well on the ultrafast timescale. By virtue of the deformation potential, the coherent exciton emission becomes strongly "chirped" when the acoustic pulse train passes the quantum well. This yields the prospect to perform pump-probe terahertz acoustic experiments with nanoacoustic waves in semiconductor nanomaterials.

\section{2:20}

4aPAb16. Phonons rectification in picosecond laser ultrasonics. Bernard Perrin (INSP - UMR 7588 CNRS \& Université Pierre et Marie Curie, 140 Rue de Lourmel, 75015 Paris, France, bernard.perrin@insp.jussieu.fr), Shuo Zhang (INSP - UMR 7588 CNRS \& Université Pierre et Marie Curie, 140 Rue de Lourmel, 75015 Paris, France, Shuo.Zhang@insp.jussieu.fr), Laurent Belliard (INSP UMR 7588 CNRS \& Université Pierre et Marie Curie, 140 Rue de Lourmel, 75015 Paris, France, lbelliar@ccr.jussieu.fr), Emmanuel Péronne (INSP - UMR 7588 CNRS \& Université Pierre et Marie Curie, 140 Rue de Lourmel, 75015 Paris, France, emmanuel.peronne @ insp.jussieu.fr)

In standard ultrafast acoustic experiments very short coherent acoustic pulses are generated by the absorption of a femtosecond laser pulse in a thin metallic transducer deposited on the sample. Subsequently the acoustic pulses and the heat generated in the transducer cross the sample and are partially transmitted in the underlaying substrate. At low temperature, heating of the metallic transducer gives rise to the emission of incoherent phonons wave packets which propagate ballistically over large distances in the substrate. We report on a series of experiments which demonstrate the acoustic rectification [1-3] of these wave packets as they propagate through large GaAs or Si substrates. [1] B. Perrin, J. de Phys. C8 (1979) 216. [2] S. M. Avanesyan, V. E. Gusev, Solid State. Commun. 54 (1985) 1065. [3] B. Perrin, E. Péronne, L. Belliard, Ultrasonics, 44 (2006) 1277.

\section{$2: 40$}

4aPAb17. Optical characterization of the acoustic response in a nanostructure using the transient reflection matrix formalism. Denis Mounier (LPEC/UMR 6087/CNRS/Université du Maine, Avenue Olivier Messiaen, 72085 Le Mans Cedex 09, France, denis.mounier@univ-lemans.fr), Pascal Picart (Laboratoire d'Acoustique de l’Université du Maine, Avenue Olivier Messiaen, 72085 Le Mans, France, pascal.picart@univ-lemans.fr), Pascal Ruello (LPEC/UMR 6087/CNRS/Université du Maine, Avenue Olivier Messiaen, 72085 Le Mans Cedex 09, France, pascal.ruello@univ-lemans.fr), Jean-Marc Breteau (LPEC/UMR 6087/CNRS/Université du Maine, Avenue Olivier Messiaen, 72085 Le Mans Cedex 09, France, jean-marc.breteau@univ-lemans.fr), Vitali Gusev (LPEC/UMR 6087/CNRS/Université du Maine, Avenue Olivier Messiaen, 72085 Le Mans Cedex 09, France, vitali.goussev@univ-lemans.fr)

The transient reflectometry and transient interferometry are the most commonly used techniques of picosecond acoustics for the study of isotropic planar stratified nanostructures. Nevertheless when anisotropy is present in the sample, the standard techniques have to be completed by transient polarimetry. The reflection properties of an anisotropic sample at oblique incidence are completely determined by the $2 \times 2$ reflection matrix $(\mathrm{RM}): \mathrm{R}=[\mathrm{rpp}, \mathrm{rps}$;rsp,rss]. Considering that the transient acoustic phenomena induce a perturbation $\Delta R$ of the reflection matrix, we demonstrate that the transient reflection matrix (TRM): $\Delta R \cdot R^{-1}$, where $R^{-1}$ is the inverse of the reflection matrix, can be completely determined experimentally using the three techniques: transient reflectometry, interferometry and polarimetry (TRIP). In particular, the off-diagonal components of the TRM can be determined by transient polarimetry measurements only. Moreover, theoretical calculations of the TRM point up the close relation between the off-diagonal components of the TRM and the presence of a shear strain wave propagating perpendicularly to the free surface of the sample. Experimental results using the transient polarimetry technique will be presented to support the theoretical prediction of the TRM formalism.

\section{3:00}

4aPAb18. Picosecond ultrasonics in a single biological cell. Bertrand Audoin (LMP, UMR CNRS 5469, Université Bordeaux I, 351, cours de la Libération, 33405 Talence, France, b.audoin@1mp.u-bordeaux1.fr), Clément Rossignol (LMP, UMR CNRS 5469, Université Bordeaux I, 351, cours de la Libération, 33405 Talence, France, c.rossignol@1mp.u-bordeaux1.fr), Nikolay Chigarev (LMP, UMR CNRS 5469, Université Bordeaux I, 351, cours de la Libération, 33405 Talence, France, n.chigarev@1mp.u-bordeaux1.fr), Marie-Christine Durrieu (INSERM U577, Université Victor Segalen Bordeaux 2, 146, rue LéoSaignat, Case 45, 33076 Talence, France, durrieumc@bordeaux.inserm.fr), Fabien Guillemot (INSERM U577, Université Victor Segalen Bordeaux 2, 146, rue Léo-Saignat, Case 45, 33076 Talence, France, fabien.guillemot@ bordeaux.inserm.fr), Guillaume Forget (Laboratoire de Biomatériaux et Reparation Tissulaire, INSERM U 577, Université Bordeaux 2, 146, rue Léo-Saignat, 33076 Bordeaux, France, guillaume.forget@bordeaux.inserm.fr)

The picosecond ultrasonic technique has been developed during the last 20 years due to permanent interest in the study of mechanical properties of nano- and micro-layers made of metals and semiconductors used in solid state physics and microelectronics. The technique relies on generation, propagation and detection of ultra-short acoustic waves by femtosecond laser pulses. Important advan- 
tages of these techniques are the absence of direct contact to the sample and extremely high frequency range. In this presentation, the picosecond ultrasonics technique is applied to generate and detect acoustic waves with a frequency as high as $15 \mathrm{GHz}$ in a single biological cell. Hypersound velocity and attenuation can thus be mapped in the cell with the small lateral resolution provided by optics, ie $1 \mu \mathrm{m}$. In addition to single cell imaging, the sensitivity of the measurements to cell compressibility suggests promising perspectives in the field of biology. An application to the analysis of mouse cells grafting on biomaterials will illustrate the potentialities for quantitative evaluation of implants bio compatibility.

\section{Contributed Paper}

3:20

4aPAb19. Parallel detection for picosecond ultrasonics. Richard Smith (University of Nottingham, School Electrical and Electronic Engineering, University Park, NG7 2RD Nottingham, UK, richard.j.smith @ nottingham.ac.uk), Mike Somekh (University of Nottingham, School Electrical and Electronic Engineering, University Park, NG7 2RD Nottingham, UK, mike.somekh@ nottingham.ac.uk), Steve Sharples (University of Nottingham, School Electrical and Electronic Engineering, University Park, NG7 2RD Nottingham, UK, steve.sharples@nottingham.ac.uk), Roger Light (University of Nottingham, School Electrical and Electronic Engineering, University Park, NG7 2RD Nottingham, UK, roger.light @ nottingham.ac.uk), Nicholas Johnston (University of Nottingham, School Electrical and Electronic Engineering, University Park, NG7 2RD Nottingham, UK, Nicholas.Johnston@nottingham.ac.uk), Mark Pitter (University of Nottingham, School Electrical and Electronic Engineering, University Park, NG7 2RD Nottingham, UK, mark.pitter@nottingham.ac.uk)
Picosecond laser ultrasonics is a powerful technique for measurement and diagnosis of micro- and nano-scale structures. Unfortunately the data acquisition speeds are slow, this is a major drawback for widespread acceptance and usually makes imaging impractical. We are engaged in a program to accelerate the data capture rate by parallel acquisition of the incoming signals. This involves electronic developments as well as optical design. In order to parallelise the electronics we have used two approaches (i) a modified commercial camera and (ii) a custom built CMOS array detector. These approaches use an integrating camera which by application of suitable algorithms can perform the task of parallel lock in detection. Modulation depths below 1 part in 106 , over 512 pixels can be readily detected with the commercial detector and smaller modulation depths are possible with the custom detector on account of the large well depth and hence high signal to noise. We also discuss the novel optical detection configurations that allow parallel detection of ultrasonic waves with ultrasonic wavelengths below the optical diffraction limit. Results are presented that show image acquisition rates orders of magnitude faster than normally possible with picosecond ultrasonic systems. Prospects for ultrahigh resolution optical resolution are discussed.

3:40-5:00 Posters

Lecture sessions will recess for presentation of poster papers on various topics in acoustics. See poster sessions for topics and abstracts.

\section{Contributed Paper}

\section{5:00}

4aPAb20. Acoustic phonon generation by intrinsically localized vibrational modes in double-helices of DNA macromolecules and transition from inter-strand energy exchange to nonlinear self-trapping. Yuriy A. Kosevich (Semenov Institute of Chemical Physics Russian Academy of Sciences, ul. Kosygina 4, 119991 Moscow, Russian Federation, yukosevich@yahoo.com), Alexander V. Savin (Semenov Institute of Chemical Physics Russian Academy of Sciences, ul. Kosygina 4, 119991 Moscow, Russian Federation, asavin@center.chph.ras.ru), Voislav L. Golo (Department of Mechanics and Mathematics, Lomonosov Moscow State University, 119992 Moscow, Russian Federation, voislav.golo@gmail.com), Yuriy S. Volkov (Department of Mechanics and Mathematics, Lomonosov Moscow State University, 119992 Moscow, Russian Federation, yu.volkov@gmail.com)

We study ultrafast dynamics of intrinsically localized vibrational modes (breathers) in a double helix of two weakly coupled chains of nonlinear oscillators. With this we model nonlinear dynamics of DNA-type macromol- ecules, which can be studied by means of femtosecond infrared pump-probe laser spectroscopy similar to the case of protein $\alpha$-helices [1]. We show that there are two regimes of coupled breathers: the time-periodic wandering of low-amplitude breather between the chains, and the one-chain-localization (self-trapping) of high-amplitude breather. We also find bound states of two breathers, localized in different chains, with different positions in the chains. The helix symmetry of the system results in a specific chiral mode which accomplishes the interaction between torsional and longitudinal acoustic modes in the constituent chains. In both nonlinear regimes, the inter-strand energy exchange gives rise to acoustic phonon generation in the coupled chains, and the generation is much stronger in the wandering-breather regime. Ultrafast acoustic phonon generation can be detected by means of optoacoustics, which can therefore provide a tool to study in time domain the inter-strand energy exchange and the transition to nonlinear self-trapping in DNA-type macromolecules. 1. J. Edler, R. Pfister, V. Pouthier, C. Falvo, and P. Hamm, 2004, Phys. Rev. Lett. v. 93, 106405.

\section{Invited Papers}

4aPAb21. Coherant acoustic excitation of nanostructures probed with asynchronous optical sampling. Thomas Dekorsy (University Konstanz, Fach M700, Fachbereich Physik, 78457 Konstanz, Germany, thomas.dekorsy@uni-konstanz.de)

We report the high-sensitivity detection of coherent acoustic excitation in semiconductor heterostructures and metallic nanostructures by using high-speed asynchronous optical sampling. Asynchronous optical sampling is based on two tunable femtosecond Ti: sapphire lasers with slightly different repetition rates close to $1 \mathrm{GHz}$. This new technique provides the performance of an all-optical oscilloscope for coherent excitations in a pump-probe set-up without any mechanically moving part. A time delay of $1 \mathrm{~ns}$ is scanned with a frequency of $10 \mathrm{kHz}$ and a time resolution of $100 \mathrm{fs}$. Investigations on coherent zone-folded phonons in semiconductor superlattices and nanoscale metallic structures are discussed. For the latter the influence of the substrate on the damping of acoustic excitations is investigated in detail. 
4aPAb22. Opto-acousto-optic evaluation of the physical properties of nanoporous materials. Charfeddine Mechri (Université du Maine, avenue O. Messiaen, 72085 Le Mans, France, cmechri@hotmail.com), Pascal Ruello (LPEC/UMR 6087/CNRS/Université du Maine, Avenue Olivier Messiaen, 72085 Le Mans Cedex 09, France, pascal.ruello@univ-lemans.fr), M Nsenkoue (Université du Maine, avenue O. Messiaen, 72085 Le Mans, France, m.nsenkoue@univ-lemans.fr), Denis Mounier (LPEC/UMR 6087/CNRS/Université du Maine, Avenue Olivier Messiaen, 72085 Le Mans Cedex 09, France, denis.mounier@univ-lemans.fr), Jean-Marc Breteau (LPEC/UMR 6087/CNRS/Université du Maine, Avenue Olivier Messiaen, 72085 Le Mans Cedex 09, France, jean-marc.breteau@univ-lemans.fr), Mathieu Edely (LPEC/UMR 6087/CNRS/Université du Maine, Avenue Olivier Messiaen, 72085 Le Mans Cedex 09, France, mathieu.edely@univ-lemans.fr), Y Minhao (Université du Maine, avenue O. Messiaen, 72085 Le Mans, France, y.minhao@univ-lemans.fr), Alain Gibaud (Université du Maine, avenue O. Messiaen, 72085 Le Mans, France, alain.gibaud@univ-lemans.fr), I Povey (Tyndall National Institute, University College Cork, Prospect Row, 115 Cork, Ireland, i.povey@tyndall.ie), M Pemble (Tyndall National Institute, University College Cork, Prospect Row, 115 Cork, Ireland, m.pemble@tyndall.ie), Sergei Romanov (Tyndall National Institute, University College Cork, Prospect Row, 115 Cork, Ireland, sergei.romanov@tyndall.ie), Mikhail Baklanov (IMEC, Kapeldreef 75, B-3001 Leuven, Belgium, baklanov@imec.be), P Verdonck (IMEC, Kapeldreef 75, B-3001 Leuven, Belgium, verdonck@imec.be), Vitali Gusev (LPEC/UMR 6087/CNRS/Université du Maine, Avenue Olivier Messiaen, 72085 Le Mans Cedex 09, France, vitali.goussev@univ-lemans.fr)

The porous materials with the characteristic dimensions of the pores from few nanometers up to a few hundred of nanometers find applications in microelectronic industry (as low-k materials), in photovoltaics and for developing of effective chemical sensors. When the pores are ordered in a spatially periodic structure, these systems present photonic and/or phononic properties which are of a prime interest in applied optics and telecommunication (light and/or phonons spectrum control). Here we report how the methods of picosecond laser ultrasonics based on the generation and detection by lasers of the acoustic waves with frequencies in the band of $10 \mathrm{GHz}$ - $1 \mathrm{THz}$ (with the lengths of hundreds of nanometers down to few nanometers) are applied for the evaluation of the mechanical and optical properties of these materials.

\section{6:00}

4aPAb23. Nanoscale objects as promising high frequency acoustic transducers in picosecond acoustics. Arnaud Devos (IEMNCNRS, Cité Scientifique - Avenue Poincaré, BP 60069, 59652 Villeneuve d'Ascq Cedex, France, arnaud.devos@isen.fr)

Twenty year ago, H. Maris opened up the field of nanoscale acoustics by demonstrating the opportunity of using ultrashort optical pulses for generating and detecting high frequency acoustic waves. Roughly, an optical pulse is converted in a picosecond acoustic pulse through the optical absorption in a thin metallic layer. Since then, this so-called picosecond ultrasonics has known a larger and larger success all around the world. Up to now, picosecond ultrasonics meets two main limitations. First it is difficult to reach the THz range using the usual way of producing the acoustic pulse. Second, in the common geometry only longitudinal waves are excited by the laser. Here we present some results showing that nanoscale objects could help in overcoming both difficulties. We first show that semiconductor quantum dots can be a very efficient emitter of coherent phonons whose frequency can be higher than those obtained in metallic thin films. Second we show that 2D arrays of nanosize metallic dots offers a way of generating and detecting high frequency surface acoustic waves.

\section{6:20}

4aPAb24. Nanoultrasonics based on piezoelectric semiconductor nanolayers. Chi-Kuang Sun (Naional Taiwan University, 1, Section 4, Roosevelt Road, 10617 Taipei, Taiwan, sun@cc.ee.ntu.edu.tw)

In this presentation, we will review our recent work on the development of nanoultrasonics based on piezoelectric semiconductor nanolayers. Through epitaxial growth of multiple or single piezoelectric semiconductor layers with a period on the order of $10 \mathrm{~nm}$, nanoacoustic waves with a frequency of 1 terahertz and a wavelength of $10 \mathrm{~nm}$ can be excited and measured with femtosecond optical pulses. Using temperal coherent and spatial nonlinear optical controls, we are able to synthesize nanoacoustic waveforms and generate a lateral acoustic spot on the order of $100 \mathrm{~nm}$ without the need of the near-field optical techniques. In this presentation, we will also discuss the potential use of this terahertz acoustic source for various nanoacoustic applications, including nanoultrasonic imaging.

\section{6:40}

4aPAb25. Photoelastic transduction in photo-phononic nanodevices. Alejandro Fainstein (Centro Atomico Bariloche \& Instituto Balseiro, C. N. E. A., 8400 S. C. de Bariloche, Argentina, afains@cab.cnea.gov.ar), Bernard Jusserand (INSP - UMR 7588 CNRS \& Université Pierre et Marie Curie, 140 Rue de Lourmel, 75015 Paris, France, Bernard.Jusserand@insp.jussieu.fr), Maria Florencia Pascual Winter (INSP - UMR 7588 CNRS \& Université Pierre et Marie Curie, 140 Rue de Lourmel, 75015 Paris, France, Florencia .PascualWinter@insp.jussieu.fr), Norberto Daniel Lanzillotti Kimura (Centro Atomico Bariloche \& Instituto Balseiro, C. N. E. A., 8400 S. C. de Bariloche, Argentina, lanzilln@ib.cnea.gov.ar), Guillermo Rozas (Centro Atomico Bariloche \& Instituto Balseiro, C. N. E. A., 8400 S. C. de Bariloche, Argentina, rozasg@ib.cnea.gov.ar), Bernard Perrin (INSP - UMR 7588 CNRS \& Université Pierre et Marie Curie, 140 Rue de Lourmel, 75015 Paris, France, bernard.perrin@insp.jussieu.fr), Agnès Huynh (INSP - UMR 7588 CNRS \& Université Pierre et Marie Curie, 140 Rue de Lourmel, 75015 Paris, France, agnes.huynh@insp.jussieu.fr), Aristide Lemaitre (LPN, CNRS route de Nozay, 91460 Marcoussis, France, Aristide.Lemaitre@lpn.cnrs.fr)

We will discuss the new possibilities that semiconductor superlattices and acoustic nanocavities open for the controlled manipulation of quasi-monochromatic acoustic waves in the terahertz range. Playing with the specific electronic properties of quantum wells constituting acoustic nanodevices allows to selectively generate or detect phonons with a specific spatial distribution of the deformation along the acoustic device propagation axis. We could for instance demonstrate the selective generation of cavity phonons at resonance with cavity excitonic transitions or the increased photoelastic coupling of folded acoustic modes in mirrors when the number of nodes of the acoustic mode coincide with the one of the dominantly resonant excitonic transition. We also used the combination of photonic and phononic cavities to ensure phase matching with cavity phonons in the standard detection scheme corresponding to transient reflectivity 
in the time domain or Raman backscattering in the frequency domain. Photonic cavities moreover provide a strong increase of the internal optical fields by quality factors up to 100 typically, resulting in high enhancements of the transduction efficiency of monochromatic phonons to the benefit of the envisioned high speed modulation of optoelectronic properties of coupled photo-phononic nanodevices.

\section{Contributed Papers}

7:00

4aPAb26. Thermomechanical behavior of surface acoustic waves in ordered arrays of nanodisks studied by near-infrared pump-probe diffraction experiments and finite element simulations. Claudio Giannetti (Università Cattolica del Sacro Cuore, via dei Musei 41, I-25121 Brescia, Italy, c.giannetti@dmf.unicatt.it), Francesco Banfi (Università Cattolica del Sacro Cuore, via dei Musei 41, I-25121 Brescia, Italy, f.banfi@dmf.unicatt.it), Damiano Nardi (Università Cattolica del Sacro Cuore, via dei Musei 41, I-25121 Brescia, Italy, dnardi@dmf.unicatt.it), Bernard Revaz (Département de Physique Theorique, Universitè de Genève, 24 Quai Ansermet, CH-1211 Genève, Switzerland, bernard.revaz@gmail.com), Gabriele Ferrini (Università Cattolica del Sacro Cuore, via dei Musei 41, I-25121 Brescia, Italy, gabriele@dmf.unicatt.it), Paolo Vavassori (Dipartimento di Fisica Univeristà di Ferrara, via dell'università, I-44100 Ferrara, Italy, vavassori@fe.infn.it), Vitali Metlushko (Department of Electrical and Computer Engineering, University of Illinois at Chicago, Chicago, IL 60607, USA, vmetlush@ece.uic.edu), Fulvio Parmigiani (Dipartimento di Fisica, Università degli Studi di Trieste and Sincrotrone Trieste, Basovizza, I-34012 Trieste, Italy, fulvio@dmf.unicatt.it)

The ultrafast thermal and mechanical dynamics of a two-dimensional lattice of metallic nanodisks has been studied by near-infrared pump-probe diffraction measurements over a temporal range spanning from 100 fs to several nanoseconds. The experiments demonstrate that in these systems a surface acoustic wave (SAW), with a wave vector given by the reciprocal periodicity of the two-dimensional array, can be excited by $\sim 120$ fs Ti: sapphire laser pulses. We unambiguously show that the observed SAW velocity shift originates from the mechanical interaction between the SAWs and the nanodisks, while the correlated SAW damping is due to the energy radiation into the substrate. In order to clarify the interaction between the nanodisks and the substrate, numerical calculations of both the elastic eigenmodes and the time-dynamics of the system, following the impulsive heating excitation by the laser, are performed. Simulations based on finite- elements analysis, together with a wavelet analysis of our experimental data, suggest the opening of a band-gap at the centre of the super-Brillouin zone. The modes at the centre of the super-Brillouin zone are excited following laser excitation, as opposed to thermal population, of the elastic modes.

\section{$7: 20$}

4aPAb27. High frequency acoustics in nanostructures by spontaneous Brillouin light scattering. Tim Still (Max Planck Institute for Polymer Research, Ackermannweg 10, 55128 Mainz, Germany, still@mpip-mainz.mpg.de), Markus Retsch (Max Planck Institute for Polymer Research, Ackermannweg 10, 55128 Mainz, Germany, retsch@mpip-mainz.mpg.de), Revekka Sainidou (Instituto de Óptica CSIC, Serrano 121, 28006 Madrid, Spain, rsainid@io.cfmac.csic.es), Ulrich Jonas (Max Planck Institute for Polymer Research, Ackermannweg 10, $55128 \quad$ Mainz, Germany, jonas@mpip-mainz.mpg.de), George Fytas (Department of Materials Science and Technology, University of Crete and Forth, 71110 Heraklion, Greece, fytas@mpip-mainz.mpg.de)

We report on the dispersion of high frequency $(\mathrm{GHz})$ acoustic excitations in three-dimensional colloidal crystalline or amorphous assemblies of sub-micron particles in different matrices by Brillouin light scattering (BLS) in order to explore the phononic and elastic properties of nanostructured materials. In air, we record eigenmodes of the individual particles, which are shown to be independent from the crystallinity and the composition of the sample but sensitively depend on the particle architecture (e.g core/shell silica/PMMA, hollow spheres) and their mechanical properties at nanoscale. In fluid matrices, the dispersion relations are recorded. We demonstrate the occurence of two hypersonic phononic band gaps of different nature. One is a Bragg gap appearing only in crystalline samples, the other one is a particle resonance-induced hybridization gap, which appears in crystalline samples along with the Bragg gap and alone in amorphous samples. Furthermore, we investigate the influence of filling fraction, crystallinity and monodispersity of size on the hypersonic behaviour of our samples. 


\title{
Session 4aPAc
}

\section{Physical Acoustics, Acoustical Oceanography, and Biomedical Ultrasound/Bioresponse to Vibration: Acoustically Activated Bubble Dynamics and Applications I}

\author{
Erich C. Everbach, Cochair \\ Swarthmore College, 500 College Avenue, Engineering Department, Swarthmore, PA 19081, USA \\ Joachim Holzfuss, Cochair \\ Institute of Applied Physics, TU Darmstadt, Schlossgartenstr. 7, Darmstadt, 64289, Germany
}

Invited Papers

8:00

4aPAc1. Bubbles, surfactants, shape oscillations, optical levitation, and light scattering: a survey. Philip L. Marston (Washington State University, Physics and Astronomy Department, Pullman, WA 99164-2814, USA, marston@wsu.edu), David B. Thiessen (Washington State University, Physics and Astronomy Department, Pullman, WA 99164-2814, USA, thiessen@wsu.edu)

Research emphasizing relatively slow aspects of bubble dynamics will be summarized. Though the attention is mainly on acoustically levitated bubbles larger than the size for monopole resonance, early experiments on stable optical levitation of gas bubbles in water [J. Acoust. Soc. Am. 83, 970-975 (1988)] will also be noted as well as fundamental aspects of light scattering by bubbles [J. Opt. Soc. Am. and/or Applied Optics (1979-1991)]. Modulated radiation pressure was demonstrated to be an effective way for mode-specific excitation of shape oscillations of acoustically levitated bubbles [J. Acoust. Soc. Am. 93, 706-713 (1993)]. The damping of shape oscillations was demonstrated to be strongly influenced by the presence of insoluble or soluble surfactants [J. Fluid Mech. 300, 149-167 (1995); Phys. Rev. Let. 75, 2686-2689 (1995); J. Acoust. Soc. Am. 102, 3372-3377 (1997)]. A convenient way of measuring the damping was to use laser beam extinction to monitor freely decaying shape oscillations. This technique was sufficiently sensitive to reveal the strong dependence of the damping on surface elasticity. Even for clean bubbles, an improved analysis of the damping was needed. [Sponsored in part by NASA and by ONR.]

4aPAc2. Measuring the Extreme Conditions Created During Cavitation. Kenneth S. Suslick (University of Illinois at UrbanaChampaign, 600 S. Mathews Av., Urbana, IL 61801, USA, ksuslick@uiuc.edu)

Extreme temperatures and pressures are produced through acoustic cavitation: the formation, growth and collapse of bubbles in a liquid irradiated with high intensity ultrasound. Single bubbles have generally been assumed to give higher temperature conditions than bubble clouds, but confirmation from the single buble sonoluminescence (SBSL) emission spectra have been problematic because SBSL typically produces featureless emission spectra that reveal little about the intra-cavity physical conditions or chemical processes. Here we present definitive evidence of the existence of a hot, highly energetic plasma core during SBSL. From a luminescing bubble in sulfuric acid, excited state to excited state emission lines are observed both from noble gas ions $(\mathrm{Ar}+, \mathrm{Kr}+$, and $\mathrm{Xe}+)$ and from neutral atoms ( $\mathrm{Ne}, \mathrm{Ar}, \mathrm{Kr}$, and $\mathrm{Xe}$ ). The excited states responsible for these emission lines range from $8.3 \mathrm{eV}$ (for $\mathrm{Xe}$ ) to $37.1 \mathrm{eV}$ (for $\mathrm{Ar}+$ ) above the ground state. Observation of emission lines allows for identification of intra-cavity species responsible for light emission; the energy levels of the emitters indicate the plasma generated during cavitation is comprised of highly energetic atomic and ionic species.

\section{$8: 40$}

4aPAc3. Nonlinear dynamics of sonoluminescing bubbles. Joachim Holzfuss (Institute of Applied Physics, TU Darmstadt, Schlossgartenstr. 7, 64289 Darmstadt, Germany, Joachim.Holzfuss@ @ physik.tu-darmstadt.de)

The current work focuses on the acoustical and nonlinear dynamical aspects of sonoluminescence (SBSL). Several hydrodynamical instabilities in parameter space are analyzed in detail numerically. Their occurrence in experiments is discussed especially in the context of period doubled unisotropic light emission. The acoustical emissions during stable and unstable oscillations show characteristics of shock waves. The emitted sound generates a complex acoustic environment in the driving cell leading to backreactions to the bubble. Characteristic dynamical effects during unstable sonoluminescence are clarified. Chemical processes during high temperature and high pressure, spatial translations, gas diffusion, the highly nonlinear bubble oscillations and acoustic emissions are attributed to oscillations and modulations of bubble dynamics outside the range of stable SBSL. In particular reasons for quasiperiodic oscillations with incommensurate frequencies in different setups are found. 
4aPAc4. Bubble-bubble interaction in disperse bubble clouds. Andrew J. Szeri (University of California, 6119 Etcheverry Hall, Berkeley, CA 94720-1740, USA, andrew.szeri@berkeley.edu), Jean O. Toilliez (University of California, 6119 Etcheverry Hall, Berkeley, CA 94720-1740, USA, toilliez@me.Berkeley.EDU), Chris Heckman (University of California, 6119 Etcheverry Hall, Berkeley, CA 94720-1740, USA, checkman@berkeley.edu), Parastou Eslami (University of California, 6119 Etcheverry Hall, Berkeley, CA 94720-1740, USA, peslami20@ berkeley.edu)

Disperse bubble fields driven by pressure waves feature effects of time delays associated with the finite speed of travel of the driving acoustic wave, and the finite travel time of pressure waves between bubbles. The relative spatial arrangement of bubble nuclei in a disperse field, and the direction of the incoming pressure wave, both influence the cavitation behavior of a cloud of nuclei. In this paper, we consider the dynamics of a disperse field of bubble nuclei driven by a strong rarefaction, such as one sees in shock wave lithotripsy. We make comparisons to experimental work published in J. I. Iloreta, A. J. Szeri, Y. Zhou, G. Sankin and P. Zhong, Assessment of shock wave lithotripters via cavitation potential, Physics of Fluids 19, 086103 (2007). Bubble-bubble interactions are the key ingredient to resolving a mystery concerning the extent of cavitation bubble growth.

4aPAc5. The effects of sonication on bacteria. Timothy J Mason (Sonochemistry Centre, Coventry University, Priory Street, CV1 5FB Coventry, UK, t.mason@ coventry.ac.uk), L Paniwnyk (Sonochemistry Centre, Coventry University, Priory Street, CV1 5FB Coventry, UK, apx077@coventry.ac.uk), E Joyce (Sonochemistry Centre, Coventry University, Priory Street, CV1 5FB Coventry, UK, apx077@ coventry.ac.uk)

High power ultrasound at frequencies around $20 \mathrm{kHz}$ is capable of killing bacteria and for many years has been standard technique in microbiology for the disruption of living cells to release their contents. So successful is this effect that ultrasound has been studied as a possible method for water disinfection. The energies required for using ultrasound alone are high but commercial equipment is available and is often used for disinfection in conjunction with other techniques such as ozonation or uv irradiation. Now evidence is emerging that is possible to induce effects on bacteria other than kill by modifying the acoustic energy entering the suspension (Ultrason. Sonochem. 10:315, 2003). This can be done by altering the duration of exposure, the acoustic power used or the frequency of the ultrasound. In this way sonication can lead to such effects as deagglommeration, enhanced reaction to biocides and gene transfer (Nucleic Acids Res. 35:e129, 2007).

\section{9:40}

4aPAc6. Cavitational activity in bacterial biofilms exposed to $1 \mathrm{MHz}$ ultrasound. Erich C. Everbach (Swarthmore College, 500 College Avenue, Engineering Department, Swarthmore, PA 19081, USA, ceverba1@ swarthmore.edu), Roby Velez (Swarthmore College, 500 College Avenue, Engineering Department, Swarthmore, PA 19081, USA, rvelez1@swarthmore.edu), Amy C. Vollmer (Swarthmore College, 500 College Avenue, Biology Department, Swarthmore, PA 19081, USA, avollme1@ swarthmore.edu)

An exposure system was previously developed to quantify destruction of bacterial biofilms by $1 \mathrm{MHz}$ c.w. ultrasound at $0.8 \mathrm{MPa}$ peak-to-peak acoustic pressure amplitude (JASA 122(5):3052, 2007). Bacterial killing is quantified via confocal microscopy using florescent E. Coli and image processing. Recently, a passive detector of inertial and stable cavitation was included, relying upon the presence and character of acoustic emissions. The detector, a PVDF array placed on the microscope slide forming the base of the exposure chamber, produces a proxy measure of cavitational activity during ultrasound exposure. Acoustic pressure thresholds for biofilm destruction and cavitational activity suggest that inertial and stable cavitation both play a role in biofilm destruction by ultrasound.

10:00-10:20 Break

10:20

4aPAc7. Bubbles in piezo-acoustic inkjet printing. Detlef Lohse (Physics of Fluids, University of Twente, P.O. Box 217, 7500 AE Enschede, Netherlands, d.lohse@utwente.nl), Roger Jeurissen (Physics of Fluids, University of Twente, P.O. Box 217, 7500 AE Enschede, Netherlands, r.j.m.jeurissen@tnw.utwente.nl), Jos De Jong (University of Twente, P.O. Box 217, Department of Science and Technology, 7500 AE Enschede, Netherlands, jos.dejong@tnw.utwente.nl), Michel Versluis (Physics of Fluids, University of Twente, P.O. Box 217, 7500 AE Enschede, Netherlands, m.versluis@utwente.nl), Herman Wijshoff (Oce Technology, P.O. Box 101, 5900 MA Venlo, Netherlands, herman.wijshoff@oce.com), Marc Van Den Berg (Oce Technology, P.O. Box 101, 5900 MA Venlo, Netherlands, marc.van.den.berg@oce.com), Hans Reinten (Oce Technology, P.O. Box 101, 5900 MA Venlo, Netherlands, hans.reinten@oce.com)

Ink-jet printing is considered as the hitherto most successful application of microfluidics. A notorious problem in piezo-acoustic ink-jet systems is the formation of air bubbles during operation. They seriously disturb the acoustics and can cause the droplet formation to stop. We could show by a combination of acoustical detection and high-speed visualization that the air-bubbles are entrained at the nozzle and then grow by rectified diffusion. Experimental results on the droplet velocity as a function of the equilibrium radius R0 of the entrained bubble are presented, too. Surprisingly, the droplet velocity shows a pronounced maximum around R $0=17$ micrometer before it sharply drops to zero around $\mathrm{R} 0=19$ micrometer. A simple one-dimensional model is introduced to describe this counterintuitive behavior which turns out to be a resonance effect of the entrained bubble. We show that the bubble counteracts the pressure buildup necessary for the droplet formation. The channel acoustics and the air bubble dynamics are modeled. It is crucial to include the confined geometry into the model: The air bubble acts back on the acoustic field in the channel and thus on its own dynamics. This two-way coupling limits further bubble growth and thus determines the saturation size of the bubble. 


\section{Contributed Papers}

10:40

4aPAc8. The acoustic excitation mechanism of bubbles released from a nozzle. Grant Deane (Scripps Inst. Oceanography, Univ. Califiornia, San Diego, La Jolla, CA 92093, USA, gdeane@ucsd.edu), Helen Czerski (Scripps Inst. Oceanography, Univ. Califiornia, San Diego, La Jolla, CA 92093, USA, hczerski@ucsd.edu)

At the moment of their formation, bubbles emit a short pulse of sound Bubble noise is associated with sound from a variety of natural processes, including whitecaps, waterfalls, breaking surf and rain. A number of acoustic excitation mechanisms for bubble noise have been proposed, including the increase in internal pressure of the bubble associated with the Laplace pressure, hydrostatic pressure effects, shape mode to volume mode coupling, and a fluid jet associated with the collapse of the neck of air formed during bubble creation. Using bubbles released from a nozzle as a model system, we have determined that sound production is excited by a sudden decrease in bubble volume driven by the collapse of the neck of gas joining the bubble to its parent. A simple analytical model of neck collapse driven by surface tension energy is in agreement with high speed photographic measurements, and sufficient to explain the details of acoustic excitation. [Work supported by ONR and NSF]

\section{1:00}

4aPAc9. Characterizing microbubble interactions with ultrasound using flow cytometry. Thomas Matula (Center for Industrial and Medical
Ultrasound, Applied Physics Lab., University of Washington, 1013 NE 40th St., Seattle, WA 98105, USA, matula@apl.washington.edu), Jarred Swalwell (Center for Industrial and Medical Ultrasound, Applied Physics Lab., University of Washington, 1013 NE 40th St., Seattle, WA 98105, USA, jarred@u.washington.edu)

Characterizing the fundamental interaction of ultrasound with microbubbles is challenging because of the small spatial and temporal scales. High speed optical imaging is perhaps the most well-known method, as it provides direct information about their response. Although the image is in a plane, the image quality can be sufficient to obtain important information about bubble response. However, high-speed cameras are expensive, data is very limited, and difficult to process. We previously showed how light scattering can be used to obtain similar information - volume oscillations, destruction, even shell properties. Light scattering can be an inexpensive method for probing microbubbles. The difficulties with light scattering (also with optical imaging) are alignment and signal/noise. In this talk we will describe a technique to use commercially-available light-scattering systems to investigate the interaction of pulsed ultrasound with microbubbles. In particular, we developed a technique to insonify microbubbles flowing through the focal region of a flow cytometer. Attached to the quadrature side of a flow cuvette is a small piezoelectric transducer, driven in pulsed mode at various voltages to induce a bubble response. The light scattered from the bubbles can be used for sizing, destruction thresholds, and to assess volume oscillations. Funded by NIH \#5R01EB000350

\section{Invited Paper}

\section{1:20}

4aPAc10. Determination of cavitation bubble lifetimes using bubble-bubble coalescence data. Franz Grieser (Department of Chemical and Biomolecular Engineering, The University of Melbourne, Parkville, Victoria, 3010 Melbourne, Australia, franz@unimelb.edu.au), Devi Sunartio (Department of Chemical and Biomolecular Engineering, The University of Melbourne, Parkville, Victoria, 3010 Melbourne, Australia, franz@unimelb.edu.au), Muthupandian Ashokkumar (Department of Chemical and Biomolecular Engineering, The University of Melbourne, Parkville, Victoria, 3010 Melbourne, Australia, masho@unimelb.edu.au)

The effect that surface-active solutes, such as aliphatic alcohols and sodium dodecylsulfate, have on the extent of bubble coalescence in liquids under different sonication conditions has been investigated by measuring the volume change of the solution following a period of sonication. The data obtained led to the conclusion that SDS does not reach equilibrium adsorption level at the bubble/solution interface. On this basis, a method is proposed for estimating nonequilibrium surface excess values for solutes that do not fully equilibrate with the bubble/solution interface during sonication. For the case of SDS in the presence of excess $\mathrm{NaCl}$, the method was further employed to estimate the maximum lifetime of bubbles in a multibubble field. Data obtained from this study suggests that an acoustic bubble in a multibubble field has a finite lifetime, and that this lifetime decreases with increasing applied frequency, ranging from up to $0.35 \pm 0.05 \mathrm{~ms}$ for $213 \mathrm{kHz}$ to $0.10 \pm 0.05 \mathrm{~ms}$ for $1062 \mathrm{kHz}$. These estimated lifetimes equate to a bubble in a multibubble field undergoing an upper limit of 50-200 oscillations over its lifetime for applied acoustic frequencies between $200 \mathrm{kHz}$ and $1 \mathrm{MHz}$.

\section{Contributed Papers}

11:40

4aPAc11. Interpretation of the pressure waves radiated by oscillating bubbles. Karel Vokurka (Technical University of Liberec, Physics Department, Studentska 6, 46117 Liberec, Czech Republic, karel.vokurka @ tul.cz), Silvano Buogo (CNR-Istituto di Acustica 'O,M.Corbino', Via del Fosso del Cavaliere, 100, 00133 Rome, Italy, silvano.buogo@idac.rm.cnr.it)

An oscillating bubble is an excellent acoustic radiator. In a pressure wave emitted by the oscillating bubble information about the bubble properties and behavior is present. Hence, when using a suitable method, this information could be extracted and used to improve our understanding of the physical processes accompanying the bubble oscillations. However, to be able to extract this information, a number of prerequisites must be met. First, the measuring apparatus should be able to record a faithful copy of the pressure wave. Second, a large, statistically representative set of pressure records must be available for the analysis. Third, a suitable method must be used to analyze the recorded waves. All these requirements will be discussed in detail at the conference. Presented results are based on experience gained during evaluation of a large set of pressure records obtained recently in ex- periments with spark generated bubbles. [Work has been partly supported (K.V.) by the Czech Ministry of Education as the research project MSM 46747878501.]

\section{2:00}

4aPAc12. Dynamics and radiation of single cavity in an abnormal compressible bubbly media. Valeriy K. Kedrinskiy (Lavrentyev Institute of Hydrodynamics, Siberian Division of the Russian Academy of Sciences, Lavrentyev prospect 15, 630090 Novosibirsk, Russian Federation, kedr @hydro.nsc.ru)

The equation of a pulsation of a single cavity in the equilibrium (on pressure) bubbly medium was suggested. The state of such medium is described by Lyakhov's equation which at the condition of pressure equilibrium in the both phases (gas/liquid) becomes essentially simpler. The numerical analysis of features of cavity dynamics and the acoustic losses was executed. The notion "acoustic losses" mean a radiation generated by cavity. The analysis of radiation parameters was restricted by the vicinity of cavity wall from a liquid side. The studies of cavity behavior, the structure and 
amplitude of a radiation have shown that the degree of a cavity compression by a stationary shock wave goes down when the volumetric concentration of gas phase $\mathrm{K}$ in the medium increases. The amplitude of pulsation essentially decrease and function $\mathrm{R}(\mathrm{t})$ (radius cavity vs. time) asymptotically (without oscillations) tends to the equilibrium state when $\mathrm{K}$ is equal approximately
$3 \%$. The equilibrium state is defined by amplitude of an incident shock wave and does not depend on K-value. The structure of a radiation wave takes the "soliton" form, its amplitude is essentially lesser and the width is much more in the comparison with corresponding parameters for a single-phase liquid. (RFBR 06-01-00317a financial support).

\section{2:20-1:40 Lunch Break}

\section{Contributed Papers}

\section{$1: 40$}

4aPAc13. Equilibrium state of a multi-size bubble population in a liquid. Svetlana Kovinskaya (Mechmath LLC, 14530 Bluebird Trail, Prior Lake, MN 55372, USA, mechmath@mechmath.com)

A propagation of a linear pressure wave in the liquid with multi-size bubble population is investigated. The wave in the bubbly mixture is modeled as wave in the waveguide interacting with distributed resonators having different resonance frequencies. Each bubble sub-population has the frequency range of effective influence on the wave propagation. This range is started from the resonance frequency of the individual bubble and ended in dependency on the partial void fraction of this sub-population. Mutual influence of sub-populations leads to additional sound attenuation that in essence is a Landau damping. The dispersive equation for the propagating wave lets introduce a criterion for the equilibrium state of the bubbly mixture with multi-size bubble population. The equilibrium distribution which meets this criterion is found analytically. It is shown that the equilibrium distribution is an exact result from the resonant acoustical absorption theory. This theory is employed to find the bubble distribution from measured attenuation of the acoustical wave with assumption that only resonating bubbles contribute attenuation at the frequency of their resonance (neglecting off-resonance contributions). The deviation from the equilibrium contributes Landau damping into the resonant absorption. A dependency of Landau damping on the bubble size distribution is presented.

\section{2:00}

4aPAc14. Optical measurements of the hot spot and incandescent shock from high pressure cavitation in water. Robert Hiller (Impulse Devices, Inc., 13366 Grass Valley Av. Unit H, Grass Valley, CA 95945, USA, asa @ roberthiller.com), D. Felipe Gaitan (Impulse Devices, Inc., 13366 Grass Valley Av. Unit H, Grass Valley, CA 95945, USA, gaitan@impulsedevices .com)

Spontaneous acoustic cavitation in water at static pressure up to 300 bar has been experimentally investigated. Cavities are initiated by negative pressure and then collapse due to both acoustic pressure and shock waves reflected from the inner surface of the spherical resonator. The implosions result in intense (Mbar) shock waves and bright $(1 \mathrm{~nJ})$ light flashes which last from 5 to 40 nanoseconds. The optical spectrum of the flash is measured with a grating monochromator and intensified array detector for high wavelength resolution $(\sim 5 \mathrm{~nm})$ but slow time resolution, and with a multipleanode microchannel plate photomultiplier tube along with bandpass filters for fast time resolution $(\sim 1 \mathrm{~ns})$ but poor wavelength resolution. The spectrum is generally broad-band and featureless, matching roughly to a Planck spectrum at 5000 to $8000 \mathrm{~K}$. The spectral and temporal structure of the flashes is matched to hydrocode simulations. The model suggest the flashes are due to a shell of hot, opaque, shocked water which surrounds and obscures the central hot core. (SMDC contract W9113M-07-C-0178)

\section{2:20}

4aPAc15. Investigation of bubble dynamics and sonoluminescence in megasonic fields. Andrea Otto (Göttingen University, Friedrich-HundPlatz 1, 37077 Göttingen, Germany, aotto@physik3.gwdg.de), Till Nowak (Göttingen University, Friedrich-Hund-Platz 1, 37077 Göttingen, Germany, tnowak@physik3.gwdg.de), Robert Mettin (Göttingen University, Drittes Physikalisches Institut, Friedrich-Hund-Platz 1, 37077 Göttingen, Germany, R.Mettin@physik3.gwdg.de), Frank Holsteyns (SEZ AG,
SEZ-Strasse 1, 9500 Villach, Austria, F.Holsteyns@at.sez.com),Alexander Lippert (SEZ AG, SEZ-Strasse 1, 9500 Villach, Austria, A.Lippert@at.sez .com)

Cavitation bubble motion and bubble structures in water are investigated for standing wave fields in the megasonic range by high-speed imaging. Larger degassing bubbles and small bubbles with high translation speeds can be resolved. Groups of bubbles arrange in lines or arrays, as reported earlier by Miller [Miller, JASA 62, 1977]. Additional, sonoluminescence is measured in overall long-term and phase-resolved (gated) long-term exposures. Several distinct luminescing islands can be detected. The findings seem to be strongly related to the standing wave nature of the pressure field in our setup. Conclusions on bubble distributions and for cleaning applications are drawn.

\section{2:40}

4aPAc16. Development of an acoustics-based instrument for bubble measurement in liquids. Georges L. Chahine (Dynaflow, Inc., 10621-J Iron Bridge Rd, Jessup, MD 20794, USA, glchahine@dynaflowinc.com), Xiongjun Wu (Dynaflow, Inc., 10621-J Iron Bridge Rd, Jessup, MD 20794, USA, xiongjun@dynaflow-inc.com), Xiaozhen Lu (Dynaflow, Inc., 10621-J Iron Bridge Rd, Jessup, MD 20794, USA, xiaozhen @ dynaflow-inc.com)

The acoustic bubble spectrometer (ABS) is an acoustics-based device that provides bubble size distribution in a bubbly liquid through measurement at various frequencies of the sound speed and attenuation and solution of an inverse problem. Acoustic bursts of varying frequencies are emitted by one hydrophone and detected by another. A PC and data boards control signal generation, detection, signal processing, inverse problem solution, and results display. Extensive validation experiments were conducted against high speed-video optical measurements. The two methods give very close results for void fractions up to $3 \mathrm{e}-3$, with the ABS possessing the significant advantage of enabling near real-time measurements. The field of application is being expanded to media other than water, and the technique improved to detect larger void fractions, with the help of numerical simulations of nonlinear behavior of bubble clouds in acoustic fields.

\section{3:00}

4aPAc17. An acoustic resonator for determining the void fraction of bubbly mercury flows. Ronald A. Roy (Boston University, Dept. of Aerosp. and Mech. Eng., 110 Cummington St., Boston, MA 02215, USA, ronroy@bu.edu), Christopher E. Ormonde (Boston University, Dept. of Aerosp. and Mech. Eng., 110 Cummington St., Boston, MA 02215, USA, chrisceo@bu.edu), Parag V. Chitnis (Boston University, Dept. of Aerosp. and Mech. Eng., 110 Cummington St., Boston, MA 02215, USA, pchitnis@bu.edu), Robin O. Cleveland (Boston University, Dept. of Aerosp. and Mech. Eng., 110 Cummington St., Boston, MA 02215, USA, robinc@bu.edu), R. Glynn Holt (Boston University, Dept. of Aerosp. and Mech. Eng., 110 Cummington St., Boston, MA 02215, USA, rgholt@bu .edu)

An acoustic resonator for measuring free-gas void fraction of a heliummercury mixture is investigated. We employ a vertical, stainless steel cylindrical waveguide with a 5.08-cm i.d., a $1.27-\mathrm{cm}$ wall thickness, a $40-\mathrm{cm}$ length, and pressure-release boundary conditions at both ends. A bubble injection flow loop produces 2-phase mixtures of varying void fraction that flows upwards through the tube, spills over, and recirculates. The resonator is driven from the top by a $2.54-\mathrm{cm}$ diameter circular piston affixed to an 
electrodynamic shaker. A hydrophone mounted $1 \mathrm{~cm}$ above the tube bottom is used to measure the frequency response of the system. Sound speed is inferred by assuming a linear dependence of axial mode number on mode frequency, and void fraction is calculated assuming a mixture sound speed for a bubble population with maximum sized much smaller than the resonant sizes in the modal frequency range (Wood's limit). The system was validated using non-bubbly water and water-air mixtures of different void fractions. Void fraction measurements for Helium-Mercury mixtures will be presented. [Supported by the ORNL Spallation Neutron Source, which is managed by UT-Battelle, LLC, under contract DE-AC05-00OR22725 for the U.S. Department of Energy.]

\section{3:20}

4aPAc18. Cavitation bubbles as microfluidic actuators. Claus-Dieter Ohl (University of Twente, P.O. Box 217, Department of Science and Technology, 7500 AE Enschede, Netherlands, c.d.ohl@utwente.nl), Rory Dijkink (University of Twente, Physics of Fluids, Building Meander, Postbus 217, 7500 AE Enschede, Netherlands, r.j.dijkink@tnw.utwente .nl), Zwaan Ed (University of Twente, Physics of Fluids, Building Meander, Postbus 217, 7500 AE Enschede, Netherlands, e.v.zwaan@casema .nl), Séverine Le Gac (University of Twente, BIOS The Lab-on-a-Chip group, 7500 AE Enschede, Netherlands, S.LeGac@ewi.utwente.nl), Albert Van Den Berg (University of Twente, BIOS The Lab-on-a-Chip group, 7500 AE Enschede, Netherlands, A.vandenBerg@ewi.utwente.nl), Kinko Tsuji (Shimadzu Europa GmbH, 47269 Duisburg, Netherlands, kts @ shimadzu.de)

In this talk we give an overview on the usage of single cavitation bubbles to pump, mix, and manipulate cells in microfluidics. The bubbles are generated with a laser pulse in optically transparent lab-on-a-chip devices. The bubble pulsations is inherently fast, thus although the characteristic dimensions are small high Reynolds numbers flow can be achieved.
Experiments show that depending on the channel height 2-dimensional or 3-dimensional fluid flow is generated. Interestingly, there exists a regime which can be described with the Laplace equation, thus it is essentially a potential inviscid flow. We will present the current work (of others and our group) on cavitation assisted pumping using the jetting effect, mixing flows through the creation of vorticity, and the interaction of a bubble with suspension cells. The first results on the latter promise a fruitful future for biologic relevant applications in integrated lab-in-chip devices.

\section{3:40}

4aPAc19. Ultrasonic synthesis of enzyme coated microbubbles. Muthupandian Ashokkumar (Department of Chemical and Biomolecular Engineering, The University of Melbourne, Parkville, Victoria, 3010 Melbourne, Australia, masho@unimelb.edu.au), Francesca Cavalieri (Department of Chemical and Biomolecular Engineering, The University of Melbourne, Parkville, Victoria, 3010 Melbourne, Australia, masho@unimelb.edu.au), Franz Grieser (Department of Chemical and Biomolecular Engineering, The University of Melbourne, Parkville, Victoria, 3010 Melbourne, Australia, franz@unimelb.edu.au)

Gas-filled polymer coated microbubbles are intrinsically ultrasound responsive systems and when tailored with targeting features are promising candidates for smart drug delivery. We have ultrasonically synthesised stable, versatile, biodegradable and biocompatible microbubbles using the enzyme, lysozyme. The synthesis of lysozyme microbubbles has been achieved by sonicating an aqueous solution containing denatured lysozyme. The microbubbbles have been characterised using a number of imaging techniques such as, SEM, AFM and light microscopy. We have observed that the experimental parameters such as, length of sonication, DTT concentration and denaturisation time deeply affect the yield and the size of the microbubbles. We have also investigated the secondary structure and the enzymatic activity of lysozyme coated microbubbles. The lysozyme microbubbles have retained their enzymatic antimicrobial activities.

Lecture sessions will recess for presentation of poster papers on various topics in acoustics. See poster sessions for topics and abstracts.

\section{Contributed Papers}

\section{$5: 20$}

4aPAc20. Modeling of the effect of boundaries on ultrasound contrast agent microbubbles response. Benjamin Dollet (Physics of Fluids, University of Twente, P.O. Box 217, 7500 AE Enschede, Netherlands, benjamin.dollet@univ-rennes1.fr), Leen Van Wijngaarden (Physics of Fluids, University of Twente, P.O. Box 217, 7500 AE Enschede, Netherlands, 1.vanwijngaarden@tnw.utwente.nl), Nico De Jong (Erasmus MC, Dr Molewaterplein 50 room Ee2302, 3015GE Rotterdam, Netherlands, n.dejong @erasmusmc.nl), Detlef Lohse (Physics of Fluids, University of Twente, P.O. Box 217, 7500 AE Enschede, Netherlands, d.lohse@utwente .nl), Michel Versluis (Physics of Fluids, University of Twente, P.O. Box 217, 7500 AE Enschede, Netherlands, m.versluis@utwente.nl)

Ultrasound contrast agents are coated microbubbles currently extensively studied to target endothelial cells, for local drug delivery. It is therefore important to distinguish acoustically free-floating bubbles from bubbles located close, or targeted to blood vessels. Here, we propose a theoretical study to understand the effect of boundaries on bubble response to ultrasound. We consider the hydrodynamic interaction of a single bubble with a wall, including all possible bubble motions: volumetric oscillations, translation, and nonspherical deformations. We also include the friction in the viscous boundary layer along the wall. We derive the coupled equations of the bubble dynamics using a Lagrangian approach. We predict the bubble response to ultrasound, as a function of various parameters (applied frequency and amplitude, bubble size and coating, bubble/wall distance). We show that our new model predicts a decrease of the resonance frequency as a bubble gets closer to a wall, in agreement with experiments. We reproduce correctly the observed decrease of oscillation amplitude for a close close to the wall, showing that it is due to the coupling between oscillation and trans- lation rather than the friction in the boundary layer. The threshold for nonspherical oscillations is also discussed and compared to experimental measurements.

\section{$5: 40$}

4aPAc21. Experimental investigation of the effect of heating rate on pre-existing gas nuclei in a viscoelastic medium. Ian Webb (University of Oxford, Medical Engineering Unit, 43 Banbury Road, OX2 6PE Oxford, UK, ian.webb@eng.ox.ac.uk), Manish Arora (University of Oxford, Medical Engineering Unit, 43 Banbury Road, OX2 6PE Oxford, UK, manish.arora@eng.ox.ac.uk), Stephen Payne (University of Oxford, Dept. of Engineering Science, Parks Road, OX1 3PJ Oxford, UK, stephen.payne@eng.ox.ac.uk), Ronald A. Roy (Boston University, Dept. of Aerosp. and Mech. Eng., 110 Cummington St., Boston, MA 02215, USA, ronroy@bu.edu), Constantin C. Coussios (University of Oxford, Medical Engineering Unit, 43 Banbury Road, OX2 6PE Oxford, UK, constantin .coussios@eng.ox.ac.uk)

Inertial cavitation is known to play a key role in thermal HIFU therapy, both from the point of view of treatment safety and delivery, and as a potential tool for treatment monitoring. However, bubble behaviour in rapidly changing temperature fields remains poorly understood. Using a theoretical model, we have previously shown that, for a given initial bubble radius, a critical heating rate exists, above which the bubble will grow, and below which it will dissolve. In order to test this hypothesis, an electrical resistor embedded in $0.5 \%$ Xanthan Gum solution is used to impose a known temperature profile, measured by an array of thermocouples, on a series of embedded bubbles. An optical arrangement employing a 10 Megapixel CCD and a macro lens is used to image the bubbles within the gel at sufficient resolution for accurate sizing. The radius-time profiles for a range of bubble 
sizes are thus extracted using image analysis techniques, allowing comparison with model predictions and subsequent refinement of the theoretical model. Future work will focus on the incorporating the effects of acoustic excitation, such as rectified diffusion, to develop a unified model of bubble behaviour in viscoelastic media under the effect of a HIFU field.

\section{6:00}

4aPAc22. Damage to single biological cells induced by laser-induced tandem microbubbles. Georgy Sankin (Duke University, Department of Mechanical Engineering and Materials Science, Durham, NC 27708, USA, gns@duke.edu), Fang Yuan (Duke University, Department of Mechanical Engineering and Materials Science, Durham, NC 27708, USA, fang.yuan@duke.edu), Pei Zhong (Duke University, Department of Mechanical Engineering and Materials Science, Durham, NC 27708, USA, pzhong@duke.edu)

Recent studies have highlighted the potential for using laser-induced micro-cavitation in lab-on-a-chip devices. Shear stress in a liquid can be controlled and significantly enhanced by bubble-bubble interaction, providing new options for in situ cell treatment. Two micro-bubbles (10 $\mu$ s life time) are generated in a $25-\mu \mathrm{m}$ liquid layer using $5 \mathrm{~ns}$ tandem laser pulses delivered through the objective of a microscope. Bubble-bubble interaction in nearly two-dimensional flow is observed using high-speed video cameras. Two liquid micro-jets moving in opposite directions can be generated when the second bubble is produced at the maximum size of the first one. The jet velocity is estimated about $35 \mathrm{~m} / \mathrm{s}$. Particle imaging velocimetry reveals vortex flow motion around the oscillating bubble lasting for about $200 \mu \mathrm{s}$. Cell lyses produced by jetting from asymmetric oscillation of tandem microbubbles are investigated at various bubble-cell distances and compared with the results from single symmetric bubble oscillation. The interaction of tandem microbubbles can produce microjetting, leading to damage of adjacent single biological cells.

\section{6:20}

4aPAc23. Introduction of a compliant gas-layer serves to mitigate damage to solid surfaces from the collapse of cavitation bubble clouds. Parag V. Chitnis (Boston University, Dept. of Aerosp. and Mech. Eng., 110 Cummington St., Boston, MA 02215, USA, pchitnis@bu.edu), Nicholas J. Manzi (Boston University, Dept. of Aerosp. and Mech. Eng., 110 Cummington St., Boston, MA 02215, USA, nmanzi@bu.edu), Robin O. Cleveland (Boston University, Dept. of Aerosp. and Mech. Eng., 110 Cummington St., Boston, MA 02215, USA, robinc@bu.edu), Ronald A. Roy (Boston University, Dept. of Aerosp. and Mech. Eng., 110 Cummington St., Boston, MA 02215, USA, ronroy@bu.edu), R. Glynn Holt (Boston University, Dept. of Aerosp. and Mech. Eng., 110 Cummington St., Boston, MA 02215, USA, rgholt@bu.edu)

The collapse of transient bubble clouds near a boundary was investigated. Transient cavitation bubbles were created using a shock-wave lithotripter. A porous ceramic disk (flow-pressure 7.5psi) was placed at the lithotripter focus. Air was forced through the disk to alter the boundary condition at the ceramic disk's proximal face. Gas pressure below 7.5psi resulted in a ceramic disk partially filled with fluid (rigid boundary); gas pressure over $7.5 \mathrm{psi}$ resulted in active bubbling at the proximal face (compliant boundary). Cavitation dynamics of bubble clouds near ceramic disks were studied for varying gas pressures (0-10psi). Images of the collapse were obtained from a high-speed camera. Additionally, a passive cavitation detector (3.5 MHz focused transducer) was aligned with the lithotripter focus. Both the images and the acoustic measurements indicated that bubble clouds near a rigid boundary collapse onto the boundary, forming a re-entrant liquid jet whose impact leads to surface erosion. When a compliant boundary is introduced, bubble clouds collapse away from the surface, thus mitigating cavitation damage. The damage to the ceramic disks was quantified using micro-CT imaging. [Supported by the ORNL Spallation Neutron Source, which is managed by UT-Battelle, LLC, under contract DE-AC0500OR22725 for the U.S. Department of Energy.]

\section{6:40}

4aPAc24. Stability and simulations of pulsating contrast agents. Nikos A. Pelekasis (Dept. Mechanical Engineering, University of Thessaly, Pedion Areos, 38334 Volos, Greece, pel@uth.gr), Kostas Tsiglifis (Dept. Mechanical Engineering, University of Thessaly, Pedion Areos, 38334 Volos, Greece, kotsigl@uth.gr)

The encapsulating membrane of ultrasound contrast agents, UCA's, is treated as a viscoelastic thin shell whose deviation from linear Hookean behavior is modeled as a strain softening or strain hardening effect via a parameter measuring the degree of membrane softness. As the amplitude of sound increases it controls the shift in resonance frequency until it hits the forcing frequency in which case an abrupt increase in the microbubble response takes place. Only strain softening shells exhibit this behavior. Deviations from sphericity are modeled via an additional parameter, namely the scalar bending modulus. This parameter controls static buckling of the shell, the onset of parametric instability and dynamic buckling. In this fashion phase diagrams can be constructed for a specific UCA that map regions of subharmonic growth of shape modes. Stability analysis and numerical simulations are employed in order to capture the onset, growth and break-up or saturation of shape modes. The above two parameters are added to the area dilatation modulus and viscosity of the membrane in order to construct a model that can be used to design new agents that behave optimally in different diagnostic or therapeutic modalities.

\section{7:00}

4aPAc25. Acoustic characterization of an ultrasound surgical transmitter in the linear and nonlinear regime of working. Antonio Petosic (Faculty of Electrical Engineering and Computing, Unska 3, 10000 Zagreb, Croatia, antonio.petosic@fer.hr), Bojan Ivančević (Faculty of Electrical Engineering and Computing, Unska 3, 10000 Zagreb, Croatia, bojan.ivancevic@fer.hr), Dragoljub Svilar (Brodarski Institut, Avenija Većeslava Holjevca bb, 10000 Zagreb, Croatia, dsvilar@hrbi.hr)

The method for measurement of a derived acoustic power of an ultrasound surgical transducer has been suggested in the free field conditions. The pressure field of the transmitter, immersed in depth of quater wavelength and vibrating at the fundamental frequency $(\approx 25 \mathrm{kHz})$, has been measured with calibrated hydrophone at different excitation levels. In the linear regime, the transmitter has been theoretically described as an acoustic dipole, the source parameters have been found and good agreement between theoretical and experimental results is obtained. When transmitter is excitated at higher excitation levels, the nonlinear behaviour in loading medium appears, with strong cavitation activity. In the averaged power spectrum of the recorded acoustic pressure signal, is evident the presence of harmonics $(n \cdot f)$, subharmonics $(f / q)$, ultraharmonics $(n \cdot f / q)$ of excitation frequency. The spatial pressure distribution of each discrete frequency component in the free acoustic field has been measured and its contribution to total acoustic power has been calculated. The total acoustic power in the cavitation noise signal is estimated integrating the averaged pressure power spectrum with appropriate contributions of each frequency component in the signal. 


\title{
Session 4aPPa
}

\section{Psychological and Physiological Acoustics: General Topics in Psychological and Physiological Acoustics IV}

\author{
Elizabeth Strickland, Cochair \\ Purdue University, 500 Oval Drive, West Lafayette, IN 47907, USA
}

Armin Kohlrausch, Cochair

Philips Research Europe, Digital Signal Processing (MS WO02), High Tech Campus 36, Eindhoven, 5656 AE, Netherlands

\author{
Alain De Cheveigne, Cochair \\ CNRS, Universite Paris 5, Ecole Normale Superieure, 29 rue d'Ulm, Paris, 75230, France
}

\section{Contributed Papers}

\section{8:00}

4aPPa1. Circumaural transducer arrays for binaural synthesis. Raphaël Greff (A-Volute, 4120 route de Tournai, 59500 Douai, France, raphael.greff@a-volute.com), Brian F. Katz (LIMSI-CNRS, B.P. 133, 91403 Orsay, France, brian.katz@limsi.fr)

Binaural cues such as the interaural time and level differences are the primary cues for estimation of the lateral position of a sound source, but are not sufficient to determine elevation or the exact position on the "cone of confusion". Spectral content of the head-related transfer function (HRTF) provides cues that permit this discrimination, notably high frequency peaks and notches created by diffraction effects within the pinnae. For high quality binaural synthesis, HRTFs need to be individualized, matching the morphology of the listener. Typical means for this are to measure or calculate the HRTF of the listener, but these lengthy and costly methods are not feasible for general public applications. This paper presents a novel approach for HRTF individualization, separating the head and torso effect from that of the pinnae. The head/torso component is numerically modelled while the pinnae component is created using a multiple transducer array placed around each pinna. The philosophy of this method consists in trying to excite the correct localization cues provided by the diffraction of the reconstructed wave front on the listener's own pinnae. Simulations of HRTF reconstruction with various array sizes and preliminary auditory localization tests are presented.

\section{$8: 20$}

4aPPa2. Interaural-time-difference sensitivity to acoustic temporally-jittered pulse trains. Matthew J. Goupell (Austrian Academy of Science / Acoustics Research Institute, Wohllebengasse 12-14, 1040 Vienna, Austria, matt.goupell@gmail.com), Piotr Majdak (Austrian Academy of Science / Acoustics Research Institute, Wohllebengasse 12-14, 1040 Vienna, Austria, piotr@majdak.com), Bernhard Laback (Austrian Academy of Science / Acoustics Research Institute, Wohllebengasse 12-14, 1040 Vienna, Austria, Bernhard.Laback@oeaw.ac.at)

Bandpass-filtered pulse trains in acoustic hearing have been used to understand the high-rate pulse trains used in electrical stimulation strategies. In a left-right discrimination test, sensitivity to interaural time differences (ITD) in 600-pulses-per-second (pps) periodic pulse trains and aperiodic (temporally-jittered) pulse trains was tested with six normal-hearing listeners. It was found that jitter significantly and systematically increased ITD performance. A second experiment using 1200-pps pulse trains was performed to show that listeners were not solely benefiting from the longest interpulse intervals and thus the instances of reduced rate by adding jitter. To better understand the effect of jitter, the output of a basilar membrane model and a higher-level physiologically-based model was observed. Results from the modeling were reminiscent of an effect called "release from binaural adaptation" where the binaural system is reactivated by a temporal irregularity and this release possibly occurs at the level of the cochlear nucleus or lower. These results help understand pulse-rate limitations of ITD in cochlearimplant listeners.

\section{8:40}

4aPPa3. From sounds to melodies: Memory for sequences of pitch and loudness. Marion Cousineau (CNRS UMR 8158; Univ. Paris Descartes; Ecole Normale Supérieure, 29 rue d'Ulm, 75005 Paris, France, mcousineau@ens.fr), Daniel Pressnitzer (CNRS UMR 8158; Univ. Paris Descartes; Ecole Normale Supérieure, 29 rue d'Ulm, 75005 Paris, France, Daniel.Pressnitzer@ens.fr), Laurent Demany (CNRS UMR 5227; Univ. Victor Segalen, 146 rue Léo Saignat, 33076 Bordeaux, France, laurent .demany@psyac.u-bordeaux2.fr)

In order to understand speech or appreciate music, listeners have to process and remember patterns of sounds that vary along many perceptual dimensions. Here we investigated the perception of pitch sequences and loudness sequences, using a psychophysical method that uncouples discriminability and memory capacity. Pitch could be produced by either resolved or unresolved harmonics. Random sequences were constructed for which a single attribute (pitch or loudness) could take only two different values. These values were selected individually for each participant to produce equal discriminability (d') for isolated sounds. The participants then had to perform Same-Different judgments on pairs of sequences of two, four or eight elements each. We found that performance decreased rapidly with the number of elements for the loudness and pitch of unresolved harmonics conditions. With sequences of four and eight elements, performance was markedly better for the pitch of resolved harmonics condition. These findings show that short-term auditory memory capacity changes with the type of attribute that is varied within a sequence. For pitch, resolved harmonics yield a higher capacity than do unresolved harmonics; this could explain part of the difficulties encountered by cochlear implant users when listening to music.

\section{9:00}

4aPPa4. Local-pitch identification accuracy depending on the trajectory of frequency-modulated tones. Yuki Hiruma (School of Media Science, Tokyo Univ. of Technology, 1404-1 Katakuracho, Hachioji, 192-0982 Tokyo, Japan, m01043572a@mss.teu.ac.jp), Kiyoaki Aikawa (School of Media Science, Tokyo Univ. of Technology, 1404-1 Katakuracho, Hachioji, 192-0982 Tokyo, Japan, aik@media.teu.ac.jp)

Local-pitch identification accuracies were analyzed for frequencymodulated (FM) tones. The problem was whether every portion of a continuous sound was perceived at the same accuracy or not. Psychophysical experiments revealed that the local-pitch identification accuracies were significantly different among the nodes of continuous FM tones. Also, the accuracies were dependent on the frequency trajectory shape. The stimuli included two types of piecewise-linear FM tones of up-down-up and downup-down glide sequences. Each tone had four nodes; the initial, two intermediate points, and the final. The duration of each linear glide was 100 ms. The frequency range was between 1000 and $1500 \mathrm{~Hz}$. A pair of FM tone was presented with one-second interval. The frequency was shifted up or down at one of the nodes in either of the tones. The shift amounts were $0 \%$, 
$4 \%$, and $8 \%$. The subjects were requested to answer whether two pitch sequences were the same or different. The pitch identification accuracy was low at the initial for both types of FM tones. The accuracy at the final was highest for the up-down-up tone. The intermediate high frequency node showed the highest accuracy for the down-up-down tone. These results indicated that the local-pitch identification accuracies were trajectorydependent.

\section{9:20}

4aPPa5. Pitch discrimination: Combination of information across frequency. Hedwig E. Gockel (MRC CBU, 15 Chaucer Rd., CB2 7EF Cambridge, UK, hedwig.gockel@mrc-cbu.cam.ac.uk), Robert P. Carlyon (MRC CBU, 15 Chaucer Rd., CB2 7EF Cambridge, UK, bob.carlyon@mrc-cbu.cam.ac.uk), Christopher J. Plack (Psychology Department, Lancaster University, LA14YF Lancaster, UK, c.plack@lancaster .ac.uk)

Performance (d') for fundamental frequency (F0) discrimination was measured for two complex tones (A and B) presented either individually or simultaneously. The objective was to investigate how information is combined in pitch processing. For most subjects, tones A and B were filtered from $1350-1650$ and $3300-4200 \mathrm{~Hz}$, respectively, such that both contained only unresolved components (components were added in sine phase). The tones had identical nominal F0s of $75 \mathrm{~Hz}$, and the difference in F0 between the two intervals of a $2 \mathrm{AFC}$ trial was the same for A and B. When A and B were tested individually, performance was equal for the two tones (d'value 1). For some subjects, to achieve equal d' for the two tones, all components were added in alternating phase, F0 was increased to $90 \mathrm{~Hz}$, and tones A and B were filtered from 1375-1875 and 3900-5400 Hz, respectively. Presenting the tones simultaneously should increase d' by a factor of 1.41 , if information from both were combined optimally. The results showed no increase in d' above that measured for the individual tones for three subjects, and only a very small improvement for another three subjects (factor of about 1.1) [supported by EPSRC Grant EP/D501571/1].

\section{9:40}

4aPPa6. The role of compression in forward masking by Schroeder-phase complexes. Magdalena Wojtczak (University of Minnesota, Department of Psychology, 75 E. River Road, Elliott Hall N218, Minneapolis, MN 55455, USA, wojtc001@umn.edu), Andrew J. Oxenham (University of Minnesota, Department of Psychology, 75 E. River Road, Elliott Hall N218, Minneapolis, MN 55455, USA, oxenham@umn .edu)

Waveforms with flat temporal envelopes can produce more forward masking than waveforms with more modulated, or peakier, temporal envelopes after auditory filtering, even when the rms amplitude of the two waveforms is equal. This has been explained in terms of basilar-membrane nonlinearity, which can result in a higher rms amplitude for the flatter than for the peakier temporal waveform after compression. Here, forward masking was measured as a function of the phase curvature of two Schroeder-phase complexes, one with components around the signal frequency (on-frequency masker) and the other with components well below the signal frequency (off-frequency masker). The experiment tested the hypothesis that since the basilar-membrane response to the off-frequency complex at the signalfrequency place is presumably linear, masking should not depend on the phase curvature of the complex, whereas compression of the on-frequency masker should produce phase-dependent thresholds, with the minimum corresponding to the peakiest internal representation of the masker. The results replicate prominent phase effects using on-frequency maskers, but also show some phase effects with off-frequency maskers, which are not predicted by our current understanding of basilar-membrane compression. Other possible influences, such as efferent effects and neural compression, are considered. [Supported by NIH grant R01DC03909].

\section{0:00}

4aPPa7. Additivity of auditory masking using Gaussian-shaped tones Bernhard Laback (Austrian Academy of Science / Acoustics Research Institute, Wohllebengasse 12-14, 1040 Vienna, Austria, Bernhard.Laback@oeaw.ac.at), Peter Balazs (Austrian Academy of Science / Acoustics Research Institute, Wohllebengasse 12-14, 1040 Vienna, Austria, peter.balazs@oeaw.ac.at), Gwenael Toupin (Austrian Academy of Science / Acoustics Research Institute, Wohllebengasse 12-14, 1040 Vienna, Austria, toupin@kfs.oeaw.ac.at), Thibaud Necciari (CNRS-LMA, 31, chemin Joseph Aiguier, 13402 Marseille, France, necciari@1ma.cnrs-mrs.fr), Sophie Savel (CNRS-LMA, 31, chemin Joseph Aiguier, 13402 Marseille, France, savel@1ma.cnrs-mrs.fr), Sabine Meunier (CNRS-LMA, 31, chemin Joseph Aiguier, 13402 Marseille, France, meunier@1ma.cnrs-mrs.fr), Solvi Ystad (CNRS-LMA, 31, chemin Joseph Aiguier, 13402 Marseille, France, ystad@1ma.cnrs-mrs.fr), Richard Kronland-Martinet (CNRS-LMA, 31, chemin Joseph Aiguier, 13402 Marseille, France, kronland@lma.cnrs-mrs.fr)

Both temporal and spectral masking have been studied extensively in the literature. Mostly, they have been regarded as separate phenomena. Very little is known about the interaction between these two effects, i.e. masking in the time-frequency domain. Data on the time-frequency spread of masking evoked by a single Gaussian-shaped tone pulse are presented in an accompanying study at the same conference (Necciari et al.). The current study gathers data on the additivity of masking by up to four, approximately equally effective Gaussian maskers $(E R B=600 \mathrm{~Hz})$, separated either along the time or the frequency axis. For temporal separation, the amount of masking increases with the number of maskers, with excess masking (exceeding linear additivity) of up to $25 \mathrm{~dB}$. For frequency separation (preliminary data) excess masking amounts up to $15 \mathrm{~dB}$, and the higher-frequency masker (relative to the target) contributes more to the additivity than the lowerfrequency maskers. Experiments with multiple maskers combining both temporal and frequency separation are underway. Combined with the single maker data, these data may serve as a basis for modeling time-frequency masking effects in complex signals. Work partly supported by OEAD (WTZ project AMADEUS) and ANR

\section{0:20-10:40 Break}

\section{0:40}

4aPPa8. Auditory masking using Gaussian-windowed stimuli. Thibaud Necciari (CNRS-LMA, 31, chemin Joseph Aiguier, 13402 Marseille, France, necciari@1ma.cnrs-mrs.fr), Sophie Savel (CNRS-LMA, 31, chemin Joseph Aiguier, 13402 Marseille, France, savel@1ma.cnrs-mrs.fr), Sabine Meunier (CNRS-LMA, 31, chemin Joseph Aiguier, 13402 Marseille, France, meunier@1ma.cnrs-mrs.fr), Solvi Ystad (CNRS-LMA, 31, chemin Joseph Aiguier, 13402 Marseille, France, ystad@1ma.cnrs-mrs.fr), Richard Kronland-Martinet (CNRS-LMA, 31, chemin Joseph Aiguier, 13402 Marseille, France, kronland@1ma.cnrs-mrs.fr), Bernhard Laback (Austrian Academy of Science / Acoustics Research Institute, Wohllebengasse 12-14, 1040 Vienna, Austria, Bernhard.Laback@oeaw.ac.at), Peter Balazs (Austrian Academy of Science / Acoustics Research Institute, Wohllebengasse 12-14, 1040 Vienna, Austria, peter.balazs@oeaw.ac.at)

This study investigates auditory masking with Gaussian-windowed tones as target and masker stimuli. On the purpose of developing a time-frequency masking model, such stimuli minimize the time-frequency uncertainty. Also, as proposed by van Schijndel et al. (1999), they activate a single spectrotemporal observation window of the auditory system. The study presented here measured auditory masking with Gaussian-windowed stimuli with an $\mathrm{ERB}$ of $600 \mathrm{~Hz}$ and an effective duration of $9.6 \mathrm{~ms}$. The masker was centered at $4 \mathrm{kHz}$. Its level was $60 \mathrm{~dB}$ SL. Four experiments were conducted. (1) Absolute thresholds for Gaussian-windowed and 300-ms-sinusoidal targets were measured and compared for 11 frequencies. (2) Masking patterns were obtained with targets of various frequency separations from the masker. (3) Forward masking functions with 4-kHz targets were measured at 5 temporal separations. (4) Forward masking was measured for different frequency separations between masker and target. These data are compared with those typically obtained with stimuli that are broader either in the frequency or time domain. A modelling attempt is made. A companying article 
on multiple masker additivity based on the present results is presented at the same conference (Laback et al.). Work partly supported by EGIDE (PAI Amadeus) and the ANR.

\section{1:00}

4aPPa9. Gaussian-noise discrimination as a tool to investigate auditory object formation. Tom L. Goossens (Technische Universiteit Eindhoven, Den Dolech 2, 5600 MB Eindhoven, Netherlands, tomgoos @ gmail.com), Steven Van De Par (Philips Research Europe, Digital Signa Processing (MS WO02), High Tech Campus 36, 5656 AE Eindhoven, Netherlands, steven.van.de.par@philips.com), Armin Kohlrausch (Philips Research Europe, Digital Signal Processing (MS WO02), High Tech Campus 36, 5656 AE Eindhoven, Netherlands, armin.kohlrausch@philips.com)

In the present study we show that, in a same/different experiment, listeners are good at discriminating 50-ms Gaussian-noise tokens with a spectral range of 350-850 Hz. However, when an identical 200-ms noise fringe, with the same statistical properties as the 50-ms target tokens, is appended to both target tokens, listeners show very poor discrimination performance. Apparently, these identical fringes cannot be ignored and these extra noninformative fringes impair the discrimination of the target tokens. It seems that a target token and the appended fringe form one auditory object and that access to subparts of these tokens is not possible. When a perceptual cue is introduced that can lead to the segregation of the target token and noise fringe, e.g., a temporal gap between target and fringe, the ability to discriminate improves implying that the non-informative noise can be (partly) ignored when it is part of a different auditory object than the target token. This method is used as a new approach to investigate the influence of cues such as spectral range, level, interaural level difference, and interaural time delay, on the formation of auditory objects.

\section{1:20}

4aPPa10. Evidence for Poisson processes in change detection Christian Kaernbach (Institut für Psychologie, Universität Kiel, Olshausenstr. 62, 24098 Kiel, Germany, chris2008@kaernbach.de)

Comparisons between two stimuli (e.g., "which stimulus was louder?") and change detection ("same or different?") are often assumed to operate on the same decision basis. In the Gaussian signal detection theory, each of the two stimuli to be compared is transformed into a number, and the comparison is then made between these two numbers. If both stimuli are well above absolute threshold, the numbers to be compared have to be large, which motivates the use of normal distributions. The present study tests this assumption by measuring same-different ROC curves for the detection of small changes in the intensity of sinusoids. In contrast to previous studies, change detection was measured not only when the possible direction of change was a priori unknown, but also in two conditions where changes had a fixed direction. The obtained ROCs are asymmetric. This points to Poisson processes with low means. Moreover, the sensitivity to increments was significantly higher than the sensitivity to decrements. The results put into question the Gaussian theory of sensory comparisons and change detection.

\section{$11: 40$}

4aPPa11. Low level auditory processing of simple and complex sounds in autism. Anna C. Bonnel (McGill University, Faculty of Educational and Counselling Psychology, 3700 McTavish Street, Montreal, QC H3A1Y2, Canada, anna.bonnel@mail.mcgill.ca), Stephen McAdams (Centre for Interdisciplinary Research in Music Media \& Technology (CIRMMT) - Schulich School of Music - McGill Univ., 555 Sherbrooke Street West, Montreal, QC H3A1E3, Canada, smc@music .mcgill.ca), Bennett K. Smith (Centre for Interdisciplinary Research in Music Media \& Technology (CIRMMT) - Schulich School of Music - McGill
Univ., 555 Sherbrooke Street West, Montreal, QC H3A1E3, Canada, bennett.smith@music.mcgill.ca), Armando Bertone (McGill University, Faculty of Educational and Counselling Psychology, 3700 McTavish Street, Montreal, QC H3A1Y2, Canada, armando.bertone@mcgill.ca), Jake A. Burack (McGill University, Faculty of Educational and Counselling Psychology, 3700 McTavish Street, Montreal, QC H3A1Y2, Canada, jake.burack@mcgill.ca), Valter Ciocca (University of British Columbia, School of Audiology and Speech Sciences, 5804 Fairview Avenue (J.M. Mather Building), Vancouver, AB V6T 1Z3, Canada, vvciocca @audiospeech.ubc.ca), Laurent Mottron (Pervasive Developmental Disorders Clinic, Riviere-des-Prairies Hospital and Fernand Seguin Research Center, University of Montreal, Montreal, QC H3C 3J7, Canada, mottronl @istar.ca)

The documented dissociation between enhanced and diminished auditory processing performance in autistic listeners may be linked to the neural complexity required to process auditory stimuli. To test this hypothesis, four discrimination experiments were designed targeting pitch, spectral envelope, vocal timbre, and loudness. A range of pure- and complex-tone stimuli, with or without frequency or amplitude modulation, varied along spectral and temporal dimensions. An adaptive procedure was used to assess the auditory discrimination thresholds of groups of high-functioning participants with autism (HFA), Asperger syndrome (ASP), and typically developing individuals (TDs). Our research question was whether increasing the level of spectral and/or temporal complexity would have a detrimental impact on autistic listeners' ability to discriminate between acoustic stimuli. Preliminary results suggest that auditory discrimination performance of the latter group is not as dependent on levels of spectro-temporal complexity as originally predicted. The results will be interpreted in the context of current perceptually based models of enhanced and diminished perceptual functioning in autism.

\section{2:00}

4aPPa12. Roughness detection in fricative-like noise and tone stimuli Jonathan Pincas (University of Surrey, GU2 7XH Guildford, UK jon@pincas.co.uk), Philip J. Jackson (University of Surrey, Centre for Vision, Speech and Signal Processing, GU2 7XH Guildford, UK, p.jackson @ surrey.ac.uk)

Audio (spectral) and modulation (envelope) frequencies both carry information in a speech signal. While low modulation frequencies $(2-20 \mathrm{~Hz})$ convey syllable information, higher modulation frequencies $(80-400 \mathrm{~Hz})$ allow for assimilation of perceptual cues, e.g., the roughness of amplitudemodulated noise in voiced fricatives, considered here. Psychoacoustic 3-interval forced-choice experiments measured AM detection thresholds for modulated noise accompanied by a tone with matching fundamental frequency at 125Hz: (1) tone-to-noise ratio (TNR) and phase between tone and noise envelope were varied, with silence between intervals; (2) as (1) with continuous tone throughout each trial; (3) duration and noise spectral shape were varied. Results from (1) showed increased threshold (worse detection) for louder tones (40-50dB TNR). In (2), a similar effect was observed for the in-phase condition, but out-of-phase AM detection appeared immune to the tone. As expected, (3) showed increased thresholds for shorter tokens, although still detectable at $60 \mathrm{~ms}$, and no effect for spectral shape. The phase effect of (2) held for the short stimuli, with implications for fricative speech tokens (40ms-100ms). Further work will evaluate the strength of this surprisingly robust cue in speech.

\section{2:20}

4aPPa13. Measurement of equal-loudness contours using eardrum pressure as reference signal. Sebastian Schmidt (Institute of Communication Acoustics, Ruhr-Universität Bochum, IC 1/142, Universitätsstr. 150, 44780 Bochum, Germany, sebastian.schmidt@rub.de), Herbert Hudde (Institute of Communication Acoustics, Ruhr-Universität Bochum, IC 1/142, Universitätsstr. 150, 44780 Bochum, Germany, herbert.hudde @ rubr-uni-bochum.de)

Equal-loudness contours represent the relationship of loudness perception and sound pressure at the ear. Usually, the reference pressure is defined with a standardised calibration procedure. Individual ear canal characteristics significantly influence the contours, resulting in peaks and notches. By 
choosing the eardrum pressure as reference for the perception measurements, individual ear canal features are cancelled, when loudness and pressure are related. Thus, flatter contours can be achieved. Finite-element simulations of the sound field in the canal have shown that signals at remote positions may differ significantly from the eardrum pressure. Thus, to achieve eardrum related measurements, it is necessary to insert probe microphones sufficiently deep into the canal. However, the desired signal has to be estimated from a distance, since measurements directly at the drum are not practical. A method is shown to determine both the probe tip distance and the transformation terms to calculate the drum pressure estimate. In this contribution, the results of equal-loudness contour measurements are discussed. The positioning and transformation processes are verified by simulations with one-dimensional and finite-element models and experiments.

\title{
Session $4 \mathrm{aPPb}$
}

\section{Psychological and Physiological Acoustics: General Topics in Psychological and Physiological Acoustics V}

\author{
Elizabeth Strickland, Cochair \\ Purdue University, 500 Oval Drive, West Lafayette, IN 47907, USA
}

Armin Kohlrausch, Cochair

Philips Research Europe, Digital Signal Processing (MS WO02), High Tech Campus 36, Eindhoven, 5656 AE, Netherlands

\author{
Alain De Cheveigne, Cochair \\ CNRS, Universite Paris 5, Ecole Normale Superieure, 29 rue d'Ulm, Paris, 75230, France
}

\section{Contributed Papers}

\section{8:40}

4aPPb1. A fast FFT-based integral-equation solver for simulation of elastoacoustic wave propagation in human head. Elizabeth Bleszynski (Monopole Research, 739 Calle Sequoia, Thousand Oaks, CA 91360, USA, elizabeth@monopoleresearch.com), Marek Bleszynski (Monopole Research, 739 Calle Sequoia, Thousand Oaks, CA 91360, USA, marek@monopoleresearch.com), Thomas Jaroszewicz (Monopole Research, 739 Calle Sequoia, Thousand Oaks, CA 91360, USA, tomek@monopoleresearch.com)

We describe formulation, implementation, and representative applications of a fast integral-equation solver for modeling wave propagation in inhomogeneous visco-elastic and visco-acoustic media (e.g. in biological tissues). The present approach is an extension of our work on fast integral equation solvers in pure acoustics. It is based on Lippmann-Schwinger (L-S) integral equations. It incorporates: (i) FFT-based compression of the stiffness matrix and the corresponding fast iterative method resulting in the solution complexity proportional to the number of unknowns, and ability to solve problems of several million unknowns, (ii) piecewise-linear basis functions supported on tetrahedra, representing displacement field, and corresponding efficient algorithms for evaluation of Galerkin matrix elements, (iii) rigorous two-stage solution scheme applicable to scatterers composed of high contrast materials and consisting of transforming the L-S equations into a system of two well-conditioned problems (conventional solution schemes, when applied to such scatterers, lead to stiffness matrix of large condition number). We present applications of the developed approach to simulation of elastic wave propagation in realistic models of the human head, with the goal of comparing sound transmission through the normal auditory airways and through bone conduction, and in the presence and absence of noiseprotective devices. This work is supported by the Air Force Office of Scientific Research.

\section{9:00}

4aPPb2. Towards a transfer function used to adjust audio for bone-conduction transducers. Raymond M. Stanley (Georgia Institute of Technology, Georgia Tech, School of Psychology, 654 Cherry St, Atlanta,
GA 30332-0170, USA, rms@ gatech.edu),Bruce N. Walker(Georgia Institute of Technology, Georgia Tech, School of Psychology, 654 Cherry St, Atlanta, GA 30332-0170, USA, bruce.walker@psych.gatech.edu)

Bone-conduction transducers may effectively replace normal airconduction headphones in cases where the ears need to be plugged, or else remain unoccluded. However, sounds designed to be presented via air conduction need to be adjusted to maintain optimal perception via bone conduction. This study sought to find bone-to-air amplitude and phase shifts, as preliminary data for a complete transfer function between the boneconduction and air-conduction pathway. The variability or stability of the shift data can indicate the feasibility of making effective adjustments to sounds to account for the bone-conduction pathway. Listeners cancelled airconducted and bone-conducted tone pairs by method of adjustment at three frequencies $(500,3150$, and $8000 \mathrm{~Hz})$. The amplitude adjustments were relatively consistent, while the phase adjustments were quite variable. Further analysis revealed that the variability in phase adjustments came from differences between people, but were relatively consistent within a person. Together, these data suggest that generalized adjustments for the boneconduction pathway may not be effective, but that individualized adjustments may be both necessary and potentially quite effective. These results can be extended to continuous transfer functions applied to sounds before they are presented via bone-conduction transducers ("bonephones") in an auditory display.

\section{9:20}

4aPPb3. Variability of hearing protection devices attenuation as a function of source location. Hugues Nelisse (IRSST, Service de la recherche, 505 Boulevard de Maisonneuve O, Montréal, QC H3A3C2, Canada, hugnel@irsst.qc.ca), Marc-André Gaudreau (École de Technologie Supérieure, Département de Génie Mécanique, 1100, rue Notre-Dame Ouest, Montreal, QC H3C 1K3, Canada, gaudream@cdrummond.qc .ca), Jérémie Voix (École de Technologie Supérieure, Département de Génie Mécanique, 1100, rue Notre-Dame Ouest, Montreal, QC H3C 1K3, Canada, jvoix@jerevox.com), Jérôme Boutin (IRSST, Service de la recherche, 505 Boulevard de Maisonneuve O, Montréal, QC H3A3C2, Canada, jerbou@irsst.qc.ca), Frédéric Laville (École de Technologie Supérieure, 
Département de Génie Mécanique, 1100, rue Notre-Dame Ouest, Montreal, QC H3C 1K3, Canada, Frederic.Laville@etsmtl.ca)

It is of common knowledge, and well documented, that laboratorymeasured noise attenuation values of most hearing protection devices (HPD) exceed significantly the attenuation values obtained in real-world workplace environments. Various reasons may explain such discrepancies (lack of training, wearing time, lack of comfort, bad fitting, noise environments, etc.) but very few of them have been studied in details due to the complexity of the problem. This study focuses on the variability of the attenuation of HPDs as a function of the location of the noise source. Laboratory measurements were performed where subjects, wearing a HPD and facing a loudspeaker, were asked to rotate slowly on a rotating chair to simulate different angular positions of the head relative to the source. The protected and unprotected sound pressure signals for both ears were recorded as time signals using miniature microphones placed respectively inside and outside the HPD (F-MIRE technique). The microphones signals were processed to obtain attenuation values for the different angular positions. Results for different type of HPD (ear-muffs and ear-plugs) are presented and discussed.

\section{9:40}

4aPPb4. Assessment of exposure to impulsive and continuous noise by auditory brainstem response. Jan Zera (Centr. Inst. for Labour Prot. Natl. Res. Inst., Czerniakowska 16, 00-701 Warsaw, Poland, jazer@ciop.pl), Krzysztof Kochanek (The Institute of Physiology and Pathology of Hearing, Pstrowskiego 1, 01-943 Warsaw, Poland, k.kochanek@ifps.org.pl), Adam Pilka (The Institute of Physiology and Pathology of Hearing, Pstrowskiego 1, 01-943 Warsaw, Poland, a.pilka@ifps.org.pl), Rafal Mlynski (Centr. Inst. for Labour Prot. - Natl. Res. Inst., Czerniakowska 16, 00-701 Warsaw, Poland, rmlynski@ciop.pl)

The aim of the work was to measure the wave V latency or thresholds in the auditory brainstem responses (ABRs) produced by impulsive and continuous noise. A forward-masking paradigm was used in which the ABR was evoked by a $4-\mathrm{kHz}$ tone pip. The tone pip was masked by a preceding 201-ms or 501-ms interval of click trains or band-pass noise presented at various levels. The inter-click interval ranged from $20 \mathrm{~ms}$ (50 clicks/s) to $100 \mathrm{~ms}$ (10 clicks/s). The center frequencies of the noise bands ranged from 250 to $4000 \mathrm{~Hz}$. Results show that changes in wave V latency may be used as an indicator of the equivalence of the effect of various kinds of noise on the human auditory system. [Work supported by the Polish Ministry of Science and Higher Education, grant 4T07B00428].

\section{0:00}

4aPPb5. Evaluation of auditory processing disorder and auditory efferent system functionality in adult dyslexics: towards a unification theory of auditory-language processing impairments? Michel Hoen (Laboratoire d'Etude des Mécanismes Cognitifs (EMC). EA 3082 CNRS, Université Lumière Lyon 2, 5, Avenue Pierre Mendès-France, 69676 Bron Cedex, France, Michel.Hoen@univ-lyon2.fr), Claire Grataloup (Laboratoire Dynamique du Langage (DDL), UMR 5596 CNRS, Université de Lyon et Lyon 2, Institut des Sciences de 1'Homme - 14 avenue Berthelot, 69363 Lyon Cedex 07, France, Claire.Grataloup@pse .unige.ch), Evelyne Veuillet (Univ. Lyon 1 - Lab. Neurosciences, Service Pr Collet, Pavillon U, Hôpital Edouard Herriot, F-69003 Lyon, France, iakhoun@olfac.univ-lyon1.fr), Hung Thai-Van (Univ. Lyon 1 - Lab. Neurosciences, Service Pr Collet, Pavillon U, Hôpital Edouard Herriot, F-69003 Lyon, France, iakhoun@olfac.univ-lyon1.fr), Lionel Collet (Univ. Lyon 1 Lab. Neurosciences, Service Pr Collet, Pavillon U, Hôpital Edouard Herriot, F-69003 Lyon, France, iakhoun@olfac.univ-lyon1.fr), Fanny Meunier (Laboratoire Dynamique du Langage (DDL), UMR 5596 CNRS, Université de Lyon et Lyon 2, Institut des Sciences de 1'Homme - 14 avenue Berthelot, 69363 Lyon Cedex 07, France, fanny.meunier@univ-lyon2.fr)

In a recent paper, Veuillet et al., (2007) suggested that some auditory processing mechanism could be impaired in children with dyslexia. They reported a link between children's ability to perceive phonemic boundaries and the physiological functionality of their medial olivocochlear system (MOC), an auditory efferent pathway functioning under central control. In the present experiment, we extended this observation by comparing speechin-speech comprehension performances in a group of control participants
$(\mathrm{N}=40)$ and a group of adults who had been diagnosed dyslexic as children $(\mathrm{N}=49)$. Confirming the idea that patients with dyslexia present auditory processing disorders (APD), we show that adult dyslexics exhibit greater difficulty in comprehending speech in noise. Data moreover suggest a link between speech-in-noise comprehension difficulty and the MOC functionality in these participants. More precisely, it appears that the absolute functionality of the left and right MOC bundles does not differ in dyslexic patients compared to controls. What appears to be differing is the functional asymmetry in the MOC functionality between both ears, normal readers showing a classical functional asymmetry, while dyslexic adults show an absence of, or reduced functional asymmetry between MOC bundles. These results will be discussed in the context of current models of APD and Dyslexia.

\section{0:20-10:40 Break}

\section{0:40}

4aPPb6. Training of English vowel perception by Finnish speakers to focus on spectral rather than durational cues. Maria Uther (Brunel University, School of Social Sciences, UB8 3PH Uxbridge, UK, maria.uther@brunel.ac.uk), Sari Ylinen (Cognitive Brain Research Unit, University of Helsinki, PO BOX 9, 00014 Helsinki, Finland, sari.ylinen@helsinki.fi), Antti Latvala (Cognitive Brain Research Unit, University of Helsinki, PO BOX 9, 00014 Helsinki, Finland, antti.latvala@helsinki.fi), Reiko Akahane-Yamada (ATR Promotions, Human Information Sciences Laboratory, -2-2 Hikaridai Keihanna Science City, Seika-cho, Soraku-gun, 619-0288 Kyoto, Japan, yamada@atr.jp), Paul Iverson (University College London, Department of Phonetics and Linguistics, 4, Stephenson Way, NW1 2HE London, UK, p.iverson@ucl.ac.uk)

This study used the High-Variability Phonetic Training (HVPT) technique to train Finnish speakers to distinguish English vowels. It was found that Finnish speakers tend to use durational cues (which are phonemically relevant in their own language) to make a vowel category distinction rather than the relevant spectral cues. We used duration-modified stimuli with a HVPT program to 'force' the use of spectral cues. We focused on the /i/ (as in 'feet') vs /I/ (as in 'fit') vowel contrast and tested behavioural performance using a perceptual identification task. We also measured the Mismatch negativity (MMN) component of auditory event-related potential (ERP) before and after the training to look at changes in brain responses. The worst pre-test performance was for the 'modified duration' condition (i.e. where the learner had no choice but to rely on spectral cues). There were also asymmetries in vowel perception in both behavioural and MMN tasks, with the detection of /i/ being more difficult compared to the detection of /I/. Nevertheless, training did result in marked improvement of the most problematic contrasts for Finnish speakers.

\section{1:00}

4aPPb7. Effects of voice familiarity and age on perceptual organization of sound from two competing talkers. Ingrid S. Johnsrude (Queen's University, Dept Psychology, 62 Arch Street, Kingston, ON K7L 3N6, Canada, ingrid.johnsrude@queensu.ca), Allison Mackey (The Rotman Research Institute, Baycrest, 3560 Bathurst Street, 938, Toronto, ON M6A 2E1, Canada, allison.mackey@hotmail.com), Elizabeth M. Alexander (Queen's University, Dept Psychology, 62 Arch Street, Kingston, ON K7L 3N6, Canada, 3ema@queensu.ca), Heather Macdonald (Queen’s University, Dept Psychology, 62 Arch Street, Kingston, ON K7L 3N6, Canada, hpmacdonald@gmail.com), Robert P. Carlyon (MRC CBU, 15 Chaucer Rd., CB2 7EF Cambridge, UK, bob.carlyon@mrc-cbu.cam.ac.uk)

We used the Coordinate Response Measure (CRM) procedure (Bolia et al, 2000) to examine whether a non-acoustic characteristic of speech -voice familiarity- can affect a listener's ability to separate competing voices. We tested 27 listeners, aged 45-79, with their spouse's and two novel voices (other listeners' spouses). Couples had been living together at least 18 years. On each trial, two different talkers produced two of four callsigns (one being the target 'Baron'), two of four colours and two of eight numbers, and the participant responded by indicating the colour-number combination to which 'Baron' was told to go. We tested three conditions: spouse-voice target phrase with a novel-voice masker phrase $(\mathrm{F} / \mathrm{N})$; novel-voice target with 
spouse-voice masker (N/F); novel-voice target with (different) novel-voice masker $(\mathrm{N} / \mathrm{N})$. Compared to $\mathrm{N} / \mathrm{N}$, performance was significantly better in $\mathrm{F} / \mathrm{N}$, and, crucially, N/F conditions, indicating that listeners can exploit learned characteristics of a masking voice to help them track a novel voice. Furthermore, whereas the younger participants (under 60) benefited from having a familiar voice as target or as masker, the older group (over 60) benefited only when the familiar voice was the focus of attention, suggesting that the ability to use voice familiarity to segregate sounds changes with age.

\section{1:20}

4aPPb8. Detection threshold and prominence of background music with interfering speech. Mark M. Houben (TNO, Kampweg 5, P.O. Box 23, 3769 ZG Soesterberg, Netherlands, mark.houben@tno.nl)

Spoken radio and television messages are often accompanied by background music. When automating identification of broadcast music for handling copyright exploitation, it may be questioned to what extent music at very low sound levels should be taken into account. Should it just be perceivable by listeners who attend to the speech, or should the music be present more prominently? We studied detection and level of prominence of music with interfering speech. For this, Dutch radio and television broadcasts were recorded and speech fragments without background music were selected. To the fragments, music was added with various level differences between speech and music. Subjects were presented with these stimuli and answered a random generic question about the speech after each stimulus. As a second task, the prominence of the background music had to be scored on a scale (including 'not present'). Results show that, on average, 50\% of the music is detected if the level of the music is $45 \mathrm{~dB}$ lower than the speech. A threshold based on a criterion of 'moderate prominence' results in a level difference of $26 \mathrm{~dB}$. This level happens to coincide with a detection threshold of $95 \%$.

\section{1:40}

4aPPb9. Emotion and meaning in interpretation of sound sources Penny Bergman (Chalmers University of Technology, Division of Applied Acoustics - Chalmers Room Acoustics Group, Sven Hultins gata 8a, 41296 Gothenburg, Sweden, penny@chalmers.se), Daniel Vastfjall (Chalmers University of Technology, Division of Applied Acoustics - Chalmers Room Acoustics Group, Sven Hultins gata 8a, 41296 Gothenburg, Sweden, daniel.vastfjall@psy.gu.se), Niklas Fransson (Chalmers University of Technology, Division of Applied Acoustics, Sven Hultinsgata 8a, 41296 Gothenburg, Sweden, niklas.fransson@psy.gu.se), Anders Sköld (Chalmers University of Technology, Division of Applied Acoustics, Sven Hultinsgata 8a, 41296 Gothenburg, Sweden, anders.skold@chalmers.se)

Research regarding the perception of sound focuses in large on the acoustical properties of the sound. We argue that, for a more complete picture of sound perception, one must take the non-physical properties into account. By changing the emotional descriptor of a sound the perception in terms of level of annoyance will change. The present study investigates how a priming picture placing the origin of the sound in either a positive or negative environment affects the level of annoyance to same sound. Three different sounds were used in the experiment, all based on pink noise. The participants were, in the beginning of each sound, exposed to a picture telling where the sound originated. The picture was either a positive environment (a picture of a waterfall) or a negative environment (a picture of a larger factory). While listening to the sounds the participants completed different performance tasks. In the end of each sound the participants rated the level of annoyance. Results show that the annoyance ratings are significantly lower when primed with a positive picture. Results also indicate that for more attention demanding tasks this correlation is stronger. The findings are discussed in relation to theories of sound perception.

\section{2:00}

4aPPb10. Fast Detection for Natural Animal Sounds. Clara Suied (CNRS - UPMC UMR 7593, Pavillon Clérambault, Hôpital de la Salpêtrière, 47, Bd de l'Hôpital, 75013 Paris, France, clara.suied@ircam.fr), Marie Magnin (IRCAM - UMR CNRS 9912, Equipe Perception et Design Sonores, 1 place Igor Stravinsky, 75004 Paris, France, magnin.marie@gmail.com), Sabine Langlois (Renault, 1 avenue du golf, 78288 Guyancourt, France, sabine.langlois@renault.com), Patrick Susini (IRCAM - UMR CNRS 9912, Equipe Perception et Design Sonores, 1, place Igor Stravinsky, 75004 Paris, France, susini@ircam.fr), Stephen McAdams (Centre for Interdisciplinary Research in Music Media \& Technology (CIRMMT) - Schulich School of Music - McGill Univ., 555 Sherbrooke Street West, Montreal, QC H3A1E3, Canada, smc@music.mcgill.ca)

Human listeners seem remarkably good at identifying complex and natural sounds. Relatively little attention, however, has been paid to the simple detection of such sounds. The present experiments measured the time course of sound detection in human listeners. Two categories of sounds were used: a train of $1-\mathrm{kHz}$ pure tone pulses varying along an Inter Onset Interval dimension (termed here "IOI" sounds) and animal call sound samples. All sounds were equalized in loudness in a preliminary experiment. The task consisted of a simple reaction time, in which listeners were presented with interleaved IOI and animal sounds and had to manually press a button as fast as possible when they heard any stimulus. Two main results were observed. First, reaction times were significantly shorter for IOIs of 50, 33 and $25 \mathrm{~ms}$ than for an IOI of $100 \mathrm{~ms}$, with no significant differences between IOIs of 33 and $25 \mathrm{~ms}$, highlighting a threshold at $33 \mathrm{~ms}$. Second, average reaction times were significantly shorter for animal sounds than for IOI sounds. Differences in terms of spectral content and temporal modulation characteristics might explain part of these effects. These results also suggest a fast detection advantage for natural animal sounds.

12:20-2:00 Lunch Break

\section{Contributed Papers}

2:00

4aPPb11. The effect of postural information on the perceived velocity of moving sound sources. Shuichi Sakamoto (R.I.E.C., Tohoku University, 2-1, Katahira, Aoba-ku, 980-8577 Sendai, Japan, saka@ais.riec .tohoku.ac.jp), William L. Martens (McGill University, Schulich School of Music, 555 Sherbrooke Street West, Montreal, QC H3A 1E3, Canada, wlm@music.mcgill.ca), Yôiti Suzuki (R.I.E.C., Tohoku University, 2-1, Katahira, Aoba-ku, 980-8577 Sendai, Japan, yoh@ais.riec.tohoku.ac.jp)

In this study, the effect of postural information on the perceived velocity of moving sound sources was investigated. Auditory information was presented via five surround speakers and five subwoofers. Two sounds were presented first in front of participants, and then these moved past them toward the rear. At the same time, rearward pitch motion was presented via a motion platform on which participants were situated. Duration of stimulus was $8 \mathrm{~s}$. Initial distance of sound sources was set to 2.0, 4.0, 6.0, 8.0 and $10.0 \mathrm{~m}$. Amplitude of pitch variation was $0,0.2,0.4,1.0$ and 2.0 degrees Participants made magnitude estimates for speed of moving sound images, and judgment of goodness of movement matching between both auditory and postural information. The resulting speed magnitude estimates showed that pivot magnitude significantly affected the estimated velocity of sound sources except when velocity of sound sources was extremely high. Moreover, participants judged the multimodal match to be poor when the velocity of the moving sound sources was extremely high or low. These results suggest that the strongest multimodal interaction occurs when auditory information and postural variation are well matched, and are consistent with self motion. 
2:20

4aPPb12. Auralization of an orchestra with phase-shifted string sections. Michelle C. Vigeant (Univ. of Nebraska - Lincoln, Architectural Eng. Program, 1110 S. 67th St., Omaha, NE 68182-0681, USA, michelle.vigeant@gmail.com), Lily M. Wang (University of Nebraska Lincoln, 1110 S. 67th St., Omaha, NE 68182-0681, USA, LWang4@UNL .edu)

Orchestra auralizations have been created in ODEON using multichannel individual instrument anechoic recordings of two symphonies; however, only one or two string instruments were recorded to represent each string section. To simulate the chorus effect of an entire string section more accurately, the anechoic tracks of the single string instruments have been mixed with other versions of the same signal, each with some phase shift in time. Two groups of phase shifts were used: one with shorter delays of up to $23 \mathrm{~ms}$, and one with longer delays of up to $47 \mathrm{~ms}$. A maximum of seven differently phase-shifted signals were combined with the original to create a final anechoic recording for use in the auralizations, depending on the number of players each source represented. Using paired comparisons, test subjects were asked to identify the auralization that sounded most similar to the experience of listening to an orchestra in an actual concert hall: one having none, short or long phase-shifts. Results show that subjects have difficulty differentiating between these three types of auralizations, indicating that phase shifting may not be required for such multi-source multi-channel orchestra auralizations. [Work supported by the National Science Foundation.]

\section{2:40}

4aPPb13. Individual differences in auditory localization of real sources in the horizontal plane. Sophie Savel (CNRS-LMA, 31, chemin Joseph Aiguier, 13402 Marseille, France, savel@1ma.cnrs-mrs.fr)

Recent studies on spatial hearing showed that individual variability is greater with virtual sources than with real ones, but that sensorial feedback provides an efficacious and rapid learning procedure. This study evaluated the degree of inter-individual variability with real sound sources in the absence of feedback. Fifty normal-hearing listeners, aged 18-62, either left- or right handed, participated. The experiment was conducted in an anechoic room. One of twelve loudspeakers placed in the frontal horizontal plane and hidden by a curtain emitted a train of low-pass pulses. Listeners had to indicate the estimated direction of that source by placing a pointer on a screen. No feedback was given to them. Each listener completed 480-720 trials. Results indicated that variability between individual was great in both resolution in bias. Precisely, individuals differed (1) in the size of their maximum error, (2) in the azimuthal region in which this maximum error occurred, and (3) in the spatial symmetry of their performance. Indeed, 25\% of the listeners showed significant left-right differences in their performance, these differences always favoring the left side of space. Such asymmetries have been noticed earlier but attributed to greater front-back confusions on the right, which cannot explain the present results.
3:00

4aPPb14. Investigating effects of spatially disparate visual stimuli on auditory localization in VR environments. Khoa-Van Nguyen (IRCAM, 1 Place Igor Stravinsky, 75004 Paris, France, nguyen@ircam.fr), Clara Suied (CNRS - UPMC UMR 7593, Pavillon Clérambault, Hôpital de la Salpêtrière, 47, Bd de l'Hôpital, 75013 Paris, France, clara.suied@ircam.fr), Isabelle Viaud-Delmon (CNRS - UPMC UMR 7593, Pavillon Clérambault, Hôpital de la Salpêtrière, 47, Bd de 1'Hôpital, 75013 Paris, France, ivd@ext.jussieu.fr), Olivier Warusfel (IRCAM, 1 Place Igor Stravinsky, 75004 Paris, France, Olivier.Warusfel@ircam.fr)

Investigating the time and spatial constraints under which visual and auditory stimuli are perceived as a unique percept or as spatially coincident has been a topic of numerous researches in neuroscience. However, these findings have been derived up to now in extremely simplified stimulation context consisting in the combination of elementary auditory and visual stimuli usually displayed in dark and anechoic conditions. The present experiment is conducted in a VR environment using a stereoscopic passive screen and binaural audio rendering. Auditory stimuli are displayed on headphones using individualized head-related transfer functions and visual stimuli are integrated in a visual background in order to convey visual perspective. The experiment investigates the effect of a spatially disparate visual stimulus on the auditory localization judgments (crossmodal bias), as well as the relation between the magnitude of the crossmodal bias and the perception of a unified bi-modal stimulus. The present study will indicate whether previous findings (Hairston et al., Journal of Cognitive Neuroscience, 2003) still hold in more complex audio-visual contexts such as those offered by VR environments.

3:20

4aPPb15. The Role of Auditory-Visual Integration in Object Recognition. Clara Suied (CNRS - UPMC UMR 7593, Pavillon Clérambault, Hôpital de la Salpêtrière, 47, Bd de l'Hôpital, 75013 Paris, France, clara.suied@ircam.fr), Nicolas Bonneel (REVES/Inria Sophia-Antipolis, 2004 route des lucioles, BP 93, FR-06902 Sophia Antipolis, France, Nicolas.Bonneel@sophia.inria.fr), Isabelle Viaud-Delmon (CNRS - UPMC UMR 7593, Pavillon Clérambault, Hôpital de la Salpêtrière, 47, Bd de l’Hôpital, 75013 Paris, France, ivd@ext.jussieu.fr)

Combining auditory and visual information about the same external event enhances perception and behavioural performance. Numerous factors have been shown to contribute to the integration of visual and auditory stimuli, like spatial or semantic relationships between the two stimuli. We studied the influence of spatial disparity between the auditory and the visual stimuli on bimodal object recognition in a go/no-go task, under realistic virtual environment. Participants were asked to react as fast as possible to a target object, presented in the visual and/or the auditory modality, and to inhibit a distractor object. Reaction times were significantly shorter for semantically congruent bimodal stimuli than would be predicted by independent processing of information about the auditory and the visual targets. Moreover, reaction times were significantly shorter for semantically congruent bimodal stimuli (i.e. visual and auditory targets) than for semantically incongruent bimodal stimuli (i.e. target represented in only one sensory modality). Importantly, these results were not altered by a large spatial disparity between the auditory and the visual targets. Altogether our findings suggest that rules governing multisensory integration vary according to the purpose for which auditory and visual stimuli are combined.

Lecture sessions will recess for presentation of poster papers on various topics in acoustics. See poster sessions for topics and abstracts. 


\section{Contributed Papers}

\section{$5: 20$}

4aPPb16. Why is sharp-limited low-frequency noise extremely annoying? Detlef Krahé (Univ. of Wuppertal, Rainer-Gruenter-Str. 21, 42119 Wuppertal, Germany, krahe@uni-wuppertal.de)

Sharp-limited low-frequency noise having only weak components of higher frequencies, such as noise of an air condition or traffic noise attenuated by a thick window, has a very annoying effect on persons also at low levels. The strong fluctuation, which is specific to this kind of sound, is a frequently used explanation for this effect possibly caused by adaptation in the inner ear. Another or additional explanation could be a strong synchronism in the activities on the nerve fibers. Computer models of the auditory system show this synchronism. If some components at higher frequencies are added, the synchronism disappears and the noise is judged less uncomfortable. This raises the question, if noise protection resulting in a sound as described can not be even counterproductive. Differently sharplimited sounds are investigated by an auditory model. The results are compared with results of judgment by hearing.

\section{$5: 40$}

4aPPb17. Comparison of subjective and objective evaluation methods for audio source separation. Josef Kornycky (I-Lab Multimedia and DSP Research Group, Centre for Communication Systems Research, University of Surrey, GU2 7XH Guildford, UK, J.Kornycky@surrey.ac.uk), Banu Gunel (I-Lab Multimedia and DSP Research Group, Centre for Communication Systems Research, University of Surrey, GU2 7XH Guildford, UK, B.Gunel@surrey.ac.uk), Ahmet Kondoz (I-Lab Multimedia and DSP Research Group, Centre for Communication Systems Research, University of Surrey, GU2 7XH Guildford, UK, A.Kondoz@surrey.ac.uk)

The evaluation of audio separation algorithms can either be performed objectively by calculation of numerical measures, or subjectively through listening tests. Although objective evaluation is inherently more straightforward, subjective listening tests are still essential in determining the perceived quality of separation. This paper aims to find relationships between objective and subjective results so that numerical values can be translated into perceptual criteria. A generic audio source separation system was modelled which provided varying levels of interference, noise and artifacts. This enabled a full spread of objective measurement values to be obtained. Extensive tests were performed utilising the output synthesised by this separation model. The relationships found were presented and the factors of prime importance were determined.

\section{6:00}

4aPPb18. An exploration of attentional monitoring of isochronous asynchronous streams in deviant detection and sensorimotor synchronization. Martine Turgeon (Lancaster University, Psychology Department, Fylde College, LA1 4YF Lancaster, UK, m.turgeon@lancaster.ac .uk)

Past studies of auditory selective attention focused on location and/or pitch cues in stream segregation. In this study, two perceptually-segregated streams competed for attention in a perceptual-judgment task based on timbre cues and a sensorimotor task (tapping to the sounds of one stream). Both streams shared the same temporal structure (isochronous 100-ms events at $1.67 \mathrm{~Hz}$ ) but were asynchronous (target-distractor asynchronies of -200 , $-100,100,200 \mathrm{~ms})$, differed in pitch $(712$ or $1000 \mathrm{~Hz})$ and could share or not their perceived location (diotic vs. dichotic). Deviants were amplitude modulated at 25 or $50 \mathrm{~Hz}$ and varied in temporal envelopes. Attentional filtering (distracting stream constant) was done using a discrimination task requiring pressing one of two keys upon hearing one of two deviants (Experiment 1) and a synchronization task requiring selective tapping to the high-pitch or low-pitch sounds (Experiment 3). Attentional monitoring (distracting stream varies) required maintaining attention to the same stream upon hearing slow-modulated deviants and switching attention to the other stream upon hearing the fast-modulated deviants. This was done for the discrimination task (Experiment 2) and synchronization task (Experiment 4). Perceptual and perceptuo-motor results suggest attentional-filtering and attentional-monitoring costs. Surprisingly, the mere presence of a nonconcurrent stream in a different frequency band interferes with deviantdiscrimination and synchronization-tapping performance.

\section{$6: 20$}

4aPPb19. Normalization in count-comparison model of interaural time difference decoding. Ville Pulkki (Helsinki University of Technology, P.O .Box 5400, 02015 TKK, Finland, Ville.Pulkki@tkk.fi)

Recent neurophysical studies suggest that binaural decoding is based on count comparison for both ITD and ILD. In such mechanisms, the neural signals are stronger in the auditory pathways leading to the ipsilateral hemisphere when a signal is presented earlier, or with higher level, to the contralateral ear. A computational model is described implementing binaural cue decoding based on count-comparison principles for ITD decoding, which is assumed to occur in medial superior olive (MSO). Pooled response of MSO is modeled as running multiplication between inputs, which are derived from ear canal signals with GTFB filtering and phase-locked impulse generator. The contralateral and ipsilateral inputs to MSO are then convolved with different responses. The model output corresponds well to neurophysiological results. In the earlier version of the model, the MSO output was normalized only with the contralateral signal, as suggested by the neuroanatomy. It has been later found out that the output of MSO mode depends on ILD, which is in contradiction with psychoacoustics studies. In this study, it is proposed that the ipsilateral input to MSO is self-normalized, which provides ILD-independent ITD decoding.

\section{6:40}

4aPPb20. Representation of Harmonic Sounds in the Helix of the Lateral Lemniscus. Gerald Langner (Neuroacoustics, TUD, Zool. Institut, Schnittspahnstr. 3, 64287 Darmstadt, Germany, gl@bio.tu-darmstadt.de), Claudia Simonis (Schnittspahnstr. 3, 64287 Darmstadt, Germany, simonis@bio.tu-darmstadt.de), Antje Sauck (Schnittspahnstr. 3, 64287 Darmstadt, Germany, antjesalg@ freenet .de), Ralf Galuske (Schnittspahnstr. 3, 64287 Darmstadt, Germany, galuske @ mpih-frankfurt.mpg.de)

The percept of pitch of harmonic sounds is based on temporal processing. This explains also our ability to recognize harmonic relationships between different sounds, because as a result of a neuronal correlation analysis in the auditory midbrain neurons are tuned not only to a certain pitch but to a certain degree also to integer multiples of that pitch. The responses to harmonics of a pitch are suppressed within $30 \mathrm{~ms}$ after signal onset by inhibition with the likely source being the ventral nucleus of the lateral lemniscus (VNLL). An investigation of spatial representation of periodicity information with the 2-Deoxyglucose method in gerbils showed that low pitch is represented dorsally and high pitch ventrally along the length-axis of the VNLL. Three-dimensional computer reconstructions of the VNLL (program AMIRA) gave evidence for a helical periodicity map with 7 to 8 turns, reminiscent of the pitch helix known from music psychology. Moreover, the spatial organization of the VNLL suggests that it is organized as a double-helix representing musical octaves and fifths. Reconstructions of the VNLL of Nissl-stained human brains gave evidence of a similar organization and therefore of a similar functional role of the VNLL for pitch processing in humans. 


\title{
Session 4aSAa
}

\section{Structural Acoustics and Vibration, Computational Acoustics, and EURONOISE: Fluid-Structure Interaction II}

\author{
Noureddine Atalla, Cochair \\ Univ. de Sherbrooke, Mechanical Engineering Depart., 2500 Boulevard de l'Université, Sherbrooke, QC J1K 2R1, Canada \\ Vicente Cutanda Henriquez, Cochair \\ Institute of Sensors, Signals and Electrotechnics, University of Southern Denmark, Niels Bohrs Allé 1, Odense S, 5230, \\ Denmark
}

Stefan Schneider, Cochair

Laboratoire de Mécanique et d'Acoustique, UPR 7051 CNRS, 31,Chemin Joseph-Aiguier, Marseille, 13402, France

\section{Contributed Papers}

\section{8:00}

4aSAa1. Fluid-structure interaction and computational aeroacoustics of the flow past a thin flexible structure. Frank Schäfer (University Erlangen-Nuremberg, Institute of Fluid Mechanics, Cauerst. 4, 91058 Erlangen, Germany, frank.schaefer@1stm.uni-erlangen.de), Thomas Uffinger (University Erlangen-Nuremberg, Institute of Fluid Mechanics, Cauerst. 4, 91058 Erlangen, Germany, thomas.uffinger@1stm .uni-erlangen.de), Stefan Becker (University Erlangen-Nuremberg, Institute of Fluid Mechanics, Cauerst. 4, 91058 Erlangen, Germany, stefan.becker@1stm.uni-erlangen.de), Jens Grabinger (Univ. ErlangenNuremberg, Dept. of Sensor Technology, Paul-Gordan-Str. 3/5, 91052 Erlangen, Germany, jens.grabinger@1se.eei.uni-erlangen.de), Manfred Kaltenbacher (Univ. Erlangen-Nuremberg, Dept. of Sensor Technology, Paul-Gordan-Str. 3/5, 91052 Erlangen, Germany, manfred.kaltenbacher@1se .eei.uni-erlangen.de)

In many technical applications the interaction between a fluid flow and a thin flexible structure leads to the generation of acoustic noise which is caused by flow induced structural vibrations. Examples for such applications are coverings and panelings of cars and airplanes. In many cases the generated noise is unwanted so that noise reduction is a topic of major interest. In the present work we investigate the acoustic field resulting from the interaction of a thin flexible structure with a turbulent flow field by means of numerical simulation. Two different model configurations are considered: one is the flow over a flexible plate, in the second case the flexible plate is located in the wake of a square cylinder. The major aim of this work is to provide a better understanding of the noise generation processes in these flow cases. The numerical methodology applied is utilized for a decomposition of the acoustic field into one part generated by the structural vibrations and another part which is due to stream noise. Finally, comparisons to experimental data available at our institute are provided.

\section{8:20}

4aSAa2. An Equivalent-Acoustic Finite Element Method for Modeling Sound Absorbing Materials. Donald J. Nefske (General Motors R\&D Center, Vehicle Development Research Laboratory, 30500 Mound Rd., Warren, MI 48090-9055, USA, DNefske@aol.com), Shung H. Sung (General Motors R\&D Center, Vehicle Development Research Laboratory, 30500 Mound Rd., Warren, MI 48090-9055, USA, Shung.H.Sung@gm.com)

An equivalent-acoustic finite element method is developed for modeling sound absorbing materials, such as seats and interior trim in the automobile passenger compartment. The equivalent-acoustic method represents the sound absorbing material using acoustic finite elements with frequencydependent material properties determined from the measured acoustic impedance of sound absorbing material samples. Solution of the equivalentacoustic model within the Nastran computer capability and coupling of the model with an acoustic finite element model of a surrounding enclosure, such as the passenger compartment, are developed. The accuracy of the equivalent-acoustic method is assessed for modeling a sound absorbing material in a one-dimensional impedance tube, a foam layer in a rectangular box enclosure, and an automotive seat in a semi-reverberant enclosure.

\section{$8: 40$}

4aSAa3. Finite element modelling of transient elastic wave propagation in an inhomogeneous anisotropic fluid/solid multilayer medium: A time-domain method. Guillaume Haiat (CNRS, Laboratoire de Recherches Orthopédiques, 10, Avenue de Verdun, 75010 Paris, France, haiat@univ-paris12.fr), Salah Naili (Université Paris 12, B2OA, 61, Avenue du Général de Gaulle, 94010 Créteil, France, naili@univ-paris12.fr), Quentin Grimal (Université Paris 6, Laboratoire d'Imagerie Paramétrique, 15, rue de l'Ecole de Médecine, 75006 Paris, France, quentin.grimal@lip.bhdc.jussieu.fr), Maryline Talmant (Université Paris 6, Laboratoire d'Imagerie Paramétrique, 15, rue de l'Ecole de Médecine, 75006 Paris, France, talmant@lip.bhdc.jussieu.fr), Christophe Desceliers (Université de Marne la Vallée, 5, Boulevard Descartes, 77454 Marne la Vallée, France, christophe.desceliers@univ-mlv.fr), Christian Soize (Université de Marne la Vallée, 5, Boulevard Descartes, 77454 Marne la Vallée, France, soize@univ-mlv.fr)

The axial transmission technique is used clinically for cortical bone assessment. However, ultrasonic propagation in this multiscale medium remains unclear, in particular because of the heterogeneous nature of cortical bone. The aim of this work is to evaluate the effect of spatial gradients of elastic moduli on the ultrasonic response of the bone structure. Therefore, a 2D finite element time-domain method is developed to simulate transient wave propagation in a three-layer medium constituted of an inhomogeneous transverse isotropic solid layer sandwiched between two acoustic fluid layers and excited by an acoustic linear source located in one fluid layer delivering broadband ultrasonic pulses. The model couples the acoustic propagation in both fluid media with the elastodynamic response of the solid. The conditions of continuity are used to model the fluid-structure interaction. A constant spatial gradient of material properties in the direction perpendicular to the layer is considered in the solid structure. In the presence of a gradient, the first arriving signal (FAS) velocity depends on the average material properties when the thickness is smaller than the wavelength (guided wave modes) whereas the FAS velocity depends on the velocity at the surface when the thickness is larger than the wavelength (lateral wave). 
9:00

4aSAa4. Dynamic behavior analysis of vehicle acoustic trim using FEM. Norimasa Kobayashi (Toyota Motor Corporation, 1, Toyota-cho, 471-8572 Toyota, Aichi, Japan, koba@nori.tec.toyota.co.jp), Yoshihisa Uno (Toyota Motor Corporation, 1, Toyota-cho, 471-8572 Toyota, Aichi, Japan, yoshi@uno.tec.toyota.co.jp), Hiroo Yamaoka (Toyota Motor Corporation, 1, Toyota-cho, 471-8572 Toyota, Aichi, Japan, yamaoka@giga.tec .toyota.co.jp)

Multilayer vehicle acoustic trim containing poroelastic materials affects noise and vibration phenomena not only in the high-frequency range but also in the low and mid-frequency range. However, there are few established technology to analyze the vehicle model which includes the characteristics of the trim. In this paper, a methodology for the analysis of multilayer acoustic trim within a vehicle FEM model is derived based on Biot theory. Using this methodology, dynamic vibration, radiation and absorption behavior of three types of multilayer trim including conventional isolative type, absorptive type and isolative type with absorptive top layer backed by rectangular metal plates are analyzed. The trim model is also applied to a vehicle FEM model and its effects on interior sound pressure level are validated with experimental data.

\section{9:20}

4aSAa5. Noise reduction prediction of Ariane 5 fairing with acoustic protection made of porous-elastic material. Slaheddine Frikha (ESI France, 99 rue des Solets, Parc des affaires SILIC - BP 80112, 94513 Rungis, France, slaheddine.frikha@esi-group.com), Mohamed Ali Hamdi (ESI GROUP \& UTC, 20, Rue Du Fonds Pernant, 60471 Compiègne, France, Mohamed-Ali.Hamdi@esi-group.com), Philippe Roux (CNES, Rond Point de L'Espace, 91023 Courcouronnes, France, philippe.roux@cnes.fr), Lassen Mebarek (ESI GROUP \& UTC, 20, Rue Du Fonds Pernant, 60471 Compiègne, France, Lassen.Mebarek @esi-group.com), Abderrazak Omrani (ESI GROUP \& UTC, 20, Rue Du Fonds Pernant, 60471 Compiègne, France, Abderrazak.Omrani@esi-group .com)

ESI Group (Formerly STRACO) has been involved during the last two decades in the numerical prediction of noise reduction index of ARIANE 5 fairing. During the 1990's, STRACO developed an axi-symmetric, boundary element model of the fairing where the fairing protection made of distributed Helmholtz Resonator is modeled by equivalent local impedance. Recently, the fairing acoustic protection has been replaced by a foam-made insulator. Such porous materials are widely used by transportation industries to improve the payload acoustic comfort. In collaboration with automotive industry, ESI group developed RAYON-VTM, a powerful tool allowing the predicting of fully trimmed vehicle vibroacoustic response up to $500 \mathrm{~Hz}$. RAYON VTM model the porous-elastic material using a 3D Finite Element (PEM), based on the Modified-Biot-Equations. This new module of RAYON software has been applied to predict the vibroacoustic response of the ARIANE 5 fairing allowing a detailed modeling of the acoustic protection. A fully 3-D model of the fairing has been developed. The results show the influence of intrinsic modeling of the porous-elastic protection as well as the influence of non-axi-symmetric details usually neglected in the axisymmetric approach.

\section{9:40}

4aSAa6. Influence of solid phase elasticity in poroelastic liners submitted to grazing flows. Benoit Nennig (Université de Technologie de Compiègne, Lab. Roberval UMR 6253, Dept. Acoustique, BP 60319, 60203 Compiègne, France, benoit.nennig@utc.fr), Jean-Daniel Chazot (Université de Technologie de Compiègne, Lab. Roberval UMR 6253, Dept. Acoustique, BP 60319, 60203 Compiègne, France, jean-daniel.chazot@utc.fr), Emmanuel Perrey-Debain (Université de Technologie de Compiègne, Lab. Roberval UMR 6253, Dept. Acoustique, BP 60319, 60203 Compiègne, France, emmanuel.perrey-debain @utc.fr), Mabrouk Ben Tahar (Université de Technologie de Compiègne, Lab. Roberval UMR 6253, Dept. Acoustique, BP 60319, 60203 Compiègne, France, mabrouk.bentahar@utc.fr)

In the present work, we study the sound propagation in a duct treated with a poroelastic liner exposed to a grazing flow. Acoustic propagation in the liner and in the fluid domain is respectively governed by Biot's model and Galbrun's equation. Here, the coupling between Galbrun's and Biot's equation is carried out with a mixed pressure-displacement FE. On one hand, a mixed formulation is used in Galbrun's equation to avoid numerical locking. And on the other hand, in poroelastic media, the description of both phases involves the displacement of the solid phase and the pressure in the fluid phase. In addition of using the complete Biot's model, simplified models are also tested. A fluid equivalent model that does not take into account solid phase elasticity and a model that neglects only the shear stress are hence used. These two simplified models enable to evaluate the contributions of the compressional and shear waves in the solid phase. Finally, validity of each simplified model in the specific case of a liner exposed to a grazing flow is discussed for different materials.

\section{0:00-10:20 Break}

\section{$10: 20$}

4aSAa7. An Evolutionary Optimization Method applied to Absorbing Poroelastic Systems. Francisco Silva (Unité de Mécanique (UME), ENSTA, Chemin de la Hunière, 91761 Palaiseau, France, francisco-ilson.dasilva@ensta.fr), Renato Pavanello (Depart. of Computational Mechanics - FEM - Unicamp, POBOX 6122, Rua Mendeleiev, s/n Cidade Universitária, 13083-970 Campinas, Brazil, pava@fem.unicamp.br)

Poroelastic materials can be used in engineering applications such as: noise control of automobiles, acoustical insulation systems for aircrafts, industrial, environmental and domestic sound quality control, etc. The insulating systems must be as light as possible and the acoustic absorption in the low frequency domain must be maximized for certain gaps. Topology Optimization is frequently used to design structures and acoustic systems in a large range of engineering applications. In this work, we propose one method to maximize the absorbing performance of insulation poroelastic systems using a coupled finite element model and Evolutionary strategies. The goal is find the best distribution of poroelastic material on insulating systems. The absorbing performance of the poroelastic material samples in a Kundt tube is simulated using a coupled poroelastic and acoustic finite element model. The Biot-Allard Coupled Model is used to represent the foam material. The porous material model is coupled to a waveguide using a modal superposition technique. A sensitivity number indicating the optimum locations for porous material removing is derived and used in a numerical hard kill scheme. The sensitivity number is used to form an evolutionary poroelastic optimization (EPO) algorithm which is verified though numerical examples.

\section{0:40}

4aSAa8. Acoustic frequency wave propagation and fluid-loading in composite sandwich panels. Jennifer F. Crossley (Swansea University Engineering Department, Singleton Park, SA2 ODS Swansea, UK, 437441@swansea.ac.uk), Johann Sienz (Swansea University Engineering Department, Singleton Park, SA2 0DS Swansea, UK, J.Sienz@swansea.ac .uk)

Composite sandwich panels comprising glass fibre reinforced epoxy skins with nomex honeycomb cores have found application as aircraft interior fixtures and partitions. This is due to their low density combined with static strength and stiffness. In this complex acoustic and vibration environment however, the same properties can lead to unwanted sound transmission through the structure. We analyse, in this paper, fluid-loading of such a panel by an acoustic fluid using an elementary theory which has been developed in the study of structural dynamics and vibration, based on a generalisation of Timoshenko theory for homogeneous beams. The dispersion relations derived from this theory, with and without fluid-loading terms, are used to quantify the effect of fluid-loading by air on shear and flexural waves a acoustic frequencies in such stiff, lightweight structures. The most appropriate method of fluid-structure coupling to be applied in modelling an internal acoustic field enclosed by such a structure is discussed. 
11:00

4aSAa9. A formulation based on modal optimization for predicting sound radiation from fluid-loaded aircraft structures. Olivier Collery (Airbus France - Acoustics and Environment Department, 316 route de Bayonne, F-31060 Toulouse Cedex 09, France, olivier.collery @insa-lyon.fr), Jean-Louis Guyader (INSA de Lyon - LVA, Bâtiment St. Exupéry, 25 bis avenue Jean Capelle, F-69621 Villeurbanne Cedex, France, jean-louis.guyader@insa-lyon.fr)

This study is led in the context of understanding sound transmission through fluid-loaded aircraft structures with non-uniform damping. The flu$\mathrm{id} /$ structure coupling is here highlighted. Classic approaches using modal radiation impedances to formulate the fluid/structure coupling do lead to complexity and are computationally time-consuming. A method defining rigorously this coupling and avoiding the calculation of the modal radiation impedances is proposed. This method aims at developing a simple formulation taking advantage of current technical progress in optimization algorithms. Sound radiation from a simply supported, baffled, fluid-loaded plate excited mechanically or acoustically is here solved in optimizing the modal amplitudes so that they fit the governing equation with fluid loading. To perform this optimization, a sampling of the plate into observation points is first done, then a modal decomposition into in vacuo modes is led. Comparison with results from the literature over $[10-1000 \mathrm{~Hz}]$ for 3 reference cases of steel plate immersed in air (critical frequencies equal to $\mathrm{fC} 1=1$ $.2 \mathrm{kHz}$ and $\mathrm{fC} 2=12 \mathrm{kHz}$ ) show excellent agreement within $1 \mathrm{~dB}$. The simplicity and computation time allow an extension to non-uniform damped aircraft structures and a prediction over a large frequency band. As perspectives, results from multi-layered plates with a local damping patch are presented.

THURSDAY MORNING, 3 JULY 2008

ROOM 252B, 11:40 A.M. TO 7:00 P.M.

Session 4aSAb

\title{
Structural Acoustics and Vibration and EURONOISE: Distributed Active Noise and Vibration Control I
}

\author{
Kenneth Cunefare, Cochair \\ Woodruff School of Mech. Eng., The Georgia Inst. of Technol., Atlanta, GA 30332-0405, USA \\ Manuel Collet, Cochair \\ FEMTO-ST UMR CNRS, 23 chemin de l'Epitaphe, Besançon, 25000, France
}

Invited Papers

\begin{abstract}
11:40
4aSAb1. Smart panels with active wedges. Paolo Gardonio (ISVR, University of Southampton, Highfield, SO17 1BJ Southampton, UK, pg@isvr.soton.ac.uk), Yohko Aoki (ISVR, University of Southampton, Highfield, SO17 1BJ Southampton, UK, ya@isvr.soton.ac.uk), Stephen J. Elliott (ISVR, University of Southampton, Highfield, SO17 1BJ Southampton, UK, sje@isvr.soton .ac.uk)

This paper presents an experimental study comparing the vibration and sound radiation control performance obtained from six different rectangular panels with various geometries of active wedge. Each panel is equipped with sixteen triangular piezoceramic actuators along the panel border and accelerometer sensors located at the top vertex of the triangular actuators. These sensor-actuator pairs are used to implement decentralized velocity feedback loops that produce active damping on the plate. The primary objective of this paper is to investigate the effect of the size and geometry of the triangular actuators on stability and control performance. Narrow frequency band measurements highlight that vibration reductions at the first few resonant frequencies are significantly improved by increasing the height of the triangular actuators. However, the increase of the height also results in spillover effects at frequencies higher than around $700 \mathrm{~Hz}$. In contrast, an increase of the base length of the triangular actuators improves the overall control performance up to $1 \mathrm{kHz}$ without increasing the spillover effect.
\end{abstract}

\section{2:00}

4aSAb2. A new hybrid passive/active cell to realize a complex impedance boundary condition. Marie-Annick Galland (Centre Acoustique du LMFA, Ecole Centrale de Lyon, 36 avenue Guy de Collongue, 69134 Ecully cedex, France, marie-annick.galland@ec-lyon.fr), Jean-Baptiste Dupont (Centre Acoustique du LMFA, Ecole Centrale de Lyon, 36 avenue Guy de Collongue, 69134 Ecully cedex, France, jean-baptiste.dupont@ec-lyon.fr)

Hybrid passive/active cells previously developed at the LMFA have proved their efficiency for global noise reduction. A hybrid cell combines passive absorbent properties of a porous layer and active control at its rear face. Active control is mainly used to increase absorption at low frequencies by cancelling the imaginary part of the surface impedance presented by the absorber. A specific decentralized control algorithm allowed realizing large panels to reduce multi-tone noise radiated by a flow duct. Generally, the design of such absorbers starts by determining the optimal impedance for the targeted application, defined as that which, for each frequency, produces the highest reduction. However, in many cases results show that this optimal impedance is complex and that both real and imaginary parts are frequency dependent. This paper investigates the potential of a new hybrid absorber intended to realize any of impedance. The new cell uses one microphone on each side of a resistive cloth. Normal velocity can then be deduced by a simple pressure difference, which allows an easy synthesis of the error signal. First experiments carried out in a standing wave tube are described and different control strategies are examined, allowing us to validate this promising concept. 
4aSAb3. The performance trade off of decentralised, distributed and centralised controllers. Oliver N. Baumann (Institute of Sound and Vibration Research, University of Southampton, Highfield, SO171BJ Southampton, UK, onb@isvr.soton.ac.uk), Kenneth Frampton (ISVR, University of Southampton, Highfield, SO17 1BJ Southampton, UK, kdf@soton.ac.uk), Paolo Gardonio (ISVR, University of Southampton, Highfield, SO17 1BJ Southampton, UK, pg @isvr.soton.ac.uk)

Direct velocity feedback control of structures is well known to increase structural damping and thus reduce vibration. In multichannel systems the way in which the velocity signals are used to inform the actuators ranges from decentralised controller through distributed or clustered controllers to the fully centralised controller. The objective of distributed controllers is to exploit the anticipated performance advantage of the centralised controller whilst maintaining the ease of implementation and robustness of the decentralised controller. It has been observed, however, that in many vibration control systems the centralised controller struggles to perform significantly better than a decentralised controller. This paper compares a number of distributed controllers and optimisation techniques for the reduction of kinetic energy and radiated sound power and identifies the conditions under which the centralised and distributed controllers offer a significant performance advantage.

\section{2:40}

4aSAb4. Reduced models for elastoacoustic problems with intelligent interfaces. Mohamed Ichchou (Centre Acoustique du LMFA, Ecole Centrale de Lyon, 36 avenue Guy de Collongue, 69134 Ecully cedex, France, mohamed.ichchou@ec-lyon.fr), Aida A. Kacem (Centre Acoustique du LMFA, Ecole Centrale de Lyon, 36 avenue Guy de Collongue, 69134 Ecully cedex, France, aida.hadjkacem@ec-lyon.fr)

Effective and predictive noise and vibration modelling and design tools as well as efficient and high-performance materials are essential to produce world-leading products with regards to the noise and vibration quality. In one hand, the modern trend towards virtual design and prototyping requires good analysis and modelling tools in the entire noise frequency band. In another hand, new passive, adaptive or active materials offer improved technologies issues for the vibration and noise treatment. However, there is still a lack of efficient numerical tools in the low frequency band, and an absence of tools in the mid-frequency range. Moreover, although new materials potential can be demonstrated in prototype structures, their performances are still insufficient in integrated applications. To bring advances in noise and vibration treatment using new materials, the challenge is to be capable of supporting specific aspects related to advanced materials and virtual prototyping methods. An essential requirement for this is a multi-disciplinary analysis and coupled simulation tools where effects on a very different scale need to be interconnected. The paper proposes reduced models of elastoacoustic problems with localised intelligent interface. The main focus is development of fast simulation tool for the design of new systems. Theoretical as well as numerical experiments are offered in this work.

\section{1:00-2:00 Lunch Break}

\section{Invited Papers}

\section{2:00}

4aSAb5. Decentralised active control of single - frequency panel vibrations using piezoelectric actuator - sensor pairs. Alain Berry (Univ. de Sherbrooke, Mechanical Engineering Depart., 2500 Boulevard de l'Université, Sherbrooke, QC J1K 2R1, Canada, alain.berry@usherbrooke.ca), Philippe Micheau (Univ. de Sherbrooke, Mechanical Engineering Depart., 2500 Boulevard de l'Université, Sherbrooke, QC J1K 2R1, Canada, Philippe.Micheau@USherbrooke.ca), Rémi Louviot (Univ. de Sherbrooke, Mechanical Engineering Depart., 2500 Boulevard de l’Université, Sherbrooke, QC J1K 2R1, Canada, remi.louviot@usherbrooke.ca), Yvonnick Brunet (Univ. de Sherbrooke, Mechanical Engineering Depart., 2500 Boulevard de l'Université, Sherbrooke, QC J1K 2R1, Canada, yvonnick.brunet@usherbrooke.ca)

This paper addresses active control of the bending response of a panel using independent PZT (piezoceramic) actuator - PVDF (piezopolymer) sensor pairs distributed on the panel. Previous work showed that under the assumption of collocated, dual actuator sensor pairs, decentralised static gain control is stable due to plant matrix passivity at all frequencies. However, duality is not guaranteed for collocated PZT - PVDF pairs because of the coupling of piezoelectric transducers with both bending and extensional modes of the panel. Moreover, the spatially local nature of PZT actuator to PVDF sensor transfers on the panel can lead to a diagonal - dominant FRF matrix but is detrimental to global control of the panel vibration or acoustic radiation; hence, a non-diagonal dominant plant matrix is more likely to result in global control for this problem. In the case of single-frequency disturbance, stability analysis shows that plant matrix passivity is only required at the disturbance frequency and that a phase - shift compensation, identical for all independent units, can ensure stable decentralized control. Guidelines for the design of decentralised PZT - PVDF pairs are provided, with the objective of global vibroacoustic control of a panel.

4aSAb6. Broad-Band Vibration Attenuation in Plates With Periodic Arrays of Shunted Piezoelectric Patches. Massimo Ruzzene (Georgia Institute of Technology, School of Aerospace Engineering, 270 Ferst Drive, Atlanta, GA 30332-0150, USA, massimo.ruzzene@ae.gatech.edu), Luca Airoldi (Dipartimento di Ingegneria Aerospaziale, Via La Masa 34, 20156 Milano, Italy, luca .airoldi@gmail.com)

Periodic arrays of shunted piezoelectric patches are employed for the control of waves propagating over the surface of plate structures. The shunted piezoelectric patches act as sources of impedance mismatch, which yield interference phenomena resulting from the interaction between incident, reflected and transmitted waves produced by the mismatch. The impedance mismatch corresponding to the shunted piezos can be tuned to achieve strong attenuation over frequency bands which are defined by the shunting circuit connected to the patches. Broad-band vibration attenuation can be achieved through the application of series and parallel of multiple resonant 
circuits, or through the implementation of negative capacitance configurations. The ability of plates with periodic shunted piezoelectric patches to attenuate vibrations over extended frequency bands is demonstrated numerically, through Finite Element models of the considered electromechanical structures. The effect of number of patches and of their periodic distributions is investigated together with the analysis and the comparison of performance achieved with various shunting strategies.

\section{2:40}

4aSAb7. Use of head mounted microphone arrays for active control. Marty Johnson (Virginia Tech, Mechanical Engineering, 143 Durham 0238, Blacksburg, VA 24061, USA, martyj@vt.edu)

There has been an effort recently to create head mounted or user worn microphone arrays. These arrays pose challenges in their design and characterization but can be used for a number of different purposes. Initially these arrays were intended for sound source localization and natural hearing restoration (where the hearing of a user whose ears are obstructed by an encapsulating helmet or headset is restored). Once in place, and assuming the user is wearing headphones, these arrays can be used for a number of different applications including voice isolation for communication, focused listening and noise cancellation. Specifically this paper investigates the use of these arrays for the active control of noise both at the user's ears and in the communications/voice signals sent from the user. A numerical study, using both data generated numerically and experimentally, demonstrates that large reductions in noise can be achieved using adaptive active control methods.

\section{3:00}

4aSAb8. Active control of multimodal tonal noise propagated in circular duct with axial subsonic mean flow until $\mathrm{M}=0.3$. Martin Glesser (CNRS - LMA, 31 Chemin Joseph Aiguier, 13009 Marseille, France, glesser@1ma.cnrs-mrs.fr), Emmanuel Friot (Laboratory for Mechanics and Acoustics CNRS, 31 chemin Joseph Aiguier, 13009 Marseille, France, friot@lma.cnrs-mrs.fr), Muriel Winninger (CNRS - LMA, 31 Chemin Joseph Aiguier, 13009 Marseille, France, winninger@lma.cnrs-mrs.fr), Cédric Pinhède (CNRS - LMA, 31 Chemin Joseph Aiguier, 13009 Marseille, France, pinhede@1ma.cnrs-mrs.fr), Alain Roure (CNRS - LMA, 31 Chemin Joseph Aiguier, 13009 Marseille, France, roure@1ma.cnrs-mrs.fr)

As new generation of aircraft engine with lower blade passing frequency appeared in the 1990's, the fan tones radiated from the inlets had become one of the dominant source of sound. Efforts have then been made to develop active noise control. Encouraging results have been obtained but the physical limitation of the fan tones reduction have not been clearly determined, owing mainly to the complexity of the experimental rigs. This paper present an experimental investigation of the control of multimodal tonal noise propagated in circular duct in presence of a mean flow $(M \leqslant 0.3)$. A laboratory wind tunnel has been implemented for this purpose. Two limiting factors for the sound reduction are underlined: (i) the degradation of the secondary transfer matrix conditioning as the number of propagating modes increases in the duct and (ii) the degradation of the hypothesis of the time-invariance of the system to control as the flow velocity is increased. The effect of those limiting factors on the control efficiency are evaluated.

\section{Contributed Paper}

\section{3:20}

4aSAb9. New prospects in implementing distributed control strategies for mechanical structures optimization. Manuel Collet (FEMTO-ST UMR CNRS, 23 chemin de l'Epitaphe, 25000 Besançon, France, manuel .collet@univ-fcomte.fr)

The research activities in the fields of smart materials and structures today are very important and represent a large potential for the technological innovations. New methods are now available which allow active transducers and their driving electronics to be directly integrated in otherwise passive structures. Today the main research challenge deals with the development of new multi-functional structures integrating their own electro-mechanical controlling systems. In the past few years, a technological revolution has occurred in the fields of integrated MEMS that offers new opportunities for smart structures design and optimization. By using such as integrated active or hybrid distributed set of electromechanical transducers, we could also control the material's intrinsic mechanical behavior for building new desired functionalities. We can also speak of "integrated distributed smart structures". Through two examples, this paper aims at showing what can be the main advantages in developing such of integrated distributed control strategies in comparison with passive and "classical" active systems. The adopted point of view takes also into consideration energy balance assessments, absolute efficiency and of course robustness. The first introduced example deals with the vibroacoustic impedance control of tube for wave power flow cancellation. The second one treats of the optimization of hybrid shunted piezoelectric distributed patches for mechanical power flow control.

3:40-5:20 Posters

Lecture sessions will recess for presentations of poster papers on various topics in acoustics. See poster sessions for topics and abstracts.

\section{Contributed Papers}

$5: 20$

4aSAb10. Online adaptive distributed control of vibro-acoustic systems. Kenneth Frampton (ISVR, University of Southampton, Highfield, SO17 1BJ Southampton, UK, kdf@soton.ac.uk)

One of the primary difficulties in distributed, real-time vibro-acoustic control is the difficulty of high-bandwidth network communication of sensor data. The necessary communications rate has proven overwhelming to existing communications protocols. In order to overcome this limitations an adaptive control technique has been developed that requires only occasional inter-node communication of sensor data in order to maintain optimal performance. The technique is based on iterative feedback tuning (IFT) which has the advantage of not requiring a system model. In this system, each control node collects its sensor data over a period of time. The data is then communicated to other nodes in the system. Once a node has received the required sensor data the local adaptation algorithm is initiated. Local feedback gains are adjusted based on an estimate of the cost function gradient and the new control law is implemented until the next adaptation cycle. This work presents the theory behind this adaptive control technique and simulation results of the control performance are presented. It is demonstrated that this approach to distributed control can perform as well as model based optimal distributed control, and nearly as well as centralized control. 
$5: 40$

4aSAb11. Damping of a device with piezoelectric materials and negative capacitance electrical circuits. Christian S. Granger (IEMN, 41 boulevard Vauban, 59046 Lille, France, christian.granger@isen .fr), Anne-Christine Hladky (IEMN, UMR CNRS 8520, avenue Poincaré, BP 60069, 59652 Villeneuve d'Ascq, France, anne-christine.hladky @ isen.fr), Jean-Claude Debus (IEMN, 41 boulevard Vauban, 59046 Lille, France, jean-claude.debus@isen.fr)

In this paper, the damping of a device is obtained by a transfer of the vibratory energy into electrical energy and then into thermal energy (dissipation in an electrical resistance). The transfer is carried out by using piezoelectric materials (PZT piezoelectric plates, macro fibre composite MFC) and it is improved by charging the piezoelectric materials by an electrical circuit having a negative capacitance impedance. Two devices are considered: a clamped plate, witch is a academic case, and a large aluminium plate $(0.85 \mathrm{~m} \times 0.78 \mathrm{~m} \times 2 \mathrm{~mm})$. The optimal position and the geometry of the ceramics are determined using an analytical method and a numerical method with the help of the ATILA finite element code. The equivalent electrical circuit of the device is conceived. Damping of the device charged by the electrical circuits is measured using a laser vibrometer. Damping can be performed on a given frequency range, covering several bending modes, by using several ceramics on the plate and several external electrical circuits, connected to the active material. Finally, tests in an anechoic chamber show the efficiency of the device for the reduction of the noise transmitted through the plate.

\section{6:00}

4aSAb12. Decentralized control of sound radiation using a high-authority/low-authority control strategy with anisotropic actuators. Noah H. Schiller (NASA Langley Research Center, 2 North Dryden Street, Hampton, VA 23681, USA, noah.h.schiller @ nasa.gov), Randolph H. Cabell (NASA Langley Research Center, 2 North Dryden Street, Hampton, VA 23681, USA, randolph.h.cabell@nasa.gov), Chris R. Fuller (Virginia Tech, 131 Durham Hall, Blacksburg, VA 24061, USA, christopher.r.fuller@nasa.gov)

This paper describes a combined control strategy designed to reduce sound radiation from stiffened aircraft-style panels. In particular, the control approach uses robust active damping in addition to high-authority LQG control. Active damping is achieved using direct velocity feedback with triangularly shaped strain actuators and point velocity sensors [P. Gardonio and S.J. Elliott, JASA 117(4), 2046-2064 (2005)]. However unlike previous work, anisotropic actuators are used since they outperform traditional isotropic actuators in this application. While active damping is simple and robust, stability is guaranteed at the expense of performance. Therefore, this approach is often referred to as low-authority control. In contrast, LQG control strategies can achieve substantial reductions in sound radiation. Unfortunately, the unmodeled interaction between neighboring control units can destabilize decentralized control systems. Numerical simulations show that combining active damping and decentralized LQG control can be mutually beneficial. In particular, augmenting the in-bandwidth damping supplements the performance of the LQG control strategy and reduces the destabilizing interaction between neighboring control units. Therefore, the performance of the combined system can be better than the sum of each individual strategy.

\section{6:20}

4aSAb13. An impedance matching technique for active-passive vibration control. Jonas L. Svensson (Chalmers University of Technology, Division of Applied Acoustics, SE-41296 Gothenburg, Sweden, jonas.svensson@chalmers.se), Patrik B. Andersson (Chalmers University of Technology, Division of Applied Acoustics, SE-41296 Gothenburg, Sweden, patrik.andersson@chalmers.se), Wolfgang Kropp (Chalmers University of Technology, Division of Applied Acoustics, SE-41296 Gothenburg, Sweden, wolfgang.kropp@chalmers.se)

Impedance matching techniques have shown potential for active vibration control of structures in bending. Such structures are commonly described by Euler-Bernoulli theory. Previous studies concerning impedance matching of these structures have only considered scalar quantities. However, for an Euler-Bernoulli beam four field variables are involved which implies that a scalar impedance is insufficient. The purpose of this study is therefore to expand the technique to include full $2 \times 2$ matrices. This is achieved by first deriving the reflection matrix as a function of the characteristic impedance matrices of an Euler-Bernoulli beam and an arbitrary termination impedance. An active impedance load is then introduced in order to manipulate the reflection matrix. A theoretical example is given where the approach is utilized to match the junction between an Euler-Bernoulli beam and a sandwich composite. This proposed active-passive damping configuration employs active control to enclose all incident power in the sandwich composite. Results show that the active impedance load is responsible for the main part of the power absorption over a broad frequency range.

\section{6:40}

4aSAb14. Hybrid Foams for passive/active control of transmission loss of panels. Morvan Ouisse (FEMTO-ST UMR CNRS, 23 chemin de l'Epitaphe, $25000 \quad$ Besançon, France, morvan.ouisse @ univ-fcomte.fr), Manuel Collet (FEMTO-ST UMR CNRS, 23 chemin de l'Epitaphe, 25000 Besançon, France, manuel.collet@univ-fcomte.fr)

Absorbing materials are widely used in transport industry. Classical foams can efficiently improve transmission loss of panels, but only in the higher frequency range. For the lower part of the frequency range, structural active control can be a good strategy to reduce the vibratory level and induced noise. Trying to take advantage of those two points is the objective of the development of hybrid foams. Many practical and scientific aspects are related to their design: which passive material should be used? Which active material, and what shapes should be better? In order to understand the basics of the hybrid foams, some specific multiphysics models are developed, including vibro-acoustic modelling of foams, coupled with elasto-dynamics for structural parts and piezo-elastic models for active parts. The objective of the strategy is to try to take advantage of distributed actuators within the foam in order to modify the energy transmission path. The physical strategy is presented in the paper, together with the full description of the model, with numerical results. The main difficulty for the numerical part is the very high number of degrees of freedom which are required by the model. Some model reduction strategies are then investigated. 


\title{
Session 4aSCa
}

\section{Speech Communication: Acoustics of Speech Production: Aeroacoustics and Phonation}

\author{
David A. Berry, Cochair \\ The Laryngeal Dynamics Laboratory, Division of Head \& Neck Surgery, David Geffen School of Medicine at UCLA, 31-24 \\ Rehab Center, 1000 Veteran Ave., Los Angeles, 90095-1794, USA \\ Xavier Pelorson, Cochair \\ Département Parole \& Cognition, GIPSA-lab, 46, avenue Félix Viallet, Grenoble Cedex, 38031, France
}

\section{Invited Papers}

\section{$8: 40$}

4aSCa1. Implications of the fluctuating drag force voice source. Richard S. McGowan (CReSS LLC, 1 Seaborn Place, Lexington, MA 02420, USA, rsmcgowan@earthlink.net), Michael S. Howe (College of Engineering, Boston Univ., 110 Cummington Street, Boston, MA 02215, USA, mshowe@bu.edu)

A recently published paper on the aeroacoustics of the voice source calculates the acoustic source of voice caused by fluctuation drag forces [Howe, M. S. and McGowan, R. S., J. Fluid Mech., 592, 367-92]. There are two extensions to this calculation that will be presented: 1) inclusion of the ventricular folds downstream of the vocal folds, and 2) the fluid-structure interaction at the vocal folds. For the ventricular folds, the effect of tissue shape on the drag forces will be investigated in terms of the shape's effect on the relation between the Lamb vector and the Kirchoff vector. Regarding the fluid-structure interaction at the vocal folds, the two-mass model will be examined in light of the recently published calculation of drag forces. [Work supported by grant NIDCD-004688 to Dr. G. S. Berke of UCLA.]

\section{9:00}

4aSCa2. Investigation of the mechanisms of voicing offset. Anna Barney (ISVR, Univ. of Southampton, SO17 1BJ Southampton, UK, ab3@soton.ac.uk), Luis Jesus (Escola Superior de Saúde da Universidade de Aveiro, Universidade de Aveiro, Campus Universitário de Santiago, 3810-193 Aveiro, Portugal, 1mtj@ua.pt), Ricardo Santos (Escola Superior de Saúde da Universidade de Aveiro, Universidade de Aveiro, Campus Universitário de Santiago, 3810-193 Aveiro, Portugal, ricardo.santos@ua.pt)

During voiced speech the source of sound production arises from the vibrations of the vocal folds within the larynx. In order to terminate these vibrations it is necessary either to adduct the vocal folds to produce a forced closure or to reduce the pressure drop across them to below a threshold level. A reduction in pressure drop might be achieved by reducing the sub-glottal pressure, by increasing the supra-glottal pressure or by abduction of the folds. Two human subjects were asked to achieve phonation offset by each of these strategies in turn. Acoustic and EGG signals measured on the subjects were compared with the output of a simple theoretical vocal fold model. A further comparison was made with corresponding signals recorded during the production of selected short phrases by a European Portuguese speaker; a language where devoicing of both vowels and voiced consonants is particularly prevalent.

$$
\text { 9:20 }
$$

4aSCa3. Numerical study of volume sources associated with displacement flow during phonation. Jong Beom Park (McGill University, 817 Sherbrooke St. West, Montreal, QC H3A 2K6, Canada, jong.b.park@mail.mcgill.ca), Luc Mongeau (McGill University, 817 Sherbrooke St. West, Montreal, QC H3A 2K6, Canada, luc.mongeau@mcgill.ca)

The glottal displacement flow is a volume flow induced by the motion of the vocal folds in the absence of transglottal pressure. This source may eventually contribute to the radiated sound, in particular during glottal opening. The volume source strength was numerically computed for a time-varying rigid M5 geometry, and decomposed into monopole and dipole components. The air mass entrained by the dipole moment was found to have a length equivalent to the glottal dimension based on the net force exerted on the fluid by the vocal folds wall. The dipole moment, associated with asymmetric geometries and/or motion, is significant even for the case of motion of a incompressible deformable solid with constant volume, but having a time-varying shape.

\section{$9: 40$}

4aSCa4. Aeroacoustic production of speech sounds. Michael Krane (ARL Penn State, PO Box 30, State College, PA 16804-0030, USA,mhk5@only.arl.psu.edu)

Speech sound production is described in terms of its essential physics by focusing on the aeroacoustics of jets in the vocal tract. Aeroacoustic theory is used to show that the primary sources of sound may be expressed in terms of unsteady aerodynamic forces on the vocal tract walls, especially where a jet is formed, and where the jet performs a strong interaction with the walls. The theory further clarifies which details of jet structure and vocal tract geometry contribute to sound production. This information is used to guide useful 
approximations concerning the aerodynamics. With the assistance of measurement, these approximations are used to develop a model of the sound source in the time and frequency domains. The contribution of jet structure and vocal tract geometry to sound source characteristics is then discussed. Model predictions of the resulting acoustic field are compared to acoustic measurements.

\section{Contributed Papers}

\section{0:00}

4aSCa5. Increasing the complexity of glottal flow models: in-vitro validation for steady flow conditions. Julien Cisonni (Département Parole \& Cognition, GIPSA-lab, 46, avenue Félix Viallet, 38031 Grenoble Cedex, France, julien.cisonni@gipsa-lab.inpg.fr), Annemie Van Hirtum (Département Parole \& Cognition, GIPSA-lab, 46, avenue Félix Viallet, 38031 Grenoble Cedex, France, annemie.vanhirtum@gipsa-lab.inpg.fr) , Xiao Yu Luo (Dept. of Mathematics, Univ. of Glasgow, University Gardens, G12 8QW Glasgow, UK, x.luo@maths.gla.ac.uk), Xavier Pelorson (Département Parole \& Cognition, GIPSA-lab, 46, avenue Félix Viallet, 38031 Grenoble Cedex, France, pelorson@icp.inpg.fr)

Quasi one-dimensional glottal flow descriptions predict vocal folds oscillations characteristics which are qualitatively relevant to in-vitro and invivo experimental data. The current paper considers the resolution of the 2D Navier-Stokes equations in order to obtain a refined description of the flow phenomena adapted to more realistic glottal geometry. The pressure and flow rate predictions obtained from quasi one-dimensional flow models and the resolution of the 2D Navier-Stokes equations are examined for steady flows within a rigid glottis. The models predictions are validated against invitro measurements performed on rigid constriction replicas comparable to the geometrical conditions of the glottis and mounted in a suitable set-up. The confrontation between the experimental and computed data tends to show that the accuracy of the estimated pressures increases with the complexity of the flow model whereas the inverse tendency can be observed for the estimated flow rates. A focus is made on the flow separation point which is predicted by the resolution of the Navier-Stokes equations and appears to be a crucial parameter of the quasi one-dimensional flow models. The use of a variable separation criterion obtained from the $2 \mathrm{D}$ flow modelling in the quasi one-dimensional models makes the different models predictions more similar.

\section{0:20}

4aSCa6. Calculation model of the influence of the vocal fold shape on the vocal fold oscillation form. Andreas Gömmel (RWTH Aachen, Civil Engineering Dept., Structural Statics and Dynamics, Mies-van-der-Rohe-Str. 1, 52064 Aachen, Germany, goemmel@lbb.rwth-aachen.de), Christoph Butenweg (RWTH Aachen, Civil Engineering Dept., Structural Statics and Dynamics, Mies-van-der-Rohe-Str. 1, 52064 Aachen, Germany, butenweg@lbb.rwth-aachen.de), Malte Kob (RWTH Aachen, Dept. of Phoniatrics, Pedaudiology, and Communication Disorders, Pauwelsstr. 30, 52074 Aachen, Germany, mkob@ukaachen.de)

Vocal fold (VF) oscillation is driven by fluid-structure interaction effects. A possible way of modeling these effects is the finite-element (FE) method. The presented FE model consists of two coupled domains: A fluid domain representing the air, and a structural domain representing the VFs. In principle, each of the domains is a stand-alone simulation model. In the current implementation a thin three-dimensional frontal slice of the vocal folds and the sub- and supraglottal areas is modeled. Flow calculation is done using the standard Navier-Stokes equations. The air is modeled as a transient, viscous, and laminar flow. Constant physiologic values of pressure are used as driving force. For structural analysis, linear volume elements are used. There are two different models which differ in the VF shape. The first one is an assumed shape of a normal voice while the second shape was measured at an excised larynx and resembles more a falsetto voice. The results support two observations and assumptions: During normal phonation the VF touch each other in a constantly changing converging and diverging shape while during the more falsetto-like phonation, no converging/diverging shape is visible and no closure occurs.

\section{0:40-11:00 Break}

\section{1:00}

4aSCa7. Intraglottal pressure distributions for oblique glottal angles. Ronald C. Scherer (Bowling Green State University, Department of Physics and Astronomy, Bowling Green, OH 43403, USA, ronalds@bgnet.bgsu.edu)

Asymmetric vocal fold oscillation occurs for both normal and pathological phonation. The pressures on the right and left glottal walls receive different pressures when the glottis is oblique, as we have shown previously. Different driving forces may affect the motion, although to what extent is not yet known. We have continued empirical modeling of various oblique glottal shapes using a Plexiglas model (M5), and report the findings of those studies. In general, an oblique glottis tends to receive higher pressures on the more convergent side, and the cross-channel pressure differences may shift polarity between upstream and downstream sections. The effects on intraglottal pressures for a wide range of obliquity, minimal glottal diameter, transglottal pressure, and included glottal angle will be shown and discussed relative to potential oscillatory effects. [supported by NIH R01DC03577]

\section{1:20}

4aSCa8. Separated flow behavior in an in-vitro rigid model of the laryngeal channel. Denisse Sciamarella (CNRS, Bâtiment 508, Université Paris-Sud, 91403 Orsay, France, denisse@limsi.fr), Elisa Chisari (LFD, Facultad de Ingenieria, Universidad de Buenos Aires, Av. Paseo Colon 850, C1063ACV Buenos Aires, Argentina, elisachisari@gmail.com), Guillermo Artana (LFD, Facultad de Ingenieria, Universidad de Buenos Aires, Av. Paseo Colon 850, C1063ACV Buenos Aires, Argentina, gartana@fi.uba.ar), Lucie Bailly (Département Parole \& Cognition, GIPSA-lab, 46, avenue Félix Viallet, 38031 Grenoble Cedex, France, Lucie.Bailly@gipsa-lab.inpg.fr), Xavier Pelorson (Département Parole \& Cognition, GIPSA-lab, 46, avenue Félix Viallet, 38031 Grenoble Cedex, France, pelorson@icp.inpg.fr)

Flow through an in-vitro rigid model of the scaled-up laryngeal channel is measured using pressure sensors and visualized using the Schlieren technique for different geometrical configurations. Three downstream flowconditions are considered: steady, quasi-impulsive and periodical using an electromechanical device controlling the inflow and producing the cyclic jet emerging from the glottis. The separated flow behavior in the presence of a ventricular constriction ("false vocal folds") is also examined. Direct theoretical flow predictions and numerical simulations are proposed to quantify the aerodynamic impact involved by the ventricular bands on the pressure distribution. Two parameters are investigated: the aperture of the ventricular bands and the distance between the vocal folds and the ventricular bands. The influence of both parameters are measured and compared to the theoretical outcome.

\section{1:40}

4aSCa9. Aeroacoustic measurements in a vocal tract model. Daniel J Leonard (ARL Penn State, PO Box 30, State College, PA 16804-0030, USA, dj1239@psu.edu), Michael Krane (ARL Penn State, PO Box 30, State College, PA 16804-0030, USA, mhk5@only.arl.psu.edu)

An experiment to clarify the relation between turbulent jet structure, vocal tract wall shape and the resulting sound is described. A life-scale model of the vocal tract ( $18 \mathrm{~cm}$ length, $2.6 \mathrm{~cm} \times 2.6 \mathrm{~cm}$ square cross-section), fabricated from clear cast acrylic is used. A jet, formed at a constriction, passes over or against a simple obstacle, generating sound. Correlated aerodynamic and acoustic measurements are used to determine the transfer function between the obstacle and the measurement location outside the model and the aeroacoustic source spectrum. For comparison the source spectrum is also estimated using the model described in Krane (JASA, 2005), using aerodynamic measurements as empirical input. 
12:00

4aSCa10. Influence of vocal fold stiffness on phonation characteristics at onset in a body-cover vocal fold model. Zhaoyan Zhang (School of Medicine, University of California, 31-24 Rehab Center, 1000 Veteran Ave., Los Angeles, CA 90095-1794, USA, zyzhang@ucla.edu), Juergen Neubauer (School of Medicine, University of California, 31-24 Rehab Center, 1000 Veteran Ave., Los Angeles, CA 90095-1794, USA, jneubauer @ mednet.ucla.edu)

The influence of the body and cover stiffnesses on phonation onset and the resulting vibration pattern was investigated in a body-cover continuum model of the vocal folds. An eigenvalue analysis was performed to obtained phonation onset characteristics. The analysis showed that, with increasing body-cover stiffness ratio, both the phonation threshold pressure and frequency (normalized by the Young's modulus and wave speed of the cover layer, respectively) first increased rapidly and then gradually approached a plateau. For a given glottal resting opening, a soft vocal fold body led to a larger prephonatory glottal opening, which had an negative effect on phonation onset pressure, and for certain vocal fold geometries, led to a local minimum in the phonation threshold pressure as a function of the body stiffness. Although the phonation threshold pressure was low for a vocal fold configuration with both a soft cover and a soft body, the vocal fold vibration at onset exhibited a significant whole-body vertical motion and a low sound production efficiency, and therefore it may not be desirable for voice production. For a large body-cover stiffness ratio, this vertical motion was suppressed and vibration was restricted to the cover layer and the medial surface, resulting in a more effective flow modulation and a better sound production efficiency.
12:20

4aSCa11. Modeling of voice registers and bifurcation theory. Marco Zemke (Institute for Theoretical Biology, Invalidenstraße 43, 10115 Berlin, Germany, marco.zemke@biologie.hu-berlin.de), Isao Tokuda (Japan Advanced Institute of Science and Technology, 1-1 Asahidai, Nomi-shi, 9231292 Ishikawa, Japan, isao@jaist.ac.jp), Hanspeter Herzel (Institute for Theoretical Biology, Invalidenstraße 43, 10115 Berlin, Germany, h.herzel @biologie.hu-berlin.de)

The exact definition of registers in the human voice is still under debate. Especially the quantitative analysis of transitions between these registers have not been investigated in much detail yet. Experiments show different kinds of voice instabilities that appear close to the transition from chest to falsetto register. These instabilities include abrupt jumps between the two registers exhibiting hysteresis, aphonic episodes, subharmonics and chaos. To model these phenomena we start with a three mass cover model of the human larynx which can vibrate in chest and falsetto like patterns. We extend our model to a four mass body-cover polygon model with a supraglottal resonator. We more realistic make use of bifurcation theory to understand how small smooth changes of parameter values can cause sudden qualitative changes in the dynamical behavior of the larynx. Experiments with excised human larynges and untrained singers show comparable coexistence of both registers in a extended parameter region. Our study suggests that voice range profiles can be generalized as two dimensional bifurcation diagrams.

12:40-2:00 Lunch Break

\section{Contributed Papers}

\section{2:00}

4aSCa12. Theoretical and experimental results of phonation threshold pressure vs. oscillation frequency of the vocal folds. Jorge $C$. Lucero (Univ. Brasilia, Dept. Mathematics, 70910-900 Brasilia - DF, Brazil, lucero@unb.br), Annemie Van Hirtum (Département Parole \& Cognition, GIPSA-lab, 46, avenue Félix Viallet, 38031 Grenoble Cedex, France, annemie.vanhirtum@gipsa-lab.inpg.fr), Nicolas Ruty (Département Parole \& Cognition, GIPSA-lab, 46, avenue Félix Viallet, 38031 Grenoble Cedex, France, nicolas.ruty@gipsa-lab.inpg.fr), Julien Cisonni (Département Parole \& Cognition, GIPSA-lab, 46, avenue Félix Viallet, 38031 Grenoble Cedex, France, julien.cisonni@gipsa-lab.inpg.fr), Xavier Pelorson (Département Parole \& Cognition, GIPSA-lab, 46, avenue Félix Viallet, 38031 Grenoble Cedex, France, pelorson@icp.inpg.fr)

The dynamical principles of the vocal fold oscillation at phonation were set forth by Titze (I. R. Titze, J. Acoust. Soc. Am. 83, 1536-1552, 1988), by representing motion of the tissues as a surface wave propagating in the direction of the airflow. An important result of his work was an equation for the phonation threshold value of lung pressure, defined as the minimum value required to initiate the vocal fold oscillation. Titze's model assumed a small time delay for the mucosal wave to travel along the vocal folds, with the consequence that the phonation threshold pressure results independent of the oscillation frequency. Here, we consider an extension of his model for an arbitrary time delay. Our results show that the threshold pressure increases with oscillation frequency following a $x / \sin (x)$ law. We investigate the validity of the theoretical equation by comparing it with pressure measures from a mechanical replica of the vocal folds, under various configurations. In general, the equation shows good agreement with the experimental data, and may find applications for building empirical relations of glottal aerodynamics, and for clinical studies of phonation. [Work supported by CAPESBrazil]

2:20

4aSCa13. Physical modelling of Vowel-Stop-Vowel sequences. Claire Brutel-Vuilmet (GIPSA-Lab, INPG, 46 Av. Felix-Viallet, 38031 Grenoble, France, claire.brutel-vuilmet@gipsa-lab.inpg.fr), Xavier Pelorson
(Département Parole \& Cognition, GIPSA-lab, 46, avenue Félix Viallet, 38031 Grenoble Cedex, France, pelorson@icp.inpg.fr), Annemie Van Hirtum (Département Parole \& Cognition, GIPSA-lab, 46, avenue Félix Viallet, 38031 Grenoble Cedex, France, annemie.vanhirtum @ gipsa-lab.inpg.fr), Susanne Fuchs (ZAS, Schuetzenstr. 18, 10117 Berlin, Germany, fuchs@zas.gwz-berlin.de)

The physical phenomena involved in the production of vowel sounds are now well described and several effective physical models have been developed. On the contrary, only a few studies concern the physical aspects of the production of consonant sounds (fricatives, stops...) or the production of vowel-consonant-vowel sequences. Indeed, more complex acoustical and aerodynamical mechanisms should be considered (generation of aeroacoustical sources, dynamical description...). In this contribution, we focus on the production of vowel-stop-vowel sequences. A stop sound is produced after a complete occlusion of the vocal tract. In an intervocalic context, the closure entails an increase of the intraoral pressure, which results in the cessation of the vocal folds vibration. The opening is quickly followed by the generation of an aeroacoustical source, and entails a decrease of the intraoral pressure, which results in the resumption of the vocal folds motion. A physical model able to describe these aerodynamical observations and to predict the evolution of the intraoral pressure during the closure gesture is first detailed. Different interesting results are discussed (influence of the closure time, influence of the closure position, coordination of the different sources...). And a confrontation between theoretical results and experimental data is presented.

\section{2:40}

4aSCa14. Analysis of frication noise modulation from a physical model. Anna Barney (ISVR, Univ. of Southampton, SO17 1BJ Southampton, UK, ab3@soton.ac.uk), Philip J. Jackson (University of Surrey, Centre for Vision, Speech and Signal Processing, GU2 7XH Guildford, UK, p.jackson @ surrey.ac.uk)

A physical model, built to investigate the aeroacoustic properties of voiced fricative speech, was used to study the amplitude modulation of the 
turbulence noise it generated. The amplitude and fundamental frequency of glottal vibration, relative positions of the constriction and obstacle, and the flow rate were varied. Measurements were made from pressure taps in the duct wall and the sound pressure at the open end. The high-pass filtered sound pressure was analyzed in terms of the magnitude and phase of the turbulence noise envelope. The magnitude and phase of the observed modulation was related to the upstream pressure. The effects of moving the obstacle with respect to the constriction are reported (representative of the teeth and the tongue in a sibilant fricative respectively). These results contribute to the development of a parametric model of the aeroacoustic interaction of voicing with turbulence noise generation in speech.

\section{3:00}

4aSCa15. Towards sibilant /s/ modelling: preliminary computational results. Xavier Grandchamp (Département Parole \& Cognition, GIPSA-lab, 46, avenue Félix Viallet, 38031 Grenoble Cedex, France, xavier.grandchamp@gipsa-lab.inpg.fr), Annemie Van Hirtum (Département Parole \& Cognition, GIPSA-lab, 46, avenue Félix Viallet, 38031 Grenoble Cedex, France, annemie.vanhirtum@gipsa-lab .inpg.fr), Xavier Pelorson (Département Parole \& Cognition, GIPSA-lab, 46, avenue Félix Viallet, 38031 Grenoble Cedex, France, pelorson@icp.inpg.fr), Kazunori Nozaki (Cyber Media Center, Osaka University Cybermedia, 5-1 Mihogaoka Ibaraki, 567-0047 Osaka, Japan, nozaki@cmc.osaka-u.ac.jp), Shinji Shimojo (Cyber Media Center, Osaka University Cybermedia, 5-1 Mihogaoka Ibaraki, 567-0047 Osaka, Japan, shimojo@cmc.osaka-u.ac.jp)

Acoustic effects of the turbulence in human sounds production is generally accepted in the case of fricatives. Nevertheless, this phenomenon is not taken into account in physical modelling of the speech production. Steady flow in a uniform two dimensional channel with a one side triangular obstacle has been simulated using Large Eddy Simulation for different Reynolds numbers. The used geometrical and flow characteristics are severe simplifications of the human articulators during the production of the sibilant $/ \mathrm{s} /$. The impact of different geometrical configurations of the downstream obstacle representing the teeth on the computational results is searched in order to detect the aeroacoustic sources produced by turbulent flow, crucial in /s/ production.

\section{3:20}

4aSCa16. The Influence of Constriction Geometry on Sound Generation in Fricative Consonants. Gordon Ramsay (Haskins Laboratories, 300 George Street, New Haven, CT 06511, USA, ramsay@haskins .yale.edu)

Sound generation in fricative consonants is traditionally supposed to depend only on the Reynolds Number, usually defined in terms of the constriction area and the volume velocity at the constriction. The potential influence of the detailed three-dimensional geometry of the constriction is often ignored, even though previous empirical studies have shown this to have an important effect on the spectral shape of the source and the overall sound strength. At present, the physical processes governing turbulent jet formation and aeroacoustic source generation in fricative consonants are not fully understood. In this paper, we use large-eddy simulations of threedimensional viscous incompressible flow to visualize the development of the turbulent flow field and aeroacoustic source distribution in an elliptical duct representing the vocal tract, for elliptical, laminar, and grooved constriction shapes that share the same cross-sectional area function. By contrasting results for these geometries, we test the hypothesis that turbulent jet formation is determined largely by the shape of the boundary layer where flow separates at the exit of the constriction, and that the perimeter of the constriction, rather than the cross-sectional area, may therefore be a more appropriate parameter for characterizing properties of the aeroacoustic source.

\title{
Session $4 \mathrm{aSCb}$
}

\section{Speech Communication: Neurobiology of Speech Perception}

\author{
Paul Iverson, Cochair \\ University College London, Department of Phonetics and Linguistics, 4, Stephenson Way, London, NW1 2HE, UK \\ Christophe Pallier, Cochair \\ Cognitive Neuroimaging Unit INSERM 562, CEA/Neurospin, bat 145, point courier 156, Gif/Yvette, 91191, France
}

\section{Invited Papers}

4aSCb1. Involvement of Auditory Cortex in Speech Production. Frank H. Guenther (Boston University, 677 Beacon Street, Boston, MA 02215, USA, guenther@cns.bu.edu)

In addition to their role in speech perception, the auditory cortical areas of the superior temporal lobe are important for the formation and maintenance of motor commands for speech production. Using a combination of neural network modeling, neuroimaging, and auditory perturbation experiments, we have characterized the network of brain regions involved in auditory feedback control of segmental aspects of speech. This network involves auditory error cells in bilateral posterior superior temporal cortex which become active when the current auditory feedback mismatches the auditory target for the current speech sound. Projections from these auditory cortical areas to the right hemisphere ventral premotor areas, then on to primary motor cortex, transform perceived auditory errors into corrective movement commands for the speech articulators. The DIVA model of speech production produces a close quantitative fit to acoustic data collected during unexpected auditory perturbation of speech and during sensorimotor adaptation to sustained auditory perturbations. Neuroimaging results motivate a modification of the model to include right ventral premotor cortical areas in the auditory feedback circuit for speech production. [Work supported by NIDCD, NSF.] 
4aSCb2. Motor regions contribute to speech perception: awareness, adaptation and categorisation. Matthew $\mathrm{H}$. Davis (MRC CBU, 15 Chaucer Rd., CB2 7EF Cambridge, UK, matt.davis@mrc-cbu.cam.ac.uk), Ingrid S. Johnsrude (Queen's University, Dept Psychology, 62 Arch Street, Kingston, ON K7L 3N6, Canada, ingrid.johnsrude@queensu.ca), Alexis G. Hervais-Adelman (Centre for the Neural Basis of Hearing, Department of Physiology, Development and Neuroscience, University of Cambridge, Downing Site, CB23EG Cambridge, UK, alexis.hervais-adelman@mrc-cbu.cam.ac.uk), Jack C. Rogers (MRC Cognition and Brain Sciences Unit, 15 Chaucer Road, CB2 7EF Cambridge, UK, jack.rogers@mrc-cbu.cam.ac.uk)

Functional imaging and TMS studies show that motor and premotor cortex responds to heard speech though the functional significance of this response is unclear. Three recent fMRI studies, showing modulation of motor responses to heard speech in the absence of overt spoken or manual responses, may shed light on how regions typically associated with speech production contribute to perception. (1) Motor activity remains robust during light anaesthetic sedation, but is obliterated for deeply sedated participants who are no longer aware of speech. (2) Motor cortex responds more to distorted yet intelligible noise-vocoded words than to clear speech or unintelligible noise. This neural correlate of listening effort is also observed for "clear-then-vocoded" presentations that enhance perceptual adaptation compared to a matched "vocoded-then-clear" condition that doesn't enhance adaptation. (3) During paired priming of audio-morphed syllables we see a greater response to acoustic changes that cross phonological category boundaries compared to acoustically-equivalent within-category changes. Additional responses to between-category pairs extend to motor regions, suggesting that neural correlates of categorical perception include regions involved in speech production. These findings illustrate how perceptual awareness, adaptation and categorisation of speech all engage motor regions. Implications for neurobiological accounts of speech perception will be discussed.

\section{9:20}

4aSCb3. From Speech to Language: Mapping the Auditory Comprehension Network. Jonas Obleser (Max Planck Institute of Human Cognitive and Brain Sciences, Stephanstrasse 1A, 04103 Leipzig, Germany, jonas@ obleser.de)

Speech comprehension is a complex perceptual and cognitive task that is fulfilled in a surprisingly robust manner. I will present a series of studies that aim at disentangling the interaction of sensory/acoustic and cognitive/linguistic factors driving the speech comprehension system: (i) How does the system in its entirety deal with noise at the auditory entry level? (ii) Which are key contextual influences that aid speech comprehension when the signal quality drops, and (iii) what is the functional circuitry within and across auditory cortex that copes with comprehension difficulties? Main results include: 1 . Comprehension of intermediate signal quality based on semantic predictability engages a left- hemispheric, widely distributed array of brain structures. Also, functional connectivity amongst these areas appears enhanced. 2. Signal intelligibility gates and enables the expected semantic effects (cloze probability; BA44 BOLD response and EEG N400), whereas semantic effects shape the intelligibility modulation in anterolateral temporal cortex. 3. The angular gyrus (BA 39) enhances difficult yet successful speech comprehension whereas left posterior STG reflects higher computational effort (either poor signal quality or low cloze probability) in speech comprehension. Behavioural, EEG and fMRI data will be presented, and themes of (bi-)laterality and bottom-up/top-down directionality will be re-visited along the way.

\section{9:40}

4aSCb4. An fMRI study of subliminal priming of spoken words. Christophe Pallier (Cognitive Neuroimaging Unit INSERM 562, CEA/Neurospin, bat 145, point courier 156, 91191 Gif/Yvette, France, christophe@pallier.org), Sid Kouider (Laboratoire de Sciences Cognitives et Psycholinguistique, Ecole Normale Supérieure, 29 rue d'Ulm, 75005 Paris, France, sid.kouider@ens.fr), Vincent De Gardelle (Laboratoire de Sciences Cognitives et Psycholinguistique, Ecole Normale Supérieure, 29 rue d'Ulm, 75005 Paris, France, gardelle@biologie.ens.fr)

Repetition priming has been widely used to study spoken and written word recognition. Its physiological counterpart is repetition suppression, a reduction in neural activity resulting in a measurable decrease of the fMRI signal. By varying the representational level at which the repetition occurs, one can determine which properties are encoded in a given brain area, and which are not. We will report on an fMRI experiment using subliminal auditory priming of spoken words. Subliminal priming has been used, for example, by Dehaene et al. to study visual word recognition. Our experiment employs a technique developped by Kouider \& Dupoux that allows subliminal presentation of auditory stimuli using temporal compression and forward masking. The participants perform a lexical decision task on the target item, which is preceded by a subliminal prime that can be phonetically identical or different from the target, and spoken or not by the same speaker. A fast-event related paradigm is used where each prime-target pair is presented during silent gaps between the acquisitions. The planned analyses will seek to identify the brain regions showing subliminal repetition suppression to word repetition regardless of speaker change, as well as other areas sensitive to speaker change regardless of linguistic content.

\section{Contributed Paper}

10:00

4aSCb5. The neural bases of normalising for accented speech: A repetition suppression functional magnetic resonance imaging study. Patti Adank (F.C. Donders Centre for Cognitive Neuroimaging, Kapittelweg 29,6525EN Nijmegen, Netherlands, patti.adank@fcdonders .ru.nl), Peter Hagoort (F.C. Donders Centre for Cognitive Neuroimaging, Kapittelweg 29, 6525EN Nijmegen, Netherlands, peter.hagoort@fcdonders .ru.nl)

A repetition suppression fMRI paradigm was employed to explore the neuroanatomical substrates of normalisation for accented speech in spoken sentence processing. Sentences were produced in two accents: in Standard Dutch and an artificial accent of Dutch. In the experiment, participants listened to two sentences presented in quick succession. The second sentence was either spoken by the same speaker in the same accent, by the same speaker in a different accent, by a different speaker in the same accent, or by a different speaker in a different accent. This design allowed us to study neural responses to a change in speaker only, a change in accent only and a change in accent and speaker. Results showed small effects for a change of speaker only in right Superior Temporal Gyrus (STG). A change of accent only showed extensive activations in left and right STG and Superior Temporal Sulcus (STS). Finally, a change of speaker and accent showed extensive activations in left and right STG and STS, and increased activity in left Inferior Frontal Gyrus (IFG). The results indicate that normalisation processes for accented speech recruit a wide neural network. The role of left IFG in normalisation processes will be discussed. 


\title{
Invited Papers
}

\author{
10:40
}

4aSCb6. Streams of processing and hemispheric asymmetries in speech perception. Sophie K. Scott (University College London, Institute of Cognitive Neuroscience, 17 Queen Square, WC1N 3AR London, UK, sophie.scott@ucl.ac.uk)

Studies in non-human primates have indicated that, as in the visual system, there are (at least) two streams of processing in the auditory system. These pathways are associated with different types of auditory processes - an anterior 'what' pathway and posterior 'how/where' pathway(s). In this talk I will use these neurophysiological theories as a framework for interpreting findings from a range of PET and fMRI studies of human speech perception and production, and present evidence that the anterior 'what' pathway in humans shows hierarchical processing of the speech signal, reflecting a move from acoustic/phonetic processing to a more abstract representation in the anterior superior temporal sulcus. In contrast, posterior auditory areas in humans are associated with sensory/motor interactions in speech, and with aspects of working memory processing. I will address how these systems are differentially recruited when speech perception is made difficult, due to different types of masking noise. Finally, I will outline differences in the processing of speech in left and right auditory areas.

\section{1:00}

4aSCb7. The temporal analysis of spoken language. David Poeppel (University of Maryland, 1401 Marie Mount Hall, College Park, MD 20742, USA, dpoeppel@umd.edu)

The (concurrent) construction of syllabic and phonemic representations forms the basis for creating interpretable representations of speech; therefore we look here to temporal attributes commensurate with their acoustic implementation. Based on a distributed model of the functional anatomy of speech perception (Hickok \& Poeppel 2007) and on the assumption that the perception of speech requires multi-time resolution analysis (Poeppel 2003), electrophysiological data are shown that illustrate how auditory cortex makes use of one specific temporal mechanism, the processing of phase (Luo \& Poeppel 2007). We hypothesized that the phase pattern of cortical rhythms associated with modulation rates mediating intelligible speech provide an encoding mechanism. We observed that the phase of the theta band response generated in auditory cortex tracks sentence-level acoustics with the sensitivity and specificity necessary for neuronal encoding. The data are consistent with the view that a $\sim 200 \mathrm{~ms}$ temporal window (period of theta oscillation) segments the incoming signal, resetting and sliding to track speech dynamics. This hypothesized mechanism for cortical speech analysis is based on the stimulus-induced modulation of inherent cortical rhythms and provides supporting evidence implicating the syllable as a computational primitive for the representation of spoken language.

\section{1:20}

4aSCb8. ERPs and speech sound perception - possibilities and restrictions. Maija S. Peltola (University of Turku, Department of Phonetics, FIN-20014 Turku, Finland, maija.peltola@utu.fi)

Speech sound perception is a complex combination of attention independent and attention dependent processes which both contribute to the final goal of understanding the spoken message. The preattentive level has become more accessible to research and event related potentials (ERPs) can easily be used to study the automatic processing of the speech signal. In particular, the mismatch negativity (MMN) response offers a tool for investigating the manner in which speech sounds are encoded as neural representations. Crosslinguistic studies revealing different kinds of representations in native speakers of different languages form the core for further studies, which have shown the plasticity of the brain in forming new representations for non-native sounds in various types of learning environments. However, despite all these promising advances there are still some restrictions connected both with the methodology available and the conclusions that can be reached on the basis of the occasionally contradicting results. Also, since results obtained by using attention independent and dependent methods are not always compatible, some potentially significant results may never reach the attention that they deserve, even if this incompatibility may be one of the keys into the understanding of the complicated mechanisms underlying speech sound perception.

\section{Contributed Paper}

\section{1:40}

4aSCb9. Cognitive control skills and speech perception after short-term second language experience during infancy. Barbara Conboy (University of Washington, Dept. of Speech \& Hearing Sciences, and Institute for Learning \& Brain Sciences, Box 357988, Seattle, WA 98195, USA, bconboy@u.washington.edu), Jessica Sommerville (University of Washington, Dept. of Speech \& Hearing Sciences, and Institute for Learning \& Brain Sciences, Box 357988, Seattle, WA 98195, USA, sommej@u.washington.edu), Patricia K. Kuhl (University of Washington, Dept. of Speech \& Hearing Sciences, and Institute for Learning \& Brain Sciences, Box 357988, Seattle, WA 98195, USA,pkkuhl@u.washington.edu)
Previous research has linked increasing cognitive abilities to reductions in sensitivity to nonnative phonemes toward the end of the first year, but found no association between cognitive skills and native speech perception (Conboy et al., 2006; Lalonde \& Werker, 1995). The present study examined cognitive abilities and brain activity to second-language (L2) phoneme contrasts in infants who had short-term experience with the L2: we predicted better cognitive skills in infants with better discrimination of the L2 contrast. Seventeen infants from monolingual English homes completed event-related potential (ERP) speech perception testing and nonlinguistic tasks requiring attentional flexibility, memory, and inhibitory control at 11 months, after twelve Spanish play sessions from 9.5 - 10.5 months. An ERP oddball paradigm assessed discrimination of English and Spanish contrasts 
(English: voiced /da/ vs. voiceless-aspirated [tha]; Spanish: prevoiced /da/ vs. voiceless-unaspirated /ta/). Infants showed broad mismatch negativity (MMN) discriminatory responses to both contrasts. Larger Spanish MMN amplitudes were linked to better performance on cognitive tasks (detour- reaching object-retrieval and the A not B task) (Fisher's exact test, $\mathrm{p}=.01$ ), suggesting a role for specific cognitive abilities in the early stages of phonetic learning. There was no association between English MMN amplitudes and cognitive skills.

\title{
Session 4aSPa
}

\section{Signal Processing in Acoustics, Acoustical Oceanography, and ECUA: Model-Based Signal Processing II}

\author{
Sean Lehman, Cochair \\ Lawrence Livermore Natl. Lab., Livermore, CA 94551, USA \\ Christian Pichot, Cochair \\ Antennas \& Telecommunications Laboratory, University of Nice-Sophia Antipolis, France
}

\section{Contributed Paper}

\section{8:00}

4aSPa1. Water column tomographic inversion with a network of drifting buoys. Sergio Jesus (ISR, Universidade do Algarve, PT-8005-139 Faro, Portugal, sjesus@ualg.pt), Cristiano Soares (ISR, Universidade do Algarve, PT-8005-139 Faro, Portugal, csoares@ualg.pt), Nelson Martins (ISR, Universidade do Algarve, PT-8005-139 Faro, Portugal, nmartins@ualg.pt)

The estimation of ocean environmental properties by means of the inversion of acoustic signals has in several occasions been performed using a single vertical array of acoustic receivers, with a towed acoustic source as an attempt to ensure a rapid spatial coverage of the area of interest, as only a single ocean transect is "seen" at each time. Ideally, one would like to obtain an instantaneous picture of the complete area (volume) under observation. However, the resulting acoustic observations, hence environmental estimates, are not simultaneous in time. Using multiple acoustic receiving arrays appears to be a natural step towards both increasing the spatial coverage, and obtaining simultaneous environmental estimates of different ocean transects. It also gives a higher chance to capture spatial transient features, as for example solitons. Using multiple receiver arrays represents the addition of a new spatial dimension at the receiving end and opens up the number of possibilities to a Nx2D or full 3D view of the ocean. Taking support on the data set of the RADAR'07 experiment (July 9 - 16, 2007) where data was simultaneously collected on three vertical arrays, this paper explores space coherent processing of the several receiving arrays and Nx2D or 3D environmental constrained water coloumn matched-field inversion.

\section{Invited Paper}

4aSPa2. Model based echo processing architectures for sonar target classification. Manell E. Zakharia (French Naval Acadamey, BP 600, 29240 Brest-Armees, France, manell.zakharia@ecole-navale.fr)

The discrimination between man-made and natural targets is faced to the problem of setting up appropriate processing architectures that extract relevant and robust parameters that could be used for classification. To be robust, signal models have to be associated to physical models and echo parameters have to be associated to physical ones. Several models already published are investigated: bright spots, generalized bright spots, resonances. Associated processing architectures are presented: matched filter, bank of filters, AR modeling. Their performance are compared on experimental data set obtained in tank. The discriminating performance are compared in the case of shells (man-made) and solid targets (natural) of the same shape insonified in a random incidence (monostatic). Following a detailed description of echo formation mechanisms in the time-frequency plane, an explicit time-frequency architecture is presented: the time-frequency filtering. Finally a new (all chirp) model based on velocity dispersion of surface waves ,is proposed that could reduce the number of discriminating parameters and be robust to minor changes of shell characteristics.

\section{Contributed Papers}

\section{8:40}

4aSPa3. Automatic Acoustics Measurement of Audible Inspirations in Pathological Voices. Eduardo Castilllo-Guerra (University of New Brunswick, P.O. Box 4400, 15 Dineen Dr., D36 Head Hall, Fredericton, NB E3B 5A3, Canada, ecastill@unb.ca), Williams Lee (University of New Brunswick, P.O. Box 4400, 15 Dineen Dr., D36 Head Hall, Fredericton, NB E3B 5A3, Canada,wlee@unb.ca)

Audible inspiration (AI) is a type of speech perturbation commonly heard in pathologic voices. This acoustic parameter is used in conjunction with other acoustic observations to assess different types of pathologic conditions of speech associated with neurological or vocal cord disorders.
However, the perception of this speech perturbation is often very subjective and difficult to appraise in a quantitative and consistent form. This work reports an algorithm to estimate the severity of this perturbation using timefrequency characteristics. The algorithm is based on a linear combination of the frequency of occurrence, the duration and the intensity of the inspirations. An algorithm to segment the AIs in conversational speech is proposed. The AI index was first evaluated with the Massachusetts Eye and Ear Infirmary Voice Database and then with two other databases containing recording from motor speech disorders. The segmentation algorithms was 90\% effective detecting the audible inspirations and the AI index showed a high correlation $(80.8 \%)$ with respect to the average perceptual judgment obtained from three judges with experience evaluating disordered speech. 
9:00

4aSPa4. Comparative Study of Wideband Subspace Direction of Arrival (DOA) estimation methods. Sheraz Khan (Laboratoire Ondes et Acoustique, ESPCI, 10 rue Vauquelin, 75231 Paris, France, sheraz.khan @ polytechnique.edu)

Signal subspace Methods like ESPRIT, MUSIC and MATRIX PENCIL, provides high resolution Direction Of Arrival (DOA) estimation in comparison to traditional Delay and Sum and Capon methods, which are limited by sensor spacing. However underwater Acoustics signals are inherently wideband in nature and most of these Subspace methods works on narrowband signals. Currently modified version of these methods for wideband signals are emerging. These methods are broadly classified as Coherent and Incoherent methods depending upon how signals of different frequencies have been merged. Performances of these methods are evaluated using extensive Monte-Carlo simulations under various protocols by comparing there Mean Square Error in DOA estimation and by their respective resolution power. This comparison study is also complemented with real acoustic data from public domain.

9:20

4aSPa5. Application of statistical methods in underwater signal classification. Brett E. Bissinger (ARL Penn State, PO Box 30, State College, PA 16804, USA, beb194@psu.edu), Richard Lee Culver (ARL Penn State, PO Box 30, State College, PA 16804, USA, rlc5@ psu.edu), Nirmal K. Bose (ARL Penn State, PO Box 30, State College, PA 16804, USA, bkn@engr.psu.edu), Colin W. Jemmott (ARL Penn State, PO Box 30, State College, PA 16804, USA, cwj112@psu.edu)

The overall goal of our work is to utilize knowledge of the ocean environment to improve sonar detection and classification performance. Source classification and localization in the underwater environment is a challenging problem in part because propagation through the space- and timevarying medium introduces multipath, variability, and decorrelation to the signal. Traditional underwater signal classification has relied on parametric methods such as the likelihood ratio tests. Recent research has explored nonparametric methods like maximum entropy and maximum likelihood with favorable results. This talk considers other, more contemporary nonparametric methods, e.g. principle component analysis, independent component analysis and support vector machines, and compares their structure and performance with previous results. Work supported by Office of Naval Research Undersea Signal Processing.

9:40

4aSPa6. Estimation of acoustic directivity from microphone array measurements using parametric models. Jean Bulté (ONERA, BP 72 29, avenue de la division Leclerc, 92322 Châtillon, France,
jean.bulte@onera.fr),Vincent Fleury(ONERA, BP 72 - 29, avenue de la division Leclerc, 92322 Châtillon, France, vincent.fleury@onera .fr), Renaud Davy (ONERA, BP 72 - 29, avenue de la division Leclerc, 92322 Châtillon, France, renaud.davy@onera.fr)

In this paper, we are interested in recovering the far-field acoustic pattern of a directive source from signals recorded in the near-field by an array of microphones with a reduced spatial extent. This question is particularly relevant in small test facilities where far-field acoustic measurements can not be carried out. A two-step approach is suggested. Firstly, the characteristics of sources are estimated from near-field measurements. Secondly, these characteristics are used to estimate the far-field radiation pattern. The main difficulty of this problem mainly resides in the first step. Due to the reduced spatial extent of the array, much information is lost about source characteristics, which mathematically leads to solve an ill-posed inverse problem. Our approach consists in using a parametric model based on physical assumptions, which has the virtue of regularizing the estimation problem. The suggested method is firstly evaluated with simulations, and then applied to experimental data recorded during aeroacoustic tests with a subsonic jet in an anechoic wind tunnel. It is shown that comparison between far-field measurements and estimated far-field pattern are in good agreement.

\section{0:00}

4aSPa7. A volumetric interferometric synthetic aperture sonar reconstruction algorithm. Michael Hayes (University of Canterbury, Private Bag 4800, 8022 Christchurch, New Zealand, michael.hayes @ canterbury.ac.nz), Peter T. Gough (University of Canterbury, Private Bag 4800, 8022 Christchurch, New Zealand, peter.gough@canterbury.ac.nz)

Interferometric synthetic aperture sonar (InSAS) bathymetric reconstruction is an inverse problem that is often simplified to a time delay estimation problem. This uses a simple system model of a continuous scattering surface with single scatterer per resolution cell. This model is violated by layover, multiple scattering, occlusions, or sea-surface multipath producing artefacts in the reconstructed image. While some artefacts, such as from occluded shadow regions, can be rejected by using a threshold on the correlation coefficient, this does not work in general. Moreover, since each pixel is reconstructed independently it is difficult to improve the reconstruction by adding prior information. In this paper we propose a reconstruction algorithm using a probabilistic volumetric model; similar to those used for photometric 3-D scene reconstruction from multiple cameras. While significantly slower than time delay estimation methods, the bathymetric reconstruction can be improved due to better scene modelling and the incorporation of priors such as surface continuity. Furthermore, an advantage of a volumetric model is that correction for the footprint shift is implicit. We demonstrate the algorithm using both simulated and real data. 


\title{
Session $4 \mathrm{aSPb}$
}

\section{Signal Processing in Acoustics, Acoustical Oceanography, and ECUA: Bayesian Signal Processing II}

\author{
Zoi-Heleni Michalopoulou, Cochair \\ Department of Mathematical Sciences, New Jersey Institute of Technology, 323 M L King Blvd, Newark, NJ 07102, USA
}

Simon J. Godsill, Cochair

Trumpington Street, Cambridge, CB21PZ, UK

\section{Invited Papers}

\section{$10: 40$}

4aSPb1. An Overview of Bayesian Computational methods for audio signal processing. Simon J. Godsill (Trumpington Street, CB21PZ Cambridge, UK, sjg@eng.cam.ac.uk), Ali-Taylan T. Cemgil (Trumpington Street, CB21PZ Cambridge, UK, atc27@eng.cam.ac.uk), Paul Peeling (Trumpington Street, CB21PZ Cambridge, UK, php23@cam.ac.uk)

In this talk we describe Bayesian computational models and methods for inference about noisy audio signals, with the aim of performing tasks such as musical trnscription, source separation, automated annotation with content descriptors, noise reduction and objectbased coding. The models are structured models of non-stationary sparsity in audio sources, usually expressed in the time-frequeny plane. The computational inference methods are based around Monte Carlo techniques including the particle filter for rapid on-line inference and Markov chain Monte Carlo for batch problems of higher compleixity. Examples will be given from the spheres of source separation, multiresolution noise reduction, denoising and intetrpolation of missing batches from audio. We will also describe their use for acoustical analysis of the properties of a musical instrument, focusing on the parameters of inharmonicity in struck or plucked strings.

\section{1:00}

4aSPb2. Bayesian approach to signal detection, source localization and ocean environmental parameter estimation. Loren W. Nolte (Duke University, Department of Electrical and Computer Engineering, Durham, NC 27708, USA, 1wn@ee.duke.edu)

From a Bayesian perspective, signal detection, source localization and ocean environmental parameter estimation algorithms can be viewed as simultaneous signal processing operation. In these cases, the likelihood function provides a mechanism for incorporating physical models of the ocean environment. In addition, one can incorporate uncertainties of source location and ocean environmental parameters a priori, rather than dealing with them "after the processing", and this approach also provides a posteriori probabilities of these source and ocean parameters as outputs of the processing. In addition to affecting the structure of optimal signal processing algorithms, these uncertainties affect how well one can spatially localize the source of sound, or the accuracy with which one can estimate the ocean environmental parameters. In passive and active sonar detection, these uncertainties, along with the knowledge of the ocean physics that has been incorporated in the likelihood function, determine detection performance. In particular, this paper will illustrate how signal detection theory can provide quantitative upper bounds of sonar detection performance on the receiver operating characteristic (ROC) as a function of the amount of uncertainty in ocean environmental parameters, source location, and signal-to-noise ratio.

\section{$11: 20$}

4aSPb3. Desoloing for musical accompaniment systems. Lawrence J. Raphael (Adelphi University, 1 South Avenue, Garden City, NY 11530, USA, raphael@adelphi.edu)

I discuss ongoing work for musical accompaniment systems in which we remove the soloist from a full recording for soloist and accompanying ensemble (e.g. a concerto), leaving just the accompaniment. I will discuss briefly the score matching problem, which generates a correspondence between a symbolic music representation and the audio. Using this score match, straightforward masking leads to usable source separation results, since the nature of the accompaniment problem partially compensates for the damage done by masking. However, I will discuss methods of improving this separation process involving imputing unobserved audio. The presentation will include a live demonstration of the accompaniment system.

\section{$11: 40$}

4aSPb4. Bayesian linear regression onto data-driven dictionaries with application to music transcription. Cédric Févotte (CNRS-Telecom ParisTech, 37-39, rue Dareau, 75014 Paris, France, fevotte@tsi.enst.fr)

In this work, we describe a linear regression technique where features of the dictionary (the collection of explanatory variables) are learnt from the data itself. Our Bayesian setting allows to add regularization constraints on both the explanatory variables and the regressors that fit physical properties of sound. More precisely, smoothness constraints can be imposed on the first ones while time- 
persistency and/or sparse constraints can be imposed on the second ones. When applied to music, we observe that the retrieved explanatory variables bear a semantic value and that the overall process yields a compact data-driven object-based transcription of the original signal.

4aSPb5. Bayesian formant tracking using conditionally linear Gaussian models. Patrick Wolfe (Harvard University, Harvard University - SEAS, 33 Oxford St Rm MD-129, Cambridge, MA 02138-2901, USA, patrick@seas.harvard.edu)

Formants play a central role in the perception and analysis of speech. In this presentation we describe Bayesian approaches to estimating vocal tract resonances from speech waveforms, formulated as a statistical model-based tracking problem. In particular, approaches by Deng and colleagues have shown the promise of an extended Kalman filtering approach based on a robust linearization of the formant-to-cepstrum map. We describe recent extensions to model inter- as well as intra-formant correlation, and detail efficient inference schemes that preserve conditional Gaussianity. A database of formant trajectories provides a notion of ground truth by which estimator performance can be evaluated, and which demonstrates the efficacy of our approach relative to contemporary benchmark tools for formant analysis.

4aSPb6. Bayesian tracking and geoacoustic inversion. Stan E. Dosso (University of Victoria, School of Earth and Ocean Sciences, Victoria, BC V8W 3P6, Canada, sdosso@uvic.ca), Michael J. Wilmut (University of Victoria, School of Earth and Ocean Sciences, Victoria, BC V8W 3P6, Canada, mjwilmut@uvic.ca)

This paper describes a Bayesian approach to two related inverse problems in underwater acoustics: localizing/tracking an acoustic source when ocean environmental properties are unknown, and determining environmental properties using acoustic data from an unknown (moving) source. The goal of this work is not simply to estimate values for source and/or environmental parameters, but to determine parameter uncertainty distributions, thereby quantifying the state of knowledge and information content of the inversion. A common formulation is applied for both problems in which source parameters (location and spectrum) and environmental parameters are considered unknown random variables constrained by noisy acoustic data and by prior information on parameter values (e.g., physical limits for environmental properties) and on inter-parameter relationships (limits on horizontal and vertical source speed). Given the strong nonlinearity of the inverse problem, marginal posterior probability densities are computed numerically using efficient Markovchain Monte Carlo importance sampling methods. Source tracking results are represented by joint marginal probability distributions over range and depth, integrated over unknown environmental parameters. The approach is illustrated with two examples representing tracking a quiet submerged source and geoacoustic inversion using noise from an unknown ship-of-opportunity. In both cases, source, seabed, and water-column parameters are unknown.

\section{2:40-2:00 Lunch Break}

\section{Invited Papers}

\section{2:00}

4aSPb7. Geoacoustic Environment Tracking Using Kalman and Particle Filters. Peter Gerstoft (Marine Physical Laboratory, Scripps Institute of Oceanography, 8602 La Jolla Shores Drive, La Jolla, CA 92093-0238, USA, gerstoft@ucsd.edu), Caglar Yardim (Marine Physical Laboratory, Scripps Institute of Oceanography, 8602 La Jolla Shores Drive, La Jolla, CA 92093-0238, USA, cyardim@ucsd.edu), William Hodgkiss (MPL, Scripps Institution of Oceanography, University of California, San Diego, La Jolla, CA 92093-0238, USA,wsh@mpl.ucsd.edu)

This paper addresses the problem of tracking the geoacoustic environmental parameters such as the sound speeds in water, sediment and the bottom, sediment attenuation, density and thickness. The tracking is based on using acoustic measurements within an extended Kalman (EKF), unscented Kalman (UKF), and particle filter (PF) framework. The acoustic field is computed using a normal mode code which introduced a varying degree of nonlinearity depending on the environmental parameter of interest. Posterior Cramer-Rao lower bounds (PCRLB) are used to compute the tracking performances of the filters, including the filter efficiencies, divergence statistics, and computational complexities. The results showed that some of the parameters such as the water column parameters can be tracked by Kalman filters, however, the tracking performance of the Kalman filters was limited by the highly nonlinear relation between the sediment/bottom parameters and the acoustic field and non-Gaussian densities of these parameters. Particle filters proved to be very promising in tracking sediment layer parameters, even in the abruptly changing environments.

4aSPb8. Inference and learning in gamma chains for Bayesian audio processing. Ali Taylan Cemgil (University of Cambridge, Trumpington street, CB2 1PZ Cambridge, UK, atc27@cam.ac.uk), Onur Dikmen (Bogazici University, Dept. of Computer Engineering, 80815 Istanbul, Turkey, onuro@boun.edu.tr)

Statistical description of complex phenomena encountered in many applications requires construction of nonstationary models. A first step in analysis of such nonstationary sources involves typically a traditional time-frequency analysis Short time Fourier transform. In all these techniques, the underlying implicit assumption is that the process is piecewise stationarity, however dependencies across frequency bands or time frames are not explicitly characterised. Here, we investigate a class of prior models, called Gamma chains, for modelling such statistical dependencies in the time-frequency representations of signals. In particular, we model the prior variance of transform coefficients using Markov chains, trees and fields of inverse Gamma random variables. This model class is Markovian and conditionally conjugate, so standard inference methods like Gibbs sampling, variational Bayes or sequential Monte Carlo can be applied 
effectively and efficiently. We also show how hyperparameters, that determine the coupling between prior variances of transform coefficients, can also be optimised. We discuss the pros and cons of various inference schemata (variational Bayes, Gibss sampler and particle filtering) in terms of complexity and optimisation performance for this model class. We illustrate the effectiveness of our approach in audio denoising and single channel audio source separation applications.

\section{2:40}

4aSPb9. A nonlinear frequency-domain beamformer for underdetermined speech mixtures. Michael Davies (University of Edinburgh, ICDOM, Kings Buildings, Mayfield Road, EH9 3JL Edinburgh, UK, mike.davies@ed.ac.uk), Mohammad Dmour (University of Edinburgh, Institute for Digital Communications, School of Engineering \& Electronics, Alexander Graham Bell Building, Kings Buildings, Mayfield Road, EH9 3JL Edinburgh, UK, m.dmour@ed.ac.uk)

Extraction of a target speech source from among multiple interfering speech sources is challenging when there are fewer microphones than sources (the underdetermined case). Existing speech source separation techniques often suffer from artifacts as well as performance deterioration in reverberant environments, and in some cases also need to estimate the number of sources present. This paper introduces a frequency-domain non-linear beamformer that can perform speech source separation of underdetermined mixtures, is reasonably artifact free and does not require prior knowledge of the number of speakers. Our approach models the data via a Gaussian mixture distribution in the observation domain, which can be learned using the expectation maximization (EM) algorithm. A non-linear distortionless beamformer is then developed, based on this model. Simulations of the non-linear beamformer in underdetermined mixtures with room reverberation confirm its capability to successfully separate speech sources with virtually no artifacts.

\section{3:00}

4aSPb10. Bayesian model selection applied to room-acoustic energy decay analysis. Tomislav Jasa (Institute fur Neuroinformatik ETH/UNIZ, Winterthurerstrasse 190, 8057 Zurich, Switzerland, jasa@ini.phys.ethz.ch), Ning Xiang (Rensselaer Polytechnic Institute, Greene Building, School of Architecture, 110 8th Street, Troy, NY 12180, USA, xiangn@ rpi.edu)

The previous work of Xiang et al. [Xiang \& Goggans, J. Acoust. Soc. Am. 110 (2001), pp. 1415-1424; 113 (2003), pp. 2685-2697; Xiang \& Jasa, J. Acoust. Soc. Am. 120 (2006) pp.3744-3749] successfully applied the Bayesian formulism to estimate multiple decay parameters from Schroeder decay functions measured or calculated in acoustically coupled spaces. In this work we consider a more difficult problem of determining the correct decay model in the presence of energy decay data within the Bayesian framework. We will compare the Annealing/Thermodynamic algorithm [Neal, R. M., Statistics and Computing 11 (2001), pp. 125-139], the Nested Sampling algorithm [Silvia \& Skilling, Data Analysis: A Bayesian Tutorial, Oxford Science Publications (2006)], and a combined Variational/Markov Chain Monte Carlo approach in order to determine the correct decay model. The advantages/shortcomings of these methods are discussed in the context of the decay model selection and parameter estimation using experimentally measured data from real coupled spaces.

\section{$3: 20$}

4aSPb11. Efficient Bayesian inference for multiple pitch estimation of music audio. Emmanuel Vincent (INRIA Rennes Bretagne Atlantique, Campus de Beaulieu, 35042 Rennes Cedex, France, emmanuel.vincent@irisa.fr)

Multiple pitch estimation consists of estimating the number of active notes and their fundamental frequencies on each time frame of a music signal. This is a core problem for several applications, including score transcription and source separation. Bayesian harmonic models are a promising approach, since they allow the joint exploitation of various priors on the model parameters. However existing Bayesian inference methods often rely on specific prior distributions and remain computationally demanding for realistic data. We propose a generic inference method based on adaptive factorization of the joint posterior that allows the application of such models to real-world data. We evaluate the results for the task of multiple pitch estimation using different levels of factorization.

\section{$3: 40$}

4aSPb12. Bayesian single channel blind speech dereverberation using Monte Carlo methods. James R. Hopgood (Institute for Digital Communications, The University of Edinburgh, School of Engineering and Electronics, Alexander Graham Bell Building, The King's Buildings, Mayfield Road, EH9 3JL Edinburgh, UK, James.Hopgood@ed.ac.uk), Christine Evers (Institute for Digital Communications, The University of Edinburgh, School of Engineering and Electronics, Alexander Graham Bell Building, The King's Buildings, Mayfield Road, EH9 3JL Edinburgh, UK, c.evers@ed.ac.uk), Judith Bell (Heriot Watt University, School of Engineering and Physical Sciences, Riccarton, EH14 4AS Edinburgh, UK, j.bell@hw.ac.uk)

Audio signals in confined spaces exhibit reverberation due to reflections off surrounding obstacles. Moreover, the signal is distorted by noise, usually modeled as an additive signal observed within the room, independent of the microphone's location, and unaffected by the acoustics. Reverberation and noise cause significant deterioration of audio quality and intelligibility to signals recorded in acoustic environments. Bayesian blind dereverberation infers knowledge about the system by exploiting the statistical properties of speech and the acoustic channel. In the Bayesian framework, the reverberant and noisy signal can be enhanced by processing it either sequentially using online methods or in a batch using offline methods. This paper compares several distinct Bayesian approaches for single-channel blind speech dereverberation. These include Markov chain Monte Carlo methods for batch processing, and sequential Monte Carlo (particle filtering) methods for online processing. In the batch method, static parametric models are used for modeling the statistics of the speech and channel. Optimal parameter estimates are then used to enhance the observed signal. In the sequential approach, the clean speech signal is considered itself an unknown state. Various dynamical models and optimal sampling strategies are discussed for state estimation. The results demonstrate the superiority of the sequential method. 


\title{
Session 4aUWa
}

\section{Underwater Acoustics, Acoustical Oceanography, and ECUA: Impact of Internal Waves on Shallow Water Propagation}

\author{
James F. Lynch, Cochair \\ Woods Hole Oceanographic Institution, 98 Water Street, Bigelow 203A, MS-11, Woods Hole, MA 02543, USA \\ Thomas Folégot, Cochair \\ NATO Undersea Research Center, Viale San Bartolomeo 400, La Spezia, 19126, Italy
}

Invited Paper

8:00

\begin{abstract}
4aUWa1. Spatial and temporal sound field fluctuations due to propagating internal waves in shallow water. Mohsen Badiey (University of Delaware, College of Marine and Earth Studies, S. College Street, Newark, DE 19716, USA, badiey@udel.edu), Boris Katsnelson (Voronezh State University, 1, Universitetskaya sq., 394006 Voronezh, Russian Federation, katz@phys.vsu.ru), James F. Lynch (Woods Hole Oceanographic Institution, 98 Water Street, Bigelow 203A, MS-11, Woods Hole, MA 02543, USA, jlynch@whoi .edu)
\end{abstract}

Fluctuating three dimensional acoustic wave propagation in shallow water presents a challenge due to the anisotropic nature of the environment. Temporal and spatial changes in the water column caused by the passage of internal waves are among the the primary causes of the anisotropy. Three main mechanisms for the acoustic field variations have been determined: 1) adiabatic propagation,. 2) horizontal refraction, and 3) mode coupling. All these mechanisms provide different characteristics of the sound fluctuations, and depend on the angle between the internal wave direction and the source receiver propagation path, as well as frequency and mode number. In a recent multi-institutional shallow water experiment (SW06) a series of source-receiver positions were created to examine the effects of $3 \mathrm{D}$ environmental fluctuations on the propagation of low to mid frequency $(0.05-3 \mathrm{kHz})$ broadband acoustic signals while extensive environmental observations were made. These recent observational results confirm the above theoretical hypothesis. Work supported by ONR-321OA and RFBR.

\section{Contributed Papers}

\section{8:20}

4aUWa2. Sound intensity variations in the presence of shallow-water internal waves passing through acoustic track. Jing Luo (University of Delaware, College of Marine and Earth Studies, S. College Street, Newark, DE 19716, USA, luojing@udel.edu), Mohsen Badiey (University of Delaware, College of Marine and Earth Studies, S. College Street, Newark, DE 19716, USA, badiey@udel.edu), Entin A. Karjadi (College of Marine and Earth Studies, University of Delaware, Newark, DE 19716, USA, karjadi@udel.edu), Boris Katsnelson (Voronezh State University, 1, Universitetskaya sq., $394006 \quad$ Voronezh, Russian Federation, katz@phys.vsu.ru), Alexander Tshoidze (Voronezh State University, 1, Universitetskaya sq., 394006 Voronezh, Russian Federation, tshoidze@phys.vsu.ru), James Moum (College of Oceanic and Atmospheric Sciences, Oregon State University, 104 COAS Administration Bldg, Corvallis, OR 93371, USA, jmoum@coas.oregonstate.edu), James F. Lynch (Woods Hole Oceanographic Institution, 98 Water Street, Bigelow 203A, MS-11, Woods Hole, MA 02543, USA, jlynch@whoi.edu)

Fluctuations of low frequency pulses (LFM signals in $270-330 \mathrm{~Hz}$ band) in the presence of internal solitary wave (ISW) packet during the SW06 experiment are analyzed to quantify the interaction of sound with ISW field. Three situations during approximately 2 hours (20:30 - 22:30 GMT of August 17, 2006) are considered: a period when ISW was absent, a period when ISW started to intersect the acoustic track, and a period when ISW occupied the acoustic track. The propagation direction, velocity, and amplitude of the ISW were estimated from the on-board radar images recorded by two research vessels along with temperature records of sensors moored at the source, at the receiver, and between the source-receiver track. Modeal and frequency filtering of received pulses was carried out as well as analysis of temporal variations of the field depth distribution. This analysis allows us to identify two different acoustic fluctuation mechanisms: horizontal refraction and adiabatic variability, and it confirms the previously proposed theory on the sound field fluctuations due to ISW. Work was supported by ONR and RFBR.

\section{8:40}

4aUWa3. Short-range acoustic propagation through non-linear internal waves. Daniel Rouseff (Applied Physics Laboratory, University of Washington, 1013 NE 40th St, Seattle, WA 98105, USA, rouseff@apl.washington.edu), Dajun Tang (Applied Physics Laboratory, University of Washington, 1013 NE 40th St, Seattle, WA 98105, USA, djtang @apl.washington.edu), Kevin L. Williams (Applied Physics Laboratory, University of Washington, 1013 NE 40th St, Seattle, WA 98105, USA, williams@apl.washington.edu), James Moum (College of Oceanic and Atmospheric Sciences, Oregon State University, 104 COAS Administration Bldg, Corvallis, OR 93371, USA, jmoum@coas.oregonstate .edu), Zhongkang Wang (Hangzhou Applied Acoustics Research Institute, PO Box 1249, 310012 Hangzhou, China, zkwang@apl.washington.edu)

During the Shallow Water 2006 Experiment (SW06), mid-frequency acoustic transmission data were collected on a vertical array over a continuous 7-hour period at range $550 \mathrm{~m}$. The relatively short range was deemed desirable for studying the effects of internal waves; individual waves in a packet of nonlinear internal waves might be isolated between the acoustic source and receiver. Of present interest are data immediately before, during and after the passage of a non-linear internal wave on 18 August 2006. Among other features, the data show a new acoustic path being generated as the internal wave passes the acoustic source. A ray-based model is developed for the observed effect that uses as input nearby oceanographic measurements. [Work supported by ONR.] 


\section{9:00}

4aUWa4. Intensity fluctuations of mid-frequency sound signals passing through moving nonlinear internal wave in experiment sw06. Boris Katsnelson (Voronezh State University, 1, Universitetskaya sq., 394006 Voronezh, Russian Federation, katz@phys.vsu.ru), Valery Grigorev (Voronezh State University, 1, Universitetskaya sq., 394006 Voronezh, Russian Federation, grig@box.vsi.ru), James F. Lynch (Woods Hole Oceanographic Institution, 98 Water Street, Bigelow 203A, MS-11, Woods Hole, MA 02543, USA, jlynch@whoi.edu), Dajun Tang (Applied Physics Laboratory, University of Washington, 1013 NE 40th St, Seattle, WA 98105 , USA, djtang@apl.washington.edu)

Fluctuations of an intensity of the broadband pulses are studied in midfrequency area $(2-4.5 \mathrm{kHz})$ propagating in the shallow water in the presence of intensive internal waves (IW) moving approximately along an acoustic track. Theory elaborated earlier predicts that in this case specific features of fluctuations are provided by modes coupling (for low frequency sound) or ray scattering (high frequency area) and depend on direction of propagation of signals relative wave front of IW. The corresponding research was carried out during multi-institutional experiment sw06 in New Jersey shelf. We analyze temporal dependence of intensity for the sequence of the sound pulses radiated from the R/V Knorr during approximately one hour - 15:31 -16:20 GMT (August 13, 2006) and received by two separate single hydrophone units (SHRUs) placed at different distance from the source $(\sim 4 \mathrm{~km}$ and $\sim 12 \mathrm{~km})$. The corresponding acoustic tracks had a little different directions relative wave front of IW. Properties of IW were established using temperature records of sensors in different locations. It is shown that frequency spectra of fluctuations for these SHRUs have different predominating frequencies in accordance with mentioned directions of acoustic tracks. Results of measurements are compared with theoretical estimations and demonstrate good consistency.

\section{9:20}

4aUWa5. Simultaneous nearby measurements of acoustic propagation and high-resolution sound speed structure containing internal waves. Frank S. Henyey (Applied Physics Laboratory, University of Washington, 1013 NE 40th St, Seattle, WA 98105, USA, frank@apl.washington.edu) , Kevin L. Williams (Applied Physics Laboratory, University of Washington, 1013 NE 40th St, Seattle, WA 98105, USA, williams @apl.washington.edu), Dajun Tang (Applied Physics Laboratory, University of Washington, 1013 NE 40th St, Seattle, WA 98105, USA, djtang@apl .washington.edu)

On two occasions during the SW06 experiment, towed CTD chain measurements were made close to an acoustic propagation path. The acoustic path was $1 \mathrm{~km}$ long, oriented roughly in the direction of propagation of large nonlinear internal waves. On the first occasion, large nonlinear internal waves were absent, and on the second occasion, they were present. The CTD chain was towed in loops around the acoustic path, roughly $200 \mathrm{~m}$ on either side of the path. On the first occasion, 17 loops were made in about $5.5 \mathrm{hr}$, and on the second occasion, 7 loops were made in about $2.5 \mathrm{hr}$. Throughout these time periods, acoustic transmissions between $2 \mathrm{kHz}$ and $10 \mathrm{kHz}$ were carried out. The acoustic environment on the path is estimated by space and time interpolation between the tows on the two sides of the path. The acoustic data is compared with propagation modeling in this environment.

\section{9:40}

4aUWa6. The effects of non-linear internal wave curvature on acoustic propagation. Timothy Duda (Woods Hole Oceanographic Institution, 98 Water Street, Bigelow 107, MS-12, Woods Hole, MA 02543, USA, tduda@whoi.edu), James F. Lynch (Woods Hole Oceanographic Institution, 98 Water Street, Bigelow 203A, MS-11, Woods Hole, MA 02543, USA, jlynch@whoi.edu), Ying-Tsong Lin (Woods Hole Oceanographic Institution, 98 Water Street, Bigelow 107, MS-12, Woods Hole, MA 02543, USA, ytlin@whoi.edu), Arthur Newhall (Woods Hole Oceanographic Institution, 98 Water Street, Bigelow 203A, MS-11, Woods Hole, MA 02543 , USA, anewhall@whoi.edu), Hans Graber (RSMAS - University of Miami, 4600 Rickenbacher Causeway, Miami, FL 33149, USA, hgraber
@ rsmas.miami.edu),Micheal Caruso(RSMAS - University of Miami, 4600 Rickenbacher Causeway, Miami, FL 33149, USA, mcaruso@rsmas.miami .edu)

It is well known that coastal nonlinear internal waves can have curved wavefronts, due to either being generated at a "point source" such as a submarine canyon or valley or due to the wave being refracted by large scale water column or bathymetric features. We will explore the first case here, with the emphasis on circular wave fronts. Three cases of acoustic propagation in the vicinity of a circular wavefront will be considered: 1) the source within a soliton train, 2) the source in front of the soliton train, and 3) the source behind the soliton train. Simple geometric forms are presented showing how the curved wavefronts can lead to mode number and frequency dependent dispersion and shadowing effects. Work sponsored by Office of Naval Research.

\section{0:00}

4aUWa7. The effects of the internal waves on the statistic characteristics of the sound fields in the 2001 ASIAX South China Sea Experiment. Lixin Wu (National Laboratory of Acoustics, Institute of Acoustics, Chinese Academy of Sciences, NO.21, Bei-Si-huan-Xi Road, 100080 Beijing, China, wlx@mail.ioa.ac.cn), Jun Wang (National Lab. of Acoustics, Institute of Acoustics, Chinese Academy of Sciences, NO.21, Bei-Si-huan-Xi Road, 100080 Beijing, China, wangj@mail ioa.ac.cn), Shaoqiang Wang (National Lab. of Acoustics, Institute of Acoustics, Chinese Academy of Sciences, NO.21, Bei-Si-huan-Xi Road, 100080 Beijing, China, wsq@mail.ioa.ac.cn), James F. Lynch (Woods Hole Oceanographic Institution, 98 Water Street, Bigelow 203A, MS-11, Woods Hole, MA 02543, USA, jlynch@whoi.edu), Timothy Duda (Woods Hole Oceanographic Institution, 98 Water Street, Bigelow 107, MS-12, Woods Hole, MA 02543, USA, tduda@whoi.edu)

Internal waves are one of primary sources of ocean variations in shallow water. The temporal-spatial stability of sound channel may be degraded by the activities of internal waves. We present analyses of statistic characteristics observed in acoustic signals transmitted by two $400 \mathrm{~Hz}$ sources moored as part of ASIAEX 2001 South China Sea (SCS) experiment. One source was $31.3 \mathrm{~km}$ offshore from the receiving array, and the other was $20.6 \mathrm{~km}$ alongshore from the array. Time series of signal intensity measured at individual phones of a 16-element vertical line array and a 32-element horizontal line array. temporal-spatial correlations were observed from 2 May to 17 May 2001. The temporal-spatial decorrelation scales are closely related to internal wave properties. The largest internal wave, especially solitary wave packets, are the principal contributors to reduction of the temporal-spatial decorrelation scales. We also present elementary analyses of higher-order statistics (HOS), such as bispectrum, bicoherency coefficients, etc.

\section{0:20-10:40 Break}

\section{0:40}

4aUWa8. Observed acoustic arrival structure and intensity variability induced by transbasin nonlinear internal waves in the South China Sea basin. Chingsang Chiu (Naval Postgraduate School, Department of Oceanography, 833 Dyer Road, Rm 328, Monterey, CA 93943-5738, USA, chiu@nps.edu), D. Benjamin Reeder (Naval Postgraduate School, Department of Oceanography, 833 Dyer Road, Rm 328, Monterey, CA 939435738, USA, dbreeder@nps.edu), Christopher Miller (Naval Postgraduate School, Department of Oceanography, 833 Dyer Road, Rm 328, Monterey, CA 93943-5738, USA, cwmiller@nps.edu), Justin Reeves (Naval Postgraduate School, Department of Oceanography, 833 Dyer Road, Rm 328, Monterey, CA 93943-5738, USA, jmreeves@nps.edu), Steve Ramp (Naval Postgraduate School, Department of Oceanography, 833 Dyer Road, Rm 328, Monterey, CA 93943-5738, USA, sramp@nps.edu), Yiing-Jang Yang (Naval Academy, Department of Marine Science, P.O. Box 90175, Tsoying Kaohsiung, Taiwan, yjyang@cna.edu.tw), Ruey-Chang Wei (National Sun Yat-sen University, 70 Lien-Hai Road, Institute of Undersea Technology, 804 Kaohsiung City, Taiwan, rcwei@mail 
.nsysu.edu.tw), Chifang Chen(National Taiwan University, Dept. of Engineering Science and Ocean Engineering, University No. 1 Roosevelt Road, Section \#4, Taipei, Taiwan, chifang@ntu.edu.tw)

During the 2005-2006 Windy Island Nonlinear Soliton Experiment (WISE), large-amplitude internal waves were observed by in situ oceanographic sensors to propagate eastward from the Luzon Strait, through the deep basin, onto the shelf and Dongsha Plateau of the South China Sea year round except winter. In a parallel effort to measure the effects of these transbasin internal waves on long-range, low-frequency sound propagation, an acoustic source with a center frequency of $400-\mathrm{Hz}$ and bandwidth of $100-\mathrm{Hz}$ was moored on the west side of the deep basin transmitting a phasemodulated m-sequence signal every 15 min from February to October 2006. These periodic transmissions were recorded by a receiver moored $\sim 166 \mathrm{~km}$ to the east of the source. The recording was processed to give the arrival structure of a pulse and its temporal changes over the eight-month period. The observed temporal variability in the arrival structure and in the acoustic intensity were analyzed using time-series techniques and models with emphasis to elucidate the connection to the observed sound speed variability induced by the nonlinear transbasin internal waves. Results from the analysis are presented and discussed. [The research is sponsored by the US ONR and the Taiwan NSC.]

\section{1:00}

4aUWa9. Spatial and temporal coherence of low-frequency acoustic field in shallow water: Experimental results. Lianghao Guo (National Laboratory of Acoustics, Institute of Acoustics, Chinese Academy of Sciences, NO.21, Bei-Si-huan-Xi Road, 100080 Beijing, China, glh2002@mail.ioa.ac.cn), Zaixiao Gong (National Laboratory of Acoustics, Institute of Acoustics, Chinese Academy of Sciences, NO.21, Bei-Sihuan-Xi Road, 100080 Beijing, China, gzx@mail.ioa.ac.cn), Lixin Wu (National Laboratory of Acoustics, Institute of Acoustics, Chinese Academy of Sciences, NO.21, Bei-Si-huan-Xi Road, 100080 Beijing, China, wlx@mail.ioa.ac.cn), Xilu Li (National Laboratory of Acoustics, Institute of Acoustics, Chinese Academy of Sciences, NO.21, Bei-Si-huan-Xi Road, 100080 Beijing, China, glh2002@mail.ioa.ac.cn)

Spatial and temporal coherence of acoustic field has very important effects on applications of underwater acoustics. In this paper, recent experimental results of low-frequency signal coherence in shallow water are presented. For signals with low frequencies of $100 \sim 500 \mathrm{~Hz}$ in shallow water, the vertical correlation has no distinct depth dependence, but it has obvious range dependence. The horizontal correlation length is greater than 40 wavelengths, the temporal correlation length is greater than $510 \mathrm{~s}$ at frequency of $475 \mathrm{~Hz}$ and greater than 1800 s at frequency of $150 \mathrm{~Hz}$. These experimental results show that low-frequency acoustic field has strong spatial coherence and temporal stability in shallow water. [Work supported by the National Natural Science Foundation of China under Grant No.10734100]

\section{$11: 20$}

4aUWa10. Temporal correlation of MFP with the presence of internal waves. Zhenglin Li (Haikou Acoustic Lab. \& National Lab. of Acoustics, Inst. of Acoustics, Chinese Academy of Sciences, 63 Binghaidadao, 570105 Haikou, China, lzhl@mail.ioa.ac.cn), Guihua Ji (Haikou Acoustic Lab. \& National Lab. of Acoustics, Inst. of Acoustics, Chinese Academy of Sciences, 63 Binghaidadao, 570105 Haikou, China, jgh@mail.ioa .ac.cn), Qiongxing Dai (Haikou Acoustic Lab. \& National Lab. of Acoustics, Inst. of Acoustics, Chinese Academy of Sciences, 63 Binghaidadao, 570105 Haikou, China, dqx66@163.com)

Internal wave is a dominant source of ocean uncertainties in shallow waters. The ability of passive source localization may be degraded due to mismatch between model predictions and measurements caused by the activities of internal waves. Using ocean environment measurements from an experiment, the effects of Garrett-Munk and solitary internal waves on the temporal correlation of matched-field processing (MFP) in shallow water for sources with different frequencies and different depths are numerically investigated. It is shown that the temporal correlation of MFP decreases as the amplitude of solitons or the average energy density of linear internal waves increased. For a source with lower frequency or located below the thermocline, the temporal correlation of MFP is less affected by internal waves, and the length of which is longer. Moreover, the effects of the range between solitons and source on the temporal correlation of MFP are relatively small.

\section{$11: 40$}

4aUWa11. Observations of noise generated by nonlinear internal waves on the continental shelf during the SW06 experiment. Andrey $\mathrm{N}$. Serebryany (Woods Hole Oceanographic Institution, 98 Water Street, Bigelow 203A, MS-11, Woods Hole, MA 02543, USA, aserebryanyy@whoi.edu), Arthur Newhall (Woods Hole Oceanographic Institution, 98 Water Street, Bigelow 203A, MS-11, Woods Hole, MA 02543, USA, anewhall@whoi.edu), James F. Lynch (Woods Hole Oceanographic Institution, 98 Water Street, Bigelow 203A, MS-11, Woods Hole, MA 02543, USA, jlynch@whoi.edu)

As part of the Shallow Water 2006 (SW06) experiment, simultaneous measurements of coastal internal wave oceanography and ocean acoustics were made over a two month period in late summer. The generation of noise by nonlinear internal waves propagating on the shelf during the SW06 experiment was observed, and is reported upon here. Three main types of noise were observed: bed noise, mid-column noise and noise from the sea surface. Surface noise is created due to an enhancement of surface wave breaking in the convergence zone created by the internal waves. Strong broadband bed noise was observed during the moments of internal wave passage above a horizontal array of hydrophones lying on the bottom. Appearance of bed noise in the form of several spikes we observed coinciding with the strongest bottom currents created by internal wave orbital currents. For the case of breaking internal waves, the near-bed spike-like noise disappeared and mid-column noise of a different character was seen instead. Mechanisms of the various types noise generation will be discussed. Work sponsored by ONR.

\section{2:00}

4aUWa12. Applying the Data Nullspace Projection Method to a Geoacoustic Bayesian Inversion in a Randomly Fluctuating Shallow-Water Ocean. James F. Lynch (Woods Hole Oceanographic Institution, 98 Water Street, Bigelow 203A, MS-11, Woods Hole, MA 02543 , USA, jlynch@whoi.edu), Ying-Tsong Lin (Woods Hole Oceanographic Institution, 98 Water Street, Bigelow 107, MS-12, Woods Hole, MA 02543, USA, ytlin@whoi.edu), Arthur Newhall (Woods Hole Oceanographic Institution, 98 Water Street, Bigelow 203A, MS-11, Woods Hole, MA 02543, USA, anewhall@whoi.edu)

Bayesian inversion techniques which are commonly used in geoacoustic inversion can suffer the effects of uncertain water-column fluctuations. To reduce these effects, one could also invert for the fluctuating water-column parameters; however, there are issues with this approach. One obvious problem is that the dimensions of parameter space will increase, so that Bayesian inversion may not be efficient. Another issue arises from the temporal and spatial randomness and variability of the water-column parameters; this requires extra effort in handling the randomness and variability in the inversion procedure. In this paper, we propose another approach to the problem. The data nullspace projection method, which has been applied to perturbative inversion, is extended to Bayesian inversion using acoustic modal wavenumbers and group velocities. The idea of this method is to project acoustic data onto a subspace that is insensitive to uncertain water-column fluctuations, and use the projected data to invert for bottom properties. The advantage of this approach is that we do not need to invert for water-column parameters, so that the inversion requires less operations than the previous approach. A numerical simulation demonstrates the feasibility of the projection approach. It is then applied to real data collected in the SW06 experiment.

\section{2:20}

4aUWa13. Acoustic propagation modeling in the presence of environmental uncertainty. Yu Yu Khine (USNRL, 4555 Overlook Ave SW, Washington, DC 20375, USA, yuyu.khine@nrl.navy.mil), Steven 
Finette(USNRL, 4555 Overlook Ave SW, Washington, DC 20375, USA, steven.finette@nrl.navy.mil), Roger Oba (USNRL, 4555 Overlook Ave SW, Washington, DC 20375, USA, roba@wave.nrl.navy.mil)

Due to incomplete knowledge of ocean environments, this research incorporates environmental uncertainty into an acoustic model representing wave propagation in order to quantitatively represent the uncertainty of the acoustic field. The waveguide considered here has a spatially varying, uncertain sound speed distribution with a known correlation length. KarhunenLoeve and polynomial chaos expansions are used to represent the uncertainty in the environment and acoustic field, respectively, in a narrow- angle parabolic wave equation. In this two-dimensional model, the water depth is $150 \mathrm{~m}$ and propagation is over a range of $20 \mathrm{~km}$. The environmental uncertainty term in the wave equation is assumed to vary randomly in the range direction, and is characterized by an exponentially decaying correlation function. An implicit finite difference scheme is used to solve a set of coupled differential equations for the stochastic envelope function at different source frequencies in the range of a few hundred Hz. The simulated results will include probability density functions at selected spatial locations in the waveguide, first and second moments of the field, and these results will be compared with those obtained independently from Monte Carlo samplings from the same ocean environment. Work supported by ONR.

12:40-1:40 Lunch Break

\section{Contributed Papers}

\section{$1: 40$}

4aUWa14. New Numerical Computation of Acoustic Propagation in the Ocean in the Presence of Internal Waves is 1000 Times Faster than Traditional Split-Step Fourier Approaches. Alfred R. Osborne (University of Torino, Dipartimento di Fisica Generale, Via Pietro Giuria 1, 10124 Torino, Italy, al.osborne@gmail.com)

A new numerical model for acoustic propagation based upon the large angle parabolic type equations is found to execute about 1000 times faster than the FFT split step algorithm. The approach is applied to the imaging and nonlinear filtering of internal waves in shallow water regions for 3-dimensional propagation of acoustic waves. For appropriate array placement one can construct the inverse problem and hence the internal wave field from the acoustic measurements themselves. The unique nonlinear filtering method allows one to filter out the internal waves from the density field and hence to realized acoustic wave propagation in the absence of the internal waves. The hyperfast acoustic model has some of the following characteristics: (1) The work can be extended to the fully 3-dimensional Helmholtz equation. (2) The method is a kind of multi-dimensional Fourier analysis which exactly solves the wave equations (PE, large angle PEs and Helmholtz)) so that the acoustic wave does not degrade with range as with more traditional numerical integrations. (4) Multi-dimensional Fourier transforms can be used to nonlinearly filter acoustic signals in a wide range of applications and hence the approach is quite robust to interference from background acoustic noise.

\section{2:00}

4aUWa15. Modeling probability density functions for acoustic propagation through internal waves in shallow water environments. Kevin D. Lepage (Naval Research Laboratory, 4555 Overlook Ave SW, Washington, DC 20375, USA, kevin.lepage@nrl.navy.mil)

Acoustic propagation through internal waves is shallow water introduces randomness to the acoustic field in that internal waves are generally incompletely measured and are therefore best understood as random realizations of a stochastic process. Due to the physics of internal waves these environmental perturbations are expressed in a finite number of modes, each of which affects acoustic propagation differently. As acoustic propagation in shallow water is itself confined into modes, a matrix of interaction strengths for both the accumulated phase and the mode coupling may be derived which can be integrated forward along the acoustic path to account for the accumulation of uncertainty. A powerful ansatz for solving this problem is a Polynomial Chaos (PC) expansion of the complex modal amplitudes in the random variables which are the internal wave amplitudes. In this work the PC technique is used to derive the probability density functions of the complex modal amplitudes in the presence of a homogeneous internal wave field, showing good agreement with Monte-Carlo results. The pdfs of the complex modal amplitudes can then be combined to obtain the pressure field pdf as well as various statistics of interest such as the scintillation index. [Work supported by ONR]

\section{2:20}

4aUWa16. A modified dnoidal model for internal solitary waves and its effect on sound transmission. Shaoqiang Wang (National Lab. of Acoustics, Institute of Acoustics, Chinese Academy of Sciences, NO.21, Bei-Sihuan-Xi Road, 100080 Beijing, China, wsq@mail.ioa.ac.cn), Lixin Wu (National Laboratory of Acoustics, Institute of Acoustics, Chinese Academy of Sciences, NO.21, Bei-Si-huan-Xi Road, 100080 Beijing, China, wlx@mail.ioa.ac.cn), Changqing Hu (Shanghai Acoustics Lab., Chinese Academy of Sciences, No.456, Xiao-Mu-Qiao Road, Xu-Hui District, 200032 Shanghai, China, hchq@mail.ioa.ac.cn)

A new solution of KDV equation in the form of dnoidal function is developed in this paper. Based on this new solution, a modified solution of KDV equation with slowly varying parameters in the form of dnoidal function is derived. Numerical simulation shows that the wave pockets scale of the modified solution is only $1 / 5$ to the solution in the reference (JASA, 108 (3), pp 957-972), and also the equilibrium positions are different. Comparing the solutions with slowly varying parameters with the published data of internal solitary waves in the ocean indicates that the modified solution is better for the description of the dnoidal wave. Based on the modified solution of KDV equation with slowly varying parameters, a modified model for dnoidal wave is given and its effect on sound transmission is discussed in this paper. 


\title{
Session $4 \mathrm{aUWb}$
}

\section{Underwater Acoustics and ECUA: Determination of Acoustic Properties of Materials for Sonar Applications I}

\author{
Kenneth G. Foote, Cochair \\ Woods Hole Oceanographic Institution, Woods Hole, MA 02543, USA \\ Stephen P. Robinson, Cochair \\ National Physical Laboratory, Hampton Road, Teddington, TW11 OLW, UK
}

Invited Papers

8:00

4aUWb1. Phase change measurement, sound speed and attenuation determination from underwater acoustic panel tests. Jean Piquette (Naval Undersea Warfare Center, 1176 Howell Street, Newport, RI 02841, USA, paoleroae@npt.nuwc.navy.mil), Anthony Paolero (Naval Undersea Warfare Center, 1176 Howell Street, Newport, RI 02841, USA, paoleroae@npt.nuwc.navy.mil), Robert Drake (Naval Undersea Warfare Center, 1176 Howell Street, Newport, RI 02841, USA, Drakerm@npt.nuwc.navy.mil)

Material measurements in underwater acoustics are employed regularly for characterization of materials used as acoustic windows. Various techniques have been developed to measure the acoustic attenuation magnitudes of materials. Recently, there has been increased interest in window configurations of complex geometries and of phase response of that material to an acoustic wave. This latter phase parameter can be of high significance for acoustic devices composed of an array of elements. Thus, the phase response of the attenuation is needed to properly characterize material performance on the overall system. This translates into a primary need to measure the complex acoustic attenuation of panels at various angles of incidence. This paper summarizes the methodology to measure the complex attenuation of materials. Case examples of test materials measured will be presented; comparisons to theoretical response will be provided. Considerations given to rigging and required acoustic settling times are discussed. Expansion of the methodology that incorporates a causal relationship between the measured phase response and attenuation to the sound speed of the material will be developed. The model relating these parameters and their implementation via an iterative least squares fitting of the parameters to the measurement data will be discussed.

\section{8:20}

4aUWb2. The underwater acoustic testing of modest sized panel materials using a multi-element array technique in a laboratory test vessel. Michael J. Martin (QinetiQ Ltd, Rm 1146, Bldg A7, Cody Technology Park, Ively Road, GU14 OLX Farnborough, UK, mjmartin1@qinetiq.com), Stephen P. Robinson (National Physical Laboratory, Hampton Road, TW11 OLW Teddington, UK, Stephen.Robinson@npl.co.uk), John Smith (DSTL, Rm 14, Bldg 352, Porton Down, SP4 0JQ Salisbury, UK, jdsmith@dstl.gov.uk), Victor F. Humphrey (Institute of Sound and Vibration, Univ. of Southampton, University Road, Highfield, SO17 1BJ Southampton, UK, vh@isvr.soton.ac.uk)

The underwater acoustic properties of materials can be assessed in panel form via a simple single hydrophone measurement technique which is ideally conducted on large test panels in open water. Alternatively measurements may be conducted in a laboratory test vessel which is capable of simulating ocean conditions. However, physical limitations imposed by the constraints of the vessel, including a reduction in test panel dimensions, require modification of the traditional technique to allow measurements to be made down to low kilohertz frequencies. This can be achieved by, in addition to a directional source, the use of a directional receiver in the form of a planar multi-hydrophone array. The technique is described and illustrated with measurements of reflection loss and insertion loss between $1 \mathrm{kHz}$ and $7 \mathrm{kHz}$ performed on a test panel in a pressure vessel through changing hydrostatic pressure from ambient to 2.8 $\mathrm{MPa}$. The efficacy of this technique is considered with simulated multi-hydrophone array measurements on a scaled panel in an open tank.

\section{$8: 40$}

4aUWb3. Panel transmission measurements: The influence of the non plane wave nature of the incident field. Victor F. Humphrey (Institute of Sound and Vibration, Univ. of Southampton, University Road, Highfield, SO17 1BJ Southampton, UK, vh@isvr.soton.ac.uk), John Smith (DSTL, Rm 14, Bldg 352, Porton Down, SP4 0JQ Salisbury, UK, jdsmith@dstl.gov.uk)

For reasons of cost and practicality, laboratory measurements of the acoustic transmission and reflection properties of materials for use in underwater applications are typically performed on samples of limited dimensions - and with the source and receiver separated by relatively short distances - resulting in a non planar measurement field. The influence of this on the resulting measurements is investigated in this paper. In particular, for low frequency measurements the influence of the evanescent wave contributions can become significant. In this paper two alternative approaches are used to evaluate the transmission properties. The first method decomposes the incident spherical wave into its plane wave components and integrates the resulting transmitted waves numerically to evaluate the transmitted field. The second approach uses an asymptotic expansion of the field in terms of wave front curvature: bounds are then 
placed on the error in this expansion at low frequency using thin plate theory. Results are compared and contrasted for measurements in the frequency range 1 to $60 \mathrm{kHz}$ for panels of simple elastic materials (steel and Perspex (polymethylmethacrylate)). In addition the nature and significance of the modes of the panel for evanescent waves are considered. The consequences for laboratory measurements are also outlined.

\section{9:00}

4aUWb4. Comparisons of the dynamic moduli of various polymers. John Smith (DSTL, Rm 14, Bldg 352, Porton Down, SP4 0JQ Salisbury, UK, jdsmith@dstl.gov.uk)

To fully characterise the elastic properties of a homogeneous, isotropic material, two independent elastic constants are needed, usually the bulk and shear moduli. For the types of polymers used in sonar applications, these moduli are typically both frequency and temperature dependent. Of particular interest is the position (in frequency and temperature) of peak loss tangent, which is related to the glass transition temperature through the free volume concept. Although there exists in the literature a lot of data on the dynamic shear (and Young's) modulus for various polymers, data on dynamic bulk modulus is scarcer, due to the difficulty of the measurement. This paper reviews the current literature on the dynamic moduli of various polymers, with particular regard to the relationship between the peak loss tangents of the bulk and shear moduli. Simple relaxation models are studied to give insight on the factors affecting the peak of the loss tangent and new measurements on a nitrile-butadiene rubber compound are presented.

\section{9:20}

4aUWb5. Pulse tube measurement of bulk modulus of visco-elastic composite materials: Theory and practice. Peter $R$ Brazier-Smith (Thales, Ocean House, Somerset, BA8 0DH Templecombe, UK, peter.brazier-smith@uk.thalesgroup.com), Allan R. Clark (Thales, Ocean House, Somerset, BA8 0DH Templecombe, UK, allan.clark@uk.thalesgroup.com)

A method for the determination of the bulk moduli and loss factors of micro-voided composite materials is presented. The method requires that the reflection and transmission coefficients of a tile of uniform thickness are determined in both amplitude and phase as functions of frequency. Reduction to the bulk modulus and loss factor then proceeds by using the analytic properties of a function of a complex variable derived from the reflection and transmission coefficients. A pulse tube is used for the determination of the complex reflection and transmission coefficients. Although other measurement techniques are available, the pulse tube has proved to be versatile in covering a large range of the frequency-temperature master curve for typical composite materials used in underwater acoustics. It achieves this versatility by using an anti-freeze/water mixture as the medium following which measurements can be made over a range of different temperatures.

\section{Contributed Papers}

\section{9:40}

4aUWb6. Determining dynamic viscoelastic properties without time-temperature shifting. Walter M. Madigosky (Catholic University of America, Department of Physics, Washington, DC, DC 20064, USA, wmadigosky@yahoo.com), Gilbert F. Lee (NSWCCD, 9500 McArthur Boulevard, Bethesda, MD 20817, USA, gilbert.f.lee@ navy.mil), Jan M. Niemiec (NSWCCD, 11798 Fox Rest Court, New Market, MD 21774, USA, jan301@ comcast.net)

A novel unbiased procedure to analyze dynamic mechanical data of rheologically simple viscoelastic polymers by modeling the data with the Havriliak and Negami (HN) equation is described. The real and imaginary parts of the HN equation are used to solve for the global frequency-time dependent parameter $(\omega \tau)$ for all the data thereby uniquely determining the time- temperature shift function. Displaying the experimental data in the form of a wicket or Argand diagram provides initial estimates for the HN parameters. An unbiased error analysis is performed to minimize the difference between experimental and calculated complex viscoelastic values. Finally the characteristic relaxation time, $\boldsymbol{t} b\rangle\langle 0\rangle$, is determined by minimizing the error between the calculated data and experimental data at the reference temperature $\langle\mathrm{T}\rangle\langle 0\rangle$. Using this procedure, the complete master curve is generated without the need for overlapping frequency data and the procedural error and operator bias associated with time-temperature shifting is eliminated. The technique can also generate a complete frequency spectrum from isochronal temperature scans such as those obtained from a torsion-pendulum, rheovibron or a Dynamic Mechanical Analyzer (DMA) apparatus, which is not otherwise possible.

\section{0:00}

4aUWb7. A new integral equation method for elastic composites. Natasha J. Willoughby (The University of Manchester, School of Mathematics, Oxford Road, M13 9PL Manchester, UK, Natasha.J.Willoughby @ student.manchester.ac.uk), William J. Parnell (The University of Manchester, School of Mathematics, Oxford Road, M13 9PL Manchester, UK, William.Parnell@manchester.ac.uk), I David Abrahams (The
University of Manchester, School of Mathematics, Oxford Road, M13 9PL Manchester, UK, David.Abrahams@manchester.ac.uk)

A new integral equation approach to elastodynamic homogenization has been proposed [1] to determine the effective properties of periodic fibre reinforced composite materials in the case of $\mathrm{SH}$ wave propagation. When all the fibres are aligned in the same direction and considered infinite in extent, the microstructure is two-dimensional. The integral equation method is based on Navier's equations of elasticity in integral equation form, and uses the notion of separation of scales and averaging to find explicit expressions for the effective properties in a convenient form. Here we discuss an extension to the in-plane problem: the response of periodic fibre reinforced composites to time harmonic (low frequency) P/SV wave propagation. For simplicity, we assume all fibres are identical and of circular cross-section, and that both host and fibre phases are isotropic; additionally we restrict attention to lattice geometries which result in, at most, orthotropic elastic symmetry. The governing equations are presented and the effective quasistatic material properties are determined via the integral equation methodology. Results are presented for the effective material properties and compared with existing methods. [1] W.J. Parnell and I.D. Abrahams. "A new integral equation approach to elastodynamic homogenization". Submitted to Proc. Roy. Soc. A, 2007.

\section{0:20-10:40 Break}

\section{0:40}

4aUWb8. Velocity dispersion analysis of acoustic scattering wave from elastic shells. Cao Zhengliang (State Key Lab Ocean Acoustics, Hangzhou Appl Acous Res Inst, Rm 1510 No 96 HuaXing Rd, 310012 Hangzhou, China, caozhengliang@yahoo.com), Du Shuanping (State Key Lab Ocean Acoustics, Hangzhou Appl Acous Res Inst, Rm 1510 No 96 HuaXing Rd, 310012 Hangzhou, China, skldusp@tom.com), Zhou Shihong (State Key Lab Ocean Acoustics, Hangzhou Appl Acous Res Inst, Rm 1510 No 96 HuaXing Rd, 310012 Hangzhou, China, shih_zhou@yahoo.com.cn), Cong 
Weihua(State Key Lab Ocean Acoustics, Hangzhou Appl Acous Res Inst, Rm 1510 No 96 HuaXing Rd, 310012 Hangzhou, China, cwhfrj@hzcnc $. \mathrm{com})$

In recent years the analysis of velocity dispersion of circumferential waves (or surface waves), excited by an incident plane wave to an elastic cylindrical or spherical shell, has theoretically provided valuable insight into the underlying mechanisms of scattering. In the present study, an approach of velocity dispersion analysis on acoustic scattering wave is advanced for the data induced by a spectral bandwidth signal. The method, based on a signal processing technique in time-frequency domain, is examined with simulating waveforms scattered from an elastic spherical and cylindrical shell respectively by linear frequency-modulated signal. In addition, the forward scattering waves of an elastic cylindrical shell filled with fluid is obtained by a line horizontal array in laboratory waveguide, and the results of velocity dispersion is compared with that of theoretical calculation. [Work supported by the National Natural Science Foundation of China (Grant No. 10704068)]

\section{1:00}

4aUWb9. Experimental study of piezoelectret foams as underwater sensors. Michael Haberman (The University of Texas, Applied Research Laboratories, 10000 Burnet Road, Austin, TX 78758, USA,
haberman@arlut.utexas.edu),Steven Embleton(The University of Texas, Applied Research Laboratories, 10000 Burnet Road, Austin, TX 78758, USA steve.embleton@arlut.utexas.edu)

This work explores the use of piezoelectret foams (PF) as acoustic sensors for underwater applications. PF material (Emfit Ltd.) provides an intriguing alternative to piezoelectric ceramics and piezopolymers due to their low density, minimal thickness, and potential for easily creating sensors of complex geometries. The foams consist of a continuous polymer containing electrically polarized elliptical voids. Typical density, thickness, and low frequency receive voltatge sensitivity (RVS) values of these foams are 300 $\mathrm{kg} / \mathrm{m} 3,80 \mu \mathrm{m}$, and $-175 \mathrm{~dB}$ re $1 \mathrm{~V} / \mu \mathrm{Pa}$, respectively. This work presents experimentally obtained RVS of piezoelectret sensors with rigid and pressure release backing. The results show PF sensor RVS is comparable to conventional sonar transducers at low ambient pressures with RVS degradation as ambient pressure increased. The results are compared with theoretical predictions using microelectromechanical mean field theory and equivalent circuit models. Theoretical predictions explain the observed sensitivity degradation due to void closure caused by the applied pressure. To overcome the performance degradation, two pressure tolerant sensor design concepts are proposed and tested. The designs aim to employ PF elements for high ambient pressure applications while leveraging foam density and thickness to create lightweight, low profile sensors.

\title{
Session 4aUWc
}

\section{Underwater Acoustics and ECUA: Geoacoustic Sediment Modeling III}

\author{
Nicholas P. Chotiros, Cochair \\ Applied Research Laboratories, University of Texas, PO Box 8029, Austin, TX 78713-8029, USA \\ Jean-Pierre Sessarego, Cochair \\ Laboratory for Mechanics and Acoustics CNRS, 31 chemin Joseph Aiguier, Marseille, 13009, France
}

\section{Contributed Papers}

\section{8:00}

4aUWc1. Measuring grain roughness for the purpose of high-frequency acoustic modeling. Kevin Briggs (Naval Research Laboratory, Marine Geosciences Division, Stennis Space Center, MS 39529 5004, USA, kbriggs@nrlssc.navy.mil), Allen Reed (Naval Research Laboratory, Marine Geosciences Division, Stennis Space Center, MS 395295004, USA, areed@nrlssc.navy.mil), Richard Ray (Naval Research Laboratory, Oceanography Division, Stennis Space Center, MS 39529-5004, USA, Ricky.Ray@nrlssc.navy.mil), Michael Richardson (Naval Research Laboratory, Marine Geosciences Division, Stennis Space Center, MS 39529. 5004, USA, Mike.Richardson@nrlssc.navy.mil)

Grain roughness and packing may be important sediment properties for newer acoustic models. We present Scanning Electron Stereomicroscopic imagery of natural sand grains of varying shape and roughness for evaluation of potential grain interactions of individual grains. The grain shape and presence of microasperities will determine the probability of the contact with surrounding grains being a point or a broader area. Moreover, the use of a micro-roughness power spectrum in characterizing the sand grain roughness may be appropriate. Grain contact information is an essential starting point for developing and evaluating acoustic models that address acoustic losses at high frequencies. This information provides the basis to understand contact mechanics, such as grain slip and frame dilation, during insonification. Because media frame stiffness depends, at the grain scale, on the number and type of grain-to-grain contacts, ultimately we would like to characterize sand sediments with imagery identifying such grain contact information. The natural shapes of grains, their variations, and their packing density present a variety of non-ideal (point) contacts. X-ray micro-focus Computed Tomography shows great promise for documenting of the number and size (area) of grain contacts within grain aggregations, and a demonstration of these data will also be presented.

\section{8:20}

4aUWc2. An in situ sediment sound speed measurement platform Design, operation and experimental results. Jie Yang (Applied Physics Laboratory, University of Washington, 1013 NE 40th Street, Seattle, WA 98105, USA, jieyang@apl.washington.edu), Dajun Tang (Applied Physics Laboratory, University of Washington, 1013 NE 40th St, Seattle, WA 98105 , USA, djtang@apl.washington.edu), Kevin L. Williams (Applied Physics Laboratory, University of Washington, 1013 NE 40th St, Seattle, WA 98105 USA,williams@apl.washington.edu)

A unique Sediment Acoustic-speed Measurement System (SAMS) was developed to directly measure sediment sound speed. The system consists of ten fixed sources and one receiver. In a typical deployment, the SAMS is deployed from a ship that is dynamically positioned. The sources are arranged just above the sea bottom and the receiver is drilled into the sediment with controlled steps by a vibro-core. The maximal sediment penetration depth is 3 meters. At each receiver depth, the 10 sources transmit to the receiver at different angles in the frequency range of $2-35 \mathrm{kHz}$, providing 10 estimates of sound speed through time-of-flight measurements from the known source-to-receiver geometry. SAMS was deployed three times during 
the recent Shallow Water Experiment 2006 (SW06) on the New Jersey shelf at $80 \mathrm{~m}$ water depth. Preliminary results of sediment sound speed are 1618 $\pm 11,1598 \pm 10$, and $1600 \pm 20 \mathrm{~m} / \mathrm{s}$ at three separate deployment locations. Little dispersion in sediment sound speed was observed. (Work supported by ONR)

\section{8:40}

4aUWc3. Estimating sediment speed and attenuation with sub-bottom reflections. Kunde Yang (Institute of Acoustic Engineering, Northwestern Polytechnical University, 710072 Xi'an, China, ykdzym@nwpu .edu.cn), Ross Chapman (University of Victoria, 3800 Finnerty Rd, Vcitoria, BC V8W 3P6, Canada, chapman@uvic.ca), Yuanliang Ma (Institute of Acoustic Engineering, Northwestern Polytechnical University, 710072 Xi’an, China, ylma@nwpu.edu.cn)

An inversion method based on sub-bottom reflection is investigated using LFM data collected by a Vertical Linear Array from the SW06 experiment. The distance between the LFM source and the array is about $230 \mathrm{~m}$. After extracting the impulse response with matched filter from the received signal, the sub-bottom reflections were found to be strong. The chirp sonar survey nearby the experiment site showed that there is a prominent shallow sub-bottom $\mathrm{R}$ reflector with about $20 \mathrm{~m}$ depth (based on 1500 $\mathrm{m} / \mathrm{s}$ ). The relative arrival time and amplitude of the sub-bottom reflection signals were used to estimate the sound speed and the attenuation of the sediment. Because the direct arrivals in the water were influenced strongly by the internal wave, the first bottom reflection was applied as the reference path to calculate the relative arrival time and absorption loss in sediment. The sediment attenuation was estimated by assuming that it had linear frequency dependence. The estimated values of the sediment sound speed and attenuation were compared with matched field geoacoustic inversion results published by other research group.

\section{9:00}

4aUWc4. Shear wave speed increases with depth to the one-sixth power in sandy/silty marine sediments. Allan D. Pierce (College of Enginering, Boston University, 110 Cummington St, Boston, MA 02215, USA, adp@bu .edu), William M. Carey (College of Enginering, Boston University, 110 Cummington St, Boston, MA 02215, USA, wcarey@bu.edu)

Gravity holds the sediment's particles in loose contact; the strength varies with depth. The distribution in shapes, sizes, and orientations is presumed independent of depth. Each particle is subject to several contact forces, and also to a buoyancy force exerted by the surrounding water. An externally imposed shear stress results in distortions in the individual grains, the nature and magnitudes of which depend on the contact areas between the grains, which in turn depend on depth. A derivation making use of fundamdental mechanics, the theory of elasticity, and Hertz's theory of contact yields shear modulus $G$ as a dimensionless quantity times $g^{1 / 3}\left(\rho_{s}-\rho_{w}\right)^{1 / 3} E^{2 / 3} d^{1 / 3}$, where $\mathrm{d}$ is depth into the sediment and $\mathrm{E}$ is the elastic modulus of the solid material in the grains. The dimensionless quantity depends on Poisson's ratio and porosity. The shear speed $\left(G / \rho_{\mathrm{av}}\right)^{1 / 2}$ consequently varies with depth as $d^{1 / 6}$. The prediction is consistent with data reported in the past by Stoll, Yamamoto, and Hamilton; the discrepancy of the theoretical prediction of 0.167 with experimentally derived exponents of the order of 0.25 , although not viewed as significant, is discussed, and it is suggested that such may be caused by the variation of porosity with depth.

\section{9:20}

4aUWc5. Measuring attenuation and velocity within unconsolidated lacustrine sediments, using high-resolution seismic data. Luke J. Pinson (National Oceanography Centre, University of Southampton Waterfront Campus, European Way, SO14 3ZH Southampton, UK, ljwp101@soton.ac.uk), Timothy J. Henstock (National Oceanography Centre, University of Southampton Waterfront Campus, European Way, SO14 3ZH Southampton, UK, then@noc.soton.ac.uk), Justin K. Dix (National Oceanography Centre, University of Southampton Waterfront Campus, European Way, SO14 3ZH Southampton, UK, jkd@noc.soton.ac.uk), Jonathan M. Bull (National Oceanography Centre, University of Southampton Waterfront Campus, European Way, SO14 3ZH Southampton, UK, jmb1@noc .soton.ac.uk), Angus I. Best (National Oceanography Centre, University of
Southampton Waterfront Campus, European Way, SO14 3ZH Southampton, UK, aib@noc.soton.ac.uk)

We present estimates of in-situ compressional-wave attenuation and velocity within the uppermost $30 \mathrm{~m}$ of unconsolidated lacustrine sediments within Lake Windermere (U.K.), using high-resolution seismic data acquired with Boomer and Chirp sources. The wide frequency bandwidth of the Chirp source allows attenuation to be examined over a frequency range of approximately $2-9 \mathrm{kHz}$, and by using a spectral ratio technique incorporating robust re-weighted least squares regression, the apparent quality factor of sediments can be accurately and precisely determined. A $60 \mathrm{~m}$ multi-channel streamer used with the Boomer source allows interval velocities between target reflectors to be obtained. Models relating quality factor to mean grainsize distinguish between coarse grain-dominated and clay-dominated sediments. The interval velocities improve the classification of the sediment sequences to clays, laminated silts \& sands, and coarse sand and gravel deposits. The results are evaluated against geological and core data.

\section{9:40}

4aUWc6. Environmental effects on frequency behavior of modal attenuation coefficients for sandy bottoms. Wendy Saintval (University of Miami, RSMAS, 4600 Rickenbacker Causeway, Miami, FL 33149, USA, wsaintval@hotmail.com), William M. Carey (College of Enginering, Boston University, 110 Cummington St, Boston, MA 02215, USA, wcarey@bu.edu), Allan D. Pierce (College of Enginering, Boston University, 110 Cummington St, Boston, MA 02215, USA, adp@bu.edu), James F. Lynch (Woods Hole Oceanographic Institution, 98 Water Street, Bigelow 203A, MS-11, Woods Hole, MA 02543, USA, jlynch@whoi.edu), William L. Siegmann (Rensselaer Polytechnic Institute, 110 8th Street, Troy, NY 12180, USA, siegmw@rpi.edu)

The modal attenuation coefficients (MACs) can be determined using a recent simplification of Biot theory [A.D. Pierce et al., J. Acoust. Soc. Am. 114, 2345 (2003)]. Numerical calculations use sandy bottom sediments and isospeed, linear, and piecewise linear water profiles, which are simplifications that preserve key features of those obtained in experiments off the New Jersey Shelf. The calculations indicate the importance of downward refracting profiles and the strength of near-interface gradients for increasing energy loss. Principal characteristics of the MACs that are observed from the calculations include: increases with interface gradient, reordering of least attenuated modes, and variations of the frequency power-law exponents of the MACs from $f^{1}$ to $f^{1}$ at frequencies up to $2 \mathrm{kHz}$. Evidence of the behavior observed in the calculations is in good agreement with previous analysis of results in Gulf of Mexico experiments [F. Ingenito, J. Acoust. Soc. Am. 53, 858--863 (1973)], for profiles that were classified as weakly downward refracting or nearly isospeed. [Work partially supported by ONR.]

\section{0:00}

4aUWc7. What is the spatial volume involved for wave reflection from flat and curved interfaces? Paul Cristini (CNRS-UMR5212 Modélisation et Imagerie en Géosciences, UPPA BP115, 64013 Pau, France, paul.cristini@univ-pau.fr), Nathalie Favretto-Cristini (CNRS-UMR5212 Modélisation et Imagerie en Géosciences, UPPA BP115, 64013 Pau, France, nathalie.favretto@univ-pau.fr), Eric De Bazelaire (11, Route du Bourg, 64230 Beyrie-en-Béarn, France, edebaz@wanadoo.fr)

The spatial region in the vicinity of the interface which actually affects the interface response, and hence the reflected wavefield, is of particular interest for the characterization of reflectors. This region represents a volume of integration of properties above and beyond the interface whose maximum lateral extent corresponds to the lateral extent of the Interface Fresnel Zone (IFZ), and whose maximum vertical extent is equal to a thickness we evaluate approximately for subcritical incidence angles and for the case of a plane homogeneous interface. The maximum vertical extent may be greater than the seismic wavelengths for subcritical incidence angles close to the critical angle and for strong impedance contrast at the interface. The whole part of reflector which actually affects the reflected wavefield is then larger than described by previous estimates which considered only the spatial region beyond the interface. In addition to the case of a flat interface, we also discuss the change in the characteristics of this part of the reflector as a function of the interface curvature. 
10:20

4aUWc8. Laboratory sound speed measurements on high water content sediment samples. Vanessa A. Martin (Laboratoire GeM (et EDF), UMR CNRS 6183 - Institut de Recherche en Génie Civil et Mécanique, IUT de St Nazaire - département Génie Civil - 58 rue Michel Ange, 44600 Saint Nazaire, France, vanessa.martin@univ-nantes.fr), Alain Alexis (Laboratoire GeM (et EDF), UMR CNRS 6183 - Institut de Recherche en Génie Civil et Mécanique, IUT de St Nazaire - département Génie Civil - 58 rue Michel Ange, 44600 Saint Nazaire, France, alain.alexis@univ-nantes.fr), Vincent Martin (Institut Jean Le Rond d'Alembert, UMR CNRS 7190, UPMC, 2 Place de la Gare de Ceinture, 78210 Saint-Cyr 1’Ecole, France, vmartin@ccr.jussieu.fr)

Laboratory measurements of sound speed in fluid viscous materials are known to be difficult, especially for frequencies of a few $\mathrm{kHz}$. An experimental set up which allows such measurements is developed. Tests are run on sediment samples of various lengths $(5 \mathrm{~cm}-20 \mathrm{~cm})$, all watersaturated but with different water contents (or densities). When sound speed only depends on the water content over a narrow frequency bandwidth, its estimation originates from time-of-flight measurements on samples of different lengths. It will appear that the water content does play a significant role on the speed of sound. When sound speed depends on frequency (dispersive waves) due to the sediment viscoelastic behaviour, the dependency can be taken into account. An analysis according to the sample length will be given to characterize the sound dispersion for different water contents. It will be shown that both the above studies on the experimental campaign yield sound speed estimations against sample lengths under various hypotheses. In these conditions, the estimation leads to information about saturated sediment behaviour.

THURSDAY MORNING, 3 JULY 2008

ROOM 362/363, 8:00 A.M. TO 1:00 P.M.

\title{
Session 4aUWd
}

\section{Underwater Acoustics and ECUA: Propagation and Reverberation}

\author{
David L. Bradley, Cochair \\ Pennsylvania State University, Post Office Box 30, State College, PA 16804-0030, USA \\ Kevin D. Lepage, Cochair \\ Naval Research Laboratory, 4555 Overlook Ave SW, Washington, DC 20375, USA
}

\section{Contributed Papers}

\section{8:00}

4aUWd1. Sound focusing and scanning in shallow water with background internal wave field. Andrey A. Lunkov (Moscow State Technical University n.a. N.E. Bauman, 2nd Baumanskaya 5, 107005 Moscow, Russian Federation, landr2004@mail.ru), Sergey A. Pereselkov (Voronezh State University, 1 Universitetskaya Sq., 394006 Voronezh, Russian Federation, pereselkov@yandex.ru)

Sound field focusing and scanning with focal spot are investigated for shallow water and long distances (up to $30 \mathrm{~km}$ ). It is studied the availability of horizontal and vertical scanning for controlling long-range low-frequency bottom reverberation as well. These researches are carried out by using numerical modeling for typical acoustic waveguides including random ones with background internal waves. The acoustic field is focused with vertical array by phase conjugation of sound wave from probe source placed at preset point. Horizontal scanning with the focal spot is performed by radiation frequency tuning. It is demonstrated that the best feasibility for scanning and hence for controlling bottom reverberation takes place for the regular waveguide under winter conditions when sound speed depends on depth only slightly and background internal waves are nonexistent practically. As an example for these conditions we can control the bottom reverberation on interval $\sim 5 \mathrm{~km}$ in the neighborhood of preset point. In summer for near bottom sound waveguide the range of distances for which it is practicable is much narrower even without internal waves. For the waveguide with intense background internal waves the control of long-range bottom reverberation becomes impossible. The work was supported by RFBR Project 05-02-16842.

\section{$8: 20$}

4aUWd2. An optimized source transmission scheme based on pressure sensitivity kernels. Kaustubha Raghukumar (MPL, Scripps Institution of Oceanography, University of California, San Diego, La Jolla, CA 92093 0238, USA, kaus@mpl.ucsd.edu), Bruce Cornuelle (MPL, Scripps Institution of Oceanography, University of California, San Diego, La Jolla, CA
92093-0238, USA, bdc@ucsd.edu),William Hodgkiss(MPL, Scripps Institution of Oceanography, University of California, San Diego, La Jolla, CA 92093-0238, USA, wsh@mpl.ucsd.edu), William A. Kuperman (MPL, Scripps Institution of Oceanography, University of California, San Diego, La Jolla, CA 92093-0238, USA, wkuperman@ucsd.edu)

A first-order Born approximation is used to obtain the pressure sensitivity of the received signal to small changes in medium sound speed. The pressure perturbation to the received signal caused by medium sound speed changes is expressed as a linear combination of single-frequency sensitivity kernels weighted by the source signal in the frequency domain. This formulation is used to optimize the pressure sensitivity kernel to give a new source transmission that can produce a focal spot and at the same time, to have less sensitivity to sound speed fluctuations than time-reversal. The formulation allows for a trade-off between quality of focal spot and sensitivity to environmental fluctuations. The optimized new source transmission uses knowledge of the medium statistics and is related to the regularized inverse filter.

\section{8:40}

4aUWd3. Passive phase conjugation processing to forward scattering waves by target in shallow water. Yoshiaki Tsurugaya (NEC @Corp., 1-10@Nissin-cho, Fuchu, 183-8501 Tokyo, Japan, y-tsurugaya@bp.jp.nec.com), Toshiaki Kikuchi (National Defence Academy, 39-21 Uhoyabe 4-chome, Yokosuka, 238-0024 Kanagawa, Japan, ADS01881@nifty.com), Koichi Mizutani (Tsukuba Univ., Tsukuba Science City, 305-8573 Ibaraki, Japan, mizutani@esys.tsukuba.ac.jp)

This paper describes the detection of an underwater target using a passive phase conjugation processing. It is assumed that a sound source and an array are disposed in shallow water, and a target exists between them. The traveling wave from the sound source and the scattered wave from the target are received by the array. However, they are received almost simultaneously. In addition, because the level of the traveling wave is considerably larger than that of the scattered wave, the detection of the scattered wave is 
difficult. Then, the traveling wave components are removed from the signals received in the array. And, the passive phase conjugation processing is given to the signals. The signal to which the passive phase conjugation is processed is similar with the signal radiated from the target. The signal radiated from the target relates to the sound wave that enters at the position of the target from the sound source for the small target. The sound wave that enters at the position of the target is uniquely decided depending on the condition of the propagation environment and the sound source. Therefore, the result that the passive phase conjugation is processed contains the position information of the target.

\section{9:00}

4aUWd4. Efficient time reversal by Lanczos iterations. Assad A. Oberai (Rensselaer Polytechnic Institute, Mechanical, Aerospace and Nuclear Engineering, 5048 JEC, 110 8th Street, Troy, NY 12180, USA, oberaa@rpi.edu), Gonzalo R. Feijoo (Woods Hole Oceanographic Institution, Woods Hole, MA 02543, USA, gfeijoo@whoi.edu), Paul E. Barbone (Boston University, 110 Cummington St, Boston, MA 02215, USA, barbone@bu.edu)

Time reversal methods have seen many applications in underwater acoustics, medical ultrasound, nondestructive evaluation, and several nonacoustics applications. Techniques to target and/or locate individual scatterers are based upon using iterative time-reversal. From a mathematical perspective, iterative time reversal is akin to the power method of extracting eigenvectors of the scattering operator. As such, standard iterative timereversal inherits the limitations of the power method. For example, iterative time-reversal converges slowly in the presence of multiple scatterers of similar strength and has difficulty identifying weaker scatterers. On the other hand, Lanczos iterations surmount these difficulties, and tend to converge faster than the power method, even when the latter works well. In this contribution we show how Lanczos iterations can be adapted to time-reversal iteration. This allows the strongest eigenvectors of the scattering operator to be measured with greater accuracy and in many fewer iterations than required by standard time-reversal. We describe how this algorithm may be implemented in a practical situation and build a new time-reversal method around it. We apply this method to some numerical examples to demonstrate its effectiveness and compare its performance with the more traditional time-reversal iterations.

\section{9:20}

4aUWd5. Specific features of surface reverberation in shallow water with focused sound field. Valeriy G. Petnikov (A.M. Prokhorov General Physics Institute, 38 Vavilov str., 119991 Moscow, Russian Federation, petniko@kapella.gpi.ru), Sergey A. Pereselkov (Voronezh State University, 1 Universitetskaya Sq., 394006 Voronezh, Russian Federation, pereselkov @ yandex.ru)

The specific features of long-range low-frequency $(230 \mathrm{~Hz})$ surface reverberation using vertical radiating array are studied in the framework of numerical experiment. We assume that the array focuses a sound field at different distances from ruffled surface in shallow water. Focusing is carried out by using a time-reversal mirror at a distance 10 - $30 \mathrm{~km}$ from radiating array. We consider sound focusing and surface reverberation in the presence of developed wind waves for acoustic waveguide common to shallow water for different seasons. The feasibility of surface reverberation suppression is estimated by sound focusing in the central region of the waveguide including the region located below thermocline. The results of numerical modeling are compared with the similar results obtained before for bottom reverberation. The work was supported by RFBR Project 05-02-16842.

\section{$9: 40$}

4aUWd6. Active sonar discrimination of water-column objects from bottom clutter using waveguide invariant modeling of distributed backscatter. Jeffrey Krolik (Duke University, ECE Dept. Hudson Hall 130, PO Box 90291, Durham, NC 27708, USA, jk@ee.duke.edu), Ryan Goldhahn (Duke University, ECE Dept. Hudson Hall 130, PO Box 90291, Durham, NC 27708, USA, rag15@ee.duke.edu), Granger Hickman (Duke University, ECE Dept. Hudson Hall 130, PO Box 90291, Durham, NC 27708,USA,gwh@ee.duke.edu)

The ability to differentiate water-column returns from clutter discretes due to backscatter from bottom features is critical to improving the detection performance in active sonar. Previous approaches include the use of statistical feature-based classifiers and physics-based depth estimation. Such methods are typically challenged by the convolutive effect of both uncertain multipath propagation and complex scattering characteristics in sonar returns. In previous work, we used waveguide invariant theory to model frequency-selective fading observed in distributed backscatter from the ocean bottom. In particular, the short-time energy spectrum of backscatter from the sea bottom at a given range can be well estimated along striations in time-frequency energy predicted by waveguide invariant theory. In this paper, we present a statistic which tests the observed frequency-selective fading of the short-time energy spectrum for a range cell under test against the spectrum predicted by waveguide invariant theory for a bottom feature using neighboring ranges and frequency bins. Results are presented for discrimination of a water column target versus bottom clutter using data simulated via a normal mode propagation model with environmental parameters from the Malta Plateau. Receiver operating characteristic analysis is performed showing good discrimination of equal energy targets from clutter. [Work supported by ONR].

\section{0:00}

4aUWd7. Using a dispersive source signal to generate a dispersive field in a nondispersive medium. Shane C. Walker (MPL, Scripps Institution of Oceanography, University of California, San Diego, La Jolla, CA 920930238, USA, shane@mpl.ucsd.edu), William A. Kuperman (MPL, Scripps Institution of Oceanography, University of California, San Diego, La Jolla, CA 92093-0238, USA, wkuperman@ucsd.edu)

In typical treatments, dispersion is considered to be a property of the medium. For example, a free space, static, homogeneous medium is dispersionless while gravity waves, particularly in the case of surface waves in a deep body of water, exhibit dispersion. Here it is shown that dispersion in the field can also result from dispersion in the source signal. As a demonstration, a dispersive source signal is shown to introduce dispersion in a dispersionless medium. Consequently, through proper design of the source signal, it is possible to tailor the resulting field dispersion to suit a variety of applications. Here potential applications to imaging in complex media are discussed. Simulation and experimental results are presented.

\section{0:20-10:40 Break}

\section{0:40}

4aUWd8. Equivalence of the waveguide invariant and two path ray theory methods for range prediction based on Lloyd's mirror patterns. Daphne Kapolka (Naval Postgraduate School, 13693 Tierra Spur, Salinas, CA 93908, USA, dkapolka@nps.edu)

For shallow, range-independent environments where the sound is dominated by low-order modes, the constant which characterizes the modal interference pattern, the waveguide invariant, is approximately equal to one. The speed of a contact which maintains a constant course and speed as it passes through its closest point of approach (CPA) can be determined from the asymptotic behavior of its tonal frequencies from the Doppler shift. This information can be used along with the change in broadband striation frequencies in a Lloyd's mirror pattern over time to extract the range of the contact as it transits through CPA. If instead of using normal mode theory, the Lloyd's mirror pattern is derived as the coherent interference between a straight-line direct and surface-reflected path, a relationship between the striation frequencies and time of a crossing contact can also be derived. This relationship can be shown to be identical to the result obtained from the nor- 
mal mode approach when the value of the waveguide invariant is equal to one. Thus, the same equations can be used to extract range information from a Lloyd's mirror pattern regardless of contact range as long as the environmental conditions support a waveguide invariant close to one.

\section{1:00}

4aUWd9. Analytical Time Domain Analysis of an Acoustic Waveguide. Hüseyin Ö. Sertlek (The Scientific and Technological Research Council of Turkey, Marmara Research Center, Information Technologies Institute, Gebze, 41400 Kocaeli, Turkey, ozkan.sertlek@bte.mam.gov.tr), Serkan Aksoy (Gebze Institute of Technology, Electronics Engineering Department, Çayırova, Gebze, 41400 Kocaeli, Turkey, saksoy@gyte.edu.tr)

A new analytical time domain Normal Mode solution for one layer acoustic channel in two dimensional Cartesian coordinates is presented in this paper. The method is based on the separation of variables technique for the time domain wave equation. Dirichlet boundary condition is applied on the upper and lower boundaries of the channel. Klein-Gordon equation is obtained and solved by using the Green function. The fundamental advantage of this new technique is not necessary to use the Fourier Transformation to obtain time domain response of arbitrary acoustical source signal in the waveguide. In order to validate the obtained results, first of all, the Fast Fourier Transform is applied to the time domain analytical data, then, the comparisons are given between KRAKEN program and a new analytical time domain Normal Mode solution in the sense of Transmission Loss relating to ranges. The excellent agreement is obtained between the results.

\section{1:20}

4aUWd10. Application of the Mode Matching Technique to Fluid Solid Layered Acoustic Problems. Hüseyin Ö. Sertlek (The Scientific and Technological Research Council of Turkey, Marmara Research Center, Information Technologies Institute, Gebze, 41400 Kocaeli, Turkey, ozkan.sertlek@bte.mam.gov.tr),Serkan Aksoy (Gebze Institute of Technology, Electronics Engineering Department, Çayırova, Gebze, 41400 Kocaeli, Turkey, saksoy@gyte.edu.tr), Deniz Bolükbas (The Scientific and Technological Research Council of Turkey, Marmara Research Center, Information Technologies Institute, Gebze, 41400 Kocaeli, Turkey, deniz.bolukbas@bte.mam.gov.tr), Sevgi Akgün (The Scientific and Technological Research Council of Turkey, Marmara Research Center, Information Technologies Institute, Gebze, 41400 Kocaeli, Turkey, sevgi.akgun @bte.mam.gov.tr)

An application of Mode Matching technique for analyzing interactions between range dependent fluid-solid layers in two dimensional cylindrical coordinates is presented by using Normal Mode method. The transmission loss for fluid-solid medium with semi-infinite bottom layer is calculated for range-dependent bottom profiles. The bottom solid half space is divided independent vertical regions due to different bottom parameters for simulation of the real acoustic environment. The transition between the independent vertical regions is ensured by using Mode Matching technique with Normal Mode method. The attenuation of acoustic wave modes in a range dependent two layered fluid-solid medium due to absorption of the lower semi-infinite solid layer is calculated. In order to validate the obtained results in the sense of Transmission Losses, Parabolic Equation program (RAM) is used for different transition cases. The good agreement is found between the results which can be used for realistic Sonar simulators.

\section{$11: 40$}

4aUWd11. The comparative analysis of two solutions of Pekeris boundary problem. Nadezhda Zlobina (Institute of Marine Technology Problems, Sukhanov str., 5à, 690950 Vladivostok, Russian Federation, zlobina@marine.febras.ru), Boris Kasatkin (Institute of Marine Technology Problems, Sukhanov str., 5à, 690950 Vladivostok, Russian Federation, bigcezar@mail.ru)

The solution of the reduced Pekeris boundary problem, satisfying to the generalized radiation condition on an impedance interface is obtained. Mode part of the solution is presented by series expansion on the total system of regular normal waves, of generalized normal waves and of leakage normal waves. The principle of the continuous continuation of the obtained solution in physical half-space with validity of boundedness condition is formulated. Expansion of the solution on physical half-space in a class of generalized functions is constructed. The obtained generalized solution includes canonical series expansion on the set of eigenfunctions of both conjugate operators corresponding an initial not self-conjugate boundary problem, the associated eigenfunctions of a waveguide with two soft boundaries, the associated eigenfunctions of a waveguide with upper soft boundary and lower rigid boundary and a set of spherical components. Results of the comparative analysis of the classical solution and generalized solution are given.

\section{2:00}

4aUWd12. Parabolic Wavelet Technique to Numerical Modelling of Sallow Water Acoustic. Mostafa Bakhoday Paskyabi (Research Engineering Institute, 16th Kilometers of Old Road Of Tehran-Karaj, Control Engineering Group, Agricultural Engineering Institute, 13445-754 Tehran, Iran, bakhoda@guilan.ac.ir), Seyed Taha Mortazavi (Research Engineering Institute, 16th Kilometers of Old Road Of Tehran-Karaj, Control Engineering Group, Agricultural Engineering Institute, 13445-754 Tehran, Iran, t_mortazavi@yahoo.com)

In this paper, parabolic equation (PE) model is applied for environmen of slowly varying with range and azimuth in a region of Persian Golf. This method is currently the most used to study propagation in non-stratified media. Due to required accuracy for a gridding step comparable with the wavelength, the computation load is very heavy. To overcome this disadvantage, we develop the weak form of PE formulation by the use of DeslauierDaubuc interpolating wavelet (DDW) basis functions. The formulation is similar to combination of conventional Finite Element Method (FEM) and split step technique except that, here DDW basis is used for approximating in depth to reduce the cost of computations and to increase the accuracy of method by the use of multi-resolution structure of wavelets. The localized and circulant representations of depth differential operators based on DDW connection coefficients allow efficient imposition of boundary values and circumvent some disadvantages of the traditional PE. Furthermore, details of bottom boundary imposing and some disadvantages of approach are presented. Numerical experiments are performed to study two layers waterbottom environment with none-smooth sediment interface to some canonical standard test problems in comparison with solutions obtained by conventional PE method.

\section{$12: 20$}

4aUWd13. Studying converted waves in shallow marine environment Nihed El Allouche (Delft University of Technology, P.O. Box Postbus 5048, 2600 GA Delft, Netherlands, n.elallouche@tudelft.nl), Guy G. Drijkoningen (Delft University of Technology, P.O. Box Postbus 5048, 2600 GA Delft, Netherlands, g.g.drijkoningen@tudelft.nl), Willem Versteeg (Ghent University, Krijgslaan 281, S8, 9000 Ghent, Belgium, willem.versteeg@ugent.be), Dick G. Simons (Delft University of Technology, P.O. Box Postbus 5048, 2600 GA Delft, Netherlands, d.g.simons @ tudelft.nl)

For many years, waves converted from compressional to shear mode have been successfully applied in hydrocarbon exploration to image and characterize the subsurface. Since shear-waves propagate with a velocity that is dependent on the shear modulus and are thus directly related to the shear strength of the sediment, they are very useful for geotechnical purposes. Generally, P-wave reflection amplitudes contain S-wave information but Riedel et al. (2001) showed that these reflections are not very sensitive to this. An alternative approach is to obtain S-wave information directly from converted waves. However, it is not clear whether these waves can be applied for geotechnical aims. The main focus will be on understanding the dependence of mode conversion on the seismic properties. In this study, we investigate the possibility of acquiring converted waves in marine unconsolidated sediments. From our numerical experiments we found that the conversion is maximal at two angles where the smallest angle appears to be more favorable in the environment of interest. Furthermore, we show how and where converted waves can be observed on a seismogram and the optimum field configuration to acquire them. 
12:40

4aUWd14. Conservative initial-boundary value problems for the Wide-Angle $P E$ in waveguides with variable bottoms. Dimitra Antonopoulou (Department of Applied Mathematics, University of Crete, 71409 Heraklion, Greece, danton@tem.uoc.gr), Vassilios Dougalis (Mathematics Department, University of Athens, 15784 Zografou, Greece, doug@math.uoa.gr), Frederic Sturm (Laboratoire de Mécanique des Fluides et d'Acoustique (UMR CNRS 5509), Ecole Centrale de Lyon, Centre acoustique, 36, avenue Guy de Collongue, 69134 Ecully Cedex, France, frederic.sturm@ec-lyon.fr), George Zouraris (Department of Applied Mathematics, University of Crete, 71409 Heraklion, Greece, zouraris @ math.uoc.gr)

We consider the third-order, Claerbout-type Wide-Angle Parabolic Equation (PE) in the context of Underwater Acoustics in a cylindrically symmetric medium consisting of water over a soft bottom $\mathrm{B}$ of range-dependent topography. There are strong indications, that the initial-boundary value problem for this equation with just a homogeneous Dirichlet boundary condition on B, may not be well-posed, for example when B is downsloping. In previous work we proposed an additional bottom boundary condition that, together with the zero field condition on B, yields a well-posed problem. In the present paper we continue our investigation of additional bottom boundary conditions that yield well-posed, physically correct problems. Motivated by the fact that the solution of the wide-angle PE in a domain with horizontal layers conserves its L2 norm in the absence of attenuation, we seek additional boundary conditions on a variable-topography bottom, that yield L2- conservative solutions of the problem. We identify a family of such boundary conditions after a range-dependent change of the depth variable that makes B horizontal. We discretize the continuous problems by secondorder accurate Crank-Nicolson type finite difference schemes, and show, by means of numerical experiments, that the new models yield accurate simulations of the acoustic field in standard, wedge-type domains with upsloping and downsloping bottoms.

\title{
Session 4aUWe
}

\section{Underwater Acoustics and ECUA: Tank Experiments I}

\author{
Raymond J. Soukup, Cochair \\ U.S. Naval Res. Lab., Acoust. Div., Code 7142, 4555 Overlook Ave. SW, Washington, DC 20375, USA \\ Jean-Pierre Sessarego, Cochair \\ Laboratory for Mechanics and Acoustics CNRS, 31 chemin Joseph Aiguier, Marseille, 13009, France
}

Invited Papers

11:40

4aUWe1. Tank experiment: The ultrasonic approach to ocean physics. Philippe Roux (LGIT - CNRS - Université Joseph Fourier, Maison des Géosciences, 1381 rue de la Piscine, BP 53, 38041 Grenoble, France, philippe.roux @ obs.ujf-grenoble.fr)

Acoustic/elastic waves ranging from a few $\mathrm{kHz}$ to a few $\mathrm{MHz}$ are nowadays easy to emit/receive through the use of "key in hands" multi-channel systems. These systems provide the instantaneous amplitude and phase of the deterministic wave propagating in the medium with a dynamic larger than $90 \mathrm{~dB}$ in some cases (16-bit amplitude sampling). The use of a large number of channels (at least 64 ) is mandatory to simultaneously investigate the spatial and temporal aspect of wave propagation in complex media. The advantage of a laboratory-scaled ocean model relies in the ease with which it can be build, modified, and/or controlled over time. Finally, the trend in ocean wave physics is now to study the dynamics of such medium (internal waves, turbulent flow, sensor motion) that requires the use of a real-time acquisition system. Examples of the types of analog experiments that could be carried out with a laboratory-scaled system are the study of nonuniform doppler shifted fields in reverberant environments, ocean acoustic tomography and similar inverse problems, coherent communications in complex moving environments, etc. After the description of the equipment, we give more specific examples of potential experiments to illustrate the versatility of the laboratory set-up.

12:00

4aUWe2. Tank experiments of sound propagation over a tilted bottom: Comparison with a 3-D PE model. Alexios Korakas (Laboratoire de Mécanique des Fluides et d'Acoustique (UMR CNRS 5509), Ecole Centrale de Lyon, Centre acoustique, 36, avenue Guy de Collongue, 69134 Ecully Cedex, France, alexis.korakas@ec-lyon.fr), Frederic Sturm (Laboratoire de Mécanique des Fluides et d'Acoustique (UMR CNRS 5509), Ecole Centrale de Lyon, Centre acoustique, 36, avenue Guy de Collongue, 69134 Ecully Cedex, France, frederic.sturm@ec-lyon.fr), Jean-Pierre Sessarego (Laboratory for Mechanics and Acoustics CNRS, 31 chemin Joseph Aiguier, 13009 Marseille, France, sessarego@lma.cnrs-mrs.fr), Didier Ferrand (Laboratoire de Mécanique et d'Acoustique (UPR CNRS 7051), 31, chemin Joseph Aiguier, 13402 Marseille Cedex 20, France, ferrand@1ma.cnrs-mrs.fr)

We present results of tank experiments of long-range acoustic propagation over a wedge-shaped oceanic bottom. Previous work investigated the propagation of broadband pulses in a range-independent configuration showing good agreement between experimental results and model predictions [Korakas et al., Proceedings of UAM 2007 (Heraklion, Crete, Greece, 25-29 June 2007)]. As a follow-up, preliminary experiments were carried out considering a tilted bottom. Non-negligible 3-D effects were observed and proved to compare favorably with numerical predictions obtained running a parabolic equation based code [Sturm et al., Proceedings of UAM 2007]. In the 
present work we continue the investigation of the wedge-like environment. Additional series of experimental measurements are performed. Received signals are recorded on a very fine spatial grid. Then, the measured data are processed using matched field processing. In our inversion algorithm, the replica are provided by a fully three-dimensional parabolic equation code. The technical aspects of the inversion procedure used (e.g., CPU time) are discussed.

\section{2:20}

4aUWe3. Laboratory investigations of the detection and characterization of buried targets by iterative, single-channel time reversal. Zachary J. Waters (Boston University, Dept. of Aerosp. and Mech. Eng., 110 Cummington St., Boston, MA 02215, USA, zjwaters@bu.edu), Benjamin R. Dzikowicz (Naval Surface Warfare Center, Panama City Division, Code HS-11, 100 Vernon Ave., Panama City, FL 32407, USA, benjamin.dzikowicz@navy.mil), R. Glynn Holt (Boston University, Dept. of Aerosp. and Mech. Eng., 110 Cummington St., Boston, MA 02215, USA, rgholt@bu.edu), Ronald A. Roy (Boston University, Dept. of Aerosp. and Mech. Eng., 110 Cummington St., Boston, MA 02215, USA, ronroy@bu.edu)

Due to the dynamic nature of the shallow water environment, targets are often buried beneath the seafloor, hindering their detection and identification by acoustic methods. Using iterative time reversal with a single channel transducer [Waters et al., J. Acoust. Soc. Am. 122,3023 (2007)], the monostatic return from a buried resonant target is enhanced, yielding convergence to a narrowband waveform characteristic of the dominant mode in the target's scattering response. Scaled laboratory experiments are performed with a broadband (Q 2) transducer operating in the 0.5-2 MHz frequency range. Solid and evacuated metallic spheres used as targets are buried beneath a layer of simulated sediment. Images generated by scanning the transducer laterally in two dimensions over an area of sediment containing multiple targets show enhancement of different modes in a single target's scattering response and test the selectivity between targets of differing type. Experiments with the transducer positioned at normal and non-normal incidence, quantify the enhancement in the signal-to-noise ratio of target returns as a function of window size and position. [Work supported by The Office of Naval Research and the Center for Subsurface Sensing and Imaging Systems (NSF ERC Award No. EEC-9986821).]

\section{2:40-2:00 Lunch Break}

\section{Invited Papers}

4aUWe4. Detection and classification of a cylindrical target partially or completely buried in thin sand/water mixture by time-frequency representation. Gerard Maze (LAUE, Université du Havre, Place Robert Schuman, F-76610 Le Havre, France, gerard.maze@univ-lehavre.fr), Dominique Decultot (LOMC FRE 3102 CNRS Groupes Ondes Acoustiques, Université du Havre (IUT), Place Robert Schuman, 76610 Le Havre, France, dominique.decultot@univ-lehavre.fr), Katia Cacheleux (LOMC FRE 3102 CNRS Groupes Ondes Acoustiques, Université du Havre (IUT), Place Robert Schuman, 76610 Le Havre, France, katia.cacheleux@univ-lehavre.fr), Romain Liétard (LOMC FRE 3102 CNRS Groupes Ondes Acoustiques, Université du Havre (IUT), Place Robert Schuman, 76610 Le Havre, France, romain.lietard@univ-lehavre.fr)

Numerous papers show that it is possible to characterize an air-filled cylindrical shell immersed in water from a time-frequency representation. The time scattered signal is constituted by echoes related to the reradiation of waves circumnavigating around the cylindrical target. For particular frequencies, these echoes make resonances which characterize this target. In first part, the scattered signal is calculated, the cylindrical shell is in water or in a medium with characteristics similar to the mixture sand/water used for the experimental measurements. In second part, the cylindrical shell is partially or completely buried in a thin sand/water mixture. It is insonified in normal or oblique incidence relatively to the interface water/mixture. The impulse time scattered signal is plotted and it is treated with a time-frequency representation. The comparison between the theoretical and experimental time-frequency representations shows us that it is possible to detect and classify the object buried in thin sand/water mixture.

4aUWe5. Acoustic radiation of low frequency flexural vibration modes in a submerged plate. Dominique Decultot (LOMC FRE 3102 CNRS Groupes Ondes Acoustiques, Université du Havre (IUT), Place Robert Schuman, 76610 Le Havre, France, dominique.decultot@univ-lehavre.fr), Romain Liétard (LOMC FRE 3102 CNRS Groupes Ondes Acoustiques, Université du Havre (IUT), Place Robert Schuman, 76610 Le Havre, France, romain.lietard@univ-lehavre.fr), Farid Chati (LOMC FRE 3102 CNRS Groupes Ondes Acoustiques, Université du Havre (IUT), Place Robert Schuman, 76610 Le Havre, France, farid.chati@univ-lehavre.fr), Gerard Maze (LAUE, Université du Havre, Place Robert Schuman, F-76610 Le Havre, France, gerard.maze@univ-lehavre.fr), Aleksander Klauson (Tallinn University of Technology, Dept. of Mechanics, Ehitajate tee 5, 19086 Tallinn, Estonia, aklauson@staff.ttu.ee)

In some submarine structures, the acoustic radiation of vibrations at the neighbourhood of sonar equipment limits their use. So the aim of this study is to understand the process of acoustic radiation of a submerged plate subjected to a vibration. For the low frequency domain, only two types of wave can propagate: the first antisymmetric Lamb wave (A0) and the first symmetric Lamb wave (S0). When the plate is immersed in water the A0 wave is modified and the new wave is named A wave. In this work, experimental and numerical analysis of vibration modes in a plate are carried out. The studied rectangular plate of thickness 10 mm is made of steel. Its length and width are respectively $1.0 \mathrm{~m}$ and $0.5 \mathrm{~m}$. The plate is partially immersed in water $(90 \%)$. Flexural vibrations are generated by a shaker normally connected to the emerging plate part. The applied signal is one sinusoidal period at a frequency which is under the critical frequency. Relations between the admittance of the plate and the radiated pressure in water are highlighted. 
4aUWe6. Inverse problems in sound radiation of complex structures from measurements in a large acoustic tank. Earl G. Williams (Naval Research Laboratory, 4555 Overlook Ave, Washington, DC 20375, USA, earl.williams@nrl.navy.mil), Brian H. Houston (Naval Research Laboratory, 4555 Overlook Ave, Washington, DC 20375, USA, houston@code7136.nrl.navy.mil), Nicolas Valdivia (Naval Research Laboratory, 4555 Overlook Ave, Washington, DC 20375, USA, valdivia@pa.nrl.navy.mil), Peter C. Herdic

(SFA Inc., Suite 405, Crofton, MD 21114, USA, herdic@ code7136.nrl.navy.mi)

The Laboratory for Structural Acoustics (LSA) at NRL consists of an indoor cylindrical tank (17 m dia. by $15 \mathrm{~m}$ deep) filled with 1 million gallons of deionized water. Key features include: 1) vibration isolation, 2) active temperature control, and 3) anechoic materials. This unique laboratory is instrumented with sophisticated mechanical, electronic and optical systems, that include large workspace in-water robotic scanners to generate nearfield acoustical holography (NAH) databases. We discuss such a database consisting of the underwater near-field pressure measured on a two-dimensional surface conformal to an internally driven complex structure floating at the air-water interface. Various inverse approaches are discussed to image the normal velocity and intensity of the structure at its interface to the fluid, as well as the total power radiated, revealing mechanisms of radiation related to the internal structure. These inverse approaches consist of the equivalent source method compared with the well established Fourier acoustics methods of NAH. This work was supported by the US Office of Naval Research.

\title{
3:00
}

4aUWe7. Broadband elastic scattering by fiberglass spherical shells and plates measured in a water tank: Acoustic inversion and wave analysis. Alessandra Tesei (NATO Undersea Research Centre, Viale San Bartolomeo 400, 19126 La Spezia, Italy, tesei@nurc.nato.int), Paul D. Fox (National Physical Laboratory, Hampton Road, TW11 OLW Teddington, UK, pf@isvr.soton.ac.uk), Gary B. Robb (National Oceanography Centre, University of Southampton Waterfront Campus, European Way, SO14 3ZH Southampton, UK, gbor199@noc.soton.ac.uk)

Spherical shells and plane plates made of different types of fiberglass (either random or textured) were measured in the backscatter direction, suspended in a water tank in a broadband frequency range between 30 and $350 \mathrm{kHz}$. The range of ka for the spheres was approximately 8 to 90 , with the fd range for the plates approximately 0.15 to $1.75 \mathrm{MHz}^{*} \mathrm{~m}$. The aim of the study was to investigate the effects of the fiber type on the object signature, as the frequency and the type of fiber layers vary. Inversion of the material parameters was conducted on the basis of the object's temporal echo. In particular, the estimate of material loss is crucial to determine at what frequency elasticity becomes irrelevant to the object's global response. The spherical shells were measured either void or filled with different materials (liquid and solid) in order to evaluate the contribution of the shell-borne elastic waves with respect to sound scattered from the interior of the object. Elastic wave analysis and analytical modeling tools were used to support the physical interpretation of the measured responses from the different objects.

4aUWe8. Modal analysis of resonances of an elliptic elastic cylinder immersed in water. Fernand Leon (LOMC FRE 3102 CNRS Groupes Ondes Acoustiques, Université du Havre (IUT), Place Robert Schuman, 76610 Le Havre, France, fernand.leon@ univ-lehavre.fr), Farid Chati (LOMC FRE 3102 CNRS Groupes Ondes Acoustiques, Université du Havre (IUT), Place Robert Schuman, 76610 Le Havre, France, farid.chati@univ-lehavre.fr), Jean-Marc Conoir (Institut Jean Le Rond d'Alembert-UMR CNRS 7190, Université Paris 6, tour 55-65, 4 place Jussieu, 75005 Paris, France, conoir@1mm.jussieu.fr)

Considerable work has been done on the scattering by cylindrical objects having a circular cross section. The modal formalism based on the theory of elasticity has been developed for studying the acoustic scattering by these elastic cylinders immersed in water. In particular, it has been demonstrated, in normal incidence, that the eigenfrequencies of a circular cylinder can be determined from a resonance spectrum. These eigenfrequencies correspond to circumferential waves that form a resonance when a phase matching along a closed path is obtained. Each eigenfrequency is characterized by a given mode n, i.e., the number of wavelengths spanning the circumference. Comparatively, little attention has been devoted to the more general case of the noncircular cylindrical cylinders such as the elliptical elastic cylinders. For these objects, we have been developed a modal formalism based on the theory of elasticity. From the results obtained theoretically and experimentally, we show how to obtain a resonance spectrum, independently of the azimuth incident angle and of the radii ratio (minor radius and major radius) so that the eigenfrequencies can be determined. We present also a modal analysis of resonances as function of the azimuth incident angle and of the radii ratio.

\author{
3:40-5:20 Posters
}

Lecture sessions will recess for the presentation of poster papers on various topics in acoustics. See poster sessions for topics and abstracts. 


\section{Invited Papers}

\section{$5: 20$}

4aUWe9. Estimation of some geoacoustic parameters of a tank experiment by match field processing. Panagiotis Papadakis (IACM-FORTH, N. Plastira 100, 70013 Vassilika Vouton, Greece, panos@iacm.forth.gr), Michael Taroudakis (University of Crete \& FORTH/IACM, Vassilika Vouton, P.O.Box 1385, 71110 Heraklion, Greece, taroud@iacm.forth.gr), Jean-Pierre Sessarego (Laboratory for Mechanics and Acoustics CNRS, 31 chemin Joseph Aiguier, 13009 Marseille, France, sessarego@lma.cnrs-mrs.fr), Patrick Sanchez (Laboratoire de Mécanique et d'Acoustique (UPR CNRS 7051), 31, chemin Joseph Aiguier, 13402 Marseille Cedex 20, France, patrick.sanchez@eoures.com)

The work deals with an inversion procedure for the estimation of geometrical and environmental parameters of a simulated shallow water environment. Two of the most significant parameters in a shallow water environment affecting the acoustic field in long range acoustic propagation are the sound speed of the sediment and the depth of the water column. The accurate estimation of these parameters is perhaps the most important task in scaled laboratory experiments. Therefore, the calibration phase of such an experiment involves the estimation of these parameters, preferably using acoustical techniques, regardless the final scope of the experiment. The present work describes an inversion procedure based on standard match field processing. The acoustic signal used was a short gaussian shaped pulse and it was recorded at a certain distance from the source and for several depths in the water column. The spectrum of the recorded signal was obtained using FFT and the complex acoustic field at the central frequency was calculated. The estimated values of the water depth and the sound speed in the sand which was used to simulate the sea-bed, were found in accordance with the observed modal structure of the acoustic field.

\section{5:40}

4aUWe10. High frequency propagation in and scattering from water-saturated granular sediments: Laboratory study. Anatoliy N. Ivakin (Applied Physics Laboratory, University of Washington, 1013 NE 40th Street, Seattle, WA 98105, USA, ivakin@apl.washington.edu), Jean-Pierre Sessarego (Laboratory for Mechanics and Acoustics CNRS, 31 chemin Joseph Aiguier, 13009 Marseille, France, sessarego@1ma.cnrs-mrs.fr)

Acoustic properties of water-saturated granular sediments at frequencies from $150 \mathrm{kHz}$ to $8 \mathrm{MHz}$ were studied in controlled laboratory conditions using broadband transducers. Two samples of medium sand sediments, with the same mean grain size, but with different width of the size distribution, were taken for the study, degassed, and their surface was flattened. Another sample of sediments was composed of glass beads of the same grain size. The main difference of glass beads from sand grains was their shape. Backscattering strength at normal and oblique incidence and reflection coefficient at normal incidence were measured for the three samples. The reflection experiments were made for different thicknesses of the samples, so that reflections from both first and second interfaces of the sediment layer were measured. This allowed also estimating sound speed and attenuation in the sediments. The results obtained for the three chosen types of the sediments were compared to demonstrate effects of the grain size distribution width and the grain shape on acoustic properties of the sediment. [Work supported by ONR and CNRS].

\section{6:00}

4aUWe11. A time domain model of scattering from small discrete volume particles: Tank validation. Gaetano Canepa (NATO Undersea Research Centre, Viale San Bartolomeo 400, 19126 La Spezia, Italy, canepa@nurc.nato.int), Jean-Pierre Sessarego (Laboratory for Mechanics and Acoustics CNRS, 31 chemin Joseph Aiguier, 13009 Marseille, France, sessarego@lma.cnrs-mrs.fr), Alessandra Tesei (NATO Undersea Research Centre, Viale San Bartolomeo 400, 19126 La Spezia, Italy, tesei@nurc.nato.int), Régine Guillermin (Laboratory for Mechanics and Acoustics CNRS, 31 chemin Joseph Aiguier, 13009 Marseille, France, guillermin@1ma.cnrs-mrs.fr), Raymond J. Soukup (U.S. Naval Res. Lab., Acoust. Div., Code 7142, 4555 Overlook Ave. SW, Washington, DC 20375, USA, soukup@abyss.nrl.navy.mil)

A model of the time domain scattering from small discrete volume particles is presented here with an experimental validation. The model was implemented on the backbone of the BORIS-3D model which originally included only surface scattering and volume scattering from small perturbation of the volume density and sound speed. The proposed model adds discrete volume scattering and simulates both monostatic and bistatic configurations. The experimental validation was performed in a tank of the CMRS/LMA Laboratory (Laboratoire de Mécanique et d'Acoustique) using an in-house produced silicon plate (with a flat upper interface) in which 10\% of the volume embeds spherical glass beads $1 \mathrm{~mm}$ in diameter. The characteristics of this plate was well known; in particular, the amplitude of the volume backscattering (as a function of the scattering angle) has already been presented. In this work, we are particularly interested in the time domain evolution of the scattered echo. The results of this study show a very good agreement between simulated and experimental data in both amplitude and time evolution shape.

\section{6:20}

4aUWe12. Backscattering From Scale Models of Elastic Ocean Bottoms with Power-law Roughness. Raymond J. Soukup (U.S. Naval Res. Lab., Acoust. Div., Code 7142, 4555 Overlook Ave. SW, Washington, DC 20375, USA, soukup@abyss.nrl.navy.mil), Edward L. Kunz (U.S. Naval Res. Lab., Acoust. Div., Code 7142, 4555 Overlook Ave. SW, Washington, DC 20375, USA, kunz@abyss.nrl.navy.mil), Gaetano Canepa (NATO Undersea Research Centre, Viale San Bartolomeo 400, 19126 La Spezia, Italy, canepa@nurc.nato.int), Harry J. Simpson (U.S. Naval Res. Lab., Acoust. Div., Code 7142, 4555 Overlook Ave. SW, Washington, DC 20375, USA, harry.simpson@ nrl.navy.mil), Jason E. Summers (U.S. Naval Res. Lab., Acoust. Div., Code 7142, 4555 Overlook Ave. SW, Washington, DC 20375, USA, jason.summers@nrl.navy.mil)

Rough surface analogs to ocean bottom interfaces with two contrasting power-law roughness spectra were fabricated from slabs of PVC. A tank experiment with a nearly monostatic geometry and transmitted signals in the band 100-300 kHz was performed, with the source and receiver positioned to produce acoustic interactions on multiple locations on the surfaces. Models of scattering strength and 
the received time series, using deterministic and stochastic representations of the surface, are used to verify the predicted dependence of the scattering, with emphasis on the sub-critical angle $(<50$ degrees grazing) region, where the predicted difference in scattering strength due to the contrast in roughness between the two fabricated surfaces is on the order of $10 \mathrm{~dB}$. The numerical models employed were perturbation theory, a second-order small-slope calculation using the power-law roughness parameters, and a fourth-order smallslope calculation using the actual grid of heights as an input. The experimental effort also focused on the near-critical angle region where the predictions of the numerical models differed markedly. [Work supported by ONR and NURC.]

\section{6:40}

4aUWe13. Shallow-water tank experiments and model comparisons over range-dependent elastic bottoms. Jon M. Collis (Boston University, Department of Aerospace and Mechanical Engineering, 110 Cummington Street, Boston, MA 02215, USA, jcollis@bu.edu), Michael D. Collins (U.S. Naval Res. Lab., Acoust. Div., Code 7142, 4555 Overlook Ave. SW, Washington, DC 20375, USA, michael.collins@nrl.navy.mil), Harry J. Simpson (U.S. Naval Res. Lab., Acoust. Div., Code 7142, 4555 Overlook Ave. SW, Washington, DC 20375, USA, harry.simpson@nrl.navy.mil), Raymond J. Soukup (U.S. Naval Res. Lab., Acoust. Div., Code 7142, 4555 Overlook Ave. SW, Washington, DC 20375, USA, soukup@abyss.nrl.navy.mil), William L. Siegmann (Rensselaer Polytechnic Institute, 110 8th Street, Troy, NY 12180, USA, siegmw@rpi.edu)

A series of tank experiments has been conducted in order to obtain high quality data for acoustic propagation in shallow-water environments with elastic bottoms. Such problems can now be solved accurately with the parabolic equation method, which is being used for comparisons with measured transmission loss. Results from the initial experiment with a flat or sloped slab of PVC demonstrated both benchmark quality agreement between computed solutions and data, and the necessity of accounting for elasticity in the bottom [J. M. Collis, et al., JASA 122]. This paper will present results of a second experiment, conducted in the same manner as the first, but modified to allow for variable bottom slopes. Time series were collected at 100-300 kHz on horizontal arrays for two source positions. Parabolic equation solutions for treating variable slopes, using coordinate transformation methods of mapping and axis rotations, will be benchmarked against the new data. [Work supported by the Office of Naval Research.]

\section{$7: 00$}

4aUWe14. The Broadband In-Water Structural Acoustics of Unexploded Ordinance. Harry J. Simpson (U.S. Naval Res. Lab., Acoust. Div., Code 7142, 4555 Overlook Ave. SW, Washington, DC 20375, USA, harry.simpson@nrl.navy.mil), Brian H. Houston (Naval Research Laboratory, 4555 Overlook Ave, Washington, DC 20375, USA, houston@ code7136.nrl.navy.mil), Michael L. Saniga (U.S. Naval Res. Lab., Acoust. Div., Code 7142, 4555 Overlook Ave. SW, Washington, DC 20375, USA, saniga@code7136.nrl.navy.mil), Joseph A. Bucaro (U.S. Naval Res. Lab., Acoust. Div., Code 7142, 4555 Overlook Ave. SW, Washington, DC 20375, USA, joseph.bucaro@nrl.navy.mil)

The Laboratory for Structural Acoustics (LSA) at the Naval Research Laboratory consists of a 1 million gallon, deionized water, indoor cylindrical tank ( $17 \mathrm{~m}$ diameter by $15 \mathrm{~m}$ deep). The key features include: 1) vibration and temperature isolation, 2) feedback controlled heating and adiabatic materials (temperature variability $<0.01{ }^{\circ} \mathrm{C}$ ), and 3 ) reverberation reducing anechoic materials. This laboratory has computer controlled robotic scanners and manipulators used for precision freefield measurements including nearfield acoustic holography and compact range scattering. The precision robotics, environmental control, and painstaking measures to insure homogeneity and stability result in a high fidelity, versatile, and unique underwater acoustic measurement laboratory. The LSA also contains an indoor rectangular tank $(10 \mathrm{~m}$ by $8 \mathrm{~m}$ ) laboratory, with a $3 \mathrm{~m}$ deep sand bottom and $4 \mathrm{~m}$ of water column. In a similar fashion to the freefield laboratory, this laboratory is used to study target scattering in a marine bottom environment. We discuss such databases focused on the challenging problem of unexploded ordinance (UXO) in water where we use the structural acoustic response measured in a series of laboratory experiments to detect and identify several common UXOs. The 360 degree broadband (1-140 kHz) compact range monostatic and bistatic measurements taken in both laboratories will be discussed.

$$
7: 20
$$

4aUWe15. Benchmarking of computational scattering models using underwater acoustic data from a corrugated wax slab. David C. Calvo (U.S. Naval Res. Lab., Acoust. Div., Code 7142, 4555 Overlook Ave. SW, Washington, DC 20375, USA, david.calvo@nrl.navy.mil), Gaetano Canepa (NATO Undersea Research Centre, Viale San Bartolomeo 400, 19126 La Spezia, Italy, canepa@nurc.nato.int), Raymond J. Soukup (U.S. Naval Res. Lab., Acoust. Div., Code 7142, 4555 Overlook Ave. SW, Washington, DC 20375, USA, soukup@abyss.nrl.navy.mil), Edward L. Kunz (U.S. Naval Res. Lab., Acoust. Div., Code 7142, 4555 Overlook Ave. SW, Washington, DC 20375, USA, kunz@abyss.nrl.navy.mil), Jean-Pierre Sessarego (Laboratory for Mechanics and Acoustics CNRS, 31 chemin Joseph Aiguier, 13009 Marseille, France, sessarego@1ma.cnrs-mrs.fr), Kevin Rudd (U.S. Naval Res. Lab., Acoust. Div., Code 7142, 4555 Overlook Ave. SW, Washington, DC 20375, USA, kevin.rudd@nrl.navy.mil)

Measured time series for underwater acoustic scattering from a $30 \mathrm{~cm}$ x $30 \mathrm{~cm}$ x $5 \mathrm{~cm}$ wax slab with a two-dimensional corrugated (rippled) surface are compared with simulation results. The experimental geometry and directionality of the sensors allowed for ensonification of the rippled surface and the appearance of shadowing effects at low grazing angles. The acoustic source transmitted impulses at 200-800 kHz (wavelengths between $0.75-0.19 \mathrm{~cm}$ ). The height and spacing of the ripples were $0.3 \mathrm{~cm}$ and $3 \mathrm{~cm}$, respectively, and the slab had negligible shear speed and a measured attenuation. We simulate the experiment with the following methods listed in increasing levels of physical accuracy and computational cost: Kirchhoff Approximation (KA), Second-order Small-Slope approximation (SSA2), the Wide-angle On-Surface Radiation Condition method (WOSRC), a Pseudo-differential Impedance Operator method (PIO), a 2-domain Integral Equation method (IE-2DOM), and an Elastodynamic Finite Integration Technique (EFIT). The range of techniques allowed us to examine effects such as reflections off the interior bottom or ends of the slab and the effectiveness of the asymptotic (KA/SSA2) and pseudo-differential (WOSRC/PIO) methods for cases that include shadowing. (Work sponsored by ONR and NURC.) 
Session 4pAAa

\title{
Architectural Acoustics: Archeological Acoustics I
}

\author{
David Lubman, Cochair \\ DL Acoustics, 14301 Middletown Lane, Westminster, CA 92683, USA \\ Jens Holger Rindel, Cochair \\ Odeon A/S, Scion DTU, Diplomvej Buildling 381, Lyngby, DK-2800, Denmark
}

\section{Invited Papers}

\author{
$1: 20$
}

\begin{abstract}
4pAAa1. Sound resonance in prehistoric times: A study of Paleolithic painted caves and rocks. Iegor Reznikoff (Université de Paris X, Département de Philosophie, 92001 Nanterre, France, dominiqueleconte@yahoo.fr)

Caves have natural properties of resonance: some parts sound very well, the sound lasts for some seconds or gives several echoes, some other parts have a dull resonance or no resonance at all. It is extremely interesting to compare in a given cave the map of the most resonant locations with the map of the locations of the paintings: are there correlations between resonance and paintings? We have studied many Paleolithic caves in France in which the answer was remarkably positive; stated shortly: the more resonant the location, the more paintings or signs are situated in this location. Here are presented some studies and results in the caves of Isturitz and Oxocelhaya in Pays Basque and in some other caves. Some considerations are given about the resonance - pictures relationship in open spaces with prehistoric painted rocks. Bibliography I. Reznikoff: Prehistoric Paintings, Sound and Rocks in Studien zur Musikarchäologie III: 2nd International Symposium on Music Archaeology, Sept. 2000, ed. E. Hickmann, Berlin, Rahden, 2002, 39-56. The Evidence of the Use of Sound Resonance from Palaeolithic to Medieval Times, Archaeoacoustics, C. Scarre \& G. Lawson ed., University of Cambridge, Cambridge, 2006, 77-84. On Primitive Elements of Musical Meaning, www.musicandmeaning.net, JMM 3 (Invited papers), 2005.
\end{abstract}

$1: 40$

4pAAa2. Architectural and acoustic restoration of the 'Benevento' Roman Theatre. Luigi Maffei (Built Environment Control Laboratory Ri.A.S., Second University of Naples, Abazia di S. Lorenzo, 81031 Aversa, Italy, luigi.maffei@ unina2.it), Gino Iannace (Built Environment Control Laboratory Ri.A.S., Second University of Naples, Abazia di S. Lorenzo, 81031 Aversa, Italy, gino.iannace@unina2.it), Leda De Gregorio (Built Environment Control Laboratory Ri.A.S., Second University of Naples, Abazia di S. Lorenzo, 81031 Aversa, Italy, ledadegreg@libero.it), Umberto Palmieri (Built Environment Control Laboratory Ri.A.S., Second University of Naples, Abazia di S. Lorenzo, 81031 Aversa, Italy, uzy@libero.it)

Acoustics as well as the extraordinary architecture are substantial part of the heritage of ancient Greek and Roman theatres. These archaeological areas are more often used for classical and modern performing activities but to emphasise the original acoustics, restoration of the original shape, reconfiguration of the space and introduction of new technologies and materials may be needed. These activities must happen taking into account the "International Charter for The Conservation and Restoration of Monuments and Sites" as main tools defining conservation, use and maintenance guidelines in matter of discipline for ancient performing places. This paper presents some proposals for the stage reconstruction of the Benevento Roman Theatre and examine the associated effects on the theatre acoustics. Design proposals, minded to preserve the place authenticity, improving the acoustics and keeping intact the surrounding atmosphere for spectators, are the result of archaeological data, contemporary performances requirements, state of the art in matter of stage setting design. Acoustic field prediction is based on numerical models and auralization aiming to reproduce the real listening conditions.

\section{Contributed Paper}

2:00

4pAAa3. Acoustic characterization of the ancient theatre at Syracuse. Marco Gullo (DREAM, Univ. of Palermo, Viale delle Scienze, Edificio 9, 90128 Palermo, Italy, mgullo@dream.unipa.it), Armando La Pica (DREAM, Univ. of Palermo, Viale delle Scienze, Edificio 9, 90128 Palermo, Italy, lapica@unipa.it), Giuseppe Rodono' (DREAM, Univ. of Palermo, Viale delle Scienze, Edificio 9, 90128 Palermo, Italy, rodon@unipa.it), Vincenzo Vinci (DREAM, Univ. of Palermo, Viale delle Scienze, Edificio 9, 90128 Palermo, Italy, vincenzo_vinci@ hotmail.it)

The ancient theatre at Syracuse, the largest open air theatre in Sicily, experienced many structural changes along its history. Today its very renowned drama festival attracts visitors from all the world that fill the entire cavea: customized seats accomodation and a prominent stage play an important role in the theatre acoustics. Because no data were available in literature on its acoustical perfomance a measurement session was carried out by our team in the framework of a wide research project on ancient theatres acoustics in the modern use. From data recorded "room criteria" parameters have been evaluated together with spectral analysis in order to gain a deeper information on the acoustic field. Main results are listed and commented and a comparison among data collected on field during the team experience in the past years is reported. 


\section{Invited Paper}

\section{$2: 20$}

4pAAa4. On the use of ancient theatres for modern performances: a scale model approach. Andrea Farnetani (Engineering Dept. - Univ. of Ferrara, Via Saragat, 1, 44100 Ferrara, Italy, andrea.farnetani@unife.it), Nicola Prodi (Engineering Dept. - Univ. of Ferrara, Via Saragat, 1, 44100 Ferrara, Italy, nicola.prodi@unife.it), Roberto Pompoli (Engineering Dept. - Univ. of Ferrara, Via Saragat, 1, 44100 Ferrara, Italy, roberto.pompoli@unife.it)

Ancient theatres are widely used today for modern performances including drama, music and ballets. Despite the state of conservation of the stage, the scenery is seldom designed with little care about its acoustical efficiency. Moreover, depending on the specific venue, a sound system can be employed in the performance. To clarify the acoustical impact of all these elements in ancient theatres, different stage settings and a sound sytem were investigated by means of scale model measurements. The scale model is a 1:20 scale reproduction of the ancient theatre of Siracusa (Italy). It is conceived as modular structure so that different configurations of the cavea and of the stage can be reproduced. To investigate the stage-set effects different groups of reflecting panels were arranged on the platform and an orchestra shell was tested too. Then, to simulate the sound system, two directional high frequency sources were assembled and optimized. The interplay of stage, sound system and theatre architecture was outlined by a comprehensive set of acoustical measurements.

\section{Contributed Paper}

\section{2:40}

4pAAa5. "Acoustics vases in ancient theatres: disposition, analysis from the ancient tetracordal musical system". Arturo Barba Sevillano (Grup d'Acustica Arquitectònica, Ambiental i Industrial, E.T.S.I.I, Univ. Politécnica de Valencia, Camino de Vera, s/n, 46022 Valencia, Spain, arbarse@doctor.upv.es), Radu Lacatis (Grup d'Acustica Arquitectònica, Ambiental i Industrial, E.T.S.I.I, Univ. Politécnica de Valencia, Camino de Vera, s/n, 46022 Valencia, Spain, rala1@doctor.upv.es), Alicia Giménez (Grup d'Acustica Arquitectònica, Ambiental i Industrial, E.T.S.I.I, Univ. Politécnica de Valencia, Camino de Vera, s/n, 46022 Valencia, Spain, agimenez@fis.upv.es), José Romero (Grup d'Acustica Arquitectònica, Ambiental i Industrial, E.T.S.I.I, Univ. Politécnica de Valencia, Camino de Vera, s/n, 46022 Valencia, Spain, romerof@fis.upv.es)
The acoustics of the ancient Greek and Roman theatres has always been rated as excellent by experts, without discussion. Beyond the purely architectural aspects, in this kind of outdoor theatres some mechanisms were used in order to improve the acoustics. In this paper we have studied the texts about "theatre's vases" of the famous book "On Architecture" by Vitruvius. Different interpretations and illustrations of these vases, that several translators carried out in the sixteenth to the eighteenth centuries, have been researched. From the wide bibliography consulted in this regard we have developed a plane with the disposition of the bronze vases in the theatres. In this plane we have specified the frequency of each one of them, and explained their disposition from the tetracordal musical system acquired from the Greek culture. Finally, an analysis of the disposition of the vases has been made. We have studied and looked for the musical intervals and harmonic relations among adjacent vases.

\section{Invited Papers}

\section{3:00}

4pAAa6. Musical granite pillars in ancient Hindu temples. Paul Calamia (Rensselaer Polytechnic Institute, Greene Bldg., 110 8th St., Troy, NY 12180, USA, calamp@rpi.edu), Jonas Braasch (Rensselaer Polytechnic Institute, Greene Bldg., 110 8th St., Troy, NY 12180, USA, braasj@rpi.edu)

In some ancient Hindu temples built in India between the 7th century and the 16th century can be found a fascinating architectural element: intricately designed musical pillars of solid granite. These pillars, clusters of which were often carved from a single piece of stone, were tuned by means of their length, width, and tension (induced by a load from above) and were played to accompany devotional readings and dance performances. Various tunings and sound characteristics were employed based on the intended purpose. In this paper, examples of extant pillars will be presented and discussed within the context of their acoustical and architectural significance.

4pAAa7. Convolution-scattering model for staircase echoes at the temple of Kukulkan. David Lubman (DL Acoustics, 14301 Middletown Lane, Westminster, CA 92683, USA, dlubman@dlacoustics.com)

Chirped echoes from staircases at the temple of Kukulkan at Chichen Itza have stimulated much interest since first reported at scientific meetings by the author in 1998. Among them are Declercq et al (J. Acoust. Soc. Am. 116, (6) 2004). They correctly observed that the echo depends strongly on the "type" of incident sound, but offered no explanation. Those authors overlooked an earlier explanation given at the First Pan-American/Iberian Meeting on Acoustics at Cancun, Mexico (Lubman, J. Acoust. Soc. Am. 112, (5) (2002). It included a mathematical model of the chirped echo effect and a full simulation of the chirped echo, including auralization. This model explicitly shows echo dependence on the time-frequency structure of the impinging sound. With it, ethnomusicologists and others can conveniently simulate the staircase echo for any recorded sound stimulus, including handclaps, voices, and ethnic sound instruments. The clap-echo system is modeled as a time-invariant linear system. The echo time series is found by convolving the stimulus (e.g., handclap) with the staircase impulse response. This earlier model is far more computationally efficient than the Declercq model, and achieves greater accuracy by avoiding the false and needless assumptions of plane wave propagation and infinite corrugated periodic surfaces. 


\section{Invited Paper}

\section{5:00}

4pAAa8. On the Acoustics of the Underground Galleries of Ancient Chavín de Huántar, Peru. Jonathan S. Abel (Stanford Univ., Center for Computer Research in Music and Acoustics (CCRMA), Dept. of Music, Stanford, CA 94305-8180, USA, abel@ccrma.stanford.edu), John W. Rick (Stanford University, Archaeology Center, P.O. Box 20446, Stanford, CA 94309, USA, johnrick@stanford.edu), Patty P. Huang (Stanford Univ., Center for Computer Research in Music and Acoustics (CCRMA), Dept. of Music, Stanford, CA 94305-8180, USA, pph@ccrma.stanford.edu), Miriam A. Kolar (Stanford Univ., Center for Computer Research in Music and Acoustics (CCRMA), Dept. of Music, Stanford, CA 94305-8180, USA, kolar@ccrma.stanford.edu), Julius O. Smith (Stanford Univ., Center for Computer Research in Music and Acoustics (CCRMA), Dept. of Music, Stanford, CA 94305-8180, USA, jos@ccrma.stanford.edu), John M. Chowning (Stanford Univ., Center for Computer Research in Music and Acoustics (CCRMA), Dept. of Music, Stanford, CA 94305-8180, USA, jc@ccrma.stanford.edu)

Chavín de Huántar is a monumental World Heritage archaeological site in the Peruvian highlands predating Inca society by over 2000 years. The importance of site acoustics is suggested by distinctive architectural features, notably an extensive network of underground galleries used in part for ritual purposes. The labyrinthine galleries are arranged in a series of small rectangular alcoves off narrow corridors. Here we focus on measuring and modeling the acoustics at Chavín to understand the implications of auditory experiences within the galleries as related to the site's role in developing religious authority. Acoustic measurements and models of a site can be used to archive site acoustics, estimate the acoustics of inaccessible or alternative site architectures, and reconstruct original site acoustics; they may also be used to corroborate aspects of rituals suggested by other archaeological data. Preliminary acoustic measurements at Chavín show a short reverberation time, dense and energetic early reflections, and a large lateral energy fraction. The short reverberation time would enable rhythmically articulated group performance using Strombus shell trumpets found onsite. The early reflections would provide strong acoustic reinforcement and consistent resonances for participants in gallery alcoves. The wide soundfield would envelop participants, contributing to the ritual experience.

\section{Contributed Paper}

$5: 20$

4pAAa9. Assessment of Acoustical Characteristics for Historical Baths (Hammams). Asuman Aydin (Middle East Technical University, Dept of Architecture, METU (ODTU), Inonu Blvd, 06531 Ankara, Turkey, aydinasuman@yahoo.com), Ayse Tavukcuoglu (Middle East Technical University, Dept of Architecture, METU (ODTU), Inonu Blvd, 06531 Ankara, Turkey, aysetavukcuoglu@yahoo.com), Mehmet Caliskan (Middle East Technical University, Dept of Architecture, METU (ODTU), Inonu Blvd, 06531 Ankara, Turkey, caliskan@metu.edu.tr)

Comprehensive studies are necessary to better understand the original acoustical characteristics of historical baths (hammams) in order to discover the historical technologies establishing acoustical properties in these structures and keep their proper functioning for long periods of time. The study was conducted on five historical Turkish hammams, belonging to the Otto- man period. Their acoustical performances were examined in terms of basic acoustical parameters, such as, reverberation time, early decay time, clarity, lateral fraction, sound transmission index. The 3D computer modelling and acoustics simulation were done for each structure by "ODEON combined $8.5^{\prime \prime}$. Their audio performance was evaluated by taking into consideration of the volume, materials use and environmental conditions. The analyses were done for the case of the unoccupied condition while one of the structures, Sengul Hammam in Ankara, was analysed both for non- and fully-occupied conditions. The joint interpretation of the results was done in order to define the acoustical features of the hammam structures. The potentials for improving the acoustical performance of interiors were also discussed. The use of 3D computer modeling and acoustical simulation, adapted for the case of historical hammam structures, provided a good opportunity for the assessment of their acoustical performance on quantitative basis

\section{Invited Papers}

4pAAa10. History and acoustics of the Asian free-reed mouth organs. James Cottingham (Coe College, 1220 First Avenue NE, Cedar Rapids, IA 52402, USA, jcotting@coe.edu)

Mouth-blown instruments employing a free reed coupled to a pipe resonator have long been known and used throughout East and Southeast Asia. Details of the origin and development of these instruments are not known, but are closely connected with the history and prehistory of a multitude of ethnic groups. Free reed instruments have been employed in a variety of ways, from simple signaling devices to use in the court music of Japan and China. The pipe resonators vary from the buffalo horn to bamboo pipes of nearly cylindrical cross section. The instruments exemplify a pipe-resonator coupling significantly different from that of the standard wind instruments of European origin. In some cases the reed is at or near one end of an open or closed pipe resonator, but in other examples the reed is mounted in the side of the resonator away from the ends. A summary of recent experimental investigations of these instruments will be presented, along with musical examples. 
4pAAa11. The mridangam: A study of the history and acoustics of an ancient South Indian drum. Rohan Krishnamurthy (Kalamazoo College, 544 Sunrise Circle, Kalamazoo, MI 49009, USA, rohan@rohanrhythm.com), Ian Hempe (Coe College, 1220 First Avenue NE, Cedar Rapids, IA 52402, USA, ikhempe@coe.edu), James Cottingham (Coe College, 1220 First Avenue NE, Cedar Rapids, IA 52402, USA, jcotting@coe.edu)

The acoustical properties of the South Indian drum, the mridangam, were studied. The barrel-shaped mridangam has been described in ancient Hindu scriptures and depicted in cave paintings and temple sculptures. With a claimed antiquity dating back to the Vedic period, it is the principal percussion instrument in South Indian classical music and dance and possesses unique tonal properties. The mridangam is comprised of three primary parts: The tonal head (valanthalai), the bass head (thoppi), and the central shell (kattai), to which the two heads are traditionally fastened by leather rope. Measurements of modes and mode frequencies were made on traditional drums, as well as on drums where the heads were remounted using a new and user-friendly design. Measurements of drumhead vibration and sound spectra were also made when the drumhead was excited by a skilled player using standard strokes. The frequencies of the first few modes of the tonal head were found, as expected, to be tuned approximately harmonic. Practical performance variables, including effects of altering mounting tension and coupling between the drumheads, were also studied. Results from the study will be followed by a practical demonstration of the instrument.

6:20

4pAAa12. Auditory capacities of human fossils: A new approach to the origin of speech. Ignacio Martínez ((1) Universidad de Alcala de Henares; (2) Centro UCM-ISCIII de Evolución y Comportamiento Humanos, Centro UCM-ISCIII de Evolución y Comportamiento Humanos, C/ Sinesio Delgado, N 4, Pabellón 14, 28029 Madrid, Spain, imartinezm@isciii.es), Rolf Michael Quam ((1) American Museum of Natural History; (2) Centro UCM-ISCIII de Evolución y Comportamiento Humanos, Division of Anthropology, American Museum of Natural History, Central Park West @ 79th St., New York, NY 10024, USA, rquam@amnh.org), Manuel Rosa (Universidad de Alcalá de Henares, Edificio de Ciencias, Campus Universitario, 28871 Alcalá de Henares, Spain, manuel.rosa@uah.es), Pilar Jarabo (Universidad de Alcalá de Henares, Edificio de Ciencias, Campus Universitario, 28871 Alcalá de Henares, Spain, mpilar.jarabo@uah.es), Carlos Lorenzo ((1) Universitat Rovira i Virgili (2) Centro UCM-ISCIII de Evolución y Comportamiento Humanos, Universitat Rovira i Virgili, Facultat de Lletres Institut de Paleoecologia Humana, Area de Prehistoria, i Evolució Social, Imperial Tarraco, 1, 43005 Tarragona, Spain, clorenzo@prehistoria.urv.es), Juan Luis Arsuaga ((1) Universidad Complutense de Madrid; (2) Centro UCM-ISCIII de Evolución y Comportamiento Humanos, Centro UCM-ISCIII de Evolución y Comportamiento Humanos, C/ Sinesio delgado, N 4, Pabellón 14, 28029 Madrid, Spain, jlarsuag@isciii.es)

The origin and evolution of human language has mainly dealt with the reconstruction of the upper respiratory tract of human fossils. After decades of controversy no clear results have arisen from these studies. We propose a new approach to this issue based on the possibility to reconstruct the sound power transmission, through the external and middle ear, in fossil specimens. The results thus obtained in the more than 500 ky old fossils from the Sima de los Huesos site (Sierra de Atapuerca, Spain) show that this hominins had the same auditory capacities as modern human, suggesting an older origin for speech than any previous study.

\section{6:40}

4pAAa13. The marvellous sound world in the 'Phonurgia Nova' of Athanasius Kircher. Lamberto Tronchin (DIENCA CIARM, University of Bologna, viale Risorgimento, 2, I-40136 Bologna, Italy, tronchin@ciarm.ing.unibo.it), Ilaria Durvilli (DIENCA - CIARM, University of Bologna, viale Risorgimento, 2, I-40136 Bologna, Italy, ilaria.durvilli@mail.ing.unibo.it), Valerio Tarabusi (DIENCA - CIARM, University of Bologna, viale Risorgimento, 2, I-40136 Bologna, Italy, ciarm02@hotmail.com), Galia Mastromatteo (DIENCA - CIARM, University of Bologna, viale Risorgimento, 2, I-40136 Bologna, Italy, gam@ ciarm.ing.unibo.it)

Athanasius Kircher, Jesuit, was born in Geisa, Thüringen, in 1608. He spent a large amount of his life in Rome, where he died in 1680. He was active in many different topics, ranging from geology to philosophy. He was the author of many books at his time, among all the Musurgia Universalis, written in 1650, and the Phonurgia Nova, of 1673. Whilst the Musurgia Universalis gathered a wide attention also in recent years, the Phonurgia Nova, which means " a new method of sound production", was only recently rediscovered. In this paper the original Latin version of the Phonurgia Nova, which comprises two different books, i.e. the "Phonosophia nova" and the "Phonosophia anacamptica", is analyzed. The first book deals with the influence of music in the human being, whereas the second book analyses the sound propagation in enclosure. This book reveals some interesting acoustic apparatus for sound production and propagation, as the "tuba stentorophonica" (the loud trumpet), the "statua citofonica" (the talking statue). Some of these phonic apparatus are described, analyzed and commented. 


\title{
Session 4pAAb
}

\section{Architectural Acoustics: Archeological Acoustics II (Poster Session)}

\author{
David Lubman, Cochair \\ DL Acoustics
}

\author{
Jens Holger Rindel, Cochair \\ Odeon $A / S$
}

\begin{abstract}
All posters will be on display from 3:40 p.m. to 5:20 p.m. To allow contributors an opportunity to see other posters, contributors of odd-numbered papers will be at their posters from 3:40 p.m. to 4:30 p.m. and contributors of even-numbered papers will be at their posters from 4:30 p.m. to 5:20 p.m.
\end{abstract}

\section{Contributed Papers}

4pAAb1. Acoustics of Unique Baroque Theatre in Český Krumlov Jana Dolejsi (Studio D - Akustika s.r.o., Zizkova 12, 37122 Ceske Budejovice, Czech Republic, jana.dolejsi@akustikad.com), Pavel Slavko (Státní hrad a zámek, 38101 Cesky Krumlov, Czech Republic, castle@ckrumlov.cz), Ladislav Pouzar (Chvalšinská 231, 38101 Cesky Krumlov, Czech Republic, ladislav@pouzar.cz), Monika Rychtarikova (Lab. ATF, Katholieke Universiteit Leuven, Celestijnenlaan 200D, B-3001 Leuven, Belgium, Monika.Rychtarikova@bwk.kuleuven.be)

The castle theater in the Český Krumlov is a unique historical site in Europe which represents a valuable example of a theater with Baroque scenes from the late 18th century. It was preserved with exceptional completeness and authenticity since it has never burned, and during the last two centuries it was not modernized. The baroque period must be understood as a universal style with many methods of expression which act in harmony, and the individual elements must complement each other and be interconnected to produce an effective unit. It was a challenge to experimentally prove these relationships and connections in as wide context as possible (i.e. relations between the architecture, contemporary scene lighting, perspectives and proportions, roomacoustic properties, contemporary musical interpretations and vocal techniques, placing of the orchestra and other. According to musicians, actors and audience is this theatre known as a hall with excellent acoustic condition. However, no room acoustic measurements were performed in the theatre in the last century. This article report on results from the acoustical measurements and simulation in the main hall of the theatre which help us to understand which acoustical values people find pleasant in the room of this character.

4pAAb2. Acoustical reconstruction of San Petronio Basilica in Bologna during the Baroque period: the effect of festive decorations. Francesco Martellotta (DAU - Politecnico di Bari, via Orabona 4, 70125 Bari, Italy, f.martellotta@poliba.it), Ettore Cirillo (DAU - Politecnico di Bari, via Orabona 4, 70125 Bari, Italy, e.cirillo@poliba.it), Sabrina Della Crociata (DAU - Politecnico di Bari, via Orabona 4, 70125 Bari, Italy, sade11984@libero.it), Emanuele Gasparini (via Centurare 33, 37062 Dossobuono, Italy, emanuele.gasparini@libero.it), Daniela Preziuso (DAMS Alma Mater Studiorum Univ. di Bologna, via Barberia, 4, 40123 Bologna, Italy, aleinad2002@libero.it)

The Basilica of San Petronio in Bologna (Italy) is a large Gothic church characterized by three naves divided by cluster piers made of brick and flanked by square chapels. It is $130 \mathrm{~m}$ long, $60 \mathrm{~m}$ wide and $44 \mathrm{~m}$ high, developing a volume of $170000 \mathrm{~m}^{3}$. The widespread use of smooth plaster and the substantial lack of decoration give rise to a reverberation time (in unoccupied conditions) which varies from about $13 \mathrm{~s}$ at $125 \mathrm{~Hz}$ to $5 \mathrm{~s}$ at $4 \mathrm{kHz}$, with an average of $10.7 \mathrm{~s}$ at mid frequencies. In occupied conditions the expected mid-frequency reverberation time should lower to about $7 \mathrm{~s}$. None- theless, these acoustic conditions appear scarcely compatible with the characteristics of the Baroque music which was composed for the "Cappella musicale" during the 17th century. However, historical research pointed out how, in that period, rich draping and curtains were often used during the major religious and civil celebrations. The analysis of the acoustic consequences of such temporary installations was performed by means of acoustic simulation based on historical records calibrated on the current configuration of the church. The paper presents the results of such reconstruction.

4pAAb3. Assessment of strong reflections in ancient theatres: Spatial information from parallel measurement data. Giuseppe Rodonò (DREAM, Univ. of Palermo, Viale delle Scienze, Edificio 9, 90128 Palermo, Italy, rodon@unipa.it), Marco Gullo (DREAM, Univ. of Palermo, Viale delle Scienze, Edificio 9, 90128 Palermo, Italy, mgullo@dream.unipa.it), Armando La Pica (DREAM, Univ. of Palermo, Viale delle Scienze, Edificio 9, 90128 Palermo, Italy, lapica@unipa.it), Vincenzo Vinci (DREAM, Univ. of Palermo, Viale delle Scienze, Edificio 9, 90128 Palermo, Italy, vincenzo_vinci@hotmail.it)

The sound field in ancient open-air theatres shows a finite time-response in a transient. The structure of the time-response depends on geometrical characteristics of the theatre and source-receiver position: upon geometry dimension of the stage area its possible to observe nearly strong reflections and quantify the associated delay time. Sampling the theatre space with a single microphone does not allow any directional information on the sound field but the parallel use of more microphones and software post-processing could add spatial information. Dataset consists of four syncronized impulse response measured along a radial direction in the theatre plan for differents source positions. A directional receiver system based on a post-processing method has been implemented and applied to measurement data. The obtained experimental results are reported and discussed.

4pAAb4. Ultrasonic quantitative strength assessment of artificially aged and archaeological wood samples. Ari Salmi (Univ. of Helsinki / Dept. of Physical Sciences, POB 64 (Gustaf Hällströmin katu 2), 00014 Helsinki, Finland, edward.haeggstrom@helsinki.fi), Kari Steffen (P.O.B 56 University of Helsinki, 00014 Helsinki, Finland, kari.steffen@helsinki.fi), Joona Eskelinen (Univ. of Helsinki / Dept. of Physical Sciences, POB 64 (Gustaf Hällströmin katu 2), 00014 Helsinki, Finland, joona.eskelinen@helsinki.fi), Edward Hæggström (Electronics Research Unit, University of Helsinki, P.O.Box 64 (Gustaf Hällströmin katu 2), FIN-00014 Helsinki, Finland, edward.haeggstrom@helsinki.fi)

A large fraction of discovered archaeological artefacts are wooden. Since materials grow brittle and their stiffness decreases over time, it is vital for archaeologists to have methods that assess the strength of the object prior to 
moving it. We present preliminary shear and elastic modulus data measured using $100 \mathrm{kHz}$ longitudinal and shear ultrasonic tone burst throughtransmission. Artificially aged wooden samples and archaeological samples of known age were used to validate the aging procedure by comparison of the shear and elastic modulus. We also measured the modulae of waterlogged samples obtained from the ship wreck Vrouw Maria sunken in the Finnish archipelago in 1771, and compared to samples artificially aged to similar age. Scanning electron microscopy (SEM) was used to compare the structure of artificially and naturally aged samples. The results presented may be used to create an artefact model for strength assessment, and to give guidelines of strength vs. age for archaeologists to support their logistics decisions.

4pAAb5. The Parpalló Cave: A singular archaeological acoustic site. Noé Jiménez González (Universidad Politécnica de Valencia, C/ La trampa
$\mathrm{N}^{\circ}$ 2, 02520 Chinchilla de Montearagón, Spain, nojigon@epsg .upv.es), Rubén Picó (EPSG - Univ. Politécnica de Valencia, c/ NazaretOliva s/n, 46780 Grau de Gandia, Spain, rpico@fis.upv.es), Javier Redondo (IGIC - Universitat Politècnica de València, Cra. Nazaret-Oliva S/N, E-46730 Gandia, Spain, fredondo@fis.upv.es)

The Parpalló Cave is located in the slopes of Montdúber, in Valencia (Spain). It is one of the most important Palaeolithic sites, not only in Spain, but in the world. It was a privileged location for its inhabitants from the Upper Palaeolithic onwards. It has one of the most spectacular collections of Palaeolithic art mobilier found to date. The Parpalló cave features a considerably large opening which, undoubtedly, influences its acoustic properties. Indeed, the sound pressure field inside the cave is not excessively reverberant and intelligibility is significantly better than in similar enclosures. In this work a study of the acoustic properties of the Parpalló cave is performed by using numerical simulation.

\title{
Session 4pAAc
}

\section{Architectural Acoustics and Psychological and Physiological Acoustics: Speech Segregation in Rooms II (Poster Session)}

\author{
Barbara Shinn-Cunningham, Cochair \\ Boston University Hearing Research Center \\ Mathieu Lavandier, Cochair \\ Cardiff University
}

\begin{abstract}
All posters will be on display from 3:40 p.m. to 5:20 p.m. To allow contributors an opportunity to see other posters, contributors of odd-numbered papers will be at their posters from 3:40 p.m. to 4:30 p.m. and contributors of even-numbered papers will be at their posters from 4:30 p.m. to 5:20 p.m.
\end{abstract}

\section{Contributed Papers}

4pAAc1. Effects of spatial factors of speech, noise, and reverberation sounds on listening difficulty. Hayato Sato (Environmental Acoustics Laboratory, Graduate School of Engineering, Kobe University, Rokkodai 1-1, Nada, 657-8501 Kobe, Japan, hayato@kobe-u.ac.jp), Tomoyasu Takaoka (Environmental Acoustics Laboratory, Graduate School of Engineering, Kobe University, Rokkodai 1-1, Nada, 657-8501 Kobe, Japan, Tomoyasu.Takaoka@mb2.seikyou.ne.jp), Masayuki

Morimoto (Environmental Acoustics Laboratory, Graduate School of Engineering, Kobe University, Rokkodai 1-1, Nada, 657-8501 Kobe, Japan, mrmt@kobe-u.ac.jp)

It is well known that the amount of auditory masking in binaural listening is affected by spatial factors of signal and masking sounds such as the degree of interaural cross-correlation and the arriving direction. The amount of auditory masking for speech signal are usually measured using speech intelligibility tests. However, in the sound fields that appears in everyday life, speech intelligibility are usually close to $100 \%$. Therefore, it was unclear how much the effects of spatial factors on speech communication in everyday life are. Listening tests were performed to clarify the effects of spatial factors on listening difficulty ratings [M. Morimoto et al., J. Acoust. Soc. Am. 116, 1607-1613 (2004)] focussing on the degree of interaural cross-correlations of speech, noise, and reverberation sounds, and on the arriving direction of speech signal. The two spatial factors were controlled by several loudspeakers which are horizontally installed in an anechoic room. The results of listening tests showed that the effects of the two spatial factors on listening difficulty ratings decreased with improving listening conditions, and the effects can be ignored in the sound fields that often appears in everyday life.

4pAAc2. Binaural enhancement of speech intelligibility metrics. Florent Deux (Chalmers University of Technology, Division of Applied Acoustics, Chalmers Room Acoustic Group, SE-41296 Göteborg, Sweden, florentdeux@gmail.com), Mendel Kleiner (Chalmers Rm. Acoust. Group, Dept. of Appl. Acoust., Chalmers Univ. of Technology, 41296 Gothenburg, Sweden, mendel.kleiner@chalmers.se)

Efficient methods of quantifying speech intelligibility are needed for designing and understanding functional architectural spaces. All current measures of speech intelligibility are based on monaural impulse response, which excludes consideration of important binaural aspects of human hearing, including dereverberation and decoloration. Acquisition of binaural data has increasingly become common practice, using dummy heads or in-ear microphones, making the development of binaural intelligibility measures especially timely. The need for such a measure is illustrated by calculations of speech transmission index (STI) with a single energy-based impulse obtained through combination of binaural data channels. Each method of 
combination produces significantly different STI values that illustrate the impact of orientation and location on intelligibility calculations. The relationship between such estimation variation and subjective experience must be studied to determine the research direction for a much needed, uniquely binaural speech intelligibility measure. The aim of this research is then to provide a guide to be used in comparing values obtained by the various methods. The project will then involve measurements of psychoacoustic metrics for speech intelligibility by objective methods as well as determination of speech intelligibility by subjective methods using tests with binaural recording and playback listening.

\title{
Session 4pAAd
}

\section{Architectural Acoustics: Architectural Acoustics Potpourri III (Poster Session)}

\author{
Ingo Witew, Cochair \\ Institute of Technical Acoustics, Templergraben 55, Aachen, 52056, Germany \\ Byron Harrison, Cochair \\ Talaske, 1033 South Boulevard, Oak Park IL 60302, USA
}

\begin{abstract}
All posters will be on display from 3:40 p.m. to 5:20 p.m. To allow contributors an opportunity to see other posters, contributors of odd-numbered papers will be at their posters from 3:40 p.m. to 4:30 p.m. and contributors of even-numbered papers will be at their posters from 4:30 p.m. to 5:20 p.m.
\end{abstract}

\section{Contributed Papers}

4pAAd1. Analysis of the impact of sound diffusion in the reverberation time of an architectural space - A proposal for the characterization of diffusive surfaces using scale models. Elisa Garay-Vargas (Program of Graduate Studies in Design, CyAD - Universidad Autónoma Metropolitana, Av. San Pablo 180, Edificio D 101, Col. Reynosa Tamaulipas, Delegacion Azcapotzalco, 02200 Mexico, D.F., Mexico, elisagaray@gmail.com) , Fausto E. Rodriguez-Manzo (Departamento de Procesos y Tecnicas, CyAD, Universidad Autonoma Metropolitana-Azcapotzalco, Av. San Pablo 180, Edificio H-PB, Col. Reynosa Tamaulipas, Delegacion Azcapotzalco, 02200 Mexico, D.F., Mexico, rfme@correo.azc.uam.mx)

The acoustic quality of architectural spaces has been related directly with the reverberation phenomenon, in order to understand more about sound in architecture, this proposal tries to conclude how diffusion acts on the reverberation time. The fact that architects deal with absorption coefficients and change the reverberation time by playing and designing with materials, opens a door to the possibility of new ways of architectural design, knowing how simple or complex and even natural diffusion surfaces will act in space. Several examples of the use of diffusion and reverberation in architectural spaces can be found in buildings like the Jewish Museum from Daniel Libeskind or the Therme Vals from Peter Zumthor, the use of big surfaces of concrete in the first and uneven surfaces in the second, transform each of these places in one with very interesting sound qualities. To create spaces like these we can take diffusion as a design argument but, How will certain diffusive surfaces influence on the reverberation time of an architectural space? To answer this question it is necessary to experiment with scale models measuring the reverberation time, and not the diffusion itself.

4pAAd2. Re-evaluating Noise Criterion in Digital Audio Recording Environments. Linda Gedemer (5930 Penfield Ave., Woodland Hills, CA 91367, USA, gedeml@rpi.edu)

The absence of audible noise in recording studios is highly desirable. For this reason, the acoustic design of recording facilities usually involves an accurate assessment of the audibility of background noise. However, noise criterion that sets levels for inaudible noise do not take in account the sensitivity of today's digital recording equipment and post production processing. Noise-level criterion and measurement techniques exist in order to created an atmosphere where noise is rated at a level of audibility with respect to the type of space being measured. In this paper these criterion will be analyzed in terms of critical recording spaces. The criterion will be considered in terms of current building practices and digital audio recording equipment used. A set of questions were sent to working sound engineers in order to received first hand opinions regarding noise issues in recording studios. It is not the intention of this report to suggest a new noise criterion but rather to better understand existing problems which could then help in the creation of a more relevant noise criterion to be developed in the future.

4pAAd3. Possible correlation between acoustic and thermal performances of building structures. Giovanni Semprini (University, DIENCA Dept. Facoltà di Ingegneria, Viale Risorgimento 2, 40136 Bologna, Italy, giovanni.semprini@mail.ing.unibo.it), Alessandro Cocchi (University, DIENCA Dept. Facoltà di Ingegneria, Viale Risorgimento 2, 40136 Bologna, Italy, alessandro.cocchi@mail.ing .unibo.it), Cosimo Marinosci (DIENCA - Univ. of Bologna, Viale Risorgimento 2, 40136 Bologna, Italy, cosimo.marinosci@mail.ing.unibo.it)

Most European standards required high performance values for sound and thermal insulation in building structures, according to Directive EEC $89 / 106$. Sound transmission and heat transfer in structures have different physical and analytical approach and specific parameters of performance (i.e. sound transmission loss or thermal transmittance) are not directly correlated each others; many kind of structures have also different behaviour depending on mechanical properties of materials, numbers of layers of materials, etc. The aim of this work is to analyse possible correlation between sound transmission performances and thermal properties values in order to evaluated common trends related to physical properties of the various building components, like for example density or surface mass.

4pAAd4. On the use of a corrugated ceiling for noise reduction in rooms. Nico F. Declercq (Georgia Tech Lorraine - G.W. Woodruff School of ME, UMI Georgia Tech - CNRS 2958, 2 rue Marconi, 57070 Metz, France, nico.declercq@me.gatech.edu), Katelijn Vanderhaeghe (Georgia Tech Lorraine - G.W. Woodruff School of ME, UMI Georgia Tech - CNRS 
2958, 2 rue Marconi, 57070 Metz, France, declercq@ieee.org),Katrien Dewijngaert (Georgia Tech Lorraine - G.W. Woodruff School of ME, UMI Georgia Tech - CNRS 2958, 2 rue Marconi, 57070 Metz, France, declercq@ieee.org), Patricia Verleysen (Ghent University, Mechanics of Materials and Structures, Sint-Pietersnieuwstraat 41, 9000 Ghent, Belgium, Patricia.Verleysen@ugent.be)

In certain halls the acoustics is dominated by distinct frequencies having disturbingly high intensities caused by yelling children, such as in an indoor swimming pool, or by industrial production processes. The use of damping materials to cover the ceiling, floor and side walls is not always permitted because of esthetic reasons or because of safety grounds or even humidity. The current study shows that in those cases the use of a corrugated surface is possible and is actually more effective in filtering out very distinct frequencies. An example is presented for frequencies at 3 to $4 \mathrm{kHz}$ and numerical results are obtained in support of the use of a corrugated structure rather than classic damping materials such as baffles.

4pAAd5. Musicians' Adjustment of Performance to Room Acoustics, Part III: Understanding the Variations in Musical Expressions. Kosuke Kato (Center for Advanced Science and Innovation, Osaka University, Yamadaoka 2-1, Suita-shi, 565-0871 Osaka, Japan, kato@casi .osaka-u.ac.jp), Kanako Ueno (Institute of Industrial Science, University of Tokyo, Komaba 4-6-1, Meguro-ku, 153-8505 Tokyo, Japan, ueno@iis.u-tokyo.ac.jp), Keiji Kawai (Graduate School of Science and Technology, Kumamoto University, Kurokami 2-39-1, Kumamoto-shi, 8608555 Kumamoto, Japan, kawai@arch.kumamoto-u.ac.jp)

This paper attempts to investigate the acoustic variations in the musical sound signals produced by professional performers under different room acoustic conditions. The sound signals produced by four professional instrumentalists and an operatic baritone singer under simulated concert hall sound fields were recorded by placing a unidirectional microphone close to either the instruments or the mouth of the opera singer. In order to quantify the extent of the variations in the resulting sound signals due to the adjustment of the musical expressions, an acoustic analysis was conducted. The results indicated that the "note-on ratio," defined as the ratio of tone duration and inter-onset interval, of several staccato tones was decreased in reverberant halls. The higher harmonics of tones of an oboist and a flutist were suppressed in reverberant sound fields, while the vibrato extent of a violinist was considerably varied as was reported in our previous study (Part II). Based on the results of the interviews with the performers during the recordings, it was inferred that the variations in the musical sound signals were produced by the performers to adjust to the room acoustic conditions.

4pAAd6. In situ measurement of acoustic parameters with smart devices. Karl Van Nieuwenhuyse (Vrije Universiteit Brussel, Acoustics and Vibration Research Group, Dept. of Mechanical Engineering, Pleinlaan 2, BE-1050 Brussels, Belgium, karl.van.nieuwenhuyse@ skynet.be), Patrick Guillaume (Vrije Universiteit Brussel, Acoustics and Vibration Research Group, Dept. of Mechanical Engineering, Pleinlaan 2, BE-1050 Brussels, Belgium, Patrick.Guillaume@vub.ac.be), Steve Van Landuit (Vrije Universiteit Brussel, Acoustics and Vibration Research Group, Dept. of Mechanical Engineering, Pleinlaan 2, BE-1050 Brussels, Belgium, svanlandui @ vub.ac.be), Cedric Vuye (Hogeschool Antwerpen, Dept. of Industrial Sciences, Paardenmarkt 92, BE-2000 Antwerpen, Belgium, c.vuye@ha.be), Gert De Sitter (Vrije Universiteit Brussel, Acoustics and Vibration Research Group, Dept. of Mechanical Engineering, Pleinlaan 2, BE-1050 Brussels, Belgium, gert.de.sitter@vub.ac.be)

Instruments for in situ analysis of the acoustic parameters of a room are, due to their high prime cost, not widely accessible. In an effort to change this, new software was developed to enable these measurements by use of highly portable consumer electronics such as PDA's and Smart phones. The newly developed software calculates the reverberation time using the impulse method and aims at delivering accurate results using the built-in microphone or an external one. This paper will discuss the accuracy of the new software in comparison with professional equipment through several case studies, even as the different techniques used to overcome the inherent problems forthcoming the equipment used, i.e. microphone quality and automatic gain control.

4pAAd7. Efficiency of two-dimensional interpolation algorithms for high-quality dynamic binaural synthesis. Karim Helwani (Department of Audio Communication, Technical University of Berlin, Sekr. EN-08, Einsteinufer 17c, 10587 Berlin, Germany, karim.helwani@gmx.de), Alexander Lindau (Department of Audio Communication, Technical University of Berlin, Sekr. EN-08, Einsteinufer 17c, 10587 Berlin, Germany, alexander.lindau@tu-berlin.de), Stefan Weinzierl (Department of Audio Communication, Technical University of Berlin, Sekr. EN-08, Einsteinufer 17c, 10587 Berlin, Germany, stefan.weinzierl@tu-berlin.de)

Binaural synthesis of acoustical environments is based on binaural room impulse responses (BRIRs) measured with an angular resolution of typically between $1^{\circ}$ and $15^{\circ}$. Considering the size of the resulting BRIR database used for auralization and the long measurement duration for its acquisition, it is reasonable to use interpolation from a lower resolution BRIR grid. Based on a mathematical formulation of the interpolation problem for BRIRs, a set of different solutions for two-dimensional spaces is described and compared with regard to efficiency and real time performance. In order to evaluate the degradation introduced by interpolation, different methods have been implemented and applied to a HRTF and a BRIR database measured in two degrees of freedom with $1 \% 1^{\circ}$ horizontal/vertical resolution. These are bilinear interpolation (i.e. nearest neighbour, inverse distance weighting), spherical spline interpolation, wavelet interpolation, and interpolation based on principal component analysis (PCA). A listening test following an $\mathrm{ABX}$ procedure has been performed to evaluate the efficiency of the different interpolation methods according to the detection rates of interpolated versus measured BRIR databases in a dynamically auralized acoustical environment.

4pAAd8. Perception of teachers and pupils to the acoustics of classrooms. Carmen L. Loro (Federal University of Parana, Centro Politécnico - Setor de Tecnologia, Bairro Jardim das Américas, 81531-990 Curitiba, Brazil, loro@arnet.com.br), Paulo Henrique Trombetta T. Zannin (Federal University of Parana, Centro Politécnico - Setor de Tecnologia, Bairro Jardim das Américas, 81531-990 Curitiba, Brazil, paulo.zannin @ pesquisador.cnpq.br)

The present survey has evaluated the acoustic characteristics of classrooms built as modular classrooms. The study has focused on the background noise, reverberation time, noise insulation and interviews with teachers and pupils. The acoustic comfort of classrooms in a Brazilian public school has been evaluated through interviews with 62 teachers and 464 pupils. Acoustic measurements have revealed the poor acoustic quality of the classrooms. The walls between the classrooms and the corridor have permanent ventilation openings on glassy bricks. The measured weighted apparent sound reduction index (ISO 140-4; ISO 717-1) for the wall, with a door and glassy brick, was R'w $=17 \mathrm{~dB}$. The low value of the weighted apparent sound reduction index $\mathrm{R}^{\prime} \mathrm{w}=17 \mathrm{~dB}$ contributes significantly to the noise transmission from one room to the other, contributing to the elevated levels of background noise inside the classrooms. Interviews with teachers and pupils have shown that the main noise sources noted in the classrooms originate inside the school: voices of students and of the teacher of the neighboring classroom.

4pAAd9. Comparing the sound absorption of different objects. Robert Hickling (Sonometrics Inc., 8306 Huntington Road, Huntington Woods, MI 48070-1643, USA, sonometrics@comcast.net), John Kopko (Kolano and Saha Engineers Inc., 3559 Sashabaw Road, Waterford, MI 48329, USA, jkopko@kandse.com), Pranab Saha (Kolano and Saha Engineers Inc., 3559 Sashabaw Road, Waterford, MI 48329, USA, prsaha@kandse.com)

Current methods of measuring sound absorption involve a piece of material. Small pieces are tested in impedance tubes. Larger pieces are tested in a reverberation room, using the reverberation-time decay method, as in ISO Standard 354. However these procedures do not determine the absorption of a complete object. It is proposed to determine the absorption of 
an object by measuring the negative sound power of the object in a reverberation room using sound intensity. It has been shown that sound power using sound intensity can be measured accurately in reverberation rooms $(R$. Hickling, Proceedings NOISE-CON 97, pages 483-488). Negative sound power on its own, however, cannot provide a satisfactory comparison indicator between objects because it depends on the room. It is necessary, there- fore, to develop a normalization procedure to make the negative soundpower measurement independent of the room. A procedure is described which was tested for the same object (a car seat) in two different reverberation rooms. The results are encouraging. It is planned to conduct further tests using a variety of objects in different rooms to check the validity of the normalization procedure.

\title{
Session 4pAAe
}

\section{Architectural Acoustics and Noise: Acoustics and Privacy in Healthcare Facilities II: Emerging Research Around the World II (Poster Session)}

\author{
William Cavanaugh, Cochair \\ Cavanaugh Tocci Associates, Inc. \\ Kerstin Persson Waye, Cochair \\ Dept. of Environ. Medicine, The Sahlgrenska Acad. of Gothenburg Univ.
}

\begin{abstract}
All posters will be on display from 3:40 p.m. to 5:20 p.m. To allow contributors an opportunity to see other posters, contributors of odd-numbered papers will be at their posters from 3:40 p.m. to 4:30 p.m. and contributors of even-numbered papers will be at their posters from 4:30 p.m. to 5:20 p.m.
\end{abstract}

\section{Contributed Paper}

4pAAe1. The noise control in laboratories and health areas: A keyword in order to pratice biosafety. Marta Ribeiro Valle Macedo (Fundaçào Oswalso Cruz, Avenida Brasil 4365 - Manguinhos, 21040-360 Rio de Janeiro, Brazil, mribeiro@ fiocruz.br)

The noise control in laboratories and health areas: a keyword in order to pratice biosafety Marta Ribeiro Valle Macedo Coordenação de Saúde do trabalhador, Fundação Oswaldo Cruz, Av. Brasil 4365, Manguinhos-Rio de Janeiro, Brasil, CEP.21040-360 The environmental noise control is an increasing preoccupation at the international level in order to observing the effects on the human health. At the outdoor environment or in habitations, in work places and health areas, these effects are verified, as mentioned by World
Health Organization $(1995 ;$ 1999) and in rich bibliography about this subject. In areas with biological and chemical risk, where the development of activities need of great concentration, noise is a stressor agent and causes direct and indirect effects on the human health as well as affecting the intelligibility of the speech, the performance of activities and causing work accidents. However, the exposition to the main sound sources found in laboratories and hospitals located at the Fundação Oswaldo Cruz (Fiocruz), a Brazilian health institution, could have been avoided if some noise control measures had been employed. This paper presents the main situations observed at the evaluation devoloped during 2007, in 744 environments of work and shows how environmental noise comes interfering at the workers performance in Fiocruz. 


\title{
Session 4pAAf
}

\section{Architectural Acoustics: Case Studies and Design Approaches II (Poster Session)}

\author{
Ingo Witew, Cochair \\ Institute of Technical Acoustics, Templergraben 55, Aachen, 52056, Germany \\ Byron Harrison, Cochair \\ Talaske, 1033 South Boulevard, Oak Park IL 60302, USA
}

\begin{abstract}
All posters will be on display from 3:40 p.m. to 5:20 p.m. To allow contributors an opportunity to see other posters, contributors of odd-numbered papers will be at their posters from 3:40 p.m. to 4:30 p.m. and contributors of even-numbered papers will be at their posters from 4:30 p.m. to 5:20 p.m.
\end{abstract}

\section{Contributed Papers}

4pAAf1. Predicting the acoustics of historic Istanbul Tunnel: Simulation, calculation methods and geometrical details. Filiz Bal Kocyigit (Karabuk University, 232. Str. No: 5/5 Ilkbahar Mah., Cankaya, 06550 Ankara, Turkey, filizbkocyigit@yahoo.com), Sertan Senturk (20th Street 17/6 Bahçelievler, 06490 Ankara, Turkey, sertansenturk@yahoo.com)

The Istanbul Tunnel, which was designed and constructed by Eugene Henry Gavand at 1875 is the 3rd. Metro and 2nd underground railway system in the world after Washington Metro (1868). In this paper, the acoustics of the Istanbul Tunnel are investigated. This is a special case which sets up a challenge to these prediction methods. The shape of the tunnel and therefore focusing the sound, reverberant wall, and therefore fluctuation effect demands high accuracy in predicting the early reflections. The energy dissipates quickly in this type of enclosures and there is little masking effect of the reverberation. Another aspect that has been shown to give very different results in this case study is the geometrical detailing of the models. When the Istanbul Tunnel is compared with modern metro systems; nowadays railway systems are controlled by modern electronic and mechanical system, but in the 19th century these electronic systems were not available. A solution could be found with the shape of the building. The aim of this paper is to clarify some of the problems that can arise in this type of constructions, and give guidelines for how they can be overcome / avoided. Another objective is to emphasize that room acoustic computer simulations although very useful, need careful consideration about the underlying calculation methods.

4pAAf2. The Royal Church of San Lorenzo in Turin: Guarino Guarini and the Baroque architectural acoustics. Marco Caniato (University of Trieste, Piazzale Europa, 34100 Trieste, Italy, caniatomarco @ vodafone.it), Federica Bettarello (Engineering Dept. - Univ. of Ferrara, Via Saragat, 1, 44100 Ferrara, Italy, federica.bettarello@unife.it), Marco Masoero (Politecnico di Torino, Corso Duca degli Abruzzi 24, 10123 Torino, Italy, marco.masoero@polito.it)

In 1666 the architect Guarino Guarini received from Carlo Emanuele II, Duke of Savoy, the appointment to build in Turin a new church dedicated to S. Lorenzo. The architect conceived a design in Baroque style with a very particular ribbed dome and this peculiarity is a very hard to find feature throughout Europe. Acoustics measurements were performed in S. Lorenzo in order to investigate how this unique architecture affects the response parameters used in architectural acoustics. Results are discussed in the paper, comparing to the methodology suggested by Cirillo and Martellotta in order to characterize the acoustics of churches.
4pAAf3. Bernardo Antonio Vittone: acoustics and architecture in the XVIII century. Marco Caniato (University of Trieste, Piazzale Europa, 34100 Trieste, Italy, caniatomarco@vodafone.it), Vilma Fasoli (University of Trieste, Piazzale Europa, 34100 Trieste, Italy, fasoli@tin.it)

Contemporary critics have identified Savoy architect Bernardo Vittone as a major contributor to the establishment of the concept and experience of architecture as the "art of construction" in the 18th century. Vittone grew and worked under the guidance of mathematician Guarino Guarini, whose mastery of theoretical and scientific aspects he was never credited to have matched. Vittone's approach to architecture was mainly practical, his plans chiefly derived from the rules of mechanics and descriptive geometry. Experience was his main source of inspiration. Designs, plans, domes and vaults are but ways to achieve balance and harmony and so are precision and control of the relation between geometry and acoustics, architecture and music. In his book "Alternative instructions on the civil Architecture" morover he included in the second appendix the "Armonic instructions", within a dissertation on the nature of sound, its propagation in open spaces, in closed ones and its relation with music. In this paper a commented overview is given matching his description of acoustics with present-day knowledge.

4pAAf4. The engineering method of sound field control for amplifications in sports arena. Jian Peng (Guangzhou Radio, 231 Huanshi Zhong Road, 510000 Guangzhou, China, zp24@drexel.edu), Zhao Peng (Drexel Univ., 3175 JFK Blvd, APT 707, Philadelphia, PA 19104, USA, zp24@drexel.edu)

This paper discusses the engineering method of sound field control for amplifications in sports arena, which includes the selection, assembly and adjustment of the loudspeaker system. It also includes the site characteristics of the sports arena, as well as the relations between the loudspeaker and the site. Through the measurements of the rated sensitivity and maximum noise power, the loudspeaker system can be determined as distributed or central system, linear or plane array. With the adjustments on parameters such as the directivity $\mathrm{D}(\Theta)$ and the directivity factor $\mathrm{Q}$, the loudspeaker system will be able to effectively control the sound field during amplification process and to achieve better sound quality in sports arena. Besides determining the effective engineering method for sound field control in the amplification process, this paper also considers the acoustical characteristics of sound sources and coupling effect in the sound field. 
4pAAf5. Correlation between architectural and acoustical parameters in Catholic churches of the colonial era in Ayacucho, Peru. Carlos R. Jimenez (Pontificia Universidad Catolica Del Peru, Av. Universitaria 1801, San Miguel, 32 Lima, Peru, cjimene@ @ucp.edu.pe)

The paper reports the analysis of the results of an acoustic survey carried out on several Catholic Churches in the city of Ayacucho, a southern city in the Peruvian Andes, built during the colonial era through centuries XVI to XVIII. The study is performed taking into account both room-average values and individual position values of many acoustical parameters measured according to ISO 3382. Effects among architectural characteristics of each church as lenght, height, room volume, interior surfaces area, total absorbing area, mean absorption coefficient, and some objective acoustical parameters as reverberation time, early decay time, clarity, definition and center time, are investigated.

4pAAf6. Simulation of the reverberation time of an existing architectural space using a 1:10 scale model. Luz Del Carmen Gonzalez-Rodriguez (Program of Graduate Studies in Design, CyAD - Universidad Autonoma Metropolitana, Av. San Pablo 180, Edificio D 101, Col. Reynosa Tamaulipas, Delegacion Azcapotzalco, 02200 Mexico, D.F., Mexico, loozie@gmail.com), Fausto E. Rodriguez-Manzo (Departamento de Procesos y Tecnicas, CyAD, Universidad Autonoma MetropolitanaAzcapotzalco, Av. San Pablo 180, Edificio H-PB, Col. Reynosa Tamaulipas, Delegacion Azcapotzalco, 02200 Mexico, D.F., Mexico,rfme@correo.azc .uam.mx)

Recently the science of acoustics has been reconsidered and valued more than just a tool concerning physics, telecommunications, and music. It is now an important topic of research for the acoustic comfort of places where people work and live, and it is also considered as an essential issue for the physical and mental health of human beings. Sound has to be integrated as a design concept in architecture, but architects need tools for understanding more the way to reach not only good acoustics but also good architectural design. It has been proven that physical scale models are very useful for the prediction of the acoustical behaviour of rooms, therefore this process is analyzed and studied at the Acoustic Design and Analysis Laboratory of the UAM-Azcapotzalco in Mexico City, in order to promote further investigations that provide useful data for the design of architectural spaces as well as the design of sound control devices. This paper shows the work of a master degree thesis where the main objective is to present the measurements of reverberation time taken in an existing room compared with those taken in the physical scale model of the same room.

4pAAf7. Architectural Acoustics Design using Diffusing Surfaces James Heddle (James Heddle Pty Ltd, Unit 2315 / 180 Grey Street, South Bank, 4101 Brisbane, Australia, acousticdesign@gmail.com)

This paper reviews the design challenges and development of acoustic treatments for three acoustical design projects in Australia. The projects that will be outlined were a surround sound widescreen lecture theatre, a contemporary council chambers and the rectification of a problematic brass band practice room. The treatments included sound diffusing surfaces of the geometric type with or without embedded amplitude reflection gratings. Where the gratings were used in multiples the designs also addressed periodicity issues using modulation of the grating patterns with the best known merit factors. The design approach and the development of the acoustical surface features and treatments to solve the design issues, including problems experienced in the building phase, will be discussed.
4pAAf8. Evaluation of existing sound system designs in mosques and alternative modern solutions. Wasim Orfali (Nordmannzeile 12, 12157 Berlin, Germany, orfaliwasim@hotmail.com), Wolfgang Ahnert (Ahnert Feistel Media Group, Arkonastr. 45-49, 13189 Berlin, Germany, wahnert @ada-acousticdesign.de)

Sound intelligibly and audibility was from the old days and will remain a central issue in any sound system design especially in mosques. In other worship spaces sound system may not be needed during specific worshiping modes like congregational prayers. In mosques all worshiping modes require professionally designed sound system to maintain the needed intelligibility and audibility levels in such spaces. Here the current used sound systems in mosques will be discussed and pro and cons will be highlighted. Also, the effect of these systems on mosques applicable sound paramete will be introduced. In the same time, modern sound system design solution for different mosques architectural form and size will be suggested. Case study examples will be used to show the advantages of the new suggested sound system configurations.

4pAAf9. Room acoustics enhancement system in a Multifunctional Cultural Centre. Géza Balogh, Jr. (Interton Electroacoustics, Major u. 63 , H-1119 Budapest, Hungary, balogh@interton.hu), Géza Balogh, Dr (Interton Electroacoustics, Major u. 63, H-1119 Budapest, Hungary, drbalogh@interton.hu)

In the lecture, we present our system called DCR (Digital Control of Reverberation), which is capable of changing the reverberation of a room by means of electroacoustical devices. Using the DCR system, we are able to change the enclosure, i.e. we create an electroacoustical enclosure on the walls and on the ceiling. The parameters of the electroacoustical enclosure can be changed in several steps. The system was installed in Hungary for the first time in the city of Debrecen, in the Kölcsey Cultural Centre. The principle of the DCR and the problems and their solutions will be presented via this specific installation. We study the effect of the distance between the microphones and the loudspeakers, and the factors that influence this effect. We also analyse the room acoustics parameters in respect to the frequency, in the different status of the auditorium. Our room acoustics enhancement DCR system is based on the non-inline principle, thus the room acoustics of the large auditorium (1100 seats) of the Kölcsey Centre remained absolutely natural. We use several numbers of independent digital channels in the system, and each channel consists of one microphone, one or two loudspeakers, a power amplifier and a DSP.

4pAAf10. Translating acoustics into architecture. Ewart A Wetherill (Ewart A. Wetherill, AIA, 28 Cove Road, Alameda, CA 94502 7416, USA, redwetherill@sbcglobal.net)

Ever since Wallace Clement Sabine's first presentation to the American Institute of Architects in 1898, a substantial gap has often existed between available information on building acoustics and its successful application to the design of buildings. Experience over at least the past 40 years suggest that one of the reasons for this has been the difficulty of adapting construction methods, that were selected to meet other criteria, to satisfy specific acoustical conditions. In a complex project it is important that requirements for individual spaces be identified and resolved early in the design process so that they can be integrated successfully into the overall building design This paper discusses a procedure that has been found very convenient for establishing acoustical requirements during the schematic design and transferring recommendations efficiently for inclusion in the construction documents. The information is compiled in a book-style format for ease of distribution and for fast reference at any time in design and construction. 


\title{
Session 4pAAg
}

\section{Architectural Acoustics: Prediction Methods in Building Acoustics III (Poster Session)}

\author{
Berndt Zeitler, Cochair \\ NRC - Institute for Research in Construction \\ Catherine Guigou-Carter, Cochair \\ CSTB
}

\begin{abstract}
All posters will be on display from 3:40 p.m. to 5:20 p.m. To allow contributors an opportunity to see other posters, contributors of odd-numbered papers will be at their posters from 3:40 p.m. to 4:30 p.m. and contributors of even-numbered papers will be at their posters from 4:30 p.m. to 5:20 p.m.
\end{abstract}

\section{Contributed Papers}

4pAAg1. Determination of unknown parameters in impervious layers by inverse method. Eva Escuder Silla (Escola Politècnica Superior de Gandia, Universitat Politècnica de València, Crtra Natzaret-Oliva s/n, 46730 Gandia, Spain, evescude@fis.upv.es), Jesús Alba Fernandez (Escola Politècnica Superior de Gandia, Universitat Politècnica de València, Crtra Natzaret-Oliva s/n, 46730 Gandia, Spain, jesalba@ fis.upv.es), Jaime Ramis Soriano (DFISTS. Univ. de Alicante, Carretera de Sant Vicent del Raspeig s/n, 03690 San Vicente del Raspeig, Spain, jramis@ua.es), Romina Del Rey Tormos (Escola Politècnica Superior de Gandia, Universitat Politècnica de València, Crtra Natzaret-Oliva s/n, 46730 Gandia, Spain, roderey@doctor .upv.es)

In this work, a novel procedure is shown for the determination of unknown parameters in impervious layers used in multilayer structures by inverse method and using scale models. Experimental pressure and velocity data are obtained by Nearfield Acoustic Holography (NAH) for the calculation of the transmission loss of the different multilayer structures mounted on the window of a wooden box designed for that end. These data are used as input data in the inverse method. The forecast model of acoustic insulation in multilayered structures used in this work was Trochidis\&Kalaroutis model based on Spatial Fourier Transform (SFT). By applying Trochidis\&Kalaroutis model and adjusting by numerical methods the variables that define the impervious layers of the system, the values of the unknown magnitudes of the layers are calculated. For validation purposes the results are compared to those obtained with Ookura\&Saito model.

4pAAg2. New Roadway noise modeling to predict noise propagation in front of urban façade. Hany Hossam Eldien (Suez Canal Univ., 25500 Suez, Egypt, hany.hossam@gmail.com), Philippe Woloszyn (UMR ESO, Université de Haute Bretagne - Rennes II, Place du Recteur Henri Le Moal, CS 24307, 35043 Rennes Cedex, France, philippe.woloszyn@univ-rennes2 .fr)

Roadway noise is the most important example of a linear noise source, since it comprises about 80 percent of the environmental noise exposure for humans worldwide. Due to the complexity of the variables, a line source acoustic model is realized by a computer model . In this paper we propose a new approach system based upon Schröder's quadratic residue diffuser modeling for modeling a linear traffic source. 1/10th scale model has been used to simulate the traffic noise propagation in front of the building façade and for a free field. The line source directivity is made quasi-uniform in the aperture angle of interest. The line source directivity measurements were made using a 1/4" microphone Larson Davis type 2530. The sequence of the quadratic residue diffusor is calculated following the Schröder's modulo for- mula and with $1.60 \mathrm{~m}$ length, $0.14 \mathrm{~m}$ height, and $0.11 \mathrm{~m}$ width. The line source has also been weighted by a full-size equivalent normalised traffic noise spectrum according to the French standard NF EN 1793-3.

4pAAg3. Influence of the conservation state of the facade elements as a variable of prediction of acoustic insulation to airborne sound. Leonardo Meza (Pontificia Universidad Católica de Chile, Av. Vicuña Mackena 4860, Macul, 7820436 Santiago, Chile, 1mezam@uc.cl)

The different prediction methods of acoustic insulation are based on the acoustic properties of the constituent materials of the buildings, however, they don't usually consider the conservation state as a variable within the prediction, ignoring the natural process of degradation that the material and the construction elements suffer which sometimes are increased by the deterioration due to the use of itself. At this paper, the behavior of some construction elements is analyzed by means of acoustic insulation measurements in-situ, such as windows and its woodwork, which presents in many cases a diminish in the acoustic performance due to the damage and deterioration. Finally, the incorporation of this variable is proposed in future prediction models of acoustic insulation.

4pAAg4. Variable Source-Directivity Using DodecahedronLoudspeakers. Gottfried K. Behler (RWTH Aachen University, Templergraben 55, D-52056 Aachen, Germany, gkb@akustik.rwth-aachen.de), Martin Pollow (RWTH Aachen University, Templergraben 55, D-52056 Aachen, Germany, martin.pollow@akustik .rwth-aachen.de)

For room-acoustical measurements dodecahedron loudspeakers are commonplace to achieve a uniform directivity. Therefore all transducers are fed with the same signal. If the signals for the twelve transducers are individually adjustable, the variation of amplitude and phase offers the possibility to achieve a predefined directivity. The goal is to calculate the twelve frequency dependent amplitude- and phase-coefficients for any given directivity with the least possible error. A simple approach like superposition unfortunately does not reveal a correct result, since all transducer interact with each other. The decomposition of spherical functions into spherical harmonics, however, leads to an analytic solution for the prediction of the sound radiation. The acoustical components - like sound pressure and sound velocity - are split up into weighted, orthogonal base functions which can be combined in a way that the mutual coupling between different membrane vibrations is respected. Under these conditions complex filter transfer functions, individually optimized for each one of the twelve transducers can be 
computed with respect to the target directivity function. Different approaches for the optimization procedure are discussed. Besides the more theoretical approach measurements with a real multichannel system are shown.

4pAAg5. In situ evaluation of the vibration reduction index $\mathrm{Kij}$ Arianna Astolfi (Politecnico di Torino, Department of Energetics, Corso Duca degli Abruzzi, 24, 10129 Turin, Italy, arianna.astolfi@polito .it), Alessandro Schiavi (Istituto Nazionale di Ricerca Metrologica, str. delle Cacce, 91, 10135 Turin, Italy, a.schiavi@inrim.it), Cristiana Taricco (Via Tapparelli 31, 12038 Savigliano (CN), Italy, c.taricco @ fastwebnet.it), Simone Geroso (Politecnico di Torino, Department of Energetics, Corso Duca degli Abruzzi, 24, 10129 Turin, Italy, geroso@alice.it), Fabrizio Bronuzzi (Politecnico di Torino, Department of Energetics, Corso Duca degli Abruzzi, 24, 10129 Turin, Italy, fabrizio.bronuzzi@polito.it), Andrea Savio (Via Madonnina, 12, 10090 Gassino Torinese (TO), Italy, andrea.savio_84@alice.it)

As stated in EN 12354-1 and 2 Standards the vibration reduction index Kij is a quantity related to the vibrational power transmission over a junction between structural elements, normalized in order to make it invariant. This quantity allows to quantify the flanking transmission both in air-borne and impact sound insulation between rooms. It is important to underline that the measurement method reported in the EN 10848-1 Standard is only referred to the laboratory measurement, anyway in the Annex E of the EN 12354-1 Standard is indicated that the same methodology is probably useble also in in situ condition. In this work several measurements of the vibration reduction index in situ are reported in order to verify if the methodology is also suitable in uncontrolled conditions as in the field. The measured results are compared with the previsional model results according to the EN 12354-1. In particular the case studies are the T-junctions and the crossjunctions between the ceilings and the vertical walls in some typical rooms in dwellings. From the first experimental results some peculiar building fea- tures, influencing the measurement and the calculation data, have been evidenced, expecially with reference to the south European building typologies in brick and concrete.

4pAAg6. High sound pressure models for microperforated panels backed by an air cavity. Rostand Tayong (Lab. de Recherche en Mécanique et Acoustique, I.S.A.T - Univ. de Bourgogne, 49, rue Mademoiselle Bourgeois, $58000 \quad$ Nevers, France, rostand.tayong@u-bourgogne .fr), Thomas Dupont (Lab. de Recherche en Mécanique et Acoustique, I.S .A.T - Univ. de Bourgogne, 49, rue Mademoiselle Bourgeois, 58000 Nevers, France, thomas.dupont@u-bourgogne.fr), Marie-Annick Galland (Centre Acoustique du LMFA, Ecole Centrale de Lyon, 36 avenue Guy de Collongue, 69134 Ecully cedex, France, marie-annick.galland@ec-lyon .fr), Philippe Leclaire (Lab. de Recherche en Mécanique et Acoustique, I.S A.T - Univ. de Bourgogne, 49, rue Mademoiselle Bourgeois, 58000 Nevers, France, philippe.leclaire@gmail.com)

When submitted to relatively high sound pressure amplitudes, Micro Perforated Panels (MPP) are influenced by certain effects, which are non negligible (vibration of the panel, end radiation and also proximity of the perforations). A model of the total impedance of the MPP is derived from the sum of the contributions of each effect in the case of relatively high sound pressure. The effect of end radiation is supposed to be independent of the propagation inside the apertures. The model is applicable for low Mach numbers. In order to validate the models, various steel MPP specimens were built with different aperture diameters, interstices (distance between two near apertures) and thickness sizes. The experimental method consists in measuring the acoustical pressure before the specimen and the velocity at the aperture entrance. The experimental setup is based on the use of an impedance circular tube. A loudspeaker capable of delivering high sound pressure is used as a source. The excitation is a white noise in a frequency range between $500 \mathrm{~Hz}$ and $5000 \mathrm{~Hz}$ and the detection is performed with microphones. The comparison between measurements and simulations for the impedance and absorption coefficient is done and discussed.

\title{
Session 4pAAh
}

\section{Architectural Acoustics: Measuring Methods and Uncertainty in Building Acoustics II (Poster Session)}

\author{
Brandon Tinianow, Cochair \\ Werner Scholl, Cochair \\ Physikalisch-Technische Bundesanstalt
}

\begin{abstract}
All posters will be on display from 3:40 p.m. to 5:20 p.m. To allow contributors an opportunity to see other posters, contributors of odd-numbered papers will be at their posters from 3:40 p.m. to 4:30 p.m. and contributors of even-numbered papers will be at their posters from 4:30 p.m. to 5:20 p.m.
\end{abstract}

\section{Contributed Papers}

4pAAh1. The examination of the influence of standing waves on reverberation time measurements in small reverberant rooms. Marko Horvat (Faculty of EE and Computing, Unska 3, Department of Electroacoustics, HR-10000 Zagreb, Croatia, marko.horvat@fer.hr), Kristian Jambrosic (Faculty of EE and Computing, Unska 3, Department of Electroacoustics, HR-10000 Zagreb, Croatia, kristian.jambrosic@fer.hr), Hrvoje Domitrovic (Faculty of EE and Computing, Unska 3, Department of Electroacoustics, HR-10000 Zagreb, Croatia, hrvoje.domitrovic@ @er.hr)

The measurements of reverberation time are performed in a small and rather reverberant shoe-box type room in order to determine the spatial distribution of reverberation time with respect to the formation of standing waves in the room itself. The measurements are made in a two-axes raster with equidistant distribution of measurement points. The goal is to investigate the conclusions made in an earlier work by Graber et al. that the distribution of reverberation time values along the fore mentioned raster reaches maximum values at the points of minimum sound pressure level andvice versa. The room in which the measurements are made is deliberately chosen to be relatively small in order to examine the lowest modes of standing waves that form in the room.

4pAAh2. Effect of the estimation of the wall/floor junction type on the acoustic isolation. Jesús Alba Fernandez (Escola Politècnica Superior de 
Gandia, Universitat Politècnica de València, Crtra Natzaret-Oliva s/n, 46730 Gandia, Spain, jesalba@fis.upv.es), Jaime Ramis Soriano (DFISTS. Univ. de Alicante, Carretera de Sant Vicent del Raspeig s/n, 03690 San Vicente del Raspeig, Spain, jramis@ua.es), Eva Escuder Silla (Escola Politècnica Superior de Gandia, Universitat Politècnica de València, Crtra Natzaret-Oliva s/n, 46730 Gandia, Spain, evescude@fis.upv.es), Romina Del Rey Tormos

(Escola Politècnica Superior de Gandia, Universitat Politècnica de València, Crtra Natzaret-Oliva s/n, 46730 Gandia, Spain, roderey@doctor.upv.es)

Deviations between the laboratory measurement and the final in situ measurement, are common in the acoustic isolation. A fundamental difference between both cases is the effect of the lateral flanks. This effect produce that the global acoustic insulation changes substantially. With the standard UNE-EN-12354-1, it can be estimated the effect of the flank, obtaining the vibration reduction indexes and evaluating the insulation of every way. It is also possible to carried out a measurement procedure with the Standard ISO 10848, in it the index is measured and then the index is calculated. This paper shows the differences of results of airborne sound insulation and impact insulation with in situ measurements of the vibration reduction index and the different estimations that the Standard allow. Different unions are studied, and the influence of these in the global result of the insulation is evaluated.

4pAAh3. Study in the measurement of noise air insulation in laboratory of the effect in the diffuse field. Romina Del Rey Tormos (Escola Politècnica Superior de Gandia, Universitat Politècnica de València, Crtra Natzaret-Oliva s/n, 46730 Gandia, Spain, roderey@doctor.upv.es), Jesús Alba Fernandez (Escola Politècnica Superior de Gandia, Universitat Politècnica de València, Crtra Natzaret-Oliva s/n, 46730 Gandia, Spain, jesalba@fis.upv.es), Jaime Ramis Soriano (DFISTS. Univ. de Alicante, Carretera de Sant Vicent del Raspeig s/n, 03690 San Vicente del Raspeig, Spain, jramis@ua.es), Eva Escuder Silla

(Escola Politècnica Superior de Gandia, Universitat Politècnica de València, Crtra Natzaret-Oliva s/n, 46730 Gandia, Spain, evescude@fis.upv.es)

We can obtain, in a transmission chamber, the air transmitted noise insulation. In the standard 140-1 the characteristics of these chambers are described. One of these characteristics tries to ensure diffuse field inside these chambers. Nevertheless, we cannot assure an incident angle between $0^{\circ}$ and $90^{\circ}$ on the test wall, there is a limit angle lower than $90^{\circ}$. In this paper, we study the valuation of the committed mistake by limit angle in transmission chambers. Expressions used for calculating the insulation in transmission chambers are obtained from transmission description in diffuse field, in which case the angle is $90^{\circ}$. In this work, for different materials commonly used in transmission chambers tests, the influence of the indetermination of a mistake and the global mistake evolution with limit angle is studied.

4pAAh4. Student project of building an impedance tube. Mia Suhanek (Faculty of EE and Computing, Unska 3, Department of Electroacoustics, HR-10000 Zagreb, Croatia, mia.suhanek@fer.hr), Kristian Jambrosic (Faculty of EE and Computing, Unska 3, Department of Electroacoustics, HR-10000 Zagreb, Croatia, kristian.jambrosic@fer.hr), Hrvoje Domitrovic (Faculty of EE and Computing, Unska 3, Department of Electroacoustics, HR-10000 Zagreb, Croatia, hrvoje.domitrovic@fer.hr)

This paper describes a student project of building an impedance tube for measuring the absorption coefficient using the transfer-function method, in accordance with the standard ISO 10534-2. This method is well-established and has many advantages compared to the older method using standing wave ratio (ISO 10354-1) in terms of measurement speed and accuracy. For the tube, only inexpensive materials and transducers were used. The tube was designed for the frequency range between 90 and $2000 \mathrm{~Hz}$. In order to achieve this range with one tube, three microphone positions have been used. The resulting absorption coefficient has been calculated using the oneand two-microphone method. Different broadband excitation signals have been used in order to compare their robustness, such as MLS, frequency sweep and white noise. Various problems with the design and construction are addressed and the optimal configuration is discussed.

4pAAh5. Uncertainty of airborne sound insulation index measurement in laboratory conditions. Tadeusz Wszolek (University of Science and Technology, Department of Mechanics and Vibroacoustics, Al.Mickiewicza 30, 30-059 Krakow, Poland, twszolek@agh.edu.pl)

In buildings, airborne sound insulation is used to define the acoustic quality of walls between rooms. However the evaluation of sound insulation is sometimes difficult or even ambiguous, both in field and laboratory measurements, in spite of the fact that there are some unified measurement procedures specified in the ISO 140 standards. There are problems with the reproducibility and repeatability of the measured results. Some difficulties may be caused by non-diffuse acoustic fields, non uniform reverberation time or large spread of the reverberation time measurements especially in low frequency band. Some minor problems are also posed by flanking transmission and the $\mathrm{S} / \mathrm{N}$ ratio. In the present work partial uncertainty analysis has been carried out for all the above mentioned factors and their influence has been evaluated on the combined uncertainty in $1 / 3$ octave bands and the $\mathrm{Rw}$ index, using the uncertainty propagation law

4pAAh6. Blind estimation method of reverberation time based on concept of modulation transfer function. Masashi Unoki (JAIST, 1-1 Asahidai, 923-1292 Nomi, Japan, unoki@jaist.ac.jp), Sota Hiramatsu (JAIST, 1-1 Asahidai, 923-1292 Nomi, Japan, s0610073@jaist .ac.jp)

This paper proposes a method for blindly estimating the reverberation time based on the concept of the modulation transfer function (MTF). This method estimates the reverberation time from the reverberant signal without measuring room acoustics. In the MTF-based speech dereverberation method, proposed by the authors, a process for estimating a parameter related to the reverberation time was incorporated. In this paper, we investigate whether the estimation process, previously presented by authors, works as a blind estimation method and point out a problem with their method. We then propose a new method for blindly estimating the reverberation time to resolve the problem. In the proposed method, the reverberation time is correctly estimated by inverse-MTF filtering in the modulation frequency domain. We evaluated the proposed method with their method using both artificial MTF-based signals and speech signals to show how well the proposed method correctly estimates the reverberation time in artificial reverberant environments. Results suggested that the proposed method correctly estimates reverberation times from the observed reverberant signals. [Work supported by a Grant-in-Aid for Science Research from the Japanese Ministry of Education (No. 18680017).]

4pAAh7. Realization of a measurement system for physical and acoustic measurements on brick walls. Luca Barbaresi (DIENCA - Univ. of Bologna, Viale Risorgimento 2, 40136 Bologna, Italy, luca.barbaresi @mail.ing.unibo.it), Massimo Garai (DIENCA - Univ. of Bologna, Viale Risorgimento 2, 40136 Bologna, Italy, massimo.garai@mail.ing.unibo .it), Paolo Guidorzi (DIENCA - Univ. of Bologna, Viale Risorgimento 2, 40136 Bologna, Italy, paolo.guidorzi@mail.ing.unibo.it), Giovanni Semprini (University, DIENCA Dept. Facoltà di Ingegneria, Viale Risorgimento 2, 40136 Bologna, Italy, giovanni.semprini@mail.ing.unibo.it)

Aim of this study is the development of a system for the measurement of frequency values of some physical variables such as the damping factor, the thickness of a structural element, the structural reverberation time and the longitudinal wave speed propagation in light brick walls. Such variables are required by the UNI EN 12354-1 normative for the analytical estimation of the sound reduction index $\mathrm{R}$ of monolithic elements in the laboratory. The results of the calculation, as a function of frequency, will be shown and compared with the measured values of the sound reduction index $\mathrm{R}$. 
4pAAh8. Swept Sine against MLS in room acoustics with music signals as background noise. Joel P. Paulo (ISEL, R. Conselheiro Emídio Navarro,1, 1959-007 Lisbon, Portugal, jpaulo@deetc.isel.ipl.pt), J. Luis Bento Coelho (CAPS, Instituto Superior Técnico, TU Lisbon, Av. Rovisco Pais, P-1049-001 Lisbon, Portugal, bcoelho@ist.utl.pt)

The Swept Sine and the MLS techniques are very popular in room acoustic measurement set-ups. Advantages and disadvantages of both methods have been well investigated and can be found in the literature. However, information regarding the performance of these techniques in the presence of high background music levels is scarce. Since the estimation of the room impulse response is based on the correlation between signals, the likelihood between the test signal and the music contents has an important role on the results accuracy. This paper explores these issues by taking into account the semantic information of the music signals when used as disturbance. The method used for the assessment of the gain between the two techniques consists on splitting each frame in segments and applying a weighting function depending on a likelihood function. The features used for the likelihood function are the rms value of each segment, spectral energy envelope relation, bandwidth and harmonic structure. Several examples are presented for comparison of the performance of the Swept Sine and the MLS techniques. Advantages and disadvantages of each technique are discussed for music signals as noise.

4pAAh9. Effects of filtering of room impulse responses on room acoustics parameters by using different filter structures. Csaba Huszty (Budapest University of Technology and Economics, BME Dept. of Telecommunications, Magyar tudósok körútja 2, H-1117 Budapest, Hungary, huszty@hit.bme.hu), Norbert Bukuli (Budapest University of Technology and Economics, BME Dept. of Telecommunications, Magyar tudósok körútja 2, H-1117 Budapest, Hungary, bukuli.norbert@gmail .com), Ákos Torma (Budapest University of Technology and Economics, BME Dept. of Telecommunications, Magyar tudósok körútja 2, H-1117 Budapest, Hungary, tormakos@gmail.com), Fülöp Augusztinovicz (Budapest University of Technology and Economics, BME Dept. of Telecommunications, Magyar tudósok körútja 2, H-1117 Budapest, Hungary, fulop@hit.bme.hu)
Room acoustic evaluation is usually based on post-processing of measured room impulse responses (RIRs), and this often requires some kind of filtering, for instance to derive fractional octave band parameters of a room. In this paper it is shown that the considerable variance of room acoustic parameters of almost any hall is partly caused by the filtering method and the filter properties used in the course of post-processing. The paper proposes new qualification methods and parameters for determining the quality of FIR filter banks, taking their use for acoustic evaluation into account. It suggests practical considerations for the design as well, and shows the analysis and comparison of effects of various filter properties -- such as filter types and topology structures -- on some room acoustics parameters. By using the suggested methods, it is possible to derive more accurate and reliable results in room acoustic evaluation.

4pAAh10. Reverberation time measuring methods. Kristian Jambrosic (Faculty of EE and Computing, Unska 3, Department of Electroacoustics, HR-10000 Zagreb, Croatia, kristian.jambrosic@fer.hr), Marko Horvat (Faculty of EE and Computing, Unska 3, Department of Electroacoustics, HR-10000 Zagreb, Croatia, marko.horvat@fer.hr), Hrvoje Domitrovic (Faculty of EE and Computing, Unska 3, Department of Electroacoustics, HR-10000 Zagreb, Croatia, hrvoje.domitrovic@fer.hr)

In this paper different well-established methods of reverberation time measurement are compared. Furthermore, the results obtained using these methods are compared to the results provided by some additional methods which could serve as an in situ tool if, for any reason, the reverberation time measurements cannot be carried out using the standardized methods. The methods compared in this paper include the standardized methods (EN ISO 3382:2000), namely the impulse response measured with pink noise, exponential sweep, MLS, but also pistol shots of different calibers, balloon bursts, gated external pink noise, and the B\&K filtered burst method. In order to make the comparison, the measurements were performed in four acoustically very different spaces - a rather small and well-damped listening room, a much bigger damped listening room, a rather reverberant atrium, and a large and very reverberant shoebox-shaped room. The results were evaluated according to signal-to-noise ratio criterion as well. Special attention has been given to the influence of room modes on measurement results.

\title{
Session 4pAAi
}

\section{Architectural Acoustics and the National Council of Acoustical Consultants: Student Design Competition}

\author{
Byron W. Harrison, Cochair \\ The Talaske Group Inc., 1033 South Boulevard, Oak Park, IL 60302, USA \\ Anders Christian Gade, Cochair \\ Dept. of Acoustic Technology, Technical University of Denmark, Building 352, DK 2800 Lyngby, Denmark
}

\begin{abstract}
The Technical Committee on Architectural Acoustics of the Acoustical Society of America, the National Council of Acoustical Consultants, and the Robert Bradford Newman Student Award Fund are sponsoring the 2008 Student Design Competition that will be professionally judged at this meeting. The purpose of this design competition is to encourage students enrolled in architecture, engineering, physics, and other university curriculums that involve building design and/or acoustics to express their knowledge of architectural acoustics in the design of a new theater performance space as part of a university building project.
\end{abstract}

This competition is open to undergraduate and graduate students from all nations. Submissions will be poster presentations that demonstrate room acoustics, noise control, and acoustic isolation techniques in building planning and room design. The submitted designs will be displayed in this session and they will be judged by a panel of professional architects and acoustical consultants. An award of USD $\$ 1250.00$ will be made to the entry judged "First Honors." Four awards of USD\$700.00 will be made to each of four entries judged "Commendation." 


\title{
Session 4pABb
}

\section{Animal Bioacoustics, Psychological and Physiological Acoustics, and ECUA: Auditory Brainstem Response and Behavior Correlation III (Poster Session)}

\author{
Elizabeth Brittan-Powell, Cochair \\ University of Maryland \\ Alexander Supin, Cochair \\ Institute of Ecology and Evolution
}

\begin{abstract}
All posters will be on display from 3:40 p.m. to 5:20 p.m. To allow contributors an opportunity to see other posters, contributors of odd-numbered papers will be at their posters from 3:40 p.m. to 4:30 p.m. and contributors of even-numbered papers will be at their posters from 4:30 p.m. to 5:20 p.m.
\end{abstract}

\section{Contributed Papers}

4pABb1. Intense sonar pings induce temporary threshold shift in a bottlenose dolphin (Tursiops truncatus). T Aran Mooney (University of Hawaii, Hawaii Institute of Marine Biology, P.O. Box 1106, Kailua, HI 96734, USA, mooneyt@hawaii.edu), Paul E. Nachtigall (University of Hawaii, Hawaii Institute of Marine Biology, P.O. Box 1106, Kailua, HI 96734, USA, nachtiga@hawaii.edu), Stephanie Vlachos (University of Hawaii, Hawaii Institute of Marine Biology, P.O. Box 1106, Kailua, HI 96734, USA, vlachos@hawaii.edu)

For over a decade it has been suggested that high intensity anthropogenic sounds, such as sonar, could induce a temporary threshold shift (TTS) in odontocetes. Although TTS has been examined in marine mammals, the temporary physiological effects of sonar sounds have yet to be established. This study explored the effects of high-intensity (up to $203 \mathrm{~dB}$ re: $1 \mu \mathrm{Pa}$ ), mid-frequency sweeps $(2-4 \mathrm{kHz})$ on the hearing of a bottlenose dolphin (Tursiops truncatus). The goal was to determine if these sounds could induce TTS and what sound exposure levels (SEL; dB re: $1 \mu \mathrm{Pa}^{2} \mathrm{~s}$ ) were necessary for TTS to be induced. Fatiguing sounds were presented to mimic that of mid-frequency sonar. Hearing thresholds were measured before and after exposures using auditory evoked potentials to determine amount of shift and rate of recovery. Temporary threshold shifts of 5-6 dB were measured using SELs of $214 \mathrm{~dB}$, in situations when 15 sonar pings were presented in series. Recovery to normal hearing was rapid, typically within 5 to $10 \mathrm{~min}$. Exposure levels required to induce TTS were high, supporting the notion that relatively short sounds must be of relatively high intensity to induce threshold shifts.

4pABb2. The auditory time resolution in bottlenose dolphins: Behavioral experiments versus auditory evoked potential methods. Gennadi L. Zaslavski (University Authority for Applied Research, RAMOT, Tel-Aviv University, str. Gordon 51 app 7, 42442 Netanya, Israel, gennadi .zaslavski@gmail.com)

Non-invasive auditory evoked potentials (AEP) methods are now widely used to study dolphins' hearing because some auditory characteristics can be obtained much faster compare to behavioral methods. The bottlenose dolphin auditory time resolution assessed using evoked potentials responses to a double click, amplitude modulated tone and periodic click is generally believed to be around 300 microseconds. This assessment is claimed to be in full agreement with behavioral measurements. The intention of this paper is to reevaluate behavioral results which are believed to support AEP methods in light of numerous behavioral results indicative of the bottlenose dolphin time resolution as high as $20-30$ microseconds. We found that as long as there are differences in waveforms, bottlenose dolphins are able to discriminate between very short (as short as a bottlenose dolphin sonar click) signals with identical energy spectra as well as between brief noise signals with random energy spectra. Auditory evoked responses do not reveal any differences between such signals whereas the differences are readily indicated by behavioral responses of the dolphins. The auditory temporal analysis of brief signals in bottlenose dolphins seems to be inaccessible by AEP methods, at least in their present form. 


\title{
Session $4 p A B c$
}

\section{Animal Bioacoustics and ECUA: Odontocete Acoustics II (Poster Session)}

\author{
David Mellinger, Cochair \\ Oregon State Univ. and NOAA \\ Michel Andre, Cochair \\ Laboratori d'Aplicacions Bioacústiques (Universitat Politècnica de Catalunya)
}

\begin{abstract}
All posters will be on display from 3:40 p.m. to 5:20 p.m. To allow contributors an opportunity to see other posters, contributors of odd-numbered papers will be at their posters from 3:40 p.m. to 4:30 p.m. and contributors of even-numbered papers will be at their posters from 4:30 p.m. to 5:20 p.m.
\end{abstract}

\section{Contributed Papers}

4pABc1. Decalcifying Protocol of Odontocete Ear Samples with RDO®. Maria Morell (Laboratori d'Aplicacions Bioacústiques (Universitat Politècnica de Catalunya), avda. Rambla Exposició s/n, 08800 Vilanova i la Geltrú, Spain,_maria.morell@lab.upc.edu), Eduard Degollada (Laboratori d'Aplicacions Bioacústiques (Universitat Politècnica de Catalunya), avda. Rambla Exposició s/n, 08800 Vilanova i la Geltrú, Spain, edmaktub @edmaktub.com), Josep Maria Alonso (Laboratori d'Aplicacions Bioacústiques (Universitat Politècnica de Catalunya), avda. Rambla Exposició s/n, 08800 Vilanova i la Geltrú, Spain, jmalonso@iim.csic.es), Thierry Jauniaux (Université de Liège, Place du 20-Aout, 9, B-4000 Liège, Belgium, T.Jauniaux@ulg.ac.be), Mardik Leopold (Imares, P.O. Box 167, 1790 AD Den Burg, Texel, Netherlands, Mardik.Leopold@wur.nl), Kees Camphuysen (Royal NIOZ (Netherlands Inst. for Sea Research), $1790 \mathrm{AB}$ Den Burg, Texel, Netherlands, camphuys@nioz.nl), Michel Andre (Laboratori d'Aplicacions Bioacústiques, Universitat Politècnica de Catalunya, avda. Rambla Exposició s/n, 08800 Vilanova i la Geltrú, Spain, michel.andre@upc.edu)

The study of the organ of Corti is essential to assess the impact of underwater noise on cetaceans. While classical histology techniques (including EDTA decalcification) have been previously considered, the process is time consuming and artifacts, probably directly deriving from the protocol, often appear and difficult the analysis. However, no matter the choice of the analysis technique, one of the challenging step after extraction and fixation of the samples is to decalcify the bone envelope to access the cochlea without damaging the soft tissues. Here, we propose to use a fast commercial decalcifier (RDO $®)$. 103 ears from 12 different odontocetes species stranded in the Mediterranean, Spanish North Atlantic and North Sea have been used to precisely determine the decalcification time. Specifically, the species processed were: Phocoena phocoena $(n=45)$, Stenella coeruleoalba $(n=12)$, Stenella frontalis $(n=12)$, Tursiops truncatus $(n=13)$, Delphinus delphis $(n=4)$, Kogia simus $(n=3)$, Kogia breviceps $(n=2)$, Globicephala macrorhynchus $(n=3)$, Globicephala melas $(n=2)$, Steno bredanensis $(n=4)$, La- genodelphis hosei $(n=2)$ and Ziphius cavirostris $(n=1)$. Depending on the species, the decalcification time ranged from several hours to a few days, allowing a subsequently faster observation of the cochlear structures through routine microscope techniques.

4pABc2. Mesoplodon densirostris transmission beam pattern estimated from passive acoustic bottom mounted hydrophones and a DTag recording. Jessica Ward (NAVSEA, Newport Undersea Warfare Center, Newport, RI RI 02841, USA, wardja@npt.nuwc.navy.mil), David Moretti (NAVSEA, Newport Undersea Warfare Center, Newport, RI RI 02841, USA, MorettiDJ@npt.nuwc.navy.mil), Ronald P. Morrissey (Naval Undersea Warfare Center Division Newport, 1176 Howell Street, Bldg 1351, 2nd Floor, Newport, RI 02841, USA, morrisseyrp@npt.nuwc .navy.mil), Nancy A. Dimarzio (Naval Undersea Warfare Center Division Newport, 1176 Howell Street, Bldg 1351, 2nd Floor, Newport, RI 02841, USA, dimarziona@npt.nuwc.navy.mil), Peter Tyack (Woods Hole Oceanographic Institution, Applied Ocean Physics \& Engineering Dept., Woods Hole, MA 02543, USA, ptyack@whoi.edu), Mark Johnson (Woods Hole Oceanographic Institution, Applied Ocean Physics \& Engineering Dept., Woods Hole, MA 02543, USA, majohnson@whoi.edu)

The transmission beam pattern of a female Mesoplodon densirostris tagged on October 23, 2006 in the Tongue of the Ocean, Bahamas is estimated using data from a Woods Hole Oceanographic Institution DTag and simultaneous recordings from broadband, bottom mounted hydrophones at the Atlantic Undersea Test and Evaluation Center, Andros Island, Bahamas. The bottom mounted hydrophones are used to localize the tagged animal and measure the received level of each click. Pitch, roll and heading from the DTag are used to determine the horizontal and vertical aspect angles relative to the hydrophone. An estimate of the M. densirostris horizontal and vertical transmission beam pattern based on four dives will be presented. 


\title{
Session 4pABd
}

\section{Animal Bioacoustics: General Topics in Animal Bioacoustics I}

\author{
Richard R. Fay, Cochair \\ Loyola University Chicago, Parmly Hearing Institute, 6525 N. Sheridan Rd., Chicago, IL 60626, USA
}

\author{
Michel Andre, Cochair \\ Laboratori d'Aplicacions Bioacústiques, Universitat Politècnica de Catalunya, avda. Rambla Exposició s/n, Vilanova i la \\ Geltrú, 08800, Spain
}

\section{Contributed Papers}

$5: 20$

4pABd1. Vocalizations of the Spotted Hyena (Crocuta crocuta): Eliciting Acoustic Variation in Groans. Frédéric E. Theunissen (UC Berkeley, Dept. of Psychology, 3210 Tolmant Hall, Berkeley, CA 94720-1650, USA, theunissen @ berkeley.edu), Steve Glickman (UC Berkeley, Dept. of Psychology, 3210 Tolmant Hall, Berkeley, CA 94720-1650, USA, glickman@berkeley.edu), Suzanne Page (UC Berkeley, Dept. of Psychology, 3210 Tolmant Hall, Berkeley, CA 94720-1650, USA, mweldele @ berkeley.edu)

Spotted hyenas (Crocuta crocuta) are highly social animals possessing a complex vocal repertoire. Vocal signals of different types correlate with distinct social circumstances. Groans constitute a very large category in the spotted hyena repertoire which is not well understood. Sounds labeled as groans vary in their acoustical quality from more growling sounds to more tonal vocalizations. Groans are also elicited in many different social interactions. To begin to decipher the meaning of these vocal signals, we examined how the variation in the acoustic properties of groans was correlated with experimentally controlled eliciting conditions. Groans were elicited in adult hyenas presented with three objects: unfamiliar spotted hyena cubs, meaty bones, and the empty transport cage used to contain bones or cubs on other trials. Cubs elicited more groans from more adults than other objects but all objects elicited vocal responses. More importantly, discriminant analysis revealed differences in the acoustic characteristics of groans elicited by cubs and those elicited by other objects. Cubs elicited more prolonged and more tonal groans with higher fundamental frequency. Our study shows that groans can be classified into different groups and that these different sounds were produced in different behavioral contexts.

\section{$5: 40$}

4pABd2. The relationship between complex vocal signaling and immunocompetence in the brown-headed cowbird (Molothrus ater). Samantha Levinson (UC Santa Barbara, 6689 El Colegio Rd. Apt. 46, Goleta, CA 93117, USA, slevinson@umail.ucsb.edu), Loren Merrill (UC Santa Barbara, 6689 El Colegio Rd. Apt. 46, Goleta, CA 93117, USA, lmerrill@lifesci.ucsb.edu)

Male brown-headed cowbirds (Molothrus ater) use song in mate attraction and in male-male competition to signal their quality to potential mates and competitors. Singing is an energetically expensive activity for birds, so repertoire size, singing rate, and complexity of songs should be honest indicators of a male's overall quality. Resistance to parasites is a major component of male quality, and some aspects of immunity are inherited by his offspring. Females obtain indirect benefits from mating with healthy males by having healthy offspring, so song may signal immune condition to females. Male cowbirds usually sing between four and six different song types when displaying to females. These songs differ in the amount of frequency modulation required during singing, which indicates that the difficulty of production of these songs by the syrinx also varies. In this study, we investigate the correlation between immune function and singing behavior, specifically the size of a bird's repertoire and the complexity of his songs.
Understanding the relationship between repertoire size, song complexity, and immune function is important to understanding the role of vocal signaling in mate attraction in songbirds.

\section{6:00}

4pABd3. The statistics of plant echoes as perceived by echolocating bats. Yossi Yovel (Eberhard-Karls-Universität Tübingen, Zool. Institut, Abt. Tierphysiologie, Auf der Morgenstelle 28, 72076 Tübingen, Germany, yossiyovel@hotmail.com), Matthias O. Franz (University of Applied Sciences, Konstanz, Germany, HTWG Konstanz, Brauneggerstr. 55, D-78462 Konstanz, Germany, mfranz@htwg-konstanz.de), Peter Stilz (Eberhard-Karls-Universität Tübingen, Zool. Institut, Abt. Tierphysiologie, Auf der Morgenstelle 28, 72076 Tübingen, Germany, peter.stilz@uni-tuebingen.de), Hans-Ulrich Schnitzler (EberhardKarls-Universität Tübingen, Zool. Institut, Abt. Tierphysiologie, Auf der Morgenstelle 28, 72076 Tübingen, Germany, hans-ulrich.schnitzler@unituebingen.de)

To explore the statistics of complex natural plant echoes, we emitted batlike downsweeps $(200-0 \mathrm{kHz})$ and recorded the echoes of various tree species. A Hilbert transform was used to calculate the envelope of the echoes impulse responses. This corresponds to a one-dimensional representation of the spatial reflector arrangement of the plant. We then calculated the envelope's power spectrum to asses the amount of periodic structures. In control experiments we compared power spectra of a single leaf, a branch and a few branches, and tested the effect of systematically decreasing the leaf density of a plant. On a bi-logarithmic plot, the averaged power spectra of all trees have a sigmoid shape with three approximately linear domains that represent different scales of structure, but differ between species. We hypothesize that the first domain is influenced by the gross skeleton of branches, while the others are associated with smaller scale structures. The control experiments showed a similar dependence between leaf density and power spectrum. Modeling plants as simple three-dimensional textures with stationary statistics was sufficient to predict the characteristics of the spectra. Our findings suggest an interpretable relation between the power spectrum of the echo's envelope and the spatial statistics of the plant.

\section{6:20}

4pABd4. Modelling of the echo generation process in bat echolocation. Timos Papadopoulos (University of Southampton, SPCG-ISVR, SO17 1BJ Southampton, UK, tp@isvr.soton.ac.uk), Robert Allen (University of Southampton, SPCG-ISVR, SO17 1BJ Southampton, UK, R.Allen@soton .ac.uk)

Very few studies exist that attempt to model the detailed shape of the echoes generated by real targets in bat echolocation. The modelling becomes even more complicated when one attempts to take into account the specific acoustic characteristics of the bat as a source and receiver. Hence, the exact physical acoustics basis that underpins the target detection and classification capabilities demonstrated by bats remains a largely open research question. We use previously published work on real target echo measurement (Simmons and Chen, JASA 1989) as a starting point but modify their ex- 
perimental method in a way that allows the incorporation of the bat's source and receiver characteristics in the modelling. Furthermore, we compare our measurements with analytically predicted results and show good agreement. We discuss how our experimental method can be used for the prediction of the binaural signals that constitute the actual input to the bat's auditory system during echolocation.

6:40

4pABd5. The simulation of bat-oriented auditory processing using the experimental data of echolocating signals. Su Yeon Kim (University of Southampton, SPCG-ISVR, SO17 1BJ Southampton, UK, syk@isvr.soton.ac.uk), Robert Allen (University of Southampton, SPCGISVR, SO17 1BJ Southampton, UK, R.Allen@soton.ac.uk), Daniel Rowan (University of Southampton, SPCG-ISVR, SO17 1BJ Southampton, UK, dr@isvr.soton.ac.uk)

There are various approaches to understanding the echolocation phenomenon of bats. A part of the echolocating process is assessed here by determining what acoustic signal a bat's ears receive during echolocation. It is simplified in an experimental rig to measure the reflections from objects in different horizontal angles which represents a sound localisation task in bats. It has been assumed in this study that the remarkable echolocating ability of bats, which is not shown in the most other mammalian species, is achieved by their different physical shape of head and ears, and specialised auditory processing of echolocating signals. In human studies in terms of sound localisation, physical characteristics are usually modelled as a head-related transfer function (or HRTF) and gammatone filter banks are widely used to simulate auditory processing in the cochlear. A modified filterbank is used here to represent the auditory processing in bats and combined with the experimental data of object reflections. Bat HRTFs will be used subsequently to determine the acoustic reflections at both ears.

\section{7:00}

4pABd6. Steady streaming near model cod otoliths. Charlotte Kotas (Georgia Institute of Technology, Mechanical Engineering, 771 Ferst Drive, Atlanta, GA 30332-0405, USA, charlotte.kotas@gatech.edu), Peter H. Rogers (Georgia Institute of Technology, Mechanical Engineering, 771 Ferst Drive, Atlanta, GA 30332-0405, USA, peter.rogers@me.gatech.edu), Minami Yoda (Georgia Institute of Technology, Mechanical Engineering, 771 Ferst Drive, Atlanta, GA 30332-0405, USA,minami.yoda@me.gatech.edu)

Typical fish distinguish sounds at about 10-1000 Hz with particle motions as small as $0.1 \mathrm{~nm}$ and angular separation near $10^{\circ}$ using their ears, which contain dense, bony otoliths weakly suspended in endolymph and tissue. The otoliths oscillate relative to incident sound, inducing flows in the surrounding fluid which are in turn sensed by the hair cells on the overlying macular membrane which project into the groovelike sulcus on the otolith These fluid flows are then sampled by the hair cells and 'heard' by the fish. The irregular geometry of the otolith shapes the flow patterns. The hair cells, which are organized into different ciliary orientation groups on the macula, preferentially sample the flow patterns along their axes. The steady component of the fluid motion near enlarged models of an actual cod otolith oscillated at 2--20 Hz along different directions was studied in the vicinity of the hair cells using phase-locked particle-image velocimetry (PIV) and pathline visualizations. The possible relationship between the flow patterns and hearing capabilities is discussed. Although the oscillation amplitudes studied are much larger than those for underwater sound, evidence suggests that the flow patterns are amplitude independent. [Supported by ONR.]

\section{$7: 20$}

4pABd7. Sound source segregation by goldfish: Two simultaneous tones. Richard R. Fay (Loyola University Chicago, Parmly Hearing Institute, 6525 N. Sheridan Rd., Chicago, IL 60626, USA, rfay@luc.edu)

In 1964, van Bergeijk asked, "...given that a fish can discriminate between sounds A and B when they are presented separately, can he still discriminate either one when both are presented simultaneously? Or do the two sounds blend to form a new entity (such as a chord)." This question was a very early query about sound source segregation by fishes. We have investigated the role that frequency separation plays in this phenomenon using classical respiratory conditioning with a stimulus generalization paradigm. Groups of animals were first conditioned to 2-tone mixtures comprised of $150 \mathrm{~Hz}$ (A) and another frequency (B) ranging from $300 \mathrm{~Hz}$ to $750 \mathrm{~Hz}$. Generalization tests were then carried out using single tones between 50 and 900 $\mathrm{Hz}$. Group mean generalization gradients showed that the two tones were segregated at the widest A-B spacings, and tended to be segregated as the A-B spacing was reduced. The limited resolution of the test procedure did not allow an answer at the narrowest spacings. Source segregation, even of abstract tones, may be a fundamental feature of the sense of hearing of all organisms, and occurs in the absence of auditory cortex and complex cognitive abilities

\section{$7: 40$}

4pABd8. Passive Acoustic Detection and Monitoring of Schools of Herring. Thomas R. Hahn (University of Miami, RSMAS, 4600 Rickenbacker Causeway, Miami, FL 33149, USA, t.hahn@miami.edu), Gary L. Thomas (University of Miami, RSMAS, 4600 Rickenbacker Causeway, Miami, FL 33149, USA, gthomas@rsmas.miami.edu)

Passive acoustic detection and monitoring of various marine fishes has recently received much attention in the literature. It has been recognized that passive acoustic techniques have the potential to complement traditional active acoustic surveys and to significantly increase their overall efficiency, if the acoustic signatures of the considered species are well understood. In this paper, the potential of passive acoustic techniques is explored for the specific case of Pacific herring (Clupea palassii). It is demonstrated that schools of herring can acoustically be detected by observing the sound of coordinated bubble release, triggered, e.g., by predator activity. This sound not only has identifiable features that can be exploited for determining the presence or absence by simple means, but could also carry abundance and size information. Work supported by ONR and the NMFS via the PWSSC. 


\title{
Session 4pAOa
}

\section{Acoustical Oceanography, Underwater Acoustics, and ECUA: Rapid Environmental Assessment}

\author{
John C. Osler, Cochair \\ Defence R\&D Canada - Atlantic, P.O. Box 1012, Dartmouth, NS B2Y3Z7, Canada \\ Yann Stephan, Cochair \\ SHOM, 13 rue du Chatellier, CS 92803, Brest cedex 2, 29228, France
}

\section{Invited Paper}

2:00

\begin{abstract}
4pAOa1. Overview of U.S. Navy Operational Oceanographic Models in Support of Acoustic Applications. Richard Allard (Naval Research Laboratory, NRL Code 7322, Stennis Space Center, MS 39571, USA, allard@nrlssc.navy.mil), Charlie Barron (Naval Research Laboratory, NRL Coded 7323, Stennis Space Center, MS 39529, USA, barron@nrlssc.navy.mil), Frank Bub (Naval Oceanographic Office, Balch Blvd, Stennis Space Center, MS 39529, USA, frank.bub@navy.mil), Emanuel F. Coelho (University of Southern Mississippi, Balch Blvd, Stennis Space Center, MS 39529, USA, coelho@nrlssc.navy.mil), James Cummings (Naval Research Laboratory, NRL Code 7322, Stennis Space Center, MS 39571, USA, cummings@nrlmry.navy.mil), J. Pacquin Fabre (Naval Research Laboratory, NRL Code 7322, Stennis Space Center, MS 39571, USA, josie.fabre@ nrlssc.navy.mil), Robert Helber (Naval Research Laboratory, NRL Code 7322, Stennis Space Center, MS 39571, USA, helber@nrlssc.navy.mil), Clark Rowley (Naval Research Laboratory, NRL Code 7322, Stennis Space Center, MS 39571, USA, rowley@nrlssc.navy.mil)
\end{abstract}

The Naval Oceanographic Office operational global $1 / 8^{\circ}$ Navy Coastal Ocean Model assimilates satellite and in-situ data to produce daily 72-hr forecasts. Output includes 3D fields of temperature, salinity, u- and v-components of ocean currents at standard depth levels, and these support derived fields including sound speed and sonic layer depth. The global model provides initial/boundary conditions for nested regional models, primarily relocatable NCOM. The relocatable NCOM modeling system can be set up quickly for areas of interest, includes river and tidal forcing, and is forced with a high-resolution atmospheric mesoscale model. Local and remote observations are incorporated into the models through the Navy Coupled Ocean Data Assimilation system, which assimilates sea surface temperature data from satellite, ships and buoys, profile data from floats and gliders, xbt's, CTD's, fixed and drifting buoys as well as altimeter-derived sea surface heights and ice concentration. In this presentation we will discuss how the operational ocean models feed into acoustic prediction models and tactical decision aids, the role glider observations will play in the modeling strategy, the use of ensembles to provide improved prediction error estimates and guide new observations, and future plans.

\section{Contributed Paper}

\section{2:20}

4pAOa2. High-frequency multibeam echosounder classification for rapid environmental assessment. Kerstin Siemes (Acoustic Remote Sensing Group, Delft Institute of Earth Observation and Space Systems, Delft University of Technology, Kluyverweg 1, 2629 HS Delft, Netherlands, k.siemes@tudelft.nl), Mirjam Snellen (Acoustic Remote Sensing Group, Delft Institute of Earth Observation and Space Systems, Delft University of Technology, Kluyverweg 1, 2629 HS Delft, Netherlands, m.snellen @ tudelft.nl), Dick G. Simons (Delft University of Technology, P.O. Box Postbus 5048, 2600 GA Delft, Netherlands, d.g.simons@tudelft .nl), Jean-Pierre Hermand (Université libre de Bruxelles (U.L.B.) - Environmental hydroacoustics lab, av. Franklin D. Roosevelt 50, CP 194/5, 1050 Bruxelles, Belgium, jhermand@ulb.ac.be), Matthias Meyer (Royal Netherlands Naval College (NLDA) - REA group, PO Box 10000, 1780 Den Helder, Netherlands, mmeyer@ulb.ac.be), Jean-Claude Le Gac (NATO Undersea Research Center, Viale San Bartolomeo 400, 19126 La Spezia, Italy, legac@nurc.nato.int)

For shallow-water naval operations, obtaining rapidly an accurate picture of the environmental circumstances often is of high importance. Hereto a multi-sensor approach is required. In this context, the MREA/BP'07 ex- periment has been carried out south of Elba (Mediterranean Sea), where several techniques of environmental characterization covering the fields of underwater acoustics, physical oceanography and geophysics have been combined [Le Gac\&Hermand, 2007]. The required information typically concerns water-column properties, sea surface roughness, and sediment geoacoustic properties. Estimating these geo-acoustic parameters from inversion of acoustic data received on drifting sparse arrays has proved to be a promising approach. Part of MREA/BP'07 was therefore dedicated to this type of measurement. For validating the resulting geo-acoustic estimates sediment samples were collected. Additionally, measurements were carried out using a multibeam-echosounder. This system provides depth information, but also allows for seafloor classification. The classification approach taken is model-based employing the backscatter data. It discriminates between sediments in the most optimal way by applying the Bayes decision rule for multiple hypotheses, implicitly accounting for backscatter-strength ping-to-ping variability. Here, results of seafloor classification using the multibeam data and a preliminary comparison with the sediment sample analysis and the geo-acoustic parameter estimates as obtained from the drifting arrays are presented. 


\section{Invited Papers}

\section{2:40}

4pAOa3. Integrated scheme of rapid environmental assessment for shallow water acoustics. Jean-Claude Le Gac (NATO Undersea Research Center, Viale San Bartolomeo 400, 19126 La Spezia, Italy, legac@nurc.nato.int), Jean-Pierre Hermand (Université libre de Bruxelles (U.L.B.) - Environmental hydroacoustics lab, av. Franklin D. Roosevelt 50, CP 194/5, 1050 Bruxelles, Belgium, jhermand@ulb.ac.be), Frans Absil (Royal Netherlands Naval College (NLDA) - REA group, PO Box 10000, 1780 Den Helder, Netherlands, fgj.absil@nlda.nl)

Predicting sound propagation in shallow or very shallow water environments requires that the frequency-dependent acoustic properties be assessed for all components of the waveguide, i.e., the water column, sea bottom and sea surface interface. During the Maritime Rapid Environmental Assessment MREA/BP'07 sea trial in April-May 2007, south of Elba Island in the Mediterranean Sea, an integrated MREA scheme has been implemented to provide a full 4D (3D+T) environmental picture that is directly exploitable by acoustic propagation models. Based on a joint multi-disciplinary effort, several standard and advanced techniques of environmental characterization covering the fields of underwater acoustics, physical oceanography and geophysics have been combined within a coherent scheme of data acquisition, processing and assimilation. The paper presents the whole architecture of the implemented scheme. Based on a preliminary analysis of MREA/BP'07 data, advantages and drawbacks of the approach will be discussed. Ways ahead for further improvement and perspectives are finally drawn.

4pAOa4. The application of rapid environmental assessment to sonar performance. Paul C. Hines (Defence R\&D Canada Atlantic, P.O. Box 1012, Dartmouth, NS B2Y3Z7, Canada, Paul.Hines@drdc-rddc.gc.ca), Sean Pecknold (Defence R\&D Canada Atlantic, P.O. Box 1012, Dartmouth, NS B2Y3Z7, Canada, sean.pecknold@drdc-rddc.gc.ca), John C. Osler (Defence R\&D Canada Atlantic, P.O. Box 1012, Dartmouth, NS B2Y3Z7, Canada, john.osler@drdc-rddc.gc.ca)

Naval sonar operations and planning in littoral environments requires information from historical databases, in situ sampling of environmental parameters, and models capable of estimating sonar performance and the uncertainty in the estimate. Defence R\&D Canada's approach to enable rapid environmental assessment (REA) for sonar incorporates three components: (1) a GIS-enabled database to manage historical environmental data, (2) measurement tools that operate while underway to provide in situ sampling of water column and seabed properties, and (3) a sensitivity model that examines the relative importance of different environmental parameters in order to quantify the impact of incomplete or degraded environmental information, and to specify the appropriate spatial and temporal scales for sampling. In this presentation, the integration of these aspects of REA will be demonstrated using transmission loss data collected in shallow water. Predictions made using REA data provide a substantially better fit to the measurements than those using historical databases.

4pAOa5. Systemic approach to performance prediction and the exploitation of environmental information in naval systems. Patrick Grenard (DGA/DET/CTSN, BP28, 83800 Toulon Armées, France, Patrick.Grenard@dga.defense.gouv.fr)

Interoperability of systems and implementation of network enabled capabilities are at the heart of defense transformation. By providing extensive capabilities for data gathering, processing and presentations, they achieve a greater tactical advantage through superior knowledge of and use of the operational environment. Within the framework of the deployment of the new French Navy information system SIC21, a system of systems is being developed in order to support the implementation of the NATO concept of Recognized Environmental Picture (REP). The metasystem established allows for the assimilation of Rapid Environmental Assessment (REA) data, and for a local or remote production of the REP. In order to support decision making and to guide actions local exploitation of the REP is carried out through embedded tactical decision aids. They rely on advanced performance prediction tools including propagation modelling capabilities called in simulation scenarios. The aims of the implemented models are to predict performances at the appropriate level of granularity, to provide a confidence level on the proposed tactical picture, to define the appropriate level of marine environmental knowledge that should be acquired, to optimise sensor settings, and finally to provide inversion tools for the REA, ensuring consistency with forward modelling used in performance prediction. 


\title{
Session 4pAOb
}

\section{Acoustical Oceanography, Signal Processing in Acoustics, and ECUA: Passive Acoustic Tomography I (Poster Session)}

\author{
Karim Sabra, Cochair \\ Sergio Jesus, Cochair \\ $I S R$
}

Georgia Institute of Technology, School of Mechanical Engineering

\begin{abstract}
All posters will be on display from 3:40 p.m. to 5:20 p.m. To allow contributors an opportunity to see other posters, contributors of odd-numbered papers will be at their posters from 3:40 p.m. to 4:30 p.m. and contributors of even-numbered papers will be at their posters from 4:30 p.m. to 5:20 p.m.
\end{abstract}

\section{Contributed Paper}

4pAOb1. Low-frequency acoustic signature of hurricane Ernesto James Traer (Marine Physical Laboratory, Scripps Institute of Oceanography, 8602 La Jolla Shores Drive, La Jolla, CA 92093-0238, USA, jtraer@ucsd.edu), Peter Gerstoft (Marine Physical Laboratory, Scripps Institute of Oceanography, 8602 La Jolla Shores Drive, La Jolla, CA 920930238, USA, gerstoft@ucsd.edu), Laura Brooks (Marine Physical Laboratory, Scripps Institute of Oceanography, $8602 \mathrm{La}$ Jolla Shores Drive, La Jolla, CA 92093-0238, USA, lbrook02@gmail.com), William Hodgkiss (MPL, Scripps Institution of Oceanography, University of California, San Diego, La Jolla, CA 92093-0238, USA, wsh@mpl .ucsd.edu), David Knobles (Applied Research Laboratories, UT at Austin, P. O. Box 8029, Austin, TX 78713, USA, knobles@arlut.utexas.edu)
The ambient noise level variations produced by Hurricane Ernesto were observed by the SWAMI32, SWAMI52 and SHARK arrays as the storm passed over the SW06 shallow water site. Microseism signals were detected in the water column near $0.1 \mathrm{~Hz}$ and were tracked with a beamformer over a period of several days observing variations that were very closely linked to measured surface waves. 5-75 $\mathrm{Hz}$ beamforming showed a sound-field dominated by local surface-noise punctuated by brief surges of noise from distant sources. Beamforming and time-domain cross-correlations showed that changes in acoustic environment on the time-scale of hours occurred at all three arrays with good correlation in time and directionality suggesting the storm induced noise-field is homogeneous over many kilometers. 


\title{
Session 4pAOc
}

\section{Acoustical Oceanography and ECUA: General Topics in Acoustical Oceanography I (Poster Session)}

\author{
Andone Lavery, Cochair \\ Woods Hole Oceanographic Institution \\ Yann Stephan, Cochair \\ SHOM
}

\begin{abstract}
All posters will be on display from 3:40 p.m. to 5:20 p.m. To allow contributors an opportunity to see other posters, contributors of odd-numbered papers will be at their posters from 3:40 p.m. to 4:30 p.m. and contributors of even-numbered papers will be at their posters from 4:30 p.m. to 5:20 p.m.
\end{abstract}

\section{Contributed Papers}

4pAOc1. The sea and ocean 3D acoustic waveguide: rays dynamics and chaos phenomena. Alexander V. Glushkov (Odessa University, P.O.Box 24a, 65009 Odessa-9, Ukraine, glushkov@paco.net), Andrey A. Svinarenko (Odessa University, P.O.Box 24a, 65009 Odessa-9, Ukraine, glushkov@paco.net), Olga Y. Khetselius (Odessa University, P.O.Box 24a, 65009 Odessa-9, Ukraine, glushkov@paco.net), Nikoly S. Serbov (Odessa University, P.O.Box 24a, 65009 Odessa-9, Ukraine, glushkov@paco.net)

It has been carried out modeling of the sea and ocean 3D acoustic waveguide. On the basis of Hamiltonian equations of rays it is studied a dynamics of rays. It has been shown that for acoustic waveguide in a shallow sea with non-level bottom under the rays propagation in a waveguide dependence of the of temporal frequency upon the output angle represents a fractal measure in accordance with Abdullaev-Zaslavsky result. For the ocean 3D acoustic waveguide on the basis of solving the eiconal equations in the Hamiltonian form it has been studied the fractal dynamics, including the chaotic one. There are presented the data of numerical solution of equations for the typical acoustic channel in the North-Atlantic region. The conditions for the Arnold diffusion effect realization are discussed.

4pAOc2. Fuzzy Clustering of Oceanographic Sound Speed Profiles for Acoustic Characterization. John Dubberley (Naval Research Laboratory, Bldg. 1005 Rm D-23, Stennis Space Center, MS 39529, USA, john.dubberley@nrlssc.navy.mil), Robert Zingerelli (Naval Research Laboratory, Bldg. 1005 Rm D-23, Stennis Space Center, MS 39529, USA, robert.zingerelli@nrlssc.navy.mil)

Historic oceanographic sound speed profiles have traditionally been grouped by area and time period, usually one degree square area and monthly time. After grading the profiles, mean profiles and standard deviations are calculated from the accepted profiles and in the acoustics community they are then used to predict the expected acoustic response of the region. Here the historic profiles in NOAA's World Ocean Database 2005 (WOD2005) will be divided into the same area and time periods, but in subsets with a sufficient number of profiles, fuzzy clustering will be employed on acoustically relevant oceanographic parameters (mixed layer depth, surface temperature, sound speed gradient, etc) to divide the population into multiple clusters. A parabolic equation acoustic transmission model is then applied on the WOD2005 statistical profiles and on the fuzzy cluster populations. Conclusions will be drawn about the suitability of this clustering to capture the variability of acoustic response at a given time and place.

4pAOc3. On the consideration of motion effects in underwater geoacoustic inversion. Nicolas Josso (GIPSA-lab, dep. DIS, 961, rue de la Houille Blanche, 38402 St Martin d'Hères, France, nicolas.josso@gispa-lab.inpg.fr), Cornel Ioana (GIPSA-lab, dep. DIS, 961, rue de la Houille Blanche, 38402 St Martin d'Hères, France, cornel.ioana@gipsa-lab.inpg.fr), Cédric Gervaise (E3I2 - EA3876, 2 rue François Verny, 29806 Brest Cedex, France, cedric.gervaise@ensieta.fr), Jérôme I. Mars (GIPSA-lab, dep. DIS, 961, rue de la Houille Blanche, 38402 St Martin d'Hères, France, jerome.mars @ gipsa-lab.inpg.fr)

The estimation of an impulse response (IR) of a propagation channel is necessary for a large number of underwater acoustic applications: underwater communication, sonar detection and localization, marine mammal monitoring, etc. Basically, it informs us about the distortions of a transmitted signal in one underwater channel. This operation is usually subject to additiona distortions due to the motion of the transmitter-channel-receiver configuration. This paper points on the effects of the motion while estimating the IR for shallow water environments in the very low frequencies bandwidth with matching filtering between the transmitted and the received signals. We propose a methodology to compare between the IR estimation in motionless and motion contexts, respectively. Using this methodology an objective criterion for motion effect analysis is proposed in order to measure the distortions due to the motion phenomena. The proposed methodology is applied to real data sets issued from PASSTIME campaign (SHOM, Bay of Biscaie, 2005) proving also its interest for motion effect analysis. 


\title{
Session 4pAOd
}

\section{Acoustical Oceanography and ECUA: Acoustic Characterization of Sea Floor Habitats I (Poster Session)}

\author{
Christian De Moustier, Cochair \\ UNH, Center for Coastal and Ocean Mapping \\ Dick Simons, Cochair \\ Delft University of Technology \\ Xavier Lurton, Cochair \\ Institut Français de Recherche pour l'Exploitation de la Mer
}

\begin{abstract}
All posters will be on display from 3:40 p.m. to 5:20 p.m. To allow contributors an opportunity to see other posters, contributors of odd-numbered papers will be at their posters from 3:40 p.m. to 4:30 p.m. and contributors of even-numbered papers will be at their posters from 4:30 p.m. to 5:20 p.m.
\end{abstract}

\section{Contributed Paper}

4pAOd1. Hierarchical spline technique application for real time 3D displaying of seafloor using multibeam sonar data. Jerzy Demkowicz (Gdansk University of Technology, ul. Narutowicza 11/12, 80952 Gdansk, Poland, demjot@eti.pg.gda.pl)

Multibeam sonar records have a high resolution raster character. Unfortunately, interpolating and approximating and eventually displaying scattered 3D raster data of high volume leads to some difficulties related to a computer processing power. Usually the problem solution leads to multi- resolution wavelet approach. The paper presents some advantages of using hierarchical splines as applied to real data from multibeam EM 3002 sonar acquired during acoustic survey on Southern Baltic. The proposed approach is two folded: firstly, all acquired multibeam sonar raw data are interpolated with high density uniform spline interpolation. The knots and control points of interpolated network are saved for defined resolution level. In the next stage, preprocessed high resolution data are combined with low resolution data sets after knot decimation process. Such approach allow real time 3D displaying of multibeam sonar data for different zoom levels 


\title{
Session 4pAOg
}

\section{Acoustical Oceanography and ECUA: Acoustic Characterization of Sea Floor Habitats II}

\author{
Christian De Moustier, Cochair \\ UNH, Center for Coastal and Ocean Mapping, Chase Ocean Engineering Lab, 24 Colovos Road, Durham, NH 03824, USA \\ Dick G. Simons, Cochair \\ Delft University of Technology, P.O. Box Postbus 5048, Delft, 2600 GA, Netherlands \\ Xavier Lurton, Cochair \\ Institut Français de Recherche pour l'Exploitation de la Mer, NSE/AS, BP 70, Plouzané, 29280, France
}

\section{Invited Paper}

$$
5: 20
$$

\begin{abstract}
4pAOg1. High frequency scattering measurements for mussel bed characterisation. M. Snellen (Delft Institute of Earth Observation and Space Systems, Delft University of Technology, Kluyverweg 1, 2629 HS Delft, Netherlands, M.Snellen@tudelft.nl), Dick G. Simons (Delft University of Technology, P.O. Box Postbus 5048, 2600 GA Delft, Netherlands, d.g.simons@tudelft.nl), Rolf Riethmueller (Institute for Coastal Research, GKSS Research Centre Geesthacht, Max-Planck-Str. 1, D-21502 Geesthacht, Germany, rolf.riethmueller@gkss.de)
\end{abstract}

Several approaches exist towards seafloor classification using high-frequency backscattering measurements. The classification approach taken in this paper is a model-based classification employing backscatter data measured by a multibeam-echosounder (MBES) system. The method discriminates between sediments in the most optimal way by applying the Bayes decision rule for multiple hypotheses, implicitly accounting for the backscatter strength ping-to-ping variability. The method's applicability for seafloor classification has been demonstrated by using $300 \mathrm{kHz}$ MBES data collected in the Cleaver-Bank area (North Sea). The area is well-known from a geological point of view due to extensive sampling campaigns and is characterized by a wide variety of seafloor types. Here we apply the classification method to MBES data acquired in the Oosterschelde estuary (the Netherlands) which is known to contain mussel culture spots. Also recordings using a video camera towed close to the seafloor and core measurements have been taken. From the video recordings estimates of mussel coverage as a function of position were derived. Analysis of the MBES results shows that they clearly reveal the presence of the mussel beds, indicating the usefulness of acoustic classification for habitat mapping. A comparison between MBES analysis results, video recordings and sample analysis will be presented.

\section{Contributed Papers}

\section{$5: 40$}

4pAOg2. Vertical echosounder versus side-scan sonar mapping of Posidonia Oceanica fields. Noela Sanchez-Carnero (Universidade da Coruña, Campus de Zapateira s/n, E-15071 A Coruña, Spain, noesanchez @udc.es), Víctor Espinosa (IGIC - Universitat Politècnica de València, Cra. Nazaret-Oliva S/N, E-46730 Gandia, Spain, vespinos@fis.upv .es), Miguel Rodilla (IGIC - Universitat Politècnica de València, Cra. Nazaret-Oliva S/N, E-46730 Gandia, Spain, mrodilla@hma.upv.es), Ester Soliveres (IGIC - Universitat Politècnica de València, Cra. NazaretOliva S/N, E-46730 Gandia, Spain, essogon@epsg.upv.es), Juan Freire (Universidade da Coruña, Campus de Zapateira s/n, E-15071 A Coruña, Spain, jfreire@udc.es)

Posidonia fields in the "Cabo de Gata" marine natural park, located in the south-east mediterranean spanish coast, have been mapped by means of two different acoustical tools: a vertical single-beam scientific echosounder and a side-scan sonar. The measured transects have been dived and recorded with a video camera in order to validate the predictions from the extracted acoustical data. We compare both the results obtained from the application of commercial software for bottom classification, and the processing with alternative algorithms in the case of the vertical echosounder, with those derived from the analysis of the side-scan sonar data.

\section{6:00}

4pAOg3. Comparison of multi-beam and single-beam seabed backscatter and sampling resolution near normal incidence. Rudy Kloser (GPO Box 1538, 7001 Hobart, Australia, rudy.kloser@csiro.au)

The seabed backscatter difference near normal incidence $\left(<30^{\circ}\right)$ is compared between two multibeams (EM300 and EM1002) and two on-axis calibrated single beam EK60 (38 and $120 \mathrm{kHz}$ ) echo sounders and found to differ by 7 to $10 \mathrm{~dB}$. A seabed backscatter model supported the calibration of the single beam backscatter near normal incidence. Potential errors due to incorrectly applied equivalent area compensation were found to contribute to this difference but not significantly. Estimating the equivalent area of sampling from multibeam and single beam echosounders highlights the complexities of sampling resolutions as a function of incidence angle and pulse length. These variable sample sizes influence the measured backscatter due to the patchy nature of seabed substrate and fauna. The current backscattering processing method for a national upper slope mapping program relies on producing metrics at the highest spatial resolution. Calibration errors on these metrics for seabed classification for a national mapping program are discussed. 
6:20

4pAOg4. Using the MBES for classification of riverbed sediments Alireza Amiri-Simkooei (Acoustic Remote Sensing Group, Delft Institute of Earth Observation and Space Systems, Delft University of Technology, Kluyverweg 1, 2629 HS Delft, Netherlands, a.amirisimkooei @tudelft.nl), Mirjam Snellen (Acoustic Remote Sensing Group, Delft Institute of Earth Observation and Space Systems, Delft University of Technology, Kluyverweg 1, 2629 HS Delft, Netherlands, m.snellen@tudelft.nl), Dick G. Simons (Delft University of Technology, P.O. Box Postbus 5048, 2600 GA Delft, Netherlands, d.g.simons@tudelft.nl)

For keeping the Dutch rivers suitable for commercial activities measures are required. For example, the bottom of the river Waal, connecting Rotterdam with German industrial areas, is subsiding. Since the subsidence varies along the river, dangerous shoals occur. Sediment suppletions are planned to counteract the subsidence. Appropriate suppletion material is expected to keep the bottom more stable. To monitor the suppletion effectiveness, multibeam-echosounder (MBES) measurements are planned, allowing for simultaneous estimation of bathymetry and sediment composition. For the latter, we apply a method employing the MBES backscatter data. It estimates the number of sediment types present in the survey area and discriminates between them by applying the Bayes decision rule for multiple hypotheses, implicitly accounting for the backscatter strength ping-to-ping variability. The method's applicability was demonstrated in a well-surveyed test area (North Sea). In 2007, MBES measurements were acquired at the Waal, accompanied with extensive sediment grabbing. Contrary to the test area, water depths are very shallow and significant bottom slopes exist, requiring corrections. The lower water depths correspond to smaller beamfootprints, resulting in a higher ping-to-ping variability. Consequently the discriminating power between sediments will decrease. The performance of the classification method for this river environment is assessed.

\section{6:40}

4pAOg5. Analysis of Backscatter and Seafloor Acoustical Properties for Geosciences and Biodiversity Mapping Studies in Cook Strait, New Zealand. Xavier Lurton (Institut Français de Recherche pour l'Exploitation de la Mer, NSE/AS, BP 70, 29280 Plouzané, France, lurton@ifremer.fr), Geoffroy Lamarche (National Institute of Water and Atmospheric Research (NIWA), Private bag 14-901, 6021 Wellington, New Zealand, g.lamarche@niwa.co.nz), Anne-Laure Verdier (National Institute of Water and Atmospheric Research (NIWA), Private bag 14-901, 6021 Wellington, New Zealand, a.verdier@niwa.co.nz), Jean-Marie Augustin (Institut Français de Recherche pour 1'Exploitation de la Mer, NSE/AS, BP 70, 29280 Plouzané, France, augustin@ifremer.fr), Ian Wright (National Institute of Water and Atmospheric Research (NIWA), Private bag 14-901, 6021 Wellington, New Zealand, i.wright@niwa.co.nz), Ashley Rowden (National Institute of Water and Atmospheric Research (NIWA), Private bag 14-901, 6021 Wellington, New Zealand, a.rowden@niwa.co.nz), Alan Orpin (National Institute of Water and Atmospheric Research (NIWA), Private bag 14-901, 6021 Wellington, New Zealand, a.orpin@niwa.co.nz), Miles Dunkin (National Institute of Water and Atmospheric Research (NIWA), Private bag 14-901, 6021 Wellington, New Zealand, m.dunkin@niwa.co.nz)

A quantitative analysis was conducted over sonar backscatter data collected on the Cook Strait region, central New Zealand, featuring multibeam ( $30 \mathrm{kHz}$ ) bathymetry and backscatter data, groundtruthed by an extensive geological database (photographs, seabed samples, high-resolution seismics). A first processing step removes the effects of the sounder, seafloor topography, and water column. A second step includes sonar image mosaicing, signal calibration and compensation, speckle noise filtering, image segmentation and textural analysis. Backscatter angular dependence is then extracted from the raw data accounting for the co-registered multibeam bathymetry; it is linked to the various facies of this geologically very active region, forming a catalogue usable for future investigation. Some local fea- tures are analysed in details, referring to the geological local context. Also the backscatter data from the Haungaroa volcano were used for a proof-ofconcept biodiversity mapping exercise. Ecological theory was utilised to predict biodiversity from the seabed substrate heterogeneity, derived from the segmentation of the backscatter data properly pre-processed. The backscatter analysis resulted in the identification of local features with geological, sedimentological, topographic, and possibly biological significance, otherwise not recognised with conventional surveying. This emphasises the potential of backscatter data in submarine seismic hazard studies and largescale biodiversity mapping.

\section{7:00}

4pAOg6. Seabed biotope mapping using multi-beam backscatter based on reference sites. Rudy Kloser (GPO Box 1538, 7001 Hobart, Australia, rudy.kloser@csiro.au)

A multibeam sonar (MBS) was used to discriminate ecological relevant seabed characteristics based on 62 reference sites spanning depths $50 \mathrm{~m}$ to $400 \mathrm{~m}$ sampled with georeferenced video, sediment grab and rock dredge. The simple ecologically derived terrain characteristics of soft, hard, smooth and rough were found to have the most predictive power for discrimination of the biota using data from video and physical sampling. The acoustic data were corrected for range and incidence angle effects and analysed based on phenomenological characteristics and inversion of a seabed scattering model. Near normal incidence $\left(<16^{\circ}\right)$ the seabed backscatter showed poor correlation to seabed characteristics (cross validation error $32 \%$ ) and was sensitive to the estimation of the correct seabed incidence angle. Using the trend in backscatter near normal incidence $\left(<30^{\circ}\right)$ greatly improved (cross validation error $4 \%$ ) the classification but also increased the spatial scale of classification. The length of biotope scales were derived from the video transects with $50 \%$ of patches less than $18 \mathrm{~m}$. Referencing the seabed backscatter to a consistent incidence angle $\left(40^{\circ}\right)$ gave the highest spatial resolution derived metric and minimised range, incident angle and beam compensation errors. Using this simple metric, high probabilities of prediction of fauna functional groups were recorded.

\section{$7: 20$}

4pAOg7. Statistics of seafloor backscatter measured with multibeam sonar systems. John D. Penrose (Curtin University of Technology, Centre for Marine Sci \& Tech, GPO Box U1987, 6845 Perth, WA, Australia, j.penrose@cmst.curtin.edu.au), Alexander Gavrilov (Curtin University of Technology, Centre for Marine Sci \& Tech, GPO Box U1987, 6845 Perth, WA, Australia, a.gavrilov@cmst.curtin.edu.au), Iain M. Parnum (Curtin University of Technology, Centre for Marine Sci \& Tech, GPO Box U1987, 6845 Perth, WA, Australia, i.parnum@cmst.curtin.edu.au)

A number of theoretical models for seafloor backscatter statistics developed for the recent years show a good agreement with experimental measurements made with sonar systems. However, methods of data collection used in multibeam systems are commonly not taken into consideration when analysing backscatter statistics. Using data collected with a Reson Seabat 8125 system and based on theoretical considerations, it is shown that the seafloor backscatter strength derived from the peak intensity measured as a single value for each beam leads to considerable backscatter overestimation at oblique angles of incidence when the beam footprint is much larger than the insonification area. This occurs because variations of the peak intensity are extreme value distributed, which can be well approximated by the Gumbel distribution. The location parameter of the Gumbel distribution depends on the ratio of the footprint and insonification areas, which results in distorted angular dependence of backscatter strength estimates. On the other hand, the average backscatter strength derived from the integral intensity, i.e. backscatter energy, is a consistent estimate of the actual seafloor backscatter strength. The Gamma distribution is demonstrated to be a good approximation for statistics of the integral intensity, even when the scattering regime is expected to be non-Rayleigh. 


\title{
Session 4pAOh
}

\author{
Acoustical Oceanography, Signal Processing in Acoustics, and ECUA: Passive Acoustic Tomography II \\ Karim G. Sabra, Cochair \\ Georgia Institute of Technology, School of Mechanical Engineering, 771 Ferst Drive, NW, Atlanta, GA 30332-0405, USA \\ Sergio Jesus, Cochair \\ ISR, Universidade do Algarve, Faro, PT-8005-139, Portugal
}

\section{Invited Papers}

\section{$5: 40$}

4pAOh1. Analysis of passive seabed imaging techniques. Thomas M. Siderius (HLS Research Inc., 3366 N. Torrey Pines Ct, Suite 310, La Jolla, CA 92037, USA, siderius@hlsresearch.com)

Recently, a passive technique has been developed to image seabed layering. The method exploits naturally occurring acoustic noise generated on the sea-surface, primarily from breaking waves. The processing exploits the noise coherence through cross-correlations between sensors to recover travel times to significant seabed reflectors. To make this a practical tool, beamforming is used with a vertical array of hydrophones and this greatly reduces the required averaging times. Several data sets using moored arrays have shown stable returns from the seabed. Imaging seabed layering over extended areas requires the array to move which has been accomplished by allowing the array to drift. This, however, introduces a number of complications. If the array moves too rapidly, there is potential for the ensonified seabed (in the beam) to change within the averaging time. Another potential problem could be caused by vertical motion of the array (e.g. by surface coupling). In this case, Doppler shifts may cause degradation in the cross-correlation peaks. In some cases, these degrading factors may be reduced through signal processing. In this presentation potential mechanisms that degrade passive seabed imaging will be described with possible mitigating signal processing. Numerical modeling and measured data sets will be analyzed.

6:00

4pAOh2. Passive tomography of the oceanic environment using ambient noise cross-correlations. Karim G. Sabra (Georgia Institute of Technology, School of Mechanical Engineering, 771 Ferst Drive, NW, Atlanta, GA 30332-0405, USA, karim.sabra@me .gatech.edu)

The random nature of noise and scattered fields tends to suggest limited utility. Indeed, acoustic fields from random sources or scatterers are often considered to be incoherent, but there is some coherence between two sensors that receive signals from the same individual source or scatterer. An estimate of the Green's function (or impulse response) between two points can be obtained from the cross-correlation of ambient noise recorded at these two points. Recent theoretical and experimental studies in ultrasonics, civil engineering, underwater acoustics and seismology have investigated this technique in various environments and frequency ranges. These results provide a means for passive tomography of the ocean environment using only the ambient noise field, without the use of active sources. The coherent wavefronts emerge from a correlation process that accumulates contributions over time from noise sources whose propagation paths pass through both receivers. We will examine the background physics of extracting these coherent structures and present experimental results confirming these theoretical arguments. Further we will present experimental results such as using noise for time synchronization and localization of unconnected acoustic receivers, and for constructing passive tomographic images of the environment.

$$
\text { 6:20 }
$$

4pAOh3. Passive Geoacoustic Inversion using broadband ship noise in Bay of Biscay shallow water environment. Cédric Gervaise (E3I2 - EA3876, 2 rue François Verny, 29806 Brest Cedex, France, cedric.gervaise@ensieta.fr)

Our communication proposes a new geoacoustic inversion method for shallow water environments (100 to $300 \mathrm{~m})$. The method relies on the inversion of broadband noise produced by ships of opportunity. The interference patterns generated by the ship movement and the propagation properties of the waveguide are exploited to extract the relative dispersion curves on a chosen bandwidth. These curves are then inverted to estimate geoacoustic properties. This inversion scheme was previously tested (with success) against real data from a very shallow water (10 to $30 \mathrm{~m}$ ) trial performed off the Southern coast of Barcelona, Spain [1]. To deal with shallow waters, our inversion scheme is improved and tests against real data from PASSTIME trial performed in Bay of Biscay during October 2005. Inversion's results are compared with ground truth and the results showed to be accurate and robust. The proposed technique, which is suitable to a small number of hydrophones and quite easy to implement, offers interesting perspectives for passive geoacoustic inversion. [1] S. Vallez, C. Gervaise, Y. Stephan, M. Andre, Inversion géoacoustique d'un canal très petits petits fonds à partir des navires en mouvement - traitement incohérent, accepté pour publication Revue Traitement du Signal 


\section{Contributed Papers}

6:40

4pAOh4. The passive mode tomography of the ocean using data from short vertical arrays bent by the ocean currents. Andrey Shurup (Department of Acoustics, Physics Faculty, Moscow State University, Leninskie Gory, 119991 Moscow, Russian Federation, burov@phys.msu.ru), Sergey Sergeev (Department of Acoustics, Physics Faculty, Moscow State University, Leninskie Gory, 119991 Moscow, Russian Federation, sergeev@su29.ru), Valentin Burov (Department of Acoustics, Physics Faculty, Moscow State University, Leninskie Gory, 119991 Moscow, Russian Federation, burov@phys.msu.ru)

The possible realization of passive ocean tomography based on the widely discussed relation between the Green's function and ambient noise cross-correlation is discussed. The problem is considered in the mode representation of acoustic field in adiabatic approximation. The estimated time of signal accumulation required to determine the Green's function with sufficient accuracy reveals the possibility of implementing the schemes of the mode tomography of the ocean based on the measuring the ambient noise field of the ocean. It is shown that the use of the vertical arrays with vector receivers allows a decrease in the accumulation time to one or several hours, depending on the conditions of experiment. The mode structure of acoustic field is determined from the cross-correlation matrix of the noise field received by the hydrophones of short vertical arrays bent by the ocean currents and covering only the part of the sound channel. The proposed algorithm differs from the commonly used mode-filtering procedure and allows a compensation of antenna declination from the vertical profile and takes into account of the finite length of antenna aperture, that is ordinary explored in ocean experiments.

\section{7:00}

4pAOh5. Emergence of the deterministic Green's function from thermal noise in inhomogeneous solids and fluid-solid structures. Oleg A. Godin (NOAA/ESRL, 325 Broadway, Mail Code R/PSD99, Boulder, CO 80305-3328, USA, Oleg.Godin@noaa.gov)

S. M. Rytov [A Theory of Electrical Fluctuations and Thermal Radiation (USSR Academy of Sciences, Moscow, 1953)] was apparently the first to establish theoretically a simple relation between deterministic Green's function and cross-correlation of fluctuations of wave fields generated by random sources. He used reciprocity considerations to analyze fluctuations of electromagnetic fields. An acoustic counterpart of the Rytov's approach was applied in [O. A. Godin, Emergence of the acoustic Green's function from thermal noise, J. Acoust. Soc. Am. 121, EL96-EL102 (2007)] to derive exact and asymptotic relations between acoustic Green's functions and crosscorrelation of thermal noise in inhomogeneous moving or motionless fluids. In this paper, these results are extended to solid and fluid-solid media and compared to experimental and theoretical results previously reported in the literature. It is shown that, with the averaging time being sufficiently large, the two-point correlation function of thermal noise contains as much information about the environment as can be obtained acoustically by placing transceivers at the two points. A possibility of retrieving parameters of a fluid motion, such as a flow in a pipe or an oceanic current, from crosscorrelations of diffuse noise fields is addressed.

\section{$7: 20$}

4pAOh6. Comparing time domain Green's functions with simulated noise and ambient noise data cross-correlation for a horizontal array. Stephanie Fried (MPL, Scripps Institution of Oceanography, University of California, San Diego, La Jolla, CA 92093-0238, USA, sefried@ucsd.edu), Karim G. Sabra (Georgia Institute of Technology, School of Mechanical Engineering, 771 Ferst Drive, NW, Atlanta, GA 30332-0405, USA, karim.sabra@me.gatech.edu), Philippe Roux (LGIT CNRS - Université Joseph Fourier, Maison des Géosciences, 1381 rue de la Piscine, BP 53, 38041 Grenoble, France, philippe.roux @obs.ujf-grenoble.fr), William A. Kuperman (MPL, Scripps Institution of Oceanography, University of California, San Diego, La Jolla, CA 920930238, USA,wkuperman@ucsd.edu)

Previous work has shown that an approximation of the Green's function can be extracted from ambient noise data through cross-correlating the received signals along an array. The resulting Green's function approximation gives accurate time-of-arrivals for the multipaths between hydrophones but can only approximate the magnitude of the arrivals in the time domain Green's function. Nevertheless, some useful information can be obtained from the relative amplitudes of the correlated returns assembled. Further, a Monte Carlo noise model simulation for a similar environment for which noise data was collected reproduces the same cross-correlation arrival structure for the processed ambient noise data and the theoretical time domain Green's function arrival structure. [Research supported by ONR].

\section{7:40}

4pAOh7. Acoustic modeshape inversion using deep water ambient noise measurements. Kathleen E. Wage (George Mason University, 4400, University Drive, Fairfax, VA 22030, USA, kwage@gmu.edu), Khalid Almuhanna (George Mason University, 4400, University Drive, Fairfax, VA 22030, USA, kalmuha1@gmu.edu)

Assuming that ambient noise can be represented by a sum of uncorrelated acoustic modes, the eigenvectors of the noise covariance matrix for a vertical line array should correspond to the sampled modeshapes. In previous work several authors have investigated using an eigendecomposition of the noise covariance to estimate the mode functions in shallow water, e.g., Wolf et al. [Proc. of the 1993 IEEE Oceans Conf.,vol. I, pp. 99-104], Hursky et al. [J. Acoust. Soc. Am., 109(4), pp. 1355-1366], and Nielsen and Westwood [J. Acoust. Soc. Am., 111(2), pp. 748-756]. While the same approach should work for deep water scenarios, only few deep water experiments have deployed arrays with sufficient aperture to resolve the modes, e.g., the work D'Spain et al. [Pure appl. geophys., Vol. 158, pp. 475-512]. This paper explores the problem of inverting for the acoustic modes of a deep water waveguide using ambient noise measurements. In particular the paper focuses on important signal processing issues, including data snapshot requirements, and the effects of array tilt. Data from a deep water propagation experiment will be used to quantify how well the empirical modes match the true modes derived from measured environmental data. [Work supported by an ONR Young Investigator Award.] 


\title{
Session 4pBBa
}

\section{Biomedical Ultrasound/Bioresponse to Vibration: Ultrasonic Characterization of Bone I}

\author{
Keith A. Wear, Cochair \\ U.S. Food and Drug Administration, Center for Devices and Radiological Health, 10903 New Hampshire Ave, Bldg 62, Rm \\ 3108, Silver Spring, MD 20993, USA \\ Kay Raum, Cochair \\ Martin Luther University of Halle-Wittenberg, Dept. of Orthopedics, Q-BAM Group, Magdeburger Str. 22, Halle, 06097, \\ Germany
}

\section{Invited Papers}

$$
\text { 2:00 }
$$

4pBBa1. Present state and future trends in ultrasonic characterization of bone. Pascal Laugier (Université Paris 6, Laboratoire d'Imagerie Paramétrique, 15, rue de l’Ecole de Médecine, 75006 Paris, France, laugier@lip.bhdc.jussieu.fr)

Although it has been over 20 years since the first recorded use of a quantitative ultrasound (QUS) technology to predict bone fragility, the field has not yet reached its maturity. QUS have the potential to predict fracture risk in a number of clinical circumstances and has the advantages of being non-ionizing, inexpensive, portable, highly acceptable to patients and repeatable. However, the wide dissemination of QUS in clinical practice is still limited and suffering form the absence of clinical consensus on how to integrate QUS technologies in bone densitometry armamentarium. There are a number of critical issues that need to be addressed in order to develop the role of QUS within rheumatology. These include issues of technologies adapted to measure the central skeleton, data acquisition and signal processing procedures to reveal bone properties beyond bone mineral quantity and elucidation of the complex interaction between ultrasound and bone structure. In this presentation, we review recent developments to assess bone mechanical properties. We conclude with suggestions of future lines and trends in technology challenges and research areas such as new acquisition modes, advanced signal processing techniques, and models.

$$
\text { 2:20 }
$$

4pBBa2. Ultrasonic guided waves in bone. Petro Moilanen (University of Jyväskylä, Department of Physics, PO. Box 35, 40014 Jyväskylä, Finland, pemoilan@cc.jyu.fi)

Recent progress in quantitative ultrasound (QUS) has shown increasing interest towards measuring long bones by ultrasonic guided waves. This technology is widely used in the field of non-destructive testing and evaluation of different waveguide structures. Cortical bone provides such an elastic waveguide and its ability to sustain loading and resist fractures is known to relate to its mechanical properties at different length scales. As guided waves could yield diverse characterization of bone's mechanical properties at the macroscopic level, the method of guided waves has a strong potential over the standardized bone densitometry as a tool for bone assessment. Despite this, development of guided wave methods is challenging, e.g., due to interferences and multiparametric inversion problem. This paper discusses the promises and challenges related to bones characterization by ultrasonic guided waves.

\section{Contributed Papers}

\section{2:40}

4pBBa3. Dual frequency ultrasound measurement of bone - a technique for elimination of soft tissue effects on pulse-echo measurements. Ossi Riekkinen (University of Kuopio, POB 1627, 70211 Kuopio, Finland, Ossi.Riekkinen@uku.fi), Mikko Hakulinen (University of Kuopio, POB 1627, $70211 \quad$ Kuopio, Finland, Mikko.Hakulinen@uku.fi), Juha Töyräs (Kuopio University Hospital, POB 1777, $70211 \quad$ Kuopio, Finland, Juha.Toyras@kuh.fi),Jukka Jurvelin (University of Kuopio, POB 1627, 70211 Kuopio, Finland, Jukka .Jurvelin@uku.fi)

Quantitative ultrasound (US) measurements have been suggested for screening of osteoporosis. However, soft tissues overlying bones affect reliability of the measurements. In this in vitro study, a novel dual frequency ultrasound (DFUS) technique is introduced for elimination of the errors induced by soft tissues on bone pulse-echo US measurements. In DFUS, US reflection from soft tissue-bone interface is measured with two different US frequencies. By knowing the frequency specific US attenuation and speed in adipose and lean tissues, the effect of soft tissue can be determined. DFUS, conducted at frequencies of $2.25 \mathrm{MHz}$ and $5.0 \mathrm{MHz}$, was validated using human trabecular bone samples $(n=25)$ covered with heterogeneous soft tissues. DFUS, reduced $(\mathrm{p}<0.01)$ the mean error induced by soft tissues from $58.6 \%$ to $-4.9 \%$ and from $127.4 \%$ to $23.8 \%$ in broadband ultrasound backscattering and integrated reflection coefficients (at $5.0 \mathrm{MHz}$ ), respectively. Our results suggest that DFUS is a technique capable to minimize the errors induced by the soft tissue overlying the bone. As no reflection information within soft tissue (adipose-lean tissue interface) is needed in pulse-echo measurements DFUS may enhance the accuracy of ultrasound measurements. Thereby, DFUS shows a significant clinical potential.

\section{3:00}

4pBBa4. Improved standardization methods for clinical measurements of BUA and SOS. Keith A. Wear (U.S. Food and Drug Administration, Center for Devices and Radiological Health, 10903 New Hampshire Ave, Bldg 62, Rm 3108, Silver Spring, MD 20993, USA, keith.wear@fda.hhs .gov)

BACKGROUND: Although calcaneal broadband ultrasound attenuation (BUA) and speed of sound (SOS) are good predictors of osteoporotic fracture risk, BUA and SOS measurements exhibit substantial inter-system 
variability. PURPOSE: 1) Compare phase insensitive (PI) detection, which suppresses phase cancellation, and conventional phase sensitive (PS) detection for measurement of BUA. 2) Test a new compensation formula for reducing variability in SOS measurements. METHODS: Data from 73 women were acquired using a GE Lunar Achilles Insight bone sonometer. Radio frequency data were processed off-line using both PI and PS algorithms. RESULTS: BUA measurements (mean $\pm \mathrm{sd}$ ) were $81.4 \pm 21.4 \mathrm{~dB} / \mathrm{MHz}$ (PS) and $67.2 \pm 9.7 \mathrm{~dB} / \mathrm{MHz}(\mathrm{PI})$. Compensation of SOS measurements reduced 1) average transit-time-marker-related SOS variability by $75 \%$ in 73 women and 2) bandwidth-related SOS variability by $80 \%$ in a bonemimicking phantom. CONCLUSION: These new methods will enable a substantial improvement in consistency in bone sonometry. The mention of commercial products, their sources, or their use in connection with material reported herein is not to be construed as either an actual or implied endorsement of such products by the Department of Health and Human Services.

\section{3:20}

4pBBa5. Characterization of Ultrasound Propagation Through Ex-vivo Human Temporal Bone. Azzdine Y. Ammi (University of Cincinnati, Biomedical Engineering, MSB, 231 Albert Sabin Way, Cincinnati, OH 45267-0586, USA, azzdine.ammia@uc.edu), Douglas T. Mast (University of Cincinnati, Biomedical Engineering, MSB, 231 Albert Sabin Way, Cincinnati, OH 45267-0586, USA, masttd@email .uc.edu), I-Hua Huang (1415 2nd Aveue, Unit 1804, Seattle, WA 98101, USA, helloihua@gmail.com), Todd A. Abruzzo (University of Cincinnati, Dept.s of Radiol., Neurosurg. \& Biomed. Engineer., Cincinnati, OH 45267,
USA, Todd.Abruzzo@Healthall.com),Constantin C. Coussios(University of Oxford, Medical Engineering Unit, 43 Banbury Road, OX2 6PE Oxford, UK, constantin.coussios@eng.ox.ac.uk), George J. Shaw (University of Cincinnati, Biomedical Engineering, MSB, 231 Albert Sabin Way, Cincinnati, OH 45267-0586, USA, SHAWGE@UCMAIL.UC.EDU), Christy K. Holland (University of Cincinnati, Biomedical Engineering, MSB, 231 Albert Sabin Way, Cincinnati, OH 45267-0586, USA, Christy.Holland@uc .edu)

Knowledge of cranial and intracranial ultrasonic properties is essential for optimal results in brain vasculature imaging and therapy. The aims of this study were to perform measurements of the intracranial acoustic pressure field, to identify ultrasound parameters that maximize penetration and minimize beam aberration, and to estimate the speed of sound and the attenuation per unit length in the temporal bone (TB). In vitro experiments were conducted on five human skulls. In a water-filled tank, two unfocused $(0.12$ and $1.03 \mathrm{MHz})$ and one focused $(2.00 \mathrm{MHz})$ transducers were consecutively placed near the TB of each skull. The acoustic pressure field was measured in a volume estimated to encompass the middle cerebral artery (MCA). For each measurement, the intracranial distance from the position of maximum acoustic pressure to the estimated MCA origin was quantified. The pressure reductions at these locations relative to the free field were also estimated. The intracranial $-3 \mathrm{~dB}$ depth of field and beam width were investigated as a function frequency. The speed of sound in TB at $1.03 \mathrm{MHz}$ was 1752.1 to $3285.3 \mathrm{~m} / \mathrm{s}$. This work provides quantitative information on the cranial and intracranial ultrasound properties, which are needed for optimal insonation of the brain vasculature.

\section{3:40-5:20 Posters}

Lecture sessions will recess for presentation of poster papers on various topics in acoustics. See poster sessions for topics and abstracts.

\section{Contributed Papers}

\section{$5: 20$}

4pBBa6. Estimation of femoral bone density from trabecular direct wave and cortical guided wave ultrasound velocities measured at the proximal femur in vivo. Reinhard Barkmann (Medizinische Physik, Diagnostische Radiologie, Universitätsklinikum Schleswig-Holstein, ArnoldHeller-Str.9, 24105 Kiel, Germany, barkmann@rad.uni-kiel.de), Stefanie Dencks (Medizinische Physik, Diagnostische Radiologie, Universitätsklinikum Schleswig-Holstein, Arnold-Heller-Str.9, 24105 Kiel, Germany, dencks@rad.uni-kiel.de), Alexander Bremer (Medizinische Physik, Diagnostische Radiologie, Universitätsklinikum Schleswig-Holstein, ArnoldHeller-Str.9, 24105 Kiel, Germany, Alexander.Bremer@gmx.net), Pascal Laugier (Université Paris 6, Laboratoire d'Imagerie Paramétrique, 15, rue de l'Ecole de Médecine, 75006 Paris, France, laugier@lip.bhdc .jussieu.fr), Frederic Padilla (Université Paris 6, Laboratoire d'Imagerie Paramétrique, 15, rue de l'Ecole de Médecine, 75006 Paris, France, Frederic.Padilla@lip.bhdc.jussieu.fr), Kim Brixen (Odense University Hospital, Department of Endocrinology, 5000 Odense, Denmark, Kim.Brixen@ouh.regionsyddanmark.dk), Jesper Ryg (Odense University Hospital, Department of Endocrinology, 5000 Odense, Denmark, Jesper.Ryg@ouh.regionsyddanmark.dk), Claus C. Glüer (Medizinische Physik, Diagnostische Radiologie, Universitätsklinikum SchleswigHolstein, Arnold-Heller-Str.9, 24105 Kiel, Germany, glueer@rad.uni-kiel .de)

Bone mineral density (BMD) of the proximal femur is a predictor of hip fracture risk. We developed a Quantitative Ultrasound (QUS) scanner for measurements at this site with similar performance (FemUS). In this study we tested if ultrasound velocities of direct waves through trabecular bone and of guided waves through cortical bone could be used to estimate BMD. In two centres, Kiel and Odense, we measured time-of-flight (TOF) of waves through the trabecular greater trochanter and cortical intertrochanter as well as a wave through soft tissue only. TOF was adjusted for leg width using ultrasound echoes reflected from the skin of the leg to yield speed-ofsound (SOS) of different wave components. Data were cross-calibrated and pooled (62 women). Bivariate correlations and a multivariate model were calculated for the estimation of femur BMD. BMD correlated both with trabecular and cortical SOS but not soft tissue SOS. Coefficient of determination, percentage residual error (RMSE) and level of significance (p) were $\mathrm{R}^{2}=0.51, \quad \mathrm{RMSE}=12.6 \%, \mathrm{p}<0.0001$ for trabecular and $\mathrm{R}^{2}=0.53$, $\mathrm{RMSE}=12.3 \%, \mathrm{p}<0.0001$ for cortical measurements. The combination of trabecular, cortical and soft tissue SOS improved the correlation to $\mathrm{R}^{2}=0.69, \mathrm{RMSE}=10.4 \%, \mathrm{p}<0.0001$. Multiwave ultrasound methods allow estimation of femoral BMD with a low residual error.

\section{$5: 40$}

4pBBa7. Estimation of in vivo cancellous bone elasticity. Takahiko Otani (Doshisha University, 1-3, Tatara Miyakodani, 610-0321 Kyotanabe, Japan, totani@oyoe.jp), Isao Mano (Oyo Electric Co., Ltd., 63-1, Nakamichiomote Hirakawa, 610-0101 Joyo, Japan, imano@oyoe.jp), Toshiyuki Tsujimoto (Horiba, Ltd., 2, Miyanohigashi-cho, Kisshoin, Minami-ku, 6018510 Kyoto, Japan, toshiyuki.tsujimoto@horiba.com)

Effect of decreasing bone density (a symptom of osteoporosis) is greater for cancellous bone than for dense cortical bone, because cancellous bone is metabolically more active. Therefore, bone density or bone mineral density at cancellous bone is generally used to estimate the onset of osteoporosis. Elasticity or elastic constant is one of fundamental mechanical parameters and directly related to the mechanical strength of bone. Accordingly, elasticity is a preferable parameter to assess the fracture risk. A novel ultrasonic bone densitometer LD-100 has been developed to obtain mass density and elasticity of cancellous bone with a spatial resolution comparable to that of the peripheral quantitative computed tomography system. Bone mass density and bone elasticity are evaluated using ultrasonic parameters based on fast and slow waves in cancellous bone using a modeling of ultrasonic wave propagation path. Elasticity is deduced from measured bone mass density and propagation speed of fast wave. Thus, elasticity of cancellous bone is approximately expressed by a cubic equation of bone mass density. 
6:00

4pBBa8. Fracture and bone defect assessment using quantitative ultrasound wave propagation. Mikey Szczupak (Stony Brook University, SUNY, Department of Biomedical Engineering, 350 Psychology-A Building, Stony Brook, NY 11794, USA, mszczupak90@yahoo.com), Yi Xia (Stony Brook University, SUNY, Department of Biomedical Engineering, 350 Psychology-A Building, Stony Brook, NY 11794, USA, xia.yi76@gmail.com), Jiqi Cheng (Stony Brook University, SUNY, Department of Biomedical Engineering, 350 Psychology-A Building, Stony Brook, NY 11794, USA, chengjiqi@yahoo.com), Yi-Xian Qin (Stony Brook University, SUNY, Department of Biomedical Engineering, 350 Psychology-A Building, Stony Brook, NY 11794, USA, yi-xian.qin@sunysb .edu)

Nondestructive evaluation of early fracture and monitoring its healing, particularly in non-typical fracture, is critical yet challenge. Quantitative ultrasound test along the long bone is largely dependant on bone density, gap size, the level of maturity of the callus, and mineralized status. We propose that the ultrasonic velocity (UV) is dependent on fracture gap size and the degree of porosity. The objective of the study was to develop an analytical and a multiple ultrasound sensing system for non-invasive fracture assessment. An analytical model was generated as a function between UV and the gap size in long bone. The ultrasound measurements were performed in the intact pig femur with controlled fracture sizes, e.g., $1 \mathrm{~mm}, 3 \mathrm{~mm}$ and $5 \mathrm{~mm}$. A serial ultrasound transducers were aligned $3 \mathrm{~cm}$ above bone with an angle approximately equal to the critical angle. The results indicated that the UV decreased with increase of fracture size in a linear manner $(r=0.96)$, in which UV decreased $6.7 \%, 16.3 \%$ and $26.8 \%$ for 1,3 and $5 \mathrm{~mm}$ gaps, respectively. The simulation result significantly matched the experiment measurement $(\mathrm{r}=0.98)$. The results demonstrated that quantitative ultrasound has potential to the diagnosis of fracture, monitoring fracture healing, and other bone disorders.

\section{6:20}

4pBBa9. Monitoring of trabecular bone induced microdamage using a nonlinear wave-coupling technique. Guillaume Renaud (LUSSI, $10 \mathrm{Bd}$ Tonellé, 37032 Tours, France, guillaume.renaud28@etu.univ-tours .fr), Samuel Callé (LUSSI, 10 Bd Tonellé, 37032 Tours, France, calle_s@med.univ-tours.fr), Jean-Pierre Remenieras (LUSSI, 10 Bd Tonellé, 37032 Tours, France, remenier@med.univ-tours.fr), David Mitton (Laboratoire de biomécanique, 151, Boulevard de l'hopital, 75013 Paris, France, david.mitton@paris.ensam.fr), Julie Blanchi (Laboratoire de biomécanique, 151, Boulevard de l'hopital, 75013 Paris, France, julieblanchi@gmail.com), Marielle Defontaine (LUSSI, 10 Bd Tonellé, 37032 Tours, France, defontai@med.univ-tours.fr)

Bone tissue contains microcracks which may affect its mechanical properties as well as the whole trabecular structure. The relationship between crack density and bone strength is nevertheless poorly understood. Efficient nonlinear (NL) ultrasound methods have been widely developed for nondestructive testing and geophysical applications to detect microdamage. Moreover it has been reported that elastic nonlinearities increase with induced damage. We propose to monitor trabecular bone microdamage using a NL wave coupling technique. Ultrasonic short bursts times of flight (TOF) are modulated as result of NL interaction with a low-frequency (LF) wave in the medium. TOF modulation (TOFM), or propagation velocity variations, are directly related to NL elasticity variations. This technique allows measuring the instantaneous TOFM as a function of the LF pressure. It is thus possible to analyze separately elasticity variations in tension and in compression, and to distinguish the tension to compression phase from the compression to tension phase (hysteresis). In several trabecular bone samples, different TOFM amplitudes in tension and in compression are observed, probably due to microdamage. For increasing damage levels progressively induced by quasistatic compression testing, linear and nonlinear ultrasound parameters are compared to biomechanical parameters.

6:40

4pBBa10. Local measurements of the diffusion constant in multiple scattering media: Application to human trabecular bone imaging. Alexandre Aubry (Laboratoire Ondes et Acoustique, ESPCI, Université Paris 7, CNRS, 10 rue Vauquelin, 75005 Paris, France, alexandre.aubry
@espci.fr),Arnaud Derode(Laboratoire Ondes et Acoustique, ESPCI, Université Paris 7, CNRS, 10 rue Vauquelin, 75005 Paris, France, arnaud.derode@ujf-grenoble.fr), Frederic Padilla (Université Paris 6, Laboratoire d'Imagerie Paramétrique, 15, rue de l'Ecole de Médecine, 75006 Paris, France, Frederic.Padilla@lip.bhdc.jussieu.fr)

Multiple scattering of ultrasound can occur in trabecular bones. A typical signature of multiple scattering is the coherent backscattering effect, and the average trajectory of a wave undergoing multiple scattering can be characterized by the diffusion constant $D$. In this work, we present local measurements of the diffusion constant for ultrasonic waves in human trabecular bone, based on near-field observation of the backscattered intensity. The experimental set up consists in an array of programmable transducers placed in front of a slice of bone that we want to image. By achieving Gaussian beamforming both at emission and reception, an array of virtual sources and receivers located in the near-field is constructed. The time evolution of the incoherent component of the intensity backscattered on this virtual array is shown to represent directly the growth of the diffusive halo as $(D t)^{1 / 2}$. A matrix treatment is proposed to separate the incoherent intensity from the coherent backscattering peak. Once the incoherent contribution is isolated, local measurements of the diffusion constant $D$ are achieved around $3 \mathrm{MHz}$ and then a $D$-map of the bone is built. These measurements are shown to be strongly correlated with the bone mineral density.

\section{7:00}

4pBBa11. Relationship Between Mechanical Properties and Acoustic Parameters Obtained from Fast and Slow Waves for Cancellous Bone. Masaki Harada (Doshisha University, 1-3, Tatara Miyakodani, 610-0321 Kyotanabe, Japan, dth0315@mail4.doshisha.ac.jp), Kazuto Tanaka (Doshisha University, 1-3, Tatara Miyakodani, 610-0321 Kyotanabe, Japan, ktanaka@mail.doshisha.ac.jp), Tsutao Katayama (Doshisha University, 1-3, Tatara Miyakodani, 610-0321 Kyotanabe, Japan, tkatayam@mail.doshisha.ac.jp), Katsunori Mizuno (Doshisha University, 1-3, Tatara Miyakodani, 610-0321 Kyotanabe, Japan, dtg0151@mail4 .doshisha.ac.jp), Hiroki Soumiya (Doshisha University, 1-3, Tatara Miyakodani, 610-0321 Kyotanabe, Japan, mmatsuka@mail.doshisha .ac.jp), Mami Matsukawa (Doshisha University, 1-3, Tatara Miyakodani, 610-0321 Kyotanabe, Japan, mmatsuka@mail.doshisha.ac.jp)

The bone-density measuring equipment using the characteristics of the ultrasound has been applied for the diagnosis of osteoporosis. The propagated ultrasonic wave in the cancellous bone changes dramatically due to its complicated structure, and is separated into fast wave and slow wave. The parameters obtained from two-waves are closely related to the structure properties and the elastic properties of bone, which is considered to provide important information related to bone strength. However, the relationship between the strength obtained by the mechanical tests and the fast and slow wave properties is not clearly investigated. In this study, compression tests of the cancellous bovine bone were conducted to determine the compressive strength in addition to the ultrasonic measurements. Our results showed that amplitude ratio of fast and slow wave significantly correlated $(\mathrm{r} 2=0.88)$ with the compressive strength, which reasonably indicates that the bone strength increases in proportional to the density of the cancellous bone.

\section{$7: 20$}

4pBBa12. Two-dimensional simulation of circumferential wave propagation in cortical bone at the femoral neck: sensitivity to bone geometry. Julien Grondin (Université Paris 6, Laboratoire d'Imagerie Paramétrique, 15, rue de l'Ecole de Médecine, 75006 Paris, France, Julien.Grondin@lip.bhdc.jussieu.fr), Quentin Grimal (Université Paris 6, Laboratoire d'Imagerie Paramétrique, 15, rue de l'Ecole de Médecine, 75006 Paris, France, quentin.grimal@lip.bhdc.jussieu.fr), Pascal Laugier (Université Paris 6, Laboratoire d'Imagerie Paramétrique, 15, rue de 1'Ecole de Médecine, 75006 Paris, France, laugier@lip.bhdc.jussieu.fr)

Assessment of bone status using ultrasound techniques has proved to be efficient to quantify the risk of osteoporotic fracture. A prototype scanner is currently developed (European FEMUS project) to assess bone quality at the hip. Circumferential waves in femoral neck cortical bone are expected to propagate in the prototype configuration. In this work, the relationships 
between time of flight (TOF) of circumferential waves and femoral neck cross-section geometrical parameters were investigated. Two-dimensional finite-difference time-domain simulations of through transmission propagation of a plane wave at $0.5 \mathrm{MHz}$ central frequency were performed on eight femoral neck cross-section models reconstructed from X-ray computed tomography data of one human femur. An ellipse with major radius (a) and minor radius (b) was fitted on the external circumference of each crosssection. The TOF was highly correlated to the ellipticity a/b $(R=-0.9607$, $\mathrm{p}<10-3)$ and to the area delineated by the endosteal surface $(\mathrm{R}=-0.9717$, $\mathrm{p}<10-4)$. These results indicate that the TOF is sensitive to the shape of the femoral neck cross-section. This is interesting insofar as previous studies highlighted the importance of the relationships between geometrical parameters and bone strength. In future works, these 2-D results will be challenged in 3-D configurations.

\section{$7: 40$}

4pBBa13. Array transducer applied to low-frequency guided wave ultrasonography: An in vivo study on human radius and tibia. Vantte Kilappa (University of Jyväskylä, Department of Physics, PO. Box 35, 40014 Jyväskylä, Finland, warma@iki.fi), Petro Moilanen (University of Jyväskylä, Department of Physics, PO. Box 35, 40014 Jyväskylä, Finland, pemoilan@cc.jyu.fi), Tianhui Chen (University of Jyväskylä, Department of Health Sciences, PO. Box 35, 40014 Jyväskylä, Finland, cth006@hotmail.com), Hongqiang Ma (University of Jyväskylä, Department of Health Sciences, PO. Box 35, 40014 Jyväskylä, Finland, mhqsir0827@hotmail.com), Jussi Timonen (University of Jyväskylä, Department of Physics, PO. Box 35, 40014 Jyväskylä, Finland, Jussi.Timonen @ phys.jyu.fi), Sulin Cheng (University of Jyväskylä, Department of Health Sciences, PO. Box 35, 40014 Jyväskylä, Finland, Sulin.Cheng@sport.jyu.fi)

Velocity $\left(V_{F A S}\right)$ of a first arriving signal for $f<0.5 \mathrm{MHz}$ is expected to have an enhanced sensitivity to endosteal osteoporotic changes as compared to using higher frequencies. In this study a group of males and females (aged 10-87 years) was measured by using a new array probe $\left(f_{c}=0.4\right.$ $\mathrm{MHz}$ ) on the radius and tibia. In addition, peripheral quantitative computed tomography was used to assess the bone mineral density (BMD) and cortical thickness $(\mathrm{CTh})$ at the same bone locations. Initial results $(\mathrm{n}=57)$ showed that the repeatability error of the $V_{F A S}$ data $\left(C V_{r m s}\right)$ was $0.5 \%$. When $V_{F A S}$ data for the radius was plotted by age it characterized well, yet better than $\mathrm{BMD}$ or $\mathrm{CTh}$, the typical growth and loss curve of bone. $V_{F A S}$ for the radius was strongly correlated with total $\mathrm{BMD}(r=0.84, p<0.001)$, cortical BMD $(r=0.77, p<0.001)$, subcortical BMD $(r=0.66, p<0.001)$ and CTh $(r=0.59, p<0.001)$. Corresponding results for the tibia were similar but clearly weaker than those for the radius. In conclusion, the lowfrequency $V_{F A S}$ had good accuracy and it predicted well both the geometry and material properties throughout the cortex.

\title{
Session 4pBBb
}

\section{Biomedical Ultrasound/Bioresponse to Vibration: Ultrasonic Characterization of Bone II (Poster Session)}

\author{
Keith Wear, Cochair \\ U.S. Food and Drug Administration, Center for Devices and Radiological Health \\ Frederic Padilla, Cochair \\ Université Paris 6
}

\begin{abstract}
All posters will be on display from 3:40 p.m. to 5:20 p.m. To allow contributors an opportunity to see other posters, contributors of odd-numbered papers will be at their posters from 3:40 p.m. to 4:30 p.m. and contributors of even-numbered papers will be at their posters from 4:30 p.m. to 5:20 p.m.
\end{abstract}

\section{Contributed Papers}

\begin{abstract}
4pBBb1. Cross-sectional ultrasonic tomography of the medullary cavity of child bones - Limits of resolution. Philippe Lasaygues (Laboratory for Mechanics and Acoustics CNRS, 31 chemin Joseph Aiguier, 13009 Marseille, France, lasaygues@lma.cnrs-mrs.fr), Régine Guillermin (Laboratory for Mechanics and Acoustics CNRS, 31 chemin Joseph Aiguier, 13009 Marseille, France, guillermin@1ma.cnrs-mrs.fr), Eric Debieu (Laboratory for Mechanics and Acoustics CNRS, 31 chemin Joseph Aiguier, 13009 Marseille, France, debieu@1ma.cnrs-mrs.fr), Jean-Pierre Lefebvre (Laboratory for Mechanics and Acoustics CNRS, 31 chemin Joseph Aiguier, 13009 Marseille, France, lefebvre@1ma.cnrs-mrs.fr), Philippe Petit (Pediatric radiology department, 'Timone' Children's Hospital, 13006 Marseille, France, philippe.petit@mail.ap-hm.fr)
\end{abstract}

In children with bone diseases, ultrasonography has proved to be a highly effective tool for assessing congenital disorders. However, with standard devices, this method of examination is limited and not suitable for diagnostic large purposes as tumors or sub-periostal infectious. Authors dealt with the imaging of more adult bones than children, and the main aim has usually been to assess the thickness of the diaphysis and/or to estimate the wave velocity crossing the structure. Our group has been focusing on the cross-sectional radial imaging process, using ultrasonic computed tomography, of child bones. Although this method is known to provide a potentially valuable means of imaging objects with similar acoustical impedance, problems arise when it is proposed to obtain quantitative tomograms of more highly contrasted media. Finding solutions involves either using non-linear schemes. In this paper, we recall the advantages and limitations of ultrasonic computed tomography methods when dealing with highly contrasted scatterers. The results obtained are promising and suggest that the geometrical and acoustical characteristics of children's bones can be efficiently determined using this ultrasonic computed tomography method. 
4pBBb2. Model study on assessment of bone by ultrasonic guided waves through a varying layer of soft tissue. Alexey Tatarinov (Artann Laboratories, 1753 Linvale-Harbourton, Lambertville, NJ 08350, USA, tatarinov@artannlabs.com), Armen Sarvazyan (Artann Laboratories, 1753 Linvale-Harbourton, Lambertville, NJ 08350, USA, armen@artannlabs .com)

Propagation parameters of ultrasonic guided waves in long bones are sensitive to changes of the cortical thickness, which is one of bone parameters affected by osteoporosis. Meanwhile, the guided waves in bone can be masked by the longitudinal waves in the overlying soft tissues, especially in obese patients. The goal of this study was to explore a possibility to minimize the effect of soft tissue on quantitative assessment of propagation parameters of axial guided waves in long bones. Phantoms and animal bone fragments modeling the axial gradients of the cortical thickness in the human proximal tibia were used. The specimens were covered by a layer of soft tissue of varied thickness. Ultrasonic signals were acquired in the pulse mode at $0.1 \mathrm{MHz}$ by the surface transmission. The specimens were scanned lengthwise and the acquired signals were plotted versus the scanned distance. Analysis of the obtained waveform profiles along the tested specimens allowed quantitative evaluation of the variations of the cortical thickness despite the presence of a 5-6 mm soft tissue layer. The ratio of the acoustic wavelength to the soft tissue layer thickness defines the level of the soft tissue effect.

4pBBb3. Using Singular Value Decomposition to analyse a low frequency contribution on human cortical bone with a $1 \mathrm{MHz}$ axial transmission probe. Magali Sasso (Echosens, R\&D department, 153 avenue d'Italie, 75013 Paris, France, magali.sasso@echosens.com), Maryline Talmant (Université Paris 6, Laboratoire d'Imagerie Paramétrique, 15, rue de l'Ecole de Médecine, 75006 Paris, France, talmant@lip.bhdc.jussieu .fr), Guillaume Haiat (CNRS, Laboratoire de Recherches Orthopédiques, 10, Avenue de Verdun, 75010 Paris, France, haiat@univ-paris12.fr), Pascal Laugier (Université Paris 6, Laboratoire d'Imagerie Paramétrique, 15, rue de l'Ecole de Médecine, 75006 Paris, France, laugier@lip.bhdc .jussieu.fr), Salah Naili (Université Paris 12, B2OA, 61, Avenue du Général de Gaulle, 94010 Créteil, France, naili@univ-paris12.fr)

The configuration of axial transmission technique dedicated to cortical bone characterization generates multiple contributions, associated with different propagation modes. The first arriving signal velocity is classically evaluated using time-of-flight measurements while the analysis of later arrivals requires the development of specific signal processing tools. We focus here on an Energetic Low Frequency (ELF) later contribution acquired by a 1-MHz multi-element bi-directional probe devised in the LIP. Using a procedure adapted from the Singular Value Decomposition (SVD), the ELF contribution was separated from the rest of the signal. The ability of the method to provide an accurate phase velocity estimate of a dispersive wave was established in a controlled-case study on the propagation of Lamb waves on plates using FDTD (Finite-Difference Time-Domain) simulations. The method applied on signals acquired previously in vitro on human radii using the bidirectional device show that the ELF is consistent with the A0 Lamb mode and that its velocity is related to cortical bone thickness $(\mathrm{R} 2=$ $0.6, \mathrm{p}<10-5)$. Identification of A0 type wave agree with results reported in the literature obtained with a mono-element device which operates in the $100-300 \mathrm{kHz}$ frequency band.

4pBBb4. Variability of velocities provided by axial transmission due to irregular geometry of cortical bones. Thiên-Ly Pham (Université Paris 6, Laboratoire d'Imagerie Paramétrique, 15, rue de l'Ecole de Médecine, 75006 Paris, France, pham@lip.bhdc.jussieu.fr), Maryline Talmant (Université Paris 6, Laboratoire d'Imagerie Paramétrique, 15, rue de l'Ecole de Médecine, 75006 Paris, France, talmant@lip.bhdc .jussieu.fr), Pascal Laugier (Université Paris 6, Laboratoire d'Imagerie Paramétrique, 15, rue de l'Ecole de Médecine, 75006 Paris, France, laugier @ lip.bhdc.jussieu.fr)

Early predictions of the velocities of axially transmitted waves on cortical bones were based on waveguides such as plate and tube of regular geometry and uniform thickness. We investigate the role of the actual irregular geometry by means of numerical simulations comparing monodirectional and bidirectional multielement modalities. Propagation was simulated using a finite difference method in a set of human radii previously examined by a bidirectional device. Individual geometry of the samples was reconstructed from X Ray tomography (pixel $=100 \mathrm{~mm}$ ). The material constituting the bone models was considered to be a transverse isotropic medium with chosen fixed elastic properties taken from literature. In addition simulations were performed on plate of either constant or variable thickness. Preliminary results perfomed on 9 samples show that bidirectional technique reduces the variability of the velocities of axially transmitted waves compared to monodirectional array when irregular geometries are involved. Whereas bidirectional velocity is significantly correlated to the velocity obtained in plates whose thickness is equal to the mean thickness of the specimen $\left(\mathrm{r}^{2}=0.77\right.$, $\mathrm{p}=0.0018, \mathrm{RMSE}=29 \mathrm{~m} / \mathrm{s}$ ), no significant correlation is found for monodirectional velocity. The effect is mainly attributed to the irregular external surface of real samples.

4pBBb5. A finite element model of the lamellar osteonal structure based on ultrahigh frequency acoustic impedance data. Kay Raum (Martin Luther University of Halle-Wittenberg, Dept. of Orthopedics, Q-BAM Group, Magdeburger Str. 22, 06097 Halle, Germany, kay.raum@medizin.uni-halle.de), Quentin Grimal (Université Paris 6, Laboratoire d'Imagerie Paramétrique, 15, rue de l'Ecole de Médecine, 75006 Paris,France, quentin.grimal@lip.bhdc.jussieu.fr), Alf Gerisch (Martin Luther University of Halle-Wittenberg, Institut für Mathematik, 06099 Halle, Germany, alf.gerisch@medizin.uni-halle.de)

Materials: A finite element (FE) model was developed, in which the osteon is considered to consist of a central Haversian canal filled with an incompressible fluid and surrounding sets of lamellar units. Each lamellar unit was further subdivided in five sublayers, whereas the orientation of the symmetry axis between adjacent sublayers was shifted clockwise. A sublayer consists of one to ten layers of parallel oriented mineralized collagen fibrils (thickness: $0.2 \mu \mathrm{m}$, constant transverse isotropic stiffness tensor). Results: A variation of the sublayer thicknesses results in either isotropic or anisotropic tissue compound properties. By changing the individual layer thicknesses various degrees of anisotropy could be produced. A good agreement with the lamellar pattern obtained in 1.2-GHz SAM images as well as with the anisotropic elastic coefficients measured at the tissue level $(50-\mathrm{MHz}$ ultrasound) was obtained by choosing an asymmetric lamellar unit Conclusions: With the proposed combination of experimentally derived microelastic and microstructural data by $1.2 \mathrm{GHz}$ SAM and a micromechanical FE the homogenized elastic stiffness tensor of lamellar bone tissue was derived. The tissue anisotropy was explained by the asymmetric twisted plywood structure.

4pBBb6. Distribution and anisotropy of fast wave speed in the cancellous bone of bovine femur. Katsunori Mizuno (Doshisha University, 1-3, Tatara Miyakodani, 610-0321 Kyotanabe, Japan, dtg0151@mail4.doshisha.ac.jp), Hiroki Soumiya (Doshisha University, 1-3, Tatara Miyakodani, 610-0321 Kyotanabe, Japan, mmatsuka @ mail.doshisha.ac.jp), Mami Matsukawa (Doshisha University, 1-3, Tatara Miyakodani, 610-0321 Kyotanabe, Japan, mmatsuka@mail .doshisha.ac.jp), Takahiko Otani (Doshisha University, 1-3, Tatara Miyakodani, 610-0321 Kyotanabe, Japan, totani@oyoe.jp), Masahiko Takada (Omihachiman Community Medical Center, 1379 Tsuchida-cho, 523*0082 Omihachiman, Japan, takada@design.kyushu-u.ac.jp), Isao Mano (Oyo Electric Co., Ltd., 63-1, Nakamichiomote Hirakawa, 610-0101 Joyo, Japan, imano@oyoe.jp), Toshiyuki Tsujimoto (Horiba, Ltd., 2, Miyanohigashi-cho, Kisshoin, Minami-ku, 601-8510 Kyoto, Japan, toshiyuki.tsujimoto@horiba.com)

The ultrasonic longitudinal wave in the cancellous bone is separated into two waves, fast and slow waves [1]. In this study, the relationship between the fast wave speed and the cancellous bone structure is experimentally investigated. A conventional ultrasonic pulse measurement was performed using a PVDF focus transmitter (Custom made, Toray) and a self-made PVDF receiver. Cylindrical specimens of cancellous bone were taken from the head of bovine femur in the distal part, along the three orthogonal directions. The distribution of fast wave speed was obtained by changing the measurement position along the cylindrical axis. The anisotropy of speed was also investigated by rotating the specimens. The structural parameters 
of each specimen were also measured by X-ray micro CT (MCT-12505MF, Hitachi), which gave us the trabecular length and alignment from MIL (mean intercept length) parameters through TRI/3D-Bon software (Ratoc). We found that the fast wave showed large distribution and strong anisotropy depending on the measurement positions and wave propagation directions in the specimens. The fast wave showed the maximum speed in case of wave propagation along the load direction. Reference [1] A. Hosokawa and T. Otani, J. Acoust. Soc. Am., 101, 558 (1997).

4pBBb7. Ultrasonic wave properties in the bone axis direction of bovine cortical bone. Kazufumi Yamamoto (Orthopaedic Surgery, Hamamatsu University School of Medicine, 1-20-1 Handayama Higasi-ku, 431-3192 Hamamatsu, Japan, kazyama2323@hotmail.com), Yuichiro Yaoi (Doshisha University, 1-3, Tatara Miyakodani, 610-0321 Kyotanabe, Japan, yuichiro_y0306@hotmail.co.jp), Yu Yamato (Orthopaedic Surgery, Hamamatsu University School of Medicine, 1-20-1 Handayama Higasi-ku, 431-3192 Hamamatsu, Japan, kazyama2323@hotmail.com), Takahiro Yanagitani (Graduate school of engineering, Tohoku University, 28 Kawauchi Aoba-ku, 980-8579 Sendai, Japan, mmatsuka@mail.doshisha .ac.jp), Takaaki Koizumi (Doshisha University, 1-3, Tatara Miyakodani, 610-0321 Kyotanabe, Japan, mmatsuka@mail.doshisha.ac.jp), Mami Matsukawa (Doshisha University, 1-3, Tatara Miyakodani, 610-0321 Kyotanabe, Japan, mmatsuka@mail.doshisha.ac.jp), Kaoru Yamazaki (Orthopaedic Surgery, Hamamatsu University School of Medicine, 1-20-1 Handayama Higasi-ku, 431-3192 Hamamatsu, Japan, kazyama2323@hotmail.com), Akira Nagano (Orthopaedic Surgery, Hamamatsu University School of Medicine, 1-20-1 Handayama Higasi-ku, 431-3192 Hamamatsu, Japan, kazyama2323@ hotmail.com)

Quantitative ultrasound (QUS) is a good method to measure elastic properties of bone (one indicator of bone quality) in vivo. Bovine cortical bone has two typical microstructures, plexiform and Haversian. In the nanoscopic level, bone consists of calcium phosphate, which forms incomplete hydroxyapatite (HAp) crystal. The preferred orientation of c-axis of HAp crystallites induces anisotropy and inhomogeneity of elastic properties in bone. In this study, relationship between speed of sound (SOS) and HAp crystallites orientation in the axial direction were investigated in two foreign age bovine cortical bones. The dependence of attenuation on the anatomical position was also investigated. Two ring shaped cortical bone samples were made from 36 and 24-month-old bovine femur. SOS was measured by a conventional ultrasonic pulse system, using self-made PVDF transducers. The integrated intensity of (0002) peak obtained using X-ray diffraction was estimated to evaluate the amount of preferred orientation. Regardless of age, a significant correlation between SOS and preferred orientation of HAp crystallite was observed in the parts of the plexiform structure, and gradient of the relation showed a similar tendency. Attenuation seemed to strongly depend on bone microstructure because of its porosity.

4pBBb8. Anisotropy of ultrasonic longitudinal wave in the cortical bone of bovine femur. Yuichiro Yaoi (Doshisha University, 1-3, Tatara Miyakodani, 610-0321 Kyotanabe, Japan, yuichiro_y0306@hotmail .co.jp), Kazufumi Yamamoto (Orthopaedic Surgery, Hamamatsu University School of Medicine, 1-20-1 Handayama Higasi-ku, 431-3192 Hamamatsu, Japan, kazyama2323@hotmail.com), Takaaki Koizumi (Doshisha University, 1-3, Tatara Miyakodani, 610-0321 Kyotanabe, Japan, mmatsuka@mail .doshisha.ac.jp), Mami Matsukawa (Doshisha University, 1-3, Tatara Miyakodani, 610-0321 Kyotanabe, Japan, mmatsuka@mail .doshisha.ac.jp), Kaoru Yamazaki (Orthopaedic Surgery, Hamamatsu University School of Medicine, 1-20-1 Handayama Higasi-ku, 431-3192 Hamamatsu, Japan, kazyama2323@hotmail.com), Akira Nagano (Orthopaedic Surgery, Hamamatsu University School of Medicine, 1-20-1 Handayama Higasi-ku, 431-3192 Hamamatsu, Japan, kazyama2323 @hotmail.com)

Quantitative ultrasound (QUS) is a good method to measure elastic properties of bone. It is known that the mammalian cortical bone shows strong anisotropy and inhomogeneity. We have investigated the distribution of ultrasonic longitudinal wave properties in bovine femur, considering the structure in the nanoscopic level [Yamato et al. Calcified Tissue International, Accepted]. In this study, the anisotropy in the axial-tangential plane of bovine cortical bone is experimentally investigated using an ultrasonic pulse technique. The ultrasonic pulse measurement was performed using a PVDF focus transmitter (Custom made, Toray) and a self-made flat PVDF receiver. Three ring-shaped cortical bone samples were made from a 32month-old bovine femur. Four cylindrical specimens were taken from one ring-shaped cortical bone sample along the radial direction. The anisotropy of speed was investigated by rotating the specimens. We found that directivities of ultrasonic longitudinal wave in these specimens were similar. In addition, the direction of the fastest wave speed was a little different from the bone axis. The results indicate the complicated anisotropy of the cortical bone.

4pBBb9. Ultrasonic velocity dispersion in bovine cortical bone. Guillaume Haiat (CNRS, Laboratoire de Recherches Orthopédiques, 10, Avenue de Verdun, 75010 Paris, France, haiat@univ-paris12.fr), Magali Sasso (Echosens, R\&D department, 153 avenue d'Italie, 75013 Paris, France, magali.sasso@echosens.com), Salah Naili (Université Paris 12, B2OA, 61, Avenue du Général de Gaulle, 94010 Créteil, France, naili@univ-paris12.fr), Kazufumi Yamamoto (Orthopaedic Surgery, Hamamatsu University School of Medicine, 1-20-1 Handayama Higasi-ku, 431-3192 Hamamatsu, Japan, kazyama2323@hotmail.com), Mami Matsukawa (Doshisha University, 1-3, Tatara Miyakodani, 610-0321 Kyotanabe, Japan, mmatsuka@mail.doshisha.ac.jp)

The evaluation of cortical bone quality has become possible in clinical practice, but the interaction between a broadband ultrasonic pulse and this complex multiscale medium remains poorly understood. Specifically, the frequency dependence of phase velocity has been sparsely investigated. This study aims at evaluating the determinants of the frequency dependence of phase velocity in bovine femoral cortical bone samples using an in vitro ultrasonic transmission device. Phase velocity is shown to vary quasi linearly in a $1 \mathrm{MHz}$ restricted bandwidth around $4 \mathrm{MHz}$, which enables dispersion evaluation. Axial dispersion is significantly higher than radial and tangential dispersions. Significant differences in dispersion are obtained according to the anatomical location. The microstructure of each sample is determined using an optical microscope, which allows assessing the dependence of dispersion on the type of bone microstructure. Mostly positive, but also negative values of dispersion are measured. Negative dispersion is obtained mostly in samples constituted of mixed microstructure, which may be explained by phase cancellation effects due to the presence of different microstructures within the same sample. Dispersion is shown to be related to broadband ultrasonic attenuation values, especially in the radial direction. This dependence is compared with results derived from the local KramersKrönig relationships.

4pBBb10. Diffuse ultrasonic backscatter from cortical bone. Joseph A. Turner (University of Nebraska, Dept. of Engineering Mechanics, W317.4 NH, Lincoln, NE 68588, USA, jaturner@unl.edu), Goutam Ghoshal (University of Nebraska, Dept. of Engineering Mechanics, W317.4 NH, Lincoln, NE 68588, USA, goutamghoshal@ rediffmail.com)

Diffuse ultrasonic backscatter techniques have been used primarily for characterization of microstructure in structural materials such as polycrystalline metals. Such measurements exploit the spatial variance of the signals from a modified pulse-echo technique. In this presentation, experiments are discussed using this technique on samples of porcine cortical bone at center frequencies of 15,20, and $25 \mathrm{MHz}$. The time domain results obtained are analyzed with respect to a single-scattering model that includes statistical information about the microstructure. In addition, the model includes a rigorous description of the transducer beam pattern as it interacts with the liquid-sample. These results provide information regarding the ability of single-scattering models to capture the ultrasound propagation in such materials. 
4pBBb11. Effects of bone marrow on the ultrasonic propagation in the cancellous bone - Comparative study on experiment and simulation. Takashi Saeki (Doshisha University, 1-3, Tatara Miyakodani, 610-0321 Kyotanabe, Japan, dth0915@mail4.doshisha.ac.jp), Masahiro Emura (Doshisha University, 1-3, Tatara Miyakodani, 610-0321 Kyotanabe, Japan, dth0915@mail4.doshisha.ac.jp), Katsunori Mizuno (Doshisha University, 1-3, Tatara Miyakodani, 610-0321 Kyotanabe, Japan, dtg0151@mail4.doshisha.ac.jp), Mami Matsukawa (Doshisha University, 1-3, Tatara Miyakodani, 610-0321 Kyotanabe, Japan, mmatsuka@mail.doshisha.ac.jp), Yoshiki Nagatani (Nara Medical University, 840 Shijo-cho, 634-8522 Kashihara, Japan, naramed-u@ nagatani.ne.jp)

Longitudinal ultrasonic wave in cancellous bone separates into fast and slow waves depending on the bone structure. This phenomenon seems useful for the diagnosis of osteoporosis. In this study, we have investigated the influences of soft tissue (bone marrow) in the cancellous bone on the propagation of waves, in order to investigate the mechanism of this phenomenon. First, we have experimentally investigated the temperature dependence of longitudinal wave velocity and attenuation in bovine bone marrow, using a conventional ultrasonic pulse method. We used the ultrasonic wave at $1 \mathrm{MHz}$. Then, we simulated the wave propagation in cancellous bone. For simulation, we used the 3 dimensional elastic FDTD (Finite Difference Time Domain) method. Here, we used the X-ray CT pictures of actual cancellous bone obtained from the head of left bovine femur as the bone model. By changing the velocity and the attenuation values in the soft tissue among trabeculae from those of marrow to the water, we have found the changes in the waveforms of both fast and slow waves. This indicates the changes in both wave properties, due to the properties of soft tissue.

4pBBb12. Measurement of the tortuosity and the viscous characteristic length of human cancellous bone via transmitted waves. Naima Sebaa (Laboratoire d'Acoustique de l'Université du Maine, Avenue Olivier Messiaen, 72085 Le Mans, France, naima.sebaa@univ-lemans.fr), Laurens Boeckx (Lab. ATF, Katholieke Universiteit Leuven, Celestijnenlaan 200D, B-3001 Leuven, Belgium, laurens.boeckx@fys.kuleuven.be), Zine Fellah (CNRS-Laboratoire de Mécanique et d'Acoustique, 31 Chemin Joseph Aiguier, 13402 Marseille, France, fellah@1ma.cnrs-mrs.fr), Mouna Naas (Laboratoire d'Acoustique de l'Université du Maine, Avenue Olivier Messiaen, 72085 Le Mans, France, mouna.naas.etu@univ-lemans.fr), Tom L. Goossens (Technische Universiteit Eindhoven, Den Dolech 2, 5600 MB Eindhoven, Netherlands, tomgoos@gmail.com), Steven Boonen (Afdeling Geriatrie, UZ Herestraat, 3000 Leuven, Belgium, Steven.Boonen@med .kuleuven.be), Georges Van Der Perre (Afd. Biomechanica en Grafisch Ontwerpen, Celestijnenlaan 300, 3000 Leuven, Belgium, Georges .VanderPerre@mech.kuleuven.be), Philippe Leclaire (Lab. de Recherche en Mécanique et Acoustique, I.S.A.T - Univ. de Bourgogne, 49, rue Mademoiselle Bourgeois, 58000 Nevers, France, philippe.leclaire@gmail .com), Claude Depollier (Laboratoire d'Acoustique de l'Université du Maine, Avenue Olivier Messiaen, 72085 Le Mans, France, claude.depollier@univ-lemans.fr), Walter Lauriks (Lab. ATF, Katholieke Universiteit Leuven, Celestijnenlaan 200D, B-3001 Leuven, Belgium, Walter.Lauriks@fys.kuleuven.be)

For a few years several authors have proposed the model of Biot to describe the ultrasonic wave propagation in cancellous bone. One drawback of this model is the number of parameters which it needs. Two significant parameters to describe the geometry of the trabeculae are tortuosity and viscous characteristics length. In this communication, we propose two methods to measure these parameters. The first method consists in estimating these two parameters by inversion from the transmission coefficient. In the second method, we get the tortuosity from direct measurement using the focused transducers. The scan of a layer of cancellous bone shows a good correlation between direct measurements and the results of the inversion.
4pBBb13. Simulation of ultrasound wave propagation through trabecular bone samples with and without bone marrow. Liesbet Goossens (Afdeling Biomechanica en Grafisch Ontwerpen, Departement Werktuigkunde, Katholieke Universiteit Leuven, Afd. Biomechanica en Grafisch Ontwerpen, Celestijnenlaan 300, 3000 Leuven, Belgium, liesbet.goossens@mech.kuleuven.be), Jef Vanderoost (K.U.Leuven, Celestijnenlaan 300c, bus 2419, 3001 Heverlee, Belgium, jef.vanderoost@mech.kuleuven.be), Siegfried Jaecques (Department of Dentistry, Oral Pathology and Maxillo-Facial Surgery, BIOMAT Research Cluster, Katholieke Universiteit Leuven, Kapucijnenvoer 7 - bus 7001, 3000 Leuven, Belgium, siegfried.jaecques@med.kuleuven.be), Steven Boonen (Afdeling Geriatrie, UZ Herestraat, 3000 Leuven, Belgium, Steven.Boonen@med.kuleuven.be), Jan D’Hooge (Division of Cardiovascular Imaging and Dynamics, Katholieke Universiteit Leuven, UZ Herestraat 49 - bus 7003, 3000 Leuven, Belgium, jan.dhooge@uz .kuleuven.be), Walter Lauriks (Lab. ATF, Katholieke Universiteit Leuven, Celestijnenlaan 200D, B-3001 Leuven, Belgium, Walter.Lauriks @ fys.kuleuven.be), Georges Van Der Perre (Afd. Biomechanica en Grafisch Ontwerpen, Celestijnenlaan 300, 3000 Leuven, Belgium, Georges .VanderPerre@mech.kuleuven.be)

For the clinical assessment of osteoporosis, ultrasound has been proposed as an alternative or supplement to the Dual-Energy X-ray Absorptiometry technique. However the interaction of ultrasound waves with (trabecular) bone remains relatively poorly understood. The aim of the present study was to improve the understanding of this interaction by simulating ultrasound wave propagation in fifteen trabecular bone samples from the human lumbar spine, using $\mu \mathrm{CT}$ based Finite Elements Modelling. The model included only the solid bone, without the bone marrow. Two structural parameters were calculated: the bone volume fraction (BV/TV) and the structural (apparent) elastic modulus $\left(\mathrm{E}_{\mathrm{s}}\right.$ ), and the ultrasound parameter Speed Of Sound (SOS). At $1 \mathrm{MHz}$, correlations between SOS and the parameters $\mathrm{BV} / \mathrm{TV}$ and $\mathrm{E}_{\mathrm{s}}$ were rather weak but the results can be explained from the specific features of the trabecular structure and the intrinsic material elastic modulus $\mathrm{E}_{\mathrm{i}}$. The correlation found between the simulated SOS values and those calculated from the simple bar equation was poor when the three directions are considered separately. However at lower frequencies (50-300 kHz), this correlation significantly improved. Currently we investigate the correlations between SOS and the structural parameters when the bone marrow is included in the FE model.

4pBBb14. Modeling the ultrasonic scattering in trabecular bone. Andrzej Nowicki (Institute of Fundamental Technological Research, Swietokrzyska 21, 00-049 Warsaw, Poland, anowicki@ippt.gov.pl), Jerzy Litniewski (Institute of Fundamental Technological Research, Swietokrzyska 21, 00-049 Warsaw, Poland, jlitn@ippt.gov.pl)

Computer simulations conducted to investigate the properties of the ultrasound pulse-echo signal, as it is received on the transducer surface after scattering in trabecular bone was developed. It can be used to yield an ideal environment in which, the effects of various parameters (scatterer mechanical and geometrical properties, scatterers' concentration), the shape of incident wave and experimental conditions influencing the scattering of ultrasonic waves in trabecular bone structure can be examined individually. The Wear's scattering model of a cancellous bone was applied with modifications that allow for changes of mechanical and geometrical properties of individual trabeculae as well as their spatial density variation. The model also enables considering the groups of scatterers with varying mean values, e.g. thick and thin trabeculae of cancellous bone. Also, the real interrogating pulses are considered, thus the pulse shape, the emitted field structure and the frequency transfer function of the transmitting-receiving transducer are applied in simulations. The results proved that the computer simulation is a useful tool for gaining a better understanding of the scattering of ultrasonic waves in biological tissue and has a particular relevance in studying scattering in cancellous bone which may be approximated as a collection of cylindrical trabeculae. 
4pBBb15. Dual frequency ultrasound technique enables determination of soft tissue composition and improves reliability of in vivo ultrasound bone densitometry. Janne P. Karjalainen (University of Kuopio, РОВ 1627, 70211 Kuopio, Finland, janne.karjalainen@uku.fi), Juha Töyräs (Kuopio University Hospital, POB 1777, 70211 Kuopio, Finland, Juha.Toyras@kuh.fi), Toni Rikkonen (University of Kuopio, POB 1627, 70211 Kuopio, Finland, toni.rikkonen@uku.fi), Jukka Jurvelin (University of Kuopio, POB 1627, $70211 \quad$ Kuopio, Finland, Jukka.Jurvelin@uku.fi), Ossi Riekkinen (University of Kuopio, POB 1627, 70211 Kuopio, Finland, Ossi.Riekkinen@uku.fi)

Soft tissues diminish reliability of the bone ultrasound backscatter measurements. In this study, the ability of a single broadband transducer dual frequency ultrasound (DFUS) technique to monitor the changes in soft tissue was investigated in a body builder during a 21 week training and dieting period, inducing a weight loss of $16.5 \mathrm{~kg}(18 \%)$. Then, DFUS was applied to correct the errors induced by soft tissues on the measurements of integrated reflection coefficient (IRC) in human distal femur. In DFUS, US reflection from soft tissue-bone interface is determined with two different US frequencies and, by knowing the frequency specific US attenuation and speed in adipose and lean tissues, their content can be determined. The dual energy X-ray absorptiometry (DXA) indicated that significant changes in quantity and composition of soft tissue, but not in bone density, took place during the diet. As compared with DXA, the single transducer DFUS could determine local soft tissue composition $\left(r^{2}=0.88, n=8, p<0.01\right)$. The change in uncorrected IRC associated significantly with the change in body composition $\left(r^{2}=0.56, n=8, p<0.05\right)$. The IRC values, corrected by DFUS, showed only minor variation $(\mathrm{SD}= \pm 1.26 \mathrm{~dB})$ during the diet.

4pBBb16. Simulation and modeling of a new quantitative ultrasound imaging device using cylindrical crossed beam forming arrays. Sylvain Haupert (Université Paris 6, Laboratoire d'Imagerie Paramétrique, 15, rue de l'Ecole de Médecine, 75006 Paris, France, sylvain.haupert@lip.bhdc.jussieu.fr), Djelloul Reguieg (Université Paris 6, Laboratoire d'Imagerie Paramétrique, 15, rue de l'Ecole de Médecine, 75006 Paris, France, reguieg@gmail.com), Frederic Padilla (Université Paris 6, Laboratoire d'Imagerie Paramétrique, 15, rue de 1'Ecole de Médecine, 75006 Paris, France, Frederic.Padilla@lip.bhdc.jussieu.fr), Marielle Defontaine (LUSSI, 10 Bd Tonellé, 37032 Tours, France, defontai@med.univ-tours.fr), Pascal Laugier (Université Paris 6, Laboratoire d'Imagerie Paramétrique, 15, rue de 1'Ecole de Médecine, 75006 Paris, France, laugier@lip.bhdc.jussieu.fr)

Real-time quantitative ultrasound imaging (QUI) produces images of bone properties with the advantages of being non-ionizing, portable and repeatable. A real-time QUI technique has been proposed, based on two 2-D arrays ( $24 \times 24$ transducer elements) to produce a confocal spherical focusing in transmit and receive modes. However, the electronics to manage beam scanning and focusing is complex and remains expensive. To overcome these disadvantages, a new device has been developed, based on two 1-D transducer arrays (72x1 transducer elements) and confocal cylindrical crossed-beam forming. The intersection of two orthogonal cylindrical focus lines replaces the spherical focused beams. Initial results obtained with this approach showed a distortion and a shift of the spectrum in the low frequency range compared to the reference confocal spherical focusing technique. The aim of the study was to explain the observed differences between spherical and cylindrical focusing techniques using numerical simulations of elementary impulse responses calculated in the confocal and reception planes. The orthogonal configuration of the transmit and receive focusing line results in orthogonal curvatures of the transmitted wavefront and receiving aperture, with summation by the receiving aperture of an outof-phase wavefront. We show that this effect is the major source for the frequency response artifacts.

4pBBb17. Longitudinal assessment of human bone quality using scanning confocal quantitative ultrasound. Yi-Xian Qin (Stony Brook University, SUNY, Department of Biomedical Engineering, 350 Psychology-A Building, Stony Brook, NY 11794, USA, yi-xian.qin@sunysb.edu), Yi Xia (Stony Brook University, SUNY, Department of Biomedical Engineering, 350 Psychology-A Building, Stony Brook, NY 11794, USA, xia.yi76@gmail.com), Wei Lin (Stony Brook University, SUNY, Department of Biomedical Engineering, 350 Psychology-A Building, Stony Brook, NY 11794, USA, wei.lin@sunysb.edu), Jiqi Cheng (Stony Brook University, SUNY, Department of Biomedical Engineering, 350 Psychology-A Building, Stony Brook, NY 11794, USA, chengjiqi@yahoo.com), Jesse Muir (Stony Brook University, SUNY, Department of Biomedical Engineering, 350 Psychology-A Building, Stony Brook, NY 11794, USA, jmuir@ic.sunysb.edu), Clint Rubin (Stony Brook University, SUNY, Department of Biomedical Engineering, 350 Psychology-A Building, Stony Brook, NY 11794, USA, crubin@sunysb .edu)

Microgravity and aging induced bone loss is a critical skeleton complication occurred particularly in the weight-supporting skeleton, which leads to osteoporosis and fracture. Advents in quantitative ultrasound (QUS) provide a unique method for evaluating both bone strength and density. Using an imaging-base confocal scanning ultrasound diagnostic system (SCAD), the goals of this work were to non-invasively characterize bone quality at proximal femur, and longitudinally monitor effectives of calcaneus bone loss in a 90-day bedrest. QUS scanning was performed at proximal femur (cadaver) and calcaneus (bedrest subjects) regions with QUS images of $80 \times 80 \mathrm{~mm}^{2}$ for hip and $40 \times 40 \mathrm{~mm}^{2}$ for calcaneus. QUS was processed to calculate the ultrasound attenuation (ATT; dB), wave ultrasound velocity (UV), and the broadband ultrasound attenuation (BUA; dB/MHz). Human cadaver proximal femurs have been measured with the SCAD, micro-CT, DXA, and mechanical strength test. Human calcaneus of bedrest subjects were measured using SCAD and DXA in day 0 (baseline), day 60 and day 90. Results demonstrated that QUS measurement has the capability to predict bone BMD, microstructure and mechanical properties in human bone, and indicated significant sensitivity to the progressive change of bone quality, particularly in the trabecular bone region with remodeling activities. 


\title{
Session $4 p B B c$
}

\section{Biomedical Ultrasound/Bioresponse to Vibration: Theoretical and Computational Models of Ultrasonic Propagation in Bones II (Poster Session)}

\author{
James Miller, Cochair \\ Washington University \\ Pascal Laugier, Cochair \\ Université Paris 6
}

\begin{abstract}
All posters will be on display from 3:40 p.m. to 5:20 p.m. To allow contributors an opportunity to see other posters, contributors of odd-numbered papers will be at their posters from 3:40 p.m. to 4:30 p.m. and contributors of even-numbered papers will be at their posters from 4:30 p.m. to 5:20 p.m.
\end{abstract}

\section{Contributed Papers}

4pBBc1. Investigation of effect of trabecular microstructure on ultrasound propagation through cancellous bone using finite-difference time-domain simulations. Atsushi Hosokawa (Akashi National College of Technology, 679-3 Nishioka, Uozumi, 674-8501 Akashi, Japan, hosokawa@akashi.ac.jp)

Using finite-difference time-domain (FDTD) numerical simulations, it was investigated how the trabecular microstructure could affect the propagation of ultrasound waves through cancellous bone. Three-dimensional (3D) numerical models of cancellous bone were reconstructed from 3D micro-computed tomography images of bovine femoral bone with oriented trabecular structure. In these models, the trabecular elements were eroded to increase porosity using an image processing technique. Three erosion procedures were given to realize different changes in the trabecular microstructure with increasing porosity. FDTD simulations of the ultrasound pulse waves propagating through the cancellous bone models were performed in two cases of the propagations parallel and perpendicular to the main trabecular orientation, and the porosity dependences of the propagation properties, attenuation and propagation speed, were derived for various trabecular changes. It was demonstrated from the simulated results that the propagation properties in both directions parallel and perpendicular to the trabecular orientation could be affected by the trabecular microstructure. In addition, the effects of the major (or plate-like) and minor (or rod-like) trabecular elements on the ultrasound propagation were respectively investigated for both the parallel and perpendicular propagations.

4pBBc2. Excess ultrasonic attenuation due to inhomogeneities in porous media. Mouna Naas (Laboratoire d'Acoustique de l'Université du Maine, Avenue Olivier Messiaen, 72085 Le Mans, France, mouna.naas.etu@univ-lemans.fr), Naima Sebaa (Laboratoire d'Acoustique de l'Université du Maine, Avenue Olivier Messiaen, 72085 Le Mans, France, naima.sebaa@univ-lemans.fr), Zine Fellah (CNRS-Laboratoire de Mécanique et d'Acoustique, 31 Chemin Joseph Aiguier, 13402 Marseille, France, fellah@lma.cnrs-mrs.fr), Mohamed Fellah (Laboratoire de Physique Théorique, Institut de Physique, USTHB, BP, 16111 Alger, Algeria, fellah1@yahoo.com), Walter Lauriks (Lab. ATF, Katholieke Universiteit Leuven, Celestijnenlaan 200D, B-3001 Leuven, Belgium, Walter.Lauriks@fys.kuleuven.be), Claude_ Depollier (Laboratoire d'Acoustique de l'Université du Maine, Avenue Olivier Messiaen, 72085 Le Mans, France, claude.depollier@univ-lemans.fr)

While Biot's theory seems well adapted to model the acoustical waves propagation in cancellous bone, some of its predictions do not agree with the experimental results. The excess of attenuation of the fast wave is one of these discrepancies. In this paper we propose a modified Biot's model which takes into account the fluctuations of the physical parameters and their correlations. As a result of this model, we show that this excess of attenuation is due to several processes: i) classical Biot's attenuation, ii) scattering leading to the extension of the wave path, iii) mode conversion. Some comparison between experimental results and numerical simulations are proposed.

4pBBc3. Relationship between QUS parameters and a cellular modelbased estimation of bone strength. Guillaume Haiat (CNRS, Laboratoire de Recherches Orthopédiques, 10, Avenue de Verdun, 75010 Paris, France, haiat@univ-paris12.fr), Frederic Padilla (Université Paris 6, Laboratoire d'Imagerie Paramétrique, 15, rue de l'Ecole de Médecine, 75006 Paris, France, Frederic.Padilla@lip.bhdc.jussieu.fr), Pascal Laugier (Université Paris 6, Laboratoire d'Imagerie Paramétrique, 15, rue de l'Ecole de Médecine, 75006 Paris, France, laugier@lip.bhdc.jussieu.fr)

The final goal of quantitative ultrasound (QUS) techniques is to assess bone strength. However, the translation of QUS results into bone strength remains elusive because the physical principles of ultrasonic propagation in bone are not fully understood yet. Here, the sensitivity of Broadband Ultrasonic Attenuation and Speed of Sound to variations of bone strength is derived. Therefore, a cellular model is combined to a multiple regression analysis resulting from the analysis of finite-difference time domain (FDTD) simulations coupled with imaging techniques. The variation of QUS variables induced by a variation of strength of $10 \%$, realized either by a change in material properties or a change in bone volume fraction (BV/TV) is investigated. Except when BV/TV is high, the variations of BUA in response to a variation in strength realized by a pure change of BV/TV is higher than the technique imprecision and thus can be detected. When the variation of strength is realized by changes of elastic properties, the response in QUS properties is dominated by the variation in C11 over C44. The interpretation of these data, however, is not straightforward due to sparse description of elastic properties at the tissue level, which is a limitation of the cellular model.

4pBBc4. Bulk waves velocities are dependent on frequency in cortical bone. Cécile Baron (Université Paris 6, Laboratoire d'Imagerie Paramétrique, 15, rue de l'Ecole de Médecine, 75006 Paris, France, norabelic@yahoo.fr), Quentin Grimal (Université Paris 6, Laboratoire d'Imagerie Paramétrique, 15, rue de l'Ecole de Médecine, 75006 Paris, France, quentin.grimal@lip.bhdc.jussieu.fr), Maryline Talmant (Université 
Paris 6, Laboratoire d'Imagerie Paramétrique, 15, rue de l'Ecole de Médecine, 75006 Paris, France, talmant@lip.bhdc.jussieu.fr), Pascal Laugier (Université Paris 6, Laboratoire d'Imagerie Paramétrique, 15, rue de 1’Ecole de Médecine, 75006 Paris, France, laugier@lip.bhdc.jussieu.fr)

Any model of ultrasound propagation in cortical bone requires the knowledge of the effective elastic properties of bone at, let's say, the mm scale. It is well known that the porous network of cortical bone interacts with ultrasonic waves and plays a major role in the mechanical behaviour. The purpose of the present work is to emphasize that, in cortical bone, there is some variation of the effective bulk wave velocities, and consequently of the elastic properties, with frequency in the $\mathrm{MHz}$ range due to the effect of the porous network. A Finite Difference Time Domain (FDTD) code is used to simulate the ultrasonic propagation of compression and shear waves, transverse to, and along the bone axis, in cortical bone volumes reconstructed from micro-computed tomography (microCT). The resolution of the microCT data allowed to model the 3D networks of resorption cavities and Haversian pores. It is found that, for porosities typically above 5-6\%, the effective phase velocity is dependent on the frequency. Preliminary results indicate that the influence of the frequency is more important in the range 1-5 MHz.

THURSDAY AFTERNOON, 3 JULY 2008

P3-B, LEVEL 3, 3:40 TO 5:20 P.M.

\title{
Session 4pBBd
}

\section{Biomedical Ultrasound/Bioresponse to Vibration and Physical Acoustics: Light and Sound for Medical Imaging and Therapy II (Poster Session)}

\author{
Ronald Roy, Cochair \\ Boston University \\ Emmanuel Bossy, Cochair \\ Laboratoire Photons et Matière, ESPCI/CNRS
}

\begin{abstract}
All posters will be on display from 3:40 p.m. to 5:20 p.m. To allow contributors an opportunity to see other posters, contributors of odd-numbered papers will be at their posters from 3:40 p.m. to 4:30 p.m. and contributors of even-numbered papers will be at their posters from 4:30 p.m. to 5:20 p.m.
\end{abstract}

\section{Contributed Papers}

4 pBBd1. Selective ultrasonic focusing towards an optical contrast agent by use of photoacoustic-guided time-reversal. Arik $\mathrm{R}$. Funke (Laboratoire Photons et Matière, ESPCI/CNRS, 10 rue Vauquelin, 75231 Paris Cedex 05, France, arik.funke@espci.fr), Emmanuel Bossy (Laboratoire Photons et Matière, ESPCI/CNRS, 10 rue Vauquelin, 75231 Paris Cedex 05, France, emmanuel.bossy@espci.fr), Jean-François Aubry (Laboratoire Ondes et Acoustique, ESPCI, Université Paris 7, CNRS, 10 rue Vauquelin, 75005 Paris, France, jean-francois.aubry@espci.fr), Mathias Fink (Laboratoire Ondes et Acoustique, ESPCI, Université Paris 7, CNRS, 10 rue Vauquelin, 75005 Paris, France, mathias.fink@espci.fr), Albert-Claude Boccara (Laboratoire Photons et Matière, ESPCI/CNRS, 10 rue Vauquelin, 75231 Paris Cedex 05, France, boccara@optique.espci.fr)

We recently demonstrated experimentally that optically absorbing regions embedded at centimetric depths in an optically diffusive medium can be used, through the photoacoustic effect, as ultrasound sources for timereversal techniques [Bossy et al., APL 89(18), 2006]. If the optically absorbing region is spatially localized, the emission of the time-reversed photoacoustic waves provides a method to automatically focus ultrasound towards the absorbing region. In biological tissue, this approach has the potential to automatically guide high-intensity focused ultrasound (HIFU) beams towards a targeted region labelled with an optically absorbing contrast agent. However, such an approach is conceivable only if the HIFU beam is guided towards the sole targeted region, and not towards other potentially strong endogenous optical absorbers (such as blood vessels). In this work, we present a method to selectively focus time-reversed photoacoustic waves towards an exogenous wavelength-dependent optical contrast agent. Experiments were performed in vitro on tissue-mimicking phantoms, with nanoparticles as contrast agents. A tunable nanosecond pulsed laser was used with different optical wavelengths to generate photoacoustic waves. A multi- channel time-reversal ultrasound system was used to emit ultrasound signals derived from time-reversed photoacoustic signals obtained with different optical wavelengths.

4pBBd2. Point spread function of the limited-view array transducers in optoacoustic tomography: numerical and experimental studies. Ivan Pelivanov (International Laser Center of Moscow State University, Vorob'yovy Gory, 1, 119992 Moscow, Russian Federation, pelivanov@ilc.edu.ru), Tatiana Khokhlova (International Laser Center of Moscow State University, Vorob'yovy Gory, 1, 119992 Moscow, Russian Federation, t_khokhlova@ilc.edu.ru), Varvara Simonova (International Laser Center of Moscow State University, Vorob'yovy Gory, 1, 119992 Moscow, Russian Federation, simvar@mail.ru), Alexei Podolsky (International Laser Center of Moscow State University, Vorob'yovy Gory, 1, 119992 Moscow, Russian Federation, uzh@yandex.ru), Alexander Karabutov (Moscow State University, MSU, 1, building 2, GSP-2, Leninskiye Gory, 119992 Moscow, Russian Federation, akarabutov@gmail.com)

Optoacoustic (OA) tomography utilizes pulsed laser radiation for thermoelastic excitation of wideband ultrasonic pulses. The distribution of laserinduced heat release can be reconstructed if the ultrasonic pulses are detected by an array of transducers. The resolution provided by an array depends on various aspects of its design - the number of elements, element size, spacing and arrangement - and is determined by the point spread function (PSF) of the array. In present work PSFs of arrays with different characteristics are calculated using the Rayleigh integral for simulating the direct problem, and backprojection algorithm for solving the inverse problem of OA tomography. Universal dependencies of the resolution provided by an array on the dimensions and arrangement of its elements are found. Numerical studies are validated by experimental measurements of PSFs of different arrays. 
4pBBd3. A combined platform for b-mode and real-time optoacoustic imaging based on raw data acquisition. Marc Fournelle (Fraunhofer IBMT, Ensheimer Strasse 48, 66386 Sankt Ingbert, Germany, marc.fournelle@ibmt.fhg.de), Kirsten Maass (Fraunhofer IBMT, Ensheimerstrasse 48, 66386 Sankt Ingbert, Germany, kirsten.maass@ibmt.fhg.de), Heinrich Fonfara (Fraunhofer IBMT, Ensheimerstrasse 48, $66386 \quad$ Sankt Ingbert, Germany, heinrich.fonfara@ibmt.fhg.de), Hans-Joachim Welsch (Fraunhofer IBMT, Ensheimerstrasse 48, $66386 \quad$ Sankt Ingbert, Germany, hansjoachim.welsch@ibmt.fhg.de), Holger J. Hewener (Fraunhofer IBMT, Ensheimer Strasse 48, 66386 Sankt Ingbert, Germany, holger.hewener@ibmt.fraunhofer.de), Christian Günther (Fraunhofer IBMT, Ensheimerstrasse 48, 66386 Sankt Ingbert, Germany, christian.guenther@ibmt.fhg.de), Robert M. Lemor (Fraunhofer IBMT, Ensheimer Strasse 48, 66386 Sankt Ingbert, Germany, robert.lemor@ibmt.fhg .de)

Optoacoustic imaging is a new promising medical imaging modality combining the benefits of optical and acoustical methods. Optoacoustics allow to make the high intrinsic optical contrast in biological tissue accessible to acoustical detection. In addition, the possibility of using nanoscaled contrast agents makes of optoacoustics an ideal candidate for molecular imaging. While optoacoustics are an emerging imaging modality with poor clinical experience, ultrasound is widely used for diagnosis. Accordingly, optoacoustic images are much harder to interpret than b-mode images. For this reason, we developed a hardware platform which allows combined b-mode and optoacoustic imaging using a 2-in-1 transducer with arrays of different frequency for the two modalities. The system supports simultaneous data acquisition of 128 channels with a sample rate of 80 MSamples allowing the usage of transducers with frequencies up to $20 \mathrm{MHz}$. The unprocessed data is transferred to a PC where the images are reconstructed with algorithms adapted to both modalities. A software for hardware control, data processing and visualization in real-time was developed. B-mode and optoacoustic images of tissue phantoms were generated and different types of nanoparticles were used as optoacoustic contrast agent. Further, first invivo measurements underlying the high potentials of the combined system were obtained.

4pBBd4. A maximum likelihood method for obtaining integrated attenuation from ultrasound transmission mode measurements. Rene G. Willemink (University of Twente, Signals and Systems Group (SaS), Faculty of Electrical Engineering, Mathematics and Computer Sciences (EWI), P.O. Box 217, 7500AE Enschede, Netherlands, G.H.Willemink@utwente.nl), Srirang Manohar (University of Twente, Biophysical Engineering Group (BPE), Faculty of Science and Engineering (TNW), P.O. Box 217, 7500 AE Enschede, Netherlands, s.manohar@tnw.utwente.nl), Kees H. Slump (Univ. of Twente, P.O. Box 217, 7500 AE Enschede, Netherlands, c.h.slump@utwente.nl), Ferdi Van Der Heijden (Univ. of Twente, P.O. Box 217, 7500 AE Enschede, Netherlands, f.vanderheijden@utwente.nl), Ton G. Van Leeuwen (Univ. of Twente, P.O. Box 217, 7500 AE Enschede, Netherlands, a.g.j.m.vanleeuwen @ tnw.utwente.nl)

In photoacoustic imaging applications an image is reconstructed of the optical absorption distribution the imaged object. The photoacoustic measurements however are also dependent on acoustic properties of the imaged object. By estimating the acoustic properties from photoacoustic measurements, we can improve the performance of the optical absorption reconstruction and allow for the imaging of separate acoustic modalities. We derive and evaluate a maximum likelihood estimator for the measurements of integrated acoustic attenuation. This estimator is applicable to media like soft tissue. In this kind of media, the attenuation due to dissipative effects obeys a frequency power law. By measuring the propagation of ultrasound signals through such a medium, the parameters that describe the attenuation can be estimated. In this paper a new method is introduced for estimating the attenuation of ultrasound media by means of transmission mode measurements. The method is based on analyzing the noise characteristics of the received signals and the formulation of a maximum likelihood estimator The new estimator is compared to existing methods and was found to be a better estimator in terms of the RMS error than previous methods.

THURSDAY AFTERNOON, 3 JULY 2008

ROOM 353, 2:00 TO 7:40 P.M.

\title{
Session 4pEAa
}

\section{Engineering Acoustics: Transducers I}

\author{
Robert M. Koch, Chair \\ NUWC, 1176 Howell Street, Code 8232, Bldg. 1302, Newport, RI 02841-1708, USA
}

\section{Contributed Papers}

2:00

4pEAa1. Analytical modeling of piezoelectric ceramic transducers based on coupled vibration analysis. Boris Aronov (UMassD, 22 Seabeds Way, apt. 12, Needham, MA 02494, USA, baronov@comcast.net)

The energy method for analyzing piezoelelctric ceramic transducers [B. S. Aronov, JASA, 117(1), 210-220.] is applied to the treatment of transducers with mechanical systems that can be cobsidered as two-dimensional. Analysis is made following the general outline of the theory of coupled vibration in two degree-of-freedom systems and its extention to calculating the resonance frequencies of elastic bodies, as suggested by Gibbe and Blechshmidt [Ann. Physik, Ser. 5, 18, No. 5, 417-485 (1933)]. The approach to the problem is illustrated with examples of piezoelectric rectangular plates, stripes and thin-walled cylinders at various orientations relative to crystallographic coordinate system. For all of the examples the resonance frquencies and effective coupling coefficients are presented as functions of the dimensional aspect ratios. Equivalent electromechanical circuits are introduced that permit calculation of the transducers performance under dif- ferent loading conditions. Practical recommendations for improving the electromechanical properties of the transducers by use of appropriate aspect ratios are discussed.

\section{$2: 20$}

4pEAa2. Resonance frequencies of the multilayered piezotransducers Francisco J. Arnold (CESET - Unicamp, R. Paschoal Marmo, 1888 - JD Nova Italia, 13484332 Limeira, Brazil, arnold@ceset.unicamp.br)

Piezoelectric transducers used in high power ultrasonic applications are composed of piezoelectric ceramics, metallic blocks and a central bolt that pre-stresses the assembly. The impedance characterization of these transducers has been done by using numerical methods but, in many cases, onedimensional simplified models are enough to a good physical interpretation of the problem. In this work was used the Mason's equivalent electric circuit for one-dimensional modeling of composed transducers. It was derived Thevénin's equivalent circuit to simplify the problem and evaluate the ef- 
fects of the central bolt on the resonances. Some transducers were mounted and the transmission line circuit was used to measure the resonance frequencies. The results show a good fitting between the experimental and simulated by electric circuit. By using the presented model it was identify other longitudinal vibration modes further that considered in previous models and, so that, it was increased the possibility of investigate the behavior of the transducers using the frequency spectrum.

\section{2:40}

4pEAa3. Integrated transducers for marine animal tags using thick film PZT. Rasmus Lou-Møller (Ferroperm Piezoceramics A/S, Hejreskovvej 18A, 3490 Kvistgaard, Denmark, rm@ferroperm.net), Erling Ringgaard (Ferroperm Piezoceramics A/S, Hejreskovvej 18A, 3490 Kvistgaard, Denmark, er@ferroperm.net), Tomasz Zawada (Ferroperm Piezoceramics A/S, Hejreskovvej 18A, 3490 Kvistgaard, Denmark, tz@ferroperm-piezo.com), Sigmar Gudbjoernsson (Star-Oddi, Vatnagardar 14, 104 Raykjavik, Iceland, sigmar@star-oddi.com), Haraldur Hilmarsson (Star-Oddi, Vatnagardar 14, 104 Raykjavik, Iceland, sigmar @ star-oddi.com)

The technology of printing PZT ceramic layers onto curved substrates using pad printing is demonstrated by the application of a transmitter/receiver system printed on alumina cylinders. The cylinder is used as housing for a Data Storage Tag (DST) for marine animals. DST's are used for monitoring behaviour and lifecycle of animals, providing valuable knowledge for marine biologists and researchers. Electrodes and PZT ceramic is printed directly onto the alumina housing using the pad printing technique, which eliminates the need for complex assembly procedures and other post processing steps. Since the PZT is situated on the external surface of the housing, direct contact between the acoustic elements and the marine animal is ensured and the elements do not take up valuable space inside the tag. The manufacturing of the active elements and the technology of pad printing PZT thick film is presented. The piezoelectric properties of the film and the acoustic properties have been tested under laboratory conditions. According to the obtained results one can conclude that the transmitter/receiver system was able to assure sufficient sensitivity in the required distance keeping the power consumption on an extremely low level. Second generation devices will be ready for commercialisation in the near future.

\section{3:00}

4pEAa4. High-Output and High-Fidelity Microsized Driver System. Keehoon Kim (Physical Optics Corporation, 20600 Gramercy Pl \#103, Torrance, CA 90501, USA, kkim@poc.com), Tao Xu (Physical Optics Corporation, 20600 Gramercy Pl \#103, Torrance, CA 90501, USA,
txu@poc.com),Reginald Daniels(Air Force Research Laboratory, 2610 Seventh St. Bldg 441, Wright Patterson AFB, OH 45433, USA, Reginald .Daniels@WPAFB.AF.MIL)

Active noise reduction (ANR) for aircrew hearing protection in harsh noise environments such as military and civilian flight operations requires a high-performance, microsized driver that provides not only high acoustic power, at least $130 \mathrm{~dB}$ SPL, but also high fidelity sound quality, especially in low-frequency ranges below $100 \mathrm{~Hz}$. A new microsized smart material actuator (MSMA) is being developed by using high-density and directconversion piezoelectric exciters combined with a unique acoustic structure, unlike conventional moving coil/magnet-type drivers. The dimensions of the MSMA are $6 \mathrm{~mm}$ in diameter by $7 \mathrm{~mm}$ long, small enough for ear-canal application as an earplug driver, maximizing ANR effectiveness. The MSMA system consists of a microscale earplug driver, a protective package made of medical-grade stainless steel, and a compact actuation amplifier easily connected to or embedded in an external ANR controller. Performance tests show the system produces over $130 \mathrm{~dB}$ SPL in a $1 \mathrm{cc}$ trapped cylindrical volume with very flat frequency responses even below $100 \mathrm{~Hz}$. The total harmonic distortion of the MSMA is lower than $4 \%$ over all audible frequency ranges, without phase delay or discontinuities.

\section{3:20}

4pEAa5. MEMS-based magnetic and electrostatic acoustic microspeakers. Michael Pedersen (Novusonic Corporation, P.O. Box 183, Ashton, MD 20861, USA, info@novusonic.com)

Acoustic microspeakers remains a challenging application area for MEMS technology. This is mostly due to the inherently low transduction factors normally achievable in MEMS technology. While established electromagnetic transduction principles may be adapted for MEMS technology, there are also other transduction methods, such as electrostatic, with high performance potential. In this paper, the design, fabrication, and testing of two different types of MEMS microspeakers is presented. Firstly, an electromagnetic microspeaker is shown consisting of a MEMS diaphragm with integrated moving and an external rare earth permanent magnet and yoke structure. Secondly, a novel MEMS electrostatic microspeaker based on rolling contact is presented. In this device, high transduction forces are achieved by the strong electrical field over a solid insulator, and the forces are translated to a MEMS diaphragm by an integrated cantilever structure. Measurements on the electromagnetic microspeakers show a sensitivity of $83 \mathrm{~dB}$ $\mathrm{SPL} / \mathrm{mW}$ at $1 \mathrm{kHz}$ in a B\&K 4153 acoustic coupler. The resonance frequency of the $6 \mathrm{~mm}$ diameter diaphragm was $3.2 \mathrm{kHz}$. Numerical simulations suggest that simple changes to the moving coil geometry and material may increase the sensitivity to $108 \mathrm{~dB} \mathrm{SPL} / \mathrm{mW}$.

\section{Contributed Papers}

\section{$5: 20$}

4pEAa6. Sound pressure estimation at an electroacoustic transducer's voicing face by way of all-electrical sensing. Florian Sandoz (Ecole Polytechnique Fédérale de Lausanne, EPFL STI LEMA, Station 11, CH 1015 Lausanne, Switzerland, florian.sandoz@epfl.ch), Hervé Lissek (Ecole Polytechnique Fédérale de Lausanne, EPFL STI LEMA, Station 11, CH 1015 Lausanne, Switzerland, herve.lissek@epfl.ch)

When it comes to designing active control disposals, sound pressure sensing is usually required. In the framework of active control of acoustic impedance, this work aims at conceiving a digital estimator that processes voltage and current measurement to compute an estimation of the pressure in the vicinity of the diaphragm of an electro-dynamic speaker. After dealing with modeling concerns of the actuator, the bilinear transform is used to obtain equations of two IIR filters. An adaptive identification using the LMS algorithm and a SVD-based identification are tested to compute filters coefficient, both leading to poor experimental results. Accordingly, the actual transfer functions of the speaker are measured and optimal IIR filters are designed using the simulated annealing algorithm. It is then shown that good results can be achieved experimentally when the speaker is loaded electrically by a short circuit or an open circuit. Finally, performances of the process are discussed regarding other operating conditions. 


\section{5:40}

4pEAa7. Virtual Prototyping of Electrodynamic Loudspeakers by Utilizing a Finite Element Method. Reinhard Lerch (Univ. ErlangenNuremberg, Dept. of Sensor Technology, Paul-Gordan-Str. 3/5, 91052 Erlangen, Germany, reinhard.lerch@1se.eei.uni-erlangen.de), Manfred Kaltenbacher (Univ. Erlangen-Nuremberg, Dept. of Sensor Technology, Paul-Gordan-Str. 3/5, 91052 Erlangen, Germany, manfred.kaltenbacher @1se.eei.uni-erlangen.de), Martin Meiler (Simetris GmbH, Am Weichselgarten 7,91058 Erlangen, Germany, martin.meiler@simetris.de)

To speed up the development of electrodynamic loudspeakers, computer tools have to be applied. With appropriate computer simulations, the costly and lengthy fabrication of prototypes, as required within conventional experimental design, can be reduced tremendously. Present computer modeling tools are mainly based on equivalent circuit representations. The main drawback of these models, however, stems from the fact that the circuit element parameters have to be measured on a prototype, first. Therefore, the need for appropriate numerical simulation tools based on finite element method (FEM) arises, since as input parameters they suffice with geometrical and material data. However, present finite element tools suffer from their incompleteness in respect to full field couplings and nonlinear features. In this paper, a new finite element scheme is introduced and its utilization within the computer-aided design of electrodynamic loudspeakers is demonstrated. This scheme allows the precise and efficient calculation of the electromagnetic, mechanical and acoustic fields, including their couplings. Furthermore, nonlinear effects in the mechanical behavior of the spider as well as magnetic nonlinearities due to the nonhomogeneity of the magnetic field are taken into account.

\section{6:00}

4pEAa8. Tactile Touch Plate with Variable Boundary Conditions. Ros Kiri Ing (Laboratoire Ondes et Acoustique, ESPCI, Université Paris 7, CNRS, 10 rue Vauquelin, 75005 Paris, France, ros-kiri.ing@espci .fr), Didier Cassereau (Laboratoire Ondes et Acoustique, 10, rue Vauquelin, 75231 Paris, France, didier.cassereau@espci.fr), Mathias Fink (Laboratoire Ondes et Acoustique, ESPCI, Université Paris 7, CNRS, 10 rue Vauquelin, 75005 Paris, France, mathias.fink@espci.fr), Jean-Pierre Nikolovski (Laboratoire Ondes et Acoustique, ESPCI, Université Paris 7 , CNRS, 10 rue Vauquelin, 75005 Paris, France, jean-pierre.nikolovski@cea fr)

The touch screen device is becoming more and more widespread because it is a very user friendly human/machine interface. In acoustic domains, several approaches are used to realize such a device. Triangulation or Rayleigh waves absorption are such classical methods. However, these approaches are limited because they need a large number of sensors and are only applicable to plates of constant thickness and homogeneous materials. To remedy these limitations, a new approach is proposed using only two sensors. In this approach, one sensor is used to excite the plate, either continuously or impulsively. The second sensor is used to detect the acoustic waves generated in the plate. When an object comes into contact with the plate, some acoustic wave characteristics change. These changes affect different frequencies and depend on the position of the contact point. Comparing these changes with pre-recorded values, it is possible to achieve a tactile touch plate that only responds to specific touch locations. Several experiments with different types of plates were conducted and the results will be presented.

\section{6:20}

4pEAa9. Personal sound system design for mobile phone, monitor, and television set: cylindrical shape approach. Ji-Ho Chang (Center for Noise and Vibration Control, Korea Advanced Institute of Science and Technology, Dep. of Mechanical Engineering, Guseong-dong, Yuseong-gu, 305701 Daejon, Republic of Korea, chang.jiho@gmail.com), Jin-Young Park (Center for Noise and Vibration Control, Korea Advanced Institute of Science and Technology, Dep. of Mechanical Engineering, Guseong-dong,
Yuseong-gu, 305-701 Daejon, Republic of Korea, jypark1979 @ kaist.ac.kr), Yang-Hann Kim (Center for Noise and Vibration Control, Korea Advanced Institute of Science and Technology, 4114, Department of Mechanical Engineering, Guseong-dong, Yuseong-gu, 305-701 Daejon, Republic of Korea, yanghannkim@kaist.ac.kr)

Personal sound system that focuses sound on the user and reduces in the other zone has great interest in these days because it has significant needs to be applied in personal devices such as mobile phone, monitor, and television set. We have shown the feasibility of the personal sound system using a line array of loudspeaker units [C.-H. Lee et. al., J. Acoust. Soc. Am., 122, 3053 (2007)] based on acoustic contrast control [J.-W. Choi, Y.-H. Kim, J. Acoust Soc. Am. 111, 1695(2002)], with the successful result of about 20dB difference between the front and the side region for $800-5 \mathrm{kHz}$ range. Continuing this research, we try to apply acoustic contrast control in cylindrical shape instead of two-dimensional planar shape that was used before in order to reduce the level of side lobes more. That is, acoustically bright zone and dark zone are determined as cylindrical shape surrounding the array of loudspeakers. Computer simulation and experimental result will be addressed and evaluated by comparing to the previous result. (This work was supported by the Korea Science and Engineering Foundation(KOSEF) through the National Research Lab. Program funded by the Ministry of Science and Technology(M10500000112-05J0000-11210).)

\section{$6: 40$}

4pEAa10. Efficient Directivity Pattern Control for Spherica Loudspeaker Arrays. Franz Zotter (Institute of Electronic Music and Acoustics, Inffeldgasse 10 / 3, 8010 Graz, Austria, zotter@iem.at), Hannes Pomberger (Institute of Electronic Music and Acoustics, Inffeldgasse 10 / 3, 8010 Graz, Austria, pom@sbox.tugraz.at), Andrew Schmeder (Center for New Music and Audio Technologies, 1750 Arch Street, Berkeley, CA 94720, USA, andy@cnmat.berkeley.edu)

With an appropriate control system, directivity pattern synthesis can be accomplished with spherical loudspeaker arrays, e.g. in the shape of Platonic solids or spheres. The application of such devices for the reproduction of natural or artificial directivity patterns poses a relatively young field of research in computer music and acoustic measurements. Using directivity measurements with microphones, the directivity patterns of the individual speakers on the array can be determined. Usually, the directivity of the whole array may be regarded as a linear combination of these patterns. In order to gain control, the measurement data of the linear system need to be inverted. Given L loudspeakers and $\mathrm{M}$ microphones, this inversion yields the desired control system, an expensive LxM multiple-input-multipleoutput (MIMO) filter. We introduce discrete spherical harmonics transform and decoder matrices to reduce the number of channels required for this control system, thus reducing the computational effort. However, this step often leads to a sparse MIMO-system, in which many off-diagonal transfer functions vanish. If applicable, the computation of the non-zero transfer functions only can be done at even much lower cost. A case study for an icosahedral loudspeaker array is given, showing the properties of the sparse MIMO-system.

\section{7:00}

4pEAa11. Effects of enclosure design on the directivity synthesis by spherical loudspeaker arrays. Alexander Mattioli Pasqual (Universidade Estadual de Campinas, Rua Mendeleiev, 200, Cidade Universitária "Zeferino Vaz", 13083-970 Campinas, Brazil, pasqual@fem.unicamp.br), José Roberto Arruda (Universidade Estadual de Campinas, Rua Mendeleiev, 200, Cidade Universitária "Zeferino Vaz", 13083-970 Campinas, Brazil, arruda@fem.unicamp.br), Philippe Herzog (Laboratoire de Mécanique et d'Acoustique - CNRS, 31 chemin Joseph Aiguier, 13402 Marseille, France, herzog@1ma.cnrs-mrs.fr)

Spherical loudspeaker arrays have been used to generate non-uniform directivity patterns. It is known that the poor radiation efficiency of spherical sources and the loudspeaker electroacoustic behavior impose constraints on the directivity synthesis at low frequencies, which are aggravated as the source volume is made smaller. In this work, the effects of the enclosure design on the loudspeaker signal powers are analyzed. Two different approaches have been reported in literature, although quantitative comparisons 
have not been provided. In the first approach, the drivers share the same enclosure volume and in the second, they have their own independent sealed cavities. Here, an analytical model that takes into account the interior and exterior acoustic coupling is used in order to evaluate the voltages that must feed the array drivers. It is shown that the signal powers can be reduced at low frequencies by letting the drivers share the same enclosure volume. However, this leads to controllability problems, since some natural frequencies of the enclosure are in the operation range of the spherical array. If controllability at natural frequencies is neglected, a simple lumped parameter model of the enclosure presents good agreement with the continuous model, indicating that heavy calculations may be unnecessary.

$$
7: 20
$$

4pEAa12. Relative calibration and characterization of $1 / 4$ " condenser microphones under different environmental conditions. Cécile Guianvarc'H (Institut National de Métrologie (LNE-INM/Cnam), 61 rue du Landy, 93210 La Plaine Saint Denis, France, cecile.guianvarch@cnam.fr) , Paolo Alberto Giuliano Albo (Istituto Nazionale di Ricerca Metrologica (INRiM), Strada delle Cacce, 91, 10135 Torino, Italy, albo@inrim .it), Roberto Gavioso (Istituto Nazionale di Ricerca Metrologica (INRiM), Strada delle Cacce, 91, 10135 Torino, Italy, r.gavioso@inrim.it), Giuliana Benedetto (Istituto Nazionale di Ricerca Metrologica (INRiM), Strada delle Cacce, 91, 10135 Torino, Italy, gbenedet@inrim.it), Laurent Pitre (Institut National de Métrologie (LNE-INM/Cnam), 61 rue du Landy, 93210 La Plaine Saint Denis, France, pitre@cnam.fr), Arnaud Guillou (Institut National de Métrologie (LNE-INM/Cnam), 61 rue du Landy, 93210
La Plaine Saint Denis, France, guillou.arnaud@gmail.com),Michel Bruneau (Laboratoire d'Acoustique de l'Université du Maine, Avenue Olivier Messiaen, 72085 Le Mans, France, michel.bruneau@univlemans.fr), Anne-Marie Bruneau (Laboratoire d'Acoustique de l'Université du Maine (LAUM, UMR CNRS 6613), Avenue Olivier Messiaen, 72085 Le Mans, France, anne-marie.bruneau@univ-lemans.fr)

Measurement condenser microphones are commonly used in air at ambient temperature and pressure. However, several applications require to use such microphones in environments which are significantly different. In particular, for the determination of the Boltzmann constant by an acoustic method, measurements take place in a cavity filled with pure argon or helium over a wide pressure range at the temperature of the triple point of water. For this application, it is important to determine the microphone frequency response and acoustic input impedance with a low uncertainty in these gas conditions. A few previous works have examined the influence of static pressure, temperature and gas composition on microphone sensitivity. In one case, these results were supported by a theoretical investigation using a lumped-element model. The aim of the present work is to compare theoretical results from different lumped-element models with experimental relative calibration results obtained using an electrostatic actuator technique. Measurements are performed on 1/4" condenser microphones maintained in argon and helium environments, at $273.16 \mathrm{~K}$, in the pressure range between $50 \mathrm{kPa}$ and $700 \mathrm{kPa}$. The results are used to test the existing theoretical models and to compare the microphone properties with the manufacturer's data.

\section{Session 4pEAb}

\section{Engineering Acoustics, Underwater Acoustics, Acoustical Oceanography, and ECUA: Acoustics in Marine Archeology}

Kenneth Walsh, Cochair

K \& M Engineering LTD, 51 Bayberry Lane, Middletown, Rhode Island 02842, USA

Tuncay Akal, Cochair

SUASIS: Underwater Systems Technology Development, Tubitak-Marmara Research Center, Tech. D. Free Zone, Block A - L4, Gebze, Kocaeli, 41470, Turkey

\section{Invited Papers}

4pEAb1. Green's theorem as the foundation of interferometry. Adriana Citlali Ramírez (WesternGeco, 10001 Richmond Ave, Houston, TX 77042, USA, aperez50@slb.com), Arthur B. Weglein (University of Houston, Physics Department, 617 S\&R Bldg1, Houston, TX 77204-5005, USA, aweglein@uh.edu)

A prerequisite for applying full wavefield theory to marine exploration is the completeness and proper sampling of recorded data, which can be satisfied with data extrapolation/interpolation techniques. Green's theorem can be applied to acoustic measurements of the Earth's subsurface to obtain exact equations that incorporate boundary conditions for the retrieval of the Earth's Green's function in positions where it was not measured. Recently, a number of papers on seismic interferometry have shown methods to reconstruct the Green's function between a pair of receivers by using data cross correlations. Current interferometry methods require dual measurements (pressure field and its normal derivative) which are not always available. The lack of dual measurements has encouraged the arrival of algorithms using high frequency and one-way wave approximations to the normal field derivative. The approximations are compromises to the exact theory and, hence, produce artifacts. We present a unifying framework for a broad class of interferometry techniques using Green's theorem. This framework and foundation allows errors and artifacts to be anticipated and fully explained as a consequence of approximations made within Green's theorem. We also develop a set of more effective interferometry methods, where fewer (or none) approximations are made and the result is improved. 
4pEAb2. Surveillance and Protection of Underwater Archaeological Sites: Sea-Guard. Tuncay Akal (SUASIS: Underwater Systems Technology Development, Tubitak-Marmara Research Center, Tech. D. Free Zone, Block A - L4, Gebze, 41470 Kocaeli, Turkey, tuakal@yahoo.com), Piero Guerrini (NURC Nato Undersea Research Centre, Viale San Bartolomeo 400, 19026 La Spezia, Italy, guerrini@nurc.nato.int), William A. Kuperman (MPL, Scripps Institution of Oceanography, University of California, San Diego, La Jolla, CA 92093-0238, USA, wkuperman@ucsd.edu), Kerem Koprulu (SUASIS: Underwater Systems Technology Development, Tubitak-Marmara Research Center, Tech. D. Free Zone, Block A - L4, Gebze, 41470 Kocaeli, Turkey, keremkpr@ yahoo.com), Philippe Roux (LGIT - CNRS - Université Joseph Fourier, Maison des Géosciences, 1381 rue de la Piscine, BP 53, 38041 Grenoble, France, philippe.roux@obs.ujf-grenoble.fr), Karim G. Sabra (Georgia Institute of Technology, School of Mechanical Engineering, 771 Ferst Drive, NW, Atlanta, GA 30332-0405, USA, karim.sabra@me.gatech.edu)

Recent advances in underwater detection and survey techniques have increased the possibility of finding the wrecks of vessels and their cargoes. Since prehistoric times, many populations have used vessels for transportation and trade and built harbors and it is now estimated that there are about a million antique wrecks that lie underwater, still to be discovered. Many of these wrecks and ancient harbours lie within shallow depths and can be discovered and accessed easily by humans using available hardware such as side-scan sonars, multi-beam echo sounders, ROVs, GPS navigation, diving gear. Even though the majority of known archaeological sites on land are well protected by guards and high tech equipment, there is no technology for surveillance and protection of underwater archeology sites. This paper presents a new system specifically adapted for the surveillance and protection of underwater archaeological sites (SEAGUARD). Options available to the acoustic sensor suite of this system are pressure hydrophones and/or vector sensors. The system is based on an underwater acoustic monitoring of the noise field near an archaeological site sending preprocessed signals as an alarm to the authorities on shore via GSM/GPRS or Satellite network.

\section{Contributed Paper}

3:00

4pEAb3. Acoustic methods in extremely shallow water for reconstruction of ancient environments. Fantina Madricardo (CNR-Istituto di Scienze Marine, Riva Sette Martiri - Castello 1364/a, 30122 Venice, Italy, fantina.madricardo@ismar.cnr.it), Sandra Donnici (CNR-Istituto di Scienze Marine, Riva Sette Martiri - Castello 1364/a, 30122 Venice, Italy, sandra.donnici@ismar.cnr.it), Alberto Lezziero (PHAROS sas, Via della Libertà 12, 30175 Venice, Italy, alezziero@gmail.com), Federica De Carli (CNR-Istituto di Acustica 'O,M .Corbino', Via del Fosso del Cavaliere, 100, 00133 Rome, Italy, federica .decarli@gmail.com)
A large area of the Venice Lagoon was surveyed through a modified traditional echosounder. For the first time, acoustic methods together with geological analysis were used systematically to investigate natural and anthropogenic morphologies buried in the lagoonal sediments. The shallowness (with depths often $<1 \mathrm{~m}$ ) of the Venice Lagoon represents a challenge for underwater acoustic methods. The results we present in this paper show that such methods are very useful for detailed geomorphological and archaeological reconstruction and can be extended to other similar environments. In this context, a general methodology of multidisciplinary data collection was developed. As a synthesis of our acoustic and geoarchaeological investigation, maps of the ancient lagoonal environment were produced.

\section{Invited Paper}

4pEAb4. Precision positioning for deep-water archaeology. Brian Bingham (Franklin W. Olin College of Engineering, 1000 Olin Way, MH 368, Needham, MA 02492, USA, bbing@olin.edu)

Underwater archaeology makes use a variety of tools for deep-water exploration, but the defining characteristic of archaeology, what distinguishes the endeavor as scientific in purpose, is the quantifiable techniques employed for precision navigation. Precise positioning enables the collocation of archaeological results into a common reference frame, creating a persistent data products with the spatial context to enable interpretation. With this common context, data products such as site-maps, micro-bathymetric maps and photomosaics afford the capability to perform measurements with known accuracy and resolution, a key differentiator of the science of archaeology. Acoustic ranging is the meter stick of marine archeology. Archaeology has leveraged standard navigation techniques: long baseline (LBL) positioning, ultra-short baseline (USBL) tracking, Doppler velocity log (DVL) dead-reckoning, etc. However, the extreme precision required for archaeology has driven the development of new precise ranging instruments. This paper presents the capabilities and limitations of today's precision ranging instruments which use discrete sequence spread spectrum (DSSS) signal processing to provide the requisite performance. In addition to a technical discussion, the paper presents the results of recent field work in the Aegean, the Mediterranean and the East Coast of the Unites States. 


\title{
Session 4pEAc
}

\section{Engineering Acoustics: Ultrasonic Acoustic MEMS II (Poster Session)}

\author{
F. Levent Degertekin, Cochair \\ Georgia Institute of Technology \\ Dominique Certon, Cochair \\ Lab. LUSSI Inserm U930 CNRS FRE 2448 Univ. François Rabelais
}

\begin{abstract}
All posters will be on display from 3:40 p.m. to 5:20 p.m. To allow contributors an opportunity to see other posters, contributors of odd-numbered papers will be at their posters from 3:40 p.m. to 4:30 p.m. and contributors of even-numbered papers will be at their posters from 4:30 p.m. to 5:20 p.m.
\end{abstract}

\section{Contributed Papers}

4pEAc1. A surface acoustic wave impedance loaded sensor for wireless humidity measurement. Rung-De Wang (Institute of Applied Mechanics, National Taiwan University, No. 1, Sec. 4, Roosevelt Road, 106 Taipei, Taiwan, r94543020@ntu.edu.tw), Yung-Yu Chen (Department of Mechanical Engineering, Tatung University, No. 40, Sec. 3, Chungshan N. Rd., 104 Taipei, Taiwan, yychen@ttu.edu.tw), Tsung-Tsong Wu (Institute of Applied Mechanics, National Taiwan University, No. 1, Sec. 4, Roosevelt Road, 106 Taipei, Taiwan,wutt@ndt.iam.ntu.edu.tw)

The control of humidity is required in various areas such as the smart living space, industry, agriculture, and medicine. To reduce cost and increase lifetime, a wireless humidity sensor without an additional power supply should be developed to fulfill their needs. Recently, there are more and more efforts on surface acoustic wave (SAW) based radio frequency identification (RFID) system. The RFID system primarily consists of a reader and transponders, composed of SAW tags and antennas. Due to the capabilities of passive operation and wireless connection, SAW tags are also suitable for remote sensing. Therefore, in this study, a wireless humidity sensor is accomplished by integrating a $433 \mathrm{MHz} 128^{\circ} \mathrm{YX}-\mathrm{LiNbO} 3 \mathrm{SAW}$ tag with a resistive humidity sensor. The SAW tag is designed as an impedance loaded sensor. Moreover, because nanostructure sensing materials possess high surface-to-volume ratio, large penetration depth, and fast charge diffusion rate, CSA-doped polyaniline nanofibers are synthesized by the interfacial polymerization method and further deposited on the resistive humidity sensor as sensitive film to enhance the sensitivity. Finally, the humidity sensor was constructed and measured. Results indicate that our proposed sensor exhibits not only good linearity but also high sensitivity. Furthermore, it is indeed capable of passive remote sensing.

4pEAc2. Periodically poled transduction structures built on thinned single crystal Lithium Niobate layers bonded onto Silicon. Emilie Courjon (CNRS FEMTO-ST, 32 Avenue de l'Observatoire, 25044 Besançon, France, emilie.courjon@femto-st.fr), Gwenn Ulliac (CNRS FEMTO-ST, 32 Avenue de l'Observatoire, 25044 Besançon, France, gwenn.ulliac@femto-st.fr), Jérôme Hauden (Photline Technologies, 16 rue Jouchoux, 25000 Besançon, France, jerome.hauden@photline .com), Sylvain Ballandras (CNRS FEMTO-ST, 32 Avenue de l'Observatoire, 25044 Besançon, France, sylvain.ballandras@femto-st.fr)

The demand for highly coupled high quality acoustic wave devices has generated a strong innovative activity, yielding the investigation of new excitation principles and wave guide structures. In a recent work, we have investigated the interest of periodically poled transducers (PPTs) on single crystal $\mathrm{LiNbO}_{3} \mathrm{Z}$-cut plates. The PPT simply consists of a periodically poled ferroelectrics layer metallized on its two faces for acoutoelectric excitation.
In this work, we have fabricated PPTs on single crystal $\mathrm{LiNbO}_{3}$ Z-cut thinned layers reported on Silicon to develop a new kind of wave guide. Their fabrication is based on a home-made wafer bonding technique based on a metal-metal adhesion, the lithium niobate being lapped and polished in order to obtain a layer exhibiting a few tenth micrometer thick. The corresponding devices have successfully fabricated and tested in the frequency range 100-300 MHz. Comparison between experimental measurements and theoretical analysis (using a combination of finite and boundary element analysis) show that the modes are well controlled and that different kind of wave polarization may be excited.

4pEAc3. Acoustical properties characterization of a composite made of SU-8 and nanoparticules for BioMEMS application. Julien Carlier (IEMN - DOAE, Université de Valenciennes, Le Mont - Houy, 59313 Valenciennes, France, julien.carlier@univ-valenciennes.fr), Pierre Campistron (IEMN - DOAE, Université de Valenciennes, Le Mont - Houy, 59313 Valenciennes, France, pierre.campistron@univ-valenciennes .fr), Dorothée Callens (IEMN - DOAE, Université de Valenciennes, Le Mont - Houy, 59313 Valenciennes, France, dorothee.callens@univvalenciennes.fr), Caroline Soyer (IEMN - DOAE, Université de Valenciennes, Le Mont - Houy, 59313 Valenciennes, France, caroline.soyer @univ-valenciennes.fr), Bertrand Nongaillard (IEMN - DOAE, Université de Valenciennes, Le Mont - Houy, 59313 Valenciennes, France, Bertrand.Nongaillard@univ-valenciennes.fr), Shengxiang Wang (IEMN DOAE, Université de Valenciennes, Le Mont - Houy, 59313 Valenciennes, France, shx_wang@126.com), Xingzhong Zhao (Department of Physics, Wuhan University, 430072 Wuhan, China, xzzhao@whu.edu.cn)

The photoresist SU-8 (often used in microtechnology) has been acoustically characterized at the frequency of $1 \mathrm{GHz}$ thanks to $\mathrm{ZnO}$ transducers. This material will be used to achieve acoustical matching between silicon and water at this frequency and a gain of about $5 \mathrm{~dB}$ has been obtained using the combination of this material with a silicon oxyde layer. The acoustical characterization of a composite material made of this photoresist SU-8 doped with nanoparticules $\left(\mathrm{TiO}_{2}\right.$ for example) is also presented. The aim is to achieve matching layers with specific mechanical impedance around 5 MRay and depending on the nanoparticules concentration. The targeted application concerns the integration of high frequency ultrasonic transducer in Lab-on-Chip for biological cell characterization, especially adhesion properties or biological cells mechanical behaviour. The mechanical impedance and the attenuation of this composite material are characterized around 1 $\mathrm{GHz}$ thanks to a standing wave ratio method measuring S11 parameter and extracting the targeted parameters thanks to signal processing. 
4pEAc4. High Overtone Bulk Acoustic Resonators Based on Thinned Single-crystal Piezoelectric Layers: Filters and Frequency Sources Applications. Dorian Gachon (CNRS FEMTO-ST, 32 Avenue de l’Observatoire, 25044 Besançon, France, dorian.gachon@femto-st .fr), Jeremy Masson (CNRS FEMTO-ST, 32 Avenue de l'Observatoire, 25044 Besançon, France, jeremy.masson@femto-st.fr), Emilie Courjon (CNRS FEMTO-ST, 32 Avenue de l'Observatoire, $25044 \mathrm{Be}-$ sançon, France, emilie.courjon@femto-st.fr), Sylvain Ballandras (CNRS FEMTO-ST, 32 Avenue de l'Observatoire, 25044 Besançon, France, sylvain .ballandras@femto-st.fr)

The thin film bulk acoustic wave resonators exploiting the thicknessextensional vibration mode of piezoelectric thin films is a key technology as alternative solutions to standard SAW resonators. Lakin have emphasized the capability of High Overtone Bulk Acoustic Resonators to present high quality factors at frequencies in the $\mathrm{GHz}$ range. HBAR spring from the conjugation of the strong coupling coefficient of deposited piezoelectric thin films and of the high intrinsic quality of used substrates. The piezoelectric film and the two electrodes on its both sides are used as transducer whereas the acoustic energy is mainly trapped in the substrate. The resonant frequency corresponds to a half wavelength in the entire thickness of the device and, in opposition to FBAR, we can utilized both odd and even harmonics. The fundamental, generally in the vicinity of $10 \mathrm{MHz}$, has no specific interest but Q.f products around $1.1 \times 10^{14}$ have already been obtained for high overtones using aluminum nitride thin films deposited onto sapphire. In view of improving the $\mathrm{Q}$ factor of thin films, it is desirable to use a single-crystal piezoelectric material such as lithium niobate. We show and compare the fabrication in both approaches. Different measurement results are exposed for both approaches for the fabrication of oscillator and filters are shown and discussed.

4pEAc5. Micromachined probe performance assessment. Mathieu Legros (Vermon SA, 180 rue Général Renault, 37000 Tours, France, m.legros@vermon.com), Cyril Meynier (Vermon SA, 180 rue Général Renault, 37000 Tours, France, c.meynier@vermon.com), Guillaume Ferin (Vermon SA, 180 rue Général Renault, 37000 Tours, France, g.ferin@vermon.com), Rémi Dufait (Vermon SA, 180 rue Général Renault, 37000 Tours, France, r.duvait@vermon.com)

In recent years, Capacitive Micromachined Ultrasound Transducer (CMUT) technology was widely investigated and functional prototypes have been released by several R\&D teams. CMUT technology offers outstanding characteristics in acoustic, interconnect packaging capabilities or in integration features that are exciting criteria for new medical applications. We propose a full acoustic characterization report of a CMUT probe. A linear array was fully packaged with electronic preamplifier boards integrated. A complete acoustic characterization of the probe is then performed and presented. In a second phase, a linear probe with piezocomposite technology is realized. The conception is done in regard to the geometric characteristics and to the acoustic response of the micromachined probe. Then an electroacoustical benchmark CMUT / piezocomposite is realized in the closest conditions. Using a commercial ultrasound imaging platform, an image assessment is performed. The images are first analysed in a quantitative way with a tissue mimicking phantom, using a computerized tool who considered imaging parameters such as contrast, resolution or signal to noise ratio. In a second way, a clinical perspective is discussed with in vivo images.

4pEAc6. CMUT membrane model based on theory of stratified plates Nicolas Senegond (Lab. LUSSI Inserm U930 CNRS FRE 2448 Univ. François Rabelais, 10, Boulevard Tonnellé, 37032 Tours, France, nicolas.senegond@univ-tours.fr), Franck Teston (Lab. LUSSI Inserm U930 CNRS FRE 2448 Univ. François Rabelais, 10, Boulevard Tonnellé, 37032 Tours, France, franck.teston@univ-tours.fr), Cyril Meynier (Vermon SA, 180 rue Général Renault, 37000 Tours, France, c.meynier@vermon .com), Dominique Certon (Lab. LUSSI Inserm U930 CNRS FRE 2448 Univ. François Rabelais, 10, Boulevard Tonnellé, 37032 Tours, France, dominique.certon@univ-tours.fr)

To optimize and characterize capacitive micromachined transducers, static and dynamic mechanical behavior of the membrane have to be considered and modeled. Most of cMUT models are based on the classical plate equation. The flexural rigidity is homogenized for multi-layer structure taking into account properties of each material. Homogenization relations are available provided the flexural plate equation can be separated from the inplane deformation equation, so-called the membrane-like behavior equation. Practically, for membrane partially covered with electrode, at the metallized/non-metallized discontinuity, these equations cannot be solved separately and homogenization procedure is no more available. Moreover, initial membrane deflection introduces supplementary coupling between inplane and flexural displacements requiring modification of homogenization relations. A complete analytical formulation of the plate equation is developed in this paper. A finite difference meshing has been used to numerically solve the new set of plate equations. First, a cMUT with simple geometry is modeled for validation and comparison with Finite Element simulation (COMSOL Multiphysics software). Then, the validity domain of the "simple" flexural plate equation is discussed. Finally, an example of membrane optimization is given, where a multi-layer structure is proposed to reduce influence of static pre-stresses in the membrane on collapse voltage and resonance frequency.

4pEAc7. Pure-shear mode BAW resonators consisting of (11-20)textured ZnO films. Takahiko Yanagitani (Faculty of Engineering Tohoku University, 6-6-05 Aramaki Aoba Aoba-ku, 610-0321 Kyotanabe, Japan, yanagi@ecei.tohoku.ac.jp), Kiuchi Masato (National Institute of Advanced Industrial Science and Technology, 1-8-31 Midorigaoka, Osaka, 563-8577 Ikeda, Japan, yanagitani@usl.doshisha.ac.jp), Mami Matsukawa (Doshisha University, 1-3, Tatara Miyakodani, 610-0321 Kyotanabe, Japan, mmatsuka@mail.doshisha.ac.jp), Yoshiaki Watanabe (Faculty of Engineering, Doshisha Univ., 1-3 Miyakodani Tatara, 610-0321 Kyotanabe, Japan, kwatanab@mail.doshisha.ac.jp)

Thickness pure-shear mode film bulk acoustic wave resonators (FBARs) made of (11-20) textured $\mathrm{ZnO}$ films have been fabricated. We have also fabricated FBAR structure consisting of two layers of the (11-20) textured $\mathrm{ZnO}$ film with opposite polarization directions. This FBAR structure operated in second overtone pure-shear mode, and allowed shear-mode FBARs at higher frequency. The effective electromechanical coupling coefficients $k_{\text {eff }}^{2}$ of pure-shear mode FBAR and second overtone pure-shear mode FBAR in this study were found to be $3.3 \%$ and $0.8 \%$, respectively. The temperature coefficient of frequency (TCF) of thickness extensional mode FBAR, pure-shear mode FBAR, and second overtone pure-shear mode FBAR were measured in the temperature range of $10-60{ }^{\circ} \mathrm{C}$. TCF values of $-63.1 \mathrm{ppm} /{ }^{\circ} \mathrm{C},-34.7$ $\mathrm{ppm} /{ }^{\circ} \mathrm{C}$, and $-35.6 \mathrm{ppm} /{ }^{\circ} \mathrm{C}$ were found for the thickness extensional mode FBAR, the pure-shear mode FBAR, and the second overtone pure-shear mode FBAR, respectively. These results demonstrated that pure-shear mode $\mathrm{ZnO}$ FBARs have more stable temperature characteristics than the conventional thickness extensional mode $\mathrm{ZnO}$ FBARs.

4pEAc8. Influence of the shape of the membrane of computed cMUT using FEA/BEM analysis. Stanislas Clatot (CNRS FEMTO-ST, 32 Avenue de l'Observatoire, 25044 Besançon, France, stanislas.clatot @femto-st.fr), Emeline Sadoulet (CNRS FEMTO-ST, 32 Avenue de l'Observatoire, 25044 Besançon, France, emeline.sadoulet-reboul @ univ-fcomte.fr), Olivia Arbey (CNRS FEMTO-ST, 32 Avenue de l'Observatoire, 25044 Besançon, France, olivia.arbey@femto-st .fr), William Daniau (CNRS FEMTO-ST, 32 Avenue de l'Observatoire, 25044 Besançon, France, william.daniau@femto-st.fr), Julien Garcia (CNRS FEMTO-ST, 32 Avenue de l'Observatoire, 25044 Besançon, France, julien.garcia@femto-st.fr), Joseph Lardies (FEMTO-ST Applied Mechanics, 24 chemin de l'épitaphe, 25000 Besançon, France, joseph.lardies@univ-fcomte.fr), Sylvain Ballandras (CNRS FEMTO-ST, 32 Avenue de l'Observatoire, 25044 Besançon, France, sylvain .ballandras@femto-st.fr), Marc Berthillier (University of Franche-Comté, Institute FEMTO - LMARC, 24 rue de 1'Epitaphe, 25000 Besançon, France, marc.berthillier@univ-fcomte.fr)

The possibility to excite and detect acoustic waves in fluids using capacitive micro-machined ultrasonic transducers (cMUT) built on silicon using clean room techniques offers attractive opportunities for manufacturing high quality low cost imaging probes. CMUTs developed for acoustic imaging exploit the first flexural mode of thin and stiff membranes, leading to 
bandwidth larger than $100 \%$. These transducers can be accurately designed using mixed finite element analysis/boundary element methods (FEA/BEM). Periodic FEA particularly allows for the simulation of devices exhibiting complicated shape interfaces and involving materials of different nature. BEM also are particularly well-suited to provide an accurate description of any stacked medium assuming flat interfaces for the radiation area and the layer interface for 2 and 3D structures as well. In this work, we have analysed the influence of the shape of the MUT membrane on the spectral response of the transducer. 3D Computations have been conducted considering radiation coniditions in the substrate on the backside (silicon) and in viscous water on the front side. we have particularly focused the computation on solutions allowing for reducing parasitic modes in the actual operation of the transducer. The efficiency of the different configuration are compared in termes of emitted pressure contributions.

\title{
Session 4pEAd
}

\section{Engineering Acoustics and Signal Processing in Acoustics: Transducers and Signal Processing for the Oil and Gas Industry II (Poster Session)}

\author{
Fernando Garcìa-Osuna, Cochair \\ Schlumberger \\ Benoit Froelich, Cochair \\ Etudes et Productions Schlumberger
}

\begin{abstract}
All posters will be on display from 3:40 p.m. to 5:20 p.m. To allow contributors an opportunity to see other posters, contributors of odd-numbered papers will be at their posters from 3:40 p.m. to 4:30 p.m. and contributors of even-numbered papers will be at their posters from 4:30 p.m. to 5:20 p.m.
\end{abstract}

\section{Contributed Papers}

4pEAd1. Multimode evaluation of cement behind steel pipe. Benoit Froelich (Etudes et Productions Schlumberger, 1, rue Becquerel, BP 202, 92142 Clamart, France, bfroelich@clamart.oilfield.slb.com)

Oil wells are usually cased with a steel pipe, and cement is injected in the annulus between pipe and rock in order to provide hydraulic isolation of the reservoir. The traditional ultrasonic technique to evaluate cement behind steel pipe has been to excite the pipe thickness mode, and to extract the acoustic impedance of the material from the pulse-echo response. However, this technique suffers from some limitations, such as a limited depth of investigation or a poor discrimination between mud and light cement. To alleviate such limitations, the thickness mode can be combined with the pipe flexural mode. Although dispersive, the flexural mode has the unique property of a quasi constant group velocity in a specific frequency domain which is high enough to provide azimuthal resolution. Such property provides the means for an accurate measurement of the time of arrival and the amplitude of the different echoes generated by a particular geometry. In particular, the echo traveling within the annulus and reflected by the rock can be easily detected from within the pipe, and provide novel information on the annulus fill.
4pEAd2. Data fusion technique applied to steam wastage estimation and fault detection in an industrial process heating application. Sivaram Nishal Ramadas (Institute of Sound and Vibration Research, University of Southampton, University Road, Highfield, S017 1BJ Southampton, UK, nishal@ieee.org)

Data fusion, the process of combining information obtained together from many heterogeneous sensors to form a single composite picture of the environment, is used widely in many applications. Modern steam heating systems consist of mechanical devices known as 'traps', which are robust and reliable but inevitably over time can wear and fail, with the possibility of leaking steam. To diagnose such faulty steam traps and the level of leakage under operating conditions in a closed system is difficult. This paper presents the preliminary work carried out to integrate together data recorded from commercial sensors (such as piezoelectric acoustic emission devices, pressure transmitters, and thermocouples) to estimate steam wastage and fault detection in a steam system. Experimental data were acquired from a purpose built steam wastage test rig (built similar to the method outlined in the British Standard for determination of steam loss from traps), capable of simulating varying condensate loads by injecting preheated water into a steam test line. The captured composite data is then used to develop a signal processing algorithm to diagnose effective trap operation and quantify the rate of steam loss in the system and the results are discussed. 


\title{
Session 4pEAe
}

\section{Engineering Acoustics: Transducers II (Poster Session)}

\author{
Robert M. Koch, Chair \\ NOWC
}

\begin{abstract}
All posters will be on display from 3:40 p.m. to 5:20 p.m. To allow contributors an opportunity to see other posters, contributors of odd-numbered papers will be at their posters from 3:40 p.m. to 4:30 p.m. and contributors of even-numbered papers will be at their posters from 4:30 p.m. to 5:20 p.m.
\end{abstract}

\section{Contributed Papers}

4pEAe1. Condenser microphone as parametric electroacoustic system and its time-domain modelling via equivalent electrical circuit in SPICE software. Michal Vlk (Czech Technical University, Faculty of Electrical Engineering, Department of Radioengineering, Technická 2, 16627 Praha 6 - Dejvice, Czech Republic, vlkm3@fel.cvut.cz)

Condenser microphone usually works with constant polarisation voltage. Where special attentions are prescribed for spectrum of self noise there may be useful to use high frequency voltage (pump) applied to transducer instead of DC charge. When frequency of pump is orders of higher than frequency of acoustic signals, large parametric gain occurs in the transducer. Method for describing such a system via equivalent electric network with approach of electronic filter design blocks was developped and special analogies were used for convergency improvement. Method was applied to time-domain simulation of pressure transducer with current-mode diode discriminator in SPICE computer analysis software. Simulations of transient behavior after switching system on was demonstrated.

4pEAe2. Modelling of radiofrequency MEMS bulk acoustic wave resonators with Legendre polynomial method. Antoine A. Raherison (IEMN - DOAE, Université de Valenciennes, Le Mont - Houy, 59313 Valenciennes, France, raherson_antoine@yahoo.fr), Faniry Emilson F. Ratolojanahary (LAPAUF, Universite de Fianarantsoa, 301 Fianarantsoa, Madagascar, faniry@univ-fianar.mg), Jean-Etienne J. Lefebvre (IEMN DOAE, Université de Valenciennes, Le Mont - Houy, 59313 Valenciennes, France, jean-etienne.lefebvre@univ-valenciennes.fr), Lahoucine L. Elmaimouni (Faculte polydisciplinaire d'Ouarzazate, Universite Ibn Zohr, BP 638, Ouarzazate, Morocco, la_elmaimouni@yahoo.fr), Tadeusz T. Gryba (IEMN - DOAE, Université de Valenciennes, Le Mont - Houy, 59313 Valenciennes, France, tadeusz.gryba@univ-valenciennes.fr)

Radio-Frequency electro-acoustic resonators and filters are key components for wireless communication devices. The next generation of mobile systems requires for these components a strict size reduction, higher operating frequency and better power resistance. So, MEMS BAW (Bulk Acoustic Wave) devices have emerged as an efficient alternative to established Surface Acoustic Wave filtering solutions. Knowledge of the electromechanical coupling coefficient and the Q-factor are of primary importance for the MEMS BAW device design. In our model, the Legendre polynomial method which describes the structure and incorporates automatically the boundary conditions in constitutive and propagation equations is used to calculate these parameters. It is the first time this method is applied to study standing rather than propagative waves. The advantage of this approach are, in a unique formulation, to take into account the presence of sources, existence of electrodes' losses and dissimilarity of the constituent materials of the resonator. To validate this approach, it is applied to a $\mathrm{Al} / \mathrm{ZnO} / \mathrm{Al}$ resonator. Through harmonic and modal analysis, the influence of electrodes properties on MEMS BAW resonator performances are illustrated; the results are compared with those published earlier. This method is an efficient tool for designing MEMS BAW filter.

4pEAe3. Design of dispersive layered SAW filters with CMOS low noise amplifier. Tadeusz T. Gryba (IEMN - DOAE, Université de Valenciennes, Le Mont - Houy, 59313 Valenciennes, France, tadeusz.gryba@univ-valenciennes.fr), Julien Carlier (IEMN - DOAE, Université de Valenciennes, Le Mont - Houy, 59313 Valenciennes, France, julien.carlier@univ-valenciennes.fr), Etienne Ntagwirumugara (IEMN DOAE, Université de Valenciennes, Le Mont - Houy, 59313 Valenciennes, France, entagwir@yahoo.fr), Victor Y. Zhang (IEMN-CNRS, Av. Poincare, Cite Scientifique, B.P. 60069, 59652 Villeneuve d'Ascq Cedex, France, victor.zhang@iemn.univ-lille1.fr), Jean-Etienne J. Lefebvre (IEMN DOAE, Université de Valenciennes, Le Mont - Houy, 59313 Valenciennes, France, jean-etienne.lefebvre@univ-valenciennes.fr)

Up to now, RF front-end surface acoustic wave (SAW) filters for mobile communication are mainly fabricated on $\mathrm{LiNbO}_{3}$ and $\mathrm{LiTaO}_{3}$ substrates. A monolithic integration of these filters on $\mathrm{Si}$ substrates is highly desirable, but $\mathrm{Si}$ is non piezoelectric. One alternative is the deposition of a piezoelectric film on the semiconductor substrate. CMOS technology is very attractive for integrating the radio frequency modules in a single chip. This paper presents the analysis and realisation of a SAW passband filter on silicon substrate based on a piezoelectric $\mathrm{ZnO}$ thin film working near $1 \mathrm{GHz}$, integrated with a CMOS low-noise amplifier. We propose a modified coupling of modes (COM) approach for a layered $\mathrm{ZnO} / \mathrm{Si}$ surface acoustic wave filter. The COM parameters in this formulation are the Rayleigh wave velocity, the electromechanical coupling coefficient, the complex reflection coefficient, the transduction coefficient and the inter-digital capacitance C. This is a dispersive SAW layered filter some parameters of which become frequency dependent due to the phase velocity dispersion. We present the theoretical and experimental results of the filter integrated with a CMOS low noise amplifier.

4pEAe4. A stepped plate transducer as ultrasonic range sensor. Yub Je (Postech, San 31, Hyoja-Dong, Namgu, 790-784 Pohang, Republic of Korea, effortjy@postech.ac.kr), Jong-Kyu Park (Postech, San 31, HyojaDong, Namgu, 790-784 Pohang, Republic of Korea, chong@ postech.ac.kr) , Dong Hoon Yi (Postech, San 31, Hyoja-Dong, Namgu, 790-784 Pohang, Republic of Korea, effortjy@hotmail.com), Haksue Lee (Postech, San 31, Hyoja-Dong, Namgu, 790-784 Pohang, Republic of Korea, jolary@postech.ac.kr), Wonkyu Moon (Postech, San 31, Hyoja-Dong, Namgu, 790-784 Pohang, Republic of Korea, wkmoon@postech.ac.kr)

A new type of highly directional ultrasonic transducer is designed and tested as an ultrasonic range sensor by using the parametric acoustic arrays. To get intensive primary waves, a modified stepped plate transducer is proposed. Gallego-juarez et al.(1978) first proposed stepped plate transducer for high power radiation at one frequency. The steps with the height of half- 
wavelength of sound in air compensate discrete phase difference on the vibrating plate. However, two collimated beams are required for the parametric acoustic array. The position and height of the steps are modified to compensate the flexural vibration for two frequencies in this transducer. The transducer diameter is $50 \mathrm{~mm}$. The optimal primary frequencies are designed as $80 \mathrm{kHz}$ and $120 \mathrm{kHz}$ to generate difference frequency of $40 \mathrm{kHz}$ efficiently. The proposed transducer has the HPBW of $5^{\circ}$ that is much higher directivity than general ultrasonic range sensor(Generally $20^{\circ}$ on same size). The maximum SPL is $130 \mathrm{~dB}$ at primary frequencies and $95 \mathrm{~dB}$ at difference frequency on $75 \mathrm{Vpk}$ input. These experimental results show that the proposed transducer can successfully improve the spatial resolution of ultrasonic sensor. [Research is partly supported by MIC/IITA Intelligent Robot Sensor and partly supported by DAPA and ADD UD070054AD]

4pEAe5. A study on the adaptive design of loudspeaker driver parameters for a given enclosure. Seokjin Lee (Applied Acoustics Lab., INMC, 132-302, Seoul National University, San 56-1, Sillim-dong, Kwanak-gu, 151-742 Seoul, Republic of Korea, sjlee@acoustics.snu .ac.kr), Junguk Noh (Applied Acoustics Lab., INMC, 132-302, Seoul National University, San 56-1, Sillim-dong, Kwanak-gu, 151-742 Seoul, Republic of Korea, junoh37@acoustics.snu.ac.kr), Koeng-Mo Sung (Applied Acoustics Lab., INMC, 132-302, Seoul National University, San 56-1, Sillim-dong, Kwanak-gu, 151-742 Seoul, Republic of Korea, kmsung@snu .ac.kr)

In the conventional methods of electro-dynamic loudspeaker design, driver units are selected first and then the enclosures are designed for the best compensation of frequency responses of the given drivers. However, in the industry of nowadays, these conventional methods are not appropriate; the enclosure design is determined first, and after that, a proper driver unit is selected by trial and error. In this paper, an adaptive design method of loudspeaker driver parameters for a given enclosure is studied. We estimated loudspeaker parameters such as diaphragm size, acoustic compliance, acoustic resistance, etc., to obtain an optimal alignment or desired response curve for a given enclosure. In the proposed method, the target frequency response curve in low-frequency range is determined first. Second, the optimal size of driver is calculated. The other driver parameters, such as driver suspension, $\mathrm{Q}$ of driver, etc., are designed lastly. Easier and faster design can be achieved by the proposed design method of electro-dynamic loudspeakers.

4pEAe6. Consideration on design of the sensitivity in piezoelectric vibratory tactile sensor. Subaru Kudo (School of Science and Engineering, Ishinomaki Senshu University, 1 Shinmito, Minamisakai, 986-8580 Ishinomaki-city, Miyagi, Japan, kudou@isenshu-u.ac.jp)
The piezoelectric vibratory tactile sensors have been used for measuring the softness and hardness of an object. They make use of changes in the resonance frequency when their vibrating sections are brought into contact with an object. In this study, the sensitivity of the frequency change on the tactile sensors is experimentally considered. The longitudinal-bar-type and the fixed-free-bar type resonators are used as the tactile sensors. Then, the experimental characteristics of the manufactured tactile sensors are shown by measuring the softness and hardness of the test pieces. The differences of the characteristics of the sensitivity are discussed from the viewpoint of the resonance frequency, vibration mode and the dimensions of the resonator. It is clarified that the sensitivity on the tactile sensor is inversely proportional to the mass of the resonator. Moreover, the effects of the resonance frequency change on the tactile sensor by the load force and the additional mass, in case of contacting with an object, are analyzed using the finite element method. The obtained results will be useful for designing the piezoelectric vibratory tactile sensor.

4pEAe7. Improvement of low frequency signal radiation performance for piezoelectric loudspeakers. Juro Ohga (2-24-3, Tamanawa, 247-0071 Kamakura, Japan, johga@nifty.com)

Piezoelectric loudspeaker is characterized by their very simple constructions, but their radiation performance at low frequency region is poor because their diaphragms are too stiff for large amplitude vibration. This paper proposes two innovative methods for improvement of low frequency radiation performance of piezoelectric direct-radiator loudspeakers. One is a flatshape loudspeaker unit with a tuck-shaped flexible diaphragm by a sheet of piezoelectric film. The other is a paper cone loudspeaker unit with a large radiator driven by revolution of piezoelectric ultrasonic motors. A loudspeaker system with satisfactorily wide frequency range for music is constructed by combination of these two sorts of loudspeaker units.

4pEAe8. Geometrical optimization of vented boxes. Guillaume Pellerin (CNAM Paris, 5, rue du Vert Bois, 75003 Paris, France, guillaume .pellerin@cnam.fr)

Today, low frequency reproduction with loudspeakers in vented box is limited by two factors: the volume of the box, and non-linear airflow through the vent. We propose a novel approach that takes into account aerodynamical parameters, leading to original profiles and an improved functioning of the vented box. For example, under certain alignment conditions, there exits a second cut-off frequency below the first one, localized on the lower impedance hump. Using this lower cut-off frequency and an adapted vent profile makes it possible to radiate frequencies under $40 \mathrm{~Hz}$ with box volumes smaller than 1 liter and small drivers. A prototype will be demonstrated. 


\title{
Session 4pEAf
}

\section{Engineering Acoustics: Sound Fields II (Poster Session)}

\author{
Raymond Kirby, Chair \\ Brunel University
}

\begin{abstract}
All posters will be on display from 3:40 p.m. to 5:20 p.m. To allow contributors an opportunity to see other posters, contributors of odd-numbered papers will be at their posters from 3:40 p.m. to 4:30 p.m. and contributors of even-numbered papers will be at their posters from 4:30 p.m. to 5:20 p.m.
\end{abstract}

\section{Contributed Papers}

4pEAf1. Application of Advanced Features in Computational Acoustics. Reinhard Lerch (Univ. Erlangen-Nuremberg, Dept. of Sensor Technology, Paul-Gordan-Str. 3/5, 91052 Erlangen, Germany, reinhard.lerch @1se.eei.uni-erlangen.de), Manfred Kaltenbacher (Univ. ErlangenNuremberg, Dept. of Sensor Technology, Paul-Gordan-Str. 3/5, 91052 Erlangen, Germany, manfred.kaltenbacher@1se.eei.uni-erlangen.de), Martin Meiler (Simetris GmbH, Am Weichselgarten 7, 91058 Erlangen, Germany, martin.meiler@simetris.de)

Modern numerical simulation tools allow the analysis of the generation and propagation of sound. However, a variety of computational features enhancing their applicability are missing in these codes. Therefore, it is sometimes cumbersome to come to practically useful results when applying such codes to real life problems. Here, some of these lacking features will be addressed and, furthermore, their implementation in a new finite element code is reported. These features are: Frequency dependent damping propagation medium, nonmatching grids for computing sound in neighbouring domains with quite different propaga-tion velocities and perfectly matched layers (for handling of open domain problems without reflections at boundaries of the computational domain). A finite element environment including pre- and post-processing has been established to support the development of electroacoustic devices. The following real life examples will be reported: electrodynamic loudspeakers, noise emission from power transformers, ultrasound devices for medical imaging such as conventional piezoceramic arrays as well as CMUTs (capacitive micromachined ultrasound transducers). It will be also shown that the code is able to handle nonlinear effects in transducing mechanisms and acoustic wave propagation, as occurring in HIFU (high intensive focused ultrasound) applications.

4pEAf2. Theoretical Study of Near 3D Sound Field Reproduction Based on Wave Field Synthesis. Toshiyuki Kimura (National Institute of Information and Communications Technology, 4-2-1, Nukui-Kitamachi, Koganei, 184-8795 Tokyo, Japan, t-kimura@nict.go.jp), Yoko Yamakata (National Institute of Information and Communications Technology, 4-2-1, Nukui-Kitamachi, Koganei, 184-8795 Tokyo, Japan, yamakata@nict.go.jp), Michiaki Katsumoto (National Institute of Information and Communications Technology, 4-2-1, Nukui-Kitamachi, Koganei, 184-8795 Tokyo, Japan, katumoto@nict.go.jp)

It is very important to develop near 3D sound field reproduction techniques in order to realize the ultra-realistic communication such as 3D TV and 3D tele-conference. In this report, the principle of the near 3D sound field reproduction technique using wave field synthesis is defined from Kirchhoff-Helmholtz integral equation and two methods (dipole control method and directional point control method) are proposed. The performance of two proposed methods is studied by computer simulation and it is shown that the dipole control method has good performance and that the directional point control method has good performance if the directivity of loudspeakers is unidirectional or shotgun.

4pEAf3. Performance Evaluation of 3D Sound Field Reproduction System with a Few Loudspeakers and Wave Field Synthesis. Munenori Naoe (National Institute of Information and Communications Technology, 4-2-1, Nukui-Kitamachi, Koganei, 184-8795 Tokyo, Japan, nm.s512.ex@nict.go.jp), Toshiyuki Kimura (National Institute of Information and Communications Technology, 4-2-1, Nukui-Kitamachi, Koganei, 184-8795 Tokyo, Japan, t-kimura@nict.go.jp), Yoko Yamakata (National Institute of Information and Communications Technology, 4-2-1, NukuiKitamachi, Koganei, 184-8795 Tokyo, Japan, yamakata @ nict.go.jp), Michiaki Katsumoto (National Institute of Information and Communications Technology, 4-2-1, Nukui-Kitamachi, Koganei, 184-8795 Tokyo, Japan, katumoto@nict.go.jp)

A conventional 3D sound field reproduction system using wave field synthesis places a lot of loudspeakers around the listener. However, since such a system is very expensive and loudspeakers come into the listener's field of vision, it is very difficult to construct an audio-visual system with it We developed and evaluated a 3D sound field reproduction system using eight loudspeakers placed at the vertex of cube and wave field synthesis. We compared the sound localization of a loudspeaker array with that of seventeen loudspeakers placed around the listener and found that their localization capabilities were equivalent except the normal direction of cube's planes.

4pEAf4. Synthesis of wave front in the sound field recording/reproduction system based on the boundary surface contro principle. Seigo Enomoto (Advanced Telecommunication Research Institute International, 2-2-2 Hikaridai, 'Keihanna Science City', 6190288 Kyoto, Japan, seigo.enomoto@atr.jp), Yusuke Ikeda (Advanced Telecommunication Research Institute International, 2-2-2 Hikaridai, 'Keihanna Science City', 6190288 Kyoto, Japan, yusuke.ikeda@atr.jp), Shiro Ise (Urban and Envronemental Engineering, Graduate School of Engeneering, Kyoto University, C1-386, Kyodai-Katsura, Nisikyo-ku, Kyoto-si, 6158540 Kyoto, Japan, ise@archi.kyoto-u.ac.jp), Satoshi Nakamura (Advanced Telecommunication Research Institute International, 2-2-2 Hikaridai, 'Keihanna Science City', 6190288 Kyoto, Japan, satoshi.nakamura@atr.jp)

Based on the boundary surface control (BSC) principle, a new recording/reproduction system is developed to realize high fidelity threedimensional sound field reproduction. Theoretically, by using this new system, physically faithful reproduction could be achieved in any acoustic environments. Sound recording/reproduction systems based on the BSC principle require many loudspeakers and many microphones. In this new system, the microphone array system to record 3D sound field consists of 70 microphones, and the loudspeaker system to reproduce the recorded $3 \mathrm{D}$ sound field consists of 62 full-range units and 8 sub-woofer units. To evaluate the ability of this system, the wave front which is measured in the sound- 
proofed room is compared with the reconstructed wave front within this system. The experiment shows that the reconstructed secondary wave front is very similar to the primary wave front in lower frequency.

4pEAf5. Three-Dimensional FDTD Method for Analysing of the acoustic characteristics of Compact Acoustic Reproduction Systems Yuichiro Koga (Kansai University, Kobe-Nishiku-Gakuenhigasimati5-1512, 651-2102 Kobe, Japan, koga0814@joho.densi.kansai-u.ac.jp)

We propose a method for analysing of the acoustic characteristics of compact acoustic reproduction systems with Three-Dimensional FDTD method. We reproduce actual structure of compact acoustic reproduction systems and take the frequency characteristic of sound pressure level at a point with Three-Dimensional FDTD method. To compare the frequency characteristic of sound pressure level of actual measurement of compact acoustic reproduction systems to measurement of the proposed method, we show availability of the proposed method for analysing of the acoustic characteristics of compact acoustic reproduction systems. The proposed method could explain actual phenomena on compact acoustic reproduction systems well.

4pEAf6. Sound localization in multiple regions: theory and applications. Yang-Hann Kim (Center for Noise and Vibration Control, Korea Advanced Institute of Science and Technology, 4114, Department of Mechanical Engineering, Guseong-dong, Yuseong-gu, 305-701 Daejon, Republic of Korea, yanghannkim@kaist.ac.kr), Ji-Ho Chang (Center for Noise and Vibration Control, Korea Advanced Institute of Science and Technology, Dep. of Mechanical Engineering, Guseong-dong, Yuseong-gu, 305701 Daejon, Republic of Korea, chang.jiho@gmail.com), Jin-Young Park (Center for Noise and Vibration Control, Korea Advanced Institute of Science and Technology, Dep. of Mechanical Engineering, Guseong-dong, Yuseong-gu, 305-701 Daejon, Republic of Korea, jypark1979@kaist.ac.kr)

It is often required to have several listening zones, which allows us to have different sounds that we select. For example, in a room, someone wants to listen sound from TV set, and other wants to listen music. In a car, a driver might wants to hear the information coming from his/her navigator system, and the passenger at the back side wants have a quiet zone so that he/she can sleep. To accomplish this kind of acoustic zones, we need to generate multiple sound zones by using multiple speakers. The performance has to be evaluated in accordance with how well one can listen the sound that is expected to have. We proposed to maximize the acoustic contrast between the zones that are defined. The basic concept associated with this approach was proposed by Choi and Kim [J. Acoust. Soc. Am., Vol.111(4), 16951700, Apr. 2002.], but this paper extend this fundamental idea to multiple zone cases. Theoretical formulation which shows what we have proposed is well addressed and several practical cases, including car audio system will be demonstrated. [This work was supported by the Korea Science and Engineering Foundation (KOSEF) through the National Research Lab. Program funded by the Ministry of Science and Technology (M1050000011205J0000-11210)]

4pEAf7. Sound focused personal audio system design: Performance improvement in acoustic contrast control by spatial weighting for obtaining spatially averaged acoustic potential energy. Jin-Young Park (Center for Noise and Vibration Control, Korea Advanced Institute of Science and Technology, Dep. of Mechanical Engineering, Guseong-dong, Yuseong-gu, 305-701 Daejon, Republic of Korea, jypark1979 @ kaist.ac.kr), Ji-Ho Chang (Center for Noise and Vibration Control, Korea Advanced Institute of Science and Technology, Dep. of Mechanical Engineering, Guseong-dong, Yuseong-gu, 305-701 Daejon, Republic of Korea, chang.jiho@gmail.com), Chan-Hui Lee (Center for Noise and Vibration Control, Korea Advanced Institute of Science and Technology, Dep. of Mechanical Engineering, Guseong-dong, Yuseong-gu, 305-701 Daejon, Republic of Korea, Chance99@kaist.ac.kr), Yang-Hann Kim (Center for Noise and Vibration Control, Korea Advanced Institute of Science and Technology, 4114, Department of Mechanical Engineering, Guseong-dong, Yuseong-gu, 305-701 Daejon, Republic of Korea, yanghannkim@kaist.ac.kr)

In acoustic contrast control [J.-W. Choi and Y.-H. Kim, J. Acoust. Soc. Am. 111. 1695 (2002)], spatially averaged acoustic potential energy is used as a representative spatial parameter because acoustic contrast control aims to maximize spatially averaged acoustic potential energy ratio between acoustically bright and dark zone. Therefore, spatial averaging process should be concerned carefully because control performance is sensitive to how to determine bright and dark zone. We have already got successful result in feasibility study for personal audio system with over $20 \mathrm{~dB}$ difference between bright (frontal) and dark (side) zone [C.-H. Lee et al., J. Acoust. Soc. Am. 111, 3053 (2007)], without any spatial weighting in spatial averaging of acoustic potential energy. Recently, we're trying to improve the control performance by giving spatial weighting in spatial averaging process because how to give spatial weighting has to do with the improvement of control performance to satisfy the original purpose of personal audio system more closely. In this paper, it will be covered the investigation for how to give spatial weighting in averaging process and shown experimental evaluations for a sound focused personal audio system. [Supported by the Korea Science and Engineering Foundation (KOSEF) through the National Research Lab. Program funded by the Ministry of Science and Technology (M10500000112-05J0000-11210)]

4pEAf8. 3D sound field rendering under non-idealized loudspeaker arrangements. Alois Sontacchi (Institute of Electronic Music and Acoustics, Inffeldgasse 10 / 3, 8010 Graz, Austria, sontacchi@iem.at), Franz Zotter (Institute of Electronic Music and Acoustics, Inffeldgasse 10 / 3, 8010 Graz, Austria, zotter@iem.at), Robert Höldrich (Institute of Electronic Music and Acoustics, Inffeldgasse 10 / 3, 8010 Graz, Austria, hoeldrich@iem.at)

The approach to realise periphonic sound field reproduction based on spherical harmonics (multi-pole theory) has already been well-known as Ambisonics and Higher Order Ambisonics, respectively. By the aid of an $\mathrm{N}$-dimensional orthogonal set of vectors any arbitrary source free sound field can be described. Reproduction is realized by projection of the encoded sound field on a regular loudspeaker distribution over a spherical surface. The used set of vectors exhibits a defined hierarchic with interesting symmetries. In the original scheme sound sources represented by plane waves (sources in far distance) can be encoded independent of the decoding process on the regular loudspeaker layout. Usually, in practice - in contrast to theory, 3D loudspeaker layouts are requested for the upper hemisphere. This restriction is caused by the physical configuration. First of all that demand bounds the reproduction of sound sources to the upper area. Furthermore caused by these facts idealized regular layouts considering the 3 dimensions are impossible. Within this contribution we will show how the symmetries of the spherical harmonics can be used to obtain optimized decoding rules and to overcome insufficient irregular loudspeaker arrangements.

4pEAf9. Measurements of head-related transfer function in sagittal and frontal coordinates. Takashi Nakado (Nagoya University, Furo-cho, Chikusa-ku, 4648603 Nagoya, Japan, nakado@sp.m.is.nagoya-u .ac.jp), Takanori Nishino (Nagoya University, Furo-cho, Chikusa-ku, 4648603 Nagoya, Japan, nishino@media.nagoya-u.ac.jp), Kazuya Takeda (Nagoya University, Furo-cho, Chikusa-ku, 4648603 Nagoya, Japan, kazuya.takeda@nagoya-u.jp)

3D sounds can be generated by using a head-related transfer function (HRTF), which is defined as the acoustic transfer function between a sound source and the entrance to the ear canal. Since HRTF depends on a subject and the sound source direction, many HRTF measurements were conducted. In most case, HRTFs were measured in horizontal coordinates. However, HRTF measurements in other coordinates are also useful. In previous researches, HRTFs measured in sagittal coordinates were used to investigate the relation between spectral cues and vertical angle perception. Although HRTF measurement in frontal coordinates is rarely conducted, there is an advantage to measure HRTFs densely in the front and rear where sound localizations are very sensitive. Therefore, we measured HRTFs for about 
2,300 directions in sagittal and frontal coordinates and constructed a database. The measurements were conducted in a soundproof chamber with two head-and-torso simulators (B\&K 4128 and KEMAR). The HRTF database can be downloaded at http://www.sp.m.is.nagoya-u.ac.jp/HRTF/

4pEAf10. A New Sound Field Reproduction Method Based on the Spatial Covariance. Yoshinori Takahashi (NHK Science \& Technical Research Laboratories, 1-10-11 Kinuta, Setagaya-ku, 157-8510 Tokyo, Japan, yoshinori@ieee.org), Akio Ando (NHK Science \& Technical Research Laboratories, 1-10-11 Kinuta, Setagaya-ku, 157-8510 Tokyo, Japan, ando.aio@nhk.or.jp)

This work investigates a sound field reproduction method based on the sound field statistics. The covariance among recorded multi-channel signals might represent the relative relationship and the mutual magnitude characteristics between each point in the sound field that time invariant. The author Y. Takahashi et al. proposed a reproduction method minimizing the difference in the spatial covariance between the original and reproduced sound fields [19th ICA, RBA15-012]. We call the method "SOund field Reproduction method based on the sPAtial Covariance (SORPAC)". However it has not been clarified that the theoretical background and the characteristics in listening. The SORPAC doesn't require the information of sound source locations and transfer functions. In this work, we described the reproduction theory of SORPAC in frequency domain. And we showed the relationship between SORPAC and the general wave surface control. Then we confirmed that the SORPAC is able to reproduce the sound source direction without the wave surface control. As a application of SORPAC, we demonstrated a multi-channel contents down-mixing experiment and evaluated the result from the binaural-listening point of view.

4pEAf11. Optimal array pattern synthesis with desired magnitude response. Alexander Mattioli Pasqual (Universidade Estadual de Campinas, Rua Mendeleiev, 200, Cidade Universitária "Zeferino Vaz", 13083-970 Campinas, Brazil, pasqual@fem.unicamp.br), José Roberto Arruda (Universidade Estadual de Campinas, Rua Mendeleiev, 200, Cidade Universitária "Zeferino Vaz", 13083-970 Campinas, Brazil, arruda@fem.unicamp.br), Philippe Herzog (Laboratoire de Mécanique et d'Acoustique - CNRS, 31 chemin Joseph Aiguier, 13402 Marseille, France, herzog@lma.cnrs-mrs.fr)

Letting Euclidean norm be the performance parameter, the task of finding the best approximation of a complex function in a finite dimension subspace leads to a convex optimization problem that can be easily solved by the least-squares method. However, this procedure leads to a sub-optimal solution in applications that have no phase requirements on the approximated function. In this case, semidefinite programming has been used to obtain optimal magnitude responses. In this work, this non-convex optimization problem is dealt with by using an iterative method based on the least-squares, which is illustrated on directivity synthesis by spherical loudspeaker arrays. Usually, instead of synthesize directly the desired pattern, the strategy adopted is to reproduce its truncated spherical harmonic representation. The truncation order is determined by the number of drivers of the spherica array. It is shown that truncation error and signal powers can be significantly reduced if phase error is neglected, providing potential means to improve directivity synthesis for applications requiring only magnitude response.

THURSDAY AFTERNOON, 3 JULY 2008

ROOM 202/203, 2:20 TO 6:20 P.M.

\title{
Session 4pED
}

\section{Education in Acoustics: Acoustics Education Software}

\author{
Ralph T. Muehleisen, Cochair \\ Illinois Institute of Technology, Civil, Architectural and Environmental Engineering, 3201 S. Dearborn St., Room 228, Chicago, \\ IL 60616, USA
}

Catherine Potel, Cochair

Laboratoire d'Acoustique de l'Université du Maine, Avenue Olivier Messiaen, Le Mans, 72085, France

\section{Invited Papers}

$$
\text { 2:20 }
$$

4pED1. Education software for numerical acoustics. Olivier Dazel (Laboratoire d'Acoustique de l'Université du Maine, Avenue Olivier Messiaen, 72085 Le Mans, France, olivier.dazel@univ-lemans.fr)

Numerical computations tools and scientific computing programs are more and more used by acousticians. This induces that higher education institutions have to include numerical techniques in their programs. Several general aspects should be considered while designing such courses; they are the initial level of the students, their ability with mathematics and/or physics and the aim of the formation. Particular aspects on the choice of a type of software and the methodology need also to be studied. This talk will detail these aspects around the numerical courses at Université du Maine in Le Mans (France) whose formations ranges are from sound and vibration technicians to $\mathrm{PhD}$ students. Some typical examples and numerical tools will be presented to illustrate the purpose.

\section{2:40}

4pED2. Computer tools for architectural acoustics education. Jian Kang (School of Architecture, University of Sheffield, Western Bank, S10 2TN Sheffield, UK, j.kang@sheffield.ac.uk)

Calculating/simulating acoustic performance of architectural spaces and building elements plays an important role in architectura education. Unfortunately, architectural students often hesitate to use theoretical formulae/models commonly applied by acousticians. This study aims to develop simple calculation/simulation tools to help architectural students to understand basic acoustic principles. 
Focusing on the effectiveness of various key parameters, as well as on scientific visualisation way of presenting teaching materials, five tools have been developed: (1) sound distribution behind an environmental noise barrier, with parameters including barrier height, source-barrier distance, and source height; (2) sound distribution in a rectangular street canyon, with parameters including street length, width, building height, boundary absorption coefficient, air absorption, and the height of receiver plane; (3) reverberation time calculation in a rectangular space, with parameters including room dimensions and boundary absorption coefficients, where a database of absorption coefficients is also included; (4) absorption of perforated panel absorbers, especially micro-perforated panel absorbers, with parameters including hole size, hole spacing, panel thickness, and depth of airspace; (5) digital audio animation for urban soundscape design, considering idealised cross-streets and squares in a 2D environment, where the sound file with multiple sources can be played back, with reverberation effects. [Work supported by the Theodore John Schultz Grant]

\section{3:00}

4pED3. Live computer-based demonstrations in musical acoustics education. Donald M. Campbell (Edinburgh University, 4201 JCMB, Kings Buildings, Mayfield Road, EH9 3JZ Edinburgh, UK, d.m.campbell@ed.ac.uk), David Skulina (Edinburgh University, 4201 JCMB, Kings Buildings, Mayfield Road, EH9 3JZ Edinburgh, UK, dskulina@ph.ed.ac.uk)

Live demonstrations play a vital pedagogical role in any science education programme. For a subject as interdisciplinary as musical acoustics, embracing as it does mathematics, physics, engineering, psychology, cognitive science, neurophysiology, musicology and music technology, demonstrations of psychoacoustical phenomena and performances on musical instruments provide invaluable methods for involving and intriguing a general audience. The recent dramatic increase in the memory and speed of laptop computers has opened new possibilities for live computer-based demonstrations. This talk explores some of these possibilities, including demonstrations of the analysis and display of musical instrument sounds using real-time spectrographic programs and the explanation of important musical instrument characteristics using physical modelling synthesis.

\section{$3: 20$}

4pED4. A computer model to study the properties of guided waves. Michael J. Lowe (Imperial College London, Department of Mechanical Engineering, SW7 2AZ London, UK, m.lowe@imperial.ac.uk)

The author's research group has a specialist interest in developing guided wave techniques for the Non Destructive Testing (NDT) of structures. Guided waves travel along the structure and so can be used to inspect large lengths or areas very much faster then the traditional point-by-point ultrasonic methods. The development of these inspection methods requires careful study and understanding of the properties of the guided waves. To address this need, the author's team has created a general purpose modelling tool. This can model waves in waveguides consiting of an arbitrary number of layers which can be flat or cylindrical, elastic or damped, isotropic or anisotropic solids, or perfect or viscous fluids, and can be immersed in a fluid or embedded in a solid. The primary output of the program is the dispersion curves, that is the frequency-velocity relationships of any modes which could travel in the structure. The program also predicts the attenuation of the modes, caused by leakage into surrounding materials or by material damping, and the mode shapes The presentation will explain the basis of the modelling tool and illustrate the way in which it can be used to understand the guided waves.

3:40-5:20 Posters

Lecture sessions will recess for presentation of poster papers on various topics in acoustics. See poster sessions for topics and abstracts.

\section{Invited Paper}

4pED5. DELTAEC is also an acoustics teaching tool. Steven Garrett (Penn State, Applied Research Laboratory, PO Box 30, State College, PA 16804, USA, sxg185@psu.edu)

A major revision of the Los Alamos thermoacoustics code, renamed DELTAEC, was released in 2007. It takes advantage of a user-friendly, menu-driven Windows environment and has indigenous plotting capabilities. DELTAEC is a differential equation solver that analyzes one-dimensional acoustical networks defined by a series of "segments" representing ducts, compliances, speakers, etc. This talk will relate experiences using this software to teach a first-year graduate core course on acoustics in fluids. Examples include illustration of effective length and quality factor of Helmholtz resonators, as well as the more challenging standing wave solutions within a resonator of variable cross-section. The plotting feature allows immediate illustration of the pressure and velocity fields as well as power flow within the resonator. DELTAEC will also adjust gas mixture concentration to match a specified frequency. Segments representing electrodynamic loudspeakers, radiation loading, and flow resistance in porous media will be used to demonstrate the coupled-oscillatory behavior of a bass-reflex enclosure's complex electrical impedance $v s$. frequency. Calculation of the modes of a catenoidal horn of finite length will be presented. The "thermo-physical property" feature provides fluid and solid properties at the students' choice of pressure, temperature and frequency, making it useful as a "handbook" for other assignments. 


\title{
Contributed Papers
}

$5: 40$

4pED6. CNAQ: a MATLAB acquisition tool for computing transfer functions over audio cards. Guillaume Pellerin (CNAM Paris, 5, rue du Vert Bois, 75003 Paris, France, guillaume.pellerin@cnam.fr)

Since transfer function measurements require very precise buffer management, it is not easy to find any free and/or educational software which processes them with consumer audio cards, cheaper than professional ones but useful. Some people often use musical sequencers to generate and acquire signals but data can't be easily analyzed in other specific engineering codes because the lengths of the samples can't be easily fixed for example. Therefore, we decided to develop a specific software for general audio acoustic measurements with these main constraints: choose a programming language compatible with existing educational and professional tools, promote multi-platform interpreted languages, be able to use consumer audio cards, publish the source code under the terms of a free software license. CNAQ provides a graphical interface under MATLAB to process sweep sine measurements for any kind of acoustical or electronic application in the audio range. The current features of the software and some development ideas, such as implementing convolution based Farina's method, will be also presented.

\section{6:00}

4pED7. Noise Metric - Environmental Noise Analysis Software. Milan Stojiljković (Faculty of Technical Sciences, Department of Environmental Engineering, Trg Dositeja Obradovića 6, 21000 Novi Sad, Yugoslavia
noisemetric@gmail.com),Vlado Delic(Faculty of Technical Sciences, Department of Tellecomunications, Laboratory for Acoustics and Speech Technologies, Trg Dositeja Obradovića 6, 21000 Novi Sad, Yugoslavia, vdelic @uns.ns.ac.yu)

Noise Metric software suite addresses the need for a modern low-cost acquisition, post-processing and analysis system that accommodates professional grade instruments used in typical environmental noise assessments. Software is designed to comply with national and international legislation and to provide a comprehensive overview of environmental acoustics theory in an interactive help file. Educational use is emphasized by providing measurement wizards and example case histories, with included demonstration measurement files for student self study. Acknowledging tight student financial budgets, the system is not limited to use with professional grade equipment. It can be configured for less expensive and less precise operation by providing integration with standard PC sound boards and low-cost microphones. Current beta version focuses on community noise and traffic noise assessment, with future modules planned for occupational noise and basic acoustic calculations. Off line analysis module is supplemented by a real time module for recording and logging the measurements and a browser for viewing the analysis results, noise rating level calculation and report generation. Every attempt is being made to automate as many analysis tasks as possible and thus minimize the human induced errors in the assessment process. Preliminary product testing was successfully completed by comparison with proprietary software products.

THURSDAY AFTERNOON, 3 JULY 2008

AMPHI MAILLOT, 1:40 TO 7:00 P.M.

\section{Session 4pMUa}

\section{Musical Acoustics: Control of Natural and Synthetic Musical Sounds I}

\author{
Marcelo M. Wanderley, Cochair \\ Centre for Interdisciplinary Research in Music Media \& Technology (CIRMMT) - Schulich School of Music - McGill Univ., 555 \\ Sherbrooke Street West, Montreal, QC H3A1E3, Canada \\ Jean Kergomard, Cochair \\ Laboratoire de Mécanique et d'Acoustique CNRS UPR-7051, 31, Chemin Joseph Aiguier, Marseille Cedex 20, 13402, France
}

\section{Invited Papers}

\section{$1: 40$}

4pMUa1. Systems control theory for sound synthesis: an overview. Brigitte D’Andréa-Novel (Ecole des Mines de Paris, 60 Bd. Saint-Michel, 75006 Paris, France, brigitte.dandrea-novel@ensmp.fr), Denis Matignon (Univ. Toulouse; ISAE. Dept. MIA, 10 av. Edouard Belin, 31055 Toulouse, France, denis.matignon@isae.fr)

The aim of this talk is to present some classical techniques and methods from control theory to deal with the problem of sound synthesis based on physical models. A first part will be devoted to the case of finite-dimensional state space, illustrated by: 1 . KellyLochbaum structure for 1D wave equations; 2 . Realization theory for passive impedance of rational type. The second part considers the infinite-dimensional case. More precisely the authors will present: 1. The operator associated to PDEs in acoustics, its diagonalization leading to "Riemann invariants" useful to study stability properties; 2. A Lyapunov method to elaborate boundary control to ensure stabilization of an equilibrium regime and some kind of robustness; 3. Diffusive realizations of fractional time derivatives, useful when modeling visco-thermal losses in acoustical ducts. These tools are well adapted to the class of "wind instruments". 
4pMUa2. Extracting Reed Control Parameters Using Acoustic Measurements and Inverse Filtering. Tamara R. Smyth (Simon Fraser University, 2728 West 5th Ave, Vancouver, BC V6K 1T4, Canada, tamaras@ cs.sfu.ca), Jonathan Abel (Stanford University, 752 College Ave, Menlo Park, CA 94025, USA, jonathan.abel@ comcast.net)

The control of virtual musical instruments often relies on either a specially-developed controller on which the performer has usually not gained sufficient virtuosity to play musically, or an existing multipurpose general controller with control parameters not always easily, or intuitively, mapped to the synthesis parameters of the virtual instrument being performed. A response to this problem is to obtain control information from a musical performance where the performer uses an instrument with which s/he is sufficiently familiar. In this work, we incorporate a previously developed measurement technique to transform a measured clarinet signal into a sequence of pulses corresponding to the reed displacement as a function of time. The measurement technique, shown to obtain accurate reflection functions from various tube structures, is used to obtain a filter modeling the bore and bell of the wind instrument used in the performance. The "reed pulse" waveform is then isolated by inverse filtering the measured clarinet signal. The characteristics of this residual waveform, which evolve with the performer's control of the instrument, may be extracted and remapped to the synthesis parameters of a physical model.

\section{Contributed Papers}

\section{2:20}

4pMUa3. Modeling and automatic control of a slide flute. Brigitte D'Andréa-Novel (Ecole des Mines de Paris, 60 Bd. Saint-Michel, 75006 Paris, France, brigitte.dandrea-novel@ensmp.fr), Benoit Fabre (Institut Jean Le Rond d'Alembert / LAM (UPMC / CNRS / Ministère Culture), 11, rue de Lourmel, 75015 Paris, France, fabreb@ccr.jussieu.fr), Jean-Michel Coron (Lab. Jacques-Louis Lions, UPMC and Institut Universitaire de France, 4 place Jussieu, 75005 Paris, France, Jean-Michel.coron@math.upsud.fr)

In this paper, we consider the problem of modeling and control of a slide flute: a kind of recorder without finger holes but which is ended by a piston mechanism to modify the length of the resonator. A previous study has been done, but with a very simple boundary condition for the mouth, corresponding to an ideal situation assuming that the acoustic pressure is zero at the entrance of the resonator. In this work, we have taken into account a more realistic model, describing the coupling effects between the jet and the pipe. The jet is obtained by blowing through a flue channel and formed by flow separation at the flue exit, and finally directed towards a sharp edge, called the labium. The resulting structure will be described by two linear PDEs coupled with nonlinear ODEs describing the boundary conditions: for the mouth, taking into account the jet dynamics, and for the piston. A modal analysis is performed using the linearized boundary conditions which can also be used to compute the suitable blowing pressure and the suitable pipe length to obtain a desired fundamental frequency or equivalently a desired pitch. This will constitute the basis of our control algorithm.

\section{2:40}

4pMUa4. Physical parameters of the violin bridge changed by active control. Henri Boutin (Institut Jean le Rond d'Alembert, Lab. d'Acoustique Musicale, 11, rue de Lourmel, 75015 Paris, France, boutin@lam.jussieu.fr), Charles Besnainou (Institut Jean le Rond d'Alembert, Laboratoire d'Acoustique Musicale, 11, rue de Lourmel, 75015 Paris, France, chbesnai@ccr.jussieu.fr)

The physical parameters of a violin bridge have a significant influence on the tonal colouration of its sound. The resonance peaks of the bridge shape the response of the violin body. Reinicke and Cremer developed a simple bridge model that shows a typical broad frequency peak around $2.5 \mathrm{kHz}$, because it incorporates the coupling to the violin body and the soundpost. By using the same model, Jim Woodhouse revealed the effect of some parameters of the bridge (mass, stiffness and foot spacing) on the instrument frequency response. Here the parameters of the violin resonance peaks are changed in real time, by applying an active control method. Such a technique, very useful in noise reduction, enabled to change separately the position and the shape of each peak of the bridge input admittance. On the bridge, 2 actuators and an accelerometer are placed at strategic positions in order to change the peak frequency and the damping factor values. The system behaviour is controlled by a Digital Signal Processor. Some sound results achieved with a real violin back up the theoretical equations.

\section{Invited Paper}

3:00-3:20 Break

4pMUa5. Between the frog and the tip - bowing gestures and bow-string interaction in violin playing. Anders Askenfelt (Dept. of Speech, Music and Hearing, Royal Institute of Technology (KTH), Lindstedtsvägen 24, SE-100 44 Stockholm, Sweden, andersa @ speech.kth.se)

The motion of the bow gives a natural visualization of a string performance. Watching the player's bowing may augment the communicative power of the music, but all relevant bow control parameters are not easy to capture by a spectator. The string player controls volume of sound and tone quality continuously by coordination of three basic bowing parameters (bow velocity, bow-bridge distance, and bow force), which set the main conditions for the bow-string interaction. At a more detailed level of description, the tilting of the bow, which among other things controls the effective width of the bow hair, enters into the model. On a longer time scale, pre-planned coordination schemes ('bowing gestures'), including the basic bowing parameters and the angles between the path of the bow and the strings, builds the performance. Systems for recording bowing parameters will be reviewed and results from old and current studies on bowing gestures presented. The player's choice and coordination of bowing parameters are constrained both in attacks and 'steady-state' according to bow-string interaction models. Recent verifications of these control spaces will be examined. Strategies for starting notes and examples of how players do in practice will be presented and compared with listeners' preferences. 
3:40-5:00 Posters

Lecture sessions will recess for presentation of poster papers on various topics in acoustics. See poster sessions for topics and abstracts.

\title{
Invited Papers
}

\author{
5:00
}

4pMUa6. The guitar as an extension of the voice - Phonetic gestures underlying guitar timbre perception and description. Caroline Traube (Laboratoire informatique, acoustique et musique, Faculté de musique, Université de Montréal, C.P. 6128, succursale Centre-Ville, Montréal, QC H3C 3J7, Canada, caroline.traube@umontreal.ca), Maryse Lavoie (Laboratoire informatique, acoustique et musique, Faculté de musique, Université de Montréal, C.P. 6128, succursale Centre-Ville, Montréal, QC H3C 3J7, Canada, maryse .lavoie@umontreal.ca)

The guitar is an instrument that gives the player great control over timbre. Different plucking techniques involve varying the finger position along the string and the inclination between the finger and the string. Guitarists perceive subtle variations of these parameters and have developed a rich vocabulary to describe the tones they produce on their instrument. Vocal imitations - onomatopoeias - is another way to intuitively describe instrumental tones. The data that we collected showed that guitar tones can be consistently associated with different types of vowels depending on instrumental gesture parameters, suggesting that guitar tones can evoke "phonetic gestures" as defined in the motor theory of speech perception. In addition, these phonetic gestures seem to be at the origin of a large subset of commonly used verbal descriptors: open, oval, round, thin, closed, nasal, hollow, etc. These analogies can be explained by the combfilter shaped spectral envelope of plucked-string instruments which emphasizes energy in the region of vocal formants. We conclude that, when technical difficulties have been surmounted, performers can use their musical instrument as an extension of their voice, the musical instruments allowing a virtuosic control of sound parameters similar to those involved in the paralinguistic elements of speech.

4pMUa7. Production and perception of goal-points and coarticulations in music. Rolf Inge Godøy (University of Oslo, Department of Musicology, P.B. 1017 Blindern, N-0315 Oslo, Norway, r.i.godoy@imv.uio.no), Alexander Refsum Jensenius (University of Oslo, Department of Musicology, P.B. 1017 Blindern, N-0315 Oslo, Norway, a.r.jensenius@imv.uio.no), Kristian Nymoen (University of Oslo, Department of Musicology, P.B. 1017 Blindern, N-0315 Oslo, Norway, krisny@hf.uio.no)

From our studies of sound-related movement (http://musicalgestures.uio.no), we have reason to believe that both sound-producing and sound-accompanying movements are centered around what we call goal-points, meaning certain salient events in the music such as downbeats, or various accent types, or melodic peaks. In music performance, these goal-points are reflected in the positions and shapes of the performers' effectors (fingers, hands, arms, torso, etc.) at certain moments in time, similar to what is known as keyframes in animation. The movement trajectories between these goal-points, similar to what is known as interframes in animation, may often demonstrate the phenomenon of coarticulation, i.e. that the various smaller movement are subsumed under more superordinate and goaldirected movement trajectories. In this paper, we shall present a summary of recent human movement research in support of this scheme of goal-points and coarticulations, as well as demonstrate this scheme with data from our ongoing motion capture studies of pianists' performance and other researchers' motion capture data.

4pMUa8. Perception, verbal description and gestural control of piano timbre. Caroline Traube (Laboratoire informatique, acoustique et musique, Faculté de musique, Université de Montréal, C.P. 6128, succursale Centre-Ville, Montréal, QC H3C 3J7, Canada, caroline.traube@umontreal.ca), Michel Bernays (Laboratoire informatique, acoustique et musique, Faculté de musique, Université de Montréal, C.P. 6128, succursale Centre-Ville, Montréal, QC H3C 3J7, Canada, michel.bernays@gmail.com), Madeleine Bellemare (Laboratoire informatique, acoustique et musique, Faculté de musique, Université de Montréal, C.P. 6128, succursale Centre-Ville, Montréal, QC H3C 3J7, Canada, madeleine.bellemare@umontreal.ca)

Musical expressivity in virtuosic pianistic performance relies heavily on timbre and performers call upon a vast vocabulary to describe the nature of their sound; examples of adjectives include velvety, metallic, bright, round and dark. The present study aims to determine whether this vocabulary, its perceptual meaning and the gesture applied to obtain the sounds it describes, stand as consensual among pianists. The relations between timbre, articulation, register and dynamics are also examined. Nearly 100 verbal descriptors were collected as well as the description of the associated gestures. Some timbres are specific to certain dynamic levels and others are the result of a combination of at least two sonic elements into one resulting sound object, where articulation, - the relation between one note to the next - plays a crucial role. A subset of these adjectives has been selected for further study. A professional pianist performed, on a computer-controlled recording acoustic piano, three short project-designed pieces with several timbres, as designated by adjectives. The excerpts were also captured with microphones to serve as stimuli for a timbre recognition task, both in free form and by selection, to which a group of 17 pianists performed with great accuracy. 


\section{Contributed Paper}

6:00

4pMUa9. Study of brass performer gestures. René E. Causse (IRCAM, 1 Place Igor Stravinsky, 75004 Paris, France, causse@ircam.fr), Vincent Freour (IRCAM -CNRS (UMR 9912 STMS), 1, Place Igor Stravinsky, 75004 Paris, France, Vincent.Freour@ircam.fr)

Brass instrument playing requires the musican to control his respiratory gesture and the elastic properties of his lips. This raises the question of musician gesture optimisation and strategy in order to complete a musical exercise. It also makes gesture characterization very hard to conduct in a non-invasive way. On the other hand, it is possible to measure some control parameters (linked to the respiratory and lip-adjustment gesture) like lip force applied on the mouthpiece and mouth air pressure. Theses parameters measurements and also the specific mouthpiece receiver developed to measure lip force with a minimum interference to the player are presented. Respiratory flows during live playing are also evaluated thanks to the calibration of respiratory belts used on the thoracial and abdominal regions of musicians. Details of the method for carrying out this type of measurement and preliminary results are reported. During this measurement sound recording and analysis are also conducted thanks to a set of audio descriptors. Links between control parameters measurements and sound characterisation are examined. Parallel development of a automated artificial mouth, used for experimental validations, is also outlined. [Work, within the CONSONNES project, is lead with the support of the French Research National Agency ANR].

\section{Invited Papers}

4pMUa10. Refining mapping strategies to improve the sound quality of physically controlled synthesis. Vincent Verfaille (Centre for Interdisciplinary Research in Music Media \& Technology (CIRMMT) - Schulich School of Music - McGill Univ., 555 Sherbrooke Street West, Montreal, QC H3A1E3, Canada, vincent@music.mcgill.ca), Arnaud Rebillout (4 le Cabut, 33390 Cars, France, arnaud.rebillout@gmail.com), Philippe Guillemain (Laboratoire de Mécanique et d'Acoustique CNRS UPR-7051, 31, Chemin Joseph Aiguier, 13402 Marseille Cedex 20, France, guillemain@lma.cnrs-mrs.fr), Marcelo M. Wanderley (Centre for Interdisciplinary Research in Music Media \& Technology (CIRMMT) - Schulich School of Music - McGill Univ., 555 Sherbrooke Street West, Montreal, QC H3A1E3, Canada, marcelo.wanderley@mcgill.ca)

A new technique, called 'physically controlled synthesis', is being developed to improve the controllability and sound quality of digital sound synthesis. It can be seen as a mapping strategy that combines a synthesis model based on a physical model (controllability) with a signal model based on additive synthesis (sound quality), and a database of pre-analyzed natural instrumental sounds. A key point is the computation of perceptually relevant timbre descriptors that interface the two synthesizers to provide additive synthesis data by navigating the database and selecting neighbour additive frames to morph. To limit the latency introduced, we developed specific algorithms to extract sound features from the physical model; the database size was increased offline by using a higher control sampling rate. In addition to previous works by the authors, devoted to the permanent regime and using a 2D indexation of the additive database, transients handling is achieved through a 3D search in a frame-by-frame basis that ignores the natural time unfolding, hence allowing to choose the best frame with the proper pitch in addition to the two other sound descriptors.

\section{6:40}

4pMUa11. Inverse problem: Recovering the full-state of a simplified model of a trumpet-like instrument from the radiated pressure. Thomas Hélie (IRCAM, 1, place Igor Stravinsky, 75004 Paris, France, thomas.helie@ircam.fr), Brigitte D’Andréa-Novel (Ecole des Mines de Paris, 60 Bd. Saint-Michel, 75006 Paris, France, brigitte.dandrea-novel@ensmp.fr)

We consider a simplified model of a trumpet-like instrument composed of a valve (including the mechanics of the lips), a jet (coupled with the valve dynamics), and an acoustic pipe excited by the jet and radiating in the air. A special care is devoted to the energy balance of the whole system and its dissipative property. This leads us to introduce a model of a non-stationary jet. In a second step, the problem of the observation of the full-state (that is, the position and the velocity of the lips, the flow and the pressure in the jet and in the acoustic pipe) from the radiated pressure is analyzed. This problem can be recasted as a problem of control engineering, using a so-called neutral system (differential system including the delayed state and its time derivative). We show how the energy balance can help to solve this problem and define a naturally well-posed observator. As a last step, this work is recasted in the context of a more general inverse problem: What control input (pressure in the mouth, parameter of the lips, etc) must be used to feed the model in order to recover a target sound? 


\title{
Session 4pMUb
}

\section{Musical Acoustics: Bowed and Keyboard Stringed Instruments II (Poster Session)}

\author{
Seiji Adachi, Cochair \\ Fraunhofer Institute for Building Physics \\ Simon Félix, Cochair \\ Laboratoire d'Acoustique de l'Université du Maine
}

\begin{abstract}
All posters will be on display from 3:40 p.m. to 5:20 p.m. To allow contributors an opportunity to see other posters, contributors of odd-numbered papers will be at their posters from 3:40 p.m. to 4:30 p.m. and contributors of even-numbered papers will be at their posters from 4:30 p.m. to 5:20 p.m.
\end{abstract}

\section{Contributed Papers}

4pMUb1. Experimental modal analysis of bows. Enrico Ravina (University of Genoa - Centre of Research on Choral and Instrumental Music (MUSICOS), Via Opera Pia 15 A, 16145 Genova, Italy, enrico.ravina@unige.it), Paolo Silvestri (Univ. of Genoa - DIMEC, Via Opera Pia 15 A, 16145 Genova, Italy, p.silvestri@unige.it), Antonino Airenti (Bow Maker, Via Marussig 13, 16100 Genova, Italy, inf @baroquebows.net)

The vibration performances of bow instruments are usually studied developing numerical and experimental modal analyses of the body of the instrument or of their parts (tailpiece, bridge, fingerboard, neck). The dynamic contribution of the bow is less considered, but the mutual actions generated between bow and strings are conditioned by the mechanical features of the bow. The paper analyzes the dynamic behaviour of different kind of bows (clip-in frog and screw-driven frog) and different geometries (baroque, modern,...) through experimetal modal analyses. Bows are instrumented with micro-accelerometers and excited by a micro-hammer. Frequency Response Functions up to $2500 \mathrm{~Hz}$ allow a good characterization of the bow and show significant differences about the modal shapes. The study is integrated with the experimental strain analysis, based on micro strain gauges glued on the body; the very small dimensions of the transducers $(2.5 \mathrm{~mm})$ allow, from one side, a not intrusive analysis but, from another side, require specific contrivances of mounting. Details on the integrated experimentations are focussed and discussed.

4pMUb2. Spherical mapping of violins. Enrico Ravina (University of Genoa - Centre of Research on Choral and Instrumental Music (MUSICOS), Via Opera Pia 15 A, 16145 Genova, Italy, enrico.ravina@unige.it), Paolo Silvestri (Univ. of Genoa - DIMEC, Via Opera Pia 15 A, 16145 Genova, Italy, p.silvestri@unige.it), Pio Montanari (Master Violinmaker, Via delle Compere 2, 16100 Genova, Italy, montanaripio@libero.it), Guido De Vecchi (Musician, Via S. Maria di Castello 37, 16100 Genova, Italy, ensemble@ilfalcone.com)

An original experimental approach oriented to the evaluation of the acoustic performances of violins is described. Starting from 14th Century the violins family have passed through significant changing, strongly influencing their sound. The "instrumental music" requires different parts for different voices: the violin family changes its mechanical structure following this requirement. The structural elements are modified in order to adequate the sound to the aesthetic taste of the historical period. Mechanical modifications involve geometry, relative positions and structural characteristics of fixed and mobile parts. The consequence is a significant alteration of vibration and acoustic responses. The paper describes a systematic approach oriented to evaluate the acoustic performances of differently mounted violins (baroque, classical, modern) by means a spherical mapping of the generated sound. A workbench based on a semicircular structure carrying an array of
10 microphones interfaced to a portable acquisition unit, has been designed and realized. The violin, played by the musician in anechoic chamber or in representation room, is located at the centre of this semicircle: changing the relative angular position between the violin and the array acoustic spherical maps describing the actual sound emission are generated. A systematic comparison among differently mounted violins is shown and discussed.

4pMUb3. On the generation of axial modes in the nonlinear vibration of strings. Alexandre Watzky (Laboratoire de Mécanique Physique, Université Paris 12 - Fac. des Sciences \& Technologie, 61 av. du Gal. de Gaulle, 94010 Créteil, France, watzky@univ-paris12.fr)

Vibrating strings are known to be nonlinear. Transverse vibrations induce axial motion as well as a modulation of the string's tension. An overview of the nonlinear models of the vibrating string has been presented at the 150th Meeting of the ASA. It has been shown that if temporal considerations are prevalent from an acoustical point of view, the involved coupling mechanisms are mainly ruled by the spatial shape of the modes. The purpose of this communication is to investigate the specific case of transverse-axial interactions and to examine the possible generation of axial modes through transverse vibrations. It also gives the opportunity to discuss the common hypotheses. Despite the complex modal couplings that can occur, this results enable to avoid or favor axial modes in the design of the string itself or of the boundary conditions i.e. the bridge.

4pMUb4. The application of thin plate theory to the time evolution of musical instruments. Christopher Gorman (Rollins College, Department of Physics, Winter Park, FL 32789, USA, cgorman@rollins.edu), David Parker (Rollins College, Department of Physics, Winter Park, FL 32789, USA, dparker@rollins.edu), Connor Ballance (Rollins College, Department of Physics, Winter Park, FL 32789, USA, ballance@vanadium.rollins.edu), Donald Griffin (Rollins College, Department of Physics, Winter Park, FL 32789, USA, griffin@vanadium.rollins.edu), Thomas Moore (Rollins College, Department of Physics, Winter Park, FL 32789, USA, tmoore@ rollins.edu)

The steady state dynamics of many musical instruments can be modeled using thin-plate theory. It has been shown that the normal-mode frequencies of systems as diverse as the orchestral crotale and piano soundboard can be accurately calculated within this approximation, and it is therefore reasonable to ask if thin-plate theory can be applied to model the time evolution of these instruments. To answer this question we have modeled a struck flat plate using finite differences and compared the decay of the eigenmodes to experimental results. We find that the time evolution of the motion of a struck thin plate is not well described under the thin-plate approximation even when the modal frequencies are predicted accurately. We propose that 
mode coupling between longitudinal and transverse modes requires that a full three dimensional model be used to predict the time evolution even when the plate is thin.

4pMUb5. Considering the effect of hammer shank flexibility using a multibody dynamic simulation model of a piano action mechanism with string contact. Chandrika P. Vyasarayani (University of Waterloo, Department of Systems Design Engineering, Waterloo, ON N2L 3G1, Canada, cpvyasar@engmail.uwaterloo.ca), Stephen Birkett (University of Waterloo, Department of Systems Design Engineering, Waterloo, ON N2L 3G1, Canada, sbirkett@real.uwaterloo.ca), John McPhee (University of Waterloo, Department of Systems Design Engineering, Waterloo, ON N2L 3G1, Canada,mcphee@real.uwaterloo.ca)

A piano action mechanism converts a pianist's mechanical input into acceleration of the piano hammer, which impacts the string for tone generation. We present a multibody dynamic model of the mechanism, considering the differences when hammer shank flexibility is included as compared to a rigid shank. The model is developed using the graph theoretic approach and includes the hammer-string interaction. A Rayleigh beam model including complete second order deformation field is used for simulating hammer shank flexibility. The governing partial differential equation is discretized using Ritz approach considering Taylor monomials as assumed modes. A convergence study confirms that two bending modes and one axial mode are sufficient to represent the hammer shank deformation. The vibrating string is modeled using a standard modal analysis procedure. The many contacts between components of the mechanism include significant sliding during contact; for these contacts a modified Hunt-Crossley law is used to represent the normal force, and interface friction is handled using a Cull and Tucker friction model. The results of parametric studies show the effect of hammer head friction on the dynamics of the mechanism during string impact, as well as the influence of hammer shank flexibility on the frequency response of the string.

4pMUb6. A complex model for piano action. José Lozada (Laboratoire de Mécanique des Solides, Ecole Polytechnique, 91128 Palaiseau Cedex, France, jose.lozada@cea.fr), Xavier Boutillon (Laboratoire de Mécanique des Solides, Ecole Polytechnique, 91128 Palaiseau Cedex, France, boutillon@1ms.polytechnique.fr), Moustapha Hafez (Laboratoire des Interfaces Sensorielles, CEA-LIST, 18 route du Panorama, BP 6, 92265 Fontenay-aux-Roses Cedex, France, moustapha.hafez@cea.fr)

Available mechanical models of piano action are extremely simplified in view of the complexity of an actual mechanism. Several arguments will be presented which indicate that more dynamical complexities than those of- fered by the current models are considered as intrinsic properties of good piano actions. A more complete model of a grand piano action during the attack of a note will be presented which includes the six degrees of freedom of the action, all considered as rigid bodies: the key, the damper, the whippen, the repetition lever, the jack, and the hammer. In this model, coupling features are represented by lumped elements: Coulomb friction, linear and nonlinear springs. Methods for characterizing each element of the model will be presented. The final result will be given in terms of dynamical equations, coupling equations, and geometrical constrains.

4pMUb7. Feature exaggeration in scale performance on the piano. Shinya Morita (Graduate School of Science and technology, Ryukoku University, 1-5, Yokotani, Oe-cho, Seta, 520-2194 Otsu, Shiga, Japan, t040550@mail.ryukoku.ac.jp), Norio Emura (Faculty of Engineering, Doshisha University, 1-3, Tataramiyakodani, 610-0321 Kyo-tanabe, Kyoto, Japan, etf1702@mail4.doshisha.ac.jp), Masanobu Miura (Graduate School of Science and technology, Ryukoku University, 1-5, Yokotani, Oe-cho, Seta, 520-2194 Otsu, Shiga, Japan, miura@rins.ryukoku.ac.jp), Seiko Akinaga (Department of Education, Shukugawa Gakuin College, 6-58, Kosikiiwa-cho, 662-8555 Nishinomiya, Hyogo, Japan, akinaga@shukugawa-c.ac.jp), Masuzo Yanagida (Faculty of Engineering, Doshisha University, 1-3, Tataramiyakodani, 610-0321 Kyo-tanabe, Kyoto, Japan, myanagid@mail.doshisha.ac.jp)

This paper proposes a set of parameters for describing features of scaleplaying on the piano. The parameter set consists of 15 parameters, among which 12 are three sets of four parameters pi1 through pi4 where $\mathrm{i}=\{1,2$, $3\}$ distinguishes three basic features; onset time, velocity, and duration. Each of these basic features is modeled as the sum of a global curve and the deviation from it, where the spline interpolation is employed using locally averaged points, or representative points, as the points to be passed. The local average is calculated for each sequence of notes played without finger crossing. The suffix $\mathrm{j}$ in pij distinguishes the standard deviations $(j=0)$, the rms deviation from the spline curve $(j=1)$, the range of the curve $(j=2)$, the rms difference between successive notes $(j=3)$, and the rms of the spline curve from the metronomic line $(\mathrm{j}=4)$. All parameters are made controllable with slider bars from $0 \%$ to $200 \%$ for synthesizing suppressed performance or exaggerated performance, where $100 \%$ represents the original performance. Proposed parameter set is expected to be useful in self-training of piano, as it can indicate the features and undesirable habits of the player by setting values above $100 \%$ in exaggerated form. 


\title{
Session 4pMUc
}

\section{Musical Acoustics: Plucked Stringed Instruments II (Poster Session)}

\author{
François Gautier, Cochair \\ Laboratoire d'Acoustique de I'Université du Maine \\ Chris Waltham, Cochair \\ University of British Columbia
}

\begin{abstract}
All posters will be on display from 3:40 p.m. to 5:20 p.m. To allow contributors an opportunity to see other posters, contributors of odd-numbered papers will be at their posters from 3:40 p.m. to 4:30 p.m. and contributors of even-numbered papers will be at their posters from 4:30 p.m. to 5:20 p.m.
\end{abstract}

\section{Contributed Papers}

4pMUc1. The physics of the American five-string banjo. Laurie Stephey (Rollins College, Department of Physics, Winter Park, FL 32789, USA, 1stephey@rollins.edu), Thomas Moore (Rollins College, Department of Physics, Winter Park, FL 32789, USA, tmoore@rollins.edu)

We report on a systematic study of the American five-string banjo, which was undertaken in an effort to understand the dynamics of this complex instrument. The deflection shapes of the membranic head were studied and catalogued using time-averaged electronic speckle pattern interferometry. The impedance presented to the strings was measured using laser Doppler vibrometry coupled with an integrated force sensor and harmonic driver. Additionally, time resolved spectral analysis of the plucked strings was used to quantify the characteristic decay of the coupled string/membrane system, while time-resolved interferometric studies of the membrane have led to a better understanding of the motion after a string is plucked. All of these investigations help to reveal the importance of the various parameters that affect the sound of this unusual instrument.

4pMUc2. The interaction between the strings and soundboard of a harp. Chris Waltham (University of British Columbia, Department of Physics \& Astronomy, Vancouver, BC V6T 1Z1, Canada, cew@phas.ubc.ca)

The harp is an instrument with a set of plucked strings that excite the sound board directly, without the medium of a bridge. The strings are positioned at an acute angle to the plane of the sound board. The quality of the sound produced depends on the motion of the string, which is non-planar, and its interaction with the resonances of the sound board. The interaction is intrinsically non-linear as the soundboard responds to changes in both the angle and the tension of the string. To avoid the difficulties of string-string interactions on a real harp, a small test "instrument" has been constructed with a single string and a variable-angle sound board. The string and sound board motions have been measured simultaneously. Preliminary results will be presented.

4pMUc3. Categorization of guitars from bridge admittance measurements. François Gautier (Laboratoire d'Acoustique de l’Université du Maine, Avenue Olivier Messiaen, 72085 Le Mans, France, francois.gautier@univ-lemans.fr), Jean-Loic Le Carrou (Laboratoire d'Acoustique de l'Université du Maine, Avenue Olivier Messiaen, 72085 Le
Mans, France, jean-loic.le_carrou@univ-lemans.fr),Maarten Hol(LAUM CNRS, Université du Maine, Lab. d'Acoustique Université du Maine, UMR CNRS 6613, 72085 Le Mans Cedex 9, France, M.Hol@student .tue.nl), Vincent Doutaut (Institut Technologique Européen des Métiers de la Musique, 71 Av. O. Messiaen, 72000 Le Mans, France, Vincent.Doutaut @itemm.fr)

The acoustical characteristics of the guitar depend on the instrument makers' choices concerning the geometry, the material and the assembly techniques. The aim of this paper is to define criteria permitting the discrimination of guitars according to their acoustic characteristics. These criteria can be used, for example, by instrument makers to test the repeatability of their making process. Evaluation of the guitars' quality from this categorization is beyond the scope of this paper. A low-cost portable system allowing bridge admittance measurements has been designed and used on 4 groups of classical guitars, each being composed of about 10 similar instruments. A statistical study shows that the tested instruments can clearly be differentiated according to (1) the modal parameters associated to the first 2 vibroacoustic modes (air mode A0 and first soundboard mode T1), and to (2) a 'merit indicator', close to the one defined by C. Barlow (Proc.I.O.A., vol 19, Pt 5, 1997, 69-78). This 'merit indicator' is computed from the mean value of the bridge admittance and an estimation of the critical frequency of the soundboard.

4pMUc4. Modal analysis and transient string response of solid body electric bass guitars with effects of the instrumentalist. Dave Woolworth (Oxford Acoustics, Inc., 356 CR 102, Oxford, MS 38655, USA, dave@oxfordacoustics.com), Henry A. Scarton (Rensselaer Polytechnic Institute, Department of Mechanical Engineering, Troy, NY 12180, USA, scarton@rpi.edu)

This paper documents modal analysis of seven solid body electric basses. The basses are modeled in one dimension of motion normal to the face of the instrument in both free-free state and with boundary conditions imposed by a musician. The results are compared and analyzed in terms of bending and torsion. Analysis is done regarding open string harmonic content of the electromagnetic output over time versus the resonant frequencies of the instruments. Results of finite element analysis modeling of a simplified bass guitar structure will be presented, and significance of damping effects on harmonic output will be considered in terms of the listener. 


\title{
Session 4pMUd
}

\section{Musical Acoustics: Wind Instruments II (Poster Session)}

\author{
Seiji Adachi, Cochair \\ Fraunhofer Institute for Building Physics \\ Simon Félix, Cochair \\ Laboratoire d'Acoustique de l'Université du Maine
}

\begin{abstract}
All posters will be on display from 3:40 p.m. to 5:20 p.m. To allow contributors an opportunity to see other posters, contributors of odd-numbered papers will be at their posters from 3:40 p.m. to 4:30 p.m. and contributors of even-numbered papers will be at their posters from 4:30 p.m. to 5:20 p.m.
\end{abstract}

\section{Contributed Papers}

4pMUd1. Controllable pitch-bending effects in the accordion playing. R. Llanos-Vazquez (Dpto. Física Aplicada 1. Escuela Técnica Superior de Ingeniería, Alameda de Urquijo s/n, 48013 Bilbao, Spain, rucurdu@orange .es), M. J. Elejalde-García (Dpto. Física Aplicada 1. Escuela Técnica Superior de Ingeniería, Alameda de Urquijo s/n, 48013 Bilbao, Spain, mariajesus.elejalde@ehu.es), E. Macho-Stadler (Dpto. Física Aplicada 1. Escuela Técnica Superior de Ingeniería, Alameda de Urquijo s/n, 48013 Bilbao, Spain, erica.macho@ehu.es)

The accordion employs reeds in which the tongue is mounted outside the reed frame in such a way that sounding is normally possible only on one direction of airflow. Under normal operating conditions the reeds behave as blown-closed. Pitch bending technique allows players to make a controllable glide, non tempered glissando, from one pitch to another. Pitch-bending frequency shift, defined as the percentage of the ratio of the frequency change and the original frequency has been measured in a series of experiments. Some of the results involving the dependence of the function on pitch, direction of the bellows movement, cassoto possibilities and harmonic number are reported here. If the player qualifies, he/she can make controllable pitchbending effects where the glissando may fall an exact semitone.

4pMUd2. Study the mouthpiece of the txistu. A. Agos-Esparza (Dpto. Física Aplicada 1. Escuela Técnica Superior de Ingeniería, Alameda de Urquijo s/n, 48013 Bilbao, Spain, bckagesa@ikasle.ehu.es), M. J. Elejalde-García (Dpto. Física Aplicada 1. Escuela Técnica Superior de Ingeniería, Alameda de Urquijo s/n, 48013 Bilbao, Spain, mariajesus.elejalde@ehu.es), E. Macho-Stadler (Dpto. Física Aplicada 1. Escuela Técnica Superior de Ingeniería, Alameda de Urquijo s/n, 48013 Bilbao, Spain, erica.macho@ehu.es)

The txistu is a three finger holed recorder from the Basque Country. The evolution of the txistu involves different kinds of wood, bore length, finger hole positioning and it being made of two or three pieces. The txistu also has a unique mouthpiece, made up by a small metallic piece of the pipe and also a metal reed which was introduced centuries ago. The distance between the pipe and the reed can be adjusted to fit the user's preferences. By using a blowing machine and data acquisition software, experiments study the influence of the shape and inclination of the reed on the values of the harmonic content and the sonority of the final note.

4pMUd3. Automated fingering services for woodwinds: development of a "virtual clarinet". Yakov Kulik (University of New South Wales, Music Acoustics, School of Physics, NSW 2052 Sydney, Australia,
ykulik@phys.unsw.edu.au),Andrew Botros(University of New South Wales, Music Acoustics, School of Physics, NSW 2052 Sydney, Australia, ABotros@phys.unsw.edu.au), John Smith (University of New South Wales, Music Acoustics, School of Physics, NSW 2052 Sydney, Australia, john .smith@unsw.edu.au)

The Virtual Flute is a popular web service that recommends alternative fingerings for difficult passages, timbre variations, intonations or multiphonics. Its database was generated by a machine-learned expert system analysing waveguide models for all 39,744 fingerings. The relatively simple geometry of the flute and its tone holes allowed a simple yet accurate model. The development of similar systems for other woodwinds faces greater modelling and computational challenges. For example, the clarinet has a more complex geometry, with tone holes whose radius and length vary by factors of 4.2 and 2.8. Further, it has several million different fingerings. To achieve the required accuracy, individual measurements of each hole separately and of mouthpiece and bell, as well as several dozen fingering examples, were used to determine parameters of a still simple waveguide model. The model uses conical and cylindrical segments with parallel and shunt impedances at junctions, representing tone holes. This approach of incrementally enhancing our waveguide model allows computational advantages: an efficient, woodwind-generic software framework is built that can adapt to the instrument of interest. We report interim results with such a system, with further potential applications in the design of woodwind instruments and other acoustic duct systems.

4pMUd4. Source-resonator modeling: a rough paradox. Laurent P. Millot (IDEAT (University Paris 1), ENS Louis-Lumière, 7 allée du Promontoire, 93161 Noisy-le-grand, France, 1.millot@ens-louis-lumiere.fr)

Using the example of a short pipe loaded by a free reed located upwards, we will underline the fact that a reflection function based modeling will not give the same results (and synthesized sounds) as an unsteady incompressible and lossless Bernoulli description while this model is commonly assumed to be a low-frequency limit case of the first one, and that the second description is the only valid one. This paradox will be explained and it will be underlined why the so-called electro-acoustical analogies do not constitute a low-frequency limit case of reflection function or input impedance descriptions, based on a waves paradigm. Extension to striking reeds and/or longer pipes, as found in saxophone, clarinet or sheng for instance, will be discussed and a strange coupling between a priori incompatible models, commonly used in Musical Acoustics notably, will be described. 
4pMUd5. Sound quality of the 16 " french bagpipe: wood influence. Mathieu Paquier (LISyC EA 3883, 6 avenue Victor Le Gorgeu, CS 93837, 29238 Brest Cedex 3, France, mathieu.paquier@univ-brest.fr), Raphaël Jeannin (LISyC EA 3883, 6 avenue Victor Le Gorgeu, CS 93837, 29238 Brest Cedex 3, France, jeanninraphael@yahoo.fr)

The most played among the bagpipes from the centre of France is the 16-inch musette, called in this way because of the length of the melodic pipe (oboe). Though these instruments are less known than the biniou from Brittany or the Great Highland Bagpipe, the number of players and makers is, nowadays, in increase because of their easy play and quasi-chromatic scale.
Whereas the Breton and Scottish bagpipes are always made of very hard woods, some 16" musettes are fabricated with softer woods. This difference is certainly related to the flexibility of the 16 " oboe double-reed. At first, we recorded some short musical sequences played on 16" musettes made of 5 different woods (African Ebony, Santos Rosewood, Boxwood, African Blackwood and Service Tree), then some listeners (specialist and naïve) were asked to give their feedback about the quality of the recorded sounds. In a second set of experiments, we recorded some single notes played with these various bagpipes and analyzed the acoustic features of the recorded signals. At last, the perceptual results were compared with the physical parameters obtained in the second experiment

\title{
Session 4pMUe
}

\section{Musical Acoustics: Control of Natural and Synthetic Musical Sounds II (Poster Session)}

\author{
Marcelo Wanderley, Cochair \\ Centre for Interdisciplinary Research in Music Media \& Technology (CIRMMT) - Schulich School of Music - McGill Univ. \\ Jean Kergomard, Cochair \\ Laboratoire de Mécanique et d'Acoustique CNRS UPR-7051
}

\begin{abstract}
All posters will be on display from 3:40 p.m. to 5:20 p.m. To allow contributors an opportunity to see other posters, contributors of odd-numbered papers will be at their posters from 3:40 p.m. to 4:30 p.m. and contributors of even-numbered papers will be at their posters from 4:30 p.m. to 5:20 p.m.
\end{abstract}

\section{Contributed Papers}

4pMUe1. Exploration of timbre variations in music performance. Mathieu Barthet (CNRS-Laboratoire de Mécanique et d'Acoustique, 31 Chemin Joseph Aiguier, 13402 Marseille, France, barthet@1ma.cnrs-mrs .fr), Philippe Guillemain (Laboratoire de Mécanique et d'Acoustique CNRS UPR-7051, 31, Chemin Joseph Aiguier, 13402 Marseille Cedex 20 France, guillemain@1ma.cnrs-mrs.fr), Richard Kronland-Martinet (CNRSLMA, 31, chemin Joseph Aiguier, 13402 Marseille, France, kronland@1ma.cnrs-mrs.fr), Solvi Ystad (CNRS-LMA, 31, chemin Joseph Aiguier, 13402 Marseille, France, ystad@1ma.cnrs-mrs.fr)

Timbre has seen during the twentieth century a growing attention from both musicians and scientists, the former to expand a traditional music system until now governed by the structures of pitches, the latter to better understand how timbre is produced and how it is processed by the perceptive and cognitive systems. In the music performance context, many studies deal with the role of timing and dynamics, but much fewer are dedicated to the one of timbre. The works presented here aim at showing the importance of timbre variations from two points of view: the production of sounds and their perception. The relations between the control parameters (mouth pressure and reed aperture) of a simplified physics-based clarinet synthesis model and the generated timbres have been investigated. Experiments have further been done to measure the within-individual consistency of timbre variations of a clarinet player repeating several instances of a musical excerpt from a Bach piece while keeping the same musical intention. Brightness variations characterized by the time-varying Spectral Centroid showed a strong consistency across the repetitions. The influence of such brightness variations on the perceived musical quality of a performance was then assessed thanks to an analysis-transformation-synthesis paradigm.
4pMUe2. Active Sound Design of a Bassoon. Frederic Konkel(TU Berlin, Institute of Fluid Mechanics and Engineering Acoustics, Einsteinufer 25, Sekr. TA 7, D-10587 Berlin, Germany, f.konkel@advacoustics.de), Andre Jakob (TU Berlin, Institute of Fluid Mechanics and Engineering Acoustics, Einsteinufer 25, Sekr. TA 7, D-10587 Berlin, Germany, kontakt@advacoustics.de), Frank Heintze (Staatskapelle Berlin, Staatsoper Unter den Linden, Unter den Linden 7, D-10117 Berlin, Germany, f.heintze@advacoustics.de), Michael Möser (TU Berlin, Institute of Fluid Mechanics and Engineering Acoustics, Einsteinufer 25, Sekr. TA 7, D-10587 Berlin, Germany, moes0338@mailbox.tu-berlin.de)

Possibilities of influencing the sound characteristics of a woodwind by means of active noise control were investigated. The woodwind used in this investigation was a bassoon. The first step of the investigation consisted of a set of measurements of the sound spectra of different tones and volumes by means of measurement microphones inside and outside the instrument. Additionally measurements of the radiation characeristics of the instrument were performed by means of the acoustic camera. An experimental setup was designed with the instrument driven mechanically by compressed air. The aim of the work was to change the sound characteristics of the bassoon by changing individual harmonics of the tones by means of a loudspeaker attached to the bassoon. The loudspeaker was attached to the bassoon via a tube. Suitable positions for the connection of the tube to the instrument were examined as well as suitable possibilites for the generation of the reference signal neccessary for the feedforward control scheme. The experimental setup will be presented here as well as results of the measurements with and without active sound design with an analog controller. 
4pMUe3. The influence of control parameters and physical parameters on reed instruments playing. Jean Kergomard (Laboratoire de Mécanique et d'Acoustique CNRS UPR-7051, 31, Chemin Joseph Aiguier, 13402 Marseille Cedex 20, France, kergomard@1ma.cnrs-mrs.fr), Philippe Guillemain (Laboratoire de Mécanique et d'Acoustique CNRS UPR-7051, 31, Chemin Joseph Aiguier, 13402 Marseille Cedex 20, France, guillemain@1ma.cnrs-mrs.fr), Fabrice Silva (CNRS-Laboratoire de Mécanique et d'Acoustique, 31 Chemin Joseph Aiguier, 13402 Marseille, France, silva@lma.cnrs-mrs.fr), Christophe Vergez (CNRS-Laboratoire de Mécanique et d'Acoustique, 31 Chemin Joseph Aiguier, 13402 Marseille, France, vergez@1ma.cnrs-mrs.fr)

An attempt to summarize the influence of both control and physical parameters on production and radiation of sound of reed instruments is given. Some parameters, such as the shape of the resonator, are fixed by the instrument maker, or chosen by the instrumentalist itself: the the reed, and the fixation of the reed on the mouthpiece. These parameters, named physical parameters, are fixed when playing. The second kind of parameters can be totally or partially controlled when playing: the fingering is an obvious one, then the way the reed is pinched by the lip, with an effect on both the reed opening and the reed dynamics, finally the mouth pressure and the shape of the vocal tract. The influence of these parameters is discussed with respect to several attributes of the sound: the various thresholds (normal sound at pianissimo level, extinction at fortissimo level), the control of different regimes, the shape of transients, the playing frequency, the spectrum. The state of present knowledge is given, with emphasis of further research that should be done, and a comparison with the cases of sound controllers and artificial mouth is discussed.

4pMUe4. A setup for measurement of bowing parameters in bowed-string instrument performance. Erwin Schoonderwaldt (Dept. of Speech, Music and Hearing, Royal Institute of Technology (KTH), Lindstedtsvägen 24, SE-100 44 Stockholm, Sweden, schoondw@kth.se), Matthias Demoucron (IRCAM, 1 Place Igor Stravinsky, 75004 Paris, France, demoucron@ircam.fr), Nicolas Rasamimanana (IRCAM, 1 Place Igor Stravinsky, 75004 Paris, France, rasamimanana@ircam.fr)

An accurate measurement of instrumentalists' actions in playing situations is the basis for several research topics such as musical performance analysis, control of sound synthesis algorithms or effective validation of theoretical results in musical acoustics. We present a setup for a complete and accurate measurement of bowing parameters in bowed-string instrument performance, with minimum interference for the player. The method is based on the combined use of motion capture and sensors attached to the bow. An optical motion capture system was used to track the position and orientation (6 DOF) of the bow and the instrument. In addition, sensors were attached to the frog of the bow for measuring bow acceleration in different axes and bow force exerted on the strings. Both sensors can be easily attached to any bow. We will describe how the data from the different systems are combined for an accurate calculation of bowing parameters, such as bow-bridge distance, bow velocity, bow acceleration, bow angles and bow pressure. Finally, we will present examples of measurements and briefly discuss some potential applications.

THURSDAY AFTERNOON, 3 JULY 2008

P2-D, LEVEL 2, 3:40 TO 5:20 P.M.

\title{
Session 4pMUf
}

\section{Musical Acoustics: Virtual Musical Instruments II (Poster Session)}

\author{
Julius Smith, Cochair \\ Stanford Univ.
}

\author{
Antoine Chaigne, Cochair \\ ENSTA
}

\begin{abstract}
All posters will be on display from 3:40 p.m. to 5:20 p.m. To allow contributors an opportunity to see other posters, contributors of odd-numbered papers will be at their posters from 3:40 p.m. to 4:30 p.m. and contributors of even-numbered papers will be at their posters from 4:30 p.m. to 5:20 p.m.
\end{abstract}

\section{Contributed Papers}

4pMUf1. Simulation of Acoustic Pressure and Flow Velocity in Human Glottis. Petr Sidlof (Academy of Sciences of the Czech Republic, Narodni 3, $11720 \quad$ Prague, Czech Republic, sidlof@it.cas.cz), Antoine Chaigne (ENSTA, Chemin de la Hunière, 91761 Palaiseau, France, antoine.chaigne@ensta.fr), Olivier Doare (ENSTA, Chemin de la Hunière, 91761 Palaiseau, France, olivier.doare@ensta.fr), Olivier Cadot (ENSTA Chemin de la Hunière, 91761 Palaiseau, France, olivier.cadot@ensta.fr)

The pressure and velocity fields in coronal plane along the vibrating vocal folds were studied using a finite element mathematical model. The shapes of the vocal folds were specified according to data measured on excised human larynges in phonation position. The mathematical model of the flow is based on 2D incompressible Navier-Stokes equations adapted to deal with the time-variable shape of the domain, caused by vocal fold vibration. The numerical simulations allow to observe closely various flow features related to phonation - flow separation in the glottis, Coanda effect or vortex shedding. The numerical results were verified experimentally by Particle Image velocimetry (PIV) on a physical vocal fold model. In addition to acoustic, subglottal pressure and impact intensity measurements, flow velocity fields were recorded in the domain immediately above glottis. Analysis of the PIV images gives good insight into the dynamics of the supraglottal flow. Among other applications, the results should be used in the future for singing voice simulations based on physical models. 
4pMUf2. A synthesis and analysis framework for wind instrument sounds based on the digital pulse forming principle. Michael Oehler (Institute for applied Musicology and Psychology, Saarstrasse 1A, 50677 Koeln, Germany, michael.oehler@iamp.info), Christoph Reuter (University of Cologne - Musicological Institute, Beethovenstrasse 4, 50674 Koeln, Germany, info@chr-reuter.de)

A digital real-time-capable analysis- and synthesis-system for wind instrument sounds, based on the pulse forming theory, has been developed. The rediscovered model for the sound generating process of wind instruments rests upon the idea, that wind instrument sounds can basically be put down to its excitation impulses, which independently of the fundamental always behave according to the same principles. First realised in the analogue wind instrument synthesizer Realton Variophon (1975), the sound synthesis method has currently been transferred onto a digital platform [supported by the German Research Foundation]. Instrument specific algorithms control the pulse width and shape according to the applied pitch and dynamic values. That way subtle sound nuances that can be produced on acoustic wind instruments as well as real timbre modulation may be synthesized by just modifying a single parameter (i.e. breath pressure). In order to validate the performance of the developed framework, several perception experiments were conducted subsequently.

4pMUf3. Perceptual effects of radiation control with a multi-louspeaker device. Nicolas Misdariis (IRCAM - UMR CNRS 9912, Equipe Perception et Design Sonores, 1, place Igor Stravinsky, 75004 Paris, France, misdarii@ircam.fr), Alexandre Lang (Université de Technologie de Compiègne, E.A. Costech - Groupe de Suppléance Perceptive, BP 60319, 60206 Compiègne Cedex, France, lang.alexandre@gmail.com), Brian F. Katz (LIMSI-CNRS, B.P. 133, 91403 Orsay, France, brian.katz@limsi.fr), Patrick Susini (IRCAM - UMR CNRS 9912, Equipe Perception et Design Sonores, 1, place Igor Stravinsky, 75004 Paris, France, susini@ircam.fr)

This study investigates the perceptual issue of acoustical radiation control with the following hypothesis: radiation control can reduce the perceptual gap between a sound coming from an acoustical source (e.g., an instrument) and a sound coming from an electro-acoustical device (e.g., the recording of an instrument played by a loudspeaker). The work is technically supported by a generic multi-loudspeaker device that allows sound reproduction with controlled directivity patterns in a given number of spatial dimensions. The conducted experiment involves two distinct sound corpus: speaking/singing voice (recorded and spatially measured) and a struck plate (synthesized and spatially computed with modeled directivity). Incremental levels of radiation pattern control, in terms of precision of the reproduction, are also considered by combining several diffusion systems and different directivity patterns. Participants are asked to evaluate the stimuli on three semantic differential scales: source width (largeur), distance (distance) and realism (réalisme). The results show a global significant effect of radiation on sound perception: the acoustical/electro-acoustical gap is significantly reduced through radiation control, especially when considering the scales of width and distance. Theoretical principles, technical elements, experimental set-up and overall results will be presented, detailed and discussed during the presentation

4pMUf4. Measuring and Modeling Violin Sound Radiation for Sound Equalization. Alfonso Perez Carrillo (Pompeu Fabra University, Ocata $1,3^{\circ}, 08003 \quad$ Barcelona, Spain, aperez@iua.upf.edu), Jordi Bonada (Pompeu Fabra University, Ocata 1,3, 08003 Barcelona, Spain, jbonada@iua.upf.edu), Esteban Maestre (Pompeu Fabra University, Ocata

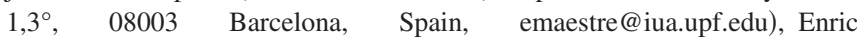
Guaus (Pompeu Fabra University, Ocata 1,3ㅇ, 08003 Barcelona, Spain, eguaus@iua.upf.edu), Merlijn Blaauw (Pompeu Fabra University, Ocata 1,3, 08003 Barcelona, Spain, mblaauw@iua.upf.edu)

During a performance a violin is put into vibration and this vibration is radiated to the air. Depending on the distance and direction of the listener, he perceives a slightly different sound. Sound radiation at each point in the space is determined by the impulse response or transfer function between vibration of the violin and sound pressure at that point. There is variety of methodologies to obtain acoustic impulse responses of violins. They differ mainly in the way of exciting the violin, the point of excitation, the position where the acoustic response is measured and how excitation and response signals are deconvolved. In this work we measure violin vibration with a bridge pickup and we 1) propose a method to calculate the transfer function between signals from the bridge pickup and a microphone, 2) use a 3D motion tracker to get the position of the microphone respect to the violin so that we can calculate the response for different directions and distances and 3) we model sound radiation as a "reference" impulse response and a parametric filter whose response depends on distance and orientation with respect to the "reference" position.

4pMUf5. Virtual electric guitars and associated audio effects in Faust and $\mathbf{C}++$. Julius O. Smith (Stanford Univ., Center for Computer Research in Music and Acoustics (CCRMA), Dept. of Music, Stanford, CA 94305-8180, USA, jos@ccrma.stanford.edu)

Advances in computing technology, both in hardware and software, are enabling new levels of performance in real-time for virtual musical instruments based on acoustic principles. This paper is concerned with software implementation technology for such instruments, written in high-level languages that compile down to efficient low-level implementations on a wide variety of platforms. Specific results will be presented for the case of virtual electric guitars and associated digital audio effects expressed in the Faust and $\mathrm{C}++$ languages, and compiled to become plugins for VST, Pure Data, and other real-time performance environments. This work builds upon a previous paper at the Linux Audio Conference (LAC-2008), and laboratory module written for the RealSimple project at CCRMA (http://ccrma.stanford .edu/realsimple/).

4pMUf6. Fine tuning of guitar sounds with changed top plate, back plate and rim geometry using a whole body 3D Finite-Difference model. Rolf Bader (University of Hamburg, Institute of Musicology, Neue Rabenstr. 13, 20354 Hamburg, Germany, R_Bader@t-online.de)

The change of radiated sounds from guitars is investigated in terms of the fine tuning of these sounds applying slight changes to the guitar body, instrument builders would do with their guitars. As changes of these geometries of real guitars can only be perceived when the instrument is put together, the question is mostly open how different the whole instrument would have sounded with slight changes. So here the virtual guitar can be of great use. In the model, the top plate thickness, fan bracing, rim thickness and back plate geometry were changed in a linear way. Then these results could be associated directly to a change in only one geometry parameter. It is not assumed, that the sounds fit one existing guitar perfectly. Rather th overall influence of these changes on the sounds is investigated to show an overall behaviour. As the eigenfrequencies of the guitar body are that many, that a more or less closed resonance curve is achieved (without so-called 'dead spots'), the changes in the sounds are measured in the following terms: changes in the initial transient of the sounds, its brightness using the spectral centroid, its density using a spectral entropy measurement and amplitude fluctuations of the different sound partials.

4pMUf7. Nonlinear vibrations of impacted rectangular plates. Comparison between numerical simulation and experiments. Cédric Camier (ENSTA, Chemin de la Hunière, 91761 Palaiseau cedex, France, cedric.camier@ensta.fr), Kevin Arcas (ENSTA, Chemin de la Hunière, 91761 Palaiseau, France, arcas@ensta.fr), Stefan Bilbao (University of Edinburgh, Room 7306B, JCMB, King's Bldgs., Mayfield Rd., EH9 3JZ Edinburgh, UK, sbilbao@staffmail.ed.ac.uk)

Large amplitude vibrations of free-edge rectangular plates, subjected to an impulse excitation is addressed. In particular, this work is devoted to the analysis and simulation of a nonlinear von Kármán plate equations and by associate experimental investigations. Time domain simulations are achieved using implicit finite differences (FD) schemes recently developed by Bilbao. These energy-conserving methods guarantee the stability of the algorithm. To compare with simulations results, an experimental setup which allows reproducible impulse excitation and measurements by laser vibrometry has been developed. The time history of the force applied to a rectangular steel plate is recorded and this signal is used as excitation term in the simulations. We perform a parametric study, with both experimental and 
numerical approaches, by increasing gradually the amplitude of forcing. Non-linear phenomena, such as pitch glide and chaotic behaviour are observed and discussed.

4pMUf8. Active control of a vibrating string. Edgar Berdahl (Stanford Univ., Center for Computer Research in Music and Acoustics (CCRMA), Dept. of Music, Stanford, CA 94305-8180, USA, eberdahl@ccrma .stanford.edu), Guenter Niemeyer (Stanford Univ., Mech. Eng., Bldg. 530, Stanford, CA 94305, USA, gunter.niemeyer@stanford.edu), Julius O. Smith (Stanford Univ., Center for Computer Research in Music and Acoustics (CCRMA), Dept. of Music, Stanford, CA 94305-8180, USA, jos @ ccrma.stanford.edu)

We discuss the specifics of applying active feedback control to a vibrating string. Using sensors, actuators, and digital controller hardware, we make the acoustics of the string programmable, yet the string retains its tangible qualities. As a consequence, fretting, bowing, and plucking controlled and uncontrolled strings have similar physical consequences. Consider that any controller emulating a network of springs, masses, and dashpots attached to the string is a passive controller. To allow the string's acoustics to be programmable over a wide range, we should be able to implement passive controllers. This means that there must be at least one linear and collocated sensor/actuator pair. We explain how to construct such a pair in the laboratory. Finally, we explain one controller particular to one-dimensional systems such as vibrating strings. Whenever the sensor detects a pulse arriving, the actuator emits a new pulse. The output spectrum consists of a harmonic series proportional to the sampling of the product of the pulse's Fourier transform and the transfer function from the actuator to the sound recording device. Sound examples are presented. Finally, we discuss an open source environment we have created for adjusting controller parameters in real time from standard computer music software.

4pMUf9. Interfacing Digital Waveguide with CORDIS ANIMA networks. Alexandros Kontogeorgakopoulos (ACROE-ICA (Institut National Polytechnique de Grenoble), 46 av. Félix Viallet, 30031 Grenoble, France, alexandros.kontogeorgakopoulos@imag.fr), Claude Cadoz (ACROE-ICA (Institut National Polytechnique de Grenoble), 46 av. Félix Viallet, 30031 Grenoble, France, claude.cadoz@imag.fr)

The aim of this article is mainly to offer a link between the Digital Waveguide and the CORDIS-ANIMA physical modeling formalism. The first one is highly a modular lumped physical modeling and simulation system based on the mass-interaction paradigm while the second one offers accurate and efficient discrete time distributed models synthesized typically by delay lines and scattering junctions, in combination with digital filters. Both of them are widely developed and used in the domain of computer music field by scientists and artists. Although Digital Waveguide models have already been combined with Wave Digital Filters, they have never been exploited and integrated with CORDIS ANIMA networks. Wave Digital Filters are lumped models which are based on a scattering theoretic formulation which simplifies interfacing to Digital Waveguide models in contrast with the CORDIS ANIMA models. This research investigates the similarities of those formalisms, as well as focuses on the advantages of each modeling technique and proposes a real time computable interface between them. Moreover it results as well in a common convenient structural representation of their computational algorithms using signal processing block diagrams. These hybrid models were designed directly by their block diagrams, simulated and run in differ time using the Simulink software package of the matlab technical computing language.

THURSDAY AFTERNOON, 3 JULY 2008

ROOM 250A, 2:00 TO 3:40 P.M.

\title{
Session 4pNSa
}

\section{Noise and EURONOISE: Vibration Perception I}

\author{
Patricia Davies, Cochair \\ Ray W. Herrick Lab., School of Mechanical Engineering, Purdue Univ., 140 S. Martin Jischke Drive, West Lafayette, IN \\ 47907-2031, USA \\ Michael J. Griffin, Cochair \\ Institute of Sound and Vibration Research, University of Southampton, Human Factors Research Unit, Southampton, SO17
} IBJ, UK

Invited Papers

4pNSa1. Recent research activities for the assessment of vibration in living environment with respect to human perception in Japan. Yasunao Matsumoto (Saitama University, 255 Shimo-Ohkubo, Sakura, 338-8570 Saitama, Japan, ymatsu@mail.saitama-u.ac.jp), Sunao Kunimatsu (National Institute of Advanced Industrial Science and Technology, Tsukuba Central 7, 1-1-1 Higashi, 305-8567 Tsukuba, Japan, s.kunimatsu@aist.go.jp), Takashige Ishikawa (Japan Women's University, 2-8-1 Mejirodai, Bunkyou, 112-8681 Tokyo, Japan, ishikawa@fc.jwu.ac.jp), Yoshihiro Hirao (Kobayashi Institute of Physical Research, 3-20-41 Higashi-Motomachi, 185-0022 Tokyo, Japan, hirao@kobayasi-riken.or.jp)

The number of complaints against vibration has been increasing gradually in recent years in Japan. This fact may imply problems of the Vibration Regulation Law that was implemented almost 30 years ago so as to regulate vibrations caused by factories and construction works and to mitigate vibration problems caused by road traffic. A group of experts has been investigating an assessment method that may be able to assess recent vibration problems reasonably since 2004 . This paper presents a part of group activity that aims at improving the understanding of human vibration perception and applying it in vibration assessment in living environment. Results obtained from experiments involving human subjects to determine perception thresholds of whole-body vibration are summarised. 
4pNSa2. Development of a social survey questionnaire of reactions to vibration in residential buildings. Henrietta Howarth (Institute of Sound and Vibration Research, University of Southampton, Human Factors Research Unit, SO17 1BJ Southampton, UK, h.howarth@soton.ac.uk), Michael J. Griffin (Institute of Sound and Vibration Research, University of Southampton, Human Factors Research Unit, SO17 1BJ Southampton, UK, M.J.Griffin@soton.ac.uk)

A social survey questionnaire has been developed to determine human responses to vibration in residential environments. The overall aim was to produce a robust methodology for obtaining responses that could be combined with vibration measurements so as to investigate dose-response relationships for vibration in residential buildings. The vibration considered included that from sources outside the control of residents (e.g., road, rail, industrial, construction). This paper describes the development of the questionnaire and explains its structure and content. A review of social surveys and field and laboratory studies of vibration and noise is included. Methods of analysing responses obtained to the questionnaire are summarised.

4pNSa3. Cross-modality matching of loudness and whole-body vibration strength. Stephan Töpken (Oldenburg University, Institute of Physics - Acoustics, Carl-von-Ossietzky Str. 9-11, 26111 Oldenburg, Germany, stephan.toepken@ uni-oldenburg.de), Michael Bellmann (ITAP GmbH, Marie-Curie Str. 8, 26129 Oldenburg, Germany, Bellmann@itap.de), Reinhard Weber (Oldenburg University, Institute of Physics - Acoustics, Carl-von-Ossietzky Str. 9-11, 26111 Oldenburg, Germany, Reinhard.Weber@ uni-oldenburg.de)

In every day live humans are often exposed to noise and vibration simultaneously. Regarding comfort issues inside a car, it is of interest to know, whether the simultaneous perceptions of noise and vibration interfere. Laboratory tests have been carried out with temporally overlapping, partially overlapping and non overlapping presentations of acoustic and whole-body vibration stimuli. Sitting on a rigid chair on a vibration test bench, participants are asked to judge the strength of the excited whole-body vibrations in comparison to the loudness of noise presented via closed headphones. An adaptive method is employed to determine the subjective point of equality (PSE) between both sensory channels. The acoustic stimulus is a 1/2-octave band-pass noise centred at $100 \mathrm{~Hz}$ with a fixed noise level, the vibration stimulus is a narrowband noise, also $1 / 2$-octave broad with a centre frequency of $31,5 \mathrm{~Hz}$. The signals have an equal duration of one second. The signal parameters are chosen with respect to specific situations in a car. The PSE's of the loudness and the vibration strength as a function of the temporal order of the acoustic and vibration stimuli exhibit no dependency on the degree of temporal overlap of the stimulus presentation.

\section{3:00}

4pNSa4. Thresholds for the perception of fore-and-aft, lateral and vertical vibration by seated persons. Miyuki Morioka (Institute of Sound and Vibration Research, University of Southampton, Human Factors Research Unit, SO17 1BJ Southampton, UK, M.Morioka@soton.ac.uk), Michael J. Griffin (Institute of Sound and Vibration Research, University of Southampton, Human Factors Research Unit, SO17 1BJ Southampton, UK, M.J.Griffin@soton.ac.uk)

Vibration experienced in transport and in buildings can yield discomfort or annoyance if the vibration exceeds the threshold for vibration perception. Knowledge of thresholds makes it possible to determine which frequencies and directions of low magnitude vibration give rise to perception. The effect vibration frequency ( 2 to $315 \mathrm{~Hz}$ ) on absolute thresholds for the perception of whole-body vibration has been determined experimentally with 12 seated persons for each of the three axes of excitation (fore-and-aft, lateral and vertical). The frequency-dependence of the thresholds differed between the three axes. At frequencies, greater than $10 \mathrm{~Hz}$, sensitivity was greatest for vertical vibration. At frequencies less than $3.15 \mathrm{~Hz}$, sensitivity was greatest to fore-and-aft vibration. In all three axes, the acceleration threshold contours at frequencies greater than $80 \mathrm{~Hz}$ were U-shaped, suggesting the same psychophysical channel mediated high frequency thresholds for fore-and-aft, lateral and vertical vibration. It is shown that the frequency-dependence of absolute thresholds for the perception of whole-body vibration are not consistent with the frequency weightings used in current standards.

\section{Contributed Paper}

3:20

4pNSa5. Is there a perceptive signature of vehicles vibrations? Maël Amari (PSA Peugeot Citroën, Centre Technique de Vélizy, Route de Gisy, 78943 Vélizy-Villacoublay, France, mael.amari@mpsa.com), Etienne Parizet (Laboratoire Vibrations Acoustique, Insa Lyon, 25 bis, av. J. Capelle, 69621 Villeurbanne Cedex, France, etienne.parizet @insa-lyon.fr), Vincent Roussarie (PSA Peugeot Citroën, Centre Technique de Vélizy, Route de Gisy, 78943 Vélizy-Villacoublay, France, vincent .roussarie@mpsa.com)

The vibro-acoustic comfort of vehicles running at low speed has been studied for several years by car manufacturers. Even if car passengers are exposed to a complex environment involving sight, hearing and touch, it is generally agreed that vibrations transmitted through the seat is a very sig- nificant parameter in such situations. Previous laboratory experimentations revealed that vehicles were strongly discriminated even when subjects were submitted to seat vibrations only. The ranking of vehicles was also identical for different tested roads. All these observations raised the question of the existence of an identifiable signature of vehicles, independent of the road type. A perceptive experiment designed to evaluate the influence of such phenomenon was conducted. Subjects were submitted to a free sorting test. The categorisation task consisted in grouping vibrations stimuli recorded in 9 cars running on 3 differents roads according to their similarity. The RMS level of stimuli was normalised so that its effect was not predominant during the experiment. Results showed that stimuli groups corresponded to the 3 tested roads, and were correlated to time envelopes of vibrations. Vehicles were not significantly discriminated. 
Session 4pNSb

\title{
Noise, Architectural Acoustics, and EURONOISE: Noise, Vibration and Acoustics for Medical and Research Facilities and Their Occupants I
}

\author{
James West, Cochair \\ Johns Hopkins University, Department of Electrical Engineering, 3400 North Charles Street, Baltimore, MD 21218, USA
}

Jack B. Evans, Cochair

JEAcoustics, 1705 West Koenig Lane, Austin, TX 78756, USA

Marc Asselineau, Cochair

Peutz \& Associés, 10 rue des Messageries, Paris, F75010, France

Erica Ryherd, Cochair

Woodruff School of Mechanical Engineering, Georgia Institute of Technology, Atlanta, GA 30332-0405, USA

\section{Invited Papers}

4pNSb1. Toward a comprehensive hospital noise reduction research program. Ilene Busch-Vishniac (McMaster University, 1280 Main Street West, Hamilton, ON L9G 4X6, Canada, provost@mcmaster.ca), James West (Johns Hopkins University, Department of Electrical Engineering, 3400 North Charles Street, Baltimore, MD 21218, USA, jimwest@ jhu.edu)

Over the last few years there has been a growing interest in the control of hospital noise. This is prompted by a number of drivers: the recognition that hospital noise is a top complaint of patients, staff, and visitors; the move to a digital hospital and the impediment of speech recognition in very noisy environments; the implications of HIPAA and speech privacy on hospitals; and concerns about safety when communication is compromised by noise. Although there are now a number of studies of various issues associated with hospital noise, each views only a small piece of the very complicated problem. This presentation outlines a comprehensive noise research program designed to link acoustical measures with noise interventions and with staff and patient outcomes. Only through such an approach will it be possible to answer important questions such as which noise interventions are most effective and what hospital design strategies are most effective in terms of noise mitigation.

4pNSb2. Describing the sound environment of the neurological intensive care unit and personnel response. Erica Ryherd (Woodruff School of Mechanical Engineering, Georgia Institute of Technology, Atlanta, GA 30332-0405, USA, erica.ryherd@me.gatech.edu), Kerstin Persson Waye (Dept. of Environ. Medicine, The Sahlgrenska Acad. of Gothenburg Univ., Box 414, 40530 Gothenburg, Sweden, kerstin.persson-waye@amm.gu.se)

The hospital soundscape is populated with a number of noise sources that may be detrimental to the occupational environment, including medical equipment, alarms, portable carts, activities, paging/communication, and ventilation systems. This paper describes a study in which sound measurements were made over five days and occupant evaluations were conducted in a neurological intensive care unit (ICU). Forty-seven nursing staff members completed questionnaires regarding general reactions to the ICU environment as well as perceived psychological and physiological reactions to the noise. Acoustical characteristics such as level distributions, restorative periods, and spectral content were explored in addition to overall equivalent, minimum, maximum, and peak sound pressure levels. Results showed the mean length of restorative periods (LAeq below $50 \mathrm{~dB}$ for more than 5 min) was 9 and 13 minutes for day and night, respectively. Sound measurements near the patients showed LAeq values of $53-58 \mathrm{~dB}$, and dosimeters worn by the personnel revealed higher noise levels. Questionnaire results indicated that $91 \%$ of those surveyed felt that the noise negatively affected them in their daily work environment. They perceived the noise as contributing to stress symptoms such as irritation, fatigue, tension headaches, and difficulties concentrating. [Work supported by ASA and Swedish FAS].

$$
\text { 2:40 }
$$

4pNSb3. Indoor environment and acoustic conditions in two Finnish hospital wards. Annu Haapakangas (Finnish Institute of Occupational Health, Lemminkäisenkatu 14-18 B, 20520 Turku, Finland, annu.haapakangas@ttl.fi), Riikka Helenius (Finnish Institute of Occupational Health, Lemminkäisenkatu 14-18 B, 20520 Turku, Finland, riikka.helenius@ttl.fi), Valtteri O. Hongisto (Finnish Institute of Occupational Health, Lemminkäisenkatu 14-18 B, 20520 Turku, Finland, valtteri.hongisto@ttl.fi)

The aim of this study was to determine the acoustic conditions of two typical Finnish wards. The methods included long-term noise measurements, building acoustical measurements and questionnaires for patients $(\mathrm{N}=33)$ and nurses $(\mathrm{N}=27)$. The average sound pressure levels were within 49 and $58 \mathrm{dBA}$ in the corridors, office and patient rooms. Personal noise exposure levels among nurses were within 56 and $70 \mathrm{dBA}$. The noise was mainly caused by people. Ventilation noise level was $28 \mathrm{dBA}$. Building acoustic measurements 
showed reasonable agreement with national directions. Indoor environment was not a problem for most patients. Some disturbance from thermal conditions, dry air and noise was experienced. From daytime noise sources, other patients' snore and groans caused annoyance in some patients. Night-time sleep was disturbed most by anxiety, pain, noise, thermal conditions and an uncomfortable bed. Patients were, on average, quite satisfied with the overall room conditions. Nurses were more bothered by environmental factors than patients, giving highest annoyance ratings to thermal conditions, air quality and noise. Noises were experienced to cause some distraction when concentrating to work tasks. Sounds of phones ringing were experienced as particularly detrimental. Lack of privacy was the most obvious problem in both staff and patient evaluations.

3:00

4pNSb4. Eliminating environmental stressors in hospitals: managing noise through different interventions. Michael Phiri (The University of Sheffield, School of Architecture, Arts Tower, Western Bank, S10 2TN Sheffield, UK, m.phiri@sheffield.ac.uk)

There is growing body of knowledge of over 1,000 scientific studies which provide evidence that patients experience positive health outcomes in an environment that incorporates natural light, elements of nature, soothing colours, meaningful and varying stimuli, peaceful sounds, pleasant views and a sense of beauty. This paper reviews the research, its practical applications in order to enhance acoustics comfort and quality of care in healthcare settings. Healthcare planners can carry out small-scale, medium- and large-scale evidencebased design interventions. Small-scale interventions which minimise cost maximise impact on acoustic comfort can be implemented relatively quickly and easily e.g. provision of rubbish bins with a damping system to close lids slowly, dimming lights on the wards etc. Control measures include strategies to reduce noise levels in healthcare facilities with or without physical environmental alterations including specification of appropriate absorbent materials (e.g. acoustic ceiling tiles). Medium-scale interventions e.g. inpatient or nursing unit design involve consideration of the shape, geometry and other characteristics of a room in order to deal with background noise, speech intelligibility and other elements which promote acoustic comfort. Large-scale interventions involve large capital works notably entire hospital complexes, assemblies of departments/specialties but have evidence extrapolated from the small- and medium-scale interventions.

3:20

4pNSb5. Improved perception of sound environments through room acoustic interventions. Marc R. Janssen (Saint-Gobain Ecophon AB, Box 500, 26061 Hyllinge, Sweden, marc.janssen@ecophon.se)

Noise in hospitals is apparently among the top complaints of patients, visitors, and staff. An increasing amount of evidence shows the negative effects of sound within health care environments, affecting the quality of care in the end. Very few hospitals have actually been able to characterize hospital noise and to find effective noise control approaches. This paper will show international results of room acoustic interventions on the patients and staff perception of the sound environment. Next to that the suggestions will be presented on the use of relevant acoustical parameters to support the performance and well-being of staff and patients. One specific example will deal with a case study at the Thorax ICU at the Karolinska University Hospital in Stockholm. The purpose of the study has been to clarify how room acoustics in a patient ward affect the staff's perception of the noise situation in that ward. Another purpose has been to develop a better understanding of how a set of requirements for room acoustics should be formulated in order to obtain a supportive health care environment. It is possible to conclude that, by adding more absorption to the room, the noise situation has been positively influenced and perceived.

3:40-5:20 Posters

Lecture sessions will recess for presentation of poster papers on various topics in acoustics. See poster sessions for topics and abstracts.

\section{Contributed Paper}

\section{5:20}

4pNSb6. Aural Connectivity: Enhancing sound environments in critical care settings for effective nurse auditory monitoring. Selen Okcu (Georgia Institute of Technology, College of Architecture, Atlanta, GA 30332-0155, USA, okcuse@ yahoo.com), Craig Zimring (Georgia Institute of Technology, College of Architecture, Atlanta, GA 30332-0155, USA, craig.zimring@coa.gatech.edu), Erica Ryherd (Woodruff School of Mechanical Engineering, Georgia Institute of Technology, Atlanta, GA 30332-0405, USA, erica.ryherd@me.gatech.edu)

In intensive care unit (ICU) settings, the sound environment is critically important to nurses accomplishing their tasks. In earlier studies by the authors, it was found that non-amplified environmental sounds such as patient bodily sounds, patient threatening/unusual sounds, and help calls from patients and other caregivers are critically important auditory cues that nurses must listen for and respond to immediately. These sounds do not exist in isolation but matter as a pattern of aural connectivity that can support a nurse's critical monitoring abilities as she moves through her workplace. Aural connectivity is a network measure that reflects the overall pattern of where users can hear and respond to different key sounds within a setting. This paper describes the sound environments of two ICU hospital settings with similar patient acuity levels but differing layout designs. Preliminary results regarding the patterns of aural connectivity and the role that layout design might play in those patterns are discussed and potential implications for layout design are proposed. 


\section{Invited Papers}

\section{5:40}

4pNSb7. Vibration Effects on Laboratory Mice during Building Construction. Richard A. Carman (Wilson, Ihrig \& Associates, 5776 Broadway, Oakland, CA 94618, USA, rcarman@wiai.com), Deborah A. Jue (Wilson, Ihrig \& Associates, 5776 Broadway, Oakland, CA 94618, USA, djue@wiai.com), Gary M. Glickman (Wilson, Ihrig \& Associates, 65 Broadway, Suite 401, New York, NY 10006, USA, gglickman@wiai.com)

Laboratory animals, in particular mice, are an integral part of medical and scientific research. Genetic research involving mice can be substantially affected by disruptions to the animals' environment. A new research facility is being built in close proximity to an existing one and the work will involve both demolition and new construction. Prior to construction, a study was conducted at the research facility to establish acceptable vibration levels in the vivaria areas. The study involved an experiment using an electrodynamic shaker to determine the effect of whole-body vibration on pregnant mice. The results of that study have been published. During construction, continuous vibration monitoring was conducted and the program and instrumentation used for monitoring are described. Results are presented, which include the researchers' data on the observed effects on the mice as well as the measured vibration levels during the construction.

\section{6:00}

4pNSb8. "Vibration Kills" and other lessons from the trenches. Hal Amick (Colin Gordon \& Associates, P. O. Box 39, San Bruno, CA 94066, USA, hal.amick@ colingordon.com), Michael L. Gendreau (Colin Gordon \& Associates, P. O. Box 39, San Bruno, CA 94066, USA, michael.gendreau@ colingordon.com)

In many areas of acoustics and vibration design, criteria are based upon parameters such as comfort, privacy, intelligibility, productivity, or machine precision. One can approach these criteria with a somewhat dispassionate attitude. However, when working with the biological research communities, one is periodically reminded by the researchers and medical practitioners that much of their work ultimately deals with life-and-death issues, either for patients for whom a drug or medical device is intended, or for organisms used in test protocols. The authors will share a collection of case studies in which these issues are illustrated. These include: Death of cells due to excessive vibration during electrophysiology. Vibration-induced nausea experienced by a surgeon using a surgical microscope for spine surgery. A life-saving medical technology resulting from a serendipitous discovery in a low-vibration lab environment. These case studies highlight the need for care in the specification, design, and construction of biological and biomedical research and healthcare facilities.

4pNSb9. Don't forget the quench pipe when installing an MRI. Carel Ostendorf (Cauberg-Huygen R.I. BV, Postbus 480, 6200 AL Maastricht, Netherlands, c.ostendorf@chri.nl)

An MRI is a useful medical device but it makes a lot of noise. A sound level of $90 \mathrm{~dB}(\mathrm{~A})$ or more in the MRI room is not unusual. Placing an MRI in an existing hospital means that extra care has to be taken to prevent the noise from the MRI causing nuisance in adjacent rooms. In this paper the situation is discussed in which complaints appeared after the installation of the MRI. First sound measurements were done to see if the sound level in the office above the MRI fulfils the noise ratings. This way it would be clearer if the acoustic measures were doing their job. Whatever the outcome, the hospital wanted to put an end to the complaints. So, more sound measurements were done to establish the cause of the nuisance and to point out what extra measures have to be taken to solve the problem. It turned out that the quench pipe played an important role in this situation.

\section{6:40}

4pNSb10. Generic noise criterion curves for sensitive equipment. Michael L. Gendreau (Colin Gordon \& Associates, P. O. Box 39, San Bruno, CA 94066, USA, michael.gendreau@colingordon.com)

Electron beam-based instruments are sensitive to the environment in which they operate. Adverse environments may limit their achievable resolution. Many equipment manufacturers provide specifications for the acceptable level of various environmental conditions, such as vibration, EMI, and acoustic noise. However, the quality of the specifications vary significantly, from well-defined to conjectural. Additionally, during the design of a facility, the specific instruments that will be used may not yet be known. Thus, it is useful to have "generic" criteria, intended to represent entire classes of instruments, to use in the design of facilities. Generic vibration criteria exist to aid in the design of laboratories, though there have been no such instrument-based generic criteria available for acoustic noise. The generic noise criteria that are currently used in lab design (NC, NR, dBA, etc.) were established to address the effect of noise on human beings. Using noise specifications for a significant number of instruments with varying resolving powers, correlation of resolution with environmental noise is demonstrated. Based on the data reviewed, generic noise criterion curves have been developed and presented for use in the design of facilities that contain noise-sensitive equipment. These are applicable when other well-defined and specific criteria are not available. 


\title{
Session $4 p N S c$
}

\section{Noise and EURONOISE: Potential to Reduce Tire/Road Noise I}

\author{
J. Stuart Bolton, Cochair \\ Ray W. Herrick Labs., School of Mech. Eng., Purdue University, 140 S. Martin Jischke Drive, West Lafayette, IN 47907-2031, \\ USA \\ Ernst-Ulrich Saemann, Cochair \\ Continental AG, Jaedekamp 30, Hannover, 30419, Germany
}

\section{Invited Papers}

4pNSc1. The influence of tyre design on tyre/road noise - some fundamental thoughts. Wolfgang Kropp (Chalmers University of Technology, Division of Applied Acoustics, SE-41296 Gothenburg, Sweden, wolfgang.kropp@chalmers.se), Patrick Sabiniarz (Chalmers University of Technology, Division of Applied Acoustics, SE-41296 Gothenburg, Sweden, patrick.sabiniarz@chalmers.se)

Tyre/road noise generation mechanisms are divided into two categories, tyre vibrations (due to time varying contact forces) and airflow related processes (e.g. air-pumping) in the contact between tyre and road. The paper only focuses on tyre vibrations. An existing model for the simulation of tyre/road interaction is used to investigate the influence of tyre design on the vibrational energy stored in the tyre structure during rolling. It can be shown that ,although design is changed substantially, very little changes can be observed with respect to input power through the contact into the tyre structure. Changes in driving point mobilities of tyres are not directly related to changes in noise generation. Geometry changes leading to different contact geometry and in this way to different modal composition of the vibrational field as well as design changes leading to changes in wave speed are of higher importance. These changes have a strong influence on the radiation efficiency of tyres.

4pNSc2. Development of low noise tyres in EC project SILENCE. Ernst-Ulrich Saemann (Continental AG, Jaedekamp 30,30419 Hannover, Germany, Ernst-Ulrich.Saemann@ conti.de)

A lot of efforts were made in the last two decades to lower the tire/road noise. The tire industry has optimized the tread pattern as the main influence parameter so that nowadays the radiated sound pressure of a modern tire in the far field is very close to that of a blank tire with the same construction. Because the tread pattern is needed to achieve the required safety level further noise reduction has to be addressed mainly by tire construction. For many years tire manufacturers have been searching for a construction, which fulfils the targets of the automotive industry and generates less noise. The research was done not only in house but also with public projects. In the EC Project SILENCE a subproject has provided design solutions and hardware solutions for noise reduction, with respect to vehicle/tyre/road integration, under typical urban and suburban traffic conditions. This improvement is based on an increased understanding of noise generation and radiation mechanisms gained by the further development of experimental and simulation techniques. A series of low noise tyres (prototypes) has been developed and tested on a selection of appropriate low noise road surfaces.

\section{$2: 40$}

4pNSc3. The ranking of rolling noise from passenger car tyres - a comparison between measurements and modelling results. Truls Berge (SINTEF ICT Dept. of Acoustics, O.S. Bragstads pl., NO-7465 Trondheim, Norway, truls.berge@ sintef.no)

Tyres are type approved with regard to rolling noise on an ISO-test track, according to the EU-directive 2001/43/EC. The test track is basically a rather smooth road surface with maximum chipping size of $8 \mathrm{~mm}$. However, most surfaces normally used on roads, especially in the northern European countries, are rougher surfaces, typically Stone Mastic Asphalt (SMA) with 11 to 16 mm stones. A project has been initiated to compare the noise levels of a selection of highly used after market summer tyres (in Norway). Noise measurements of 12 tyres have been performed on a selection of new and old SMA-road surfaces. The 3D texture of the same road surfaces has been measured with laser profile equipment. In addition, the point mobility and other design features of the tyres have been measured to be used as input data to the SPERoN tyre/road noise model. Then, comparison will be made between measurements and modelling results. Preliminary results from the noise measurements show a difference of 2.5-3 dB(A) on SMA-surfaces, between the tyres. The project is a co-operative between SINTEF (Norway), MüllerBBM (Germany), and Chalmers University (Sweden). 
4pNSc4. Sound Radiation Modes of a Tire on a Reflecting Surface. J. Stuart Bolton (Ray W. Herrick Labs., School of Mech. Eng., Purdue University, 140 S. Martin Jischke Drive, West Lafayette, IN 47907-2031, USA, bolton@purdue.edu), Kiho Yum (Hyundai Motor Company, Chassis \& Powertrain Engineering Team 3, Hyundai-Kia Motors Corporate R\&D Division, Hwasung-Si, Gyeonggi-Do, 772-1, 445-706 Jangduk-Dong, Republic of Korea, kihoyum@hyundai-motor.com)

Wave number decomposition of a tire's radial vibration can be used to reveal the wave propagation characteristics of tires. In this paper, the relationship between the structural wave propagation characteristics of a tire excited at one point and its sound radiation is considered. The sound radiation resulting from structural vibration of a tire in contact with the ground was investigated by using boundary element analysis. In particular, the orthogonal radiation modes of a tire in the presence of a reflecting surface, along with their radiation efficiency characteristics, were calculated by applying an eigenvector analysis to the tire's radiation resistance matrix. The latter analysis made use of acoustic transfer vectors and a recovery surface appropriate for a pass-by noise test. The radiation mode results reveal that it is the vibration in the region close to the contact patch that primarily controls sound radiation. In particular, to reduce pass-by noise levels, it is necessary to mismatch the tire's structural ring mode and the radiation modes with high radiation efficiencies. It has also been found that the radiation from a tire is controlled by a relatively small number of radiation modes (although the number of contributing modes increases with frequency).

$$
3: 20
$$

4pNSc5. Analysis of truck tire rolling noise and structural vibrations. Samuel Blanc (Goodyear, Technical Center, L - 7750 Colmar-Berg, Luxembourg, samuel_blanc@goodyear.com), Jan Leyssens (Goodyear, Technical Center, L - 7750 Colmar-Berg, Luxembourg, jan.leyssens@goodyear.com)

Legislators are increasingly demanding towards the vehicle and tire industry for further reduction of tire/road noise emissions. With the need to develop quieter tires, noise has become one of the driving performance criteria in the design process, together with safety, wear and rolling resistance. Next generation truck tires will have to meet more severe legal requirements. In this context, noise has to be improved both at constant speed for the tire regulation and under acceleration for the vehicle legislation. Field test results of different tire tread designs under accelerated and constant speed conditions are given in this presentation. Noise mechanisms and effects of rolling conditions are discussed based on pass-by and near-field microphones. In addition, the dynamic vibration of a truck tire casing is analysed using both detailed FEA modelling and experimental results.

\author{
3:40-5:20 Posters
}

Lecture sessions will recess for presentation of poster papers in various topics in acoustics. See poster sessions for topics and abstracts.

\title{
Invited Paper
}

\section{$5: 20$}

4pNSc6. Simulation of tire tread block dynamics with respect to complex contact phenomena. Gunnar Gäbel (Institute of Dynamics and Vibration Research, Leibniz University Hannover, Appelstr. 11, 30167 Hannover, Germany, gaebel@ids.uni-hannover.de), Patrick Moldenhauer (Institute of Dynamics and Vibration Research, Leibniz University Hannover, Appelstr. 11, 30167 Hannover, Germany, moldenhauer@ids.uni-hannover.de), Matthias Kröger (Institute of Dynamics and Vibration Research, Leibniz University Hannover, Appelstr. 11, 30167 Hannover, Germany, kroeger@ids.uni-hannover.de)

Vibrations of the tire structure are caused by the interaction between the rolling tire and the road surface. The tread block is the only tire component which is directly in contact with the cleft road surface texture and therefore of special interest. The contact due to the rough surface leads to complex contact phenomena e.g. friction characteristics depending on normal contact pressure and relative velocity, non-linear contact stiffness and wear effects changing the tread block geometry and in hence the local contact forces. These contact phenomena strongly influence the dynamical behaviour of the tread block and the whole tire. A modular model based on a modal reduction method will be presented to investigate the tread block dynamics under consideration of the local friction characteristic, the non-linear contact stiffness and wear which are also analyzed experimentally on a concrete road surface to identify the model parameters.

\section{Contributed Papers}

\section{$5: 40$}

4pNSc7. Transmissibility of a deformed rotating tyre. Ruud Van Doorn (Eindhoven University of Technology, Dept. of Mechanical Engineering, Dynamics \& Control, P.O. Box 513, 5600MB Eindhoven, Netherlands, r.r.j.j.v.doorn@student.tue.nl), Ines Lopez (Eindhoven University of Technology, Dept. of Mechanical Engineering, Dynamics \& Control, P.O. Box 513,5600MB Eindhoven, Netherlands, i.lopez@tue.nl), René Van Der Steen (Eindhoven University of Technology, Dept. of Mechanical Engineering, Dynamics \& Control, P.O. Box 513, 5600MB Eindhoven, Netherlands, r.v.d.steen@tue.nl), N.b. Roozen (Eindhoven University of Technology, Dept. of Mechanical Engineering, Dynamics \& Control, P.O. Box 513, 5600MB Eindhoven, Netherlands, n.b.roozen@tue.nl), Henk
Nijmeijer(Eindhoven University of Technology, Dept. of Mechanical Engineering, Dynamics \& Control, P.O. Box 513, 5600MB Eindhoven, Netherlands, h.nijmeijer@tue.nl)

The major source of environmental noise exposure is road traffic noise. Of all noise sources, tyre rolling noise is dominant for speeds above 30 $\mathrm{km} / \mathrm{h}$ for passenger cars. Tyre rolling noise can be subdivided into interior and exterior noise. For the interior noise to which the passengers are exposed to, the tyre transmissibility is essential since it relates the contact forces with the axle forces. These axle forces are responsible for the structure borne interior noise. Here, a Finite Element tyre model, including a fully coupled air column, is used to examine the transmissibility in the frequency domain 0-300 Hz. It is shown that three aspects are essential in mod- 
eling the axle forces resulting from tyre-road interaction: 1) the tyre deformation since it leads to a set of non-axisymmetric eigenmodes, 2) the relatively low-damped non-axisymmetric acoustic resonance, and 3) rotation. A methodology using substructuring techniques is presented to include rotational effects both in the case of an undeformed and deformed tyre. These effects of rotation on the transmissibility differ in the deformed and undeformed case: frequency loci veering occurs in the deformed case, while in the undeformed case rotation results in a pure split of the eigenfrequencies.

\section{6:00}

4pNSc8. Simulation and analysis of tire road noise, finite element results and validation. Maik Brinkmeier (Institut für Baumechanik und Numerische Mechanik, Appelstraße 9A, 30167 Hannover, Germany, brinkmeier@ibnm.uni-hannover.de), Udo Nackenhorst (Institut für Baumechanik und Numerische Mechanik, Appelstraße 9A, 30167 Hannover, Germany, nackenhorst@ibnm.uni-hannover.de), Jan Biermann (Institut für Modellierung und Berechnung, Denickestraße 17, 21073 Hamburg, Germany, biermann@tu-harburg.de), Otto Von Estorff (Institut für Modellierung und Berechnung, Denickestraße 17, 21073 Hamburg, Germany, estorff@tu-harburg.de)

This presentation shows the methods and results of the German research project "Silent Traffic". The main topics are the simulation of tire road noise as well as the validation of the methods and finite element models. The target is to understand the mechanisms of sound generation and to get suggestions to reduce the traffic noise resulting from the virtual system. The investigations are based on a physical modeling of the tire road system rather then on the processing of statistical data. The simulation procedure can be decomposed into four steps: The computation of non-linear stationary rolling, the eigenvalue analysis in the deformed state, the analysis of road surface textures, and the calculation of the noise radiation including a modal super- position approach with an excitation by deterministic functions. Thereby, the numerical model enables for a detailed analysis of certain effects of the sound generation and radiation that contribute to the overall tire road noise The simulation results are compared to measurements, both for structural dynamics and acoustics, to show the quality of the model und to indicate possible improvements for further development.

\section{6:20}

4pNSc9. A contact solver suitable for tyre/road noise analysis. Arjan Schutte (University of Twente, Dept. Mechanical Engineering, P.O. Box 217, 7500 AE Enschede, Netherlands, j.h.schutte@utwente.nl), Ysbrand H. Wijnant (University of Twente, Dept. Mechanical Engineering, P.O. Box 217, 7500 AE Enschede, Netherlands, Y.H.Wijnant@ctw.utwente.nl), André De Boer (University of Twente, Dept. Mechanical Engineering, P.O. Box 217, 7500 AE Enschede, Netherlands, a.deboer@utwente.nl)

Road traffic noise is a major environmental problem in modern society. The interaction between tyre and road surface, the major noise source, is non-linear and is best described in the time domain. The currently used contact models for acoustic analyses have problems with either accuracy or calculation speed. At the Structural Dynamics and Acoustics group of the University of Twente an alternative contact algorithm has been developed. The characteristic feature of this algorithm is that, while solving the set of equations, the contact condition, i.e. the condition stating that there is no overlap between the bodies, is satisfied exactly. Hence, there is no need for contact elements or contact parameters. The possibility to optimize and speed up the algorithm, using multigrid is the major advantage of the new approach. In this paper the contact algorithm is applied to a two-dimensional finite element model. Coulomb friction is taken into account. Some test simulations illustrate the ease of the algorithm. The model will be extended and nonlinear material behaviour will be added. Multigrid and multilevel will be used to speed up the calculation. The goal is to compute the vibrations and radiated noise pattern of a profiled tyre rolling on a road.

\section{Invited Paper}

\section{$6: 40$}

4pNSc10. The effect of porous road surfaces on radiation and propagation of tyre noise. Bert Peeters ( $\mathrm{M}+\mathrm{P}-\mathrm{consulting}$ engineers, PO Box 2094, 5260 CB Vught, Netherlands, BertPeeters@mp.nl), Ard Kuijpers (M+P - consulting engineers, PO Box 2094, 5260 CB Vught, Netherlands, ArdKuijpers@mp.nl)

It is well-known that porous road surfaces are very effective for the abatement of tyre/road noise. However, the physical principles behind the noise-reducing properties of these surfaces are not well understood and often even misinterpreted. Lack of understanding becomes a problem when developing a prediction model for tyre/road noise to be able to optimize the road surface for noise abatement. In the framework of the EU SILENCE project and the Dutch IPG program a model has been developed to predict the influence of road surface porosity on the radiation and propagation of tyre/road noise. First step in the model development was to gain physical insight from stationary and vehicle measurements with passenger car and truck tyres on both dense and porous surfaces. Next step was to qualitatively and quantitatively describe the observed physical phenomena in a mathematical model according to the KISS principle: avoid unnecessary complexity, both in input parameters and in the mathematical model. The end result is a mathematical model that describes the noise reduction potential for a standard tyre on various (porous) road surfaces, using a sound absorption spectrum as input. This model is available for a broad audience in the recently launched SPERoN acoustic optimization tool. 


\title{
Session 4pNSd
}

\section{Noise and EURONOISE: Cultural Variations in Sound/Noise Assessment I (Poster Session)}

\author{
Catherine Guastavino, Cochair \\ Centre for Interdisciplinary Research in Music Media \& Technology (CIRMMT) - School of Information Studies - McGill Univ. \\ Danièle Dubois, Cochair \\ CNRS
}

\begin{abstract}
All posters will be on display from 3:40 p.m. to 5:20 p.m. To allow contributors an opportunity to see other posters, contributors of odd-numbered papers will be at their posters from 3:40 p.m. to 4:30 p.m. and contributors of even-numbered papers will be at their posters from 4:30 p.m. to 5:20 p.m.
\end{abstract}

\section{Contributed Papers}

4pNSd1. Noise exposition in the daily life. José Romero(Grup d'Acustica Arquitectònica, Ambiental i Industrial, E.T.S.I.I, Univ. Politécnica de Valencia, Camino de Vera, s/n, 46022 Valencia, Spain, romerof@fis.upv .es), Alicia Giménez (Grup d'Acustica Arquitectònica, Ambiental i Industrial, E.T.S.I.I, Univ. Politécnica de Valencia, Camino de Vera, s/n, 46022 Valencia, Spain, agimenez@fis.upv.es), Salvador Cerdá (Grup d'Acustica Arquitectònica, Ambiental i Industrial, E.T.S.I.I, Univ. Politécnica de Valencia, Camino de Vera, s/n, 46022 Valencia, Spain, salcerjo@mat.upv .es), Joaquin Navasquillo (Facultad de Ciencias, Univ. de Valencia, Blasco Ibañez, 15, 46010 Valencia, Spain, joaquin.navasquillo@uv.es), Radu Lacatis (Grup d'Acustica Arquitectònica, Ambiental i Industrial, E.T.S.I.I, Univ. Politécnica de Valencia, Camino de Vera, s/n, 46022 Valencia, Spain, rala1@doctor.upv.es), Arturo Barba Sevillano (Grup d'Acustica Arquitectònica, Ambiental i Industrial, E.T.S.I.I, Univ. Politécnica de Valencia, Camino de Vera, s/n, 46022 Valencia, Spain, arbarse@doctor.upv.es)

Nowadays, the man lives exposed in a world of sounds (pleasant - disagreeable, nonannoying - annoying) throughout the time, during the 24 hours a day. The man is exposed to the noise in his daily life, since he awakes, moves, works, entertains itself, rests, and to sleep. We have made a survey of levels of exhibition to the noise, having distinguished they are leisure, work or rest, in a amog young population of 20-35 years without hearing problems, and of both sexes. The results show us and agree with other authors, that the exhibition levels are highest in the activities chosen, like for example the leisure. In addition, the levels of exhibition, to the noise are related with the diverses activities of the population.
4pNSd2. Noise(s) and sound(s): comparing various conceptualizations of acoustic phenomena across languages. Danièle S. Dubois (CNRS, LCPE/LAM 11 rue de Lourmel, 75015 Paris, France, ddubois @ ccr.jussieu.fr), Catherine Guastavino (Centre for Interdisciplinary Research in Music Media \& Technology (CIRMMT) - School of Information Studies - McGill Univ., 3459 McTavish, Montreal, QC H3A1Y1, Canada, Catherine.Guastavino@mcgill.ca)

Musical listening focuses on perceptual attributes of the sound itself (e.g. pitch, loudness), whereas everyday listening focuses on events to gather relevant information about what happens in our environment (e.g. car approaching), that is, not about the sound itself but rather about noise as produced by sources and actions, and the effect of sound/noise on listeners. Previous linguistic analyses of discourses identified different conceptualizations of everyday/environmental noises and of musical sounds, as well as different conceptualizations for acousticians and non-acousticians. A more extensive psycholinguistic investigation was conducted to evaluate how different languages (not only consensual vocabularies) differently structure the semantic space of acoustic phenomenon. An open questionnaire was administered to expert listeners (acousticians) and naïve listeners from 5 different languages (French, Italian, Spanish, German and English) to collect linguistic resources available in each language and used in discourse to describe acoustic phenomena in their native languages. Presented in this paper are the first results of the comparative psycholinguistic analysis, in terms of linguistic devices and correlated conceptualizations, focusing on - the distinction between noise(s) and sound(s) across languages - the difference between scientific discourses and common sense discourses within and across languages. 


\title{
Session 4pNSf
}

\section{Noise and EURONOISE: Car Acoustics III (Poster Session)}

\author{
Luc Mongeau, Cochair \\ McGill University \\ Virginie Maillard, Cochair \\ RENAULT
}

\begin{abstract}
All posters will be on display from 3:40 p.m. to 5:20 p.m. To allow contributors an opportunity to see other posters, contributors of odd-numbered papers will be at their posters from 3:40 p.m. to 4:30 p.m. and contributors of even-numbered papers will be at their posters from 4:30 p.m. to 5:20 p.m.
\end{abstract}

\section{Contributed Papers}

4pNSf1. New vehicule noise emission values to update the French 'Guide du bruit'. Sonia Doisy (Laboratoire des Ponts et Chaussées, 11, rue Jean Mentelin, BP 9,67035 Strasbourg, France, sonia.doisy@equipement .gouv.fr), Joel Lelong (INRETS, 25 av. F. Mitterrand, case 24, 69675 Bron, France, lelong@inrets.fr), Jean-François Hamet (INRETS, 25 av. F. Mitterrand, case 24, 69675 Bron, France, hamet@inrets.fr)

French traffic noise prediction models are based on vehicle noise emission values defined by the "Guide du Bruit des Transports Terrestres", issued in 1980. A research programme was established in order to update these values and take into account developments in car and road technology. The methodology followed to output emission values has been developed by successive steps, as the processing performed on the collected data went along. After several attempts the shape adopted for the emission formulas considers the vehicle pass-by LAmax as the sum of two subsource contributions: the power unit contribution which varies with acceleration and the road gradient, and the tyre/road contribution which varies with speed and the road surface type. New emission values are now completely defined, and ready to be used in noise prediction models.

4pNSf2. Acoustical Parameters of Automotive Interiors using Hybrid Fleeces basing on natural fibres. Christian R. Koenig (University of Bremen, Badgasteiner Str. 1, FZB - Room 2140, 28359 Bremen, Germany, chkoenig@uni-bremen.de), Dieter H. Müller (University of Bremen, Badgasteiner Str. 1, FZB - Room 2140, 28359 Bremen, Germany, ml@biba.uni-bremen.de), K.-D. Thoben (University of Bremen, Badgasteiner Str. 1, FZB - Room 2140, 28359 Bremen, Germany, tho@ biba.unibremen.de)

Hybrid fleeces are often used to produce composites and layered structures for car interiors. The fleeces consist of reinforcement fibres and polymeric fibres as matrix material. The utilization of natural fibres as a reinforcement for composite may be regarded as an emerging research area in polymer science. An important range of application can be found in the automotive industry. Despite of ecological gains like less environmental impact of the later product within the formation, usage and disposal period further technical and economical advantages result from this strategy. In addition to a reduction of the component's cost and net weight or an improvement of driving safety due to the crash behaviour of the composite material, natural fibre reinforced polymers offer increased recycling capabilities over conventional polymers used in that area. The presentation concentrates on the possibilities by using different materials, different ratios of thermoplastic and natural fibre material and different process parameters. Layers are especially investigated to demonstrate how the fleeces and the tech- niques to bond the fleeces can influence the acoustical behaviour and mechanical properties. Based on the results of the measurements optimised, multi-layered sandwiches were developed and will be presented.

4pNSf3. Statistical energy analysis limits for acoustic radiation car: an alternative approach. Gérard Borello (InterAC, 10 impasse Borde-Basse, ZA. La Violette, 31240 L'Union, France, gerard.borello@interac.fr), Alex Borello (InterAC, 10 impasse Borde-Basse, ZA. La Violette, 31240 L’Union, France, info@interac.fr), Julien Primus (InterAC, 10 impasse Borde-Basse, ZA. La Violette, 31240 L’Union, France, info@interac .fr), Laurent Gagliardini (PSA Peugeot Citroën, Route de Gisy, 78943 Vélizy-Villacoublay Cedex, France, laurent.gagliardini@mpsa.com)

Due to a new pass-by noise regulation, Vehicle exterior noise will have to be reduced in the coming years. This may be achieved by optimizing underbody and underhood absorption and screening apertures. There is then a need for numerical techniques able to predict sound reduction related to acoustic absorption and transmission loss changes. Through a work supported by ADEME and headed by PSA, energy-based predictive techniques such as Analytical Statistical Energy Analysis (ASEA) and discretized Energy Flow Analysis (DEFA) were tested against the actual physical problem to be solved through a series of benchmarks. Both theories are compared across several simple acoustic problems. It is concluded that both methods do not fit to the initial acoustic optimization requirement due to their intrinsic assumptions that restrict their applicative range. More fitted numerical techniques are now investigated: among new candidates, the Virtual SEA (VSEA) technique that allows the creation of a numerical model of coupled acoustic cavities from the finite element global modes without the serious limitations of ASEA and a matrix approach based on Craigh-Bampton substructuration of the cavities.

4pNSf4. A multiple regression model for predicting rattle noise subjective rating from in-car microphones measurements. Benoît Gauduin (Genesis S.A., Bâtiment Gérard Mégie, Domaine du Petit Arbois BP 69, 13545 Aix-en-Provence Cedex 4, France, benoit.gauduin @ genesis.fr), Christophe Noel (Genesis S.A., Bâtiment Gérard Mégie, Domaine du Petit Arbois - BP 69, 13545 Aix-en-Provence Cedex 4, France, christophe.noel@free.fr), Jean-Louis Meillier (Renault, Centre Technique d'Aubevoye, Parc de Gaillon, 27940 Aubevoye, France, jean-louis.meillier@renault.com), Patrick Boussard (Genesis S.A., Bâtiment Gérard Mégie, Domaine du Petit Arbois - BP 69, 13545 Aix-enProvence Cedex 4, France, patrick.boussard@genesis.fr)

In some situations when the road is deformed, the shock absorbers of vehicles may produce a specific sound, called rattle noise. It may be perceived by the driver and wrongly considered as a malfunction of the vehicle. 
This sound is part of the global acoustic comfort of the vehicle and hence is studied by RENAULT. The approach presented here aims at predicting the rattle noise subjective rating given by a RENAULT expert on a scale from 0 to 10 , by developing a model based on in-car binaural microphones measurements in the ears if the driver. First, a set of 11 metrics has been build, related to temporal aspects, spectral components and time-frequency information of the rattle noise recorded. The corpus is made of 19 different configurations of shock absorbers of a given car. The method used to select the most relevant metrics for the multiple regression model is presented. This selection is based on a statistical robustness estimation of the model. Hence, it appears that only 6 metrics are sufficient to build the model. Finally, the performance of the model is evaluated on 5 new configurations of shock absorbers.

4pNSf5. Structure borne noise inside a coach. Joanes Berasategi (Mondragon University, Loramendi 4, Apdo 23, 20500 Arrasate, Spain, joanes.berasategui@alumni.eps.mondragon.edu), Unai Galfarsoro (Mondragon University, Loramendi 4, Apdo 23, 20500 Arrasate, Spain, ugalfarsoro@eps.mondragon.edu), María Jesús Elejabarrieta (Mondragon University, Loramendi 4, Apdo 23, 20500 Arrasate, Spain, mjelejabarrieta@eps.mondragon.edu), Igor Insausti (Mondragon University, Loramendi 4, Apdo 23, 20500 Arrasate, Spain,ugalfarsoro@eps.mondragon.edu)

Nowadays the use of coaches as ground collective transport is generalizing in society and besides, its use is encouraged by all public institutions. Users of this type of vehicles request that they are comfortable, even more in long trips. Therefore, decreasing noise and vibrations inside the coach is a essential requirement to obtain a good quality of the vehicle and the satisfaction of the traveller. To increase the vibroacoustic comfort it is neces- sary to know qualitatively and quantitatively the noise and vibration sources, as well as their transmission paths. Thus, in this work a procedure has been defined and applied to measure and analyse the vibration and acoustic behaviour of a coach in different operating modes: idling, three constant speeds, acceleration and deceleration. 32 acceleration and sound pressure signals have been acquired, corresponding to different interior and exterior points of the coach. The analysis of the acceleration autospectra has allowed to determine the contribution of elements like floor, glasses and lateral panels to the sound pressure perceived by the traveller in the passenger compartment in the different studied operating modes.

4pNSf6. Experimental approach for reducing uncertainties associated with road vehicle noise according to ISO 362. Louis-Ferdinand Pardo (UTAC, Autodrome de Linas-Montlhéry, BP 20212, 91311 Montlhéry, France, louis-ferdinand.pardo@utac.com), Thierry Ageron (UTAC, Autodrome de Linas-Montlhéry, BP 20212, 91311 Montlhéry, France, ageron@utac.com), Serge Ficheux (UTAC, Autodrome de LinasMontlhéry, BP 20212, 91311 Montlhéry, France, ficheux@utac .com), Celine Berthou (UTAC, Autodrome de Linas-Montlhéry, BP 20212, 91311 Montlhéry, France, berthou@utac.com)

This paper proposes an approach for reduction uncertainties related to sound pressure levels measured in accordance with procedures given in ISO 362 for noise emitted by road vehicles under acceleration This approach is based on an experimental assessing of several disturbing factors that lead to variation in the resulting level observed day to day and site to site for the same vehicle. The assessment of corrections to build the model is based on an independent analysis on each influent factor (test track, noise measuring device and temperature) taking into account of vehicle noise behaviour depending on power unit and tyres. 


\title{
Session $4 p N S g$
}

\section{Noise, Architectural Acoustics, and EURONOISE: Noise, Vibration and Acoustics for Medical and Research Facilities and Their Occupants II (Poster Session)}

\author{
James West, Cochair \\ Johns Hopkins University \\ Jack Evans, Cochair \\ JEAcoustics \\ Marc Asselineau, Cochair \\ Peutz \& Associés \\ Erica Ryherd, Cochair \\ Georgia Institute of Technology
}

\begin{abstract}
All posters will be on display from 3:40 p.m. to 5:20 p.m. To allow contributors an opportunity to see other posters, contributors of odd-numbered papers will be at their posters from 3:40 p.m. to 4:30 p.m. and contributors of even-numbered papers will be at their posters from 4:30 p.m. to 5:20 p.m.
\end{abstract}

\section{Contributed Papers}

4pNSg1. Noise control of laboratories: case studies. Marc Asselineau (Peutz \& Associés, 10 rue des Messageries, F75010 Paris, France, m.asselineau@peutz.fr)

Laboratories for research and production usually feature work areas complete with exhaust equipment as well as specific equipment (e.g. heaters, shakers, etc.). More to the point, such research and production areas are often linked to open plan offices where workers process their results, as well as partitioned offices where supervising engineers or researchers work. Suchfitting out is supposed to help circulate information around the team and save valuable space. Unfortunately, it easily can be noisy and rather uncomfortable, especially when coming from older fully partitioned laboratories and offices. This paper aims to illustrate a few acoustical highlights of laboratories through a few cases studies, looking at such parameters as spatial sound level decay, background noise level and reverberation time, but also at such additional factors as general background and psychological aspects, and looking at tentative standards. It turns out that both the acoustical quality of the room and the space planning must be developed according to the users' needs.

4pNSg2. Noise reduction in an operating room: A case study. James West (Johns Hopkins University, Department of Electrical Engineering, 3400 North Charles Street, Baltimore, MD 21218, USA, jimwest@jhu.edu), Ilene Busch-Vishniac (McMaster University, 1280 Main Street West, Hamilton, ON L9G 4X6, Canada, provost@mcmaster.ca), Joseph King (Shamoon College of Engineering, Math. Department, Bialik/Basel Sts., 84100 Beer Sheva, Israel, joseph.a.king@usa.dupont.com), Natalia Levit (DuPont, 5401 Jefferson Davis Highway, Richmond, VA 23234, USA, natalia.v.levit@usa.dupont .com)

In our previous study reported in INTER-NOISE 2006 we found that operating rooms are among the most problematic areas in the healthcare industry. The maximum peak levels measured during various surgical procedures are extremely high - 100-120 dB, which can potentially lead to the hearing damage and interfere with the speech communication during surgery. Neurosurgery and orthopedic operating rooms are found to be among the noisiest overall. However, introduction of the acoustical materi- als to the operating room is very difficult due to the strict infectious control requirements. We report here a case study on using sound absorptive panels protected by DuPont Tyvek®, a unique flash spun plexifilamentary filmfibril sheet, combining excellent barrier properties with distinctive porous structure to make it acoustically transparent in the voice frequency range.

4pNSg3. Case Study of MRI Installation in Existing Hospitals. James Perry (Cerami \& Associates, 404 Fifth Avenue, New York, NY 10018, USA, jperry@ceramiassociates.com)

Medical facilities frequently seek to add function and capacity to their imaging facilities. The addition of new MRI units into existing hospital spaces is quite common, however many manufacturers impose and stringent low-frequency vibration limits which must be met before a unit can be delivered and installed. Vibration sources such as mechanical systems and external transit can degrade the achievable imaging resolution at levels undetectable by unaided humans. A case study is presented for one such project which required significant vibration controls to existing ventilation, water, and electrical systems to ensure acceptable vibration levels at an unlikely equipment installation.

4pNSg4. Nanotechnology research facility- A vibration and noise control design case study. Jack B. Evans (JEAcoustics, 1705 West Koenig Lane, Austin, TX 78756, USA, Evans@JEAcoustics.com), Chad N. Himmel (JEAcoustics, 1705 West Koenig Lane, Austin, TX 78756, USA, Himmel@JEAcoustics.com), Daniel J. Kupersztoch (JEAcoustics, 1705 West Koenig Lane, Austin, TX 78756, USA, Kupersztoch@JEAcoustics .com)

Vibration and noise control would be critical to the success of a proposed nanotechnology and molecular research facility. Roadway traffic, a nearby power generation plant and buildings in the vicinity were potential sources of ground borne vibration disturbances. Mechanical equipment, occupant installed support apparata and occupant activities were potential sources of internally generated vibration disturbances. On-site ground borne vibration was measured for comparison with generic floor vibration criteria for sensitive installations. Results were analyzed relative to criteria for 
potential disturbance of sensitive equipment, perception by occupants, audible radiated structure borne noise in acoustically sensitive spaces and resolution-degrading motion for scanning and for transmission electron microscopes and other nanotechnology clean room equipment. Design guidelines and structural vibration control concepts were recommended to the structural engineers, including de-tuning, damping and isolation methods. Recommendations were provided for mechanical noise control and vibration isolation. Architectural noise control, sound isolation and room acoustics guidelines were provided for research, office and meeting spaces. This case study will discuss the desired vibration and noise control objectives and the design solutions that were implemented. Building photographs will be presented. Post-construction measurement results will be graphically compared with pre-construction conditions to demonstrate apparent degree of success in mitigating vibration.

\title{
Session 4pNSh
}

\section{Noise, ASA Committee on Standards, and EURONOISE: Measurement of Occupational Noise Exposure II (Poster Session)}

\author{
William Murphy, Cochair \\ National Institute for Occupational Safety and Health \\ Beat Hohmann, Cochair \\ Suva, Physics Section
}

\begin{abstract}
All posters will be on display from 3:40 p.m. to 5:20 p.m. To allow contributors an opportunity to see other posters, contributors of odd-numbered papers will be at their posters from 3:40 p.m. to 4:30 p.m. and contributors of even-numbered papers will be at their posters from 4:30 p.m. to 5:20 p.m.
\end{abstract}

\section{Contributed Papers}

4pNSh1. New findings on noise exposure in the construction trades. Reimer Paulsen (BGIA - Institut für Arbeitsschutz, Alte Heerstr. 111, 53757 Sankt Augustin, Germany, reimer.paulsen@dguv.de)

The degree to which employees on construction sites are exposed to noise often depends on a variety of individual activities. The calculation of the average noise exposure for different construction trades in this sector calls for an enhanced level of work analysis and measurement. Recommended here are personal measurements with simultaneous recording of the various activities and machines employed. With the subsequent assignment of average sound pressure levels and activities, it is then possible to draw conclusions about part exposures and mean exposure. At euronoise 2006 in Tampere, findings for a series of construction trades were presented. In the meantime, this series of measurements has been extended to include further trades. Furthermore subsequent measurements have been performed for individual trades, this was considered necessary because certain machines and working methods have changed in these fields in the last 20 years. The findings show that technical progress does not necessarily result in a reduction in noise exposure.

4pNSh2. Who can guaranty compliance with the Exposure Limit Value of $87 \mathbf{d B}(\mathbf{A})$ ? Fabien Krajcarz (Gamba Acoustique, 2 rue de la Découverte, BP 163, 31676 Labege Cedex, France, fabien.krajcarz@acoustiquegamba.fr)

The level of daily exposure (Lex8h) of workers has to be measured in accordance with French standard NFS 31084. The attenuation of personal noise protection has to be determined according to standard NF EN ISO 4869-2. In addition to the difficulties of applying these methods, one has to be aware that the actual effectiveness of the protection can deviate considerably from its theoretical effectiveness and, ultimately, it is the effective duration of wearing the protection that really determines the protection of workers. We have provided modest evidence, more than a survey in the strictest sense of the term, of the conditions in which the protectors are worn (or not) at a large French industrial company. The results have shown that the wearing of hearing protectors is far from being routine, even when the noise levels are high. Thus, the question then arises as to the guarantee which the entity or person responsible for measuring bears for compliance with the Exposure Limit Value of $87 \mathrm{~dB}(\mathrm{~A})$, when wearing the protection.

4pNSh3. Measurement Method for Noise Exposure of Jobs of the Construction Sector. Samuel Quintana (Universidad de Castilla-La Mancha, Campus Universitario, 16071 Cuenca, Spain, Samuel.Quintana @uclm.es), Marcos D. Fernandez (Universidad de Castilla-La Mancha, Campus Universitario, 16071 Cuenca, Spain, Marcos.Fernandez @uclm.es), Noelia Chavarria (Universidad de Castilla-La Mancha, Campus Universitario, 16071 Cuenca, Spain,noe_chava@hotmail.com), Jose A. Ballesteros (Universidad de Castilla-La Mancha, Campus Universitario, $16071 \quad$ Cuenca, Spain, Josea.Ballesteros@uclm.es), Isabel Gonzalez (Universidad de Castilla-La Mancha, Campus Universitario, 16071 Cuenca, Spain, Isabel.Gonzalez@uclm.es)

Noise is one of the physical contaminants with a high presence in the construction sector. Nowadays, several negative effects produced by the exposure to noise are known, mainly regarding hearing. Although there is evidence of the existence of many other effects, some of them are not characterized precisely yet. Due to the importance that these effects have on the worker's health and well-being, it is necessary to develop some mechanisms to study and suggest preventive solutions on these questions. In this work, it has been studied the most appropriate measurement method for taking, as precisely as possible, the noise levels that the workers of the construction sector are exposed to. Several measures have been taken and analyzed to determine the best indexes and parameters to characterize the noise in the construction. For which, the current European regulations regarding the noise exposure (Directive 2003/10 of the EU) have always been taken into account. 
4pNSh4. Impulsive occupational exposure for workers in drop-forge foundries. Edward L. Zechmann (National Institute for Occupational Safety and Health, 4676 Columbia Parkway, Mailstop C-27, Cincinnati, OH 45226-1998, USA, cri6@cdc.gov), Scott Brueck (National Institute for Occupational Safety and Health, 4676 Columbia Parkway, Mailstop C-27, Cincinnati, OH 45226-1998, USA, zcd6@cdc.gov), William J. Murphy (National Institute for Occupational Safety and Health, 4676 Columbia Parkway, Mailstop C-27, Cincinnati, OH 45226-1998, USA, wjm4 @ cdc.gov)

Occupational noise exposure limits for impulsive sounds in the United States are established by the Occupational Safety and Health Administration and recommended by the National Institute for Occupational Safety and
Health to permit no impulsive exposure in excess of $140 \mathrm{~dB}$ peak SPL. Peak pressure levels in excess of this limit can be found in industrial sectors such as construction, law enforcement and manufacturing. Taylor et al. [J Acoust. Soc. Am 76:807-819, 1984] reported equivalent A-weighted levels, $L_{\text {Aeq }}$, of $108 \mathrm{~dB}$ for forge operators sampled in seven drop-forge foundries in the United Kingdom. In a recent study of noise exposure at two drop-forge manufacturing plants in the U.S., peak impulse levels were measured in a range of 117 to $154 \mathrm{~dB}$ peak SPL and 8-hour equivalent A-weighted levels, $L_{A e q 8}$, for forge operators between 95 and $116 \mathrm{~dB}$ and for press operators between 95 and $105 \mathrm{~dB}$. Kurtosis, A, B C and D-durations were assessed for the impulsive and continuous noise samples. This paper will present the results of measurement and analysis of noise exposures typical for a dropforge facility.

\title{
Session 4pNSi
}

\section{Noise, Structural Acoustics and Vibration, Signal Processing in Acoustics, and EURONOISE: Airframe Noise Measurement, Prediction, and Control II (Poster Session)}

\author{
Joe Posey, Cochair \\ NASA \\ Denis Gely, Cochair \\ ONERA
}

\begin{abstract}
All posters will be on display from 3:40 p.m. to 5:20 p.m. To allow contributors an opportunity to see other posters, contributors of odd-numbered papers will be at their posters from 3:40 p.m. to 4:30 p.m. and contributors of even-numbered papers will be at their posters from 4:30 p.m. to 5:20 p.m.
\end{abstract}

\section{Contributed Papers}

4pNSi1. Overview of revolutionary aircraft for quiet communities workshop. Joe W. Posey (NASA, Langley Research Center, MS 461, Hampton, VA 23681, USA, joe.w.posey@ nasa.gov)

At some time in the future, technical advances, environmental imperatives, societal expectations for mobility, and economic drivers will dictate that radically different aircraft will be built and flown. Therefore, aircraft designs will change even if low noise were not one of the environmental imperatives, and acousticians must be aware of the possible directions for aircraft design and the resulting opportunities and challenges for noise control. To address this need, the National Aeronautics and Space Administration sponsored a workshop entitled Revolutionary Aircraft for Quiet Communities in Hampton, VA, 24-26 July 2007. Twenty-six talks covered aircraft design, interior noise challenges, airframe noise, propulsion, and aircraft noise prediction. Revolutionary aircraft will employ dramatically improved materials, propulsion systems, and flow control technology to improve efficiency and enhance mobility. Five hours of discussion surfaced many concerns and recommendations, including the increasing need for acousticians to be involved on highly integrated teams throughout the vehicle design process. Also, other discipline experts participating in aircraft design need some education in acoustics to increase their sensitivity to noise control issues. The National Institute of Aerospace hosted the event, and the Council of European Aerospace Societies and the American Institute of Aeronautics and Astronautics were co-sponsors.
4pNSi2. Adaptive closed-loop control of cavity flows. Louis Cattafesta (University of Florida, 231 MAE-A, P.O. Box 116250, Gainesville, FL 32611, USA, cattafes@ufl.edu), Srinivasan Arunajatesan (Combustion Research and Flow Technology, Inc., 6210 Keller's Church Road, Pipersville, PA 18947, USA, ajs@craft-tech.com), Qi Song (University of Florida, 231 MAE-A, P.O. Box 116250, Gainesville, FL 32611, USA, song@ufl.edu), Cesar Moreno (University of Florida, 231 MAE-A, P.O. Box 116250, Gainesville, FL 32611, USA, cesargm@ufl.edu), Miguel Palaviccini (University of Florida, 231 MAE-A, P.O. Box 116250, Gainesville, FL 32611, USA, mrp1124@ufl.edu)

Results from a combined experimental and computational study are presented on the development of an adaptive feedback controller for the suppression of cavity pressure loads. The experiments are performed in a variable-sized cavity in a high-speed wind tunnel, while the computations are performed using the CRAFT CFD flow solver. The adaptive control system incorporates recursive algorithms for system identification with disturbance rejection algorithms for feedback control. Results are presented using unsteady surface pressure sensors on the cavity walls and an array of zeronet mass-flux (ZNMF) actuators at the leading edge. The experimental data are used to compare with and validate the computations. These novel simulations form a virtual experiment testbed that is used to assess, for example, actuator type, placement, and requirements and also candidate identification and control algorithms. 


\title{
Session 4 pNSj
}

\section{Noise and EURONOISE: Soundscape in the Heritage of Urban and Natural Areas II (Poster Session)}

\author{
Bennett Brooks, Cochair \\ Brooks Acoustics Corp. \\ Giovanni Brambilla, Cochair \\ CNR Institute of Acoustics
}

\begin{abstract}
All posters will be on display from 3:40 p.m. to 5:20 p.m. To allow contributors an opportunity to see other posters, contributors of odd-numbered papers will be at their posters from 3:40 p.m. to 4:30 p.m. and contributors of even-numbered papers will be at their posters from 4:30 p.m. to 5:20 p.m.
\end{abstract}

\section{Contributed Papers}

4pNSj1. Noise masking as a soundscaping measuring procedure Mohammed Boubezari (CAPS, Instituto Superior Técnico, TU Lisbon, Av Rovisco Pais, P-1049-001 Lisbon, Portugal, boubezari@gmail.com), J. Luis Bento Coelho (CAPS, Instituto Superior Técnico, TU Lisbon, Av. Rovisco Pais, P-1049-001 Lisbon, Portugal, bcoelho@ist.utl.pt)

Loudness is the perceived intensity of a sound that could solve the problem of measuring a sound that is already filtered by the listening procedure. However, technically it is impossible to separate physically the sources of a noise into its components as well as human perception does. Loudness cannot be operative if a sound is merged with other noises. The principle of the proposed solution consists on gradually mixing a white noise during listening until the selected sound that one wants to measure is totally masked. The level of the masking white noise is controlled independently from the listener and measured with $\mathrm{dB}(\mathrm{A})$. The listener controls the limit of audibility of the selected sound, which is masked by the white noise. This paper describes the patent deposited around this method and shows the results obtained so far, and how this technique can be helpful in a visual translation of the sound space composition.

4pNSj2. Adaptive Characterization of Near and Far field Elements in the Soundscape. David R. Barclay (Marine Physical Lab, Scripps Institution of Oceanography, UCSD, 9500 Gilman Dr. M/C 0238, La Jolla, CA 92093-0238, USA, dbarclay@mpl.ucsd.edu)

Characterization of a soundscape through objective parameters relies on our understanding of psychoacoustics and ability to model the complex signal processing of the mind. Certain physical parameters such as loudness and timbre are easily retrieved from data while other descriptive parameters are more difficult to measure objectively. Several signal processing algorithms are presented here in the context of describing a soundscape in terms of keynote sounds (background noise) and sound signals (foreground sounds). Simulations and stereo field data recorded in San Diego are analyzed. Adaptive matched field processing is used in conjunction with conventional spectral analysis for the detection and categorization of near field events. These sounds are then removed to provide a more accurate description of keynote sounds. Spatial distribution of the soundscape is measured using conventional beamforming algorithms.

4pNSj3. Internet and mobile technologies for a public role in noise surveying. Charlie Mydlarz (The University of Salford, C.S.E., Salford University, The Crescent, M5 4WT Manchester, UK, c.mydlarz@salford.ac
.uk),Ian Drumm(The University of Salford, C.S.E., Salford University, The Crescent, M5 4WT Manchester, UK, i.drumm@salford.ac.uk), Trevor J. Cox (University of Salford, Acoustics Research Centre, Newton Building, M5 4WT Salford, UK, t.j.cox@ salford.ac.uk)

The traditional method of noise surveying is to use trained professionals to go to a specific site to measure and assess noise levels using dedicated and expensive equipment. This project aims to enfranchise the public by providing them with the opportunity to play an active role in noise measurement and assessment, as well as how their soundscapes are shaped. With the implementation of mobile phone, PDA and PC applications, alongside web based collation techniques; we aim to empower the public in the gathering of context specific data on soundscapes. The methodology will provide a case study for the wider research community in developing public participation-based research activities of this kind. This will provide a better understanding of the public's relationship with their soundscape and how this relationship varies with location and demographic data. Inferences will be compared from the analysis of data sets generated with other soundscape research with a view towards validating the techniques and gaining new insights into the field.

4pNSj4. From Descriptive to Predictive Soundscape Representation. Mohammed Boubezari (CAPS, Instituto Superior Técnico, TU Lisbon, Av. Rovisco Pais, P-1049-001 Lisbon, Portugal, boubezari@gmail.com), J. Luis Bento Coelho (CAPS, Instituto Superior Técnico, TU Lisbon, Av. Rovisco Pais, P-1049-001 Lisbon, Portugal, bcoelho@ist.utl.pt)

Regarding the knowledge on psychoacoustics (Zwickers), on phenomenology of perception (Gibson. J. J.) and the results obtained at CAPS-IST (Boubezari-Bento Coelho), a descriptive and qualitative sound map was drawn and a predictive procedure was developed. The method shows that the introduction of human perception during the process of analysis and signal processing makes it possible to target measurements on one or more noise sources selected separately from their background noise. Contrary to conventional measurements, which yield overall values of LAeq, without distinguishing the sources, the method presented here allows a space description of a sound space by making each sound stand out from its context. The method allows the measurement of the range of a noisy source in a given place, the testing of the range or the masking of an urban device (fountain) or contribute to the decision and the design of a specific architectural project, for example. This paper describes the method (and its limits) and the results obtained and shows how a predictive qualitative sound map with low cost is now feasible. 


\title{
Session 4pNSk
}

\section{Noise and EURONOISE: Potential to Reduce Tire/Road Noise II (Poster Session)}

\author{
J. Stuart Bolton, Cochair \\ Ray W. Herrick Labs., School of Mech. Eng., Purdue University \\ Ernst-Ulrich Saemann, Cochair \\ Continental AG
}

\begin{abstract}
All posters will be on display from 3:40 p.m. to 5:20 p.m. To allow contributors an opportunity to see other posters, contributors of odd-numbered papers will be at their posters from 3:40 p.m. to 4:30 p.m. and contributors of even-numbered papers will be at their posters from 4:30 p.m. to 5:20 p.m.
\end{abstract}

\section{Contributed Papers}

4pNSk1. Frequency loci veering in deformed rotating tyres. Ruud Van Doorn (Eindhoven University of Technology, Dept. of Mechanical Engineering, Dynamics \& Control, P.O. Box 513, 5600MB Eindhoven, Netherlands, r.r.j.j.v.doorn@student.tue.nl), Ines Lopez (Eindhoven University of Technology, Dept. of Mechanical Engineering, Dynamics \& Control, P.O. Box 513, 5600MB Eindhoven, Netherlands, i.lopez@tue.nl), René Van Der Steen (Eindhoven University of Technology, Dept. of Mechanical Engineering, Dynamics \& Control, P.O. Box 513, 5600MB Eindhoven, Netherlands, r.v.d.steen@tue.nl), N.b. Roozen (Eindhoven University of Technology, Dept. of Mechanical Engineering, Dynamics \& Control, P.O. Box 513, 5600MB Eindhoven, Netherlands, n.b.roozen@tue.nl), Henk Nijmeijer (Eindhoven University of Technology, Dept. of Mechanical Engineering, Dynamics \& Control, P.O. Box 513, 5600MB Eindhoven, Netherlands, h.nijmeijer@tue.nl)

In previous work [1] a methodology to model tyre vibrations has been developed, which exploits a modal base determined in a standard FE package and includes rotational effects by a coordinate transformation. In the present paper, the effect of rotation on the eigenfrequencies of a deformed tyre is examined. It is well-known that rotation splits the eigenfrequencies of an undeformed tyre symmetrically around the eigenfrequencies of the nonrotating tyre, where the slope of the eigenfrequency-lines is determined by the circumferential wave number and tyre radius only. However, the eigenfrequency-lines of a deformed tyre demonstrate a fascinating mutual repulsion behaviour if the velocity is increased. This phenomenon is known as frequency loci veering and is induced by the a-periodicity resulting from the tyre deformation. Besides the effect of veering on the eigenfrequencies, the corresponding eigenmodes interact in the transition zones and finally interchange. The effects of veering are extremely intensified by the high modal density of the tyre structure, which results in a decreasing eigenfrequency distribution when the rotational velocity increases. [1] I. Lopez et al, JSV 307, 481-494, (2007)

4pNSk2. A model to evaluate the importance of tangential contact forces for tyre/road noise generation. Patrick Sabiniarz (Chalmers University of Technology, Division of Applied Acoustics, SE-41296 Gothenburg, Sweden, patrick.sabiniarz@ chalmers.se), Wolfgang Kropp (Chalmers University of Technology, Division of Applied Acoustics, SE-41296 Gothenburg, Sweden, wolfgang.kropp@chalmers.se)

The interaction between tyre and road is a complex non-linear process including radial and tangential contact forces between tyre and road surface. During recent years models have been developed which allow for predicting radial forces as function of surface and tyre properties. These models can be used to quantify the influence of radial forces on the noise generation Whereas in many cases time varying radial forces are the main reason for the generation of tyre noise, in some cases other mechanisms seem to be dominant. This paper aims on understanding the influence of tangential contact on tyre/road noise generation. For this reason the model developed by the Chalmers Tyre Road Noise Group has been extended to also include tangential interaction. The model is based on the same concept as that used by McIntyre and Woodhouse for modelling string/bow contact of musical instruments. It computes the time varying normal and tangential contact forces for a tyre rolling over a rough surface at constant speed. A small selection of simulation results are presented aiming at showing the effect of different parameters, such as friction coefficient and normal load, on the occurrence of instability phenomena such as stick/slip vibrations. 


\title{
Session 4pNSI
}

\author{
Noise and EURONOISE: Noise from Wind Power Projects II (Poster Session) \\ Eddie Duncan, Cochair \\ Resource Systems Group (RSG) \\ Kerstin Persson Waye, Cochair \\ Dept. of Environ. Medicine, The Sahlgrenska Acad. of Gothenburg Univ.
}

\begin{abstract}
All posters will be on display from 3:40 p.m. to 5:20 p.m. To allow contributors an opportunity to see other posters, contributors of odd-numbered papers will be at their posters from 3:40 p.m. to 4:30 p.m. and contributors of even-numbered papers will be at their posters from 4:30 p.m. to 5:20 p.m.
\end{abstract}

\section{Contributed Papers}

4pNSI1. A rational approach for regulating windturbine noise. Martin Van Den Berg (Ministry VROM, Po. Box 30945, IPC 635, 2500GX Den Haag, Netherlands, martin.vandenberg@minvrom.nl)

Windturbines have evolved from the cosy, wooden Dutch windmill type to large industrial generators of sustainable energy. Their noise production luckily did not evolve in step, but in some cases they can be a perhaps unsustainable nuisance for population living nearby. Presently the local and national authorities are trying to get a hold on the problem, often still using methods from the wooden machine age. The paper describes a modern approach for dealing with windturbine noise, using harmonized methods and recently acquired insights in the reaction of people to this particular noise. Tentative dose-effect relations in terms of Lden and Lnight enable comparisons with noise from transport.

4pNSI2. Wind farm aural and visual impact in the Netherlands. Frits Van Den Berg (University of Groningen - Science \& Society Group, Nijenborgh 4, 9747AG Groningen, Netherlands, fvdberg@ggd.amsterdam .nl), Eja Pedersen (Occupational and Environmental Medicine, Göteborg University, PO Box 100, SE-405 30 Göteborg, Sweden, eja.pedersen @ set.hh.se), Roel Bakker (Northern Centre for Healthcare Research, University Medical Centre Groningen, PO Box 30001, 9700 RB Groningen, Netherlands, R.H.Bakker@med.umcg.nl), Jelte Bouma (Northern Centre for Healthcare Research, University Medical Centre Groningen, PO Box 30001, 9700 RB Groningen, Netherlands, j.bouma@med.umcg.nl)

The WINDFARM perception project, carried out in 2006 in the Netherlands, aimed to explore the impact of wind farms on people living close to wind farms. The study sample was selected in three types of area (countryside, countryside with major road, built up area) by means of a Geographic Information System (GIS). Each selected address was within $2.5 \mathrm{~km}$ of a wind turbine of at least $500 \mathrm{~kW}$ electric power and a similar turbine within $500 \mathrm{~m}$ of the first. Aural impact was calculated according to three different sound propagation models: the international ISO-9613 standard, the model legally required in the Netherlands, and a simplified model as in the New Zealand Standard NZS-6808. Visual impact was quantified in two ways: the vertical angle determined by the height of the apparently tallest turbine, and the solid angle determined by all turbines where each turbine was replaced by a vertical rectangle just enclosing the turbine. Immission sound levels from the wind farms at 1948 receiver locations varied from 21 to $54 \mathrm{~dB}(\mathrm{~A})$, relative size from $0.01 \%$ to $30 \%$ of the total field of view. Results show that all impact measures are highly correlated with distance to the nearest wind turbine.

4pNSI3. Dispersal of measured sound power levels for wind turbines. René Gamba (Gamba Acoustique, 2 rue de la découverte, BP 163, 31676 Labege Cedex, France, rene.gamba@acoustique-gamba.fr), Sébastien Garrigues (Gamba Acoustique, 2 rue de la découverte, BP 163, 31676 Labege Cedex, France, sebastien.garrigues@acoustique-gamba.fr)

The standard IEC $61400-11$ provides guidance in the measurement, analysis and reporting of acoustic emissions (sound power levels) from wind turbine generator systems. The application of this standard aims to provide accurate results that can be replicated by others. We did several measurement operations according to this standard on various wind farms fitted with many turbine manufacturers on different ground types. Important differences have been noticed with equal working conditions between the most and the less noisy wind turbine on a single farm. We will present these results compared to the manufacturers' guaranteed values and initiate explanations (like the difficulties to link the wind speed at $10 \mathrm{~m}$ above ground with the wind speed received at hub height; or the influence of wind incidence on blades). 


\title{
Session 4pNSm
}

\section{Noise and EURONOISE: Vibration Perception II (Poster Session)}

\author{
Patricia Davies, Cochair \\ Ray W. Herrick Lab., School of Mechanical Engineering, Purdue Univ. \\ Michael Griffin, Cochair \\ Institute of Sound and Vibration Research, University of Southampton
}

\begin{abstract}
All posters will be on display from 3:40 p.m. to 5:20 p.m. To allow contributors an opportunity to see other posters, contributors of odd-numbered papers will be at their posters from 3:40 p.m. to 4:30 p.m. and contributors of even-numbered papers will be at their posters from 4:30 p.m. to 5:20 p.m.
\end{abstract}

\section{Contributed Papers}

4pNSm1. Hand-arm equal sensation curves for steering wheel translational and axial vibration. Mickael Sauvage (PSA Peugeot Citroën, Centre Technique de Vélizy, Route de Gisy, 78943 VélizyVillacoublay, France, mickael.sauvage@mpsa.com), Elise Gressant (PSA Peugeot Citroën, Centre Technique de Vélizy, Route de Gisy, 78943 VélizyVillacoublay, France, elise.gressant@u-cergy.fr), Olivier Lescop (PSA Peugeot Citroën, Centre Technique de Vélizy, Route de Gisy, 78943 VélizyVillacoublay, France, olivier.lescop@mpsa.com), Vincent Roussarie (PSA Peugeot Citroën, Centre Technique de Vélizy, Route de Gisy, 78943 VélizyVillacoublay, France, vincent.roussarie@mpsa.com)

The aim of the study was to determine the hand-arm equal sensation curves for steering wheel translational and axial vibration. A sensory panel of 10 trained judges performed a two-step procedure. The test stimuli used were sinusoidal vibrations in the range from 4 to $60 \mathrm{~Hz}$, with amplitude of $0.2 \mathrm{~m} / \mathrm{s}^{2} \mathrm{rms}$. The first step was to determine perceived sensations for each frequency. Four families of vibration were defined (pumping movement, shaking sensation, trembling sensation and prickling sensation). The second step was a three-down-one-up test based on these families to determine the equal sensation level. Results showed that perceived intensity depends on vibration family, frequency and excitation direction (translational or axial).

4pNSm2. A new instrument for the measurement of occupational vibration. Laurent Faiget (01dB-Metravib, 200 Chemin des Ormeaux, F-69578 Limonest, France, laurent.faiget@01db-metravib.com), Fernand
Dupont(01dB-Metravib, 6 Avenue Louis Blériot, F-31570 Ste Foy D’Aigrefeuille, France, fernand.dupont@01db-metravib.com), Christine Aujard (01dB-Metravib, 200 Chemin des Ormeaux, F-69578 Limonest, France, christine.aujard@01db-metravib.com)

Directive 2002/44/CE relative to the risks arising from vibration has been transcribed into national laws of most Member States. This directive deals with the determination of limits and action values for the daily exposure to vibration. Two physiological domains are addressed: the "hand-arm" and the "whole-body" domains, the acceptable statutory values of which are specified in the text. The experimental protocol, as well as indicators relevant for assessment, is defined in Standards ISO 5349-2 and ISO 2631-1. The equivalent frequency-weighted acceleration shall be measured on the 3 axes $\mathrm{x}, \mathrm{y}$ and $\mathrm{z}$, the bandwidth of which is defined for each domain. $01 \mathrm{~dB}$ Metravib introduces a new portable instrument that perfectly meets the requirements of this statutory application. A blind metrological instrument connected to a triaxial accelerometer is installed on site. The instrument is remotely controlled by the operator using a wireless remote control of the Pocket PC type. This remote control is used to manage measurement configurations ("whole-body", hand-arm"), to start acquisitions (immediate, delayed mode) and to collect measured data for post-processing and archiving purposes. We will present metrological and operational advancements of this new instrument and describe a real case study dealing with the assessment of daily exposure to vibration. 


\title{
Session 4pNSn
}

\section{Noise, Computational Acoustics, and EURONOISE: Source Identification in Radiation and Scattering II (Poster Session)}

\author{
J. Stuart Bolton, Cochair \\ Ray W. Herrick Labs., School of Mech. Eng., Purdue University \\ Peter Juhl, Cochair \\ Institute of Sensors, Signals and Electrotechnics, University of Southern Denmark
}

\begin{abstract}
All posters will be on display from 3:40 p.m. to 5:20 p.m. To allow contributors an opportunity to see other posters, contributors of odd-numbered papers will be at their posters from 3:40 p.m. to 4:30 p.m. and contributors of even-numbered papers will be at their posters from 4:30 p.m. to 5:20 p.m.
\end{abstract}

\section{Contributed Papers}

4pNSn1. Adaptation of the propagator for numerical acoustic holography of a wheel type object. Thibault Le Bourdon (Institut Jean Le Rond d'Alembert, UMR CNRS 7190, UPMC, 2 Place de la Gare de Ceinture, 78210 Saint-Cyr 1'Ecole, France, lbourdon@ccr.jussieu.fr), Vincent Martin (Institut Jean Le Rond d'Alembert, UMR CNRS 7190, UPMC, 2 Place de la Gare de Ceinture, 78210 Saint-Cyr l'Ecole, France, vmartin@ccr .jussieu.fr)

Holography procedure of a vibrating object can be geometrically interpreted resulting in a guarantee of its identified vibratory velocity, the quality of which depends on the pressure measurement at an array of microphones and on the propagation model. In case of a wheel with the panel body, a plane array parallel to the visible side is the only one possible. The velocity is then accessible on the side concerned and needs a propagation model with adequate acoustic conditions over the whole plane with the visible side (source plane). Having in mind such a configuration, a theoretical work in the $3 \mathrm{D}$ space has shown the surrounding influence on the reconstructed velocity on the visible side. It has appeared that vibrations other than the ones of the front side and the rear acoustic load can be described with an admittance on the source plane. We present here an exhaustive search for the adequate admittance concerned leading to a correct propagator and a correct velocity identified. The success achieved in the procedure may rely on the single velocity and single model liable to radiate a given pressure on a sufficiently large antenna.
4pNSn2. Exact solutions to the acoustic source reconstruction problem Cédric Maury (Université de Technologie de Compiègne, Centre de Recherche Royallieu, BP20529, 60205 Compiègne, France, cedric.maury@utc .fr), Teresa Bravo (Université de Technologie de Compiègne, Centre de Recherche Royallieu, BP20529, 60205 Compiègne, France, teresa.bravo-maria @utc.fr)

In this study analytical solutions are derived for the singular radiation and velocity patterns of a baffled elastic beam, thus leading to closed-form expressions for the singular value expansion of a number of integral operators which map a boundary velocity onto the acoustic pressure distribution radiated in far-field or intermediate regions. Exact solutions to this problem involve prolate spheroidal wave functions which correspond to a set of independent distributions with finite spatial support and with maximal energy concentration in a given bandwidth in the wavenumber domain. A stable solution to the inverse source reconstruction problem is obtained by decomposing the unknown boundary velocity into a number of efficiently radiating singular velocity patterns which corresponds to the number of degrees of freedom of the radiated field. It is found that the degree of ill-posedness of the inverse problem is significantly reduced when considering a hemicircular observation arc with respect to a linear array of sensors, by a factor scaling on the small angular aperture subtended by the observation line. Estimates are derived of the spatial resolution limits that can be achieved in the source reconstruction problem from the dimension of the efficiently radiating subspace. 


\title{
Session 4pNSo
}

\section{Noise, ASA Committee on Standards, and EURONOISE: Prominent Discrete Tones II (Poster Session)}

\author{
Robert Hellweg, Cochair \\ Wellesley, MA \\ Lothar Schmidt, Cochair \\ Currenta GmbH \& Co. OHG
}

\begin{abstract}
All posters will be on display from 3:40 p.m. to 5:20 p.m. To allow contributors an opportunity to see other posters, contributors of odd-numbered papers will be at their posters from 3:40 p.m. to 4:30 p.m. and contributors of even-numbered papers will be at their posters from 4:30 p.m. to 5:20 p.m.
\end{abstract}

\section{Contributed Papers}

4pNSo1. Tonalness perception of harmonic complex tones. Sebastian Fingerhuth (Institute of Technical Acoustics, RWTH Aachen University, Neustrasse 50, 52066 Aachen, Germany, sfi@akustik.rwth-aachen .de), Etienne Parizet (Laboratoire Vibrations Acoustique, Insa Lyon, 25 bis av. J. Capelle, 69621 Villeurbanne Cedex, France, etienne.parizet@insa-lyon fr)

Many everyday sounds have a more or less tonal characteristic. This normally means they have some peaks in the spectrum and generate one or more of the pitch sensations. In this work we present the results of a listening test which deals with the perception of tonalness of harmonic complex sounds. It consisted in presenting sounds via headphones to the 30 listeners who had to evaluate the tonalness using magnitude estimation with a reference sound. The sounds presented to the listeners varied in some parameters which modified the perception of tonalness. The parameters are: i) tone to noise level (3dB steps), ii) number of harmonics (1, 2, 4, 8 and 8 with f0 and $\mathrm{f} 1$ removed) and iii) spectral shape of the harmonics (constant, $-5 \mathrm{~dB} /$ oct and $5 \mathrm{~dB} /$ oct). The loudness of the stimuli were equalized. Tonalness increases for higher tone to noise ratio. A change in the spectral shape is most important for a high number of harmonics. More harmonics also increase tonalness mostly for constant and increasing spectral shape. The results of the test were also compared with the tonalness calculation algorithm from Aures' and the german DIN45681 model. Both gave a correlation coefficient > 0.95
4pNSo2. Methods for automating prominent tone evaluation and for considering variations with time or other reference quantities. Wade $\mathrm{R}$ Bray (HEAD acoustics, Inc., 6964 Kensington Road, Brighton, MI 48116, USA, wbray@headacoustics.com)

Information Technology acoustic protocols include identifying prominent tones according to likely subjective importance. Most existing methods for calculating tone-to-noise ratio (TNR) require a suspect tone to be selected by the analyst, who must also mark the width; both are potential sources of uncertainty and variability. One purpose of this paper is to present an automatic tone-detection and width-assessment methodology for more robust, less operator-intensive TNR calculation in accordance with ECMA74. The paper will also present a process giving a complete spectral representation of the prominence ratio (a specific prominence ratio) by iterating the calculation at each frequency bin of the Fourier transform of the timesignal. The ECMA-74 tone-detection procedure for TNR is also applicable to the specific prominence ratio and is automatable, yielding tones-only prominence results without user intervention for any or all tones qualifying as prominent. The conventional average prominent tone evaluation over an operating interval can cause mis-assessment or no assessment of subjectively prominent tones which change frequency and/or level with time. We will therefore also discuss applying the described procedures as functions of time or other reference quantities, and suggest considering the subjective significance of time-domain effects such as modulation occurring from certain multiple prominent tone situations. 


\title{
Session 4pNSp
}

\section{Noise and EURONOISE: Tire-Road Noise from the Road Perspective II (Poster Session)}

\author{
Paul Donavan, Cochair \\ Illingworth \& Rodkin, Inc. \\ Fabienne Anfosso-Ledee, Cochair \\ Laboratoire Central des Ponts et Chaussées
}

\begin{abstract}
All posters will be on display from 3:40 p.m. to 5:20 p.m. To allow contributors an opportunity to see other posters, contributors of odd-numbered papers will be at their posters from 3:40 p.m. to 4:30 p.m. and contributors of even-numbered papers will be at their posters from 4:30 p.m. to 5:20 p.m.
\end{abstract}

\section{Contributed Papers}

4pNSp1. Dense road surfaces with small aggregate size - tyre/road noise reduction after repaving. Nils-Åke Nilsson (Acoustic Control AB, Tumstocksvägen 1, SE-187 66 Taeby, Sweden, na.nilsson@acoustic.se), Nils Ulmgren (NCC Roads Sweden AB, R\&D Center, Bryggervägen 13, SE194 36 Upplands Väsby, Sweden, nils.ulmgren@ncc.se)

Open, porous road surfaces normally suffers from pore clogging particularly in inner city situations with low vehicle speed. After a relatively short time pore clogging could almost eliminate the noise reduction effect. A dense low noise road surface with smaller aggregate stone size could here offer substantial noise reduction due to reduced surface roughness, despite its low sound absorption factor. In this paper is presented data on the "repaving insertion loss" in $\mathrm{dB}(\mathrm{A})$ when exchanging a worn older SMA16 by a newly paved dense SMA8. Comparative results will also be shown for various types of newly paved SMA16 in comparison to the a newly paved SMA8. The technique for noise reduction mentioned above could be believed to have an "improved long term endurance" as compared to the same degree of noise reduction achieved by open graded technique particularly in city centres. For the Scandiavian countries where studded tyres are allowed, further reduction of stone size could be possible at acceptable wear rate if studded tyres are taxed or prohibited e.g. in environmental zones. This could lead to a further increased reduction of tyre/road noise in such zones. Grant from European Commission to Project Quiet City Transport (QCITY) is acknowledged.

4pNSp2. Measurement of pavement absorption using ISO 13472-2 Andrew F. Seybert (University of Kentucky, Dept. of Mech. Engineering, 151 RGAN Bldg., Lexington, KY 40506, USA, seybert@engr.uky.edu), Jun Han (University of Kentucky, Dept. of Mech. Engineering, 151 RGAN Bldg., Lexington, KY 40506, USA, jhan@engr.uky.edu)

This paper discusses some recent experience with ISO draft standard 13472-2 for the measurement of the absorption of pavements. The draft standard is meant to be used for the measurement of the sound absorption coefficient of "reflective" pavements, e.g., pavements of test tracks used for passby noise measurements of vehicles, tires, etc. An impedance tube has been constructed based on the requirements of the draft standard, and the device has been used in the laboratory (with pavement samples) and in situ to measure pavement absorption. The papers discusses these results and other important features of the draft standard including microphone calibration, parasitic tube absorption, and the construction features of the impedance tube and source.
4pNSp3. A first step toward a close proximity noise map. Moises Bueno (Laboratory of Acoustics Applied to Civil Engineering (LA2IC), Universidad de Castilla-La Mancha, Department of Applied Physics, 13071 Ciudad Real, Spain, moises.bueno@uclm.es), Urbano Viñuela (Laboratory of Acoustics Applied to Civil Engineering (LA2IC), Universidad de Castilla-La Mancha, Department of Applied Physics, 13071 Ciudad Real, Spain, urbano.vinuela@uclm.es), Fernando Terán (Laboratory of Acoustics Applied to Civil Engineering (LA2IC), Universidad de Castilla-La Mancha, Department of Applied Physics, 13071 Ciudad Real, Spain, fernando.teran @uclm.es), S. E. Paje (Laboratory of Acoustics Applied to Civil Engineering (LA2IC), Universidad de Castilla-La Mancha, Department of Applied Physics, 13071 Ciudad Real, Spain, santiago.exposito@uclm.es), Jeane Luong (Mecanique d'ingenierie des systemes, Université Pierre et Marie Curie, 75252 Paris, France, jeanne.luong@etu.upmc.fr)

In recent years, environmental noise has become a serious issue for civil infrastructure and environmental administration due to public concern over the subject of noise pollution. The most significant deterioration of environmental acoustics conditions comes from road traffic transportation. The predominant noise source is the combination of the tire/pavement interaction and the propulsion systems of the vehicles. Generally, tire/pavement interaction is the principal source of noise for speeds above $40 \mathrm{~km} / \mathrm{h}$ in the case of most modern cars. In this research, geo-referenced close proximity rolling noise is used for acoustical characterization of asphalt concrete surfaces in an urban environment. A close proximity noise map of streets with low speed limits is presented for a reference speed of $50 \mathrm{~km} / \mathrm{h}$. Different pavements and pavement conditions, common in urban streets, are analyzed: dense and semidense asphalt concrete, with Spanish denomination D-8 and S-12, respectively, and on the other hand, dense pavement at the end of its service life (D-8*). Noise levels from dense surfaces (D-8) increase significantly over time, principally due to the appearance of surface defects such as cracks and ruts.

4pNSp4. Road pavement classification based on noise emission characteristics. Joel P. Paulo (ISEL, R. Conselheiro Emídio Navarro,1, 1959-007 Lisbon, Portugal, jpaulo@deetc.isel.ipl.pt), J. Luis Bento Coelho (CAPS, Instituto Superior Técnico, TU Lisbon, Av. Rovisco Pais, P-1049001 Lisbon, Portugal, bcoelho@ist.utl.pt)

The measurement procedure to evaluate the influence of road surface characteristics on vehicle and traffic noise is designated by Close-Proximity (CPX) method, as described in the ISO 11819-2 draft. This procedure consists on acquiring the vehicle rolling noise signal near the tires and close to the surface by means of at least two microphones, in a special arrangement for the determination of the Close-Proximity Sound Index (CPXI). Road 
traffic noise is estimated by taking into account the absorption characteristics of road surface on the propagation of sound and the speed and type of vehicles. However, the particular characteristics of the different pavement types, which may influence the sound radiation, are not considered. The main goal of this research is to identify and classify different types of road pavements, for different stress conditions, using the CPX method. Such information can be used as a guideline for calibrating noise mapping models in order to achieve more realistic and accurate results. The classification of the different road surfaces consists on a supervised learning technique based on the Support Vector Machine, SVM, algorithms. Results based on error analysis are presented and discussed.

4pNSp5. A single wheel trailer for tire/road noise measurements enabling both the CPX- and pass-by methods. Martin Höjer (Acoustic Control AB, Tumstocksvägen 1, SE-187 66 Taeby, Sweden, martin.hojer@acoustic.se), Nils-Åke Nilsson (Acoustic Control AB, Tumstocksvägen 1, SE-187 66 Taeby, Sweden, na.nilsson@acoustic.se)

A single wheel trailer for tyre/road noise measurements has been developed. It has a towing beam, up to $8 \mathrm{~m}$ long, which ensures low background noise from support wheels and towing vehicle. Shielding hood is not necessary. The single wheel trailer can thus be used both for CPXmeasurements with on-board carried microphones and pass-by measurements using stationary road-side microphones. The advantage of measuring in the free field with a minimum influence from the suspension attachments etc. is that the emission frequency spectra will be almost undisturbed. It can thus be more easily compared to the sound levels at road-side residents. Due to the long towing beam, tyre prototypes $>10 \mathrm{~dB}$ quieter can be studied without disturbing background noise. The trailer has been specially designed to ensure stable vehicle dynamics, even though the normally loaded measurement tyre is rolling up to 8 meters behind the support trailer. Another interesting feature is the telescopic design of the towing beam, which enable compaction of the trailer so that the measurement wheel is landed on the trailer support part, enabling convenient transportation to the measurement site.

4pNSp6. A noise classification system for low-noise road surfacings experiences and status. Bent Andersen (Danish Road Institute/Road Directorate, Guldalderen 12, 2640 Hedehusene, Denmark, bea@ vd.dk), Jørgen Kragh (Danish Road Institute/Road Directorate, Guldalderen 12, $2640 \mathrm{He}$ dehusene, Denmark, kragh@vd.dk), Sigurd N. Thomsen (Danish Road Institute/Road Directorate, Guldalderen 12, 2640 Hedehusene, Denmark, snt @ vd.dk)

As a result of an increasing pressure from the population as well as from road administrations a noise classification system for road surfacings was put into operation in the autumn 2006. The so-called SRS-system (a Danish abbreviation for noise reducing wearing courses) is provisional and noncommittal for a couple of years until sufficient experience has been collected. It is a comprehensive system including: 'Guidelines on the use of low-noise asphalt surfacings for noise abatement. 'Method for declaration and documentation of noise reduction based on the CPX (close proximity)method. Paradigm for use in preparation of tendering documents and contracts. The measurement method is based upon the ISO draft standard describing the CPX-method, but includes further specification. Reference values for dense asphalt concrete are defined, and $2 \mathrm{~dB}$ wide noise reduction classes A, B, and C are defined at 50 and $80 \mathrm{~km} / \mathrm{h}$. The method also specifies an inter-calibration procedure (including definition of reference road sections) to be applied for different CPX measurement devices. Each year a specific calibration constant is issued for each device. The paper will also discuss the recent developments in the selection of future reference tyres for CPX measurements. Finally, a status of the classified surfacings will be presented.

\title{
Session $4 p N S q$
}

\section{Noise and EURONOISE: Fan Noise and Low-Mach Number Rotating Blade Noise II (Poster Session)}

\author{
Scott Morris, Cochair \\ Notre Dame \\ Michel Roger, Cochair \\ Ecole Centrale de Lyon
}

\begin{abstract}
All posters will be on display from 3:40 p.m. to 5:20 p.m. To allow contributors an opportunity to see other posters, contributors of odd-numbered papers will be at their posters from 3:40 p.m. to 4:30 p.m. and contributors of even-numbered papers will be at their posters from 4:30 p.m. to 5:20 p.m.
\end{abstract}

\section{Contributed Papers}

4pNSq1. Prediction of blade trailing-edge noise of an axial flow fan. Alain Guedel (CETIAT, BP 2042, 69603 Villeurbanne Cedex, France, alain.guedel@cetiat.fr), Arthur Finez (Ecole Centrale de Lyon, 36 Avenue Guy de Collongue, Centre Acoustique, 69134 Ecully, France, arthur.finez@gmail.com), Gérald Perrin (CETIAT, BP 2042, 69603 Villeurbanne Cedex, France, gerald.perrin@cetiat.fr), Michel Roger (Ecole Centrale de Lyon, 36 Avenue Guy de Collongue, Centre Acoustique, 69134 Ecully, France, michel.roger@ec-lyon.fr)

Broadband noise is a major part of the noise radiated by low-speed fans such as industrial and domestic fans. One of the main mechanisms of broadband noise is blade trailing-edge noise, which is due to the convection of the turbulent flow of the boundary layer past the blade trailing edge. The objective of this work is to predict trailing-edge noise of an axial flow-fan with an analytical model adapted from Amiet's formulation. The input data of the model are the spectra and correlation length scales of the wall pressure fluctuations on the blade suction side close to the trailing edge. These data are measured with small pressure transducers flush mounted on the blade suction side. A first study was performed on a 800 -mm axial fan without shroud. The comparison between the predicted and measured far-field sound pressure spectra proved quite good, which validated the model in this impeller configuration. The next stage in progress is to insert a shroud on the 
same impeller and validate the noise prediction in this case for different fan operating points. The importance of additional broadband noise sources, such as blade tip noise, could also be assessed on this fan configuration.

4pNSq2. Fan Blade Trailing-Edge Noise Prediction Using RANS Simulations. Yannick Rozenberg (ONERA, BP 72 - 29 avenue de la Division Leclerc, 92322 Chatillon Cedex, France, yannick.rozenberg@onera.fr), Michel Roger (Ecole Centrale de Lyon, 36 Avenue Guy de Collongue, Centre Acoustique, 69134 Ecully, France, michel.roger@ec-lyon.fr), Stéphane Moreau (Valeo Thermal Systems, rue Louis Normand, 8, 78321 La Verrière, France, stephane.moreau@valeo .com)

An analytical model based on Paterson \& Amiet's work dealing with the trailing-edge noise of a blade has been previously validated thanks to a dedicated experiment on a low speed axial fan. Wall-pressure spectra near the trailing-edge of the blade and at different radii are needed for an accurate prediction. Only experiments and LES simulations are able to provide them. In an industrial context, both methods can not be applied since they are too expensive and time-consuming. To overcome this difficulty, RANS simulations are combined with semi-empirical wall-pressure spectra to obtain the needed input data. The effect of the mean-pressure gradient is taken into account. The model is applied first to the noise radiated by an airfoil placed in the open-jet of an anechoic wind tunnel, then to an automotive cooling fan and finally to an aircraft engine fan. RANS simulations are postprocessed to run the analytical model with appropriate input data. The noise predictions are then compared with experimental results.

4pNSq3. Experimental investigation of wind turbine noise. Maud Leroux (Laboratoire d'Etudes Aérodynamiques (LEA), Université de Poitiers - ENSMA - CNRS, Bâtiment K, 40 Avenue du Recteur Pineau, 86022 Poitiers, $\quad$ France, maud.leroux@lea.univ-poitiers.fr), Yves Gervais (Laboratoire d'Etudes Aérodynamiques (LEA), Université de Poitiers - ENSMA - CNRS, Bâtiment K, 40 Avenue du Recteur Pineau, 86022 Poitiers, France, yves.gervais@lea.univ-poitiers.fr), Jacques Borée (Laboratoire d'Etudes Aérodynamiques (LEA), Université de Poitiers - ENSMA - CNRS, Bâtiment K, 40 Avenue du Recteur Pineau, 86022 Poitiers, France, jacques.boree@lea.ensma.fr), Arnaud Ménoret (Signal Developpement, 12 Boulevard Chasseigne, 86000 Poitiers, France, a.menoret @ signal-developpement.com)

Broadband noise is nowadays the major contribution to the total spectrum of noise generated by wind turbines. The mechanisms that generate airfoil self-noise have been studied through years and many authors agree that dominant noise comes from inflow turbulence, and interaction between tur- bulent boundary layer and trailing edge of the airfoil. This study presents results from combined experimental techniques in order to better identify and predict noise issuing from a NACA 0012. Noise from trailing edge is most investigated in a 2D configuration in an anechoic wind tunnel, using microphone array (far field measurements), wall pressure fluctuations, hot wire. The data base turns out to be useful in order to improve extensions of Amiet and Brooks models. Some aspects of noise mechanisms and their characteristics are better identified and refined when wind tunnel results are compared to measurements of noise on full scale wind turbines. Measurements in situ achieved with a microphone array of 30 meters wide are used to provide complementary informations on $3 \mathrm{D}$ rotating source in terms of localisation and specific directivity.

4pNSq4. Measurement of wall pressure fluctuations for noise prediction in axial flow fans. Jérémy Hurault (Lab. d'Energétique et de Mécanique des Fluides Interne, Arts et Métiers ParisTech, 151 Boulevard de l'Hôpital, 75013 Paris, France, jeremy.hurault@ paris.ensam.fr), Smaine Kouidri (LIMSI-CNRS, BP 133, 91403 Orsay Cedex, France, smaine.kouidri@limsi.fr), Farid Bakir (Lab. d’Energétique et de Mécanique des Fluides Interne, Arts et Métiers ParisTech, 151 Boulevard de l'Hôpital, 75013 Paris, France, farid.bakir@paris.ensam.fr), Robert Rey (Lab. d'Energétique et de Mécanique des Fluides Interne, Arts et Métiers ParisTech, 151 Boulevard de l'Hôpital, 75013 Paris, France, robert .rey@paris.ensam.fr)

The unsteady pressure field on fan blades is an important investigation topic. Both numerical simulation and experimental techniques are used in order to achieve this purpose. However neither has given yet entire satisfaction. The CFD tools using the resolution of the averaged Navier Stokes equations do not really give the unsteady aerodynamic characteristics of the flow needed for an accurate noise prediction. In addition, tools using large eddy simulation are still expensive for industrial users in the case of a complex geometry such as turbomachinery. When a lifting surfaces goes through turbulence, pressure fluctuations occur on their surfaces that can radiate noise. To calculate these fluctuations and thus the noise requires a theoretical model of the unsteady aerodynamics. The validation and development of these models require data and understanding from experiments. Unsteady surface pressure measurements were carried out on one fan blade with an array of pressure transducers with high sensitivity. The fan studied is a low pressure and low Mach number axial flow fan. Investigations of unsteady surface pressure are carried out in different configuration, spanwise, chordwise, pressure side and suction side. Data are gathered through a slip ring by an analyser. The unsteady wall pressure spectra is used as an input for trailing edge noise analytical prediction model. 


\title{
Session $4 \mathrm{pNSr}$
}

\section{Noise and EURONOISE: Cultural Variations in Sound/Noise Assessment II}

\author{
Catherine Guastavino, Cochair \\ Centre for Interdisciplinary Research in Music Media \& Technology (CIRMMT) - School of Information Studies - McGill Univ., \\ 3459 McTavish, Montreal, QC H3A1Y1, Canada \\ Danièle S. Dubois, Cochair \\ CNRS, LCPE/LAM 11 rue de Lourmel, Paris, 75015, France
}

\section{Invited Papers}

$5: 20$

4pNSr1. Acoustical Variety of Soundscapes - Comparison of Soundscapes. Klaus Genuit (HEAD acoustics GmbH, Ebertstrasse 30a, 52134 Herzogenrath, Germany, klaus.genuit@head-acoustics.de), André Fiebig (HEAD acoustics GmbH, Ebertstrasse 30a, 52134 Herzogenrath, Germany, andre.fiebig@head-acoustics.de)

Noise of urban places varies not only with regard to its physical parameters, but also it is perceived and assessed differently because of cultural, sociological, historical and economic influences. The physical description of a soundscape does not cover the complexity of human perception in a specific environmental setting. Therefore, the task of soundscape researchers has to consider more aspects than the measurement and interpretation of the acoustical differences caused by location-specific noise sources. Moreover, semantic and cognitive aspects relating to culture, tradition and economy must be extensively analyzed. Cultural and sociological conditions influence the people's evaluations of their surroundings. A deeper understanding is necessary to adequately analyze soundscapes, especially where specific noise phenomena - e.g. temporal and spectral effects - are of more importance to the listeners' well-being than an averaged SPL value. The presented paper compares and analyses environmental sounds of different cities scattered all over the world by means of conventional and advanced acoustical analyses. The results could provide reliable data for further investigations covering sociological and cultural issues.

\section{$5: 40$}

4pNSr2. Naïve and expert listeners use different strategies to categorize everyday sounds. Guillaume Lemaitre (IRCAM UMR CNRS 9912, Equipe Perception et Design Sonores, 1, place Igor Stravinsky, 75004 Paris, France, lemaitre@ircam.fr), Olivier Houix (IRCAM - UMR CNRS 9912, Equipe Perception et Design Sonores, 1, place Igor Stravinsky, 75004 Paris, France, olivier.houix@ircam.fr), Nicolas Misdariis (IRCAM - UMR CNRS 9912, Equipe Perception et Design Sonores, 1, place Igor Stravinsky, 75004 Paris, France, misdarii@ircam.fr), Patrick Susini (IRCAM - UMR CNRS 9912, Equipe Perception et Design Sonores, 1, place Igor Stravinsky, 75004 Paris, France, susini@ircam.fr)

We report an experiment investigating the influence of the expertise of listeners on the strategy used to categorize sounds. A set of sixty kitchen sounds was selected, based on their causal uncertainty (ranging from very well identified to ambiguous). Thirty listeners were selected on the basis of their expertise in sound and music: fifteen "experts" and fifteen "naïves". First, they had to group together the sounds. Second, they had to describe the properties shared by the sounds in each category. Finally, they were provided with a description of different strategies of classification previously identified (acoustical, causal or semantic similarity), and required to indicate, for each category, which one they had used. The results show a strong influence of the expertise of the participants: while naive listeners made categories mainly on the basis of the events that they identified as having caused the sounds, experts made mainly categories of sounds on the basis of the perceived acoustical properties (timbre, time patterns, etc.). This result is coherent with the available literature demonstrating the coexistence of different strategies of listening, and links these strategies to the skills of the listeners. [This work is founded by the FP6 NEST Pathfinder European project CLOSED]

\section{Contributed Paper}

\section{6:00}

4pNSr3. Cultural variations and constants in emotional reactions to sounds. Daniel Vastfjall (Chalmers University of Technology, Division of Applied Acoustics - Chalmers Room Acoustics Group, Sven Hultins gata 8a, 41296 Gothenburg, Sweden, daniel.vastfjall@psy.gu.se)

In this talk I will argue that emotional reactions to sounds can be both constant and vary between different cultures. A model of sound perception that was developed by Chalmers room acoustics group, the Emotional
Reaction Model (ERM) will be reviewed. The ERM predicts that emotional reactions to auditory events can be both elicited by 1) certain form features (eg. a steep rise time, a loud sound, a rough sound) and 2) content features (eg. the qualitative experience of the sound source; the loud, rough sound is a tiger roaring). The influence of form features are expected to be rather constant across people and cultures, while content features are expected to vary more between people. Empirical evidence supporting these predicitions will be reviewed and the implications for our understanding sound perception will be discussed. 


\title{
Invited Papers
}

$6: 20$

4pNSr4. When exposed to sounds, would perceived loudness not be affected by social context? Pieter Jan Stallen (Leiden University, PX Box 9555, 2300 RB Leiden, Netherlands, stallen@fsw.leidenuniv.nl)

After decades of predominantly correlation studies of non-auditory factors and environmental noise annoyance, Maris, Stallen, Vermunt and Steensma (2007a,2007b) have demonstrated experimentally that a negative and positive relationship between producer and receiver of environmental sounds can cause the receiver to be more and less annoyed by the sound, respectively. This finding raises the question whether the context of environmental sound, and social context in particular, could already be determining responses to environmental sounds at earlier stages of auditory processing. This paper will present answers regarding perceived loudness, which is generally considered to be a more immediate (and less evaluative) response to sound exposure than the feeling of annoyance. From a statistics point of view, there is room for early influences as isophones which are based upon equal loudness judgments seem to hide no less, if not more, variation than the mean annoyance score at various sound pressure levels (cf. Berglund and Preis, 1997). It will be argued that seemingly conflicting empirical findings could be reconciled by postulating different attentional mechanisms as they vary with task characteristics and demands. Ideas will be presented by which the presumed theoretical model could be tested experimentally.

\section{6:40}

4pNSr5. Comparison of Japanese and English language descriptions of piano performances captured using popular multichannel microphone arrays. William L. Martens (McGill University, Schulich School of Music, 555 Sherbrooke Street West, Montreal, QC H3A 1E3, Canada, wlm@music.mcgill.ca), Sungyoung Kim (Yamaha, 203 Matsunokijima, 438-0192 Iwata, Japan, sungyoung_kim@gmx.yamaha.com), Atsushi Marui (Tokyo University of the Arts, 1-25-1 Senju, Adachi, 120-0034 Tokyo, Japan, marui@ms.geidai.ac.jp)

In a cross-cultural comparison of musical sound evaluations, the way in which bipolar adjective pairs are used by native speakers of Japanese and English language was studied via a subjective rating task. These ratings were collected in response to eight solo piano performances that had been captured using four popular multichannel microphone arrays, reproduced via a standard 5-channel loudspeaker array, re-recorded binaurally, and finally presented via headphones. This allowed nearly identical stimuli to be presented to all listeners, without any modulation of the loudspeaker signals via listener head movements. Average ratings were compared to acoustical measures made on the 32 binaural stimuli, and to salient perceptual dimensions that previously had been derived from pairwise dissimilarity ratings between the stimuli. Results showed close agreement in how the selected terms were used by native speakers of Japanese and English language in the context of this study.

\section{Session 4pPAa}

\section{Physical Acoustics: Ultrasonics: Material Characterization II (Poster Session)}

\author{
Jim A. Tencate, Cochair \\ Los Alamos National Laboratory \\ Koen Van Den Abeele, Cochair \\ K. U. Leuven, Campus Kortrijk
}

\begin{abstract}
All posters will be on display from 3:40 p.m. to 5:20 p.m. To allow contributors an opportunity to see other posters, contributors of odd-numbered papers will be at their posters from 3:40 p.m. to 4:30 p.m. and contributors of even-numbered papers will be at their posters from 4:30 p.m. to 5:20 p.m.
\end{abstract}

\section{Contributed Papers}

4pPAa1. Structure and ultrasonic properties of vanadium tellurite glasses containing cupper oxide. Nadia Abd El-Aal (National Institute of Standards, 136 Tersa St, El-Haram, 12211 Giza, Egypt, n_nadia_99@yahoo.com), Hisham Afifi (National Institute of Standards, 136 Tersa St, El-Haram, 12211 Giza, Egypt, hmafifi@hotmail.com)

The elastic properties of vanadium tellurite (65TeO2-(35-x) V2O5$\mathrm{xCuO})$ glasses with different compositions of cupper ( $\mathrm{x}=7.5$ to $17.5 \mathrm{~mol} \%$ in steps of $2.5 \mathrm{~mol} \%$ ) have been studied at room temperature $(300 \mathrm{~K})$. The ultrasonic velocity measurements have been made using a transducer having resonating frequency of $4 \mathrm{MHz}$ (both longitudinal and shear). The density, molar volume, and ultrasonic velocities show an interesting observations, which are used to explore the structural changes in the network. Elastic moduli, Poisson ratio, crosslink density, microhardnes, and Debye temperature of the glasses have been determined using the experimental data. The composition dependence of the elastic properties explores useful information about the physical properties of the vanadium tellurite glasses doped with cupper. Quantitative analysis has been carried out in order to obtain more informations about the structure of the glass under the study, based on bond compression model, and ring deformation model, i.e., the cation-anion bond of each oxide. The observed results through ultrasonic non-destructive evaluation investigate the structural changes and mechanical properties of the glass. 
4pPAa2. Elastic characterization of ceramic balls using resonant ultrasound spectroscopy of spheroidal modes. François Deneuville (Institut d'Electronique de Microélectronique et de Nanotechnologie, Département Opto-Acousto-Electronique (UMR CNRS 8520), Université de Valenciennes et du Hainaut-Cambrésis, Le Mont-Houy, 59313 Valenciennes cedex 9, France, francois.deneuville@gmail.com), Marc Duquennoy (Institut d'Electronique de Microélectronique et de Nanotechnologie, Département Opto-Acousto-Electronique (UMR CNRS 8520), Université de Valenciennes et du Hainaut-Cambrésis, Le Mont-Houy, 59313 Valenciennes cedex 9, France, Marc.Duquennoy@univ-valenciennes .fr), Mohammadi Ouaftouh (Institut d'Electronique de Microélectronique et de Nanotechnologie, Département Opto-Acousto-Electronique (UMR CNRS 8520), Université de Valenciennes et du Hainaut-Cambrésis, Le Mont-Houy, 59313 Valenciennes cedex 9, France, mohammadi .ouaftouh@univ-valenciennes.fr), Frédéric Jenot (Institut d'Electronique de Microélectronique et de Nanotechnologie, Département Opto-AcoustoElectronique (UMR CNRS 8520), Université de Valenciennes et du HainautCambrésis, Le Mont-Houy, 59313 Valenciennes cedex 9, France, frederic.jenot@univ-valenciennes.fr), Mohamed Ourak (Institut d'Electronique de Microélectronique et de Nanotechnologie, Département Opto-Acousto-Electronique (UMR CNRS 8520), Université de Valenciennes et du Hainaut-Cambrésis, Le Mont-Houy, 59313 Valenciennes cedex
9, France, Mohamed.Ourak@univ-valenciennes.fr),Sébastien Desvaux(SKF Group, Z.I. n`2 Batterie 900 Rouvignies, 59309 Valenciennes, France, sebastien.desvaux@snfa.com)

The use of ceramic balls, in particular silicon nitride balls, allows a substantial improvement of bearing performances. For critical aerospace and space applications, there is a need for developing new nondestructive techniques for the characterization of these balls. We propose in this work to study the possibilities offered by a specific technique of resonant ultrasound spectroscopy of spheroidal modes. As shown by the theoretical study on elastic sphere vibrations, these modes allow to characterize the whole volume of balls or only the close-to-surface layers, according to the considered frequency range. To acquire the resonance spectra of these modes, a specific measurement system composed of a piezoelectric ultrasonic probe and an optical interferometer was developed. A self-implemented numerical processing of measured spectra allows to detect the resonance frequencies and to deduce from them the propagation velocity of the spheroidal waves in each inspected subsurface layers. Then, we propose a method based on these results that permit to estimate the elastic coefficients of the balls according to various inspection depths. This method has the advantage of providing very high precision evaluations of the elastic coefficients over a wide frequency range.

\title{
Session 4pPAb
}

\section{Physical Acoustics and Computational Acoustics: Combustion Noise and Thermo-Acoustics II (Poster Session)}

\author{
Tim Lieuwen, Cochair \\ Georgia Institute of Technology \\ Maria Heckl, Cochair \\ Keele University \\ Rafael Piscoya, Cochair
}

Technische Fachhochschule Berlin, Univ. of Applied Sciences

\begin{abstract}
All posters will be on display from 3:40 p.m. to 5:20 p.m. To allow contributors an opportunity to see other posters, contributors of odd-numbered papers will be at their posters from 3:40 p.m. to 4:30 p.m. and contributors of even-numbered papers will be at their posters from 4:30 p.m. to 5:20 p.m.
\end{abstract}

\section{Contributed Papers}

4pPAb1. Inversion of the bulk viscosity in nonequilibrium media with heat release and new acoustical properties of such media. Nonna Molevich (P.N. Lebedev Physical Institute Samara branch, Pervomaiskaya Str 21-36, 443100 Samara, Russian Federation, molevich@fian.smr.ru)

New acoustical properties, which are caused by the inversion of the bulk (second) viscosity in nonequilibrium media, are investigated. Negative bulk viscosity can take place due to the positive feedback between the sound perturbation and the nonequilibrium heat release. During previous years, the conditions for the negative bulk viscosity existence were found in a large number of nonequilibrium media such as a vibrationally excited gas with stationary nonequilibrium, nonisothermal plasma, chemical active mixtures with irreversible reactions, media with nonequilibrium phases, upper atmosphere layers, and earth magma with bubbles. The following phenomena are discussed for gaseous media with negative viscosity: (1) New dispersion characteristics (in particular, the low-frequency sound speed can exceed the high-frequency one); (2) Acoustically instability of nonequilibrium media, nonlinear mechanisms of the wave growth stabilization and stationary self-sustaining structures; (3) Linear and non-linear sound beam refraction including the self-focusing (due to two self-action mechanisms in acoustically active media: the gas cooling by sound and the excitation of acoustical streaming in direction opposite to sound propagation) and the anomalous reflection (with reflection coefficient $\mathrm{R}>1$ ) on a boundary between equilibrium and nonequilibrium media; (4) The vortex and thermal wave amplification due to intensive parametric interactions in acoustically active media. 
4pPAb2. Stationary structures in acoustically active nonequilibrium media with one relaxation process. Rinat Galimov (Samara State Aerospace University, Magnitnaya st. 15, 443017 Samara, Russian Federation, renrk@mail.ru), Nonna Molevich (P.N. Lebedev Physical Institute Samara branch, Pervomaiskaya Str 21-36, 443100 Samara, Russian Federation, molevich@fian.smr.ru)

Chemical active mixtures with irreversible reactions, vibrationally excited gases, and nonisothermal plasmas are examples of acoustically active nonequilibrium media. In such media it is possible the existence of stationary nonlinear structures that are different from the step-wise shock wave structures. In the first part of the present paper it is investigated the solutions of a general acoustical equation, describing in the second order perturbation theory a nonlinear evolution of wide spectrum acoustical disturbances in nonequilibrium media with one relaxation process. Its low- and high- frequency limits correspond to Kuramoto-Sivashinsky equation and the Burgers equation with a source, respectively. Stationary structures of general equation, the conditions of their establishment and all their parameters are found analytically and numerically. In acoustically active media it is predicted the existence of the stationary solitary pulse. Then, we consider 1-D relaxing gas dynamics system of equations with simple Landau-Teller model of relaxation. The possible stationary profiles are shown in nonequilibrium degree- stationary wave speed bifurcation diagram. The boundaries of this diagram are obtained in analytical forms. The field of weak shock wave instability is shown in this bifurcation diagram. Unstable shock wave disintegrates into the sequence of solitary pulses described by the general acoustical equation.

4pPAb3. Thermoacoustic waves near the liquid-vapor critical point Pierre Carles (Université Pierre et Marie Curie, 4 Place Jussieu, 75005 Paris, France, carles@ccr.jussieu.fr)

The thermal relaxation in a fixed-volume cell filled with a near-critical fluid is governed by the rapid expansion of thermal boundary layers, which drive a series of thermo-acoustic waves in the bulk fluid. The long-term cumulative effect of these waves is to increase the pressure in the cell, which in turn leads to a global temperature increase (a process named the Piston Effect). Recently and for the first time, the thermo- acoustic waves produced by the Piston Effect have been measured experimentally using interferometric methods [Y. Miura et al., to appear in Phys. Rev. E (2006)]. In the present work, we use asymptotic methods in order to derive a complete theoretical model of the Piston-Effect-driven acoustic waves, applicable to real fluid equations of state and to arbitrary reduced temperatures. The predictions of this model are compared to the above-mentioned experimental data, and an excellent agreement is observed without any fitting parameter. This result confirms the high precision of the data in question, and shows that asymptotic models such as ours can be a powerful tool for analyzing the results of such experiments.
4pPAb4. Prediction of noise emissions from industrial flares Carl-Christian Hantschk (Müller-BBM GmbH, Robert-Koch-Str. 11, 82152 Planegg, Germany, Carl-Christian.Hantschk@MuellerBBM.de), Edwin Schorer (Müller-BBM GmbH, Robert-Koch-Str. 11, 82152 Planegg, Germany, Edwin.Schorer@MuellerBBM.de)

In many industries where combustible waste gases are obtained, flares are used to burn these gases in a controlled manner. Among other environmental aspects, the noise emissions associated with flaring are becoming increasingly important in many countries as population density goes up and residential and industrial areas move closer together. Installing noise control equipment on flares is almost impossible while they are in service, since flares are typically a safety related plant component that can only be turned off after the connected plant has been shut down. Accordingly, in order to plan appropriate noise control measures in time and to avoid unnecessary costs, predicting the noise emissions of flares as early in the design process as possible is crucial. This requires knowledge of the relevant individual noise sources associated to the flare system and the ability to calculate their respective contribution - in the operating condition in question - to the overall noise emission, based on the data available in the planning stage. The present paper summarizes these sources and outlines some of the individual effects and parameters having an influence on the acoustical characteristics of flares.

4pPAb5. Modelling of acoustic losses with the wave equation for the analysis of combustion instabilities. Elke Wanke (Lehrstuhl für Thermodynamik, Technische Universität München, Boltzmannstrasse 15, 85747 Garching, Germany, wanke@td.mw.tum.de), Fabian Weyermann (Lehrstuhl für Thermodynamik, Technische Universität München, Boltzmannstrasse 15, 85747 Garching, Germany, weyermann@td.mw.tum.de), Christoph Hirsch (Lehrstuhl für Thermodynamik, Technische Universität München, Boltzmannstrasse 15, 85747 Garching, Germany, hirsch@td.mw.tum.de), Thomas Sattelmayer (Lehrstuhl für Thermodynamik, Technische Universität München, Boltzmannstrasse 15,85747 Garching, Germany, sattelmayer@td .mw.tum.de)

A numerical design tool for the assessment of the stability of combustion chambers has been developed, which is able to compute geometrically complex systems with thermoacoustic feedback in the time domain. It is shown that internal acoustic losses can be considered although the method is based on the solution of the wave equation. The presented method overcomes a serious limitation of the original approach and allows to make quantitative predictions. The model is based on the Bernoulli equation and derives the required information from the spatial distribution of the loss of total pressure. For the purpose of a comprehensive validation of the model, simulations were carried out in the frequency domain before the model was implemented. As the internal acoustic losses in combustors stem almost completely from the flow separation at the exit of the burners the losses are independent from temperature. For this reason the influence of the flame was neglected in the study to only focus on the modelling of acoustic losses. The numerical results are validated with single burner test rig experiments. 


\title{
Session 4pPAc
}

\section{Physical Acoustics: Quantum Acoustics II (Poster Session)}

\author{
Michel DeBilly, Cochair \\ Institut Jean le Rond d'Alembert \\ Walter Lauriks, Chair \\ Katholieke Universiteit Leuven
}

\begin{abstract}
All posters will be on display from 3:40 p.m. to 5:20 p.m. To allow contributors an opportunity to see other posters, contributors of odd-numbered papers will be at their posters from 3:40 p.m. to 4:30 p.m. and contributors of even-numbered papers will be at their posters from 4:30 p.m. to 5:20 p.m.
\end{abstract}

\section{Contributed Papers}

4pPAc1. Acoustic studies of the martensite phase transition in $\mathrm{Ti}-\mathrm{Ni}$ alloys. Andrew A. Abramovich (St. Petersburg State Technological University of Plant Polymers, Chernykh str., 4, Vasenko str., 5/15, apt.32, 195197 St. Petersburg, Russian Federation, andrew@ns2740 .spb.edu), Elena V. Charnaya (Department of Physics, St. Petersburg State University, Petrodvorets, Ulianovskaya, 1, 198504 St. Petersburg, Russian Federation, charnaya@paloma.spbu.ru), Sergei P. Belyaev (Department of Mathematics and Mechanics, St. Petersburg State University, Petrodvorets, Ulianovskaya, 1, 198504 St. Petersburg, Russian Federation, belyaev@paloma.spbu.ru), Aleksander E. Volkov (Department of Mathematics and Mechanics, St. Petersburg State University, Petrodvorets, U1ianovskaya, 1, 198504 St. Petersburg, Russian Federation, volkov@paloma .spbu.ru)

Acoustic studies of elastic properties and attenuation of ultrasonic waves (longitudinal and transverse) at the martensite phase transition were carried out in the Ti-Ni based polycrystalline alloys. Measurements were carried out using a pulse ultrasonic technique within a temperature range of 190 to 440 $\mathrm{K}$ upon continuous warming and cooling the samples after various hardening and annealing treatment. Anomalies of ultrasound velocity and attenuation were observed through the martensite phase transition which depended on wave polarization and thermal history of the samples, peaks of attenuation were seen only for transverse waves. A pronounced thermal hysteresis of acoustic features was also observed upon warming and cooling. The obtained results were treated on the basis of the phenomenological Landau theory for the B2-B19' ferroelastic phase transition.

4pPAc2. Observation of induced shear acoustic phonons by Brillouin scattering. Taisuke Yoshida (Faculty of Engineering Doshisha University, 1-3 Tatara Miyakodani, 610-0321 Kyotanabe, Japan, dth0186@mail4 .doshisha.ac.jp), Sigeo Murata (Faculty of Engineering Doshisha University, 1-3 Tatara Miyakodani, 610-0321 Kyotanabe, Japan, dth0186@mail4.doshisha.ac.jp), Takahiko Yanagitani (Faculty of Engineering Tohoku University, 6-6-05 Aramaki Aoba Aoba-ku, 610-0321 Kyotanabe, Japan, yanagi@ecei.tohoku.ac.jp), Mami Matsukawa (Doshisha University, 1-3, Tatara Miyakodani, 610-0321 Kyotanabe, Japan, mmatsuka @ mail.doshisha.ac.jp)

The Brillouin scattering measurement is an efficient nondestructive and noncontact method which enables the simultaneous measurement of longitudinal and shear wave velocities at hypersonic frequencies. However, the measurement accuracy of shear wave velocities is low because of the weak
Brillouin scattering from the thermal phonons. In this study, therefore, we have tried to overcome this problem by making use of the induced coherent shear phonons. We have adopted the Reflection Induced $\theta$ A (RI $\theta$ A) scattering geometry for Brillouin scattering measurement. To induce shear acoustic phonons, we used an uniaxially aligned $\mathrm{ZnO}$ film transducer developed in our laboratory, which can be fabricated on any solid materials without the epitaxy technique. As a result, we obtained the intense Stokes peak in case of silica glass sample which is larger than that obtained from the thermal phonons. It means that observation of induced shear acoustic phonons was achieved. Because the RI $\theta$ A geometry enables the simultaneous measurement of longitudinal and shear phonons in plane, this technique opens the new feature for the nondestructive elasticity measurement.

4pPAc3. Study of acoustical phonon modes in superlattices with SiGe QDs. Anatoliy Yaremko (Lashkaryov Institute of Semiconductor Physics, NAS of Ukraine, Prospekt Nauky 45, 03028 Kyiv, Ukraine, yaremko@isp.kiev.ua), Volodymyr Yukhymchuk (Lashkaryov Institute of Semiconductor Physics, NAS of Ukraine, Prospekt Nauky 45, 03028 Kyiv, Ukraine, yukhym@isp.kiev.ua), Volodymyr Dzhagan (Lashkaryov Institute of Semiconductor Physics, NAS of Ukraine, Prospekt Nauky 45, 03028 Kyiv, Ukraine, dzhagan@isp.kiev.ua), Mykhailo Valakh (Lashkaryov Institute of Semiconductor Physics, NAS of Ukraine, Prospekt Nauky 45, 03028 Kyiv, Ukraine, valakh@isp.kiev.ua)

Multilayers with SiGe nanoislands (QD's) grown in a broad temperature range are studied using Raman spectroscopy, HRXRD and compared with similar multilayers without islands. As the growth temperature increases, $\mathrm{Si}$ content in the islands increases, partially relieving strain. These structural transformations manifest themselves in both the intensity and frequency of the low-frequency Raman peaks. Due to composition- and strain-induced changes in the island band structure, excitation conditions come out of resonance, reducing Raman peak intensity. We have shown that at the interpretation of the Raman scattering by folded acoustic phonons for structures with nanoislands the real morphology of the island layer should be considered. The observed series of the low-frequency Raman peaks, for the multilayered structures with the number of QD layers below ten, is due to the acoustic phonon modes within the islands. The enhancement of the scattering intensity due to resonance of the excitation light with the electronic transitions within the islands plays a significant role. Theoretical analysis was fulfilled in the framework of microscopic approach with using Green function method and taking into account the real structure of QD's and their interaction with surrounding matrix. 
4pPAc4. Evolution of ultrasound transmission during the sol-gel transition. Bożena Gadomska (University of Warsaw, Department of Chemistry, Laboratory of Physicochemistry of Dielectrics and Magnetics, ul. Żwirki i Wigury 101, 01-905 Warsaw, Poland, bogad@chem.uw.edu.pl), Wojciech Gadomski (University of Warsaw, Department of Chemistry, Laboratory of Physicochemistry of Dielectrics and Magnetics, ul. Żwirki i Wigury 101, 01-905 Warsaw, Poland, gado@chem .uw.edu.pl)

The velocity of the ultrasound wave has been detected during the gelation process of the aqueous gelatin solution. We observe monotonic decrease of the ultrasound transmission in train of the gel network formation. In order to find the dependance of the ultrasound velocity on the number of the intermolecular bonds, responsible for gel formation, we have performed the simultaneous measurements of the optical activity evolution. The experiments have been performed in the gelatin gel and in the silica gel. We have described the process in terms of the percolation theory by power law, according to Landau theory of the second order phase transitions, and by fitting the theoretical results to the experimental curves we have found the appropriate critical exponents. We have also shown how the ultrasound velocity depends on the concentration of the gelling substance and on the temperature, in which the process of the sol-gel transition has been performed.

4pPAc5. Acoustic wave propagation in cubic piezoelectric semiconductor plates. Bernard Collet (Université Pierre et Marie Curie Institut Jean le Rond d'Alembert-UMR-CNRS 7190, T65-Case 162, 4 place Jussieu, 75252 Paris Cedex 05, France, bc@ccr.jussieu.fr)

Piezoelectric materials can be either dielectric or semiconductors. An acoustic wave propagating in a piezoelectric crystal is usually accompanied by an electric field. When the crystal is also a semiconductor, the electric field produces currents and space charges, resulting in dispersion and acoustic loss. The interaction between a travelling acoustic wave and mobile charges in piezoelectric semiconductors is currently called the acoustoelectric effect. It was shown experimentally and proved theoretically that an acoustic wave travelling in a piezoelectric semiconductor can be amplified by the application of an initial dc electric field. Piezoelectric semiconductors devices often have structural design of plates or rods. Here we study the thin piezoelectric semiconductor plates. Two-dimensional equations for coupled extensional, flexural and thickness-shear motions are obtained systematically from the three-dimensional equations by retaining lower order terms in power series expansions in the plate thickness coordinate. The twodimensional equations are specialized to crystals of cubic $(43 \mathrm{~m})$ symmetry. Propagation of extensional, flexural and thickness-shear waves and their amplification by a dc electric field are analyzed.

4pPAc6. Ultrasonic annealing of radiation defects in silicon. Artem Podolian (T Shevchenko Kiev National University, Dept of Physics, 03680 Kiev, Ukraine, gogi@univ.kiev.ua), Oleg Korotchenkov (T Shevchenko Kiev National University, Dept of Physics, 03680 Kiev, Ukraine, olegk @ univ.kiev.ua)

Cold annealing of radiation defects in silicon is reported and the likely origin of the effect is suggested. The data on photocurrent transients and photoluminescence spectra are contrasted in $\gamma$-irradiated float zone and Czochralski silicon. The ultrasonic load is shown to slow down the current decay, indicative of the decreased defect densities. The microscopic model includes the likely pathways for E-center annealing in zone and $\mathrm{C}-\mathrm{O}-\mathrm{V}_{2}$ complex redistribution in Czochralski materials. The carbon involvement into the annealing effect is further evidenced by taking the photoluminescence spectral evolution with applied ultrasonic loads. The usage of the presented technique in improving the performance of radiatively damaged silicon detectors is furthermore discussed.

\title{
Session 4pPAd
}

\section{Physical Acoustics: General Topics in Nonlinear Acoustics II (Poster Session)}

\author{
Thomas Matula, Cochair \\ University of Washington \\ Murray Korman, Cochair \\ U.S. Naval Academy
}

\begin{abstract}
All posters will be on display from 3:40 p.m. to 5:20 p.m. To allow contributors an opportunity to see other posters, contributors of odd-numbered papers will be at their posters from 3:40 p.m. to 4:30 p.m. and contributors of even-numbered papers will be at their posters from 4:30 p.m. to 5:20 p.m.
\end{abstract}

\section{Contributed Papers}

4pPAd1. Parametric audible sounds by phase-cancellation excitation of primary waves. Tomoo Kamakura (Dept. of Electronic Eng., Univ. of Electro-Communications, 1-5-1, Chofugaoka, 182-8585 Chofu-shi, Japan, kamakura@ee.uec.ac.jp), Shinichi Sakai (Dept. of Electronic Eng., Univ. of Electro-Communications, 1-5-1, Chofugaoka, 182-8585 Chofu-shi, Japan, sakashin@ew3.ee.uec.ac.jp), Hideyuki Nomura (Dept. of Electronic Eng., Univ. of Electro-Communications, 1-5-1, Chofugaoka, 182-8585 Chofu-shi, Japan, nomu@ee.uec.ac.jp), Masahiko Akiyama (Dept. of Electronic Eng., Univ. of Electro-Communications, 1-5-1, Chofugaoka, 1828585 Chofu-shi, Japan, akiyama@ew3.ee.uec.ac.jp)
An ultrasound source with a simple configuration is considered as a theoretical model. The source with a circular aperture consists of two coaxially arranged planar emitters: i.e., one is an inner disc emitter and the other is an outer ring emitter. The active areas of these emitters are the same. The outer diameter of the source is $20 \mathrm{~cm}$. Both the emitters are driven individually at the same frequencies of 40 and $42 \mathrm{kHz}$ but different phase angles. Especially, we focus on two extreme cases of the usual in-phase driving and outof-phase driving. Numerical computation using the KZK equation demonstrates that when the driving signals are in phase the difference frequency beam of a $2-\mathrm{kHz}$ wave has a candle-flame-like directivity. The beam has a 
similar directivity when the signals are out-of-phase by 180 degrees, although the peak of the sound pressure level decreases by few decibels. Interestingly, the second harmonic pressure level of the difference frequency reduces by ten decibels and more. Needless to say, the pressure amplitudes of the primary waves are suppressed considerably near the beam axis. Experimental verification is done using an airborne ultrasound source with a 19.2-cm circular aperture.

4pPAd2. The Generation of Acoustic Waves and Cavitation Processes in Regime of Phase Synchronization During Multichannel Discharges in Electrolyte. Vyacheslav S. Teslenko (Lavrentyev Institute of Hydrodynamics SB RAS, Lavrentyev ave., 15, 630090 Novosibirsk, Russian Federation, vteslenko@academ.org), Alexey P. Drozhzhin (Lavrentyev Institute of Hydrodynamics SB RAS, Lavrentyev ave., 15, 630090 Novosibirsk, Russian Federation, ruslan@hydro.nsc.ru), Ruslan N. Medvedev (Lavrentyev Institute of Hydrodynamics SB RAS, Lavrentyev ave., 15, 630090 Novosibirsk, Russian Federation, drozh@hydro.nsc.ru)

In the paper the problems of acoustic waves generation of required frequency, amplitude and profile by the phase synchronization of multichannel discharges are considered. The phase synchronization of discharges is supplied by the implementation in the discharge circuit of additional inductance which acts as a dynamic feedback and ensures the selfsynchronization of $\mathrm{N}$ generated bubbles and acoustic wave's radiation [1]. The devices of such types may be used to form acoustic fields with required frequency and shape. References: 1. V. S. Teslenko, R. N. Medvedev, and A. P. Drozhzhin, Self-Synchronization of Electrohydrodynamic Autooscillations during Multichannel Discharges in Electrolyte // ISSN 1063-7850, Technical Physics Letters, 2007, Vol. 33, No. 10, pp. 833-836. http://www.swsl.newmail .ru/publ/TEPL833.pdf

4pPAd3. Acoustic properties of TaCl - TaBr mixed crystals. Farkhad Akhmedzhanov (Navoi State Mine Institute, 27a Yuzhnaya Street, 210100 Navoi, Uzbekistan, farkhad2@yahoo.com)

Attenuation coefficient and phase velocity of transversal and longitudinal acoustic waves in $\mathrm{TaCl}$ - $\mathrm{TaBr}$ mixed crystals have been investigated by Bragg light diffraction on the acoustic waves. The measurements were carried out at the frequencies from 200 to $1200 \mathrm{MHz}$ at home temperature. Moreover, the phase velocity was determined by using Raman-Nath diffraction and optical heterodyning at $10 \mathrm{MHz}$. The investigations shown, that the change of acoustic properties in the investigated mixed crystals is nonadditive. Nonlinear interaction constants have been calculated taking into consideration various factors, which can influence on the propagation of acoustic waves in mixed crystals. The results were compared with similar investigations in $\mathrm{NaCl}-\mathrm{NaBr}$ mixed crystals.

4pPAd4. Adjusting the phase of the signals transmitted from dual frequency probe for reducing second harmonic during propagation. Pasovic Mirza (Université de Lyon, 43 boulevard du 11 Novembre 1918, 69622 Villeurbanne, France, mirza.pasovic@creatis.insa-lyon.fr), Christian Cachard (Université de Lyon, 43 boulevard du 11 Novembre 1918, 69622 Villeurbanne, France, christian.cachard@creatis.insa-lyon.fr), Guillaume Matte (Biomedical Engineering, Erasmus MC, P.O. Box 2040, 3000 CA Rotterdam, Netherlands, g.matte@erasmusmc.nl), René Van Der Steen (Eindhoven University of Technology, Dept. of Mechanical Engineering, Dynamics \& Control, P.O. Box 513, 5600MB Eindhoven, Netherlands, r.v.d.steen@tue.nl), Nico De Jong (Erasmus MC, Dr Molewaterplein 50 room Ee2302, 3015GE Rotterdam, Netherlands, n.dejong@erasmusmc.nl), Olivier Basset (Université de Lyon, 43 boulevard du 11 Novembre 1918, 69622 Villeurbanne, France, olivier.basset @ creatis.insa-lyon.fr)

During propagation of the ultrasound wave, nonlinearities of the medium, causes rise of higher harmonics that are seen in amplitude spectrum. In ultrasound contrast harmonic imaging this is unwanted effect, since it is expected to image only higher harmonics produced by contrast agents. In previous studies Christopher, Krishnan and Shen proposed transmitting either an inverted signal (collected by hydrophone), transmitting 2nd harmonic with broadband transducer or transmitting third harmonic to reduce the second harmonic at measurement point. Our study uses a dual frequency probe which transmits one wave at frequency f0 and one wave at double frequency $2 \mathrm{f0}$ named second harmonic reduction signal (SHRS) for reducing the second harmonic in the propagating signal. The hardest task is the appropriate adjustment of the phase of the SHRS to reduce second harmonic during propagation in the focal area. We outline how to set phase and established that the phase of the SHRS in not affected by the nonlinear parameter $\mathrm{B} / \mathrm{A}$ and excitation pressure $\mathrm{P} 0$ but rather by the excitation frequency and the ultrasound system geometry (transducer size and distance to focal area). A reduction of $25 \mathrm{~dB}$ has been obtained in simulation $(\mathrm{Po}=100 \mathrm{kPa}$, fo $=2.25 \mathrm{MHz})$. Experiments in water tank, have shown the reduction of 2 nd harmonic by $30 \mathrm{~dB}$.

4pPAd5. Energy pumping in acoustics. Romain Bellet (Laboratoire de Mécanique et d'Acoustique - CNRS, 31 chemin Joseph Aiguier, 13402 Marseille, France, bellet@1ma.cnrs-mrs.fr), Bruno Cochelin (Laboratoire de Mécanique et d'Acoustique - CNRS, 31 chemin Joseph Aiguier, 13402 Marseille, France, bruno.cochelin@ec-marseille.fr), Pierre-Olivier Mattei (Laboratoire de Mécanique et d'Acoustique - CNRS, 31 chemin Joseph Aiguier, 13402 Marseille, France, mattei@1ma.cnrs-mrs.fr), Philippe Herzog (Laboratoire de Mécanique et d'Acoustique - CNRS, 31 chemin Joseph Aiguier, 13402 Marseille, France, herzog@1ma.cnrs-mrs.fr)

Energy pumping corresponds to a particular vibratory regime of a nonlinear system coupled to a linear primary structure. Its non-linear behaviour allows to reduce vibrations of the primary structure, and is thus a new passive vibration control technique. This phenomenon has mainly been studied in mechanical engineering, so we transposed its principle to noise control in an acoustic medium. The presentation will focus on results about acoustic energy pumping that we observed both experimentally and numerically, in the time and frequency domains. These results highlight two main points: physically, energy pumping corresponds to an irreversible transfer of energy from the primary system to the non-linear absorber, and practically, its effects are a noise level limitation in the acoustic medium in permanent regime and a much faster sound extinction in transient regime.

4pPAd6. Shock wave propagation in heterogeneous medium, from ultrasound to sonic boom. Lili Ganjehi (Institut Jean Le Rond d'Alembert, Université Pierre et Marie Curie, Boites 161 et 162, 4 place Jussieu, 75252 Paris Cedex 05, France, ganjehi@lmm.jussieu.fr), François Coulouvrat (Institut Jean Le Rond d'Alembert, Université Pierre et Marie Curie, Boites 161 et 162, 4 place Jussieu, 75252 Paris Cedex 05, France, coulouvr@ccr.jussieu.fr), Jean-Louis Thomas (Centre National de la Recherche Scientifique, Institut des NanoSciences de Paris, Université Pierre et Marie Curie, 4 place Jussieu, 75252 Paris Cedex 05, France, thomasj1@ccr.jussieu.fr), Régis Marchiano (Institut Jean Le Rond d'Alembert, Université Pierre et Marie Curie, Boites 161 et 162, 4 place Jussieu, 75252 Paris Cedex 05, France, marchi@1mm.jussieu.fr)

Strong variability of sonic boom due to the atmospheric turbulence is known since the first test flight recordings in the 1960's. To simulate this, a laboratory scale experiment is conducted with ultrasonic shock waves in water at $1 \mathrm{MHz}$. The experiment is designed for an optimal 1:100,000 scaling with sonic boom. It includes single or multiple heterogeneities of varying sizes but comparable to the acoustical wavelength. Its deterministic aspect allows detailed comparisons with the results of a numerical model based on a nonlinear wide angle parabolic approximation. The experiments show the following features of the shock wave propagation : wavefront folding, local amplification (acoustical lens effect), increase of the rise time, strong variability of the time waveforms. All these features are in qualitative agreement with sonic boom observations. They are observed for a single heterogeneity provided this one is sufficiently large, but are amplified in case of multiple heterogeneities. Comparisons with results of numerical simulations show good agreement in various configurations. Improvements provided by the wide angle approach with respect to the standard parabolic approximation will also be discussed. Preliminary simulations for $\mathrm{N}$ wave propagation in a randomly heterogeneous medium will finally be presented. 
4pPAd7. A route to chaotic state on an electrodynamic loudspeaker Antonio Petosic (Faculty of Electrical Engineering and Computing, Unska 3, 10000 Zagreb, Croatia, antonio.petosic@fer.hr), Ivan Djurek (Faculty of Electrical Engineering and Computing, Unska 3, 10000 Zagreb, Croatia, ivan.djurek@fer.hr), Djurek Danijel (AVAC, Kesten brijeg 15, 10000 Zagreb, Croatia, danijel.djurek@zg-net.hr)

The low frequency electrodynamic loudspeaker (EDL) unit has been measured and analyzed in terms of chaotic behavior. It was found that an electrodynamic loudspeaker can function as a chaotic system. Loudspeaker impedance and vibration amplitude as function of driving frequency were measured at various driving currents, and well-know cut-off effect from nonlinear dynamical systems has been noticed. In the frequency region near cut-off and at higher driving currents the period doubling and later chaotic state occur. The experimentally obtained chaotic state was confirmed theoretically solving nonlinear equation of motion with strong nonlinear effective stiffness spatial dependency. It was found that statically measured suspension effective stiffness does not enables chaotic state when included in differential equation, and it has been concluded that membrane viscoelastic properties enhance the restoring force far enough to obtain chaos. The nonlinear equation describing anharmonic periodically driven oscillator has been solved numerically and the theoretical results were compared to experimental results.

4pPAd8. Analysis of the effects of the oscillations of a rigid sphere inside a cylindrical cavity containing a standing acoustic wave. Edgar A. Torres (Universidad Nacional Autonoma de Mexico, Centro de Ciencias Aplicadas y Desarrollo Tecnologico, CCADET-UNAM, Circuito Exterior s/n, Cd. Universitaria, A. P. 70-186, 02510 Mexico, D.F., Mexico, edgar.augusto.torres.gallegos@gmail.com), Arturo Santillan (Universidad Nacional Autonoma de Mexico, Centro de Ciencias Aplicadas y Desarrollo Tecnologico, CCADET-UNAM, Circuito Exterior s/n, Cd. Universitaria, A. P. 70-186, 02510 Mexico, D.F., Mexico, arturo.orozco@ccadet.unam.mx)

Under certain driving conditions of a single-axis acoustic levitation device, a suspended sample leaves its stability state and starts to oscillate vertically around the initial equilibrium position. A published theory on such instabilities [J. Rudnick and M. Barmatz, "Oscillational instabilities in single-mode acoustic levitators," J. Acoust. Soc. Am., 87(1), 81-92, 1990] predicts the occurrence of time delays between the response of the cavity of the device and the motion of the sample inside it. In this paper, a theoretical and experimental investigation on similar time delay effects will be described. A solid sphere was moved in a controlled way inside a closed cylindrical cavity by means of a rod connecting the object to the outside of the system. A standing wave was generated inside the cavity by using a speaker. In this way, oscillations of the sphere were produced and the response of the sound field to such movement was studied. The effect of the frequency of the oscillations of the sphere on the time delay between the sound pressure and the movement of that object will be reported. In addition, the relations between the obtained results and the published theory on oscillational instabilities will be discussed.

4pPAd9. Manipulation of the behavior of $\mathrm{SiC}$ particles and oil bubbles using ultrasonic standing wave field. Seung Hyun Cho (Korea Research Institute of Standards and Science, Doryong dong 1, Yuseong gu, 305-340 Daejon, Republic of Korea, seungcho@kriss.re.kr), Dae-Cheol Seo (Korea Research Institute of Standards and Science, Doryong dong 1, Yuseong gu, 305-340 Daejon, Republic of Korea, dcseo@kriss.re.kr), Bong Young Ahn (Korea Research Institute of Standards and Science, Doryong dong 1, Yuseong gu, 305-340 Daejon, Republic of Korea, ahnby@kriss.re.kr)

Using ultrasound, particles submerged or flowing in fluid can be manipulated since ultrasound has an effect on the behavior of particles. Specifically, in standing wave field, particles generally move to pressure nodes or pressure antinodes due to acoustic radiation force. In this work, the behavior of SiC particles and oil bubbles in flowing water by standing wave field was investigated. Standing wave field in frequencies between 2 and 2.5 $\mathrm{MHz}$ was formed in a few mm narrow flow channel using a water coupled ultrasonic transducer and a steel reflector. We observed the effect of the standing wave parameters such as frequency, flow channel width, or sound intensity on the behavior of the particles. Various interesting results were obtained through some experiments. We separated SiC particles and oil bubbles. It was shown that the operating frequency of standing wave can control the particle moving location. Sound intensity increase also leads to the entrapment of moving particles. The resulted observations reveal the possibility of various applications of the ultrasonic standing wave to the manipulation of particles. 


\title{
Session 4pPAe
}

\section{Physical Acoustics: Ultrasonics: NDT and Layered Systems II (Poster Session)}

\author{
Vincent Pagneux, Cochair \\ Laboratoire d'Acoustique de l'Université du Maine \\ Claire Prada, Cochair \\ Université Paris 7
}

\begin{abstract}
All posters will be on display from 3:40 p.m. to 5:20 p.m. To allow contributors an opportunity to see other posters, contributors of odd-numbered papers will be at their posters from 3:40 p.m. to 4:30 p.m. and contributors of even-numbered papers will be at their posters from 4:30 p.m. to 5:20 p.m.
\end{abstract}

\section{Contributed Papers}

4pPAe1. Dual frequency ultrasound- A pulse-echo technique for analysis of layered material. Ossi Riekkinen (University of Kuopio, POB 1627, $70211 \quad$ Kuopio, Finland, Ossi.Riekkinen@uku.fi), Mikko Hakulinen (University of Kuopio, POB 1627, 70211 Kuopio, Finland, Mikko.Hakulinen@uku.fi), Juha Töyräs (Kuopio University Hospital, POB 1777, $70211 \quad$ Kuopio, Finland, Juha.Toyras@kuh.fi), Jukka Jurvelin (University of Kuopio, POB 1627, 70211 Kuopio, Finland, Jukka .Jurvelin@uku.fi)

Ultrasound (US) thickness gauges typically analyse layered materials by utilizing ultrasound reflections between different layers and prior knowledge for the material order within the layered structure. In this study, a dual frequency ultrasound (DFUS) technique is applied to eliminate the effect of overlying layered structure on the measurements of the object of interest without prior knowledge of the order of materials within the multilayered structure. DFUS technique utilizes prior knowledge on US attenuation coefficient and speed at two frequencies in multilayered materials, consisting of two different material types. Then, US reflection from the front (first) and the back (last) surfaces of the multilayered structure is measured using two different US frequencies. No reflections from the internal interfaces are needed. The technique was validated using several elastomer samples and their combinations, measured at $2.25 \mathrm{MHz}$ and 5.0 MHz. DFUS reduced the mean error, induced by the overlying elastomers, in reflection from the object of interest from $103.6-289.4 \%$ to $-15.9-5.6 \%$ and from $37.5-77.5 \%$ to $-12.0-4.9 \%$ with $5.0 \mathrm{MHz}$ and $2.25 \mathrm{MHz}$, respectively. Based on these results, DFUS is a straightforward technique to analyse the multilayered structure without the need for echoes from internal interfaces.

4pPAe2. Ultrasonic pulse propagation in a bonded three-layered structure. Jose Luis San Emeterio (Instituto de Acustica CSIC, Serrano 144, $28006 \quad$ Madrid, Spain, jluis@ia.cetef.csic.es), Antonio Ramos (Instituto de Acustica CSIC, Serrano 144, 28006 Madrid, Spain, aramos@ia.cetef.csic.es), Emilia Pardo (Instituto de Acustica CSIC, Serrano 144, 28006 Madrid, Spain, empargo@ia.cetef.csic.es), Julio C. Leite (COPPE, Universidad Federal Rio de Janeiro, 68501 Rio de Janeiro, Brazil, juliobastosleite@jahoo.com.br), Jose Miguel Alvarez (INTA, Carretera Ajalvir, Km 4, 28850 Madrid, Spain, miguelalj@inta.es), Carlos Perez Trigo (INTA, Carretera Ajalvir, Km 4, 28850 Madrid, Spain, pereztrc @inta.es)

Bonded layers are used in the assembly of many critical functional parts of industrial equipment. In this work, ultrasonic pulse propagation in a steelrubber-rubber bonded composite structure is investigated by means of computer simulation and pulse echo experimental evaluation. Ultrasonic pulse propagation is modelled using a 2D time domain finite-difference software. For the experimental measurements, two test samples were fabricated by bonding a thin layer of steel and two thin layers of rubber, including deb- onded areas at marked regions of each interface. Several ultrasonic traces were acquired by contact pulse-echo testing, using a $5 \mathrm{MHz}$ wideband transducer, from the external steel surface. The large acoustic impedance mismatch existing between steel and rubber layers makes that only a very small part of the ultrasonic energy is transmitted through the first (steel-rubber) interface. The high attenuation in rubber materials and the possible overlapping of multiple echoes are additional characteristics of the complex ultrasonic pulse propagation in this flat structure. Some differences in time and frequency domains, between the received signals from normal bonded areas and completely debonded areas are discussed, looking for defect detection at the first (steel-rubber) and second (rubber-rubber) interfaces.

4pPAe3. Inverse Problem to Determine an Epoxy Film Parameters in a Tri-Layer Metal/Adhesive/Metal Structure. Naima Ismaili Alaoui (Laboratoire d'Electronique Signaux-Systemes et Informatique LESSI, Université Sidi Mohammed Ben Abdellah, Faculté des Sciences Dhar el Mehraz, 30000 Fes, Morocco, nanialaoui@ hotmail.com), Mounsif Ech-Cherif El-Kettani (LOMC FRE-3102 CNRS, Groupe Ondes Acoustiques, University of Le Havre, Place Robert Schuman, BP 4006, 76610 Le Havre, France, elkettani@univ-lehavre.fr), Driss Chenouni (Laboratoire d'Electronique Signaux-Systemes et Informatique LESSI, Université Sidi Mohammed Ben Abdellah, Faculté des Sciences Dhar el Mehraz, 30000 Fes, Morocco, d_chenouni@yahoo.fr), Zakia_Lakhlii (Laboratoire d'Electronique Signaux-Systemes et Informatique LESSI, Université Sidi Mohammed Ben Abdellah, Faculté des Sciences Dhar el Mehraz, 30000 Fes, Morocco, zlakhliai@hotmail.fr), Jean-Louis Izbicki (LOMC FRE-3102 CNRS, Groupe Ondes Acoustiques, University of Le Havre, Place Robert Schuman, BP 4006, 76610 Le Havre, France, jean-louis.izbicki @ univ-lehavre.fr), Bruno Morvan (LOMC FRE-3102 CNRS, Groupe Ondes Acoustiques, University of Le Havre, Place Robert Schuman, BP 4006, 76610 Le Havre, France, bruno.morvan@univ-lehavre.fr)

The aim of this work is to give the elastic parameters and the thicknes of a slim epoxy film of a tri-layer metal/adhesive/metal structure, by inverse problem on the experimental data, knowing the characteristic equation of the structure. This equation is firstly solved and the sensitivity of some modes to the parameters of interest is studied. The couple of solutions wavenumberfrequency obtained from the direct problem are reintroduced in the characteristic equation, looking for the longitudinal and shear waves velocities, and the epoxy thickness that minimise this equation. A criterion that takes into account the averaged and the variance values of each parameter is deduced and allows these parameters determination in $\pm 3 \%$ of the used values. To test the reliability of the method, the wavenumber solution is randomly perturbed in $\pm 5 \%$ range, as the experimental wavenumber is expected to be weakly different from the theoretical one. The velocities and the thickness obtained still in the $\pm 3 \%$ range around the used values. This 
method is then applied on experimental data obtained from a Duraluminu$\mathrm{m} /$ epoxy/Duraluminum structure. The longitudinal and shear velocities and the thickness of the epoxy film obtained are in $\pm 3 \%$ around the given values.

4pPAe4. The analysis of diffraction effects of acoustic waves on the crack's top. Yulia V. Zhitlukhina (Institute of Metal Physics, 18, Sofia Kovalevskaya St., GSP-170, 620041 Ekaterinburg, Russian Federation, julika_sun@mail.ru), Dmitry V. Perov (Institute of Metal Physics, 18, Sofia Kovalevskaya St., GSP-170, 620041 Ekaterinburg, Russian Federation, peroff@imp.uran.ru), Anatoly B. Rinkevich (Institute of Metal Physics, 18, Sofia Kovalevskaya St., GSP-170, 620041 Ekaterinburg, Russian Federation, rin@imp.uran.ru)

Diffraction effects and features of acoustic wave propagation in elastic media with microcrack were investigated in detail for pulse probing signals. The crack's plane was oriented across the direction of longitudinal ultrasonic wave incidence so that the detection of such a crack with so "inconvenient" spatial location is difficult enough by using traditional acoustic techniques. By using laser interferometer, the set of instantaneous pictures of acoustic field on the specimen's surface, corresponding to different time moments was obtained what allowed investigating and visualizing of acoustic field propagation and diffraction's effects on the crack's top in dynamics. Using various methods of numerical modeling of diffraction processes of acoustic wave on the crack's edge and top for pulse signals the origin of V-like structures on the snapshots of acoustic fields was explained and analyzed.

4pPAe5. Structural Health Monitoring using cross-correlation of an ambient noise field. Najib Abou Leyla (UVHC, IEMN-DOAE, Le Mont Houy, 59313 Valenciennes, France, najib.abouleyla@univ-valenciennes .fr), Emmanuel Moulin (UVHC, IEMN-DOAE, Le Mont-Houy, 59313 Valenciennes, France, emmanuel.moulin@univ-valenciennes.fr), Jamal Assaad (UVHC, IEMN-DOAE, Le Mont-Houy, 59313 Valenciennes, France, jamal.assaad@univ-valenciennes.fr), Sébastien Grondel (UVHC, IEMN-DOAE, Le Mont-Houy, 59313 Valenciennes, France, sebastien.grondel@univ-valenciennes.fr), Pascal Poussot (UVHC, IEMNDOAE, Le Mont Houy, 59313 Valenciennes, France, pascal.poussot@univvalenciennes.fr)

Theoretical and experimental studies in underwater acoustic, seismology and more recently ultrasonic have demonstrated that an estimate of the Time Domain Green Function (TDGF) between two receivers could be obtained from the cross-correlation of a diffuse acoustic noise field of these two receivers. The aim of the work is to exploit this technique in order to characterize Structural Health Monitoring (SHM) of aeronautic structures without the use of active sources. In this case, the aero-acoustic and/or mechanical sources (engine) generate an ambient noise field with some imperfections for the application. Indeed, source concentrations, source directivity and non-random components in time can appear which leads to an erroneous estimate of the TDGF. A key point to the study is thus to understand the influence of such imperfections. Therefore, experimental measurements have been performed using different types of acoustic noise sources (localized or diffuse). Cross-correlation results obtained in each case are then compared and theoretically interpreted. Finally, the potential of this technique in terms of damage detection is verified.

4pPAe6. Prediction of ultrasonic noise and attenuation for the simulation of non destructive testing. Frédéric Jenson (CEA-LIST, Centre de Saclay, 9119 Gif-sur-Yvette, France, frederic.jenson@cea.fr), Vincent Dorval (CEA-LIST, Centre de Saclay, 9119 Gif-sur-Yvette, France, vincent.dorval@cea.fr), Gilles Corneloup (LCND - Université de la Méditerranée, IUT Aix Provence, Avenue Gaston Berger, 13625 Aix en Provence Cedex, France, gilles.corneloup@univmed.fr)

Ultrasonic non destructive testing of some polycrystalline materials can be significantly affected by their microstructure. In such materials a fraction of the acoustic energy is redirected in all directions, which leads to both attenuation and structural noise and causes significant loss in detection performances. Consequently, being able to predict these phenomena would help in designing better testing procedures. During previous works at CEALIST, noise and attenuation models have been developped and implemented into the simulation software for non destructive testing CIVA. The noise model describes the microstructure of the material as a set of point-like scatterers and the attenuation model uses a filtering approach. They both require reference ultrasonic measurements to reproduce the behaviour of a given material. The connection of this approach to a scattering model relating noise and attenuation to microstructural characteristics is studied in this work. The selected model is based on the Born approximation and allows one to relate physical quantities such as the scattering cross section and the attenuation coefficient to second order statistical properties of the microstructure and to elastic properties of a single crystallite. This model accounts for important effects such as anisotropic scattering and mode conversions, but neglects multiple scattering events. Simulation results obtained with this approach are compared to experimental results.

4pPAe7. Ultrasonic control of the adhesion quality of two aluminium sheets. Naima Taifi (Fac. des sciences; Univ. Chouaib Doukkali, B.P: 20, 24000 El Jadida, Morocco, taifinaima@yahoo.fr), Bouazza Faiz (Ibn Zohr University, FS Agadir, 80000 Agadir, Morocco, faizbou@ hotmail.com), Ali Moudden (Ibn Zohr University, FS Agadir, 80000 Agadir, Morocco, ali_moudden@yahoo.fr), Gerard Maze (LAUE, Université du Havre, Place Robert Schuman, F-76610 Le Havre, France, gerard.maze @ univ-lehavre.fr), Dominique Decultot (LOMC FRE 3102 CNRS Groupes Ondes Acoustiques, Université du Havre (IUT), Place Robert Schuman, 76610 Le Havre, France, dominique.decultot@univ-lehavre.fr), Driss Izbaim (Ibn Zohr University, FS Agadir, 80000 Agadir, Morocco, driss _izbaim@yahoo.fr)

In this work, we present two ultrasonic methods allowing to control the quality of adhesion of two aluminium sheets with the same thickness. These methods are based on the analysis of the ultrasonic signals retrodiffused by the $\mathrm{Al} / \mathrm{glue} / \mathrm{Al}$ structure. The first method consists in controlling the behaviour of the mode of the sheets which is splitted. Two parameters controlling the transfers were allowed to characterize any type of adhesion: good, bad and intermediate. The second method is based on control of the width of the mode of the adhesive. The representation of this width by Argan diagram allows to control the quality of adhesion from the measurement of the diagram diameter. 


\title{
Session 4pPAf
}

\section{Physical Acoustics: Ultrasonics: Industrial NDT II (Poster Session)}

\author{
Bertrand Nongaillard, Cochair \\ IEMN-DOAE \\ Christophe Aristegui, Cochair \\ Université Bordeaux
}

\begin{abstract}
All posters will be on display from 3:40 p.m. to 5:20 p.m. To allow contributors an opportunity to see other posters, contributors of odd-numbered papers will be at their posters from 3:40 p.m. to 4:30 p.m. and contributors of even-numbered papers will be at their posters from 4:30 p.m. to 5:20 p.m.
\end{abstract}

\section{Contributed Papers}

4pPAf1. Simultaneous Sum-Frequency and Vibro-Acoustography Imaging for Nondestructive Evaluation (NDE) and Testing (NDT) Applications. Farid Mitri (Dep. of Physiology and Biomed. Eng., Mayo Clinic College of Medicine, 200 First Street SW, Rochester, MN 55905, USA, mitri.farid@mayo.edu), Glauber T. Silva (Instituto Nacional de Matematica Pura e Aplicada, 22460 Rio de Janeiro, Brazil, mitri@ieee.org), James Greenleaf (Dep. of Physiology and Biomed. Eng., Mayo Clinic College of Medicine, 200 First Street SW, Rochester, MN 55905, USA, jfg@mayo.edu), Mostafa Fatemi (Dep. of Physiology and Biomed. Eng., Mayo Clinic College of Medicine, 200 First Street SW, Rochester, MN 55905, USA, fatemi@mayo.edu)

High-resolution ultrasound imaging systems for inspection of defects and flaws in materials are of great demand in many industries. Among these systems, Vibro-acoustography (VA) has shown excellent capabilities as a non-contact method for non-destructive high-resolution imaging applications. This method consists of mixing two confocal ultrasound beams, slightly shifted in frequency, to produce an acoustic emission field at the difference frequency of the primary incident ultrasound beams. In addition to the difference frequency signal, there exists another signal at the sum frequency, formed in the intersection region of the two primary beams. The goal of this study is to investigate the formation of high-resolution images using the sum frequency of ultrasound waves in VA while concurrently forming the conventional difference-frequency VA image, thereby increasing the amount of information acquired during a single scan. A theoretical model describing the sum frequency wave propagation, including beam forming and image formation in the confocal configuration is developed and verified experimentally. Moreover, sample experiments are performed on a flawed fiber-reinforced ceramic composite plate. Images at both the difference and sum frequencies are compared and discussed. Results show that the sum frequency image produces a high-resolution C-scan of the plate by which the flaws and structural details of the plate can be detected.

4pPAf2. Ultrasonic polar c-scan system for range of material sizes and its capabilities for non-destructive testing. Kyle Barbour (Georgia Tech Lorraine - G.W. Woodruff School of ME, UMI Georgia Tech - CNRS 2958, 2 rue Marconi, 57070 Metz, France,nfdaaa@gmail.com), John M. Vander Weide (Georgia Tech Lorraine - G.W. Woodruff School of ME, UMI Georgia Tech - CNRS 2958, 2 rue Marconi, 57070 Metz, France, nico.declercq@me.gatech.edu), Sarah W. Herbison (Georgia Tech Lorraine - G.W. Woodruff School of ME, UMI Georgia Tech - CNRS 2958, 2 rue Marconi, 57070 Metz, France, sherbison@gatech.edu), Nico F.
Declercq(Georgia Tech Lorraine - G.W. Woodruff School of ME, UMI Georgia Tech - CNRS 2958, 2 rue Marconi, 57070 Metz, France, nico .declercq@me.gatech.edu)

The principle of multi-directional incident ultrasound has already shown to be a promising technique for the nondestructive evaluation of composites and other materials. The advantage is the correspondence between stiffness, damage and the registered double through transmission patterns. $\mathrm{C}$-scans are widely used as a tool for the detection of defects in materials. A new ultrasonic scanner has been developed, called the Polar C-Scan, which enables efficient polar scan measurements in combination with $\mathrm{C}$-scan capabilities The scanner opens many possibilities for nondestructive testing by means of polar scans, C-scans, single transmission, double through transmission and even reflection. Furthermore the system produces almost no noise, which enables highly sensitive measurements in the time domain and the frequency domain. The presented work shows a thorough investigation of all the capabilities of the system and presents results for fiber reinforced composites after different fatigue cycles.

4pPAf3. Ultrasonic and acoustic method for viscoelastic complex media characterization. Georges Nassar (IEMN - DOAE, Université de Valenciennes, Le Mont - Houy, 59313 Valenciennes, France, gnassar@univ-valenciennes.fr), Fabrice Lefebvre (IEMN - DOAE, Université de Valenciennes, Le Mont - Houy, 59313 Valenciennes, France, Fabrice.Lefebvre@univ-valenciennes.fr), Alain Skaf (IEMN - DOAE, Université de Valenciennes, Le Mont - Houy, 59313 Valenciennes, France, alain.skaf@univ-valenciennes.fr), Bertrand Nongaillard (IEMN - DOAE, Université de Valenciennes, Le Mont - Houy, 59313 Valenciennes, France, Bertrand.Nongaillard@univ-valenciennes.fr)

In this paper, the potentialities of a low frequency ultrasonic/acoustic technique devoted to the study and characterization of the viscoelastic complex media is investigated. This work shows the limit of the use of ultrasound in a viscoelastic media with a complex matrix. In this context the cheese was indicated as a model of propagation medium, such a product having a very complex matrix in term of texture, openings, crystallization, moisture,... . Theoretical basis of sound attenuation in cheese is recalled, especially the effects of the matrix viscoelasticity and the scattering of ultrasonic energy by holes and cracks. Depending on the degree of openness, ultrasonic velocity or attenuation is chosen to represent the evolution of the cheese. For very high degree of openness, ultrasounds are no longer usable and a tap-test acoustic technique is employed and allows a quality indicator to be constructed. Experimental validations were done with optical images of cut cheeses and rheological measurements. The results indicate that a high degree of sensitivity can be reached with ultrasonic / acoustic non destructive technique. 


\title{
Session 4pPAg
}

\section{Physical Acoustics: Nonlinear Acoustics of Consolidated Materials and Non Destructive Testing II (Poster Session)}

\author{
James Tencate, Cochair \\ Koen Van Den Abeele, Cochair \\ K.U.Leuven Campus Kortrijk
}

EES-11 (Geophysics) - Los Alamos National Laboratory

\begin{abstract}
All posters will be on display from 3:40 p.m. to 5:20 p.m. To allow contributors an opportunity to see other posters, contributors of odd-numbered papers will be at their posters from 3:40 p.m. to 4:30 p.m. and contributors of even-numbered papers will be at their posters from 4:30 p.m. to 5:20 p.m.
\end{abstract}

\section{Contributed Papers}

4pPAg1. Theoretical investigations into the use of linear and nonlinear inhomogeneous waves for non-destructive testing. John M. Vander Weide (Georgia Tech Lorraine - G.W. Woodruff School of ME, UMI Georgia Tech - CNRS 2958, 2 rue Marconi, 57070 Metz, France, nico.declercq@me.gatech.edu), Nico F. Declercq (Georgia Tech Lorraine G.W. Woodruff School of ME, UMI Georgia Tech - CNRS 2958, 2 rue Marconi, 57070 Metz, France, nico.declercq@me.gatech.edu)

Following the shift to incorporate more materials by design into modern engineering is the theoretical need to model an increasingly more complicated material structure and predict a design life for complex engineered materials. The presence of defects, interface cracks and delaminations adds to the inherent nonlinearity of a structure by degrading material stiffness and other physical properties contributing to fracture. The presence of local defects either at interfaces between two different materials or grain boundaries within the bulk material provide a natural location for the formation and subsequent propagation of cracks. This work provides initial theoretical investigations into the use of ultrasonic inhomogeneous waves to nondestructively probe such interface defects. Characterized theoretically by a complex valued wave number, the inhomogeneous wave is the more general plane wave solution of the wave equation. The complex valued wave number allows for the material effects of damping along the propagation direction and wave inhomogeneity or amplitude decay along the wavefront. To study the interaction with cracks, a singular integral equation formulation like that of Krenk and Schmidt is extended to farfield simulations with ultrasonic inhomogeneous waves.

4pPAg2. Acoustic emission of structures basis on the A3B5 compounds Vitaly V. Veleschuk (Institute of Semiconductors Physics, NASU, Prospect Nauki 45, 03028 Kyiv, Ukraine, vvvit@ukr.net), Oleg V. Lyashenko (Institute of Semiconductors Physics, NASU, Prospect Nauki 45, 03028 Kyiv, Ukraine, lyashenk@mail.univ.kiev.ua), Olexander I. Vlasenko (Institute of Semiconductors Physics, NASU, Prospect Nauki 45, 03028 Kyiv, Ukraine, vvvit@pochta.ru)

Failure of internal mechanical pressure at origin and movement of dislocations in processes of fast degradation and defect formation in LED and semiconductor lasers lead to occurrence of acoustic emission (AE) - to the phenomenon of radiation of pulse spontaneous acoustic wave's noise character. This failure of internal mechanical pressure in local volumes with occurrence AE is possible only at action of external fields (influences) of the certain physical nature, in our case - non-uniform fields of the thermomechanical pressure created by a constant direct current. In work were investigated n+-n-p- structures basis on the GaP:N, GaP0,85As0,15:N, Zn-O, In$\mathrm{GaN} / \mathrm{GaN}$ and p+-p-n- structures basis on the Ga0,7A10,3As and Ga0,65A10,35As. During step-by-step increase of a current 10-25 multiple change of AE occurrence threshold and a destruction threshold of structures was revealed at natural ageing structures after $6 * 108$ s. For some samples of structures at low temperatures (77) AE occurrence threshold came nearer or even corresponded to a destruction threshold. The given effect explains gradual saturation of dislocations by atoms of impurity with formation of Cottrell cloud, that considerably lowers their mobility and increases activation energy and accordingly a AE occurrence threshold

4pPAg3. Polar scans as a nonlinear acoustics tool. John M. Vander Weide (Georgia Tech Lorraine - G.W. Woodruff School of ME, UMI Georgia Tech - CNRS 2958, 2 rue Marconi, 57070 Metz, France, nico.declercq@me.gatech.edu), Nico F. Declercq (Georgia Tech Lorraine G.W. Woodruff School of ME, UMI Georgia Tech - CNRS 2958, 2 rue Marconi, 57070 Metz, France, nico.declercq@me.gatech.edu)

Nonlinear acoustic wave response provides a powerful means of assessing material properties. One very interesting area of application is testing for material damage. While an undamaged material may have a very linear acoustic response, damage such as crack formation creates strong nonlinearity with acoustic excitation amplitude. Many nonlinear acoustics techniques for nondestructive testing analyze the harmonic content in the wave scattered from a crack. A planar c-scan can be used in conjunction with the nonlinear acoustics techniques to provide defect detection and imaging. Polar scans are a complementary technique for damage assessment which has not yet found application in nonlinear acoustics. In the polar scan, the sound field in the hemisphere around a material point is measured and plotted to display a unique signature of local material properties. This work provides initial investigations into the use of the polar scan technique in nonlinear acoustics by detection of the second harmonic.

4pPAg4. Detection of the nonlinearity evolution in concrete samples subject to quasi-static loadings. Paola Antonaci (Politecnico di Torino, Corso Duca degli Abruzzi 24, 10129 Torino, Italy, paola.antonaci@polito .it), Pietro Bocca (Politecnico di Torino, Structural Engineering Department, 10129 Torino, Italy, pietro.bocca@polito.it), Caterina Bruno (Politecnico di Torino, Structural Engineering Department, 10129 Torino, Italy, caterina.bruno@polito.it), Antonio S. Gliozzi (Politecnico di Torino, Corso Duca degli Abruzzi 24, 10129 Torino, Italy, antonio.gliozzi@polito.it), Davide Masera (Politecnico di Torino, Structural Engineering Department, 10129 Torino, Italy, davide.masera @polito.it), Marco Scalerandi (Politecnico di Torino, Corso Duca degli Abruzzi 24, 10129 Torino, Italy, marco.scalerandi@infm.polito.it) 
The signature of nonlinearity in the elastic response of a specimen to an impingent ultrasonic wave is usually determined through Fourier analysis, which provides low amplitude signals, often below noise level. We suggest here an alternative, based on the amplitude dependence of the response of the system. Our procedure is conceptually simple and easy to implement. In addition, it keeps simultaneously into account the nonlinear signature effects on phases, amplitudes and frequencies of the response. The procedure is described and used to analyse the variation of the nonlinearity in a concrete bar subject to quasi-static loadings of increasing amplitude. The sensitivity of the approach allows to distinguish the compaction phase (up to a load of $30 \%$ of the rupture loading) from a microdamage progression (up to a load of $60 \%$ ) and the pre-rupture phases.

4pPAg5. Nondestructive tests of cylindrical steel samples using the ultrasonic projection method and the ultrasound transmission tomography method. Krzysztof J. Opielinski (Wroclaw University of Technology/Institute of Telecommunications, Teleinformatics and Acoustics, Wybrzeze Wyspianskiego 27, 50-370 Wroclaw, Poland, krzysztof.opielinski@pwr.wroc.pl), Tadeusz Gudra (Wroclaw University of Technology/Institute of Telecommunications, Teleinformatics and Acoustics, Wybrzeze Wyspianskiego 27, 50-370 Wroclaw, Poland, Tadeusz.Gudra @ pwr.wroc.pl)

The paper presents some methods of NDT of cylindrical steel samples by means of ultrasonic projection (UP) method and ultrasound transmission tomography (UTT) method. Some ways of scanning were proposed, using different measurement geometries and rendering possible the characterization and visualization of the inner structure of steel samples by projection and tomographic images of measured acoustic parameters. The measurements proposed in the paper allow us to obtain at the same time the distributions of mean values of a number of acoustic parameters characterizing the structure of samples: the mean amplitude of the ultrasonic wave after running through the sample, the mean runtime on the transmitter-receiver path, and the mean decrease of the ultrasonic wave frequency after running through the sample. These parameters measured in the tomographic scanning setup from many directions around the samples allow us to reconstruct the distributions of the local values of acoustic parameters such as respectively: the ultrasonic wave attenuation coefficient, the group velocity of the ultrasonic wave, the derivative of attenuation coefficient along the frequency. The reconstructed distributions of the local values of acoustic parameters render possible the imaging of the samples' internal structure cross-sections, each of the parameters characterizing different features of the structure.

4pPAg6. Use of point-source/point-receiver elastic waves in NDT-application. Alexandr I. Korobov (Dept. of Acoustics, Physics Faculty, M.V. Lomonosov Moscow State University, Leninskie gory 1, 119991 Moscow, Russian Federation, akor@acs465a-1.phys.msu.ru), Natalya I. Odina (Dept. of Acoustics, Physics Faculty, M.V. Lomonosov Moscow State University, Leninskie gory 1, 119991 Moscow, Russian Federation, niodina@mail.ru), Anna V. Abramova (Dept. of Acoustics, Physics Faculty, M.V. Lomonosov Moscow State University, Leninskie gory 1, 119991 Moscow, Russian Federation, akor@acs465a.phys.msu.ru)

The ultrasonic automated experimental setup and technique for research of anisotropy of elastic properties of micro- and nanocrystalline metals and residual stresses are developed. Setup works in range of frequencies of 0.2-5 $\mathrm{MHz}$. Accuracy of measurement of propagation time of elastic wave is equal to $0.2 \mathrm{~ns}$, amplitude - $1 \%$. Setup allows to make diagnostics of metals using volume, Raleigh and Lamb waves and to carry out scanning on linear and angular coordinates. Use of point source and receiver of acoustic waves and the high time resolution allows to carry out research of anisotropy and residual pressure with high spatial resolution. The $\mathrm{x}$-y-coordinate device operated by personal computer, allows to carry out two-dimensional scanning of the sample by elastic waves with step of 10 micron. Experimental results of diagnostics of anisotropy of elastic properties in a number of microcrystalline constructional materials with residual stresses (alloys of aluminum and steel), and also a steel plate in the field of welded seam are presented. Significant anisotropy of elastic properties caused by these defects is revealed in investigated samples. The work is supported by RFBR.
4pPAg7. Acoustic attenuation in silicon and silicon oxide. Anatolij P Onanko (Taras Shevchenko Kyiv National University, physics faculty, prosp. Glushkova, 2/1, 03028 Kyiv, Ukraine, onanko@univ.kiev.ua), Oleg V. Lyashenko (Institute of Semiconductors Physics, NASU, Prospect Nauki 45, 03028 Kyiv, Ukraine, lyashenk@mail.univ.kiev.ua), Inna O. Lyashenko (Taras Shevchenko Kyiv National University, physics faculty, prosp. Glushkova, 2/1, 03028 Kyiv, Ukraine, lyashik@ukr.net), Yurij A. Onanko (Taras Shevchenko Kyiv National University, physics faculty, prosp. Glushkova, 2/1, 03028 Kyiv, Ukraine, onanko@univ.kiev.ua)

In the present work the non-destructive acoustic attenuation (AA) method of the technological control the structure defects of semiconductor plates after various dozes of the X-ray irradiation is developed. For measurement of temperature dependences of AA the method of resonant fluctuations of a plate on frequency $1.5 \mathrm{kHz}$ was used at elastic deformation in vacuum. Measurements of temperature dependences AA in silicon plate a 460-470 micron thick after drawing a $600 \mathrm{~nm}$ layer of silicon oxide. Disk of a p-type silicon, doped B, orientation (100) with specific electroresistance 7,5 $\mathrm{ohm} \cdot \mathrm{cm}$. The small maximums of AA were observed at temperature $346 \mathrm{~K}$ and $380 \mathrm{~K}$. The main maximum of AA was observed at temperature $510 \mathrm{~K}$. The affinity received by us of value of energy of activation $0,8 \mathrm{eV}$ of AA at $510 \mathrm{~K}$ to energy of migration interstitial atoms $0,85 \mathrm{eV}$ of silicon allows to assume the relaxation mechanism caused by reorientation interstitial atoms of silicon in dumbbell configurations.

4pPAg8. Nondestructive Evaluation of heterogeneous materials using acoustic emission and ultrasound. Adil Faiz (ENSIM - LAUM, Université du Maine, rue Aristote, 72085 Le Mans, France, adil.faiz@univ-lemans.fr), Rachid El Guerjouma (ENSIM - LAUM, Université du Maine, rue Aristote, 72085 Le Mans, France, Rachid.El_Guerjouma@univ-lemans.fr), Mouras Bentahar (Université du Maine LAUM, Lab. d'Acoustique Université du Maine, UMR CNRS 6613 72085 Le Mans Cedex 9, France, mourad.bentahar@univ-lemans.)

Heterogeneous materials as Composites and concrete are advantageous as structural components in many applications. However, damage detection in such materials is difficult due to their heterogeneity and anisotropy. Furthermore, conventional non destructive technique as X-radiographic is mostly not very sensitive to early damage and very time consuming and expensive. The purpose of this contribution is to study the capabilities of several methods, non destructive and very sensitive for damage characterisation, as Acoustic Emission (AE) and ultrasonic for the structural health monitoring of heterogeneous materials as polymer based composite materials and concrete. These materials are instrumented by piezoelectric sensors in order to detect acoustic emission and to measure the ultrasonic velocity. The mechanisms of the damage events and their space-time localizations are identified from AE. Simultaneously, the longitudinal ultrasonic velocity is measured in situ by transmission through the composites thickness. The AE is very well correlated with the loss of stiffness determined from ultrasonic velocity measurements showing the potentiality of this combined approach for in situ structural health monitoring.

4pPAg9. In-situ measured Q- factor dependence from load in short and long time periods. Nora A. Vilchinska (LAA-Latvian Acoustics Association, 3 Kurzemes pr, LV-1064 Riga, Latvia, vilcinska@hotmail.com)

Dynamic loaded large object is under research. Long time periods (8 years) measurements are made by variety loads: from quasi static till strong motion. Short time measurements start from quasi static and load goes stepby-step till strong motion- maximum loaded- and return in the same way to quasi static. This experiment longs 60 minutes. An assessment of material quality factor (Q-factor) in places of measurements was made taking into account the absorbed and emitted energy. The Q-factor for energy is calculated from RMS response spectra curves. The Q-factor and its changes in long time and short time experiments in some MPs are compared. The smaller is the Q-factor, the higher is the concentration of interior invisible cracks. Structural alterations, opening of fractures and their closure under load, and transitional processes are reflected in the spectra of emitted acoustic signals and in the nonlinearity of response Q-factor. 


\title{
Session 4pPAh
}

\section{Physical Acoustics: Acoustic Probes of Planetary Environments II (Poster Session)}

\author{
Andi Petculescu, Cochair \\ University of Louisiana \\ Martin Towner, Cochair \\ PSSRI
}

\begin{abstract}
All posters will be on display from 3:40 p.m. to 5:20 p.m. To allow contributors an opportunity to see other posters, contributors of odd-numbered papers will be at their posters from 3:40 p.m. to 4:30 p.m. and contributors of even-numbered papers will be at their posters from 4:30 p.m. to 5:20 p.m.
\end{abstract}

\section{Contributed Papers}

4pPAh1. An ultrasonic anemometer for Mars. Colin Wilson(University of Oxford, Department of Atmospheric, Oceanic and Planetary Physics, OX1 3PU Oxford, UK, wilson@atmos.ox.ac.uk), David Hutchins (University of Warwick, School of Engineering, CV4 7AL Coventry, UK, D.A.Hutchins@warwick.ac.uk), Lee A. Davis (University of Warwick, School of Engineering, CV4 7AL Coventry, UK, lee.davis@warwick.ac.uk), Martin C. Towner (PSSRI, The Open University, Walton Hall, MK7 6AA Milton Keynes, UK, m.c.towner@open.ac.uk)

Ultrasonic anemometers are often used for studies of 3-D atmospheric turbulence on Earth, due to their robust calibration and fast operation $(>10$ $\mathrm{Hz}$ ). The same qualities make ultrasonic anemometry attractive for use on Mars, where similar atmospheric turbulence is found. The low density of Martian atmosphere - a hundredth that of the Earth's - is problematic, because of the large acoustic impedance mismatch between the atmosphere and piezoelectric transducers. One solution to this problem is to use piezoelectric transducers with a hornlike resonator bonded to their front surface. Another solution is to use a capacitive membrane transducer instead. We report on performance of various ultrasonic transducers in carbon dioxide Martian pressures, and present an instrument design capable of meeting the stringent mass, power, and environmental requirements of the European ExoMars lander.

4pPAh2. Acoustic tomography of the internal wave-ssociated fluctuations in the lower atmosphere. Igor Chunchuzov (Obukhov Institute of Atmospheric Physics, 3 Pyzhevskii Per., 119017 Moscow, Russian Federation, igor.chunchuzov@gmail.com), Sergey Kulichkov (Obukhov Institute of Atmospheric Physics, 3 Pyzhevskii Per., 119017 Moscow,
Russian Federation, snk@ifaran.ru),Vitaly Perepelkin(Obukhov Institute of Atmospheric Physics, 3 Pyzhevskii Per., 119017 Moscow, Russian Federation, vitaliper54@gmail.com), Astrid Ziemann (Leipzig Institute for Meteorology, Stephanstr. 3, 04103 Leipzig, Germany, ziemann@uni-leipzig .de), Klaus Arnold (Leipzig Institute for Meteorology, Stephanstr. 3, 04103 Leipzig, Germany, Arnold@uni-leipzig.de), Anke Kniffka (Leipzig Institute for Meteorology, Stephanstr. 3, 04103 Leipzig, Germany, kniffka@rz .uni-leipzig.de)

The two different schemes of acoustic tomography of the atmospheric boundary layer $(\mathrm{ABL})$ were used in the field experiments conducted near Melpitz (Germany) and Zvenigorod (Russia). The mesoscale effective sound speed fluctuations (periods $1 \mathrm{~min}-1 \mathrm{~h}$ ) averaged over different acoustic ray paths were retrieved from the fluctuations of sound travel time between sources and receivers. It was found that a major contribution to the retrieved fluctuations comes from the wind speed fluctuations. By using a coherence analysis of the retrieved and measured wind speed fluctuations in the spatially distanced points the wave like fluctuations with periods of $16-20 \mathrm{~min}$, 8-10min, 4-5min, 1-2min have been filtered, and their horizontal translation velocities and scales have been estimated. Similar periods were also found in the variations of the vertical turbulent fluxes of momentum and heat near ground. The mechanism of origination of these periods in the observed fluctuations is proposed. The effect of the wind shear variations induced by internal waves on the turbulence intensity was observed. This effect showed a substantial role played by internal waves in the origin of an intermittency of turbulence in the stably stratified ABL. This work was supported by RFBR, grants 06-05-64229, 05-05-64973, 07-05-91555. 


\title{
Session 4pPAi
}

\section{Physical Acoustics, Acoustical Oceanography, and Biomedical Ultrasound/Bioresponse to Vibration: Acoustically Activated Bubble Dynamics and Applications II (Poster Session)}

\author{
Erich Everbach, Cochair \\ Swarthmore College \\ Joachim Holzfuss, Cochair \\ Institute of Applied Physics, TU Darmstadt
}

\begin{abstract}
All posters will be on display from 3:40 p.m. to 5:20 p.m. To allow contributors an opportunity to see other posters, contributors of odd-numbered papers will be at their posters from 3:40 p.m. to 4:30 p.m. and contributors of even-numbered papers will be at their posters from 4:30 p.m. to 5:20 p.m.
\end{abstract}

\section{Contributed Papers}

4pPAi1. Sound propagation in viscoelastic pipe with liquid-bubble mixture. Semyon Levitsky (Shamoon College of Engineering, Math. Department, Bialik/Basel Sts., 84100 Beer Sheva, Israel, levits@sce .ac.il), Rudolf Bergman (Shamoon College of Engineering, Math. Department, Bialik/Basel Sts., 84100 Beer Sheva, Israel, rudolf@sce.ac.il), Jehuda Haddad (Shamoon College of Engineering, Math. Department, Bialik/Basel Sts., 84100 Beer Sheva, Israel, jehuda@sce.ac.il)

Propagation of acoustic waves in thin-walled polymeric tube with viscous liquid is investigated. Dynamics of the tube - liquid interaction is studied within conjugated quasi-one-dimensional formulation; the tube material is supposed to follow linear viscoelastic model with appropriate choice of the compliance function. It is assumed that the liquid contains fine air bubbles; the concentration of free gas is supposed to be small. Compressibility of liquid in the wave in the presence of bubbles can be almost entirely attributed to compressibility of the gas phase; it is accounted for within dispersion equation for bubbly liquid. Both heat and viscous losses are included in the phase interaction description at the liquid-gas interface. The resulting dispersion equation for the waveguide with liquid-gas mixture is studied in the long-wave range, where sound length is larger from the waveguide diameter. Results of simulations illustrate strong influence of the pipe mechanical properties and parameters of the gas phase on sound dispersion and attenuation.

4pPAi2. Acoustic measurement of bubble size and position in an ink jet printhead. Arjan Van Der Bos (Physics of Fluids, University of Twente, P.O. Box 217, 7500 AE Enschede, Netherlands, j.a.vanderbos@tnw.utwente .nl), Roger Jeurissen (Physics of Fluids, University of Twente, P.O. Box 217, 7500 AE Enschede, Netherlands, r.j.m.jeurissen@tnw.utwente.nl), Jos De Jong (Oce Technology, P.O. Box 101, 5900 MA Venlo, Netherlands, jos.dejong@oce.com), Detlef Lohse (Physics of Fluids, University of Twente, P.O. Box 217, 7500 AE Enschede, Netherlands, d.lohse@utwente .nl), Michel Versluis (Physics of Fluids, University of Twente, P.O. Box 217, 7500 AE Enschede, Netherlands, m.versluis@utwente.nl), Hans Reinten (Oce Technology, P.O. Box 101, 5900 MA Venlo, Netherlands, hans.reinten@oce.com), Marc Van Den Berg (Oce Technology, P.O. Box 101, 5900 MA Venlo, Netherlands, marc.van.den.berg@oce.com), Herman Wijshoff (Oce Technology, P.O. Box 101, 5900 MA Venlo, Netherlands, herman.wijshoff@oce.com), Richard Stevens (Physics of Fluids, University of Twente, P.O. Box 217, 7500 AE Enschede, Netherlands, r.j.a.m .stevens@student.utwente.nl)
An acoustic measurement method of the volume and position of a bubble in an ink jet print head, is presented. The system is driven by a piëzo actuator. The actuator is also used as a sensor by measuring the current through the piëzo. The method used to determine the volume and position of the bubble is based on a linear model of the investigated system. This model predicts the current for a given position and volume of the bubble. The inverse problem is to infer the position and volume of the bubble from the measured current through the piëzo actuator. The solution of the inverse problem is demonstrated. Thus, an acoustical measurement method of these properties is obtained. The results from the acoustical measurement method correspond closely with results from optical measurements. This indicates validity of the presented method.

4pPAi3. Single bubble oscillations in viscoelastic media. Anatoliy Strybulevych (Dept. of Physics and Astronomy, Univ. of Manitoba, Winnipeg, MB R3T 2N2, Canada, anatoliy@physics.umanitoba.ca), Valentin Leroy (Univ. of Manitoba, Dept. of Physics and Astronomy, Univ. of Manitoba, Winnipeg, MB R3T 2N2, Canada, valeroy77@yahoo.fr), Martin G. Scanlon (Univ. of Manitoba, Dept. of Food Science, 250 Ellis Bldg., Winnipeg, MB R3T 2N2, Canada, scanlon@cc.umanitoba.ca), John H. Page (Dept. of Physics and Astronomy, Univ. of Manitoba, Winnipeg, MB R3T 2N2, Canada, jhpage@cc.umanitoba.ca)

We report a new ultrasonic technique for determining the viscoelasticity of soft materials based on the oscillations of single bubbles injected into the material of interest. It is known that bubbles in a liquid act as strong acoustic scatterers that exhibit a low frequency resonance known as the Minnaert resonance. In the case of viscoelastic media, the complex frequencydependent shear modulus causes the Minnaert frequency to be shifted to a higher value and leads to additional ultrasonic absorption. Therefore, both storage and loss shear moduli can be determined from the resonance frequency and the damping rate of the acoustic oscillations of a single bubble that has been injected into the sample. Experiments were performed on optically transparent commercial hair gel, agar gel, and PDMS rubber, allowing independent measurements of the bubble sizes to be made by an optical imaging technique. The acoustical properties of the samples were measured by sweeping the frequency of a continuous sinusoidal signal from 4 to 50 $\mathrm{kHz}$. Because the Minnaert frequency is inversely proportional to the radius of the bubble, experiments on bubbles of different sizes enabled the frequency dependence of the complex shear moduli of the materials to be determined. 
4pPAi4. Experimental examination on the interactive force between two bubbles under ultrasound irradiation: Influence of the distance between two bubbles on bubble behavior. Takaaki Fujikawa (Faculty of Engineering, Doshisha Univ., 1-3 Miyakodani Tatara, 610-0321 Kyotanabe, Japan, dth0106@mail4.doshisha.ac.jp), Kenji Yoshida (Faculty of Engineering, Doshisha Univ., 1-3 Miyakodani Tatara, 610-0321 Kyotanabe, Japan, etf1103@mail4.doshisha.ac.jp), Yoshiaki Watanabe (Faculty of Engineering, Doshisha Univ., 1-3 Miyakodani Tatara, 610-0321 Kyotanabe, Japan, kwatanab@mail.doshisha.ac.jp)

The interactive force called the secondary Bjerknes force works among multiple bubbles under ultrasound irradiation, which results in the complicated behaviors of bubbles. In this study, we have experimentally examined the direction of the interactive force depending on the distance between two bubbles. When two bubbles vibrate individually, the direction of the interactive force depends on the phase difference between vibrations of these bubbles. In addition to this theory, considering the influence of the radiated acoustic wave from a bubble vibration on the other bubble vibration, Ida has pointed that this influence induces a change in the direction of the interactive force [M. Ida et al., Phys. Rev. E 67, 056617 (2003)]. In order to examine this theoretical prediction, the behaviors of both a free bubble and a bubble adhered to the polymer were observed under ultrasound irradiation, using a high-speed video camera. From the results, we found the reversal of the direction of the interactive force due to the variation in the distance between two bubbles. These innovative experimental results give an interesting point of view to understand the dynamics of multiple bubbles.

4pPAi5. Destruction of the gas-filled capsule using effects of the collapsing bubble near the capsule. Jun Miyabe (Faculty of Engineering, Doshisha Univ., 1-3 Miyakodani Tatara, 610-0321 Kyotanabe, Japan, dth0144@mail4.doshisha.ac.jp), Kenji Yoshida (Faculty of Engineering, Doshisha Univ., 1-3 Miyakodani Tatara, 610-0321 Kyotanabe, Japan, etf1103@mail4.doshisha.ac.jp), Daisuke Koyama (Tokyo Institute of Technology, 4259 Nagatsuda, 226-8503 Yokohama, Japan, dkoyama@sonic.pi.titech.ac.j), Yoshiaki Watanabe (Faculty of Engineering, Doshisha Univ., 1-3 Miyakodani Tatara, 610-0321 Kyotanabe, Japan, kwatanab@mail.doshisha.ac.jp)

A new technique to destroy the gas-filled capsule is proposed, making use of the collapsing bubble near the capsule in the ultrasound field. At the moment of collapses under the ultrasound irradiation, the bubble causes flow and acoustic wave radiation. These phenomena are expected to assist the destruction of the capsule. We observed the effect of the bubble on this destruction, using a high-speed video camera which enables the real-time imaging. In this experiment, we use gas-filled capsule made of polyvinyl chloride. When no bubble was near the capsule at sound pressure of $60 \mathrm{kPa}$, the capsule showed no vibration. However, in the presence of neighboring bubble, the capsule showed the destruction behavior of the deformation and the emission of internal gas. At higher sound pressure such as over $100 \mathrm{kPa}$, the capsule showed above destruction behavior even if the bubble was not located near the capsule. In the presence of the bubble, however, the capsule was destructed in a shorter time. These experimental results give significant knowledge to a technique manipulating the gas-filled capsule destruction in the drug delivery system.

4pPAi6. Acoustic transmission through one plane of bubbles. Valentin Leroy (Univ. of Manitoba, Dept. of Physics and Astronomy, Univ. of Manitoba, Winnipeg, MB R3T 2N2, Canada, valeroy77@yahoo.fr), Anatoliy Strybulevych (Dept. of Physics and Astronomy, Univ. of Manitoba, Winnipeg, MB R3T 2N2, Canada, anatoliy@physics.umanitoba.ca), Martin G. Scanlon (Univ. of Manitoba, Dept. of Food Science, 250 Ellis Bldg., Winnipeg, MB R3T 2N2, Canada, scanlon@cc.umanitoba.ca), John H. Page (Dept. of Physics and Astronomy, Univ. of Manitoba, Winnipeg, MB R3T2N2, Canada,jhpage@cc.umanitoba.ca)

We measured the transmission of ultrasonic waves through one layer of bubbles, for frequencies ranging from 30 to $250 \mathrm{kHz}$. The layer was a true 2D structure obtained by injecting very monodisperse bubbles (with radius $\mathrm{r}=100 \mu \mathrm{m}$ ) into a yield-stress polymer gel. Even for layer with a low concentration of bubbles (areal fraction, $n \pi r^{2}$, of $10-20 \%$, where $n$ is the number of bubbles per unit area), the transmission was found to be significantly reduced by the presence of bubbles $(-20$ to $-50 \mathrm{~dB})$ and showed a sharp minimum at a particular frequency. Interestingly, this frequency did not correspond to the individual Minnaert resonance of the bubbles, but depended on the concentration, which we interpret as an indication of strong coupling between the bubbles. We propose a simple model, based on a self-consistent relation, which takes into account the coupling between the bubbles and gives good agreement with the measured transmission.

4pPAi7. Nonlinear ultrasonic waves in water-air mixtures. Christian Vanhille (Universidad Rey Juan Carlos, Tulipán, s/n., 28933 Móstoles, Madrid, Spain, christian.vanhille@urjc.es), Cleofé Campos-Pozuelo (Instituto de Acústica, CSIC, Serrano, 144, 28006 Madrid, Spain, ccampos@ia.cetef.csic.es)

In this paper we present some features of nonlinear ultrasonic waves in water-air mixtures. This analysis is based on the coupling of the linear wave equation to the bubble equation in a volume formulation. The system is solved via the development of a numerical model (SMOW-BL code). The main restrictions of the model are: the nonlinear behaviour comes exclusively from the bubble vibration, all the bubbles have the same size, air inside the bubbles is adiabatic. Continuous waves at low ultrasonic frequency and wide band pulses propagation in an open domain are analysed. Results obtained by considering several bubbly layers in water are given. The existence of soliton in a water-air mixture is demonstrated. Some results corresponding to standing waves are also presented. A model which allows us to consider the self-generation of air bubbles in water, i.e., cavitation, is proposed. 


\title{
Session 4pPAj
}

\section{Physical Acoustics: Ultrafast Acoustics II (Poster Session)}

\author{
David Hurley, Cochair \\ Materials Characterization Department, Idaho National Laboratory
}

\author{
Clément Rossignol, Cochair \\ LMP, UMR CNRS 5469, Université Bordeaux I
}

\begin{abstract}
All posters will be on display from 3:40 p.m. to 5:20 p.m. To allow contributors an opportunity to see other posters, contributors of odd-numbered papers will be at their posters from 3:40 p.m. to 4:30 p.m. and contributors of even-numbered papers will be at their posters from 4:30 p.m. to 5:20 p.m.
\end{abstract}

\section{Contributed Papers}

4pPAj1. Complete characterization in thin film using picosecond ultrasonics and nanostructured transducer. Pierre-Adrien Mante (IEMN-CNRS, Cité Scientifique - Avenue Poincaré, BP 60069, 59652 Villeneuve d'Ascq Cedex, France, pierre-adrien.mante @isen.fr), Jean-François Robillard (IEMN-CNRS, Cité Scientifique - Avenue Poincaré, BP 60069, 59652 Villeneuve d'Ascq Cedex, France, jean-francois.robillard@isen.fr), Arnaud Devos (IEMN-CNRS, Cité Scientifique - Avenue Poincaré, BP 60069, 59652 Villeneuve d'Ascq Cedex, France, arnaud.devos@isen.fr), Isabelle Roch-Jeune (IEMN-CNRS, Cité Scientifique - Avenue Poincaré, BP 60069, 59652 Villeneuve d'Ascq Cedex, France, isabelle.roch-jeune@iemn.univ-lille1.fr)

Picosecond ultrasonics is an efficient method to excite and detect vibrations within a thin film. A strong optical pulse warms a material surface, which leads to the creation of an acoustic wave propagating at the sound velocity. The waves propagation modifies the optical properties of the material that can be detected by a second time-shifted optical pulse. With an usual metallic transducer, only longitudinal waves can be generated. The use of this technique on a nanostructured transducer adds in-plane propagating waves. In the case of an isotropic medium, we have access to all acoustic properties. In order to confirm this statement, we realized and studied 2D lattices of metallic nanostructures. We used e-beam lithography to obtain defect free lattices of aluminum nanocubes. Both cubes width and lattice parameters were chosen to optimize the properties measurements [1]. This nanostructuration allows us to get information about longitudinal and inplane waves, in the gigahertz frequency range. The experiments where carried out with an aluminum lattice on a $600 \mathrm{~nm}$-thick silica film. This method, demonstrated on a well-known material, is suitable for any thin film. [1] J.-F. Robillard, A. Devos and I. Roch-Jeune "Time-resolved vibrations of two-dimensional hypersonic phononic crystals", Phys. Rev. B. 76, 092301 (2007)

$4 p P A j 2$. Generation of acoustical phonons by femtosecond laser pulses in GaAs in the presence of external electric field. Philippe Babilotte (LPEC/UMR 6087/CNRS/Université du Maine, Avenue Olivier Messiaen, 72085 Le Mans Cedex 09, France, philippe.babilotte.etu @univ-lemans.fr), Pascal Ruello (LPEC/UMR 6087/CNRS/Université du Maine, Avenue Olivier Messiaen, 72085 Le Mans Cedex 09, France, pascal.ruello@univ-lemans.fr), Denis Mounier (LPEC/UMR 6087/CNRS/Université du Maine, Avenue Olivier Messiaen, 72085 Le Mans Cedex 09, France, denis.mounier@univ-lemans.fr), Daniel Pugliese (LPEC/UMR 6087/CNRS/Université du Maine, Avenue Olivier Messiaen, 72085 Le Mans Cedex 09, France, Daniel.Pugliese@univ-lemans.fr), Mathieu Edely (LPEC/UMR 6087/CNRS/Université du Maine, Avenue Olivier
Messiaen, 72085 Le Mans Cedex 09, France, mathieu.edely@univ-lemans .fr), Alain Bulou (LPEC/UMR 6087/CNRS/Université du Maine, Avenue Olivier Messiaen, 72085 Le Mans Cedex 09, France, alain.bulou @ univ-lemans.fr), Jean-Marc Breteau (LPEC/UMR 6087/CNRS/Université du Maine, Avenue Olivier Messiaen, 72085 Le Mans Cedex 09, France, jean-marc.breteau@univ-lemans.fr), Vitali Gusev (LPEC/UMR 6087/CNRS/Université du Maine, Avenue Olivier Messiaen, 72085 Le Mans Cedex 09, France, vitali.goussev@univ-lemans.fr)

Experimental results on the generation and the detection by fs laser pulses of the acoustical phonons at frequencies from tens to hundreds $\mathrm{GHz}$ in GaAs in the presence of external electric field are presented. The influence of the magnitude and the direction of the applied electric field on the parameters of ps ultrasound is investigated. Results obtained in GaAs and low-temperature GaAs are compared. The experimental opportunities to discriminate the acoustical phonons produced by laser-induced inverse piezoelectrical effect and the acoustical phonons due to the thermoelastic mechanism and to the mechanism of electron-phonon deformation potential are discussed. This study was supported by ANR project BLAN06-3-136284.

4pPAj3. Temperature Dependence of Elastic Constant Measurements on Thin Films by Picosecond Ultrasonics. Patrick Emery (IEMN-CNRS, Cité Scientifique - Avenue Poincaré, BP 60069, 59652 Villeneuve d'Ascq Cedex, France, patrick.emery@isen.fr), Arnaud Devos (IEMN-CNRS, Cité Scientifique - Avenue Poincaré, BP 60069, 59652 Villeneuve d'Ascq Cedex, France, arnaud.devos@isen.fr)

The temperature dependence of mechanical parameters is well-known for bulk materials. New methods have to be developed to access such characteristics on thin films. These measurements are needed for understanding the temperature behavior of acoustic components in microelectronics, as for Bulk Acoustic Wave (BAW) resonators used in wireless communication. A BAW resonator uses the thickness mode resonance of a piezoelectric layer (Aluminium Nitride). In the radio-frequency range, a BAW resonator is a complex stack of various materials in thin film. The temperature dependences measurements offer a way to regulate the performances' drift induced by the warming of the device and to design temperature-independent components. Here, we present a method based on a variable temperature picosecond ultrasonics setup. The procedure is first validated on silica, then applied on various BAW technology materials (AlN, Mo, SiN, W)

4pPAj4. Photothermal and photoacoustic imaging by ultrafast optical sampling. Stefan Dilhaire (LMP, UMR CNRS 5469, Université Bordeaux I, 351, cours de la Libération, 33405 Talence, France, stefan.dilhaire @ u-bordeaux1.fr), Jean-Michel Rampnoux (LMP, UMR CNRS 5469, 
Université Bordeaux I, 351, cours de la Libération, 33405 Talence, France, jm.rampnoux@cpmoh.u-bordeaux1.fr), Gilles Pernot (LMP, UMR CNRS 5469, Université Bordeaux I, 351, cours de la Libération, 33405 Talence, France, g.pernot@cpmoh.u-bordeaux1.fr), Gaetan Calbris (LMP, UMR CNRS 5469, Université Bordeaux I, 351, cours de la Libération, 33405 Talence, France, g.calbris@cpmoh.u-bordeaux1.fr), Stephane Grauby (LMP, UMR CNRS 5469, Université Bordeaux I, 351, cours de la Libération, 33405 Talence, France, s.grauby@cpmoh.u-bordeaux1.fr), Clément Rossignol (LMP, UMR CNRS 5469, Université Bordeaux I, 351, cours de la Libération, 33405 Talence, France, c.rossignol@lmp.u-bordeaux1 .fr), Sebastien Ermeneux (Alphanov, 351 cours de la Libération, 33405 Talence, France, sebastien.ermeneux@alphanov.com), Eric Mottay (Amplitudes Systèmes, 6, allée du doyen Georges Brus, 33600 Pessac, France, emottay@amplitude-systemes.com)

We describe a new ultrafast imaging technique based on the use of two new generation Ytterbium lasers emitting at $1030 \mathrm{~nm}$ at $50 \mathrm{MHz}$ repletion rate. Ultrafast acquisition is achieved by slightly shifting the repetition rate of the "pump" and the "probe" beams. In that conditions a single shot response is acquired in $1 \mathrm{~ms}$ that allows sweeping the surface of the device or the material and obtain movies of the reflectivity field of the surface. This technique allows filming the reflectance changes of a material at very high speed typically 1 Tera frame per second during $20 \mathrm{~ns}$. We will show applications of this technique in acoustic imaging of surface waves and non destructive evaluation of microelectronic materials.

4pPAj5. The role of coherent phonons in the vibronic laser. Wojciech Gadomski (University of Warsaw, Department of Chemistry, Laboratory of Physicochemistry of Dielectrics and Magnetics, ul. Żwirki i Wigury 101, 01-905 Warsaw, Poland, gado@chem.uw.edu.pl), Bożena Gadomska (University of Warsaw, Department of Chemistry, Laboratory of Physicochemistry of Dielectrics and Magnetics, ul. Żwirki i Wigury 101, 01905 Warsaw, Poland, bogad@chem.uw.edu.pl)

Herewith we present the quantum theory of the vibronic laser, which is based on the crucial role played by the host crystal phonons in the laser dynamics. In the solid state transition-metal ion laser the operation takes place between the vibronically broadened electronic levels of the gain center, thus the laser action is accompanied by the creation or annihilation of the host lattice phonons. The nonequilibrium coherent phonons, produced in the process of the nonradiative energy transition from the photoexcited impurity ion, pump the energy level, from whivh the laser action takes place.
One can say that the laser photons are produced at the cost of lattice phonons. This is the reason why the coherent phonons strongly influence the character of the laser action and govern the complicated laser dynamics. In the laser equations, derived by us, the number of coherent phonons is treated as one of dynamical variables coupled with the number of photons. In solution we observe the effect of energy pulling between photons and phonons. Phonons follow all forms of the photon dynamics including selfpulsations and chaotic dynamics.

4pPAj6. Picosecond ultrasonics signal in biological materials: Comparison between predictions and experiments. Mathieu Ducousso (LMP, UMR CNRS 5469, Université Bordeaux I, 351, cours de la Libération, 33405 Talence, France, m.ducousso@1mp.u-bordeaux1 .fr), Clément Rossignol (LMP, UMR CNRS 5469, Université Bordeaux I, 351, cours de la Libération, 33405 Talence, France, c.rossignol @1mp.u-bordeaux1.fr), Bertrand Audoin (LMP, UMR CNRS 5469, Université Bordeaux I, 351, cours de la Libération, 33405 Talence, France, b.audoin@1mp.u-bordeaux1.fr), Fabien Guillemot (INSERM U577, Université Victor Segalen Bordeaux 2, 146, rue Léo-Saignat, Case 45, 33076 Talence, France, fabien.guillemot@bordeaux.inserm.fr), Marie-Christine Durrieu (INSERM U577, Université Victor Segalen Bordeaux 2, 146, rue Léo-Saignat, Case 45, 33076 Talence, France, durrieumc@bordeaux.inserm .fr)

Picosecond ultrasonics is a non-destructive method for measuring mechanical properties such as velocity or stiffness coefficients for nanometric materials [1]. This technique uses femtosecond laser pulses for generating and detecting acoustics waves from $\mathrm{GHz}$ to $\mathrm{THz}$. Its resolution is about nanometers in depth and a few micrometers laterally. For transparent materials it allows generating the so-called Brillouin oscillations, which frequencies are determined by the material sound velocity and the light beam wavelength. In this paper this technique is applied to biological cells. Measurements suggest promising perspectives for the imaging inside a single living cell; frequency content is from 5 to $20 \mathrm{GHz}$. A theoretical model based on Fourier heat and acoustic wave equations has been developed. These equations include heat diffusion and acoustic propagation respectively. It permits numerical simulations in time domain. First experimental results on animal and vegetal cells are presented and confronted with these calculated waveforms. [1] C. Thomsen, H. T. Grahn, H. J. Maris, J. Tauc, Phys. Rev. B 34, 4129, 1986 


\title{
Session 4pPAk
}

\section{Physical Acoustics: Thermoacoustics II (Poster Session)}

\author{
Steven Garrett, Cochair \\ Penn State University \\ Philippe Blanc-Benon, Cochair \\ Ecole Centrale de Lyon
}

\begin{abstract}
All posters will be on display from 3:40 p.m. to 5:20 p.m. To allow contributors an opportunity to see other posters, contributors of odd-numbered papers will be at their posters from 3:40 p.m. to 4:30 p.m. and contributors of even-numbered papers will be at their posters from 4:30 p.m. to 5:20 p.m.
\end{abstract}

\section{Contributed Papers}

4pPAk1. Experimental evaluation of the wavenumber in stacked screen regenerators. Yuki Ueda (Tokyo University of Agriculture and Technology, Nakacho 2-24-16, 187-8588 Koganei, Tokyo, Japan, uedayuki@cc.tuat .ac.jp)

The experimental method to evaluate the wavenumber of the acoustic wave propagating in pores media is proposed. The method was applied to four types of stacks composed of square channels and seven types of regenerators consisting of stacked mesh screens. The experimental results of the stacks agreed well with the theoretical results. The experimentally obtained wavenumber in the stacked screen regenerators were found to be similar to that in circular channels. However, it was found that they depend on the types of regenerators. Based on the dependence on the regenerator types of the evaluated wavenumber, the effective radius in the stacked screen regenerators was addressed.

4pPAk2. A thermoacoustic device for sound reproduction. Fotios Kontomichos (Audio and Acoustic Technology Group, Wire Communications Laboratory, Electrical Engineering \& Computer Technology Department, University of Patras, 26500 Patras, Greece, fotkon@wcl.ee .upatras.gr), Alexandros Koutsioubas (University of Patras Dept. of Physics, Rio, 26500 Patras, Greece, alexandk@physics.upatras.gr), John Mourjopoulos (Audio and Acoustic Technology Group, Wire Communications Laboratory, Electrical Engineering \& Computer Technology Department, University of Patras, 26500 Patras, Greece, mourjop@wcl.ee .upatras.gr), Nikolaos Spiliopoulos (University of Patras Dept. of Physics, Rio, 26500 Patras, Greece, nspiliop@physics.upatras.gr), Alexandros Vradis (University of Patras Dept. of Physics, Rio, 26500 Patras, Greece, vradis@physics.upatras.gr)

Many current research efforts focus on alternative electroacoustic transduction devices having no moving parts, in order to achieve sufficient audio performance from compact solid state devices. Thermoacoustic loudspeakers are transducers based on the conversion of A/C current signals to thermal energy, causing a local fluctuation of air pressure which generates acoustic waves. A thermoacoustic actuator does not involve any movement of solid components in order to generate an acoustic wave and it is based on a mechanism of a "virtual" piston produced by vibrating air molecules via alternating heat transfer to the medium. This work examines such novel and alternative audio transduction technologies based on a novel hybrid thermoacoustic transducer prototype which was developed at the University of Patras through the cooperation between Audio and Acoustic Technology Group and Solid State Physics Laboratory. This hybrid solid state device without moving parts is based on the thermoacoustic method of sound re- production and preliminary measurements of its performance are presented The theoretical principles of these systems are also simulated, resulting into comparisons with the measured performance of the prototype.

4pPAk3. Numerical modelling of acoustic streaming in resonators Abdennour Boufermel (Laboratoire d'Acoustique de l'Université du Maine, Avenue Olivier Messiaen, 72085 Le Mans, France, boufermel@hotmail .fr), Nicolas Joly (Laboratoire d'Acoustique de l'Université du Maine, Avenue Olivier Messiaen, 72085 Le Mans, France, nicolas.joly@univ-lemans .fr), Pierrick Lotton (Laboratoire d'Acoustique de l'Université du Maine, Avenue Olivier Messiaen, 72085 Le Mans, France, Pierrick.Lotton@univlemans.fr)

The acoustic wave propagation in thermoviscous fluid can generate slow phenomena, such as streaming and thermoacoustic effects, by nonlinear processes localised mainly in the viscous and thermal boundary layers. The model presented consists in computing numerically by the finite element method, (i) first, the harmonic solution for linear acoustics in thermoviscous fluid including the boundary layers, and (ii) second, the unsteady solution for the acoustic streaming and heat transfer. The model formulation is based on the mass and momentum conservation equations for the streaming, and the energy conservation equation for heat transfer. The streaming is presented as a standard form of an incompressible flow for velocity vector for mass transport, where the nonlinear effects of acoustics are considered as excitation forces for the streaming and sources for heat transfer. As the performance of thermoacoustic devices is limited by the convective heat transfer of the streaming, this study is suited for the optimisation of these systems. Another application is the development of microfluidic devices.

4pPAk4. Experimental validations of a new thermoacoustic simulation software CRISTA. Adrien Bétrancourt (LIMSI-CNRS, BP 133, F-91403 Orsay, France, adrien.betrancourt@limsi.fr), Thierry Le Pollès (Hekyom SARL, 2 rue Jean Rostand, 91400 Orsay, France, thlepolles@hekyom .com), Gérard Defresne (LIMSI-CNRS, BP 133, F-91403 Orsay, France, defresne@limsi.fr), Diana Baltean-Carlès (LIMSI-CNRS, BP 133, F-91403 Orsay, France, baltean@limsi.fr), Patxi Duthil (Institut de Physique Nucléaire d'Orsay, 15 rue Georges Clémenceau, 91400 Orsay, France, duthil@ipno.in2p3.fr), Jean-Pierre Thermeau (Institut de Physique Nucléaire d'Orsay, 15 rue Georges Clémenceau, 91400 Orsay, France, thermeau@ipno.in2p3.fr), Maurice-Xavier François (LIMSI-CNRS, BP 133, F-91403 Orsay, France, mxf@limsi.fr)

A new simulation software CRISTA has been developed at LIMSICNRS. It is based on the Rott's equations approximation. It computes all thermal and acoustic parameters of a given thermoacoustic device whose geometry is previously designed with another program TADESIGN. To realize 
the simulation, the user needs only to define a drive ratio at some point of the system and the heat exchanger temperatures. Note that for a prime mover the hot heat exchanger temperature is a simulation result. Every converged solution guarantees the physical principles. Moreover, CRISTA allows computing the quality factor of the resonator. The experimental validations have been successfully performed on different devices coupled to the same prime mover: a simple RLC load, an acoustic amplifier, a pulse tube refrigerator and a lumped boost pulse tube refrigerator.

4pPAk5. Numerical simulation of a thermoacoustic wave amplification Omar Hireche (LIMSI-CNRS, BP 133, F-91403 Orsay, France, hireche@limsi.fr), Catherine Weisman (LIMSI-CNRS, BP 133, F-91403 Orsay, France, weisman@limsi.fr), Diana Baltean-Carlès (LIMSICNRS, BP 133, F-91403 Orsay, France, baltean@limsi.fr), Luc Bauwens (University of Calgary, Departement of Mechanical and Manufacturing Engineering, 2500 University Drive NW, Calgary, AB AB T2N 1N4, Canada, bauwens@ucalgary.ca), Maurice-Xavier François (LIMSI-CNRS, BP 133, F-91403 Orsay, France, mxf@limsi.fr), Patrick Le Quere (LIMSICNRS, BP 133, F-91403 Orsay, France, plq@limsi.fr)

We performed a numerical study of the thermal and physical phenomena occurring in thermoacoustic wave generators. The goal of the simulation is to predict the amplification due to thermoacoustics of a wave initially of small amplitude. Therefore, we focus on the stack and the two heat exchangers, which we call the active cell, which is acoustically compact. The resonator area is split into two parts: the active cell, in which heat transfer takes place, and a resonator, in which the flow is acoustic. The flow in the twodimensional active cell can be approximated as a low Mach number viscous, conductive flow, subjected to spatially uniform pressure fluctuations. This model is formally derived using asymptotic expansions in terms of Mach number. The focus here is heat transfer between two successive stack plates. The two-dimensional time-dependent problem resulting from this model is solved numerically. Outside the active cell, flow in the resonator is described by a reversible acoustic one-dimensional model. The coupling between the two zones is obtained by matching the velocity at the interface. The acoustics in the resonance tube can be solved using the d'Alembert solution, relating velocities at the interface to velocity values at an earlier time.

4pPAk6. Green chillies: a practical thermoacoustic refrigerator in day-to-day use since February 2007. Philip Spoor (CFIC-Qdrive, 302 Tenth St., Troy, NY 12180, USA, pspoor@cficinc.com)

CFIC, Inc. recently designed and built a thermoacoustic food refrigerator for the U.S. Army's Combat Feeding program (based at the Natick Soldier Center in Natick, MA). The Army wants a more combat-hardy alternative to standard vapor-compression devices, which have many leak-prone braze joints. However, the thermoacoustic "Army Fridge" has generated increasing commercial and public interest as the search for environmentally benign alternatives to conventional refrigeration has intensified. The Army fridge uses helium as its working fluid, and it has no pumps or cirulating fluids, only heat pipes and fans for heat exchange with the air. Unlike all other thermoacoustic prototypes known to us, this device is expressly designed like a product, with all the thermoacoustic hardware and controls confined to a relatively small enclosure on top of a large (17 cubic foot) cabinet, and operation accomplished by a single power switch and a thermostat. In early 2007 the Army fridge went into long-term testing at CFIC, Inc. as our auxiliary refrigerator. By March 2007 it will have surpassed 1 year (8760 hours) of continuous running. We will present details of construction, performance history, and recommendations for higher efficiency and lower cost.

4pPAk7. Effect of an obstacle on Rayleigh acoustic streaming cells. Solenn Moreau (Laboratoire d'Etudes Aérodynamiques (LEA), Université de Poitiers, ENSMA, CNRS, Bat K, 40 avenue du recteur Pineau, 86022 Poitiers, France, solene.moreau@lea.univ-poitiers.fr), Helene Baillet (Laboratoire d'Etudes Aérodynamiques (LEA), Université de Poitiers, ENSMA, CNRS, Bat K, 40 avenue du recteur Pineau, 86022
Poitiers, France, helene.baillet@lea.univ-poitiers.fr),Jean-Christophe Valière (Laboratoire d'Etudes Aérodynamiques (LEA), Université de Poitiers, ENSMA, CNRS, Bat K, 40 avenue du recteur Pineau, 86022 Poitiers, France, jean-christophe.valiere@lea.univ-poitiers.fr)

Acoustic streaming has harmful consequences on thermoacoustic machines behaviour because of the associated heat transfers. A preliminary study was carried out in order to study the effect of an obstacle on the Rayleigh cells to help in understanding the role of such phenomena in thermoacoustic machines. An obstacle was introduced in a half-wavelength cylindrical wave guide to study its effects on acoustic streaming. The obstacle was placed at various positions along the wave guide axis and experiments were carried out at various acoustic levels. The axial streaming velocity was measured using Laser Doppler Velocimetry (LDV). It was observed that adding an obstacle in the streaming pattern modifies the latter and that new streaming vortices appear in the vicinity of the obstacle. When the obstacle position approaches a maximum of the Rayleigh streaming velocity the number and the amplitude of acoustic streaming vortices at the ends of the obstacle increase. Similar tendencies were observed when the acoustic velocity amplitude was increased. Because streaming in the vicinity of the obstacle end is complex and has a high amplitude, heat effects can be expected to be important and complex at the ends of the thermaoacoustic stack where heat exchangers are located.

4pPAk8. Experimental study of the thermoacoustic effect using infrared thermography. Vincent Feuillet (Laboratoire d'Acoustique de l'Université du Maine, Avenue Olivier Messiaen, 72085 Le Mans, France, vincent.feuillet@univ-lemans.fr), Guillaume Penelet (Laboratoire d'Acoustique de l'Université du Maine, Avenue Olivier Messiaen, 72085 Le Mans, France, guillaume.penelet@univ-lemans.fr), Pierrick Lotton (Laboratoire d'Acoustique de l'Université du Maine, Avenue Olivier Messiaen, 72085 Le Mans, France, Pierrick.Lotton@univ-lemans.fr), Lionel Camberlein (Laboratoire d'Acoustique de l'Université du Maine, Avenue Olivier Messiaen, 72085 Le Mans, France, lionel.camberlein@univ-lemans fr)

The thermal metrology used to study experimentally the thermoacoustic effect is almost always based on thermoelectric junctions. This kind of measurement is intrusive and provides a few information in space. This paper presents an experimental set-up where temperature measurement on the thermoacoustic stack is acquired by infrared thermography. This measurement provides more information in space and time to study complex physical phenomena, such as heat transfer through both ends of the stack, non linear edge effects, or optimal spacing between stack and heat exchangers. The experimental set-up consists of a half-wavelength resonator with a square cross section closed by a rigid wall at one end and coupled to an electrodynamic loudspeaker at the other end. The thermoacoustic core is either a single plate or a stack of parallel plates made of Kapton. Temperature measurements are carried out along the stack by the use of an infrared camera. The acoustic pressure is measured by a microphone flush-mounted on the wall at the exit of the resonator. First results show the essential role played by the edge effects and the heat generated by viscous losses.

4pPAk9. LDV measurements of acoustic streaming in a traveling wave, closed-loop resonator. Cyril Desjouy (Laboratoire d'Acoustique de l'Université du Maine, Avenue Olivier Messiaen, 72085 Le Mans, France, cyril.desjouy@univ-lemans.fr), Pierrick Lotton (Laboratoire d'Acoustique de l'Université du Maine, Avenue Olivier Messiaen, 72085 Le Mans, France, Pierrick.Lotton@univ-lemans.fr), Guillaume Penelet (Laboratoire d'Acoustique de l'Université du Maine, Avenue Olivier Messiaen, 72085 Le Mans, France, guillaume.penelet@univ-lemans.fr), James Blondeau (Laboratoire d'Acoustique de l'Université du Maine, Avenue Olivier Messiaen, 72085 Le Mans, France, james.blondeau@univ-lemans.fr)

The first part of this work deals with the study of an annular acoustic resonator, where the acoustic field is controlled by two loud-speakers in order to generate a purely traveling wave. The results of a complete electroacoustic modeling of this device, where the acoustic field is accurately defined, are compared to the experimental results. The second part deals with the characterization of various non-linear effects occurring in such a device. The generation of acoustic streaming is especially investigated in our study. 
Laser Doppler Velocimetry measurements are performed in order to characterize both first order (acoustic) and second order (acoustic streaming) velocity fields. Works are now in progress in order to improve accuracy of streaming measurement and to compare the experimental data to the available theories. This study should contribute to enhance the designing of thermoacoustic devices and should be also useful for its potential applications in microfluidics, especially for the development of micro-pumps and micromixers.

4pPAk10. Weakly non-linear thermoacoustics for general porous media. Peter In 'T Panhuis (Eindhoven University of Technology, P.O. Box 513, 5600 MB Eindhoven, Netherlands, p.h.m.w.panhuis @ tue.nl), Sjoerd Rienstra (Eindhoven University of Technology, P.O. Box 513, 5600 MB Eindhoven, Netherlands, s.w.rienstra@tue.nl), Han Slot (Eindhoven University of Technology, P.O. Box 513, 5600 MB Eindhoven, Netherlands, j.j.m.slot@tue.nl), Jos Zeegers (Eindhoven University of Technology, Den Dolech 2, 5612 AZ Eindhoven, Netherlands, j.c.h .zeegers@tue.nl)

A weakly nonlinear theory for thermoacoustics, including acoustic streaming, a temperature-dependent viscosity and slowly varying pores with arbitrarily shaped cross-sections, has been constructed by systematically applying dimensional analysis and small-parameter asymptotics. In this way a set of equations for the acoustic and streaming variables can be derived. For some simple cases explicit solutions can be found, such as the short-stack approximation, but for the more advanced applications we have to resort to a numerical solution. The theory has been implemented both for standingwave and traveling-wave applications. For the case of a standing-wave system we have compared our computations with experimental data and found a remarkable agreement.

4pPAk11. Viscous and thermal effects in acoustic radiation problems. Husnain Inayat Hussian (INSA de Lyon - LVA, Bâtiment St. Exupéry, 25 bis avenue Jean Capelle, F-69621 Villeurbanne Cedex, France, husnain .inayat-hussain@insa-lyon.fr), Jean-Louis Guyader (INSA de Lyon - LVA, Bâtiment St. Exupéry, 25 bis avenue Jean Capelle, F-69621 Villeurbanne Cedex, France, jean-louis.guyader@insa-lyon.fr)
The thermal and viscous effects are generally neglected in acoustic radiation problems. In the present work we observe if these effects have any significance on acoustic radiation characteristics. Several researches have been materialised in this respect, notably, Bruneau, Beltman et al. These models study factors like inertial and viscous terms for example and then keep some while neglect others based on their significance. Our approach is quite general and we take all the factors into consideration. Our model not only deals with the thermal and viscous effects but compressibility and all other terms are taken into consideration. A system of differential equations issuing from conservation principles is linearized. Next harmonic dependence is assumed and a linear system is obtained. This linear system generates different waves among which only those are kept, which follow the sommerfeld condition. These waves produce an equal number of linear systems, and consequently; pressure, temperature, and the normal and tangential acoustic veocities are determined, using the boundary conditions of no slip, isothermal wall and matching normal acoustic velocity. Our aim is to apply these findings to a viscothermal fluid within a double wall to see if acoustic transmission is improved.

4pPAk12. Physical mechanism and theoretical model of thermoacoustic heat exchangers. Ercang Luo (Technical Institute of Physics and Chemistry, CAS, Beiyitiao Rd., Zhongguancun St., P.O.Box 2711, 100080 Beijing, China, Ecluo@cl.cryo.ac.cn), Bo Gao (Graduate University of Chinese Academy of Sciences, Zhongguancun St., 100049 Beijing, China, gaobozaihit@163.com)

Unlike a regenerator, there are both oscillating heat exchange flux (first order) and non-zero time-averaged heat exchange flux (second order) in the hot and cold end heat exchangers of thermoacoustic systems. The non-zero time-averaged heat flux just reflects the net heat exchange between the working substance and external heat sinks, which is more important and interesting for design. In this paper, the physical mechanism of oscillating flow heat exchanger is first analyzed. Based on understanding of the heat transfer mechanism, the theoretical model of heat transfer is then developed; in this part, the key of the problem is to obtain transversal distribution of the second order time-averaged temperature, T20(y). Eventually, an analytical expression of Nusselt number for the thermoacoustic heat exchangers is obtained under the assumption of laminar flow. 


\title{
Session 4pPPa
}

\section{Psychological and Physiological Acoustics: Role of Temporal Fine Structure in Speech and Non-Speech Perception for Normal and Hearing-Impaired People I}

\author{
Christian Lorenzi, Cochair \\ Univ Paris Descartes, CNRS, Ecole Normale Superieure, DEC, 29 rue d'Ulm, Paris, 75005, France \\ Brian Moore, Cochair \\ University of Cambridge, Department of Experimental Psychology, Downing Street, Cambridge, CB2 3EB, UK
}

\section{Invited Papers}

4pPPa1. Role of temporal fine structure cues in speech intelligibility. Christian Lorenzi (Univ Paris Descartes, CNRS, Ecole Normale Superieure, DEC, 29 rue d'Ulm, 75005 Paris, France, lorenzi@ens.fr)

A number of studies have investigated the role of two temporal features of the narrowband speech signals at the output of auditory filters in speech identification: temporal envelope (E) and temporal fine structure (TFS) cues. To assess the contribution of each feature to speech identification, speech stimuli were split into an array of contiguous analysis bands and processed using several techniques to remove, as far as possible, either E or TFS cues within each band. Overall, the outcome of these studies indicated that, after moderate to subtantial training, high levels of speech identification measured in quiet could be obtained for normal-hearing listeners on the basis of E cues or TFS cues alone. The results obtained with TFS cues only may appear surprising, because it is generally considered that, at least for non-tonal languages, E cues carry most of the phonetic information required for speech identification in quiet whereas TFS cues play mainly a role in conveying the pitch cues required for the segregation of speech and background sounds. Further work assessing the extent to which TFS cues alone can convey useful linguistic information in addition to these pitch cues will be reviewed.

4pPPa2. Role of temporal fine structure in speech perception. Fan-Gang Zeng (University of California Irvine, 364 Med Surge II, Irvine, CA 92697, USA, fzeng@uci.edu)

Recent studies have shown that lack of access to the temporal fine structure cue is a major reason for the difficulty in speech perception in noise by hearing-impaired listeners. To further understand the role of temporal fine structure, we need to define the temporal fine structure and to delineate its relationship to the temporal envelope in both acoustical and perceptual domains. This talk will first examine the relationship between temporal envelope and temporal fine structure in signal processing terms and then relate it to speech production and perception. Acoustically, the temporal fine structure primarily contributes to changes in fundamental frequency, harmonics, and formant transition. Perceptually, while the temporal fine structure can contribute to speech intelligibility via the formant transition cue, it contributes to speech perception in noise by enhancing auditory objection formation rather than increasing speech intelligibility directly.

$$
\text { 2:40 }
$$

4pPPa3. Temporal fine structure coding for pitch and speech perception. Andrew J. Oxenham (University of Minnesota, Department of Psychology, 75 E. River Road, Elliott Hall N218, Minneapolis, MN 55455, USA, oxenham@umn.edu)

Temporal fine structure can be defined mathematically with relative ease. Understanding its perceptual importance, or even how it is coded in the peripheral auditory system, is another matter. This talk will review recent work from our lab that addresses some of these issues. The focus will be on the use and representation of temporal fine structure in complex pitch perception with spectrally resolved and unresolved components, and on the importance of temporal fine structure when using F0 information in speech to segregate target speech for competing talkers and other complex interfering sounds. [Supported by National Institutes of Health grant R01 DC 05216.]

$$
\text { 3:00 }
$$

4pPPa4. The contribution of temporal fine structure information to the intelligibility of speech in noise. Kathryn Hopkins (University of Cambridge, Department of Experimental Psychology, Downing Street, CB2 3EB Cambridge, UK, kh311@cam.ac.uk), Brian Moore (University of Cambridge, Department of Experimental Psychology, Downing Street, CB2 3EB Cambridge, UK, bcjm@cam.ac.uk)

Temporal fine structure (TFS) information in speech may be particularly useful when listening to a target in a background that contains temporal 'dips'. A change in TFS may allow identification of signal portions where the target-to-background ratio is high. This hypothesis was tested. Speech reception thresholds were measured with steady and an 8-Hz amplitude-modulated noise background for signals processed to contain variable amounts of TFS information. Signals were filtered into channels and channel signals for channel numbers above a 'cut off channel' (CO) were tone-vocoded to remove TFS information, while channel signals with channel numbers of $\mathrm{CO}$ and below were left unprocessed. Signals from all channels were combined. Five values of $\mathrm{CO}$ were tested for each noise type, with 
ten normal-hearing subjects. Subjects benefited more from TFS information when listening in the modulated masker than the steady masker. For steady noise, addition of TFS information above $548 \mathrm{~Hz}$ did not improve performance, whereas for modulated noise, addition of TFS at high frequencies did improve performance. The greater benefit from TFS information when listening in modulated noise is consistent with the idea that TFS information is important for listening in the dips of a fluctuating masker.

4pPPa5. Discrimination of stochastic patterns of frequency modulation relevant to speech perception. Stanley Sheft (Parmly Hearing Inst., Loyola Univ. Chicago, 6525 N. Sheridan Road, Chicago, IL 60626, USA, ssheft@luc.edu), Christian Lorenzi (Univ Paris Descartes, CNRS, Ecole Normale Superieure, DEC, 29 rue d’Ulm, 75005 Paris, France, lorenzi@ens.fr)

Recent work has suggested involvement of temporal fine structure (TFS) information in speech perception, especially in adverse listening conditions. However, little is known regarding discrimination of pattern of TFS modulation, an ability that would underlie robust contribution to speech processing. The present study evaluated the ability to discriminate among stochastic patterns of frequency modulation (FM). Contrasting modulators were different samples of lowpass noise that shared a common bandwidth and resulted in the same maximum frequency excursion. Performance levels declined in an orderly manner with modulator bandwidth, similar to results obtained when lowpass noise is used to modulate amplitude (i.e., AM) rather than fine structure. Modeling, however, indicated that the similarity was not simply a result of FM-to-AM conversion at the output of auditory filters. In additional conditions, discrimination of stochastic FM was measured in the presence of wideband noise which in some cases was sinusoidally amplitude modulated at either 4 or $20 \mathrm{~Hz}$. With both unmodulated and modulated maskers, little effect on performance was noted, even with a signal carrier level as low as $15-20 \mathrm{~dB}$ above detection threshold. Absence of a substantial masking effect indicates availability of TFS cues for speech perception in noise. [Work supported by NIH.]

3:40-5:20 Posters

Lecture sessions will recess for presentation of poster papers on various topics in acoustics. See poster sessions for topics and abstracts.

\section{Invited Paper}

4pPPa6. The role of temporal fine structure in speech source segregation. Joshua G. Bernstein (Walter Reed Army Medical Center, Army Audiology and Speech Center, 6900 Georgia Ave. NW, Washington, DC 20307-5001, USA, joshua.bernstein@amedd.army.mil), Kenneth W. Grant (Walter Reed Army Medical Center, Army Audiology and Speech Center, 6900 Georgia Ave. NW, Washington, DC 20307-5001, USA, grant@tidalwave.net)

Normal-hearing $(\mathrm{NH})$ listeners show better speech recognition when a stationary noise masker is replaced by an opposite-gender competing talker at the same signal-to-noise ratio (SNR). Hearing-impaired (HI) listeners often do not show this interfering-talker benefit (ITB). This may be due to a reduced ability to use temporal fine structure (TFS). Consistent with this idea, NH listeners also show little ITB when TFS is removed. We hypothesized that TFS underlies the ITB by providing source-segregation cues. To test this hypothesis, non-auditory segregation cues were introduced in the form of a video of the talker's face. Speech intelligibility was estimated in NH listeners as a function of SNR for sentences spoken by a female talker and masked by speech-spectrum shaped stationary noise or a single male talker. Target and masker were summed before processing by a 15-channel noise vocoder to remove TFS, and presented with or without accompanying video. Without video, listeners received little ITB, consistent with previous results. Auditory-visual conditions yielded as much as $9 \mathrm{~dB}$ of ITB, supporting the hypothesis that a diminished ability to perceptually segregate sources contributes to the lack of ITB in the absence of TFS. Similar results were obtained for unprocessed speech in HI listeners.

\section{Contributed Paper}

\section{5:40}

4pPPa7. Relative importance of $E$ and TFS speech cues in low and high-frequency channels. Marine Ardoint (Univ Paris Descartes, CNRS, Ecole Normale Superieure, DEC, 29 rue d'Ulm, 75005 Paris, France, marine.ardoint@ens.fr), Christian Lorenzi (Univ Paris Descartes, CNRS, Ecole Normale Superieure, DEC, 29 rue d'Ulm, 75005 Paris, France, lorenzi @ens.fr)

Previous studies have shown that perfect speech identification in quiet could be obtained for broadband speech processed using the Hilbert transform to preserve either temporal envelope (E) or temporal fine structure (TFS) cues only in narrow frequency bands. However, little is known regarding the respective contribution of each cue to speech intelligibility in low and high frequency bands. The goal of the present study was to assess the contribution of E and TFS cues of the low- and high-frequency channels to consonant identification. Vowel-consonant-vowel stimuli were split into an array of 16 analysis bands spanning the range $80-8,020 \mathrm{~Hz}$ and processed using the Hilbert transform to keep either E or TFS cues only within each band. Identification scores were measured for low-pass and high-pass filtering of the processed stimuli for a group of normal-hearing listeners. Results will be discussed in light of phase-locking properties to E and TFS. In most mammals, phase-locking of auditory-nerve fibers begins to decline above 1 $\mathrm{kHz}$ and disappears above $4-5 \mathrm{kHz}$. TFS-coded speech intelligibility should therefore drop when stimuli are high-pass filtered above $1 \mathrm{kHz}$ and be at chance level for high-pass filtering above $5 \mathrm{kHz}$. No such drop should be expected for E-coded speech. 


\section{Invited Papers}

\section{6:00}

4pPPa8. The importance of temporal fine structure coding for speech perception in listeners with sensorineural hearing impairment as compared to normal hearing listeners. Emily Buss (University of North Carolina, School of Medicine, 1115 Bioinformatics. Bldg., CB7070, 130 Mason Farm Rd., Chapel Hill, NC 27599, USA, ebuss@med.unc.edu), Joseph W. Hall (University of North Carolina, School of Medicine, 1115 Bioinformatics. Bldg., CB7070, 130 Mason Farm Rd., Chapel Hill, NC 27599, USA, jwh@med.unc.edu), John H. Grose (University of North Carolina, School of Medicine, 1115 Bioinformatics. Bldg., CB7070, 130 Mason Farm Rd., Chapel Hill, NC 27599, USA, jhg@med.unc.edu)

Physiological data suggest that the neural representation of temporal fine structure could benefit speech recognition in normal hearing listeners. Conversely, it has been hypothesized that deficits in the encoding of fine structure could play a role in the poor speech perception of patients with sensorineural hearing loss, particularly for speech presented at moderate to high levels and speech presented in background noise. Experiments on the psychoacoustical abilities of adults with hearing loss have shown a correlation between performance on tasks thought to rely on fine structure cues (e.g., low rate FM detection) and ability to understand speech or lowpass filtered speech, lending support to this hypothesis. This talk will review these data, as well as the mechanisms proposed to account for this result, and will consider the relation of these findings to experiments with normal-hearing listeners.

\section{6:20}

4pPPa9. Fine-structure processing, frequency selectivity and speech perception in hearing-impaired listeners. Olaf Strelcyk (Centre for applied hearing research, Technical University of Denmark, DTU, Bygn. 352, 2800 Lyngby, Denmark, os@oersted.dtu.dk), Torsten Dau (Centre for applied hearing research, Technical University of Denmark, DTU, Bygn. 352, 2800 Lyngby, Denmark, tda@oersted.dtu.dk)

Hearing-impaired people often experience great difficulty with speech communication when background noise is present, even if reduced audibility has been compensated for. Other impairment factors must be involved. In order to minimize confounding effects, the subjects participating in this study consisted of groups with homogeneous, symmetric audiograms. The perceptual listening experiments assessed the intelligibility of full-spectrum as well as low-pass filtered speech in the presence of stationary and fluctuating interferers, the individual's frequency selectivity and the integrity of temporal fine-structure processing. The latter was addressed in a binaural and a monaural experiment. In the binaural experiment, the lateralization threshold was measured for low-frequency tones with ongoing interaural phase delays. In the monaural experiment, detection thresholds for low-rate frequency modulation were obtained. In addition, these binaural and monaural thresholds were measured in a stationary background noise in order to assess the persistence of the finestructure processing to interfering noise. Apart from elevated speech reception thresholds, the hearing impaired listeners showed poorer performance than the normally hearing in terms of frequency selectivity and fine-structure processing, despite normal audiometric thresholds at the test frequencies. However, the binaural fine-structure processing was not found to be particularly vulnerable to interfering noise in these listeners. 


\title{
Session $4 p P P b$
}

\section{Psychological and Physiological Acoustics: Role of Temporal Fine Structure in Speech and Non-Speech Perception for Normal and Hearing-Impaired People II (Poster Session)}

\author{
Christian Lorenzi, Cochair \\ Brian Moore, Cochair \\ University of Cambridge
}

Univ Paris Descartes, CNRS, Ecole Normale Superieure

\begin{abstract}
All posters will be on display from 3:40 p.m. to 5:20 p.m. To allow contributors an opportunity to see other posters, contributors of odd-numbered papers will be at their posters from 3:40 p.m. to 4:30 p.m. and contributors of even-numbered papers will be at their posters from 4:30 p.m. to 5:20 p.m.
\end{abstract}

\section{Contributed Papers}

4pPPb1. Facilitation of intensity discrimination of short stimuli in noise occurs not far from a stimuli detection threshold. Liudmila Rimskaya-Korsakova (N. N. Andreyev Acoustics Institute, Shvernika 4, 117036 Moscow, Russian Federation, lkrk@akin.ru)

Adaptation is a main property of sensory receptors. In a basis of adaptation are properties of peripheral receptors and central neuron. There is a question: what is the auditory adaptation mechanism to short high frequency stimuli? How the auditory system can trace stimulus amplitude changes? The probable answer is: stimuli are mixed with noise of different origin. Noise adapts hearing and improves discrimination of stimulus. Simulation researches of peripheral encoding were shown the amplitude structure of short stimulus acting in isolation or in noise could be preserved when intensity are not far from thresholds of fibers. For each stimulus level there is the certain noise level, when amplitude structure can be detected without losses. In this work the validity of these statements was checked up in auditory experiments. The thresholds of intensity discrimination (ID) for short stimulus presented in silence and in conditions of simultaneous and forward masking have been estimated. In a range of average stimulus intensity and for each stimulus level there is the certain noise level when ID is better in noise but not in silence. The ID facilitation is registered near to the stimulus detection thresholds and after adaptation of hearing by noise. Results of auditory researches correspond to the simulation results.

4pPPb2. Intelligibility of temporal fine structure speech signals with restricted FM excursion. Gaëtan Gilbert (Equipe audition LPP UMR CNRS 8158, 29, rue d'Ulm, 75005 Paris, France, gaetan.gilbert@ens .fr), Agnes Leger (Equipe audition LPP UMR CNRS 8158, 29, rue d'Ulm, 75005 Paris, France, legeragnes@gmail.com), Christian Lorenzi (Univ Paris Descartes, CNRS, Ecole Normale Superieure, DEC, 29 rue d'Ulm, 75005 Paris, France, lorenzi@ens.fr)

The Hilbert transform is the most common demodulation technique to derive temporal fine structure (TFS) signals. However, for speech stimuli, the Hilbert transform generally leads to results that have no clear physical meaning, generating undesired artefacts; for instance, instantaneous frequency may vary well beyond the analysis filters bandwidth. This study examined the intelligibility in quiet of TFS-coded Vowel-Consonants-Vowel signals generated with a demodulation technique minimizing these artefacts. Speech items were passed through a 16 FIR filters (750th order) filterbank. A Greenwood mapping was used to set filters bandwidth between 80-8020 $\mathrm{Hz}$ (approximately 2 ERBs wide). A frequency-modulation function was extracted at the output of each filter, hard limited within the analysis bandwidth and lowpass filtered. Spectral cues were removed by equating the rms across bands. Identification scores ranged between chance level $(6.25 \%)$ with no improvement across sessions for the least experienced listeners and $50 \%$ correct for the most experienced listeners. Further experiments will investigate these between-listeners differences.

4pPPb3. Hard times for the pitch of complex sounds in reverberant environments. Mark Sayles (Centre for the Neural Basis of Hearing, The Physiological Laboratory, Downing Street, CB2 3EG Cambridge, UK, ms417@cam.ac.uk), Ian M. Winter (Centre for the Neural Basis of Hearing, The Physiological Laboratory, Downing Street, CB2 3EG Cambridge, UK, imw1001@cam.ac.uk)

Reverberation is present in most everyday listening environments, with sound reaching our ears directly from the source(s), and indirectly after reflecting from nearby surfaces. Reverberation smears spectral transitions through time and disrupts temporal envelope modulation in harmonic complex sounds such as voiced portions of speech. Envelope modulation can provide a cue to the pitch of complex sounds and is relied upon by hearing impaired listeners. We have examined the effects of reverberation on the temporal representation of the dynamic pitch of frequency-modulated harmonic complex sounds in the responses of single units from the ventral cochlear nucleus. Without reverberation most units provide a strong representation of the modulated pitch in their short-term interspike interval distributions. Relatively mild reverberation degrades the representation based on envelope modulation, whilst more severe reverberation removes it. The representation of pitch based on fine structure is more robust to the effects of reverberation; however, this representation is smeared in time by indirect sound energy. The effects of reverberation are critically dependent on the neuron's best-frequency, source-to-receiver distance, fundamental frequency and frequency modulation rate. We observe comparable results in human listeners using the same sounds.

4pPPb4. Effect of speech rate on speech-on-speech masking. Jing Chen (Dept. of Machine Intelligence, Speech and Hearing Research Center, 2 Science Building, Peking Univ., 5 Yeheyuan Road, Haidian District, 100871 Beijing, China, chenj@cis.pku.edu.cn), Xihong H. Wu (Dept. of Machine Intelligence, Speech and Hearing Research Center, 2 Science Building, Peking Univ., 5 Yeheyuan Road, Haidian District, 100871 Beijing, China,wxh@cis.pku.edu.cn), Xuefei F. Zou (Dept. of Machine Intelligence, Speech and Hearing Research Center, 2 Science Building, Peking Univ., 5 Yeheyuan Road, Haidian District, 100871 Beijing, China, zouxf@cis.pku.edu.cn), Zhiping P. Zhang (Dept. of Machine Intelligence, Speech and Hearing Research Center, 2 Science Building, Peking Univ., 5 
Yeheyuan Road, Haidian District, 100871 Beijing, China, zhangzp@cis.pku.edu.cn), Lijuan J. Xu (Dept. of Psychology, Peking Univ., 5 Yeheyuan Road, Haidian District, 100871 Beijing, China, lijuan179021@yahoo.com.cn), Mengyuan Y. Wang (Dept. of Psychology, Peking Univ., 5 Yeheyuan Road, Haidian District, 100871 Beijing, China, motoluto@163.com), Liang Li (Dept. of Psychology, Peking Univ., 5 Yeheyuan Road, Haidian District, 100871 Beijing, China, liangli@pku.edu.cn), Huisheng S. Chi (Dept. of Machine Intelligence, Speech and Hearing Research Center, 2 Science Building, Peking Univ., 5 Yeheyuan Road, Haidian District, 100871 Beijing, China, chi@pku.edu.cn)

Target speech can be better recognized under speech-on-speech masking conditions if certain differences between target and masker (e.g. in loudness, pitch, location) can be used as cues for streaming. This study examined whether the speech rate can be used by listeners as a cue for unmasking target speech. The rate difference between target and masking speech was manipulated by changing the rate of masking speech using the Synchronized Overlap-Add Fixed Synthesis (SOLAFS) algorithm, and consequently, the ratio of target speech to masking speech (the speech rate ratio, SRR) was quantified. Both target and masker speech were Chinese nonsense sentences and they were co-presented with the signal-to-masker ratio of $-7 \mathrm{~dB}$. The results show that speech recognition was significantly increased with the SRR increase from 1 to 1.5 or the SRR decreased from 1 to 0.5 . Moreover, the unmasking effect of precedence-induced perceived spatial separation on target-speech recognition was increased monotonically with the increase of the SRR from 0.5 to 1.5 . These results suggest that the speech rate is one of the factors influencing both energetic masking and informational masking of Chinese speech. [Supported by the NSFC 60435010; 60535030; 60605016; 30670704]

4pPPb5. Charactering the individual ear by the "Auditory Profile". Wouter A. Dreschler (AMC, Clinical and Experimental Audiology, 1105 Amsterdam, Netherlands, w.a.dreschler@amc.uva.nl), Thamar E. Esch Van

(AMC - Dept. of Clinical and Experimental Audiology, Meibergdreef 9, 1105AZ Amsterdam, Netherlands, t.e.vanesch@amc.nl), Birgitta Larsby (Linkoepings Universitet, Hus Origo Campus Valla, 58183 Linkoeping, Sweden, birgitta.larsby@inr.liu.se), Mathias Hallgren (Linkoepings Universitet, Hus Origo Campus Valla, 58183 Linkoeping, Sweden, mathias.hallgren@inr.liu.se), Mark E. Lutman (University of Southampton, University Road, Highfield / ISVR, SO17 iBJ Southampton, UK, mel@isvr.soton.ac.uk), Johannes Lyzenga (Vrije Universiteit Medical Center, Boelelaan 1117, 1081 HV Amsterdam, Netherlands, j.lyzenga@vumc.nl), M Vormann (Hoerzentrum Oldenburg, Hoerzentrum Oldenburg, 26129 Oldenburg, Germany, matthias.vormann@web.de), Birger Kollmeier (Universität Oldenburg, Medizinische Physik, Carl-von-Ossietzky Str. 9-11, 26111 Oldenburg, Germany, birger.kollmeier@uni-oldenburg.de)

This paper describes a new approach to auditory diagnostics, which is one of the central themes of the EU-project HEARCOM. For this purpose we defined a so-called "Auditory Profile" that can be assessed for each in- dividual listener using a standardized battery of audiological tests that - in addition to the pure-tone audiogram - focus on loudness perception, frequency resolution, temporal acuity, speech perception, binaural functioning, listening effort, subjective hearing abilities, and cognition. For the sake of testing time only summary tests are included from each of these areas, but the broad approach of characterizing auditory communication problems by means of standardized test is expected to have an added value above traditional testing in understanding the reasons for poor speech reception. The Auditory profile may also be relevant in the field of auditory rehabilitation and for design of acoustical environments. The results of an international 5 -center study (in 4 countries and in 4 languages) will be presented and the relevance of a broad but well-standardized approach will be discussed.

4pPPb6. Evidence of neural processing of the interaural correlation between long-duration noises in humans. Wenjie J. Wang (Dept. of Psychology, Peking Univ., 5 Yeheyuan Road, Haidian District, 100871 Beijing, China, wenjie@pku.edu.cn), Lingzhi Z. Kong (Dept. of Psychology, Peking Univ., 5 Yeheyuan Road, Haidian District, 100871 Beijing, China, konglingzhi@gmail.com), Jingyu Y. Li (Dept. of Machine Intelligence, Speech and Hearing Research Center, 2 Science Building, Peking Univ., 5 Yeheyuan Road, Haidian District, 100871 Beijing, China, lijy@cis .pku.edu.cn), Qiang Huang (Dept. of Machine Intelligence, Speech and Hearing Research Center, 2 Science Building, Peking Univ., 5 Yeheyuan Road, Haidian District, 100871 Beijing, China, QIANG.HUANG @SPREADTRUM.COM), Xihong H. Wu (Dept. of Machine Intelligence, Speech and Hearing Research Center, 2 Science Building, Peking Univ., 5 Yeheyuan Road, Haidian District, 100871 Beijing, China, wxh@cis.pku.edu.cn), Liang Li (Dept. of Psychology, Peking Univ., 5 Yeheyuan Road, Haidian District, 100871 Beijing, China, liangli@pku.edu.cn)

When the delay between two correlated sounds is sufficiently short, attributes of the lagging sound are perceptually captured by the leading sound, causing a fused image as coming from the leading-sound location. To investigate neural processing of the interaural correlation between long-duration sounds in humans, we recorded scalp event-related potentials (ERPs) to a transient silent gap that was inserted in long-duration broadband noises presented via headphones. The noises at the two ears were either correlated or uncorrelated. When the noises were correlated but not uncorrelated, participants mainly perceived only one gap image whose inside-head position was modulated by the interaural time difference (ITD). Compared to ERPs when the noises were uncorrelated, the amplitude of the N1/P2 component to the gap was smaller and amplitudes in the latency range of 100 to $200 \mathrm{~ms}$ were more imbalanced between the two hemispheres when the noises were correlated. When the ITD for the correlated noises was increased to $16 \mathrm{~ms}$, which was beyond the ITD range for fusion, two gap images were perceived, and the amplitude of the N1/P2 component to the gap became larger and the sustained potentials during 300-500 ms after the gap onset became more negative. [Supported by NSFC]. 


\title{
Session 4pPPc
}

\section{Psychological and Physiological Acoustics: General Topics in Psychological and Physiological Acoustics VI (Poster Session)}

\author{
Elizabeth Strickland, Cochair \\ Purdue University
}

\author{
Armin Kohlrausch, Cochair \\ Philips Research Europe
}

Alain De Cheveigne, Cochair

CNRS, Universite Paris 5, Ecole Normale Superieure

\begin{abstract}
All posters will be on display from 3:40 p.m. to 5:20 p.m. To allow contributors an opportunity to see other posters, contributors of odd-numbered papers will be at their posters from 3:40 p.m. to 4:30 p.m. and contributors of even-numbered papers will be at their posters from 4:30 p.m. to 5:20 p.m.
\end{abstract}

\section{Contributed Papers}

4pPPc1. A computational model of binaural speech intelligibility level difference. Kalle J. Palomäki (Adaptive Informatics Research Centre, Helsinki University of Technology, P.O. Box 5400, 02015 Espoo, Finland, kalle.palomaki@tkk.fi), Guy J. Brown (University of Sheffield, Dept. of Computer Science, Regent Court, 211 Portobello Street, S1 4DP Sheffield, UK, g.brown@dcs.shef.ac.uk)

This study addresses two questions relating to the binaural intelligibility level difference (BILD). First, we ask whether the BILD is underlain by an equalization-cancellation (EC) mechanism, in which a disparity between the interaural time difference of the target and masker is exploited within each frequency channel, rather than across channels. Second, we consider the effects of three sources of internal noise on the EC mechanism: jitter in neural delays, noise in the equalization process and nonlinearities in the auditory pathway. These issues are investigated using a computational model consisting of peripheral auditory model, binaural processor, auditory scene processor and automatic speech recognition system. The binaural model is based on EC processing, with performance limited by internal noise. The auditory scene processor groups speech harmonics by common F0 and identifies 'glimpses' in which the signal-to-noise ratio is favorable for speech. The performance of human listeners and the computational model are compared on the same speech intelligibility test (Edmonds \& Culling, 2005, JASA 117 (5), 3069-3078). The BILD of human listeners can be replicated by adjusting parameters that determine the internal noise in the EC model; however, the speech reception threshold of the model is lower than that of human listeners.

4pPPc2. Bernoulli coding on the auditory nerve and its implications for central auditory processing. Robert A. Houde (Center for Communications Research, 125 Tech Park Drive, Rochester, NY 14623, USA, rahoude@gmail.com), James M. Hillenbrand (Western Michigan University, Dept of Speech Path \& Aud., 1903 W. Michigan Ave., Kalamazoo, MI 49008, USA, james.hillenbrand@wmich.edu)

The auditory periphery is well represented as a bank of band pass filter/inner hair cell (IHC) channels, with each IHC providing half wave rectification, amplitude compression, and conversion to firing probability on the auditory nerve (AN) fibers innervating that IHC. Frequency resolution varies dramatically with sound intensity, ranging from sharp tuning near threshold to very broad at high intensities. Cochlear filtering provides a satisfactory representation of broadband characteristics such as timbre but not the fine frequency resolution required for perceptual frequency discrimination.
High resolution frequency analysis must, therefore, be provided by post-AN processes. We present a model of AN coding in which fine frequency analysis is carried out at central auditory stages. By this model the stochastic process on each AN fiber resulting from the IHC's firing probability is modeled by a Bernoulli process. As a result, the IHC output signal is transferred to the cochlear nucleus $(\mathrm{CN})$ without further filtering, where it can be recovered by a simple summation over those AN fibers from the region of that IHC. We present a neurally plausible process for narrow band analysis at the $\mathrm{CN}$ using the regular pulses of chopper cells.

4pPPc3. The first effect of pitch shift as a function of component spacing. Adam Mielczarek (Acoustics Division, Wroclaw University of Technology, Wybrzeze Wyspianskiego 27A, 50-370 Wroclaw, Poland, adam .mielczarek@pwr.wroc.pl)

The paper presents the results of an experiment regarding the influence of component spacing on the first effect of pitch shift. During the adjustment procedure, the listeners matched the pitch of the three-component complex to the same sensation produced by pure tone. The stimuli were composed of the 3rd, 4th and 5th harmonics of 100, 200 or $400 \mathrm{~Hz}$ shifted up in the frequency domain by $30 \mathrm{~Hz}$. The level of each component was $50 \mathrm{~dB}$ SPL. The subject was presented with a 5-s sample of the test complex, and after a 500 ms break he had to define the pitch of the three-component complex using the matching tone. The results of the experiment suggest that the pitch shift phenomenon is based on the relative frequency rather than on the absolute frequency or the dominant component number.

4pPPc4. Neuronal representation of pitch ambiguity. Mark Sayles (Centre for the Neural Basis of Hearing, The Physiological Laboratory, Downing Street, CB2 3EG Cambridge, UK, ms417@cam.ac.uk), Ian M. Winter (Centre for the Neural Basis of Hearing, The Physiological Laboratory, Downing Street, CB2 3EG Cambridge, UK, imw1001@cam.ac .uk)

Iterated rippled noise (IRN) is produced by delaying a broadband noise by time $d$, multiplying by gain $g$, adding the delayed noise to the original, and repeating this process for $n$ iterations. When $g=+1$ IRN has a welldefined pitch at $1 / d \mathrm{~Hz}$. If $g=-1$ the pitch can be ambiguous. A gain of -1 is equivalent to applying a frequency-independent phase shift $\phi$ of $\pi$ rads to the delayed noise $(g=+1 \equiv \phi=0)$. We recorded spike-trains from single units in the ventral cochlear nucleus in response to IRN with varying $\phi$ Units with high best frequencies represented waveform envelope modula- 
tion (independent of $\phi$ ), however, units in the phase-locking range of best frequencies represented stimulus fine structure (which varies with $\phi$ ). Fine structure responders show a gradual transition from a well-defined peak in the interspike interval distribution at $d$ when $\phi=0$ to two equal-amplitude peaks flanking $d$ when $\phi=\pi$, and a gradual shift back to a well-defined peak at $d$ as $\phi$ approaches $2 \pi$. Within the dominance region for pitch interspike interval distributions account for psychophysical pitch matches of $1.07 / d$ and $0.94 / d \mathrm{~Hz}$ for $\phi=\pi / 2$ and $3 / 2 \pi$ respectively, as well as the ambiguous pitches of $0.88 / d, 1.14 / d$, and $1 / 2 d \mathrm{~Hz}$ heard when $\phi=\pi$ rads.

4pPPc5. The effect of regional dialect on the psychometric reliability and validity of two sets of Mandarin speech audiometry materials Shawn L. Nissen (Brigham Young University, 138 TLRB, 1190 North 900 East, Provo, UT 84602, USA, shawn_nissen@byu.edu), Richard W. Harris (Brigham Young University, 138 TLRB, 1190 North 900 East, Provo, UT 84602, USA, richard_harris@byu.edu), Jamie Garlick (Brigham Young University, 138 TLRB, 1190 North 900 East, Provo, UT 84602, USA, jamie.garlick@gmail.com), Nathan Richardson (Brigham Young University, 138 TLRB, 1190 North 900 East, Provo, UT 84602, USA, nathan000000@yahoo.com)

Previous research has shown conflicting evidence on the effect of testing an individual's hearing acuity with speech perception materials created in a mutually intelligible, yet non-regional dialect. Thus, the aim of this study is to examine the validity and reliability of using previously developed psychometrically equivalent speech audiometry materials in Mainland Mandarin and Taiwan Mandarin to evaluate the speech perception abilities (word recognition and speech reception threshold) of regional and non-regional listeners of the presented dialects. In addition, this study will investigate whether a native speaker of one Mandarin dialect is able to accurately administer and score the results from listeners of a different regional dialect. Some aspects of the listeners' performance on materials from a non-regional Mandarin dialect were found to be significantly different statistically. However, it is unclear if such differences are large enough to make a difference in the clinical testing of speech perception. In terms of scoring accuracy, a high percentage of agreement was found between the two interpreters from different dialectal backgrounds. [Work supported by research funding from Brigham Young University School of Education]

4pPPc6. Estimating the effective frequency of cochlear implant electrodes using contralateral residual acoustic hearing. Tim Green (UCL, Wolfson House, 4, Stephenson Way, NW1 2HE London, UK, tim.green@ucl.ac.uk), Andrew Faulkner (UCL, Wolfson House, 4, Stephenson Way, NW1 2HE London, UK, andyf@phon.ucl.ac.uk), Stuart Rosen (UCL, Wolfson House, 4, Stephenson Way, NW1 2HE London, UK, stuart@phon.ucl.ac.uk)

For some cochlear implant (CI) users a contralateral hearing aid provides significantly improved speech perception. Important factors in the bimodal transmission of speech spectral information are likely to include the extent to which the frequency selectivity of residual hearing allows additional place-coded channels, and mismatches between frequency-to-place maps across modalities. When acoustic place coding extends above around $500 \mathrm{~Hz}$ an overlap of frequency coverage between acoustic and electric hearing may result in interaural conflicts. However, addressing this issue requires accurate knowledge of CI frequency-to-place maps. Effective characteristic frequencies of CI electrodes have previously been estimated using comparisons of the pitch produced by electrical stimulation with that produced by contralateral acoustic sinusoids In the present work, the acoustic stimuli used for pitch comparisons are either sinusoids or $1 / 3$ octave bands of noise. The latter minimize temporal pitch cues and may reduce differences in perceived quality between electrical and acoustical auditory sensations. Electrical stimuli are high-rate (900 pps or greater) singleelectrode pulse trains. Comparisons are performed at different levels spaced over the dynamic range and both paired-comparison and adjustment tasks are used. Results will be discussed in relation to speech processing approaches for optimally combining an implant and contralateral hearing aid.
4pPPc7. Performance on auditory temporal-processing tasks for speech and non-speech stimuli by young and elderly listeners. Diane Kewley-Port (Indiana University, Speech and Hearing Sciences, 200 S. Jordan, Bloomington, IN 47405, USA, kewley@indiana.edu), Larry Humes (Indiana University, Speech and Hearing Sciences, 200 S. Jordan, Bloomington, IN 47405, USA, humes@indiana.edu), Daniel Fogerty (Indiana University, Speech and Hearing Sciences, 200 S. Jordan, Bloomington, IN 47405, USA, dfogerty@indiana.edu), Dana Kinney (Indiana University, Speech and Hearing Sciences, 200 S. Jordan, Bloomington, IN 47405, USA, danakin@indiana.edu)

Results from three auditory tasks are presented from a larger series of temporal-processing tasks completed in three sensory modalities by young and older adults. The first task measured temporal gap detection in noise bands. The second and third tasks used digitally processed vowels in four words (pit, pet, pot, put) as the stimuli. The second task required listeners to identify the order of either two- or four-vowel sequences presented monaurally or dichotically. The third task measured the identification of these four vowels when presented either before or after a noise or vowel-like masker (forward- or backward-masking tasks). Altogether, performance was obtained for 14 auditory temporal-processing measures. Young $(\mathrm{N}=20)$ and older $(\mathrm{N}=50)$ adults participated. Preliminary analyses (based on data from 50 of the 70 subjects) indicate that young listeners performed significantly better and with less variability than elderly listeners on all tasks. For most tasks, there was considerable overlap between the data from young and elderly listeners, indicating a modest negative impact of aging. At the individual level, correlational analyses among the older adults indicated that pure-tone thresholds were not predictive of temporal-processing performance and that performance on many of the temporal-processing tasks was moderately correlated. [Supported by NIA R01 AG022334.]

4pPPc8. MEG measures of the auditory steady-state response: Sinusoidal and non-sinusoidal stimuli. Garreth Prendergast (The University of York, Heslington, YO10 5DD York, UK, garreth.prendergast @ynic.york.ac.uk), Sam R. Johnson (The University of York, Heslington, YO10 5DD York, UK, sam@ynic.york.ac.uk), Gary G. Green (The University of York, Heslington, YO10 5DD York, UK, gary.green@ynic.york.ac .uk)

Human sensitivity to amplitude modulation has long been of interest to researchers, both in behavioural and neurological measures. Processing of amplitude modulation is implicated in the process of speech perception and sinusoidal amplitude modulation is used extensively to probe the mechanisms involved in encoding this information. The temporal envelope of speech is more accurately described as bursts of modulation rather than continuous modulation and the current work exposes participants to a continuum of modulation waveforms; from sinusoidal to pulsatile. Waveforms were amplitude modulated at $4 \mathrm{~Hz}$ and imposed upon a $500 \mathrm{~Hz}$ pure-tone carrier. The waveforms were generated using raised-cosine pulses with different half-durations. Half-durations of 8, 16, 24, 32, 64 and $125 \mathrm{~ms}$ were used (125 ms producing sinusoidal amplitude modulation at $4 \mathrm{~Hz}$ ). Stimuli were 240 seconds in duration and responses were collected on a 248 channel whole-head MEG scanner. The frequency domain steady-state response was analysed from each condition in 14 participants, and results confirmed that the response to sinusoidal amplitude modulation was significantly lower than to modulations more representative of those found in speech signals. This suggests that non-sinusoidal stimuli may be more effective when investigating these auditory mechanisms.

4pPPc9. Laboratory synthesis of industrial noise environments with predetermined statistical properties. Wei Qiu (State University of New York, 101 Broad Street, Plattsburgh, NY 12901, USA, wei.qiu@plattsburgh .edu), Bob Davis (State University of New York, 101 Broad Street, Plattsburgh, NY 12901, USA, davisri@plattsburgh.edu), Roger P. Hamernik (State University of New York, 101 Broad Street, Plattsburgh, NY 12901, USA, roger.hamernik@plattsburgh.edu)

High-level nonGaussion noise is commonly found in a variety of industrial environments. Recent experiments have shown that for a given energy level, the statistical properties of a noise can have a strong effect on the extent of hearing loss produced in exposed individuals. In order to study, in an 
animal model, the effects on hearing of such noise environments, the statistical properties of the noise as embodied in the kurtosis metric must be under experimental control. For a fixed value of kurtosis and energy level the following four variables will have a strong effect on hearing loss: (1) peak histogram; (2) interval histogram; (3) duration of noise transients; and (4) level of any background Gaussian noise. Simulations have shown that the relations among kurtosis and these variables are nonlinear. However, under certain restricted conditions, these relations may be linear. Accordingly, two strategies for designing controlled industrial noise exposures are presented: (1) the interval-priority model and (2) the duration-priority model. Computer simulations and measurements of actual acoustic environments showed that these two models could be effectively used to simulate a wide variety of realistic industrial noises.

4pPPc10. Intervention for restricted dynamic range and reduced sound tolerance. Charles Formby (University of Alabama, 700 University Boulevard East Suite 315, Tuscaloosa, AL 35487, USA, cformby@as.ua .edu), Monica Hawley (University of Maryland, 16 S. Eutaw St, Suite 500, Baltimore, MD 21201, USA, moncia@hawleyonline.net), Laguinn Sherlock (University of Maryland, 16 S. Eutaw St, Suite 500, Baltimore, MD 21201, USA, gsherlock@smail.umaryland.edu), Susan Gold (University of Maryland, 16 S. Eutaw St, Suite 500, Baltimore, MD 21201, USA, sgold@smail.umaryland.edu), Allyson Segar (University of Maryland, 0100 Lefrak Hall, College Park, MD 20742, USA, asegar@hesp.umd.edu), Christine Gmitter (University of Maryland, 0100 Lefrak Hall, College Park, MD 20742, USA, cgmitter@hesp .umd.edu), Justine Cannavo (University of Maryland, 0100 Lefrak Hall, College Park, MD 20742, USA, jcannavo@hesp.umd.edu)

Hyperacusis is an abnormal condition of sound intolerance that may cause some persons to reject amplified sound from their hearing aids. A significant secondary benefit reported for many patients receiving Tinnitus Retraining Therapy (TRT) is increased Loudness Discomfort Levels (LDLs). TRT involves both counseling and sound therapy (i.e., daily exposure to soft sound from bilateral noise generators (NGs)). We implemented a randomized, double-blind, placebo-controlled clinical trial to assess the efficacy of TRT as an intervention to improve sound tolerance in hearing-aid eligible persons with hyperacusis and/or restricted dynamic ranges. Subjects were assigned to one of four treatment groups: 1) full treatment, both counseling and NGs, 2) counseling and placebo NGs, 3) NGs without counseling, and 4) placebo NGs without counseling. They were evaluated at least monthly, typically for five months or more, on a variety of audiometric tests, including LDLs, the Contour Test for Loudness, and word recognition measured at comfortable and loud levels. Over $80 \%$ of the subjects assigned to full treatment achieved significant benefit (defined as shifts of greater than $10 \mathrm{~dB}$ in LDLs or the Contour Test uncomfortable level); whereas, most subjects assigned to a partial treatment group did not benefit from their treatment. [Supported by NIH]

4pPPc11. Relationship between a visual stimulus with a feeling of depth and its equivalent sound pressure level (ESPL). Hiroshi Hasegawa (Utsunomiya Univ., 7-1-2 Yoto, Tochigi-ken, 321-8585 Utsunomiya-shi, Japan, hasegawa@is.utsunomiya-u.ac.jp), Hirotaka Ono (Utsunomiya Univ., 7-1-2 Yoto, Tochigi-ken, 321-8585 Utsunomiyashi, Japan, hasegawa@is.utsunomiya-u.ac.jp), Takumi Ito (Utsunomiya Univ., 7-1-2 Yoto, Tochigi-ken, 321-8585 Utsunomiya-shi, Japan, hasegawa @ is.utsunomiya-u.ac.jp), Ichiro Yuyama (Utsunomiya Univ., 7-1-2 Yoto, Tochigi-ken, 321-8585 Utsunomiya-shi, Japan, yuyama@ is.utsunomiya-u .ac.jp), Masao Kasuga (Utsunomiya Univ., 7-1-2 Yoto, Tochigi-ken, 3218585 Utsunomiya-shi, Japan, kasuga@is.utsunomiya-u.ac.jp), Miyoshi Ayama (Utsunomiya Univ., 7-1-2 Yoto, Tochigi-ken, 321-8585 Utsunomiya-shi, Japan, nakatsue@ is.utsunomiya-u.ac.jp)

This study investigated the equivalent perception between a visual stimulus and its associated sound. Experiments of an auditory-visual stimulus presentation using an audio-video clip of a man beating a drum on a road were performed. The visual stimulus had a feeling of depth with a perspective view of the road. The visual stimulus was projected onto a screen that had the viewing angles of 43.8 deg. $(\mathrm{W}) * 25.4$ deg. $(\mathrm{H})$. Four kinds of distance between the subject and the visual stimulus from 5 to $40 \mathrm{~m}$, seven kinds of the delay time between auditory and visual stimulus from $-8 \mathrm{~F}$ to 8 $\mathrm{F}(1 \mathrm{~F}=1 / 30 \mathrm{~s})$, where " $+"$ indicates that the visual event preceded the sound, and nine levels of the sound stimulus from $-12 \mathrm{~dB}$ to $12 \mathrm{~dB}$ of the standard sound pressure level (SPL) were combined and presented. We evaluated the sound pressure level matching with each presentation pattern (equivalent sound pressure level; ESPL). As a result, we obtained that the ESPL tended to decrease when the delay time increased (the sound was delayed). This result shows a possibility that the visual stimulus was a little shifted to the direction of the sound.

4pPPc12. Temporal dynamics of stimulus specific processing in the human auditory cortex as revealed by electroencephalography. Paul M Briley (MRC Institute of Hearing Research, University Park, NG7 2RD Nottingham, UK, paul@ihr.mrc.ac.uk), Katrin Krumbholz (MRC Institute of Hearing Research, University Park, NG7 2RD Nottingham, UK, katrin @ihr.mrc.ac.uk)

When the same sound is presented repeatedly, the electrical brain response recorded over the scalp decreases in amplitude, an effect known as adaptation. Adaptation is dependent on both the similarity of the sounds and the time between them. It has been particularly well studied for a deflection of the electrical response known as the N100, which peaks about $100 \mathrm{~ms}$ after sound onset and receives major contributions from auditory cortica sources. Adaptation may reflect decreased sensitivity to repetitive stimuli, but could also indicate more efficient processing of familiar events. Research on adaptation has often employed an alternating tone paradigm (AB-A-B), examining the effects of changing inter-stimulus interval (ISI) or the frequency separation between A and B tones. Decreasing the frequency separation leads to an increase in N100 adaptation, and it has been suggested that the frequency specificity of this adaptation sharpens with decreasing ISI. In contrast, some studies have used A-B pairs with long inter-pair gaps and have found an enhancement of the N100 response to the B tone at shor ISIs. In order to gain a better understanding of the processes contributing to adaptation and enhancement, this study investigates the temporal dynamics and the frequency selectivity of these effects.

4pPPc13. An Investigation of Width and Depth Perception toward a Sound Image Constructed of Multiple Variant Sound Waves Emitted from a Loudspeaker Array. Yoko Yamakata (National Institute of Information and Communications Technology, 4-2-1, Nukui-Kitamachi, Koganei, 184-8795 Tokyo, Japan, yamakata@nict.go.jp), Toshiyuki Kimura (National Institute of Information and Communications Technology, 4-2-1, Nukui-Kitamachi, Koganei, 184-8795 Tokyo, Japan, t-kimura@nict.go.jp), Munenori Naoe (National Institute of Information and Communications Technology, 4-2-1, Nukui-Kitamachi, Koganei, 184-8795 Tokyo, Japan, nm.s512.ex@ nict.go.jp), Michiaki Katsumoto (National Institute of Information and Communications Technology, 4-2-1, Nukui-Kitamachi, Koganei, 184-8795 Tokyo, Japan, katumoto@nict.go.jp)

Many musical instruments, including violins and guitars, vibrate their resonant bodies differently over their surface when they make a sound. This paper aims to reveal the influences of such vibration variation of a soundboard surface on the width and depth perception of the sound image when listeners were in a near-field $50 \mathrm{~cm}$ or $1 \mathrm{~m}$ away from the soundboard. In this paper, a loudspeaker array mimicked the surface vibration as each loudspeaker makes a corresponding sound independently and cooperatively Three types of sounds, synthesized single-tone, multi-tone, and instrumental, were used as sources. To know what factors affect the perception of the sound image, various test sound sets were prepared by varying an origina sound set in amplitude or delay for each frequency for each loudspeaker. Eight subjects were asked to identify which sound image in a pair of test sounds was wider or farther than the other according to Scheffe's pair comparison method. The results shows that a test sound set with a delay varia- 
tion, which mimics sounds emitted by bending vibrations propagating on a soundboard, obviously influences the perception of sound image width and that the amplitude variation does not have much influence.

4pPPc14. MEG Recordings of Amplitude-modulated Noise and Tonal Stimuli in Healthy Adult Listeners. Yang Zhang (University of Minnesota, Dept. of Speech-Language-Hearing Sci. \& Center for Neurobehavioral Development, Minneapolis, MN 55455, USA, zhang470@umn .edu), Yingjiu Nie (University of Minnesota, Dept. of Speech-LanguageHearing Sci. \& Center for Neurobehavioral Development, Minneapolis, MN 55455, USA, niex0008@umn.edu), Toshiaki Imada (University of Washington, Dept. of Speech \& Hearing Sciences, and Institute for Learning \& Brain Sciences, Box 357988, Seattle, WA 98195, USA, imada@u.washington.edu), Keita Tanaka (Tokyo Denki University, Research Center for Advanced Technologies, 270-1382 Inzai, Japan, ktanaka@rcat.dendai.ac.jp), Masaki Kawakatsu (Tokyo Denki University, School of Information Environment, 270-1382 Inzai, Japan, kawakatu@asrl .dendai.ac.jp)

Amplitude modulation (AM) provides very important auditory information for the perception of complex sounds by normal listeners as well as cochlear implant users. The present study used a 122-channel whole-head magnetoencephalography (MEG) system to record auditory responses to amplitude-modulated pure tones and broadband noises in six healthy male adult subjects. The stimuli were presented in blocks of twenty with a brief silence in between, and the AM rates for both types of stimuli were at 20, 40, and $80 \mathrm{~Hz}$. At least 80 artifact-free trials were collected for each stimulus. As expected, the MEG data showed a significant bilateral effect of AM rate in the N1m component. There was also strong evidence that the neural representations of both the unmodulated pure tone and noise stimuli in the auditory regions of both hemispheres could be significantly affected by the global context of block design stimulus presentation.

4pPPc15. Critical-band compression method of speech enhancement for elderly people: Investigation of syllable and word intelligibility. Keiichi Yasu (Dept. of Electrical and Electronics Engineering, Sophia University, 7-1 Kiyoi-cho, Chiyoda-ku, 102-8554 Tokyo, Japan, k-yasu@sophia.ac.jp), Hideki Ishida (Dept. of Electrical and Electronics Engineering, Sophia University, 7-1 Kiyoi-cho, Chiyoda-ku, 102-8554 Tokyo, Japan, ishida-h@sophia.ac.jp), Ryosuke Takahashi (Dept. of Electrical and Electronics Engineering, Sophia University, 7-1 Kiyoi-cho, Chiyoda-ku, 102-8554 Tokyo, Japan, ryo.t.sust@gmail.com), Takayuki Arai (Dept. of Electrical and Electronics Engineering, Sophia University, 7-1 Kiyoi-cho, Chiyoda-ku, 102-8554 Tokyo, Japan, arai@sophia.ac.jp), Kei Kobayashi (Dept. of Electrical and Electronics Engineering, Sophia University, 7-1 Kiyoi-cho, Chiyoda-ku, 102-8554 Tokyo, Japan, kei-koba @ba2.so-net.ne.jp), Mitsuko Shindo (Sophia Univ. Research Center for Communication Disorders, 7-1 Kiyoi-cho, Chiyoda-ku, 102-8554 Tokyo, Japan, shindo-m@sophia.ac.jp)

Auditory filters for the hearing impaired tend to be wider than those of normal hearing people. Thus, the frequency selectivity decreases because of increased masking effects [Glasberg and Moore, J. Acoust. Soc. Am., 79(4), 1020-1033, 1986]. We have developed a method, called "critical-band compression," in which the critical band is compressed along the frequency axis [Yasu et al., Handbook of the International Hearing Aid Research Conference (IHCON), 55, Lake Tahoe, 2004]. We investigated whether our method improves syllable and word intelligibility. Thirty one elderly people participated in experiments. First, we measured the auditory filter bandwidth using a notched noise method [Patterson, J. Acoust. Soc. Am., 59(3), 640-654, 1976]. Second, we conducted syllable and word intelligibility tests. The compression rates of critical-band compression were set to $0 \%$ for the original, and $25 \%, 50 \%$, and $75 \%$. The results were that the percentages of correct responses were almost the same at $0 \%, 25 \%$ and $50 \%$ compression rates for syllable and word intelligibility. A significant correlation was not obtained between the compression rate of processing and the auditory filter bandwidth. [Work supported by JSPS.KAKENHI (16203041) and Sophia University Open Research Center from MEXT.]

4pPPc16. Speaker size discrimination for acoustically scaled versions of whispered words. Yoshie Aoki (Faculty of Systems Engineering, Wakayama University, 930 Sakaedani, 640-8510 Wakayama, Japan, s085065@sys.wakayama-u.ac.jp), Toshio Irino (Faculty of Systems Engineering, Wakayama University, 930 Sakaedani, 640-8510 Wakayama, Japan, irino@sys.wakayama-u.ac.jp), Hideki Kawahara (Faculty of Systems Engineering, Wakayama University, 930 Sakaedani, 640-8510 Wakayama, Japan, kawahara@sys.wakayama-u.ac.jp), Roy D. Patterson (Centre for the Neural Basis of Hearing, Department of Physiology, Development and Neuroscience, University of Cambridge, Downing Site, CB23EG Cambridge, UK, rdp1@ cam.ac.uk)

Humans can extract the message from the voices of men, women, and children without being confused by the size information, and they can extract the size information without being confused by the message. This suggests that the auditory system can extract and separate information about vocal tract shape from information about vocal tract length. Smith et al. [J. Acoust. Soc. Am. 117(1), 305-318 (2005)], Ives et al. [J. Acoust. Soc. Am. 118(6), 3816-3822 (2005)], and Aoki et al. [ARO, 31st Midwinter meeting (2008)] performed discrimination experiments with acoustically scaled vowels, syllables, and naturally spoken words, respectively, and demonstrated that the ability to discriminate speaker size extends beyond the normal range of speaker sizes. Smith and Patterson [BSA Cardiff (2005)] demonstrated that performance on the size-discrimination task is only marginally reduced when the vowels are unvoiced. We extended these size-discrimination experiments to whispered versions of naturally spoken, four-mora Japanese words. The just-noticeable-difference for the whispered words was about 6 $\%$, which is roughly the same as that for voiced words. The results show that voicing is not required for effective extraction of the size information. Research supported by JSPS Grant-in-Aid [B18300060] and the UK-MRC [G0500221].

4pPPc17. Effects of modality-dependent cuing and eye movements on sound localization. Beáta Tomoriová (Laboratory of Perception and Cognition, Technical University of Košice, Letná 9, 04200 Košice, Slovakia, beata.tomoriova@gmail.com), Rudolf Andoga (Laboratory of Perception and Cognition, Technical University of Košice, Letná 9, 04200 Košice, Slovakia, rudolf.andoga@tuke.sk), Norbert Kopčo (Laboratory of Perception and Cognition, Technical University of Košice, Letná 9, 04200 Košice, Slovakia,kopco@tuke.sk)

A previous study of visual and auditory hemispheric cuing in horizontal sound localization found modality-dependent effects of cuing resulting in biases in responses [Kopco, Tomoriova, Andoga, J. Acoust. Soc. Am. 121, 3094, 2007]. The previous study also suggested that some of the effects might be due to eye movements as eye fixation was not controlled. The goal of the current study was to isolate the attentional effects from the eye movement effects. An experiment identical to the previous one was performed, with the exception that the subjects were fixating the center of the audiovisual display. Localization performance was measured for transient auditory stimuli originating in the frontal horizontal plane. In most runs, a cue preceded the stimulus and indicated (correctly or incorrectly) the hemisphere (left vs. right) from which the subsequent target arrived. The cues differed by modality and the cue-to-target onset asynchrony. The listeners were instructed to focus their attention to the cued side. Compared to the previous study, a reduction in some effects was observed. However, modalitydependent biases in performance persisted, confirming that auditory spatial attentional control is modality dependent and operating on time scale of seconds. [Supported by the Slovak Science Grant Agency.] 
4pPPc18. Synchrony-asynchrony discrimination of audio-visual signals in auditory streaming. Maori Kobayashi (R.I.E.C., Tohoku University, 2-1, Katahira, Aoba-ku, 980-8577 Sendai, Japan, maori@ais.riec.tohoku.ac.jp), Shuichi Sakamoto (R.I.E.C., Tohoku University, 2-1, Katahira, Aoba-ku, 980-8577 Sendai, Japan, saka@ais.riec.tohoku.ac.jp), Yôiti Suzuki (R.I.E.C., Tohoku University, 2-1, Katahira, Aoba-ku, 980-8577 Sendai, Japan, yoh@ ais.riec.tohoku.ac.jp)

Temporal synchrony is a critical condition for integrating information presented in different sensory modalities. In this study, the effect of tonal organization on synchrony-asynchrony discrimination was examined. The auditory sequences were four repetitions of a triplet pattern comprising a low-frequency tone $(\mathrm{L})$ and a high-frequency tone $(\mathrm{H})$. The frequency difference $(\Delta \mathrm{F})$ between $\mathrm{L}$ and $\mathrm{H}$ was either approximately $1 / 12,1 / 6,1 / 3,1 / 2$, or 1 octave, centered at $1 \mathrm{kHz}$. Each tone was of $33.2 \mathrm{~ms}$ duration including rising and falling raised-cosine ramps of $5 \mathrm{~ms}$. The stimulus onset asynchrony (SOA) of adjacent tones was randomized between 33.2 and $332 \mathrm{~ms}$. The tone sequences were presented diotically via headphones at $65 \mathrm{~dB}$ SPL. The visual stimulus was a luminance-modulated Gaussian blob presented on a CRT monitor. The visual stimulus duration was $8.3 \mathrm{~ms}$. Synchronyasynchrony discrimination thresholds of visual-auditory stimulus onsets were measured using the 2IFC paradigm with a 2-up 1-down method under six $\Delta \mathrm{F}$ conditions. The results demonstrated that synchrony-asynchrony discrimination improved for audio-visual pulse trains at $\Delta \mathrm{F}$ between $\mathrm{L}$ and $\mathrm{H}$ greater than $1 / 3$ octave, suggesting that audio-visual synchrony perception is influenced by the build-up of auditory streaming.

4pPPc19. Voice quality of emphatics in comparison with non-emphatics in Morrocan Arabic. Karim Shoul (Lab. LPP UMR 7018 CNRS, 19, rue des bernardins, 75005 Paris, France, shoulkarim@ hotmail .com)

Based on acoustic and physiological data, this study examines the voice quality of emphacized (also called pharyngealized) vowels in Moroccan Arabic. The aim is to determine whether, as argued by some authors (Heath 1987, Fre woldu 1986), these vowels are creaky or glottalized. For this purpose, the /a/ vowel is considred in syllables after initial and intervocalic /t, $\mathrm{d}, \mathrm{s} /$ and their emphatic counterparts for an acoustic study as well as a physiological one (Fourcin's EGG 1974). The cues axamined inculde F0 values, duration and amplitude of the acoustic and glottalic signals, as well as the open quotien (0q) (as seen by Henrich 2001). Results of the acoustic analysis show no significant differences between emphacized and nonemphacized vowels as far as F0 values, duration and amplitude of the acoustic signal are concerned. The same absence of difference is observed from the EGG experiment, which indicate that 0q represents half of the whole glottal phase. These findings sugget that emphacized vowels, just like the corresponding non-emphacized counterparts, are characterized by a modal voice quality. They imply that 'secondary' pharyngealization does not require a narrowing of the supra-glottic cavity which would affect the mode of vocal-fold vibrations.

4pPPc20. Influence of multi-channel dynamic range compression on intelligibility: effect of envelope modulation bandwidth. Michael A. Stone (University of Cambridge, Department of Experimental Psychology, Downing Street, CB2 3EB Cambridge, UK, mas19@cam.ac.uk), Christian Füllgrabe (University of Cambridge, Department of Experimental Psychology, Downing Street, CB2 3EB Cambridge, UK, cf277@cam.ac.uk), Brian Moore (University of Cambridge, Department of Experimental Psychology, Downing Street, CB2 3EB Cambridge, UK, bcjm@cam.ac.uk)

Stone and Moore [J. Acoust. Soc. Am. (in press)] showed that, as the speed and number of channels in a multi-channel compressor increased, intelligibility of noise-vocoded signals in a competing speech task decreased. The noise vocoder is often used to simulate the information conveyed by cochlear implants. However, the vocoder of Stone and Moore preserved only low-rate $(<45 \mathrm{~Hz})$ envelope modulations whereas some implantees show sensitivity to envelope modulation rates up to about $300 \mathrm{~Hz}$. Furthermore, intrinsic fluctuations in the noise carriers affect the reception of lowrate modulations of the signal (Whitmal et al. [J. Acoust. Soc. Am. 122: 2376-2388, (2007)]). Here, a tone vocoder with $\mathrm{N}=8$ or $\mathrm{N}=16$ channels was used. Vocoding was preceded by N/8, N/4, or N/2 channels of com- pression, each using one of three speeds, affecting modulation rates up to about 2,6 , or $18 \mathrm{~Hz}$, respectively. The lowpass filters extracting the channel envelopes had corner frequencies of 45 or $180 \mathrm{~Hz}$. Intelligibility was measured using IEEE sentences with a competing speaker. The deleterious effect of compression with increasing channel number and speed was greater for the lower corner frequency. Compression of rates below $6 \mathrm{~Hz}$ affected intelligibility, independent of the presence of higher modulation rates.

\section{4pPPc21. Characterizing lexical interferences in informational} masking during speech-in-speech comprehension. Michel Hoen (Laboratoire d'Etude des Mécanismes Cognitifs (EMC). EA 3082 CNRS, Université Lumière Lyon 2, 5, Avenue Pierre Mendès-France, 69676 Bron Cedex, France, Michel.Hoen@univ-lyon2.fr), Claire Grataloup (Laboratoire Dynamique du Langage (DDL), UMR 5596 CNRS, Université de Lyon et Lyon 2, Institut des Sciences de l'Homme - 14 avenue Berthelot, 69363 Lyon Cedex 07, France, Claire.Grataloup@pse.unige.ch), François Pellegrino (Laboratoire Dynamique du Langage (DDL), UMR 5596 CNRS, Université de Lyon et Lyon 2, Institut des Sciences de l'Homme - 14 avenue Berthelot, 69363 Lyon Cedex 07, France, Francois.Pellegrino@univ-lyon2.fr), Lionel Collet (Univ. Lyon 1 - Lab. Neurosciences, Service Pr Collet, Pavillon U, Hôpital Edouard Herriot, F-69003 Lyon, France, iakhoun@olfac.univ-lyon1.fr), Fanny Meunier (Laboratoire Dynamique du Langage (DDL), UMR 5596 CNRS, Université de Lyon et Lyon 2, Institut des Sciences de l'Homme - 14 avenue Berthelot, 69363 Lyon Cedex 07, France, fanny.meunier@univ-lyon2.fr)

Results from our former research on the characterization of informational masking effects occurring during speech-in-speech comprehension showed that phonological and lexical information create specific informational masking effects, depending on the number of speakers involved (Hoen et al., 2007). The goal of the present study was to better characterize purely lexical factors potentially participating into informational masking phenomena. We evaluated speech-in-speech comprehension performances of 40 normal hearing participants listening to isolated lexical items presented together with different speech babble sounds. Lexicality of target items was controlled by using words of variable lexical frequency, high- vs. lowfrequency items, as well as pseudowords. The interaction between lexicality of target items and lexicality of words in background noise was controlled by creating babble sounds made of high- or low-frequency words. Results show that, as in silence, lexicality of target items plays a determinant role, high-frequency words being always more intelligible $(70 \%)$ than lowfrequency words $(50 \%)$, or pseudowords $(37 \%)$. Conversely, the frequency of lexical items present in the babble had an effect only on the comprehension of pseudowords, the latter being more intelligible in a background of low-frequency items. Together, these results give new precisions on the detailed informational masking effects occurring during speech-in-speech comprehension.

4pPPc22. Perceived plausibility of a multi-modal musical performance with introduced auditory-visual spatial and temporal mismatch. Danie Valente (Rensselaer Polytechnic Institute, Greene Bldg., 110 8th St., Troy, NY 12180, USA, danvprod@gmail.com), Jonas Braasch (Rensselaer Polytechnic Institute, Greene Bldg., 110 8th St., Troy, NY 12180, USA, braasj @ rpi.edu)

One of the biggest problems for multi-modal virtual musical performance is that of auditory-visual mismatch. Often such performances place musicians in a dry, studio-like environment and rely on acoustic room modeling techniques to place the musician's sound in an environment more conducive to musical performance. The problem, for example occurs when the musician sounds as if he/she is in a large performance space but from a visual standpoint appears to be located in a very small studio environment. This experiment aimed to reveal the impact of such mismatches on the perceived plausibility of a multi-modal musical performance. Listeners were confronted with three contrasting musical excerpts presented in a virtual environment with varying degrees of auditory visual mismatch. In the first phase, listeners were able to adjust the acoustic modeling algorithm using the salient parameters of direct to reverberant ratio and reverberation time. As the visual space volume that the performance was presented in increased, the listeners repeatedly increased reverberation time and decreased direct to reverberant ratio. During the second phase, where varying levels of spatial 
and temporal mismatch were purposefully introduced, the level of repeatability and accuracy of the listening group decreased despite subjective responses indicating accurate perceived performance realism.

4pPPc23. Haircell non-functionality and dead regions in the cochlea: an exploring study. Bastiaan Warnaar (AMC - Dept. of Clinical and Experimental Audiology, Meibergdreef 9, 1105AZ Amsterdam, Netherlands, b.warnaar@amc.nl), Wouter A. Dreschler (AMC, Clinical and Experimental Audiology, 1105 Amsterdam, Netherlands, w.a.dreschler@amc.uva.nl)

Dead regions (DRs) refer originally to regions in the cochlea without evoked electrical potentials due to non-functional inner hair cells and/or fibers of the auditory nerve. The focus of this study is to characterize hair cell non-functionality in the cochlea by means of psychophysical tests. A battery of tests was administered to a group of 13 subjects with a steep sloping tone threshold ( $>50 \mathrm{~dB} /$ octave) between 1 and $2 \mathrm{kHz}$ and severe loss $(>60 \mathrm{~dB}$ $\mathrm{HL})$ at high frequencies. Psychophysical Tuning Curves (PTC) and Threshold Equalizing Noise (TEN) are the classical tests to diagnose dead regions. Both use as criteria the phenomenon of off-frequency listening. Based on the results of complementary tests like Notched Noise measurements (NN) and Otoacoustic Emissions (OAE), it is argued that off-frequency listening is not necessarily connected to loss of inner hair cells and/or nerve fibers. Furthermore, combination tones produced by well functioning outer hair cells at places of severe hearing loss ( $>60 \mathrm{~dB}$ HL) are found. This may be explained by the presence of a dead region, which is verified with PTC and/or TEN measurements in 3 out of 4 of the cases.

4pPPc24. Audio-visual quality model for internet protocol television services. Marieneige Garcia (Deutsche Telekom Laboratories, Berlin Institute of Technology, Ernst-Reuter-Platz 7, 10587 Berlin, Germany, marie-neige.garcia@telekom.de), Alexander Raake (Deutsche Telekom Laboratories, Berlin Institute of Technology, Ernst-Reuter-Platz 7, 10587 Berlin, Germany, alexander.raake@telekom.de)

This paper presents a model for predicting the perceived audio-visual quality of IPTV services. Our model follows a modular approach and audiovisual quality is deduced from the perceived audio quality, the perceived video quality, the interaction between the audio quality and the video quality and the quality of the interaction between audio and video (lipsynchronization). In its current form, the model covers H.264 video codec, Standard Definition and High Definition video resolutions, MP2 audio codec and wav audio format. Addressed degradations, which generate different visual and auditory perceptual dimensions, are compression artifacts, packet losses, reduced bandwidth and delay between audio and video. Results demonstrate a mutual influence of the perceived audio and video qualities and the predominance of the video quality on the overall audio-visual quality. We analyze the interaction between visual perceptual dimensions, like blockiness, blurriness, slicing and freezing, and auditory perceptual dimensions, like frequency content (brightness), interruptedness, and freezing. We also study the influence of the type of the degradation on the interaction between perceived audio and video qualities. At last, we examine the influence of the audio-visual content (music video, news, etc.) on the perceived audiovisual quality. An outlook highlights future model extensions.

4pPPc25. Neural coding of envelope and fine structure in noise degraded speech. Jayaganesh Swaminathan (Purdue University, 500 Oval Drive, West Lafayette, IN 47907, USA, jswamy@purdue.edu), Michael Heinz (Purdue University, 500 Oval Drive, West Lafayette, IN 47907, USA, mheinz@purdue.edu)

Numerous perceptual studies have revealed that envelope is sufficient for speech perception in quiet, but that temporal fine structure (TFS) is required for speech perception in noise. However, the neural correlates of these perceptual observations remain unknown. The primary focus of the present work was to develop and evaluate neural cross correlation coefficient (CCC) metrics to quantify envelope and TFS coding in auditory-nerve responses to noise degraded speech. Shuffled auto- and cross-correlogram analyses were used to compute separate CCCs to quantify stimulus-related envelope and fine structure based on neural spike train data from a computational auditory-nerve model. The neural CCCs have a wide dynamic range as revealed by near-zero values for uncorrelated conditions and near-one values for correlated conditions based on broadband noise responses. Spectrally matched noise was systematically added to a speech sentence at different signal-to-noise ratios (SNRs). Initial analyses reveal that CCC_ENV $>$ CCC_TFS for positive SNRs, whereas CCC_TFS $>$ CCC_ENV for negative SNRs. Predicted effects of hearing loss on envelope and TFS coding will also be discussed. These neural metrics can be used to evaluate temporal coding of speech with implications for cochlear-implant and hearing-aid strategies. Supported by NIH-NIDCD.

4pPPc26. The effect of masker type and word-position on word recall and sentence understanding. Payam Ezzatian (University of Toronto Mississauga, 3359 Mississauga rd N., Mississauga, ON L5L 1C6, Canada, payam.ezzatian@utoronto.ca), Liang Li (University of Toronto Mississauga, 3359 Mississauga rd N., Mississauga, ON L5L 1C6, Canada, liang.li@utoronto.ca), Kathy Pichora-Fuller (University of Toronto Mississauga, 3359 Mississauga rd N., Mississauga, ON L5L 1C6, Canada, k.pichora.fuller@utoronto.ca), Bruce Schneider (University of Toronto Mississauga, 3359 Mississauga rd N., Mississauga, ON L5L 1C6, Canada, bruce.schneider@utoronto.ca)

Speech understanding is influenced by not only the presence, but also the specific nature of maskers. Noise maskers primarily result in energetic masking, whereas speech maskers create additional interference due to linguistic and acoustic similarities to the target. The present study examined the influence of different types of maskers and target word position on the immediate recall of words in sentences by normal-hearing younger adults. In Experiment 1, nonsense sentences with 3 keywords (e.g., A "house" should "dash" to the "bowl".) were presented against a background of speechspectrum noise or two-talker nonsense speech. With the speech masker, accuracy increased with word position. With the speech-spectrum noise masker, performance was highest for the first word and did not vary linearly with word position. In Experiment 2, when the speech-masker was noisevocoded to preserve envelope information while disrupting fine structure cues and minimizing semantic content, performance was similar to that found with the speech-spectrum masker. The results suggest that the ability to track a target sentence in conditions of informational masking improves as the target utterance unfolds over time.

4pPPc27. Training-induced auditory plasticity measured using auditory steady-state responses. Karolina Kluk (The University of Manchester, Human Communication and Deafness Research Group, Ellen Wilkinson Building, Oxford Road, M13 9PL Manchester, UK, karolina.kluk@manchester.ac.uk), Christine M. Tan (University of Essex, Department of Psychology, Wivenhoe Park, CO4 3SQ Colchester, UK, ctan@essex.ac.uk), Michael S. John (Rotman Research Institute, Baycrest Centre for Geriatric Care, 3560 Bathurst Street, Toronto, AB M6A 2E1, Canada, sjohn@rotman-baycrest.on.ca), Terence W. Picton (Rotman Research Institute, Baycrest Centre for Geriatric Care, 3560 Bathurst Street, Toronto, AB M6A 2E1, Canada, tpicton@rotman-baycrest.on.ca)

Re-mapping of the primary auditory cortex may be induced by extensive training. For example, training of monkeys to perform frequency discrimination (FD) at one carrier frequency expands the representation of that frequency region in the auditory cortex. This study was intended to demonstrate training-induced auditory plasticity using auditory steady-state responses (ASSRs) in humans. Right-handed, non-musicians underwent FD training in their left ear only at $1 \mathrm{kHz}$. ASSRs were recorded to 1 - and $2-\mathrm{kHz}$ amplitude modulated tones (100\% AM depth at rates of 41, 83 and $45,87 \mathrm{~Hz}$, across two conditions). ASSRs recorded at the start of the experiment were compared with the ASSRs recorded after three two-hour sessions of FD training scheduled 24 hours apart. The results revealed significant increase in the amplitude of ASSRs recorded to 41- and 45- Hz AM tones (at $1 \mathrm{kHz}$ only) presented to the trained left ear. There was no significant change in the amplitude of ASSRs recorded to the $2-\mathrm{kHz}$ tones or to any stimuli presented to the un-trained right ear. As expected FD training 
had no effect on 83- and 87-Hz ASSRs (which are generated mainly in the brainstem). These results support the idea of training-induced reorganization of the auditory cortex.

4pPPc28. Hearing-screening tests based on filtered sounds and on speech-in-noise intelligibility tests. Bozena Kostek (Gdansk University of Technology, Multimedia Systems Department, 11/12 Gabriela Narutowicza Street, 80-952 Gdansk, Poland, bozenka@sound.eti.pg.gda.pl), Andrzej Czyzewski (Gdansk University of Technology, Multimedia Systems Department, 11/12 Gabriela Narutowicza Street, 80-952 Gdansk, Poland, andcz@sound.eti.pg.gda.pl), Lukasz Kosikowski (Gdansk University of Technology, Multimedia Systems Department, 11/12 Gabriela Narutowicza Street, 80-952 Gdansk, Poland, kosiq@sound.eti.pg.gda.pl), Krzysztof Kochanek (The Institute of Physiology and Pathology of Hearing, Pstrowskiego 1, 01-943 Warsaw, Poland, k.kochanek@ifps.org.pl), Henryk Skarzynski (The Institute of Physiology and Pathology of Hearing, Pstrowskiego 1, 01-943 Warsaw, Poland, h.skarzynski@ifps.org.pl)

A hearing-screening system dedicated to small-children in pre-schools and primary schools is described in the paper. It uses as a hardware a palmtop computer supplemented with a small sound calibrating device. The described application provides tests that employ automatic questionnaire analysis, audiometric test procedures, and assessment of speech intelligibility in noise. In the speech-in-noise intelligibility tests, pictures are used for young children, and the screening tests are supervised by adults. Apart from the standardized audiometric tests, the screening tests employ environmental sounds filtered in audiometric frequency bands and calibrated as to their levels. When all the testing is completed, the system automatically analyzes the results for each child examined. The decision is made automatically by the expert system taking into account the number of incorrect answers. Children whose hearing impairment is confirmed are referred to treatment in rehabilitation centers. The project presented is a part of the large-scale "I can hear..." screening tests program carried out in Poland for the last few years. This may help to increase awareness and inspire action against noise at a very early age. The methods employed for filtering and calibration environmental sounds and results achieved are presented in the paper.

4pPPc29. The effect of masker type and word-position on word recall and sentence understanding. Payam Ezzatian (University of Toronto Mississauga, 3359 Mississauga rd N., Mississauga, ON L5L 1C6, Canada, payam.ezzatian@utoronto.ca), Liang Li (University of Toronto Mississauga, 3359 Mississauga rd N., Mississauga, ON L5L 1C6, Canada, liang.li@utoronto.ca), Kathy Pichora-Fuller (University of Toronto Mississauga, 3359 Mississauga rd N., Mississauga, ON L5L 1C6, Canada, k.pichora.fuller@utoronto.ca), Bruce Schneider (University of Toronto Mississauga, 3359 Mississauga rd N., Mississauga, ON L5L 1C6, Canada, bruce.schneider@utoronto.ca)

Speech understanding is influenced by not only the presence, but also the specific nature of maskers. Noise maskers primarily result in energetic masking, whereas speech maskers create additional interference due to linguistic and acoustic similarities to target speech. The present study examined the influence of different types of maskers and target word position on the immediate recall of words in sentences by normal-hearing younger adults. In Experiment 1, nonsense sentences with 3 keywords (e.g., A "house" should "dash" to the "bowl".) were presented against a background of speech-spectrum noise or two-talker nonsense speech. With the speech masker, accuracy increased with word position in a linear fashion. With the speech-spectrum noise masker, performance was highest for the first word but the same for the second keyword and third keyword. In Experiment 2, when the speech-masker was noise-vocoded to preserve envelope information while disrupting fine structure cues and minimizing semantic content, performance was similar to that found with the speech-spectrum noise masker. The results suggest that the ability to track a target sentence in conditions of informational masking improves as the target utterance unfolds over time.
4pPPc30. Empirical comparisons of pitch patterns in music, speech, and birdsong. Adam T. Tierney (UC San Diego Dept. of Cognitive Science, Neurosciences Institute, 9500 Gilman Drive, La Jolla, CA 92093-0515, USA, AdamTierney@gmail.com), Frank A. Russo (Ryerson University Department of Psychology, 350 Victoria Street, Toronto, ON M5B 2K3, Canada, russo@ryerson.ca), Aniruddh D. Patel (Neurosciences Institute, 10640 John Jay Hopkins Drive, La Jolla, CA 92121, USA, apatel@nsi.edu)

In music, large intervals ("pitch skips") are often followed by reversals, and phrases often have an arch-like shape and final durational lengthening. These regularities could reflect motor constraints on pitch production or could reflect the melodic characteristics of speech. To distinguish between these possibilities we compared pitch patterns in instrumental musica themes, sentences, and birdsongs. Patterns due to production-related constraints should be common to all three domains, whereas patterns due to statistical learning from speech should be present in speech but not birdsong Sequences were taken from English and French instrumental classical music, sentences from 4 languages, and songs of 56 songbird families. For sentences and birdsongs each syllable/note was assigned one pitch. For each sequence, we quantified patterns of post-skip reversals, the direction of the initial and final interval, the relative duration of the final syllable/note, and the pitch contour shape. Post-skip reversals predominated in all domains, likely reflecting a shared constraint: skips frequently take melodies toward the edges of the pitch range, forcing a subsequent reversal (as suggested by Von Hippel \& Huron, 2000). Arch-like contours and final lengthening were found in music and speech but not birdsong, possibly reflecting an influence of speech patterns on musical structure.

4pPPc31. Form and content in emotional reactions to sounds. Danie Vastfjall (Chalmers University of Technology, Division of Applied Acoustics - Chalmers Room Acoustics Group, Sven Hultins gata 8a, 41296 Gothenburg, Sweden, daniel.vastfjall@psy.gu.se), Erkin Asutay (Chalmers University of Technology, Division of Applied Acoustics, Sven Hultinsgata 8a 41296 Gothenburg, Sweden, erkin@student.chalmers.se), Anders Genell (Chalmers University of Technology, Division of Applied Acoustics, Sven Hultinsgata 8a, 41296 Gothenburg, Sweden, Anders.Genell@ ta .chalmers.se), Ana Tajadura (Chalmers University of Technology, Division of Applied Acoustics, Sven Hultinsgata 8a, 41296 Gothenburg, Sweden, ana .tajadura@chalmers.se)

People react emotionally to auditory stimuli. Despite this fact relatively little is known how sounds can create emotional reactions in listeners. We have developed a framework, the Emotion Reaction Model (ERM), that predict that both form features (i.e classical psychophysical attributes such as loudness, sharpness etc) and content feratures (i.e. psychological associations to the sound producing source). Using ERM we tested the relative contribution of form vs. content in producing emotional reactions to sounds. In a first experiment, participants rated their emotional reactions to sounds from qualitatively different categories (animals, humans, machine noise) and to same sounds with time or frequency scrambling applied (thus rendering them difficult to identify, but with retained psychoacoustical properties). Experiments 2 used the same sounds but with a priming procedure and experiment 3 assessed emotional reactions using physiological measures. Overall, content, rather than form, appeared to have the biggest impact on emotional reactions. This research may complement traditional psychoacoustical theories that focus solely on form features.

4pPPc32. Learning to read; attaching your ear to your tongue. Eugene Galanter (Children's Progress, Inc./ Columbia University, 460 Riverside Drive, New York, NY 10027, USA, eg53@columbia.edu)

Really early learning predicts later success in school, life, and society (1), (2). Speech fluency, measured by accuracy, rate, and prosody (3), predicts reading comprehension (4) and reading proficiency (5). Speech fluency may be improved by one-on-one teacher interactions. When properly administered they give teachers useful information, but are time-consuming and subject to teacher's expectations. The results are commonly normreferenced; a "wait to fail" model where teachers find deficits later in "highstakes tests" (6). These failures led us to develop "voice mirroring (VM)." (7) $\mathrm{V}-\mathrm{M}$ lets children self-correct their speech by hearing it mirrored after amplification. The child hears discrepancies between their own speech and 
their teacher's to self-correct. Children supported by V-M demonstrate better reading skills as estimated by their teachers; (single-blind). Well-spoken children read well

4pPPc33. Temporal response of a simplified bidimensional numerical model of the cochlea. Christine Lepine (Université Paul Sabatier, PHASE, 118, route de Narbonne, 31062 Toulouse cedex 9, France, salmets@aol.com), Vincent Gibiat (Université Paul Sabatier, PHASE, 118, route de Narbonne, 31062 Toulouse cedex 9, France, gibiat@cict.fr)

Within the framework of a study related to bone conduction, numerical simulations have been performed in the time domain, with the aim of comparing the cochlear partition displacement in the case of different places of stimulation. An oversimplified 2D model of the cochlea is used. It is first excited with pulses centered on various audible-range frequencies with a localisation of the source which is analogous to the position of the oval window. Secondly, new sets of calculations introduce different localisations and/or spatial extensions of the sources. An analogy with seismology being adequate to simulate the solid-fluid (cochlear partition-perilymph) coupling, a finite difference numerical simulation based upon the Virieux scheme for elastic waves propagation has been used. The movement of the simplified basilar membrane is observable when excited via air or bone conduction. Results of the propagation of a single pulse within the model will be presented and discussed through information available in literature.

4pPPc34. Modelling of the cochlea response as a versatile tool for acoustic signal processing. Marinus $\mathrm{M}$. Boone (University of Technology Delft, Lorentzweg 1, 2628 CJ Delft, Netherlands, m.m.boone @ tudelft.nl), Diemer De Vries (University of Technology Delft, Lorentzweg 1, 2628 CJ Delft, Netherlands, d.devries@tudelft.nl), Tjeerd C. Andringa (University of Groningen, Dept. Artificial Intelligence, P.O. Box 407, 9700 AK Groningen, Netherlands, t.andringa@ai.rug.nl), Anton Schlesinger (University of Technology Delft, Lorentzweg 1, 2628 CJ Delft, Netherlands, A.Schlesinger@tudelft.nl), Jasper Van Dorp Schuitman (University of Technology Delft, Lorentzweg 1, 2628 CJ Delft, Netherlands, J.vanDorpSchuitman@tudelft.nl), Bea Valkenier (University of Groningen, Dept. Artificial Intelligence, P.O. Box 407, 9700 AK Groningen, Netherlands, bea@ai.rug.nl), Hedde Van De Vooren (University of Groningen, Dept. Artificial Intelligence, P.O. Box 407, 9700 AK Groningen, Netherlands, h.van.de.vooren@ai.rug.nl)

The inner ear or cochlea processes the acoustic signals that enter the oval window into a specific time-frequency pattern. Many acoustic signal processing methods are based on this behaviour. A fundamental method is to calculate this time-frequency response by solving the differential equation of the movement of the basilar membrane, followed by a visualisation of the excitation patterns in a time-frequency plot. For that purpose Continuity Preserving Signal Processing (CPSP) is a promising method. In the presentation an overview will be given of a project that is carried out by TUD (University of Technology Delft) together with RUG (University of Groningen) being sponsored by STW (Dutch Technology Foundation). The project divides into four sub-projects which are closely related: Automatic Keyword Spotting, Machine Analysis and Diagnostics, Speech Intelligibility Enhancement for Hearing Aids and Quality Assessment of Room Acoustics. Results that have been obtained in the project will be summarised. Detailed results of the sub-projects will be presented in separate presentations.

4pPPc35. Improving speech intelligibility based on a conjunction of multiple perceptual models. Anton Schlesinger (University of Technology Delft, Lorentzweg 1, 2628 CJ Delft, Netherlands, A.Schlesinger @ tudelft.nl), Marinus M. Boone (University of Technology Delft, Lorentzweg 1, 2628 CJ Delft, Netherlands, m.m.boone@tudelft.nl)

The enhancement of speech intelligibility is crucial to the success of hearing aids and other speech transmission systems. Such systems often have to operate in real-world situations, in which noise, reverberation and competing talkers impair the signal-to-noise ratio and therewith the degree of intelligibility. Psychoacoustic and neuropsychological models progressively explain the working principle of the auditory path. In order to technologically achieve the mammalian hearing-performance, Computational Auditory Scene Analysis (CASA), a vivid field of research in acoustics, con- verts these models into technical approaches. Asking for neuropsychological evidence that underlies the extraction of sound-objects in reverberation and noise, two models are essential. These are echo-suppression, which is subject to the precedence effect, and modulation perception, performed by a neural mapping of time-information of the auditory input into a periodotopical organization. The models have been applied and successfully tested in enhancing the signal-to-noise ratio, respectively. In this work, we conjunct both models and obtain a temporal and lateral inhibition system, which successfully suspends interferences. An evaluation of the proposed approach verifies the consistency with psychoacoustical experiments of the precedence effect and modulation perception; the resulting speech intelligibility benefits considerably in acoustically adverse situations.

4pPPc36. Tinnitus: one problem that can be solved. G. Mario Mattia (Bruel Acoustics S.r.1. - EuroAcustici, Viale Cesare Pavese 304, 00144 Roma, Italy, m.mattia@euroacustici.org)

The author axplains the origin of idiopathic or subjective tinnitus. After more than ten years of research (at a personal centre and at Rome University "La Sapienza" Faculty of Medicine ...) we have solved more than $80 \%$ of tinnitus problems for a thousand people. We have found that the perceived sound can be a source of stress and can be recorded in the brain as "dangerous". We illustrate that a psychological and neurological rehabilitation implemented in a multifactors approach can give health and offer a new normal quality of life. With biofeedback EEG, EMG and GSR we are implementing our therapy. The biofeedback helps people understand the effects of stress on tinnitus and how to manage this stress to optimize performance and improve health. Previous studies have shown an enhancement of human performance and faster rehabilitation when physiological measures (respiration rate, heart rate, skin conductance, temperature, and surface electromyography) were fed back in sessions of Autogenic Training.

4pPPc37. Subjective evaluation of accelerating car interior noise using brain magnetic field. Kenji Takami (Univ. of Hyogo, 2167 Shosha, 6712201 Himeji, Japan, hyogo.takami@aist.go.jp), Shunsuke Ishimitsu (Hiroshima City University / National Institute of Advanced Industrial Science and Technology, 3-4-1 Ozuka-Higashi, Asa-Minami-Ku, 731-3194 Hiroshima, Japan, ishimitu@hiroshima-cu.ac.jp), Seiji Nakagawa (National Institute of Advanced Industrial Science and Technology (AIST), 1-8-31 Midorigaoka, 563-8577 Ikeda, Osaka, Japan, s-nakagawa@aist.go.jp), Toshihiko Asami (Univ. of Hyogo, 2167 Shosha, 671-2201 Himeji, Japan, asami@eng.u-hyogo.ac.jp)

Recently, the production concept of car engine sound has been changing to the sound design from the cure against noise. Although most work has concentrated on comfortable engine sound, the psychoacoustic effects of time-varying rate of frequency in accelerating engine sound have not been sufficiently studied. Thus we investigated the effects of time-frequencychanging rate in car interior noise on auditory impression using psychological and neurophysiological methods. Harmonic complex tones which simulated accelerating car noise were used as stimuli. Frequencies of the stimuli were time-varying by $15,25,35,50$, and $70 \mathrm{~Hz} / \mathrm{s}$, like accelerating-engine sounds. First, subjective evaluations were examined using a SD method. Second, neuronal activities of the auditory cortex evoked by these stimuli were measured by magnetoencephalography(MEG). The results indicated that time-varying rate of frequency has significant effects on subjective impression and neuronal activities of the auditory cortex.

4pPPc38. Subjective experiment on auditory localization for traffic alarm sounds. Sakae Yokoyama (Chiba Institute of Technology, 2-17-1, Tsudanuma, Narashino, 275-0016 Chiba, Japan, sakae@iis.u-tokyo .ac.jp), Hideki Tachibana (Chiba Institute of Technology, 2-17-1, Tsudanuma, Narashino, 275-0016 Chiba, Japan, tachibana@acoust.cs.itchiba.ac.jp)

When riding a motorbike with a helmet or driving a car, it is often experienced that the judgment of directions of alarm sounds like a horn and sirens of engine truck and ambulance car becomes difficult. This kind of auditory localization is seriously related to traffic safety. In this study, therefore, the effects of covering the driver' $s$ head with a helmet and sound transmission into a car on the auditory localization for traffic alarm sounds 
were examined by laboratory tests. To simulate a 3-dimensional sound field, the 6-channel recording/reproduction technique was applied and the incident direction of the test sounds was varied in twelve horizontal directions.

4pPPc39. Development of pure-tone auditory threshold in school children. Reinhard Müller (University of Giessen, Aulweg 123, 35392 Giessen, Germany, reinhard.mueller@audio.med.uni-giessen.de)

The overwhelming majority of publications concerning hearing in children is related to diseases, but normal development of hearing attracts little attention. Normal hearing, as defined by ISO 7029, refers to persons at the age of 18. While aging effects of hearing may be estimated by the use of formulas, children are not included. A field-study at a primary school in Germany showed a notably lower hearing sensitivity for children than for young adults. First graders did not hear well, but auditory performance improved with rising age. For validating this result, the first graders of the field study were again tested 3 years later. The second tests showed the expected improvement of their hearing threshold. So the hearing sense starts not with the full capability but underlies a certain development. Maybe training effects are the key to understanding this topic, as in other human skills.
4pPPc40. Problems in sound quality evaluation in Brazil: general or cultural ones? Stephan Paul (Lab. of Vibrations and Acoustics, Fed. Univ. of Santa Catarina, CTC, EMC, Campus Trindade, 88040 Florianópolis, Brazil, stephan.paul.acoustic@gmail.com)

Within modern sound engineering the individual perception of sound events has to be considered. Within sound and vibration related research all over the world several instruments were already developed, but this instruments are subject to several restrictions, especially their language. When sound evaluations are to be made in Brazil adequate evaluation instruments are necessary. This article is intended to discuss some of the problems encountered by the author and its co-workers when developing assessment procedures and especially instruments for evaluation of sound quality with Brazilian subjects. Especially the work undertaken to study descriptors for sound and vibration and the problems resulting in this work will be discussed. We consider the present congress, with participants from all over the world, to be a suitable platform to discuss some of the problems and results obtained.

THURSDAY AFTERNOON, 3 JULY 2008

P2-C, LEVEL 2, 3:40 TO 5:20 P.M.

\title{
Session 4pPPd
}

\section{Psychological and Physiological Acoustics: Auditory Perception of Sound Source Properties II (Poster Session)}

\author{
John Neuhoff, Cochair \\ The College of Wooster \\ Anna Preis, Cochair \\ Institute of Acoustics, Adam Mickiewicz University
}

\begin{abstract}
All posters will be on display from 3:40 p.m. to 5:20 p.m. To allow contributors an opportunity to see other posters, contributors of odd-numbered papers will be at their posters from 3:40 p.m. to 4:30 p.m. and contributors of even-numbered papers will be at their posters from 4:30 p.m. to 5:20 p.m.
\end{abstract}

\section{Contributed Papers}

4pPPd1. Auditory-guided reaching movements in the peripersonal frontal space. Florian Dramas (IRIT, Univ. Toulouse 3 - INPT - Univ. Toulouse 1 - CNRS Equipe Diamant, Univ. Paul Sabatier, 31062 Toulouse, France, florian.dramas@irit.fr), Brian F. Katz (LIMSI-CNRS, B.P. 133, 91403 Orsay, France, brian.katz@limsi.fr), Christophe Jouffrais (IRIT, Univ. Toulouse 3 - INPT - Univ. Toulouse 1 - CNRS Equipe Diamant, Univ. Paul Sabatier, 31062 Toulouse, France, jouffrais@irit.fr)

Previous studies on auditory localization processes have shown that humans can localize sound sources accurately, including distance in certain situations. Few studies have examined auditory localization by binaural mechanisms in the peripersonal space. Numerous studies have examined auditory localization through verbal report or various pointing movements. This study examines the precision of hand "reaching" movement towards an auditory object. An experimental platform (semicircle, radius $1 \mathrm{~m}$ ) was constructed with 35 small loudspeakers placed under an acoustically transparent grid. Blindfolded subjects were seated within the platform at table height. Test protocol consisted of a brief audio stimulus presented via a single loudspeaker followed by the subject placing their index finger (preferred hand) at the location of the sound object. Optical finger tracking was used during the course of the experiment. Two test variables were investigated: different au- dio stimuli, Gaussian noise bursts varying the number and the duration of each burst; room acoustic conditions, with and without acoustical damping for reflection suppression. Preliminary results show precision of localization does not grow indefinitely with the number of burst repetitions but reaches a limit. Azimuth precision remains accurate, even with short burst conditions, contrary to the distance perception which increases with the stimuli duration.

4pPPd2. In-situ observation of the perceptive process linked to dashboard tapping sounds. Francois Montignies (Renault Technocentre, FR TCR LAB 252, 1 avenue du Golf, 78288 Guyancourt Cedex, France, francois.montignies@insa-lyon.fr), Valery Nosulenko (Institute of Psychology, Russian Academy of Science, 129366 Moscow, Russian Federation, valery.nosulenko@gmail.com), Etienne Parizet (Laboratoire Vibrations Acoustique, Insa Lyon, 25 bis, av. J. Capelle, 69621 Villeurbanne Cedex, France, etienne.parizet@insa-lyon.fr)

It is well-known that in show-rooms some people might tap on the dashboard of vehicles. The aim of this study was to determine the importance of this phenomenon and to identify which properties of the vehicle are perceived through the sound thus produced. An ethomethodological observation 
was conducted to collect data about the action/perception process of a subject exploring a static vehicle. The work was based on the methodology developed by Nosulenko and Saymolenko to evaluate perceived quality using free verbalisations in a comparison task. 52 naive subjects were placed in ecologically valid conditions. Their task consisted in freely exploring two vehicles and selecting their preferred one. From a qualitative analysis of audiovisual recordings, a data base was built. It linked verbalisations, operations and perceived objects, and allowed the quantification of indicators related to activity and perception. The analysis of operations validated that the tapping operation was not anecdotal. Moreover, dashboard was one of the main perceived objects linked to to the auditive dimension. Finally, a significative effect of the tapping operation on the evaluation of dashboard material quality was observed, suggesting an implicite influence of sound on this perceived property.

4pPPd3. Hemispheric Differences in the Recognition of Environmental Sounds. Julio Gonzalez (Universitat Jaume I, Dept. Psicologia Basica, Clinica y Psicobiologia, Campus Riu Sec. Facultad CC. Humanas y Sociales, 12071 Castellon de la Plana, Spain, gonzalez@psb.uji.es), Conor T. McLennan (Cleveland State University, Dpt. Psychology, 2121 Euclid Ave. CB 175, Cleveland, OH 44115, USA, c.mclennan@csuohio.edu)

In the visual domain, Marsolek and colleagues have provided support for their claim that two dissociable and parallel neural subsystems underlie abstract and specific object recognition [Marsolek, 1999; Marsolek \& Burgund, 2003]. According to their dissociable subsystems theory, an abstractcategory subsystem operates more effectively in the left hemisphere (LH) and is less sensitive to the specific surface characteristics of the stimuli, whereas a specific-exemplar subsystem operates more effectively in the right hemisphere $(\mathrm{RH})$ and is more sensitive to specific stimulus characteristics. In the present study, we tested this hypothesis in the auditory domain by conducting 2 long-term repetition-priming experiments on the recognition of environmental sounds. Participants attempted to identify target sounds from an initial $750 \mathrm{~ms}$ sound stem. Target stems were primed by either an identical or a different exemplar sound (e.g., the same or different tokens of a bagpipe). Target stems were presented monaurally in both experiments; however, in Exp. 2 white noise was simultaneously administered to the opposite ear. Our results are consistent with Marsolek's framework. In particular, in both experiments an exemplar specificity effect was obtained when the sounds were presented to the left ear (RH), but not when the sounds were presented to the right ear ( $\mathrm{LH})$.

4pPPd4. Perception of speech properties from extremely brief segments. Sue Harding (Sheffield University, Computer Science Department, Regent Court, 211 Portobello St., S1 4DP Sheffield, UK, s.harding@dcs.shef.ac.uk), Martin Cooke (Sheffield University, Computer Science Department, Regent Court, 211 Portobello St., S1 4DP Sheffield, UK, m.cooke@dcs.shef.ac.uk)

A glance at a visual scene enables observers to become rapidly aware of its most important characteristics. Here, we describe experiments using very brief segments of natural speech which demonstrate that a surprising amount of information can be determined from only a few milliseconds of the auditory signal. Segments with durations ranging from 2.5 to $80 \mathrm{~ms}$ were extracted from six vowels and six fricatives spoken by males and females. Listeners identified the phoneme and/or gender, or whether a vowel or consonant had been presented. While listeners' performance dropped close to chance for the $2.5 \mathrm{~ms}$ stimuli for most tasks, for the vowel/fricative distinction listeners obtained scores above $70 \%$ even for such short segments. Listeners performed well above chance for the $10 \mathrm{~ms}$ stimuli for three out of four tasks. Combining results within tasks showed that listeners also distinguished voiced from unvoiced phonemes in less than $10 \mathrm{~ms}$. Threshold values from logistic fits indicate the order in which information becomes available: vowel/fricative distinction $(3.0 \mathrm{~ms})$, voicing distinction $(6.7 \mathrm{~ms})$, phoneme identification (11.9 ms) and gender identification (15.3 ms). By exploiting the "gist" of an auditory scene, listeners may be able to deploy prior knowledge rapidly to constrain further interpretation.

4pPPd5. Comparison of headphones and equalization for virtual auditory source localization. David Schonstein (Arkamys, 5 rue Frédéric Bastiat, 75008 Paris, France, dschonstein@arkamys.com), Laurent Ferré (LIMSI-CNRS, B.P. 133, 91403 Orsay, France, laurent.ferre @limsi.fr), Brian F. Katz (LIMSI-CNRS, B.P. 133, 91403 Orsay, France, brian.katz@limsi.fr)

This study investigates the variation in localization performance between different headphone styles. Eight different headphones (including various in-ear, circumaural open and closed, and bone conduction headphones) were tested. In addition, the effect of headphone equalization (aiming to produce an approximately flat frequency response) was investigated. Localization was examined for 24 locations distributed on a sphere surrounding the listener. A single subject participated in the study using a single chosen non-individual HRTF set. Each location was repeated 6 times, resulting in a total of 144 localization reports. Overall, results were relatively consistent for 3 out of the 8 headphones tested. For these headphones, there was no significant difference in lateral angle error, associated with ITD and ILD cues. Polar angle errors, associated with the cone of confusion, however did vary significantly for these headphones. The headphone equalization had varying effects on localization accuracy depending on the headphone. Globally, headphone equalization showed no significant effect on localization accuracy. The results serve as a preliminary investigation, highlighting consistent results for only a select group of headphones tested for effective sound rendering in virtual auditory space. In addition, the results suggest that headphone equalization has a minimal influence on localization accuracy under these conditions.

4pPPd6. Perception of Sound Source Distance and Loudness in a Coherent Field of a Reverberant Field. Yoshifumi Hara (Kogakuin University, 1-24-2, Nishi-Shinjuku, Shinjuku-ku, 185-0012 Tokyo, Japan, kuro5hiron@ymail.plala.or.jp), Yoshinori Takahashi (Kogakuin University, 1-24-2, Nishi-Shinjuku, Shinjuku-ku, 185-0012 Tokyo, Japan, yoshinori@ieee.org), Hiroaki Nomura (Kure National College of Technology, 2-2-11, Aga-Minami Kure City, 737-8506 Hiroshima, Japan, hnomura@kure-nct.ac.jp), Mikio Tohyama (Waseda University, 1-3-10, Bldg. 29-7, Nishi-Waseda, Shinjuku-ku, 169-0051 Tokyo, Japan, m_tohyama@waseda.jp), Kazunori Miyoshi (Kogakuin University, 1-24-2, Nishi-Shinjuku, Shinjuku-ku, 185-0012 Tokyo, Japan, miyoshi@cc .kogakuin.ac.jp)

Perception of reverberant sound field changes with a sound source distance (SSD). This article describes SSD perception in a coherent region close to the sound source in the reverberation field. We performed listening tests for SSD perception and loudness of speech and carried out transfer functions analysis using a reverberant room. We confirm that both SSD perception and loudness are correlated to the standard deviation of the magnitude frequency response of the transfer function in the coherent region. That is SSD erception and loudness increase as SSD becomes long in the coherent region. However loudness decreases in an incoherent region. Consequently, we surmise SSD perception in the coherent region might be due to increase in loudness.

4pPPd7. The influence of pinna position on head-related transfer function. Przemyslaw Plaskota (Wroclaw University of Technology, Wybrzeze Wyspianskiego 27, 50-370 Wroclaw, Poland, przemyslaw.plaskota@pwr.wroc.pl), Andrzej B. Dobrucki (Wroclaw University of Technology, Wybrzeze Wyspianskiego 27, 50-370 Wroclaw, Poland, andrzej.dobrucki@pwr.wroc.pl)

The changes of spectrum of sound at listener ear are one of the major cues for sound source localization. Head Related Transfer-Function (HRTF) describes the influence of torso, head and pinna on sound spectrum. It is possible to recognize HRTF using computational method, e.g. Boundary Elements Method (BEM). The numerical model used for calculation of HRTF is constructed by transfer geometrical shape of head and pinna into numerical domain. Important question during geometry reconstruction process is 
the accuracy of shape mapping. The pinna has significant influence on HRFT. In the paper, the influence of accuracy of pinna geometry transformation and pinna position on HRTF is presented. Particularly, the pinna flare angle, pinna rotation angle and position of ear entrance were taking into considerations. Measurements have been done on numerical model with the invariable pinna and head shapes, using BEM method.

4pPPd8. Environmental Enrichment Increases Response Strength And Paired-Pulse Depression Of Auditory Cortex Neurons. Cherie R. Percaccio (Univ. of Texas, 800 W. Campbell Rd, Richardson, TX 75080, USA, cheriep@u.washington.edu), Autumn L. Pruette (Univ. of Texas, 800 W. Campbell Rd, Richardson, TX 75080, USA, autumn.pruette @ utsouthwestern.edu), Shilpa T. Mistry (Univ. of Texas, 800 W. Campbell Rd, Richardson, TX 75080, USA, shilpa.mistry@utsouthwestern .edu), Yeting H. Chen (Univ. of Texas, 800 W. Campbell Rd, Richardson, TX 75080, USA, superhelen@gmail.com), Daniel L. Rathbun (Univ. of Texas, 800 W. Campbell Rd, Richardson, TX 75080, USA, dlrathbun@ucdavis.edu), Michael P. Kilgard (Univ. of Texas, 800 W. Campbell Rd, Richardson, TX 75080, USA, kilgard@utdallas.edu)

A wide variety of sensory gating impairments have been associated with autism. Abnormal brain development may alter patterns of interaction between the child and the environment and hinder the acquisition of critical language skills. After several months of therapy, autistic symptoms may subside as children advance to higher cognitive stages. This study modeled the physiological changes associated with therapy-related gains in children by investigating enrichment-induced plasticity in rat auditory cortex. Evoked potential response strength and paired-pulse depression were enhanced by exposure to an enriched environment and degraded by exposure to a standard environment. While neither exercise nor social stimulation, specifically, resulted in any plasticity, rats that heard the enriched environment from a distance also exhibited enhanced responses. The degree of enrichment-induced plasticity was not reduced by a substantial and persistent cholinergic deficit. The finding that enrichment increases response strength and paired-pulse depression in the auditory cortex of rats is consistent with earlier clinical observations, suggesting that proper sensory development is necessary for higher cognitive processes. In the future we will investigate if clinical gains during and after therapy are associated with increased event-related potential discrimination and hemispheric localization of speech stimuli in children with autism.

4pPPd9. Directional loudness measurements for a multichanne system. Densil Cabrera (University of Sydney, Faculty of Architecture Design and Planning, NSW 2006 Sydney, Australia, densil@usyd.edu .au), Luis Miranda (University of Sydney, Faculty of Architecture, Design and Planning, NSW 2006 Sydney, Australia, lmir9852@mail.usyd.edu .au), Ian Dash (Australian Broadcasting Corporation, Technology Research \& Standards, Level 11, Ultimo Building, 2001 Sydney, Australia, Dash.Ian @ abc.net.au)

Loudness matching listening tests were conducted to quantify the difference in loudness level from a constant signal played from various horizontal directions. The multichannel system used for this tests was a 5-channel system, set up according to the ITU Recommendation BS.116-1 Methods for the subjective assessment of small impairments in audio systems including multichannel sound systems and the test signals were octave bands of noise with centre frequencies from $63 \mathrm{~Hz}$ to $8000 \mathrm{~Hz}$. These tests were conducted as part of ongoing research for the ITU Recommendation BS.1770 Algorithms to measure audio programme loudness and true-peak level. The aim of this experiment is to contribute to the design of a loudness meter by providing channel weightings, and results indicate that listeners are more sensitive to the surround channels than the other channels in the mid and high frequency range.
4pPPd10. Investigating the potential of human echolocation in virtual sonic trigonometry. Flaithri E. Neff (University College Cork, Western Road, IRL Cork, Ireland, fn2@cs.ucc.ie), Ian Pitt (University College Cork, Western Road, IRL Cork, Ireland, i.pitt@cs.ucc.ie)

Describing a mathematical problem often involves visual diagrams. For blind students this accentuates the challenges they face. Projects such as LAMBDA have used linear speech and Braille to convey algebraic equations. However, spatial features, for example in trigonometry, are difficult to map to a linear-based system. Traditional tactile methods (e.g. German film) convey simple shapes but need Braille support and speech-tactile interfaces (e.g. NOMAD) require unconventional equipment. Cognitive issues regarding tactile interpretation of $3 \mathrm{D}$ shapes also persist. Blind students interact regularly with speech technology and audio games. This exposure means that the auditory system is potentially becoming accustomed to sonic interpretation of computer-based information. Some of our research has looked at expanding the sonic environment to include spatial information aimed at trigonometry. The next stage is to provide interactive user control. Our system is based on a user interface model in order to consider the cognitive issues involved. We use Microsoft's XNA/XACT environment to create our auditory scene. In this paper we discuss how to implement sonicbased user interaction while further simplifying our auditory scene. In order to achieve this, we examine the potential of human echolocation to orient within the virtual walls and corners of a triangle.

4pPPd11. Toward synthesis tools using 'evocation' as control parameters. Adrien Merer (CNRS-LMA, 31, chemin Joseph Aiguier, $13402 \quad$ Marseille, France, merer@lma.cnrs-mrs.fr), Mitsuko Aramaki (CNRS - INCM and Université de Provence, 31, chemin Joseph Aiguier, 13402 Marseille, France, aramaki@lma.cnrs-mrs.fr), Richard Kronland-Martinet (CNRS-LMA, 31, chemin Joseph Aiguier, 13402 Marseille, France, kronland@1ma.cnrs-mrs.fr), Solvi Ystad (CNRS-LMA, 31, chemin Joseph Aiguier, 13402 Marseille, France, ystad@lma.cnrs-mrs .fr)

This study addresses the design of synthesis tool controlled by highlevel parameters, such as mental evocations induced by sounds. As a first approach, we considered sounds evoking motions and we addressed 3 main questions: What are the different categories of motion? What are the common acoustic features of sounds within a category? How to synthesize sounds that evokes specific motions? We gathered samples used by electroacoustic music composers as a framework for their compositions and synthesized sounds. Then we effectuated a two-steps listening test. The first part aims at determining these different motion categories. It consisted in a free categorization task in which listeners build their own groups of sounds as function of evoked motions. The second part aims at determining a set of sounds characteristic of each of these categories. It consisted in a constrained categorization task with predefined categories represented by prototypical sounds (deduced from free categorization task). We used a feature selection method to highlight most relevant signal descriptors for each category. Finally, designing a synthesis tool implies the calibration of these descriptors (a specific range of values for each category) and their control (leading to address the inverse problem). These aspects are currently being investigated.

4pPPd12. Human recognition by active and passive acoustic signatures. Alexander Ekimov (University of Mississippi, NCPA, 1 Coliseum Drive, University, MS 38677, USA, aekimov@olemiss.edu), James M. Sabatier (University of Mississippi, NCPA, 1 Coliseum Drive, University, MS 38677, USA, sabatier@olemiss.edu)

Recognition of different sensed objects is a problem that often appears in practice. One of the solutions is based on analysis of the signatures of the specific objects. This method was applied for the acoustic detection of walking humans. Human footsteps excite envelopes of broadband acoustic signals in the air due to periodic friction forces between the foot and the ground/floor. The repetition frequency of these envelopes is equal to the footstep rate and usually lies below $3 \mathrm{~Hz}$. High frequencies in these envelopes allow detection and localization of a walker using a narrowband ultrasonic receiver with a high directivity pattern. Consequently, periodic low frequency human motion results in passive ultrasonic detection using this 
method. This motion has also unique Doppler signatures and is measured using ultrasonic sonar. Common analyses of passive and Doppler signatures allow the extraction of the specific cadence in human motion and recognition of a human while rejecting other moving or stationary objects. [Work supported by Department of the Army, Army Research Office contract W911NF-04-1-0190].

4pPPd13. Strategic listener movement in a model of auditory distance perception. Yan-Chen Lu (Sheffield University, Computer Science Department, Regent Court, 211 Portobello St., S1 4DP Sheffield, UK, y.c.lu@dcs.shef.ac.uk), Martin Cooke (Sheffield University, Computer Science Department, Regent Court, 211 Portobello St., S1 4DP Sheffield, UK, m.cooke@dcs.shef.ac.uk)

A mobile listener has the potential to exploit dynamic auditory cues to judge sound source distance. One such cue is motion parallax, which employs a sequence of azimuth estimates from interaural time differences to triangulate sound source location. However, distortions due to reverberation and competing sources complicate matters, so it is of interest to know what active strategies listeners might adopt to arrive at robust location estimates. One hypothesis is that not all listener motion trajectories are equallybeneficial for distance estimation. Trajectories designed via certain optimisation criteria might lead to faster and more robust estimates in a wider range of environments. Eight listener motion strategies were tested, from naive approaches such as random walks and head-rotation only to more sophisticated techniques based on sequential Monte Carlo methods. In the latter case, strategies included movement towards the expected source location, or in the most informative direction, or movement reducing overall uncertainty. Evaluation in a simulated acoustic environment with single sources under both anechoic and reverberant conditions demonstrated that moving towards the most likely source location led to the most accurate estimation of distance and subsequent tracking of a moving source. Significant problems remain in estimating distance in multi-source conditions.

THURSDAY AFTERNOON, 3 JULY 2008

P2-B, LEVEL 2, 3:40 TO 5:20 P.M.

\title{
Session 4pSAa
}

\section{Structural Acoustics and Vibration, Computational Acoustics, and EURONOISE: Efficient Boundary Element Methods II (Poster Session)}

\author{
Ramani Duraiswami, Cochair \\ University of Maryland Institute for Advanced Computer Studies \\ Haike Brick, Cochair \\ TFH Berlin - University of Applied Sciences
}

\begin{abstract}
All posters will be on display from 3:40 p.m. to 5:20 p.m. To allow contributors an opportunity to see other posters, contributors of odd-numbered papers will be at their posters from 3:40 p.m. to 4:30 p.m. and contributors of even-numbered papers will be at their posters from 4:30 p.m. to 5:20 p.m.
\end{abstract}

\section{Contributed Papers}

4pSAa1. Application of the fast multipole method for solving very large acoustic radiation problems. Raphael Hallez (LMS International, Researchpark Z1, Interleuvenlaan 68, 3001 Leuven, Belgium, raphael.hallez @ Imsintl.com), Koen De Langhe (LMS International, Researchpark Z1, Interleuvenlaan 68, 3001 Leuven, Belgium, koen.delanghe@1msintl .com), Michel Tournour (LMS International, Researchpark Z1, Interleuvenlaan 68, 3001 Leuven, Belgium, michel.tournour@1msintl.com), Toufic Abboud (IMACS, Ecole Polytechnique, 91128 Palaiseau Cedex, France, abboud@imacs.polytechnique.fr)

Boundary element method is well known and extensively used to solve acoustic radiation problems. It is especially appropriated for exterior radiation since the fluid domain does not need to be meshed, as opposed to the finite element method. However, the mathematical formulation leads to a dense matrix system of equations. Therefore, the size of the model increases drastically as the frequency of analysis increases and huge computer resources are required to solve complex models in the mid-frequency range. The fast multipole method can be used to extend the boundary element model and solve such problems. For a model with $\mathrm{N}$ nodes, this technique brings the number of operations down to $\mathrm{O}\left(\mathrm{N}^{*} \log \mathrm{N}\right)$ instead of $\mathrm{O}\left(\mathrm{N}^{*} * 3\right)$ for conventional boundary elements. This new methodology has been applied here to study the acoustic radiation of a complete car in the midfrequency range. The accuracy of the results as well as the computation time demonstrate the great potential of this new method to solve very large acoustic radiation problems.
4pSAa2. FE-Model Reduction for FE-BE Coupling with Large Fluid-Structure Interfaces. Michael Junge (Institute of Applied and Experimental Mechanics, University of Stuttgart, Pfaffenwaldring 9, 70550 Stuttgart, Germany, junge@iam.uni-stuttgart.de), Jens Becker (Institute of Applied and Experimental Mechanics, University of Stuttgart, Pfaffenwaldring 9, 70550 Stuttgart, Germany, becker@iam.uni-stuttgart.de), Dominik Brunner (Institute of Applied and Experimental Mechanics, University of Stuttgart, Pfaffenwaldring 9, 70550 Stuttgart, Germany, brunner@iam.uni-stuttgart.de), Lothar Gaul (Institute of Applied and Experimental Mechanics, University of Stuttgart, Pfaffenwaldring 9, 70550 Stuttgart, Germany, gaul@iam.uni-stuttgart.de)

For the finite element method model, reduction techniques exist to represent the dynamic behavior of component substructures. Depending on the type of reduction method, the reduction basis contains constraint or attachment modes, which are computed for all structural degrees of freedom on an interface. The interface can either be defined by adjacent substructures or by coupling interfaces to other physical domains, as it is the case for FE-BE coupled systems. A large interface thus leads to an increased size of the reduced order model and limits standard model reduction techniques to applications with small interfaces. In this work, interface reduction methods are investigated. Here, the size of the reduced order model is decreased by reducing the number of retained interface modes, while marginally increasing the reduction error. A direct reduction method based on strain--energy considerations is presented. Additionally, an iterative reduction scheme is pro- 
posed which only adds a basis vector to the reduction basis, if the spanned subspace is sufficiently enlarged. The applicability of the proposed methods is shown for an example structure.

4pSAa3. Modeling of the acoustic eigenproblem with sound absorption using boundary element method. Antoine Lavie (Univ. d'Artois, Faculté des Sciences Appliquées - Lab. LAMTI, Technoparc Futura, 62400 Bethune, France, antoine.lavie@univ-artois.fr), Alexandre Leblanc (ISAE, 10, av Edouard Belin, 31055 Toulouse, France, alexandre.leblanc@isae .fr), Abdelkader Haddi (Univ. d'Artois, Faculté des Sciences Appliquées Lab. LAMTI, Technoparc Futura, 62400 Bethune, France, abdelkader.haddi @univ-artois.fr)
This paper deals with determination of resonant frequencies for absorbant 3D acoustic cavities. The behaviour of the sound absorbing boundary can be described with a Robin condition as proposed by Rajakumar et al [Int. J. Numer. Methods Eng., 36, 3957-3072 (1993)]. This approach is inaccurate, especially for low frequencies because the absorption coefficient is assumed to be constant. We observed the acoustic admittance for foam and fibrous type materials varies linearly for low frequencies. The introduction of a new absorption coefficient allows to take into account this behavior in order to improve the accuracy in the determination of the first modes (typically less than $500 \mathrm{~Hz}$ in car interior). This formulation has been implemented in a boundary element program we have developed. The results are compared with those given by the finite element program ANSYS. Computations are carried out for rectangular parallelepiped and Sedan car interior

\title{
Session 4pSAb
}

\section{Structural Acoustics and Vibration and EURONOISE: Distributed Active Noise and Vibration Control II (Poster Session)}

\author{
Kenneth Cunefare, Cochair \\ Georgia Institute of Technology \\ Manuel Collet, Cochair \\ FEMTO-ST UMR CNRS
}

\begin{abstract}
All posters will be on display from 3:40 p.m. to 5:20 p.m. To allow contributors an opportunity to see other posters, contributors of odd-numbered papers will be at their posters from 3:40 p.m. to 4:30 p.m. and contributors of even-numbered papers will be at their posters from 4:30 p.m. to 5:20 p.m.
\end{abstract}

\section{Contributed Papers}

4pSAb1. Active noise control in turbofan aircrafts: theory and experiments. Ernesto Monaco (Dept. of Aerospace Engineering - University of Naples, Via Claudio, 21, 80125 Naples, Italy, ermonaco @ unina.it), Leonardo Lecce (Dept. of Aerospace Engineering - University of Naples, Via Claudio, 21, 80125 Naples, Italy, leonardo@unina.it), Ciro Natale (Dipartimento di Ingegneria dell'Informazione - Seconda Università degli Studi di Napoli, Via Roma 29, I-81031 Aversa (CE), Italy, ciro.natale@unina2.it), Salvatore Pirozzi (Dipartimento di Ingegneria dell'Informazione - Seconda Università degli Studi di Napoli, Via Roma 29, I-81031 Aversa (CE), Italy, salvatore.pirozzi@unina2.it), Chris May (Laboratory of Process Automation (LPA) - Saarland University, Gebäude A5 1, D-66123 Saarbrücken, Germany, c.may@zip.uni-sb.de)

This paper presents the activities developed by the authors within the research project M.E.S.E.M.A. funded by the European Commission. A noise and vibration control system using magnetostrictive actuators has been designed and experimentally tested on a large scale (fuselage mock-up) test article, for controlling noise and vibrations between $150-500 \mathrm{~Hz}$. The environmental noise and vibration excitation was representative of a small/medium turbofan aircraft. Firstly a numerical model of the test article has been developed in MSC/NASTRAN coupling the structural part with the interior acoustic volume. Furthermore the experimental characterisation of the testarticle has been carried on. The model, updated by the mean of the experimental results, was employed to derive the required control actuators performances in order to achieve the best control predicted using a well consolidated "feed-forward" approach. Genetic Algorithms have been employed in order to optimise the positioning of the actuators. Dedicated magnetostrictive actuators have been designed together with light power amplifiers meeting the specifications; on each actuator an optoelecronic sensor, based on Bragg grating, has been integrated to optimize the actuator performance. A two-level ANVC system has been designed and tested on a full scale fuselage mock-up. The paper present an overview of the activities developed as well as of the achieved results.

4pSAb2. Active vibration control for the identification-based model of a circular plate. Lucyna Leniowska (University of Rzeszow, Rejtana 16, PL35-310 Rzeszow, Poland, lleniow@univ.rzeszow.pl), Pawel Kos (University of Rzeszow, Rejtana 16, PL35-310 Rzeszow, Poland, pkos @univ.rzeszow.pl)

An active vibration control system is proposed for suppressing the small amplitude vibration of circular plate. An experimental set-up consists of a hard-walled cylinder with a thin metallic plate at one end. Primary excitation is provided by a low frequency loudspeaker installed centrally at the bottom of the cylinder. The vibrations of the plate are measured by the application of strain sensors and accelerometers. Intelligent materials such as 2-layer piezo disk elements are used as the actuators. For the considered system the OE (Output Error) method of discrete-time model identification for real-time active vibration control have been applied. The mathematical model obtained by this method identification is then employed for the linear pole placement controller design. Numerical simulations describing the at tenuation effects are presented and discussed. 
4pSAb3. Adaptive predictive feedback control of circular plate vibrations. Lucyna Leniowska (University of Rzeszow, Rejtana 16, PL35310 Rzeszow, Poland, lleniow@univ.rzeszow.pl), Pawel Kos (University of Rzeszow, Rejtana 16, PL35-310 Rzeszow, Poland, pkos@univ.rzeszow.pl)

Adaptive predictive controller, consists of an on-line identification technique coupled with a control scheme, is used in this paper for a plate vibration suppression. It is assumed, that the system to be regulated is unknown and the control schemes presented have the ability to identify and suppress the plate vibrations with only an initial estimate of the system order. The choice of structure is motivated by its representative nature. This configuration has also been well studied by the authors both analytically and experimentally, using several kind of controllers (PID, PI2D, fuzzy, LQR). There are two fundamental steps involved in the closed-loop system. The first step is to identify a mathematical model. The second step is to use the identified model to design a controller. One drawback of this approach is that, the control signal is fed to the actuator after updates of the control law expression, which always leads to some delay. In order to align better the updating process, the authors introduce the prediction of plant output with established error convergence. The one-step ahead system output prediction is calculated from the recursive formulas of the interpolation functions chosen. Simulations are included and discussed.
4pSAb4. Vibration control of flexible 3D robot arm with join and distributed actuators. Ryszard Leniowski (Rzeszow University of Technology, W.Pola 2, PL 35-902 Rzeszow, Poland, lery@prz-rzeszow.pl)

An active vibration control system is proposed for suppressing amplitude vibration of flexible 3D robot arm. This system integrates control algorithms, intelligent materials and software technologies. The mathematical model of physical system is based upon the geometry and properties of an experimental set-up consisting of a Flex3D robot with a flexible joints and flexible arm. The tip of the arm is loaded by eccentric mass. The vibrations of the plate are measured by the application of a grid of strain sensors and pair of coupled gyroscope-accelerometer. Two kinds of actuators are used. The first is a grid of PZT elements which form a local segments of compensators. Second is a standard BLDC motor located in the join. For the considered system the linear and non-linear (Neural Network of RungeKutta type models) of discrete-time model identification for real-time active vibration control have been applied. The mathematical model obtained by this method identification is then employed for the two class of controllers: linear pole placement controller for local segments compensators and nonlinear reduced model reference for servo-controller. Virtual simulations are included and discussed.

\title{
Session 4pSAc
}

\section{Structural Acoustics and Vibration, Computational Acoustics, and EURONOISE: Fluid-Structure Interaction III (Poster Session)}

\author{
Noureddine Atalla, Cochair \\ Univ. de Sherbrooke
}

Vicente Cutanda Henriquez, Cochair

Institute of Sensors, Signals and Electrotechnics, University of Southern Denmark

\author{
Stefan Schneider, Cochair \\ Laboratoire de Mécanique et d'Acoustique, UPR 7051 CNRS
}

\begin{abstract}
All posters will be on display from 3:40 p.m. to 5:20 p.m. To allow contributors an opportunity to see other posters, contributors of odd-numbered papers will be at their posters from 3:40 p.m. to 4:30 p.m. and contributors of even-numbered papers will be at their posters from 4:30 p.m. to 5:20 p.m.
\end{abstract}

\section{Contributed Papers}

4pSAc1. Modified amplitude and strouhal number scaling for correction of turbulent wall pressure fluctuations. Thomas Galib (NUWC, Howell St, Newport, RI 02841, USA, galibta@npt.nuwc.navy.mil)

Pressure fluctuations were measured in an external turbulent boundary layer over a buoyantly propelled axisymmetric body of revolution. Data were measured for three cases, resulting in axial length Reynolds numbers of $6.88 \times 10,4.27 \times 10$, and $3.21 \times 10$ at the measurement locations. The fresh water measurements were made in a fully developed turbulent boundary layer, following natural transition, with a near zero (very mildly adverse) pressure gradient. The salt water measurements were made in a favorable pressure gradient following a flow trip to force transition. The momentum thickness Reynolds number was greater than 4400 for all measurements, and the data were scaled with outer variables. The turbulence data were corrected using Corcos correction factors and then further scaled in both am- plitude and frequency by the square root of (transducer radius/displacement thickness), in an attempt to resolve spatial resolution effects. This resulted in excellent agreement among the spectra to a modified Strouhal number of 1 , which was the range of validity for the data. A second data set (constant freestream velocity and increasing displacement thickness with downstream measurement location), scaled somewhat better with the ratio of transducer radius to displacement thickness.

4pSAc2. The stability of nonequilibrium supersonic boundary layer. Igor Zavershinsky (Samara State Aerospace University, 34 Moskow Str., 443086 Samara, Russian Federation, zav@smr.ru)

The present work looks at the influence of molecular vibrational energy nonequilibrium on the instability of boundary layer. The boundary layer loses its stability at supercritical Reinolds numbers. At small Mach numbers 
$\mathrm{M}<2$ can be unstable only Tollmien-Schlichting waves, which phase velocity in relation of mean flow is subsonic. The recent studies were shown that in the acoustically active gas the critical Reynolds number for subsonic disturbances is decreased in comparison with equilibrium media [1,2]. At large Mach numbers $M>2$ in addition to Tollmien-Schlichting waves we must take into account both falling and reflecting acoustic waves (Mach waves). As a result the interaction between vortical structure and acoustic waves leads to shift of critical Reinolds number in comparison to subsonic case. Here the dependence of critical Reynolds number on thin flat plane from degree of nonequilibrium for spatial supersonic disturbances is founded. It was shown that acoustical activity of media has a large destabilizing influence. The increment of this instability is raised with growth of the degree of non-equilibrium. 1. Bertolotti F.P. J. Fluid Mech., 1998, V. 372. P. 93. 2. Knestyapin V.N., Kogan E.Ya., Zavershinsky I.P. High Temperatures. 2007. V.45. N2. P.1.

4pSAc3. Coupled wavenumbers of structural acoustic waveguides: A unified asymptotic approach. Abhijit Sarkar (Indian Institute of Science, FRITA Lab, Dept. of Mechanical Engg., Indian Institute of Science, 560012 Bangalore, India, asarkar@mecheng.iisc.ernet.in), Venkata R. Sonti (Indian Institute of Science, FRITA Lab, Dept. of Mechanical Engg., Indian Institute of Science, 560012 Bangalore, India, sonti@mecheng.iisc.ernet.in)

In the literature, the coupled wavenumbers in flexible-walled acoustic waveguides have been found mainly using numerical methods for a fixed set of parameters. These solutions, although useful, do not continuously track the coupled wavenumbers as the fluid-loading parameter is varied from small to large values. Such a continuous tracking is possible by applying the asymptotic methods to the coupled dispersion relation. Analytical formulae for the coupled wavenumbers can also be found. In this work, we present a consistent, unified and physically insightful view of structural acoustic coupling in the context of a planar structural-acoustic waveguide (with two different BCs), the axisymmetric and beam modes of a fluid-filled circular cylindrical shell and finally a fluid-filled elliptic cylindrical shell. In all the five cases, we use a single fluid-structure-coupling asymptotic parameter $(\epsilon)$. The regular perturbation method is used to solve the coupled dispersion relation for small and large values of $\epsilon$. The circular and the elliptic cylinders necessitate the use of additional asymptotic parameters. A general trend in all systems is that a given wavenumber branch transits from a rigid-walled solution to a pressure-release solution with increasing $\epsilon$. Also, the wavenumber curves veer where the earlier uncoupled wavenumbers intersected.

4pSAc4. Boundary element model to study sound transmission provided by a single panel in the presence of an elastic interlayer. Andreia Pereira (University of Coimbra, Department of Civil Engeneering Pólo II da Universidade - Rua Luís Reis Santos, 3030-788 Coimbra, Portugal, apereira@dec.uc.pt), António Tadeu (University of Coimbra, Department of Civil Engeneering - Pólo II da Universidade - Rua Luís Reis Santos, 3030-788 Coimbra, Portugal, tadeu@dec.uc.pt)

In this paper the Boundary Element Method is applied to develop a numerical model which may be used to study the airborne and impact sound insulation provided by a single panel of infinite extent bounded by two fluid media, with an elastic interlayer (joint) inserted into the partition. The elastic interlayer is placed in the panel, perpendicular to the its surfaces and fully occupying the panel thickness. When the interlayer assumes the properties of a resilient material, it is able to reduce the wave propagation through the elastic medium of the partition. The model is excited by a harmonic line load that acts either in the fluid medium or in the elastic medium in the direction perpendicular to the panel's surface. The model is developed following a direct frequency domain formulation which assumes full coupling between the fluid media and the elastic media. Analytical Green's functions for an elastic single layer bounded by fluid media are used to avoid having to discretize the horizontal surfaces of the partition. Numerical simulations are displayed in order to illustrate the applicability of the proposed model to the analysis of airborne and impact sound insulation when an elastic interlayer is inserted into a single partition.
4pSAc5. Numerical analysis of airborne and impact sound insulation between non-contiguous acoustic spaces using the Boundary Element Method. Andreia Pereira (University of Coimbra, Department of Civil Engeneering - Pólo II da Universidade - Rua Luís Reis Santos, 3030-788 Coimbra, Portugal, apereira@dec.uc.pt), António Tadeu (University of Coimbra, Department of Civil Engeneering - Pólo II da Universidade - Rua Luís Reis Santos, 3030-788 Coimbra, Portugal, tadeu@dec.uc.pt), Paulo Santos (University of Coimbra, Department of Civil Engeneering - Pólo II da Universidade - Rua Luís Reis Santos, 3030-788 Coimbra, Portugal, pfsantos@dec.uc.pt)

In this paper a Boundary Element Method (BEM) is used to predict the acoustic behaviour provided by four two-dimensional acoustic closed spaces separated by slabs and walls, surrounded by an elastic infinite medium. The walls and slabs are modelled as single partitions. The model is excited by cylindrical loads in the form of an airborne sound source placed in the acoustic space or an impact sound source acting on the slab, perpendicular to its surface. The formulation is developed following a direct BEM approach which assumes full coupling between the fluid medium and the elastic medium. The model requires the discretization of all interfaces and allows the analysis in the low and medium frequency range. A numerical analysis is performed to study airborne and impact sound insulation between acoustic non-contiguous spaces, where the sound pressure level which is established in the receiving room is due to flanking transmission. The influence of the structure's stiffness on the sound insulation is discussed for varying thicknesses of slabs and walls. The acoustic behaviour of the structure is described by sound insulation curves and average vibration velocity level curves of the walls and slabs and the results are discussed.

4pSAc6. Influence of temperature on sound transmission through viscoelastic sandwich plates. Samir Assaf (ESTACA, 34 rue Victor Hugo, 92300 Levallois Perret, France, sassaf@estaca.fr), Mohamed Guerich (ESILV, 92916 Paris la Défense, France, mohamed.guerich @ devinci.fr)

A numerical study to investigate the effects of temperature on the diffuse sound transmission loss (TL) of sandwich plates is presented. The numerical prediction tool used is based on a finite element formulation for the sandwich plate coupled to a boundary element method for the acoustic medium. The plate formulation is derived from Kirchhoff's theory for the elastic faces and Mindlin's theory for the core. The frequency-temperature dependence of the viscoelastic material properties are taken into account using an experimentally derived viscoelastic constitutive law. The results presented deal with a laminated glass subjected to a diffuse sound field. It is found that the dip of the TL curve at the coincidence frequency of the plate is totally removed for temperatures where this frequency is in the transition region of the used viscoelastic material. Indeed, the relative low value of the storage modulus and the high value of the loss factor in this region induce high transverse shear deformations of the viscoelastic interlayer and thus high energy losses.

4pSAc7. A transmission loss definition based on the root mean squared intensity. Ysbrand H. Wijnant (University of Twente, Dept. Mechanical Engineering, P.O. Box 217, 7500 AE Enschede, Netherlands, Y.H.Wijnant @ ctw.utwente.nl)

Numerical simulation enables the calculation of the sound power transmitted through any structure for any arbitrary source but it can not be used to determine transmission loss, which is based on the transmitted power divided by the incident power. One can calculate the active power (= incident minus reflected power), but one generally can not determine the incident and reflected power themselves. In fact, apart from 1 dimensional analysis, transmission loss is only defined for diffuse sound fields and it only quantifies the acoustical performance of the structure for this field and not for the actual, in-situ, source. While the incident power can not be determined, the active and root mean squared power can. The latter quantity is introduced and is a measure for the total energy flow per period, i.e. the net energy flow and the energy flowing back and forth. A transmission loss definition based on the ratio between the active power and the root mean squared power is 
shown to closely resemble the transmission loss in the 1 dimensional case Results for a 2 dimensional case show that the ratio is a quantity which does characterize the transmission of the structure as it is excited by the source.

4pSAc8. Fast solutions in FSI-Problems using CMS-Methods Johannes Guggenberger (Mueller-BBM， Robert-Koch-Str. 11， 82152 Planegg, Germany, johannes.guggenberger@muellerbbm.de)

Parameter studies in FSI-problems may often become quite time consuming. In most cases the fluid parameters are well defined and only the influence of the parameters of the solid is subject to investigate. Therefore it would be desirable to investigate the fluid and solid part separately and fi- nally combine them using CMS-Methods. This approach would also provide a good physical insight into the individual and combined behaviour of the components fluid and solid. Generally for each interface DOF one constraint mode must be added to the modal base. Since in most problems in FSI the fluid-structure interface involves many DOF the general CMS approach becomes inefficient. To reduce the number of constraint modes it is proposed to use the mode shapes of each component as a load function on the other domain. The static solution provides the modal based constraint modes. Their number corresponds to the number of total component modes which is in most cases much less then in the classic approach. The application is shown in optimization, updating, and monte-carlo-simulation problems.

\title{
Session 4pSCa
}

\section{Speech Communication: Articulatory Modeling and Control of Speech and Singing Organs}

\author{
Maureen Stone, Cochair \\ Vocal Tract Visualization Lab, Depts of Biomedical Sciences and Orthodontics, University of Maryland Dental School, 650 W. \\ Baltimore St., Baltimore, MD 21201, USA \\ Phil Hoole, Cochair \\ Institut für Phonetik und Sprachverarbeitung, Schellingstr. 3, München, 80799, Germany
}

\section{Invited Papers}

4pSCa1. Issues in the acoustic modeling of the vocal tract - a progress report on APEX. Björn Lindblom (Stockholm University, Universitetsvägen 10C, SE10691 Stockholm, Sweden, lindblom@ling.su.se), Johan Sundberg (KTH, Department of Speech, Music and Hearing, Lindstedtsvägen 24, SE-100 44 Stockholm, Sweden, jsu@csc.kth.se)

Faced with an acoustic record of the human voice an investigator is often led to wonder: How was this sample produced? Direct articulatory measurements lacking, the researcher may nonetheless find information of value by resorting to numerical models that relate cavity shapes to acoustic parameters. The classical 'three-parameter models' exemplify this type of tool. The APEX model is another example, developed to help answering questions about the acoustic consequences of articulatory movements. Based on X-ray measurements from a single speaker, APEX converts input specifications for articulatory parameters such as jaw, lips, larynx, tongue tip and tongue body into formant frequencies. The introduction of independent control of the jaw and the tongue and the fact that possible tongue shapes are specified relative to a neutral reference tongue have made significant insights into various topics possible (e.g., coordination jaw/tongue in singing, compensatory articulations). Recently we have been able to increase the physiological realism of APEX representations using MRI and X-ray data. We have also investigated physical models to improve the treatment of certain 3-D front cavity configurations such as raised tongue blade and spread lips. The goal of our paper is to present an overview of the most recent version of APEX.

$$
2: 20
$$

4pSCa2. Articulatory comparison of spoken and sung vowels based on MRI. Pierre Badin (Département Parole \& Cognition, GIPSA-lab, 46, avenue Félix Viallet, 38031 Grenoble Cedex, France, pierre.badin@ gipsa-lab.inpg.fr), Nathalie Henrich (Département Parole \& Cognition, GIPSA-lab, 46, avenue Félix Viallet, 38031 Grenoble Cedex, France, Nathalie.Henrich@ gipsa-lab.inpg.fr), Laurent Lamalle (Plateforme régionale IRM 3Tesla, IFR nº 1, RMN Biomédicale : de la cellule à l'homme, CHU de Grenoble, BP 217,38043 Grenoble Cedex 9, France, Laurent.Lamalle@ujf-grenoble.fr)

Understanding the differences of articulatory strategies between spoken and sung vowels is of interest to both speech and singing research. We have thus used MRI to record midsagittal images from three subjects producing sustained vowels with various characteristics. The subjects were a professional lyric soprano, a semi-professional soprano, and a semi professional bass. They were instructed to produce combinations of (1) the ten French or the five Italian oral vowels, (2) speaking, amateur singing, or professional singing modes, (3) chest or falsetto registers, (4) pitch levels varying from B2 (120 Hz) to F5 (700 Hz). Any combination that the subject would not feel comfortable with was excluded from the corpus. The midsagittal contours of the vocal organs (jaw, lips, tongue, velum, pharyngeal wall, hyoid bone, etc.) were manually traced on each image, and a number of articulatory measurements (jaw or hyoid bone height, lip aperture, tongue position, etc.) were automatically derived. Our contribution analyses the influence of the various production conditions on these articulatory characteristics, such as the jaw aperture increase related to pitch increase, or the lower position of the larynx for singing in comparison to speech. Some acoustics considerations will be discussed as well. 
4pSCa3. Resonance characteristics of the hypopharyngeal cavities. Kiyoshi Honda (Phonetics \& Phonology Laboratories, UMR7018-CNRS \& University of Paris III, 19, rue des Bernardins, 75005 Paris, France, honda@atr.jp), Tatsuya Kitamura (Konan University, Okamoto 8-9-1, Higashinada, 658-8501 Kobe, Japan, t-kitamu@konan-u.ac.jp), Hironori Takemoto (ATR Cognitive Information Science Laboratories, 2-2-2 Hikaridai, Seika-cho Soraku-gun, 619-0288 Kyoto, Japan, takemoto@atr.jp), Parham Mokhtari (ATR Cognitive Information Science Laboratories, 2-2-2 Hikaridai, Seika-cho Soraku-gun, 619-0288 Kyoto, Japan, parham@atr.jp), Seiji Adachi (Fraunhofer Institute for Building Physics, Nobelstrasse 12, 70569 Stuttgart, Germany, seiji.adachi@ibp.fraunhofer.de)

The hypopharyngeal cavities are the narrow, complex parts of the lower vocal tract that include the supraglottal laryngeal cavity and bilateral cavities of the piriform fossa. These small regions exhibit rather strong acoustic influence on vowel spectra in the higher frequencies and contribute to determining voice quality and speaker characteristics. The laryngeal cavity functions nearly as a Helmholtz resonator to generate an extra formant in the vicinity of $3 \mathrm{kHz}$, and the piriform fossa forms a pair of side-branches of the vocal tract to cause spectral zeros in the vicinity of $4-5 \mathrm{kHz}$. Vocal-tract models without employing these acoustic effects can hardly simulate natural-sounding voices of a particular speaker. Therefore, realistic acoustic models of vowel production must include the three functional components: glottal source sounds, hypopharyngeal-cavity coupling, and resonance of the vocal-tract proper. This presentation demonstrates the results from acoustic experiments on solid vocal-tract models and computer simulations of the cavities' effects based on our MRI-based visualization of the vocal tract. A possible control for singing voice qualities will be discussed based on the threecomponent model.

\section{3:00}

4pSCa4. Observation of voice registers. Ken-Ichi Sakakibara (Health Sciences University of Hokkaido, 2jo-5chome, Kita-ku, 0028072 Sapporo, Japan, quesokis@gmail.com), Hiroshi Imagawa (Department of Otolaryngology, University of Tokyo, 7-3-1, Hongo, Bunkyo-ku, 113-8655 Tokyo, Japan, imagawa@m.u-tokyo.ac.jp), Miwako Kimura (Department of Otolaryngology, University of Tokyo, 7-3-1, Hongo, Bunkyo-ku, 113-8655 Tokyo, Japan, mkimu-tky@umin.ac.jp), Isao Tokuda (Japan Advanced Institute of Science and Technology, 1-1 Asahidai, Nomi-shi, 923-1292 Ishikawa, Japan, isao@jaist.ac.jp), Takaharu Nito (Department of Otolaryngology, University of Tokyo, 7-3-1, Hongo, Bunkyo-ku, 113-8655 Tokyo, Japan, taka.nito@nifty.com), Niro Tayama (International Medical Center of Japan, 1-21-1, Toyama, Shinjuku-ku, 162-8655 Tokyo, Japan, ntayama@imcj.hosp.go.jp)

In singing, voice register is one of the most salient aspects of voice quality, and it has therefore generated a lot of debates, acoustically, physiologically, and pedagogically. In singing, the voice registers can be physiologically classified into four categories: vocal fry, modal, falsetto, and whistle. In this study, vocal fold vibratory patterns appeared in each register were observed using high-speed images and simulated using the two-mass model. In vocal fry, three different vibratory patterns (aperiodic, subharmonic, and periodic with small open quotient) were observed. In addition, the simulation showed that transitions between the three different vibratory patterns are easy. In whistle, closure of the posterior part of glottis and rapid vibration of the anterior part were observed.

4pSCa5. Voice production modes and vocal tract shape in South-Siberian throat-singing. Sven Grawunder (Max Planck Institute for Evolutionary Anthropology, Deutscher Platz 6, 04103 Leipzig, Germany, grawunder@eva.mpg.de)

South-Siberian throat-singing features reinforced harmonics as carrier of sung melodies and enforced phonation modes. Available articulatory studies of throat-singers suggest that throat-singing makes use of three voice production mechanisms which result in two basic voice modes (mid tensed vs. low rough). Thus all three mechanisms share an excessive constriction of the larynx entrance i.e. approximation of the aryepiglottic folds and the epiglottis. The current study comprises acoustic data from 69 male singers. 25 singer were recorded by use of a field setting for acoustic, electroglottographic and subglottal resonance signal acquisition. Perturbation measures show dominance of individual variability over areal (cultural) factors, but strong influence of articulatory reinforcement strategies. The data also provide evidence for a model of reinforcement of harmonics by means of (1) voice source variation (closing phase, excitation strength), i.e. increased subglottal pressure, while air flow remains constant or lowered for the tensed mode; and double cycle modes involving mass bodies of upper laryngeal structures for the low mode; (2) formant merging due to multiple vocal tract constrictions including a coupling of source to the adjacent epilaryngeal tube of 1/6 vocal-tract length and bandwidth tuning as a result of adjustment of lip radiation. 
Session $4 \mathrm{pSCb}$

\title{
Speech Communication: General Topics in Speech Communication III (Poster Session)
}

\author{
Christine Shadle, Cochair \\ Haskins Laboratories \\ Sharon Coffey-Corina, Cochair \\ Center for Mind and Brain UC Davis \\ Bart De Boer, Cochair \\ Amsterdam, Netherlands
}

\begin{abstract}
All posters will be on display from 3:40 p.m. to 5:20 p.m. To allow contributors an opportunity to see other posters, contributors of odd-numbered papers will be at their posters from 3:40 p.m. to 4:30 p.m. and contributors of even-numbered papers will be at their posters from 4:30 p.m. to 5:20 p.m.
\end{abstract}

\section{Contributed Papers}

4pSCb1. Feature extraction for vowel recognition using mellin transform. Mahdi Jamaati (Technical University of Shahrood, 12345 Shahrood, Iran, mahdi.jamaati@gmail.com), Milad Lankarany (Technical University of Shahrood, 12345 Shahrood, Iran, milad.lankarany@gmail .com), Hossein Marvi (Technical University of Shahrood, 12345 Shahrood, Iran, h_marvi@shahroodut.ac.ir)

This paper proposed a new feature extraction algorithm for vowel recognition using Mellin transform and MFCC. The scale transform is a particular restriction of the Mellin transform. The key property of the scale transform is the scale invariance. This algorithm consists of 3 main stages which are as follow. First stage contains, Extraction spectral envelope from vowels, by using cepstrum method. In the second stage, Mellin transform is used for mapping same vowels with different pitch and different vocal tract to the same waveforms. In third stage, we used a new MFCC algorithm in order to extract main parameters from the output of second stage. The new MFCC algorithm contains third and fourth order cumulant of log-melamplitudes. This new MFCC coefficients are robust in presence of additive white noise. Moreover, The LPC method has been used instead of MFCC method in third stage, Experimental result indicates that the new MFCC algorithm produced better result than LPC and original MFCC.

4pSCb2. Empirical articulatory-acoustic relations for vowels. Richard S. McGowan (CReSS LLC, 1 Seaborn Place, Lexington, MA 02420, USA, rsmcgowan@earthlink.net), Michael A. Berger (Dept. of Linguistics, Univ. of Rochester, 503 Lattimore Hall, Rochester, NY 14627-0096, USA, m.a .berger@sms.ed.ac.uk)

Vowels tokens were extracted from four talkers in the Wisconsin X-ray Microbeam Speech Production Database. The neighboring phonemes of these vowels were restricted to be non-nasal and non-liquid. The first three formant frequencies were measured using LPC analysis with manual corrections at a rate corresponding to the pellet trajectory sampling rate, thus yielding large amounts of simultaneous formant frequency and pellet position data points (between 11,000 and 20,000 for each talker.) Principal components analysis was performed for both the formant frequencies and the pellet position data, to produce three orthogonal acoustic components and four orthogonal articulatory components. A local linear regression technique, known as loess [Cleveland, W. S. and Devlin, S. J. (1988), J. Amer. Stat. Assoc., 83, 596 - 610], was applied to orthogonal components to map between the acoustic and articulatory domains. This technique permits regression slopes to vary within the domain of the independent variables. The results will be discussed in terms of optimization of loess parameters (e.g. size of local neighborhoods), goodness of fit of the mappings, and the degree to which slopes in the mappings vary. Manually corrected formant frequencies will be compared with fully automatic Line Spectral Frequencies. [Supported by NIDCD-001247 to CReSS LLC]

4pSCb3. Cross-language study of age perception by elderly listeners. Kyoko Nagao (Center for Pediatric Auditory and Speech Sciences, Nemours Biomedical Reserach, A.I. du Pont Hospital for Children, 1600 Rockland Road, Wilmington, DE 19803, USA, nagao@asel.udel.edu), Amanda K. Riley (Indiana University, Department of Speech and Hearing Sciences, 200 S Jordan Avenue, Bloomington, IN 47405, USA, mandieriley@gmail .com)

Recent studies show that perception of speaker's age is influenced by a listener's familiarity with the speaker's language. However, these results were based on young adult listeners. The current study examines whether language familiarity influences the perception of speaker's age in elderly listeners. The vowel stimuli was prepared from the sustained vowel /i/ collected from 30 native speakers of American English and 30 native speaker of Japanese. The sentence stimuli was taken from the reading of the North Wind and the Sun by these 60 speakers (in their native language). Fifteen elderly native speakers of English (mean age 73.5 years, range from 67 to 84 years) listened to both stimuli types and estimated the age of speakers. Correlation between perceived age and chronological age was moderate for the sentence stimuli, but weak for the vowel stimuli. Correlation between perceived age and chronological age was stronger when the listeners judged sentence stimuli in their native language than when they judged sentence stimuli in the foreign language $(r=0.74$ versus $r=0.63)$. The vowel stimuli did not show the effect of speaker language. The results suggest that linguistic information has a critical role in age perception, regardless of listener's age.

4pSCb4. Acoustic tubes with maximal and minimal resonance frequencies. Bart De Boer (Spuistraat 210, 1012VT Amsterdam, Netherlands, b.g.deboer@uva.nl)

This paper presents a theoretical derivation of acoustic tract shapes that minimize and maximize resonance frequencies. The derivation is based on a symmetry of Webster's horn equation and on Ehrenfest's adiabatic invariance hypothesis. It is shown that for minimizing formant frequencies, abrupt transitions are necessary, while for maximizing resonance frequencies, gradual transitions are needed. It is argued that this has implications for modeling human, animal and prehistoric vocal tracts. Such models should 
represent the anatomic (in)ability to produce abrupt and gradual transitions correctly, otherwise they would have biases towards different sets of formant frequencies than real vocal tracts.

4pSCb5. Rhythmic characteristics of prose and verse in varieties of Portuguese. Zinny Bond (Ohio University, 18 Maplewood Drive, Athens, OH 45701, USA, bond@ohio.edu), Verna Stockmal (Ohio University, 384 Gordy Hall, Athens, OH 45701, USA, grannymeem@hotmail.com), Emilia A. Marks (Ohio University, Department of Modern Languages, Athens, $\mathrm{OH}$ 45701, USA, markse@ohio.edu), Audra Woods (Ohio University, Speech and hearing sciences, Athens, OH 45701, USA, aw365402@ohio.edu)

Whether varieties of Portuguese differ in their rhythmic classification is not entirely clear. European Portuguese is generally considered to employ stress-based rhythm whereas the rhythmic classification of Brazilian Portuguese is disputed. Acoustically-based measures of rhythm have usually employed spoken prose passages for language samples. Possibly, the apparent rhythmic characteristics of language varieties may be clarified by employing different types of language materials. We selected spoken prose and traditional verse for investigation. Five native speakers of European Portuguese and five native speakers of Brazilian Portuguese recorded a sonnet consisting of 154 syllables and a short prose passage consisting of 270 syllables. From these recordings, acoustically based rhythm metrics, as suggested by Ramus, et al. [Cognition 73, 1999, 265-292] and Ling, et al. [Language and Speech 43, 2000, 377-401] were calculated. Measures of consonantal duration variability increased from spoken prose to spoken verse, suggesting that the talkers employed slower, more clearly articulated speech in reading the poem than in reading the prose passage. However, the two tasks did not clearly distinguish between the two varieties of Portuguese.

4pSCb6. Experimental study of turbulent flow sound production in presence of a simplified vocal tract constriction. Olivier Estienne (Département Parole \& Cognition, GIPSA-lab, 46, avenue Félix Viallet, $38031 \quad$ Grenoble Cedex, France, olivier.estienne @ gipsa-lab.inpg.fr), Annemie Van Hirtum (Département Parole \& Cognition, GIPSA-lab, 46, avenue Félix Viallet, 38031 Grenoble Cedex, France, annemie.vanhirtum@gipsa-lab.inpg.fr), Xavier Pelorson (Département Parole \& Cognition, GIPSA-lab, 46, avenue Félix Viallet, 38031 Grenoble Cedex, France, pelorson@icp.inpg.fr), Helène Bailliet (Laboratoire d'Etudes Aerodynamiques - CNRS, Bat K, 40 avenue du recteur Pineau, 86022 Poitiers, France, helene.bailliet@lea.univ-poitiers.fr)

Sound production due to turbulence is widely shown to be an important phenomenon involved in a.o. fricatives, singing, whispering and speech pathologies. In spite of it relevance, only a few recent models are dealing with turbulence consequences during voiced sound production. The current study presents preliminary results of an experimental characterisation of the aeroacoustical effects of a turbulent flow in case of a constricted channel flow, by means of measuring the velocity fluctuations and the acoustic field downstream of the constriction. Aiming a future application in speech production, the influence of typical vocal tract shape parameters on the velocity distribution and sound field is explored: the tube shape and length as well as the degree, geometry and position of the constriction. The influence of the Reynolds number of the upstream flow is also observed. Results are discussed with respect to the upper airways and human sound production and will be exploited with respect to simplified models for velocity fluctuations and sound production.

4pSCb7. Influences of perceived racial identity on human talker identification. Tyler K. Perrachione (Massachusetts Institute of Technology, 77 Massachusetts Avenue, Cambridge, MA 02139, USA, tkp@mit.edu), Joan Y. Chiao (Northwestern University, 2029 Sheridan Road, Evanston, IL 60208, USA, jchiao@northwestern.edu), Patrick C. Wong (Northwestern University, 2240 Campus Drive, Evanston, IL 60208, USA,pwong@northwestern.edu)

Talker identification, the process by which human listeners recognize individuals by their voice, is one of the most poorly understood abilities of the human auditory system. Current psychological models of talker identification rely on strict analogies to face perception and the visual system, despite differences in the objects of perception in these two modalities. Here we investigate the existence of an own-race bias in voice perception - a phenomenon which has been pivotal in the study of face perception. Our results demonstrate an own-race bias in talker identification: listeners of different ethnic backgrounds show an advantage for identifying individual voices of the same race as themselves. However, unlike in vision, the own-race bias in talker identification manifests based specifically on the perceived, but not actual, race of a talker. The influence of perceived race suggests physical (voice structural) cues do not give rise to this effect. Instead, the own-race bias in talker identification is a result of listeners' asymmetric exposure to talkers' socially-acquired manners of expression (i.e. the dynamic features of voice and speech). Such manners of expression may be stereotypically associated with a particular ethnic group, although not actually exhibited by all members of that group. [Work supported by NIH]

4pSCb8. Signal densities and criterion variance in speech and nonspeech perception. Luis E. Lopez-Bascuas (Universidad Complutense Madrid, Facultad Psicologia, Campus Somosaguas, 28223 Madrid, Spain, lelopezb@psi.ucm.es)

The actual shape of signal densities has become an important issue when studying speech perception within the framework of Signal Detection Theory (SDT). Using an SDT model that allowed unequal criterion variances, López-Bascuas [Proc. Eurosp. 3, 2281-2283 (1995)] found that speech signals did not accommodate to the standard Gaussian assumption. However, Schouten and van Hessen [J. Acoust. Soc. Am. 104, 2980-2990 (1998)] measured response distributions directly and, assuming an interval scale, concluded that the Gaussian assumption held for both continua. Nevertheless, Pastore and Macmillan [J. Acoust. Soc. Am. 111, 2432 (2002)] applied ROC analysis to Schouten and van Hessen's data and their curves supported the Gaussian assumption for the nonspeech signals only. Later, López-Bascuas et al. [J. Acoust. Soc. Am. 115, 2465 (2004)] showed that non-linear z-transformed ROCs are not sufficient evidence for postulating non-gaussian signal densities. In this paper we try to figure out whether unequal criterion variances could underlie non-linear z-transformed ROCs by fitting a restricted Thurstonian SDT-like model to a nonspeech continuum composed by white noise and a square wave. The results indicate that unequal criterion variances is the possible cause of the deviant ROCs for nonspeech.

4pSCb9. Perception of reduced speech: Approximated stops. Natasha Warner (University of Arizona, MPI NIjmegen, Box 210028, Dept. of Linguistics, Tucson, AZ 85721-0028, USA, nwarner@u.arizona.edu), Tucker V. Benjamin (University of Alberta, Dept. of Linguistics, 4-32 Assiniboia Hall, Edmonton, AB T6G 2E7, Canada, bvtucker@ualberta.ca), Amy Fountain (University of Arizona, MPI NIjmegen, Box 210028, Dept. of Linguistics, Tucson, AZ 85721-0028, USA, avf@u.arizona.edu)

Natural, spontaneous speech often shows extreme reductions of many speech segments, to the point of apparent deletion. Where the flap allophone of $/ \mathrm{t} /$ and $/ \mathrm{d} /$ is expected in American English, one frequently sees an approximant-like or even vocalic pattern, rather than a clear flap. Still, such tokens are usually perceived as containing a /t/ or /d/ (e.g. 'needle' even with a very reduced /d/ is usually not perceived as 'kneel'). The current work identifies acoustic characteristics of reduced 'flaps' and presents phonetic identification data for continua that manipulate these characteristics. Presence vs. absence of a dip in intensity, duration of that dip, and degree of that dip in decibels are manipulated. Degree of intensity dip has the strongest effect, with a minimal dip in intensity more likely to be perceived as 'kneel' and a strong dip in intensity more likely to be perceived as 'needle.' The results indicate that all three of these characteristics do affect listeners' percept of a consonant, but not sufficiently to completely account for the percept. Listeners are sensitive to the acoustic characteristics of consonant reduction, but they are also very skilled at evaluating variability along the acoustic dimensions that realize reduction. 
4pSCb10. Amplitude modulation shape and speech intelligibility Garreth Prendergast (The University of York, Heslington, YO10 5DD York, UK, garreth.prendergast@ynic.york.ac.uk), Sam R. Johnson (The University of York, Heslington, YO10 5DD York, UK, sam@ynic .york.ac.uk), Gary G. Green (The University of York, Heslington, YO10 5DD York, UK, gary.green@ynic.york.ac.uk)

Research has demonstrated that low frequency amplitude modulations in speech signals are crucially important to maintaining intelligibility. The current work demonstrates a flexible way of characterising the pulsatile bursts of energy found in the temporal envelopes of sub-band filtered speech. Speech was passed through a 128-filter Gammatone filterbank and the temporal envelope of each filter extracted using the Hilbert transform. Thirtyfive raised cosine pulses were fitted to model the envelope of each filter and each pulse was defined by its amplitude, half-duration and centre position. The distribution of these pulses demonstrates that the most commonly found pulse half-duration in speech is around $10 \mathrm{~ms}$ and few pulses have halfdurations longer than $25 \mathrm{~ms}$. Highly intelligible vocoded speech is generated using the extracted pulses and these measures suggest that the auditory system may signal the position in time of the amplitude modulations rather than representing low-frequency information. This method creates a flexible framework within which to further probe the mechanisms involved and allows the ability to focus on cross-channel information in the time domain.

4pSCb11. Training English vowels for French speakers with varying English experience. Paul Iverson (University College London, Department of Phonetics and Linguistics, 4, Stephenson Way, NW1 2HE London, UK, p.iverson@ucl.ac.uk), Melanie Preece-Pinet (University College London, Department of Phonetics and Linguistics, 4, Stephenson Way, NW1 2HE London, UK, m.pinet@ucl.ac.uk)

It is clear that high-variability phonetic training can improve speech perception for adult second-language learners, but it is uncertain what levels of processing are responsible for this change and whether this interacts with the previous experience of the learners. The present study investigated these issues by giving auditory training to French speakers with varying degrees of English experience. Listeners completed 8 sessions of high-variability identification training with clusters of minimal-pair words (e.g., sleet, slit, slight, slate) and a different speaker in each session. The pre/post tests evaluated changes in vowel perception using open-set identification in quiet and noise, a 3-interval category discrimination task, and a task in which individuals mapped their best exemplars for English vowels in a space that included formant movement and duration. The preliminary results suggest that French speakers improved substantially in their identification performance (about 20 percentage points), without having large changes in their ability to discriminate these categories. The results support a view that training improves the process of applying phonetic category knowledge, without changing the underlying categories themselves.

4pSCb12. Computerized assessment and training of the perception of American English (AE) speech sounds by adult learners of English. James Miller (Communication Disorders Technology, Inc., Indiana Univeristy Research Park, 501 N. Morton Street Sta 215, Bloomington, IN 47404, USA, jamdmill@indiana.edu), Roy Sillings (Communication Disorders Technology, Inc., Indiana Univeristy Research Park, 501 N. Morton Street Sta 215, Bloomington, IN 47404, USA, rsillings@comdistec.com), Charles S. Watson (Communication Disorders Technology, Inc., Indiana Univeristy Research Park, 501 N. Morton Street Sta 215, Bloomington, IN 47404, USA, watson@indiana.edu), Isabelle Darcy (Indiana University, Dept. of Second Language Studies, Memorial Hall 315, 1021 E. Third Street, Bloomington, IN 47405, USA, idarcy@indiana.edu), Kathleen Bardovi-Harlig (Indiana University, Dept. of Second Language Studies, Memorial Hall 315, 1021 E. Third Street, Bloomington, IN 47405, USA, bardovi@indiana.edu)

Fourteen volunteer students, with first languages other than English and enrolled in English pronunciation classes, used a specialized software program, the "Speech Perception Assessment and Training System (SPATS)." The software was made available in a language laboratory for seven weeks. Students used the program between 3 and 19 hours, the mean being 13 hours. The goal of SPATS is to train the perception of English words as they are produced in naturally spoken, fluent sentences. The average student correctly identified 1723 words in 313 different sentences as spoken by 10 different talkers and also attempted to identify a total of 7530 presentations of syllable onsets, nuclei, or codas as spoken by 8 different talkers in several phonetic contexts. These syllable constituents were presented in quiet, the sentences in moderate amounts of 12-talker babble. All showed significant progress in perception of $\mathrm{AE}$ speech sounds, a few approaching the levels of native speakers. By extrapolation, with 20 to 30 hours of program use nearly all might approach the perceptual performance of native speakers. Those that returned post-training questionnaires viewed the program favorably and recommended its use in regular classes. Speech samples were collected. Accent ratings and spectrographic analyses will be presented.

4pSCb13. Integrated magnetic resonance imaging methods for speech science and technology. Shinobu Masaki (ATR Cognitive Information Science Laboratories, 2-2-2 Hikaridai, Seika-cho Soraku-gun, 619-0288 Kyoto, Japan, masaki@atr.jp), Yukiko Nota (National Institute of Information and Communications Technology/ATR Cognitive Information Science Laboratories, 2-2-2 Hikaridai, Seika-cho, 619-0288 Kyoto, Japan, ynota@atr.jp), Sayoko Takano (ATR Cognitive Information Science Laboratories, 2-2-2 Hikaridai, Seika-cho Soraku-gun, 619-0288 Kyoto, Japan, tsayoko@atr.jp), Hironori Takemoto (ATR Cognitive Information Science Laboratories, 2-2-2 Hikaridai, Seika-cho Soraku-gun, 619-0288 Kyoto, Japan, takemoto@atr.jp), Tatsuya Kitamura (Konan University, Okamoto 8-9-1, Higashinada, 658-8501 Kobe, Japan, t-kitamu@konan-u.ac .jp), Kiyoshi Honda (Phonetics \& Phonology Laboratories, UMR-7018CNRS \& University of Paris III, 19, rue des Bernardins, 75005 Paris, France, honda@atr.jp)

This presentation introduces our integration of magnetic resonance imaging (MRI) techniques at ATR Brain Activity Imaging Center (Kyoto, Japan) toward research into speech science and technology. The first breakthrough in our application of MRI to speech research was the motion imaging of the speech organs in articulation using a cardiac cine-MRI method. It enables us to acquire information in the time-space domain to reconstruct successive image frames using utterance repetitions synchronized with MRI scans. This cine-technique was further improved for high-quality imaging and expanded into three-dimensional (3D) visualization of articulatory movements. Using this technique, we could successfully obtain temporal changes of vocal-tract area function during a Japanese five-vowel sequence. This effort also contributed to developing other techniques to overcome the limitations of MRI, such as the post-hoc inclusion of teeth images in 3D volumes or the phonation-synchronized scan for crystal-sharp static imaging. Further, a custom high-sensitivity coil was developed to visualize the fine structures of the lip muscles and laryngeal airway. The potentials of new MRI approaches such as ultra-high-resolution imaging with a higherfield scanner or real-time motion imaging during a single utterance will be discussed toward future contributions to speech science and technology.

4pSCb14. Acoustic characteristics of solid vocal tracts modeled from ATR MRI database of Japanese vowel production. Tatsuya Kitamura (Konan University, Okamoto 8-9-1, Higashinada, 658-8501 Kobe, Japan, t-kitamu@konan-u.ac.jp), Hironori Takemoto (ATR Cognitive Information Science Laboratories, 2-2-2 Hikaridai, Seika-cho Sorakugun, 619-0288 Kyoto, Japan, takemoto@atr.jp), Kiyoshi Honda (Phonetics \& Phonology Laboratories, UMR-7018-CNRS \& University of Paris III, 19, rue des Bernardins, 75005 Paris, France, honda@atr.jp)

"ATR MRI database of Japanese vowel production" provides volumetric magnetic resonance images and speech data of the five Japanese vowels produced by a male native Japanese speaker. In this study, the database was used to evaluate acoustic characteristics of vocal tracts for five Japanese vowels; we measured frequency responses of realistic vocal tract solid models formed by a stereo-lithographic technique. The model's glottis was sealed with a plastic plane with a 1.2-mm hole. A time-stretched pulse signal generated from a horn driver unit was introduced into the solid model at the lip end. The response signals of the models were recorded at the model's glottis from the hole by a probe microphone. This method permits accurate measurement of acoustic characteristics of the vocal tract including the laryngeal cavity, which generates the laryngeal cavity resonance during closed-glottis periods of phonation [H. Takemoto et al., JASA, 120, 2228- 
2238 (2006), T. Kitamura et al., JASA, 120, 2239-2249 (2006)]. The results provide a benchmark for evaluating numerical analysis methods, such as transmission line models, finite element methods, and finite difference-time domain-methods, which have been used to study vocal tract acoustics. [Work supported by SCOPE (071705001) of Ministry of Internal Affairs and Communications, Japan.]

4pSCb15. Linguistic versus non-linguistic processing of speech prosody in dichotic listening. Ritsu Kanamura (Graduate School of Comprehensive Scientific Research, Prefectural Univ. of Hiroshima at Mihara, 1-1 Gakuen-machi, 723-0053 Mihara, Japan, bulle.de.savon.von.von@gmail .com), Satoshi Imaizumi (The Prefectural Univ. of Hiroshima, 1-1 Gakuenmachi, 723-0053 Mihara, Japan, imaizumi@pu-hiroshima.ac.jp)

Linguistic and non-linguistic information processing of speech prosody are studied using two dichotic listening tasks, a Word task and a F0 task During the Word task, subjects were required to identify either right- or leftear stimulus from two-syllable homophonic words presented with different pitch accents. During the F0 task, subjects were required to identify either right- or left-ear stimulus from F0 partials extracted from the words used in the Word task. The correct percent of responses was high and RT was short for high familiarity words presented to the right ear rather than the others under the Word task, while no such differences were found under the F0 task. RT of the Word task was shorter than the F0 task. These results suggest that the processing of linguistic speech prosody under dichotic listening conditions is based on the interactive auditory and linguistic neural resources with right- ear or left-hemispheric dominance, and are faster than auditory F0 pattern identification. The tasks developed can be used to detect central auditory processing disorders.

4pSCb16. Broader range of training voices improves performance of HMM model of phonemic identification. J Parchment (University of Arizona, Dept. of Linguistics, Douglass Building, Room 200E, Tucson, AZ 85721, USA,jparch@u.arizona.edu)

A model proposed by Lin (2005) learns phonetic categories from waveform input. Recorded speech from a set of male talkers is divided into training and test sets. The training set is separated into phonemes, subjected to cepstral analysis, and used as the input to a Hidden Markov Model, which clusters the phonemes into phonemic categories. After this unsupervised learning process, the model is then able to accurately identify speech segments in the test set, showing that relevant acoustic information is captured by the model. The current study explores the outcome when a model of this type is trained on a range of talkers differing in sex and vocal tract configuration. Preliminary results suggest that this approach can improve performance when testing is generalized to a wider range of new talkers. However, too wide a range of training voices reduces accurate categorization, while too narrow a range reduces generalizability. Continuing efforts seek to quantify the optimum range of training voices and to identify the variables that can predict the degree of improvement in performance on test voices. This work has implications for automatic speech recognition models as well as for issues of speaker normalization.

4pSCb17. A Method of Co-registering Multiple Magnetic Resonance Imaged Vocal Tract Volumes for Fricatives. Michael I. Proctor (Haskins Laboratories, 300 George St, New Haven, CT 06511, USA, proctor@haskins.yale.edu), Christine Shadle (Haskins Laboratories, 300 George St, New Haven, CT 06511, USA, shadle@haskins.yale.edu), Khalil Iskarous (Haskins Laboratories, 300 George St, New Haven, CT 06511, USA, iskarous@haskins.yale.edu)

In a study of fricative production, Magnetic Resonance Imaging was used to acquire three-dimensional tract geometries of fricatives produced in four different vowel contexts. Short scan sequences were used to image multiple productions of each fricative in a single session. Although minimizing the number of tokens, this resulted in a coarser spatial resolution in some parts of the tract, which required a different approach to assembling vocal tract volumes. Sagittal, axial and oblique-coronal imaging orientations were chosen to best resolve the tract in the mid-sagittal plane, the pharynx, and around the fricative constriction. Each fricative token was acquired using a 37 second imaging sequence. Dental impressions were imaged separately.
Three corresponding point clouds were created by sampling air-tissue boundaries in each image stack, and aligned using anatomical landmarks on the face and spinal column, creating a single, multiply-sampled volume. Area functions were estimated using the most suitable data source at each region of the tract. Vocal tract models were constructed for eight fricatives produced in four vowel contexts by four speakers of American English. The advantages of this method of tract modeling, and its automatic application to potentially variable MRI data sources will be discussed. [Funded by NIHNIDCD-RO1-DC006705]

4pSCb18. Acoustical analysis of Canadian and Parisian French word-final vowel productions in varying phonetic contexts. Franzo Law (CUNY Graduate Center, 365 5th Avenue, Program in SpeechLanguage-Hearing Sciences, New York, NY 10016, USA, flaw@gc.cuny.edu), Winifred Strange (CUNY Graduate Center, 365 5th Avenue, Program in Speech-Language-Hearing Sciences, New York, NY 10016, USA, strangepin@aol.com)

Dialects can differ greatly in their phonetics and phonology, and can diverge to a great degree in vowel production. This study explores the acoustic nature of Canadian French $(\mathrm{CF})$ vowels in word-final position, relative to those of Standard Parisian French (PF). CF and PF male participants were recorded producing minimal sets of words, differing in the final vowels /i y e "epsilon" a "o-bar" o u/, embedded in carrier phrases. Real-word minimal sets were constructed using words with final vowels preceded by labial, coronal, and back stops and fricatives. (Monosyllabic CV words were used whenever possible; VCV and CCV words were used when no CV word was available.) Nonsense disyllabic minimal sets were also recorded with vowels preceded by labial, coronal, and back stops in the following context: /gisCV/. Of particular interest was the stability of the /e - "epsilon"/ distinction in word-final position for Parisian French, which was maintained in preliminary results. Across-syllable boundary coarticulatory effects are also discussed. This is the first part of a larger study investigating secondlanguage lexical and morphosyntactic $\mathrm{CF}$ vowel perception by English learners of French. [Work supported by NIH F31DC008075]

4pSCb19. An MRI Study of the Effect of Vowel Context on English Fricatives. Christine Shadle (Haskins Laboratories, 300 George St, New Haven, CT 06511, USA, shadle@haskins.yale.edu), Michael I. Proctor (Haskins Laboratories, 300 George St, New Haven, CT 06511 , USA, proctor@haskins.yale.edu), Khalil Iskarous (Haskins Laboratories, 300 George St, New Haven, CT 06511, USA, iskarous@haskins.yale.edu)

To gain a better understanding of the long-observed effects of vocalic context, the articulation of fricatives was investigated using Magnetic Resonance Imaging. Five speakers of American English were imaged while producing eight fricatives in the contexts [i-a-u-schwa]. Sagittal, axial and oblique-coronal volumes were acquired for each vowel-fricative combination. Acoustic recordings were made during scans and separately in an anechoic chamber. Vocal tract models were generated by aligning and superimposing all three stack orientations. The models reveal that a variety of articulatory strategies are employed in the production of English fricatives, and that vocalic context is significant. For some subjects, tongue shape differs little with vowel context; other subjects show highly varied tongue shape differences but little difference in lip rounding. Two subjects show significant variation with vowel context for every fricative, including $[\theta]$; two show very little difference, even for [f]. The sublingual cavity in [sh] is extensive for two subjects, but only slightly bigger than in [s] for two other subjects. Tongue grooving in and behind the constriction also varies, and is especially evident in the oblique scans. Overall, more variation and effect of vowel context was observed than expected from the literature. [Funded by NIH-NIDCD-RO1-DC006705]

4pSCb20. F0 contour estimation based on time-varying complex speech analysis. Keiichi Funaki (Univ. of the Ryukyus, Senbaru 1, Nishihara, 903-0213 Okinawa, Japan, funaki@cc.u-ryukyu.ac.jp)

Robust F0 (Fundamental frequency) estimation plays an important role in speech processing such as speech coding and tonal speech recognition. We have already proposed robust F0 estimation algorithm based on timevarying complex AR (TV-CAR) speech analysis for analytic signal, in 
which the weighted autocorrelation function is calculated using the complex residual and then the F0 is searched as the peak sample for each frame [IEICE Trans. on Fundamentals, Vol.E91-A.No.3] [IEICE Trans. on Fundamentals, Vol.E90-A, No.8]. Although the algorithm can estimate more robust F0 estimation for IRS filtered speech corrupted by additive noise, the algorithm cannot perform better for non-IRS filtered speech or slightly contaminated IRS-filtered speech. In addition, the frame-based F0 estimation cannot extract the F0 trajectories in the time-domain. In order to cope with the drawbacks, this paper proposes quite simple F0 contour estimation algorithm based on the TV-CAR speech analysis, in which the F0 contour is estimated by peak-picking for the estimated time-varying spectrum that is the same manner as formant frequency estimation. The experimental results demonstrate that the proposed method leads to more accurate continuous F0 estimation than the conventional one for high-pitched speech due to the nature of analytic signal for non-IRS filtered high-pitched female speech.

4pSCb21. Extraction of vocal tract area function from three-dimensional magnetic resonance images using digital waveguide mesh. Kenji Inoue (Osaka Institute of Technology, 4-18-15, Tanabe, Higashisumiyosi-ku, 546-0031 Osaka, Japan, chikuwa.bushi@gmail .com), Hironori Takemoto (ATR Cognitive Information Science Laboratories, 2-2-2 Hikaridai, Seika-cho Soraku-gun, 619-0288 Kyoto, Japan, takemoto@atr.jp), Tatsuya Kitamura (Konan University, Okamoto 8-9-1, Higashinada, 658-8501 Kobe, Japan, t-kitamu@konan-u.ac.jp), Shinobu Masaki (ATR Cognitive Information Science Laboratories, 2-2-2 Hikaridai, Seika-cho Soraku-gun, 619-0288 Kyoto, Japan, masaki@atr.jp), Hirotake Nakashima (Osaka Institute of Technology, 1-79-1, Kitayama, Hirakata, 573-0196 Osaka-fu, Japan, nakas@is.oit.ac.jp)

A method is proposed in this paper to extract the vocal tract area function from the three-dimensional magnetic resonance images. The proposed method uses the digital waveguide mesh, an implementation of the finitedifference time-domain (FDTD) method, to simulate wave propagation in the vocal tract from the glottis to the lips. The dimensions of the vocal tract areas are then calculated along the traveling wavefront that emerges from the simulation. Formant analysis has been conducted for Japanese vowels to show the validity of the proposed method. The calculated formant frequencies of the area functions obtained by the proposed method and other existing methods have been compared to those measured from the acoustic utterance of the imaged person.

4pSCb22. Increasing speech alignment through crossmodal speaker familiarity. Rachel M. Miller (University of California, Riverside, Department of Psychology, 900 University Ave., Riverside, CA 92521, USA, rmill002@ucr.edu), Kauyumari Sanchez (University of California, Riverside, Department of Psychology, 900 University Ave., Riverside, CA 92521, USA, ksanc004@student.ucr.edu), Lawrence D. Rosenblum (University of California, Riverside, Department of Psychology, 900 University Ave., Riverside, CA 92521, USA, rosenblu@ citrus.ucr.edu)

In speech alignment phenomena, individuals inadvertently imitate aspects of another talker's utterances. Recent research has shown that when asked to shadow words, subjects not only align to the speech they hear, they also align to the speech they see when shadowing words by lipreading [Miller, et al., J. Acoust. Soc. Am., 120, 5, Pt. 2 (2006)]. This research also showed that some of the dimensions to which subjects align are the same whether based on shadowing of auditory or visual speech stimuli. This might mean that subjects can align to a speaker's idiolectic dimensions available in both modalities. To examine this possibility, an experiment was conducted to see if alignment increased with exposure to the same or a different speaker, across two blocks of presentations that were: a) both auditory; b) both visual; or c) one auditory and one visual. If subjects align to amodal, idiolectic speaker style, then alignment should be comparable across presentation types in the same speaker condition. Results revealed that alignment increased when the speaker was the same over the course of the two blocks regardless of presentation type. These results suggest that alignment can be based on amodal, idiolectic dimensions which are available across modalities.

4pSCb23. Perception of Japanese consonants by native speakers of American English. Takeshi Nozawa (Ritsumeikan University, College of Economics, 1-1-1 Nojihigashi, Kusatsu, 525-8577 Shiga, Japan, t-nozawa @ec.ritsumei.ac.jp)

Four native speakers of Japanese produced Japanese multi-syllable words and non-words, which include stops, fricatives, affricates, nasals and the liquid. Twelve native speakers of American English, who had never learned Japanese, heard their utterances and spelled out what they heard in English alphabet. What was of interest was whether native speakers of American English would perceive Japanese consonants in a way they are transcribed in English alphabet. The results revealed that Japanese stops were equated with generally the phonetically closest English stops, but voiceless stops were more likely to be equated with voiced stops of the same place of articulation than the other way around. Among voiced stops, /b/ and /d/ respectively were equated with $/ \mathrm{v} /$ and /1/. The word-initial /ts/, though words like "tsunami" have become part of English, was predominantly equated with /s/ rather than /ts/. The Japanese liquid, which is usually transcribed as /r/, was predominantly equated with /1/ rather than /r/. This agrees with results of previous research that demonstrate that English $/ \mathrm{r} /$ is more dissimilar from Japanese /r/ than English /1/ (See Aoyama et al. 2004).

4pSCb24. Synchronous speech and speech rate. Miran Kim (Suny, Dept. Linguistics, S201, SBS building, Stony Brook, NY 11794-4376, USA, mrkim@ic.sunysb.edu), Hosung Nam (Haskins Laboratories, 300 George St., Suite 900, New Haven, CT 06511, USA, nam@haskins.yale.edu)

Synchronously read speech has shown to reduce a high degree of speaker variability of reading exhibited by speakers in laboratory recording; e.g., pause placement and duration, and speech rate. However, quantitative analysis of speech rate has rarely been found in studies on synchronous speech. This study examines Mandarin Chinese (2 dialects from Taiwan and Shanghai), which is a syllable-time language and thus expected to exhibit a relatively stable speech rate, in both read-alone and read-together speech. Consistency and variability of speech rate are compared in both reading types across repetitions within a subject, across subjects, and across dialects. The results show that speech rate is more stable in read-together than in read-alone speech, and that speech rate in synchronous reading falls on a constant value of speech rate rather than on the average between the speakers' rates in pair. This global pattern is consistent across dialects, and stylized local variation of speech rate over prosodic units (Intonational phrase) is also observed unique to each dialect. We discuss how timing in synchronous speech cannot be accounted for only by dynamic entrainment of speakers with different speech rates and how there should also be rhythmic information shared by speakers of a language.

4pSCb25. Effects of auditory, visual, and audio-visual training on nonnative perception of English fricatives. Yue Wang (Simon Fraser Univ., RCB 9224, 8888 Univ. Dr., Burnaby, BC V5A 1S6, Canada, yuew@sfu.ca), Dawn Behne (Norwegian University of Science and Technology, Psychology Dept, NO 7491 Trondheim, Norway, dawn.behne@svt.ntnu.no), Angela Cooper (Simon Fraser Univ., RCB 9224, 8888 Univ. Dr., Burnaby, BC V5A 1S6, Canada, akcooper@sfu.ca), Haisheng Jiang (Simon Fraser Univ., RCB 9224, 8888 Univ. Dr., Burnaby, BC V5A 1S6, Canada, hdjiang@sfu.ca), Nina Leung (Simon Fraser Univ., RCB 9224, 8888 Univ. Dr., Burnaby, BC V5A 1S6, Canada, nhleung@alumni.sfu.ca), Jung-Yueh Tu (Simon Fraser Univ., RCB 9224, 8888 Univ. Dr., Burnaby, BC V5A 1S6, Canada, jta31@sfu.ca)

This study examines the effects of auditory (A), visual (V), and audiovisual (AV) training on nonnative speech perception. Mandarin Chinese natives were trained to perceive English voiceless fricatives (in monosyllabic words and nonwords) of three visually distinct places of articulation: interdentals nonexistent in Mandarin, labiodentals and alveolars common in both languages. Participants were randomly assigned to a control group or one of three 2-week (six sessions, 40 minutes/session) training groups with a dif- 
ferent input modality: A, V, or AV. In pre- and post-tests, the fricatives are presented in four ways for an identification task: A-only, V-only, AV congruent $(\mathrm{AVc})$, and $\mathrm{AV}$ incongruent $(\mathrm{AVi})$. Additionally, three generalization posttests are administered testing voiced fricatives, new real words, and a new speaker. Results show that post-training, the trainees reveal: (1) improvements corresponding to training type (e.g., the V-training group improves most for the V-only stimuli), (2) greater improvements for the familiar (but less visually distinct) alveolars than for the new interdentals, (3) decreased AV-fusion for the AVi stimuli, and (4) consistent patterns in the generalization tests. Results are discussed in terms of the effects of speech input modality, experience, and L1 on L2 AV speech learning. [Research supported by SSHRC]

4pSCb26. Comparison of the brain regions for consonant processing in Japanese and English subjects. Yoshikazu Oya (Faculty of Systems Engineering, Wakayama University, 930 Sakaedani, 640-8510 Wakayama, Japan, s085067@sys.wakayama-u.ac.jp), Toshio Irino (Faculty of Systems Engineering, Wakayama University, 930 Sakaedani, 640-8510 Wakayama, Japan, irino@sys.wakayama-u.ac.jp), Alexis G. Hervais-Adelman (Centre for the Neural Basis of Hearing, Department of Physiology, Development and Neuroscience, University of Cambridge, Downing Site, CB23EG Cambridge, UK, alexis.hervais-adelman@mrc-cbu.cam.ac.uk), David T. Ives (Centre for the Neural Basis of Hearing, Department of Physiology, Development and Neuroscience, University of Cambridge, Downing Site, CB23EG Cambridge, UK, dti20@cam.ac.uk), Hideki Kawahara (Faculty of Systems Engineering, Wakayama University, 930 Sakaedani, 640-8510 Wakayama, Japan, kawahara@sys.wakayama-u.ac.jp), Roy D Patterson (Centre for the Neural Basis of Hearing, Department of Physiology, Development and Neuroscience, University of Cambridge, Downing Site, CB23EG Cambridge, UK, rdp1@ cam.ac.uk)

A recent fMRI study on speech-sound processing by Uppenkamp et al. [Neuroimage, 31(3), 1284-1296 (2006)] revealed that regions of the left and right, superior temporal gyri (STG) and anterior, superior temporal sulci (STS) respond preferentially to speech-like stimuli. Hervais-Adelman et al. [BSA London (2007)] extended this research to investigate the processing of consonant-vowel (CV) and vowel-consonant (VC) syllables and determine the locus of consonant-processing in the brain of English speakers. This paper reports an experiment with the same stimuli but with Japanese subjects for whom VC syllables are a novelty. In both the English and Japanese subjects, there was enhanced activity in left STS for vowels over non-speech sounds, as in Uppenkamp et al. (2006). A significant difference was observed between the responses to CV and VC syllables in left STS and planum temporale (PT). There was almost no CV-VC difference in the English subjects. The activity regions for VC syllables were larger than for CV syllables in Japanese subjects, probably because the Japanese subjects would have heard the VCs as two syllable speech sounds. Research supported by JSPS Grant-in-Aid [B18300060] and the UK-MRC [G0500221, G9900369].

4pSCb27. Influence of music education on second language acquisition. Barbara Pastuszek-Lipinska (Adam Mickiewicz University, al .Niepodleglosci 4, 61-874 Poznan, Poland, energin@wp.pl)

To explore the extent to which music education influences secondlanguage acquisition, two groups of native Polish speakers, musicians and non-musicians, were asked to reproduce sentences in six languages: English, French, Italian, Spanish, Japanese, and Belgian Dutch. The speech stimuli were developed with a text-to-speech application and differed phonemically, phonostylistically, and in length. The paper includes results of a general auditory analysis of subjects' productions as well as the results of a web-based listening test with a panel of native speakers of the involved languages. All collected data were also analyzed with statistical tools. The results revealed that music education exerted a measurable impact on speech perception and production. Musicians outperformed non-musicians in the study. From the results, it appears that the influence of musical expertise extends beyond music processing to speech processing, and the strength of this influence is connected not only to auditory training. Therefore, the superior performance of the musicians in the task may be interpreted as evidence that music edu- cation is an enabling factor in the successful acquisition of a second language. It also indicates that the impact of musical training is not a myth, but has a scientific basis.

4pSCb28. The relation between articulatory and aerodynamic properties of single $/ \mathbf{t} /, / \mathrm{S} /$ and their combination in $/ \mathbf{t S} /$ and $/ \mathbf{S t} /$ Susanne Fuchs (ZAS, Schuetzenstr. 18, 10117 Berlin, Germany, fuchs@zas.gwz-berlin.de), Laura L. Koenig (Haskins Labs. and Long Island Univ., Brooklyn, New York, NY 11201-8423, USA, koenig@haskins .yale.edu)

Intraoral pressure changes during speech production are a result of different factors, such as articulatory movements (place and manner of articulation), subglottal pressure, and laryngeal-oral coordination. This study attempts to provide a better understanding of the relation between aerodynamics and articulation in single voiceless consonants and their combinations. We gathered electropalatographic data simultaneously with intraoral pressure data for 9 native speakers of German. During voiceless aspirated stop production intraoral pressure equalizes with subglottal pressure since an oral closure is formed and the glottis is open. During voiceless fricative production intraoral pressure is lower than in stop production since the air can escape through the oral constriction. In /t, S, St/ the intraoral pressure changes are highly correlated with the articulatory behaviour. However, the affricate /tS/ shows an unusual relationship with aerodynamics and articulation. Although an oral closure is formed, pressure rises at a later point and reaches its peak during the fricative. We interpret these findings with respect to a special laryngeal - oral coordination in affricates as reported in Hoole et al. (2003) who found a relatively late glottal opening during the stop portion of the affricate.

4pSCb29. Experimental study of the fluid-structure-acoustic interaction in a human voice model. Stefan Kniesburges (University Erlangen-Nuremberg, Institute of Fluid Mechanics, Cauerst. 4, 91058 Erlangen, Germany, stefan.kniesburges@1stm.uni-erlangen.de), Stefan Becker (University Erlangen-Nuremberg, Institute of Fluid Mechanics, Cauerst. 4, 91058 Erlangen, Germany, stefan.becker@1stm.uni-erlangen .de), Stefan Mueller (University Erlangen-Nuremberg, Institute of Fluid Mechanics, Cauerst. 4, 91058 Erlangen, Germany, stefan.mueller@1stm .uni-erlangen.de), Antonio Delgado (University Erlangen-Nuremberg, Institute of Fluid Mechanics, Cauerst. 4, 91058 Erlangen, Germany, adelgado@1stm.uni-erlangen.de), Gerhard Link (Univ. ErlangenNuremberg, Dept. of Sensor Technology, Paul-Gordan-Str. 3/5, 91052 Erlangen, Germany, gerhard.link@1se.eei.uni-erlangen.de), Manfred Kaltenbacher (Univ. Erlangen-Nuremberg, Dept. of Sensor Technology, Paul-Gordan-Str. 3/5, 91052 Erlangen, Germany, manfred.kaltenbacher @1se.eei.uni-erlangen.de), Michael Doellinger (University Hospital Erlangen, Department of Phoniatrics and Pediatric Audiology, Medical School, Bohlenplatz 21, 91054 Erlangen, Germany, michael.doellinger@ukerlangen.de)

A fluid-structure-coupled in-vitro model was developed for the investigation of the physical processes of the human phonation. The setup enables to simulate self-sustained vocal fold oscillation and to analyse the resulting supraglottal flow field. Measurement techniques like high-speed flow visualization, Particle Image Velocimetry (PIV) of the time-dependent flow field, unsteady pressure measurement, vibration measurement by a LaserScanning-Vibrometer were applied. The acoustic field was simultaneously recorded. Analysis was performed regarding correlations between the acoustic field, the flow velocity and the displacement of the vocal folds. The results support the existence of the Coanda-effect during phonation as assumed in previous work. The flow attaches to one vocal fold just past the glottis and forms a spacious vortex behind the vocal folds. This behaviour is not linked to one vocal fold and changes stochastically from cycle to cycle. The analysis indicates, that the acoustic sound is primarily produced by the pressure fluctuation caused by the pulsating flow rate due to the opening and closing process of the vocal folds. We assume, that the structural sound and the turbulence-induced sound do have minor acoustical implication. 
4pSCb30. FEM simulation of tongue deformation for $/ i /$ with a four-cube model applied to tagged cine-MRI data. Sayoko Takano (ATR Cognitive Information Science Laboratories, 2-2-2 Hikaridai, Seika-cho Soraku-gun, 619-0288 Kyoto, Japan, tsayoko@atr.jp), Hiroki Matsuzaki (Hokkai-Gakuen University, 1-1, Minami-26, Nishi-11, Chuouku, 064-0926 Sapporo, Japan, matsu@eli.hokkai-s-u.ac.jp), Kunitoshi Motoki (Hokkai-Gakuen University, 1-1, Minami-26, Nishi-11, Chuou-ku, 064-0926 Sapporo, Japan, motoki@eli.hokkai-s-u.ac.jp)

Roles of extrinsic and intrinsic tongue muscles in the production of vowel /i/ were examined using a finite element method (FEM) applied to the tagged cine-MRI data. It has been thought that tongue tissue deformation for $/ \mathrm{i} /$ is mainly due to the combined actions of the genioglossus muscle bundles advancing the tongue root to elevate the dorsum with a mid-line grooving. A recent study with the tagging-MRI revealed earlier, faster and greater tissue deformation at anterior top of the tongue than posterior part during /ei/ production. This result implies the contribution of the intrinsic tongue muscle (transverse anterior) with an independent hydrostat mechanism from that of the genioglossus muscle bundles. In this study, a simple four-cube model is built to examine the co-contraction effect of the genioglossus and transverse muscles using using the FEM. The simulation result with the anterior transverse muscle ( $\mathrm{Ta}$ ) showed good agreement with the pattern of the tongue deformation obtained from the tagged-MRI data, suggesting that transverse anterior also plays an important role for the realization of the tongue shape for the production of vowel $/ \mathrm{i} /$.

4pSCb31. Classification of normal and pathological vocal fold vibrations using Phonvibrograms. Jörg Lohscheller (University Hospital Erlangen, Department of Phoniatrics and Pediatric Audiology, Medical School, Bohlenplatz 21, 91054 Erlangen, Germany, joerg.lohscheller@uk-erlangen.de), Daniel Voigt (Dept. Phoniatrics and Pediatric Audiology, Bohlenplatz 21, 91054 Erlangen, Germany, daniel.voigt@uk-erlangen.de), Michael Doellinger (University Hospital Erlangen, Department of Phoniatrics and Pediatric Audiology, Medical School, Bohlenplatz 21, 91054 Erlangen, Germany, michael.doellinger@ukerlangen.de)

Clinical examination of voice disorders demands an endoscopical observation of vocal fold vibrations. High-speed endoscopy is the state-of-the-art technology for investigation of vocal fold vibrations. A novel visualization strategy is proposed which transforms the segmented contours of vocal fold edges into a set of two dimensional images, denoted Phonovibrograms (PVG). Within PVGs the individual type of vocal fold vibration becomes uniquely characterized by specific geometric patterns which can be seen as fingerprints of vocal fold vibration. The PVGs give an intuitive access on the type and degree of the laryngeal asymmetry which is essential to quantify the effects of functional and organic voice disorders. To determine the vibration characteristics within the computed PVG pattern recognition algorithms are applied. Thus, for each vocal fold the vibration type can be quantified and classified. The results of the PVG classification will be presented in 80 subjects (normal and pathological voices). It will be shown, that a classification of the vibration type can be performed very precisely even in disturbed vocal fold vibrations. The obtained PVG images can be documented and stored on a hard-disc using a lossless image data-format. The quantitative description of PVG patterns has the potential to realize a novel classification of vocal fold vibrations.

4pSCb32. Glottal-opening and airflow pattern during production of voiceless fricatives: a new non-invasive instrumentation. Kiyoshi Honda (Phonetics \& Phonology Laboratories, UMR-7018-CNRS \& University of Paris III, 19, rue des Bernardins, 75005 Paris, France, honda@atr.jp), Shinji Maeda (CNRS \& ENST, 46, rue Barrault, 75634 Paris, France, maeda@tsi.enst.fr)

In the production of voiceless fricatives, the airflow passing through the vocal tract is controlled by reciprocal open-close patterns of the glottal and oral constriction. In order to observe such coordinated patterns, the authors have developed a combined method using a non-invasive (external-lighting and sensing) photoglottographic (ePGG) technique and a pressure-difference airflow mask. The former technique has the advantage of no restriction of phonetic environments (improvement from the standard PGG) and can be further combined with instrumentation of intraoral air pressure and/or of articulatory movements. Our PGG-airflow data are examined to address a question: It has been known that the glottis opens always wider for wordinitial fricatives than for word-medial ones despite no obvious difference in acoustic and physiological requirements. It will be discussed whether the degrees of glottal opening during a fricative co-vary with other aerodynamic and articulatory controls in CVCV utterances with $\mathrm{V}=$ nonclose vowels.

4pSCb33. Mucosal wave analysis of a human vocal fold in the hemilarynx experiment. Michael Doellinger (University Hospital Erlangen, Department of Phoniatrics and Pediatric Audiology, Medical School, Bohlenplatz 21, 91054 Erlangen, Germany, michael.doellinger @ uk-erlangen.de), David A. Berry (The Laryngeal Dynamics Laboratory, Division of Head \& Neck Surgery, David Geffen School of Medicine at UCLA, 31-24 Rehab Center, 1000 Veteran Ave., Los Angeles, 90095-1794, USA, daberry@ucla.edu), Jörg Lohscheller (University Hospital Erlangen, Department of Phoniatrics and Pediatric Audiology, Medical School, Bohlenplatz 21, 91054 Erlangen, Germany, joerg.lohscheller@uk-erlangen de)

The mucosal wave propagation was investigated in laboratory experiments across a variety of phonatory conditions. The focus was on the medial and superior surface dynamics of the vocal fold, which quantify mucosal wave propagation, but have been relatively little studied. High-speed, digital imaging of the entire surface of the vocal fold was performed using an excised human hemilarynx setup. Surface dynamics were characterized and differentiated across a variety of phonatory conditions. During sustained, flow-induced oscillation, the local maxima of vocal fold mucosal displacements, velocities and acceleration and their particular phase delays in the glottal cycle were investigated. Statistical analysis was performed, examining the influence of applied stimulations. Increasing the airflow yielded higher values for lateral displacements as well as higher velocity/acceleration values. Elongating the vocal fold resulted in decreased lateral displacements. The mucosal wave propagation apparently increased for higher flow, elongated folds, and higher adduction forces. While an understanding of the correlation between vocal fold dynamics and phonatory physiology/pathology is still in its infancy, the data presented here help to establish such connections. The data are also useful for the development and evaluation of physical and numerical models of vocal fold vibration.

4pSCb34. Categorical and non-categorical perception of speech: Behavioural and neural evidence. Jack C. Rogers (MRC Cognition and Brain Sciences Unit, 15 Chaucer Road, CB2 7EF Cambridge, UK, jack.rogers@mrc-cbu.cam.ac.uk), Matthew H. Davis (MRC CBU, 15 Chaucer Rd., CB2 7EF Cambridge, UK, matt.davis@mrc-cbu.cam.ac.uk)

What effects do within- and between-category variations have on the perception of speech? Using audio-morphing and the "Straight" channel vocoder (Kawahara, 2004), we produced 320 high-quality phonetic continua varying in place, manner and voicing including word/word (blade/glade), word/pseudo (blouse/glouse), pseudo/word (bown/gown) and pseudo/pseudo (blem/glem) pairs. A 2AFC task confirmed the category boundary shift for word/pseudo and pseudo/word pairs (Ganong, 1980), equivalent for onset (bench/gench) and offset (flad/flag) pairs. This suggests that lexical influences on categorical perception are not produced on-line but rather occur post-perceptually, consistent with top-down effects. Sensitivity to within- and between-category phonological variation was investigated using sparse fMRI in a paired auditory repetition priming paradigm. Minimal pairs (48 across the 4 stimulus groups) were presented to participants who listened in the context of a semantic monitoring task. Between-category pairs with a phonological change produced a greater neural response compared to within-category same pairs with the same magnitude of acoustic difference. This response to phonologically different pairs provides a neural correlate of categorical perception in left middle temporal, inferior frontal and pre-central regions. These responses in inferior frontal regions may contribute towards top-down influences on categorical perception of speech (cf Ganong Effect). 
4pSCb35. Distinguishing Place of Consonant Articulation using the Aurora System. Yana Yunusova (University of Toronto, 160-500 University Ave, Toronto, ON M5G 1V7, Canada, yana.yunusova@utoronto .ca), Jeff Stanley (Northern Digital Inc, 103 Randall Drive, Waterloo, ON N2V 1C5, Canada, jstanley@ndigital.com), Jordan R. Green (University of Nebraska, 3180 Barkley Center, Lincoln, NE 68583-0738, USA, jgreen4 @unl.edu)

The goal of this project is to determine the effect of bite-block and speaking rate manipulation on lingual targets for consonants using a recently developed Aurora, a 3-D electromagnetic movement tracking system (NDI). Bite-block and speaking rate manipulations are common intervention techniques with speakers with dysarthria and apraxia of speech. Therefore, understanding their effect on consonant target regions is essential for predicting the outcomes of articulatory training in these clinical populations. In this project, articulatory positions of two sensors on the tongue tip and dorsum will be recorded independent of the head. Stops $/ \mathrm{t}, \mathrm{k} /$ and fricatives $/ \mathrm{s}, \mathrm{sh} /$ will be embedded in aCa syllables. A bite block condition will be used to eliminate jaw contribution to tongue movements. The location and size of articulatory regions associated with each consonant will be compared in bite block and no-block conditions. Additionally, the rate effect on the location and size of the consonant articulatory regions will be examined. Potential clinical implication of the finding on articulatory intervention will be considered.

4pSCb36. Discrimination of Mandarin tone 1 vs. tone 4 in disyllables by adult speakers of English. Shari Berkowitz (CUNY Graduate Center, Speech Acoustics and Perception Lab, 365 Fifth Avenue, New York, NY 10016, USA, shariellen@nyc.rr.com)

Previous research on cross-language perception of lexical tone has mainly used monosyllabic stimuli; however disyllables may be more difficult for non-natives to discriminate due to coarticulation and context effects (Berkowitz \& Strange, 2007). Preliminary work suggests that there is an effect of context on discrimination of tone 1 vs. 4 , despite the fact that this is usually considered an easy contrast when tested with monosyllabic stimuli. Disyllabic Mandarin nonsense words served as stimuli in a categorial same/different task. Pairings of tone 1 and tone 4 in initial and final position were tested in the context of all four tones. American English listeners with no background in tone languages completed the experiment without feedback. Data was scored with A' and was analyzed for effect of tone, initial vs. final position in the disyllable, and height and contour of the fundamental frequency. The results of this paradigm will be used to design a study of tone perception in preschool groups who speak Mandarin as their L1, English as their L1, and internationally adopted children who were previously exposed to Mandarin.

4pSCb37. Articulatory features influencing regressive place assimilation across word-boundaries in German. Marion Jaeger (Institut für Phonetik und Sprachverarbeitung, Schellingstr. 3, 80799 München, Germany, jaeger@phonetik.uni-muenchen.de), Phil Hoole (Institut für Phonetik und Sprachverarbeitung, Schellingstr. 3, 80799 München, Germany, hoole@phonetik.uni-muenchen.de)

Within current phonological theories the greater tendency of $\mathrm{C} 1$ nasals vs. C1 plosives to undergo regressive place assimilation is often treated as the consequence of acoustic-perceptual properties of nasality (e.g. Steriade, 2001). Little is known about the articulatory patterns underlying this asymmetry. Our current EMA study aims to test and compare the effects of manner of articulation of C1 (alveolar nasal vs. alveolar plosive), place of articulation of C2 (labial vs. dorsal plosive), vowel context (palatal/i/ vs. non-palatal vowel /a/), and word frequency upon the intra- and intergestural timing and movement magnitude of various articulators in $\mathrm{C} 1 \mathrm{C} 2$ sequences across word-boundaries in German subjects. Our analyses of non-palatal vowel contexts in three speakers showed a greater likelihood of reduction of the tongue tip both in words with a nasal $\mathrm{C} 1$ and in high frequency words. For those word pairs in which tongue tip displacement was measurable, tongue tip - tongue back overlap was significantly greater in word pairs with a nasal $\mathrm{C} 1$ and in word pairs with high frequency words. On the other hand, tongue tip - lower lip overlap was only significantly greater in word pairs with high frequency words.

4pSCb38. Time-frequency detection of stridence in fricatives and affricates. Slobodan Jovicic (School of Electrical Engineering, University of Belgrade, Bulevar kralja Aleksandra 73, 11000 Belgrade, Serby, jovicic@etf.bg.ac.yu), Silvana Punisic (Institute for Experimental Phonetics and Speech Pathology, Gospodar Jovanova 35, 11000 Belgrade, Serby, iefpgmir@eunet.yu), Zoran Saric (Institute for Experimental Phonetics and Speech Pathology, Gospodar Jovanova 35, 11000 Belgrade, Serby, sariczoran@yahoo.com)

As wheezes in abnormal breath sounds observed in patients with obstructive pulmonary diseases, the stridence in voice is manifested as excessively sharp, conspicuous, usually habitual hiss that is especially distinct with whispering. This paper reviews the articulator and acoustics features of stridence in unvoiced fricatives and affricates, and presents an algorithm for detection of stridence. Detection of stridence was based on: time-frequency representation by FFT power spectra, time-frequency representation by ARBurg power spectra, and power trajectory of signals in characteristic frequency bands. Many features are extracted from this analysis, as: the local power spectra maximum to average surrounding power ratio, the correlation coefficient between spectral and power maxima in signal, the spectral power slope in selected frequency band, spectral entropy in selected frequency band, and phoneme duration. The extracted set of features is input to the nonlinear classificator that decides about stridence in voice and the level of pathology. The algorithm was tested with speech database of normal and pathology voices. The speakers were both sex and the different ages. The results of automatic stridence detection showed high level of coincidence with the judgment of speech therapists.

4pSCb39. Adaptive microphone array free of the desired speaker cancellation combined with postfilter. Slobodan Jovicic (School of Electrical Engineering, University of Belgrade, Bulevar kralja Aleksandra 73, 11000 Belgrade, Serby, jovicic@etf.bg.ac.yu), Zoran Saric (Institute for Experimental Phonetics and Speech Pathology, Gospodar Jovanova 35, 11000 Belgrade, Serby, sariczoran@yahoo.com)

The optimal microphone array includes two processing blocks - minimum variance distortionless response (MVDR) beamformer and the singlechannel Wiener filter, which acts as post-filter. The main drawback of MVDR beamformer is the cancellation of the desired speech signal and its degradation in multi-path wave propagation environment. To make the adaptive algorithm robust against room reverberation and to prevent desired signal cancellation, an estimation of the unknown desired speaker's transfer function was proposed. The estimation is based on the imperfect signal and the interference covariance matrices estimated from available microphone signals during speaker activity and pause of speech respectively. As MVDR beamformer suppresses coherent interference, post-filter has to reduce diffuse acoustic noise. The post-filter proposed in this paper is developed under assumption that complex coherence function is unknown but time invariant. The additional improvement of the post-processing algorithm on low frequencies is obtained by combining a priori noise power attenuation factor for diffuse noise field with estimated one. The proposed algorithm is tested on simulated room with reverberation, and compared with some known post-processing algorithms with rather good results.

4pSCb40. The role of source and filter cues in emotion recognition in speech. Disa Sauter (Birkbeck College London, Henry Wellcome Building, Malet Street, WC1E 7HX London, UK, d.sauter@bbk.ac.uk), Frank Eisner (University College London, Institute of Cognitive Neuroscience, 17 Queen Square, WC1N 3AR London, UK, f.eisner@ucl.ac.uk), Stuart Rosen (Department of Phonetics and Linguistics, University College London, Gower Street, WC1E 6BT London, UK, s.rosen@ucl.ac.uk), Sophie K. Scott (University College London, Institute of Cognitive Neuroscience, 17 Queen Square, WC1N 3AR London, UK, sophie.scott@ucl.ac.uk)

In the context of the source-filter theory of speech, it is well established that intelligibility is heavily reliant on information carried by the filter, that is, spectral cues (e.g., Faulkner et al., 2001; Shannon et al., 1995). However, 
the extraction of other types of information in the speech signal, such as emotion and identity, is less well understood. In this study we investigated the extent to which emotion recognition in speech depends on filterdependent cues, using a forced-choice emotion identification task at ten levels of noise-vocoding ranging between one and 32 channels. In addition, participants performed a speech intelligibility task with the same stimuli. Our results indicate that compared to speech intelligibility, emotion recognition relies less on spectral information and more on cues typically signaled by source variations, such as voice pitch, voice quality, and intensity. We suggest that, while the reliance on spectral dynamics is likely a unique aspect of human speech, greater phylogenetic continuity across species may be found in the communication of affect in vocalizations.

4pSCb41. Model of the configurations of intonation movements in Danish - suggesting an 'intoneme'. Sophia Frovin (SLK, English Department, University of Aarhus, Jens Chr. Skous Vej 5, 8000 Aarhus C, Denmark, sophiafrovin@yahoo.com)

This study examines the inventory of intonation movements in Danish. The aim is to identify the micro intonation and to produce a model of the movement patterns in Danish, which would predict the intonation of synthetic speech in order to establish a close-to-natural intonation. The theoretical background is a modified version of the "close copy theory" ('t Hart et al.), in which movements are tendency lines in the intonation pattern. A new movement starts when the tendency line shifts direction. The acoustic analysis comprises measurements of time, semitones and slope (semitones/second = slope) and a description of the (parts of) phones in intonation movements of actual speech. The analysis suggests that the slope of the movement is one of the most important features of intonation, and that speakers are aiming to produce one of a set of 5 standard slopes, depending mainly on the phonetic segment, suggesting an underlying unit - an "intoneme"- displaying the same relationship as that of phoneme and phone.

4pSCb42. Influences of manner and voicing on articulatory coordination in German and French initial consonant clusters. Barbara Kuehnert (Laboratoire de Phonétique et Phonologie - UMR 7018 CNRS Paris 3, 19, rue des Bernadins, 75005 Paris, France, barbara.kuhnert @ univ-paris3.fr), Phil Hoole (Institut für Phonetik und Sprachverarbeitung, Schellingstr. 3, 80799 München, Germany, hoole@phonetik.uni-muenchen .de), Christine Mooshammer (Haskins lab and MIT Research lab of electronics, 300 George street suite 900, New Haven, CT 06511, USA, tine@haskins.yale.edu), Lasse Bombien (Institut für Phonetik und Sprachverarbeitung, Schellingstr. 3, 80799 München, Germany, lasse@phonetik .uni-muenchen.de)

This study aims for improved understanding of whether and how coordination patterns of supraglottal gestures in complex syllable onsets are driven by competing demands of motor economy for the speaker and high recoverability for the listener. Specifically, EMA data for four German and three French speakers was acquired for $\mathrm{C} 1 \mathrm{C} 2$ clusters where manner of articulation was varied for $\mathrm{C} 2(/ \mathrm{l} / \mathrm{vs}$. /n/) and voicing for $\mathrm{C} 1$ (/p/ vs. /b/, /k/ vs. $/ \mathrm{g} /$ ). Results (1): A robust effect of less overlap of the constriction gesture for $\mathrm{C} 1$ and $\mathrm{C} 2$ when $\mathrm{C} 2$ is nasal. Clusters with nasal $\mathrm{C} 2$ may require less overlap in order to avoid compromising the acoustic characteristics of the $\mathrm{C} 1$ burst by early velar lowering. Interestingly, such clusters appear to be less stable diachronically and may be less favoured for the formation of complex onsets because of reduced scope for efficient parallel transmission of segmental information. Results (2): For German, a consistent effect of less overlap for voiceless compared to voiced C1. Discussion here will centre on whether possible cross-language differences between German and French can be related to differences in timing of voice onset and resulting differences in the acoustic properties of the C1-C2 transitions.

4pSCb43. Characterisation of the velocity distribution on rigid in-vitro upper airway replicas. Annemie Van Hirtum (Département Parole \& Cognition, GIPSA-lab, 46, avenue Félix Viallet, 38031 Grenoble Cedex, France, annemie.vanhirtum@gipsa-lab.inpg.fr), Xavier Grandchamp (Département Parole \& Cognition, GIPSA-lab, 46, avenue Félix Viallet, 38031 Grenoble Cedex, France, xavier.grandchamp
@ gipsa-lab.inpg.fr),David Marx(Laboratoire d'Etudes Aerodynamiques CNRS, Bat K, 40 avenue du recteur Pineau, 86022 Poitiers, France, david.marx@lea.univ-poitiers.fr), Xavier Pelorson (Département Parole \& Cognition, GIPSA-lab, 46, avenue Félix Viallet, 38031 Grenoble Cedex, France, pelorson@icp.inpg.fr), Helène Bailliet (Laboratoire d'Etudes Aerodynamiques - CNRS, Bat K, 40 avenue du recteur Pineau, 86022 Poitiers, France, helene.bailliet@lea.univ-poitiers.fr)

Qualitative and quantitative characterisation of the velocity distribution is an important aspect in order to study respiration related flow phenomena in the upper airways as well as for human speech production. Classical phonation models exploit one-dimensional flow descriptions in order to estimate the pressure forces exerted on the vocal folds tissues. In this case, the velocity distribution is expressed as an analytical relationship depending on volume velocity airflow, geometry and pressure distribution. Therefore simplified flow models can be validated by measuring the relevant quantities. However, more detailed and quantitative velocity predictions, aiming e.g. to improve phonation modelling or to study turbulent sound production, require increased precision for both the qualitative and quantitative characterisation of the velocity distribution. The current paper presents preliminary in-vitro measurements of the velocity distribution obtained by Particle Image Velocimetry combined with hot film anemometry. Several simplified rigid geometries are assessed in order to represent different portions of the upper airway. Besides qualitative results a first quantitative comparison between simulated and measured velocities is provided and discussed.

4pSCb44. Experimental analysis of the relationship between the glottal flow and glottal area waveforms. Raphael Schwarz (University of South Carolina, 1621 Greene St, Williams Brice Building, 6th Fl, Columbia, SC 29208, USA, rschwarz@gwm.sc.edu), Dimitar D. Deliyski (University of South Carolina, 1621 Greene St, Williams Brice Building, 6th Fl, Columbia, SC 29208, USA, deliyski@gwm.sc.edu)

A very important but little studied aspect of human voice production is the relationship between the vocal fold vibration and the transglottal airflow. To analyze this relationship, in this study we combined high-speed videoendoscopy of the glottis for determining the glottal area waveform (GAW) with inverse filtering of the acoustic signal for estimating the glottal flow waveform (GFW). The high-speed camera system, recording at 20,000 pps, and the audio recording hardware were triggered by the same quartz oscillator to achieve synchronization with an unprecedented accuracy within 25 $\mu$ s. We developed an image processing algorithm for automatic extraction of the GAW from the high-speed images. The high-speed video samples and the corresponding acoustic signals were obtained from 12 normophonic individuals (6 male, 6 female) for different voicing conditions: register (pulse, modal, falsetto); adductory adjustment (loose, normal, pressed); longitudinal tension within modal register (low, comfortable, high pitch); non-stationary phonation (variation in pitch and loudness). To compare the resulting GAWs and GFWs, the waveforms were parameterized concerning their temporal and spectral features. It is shown, that the revealed relationships between the vocal fold vibrations and the transglottal flow are comprehensible by accounting the different phonation conditions. [Work supported by NIH.]

4pSCb45. Pitch production by bilingual Mandarin-Min speakers and monolingual Mandarin speakers in speaking Taiwan Mandarin and Min. E-Chin Wu (Graduate Institute of Linguistics, National Taiwan Univ, Grad. Inst. of Linguistics, National Taiwan Univ., 1, Roosevelt Rd. Sec. 4, 106 Taipei, Taiwan, r95142002@ntu.edu.tw), Janice Fon (Grad. Inst. of Linguistics, National Taiwan Univ., 1, Roosevelt Rd. Sec. 4, 106 Taipei, Taiwan, jfon@ntu.edu.tw)

In this study, Mandarin produced by bilingual Mandarin-Min speakers and monolingual Mandarin speakers were compared to see whether the presence of Min would pose influence on the pitch range utilized by bilingual Mandarin-Min speakers in speaking Mandarin. The results showed that bilingual Mandarin-Min speakers and monolingual Mandarin speakers did indeed differ in their pitch values when speaking Mandarin. Intriguingly, however, the difference did not go in the same direction for males and females. 
It is proposed here that the difference found in the male group was due to influence of Min, while the difference found in the female groups was due to social factors. The results of this study indicate that both language experiences and social constraints are important factors that influence a person's use of pitch.

4pSCb46. Probing the independence of formant control. Ewen Macdonald (Queen's University, 62 Arch St, Dept. of Psychology, Humphrey Hall, Kingston, ON K7L 3N6, Canada, ewen.macdonald@queensu .ca), Bryan P. Burt (Queen's University, 62 Arch St, Dept. of Psychology, Humphrey Hall, Kingston, ON K7L 3N6, Canada, bryan.burt@queensu .ca), Kevin G. Munhall (Queen's University, 62 Arch St, Dept. of Psychology, Humphrey Hall, Kingston, ON K7L 3N6, Canada, kevin.munhal @ queensu.ca)

Previous experiments in speech motor learning have demonstrated that acoustic feedback is used to control formant frequencies (Houde and Jordan 1998; Purcell and Munhall 2006; Villacorta, Perkell, and Guenther 2007). In these studies, the formants of a vowel were shifted using a real-time signal processing system. When subjects spoke a vowel, they heard themselves saying a different vowel. The talkers spontaneously compensated for this auditory feedback perturbation by producing formants in the opposite direction in frequency to the perturbation. The purpose of the present study was to investigate if compensating for a perturbation in either the first or second formant also leads to a change in production of the other formant. A between-subjects experiment was conducted where half the participants had a perturbation applied only to the first formant and the other half had a perturbation applied only to the second formant. As in previous experiments, both groups compensated for the perturbed formant. In compensating for the perturbed formant, the production of the other, unperturbed, formant was also affected. However, the change in frequency was quite small. The results will be discussed in terms of the independent variables of speech motor planning

4pSCb47. Pitch Tracking using the Generalized Harmonicity Indicator. Darren Haddad (Air Force Research Lab, 525 Brooks Road, Rome, NY 13441, USA, darren.haddad@rl.af.mil), Andrew Noga (525 Brooks Road, Rome, NY 13441, USA, Andrew.Noga@rl.af.mil), Tappan Sarkar (323 Link Hall, Syarcuse University, Syracuse, NY 13244-1240, USA, tksarkar@syr.edu)

For many audio applications, a process is required to obtain an accurate estimate of the fundamental and harmonics of periodic sections of the audio signal. The Generalized Harmonicity Indicator (GHI) is to determine, assess and track the fundamental and harmonic frequencies of consecutive time segments of a speech signal using the Matrix Pencil (MP) technique[i]. Various methods of fundamental and harmonic frequency tracking have been proposed and developed, but most have been based on other low resolution techniques such as FFT and cepstral analyses. This is opposed to using a super-resolution frequency estimation technique as provided by the MP. The prior art in the area of super-resolution speech fundamental determination consists of the "super resolution pitch determinator" (SRPD)[ii] and the "enhanced SRPD" (eSRPD) methods. Because these prior methods do not explicitly process a spectral representation or decomposition of the input audio signal, they are not considered to be in the same class as the MP GHI, although they provide a baseline for comparing different estimation techniques. [i] Haddad, D.M., Sarkar, T.K., Noga, A.J.; "Speech Compression Using the Matrix Pencil Technique"; IEEE 12th DSP Workshop; Sept. 2006; Page(s):218-221. [ii] Y. Medan, E., Yair, D., Chazan, "Super Resolution Pitch Determination of Speech Signals," IEEE Trans. On Signal Processing, ASSP-39(1):40-48, 1991.

4pSCb48. Intraglottal pressures in a static physical model of the converging glottis: entrance loss coefficients, exit coefficients, Bernoulli effects, and viscous effects. Lewis P. Fulcher (Bowling Green State University, Department of Physics and Astronomy, Bowling Green, OH 43403, USA, fulcher@bgsu.edu), Ronald C. Scherer (Bowling Green State University, Department of Physics and Astronomy, Bowling Green, OH 43403, USA,ronalds@bgnet.bgsu.edu)
Pressure distributions were obtained with a static physical model (M5) at diameters $\mathrm{d}=0.005,0.01,0.02,0.04,0.08,0.16$, and $0.32 \mathrm{~cm}$ for converging angles of 5, 10, 20, and 40 degrees. At each diameter and angle, transglottal pressures typically ranged from 3 to $15 \mathrm{~cm} \mathrm{H}_{2} \mathrm{O}$. For each angle, diameter, and transglottal pressure, the measured pressure at the glottal entrance was used to calculate an entrance loss coefficient, and the measured pressure near the glottal exit was used to determine an exit coefficient. Previous work with the uniform glottis, where the only important physical effect was viscosity, found linear fits to the intraglottal pressures to be excellent approximations. Since the widening channel of the converging glottis produces Bernoulli effects as well as viscous effects, a parabolic form for intraglottal pressures is explored. Such an analytic form for the intraglotta pressures when accompanied by tables of entrance loss and exit coefficients would be a useful tool for researchers needing expressions easily included in numerical models of phonation. The validity of the new analytic treatment will be assessed by comparing the calculated pressures with the observed M5 pressures. [Work supported by NIH R01DC03577.]

4pSCb49. Mitigation of Nonlinear Distortion in Speech Signals Using Histogram Matching. Brett Smolenski (RADC, 2433 Forest Lane, Schwenksville, PA 19473, USA, bsmolens@gmail.com)

Nonlinear distortion is a common artifact in audio communication equipment. In addition, it is well know that the normalized amplitude distribution of speech signals converges to approximately a gamma distribution after a few seconds. Hence, the transfer function of any memoryless nonlinearity distorting the speech signal can easily be estimated, provided one has buffered enough data. This research shows how both parametric and nonparametric histogram matching algorithms can be employed to remove the effects of these types of distortions. Further, the improvement these algorithms have on speaker identification performance is also studied. This approach represents a radical departure from the traditional approach taken in speech enhancement. The traditional approach has been to first acquire a model or representation of the distortion, noise, or interference that is corrupting the signal, and then attempt to remove this from the signal, usually introducing other forms of distortion in the process. With this approach a model of clean undistorted speech is used that the distorted speech is then matched to.

4pSCb50. Fricative synthesis investigations using the transmission line matrix method. Athanasios Katsamanis (National Technical University of Athens, School of Electrical and Computer Engineering, Zografou campus, 15773 Athens, Greece, nkatsam@cs.ntua.gr), Petros Maragos (National Technical University of Athens, School of Electrical and Computer Engineering, Zografou campus, 15773 Athens, Greece, maragos@cs.ntua.gr)

We investigate the potential of properly applying the Transmission Line Matrix method to simulate the 3D acoustic field in the vocal tract especially for the synthesis of fricatives. For fricatives, we are mainly interested in the higher end of the spectrum where the planar wave propagation assumption that is accepted in the one-dimensional simulation cannot provide accurate results. This is the main reason why we explore $3 \mathrm{D}$ acoustic field simulation. Proper incorporation of noise sources is considered to account for frication. Their placement and acoustic properties are investigated. Motivated by measurements of the airflow during fricative production, we also explore the influence of mean flow to acoustics in the tract. Sound propagation in moving medium is considered for this purpose. The applied vocal tract geometry is determined from 3D MRI images. We present computational considerations for the analyzed framework in parallel to potential benefits compared to the one-dimensional vocal tract simulation.

4pSCb51. Acquisition of the production of 'new' and 'similar' vowels: the case of $/ \mathbf{u} /$ and $/ \mathbf{y} /$ in French by Japanese-speaking learners. Takeki Kamiyama (Laboratoire de phonétique et phonologie (UMR 7018), 19, rue des Bernardins, 75005 Paris, France, takekik@phiz.c.u-tokyo.ac.jp)

French /y/ (F2/F3 close around $2000 \mathrm{~Hz}$ for males) does not have an equivalent phoneme in Japanese and English, whereas /u/ (F1/F2 close $<1000 \mathrm{~Hz}$ ) has a phonemic counterpart (high back) in both Japanese and English, but its phonetic realization is different from French /u/, with a higher F2. Flege (1987) found out that it was easier for American English 
speakers to acquire a native-like production of French /y/ ("new" phone) than that of /u/ ("similar" phone). Our recording of two groups of adult Japanese-speaking learners of French (JLF) shows a similar tendency. We measured the formants of isolated vowels embedded in a carrier sentence read 3 times by JLFs. In the first group, consisting of 3 elementary learners who volunteered for the task, 2 learners pronounced /y/ with close F2/F3, but none of them produced a low F2 $(<1000 \mathrm{~Hz})$ for $/ \mathrm{u} /$. In the second group, consisting of $50 \mathrm{JLFs}$ in 4 different classes and levels (elementary to upper intermediate), only 4 of them, who had all lived in a French-speaking country except one, produced /u/ with a low F2 $(<1000 \mathrm{~Hz})$, whereas a dozen of them pronounced /y/ with close F2/F3.

4pSCb52. On the influence of vocal fold collision on phonation Juergen Neubauer (School of Medicine, University of California, 31-24 Rehab Center, 1000 Veteran Ave., Los Angeles, CA 90095-1794, USA, jneubauer@mednet.ucla.edu), Zhaoyan Zhang (School of Medicine, University of California, 31-24 Rehab Center, 1000 Veteran Ave., Los Angeles, CA 90095-1794, USA, zyzhang@ucla.edu)

For laryngoscopic observations show that the closed time of the glottis is significantly long, especially for low-frequency vocal fold vibrations in the vocal fry and chest register. Vocal fold contact appears to be a major part of the phonatory cycle and may play an important role for self-sustained vocal fold oscillations. Using a 2D, finite element, self-oscillating model of the coupled vocal fold-glottal flow system, we studied the influence of the mechanical impact on phonation onset mechanisms and vocal fold vibratory behavior. The air flow was assumed to be laminar and the compressible Navier-Stokes equations were solved for the flow domain. For fixed values of the Young's modulus of the vocal fold we found that vocal fold contact significantly increased the vibration frequency as compared to the case of no contact. The changed total pressure forces on the vocal fold in medial-lateral (lift force) and inferior-superior (drag force) directions resulted in different phonation threshold pressure values and vocal fold vibration patterns. The increase in phonation frequency due to contact will be discussed based on the theory of impact oscillators.

4pSCb53. ERPs to words correlate with behavioral measures in children with Autism Spectrum Disorder. Sharon Coffey-Corina (Center for Mind and Brain UC Davis, 267 Cousteau Pl, Davis, CA 95618, USA, sccorina@ucdavis.edu), Denise Padden (I-LABS, University of Washington, Box 357920, Seattle, WA 98195, USA, dpadden@u.washington.edu), Patricia K. Kuhl (University of Washington, Dept. of Speech \& Hearing Sciences, and Institute for Learning \& Brain Sciences, Box 357988, Seattle, WA 98195, USA, pkkuhl@u.washington .edu)

Children with Autism Spectrum Disorder (ASD) participated in a research study that involved both electrophysiological and behavioral measures. Event related brain potentials (ERPs) were recorded during auditory presentation of known and unknown words. Behavioral measures of language/cognitive function and severity of autism symptoms were also collected at the time of ERP testing and again one year later. In general, higher functioning children with ASD exhibited more localized brain effects for differences between known and unknown words. Lower functioning children with ASD had more diffuse patterns of response to the different word classes and also exhibited a stronger right hemisphere lateralization. That is, they showed differences between known and unknown words at many electrode sites and larger differences in the right hemisphere. In addition, significant correlations were obtained between specific brain wave measurements for both known and unknown words and the various behavioral measures. Patterns of ERPs effectively predicted later behavioral scores.

4pSCb54. Magnetoencephalography as a tool to study speech perception in awake infants. Toshiaki Imada (University of Washington, Dept. of Speech \& Hearing Sciences, and Institute for Learning \& Brain Sciences, Box 357988, Seattle, WA 98195, USA, imada@u.washington .edu), Alexis N. Bosseler (University of Washington, Dept. of Speech \& Hearing Sciences, and Institute for Learning \& Brain Sciences, Box 357988, Seattle, WA 98195, USA, bosseler@u.washington.edu), Samu Taulu (Elekta-Neuromag Oy, 22 Elimäenkatu, 00510 Helsinki, Finland,
samu@squid.neuromag.fi),Elina Pihko(BioMag Laboratory, Helsinki University Central Hospital, 00029 Helsinki, Finland, pihko@biomag.hus.fi), Jyrki Mäkelä (BioMag Laboratory, Helsinki University Central Hospital, 00029 Helsinki, Finland, jyrki.makela@hus.fi), Antti Ahonen (Elekta-Neuromag Oy, 22 Elimäenkatu, 00510 Helsinki, Finland, antti.ahonen@elekta.com), Patricia K. Kuhl (University of Washington, Dept. of Speech \& Hearing Sciences, and Institute for Learning \& Brain Sciences, Box 357988, Seattle, WA 98195, USA, pkkuhl@u.washington.edu)

Magnetoencephalography (MEG) provides a safe, noninvasive method for studying the developing brain by offering reliable localization of the brain regions activated during speech processing. However technical challenges make recording awake infants difficult. The small size of the infant head in the adult-sized helmet results in a low signal-to-noise ratio. Head and limb movement, which is typical of young infants, produces signal artifact that is difficult to overcome during signal processing. This study used MEG to study phonetic processing in awake, non-sedated typically developing infants from 5 to 16 months. The recordings were made using the Elekta Neuromag® 306-channel instrument at BioMag Laboratory, Helsinki University Central Hospital, Finland. Infants listened to speech syllables produced by a loudspeaker inside the magnetically shielded room. Newly developed signal processing methods and behavioral entertainment greatly improved the quality of the data, producing 29 successful infant recordings out of 35 attempts. We describe the methods, as well as removal of movement-modulated artifacts, efficient interference suppression, and movement compensation during data analysis. Whole-head MEG recordings in awake babies a few months old are now feasible.

4pSCb55. Adapting second language phonemic perception training to common instructional situations: A progress report. Thomas R. Sawallis (Univ. of Alabama, English Dept., Box 870244, Tuscaloosa, AL 35487, USA, tsawalli@bama.ua.edu), Michael W. Townley (Univ. of Alabama, English Dept., Box 870244, Tuscaloosa, AL 35487, USA, town1003 @bama.ua.edu)

Although current L2 pedagogy de-emphasizes phoneme-level pronunciation training, laboratory experiments demonstrate benefits from training non-natives in perception of difficult target-language phonemic contrasts. Specifically, evidence shows that: learners' perceptual performance improves (Jamieson \& Morosan, 1986; Flege, 1995), improvements generalize to new talkers and words (Lively, Logan, \& Pisoni, 1993), perceptual training triggers production improvements (i.e., without production training, Bradlow et al., 1997), and both perceptual (Lively et al., 1994) and production improvements (Bradlow et al., 1999) are maintained over several months. We are adapting perceptual training methods from such studies for use in common instructional situations, starting with Japanese students learning the English /1-r/ contrast. This paper addresses three practical concerns. First, studies show that the perceptual training needs tokens with multiple kinds of variation. We discuss phonological variation in corpus design, sociolinguistic variation in talker recruitment, and inducement of within-talker variations. Second the training must be usable both independently and in classrooms. We discuss the design of sessions short enough for inclusion as part of daily classroom activities. Third, the training must be computercontrolled. We discuss necessary functions, available programs, and our choice. Finally, we briefly demonstrate the training, and give a sketch of interim results.

4pSCb56. "Polyaural" array processing for robust automatic speech recognition in noisy and reverberant environments. Richard $M$. Stern (Carnegie Mellon University, Department of Electrical and Computer Engineering and Language Technologies Institute, 5000 Forbes Avenue, Pittsburgh, PA 15213, USA, rms@cs.cmu.edu), Evandro B. Gouvea (Carnegie Mellon University, Department of Electrical and Computer Engineering and Language Technologies Institute, 5000 Forbes 
Avenue, Pittsburgh, PA 15213, USA, egouvea@cs.cmu.edu),Kshitiz Kumar (Carnegie Mellon University, Department of Electrical and Computer Engineering and Language Technologies Institute, 5000 Forbes Avenue, Pittsburgh, PA 15213, USA, kshitizk@cs.cmu.edu)

It is well known that human binaural processing is very useful for separating incoming sound sources as well as for improving the intelligibility of speech in reverberant environments. In this paper we present a new method of signal processing for robust speech recognition using multiple microphones. The method, loosely based on the human binaural hearing system, consists of passing the speech signals detected by multiple microphones through bandpass filtering and nonlinear halfwave rectification operations, and then cross-correlating the outputs from each channel within each frequency band. These operations provide rejection of off-axis interfering signals. These operations are repeated (in a non-physiological fashion) for the negative of the signal, and an estimate of the desired signal is obtained by combining the positive and negative outputs. We demonstrate that the use of this approach provides substantially better recognition accuracy than delay-and-sum beamforming using the same sensors for target signals in the presence of additive broadband and speech maskers, and it provides substantial improvements in specific reverberant environments as well. [Supported by NSF and DARPA]

4pSCb57. Coupled 2D-Fluid-Structure-Acoustic Simulation of the Human Voice. Gerhard Link (Univ. Erlangen-Nuremberg, Dept. of Sensor Technology, Paul-Gordan-Str. 3/5, 91052 Erlangen, Germany, gerhard.link @1se.eei.uni-erlangen.de), Manfred Kaltenbacher (Univ. Erlangen-
Nuremberg, Dept. of Sensor Technology, Paul-Gordan-Str. 3/5, 91052 Erlangen, Germany, manfred.kaltenbacher@1se.eei.uni-erlangen.de), Michael Doellinger (University Hospital Erlangen, Department of Phoniatrics and Pediatric Audiology, Medical School, Bohlenplatz 21, 91054 Erlangen, Germany, michael.doellinger@uk-erlangen.de), Reinhard Lerch (Univ. Erlangen-Nuremberg, Dept. of Sensor Technology, Paul-Gordan-Str. 3/5, 91052 Erlangen, Germany, reinhard.lerch@1se.eei.uni-erlangen.de)

The human voice is a key factor in social life. If the phonation process is disturbed due to a disease as e.g. hoarseness, communication and social life are strongly affected. Therefore, it is necessary to enhance therapies in order to minimize affliction caused by a disease. There exist different approaches to improve therapies. Experimental field studies of different physical parameters, like acoustic pressure or vocal fold displacements are one possibility. Another promising approach to advance insight into laryngeal dynamics is given by numerical simulations. Due to the growing computing power the complexity of numerical phonation models is steadily increasing and full fluid-structure-acoustic interacting models are now feasible. Therefore, we have developed a two-dimensional numerical model for the human phonation process, which includes the complete fluid-structure-acoustic interaction. As discretization method the finite-element method was applied for all possible three physical fields. The fluid-structure and the structureacoustic interactions are based on general continuum mechanical principles; the fluid-acoustic interaction is based on Lighthill's acoustic analogy. Therewith, the analysis of all sound mechanisms, which consist of the eddyinduced, the volume-induced, and the mechanical-induced sound can be performed. First simulation results will be presented and discussed.

\title{
Session $4 \mathrm{pSPa}$
}

\section{Signal Processing in Acoustics, Acoustical Oceanography, and ECUA: Model-Based Signal Processing III (Poster Session)}

\author{
Sean Lehman, Cochair \\ Pleasanton, $C A$
}

\section{Christian Pichot, Cochair}

\begin{abstract}
All posters will be on display from 3:40 p.m. to 5:20 p.m. To allow contributors an opportunity to see other posters, contributors of odd-numbered papers will be at their posters from 3:40 p.m. to 4:30 p.m. and contributors of even-numbered papers will be at their posters from 4:30 p.m. to 5:20 p.m.
\end{abstract}

\section{Contributed Papers}

4pSPa1. Detection and classification using the Estimated Ocean Detector. Richard Lee Culver (ARL Penn State, PO Box 30, State College, PA 16804, USA, rlc5@psu.edu), Colin W. Jemmott (ARL Penn State, PO Box 30, State College, PA 16804, USA, cwj112@psu.edu), Brett E. Bissinger (ARL Penn State, PO Box 30, State College, PA 16804, USA, beb194@psu.edu), Nirmal K. Bose (ARL Penn State, PO Box 30, State College, PA 16804, USA, bkn@engr.psu.edu)

We have developed the Estimated Ocean Detector, a likelihood ratio receiver with an estimator-correlator structure, and applied it to detection and classification of underwater acoustic signals. The receiver requires that the noise probability density function (pdf) to belong to the exponential class but need not be Gaussian. A composite hypothesis is employed in order to incorporate knowledge (or predictions) of the signal parameter statistics. Previously, receiver performance was demonstrated for Gaussian noise and sinusoidal signals with known frequency and phase and whose amplitude pdfs were predicted using knowledge of the ocean environment and an acoustic propagation program. In order for the receiver to be useful operationally, it must be able to accommodate unknown signal phase and incorporate numerical estimates of noise and signal parameter pdfs. Progress toward satisfying these requirements is reported in this talk. Work supported by the Office of Naval Research Undersea Signal Processing. 
4pSPa2. Fractal analysis of signals of the seismic acoustic emission Alexander V. Glushkov (Odessa University, P.O.Box 24a, 65009 Odessa-9, Ukraine, glushkov@paco.net), Andrey A. Svinarenko (Odessa University, P.O.Box 24a, 65009 Odessa-9, Ukraine, glushkov@paco.net), Yaroslav I. Lepikh (Odessa University, P.O.Box 24a, 65009 Odessa-9, Ukraine, glushkov@paco.net)

Paper is devoted to analysis fractal properties of signals of the seismic acoustic emission in periods between earthquakes. Earlier it has been carried out an analysis of natural data on distribution of the earthquake hypercenters (Mukhamedov, 1992; Salimi et al, 1993). To reveal the fractal properties for signals of the seismic acoustic emission the joint wavelet analysis is carried out by using the non-decimated wavelet transform (Glushkov et al, 2004, 2005). We present the fractal processing data for envelopes of signal of the seismic acoustic emission in different ranges of frequencies. The dependences of the Herst indicator and fractal dimension curve on the lengths of considered intervals are presented. It takes a place crossover upon a behaviour with the Herst indicator $\mathrm{H}=0,4-0,6$. It is given the physical interpretation of the seismic acoustic emission. References: Mukhamedov V.A., Izv. Russian Acad.Sci. 3, 39 (1992). Salimi M., Robertson M., Sammis S., Phys .Rev.Lett. 70, 2186 (1993). Glushkov A.V. etal, Nonlinear Processes in Geophys. 11, 285 (2004). Glushkov A.V. et al, Atmospheric Res. (Elseiver). 77, 100 (2005).

4pSPa3. A torpedo detection and 3-D tracking system. Nico Roosnek (Roosnek Research \& Development, Vlaskamp 82, 2592 AC The Hague, Netherlands, nico@ roosnek.nl)

Ship torpedo defence systems are ideally based on 3D information of incoming torpedoes. Therefore a Torpedo Detection and Location (TDL) sonar system has been designed based on a transmitting transducer, two receiving arrays and optimal signal processing with 3D-tracking capabilities with optimal estimation. The transmitted signal is a chirp. The signal processing with intrinsic beamforming is based on the phase of the signal and on optimal estimation (Kalman filtering). For testing the capabilities of such a design a test system on detection and tracking of a torpedo-like object in the atmosphere has been developed. Some figures about its capability, which can be extrapolated to underwater situations, will be given.

4pSPa4. Chirplet Transform Analysis for Ultrasonic Inspection of Composite Materials. Abdessalem Benammar (Image and signal processing laboratory. Welding and NDT Centre, Route de Dely-Ibrahim, BP 64, Chéraga, 16035 Alger, Algeria, Abs_benammar@yahoo.fr), Redouane Drai (Image and signal processing laboratory. Welding and NDT Centre, Route de Dely-Ibrahim, BP 64, Chéraga, 16035 Alger, Algeria, drai_r@yahoo.fr), Ahmed Kechida (Image and signal processing laboratory. Welding and NDT Centre, Route de Dely-Ibrahim, BP 64, Chéraga, 16035 Alger, Algeria, Abs_benammar@yahoo.fr), Abderrezak Guessoum (Image and signal processing laboratory. Welding and NDT Centre, Route de Dely-Ibrahim, BP 64, Chéraga, 16035 Alger, Algeria, Abs _benammar@yahoo.fr)

In this work, a successive parameter estimation algorithm based on the chirplet transform is presented. The chirplet transform is used not only as a means for time frequency representation, but also to estimate the echo parameters, including the amplitude, time-of-arrival, center frequency, bandwidth, phase, and chirp rate. We initially apply this method to simulated signals with additional structural noise. These signals contain several echo defects, closer between them. This stage permits to see the robustness of the developed algorithm. Thereafter, we validate all simulated results by experimental results obtained on composite material with and without delamination defects.

4pSPa5. Source localisation on a single hydrophone. Grégoire Le Touzé (GIPSA-lab, dep. DIS, 961, rue de la Houille Blanche, 38402 St Martin d'Hères, France, gregoire.letouze@lis.inpg.fr), Barbara Nicolas (GIPSA-lab, dep. DIS, 961, rue de la Houille Blanche, 38402 St Martin d'Hères, France, barbara.nicolas@gipsa-lab.inpg.fr), Jérôme I. Mars (GIPSA-lab, dep. DIS, 961, rue de la Houille Blanche, 38402 St Martin d'Hères, France, jerome.mars@gipsa-lab.inpg.fr)

The aim of the study is to localise an undewater Ultra Low Frequency (ULF) source in a shallow water environement. The acoustic signal is recorded on a single hydrophone and the source, which has to be short in time, is unknown. To perform the localisation, we have developed modal filters based on time-frequency techniques. Different localisation techniques are proposed: - Conventional Matched Mode Processing: results are good for range estimation but contain error on source depth estimation. We show that those errors are due to the ULF band. - Source depth estimation technique based on mode amplitude estimation: this estimation is precise but presents ambiguities. - Range estimation technique using mode phase estimation: we recently developed this method which also estimates mode signs. The source range is precisely estimated and mode sign estimation avoids ambiguity on source depth estimation combined with the previous method. These methods are validated on real data comming from the North Sea.

4pSPa6. Analyze effects of the flow on the vocalic reduction and the coarticulation in sequences $\mathrm{CV}$ of pharyngal Arabic. L Falek (Université des sciences et de la technologie Houari Boumédiène (USTHB), Laboratoire de communication parlée et de traitement du signa, Faculté d'Electronique et d'Informatique, BP 32, El Alia, Alger, Algeria, lilalcpts@yahoo.fr), O. Bouferroum (Université des sciences et de la technologie Houari Boumédiène (USTHB), Laboratoire de communication parlèe et de traitement du signa, Faculté d'Electronique et d'Informatique, BP 32, El Alia, Alger, Algeria), A. Djeradi (Université des sciences et de la technologie Houari Boumédiène (USTHB), Laboratoire de communication parlèe et de traitement du signa, Faculté d'Electronique et d'Informatique, BP 32, El Alia, Alger, Algeria)

The degree of coarticulation and the vocalic reduction (RV) are indices related to good engine control (Gay 1978). Fowler (1998) explains why locus equation (LE) is used to characterize, at the same time, the place of articulation and the degree of coarticulation between consonants and vowels: a strong slope $(\mathrm{m}=1)$ indicates a maximum coarticulation between consonants and vowels (i.e. minimal resistance of the coarticulation), while a weak slope $(\mathrm{m}=0)$ indicates absence of coarticulation between consonants and vowels (maximum resistance of the coarticulation). The bond between the degree of coarticulation and the RV can be explained according to the linear relation between F2onset and F2milieu: the modifications of values of F2milieu will affect those of F2onset and consequently those of the slopes. In this study, the analysis of the vocalic reduction and slopes of the equations of locus, carried out on CV (extracts starting from sentences) in standard Arabic pronounced by speakers having different mother tongues (near to Arabic standard and very far away from standard Arabic), and at speed of variable elocution, revealed a vocalic reduction and a variation of the slope of the locus equation, specific to each speaker, who seems to be related to his mother tongue. El Tamimi (2006) carried out a similar study with normal flow, in dialectical Arabic and in French, with normal flow, an influence of the mother tongue showed on the vocalic reduction and the slope of the equation of locus. 


\title{
Session $4 \mathrm{pSPb}$
}

\section{Signal Processing in Acoustics: Advances in Acoustic Sensors and Networks for Defense Applications II (Poster Session)}

\author{
Brian Ferguson, Cochair \\ Defence Science and Technology Organisation
}

Pierre Naz, Cochair

ISL

\begin{abstract}
All posters will be on display from 3:40 p.m. to 5:20 p.m. To allow contributors an opportunity to see other posters, contributors of odd-numbered papers will be at their posters from 3:40 p.m. to 4:30 p.m. and contributors of even-numbered papers will be at their posters from 4:30 p.m. to 5:20 p.m.
\end{abstract}

\section{Contributed Papers}

4pSPb1. Aircrafts localisation and tracking with arrays of microphones. Gaetano Caronna (Università La Sapienza - Dept. Fisica Tecnica, Via Eudossiana 18, 00184 Roma, Italy, gaetano.caronna@uniroma1.it), Pierluigi Testa (Università La Sapienza - Dept. Fisica Tecnica, Via Eudossiana 18, 00184 Roma, Italy, pierluigi.testa@uniroma1.it)

University of Roma "La Sapienza" will participate in a project, financed by the Italian Ministry of Research, aiming to detect and to track aircrafts near the airport using the acoustic emissions of the aircraft and the technique of acoustic beam-forming. Although the principle is well known and some applications are reported in literature and explored also by our group in a previous European FP6 project ("Safe Airport"), the final performance and the engineering value are not assessed. In this reseach effort the problem is again raised aiming to create a more realistic model including wind and temperature gradient that, in particular conditions, can drastically impair the validity of the results, estimating the related errors. A simulation software was written considering the steering drive on the microphones outputs, being the mechanical rotation of the array prohibited, due to the aerodynamic noises induced. At least two arrays, opportunely located, are requested in order to perform a triangulation and detect the source of the acoustic wave emitted by the aircraft. The precision in the localisation of the acoustic source was estimated in a simulation where real aircraft acoustic emissions and environmental noise were used as imput.

4pSPb2. Acoustic Source Localization via Distributed Sensor Networks using Tera-scale Optical-Core Devices. Neena Imam (Oak Ridge National Laboratory, 1 Bethel Valley Road, Oak Ridge, TN 37831-6015, USA, imamn@ornl.gov), Jacob Barhen (Oak Ridge National Laboratory, 1 Bethel Valley Road, Oak Ridge, TN 37831-6015, USA, barhenj@ornl.gov)

For real-time acoustic source localization applications, one of the primary challenges is the considerable growth in computational complexity associated with the emergence of ever larger, active or passive, distributed sensor networks. The complexity of the calculations needed to achieve accurate source localization increases dramatically with the size of sensor arrays, resulting in substantial growth of computational requirements that cannot be met with standard hardware. One option to meet this challenge builds upon the emergence of digital optical-core devices. The objective of this work was to explore the implementation of key building block algorithms used in underwater source localization on optical-core digital processing platform recently introduced by Lenslet Inc. We investigate key concepts of threat-detection algorithms such as Time Difference Of Arrival (TDOA) estimation via sensor data correlation in both time and frequency domains with the purpose of implementation on the optical-core processor. We illustrate our results with the aid of numerical simulation and actual optical hardware runs. The major accomplishments of this research, in terms of computational speedup and numerical accuracy achieved via the deployment of optical processing technology, should be of substantial interest to the acoustic signal processing community.

4pSPb3. Performance of high-resolution sensor array processing algorithms in the localization of acoustic sources. Joseph Lardies (FEMTO-ST Applied Mechanics, 24 chemin de l'épitaphe, 25000 Besançon, France, joseph.lardies@univ-fcomte.fr), Hua Ma (University of Franche-Comté, Institute FEMTO - LMARC, 24 rue de l'Epitaphe, 25000 Besançon, France, rosyhorse@hotmail.fr), Marc Berthillier (University of Franche-Comté, Institute FEMTO - LMARC, 24 rue de l'Epitaphe, 25000 Besançon, France, marc.berthillier@univ-fcomte.fr), Emmanuel Foltete (FEMTO-ST Applied Mechanics, 24 chemin de l'épitaphe, 25000 Besançon, France, emmanuel.foltete@univ-fcomte.fr)

The localization of noise sources from a specified direction may often be accomplished with an array of sensors. One commonly used processor consists of delay and add networks: a conventional beamformer, however its spectrum suffers from the Rayleigh resolution and its performance is highly degraded, specially in lower frequency range. In the communication, the performance of some typical high-resolution sensor array processing algorithms: Minimum Variance, MUSIC, Mini-Norm algorithms are investigated for wideband source location. Their performances are compared with a new source localization algorithm which is based on a sparse representation of sensor measurements with an overcomplete basis composed of samples from the array manifold. The key of the method is the use of the SVD for data reduction and the formulation of a joint multiple-sample sparse representation problem in the signal subspace domain. Increased resolution and improved robustness to noise is obtained with this algorithm applied to various numerical examples. 


\title{
Session 4pSPc
}

\section{Signal Processing in Acoustics: Filter Design, Detection, and Estimation II (Poster Session)}

\author{
Michael Roan, Cochair \\ Virginia Tech \\ Jorge Quijano, Cochair \\ NEAR Lab-Portland State University
}

\begin{abstract}
All posters will be on display from 3:40 p.m. to 5:20 p.m. To allow contributors an opportunity to see other posters, contributors of odd-numbered papers will be at their posters from 3:40 p.m. to 4:30 p.m. and contributors of even-numbered papers will be at their posters from 4:30 p.m. to 5:20 p.m.
\end{abstract}

\section{Contributed Papers}

4pSPc1. Averaged Lagrange Method for interpolation filter. Jonathan Andrea (LIEN - BP 239, Université Henri Poincaré, 54506 Vandoeuvre, France, jonathan.andrea@lien.uhp-nancy.fr), Frederic Coutard (LIEN - BP 239, Université Henri Poincaré, 54506 Vandoeuvre, France, frederic.coutard@lien.uhp-nancy.fr), Patrick Schweitzer (LIEN - BP 239, Université Henri Poincaré, 54506 Vandoeuvre, France, patrick.schweitzer@lien.uhp-nancy.fr), Etienne Tisserand (LIEN - BP 239, Université Henri Poincaré, 54506 Vandoeuvre, France, etienne.tisserand @lien.uhp-nancy.fr)

This paper presents a new method for Lagrange interpolation for reducing distortions without introducing any complexity. The aim is to improve the linearity of the phase and the gain responses of the interpolation filter by an averaging method. A first FIR interpolation filter of the second order computes the values between three successive samples of the input signal. At the same time, a second filter, identical to the first, computes the values between (xk-2, xk-1, xk ). Finally, the common values between (xk-2, xk-1 ) provided by the two filters are averaged two by two. This double interpolation can be simply done with a single third order filter and with a Farrow structure filter. Compared to the usual Lagrange's third order interpolation filter, the behaviour of the filter we propose is more regular especially in the high frequencies of the Nyquist band. More, the filter coefficients are easier to calculate. The designed filter is tested on a FPGA of Altera. The results shows that the method significantly reduces distortions and improves quality of the frequency response.

4pSPc2. Application of digital filters for measurement of nonlinear distortions in loudspeakers using Wolf's method. Rafal Siczek (Wroclaw University of Technology, Wybrzeze Wyspianskiego 27, 50-370 Wroclaw, Poland, rafal.siczek@pwr.wroc.pl)

The design of digital filters used for measurement of nonlinear distortions in loudspeakers by the Wolf's method is presented in the paper. The Wolf's method has been developed in 1953 and originally the analog filters was applied. This method requires particularly steep slopes of both bandreject filters (at the transmitting part of the measurement system) and bandpass filters (at the receiving part). The digital filters allows for achievement of such steep slopes with a relatively small cost in comparison with analog ones. The very high attenuation in the barrage bands can be achieved. In the very narrow transition band any irregularities do not appear. The design procedures using MATLAB package have been described. Then, the digital filter allow for achievement of a high accuracy of measurement.
4pSPc3. Objective quality measurement of the excitation of impact sounds in a source/filter model. Mathieu Lagrange (Centre for Interdisciplinary Research in Music Media \& Technology (CIRMMT) - Schulich School of Music - McGill Univ., 555 Sherbrooke Street West, Montreal, QC H3A1E3, Canada, mathieu.lagrange@mcgill.ca), Bruno L. Giordano (Centre for Interdisciplinary Research in Music Media \& Technology (CIRMMT) - Schulich School of Music - McGill Univ., 555 Sherbrooke Street West, Montreal, QC H3A1E3, Canada, bruno.giordano@music.mcgill.ca), Philippe Depalle (Centre for Interdisciplinary Research in Music Media \& Technology (CIRMMT) - Schulich School of Music - McGill Univ., 555 Sherbrooke Street West, Montreal, QC H3A1E3, Canada, depalle@music.mcgill.ca), Stephen McAdams (Centre for Interdisciplinary Research in Music Media \& Technology (CIRMMT) Schulich School of Music - McGill Univ., 555 Sherbrooke Street West, Montreal, QC H3A1E3, Canada, smc@music.mcgill.ca)

For the modeling of percussive (non-sustained) sounds, the excitation signal can be estimated from an original sound in several ways, usually by a time-domain deconvolution process. The source signal obtained by such a process cannot be compared with the original excitation because it is usually unknown. Hence in most of the approaches available in the literature, the validation of the deconvolution process is quantifed in terms of spectral flatness, i.e. a source signal is considered as a good estimation of the excitation when most of the resonant content has been removed. However, the excitation signal is usually a percussive burst, the time domain properties of which are known to be very important, at least perceptually speaking. To evaluate the time domain properties of the estimated excitation, we propose in this paper to compare the estimated excitation to the recording of the acceleration of the hammer hitting a plate. In the recordings considered, the evolution of the acceleration of the hammer has a specifc pattern with several peaks due to the bouncing of the hammer on the suspended plate. This specifc pattern allows us to propose a metric that can be useful for objectively measuring the quality of the estimation process.

4pSPc4. Detecting Scenes in Lifelog Videos based on Probabilistic Models of Audio data. Kiichiro Yamano (Hosei University, 3-7-2 Kajinocho, 184-8584 Koganei, Japan, n04k1035@cis.k.hosei.ac.jp), Katunobu Itou (Hosei University, 3-7-2 Kajino-cho, 184-8584 Koganei, Japan, itou @ hosei.ac.jp)

Lifelog videos are recorded every activity in everyday lives. To utilize them efficiently, it is required to be indexed automatically. To index continuous shots of the lifelog, significant scenes are detected automatically. For detection, many researches employ image features such as color and edge, however, the accuracy is insufficient. In this study, we propose 
probabilistic models for scene detection from lifelog video. In this method, mel-frequency filter bank output of audio tracks of the lifelog videos is modeled statistically with hand-labeled training data. We tested the proposed method to use train station scene. We collected 11 hour sound data for such scenes. To analyze them, we defined seven categories, such as stopping trains, passing trains, starting trains, waiting, and so on. Our method achieved to $100 \%$ for waiting scene and $18.4 \%$ in average.

4pSPc5. Estimation of reflection location by the correlation coefficient function. Hideo Shibayama (Shibaura Institute of Technology, 3-7-5, Toyosu, Koto-ku, 135-8548 Tokyo, Japan, sibayama@sic.shibaura-it.ac .jp), Takeshi Araya (Hitachi Information \& Communication Engineering, Ltd, 393 Totsukamati Totsuka-ku, 244-8502 Yokohama Kanagawa, Japan, takeshi.araya.tx@hitachi.com), Yoshiaki Makabe (Hitachi Information \& Communication Engineering, Ltd, 393 Totsukamati Totsuka-ku, 244-8502 Yokohama Kanagawa, Japan, yoshiaki.makabe.hd@hitachi.com), Eiji Okarura (Hitachi Information \& Communication Engineering, Ltd, 393 Totsukamati Totsuka-ku, 244-8502 Yokohama Kanagawa, Japan, eiji.okamura .mh@hitachi.com)

Gas is supplied to households through gas pipes that branch out from a main pipe. It is essential to carry out maintenance of these branch pipes to ensure the safe supply of gas. However, there are many cases in which the state of buried gas pipes and the connection condition of a large number of pipes are unknown because no detailed information is included in piping drawings or only plan views are available. If the piping arrangement can be estimated on the basis of response waves originating from an acoustic wave travelling in a gas pipe emitted by a loudspeaker, which is placed at one end of the pipe, this method will be important for the maintenance of branch pipes. To realize this, we report a method of estimating the length of gas pipes using correlation coefficient between a driving signal and measured waves. And, we show the experimental results with high accuracy.

4pSPc6. An experiment for signal identification of the MIMO communication by sonic waves. Daisuke Hayashi (Shibaura Institute of Technology, 3-7-5, Toyosu, Koto-ku, 135-8548 Tokyo, Japan, m107077 @ sic.shibaura-it.ac.jp), Yasukazu Maeda (Shibaura Institute of Technology, 3-7-5, Toyosu, Koto-ku, 135-8548 Tokyo, Japan, m107092@sic.shibaura-it
.ac.jp),Toru Itakura(Shibaura Institute of Technology, 3-7-5, Toyosu, Kotoku, 135-8548 Tokyo, Japan, m106009@sic.shibaura-it.ac.jp), Hideo Shibayama (Shibaura Institute of Technology, 3-7-5, Toyosu, Koto-ku, 1358548 Tokyo, Japan, sibayama@sic.shibaura-it.ac.jp)

Recently, the communication method by MIMO works in a field of the wireless communication. We do research on application to the MIMO system in an acoustic field by sonic wave. As the transmission method, we use the space division multiplexing (SDM) that is the method for the purpose of the improvement of the transmission rate that accepted the number of the transmission elements by sending plural signals at the same time. It is important to identify each signal from several different signals that are transmitted at the same frequency band. And, we study method for detecting each signal. This paper describes influence of the signal detection for the different conditions of the multi-path propagation.

4pSPc7. Transverse vectorization of fast Fourier transforms on multicore architectures. Travis Humble (Oak Ridge National Laboratory, 1 Bethel Valley Road, Oak Ridge, TN 37831-6015, USA, humblets@ornl .gov), Jacob Barhen (Oak Ridge National Laboratory, 1 Bethel Valley Road, Oak Ridge, TN 37831-6015, USA, barhenj@ornl.gov), Michael Traweek (Office of Naval Research, 875 North Randolph Street, Arlington, VA 22203, USA, Mike.Traweek@navy.mil)

Single-instruction, multiple-data (SIMD) multicore computing architectures, such as the IBM Cell Broadband Engine Architecture, offer new opportunities for quickly and efficiently calculating the 1D-FFT of acoustic signals, as time-sampled data arrays can be naturally partitioned across the multiple cores on which vectorized implementations of the FFT operate. Building on this parallel pipeline model, we consider the case that $M$ data arrays of length $N$ reside within each core. Whereas the cost of sequentially executing these $M$ FFT's conventionally scales as $\alpha M N \log _{2} N$, we demonstrate a transverse vectorization solution whose cost scales as $\alpha \beta N \log _{2} N$, where $\alpha$ and $\beta$ are constant scaling factors. Our approach makes use of the SIMD instruction set and large vector register file inherent to each core of the IBM Cell in order to calculate the FFT of $M$ data arrays simultaneously. By efficiently using all the available vector registers in performing the FFT, this transverse SIMD vectorization solution further reduces the computational complexity of the conventional parallel pipeline model. 
Session 4pUWa

\title{
Underwater Acoustics and ECUA: Image and Signal Processing
}

\author{
Ivars Kirsteins, Cochair \\ NUWC, 1176 Howell St, Newport, RI 02841, USA \\ Ioannis Koukos, Cochair \\ Hellenic Naval Academy, Telecommunications Lab, Terma Hatzikyriakou Street, Piraeus, 18539, Greece
}

\section{Contributed Papers}

2:00

4pUWa1. Physics-based signal processing methodologies for separating target echoes into their constituent elastic and geometric components. Ivars Kirsteins (NUWC, 1176 Howell St, Newport, RI 02841, USA, kirsteinsip@npt.nuwc.navy.mil), Alessandra Tesei (NATO Undersea Research Centre, Viale San Bartolomeo 400, 19126 La Spezia, Italy, tesei @ nurc.nato.int)

Echoes from even simple canonical symmetric shapes such as solid elastic spheres are highly complex, consisting of a superposition of specular, elastic, and geometric diffractive components closely spaced in time and frequency. Although measurement of the individual elastic and geometric components is important for analysis of scattering physics and object identification, in practice individual components are often hard to measure because of mutual interference between components. When an object interacts with a boundary, such as the sea floor, additional interfering echoes make the object signature even more complicated to interpret. Motivated by this problem, a frequency-domain technique is developed for separating an echo into its constituent elastic and geometric components, and for isolating specular reflections from a possibly interacting boundary. It is based on modelling the echo components in the frequency domain as amplitudemodulated piecewise-linear chirps, utilizing physically-inspired group delay models and constraints, and then using a modified Costas' residual signal analysis (RSA) algorithm in combination with chirp transform analysis to decompose the echo. The RSA scheme is demonstrated on actual echoes collected in the NURC EVA-06 sea trial from spherical and cylindrical target shapes and is shown to work well with the separated components verified against modelling predictions.

\section{2:20}

4pUWa2. Two Dimensional Wavelet Coefficient Statistics for Sea Bottom Classification. Ioannis Koukos (Hellenic Naval Academy, Telecommunications Lab, Terma Hatzikyriakou Street, 18539 Piraeus, Greece, jalex_14u@hotmail.com), Theodoros Mavroidis (Hellenic Naval Academy, Telecommunications Lab, Terma Hatzikyriakou Street, 18539 Piraeus, Greece, tmavroid@tellas.gr), Georgios Vardoulias (Hellenic Naval Academy, Telecommunications Lab, Terma Hatzikyriakou Street, 18539 Piraeus, Greece,ggvard@gmail.com)

In this paper we examine the classification of different seafloors based on the analysis of images obtained by side-scan sonar. For this purpose, we apply various two dimensional multilevel wavelet decomposition schemes on images obtained from three different seafloor types, i.e., sand ripples, rocks and sands, and then we examine the statistics of the corresponding wavelet coefficients. The observed Probability Density Functions (pdf) are modeled using various theoretical distributions such as the Alpha Stable, Sum of Gaussians, Log-normal. The parameters of the fit are subsequently used to classify the side scan sonar images according to well known cluster analysis techniques. The use of the energy of the wavelet coefficients as a tool for side scan sonar image classification is also evaluated. A new unsupervised classification scheme based on the pdf fitting parameters is proposed.
2:40

4pUWa3. Computer Vision Techniques Applied for Reconstruction of Seafloor 3D Images from Side Scan and Synthetic Aperture Sonars Data. Krzysztof Bikonis (Gdansk University of Technology, Department of Geoinformatics, Narutowicza 11/12, 80-952 Gdansk, Poland, binio@eti.pg.gda.pl), Andrzej Stepnowski (Gdansk University of Technology, Department of Geoinformatics, Narutowicza 11/12, 80-952 Gdansk, Poland, astep@pg.gda.pl), Marek Moszynski (Gdansk University of Technology, Department of Geoinformatics, Narutowicza 11/12, 80-952 Gdansk, Poland, marmo@eti.pg.gda.pl)

The Side Scan Sonar and Synthetic Aperture Sonar are well known echo signal processing technologies that produce $2 \mathrm{D}$ images of the seafloor. Both systems combines a number of acoustic pings to form a high resolution image of seafloor. It was shown in numerous papers that 2D images acquired by such systems can be transformed into 3D models of seafloor surface by algorithmic approach using intensity information, contained in a grayscaled images. The paper presents the concept of processing the Side Scan Sonar and Synthetic Aperture Sonar records for detailed reconstruction of 3D seafloor using Shape from Shading techniques. Shape from Shading is one of the basic techniques used in computer vision for the objects reconstruction. The algorithms proposed in the paper use the assumed Lambert model of backscattering strength dependence on incident angle and utilize additionally the information from shadow areas for solving obtained set of equations. The idea was verified by simulation study. The obtained results of $3 \mathrm{D}$ shape reconstruction are presented and the performance of the algorithms is discussed.

\section{3:00}

4pUWa4. Underwater vehicle attitude estimation using Hough transformation. Hisashi Shiba (Radio Application Division, NEC Corporation, 1-10, Nisshin-cho, Fuchu, 183-8501 Tokyo, Japan, h-shiba@aj.jp.nec .com)

In these decades various imaging sonar systems have been developed. They are very effective for underwater investigations such as geologies, fisheries, resource surveys and securities. To extend these system abilities we studied new imaging sonar applications and found they are also useful in underwater vehicle navigations. In this presentation I propose a new method of estimating vehicle attitude parameters relative to the ocean surface or the seafloor by the onboard imaging sonar. The ocean surface or the seafloor is often obtained as a plane in sonar images. However, the images are sometimes too noisy to identify the plane locations because of ambient noise and reverberations by texture patterns on the planes. The new method describes these planes with plane equation parameters connected to attitude parameters, and searches the most appropriate parameter sets using a voting procedure which is a three dimensional expansion of the Hough transformation widely applied in line detections on noisy two dimensional images. The method provides the plane location and attitude parameters simultaneously without other sensors. After the algorithm explanation multi-beam imaging sonar simulations are shown and accuracies are evaluated. 
3:20

4pUWa5. Adaptive coding/modulation for shallow-water UWA communications. Sanjay Mani (Arizona State University, Dept. of Electrical Engineering, Tempe, AZ 85287-5706, USA, sanjay.mani@asu.edu), Tolga M. Duman (Arizona State University, Dept. of Electrical Engineering, Tempe, AZ 85287-5706, USA, duman@asu.edu), Paul Hursky (HLS Research, Inc., 3366 N. Torrey Pines Ct., Ste. 310, La Jolla, CA 92037, USA, paul.hursky@hlsresearch.com)

We consider adaptive modulation and coding techniques for Phase Shift Keying (PSK) transmission schemes over underwater acoustic (UWA) channels. Adaptive modulation and coding can be an effective means of obtaining higher data rates while retaining acceptable error levels by exploiting knowledge of the channel state. This is particularly significant in the UWA scenario, where attained spectral efficiencies are critical since usable bandwidth is a severe limitation. We examine the use of channel capacity and post-equalization signal to noise ratio (SNR) as adaptation metrics. We illustrate the ideas both through simulations and using the results of a recent experiment (AUVfest 2007). In the experiment, in order to evaluate effectiveness of adaptive coding/modulation for UWA communications, we have transmitted a bank of signals with varying spectral efficiencies (obtained by changing the number of transmit elements, modulation scheme, and code rate) back to back. Using the received signals, we illustrate the relevance of the channel condition metrics under consideration, that is, we evaluate the proposed metrics for various scenarios, and provide indications of the transmission rates that could have been achieved reliably.

THURSDAY AFTERNOON, 3 JULY 2008

AMPHI BORDEAUX, 3:00 TO 5:40 P.M.

\title{
Session $4 p U W b$
}

\section{Underwater Acoustics and ECUA: High Frequency Variability II}

\author{
Marcia Isakson, Cochair \\ Applied Research Laboratories, University of Texas, PO Box 8029, Austin, TX 78713-8029, USA \\ Thomas Folégot, Cochair \\ NATO Undersea Research Center, Viale San Bartolomeo 400, La Spezia, 19126, Italy
}

\section{Invited Papers}

3:00

4pUWb1. Examples of high frequency variability in underwater acoustic systems. Peter Stein (Scientific Solutions, Inc., 99 Perimeter Rd., Nashua, NH 03063, USA, pstein@scisol.com)

Invited paper for the structured (special) session UW09 High Frequency Variability In this paper we will look at examples of high frequency variability encountered by the author during the design and implementation of underwater acoustic systems over the last 20 years. These systems include those for radiated noise measurements, short baseline tracking, tomographic correction of numerical ocean models, marine mammal detection, and diver detection. The examples will highlight the different physical sources of variability and their effects on system performance. Methods used for mitigating the effects both in the physical design of the sensor system and in the signal processing will also be discussed.

4pUWb2. Gaussian beam tracing for high-frequency acoustics. Michael B. Porter (HLS Research, Inc., 3366 N. Torrey Pines Ct., Ste. 310, La Jolla, CA 92037, USA, michael.porter@hlsresearch.com), Martin Siderius (HLS Research, Inc., 3366 N. Torrey Pines Ct., Ste. 310, La Jolla, CA 92037, USA, martin.siderius@hlsresearch.com), Paul Hursky (HLS Research, Inc., 3366 N. Torrey Pines Ct., Ste. 310, La Jolla, CA 92037, USA, paul.hursky@hlsresearch.com)

Gaussian beam tracing is an approach that constructs full-wave beams around the skeleton of conventional ray theory. This Gaussian beam approach leads to a very simple algorithm and provides remarkable accuracy and speed. For high-frequency, broadband applications, Gaussian beams are often the only practical approach, as the standard full-wave modeling alternatives are often thousands of times slower. Gaussian beams are derived using high-frequency asymptotics, and therefore fit naturally to certain current areas of interest in HF acoustics, such as acoustic communications. However, what often surprises people who are not familiar with the technique is that it works quite well at lower frequencies, depending on the water depth. That fact, is really a consequence of 20 years of continued advances in the Gaussian beam method. We will review those developments in the context of HF variability, considering effects of boundary, volume, and source/receiver dynamics. 


\title{
Invited Papers
}

5:00

4pUWb3. Field-calibration: exploiting high-frequency mobile platform transmissions for source localization at lower frequency with arrays. Paul Hursky (HLS Research, Inc., 3366 N. Torrey Pines Ct., Ste. 310, La Jolla, CA 92037, USA, paul.hursky@hlsresearch.com), Michael B. Porter (HLS Research, Inc., 3366 N. Torrey Pines Ct., Ste. 310, La Jolla, CA 92037, USA, michael.porter@hlsresearch.com)

Multipath arrivals in an ocean waveguide with a reflective enough bottom can be used as a fingerprint for source range and depth and this has been demonstrated by many researchers using matched field processing. However, MFP relies upon acoustic propagation models to produce the Green's functions to be used as steering vectors for this processing. We have proposed to instead directly measure these Green's functions using wideband acoustic comms signals from mobile platforms such as AUVs that are increasingly part of naval applications. Thus we measure the multipath arrival pattern at $8-16 \mathrm{kHz}$ from the many locations AUVs are visiting, and then apply such measurements for locating other sources at low frequency. A key factor in this process is being able to capture the essential features of the impulse response function at high frequency, where fluctuations are much more severe than at low frequency. We have previously presented experimental results of applying this technique using a single hydrophone receiver. Here we will report on a continuation of that work, using vertical arrays and data from the RADAR '07 experiment.

\section{$5: 20$}

4pUWb4. The effects of sediment variability on reflection coefficient measurements. Marcia Isakson (Applied Research Laboratories, University of Texas, PO Box 8029, Austin, TX 78713-8029, USA, misakson@arlut.utexas.edu), Nicholas P. Chotiros (Applied Research Laboratories, University of Texas, PO Box 8029, Austin, TX 78713-8029, USA, chotiros@arlut.utexas.edu)

The statistical distribution of specularly scattered acoustic energy, commonly known as the reflection coefficient, is an important parameter when developing models for shallow water propagation and acoustic communications. The distribution of measured reflection coefficient data from 5 to $50 \mathrm{kHz}$ and 10 to 70 degrees grazing angles was taken from a sea bottom recently perturbed by hurricane off the coast of Florida at the Sediment Acoustic Experiment 2004 (SAX04). The width and shape of the distributions are attributed to varying sediments in the experimental area and interface roughness. These distributions are analyzed to determine the scattering from different types of sediments including sand and mud. The effects of both roughness scattering and sediment variability on the mean

value and distribution of the measured reflection coefficient will be explored. [Work sponsored by ONR Ocean Acoustics.]

THURSDAY AFTERNOON, 3 JULY 2008

P3-C, LEVEL 3, 3:40 TO 5:20 P.M.

\section{Session 4pUWc}

\section{Underwater Acoustics and ECUA: Tank Experiments II (Poster Session)}

\author{
Raymond Soukup, Cochair \\ U.S. Naval Res. Lab. \\ Jean-Pierre Sessarego, Cochair \\ Laboratory for Mechanics and Acoustics CNRS
}

\begin{abstract}
All posters will be on display from 3:40 p.m. to 5:20 p.m. To allow contributors an opportunity to see other posters, contributors of odd-numbered papers will be at their posters from 3:40 p.m. to 4:30 p.m. and contributors of even-numbered papers will be at their posters from 4:30 p.m. to 5:20 p.m.
\end{abstract}

\section{Contributed Papers}

4pUWc1. Acoustical and optical measurements on a mixture of air microbubbles in water. Vincent Duro (LOMC FRE 3102 CNRS Groupes Ondes Acoustiques, Université du Havre (IUT), Place Robert Schuman, 76610 Le Havre, France, vincent.duro421@univ-lehavre.fr), Dominique Rajoana (LOMC FRE 3102 CNRS Groupes Ondes Acoustiques, Université du Havre (IUT), Place Robert Schuman, 76610 Le Havre, France, raphael.rajaona@univ-lehavre.fr), Dominique Decultot (LOMC FRE 3102 CNRS Groupes Ondes Acoustiques, Université du Havre (IUT), Place Robert Schuman, 76610 Le Havre, France, dominique.decultot@univ-lehavre .fr), Gerard Maze (LAUE, Université du Havre, Place Robert Schuman, F-76610 Le Havre, France, gerard.maze@univ-lehavre.fr)
An important challenge for current naval research is the modernization of battleships. Their target detection system must be increasingly efficient and they must be increasingly undetectable. Due to turbulent flows and bubbles, ship wakes are a detectable acoustic signature and ship bow waves disturb sonar detection. In this work, we study sound propagation through bubble clouds in water. We have developed an experimental set-up which permits us to acquire, in synchronization, acoustical signals and optical images. The phenomenon of bubble monopole resonance in very low frequency, related to bubble size, provokes effects of strong sound damping and sound speed dispersion. These experimental results, related to theoretical results, permit to estimated sizes and concentrations of bubbles. The ac- 
quired bubble images permit to know the real bubble sizes and concentrations, in order to correlate with the experimental acoustical results. Air bubbles are generated with a high pressure water jet introduced into the host liquid medium. A hydrodynamic study is done to characterize the bubble jet. We present in this work, theoretical results establishing a complex effective wave number characterizing the sound propagation in an effective medium. All results are discussed and compared with results of others papers on this subject.

4pUWc2. Acoustic methods in extremely shallow water: validity of the far field approximation. Fantina Madricardo (CNR-Istituto di Scienze Marine, Riva Sette Martiri - Castello 1364/a, 30122 Venice, Italy, fantina.madricardo@ismar.cnr.it), Silvano Buogo (CNR-Istituto di Acustica 'O,M.Corbino', Via del Fosso del Cavaliere, 100, 00133 Rome, Italy, silvano.buogo@idac.rm.cnr.it), Paola Calicchia (CNR-Istituto di Acustica 'O,M.Corbino', Via del Fosso del Cavaliere, 100, 00133 Rome, Italy, paola.calicchia@idac.rm.cnr.it), Emiliano Boccardi (CNR-Istituto di Scienze Marine, Riva Sette Martiri - Castello 1364/a, 30122 Venice, Italy, E.Boccardi@1se.ac.uk)

In this paper we present some experimental results concerning the possibility of using traditional echosounder in extremely shallow water environments. In the framework of the Echos Project, a collaboration between ISMAR and IA, a wide area of the Venice Lagoon has been explored with a traditional echosounder. Since the explored area was extremely shallow (up to $50 \mathrm{~cm}$ ), it became necessary to check experimentally whether the far field approximation was still valid in such conditions. In this work the acoustic field of the echosounder ELAC LAZ72 used for the sub bottom in vestigation is experimentally characterized and the validity of the far field approximation is verified.
4pUWc3. Acoustic measurements of bubbles in the wake of ship model in tank. Alexander Sutin (Stevens Institute of Technology, Castle Point on Hudson, Hoboken, NJ 07030, USA, asutin@stevens.edu), Alexander Benilov (Stevens Institute of Technology, Castle Point on Hudson, Hoboken, NJ 07030, USA, Alexander.Benilov@stevens.edu), Heui-Seol Roh (Stevens Institute of Technology, Castle Point on Hudson, Hoboken, NJ 07030, USA, Heui-Seol.Roh@stevens.edu), Young In Nah (Agency of Defense Development, P.O.Box, ChinHae, 645-600 Kyung Nam, Republic of Korea, yinah@add.re.kr)

The interest to bubble generation by moving ships is connected with large area of bubble wake reaching several kilometers that can be used for ship detection. We developed acoustic system for the measurements of bubble density and conducted measurements in $100 \mathrm{~m}$ long towing tank. The developed system measured attenuation of ultrasound in wide frequency band from 50 to $800 \mathrm{kHz}$ between two acoustic sensors placed on the distance $20 \mathrm{~cm}$. The attenuation of sound produced by bubbles was observed during several minutes after model of ship passed the point of measurement. The attenuation was recalculated to the bubble size distribution for bubbles from 4 to 65 microns using theory of resonance bubble attenuation. The measured bubble size distribution can be interpolated by power dependence $n(R) \sim R(-3.5)$ that is typical for bubbles at sea subsurface layer. The dependencies of bubble concentration of model ship speed and type of propeller were investigated. The generation of bubbles was observed when the model speed exceeds definite threshold that can be connected with the cavitation threshold. The theory describing dynamics of wake turbulence based on the shear-free turbulent wake was developed. The measured bubble concentration was in good agreement with the developed theory.

THURSDAY AFTERNOON, 3 JULY 2008

P3-C, LEVEL 3, 3:40 TO 5:20 P.M.

\title{
Session 4pUWd
}

\section{Underwater Acoustics and ECUA: Determination of Acoustic Properties of Materials for Sonar Applications II (Poster Session)}

\author{
Kenneth Foote, Cochair \\ Woods Hole Oceanographic Institution
}

Stephen Robinson, Cochair

National Physical Laboratory

All posters will be on display from 3:40 p.m. to 5:20 p.m. To allow contributors an opportunity to see other posters, contributors of odd-numbered papers will be at their posters from 3:40 p.m. to 4:30 p.m. and contributors of even-numbered papers will be at their posters from 4:30 p.m. to 5:20 p.m.

\section{Contributed Paper}

4pUWd1. Experimental study on resistance and noise reduction of low functional surfaces hydrophobic coatings. Lin Zhang (Harbin Engineering University, 31st Building Wenmiao Street, 150001 Harbin, China, zhanglin8624@yahoo.com.cn), Ting Jiang (Harbin Engineering University, 31st Building Wenmiao Street, 150001 Harbin, China, jianfly@163.com)

The object moves in viscous fluid will accepting two kinds of resistance, one kind is the friction drag that the fluid applied to the object directly, another kind is the pressure drag that because of separating the fluid and taking from, and this drag force can create the flow noise. On theoretical aspect, this article analyses the relation between physical properties of the material and flow resistance and flow noise. The conclusion is that the surface material has the effect of reducing the flow resistance and noise because of its experimental on reducing the mutual action between fluid and solid surface under given conditions. On experimental aspect, use the gravitational low noise water tunnel test condition and adopt torpedo model, many times have the test on the drag and noise reduction of the torpedo model under diversity flow rate, have coating and not coating situation. Indicate by analysis that the low surface energy dewatering coatings used in experiment have the certain drag and noise reduction effect under the high flow rate, and with the flow rate power-up, the effect of the drag and noise reduction becomes better and better. 


\title{
Session 4pUWe
}

\section{Underwater Acoustics and ECUA: General Topics in Underwater Acoustics (Poster Session)}

\author{
David L. Bradley, Cochair \\ Pennsylvania State University \\ Kevin LePage, Cochair \\ Naval Research Laboratory
}

\begin{abstract}
All posters will be on display from 3:40 p.m. to 5:20 p.m. To allow contributors an opportunity to see other posters, contributors of odd-numbered papers will be at their posters from 3:40 p.m. to 4:30 p.m. and contributors of even-numbered papers will be at their posters from 4:30 p.m. to 5:20 p.m.
\end{abstract}

\section{Contributed Papers}

4pUWe1. A solution to the problem of simultaneous classification and localization of underwater objects from their acoustic field. Andrey I. Mashoshin (Central Scientific and Research Inst. 'Elektropribor', 30, Malaya Posadskaya Street, 197046 St. Petersburg, Russian Federation, amashoshin@eprib.ru)

This paper presents one of the solutions to the problem of simultaneous classification and localization of underwater objects from its acoustical field. It was shown that both problems (classification and localization) have some common features that allow to consider them as one mutual problem which can be solved by maximum likelihood method. The solution takes into account: - the fundamental physical representations of the transmission, propagation, reception of signals, and the measurement of their parameters against the noise background; - the target parameters vector describing features of its noise radiation and motion; - the parameters vector characterizing the observation conditions; - the errors vector of the signal parameter measurements. The theoretical solution is illustrated by solving a relatively simple but practically important problem of classification and ranging of an underwater object from passive underwater acoustic observation data.

4pUWe2. On acoustic tomography method physical advantages in long range ocean inhomogeneities control. Andrew Semenov (Acad. N.N Andreev's Acoustics Institute, Russ. Acad. of Sci., 4 Shvernik Street, 117036 Moscow, Russian Federation, asemen@akin.ru)

An acoustic tomography method based on observation of signals transecting an inhomogeneity is discussed. While for various inhomogeneities (ships, underwater objects, sea mammals etc.) short range monitoring of back scattered sonar reflections could be used, their monitoring at longer ranges is still a problem. This is due to the fast decay of sonar signals with distance, especially in unfavorably absorbing shallow water regions where there is functional distance dependence of optimum frequency. New perspectives on ocean climate change measurement (ATOC) require monitoring of extra long (500 - $1000 \mathrm{~km}$ ) tomography traces, where ocean vortices and icebergs could hamper the progress. The physical limits (optimum frequencies and efficiencies) for two tomographic methods for deep and, most unfavorable in the author's view, shallow ocean regions are compared. For several types of inhomogeneities tomorgaphic measurement optimization estimates are discussed. For ranges up to $500 \mathrm{~km}$ the acoustic tomography method has substantial advantages with respect to conventional sonar monitoring even for unfavorable shallow water regions at least up to $300 \mathrm{~km}$ distance and in all deep water regions. Tomographic measurements are also indispensable in long-range ocean vortices monitoring.
4pUWe3. A hydrophone calibration centre for the Mediterranean area Giovanni Bosco Cannelli (CNR-Istituto di Acustica 'O,M.Corbino', Via del Fosso del Cavaliere, 100, 00133 Rome, Italy, giovannibosco.cannelli@idac .rm.cnr.it), Silvano Buogo (CNR-Istituto di Acustica 'O,M.Corbino', Via del Fosso del Cavaliere, 100, 00133 Rome, Italy, silvano.buogo@idac.rm .cnr.it), Paola Calicchia (CNR-Istituto di Acustica 'O,M.Corbino', Via del Fosso del Cavaliere, 100, 00133 Rome, Italy, paola.calicchia@idac.rm.cnr .it)

The Underwater Acoustics Laboratory of the Institute of Acoustics "O. M. Corbino" in Rome, Italy, has recently achieved accreditation for hydrophone calibration. It can offer hydrophone calibration services operating under a management system compliant with UNI EN ISO/IEC standard and traceability of measurements to NPL primary standards. Precise, traceable measurements are needed today in all applications of underwater acoustics, where a faithful response of measuring devices is essential for a correct evaluation of the system performance and for a rigorous assessment of the environmental impact of man-made activities at sea. To attain these requirements the Laboratory has a water tank $6 \mathrm{~m}$ long, $4 \mathrm{~m}$ wide and 5,5 $\mathrm{m}$ deep, equipped with a motorized two-trolley positioning system capable of handling loads of up to $100 \mathrm{~kg}$. A variety of test and measurement equipment is available, controlled by a computer acoustic calibration system, and standalone instruments for signal generation, acquisition and analysis. Besides, two windows are provided on one of the tank walls for combined acoustical and optical measurements, which can be performed using the available Laser Doppler Vibrometer (up to $100 \mathrm{kHz}$ ) and the 16-mm film rotating prism high-speed camera (up to 10000 frames per second).

4pUWe4. Characteristics of sound pressure field focused by an acoustic aplanat lens. Toshiaki Nakamura (National Defense Academy, 1-10-20 Hashirimizu, 239-8686 Yokosuka, Japan, toshiaki@nda.ac.jp), Yuji Sato (Tsukuba Univ., Tsukuba Science City, 305-8573 Ibaraki, Japan, yuji@aclab.esys.tsukuba.ac.jp), Ayano Miyazaki (National Defense Academy, 1-10-20 Hashirimizu, 239-8686 Yokosuka, Japan, g44044@nda.ac .jp), Kazuyoshi Mori (National Defense Academy, 1-10-20 Hashirimizu, 239-8686 Yokosuka, Japan, kmori@nda.ac.jp)

In this paper, we describe the characteristics of an acoustic aplanat lens which can eliminate both spherical and coma aberrations. A singlet aplanat lens was designed using a ray theory in paraxial area. Sound pressure fields of the bi-concave aplanat lens and a spherical lens with $160 \mathrm{~mm}$ in diameter for the frequency of $500 \mathrm{kHz}$ were evaluated by a three-dimensional finite difference time domain (3-D FDTD) method. The aplanetic lens showed better convergence characteristics than spherical lens. Two bi-concave lenses of aplanat and spherical lenses were made by acrylic resin and water tank experiment was conducted to compare the on-axis characteristics and 
beam patterns at the focus of them. As a result, the aplanat lens could focus higher sound pressure than the spherical one within the incident angle of 10 degrees.

4pUWe5. Echo analysis of objects on the seafloor. Xiukun Li (College of Underwater Acoustic Engineering, Harbin Engineering University, 150001 Harbin, China, xiukun_li@yahoo.com.cn), Huiguang Chi (College of Underwater Acoustic Engineering, Harbin Engineering University, 150001 Harbin, China, xiukun_li@yahoo.com.cn)

Echoes from elastic objects on the seafloor comprise two kinds of acoustic components as well as bottom reverberation. One is elastic scattering echoes, and the other is geometric echoes. These echoes are called highlights, which can be used for identification of objects. Because time delay among highlights from small objects is very short, neither the wavelets resolved highlights structure nor suppressed bottom reverberation according to research. In order to obtain highlights structure and decay reverberation, the Hilbert Huang Transform (HHT), which is a new idea for analyzing nonlinear and nonstationary is applied in the paper. The HHT can describe the data from the instantaneous frequency and energy rather than the global frequency and energy defined by the Fourier spectral analysis, and the adjustable window Fourier spectral analysis defined by the wavelet. The results from both extensive simulations and real data show that the HHT has the highest resolution in time and frequency domain, and may prove to be a vital method for identification of objects on the sea floor.

4pUWe6. Tidal Effect of Reciprocal Sound Propagations at the Experiment in Hashirimizu Port. Hanako Ogasawara (National Defense Academy, 1-10-20 Hashirimizu, 239-8686 Yokosuka, Japan, ogasawar@nda.ac.jp), Toshiaki Nakamura (National Defense Academy, 1-10-20 Hashirimizu, 239-8686 Yokosuka, Japan, toshiaki@nda.ac .jp), Kazuyoshi Mori (National Defense Academy, 1-10-20 Hashirimizu, 239-8686 Yokosuka, Japan, kmori@nda.ac.jp), Koichi Mizutani (Tsukuba Univ., Tsukuba Science City, 305-8573 Ibaraki, Japan, mizutani@esys .tsukuba.ac.jp)

Acoustical monitoring method could monitor wide area such as ocean because it can spatially measure the measurement object with a few sensors. It is important to monitor ocean structure changes for understanding global climate changes and for controlling aquatic resources. We investigated sound propagation characteristics using sound propagated data measured at Hashirimizu port of Yokosuka, Japan in August 2006 and 2007. The experimental area was very shallow water with the average depth of $5 \mathrm{~m}$ and travel distance of $110 \mathrm{~m}$. M-sequence signal alternately propagated every $30 \mathrm{sec}$ onds from each bank side. Water depth change caused by tide affected received signal amplitude traveled with the frequency of $12 \mathrm{kHz}$ and $80 \mathrm{kHz}$.
It was also confirmed from simulated sound pressure by FDTD method through the change of assumed water depth. Furthermore, water temperature fluctuation could be confirmed from the travel time changes.

4pUWe7. Reduced Scale Experiment of Frequency Dependence of Single Spherical Biconcave Acoustic Lens for Ambient Noise Imaging. Kazuyoshi Mori (National Defense Academy, 1-10-20 Hashirimizu, 2398686 Yokosuka, Japan, kmori@nda.ac.jp), Hanako Ogasawara (National Defense Academy, 1-10-20 Hashirimizu, 239-8686 Yokosuka, Japan, ogasawar@nda.ac.jp), Toshiaki Nakamura (National Defense Academy, 1-10-20 Hashirimizu, 239-8686 Yokosuka, Japan, toshiaki@nda.ac.jp)

By the numerical analysis results using the Finite Difference Time Domain method in our previous studies, it was supposed that the spherical biconcave lens with an aperture diameter of $2.0 \mathrm{~m}$ has sufficient directional resolution (for example, the beam width is $1 \mathrm{deg}$ at $60 \mathrm{kHz}$ ) for realizing the Ambient Noise Imaging (ANI) system. In this study, to confirm the directional resolution of the lens in a wide frequency band of $20-100 \mathrm{kHz}$, we performed a reduced scale experiment of one-fifth space in a water tank. The lens, made of acrylic resin, has an aperture diameter of $400 \mathrm{~mm}$ and a radius of curvature of $500 \mathrm{~mm}$. A burst pulse of 25 cycles at 100, 200, 300, and 500 $\mathrm{kHz}$, in which the frequency increases 5 times, was radiated from the sound source to the lens. The results show that the $-3 \mathrm{~dB}$ area, whose pressure is 3 $\mathrm{dB}$ lower than the maximum at the image point, does not overlap each other at 300 and $500 \mathrm{kHz}$. It is supposed that the lens of $2.0 \mathrm{~m}$ aperture has the fine resolution over $60 \mathrm{kHz}$ in the original scale.

4pUWe8. Suppression of side lobe level on the cone characteristics of the directivity pattern of an antenna as an important factor in its directivity index and effective aperture. Zvonimir M.S Milosic (MORH, Trg kralja Petra Kresimira IV, HR-10000 Zagreb, Croatia, zvonimir.milosic@morh.hr)

This paper will present a universal procedure for precise de-embedding of the directivity index and effective aperture dependence of the suppressed side lobes on measured cone directivity patterns of sonar antennas. The procedure is derived on an idealized model of cone characteristics and directivity patterns of antennas. It is universally applicable to any contemporary sonar or hydroacoustic communication system with a cone directivity pattern. In accordance with the given expressions of directivity index and effective aperture of circle baffled pistons, this paper will present substitute analytical functions for hypothetical circle antennas with suppressed minor lobes. The presented graphs of functional behavior in a two-dimensional system, and in a three-dimensional coordinate system, are an excellent base for standardization, unification and quality evaluation of sonar or radar systems and also medical scanners. Key words: directivity index of sonar antenna, idealized model of cone directivity pattern, level of suppressed side lobes, effective aperture 


\title{
Session 4pUWf
}

\section{Underwater Acoustics and ECUA: Sound Propagation in 3-Dimensional Environments I (Poster Session)}

\author{
David Calvo, Cochair \\ U.S. Naval Res. Lab. \\ Michael Taroudakis, Cochair \\ University of Crete \& FORTH/IACM
}

\begin{abstract}
All posters will be on display from 3:40 p.m. to 5:20 p.m. To allow contributors an opportunity to see other posters, contributors of odd-numbered papers will be at their posters from 3:40 p.m. to 4:30 p.m. and contributors of even-numbered papers will be at their posters from 4:30 p.m. to 5:20 p.m.
\end{abstract}

\section{Contributed Paper}

4pUWf1. On a new approach to numerical modeling of a low-frequency underwater sound in $\mathbf{2}$ and 3-dimensional oceanic waveguides. Oleg E. Gulin (Il'ichev Pacific Oceanological Institute of Far-East Branch of Russian Academy of Sciences, 43, Baltiyskaya st., 690068 Vladivostok, Russian Federation, gulinoe@rambler.ru)

A new method to compute underwater sound fields in irregular planar waveguides is proposed. It realizes the full two-way propagation approach and exploits an idea of a problem solution dependence on a certain variable parameter that is the position of a boundary of the irregular region. With respect to this parameter for waveguide modes, an initial value problem can be formulated in the horizontal plane that is completely equivalent to the boundary value problem for the original wave equation (Helmholtz equation). This fact allows simulation of sound fields in waveguides based on ordinary differential equations with traditional approximations and for arbitrary source distance from the irregular region and the degree of irregularities. Examples of a simulation for a 2-D irregular waveguide model with an upslope rigid or absorbing penetrable bottom are presented for low frequencies and shallow sea conditions. They illustrate the strong difference between our solution and approximate solutions that arise due to both mode coupling and considerable backscattering within the considered irregular waveguide models

\section{Session 4pUWg}

\section{Underwater Acoustics and ECUA: Nonlinear Acoustic Methods in Searching for Buried Objects}

\author{
Benjamin R. Dzikowicz, Cochair
}

Naval Surface Warfare Center, Panama City Division, Code HS-11, 100 Vernon Ave., Panama City, FL 32407, USA

Eugeniusz Kozaczka, Cochair

Univ., Narutowicza 11/12, Gdansk, 80-952, Poland

\section{Invited Papers}

$5: 20$

4pUWg1. Parametric sonars in searching of buried objects. Eugeniusz Kozaczka (Univ., Narutowicza 11/12, 80-952 Gdansk, Poland, kozaczka@pg.gda.pl)

The subject of this paper is the description of usage of the parametric sonar for searching of the objects that are on the surface of the seabed or very close to it. Searching of underwater objects, especially these ones buried in the seabed has a very practical meaning. Presently more often mass destruction weapon is placed in the very difficult way to find it. Moreover searching of the objects of the archaeological character at sea requires usage of devices those have possibility of penetration of sediment which covers the searched object. In this case the most useful of acoustic means are parametric sonars that due to their specific features are good tools for underwater searching. The principle of detection of buried objects is similar to detection in the case of usage of the classical sonars. The dispersion of the sound field enables to track the object and sometimes enables to define the shape of the searched object. The measurement equipment can be connected to the Global Positioning System (GPS) and complementary equipment that is necessary in such kind of investigations. There will be presented experimental results that were obtained during the trials in the Gdansk Bay. Also will be shown the typical images for chosen objects. 
4pUWg2. Observation of sub-bottom sediments in the Southern Baltic by means of nonlinear acoustic method. Grazyna Grelowska (Polish Naval Academy, Smidowicza 69, 81-103 Gdynia, Poland, ggrel@wp.pl), Eugeniusz Kozaczka (Univ., Narutowicza 11/12, 80-952 Gdansk, Poland, kozaczka@pg.gda.pl), Ignacy Gloza (Polish Naval Academy, Smidowicza 69, 81-103 Gdynia, Poland, i.gloza@amw.gdynia.pl)

The aim of the paper is to present results of preliminary experimental investigation using parametric echosounder in natural conditions for profiling the subbottom sediments in the Gulf of Gdansk. The ability to predict seabed properties: seafloor roughness, subbottom structure and discrete scatterers laying on the seafloor or buried into sediments, from remotely sensed data is important especially in regions that need permanent monitoring. Precise determination of seabed structure or localisation of buried objects in the sand requires the use of a low frequency signal to penetrate the sediment and a narrow beam to provide high-resolution data. Both requirements can be achieved with a parametric (nonlinear) technique. The special experimental setup has been arranged allowing penetration of bottom sediments as well as precise positioning and following a given route. Measurements were conducted exactly along given routes, for that geological profiles have bean taken by means of another method. It allow as to compare detailed date obtained by means of parametric echosounding to ones given at geological map. In all investigations the primary frequency of the array was of $100 \mathrm{kHz}$, whereas the secondary frequency changed in range $5 \mathrm{kHz}-15 \mathrm{kHz}$.

6:00

4pUWg3. Transmission of low frequency sounds from ships into water environment. Ignacy Gloza (Polish Naval Academy, Smidowicza 69, 81-103 Gdynia, Poland, i.gloza@amw.gdynia.pl)

The main goal is presentation the broad experimental research associated with the transmission of acoustic energy generated by moving objects into the water. I present the methodology of evoluation of the transmission of low frequency vibration from the mechanisms of ship into the water environment. For that purpose the research of vibration distribution over ship's hull were conducted along with the hydroacoustic field. The underwater sound measurements were performed both for sailing condition and during anchorage. The hull is source of complex surface shape inducing waves of different frequencies depending on the working parameters of ship's machinery. As a result of mechanical incitement of the hull's surface a complex distribution of vibration is created depending on vibration spectra of the primary sources as for the main propulsion units, generators or other mechanisms. This research allowed to determine the transmission coefficient for the mechanical vibration energy going through the ship's hull and the evaluation of influence on the hydroacoustic field of a ship. The values signaling the changes of the technical condition of machinery and propulsion system.

\section{6:20}

4pUWg4. Detection of a resonant target buried in sediment using iterative time reversal: mid-frequency pond experiments. Benjamin R. Dzikowicz (Naval Surface Warfare Center, Panama City Division, Code HS-11, 100 Vernon Ave., Panama City, FL 32407, USA, benjamin.dzikowicz@navy.mil), Zachary J. Waters (Boston University, Dept. of Aerosp. and Mech. Eng., 110 Cummington St., Boston, MA 02215, USA, zjwaters@bu.edu), R. Glynn Holt (Boston University, Dept. of Aerosp. and Mech. Eng., 110 Cummington St., Boston, MA 02215, USA, rgholt@bu.edu), Ronald A. Roy (Boston University, Dept. of Aerosp. and Mech. Eng., 110 Cummington St., Boston, MA 02215, USA, ronroy@bu.edu)

Iterative time reversal techniques developed at smaller scales, [Waters et al., J. Acoust. Soc. Am. 122, 3023 (2007)], are applied to the detection of a $15 \mathrm{~cm}$ diameter stainless steel shell buried in sandy sediment at the acoustic test pond at the Naval Surface Warfare Center - Panama City Division. A mid-frequency, directional projector is located $1.5 \mathrm{~m}$ above the sediment and directed normally to it. A hydrophone is located midway between the sediment and the projector. This system gives a response between $20 \mathrm{kHz}$ and $200 \mathrm{kHz}$. A calibration filter is designed using the direct path response between the projector and hydrophone. This filter is applied at each time reversal iteration to prevent the time reversal technique from converging to the transducer resonance. Application of iterative time reversal allows the detection of the target at greater depths than otherwise possible due to the resonance scattering of the target. Additional experiments explore the application of filters, and the effect of different window sizes. Also, comparisons with similar laboratory experiments and comments on real world applications are discussed. [Work supported by the Office of Naval Research.]

4pUWg5. Sonar detection of targets buried under seafloor ripple at shallow grazing angles. Joseph Lopes (Naval Surface Warfare Center - Panama City Division, 110 Vernon Ave, Panama City, FL 32407, USA, joseph.1.lopes@navy.mil), Raymond Lim (Naval Surface Warfare Center - Panama City Division, 110 Vernon Ave, Panama City, FL 32407, USA, raymond.lim@ navy.mil), Carrie Dowdy (Naval Surface Warfare Center - Panama City Division, 110 Vernon Ave, Panama City, FL 32407, USA, carrie.dowdy@ navy .mil), Kevin L. Williams (Applied Physics Laboratory, University of Washington, 1013 NE 40th St, Seattle, WA 98105, USA, williams@apl.washington.edu), Eric Thorsos (Applied Physics Laboratory, University of Washington, 1013 NE 40th St, Seattle, WA 98105, USA, eit@apl.washington.edu)

This paper summarizes results from modeling and measurement efforts investigating shallow grazing angle reverberation levels from a rippled bottom and subcritical detection of targets buried under such interfaces. The focus of this work is associated with frequencies less than $10 \mathrm{kHz}$ where evanescent transmission is important. Measurements were performed in a 13.7-m deep, 110-m long, 80-m wide test-pool with a $1.5-\mathrm{m}$ layer of sand on the bottom. Rippled contours were artificially formed with the aid of a sand scraper. A parametric sonar that generated difference frequency signals in the 1 to $20 \mathrm{kHz}$ frequency range was placed onto a rail system permitting acquired data to be processed and displayed similar to that of a side scan sonar. The buried target was a solid aluminum cylinder. The seabed roughness was measured to assess ripple fidelity and to estimate the small-scale roughness spectrum which was used in scattering models to calculate the backscattered signal levels from the target and bottom. Acoustic backscatter data obtained for various ripples parameters (wavelengths, heights, orientation, etc.) were compared to model predictions based on perturbation theory. [Work supported by the Office of Naval Research and the Strategic Environmental Research and Development Program, USA]. 
4pUWg6. Changing of scattering properties of underwater objects covered by elastic shell. Grazyna Grelowska (Polish Naval Academy, Smidowicza 69, 81-103 Gdynia, Poland, ggrel@wp.pl), Ignacy Gloza (Polish Naval Academy, Smidowicza 69, 81-103 Gdynia, Poland, i.gloza@amw.gdynia.pl)

In certain situations it is of a great importance to decrease reflection properties of underwater objects positioned into water, deployed on a seabed or buried into sediments. This effect could be achieved by means of covering shell changing acoustic parameters of considered object. The aim of the paper is to present the idea of performing the covering material for underwater objects as well as results of experimental investigation of its scattering characteristics. Acoustic scattering measurements were performed in laboratory condition on solid air-filled objects of sphere or ellipsoidal shape with or without covering shell. The objects were insonified by acoustic source at frequency in range of $60 \mathrm{kHz}-150 \mathrm{kHz}$.

\title{
Contributed Paper
}

$7: 20$

4pUWg7. Experimental study of parametric transmission: Simultaneous generation of two beams. Maud Amate (GESMA, BP42, 29240 Brest Armées, France, maud.amate@dga.defense.gouv.fr), Pierre Cervenka (Institut Jean le Rond D'Alembert, UMR 7190, 2 Place de la Gare de Ceinture, 78210 Saint Cyr L'Ecole, France, cvk@ccr.jussieu.fr), Jacques Marchal (Institut Jean le Rond D’Alembert, UMR 7190, 2 Place de la Gare de Ceinture, 78210 Saint Cyr L'Ecole, France,jmarchal@ccr.jussieu.fr)

In order to respond to the buried mines threat, an approach of GESMA (Groupe d'Etudes Sous-Marines de l'Atlantique) is to take advantage of the parametric transmission in sonar imagery. This technique allows producing narrow beamwidths at low frequencies. Such a parametric transmitter has been designed and calibrated in collaboration with an academic laboratory of Paris VI, and the targeted benchmark is fulfilled: $2^{\circ} @ 20 \mathrm{kHz}$, with sufficient source level. The design of the complete sonar system is based on a sequential multibeam transmission associated with a synthetic aperture technique at receive. However, the shortfall of the sweeping technique lies in the timing limitation. One solution to increase the coverage rate is the simultaneous generation of several parametric beams in different directions. Experimentations have been conducted in a tank with different signals: CW, LFM, Ricker. This presentation addresses the results of these experimentations in the context of buried mines detection and classification.

THURSDAY AFTERNOON, 3 JULY 2008

ROOM 362/363, 5:20 TO 7:40 P.M.

\section{Session 4pUWh}

\section{Underwater Acoustics, Computational Acoustics, and ECUA: Numerical Methods in Underwater Scattering}

\author{
Andrew F. Seybert, Cochair \\ University of Kentucky, Dept. of Mech. Engineering, 151 RGAN Bldg., Lexington, KY 40506, USA \\ Martin Ochmann, Cochair \\ Technische Fachhochschule Berlin, Univ. of Applied Sciences, Luxemburger Str. 10, Berlin, 13353, Germany \\ Bodo Nolte, Cochair \\ Forschungsanstalt der Bundeswehr für Wasserschall und Geophysik, Klausdorfer Weg 2-24, Kiel, 24148, Germany
}

\section{Invited Papers}

4pUWh1. BIE and coupled BIE-FEM methods for scattering from underwater objects. Ilkka Karasalo (FOI, Gullfossgatan 6, SE 16490 Stockholm, Sweden, ilkka.karasalo@foi.se)

Two techniques are described for computation of acoustic scattering by a smooth elastic object in a fluid space or a fluid layer of a layered fluid-solid medium. Both techniques use a frequency-domain boundary integral equation (BIE) method for the exterior layered medium, with the Green's function computed numerically by adaptive high-order transform integration and exact finite elements. The surface of the object is parameterized by a smooth map of the unit sphere, and the BIE is discretized by B-splines, point collocation and numerical integration in polar-like coordinates centered at the collocation point. Two approaches for handling the interior dynamics of the object are described. In the first, applicable to objects structured into homogeneous layers separated by closed smooth interfaces, the interior dynamics is formulated as a system of BIEs with displacements and tractions at the interfaces as unknowns. In the second, the interior is discretized by a general-purpose finite element (FEM) method, and an impedance matrix connecting tractions and displacements on the exterior surface is computed by eliminating interior degrees of freedom by Gaussian elimination. Computational results on test cases of the 2006 target strength benchmark workshop at NURC are presented. 
4pUWh2. Calculation of the acoustic target strength of elastic objects based on BEM-BEM-coupling. Ralf Burgschweiger (Technische Fachhochschule Berlin, Univ. of Applied Sciences, Luxemburger Str. 10, 13353 Berlin, Germany, burgi@tfh-berlin.de), Martin Ochmann (Technische Fachhochschule Berlin, Univ. of Applied Sciences, Luxemburger Str. 10, 13353 Berlin, Germany, ochmann@tfh-berlin.de), Bodo Nolte (Forschungsanstalt der Bundeswehr für Wasserschall und Geophysik, Klausdorfer Weg 2-24, 24148 Kiel, Germany, bodonolte@bwb.org)

Based on a BEM-BEM-coupling method, the scattered pressure from elastic objects placed in water and partially buried in the sediment is calculated. For this reason, a special variant of the boundary element method (BEM) is implemented. It contains a pre- and a postprocessor with 3D visualization, in order to define the geometry of the scattering objects in the interface layer between fluid and sediment and the parameters needed for characterizing the fluid and the elastic material. The solver is able to perform numerical calculations in a multiple parallel manner. For the solution of the underlying system of linear equations, we use different kinds of approximate and direct solution techniques. Simple acoustical exterior problems, for example, the sound scattering by elastic solid cylinders and spheres placed in a fluid are treated by the BEM-BEM-coupling method. The results will be compared with analytical solutions or solutions obtained from other numerical methods.

\section{Contributed Papers}

\section{6:00}

4pUWh3. Modeling of scattering from targets in an oceanic waveguide using Kirchhoff/diffraction method. Keunhwa Lee (Seoul National University, San 56-1, Silim, Kwanak, 11111 Seoul, Republic of Korea, nasalkh2@snu.ac.kr), Woojae Seong (Seoul National University, Room. 306, Bd. 34, San 56-1, Sillim-dong, Kwanak-gu, College of Engineering, Dept. of Naval Architecture and Ocean Engineering, 151-744 Seoul, Republic of Korea, wseong@snu.ac.kr), Yongtaek Joo (Daewoo shipbuilding \& marine engeineering CO.,LTD., Aju-dong, Geoje city, 11111 Geoje, Republic of Korea, yhsaga@dsme.co.kr)

The target scattering model in an oceanic waveguide is presented. The target scattered pressure field is formulated using the generalized Green's function method [F. Ingenito, J. Acoust. Soc. Am. 82, 2051-2059 (1987)]. The concept of Kirchhoff/diffraction method is introduced in order to simplify the Fredholm integral equation. In numerical analysis, complex target is divided into numerous polygon facets, whose analytic solution for scattered field is derived based on the waveguide solution by the ray or normal mode theory. This solution is used in constructing the target scattered field for complex target. Comparison between ray and normal mode based target scattering model is shown. Finally discussion for conditions of the source/receiver and target which improves the numerical efficiency is given.

\section{6:20}

4pUWh4. Modeling ocean reverberation under short pulse conditions. Henry Weinberg (Alion Science Incorporated, 23 Colonial Drive, Waterford, CT 06385, USA, chic@ct.metrocast.net)

The Comprehensive Acoustic System Simulation (CASS) is a standard model for predicting ocean reverberation. However, the current version, CASS V4.1, is known to have theoretical and numerical difficulties when investigating short pulse lengths. This is due to two model requirements: (1) the time increment for sampling reverberation should not exceed the pulse length; and (2) the range increment for sampling the environment should not exceed half the sound speed-pulse length product. Unless these requirements are met, certain phenomena, such as time splitting, may not be accurately modeled. In addition, the predicted results may have an unrealistic step function appearance. On the other hand, very small time and range increments often lead to excessive computational requirements. A simple modification to CASS V4.1 appears to have relaxed the current increment requirements substantially. The range increment must still be small enough to sample environmental features, but not to the extent dictated by small pulse lengths. Although the modification is based on a well known mathematical method for accelerating convergence, its success in modeling reverberation was unexpected.

\section{6:40}

4pUWh5. Comparison of two monostatic reverberation models based on ray theory. Youngmin Chu (Seoul National University, Room. 306, Bd. 34, San 56-1, Sillim-dong, Kwanak-gu, College of Engineering, Dept. of Naval Architecture and Ocean Engineering, 151-744 Seoul, Republic of Korea,sks655@snu.ac.kr), Woojae Seong (Seoul National University,
Room. 306, Bd. 34, San 56-1, Sillim-dong, Kwanak-gu, College of Engineering, Dept. of Naval Architecture and Ocean Engineering, 151-744 Seoul, Republic of Korea, wseong@snu.ac.kr), Won Tchon Oh (Agency for Defense Development, 645-016 Jinhae, Republic of Korea, wontchon @ chollian.net)

Two monostatic reverberation models applicable for range dependent environment are presented. Considering the roughness of both the sea surface and bottom, we calculate the reverberation signal, based on the ray propagation model and scattering model using the composite-roughness theory and/or other empirical formulae. First model computes the reverberation time signal using two-way eigenray searching between source/receiver position along the discrete scattering area (dA). The other model uses one-way eigenray searching along the discrete ray angle $(\mathrm{d} \theta)$ which generates a nonuniform scattering area providing less accurate solution at a reduced computational burden. The time series calculated from both models are shown and compared with the solutions presented in the Reverberation Modeling Workshop (Nov. 2006, Austin, TX).

\section{7:00}

4pUWh6. A novel modelling approach for sound propagation analysis in a multiple scatterer environment. Bert Van Genechten (K.U.Leuven Dept. of Mechanical Engineering, Celestijnenlaan 300B - bus 2420, 3001 Heverlee, Belgium, Bert.VanGenechten@mech.kuleuven.be), Bart Bergen (K.U.Leuven - Dept. of Mechanical Engineering, Celestijnenlaan 300B - bus 2420, 3001 Heverlee, Belgium, Bart.Bergen@mech.kuleuven .be), Bert Pluymers (K.U.Leuven - Dept. of Mechanical Engineering, Celestijnenlaan 300B - bus 2420, 3001 Heverlee, Belgium, Bert.Pluymers @ mech.kuleuven.be), Dirk Vandepitte (K.U.Leuven - Dept. of Mechanical Engineering, Celestijnenlaan 300B - bus 2420, 3001 Heverlee, Belgium, Dirk.Vandepitte@mech.kuleuven.be), Wim Desmet (K.U.Leuven - Dept. of Mechanical Engineering, Celestijnenlaan 300B - bus 2420, 3001 Heverlee, Belgium,Wim.Desmet@mech.kuleuven.be)

In the past decades element-based numerical modelling techniques have become a commonly used and invaluable tool for the analysis of acoustic radiation and scattering problems. However, the pollution errors associated with the element discretisation inherent to these methods increase with the increasing size of the problem domain. As a result, the applicability of these methods for radiation and scattering problems in which the source and receiver positions are located far from each other is often prohibited. The Wave Based Method (WBM) is an alternative deterministic prediction method for the analysis of steady-state acoustic problems. It is based on an indirect Trefftz approach in that wave functions, which are exact solutions of the underlying differential equation, are used to describe the dynamic response. The enhanced computational efficiency of the WBM as compared to the element based methods has been shown already for the analysis of both finite and (semi-)infinite acoustic problems. This paper introduces a novel WBM-based methodology to model the acoustic source-receiver transfer path functions in a multiple scatterer environment. A sound propagation validation case illustrates the potential of the proposed approach. 
$7: 20$

4pUWh7. The basis choice and the reconstruction of combined refractive-kinetic inhomogeneities in the problems of ocean acoustic tomography. Andrey Shurup (Department of Acoustics, Physics Faculty, Moscow State University, Leninskie Gory, 119991 Moscow, Russian Federation, burov@phys.msu.ru), Sergey Sergeev (Department of Acoustics, Physics Faculty, Moscow State University, Leninskie Gory, 119991 Moscow, Russian Federation, sergeev@su29.ru), Valentin Burov (Department of Acoustics, Physics Faculty, Moscow State University, Leninskie Gory, 119991 Moscow, Russian Federation, burov@phys.msu.ru)

The characteristics of the acoustical signal in ocean are determined by both the refractive inhomogeneities (the perturbation of sound velocity) and the presence of ocean currents. The methods of acoustical tomography can be applied to the simultaneous reconstruction of both refractive and kinetic inhomogeneities. In this paper the problem of the combined refractivekinetic inhomogeneites reconstruction by the tomography methods is considered. For the realization of proposed scheme so called band basis consisted of a number of intersected stripes is applied. The advantage of the band basis in tomographic applications is conditioned by the simplicity of solving the direct problem and the possibility of describing all types of inhomogeneities in a unified manner. The comparison of the band basis with the commonly used basis composed of nonoverlapping squares is considered. The results of the reconstruction of model kinetic and combined refractive-kinetic inhomogeneities in band basis are presented. The possibility of complete tomography reconstruction of two-dimensional flows based on the scattering data only is illustrated. 


\title{
Session 5aAAa
}

\section{Architectural Acoustics: New Frontiers in Room Acoustical Modeling I}

\author{
Murray Hodgson, Cochair \\ The University of British Columbia, Department of Electrical and Computer Engineering, 2332 Main Mall, Vancouver, \\ BC V6T 1Z4, Canada \\ Vincent Valeau, Cochair \\ Laboratoire d'Etudes Aérodynamiques (LEA), Université de Poitiers - ENSMA - CNRS, Bâtiment K, \\ 40 Avenue du Recteur Pineau, Poitiers, F-86022, France
}

\section{Contributed Papers}

\section{8:00}

5aAAa1. Multiresolution geometrical-acoustics modeling. Benjamin Markham (Rensselaer Polytechnic Institute, Greene Bldg., 110 8th St., Troy, NY 12180, USA, markhb@rpi.edu), Paul Calamia (Rensselaer Polytechnic Institute, Greene Bldg., 110 8th St., Troy, NY 12180, USA, calamp @ rpi.edu)

Geometrical-acoustics (GA) modeling techniques assume that surfaces are large relative to the wavelengths of interest. For a given scenario, practitioners typically create a single 3D model with large, flat surfaces that satisfy the assumption over a broad range of frequencies. Such geometric approximations lead to errors in the spatial distribution of the simulated sound field because geometric details that influence reflection and scattering behavior are omitted. To compensate for the approximations, modelers typically estimate scattering coefficients for the surfaces to account stochastically for the actual, wavelength-dependent variations in reflection directionality. A more deterministic approach could consider a series of models with increasing geometric detail, each to be analyzed at a corresponding frequency band for which the requirement of large surface dimensions is satisfied. Thus, to improve broadband spatial accuracy for GA simulations, we propose a multiresolution modeling approach. Using scale model measurements of a corrugated wall, comparisons of our method with non-GA techniques, and some simple listening tests, we will demonstrate that multiresolution geometry provides more spatially accurate results than single-resolution approximations when using GA techniques, and that this improved accuracy is aurally significant.

\section{8:20}

5aAAa2. On the analysis of the time spreading of sound diffusers Javier Redondo (IGIC - Universitat Politècnica de València, Cra. NazaretOliva S/N, E-46730 Gandia, Spain, fredondo@fis.upv.es), Rubén Picó (EPSG - Univ. Politécnica de Valencia, c/ Nazaret-Oliva s/n, 46780 Grau de Gandia, Spain, rpico@fis.upv.es), Mark R. Avis (University of Salford, Acoustics Research Centre, Newton Building, M5 4WT Salford, UK, m.r.avis@salford.ac.uk)

Since the invention of sound diffusers three decades ago a substantial effort has been made to predict the acoustic behaviour of these structures. BEM methods are well established for this purpose after a systematic comparison between simulations and experimental data. Volumetric methods such as finite element methods (FEM) or the finite difference time domain method (FDTD) are not often used, due to their large computational cost. However, near to far field transformations (NFFT) can overcome that problem. Recently some of the authors have shown that the FDTD method is a useful technique to analyse the time domain signature of sound diffusers. In this paper a careful analysis of the performance of diffusers in the time domain ("time spreading") are reported, opening a new field of research.

\section{Invited Papers}

\section{8:40}

5aAAa3. Diffraction modeling in acoustic radiance transfer method. Samuel Siltanen (Helsinki University of Technology, P.O. Box 5400, 02015 TKK, Finland, Samuel.Siltanen@tml.hut.fi), Tapio Lokki (Helsinki University of Technology, P.O. Box 5400, 02015 TKK, Finland, Tapio.Lokki@tkk.fi)

The room acoustic radiance transfer method is a solution to recently presented room acoustic rendering equation which formulates the mathematical basis for all the ray-based (geometrical) room acoustic modeling algorithms. The basic acoustic transfer method gives as accurate results as the state-of-the-art commercial room acoustic modeling software. However, the basic method still lacks, e.g., diffraction modeling and modeling of complex reflections from surfaces. In this paper we discuss different diffraction modeling methods in the light of the acoustic radiance transfer method. The problems as well as benefits of each diffraction modeling method are summarized to understand which one of them can be implemented together with acoustic radiance transfer. Finally, some implementation examples are given.

\section{9:00}

5aAAa4. Can also diffracted sound be handled as flow of particles? Some new results of a beam tracing approach based on the uncertainty principle. Uwe M. Stephenson (Hafen City University Hamburg, Nelkenweg 10, 23843 Bad Oldesloe, Germany, post @umstephenson.de)

In computational room acoustics as well as noise immission prognosis efficient ray or beam tracing methods are well approved - but the problem of the neglected diffraction is still unsolved in general. The author's successful approach of 1986 based on Heisenbergs uncertainty principle was extended to the more efficient beam tracing technique and presented at the ICA 2007. The algorithm has now been generalized to recursive higher order diffraction. Now, not only single edge, but also multiple edge diffraction could be simulated 
(slit and "wide obstacle" with two edges). The results have again been compared with Svensson's exact wave-theoretical secondary edge source model. With some restrictions, they seem to indicate, that indeed even diffraction of sound - like light - may be handled as flow of particles. To avoid the feared explosion of computation time with higher order diffraction, a beam reunification may now be achieved by Quantized Pyramidal Beam Tracing.

\section{Contributed Paper}

\section{9:20}

5aAAa5. General impedance boundary conditions in pseudospectral time-domain methods for room acoustics. Carlos Spa (Universitat Pompeu Fabra - Fundació Barcelona Media, Ocata, 1, 08003 Barcelona, Spain, carlos.spa@upf.edu), Toni Mateos (Universitat Pompeu Fabra Fundació Barcelona Media, Ocata, 1, 08003 Barcelona, Spain, toni.mateos@barcelonamedia.org), Adan Garriga (Universitat Pompeu Fabra - Fundació Barcelona Media, Ocata, 1, 08003 Barcelona, Spain, adan .garriga@barcelonamedia.org)

Finite-differences in the time domain (FDTD) are among the most accurate numerical techniques to simulate wave phenomena. The main drawback of FDTD numerical schemes is their computational cost in large scale simulations. The recently developed Fourier pseudospectral time-domain (PSTD) techniques, by approximating the spatial derivatives more efficiently, have improved significantly the accuracy and time costs of the simulations of electromagnetic fields. As a step towards applying PSTD techniques to room acoustic problems, we present here a framework to properly deal with material modeling in terms of generic impedance boundary conditions, beyond the common perfectly matched layer absorbing boundaries. We apply our results to a few representative cases (simple but reverberant rooms) and analyse its performance in terms of accuracy and computational resources. In particular we analyse whether the mild constraints that PSTD imposes on space-time discretization lead to good enough results in room acoustic simulations.

9:40-11:00 Posters

Lecture sessions will recess for presentation of poster papers on various topics in acoustics. See poster sessions for topics and abstracts.

Invited Papers

11:00

5aAAa6. Ray-tracing prediction of sound-pressure and sound-intensity fields in empty and fitted rooms. Murray Hodgson (The University of British Columbia, Department of Electrical and Computer Engineering, 2332 Main Mall, Vancouver, BC V6T 1Z4, Canada, mhodgson@interchange.ubc.ca), Owen Cousins (The University of British Columbia, Department of Electrical and Computer Engineering, 2332 Main Mall, Vancouver, BC V6T 1Z4, Canada, owencousins@ hotmail.com), Gary Chan (The University of British Columbia, Department of Electrical and Computer Engineering, 2332 Main Mall, Vancouver, BC V6T 1Z4, Canada, gakachan@interchg.ubc.ca), Vincent Valeau (Laboratoire d'Etudes Aérodynamiques (LEA), Université de Poitiers - ENSMA - CNRS, Bâtiment K, 40 Avenue du Recteur Pineau, F-86022 Poitiers, France, vincent.valeau@lea.univ-poitiers.fr)

A Monte-Carlo ray-tracing model has been adapted to the prediction of sound-pressure and sound-intensity fields in rooms with surfaces of arbitrary surface impedance, and containing parallelepiped obstacles. Phase changes due to propagation distance and wall reflection were accounted for. Diffraction around obstacle edges was modeled by the Unified Theory of Diffraction. The model was also extended to include the out-of-phase secondary sound source of a single-channel, global active-noise-control (ANC) system. The new model was validated in comparison with predictions by reference models (e.g. FEM) and with the results of experiments in an anechoic chamber and in real rooms. It was used to predict the effectiveness of ANC. This paper discusses the development of the new models, the results of the validation tests and ANC predictions.

11:20

5aAAa7. The intensity in a reverberant field as an acoustic energy-density gradient. Vincent Valeau (Laboratoire d'Etudes Aérodynamiques (LEA), Université de Poitiers - ENSMA - CNRS, Bâtiment K, 40 Avenue du Recteur Pineau, F-86022 Poitiers, France, vincent.valeau@lea.univ-poitiers.fr), Matthieu Boirlaud (ESIP, Av. du Recteur Pineau, 86022 Poitiers Cedex, France, matthieu.boirlaud@etu.univ-poitiers.fr), Judicaël Picaut (Lab. Central des Ponts et Chaussées, Division Entretien, Sécurité et Acoustique des Routes, Route de Bouaye - BP 4129, 44341 Bouguenais Cedex, France, Judicael.Picaut@lcpc.fr), Murray Hodgson (The University of British Columbia, Department of Electrical and Computer Engineering, 2332 Main Mall, Vancouver, BC V6T 1Z4, Canada, mhodgson@interchange.ubc.ca)

The classical assumption for diffuse reverberant sound fields is that the acoustic energy flow at any location in a room -- i.e., the acoustic intensity -- is null. For rooms with disproportionate dimensions and/or uneven absorption distributions, this assumption no longer holds. Over the past few years, a room-acoustic diffusion model has been developed that can be seen as an extension of the statistical theory to nondiffuse sound fields. This work investigates the basic gradient-equation underlying this diffusion theory: it states that the energy-flow vector is proportional to both the gradient of the acoustic energy density, and to the room diffusion constant. The gradient equation for the acoustic intensity is confirmed numerically by comparison with calculations of the intensity vector using a particle-tracing model and a ray-tracing model. Examples are presented for a room with a diffuse sound field, and for elongated rooms where the sound field is strongly non-diffuse, in the case of both diffuse and specular reflections. 
5aAAa8. Prediction and tailoring of steady-state broadband sound fields in enclosures using absorption scaling and energy-intensity boundary elements. Donald B. Bliss (Duke University, Mechanical Engineering and Materials Science, 148B Hudson Hall, Durham, NC 27708, USA, dbb@duke.edu), Krista A. Michalis (Duke University, Mechanical Engineering and Materials Science, 148B Hudson Hall, Durham, NC 27708, USA, kam49@duke.edu), Linda P. Franzoni (Duke University, Mechanical Engineering and Materials Science, 148B Hudson Hall, Durham, NC 27708, USA, franzoni@ duke.edu)

Enclosures with diffuse reflection boundaries are modeled with an energy-intensity boundary element method using uncorrelated broadband directional sources. An absorption-based perturbation analysis shows the spatial variation of the acoustic field obeys certain scaling laws. A series expansion in terms of average absorption gives separate boundary integral problems at each order. The lowestorder solution has a uniform level proportional to the reciprocal of the average absorption. The next-order solution is independent of average absorption and primarily responsible for spatial variation of the acoustic field. This solution depends on the spatial distribution of absorption and input power sources, but not their overall level. For the primary spatial variation, the effects of the relative distributions of absorption and input power are linear and uncoupled. These distributions can be expressed in terms of constituent spatial modes corresponding to the ways absorption and input power can be distributed. Solved numerically once for each mode, the acoustic field can be expressed in terms of the modal amplitudes in closed form. These amplitudes can be adjusted to tailor the spatial variation. Examples include how to distribute absorption to minimize sound levels in one location, or how to achieve a uniform interior field. (Sponsor: NSF)

\section{Contributed Papers}

\section{2:00}

5aAAa9. Analysis of room transfer function and reverberant signal statistics. Eleftheria Georganti (Acoustic Technology Department, Technical University of Denmark, Ørsted Plads, B352, DK-2800 Lyngby, Denmark, ege@oersted.dtu.dk), John Mourjopoulos (Audio and Acoustic Technology Group, Wire Communications Laboratory, Electrical Engineering \& Computer Technology Department, University of Patras, 26500 Patras, Greece, mourjop@wcl.ee.upatras.gr), Finn Jacobsen (Acoustic Technology Department, Technical University of Denmark, Ørsted Plads, B352, DK2800 Lyngby, Denmark, fja@oersted.dtu.dk)

For some time now, statistical analysis has been a valuable tool in analyzing room transfer functions (RTFs). This work examines existing statistical time-frequency models and techniques for RTF analysis (e.g., Schroeder's stochastic model and the standard deviation over frequency bands for the RTF magnitude and phase). RTF fractional octave smoothing, as with $1 / 3$ octave analysis, may lead to RTF simplifications that can be useful for several audio applications, like room compensation, room modeling, auralisation purposes. The aim of this work is to identify the relationship of optimal response smoothing (e.g., as in complex smoothing) with respect to the original RTF statistics. More specifically, the RTF statistics, derived after the complex smoothing calculation, are compared to the original statistics across space inside typical rooms, by varying the source, the receiver position and the corresponding ratio of the direct and reverberant signal. In addition, this work examines the statistical quantities for speech and audio signals prior to their reproduction within rooms and when recorded in rooms. Histograms and other statistical distributions are used to compare RTF minima of typical "anechoic" and "reverberant" audio speech signals, in or- der to model the alterations due to room acoustics. The above results are obtained from both in-situ room response measurements and controlled acoustical response simulations.

\section{$12: 20$}

5aAAa10. On the implementation of room acoustics modeling software using Digital Waveguide Mesh. José J. Lopez (Tech. Univ. of Valencia, Camino de Vera S/N, 46021 Valencia, Spain, jjlopez@dcom.upv.es), José Escolano (University of Jaén, Alfonso X, 28, E-23700 Linares, Spain, escolano@ujaen.es), Basilio Pueo (University of Alicante, Signals, Systems and Telecommunications, Cta San Vicente del Raspeig s/n, E-03690 Alicante, Spain,basilio@ua.es)

The Digital Waveguide Mesh (DWM) method for room acoustic simulation has been introduced in the last years to solve sound propagation problems numerically. However, the huge computer power needed in the modeling of large rooms and the complexity to incorporate realistic boundary conditions has delayed their general use, being restricted to the validation of theoretical concepts using simple and small rooms. This paper presents a complete DWM implementation where all the stages needed to analyze a room are discussed. The software starts the analysis from the architectural model of the room importing its geometry directly from a CAD file. After that, it generates the rectangular mesh of individual cells that conforms the volume to be simulated. Next, the time domain recursion is carried out using parallel computer techniques. Also the software includes a serious treatment of boundary conditions using different material with frequency dependence characteristics. Finally, the software can export the results. Additionally, some room simulation examples are presented and analyzed in detail. The work carried out demonstrate how it is possible, with the current power of the personal computer, to start to simulate real rooms with high amount of geometric details and frequency dependent boundary.

\section{2:40-2:00 Lunch Break}

\section{2:00}

5aAAa11. Defining perceptual requirements of dynamic (real-time) acoustic modeling and auralization. Linda Gedemer (5930 Penfield Ave., Woodland Hills, CA 91367, USA, gedeml@ @pi.edu)

Acoustic modeling is evolving from static sources and receivers to dynamic sources and receivers as computational speeds allow for faster renderings. Current research has focused on creating highly accurate dynamic models and auralizations but still struggle with the trade-off between accuracy and available processing speed. The question remains as to how accurate do these models need to be if the receiver (listener) is moving dynamically through the acoustic model. The focus of this paper is to address the accuracy required of dynamic models and/or auralizations from a perceptual standpoint. From this, the necessity for creating models that are perhaps more accurate than required will be considered.

\section{2:20}

5aAAa12. The scanning and voxellisation of complex 3D objects for incorporation within finite difference time domain based acoustic prediction. Ian Drumm (The University of Salford, C.S.E., Salford University, The Crescent, M5 4WT Manchester, UK, i.drumm@salford.ac.uk)

This paper presents techniques developed to incorporate complex 3D objects within the author's own finite difference time domain based acoustic prediction software application. Using a combination of 3D laser scanning, ray based voxellisation and a least pth norm based filter design approach to emulate the absorption profiles of non-rigid boundaries; complex objects can be incorporated into a variety of FDTD based acoustic prediction scenarios. The paper will evaluate the success of the approach and explore its application. Of particular interest is the emulation of furniture, people, acoustic modifiers and other complex non planer structures within room acoustics predictions. 


\title{
Invited Paper
}

$2: 40$

5aAAa13. Localization and mesoscopics in structures and rooms II. Richard Weaver (Dept. of Physics, University of Illinois, Urbana, IL 61801, USA, r-weaver@uiuc.edu)

A new theory is developed which makes quantitative predictions for energy transport that are consistent with localization. The theory promises ultimately to apply to a wide variety of systems. It is based on an assumption of S-matrices that are incoherent - as conventional diffuse field theories would have it - but then corrected to assure exact energy conservation. It is shown that the resulting system responses (they are concatenations of S matrices) have coherence, are consistent with localization, and predict Greens function retrieval. Localization emerges naturally from competition between rates of transport and rate of eigen-mode resolution. Examples are presented corresponding to coupled rooms and to diffusion in a multiply scattering medium. Future generalizations are discussed and the mathematical challenges are outlined. This is the second talk in a pair begun in a different session. Work supported by NSF CMS 05-28096.

FRIDAY MORNING, 4 JULY 2008

ROOM 243, 8:00 A.M. TO 3:20 P.M.

\section{Session 5aAAb}

\section{Architectural Acoustics and Noise: Airborne and Impact Sound Insulation I}

\author{
Berndt Zeitler, Cochair \\ NRC - Institute for Research in Construction, 1200 Montreal Road, Building M-27, Ottawa, ON K1A OR6, Canada \\ Patrizio Fausti, Cochair \\ Engineering Dept. - Univ. of Ferrara, Via Saragat, 1, Ferrara, 44100, Italy
}

\section{Contributed Paper}

8:00

5aAAb1. Maximizing field performance of thin, light-weight partition and floor assemblies. Ronald Eligator (Acoustic Dimensions, 145 Huguenot Street, New Rochelle, NY 10801, USA, religator@acousticdimensions .com)

Laboratory test reports are an important source of information regarding anticipated performance of a sound barrier construction. However, differences in construction conditions between the laboratory and a real building can greatly affect, usually negatively, the performance of a sound barrier assembly in the field. This reality, combined with the typical client's focus on reducing construction costs, requires that for a sound barrier construction to be successful, the effect of abutting constructions must be well understood. This paper reports on our firm's experience in translating lab-tested lightweight sound barrier assemblies to the field, relating design conditions to field results in terms of physical construction and noise reduction results.

\section{Invited Paper}

5aAAb2. Monitoring field and laboratory performance for airborne and impact sound insulation for $110,000 \mathrm{homes}$ per annum. Sean Smith (BPC, Napier University, 42 Colinton Road, EH10 5BT Edinburgh, UK, se.smith@ napier.ac.uk), Dave Baker (Robust Details Ltd, Davy Avenue, Knowlhill, MK5 8NB Milton Keynes, UK, dbaker@robustdetails.com), David Panter (Robust Details Ltd, Davy Avenue, Knowlhill, MK5 8NB Milton Keynes, UK, dpanter@robustdetails.com)

This paper provides an overview of a unique monitoring system used in England and Wales for tracking the sound insulation performance for 110000 new build homes per annum. The regulatory approach, known as Robust Details was established within the building regulations and passed by parliament in 2004 after an 18 month initial research project. Annually over 5000 construction sites now use this system approach for separating walls and floors for attached houses and apartments. Acoustic site inspections and sound insulation field testing reports provide systematic feedback to the design and performance review. Since the RD systems inception on-site sound insulation compliance rates have risen from $40 \%$ to $97 \%$. This paper will discuss the role of laboratory benchmark testing for products and also the role of acoustic "field" inspectors in tracking the airborne and impact performance. In addition the role of intervention and statistical analysis in upgrading or withdrawing specific wall and floor constructions will be presented. 


\section{Contributed Paper}

8:40

5aAAb3. Changed sound properties due to minor construction changes in a lightweight building. Fredrik Ljunggren (Luleå Univ. of Technology, Div. of Sound and Vibration, S-97187 Luleå, Sweden, fredrik.ljunggren@1tu se)

This paper relates to building acoustic measurement inside a two-story office house. The construction, which is known as lightweight, is prefabricated in volumes at a factory and is then transported to the building yard for assembling. It is build up of a wooden frame with particle boards and plaster boards attached. The building consists of a number of nominally, or almost nominally, identical rooms with assumed identical sound properties. In the projection stage, the construction was slightly modified in some of the rooms in order to see in what way the sound properties would be affected. In total eight impact sound pressure level measurements and eight sound reduction index measurements were performed and analysed for the different setups.

\section{Invited Papers}

\section{9:00}

5aAAb4. The drying process influence on the brick walls sound reduction index: laboratory evaluations and theoretical analysis. Chiara Scrosati (Construction Technologies Institute of Italian National Research Council, Viale Lombardia, 49, 20098 San Giuliano Milanese (MI), Italy, labacustica@itc.cnr.it), Fabio Scamoni (Construction Technologies Institute of Italian National Research Council, Viale Lombardia, 49, 20098 San Giuliano Milanese (MI), Italy, Fabio.Scamoni@itc.cnr.it), Fabrizio Valentini (Construction Technologies Institute of Italian National Research Council, Viale Lombardia, 49, 20098 San Giuliano Milanese (MI), Italy, Fabrizio.Valentini@itc.cnr.it)

This paper presents experimental results showing the sound reduction index of different types of brick walls; the analysis was in particular carried out on double walls, single walls, and single walls with external lining. Its main purpose is to find the minimum drying time of the structure necessary to obtain the sound reduction indexes of brick walls as close as possible to laboratory real values. Moreover, it aims at making results of different laboratories as comparable as possible in terms of repeatability and reproducibility. All the fundamental quantities, such as the sound reduction index $(\mathrm{R})$, the weighted sound reduction index (R_ $\{w\})$, the spectrum adaptation terms C and C_ $\{\operatorname{tr}\}$, and temperature $(\mathrm{T})$ and relative moisture, have been analyzed. A qualitative analysis of the curves slope of the sound reduction index versus frequency has been conducted for each drying time for each type of evaluated wall. The measurements were carried out in the acoustic laboratory of ITC-CNR (the Construction Technologies Institute of the Italian National Research Council) in Milan, according to the procedures of ISO 140 part 3.

5aAAb5. Acoustical proprieties of light brick walls and its effects on flanking transmission. Giovanni Semprini (University, DIENCA Dept. Facoltà di Ingegneria, Viale Risorgimento 2, 40136 Bologna, Italy, giovanni.semprini@mail.ing.unibo.it), Luca Barbaresi (DIENCA - Univ. of Bologna, Viale Risorgimento 2, 40136 Bologna, Italy, luca.barbaresi@mail.ing.unibo.it)

Light brick walls 8-10 $\mathrm{cm}$ thick are typical structures frequently used in Italian building constructions as a internal partitions between dwellings and as internal layer of double façade walls. Due to low surface mass and rigid connection to other horizontal and vertical structures, light brick walls are often responsible of high flanking transmission. The simplest acoustic modeling of this structure for the evaluation of sound transmission in buildings, is the approach proposed by the EN 12354 standards, where the brick wall can be considered as a homogeneous structure and main acoustic parameters required are the sound reduction index and the vibration reduction index Kij. The aim of this paper is to analyse results of physical and acoustical properties (sound reduction index, structural reverberation time, radiation efficiency) of a typical $8 \mathrm{~cm}$ thick brick wall measured in laboratory testing facility, used as a partition in different test configuration: single layer wall, double brick layer wall with internal thermal layer and as a component of lateral wall, where the Kij index flanking transmission were also evaluated. Experimental results are then compared with theoretical values in order to analyse difference with homogeneous structures.

9:40-11:00 Posters

Lecture sessions will recess for presentation of poster papers on various topics in acoustics. See poster sessions for topics and abstracts.

\section{Contributed Paper}

\section{1:00}

5aAAb6. Lightweight system Technostar specified in the CCTV tower Beijing. Andrew Koster (Saint-Gobain Insulation, Parallelweg 20, 4878AH Etten-Leur, Netherlands, andrew.koster@saint-gobain.com)

The role and possibilities of lightweight mineral wool is known for quite some years, acting as the optimum spring in a mass spring mass system one can reach high acoustic values. Compared to the massive constructions (e.g., concrete) the use of a mass spring mass construction has also other advantages (e.g., logistic, dimension of foundation and labour costs). SaintGobain Isover developed a wall system called Technostar, originally defined for cinema walls. In this construction an optimal mass spring mass construction is created. The theory of mass spring mass systems will shortly come up. All elements of the system are described. The acoustical performance of the Technostar construction is calculated with acoustic software (Stiff) and compared with laboratory measurements; the influence of different parameters on the measured acoustical performance will be shown. The practical use of the Technostar wall will be shown on the basis of the project CCTV tower in Beijing. For this project the acoustic requirements, the translation in terms of the wall construction with the specific details will be shown. The system will be build in March 2008 . 


\section{Invited Paper}

$11: 20$

5aAAb7. Influence of static-load on airflow resistivity determination. Alessandro Schiavi (Istituto Nazionale di Ricerca Metrologica, str. delle Cacce, 91, 10135 Turin, Italy, a.schiavi@inrim.it), Claudio Guglielmone (Istituto Nazionale di Ricerca Metrologica, str. delle Cacce, 91, 10135 Turin, Italy, c.guglielmone@inrim.it), Piercarlo Miglietta (FIAT Group Automobiles S.p.A., Corso G. Agnelli, 220, 10135 Turin, Italy, piercarlo.miglietta@ fiat.com), Franco Alasia (Istituto Nazionale di Ricerca Metrologica, str. delle Cacce, 91, 10135 Turin, Italy, f.alasia@inrim.it)

Dynamic stiffness of resilient materials used as underlayer in floating floors give useful knowledge on the acoustical behaviour of a floor in impact sound insulation. In previous works the influence of the static load and the compressive behaviour of the resilient layers on dynamic stiffness evaluation has been shown. A new measurement technique of the airflow resistance (according to ISO 9053) in terms of static load applied on the resilient materials with open cells is proposed. Usually the airflow resistance of porous materials used as underlayers is evaluated on unloaded samples of material. In dynamic stiffness measurements the material is subject to a static load of $2 \mathrm{kPa}$. Because the dynamic stiffness value depends on airflow resistivity $\mathrm{r}\left[\mathrm{Pa} \times \mathrm{s} / \mathrm{m}^{2}\right]$ (in particular when $10 \mathrm{kPas} / \mathrm{m}^{2}<\mathrm{r}<100$ $\mathrm{kPas} / \mathrm{m}^{2}$ ) through the material, it is important to evaluate the airflow resistivity behaviour in the same condition of the dynamic stiffness measurement, i.e., under a static load of $2 \mathrm{kPa}$. A new apparatus to evaluate the airflow resistivity in terms of different static loads has been designed and realized at I.N.RI.M. of Turin. The first experimental results show evidence of the dependence of the airflow resistivity of the resilient materials on the applied load.

\section{Contributed Papers}

11:40

5aAAb8. Compression of felt-type thermal insulation layer for underfloor heating system and floor impact sound. Tongjun Cho (SKEC, Jung-Gu, Soonhwa-Dong 66, SK Soonhwa B/D, R\&D Center, 100130 Seoul, Republic of Korea, tjc@skec.co.kr), Hyun-Min Kim (SKEC, Jung-Gu, Soonhwa-Dong 66, SK Soonhwa B/D, R\&D Center, 100130 Seoul, Republic of Korea, thek80@skec.co.kr)

In Korea, almost every house uses underfloor heating, which has advantages of thermal comfort and energy efficiency. However, when it is constructed for high-rise apartment houses, it yields a problem in floor impact sound insulation. It accounts for the fact that a foam-type thermal insulator sandwiched between structural slab and heating floor functions as a spring and easily transmits impacts on the floor to the slab. In that case, the system's transmissibility is determined by dynamic stiffness of the thermal insulation layer and the lower the dynamic stiffness is, the more the floor impact is isolated. For that reason, apartments construction companies are attempting to lower the dynamic stiffness of the thermal insulation layer for impact sound reduction. As part of the attempt, felt-type materials with relatively low dynamic stiffness such as glass wool or polyester felt are considered as a substitution for the foam-type thermal insulator. However, there is a possibility that compression of the felt-type materials would increase the dynamic stiffness and the impact sound insulation effect at early stage might be weakened in the long term. This paper investigates the correlation between gradual compression of the felt-type thermal insulation layer and the impact sound variation.

\section{2:00}

5aAAb9. Acoustic and vibration characteristics of floated floors concrete structures. Seungyup Y. Yoo (Hanyang University, 133-791 Seoul, Republic of Korea, syrus81@hanyang.ac.kr), Jin Yong Jeon (Hanyang University, Department of Architectural Engineering, 133791 Seoul, Republic of Korea, jyjeon@hanyang.ac.kr)

The floating floor with multilayers of resilient materials was investigated for the isolation of floor impact sound. The floor structures were evaluated in the reduction of the heavy-weight impact sound, which is mainly affected by the boundary conditions of the tested floors. The values of single-number ratings for floor impact sounds decrease dramatically for floated floors. Insertion loss is determined by the vibration characteristics of a bare slab, such as transmissibility. The logarithmic decrement $(\xi)$ of the vibration responses was 4 times higher than that of the bare slab. Above $125 \mathrm{~Hz}$, the vibration between the slab structure and floating floor is isolated effectively. It was revealed that the resonant frequency of the slab structure and the damping factor of the floating floor are important aspects for impact sound isolation.

\section{Invited Paper}

5aAAb10. Interactions between floor and ceiling panels in presence of a fibrous material in the cavity. Thomas Scelo (Marshall Day Acoustics LTD, P. O. Box 5811, Wellesley St., 1000 Auckland, New Zealand, thomas.scelo@marshallday.co.nz)

The mechanisms governing the acoustic transmission through building elements such as floor/ceiling assemblies are complex. The vibroacoustic interactions between the finite size structure and the enclosed volume are, in particular, rendered more complex at low frequencies when the finite dimensions of the building are taken into consideration. A model developed to predict the low frequencies vibroacoustics response of mutliplates systems offers the opportunity to explore, among other geometrical and material parameters, the effects of a fibrous material in the ceiling plenum on the acoustics behaviour of the whole structure. This paper presents the theoretical approach employed to predict the interactions between the floor, the ceiling panel and the enclosed volume. It also explores the effects of varying the material characteristics and overall thickness on these interactions.

12:40-1:40 Lunch Break 


\section{Invited Paper}

\section{$1: 40$}

5aAAb11. Methods to control low frequency impact noise in wood-frame construction. Berndt Zeitler (NRC - Institute for Research in Construction, 1200 Montreal Road, Building M-27, Ottawa, ON K1A 0R6, Canada, berndt.zeitler@nrc-cnrc.gc.ca), Trevor Nightingale (NRC - Institute for Research in Construction, 1200 Montreal Road, Building M-27, Ottawa, ON K1A 0R6, Canada, Trevor.Nightingale@nrc-cnrc.gc.ca), Frances King (NRC - Institute for Research in Construction, 1200 Montreal Road, Building M-27, Ottawa, ON K1A 0R6, Canada, Frances.King@nrc-cnrc.gc.ca)

The control of low frequency impact noise is of great importance in Japan and Korea. For impact noise rating, both countries use standardised heavy and soft impactors that inject high levels of low frequency power into a wood-frame floor due to the high force applied and "good" impedance match between the source and the floor. This paper reports on a parametric study designed to confirm the important parameters for controlling low frequency impact noise and how these parameters are affected by changes to construction details. The paper shows that increasing the drive point impedance floor surface while minimising the structural coupling between the gypsum board ceiling and the structural framing (joists) are important elements. Additionally, a well-designed floor topping can be very effective when applied to a suitable floor. The paper presents data to show the relative importance of direct and flanking transmission paths. Furthermore, it is shown that repeated impacts by the Bang Machine physically change the floor assembly and as a consequence resulting impact sound pressure levels in the receive room also change. The paper concludes with a general discussion and recommendations.

\section{Contributed Papers}

2:00

5aAAb12. Field airborne and impact sound insulation of wood truss floor systems. Chad N. Himmel (JEAcoustics, 1705 West Koenig Lane, Austin, TX 78756, USA, Himmel@JEAcoustics.com), Daniel J. Kupersztoch (JEAcoustics, 1705 West Koenig Lane, Austin, TX 78756, USA, Kupersztoch@JEAcoustics.com)

A series of field measurements on wood truss floors of various types was recently completed at four residential apartment complexes. About 40 floors with different floor finishes, gypsum concrete underlayment, noise control underlayment systems, truss span lengths, ceiling types, and resilient metal channel types were constructed and measured. Trusses were all similar engineered prefabricated parallel chord wood trusses. Room volumes and room absorptive characteristics are varied and nonstandard. Measurements are normalized according to ASTM E 1007 and proposed normalized impact sound rating (NISR) procedures to provide a reasonably consistent set for analysis. Low frequency measurements were conducted to $12.5 \mathrm{~Hz}$ one-third octave band. For both transmission loss and impact sound, many of the results compare well with predictions using simple regression analysis developed by others using variables such as the mass of the layers, truss depth and spacing, insulation thickness and density, and resilient metal channel spacing. The measurements and results will be presented in the paper.
2:20

5aAAb13. Impact sound insulating performance of access floors Francesco Asdrubali (Università degli studi di Perugia, Via G. Duranti 67, 06125 Perugia, Italy, fasdruba@unipg.it), Francesco D’Alessandro (Università degli studi di Perugia, Via G. Duranti 67, 06125 Perugia, Italy, dalessandro.unipg@ciriaf.it)

Access floor are raised floor systems consisting of modular panels supported by posts at a certain height to create a gap below the floor surface. Electrical wires and pipes can be placed inside the gap in order to avoid exposed installations and to simplify operations involving the inspection, repair, changing, or adding of system elements. This paper presents the results of the measurement campaign carried out on seven different access floor configurations, obtained by changing different floor elements: panels, surface finishes, damping materials under the posts base. Impact sound insulating properties of access floors combined with false ceilings were also tested and are reported in the paper. The tests were executed in two overlapping reverberating rooms available at the Acoustics Laboratory of the University of Perugia using the procedures given by ISO 140-6 Standard. The results are expressed in terms of normalized sound impact pressure level Ln and of the corresponding single number index Ln,W.

\section{Invited Papers}

\section{2:40}

5aAAb14. Measurement of room-to-room airborne sound insulation with an access floor in a dwelling unit of condominium. Takashi Koga (Kajima Technical Research Institute, 2-19-1 Tobitakyu, Chofu, 182-0036 Tokyo, Japan, tkoga@kajima.com)

It is preferred covering a wooden access floor on the concrete floor for condominium apartments in Japan. The access floor usually installed after every partition in each dwelling unit. If access floor and ceiling were done before partition, one can obtain accuracy of construction and reduce waste material. Therefore, one can provide building more green. But, there is concern that may deteriorate sound insulation because of flanking path through under the access floor. On the other hand, there is also another dominant flanking path irrespectively to these construction methods: via doors leading into hallway. A number of field measurements of sound insulation were done with opening/closing doors to evaluate the flanking transmission via the doors. These results show that the doors affect especially in high frequency, but not significant in low-mid frequency. Next, the sound transmission performances of access floors and ceilings measured at laboratory are compared with direct sound transmission through wall itself. As a conclusion, field measurement results show that this construction method difference less affect to airborne sound insulation performance.

\section{3:00}

5aAAb15. The acoustical effect of reveal blocks, from measuring method to prediction. Frigyes Reis (Budapest University of Technology and Economics, Lab. of Building Acoustics, Mûegyetem rakpart 3., 1111 Budapest, Hungary, reis@mail.bme.hu), Balázs Tóth (Wienerberger Co., Bártfay str. 34, 1119 Budapest, Hungary, balazs.toth@ wienerberger.hu)

The analysis of the efforts to create high quality building from the point of view of energy-efficiency, and the resulting variety of new products, building constructions and systems leads to the conclusion: there are characteristic deficiencies in the field of measuring and prediction methods related to sound insulation, and there is a need to stop the gap. The example on which the problem is shown is 
the acoustical effects of reveal blocks, used around window openings, to reduce heat propagation and increase energy efficiency. The reveal blocks include more sound propagation paths, the sound insulation of which can not be determined using the existing measuring standards. The paper describes new measuring concept and method to characterise the sound insulation characteristics of reveal blocks in their built-in form. The methods are fitted to the standardised measuring methods of sound insulation. Proposal is presented for the modification in the prediction procedure of sound insulation of façade constructions, utilising the results of the new measuring method.

FRIDAY MORNING, 4 JULY 2008

P2-B, LEVEL 2, 9:40 TO 11:20 A.M.

\title{
Session 5aAAc
}

\section{Architectural Acoustics and Noise: Airborne and Impact Sound Insulation II (Poster Session)}

\author{
Berndt Zeitler, Cochair \\ NRC - Institute for Research in Construction \\ Patrizio Fausti, Cochair \\ Engineering Dept. - Univ. of Ferrara
}

\begin{abstract}
All posters will be on display from 9:40 a.m. to 11:20 a.m. To allow contributors an opportunity to see other posters, contributors of odd-numbered papers will be at their posters from 9:40 a.m. to 10:30 a.m. and contributors of even-numbered papers will be at their posters from 10:30 a.m. to 11:20 a.m.
\end{abstract}

\section{Contributed Papers}

5aAAc1. Enhancement of impact sound insulation for "Gründerzeit-Häuser" build around 1900 during refurbishment using concrete-wood composite floors or suspended ceilings. Thomas Bednar (University of Technology, Karlsplatz 13/206, A-1040 Vienna, Austria, thomas.bednar@tuwien.ac.at)

During refurbishment of Viennese buidlings that have been erected around 1900 an important aspect is to enhance the impact sound insulation of the old wooden floors. Usually the floors are wood beam floors and the uppermost floor is a massive wooden floor called Dippelbaumdecke. Two important measures are taken to increase the impact sound insulation to achieve the minimum requirement of $\operatorname{LnTw}<=48 \leqslant \mathrm{~dB}$. The paper describes the design of an optimal suspended ceiling to avoid additional measures and as an alternative the impact of an additional thin concrete floor on top of the Dippelbaum-floor to achive minimum floor heights. As the thin concrete floor has a much larger area than the rooms below the measured results showed a big influence of concrete floor area on the impact sound level.

5aAAc2. Sound insulation properties of building elements, considering the frequency range below $100 \mathrm{~Hz}$. Herbert Muellner (TGM Federal Institute of Technology, Wexstrasse 19-23, 1200 Vienna, Austria, herbert.muellner@tgm.ac.at), Anna Frey (University of Applied Sciences, FH Campus Vienna, Daumegasse 3, 1100 Vienna, Austria, anna.frey@chello.at), Catrin Humer (TGM Federal Institute of Technology, Wexstrasse 19-23, 1200 Vienna, Austria, catrin.humer@tgm.ac.at)

The constantly increasing standard of comfort in general and the rising number of apartment buildings in lightweight mode of construction directed the focus of research to the sound insulation properties of timber frame and massive wood element buildings. The complaints of the residents of terraced houses and multiple dwellings in lightweight buildings mainly concern noise events characterised by dominant low frequency. This problem regards airborne sound insulation as well as impact sound insulation. Based on empirical data the comparison of the sound insulation properties of the building elements investigated shows the specific problem of each category of construction mode in the frequency range below $100 \mathrm{~Hz}$. The typical sound insulation characteristics regarding the low frequency range are discussed in the light of the current applied sound insulation requirements as well as in relation to the problem to comply with the resident's apperception of sound insulation quality.

5aAAc3. Effect of the frequency spectrum trend on the determination of the weighted normalized impact sound pressure level for floor structures. Federica Bettarello (Engineering Dept. - Univ. of Ferrara, Via Saragat, 1, 44100 Ferrara, Italy, federica.bettarello@unife.it), Patrizio Fausti (Engineering Dept. - Univ. of Ferrara, Via Saragat, 1, 44100 Ferrara, Italy, patrizio.fausti@unife.it)

The most common floor constructions in Italian buildings are composed of two parts: the structural one, made of concrete beams and perforated bricks, and the floating floor. The results of the in situ measurements of the impact sound pressure level, realized on the structural part of the floors, show a frequency spectrum trend that increases with the frequency. The spectrum is also characterized by many peaks and dips at the high frequency range that strongly influence the determination of the weighted normalized impact sound pressure level, also for structures with similar mass and geometry. The frequency trend of the beam and brick structures is completely different from the characteristic spectrum of homogeneous concrete slabs or lightweight structures. Considerations on how those differences can influence the obtainable results in terms of the reduction of impact sound pressure level referred to the same floating floor typology, the spectrum adaptation terms and the other acoustic quantities described in the standards are reported.

5aAAc4. Influence of flanking transmission in typical Italian constructions. Simone Secchi (Univ. of Florence, Via San Niccolò 89/a, 50125 Florence, Italy, simone.secchi@taed.unifi.it), Gianfranco Cellai (Univ. of Florence, Via San Niccolò 89/a, 50125 Florence, Italy, gianfranco.cellai@unifi.it), Elisa Nannipieri (Univ. of Florence, Via San Niccolò 89/a, 50125 Florence, Italy, elisa.nannipieri@taed.unifi.it)

Actually acoustic performance of building components are analysed with reference to standardised conditions, in laboratories with suppressed flanking transmission. Laboratory results often show values of the performances quite different from those measured in real buildings, as a consequence of flanking transmission and of different conditions of realisation in 
situ. Moreover, in spite of all efforts aimed to standardise laboratory test conditions, often we find different certified performances of same building components obtained in different laboratory. In some cases, these differences may be very relevant. Besides, the Italian decree on acoustic requirement of buildings prescribes limiting values to be measured in situ. For these reasons, many producers of building materials and components more frequently presents in their depliants both laboratory values and in situ values of acoustic performances of their products. The paper shows the preliminary results of an research aimed to verify acoustic performances of partition walls and floors in a laboratory which respect conditions of ISO 140-1, but with the presence of relevant flanking transmission, similar to real buildings. The relevance of the flanking transmission has also been modified by means of a lining applied to the ceiling of the receiving room of the laboratory.

5aAAc5. Survey on the insulation performance of acoustic louvres. Lieven De Geetere (Belgian Building Research Institute, Lombardstraat 42, B-1000 Brussel, Belgium, lieven.de.geetere@bbri.be), Bart Ingelaere (BBRI, rue du Lombard, 42, B-1000 Brussel, Belgium, bart .ingelaere@bbri.be)

Machinery such as compressors, boilers and generators require adequate ventilation and extract. To avoid excessive noise breakout, acoustic louvres are frequently used. These are usually made of tilted hollow metallic blades filled with absorptive materials and perforated at the inside face. This study investigates the important characteristics of common shaped acoustic louvres with regard to sound insulation. First, a market survey on 109 existing acoustic louvres of 24 producers worldwide is made. Second, laboratory measurements of sound insulation on 38 prototypes of $1 \mathrm{~m}^{2}$ acoustic louvres are compared. The influence of several parameters are studied such as louvre depth, blade angle, blade shape, blade thickness, blade spacing, kind of absorptive material, perforation degree, and edge filling.

5aAAc6. Sound insulation characteristics of shipboard windows Sangryul R. Kim (Acoustics Team, Korea Institute of Machinery and Materials, 171 Jang-dong, Yuseong-gu, 305-343 Daejeon, Republic of Korea, srkim@kimm.re.kr), Hyun-Sil Kim (Acoustics Team, Korea Institute of Machinery and Materials, 171 Jang-dong, Yuseong-gu, 305-343 Daejeon,
Republic of Korea, hskim@kimm.re.kr),Hyun-Ju Kang(Acoustics Team, Korea Institute of Machinery and Materials, 171 Jang-dong, Yuseong-gu, 305-343 Daejeon, Republic of Korea, kanghj@kimm.re.kr)

Although small-size windows are used in a ship, shipboard windows are a multilayered structure with glasses and air-gaps for high sound insulation. This paper discusses how to improve the sound insulation performance of shipboard windows. First, the sound transmission loss (STL) obtained from various experiments with shipboard windows are introduced and studied. The results show the layer arrangement as well as the material property of each layer makes an effect on the window's STL. It is also found that the higher the sound insulation performance of the window is, the more important the effect of the window frame is. Next, theoretical investigations are carried out and discussed in comparison with the experimental results. The comparison provides possible clues to increase the STL of the window.

5aAAc7. Acoustic analysis of the framework and walls stage in the construction of a housing block. Maria Jesus Ballesteros (Universidad de Castilla-La Mancha, Campus Universitario, 16071 Cuenca, Spain, MJesus.Ballesteros1@alu.uclm.es), Samuel Quintana (Universidad de Castilla-La Mancha, Campus Universitario, 16071 Cuenca, Spain, Samuel.Quintana@uclm.es), Marcos D. Fernandez (Universidad de Castilla-La Mancha, Campus Universitario, 16071 Cuenca, Spain, Marcos.Fernandez@uclm.es), Jose A. Ballesteros (Universidad de Castilla-La Mancha, Campus Universitario, 16071 Cuenca, Spain, Josea.Ballesteros@uclm.es), Laura Rodriguez (Universidad de Castilla-La Mancha, Campus Universitario, 16071 Cuenca, Spain, Laura.Rodriguez @uclm.es)

The noise in the construction process is one of the main environmental and industrial noise sources. There are no specific regulations in several $\mathrm{Eu}-$ ropean countries for assessing such kind of noise, neither reference indexes for its evaluation. Therefore, the limits adopted are those for environmenta and industrial noise, although they do not take into account the intrinsic characteristics of this noise. A measurement procedure has been stated for assessing the noise in building sites in which, the most appropriate indexes for this noise have been analyzed and used to derive the acoustic characteristics of the framework and walls stage. This stage is considered as one of the most annoying in the construction process of a housing block. 


\title{
Session 5aAAd
}

\section{Architectural Acoustics: Coupled Volume Acoustics I (Poster Session)}

\author{
Jason Summers, Cochair \\ U.S. Naval Res. Lab. \\ Alexis Billon, Cochair \\ Universite de Liege
}

\begin{abstract}
All posters will be on display from 9:40 a.m. to 11:20 a.m. To allow contributors an opportunity to see other posters, contributors of odd-numbered papers will be at their posters from 9:40 a.m. to 10:30 a.m. and contributors of even-numbered papers will be at their posters from 10:30 a.m. to 11:20 a.m.
\end{abstract}

\section{Contributed Paper}

5aAAd1. Investigations on real-size coupled rooms based on Bayesian estimation method. Zuhre Su (Russ Berger Design Group, 4006 Belt Line Road Suite 160, Addison, TX 75001, USA, tayasu7@yahoo.com), Dave Woolworth (Oxford Acoustics, Inc., 356 CR 102, Oxford, MS 38655, USA, dave@oxfordacoustics.com), Ning Xiang (Rensselaer Polytechnic Institute, Greene Building, School of Architecture, 110 8th Street, Troy, NY 12180, USA, xiangn@rpi.edu)

As a case study, this work aims to experiment coupled-volume systems with scientifically grounded quantifiers using Bayesian analysis, a reliable analysis method in evaluating effects of different parameters on sound energy decays of coupled rooms [Xiang \& Goggans, J. Acoust. Soc. Am. 110,
1415 - 1424 (2001)]. This research uses the well-defined indicators developed by Xiang and Goggans to assess sound energy decays in a real-size hall. Derived from the model-based Bayesian inference, level differences $(\Delta \mathrm{L})$, diverse decay times, and decay time ratios are the quantitative descriptors of sound energy decays in acoustically coupled spaces. The room impulse responses of Howorth Theater in Oxford, Mississippi coupled to the adjacent lobby are experimentally measured in the case study. The two rooms are connected with two separate apertures and have distinct natural reverberation times that allow experimenting different setups for probable acoustical coupling at certain locations. In this paper, frequency, and location dependence of double-slope characteristics of sound energy decays are discussed. 


\title{
Session 5aAAe
}

\section{Architectural Acoustics: New Frontiers in Room Acoustical Modeling II (Poster Session)}

\author{
Murray Hodgson, Cochair \\ University of British Columbia \\ Vincent Valeau, Cochair \\ Laboratoire d'Etudes Aérodynamiques (LEA)
}

\begin{abstract}
All posters will be on display from 9:40 a.m. to 11:20 a.m. To allow contributors an opportunity to see other posters, contributors of odd-numbered papers will be at their posters from 9:40 a.m. to 10:30 a.m. and contributors of even-numbered papers will be at their posters from 10:30 a.m. to 11:20 a.m.
\end{abstract}

\section{Contributed Papers}

5aAAe1. Predicting the acoustics of historic Istanbul Tunnel: Simulation, calculation methods and geometrical detaıls. Filiz Bal Kocyigit (Karabuk University, 232. Str. No: 5/5 Ilkbahar Mah., Cankaya, 06550 Ankara, Turkey, filizbkocyigit@yahoo.com)

Istanbul Tunnel was designed and constructed by Eugene Henry Gavand in 1875 is the third Metro and second underground railway system in the world after Washington Metro (1868). In this paper, the acoustics of Istanbul Tunnel is investigated. This is a special case which sets up a challenge to these prediction methods. The shape of the tunnel and therefore focusing the sound, reverberant wall, and therefore fluctuation effect demands high accuracy in predicting the early reflections. The energy dissipates quickly in this type of enclosures and there is little masking effect of the reverberation. Another aspect that has been shown to give very different results in this case study is the geometrical detailing of the models. When Istanbul Tunnel compared with modern metrosystems; nowadays railway systems are controlled by modern electronic and mechanical system, but in the 19th century this electronic systems could not used. Solutions could be with the shape of the building. The aim of this paper is to clarify some of the problems that can arise in this type of constructions, and give guidelines for how they can be overcome/avoided. Another objective is to emphasize that room acoustic computer simulations, although very useful, need careful consideration about the underlying calculation methods.

5aAAe2. Size-adaptive spherical receptor acceleration method for acoustical ray tracing. Stéphane Lesoinne (University of Liège - INTELSIG, Institut Montéfiore, Sart-Tilman, Batiment 28, 4000 Liège, Belgium, s.lesoinne@ulg.ac.be), Jean-Jacques Embrechts (University of Liège - INTELSIG, Institut Montéfiore, Sart-Tilman, Batiment 28, 4000 Liège, Belgium,jjembrechts@ulg.ac.be)

Randomized ray tracing in room acoustics can be used to compute echograms, but the results computed at the receptor are affected by statistical errors [1]. To decrease these statistical errors, the usual solution is to increase the number of rays, but this also increases the computation time. Another solution is to increase the receptor size, but this lowers the spatial resolution of the computed sound field, which is particularly important for the early part of the echogram. In order to decrease the computation time and keep a sufficient resolution, the method proposed in this paper is based on a progressive modification of the receptor size (spherical type) as long as the ray's travel grows. At the same time, the number of rays into the room can be decreased as the sound ray lengths increase while keeping the statistical errors more or less constant. The consequence is that the sound field spatial resolution evolves from "precise" at the beginning of the echogram to "rough" at its end. The first applications of this new method show a significant gain of CPU time. [Broad spectrum diffusion model for room acous- tics ray-tracing algorithms, J. J. Embrechts, Jnl. Acoust. Soc. Am. 107(4) 2068-2081 (2000)].

5aAAe3. Variation of mean free path length over time and its effect on room impulse response. Miomir Mijic (Faculty of Electrical Engineering, Bulevar Kralja Aleksandra 73, 11000 Belgrade, Serby, emijic@etf.bg.ac.yu), Dragana Sumarac Pavlovic (Faculty of Electrical Engineering, Bulevar Kralja Aleksandra 73, 11000 Belgrade, Serby, dsumarac@etf.bg.ac.yu)

In their previous paper (Forum acusticum 2005, Proceedings, 23992404) the authors presented the local effect found in a large sports hall, described as significantly shorter reverberation time in the first $400-500 \mathrm{~ms}$ than that in the rest of the hall's long impulse response. To analyse this phenomenon, a new measure - mean free path length (MFPL) variation in time over the impulse response - was introduced. It is identified as the function of time obtained as an ensemble average in some fixed interval traced along the impulse response duration. The ray tracing simulation was used to calculate ray paths approaching the receiver in time. Analyzing MFPL variations over time is a new insight into the structure of room impulse response, and this paper presents its main characteristics, its dependence on geometrical properties of rooms, and also its relation to statistically defined mean free path length $(4 \mathrm{~V} / \mathrm{S})$. The paper also presents a correlation between MFPL variation over time and the sound decay curve. The MFPL variation over time reveals the causes of the local effect introduced in the previous paper, but it is generally useful in explaining the sound decay monotony deviations.

5aAAe4. Towards a realistic ray tracing for room acoustics. Jaume Durany (Universitat Pompeu Fabra - Fundació Barcelona Media, Ocata, 1, $08003 \quad$ Barcelona, Spain, jaume.durany@upf.edu), Adan Garriga (Universitat Pompeu Fabra - Fundació Barcelona Media, Ocata, 1, 08003 Barcelona, Spain, adan.garriga@barcelonamedia.org), Toni Mateos (Universitat Pompeu Fabra - Fundació Barcelona Media, Ocata, 1, 08003 Barcelona, Spain, toni.mateos@barcelonamedia.org)

We present a recently developed ray tracing engine for room acoustics computations whose improved physics provide more realistic results than present state of the art methods. Ray tracing has been widely applied within the graphics community to obtain realistic shadings, but the computational cost reduction has oriented efforts towards fast computations of apparently realistic images rather than physically correct results. In the case of audio ray tracers, a similar tendency has led to relatively fast shading algorithms that often miss its physical basis, preventing the resulting impulse responses from converging to physically correct solutions. Our engine improves this situation by focusing on two main issues. First, it ensures the mathematical convergence of the algorithms by maintaining the coherence between the 
computation of the direction of the reflected rays and the computation of the contribution of the sources to a given point in the environment. Second, the engine works directly in the frequency domain. This allows for the implementation of frequency-dependent phenomena, from surface impedance and reflections to frequency-dependent source directivity patterns. We present a comparative study of the proposed ray tracing engine with the state of the art, emphasizing properties like realism, coherence and convergence.

5aAAe5. A geometric acoustics simulation proposal for curved geometry. Arthur Van Der Harten (Rensselaer Polytechnic Institute, Greene Bldg., 110 8th St., Troy, NY 12180, USA, Arthur .vanderharten@gmail.com), Paul Calamia (Rensselaer Polytechnic Institute, Greene Bldg., 110 8th St., Troy, NY 12180, USA, calamp@rpi.edu)
Current methods for acoustical simulations based on geometrical acoustics are designed to ascertain the properties of rooms using models comprising large flat polygons. Typically these same methods are used on models of spaces with curved surfaces in which the curves are approximated using planar facets. In such cases, errors are introduced in the simulation when the infinitely varying normal of a curve is replaced with a finite number of piecewise constant normals, one for each facet. NURBS - nonuniform rational B-splines - offer an alternative geometric representation that allows curves to be represented with precision. Using Rhinoceros, a commonly used NURBS-based CAD program, as a platform for an acoustic simulation tool for models of both NURBS and polygon geometry, we can begin to discover whether it is possible to conceptualize a geometrical acoustics method that is more accurate for curved surfaces. This talk will cover the implementation and early testing of an acoustic-simulation plug-in for Rhinoceros.

FRIDAY MORNING, 4 JULY 2008

P2-B, LEVEL 2, 9:40 TO 11:20 A.M.

\title{
Session 5aAAf
}

\section{Architectural Acoustics and Musical Acoustics: New Measurement Parameters in Performing Arts Spaces I (Poster Session)}

\author{
Lily Wang, Cochair \\ University of Nebraska - Lincoln \\ Brian Katz, Cochair \\ LIMSI-CNRS
}

\begin{abstract}
All posters will be on display from 9:40 a.m. to 11:20 a.m. To allow contributors an opportunity to see other posters, contributors of odd-numbered papers will be at their posters from 9:40 a.m. to 10:30 a.m. and contributors of even-numbered papers will be at their posters from 10:30 a.m. to 11:20 a.m.
\end{abstract}

\section{Contributed Papers}

5aAAf1. Converging evidence in evaluating acoustical intimacy. Bobby E. Gibbs (Rensselaer Polytechnic Institute, Greene Bldg., 110 8th St., Troy, NY 12180, USA, gibbsb@rpi.edu), Jonas Braasch (Rensselaer Polytechnic Institute, Greene Bldg., 110 8th St., Troy, NY 12180, USA, braasj@rpi.edu), Ted Krueger (Rensselaer Polytechnic Institute, Greene Bldg., 110 8th St., Troy, NY 12180, USA, krueger@rpi.edu)

Previously, we presented a novel interactive pilot experiment in which participants modified parameters in a virtual acoustical environment so that they corresponded to three intimacy settings: low, medium, and high. Additionally, participants were asked to rank the -- yet unknown -- parameters (volume, direct-to-reverberant energy ratio, frequency attenuation, and room size) in terms of the importance in making their judgments. Based on a larger body of data, the direct-to-reverberant energy ratio emerges as the strongest acoustical correlate of intimacy. A repeated-measures test revealed that the "preservation" of this parameter (the degree to which a participant changed the value from an optimum setting) varies the least across intimacy levels and across levels of previous training. Additionally, this parameter is consistently ranked as being the most important in the ranking portion of the test. We also found that salience of some of the other parameters varied significantly across intimacy levels, possibly suggesting a weighting system is warranted. These results are compared with binaural measurements of the virtual system. In particular, we will address disparities from the pilot experiment and recent data that resulted from differences in measuring the direct-to-reverberant energy ratio. [Work supported by RPI HAAS Fellowship and ASA Minority Fellowship.]
5aAAf2. Effects of multiple directional sources on quantitative and qualitative analyses of concert hall acoustics. Youngmin Kwon (Acoustic Dimensions, 15505 Wright Brothers Drive, Addison, TX 75001, USA, ykwon@acousticdimensions.com), Gary W. Siebein (Univ. of Florida, 231 Arch, PO Box 115702, Gainesville, FL 32611, USA, gsiebein@siebeinacoustic.com), Martin A. Gold (Univ. of Florida, 231 Arch, P.O. Box 115702, Gainesville, FL 32611, USA, mgold@ufl.edu)

The "orchestral impulse reponse" measurement technique using multiple directional sources and its effects on quantitative analysis of concert hall acoustics were discussed earlier [Kwon and Siebein, J. Acoust. Soc. Am. 120, 3263(A) (2006)]. An array with 16 directional loudspeakers approximating overall sound radiation patterns of each instrumental group of an orchestra was incorporated for room acoustical measurements and analyses. As a continuous study, this paper discusses qualitative assessments of concert hall acoustics over room subjective parameters including reverberance, clarity, warmth, spaciousness, envelopment, etc. The music signals binaurally recorded in a performance hall with the same source array were evaluated by means of subjective listening tests in comparison to those recorded with the single omni-directional source (dodec). The results showed that some perceived differences are present between the array with multiple directional sources and the one with a single omni-directional source. In the main orchestra seating, in particular, reverberance and clarity are perceived more but warmth is perceived less when compared to the recordings through the single omni-source. The results will be further discussed in conjunction with their quantitative measures resulted from the above measurement technique. 
5aAAf3. Room acoustics measurements with an approximately spherical source of 120 drivers. Roger Schwenke (Meyer Sound Laboratories, 2832 San Pablo Ave, Berkeley, CA 94702, USA, rogers@meyersound.com), Franz Zotter (Institute of Electronic Music and Acoustics, Inffeldgasse 10/3, 8010 Graz, Austria, zotter@iem.at), David Wessel (Center for New Music and Audio Technologies, 1750 Arch Street, Berkeley, CA 94720, USA, wessel@cnmat.berkeley.edu.), Andrew Schmeder (Center for New Music and Audio Technologies, 1750 Arch Street, Berkeley, CA 94720, USA, andy@cnmat.berkeley.edu)

An approximately spherical source of 120 individually controlled drivers is used to perform impulse response measurements in a room with a $1.4 \mathrm{~s}$ reverberation time and a distinct echo. The signal to the drivers is processed to produce both omni-directional and unidirectional patterns. The omnidirectional pattern is compared with measurements made with a traditional 12 sided source. The unidirectional patterns are measured both pointed towards and away from the listener position. Intelligibility metrics for the different directionalities and orientations are measured. The unidirectional pattern is aimed in different directions to minimally and maximally excite the distinct echo in the room, and locate it's origin.

5aAAf4. Measurement-based fuzzy interpolation of room impulse responses. Csaba Huszty (Budapest University of Technology and Economics, BME Dept. of Telecommunications, Magyar tudósok körútja 2, H-1117 Budapest, Hungary, huszty@hit.bme.hu), Bottyán Németh (Budapest University of Technology and Economics, BME Dept. of Telecommunications, Magyar tudósok körútja 2, H-1117 Budapest, Hungary, bottyan@tmit.bme.hu), Péter Baranyi (Budapest University of Technology and Economics, BME Dept. of Telecommunications, Magyar tudósok körútja 2, H-1117 Budapest, Hungary, baranyi@tmit.bme.hu), Fülöp Augusztinovicz (Budapest University of Technology and Economics, BME Dept. of Telecommunications, Magyar tudósok körútja 2, H-1117 Budapest, Hungary, fulop@hit.bme.hu)

Application of room impulse responses (RIRs) to acoustic evaluation and auralization often requires many measurements to get enough information about the hall, or to provide enough flexibility for virtual sound source placements in convolution reverberation. In this paper we propose a measurement-based fuzzy modeling method to approximate the RIR function at an arbitrary location between available measured points, without a priori information on the hall geometry or wall reflection parameters. For the fuzzy model identification we define an accuracy indicator of the spatial density of the source positions and predict the required number of them in a selected hall. This indicator quantifies the relationship of the early reflections, determined for various measured positions. This paper also proposes a method that treats nonuniform spatial sampling of the measurement positions, and its implementation for 2D cases is shown. Nonuniform spatial sampling can be useful when RIRs at some source positions -- e.g. positions of musicians on a stage of a concert hall -- are known or have to be measured precisely, but RIRs at locations in between require an approximation only. The proposed fuzzy model of RIRs actually transforms the measured information into a uniform and tensor product form, enabling the analyst to use further matrix and tensor algebra based numerical methods.

FRIDAY MORNING, 4 JULY 2008

AMPHI BLEU, 8:00 A.M. TO 5:40 P.M.

\title{
Session 5aABa
}

\section{Animal Bioacoustics: General Topics in Animal Bioacoustics II}

\author{
Richard R. Fay, Cochair \\ Loyola University Chicago, Parmly Hearing Institute, 6525 N. Sheridan Rd., Chicago, IL 60626, USA \\ Michel Andre, Cochair \\ Laboratori d'Aplicacions Bioacústiques (Universitat Politècnica de Catalunya), avda. Rambla Exposició s/n,
} Vilanova i la Geltrú, 08800, Spain

\section{Contributed Papers}

\section{8:00}

5aABa1. Acoustic production mechanisms in pinnipeds. Marija Spasikova (Centre for Social Learning and Cognitive Evolution, University of St. Andrews, School of Psychology, KY16 9JP St. Andrews, Fife, UK, marija.spasikova@gmail.com), William T. Fitch (Centre for Social Learning and Cognitive Evolution, University of St. Andrews, School of Psychology, KY16 9JP St. Andrews, Fife, UK, wtsf@st-andrews.ac.uk), Colleen Reichmuth (UCSC Institute of Marine Sciences, Long Marine Lab - University of California, 100 Shaffer Road, Santa Cruz, CA 95060, USA, coll@ucsc.edu), Ronald J. Schusterman (UCSC Institute of Marine Sciences, Long Marine Lab - University of California, 100 Shaffer Road, Santa Cruz, CA 95060, USA, rjschust@ucsc.edu)

The ability to develop articulate speech relies on capabilities that became available to our ancestors through changes in both peripheral mechanisms (vocal acoustics and anatomy) and neural mechanisms (vocal control and imitation). Few distantly related species (cetaceans, birds and pinnipeds) have shown the ability of vocal imitation, but of the three, only the pinnipeds use the same vocal tract as us humans. For this reason we choose to study two closely related pinniped species: the harbour seal (Phoca Vitulina), a good vocal imitator (capable of complex vocal learning), and the
Californian sea lion (Zalophus californicus), a close relative with very limited vocal imitation capabilities (no evidence of complex vocal learning) to look at the acoustical production mechanisms of the species as well as the neural mechanisms underlying the same. Using previously acquired data gathered in California at the Long Marine Lab, we performed video and acoustic analysis to show correlation between jaw opening and formants frequencies in the harbour seal (Phoca Vitulina), in speech like vocalisations We are examining the involvement of tongue movement during these vocalisations, hypothesising that it will account for the remaining residuals in the correlation between the formants excursions and the jaw movements.

\section{8:20}

5aABa2. Habitat occupation time-series of St. Lawrence belugas from passive acoustic monitoring. Nathalie Roy (Fisheries and Oceans Canada \& ISMER-UQAR, 850 route de la Mer, P.O. Box 1000, Mont-Joli, QC G5H-3Z4, Canada, royn@dfo-mpo.gc.ca), Yvan Simard (Fisheries and Oceans Canada \& ISMER-UQAR, 850 route de la Mer, P.O. Box 1000, Mont-Joli, QC G5H-3Z4, Canada, simardy@dfo-mpo.gc.ca), Catherine 
Bédard(Marine Science Institute, University of Québec, 310 Allée des Ursulines, P.O. Box 3300, Rimouski, QC G5L-3A1, Canada, catherine.becard01@uqar.qc.ca), Elisabeth Leblanc (Marine Science Institute, University of Québec, 310 Allée des Ursulines, P.O. Box 3300, Rimouski, QC G5L-3A1, Canada, elisabeth.leblanc@uqar.qc.ca)

Passive acoustic monitoring (PAM) was used to generate a six-week continuous time-series of occupation of a segment of the St. Lawrence Estuary by belugas. The PAM data were acquired from a cabled hydrophone deployed at mid-depth into the 300-m deep Laurentian channel off Cap-deBon-Desir during summer 2003. Beluga vocalisation activity time-series was obtained from the $[0.5-5.0 \mathrm{kHz}]$ bandpassed PAM data that were processed to filter out noise from the heavy shipping on the nearby St. Lawrence Seaway and from occasional whale watching boats, and lowfrequency traces of clicks, via adaptive spectral subtraction and image processing methods applied to the spectrogram. The remaining traces on the binary image of the spectrogram were summed up to generate a vocalisation index, which was essentially due to belugas in this region. The vocalisation index time-series was then analysed for presence of circadian rhythms and correlations with the semi-diurnal tidal cycle, currents from an acoustic Doppler current profiler and wind. Results are interpreted from the knowledge of the biological and physical oceanographic processes occurring in the monitored area.

\section{8:40}

5aABa3. Comparison of automatic classification methods for beluga whales vocalizations. Elisabeth Leblanc (Marine Science Institute, University of Québec, 310 Allée des Ursulines, P.O. Box 3300, Rimouski, QC G5L-3A1, Canada, elisabeth.leblanc@uqar.qc.ca), Mohammed Bahoura (Eng. Dept., Université du Québec, 300 Allée des Ursulines, P.O. Box 3300, Rimouski, QC G5L-3A1, Canada, mohammed_bahoura@uqar.qc.ca), Yvan Simard (Fisheries and Oceans Canada \& ISMER-UQAR, 850 route de la Mer, P.O. Box 1000, Mont-Joli, QC G5H-3Z4, Canada, simardy@dfo-mpo.gc.ca)

The beluga whale is a loquacious marine mammal with a complex vocal repertory. It produces a wide variety of whistles and pulsed tones as well as high-frequency echolocation clicks. Automatic detection of the longdistance propagating communication calls are desirable for implementing passive acoustic monitoring (PAM) systems in their environment, for habitat utilisation studies and real-time early warning devices in anthropogenic impact mitigation systems. Signal processing methods were developed to classify beluga vocalizations from a PAM data set recorded in Cap-de-BonDésir in the Saguenay-St. Lawrence Marine Park in summer 2003 using a 6-hydrophones array with a 10-100 kHz sampling rate. The performance of an MFCC-based HMM system and a polynomial model applied to extracted time-frequency contours of vocalizations will be evaluated. The feasibility of real-time implementation will then be analysed for both methods.

\section{9:00}

5aABa4. One plus one equals one: The beluga whale (Delphinapterus leucas) produces two pulses to form its echolocation click. Marc $\mathrm{O}$. Lammers (Hawaii Institute of Marine Biology, P. O. Box 1106, Kailua, HI 96734, USA, lammers@hawaii.edu), Manuel Castellote (L'Oceanografic, C/. Junta de Murs i Valls, s/n, 46013 Valencia, Spain, mcastellote @oceanografic.org)

Empirical results have shown that two sound generators exist in the nasal system of delphinids. It had been previously assumed that these function independently, with one primarily involved in pulse production and the other in the generation of tonal signals. Here we report that in the beluga whale both generators appear to be simultaneously involved in the production of the echolocation click measured in front of the animal. A study was conducted that examined the propagation of the echolocation click of a captive 12 year-old female beluga whale at $15^{\circ}, 30^{\circ}, 45^{\circ}, 60^{\circ}, 75^{\circ}$, and $90^{\circ}$ off the center axis of both sides of the outgoing beam. The results reveal that at angles greater than $30^{\circ}$ two distinct pulses are measured that cannot be attributed to multipath reflections from the surface or any structure in the tank. The two pulses are maximally separated in time at $90^{\circ}$ off-axis and gradually converge with each $15^{\circ}$ measurement closer to the beam center. At $30^{\circ}$ the two pulses begin to form a single, albeit distorted pulse. The phenomenon is symmetrical on both sides of the animal. These findings shed new light on how beluga whales and perhaps other odontocetes form their echolocation pulse.

$$
\text { 9:20 }
$$

5aABa5. The nanomechanics of mechanosensory neurones in vivo. James F. Windmill (University of Bristol, D34 School of Biological Sciences, Woodland Road, BS8 1UG Bristol, UK, james.windmill@bristol.ac.uk), Victoria G. Pook (University of Bristol, School of Biological Sciences, Woodland Road, BS8 1UG Bristol, UK, vicky.pook@bristol.ac.uk), Daniel Robert (University of Bristol, D34 School of Biological Sciences, Woodland Road, BS8 1UG Bristol, UK, d.robert@bris.ac.uk)

The ability to detect and process sound is a sense particularly important in many animals, including insects, playing a key role in predator, prey and mate detection. Acute hearing, both in the sense of extreme sensitivity to sound and sharp frequency selectivity, relies on the active participation of auditory mechanoreceptors. In insects, active auditory mechanics was first demonstrated in mosquitoes, whereby auditory sensitivity is enhanced by the action and reaction of mechanosensory neurones to sound-induced vibrations. The mosquito's auditory neurones can generate motions that mechanically drive the antenna and tune it to biologically relevant sounds. The mechanosensory neurones are capable of detecting exquisitely small mechanical displacements, down to 100 picometres. In the mosquito's Johnston's organ ( $300 \mu \mathrm{m}$ in diameter) there is a high density of these neurones (16 000 units). The mechanical response of the mechanoreceptors was measured in vivo using an atomic force microscope, in response to stimulation of the external antenna. The work establishes the link between the previously measured non-linearities of the mosquito's antennal vibrations and the nanoscale mechanics of the mechanosensory neurones.

9:40-11:00 Posters

Lecture sessions will recess for the presentation of poster papers on various topics in acoustics. See poster sessions for topics and abstracts

\section{Contributed Paper}

\section{1:00}

5aABa6. Computer aided design of audio signal classifier systems. Sebastian V. Huebner (Pestalozzistrasse 5, 14482 Potsdam, Germany, sebastian@sejona.de)

The problem of programming classifier systems for deterministic events in bioacoustic signals is seen as a knowledge engineering problem. Advantages and disadvantages of traditional approaches are discussed. A knowledge engineering approach for interactive visual classifier system design is described and advantages of the approach are highlighted. The basic archi- tecture of a general purpose knowledge engineering framework for the acoustics domain is outlined. Emphasis is placed on both expert knowledge and on the role of data mining within the classifier system design process. Several examples illustrate how the approach can be used to create classifier systems for bioacoustic patterns in the time-frequency domain. Examples include multiscale spectrographic visualization, techniques to create and interactively modify subsymbolic and symbolic classifier systems for timefrequency patterns as well as the usage of three-dimensional visualizations to display hierarchical dependencies within complex classifier systems. 


\section{Invited Paper}

$11: 20$

5aABa7. Sounds from a neonate harbour porpoise and their potential use in acoustic monitoring. Magnus Wahlberg (Fjord\&Bælt / University of Southern Denmark, Margrethes Plads 1, 5300 Kerteminde, Denmark, magnus@fjord-baelt.dk)

Harbour porpoises (Phocoena phocoena) emit ultrasonic $(130 \mathrm{kHz})$ clicks with a stereotyped source parameters for echolocation and communication. Recently it was observed that the first days of living, a neonate harbour porpoise emitted contact calls of much a much lower frequency emphasis, around a few $\mathrm{kHz}$. The echolocation clicks produced by the calf significantly differed in duration and band width during the first four months postpartum. The acoustic signals from harbour porpoise calves are ideal for automated detection and therefore as a tool in population studies of this species. Both hydrophone array systems towed from boats, and automated click detectors (such as T-PODs) may incorporate routines for automated detection of young calves. These findings may have a large potential for facilitating biologists in gathering important biological data from this otherwise very difficult-to-study species of marine mammals.

\section{Contributed Papers}

\section{$11: 40$}

5aABa8. Two-dimensional sonar beam characteristics of single harbour porpoise (Phocoena phocoena) echolocation clicks measured with a 16 hydrophone array. Jens C. Koblitz (Eberhard-Karls-Universität Tübingen, Zool. Institut, Abt. Tierphysiologie, Auf der Morgenstelle 28, 72076 Tübingen, Germany, Jens.Koblitz@uni-tuebingen.de), Magnus

Wahlberg (Fjord\&Bælt/University of Southern Denmark, Margrethes Plads 1, 5300 Kerteminde, Denmark, magnus@fjord-baelt.dk), Peter T. Madsen (University of Aarhus, Biological Sciences, Zoophysiology, C. F. Møllers Allé, Building 1131, DK-8000 Aarhus, Denmark, peter.madsen@biology.au.dk), Peter Stilz (Eberhard-Karls-Universität Tübingen, Zool. Institut, Abt. Tierphysiologie, Auf der Morgenstelle 28, 72076 Tübingen, Germany, peter.stilz@uni-tuebingen.de), Hans-Ulrich Schnitzler (Eberhard-Karls-Universität Tübingen, Zool. Institut, Abt. Tierphysiologie, Auf der Morgenstelle 28, 72076 Tübingen, Germany, hansulrich.schnitzler@uni-tuebingen.de)

For the first time, the beam structure of single harbour porpoise echolocation clicks was simultaneously measured in both the vertical and horizontal plane. Echolocation clicks of a stationed animal and free-swimming animals catching fish were recorded with a "plus-shaped" array consisting of 16 hydrophones. The $-3 \mathrm{~dB}$ beam width in the horizontal and vertical planes is in most cases similar to what has been previously measured by Au et al. (1999). However, individual clicks are sometimes broader or narrower than what has been previously reported. This may be caused by either voluntary control of the animal's beam width, or it may be a secondary effect of variations in intensity and air content within the sound production system.

\section{2:00}

5aABa9. Dolphin modulation rate transfer functions resulting from exposure to frequency modulated signals. Dorian $\mathrm{S}$. Houser (Biomimetica, 7951 Shantung Dr., Santee, CA 92071, USA, dhouser@spawar.navy.mil), James J. Finneran (US Navy Marine Mammal Program, Space and Naval Warfare Systems Center, 53560 Hull St., Code 71510, San Diego, CA 92152, USA, james.finneran@navy.mil)

Frequency modulated (FM) signals are useful in audiometric studies where the test environment contributes to multipath interference and produces spatial variations in the sound pressure field. Rhythmic FM stimuli are useful in evoked potential audiometry because of the auditory steady state response (ASSR) that is created within the auditory system. Although some research on dolphin ASSRs resulting from rhythmic FM signals has been performed, relationships between modulation depth, modulation rate, and ASSR amplitude have not been fully explored. To address this, two dolphins were exposed to rhythmic FM signals (120 dB SPL and center frequency $=40 \mathrm{kHz}$ ) and the resulting ASSR recorded. The modulation depth (peak-to-peak) and modulation rate were varied from $0.1-10 \%$ and $200-2500$ $\mathrm{Hz}$, respectively. A total of 512 epochs were recorded for each stimulus presentation and the averaged waveform was analyzed in the frequency domain. The amplitude peak corresponding to the modulation rate was used to create the modulation rate transfer function (MRTF). Both dolphins showed a MRTF peak at a $1 \mathrm{kHz}$ modulation rate, while one dolphin showed a second peak at $500 \mathrm{~Hz}$. The use of rhythmic FM signals in ASSR studies may be a useful for testing in less than ideal acoustic environments.

12:20-1:40 Lunch Break

\section{Contributed Papers}

$1: 40$

5aABa10. Correcting gray whale (Eschrichtius robustus) call rates in San Ignacio Lagoon, using sound exposure level measurements of ambient noise. Melania Guerra (Marine Physical Laboratory, Scripps Institution of Oceanography, 9500 Gilman Dr, MC 0238, La Jolla, CA 92093 0238, USA, meguerra@ucsd.edu), Aaron Thode (Marine Physical Laboratory, Scripps Institution of Oceanography, 9500 Gilman Dr, MC 0238, La Jolla, CA 92093-0238, USA, athode@ucsd.edu), Jorge Urban (Laboratorio de Mamiferos Marinos, Universidad Autonoma de Baja California Sur, La Paz, Apartado Postal 19-B, 23080 La Paz, Mexico, jurban@uabcs .mx), Sergio Gonzalez (Laboratorio de Mamiferos Marinos, Universidad Autonoma de Baja California Sur, La Paz, Apartado Postal 19-B, 23080 La Paz, Mexico, sergiog@uabcs.mx)

Autonomous acoustic recordings of gray whales (Eschrichtius robustus) were made in San Ignacio Lagoon, in February 2005-2008, while animals were present to breed and raise calves. Counts were made of the gray whales' most common vocalization, type S1. A sequence of semi-automated procedures was implemented to assist with call detection. Hourly call rates were computed for all seasons and adjusted for expected changes in detection range, caused by variations in the ambient background noise level. In this environment, the underwater acoustic background combines biological, oceanographic and man-made sources and can present changes of $10 \mathrm{~dB}$ above the average base level of $96 \mathrm{~dB}$ re $1 \mathrm{uPa} 2$-s between 350 and $750 \mathrm{~Hz}$ over semidiurnal scales. The relative changes in call rates in 2006 and 2008 are compared with visual survey counts conducted over the same period. The definition of SNR in the present study develops from energy flux densities or sound exposure levels (SEL). SEL were calculated experimentally through even sampling in time and individual sampling for each call. By assuming that the background masks a proportion of the detected calls, corrections were applied to determine the vocal activity within a fixed detection range. 


\section{2:00}

5aABa11. The acoustic field on the melon of echolocating Atlantic bottlenose dolphin (Tursiops truncatus). Whitlow W. Au (Univ. of Hawaii, P.O. Box 1106, Kailua, HI 96734, USA, wau@hawaii.edu), Dorian S. Houser (Biomimetica, 7951 Shantung Dr., Santee, CA 92071, USA, dhouser@spawar.navy.mil), James J. Finneran (US Navy Marine Mammal Program, Space and Naval Warfare Systems Center, 53560 Hull St., Code 71510, San Diego, CA 92152, USA, james.finneran@navy.mil), Lois Dankiewicz (SAIC, 4065 Hancock Street, MS: Q1-A, San Diego, CA 92110, USA, lois.dankiewicz@saic.com), Wu-Jung Lee (MIT-WHOI Joint Program, 266 Woods Hole Road, Woods Hole, MA 02543, USA, wjlee@whoi.edu), Patrick W. Moore (US Space \& Naval Warfare Systems Center, 49620 Beluga Road, San Diego, CA 92151, USA, pmoore@spawar .navy.mil)

An array of five broadband suction cup hydrophones were placed on the melon of two bottlenose dolphins to determine where on the melon the echolocation beam emerges and to examine how signals in the acoustic near-field relate to signals in the far-field at $1 \mathrm{~m}$. Four different array geometries were used: a linear one with hydrophones arranged along the midline of the melon between 2.8 and $3.7 \mathrm{~cm}$ apart, and two around the front of the melon at 1.4 and $4.2 \mathrm{~cm}$ above the melon-rostrum crease and one across the melon in certain locations not measured by other configurations. The beam axis was found to be close to the mid-line of the melon, approximately 5.4 $\mathrm{cm}$ above the melon-rostrum crease for both animals. The signal path coincided with the low-density, low-velocity core of the melon supporting the melon hypothesis postulated many years ago by Kenneth Norris. Slight asymmetry in the signal was found with higher amplitudes on the starboard side of the melon. Although the signal waveform measured on the melon appeared distorted, when they were mathematically summed in the far-field while preserving the relative time of arrival, the resultant waveform matched that measured by the hydrophone located at $1 \mathrm{~m}$.

\section{2:20}

5aABa12. Detection of beaked whales using near surface towed hydrophones: prospects for survey and mitigation. Douglas Gillespie (Sea Mammal Research Unit, Gatty Marine Laboratory, University of St. Andrews, KY16 8LB St. Andrews, UK, dg50@st-andrews.ac.uk), Jonathan Gordon (Sea Mammal Research Unit, Gatty Marine Laboratory, University of St. Andrews, KY16 8LB St. Andrews, UK, jg20@st-andrews.ac.uk), Marjolaine Caillat (Sea Mammal Research Unit, Gatty Marine Laboratory, University of St. Andrews, KY16 8LB St Andrews, UK, marjolaine.caillat@club-internet.fr), Diane Claridge (Bahamas Marine Mammal Research Organisation, P.O. Box AB20714, Abaco, 0 Marsh Harbour, Bahamas, bmms@oii.net), David Moretti (NAVSEA, Newport Undersea Warfare Center, Newport, RI RI 02841, USA, MorettiDJ@npt.nuwc.navy.mil), Ian Boyd (Sea Mammal Research Unit, Gatty Marine Laboratory, University of St Andrews, KY16 8LB St Andrews, UK, ilb@st-andrews.ac.uk)

Beaked whales are extremely difficult to sight at sea and this hampers attempts to study them, and makes real time mitigation difficult. Passive acoustic monitoring could improve detection efficiency. Blainville's beaked whales, (Mesoplodon densirostris) are known to produce most of their vocalizations at depth. They are routinely detected on bottom mounted hydrophones arrays but the extent to which they can be detected using nearsurface hydrophones is not known. Continuous recordings were made at a sampling rate of $192 \mathrm{kHz}$ from towed hydrophone arrays during line transect surveys in the Bahamas in conjunction with teams monitoring bottommounted hydrophones at the AUTEC Tongue of the Ocean navy range. A beaked whale click detector and classifier was developed within Rainbow Click and PAMGUARD and this was both run in real time and used to analyze recordings to pick out beaked whale click trains. Detected click trains correlated well with detection of beaked whales on bottom-mounted hydrophones. Three species of beaked whale were encountered visually and detected acoustically: Mesoplodon densirostris, Ziphius cavirostris and Mesoplodon europaeus. Target motion analysis of bearings to sequences of clicks suggests a maximum detection range of approximately three kilometers and preliminary results indicate that clicks can be identified to species.

\section{2:40}

5aABa13. Acoustic localization of two distinct blue whale (Balaenoptera musculus) subspecies in the South-West Indian Ocean. Flore Samaran (CEBC-CNRS, Centre d'Etude Biologique de Chizé, 79360 Villiers en Bois, France, samaran@cebc.cnrs.fr), Olivier Adam (Université Paris Est, 61, avenue de Gaulle, 94000 Creteil, France, adam@univ-paris12.fr), Jean-François Motsch (Université Paris Est, 61, avenue de Gaulle, 94000 Creteil, France, motsch@univ-paris12.fr), Yves Cansi (Commissariat à l'Energie Atomique, Centre DAM - Ile de France, Bruyères-le-Châtel, 91297 Arpajon Cedex, France, yves.cansi@cea.fr), Gérard Ruzié (Commissariat à l'Energie Atomique, Centre DAM - Ile de France, Bruyères-le-Châtel, 91297 Arpajon Cedex, France, gerard.ruzie@cea.fr), Christophe Guinet (CEBC-CNRS, Centre d'Etude Biologique de Chizé, 79360 Villiers en Bois, France, guinet@cebc .cnrs.fr)

Analysis of one year of acoustic signal recordings from the five permanent autonomous hydrophones of the International Monitoring System in the South-West Indian Ocean reveals low frequency with high intensity calls produced by two blue whale subspecies. The "Antarctic" or "true" blue whale (B. m. intermedia) calls and the "Madagascar-type" Pygmy blue whale calls (B. m. brevicauda) were automatically detected through the matched filtering method. The potential movements were investigated by using the time difference of arrival (TDOA) of calls to assess the bearing of the sound source. The fully range dependent parabolic equation code (RAM - range-dependent acoustic model) and the PMCC code (progressive multichannel correlation) are applied to estimate the range between our system and the vocalising animals. Our results show that (1) the variation of call number revealed two distinct patterns of seasonal whale occurrences and (2) the distances from the hydrophones to the blue whales reached up to $50 \mathrm{~km}$. Tracking whales is possible when whales are concentrated of the hydrophone array.

\section{3:00}

5aABa14. Biosonar performance of a false killer whale (Pseudorca crassidens) improved with practice. Robert Gisiner (Marine Mammal Commission, 4340 East-West Highway, Room 700, Bethesda, CA 20814, USA, bgisiner@mmc.gov)

Cylinder wall thickness discrimination tasks have been used to assess the limits of dolphin biosonar. An attempt to replicate the benchmark data from a bottlenose dolphin (Tursiops truncatus) resulted in a surprising order of magnitude better performance by a false killer whale. The improvement came over multiple testing episodes, which suggests that initial limits to discrimination were overcome by learning; either through better understanding of the reinforcement contingencies, attention to novel sources of information within the returning echoes, or both. These results offer important insights into methodological considerations for testing animal psychophysical performance, especially for sensory performances like echolocation in which the subject exercises active control over the sensory input. The ability to improve sensory performance with practice also offers insights into the way the echo information is processed into an internal representation of external physical reality by the central nervous system, a process that is very likely open to improvement through experience, or learning, throughout the individual's life.

\section{3:20}

5aABa15. An analysis of Humpback whale songs for individual classification. Suleman Mazhar (URA Laboratory, Institute of Industrial Science, The University of Tokyo, 4-6-1, Komaba, Meguro, 153-8505 Tokyo, Japan, suleman@iis.u-tokyo.ac.jp), Tamaki Ura (URA Laboratory, Institute of Industrial Science, The University of Tokyo, 4-6-1, Komaba, Meguro, 153-8505 Tokyo, Japan, ura@iis.u-tokyo.ac.jp), Rajendar Bahl (Indian Institute of Technology, Delhi, Hauz Khas, 110016 New Delhi, India, rbahl@care.iitd.ernet.in)

Acoustics based tracking systems are in use for studying cetacean behaviour. Such noninvasive techniques can also prove efficient for population assessment of vocally active species. One problem in this regard is to distinguish already counted reappearing cetaceans, even if reappearance occurs after a brief interval. In this connection we propose development of in- 
dividual identification system for humpback whales (Megaptera novaeangliae) based on unique acoustic features underlying a song. Earlier we analyzed recognition performance of cepstrum based voice signature in seven humpback whales. The results were based on training and test data sampled from the same song. In this work, we test our hypothesis using data from different songs (i.e., recording timings of training and test data sets do not overlap) for two different individuals. Recognition rates for two individuals are above $80 \%$ and $95 \%$, respectively. However an eight years old song gives degraded recognition rate of around $58 \%$. The results give an insight into the effects of temporal song evolution on accuracy of our system. Our observations are followed by harmonic analysis of song units in two individuals. Cepstral coefficients and support vector machine (SVM) were used for classification and signal band-crossing rate was used for segmenting song units.

\section{3:40}

5aABa16. Phonemic segment characterization of Norwegian killer whale call types. Ari D. Shapiro (Woods Hole Oceanographic Institution, Applied Ocean Physics \& Engineering Dept., Woods Hole, MA 02543, USA, ashapiro@whoi.edu), Stephanie Seneff (Massachusetts Institute of Technology, Computer Science and Aritifical Intelligence Laboratory, Spoken Language Systems Group, Cambridge, MA 02139, USA, seneff@csail.mit.edu), Peter Tyack (Woods Hole Oceanographic Institution, Applied Ocean Physics \& Engineering Dept., Woods Hole, MA 02543, USA, ptyack@whoi.edu)

An important issue in animal vocal communication concerns the fundamental unit used to build signals into a repertoire and the syntactic rules associated with that assembly. Killer whale vocal production has traditionally been categorized by human observers into a set of discrete call types. These call types often contain internal spectral shifts, silent gaps and synchronously produced low and high frequency components. Such features motivated the analysis here which tested whether call types could be represented by a set of flexibly arranged and smaller phonemic segments. We evaluated whether segmented characterizations of stereotyped Norwegian killer whale calls yielded automated classification results of contour traces that paralleled a classification scheme using whole call type designations. Representations of calls in their entirety or as sets of either distinct or shared syllables did achieve similar performance. Calls composed of shared segments may provide a more parsimonious approach to parsing the vocal stream since there were fewer segments than call types, nearly $75 \%$ of all call types contained at least one shared syllable, and some syntactic patterns were evident. Such a system could flexibly generate new call types and contain the killer whale vocal repertoire within a subset of the possible combinations of segments.

\section{4:00}

5aABa17. AFM and BSEM: Novel approaches to the Basilar Membrane. Darlene R. Ketten (NIH/NIDCD and WHOI, Section on Auditory Mechanics, Bldg 10-5D49, Bethesda, MD 20892, USA, dketten@whoi.edu), Emilios K. Dimitriadis (NIH/NIDCD and WHOI, Section on Auditory Mechanics, Bldg 10-5D49, Bethesda, MD 20892, USA, dimitria@helix.nih.gov), Richard S. Chadwick (NIH/NIDCD and WHOI, Section on Auditory Mechanics, Bldg 10-5D49, Bethesda, MD 20892, USA, chadwick@helix.nih.gov), David Mountain (Boston University, Biomedical Engineering Dept., 44 Cummington St., Boston, MA 02215, USA, dcm @bu.edu)

For over a half century, researchers have probed cochlear biophysics with increasingly sophisticated technologies. Recently, we brought two new approaches, atomic force microscopy (AFM) and backscatter electron microscopy (BSEM), to bear on the question of how basilar membrane structural variations affect mechanical responses. Hemi-cochleae and basilar membrane segments were obtained from rat, guinea pig, and gerbil ears by temporal bone microdissection. Hemi-cochleae in PBS or formalin were imaged in a hydrated, uncoated state (VP wet mode) in Petri dishes mounted on boutons using an Hitachi S3400 N-1 SEM. Membrane segments were excised, trimmed of laminae and ligament remnants, and mounted on polylysine/albumin-coated glass for AFM imaging and force measurements. Both BSEM and AFM data indicate two distinct regions consistent with softer ground substance separating relatively stiff, ordered fiber bundles ori- ented radially in pectinate regions. Fibers and bundles ranged 0.3 to $1 \mu \mathrm{m}$ in diameter with elasticity values, based on a Hertzian contact model, in the hundreds of $\mathrm{kPa}$ range, consistent with mixed elastin and collagen. Greatest variations occurred in matrix spacing, suggesting differences are attributable largely to packing density. Middle turn ground substance spacing averaged $1.5 \mu \mathrm{m}$, consistent with previously reported values (Naidu and Mountain, 2007; Fung, 1993).

\section{4:20}

5aABa18. Depth, orientation, and acoustics of sperm whales (Physeter macrocephalus) under natural and depredation foraging conditions in the Gulf of Alaska. Delphine Mathias (Marine Physical Laboratory, Scripps Institution of Oceanography, 9500 Gilman Dr, MC 0238, La Jolla, CA 92093-0238, USA, delphine.mathias@gmail.com), Aaron Thode (Marine Physical Laboratory, Scripps Institution of Oceanography, 9500 Gilman Dr, MC 0238, La Jolla, CA 92093-0238, USA, athode@ucsd.edu), Jan Straley (University of Alaska SE, 1332 Seward Ave, Sitka, AK 99835, USA, jan.straley@uas.alaska.edu), Kendall Folkert (PO Box 6497, Sitka, AK 99835, USA, kendallcobra@yahoo.com), John Calambokidis (Cascadia Research Collective, $2181 / 2$ W. 4th Ave., Olympia, WA 98501, USA calambokidis@cascadiaresearch.org), Greg Schorr (Cascadia Research Collective, 218 1/2 W. 4th Ave., Olympia, WA 98501, USA, GSchorr@cascadiaresearch.org), William C. Burgess (Greeneridge Sciences Inc, 6060 Graham Hill Rd, suite f, Felton, CA 95018, USA, burgess@greeneridge.com), Chris Lunsford (National Marine Fisheries Service Auke Bay Laboratory, 11305 Glacier Hwy, Juneau, AK 99801-8626, USA, chris.lunsford@noaa.gov)

In July 2007 bioacoustic tags were attached to adult sperm whales in the Gulf of Alaska under both natural foraging conditions, and situations wherein the animals were depredating sablefish from commercial longlining vessels. A small Rigid-Hull Inflatable was used to approach and attach a suction-cup acoustic recording tag on 13 occasions and stayed on animals for a total of 168 hours, yielding $80 \mathrm{~h}$ of depth, orientation, and acoustic data. These results, combined with passive acoustic tracking and underwater video-camera data, indicate that sperm whales depredate at depths shallower than $50 \mathrm{~m}$, compared to natural foraging depths of $300-400 \mathrm{~m}$ in the area. During depredation the animals demonstrate changes in pitch and roll that are greater when compared with normal foraging behavior. Observations on the acoustic behavior of the animals are noted as well. The fact that these normally deep-diving animals depredate so close to the surface has interesting implications for both depredation-reduction strategies and biosonar research. [Work conducted under the SEASWAP program, supported by the North Pacific Research Board and the National Geographic Society.]

\section{4:40}

5aABa19. Fin whale (Balaenoptera physalus) movements along the Spanish mediterranean coast. Manuel Castellote (L'Oceanografic, C/. Junta de Murs i Valls, s/n, 46013 Valencia, Spain, mcastellote @oceanografic.org), José-Antonio Esteban (Research Department, Parques Reunidos Valencia S. A. L'Oceanogràfic, Ciudad de las Artes y las Ciencias, 46013 Valencia, Spain, investigacion@oceanografic.org), Christopher W. Clark (Cornell University Laboratory of Ornithology, Bioacoustics Research Program, 159 Sapsucker Woods Road, Ithaca, NY 14850, USA, cwc2 @ cornell.edu)

The Ligurian-Corsican-Provençal Basin is a fin whale summer feeding ground, but little is known about the seasonal movement patterns of this population. We acoustically monitored two regions off Spain (eastern and southern) during summer-fall-winter 2006 using seafloor autonomous recording units. Long patterned sequences of $20 \mathrm{~Hz}$ pulses and back-beats from Mediterranean fin whales were identified in both study regions using acoustic features as indicators of population identity. Eastern area: fin whale sounds were detected at very high rates (122 pulses/hour) and between 40$80 \%$ of time/day. The detection pattern can be explained by whales moving gradually through the acoustic recorder's detection area, suggesting that the area is probably used as a passage between summer and winter grounds Southern area: detections from the same population were less abundant (21 pulses/hour) and between 10-35\% of time/day. The detection pattern shows a highly variable distribution with no clear trend, suggesting that Mediter- 
ranean fin whales continuously remained in and moved out of the detection area of the recorder from November to January. These results show that this southern area is potentially used as a winter ground. This work documents a new dispersion path towards the Alboran sea, a new potential winter ground.

\section{5:00}

5aABa20. Accurate estimation of the duration of tonal signals emitted by marine mammals. Nicolas Sentenac (Thales Underwater Systems, 525, route des dolines, BP 157, 06903 Sophia Antipolis, France, nicolas .sentenac@fr.thalesgroup.com)

Passive acoustic marine mammals detection and classification is of great importance nowadays. The goal of this article is to present a tool that can be used to accurately measure the duration of tonal emissions from marine mammals, helping to classify them. Marine mammals emissions can be split into two different kinds and one of them is tonal emission. It can be defined by its narrow band frequency pattern. Narrow band signals can be detected in the time/frequency space thanks to the use of the spectrogram and thanks to a quadratic contrast criterion. The expression of this criterion for signalonly and noise-only is straightforward. This article will introduce the expression of a lower bound of the expectation of the criterion when signal and noise are mixed. As this lower bound is a function of signal to noise ratio, it can be used to threshold the criterion with the desired signal to noise ratio value. When the criterion is computed along time on a sliding window, narrow band signals with a higher snr than the thresholding one are detected as soon as they appear and until they vanish, giving an accurate estimation of the duration of such signals.

\title{
Invited Paper
}

5aABa21. Ultrasonic production and reception in frogs: Lessons from Asia. Peter Narins (UCLA, Dept. of Physiological Science, 621 Charles E. Young Drive S., Los Angeles, CA 90095-1606, USA, pnarins@ucla.edu)

Among the vertebrates, only microchiropteran bats, cetaceans and some rodents are known to produce and detect ultrasonic (US) frequencies for the purpose of communication and/or echolocation, suggesting that this capacity may be restricted to mammals. We have recently provided the first evidence of ultrasonic communication in an amphibian - the concave-eared torrent frog, Amolops tormotus (Ranidae) from Huangshan Hot Springs, China. Males of A. tormotus produce diverse birdlike melodic calls with pronounced frequency modulations that often contain spectral energy in the US range. Acoustic playback experiments conducted in the animal's natural habitat confirmed that the audible as well as the US components of an A. tormotus call could effectively evoke males' vocal responses. Electrophysiological recordings from the auditory midbrain confirmed the US hearing capacity of these frogs and that of a sympatric species facing similar environmental constraints. This extraordinary upward extension into the ultrasonic range of both the harmonic content of the advertisement calls and the frog's hearing sensitivity is likely to have coevolved in response to the intense, predominately lowfrequency ambient noise from local streams. Supported by NIH grant DC-00222.

FRIDAY MORNING, 4 JULY 2008

P3-C, LEVEL 3, 9:40 TO 11:20 A.M.

\section{Session 5aABb}

\section{Animal Bioacoustics: General Topics in Animal Bioacoustics III (Poster Session)}

\author{
Richard Fay, Cochair \\ Loyola University Chicago \\ Michel Andre, Cochair \\ Laboratori d'Aplicacions Bioacústiques (Universitat Politècnica de Catalunya)
}

\begin{abstract}
All posters will be on display from 9:40 a.m. to 11:20 a.m. To allow contributors an opportunity to see other posters, contributors of odd-numbered papers will be at their posters from 9:40 a.m. to 10:30 a.m. and contributors of even-numbered papers will be at their posters from 10:30 a.m. to 11:20 a.m.
\end{abstract}

\section{Contributed Papers}

5aABb1. A brownian energy depot model of the basilar membrane oscillation. Chul Koo Kim (Yonsei University, Department of Physics, Shinchon-dong, 120-749 Seoul, Republic of Korea, ckkim@yonsei.ac.kr)

Response of a living basilar membrane (BM) is significantly different from that of the cadaver and is known to be essentially active. The best candidate for a generator of the active force in mammals is the outer hair cells. By employing the outer hair cells as the energy depot, we newly propose an interactive energy depot model for the basilar membrane. In this model, the nonlinear responses and the spontaneous basilar membrane oscillation are obtained. In the regime of small vibration, this model is reduced to the wellknown Hopf bifurcation model. Our model suggests two-fold roles of the outer hair cells in hearing: an amplification for a weak stimulation and a protection for a strong stimulation.
$5 a A B b 2$. Slow chaotic changes in the excitability of the auditory units. Nikolay G. Bibikov (N.N. Andreyev Acustical Institute, Shvernik st. 4, 117036 Moscow, Russian Federation, bibikov@akin.ru), Alexandre B. Dymov (N.N. Andreyev Acustical Institute, Shvernik st. 4, 117036 Moscow, Russian Federation, dymov@mail.ru)

We explored the statistical properties of the spontaneous and evoked firing activity in the brainstem auditory units of the grass frog (Rana $t$ temporaria). We estimated the properties of spontaneous firing using the following functions: interspike interval distribution, hazard function, autocorrelation functions for original and shifted sequence of interspike intervals, function of interdependence of neighboring intervals. The dependences of Fano and Allans factors upon the values of counting time (window size) were also obtained. We observed a considerable deviation of the spontane- 
ous activity from the renewal process for the majority of units located both in the dorsal medullar nucleus (homolog of cochlear nucleus in the mammals) and in torus semicircularis (homolog of inferior colliculus). The negative correlation between the neighboring short interspike intervals was typical for some (mainly toral) units. However, generally week but quite reliable positive correlation between neighboring intervals was typical for the great majority of neurons. The values of Fano and Allan factors were close to 1 at window size less than $100-500 \mathrm{~ms}$ and increased in proportion to some power for larger window sizes. The exponents of these power dependences were different for different units.

5aABb3. Synchrony and neuromechanical sensitivity in the mosquito hearing organ. Joseph C. Jackson (University of Bristol, D34 School of Biological Sciences, Woodland Road, BS8 1UG Bristol, UK, j.c.jackson@bris.ac.uk), Daniel Robert (University of Bristol, D34 School of Biological Sciences, Woodland Road, BS8 1UG Bristol, UK, d.robert @bris.ac.uk)

Sound detection is a fundamental tool for many biological organisms to sense their environment. Sensing the particle velocity component of sound waves has led to the evolution of exquisitely sensitive auditory organs: small oscillators that are driven resonantly by the sound field. The sound detection threshold is often indistinguishable from Brownian motion. To achieve such low thresholds, the mammalian cochlea incorporates active mechanisms, adding energy to the sound oscillations, and so enhancing sensitivity and selectivity. Mosquito antennae are also endowed with active auditory mechanics. Males use these highly sensitive external antennae to detect the flight sound of females. Sound-induced oscillation of the antenna stimulates thousands of mechanosensory neurones. The antenna exhibits dynamic responses remarkably similar to vertebrate auditory systems: self-oscillation and amplitude-dependent bandwidth both occur. They also exhibit hitherto unseen nonlinear responses including mid-level amplification and hysteresis. The mechanisms for these effects are thought to derive from ciliary motility in the mechanosensory neurones. We show that synchronization in the motile neurones generates large stable coherent forces that provide the antenna with its nonlinear response characteristics. Synchronization ensures a coherent neuronal output, improving signal fidelity. Finally, coherent force modifies the dissipation of antennal energy, changing the bandwidth and enhancing sensitivity.

5aABb4. Changes in structure of Redwing's (Turdus iliacus) local dialect in time. Ludmila Osipova (St. Petersburg State University, Universitetskaya emb., 7/9, 199034 St. Petersburg, Russian Federation, osipoval @yandex.ru)

The question of the period of existance of a local dialect is yet to be answered. Were there a changes in time-and-frequency parameters of dialects with time? If there were, what parameters had changed? On the basis of original data for 2005 - 2007 period, and archives of Department of Vertebrate Zoology of Saint-Petersburg State University, the history of local dialect of Redwing (Turdus iliacus) from Saint-Petersburg's outskirt (Peterhof) was investigated.The archive contains recordings of local dialects of Peterhof for 1960-1991 period. Until now, the longest period of local dialect observation for this species did not exceed 10 years (Bjerke, 1981). In observed period of 48 years several changes were found. Number of elements in phrase, it's duration, frequency modulation of all elements, their configuration, duration and frequency range. The major changes occurred to the first element of song. It is gradual reduction with time is obvious.

5aABb5. Microacoustics: maintaining an ecologically relevant scale in insect bioacoustics. Erica L. Morley (University of Bristol, D34 School of Biological Sciences, Woodland Road, BS8 1UG Bristol, UK, Erica.Morley@bristol.ac.uk), Thorin Jonsson (University of Bristol, School of Biological Sciences, Woodland Road, BS8 1UG Bristol, UK, t.jonsson@bristol.ac.uk), Daniel Robert (University of Bristol, D34 School of Biological Sciences, Woodland Road, BS8 1UG Bristol, UK, d.robert @bris.ac.uk)

A major challenge when studying an organism is to maintain its environment and context as reliably as possible. When looking at sensory systems it is crucial not to assume the animal's perception the same as ours and instead measure and mimic more accurately the natural stimuli driving the sensors. In insect bioacoustics the usual practice in playback and recording techniques overlooks differences in scale and context in which the organism's sensory system evolved. Here we present an approach to emit and record low-amplitude near-field sound, while maintaining an ecologically relevant scale, in Drosophila melanogaster. To mimic the effect of male courtship song on the female sound receiver (antennae) a mechanical microwing was constructed simulating, in power and geometry, particle velocity signals emitted by singing males, with its efficacy tested by behavioural assay. Development of a miniature particle velocity microphone was also initiated for recording signals in the fly's immediate vicinity $(<2 \mathrm{~mm})$ to elucidate the magnitude, temporal and radiation characteristics of the produced sound-field.

5aABb6. The effect of courtship song components in Drosophila melanogaster. Yu-Po Chen (University of Bristol, D34 School of Biological Sciences, Woodland Road, BS8 1UG Bristol, UK, Yu-po.Chen@bristol.ac.uk), Joseph C. Jackson (University of Bristol, D34 School of Biological Sciences, Woodland Road, BS8 1UG Bristol, UK, j.c.jackson@bris.ac.uk), James F. Windmill (University of Bristol, D34 School of Biological Sciences, Woodland Road, BS8 1UG Bristol, UK, james.windmill@bristol.ac.uk), Daniel Robert (University of Bristol, D34 School of Biological Sciences, Woodland Road, BS8 1UG Bristol, UK, d.robert@bris.ac.uk)

Courtship between male and female Drosophila melanogaster involves a complex emission of sounds produced by the male. This song comprises two aspects: a low-frequency sine song followed by a series of intense pulses. These songs increase the chances of the female accepting the male. However, the reason for the complexity of the song, and the effect on the female of the song's components, is poorly understood. Using measurements of the male courtship song for a canonical stimulus, we investigate the importance of the sine song on both the mechanical and electrophysiological responses of the female antenna: the nonlinear auditory sensor. While stimulating the antenna with modified courtship songs, antennal motion was measured using laser Doppler vibrometry, and compound potentials were simultaneously measured from the auditory neurones. Results show that, even at the periphery, there exists a significant change in the way the antenna responds to the pulses as a function of the sine song intensity, most emphatically through the neurophysiological signals. Results indicate that the sine song is an advantageous trait used to increase the female perception of the pulses in both time and amplitude sensitivity, and therefore improve the male's chance of successfully courting a female.

5aABb7. The Locust's tympanal mechanics. James $F$. Windmill (University of Bristol, D34 School of Biological Sciences, Woodland Road, BS8 1UG Bristol, UK, james.windmill@bristol.ac.uk), Samuel D. Bockenhauer (Stanford University, 208 Rosse Ln, \#305, Stanford, 94305, USA, sbockenhauer@gmail.com), Thomas R. McDonagh (University of Bristol, School of Biological Sciences, Woodland Road, BS8 1UG Bristol, UK, tm3377@bristol.ac.uk), Daniel Robert (University of Bristol, D34 School of Biological Sciences, Woodland Road, BS8 1UG Bristol, UK, d.robert@bris.ac.uk)

In the ear of the desert locust frequency analysis arises from the mechanical properties of the tympanal membrane. Incident sound is spatially decomposed into discrete frequency components through a tympanal travelling wave that funnels mechanical energy to specific tympanal locations, where distinct groups of mechanoreceptor neurones project. Initial analysis of the travelling waves employs conventional, steady state FFT, allowing a detailed analysis of the spatial composition of different frequencies onto the membrane. To further understand the exact mechanics of the tympanal travelling wave, its motion was also measured in the time domain to characterise its response to single impulse and single frequency stimuli, with a resolution of $390 \mathrm{~ns}$. This allows the measurement of instantaneous wave velocity and the direct observation of wave compression across the tympanum. The locust tympanal membrane locust exploits tonotopic frequency analysis, in a similar sense to that of the travelling waves of von Békésy on the mammalian basilar membrane. However, von Békésy's wave is born from interactions between the anisotropic basilar membrane and surrounding incom- 
pressible fluids, whereas the locust's wave rides on an anisotropic membrane suspended in air. The locust's tympanum thus combines the functions of both sound reception and frequency analysis.

$5 \mathrm{AABb8}$. Automated acoustic identification of beetle larvae in imported goods using time domain analysis. James Schofield (University of York, Department of Electronics, Heslington, YO10 5DD York, UK, js517@york.ac.uk), David Chesmore (University of York, Department of Electronics, Heslington, YO10 5DD York, UK, edc1@ohm.york.ac.uk)

The detection of insect pests in imported goods is of considerable economic importance and the automation of this process is becoming more viable both technologically and financially. As a result, the Department for Environment, Food and Rural Affairs in the UK has funded a research project to develop instrumentation facilitating real-time acoustic detection of the feeding activity of insect larvae inside imported goods, such as timber. The instrumentation will also be capable of species-level identification. Previous work at York has shown that detection of beetle larvae in wood is possible using low cost piezoelectric sensors. The project described here extends this work by investigating a number of signal analysis methods for robust detection of biting events, including fractal dimension analysis. Identification is currently being carried out using time domain signal coding and artificial neural networks. This paper will concentrate on the results of various algorithms for the estimation of fractal dimension and their relative suitability for bite detection. The effects of varying sampling rates, threshold levels and signal-to-noise ratio on the detection rate will be demonstrated.

$5 \mathrm{AABb9}$. Sound insulation of artificial and natural sound signals in reeds habitats. Igor Ianovschi (Universitetskaja nab. 7/9, Dep. Vertebrate Zoology, 199034 St. Petersburg, Russian Federation, igorul@mail.ru), Ludmila Osipova (St. Petersburg State University, Universitetskaya emb., 7/9, 199034 St. Petersburg, Russian Federation, osipoval @ yandex.ru)

From the point of view of acoustical communication, dense fields of reeds are strong acoustical filtre which distorts original sound signal. In this research the rate of sound signals sound insulation in massives of reeds was estimated. As a models were used artificially synthesized, modulated by frequency signals of three frequency lines: $1-2 \mathrm{kHz}, 2-4 \mathrm{kHz}$, and $4-8 \mathrm{kHz}$ with duration of 0.1 and $0.25 \mathrm{msec}$. For comparison were also used elements of bird songs. The least damping was noted in signals with descending frequency modulation in $8-4 \mathrm{kHz}$ range in the upper level of reeds $(6 \mathrm{db}$ on $10 \mathrm{~m}$ ). The most damping were noted in signals with descending frequency modulation in $2-1 \mathrm{kHz}$ in the middle level of juncaceous massive(18 $\mathrm{db}$ to $10 \mathrm{~m})$. The signals with frequency modulation of $4-2 \mathrm{kHz}$ were extending better in the upper level of reeds (7-8 db), whereas in the lower and upper levels were noted dependence of damping rate on duration of sound impulse - the signals with duration of $0.25 \mathrm{mec}$ were damping less than signals with duration of $0.1 \mathrm{msec}$. The elements of bird songs during their emission in the middle level of juncaceous massive were damping by $15-20 \mathrm{db}$ on $10 \mathrm{~m}$.

5aABb10. Echolocation and flight strategies of Japanese house bats (Pipistrellus abramus) to attack a prey in the field revealed by a microphone array. Emyo Fujioka (Faculty of Engineering, Doshisha Univ., 1-3 Miyakodani Tatara, 610-0321 Kyotanabe, Japan, dth0902@mail4.doshisha.ac.jp), Shigeki Mantani (Faculty of Engineering, Doshisha Univ., 1-3 Miyakodani Tatara, 610-0321 Kyotanabe, Japan, bte2058@mail4.doshisha.ac.jp), Michihiro Fukuda (Faculty of Engineering, Doshisha Univ., 1-3 Miyakodani Tatara, 610-0321 Kyotanabe, Japan, dtg0108@mail4.doshisha.ac.jp), Shizuko Hiryu (Faculty of Engineering, Doshisha Univ., 1-3 Miyakodani Tatara, 610-0321 Kyotanabe, Japan, shiryu@mail.doshisha.ac.jp), Hiroshi Riquimaroux (Faculty of Engineering, Doshisha Univ., 1-3 Miyakodani Tatara, 610-0321 Kyotanabe, Japan, hrikimar@mail.doshisha.ac.jp), Yoshiaki Watanabe (Faculty of Engineering, Doshisha Univ., 1-3 Miyakodani Tatara, 610-0321 Kyotanabe, Japan, kwatanab@mail.doshisha.ac.jp)

Bats are supposed to have effective strategies for achieving a good balance between echolocation and flight behaviors while capturing small moving insects in the field. To reveal their strategies for catching insects, we successfully reconstructed 3-D flight trajectories for the bat to forage in the field by a four-microphone array system, and conducted both acoustical and behavioral analyses for capturing behavior. Data show that the flying bats changed their flight direction flexibly, and sometime repeated capturing insects every two to three seconds. During the search phase, the bat moved 0.5-0.8 $\mathrm{m}$ during an interval between successive pulses (IPI) and then decreased that the moving distance during an IPI up to $0.1 \mathrm{~m}$ just before capturing a prey. Interestingly, we found that the bat tended to descend toward a prey from above when the approach phase started. This suggests that foraging bats may effectively utilize gravity for an easy acceleration toward the prey to concentrate on the complex echolocation for capturing moving insects. [Supported by a grant to RCAST at Doshisha Univ. from MEXT of Japan: Special Research Grants for Development of Characteristic Education from the Promotion and Mutual Aid Corporation for Private Schools Japan, Innovative Cluster Creation Project.]

5aABb11. Optimization of the characteristics of receiving echoes by free-flying echolocating bats, revealed by onboard pulse-echo recordings. Shizuko Hiryu (Faculty of Engineering, Doshisha Univ., 1-3 Miyakodani Tatara, 610-0321 Kyotanabe, Japan, shiryu@mail.doshisha.ac.jp), Yu Shiori (Faculty of Engineering, Doshisha Univ., 1-3 Miyakodani Tatara, 610-0321 Kyotanabe, Japan, dtg0165@mail4.doshisha.ac.jp), Tatsuro Hosokawa (Faculty of Engineering, Doshisha Univ., 1-3 Miyakodani Tatara, 610-0321 Kyotanabe, Japan, dtg0119@mail4.doshisha.ac.jp), Hiroshi Riquimaroux (Faculty of Engineering, Doshisha Univ., 1-3 Miyakodani Tatara, 610-0321 Kyotanabe, Japan, hrikimar@mail.doshisha.ac.jp), Yoshiaki Watanabe (Faculty of Engineering, Doshisha Univ., 1-3 Miyakodani Tatara, 610-0321 Kyotanabe, Japan, kwatanab@mail.doshisha.ac.jp)

To understand complex sensory-motor behavior underlying echolocation by bats, we need to precisely measure acoustic characteristics of echoes that the bats actually listen to during flight. However, it has been technicallydifficult to develop a small and light enough microphone set at the position of the bat's ear, and information about the echoes is so far virtually lacked. Here, we have successfully monitored these returning echoes by means of an onboard telemetry microphone mounted on the bats, and we confirmed that the free-flying horseshoe bats compensated for Doppler-shifts by adjusting their call frequency, thus maintaining the echo frequency at constant. Furthermore, we found that the bats also adjusted pulse amplitude with the distance to maintain the echo intensity within the narrow range. Receiving echoes with stable amplitude could help the bats to sustain consistent analysis of successive echoes, and combined frequency and amplitude compensation may be for optimization of successive echoes for target range estimation to control approach and landing. [Supported by a grant to RCAST at Doshisha Univ. from MEXT of Japan: Special Research Grants for the Development of Characteristic Education from the Promotion and Mutual Aid Corporation for Private Schools of Japan and the Innovative Cluster Creation Project.]

5aABb12. Some problems of analyzing bio-sonar echolocation signals generated by echolocating animals living in the water and in the air. Tadeusz Gudra (Wroclaw University of Technology/Institute of Telecommunications, Teleinformatics and Acoustics, Wybrzeze Wyspianskiego 27, 50-370 Wroclaw, Poland, Tadeusz.Gudra@pwr.wroc.pl), Krzysztof Herman (Wroclaw University of Technology/Institute of Telecommunications, Teleinformatics and Acoustics, Wybrzeze Wyspianskiego 27, 50-370 Wroclaw, Poland, krzysztof.herman@pwr.wroc.pl)

In this paper some similarities and differences of bio-sonar echolocation signals generated in the water and in the air are presented. The echolocation cues of marine mammals and bats are usually frequency and amplitude signals modulated simultaneously. The main problems of the analysis of such type of signals are envelope detecting and time-frequency decomposition. In this paper some digital signal processing (DSP) algorithms that may be used to analyze this type of signals are presented. The basic linear spectrogram (STFS), the nonlinear Winger-Vill'e spectrogram and some time-scale representations of signals using wavelets methods of estimation of the fre- 
quency modulation function have been compared. An analysis of possibilities for acoustic identification of particular species of bats by means of echolocation is also presented.

5aABb13. Blue whale calls characterization using chirplet transform. Mohammed Bahoura (Eng. Dept., Université du Québec, 300 Allée des Ursulines, P.O. Box 3300, Rimouski, QC G5L-3A1, Canada, mohammed_bahoura@uqar.qc.ca), Yvan Simard (Fisheries and Oceans Canada \& ISMER-UQAR, 850 route de la Mer, P.O. Box 1000, Mont-Joli, QC G5H-3Z4, Canada, simardy@dfo-mpo.gc.ca)

The blue whale, Balaenoptera musculus, frequently produce distinctive low-frequency $(<100 \mathrm{~Hz})$ signature calls that propagate over large distances in deep oceans. Their efficient detection and identification under variable noise conditions in long-term recordings is a basic requirement of passive acoustic monitoring systems used for studying distributional ecology and habitat selection of these large-scale migrant animals. In North Atlantic, blue whale signature calls are the A and B infrasounds $(15-20 \mathrm{~Hz})$, which often occur together in $\mathrm{AB}$ phrases, and the audible D-call $(35-120 \mathrm{~Hz})$, also known as arch sound. Given the distinction of these calls by their frequency band and rate of change in frequency, the chirplet transform appears well adapted to characterize such calls. We test here this new approach from subset of vocalizations of blue whale sounds recorded in St. Lawrence Estuary. The recordings are band-pass filtered and segmented to isolate individual calls. Then, a feature vector based on the chirplet transform is extracted from each call. Finally, vector quantization (VQ) is used to classify the calls into A, B, and D vocalizations. The performance of the method is compared for various VQ code book sizes. Small code book sizes using only 3 features produced classification rates exceeding $92 \%$.

5aABb14. Burst pulses produced by free-ranging bottlenose dolphins in Tampa Bay, Florida and Mississippi Sound, Mississippi. Natalija Lace (University of Southern Mississippi, 118 College Drive, Box 5025, Hattiesburg, MS 39406, USA, kodzaks@yahoo.com), Stan A. Kuczaj (University of Southern Mississippi, 118 College Drive, Box 5025, Hattiesburg, MS 39406, USA, s.kuczaj@usm.edu), Marc O. Lammers (Hawaii Institute of Marine Biology, P.O. Box 1106, Kailua, HI 96734, USA, lammers@hawaii.edu)

Investigations of the bottlenose dolphin's acoustic repertoire have mainly focused on whistles and echolocation clicks. However, despite their widespread occurrence, burst pulses have not received much attention. The primary function of burst pulses remains unknown and a comparative analysis can be used to advance our knowledge of the function of burst pulses. We recorded the acoustic repertoires of free-ranging bottlenose dolphins in the Tampa Bay and Mississippi Sound areas using broadband recording equipment. The two habitats differ in their environmental features, including water temperature, ambient noise levels and water turbidity. Our preliminary data show that several acoustic parameters such as peak frequency and center frequency of burst pulses differ among habitats. We also report other acoustic parameters, such as the number of clicks, inter-click interval, 3-dB and rms bandwidth. In our ongoing study, we will examine relationships between environmental features of the habitats and the acoustic characteristics of burst pulses.

5aABb15. First report of burst-pulse vocalizations from white-beaked dolphins (Lagenorhynchus albirostris). Peter Simard (University of South Florida, College of Marine Science, 140 7th Ave. S., St. Petersburg, FL 33701, USA, psimard@marine.usf.edu), David Mann (University of South Florida, College of Marine Science, 140 7th Ave. S., St. Petersburg, FL 33701, USA,dmann@marine.usf.edu), Shannon Gowans (Eckerd College, Galbraith Marine Science Laboratory, 4200 54th Ave South, St. Petersburg, FL 33711, USA, gowanss@eckerd.edu)

Dolphin vocalizations are generally categorized as tonal whistles or pulsed clicks. Pulsed signals in dolphins are usually associated with echolocation, however an increasing number of species are found to produce burst pulse signals which may be used for communication. Groups of whitebeaked dolphins (Lagenorhynchus albirostris) were recorded from a $4.2 \mathrm{~m}$ rigid inflatable boat near Halifax, Canada with a hydrophone towed $25 \mathrm{~m}$ behind the boat at a depth of approximately 5m, and an M-Audio 24/96 digital recorder (16 bit, $96 \mathrm{kHz}$ ). During one 23-minute encounter, 11 burst pulse segments were recorded. Mean burst pulse duration was $0.83 \mathrm{~s}$ (SD 0.51 , range $0.22 \mathrm{~s}-1.74 \mathrm{~s}$ ). Seven segments with high signal to noise ratios were manually selected for analysis in Matlab. Mean pulse rate was $765 \mathrm{~Hz}$ (SD 182.2, range $552 \mathrm{~Hz}-941 \mathrm{~Hz}$ ). These durations and pulse rates are similar to those reported for other dolphin species. These vocalizations were made immediately before the group began actively swimming away from the boat at high speed, suggesting that these vocalizations are used in agonistic encounters as suggested for other species of dolphins. This is the first time burst-pulse vocalizations have been reported in this species.

5aABb16. Real-time acoustic monitoring of the deep-ocean environment. Michel Andre (Laboratori d'Aplicacions Bioacústiques (Universitat Politècnica de Catalunya), avda. Rambla Exposició s/n, 08800 Vilanova i la Geltrú, Spain, michel.andre@upc.edu), Mike Van Der Schaar (Laboratori d'Aplicacions Bioacústiques (Universitat Politècnica de Catalunya), avda. Rambla Exposició s/n, 08800 Vilanova i la Geltrú, Spain, mike.vanderschaar@upc.edu), A. Mas (Laboratori d'Aplicacions Bioacústiques (Universitat Politècnica de Catalunya), avda. Rambla Exposició s/n, 08800 Vilanova i la Geltrú, Spain, alex.mas@lab.upc.edu), A. Roma (Laboratori d'Aplicacions Bioacústiques (Universitat Politècnica de Catalunya), avda. Rambla Exposició s/n, 08800 Vilanova i la Geltrú, Spain, agnes.roma@lab.upc.edu), J.v. Castell (Laboratori d'Aplicacions Bioacústiques (Universitat Politècnica de Catalunya), avda. Rambla Exposició s/n, 08800 Vilanova i la Geltrú, Spain, joan.v.castell@lab.upc.edu), Maria Morell (Laboratori d'Aplicacions Bioacústiques (Universitat Politècnica de Catalunya), avda. Rambla Exposició s/n, 08800 Vilanova i la Geltrú, Spain maria.morell@lab.upc.edu), M. Solè (Laboratori d'Aplicacions Bioacústiques (Universitat Politècnica de Catalunya), avda. Rambla Exposició s/n, 08800 Vilanova i la Geltrú, Spain, marta.sole@lab.upc.edu), J.f. Rolin (IFREMER, Centre de Brest, BP 70, 29280 Plouzane, France, jean.francois.rolin@ifremer.fr), Roland Person (IFREMER, Centre de Brest, BP 70, 29280 Plouzane, France, roland.person@ifremer.fr)

ESONET is a European Network of Excellence (NoE) associating 50 partners (research centres, universities, industrials and SMEs) from 14 countries: France, Germany, Italy, UK, Spain, Portugal, Greece, Belgium, Ireland, the Netherlands, Norway, Sweden, Bulgaria, and Turkey. More than 300 scientists and engineers will participate to its activities. The aim of the ESONET NoE is the lasting integration of European research on deep-sea multidisciplinary observatories. ESONET is particularly sensitive on the effects of noise on marine organisms. Because our knowledge is still quite limited, ESONET is developing a Demonstration Mission, called LIDO, Lis tening to the Deep-Ocean Environment, a research program that will help establishing a scientific base to allow (1) the real-time automatic identification and classification of nonbiological and biological sounds, (2) the monitoring of marine organisms and population dynamics, (3) the assessment and control of the long term effects of anthropogenic sources on marine organisms, (4) the education of the public, end-users and the administration, and (5) the approval of ethical guidelines and procedures. This latter point constitutes a major criterion to award the ESONET LABEL to the observatories. This paper presents the data management architecture and the RT analysis processes that will be carried out at the observatories.

5aABb17. The acoustic role of supralaryngeal air sacs. Bart De Boer (Spuistraat 210, 1012VT Amsterdam, Netherlands, b.g.deboer@uva.nl)

This paper investigates the acoustic effect of supralaryngeal air sacs on the range of sounds that a given vocal tract can generate. Humans do not have supralaryngeal air sacs, whereas apes (chimpanzees, orangutans and gorillas) do. It has also been found that the anatomy of the hyoid bone of Neanderthals (the Kebara hyoid) is consistent with the absence of air sacs, while that of Australopithecines (the Dikika baby) is consistent with their presence. As an important difference between humans and all other primates is that humans speak, it has been suggested that the loss of air sacs has something to do with the evolution of speech. Here an articulatory model is used to compare the acoustic properties of a humanlike vocal tract with and without a chimpanzeelike air sac. The articulatory model is based on the Mermelstein model, and the air sac is a simplified model of a side branch with the approximate size of a chimpanzee air sac. It is found that the air sac reduces the range of articulations, and decreases the average formant 
frequency. This would provide an explanation for the presence of air sacs in apes (size exaggeration) and their disappearance in humans (increased articulatory range).

5aABb18. Quantitative assessment of bone properties during defect healing in an animal defect model after augmentation with different bone graft materials using scanning acoustic microscopy. Mathias Schulz (ZHBO Universitätsklinikum Halle, Dept. of Traumatology and Reconstructive Surgery, Ernst-Grube-Str. 40, 06120 Halle, Germany, mathias.schulz@medizin.uni-halle.de), Kay Raum (Martin Luther University of Halle-Wittenberg, Dept. of Orthopedics, Q-BAM Group, Magdeburger Str. 22, 06097 Halle, Germany, kay.raum@medizin.uni-halle.de), Joerg Brandt (Martin Luther University of Halle-Wittenberg, Dept. of Orthopedics, Q-BAM Group, Magdeburger Str. 22, 06097 Halle, Germany, joerg.brandt@medizin.uni-halle.de), Kay Brehme (ZHBO Universitätsklinikum Halle, Dept. of Traumatology and Reconstructive Surgery, Ernst-Grube-Str. 40, 06120 Halle, Germany, kay.brehme@medizin.uni-halle .de)

The aim of this study was to evaluate the dynamic process of bone healing and bone remodeling in an animal defect model. A nanocrystalline hydroxyapatite in an aqueous suspension paste and mixed with either autogenous or allogenic bone was implanted. The investigation was performed using quantitative acoustic microscopy at a frequency of $50 \mathrm{MHz}$. 4-mm diameter defects were prepared on each femur at distal metaphysis in 60 white New Zealand rabbits. The animals were sacrificed after 2, 4, 6, 8, or 12 weeks. The influences on the acoustic impedance values only in the newly formed bone were analyzed by two-factor analysis of variance and post-hoc multiple comparison tests. Moreover, the kinetics of bone stiffening was evaluated by fitting the impedance data to an exponential growth model. In all treatment groups the impedance increased with healing time. Significant differences between the treatment groups were observed 4,6 , and 8 weeks after treatment $(\mathrm{p}<0.05)$. The experimental results agreed with the exponential growth model with coefficients of correlation (R2) between 0.6 and 0.8 . Nanocrystalline hydroxyapatite paste in combination with autogenous bone was found to be superior to the other evaluated treatment strategies.

5aABb19. An opportunistic passive acoustics study of the spatial and temporal distribution and vocal behavior of Blainville's beaked whale ("Mesoplodon densirostris") in the presence of mid-frequency active sonar. David Moretti (NAVSEA, Newport Undersea Warfare Center, Newport, RI RI 02841, USA, MorettiDJ@npt.nuwc.navy.mil), Ronald P. Morrissey (Naval Undersea Warfare Center Division Newport, 1176 Howell Street, Bldg 1351, 2nd Floor, Newport, RI 02841, USA, morrisseyrp@npt.nuwc.navy.mil), Nancy A. Dimarzio (Naval Undersea Warfare Center Division Newport, 1176 Howell Street, Bldg 1351, 2nd Floor, Newport, RI 02841, USA, dimarziona@npt.nuwc.navy.mil), Jessica Ward (NAVSEA, Newport Undersea Warfare Center, Newport, RI RI
02841, USA, wardja@npt.nuwc.navy.mil),Susan Jarvis(Naval Undersea Warfare Center Division Newport, 1176 Howell Street, Bldg 1351, 2nd Floor, Newport, RI 02841, USA, Sjarvis@wpi.edu), Elena McCarthy (Naval Undersea Warfare Center Division Newport, 1176 Howell Street, Bldg 1351, 2nd Floor, Newport, RI 02841, USA, mccarthy@nurc .nato.int), Annamaria Izzi (Naval Undersea Warfare Center Division Newport, 1176 Howell Street, Bldg 1351, 2nd Floor, Newport, RI 02841, USA, izzia.ctrmccarthy@ nurc.nato.int)

The effect of mid-frequency active sonar, has increasingly become an issue with navies worldwide. The U.S. navy ranges have been used to develop passive acoustic algorithms and tools to detect, classify, and localize marine mammal vocalizations which have been applied to an opportunistic passive acoustic study of Blainville's beaked whales. Based on Woods Hole Oceanographic Digital Tags (Dtags), these animals are known to produce echo-location clicks only during deep foraging dives. Using passive acoustics detection of vocalizations, foraging groups of animals were isolated and the duration of vocalizations was used as a measure of foraging behavior. The animals' vocal behavior and spatial and temporal distribution were characterized during periods with no active sonar on range. These results are compared to those derived from opportunistic data obtained during multiship active mid-frequency sonar operations.

$5 a A B b 20$. Testing the acoustic tolerance of harbour porpoise hearing for impulsive sounds. Klaus Lucke (FTZ Westkueste/University of Kiel, Hafentoern 1, 25761 Buesum, Germany, lucke@ftz-west.uni-kiel.de), Paul A. Lepper (Loughborough University, Electronic \& Electrical Engineering, LE113TU Leicestershire, UK, p.a.lepper@lboro.ac.uk), Marie-Anne Blanchet (Fjord\&Bælt / University of Southern Denmark, Margrethes Plads 1, 5300 Kerteminde, Denmark, marie@fjord-baelt.dk), Ursula Siebert (FTZ Westkueste / University of Kiel, Hafentoern 1, 25761 Buesum, Germany, ursula.siebert@ftz-west.uni-kiel.de)

The planned construction of offshore wind turbines in the North and Baltic Seas involves the emission of high numbers of intense impulsive sounds when the foundations of the turbines are being driven into the ground by pile driving. Based on information from other odontocete cetaceans it can be assumed that the source levels which will on average exceed $225 \mathrm{~dB}$ re $1 \mu \mathrm{Pa}$ pose a risk at least for temporary threshold shift (TTS) on harbour porpoises which inhabit these waters. In order to base the definition of noise exposure criteria on information on the tolerance of the hearing of this species a TTS study was conducted on one of the harbour porpoises held at the Fjord and Baelt in Kerteminde, Denmark. The hearing data were collected by using the AEP method. An airgun was chosen as sound source for the fatiguing sound stimulus to simulate the impulsive sounds at sufficiently high levels. This study comprises the testing of the animals normal hearing sensitivity and subsequent repetitions of these tests after an exposure to single impulsive sounds from the airgun at increasing levels. The baseline hearing data, thresholds for behavioural reactions and the resulting TTS levels will be presented. 


\title{
Session 5aAO
}

\section{Acoustical Oceanography and ECUA: General Topics in Acoustical Oceanography II}

\author{
Andone Lavery, Cochair \\ Woods Hole Oceanographic Institution, Applied Ocean Physics \& Engineering Department, 98 Water Street, MS \#11, \\ Woods Hole, MA 02543, USA \\ James H. Miller, Cochair \\ University of Rhode Island, Department of Ocean Engineering, Narragansett Bay Campus, Narragansett, RI 02882, USA
}

\section{Contributed Papers}

\section{1:00}

5aA01. Analysis of Munk waveguide normal modes based on the Rayleigh-Schrödinger perturbation and adiabatic invariant theories. Luiz Guimarães (Dept. Física Nuclear, Instituto de Física, Universidade Federal do Rio de Janeiro, Cx Postal 68528, 21945-970 Rio de Janeiro, Brazil, LULA@IF.UFRJ.BR)

This work concerns to obtain accurate explicit formulas related to discrete spectrum of the Munk deepwater acoustic wave guide problem. To this end, based on the Rayleigh-Schrödinger perturbation theory, we developed analytic results related to the discrete values of the radial wave numbers as well as to the eigen pressure felds. We compare these previous results with JWKB ones. In addition, based on invariant adiabatic theory to wave equation $[1,2]$, we tried to improve the accuracy of the JWKB calculation applying Olver's uniform asymptotic expansion (UAE) theory for the solution of the second-order differential equation with two turning points [3-5]. Comparing these three above particular frameworks, we conclude that UAE theory is accurate and it well describes the Munk wave guide normal modes. [1] L. M. Brekhovskikh and Yu. P. Lysanov, Fundamentals of Ocean Acoustics, Spinger, NY, 2001. [2] B. G. Katsnelson and V. G. Petnikov, Shallow Water Acoustics. Spinger, UK, 2002. [3] F. W. J. Olver, Asymptotics and special functions, Academic Press, London, 1974. [4] L. G. Guimarães and H. M. Nussenzveig, J. Mod. Optic., 41, 625 (1994). [5] P. C. G. de Moraes and L. G. Guimarães, JQSRT, 74757 (2002).

\section{1:20}

5aAO2. High-resolution population density imaging of random scatterers through cross-spectral coherence in matched filter variance. Mark Andrews (Northeastern University, 302 Stearns Center, Rm 311, 360 Huntington Ave, Boston, MA 02115, USA, Andrews.mar@ neu.edu), Zheng Gong (Northeastern University, 302 Stearns Center, Rm 311, 360 Huntington Ave, Boston, MA 02115, USA, zgong@ece.neu.edu), Daniel Cocuzzo (Northeastern University, 302 Stearns Center, Rm 311, 360 Huntington Ave, Boston, MA 02115, USA, dcocuzzo@ece.neu.edu), Purnima Ratilal (Northeastern University, 302 Stearns Center, Rm 311, 360 Huntington Ave, Boston, MA 02115, USA, purnima@ece.neu.edu)

The matched filter enables imaging with high spatial resolution and high signal-to-noise ratio by coherent correlation with the expected field from what is assumed to be a discrete scatterer. In many imaging systems, however, returns from large numbers of scatterers are received together and the coherent or expected field vanishes. This is the case when imaging schools of fish, other groups of marine life, or other diffuse scatterers in sonar or ultrasound applications. Here we show that despite the absence of an expected field, cross spectral coherence in the matched filter variance retains a pulse compression property that enables high-resolution imaging of scatterer population density. Both analytic and numerical models are developed for active imaging systems. We show the conditions for when the coherent intensity can be neglected. The model is implemented for several scenarios where single scattering dominates and also for cases where multiple scattering is important. It can applied to imaging in both free space and waveguide environments.

\section{1:40}

5aA03. Using angular dependence of multibeam echo features in seabed classification. Zbigniew Lubniewski (Gdansk University of Technology, Department of Geoinformatics, Narutowicza 11/12, 80-952 Gdansk, Poland, lubniew@eti.pg.gda.pl), Andrzej Chybicki (Gdansk University of Technology, Department of Geoinformatics, Narutowicza 11/12, 80-952 Gdansk, Poland, andrzej.chybicki@eti.pg.gda.pl)

The approach to seabed classification based on processing multibeam sonar echoes is presented. The multibeam sonars, besides their well verified and widely used applications like high resolution bathymetry measurements or underwater object imaging, are also the promising tool in seafloor identification and classification, having several advantages over conventional single beam echosounders. The proposed seabed classification method assumes calculation of a set of parameters of an echo envelope, similarly as in single beam classification. These parameters include echo energy, echo length, statistical moments of echo energy and the set of echo shape descriptors. They are extracted for each consecutive beam allowing the estimation of their dependence on seafloor incident angle. The characteristic features of this dependence are described quantitatively and constitute the input information for an automatic supervised seabed classifier. The results of the simple classification procedure applied for multibeam data records acquired from several bottom types in Gdańsk Bay region are presented and discussed.

\section{2:00}

5aAO4. Applications of compression techniques for reducing the size of multibeam sonar records. Andrzej Chybicki (Gdansk University of Technology, Department of Geoinformatics, Narutowicza 11/12, 80-952 Gdansk, Poland, andrzej.chybicki@eti.pg.gda.pl), Marek Moszynski (Gdansk University of Technology, Department of Geoinformatics, Narutowicza 11/12, 80-952 Gdansk, Poland, marmo@eti.pg.gda.pl)

High efficiency of multibeam sonar system (MBS) hardware due to operational requirements (i.e., high frequency, high ping rate, and high resolution of collected data) results in very large volumes of datasets stored on local hard drives of operator's station. In this context, the process of archiving of such warehouse of data collected in previous surveys becomes crucial problem. The paper investigates various lossy and lossless compression methods that can be applied to multibeam sonar data to reduce the size of acquired files without loosing relevant information. The specific character of MBS data allows applying various signal, image, and video compression methods to achieve better results than when using standard ones. Various techniques of reordering the data were analysed to achieve best possible compression ratio. 


\title{
Session 5aBBa
}

\section{Biomedical Ultrasound/Bioresponse to Vibration and Engineering Acoustics: Transducers for Medical Imaging and Therapy I}

\author{
Jeffrey A. Ketterling, Cochair
}

Frederic L. Lizzi Center for Biomedical Engineering, Riverside Research Institute, 156 William St., New York, NY 10038, USA

\author{
Marc Lethiecq, Cochair \\ LUSSI, 10 Bd Tonellé, Tours, 37032, France
}

\section{Invited Papers}

\section{8:00}

5aBBa1. Designing advanced piezoelectric ceramics for novel ultrasonic applications. Erling Ringgaard (Ferroperm Piezoceramics A/S, Hejreskovvej 18A, 3490 Kvistgaard, Denmark, er@ferroperm.net), Wanda W. Wolny (Ferroperm Piezoceramics A/S, Hejreskovvej 18A, 3490 Kvistgaard, Denmark, ww@ferroperm.net), Torsten Bove (Ferroperm Piezoceramics A/S, Hejreskovvej 18A, 3490 Kvistgaard, Denmark, tb@ferroperm.net)

As new applications of medical ultrasound continue to emerge and the development of electronics continues, there is an increased interest in specialised piezoelectric ceramics with optimised properties. With the present level of knowledge about piezoceramics it is possible to design materials combining selected characteristics - although of course the laws of physics prevent changing certain properties independently. The main parameters in the design process are composition modification and controlled porosity. One of the new types of materials developed is especially intended for high-intensity focused ultrasound (HIFU) applications, where the ability to continuously generate high acoustic power should be combined with specifications for electrical impedance matching. Since the first requirement calls for very low dielectric and mechanical losses (hard doping), whereas for typical applications the second one calls for a high permittivity (soft material), a new combination was needed. The new PZT-based materials Ferroperm Pz52 and Pz54 fulfil these requirements and are already being extensively used for HIFU applications. Another example of new materials is the commercial family of porous PZT. The introduction of porosity affects all properties, and by optimising composition and porosity level it is possible to design materials that can replace lead metaniobate-based ceramics for a number of applications.

\section{8:20}

5aBBa2. Dual-mode ultrasound array (DMUA) systems for noninvasive surgery. John Ballard (University of Minnesota, 200 Union St SE, Rm 4-174 EECS Bldg, Minneapolis, MN 55455, USA, bal1250@umn.edu), Yayun Wan (University of Minnesota, 200 Union St SE, Rm 4-174 EECS Bldg, Minneapolis, MN 55455, USA, wanx0028@umn.edu), Emad S. Ebbini (University of Minnesota, 200 Union St SE, Rm 4-174 EECS Bldg, Minneapolis, MN 55455, USA, emad@umn.edu)

Advances in microelectronics and piezocomposite transducer technology have made it possible to design, fabricate, and test therapeutic arrays with imaging capabilities suitable for image-guided noninvasive surgery. Prototype DMUAs have been recently tested and were shown to be capable of generating therapeutic HIFU beams suitable for tissue ablation while intermittently imaging the target volume before, during, and after lesion formation. An additional advantage of imaging with DMUAs is the potential for identifying critical regions in the treatment field for targeting or avoidance by the HIFU beam. We have developed an image-based refocusing algorithm that allows for maximizing the power deposition at the location of HIFU focus while minimizing the power deposition at critical targets to be avoided. Grayscale images obtained using the DMUA are used to define the coordinate of the target and the grid points in the treatment region. A major application of this capability is the targeting of liver tumors by large-aperture arrays in the presence of the rib cage. In this paper, we present experimental validation of this algorithm in vitro. Quantitative analysis of the improvements in the quality of the HIFU beam at the target will be presented and discussed.

\section{$8: 40$}

5aBBa3. High power sources for ultrasound thermal therapy and shock wave lithotripsy. Neil Owen (INSERM, U556, 151 Cours Albert Thomas, 69003 Lyon, France, Neil.Owen@lyon.inserm.fr), Dominique Cathignol (INSERM, U556, 151 Cours Albert Thomas, 69003 Lyon, France, cathignol@lyon.inserm.fr), David Melodelima (INSERM, U556, 151 Cours Albert Thomas, 69003 Lyon, France, melodelima@lyon.inserm.fr), Alain Birer (INSERM, U556, 151 Cours Albert Thomas, 69003 Lyon, France, birer@lyon .inserm.fr), Jean Yves Chapelon (INSERM, U556, 151 Cours Albert Thomas, 69003 Lyon, France, chapelon@lyon.inserm.fr), Cyril Lafon (INSERM, U556, 151 Cours Albert Thomas, 69003 Lyon, France, lafon@lyon.inserm.fr)

We present a summary of Inserm's experience with high power sources, which are necessary for ultrasound thermal therapy and lithotripsy. Moreover, generating high intensity pseudo-continuous waveforms or high pressure pulses imposes different constraints on the transducer materials, specifically heat and mechanical stress. For thermal therapy, miniature piezoceramic transducers were used for interstitial, intratumoral, and endoluminal applicators. These probes operated at surface intensities up to $50 \mathrm{~W} / \mathrm{cm}^{2}$ and generated elementary lesions in vivo within tens of seconds. Piezocomposite transducers were developed for large-aperture, highly-focused beams used in extracorporeal or intraoperative treatments. Focal intensities were $1000 \mathrm{~W} / \mathrm{cm}^{2}$ or higher and up to 256 elements were utilized. Miniaturized piezocomposite transducers are currently being developed for dual-mode imaging and therapy. For lithotripsy, piezoelec- 
tric shock wave generators were developed as alternatives to electrohydraulic or electromagnetic generators. Using piezocomposite materials and a novel prestraining method increased transducer surface pressure compared to a multielement piezoceramic design, and therefore halved the aperture diameter. In in vitro tests, plaster kidney stone models were comminuted with $\sim 200$ shock waves, a number comparable to values published for electrohydraulic generators, the current "gold" standard. This work contributes to the advancement of transducer performance in therapeutic ultrasound. [Supported by Inserm Post-doctoral Fellowship.]

\section{9:00}

5aBBa4. An eight-element annular array for image-guided high intensity focused ultrasound therapy. Peter Kaczkowski (Center for Industrial and Medical Ultrasound, Applied Physics Lab., University of Washington, 1013 NE 40th St., Seattle, WA 98105, USA, peter@apl.washington.edu), Bryan Cunitz (Center for Industrial and Medical Ultrasound, Applied Physics Lab., University of Washington, 1013 NE 40th St., Seattle, WA 98105, USA, bwc@apl.washington.edu), George Keilman (Sonic Concepts, 11807 North Creek Parkway South - Suite 111, Bothell, WA 98011, USA, gkeilman@sonicconcepts.com)

The investigation of high intensity focused ultrasound (HIFU) as a tool for noninvasive thermally ablative therapy has required deeper understanding of the relative roles of nonlinear mechanisms involved in heat deposition. Attempts at quantifying the dose response to particular exposure conditions in vitro are complicated by the interplay of several mechanisms. These include microbubble cavitation, nonlinear acoustic propagation and attenuation, dependence of tissue parameters on temperature and temperature history, and formation and evolution of vapor bubbles due to boiling. One immediately evident consequence of such effects is distortion of coagulative lesion shape and size, colloquially evolving from "cigars" to "tadpoles". Developing a quantitative understanding of the relative roles of relevant nonlinear mechanisms is not straightforward, yet is desirable for design of algorithms for therapy planning and real time monitoring using ultrasound. A historical perspective of research toward this end will be presented along with a recommendation for suitable terminology for the various physical acoustic regimes encountered in HIFU therapy. [Work supported by Army MRMC, NIH DK43881, NSBRI SMS00402, and RFBR.]

\section{9:20}

5aBBa5. Transducers for reduced aberration in HIFU by nonlinear harmonic focusing. Gregory Clement (Harvard Medical School, Focused Ultrasound Lab - BWH Radiology, 221 Longwood Ave RM 521, Boston, MA 02115, USA, gclement@hms.harvard.edu), Jason White (Harvard Medical School, Focused Ultrasound Lab - BWH Radiology, 221 Longwood Ave RM 521, Boston, MA 02115, USA, white@bwh.harvard.edu)

We have been investigating a low-frequency transducer design that promotes the divergence of the low frequency beam while promoting the focusing of nonlinear-induced higher harmonic frequencies. In this manner, a low frequency - and thus less absorbed and less distorted - beam is passed through the near field, peaking before the buildup of higher-frequency components. The high frequency components are then focused by means of the wavefront design. Using this approach, we expect reduced distortion of the ultrasound focus, while allowing a beam that uses mechanical or thermal effects for ablation, as opposed to cavitation. In our preliminary study we have been working to establish feasibility of the approach. Two source transducers $(0.272 \mathrm{MHz}$ and $0.272 \mathrm{MHz})$ with identical geometries were used to propagate through ex vivo human skull, representing strong nearfield aberration. Transmitted fields were scanned after transsskull propagation with a pvdf needle hydrophone. Reconstructions were performed at the fundamental frequencies for both scans, and for the $0.272 \mathrm{MHz}$ transducer, an additional reconstruction was performed at its second harmonic (by design $0.544 \mathrm{MHz}$ ) The harmonic signal was observed to be significantly less distorted than the same frequency directly propagated from the transducer. A numeric study for optimizing the method will also be presented.

9:40-11:00 Posters

Lecture sessions will recess for presentation of poster papers on various topics in acoustics. See poster sessions for topics and abstracts.

\section{Contributed Papers}

\section{1:00}

5aBBa6. Combined two frequency array for optoacoustics and acoustics. Kirsten Maass (Fraunhofer IBMT, Ensheimerstrasse 48, 66386 Sankt Ingbert, Germany, kirsten.mass@ibmt.fhg.de), Christian Degel (Fraunhofer IBMT, Ensheimer Strasse 48, 66386 Sankt Ingbert, Germany, christian.degel@ibmt.fraunhofer.de), Marc Fournelle (Fraunhofer IBMT, Ensheimer Strasse 48, 66386 Sankt Ingbert, Germany, marc.fournelle@ibmt.fhg.de), Heinrich Fonfara (Fraunhofer IBMT, Ensheimerstrasse 48, 66386 Sankt Ingbert, Germany, heinrich.fonfara@ibmt .fhg.de), Robert M. Lemor (Fraunhofer IBMT, Ensheimer Strasse 48, 66386 Sankt Ingbert, Germany, robert.lemor@ibmt.fhg.de)

A combined transducer for optoacoustics and b-mode ultrasound has to be built according to the specific requirements of both modalities. In order to combine the two tasks in one transducer, we have designed a probe consisting of a PZT array and a PVDF array in a sandwich assembly. The $20 \mathrm{MHz}$ PZT array is a linear array with 128 elements bearing a $150 \mu \mathrm{m}$ pitch. It operates as a high resolution transmit and receive array for the visualization of geometrical structures in biological tissue. A thin piezoelectric polymer film (PVDF) that is structured as receiving array is superposed to the PZT array. The PVDF array allows to detect the small optoacoustic pressure transients resulting of laser irradiation of small biological structures. Its acoustic impedance of approximately 4-4,5 MRayl allows to use it in a double function as optoacoustic receiving array and matching layer for the PZT array The combined array is driven by a custom made multichannel read-out system supporting sampling frequencies of $80 \mathrm{MHz}$ and combined imaging with b-mode and optoacoustics. This combined probe integrates the benefits of a high resolution PZT array and gives access to the specificity of optoacoustic signal generation.

\section{1:20}

5aBBa7. Dual-frequency driving transducer for ultrasonic echography Iwaki Akiyama (Shonan Institute of Technology, 1-1-25 Tsujidonishikaigan, 251-8511 Fujisawa, Japan, akiyama@iwaki.org), Natsuki Yoshizumi (Shonan Institute of Technology, 1-1-25 Tsujido-nishikaigan, 251-8511 Fujisawa, Japan, yoshizumi@pari.go.jp), Shigemi Saito (Tokai University, 3-20-1 Orito, Shimizu-ku, 424-8610 Shizuoka, Japan, ssaito@scc.u-tokai.ac.jp), Daisuke Koyama (Tokyo Institute of Technology, 4259 Nagatsuda, 226-8503 Yokohama, Japan, dkoyama@sonic 
.pi.titech.ac.j),Kentarou Nakamura(Tokyo Institute of Technology, $4259 \mathrm{Na}$ gatsuda, 226-8503 Yokohama, Japan, knakamur@sonic.pi.titech.ac.jp)

We propose a novel ultrasonic imaging technique by transmitting ultrasonic pulse of dual-frequency and receiving multifrequency echoes from the biological tissues. When the ultrasonic pulse of two frequencies is transmitted from a transducer, the secondary waves are generated during the nonlinear propagation through the biological media. A choice of $\mathrm{f}_{0}$ and $4 \mathrm{f}_{0}$ as the dual-frequency results in the generation of $2 \mathrm{f}_{0}, 3 \mathrm{f}_{0}$, and $5 \mathrm{f}_{0}$ components as the secondary waves. Multifrequency echoes are capable of improving the image quality by reducing the speckle noise. We have developed the following annular type transducer. The PZT disk of $7 \mathrm{~mm}$ in diameter is coaxially arranged in the PZT ring of $9 \mathrm{~mm}$ in inner diameter and $17 \mathrm{~mm}$ in outer diameter. The ring and circular transducers transmit the pulses of 2 and 8 $\mathrm{MHz}$, respectively. It was confirmed that this transducer formed the ultrasonic beams of 4,6 , and $10 \mathrm{MHz}$ as well as the beams of 2 and $8 \mathrm{MHz}$ in degassed water. Then the experiments of imaging the agar-gel phantom and pork meat were carried out by mechanical scanning. As a result, we also confirmed the improvement of image quality by reducing the speckle noise.

\section{1:40}

5aBBa8. Progress towards transducers and arrays for real-time high frequency biomedical ultrasound imaging. Sandy Cochran (Institute for Medical Science and Technology, University of Dundee, Wilson House, 1 Wurzburg Loan, DD2 1FD Dundee, UK, s.cochran@dundee.ac.uk), Jeff Bamber (Institute of Cancer Research, 15 Cotswold Road, Belmont, Sutton, SM2 5NG Surrey, UK, jeff.bamber@icr.ac.uk), Anne L. Bernassau (Institute for Medical Science and Technology, University of Dundee, Wilson House, 1 Wurzburg Loan, DD2 1FD Dundee, UK, a.l.bernassau@dundee.ac.uk), Nigel Bush (Piezo Composite Transducers Ltd, Aberdeen Science Park, Balgownie Drive, Bridge of Don, AB22 8GU Aberdeen, UK, nigel.bush@icr.ac.uk), Tim W. Button (Functional Materials Group, IRC in Materials Processing, The University of Birmingham, Edgbaston, B15 2TT Birmingham, UK, t.w.button@bham.ac.uk), Christine E. Demore (Institute for Medical Science and Technology, University of Dundee, Wilson House, 1 Wurzburg Loan, DD2 1FD Dundee, UK, c.demore@dundee.ac.uk), Luis Garcia-Gancedo (Tritech International Ltd, Peregrine Road, Westhill Business Park, Westhill, AB32 6LJ Aberdeen, UK, 1.garciagancedo@bham.ac.uk), David Hutson (Institute for Medical Science and Technology, University of Dundee, Wilson House, 1 Wurzburg Loan, DD2 1FD Dundee, UK, d.hutson@dundee.ac.uk), Duncan P. Maclennan (University of Strathclyde, DTC in Medical Devices, Bioengineering Department, Wolfson Building, 106 Rottenrow, G4 0NW Glasgow, UK, duncan.p.maclennan@strath.ac.uk), Paul M. Maher (Tritech International Ltd, Peregrine Road, Westhill Business Park, Westhill, AB32 6LJ Aberdeen, UK, paulmichaelmaher@hotmail.com), Carl Meggs (Tritech International Ltd, Peregrine Road, Westhill Business Park, Westhill, AB32 6LJ Aberdeen, UK, c.meggs@bham.ac.uk), Rhiannon A. Webster (Tritech International Ltd, Peregrine Road, Westhill Business Park, Westhill, AB32 6LJ Aberdeen, UK, raw160@bham.ac.uk)

As research into transducers and arrays for real-time high frequency biomedical ultrasound imaging continues, it is becoming increasingly clear that major applications exist for systems with significantly higher spatial resolution than those already available for human clinical imaging. A key area of research remains the design and fabrication of the transducer or array. It is now accepted that piezocomposite is the material of choice and composite design and conventional dice-and-fill fabrication techniques have been optimised to allow $40 \mathrm{MHz}$ operation, corresponding to wavelengths around $40 \mathrm{um}$. This expands the range of applications of piezocomposites but is still limited in terms of resolution of fine structures, for example at cellular level and to explore harmonic imaging. In this paper, we report continuing progress in work on new design techniques and fabrication processes with the potential for fabricating arrays that operate up to $100 \mathrm{MHz}$. The netshape micromoulding fabrication technique for the ceramic within our composites is outlined, new single element transducer designs and array fabrication based on advanced surface finishing and photolithographic processes are described, and results are presented illustrating key performance data such as the point spread function, insertion loss and imaging of post mortem human tissue.

\section{2:00}

5aBBa9. Micromachined linear array with $100 \mathrm{MHz}$ center frequency. Eike C. Weiss (Fraunhofer IBMT, Ensheimer Strasse 48, 66386 Sankt Ingbert, Germany, eike.weiss@ibmt.fraunhofer.de), Anette Jakob (Fraunhofer IBMT, Ensheimerstrasse 48, 66386 Sankt Ingbert, Germany, anette.jakob @ibmt.fraunhofer.de), Steffen H. Tretbar (Fraunhofer IBMT, Ensheimerstrasse 48, 66386 Sankt Ingbert, Germany, steffen.tretbar@ibmt.fraunhofer .de), Werner Haberer (Fraunhofer IBMT, Ensheimerstrasse 48, 66386 Sankt Ingbert, Germany, werner.haberer@ibmt.fraunhofer.de), Thorsten Knoll (Fraunhofer IBMT, Ensheimerstrasse 48, 66386 Sankt Ingbert, Germany, thorsten.knoll@ibmt.fraunhofer.de), Frank Bauerfeld (Fraunhofer IBMT, Ensheimerstrasse 48, 66386 Sankt Ingbert, Germany, frank.bauerfeld @ibmt.fraunhofer.de), Joerg Herrmann (Fraunhofer IBMT, Ensheimerstrasse 48, 66386 Sankt Ingbert, Germany, joerg.herrmann@ibmt.fraunhofer .de), Robert M. Lemor (Fraunhofer IBMT, Ensheimer Strasse 48, 66386 Sankt Ingbert, Germany, robert.lemor@ibmt.fhg.de)

High-frequency ultrasound systems based on single element transducers in the frequency range of 50-120 $\mathrm{MHz}$ and mechanical scanning of the transducer. Linear arrays with electrical scanning can be used to increase the speed of scanning and reduce the size of the transducers. We present a linear array combined with multiplexer and single channel electronics. Working at $100 \mathrm{MHz}$, lateral resolution better than $100 \mu \mathrm{m}$ is possible with an aperture of $1 \mathrm{~mm}^{2}$. The $100 \mathrm{MHz}$ array is based on silicon micro machining with a $\mathrm{ZnO}$ membrane as active material. The most important steps are the deposition of a $26 \mu \mathrm{m}$-thick $\mathrm{ZnO}$ thin film by magnetron sputtering and the anisotropic etching of the backside of the silicon wafer to fabricate the $\mathrm{ZnO}$ membrane. The individual elements of the transducer are defined by patterning a gold electrode with the desired array structure via photolithography and subsequently wet etching. Results from 32 element arrays with an 8 element 500 by $500 \mu \mathrm{m}$ aperture agree well with numerical simulations and the shifting of the aperture works as well. Due to the cost-effectiveness of the process, a large number of applications have come now into reach for high-frequency ultrasound imaging.

\section{2:20}

5aBBa10. Lead magnesium niobate-lead titanate single crystal thick films on silicon substrate for high-frequency micromachined ultrasonic transducers. Jue Peng (Department of Applied Physics and Materials Research Centre, The Hong Kong Polytechnic University, Hunghom, Kowloon Hong Kong, China, aperica@inet.polyu.edu.hk), Chen Chao (Department of Applied Physics and Materials Research Centre, The Hong Kong Polytechnic University, Hunghom, Kowloon Hong Kong, China, rikeynes@inet .polyu.edu.hk), Jiyan Dai (Department of Applied Physics and Materials Research Centre, The Hong Kong Polytechnic University, Hunghom, Kowloon Hong Kong, China, apdaijy@inet.polyu.edu.hk), Helen L. W. Chan (Department of Applied Physics and Materials Research Centre, The Hong Kong Polytechnic University, Hunghom, Kowloon Hong Kong, China, apahlcha@inet.polyu.edu.hk), Haosu Luo (The Shanghai Institute of Ceramics, Chinese Academy of Sciences, 201800 Shanghai, China, hsluo @ mail.sic.ac.cn)

Some new clinical applications requiring ultrasonic frequencies higher than $30 \mathrm{MHz}$ are emerging, such as ophthalmological and dermatological imaging and intravascular imaging with probes mounted on catheter tips. High frequency ultrasonic transducer (HFUT) has thus been a growing research area in recent years. Most of the HFUTs reported are using piezoelectric PZT ceramic thin plates, $\mathrm{ZnO}$ films or PVDF films as the transducer materials. The PMN-PT ((1-x) Pb $\left.\left(\mathrm{Mg}_{1 / 3} \mathrm{Nb}_{2 / 3}\right) \mathrm{O}_{3-\mathrm{x}} \mathrm{PbTiO}_{3}\right)$ single crystal, although possessing the highest piezoelectric coefficients, has seldom been used for this purpose because it is difficult to lap down the brittle single crystals to thin plates and handle them in conventional way of transducer fabrication. In this work, a novel high-frequency ultrasonic transducer structure is realized by using PMN-PT-on-silicon technology and silicon micromachining. A hybrid processing method involving wafer bonding, mechanical lapping and wet chemical thinning is successfully developed. PMN-PT thick films with thickness ranging from several to tens of microns have been demonstrated and they have properties comparable to those of PMN-PT bulk samples. A prototype high frequency ultrasonic transducer is being fabricated and characterized. Acknowledgement Financial support from the Innovation and Technology Fund (K-ZP21) is acknowledged. 


\title{
Session 5aBBb
}

\section{Biomedical Ultrasound/Bioresponse to Vibration: Ultrasonic Characterization of Bone III}

\author{
Keith A. Wear, Cochair \\ U.S. Food and Drug Administration, Center for Devices and Radiological Health, 10903 New Hampshire Ave, Bldg 62, \\ Rm 3108, Silver Spring, MD 20993, USA \\ Emmanuel Bossy, Cochair \\ Laboratoire Photons et Matière, ESPCI/CNRS, 10 rue Vauquelin, Paris Cedex 05, 75231, France
}

\section{Invited Paper}

8:20

\begin{abstract}
5aBBb1. Microelastic imaging of mineralized tissues - Principles and applications in musculoskeletal research. Kay Raum (Martin Luther University of Halle-Wittenberg, Dept. of Orthopedics, Q-BAM Group, Magdeburger Str. 22, 06097 Halle, Germany, kay.raum@medizin.uni-halle.de)
\end{abstract}

High frequency ultrasound has become one of the most powerful tools for microelastic characterization of hard biological materials. A major advantage is the possibility to map not only the microstructure, but also the heterogeneous anisotropic elasticity of mineralized tissues. Quantitative concepts for deriving anisotropic elastic parameters with frequencies from $50 \mathrm{MHz}$ to the $\mathrm{GHz}$ range will be presented. Due to the scalability ultrasound can be applied for large animal studies, e.g., to predict the mechanical stability in sheep tibiae after callus distraction, as well as for small animal models, e.g., to study the effects of genetic differences in inbred strain mice. Moreover, SAM data are suitable for numerical deformation or sound propagation analyses on "real- life" models. Such models are crucial for the development and validation of new non-invasive diagnostic tools dedicated to the prediction of an individual fracture risk. For example, it has been shown that the velocity of the first arriving signal - measured with the axial transmission technique at the radius - can be predicted by a nonlinear combination of fracture determining parameters, i.e., porosity, cortical thickness and tissue elasticity, assessed by $50-\mathrm{MHz}$ SAM. These findings may lead to the establishment of pathology specific treatment and regeneration monitoring strategies.

\section{Contributed Papers}

\section{$8: 40$}

5aBBb2. Assessment of cortical bone density and anisotropy in human femur using ultrasound and x-ray. Dorian Cretin (Furuno Electric Co., Ltd, 9-52 Ashihara-cho, 662-8580 Nishinomiya, Japan, dorian.cretin@furuno.co.jp), Ryouichi Suetoshi (Furuno Electric Co., Ltd, 9-52 Ashihara-cho, 662-8580 Nishinomiya, Japan, ryohichi.suetoshi@furuno.co.jp), Atsushi Uodome (Furuno Electric Co., Ltd, 9-52 Ashihara-cho, 662-8580 Nishinomiya, Japan, atsushi.uodome@furuno.co.jp), Shinji Ogawa (Furuno Electric Co., Ltd, 9-52 Ashihara-cho, 662-8580 Nishinomiya, Japan, shinji.ogawa@furuno.co.jp), Sakaya Miyabe (Graduate School of Engineering, Osaka Univ., Division of Materials and Manufacturing Science, 2-1 Yamadaoka, Suita, 565-0871 Osaka, Japan, miyabe@mat.eng.osaka-u.ac.jp), Takayoshi Nakano (Graduate School of Engineering, Osaka Univ., Division of Materials and Manufacturing Science, 2-1 Yamadaoka, Suita, 565-0871 Osaka, Japan, nakano@mat.eng .osaka-u.ac.jp)

The purpose of this research is to investigate the contribution of the intrinsic properties of human bone to the ultrasound velocities in the axial, radial, and tangential directions. Samples of cortical bone were cut from 19 human medial femurs and polished to a rectangular parallelepiped shape. The orientation of apatite crystallites was assessed by microbeam $\mathrm{x}$-ray diffraction, the density with a densitometer using the Archimedes' principle. Bone mineral density (BMD) was also measured with peripheral quantitative computed tomography (pQCT) and dual-X-ray absorptiometry (DXA). The speed of sound (SOS) for each three directions was measured with 3 $\mathrm{MHz}$ ultrasonic broadband transducers. Radial and tangential SOS demonstrated a strong correlation with density $(\mathrm{R}=0.83, \mathrm{p}<0.0001$ and $\mathrm{R}=0$ $.85, \mathrm{p}<0.0001$, respectively). In the axial direction, the correlation between density and SOS was moderate $(\mathrm{R}=0.59)$. However, a significant correlation was found by using density and apatite orientation of c-axis in a multiple regression analysis $(\mathrm{R}=0.85, \mathrm{p}<0.001)$. Axial SOS can be explained by contributions of both density and crystal orientation.

\section{9:00}

5aBBb3. Experimental determination of Young modulus and Poisson ratio in cortical bone tissue using high resolution scanning acoustic microscopy and nanoindentation. Fabienne Rupin (Université Paris 6, Laboratoire d'Imagerie Paramétrique, 15, rue de l'Ecole de Médecine, 75006 Paris, France, fabienne.rupin@lip.bhdc.jussieu.fr), Amena Saied (Université Paris 6, Laboratoire d'Imagerie Paramétrique, 15, rue de l'Ecole de Médecine, 75006 Paris, France, saied@lip.bhdc.jussieu.fr), Davy Dalmas (CNRS/Saint-Gobain, SVI Saint-Gobain Recherche, 93303 Aubervilliers Cedex, France, Davy.Dalmas@saint-gobain.com), Françoise Peyrin (Creatis, UMR CNRS 5515, INSERM U630, 69621 Villeurbanne Cedex, France, peyrin@esrf.fr), Sylvain Haupert (Université Paris 6, Laboratoire d'Imagerie Paramétrique, 15, rue de l'Ecole de Médecine, 75006 Paris, France, sylvain.haupert@lip.bhdc.jussieu.fr), Etienne Barthel (CNRS/Saint-Gobain, SVI Saint-Gobain Recherche, 93303 Aubervilliers Cedex, France, Etienne.Barthel@saint-gobain.com), Georges Boivin (Unité INSERM U831, Faculté de Médecine R. Laennec, 69372 Lyon Cedex 08, France, Georges.boivin@sante.univ-lyon1.fr), Pascal Laugier (Université Paris 6, Laboratoire d'Imagerie Paramétrique, 15, rue de l’Ecole de Médecine, 75006 Paris, France, laugier@lip.bhdc.jussieu.fr)

Nanoindentation allows measurements of local mechanical properties of bone tissue. Scanning acoustic microscopy (SAM) provides images related to bone density and elasticity. In both techniques, the estimation of Young modulus (E) relies on the accuracy of Poisson's ratio value $(\sigma)$. In cortical bone, $\sigma$ varies between 0.15 and 0.45 but, is classically set to 0.3 , resulting in an approximate value of $\mathrm{E}$. This study describes a new method combining SAM and nanoindentation techniques to locally evaluate $\sigma$ in human femo- 
ral cortex. A $200 \mathrm{MHz}$ SAM-based acoustic impedance (8 $\mu \mathrm{m}$ lateral resolution) was combined with synchrotron microtomography (to provide local bone mineral density) to map the distribution of near surface elastic modulus. Whereas, nanoindentation modulus was calculated on several osseous regions. Assuming the equalization rule, the intersection of both modulus curves versus $\sigma$ permits to accurately derive $\sigma$. The method was tested on aluminium, PMMA and polycarbonate samples of known $\sigma$ and provided experimental $\sigma$ values with a precision better than $3 \%$. In bone, $\sigma$ was $0.42 \pm 0.01$ corresponding to $\mathrm{E}=20 \pm 1 \mathrm{GPa}$. Our preliminary results indicate that combination of high-resolution SAM and nanoindentation may be relevant to accurately determine both Poisson ratio and Young modulus of bone tissue.

\section{9:20}

5aBBb4. Vibration spectroscopy and guided wave propagation data as indicators of structural and mechanical degradation of human bones. Erick Ogam (Laboratoire de Mécanique et d'Acoustique CNRS UPR-7051, 31, Chemin Joseph Aiguier, 13402 Marseille Cedex 20, France, ogam@1ma.cnrs-mrs.fr), Armand Wirgin (CNRS-Laboratoire de Mécanique et d'Acoustique, 31 Chemin Joseph Aiguier, 13402 Marseille, France, wirgin@1ma.cnrs-mrs.fr), Zine Fellah (CNRS-Laboratoire de Mécanique et d'Acoustique, 31 Chemin Joseph Aiguier, 13402 Marseille, France, fellah@1ma.cnrs-mrs.fr), Catherine Masson (INRETS - Laboratoire de Biomécanique Appliquée- UMRT 24, Faculté de Medecine, Bd. Pierre Dramard, 13916 Marseille, France, catherine.masson@inrets.fr), Philippe
Guillemain(Laboratoire de Mécanique et d'Acoustique CNRS UPR-7051, 31, Chemin Joseph Aiguier, 13402 Marseille Cedex 20, France, guillemain@lma.cnrs-mrs.fr), François Gabrielli (INRETS - Laboratoire de Biomécanique Appliquée- UMRT 24, Faculté de Medecine, Bd. Pierre Dramard, 13916 Marseille, France, francois.gabrielli@inrets.fr), Jean-Philippe Groby (DRE/L2S - UMR8506 CNRS/Supelec/Univ. Paris Sud 11, Département de Recherche en Electromagnétisme/Laboratoire des signaux et systèmes, 3 rue Joliot-Curie, 91192 Gif-sur-Yvette cedex, France, groby@1ss.supelec.fr), Robert Gilbert (University of Delaware, Department of Mathematics, 317 Ewing Hall, Newark, DE 19716, USA, gilbert @math.udel.edu)

Vibration spectroscopy and guided wave modes analysis of long bones are investigated as tools to assess bone quality or integrity. Two different methods of vibroacoustic experiments are undertaken to determine the health of dry human tibia bones. The first method involves vibratory modes of the bone, excited and measured by piezoelectric transducers and sensors respectively. The measured frequency response is validated using finite element simulation (FES), also used in the inverse problem for retrieval of the bone material properties. The principle of in-plane (IP) and out-of-plane (OP) mode splitting to determine the degree of the damage, that we developed in an earlier study, is applied to classify the bones according to their degree of damage. The second method involves the analysis of the guided wave phase velocities recovered using the reassigned Gabor spectrogram from signals measured along the diaphysis of the tibias. The frequency difference between the peaks of the split modes are then correlated to the phase velocities of the retrieved Lamb modes.

\title{
Session 5aBBc
}

\section{Biomedical Ultrasound/Bioresponse to Vibration and Engineering Acoustics: Transducers for Medical Imaging and Therapy II (Poster Session)}

\author{
Jeffrey Ketterling, Cochair \\ Marc Lethiecq, Cochair \\ LUSSI
}

Frederic L. Lizzi Center for Biomedical Engineering

\begin{abstract}
All posters will be on display from 9:40 a.m. to 11:20 a.m. To allow contributors an opportunity to see other posters, contributors of odd-numbered papers will be at their posters from 9:40 a.m. to 10:30 a.m. and contributors of even-numbered papers will be at their posters from 10:30 a.m. to 11:20 a.m.
\end{abstract}

\section{Contributed Papers}

5aBBc1. High performance ultrasound arrays assessment through in vitro imaging performance. Mathieu Legros (Vermon SA, 180 rue Général Renault, 37000 Tours, France, m.legros@ vermon.com), Guillaume Ferin (Vermon SA, 180 rue Général Renault, 37000 Tours, France, g.ferin@vermon.com), Leong Ratsimandresy (Vermon SA, 180 rue Général Renault, 37000 Tours, France, 1.ratsi@vermon.com), Rémi Dufait (Vermon SA, 180 rue Général Renault, 37000 Tours, France, r.duvait @ vermon.com)

Probes are well-known to be a capital element for ultrasound image quality. During design, many parameters can be tuned on the acoustic stack to optimise the electrical, electroacoustical and acoustical performance measurements. But the effects on image quality of these performances are not so well identified. To overcome such a limitation, we developed a quan- titative method for image quality assessment. A set of algorithms was developed to assess in vitro images. The goal of this investigation is to link the electroacoustical, acoustical performances and transducer parameters to the imaging performance. Ultrasound test objects were used to quantify the ultrasound images. The acquisition was carried out on a commercial scanner and imaging parameters were set constant in order to benchmark the probes in the same environment. From B-mode images, key parameters such as axial and lateral resolutions, contrast, statistic or metric parameters, and signal to noise ratio are established. Data from transducers, exhibiting different trade-offs on their performances (bandwidth, bandwidth shape, center frequency, elementary directivity) were characterized. Using the algorithms developed, all identified image properties were analysed with regard to these performances. The impact of each electroacoustical parameter on image quality have been identified and discussed. 
5 aBBc2. Modeling of different transducer configurations with combined pseudospectral and finite-difference time-domain methods. Erwan Filoux (LUSSI, 10 Bd Tonellé, 37032 Tours, France, erwan.filoux@etu.univ-tours.fr), Franck Levassort (LUSSI, 10 Bd Tonellé, 37032 Tours, France, franck.levassort@univ-tours.fr), Samuel Callé (LUSSI, 10 Bd Tonellé, 37032 Tours, France, calle_s@med .univ-tours.fr), Marc Lethiecq (LUSSI, 10 Bd Tonellé, 37032 Tours, France, lethiecq@univ-tours.fr)

The numerical simulation of acoustic waves propagating in inhomogeneous media is often achieved using pseudospectral (PS) algorithms, which require few nodes per wavelength to converge, while complex piezoelectric structures are simulated with finite-difference (FD) or finite-element (FE) methods. A combination of the PS and FD algorithms, retaining their advantages, is presented in order to simulate the behavior of various piezoelectric transducers used in ultrasonic imaging with one single model. The theory is exposed and the algorithm is applied to simulate PZT resonators flooded into water. Perfectly matched layers are developed to absorb the mechanical waves at the borders of the computational domain, and space-shifted grids are used to reduce Gibbs phenomenon. The electrical impedance and various physical parameters (displacements, electric potentials) are calculated. Different high frequency transducer configurations have been modeled. In the case of a simple two-dimensionnal plate, described in Cartesian coordinates, the results are satisfactorily compared to those obtained with a commercial FE software. Then, simulations of an axisymmetrical single-element transducer are favorably compared to FE simulations and experimental measurements. The hybrid algorithm is also used to calculate the large radiation pattern of an annular array with little time-processing, which illustrates the efficiency of the method.

\title{
Session 5aBBd
}

\section{Biomedical Ultrasound/Bioresponse to Vibration: General Topics in Biomedical Ultrasound/Bioresponse to Vibration I (Poster Session)}

\author{
Michael Bailey, Cochair \\ Center for Industrial and Medical Ultrasound, Applied Physics Lab. \\ Oleg Sapozhnikov, Cochair \\ Center for Industrial and Medical Ultrasound, Applied Physics Lab.
}

\begin{abstract}
All posters will be on display from 9:40 a.m. to 11:20 a.m. To allow contributors an opportunity to see other posters, contributors of odd-numbered papers will be at their posters from 9:40 a.m. to 10:30 a.m. and contributors of even-numbered papers will be at their posters from 10:30 a.m. to 11:20 a.m.
\end{abstract}

\section{Contributed Papers}

5aBBd1. $1 \mathrm{kHz}$ sound stimulates nitric oxide and prostaglandin E2 production by rat mesenchymal stem cells. Dorothée Bossis (Université Paris 6, Laboratoire d'Imagerie Paramétrique, 15, rue de l'Ecole de Médecine, 75006 Paris, France, dorothee.bossis@lip.bhdc.jussieu.fr), Frederic Padilla (Université Paris 6, Laboratoire d'Imagerie Paramétrique, 15, rue de l'Ecole de Médecine, 75006 Paris, France, Frederic.Padilla @ lip.bhdc.jussieu.fr), Bertrand David (Faculté de Médecine Paris 7- Denis Diderot, Laboratoire Biomécanique et Biomatériaux Ostéo-Articulaires B2OA, 10 avenue de Verdun, 75010 Paris, France, bertrand.david @ paris7.jussieu.fr), Hervé Petite (Faculté de Médecine Paris 7- Denis Diderot, Laboratoire Biomécanique et Biomatériaux Ostéo-Articulaires B2OA, 10 avenue de Verdun, 75010 Paris, France, hpetite@infobiogen .fr), Pascal Laugier (Université Paris 6, Laboratoire d'Imagerie Paramétrique, 15, rue de l'Ecole de Médecine, 75006 Paris, France, laugier @lip.bhdc.jussieu.fr)

Pulsed ultrasound has become a common therapy for delayed unions and non unions. Currently available clinical devices use modulated $(1 \mathrm{kHz}) 1.5$ $\mathrm{MHz}$ ultrasound stimulation that produces a $1 \mathrm{kHz}$ radiation force vibration. It was hypothesized that this radiation force is responsible for the stimulatory effect on bone formation. Therefore, we have investigated the effect of $1 \mathrm{kHz}$ stimulation in rat mesenchymal stem cells (MSCs) by measuring the production of on nitric oxide (NO) and prostaglandin E2 (PGE2). Continuous wave $1 \mathrm{kHz}$ low power sound was applied to cultured rat MSCs for 20 min. NO concentration was determined by amperometry and PGE2 was assayed by ELISA in the supernatant. The stimulation produced a significant increase in both NO and PGE2 concentration compared to controls. We conclude that rat MSCs respond to the mechanical stress produced by $1 \mathrm{kHz}$ continuous wave as evidenced by the increase in NO and PGE2 production. This study provides evidence for the action of $1 \mathrm{kHz}$ stimulation on bone cells.

5aBBd2. Accurate ultrasonic measurement of two-dimensional displacement of heart wall motion for estimation of myocardial regional strain rate. Yasunori Honjo (Department of Electronic Engineering, Graduate School of Engineering, Tohoku University, 6-6-05 Aramaki-azaAoba, Aoba-ku, 980-8579 Sendai, Japan, honjo@us.ecei.tohoku .ac.jp), Hideyuki Hasegawa (Department of Electronic Engineering, Graduate School of Engineering, Tohoku University, 6-6-05 Aramaki-azaAoba, Aoba-ku, 980-8579 Sendai, Japan, hasegawa@us.ecei.tohoku .ac.jp), Hiroshi Kanai (Department of Electronic Engineering, Graduate School of Engineering, Tohoku University, 6-6-05 Aramaki-aza-Aoba, Aoba-ku, 980-8579 Sendai, Japan, hasegawa@us.ecei.tohoku.ac.jp)

Methods for imaging of strain rate in the heart wall are useful for quantitative evaluation of regional heart function. We developed a method which can accurately measure the heart wall motion along an ultrasonic beam based on phase changes in $\mathrm{rf}$ echoes. However, there are some components in the wall motion which are not along each ultrasonic beam. Therefore, the measurement of motion in the direction perpendicular (lateral) to the beam has been required in addition to that in the axial direction, but some unsolved problems remain in estimation of lateral motion of the wall. In this study, two-dimensional displacement was estimated by $2 \mathrm{D}$ cross-correlation between rf echoes. Important parameters, the sizes of a region-of-interest and search region, which determine tracking accuracy, were adaptively op- 
timized by referring to instantaneous wall velocities, in the respective cardiac phases. The correlation coefficient between the lateral displacement estimated by the $2 \mathrm{D}$ tracking with optimized parameters in longitudinal-axis view and axial displacement in apical view (corresponding to lateral displacement in longitudinal-axis view) separately and accurately estimated by the $1 \mathrm{D}$ phase-based method was 0.93 . These results show possibility of this method for accurate measurement of two-dimensional heart motion to assess the regional myocardial strain rate.

5aBBd3. Flow-mediated change in viscoelasticity of radial arterial wall measured by 22-MHz ultrasound. Kazuki Ikeshita (Department of Electronic Engineering, Graduate School of Engineering, Tohoku University, 6-6-05 Aramaki-aza-Aoba, Aoba-ku, 980-8579 Sendai, Japan, ikeshita@us.ecei.tohoku.ac.jp), Hideyuki Hasegawa (Department of Electronic Engineering, Graduate School of Engineering, Tohoku University, 6-6-05 Aramaki-aza-Aoba, Aoba-ku, 980-8579 Sendai, Japan, hasegawa@us.ecei.tohoku.ac.jp), Hiroshi Kanai (Department of Electronic Engineering, Graduate School of Engineering, Tohoku University, 6-6-05 Aramaki-aza-Aoba, Aoba-ku, 980-8579 Sendai, Japan, hasegawa@us.ecei .tohoku.ac.jp)

The endothelial dysfunction is considered to be an initial step of atherosclerosis. Moreover, it was reported that the smooth muscle, which constructs the media of the artery, changes its characteristics due to earlystage atherosclerosis. Therefore, it is essential to develop a method for assessing the regional endothelial function and mechanical property of the arterial wall. There is an ultrasound-based conventional technique to measure the change in inner diameter of the brachial artery caused by flow-mediated dilation (FMD) after release of avascularization. In this study, the transient change in the mechanical property of the arterial wall was further revealed by measuring the stress-strain relationship during each heartbeat. For this measurement, the minute change in thickness (strain) of the radial artery was measured using the ultrasonic phased tracking method, together with the waveform of blood pressure (stress) which was continuously measured at the radial artery. From in vivo experiments, it has been founded the slope of the stress-strain hysteresis loop decreases due to FMD and the area increase depends on the ratio of elastic modulus and loss modulus (depends on viscosity). These results show a potential of the proposed method for thorough analysis of the transient change in viscoelasticity due to FMD.

5aBBd4. Experimental investigation of the scattering of sound by solid spheres in a liquid. Jason Bach (Georgia Tech Lorraine - G.W. Woodruff School of ME, UMI Georgia Tech - CNRS 2958, 2 rue Marconi, 57070 Metz, France, jsbach82@yahoo.com), Nico F. Declercq (Georgia Tech Lorraine - G.W. Woodruff School of ME, UMI Georgia Tech - CNRS 2958, 2 rue Marconi, 57070 Metz, France, nico.declercq@me.gatech.edu), David $\mathrm{Ku}$ (Georgia Tech Lorraine - G.W. Woodruff School of ME, UMI Georgia Tech - CNRS 2958, 2 rue Marconi, 57070 Metz, France, david.ku@me .gatech.edu)

The framework of this investigation is the characterization of spheres in a liquid by means of ultrasound. Experiments are performed to study the effectiveness of the use of bounded ultrasonic pulses in single transmission and in double through transmission to characterize size and shape of spheres. Special attention is paid to the influence of the beam width and frequency in comparison with the size of the sphere. The research is performed by means of a new generation polar c-scan apparatus. In a first step the interaction of sound with a single sphere is studied. In a second step two spheres are studied where one sphere crosses the sound path through the other sphere. Limitations for detection and characterization of spheres depending on their relative position and on the characteristics of the applied ultrasonic pulse are described in detail. The research is performed in the framework of the use of ultrasound for biomedical applications.

FRIDAY MORNING, 4 JULY 2008

P3-B, LEVEL, 9:40 TO 11:20 A.M.

\title{
Session 5aBBe
}

\section{Biomedical Ultrasound/Bioresponse to Vibration: Biomedical Applications of Acoustic Radiation Force I (Poster Session)}

\author{
Mostafa Fatemi, Cochair \\ Dep. of Physiology and Biomed. Eng., Mayo Clinic College of Medicine \\ Mathias Fink, Cochair \\ Laboratoire Ondes et Acoustique
}

\begin{abstract}
All posters will be on display from 9:40 a.m. to 11:20 a.m. To allow contributors an opportunity to see other posters, contributors of odd-numbered papers will be at their posters from 9:40 a.m. to 10:30 a.m. and contributors of even-numbered papers will be at their posters from 10:30 a.m. to 11:20 a.m.
\end{abstract}

\section{Contributed Papers}

5aBBe1. Comparative study of vibro-acoustography and B-mode ultrasound in prostate imaging. Azra Alizad (Dept. of Physiology and Biomed. Eng., Mayo Clinic College of Medicine, 200 First Street SW, Rochester, MN 55905, USA, aza@mayo.edu), Farid Mitri (Dept. of Physiology and Biomed. Eng., Mayo Clinic College of Medicine, 200 First Street SW, Rochester, MN 55905, USA, mitri.farid@mayo.edu), Brian Davis (Dep. of Radiation Oncology, Mayo Clinic College of Medicine, 200 1st St. SW, Rochester, MN 55905, USA, davis.brian@mayo.edu), James Greenleaf (Dept. of Physiology and Biomed. Eng., Mayo Clinic College of Medicine, 200 First Street SW, Rochester, MN 55905, USA, jfg@mayo.edu), Mostafa Fatemi (Dept. of Physiology and Biomed. Eng.,
Mayo Clinic College of Medicine, 200 First Street SW, Rochester, MN 55905, USA, fatemi@mayo.edu)

Vibro-acoustography (VA) is an imaging modality that uses the radiation force of ultrasound to vibrate tissue at a low frequency and records the resulting acoustic field to produce an image that is represents object characteristics at both ultrasound as well as low (audio) frequencies. Currently, B-mode ultrasound is used for prostate imaging. This imaging method has some limitations, including the speckle noise, which limit its ability in detection of lesions and calcifications. The purpose of this study is to explore the potentials of VA for prostate imaging. VA, B-mode ultrasound, and radiography were performed on 12 excised human prostate samples and the 
resulting images were compared. VA system parameters were, center frequency $3 \mathrm{MHz}, \Delta \mathrm{f}=50 \mathrm{kHz}$, image area $5 \times 5 \mathrm{~cm}$, pixel size $0.2 \times 0.2 \mathrm{~mm}$. Images evaluated in terms of visibility of anatomical and pathological structures and contrast. Results showed that prostate anatomical structures, mass lesions, and calcifications were visible in the VA images taken at different depths, and VA image quality was superior to B-mode. VA images exhibited significant contrast between the central and peripheral zones with enhanced borders. It is concluded that, with further development, VA may become suitable modality for in prostate imaging.

5aBBe2. Acoustic radiation forces in monitoring of milk composition Diana Priev (Hebrew University, HaGomeh 16/4, 98537 Maale Adumim, Israel, diana.priev@mail.huji.ac.il), Victor Ponomarev (NDT Instruments Ltd, HaGomeh 16/4, 98537 Maale Adumim, Israel, viktora @aaanet.ru), Aba Priev (Hebrew University, Hadassah Medical School, Ein Kerem, 91120 Jerusalem, Israel, abbap@ekmd.huji.ac.il)

High sensitivity of ultrasonic velocity and attenuation to composition and state of milk and other liquid food products is now well established. Unfortunately, existing devices include measurements of the acoustic properties of milk at different temperatures and therefore require waiting for temperature equilibration of the milk when the sample is heated or cooled and cannot be used for real-time monitoring. In this paper ultrasonic particles analyzer of NDT Instruments, AcoMilk-02, used for continuous monitoring of fat globules and somatic cells of raw milk in computerized milking station of cowsheds has been described. This device is based on high intensity standing wave for preliminary separation and concentration of the fat globules and somatic cells by the acoustic radiation forces and low intensity standing wave to measure their content. Testing of analyzer was carried out on 5 cows during one-month period. It was found that milk production level, stage of lactation, and outside temperature have significant influence on the milk composition. Continuous monitoring of milk fat and somatic cells count that typically have high day-to-day variation, provide a much-needed tool for dairy management and for veterinary diagnostic purposes.

5aBBe3. Ultrasound-enhanced latex immunoassay of pathogens in water. Danny Bavli (Hebrew University, Hadassah Medical School, Ein Kerem, 91120 Jerusalem, Israel, danny.bavli@mail.huji.ac.il), Yechezkel Barenholz (Hebrew University, Hadassah Medical School, Ein Kerem, 91120 Jerusalem, Israel, yb@cc.huji.ac.il), Noam Emanuel (Hebrew University, Hadassah Medical School, Ein Kerem, 91120 Jerusalem, Israel, noam.emanuel@mail.huji.ac.il), Victor Ponomarev (NDT Instruments Ltd,
HaGomeh 16/4, 98537 Maale Adumim, Israel, viktora@aaanet.ru),Aba Priev (Hebrew University, Hadassah Medical School, Ein Kerem, 91120 Jerusalem, Israel, abbap@ekmd.huji.ac.il)

Real-time knowledge of contaminants in water is an essential component of any potable water security system. Latex immunoassay is the basic technique in rapid identification of pathogens. We used acoustic radiation to accelerate the latex immunoassay and to bring about separation between free and bound antigen (rotavirus SA-11) in less than one minute. The ultrasonic cylindrical standing-wave device of NDT Instruments, UltraAssay 101, creates areas of maximum and minimum potential energy (nodes). Acoustic radiation forces acting on the pathogens drive them directly to the central node. In this manner, antibody-antigen complexes accumulate in the nodes in 40-60 sec. The nonbound antibodies are washed out of the separation area by the buffer flow. The forces responsible for separation of particles in the UltraAssay 101 depend on the frequency of the standing-wave resonator and on the density, compressibility and size of the immune complexes. Additionally, UltraAssay 101 is able to directly monitor water salinity, turbidity and specific gravity.

5aBBe4. Wave propagation modes and arterial stiffness. Miguel Bernal (Dept. of Physiology and Biomed. Eng., Mayo Clinic College of Medicine, 200 First Street SW, Rochester, MN 55905, USA, bernalrestrepo.miguel@mayo.edu), James Greenleaf (Dept. of Physiology and Biomed. Eng., Mayo Clinic College of Medicine, 200 First Street SW, Rochester, MN 55905, USA, jfg@mayo.edu)

Arterial elasticity is an independent predictor of cardiovascular disease and mortality. Measurment of waves in thin shells can be used to estimate the circumferential and the longitudinal elastic moduli. Latex tubes and excised pig arteries were used as isotropic and anisotropic models. Waves were generated using focused ultrasound $(1 \mathrm{~ms}, 3 \mathrm{MHz})$ in different locations of the wall. The modes of propagation of the waves were detected with a laser vibrometer or an ultrasound pulse echo tool while the excitation was moved known distances. Bending waves were predominant when exciting in the center of the wall, while a combination of torsion and bending waves resulted from exciting off center. Wave speeds of $15 \mathrm{~m} / \mathrm{s}$ and $5 \mathrm{~m} / \mathrm{s} \mathrm{respec}$ tively, were measured for the bending waves. Using the Moens-Korteweg equation, values of 1.1 and $0.350 \mathrm{MPa}$ were found for the longitudinal elastic modulus in the tube and artery respectively. Generation of differen modes in the tubes and arteries is possible using focused ultrasound. Elastic components of the tubes and vessels in the longitudinal and the circumferential direction can be estimated from the speed of propagation and the frequency of the bending and the torsional waves. 


\title{
Session 5aBBf
}

\section{Biomedical Ultrasound/Bioresponse to Vibration: Biomedical Applications of Acoustic Radiation Force II}

\author{
Mostafa Fatemi, Cochair \\ Dep. of Physiology and Biomed. Eng., Mayo Clinic College of Medicine, 200 First Street SW, Rochester, MN 55905, USA \\ Mathias Fink, Cochair \\ Laboratoire Ondes et Acoustique, ESPCI, Université Paris 7, CNRS, 10 rue Vauquelin, Paris, 75005, France
}

\section{Invited Paper}

\section{1:00}

5aBBf1. Biomedical applications of radiation force generated in standing ultrasonic waves. Lev Ostrovsky (Zel Technol./Univ. of Colorado, 325 Broadway, R/PSD99, Boulder, CO 80305, USA, Lev.A.Ostrovsky@noaa.gov), Armen Sarvazyan (Artann Laboratories, 1753 Linvale-Harbourton, Lambertville, NJ 08350, USA, armen@artannlabs.com)

This talk presents an overview of physical basis for the action of acoustic radiation force on particles in standing waves in relation to biomedical applications. The effect itself is known since the 19th century (Kundt, 1974) and its biomedical significance was demonstrated in 1971 by Dyson et al. However, despite a long history, extensive studies of particle behavior in standing ultrasonic waves have started only during the last decade due to numerous emerging biomedical applications. The range of currently explored applications is broad: targeted drug and gene delivery, increasing sensitivity of biosensors and immunochemical tests, manipulating cells in suspensions, microstirring, and others. The presentation will focus on theoretical analysis and applications of radiation force acting on particles and bubbles in the standing ultrasonic fields. Dynamics of particle motion induced by ultrasonic radiation force as a function of frequency, intensity, and other variables will be considered. The principal difference in interaction of ultrasonic standing wave field with solid particles and with bubbles due to resonant properties and high compressibility of the latter will be quantitatively analyzed. Several specific biomedical applications of the analyzed phenomena will be discussed.

\section{Contributed Papers}

11:20

5aBBf2. Radiation force on spheres in acoustic beams and related aspects of scattering. Philip L. Marston (Washington State University, Physics and Astronomy Department, Pullman, WA 99164-2814, USA, marston@wsu.edu), David B. Thiessen (Washington State University, Physics and Astronomy Department, Pullman, WA 99164-2814, USA, thiessen@wsu.edu)

The close connection between the acoustic radiation force on objects in fluids and the angular distribution of the farfield scattering is especially useful in cases where dissipative effects are weak in the surrounding fluid. This connection also applies to objects illuminated by acoustic beams and has been used to extend the analysis of scattering by spheres in Bessel beams [P. L. Marston, J. Acoust. Soc. Am. 121, $753-757$ (2007); 122, 247-252 (2007)] to the evaluation of the radiation force on spheres [P. L. Marston, J. Acoust. Soc. Am. 120, 3518-3524 (2006); 122, 3162-3165 (2007)]. The quantitative predictions (which include situations of negative radiation force) have been verified by nearfield analysis of the radiation force using the finite element method. We have also examined the radiation force on a sphere for a special case of co-propagating Bessel beams having unequal beam parameters. The scattering of a higher order Bessel beam by a sphere placed on the axis has also been analyzed. The backscattering in that case vanishes in agreement with prior predictions [B. T. Hefner and P. L. Marston, J. Acoust. Soc. Am. 106, 3313-3316 (1999)] for a general class of helicoidal acoustic beams. [Supported by NASA and ONR.]

11:40

5aBBf3. Modeling the modulated acoustic radiation force distribution in a viscoelastic medium driven by a spherically focused ultrasound transducer. Faik Can Meral (University of Illinois, 842 West Taylor St. MC 251, Chicago, IL 60607, USA, fmeral2@uic.edu), Thomas J. Royston (University of Illinois at Chicago, 842 W. Taylor St. ERF
1072, Chicago, IL 60607, USA, troyston@uic.edu),Richard L. Magin (University of Illinois, 842 West Taylor St. MC 251, Chicago, IL 60607, USA,rmagin@uic.edu)

Acoustic radiation force created by focused ultrasound transducers is gaining great interest in medical diagnosis. This study aims to clarify the acoustic power delivery by means of a modulated focused transducer and to predict the performance of such systems. A spherical-cap shaped transducer, made of piezoelectric material, is used to create ultrasonic waves at a focal point. Different modulation methods, given in the literature and reviewed here, are available for creating a concentrated alternating force due to the acoustic radiation pressure. The relationship between the voltage input to the piezoelectric transducer and its resulting mechanical deformation is examined using a finite element model (FEM) for high frequency harmonic excitation $(3-10 \mathrm{MHz})$. The oscillating surface of the transducer drives the contacting media, which exerts an acoustic load on the transducer that is also considered in the FE analysis. Also, the motion of resulting acoustic waves in a lossy medium is studied for a more accurate estimation of the induced force distribution and energy dissipation within the medium. Ultimately, the intention of this work is to relate the electrical power input to the transducer to the resulting dynamic force generated in the coupling medium. [Research supported by NIH Grant \# EB004885.]

\section{2:00}

5aBBf4. Biomedical applications of acoustic radiation force based on somatosensory reception. Leonid R. Gavrilov (N.N. Andreyev Acoustics Institute, 4, Shvernik Street, 117036 Moscow, Russian Federation, gavrilov@akin.ru), Jeffrey W. Hand (Imperial College London, Hammersmith Campus, Ducane Road, W12 0NN London, UK, 
j.hand@imperial.ac.uk),Efim M. Tsirulnikov(I.M. Sechenov Institute of Evolutionary Physiology and Biochemistry, 44 Torez ave., 194223 St. Petersburg, Russian Federation, tsiruln@iephb.ru)

We have previously shown that short pulses of focused ultrasound may be used to stimulate locally receptor neural structures and thereby induce sensations that humans can perceive through the skin (for example tactile, warmth and cold, tickling, pain, etc.). Furthermore, ultrasound modulated by sound signals (tone, speech, music, etc.) can induce sound sensations corre- sponding to the nature of the modulation in persons with normal hearing. The mechanism underlying these effects that have been used in clinical diagnostic practice is related to the radiation force. We also showed recently that the phenomenon of the radiation force could be used in perspective robotic systems, sensors, and automated control systems, based on the use of tactile sensations in the human-machine interface, as well as in devices that may allow blind and visually impaired persons to perceive textual information presented on a tactile display. The research was supported by INTAS (05-1000008-7841) and RFBR (06-02-16079).

\section{Invited Paper}

\section{2:20}

5aBBf5. Ultrasound stimulated vibrometry for measuring tissue properties. James Greenleaf (Dept. of Physiology and Biomed. Eng., Mayo Clinic College of Medicine, 200 First Street SW, Rochester, MN 55905, USA, jfg@mayo.edu), Shigao Chen (Dept. of Physiology and Biomed. Eng., Mayo Clinic College of Medicine, 200 First Street SW, Rochester, MN 55905, USA, chen.shigao@mayo.edu), Xiaoming Zhang (Dept. of Physiology and Biomed. Eng., Mayo Clinic College of Medicine, 200 First Street SW, Rochester, MN 55905, USA, Zhang.xiaoming@mayo.edu)

Harmonic or pulsed radiation pressure and subsequent measurement of the tissue response can be used to accurately quantitatively measure fundamental material properties of tissue. The measurement of shear wave dispersion can be used to estimate elastic shear moduli of tissue using an appropriate model. Fundamental model free properties such as complex modulus can also be measured. Ultrasound radiation pressure is used to induce free propagating shear waves. The measurable properties of the shear waves such as speed are sensitive to only the local material properties of the tissue under certain circumstances. A model relating the shear wave speed as a function of frequency is related to the elastic and viscous moduli within small regions of tissue according to, for instance, the Voigt model. Shear and elastic moduli in tissue are measured with high accuracy and precision given appropriate models of wave propagation within the geometry of the tissue. Complex modulus in liver of live pig and other tissues have been made. Careful use of shear wave propagation and subsequent measurements can provide fundamental quantitative measurements of tissue mechanical properties if models are accurate. An application of this method is the noninvasive measurement of liver stiffness as a surrogate for fibrosis.

\section{2:40-2:00 Lunch Break}

\section{Invited Papers}

\section{2:00}

5aBBf6. Supersonic shear imaging: a multiwave imaging example. Mathias Fink (Laboratoire Ondes et Acoustique, ESPCI, Université Paris 7, CNRS, 10 rue Vauquelin, 75005 Paris, France, mathias.fink@espci.fr), Mickael Tanter (Laboratoire Ondes et Acoustique, ESPCI, Université Paris 7, CNRS, 10 rue Vauquelin, 75005 Paris, France, michael.tanter@espci.fr), Jeremy Bercoff (Supersonic Imagine, 13857 Aix en Provence, France, jeremy.bercoff@supersonicimagine.fr)

A new imaging method that relies on the simultaneous use of both a low frequency shear wave and a high frequency ultrasound is described. The shear waves have typically centimetric wavelengths and they propagated at low velocity in tissues. They are progressively distorted by the viscoelastic inhomogeneities of encountered tissues. When coupled to an ultrafast ultrasound scanner (5000 images per second), it allows for the follow up of the propagation of these waves with a millimetric resolution over a large zone of interest. From the spatiotemporal evolution of the shear displacement fields, inversion algorithms are used to recover the shear modulus and viscosity map with submillimetric resolution. These techniques are no more diffraction limited because, the near field of the transient waves is directly observed. In this multivave technique, the shear wave gives the contrast while the ultrasonic wave gives the spatial resolution. Shear waves are generated leveraging the innovative use of the ultrasonic radiation force generated by an ultrasound probe. A supersonic shear source is remotely created in tissues. Such a moving source creates two plane shear waves propagating in a Mach cone. In vivo images obtained in breast, liver, muscles will be presented that show the great interest of this quantitative imaging technique.

5aBBf7. Image quality assessment: Vibro-acoustography versus B-mode. Mostafa Fatemi (Dept. of Physiology and Biomed. Eng., Mayo Clinic College of Medicine, 200 First Street SW, Rochester, MN 55905, USA, fatemi@mayo.edu)

Vibro-acoustography (VA) is based on conversion of ultrasound energy from high frequency to a low frequency. This conversion, which results from the nonlinear nature of wave equation, gives VA some key advantages over linear ultrasound imaging such as B-mode. Two key features of VA are analyzed: lack of speckle noise, and angle-independency, which is the ability of imaging specular reflectors regardless of their orientation. Both the speckle noise and angle dependency are major artifacts in B-mode. Lack of speckle noise results from the fact that VA images are constructed from the low frequency acoustic signal. Using an acoustical model, it is shown that the random scatterers within soft tissue do not promote speckle noise in VA as they do in B-mode; thus VA generally produces high contrast images. Angle-independency results from the broad beam pattern of the low-frequency acoustic field. A model is constructed describing the amplitude of the acoustic signal versus object orientation angle. It is shown that the signal amplitude is relatively insensitive to object orientation; thus VA may be suitable for imaging specular objects such as implants. It is concluded that VA may be advantageous in applications where speckle noise and angle dependency are of concern. 
5aBBf8. In vivo feasibility of local harmonic motion imaging and its use for thermal surgery control. Kullervo Hynynen (University of Toronto, Sunnybrook Health Sciences Centre, Suite S6 65b, 2075 Bayview Ave, Toronto, ON M4N 3M5, Canada, khynynen@sri.utoronto.ca), Laura Curiel (University of Toronto, Sunnybrook Health Sciences Centre, Suite S6 65b, 2075 Bayview Ave, Toronto, ON M4N 3M5, Canada, lcuriel@sri.utoronto.ca), Rajiv Chopra (University of Toronto, Sunnybrook Health Sciences Centre, Suite S6 65b, 2075 Bayview Ave, Toronto, ON M4N 3M5, Canada, chopra@sri.utoronto.ca)

A local harmonic motion can be generated within the tissues by the periodic induction of radiation force using a focused ultrasound (FUS) transducer. Tissue motion can then be tracked by collecting rf signals during the excitation using a separate transducer. Finally, displacement estimates can be obtained by cross-correlating the collected rf signals. The characteristics of the induced LHM depend on the local elastic properties of the tissues making it an attractive tool for imaging and therapy control applications. LHM measurements have been obtained in vivo on rabbit muscle and it was observed that the amplitude of the motion was significantly reduced after coagulation. LHM was successfully used to spatially detect the presence of the coagulation lesions within the tissues as a drop in LHM amplitude. It was also possible to detect the location of an implanted VX2 tumor when a spatial scan was performed as the LHM amplitude was lower inside the tumor because of an increased stiffness. Measurements of LHM during tissue heating using FUS reflected the changes in stiffness and revealed the apparition of coagulation showing the potential of these measurements as an alternative control for the FUS exposure. [Work supported by NIH Grant R33 CA102884 and the Canadian Research Chair Program.]

\section{3:00}

5aBBf9. Impulsive acoustic radiation force: imaging approaches and clinical applications. Kathryn Nightingale (Biomedical Engineering, Duke Univ., 136 Hudson Hall, Durham, NC 27708, USA, kathy.nightingale@ duke.edu), Mark Palmeri (Duke University, P.O. Box 90281, Durham, NC 27708, USA, mlp6@duke.edu), Liang Zhai (Duke University, P.O. Box 90281, Durham, NC 27708, USA, liang.zhai@duke.edu), Kristin Frinkley (Biomedical Engineering, Duke Univ., 136 Hudson Hall, Durham, NC 27708, USA, kdf2@duke.edu), Michael Wang (Duke University, P.O. Box 90281, Durham, NC 27708, USA, michael.h.wang@duke.edu), Jeremy Dahl (Duke University, P.O. Box 90281, Durham, NC 27708, USA, jjd@duke.edu), Brian Fahey (Duke University, P.O. Box 90281, Durham, NC 27708, USA, bjf8@duke.edu), Stephen Hsu (Duke University, P.O. Box 90281, Durham, NC 27708, USA, sjh6@duke.edu), David Bradway (Duke University, P.O. Box 90281, Durham, NC 27708, USA, david.bradway@duke.edu), Gregg Trahey (Duke University, P.O. Box 90281, Durham, NC 27708, USA, gregg.trahey@duke.edu)

Focused acoustic radiation force can be used to locally mechanically excite tissue, and the tissue response can be monitored with conventional ultrasonic displacement estimation methods. Many groups are currently exploring the potential for radiation force based methods to derive information about tissue stiffness associated with different pathologies. These techniques can be implemented on a modified diagnostic ultrasound scanner, using the same transducer for both generating the radiation force excitation and monitoring the tissue response. Multiple locations within an imaging field of view can be interrogated sequentially, by electronically controlling the push and track apertures and beam locations. A variety of data processing and imaging approaches are under investigation. Images are generated of the tissue displacement magnitude within the region of excitation at a given time after radiation force application. These images portray relative differences in tissue stiffness, and provide interesting structural information that is well correlated with, and often exhibits improved contrast over, matched B-mode images. Methods for quantifying tissue stiffness through monitoring shear wave propagation, as originally proposed by Sarvazyan, are also under investigation. Results from ongoing clinical studies using these methods in a variety of organs (e.g., liver, prostate, breast, and heart) will be presented.

5aBBf10. Experimental validation of the amplitude-modulated harmonic motion imaging for tissues stiffness estimation. Caroline Maleke (Columbia University Dept. of Biomedical Engineering, 622 W 168th SSt, PH-7, Room 200 Center, New York, NY 10032, USA, cm2243@ columbia.edu), Jianwen Luo (Columbia University Dept. of Biomedical Engineering, 622 W 168th St, PH-7, Room 200 Center, New York, NY 10032, USA, j12767@columbia.edu), Viktor Gamarnik (Columbia University Dept of Biomedical Engineering, 622 W 168th st, PH-7, room 200 center, New York, NY 10032, USA, vg2125@columbia.edu), Elisa Konofagou (Dept. of Biomedical Engineering, Columbia Univ., 351 Engineering Terrace MC 8904, 1210 Amsterdam Ave., New York, NY 10027, USA, ek2191@ columbia.edu)

It has been previously shown that amplitude-modulated harmonic motion imaging (AM-HMI) has the capability of induce and image tissue displacement during the application of an oscillatory radiation force. Here, we aim at validating theoretical HMI findings with experimental results on similar phantoms. A finite-element-method (FEM) was first used to model a dynamic response of phantoms with inclusions at different stiffnesses and sizes. The FEM and experimental results were compared and used to describe the behavior of the locally displaced tissue. The radiation force was generated by a $4.68 \mathrm{MHz}$ FUS transducer modulated at $50 \mathrm{~Hz}$ with acoustic pressure levels varied between 1.2 and $4 \mathrm{MPa}$. A 7.5 MHz pulse-echo transducer was placed through the center of the FUS transducer and used to image the displaced tissue. A 1D-cross-correlation method on successive RF signals was used to estimate the axialdisplacement. The FEM and experimental results displayed good agreement in displacement patterns, i.e., the highest localized displacement occurred at the focus and was symmetrically distributed. In addition, mechanical testing was performed to estimate the phantom-gels moduli. A linear relationship between HMI displacements and tissue Young's moduli was established within the range of 13-to-50kPa. Further studies will involve the implementation of 1D linear-array transducer for full-view imaging. 


\section{Contributed Papers}

3:40

5aBBf11. An integrated system to deliver impulsive radiation force and to image induced transient strain for monitoring focused ultrasound surgery. Gearoid Berry (Institute of Cancer Research, 15 Cotswold Road, Belmont, Sutton, SM2 5NG Surrey, UK, gearoid.berry@icr.ac.uk), Jeff Bamber (Institute of Cancer Research, 15 Cotswold Road, Belmont, Sutton, SM2 5NG Surrey, UK, jeff.bamber@icr.ac.uk), Yixin Ma (Institute of Cancer Research, 15 Cotswold Road, SM2 5NG Sutton, UK, yixin.ma@icr.ac.uk), Ian Rivens (Institute of Cancer Research, 15 Cotswold Road, SM2 5NG Sutton, UK, ian.rivens@icr.ac.uk), Gail Ter Haar (Institute of Cancer Research, 15 Cotswold Road, Belmont, Sutton, SM2 5NG Surrey, UK, gail.terhaar@icr.ac.uk)

Thermal coagulation of tissue causes an approximate three-fold increase in stiffness, which can be easily detected by various elasticity imaging methods. Advantages have been reported, for application to breast cancer diagnosis, of an elasticity imaging method that applies a highly localised transient stress deep within the tissue using a low frequency focused ultrasound radiation force impulse, and uses relatively high frequency echo imaging to measure the transient strain generated in the tissue placed between the transducers. In this paper we describe a new system that implements this concept using a focused ultrasound surgical transducer to apply the transient $(<10 \mathrm{~ms})$ load, synchronized by a customised field programmable gate array to a Zonare C5-2 probe to image the strain. Both transducers are on the same side of the tissue and locked together for scanning to create transient strain elastograms. Performance measurements, obtained using gelatine phantoms and ex vivo thermally ablated liver tissue, indicate an elastogram spatial resolution of $2 \mathrm{~mm}$, and acceptable contrast for detecting regions of thermally coagulated tissue. An advantage of this configuration is that it may be easily integrated into focused ultrasound therapy.

\section{4:00-4:20 Break}

\section{4:20}

5aBBf12. Ultrasonic imaging of displacements inside objects induced by continuously applied fluctuating acoustic radiation forces. Hideyuki Hasegawa (Department of Electronic Engineering, Graduate School of Engineering, Tohoku University, 6-6-05 Aramaki-aza-Aoba, Aoba-ku, 9808579 Sendai, Japan, hasegawa@us.ecei.tohoku.ac.jp), Yoshitaka Odagiri (Department of Electronic Engineering, Graduate School of Engineering, Tohoku University, 6-6-05 Aramaki-aza-Aoba, Aoba-ku, 9808579 Sendai, Japan, hasegawa@us.ecei.tohoku.ac.jp), Hiroshi Kanai (Department of Electronic Engineering, Graduate School of Engineering, Tohoku University, 6-6-05 Aramaki-aza-Aoba, Aoba-ku, 980-8579 Sendai, Japan, hasegawa@us.ecei.tohoku.ac.jp)

Recently, many studies are conducted to measure the response of soft tissue to applied ultrasonic acoustic radiation force. However, the change in position of a target is dominantly induced by a radiation force applied from only one direction, particularly when the target is much harder than surrounding medium. In this case, elasticity of the target cannot be evaluated because the change in position does not relate to target's elasticity. In this study, 1-MHz continuous-wave ultrasound whose envelope fluctuated at 5 $\mathrm{Hz}$ was used to apply the radiation force fluctuating at $5 \mathrm{~Hz}$. By applying two radiation forces from two opposite horizontal directions, the object would be effectively deformed (compressed) in the horizontal direction and vertically expanded due to its incompressibility. Resultant vertical displacements were measured by ultrasonic pulses at $22 \mathrm{MHz}$ in center frequency which can be easily separated from $1-\mathrm{MHz}$ ultrasound by filtering. In in vitro experiments using chicken muscle and porcine liver, displacements at $5 \mathrm{~Hz}$ were clearly imaged. Amplitudes of displacements in liver are similar to those in muscle, whereas applied radiation force was one-fifth. Furthermore, shear wave propagating in liver was clearly visualized because liver was much softer than muscle, i.e., the wavelength was much shorter.

\section{4:40}

5aBBf13. Viscoelastic properties of myocardium tissue with surface and shear wave methods. Xiaoming Zhang (Dep. of Physiology and Biomed. Eng., Mayo Clinic College of Medicine, 200 First Street SW, Rochester, MN 55905, USA, Zhang.xiaoming@mayo.edu), Shigao Chen (Dep. of Physiology and Biomed. Eng., Mayo Clinic College of Medicine, 200 First Street SW, Rochester, MN 55905, USA, chen.shigao@mayo.edu), Matthew Urban (Dep. of Physiology and Biomed. Eng., Mayo Clinic College of Medicine, 200 First Street SW, Rochester, MN 55905, USA, urban.matthew@mayo.edu), Randall Kinnick (Dep. of Physiology and Biomed. Eng., Mayo Clinic College of Medicine, 200 First Street SW, Rochester, MN 55905, USA, kinnick.randall@mayo.edu), James Greenleaf (Dep. of Physiology and Biomed. Eng., Mayo Clinic College of Medicine, 200 First Street SW, Rochester, MN 55905, USA, jfg@mayo.edu)

Considerable interest in the elastic properties of soft tissue has increased in medicine due to their clinical relevance for monitoring various diseases as well as a biomarker for cancer. A recent emerging field is the use of acoustic radiation force for imaging and estimating the elastic properties of tissue. Newly developed imaging modalities include shear wave elasticity imaging, supersonic shear wave imaging, acoustic radiation force impulse imaging, and vibro-acoustography. In these new methods, the shear wave is used to estimate the elasticity of tissue. We have developed a novel surface wave method for noninvasively estimating the elasticity of tissue [X. Zhang et al., J. Acoust. Soc. Am., 122, 2522-2525 (2007)]. In this method, a localized ultrasound radiation force is remotely and noninvasively applied inside the tissue. The surface wave speed is used to estimate the elasticity of tissue This method has been further developed and applied to estimate the viscoelasticity of animal myocardium tissue. In this abstract, we report our recent results of in vitro studies on animal myocardium tissue. Both the surface wave and shear wave are measured and analyzed. Noninvasive characterization of viscoelastic properties of myocardium tissue may be a very important tool for assessing heart function.

\section{Invited Papers}

\section{5:00}

5aBBf14. Use of radiation force for enhancing efficacy of molecular targeted ultrasound contrast agents - in vitro and in vivo. John A. Hossack (University of Virginia, Biomedical Engineering, MR5, 415 Lane Rd, Charlottesville, VA VA 22908-0759, USA, jh7fj@virginia.edu)

Intravenously injected modified ultrasound microbubbles possessing molecule specific targeting ligands hold significant promise as a method for "molecular imaging." Unfortunately, although microbubbles can achieve specific adhesion in regions of vascular pathology, the method breaks down in high blood flow regions where the bond formation process is more problematic. I present results indicating that acoustic radiation does increase the specific targeted adhesion of microbubbles by pushing the bubbles towards the periphery of a vessel. During in vitro studies, radiation force enhanced microbubble adhesion up to 60-fold. Microbubble adhesion is observed to increase approximately with the square of acoustic pressure between 25 and $122 \mathrm{kPa}$, but decreases at higher pressures as the bubbles rupture. Our in vivo studies involved using intravital microscopy to assess the adherence of P-selectin targeted microbubbles in the mouse cremaster microcirculation and femoral vessels. Acoustic radiation force enhanced microbubble retention four-fold in cremaster venules and in the femoral vein. A 20 -fold enhancement was observed in the femoral artery. I discuss the potential for derivatives of the technique to provide molecular targeted therapeutic contribution. 
5aBBf15. Assessment of tissue viscoelasticity using acoustically interrogated laser-induced microbubble. Stanislav Y. Emelianov (Applied Research Laboratories, The University of Texas, P.O. Box 8029, Austin, TX 78713-8029, USA, emelian@mail.utexas.edu), Salavat R. Aglyamov (Applied Research Laboratories, The University of Texas, P.O. Box 8029, Austin, TX 78713-8029, USA, aglyamov@mail.utexas.edu), Andrei B. Karpiouk (Applied Research Laboratories, The University of Texas, P.O. Box 8029, Austin, TX 78713-8029, USA, Andrei.Karpiouk@engr.utexas.edu), Yurii A. Ilinskii (Applied Research Laboratories, The University of Texas, P.O. Box 8029, Austin, TX 78713-8029, USA, yura@arlut.utexas.edu), Evgenia A. Zabolostkaya (Applied Research Laboratories, The University of Texas, P.O. Box 8029, Austin, TX 78713-8029, USA, zhenia@arlut.utexas.edu)

Lasers are used in many biomedical and clinical applications, ranging from diagnosis to therapy. In eye microsurgery, for example, a pulsed laser beam produces a localized surgical effect through the formation of a cavitation microbubble as a result of tissue photodisruption occurring in the focal zone. However, to insure successful presurgical planning, surgical procedure, and postoperative stages of pathology treatment, the mechanical properties of the tissue must be analyzed before selective laser intervention. Since microbubbles are already produced during laser surgery, we have developed an integrated approach utilizing these laser-induced microbubbles as reporters of tissue viscoelasticity. Specifically, we have derived a general model of gas bubble dynamics in viscoelastic media to describe both translation and oscillations of the microbubble exposed to the acoustic radiation force. Furthermore, an ultrasound method based on temporal measurement of passive acoustic emission from cavity during laser-tissue interaction and simultaneous active pulseecho ultrasound probing of the cavitation bubble was developed to detect and characterize the laser-induced microbubbles. The results of our theoretical, numerical, and experimental studies demonstrate that measurements of gas bubble behavior exposed to acoustic radiation force can be used to assess the mechanical properties of the surrounding tissue.

\section{Contributed Papers}

\section{5:40}

5aBBf16. Acoustic radiation force manipulation of PC12 cells In vitro. Robert Muratore (Frederic L. Lizzi Center for Biomedical Engineering, Riverside Research Institute, 156 William St. Fl 9, New York, NY 100382609, USA, muratore@ @rinyc.org), Erin Szulman (Frederic L. Lizzi Center for Biomedical Engineering, Riverside Research Institute, 156 William St. Fl 9, New York, NY 10038-2609, USA, erin.szul@gmail.com), Nina Xu (Dept. of Biomedical Engineering, Columbia Univ., 351 Engineering Terrace MC 8904, 1210 Amsterdam Ave., New York, NY 10027, USA, xu.nina@gmail.com), Melissa J. Simon (Dept. of Biomedical Engineering, Columbia Univ., 351 Engineering Terrace MC 8904, 1210 Amsterdam Ave., New York, NY 10027, USA, mjs2150@columbia.edu), Barclay Morrison (Dept. of Biomedical Engineering, Columbia Univ., 351 Engineering Terrace MC 8904, 1210 Amsterdam Ave., New York, NY 10027, USA, bm2119@columbia.edu)

To understand and prevent brain injuries from head trauma, researchers study mechanically stressed neuronal tissue. To anticipate future application of controllable ultrasonic in-vivo stress, we investigated the effects of acoustic radiation force on in-vitro PC12 cells. Undifferentiated PC12 cells were serum-cultured in DMEM/F12 on poly-L-lysine-coated polystyrene. Some cultures were DAPI stained. The culture plates were placed on an inverted phase-contrast microscope. An f/1.1 ultrasound transducer with a waterfilled coupling cone was focused on the culture at a 45-degree angle-ofincidence, and excited with $30-\mathrm{ms} 4.7-\mathrm{MHz}$ pulses. Acoustic power was 8 $\mathrm{W}$, and peak pressure was estimated at $300 \mathrm{kPa}$. Digital images were recorded before, during, and after insonification. Incident-light and fluorescence images revealed three populations: cells that were stationary (apparently outside the effective force field region), cells that elongated about $2 \mu \mathrm{m}$ under radiation force and returned to approximately their original shapes when the force was removed (apparently adhered to the substrate), and cells that moved about $50 \mu \mathrm{m}$ with each pulse and did not return (apparently free-floating). We conclude that cell morphology can be influenced reversibly with acoustic radiation force. (Support was provided by the Riverside Research Institute Fund for Biomedical Engineering Research and the Gatsby Initiatives in Brain Circuitry.)

\section{6:00}

5aBBf17. Enhancement of bead-based immunoassays by the use of acoustic radiation force. Martin Wiklund (Royal Institute of Technology, Biomedical and X-Ray Physics, KTH - AlbaNova, Roslagstullsbacken 21, SE-106 91 Stockholm, Sweden, martin@biox.kth.se)

Radiation forces generated by ultrasonic standing waves have long been used for increasing the speed and sensitivity of bead-based immunoagglutination tests. More recently, detection methods based on confocal laser-scanning fluorometry and single-step homogeneous bead-based immunoassays show promise for fast, easy and sensitive bioanalytics. If such methods are combined with ultrasonic enhancement, very high sensitivity can be obtained. Here, we analyze and compare the characteristics, performance and limitations of ultrasonic enhancement used in agglutinationbased and fluorescence-based immunoassays. Both radiation force aspects, as well as reaction kinetics aspects, are discussed. Finally, we report on novel results where ultrasonic enhancement is performed in microtiter plates, using a fluorescence-based assay. Here, the ultrasound enriches the sample a factor $10^{6}-10^{7}$, and a detection limit of 60 femtomolar is obtained for a thyroid stimulating hormone (TSH) assay. Our results are compared with the results from a high-performance commercial instrument (Luminex xMAP technology). 


\title{
Session 5aED
}

\section{Education in Acoustics: General Topics in Education in Acoustics}

\author{
William M. Hartmann, Chair \\ Michigan State University, Dept. of Physics and Astronomy, East Lansing, MI 48824, USA
}

\section{Contributed Papers}

\section{8:00}

5aED1. Education for professional career in acoustics. Marion Burgess (Acoustics and Vibration Unit, University of NSW @ Aust Defence Force Academy, 2600 Canberra, Australia, m.burgess@adfa .edu.au), Matthew Stead (Bassett Acoustics, Level 6, 100 Pirie St, 5000 Adelaide, Australia, m.stead@bassett.com.au)

There is a demand around the world for staff for acoustical consulting companies as evidenced by the "positions available" listings. Companies would ideally like to hire staff with a good engineering, physics or building background plus an understanding of acoustics. Programs of study at University may deal with the principles of acoustics and vibration but there is usually a need for supplementary education and training for new staff in practical applications of those principles. While larger companies may provide this "in house" smaller companies do not have such resources and seek to supplement the basic knowledge with short courses or other educational opportunities. Most of these are available at specific times which may limit their applicability. In this paper we will discuss an approach to address this educational need with a fully flexible distance learning program. The program is loosely based on the UK Institute of Acoustics Diploma program. The modules comprising the program have been developed with the assistance of the senior consultants and from the acoustic consulting industry body. The early experiences with implementing the program will be discussed

\section{8:20}

5aED2. The need to integrate speech acoustics course work across the speech-language pathology curriculum. Kathleen Siren (Loyola College in Maryland, 4501 North Charles Street, Department of Speech-Language Pathology/Audiology, Baltimore, MD 21210, USA, ksiren@loyola.edu)

The profession of speech-language pathology faces two critical challenges: (1) the lack of sufficient evidence-based practice due to limited clinical research in the field and (2) the dramatic decline in recent years in the number of students pursuing PhDs. Although speech acoustics courses are a part of most undergraduate programs in speech-language pathology, these courses are often disassociated from students' additional undergraduate and graduate course work and clinical training. These isolated speech science courses may inadequately prepare speech-language pathology students for future research-based course work and clinical practice. This paper reports results of a survey of 140 former undergraduate students who took a speech acoustics course between 1994 and 2003. The survey asks students about additional speech acoustics course work, related research experience, and use of speech science information in clinical practice. The majority of students surveyed report that speech acoustics information is largely isolated in one undergraduate course. This paper concludes that programs must consider better harmonizing speech science course work with other undergraduate and graduate course work and clinical training to meet the current research related challenges to the profession.

\section{$8: 40$}

5aED3. Interdisciplinary teamwork, "Soundscaping”: A pedagogical challenge. Jan Tro (Norwegian University of Science and Technology, O.S. Bragstad plass 2B, NO-7491 Trondheim, Norway, tro@iet.ntnu.no)

For seven years the Norwegian University of Science and Technology, NTNU, has offered the Master course Interdisciplinary Teamwork
("Eksperter i Team", EiT). EiT is organized into "villages" (consisting of up to thirty students). The Acoustics Group, NTNU, is in charge of a village named "Soundscaping" mainly dealing with sound design in public areas. The students in each village are divided into teams of five. The team members may represent very different fields of study like history, philosophy, literature, religion, music, electronics, architecture, physics, medicine, chemistry, computer science and more. The paper will discuss aspects of interdisciplinary teamwork training in acoustics and gives visual and sound examples of very different and interesting results and sound products ranging from pure sound art installations and interactive educational programs to talking waste basket and new developed sound absorbers.

\section{9:00}

5aED4. Noise induced hearing loss amongst classical music students An audiometry study of students of the Royal Academy of Music Georgia Zepidou (London South Bank University, FESBE, Borough Road, SE1 0AA London, UK, zepidoug@1sbu.ac.uk), Stephen Dance (London South Bank University, FESBE, Borough Road, SE1 0AA London, UK, dances@1sbu.ac.uk), Salvador Morant (London South Bank University, FESBE, Borough Road, SE1 0AA London, UK, salpelmo@hotmail.com)

Under the new UK guidance for Control of Noise at Work, entertainment noise now has to be considered for all employees. Health surveillance has been undertaken as part of the induction for all 1st year students studying at the Royal Academy of Music in London, as of September 2007. Seminars were arranged and details of the audiometric tests given prior to the health surveillance, so that only valid data was collected. A total of 350 students were asked to fill out questionnaires on their particular habits. Analysis of the audiograms showed double the incidence of health surveillance warning levels of hearing loss amongst men compared to women, although both had significantly better hearing than general population for their age. When early signs of noise induced hearing loss were considered, a loss of more than 20 $\mathrm{dB}$ at any high frequency, was indicated for more than half of the brass musicians.

\section{9:20}

5aED5. Problem based Masters Programme in Acoustics - ten years of experience. Sofus Birkedal Nielsen (Aalborg University, Fredrik Bajers Vej 7 B, 9220 Aalborg Ø, Denmark, sbn@es.aau.dk)

The Section of Acoustics at Aalborg University in Denmark has more that ten years of experience in offering a masters programme in Acoustics. The programme provides students with classical and modern aspects of physical acoustics, psychoacoustics and electro acoustics. Also signal processing which today is a major tool in understanding and utilizing of the newest sound technology is a compulsory part of the study. The language is English and the programme is open for all students with the appropriate background. So far half of the students have been non-Danish citizens coming from four continents. The pedagogical concept is problem based learning, with all students organized in groups of 4 - 6 students (having their own group rooms). Each semester has its own theme with relevant courses and suggested projects from the supervisors, industry or the students themselves. The project work is a considerable part of the workload at a semester, one could say that the courses give the theoretical background for the projects which are "learning by doing" with access to the best facilities, rooms and equipment. 


\title{
Session 5aMUa
}

\section{Musical Acoustics: Control of Natural and Synthetic Musical Sounds III}

\author{
Marcelo M. Wanderley, Cochair \\ Centre for Interdisciplinary Research in Music Media \& Technology (CIRMMT) - Schulich School of Music - McGill Univ., \\ 555 Sherbrooke Street West, Montreal, QC H3AlE3, Canada \\ Jean Kergomard, Cochair \\ Laboratoire de Mécanique et d'Acoustique CNRS UPR-7051, 31, Chemin Joseph Aiguier, Marseille Cedex 20, 13402, France
}

\section{Invited Papers}

5aMUa1. Indirect acquisition of flutist gestures: a case study of harmonic note fingerings. Vincent Verfaille (Centre for Interdisciplinary Research in Music Media \& Technology (CIRMMT) - Schulich School of Music - McGill Univ., 555 Sherbrooke Street West, Montreal, QC H3A1E3, Canada, vincent@music.mcgill.ca), Marcelo M. Wanderley (Centre for Interdisciplinary Research in Music Media \& Technology (CIRMMT) - Schulich School of Music - McGill Univ., 555 Sherbrooke Street West, Montreal, QC H3A1E3, Canada, marcelo.wanderley@mcgill.ca), Philippe Depalle (Centre for Interdisciplinary Research in Music Media \& Technology (CIRMMT) - Schulich School of Music - McGill Univ., 555 Sherbrooke Street West, Montreal, QC H3A1E3, Canada, depalle @ music.mcgill.ca)

The indirect acquisition of musicians' gestures consists in retrieving information about gestures from the sound. Inspired by previous studies investigating clarinetists' gestures, we focus on the flute, which features numerous playing modes and a rich palette of sounds. Our goal is to provide musicians with guidelines for using indirect acquisition. For a variety of playing modes, we collected information about gestures, body parts involved, and effects on the acoustical signal. We then designed signal processing algorithms for recognizing which gesture is involved in a given sound; later we will quantize related movement by combining direct acquisition, indirect acquisition and motion capture. Focusing on harmonic note fingerings, a first experiment investigated octave-related harmonic note fingerings with 1 nonexpert performer (ff dynamics and normal articulation). A target $F_{0}$-guided PCA on the amplitude of the first six harmonics of $F_{0} / 4$ from 1 semi-tone frequency bands retrieved the correct fingering with two principle components in all trials. A second experiment investigated octave and non-octave related harmonic note fingerings with four expert performers. A target $F_{0}$-guided PCA on the sum of energies around $i F_{0} / k(k=2, \ldots, 6 ; i=1, \ldots, 12)$ provided wider clusters, making more difficult to retrieve the correct fingering in some specific cases that we will discuss.

\section{8:20}

5aMUa2. Control parameters of a generalized vibrato model with modulations of harmonics and residual. Vincent Verfaille (Centre for Interdisciplinary Research in Music Media \& Technology (CIRMMT) - Schulich School of Music - McGill Univ., 555 Sherbrooke Street West, Montreal, QC H3A1E3, Canada, vincent@music.mcgill.ca), Catherine Guastavino (Centre for Interdisciplinary Research in Music Media \& Technology (CIRMMT) - School of Information Studies - McGill Univ., 3459 McTavish, Montreal, QC H3A1Y1, Canada, Catherine.Guastavino@mcgill.ca), Philippe Depalle (Centre for Interdisciplinary Research in Music Media \& Technology (CIRMMT) - Schulich School of Music - McGill Univ., 555 Sherbrooke Street West, Montreal, QC H3A1E3, Canada, depalle@music.mcgill.ca)

Our previous research focused on vibrato modeling of the harmonics of musical sounds. We demonstrated the need to account for fundamental frequency modulation, global amplitude modulation, but also spectral envelope modulation linked to brightness modulations. This may result from nonlinearities between the excitation signal and the resonating body, and/or from variable spectral sampling of the impedance function as $F_{0}$ varies. For instance on flute, vibrato results from air pressure modulations. We also observed in sounds with vibrato of wind instruments that the residual also exhibits amplitude modulation, somehow due to air pressure modulations that are responsible vibrato. In a pilot listening test on shakuhashi sounds (for which the residual is obviously modulated), we asked participants to discriminate between the original modulated harmonics plus the amplitude modulated residual, and the original modulated harmonics plus a non-modulated residual, in a $\mathrm{AB}$ comparison task (same/different test). Preliminary results show that participants can hear the difference, suggesting that the amplitude modulation on the residual is audible. Subsequently, we developed a generalized vibrato model incorporating modulations on harmonics and residual. This model is presented here with an emphasis on its control parameters. 


\title{
Contributed Papers
}

8:40

5aMUa3. Why practice scales? Details of fingering transitions in flute playing. André Almeida (University of New South Wales, Music Acoustics, School of Physics, NSW 2052 Sydney, Australia, aalmeida@phys.unsw.edu.au), Renee Chow (University of New South Wales, Music Acoustics, School of Physics, NSW 2052 Sydney, Australia, z3130148@student.unsw.edu.au),John Smith (University of New South Wales, Music Acoustics, School of Physics, NSW 2052 Sydney, Australia, john.smith@unsw.edu.au)

Mastering a wind instrument requires not only expert blowing and embouchure, but also many hours of practice of exercises that improve the fingering speed, regularity and coordination. Many note transitions involve the motion of several fingers, often in opposite directions. The order of finger movement can sometimes affect the transitions between notes and produce short, spurious notes between the original and destination notes. We adapted a transverse flute for real time measurement (with a resolution of $3 \mathrm{~ms}$ ) of all key positions using reflected light sensors. Beginner and advanced players were asked to perform a phrase including multifinger transitions. The detailed motion, average speed and intervals between finger motion were analysed. The comparison among players is not surprising: an experienced player was found to have more regular time intervals between fingers moving in a same direction (within $10 \mathrm{~ms}$ ) than an amateur (within $25 \mathrm{~ms}$ ). Contrary fingering motions are slightly less regular $(15 \mathrm{~ms}$ for an experienced player against $50 \mathrm{~ms}$ for an amateur), but still unperceptible to the ear in the case of the experienced musician. A range of details will be reported, for instance the influence of scale exercises.
9:00

5aMUa4. Gesture synthesis: basic control of a flute physical model. Nicolas Montgermont (Institut Jean Le Rond d'Alembert/LAM (UPMC/CNRS/Ministère Culture), 11, rue de Lourmel, 75015 Paris, France, montgermont@lam.jussieu.fr), Benoit Fabre (Institut Jean Le Rond d'Alembert/LAM (UPMC/CNRS/Ministère Culture), 11, rue de Lourmel, 75015 Paris, France, fabreb@ccr.jussieu.fr), Patricio De La Cuadra (Centro de Investigación en Tecnologias de Audio (CITA), Universidad Católica de Chile, Alameda 340, Oficina 13, Casilla 114-D Santiago, Chile, pcuadra@uc .cl)

In the flute family, the oscillation is due to the instability of a jet at the output of a channel coupled with an acoustic resonator. Recent physical models allows to simulate the behavior of the complete instrument, but we still lack a convincing way to drive them. The simulation of the isolated instrument must be completed with a modelization of the control exerted by the flutist. Depending of the instrument of the flute family, the number and type of control parameters are differents. For example, in a recorder the player blows inside a fixed channel built by the instrument maker and in the case of the transverse flute, the channel is shaped by the player's lips during the playing. This paper presents a simple model of flute player, based on measurements carried on instrumentalists playing on a recorder or a transverse flute. The model is generating the basic features of the instrument control in order to produce given pitches and dynamics. The coupling with a flute physical model allows to study its validity.

\section{Session 5aMUb}

\section{Musical Acoustics: General (Mode Conversion, Radiation and Perception of Musical Sound) (Poster Session)}

\begin{abstract}
All posters will be on display from 9:40 a.m. to 11:20 a.m. To allow contributors an opportunity to see other posters, contributors of odd-numbered papers will be at their posters from 9:40 a.m. to 10:30 a.m. and contributors of even-numbered papers will be at their posters from 10:30 a.m. to 11:20 a.m.
\end{abstract}

\section{Contributed Papers}

5aMUb1. Subjective evaluations of the performance proficiency for fluctuating musical sounds using fluctuation strength or roughness. Nozomiko Yasui (Graduate School of Science and Technology, Ryukoku University, 1-5, Yokotani, Oe-cho, Seta, 520-2194 Otsu, Shiga, Japan, n-yasui@mail.ryukoku.ac.jp), Masafumi Kinou (Graduate School of Science and Technology, Ryukoku University, 1-5, Yokotani, Oe-cho, Seta, 520-2194 Otsu, Shiga, Japan, kino@ rins.ryukoku.ac.jp), Masanobu Miura (Graduate School of Science and Technology, Ryukoku University, 1-5, Yokotani, Oe-cho, Seta, 520-2194 Otsu, Shiga, Japan, miura@rins .ryukoku.ac.jp)

The "Fluctuation Strength (FS)" and the "Roughness" were suggested as evaluation indices of hearing sensation concerning fluctuated sounds of low or high frequencies. Past studies have been investigated FS and roughness of modulated pure tones and broadband noise. However, few studies have been reported concerning FS and roughness of attenuating sounds produced by musical instruments and performance proficiency for them. Therefore, the tremolo played on the mandolin and the closed roll played on the snaredrum are employed here as fluctuating musical sounds of low or high frequencies. Introduced here is the FS and roughness as indices for the evaluation of the performance proficiency for tremolo and roll. Specifically, experts of mandolin or snare-drum are asked to play tremolo of various plucking rate (number of plucking per second) and closed roll of various stroking rate (number of stroking per second). An evaluation experiment using the $2 \mathrm{AFC}$ method is conducted to evaluate performance proficiency for tremolo and roll. Aesthetic performances of tremolo and roll are described as "smooth, or not fluctuated" by trained players. The amount of physical FS for tremolo and physical roughness for roll are calculated by an original procedure to investigate relations between physical indices and evaluation results.

5aMUb2. Musical perception within a highly reverberant room. Nelia Valverde (Universidad de Castilla-La Mancha, Campus Universitario, 16071 Cuenca, Spain, Nelia.Valverde@uclm.es), Marcos D. Fernandez (Universidad de Castilla-La Mancha, Campus Universitario, $16071 \quad$ Cuenca, Spain, Marcos.Fernandez@uclm.es), Javier Ariza (Universidad de Castilla-La Mancha, Campus Universitario, 16071 Cuenca, Spain, Javier.Ariza@uclm.es), Jose A. Ballesteros (Universidad de Castilla-La Mancha, Campus Universitario, 16071 Cuenca, Spain, Josea.Ballesteros@uclm.es), Juan P. Ruiz (Universidad de Castilla-La 
Mancha, Campus Universitario, 16071 Cuenca, Spain, Juanpedro.Ruiz @uclm.es)

The listening of a musical composition generates a unique and different perception in every listener, but, at the same time, the specific acoustic conditions of the room chosen for its performance have a decisive influence in that perception, which can fit in, almost completely, with the acoustic qualification of the room. The aim of this work is to find out how the high reverberance of a room can have a strong influence on the perception of a musical composition. For that aim, the St. Peter's church (in Cuenca, Spain) with a circular plan, a reduced volume and a high reverberance time has been chosen for the performance. A musical composition for voice and electroacoustics has been created specifically for this church. This has been recorded within the church using a HATS in the listener position and a set of music experts have been asked a survey about their impressions on perception after listening the recording using headphones. The results of the psychoacoustic analysis reveal that the high reverberance of this church leads to an extremely low intelligibility and an unclear sound of the recording. This, together with an unbereable background noise, gives a general acoustic perception not very attractive.

5aMUb3. Foundations of sound therapy. Iegor Reznikoff (Université de Paris X, Département de Philosophie, 92001 Nanterre, France, dominiqueleconte@yahoo.fr)

In the practice of music therapy, the use of the sounds of a live naturally singing voice appears to be the most effective; in some cases, results are obtained whereas there are no results using musical sounds, and generally results are obtained in a much shorter time. Sounds and singing in just intonation are particularly efficient. This practice introduces to a deep understanding of sound therapy. Sketched here are the vocal sound/body relationship and the vocal sound / consciousness relationship, which are relevant in this therapy. Finally clinical examples are given (coma states, loss of speech, old persons, states close to death, mind handicapped persons, depression, etc.). Bibliography I. Reznikoff: On Primitive Elements of Musical Meaning, www.musicandmeaning.net, Journal of Music and Meaning 3 (Invited papers), 2005.

5aMUb4. Broadband directive sources modeling for acoustic discrete-time domain methods. José Escolano (University of Jaén,
Alfonso X, 28, E-23700 Linares, Spain, escolano@ujaen.es),José J. Lopez (Tech. Univ. of Valencia, Camino de Vera S/N, 46021 Valencia, Spain, jjlopez@dcom.upv.es)

The use of sources with complex directivities in discrete-time domain methods, such as the finite-difference time-domain method or the digital waveguide mesh, is a recently open topic. However, so far, the provided solutions work for discrete frequencies or frequency-independent sources. Both solutions are useful for very particular cases and in most cases, far from real sources. This paper provides an extension in the use of a monopole source combination for broadband directive sources in discrete-time domain simulations. This method results very effective for frequency-independent and dependent sources, even in cases where the directivity diagrams have considerable lobules. Finally, this method will be tested through several examples.

5aMUb5. Auditory resolution in virtual environments: Effects of spatialization algorithm, off-center listener positioning and speaker configuration. Georgios Marentakis (Centre for Interdisciplinary Research in Music Media \& Technology (CIRMMT) - Schulich School of Music - McGill Univ., 555 Sherbrooke Street West, Montreal, QC H3A1E3, Canada, gmarentakis@music.mcgill.ca), Nils Peters (Centre for Interdisciplinary Research in Music Media \& Technology (CIRMMT) - Schulich School of Music - McGill Univ., 555 Sherbrooke Street West, Montreal, QC H3A1E3, Canada, nils.peters@mcgill.ca), Stephen McAdams (Centre for Interdisciplinary Research in Music Media \& Technology (CIRMMT) Schulich School of Music - McGill Univ., 555 Sherbrooke Street West, Montreal, QC H3A1E3, Canada, smc@music.mcgill.ca)

We present the results of an empirical study on the effects of room and off-center listener positioning on sound localization in two virtual environments, VBAP and Ambisonics. Localization accuracy has been assessed by estimating Minimum Audible Angles and Minimum Audible Movement Angles for the two spatialization algorithms and for three directions of sound incidence, in the studio and a concert hall for listeners in off-center listener positions, with two loudspeaker setups. A model of localization accuracy as a function of the experimental variables is presented, that can be used to quantify the effects of the room and off-center listening positions on sound localization and auditory movement detection. 


\title{
Session 5aMUc
}

\section{Musical Acoustics: Plucked and Struck Idiophones I (Poster Session)}

\author{
Thomas D. Rossing, Cochair \\ Stanford University \\ Charles Besainou, Cochair \\ Institut Jean le Rond d'Alembert
}

\begin{abstract}
All posters will be on display from 9:40 a.m. to 11:20 a.m. To allow contributors an opportunity to see other posters, contributors of odd-numbered papers will be at their posters from 9:40 a.m. to 10:30 a.m. and contributors of even-numbered papers will be at their posters from 10:30 a.m. to 11:20 a.m.
\end{abstract}

\section{Contributed Papers}

5aMUc1. Analysis of drum player's motion. Hiroshi Kawakami(Nihon University, 2-42-1 Asahigaoka, Nerima, 176-8525 Tokyo, Japan, hkawakami@eva.hi-ho.ne.jp), Yuki Mito (Nihon University, 2-42-1 Asahigaoka, Nerima, 176-8525 Tokyo, Japan, mitotic@hotmail.com), Reiji Watanuma (Nihon University, 2-42-1 Asahigaoka, Nerima, 176-8525 Tokyo,Japan,VFE16733@nifty.ne.jp), Mieko Marumo (Nihon University, 2-42-1 Asahigaoka, Nerima, 176-8525 Tokyo, Japan, marumo@rg8.so-net .ne.jp)

In this experiment, the motion of a drum player's arm was measured by the optical motion capture system in various performance types. From these actual movement data, we analyzed about the acceleration and sound energy flux of the stick before and after excitation. Moreover, the snap of a wrist which is very important at the time of a high drum performance and the relation of acceleration, and the relation of sound energy flux were also analyzed. From those results, it was suggested that the snap of the wrist was important at the time of a high drum performance, and it was concerned with sound power. Especially, the performance which player hit the drum speedy and repeatedly was concerned with the acceleration of stick before and after excitation.

5aMUc2. Dampening vibration modes of the snare drum batter head Barry Larkin (Iowa State University, Department of Music, Music Hall, Ames, IA 50011, USA, blarkin@iastate.edu), Andrew Morrison (Illinois Wesleyan University, Department of Physics, 1312 N. Park St., Bloomington, IL 61701, USA, amorriso@iwu.edu)

Percussionists have always had to contend with an undesirable ringing sound while performing on the snare drum. It is usually referred to as the "edge ring." A common method to eliminate this sound comes from placing some type of dampening material on the edge of the drum head.
Manufacturers of drums have provided many ways of dealing with this problem including internal dampening devices, customized drums heads or material designed to be placed on the drum head. Using electronic television holography, it was revealed the source of this "ring" to be the third mode of vibration that produces a pitch approximately one octave and a half step above the fundamental [Larkin and Morrison, JASA, 122, \#5, p 3056]. In this update to the previous work, we will show the effect of dampening devices on the third and other modes.

5aMUc3. Finite element modelling of steelpan acoustics. Derek A Gay (The University of The West Indies, Department Civil Engineering, St Augustine, Trinidad and Tobago, derek.gay@sta.uwi.edu)

In this paper the finite element method is used to model acoustic vibrations of steelpan shells. The steelpan surface is characterized as a threedimensional compound shell, comprising notes (surfaces with reverse curvature) on a concave ellipsoidal surface attached to a cylindrical shell (the skirt). In this model note and inter-note surfaces are defined by geometric parameters which can be varied to define complex surface geometries. The geometric mesh model is used develop tenor, cello and bass steelpans instruments and a 3D finite element shell vibration algorithm is used to demonstrate their vibration characteristics. Modes shapes and frequencies of the composite shell structures are computed for typical configurations of note and skirt geometry. The model demonstrates that there exist many composite natural modes of a playing surface involving the interaction between two or more notes. In addition, it is found that the frequency range of mode shapes associated primarily within skirt vibration overlaps with the musical range of the notes underscoring the potential for "skirt-note" coupling. The degree of frequency overlap was found to be largely dependent on skirt length and configuration. 


\title{
Session 5aMUd
}

\section{Musical Acoustics and Signal Processing in Acoustics: Signal Representations and Models of Musical Sounds I (Poster Session)}

\author{
James Beauchamp, Cochair \\ University of Illinois \\ Bertrand David, Cochair \\ Télécom Paristech (ENST) / TSI - CNRS LTCI
}

\begin{abstract}
All posters will be on display from 9:40 a.m. to 11:20 a.m. To allow contributors an opportunity to see other posters, contributors of odd-numbered papers will be at their posters from 9:40 a.m. to 10:30 a.m. and contributors of even-numbered papers will be at their posters from 10:30 a.m. to 11:20 a.m.
\end{abstract}

\section{Contributed Papers}

5aMUd1. The perceptual and cognitive nonlinearities underlying musical preference across multiple exposures. Elizabeth $\mathrm{H}$. Margulis (University of Arkansas, Department of Music - MUSC 109, Fayetteville, AR 72701, USA, ehm@uark.edu), Anil K. Roy (Northwestern University, 2240 Campus Drive, Evanston, IL 60208, USA, a-roy@u.northwestern.edu), Patrick C. Wong (Northwestern University, 2240 Campus Drive, Evanston, IL 60208, USA, pwong@northwestern.edu)

Repetition is a fundamental part of engagement with the auditory world. Repeated exposures influence what gets perceived as important and what features are singled out for attention. That repetition can also influence the mysterious phenomenon of aesthetic enjoyment is a longstanding observation to which much intellectual attention has been paid. A special case of repetition and perceptual learning is music, a domain in which people choose to be exposed to the identical stimulus again and again. This apparent preference for repeated exposures connects fundamentally to notions of human identity, affect, cognitive processing, and neural function. We report on a set of experiments that uses behavioral measures to assess perceptual changes across multiple exposures (five over one week) of a 20-minute piece for orchestra. Across the five exposures, we found an inverted U-shape preference response, which possibly is decomposable to the complex interaction of multiple nonlinear components, represented by performance on tasks relating to memory, attention, affect, syntax, and error detection We argue that musical preference has remained a puzzle because the behavior depends on an underlying complex system with numerous nonlinear cognitive components revealed by our results. [Work supported by NIH, NSF, and Northwestern University.]

5aMUd2. Single sensor singer/music separation using a source/filter model of the singer voice. Jean-Louis Durrieu (Télécom Paristech (ENST) / TSI - CNRS LTCI, 46, rue Barrault, 75634 Paris Cedex 13, France, durrieu@enst.fr), Bertrand David (Télécom Paristech (ENST) / TSI - CNRS LTCI, 46, rue Barrault, 75634 Paris Cedex 13, France, bertrand.david@enst.fr), Gaël Richard (Télécom Paristech (ENST) / TSI CNRS LTCI, 46, rue Barrault, 75634 Paris Cedex 13, France, gael.richard @enst.fr)

Separating the singer voice from polyphonic music signals has many useful applications, such as demixing/remixing, desoloing or audio indexing. In the works of Benaroya on single sensor blind source separation, the signals are modeled by Gaussian mixtures (GMM) such that each state is characterized by a spectral shape. The separation itself is then done by adaptive Wiener filtering. However, to better fit general signals, the number of states for the vocal model should be equal to the number of notes multiplied by the number of vowels (or canonical vocal tract shapes) that the singer uses. Therefore, in order to separate a singer voice from background music, we suggest a source/filter model for the singer signal, keeping the same models as used by Benaroya for the background music signal. Assuming the presence of only one singer, we separate the desired part from the rest by first estimating the sung melody thanks to the source part of our model and then re-evaluating the parameters of our model. This research is partly supported by the European Commission under contract FP6-027026-K-SPACE and by the French AII (Quaero project).

5aMUd3. What/when causal expectation modelling applied to percussive audio. Amaury Hazan (Pomeu Fabra University, Ocata 1, 08003 Barcelona, Spain, ahazan@iua.upf.edu), Paul Brossier (Pomeu Fabra University, Ocata 1, 08003 Barcelona, Spain, piem@piem .org), Ricard Marxer (Pomeu Fabra University, Ocata 1, 08003 Barcelona, Spain, rmarxer@iua.upf.edu), Hendrik Purwins (Pomeu Fabra University, Ocata 1, 08003 Barcelona, Spain, hpurwins@iua.upf.edu)

A causal system for representing a musical stream and generating further expected events is presented. Starting from an auditory front-end which extracts low-level (e.g., spectral shape, mel frequency cepstral coefficients) and midlevel features such as onsets and beats, an unsupervised categorisation process builds and maintains a set of symbols aimed at representing musical stream events using both timbre and time descriptions. The time events are represented using inter-onset intervals relative to the beats. These symbols are then processed by an expectation module based on predictive partial match, a multiscale technique derived from $\mathrm{N}$-grams. The system capacity to generate an expectation that matches its transcription is evaluated using drum recordings from the ENST-drums database. We show that the MFCC-based representation leads to a more compact set of symbols and a better match between transcription and expectation. Also, we suggest that the system is sensitive to exposure and illustrate some properties of the expectation entropy while attending percussive audio patterns.

5aMUd4. Dynamical hierarchical self-organization of harmonic and motivic musical categories. Ricard Marxer (Pomeu Fabra University, Ocata 1, 08003 Barcelona, Spain, rmarxer@iua.upf.edu), Piotr Holonowicz (Pomeu Fabra University, Ocata 1, 08003 Barcelona, Spain, pholonow@iua.upf.edu), Amaury Hazan (Pomeu Fabra University, Ocata 1, 08003 Barcelona, Spain, ahazan@iua.upf.edu), Hendrik Purwins (Pomeu Fabra University, Ocata 1, 08003 Barcelona, Spain, hpurwins@iua.upf.edu)

We introduce a generic model of emergence of musical categories during the listening process. The model is based on a preprocessing and a categorization module. Preprocessing results in a perceptually plausible representation of music events extracted from symbolic input. The categorization 
module lets a taxonomy of musical entities emerge according to a cognitively plausible online learning paradigm. We show the advantages of using a conceptual clustering method in the musical domain. The system extracts multilevel hierarchies and can be tuned to clustering at various resolutions. The potential of the model is exemplified by exposing it to two different datasets resulting in music harmonic and motivic categorization consistent with music theory.

5aMUd5. A comparison of molecular approaches for generating sparse and structured multiresolution representations of audio and music signals. Bob Sturm (University of California, Box 117, Department of Electrical and Computer Engineering, Santa Barbara, CA 93106, USA, boblsturm@ece.ucsb.edu), John J. Shynk (University of California, Box 117, Department of Electrical and Computer Engineering, Santa Barbara, CA 93106, USA, shynk@ece.ucsb.edu), Aaron McLeran (University of California, Media Arts and Technology Program, Santa Barbara, CA 93106 , USA, amcleran@gmail.com), Curtis Roads (University of California, Media Arts and Technology Program, Santa Barbara, CA 93106, USA, clang@mat.ucsb.edu), Laurent Daudet (UPMC Univ Paris 06, LAM / IJLRA, 11 rue de Lourmel, 75015 Paris, France, daudet@lam.jussieu.fr)

The authors investigate the characteristics and performance of joint (single-step) and sequential (two-step) approaches to creating sparse and structured multiresolution representations of audio and music signals derived using sparse overcomplete methods. A joint approach, such as molecular matching pursuit, attempts to find structures in a signal as part of the decomposition process, while a sequential approach, such as agglomerative clustering, attempts to find structures in the completed decomposition of a signal. Each of these approaches have different benefits and drawbacks. For a joint approach, it is computationally convenient that the decomposition and structuring are done simultaneously, but usually only simple structural relations are possible. For a sequential approach, one is working in a parameter space of much smaller dimension than the original signal, but the computation is higher since the decomposition and the structure building are two separate processes. Results from these approaches using real audio and music signals will be compared and contrasted, and will contribute to our goal of creating an enhanced interface between the content of audio and music signals, e.g., onsets, notes, voices, and their multiresolution sparse atomic decompositions

5aMUd6. Initialization, distances and local minima in audio applications of the non-negative matrix factorization. Nancy Bertin (Télécom Paristech (ENST) / TSI - CNRS LTCI, 46, rue Barrault, 75634 Paris Cedex 13, France, nbertin@enst.fr), Roland Badeau (Télécom Paristech (ENST) / TSI - CNRS LTCI, 46, rue Barrault, 75634 Paris Cedex 13, France, roland.badeau@enst.fr)

The use of the non-negative matrix factorization (NMF) as a decomposition technique has dramatically grown in various signal processing applications over the last years. Its computation, based on the iterative minimization of a cost function, relies on several choices, among which the distance involved in the cost function itself but also the initialization of the algorithm. These choices are all the more crucial as the usual algorithms, iterative, only ensure convergence to a local minimum. In this work, we compare three typical distances in the NMF problem (namely Euclidian, Kullback-Leibler divergence and Itakura-Saito distance) and their combination with different initializations, in an audio context: decomposition of the time-frequency representation for the transcription of polyphonic music. Both the existence of global and local minima, and the efficiency of transcription are examined. Moreover, NMF update rules can be formulated in a unified framework for the three aforementioned cost functions. This formulation allows figuring out new NMF algorithms which could address the local minima question.
5aMUd7. Automatic morphological description of sounds. Geoffroy G. Peeters (Ircam, 1, pl. Igor Stravinsky, 75004 Paris, France, peeters@ircam .fr), Emmanuel Deruty (Ircam, 1, pl. Igor Stravinsky, 75004 Paris, France, emmanuel.deruty@gmail.com)

Morphological description has been proposed by Pierre Schaeffer. It consists in describing sounds by identifying the temporal evolution of their acoustical properties to a set of profiles. This kind of description is especially useful for indexing sounds with unknown cause such as SoundFX. The present work deals with the automatic estimation of this morphological description from audio signal analysis. In this work, three morphological descriptions are considered: - dynamic profiles (ascending, descending, ascending/descending, stable, impulsive), - melodic profiles (asc., desc. fixed, up/down, down/up) - repetition profiles. For each case we present the most appropriate audio features (loudness, pitch, pitch salience, temporal increase/decrease, lag-matrix-periodicity, ...) and mapping algorithm (slope computed from spline approximations of temporal profiles, ...) used to automatically estimate the profiles. We demonstrate the use of these descriptions for automatic indexing (using decision trees) and search-by-similarity of SoundFX.

5aMUd8. Sound feature extraction to distinguish between a grand and an upright piano. Grigorios Plitsis (Greece, grigoriosplitsis@merseymail .com)

The sound of a grand piano differs from that of an upright piano. In order to find what characterizes the produced sound, an instrument recognition system capable of producing a model of the sound features should be created. Feature extraction can be performed with the use of the Mel frequency cepstral coefficients (MFCCs). This method is useful as it approximates the mechanism of human hearing. Statistical modeling can be further applied using the Gaussian mixture model (GMM) so as to train the system. In order to decide whether the under test instrument is a grand or an upright piano the log-likelihood should be calculated. This study could assist in the identification of the parameters defining the sound of a grand and an upright piano.

5aMUd9. Instrument sound description and modelisation in the context of computer aided orchestration. Damien Tardieu (Ircam, 1, pl Igor Stravinsky, 75004 Paris, France, Damien.Tardieu@ircam.fr), Grégoire Carpentier (Ircam, 1, pl. Igor Stravinsky, 75004 Paris, France, Gregoire .Carpentier@ircam.fr), Geoffroy G. Peeters (Ircam, 1, pl. Igor Stravinsky, 75004 Paris, France, peeters@ircam.fr)

We present a work related to the description of instrumental sound in the context of computer aided orchestration. We define the orchestration problem as the search of instrument sound combinations that sound close to a given target. In the presented approach, instrument sounds are described by a set of acoustic features that are extracted from large sound sample databases. The features are then modeled by a probabilistic distribution. Finally the models are combined to approximate the description of a combination of instrument sounds. We discuss the requirement induced by the orchestration problem on the choice of the acoustic features and detail the chosen set of features. In a second part we describe the method used to learn the probabilistic model of different instruments and playing techniques and show that it allows to learn the models from few samples and to deduce the models of some playing techniques, pitches or dynamics that are not available in the training set. Finally we evaluate the proposed method on classification tasks and describe its integration in a computer assisted orchestration program. 


\title{
Session 5aMUe
}

\section{Musical Acoustics and Signal Processing in Acoustics: Signal Representations and Models of Musical Sounds II}

\author{
James W. Beauchamp, Cochair \\ University of Illinois, 1002 Eliot Drive, Urbana, IL 61801, USA \\ Bertrand David, Cochair \\ Télécom Paristech (ENST) / TSI - CNRS LTCI, 46, rue Barrault, Paris Cedex 13, 75634, France
}

\section{Contributed Paper}

\begin{abstract}
11:00
5aMUe1. Early use of the Scott-Koenig phonautograph for documenting performance. George Brock-Nannestad (Patent Tactics, Resedavej 40, DK-2820 Gentofte, Denmark, pattac@image.dk), Jean-Marc Fontaine (Université UPMC - Ministère de la Culture - CNRS - IJRA LAM, 11, rue de Lourmel, F-75015 Paris, France, jmfontai@ccr.jussieu.fr)

blackened glass plate, the scientific instrument maker Rudolph Koenig contributed by devising a very long axis representing time, because now blackened paper was wrapped around a cylinder on a threaded shaft. A twodimensional representation of the individual vibrations was obtained. Scott both deposited a sealed letter with the Paris Academy of Sciences in January, 1857 and filed a patent application in April, 1857. Later he deposited a further sealed letter and in 1859 he filed an application for patent of addition. Following the thoughts expressed and documented in his manuscripts here analyzed in context and comparing with Koenig's production it is feasible to see how they were dependent on each other, although they had different purposes in mind. The paper concentrates on Scott's interests in performance, and a number of original tracings are discussed.

Acoustics in the 1850s combined listening, observation and notation. This was "real-time", catching any phenomenon as it appeared. If it was repeatable, one could prepare for it. Continuous data rather than observation data enabled a very different analysis from observation and notebooks. Édouard-Léon Scott's invention of the phonautograph enabled this. A surface moved below a stylus that was vibrated by sound in air. Originally a
\end{abstract}

\section{Invited Papers}

\section{1:20}

5aMUe2. Sparse representations of audio: from source separation to wavefield compressed sensing? Remi Gribonval (INRIA, IRISA, Campus de Beaulieu, 35042 Rennes Cedex, France, remi.gribonval@ irisa.fr)

Sparse signal representations, which are at the heart of today's coding standards (JPEG, MPEG, MP3), are known to have had a substantial impact in signal compression. Their principle is to represent high-dimensional data by a combination of a few elementary building blocks, called atoms, chosen from a large collection called a dictionary. Over the last five years, theoretical advances in sparse representations have highlighted their potential to impact all fundamental areas of signal processing. We will discuss some current and emerging applications of sparse models in musical sound processing including: signal acquisition (Compressed Sensing - sampling wave fields at a dramatically reduced rate) and signal manipulation (e.g., source separation and enhancement for digital remastering). We will conclude by discussing the new algorithmic and modeling challenges raised by these approaches.

\section{1:40}

5aMUe3. Towards a hierarchically sparse model for audio signals. Laurent Daudet (UPMC Univ Paris 06, LAM / IJLRA, 11 rue de Lourmel, 75015 Paris, France, daudet@lam.jussieu.fr)

In this paper we discuss a major issue that arises when building sparse atomic models for music signals: in which space (/ dictionary) should we represent the signals? Having extremely redundant dictionaries is very informative for the locally most important atoms, but is irrelevant for the detail atoms that represent residual signals. Also, what are the advantages of dictionaries composed of learned atoms (which brings the issue of representativeness) compared to generic dictionaries? We here advocate for non-fixed dictionaries, with a multi-layered hierarchical decomposition: the first layer roughly describes the signal, in an extremely redundant, signaltailored, structured dictionary. This layer is also very sparse, most of the information being carried by the atoms' parameters, similarly to parametric representations. Subsequent layers give more and more details, increasing the data amount while reducing overcompleteness and structural model constraints. These techniques have obvious applications for audio coding, but are also useful for scalable music data mining. This research is supported by the French ANR (DESAM project).

\section{2:00}

5aMUe4. Bayesian inference in hierarchical non-negative matrix factorisation models of musical sounds. Ali Taylan Cemgil (University of Cambridge, Trumpington Street, CB2 1PZ Cambridge, UK, atc27@ cam.ac.uk), Tuomas Virtanen (Tampere University of Technology, Inst. of Signal Processing, FI-33101 Tampere, Finland, tuomas.virtanen@tut.fi)

There has been a surge of interest to efficient audio and music modeling using tools from statistical machine learning. One such technique, that has been particularly successful, is non-negative matrix factorisation (NMF). However, a detailed theoretical understanding of this success is missing, as well as links to other modeling strategies such as sinusoidal or harmonic models. To fill this gap, 
we describe NMF in a statistical framework, as a hierarchical generative model consisting of an observation and a prior component. We show that particular choices lead to standard NMF algorithms as special cases, where parameter estimation is carried out via maximum likelihood. Starting from this view, we develop extensions that facilitate more realistic acoustic modeling (such as spectral smoothness or harmonicity of natural sounds) and alternative inference techniques via Gibbs sampling and variational Bayes, which allow us to do principled comparisons between alternative models via Bayesian model selection. Our novel construction, where we make use of Markov chains of Gamma random variables, retains conjugacy and enables us to develop models that fit better to real data while retaining attractive features of standard NMF such as fast convergence and easy implementation. We illustrate our approach on polyphonic pitch estimation.

\section{$12: 20$}

5aMUe5. On sinusoidal modeling of nonstationary signals. Axel Roebel (IRCAM, 1, pl. Igor-Stravinsky, 75004 Paris, France, roebel@ircam.fr)

In this presentation we are going to give an overview over a number of techniques that have been developed in our group to improve the modeling of musical (nonstationary) signals using sinusoidal models. One of the basic problems with sinusoidal models is the fact that the underlying theory is derived assuming stationary sinusoids, while in the real world all sinusoidal components are non stationary. The two topics that will be covered main are, first a technique that allows to distinguish between nonstationary sinusoidal components, noise and transients, and second a new technique for parameter estimation of nonstationary sinusoids using frequency domain demodulation.

\section{2:40-1:40 Lunch Break}

\section{Invited Papers}

\section{$1: 40$}

5aMUe6. Advances in the tracking of partials for the sinusoidal modeling of musical sounds. Sylvain Marchand (LaBRI CNRS, University of Bordeaux 1, 351 cours de la Liberation, F-33405 Talence, France, sylvain.marchand@labri.fr)

Whereas sinusoidal modeling is widely used for sounds, polynomial models are still used for the model parameters, which can hardly handle modulations (vibrato and tremolo) present in musical sounds. Moreover, the partial tracking algorithms are often designed under stationarity assumptions. Advances in partial tracking may come out of the modeling of the partials themselves. We consider their parameters (frequencies and amplitudes) as predictable and slow time varying: First, the future of any partial can be determined from its past evolutions; second, no audible frequency should appear in the spectral content of these evolutions, otherwise it would question the perceptive consistency of the model. We then choose to handle nonstationary sinusoidal modeling by a deterministic approach based on linear prediction of the partial evolutions and partial discrimination based on the spectral properties of these evolutions. The underlying model for each partial is now a sum of sinusoids, thus leading to a two-level sinusoidal modeling, well suited for musical sounds, where modulations are important. The enhanced partial tracking algorithm also handles the case of crossing partials, without the need for any probabilistic approach. Better modeling the deterministic part of polyphonic sounds leads to enhanced source separation and time scaling algorithms.

5aMUe7. Adaptive subspace methods for high resolution analysis of music signals. Roland Badeau (Télécom Paristech (ENST) /TSI - CNRS LTCI, 46, rue Barrault, 75634 Paris Cedex 13, France, roland.badeau@enst.fr), Bertrand David (Télécom Paristech (ENST)/TSI - CNRS LTCI, 46, rue Barrault, 75634 Paris Cedex 13, France, bertrand.david@enst.fr)

In the field of music signals analysis, the tonal part of a broad variety of sounds is often represented as a sum of slowly varying sinusoids. The Fourier transform remains a prominent tool for estimating the parameters of this model, due to its robustness and to the existence of fast algorithms. Its main drawback relies in its spectral resolution, bounded by the length of the analysis window. Subspacebased high resolution (HR) methods are conversely not constrained by this limit, since they rely on the particular geometrical structure of the signal model. Nevertheless, they have been seldom used in audio signal processing, mainly due to their high computational cost. Based on recent advances in the field of subspace tracking, enhanced adaptive algorithms for HR analysis have thus been developed, leading to a high resolution time-frequency representation of the signal, called HR-ogram. The application of these algorithms to music signals, made difficult by the high dynamics and the presence of colored noise, has required the tuning of well-adapted preprocessing techniques. The whole tool is now mature, and allows a high quality separation of the tonal part of various musical sounds. This research is supported by the French ANR under contract ANR-06-JCJC-0027-01 (DESAM).

5aMUe8. Towards an adaptive subspace-based representation of musical spectral content. Bertrand David (Télécom Paristech (ENST)/TSI - CNRS LTCI, 46, rue Barrault, 75634 Paris Cedex 13, France, bertrand.david@enst.fr), Roland Badeau (Télécom Paristech (ENST)/TSI - CNRS LTCI, 46, rue Barrault, 75634 Paris Cedex 13, France, roland.badeau@enst.fr)

This study presents an algorithm based on an adaptive framework model for musical sound signals assumed to be composed of slowly varying frequency components surrounded by additive noise. These components appear as contours in a time-frequency representation, as for instance, a spectrogram. To extract these contours, an often used solution is to estimate the parameters (amplitude, frequency, and phase) of each component at each frame and then to link them from one to the next with the help of a distance measure or an HMM. Conversely, our method attempts to update the estimated values from one time instant to the next. It relies on principal subspace tracking (with respect to time) together with gradient descent to individually update each of the component parameters. Finally, each extracted contour, which represents the frequency and amplitude variation of a single component, is available for subsequent 
processing. Applications are demonstrated in the fields of harmonic plus noise decomposition and analysis/transformation/synthesis. This research is supported by the french Institut Telecom, TAMTAM project.

\section{2:40}

5aMUe9. Damped sinusoids and subspace based approach for lossy audio coding. Olivier Derrien (Université de Toulon, Av Georges Pompidou, BP 56, 83162 La Valette du Var, France, olivier.derrien@univ-tln.fr), Gaël Richard (Télécom Paristech (ENST) /TSI - CNRS LTCI, 46, rue Barrault, 75634 Paris Cedex 13, France, gael.richard@enst.fr), Roland Badeau (Télécom Paristech (ENST)/TSI - CNRS LTCI, 46, rue Barrault, 75634 Paris Cedex 13, France, roland.badeau@enst.fr)

The new subspace-based techniques recently introduced appear to be well adapted for the parameters estimation of a damped sinusoids + noise signal model. These high-resolution (HR) methods have a better frequency resolution than the Fourier analysis, but they are rarely used in audio coding. Although HR methods would be suitable for parametric coding at low bitrates, we show that they are also efficient for high-bitrate coding where state-of the art codecs are usually transform-based. Our coding scheme first includes a 8-band PQMF filter-bank decomposition. Then, each subband signal is segmented in onsets and a maximum-order HR analysis is performed on each segment with the ESPRIT algorithm. For each component of the model, frequency, damping, amplitude and phase are quantized. The residual signal is not coded. This codec is compared to a MDCT framework, where transform and quantization are the same as in a MPEG-AAC but without inclusion of perceptual modelling and entropy coding. Preliminary objective and subjective tests show the potential of this approach which requires, on mostly tonal signals, significantly less bits than the traditionnal MDCT method for a given quality.

\section{3:00}

5aMUe10. Auditory model based analysis of polyphonic music. Anssi Klapuri (Tampere University of Technology, Korkeakoulunkatu 1, 33720 Tampere, Finland, anssi.klapuri@tut.fi)

This study is about the use of an auditory model to extract multiple pitches from polyphonic music signals. One goal was to identify the conditions where pitch analysis using an auditory model is advantageous over more conventional time or frequency domain approaches. It is shown that these conditions include especially the processing of band-limited signals or signals where important parts of the audible spectrum are corrupted by band-limited interference. An efficient implementation strategy is described which reduces the computational complexity of the auditory model roughly by factor 10 . Further prospects of bandwise processing and redundant signal representations in general are discussed.

5aMUe11. On perceptual distortion measures and parametric modeling. Mads G. Christensen (Aalborg University, Niels Jernes Vej 12 A, DK-9220 Aalborg, Denmark, mgc@es.aau.dk)

Over the past two decades, there has been much interest in incorporating human sound perception in signal processing algorithms, often in the form of perceptual distortion measure or an approximation thereof. An example of this is MDCT-based audio coding where a good quality can be achieved at very low bit-rates by taking masking effects into account. More recently, the same principles have been applied to parametric modeling and coding of audio signals. We discuss the inherent tradeoffs in choosing a perceptual distortion measure and a parametric model and the pros and cons of various ways of implementing such perceptual distortion measures is discussed. An important question that we seek to answer is whether perception should be taken into account in the estimation of model parameters or this should be done in a separate step.

\section{Contributed Paper}

3:40

5aMUe12. Efficient coding of a xylophone sound using spikogram nonredundent coding. Rolf Bader (University of Hamburg, Institute of Musicology, Neue Rabenstr. 13, 20354 Hamburg, Germany, R_Bader@tonline.de)

Sensory systems try to use the incoming data most efficiently. Studies of Lewicki et al. lately showed, that a representation of a spike train just representing the sound and not having any redundancy is how the auditory system of the cat represents incoming sounds. To compare this theory with musical instruments, a xylophone sound was analyzed in terms of a spikogram.
Here, gammatones of a 64 channel filterbank with different attack and decay values are superposed in a way to reconstruct the original sound. Only those gammatones were used which are needed to result in the sound used as input and so no redundancy is present in the analysis of the sound. It was found, that the most reasonable fit of the gammatone shape with the empirical data indeed made the representation most efficient and so the xylophone sound is most easily represented by the auditory system. Although more analysis are needed here, musical instruments could show up to be built in a way to fit a most efficient coding by listeners and so fulfill a middle-of-the-road rule of not too much and not too few information so that listeners are interested in but not overtaxed by the sounds. 


\section{Invited Papers}

\section{4:20}

5aMUe13. A frequency shifting model of pitch. Paris Smaragdis (Adobe Systems Inc., 275 Grove St., Newton, MA 02466, USA, paris@media.mit.edu)

We present a model useful for tracking of melodies of sounds that can have arbitrary harmonic structure (including inharmonic instruments and noise sources). This model is based on a spectral shift assumption which is capable of tracking melodic movements of an instrument regardless of the irregularity of its spectrum. This technique can be used to simultaneously estimate the spectral character of the instrument to be analyzed in addition to its melody. It is capable of dealing with multiple instances of the same instrument, thereby recognizing chords as well as notes, and can also extract multiple melodies in audio signals composed out of many instruments.

5aMUe14. Modeling vocal sounds in polyphonic musical audio signals. Masataka Goto (National Institute of Advanced Industrial Science and Technology (AIST), IT, 1-1-1 Umezono, Tsukuba, 305-8568 Ibaraki, Japan, m.goto@aist.go.jp), Hiromasa Fujihara (National Institute of Advanced Industrial Science and Technology (AIST), IT, 1-1-1 Umezono, Tsukuba, 305-8568 Ibaraki, Japan, h.fujihara@aist.go.jp)

This paper describes our research aimed at modeling vocal sounds (singing voices) in available music recordings and its applications to singer identification, singer similarity, and lyrics synchronization. Our predominant-F0 estimation method, PreFEst, can obtain the melody line by modeling the input sound mixture as a weighted mixture of harmonic-structure tone models (probability density functions) of all possible F0s and estimating their weights and the tone models by MAP estimation. Since the PreFEst was designed for general melodies, we extended it to specialize in vocal melodies by using vocal timbre models --- vocal and nonvocal GMMs. Those GMMs are trained beforehand and used to evaluate the vocal probability. The GMMs are also used to identify vocal regions, but its strategy should depend on applications. For singer identification and singer-similarity calculation, since the purpose is to model singer's identity by training each singer's vocal GMM, certainly reliable vocal regions should be identified even if most true regions were missed. On the other hand, for lyrics synchronization, since the purpose is to align each phoneme to the estimated vocal melody, vocal regions should be identified without missing any true regions. We achieved this by biasing log likelihoods provided by vocal and nonvocal GMMs.

\section{5:00}

5aMUe15. Music and speech signal processing using harmonic-temporal clustering. Jonathan Le Roux (University of Tokyo, Sagayama/Ono Laboratory, 7-3-1 Hongo, Bunkyo-ku, 113-8656 Tokyo, Japan, leroux @ hil.t.u-tokyo.ac.jp), Hirokazu Kameoka (NTT Communication Science Laboratories, NTT Corporation, 3-1 Morinosato wakamiya, 243-0198 Atsugi, Kanagawa, Japan, kameoka@eye.brl.ntt.co.jp), Nobutaka Ono (University of Tokyo, Sagayama/Ono Laboratory, 7-3-1 Hongo, Bunkyo-ku, 113-8656 Tokyo, Japan, onono@ hil.t.u-tokyo.ac.jp), Alain De Cheveigne (CNRS, Universite Paris 5, Ecole Normale Superieure, 29 rue d’Ulm, 75230 Paris, France, alain.de.cheveigne@ens.fr), Shigeki Sagayama (University of Tokyo, Sagayama/Ono Laboratory, 7-3-1 Hongo, Bunkyo-ku, 113-8656 Tokyo, Japan, sagayama@ hil.t.u-tokyo.ac.jp)

We present here the principle of the recently introduced harmonic-temporal clustering (HTC) framework and its applications in both music and speech signal processing. HTC relies on a precise parametric description of the harmonic parts of the power spectrum through constrained Gaussian mixture models. The model parameters of all the elements of the acoustical scene are estimated jointly by an unsupervised 2D time-frequency clustering of the observed power density. HTC is effective for multi-pitch analysis of music signals and F0 estimation of single and multiple speaker speech signals in various noisy environments. It also enables to perform extra processing of monaural music and speech signals, such as isolation or cancellation of a particular part, noise reduction and source separation.

5aMUe16. Timbre transposition based on time-varying spectral analysis of continuous monophonic audio and precomputed spectral libraries. James W. Beauchamp (University of Illinois, 1002 Eliot Drive, Urbana, IL 61801, USA, jwbeauch@uiuc.edu), Mert Bay (University of Illinois, 212 W. Healey, Apt. 303, Champaign, IL 61820, USA, mertbay@uiuc.com)

A sinusoidal model for solo musical sounds consisting of time-varying harmonic amplitudes and frequencies allows for convenient temporal and spectral modifications. With a harmonic model, analysis frames can be grouped by fundamental frequency (F0) and then clustered in terms of their harmonic spectra. The resulting cluster centroid spectra are used as spectral libraries. When continuous audio monophonic passages are analyzed in the form of harmonic components, F0 vs. time data are used to guide the extraction of parameters from the sound in order to find appropriate library spectra for resynthesis. Two methods for finding appropriate spectra are: (1) best rms match with the incoming spectra and (2) best spectral centroid match. These give similar results, but centroid matching yields smoother spectra over time. Timbre transposition is performed by using a library that belongs to another instrument. We have found that when the target instrument has a unique timbral quality based on its spectrum, the synthesis sounds mostly like that instrument. However, if the target instrument's spectral characteristic is not sufficiently differentiated from the source, the source timbral quality may dominate, probably due to its temporal behavior being transmitted. Results will be demonstrated by audio examples. 


\title{
Contributed Papers
}

\section{$5: 40$}

5aMUe17. Comparison of the sound of a grand and an upright piano using wavelets. Grigorios Plitsis (Greece, grigoriosplitsis@merseymail .com)

Wavelet analysis is useful for extracting patterns and thus analyzing signals. Although the Fourier analysis can reveal different features of a signal, it is less appropriate for describing transient phenomena and sudden sound changes. As a result, Fourier-based music reconstruction cannot exactly imitate the physical sound, as it is not possible to know at the same time a specific frequency as well as the time of occurrence of this frequency. Wavelet analysis is capable of highlighting different attributes of a signal. Different types of wavelets are thus used in the present study in order to compare the sound produced by a grand piano with that produced by an upright piano.

\section{6:00}

5aMUe18. Modeling of piano sounds using FEM simulation of soundboard vibration. Luis I. Ortiz-Berenguer (Universidad Politecnica de Madrid, Ctra.Valencia km7, 28031 Madrid, Spain, lortiz@diac .upm.es), Francisco J. Casajus-Quiros (Universidad Politecnica de Madrid,
Ctra.Valencia km7, 28031 Madrid, Spain, javier@gaps.ssr.upm.es),David Ibanez-Cuenca (Universidad Politecnica de Madrid, Ctra.Valencia km7, 28031 Madrid, Spain, dibanez@alumnos.euitt.upm.es)

Pattern-matching methods for polyphonic transcription of piano sounds require a set of patterns that can be obtained by modeling the piano-sound spectra. The modeling should take into account not only the string stiffness but also the effect of the soundboard impedance on the string vibration. Studies on that effect corresponding to a wide range of impedance values have previously been carried out by the authors. However, actual impedance values for real pianos must be used in the model. Although the impedance value of a few grand-pianos have been measured by the authors, these results are not significative enough to create a model. Thus, a FEM simulation of soundboard vibration is proposed to obtain nearly-actual impedance values. The simulation considers several cases of vibrating plates from the simplest rectangular one and increasing the similarity to real piano soundboards. The quality of the simulation is verified comparing the obtained results with either recognized theoretical results for the simplest cases or measured values for the more complex ones. The complexity of the simulated soundboard is limited to the case that produces only slight variations in the modeled spectrum. [This work has been supported by Spanish National Project TEC2006-13067-C03-01/TCM.]

FRIDAY MORNING, 4 JULY 2008

ROOM 202/203, 11:00 A.M. TO 12:00 NOON

\section{Session 5aMUf}

\section{Musical Acoustics: Plucked and Struck Idiophones II}

\author{
Thomas D. Rossing, Cochair \\ Stanford University, CCRMA, Department of Music, Stanford, CA 94305, 26464 Taaffe Rd, Los Altos Hills, CA 94022, USA \\ Charles Besnainou, Cochair \\ Institut Jean le Rond d'Alembert, Laboratoire d'Acoustique Musicale, 11, rue de Lourmel, Paris, 75015, France
}

\section{Contributed Papers}

\section{1:00}

5aMUf1. Characterizing the sound of an African thumb piano (kalimba). David M.f. Chapman (Scientific Consultant, 8 Lakeview Avenue, Dartmouth, NS B3A 3S7, Canada, dave.chapman@ns.sympatico.ca)

The kalimba is an African percussion instrument whose notes are generated by vibrating metal tines of various lengths attached to sound board or sound box. Unlike a vibrating string or organ pipe, the overtone structure is anharmonic, that is, the overtone frequencies (which determine sound quality) are not simple integer multiples of the fundamental frequency (which determines pitch). The ratio of the first overtone to the fundamental is in the range 5.3-5.9, depending on the tine geometry. The vibrating tines are modeled as a clamped-supported-free beam and the observed overtone structure is shown to be in accordance with this model. Audio examples will be provided.

\section{1:20}

5aMUf2. The jew's harp, experimental study and modeling. Charles Besnainou (Institut Jean le Rond d'Alembert, Laboratoire d'Acoustique Musicale, 11, rue de Lourmel, 75015 Paris, France, chbesnai@ccr jussieu.fr), Joel Frelat (Institut Jean le Rond d'Alembert, Lab. d'Acoustique Musicale, 11, rue de Lourmel, 75015 Paris, France,
frelat@1mm.jussieu.fr),Adrien Mamou-Mani(Institut Jean le Rond d'Alembert, Lab. d'Acoustique Musicale, 11, rue de Lourmel, 75015 Paris, France, mamou-mani@1mm.jussieu.fr)

Under its archaic aspects jew's harp is a musical instrument highly subtle. Indeed, a metal blade (or wooden) attached to a rigid frame put into vibration by the musician, and coupled to the buccal resonator allow nice tune. The skilt of the jew's harp focuses on the conformations of this cavity whose function is to select the right components of the vibration to be amplify. In our study, we have modelled a playing technique which involves blowing during the blade vibrates. In the lake of breath, the spectre of sound produced by the blade is odd, i.e., it includes at first approximation odd components $(n+1)$ multiple the fundamental. Whereas when the musician adds breath the spectrum turns into a spectrum containing all components of basic integer multiples (n). This work takes place in the context of studies of vibrating systems under prestress and loaded. In that case the load, and the prestress are generated by the musician breath by bending the blade On the other hand, experimental studies are compare with the model results.

\section{1:40}

5aMUf3. Sound of the HANG. Thomas D. Rossing (Stanford University, CCRMA, Department of Music, Stanford, CA 94305, 26464 Taaffe Rd, Los Altos Hills, CA 94022, USA, rossing@ccrma.stanford.edu), David 
Wessel(Center for New Music and Audio Technologies, 1750 Arch Street, Berkeley, CA 94720, USA, wessel@cnmat.berkeley.edu.)

Previous studies of the HANG [T. D. Rossing, U. Hansen, F. Rohner, and S. Schärer, 142nd ASA meeting, December 2001; T. D. Rossing, A. Morrison, U. Hansen, F. Rohner, and S. Schärer, Proceedings of ISMA07, Barcelona, 2007] have discussed the modes of vibration and the sound radiation field of the HANG when note areas are excited by sinusoidal force. Since the HANG is a hand-played steel instrument, a wide variety of playing techniques are used by various performers. We report on the sound of the
HANG when played in various ways. Recordings of the HANG were made with a hemispheric microphone array. The instrument was excited by taking multiple samples of each of a number of striking techniques with the hand. These include single strokes from the soft part of the finger, as well as, the finger nail itself. Various grace note techniques like flams were also investigated. Damping was controlled by the time the finger remained in contact with the instrument, as well as, by using the palm. Force hammer measurements were also included. We report on these measurements and incorporate them in to an excited-resonance model of the instrument. Demonstrations of the real and modeled instrument will be provided.

FRIDAY MORNING, 4 JULY 2008

ROOM 250A, 8:00 A.M. TO 6:20 P.M.

\title{
Session 5aNSa
}

\section{Noise and EURONOISE: Soundscape \& Community Noise I}

\author{
Bennett Brooks, Cochair \\ Brooks Acoust. Corp., 27 Hartford Turnpike, Vernon, CT 06066, USA
}

Brigitte Schulte-Fortkamp, Cochair

TU Berlin, Institute of Fluid Mechanics and Engineering Acoustics, Einsteinufer 25, Sekr. TA 7, Berlin, D-10587, Germany

\section{Invited Papers}

\section{8:00}

5aNSa1. Soundscapes: from noise annoyance to the music of urban life. Catherine Guastavino (Centre for Interdisciplinary Research in Music Media \& Technology (CIRMMT) - School of Information Studies - McGill Univ., 3459 McTavish, Montreal, QC H3A1Y1, Canada, Catherine.Guastavino@mcgill.ca), Danièle S. Dubois (CNRS, LCPE/LAM 11 rue de Lourmel, 75015 Paris, France, ddubois@ccr.jussieu.fr)

Through an overview of empirical research over the past ten years, we present an interpretation of the evolution of the concept of soundscape. From a strictly acoustic definition in terms of physical descriptors, it evolved into a more complex notion integrating the effects of noise on citizens. Soundscape research therefore requires the contribution of human sciences (psychology of perception, cognitive psychology, sociology, anthropology of senses). There is converging evidence that people judgments of noise is related to the meaning given to the activities producing the noise. Consequently, physical descriptions in acoustics have to face the diversity of human reactions to noise correlated with the diversity of activities, the diversity of source producing noises within a diversity of physical environments (natural, architectural). This paper focuses on major emerging issues in soundscapes research, namely - the integration of such a diversity of pieces of knowledge within a general knowledge - the translation of these diverse conceptualizations into physical descriptions - the elaboration of convincing physical measurements for decision makers. We will draw consequences for further developments in the cooperative and pluridisciplinary research and for producing guidelines for new orientations in community policies.

\section{8:20}

5aNSa2. The soundscape of church bells - sound community or culture clash. Brenda H. Kiser (DL Acoustics, 14301 Middletown Lane, Westminster, CA 92683, USA, brendakiser@ix.netcom.com), David Lubman (DL Acoustics, 14301 Middletown Lane, Westminster, CA 92683, USA, dlubman@dlacoustics.com)

Church bells have long communicated important information to the surrounding community, creating a sound neighborhood. The community relied on the sound neighborhood to learn of events such as weddings, births and deaths, impending danger, church services, and the curfew. The bells thus provided communal identity. This paper reviews the sound neighborhood created by the bells of the church of St. Mary-le-Bow in the City of London, popularly known as "Bow Bells." The ringing of these bells has for centuries defined London's Cockneys and has featured in London's folklore and history since the 14th century. The current status of the soundscape is presented. Sound examples are given. This paper also discusses the future of the soundscape of church bells in light of current societal changes.

\section{8:40}

5aNSa3. How is hearing heard in second year architectural design education? Juergen Bauer (Waterford Institute of Technology, Cork Road, IRE - 00000 Waterford, Ireland, jbauer@wit.ie)

Architects are often accused of "not being interested in acoustics because it is invisible". Surely, architectural design, particularly at preliminary stage, has always been driven visually: architects usually develop a project by vision, not by hearing. On the other hand, designing architects have to deal creatively with more or less invisible parameters: sociocultural demands, climatic aspects as wind or temperature, or even daylight which can be considered to be visible but certainly is everything but constant. If an architect's design 
ability is about observation and awareness, and to give an example, if light is considered to be much more than just being bright or dark, how then could we implement early awareness that sound is much more than noise or silence, and that sound planning is much more than just using anti-noise-panels? We asked second year architecture students at Waterford Institute of Technology to (re-)think the architecture of schools and to question the sound qualities and their own sound recollection of such an environment. The paper will outline the teaching approach and present some student's responses to the given aims and brief. Finally, the lessons learnt by teaching staff will be summarized.

5aNSa4. Using the soundscape approach to develop a public space in Berlin - perception and evaluation. Brigitte Schulte-Fortkamp (TU Berlin, Institute of Fluid Mechanics and Engineering Acoustics, Einsteinufer 25, Sekr. TA 7, D-10587 Berlin, Germany, brigitte.schulte-fortkamp@tu-berlin.de), Rudi Volz (Advacoustics, Leibnizstr.21, Dr. Rudi Volz, 10625 Berlin, Germany, volz@advacoustics.de), Andre Jakob (TU Berlin, Institute of Fluid Mechanics and Engineering Acoustics, Einsteinufer 25, Sekr. TA 7, D-10587 Berlin, Germany, kontakt@advacoustics.de)

In Berlin the Soundscape Project "Nauener Platz" is carried out to rebuild a difficult public space into a space which is dominated by social freedom. A designed space related procedure is developed following the soundscape approach, which will provide acoustical indicators and parameters describing urban and other outdoor living areas with respect to physical conditions and their relevance for life. The measurement of outdoor sound quality will take into consideration not only the sound pressure but also the characteristics of the sounds which lead to specific human reactions, both positive and negative: calmness, inspiration, annoyance, discontentment, anxiety, etc. and the pathogenic effects. Qualitative evaluations through soundwalks and open interviews have been conducted introducing the new experts. Framework and procedure will be discussed. The Soundscape Project is a module of the Project "Nauener Platz - Remodelling for Young and Old"in the framework of the research program "Experimental Housing and Urban Development (ExWoSt)" of the "Federal Ministry of Transport, Building, and Urban Affairs (BMVBS)" by the "Federal Office for Building and Regional Planning (BBR)". It is related to the fields of research (ExWoSt) concerned with "Innovation of Urban Neighbourhoods for Families and the Elderly". The project executing organization is the Regional Office Berlin-Mitte.

\section{$9: 20$}

5aNSa5. Using the soundscape approach to develop a public space in Berlin - measurement and calculation. Rudi Volz (Advacoustics, Leibnizstr.21, Dr. Rudi Volz, 10625 Berlin, Germany, volz@advacoustics.de), Andre Jakob (TU Berlin, Institute of Fluid Mechanics and Engineering Acoustics, Einsteinufer 25, Sekr. TA 7, D-10587 Berlin, Germany, kontakt@advacoustics.de), Brigitte Schulte-Fortkamp (TU Berlin, Institute of Fluid Mechanics and Engineering Acoustics, Einsteinufer 25, Sekr. TA 7, D-10587 Berlin, Germany, brigitte.schulte-fortkamp@tu-berlin.de)

In this contribution the Soundscape Project "Nauener Platz" in Berlin is subject to different measurement- and calculation methods to develop acoustical indicators and parameters into a database describing urban and other outdoor living areas with respect to physical conditions and their relevance for life. The "Nauener Platz" is carried out to rebuilt a difficult open space into a space which is dominated by social freedom. To obtain the physical quantities sound pressure levels in 1/3-octave bands were measured and artificial head recordings were taken to describe the current state. Combined with calculations using noise prediction software it was possible to verify the sound propagation data outside according to the measurements and allow predictions about planned arrangements. First estimations of these measurements and calculations will be presented. The Soundscape Project is a module of the Project "Nauener Platz - remodelling for young and old". This project is in the framework of the research program "Experimental Housing and Urban Development (ExWoSt)" of the "Federal Ministry of Transport, Building, and Urban Affairs (BMVBS)" by the "Federal Office for Building and Regional Planning (BBR)". It is related to the fields of research (ExWoSt) concerned with "Innovation of Urban Neighbourhoods for Families and the Elderly". The project executing organization is the Regional Office Berlin-Mitte.

9:40-11:00 Posters

Lecture sessions will recess for presentation of poster papers on various topics in acoustics. See poster sessions for topics and abstracts.

\section{Invited Papers}

\section{1:00}

5aNSa6. Applicability of advanced measurement techniques to soundscape studies. André Fiebig (HEAD acoustics GmbH, Ebertstrasse 30a, 52134 Herzogenrath, Germany, andre.fiebig@head-acoustics.de), Klaus Genuit (HEAD acoustics GmbH, Ebertstrasse 30a, 52134 Herzogenrath, Germany, klaus.genuit@head-acoustics.de)

A wide range of measurement systems and analysis techniques were applied in previous soundscape studies to document and describe the physical conditions of the scrutinized area. Monaural and binaural recording systems positioned at different measurement points are frequently used, and the collected recordings are played back with headphones or sophisticated loudspeaker arrangements in specific surroundings for further analyses. In the paper, an overview of measurement and playback technologies applied in soundscape studies will be given and the immanent problems related to certain recording and playback systems will be discussed, e.g., measurement accuracy and expense, costs, etc. Furthermore, soundscape researchers have started to establish more and more a pool of specific analyses, which allows - at least partially - the detection of relevant (acoustical) patterns and features as well as a first classification of soundscapes. This allows even the detection of positively experienced environments. However, varying measurement technologies and procedures used in different soundscape studies lead to the problem that the studies and the obtained analysis results are often difficult to compare. The present paper takes up this problem and deals with developing a common basis of measurement techniques and procedures. 
5aNSa7. Analysis and evaluation of traffic sounds. Jens Guettes (TU Berlin, Institute of Fluid Mechanics and Engineering Acoustics, Modersohnstr.64, 10245 Berlin, Germany, jensguettes@gmx.de)

This work focuses on a residential area in Friedrichshain, a district close to the centre of Berlin. A soundscape analysis was made in this area, where the neighbours spent most of their free time, especially their leisure time. Basically, our measurements pointed out that immissions into the flats are mainly due to the low-frequency range under $200 \mathrm{~Hz}$, in particular in the case of closed windows. In addition, this low-frequency range initiates standing waves and vibrations within the flats, causing annoying secondary sounds. Furthermore, effects arose, caused by an impulsive signal structure and a discontinuity in the time structure of the source signals, depending on the street pavement and on the traffic situation. With regard to that point, different physical and perceptive measurement and analysis methods were compared and interviews within the neighbourhood were conducted. Moreover, we tried to correlate the results of the survey with those of our measurements in order to see advantages and disadvantages of the applied methods. The scope of A-weighted measurements was then critically considered. Different ways will be discussed about how the complex evaluation of traffic sounds in the future could be based more on neighbours' perception and needs.

\section{1:40}

5aNSa8. Soundscape design methods to acheive net zero environmantal noise impacts. Gary W. Siebein (Univ. of Florida, 231 Arch, P.O. Box 115702, Gainesville, FL 32611, USA, gsiebein@ siebeinacoustic.com), Robert M. Lilkendey (Siebein Associates, Inc., 625 NW 60th Street, Suite C, Gainesville, FL 32607, USA, rlilkendey@siebeinacoustic.com), Hyun Paek (Siebein Associates, Inc., 625 NW 60th Street, Suite C, Gainesville, FL 32607, USA, hpaek@siebeinacoustic.com)

Soundscape design methods were developed to approach net zero environmental noise impacts for significant building projects in complex settings. Auralization methods for various design alternatives were presented for stake holder review in a large auditorium and in a full size demonstration in the actual environment. Innovative acoustical design and analysis methods were developed to economically reduce source noise levels to the vicinity of the ambient sound level. Methods to determine the nature of the ambient sound level as it varies with time of day, time of year, and other natural and community factors included a series of long term average A-weighted sound levels as well as more detailed octave band measurements and calibrated aural recordings of specific acoustic events. Sound walks were conducted at various times of day to understand the dynamics of the acoustical environment and to identify issues. Focus group discussions among stakeholders and team members developed the long term plans for the community and appropriate architectural and acoustical design criteria for the project.

\section{2:00}

5aNSa9. The influence of subjective response on the choice of measurement for aircraft arrival noise at Nantucket Airport. Nancy Timmerman (Nancy S. Timmerman, P.E., 25 Upton Street, Boston, MA 02118-1609, USA, nstpe@ hotmail.com)

The Nantucket Airport in Massachusetts serves primarily turboprop commuter aircraft, and private or charter jets, with most of its operations in the summer. A seasonal homeowner was severely affected by arrival flight noise and requested noise measurements and noise control recommendations for this architect-designed residence. Time was spent with the homeowner to understand steps already taken, and problems still causing trouble. Standard measurement procedures for aircraft operations in the United States call for the use of A-weighted, slow response for the assessment of noise impact. Since the noise sources at this airport have strong tonal components (from the propellers), and noise control recommendations were sought, one-second 1/3-octave band levels were used to both document the observed problem, and to estimate the field transmission loss of the windows and doors. Measurements were made during a Friday afternoon, when arriving aircraft were mostly 1.5 to 2 min apart. A-weighted maximum and single event levels (SELs) were also measured for each overflight, for comparison with published aircraft data. A "short circuit" was found due to an exposed vent pipe, which resulted in no transmission loss in the $100 \mathrm{~Hz}$ band. Appropriate recommendations were made.

\section{Contributed Paper}

12:20

5aNSa10. Are perceived soundscapes within urban parks restorative? Sarah R. Payne (The University of Manchester, SED, MARC, Oxford Road, M13 9PL Manchester, UK, sarah.payne@postgrad.manchester.ac.uk)

The perceived quality of urban park soundscapes is starting to be explored, and attention restoration research has shown which environments are generally restorative. Yet the effect of perceived soundscapes on individuals' restorative experiences is hardly known. Natural environments, in general, provide restoration for people, including recovery from attentional fatigue and enabling reflection. Therefore the visually "natural" environment of ur- ban parks provides a useful setting to explore the role of varying soundscapes in restoration. Four hundred park users within Sheffield, UK, were surveyed as they left two urban parks. Data were collected on aspects of their park visit, along with measures of their current perceived restoration. Participants' perceived soundscapes were described by the amount of time they heard certain sound types in the park, and the volume at which they heard them. Sound levels [LAeq, dB(A)] within the parks were also monitored on a number of days to provide contextual information. Results of the study will be discussed along with the importance of soundscape quality and individuals' restorative experiences in helping to provide a productive and positive quality of urban life. 


\section{Contributed Papers}

\section{2:00}

5aNSa11. Perceptive relevance of soundscape descriptors: a morpho-typological approach. Jean-Dominique Polack (Institut Jean le Rond d'Alembert, Laboratoire d'Acoustique Musicale, 11, rue de Lourmel, 75015 Paris, France, polack@ccr.jussieu.fr), Jacuqes Beaumont (INRETS Laboratoire Transports et Environnement, 25, avenue François Mitterrand, 69675 Bron Cedex, France, jacques.beaumont@inrets.fr), Christine Arras (Acouphen, 33 route de Jonage - BP 30, 69891 Pusignan Cedex, France, christine.arras@acouphen.fr), Mikael Zekri (Institut Jean le Rond d'Alembert, Laboratoire d'Acoustique Musicale, 11, rue de Lourmel, 75015 Paris, France, mikael.zekri@gmail.com), Benjamin Robin (INRETS - Laboratoire Transports et Environnement, 25, avenue François Mitterrand, 69675 Bron Cedex, France, benjamin.robin@inrets.fr)

For assessing the validity of a morpho-typological classification of urban sounds with respect to sound assessment, factor analysis was applied to a database compiled in the city of Lyon and lead to a four-classes classification based on the number of lanes and whether a street is one-way or not. Free categorisation was then carried out on sound excerpts recorded in different sites corresponding to different classes. The main results are: a corpus of $42 \mathrm{~h}$ of five-channel recordings of a choice of sites from the classification at different times of the day; the validation of the relevance of the morphotypological classification for perception; and a confirmation of the semantic oppositions between sources and background noise, as well as holistic and event sequences. Further, annoyance is controlled by the absence or the presence of human or nature sound sources, pleading for research toward automatic identification of sources. Last, categorisation confirms earlier investigations based on variance analysis: in narrow and large streets, traffic increase differently governs the increase of both noise level and annoyance.

\section{2:20}

5aNSa12. Environmental management of a large construction site in a densely populated urban area: an innovative approach to noise and vibration mitigation. Marco Masoero (Politecnico di Torino, Corso Duca degli Abruzzi 24, 10123 Torino, Italy, marco.masoero@polito.it), Carlo Alessandro Bertetti (Studio Progetto Ambiente s.r.l., Corso Rosselli 44, 10128 Torino, Italy, ac.bertetti@progambiente.it)

An innovative approach to noise and vibration mitigation has been applied to the construction of a new underground railway. The aim is to overcome the difficulties in respecting noise level limits in densely populated areas, as specified by current legislation, even with state-of-the-art technology (e.g., low-emission machinery, noise barriers, etc.). The proposed approach aims at integrating the construction activity in the urban soundscape, placing maximum emphasis on construction organisation measures (e.g., construction activity scheduling and execution, training and information of contractor's personnel, etc.) and on communication and involvement of the exposed population. In this respect, two key figures have been identified: the first is an environmental engineer permanently assisting the construction manager in all the environmental impact issues; the second consists of a sample of population for which the subjective reaction to noise is monitored and fed back to the construction manager. Results are presented and discussed for one of the main construction sites in central Turin.

\section{2:40}

5aNSa13. The application of the soundscape approach in the evaluation of the urban public spaces. Monika Rychtarikova (Lab. ATF, Katholieke Universiteit Leuven, Celestijnenlaan 200D, B-3001 Leuven, Belgium, Monika.Rychtarikova@bwk.kuleuven.be), Gerrit Vermeir (Lab. ATF, Katholieke Universiteit Leuven, Celestijnenlaan 200D, B-3001 Leuven, Belgium, Gerrit.Vermeir@bwk.kuleuven.be), Markieta Domecka (Centre for Sociological Research, K.U. Leuven, Parkstraat 45, 3001 Leuven, Belgium, markieta.domecka@soc.kuleuven.be)

An important issues in the development of European cities is the design and renovation of the urban public areas. Typically, broad variety of approaches (sociological, ecological, environmental, physical, etc.) is needed. Earlier studies show the necessity of the transversal multidisciplinary approach in this issue. In order to study the acoustical dimension, the concept of soundscape needs to be proposed and elaborated. Soundscape approach differs from the classical statistical noise analysis in the evaluation of a context-related noise and in the extrapolation of environmental sounds in its complexity. Nowadays, even by using recently developed sophisticated acoustical and psycho-acoustical measurable and quantifiable parameters, it still remains difficult to grasp the complete meaning of a soundscape in words only or by numbers only. Our hypothesis is that the description of the city soundscape might be successfully done by combination of acoustical numbers and words. This article will propose a method for acoustical characterization of urban areas in the framework of the Belgian federal project: $\mathrm{SD} / \mathrm{TA} / 05 \mathrm{~A}$ Design and renovation of urban public spaces towards sustainable cities (DRUPSSuC).

\section{3:00}

5aNSa14. Subjective evaluation of community noise in Canada's National Capital Region and its relation to waking levels of salivary biomarkers. David S. Michaud (Health Canada, 775 Brookfield Road, Ottawa, ON, Ottawa, ON K1A1C1, Canada, dmichaud@hc-sc.gc.ca), Stephen E. Keith (Health Canada, 775 Brookfield Road, Ottawa, ON, Ottawa, ON K1A1C1, Canada, skeith@hc-sc.gc.ca), Jason Tsang (Health Canada, 775 Brookfield Road, Ottawa, ON, Ottawa, ON K1A1C1, Canada, jtsang@hc-sc.gc.ca), Anne Konkle (Health Canada, 50 Columbine Driveway, Tunney's Pasture, Ottawa, ON K1AOK9, Canada, akonkle@hc-sc.gc .ca)

Some research has suggested an association between long-term exposure to traffic noise and relative risk of cardiovascular disease (Babisch et al., 2005; Willich et al., 2006). It has been assumed that noise may act as a nonspecific stressor. Acute exposure to noise can evoke physiological and behavioural changes reminiscent of a stress response in rodents (Michaud et al., 2003), but it is equivocal that this occurs in humans chronically exposed to traffic noise. This pilot project examined annoyance to community noise and salivary biomarkers known to be influenced by stressor exposure. A face-to-face interview subjectively assessed community noise for 60 residents (30 males, 30 females; mean age 41.3, SD = 14.98). Traffic sound levels will be determined and respondents categorized into high $(>65 \mathrm{dBA}$, Leq24) and low $(<50 \mathrm{dBA})$ noise areas. Participants also provided saliva samples upon awakening, 30-min after awakening, and prior to bedtime. Concentrations of salivary biomarkers of alpha-amylase and cortisol were spectrophotometrically determined using commercial enzyme-linked immunosorbant assays. Two-way (high-noise versus low-noise) mixed-model analyses of variance will examine differences in questionnaire and salivary responses. Sex differences will be evaluated with independent t-tests, and polynomial regression analyses will relate salivary biomarker levels to highor low-noise areas.

\section{3:20}

5aNSa15. Processing of long-term acoustic measurements. Laurent Faiget (01dB-Metravib, 200 Chemin des Ormeaux, F-69578 Limonest, France, laurent.faiget@01db-metravib.com), Benoit Plassat (01dBMetravib, 200 Chemin des Ormeaux, F-69578 Limonest, France, benoit.plassat@01db-metravib.com), Stéphane Bloquet (01dB-Metravib, 200 Chemin des Ormeaux, F-69578 Limonest, France, stephane .bloquet@01db-metravib.com), Christine Aujard (01dB-Metravib, 200 Chemin des Ormeaux, F-69578 Limonest, France, christine.aujard@01dbmetravib.com)

Many standards and European directives relative to noise are used to assess an acoustic situation, based on long-term indicators such as Lday, Lnight or Levening. These indicators are defined mainly as the mean yearly equivalent acoustic level on a particular part of the day. Acoustic measurements are performed on reference periods representative of the typical acoustic situation. An Oper@ monitoring system performed measurements for two years on several points. An acoustic database of the one-second integration time measurement was created. Different kinds of calculations were performed on these data, the variant being the measurement time considered. This study evaluates the convergence of the classic equivalent level with these long-term indicators. 
3:40

5aNSa16. The association between residential quality of life and aircraft noise annoyance around Frankfurt Airport. Dirk Schreckenberg (ZEUS GmbH, Sennbrink 46, 58093 Hagen, Germany, schreckenberg@zeusgmbh.de)

A large field study on aircraft noise annoyance with 2312 residents from 66 areas within a radius of $40 \mathrm{~km}$ around Frankfurt Airport was performed between the announcement and implementation of the airport extension (4th runway). For the address of each participant aircraft noise levels were calculated. This presentation concentrates on results with regard to the relationship between perceived - not noise-related - environmental situation and social quality of life in the residential area (i.e. infrastructure, economic development, neighbours, attractiveness of the area) and aircraft noise annoyance. Furthermore the impact of actual perceived residential situation and the expected future situation after airport extension on noise annoyance before the change in aircraft noise exposure occurs is compared. Results of the analyses will be presented and discussed.

\section{4:00-4:20 Break}

\section{4:20}

5aNSa17. Ergonomics of intern environments: privacy and reactions to noise pollution. G. Mario Mattia (Bruel Acoustics S.r.l. - EuroAcustici, Viale Cesare Pavese 304, 00144 Roma, Italy, m.mattia@euroacustici.org) , Per V. Bruel (Bruel Acoustics S.r.l. - EuroAcustici, Viale Cesare Pavese 304, 00144 Roma, Italy, bruel@mclink.it)

The acoustical ergonomic is an important target for any room acoustics, intelligibility and community noise. The authors explain the experience on metrological and neuropsychological approach to the human reaction to noise. Environmental sound quality, room acoustics and interference of perceived sound (interference with the privacy) can start subjective effects and strong reaction by humans. The author explains experience and research of human reactions on noise interference, privacy, stress, ... annoying sounds (also with very low levels), rooms with stationary waves, long reverberation time, low acoustical building insulation, are the first cause of complain from the community. We suggest noise limits, international standards, neuropsychological interpretation of the acoustical annoyance.

\section{4:40}

5aNSa18. Noise annoyance indicators for various industrial noise sources: Results and comparisons. Marion Alayrac (EDF R\&D, 1 avenue général de Gaulle, 92141 Clamart Cedex, France, marion.alayrac@edf.fr), Stéphanie Viollon (EDF R\&D, 1 avenue général de Gaulle, 92141 Clamart Cedex, France, stephanie.viollon @edf.fr), Catherine Marquis-Favre (Université de Lyon, Ecole Nationale des Travaux Publics de l'Etat, CNRS, URA 1652, Département Génie Civil et Bâtiment, 3, rue Maurice Audin, 69120 Vaulx-en-velin, France, Catherine .MARQUIS@entpe.fr)

Characterizing the environmental impact of an industrial plant goes through a better understanding of the noise annoyance caused by industrial noise sources, which are numerous and various. A first step was to construct a perceptive typology of various industrial noise sources from a categorization test and based on perceived similarities. Now, laboratory tests are carried out, for each perceptive category separately, through the same experimental procedure, by testing the influence of the sound pressure level and of the noise spectral characteristics (low frequency noise, tonal noiseœ) on noise annoyance. The effect of spectral modifications due to the noise propagation on noise annoyance is also assessed. Subjects have also to judge the annoyance induced by an ambient noise exposure, built with a background noise and an industrial noise for which the emergence level is controlled. Different types of background noises are studied. Through a statistical analysis, indicators are developed for each perceptive category, based on classical indicators such as sound pressure level or loudness (for instance), or on indicators improved to suit spectral characteristics noticed. In this paper, the results of those listening tests are detailed and compared.

\section{5:00}

5aNSa19. Soundscape characterization in selected areas of Central London. Gianluca Memoli (Imperial College London, Department of Chemical Engineering, Exhibition road, SW7 2AZ London, UK, g.memoli@imperial.ac.uk), Alan Bloomfield (Greater London Authority (GLA), City Hall, The Queen's Walk, SE1 2AA London, UK, Alan.Bloomfield@london.gov.uk), Max Dixon (Greater London Authority (GLA), City Hall, The Queen's Walk, SE1 2AA London, UK, max.dixon @ london.gov.uk)

The Mayor of London's Ambient Noise Strategy 'Sounder City' is the first UK public policy document to promote, not just noise reduction, but positive soundscape management. For its prescriptions to be put into full effect, psychoacoustic methods are needed to characterize existing areas where action may be needed to counteract existing noise pollution. To this aim, the soundscape characterization of two different areas in Central London will be presented in this work, where the two selected parks have in common the presence of a heavily trafficked road nearby. The acoustical experience of passers-by will be mapped using an indicator related to the time history of sound energy, related in previous studies to people's perceptions. Comparison with the same description performed by classical psychoacoustic parameters and perspectives for innovative, positive soundscape based actions will be discussed.

\section{$5: 20$}

5aNSa20. Social survey on the road traffic noise effect on the quality of life in Moroccan cities. Weam Kharbaoui (Ecole Supérieure de Technologie de Salé, Laboratoire Energétique Matériaux et Environnement, Avenue du Prince Héritier, BP 227, 10000 Salé, Morocco, alex_weam @yahoo.es), Mohammed Garoum (Ecole Supérieure de Technologie de Salé, Laboratoire Energétique Matériaux et Environnement, Avenue du Prince Héritier, BP 227, 10000 Salé, Morocco, garoum1 $@$ yahoo.fr), Abdelaziz Bahoussa (Ecole Supérieure de Technologie de Salé, Laboratoire Energétique Matériaux et Environnement, Avenue du Prince Héritier, BP 227, Salé, Morocco, abahoussa@yahoo.fr), Mohammed Rhachi (Ecole Supérieure de Technologie de Salé, Laboratoire Energétique Matériaux et Environnement, Avenue du Prince Héritier, BP 227, 10000 Salé, Morocco,mrhachi@hotmail.com)

Because of the fast growth of the urbanisation, the noise road traffic causes annoyance, and a wide range of negative effects on the health in many Moroccan urban areas. This problem becomes so serious that it is necessary to develop an adequate global noise policies, regulations, and laws. Unfortunately there is a lack of sufficiently technical, scientific, and social data allowing a precise evaluation of this pollution. The objective of this work is to complete our preliminary social survey and make a further step in the evaluation of the Moroccan urban community response to road traffic noise. The questionnaire used contains 55 closed and open questions and was performed using the ISO-15666 recommendations. The sample was composed of 1200 adults chosen randomly in Rabat, Salé and Tetouen cities. The targeted population consisted on persons of both sexes of 18 and more years old. According to the road traffic condition in Moroccan cities, results were analysed and compared with those obtained in several European cities.

\section{$5: 40$}

5aNSa21. Advanced acoustical material tuned for low frequency noise reduction: a case study. Kathleen Kondylas (NEVA Associates Noise Control, 15 Beck Street, Newburyport, MA 01950, USA kondylas@earthlink.net), Natalia Levit (DuPont, 5401 Jefferson Davis Highway, Richmond, VA 23234, USA, natalia.v.levit@usa.dupont .com), Joseph A. King (DuPont, 5401 Jefferson Davis Highway, Richmond, VA 23234, USA, joseph.a.king@usa.dupont.com), Chris R Fuller (Virginia Tech, 131 Durham Hall, Blacksburg, VA 24061, USA, christopher.r.fuller@nasa.gov)

Low frequency noise is a significant annoyance at very low amplitudes, particularly when outdoor community noise from mechanical equipment penetrates commercial and residential building structures. Mid and high frequencies are selectively attenuated by the exterior walls. However, mitigation of the low frequency noise has been very challenging due to the longer wavelength and higher energy. This work presents case studies of an ad- 
vanced acoustical material in typical metal panels to mitigate community noise from outdoor mechanical equipment. These advanced materials were developed with a unique combination of patented acoustical and vibration absorbing technology, based of distributed absorbers, layered with standard acoustic materials. The materials provide enhanced low frequency noise (below $300 \mathrm{~Hz}$ ) over state of the art $10 \mathrm{~cm}$ metal panels filled with the fiberglass absorber. The case studies demonstrate superior acoustic performance in low frequency without substantial increase in weight.

\section{6:00}

5aNSa22. A study on the adaptation of soundscape to covered spaces: Part 2. Asli Ozcevik (Yildiz Technical University Faculty of Architecture, Chair of Building Physics, Besiktas, 34349 Istanbul, Turkey, aozcevik@hotmail.com), Zerhan Yuksel Can (Yildiz Technical University
Faculty of Architecture, Chair of Building Physics, Besiktas, 34349 Istanbul, Turkey, karabi@yildiz.edu.tr)

This study is the second part of a research on the Adaptation of soundscape to covered spaces. A modern and a historical shopping center were compared and evaluated by means of subjective and objective evaluations. The consistency of the objective (time histories) and subjective (listening tests) findings showed that covered commercial areas may have a specific sound environment. The formation of streets is generally the basic spatial design concept that connects open and closed shopping areas. Therefore one of the studies that authors proposed at the previous paper was the comparison of open and closed shopping areas. In this study the soundscapes of a modern and a traditional street having shopping as a basic function will be determined by soundwalks and listening tests, and the findings will be compared with those of the previous studies.

FRIDAY MORNING, 4 JULY 2008

ROOM 251, 8:00 TO 10:00 A.M.

\title{
Session 5aNSb
}

\section{Noise, Architectural Acoustics, and EURONOISE: Noise, Vibration and Acoustics for Medical and Research Facilities and Their Occupants III}

\author{
James West, Cochair \\ Johns Hopkins University, Department of Electrical Engineering, 3400 North Charles Street, Baltimore, MD 21218, USA
}

Jack B. Evans, Cochair

JEAcoustics, 1705 West Koenig Lane, Austin, TX 78756, USA

Marc Asselineau, Cochair

Peutz \& Associés, 10 rue des Messageries, Paris, F75010, France

Erica Ryherd, Cochair

Woodruff School of Mechanical Engineering, Georgia Institute of Technology, Atlanta, GA 30332-0405, USA

\section{Invited Papers}

\section{8:00}

5aNSb1. Realistic floor vibration criteria for MRI operation: A compilation of case studies. Basel Jurdy (Sparling, 720 Olive Way Suite 1400, Seattle, WA 98101-1853, USA, bjurdy@sparling.com), Michael Yantis (Sparling, 720 Olive Way Suite 1400, Seattle, WA 98101-1853, USA, myantis@ sparling.com)

Vibration criteria published by various magnetic resonance imaging (MRI) manufacturers have been found to be too conservative with respect to allowable floor vibration resulting from MRI operation. The floor vibration produced by the MRI operation is greater than the published criteria. Previous experience by the authors has shown that there is an allowable threshold for MRI operation induced vibration, above which the MRI will not operate properly. This paper reports results of a survey of structural floor systems for $1.5 \mathrm{~T}$ and 3.0T MRIs that are working satisfactorily. The most common floor systems have been analyzed structurally using finite element analysis to determine the forced response to a standardized input. Measurement results for some of the floors during MRI operation is also included.

5aNSb2. Vibration case study - design challenges for meeting medical imaging manufacturer specifications. Chris Papadimos (Papadimos Group, 818 Fifth Avenue, Suite 207, San Rafael, CA 94901, USA, chris@ papadimosgroup.com)

Manufacturer vibration specifications for medical imaging equipment impose formidable challenges particularly when locating such equipment on elevated floors, which is increasingly desirable for work flow and proximity to operating rooms and other support spaces in healthcare facilities. Urban sites can further complicate matters as such sites tend to often be near busy roads that are sources of vibration. Other internal sources of vibration that must be taken into consideration include occupant activities and operating building mechanical systems. All these conflicting interests need to be properly balanced and clearly evaluated; developing solutions requires a diligent and experienced design team. Some of the challenges that had to be overcome in order to develop vibration designs to accom- 
modate medical imaging equipment such as CT Scanners and MRI's on elevated floors of a new healthcare facility are presented in this paper. Extensive site testing to evaluate design constraints and pre-existing conditions, avoidance of incompatible adjacencies and proper considerations in terms of space layouts, development of specialized floor designs and a clear understanding of imaging equipment manufacturer specifications are the key elements addressed in this paper.

\section{Contributed Paper}

8:40

5aNSb3. Noise in an Emergency treatment ward. Sergio Beristain (Mexican Institute of Acoustics, P.O. Box 12-1022, Narvarte, 03001 Mexico, D.F., Mexico, sberista@ hotmail.com)

It is beign assessed the noise inside a typical social service Hospital in Mexico City, several sources and relevant areas have been identified and evaluated. This paper presents a detailed description of the Adults Urgency
Room, where all the emergency cases are received and attended arround the clock. In this section of the hospital, forty to sixty patients are attended at the same time in a large open plan room, separated only by curtains, with only a few private rooms for doctors in order to attend incoming patients, trying to solve each case immediatelly, allow him/her entrance into the main room for full emergency treatment, or find a bed inside the hospital for a longer treatment. Noise sourcees are all over the place, and noise variablity is large.

\section{Invited Papers}

\section{9:00}

5aNSb4. Study case of a centre for biomedical analyses: the importance of an acoustic analysis of the current situation and definition of the targets to be achieved. Fabien Krajcarz (Gamba Acoustique, 2 rue de la Découverte, BP 163, 31676 Labege Cedex, France, fabien.krajcarz@acoustique-gamba.fr)

The French regulations concerning the limits on noise levels in medical establishments include the acoustic performances of existing buildings used to accommodate defined medical activities and in which the regulations are normally observed. Nevertheless, there are cases which are either outside the list of the buildings covered, or merit special treatment. One example is described here: a centre for biomedical analyses in a large regional teaching hospital covering a technical area of several hundreds square meters. This concentration of human activities and technical equipment without any acoustic attenuation measures was the spark for a considerable labour movement. In this article we describe the challenges of these situations, the pitfalls to be avoided and any post-project and design stage measures to be implemented.

\section{9:20}

5aNSb5. Use of a versatile acoustic room for audiology applications. Chantal Laroche (University of Ottawa, 451 Smyth Road, room 3062, Guindon Hall, Ottawa, ON K1H 8M5, Canada, claroche@uottawa.ca), Christian Giguère (University of Ottawa, 451 Smyth Road, room 3062, Guindon Hall, Ottawa, ON K1H 8M5, Canada, cgiguere@uottawa.ca), Véronique Vaillancourt (University of Ottawa, 451 Smyth Road, room 3062, Guindon Hall, Ottawa, ON K1H 8M5, Canada, vaillancourt@mail.health.uottawa.ca)

For several decades, hearing tests have been carried out in audiometric test rooms which must meet acoustical requirements set forth in standards on ambient noise levels (i.e., ANSI S3.1-1999 (R2003)). It is however generally accepted that acoustical environments in which individuals with hearing loss function daily seldom correspond to environments found in audiometric rooms. Constraints regarding the sound attenuation by room walls (i.e., costs) can thus be minimized with a more rigorous control over certain acoustical characteristics within the sound room (i.e., reverberation time), thereby allowing to recreate more realistic environments of daily living. During the planning stage of the Research Unit on Noise and Communication at the University of Ottawa, the researchers opted for a versatile acoustic room with reversible panels that not only offers adequate sound attenuation by walls but also some control over reverberation time, thereby allowing to recreate more typical acoustic environments. The characteristics of this versatile acoustic room, as well as examples describing applications of use (effect of repetition on speech recognition, attenuation by headphones used with portable audio systems, effect of hearing protection on speech recognition within military noise environments) will be presented.

\section{Contributed Paper}

\section{9:40}

5aNSb6. NMR sensitivity to floor vibration. Yuval Ben-Dov (IDE, 68 Mazzeo Drive, Randolph, MA 02368, USA, yuval.ben-dov@ideworld .com), Peter Heiland (IDE, 68 Mazzeo Drive, Randolph, MA 02368, USA, peter.heiland@ideworld.com), Srinivasan Rajan (Novartis Institutes for BioMedical Research, 250 Massachusetts Avenue, Building \#600 Room \# 6C-172, Cambridge, MA 02139, USA, srinivasan.rajan@novartis.com)

NMR is widely used in the determination of molecular structures in solution. Floor vibrations interfere with the NMR measurements and are coupled with the signal of interest. To minimize the effect of floor vibra- tions, NMR spectrometers are typically installed on a solid floor with minimal structurally induced vibrations. However, in some cases this is not possible, and the tool is exposed to structural vibrations. We study the NMR sensitivity to floor vibrations. A Bruker $500 \mathrm{MHz}$ installed on IDE active isolation platform is used. By exciting the platform in a controlled manner we can measure the noise as observed in the NMR spectrum due to the platform vibrations. The sensitivity highly depends on the excitation frequency, and is not well reflected in a typical requirements specification. Revised requirements are presented for the floor vibration both for a tool installed as is, and for a tool on top of our isolation platform. 


\title{
Session 5aNSc
}

\section{Noise and EURONOISE: Acoustic Performance of Energy Efficient Building Products I}

\author{
Anthony Hoover, Cochair \\ McKay Conant Hoover, Inc., 5655 Lindero Canyon Road, Suite 325, Westlake Village, CA 91362, USA \\ Jacques Roland, Cochair \\ Centre Scientifique et Technique du Bâtiment, 24 rue Joseph Fourier, Saint Martin d'Hères, 38400, France
}

\section{Invited Papers}

\section{8:00}

5aNSc1. Buildings thermal and acoustics insulation: friends or enemies. Jacques Roland (Centre Scientifique et Technique du Bâtiment, 24 rue Joseph Fourier, 38400 Saint Martin d’Hères, France, jacques.roland@cstb.fr)

The increasing concern on climate changes and greenhouse gas accumulation has urged most of countries to enforce new thermal regulation to improve the energy efficiency of existing and new buildings. This improvement shall not at least induce a reduction of the acoustic comfort, and possibly increase it by a careful choice of products and a relevant design. The new products and technologies involve the building envelop insulation, the thermal bridges suppression, the improvement of air conditioning efficiency, and the renewable energy sources attached to the building. The author will review these new products and technologies and show what are the expected benefit or threat for the acoustic performance of buildings. This will include triple glazing, bulk insulating materials, natural fibrous materials, vacuum insulation panels, phase change materials, thermal bridge isolators, integrated photovoltaic cells, light weight construction, passive house concept, heat pumps, and air conditioning with heat exchangers.

\section{$8: 20$}

5aNSc2. Acoustic behavior of triple glazings. Marc Rehfeld (Saint Gobain Glass, CRDC, B.P. 40103, 60777 Thourotte Cedex, France, marc.rehfeld@saint-gobain.com), David Fournier (Saint Gobain Glass, CRDC, B.P. 40103, 60777 Thourotte Cedex, France, david.fournier@saint-gobain.com)

Making of triple glazings is the only way to still improve thermal performances of Insulating Glass Units. Possible ways with double glazings are already in use: increase the space between glasses, use low emissivity coatings and special gas with lower thermal conductivity as argon or krypton. Specific acoustic weak point of double glazings is the resonance between the two panes which works as a mass spring mass system, and coupling of eigenmodes of panes through the air (gas) cavity. These phenomena are of course still more important with triple glazings, as there are two resonances. The paper will give all comparative data concerning thermal and acoustic performances, and describe a way to achieve the same single number values of sound transmission loss with triple glazing that with double glazing by adding absorption in the gas cavities.

\section{Contributed Paper}

\section{8:40}

5aNSc3. Acoustic design criteria for naturally ventilated buildings. Chris D. Field (Arup Acoustics, 901 Market Street, Suite 260, San Francisco, CA 94103, USA, chris.field@arup.com)

This paper proposes satisfactory indoor noise level criteria for office buildings which are compatible with achieving minimum natural ventilation standards set out in green building rating systems for sustainable building design. Indoor air quality standards related to the use of natural ventilation in buildings conflict with the control of ingress of external noise through ventilation openings to meet internationally recognized background noise limits for building use. These standards generally assume, however, that buildings are sealed and air conditioned to meet the stated recommended indoor noise levels. It is not feasible, however, that these noise standards can be expected or are appropriate to be achieved in naturally ventilated buildings. Therefore, to account for the thermal comfort benefit of natural ventilation and the ability to locally control natural ventilation and noise levels by closing of windows, an allowable exceedance of the recommended indoor noise levels is explored. The allowable deviation from existing background noise level guidelines is determined, which is considered to be an acceptable compromise for increased thermal comfort.

\section{Invited Paper}

\section{9:00}

5aNSc4. $\mathbf{R}+\mathrm{D}$ for the integration of high acoustic-thermal performances in spanish building products. Marta Fuente (LabeinTecnalia, C/Geldo - Parque Tecnológico de Bizkaia, Edificio 700, 48160 Derio, Spain, mfuente@labein.es)

Many EU countries are introducing new national regulations aimed at complying with the European Directive "Energy Performance of Buildings" (EPBD). Subsequently, in Spain a new normative has been recently approved (CTE-HE), compiling new requirements for energy saving in buildings. In this framework, energy efficiency considerations will have an increasing impact on the design of buildings and the choice of materials and products. In the same way, the acoustical quality of dwellings in Spain is going to be guaranteed with the compliance of the new Spanish Building Regulation (CTE-HR). Searching a higher level of comfort in dwellings the CTE is in- 
creasing its requirements and is considering the building as a product itself. These new developments involve efforts and resources for the upgrading of the constructive materials and systems, demanding investments for R\&D activities. Therefore manufacturers of the constructive sector in Spain are launching many innovative research strategies for the development of better products, addressing key factors for the energy efficiency of buildings.

\section{Contributed Paper}

9:20

5aNSc5. Sound insulation of traditional roof constructions considering energy efficiency requirements. Beáta Mesterházy (Budapest University of Technology and Economics, Lab. of Building Acoustics, Mûegyetem rakpart 3., 1111 Budapest, Hungary, bmester@mail.bme.hu), Frigyes Reis (Budapest University of Technology and Economics, Lab. of Building Acoustics, Mûegyetem rakpart 3., 1111 Budapest, Hungary, reis@mail.bme.hu), Gábor Szlávik (URSA Salgótarján zRt., Budapesti út 31, 3104 Salgótarján, Hungary, gabor.szlavik@uralita.com)

The efforts for energy efficiency result changes in building constructions, also in case of roof constructions. The most important constructional conclusion is the growth of the thickness of the heat insulating layer. Beside heat insulation, inner ventilation between the layers is the other important parameter, which has a great effect on sound insulation behaviour. In regular product information sheets, either in internet or in printed forms, the effect of these later parameter is not present and this way the laboratory data do not show the reality. For the purpose of modeling the reality, a new installation method has been developed, which considers the effects of ventilation usually used in our climate conditions. This new installation method has been realised and used in laboratory experiments. The results based on the new installation method approach the reality better and reflect the acoustical importance of the certain constructional components.

9:40-10:40 Posters

Lecture sessions will recess for presentation of poster papers on various topics in acoustics. See poster sessions for topics and abstracts.

\section{Invited Paper}

\section{0:40}

5aNSc6. Acoustic performance of membrane based multilayered systems with improved thermal inertia characteristics. Catherine Guigou-Carter (CSTB, 24, rue Joseph Fourier, 38400 Saint Martin D’Hères, France, catherine.guigou@cstb.fr), Hebert Sallee (CSTB, 24, rue Joseph Fourier, 38400 Saint Martin D’Hères, France, hebert.sallee@cstb.fr), Xavier Normand (IFTH, Avenue Guy de Collongue, 69134 Ecully Cedex, France, xnormand@ifth.org)

In this paper the development of double membrane based systems, including phase change materials (denoted-PCM) is discussed. The cavity between the two membranes is filled with a new type of absorbing material: a 3D, nonwoven, complex structure made of two or three fibrous mats that allows the inclusion of small granulated particles while being fabricated. Granulated phase change materials are therefore included in this $3 \mathrm{D}$, nonwoven structure in order to increase thermal properties by the heat storage/restoring principle. The PCMs introduced in this nonwoven structure act to store part of the heat when it is in excess, and then, to restore it when the temperature inside the room is too low. The intermediate temperature chosen to correspond to a desired comfort temperature (for example around 20 ${ }^{\circ} \mathrm{C}$ ) corresponds to the phase transition temperature of the PCM. Lightweight membranes are then fixed on each side of this complex 3D, nonwoven structure, including the PCMs. The acoustic performance of such systems is investigated in terms of the sound transmission loss, both experimentally and analytically. The thermal performance is also briefly presented.

\section{Contributed Paper}

11:00

5aNSc7. Sound transmission loss of vacuum insulation panels. Waldemar Maysenhölder (Fraunhofer Institute for Building Physics, Dept. of Acoustics, Nobelstraße 12, 70569 Stuttgart, Germany, maysenhoelder @ibp.fraunhofer.de)

The sound transmission loss of a variety of vacuum insulation panels (VIP) has been measured in the laboratory. Core materials of the VIP include silica, polyurethane, micro fleece, and glass fibers. Mass law behavior and minima due to bending-wave coincidence and thickness resonance have been observed. Destroying the vacuum may reduce the sound insulation drastically. Since in practical applications the VIP need protection, the predominant interest lies in the transmission loss of multilayered assemblies, e.g., VIP sandwiched between aluminum plates. Calculations performed by the software LAYERS show that with additional "decoupling" layers (air, rubber) the coincidence dip can be avoided or shifted to higher frequencies, where it is less critical. Preliminary experimental realizations indicate the important role of a proper gluing of the layers. According to further LAYERS calculations, attachment of VIP to massive walls and protection by a plaster layer does not change the transmission loss of the wall appreciably, except for the VIP with the soft polyurethane core material.

\section{Invited Papers}

\section{$11: 20$}

5aNSc8. Vibration reduction of thermal break balcony connections. Martin Schneider (University of Applied Sciences, Schellingstr. 24, 70174 Stuttgart, Germany, martin.schneider@hft-stuttgart.de), Heinz-Martin Fischer (University of Applied Sciences, Schellingstr. 24, 70174 Stuttgart, Germany, heinz-martin.fischer@hft-stuttgart.de)

In energy efficient buildings concrete balconies have to be thermally insulated from the concrete floor to reduce heat loss and to increase surface temperature. This thermal break is obtained by polystyrene in combination with high-strength concrete thrust-force bearings and an arrangement of stainless steel straight and bent bars to act as tension and shear reinforcement. The acoustic performance of such thermal break units was investigated under laboratory conditions. Therefore the vibration level difference at different junctions 
between balcony and the thermal isolated concrete floor as well as the normalized impact sound pressure level of the floor when the balcony is excited with the standard tapping machine are measured. The influence of the number of tension and shear steel bars and the type of thermal insulating material was studied on typical sized balconies connected to small test floors. The vibration isolation at low frequencies was determined using modal testing.

\section{1:40}

5aNSc9. Using high light reflectance acoustical ceilings to increase the energy efficiency of buildings. Marsha S. Bischel (Armstrong Building Products, 2500 Columbia Ave, Lancaster, PA 17603, USA, msbischel@armstrong.com), William E. Beakes (Armstrong Building Products, Retired, 4057 Woodcrest Lane, Columbia, 17512, USA, beakeswe@embarqmail.com)

Acoustical ceilings with white, highly light-reflecting surfaces can be used as an effective, passive means to significantly improve the energy efficiency of a space. When a ceiling with high light reflectance is coupled with an indirect lighting system, illumination can be increased by $20 \%$, or the energy directly associated with lighting can be reduced by $20 \%$. In addition, lower lighting loads can reduce cooling costs by $7 \%$. A highly light reflective ceiling can also be used to reflect daylight into a space, increasing the amount of light in interior areas away from the windows by as much as $20 \%$; alternatively, the surface area of windows may be reduced by $14 \%$ while maintaining the same illumination levels. In all cases, the increased reflectance of light allows the number of fixtures to be decreased, further increasing the savings related to both energy and maintenance. The percentage of energy savings associated with lighting is proportional to the percentage increase in the light reflectance value; therefore, even small increases in light reflectance provide increases in energy efficiency. When the acoustical performance of two products is comparable, light reflectance could be a critical differentiator in determining total building performance and occupant comfort.

\section{2:00}

5aNSc10. High sound and thermal insulation constructions with dB-deck. Bart Ingelaere (BBRI, rue du Lombard, 42, B-1000 Brussel, Belgium, bart.ingelaere@bbri.be), Gerrit Vermeir (Lab. ATF, Katholieke Universiteit Leuven, Celestijnenlaan 200D, B-3001 Leuven, Belgium, Gerrit.Vermeir@bwk.kuleuven.be), Debby Wuyts (BBRI, rue du Lombard, 42, B-1000 Brussel, Belgium, debby .wuyts@bbri.be)

The new Belgian requirements demand DnT,w $\geqslant 58 \mathrm{~dB}$ and L'nT,w $\leqslant 50 \mathrm{~dB}$ between apartments for enhanced acoustic comfort. At the same time the Energy Performance Regulations impose strict requirements for the thermal insulation between apartments. Higher demands on sound insulation can be realized in practice by using higher surface weights for walls and floors and by applying failure free floating floors. However requiring higher weights for floors is in contradiction with the technique of concrete hollow floor elements. A thermal and acoustical solution was found in the development of dB-deck, a prefab double floor element with intermediate supporting elastic pads, cutting structural vibration paths between superposed apartments. In combination with double walls without anchors (allowing for a high sound reduction index and no structural transmission in the horizontal sense), this construction technique allowed for DnT,w $>63 \mathrm{~dB}$ and $\mathrm{L}$ 'nT,w $<47 \mathrm{~dB}$ without a floating floor in experimental setups This solution was developed by a team of the BBRI, KULeuven, and CDM for an important producer of hollow floor elements. This technique has now been used for real constructions. The paper presents technical details, the results of the acoustical measurements and the acoustical advantages and difficulties met in the construction of this apartment block.

\section{2:20}

5aNSc11. The effect of additional thermal lining on the acoustic performance of a wall. Jacek Nurzynski (Building Research Institute, Filtrowa Str. 1, 00-611 Warsaw, Poland, j.nurzynski@itb.pl)

External thermal insulation systems (ETICS) are commonly used in Poland mainly for thermorenovation of multifamily residential buildings constructed in seventies using industrialized methods. Additional layer improves thermal performance of a wall but also causes decrease of sound insulation in certain frequency range. Usually the acoustic effect of thermorenovation is unnoticed by inhabitants, but in the case of noisy locations it is sometimes perceived as an appearance of a new tonal outside noise source. The paper presents results of investigation on the influence of additional lining on the acoustic performance of massive wall based on laboratory tests. Resonance frequency, its location and influence on single number quantities is discussed. The problem of sound reduction index improvement prediction acc. to EN 12354-1, separate acoustical characteristics of lining and testing acc. to ISO 140-16 is also considered.

\section{2:40}

5aNSc12. Development of thermoacoustic floating floors for use between parking and dwellings. Catherine Guigou-Carter (CSTB, 24, rue Joseph Fourier, 38400 Saint Martin D’Hères, France, catherine.guigou@cstb.fr), Jean-Baptiste Chene (CSTB, 84, Avenue Jean-Jaurès, 77447 Marne-la-Vallée Cedex 2, France, jean-baptiste.chene@ cstb.fr)

The French thermal regulation (RT2005) is favoring thermal floating floor between spaces such as parking or stores, and dwelling units. However, these solutions do not fulfill the French acoustic regulation with regards to airborne noise. This paper discusses the development of solutions allowing fulfilling both the thermal and the acoustic regulation. To achieve this goal, a mixed approach combining measurements and numerical predictions is used. Furthermore, the laboratory characterization of such floating systems usually involves a concrete base floor $140 \mathrm{~mm}$ in thickness (following the NF EN 140-8 standard) and a floating concrete layer $40 \mathrm{~mm}$ in thickness. However, in situ the concrete base floor as well as the floating concrete layer can be thicker. Therefore, some laboratory measurements are performed on a thermal floating floor system combining a $200 \mathrm{~mm}$ thick concrete base floor, a thermal insulation layer (polystyrene or polyurethane based foam for example) and a $60 \mathrm{~mm}$ thick floating concrete layer. The prediction method shows that this type of thermal floating systems is not acceptable with respect to the acoustic regulation. The behaviour of such multilayered systems is investigated using a wave approach based prediction tool in order to develop solutions allowing fulfilling the acoustic regulation. 


\title{
Session 5aNSd
}

\section{Noise, ASA Committee on Standards, Architectural Acoustics, and EURONOISE: Classroom Acoustics I (Poster Session)}

\author{
Louis Sutherland, Cochair
}

Consultant in Acoustics

Luigi Maffei, Cochair

Built Environment Control Laboratory Ri.A.S., Second University of Naples

\begin{abstract}
All posters will be on display from 9:40 a.m. to 11:20 a.m. To allow contributors an opportunity to see other posters, contributors of odd-numbered papers will be at their posters from 9:40 a.m. to 10:30 a.m. and contributors of even-numbered papers will be at their posters from 10:30 a.m. to 11:20 a.m.
\end{abstract}

\section{Contributed Papers}

5aNSd1. Comparisons of predicted and measured values of speech, noise, reverberation and early reflections in optimized classrooms. Sooch San Souci (AiA - Audition, Intelligibility, Acoustics, 35 bis rue de Fay, 77140 St Pierre Les Nemours, France, s.sansouci@wanadoo.fr), Line Guerra (AiA - Audition, Intelligibility, Acoustics, 47 rue Le Corbusier, 92100 Boulogne, France, line.guerra@aiacoustique.com), Richard Campbell (Bang-Campbell Associates, 26-G Chilmark Drive, East Falmouth, MA 02536, USA, rhcamp@rcn.com)

12 newly renovated classrooms have been studied to determine the accuracy of prediction software used to guide architects, acousticians and interior designers. Several variations in renovation techniques based on traditional precepts were compared. After the renovations were complete, measurements and recordings were made of speech, noise, and reverberation when the rooms were both occupied and nonoccupied by students. A systematic map of the results was compiled, which when presented on a seat by seat basis, defined values of merit for the presumed optimizations and were compared to the predicted values. The two principle indices chosen as targets were increases in total speech level and decreases in total noise level (including late reverberation). When referred to these targets, several novel strategies proved to offer higher value/cost ratios while a few traditional strategies proved to be deficient and in some cases detrimental to at least one of the principle indices. Emerging from this study is what appears to be evidence for two points: (1) that some common acoustic criteria are inadequate for classrooms and (2) there are several strategies that might offer significant advantages over some of the traditional guidelines for acoustic treatments for learning spaces.

5aNSd2. The use of U50 to calculate the optimal absorption in a classroom. Lau Nijs (Delft University of Technology, Berlageweg 1, 2628 BG Delft, Netherlands, L.Nijs@tudelft.nl), Monika Rychtarikova (Lab. ATF, Katholieke Universiteit Leuven, Celestijnenlaan 200D, B-3001 Leuven, Belgium, Monika.Rychtarikova@bwk.kuleuven.be), Gerrit Vermeir (Lab. ATF, Katholieke Universiteit Leuven, Celestijnenlaan 200D, B-3001 Leuven, Belgium, Gerrit.Vermeir@bwk.kuleuven.be)
Common measures for the speech intelligibility predict increasing values with increasing absorption in empty classrooms. Measurements confirm this relation, so it seems as if an anechoic room is the best place to teach. However, there are two problems: the sound pressure level at the back row is too low when compared to the levels on the front row or when compared to the noise level caused by pupils plus ventilation systems. A simple method is proposed based on U50, which gives the relation between the sound energy from direct sound (from a teacher or a pupil) plus early reflections on one side and late reflections plus noise on the other. Barron's formula for the sound level decrease in a concert hall is included to predict the sound level drop through the classroom. Model results indicate that the absorption coefficient should be in the order of 20 to $30 \%$ if the signal to noise ratio is in the range between +5 to $-5 \mathrm{~dB}$. These values seem low, but measurements in existing classrooms show low signal to noise levels as well.

5aNSd3. The Nordic voice ergonomic group: legislation and vocal health. Valdis Inigbjörg Jonsdottir (Tad er Malid, Furuvellir 13, 601 Akureyri, Iceland, valdisj@ismennt.is)

Research has revealed a number of factors which have a negative impact on voice function (e.g., activity noise, background sound, poor acoustics, long duration of voice usage, speaking distance). In these circumstances vocal loading increases, potentially giving rise to voice disorders. Currently, practical measures within society for the improvement of speakers' working conditions and the prevention of voice loading are not satisfactory. Voice experts from the five Nordic countries met in Reykjavik in May 2006 to inaugurate the Nordic Voice Ergonomic Group, with the aim of investigating the current situation within voice ergonomics, with a particular focus on sound-related problems. The group found that occupational safety and health legislation relating to speech communication in educational working environment differs between Nordic countries. Moreover, this legislation does not specifically deal with speech communication or the risk of voice disorders. This presentation will report on the details of the current legislation and the Group's suggestions for legislative change which might prevent disturbance in speech communication and decrease the risk of vocal disorder as an occupational hazard. 


\title{
Session 5aNSe
}

\section{Noise and EURONOISE: Examples of Noise Control I (Poster Session)}

\author{
Brigitte Schulte-Fortkamp, Cochair \\ Pierre Etienne Gautier, Cochair \\ SNCF DIR
}

TU Berlin, Institute of Fluid Mechanics and Engineering Acoustics

\begin{abstract}
All posters will be on display from 9:40 a.m. to 11:20 a.m. To allow contributors an opportunity to see other posters, contributors of odd-numbered papers will be at their posters from 9:40 a.m. to 10:30 a.m. and contributors of even-numbered papers will be at their posters from 10:30 a.m. to 11:20 a.m.
\end{abstract}

\section{Contributed Papers}

5aNSe1. Jet noise prediction using RANS CFD input. Magdi Omais (Airbus/ONERA, 64 avenue de Paris, 92320 Châtillon, France, magdi.omais@onera.fr), Stephane Redonnet (ONERA (French aerospace Center), CFD \& Aeroacoustics Department, BP 72, 29 avenue de la division Leclerc, 92322 Chatillon Cedex, France, stephane.redonnet @ onera.fr), Bastien Caruelle (Airbus S.A.S, Department of Acoustic \& Environment, 316 route de Bayonne, 31000 Toulouse, France, bastien.caruelle@airbus.com), Eric Manoha (ONERA (French aerospace Center), CFD \& Aeroacoustics Department, BP 72, 29 avenue de la division Leclerc, 92322 Chatillon Cedex, France, eric.manoha@onera.fr)

In the present work, a volume source model based on the SNGR approach, first proposed by Bailly, has been implemented and developed for jet noise prediction purposes. This method presenting the advantage of providing unsteady three-dimensional turbulent data from a RANS computation, it shall be compatible with industrial development processes. First, the theoretical aspects of the original SNGR model were revisited. More particularly, analytical developments showed that considerable modifications were needed to take local flow effects into account while generating unsteady turbulent data. Moreover, the way to include anisotropy effects in the model, as originally proposed by Billson, was improved by using a nonlinear extension of Boussinesq approximation to recover the local Reynolds stress tensor. In a second step, the potentialities of the stochastic tool for jet noise simulations have been evaluated against existing numerical and experimental databases. In addition to that concern, its sensitivity to a slight modification of the RANS input data was studied. As a last step, the possibility of accounting for high frequency dynamics in reasonable CPU times has been deeply investigated. The main results obtained from this study show that the stochastic approach should be a promising method for future applications to industrial nozzle configurations.

5aNSe2. The primary noise sources associated with turbulent Coanda wall jets. Caroline Lubert (James Madison University, Department of Mathematics \& Statistics, MSC 1911, Harrisonburg, VA 22807, USA, lubertcp@jmu.edu)

A wide variety of aeronautical and aerospace applications utilize the Coanda effect. However, due to the enhanced turbulence levels observed with devices using this effect, turbulent mixing noise is a primary noise source. For supersonic jets close to Coanda surfaces, shock-associated noise (both broadband and screech tones) is also a significant source of sound. A theory has been developed to predict the turbulent mixing noise emitted by unit-volume of jet-type shear-layer turbulence close to a rigid plane, and extended to a plane two-dimensional wall-jet. However, most flows of practical interest are three-dimensional and, in the case of the Coanda jets, exhibit significant curvature. This paper extends the previous models for turbulent mixing noise to predict the aeroacoustic characteristics of a three- dimensional turbulent flow over a Coanda surface. Details of experimental observations of the shock-cell structure associated with supersonic Coanda wall jets, and a method of modeling the corresponding shock-associated noise, is also discussed.

5aNSe3. Efficiency of shielding effect on installed double stream nozzles. Sébastien Aeberli (SNECMA - groupe SAFRAN, Villaroche Rond-point R. Ravaud, 77550 Moissy-Cramayel, France, sebastien.aeberli @ snecma.fr)

In spite of acoustic improvements on jet noise brought by innovative technologies such as chevrons, a way to ensure important acoustic gains is to optimize the power plant integration on aircrafts in order to benefit of the shielding effect due to wing, empennage and fuselage surfaces. In this presentation, these effects are illustrated thanks to a jet noise test campaign at 1/10th scale in an anechoic chamber as part of a European project. This campaign gives a better understanding of shielding effect by a wing and also of the acoustic impact of pylon azimutal direction on two representative BPR 9 nozzles (Baseline - smooth - and Low Noise - with chevrons). The efficiency of shielding effect on jet noise sources in these configurations is assessed.

5aNSe4. Automatic segmentation of traffic noise. Fernando José Mato-Méndez (Universidad de Vigo, E.T.S.I.Telecomunicación C./ Maxwell S/N, 36200 Vigo (Pontevedra), Spain, fmato@tsc.uvigo.es)

The state of the art in audio source separation reflects a growing interest towards developing tools oriented to speech and music applications. However, its application to the study of noise, and more specifically to the study of traffic noise, is scarce. The source separation in this work environment is a key process for subsequent classification and analysis, and has motivated the present research carried out. This paper is intended to present, therefore, the different strategies followed in the implementation of an automatic segmentator for traffic noise, focusing the research on two main aspects: The removal of components of noise unrelated with the sources of study and, by other side, the detection and separation of audio sources acquired. For this purpose, measures of traffic noise are made and results are analyzed for the running system, achieving a high level of performance in real work conditions.

5aNSe5. Real time evaluation of soft microphones on a local active noise control system. Stelios M. Potirakis (Intracom Defense Electronics, $21 \mathrm{~km}$ Peania-Markopoulo Street, GR-19400 Koropi - Attica, Greece, spoti@intracomdefense.com), Maria Rangoussi (T.E.I. Piraeus/Department of Electronics, 250, Thivon str., GR-12244 Athens-Aigaleo, Greece, 
mariar@teipir.gr),Stylianos Savvaidis(T.E.I. Piraeus/Department of Electronics, 250, Thivon str., GR-12244 Athens-Aigaleo, Greece, ssavaid@teipir.gr), Nikos Zafeiropoulos (T.E.I. Piraeus / Department of Electronics, 250, Thivon str., GR-12244 Athens-Aigaleo, Greece, nikoszafeiropoulos@yahoo.gr)

Systems for the active control of acoustic noise (ANC) rely on measurements of the noise signal in order to produce the required "quiet zone." Soft microphones (SM) are a virtual microphone alternative already proposed by the authors, employing estimates rather than measurements of the noise signal obtained from room impulse responses based on measurements. Therefore, a single microphone can be used to estimate the noise signal at multiple points. This has already been successfully applied on single microphone noise mapping. In the present work, the SM method is evaluated on a real time local ANC system. The proposed scheme is a filtered-s least mean square (FXLMS) based control structure, incorporating SM. Following the proposed system design-simulation with Matlab-Simulink, a three-dimensional enclosure (office-simulating) and a real electroacoustic ANC system based on a TI ${ }^{\circledR}$ TMS320C6713 DSP, developed for this purpose, were built in the laboratory to carry out the experiments. Different options of estimating the SM signals and exploiting them into the control structure are experimentally evaluated as to their ability to enlarge the silence zone and their noise reduction performance around the listener ear zone (using a head and torso simulator) and compared to the standard FXLMS solution.

5aNSe6. Application examples of beamforming method. Andrea Cerniglia (Euroacoustic srl, via Gandhi, 13, 10013 Avigliana, Italy, a.cerniglia@euroacoustic.com)

Acoustical beamfoming technique is a powerful method for noise source investigation, suitable for many different applications. Thanks to this technology is possible to identify where the noise is coming from, in a very clear and understandable form, both in static or dynamic situations. In addition on standard features, some advanced functions can helps to make correlation between subjective and objective parameters, for better understanding of acoustical phenomena. The paper describes some real applications of a very innovative beamforming system, based on a spherical antenna. The described measurements coming from several fields as automotive, enviromental noise, occupational noise, architectural acoustic, noise barriers investigation.

\title{
Session 5aNSf
}

\section{Noise and EURONOISE: Soundscape \& Community Noise II (Poster Session)}

\author{
Bennett Brooks, Cochair \\ Brigitte Schulte-Fortkamp, Cochair \\ TU Berlin, Institute of Fluid Mechanics and Engineering Acoustics
}

\begin{abstract}
All posters will be on display from 9:40 a.m. to 11:20 a.m. To allow contributors an opportunity to see other posters, contributors of odd-numbered papers will be at their posters from 9:40 a.m. to 10:30 a.m. and contributors of even-numbered papers will be at their posters from 10:30 a.m. to 11:20 a.m.
\end{abstract}

\section{Contributed Papers}

5aNSf1. The influence of meteorological conditions on noise propagation outdoors and sustainable planning of roads and motorways. Barbara Joanna Lebiedowska (Warsaw University of Technology, ul. Lukasiewicza 17, 09-400 Plock, Poland, barbara_lebiedowska@yahoo.fr)

Adequate road planning that allows for the separation of built-up areas and travel routes is one of the basic prevention strategies of hazards resulting from road usage. If possible, the roads should never be routed across cities and residential districts. However, if for some reason a busy road must be located near a built-up area, long-term meteorological conditions, characteristic of the area, must be examined prior to the execution of any work. Road planning on the basis of the analysis of the "rose of conditions favourable" to noise propagation will help minimise the road's negative acoustic influence on the adjacent buildings and facilities. The acoustic profile of two selected sites (in Poland and in France) is examined in the paper. Conditions favourable to propagation differ significantly in both cases depending on the position of the source-to-receiver direction in relation to the directions of the world

5aNSf2. Community response to military shooting noise immissions. Mark Brink (ETH Zürich, Center for Organizational and Occupational Sciences, LEO B 9.1, 8092 Zürich, Switzerland, brink@ethz.ch), Jean Marc Wunderli (EMPA - Materials Science and Technology, Ueberlandstr. 129, CH-8600 Duebendorf, Switzerland, jean-marc.wunderli@empa.ch), Hans Boegli (Federal Office for the Environment, BAFU, 3003 Bern, Switzerland, hans.boegli@bafu.admin.ch)
The assessment of the impact of any kind of noise exposure on the population is a fundamental step in the process of noise abatement. Data about the exposure-response relationship for annoyance due to noise emissions from military shooting grounds are rather scarce at the time. In order to establish new legal exposure limit values for shooting noise from heavy and light weapons, we conducted an extensive telephone interview survey in several - mostly rural - municipalities affected by military shooting noise in Switzerland. The exposure assessment for each respondent was calculated based on the source models of a range of weapons and ammunitions of the Swiss army. Preliminary results suggest that although the responses of the population to noise from military weapons are rather dispersed, data should be sufficiently consistent to establish an exposure-response relationship which can help in defining a binding exposure limit value.

5aNSf3. Investigation of traffic noise from additional tolling lanes at Portage toll plaza. Silas Bensing (Columbia College Chicago, 370 Millport Drive, Valparaiso, IN 46385, USA, silas.bensing@loop.colum.edu)

Electronic toll collecting systems quickly and efficiently move traffic through toll facilities by automatically collecting tolls from individual vehicle transponders. An ongoing study investigates the addition of three electronic toll lanes to the existing twelve lanes at the Portage toll plaza on the Indiana Toll Road (Portage, Indiana, USA), scheduled to be completed by December 2008. The study aims to predict noise levels of the plaza after the addition of the lanes, the hypothesis being that the additional lanes will have little or no effect on the toll plaza noise levels. Noise measurements are be- 
ing made at locations in the vicinity of the existing plaza with consideration for a nearby residential area. An outdoor noise propagation software (SoundPlan) is used to create a comprehensive traffic noise model for the area under investigation. Test data from the existing site will be compared to the model and used to predict future noise levels.

5aNSf4. Assessment of military shooting noise. Hans Boegli (Federal Office for the Environment, BAFU, 3003 Bern, Switzerland, hans.boegli @bafu.admin.ch), Jean Marc Wunderli (EMPA - Materials Science and Technology, Ueberlandstr. 129, CH-8600 Duebendorf, Switzerland, jean-marc.wunderli@empa.ch), Mark Brink (ETH Zürich, Center for Organizational and Occupational Sciences, LEO B 9.1, 8092 Zürich, Switzerland, brink@ethz.ch)

The assessment of the impact of noise exposure on the population is a fundamental step in noise abatement. It includes the establishing of an exposure-response relationship and the setting of an impact threshold that specifies the protection level for the population and triggers eventually mitigating measures to reduce noise exposure. In Switzerland, the impact thresholds should be set so that, in the light of current scientific knowledge and experience, noise exposure below these thresholds will not seriously disturb the well-being of the population. For most current noise sources such as roads, railways and airports there already exist impact thresholds as part of the noise abatement legislation. Yet, no impact thresholds for military shooting grounds have been specified so far. Therefore a study was carried out in order to assess the impact of military noise exposure. The research included the calculation of noise exposure of eight military shooting grounds ranging from small infantry shooting ranges to expanded artillery and tank training facilities and a survey at over 1000 residents in the neighbourhood of these installations. Preliminary results suggest that although the responses of the population to military noise are rather dispersed, data should be sufficiently consistent to establish an exposure-response relationship.

5aNSf5. Design and evaluation of novel barriers. George Bamnios (Electronics Dept. ATEI of Thessaloniki, P.O. Box 141, Sindos, $57400 \quad$ Thessaloniki, Greece, bamnios@el.teithe.gr), Ioannis Rekanos (Physics Devision, Dept. of Mathematics, Physics and Computational Sciences, School of Engineering, Aristotle Univ. of Thessaloniki Campus, 54124 Thessaloniki, Greece, rekanos@auth.gr), Konstantinos Zacharias (Physics Devision, Dept. of Mathematics, Physics and Computational Sciences, School of Engineering, Aristotle Univ. of Thessaloniki Campus, 54124 Thessaloniki, Greece, zachark@gen.auth.gr), Athanasios Trochidis (Physics Devision, Dept. of Mathematics, Physics and Computational Sciences, School of Engineering, Aristotle Univ. of Thessaloniki Campus, 54124 Thessaloniki, Greece, trohidis@gen.auth.gr)

This work presents the results of a project for the design of novel noise barriers with improved performance. The aim of the project was twofold. First, to optimize the performance of barriers by appropriately modifying both the shape and the acoustical conditions at the edge to suppress the sound pressure. Second, to quantify the acoustic performance of the novel barriers both mathematically and experimentally in a standardized, well controlled process. The insertion loss of different types of barriers with modified edge shapes and acoustical conditions were investigated systematically in comparison with conventional ones using a method based on BEM. The comparison highlighted the influence of shape and edge configuration on barrier efficiency and allowed the design of novel barriers with optimal performance. The novel barriers were tested and their efficiency was quantified experimentally using the Maximum Length Sequence (MLS) technique, which offers the advantage of in-situ measurements in the presence of extraneous noise. The experimental results are shown to be in good agreement with the experiments. Based on the aforementioned procedure, a unified framework was established, witch allows the design of potentially improved noise barriers.
5aNSf6. Nuisance control of leisure activities. Robert Barti(LEM Laboratory, Pol. Ind. Cova Solera, Av. Can Sucarrats, 110 nau 11, 08191 Rubi, Spain, robert@lem-sl.com)

In Spain there are more than 345000 leisure activities with more than $1.2 \mathrm{M}$ jobs. This economical sector produces the $2.2 \%$ of the PIB overall Spanish economic production. Tourism is a big source of money for many Spanish cities. Leisure activities is the most common economic activity related with this sector. Typical Spanish good weather let's people to remain for a long time up to early morning in the streets. Human activity produces always noise. Acoustic noise control measurements uses $\mathrm{dB}(\mathrm{A})$ scale for evaluation. Low noise levels $\mathrm{dB}(\mathrm{A})$ has no correlation with acoustic perception of people because people can "follow" the rhythm of the music. Increase the TL of structures let's to reduce the noise from activities, but increasing the power amplifier the problem remains still in the same point. The solution checked is to use an acoustic limiter as a warranty of no contamination to neighbours from the activity. Some results about this technique are discussed in this paper. In order to solve a complain between neighbours an leisure activity, a political solution is more effective than technical solution is. The main objective is to set the correct balance between perception of sounds and acoustic comfort at home.

5aNSf7. Perspectives for a strategical mapping of soundscapes. Gianluca Memoli (Imperial College London, Department of Chemical Engineering, Exhibition road, SW7 2AZ London, UK, g.memoli@imperial .ac.uk), Gaetano Licitra (ARPAT - Dept. Firenze, Via Porpora, 22, 50144 Firenze, Italy, g.licitra@arpat.toscana.it), Mauro Cerchiai (ARPAT - Dept. Pisa, Via V. Veneto, 27, 56127 Pisa, Italy, m.cerchiai@arpat.toscana.it)

The transposition of the European Noise Directive by Member States gives a new way to action plans of urban areas, underlying (article 6 and Annex III) that dose-effect relations should be used to assess the effect of noise on populations. Particular interest, in this sense, has the relationship between annoyance and perceived soundscape: it is now almost accepted that a higher annoyance is not always proportional to higher noise levels. This paper will demonstrate that a way to move from mapping noise exposure to a map of annoyance passes through the characterization of the soundscapes present on the territory. A numerical indicator ("slope"), derived from the time history of the measured sound/noise, will in fact be used to characterize the evolution along the day of the soundscapes present in the city of Pisa (I). In particular, "slope" (correlated to annoyance in previous studies) will be used to prepare a map of the annoyance in selected areas of Pisa: predicted annoyance will be to be compared with the one extrapolated by noise levels. Differences, similarities and their effects on action plans will be discussed.

5aNSf8. Analysis and evaluation of noise reaction in open public spaces in Mexico City. Miriam German (Universidad Nacional Autonoma de Mexico-Facultad de Arquitectura, Circuito Interior S/N, Ciudad Universitaria, Delegacion Coyoacan, 02410 Mexico D.F., Mexico, lydmgg@yahoo .com.mx)

Mexico City, with a population estimated in 19 millions, is part of one of the largest metropolitan areas in the world. Unfortunately, scientific work on urban noise in Mexico City is scarce. A study on people reaction to urban noise carried out in open public spaces of two different zones of Mexico City will be presented. A previous survey in those zones showed that the noise levels exceeded the values recommended by international organizations to protect public health and welfare. The general objective of the study to be presented was to identify the sound sources perceived by the pedestrians, and to evaluate their response to urban noise pollution in the two mentioned areas. The results show that for most of the people (a) urban noise is not considered a relevant aspect to improve the quality in the city, although urban noise has a negative effect on the decision of using open public spaces; (b) the people with knowledge of the negative effects of noise pollution are more annoyed; (c) vehicular traffic is the most annoying noise source, contributing significantly the public transportation; (d) one of the principal strategies to confront urban noise is to get used to it. 


\title{
Session 5aNSg
}

\author{
Jérôme Defrance, Chair \\ $C S T B$
}

\section{Noise and EURONOISE: Propagation and Urban Noise I (Poster Session)}

\begin{abstract}
All posters will be on display from 9:40 a.m. to 11:20 a.m. To allow contributors an opportunity to see other posters, contributors of odd-numbered papers will be at their posters from 9:40 a.m. to 10:30 a.m. and contributors of even-numbered papers will be at their posters from 10:30 a.m. to 11:20 a.m.
\end{abstract}

\section{Contributed Papers}

5aNSg1. Perception of noise in suburban and urban areas. Elaine C. Paz (Federal University of Parana, Centro Politécnico - Setor de Tecnologia, Bairro Jardim das Américas, 81531-990 Curitiba, Brazil, epaz@bol.com .br), Paulo Henrique Trombetta T. Zannin (Federal University of Parana, Centro Politécnico - Setor de Tecnologia, Bairro Jardim das Américas, 81531-990 Curitiba, Brazil, paulo.zannin@pesquisador.cnpq.br)

This work analyses, using a comparative approach, the environmental noise perception in the daily lives of inhabitants of a residential area, with that of a mixed (residential and commercial) area (downtown), in a large Latin America city. The goal was to confront an ideal urban environment with one acoustically polluted, as function of noise descriptors. Concomitant with the evaluation of noise perception (subjective analysis), noise levels were measured (objective analysis) in both areas. The single average equivalent noise level (Leq) found for downtown was $73 \mathrm{~dB}(\mathrm{~A})$, and $53 \mathrm{~dB}(\mathrm{~A})$ for the residential area. A random sample of the populations of both areas was taken, through a questionnaire. Questionnaire data was treated statistically through a factorial multivariate analysis. This analysis has generated three statistical indicators: time perception, atypical noise perception, and sources and disturbances. Over $50 \%$ of both populations sampled have the perception that high frequency noise levels are gradually increasing, with higher contribution from traffic noise. The dominant organic effects reported were irritability and loss of concentration ability, these last being precursors of hearing loss.

5aNSg2. Traffic noise in shielded urban areas: comparison of experimental data with model results. Ando Randrianoelina (TNO Science and Industry, Stieljesweg 1, 2628CK Delft, Netherlands, ando.randrianoelina@tno.nl), Erik Salomons (TNO Science and Industry, Stieljesweg 1, 2628CK Delft, Netherlands, erik.salomons@tno.nl)

Noise maps of cities are commonly produced with rather simple engineering models for sound propagation. These models may be inaccurate in complex urban situations, in particular in situations with street canyons. Street canyons are urban areas that are partly or completely enclosed by buildings, for example a street between two buildings or a backyard surrounded by buildings. In this paper we study sound propagation from a city bus in a street canyon to a receiver in a nearby street canyon. Multiple reflections of sound waves in both the source canyon and the receiver canyon play an important role in the sound propagation. Experimental data are compared with three types of model results: (i) results of a numerical boundary element model, (ii) results of a 1:30 scale model experiment in a semianechoic room, and (iii) results of the Dutch standard engineering model, which is similar to the international standard ISO 9613-2. The data are in reasonable agreement with model results (i) and (ii), while the engineering model yields sound levels that are about $10 \mathrm{~dB}$ too low.

5aNSg3. Reflection of sound from a forest: comparison of experimental data to scattering theory. Erik Salomons (TNO Science and Industry,
Stieljesweg 1,2628CK Delft, Netherlands, erik.salomons@tno.nl),Jean Marc Wunderli (EMPA - Materials Science and Technology, Ueberlandstr. 129, CH-8600 Duebendorf, Switzerland, jean-marc.wunderli@empa.ch)

Reflection of sound by a forest has been investigated by means of measurements near Thun in Switzerland. Sound pulses were generated at 50 and $150 \mathrm{~m}$ from the forest, and reflected sound was recorded at distances of 100 , 200 , and $300 \mathrm{~m}$ from the forest. By comparison with numerical calculations of reflection of sound from a hard wall, using a parabolic-equation model for sound propagation in an atmosphere with wind and temperature as recorded during the measurements, the attenuation of sound waves upon reflection at the forest was determined. It was found that the reflection attenuation decreases from about $20 \mathrm{~dB}$ at low frequency to about $10 \mathrm{~dB}$ at high frequency. The experimental results for the reflection attenuation are compared with results of a theoretical model for scattering of spherical sound waves by a single row of cylinders. The model employs an effective spacing between the cylinders, to account for trees that are not at the edge of the forest, i.e., inside the forest. The model results are in good agreement with the experimental results. The comparison indicates that the effective spacing decreases with decreasing frequency, as low-frequency waves penetrate deeper into a forest than high-frequency waves do.

5aNSg4. Reflection of sound from a forest: Effective tree spacing for a scattering model with a single line of cylinders. Jean Marc Wunderli (EMPA - Materials Science and Technology, Ueberlandstr. 129, CH-8600 Duebendorf, Switzerland, jean-marc.wunderli@empa.ch), Erik Salomons (TNO Science and Industry, Stieljesweg 1, 2628CK Delft, Netherlands, erik.salomons@tno.nl)

A scattering model for the reflection of sound at forest rims is presented by the authors in another contribution to this conference. The forest is thereby modeled by a single row of cylinders with the effective spacing as single free parameter. In the present article an engineering scheme for this effective tree spacing is presented. The scheme is based on results of numerical calculations with the Euler model for sound propagation in a model forest consisting of cylinders. The numerical results show that forests are widely transparent for incoming as well as reflected sound waves. Multiple reflections can be neglected. Reflections from trees deep inside the forest experience additional propagation attenuation and are therefore weakened relative to contributions from trees in the first row. Apart from geometrical spreading foliage attenuation is the most prominent effect. The penetration depth of the forest reflection is proportional to the wavelength. The frequency dependent penetration depth is derived by means of a statistical analysis for numerous situations. Assuming the effective tree spacing to be inversely proportional to the penetration depth, the engineering scheme for the effective tree spacing is derived. Comparisons of the frequency response with measurements show good agreement. 


\title{
Session 5aNSh
}

\section{Noise, Structural Acoustics and Vibration, Physical Acoustics and EURONOISE: Sound and Vibration from Explosions in Air I (Poster Session)}

\author{
Roger Waxler, Cochair \\ University of Mississippi \\ Keith Attenborough, Cochair \\ Open University
}

\begin{abstract}
All posters will be on display from 9:40 a.m. to 11:20 a.m. To allow contributors an opportunity to see other posters, contributors of odd-numbered papers will be at their posters from 9:40 a.m. to 10:30 a.m. and contributors of even-numbered papers will be at their posters from 10:30 a.m. to 11:20 a.m.
\end{abstract}

\section{Contributed Papers}

5aNSh1. Environmental noise reduction means of weapons. Rauno Pääkkönen (Finnish Institute of Occupational Health, P.O. Box 486, 33101 Tampere, Finland, rauno.paakkonen@ttl.fi)

Noise from hand-held weapons is generated by their muzzle blast, the noise of the bullet in flight and on impact. The muzzle blast of almost all powder guns exceeds $140 \mathrm{~dB}$ near the ear of the shooter, which is considered to be able to generate a risk of hearing defects. The muzzle brake decreases recoil, but at the same time it forces the sound sideways and backwards. One novel aspect of noise attenuation is the use of active noise cancelling system at shooting ranges and in hearing protectors. The noise data has been collected on different shooting ranges in Finland. Both front and reflection types of suppressors were used. Silencers that attenuate noise to the sides and back have a significant effect, where as the noise to the front is not significantly attenuated because of the bullet noise. It is also possible to reach an attenuation of a few decibels of the maximum level of the muzzle blast with ANC headsets. The noise created by shotguns is the most significant source of environmental noise at many shooting ranges. It would be especially important to attain new ideas and innovations in the noise prevention of shotgun ranges.

5aNSh2. The development of a bespoke gunfire noise model for a military training area and the vibration impacts through the air on ancient structures. Graham Parry (ACCON UK Limited, Unit B, Fronds Park, Frouds Lane, Aldermaston, RG7 4LH Reading, UK, graham.parry @ accon-uk.com)

This paper describes the development of a bespoke weapons system noise model known as the gunfire noise analysis tool (GNAT). The requirements for such a model came out of an expansion of military training within a National Park and the need to demonstrate that noise impacts could be modeled with reasonable accuracy out to distances of $10 \mathrm{kms}$ and without the need for specialist acousticians or meteorologists to run the model. Additionally, the requirement was to ensure that noise modeling could be carried out within a matter of minutes thus allowing military training decisions to be made while protecting sensitive receptor locations from very high noise levels. The model utilised best practice and acoustic theory available at the time within the specific requirements of noise modeling outlined above. The model was validated against a number of noise measurements exercises carried out using controlled military noise events and for a live military exercise.
5aNSh3. Transducers for acoustical impulse measurements. Per Rasmussen (G.R.A.S. Sound \& Vibration A/S, Skovlytoften 33, 2840 Holte, Denmark,pr@gras.dk)

The measurement of impulsive noise signals as for example from gunshots or airbag deployments requires special considerations when selecting transducer. The high frequency content in short impulses requires high bandwidth to correctly capture high rise times and small dimensions of the transducer to avoid diffraction. For high level impulses with very rapid rise time, the wide frequency range requires the use of preamplifiers with high slew rate capabilities and the capacitance and length of cables must be considered carefully. Slew rate limitations and bandwidth limitations can drastically change the peak value of an impulse. It is thus necessary to consider the whole measurement chain from transducer to $\mathrm{AD}$-converter to ensure the correct measurement of the impulse.

5aNSh4. Large weapon source emission data protocols and results. Michael James (Blue Ridge Research and Consulting, 13 1/2 W. Walnut St., Asheville, NC 28801, USA, michael.james@blueridgeresearch .com), Micah Downing (Blue Ridge Research and Consulting, 13 1/2 W. Walnut St., Asheville, NC 28801, USA, micah.downing@blueridgeresearch .com), Pater Larry (U.S. Army Engineer Research and Development Center, 2902 Farber Drive, Champaign, IL 61821, USA, Larry.L.Pater@erdc .usace.army.mil)

The U.S. Army needs detailed information about the blast pressure field created by large weapons to avoid negative impacts on testing and training mission capabilities due to noise. The noise software tools BNOISE2 ${ }^{\mathrm{TM}}$ and RMTK Noise Tool, which the Department of Defense (DoD) uses to predict and assess impacts of large weapon noise, require accurate acoustic source emission models. A free-field representation of the sound source acoustical emission, containing no waveform signal perturbations due to the ground, other reflecting surfaces, ballistic waves, or propagation anomalies, is required. The measurement protocols have been developed to collect the free-field source emission data. These protocols are described along with acoustical data and emission source results for the $120 \mathrm{~mm} \mathrm{M}-1$ tank gun, the $105 \mathrm{~mm}$ Stryker gun, and various weights of C-4 explosives. In addition, the measured acoustic data are compared with an idealized Friedlander curve. The overall results demonstrate that the protocols do provide sufficient free-field blast waveforms. 


\title{
Session 5aNSi
}

\section{Noise and EURONOISE: Acoustic Performance of Energy Efficient Building Products II (Poster Session)}

\author{
Anthony Hoover, Cochair \\ McKay Conant Brooks, Inc. \\ Jacques Roland, Cochair \\ Centre Scientifique et Technique du Bâtiment
}

\begin{abstract}
All posters will be on display from 9:40 a.m. to 11:20 a.m. To allow contributors an opportunity to see other posters, contributors of odd-numbered papers will be at their posters from 9:40 a.m. to 10:30 a.m. and contributors of even-numbered papers will be at their posters from 10:30 a.m. to 11:20 a.m.
\end{abstract}

\section{Contributed Paper}

5aNSi1. Roof-top urban wind turbines in London. Stephen Dance (London South Bank University, FESBE, Borough Road, SE1 0AA London, UK, dances@1sbu.ac.uk), Linda Liviani (RBA Acoustics, 104 The Foundry Annexe, 65 Glasshill Street, SE1 0QR London, UK, lindalive2 @ hotmail.com)

With the Mayor of London's office decision to allow planning permission only if $10 \%$ of the building's energy consumption is from renewable sources, wind turbines are starting to be erected on rooftops of residential blocks. A Proven $6 \mathrm{~kW}, 6 \mathrm{~m}$ horizontal axis turbine was positioned on an 11 story 1960s concrete construction block of flats in the summer of 2007 Noise and vibration measurements were taken on the roof-top and in the nearest flat. In addition, energy performance was monitored, wind speed and direction recorded and weather noted. Results confirm that central London is a $24 \mathrm{~h}$ city and, given the right conditions, the turbine generators meaningful amounts of electricity.

\section{Session 5aNSj}

\section{Noise and EURONOISE: Examples of Noise Control II}

\author{
Karl Hirsch, Cochair \\ Cervus Consult, Kolpingstrasse 13, Wuerselen, D-52146, Germany \\ Pierre Etienne Gautier, Cochair \\ SNCF DIR, 45, rue de Londres, Paris, 75379, France
}

\section{Contributed Papers}

11:00

5aNSj1. Monitoring of noise reduction from traffic speed control. $\mathrm{J}$. Jabben (RIVM, A. van Leeuwenhoeklaan 9, 3720 BA Bilthoven, Netherlands, jan.jabben@rivm.nl), C. Potma (RIVM, A. van Leeuwenhoeklaan 9, 3720 BA Bilthoven, Netherlands, charlos.potma@rivm.nl)

In November 2005, at the Hague, Amsterdam, Rotterdam and Utrecht, a $80 \mathrm{~km} / \mathrm{u}$ speed limit was introduced on sections of motorways in the Netherlands. The aim was to evaluate the effects on noise, air quality and the circulation of the traffic. RIVM investigated the effects of noise emission by direct measurement at the sections with speed limit. This paper gives the results from continuous monitoring of noise levels that was started at July 2005 and continued up to November 2006. In the presentation the average measured effects on the equivalent noise emissions at the different sites will be given. Also the effects of traffic speed reduction on dwellings in the neighborhood of the motorways were evaluated using noise maps. Furthermore, apart from the average equivalent noise levels, the statistical noise indicators L5, L10, L70, and L95 were continuously monitored. It was found that speed reduction induces more reduction on maximum noise levels than on the average (equivalent) noise levels, probably due to a more steady and even distribution of traffic speeds.

11:20

5aNSj2. Automatic classification of traffic noise. Manuel A Sobreira-Seoane (University of Vigo, E.T.S.I de Telecomunicación, Rúa Maxwell s/n, 36310 Vigo, Spain, msobre@gts.tsc.uvigo.es), Alfonso Rodríguez Molares (University of Vigo, E.T.S.I de Telecomunicación, Rúa Maxwell s/n, 36310 Vigo, Spain, amolares@gts.tsc.uvigo.es), José Luis Alba Castro (University of Vigo, E.T.S.I de Telecomunicación, Rúa Maxwell s/n, 36310 Vigo, Spain, jalba@gts.tsc.uvigo.es)

When modeling a city or a secondary road to calculate a noise map, the information about the number of heavy/light vehicles and the average speed it is not always available. In this paper, a first approach to get an automatic classification of vehicles is presented. The system is based on the classifi- 
cation of the audio signal that a noise source produces. Some basic classifiers have been tested (k-nearest neighbours, FLD (Fischer linear discriminator) and principal components. As first approach, the aim of the job was to determine if the different classes (trucks, cars, and motorbikes) could be separable using different time and frequency characteristics: zero crossing ratios, spectral centroids, spectral rolloff, subband energies and mel frequency cepstral coefficients. The results shows that for some of the characteristics tested, the signals are separable, so a continuous traffic noise signal could be processed to get the information of the number of heavy trucks, cars, and motorbikes that passed by during the recording time. Information of a stereo recording could be used to get information of the direction of the vehicle. At this moment, combining three characteristics and FLD, errors bellow $9 \%$ can be reported.

\section{1:40}

5aNSj3. Remarks on a blast sound propagation model for noise management purposes at military training areas. Karl Hirsch (Cervus Consult, Kolpingstrasse 13, D-52146 Wuerselen, Germany, hirsch@cervus .de)

Germany will introduce a daily shooting noise management at its military training areas. Roughly speaking, this management adopts the basic ideas of ISO 17201-5 but uses in addition a rating scheme that allows 18 exceedance days per year $(5 \%)$. For management purposes, the underlying sound prediction scheme must support two decision (1) Whether or not, the noise load of the daily planned occupancy is within certain noise limits. (2) Whether or not, the daily changing occupancy is close to a low noise minimum for the requested training options. The propagation model must consider particular weather conditions and the terrain shielding to enable the operator to optimise the occupancy with respect to these important conditions on a daily basis. The prediction must be fast enough to run several alternatives within minutes. Therefore, the prediction could not base on a sophisticated physical propagation model but must be done along a reasonable technical scheme. This paper discusses a ray tracing approach that is expected to substitute the currently in-use formula based on fitted empirical parameters. This model will at least provide one parameter to describe the sound refraction (radius of curvature) and a rule on how to estimate up wind propagation.

\section{2:00}

5aNSj4. Simulation of highway traffic noise with utilization of equivalent sound level of pass-by vehicle. Pichai Pamanikabud (King Mongkut U. of Technology-Thonburi, Dept. of Civil Engineering, Faculty of Engineering, Pracha-u-tid Road, Thungkru, 10140 Bangkok, Thailand, pichai.pam@kmutt.ac.th), Marupong Tansatcha (Dept. of Public Works and Town \& Country Planning, Ministry of Interior, Rama 9 Rd., Huai-Khwang, 10320 Bangkok, Thailand, t_marupong@yahoo.com)

This paper presents study and building of a highway traffic noise simulation model by utilizing equivalent sound level over $20 \mathrm{~s}$ of passing-by vehicular noise in the analysis of nine types of vehicular basic noise. The basic noise of each vehicle type is applied into the formulation of the main highway traffic noise simulation model. The coefficient of propagation and ground effect for this model is also estimated and applied to the highway traffic noise model. This highway traffic noise model is then tested for its goodness-of-fit to field observations. The testing result shows that this model provides an effective prediction for highway noise in Thailand.

\section{$12: 20$}

5aNSj5. Identification of noise sources in centrifugal blower with acoustic camera. Jurij Prezelj (University of Ljubljana, Faculty of Mechanical Engineering, Aškerčeva 6, 1000 Ljubljana, Slovenia, jurij.prezelj@fs.uni-lj.si), Mirko Čudina (University of Ljubljana, Faculty of Mechanical Engineering, Aškerčeva 6, 1000 Ljubljana, Slovenia, mirko .cudina@fs.uni-lj.si)

A centrifugal blower represents the main noise source in a vacuum cleaner. Noise emitted by the centrifugal blower working as a suction unit has aerodynamic, mechanical, and electromagnetic noise origins. Noise emitted by a suction unit consists of airborne and structure-borne noise. The contribution of the individual noise source to the total noise level depends on the geometry of the suction unit, rotational speed and on the operating conditions. Operating conditions of a suction unit depend on the amount of dust particles in the dust bag and are constantly changing during operation until a zero flow rate is reached. In order to reduce noise of suction unit at broad range of operating conditions, an identification of noise sources needs to be performed. Identification of most important noise sources on the centrifugal blower was performed with an acoustic camera at the design and off-design operation conditions. From the analyses we can conclude that the rotational noise usually prevails at the design point of operation, and that the nonrotational noise prevails at off-design operation. The main source of noise within a suction unit can be attributed to the aerodynamically generated noise, at the design as well as at off-design operation.

\section{2:40-2:00 Lunch Break}

\section{Contributed Papers}

\section{2:00}

5aNSj6. Buzz-saw noise : propagation of shock waves in aero-engine inlet ducts. Rasika Fernando (Institut Jean Le Rond d'Alembert, Université Pierre et Marie Curie, Boites 161 et 162, 4 place Jussieu, 75252 Paris Cedex 05, France, fernando@1mm.jussieu.fr), Régis Marchiano (Institut Jean Le Rond d'Alembert, Université Pierre et Marie Curie, Boites 161 et 162, 4 place Jussieu, 75252 Paris Cedex 05, France, marchi@1mm .jussieu.fr), François Coulouvrat (Institut Jean Le Rond d'Alembert, Université Pierre et Marie Curie, Boites 161 et 162, 4 place Jussieu, 75252 Paris Cedex 05, France, coulouvr@ccr.jussieu.fr), Yann Druon (Airbus S.A.S, Department of Acoustic \& Environment, 316 route de Bayonne, 31000 Toulouse, France, yann.druon@airbus.com)

When high bypass ratio aircraft engines run at takeoff operating conditions, blade relative flow velocities can exceed sonic speed, thus generating forward propagating shock waves that spiral inside the intake before being radiated. "Buzz-saw" or "multiple pure tone" noise then occurs, and measured acoustic spectra close to the fan display tones at the blade passing frequency and its harmonics, along with those of the engine shaft rotation frequency. This work first attempts to reformulate McAlpine and Fisher's frequency domain model for the propagation of a sawtooth waveform spi- ralling inside a hard-walled and lined cylindrical duct with uniform flow. The nondissipative Burgers equation is solved, and modal attenuation and dispersion are added using a split-step computational method. In practice, shocks do not only occur at blade tips, but on a significant portion of the blade span. The plane wave hypothesis being no longer valid, a new threedimensional model is presented for a no-flow case. This model is based on the computation of the axial variations of the mode amplitudes under the influence of the nonlinear modal interactions. The formalism of this new model, along with the preliminary results are also presented.

$$
\text { 2:20 }
$$

5aNSj7. Numerical simulation of aerodynamical noise generated by a cylindrical cavity. Daniel-Ciprian Mincu (ONERA (French aerospace Center), CFD \& Aeroacoustics Department, BP 72, 29 avenue de la division Leclerc, 92322 Chatillon Cedex, France, dmincu@onera.fr), Stephane Redonnet (ONERA (French aerospace Center), CFD \& Aeroacoustics Department, BP 72, 29 avenue de la division Leclerc, 92322 Chatillon Cedex, France, stephane.redonnet@onera.fr), Ivan Mary (ONERA (French aerospace Center), CFD \& Aeroacoustics Department, BP 72, 29 avenue de la 
division Leclerc, 92322 Chatillon Cedex, France, ivan.mary @ onera.fr), Lionel Larcheveque (Universite de Provence, 29, avenue Robert Schuman, 13621 Aix-en-Provence, France, lionel.larcheveque @ polytech.univ-mrs.fr), Eric Manoha (ONERA (French aerospace Center), CFD \& Aeroacoustics Department, BP 72, 29 avenue de la division Leclerc, 92322 Chatillon Cedex, France, eric.manoha@onera.fr)

Cylindrical cavities are mounted at some positions on fuselage and wings of commercial transport aircraft for various service functions. Such cavities were identified as potential airframe noise sources generating high intensity whistles at frequencies situated in the audible range of $0.5-0.8 \mathrm{kHz}$. The present paper focuses on the numerical simulation of the far field noise generated by the unsteady flow passing over a cylindrical cavity with an aspect ratio (diameter/height) equal to unity, using a numerical methodology combining a LES and a Kirchhoff integration method. The LES computation was done using ONERA's cell-center/finite-volume/CFD code named FLU3M. A laminar boundary layer profile was applied upstream the computational domain, without forcing any turbulence. The LES results were coupled with a 3D Kirchhoff method in the frequency domain. The computed far field noise was compared to dedicated experimental data, showing very good agreement, especially regarding the emission frequency. The final paper will present in detail the involved numerical methods, the computational procedure, and several comparisons to experimental data. This study was completed in the framework of the AEROCAV (AEROacoustique d'une CAVité cylindrique) project funded by the FRAE (Fondation Recherche Aéronautique et Espace).

\section{2:40}

5aNSj8. Efficient method for harmonics estimation of car engine sounds: application to high quality real-time synthesis. Stephane Molla (Genesis S.A., Bâtiment Gérard Mégie, Domaine du Petit Arbois BP 69, 13545 Aix-en-Provence Cedex 4, France, stephane.molla @ genesis.fr), Florent Jaillet (Genesis S.A., Bâtiment Gérard Mégie, Domaine du Petit Arbois - BP 69, 13545 Aix-en-Provence Cedex 4, France, florent.jaillet@gmail.com), Benoît Gauduin (Genesis S.A., Bâtiment Gérard Mégie, Domaine du Petit Arbois - BP 69, 13545 Aix-en-Provence Cedex 4, France, benoit.gauduin@genesis.fr)

Additive synthesis is the most popular method for the resynthesis of sounds containing slowly evolving harmonics or partials, such as car engine noise and more generally rotating machines sounds. The most important step lies in the estimation of the amplitude and frequency of each sinusoidal component. It appears that insufficiently precise estimation often leads to unsatisfactory results in terms of sound fidelity. A method providing the time and frequency evolution of the amplitude of the harmonics of a recorded sound is presented. This technique is based on a joint time and frequency chaining of amplitude's extrema of amplitudes coming from a short-time Fourier transform, using forward and backward estimation. The proposed method is compared to a rough estimation, and to an estimation based on measured tachometric data, all carried out over the same car engine sound. This estimation method is implemented into the LEA software, which thus allows fast and precise computations of the parameters. Moreover, it also helps in estimating the frequency content of the "background" noise, which depends on the speed, allowing the extraction of the partials from the original sound in an easy and efficient way. Once these characteristics are found, a high quality real-time synthesis is possible.

\section{3:00}

5aNSj9. A simple acoustic model to characterize the internal sound field in centrifugal pumps originated by blade-tongue interaction. Jorge Parrondo (Universidad de Oviedo, Dep. de Energía, Campus de Viesques s/n, 33203 Gijón, Spain, parrondo@uniovi.es), Javier Perez (Universidad de Oviedo, Dep. de Energía, Campus de Viesques s/n, 33203 Gijón, Spain, javier.perez_castillo@siemens.com), Raul Barrio (Universidad de Oviedo, Dep. de Energía, Campus de Viesques s/n, 33203 Gijón, Spain, barrioraul @uniovi.es)

Conventional centrifugal pumps with volute casing generate fluiddynamic noise particularly at the so-called blade-passing frequency, due to the interaction of the flow exiting the pump impeller with the volute tongue. The amplitude of the sound generated is very dependent on the pump operating point. Following previous studies by the authors, a methodology has been applied to quantify the generation of tonal noise for a given centrifugal pump, previously tested in laboratory. The procedure is based on a simple acoustic model for the pump, in which one or several ideal point sources are located at some arbitrary position in the volute. These ideal sources are assumed to radiate plane sound waves along the volute, which was considered to be composed by a succession of slices, each of them equivalent to a linear three-port acoustic system with sound transmission and reflexion coefficients according to the corresponding port areas. A series of tests was conducted to check the assumptions of the acoustic model, by applying external acoustic loads onto the pump outlet duct and measuring the noise reflected. The resulting reflection coefficient was in good agreement with the predictions of the acoustic model.

\section{3:20}

5aNSj10. Performance evaluation method for high noise environment intercom headsets. Stelios M. Potirakis (Intracom Defense Electronics, $21 \mathrm{~km}$ Peania-Markopoulo Street, GR-19400 Koropi - Attica, Greece, spoti@intracomdefense.com), Yiannis Moisiadis (Intracom Defense Electronics, 21km Peania-Markopoulo Street, GR-19400 Koropi - Attica, Greece, gmoy@intracomdefense.com), Antonis Varagis (Intracom Defense Electronics, 21km Peania-Markopoulo Street, GR-19400 Koropi - Attica, Greece, avar@intracomdefense.com)

Intercoms' use in high noise environments (HNE) is typical since direct speech communication of individuals is difficult or even impossible. The headset selection for such an application is crucial since it serves both for noise attenuation and voice reproduction. It has been recently acknowledged in telecommunications (ITU-T/P.380) that headset electroacoustic measurements should be performed on head and torso simulator (HATS). However, in military applications where headsets are mainly used in HNE there is not a standard performance evaluation method but only for the earphone elements (MIL-PRF-25670B), thus excluding the acoustics of the earcup cavity, absorbing materials and face fitting quality. It is well known that the reproduced speech level should be of about $10 \mathrm{~dB}$ above noise level to achieve good intelligibility, so both sensitivity and noise reduction capability should be measured on a HATS to evaluate such a headset. In this work, a systematic methodology for measurement and performance evaluation of HNE headsets is proposed based on HATS. Critical aspects that may affect the test procedure, such as HATS response and the right headset placement are examined. Finally, a series of measurements prove the value of the proposed method. Large differences are revealed even for headsets following the same standard. 


\title{
Session 5aPAa
}

\section{Physical Acoustics: Infrasound I}

\author{
Henry E. Bass, Cochair \\ The University of Mississippi - NCPA, 1 Coliseum Drive, University, MS 38677, USA \\ Alexis Le Pichon, Cochair \\ CEA-DASE, Arpajon Cedex, Bruyères-le-Châtel, 91297, France
}

\section{Invited Paper}

\begin{abstract}
8:00
5aPAa1. Monitoring hurricanes using infrasound. Carrick L. Talmadge (The University of Mississippi - NCPA, 1 Coliseum Drive, University, MS 38677, USA, clt@olemiss.edu), Claus Hetzer (National Center for Physical Acoustics, University of Mississippi, University, MS 38677, USA, claus@olemiss.edu), Henry E. Bass (The University of Mississippi - NCPA, 1 Coliseum Drive, University, MS 38677, USA, pabass@olemiss.edu)

In spite of recent technological advances, forecasting the intensity of a hurricane remains a challenging problem. Knowledge of the location and the strength of the storm at the moment where it makes landfall is critical for civil defense planning and public safety. Part of the reason for this is that storms that are well away from land can only be monitored intermittently (e.g., three times per day). Infrasound signals are produced by the interaction of the hurricane with the sea-state and hold promise for characterizing sudden changes in hurricane strength on a time scale not possible with current sensing technologies. The University of Mississippi is leading a US national collaboration to monitor hurricanes using infrasound in the Gulf of Mexico and in the Western Atlantic Ocean. The results of measurements by from our group will be summarized, and the technical challenges associated with these measurements will be discussed.
\end{abstract}

\section{Contributed Papers}

\section{8:20}

5aPAa2. Infrasonic precursor of tropical cyclone. Konstantin A. Naugolnykh (University of Colorado/Zeltech, 325 Broadway, Boulder, CO 80305, USA, konstantin.naugolnykh@noaa.gov), Samuil A. Rybak (N. Andreev Acoustics Institute, 4 Schwernik St., 117420 Moscow, Russian Federation, am_rybak@hotmail.com)

Intense infrasonic emission was observed prior to the birth of a tropical cyclone (Netreba, 1991). It is connected apparently with instability of atmospheric layers in cyclone area. There are different mechanisms of this effect. Stratified compressional flow is unstable (Rybak, 2002), providing amplification of its potential component. Cooling of air in upward convection flow makes water vapor supersaturated. This is a nonequilibrium state of fluid where effect of instability can be developed. The equation of infrasonic propagation in such a medium is developed. (Naugolnykh, Rybak, 2006). Solutions of instability equation indicates the effect of radiation instability which produces infrasonic wave generation and its nonlinear evolution. Corresponding analyses of this equation make it possible to clarify the principally important aspects of cyclone infrasonic precursors. [Work supported by ESP.NR.NRCLG982524.]

\section{8:40}

5aPAa3. Origin of the sound prduced by volcanic eruptions. Sylvie Vergniolle (Institut de Physique du Globe, 4 Place Jussieu, 75252 Paris, France, vergniolle@ipgp.jussieu.fr)

Volcanic eruptions present different regimes, which can be understood and classified in the framework of a two-phase flow, either an annular flow such as in hawaiian fire fountains or a slug flow in strombolian explosions. The gas, exsolved at depth, carries physical information about the dynamics of strombolian activity, which in turn may lead to a better understanding of volcanic systems. The sound produced by volcanic eruptions is mostly infrasonic, although the landing of magma fragments on the ground is in the audible range. The first cause for sound generation is related to the gas overpressure when the sound waves are produced by a slug breaking at the surface. Gas overpressure induces oscillations of the gas volume and pushes passively the liquid film above the slug, and has been modeled accordingly. Sometimes the overpressure is small and the bubble vibration prior to breaking does not exist. In that case, the sound is produced by the gas escaping through a small hole on the bubble nose and is modeled as an Helmholtz resonator. The second class of sound source is related to the expulsion of a gas-ejecta mixture at a large velocity. Examples of volcanic sound waves will be presented.

\section{9:00}

5aPAa4. Implications from the analysis of meteoric infrasound. Laeslo G. Evers (Royal Netherlands Meteorological Institute (KNMI), PO Box 201, 3730 AE De Bilt, Netherlands, evers@knmi.nl), Hein Haak (Royal Netherlands Meteorological Institute (KNMI), PO Box 201, 3730 AE De Bilt, Netherlands, haak@knmi.nl), Alexis Le Pichon (CEA-DASE, Arpajon Cedex, 91297 Bruyères-le-Châtel, France, alexis.le-pichon@cea.fr), Karl Antier (CEA-DASE, Arpajon Cedex, 91297 Bruyères-le-Châtel, France, karl.antier@cea.fr)

Monitoring of infrasound has been widely applied in the past to detect nuclear tests. The Limited (Partial) Test Ban Treaty of 1963 confined nuclear test explosions to the underground marking the end of the global interest in infrasound. During the negotiation of the Comprehensive Nuclear-Test-Ban Treaty, in 1995-1996, it became gradually clear that infrasound monitoring should be become one of the four techniques for the treaty's verification system. The global interest in infrasound has increased significantly since the signature of the CTBT. Meteors are one of the few natural sources mimicking a nuclear. Therefore, it is an ideal object to test detection capability, phase identifiers, localization procedures and propagation models of infrasound and their processing techniques. In this presentation, we will show the results of studies on meteoric-generated infrasound and their implications for infrasound as verification technique. In addition, results of the 2007 .09 .15 meteor in Peru will be discussed which is one of the few meteoroids in recent times leaving an impact crater. 


\section{Invited Paper}

9:20

5aPAa5. Infrasound monitoring and global atmospheric dynamics. Elisabeth Blanc (Commissariat à l'Energie Atomique, DASE/SLDG/LSEG, Centre DAM-Ile de France, 91297 Arpajon Cedex, France, elisabeth.blanc@cea.fr), Alexis Le Pichon (CEA-DASE, Arpajon Cedex, 91297 Bruyères-le-Châtel, France, alexis.le-pichon@cea.fr), Lars Ceranna (Federal Institute of Geosciences and Natural Ressources, Section B3.11 Seismology, Stilleweg 2, 30655 Hannover, Germany, ceranna@sdac.hannover.bgr.de)

The development of the Infrasound International Monitoring System, used for the verification of the Comprehensive Test Ban Treaty, offers a powerful way to measure, atmospheric waves permanently and at a global scale. Infrasonic waves propagate in the channel formed by the temperature and wind gradients of the atmosphere. Long term observations provide information about the evolution of the propagation conditions and then of atmospheric parameters. The monitoring of continuous sources, as ocean swell, gives the characteristics of the stratospheric wave duct submitted to stratospheric warming effects. Large scale gravity waves, which are also observed by the network, produce a forcing of the stratosphere at low and middle latitudes and long-lived changes in the stratospheric circulation towards high latitudes, leading to fluctuations in the strength of the polar vortex. These fluctuations move down to the lower stratosphere with possible effects on the tropospheric temperature. Gravity wave monitoring in Antarctica reveals a gravity wave system correlated with the wind and the temperature gradients in the stratosphere. Gravity waves associated with magnetic storms are generally not observed. However, wave systems coming from North could reveal other processes related with the global dynamics of the stratosphere.

9:40-10:40 Posters

Lecture sessions will recess for presentation of poster papers on various topics in acoustics. See poster sessions for topics and abstracts.

\section{Contributed Paper}

10:40

5aPAa6. Locating infrasound events in wind with dense distributed arrays. Wheeler Howard (Mil-tec Research and Technology, 9 Industrial Park Dr, Oxford, MS 38655, USA, whoward@mil-tec.com), Kevin Dillion (Mil-tec Research and Technology, 9 Industrial Park Dr, Oxford, MS 38655, USA, kdillion@mil-tec.com), F. Douglas Shields (University of Mississippi/ NCPA, 1 Coliseum Dr, University, MS 38677, USA, dshields @olemiss.edu)
Applications for infrasound suffer due to the presence of wind noise in the sensor data. Several techniques have been and are being used to mitigate the influence of wind noise in locating infrasonic signals of interest. One of these methods is the averaging of data from multiple sensors in a distributed array. A distributed array was employed to study infrasonic signals from airborne sources. Wind noise during testing masked the location of the infrasonic signals in the time domain. Postprocessing techniques using statistical measures for dense arrays were employed to recover the onset of the infrasonic events. Data from these tests and an explanation of the postprocessing techniques will be discussed.

\section{Invited Papers}

\section{1:00}

5aPAa7. Detection and localization capability of the European infrasound network. Lars Ceranna (Federal Institute of Geosciences and Natural Ressources, Section B3.11 Seismology, Stilleweg 2, 30655 Hannover, Germany, ceranna@sdac.hannover .bgr.de), Alexis Le Pichon (CEA-DASE, Arpajon Cedex, 91297 Bruyères-le-Châtel, France, alexis.le-pichon@cea.fr), Julien Vergoz (CEA-DASE, Arpajon Cedex, 91297 Bruyères-le-Châtel, France, julien.vergoz@cea.fr), Laslo Evers (KNMI, PO Box 201, 3730 De Bilt, Netherlands, laslo.evers@knmi.nl)

The infrasound network of the International Monitoring Network (IMS) for the compliance with the Comprehensive Nuclear-TestBan Treaty (CTBT) is currently not fully established. However, it has demonstrated its capability for detecting and locating infrasonic sources like meteorites, as well as volcanic eruptions on a global scale. Such ground-truth events are rare; therefore regions providing a dense network of infrasound stations have to be considered to test and to calibrate detection and location procedures. In central Europe, several years of continuous infrasound waveform data are available for nine stations in Sweden, the Netherlands, France, and Germany, whereas only one of them is part of the IMS. This exquisite setting with an average interstation distance below $500 \mathrm{~km}$ allows the analysis of natural and artificial infrasonic activity in Europe. The results of the association of multiple arrays demonstrate the need of continuous infrasound monitoring on a regional scale to advance the development of automatic location procedures. Beside the seasonal variation of the network's detection capability, which is dominated by the prevailing stratospheric winds, dominant source regions showing repeating events will be discussed in detail considering the next recording stations.

\section{1:20}

5aPAa8. Continuous infrasound monitoring for atmospheric studies. Alexis Le Pichon (CEA-DASE, Arpajon Cedex, 91297 Bruyères-le-Châtel, France, alexis.le-pichon@cea.fr), Nicolas Brachet (CTBTO PTS/IDC Vienna International Centre, P.O. Box 1200, A-1400 Vienna, Austria, Nicolas.Brachet@ctbto.org), Lars Ceranna (Federal Institute of Geosciences and Natural Ressources, Section B3.11 Seismology, Stilleweg 2, 30655 Hannover, Germany, ceranna@sdac.hannover.bgr.de)

Infrasound data processing on a routine-basis provides a powerful tool to quantify relationships between infrasonic observables and atmospheric specification problems. In particular, studies of continuous sources of infrasonic waves provide further evidence that longrange propagation primarily depends on the seasonal variability of the prevailing zonal winds. It confirms that most of the detected infrasound energy propagates in the stratospheric waveguide. The observed detection capability of the IMS network is compared to the 
predicted one using near-real time atmospheric updates. Moreover, on regional scales, signals from volcanic activities open up new fields for investigations into inverse problems for atmospheric remote sensing in a range of altitude where routine ground based or satellite measurements are rare. With the increasing number of IMS and experimental arrays deployed around the globe, a valuable contribution can be anticipated in the understanding of the dynamics of the upper atmosphere. In return, such studies will help to optimize the siting of infrasound arrays with respect to both the number and configuration in order to monitor infrasonic sources of interest.

\section{$11: 40$}

5aPAa9. Inversion of infrasound signals for atmospheric remote sensing. Douglas Drob (Naval Research Laboratory, Space Science Division, 4555 Overlook Avenue, Washington, DC 20375, USA, douglas.drob@nrl.navy.mil), Milton Garces (Infrasound Laboratory, University of Hawaii, 73-4460 Queen Kaahumanu Highway \#119, Kailua-Kona, HI 96740, USA, milton@isla.hawaii.edu), Robert Meier (Naval Research Laboratory, Space Science Division, 4555 Overlook Avenue, Washington, DC 20375, USA, robert.meier@nrl.navy.mil), Michael Picone (Naval Research Laboratory, Space Science Division, 4555 Overlook Avenue, Washington, DC 20375, USA, j.picone@nrl.navy.mil)

This paper investigates the utilization of observed long-range infrasound signals to improve our knowledge of upper atmospheric winds and temperatures. A series of numerical experiments designed to provide a mathematical framework for implementation of this idea is presented. The statistical performance of a realistic infrasonic upper atmospheric remote sensing network over a range of different environmental and propagation modeling assumptions, source types, and geophysical variables (season, local-time, latitude, etc) is quantified. For a series of fictitious events, infrasonic observables are calculated with 3D Cartesian ray tracer and a series of atmospheric profiles spanning the range of geophysical parameters. These synthetic measurements are then inverted to estimate the original atmospheric background fields.

\section{Contributed Papers}

12:00

5aPAa10. The influence of shallow atmospheric structure on tropospheric ducted infrasound from the Buncefield oil depot explosion. David N. Green (AWE Blacknest, Brimpton, RG7 4RS Reading, UK, dgreen@blacknest.gov.uk), Matthew Hort (Met Office, FitzRoy Road, EX1 3PB Exeter, UK, matthew.hort@metoffice.gov.uk), David Bowers (AWE Blacknest, Brimpton, RG7 4RS Reading, UK, bowers@blacknest.gov.uk)

The vapour cloud explosion which destroyed the Buncefield oil depot, UK, on 11th December 2005, has proven to be a benchmark ground truth event for infrasonic studies. The regional infrasonic returns, those that propagated in the stratosphere and thermosphere, have been analysed in detail elsewhere. Here, we present the results of a study into infrasound ducted in the troposphere, recorded within $250 \mathrm{~km}$ of the source as air-to-ground coupled waves by a dense seismometer network. These tropospheric arrivals exhibit large waveform differences across the UK, both in amplitude and waveform shape. We numerically model these infrasound arrivals using a wave number integration method, incorporating a velocity profile derived from the UK Met Office numerical weather prediction model. Although some of the waveform variability is due to ground conditions at the recording site, we show that consistent changes in waveform shape across a 200 $\mathrm{km}$ swath of stations are correlated with a change in the wind vector in the lowermost $2 \mathrm{~km}$ of the atmosphere. Also, small amplitude, high-frequency precursors to the dominant acoustic signal, which might be misinterpreted as evidence of a small initial explosion, are shown to be consistent with the dispersion expected from a thin, shallow wind jet.
12:20

5aPAa11. Regional and telesonic propagation of volcano-acoustic signals. Milton Garces (Infrasound Laboratory, University of Hawaii, 734460 Queen Kaahumanu Highway \#119, Kailua-Kona, HI 96740, USA, milton@isla.hawaii.edu), David Fee (Infrasound Laboratory, University of Hawaii, 73-4460 Queen Kaahumanu Highway \#119, Kailua-Kona, HI 96740, USA, dfee@isla.hawaii.edu)

Volcanic explosions are reliable sources for studying infrasonic propagation. Large amplitude acoustic explosions with known locations and onset times provide useful constraints for the signal changes induced by atmospheric variability. As part of the Acoustic Surveillance of Hazardous Eruptions (ASHE) project, two four-element infrasound arrays with collocated seismometers have been deployed 37 and $251 \mathrm{~km}$ from Tungurahua Volcano, Ecuador since February 2006. During this period Tungurahua has been in near-constant eruption, with energetic tremor signals and a multitude of energetic explosions. A combination of array processing and energy threshold detectors have been used to identify over 12000 explosions at the nearby array (RIOE, $37 \mathrm{~km}$ ), with the peak pressure of the largest explosion around $24 \mathrm{~Pa}$. Many of the larger explosions are recorded at the more distant LITE $(251 \mathrm{~km})$ array as well. To further supplement the dataset, select data from infrasonic sensors at distances of 3-5 km from the crater will be used. State-of-the-art wave propagation and atmospheric models will be used to assess the influence of long-range propagation on the volcanic signals. By selecting a data subset with high signal to noise ratios, it should be possible to infer statistically significant atmospheric propagation effects.

12:40-1:40 Lunch Break

\section{Contributed Papers}

\section{$1: 40$}

5aPAa12. Gravity and rumble of distant sources. Philippe Delorme (ONERA/DSNA, 29, avenue de la Division Leclerc, 92322 Chatillon, France, Philippe.delorme@onera.fr), Geraldine Menexiadis (ONERA/DSNA, 29, avenue de la Division Leclerc, 92322 Chatillon, France, geraldine.menexiadis@onera.fr)

The subject of this paper is the comprehension of this physical phenomenon: Let an atmospheric source emiting an impulsive signal (such as thunder, explosion...). The received signal at short distance is itself impulsive but at long distance one can hear a rumble which can last up to one minute. If one models the propagation of the sound by the traditional equation of the waves, as the Green function is a distribution whose support is the sphere of radius CT (C: speed of sound, T: the travel time), the received signal (calculated by convolution) should last only the duration of emission. If one uses ray tracings to take into account the heterogeneity of the medium, one obtains the same conclusion (only the time of arrival is modified) in contradiction with the experimental results such as those obtained for example during the First World War to detect the position of the enemy batteries. A similar phenomenon can be observed in the study of the propagation of a sonic boom (although less obvious because the support of the 
two-dimensional Green function is not a circle). We thus propose a critic of the physical models, and our conclusion lays on the need for taking into account gravity.

\section{2:00}

5aPAa13. Study of Infrasound Propagation from the Shuttle Atlantis using a large Seismic Network. Michael A. Hedlin (University of San Diego California, Scripps Institute of Oceanography, 9500 Gilman Drive, La Jolla, CA 92093, USA, hedlin@ucsd.edu), Catherine D. Degroot-Hedlin (University of San Diego California, Scripps Institute of Oceanography, 9500 Gilman Drive, La Jolla, CA 92093, USA, chedlin@ucsd.edu), Kris Walker (University of San Diego California, Scripps Institute of Oceanography, 9500 Gilman Drive, La Jolla, CA 92093 , USA, walker@ucsd.edu), Douglas Drob (Naval Research Laboratory, Space Science Division, 4555 Overlook Avenue, Washington, DC 20375, USA, douglas.drob@nrl.navy.mil), Mark A. Zumberge (University of San Diego California, Scripps Institute of Oceanography, 9500 Gilman Drive, La Jolla, CA 92093, USA, mzumberge@ucsd.edu)

Inclement weather in Florida forced the shuttle Atlantis to land at Edwards Air Force base in southern California on June 22, 2007, passing near three infrasound stations and several hundred seismic stations in northern Mexico, southern California, and Nevada. The high signal-to-noise ratio, broad receiver coverage, and Atlantis' positional information allowed us to test our ability to model infrasound propagation through the atmosphere to regional distances. We predicted shadow zones and arrival times using rays launched at right angles to the conical shock front surrounding the shuttle, and traced through a standard atmospheric model, as well as a global G2S model. Comparison of predictions vs observations shows agreement over much of the study area for both atmospheric specifications. To the east of the shuttle trajectory, there were no detections beyond the primary acoustic carpet. Infrasound energy was detected hundreds of kilometers to the west and northwest of the shuttle trajectory, consistent with the predictions of ducting due to the westward summer-time stratospheric jet. Both atmospheric models predict regions of ensonification alternating with shadow zones to the northwest. However, infrasound energy was detected tens of kilometers beyond predicted zones of ensonification, possibly due to uncertainties in stratospheric wind speeds.

\section{2:20}

5aPAa14. Nonlinear propagation modeling of infrasound. David Norris (BBN Technologies, 1300 N. 17th St., Ste. 400, Arlington, VA 22209, USA, dnorris@bbn.com), Kevin Bongiovanni (BBN Technologies, 4 John Clarke Rd, Middletown, RI 02842, USA, kbongiov @ bbn.com), James Masi (BBN Technologies, 4 John Clarke Rd, Middletown, RI 02842, USA, jmasi@bbn.com)

The significance of nonlinear propagation effects on infrasound is studied using the Nonlinear Progressive Wave Equation (NPE) [B. E. McDonald and W. A. Kupperman, J. Acoust. Soc. Am., 81, 1406--1417 (1987)]. The NPE model accounts for nonlinear effects associated with a weak shock front, including shock-driven energy loss and self refraction. Numerical implementation is accomplished using a pseudospectral approach, which provides excellent computational efficiency while still maintaining acceptably small numerical errors. The nonlinear effects are isolated by generating NPE predictions with the nonlinear terms turned on and off. With the nonlinear terms off, the NPE reduces to a standard linear PE formulation. Waveform predictions through a realistic atmosphere are compared to ground truth observations to evaluate the NPE model performance and assess the influence of the nonlinear effects along the propagation path.

\section{2:40}

5aPAa15. Misty picture: A unique experiment for the interpretation of the infrasound propagation from large explosive sources. Olaf Gainville (Commissariat à l'Energie Atomique, DASE/SLDG/LSEG, Centre DAM-Ile de France, 91297 Arpajon Cedex, France, olaf.gainville@ec-lyon.fr), Philippe Blanc-Benon (Ecole Centrale de Lyon, LMFA, UMR CNRS 5509, Ecully, 69134 Lyon, France, Philippe.Blanc-Benon@ec-lyon.fr), Elisabeth Blanc (Commissariat à l'Energie Atomique, DASE/SLDG/LSEG, Centre DAM-Ile de France, 91297 Arpajon Cedex, France, elisabeth.blanc@cea.fr), Roger Roche (Commissariat à l'Energie Atomique, DASE/SLDG/LSEG, Centre DAM-Ile de France, 91297 Arpajon Cedex, France, roger.roche @ cea.fr), Christophe Millet (Commissariat à l'Energie Atomique, DASE/SLDG/LSEG, Centre DAM-Ile de France, 91297 Arpajon Cedex, France, christophe.millet@cea.fr), Francoise Le Piver (Commissariat à l'Energie Atomique, DASE/SLDG/LSEG, Centre DAM-Ile de France, 91297 Arpajon Cedex, France, francoise.lepiver@cea.fr), Bruno Despres (Commissariat à l'Energie Atomique, DSSI, Centre DAM-Ile de France, 91297 Arpajon Cedex, France, bruno.despres@cea.fr), Pierre-Franck Piserchia (Commissariat à l'Energie Atomique, DASE/SLDG/LSEG, Centre DAM-Ile de France, 91297 Arpajon Cedex, France, pierre-franck .piserchia@cea.fr)

In the framework of the Comprehensive Nuclear-Test-Ban Treaty, the International Monitoring System develops a 60 micro-barometric stations network. These stations, which records infrasound, detect various powerful natural and artificial sources like long range explosions, oceanic swell, and volcano eruptions. For data analysis, the CEA, in collaboration with the LMFA, develops specific methods based on measurements, data processing and numerical simulation. The Misty Picture experiment is a high explosive event (4685 Tons of ANFO) realized in 1987 in New Mexico (US). Infrasounds were recorded by 22 sensors until a distance of $1000 \mathrm{~km}$ in a quiet background noise condition. Multireflected tropospheric, stratospheric and thermospheric phases are detected. Signals recorded near the source $(1 \mathrm{~km}$ away) and observed in the geometrical shadow zone (between $150 \mathrm{~km}$ and $250 \mathrm{~km}$ from the point source) are of particular interest. This reference experiment very well documented is used to improve our understanding of the atmospheric propagation of infrasound as well as to evaluate our models Using various methods such as ray tracing, parabolic equation and finite differences, we investigate effects of the wind, atmospheric absorption, nonlinearity, refraction, (2D and 3D) and scattering by small atmospheric scales on observed phase kinds, their travel time and their waveform.

\section{Invited Paper}

5aPAa16. A low power and low noise infrasound sensor for temporary measurements. Damien Ponceau (CEA/DASE, BP12, Service TMG, 91680 Bruyères le Chatel, France, damien.ponceau@cea.fr)

Temporary infrasound measurements require low power consumption, reliable, and compact equipments. As a consequence, these equipments are not always as accurate as equipments operating on long term stations. Measurements quality can be affected. DASE has developed a portable infrasound sensor from MB2005 microbarometer. This new microbarometer consists in improved mechanics from MB2005 and in a movement transducer providing a voltage directly proportional to pressure derivate all over infrasound bandwidth and even more: sensor response phase is purely independent from environment (temperature, condensationœ) all over the same bandwidth. Moreover, thanks to its very simple design this sensor needs very low power and is lighter, smaller and cheaper than previous generation sensors. It reaches Infrasound Low Noise Model (IS LNM) down to $0.02 \mathrm{~Hz}$ and is very portable as it weights less than $3 \mathrm{~kg}$ with less than $1 \mathrm{~L}$. A batch of prototypes has been manufactured and evaluated in lab thanks to an infrasound generator used for routine calibration of microbarometers installed by DASE on some International Monitoring System stations (IMS). Sensors field evaluation is running. First results are promising. 


\title{
Session 5aPAb
}

\section{Physical Acoustics: Time Reversal Acoustics for Nonlinear Imaging I}

\author{
Alexander Sutin, Cochair \\ Stevens Institute of Technology, Castle Point on Hudson, Hoboken, NJ 07030, USA
}

Serge Dos Santos, Cochair

ENI Val de Loire, Université François Rabelais de Tours, LUSSI CNRS, rue de la Chocolaterie, Blois, 41034, France

\section{Invited Papers}

\section{8:00}

5aPAb1. Time reversal of acoustic waves in the nonlinear regime: basic physics and application to ultrasound contrast imaging Mickael Tanter (Laboratoire Ondes et Acoustique, ESPCI, Université Paris 7, CNRS, 10 rue Vauquelin, 75005 Paris, France, michael.tanter@espci.fr), Olivier Couture (Laboratoire Ondes et Acoustique, ESPCI, Université Paris 7, CNRS, 10 rue Vauquelin, 75005 Paris, France, olivier.couture@espci.fr), Mathias Fink (Laboratoire Ondes et Acoustique, ESPCI, Université Paris 7, CNRS, 10 rue Vauquelin, 75005 Paris, France, mathias.fink@espci.fr)

Time reversal invariance of acoustic waves propagation remains valid even in the nonlinear regime in nondissipative media. This very interesting property that was extensively used in linear acoustics can also find potential applications in the nonlinear regime. Harmonic imaging coupled to the use of contrast agents is a topic of wide interest in ultrasonic medical imaging. Images are built at twice the excitation frequency that corresponds to the resonance frequency of the bubbles embedded in the medium. Consequently, the contrast becomes important between areas of low and high concentration of bubbles. However, at a high mechanical index, the harmonic components of backscattered echoes depends on the intrinsic nonlinear properties of tissues as well as the bubble's resonance. The focused beam is generating harmonics during its nonlinear propagation. It results in a degradation of the harmonic image contrast. Time reversal is an elegant way to find the emission codes allowing us to cancel the harmonic components due to nonlinear propagation of the ultrasonic beam. In the absence of bubbles, if the backscattered echoes are time-reversed and reemitted by the array, the harmonic components of the resulting wavefront are transferred back to the fundamental frequency during propagation. Experiments conducted with 1D-linear arrays illustrate these cancellation techniques.

\section{$8: 20$}

5aPAb2. Localized nonlinearity time reversal imaging with chaotic cavities. Olivier Bou Matar (IEMN, UMR CNRS 8520, avenue Poincaré, BP 60069, 59652 Villeneuve d'Ascq, France, olivier.boumatar@iemn.univ-lille1.fr), Yifeng Li (IEMN - LEMAC UMR CNRS 8520, Ecole Centrale de Lille, BP 48, Cite scientifique, 59651 Villeneuve D’Ascq, France, Yifeng.li@iemn.univ-lille1.fr), Vladimir L. Preobrazhensky (IEMN, UMR CNRS 8520, avenue Poincaré, BP 60069, 59652 Villeneuve d'Ascq, France, vladimir.preobrajenski@iemn.univ-lille1.fr), Philippe Pernod (IEMN, UMR CNRS 8520, avenue Poincaré, BP 60069, 59652 Villeneuve d'Ascq, France, philippe.pernod@iemn.univ-lille1.fr)

Recent results have shown that nonlinear elastic wave spectroscopy (NEWS) and time reversal (TR) techniques can be combined to localize defects. NEWS techniques can be used either as a post-treatment of TR used as a tool for generating strong localized stress (TR-NEWS), or as a pretreatment, of TR used as a tool for defect identification (NEWS-TR). The TR process can be implemented with a programmable multielements ultrasonic system. But recently, it has been demonstrated that a single PZT ceramic glued on the sample can also be used as a TR mirror. In this case the quality of the retrofocusing process, and so the microdamage localization, depends on the sample geometry. In this study, numerical and laboratory studies, performed in order to determine and enhance the possibility to localize microdamage based on a combination of a time reversal technique using an emitter with a chaotic shape and nonlinear elastic wave response, will be presented. The benefit of using such emitters is clearly found in breaking the symmetry of the problem, leading to an unambiguous retrofocusing. Moreover, chirp-coded excitation, instead of short pulses, is studied in order to transmit more energy on the defect without increasing the peak intensity of the excitation.

\section{$8: 40$}

5aPAb3. Landmine detection using nonlinear vibrations excited by time reversal techniques. Brad Libbey (U.S. Army RDECOM CERDEC NVESDS, 10221 Burbeck Rd, Fort Belvoir, VA 22060, USA, brad.libbey@us.army.mil), Alexander Sutin (Stevens Institute of Technology, Castle Point on Hudson, Hoboken, NJ 07030, USA, asutin@stevens.edu), Armen Sarvazyan (Artann Laboratories, 1753 Linvale-Harbourton, Lambertville, NJ 08350, USA, armen@artannlabs.com)

Time reversal focuses seismic waves and excites nonlinear surface vibrations that are large in the presence of a landmine. These soil vibrations provide an accurate localization cue when contrasted with measurements made without a mine. Traditionally nonlinear effects were investigated by measuring the interaction of harmonic waves and nonlinear resonance frequency shifts. These methods provide high signal to noise ratios, but required tuning of excitation frequencies resulting in test times unsuitable for field applications. Time reversal is capable of providing broad band excitation at amplitudes large enough to drive the nonlinear mechanisms at measurable levels while reducing the overall measurement time. Spatial and temporal focusing data will be presented for three different time reversal systems compromising speakers and vibrators for seismic excitation and a Doppler laser vibrometer and geophones for signal 
recording. The high amplitude signals generated by these systems adequately drive the nonlinear responses. Special processing techniques, phase inversion and varied amplitude, have been developed to extract the nonlinear responses from the broadband response by cancelling the linear part. The remaining nonlinear response shows greater mine/no mine contrast than the linear data. Practical implementations of the developed methods will be discussed.

\section{9:00}

5aPAb4. Separation of multiple scatterers in NEWS-TR experiments. Sigfried Vanaverbeke (K.U.Leuven Campus Kortrijk, E. Sabbelaan 53, 8500 Kortrijk, Belgium, Sigfried.Vanaverbeke@kuleuven-kortrijk.be), Lieven De Lathauwer (K.U.Leuven Campus Kortrijk, E. Sabbelaan 53, 8500 Kortrijk, Belgium, Lieven.DeLathauwer@kuleuven-kortrijk.be), Herbert De Gersem (K.U.Leuven Campus Kortrijk, E. Sabbelaan 53, 8500 Kortrijk, Belgium, Herbert.DeGersem@kuleuven-kortrijk.be), Koen Van Den Abeele (K.U .Leuven Campus Kortrijk, E. Sabbelaan 53, 8500 Kortrijk, Belgium, koen.vandenabeele@kuleuven-kortrijk.be)

Nonlinear elastic wave spectroscopy combined with acoustic time reversal (NEWS-TR) is a promising new methodology for detecting microdamage at an early stage. When dealing with structures which could potentially contain many pointlike nonlinear scatterers, there is a need to develop techniques for separately imaging the defects using a distributed sensor network which acts as a timereversal mirror. In this contribution, we present numerical simulations of a newly developed version of the DORT method for nonlinear imaging and also discuss the possibility of applying PARAFAC (parallel factor analysis) and ICA (independent component analysis) methods to solve the problem of separating multiple nonlinear scatterers in the time-frequency domain.

9:20

5aPAb5. Wide band time reversal acoustic nonlinear diagnostics. Tj Ulrich (EES-11 (Geophysics) - Los Alamos National Laboratory, MS D443, Los Alamos, NM 87545, USA, tju@lanl.gov), Alexandr Sutin (Stevens Institute of Technology, Castle Point on Hudson, Davidson Laboratory, Hoboken, NJ 07030, USA, asutin@stevens-tech.edu), Jim A. Tencate (EES-11 (Geophysics) - Los Alamos National Laboratory, MS D443, Los Alamos, NM 87545, USA, tencate@lanl.gov)

Time reversal acoustics (TRA) techniques provide a means to focus energy at a localized time and space, or at an unknown location (i.e., source or scatterer not known a priori) without the need to calculate time delays, as is necessary for phased arrays. The experiments demonstrate that time reversal focusing provides localized surface vibration with amplitude sufficient to induce measurable nonlinear effects and does so over a wide frequency band. Because the amplitude is spatially focused, time reversal enables local probing of this nonlinearity that can be used for nonlinear imaging. The nonlinear effects for wide band frequency signals were measured by phaseinversion method combining two signals of opposite sign in order to cancel the linear response and preserve quadratic nonlinearities. The method works effectively for nonlinear NDE of thin plates where laser Doppler Vibrometer can be used for measurements of the surface vibration and TRA focusing of acoustic waves. Presented results include crack and delamination diagnostic is epoxy graphite composites, diffusion bonded metallic disks and impact surface damage in glass. Scanning electron microscopy and optical images are also shown to demonstrate the types of damage features detected using this method.

9:40-11:00 Posters

Lecture sessions will recess for presentation of poster papers on various topics in acoustics. See poster sessions for topics and abstracts.

\section{Contributed Papers}

\section{1:00}

5aPAb6. Time reversal use in detection of buried cracks. Pierre-Yves Le Bas (EES-11 (Geophysics) - Los Alamos National Laboratory, MS D443, Los Alamos, NM 87545, USA, pylb@lanl.gov), Brian E. Anderson (EES-11 (Geophysics) - Los Alamos National Laboratory, MS D443, Los Alamos, NM 87545, USA, bea@lanl.gov), Michele Griffa (EES-11 (Geophysics) - Los Alamos National Laboratory, MS D443, Los Alamos, NM 87545, USA, mgriffa@lanl.gov), Tj Ulrich (EES-11 (Geophysics) - Los Alamos National Laboratory, MS D443, Los Alamos, NM 87545, USA, tju@lanl.gov), Paul A. Johnson (EES-11 (Geophysics) Los Alamos National Laboratory, MS D443, Los Alamos, NM 87545, USA, paj@lanl.gov)

Time reversal has the potential to become a powerful tool in nondestructive evaluation. Coupled with nonlinear properties of cracks in a technique known as time reverse nonlinear elastic wave spectroscopy (TRNEWS), it provides the means to detect defects in complex structures. This experimental study explores the capabilities of TR to focus energy inside a 3D medium in order to activate nonlinear features or defects. Special attention is given to buried cracks. The current challenge is introducing sufficient energy in order to excite the buried feature and produce nonlinear scattering. We will provide an overview of the problem.

\section{1:20}

5aPAb7. Computational time reversal acoustics imaging of embedded defects in solid media. Michele Griffa (EES-11 (Geophysics) - Los
Alamos National Laboratory, MS D443, Los Alamos, NM 87545, USA, mgriffa@lanl.gov), Brian E. Anderson (EES-11 (Geophysics) - Los Alamos National Laboratory, MS D443, Los Alamos, NM 87545, USA, bea@lanl.gov), Tj Ulrich (EES-11 (Geophysics) - Los Alamos National Laboratory, MS D443, Los Alamos, NM 87545, USA, tju@lanl.gov), Pierre-Yves Le Bas (EES-11 (Geophysics) - Los Alamos National Laboratory, MS D443, Los Alamos, NM 87545, USA, pylb@lanl.gov), Paul A. Johnson (EES-11 (Geophysics) - Los Alamos National Laboratory, MS D443, Los Alamos, NM 87545, USA, paj@lanl.gov), Lianjie Huang (EES-11 (Geophysics) - Los Alamos National Laboratory, MS D443, Los Alamos, NM 87545, USA, ljh@lanl.gov)

Time reversal acoustics (TRA) techniques can exploit the nonlinear processes emerging from the interaction between the incident waves and nonlinear scatterers in solid media in order to localize and characterize the scatterers themselves. When nonlinear scatterers are embedded, their localization can be obtained through a mixed experimental/numerical TRA procedure: the forward propagation is performed experimentally, while the time reversal (TR) backward propagation is simulated using computational codes and a reference model of the solid. The synergetic use of dedicated processing of the forward propagation signals collected at the time reversal mirror (TRM), for example with nonlinear elastic wave spectroscopy (NEWS) techniques, and the calculation of the backpropagated wave fields at each point within the specimen leads to imaging of the nonlinear scatterers. We show examples of nonlinear scatterer (macroscopic cracks, distributed microcracks) imaging by such a procedure, exploiting high per- 
formance (parallel) computational codes. We address issues in the imaging method related to the discrepancy between the reference model of the specimen and the real specimen itself. Finally, we address the implementation of the technique for non-destructive Evaluation (NDE) real-world applications using multi-threaded computational codes to be run on common multicore desktop computers.

\section{Invited Paper}

\section{1:40}

5aPAb8. Improving signal analysis for nonlinear time reversed acoustics: simulation results. Marco Scalerandi (Politecnico di Torino, Corso Duca degli Abruzzi 24, 10129 Torino, Italy, marco.scalerandi@infm.polito.it), Antonio S. Gliozzi (Politecnico di Torino, Corso Duca degli Abruzzi 24, 10129 Torino, Italy, antonio.gliozzi@polito.it)

Nonlinear time reversed acoustics have attracted attention as a tool to localize and identify nonlinear defects in solids [1-4]. The procedure is based on extracting the features due to the nonlinear scatterers from the signals received at the transducer array and backpropagate only the resulting portion of the signals. The usual approach is to filter the received signals to keep only contributions due to higher order harmonics or sidebands. As a result, the signals are very small in amplitude and can be not detectable if transducers are located far from the scatterers. It is evident the need for novel signal processing tools to increase the signal-to-noise ratio of the nonlinear contributions. We have recently proposed a scaling subtraction method [5], which is applied here to nonlinear time reversal virtual experiments, showing the improvements in the signal-to-noise ratio, with resulting better imaging of the nonlinear scatterer. Results will be presented for two approaches to nonlinear time reversal. [1] A. S. Gliozzi et al., J. Acoust. Soc. Am. 120, 2506-2517 (2006). [2] T. J. Ulrich et al., J. Acoust. Soc. Am. 119, 1514-18 (2006). [3] T. J. Ulrich et al., Phys. Rev. Lett. 98, 10430 (2007). [4] T. Goursolle et al., J. Acoust. Soc. Am. 122, 3220 (2007). [5] M. Scalerandi et al., submitted to Appl. Phys. Lett. (2008).

\section{Contributed Papers}

\section{2:00}

5aPAb9. 3D numerical study for defect detection with nonlinear elastic wave spectroscopy and time reversal techniques. Thomas Goursolle (ENI Val de Loire, Université François Rabelais de Tours, LUSSI CNRS, rue de la Chocolaterie, 41034 Blois, France, thomas.goursolle @univ-tours.fr), Samuel Callé (LUSSI, 10 Bd Tonellé, 37032 Tours, France, calle_s@med.univ-tours.fr), Serge Dos Santos (ENI Val de Loire, Université François Rabelais de Tours, LUSSI CNRS, rue de la Chocolaterie, 41034 Blois, France, serge.dossantos@univ-tours.fr), Olivier Bou Matar (IEMN, UMR CNRS 8520, avenue Poincaré, BP 60069, 59652 Villeneuve d'Ascq, France, olivier.boumatar@iemn.univ-lille1.fr)

Nonlinear elastic wave spectroscopy (NEWS) techniques have been developed to study the anomalously high level of "nonclassical" nonlinearity of cracked materials. Within AERONEWS project (http://www.kuleuvenkortrijk.be/aeronews), more recent innovative nondestructive NEWS techniques have been proposed to detect microinhomogeneities like cracks. It has been notably shown that NEWS and time reversal (TR) techniques can be combined to precisely localize cracks with a high sensitivity. NEWS techniques can be used either as a post-treatment of TR used as a tool for strong localized stress generation (TR-NEWS), or as a pre-treatment of TR used as a tool for defect (nonlinear source) identification (NEWS-TR). A 3D multiscale pseudo-spectral time domain (PSTD) code has been developed to simulate nonlinear acoustic propagation in heterogeneous nonlinear hysteretic solids. The hysteretic nonlinearity is introduced owing to a PM space model, based on a multiscale approach, extended in 3D owning to Kelvin notations. Using this algorithm, the influence of various parameters such as the defect position or the filtering method is particularly described for NEWS-TR and TR-NEWS methods. Finally, the sensitivity and feasibility of both methods are discussed.

\section{2:20}

5aPAb10. Varied amplitude method in time reversal acoustic land mine. Laurent Fillinger (Artann Laboratories/ Stevens Institute of Technology, 711 Hudson St, Hoboken, NJ 07030, USA, laurent.fillinger @artannlabs.com), Alexander Sutin (Stevens Institute of Technology, Castle Point on Hudson, Hoboken, NJ 07030, USA, asutin@stevens.edu), Brad Libbey (U.S. Army RDECOM CERDEC NVESDS, 10221 Burbeck Rd, Fort Belvoir, VA 22060, USA, brad.libbey@us.army.mil), Armen Sarvazyan (Artann Laboratories, 1753 Linvale-Harbourton, Lambertville, NJ 08350, USA, armen@artannlabs .com)

Acoustic methods of land mine detection are being developed for nonmetal mines where conventional electromagnetic methods fail. One such method, based on time reversal acoustic principles, is the hhase-inversion method; which detects quadratic and even ordered harmonic nonlinearities in the surface vibration above a mine. While this has shown higher sensitivity than linear methods it cannot detect all nonlinear components. These components can be detected by the Varied Amplitude method that is based on wide band excitation of surface vibration using the principles of time reversal acoustics. The nonlinear effects are measured for surface vibrations with different amplitudes and the normalized received signals can then be subtracted to reveal all the nonlinear components. We conducted a set of experiments using a box with six loudspeaker and an array of spatially distributed geophones to measure the linear and nonlinear components for various types of mines in different soils. Information about nonlinear components' spatial distribution allows one to observe greater mine/no mine contrast than with linear and other nonlinear techniques. [Work was supported by the U.S. Army RDECOM CERDEC Night Vision and Electronic Sensors Directorate.] 


\title{
Session 5aPAc
}

\section{Physical Acoustics: Vibrations in Plates I}

\author{
Daniel Royer, Cochair \\ Laboratoire Ondes et Acoustique, ESPCI, Université Paris 7, CNRS, 10 rue Vauquelin, Paris, 75005, France \\ Olivier Poncelet, Cochair \\ LMP, UMR CNRS 5469, Université Bordeaux I, 351, cours de la Libération, Talence, 33405, France
}

\section{Contributed Papers}

\section{8:00}

5aPAc1. Discussion on the validity domain of SEA. Alain Le Bot (CNRS, LTDS Ecole centrale de Lyon 36, av. Guy de Collongue, 69134 Ecully, France, alain.le-bot@ec-lyon.fr), Vincent Cotoni (ESI Group, 12555 High Bluff Dr., suite 250, San Diego, CA 92130, USA, vincent.cotoni @esi-group-na.com)

This paper is concerned with the validity domain of SEA which is defined in terms of four criteria. The mode count $\mathrm{N}$ and the modal overlap $\mathrm{M}$ must be high, the normalized attenuation factor $\mathrm{m}$ and the coupling strength gamma must be small. The application of the dimensional analysis on the governing equations of plates gives the space of dimensionless parameters in which the validity domain of SEA must be delimited. This domain is discussed on the basis of the geometric properties of the surfaces delimiting it. The diagrams of validity of SEA are introduced and proposed as a tool for a practical use of SEA.

\section{8:20}

5aPAc2. Lamb waves filtering by waveguides with linear varying section. Zahia Hamitouche (LOMC FRE-3102 CNRS, Groupe Ondes Acoustiques, University of Le Havre, Place Robert Schuman, BP 4006, 76610 Le Havre, France, zahia.hamitouche@univ-lehavre.fr), Mounsif Ech-Cherif El-Kettani (LOMC FRE-3102 CNRS, Groupe Ondes Acoustiques, University of Le Havre, Place Robert Schuman, BP 4006, 76610 Le Havre, France, elkettani@univ-lehavre.fr), Hakim Djelouah (Faculty of Physics, University of Science and Technology Houari Boumedienne, BP 32 El Allia, Bab-Ezzouar, 16000 Algiers, Algeria, djelouah_hakim @ yahoo.fr), Jean-Louis Izbicki (LOMC FRE-3102 CNRS, Groupe Ondes Acoustiques, University of Le Havre, Place Robert Schuman, BP 4006, 76610 Le Havre, France, jean-louis.izbicki@univ-lehavre.fr)

This experimental and numerical work deals with the study of Lamb modes propagating in a plate with a linear variation of thickness. The varying section area exhibits a filtering phenomenon for Lamb modes having a frequency-thickness cutoff. If the incident Lamb wave, excited at a given frequency and propagating downslope, reaches its thickness cutoff in the varying section area, it is reflected and propagates upslope. Otherwise, it is transmitted outside the varying section domain with modes conversion. The S2 incident mode is a particular case: when it reaches its cutoff, it is converted into the $\mathrm{S} 1$ mode with negative groupe velocity and a downslope propagation; then the S1 Lamb wave is reflected at its cutoff. The experimental and numerical studies are in good agreements.

\section{$8: 40$}

5aPAc3. Experimental study of lamb mode conversions at the junction of two joined plates. Taoufiq Belhoussine Drissi (LOMC FRE-3102 CNRS, Groupe Ondes Acoustiques, University of Le Havre, Place Robert Schuman, BP 4006, 76610 Le Havre, France, taoufiq_belhoussine_drissi@yahoo.fr), Bruno Morvan (LOMC FRE-3102
CNRS, Groupe Ondes Acoustiques, University of Le Havre, Place Robert Schuman, BP 4006, 76610 Le Havre, France, bruno.morvan@univ-lehavre.fr), Pascal Pareige (LOMC FRE-3102 CNRS, Groupe Ondes Acoustiques, University of Le Havre, Place Robert Schuman, BP 4006, 76610 Le Havre, France, pascal.pareige@univ-lehavre.fr)

In this study we are interested in a welded junction of two plates. Plates have the same thickness and are joined along their edges. Some previous theoretical studies have shown that guided Lamb waves are suitable for the characterisation of the interface between two plates [C. Scandrett and N. Vasudevan \& M.V Predoi and M. Rousseau]. In a first time a junction perpendicular to the plates is experimentally investigated. Aluminum, copper and steel plates are used. Incident S0 Lamb wave is excited by a contact piezocomposite transducer and the surface displacements of the plate are detected by use of a laser vibrometer on both side of the junction. Converted waves are observed. From the measured normal surface displacements, reflection and transmission energy coefficients of the incident S0 wave through the junction are calculated. These coefficients are in a good agreement with the theoretically predicted ones. In a second time, the studied junction is not perpendicular to the plate. Experimental results are presented and compare to numerical ones obtained with the $\mathrm{Comsol}^{\circledR}$ Finite Element code

\section{9:00}

5aPAc4. Second order dispersive effect on zero-group velocity Lamb modes. Daniel Royer (Laboratoire Ondes et Acoustique, ESPCI, Université Paris 7, CNRS, 10 rue Vauquelin, 75005 Paris, France, daniel.royer@espci.fr), Dominique Clorennec (Laboratoire Ondes et Acoustique, ESPCI, Université Paris 7, CNRS, 10 rue Vauquelin, 75005 Paris, France, dominique.clorennec@espci.fr), Claire Prada (Laboratoire Ondes et Acoustique, ESPCI, Université Paris 7, CNRS, 10 rue Vauquelin, 75005 Paris, France, claire.prada-julia@espci.fr)

After a local and transient loading of a plate, the acoustic energy decay in the source area can be explained by three mechanisms: the energy transport phenomenon at the Lamb wave group velocity, the material damping and the second-order dispersive effect. Generally, the first mechanism dominates the other two. However, some dispersion curves of Lamb modes present a minimum frequency for a nonzero wave number. Since at these points the group velocity vanishes, no energy transport occurs, and the slower other two phenomena can be observed. Using laser-based ultrasonic techniques, we show that the mechanical response of various thin metallic plates to a laser pulse impact is dominated by the resonance of the zero group velocity $\mathrm{S}_{1}$-Lamb mode. In the first microseconds, we observed a $\mathrm{t}^{-1 / 2}$ decay of the local vibration amplitude, which cannot be explained by the material damping. We demonstrated that this effect is ascribed to the second-order term in the dispersion relation. After this power law decay, the 
mechanical displacement undergoes an exponential decay corresponding to the wave damping. Then, we show that the local attenuation of the plate material can be measured without any mechanical contact.

\section{9:20}

5aPAc5. Guided waves in plates with linear variation of thickness. Mihai Valentin M. Predoi (University Politechnica of Bucharest, Department of Mechanics, 060032 Bucharest, Romania, predoi @cat.mec.pub.ro), Mounsif Ech Cherif El Kettani (LOMC FRE-3102 CNRS, Groupe Ondes Acoustiques, University of Le Havre, Place Robert Schuman, BP 4006, 76610 Le Havre, France, mounsif.elkettani @ univ-lehavre.fr), Zahia Hamitouche (LOMC FRE-3102 CNRS, Groupe Ondes Acoustiques, University of Le Havre, Place Robert Schuman, BP 4006, 76610 Le Havre, France, zahia.hamitouche@univ-lehavre.fr) , Cristian Catalin Petre (University Politechnica of Bucharest, Department of Mechanics, 060032 Bucharest, Romania, cristian.petre@promteh.ro)

Guided waves represent promising nondestructive evaluation (NDE) techniques. Their advantage of long distance propagation is however hampered by complex wave scattering at each discontinuity along the investigated structure. These scattered waves can be used to locate and size possible defects. Theoretical investigation is a prerequisite step in the development of a NDE technique. Planar metallic structures with localized thickness variation imposed by design represent possible new industrial structures that could be tested using guided waves. The waves scattered at the region of variable thickness must be theoretically predicted and experimentally measured before any defect could be detected. Very few authors have investigated waveguides with continuous variation of thickness. Our previous numerical and experimental studies have proven the complexity of the scattering phenomenon in such cases. Among other remarks is the curved shape of the wave front. The present work investigates properties of the waves in the linear thickness variation region of the waveguide. A plain strain model, developed in polar coordinates is theoretically developed and numerically tested using specialized finite elements based algorithms. Specific properties such as stress and displacements fields are determined by numerical simulation at several frequencies and dihedral angles.

9:40-10:40 Posters

Lecture sessions will recess for presentation of poster papers on various topics in acoustics. See poster sessions for topics and abstracts.

\section{Contributed Papers}

$10: 40$

5aPAc6. Refracting characteristics of backward propagating Lamb modes. Massimo Germano (Sapienza Università di Roma, Via A. Scarpa, 14, 00161 Rome, Italy, massimo.germano@uniroma1.it), Adriano Alippi (Sapienza Università di Roma, Via A. Scarpa, 14, 00161 Rome, Italy, adriano.alippi@uniroma1.it), Andrea Bettucci (Sapienza Università di Roma, Via A. Scarpa, 14, 00161 Rome, Italy, andrea.bettucci@uniroma1 it), Annunziata D’Orazio (Sapienza Università di Roma, Via A. Scarpa, 14,00161 Rome, Italy, annunziata.dorazio@uniroma1.it)

Lamb waves are acoustic modes propagating along thin plates or shells and result from the coupling of longitudinal waves and shear vertical waves (polarized along the thickness direction), taking place at the surface boundaries for satisfying the boundary conditions of the waveguide. Their nature being a composition of two differently polarized components, it permits that, for some combination of frequency and wavenumber, a Lamb mode is propagating which has its group velocity contradirected with respect to the phase velocity. These are the so-called "backward" Lamb modes, and one of them takes place at the starting region (low wave number) of the $S_{1}$ mode dispersion curve in aluminum. Peculiar features of a wave packet of a symmetric Lamb mode $S_{1}$, backward propagating in an aluminum waveguide, are observed and anomalous behavior in refraction, diffraction and interference of this peculiar mode is experimentally put in evidence. Particularly, contradirected refraction is put in evidence, as it takes place at the boundary where a forward propagating mode is coupled to a backward propagating one, and change from forward to backward mode is obtained in case of a wedge shaped laminar plate, which goes through a zero point of the group velocity.

\section{1:00}

5aPAc7. Conversion of a Scholte - Stoneley wave at the extremity of a fluid loaded plate: comparison between finite element method, radiation mode theory and experiments. Ebrahim Lamkanfi (Ghent University, Sint-Pietersnieuwstraat 41, 9000 Ghent, Belgium, Ebrahim.Lamkanfi @ugent.be), Nico F. Declercq (Georgia Tech Lorraine - G.W. Woodruff School of ME, UMI Georgia Tech - CNRS 2958, 2 rue Marconi, 57070 Metz, France, nico.declercq@me.gatech.edu), Wim Van Paepegem (Ghent University, Sint-Pietersnieuwstraat 41, 9000 Ghent, Belgium, wim.vanpaepegem@ugent.be), Joris Degrieck (Ghent University, SintPietersnieuwstraat 41,9000 Ghent, Belgium, joris.degrieck@ugent.be)

The radiation theory has proved to produce results in agreement with experiments when the conversion is investigated of a Scholte - Stoneley wave at the extremity of a fluid loaded plate. The drawback of the radiation mode theory is that it is not universally applicable and is also very cumbersome. The advantage is that it is an exact method. There is a trend in acoustics to develop finite element models to describe the interaction of sound waves with materials. This investigation compares a newly developed finite element model to simulate the considered effect. Results are compared with the exact results obtained by the radiation mode theory [J. Acoust. Soc. Am. 101(3), 1347-1357, 1997] and experimental observations [J. Acoust. Soc. Am. 95(1), 13-20, 1994]. The study shows correspondence between the finite element analysis and earlier obtained experimental and theoretical results as a function of a relationship between mesh properties and the evanescence (rather than the wave length) of the considered Stoneley waves. 


\title{
Session 5aPAd
}

\section{Physical Acoustics: Time Reversal Acoustics for Nonlinear Imaging II (Poster Session)}

\author{
Alexander Sutin, Cochair \\ Stevens Institute of Technology \\ Serge Dos Santos, Cochair \\ ENI Val de Loire, Université François Rabelais de Tours, LUSSI CNRS
}

\begin{abstract}
All posters will be on display from 9:40 a.m. to 11:20 a.m. To allow contributors an opportunity to see other posters, contributors of odd-numbered papers will be at their posters from 9:40 a.m. to 10:30 a.m. and contributors of even-numbered papers will be at their posters from 10:30 a.m. to 11:20 a.m.
\end{abstract}

\section{Contributed Papers}

5aPAd1. Nonlinear phase conjugate ultrasonic waves in moving media. Vladimir L. Preobrazhensky (IEMN, UMR CNRS 8520, avenue Poincaré, BP 60069, 59652 Villeneuve d'Ascq, France, vladimir.preobrajenski @iemn.univ-lille1.fr), Philippe Pernod (IEMN, UMR CNRS 8520, avenue Poincaré, BP 60069, 59652 Villeneuve d'Ascq, France, philippe .pernod@iemn.univ-lille1.fr), Yuri Pyl’Nov (Moscow Institute of Radio Engineering, Electronics \& Automation, Vernadsky prosp., 78, 119454 Moscow, Russian Federation, pylnov@yandex.ru), Nikolay Smagin (Moscow Institute of Radio Engineering, Electronics \& Automation, Vernadsky prosp., 78, 119454 Moscow, Russian Federation, pylnov@yandex.ru)

The basic principle of acoustic wave phase conjugation provides compensation of phase incursions during back propagation of the phase conjugate waves $(\mathrm{PCW})$ towards the source of the primary emission. This feature of PCW results from time reversal invariance of acoustic field in a stationary medium. In moving media or in the presence of flows the time reversal invariance is broken resulting in essential modification of PCW properties. The incident wave spatial distribution is not reproduced completely by the phase conjugate wave and the phase incursions are not compensated any more. In the present paper the general theory of PCW propagation in moving nonlinear media is developed. The theory is applied for description of PCW acoustic rays and calculation of noncompensated phase shift of PCW on the source of the primary probing wave. The treatment of the phase shift of the PCW is developed as a new method of diagnostics and imaging of flow velocity distributions in liquid. The method is approved experimentally on examples of laminar and vortex flows and immersed fluid jet in water. Improvement of quality of the images of flows using nonlinear modes of propagation and interaction of phase conjugate waves is demonstrated.
5aPAd2. Evaluation of local nonlinearity using time reversal acoustic focusing. Serge Dos Santos (ENI Val de Loire, Université François Rabelais de Tours, LUSSI CNRS, rue de la Chocolaterie, 41034 Blois, France, serge.dossantos@univ-tours.fr), Alexander Sutin (Stevens Institute of Technology, Castle Point on Hudson, Hoboken, NJ 07030, USA, asutin @ stevens.edu), Armen Sarvazyan (Artann Laboratories, 1753 LinvaleHarbourton, Lambertville, NJ 08350, USA, armen@artannlabs .com), Thomas Goursolle (ENI Val de Loire, Université François Rabelais de Tours, LUSSI CNRS, rue de la Chocolaterie, 41034 Blois, France, thomas.goursolle@univ-tours.fr)

Time reversal acoustic focusing can be used for local assessment of nonlinear properties of media [Sarvazyan A., Sutin A. , JASA, 115 (5): 2595 (2004)]. Time Reversal focusing provides high acoustic pressure leading to a nonlinear response localized in space and time. Interaction area of two overlapping focused beams acts as a virtual localized source generating ultrasonic waves with combination frequencies. Measurements of the intensity of this signal allow estimation of the nonlinear parameter in the focal region. The first experiment was conducted in water with spatially varying acoustical nonlinearity produced by a microbubble column or contrast agent Optison. We used two synchronized TRA aluminium reverberators to focus ultrasonic waves with frequencies $660 \mathrm{kHz}$ and $776 \mathrm{kHz}$. Various parameters such as amplitude and frequency of the primary field, the position and number of emitters, choice of initial excitation (tone burst, binary, shirpcoded) were optimized for the nonlinear signature extraction. The second experiment was conducted in steel samples where surface vibration were measured by laser vibrometer. Excitation frequencies were between $490 \mathrm{kHz}$ and $860 \mathrm{kHz}$. These experiments show again that amplitude dependence of nonlinear generated components was slightly less than the product of primary component that is predicted by classical nonlinear theory. 


\title{
Session 5aPAe
}

\author{
Albert Migliori, Cochair \\ Los Alamos National Laboratory \\ Claude Boutin, Cochair \\ École Nationale des Travaux Publics de État
}

\section{Physical Acoustics: Ultrasonics: Transducers and Instruments I (Poster Session)}

\begin{abstract}
All posters will be on display from 9:40 a.m. to 11:20 a.m. To allow contributors an opportunity to see other posters, contributors of odd-numbered papers will be at their posters from 9:40 a.m. to 10:30 a.m. and contributors of even-numbered papers will be at their posters from 10:30 a.m. to 11:20 a.m.
\end{abstract}

\section{Contributed Papers}

5aPAe1. An ultrasonic technique using shear waves interactions to characterize new hybrid gels. Philippe Banet (ECIME Université de Cergy-Pontoise, rue d'Eragny, 95031 Neuville-sur-Oise, France, philippe.banet@u-cergy.fr), Pascal Griesmar (ECIME Université de CergyPontoise, rue d'Eragny, 95031 Neuville-sur-Oise, France, pascal.griesmar @u-cergy.fr), Frederic Vidal (LPPI - Université de Cergy-Pontoise, rue d'Eragny, 95031 Neuville-sur-Oise, France, frederic.vidal@u-cergy .fr), Jean-Yves Le Huerou (ECIME Université de Cergy-Pontoise, rue d'Eragny, 95031 Neuville-sur-Oise, France, jean-yves.lehuerou@u-cergy .fr), Claude Chevrot (LPPI - Université de Cergy-Pontoise, rue d'Eragny, 95031 Neuville-sur-Oise, France, claude.chevrot@u-cergy.fr), Dominique Teyssie (LPPI - Université de Cergy-Pontoise, rue d'Eragny, 95031 Neuville-sur-Oise, France, dominique.teyssie@u-cergy.fr), Stéphane Serfaty (ECIME Université de Cergy-Pontoise, rue d'Eragny, 95031 Neuville-sur-Oise, France, stephane.serfaty@u-cergy.fr)

Thickness shear mode transducer techniques using AT-cut quartz crystal can be used to measure viscoelastic parameters of soft materials (gels, colloidal suspensions $œ)$ during their formation. Today the control of hybrid materials synthesis and evolution has a great interest, particularly for biomedical applications such as drug delivery. With those materials, a challenge is to obtain new structures constituted by interpenetrating organic- inorganic polymers networks (IPN) to get novel therapeutic behaviours. In this paper, the monitoring of ultrasonic shear waves propagation in the material at 6 $\mathrm{MHz}$ is presented for different thermosensible hybrid (poly-Nisopropylacrylamide (PNIPAM) - silica). We show that at a mesoscopic scale it is possible to analyze mechanical interactions between different networks related to chemical bounds. The network evolution observed with our technique is in good agreement with the Fourier transform infrared analysis of NIPAM polymerization. As shown in previous work for silica gels, a precursor characteristic time of gelation process (in liquid phase) can be determined to describe the polymerization kinetic. By its nondestructive character and its simplicity of measurement, this technique allows an online optimization of new hybrid materials.

5aPAe2. Effects of materials conductivity on the viscosity measurement using a QCM. Stéphane Serfaty (ECIME Université de Cergy-Pontoise, rue d'Eragny, 95031 Neuville-sur-Oise, France, stephane.serfaty@u-cergy .fr), Emmanuel Caplain (ECIME Université de Cergy-Pontoise, rue d'Eragny, 95031 Neuville-sur-Oise, France, emmanuel.caplain@u-cergy fr), Pascal Griesmar (ECIME Université de Cergy-Pontoise, rue d'Eragny, $95031 \quad$ Neuville-sur-Oise, France, pascal.griesmar@u-cergy .fr), Jean-Yves Le Huerou (ECIME Université de Cergy-Pontoise, rue
d'Eragny, 95031 Neuville-sur-Oise, France, jean-yves.lehuerou@u-cergy .fr), Marcel Gindre (ECIME Université de Cergy-Pontoise, rue d'Eragny, 95031 Neuville-sur-Oise, France, marcel.gindre@u-cergy.fr)

Quartz crystal microbalance (QCM) is commonly used to characterize the viscosity of soft materials. For biomedical applications the modified BVD model of QCM is unsuitable due to the conductivity of the biomaterial. In order to take into account the electrical effects, a new model including a static lossy capacitor is proposed. A theoretical study of the shear wave propagation in the quartz shows that these effects modify the static and the motional branches of the BVD circuit. The conductivity effects of the material at the surface of the QCM can be modeled by same parallel elements added in both branches. In the motional branch the electromechanical coupling factor is applied to these elements. To validate the new lumped element model measurements for $\mathrm{KCl}$ mixtures are achieved. The results show that an accurate extraction of viscosity $(<5 \%)$ can be obtained for a middle of conductivity less than $0.3 \mathrm{~S} / \mathrm{m}$. In addition for water/glycerol mixtures the resonant frequency shift and damping follow an accurate linear shape $(<5 \%)$ according to the square root of the liquid viscosity and density product. These results increase (up to 200 times) the validity domain of viscosity compared to the classical modified BVD model.

5aPAe3. Viscosimetry using a new electromagnetic-acoustic microbalance. Emmanuel Caplain (ECIME Université de Cergy-Pontoise, rue d'Eragny, 95031 Neuville-sur-Oise, France, emmanuel.caplain @u-cergy.fr), Stéphane Serfaty (ECIME Université de Cergy-Pontoise, rue d'Eragny, $95031 \quad$ Neuville-sur-Oise, France, stephane.serfaty @u-cergy.fr), Pascal Griesmar (ECIME Université de Cergy-Pontoise, rue d'Eragny, 95031 Neuville-sur-Oise, France, pascal.griesmar@u-cergy .fr), Jean-Yves Le Huerou (ECIME Université de Cergy-Pontoise, rue d'Eragny, 95031 Neuville-sur-Oise, France, jean-yves.lehuerou @u-cergy.fr), Nicolas Wilkie-Chancellier (ECIME Université de CergyPontoise, rue d'Eragny, 95031 Neuville-sur-Oise, France, Nicolas.WilkieChancellier@u-cergy.fr)

The nanostructure evolution of gels, biomaterials or porous media can be characterized by its mechanical properties. Few nondestructive techniques are developed to investigate the viscosity evolution. This paper present a new electromagnetic-acoustic technique using a wireless thickness shear mode transducer. A suitable model of the measurement is also presented to characterize the viscosity of the nanostructure in contact with the transducer. This transducer is a double copper-clad PVDF substrate resonator, designed to operate over a wide radiofrequency range without lumped tuning capacitors. This architecture constitutes an alternative solution to design a high-Q ultrasonic microbalance. To characterize the material at the surface of the transducer, the evolution of the induced complex impedance is measured. From this evolution, the mechanical energy storage and dissipa- 
tion in the material can be extracted. In order to validate the lumped element model used, a series of glycerol/water mixtures is studied. We show that the resonant frequency shift and damping follow an accurate linear shape $(<5 \%)$ according to the square root of the liquid viscosity and density product. This result is in good agreement with the classical prediction of Martin and Kanazawa obtained with a quartz crystal microbalance.

5aPAe4. Functionally graded plates studied by laser ultrasonic technique. Yongdong Pan (Institute of Acoustics, Tongji University, 1239 Siping Road, 200092 Shanghai, China, ypan@mail.tongji.edu.cn), Qian Wang (Institute of Acoustics, Tongji University, 1239 Siping Road, 200092 Shanghai, China, wang_qian_@126.com), Zheng Zhong (School of Aerospace Engineering and Applied Mechanics, Tongji University, 1239 Siping Road, 200092 Shanghai, China, zhongk@mail.tongji.edu.cn), Clément Rossignol (LMP, UMR CNRS 5469, Université Bordeaux I, 351, cours de la Libération, 33405 Talence, France, c.rossignol@1mp.u-bordeaux1 .fr), Bertrand Audoin (LMP, UMR CNRS 5469, Université Bordeaux I, 351, cours de la Libération, 33405 Talence, France, b.audoin@1mp.ubordeaux1.fr)

In a functionally graded plate, the composition and structure change continuously along the thickness direction, resulting in corresponding changes of the acoustic impedance of the material. The study of the wave propagation in such a plate is important for both the nondestructive evaluation of properties of functionally gradient materials and engineering. In this work, the experimental system of laser ultrasonic measurement is introduced, and a theoretical model is proposed to predict the transient displacement field generated by laser in a functionally graded plate (FGP). After the laser line source is assumed as an idea transient force, and the FGP is considered as an inhomogeneous plate along its thickness direction, the transient displacement field is numerically solved by the Peano expansion method. Laser ultrasonic measurement was carried on several FGPs, and the direct arrivals of longitudinal and shear waves were observed in the experimental displacement field. The agreement between experimental and calculated theoretical waveforms provides a promise for both experimental and theoretical methods.

5aPAe5. Genetic manipulation engineers the elasticity of arabidopsis thaliana. Ari Salmi (Univ. of Helsinki / Dept. of Physical Sciences, POB 64 (Gustaf Hällströmin katu 2), 00014 Helsinki, Finland, edward.haeggstrom@helsinki.fi), Kimmo Mustonen (University of Helsinki, Gustaf Hällströmin Katu 2, 00015 Helsinki, Finland, kimmo.mustonen@helsinki.fi), Juha Immanen (Dept. of Biological and Environmental Sciences, P.O.B 65, 00014 Helsinki, Finland, juha.immanen@helsinki.fi), Yrjö Helariutta (Dept. of Biological and Environmental Sciences, P.O.B 65, 00014 Helsinki, Finland, yrjo.helariutta@helsinki.fi), Edward Hæggström (Electronics Research Unit, University of Helsinki, P.O.Box 64 (Gustaf Hällströmin katu 2), FIN00014 Helsinki, Finland, edward.haeggstrom@helsinki.fi)

Arabidopsis thaliana is a model species widely used in plant science studies. It grows fast and exhibits wood formation in its stem and root resembling softwood produced by angiosperm trees. The Arabidopsis model allows rapid testing of methods for wood composition modification possibly adaptable for tree breeding. We estimated ultrasonically the modulus of elasticity in Arabidopsis stems and roots in both radial and in-plane direction for two commonly used ecotypes (Columbia and Landsberg erecta). Plants were grown in greenhouse conditions and six inflorescence stems per ecotype were measured in vivo applying $4 \mathrm{MHz}$ out-of-plane and $2 \mathrm{MHz}$ in-plane ultrasonic tone bursts. The effects of AtCAD1 gene expression silencing in Col ecotype were tested using the same method. We recorded a $(10 \%$, $\mathrm{p}=0.1)$ decrease in the elastic modulus of the lignin-decreased Atcad 1 samples (10-20\% decrease in lignin content), and a significant $(29 \%, \mathrm{p}=0$ .02) difference between the sound velocities in the two ecotypes. In-plane elasticity results are also presented. The results are the first ultrasonically measured modulus values for this commonly used plant model species, creating a quantitative mechanical measure for the effects of wood composition modification.

5aPAe6. Nondestructive evaluation of heterogeneous materials using acoustic emission and ultrasound. Adil Faiz (ENSIM - LAUM, Université du Maine, rue Aristote, 72085 Le Mans, France, adil.faiz@univ-lemans.fr), Rachid El Guerjouma (ENSIM - LAUM, Université du Maine, rue Aristote, 72085 Le Mans, France, Rachid.El_Guerjouma@univ-lemans.fr), Mouras Bentahar (Université du Maine LAUM, Lab. d'Acoustique Université du Maine, UMR CNRS 6613 72085 Le Mans Cedex 9, France, mourad.bentahar@univ-lemans.)

Heterogeneous materials as composites and concrete are advantageous as structural components in many applications. However, damage detection in such materials is difficult due to their heterogeneity and anisotropy. Furthermore, conventional non destructive technique as $\mathrm{x}$-radiographic is mostly not very sensitive to early damage and very time consuming and expensive. The purpose of this contribution is to study the capabilities of several methods, nondestructive and very sensitive for damage characterisation, as acoustic emission (AE) and ultrasonic for the structural health monitoring of heterogeneous materials as polymer based composite materials and concrete. These materials are instrumented by piezoelectric sensors in order to detect acoustic emission and to measure the ultrasonic velocity. The mechanisms of the damage events and their space-time localizations are identified from AE. Simultaneously, the longitudinal ultrasonic velocity is measured in situ by transmission through the composite thickness. The AE is very well correlated with the loss of stiffness determined from ultrasonic velocity measurements showing the potentiality of this combined approach for in situ structural monitoring.

5aPAe7. Development of a system using ultrasonic waves for assessing the quality of frying oil and salad oil during frying. Driss Izbaim (Ibn Zohr University, FS Agadir, 80000 Agadir, Morocco, driss_izbaim@yahoo.fr), Bouazza Faiz (Ibn Zohr University, FS Agadir, 80000 Agadir, Morocco, faizbou@hotmail.com), Adil Hamine (Ibn Zohr University, FS Agadir, 80000 Agadir, Morocco, adil.hamine @Gmail.com), Naima Taifi (Fac. des sciences; Univ. Chouaib Doukkali, B.P: 20, 24000 El Jadida, Morocco, taifinaima@yahoo.fr), Ali Moudden (Ibn Zohr University, FS Agadir, 80000 Agadir, Morocco, ali _moudden@yahoo.fr)

The design and construction of a real-time system is described with reference to applications in quality control of frying oil. This system, developed using the LabVIEW ${ }^{\mathrm{TM}}$ software, controls the ultrasonic velocity by receiving the signals backscattered by a recipient full of oil. Measurements on samples of frying oil and salad oil are described and the results of velocity measurements on a group of samples of varying frying time taken in $160{ }^{\circ} \mathrm{C}$ are reported. The new system is built around three main elements: acquiring the oil signals, automating the signals control, and several calculations to determine in real-time the phase velocity of the oil. The results obtained show the feasibility of assessing the quality of oils using ultrasonic waves through monitoring the frying process. This system can be used also to control other industry products such as the canned fish oil, milk, and the cement.

5aPAe8. Influence of polymeric nanocomposite film on acoustic waves in piezoelectrics. Iren E. Kuznetsova (Institute of Radio Engineering and Electronics of RAS, Saratov Branch, Zelyonaya str., 38, 410019 Saratov, Russian Federation, kuziren@yandex.ru), Boris D. Zaitsev (Institute of Radio Engineering and Electronics of RAS, Saratov Branch, Zelyonaya str., 38 , 410019 Saratov, Russian Federation, zai-boris@yandex.ru), Anastasia S. Kuznetsova (Institute of Radio Engineering and Electronics of RAS, Saratov Branch, Zelyonaya str., 38, 410019 Saratov, Russian Federation, tigrnasya@yandex.ru)

At present the attention of researchers is attracted by investigation of multilayer acoustical waveguides with using of well known as well as novel materials. Also in present time there exist a lot of papers devoted to novel nanocomposite polymeric materials. But the influence of such materials on parameters of acoustic waves in piezoelectrics did not study. In this connec- 
tion the paper is devoted to theoretical investigation of acoustic waves in structure "piezoelectric - polymeric nanocomposite film." The analysis was carried out by the example of polymeric nanocomposite film with various contents of $\mathrm{Fe}$ and $\mathrm{CdS}$ nanoparticles and lithium niobate plate or substrate. As a result of conducted calculation the velocity and attenuation per wavelength have been found for acoustic waves propagating in aforementioned structure. The conducted analysis has showed that for certain value of nano- composite film thickness $d$ the attenuation of investigated waves has the resonant behavior. The obtained results allow choose such values of $\mathrm{d}$ for which the acoustic wave does not practically attenuate in the presence of the film. This opens, for example, the prospects of development of undisturbed substrates of nanocomposite materials for thin piezoelectric plates. This work is supported by RFBR 06-08-01011, RNP.2.1.1.8014 (Russia), contract 2007-3-1.3-07-15-036 (Russia).

FRIDAY MORNING, 4 JULY 2008

P3-A, LEVEL 3, 9:40 TO 11:20 A.M.

\title{
Session 5aPAf
}

\section{Physical Acoustics: Infrasound II (Poster Session)}

\author{
Henry Bass, Cochair \\ The University of Mississippi - NCPA \\ Alexis Le Pichon, Cochair \\ $C E A-D A S E$
}

\begin{abstract}
All posters will be on display from 9:40 a.m. to 11:20 a.m. To allow contributors an opportunity to see other posters, contributors of odd-numbered papers will be at their posters from 9:40 a.m. to 10:30 a.m. and contributors of even-numbered papers will be at their posters from 10:30 a.m. to 11:20 a.m.
\end{abstract}

\section{Contributed Papers}

5aPAf1. Close listening of a permanently degassing volcano: Yasur (Vanuatu). Christelle Zielinski (Institut de Physique du Globe, 4 Place Jussieu, 75252 Paris, France, zielinski@ipgp.jussieu.fr), Sylvie Vergniolle (Institut de Physique du Globe, 4 Place Jussieu, 75252 Paris, France, vergniolle@ipgp.jussieu.fr), Michel Lardy (IRD Center, BPA5, Cedex, 98848 Noumea, New Caledonia, lardy@noumea.ird.nc), Alexis Le Pichon (CEA-DASE, Arpajon Cedex, 91297 Bruyères-le-Châtel, France, alexis.le-pichon@cea.fr), Michel Frogneux (IPGS, 5, rue René Descartes, 67084 Strasbourg Cedex, France, michel.frogneux@eost.u-strasbg.fr)

Acoustic measurement is a very powerful technique to monitor gas volume and pressure at a safe distance of a volcano. Since eruptions are driven by a gas phase, acoustic recordings of eruptions can provide insights into the degassing processes. The permanent activity of Yasur volcano (Vanuatu) has been acoustically monitored continously to detect and understand the sudden increases in the volcanic degassing. In the absence of appropriate modeling of the sound source, we have used a dimensionless analysis of our infrasonic records close to the source to relate acoustic power and the velocity of the gas - ejecta mixture. Our one-year dataset at the source shows several sudden increases in gas flux over one week as well as more progressive evolution, over several months. Explosions are also detected using a method based on wavelet decomposition. This shows that most of the changes in gas flux are related to strong changes in the number of explosions. Understanding the origin of these variations has strong implications for hazards assessments.

5aPAf2. Low frequency airplane noise measurement. Xiao Di (National Center for Physical Acoustics, University of Mississippi, University, MS 38677, USA, xiaodi@olemiss.edu), Claus Hetzer (National Center for Physical Acoustics, University of Mississippi, University, MS 38677, USA, claus@olemiss.edu), Ronald A. Wagstaff (National Center for Physical Acoustics, University of Mississippi, University, MS 38677, USA, rwagstaf@olemiss.edu), Ali M. Mir (National Center for Physical Acoustics, University of Mississippi, University, MS 38677, USA, mali1 @olemiss.edu)
Low frequency airplane noise during takeoff and landing periods is a serious environment hazard. Also, the low frequencies originating from the airplanes could potentially be an easy target for detection. Since there is very little attenuation at the low frequencies, the low frequency sound can propagate to relatively long distances. Several field experiments were conducted to identify the low frequency signal and noise content from many different airplanes. What is different in our experiment from most other noise experiments is that we will identify the very low frequency content of the airplane noise spectrum, from $200 \mathrm{~Hz}$ down to the infrasound range, $0.02 \mathrm{~Hz}$. During the experiments, two infrasound microphone systems were used: Chaparral 50, which has a bandwidth from 0.02 to $50 \mathrm{~Hz}$, and Chaparral 2.5, which has a bandwidth from 0.1 to $200 \mathrm{~Hz}$. In addition to the infrasound systems, a recording system with $\mathrm{B} \& \mathrm{~K}$ microphones was also used. The data were collected before, during, and after landing and takeoff periods. The low frequency signal and noise content during each of those periods will be analyzed and reported on.

5aPAf3. Experimental study on the effects of $\mathrm{N}$-wave sonic-boom signatures on window vibration. Yusuke Naka (Japan Aerospace Exploration Agency, 6-13-1 Osawa, Mitaka, 181-0015 Tokyo, Japan, ynaka@chofu.jaxa.jp), Yoshikazu Makino (Japan Aerospace Exploration Agency, 6-13-1 Osawa, Mitaka, 181-0015 Tokyo, Japan, makino@chofu.jaxa.jp), Takeshi Ito (Japan Aerospace Exploration Agency, 6-13-1 Osawa, Mitaka, 181-0015 Tokyo, Japan, ito@chofu.jaxa.jp)

For fulfillment of overland supersonic flight, it is important to understand the effects of sonic booms on buildings, since these effects, including vibration of walls and rattling noise from windows, are believed to be the main causes of annoyance in indoor spaces. In this study, the vibration of windows impacted by sonic booms is investigated through in-laboratory experiments by using a device developed at Japan Aerospace Exploration Agency (JAXA). Rattle is captured as high-frequency components of the acceleration of the windows. The effects of sonic booms having $N$-shape signatures with various values of peak overpressure, rise time, and duration are investigated. Results show general tendency of increase of rattle with increasing peak overpressure and decreasing rise time, as expected. However, 
the results also indicate a possibility that these parameters, as well as duration, affect the time-frequency structure of the acceleration (and hence rattle) of windows in a somewhat complicated manner.

5aPAf4. Correlating electromagnetical and infrasonic signals from thunderstorms. Jelle Assink (Royal Netherlands Meteorological Institute (KNMI), PO Box 201, 3730 AE De Bilt, Netherlands, jelle.assink@gmail.com), Laeslo G. Evers (Royal Netherlands Meteorological Institute (KNMI), PO Box 201, 3730 AE De Bilt, Netherlands, evers@knmi.nl), Iwan Holleman (Royal Netherlands Meteorological Institute (KNMI), PO Box 201, 3730 AE De Bilt, Netherlands, holleman@knmi $. \mathrm{nl})$

During thunderstorm activity, electromagnetical and infrasonic signals are emitted due to the process of lightning and thunder. It is shown that correlating infrasound detections with results from a electromagnetical lightning detection network is successful up to distances of $50 \mathrm{~km}$ from the infrasound array. Infrasound recordings clearly show blast wave characteristics, relatable to $\mathrm{CG}$ discharges, with a dominant frequency between 1-5 Hz. Amplitude measurements can partly be explained by the beam pattern of a line source. The ability to measure thunderstorm activity with infrasound arrays has both positive and negative implications for infrasound verification purposes.

5aPAf5. Coherency of infrasound studied with a large aperture infrasound array in the Netherlands. Johan Mentink (Royal Netherlands Meteorological Institute (KNMI), PO Box 201, 3730 AE De Bilt, Netherlands, mentink@knmi.nl), Laeslo G. Evers (Royal Netherlands Meteorological Institute (KNMI), PO Box 201, 3730 AE De Bilt, Netherlands, evers $@$ knmi.nl)

A large aperture infrasound array (LAIA) is being constructed in the Netherlands as geophysical part of an astronomical low frequency array, i.e., the LOFAR project. This array extends the detection capabilities of infrasound up to the $\mathrm{mHz}$ range, including acoustic-gravity and gravity waves as well. Owing to its large aperture $(80 \mathrm{~km})$ and number of interstation distances, this 30 element microbarometer array is ideally suited to study the coherency of infrasound as function of frequency under various atmospheric conditions. Based on conventional and modern correlation techniques, the ultimate aim is to experimentally determine and quantify infrasound propagation characteristics. As a result, we gain understanding in the physical mechanisms responsible for decoherence, which in turn will be used to develop infrasound as a passive atmospheric probe. Knowledge on the coherency length as function of the state of the atmosphere also has direct implications for the design of optimal infrasound arrays. This study will outline the characteristics of LAIA and present the first results of the coherency studies.
5aPAf6. Modeling real porous tube infrasonic arrays. Timothy Marston (The Graduate Program in Acoustics, The Pennsylvania State University, PO Box 30, State College, PA 16804, USA, tmm357@psu.edu), Thomas Gabrielson (The Graduate Program in Acoustics, The Pennsylvania State University, PO Box 30, State College, PA 16804, USA, tbg3@psu.edu)

Measured values of acoustic resistance in porous hoses have been implemented into a previously developed porous tube model for infrasonic arrays [J. Acoust. Soc. Am. 122, 2960 (2007)]. Garden soaker hoses are commonly used in porous infrasonic arrays, and a simple method for the measurement the acoustic resistance of a segment of soaker hose will be presented. This experimentally determined resistance value can be implemented into the porous tube model for an improved array response prediction. It is anticipated that this analysis will give rise to improved phase and magnitude predictions for this type of array. Applications include design optimization of porous tube arrays, and infrasonic propagation through porous ducts. [Research funded by the Applied Research Laboratory Educational and Foundational Fund.]

5aPAf7. Finite difference time domain synthesis of infrasound propagation through an absorbing atmosphere. Catherine D. Degroot-Hedlin (University of San Diego California, Scripps Institute of Oceanography, 9500 Gilman Drive, La Jolla, CA 92093, USA, chedlin @ucsd.edu)

Equations applicable to finite difference time domain (FDTD) computation of infrasound propagation through an absorbing atmosphere are derived and examined in this paper. It is shown that over altitudes up to $160 \mathrm{~km}$, and at frequencies relevant to global infrasound propagation, i.e., $0.02-5 \mathrm{~Hz}$, the acoustic absorption in $\mathrm{dB} / \mathrm{m}$ varies approximately as the square of the propagation frequency plus a small constant term. A second-order differential equation is presented for an atmosphere modeled as a compressible Newtonian fluid with low shear viscosity, acted on by a small external damping force. It is shown that the solution to this equation represents pressure fluctuations with the observed form for infrasound propagation, i.e., sound is attenuated as $\left(\beta+\gamma f^{2}\right)$. Increased dispersion is predicted at altitudes over $100 \mathrm{~km}$. The equation is separated into two partial differential equations that are first order in time for FDTD implementation. A numerical analysis of errors inherent to this FDTD method shows that the attenuation term imposes additional stability constraints on the FDTD algorithm. Comparison of FDTD results for models with and without attenuation shows that the predicted transmission losses for the attenuating media agree with those computed from the synthesized waveforms. 


\title{
Session 5aPAg
}

\section{Physical Acoustics: Ultrasonics Under Extreme Conditions I (Poster Session)}

\author{
Albert Migliori, Cochair \\ $L A N L$
}

Frédéric Decremps, Cochair

IMPMC, Université Paris VI

\begin{abstract}
All posters will be on display from 9:40 a.m. to 11:20 a.m. To allow contributors an opportunity to see other posters, contributors of odd-numbered papers will be at their posters from 9:40 a.m. to 10:30 a.m. and contributors of even-numbered papers will be at their posters from 10:30 a.m. to 11:20 a.m.
\end{abstract}

\section{Contributed Papers}

5aPAg1. Guided modes modeling in multilayered composites plates. Morched Ben Amor (Faculté des Sciences de Sfax, Laboratoire de Physique des Matériaux, BP 802, 3018 Sfax, Tunisia, morched_benamor@yahoo.fr)

Due to the periodicity of the composite structure the Floquet wave approach seems well suited for medium characterization. The pass and stop band domains for [0/45/90/-45] cross ply composites have been plotted Dispersive guided wave propagation through a lossy composite laminates immersed in water have been investigated. Our interest is focused on a guided mode in the frequency range from $1.6 \mathrm{MHz}$ to $5 \mathrm{MHz}$. This mode has been pointed out by both reflection coefficient and energy vector analysis in term of incident angle. In this paper it is investigated how by finetuning the frequency and the incident angle, internal displacement and stress field vary in the multilayer. When the frequecy is increased the mode changes from a plate mode to a surface mode. The description includes real Floquet wave numbers as well as complex wave numbers.

5aPAg2. Influence of the calorimetric system setup on the output acoustic power measurement results. Antonio Petosic (Faculty of Electrical Engineering and Computing, Unska 3, 10000 Zagreb, Croatia, antonio.petosic@fer.hr), Bojan Ivančević (Faculty of Electrical Engineering and Computing, Unska 3, 10000 Zagreb, Croatia, bojan.ivancevic@fer
.hr),Dragoljub Svilar(Brodarski Institut, Avenija Većeslava Holjevca bb, 10000 Zagreb, Croatia, dsvilar@hrbi.hr)

The problem of measuring an output acoustic power using calorimetric method has been considered in different regimes of transmitter working. The transmitter has been driven at different excitation electrical power levels and the influence of sonotrode tip position in the different calorimetric systems on the measurement results is considered. Two calorimetric systems with different volumes of loading liquid (water) and different geometries (having influence on thermodynamical losses) have been used in the experiments. The exponential experimental temperature-time curves have been fitted with theoretical and time constants, losses, output acoustic power and electroacoustic efficiency factor have been found assuming that all ultrasound energy is absorbed in the system. The pressure field in the box is complicated and radiation impedance (radiated power) depends on the position in the complex ultrasound field where the point source is located. In the nonlinear regime of working strong cavitation activity occur with oscillating bubles in the front of vibrating tips and the radiated power is decreased because the tip is only partially radiating due to radiation impedance variations. The electroacustic efficiency factor has been compared with equivalent circuit approach where parameters of transmitter have been found for unloaded and loaded transmitter. 


\title{
Session 5aPAh
}

\section{Physical Acoustics: Ducts and Waveguides I (Poster Session)}

\author{
Marc Deschamps, Cochair \\ $L M P$ \\ Olivier Dazel, Cochair \\ Laboratoire d'Acoustique de I'Université du Maine
}

\begin{abstract}
All posters will be on display from 9:40 a.m. to 11:20 a.m. To allow contributors an opportunity to see other posters, contributors of odd-numbered papers will be at their posters from 9:40 a.m. to 10:30 a.m. and contributors of even-numbered papers will be at their posters from 10:30 a.m. to 11:20 a.m.
\end{abstract}

\section{Contributed Papers}

5aPAh1. The design and modeling of a lateral acoustic particle manipulator exhibiting quarter-wave operation. Peter Glynne-Jones (University of Southampton, School of Engineering Sciences, University Road, SO17 1BJ Southampton, UK, p.glynne-jones@soton ac.uk), Martyn Hill (University of Southampton, School of Engineering Sciences, University Road, SO17 1BJ Southampton, UK, m.hill@soton.ac.uk), Nicholas R. Harris (University of Southampton, Electronics and Computer Science, SO17 1BJ Southampton, UK, nrh@ecs.soton.ac.uk), Rosemary J. Townsend (University of Southampton, School of Engineering Sciences, University Road, SO17 1BJ Southampton, UK, R.J.Townsend@soton.ac.uk), Surendra K. Ravula (Sandia National Laboratories, Eubank Blvd, Albuquerque, NM 87123, USA, skravul@ sandia .gov)

Prior work [Petersson et al., 2004] has demonstrated the operation of a half-wave acoustic particle manipulator, whose forces act in the plane of a silicon substrate. In a half-wave device particles are directed to the centre of a channel. Devices acting in plane have manufacturing advantages, and lend themselves to many microfluidic applications. We demonstrate, for the first time, such a device with a quarter-wave mode that is able to manipulate particles to the side of a channel in addition to a near half wave mode. The design utilises resonant "islands" to create the necessary pressure release boundary condition. The device is conventionally milled in brass, permitting cheaper and quicker fabrication than in silicon. Finite element modelling is presented to elucidate the operation of both half and quarter-wave devices. In contrast to essentially one-dimensional planar devices, the twodimensional distribution of the velocity and pressure fields result in particles being constrained to a line within the channel.

5aPAh2. A semianalytical finite element method for elastic guided waves propagating in helical structures. Fabien Treyssède (Nantes Atlantique University - LCPC, Division for Metrology and Instrumentation, Route de Pornic - BP 4129, 44341 Bouguenais, France, fabien.treyssede @1cpc.fr), Ahmed Frikha (Nantes Atlantique University - LCPC, Division for Metrology and Instrumentation, Route de Pornic - BP 4129, 44341 Bouguenais, France, ahmed.frikha@lcpc.fr)

Steel multiwire cables are widely used in civil engineering as loadcarrying members. The basic element of these cables is usually a simple straight strand made of a straight core and one layer of helical wires. Several difficulties arise in the understanding of guided ultrasonic waves in such structures, partly due to the helical geometry and the interwire coupling effects. In the context of nondestructive evaluation, this paper aims at theoretically investigating the propagation of elastic waves in helical waveguides. A numerical method is chosen based on a semianalytical finite element technique that relies on a specific nonorthogonal curvilinear coordinate system. This system is shown to be translationally invariant along the helix centerline so that a spatial Fourier transform can be explicitly performed along the axis and the problem is reduced to two dimensions. A single helical wire is first considered. The convergence and accuracy of the proposed method are assessed by comparing finite element results with reference solutions. The method is then extended to study a seven wire cable with simplified contact conditions. Dispersion curves are presented for wave numbers as well as energy velocities. Significant differences with the infinite straight cylinder are observed.

5aPAh3. Numerical simulation of acoustic wave propagation via a liquid with gas bubbles. Nikolai Kudryashov (Department of Applied Mathematics, Moscow Engineering and Physics Institute (State University), 31, Kashirskoe Shosse, 115409 Moscow, Russian Federation, kudryashov@mephi.ru), Nikolai Teterev (Department of Applied Mathematics, Moscow Engineering and Physics Institute (State University), 31 Kashirskoe Shosse, 115409 Moscow, Russian Federation, us_tet@mail.ru)

Wave propagation through a liquid with gas bubbles is numerically investigated, in $1 \mathrm{D}$ and $2 \mathrm{D}$ cases. The mathematical model is constructed in one-velocity, two-temperature approximation. The liquid is described by the empiric Tait equation. The bubble oscillations are described by RayleighPlesset-type equation. The heat exchange between bubbles and the liquid is taking into account. Comparison of analytical solutions to results of numerical simulation has shown, that the developed model adequately describes dynamics of the wave propagation in a gas-liquid mixture. In the $2 \mathrm{D}$ case different regimes of wave propagation through a liquid containing gas bubbles are found. The leading wave with damped wave train is formed in a gas-liquid mixture out of bell-shaped impulse. The pressure splash can appear behind, on and before initial solitary wave in a liquid with a bubble channel and exist for a long time. The heat exchange effect on wave propagation is investigated. It is shown that properties of the wave propagation and it structure depend on the gas volume content, wavelength and on the bubble area width.

5aPAh4. Transmission gaps and Fano resonances in a simple acoustic waveguide: Analytical model. El Houssaine El Boudouti (Faculté des Sciences, Département de Physique, Université Mohamed I, 60000 Oujda, Morocco, elboudouti@yahoo.fr), Tarik Mrabti (Faculté des Sciences, Département de Physique, Université Mohamed I, 60000 Oujda, Morocco, t.mrabti@yahoo.fr), Hosni Al-Wahsh (Faculty of Engineering, Benha University, 11241 Cairo, Egypt, alwahsh2003@yahoo.com), Bahram Djafari-Rouhani (IEMN, UMR CNRS 8520, avenue Poincaré, BP 60069 , 59652 Villeneuve d'Ascq, France, Bahram.Djafari-Rouhani@univ-lille1 
.fr),Abdellatif Akjouj(IEMN, UMR CNRS 8520, avenue Poincaré, BP 60069, 59652 Villeneuve d'Ascq, France, abdellatif.akjouj@univ-lille1 .fr), Leonard Dobrzynski (IEMN, UMR CNRS 8520, avenue Poincaré, BP 60069, 59652 Villeneuve d'Ascq, France, leonard.dobrzynski@univ-lille1 .fr)

A simple acoustic device consisting of two dangling side resonators grafted at two sites on a slender tube is designed to obtain possibly transmission stop bands where the propagation of longitudinal acoustic waves is forbidden. Contrary to all known systems of this kind, a spectral transmission gap of nonzero width occurs here even with this simple structure. This is obtained by combining appropriately the zeros of transmission of the side resonators. Sharp resonant states inside the gaps can be achieved without introducing any defects in the structure. This results from an internal resonance of the structure when such a resonance is situated in the vicinity of a zero of transmission or placed between two zeros of transmission, the socalled Fano resonances. A general analytical expression for the transmission coefficient is given for these systems within the framework of the Green's function method. The amplitude and the phase time of the transmission are discussed as a function of frequency and it is shown that the width of the stop bands is very sensitive to the number of the side resonators. These results should have important consequences for the suppression of lowfrequency noise and for designing filters.

5aPAh5. Complex edge resonance in elastic waveguides. Vincent Pagneux (Laboratoire d'Acoustique de l'Université du Maine, UMR CNRS 6613, AV. O. Messiaen, 72085 Le Mans, France, vincent.pagneux@univlemans.fr)

The resonance for different configurations of elastic waveguides with a free edge is studied from the point of view of complex resonance. We determine numerically the variations of the real part and of the imaginary part of the complex resonance frequency as a function of the Poisson ratio. For three different configurations, semi-infinite 2D plates, elastic cylinder and circular hole through a plate, the results show similar behaviours which are: a real resonance frequency for a zero Poisson ratio and a real resonance frequency that corresponds to a Lamé mode at one positive value of the Poisson ratio. In all the configurations, these free edges have complex resonances which have very high quality factors. The two Poisson ratio where the complex resonance frequency has a zero imaginary part (infinite quality factors) corresponds to trapped modes for which there is a decoupling between the propagating waves and the evanescent waves. The first decoupling at zero Poisson ratio is due to the special structure of the elasticity equations there and the second decoupling is due to the decoupling between the propagating Lamé mode and the higher order evanescent modes.

5aPAh6. Measurements on quarterwavelength tubes and Helmholtz resonators. Alberto Soto-Nicolas (Nokia Co, Sinivaival 5, 33720 Tampere, Finland, alberto.soto-nicolas@ nokia.com)

The aim of this work is to determine what are factors limiting the prediction of the resonance frequency in unflanged quarter wavelength tubes and cylindrical Helmholtz resonators. When Helmholtz resonators are cylindrical, the frequency resonance may be predicted by solving a frequency 4 th order polynomial equation. This formula may be used in quarter wavelength tubes too by just eliminating the value of the internal end correction. Some measurements were carried out showing that the accuracy in the prediction of the frequency resonance is limited by three factors: The uncertainty of values of end corrections, the visco-thermal effects not considered by the formula and the error in the estimation of the sound speed.

FRIDAY MORNING, 4 JULY 2008

P3-A, LEVEL 3, 9:40 TO 11:20 A.M.

\title{
Session 5aPAi
}

\section{Physical Acoustics: General Topics in Physical Acoustics I (Poster Session)}

\author{
Walter Lauriks, Cochair \\ Katholicke Univsiteit Leuven \\ Andi Petsculescu, Cochair \\ University of Louisiana
}

\begin{abstract}
All posters will be on display from 9:40 a.m. to 11:20 a.m. To allow contributors an opportunity to see other posters, contributors of odd-numbered papers will be at their posters from 9:40 a.m. to 10:30 a.m. and contributors of even-numbered papers will be at their posters from 10:30 a.m. to 11:20 a.m.
\end{abstract}

\section{Contributed Papers}

5aPAi1. Focused borehole radiator. Dmitry Kas'Yanov( Radiophysical Research Inst., 25 Bolshaya Pecherskaya, 603950 Nizhny Novgorod, Russian Federation, vo-kak@yandex.ru)

A promising type of borehole acoustic radiators intended for both stimulating borehole geotechnological processes and investigating the borehole environment is considered. The basic difference of the radiators under consideration from the existing ones is the possibility of focusing the acoustic field into a preset region of borehole environment. Elastic fields produced by focusing borehole radiators in rock are studied. Limiting abilities of focus- ing borehole systems are investigated. Optimal type of focusing borehole radiator is shown for well stimulation. It is demonstrated that borehole radiators with a variable focal distance can be designed, which provides a real opportunity to control the distribution of elastic fields in the borehole environment. This property may be very important in developing the acoustic technologies for stimulating productive reservoirs of geotechnological boreholes.

5aPAi2. Experimental study of the convergence of two-point cross-correlation toward the Green's Function. Pierre Gouedard (LGIT 
- CNRS - Université Joseph Fourier, Maison des Géosciences, 1381 rue de la Piscine, BP 53, 38041 Grenoble, France, pierre.gouedard @ujf-grenoble.fr), Philippe Roux (LGIT - CNRS - Université Joseph Fourier, Maison des Géosciences, 1381 rue de la Piscine, BP 53, 38041 Grenoble, France, philippe.roux@obs.ujf-grenoble.fr), Michel Campillo (LGIT - CNRS - Université Joseph Fourier, Maison des Géosciences, 1381 rue de la Piscine, BP 53, 38041 Grenoble, France, Michel.Campillo@obs.ujf-grenoble.fr), Arie Verdel (Shell International Exploration and Production B.V., Kessler Park 1, 2288 Rijswijk, Netherlands, arie.verdel@shell.com), Xander Campman (Shell International Exploration and Production B.V., Kessler Park 1, 2288 Rijswijk, Netherlands, xander.campman@shell.com)

Cross-correlation of seismic noise recorded at two points yields the Green's Function (GF) between the two points when seismic noise is spatially and temporally diffuse. Seismic events yield the same result (1) when direct waves are cross-correlated from sources that are azimuthally distributed or (2) when coda waves are cross-correlated whatever the source distribution. In this work, the question of convergence toward the GF is investigated with real data. From a high-resolution survey with dense arrays of geophones and sources, we select different sets of sources and time windows to compute the cross-correlation between two receivers. We compare correlations derivative with the actual GF, and show the influence of source locations and scattering on the GF reconstruction. For direct waves, sources located in the endfire lobes of the receiver pair have an essential contribution. With sources located outside the endfire lobes, the GF can nevertheless be retrieved using coda waves.

5aPAi3. Spectral properties of the surface $\mathrm{SH}$ waves in a vertically periodic half-space. Stanislav V. Golkin (LMP, UMR CNRS 5469, Université Bordeaux I, 351, cours de la Libération, 33405 Talence, France, s.golkin@1mp.u-bordeaux1.fr), Olivier Poncelet (LMP, UMR CNRS 5469, Université Bordeaux I, 351, cours de la Libération, 33405 Talence, France, o.poncelet@1mp.u-bordeaux1.fr), Alexander Shuvalov (LMP, UMR CNRS 5469, Université Bordeaux I, 351, cours de la Libération, 33405 Talence, France, a.shuvalov@1mp.u-bordeaux1.fr)

We consider shear horizontal ( $\mathrm{SH}$ ) waves propagating under a free surface of semi-infinite medium with vertically periodical continuous or discrete variation of material properties. This case is marked by certain special features. It is known that the dispersion spectrum embracing 'physical' and "non-physical" (in the sense of radiation condition) surface SH waves precisely coincides with another spectrum, which describes the SH waves in a single period with traction-free edges and which is relatively straightforward to calculate [1]. Of a particular interest for possible applications is that the distribution of those spectral bands, where the surface SH waves are "physical" and hence do exist, depends essentially on the profile of variation of material properties across the unit cell. In the present paper, we analyze the link between the periodic-inhomogeneity profile and the existence of surface $\mathrm{SH}$ waves in the frequency-wave number domain, and we show how this feature manifests itself in the time-space response. [1] A. L. Shuvalov, O. Poncelet, and A. N. Podlipenets, "On the guided and surface shear horizontal waves in monoclinic transversely periodic layers and halfspaces with arbitrary variation of material properties across the unit cell," Stud. Geophys. Geod. 50 381-398 (2006)

5aPAi4. SH surface wave in multicracked solids. Olivier Poncelet (LMP, UMR CNRS 5469, Université Bordeaux I, 351, cours de la Libération, 33405 Talence, France, o.poncelet@lmp.u-bordeaux1 .fr), Stanislav V. Golkin (LMP, UMR CNRS 5469, Université Bordeaux I, 351, cours de la Libération, 33405 Talence, France, s.golkin @1mp.u-bordeaux1.fr), Christophe Aristegui (LMP, UMR CNRS 5469, Université Bordeaux I, 351, cours de la Libération, 33405 Talence, France, c.aristegui@1mp.u-bordeaux1.fr), Mihai Caleap (LMP, UMR CNRS 5469, Université Bordeaux I, 351, cours de la Libération, 33405 Talence, France,
m.caleap@1mp.u-bordeaux1.fr),Alexander Shuvalov(LMP, UMR CNRS 5469, Université Bordeaux I, 351, cours de la Libération, 33405 Talence, France, a.shuvalov@1mp.u-bordeaux1.fr)

We focus on the dispersion spectra of surface coherent waves in damaged semi-infinite elastic solids. The damage region is located within the surface layer containing depth-depending concentration of parallel closed cracks. The dispersion equation is similar to that for the Love waves in a coated substrate, except that the layer's effective elastic properties, being deduced from multiple scattering theory, are frequency-dependent and complex. For healthy elastic solids, the grazing $\mathrm{SH}$ wave is nondispersive. The dispersion under study is induced by the presence of cracks. The effect of both the magnitude of crack-concentration and its profile into the depth is investigated. The reliability of the semianalytical predictions is also discussed through a comparison with the numerical results obtained by using a finite-difference code.

5aPAi5. The acoustical research of shear viscoelastic properties of nanoparticles suspensions. Bair Damdinov (Buryat State University, Smolina Str., 24A, 670000 Ulan-Ude, Russian Federation, dababa@rambler.ru), Tuyana Dembelova (Buryat State University, Smolina Str., 24A, 670000 Ulan-Ude, Russian Federation, 1mf@ofpsrv .bsc.buryatia.ru), Badmaev Badma (Buryat State University, Smolina Str. 24A, 670000 Ulan-Ude, Russian Federation, 1mf@ofpsrv.bsc.buryatia.ru)

The acoustical research of some physical properties of nanoparticles suspensions has been carried out in this work. Mechanical properties of the $\mathrm{SiO}_{2}$ and YAG particles suspensions have been investigated. Resonance method is used for measurement of viscoelastic properties (shear modulus and shear viscosity) of the suspensions. It was shown that the viscosity and elasticity depend on size and concentration of particles. Colloid suspension of nanoparticles of silica dioxide $\left(\mathrm{SiO}_{2}\right)$ and yttrium alumina garnet doped by neodymium (Nd:YAG) are synthesized. The shear viscoelastic properties of the colloid suspensions have been measured by acoustical method. The researching of colloid suspension of $\mathrm{SiO}_{2}$ nanoparticles of different concentration by the acoustical resonance method is shown that viscosity and shear modulus decrease with nanoparticle concentration increasing. The nonlinear behavior of viscosity and elasticity in depending on shear influence amplitude has been shown. Investigation of viscosity of suspensions of Nd:YAG nanoparticles in ethanol in depending on concentration has been carried out The decreasing of shear modulus of the suspensions with concentration decrease has been shown. The work was supported by RFBR 05-02-16584a and 07-02-90103-Mong-a.

5aPAi6. Modelization of acoustic waves radiation from sources of complex geometry aperture. Rafik Serhane (Université des Sciences et de la Technologie Houari Boumediene; Faculté de Physique, BP 32, El-Alia, DZ-16111 Alger, Algeria, rafik_serhane@hotmail.com), Tarek Boutkedjirt (Université des Sciences et de la Technologie Houari Boumediene; Faculté de Physique, BP 32, El-Alia, DZ-16111 Alger, Algeria, tarek_boutkedjirt@hotmail.com)

The Rayleigh integral giving the impulse response for the acoustic velocity potential cannot always be determined analytically for all types of transducer apertures. The shape of the transducer surface and the spatial distribution of the excitation on its surface can complicate the calculation. This makes using numeric methods indispensable. One of these methods consists in discritizing the aperture surface in polygonal shape elements. Our work makes use of both methods of Jensen and Faure [IEEE UFFC 39-2(1992) 262, Acta Acustica 2(1994)257]. In the former, the transducer surface is subdivided in triangles. The latter one uses additional virtual triangles one side of which is that of the physical element and its summit is the field point projection on the plane containing the considered physical element. Additionally, a rotation of the virtual triangle around the field point projection is performed. In this work, the orientations of those virtual triangles are considered according to Jensen method and their contributions to the impulse response are calculated according to the two situations described by Faure The combination of the two methods is, then, generalized to the case of nonplanar complex surfaces such as concave phased arrays. 


\title{
Session 5aPAj
}

\section{Physical Acoustics: Scattering and Diffraction I (Poster Session)}

\author{
Michael L. Oelze, Cochair \\ University of Illinois at Urbana-Champaign \\ Jean-Marc Conoir, Cochair \\ Université Paris 6
}

\begin{abstract}
All posters will be on display from 9:40 a.m. to 11:20 a.m. To allow contributors an opportunity to see other posters, contributors of odd-numbered papers will be at their posters from 9:40 a.m. to 10:30 a.m. and contributors of even-numbered papers will be at their posters from 10:30 a.m. to 11:20 a.m.
\end{abstract}

\section{Contributed Papers}

5aPAj1. Time-domain acoustic pressure fields from axisymmetric impulse sources by Rayleigh's Integral. Stephen I. Warshaw (40 West 15th St. Loft 1C, New York, NY 10011, USA, siw1939@yahoo.com)

This paper shows how Rayleigh's Integral is used to efficiently and rapidly calculate time-domain pressure waveforms and wavefronts radiated from a planar baffle due to axisymmetric normal impulse accelerations that converge toward or expand away from a fixed center of symmetry. These accelerations are prescribed with simple functional forms and histories. The Rayleigh Integrals are evaluated by Gaussian integrations that seem peculiarly suited to the integrands. The acoustic fields are presented as "snapshots" of pressure contours of the evolving wavefront structures emerging from the baffle surface, and as functions of time at selected key points. As a bonus, significant insights are provided by graphs of slant range versus time that clearly identify key signal paths from the source points to the field points. The presented methods avoid the complexities inherent in more standard transform and harmonic source treatments. They also provide insights into actual acoustic fields generated by impulsive sources, as, e.g., above the ground surface over a deeply buried explosion. [J. R. Banister and W. V. Hereford, J. Geophys. Res. 96(D3), 5185-5193 (1991)] Simpler examples were reported earlier. [S. I. Warshaw, JASA 77, S60 (1985); JASA 79, S90 (1986); JASA 80, S106 (1986), and 12 ICA Toronto, L3-6 (1986)]

5aPAj2. Unified and stable scattering matrix formalism for acoustic waves in piezoelectric stacks. Victor Y. Zhang (IEMN-CNRS, Av. Poincare, Cite Scientifique, B.P. 60069, 59652 Villeneuve d'Ascq Cedex, France, victor.zhang@iemn.univ-lille1.fr), Vincent Laude (Institut FEMTOST/CNRS, 32 avenue de l'Observatoire, 25044 Besançon cedex, France, vincent.laude@femto-st.fr)

This paper presents a unified and full scattering matrix (s-matrix) formalism. A stable recursive algorithm is derived for computation of the total s-matrix of a multilayered stack in terms of the interface s-matrix, both referring to the eigen-modes amplitudes. The derivation is direct and succinct, the derived expressions of the s-matrix are terse and concise, and the recursion is efficient and convenient for implementation. By combining the s-matrix formalism and the surface impedance matrix of the external media, various terminations of the stack can be easily handled. Numerical examples are given to show its numerical superiority over other currently used matrix formalisms: including unconditional stability throughout large and small thicknesses and pole-free feature.

5aPAj3. Temperature-dependent diffusing acoustic wave spectroscopy with resonant scatterers. Valentin Leroy (Laboratoire Ondes et Acoustique, ESPCI, Université Paris 7, CNRS, 10 rue Vauquelin, 75005 Paris,
France, valeroy77@yahoo.fr),Arnaud Derode(Laboratoire Ondes et Acoustique, ESPCI, Université Paris 7, CNRS, 10 rue Vauquelin, 75005 Paris, France, arnaud.derode@ujf-grenoble.fr)

The influence of a slight temperature change on the correlation of multiply scattered acoustic waves is studied, and experimental results are discussed. The technique presented here, similar to diffuse acoustic wave spectroscopy, is based on the sensitivity of a multiple scattering medium to a slight change. Ultrasonic waves around $3 \mathrm{MHz}$ are transmitted through a sample made of steel rods in water and recorded by an array of transducers at different temperatures. The cross-correlations between highly-scattered signals are computed. The main effect of the temperature change is a simple dilation of the times of arrival, due to a change of the sound velocity in water. But the scatterers also play a role in the progressive decorrelation of waveforms. An analysis resolved in both time and frequency shows that at some particular frequencies, the resonant behavior of the scatterers are responsible for a significantly larger decorrelation. Interestingly, the experimental results allow one to detect the presence of a small resonance that was not detected earlier on the same scatterers with classical measurement of the scattering mean freepath. A simple model is proposed to interpret the experimental results.

5aPAj4. Ultrasonic wave propagation in heterogeneous solid media. Mathieu Rossat (LCND - Université de la Méditerranée, IUT Aix Provence, Avenue Gaston Berger, 13625 Aix en Provence Cedex, France, mathieu.rossat@univmed.fr), Jean-François Chaix (LCND - Université de la Méditerranée, IUT Aix Provence, Avenue Gaston Berger, 13625 Aix en Provence Cedex, France, jean-francois.chaix@univmed.fr), Vincent Garnier (LCND - Université de la Méditerranée, IUT Aix Provence, Avenue Gaston Berger, 13625 Aix en Provence Cedex, France, vincent.garnier @univmed.fr)

To check up concrete structures "in situ" engineers often employ ultrasonic waves. The implementation of tests and analysis of results even inversion of the problem need good knowledge about propagation phenomena. These phenomena require understanding interaction between ultrasonic waves and scatters, such as granular, porosity, and cracks. This defect can come from fabrication or mechanical damage. The size and the shape of obstacle have an influence on the spatial scattering of ultrasonic waves. We propose to show you the possible using multiple scattering in homogeneous models of propagation and the last work on a sphere in a hard media. Our objective is to bring obstacle geometries closer to the reality. Consequently, we study spheroidal scatters with various aspect ratios. 
5aPAj5. Comparisons between multiple scattering methods and time-domain numerical simulations for elastic waves. Mathieu Chekroun (EDF R\&D - LCPC, LCPC centre de Nantes BP4129, 44341 Bouguenais, France, mathieu.chekroun@1cpc.fr), Loic Le Marrec (Institute of Mathematical Research of Rennes IRMAR, Campus de Beaulieu, 35042 Rennes, France, loic.lemarrec@univ-rennes1.fr), Bruno Lombard (Laboratoire de Mécanique et d'Acoustique LMA, 31 chemin Joseph Aiguier, 13402 Marseille, France, lombard@1ma.cnrs-mrs.fr), Odile Abraham (LCPC, Centre de Nantes BP4129, 44341 Bouguenais, France, odile.abraham@lcpc.fr), Joel Piraux (Laboratoire de Mécanique et d'Acoustique LMA, 31 chemin Joseph Aiguier, 13402 Marseille, France, piraux@1ma.cnrs-mrs.fr)

Propagation of elastic waves in heterogeneous medium composed of scatterers embedded in a homogeneous matrix is considered. Both matrix and scatterers are isotropic elastic media. The multiple scattering regime is assumed, and the focus is put on the coherent field obtained by averaging several equivalent realizations of disorder. Classical methods, such as Independent Scattering Approximation, Foldy or Waterman-Truell's model, provide expressions of the complex effective wave number of the coherent field, leading to an effective phase velocity and effective damping factor. Two-dimensional time-domain numerical simulations are performed for studying the validity of these analytical or semianalytical methods. To reduce spurious effects, such as numerical diffraction, to a negligible level, a high-order numerical scheme and an immersed interface method are used together. Comparisons between theoretical and numerical values of the effective phase velocity and damping factor are proposed and analyzed in terms of the frequency, the scatterer density, and the propagation length.

5aPAj6. Coherent elastic wave propagation through nonuniform spatial distributions of cracks. Christophe Aristegui (LMP, UMR CNRS 5469, Université Bordeaux I, 351, cours de la Libération, 33405 Talence, France, c.aristegui@1mp.u-bordeaux1.fr), Mihai Caleap (LMP, UMR CNRS 5469, Université Bordeaux I, 351, cours de la Libération, 33405 Talence, France, m.caleap@1mp.u-bordeaux1.fr), Olivier Poncelet (LMP, UMR CNRS 5469, Université Bordeaux I, 351, cours de la Libération, 33405 Talence, France, o.poncelet@1mp.u-bordeaux1.fr), Stanislav V. Golkin (LMP, UMR CNRS 5469, Université Bordeaux I, 351, cours de la Libération, 33405 Talence, France, s.golkin@lmp.u-bordeaux1 .fr), Alexander Shuvalov (LMP, UMR CNRS 5469, Université Bordeaux I, 351, cours de la Libération, 33405 Talence, France, a.shuvalov@1mp.ubordeaux1.fr)

Models for multiple scattering of elastic waves usually concern uniform distributions of scatterers. The aim of this work is to predict the propagation of SH coherent waves through nonuniform distribution of parallel closed cracks. The spatial variation of the distribution properties is taken into account via replacing heterogeneous media by stacks of effective homogeneous layers. Propagation in each layer is then governed by the effective acoustic impedance and the effective wave number, derived in the framework of Waterman and Truell approach. On this basis, the reflection and transmission coefficients of the nonuniform distributions are calculated by using the transfer matrix method. We focus especially on distributions of crack size and concentration. Impact of the distribution profiles is also investigated. The semianalytical predictions are compared with numerical results obtained by using a finite-difference code.

5aPAj7. New approach to the problem on long-wave sound scattering by a Rankine vortex. Ivan Belyaev (Central Aerohydrodynamics Institute (TsAGI), Acoustic Division, 17 Radio str., 105005 Moscow, Russian Federation, belyaev_iv@bk.ru), Victor Kopiev (Central Aerohydrodynamics Institute (TsAGI), Acoustic Division, 17 Radio str., 105005 Moscow, Russian Federation, vkopiev@mx.iki.rssi.ru)

The well-known problem of sound scattering by a Rankine vortex at small Mach number M is considered. Despite the long history of the problem (Pitaevskii 1958, O’Shea 1975 etc.), solutions obtained by different authors still are not free from essential objections. The main difficulty is that the slow decay of the mean velocity field at infinity hinders a correct for- mulation of the problem. Most authors use a plane wave as the incident field in the leading approximation in M (Sakov 1993, Ford and Smith 1999, Howe 1999), however to obtain a physically reasonable solution the second approximation in the incident field is to be chosen beforehand. It is shown that instead of the plane wave condition, the condition of a point source far from the vortex should be posed. In the new formulation the correct solution of the problem of nonresonant sound scattering by the Rankine vortex is determined from the matching procedure in $\mathrm{M}$. This solution is compared with the previous results, and areas where these results hold are revealed. Existing solutions for resonant scattering (Kopiev and Leontiev1987, Sozou 1990) are analyzed too and a result unifying the previous ones is found.

5aPAj8. Scattering of seismic waves by a fracture zone containing randomly distributed frictional cracks. Mihai Caleap (LMP, UMR CNRS 5469, Université Bordeaux I, 351, cours de la Libération, 33405 Talence, France, m.caleap@1mp.u-bordeaux1.fr), Christophe Aristegui (LMP, UMR CNRS 5469, Université Bordeaux I, 351, cours de la Libération, 33405 Talence, France, c.aristegui@1mp.u-bordeaux1.fr)

An investigation is conducted how the geometrical properties of a crack distribution in a fault fracture zone and the frictional characteristics of the crack surface are reflected in the attenuation and dispersion of incident seismic waves. All cracks are assumed to be either aligned or randomly oriented. The crack width is assumed to obey a power law distribution, according to seismological knowledge. The crack surface is assumed to be stress-free, or to undergo viscous friction. To deal with cracks under high confining pressure, the latter case will be more realistic than the stress-free crack, due to the existence of fluid in the earth's crust and the viscoelastic response of contacting solid material to seismic waves under high confining pressure. When the crack distribution is statistically homogeneous, the calculated dispersion and attenuation exhibit that the variance of crack size affects in different way the coherent wave. The analysis on the effect of the friction shows that the crack scattering decreases as the viscosity increases, which is expected since for high viscosity, the crack faces remain almost welded to each other. The results obtained in this work will be applicable to the state close to the occurrence time in large earthquakes.

5aPAj9. Identification and characterisation of the scholte $A$ and circumferential So waves from the time-frequency analysis of an acoustic experimental signal backscattered by a tube. Rachid Latif (ESSI - ENSA, BP 1136, Ibn Zohr University, 80000 Agadir, Morocco, latif@ensa-agadir.ac.ma), Elhoucien Aassif (Ibn Zohr University, FS Agadir, 80000 Agadir, Morocco, aassif@hotmail.com), Mustapha Laaboubi (Ibn Zohr University, FS Agadir, 80000 Agadir, Morocco, laaboubi@gmail.com), Ali Moudden (Ibn Zohr University, FS Agadir, 80000 Agadir, Morocco, ali_moudden@yahoo.fr), Gerard Maze (LAUE, Université du Havre, Place Robert Schuman, F-76610 Le Havre, France, gerard.maze@univ-lehavre.fr)

The problem of the acoustic diffusion by a tube is a theoretical problem which had known since few years a considerable interest. A good comprehension of the acoustic diffusion makes it possible the study of the opposite problem. Many theoretical and practical works were made by a tube in the acoustic diffusion field. These works showed in particular that the resonance phenomenons are directly related to the physical and geometrical properties of the target. The interest of the study presented in this paper is the identification of the circumferential wave. Several frequential analysis techniques were applied to characterize the target. These analysis techniques currently used present the major disadvantage to not be able to highlight the temporal structure of the field diffused by the target. Reciprocally the acoustic temporal analysis of the target ca not always separate the successive arrivals of the various type waves in the signal and to have information on their frequential contents. The joint time-frequency representations provide this need. In this paper, the time-frequency of Wigner-Ville is applied to analyse the acoustic experimental signal backscattered by a steel tube with radii radio $\mathrm{b} / \mathrm{a}=0.97$ (a: external radius, b: internal radius) immersed in water. Through the Wigner-Ville image we have identified the scholte A and the circumferential S0 waves. 


\title{
Session 5aPAk
}

\section{Physical Acoustics: Vibrations in Plates II (Poster Session)}

\author{
Daniel Royer, Cochair \\ Université Paris 7 \\ Oliver Poncelet, Cochair \\ Université Bordeaux
}

\begin{abstract}
All posters will be on display from 9:40 a.m. to 11:20 a.m. To allow contributors an opportunity to see other posters, contributors of odd-numbered papers will be at their posters from 9:40 a.m. to 10:30 a.m. and contributors of even-numbered papers will be at their posters from 10:30 a.m. to 11:20 a.m.
\end{abstract}

\section{Contributed Papers}

5aPAk1. Study and development of a low-frequency acoustic sensor dedicated to the vibratory analysis and the mechanical characterization of the plates. Georges Nassar (IEMN - DOAE, Université de Valenciennes, Le Mont - Houy, 59313 Valenciennes, France, gnassar @univ-valenciennes.fr), Bassam Merheb (IEMN - DOAE, Université de Valenciennes, Le Mont - Houy, 59313 Valenciennes, France, bassam.merheb@univ-valenciennes.fr), Alain Skaf (IEMN - DOAE, Université de Valenciennes, Le Mont - Houy, 59313 Valenciennes, France, alain.skaf@univ-valenciennes.fr), Fabrice Lefebvre (IEMN - DOAE, Université de Valenciennes, Le Mont - Houy, 59313 Valenciennes, France, Fabrice.Lefebvre@univ-valenciennes.fr), Bertrand Nongaillard (IEMN DOAE, Université de Valenciennes, Le Mont - Houy, 59313 Valenciennes, France, Bertrand.Nongaillard@univ-valenciennes.fr)

A low-frequency acoustic method was implemented for the purpose of nondestructive control and evaluation of metal plates coating. In this method, a mechanical pulse (acoustic impact) is used to generate acoustic vibrations on a frequency band between $100 \mathrm{~Hz}$ to $20 \mathrm{kHz}$, and a compact acoustic sensor, constructed with by composite materials with an embedded ferroelectric disk, is used to receive the acoustic vibrations. The technique consists in setting in resonance the integrality of a reduced size mechanical structure. The modal frequencies of plates are calculated by two methods: analytically and by finite element method. Then, a numerical modeling of the acoustic behaviour of the sensor is presented. Finally, experimental trials are described and results showing the sensitivity of the method to evaluate coating of metal plates are analyzed.

5aPAk2. Construction of symmetrical and asymmetrical notches from elementary steps in isotropic plates. Farouk Benmeddour (UVHC, IEMN-DOAE, Le Mont-Houy, 59313 Valenciennes, France, farouk_benmeddour@yahoo.fr), Sébastien Grondel (UVHC, IEMNDOAE, Le Mont-Houy, 59313 Valenciennes, France, sebastien.grondel @ univ-valenciennes.fr), Jamal Assaad (UVHC, IEMN-DOAE, Le MontHouy, 59313 Valenciennes, France, jamal.assaad@univ-valenciennes .fr), Emmanuel Moulin (UVHC, IEMN-DOAE, Le Mont-Houy, 59313 Valenciennes, France, emmanuel.moulin@univ-valenciennes.fr), Lucie Dupont (UVHC, IEMN-DOAE, Le Mont Houy, 59313 Valenciennes, France, lucie.dupont@univ-valenciennes.fr)

The interactions of the fundamental Lamb modes with discontinuities in an isotropic structure will be presented and analyzed in a simple and a fast way. The key point is to decompose the symmetrical or asymmetrical notch into two elementary steps. The fundamental antisymmetrical and symmetrical Lamb modes contributions are separated by means of the simple arithmetic operations of addition and subtraction. The power reflection and transmission coefficients are then computed with the well-known average power flow equation described in a previous work [NDT\&E international, Vol. 41, p.1-9 (2008)]. Using these coefficients, the symmetrical notch can be constructed from one elementary symmetrical step while the asymmetrical notch is constructed from the superposition of asymmetrical down- and upsteps. Mode conversions phenomena are observed and analyzed for the asymmetrical steps and notches case. Finally, an experimental device is realized to test aluminum plates with different notch depths. Good agreement is found between experimental and numerical results.

5aPAk3. Lamb and Stoneley wave interactions with a fluid droplet. Loic Martinez (ECIME Université de Cergy-Pontoise, rue d'Eragny, 95031 Neuville-sur-Oise, France, loic.martinez@u-cergy.fr), Nicolas Wilkie-Chancellier (ECIME Université de Cergy-Pontoise, rue d'Eragny, 95031 Neuville-sur-Oise, France, Nicolas.Wilkie-Chancellier@u-cergy .fr), Christ Glorieux (Lab. ATF, Katholieke Universiteit Leuven, Celestijnenlaan 200D, B-3001 Leuven, Belgium, christ.glorieux@fys .kuleuven.be)

In order to analyse wetting aspects from an acoustical point of view, the present work investigates the interaction of a liquid droplet with Lamb and Stoneley waves propagating on a plane plate. A horizontal plane plate is in contact with a liquid on its lower interface, air in contact with its upper interface, the droplet lying on the top interface. Lamb waves are generated by a pulse trough a contact transducer placed at one edge of the plate. This generates in a wide range the high wave numbers of the Lamb modes A0, A1, SO and the fluid born Sholte Stoneley wave (A-wave). For low wave numbers, the transducer is placed at normal incidence, under both the plate and the droplet. Signals obtained by emission/reception with the same transducer $(2 \mathrm{MHz}$ ) clearly exhibits a coupling effect between acoustic modes inside the droplet and Lamb modes. Laser Doppler scans around the droplet show reflection/refraction/diffraction patterns of the incident line wavefront through the droplet. These experiments carried out with various wetting conditions point out a good correlation between the parameters of the acoustical resonances and the wetting properties, enabling an acoustic estimation of the wetting in a wide frequency range. 


\title{
Session 5aPAl
}

\section{Physical Acoustics: Ultrasonics: Transducers and Instruments II}

\author{
Albert Migliori, Cochair \\ Los Alamos National Laboratory, Los Alamos, NM, 87545, USA \\ Claude Boutin, Cochair \\ École nationale des travaux publics de l'État, Rue Maurice Audin, F- 69518 Vaulx-en-Velin Cedex, FRANCE
}

\section{Contributed Papers}

\section{1:20}

5aPAl1. Analysis of wavefronts for the piston source acoustic fields. Dmitry V. Perov (Institute of Metal Physics, 18, Sofia Kovalevskaya St., GSP-170, 620041 Ekaterinburg, Russian Federation, peroff@imp.uran .ru), Anatoly B. Rinkevich (Institute of Metal Physics, 18, Sofia Kovalevskaya St., GSP-170, 620041 Ekaterinburg, Russian Federation, rin@imp .uran.ru)

The development of modern acoustic methods is based to a great extent on a thorough investigation of the structure of acoustic fields and on the refinement of methods of their analysis and calculation. The study of the local features of acoustic field and its space-time structure is an urgent problem of physical acoustics. This work is dedicated to analytical investigation of space-time structure of wavefronts corresponding to the acoustic fields which are radiated by the piston source into elastic media. It is assumed that the spatial distributions. Various spatial distributions of acoustic field over the surface of the transducer are taken into consideration.

\section{$11: 40$}

5aPAl2. Miniature, high efficiency transducers for ultrasonic flow meter applications. Shrinivas G. Joshi (Marquette University, EECE Department, College of Engineering, P. O. Box 1881, Milwaukee, WI 532011881, USA, shri.joshi@mu.edu), Boris D. Zaitsev (Institute of Radio Engineering and Electronics of RAS, Saratov Branch, Zelyonaya str., 38, 410019 Saratov, Russian Federation, zai-boris@yandex.ru), Iren E. Kuznetsova (Institute of Radio Engineering and Electronics of RAS, Saratov Branch, Zelyonaya str., 38, 410019 Saratov, Russian Federation, kuziren @ yandex.ru)

This paper is concerned with the development of novel, miniature, high efficiency transducers for use in ultrasonic flow meters. Theoretical and experimental work done in our laboratory has shown that a flat, planar transducer that has minimal protrusion into the flow pipe can be realized based on conversion of plate acoustic waves (PAWs) to bulk acoustic waves (BAWs) and vice versa. The transducer is essentially a thin plate of a suitable piezoelectric material on which intedigital transducers (IDTs) are fabricated to generate and detect plate acoustic waves. With proper design very efficient conversion of energy from plate waves in the piezoelectric material to bulk waves in the fluid (and vice versa) can be achieved. For example, using lithium niobate as the piezoelectric material and water as the fluid, total conversion loss (PAW to BAW and back from BAW to PAW) of less than $2.5 \mathrm{~dB}$ has been obtained. An important advantage of plate waves is that since wave energy is present on both plate surfaces, the IDT can be on the surface opposite from that which is in contact with the fluid. Details of the theoretical analysis used, experimental results obtained, and potential applications will be presented.

\section{2:00}

5aPAl3. Free-field calibration of microphones: theoretical and experimental determination of the acoustic center. Dominique Rodrigues (Laboratoire Nationale de métrologie et d'Essais (LNE), 29 avenue Roger Hennequin, 78197 Trappes Cedex, France, dominique
.rodrigues@lne.fr),Jean-Noel Durocher(Laboratoire Nationale de métrologie et d'Essais (LNE), 29 avenue Roger Hennequin, 78197 Trappes Cedex, France, jean-noel.durocher@lne.fr), Michel Bruneau (Laboratoire d'Acoustique de l'Université du Maine, Avenue Olivier Messiaen, 72085 Le Mans, France, michel.bruneau@univ-lemans.fr), Anne-Marie Bruneau (Laboratoire d'Acoustique de l'Université du Maine (LAUM, UMR CNRS 6613), Avenue Olivier Messiaen, 72085 Le Mans, France, anne-marie.bruneau@univ-lemans.fr)

The concept of acoustic center is closely linked to the free-field calibration of microphones and have a great influence on the final results for the free-field sensitivity of the calibrated microphones. The international standard IEC 1094-3 defines the acoustic center of a reciprocal transducer as the point from which spherical waves seem to be diverging when the transducer is acting as a source. In the literature, the principal procedure used to measure the acoustic center of a reciprocal transducer is based on the measurement of the modulus of electrical transfer impedance at various distances of three pairs of microphones coupled by a free field. This paper presents a new procedure to determine the acoustic center of a reciprocal transducer assuming a source without any acoustic center and measuring the modulus of the electrical transfer impedance at various distances between this source and the transducer to be tested. Finally, the position of the acoustic center is deduced from the inverse distance law. Experimental values are presented and compared with theoretical values obtained using a theoretical model of the laboratory standard microphones (mounted on a semi-infinite rod) derived from those available in the literature.

\section{$12: 20$}

5aPA14. Study of acoustoelasticity behavior of concrete material under uniaxial compression. Jean-François Chaix (LCND - Université de la Méditerranée, IUT Aix Provence, Avenue Gaston Berger, 13625 Aix en Provence Cedex, France, jean-francois.chaix@univmed.fr), Ivan Lillamand (LCND - Université de la Méditerranée, IUT Aix Provence, Avenue Gaston Berger, 13625 Aix en Provence Cedex, France, ivan.lillamand@univmed.fr), Marie-Aude Ploix (LCND - Université de la Méditerranée, IUT Aix Provence, Avenue Gaston Berger, 13625 Aix en Provence Cedex, France, marie-aude.ploix@univmed.fr), Vincent Garnier (LCND - Université de la Méditerranée, IUT Aix Provence, Avenue Gaston Berger, 13625 Aix en Provence Cedex, France, vincent.garnier@univmed.fr), Gilles Corneloup (LCND - Université de la Méditerranée, IUT Aix Provence, Avenue Gaston Berger, 13625 Aix en Provence Cedex, France, gilles.corneloup@univmed.fr)

The general subject concerns the non destructive testing of prestressed structures in civil engineering. In this topic we are interested in the feasibility of stress evaluation in concrete by ultrasonic methods. To do that, we use the acoustoelasticity theory which exploits the nonlinear behaviour of media to establish the link between stress and ultrasonic velocity. This theory has been validated in homogeneous media and used in steel (to evaluated stresses in steel bolt for example). Concrete is a heterogeneous medium where the ultrasonic velocity measurement is more difficult than in steel. The scattered waves induce uncertainty in the measured values but the nonlinear behaviour of concrete is more important than the one of steel. We 
show an application in high performance concrete submitted to uniaxial compression. We measure velocities of longitudinal and transversal transmitted ultrasonic waves in the direction and perpendicularly to the stress at different level of the stress test. The sensibility of the different velocities is observed and the potential to evaluate stress in concrete is shown. Prospects particularly concern extension of tested formulations and in situ measurements. This study is supported by ANR-ACTENA, a French research program.

\section{2:40-2:00 Lunch Break}

\section{Contributed Papers}

\section{2:00}

5aPA15. Analysis of the backscattered waves in an heterogeneous material: Application on concretes. Vincent Garnier (LCND - Université de la Méditerranée, IUT Aix Provence, Avenue Gaston Berger, 13625 Aix en Provence Cedex, France, vincent.garnier@univmed.fr), Stéphane Morières (LCND - Université de la Méditerranée, IUT Aix Provence, Avenue Gaston Berger, 13625 Aix en Provence Cedex, France, stephane.moriere@gadz.org), Jean-François Chaix (LCND - Université de la Méditerranée, IUT Aix Provence, Avenue Gaston Berger, 13625 Aix en Provence Cedex, France, jean-francois.chaix@univmed.fr), Gilles Corneloup (LCND - Université de la Méditerranée, IUT Aix Provence, Avenue Gaston Berger, 13625 Aix en Provence Cedex, France, gilles.corneloup @ univmed.fr)

In heterogeneous materials, ultrasonic waves can be scattered forward or backward. The set of the backscattered contribution generates a wave that is attenuated over time. We make the measurement of this attenuation with the envelope of the backscattered signal. This information evolves with the nature, shape, and size of the scatterers so with the material state. First, we propose the simulation of the signal in which we introduce the attenuation dependence on the frequency. Second, we develop this application on concretes in which many scatterers contribute to the signal's generation. We apply the technique with differents tools to generate and exploit the backscattered waves. The attenuation evolutions with the material's nature, porosity, damage and saturation rate of the concrete are analysed.

\section{2:20}

5aPAl6. Airborne phased array for airborne applications based on cellular polymer. Christian Degel (Fraunhofer IBMT, Ensheimer Strasse 48, 66386 Sankt Ingbert, Germany, christian.degel@ibmt.fraunhofer.de) , Franz Josef Becker (Fraunhofer IBMT, Ensheimer Strasse 48, 66386 Sankt Ingbert, Germany, franz.becker@ibmt.fraunhofer.de), Martin Heinz (Fraunhofer IBMT, Industriestrasse 5, 66280 Sulzbach, Germany, martin.heinz@ibmt.fraunhofer.de), Heinrich Fonfara (Fraunhofer IBMT, Ensheimerstrasse 48, 66386 Sankt Ingbert, Germany, heinrich.fonfara@ibmt.fhg.de), Robert M. Lemor (Fraunhofer IBMT, Ensheimer Strasse 48, 66386 Sankt Ingbert, Germany, robert.lemor@ibmt.fhg .de)

Object recognition, advanced distance measurements and other inspection scenarios have an increasing demand in versatile airborne ultrasonic phased arrays for acoustic scanning without moving parts. Based on cellular polymer film with high piezoelectric effect we have realized an array structure with a pitch of $0.5 \mathrm{~mm}$ and element length of $10 \mathrm{~mm}$. The working frequency of the material was measured to $250 \mathrm{kHz}$. From pointspread simulation with the small ratio of pitch/wavelength of 0.35 we could expect good beam steering and focusing characteristics. A first test array was realized and characterized. There was good agreement between measurement results and simulations. Additionally a low frequency electronic beamformer system was developed for generating the first B-image of an airborne phased array. Measurements showed that cellular polymer is a well suitable material for airborne applications. It can easily be structured to the desired shape. It allows especially the realization of phased arrays for applications like surface or profile measurement, access control, attendance check, robot guidance etc. New airborne array types like linear, phased, curved, or circular arrays are now possible. Also single element transducers with varying apertures (rectangular, oval), shaped apertures [focusing (line- or pointfocusing), defocusing] or combinations of both are possible.

\section{2:40}

5aPA17. Nonlinear focal shift in focused ultrasonic transducers and its dependence on the Fresnel number. Yuri Makov (Moscow State University, Dept. of Acoustics, 46730 Moscow, Russian Federation, yuri_makov@mail.ru), Victor Sánchez-Morcillo (IGIC - Universitat Politècnica de València, Cra. Nazaret-Oliva S/N, E-46730 Gandia, Spain, victorsm@fis.upv.es), Francisco Camarena (IGIC - Universitat Politècnica de València, Cra. Nazaret-Oliva S/N, E-46730 Gandia, Spain, fracafe@ fis.upv.es), Víctor Espinosa (IGIC - Universitat Politècnica de València, Cra. Nazaret-Oliva S/N, E-46730 Gandia, Spain, vespinos@fis .upv.es)

In this work we present a systematic study of the on-axis location of the maximum pressure and intensity points in the field radiated by a focused transducer. We report the motion of these characteristic points as the transducer voltage is increased. Different initial distributions, ranging from uniform to Gaussian cases, are considered. The numerical analysis is based on the solutions of the KZK equation with different initial conditions. Experimental results have been obtained for three different Fresnel number focused transducers in linear and nonlinear regime in order to analyze the focal shift effect. An analytical expression of the initial (linear) shift of the maximum pressure position is given. This expression, and the results of the numerical simulations, shows a good agreement with the experimental data. As a main conclusion, we demonstrate that the axial range of the nonlinear shift of pressure is larger for strong initial focal shifts, occurring for small Fresnel number transducers. Theoretical and numerical predictions of the focal shift effect in Gaussian beams are also presented. In this work we have established the relation between the focal shift (both in linear and nonlinear regime) with the Fresnel number of the transducer.

\section{3:00}

5aPAl8. Linking static and dynamic NDT through fractional derivatives. Ari Salmi (Univ. of Helsinki / Dept. of Physical Sciences, POB 64 (Gustaf Hällströmin katu 2), 00014 Helsinki, Finland, edward.haeggstrom@helsinki.fi), Tuomas Hintikka (Electronics Research Unit, University of Helsinki, P.O. Box 64 (Gustaf Hällströmin katu 2), FIN00014 Helsinki, Finland, tuomas.hintikka@yahoo.com), Pekka Saranpaa (Finnish Forest Research Institute METLA, Vantaa Research Unit, Vantaa, FI-01301 Helsinki, Finland, pekka.saranpaa@metla.fi), Timo Karppinen (Electronics Research Unit, University of Helsinki, P.O. Box 64 (Gustaf Hällströmin katu 2), FIN-00014 Helsinki, Finland, timo.karppinen@helsinki.fi), Edward Hæggström (Electronics Research Unit, University of Helsinki, P.O. Box 64 (Gustaf Hällströmin katu 2), FIN00014 Helsinki, Finland, edward.haeggstrom@helsinki.fi), Ritva Serimaa (Univ. of Helsinki / Dept. of Physical Sciences, POB 64 (Gustaf Hällströmin katu 2), 00014 Helsinki, Finland, ritva.serimaa@ @elsinki.fi)

Static testing of mechanical properties of materials is widely used and well documented. Several extensively employed NDT techniques relying on induced mechanic wave motion (dynamic strain) exist. Comparing results obtained with dynamic NDT techniques to each other and to certified static tests is problematic especially for polymer materials whose absolute mechanical moduli depend on loading frequency. We present a study performed on 102 block-shaped dry samples of Norwegian spruce wood (Picea Abies [L.] Karst., a complex natural polymer) using ultrasound propagated in the longitudinal wood direction with frequencies ranging from $50 \mathrm{kHz}$ to 8 $\mathrm{MHz}$. The samples were also tested using a standardized three-point bending test providing longitudinal elasticity estimates at near-zero frequency (static MOE). We validate experimentally the Pritz[1] five-parameter fractional derivative model and we link together the static test and the ultrasonic NDT 
technique. Our results allow predicting the static MOE from the dynamic MOE obtained with ultrasonic test equipment, and permit comparison of data obtained with ultrasonic methods applying different frequencies. [1] T. Pritz, Five-parameter fractional derivative model for polymeric damping materials, Journal of Sound and Vibration, 265(5), 935-952 (2003)

\section{$3: 20$}

5aPA19. Influence of low temperature cycles on the integrity of pretreated 2024 T3 aluminium joints by an ultrasonic method. Martine Rousseau (Institut Jean Le Rond d'Alembert Université Paris 6 CNRS UMR 7190, 4, Place Jussieu, Case 162, 75252 Paris cedex 05, France mrousse@ccr.jussieu.fr), Valentina Vlasie Belloncle (Institut Jean Le Rond d'Alembert Université Paris 6 CNRS UMR 7190, 4, Place Jussieu, Case 162, 75252 Paris cedex 05, France, vlasie_valentina@yahoo.fr)

Many authors have considered the problem of adhesive bond durability over a long period of time or under special environmental conditions, and all have conclued that surface preparation is of paramount importance. Here, 2024 T3 aluminium alloy bonded joints, whose metallic plates underwent phosphoric acid pretreatment prior to bonding, are investigated before and after exposure to $-40{ }^{\circ} \mathrm{C}$ for $67 \mathrm{~h}$. This procedure of ageing is repeated several times and the samples are examined by ultrasonic Lamb waves after each cycle. In particular, the position of the cutoff-frequencies of ultrasonic guided waves which propagate into the bonded aluminum/epoxy/aluminium structure allows us to calculate the stiffnesses of the metal/adhesive interfaces with a rheological model. Thus, after exposure, a progressive decrease in the value of the stiffnesses is observed even for pretreated surfaces. Two complementary mechanical explanations are proposed. First, the cold exposure of the sample and the difference between thermal dilatation coefficients of aluminum and epoxy produce a damage of the interfacial zone. Second, the cooling introduces microcracks in the epoxy then caracterized by a smaller Young modulus.

\title{
Session 5aPPa
}

\section{Psychological and Physiological Acoustics: Cross-Spectral Auditory Integration: Physiological, Psychophysical, and Clinical Evidence I}

\author{
Blas Espinoza-Varas, Cochair \\ CommunicationSciences \& Disorders, OU Health Sciences Ctr., 825 NE 14th St., Oklahoma City, OK 73126-0901, USA
}

\author{
Alain De Cheveigne, Cochair \\ CNRS, Universite Paris 5, Ecole Normale Superieure, 29 rue d'Ulm, Paris, 75230, France
}

\section{Contributed Paper}

8:00

5aPPa1. Sensitivity of high-frequency inferior colliculus neurons to sinusoidal amplitude-modulation of low-frequency tones. Laurel $\mathrm{H}$. Carney (University of Rochester, Depts. of Biomedical Engineering and Neurobiology \& Anatomy, 601 Elmwood Ave., Box 603, Rochester, NY 14642, USA, Laurel.Carney@Rochester.edu)

Responses of neurons in the inferior colliculus of awake rabbit were studied using sinusoidally amplitude-modulated (SAM) tones with a wide range of carrier and modulation frequencies. Neurometric thresholds for detection of modulation were estimated by applying both average rate and temporal metrics to neural responses. For neurons tuned to high frequencies, neurometric thresholds for SAM detection were substantially lower for mid- to high-level carriers at frequencies in the tail of the tuning curves than for carriers near the characteristic frequency $(\mathrm{CF})$. Sensitivity to SAM for low carrier frequencies was often superior to sensitivity for near-CF carriers at low sound levels. These results suggest that psychophysical SAM detection thresholds at sound levels above approximately $60 \mathrm{~dB}$ SPL may be supported by "tail" responses of neurons tuned octaves above the carrier frequency. Improvement of psychophysical SAM detection thresholds with sound level is thus consistent with the contribution of a large population of responsive and sensitive "off-CF" neurons as level is increased. The fact that many auditory neurons, in both the peripheral and central nervous system, respond across several octaves at mid-to-high SPLs must be taken into account when considering "across-channel" interactions in tasks that involve both simple and complex sounds.

\section{Invited Paper}

5aPPa2. Role of peripheral nonlinearities in across-frequency processing of envelope fluctuations in normal hearing and hearing impaired listeners. Jesko Verhey (Universität Oldenburg, Medizinische Physik, Carl-von-Ossietzky Str. 9-11, 26111 Oldenburg, Germany, jesko.verhey@uni-oldenburg.de), Jesko Rennies (Universität Oldenburg, Medizinische Physik, Carl-von-Ossietzky Str. 9-11, 26111 Oldenburg, Germany, jan.rennies@ uni-oldenburg.de), Stephan M. Ernst (Universität Oldenburg, Medizinische Physik, Carl-von-Ossietzky Str. 9-11, 26111 Oldenburg, Germany, stephan.ernst@uni-oldenburg.de)

A psychoacoustical phenomenon related to the ability of the auditory system to compare information across frequency is comodulation masking release (CMR). CMR describes the effect that signal detectability of a sinusoidal signal embedded in a masker at the signal frequency can be improved by simultaneously presenting additional maskers in off-frequency regions if the additional maskers and the on-frequency masker (OFM) component have the same temporal envelope. Recently it was proposed that peripheral nonlinear processes such as suppression may account for large proportions of the CMR in conditions with a large spectral distance between the masker components. The aim of the present study was to test this hypothesis by measuring suppression and CMR within the same subjects for various frequency-level combinations of the off-frequency masker component. Average data for normal hearing listeners 
show a large overlap between the existence regions for suppression and CMR. The hearing impaired subjects with a sensorineural hearing loss show a negligible suppression and CMR. The data support the hypothesis that peripheral nonlinearities play an important role in CMR experiments with large spectral distances and large level differences between the masker components.

\section{Contributed Paper}

8:40

5aPPa3. Frequency modulated harmonics induces informational masking of Chinese speech. Xihong H. Wu (Dept. of Machine Intelligence, Speech and Hearing Research Center, 2 Science Building, Peking Univ., 5 Yeheyuan Road, Haidian District, 100871 Beijing, China, wxh@cis.pku.edu.cn), Jing Chen (Dept. of Machine Intelligence, Speech and Hearing Research Center, 2 Science Building, Peking Univ., 5 Yeheyuan Road, Haidian District, 100871 Beijing, China, chenj@cis.pku.edu.cn), Xuefei F. Zou (Dept. of Machine Intelligence, Speech and Hearing Research Center, 2 Science Building, Peking Univ., 5 Yeheyuan Road, Haidian District, 100871 Beijing, China, zouxf@cis.pku.edu.cn), Jingyu Y. Li (Dept. of Machine Intelligence, Speech and Hearing Research Center, 2 Science Building, Peking Univ., 5 Yeheyuan Road, Haidian District, 100871 Beijing, China, lijy@cis.pku.edu.cn), Lijuan J. Xu (Dept. of Psychology, Peking Univ., 5 Yeheyuan Road, Haidian District, 100871 Beijing, China, lijuan179021@yahoo.com.cn), Liang Li (Dept. of Psychology, Peking Univ., 5 Yeheyuan Road, Haidian District, 100871 Beijing, China, liangli@pku.edu.cn), Huisheng S. Chi (Dept. of Machine Intelligence,
Speech and Hearing Research Center, 2 Science Building, Peking Univ., 5 Yeheyuan Road, Haidian District, 100871 Beijing, China, chi@pku.edu.cn)

Informational masking (IM) occurs when target speech is co-presented with competing speech. It is important to know what acoustic characteristics of a speech masker are essential to induce IM. This study investigated whether frequency modulation (FM) of speech is associated with IM. Nonsense Chinese Mandarin sentences were spoken by a young female as target stimuli and maskers were tone harmonics whose fundamental frequency (F0) was modulated sinusoidally and number of harmonic streams was varied. The magnitude of IM was evaluated by measuring the precedenceinduced releasing effect of perceived spatial separation on target speech. The results show that the releasing effect reached the highest level when F0 became $252 \mathrm{~Hz}$, which was the mean pitch of target speech. In addition, the releasing effect was significantly higher when the masker contained two harmonic streams than when one stream. These results suggest that because frequency-modulated harmonics can be used to inducing IM of speech, the FM component of speech is an important acoustic factor underlying IM. [Supported by the NSFC 60435010; 60535030; 60605016; 30670704.]

\section{Invited Papers}

\section{9:00}

5aPPa4. Cross-spectral synergy and consonant identification. Thomas Ulrich Christiansen (Technical University of Denmark, Center for Applied Hearing Research, Ørsteds Plads, Building 352, 2800 Lyngby, Denmark, tuc@oersted.dtu.dk), Steven Greenberg (Technical University of Denmark, Center for Applied Hearing Research, Ørsteds Plads, Building 352, 2800 Lyngby, Denmark, steveng @ silicon-speech.com)

The auditory processing of consonants was investigated using an information-theoretic approach. Listeners identified eleven different Danish consonants spoken in a Consonant + Vowel $+[1]$ environment. Each syllable was processed so that only a portion of the original audio spectrum was present. Three-quarter-octave bands of speech, with center frequencies of 750,1500 and $3000 \mathrm{~Hz}$, were presented individually and in combination with each other. Confusion matrices were computed, and from these the amount of information transmitted for each of three phonetic-features - voicing, manner and place of articulation - was computed for each condition. From such analyses one can determine whether information associated with any given phonetic feature combines linearly across the acoustic spectrum or not. Our results indicate that information associated with voicing and manner-of-articulation combines in quasilinear fashion across the frequency spectrum. In contrast, place-of-articulation cues are integrated synergistically - information associated with two or three channels combined is far greater than predicted from the amount of information associated with individual spectral bands. Because consonants are essential for understanding speech, and place-of articulation information is crucial for decoding consonants, spoken language perception is likely to reflect highly non-linear processes.

5aPPa5. Across-frequency integration of speech information in listeners with sensorineural hearing loss. Joseph W. Hall (University of North Carolina, School of Medicine, 1115 Bioinformatics. Bldg., CB7070, 130 Mason Farm Rd., Chapel Hill, NC 27599, USA, jwh@med.unc.edu), Emily Buss (University of North Carolina, School of Medicine, 1115 Bioinformatics. Bldg., CB7070, 130 Mason Farm Rd., Chapel Hill, NC 27599, USA, ebuss@ med.unc.edu), John H. Grose (University of North Carolina, School of Medicine, 1115 Bioinformatics. Bldg., CB7070, 130 Mason Farm Rd., Chapel Hill, NC 27599, USA, jhg@med.unc.edu)

At very poor signal-to-noise ratios, good speech perception may depend critically upon the ability of the listener to combine relatively brief glimpses of the signal that are separated both in terms of frequency and time. This presentation will review past research that has addressed the ability of normal-hearing and hearing-impaired listeners to integrate speech information in this way. The presentation will also consider recent studies from our laboratory that have investigated spectral/temporal integration of speech signals in listeners with mild-to-moderate sensorineural hearing losses. One of these studies focused upon the integration of speech information that was asynchronous across frequency and the other focused upon the integration of synchronous information arising from two relatively narrow bands that were widely separated in frequency. The results from these studies indicated that although the hearing- impaired listeners exhibited some characteristics suggestive of poor processing of speech, there was little indication of essential deficits in the ability to integrate synchronous or asynchronous across-frequency speech information. 


\title{
Invited Papers
}

\section{1:00}

5aPPa6. Cross-spectral integration of speech patterns by normal and impaired listeners. Eric W. Healy (University of South Carolina, Speech Psychoacoustics Laboratory, Department of Communication Sciences and Disorders, William Brice Bldg., 1621 Greene St., Columbia, SC 29208, USA, ewh@sc.edu)

According to the current session description, "Recent evidence suggests that speech recognition depends importantly on the ability to integrate information across the frequency spectrum, which is not accounted for by the sensitivity (information content) of the respective spectral regions." Indeed, temporal speech patterns that yield near zero intelligibility when presented individually can provide intelligibility in excess of $80 \%$ when presented in pairs. These and other recent data involving the integration of speech patterns across the spectrum by the normal auditory system will be described. In addition, "speech-recognition data supporting the existence of a crossspectral integration deficit in hearing-impaired patients" will be discussed and contrasted against similar experiments involving nonspeech stimuli.

11:20

5aPPa7. Integration of acoustic cues for consonant identification by cochlear implant users. Mario Svirsky (New York University, Dept. of Otolaryngology, NYUSOM, 550 First Ave., NBV-5E5, New York, NY 10016, USA, mario.svirsky@nyumc.org), Elad Sagi (New York University, Dept. of Otolaryngology, NYUSOM, 550 First Ave., NBV-5E5, New York, NY 10016, USA, elad.sagi @ med.nyu.edu)

Users of cochlear implants (CIs) obtain substantial benefit from their devices, but their speech perception is (on average) less than perfect and there are significant individual differences among patients. In particular, their consonant identification levels are lower than those of normal hearing listeners, or even most users of hearing aids. We have developed a simple quantitative model (multidimensional Phoneme Identification or MPI model) to predict consonant confusion matrices for individual cochlear implant users based on their discrimination of three consonantal acoustic cues: place of stimulation in the cochlea, silent gap duration, and percentage of energy above $800 \mathrm{~Hz}$. Despite using only three degrees of freedom (i.e., JND for each cue) the model can explain most of the consonant pairs that are confused (or not confused) by individual CI users. However, when a listener's measured JNDs are used as inputs to the model, the predictions that result tend to have a higher percentage of correct responses than shown by the listener. One possible explanation is that CI users' speech perception may be limited not only by their basic psychophysical capabilities but also by their imperfect integration of different acoustic cues. [Work supported by NIDCD (R01-DC03937).]

\section{Session 5aPPb}

\section{Psychological and Physiological Acoustics: Otoacoustic Emissions, from Cochlear Modeling to Experimental Techniques, and Back I}

\author{
Christopher A. Shera, Cochair
}

Eaton-Peabody Laboratory, 243 Charles St, Boston, MA 02114, USA

Arturo Moleti, Cochair

University of Roma Tor Vergata - Physics Department, Via della Ricerca Scientifica, 1, Roma, 00133, Italy

\section{Invited Papers}

5aPPb1. Testing coherent reflection in chinchilla. Christopher A. Shera (Eaton-Peabody Laboratory, 243 Charles St, Boston, MA 02114, USA, shera@epl.meei.harvard.edu), Arnold Tubis (Institute for Nonlinear Science, University of California, San Diego, La Jolla, CA 92093, USA, tubisa@ aol.com), Carrick L. Talmadge (The University of Mississippi - NCPA, 1 Coliseum Drive, University, MS 38677, USA, clt@olemiss.edu)

Coherent-reflection theory explains the generation of stimulus-frequency and transient-evoked otoacoustic emissions by showing how they emerge from the coherent "backscattering" of forward-traveling waves by mechanical irregularities in the cochlear partition. Recent published measurements of stimulus-frequency otoacoustic emissions (SFOAEs) and estimates of near-threshold basilarmembrane (BM) responses derived from Wiener-kernel analysis of auditory-nerve responses allow for comprehensive tests of the theory in chinchilla. Model predictions are based on: (1) an approximate analytic expression for the SFOAE signal in terms of the BM traveling 
wave and its complex wave number; (2) an inversion procedure that derives the wave number from BM traveling waves; and (3) estimates of BM traveling waves obtained from the Wiener-kernel data and local scaling assumptions. At frequencies above $4 \mathrm{kHz}$, predicted median SFOAE phase-gradient delays are in excellent agreement with the SFOAE measurements. At frequencies below $4 \mathrm{kHz}$, chinchilla SFOAEs show strong evidence of interference between short- and long-latency components. Approximate unmixing of these components, and association of the long-latency component with the predicted SFOAE, yields close agreement throughout the cochlea.

\section{8:20}

5aPPb2. Two-tone suppression of stimulus frequency otoacoustic emissions in human ears with sensorineural hearing loss. Douglas H. Keefe (Boys Town National Research Hospital, 555 North 30th Street, Omaha, NE 68131, USA, keefe@boystown.org), John C. Ellison (Boys Town National Research Hospital, 555 North 30th Street, Omaha, NE 68131, USA, ellisonj@boystown.org), Denis F. Fitzpatrick (Boys Town National Research Hospital, 555 North 30th Street, Omaha, NE 68131, USA, fitzpatrickd@boystown.org)

Two-tone suppression (2TS) of stimulus frequency otoacoustic emissions (SFOAEs) recorded in normal-functioning human ears is a noninvasive measure of cochlear nonlinearity. This study investigates 2TS SFOAE response properties in 63 subjects with sensorineural hearing loss and normal middle-ear function. SFOAEs were measured using tones at frequencies $\left(f_{p}\right)$ from 0.5 to $8 \mathrm{kHz}$ and levels from 40-60 dB SPL. For interpreting SFOAEs, hearing status was classified at each frequency according to degree of hearing loss, with losses ranging from within normal limits to $95 \mathrm{~dB}$ HL. SFOAE suppression was measured as a function of suppressor frequency $\left(f_{s}\right)$ and level $\left(L_{s}\right)$, with results analyzed in terms of a SFOAE suppression tuning curve. A tip-to-tail difference (TTD) was defined between the $L_{s}$ needed to produce a criterion decrement in SFOAE level at $f_{s}$ one octave below $f_{p}$ and the $L_{s}$ needed to produce a criterion decrement near $f_{p}$. The TTD estimates cochlear-amplifier gain in normal-functioning ears, but its properties in impaired ears based on SFOAEs have not been examined. Results will be analyzed as a function of the hearing status to assess the relative impact of outer-hair cell dysfunction in the SFOAE source region at tonotopic place. [Work supported by NIH.]

\section{8:40}

5aPPb3. Species differences in low-level otoacoustic emissions may be explained by "hot regions" in the cochlea. Jon Siegel (Northwestern Univ, 2240 Campus Drive, Evanston, IL 60208, USA, j-siegel@ northwestern.edu)

Otoacoustic emissions evoked by low-level transients (TEOAE) and single tones (SFOAE) appear to excite the same emission mechanism, a concept originally formulated by Kemp and others and confirmed quantitatively by Kalluri and Shera (JASA 121, 2097-2110 (2007)). We have studied emissions evoked by single tones (SFOAE) measured using the suppression method. In humans, 50-70 dB SPL tones most readily suppress SFOAE evoked by lower-level tones when the suppressor is near the frequency of the evoking tone, suggesting that most of the emission originates near the peak of mechanical activity induced by the evoking tone. However, in chinchillas, Mongolian gerbils and mice, emission components originating basal to the peak appear to be relatively larger than in humans. The range of frequencies evoking the largest SFOAE correspond to those where spontaneous emissions are detected, from roughly $0.5-6 \mathrm{kHz}$ in humans, $4-12 \mathrm{kHz}$ in chinchillas, $15-30 \mathrm{kHz}$ in mice and still higher in bats. Stimulus tones below this "hot" frequency range appear to generate the largest SFOAE components basal to the peak. The existence of "hot regions" in the cochlea may explain the apparent species differences in emission behavior between humans and small mammals. (Supported by NIH grant DC-00419 and Northwestern University.)

\section{9:00}

5aPPb4. $5 \mathrm{mHz}$ oscillations in OAE intensity following sound exposure. David Kemp (UCL Ear Institute, UCL Centre for Auditory Research, 332 Gray's Inn Road, WC1X8EE London, UK, d.kemp@ UCL.ac.uk), Oliver J. Brill (UCL Ear Institute, UCL Centre for Auditory Research, 332 Gray’s Inn Road, WC1X8EE London, UK, Oliver@otodynamics.com)

Otoacoustic emission (OAE) intensity is highly stable in healthy ears but can be temporarily reduced following noise exposure. This reduction in OAE intensity is well correlated with temporary noise induced hearing threshold elevation and is presumed to be due to metabolic fatigue in the outer hair cells. These cells are responsible for the generation of OAEs and are essential for the maintenance of normal hearing threshold. The severity and recovery of post exposure OAE reduction in an individual is therefore of interest for hearing protection purposes. This paper concerns the regulation of outer hair cell status and the effect of overstimulation on that regulation. We measured OAE level fluctuations following mild over stimulation (e.g., $100 \mathrm{dBSPL}$ for $2 \mathrm{~ms}$ ) in order to observe the dynamics of OAE regulation. We propose this parallels outer hair cell physiological regulation. Under-damped oscillations in OAE level occur typically at a frequency of 5 milliHertz after sound exposure. We find oscillations are excited by both the onset and offset of overstimulation and a linear model explains our observations. The oscillation is clearly not itself a fatigue process but fatigue is revealed by the need for a small depression of the baseline of the OAE oscillations.

\section{9:20}

5aPPb5. Several properties of distortion product otoacoustic emissions can be modeled by simple input/output functions. Paul Fahey (University of Scranton, Dept. of Physics/Electrical Engineering, Scranton, PA 18510, USA, faheyp1@scranton.edu), Glen K. Martin (Jerry Pettis Memorial Veterans Medical Center, Research Service (151), 11201 Benton Street, Loma Linda, 92357, USA, glen.martin2@va.gov)

Distortion product otoacoustic emissions (DPOAEs) often exhibit regular patterns in both amplitude and phase as functions of both the levels and frequencies of the generating primary tones. Many of these patterns can be approximated when modeled by simple input/output functions. In particular, nulling patterns in rabbit DPOAEs and their modifications upon noise damage or fatiguing tonal exposures can be modeled at the level of input/output functions. Also, input/output functions can be used to propose the possible physics that underlies some complicated DPOAE patterns in level/frequency space that occur in the presence of a third tone. Both data and models will be reviewed. 
9:40-11:00 Posters

Lecture sessions will recess for presentation of poster papers on various topics in acoustics. See poster sessions for topics and abstracts.

\section{Invited Papers}

\section{1:00}

5aPPb6. Using the frequency dispersive properties of otoacoustic emissions to facilitate separation of different components. Glenis R. Long (Graduate Center of the City University of New York, Speech-Language-Hearing Program, 365 Fifth Ave, New York, NY 10016, USA, glong@gc.cuny.edu), Carrick L. Talmadge (The University of Mississippi - NCPA, 1 Coliseum Drive, University, MS 38677, USA, clt@olemiss.edu)

Otoacoustic emissions (OAE) are frequency dispersive (different frequencies have different latencies), because of the properties of the basilar membrane. These frequency-dependent latency differences, are used to separate the transient otoacoustic emissions (TEOAE) from the stimulus, but have not been used with stimulus frequency (SFOAE) and distortion product (DPOAE) otoacoustic emissions. If continuously-sweeping tones are used to generate DPOAE and SFOAE, the frequency dispersive properties of these OAE cannot only be used to separate the evoking stimuli from the OAE, but also to separate OAE coming from different cochlear regions. Modifications of the LSF procedure to extract OAE (Long \&Talmadge, 1997: JASA, 102:2831-2848; Talmadge et al. 1999: JASA,105:275-292) permit not only the extraction of the different OAE components, but also provide an estimate of the latency of the OAE, which does not depend on group delay. DPOAE and SFOAE extracted using sweeping tones in normal and impaired adults and infants provide information about the nature of cochlea function in these populations

\section{$11: 20$}

5aPPb7. Does overexposure modify the fine structure of distortion product otoacoustic emissions? Karen Reuter (Acoustics, Aalborg University, Fredrik Bajers Vej 7 B5, 9220 Aalborg Ø, Denmark, kr@es.aau.dk), Dorte Hammershøi (Acoustics, Aalborg University, Fredrik Bajers Vej 7 B5, 9220 Aalborg Ø, Denmark, dh@es.aau.dk)

It is investigated, whether the pattern of distortion product otoacoustic emission (DPOAE) fine structure (quasiperiodic variations across frequency) is altered by an acoustical overexposure. DPOAE fine structures are determined in 16 normal-hearing humans using a high frequency-resolution and primary levels of L1/L2 $=65 / 45 \mathrm{~dB}$. DPOAEs are measured both before and after the subjects are monaurally exposed to a $1 \mathrm{kHz}$ tone lasting for $3 \mathrm{~min}$ at an equivalent threshold sound pressure level of $105.5 \mathrm{~dB}$. After the exposure the DPOAE levels are shifted to lower values and recover to the initial levels within 20 min. The DPOAE fine structure pattern is analyzed by an automatic classification algorithm, which determines ripple center frequency, ripple spacing, ripple height, and ripple prevalence. For some individuals, an alteration of some of the parameters could be observed after the exposure, i.e., either a more pronounced or a less pronounced fine structure could be observed after the exposure. Across subjects, no shift of the fine structure pattern in frequency could be observed, also none of the calculated parameters changed consistently after the exposure. The results do not support the idea that the DPOAE fine structure might contain more information about the state of hearing than the DPOAE level.

\section{1:40}

5aPPb8. Changes in distortion product otoacoustic emission (DPOAE) fine structure due to contralateral acoustic stimulation. Manfred Mauermann (Universität Oldenburg, Medizinische Physik, Carl-von-Ossietzky Str. 9-11, 26111 Oldenburg, Germany, manfred.mauermann@uni-oldenburg.de), Birger Kollmeier (Universität Oldenburg, Medizinische Physik, Carl-von-Ossietzky Str. 9-11, 26111 Oldenburg, Germany, birger.kollmeier@uni-oldenburg.de)

Contralateral acoustic stimulation (CAS) can cause changes in the amplitude of the $2 \mathrm{f}_{1}-\mathrm{f}_{2}$ DPOAE in humans - most probably mediated by the medial olivocochlear reflex. DPOAE amplitude changes due to CAS show large interindividual variability and large changes from suppression to enhancement for small changes of the primary levels. The underlying mechanisms of these effects are still not fully understood. We hypothesize that the two interacting DPOAE sources might be differently affected by the CAS. If so, CAS will cause specific changes in DPOAE fine structure. Therefore, DPOAE fine structures were measured using frequency-modulated primaries ( $\mathrm{f}_{2}: 1500-3000 \mathrm{~Hz}, \mathrm{f}_{2} / \mathrm{f}_{1}: 1.2 ; \mathrm{L}_{2}: 60 \mathrm{~dB}$ SPL; $\mathrm{L}_{1}: 58,63,68 \mathrm{~dB} \mathrm{SPL}$ ) without and with a broadband CAS (50 dB SPL). The fine structure changes and shifts according to CAS were analyzed in detail - including latency windowing to separate the contributions from the two interacting DPOAE sources. The results indicate, e.g., that there is no "true" enhancement in terms of enhanced cochlear activity during broadband CAS. Rather there are slight frequency shifts in DPOAE fine structure due to changes in the relative contribution of the two DPOAE sources. This shift can lead to observations of "pseudo" enhancement of up to $20 \mathrm{~dB}$ for isolated frequencies.

\section{Contributed Paper}

12:00

5aPPb9. Otoacoustic emission latency and cochlear mechanics. Arturo Moleti (University of Roma Tor Vergata - Physics Department, Via della Ricerca Scientifica, 1, 00133 Roma, Italy, moleti@ roma2.infn.it), Renata Sisto (ISPESL - Occupational Hygiene Department, Via di Fontana Candida, 1, 00040 Monte Porzio Catone (RM), Italy, renata.sisto@ispesl.it)

Otoacoustic emission (OAE) latency data provide important information about several aspects of cochlear mechanics. Time-frequency analysis techniques, euther based on the wavelet transform or on Matching Pursuit algorithms, permit to get accurate estimates of the latency of transient evoked OAEs (TEOAEs). Wavelet estimates of the TEOAE latency of 14 young subjects, recorded at different stimulus levels from 60 to $90 \mathrm{~dB}$ pSPL, are used here to test several predictions of transmission line cochlear models. The experimental relation between latency, frequency, and stimulus level, is used to get estimates of cochlear tuning at different stimulus levels, which can be compared with behavioral and other OAE-based estimates. 
These estimates can be used to get information about the functional form of the cochlear amplifier nonlinearity. The comparison between the TEOAE latency and the auditory brainstem response (ABR) latency may help discriminating between different models for the OAE backward propagation.
Wavelet latency and phase-gradient delay may be estimated on the same TEOAE waveforms. The comparison between these two characteristic times permits, in principle, to identify the place-fixed or wave-fixed nature of the TEOAE generation mechanisms.

\title{
Session 5aPPc
}

\section{Psychological and Physiological Acoustics: Otoacoustic Emissions, from Cochlear Modeling to Experimental Techniques, and Back II (Poster Session)}

\author{
Christopher Shera, Cochair \\ Eaton-Peabody Laboratory \\ Arturo Moleti, Cochair \\ University of Roma Tor Vergata - Physics Department
}

\begin{abstract}
All posters will be on display from 9:40 a.m. to 11:20 a.m. To allow contributors an opportunity to see other posters, contributors of odd-numbered papers will be at their posters from 9:40 a.m. to 10:30 a.m. and contributors of even-numbered papers will be at their posters from 10:30 a.m. to 11:20 a.m.
\end{abstract}

\section{Contributed Papers}

5aPPc1. Hemispheric Laterality and Peripheral Auditory Asymmetry studied by Transiently Evoked Otoacoustic Emissions. Annie M. Moulin (Neurosciences Sensorielles Comportement Cognition UMR5020 CNRS, Université de Lyon, 50, avenue Tony Garnier, 69366 Lyon Cedex 07, France, amoulin@olfac.univ-lyon1.fr), Jean-Bertrand Nottet (Hôpital du service de santé des Armées Desgenettes, Boulevard Pinel, 69000 Lyon, France, jean-bertrand.nottet@wanadoo.fr)

A peripheral left-right assymetry has been shown at the cochlear level: Transiently evoked otoacoustic emissions (TEOAEs) amplitudes are greater in the right ear than in the left ear of right-handed subjects, whereas no difference is obtained in left-handed subjects (Khalfa et al., 1998). This study sought to investigate a possible link between peripheral auditory assymetry and hemispheric laterality assessed by a dichotic listening task. Peripheral auditory laterality was investigated by comparing TEOAEs from both ears of each subject in 289 normally hearing subjects, with 94 left-handed subjects (52 women), and 194 right-handed subjects (129 women). Results show significantly greater OAE amplitude in right ear than in left ears for both right-handers $(12.93 \mathrm{~dB}, \mathrm{SD}=4.46$ versus $11.89, \mathrm{SD}=4.6)$ and lefthanders $(11.62 \mathrm{SD}=3.8$ versus $10.44, \mathrm{SD}=3.8$, whereas average hearing loss across frequencies did not show any significant difference. However, in the left-handers, only the subgroups of subjects presenting a right-ear advantage at the dichotic tasks, presented a significantly greater TEOAE amplitude in the right ear versus the left ear. Those results suggest a definite link between peripheral auditory assymetry and hemispheric laterality.

5aPPc2. Temporal suppression and augmentation of click-evoked otoacoustic emissions. Sarah Verhulst (Centre for applied hearing research, Technical University of Denmark, DTU, Bygn. 352, 2800 Lyngby, Denmark, sv@oersted.dtu.dk), James M. Harte (Centre for applied hearing research, Technical University of Denmark, DTU, Bygn. 352, 2800 Lyngby, Denmark, jha@oersted.dtu.dk), Torsten Dau (Centre for applied hearing research, Technical University of Denmark, DTU, Bygn. 352, 2800 Lyngby, Denmark,tda@oersted.dtu.dk)

This study investigates and models temporal suppression of clickevoked otoacoustic emissions (CEOAEs). This suppression-effect is created when a suppressor-click is presented close in time to a test-click. The analy- sis was carried out for short time-frames of short- and long-latency CEOAEs. The latter is defined as a CEOAE with duration greater than 20 ms, typically observed for test subjects with spontaneous otoacoustic emissions (SOAEs). Previous studies have tended to exclude these test subjects but they are incorporated here. The results from six exemplary subjects demonstrate that temporal suppression is present in all CEOAEs for inter-click intervals (ICIs) less than $8 \mathrm{~ms}$. The long-latency CEOAEs showed augmentation (i.e., negative suppression) for ICIs of 6-7 $\mathrm{ms}$ which was not reported for the short-latency CEOAE at these ICIs. A phenomenological approach is adopted here to explain both temporal suppression and augmentation of CEOAEs. Recently, a feedback automatic gain control (AGC) system has been used to model CEOAE suppression. However, this system cannot account for augmentation and therefore another, dynamic nonlinear model was developed. Suppression and augmentation were modeled phenomenologically using compression or expansion of the system output. This was obtained by shifting the operating-point on the input/output-characteristic in relation to the ICI.

5aPPc3. A psychophysical test of the Kummer primary-level rule for measuring distortion-product otoacoustic emission input/output functions. Enrique A. Lopez-Poveda (University of Salamanca, Instituto de Neurociencias de Castilla y León, Avda. Alfonso X 'El Sabio' s/n, 37007 Salamanca, Spain, ealopezpoveda@usal.es), Peter T. Johannesen (University of Salamanca, Instituto de Neurociencias de Castilla y León, Avda. Alfonso X 'El Sabio's/n, 37007 Salamanca, Spain, peter@usal.es)

The standard procedure for measuring distortion-product otoacoustic emission (DPOAEs) input/output (I/O) curves involves using primaries whose levels conform to the rule of Kummer [Kummer et al., J. Acoust. Soc. Am. 103, 3431-44 (1998)]. The assumption is that the DP originates at the F2 cochlear site and primaries that conform to this rule produce equal excitation at that site, which maximizes the DP amplitude. These ideas were tested psychophysically by measuring temporal masking curves (TMCs) for masker frequencies equal to the primary frequencies. A TMC-based level rule was obtained by plotting the levels of the F1 masker against those for the F2 one for corresponding masker-probe intervals. DPOAE I/O curves were then measured using the Kummer rule, the TMC-based rule, and pri- 
mary levels optimized individually to maximize DP amplitude. DPOAEs for the TMC-based and the Kummer rule had statistically similar amplitudes, but they were both lower than those measured with individually-optimized levels. This undermines the assumption that maximum DP responses occur for primaries that produce equal excitation at the F2 cochlear site. Individually-optimized levels showed that L1 should be higher than prescribed by the Kummer rule. [Work supported by IMSERSO-131/06, PROFIT-CIT-390000-2005-4, and MEC-BFU-2006-07536, and The Oticon Foundation.]

5aPPc4. Cochlear nonlinearity in normal-hearing subjects as inferred psychophysically and from distortion-product otoacoustic emissions. Peter T. Johannesen (University of Salamanca, Instituto de Neurociencias de Castilla y León, Avda. Alfonso X 'El Sabio's/n, 37007 Salamanca, Spain, peter@usal.es), Enrique A. Lopez-Poveda (University of Salamanca, Instituto de Neurociencias de Castilla y León, Avda. Alfonso X 'El Sabio’s/n, 37007 Salamanca, Spain, ealopezpoveda@usal.es)

The aim was to investigate the correlation between estimates of compression ratio, compression threshold, and cochlear gain for normal-hearing subjects inferred from temporal masking curves (TMC) and distortionproduct otoacoustic emission (DPOAEs) input-output (I/O) curves. Special care was given to reduce the influence of DPOAE fine structure on the I/O curves. Data were collected for 10 subjects and for cochlear places with characteristic frequencies (CFs) of $0.5,1,2$, and $4 \mathrm{kHz}$. Except at $4 \mathrm{kHz}$, little correlation was found between ratio estimates obtained with the two methods. One reason is that the DPOAE I/O curves show plateaus or dips which result in unexpectedly high compression estimates. Moderately high correlation was found between the compression-threshold estimates obtained with the two methods, although DPOAE-based values were systematically lower than those based on the TMCs. Both methods confirmed that compression ratio and threshold are approximately constant across the $\mathrm{CF}$ range considered. Cochlear gain could be estimated from TMCs only and it was found to increase slightly with increasing CF. It is discussed that DPOAE I/O curves may be inappropriate to infer precise individual nonlinear cochlear characteristics in normal-hearing listeners, particularly at low CFs [Work supported by IMSERSO 131/06, PROFIT CIT-390000-2005-4, MEC BFU-2006-07536, and The Oticon Foundation.]

5aPPc5. Recovery of distortion product otoacoustic emissions (DPOAE) with high time-resolution from a moderate monaural-exposure to $\mathbf{2}-\mathbf{k H z}$ in human subjects. Miguel Angel Aranda De Toro (Acoustics, Aalborg University, Fredrik Bajers Vej 7 B5, 9220 Aalborg Ø, Denmark, maat@es.aau.dk), Rodrigo Ordoñez (Acoustics, Aalborg University, Fredrik Bajers Vej 7 B5, 9220 Aalborg Ø, Denmark, rop@es.aau.dk), Karen Reuter (Acoustics, Aalborg University, Fredrik Bajers Vej 7 B5, 9220 Aalborg Ø, Denmark, kr@es.aau.dk), Dorte Hammershøi (Acoustics, Aalborg University, Fredrik Bajers Vej 7 B5, 9220 Aalborg $\varnothing$, Denmark, dh@es.aau.dk

The amplitude of distortion product otoacoustic emissions (DPOAE) decreases temporarily after exposure to a sound of moderate level. These changes show similarities to the changes observed in absolute hearing thresholds after similar sound exposures. This paper presents the experimental protocol to study how DPOAEs in human subjects are affected after a monaural exposure of ten minutes to a pure tone of $2 \mathrm{kHz}$. The experimental protocol allows to measure fine structures of the DPOAE with high timeresolution in a limited frequency range. Thus, the results give a detailed description of the DPOAE recovery process and can be used to develop a mathematical model of the recovery. This is the first approximation to study the recovery of more complex exposures. [Work supported by the Danish Research Council for Technology and Production.]

5aPPc6. Analysis of time-frequency fine structure of transiently evoked otoacoustic emissions to study the effects of exposure to GSM radiofrequency fields. Alessia Paglialonga (Institute of Biomedical
Engineering CNR - Polytechnic Univ. Milan, Piazza Leonardo da Vinci, 32, I-20133 Milan, Italy, alessia.paglialonga@polimi.it), Gabriella Tognola (Institute of Biomedical Engineering CNR - Polytechnic Univ. Milan, Piazza Leonardo da Vinci, 32, I-20133 Milan, Italy, gabriella.tognola@polimi.it), Marta Parazzini (Institute of Biomedical Engineering CNR - Polytechnic Univ. Milan, Piazza Leonardo da Vinci, 32, I-20133 Milan, Italy, marta.parazzini@polimi.it), Federica Sibella (Institute of Biomedical Engineering CNR - Polytechnic Univ. Milan, Piazza Leonardo da Vinci, 32, I-20133 Milan, Italy, federica.sibella@polimi.it), Steven L. Bell (University of Southampton, University Road, Highfield/ISVR, SO17 iBJ Southampton, UK, alessia.paglialonga@polimi.it), Mark E. Lutman (University of Southampton, University Road, Highfield / ISVR, SO17 iBJ Southampton, UK, mel@isvr.soton.ac.uk), Gyorgy Thuroczy (National Research Institute for Radiobiology and Radiohygiene NIRR, Department of Non-Ionizing Radiation, Anna u. 5, 1775 Budapest, Hungary, alessia.paglialonga@polimi .it), Paolo Ravazzani (Institute of Biomedical Engineering CNR - Polytechnic Univ. Milan, Piazza Leonardo da Vinci, 32, I-20133 Milan, Italy, paolo .ravazzani@polimi.it)

Mobile phones have become very commonly used throughout the world within a short period of time. To date there is only limited knowledge about interaction between electromagnetic fields (EMFs) emitted by mobile phones and the auditory function. Moreover, there is widespread concern that there may be potential for harm. The aim of this study, performed in the framework of the European Commission Project GUARD "Potential adverse effects of GSM cellular phones on hearing" (5FP, QLK4-CT-200100150, 2002-2004), was to assess potential subtle changes in cochlear function of normal hearing subjects by measuring the time-frequency fine structure of TEOAEs after exposure to low-intensity EMFs emitted by GSM mobile phones. TEOAEs were recorded in 27 healthy young adults before and after $10 \mathrm{~min}$ of real or sham exposure in a double-blind design. TEOAE data were analyzed both globally (broadband analysis) and using the wavelet transform (analysis of time-frequency fine structure). The broadband analysis revealed no significant effect on TEOAEs related to exposure, confirming results of previous studies; in addition, no significant change was detected in the analysis of the elementary wavelet components, suggesting that a 10-min exposure to EMFs emitted by GSM mobile phones has no immediate after-effect on TEOAE time-frequency fine structure.

5aPPc7. Simulating electrically evoked emissions in the cochlea. Julien Meaud (University of Michigan, 2250 G G Brown Bldg, 2350 Hayward St., Ann Arbor, MI 48109, USA, jmeaud@umich.edu), Karl Grosh (University of Michigan, 2250 G G Brown Bldg, 2350 Hayward St., Ann Arbor, MI 48109, USA, grosh@umich.edu)

Electrical stimulation of the cochlea is a means for investigating the electromechanics of the organ. In this study, we use a mechanical-electricalacoustic cochlear model to make predictions of the effect of intracochlear bipolar electrical stimulation on the basilar membrane velocity and stapes velocity. Bipolar stimulation provides for a localized means of stimulating the outer hair cells $(\mathrm{OHC})$ and is a well-established technique for evoking otoacoustic emissions. We show that both fast, fluid-borne, and slow, fluidstructure waves are possible. Whether the fast or slow waves dominate the overall response depends on the level of activity in the cochlea, the frequency and location of excitation, and the boundary conditions at the stapes and round window. We show that forward propagating traveling waves may dominate the basilar membrane response even with intracochlear stimulation apical to the measurement site. These results bear direct impact on the interpretation of noninvasive measurements using otoacoustic emissions.

5aPPc8. Otoacoustic emissions in sheep (Ovis aries): Sex differences and prenatal androgen effects. Dennis McFadden (University of Texas, Psychology Dept, SEA 4.110, Austin, TX 78712-0187, USA, mcfadden@psy.utexas.edu), Edward Pasanen (University of Texas, Psychology Dept, SEA 4.110, Austin, TX 78712-0187, USA, pasanen 
@ psy.utexas.edu),Michelle D. Valero(University of Texas, Department of Biology, San Antonio, TX 78249, USA, bonfire_2002@hotmail.com), Eila K. Roberts (University of Michigan, Department of Psychology and Reproductive Sciences Program, Ann Arbor, MI 48109, USA, ekrobert@umich.edu), Theresa M. Lee (University of Michigan, Department of Psychology and Reproductive Sciences Program, Ann Arbor, MI 48109, USA, terrilee@umich.edu)

Otoacoustic emissions (OAEs) were measured in Suffolk sheep (Ovis aries), the first ruminant species tested for OAEs to our knowledge. Some sheep had been administered testosterone or estradiol during prenatal development, some had been gonadectomized after birth, and some had been allowed to develop normally. The click-evoked otoacoustic emissions (CEOAEs) exhibited by the female control group $(\mathrm{N}=11)$ were stronger than those in the male control group $(\mathrm{N}=15)$, which is the same direction of effect reported for other mammals, including humans. The females administered testosterone prenatally $(\mathrm{N}=13)$ had substantially weaker (masculinized) CEOAEs than control females. Both outcomes suggest that prenatal exposure to androgens weakens the cochlear amplifiers. The CEOAEs of males administered testosterone prenatally $(\mathrm{N}=5)$ were not different from those of control males, which is contrary to expectation, but in accord with data from similarly treated rhesus monkeys. Seven males (of 24) and seven females (of 34) had no measurable DPOAEs at any frequency or level of primary tones tested, even though all did have normal-appearing CEOAEs. No spontaneous otoacoustic emissions (SOAEs) were found in any ears, a common finding in non-human species. [Supported by NIDCD (DM) and NICHD (TML).]

5aPPc9. Potential adverse effects of UMTS electromagnetic fields generated by mobile phones on the human inner ear: A European project (EMFNEAR). Annie M. Moulin (Neurosciences Sensorielles Comportement Cognition UMR5020 CNRS, Université de Lyon, 50, avenue Tony Garnier, 69366 Lyon Cedex 07, France, amoulin@olfac.univ-lyon1 .fr), Marta Parazzini (Institute of Biomedical Engineering CNR - Polytechnic Univ. Milan, Piazza Leonardo da Vinci, 32, I-20133 Milan, Italy,
marta.parazzini@polimi.it),Mark E. Lutman(University of Southampton, University Road, Highfield/ISVR, SO17 iBJ Southampton, UK, mel@isvr.soton.ac.uk), Gyorgy Thuroczy (National Research Institute for Radiobiology and Radiohygiene NIRR, Department of Non-Ionizing Radiation, Anna u. 5, 1775 Budapest, Hungary, alessia.paglialonga@polimi .it), Mariola Sliwinska-Kowalska (Nofer Institute of Occupational Medicine, PL-90-950 Lodz, Poland, marsliw@imp.lodz.pl), Georgy Tavarkiladze (National Research Centre for Audiology and Hearing Rehabilitation, 117513 Moscow, Russian Federation, gtavartkiladze@audiology .ru), Ingrida Uloziene (Kaunas University of Medicine, LT44307 Kaunas, Lithuania, uloziene@kmu.lt), Virgilius Uloza (Kaunas University of Medicine, LT44307 Kaunas, Lithuania, uloza@kmu.lt), Shrikanta Mishra (University of Southampton, University Road, Highfield / ISVR, SO17 iBJ Southampton, UK, sm2@isvr.soton.ac.uk), Lionel Collet (Univ. Lyon 1 - Lab. Neurosciences, Service Pr Collet, Pavillon U, Hôpital Edouard Herriot, F-69003 Lyon, France, iakhoun@olfac.univ-lyon1.fr), Paolo Ravazzani (Institute of Biomedical Engineering CNR - Polytechnic Univ. Milan, Piazza Leonardo da Vinci, 32, I-20133 Milan, Italy, paolo.ravazzani @polimi.it)

To answer concerns as to the potential effects of electromagnetic fields (EMF) generated by third generation mobile phones on the inner ear, the European Commission funded project "EMFNEAR" assessed potential changes in auditory function induced by UMTS field both in humans and in laboratory animals. The purpose of this paper is to present the final outcomes of the project in humans. Normally hearing subjects (18-30 years old) were exposed to 20 min UMTS or sham exposure in a double-blind controlled study. Two different EMF field strengths were used: SAR of 0.069 $\mathrm{W} / \mathrm{kg}$ using a commercially available phone and SAR of $1.75 \mathrm{~W} / \mathrm{kg}$ using a patch antenna. Audiological assessment, involving pure tone audiometry, distortion product otoacoustic emissions, effects of contralateral acoustic stimulation on Transiently evoked otoacoustic emissions and late cognitive potentials (P300), were performed before and immediately after real or sham exposures. Results showed no effects of acute UMTS electromagnetic fields exposure on the inner auditory system of humans. 


\title{
Session 5aPPd
}

\section{Psychological and Physiological Acoustics: General Topics in Psychological and Physiological Acoustics VII (Poster Session)}

\author{
Elizabeth Strickland, Cochair \\ Purdue University
}

\author{
Armin Kohlrausch, Cochair \\ Philips Research Europe
}

Alain De Cheveigne, Cochair

CNRS, Universite Paris 5, Ecole Normale Superieure

\begin{abstract}
All posters will be on display from 9:40 a.m. to 11:20 a.m. To allow contributors an opportunity to see other posters, contributors of odd-numbered papers will be at their posters from 9:40 a.m. to 10:30 a.m. and contributors of even-numbered papers will be at their posters from 10:30 a.m. to 11:20 a.m.
\end{abstract}

\section{Contributed Papers}

5aPPd1. Phase interactions between auditory filter and complex stimuli: psychophysical evidence for level dependence in cochlear phase response. Yi Shen (Indiana Univ., 200 S. Jordan Ave., Dept. of Speech and Hearing Sciences, Bloomington, IN 47405-7000, USA, shen2@indiana.edu), Jennifer Lentz (Indiana Univ., 200 S. Jordan Ave., Dept. of Speech and Hearing Sciences, Bloomington, IN 47405-7000, USA, jjlentz@indiana.edu)

Harmonic complexes with the same power spectrum and different phase spectra lead to significant differences in their efficiency in masking puretone signals. This process is presumably due to phase interactions between the masker and the cochlea, leading to internally represented waveforms with different amounts of modulation. The present study applied this idea to estimate the phase characteristic of the auditory system at different stimulus levels. Two masking experiments were conducted for estimating whether auditory-filter phase curvatures are level dependent at 2 and $4 \mathrm{kHz}$. Curvatures were measured at fixed masker levels ranging between 50 and $90 \mathrm{~dB}$ SPL in one experiment and at fixed signal levels (25 - $50 \mathrm{~dB}$ SPL) in a second experiment. Results from both experiments suggest a level dependence in the auditory-filter phase curvature at both frequencies, which shifts from a negative value towards zero with increasing excitation level. A plausible explanation for this observed level dependence was proposed with the use of a nonlinear cochlear model. This modeling approach indicated that the behaviorally measurable phase curvature is affected by level dependent alterations in the magnitude and phase response of the basilar membrane.

5aPPd2. Is auditory intensity discrimination a comparison of entropy changes? Iftikhar Nizami (1312 Grayson Place, Decatur, GA 30030, USA, nizamii2@aol.com)

In JASA $(97,1995)$, Wong \& Norwich presented a Weber-fraction equation derived from McConville, Norwich, \& Abel (Int J Biomed Comput 27, 1991). The latter modeled two-alternative forced-choice discrimination between stimuli of identical spectra and identical durations " $\mathrm{t}$ " but differing intensities I and $\mathrm{I}+\Delta \mathrm{I}$. Discrimination depended on $\Delta \mathrm{H}(\mathrm{I}, \mathrm{t})$, the change in the information-theoretic entropy over $\mathrm{t}$ and $\Delta \mathrm{I}$. $\Delta \mathrm{H}(\mathrm{I}, \mathrm{t})$, assumed constant over intensities, emerged as one of five unknowns in a Weber-fraction equation, $(\Delta \mathrm{I}) / \mathrm{I}=f\left(\mathrm{t}_{0}, \mathrm{t}_{\mathrm{W}}, \mathrm{n}, \beta, \Delta \mathrm{H}(\mathrm{I}, \mathrm{t})\right)$. McConville et al. presumed values for $\mathrm{n}$ and $\mathrm{t}_{\mathrm{W}}$. Using $(\Delta \mathrm{I}) / \mathrm{I} \approx \Delta \mathrm{H}(\mathrm{I}, \mathrm{t}) /\left(\mathrm{I} \Delta \mathrm{t}\left[\partial^{2} \mathrm{H} / \partial \mathrm{I} \partial \mathrm{t}\right]\right)$ and setting $\Delta \mathrm{t}=\mathrm{t}$, $f\left(\mathrm{t}_{0}, \mathrm{t}_{\mathrm{W}}, \mathrm{n}, \beta, \Delta \mathrm{H}(\mathrm{I}, \mathrm{t})\right) \quad$ was approximated as $g(\mathrm{n},(\beta / \mathrm{t}), \Delta \mathrm{H}(\mathrm{I}, \mathrm{t}))$ $=\Delta \mathrm{H}(\mathrm{I}, \mathrm{t}) /\left(\mathrm{It}\left[\partial^{2} \mathrm{H} / \partial \mathrm{I} \partial \mathrm{t}\right]\right)$, which was then curvefitted to one listener's Weber fractions. The obtained $\Delta \mathrm{H}(\mathrm{I}, \mathrm{t})$ alone was substituted back into $f\left(\mathrm{t}_{0}, \mathrm{t}_{\mathrm{W}}, \mathrm{n}, \beta, \Delta \mathrm{H}(\mathrm{I}, \mathrm{t})\right)$, which was then substantiated by curvefitting to the data to reveal the remaining unknowns, $\mathrm{t}_{0}$ and $\beta$. McConville et al. had to curvefit, because they model only a single, unspecified forced-choice trial, making $\Delta \mathrm{I}$ any intensity change; and none of the unknowns could legitimately be presumed. The curvefitting was flawed: the assumption $\Delta \mathrm{H}(\mathrm{I}, \mathrm{t})=$ constan affirms Fechner's postulate; and $\partial^{2} \mathrm{H} / \partial \mathrm{I} \partial \mathrm{t}<0$, giving a negative Weber fraction. McConville et al. fail to explain auditory intensity discrimination as a comparison of entropy changes, casting doubt on Wong \& Norwich (1995).

5aPPd3. Does Norwich's Entropy Theory of Perception avoid the use of mechanisms, as required of an information-theoretic model of auditory primary-afferent firing? Iftikhar Nizami (1312 Grayson Place, Decatur, GA 30030, USA, nizamii2@aol.com)

Norwich et al.'s Entropy Theory (1975-present) stipulates that (1) the auditory receptor is uncertain about the stimulus' intensity, (2) uncertainty, given by the "entropy" $\mathrm{H}$, relates to primary afferent neuronal firing-rate $\mathrm{F}$ as $\mathrm{F}=$ constant $\times \mathrm{H}$. $\mathrm{H}$ was calculated according to Garner \& Hake (Psychological Review 58, 1951; after Shannon, Bell System Technical Journal 27, 1948) using Information Theory. $\mathrm{H}$ is available any time events, their outcomes, and the appropriate probabilities of occurrence are known, precisely because Information Theory ignores mechanisms, such as sensory transduction. Hence, any "information-theoretic" hearing model must avoid physiological mechanisms, and Norwich et al. claim to comply. But do they? The Entropy Theory was scrutinized for avoidance of physiological mechanisms. Norwich et al. stipulated throughout that (1) "We can envisage a steady sensory stimulus as a stationary stochastic sequence of microscopic sensory events" (Norwich \& Wong, Annales de la Fondation Louis de Broglie 22, 1997), (2) those events (representing different intensities) are repeatedly sampled by the receptor. Sampling is a mechanism, not separable from transduction, the event's (presumed) "outcome". Also, Norwich et al.'s attempts to separate events from outcomes produces inconsistent identification of "events." The Entropy Theory is not an information-theoretic model of primary-afferent firing.

5aPPd4. Slopes of psychometric functions for forward-masked tones with and without a suppressor. Ifat Yasin (UCL Ear Institute, 332 Gray's Inn Road, WC1X 8EE London, UK, i.yasin@ucl.ac.uk), Christopher J. Plack (Psychology Department, Lancaster University, LA1 4YF Lancaster, UK, c.plack@lancaster.ac.uk)

The compressive nonlinearity defined by the basilar membrane (BM) input-output $(\mathrm{I} / \mathrm{O})$ function is evident in the slopes of psychometric 
functions for forward-masked tones. A suppressor tone may reduce compression by linearizing the compressive portion of the BM I/O function, or reduce cochlear gain without affecting the value of maximum compression. The present study compared slopes of psychometric functions obtained for a fixed low-level signal in the presence of a variable masker with or without a suppressor gated with the masker. Thresholds and psychometric functions were obtained for four normal-hearing listeners using a 2I-AFC task. The sinusoidal signal was a low-level $4-\mathrm{kHz}$ tone $\left(0-\mathrm{ms}\right.$ steady state, $2-\mathrm{ms} \cos ^{2}$ ramps) masked by a sinusoidal masker (100-ms steady state, 2-ms $\cos ^{2}$ ramps). The maskers were either on frequency $(4 \mathrm{kHz})$ or off frequency $(2.2$ $\mathrm{kHz}$ ) and were presented at masker-signal intervals of 20-100 ms. Initial analyses suggest that as masker levels increase, the slopes of psychometric functions become increasingly shallow (on-frequency masker) or remain relatively steep (off-frequency masker). The effect of a suppressor on the slopes of the psychometric functions appears to be minimal. Results will be discussed in the context of a cochlear model of peripheral nonlinearity.

5aPPd5. An effect of top-down processing on intensity discrimination in the sensory-trace mode. Ervin R. Hafter (University of California, Department of Psychology, 3210 Tolman Hall, Berkeley, CA, CA 94720-1650, USA, hafter@berkeley.edu), Anne-Marie Bonnel (University of California, Department of Psychology, 3210 Tolman Hall, Berkeley, CA, CA 947201650, USA, AMBonnel@berkeley.edu), Swapan Gandhi (University of California, Department of Psychology, 3210 Tolman Hall, Berkeley, CA, CA 94720-1650, USA, swapangandhi@berkeley.edu)

A traditional measure of intensity discrimination presents a standard stimulus followed by a test. In one perceptual strategy, the subject compares the test to a coded version of the standard in long-term "context-coded memory" while, in another, the comparison is to a copy of the standard held in short-term "trace memory" [Durlach and Braida, J. Acoust. Soc. Amer, 46, 372-383 (1969)]. Subjects can be induced to use trace memory by roving the level of the standard randomly from trial to trial. It is well known that roving lowers overall performance, but by parsing individual standards we find that the loss is not uniform. Rather, an inverse correlation with the roved levels shows a severe departure from Weber's Law, with slopes of about $-6 \mathrm{~dB}$ over $20 \mathrm{~dB}$ of roving. This can be modeled in terms of an additive noise that, unlike the multiplicative noise implied by Weber's Law, is constant across levels. We believe this represents top-down processes involved in the use of stimuli held in rehearsal memory.

5aPPd6. Contribution of very low modulation frequencies to speech intelligibility in a competing speech task. Christian Füllgrabe (University of Cambridge, Department of Experimental Psychology, Downing Street, CB2 3EB Cambridge, UK, cf277@cam.ac .uk), Michael A. Stone (University of Cambridge, Department of Experimental Psychology, Downing Street, CB2 3EB Cambridge, UK, mas19@cam.ac.uk), Brian Moore (University of Cambridge, Department of Experimental Psychology, Downing Street, CB2 3EB Cambridge, UK, bcjm@cam.ac.uk)

Vocoder studies have demonstrated that low-rate temporal-envelope cues (below $\sim 50 \mathrm{~Hz}$ ) in a few frequency bands are sufficient for good speech identification in quiet. From the effects of low pass and high pass filtering of the temporal envelopes extracted from band pass filtered sentences, Drullman et al. [J. Acoust. Soc. Am. 95, 2670-2680 (1994); J. Acoust. Soc. Am. 95, 1053-1064 (1994)] argued that modulation frequencies between 4 and 16 $\mathrm{Hz}$ are the most important for speech identification in a steady background noise. However, Stone and Moore (JASA, in press) found that modulation frequencies below $2 \mathrm{~Hz}$ were also beneficial when the speech signal was processed through an 8-channel (but not a 12-channel) noise vocoder. The present study investigated speech identification for nine normal-hearing listeners using IEEE sentences presented with a competing talker; the mixed signal was processed using a 6-, 9-, or 15-channel noise vocoder. Low-rate temporal-envelope cues were manipulated using a compressor for each channel. Compression speed varied from slow to fast, so as to progressively degrade modulation frequencies below $4 \mathrm{~Hz}$. Independent of the spectral resolution of the vocoder, mean intelligibility decreased as the compression speed increased; even modulation frequencies below $1.3 \mathrm{~Hz}$ contributed significantly to speech intelligibility.

5aPPd7. Comodulation masking release and its representation in human auditory cortex. Stephan M. Ernst (Universität Oldenburg, Medizinische Physik, Carl-von-Ossietzky Str. 9-11, 26111 Oldenburg, Germany, stephan.ernst@ uni-oldenburg.de), Jesko Verhey (Universität Oldenburg, Medizinische Physik, Carl-von-Ossietzky Str. 9-11, 26111 Oldenburg, Germany, jesko.verhey@uni-oldenburg.de), Stefan Uppenkamp (Universität Oldenburg, Medizinische Physik, Carl-vonOssietzky Str. 9-11, 26111 Oldenburg, Germany, stefan.uppenkamp@unioldenburg.de)

The aim of the present study was to find a correlate of the psychoacoustical effect of comodulation masking release (CMR) in human auditory cortex using functional magnetic resonance imaging (fMRI). The target stimulus was a sinusoidal signal embedded in a band limited white noise which was either unmodulated or multiplied with an irregular square-wave modulator with mean modulation frequency of $40 \mathrm{~Hz}$ (comodulated condition). For all listeners participating in the fMRI study, the average psychoacoustic threshold difference between the unmodulated and the comodulated condition (i.e., the CMR) was $20 \mathrm{~dB}$. Similar to the results from a previous fMRI study on pure-tone masking (Ernst, Verhey, Uppenkamp, ARO abstracts 30 (2007), p. 302(A)), a spatial dissociation of changes of overall level and signal-to-noise ratio in auditory cortex was found. The comparison of the fMRI activation maps for a signal presented in modulated and in unmodulated noise reveals that those regions in the antero-lateral part of Heschl's gyrus previously shown to represent the audibility of a target (rather than overall level) exhibit a stronger activation for the modulated than for the unmodulated conditions. This result is interpreted as a physiological correlate of the psychoacoustical CMR effect at the level of the auditory cortex.

5aPPd8. Behavioral evidence for off-frequency compression at $4 \mathbf{k H z}$ Dhany Arifianto (University of Zagreb, Psychology Department, Lancaster University, LA1 4YF Lancaster, UK, arifianto@ieee.org), Christopher J. Plack (Psychology Department, Lancaster University, LA1 4YF Lancaster, UK, c.plack@lancaster.ac.uk)

Most behavioral techniques for estimating the basilar-membrane response rely on the assumption that the response to an off-frequency masker is linear at the signal place. To test this assumption, we modified the additivity of forward masking technique. The signal was a $4-\mathrm{kHz}, 10-\mathrm{ms}$ pure tone, presented at $10 \mathrm{~dB}$ sensation level. The maskers were $500-\mathrm{Hz}$-wide bands of noise centered on 1.5, 2, or $4 \mathrm{kHz}$. Masker 1 had a duration of 200 $\mathrm{ms}$ and was followed immediately by masker 2 with a duration of $20 \mathrm{~ms}$. The masker 2-signal silent interval ranged from 10 to $60 \mathrm{~ms}$. For each interval, the masker level required to mask the signal was determined, for each masker presented individually, and for the two maskers combined. In the combined case, the levels of the two maskers were set to be roughly equally effective, and adaptively varied together. Combining two equally effective maskers should produce a 3-dB reduction in masker level at threshold if the system is linear. A reduction greater than this indicates compression. Although compression was greatest for the 4-kHz maskers, compression was also observed at 1.5 and $2 \mathrm{kHz}$, suggesting that the assumption of a linear off-frequency response may not valid.

5aPPd9. Multiple looks in spectrotemporal integration. Evelyn Hoglund (The Ohio State University, 110 Pressey Hall, 1070 Carmack Road, Columbus, OH 43210, USA, hoglund.1@osu.edu), Lawrence Feth (The Ohio State University, 110 Pressey Hall, 1070 Carmack Road, Columbus, OH 43210, USA, feth.1@osu.edu)

The multiple looks hypothesis was originally developed as an explanation for temporal integration and resolution. Our preliminary work supports its extension to spectral integration as well spectrotemporal integration. The current study was designed to measure detection of tonal signals that vary in both spectral and temporal dimensions. Preliminary work began by measuring thresholds in quiet. Elementary signals consisted of $10 \mathrm{~ms}$ tone bursts centered on one of eight frequencies spaced 1 ERB apart. Complexes were constructed by adding two to eight individual tone bursts along the temporal or spectral dimension, or both. The signals varied along both dimensions in several ways to focus on the relative salience of each dimension for 
detection. In quiet, the spectrotemporal integration conditions showed threshold improvement to be limited by spectral integration. Surprisingly, even trial-by-trial randomization of frequencies did not negatively influence detection. For this study, the same tone complexes from the preliminary work were used to measure thresholds in a wide band energetic masker. As expected, threshold improvement was most dependent on increasing the number of tones, with less effect from other variables. Subtle differences between quiet and masked integration performance will be discussed. Research supported by a grant from NIH/NIDCD R01-DC006879.

5aPPd10. Estimating the basilar-membrane input/output-function in normal-hearing and hearing-impaired listeners using forward masking. Morten L. Jepsen (Centre for Applied Hearing Research, Technical University of Denmark, DTU, Bygn. 352, 2800 Lyngby, Denmark, mlj@oersted.dtu.dk), Torsten Dau (Centre for Applied Hearing Research, Technical University of Denmark, DTU, Bygn. 352, 2800 Lyngby, Denmark, tda@oersted.dtu.dk)

To partly characterize the function of cochlear processing in humans, the basilar membrane (BM) input-output (I/O) function can be estimated. In recent studies, forward masking has been used to estimate BM compression. If an on-frequency masker is processed compressively, while an off-frequency masker is transformed more linearly, the ratio between the slopes of growth of masking (GOM) functions provides an estimate of BM compression at the signal frequency. In this study, this paradigm is extended to also estimate the knee-point of the I/O-function between linear processing at low levels and compressive processing at medium levels. If a signal can be masked by a low-level on-frequency masker such that signal and masker fall in the linear region of the I/O-function, then a steeper GOM function is expected. The knee-point can then be estimated in the input level region where the GOM changes significantly. Data were collected from eight normal-hearing $(\mathrm{NH})$ and five hearing-impaired (HI) listeners with mild to moderate sensorineural hearing loss. Both groups showed large inter-subject but low intrasubject variability. When the knee-point could be estimated for the HI listeners it was shifted towards higher input levels and compression was similar to that of $\mathrm{NH}$ listeners.

5aPPd11. Unpredictable interruption can enhance the auditory continuity illusion. Maori Kobayashi (R.I.E.C., Tohoku University, 2-1, Katahira, Aoba-ku, 980-8577 Sendai, Japan, maori@ais.riec.tohoku .ac.jp), Makio Kashino (NTT Communication Science Laboratories, NTT Corporation, 3-1, Morinosato Wakamiya, 243-0198 Atsugi, Japan, kashino @avg.brl.ntt.co.jp)

It was examined whether the predictability of interruption timing affects the limit of perceiving the continuity illusion. The stimulus consisted of a $500-\mathrm{Hz}$ sinusoidal inducee and a 200-ms, 500-Hz, 1/3-octave noise band inducer, which were alternated repeatedly, with a fixed interval $(400 \mathrm{~ms})$ between adjacent inducers (regular condition) or with random intervals (irregular condition). The continuity limit (CL) was measured in terms of the maximum level of the inducee for the illusory continuity to be perceived while maintaining the inducer level at $60 \mathrm{~dB}$ SPL. In Experiment 1, the listeners' task was to judge whether the inducee appeared continuous or discontinuous. In Experiment 2, the task was to discriminate illusory and physical continuity in a two-interval forced choice paradigm. In both experiments, the mean CLs in the irregular condition were significantly higher than those in the regular condition. In Experiment 3, when an identical irregular interval pattern was used repeatedly in successive trials, the mean CL gradually declined, and reached the level of the regular condition in approximately 80 trials. These results suggest that unpredictability, not mere irregularity, in interruption timing promotes the continuity illusion, implying the involvement of the short-term plasticity of the auditory system.

5aPPd12. A filterbank model of human nonlinear auditory frequency selectivity. Almudena Eustaquio-Martín (University of Salamanca, Instituto de Neurociencias de Castilla y León, Avda. Alfonso X 'El Sabio' s/n, 37007 Salamanca, Spain, aeustaquio@usal.es), Enrique A.
Lopez-Poveda(University of Salamanca, Instituto de Neurociencias de Castilla y León, Avda. Alfonso X 'El Sabio's/n, 37007 Salamanca, Spain, ealopezpoveda@usal.es)

Filterbank models of human nonlinear auditory frequency selectivity are useful in a variety of applications. Existing filterbanks are almost certainly flawed because they do not account for the recent observation that compression extends to a wider range of stimulus frequencies in apical than in basal cochlear sites [Lopez-Poveda et al. (2003), J. Acoust. Soc. Am. 113, 951960]. Here, we present a filterbank based on the forward-masking data from which this observation was made. A forward-masking model was constructed by cascading a linear "outer/middle-ear" filter, followed by a dualresonance nonlinear (DRNL) filter, followed by a linear temporal window. The temporal window was assumed to be identical across listeners and cochlear places. Only the parameters of the DRNL filter were adjusted to simulate the absolute threshold and the masking data of three listeners for probe frequencies of $0.5,1,2,4$, and $8 \mathrm{kHz}$. A bank of DRNL filters was then constructed by linear or quadratic regression of the optimum parameters. It is discussed that this filterbank is the best possible approximation of human level-dependent auditory frequency selectivity to date. Additionally, the results support the linearity of the temporal window. [Work supported by IMSERSO 131/06, PROFIT CIT-390000-2005-4, MEC BFU-2006-07536.]

5aPPd13. Object's width and distance distinguished by the blind using auditory sense while they are walking. Takahiro Miura (Graduate School of Information Science and Technology, University of Tokyo, 7-3-1 Hongo, Bunkyo-ku, 113-8654 Tokyo, Japan, miura@human.rcast.u-tokyo .ac.jp), Kazutaka Ueda (Research Center for Advanced Science and Technology, University of Tokyo, 4-6-1 Komaba, Meguro-ku, 153-8904 Tokyo, Japan, ueda@human.rcast.u-tokyo.ac.jp), Teruo Muraoka (Research Center for Advanced Science and Technology, University of Tokyo, 4-6-1 Komaba, Meguro-ku, 153-8904 Tokyo, Japan, muraoka@human.rcast.u-tokyo .ac.jp), Shuichi Ino (Research Center for Advanced Science and Technology, University of Tokyo, 4-6-1 Komaba, Meguro-ku, 153-8904 Tokyo, Japan, ino@human.rcast.u-tokyo.ac.jp), Tohru Ifukube (Research Center for Advanced Science and Technology, University of Tokyo, 4-6-1 Komaba, Meguro-ku, 153-8904 Tokyo, Japan, ifukube@human.rcast.u-tokyo.ac.jp)

Mobility aid has recently become important for the blind because of increasing their outgoing opportunity. It is necessary for the blind to acquire "obstacle sense" by which they can recognize surrounding objects auditorily. In particular, it is indispensable to investigate the characteristics of the obstacle sense while they are walking. However, training method for the mobility has not been systematically proposed yet because factors regarding the obstacle sense while walking remain unknown. Final goal of this study is to propose a systematic training method for their mobility using the obstacle sense. In this study, the authors particularly focused on a relation of interaural differences between both ears to the ability of the obstacle sense while walking toward the obstacles. In the experiments, the blind subjects were asked to answer when they find the obstacle made of wooden plate with various widths in both conditions of head moving and head fixing. Auditory discrimination ability of the obstacles was measured as a function of the obstacle's width and the distance from the blind subject. From the experimental results, it was found that blind people generally can recognize the obstacles better in the head moving case than the head fixing case.

5aPPd14. Amplitude modulation depth discrimination in hearing-impaired and normal-hearing listeners. Stephan D. Ewert (Carl von Ossietzky Universität Oldenburg, Ammerländer Heerstraße 114-118, 26111 Oldenburg, Germany, stephan.ewert@uni-oldenburg.de), Jutta Volmer (Universität Oldenburg, Medizinische Physik, Carl-von-Ossietzky Str. 9-11, 26111 Oldenburg, Germany, jutta.volmer@uni-oldenburg .de), Torsten Dau (Centre for applied hearing research, Technical University of Denmark, DTU, Bygn. 352, 2800 Lyngby, Denmark, tda@oersted.dtu.dk), Jesko Verhey (Universität Oldenburg, Medizinische Physik, Carl-von-Ossietzky Str. 9-11, 26111 Oldenburg, Germany, jesko .verhey@uni-oldenburg.de)

The processing of amplitude modulations (AM) of sounds is assumed to be crucial for decoding and understanding of speech in humans. Since hearing-impaired (HI) listeners often suffer from severely hampered speech intelligibility, particularly in reverberant or noisy environments, they might also show degraded performance in AM processing tasks. However, several 
studies indicated a similar or even better performance in AM detection tasks for sensorineural HI listeners than for normal hearing $(\mathrm{NH})$ listeners when reduced audibility was compensated. In addition to AM detection, this study investigates the differential processing of amplitude modulation depth in $\mathrm{HI}$ and NH listeners. AM-depth discrimination of a 4-, 8-, and 30-Hz sinusoidal $\mathrm{AM}$, imposed on a 1- or 4-kHz pure-tone carrier, was measured. The AM of the standard ranged from being well detectable to near threshold. AM-depth discrimination thresholds strongly varied among HI listeners and were elevated in comparison to $\mathrm{NH}$ for high standard depths. A model of AM processing is suggested incorporating an individually adjusted simulation of the auditory periphery. To account for the data of HI listeners, however, the key element appeared to be an increased internal noise in the AM-depth domain. Consequences for speech perception are discussed.

5aPPd15. Exploring the psychophysical temporal effect in masking using a model of the auditory periphery. Skyler Jennings (Purdue University, 500 Oval Drive, West Lafayette, IN 47907, USA, sgjennin@purdue.edu), Michael Heinz (Purdue University, 500 Oval Drive, West Lafayette, IN 47907, USA, mheinz@purdue.edu), Elizabeth Strickland (Purdue University, 500 Oval Drive, West Lafayette, IN 47907, USA, estrick@purdue.edu)

Under some conditions, normal hearing individuals can detect a short duration signal in a simultaneous masker at a lower signal-to-masker ratio when there is preceding stimulation (either a precursor or an extension of the masker). This effect has been referred to as overshoot or the temporal effect. It has been hypothesized the neural adaptation may account for some but not all of the temporal effect. Recent psychophysical data suggest that cochlear gain may decrease over the course of the masker and improve detection. This could be mediated by the medial olivocochlear reflex (MOCR). In the present study, a model of peripheral auditory processing [Zilany and Bruce, J. Acoust. Soc. Am. 120, 1446-1466 (2006)] using a d'type metric was used to assess the relative contributions of these processes. Although the model includes neural adaptation, initial results indicate that it does not predict a temporal effect. The potential role of the MOCR will be simulated by decreasing the gain of the cochlear amplifier, to see if this will produce a temporal effect in the model predictions. [Work supported by NIH.]

5aPPd16. Intelligibility of whispered speech interleaved with speech or noise maskers. Nandini Iyer (AFRL, 2610 Seventh Street, WPAFB, OH 45433, USA, nandini.iyer@wpafb.af.mil), Douglas S. Brungart (AFRL, 2610 Seventh Street, WPAFB, OH 45433, USA, douglas.brungart @wpafb.af.mil), Brian D. Simpson (AFRL, 2610 Seventh Street, WPAFB, OH 45433, USA, Brian.Simpson@wpafb.af.mil)

When an interrupted speech signal is alternated with a noise masker, performance is generally improved relative to the condition where the speech and noise are presented continuously. However, when two interrupted speech signals are alternated, performance declines relative to the continuous presentation condition. One possible explanation for this result is that listeners in the alternated speech and noise condition use the contrast between the periodic temporal structure of voiced speech and the random temporal structure of noise to segregate the time intervals associated with the speech target and noise masker. In the current experiment, a whispered speech signal was either presented continuously in the presence of a noise masker or alternated with a noise masker at an $8-\mathrm{Hz}$ rate. The results show near perfect performance in the alternated whisper and noise condition, suggesting that harmonic structure due to voicing is not necessary to segregate a speech signal from an interleaved random-noise masker. Indeed, when whispered speech was interleaved with voiced speech, performance decreased relative to the continuous condition when the target talker was voiced but not when it was whispered, suggesting that listeners are better at selectively attending to unvoiced intervals and ignoring voiced intervals than the converse.

5aPPd17. Temporal integration functions of amplitude modulation depth discrimination: can multiple-looks model explain this? Jungmee Lee (Univ. of Arizona, 1131 E. 2nd Street, Tucson, AZ 85721, USA, jmlee6@email.arizona.edu), Derek Edwards (Univ. of Arizona, 1131 E. 2nd Street, Tucson, AZ 85721, USA, derek09@email.arizona.edu), Jennifer Andrews (Univ. of Arizona, 1131 E. 2nd Street, Tucson, AZ 85721, USA, andrewsj@email.arizona.edu), Heather Murray (Univ. of Arizona, 1131 E. 2nd Street, Tucson, AZ 85721, USA, hmurray@email.arizona.edu)

Lee and Bacon (1994) applied a multiple looks model (Viemeister and Wakefield, 1990) as a mechanism of AM depth discrimination when the carrier and the modulator were presented at the same time (gated condition). The model provided a reasonable prediction, but the performance (d') was underpredicted when the number of modulation cycles changed from two to four. They speculated that onset information loss due to gating with two modulation cycles lead to a greater improvement with four modulation cycles. Following this, one would expect that AM depth discrimination can be better predicted by the multiple-looks model for the condition where there is no loss of modulation information at onset. This study compared psychometric functions of AM depth discrimination with different modulation cycles for the gated and onset-only conditions (carrier was presented for $250 \mathrm{~ms}$ prior to the modulation to preserve onset information). The slop of the psychometric functions with two modulation cycles was shallower than those with greater modulation cycles for the gated condition, while the slop of the psychometric functions was similar for all modulation cycles for the onset-only condition. The data will be discussed with multiple looks model. [This work was supported by NIDCD Grant No. R03 DC06605-02.] 


\title{
Session 5aPPe
}

\section{Psychological and Physiological Acoustics and Computational Acoustics: Computational Auralization I (Poster Session)}

\author{
Durand Begault, Cochair \\ NASA Ames Research Center \\ Lauri Savioja, Cochair \\ Helsinki University of Technology
}

\begin{abstract}
All posters will be on display from 9:40 a.m. to 11:20 a.m. To allow contributors an opportunity to see other posters, contributors of odd-numbered papers will be at their posters from 9:40 a.m. to 10:30 a.m. and contributors of even-numbered papers will be at their posters from 10:30 a.m. to 11:20 a.m.
\end{abstract}

\section{Contributed Papers}

5aPPe1. Effect of sound spatialisation on multitasking in remote meetings. Stuart N. Wrigley (University of Sheffield, Regent Court, Dept. of Computer Science, 211 Portobello Road, s1 4dp Sheffield, UK, s.wrigley@dcs.shef.ac.uk), Simon Tucker (University of Sheffield, Dept. of Information Studies, Regent Court, 211 Portobello Street, S1 4DP Sheffield, UK, s.tucker@dcs.shef.ac.uk), Guy J. Brown (University of Sheffield, Dept. of Computer Science, Regent Court, 211 Portobello Street, S1 4DP Sheffield, UK, g.brown@dcs.shef.ac.uk), Steve Whittaker (University of Sheffield, Dept. of Information Studies, Regent Court, 211 Portobello Street, S1 4DP Sheffield, UK, s.whittaker@shef.ac.uk)

Participants in a teleconference often multitask during meetings, e.g., they work on a text-based "foreground" task whilst listening in the 'background' for a topic of interest to appear. Audio material should therefore be presented in a manner that has the smallest possible impact on the foreground task without affecting topic detection. Here, we ask whether spatialised audio presentation of a meeting is less disruptive than the singlechannel mixture of talkers that is normally used in teleconference audio. Head-related impulse responses are used to synthesise a stereo signal in which each meeting participant is placed at a spatially distinct location. A number of talker location configurations are used, and we examine how these impact upon a text-based foreground task: finding all letter "e" occurrences in a block of text as quickly as possible. We also examine the effect of listening task (e.g., "listen for keyword x" versus "listen for keyword x spoken by talker on left") and record listener preferences for audio presentation style. Our results suggest that single-channel and spatialised audio are equally disruptive when listeners are unaware of the audio presentation style. We also report studies in which presentation type and target direction/gender are cued.

5aPPe2. The use of virtual acoustics in the evaluation and development of binaural hearing aid algorithms. Monika Rychtarikova (Lab. ATF, Katholieke Universiteit Leuven, Celestijnenlaan 200D, B-3001 Leuven, Belgium, Monika.Rychtarikova@bwk.kuleuven.be), Tim Van Den Bogaert (ExpORL, Dept. Neurosciences, K.U. Leuven, Herestraat 49 bus 721, O. \& N2, B-3000 Leuven, Belgium, tim.vandenbogaert@med.kuleuven .be), Gerrit Vermeir (Lab. ATF, Katholieke Universiteit Leuven, Celestijnenlaan 200D, B-3001 Leuven, Belgium, Gerrit.Vermeir@bwk .kuleuven.be), Koen Eneman (ExpORL, Dept. Neurosciences, K.U. Leuven, Herestraat 49 bus 721, O. \& N2, B-3000 Leuven, Belgium, koen.eneman@med.kuleuven.be), Walter Lauriks (Lab. ATF, Katholieke Universiteit Leuven, Celestijnenlaan 200D, B-3001 Leuven, Belgium,
Walter.Lauriks@fys.kuleuven.be),Marc Moonen(ESAT/SISTA, K.U. Leuven, Kasteelpark Arenberg 10, B-3001 Leuven, Belgium, marc.moonen@esat.kuleuven.be), Jan Wouters (ExpORL, Dept. Neurosciences, K.U. Leuven, Herestraat 49 bus 721, O. \& N2, B-3000 Leuven, Belgium, jan.wouters@med.kuleuven.be)

The development of noise reduction algorithms for hearing aids (HA) is not longer only related to the improvement of signal to noise ratio, but also to the quality of hearing, e.g., binaural aspects of hearing. This is very important for the recognition of the localization of sound sources but also for an improved speech intelligibility in noisy situations due to spatial release from masking effects. New design and signal processing algorithms for binaural HA's need to be tested and validated in different acoustical scenarios. As it is too laborious and time consuming to perform sufficient numbers of perceptual evaluations in different rooms with different acoustical parameters, advanced acoustic modeling of different virtual acoustical environments might be needed. Virtual acoustics in our research relates to the convolution of the measured or simulated binaural signals (head related transfer functions - HRTF's) with the impulse response generated from a computer model of a room (using ODEON ${ }^{\circledR}$ software) to simulate binaural sounds. This study investigates the usage of virtual acoustics in the framework of developing algorithms for binaural hearing aids. It evaluates and quantifies the fidelity of binaural signals generated by commercially available virtual acoustics software with respect to the localization of sound and speech intelligibility in different acoustical scenarios.

5aPPe3. Auralization of urban soundscaping designs using the Arup SoundLab. Seb Jouan (Arup Acoustics, Bergius House, 11-12 Claremont Terrace, G3 7XR Glasgow, UK, seb.jouan@arup.com)

Noise is unwanted sound (from an urban planning point of view). The standard practice to control noise, while valuable, is a negative process (i.e. reducing unwanted "noise"). In response, there is a drive by several authorities to take a more positive approach to improve and manage soundscapes in cities and civic spaces. Urban soundscape design consists of planning, shaping and managing the sound to fit each area of the masterplan in terms of civic, cultural, and social character. Arup uses its SoundLab, a 3D sound facility (ambisonic), to design and auralise (the sound equivalent to visualisation) soundscapes with planners, architects or artists and demonstrate to Local Planning Authorities or clients the results of the design. An example of urban soundscaping design is Dark Neville Street, Leeds for Leeds City Council where architectural, lighting design, acoustical soundscaping design were integrated together with an artistic sonic piece by HansPeter Kuhn which was auralised in the Arup SoundLab. An example of "indoor" sonic art and soundscape design installation is "Harmonic Bridge" by 
Bill Fontana and Arup Acoustics in the Turbine Hall of the Tate Modern (June-August 2006) where the sonic art piece was composed in the SoundLab.

5aPPe4. Salience of spatial attributes on quality evaluation of multichannel audio processing devices. Inyong Choi (Seoul National University, ENG 420-025, Gwanak PO Box 34, 151-600 Seoul, Republic of Korea, inyongchoi@gmail.com), Jung-Hun Seo (Seoul National University, ENG 420-025, Gwanak PO Box 34, 151-600 Seoul, Republic of Korea, pollini@acoustics.snu.ac.kr), Koeng-Mo Sung (Applied Acoustics Lab., INMC, 132-302, Seoul National University, San 56-1, Sillim-dong, Kwanak-gu, 151-742 Seoul, Republic of Korea, kmsung@snu.ac.kr)

Objective quality assessment methods for audio signal processing devices, e.g. ITU-R BS.1387-1, measure perceived quality by using various types of test excerpts and incorporating a number of psychoacoustic metrics for evaluating various attributes of quality degradations. As many multichannel audio processing devices are being developed, recent quality assessment methods include not only timbral features but also spatial features, such as analysis on interaural disparities. In this paper, the importance of the newly proposed spatial - interaural - features are studied for different types of multichannel test signals. The spatial features have bigger salience on directly recorded sound sources than artificially manipulated multichannel contents.

5aPPe5. Assessment of simulation quality of three different auralization procedures. Stephan Paul (Lab. of Vibrations and Acoustics, Fed. Univ. of Santa Catarina, CTC, EMC, Campus Trindade, 88040 Florianópolis, Brazil, stephan.paul.acoustic@gmail.com)

When acoustical problems occur in architectural spaces acousticians are often requested to help. The help is often complicated by very different knowledge in acoustics and problems in communication between architect, musicians and acousticians. Auralization of sound sources is considered to be a powerful tool to solve the problem, making acoustic information accessible for hearing. The present research assessed the quality of the output of different simulation procedures by subjective assessment of similarity with a reference sound, considering different perceptual dimensions (localization, reverberation, and timbre). The reference sound was obtained by binaural recording in the room that was auralized using ray-tracing, mirror sources or a hybrid pyramid tracing method. Two different types of sound sources, human speech and a guitar, were auralized. The results show that the subjective quality of the simulation procedure depends on the type of sound source and the perceptive dimension that was assessed. In general the mirror source method performed best for localization of speech, whereas ray-tracing was the preferred method for the guitar sound. Overall mirror sources also outperform the other methods when good localization is required. Independent from the source the hybrid method was considered the best for the reverberation dimension.

5aPPe6. Effects of hardware on optimal filter segmentations for the segmented convolution. Frank Wefers (Institute of Technical Acoustics, RWTH Aachen University, Neustrasse 50, 52066 Aachen, Germany, Frank .Wefers@akustik.rwth-aachen.de)

The segmented convolution is a commonly used method for the auralisation in interactive virtual acoustic environments. Typically the auralisation of a virtual scene is done by convolution of the scenes' sound sources signals with impulse responses, that describe the sound transmission paths. The segmented convolution algorithm allows an efficient computation of the convolution in real-time, by segmentation of the impulse response into several parts. In order to allow the realistic simulation of complex acoustical scenes, the convolutions' computational effort must be minimised. It is a fact that the segmentation of the impulse response is a key parameter to the algorithm and has great effects on its computational load, stability and even realisability in real-time. Considerations on optimal filter segmentations mainly founded on a theoretical point, like the runtime complexity of operations or the number of required floating point operations. However, practical systems behave differently: Calculations on real hardware suffer cache losses and memory bandwidth. In this contribution, the influence of real hardware on optimal filter segmentations is examined. An optimisation algorithm is presented, that allows to gain optimal filter segmentations for a dedicated target system. The consequences of efficiency losses to the structure of optimal filter segmentations are discussed. 


\title{
Session 5aPPf
}

\section{Psychological and Physiological Acoustics and Speech Communication: Acoustic Features and Speech Perception I (Poster Session)}

\author{
Jont Allen, Cochair \\ University of Illinois \\ Sarah Hawkins, Cochair \\ University of Cambridge
}

\begin{abstract}
All posters will be on display from 9:40 a.m. to 11:20 a.m. To allow contributors an opportunity to see other posters, contributors of odd-numbered papers will be at their posters from 9:40 a.m. to 10:30 a.m. and contributors of even-numbered papers will be at their posters from 10:30 a.m. to 11:20 a.m.
\end{abstract}

\section{Contributed Papers}

5aPPf1. English speech intelligibility test among persons of Arabic native language. Mohammed Abd-Elbasseer (National Institute of Standard (NIS) - Acoustics Department, Teras Street/Al Ahram Street/Giza, 202 Cairo, Egypt, mbacir@yahoo.com), Sanaa Barakat (National Institute of Standard (NIS) - Acoustics Department, Teras Street/Al Ahram Street / Giza, 202 Cairo, Egypt, s43223@yahoo.com)

English language as a non-native language is the most common language over the world, it is the language of conferences, business and the airports. While the Arabic language is the native language of Egypt, the English language is considered as the second language. This research will conduct to investigate the effect of speech level, rate and repetition on the intelligibility of English language among some highly educated Egyptian persons. 34 males and females subjects are participated in this test. 40 sentences of BKB sentences were used with three scored words per sentence. First, they are subjected to the speech rate of about 220, 190, 160, and 100 words per minute at the speech level of about $60 \mathrm{dBA}$, which is considered as the normal level. Second,they are subjected to the speech level of 60,70 , and $80 \mathrm{dBA}$ at speech rate of 190 words per minute, which is considered as the normal rate. It was found that the intelligibility score increased with decreasing the rate and increasing the level respectively. It was found that scores of the worst scored sentence for the first 15 subjects did not change, whatever we repeat the tested sentence at normal rate and level.

5aPPf2. Spectral masking release with HiRes120 sound processing in cochlear implant users. Julie Bestel (Advanced Bionics, 76 rue de Battenheim, 68170 Rixheim, France, julieb@abionics.fr), Olivier Sterkers (Hôpital Beaujon, service ORL, 100 bd du Général Leclerc, 92118 Clichy, France, olivier.sterkers@bjn.ap-hop-paris.fr), Bruno Frachet (Hôpital Avicenne, Service ORL et de chirurgie cervico-faciale, 125 rue de Stalingrad, 93009 Bobigny, France, bruno.frachet@club-internet .fr), Bernard Meyer (Hôpital Saint-Antoine, Service ORL, 184 rue du Faubourg St Antoine, 75012 Paris, France, bernard.meyer@sat .ap-hop-paris.fr), Eric Truy (Hôpital Edouard Herriot, Service ORL, Place d'Arsonval, 69003 Lyon, France, eric.truy@chu-lyon.fr), Patrick Boyle (Advanced Bionics, 76 rue de Battenheim, 68170 Rixheim, France, patrickb@abionics.fr), Christian Lorenzi (Univ Paris Descartes, CNRS, Ecole Normale Superieure, DEC, 29 rue d'Ulm, 75005 Paris, France, lorenzi @ens.fr)

Current sound processors in cochlear implants show poor frequency resolution compared to normal auditory processes. They smooth spectral gaps within background maskers, thereby preventing implant users from experiencing release from masking (i.e., improved speech identification in maskers showing spectral gaps compared to maskers with continuous spectra). The Advanced Bionics' HiRes 120 coding scheme aims to increase the transmis- sion of fine-grain spectral information using a strategy based upon curren steering. If HiRes120 improves the precision of spectral coding compared to the standard HiRes strategy, listeners should show improved release from masking for the slowest spectral modulations in a noise masker. This hypothesis is tested in newly implanted adults who alternately use HiRes and HiRes120 sound processing for a period of 10 months. Every two or three months, consonant identification is measured in each implantee in the presence of a stationary speech-shaped noise (SSN) masker at a fixed signal-tonoise ratio yielding 30-40\% correct identification with unmodulated noise. The SSN masker is either unmodulated or spectrally modulated to produce periodic, three ERB-wide "spectral gaps" in the noise masker. Masking release scores defined as the change in consonant identification performance in modulated versus unmodulated noise will be presented and discussed.

5aPPf3. Comparison of speech vs speech-analogue temporal encoding in human: An auditory brainstem response and categorical perception assessment. Idrick Akhoun (Univ. Lyon 1 - Lab. Neurosciences, Service Pr Collet, Pavillon U, Hôpital Edouard Herriot, F-69003 Lyon, France, iakhoun@olfac.univ-lyon1.fr), Céline Richard (Univ. Lyon 1 - Lab. Neurosciences, Service Pr Collet, Pavillon U, Hôpital Edouard Herriot, F-69003 Lyon, France, iakhoun@olfac.univ-lyon1.fr), Annie Moulin (Univ. Lyon 1 - Lab. Neurosciences, Service Pr Collet, Pavillon U, Hôpital Edouard Herriot, F-69003 Lyon, France, iakhoun@olfac.univ-lyon1.fr), Evelyne Veuillet (Univ. Lyon 1 - Lab. Neurosciences, Service Pr Collet, Pavillon U, Hôpital Edouard Herriot, F-69003 Lyon, France, iakhoun@olfac.univ-lyon1 .fr), Stéphane Gallégo (Univ. Lyon 1 - Lab. Neurosciences, Service Pr Collet, Pavillon U, Hôpital Edouard Herriot, F-69003 Lyon, France, iakhoun@olfac.univ-lyon1.fr), Christian Berger-Vachon (Univ. Lyon 1 Lab. Neurosciences, Service Pr Collet, Pavillon U, Hôpital Edouard Herriot, F-69003 Lyon, France, iakhoun@olfac.univ-lyon1.fr), Lionel Collet (Univ Lyon 1 - Lab. Neurosciences, Service Pr Collet, Pavillon U, Hôpital Edouard Herriot, F-69003 Lyon, France, iakhoun@olfac.univ-lyon1.fr), Hung Thai-Van (Univ. Lyon 1 - Lab. Neurosciences, Service Pr Collet, Pavillon U, Hôpital Edouard Herriot, F-69003 Lyon, France, iakhoun@olfac.univlyon1.fr)

Temporal speech cues, such as periodicity and voice onset time (VOT), are particularly salient information for speech intelligibility. In addition, speech elicited auditory brainstem response (Speech ABR) to consonant vowels $(\mathrm{CV})$ has been shown to mimic the temporal periodicity of the acoustic signal very accurately. Consequently, Speech ABR has been described as a potential objective tool to explore the temporal integration of speech excerpts in human brainstem. We aimed at putting in evidence the specialization of temporal encoding of speech vs nonspeech signals through clinical and behavioral measurements. A sum of five sinusoids, at the five 
formant frequencies, modulated by the corresponding $\mathrm{CV}$ temporal envelope, was taken as speech-analogue. Accordingly, Speech and Analogue ABRs components were compared, although a priori due to be similar. Furthermore, categorical perception assessment was processed to both analogue and full-speech continuums. If confirmed, these preliminary results (seven normal hearing adults for physiological ABRs and behavioral categorical perception) would bring further evidence of an early specialization of temporal processing of speech, in line with previous research [Kraus and Nicol, Trends in Neuroscience 28(4), 176--81 (2005)]. [Work supported by CNRS, Cochlear \& Univ.Lyon 1.]

5aPPf4. Modulation of the voice-cuing effect on releasing speech from informational masking. Lijuan J. Xu (Dept. of Psychology, Peking Univ., 5 Yeheyuan Road, Haidian District, 100871 Beijing, China, lijuan179021@yahoo.com.cn), Jingyu Y. Li (Dept. of Machine Intelligence, Speech and Hearing Research Center, 2 Science Building, Peking Univ., 5 Yeheyuan Road, Haidian District, 100871 Beijing, China, lijy@cis.pku.edu.cn), Ying Huang (Dept. of Psychology, Peking Univ., 5 Yeheyuan Road, Haidian District, 100871 Beijing, China, innhuang@gmail.com), Xihong H. Wu (Dept. of Machine Intelligence, Speech and Hearing Research Center, 2 Science Building, Peking Univ., 5 Yeheyuan Road, Haidian District, 100871 Beijing, China, wxh@cis.pku.edu.cn), Liang Li (Dept. of Psychology, Peking Univ., 5 Yeheyuan Road, Haidian District, 100871 Beijing, China, liangli@pku.edu.cn)

In cocktail-party environments, familiarity or knowledge of target talker's voice is useful for reducing speech-on-speech masking (Yang et al., Speech Communication, 49, 892-904, 2007). In addition, the onset asynchrony between target speech and masking speech is an effective cue for unmasking target speech. The present study examined whether the voicecuing effect can be modulated by either the degree of familiarity/knowledge of target talker's voice or the speech onset asynchrony. When target speech started one second after masking speech, prepresenting a priming sentence voiced by the target talker significantly improved the recognition of the target speech which was copresented with masking speech. However, reinforcing the familiarity/knowledge of the target-talker's voice did not further improve the recognition. When target speech and masking speech started at the same time, a single presentation of voice-priming speech did not change participants' speech recognition against masking speech unless the familiarity/knowledge of target-talker's voice was reinforced by either a learning procedure or repeated presentation of the target-talker's voice before testing. These results suggest that the voice-cuing effect on releasing speech from informational masking is graded, depending on both the degree of familiarity/knowledge of the target-talker's voice and the modulation by other cues such as speech-onset asynchrony.

5aPPf5. Consequences of regional accent differences for speech perception studies. Richard Wright (University of Washington, $1417 \mathrm{NE}$ 42nd Street, Seattle, WA 98105, USA, rawright@u.washington .edu), Pamela Souza (University of Washington, 1417 NE 42nd Street, Seattle, WA 98105, USA, psouza@u.washington.edu), Stephanie Bor (University of Washington, 1417 NE 42nd Street, Seattle, WA 98105, USA, stebor@u.washington.edu), Star Reed (University of Washington, 1417 NE 42nd Street, Seattle, WA 98105, USA, starreed@u.washington .edu)

Sociophonetic research has demonstrated effects of regional accent on vowels. However, little attention is paid to regional accent in hearing and speech perception studies that use standard vowel stimuli. This poster illustrates the importance of these factors to perception. A library of synthetic vowels from nine different regions of the U.S. (e.g., southern California, upper Midwest) was generated according to published formant values. The vowels were categorized into three groups (near, medium, far) according to Euclidean distance in an F1 by F2 space relative to Pacific Northwest (PNW) vowels. Based on several experimental factors, two vowel pairs representing the high back and low front vowels were chosen as stimuli. The near, medium and far exemplars and PNW exemplars were presented to native PNW listeners in a forced-choice identification task. Dependent variables were identification accuracy and reaction time. Task difficulty was manipulated using background noise level. Results indicate that recognition scores were poorer, and reaction time was longer, for vowels that were spec- trally distant from the listener's regional accent. These data suggest that researchers should take into account regional accent in selecting vowel stimuli or generating synthetic vowels for general-use speech perception tests. Supported by NIDCD (DC00033, RO1 DC006014).

5aPPf6. Factors affecting recognition of vocoded speech: Effect of envelope cutoff frequency and carrier type. Pamela Souza (University of Washington, 1417 NE 42nd Street, Seattle, WA 98105, USA, psouza@u.washington.edu), Stuart Rosen (UCL, Wolfson House, 4, Stephenson Way, NW1 2HE London, UK, stuart@phon.ucl.ac.uk)

Multiband vocoded signals are used to study temporal cues as well as to simulate information available to cochlear implant wearers, but different implementations of these signals may affect results. This series of experiments varied envelope cutoff frequency (30 or $300 \mathrm{~Hz}$ ), carrier type (tone or noise) and number of frequency bands (2-5) for vocoded speech presented to normal-hearing subjects. Dependent measures were consonant recognition (vowel-consonant-vowel syllables), vowel recognition (/b/-vowel-/d/ words), and sentence recognition. Results indicated better performance with a high envelope cutoff for tone-vocoded signals, and no effect of envelope cutoff for noise-vocoded signals. When only low-frequency envelope cues were available, performance was better for noise-vocoded than for tonevocoded signals. When higher-frequency envelope cues were available, performance was better for tone-vocoded than for noise-vocoded signals. To understand the source of the observed differences, a follow-up study tested the relative contributions of comodulation across bands (as in the 30- and $300-\mathrm{Hz}$ tone-vocoded conditions) and spectral sidebands (as in the $300-\mathrm{Hz}$ tone-vocoded conditions). Those data indicated that comodulation across bands did not contribute to performance. The presence of sidebands which provided more spectral detail improved performance over a sparser spectrum. [Supported by the Bloedel Hearing Research Center and NIH RO1 DC006014.]

5aPPf7. When and how disyllables are contracted into monosyllables in Taiwan Mandarin? Chierh Cheng (University College London, Department of Phonetics and Linguistics, Gower Street, WC1E 6BT London, UK, chiacheng.fl86@nctu.edu.tw), Yi Xu (University College London, Wolfson House, 4 Stephenson Way, NW1 2HE London, UK, yi.xu@ucl.ac.uk)

In Taiwan Mandarin, a disyllabic word or phrase is often contracted into a monosyllabic unit in conversational speech, just as "do not" is often contracted into "don't" in English. The goal of this research is to a) test the hypothesis that contraction is directly related to time pressure, and so it can be elicited in the laboratory from nonsense disyllabic units, and b) search for the underlying mechanisms of contraction. We devised four groups of nonsense disyllabic combinations according to the level of obstruction in the intervocalic consonant: (1) zero obstruction - $\mathrm{CV}+\mathrm{V}$; $\mathrm{CV}+\mathrm{VN}$; $\mathrm{CV}+\mathrm{VV}$, (2) nasal consonant - $\mathrm{CVN}+\mathrm{V}$; $\mathrm{CV}+\mathrm{NV}$, (3) non-nasal consonant $\mathrm{CV}+\mathrm{CV}$, where $\mathrm{C}$ is a stop, fricative or affricate, and 4) nasal + non-nasal consonant-CVN $+\mathrm{CV}$. These disyllabic units were embedded in carriers that would impose different degrees of time pressure on them. Four speakers of Taiwan Mandarin read aloud these sentences at three articulatory modes: clear, natural and fast. Preliminary examinations showed that contraction occurred more frequently as speech rate increased and as intervocalic obstruction decreased. Detailed acoustic analyses are being conducted to search for evidence of the underlying mechanism of such contraction, and the results will be reported.

5aPPf8. Estimation model for the speech-quality dimension "Noisiness." Lu Huo (Institute for Circuit and System Theory, ChristianAlbrechts-University of Kiel, Kaiserstrasse 2, 24143 Kiel, Germany, lhu@tf.uni-kiel.de), Marcel Wältermann (Deutsche Telekom Laboratories, Berlin Institute of Technology, Ernst-Reuter-Platz 7, 10587 Berlin, Germany, marcel.waeltermann@telekom.de), Ulrich Heute (Institute for Circuit and System Theory, Christian-Albrechts-University of Kiel, Kaiserstrasse 2, 24143 Kiel, Germany, uh@tf.uni-kiel.de), Sebastian 
Möller(Deutsche Telekom Laboratories, Berlin Institute of Technology, Ernst-Reuter-Platz 7, 10587 Berlin, Germany, sebastian.moeller@telekom .de)

State-of-the-art assessment method of speech-transmission quality (e.g., PESQ or TOSQA) predict the mean-opinion score (MOS) quite accurately, but cannot provide diagnostic information, which is, however, highly desirable for system developers. In our research project, we aim at the development of an attribute-based speech-quality measure, which provides estimates of different attributes of speech samples and then maps them to one integralquality estimate. Three dominant, mutually orthogonal perceptual dimensions were firstly identified by auditory experiments and multidimensional analysis (MDA) for narrow-band speech transmission: "directness/ frequency content," "continuity," and "noisiness." The present paper focuses on the further decomposition and measurement of the global dimension "Noisiness." Therefore, an auditory test including samples degraded by different kinds of noises has been conducted. The subsequent MDA indicates that at least two sub-dimensions (SD), "Speech Contamination" and (perceived) "Additive-Noise Level," are further describing the global dimension "Noisiness." The first SD characterizes the degree the noise distorts the speech signal as such, whereas the second SD reflects the degree the additive circuit or background noise itself annoys the listener. The instrumental estimation methods for both SDs and the mapping to the integralquality ratings are presented in this paper.

5aPPf9. Across-ear grouping of speech bands in quiet and in the presence of interference. Erol Ozmeral (Boston University Hearing Research Center, 677 Beacon Street, Boston, MA 02215, USA, ozmeral@bu.edu), Virginia Best (Univ. of Sydney, Dept. of Physiology, NSW 2006 Sydney, Australia, ginbest@physiol.usyd.edu.au), Jaime McCoin (Boston University Hearing Research Center, 677 Beacon Street, Boston, MA 02215, USA, jmsushigirl@gmail.com), Barbara Shinn-Cunningham (Boston University Hearing Research Center, 677 Beacon Street, Boston, MA 02215, USA, shinn@cns.bu.edu)

Speech was filtered into 16 frequency bands. Three bands were randomly selected on each trial as the LOW (two low-frequency bands) and HIGH (one high-frequency band) target components. LOW and HIGH were either presented to the same (SAME) or opposite (SPLIT) ears. Four randomly selected, nonoverlapping frequency bands from a different utterance were time reversed, and summed. When present, this masker was either played to the same ear as LOW and HIGH (SAME-MASK), same ear as LOW with HIGH opposite (SPLIT-MASKLOW), or same ear as HIGH with LOW opposite (SPLIT-MASKHIGH). Intelligibility was best in the SAME and SPLIT conditions and much better than with either LOW or HIGH presented alone. Thus, in quiet, listeners can perceptually integrate information across target components with little spectro-temporal correlation, even when spatial cues promote segregation of these components. Performance in the SAME-MASK condition was generally better than either SPLITMASKLOW or SPLIT-MASKHIGH conditions (although worse than in SAME and SPLIT conditions). Furthermore, performance in the two SPLITMASK conditions was comparable to performance with LOW or HIGH alone. Thus, when spatial cues oppose grouping of the target and there is a competing off-frequency interference, listeners have difficulty integrating information across target components.

5aPPf10. The role of consonant duration and amplitude processing on speech intelligibility in noise. Jeffrey J. Digiovanni (Ohio University, W218 Grover Center, Athens, OH 45701, USA, digiovan@ohio.edu), Ashley K. Stover (Ohio University, W218 Grover Center, Athens, OH 45701, USA, as357803@ohio.edu)

The effects of hearing loss on speech intelligibility have led researchers to search for methods to ameliorate these effects. Clear speech has been studied extensively to determine the specific acoustic and phonetic differences between speech spoken in a conversational versus a clear-speech mode. Two characteristics of clear speech, increased consonant duration and selective-consonant amplification were investigated. These components were applied individually to Hearing-in-Noise Test sentences and consonant-vowel $(\mathrm{CV})$ pairs. Stimuli were presented in sound field to hearing-impaired and normal-hearing individuals in the presence of speech- shaped noise. Percent correct was measured for HINT sentences and confusion matrices were developed for the CV pair intelligibility tests. An information transmission analysis was performed. During HINT sentence presentations, subjects benefited most from lengthening the duration of the consonant, maximally in the $30 \mathrm{~ms}$ condition, beyond which significant degradation in intelligibility was observed. Consonant specific amplification of $10 \mathrm{~dB}$ resulted in improved intelligibility for sentences. These data suggest that the two processing components are viable to improve speech intelligibility and they, however, also suggest that there is a limit to the processing whereby benefits are no longer observed.

5aPPf11. The role of combined consonant duration and amplitude processing on speech intelligibility in noise. Jeffrey J. Digiovanni (Ohio University, W218 Grover Center, Athens, OH 45701, USA, digiovan@ohio.edu), Jessica A. Wolfanger (Ohio University, W218 Grover Center, Athens, OH 45701, USA, jw241702@ohio.edu)

Reduced speech intelligibility in noise, especially consonant perception, is a well documented phenomenon. Research in clear speech has revealed many acoustic and phonetic differences between speech spoken in a conversational versus clear-speech mode. In the present study two components were tested in combination, namely increases in consonant duration (and respective decreases in adjacent vowel duration) and selective-consonant amplification. Conditions in which intelligibility improvements were observed for the individual processing of duration and amplitude modifications were chosen and tested in combination. The components were applied to Hearing-in-Noise Test sentences and consonant-vowel (CV) pairs. Stimuli were presented in sound field to normal-hearing individuals in the presence of speech-shaped noise. Percent correct was measured for HINT sentences and confusion matrices were developed for the CV pair intelligibility tests. An information transmission analysis was performed. Results will be discussed with regard to the greater body of research in clear speech.

5aPPf12. Effect of amplification on the intelligibility of speech in hearing impaired children with and without dead regions in the cochlea. Alicja N. Malicka (The University of Manchester, School of Psychological Sciences, Ellen Wilkinson Building, M13 9PL Manchester, UK, alicja.malicka@manchester.ac.uk), Kevin J. Munro (The University of Manchester, School of Psychological Sciences, Ellen Wilkinson Building, M13 9PL Manchester, UK, kevin.j.munro@manchester.ac.uk), Thomas Baer (University of Cambridge, Department of Experimental Psychology, Downing Street, CB2 3EB Cambridge, UK, tb107@cus.cam.ac.uk)

Adults with high-frequency (HF) sensorineural hearing impairment with and without dead regions (DRs) in the cochlea differ in benefit from amplification of speech presented in quiet [Vickers et al., J. Acoust. Soc. Am. 110, 1164-1175 (2001)]. Subjects with HF DRs showed no improvement in speech intelligibility when spectral components of the speech above about 1.7 times the edge frequency of the DR were amplified according to a hearing-aid prescription formula while performance of those without DRs showed improvement with addition of amplified frequency components up to $7.5 \mathrm{kHz}$. In the present study we tested a group of six children (8-12 years old) who were experienced hearing-aid users with moderate-to-severe sensorineural hearing impairment. The presence of DRs was diagnosed using the TEN(HL) test and "fast" psychophysical tuning curves. Four children showed evidence for DRs (two unilateral and two bilateral). The vowelconsonant-vowel stimuli (65-dB SPL) were subjected to the frequency-gain characteristic prescribed by the DSL prescription formula then low-pass filtered with various cutoff frequencies and presented via headphones. The results showed that in ears with or without DRs the performance improved with increasing cutoff frequency up to $7.5 \mathrm{kHz}$.

5aPPf13. Acoustic correlates of fricative perception. Shira Katseff (University of California, Department of Linguistics, 1203 Dwinelle Hall, Berkeley, CA 94720, USA, skatseff@ berkeley.edu)

A number of spectral attributes have been proposed as correlates of fricative place of articulation. However, these attributes have difficulty distinguishing fricatives in noncanonical speech, e.g., from children. Because child speech is intelligible, it is possible that some of these characteristics 
are not used in speech perception. This study collected a corpus of /s/ and /S/-containing words from children between 3 and 5. Adults categorized fricatives extracted from this corpus. A series of regressions are used to compare the ability of spectral attributes to predict (1) human categorization of /s/ and /S/ productions, and (2) the intended fricative production. The analysis reveals that mistakes in perception are driven by overuse of a single spectral attribute, spectral slope. This result suggests caution in equating methods of computational separation with methods of human perception, and provides a simple method for investigating relationships between the two.

FRIDAY MORNING, 4 JULY 2008

P2-C, LEVEL 2, 9:40 TO 11:20 A.M.

\title{
Session 5aPPg
}

\section{Psychological and Physiological Acoustics: Cross-spectral Auditory Integration: Physiological, Psychophysical, and Clinical Evidence II (Poster Session)}

\author{
Blas Espinoza-Varas, Cochair \\ CommunicationSciences \& Disorders, OU Health Sciences Ctr. \\ Alain De Cheveigne, Cochair \\ CNRS, Universite Paris 5, Ecole Normale Superieure
}

\begin{abstract}
All posters will be on display from 9:40 a.m. to 11:20 a.m. To allow contributors an opportunity to see other posters, contributors of odd-numbered papers will be at their posters from 9:40 a.m. to 10:30 a.m. and contributors of even-numbered papers will be at their posters from 10:30 a.m. to 11:20 a.m.
\end{abstract}

\section{Contributed Papers}

5aPPg1. Stimulus effects on spectral shape discrimination. Mini N. Shrivastav (University of Florida, 336 Dauer Hall, Dept. of Communication Sciences and Disorders, Gainesville, FL 32611, USA, mnarendr@csd.ufl.edu), David A. Eddins (University of Rochester, Department of Otolaryngology, Rochester, NY 14618, USA, David_Eddins @URMC.Rochester.edu)

For a single stimulus such as shaped broadband noise, spectral-shape discrimination can vary based on the nature of the spectral feature that is being detected. For example, Shrivastav et al. (2006) found a significant difference in thresholds for broadband noise with a narrow localized spectral peak and those with a broad overall spectral change. They also found a moderate association between discrimination thresholds and speechidentification scores in older hearing-impaired listeners, but only for the stimuli with a narrow spectral peak. It is not clear whether these stimulusdependent effects were associated with the bandwidth of the spectral peak, the slope of the peak, or a combination of these features. In the present study, spectral shape discrimination thresholds and speech recognition scores will be measured for ten young normal-hearing and ten older hearingimpaired listeners. The broadband noise will vary systematically in the bandwidth and the slope of a single spectral peak. The results will determine if and how spectral-shape perception changes with the nature of the stimulus paradigm used to measure it. Further, the results will also indicate if the association between spectral shape perception and speech identification is observed for all stimuli or only to a subset.

5aPPg2. Resolution, spectral weighting, and integration of information across tonotopically remote cochlear regions: hearing-sensitivity, sensation level, and training effects. Blas Espinoza -Varas (CommunicationSciences \& Disorders, OU Health Sciences Ctr., 825 NE 14th St., Oklahoma City, OK 73126-0901, USA, blas-espinozavaras@ouhsc.edu)

This paper examines how listeners resolve, weight, and integrate redundant sensory information from tonotopically remote cochlear regions, one in the mid $(\mathrm{m})$ the other in the high (h) frequencies. Subjects listened to twocomponent complexes and attempted to resolve and integrate simultaneous differences in frequency $(\mathrm{DFm})$ at $\approx 1000 \mathrm{~Hz}$ and in duration $(\mathrm{DTh})$ at $\approx 3500 \mathrm{~Hz}$. Discrimination performance was studied as a function of hearing sensitivity, sensation level (SL) and training. Normal hearing listeners tend to resolve both DFm and DTh and integrate the information if the component SLs are equal, but resolve only the louder component difference if the SLs are very unequal; i.e., spectral weighting is biased and integration is limited or nil. Much the same pattern obtains when the low SL in H results from high-frequency impaired sensitivity (IS). Once established, equating the component SLs (e.g., by high-frequency amplification) is often ineffective to rectify the weighting bias; doing so requires extensive discrimination training with the low SL component. With IS, an anomalous trend obtains when the SL is much lower for (m) than for (h): the resolution of DFm is equal to or better than that of DTh, and integration is quite efficient, possibly reflecting tonotopic map reorganization.

5aPPg3. Pitch discrimination of simultaneous and nonsimultaneous complexes across spectral regions. Elizabeth Borchert (University of Minnesota, Department of Psychology, 75 E. River Road, Elliott Hall N218, Minneapolis, MN 55455, USA, olsen064@umn.edu), Andrew J. Oxenham (University of Minnesota, Department of Psychology, 75 E. River Road, Elliott Hall N218, Minneapolis, MN 55455, USA, oxenham@umn.edu), Christophe Micheyl (University of Minnesota, Department of Psychology, 75 E. River Road, Elliott Hall N218, Minneapolis, MN 55455, USA, cmicheyl@umn.edu)

Detecting differences in fundamental frequency (F0) across different spectral regions may be important in the perceptual organization of both simultaneous and sequential sounds, but it is unknown whether the same mechanisms are involved in these two cases. Here we directly compare the detection of pitch differences between pairs of complex tones presented either simultaneously or sequentially. 28 normal-hearing listeners heard two pairs of complex tones in each trial and indicated the pair in which the pitches of the two tones differed. The complex tones in each pair were bandpass filtered to have nonoverlapping spectra, but at least some resolved harmonics in all cases. Performance was generally better in the simultaneous than in the sequential condition. In a follow-up condition, it was found that introducing an onset asynchrony between the two spectral regions in the si- 
multaneous condition resulted in impaired performance, approaching that found in the sequential case, despite the duration of the simultaneous portion remaining the same. The results suggest that perceived fusion, rather than an explicit F0 extraction and comparison may underlie the detection of F0 differences between simultaneous groups of harmonics in distinct spectral regions. [Work supported by NIH grant R01 DC 05216.]

5aPPg4. Across-frequency processes involved in auditory detection of coloration. Jörg M. Buchholz (CAHR, Department of Electrical Engineering, DTU, Ørsteds Plads, Bygning 352, 2800 Kgs. Lyngby, Denmark, jb@oersted.dtu.dk), Paris Kerketsos (CAHR, Department of Electrical Engineering, DTU, Ørsteds Plads, Bygning 352, 2800 Kgs. Lyngby, Denmark, paris_kerketsos@hotmail.com)

When an early wall reflection is added to a direct sound, a spectral modulation is introduced to the signal's power spectrum. This spectral modulation typically produces an auditory sensation of coloration or pitch. Throughout this study, auditory spectral-integration effects involved in coloration detection are investigated. Coloration detection thresholds were therefore measured as a function of reflection delay and stimulus bandwidth. In order to investigate the involved auditory mechanisms, an auditory model was employed that was conceptually similar to the peripheral weighting model [Yost, JASA, 1982, 416-425]. When a "classical" gammatone filterbank was applied within this spectrum-based model, the model largely underestimated human performance at high signal frequencies. However, this limitation could be resolved by employing an auditory filterbank with narrower filters. This novel filterbank was designed to approximate auditory filter-shapes measured by Oxenham and Shera [JARO, 2003, 541-554], derived from forward masking data. The results of the present study demonstrate that a "purely" spectrum-based model approach can successfully describe auditory coloration detection even at high signal frequencies.

5aPPg5. Phoneme recognition as a function of the number of auditory filter outputs. Frederic Apoux (University of South Carolina, Speech Psychoacoustics Laboratory, Department of Communication Sciences and Disorders, William Brice Bldg., 1621 Greene St., Columbia, SC 29208, USA, apoux@sc.edu), Eric W. Healy (University of South Carolina, Speech Psychoacoustics Laboratory, Department of Communication Sciences and Disorders, William Brice Bldg., 1621 Greene St., Columbia, SC 29208, USA, ewh@sc.edu)

It has been proposed that listeners take advantage of brief "coups d'oeil" when processing speech in noise. These glimpses can be characterized both in time and frequency. The obligatory role of the auditory filters in determining the nature of any further processing suggests that the frequency extent of a glimpse should be equivalent to that of an auditory filter. Accordingly, it is hypothesized that the spectral characteristics of glimpses primarily relate to the available number of auditory channels. The present study investigated the number of auditory filter outputs needed to identify phonemes in quiet. Stimuli were first restricted to $80-8000 \mathrm{~Hz}$ and then split into 30 contiguous auditory filter width bands. Normal-hearing listeners were presented with $\mathrm{N}$ bands whose spectral location was selected randomly from trial to trial. No signal was presented in the other bands. Consistent with previous studies, performance gradually increased with the number of bands. An asymptote was reached with 24 and 16 bands for vowels and consonants, respectively. While high levels of speech understanding are typically observed with as few as 4 channels of spectral information, our results indicate that accurate phoneme recognition requires combination of a much larger number of auditory filter outputs.

\title{
Session 5aSAa
}

\section{Structural Acoustics and Vibration, ASA Committee on Standards, and EURONOISE: Ground Vehicle Noise and Vibration I}

\author{
Donald B. Bliss, Cochair \\ Paul De Vos, Cochair \\ DHV BV, Postbus 1132, Amersfoort, NL 3800 BC, Netherlands
}

Duke University, Mechanical Engineering and Materials Science, 148B Hudson Hall, Durham, NC 27708, USA

\section{Invited Papers}

\section{8:00}

5aSAa1. Contribution of the tyre to further lowering tyre/road noise. Ernst-Ulrich Saemann (Continental AG, Jaedekamp 30, 30419 Hannover, Germany, Ernst-Ulrich.Saemann@conti.de)

The tyre is the only part of a vehicle that must be originally and primarily designed to transmit forces to outside the vehicle. At the same time the amount of energy a tyre consumes per kilometre in operation and for production has to be minimized. On dry, wet, and snow-covered roads, the safety of traffic can only be ensured by a sufficient capability for acceleration and braking deceleration, cornering stability, tracking on acceleration and braking, steering precision and direction stability. The high requirements on safety, economy, and durability limit the possible reduction in the emission of tyre/road noise. Nevertheless, nowadays patterned tires can be constructed which are not more than 1-3 $\mathrm{dB}(\mathrm{A})$ louder than smooth tires. As a further reduction of tire excitation by tread pattern optimization cannot be expected and a soft tread compound cannot be used due to the customer's requirements, the only possibility to build tyres with less sound radiation is to lower the noise already from the blank tyre. Considerably more tyre/road noise reduction can be gained if the excitation by road is addressed, too. 
5aSAa2. Prediction and audio synthesis of vehicle pass-by noise. Estelle Bongini (SNCF DIR, 45, rue de Londres, 75379 Paris, France, estelle.bongini@sncf.fr), Stephane Molla (Genesis S.A., Bâtiment Gérard Mégie, Domaine du Petit Arbois - BP 69, 13545 Aix-en-Provence Cedex 4, France, stephane.molla@genesis.fr), Cédric Herviou (SNCF DIR, 45, rue de Londres, 75379 Paris, France, cedric.herviou@gmail.com), Dominique Habault (LMA CNRS, 31 chemin Joseph Aiguier, 13402 Marseille, France, habault@1ma.cnrs-mrs.fr), Franck Poisson (SNCF DIR, 45, rue de Londres, 75379 Paris, France, franck.poisson@sncf.fr)

The European project SILENCE is dedicated to the reduction of railway and roadway noise in urban areas. Within this context, SNCF and LMA (Laboratoire de mécanique et d'acoustique, Marseille) collaborate into the subproject B to develop a pass-by sound simulation software. This global modelling tool will support parametric studies on the reduction of the noise of a train or a car pass-by, by providing standard indicators (time signature, equivalent sound pressure level) and sound samples. These sound samples are computed in B-Format, which allows, after a short postprocessing, to provide various listening formats (mono-aural, binaural, œ). The software is now operational and the sources characterisation has been carried out on an AGC bi-mode BOMBARDIER train and a passenger car. Equivalent sources have been defined in various operating conditions. Simulated pass-by indicators (signature, Leqtp(A)) in various configurations have been compared to measured one and show very good agreement. A perceptive validation has been carried out with listening tests, by indirectly comparing recorded samples corpus and corresponding simulated samples corpus. It shows that the arrangement made by the listeners of the simulated corpus and the arrangement of the recorded corpus are closely the same and are based on the same perceptive criterion.

\section{Contributed Papers}

\section{8:40}

5aSAa3. Dependence of the contact area on the velocity of a rolling tire Hong Hai Nguyen (ENPC, UR Navier, 6 et 8 Avenue Blaise Pascal, Cité Descartes, Champs sur Marne, 77455 Marne la Vallée, France, nguyen.hong-hai@lami.enpc.fr), Julien Cesbron (Université d'Evry - Val d'Essonne, Laboratoire de Mécanique d'Evry, EA3332, 40, rue du Pelvoux, 91020 Evry Cedex, France, cesbron.julien@neuf.fr), Fabienne Anfosso-Ledee (Laboratoire Central des Ponts et Chaussées, BP 4129, 44341 Bouguenais Cedex, France, fabienne.anfosso@lcpc.fr), Hai Ping Yin (ENPC, UR Navier, 6 et 8 Avenue Blaise Pascal, Cité Descartes, Champs sur Marne, 77455 Marne la Vallée, France, yin@lami.enpc.fr), Silvano Erlicher (Université Paris-Est, 6 et 8 avenue Blaise Pascal, Cité Descartes - Champs sur Marne, 77455 Marne la Vallée Cedex 2, France, erlicher@lami.enpc.fr), Denis Duhamel (ENPC, UR Navier, 6 et 8 Avenue Blaise Pascal, Cité Descartes, Champs sur Marne, 77455 Marne la Vallée, France, duhamel@lami.enpc.fr)

It is known that the eigenfrequencies of a rolling tire depend on the velocity of rotation. We distinguish two causes for the stiffness increase: the frequency dependence of the complex modulus of the materials and the geometrical stiffness. The real part of the Young's modulus is monotonic according to the frequency. It contributes for an important part to the stiffening. The geometrical stiffness also increases with the rotational velocity. A consequence of these effects is the modification of the size of the contact area for different velocities of a rolling tire. Here we first present experimental results estimating the size of the contact area for a tire in statics and for different rolling velocities. Differences of $20 \%$ can be observed. Then the viscoelastic behaviours of the tire materials are presented and experimental results showing the frequency dependence of the complex modulus of the tire constitutive materials are given. Then finite element computations are presented with a real distribution of materials in the tire section and the size of the contact area is estimated and compared to experimental measurements. These results could improve the modelling of tire road interaction for tire noise predictions.

\section{9:00}

5aSAa4. Deploying successfully Laser Doppler Vibrometry techniques within the automotive NVH process. Mehdi Batel (Polytec, 32 rue Delizy, 93694 Pantin Cedex, France, 1.korn@ polytec.fr)

Laser Doppler Vibrometry is becoming today an established technique and the gold-standard for noncontact measurements in the automotive industry. These methodologies are widely used to characterize the mechani- cal behaviour of structures in vibration or strain, with always zero mass loading. They provide high precision results with very low operating costs as well as optimized setup times. The applications of laser vibrometry in the automotive industry are extremely various, and are helping today automotive professionals to improve dramatically the $\mathrm{NVH}$ performance of components and full vehicles. This paper explains how these methodologies are fitting today in the everyday challenge of automotive NVH Test \& CAE engineers: providing best in class noise and vibration quality with mass reductions constraints. A new laser scanning technique including the use of a $3 \mathrm{D}$ robot is also presented, providing an innovative way to characterize the dynamics of body structures with very high precision and rapidity. The paper will present a measurement performed on a full vehicle (43 robot positions done in $1 \mathrm{~h} 30$ hour), with 1094 points of vibration data at $1 \mathrm{~Hz}$ resolution.

\section{9:20}

5aSAa5. Application of digital speckle interferometry for vibration analysis of a statically loaded vehicle tyre. Dan Borza (National Institute of Applied Sciences of Rouen, INSA Rouen, LMR, Ave l'Universite, BP8 76800 Rouen, France, dan.borza@insa-rouen.fr), Ioana Nistea (Institut National des Sciences Appliquées de Rouen, BP8 avenue de l'Université, 76801 Saint-Etienne du Rouvray, France, ioana.nistea@insa-rouen.fr)

Vehicle noise has, as one of its main sources, tyres and their interaction with the road. Experimental analysis of tyre vibroacoustic behaviour may provide useful information on the contributions of various regions of the tyre to the resultant noise. Digital speckle interferometry represents a reliable and efficient choice for vibration analysis, providing full field and real time information on surface displacement. The optical configuration used for this study is a standard out-of-plane sensitive setup, based on a continuous wave YAG laser. A 4 bucket phase stepping algorithm is applied, with the use of a piezo actuator, in order to eliminate the random phase difference between the object and the reference wave. The measurement results are images of the tested tyre, covered with interference fringes, representing contour maps of out of plane vibration amplitudes. This paper presents measurements for the case of a tyre subjected to a point excitation at the limit of the contact surface with the road, under statical load of $4 \mathrm{kN}$. The principal resonant modes and frequencies are presented, then difficulties related to sensibility vector variation over the test surface and coupled mode resonances are discussed. 
9:40-11:00 Posters

Lecture sessions will recess for presentation of poster papers on various topics in acoustics. See poster sessions for topics and abstracts.

\section{Contributed Papers}

11:00

5aSAa6. An energy-based updated modal approach for the efficient analysis of large trimmed models. Gregory Lielens (Free Field Technologies, Rue Emile Francqui 1 - batiment B - AXIS PARC LLN, 1435 Mont-Saint-Guibert, Belgium, gregory.lielens@fft.be), Benoit Van Den Nieuwenhof (Free Field Technologies, Rue Emile Francqui 1 - batiment B AXIS PARC LLN, 1435 Mont-Saint-Guibert, Belgium, benoit.vandennieuwenhof@fft.be), Fabien Acher (Free Field Technologies, Rue Emile Francqui 1 - batiment B - AXIS PARC LLN, 1435 Mont-SaintGuibert, Belgium, fabien.acher@fft.be), Jean-Pierre Coyette (Free Field Technologies, Rue Emile Francqui 1 - batiment B - AXIS PARC LLN, 1435 Mont-Saint-Guibert, Belgium, jean-pierre.coyette@ fft.be)

Large trimmed models, widely encountered in the automotive industry, generally involve a body-in-white structure coupled to an acoustic cavity and covered by a set of trim components. Due to the large number of degrees of freedom of such models, the direct analysis in physical coordinates, though theoretically correct, is not feasible on current computers. The paper presents an alternative and efficient solution strategy in modal coordinates that relies on an update of the modal parameters of the car body and the acoustic cavity, based on the energetic behaviour of the trim components. The description of the trim component in terms of an energetic database simplifies the data exchange between the automotive manufactor and the trim provider. Furthermore, it enables a fast frequency analysis of various loadcases/trim scenarios and makes optimisation possible. The updated modal approach implemented in Actran/Trim is applied on a simplified car model on which various trim components are applied. The application shows how the stiffening, added mass and damping effects of each trim component can be derived from the energetic database and can guide an optimisation process.

\section{$11: 20$}

5aSAa7. Critical assessment of operational path analysis: mathematical problems of transmissibility estimation. Peter Gajdatsy (LMS International, Interleuvenlaan 68, 3001 Leuven, Belgium, peter.gajdatsy@1msintl.com), Karl Janssens (LMS International, Interleuvenlaan 68, 3001 Leuven, Belgium, Karl.Janssens@1msintl.com), Ludo Gielen (LMS International, Interleuvenlaan 68, 3001 Leuven, Belgium, ludo.gielen@lms.be), Peter Mas (LMS International, Interleuvenlaan 68, 3001 Leuven, Belgium, peter.mas@1ms.be), Herman Van Der Auweraer (LMS International, Interleuvenlaan 68, 3001 Leuven, Belgium, Herman .VanderAuweraer@1msintl.com)

Classical transfer path analysis (TPA) is a widely used and reliable method for tackling noise and vibration problems. But due to its complexity and time-consuming measurement procedure there is an ongoing research for simpler and faster methods. One such method, most often referred to as operational path analysis (OPA), was presented two years ago and has quickly gained popularity in the NVH field for its speed and ease of use. To estimate path contributions transmissibilities are calculated from the measured operational reference and target accelerations and sound pressures. This new method is now critically examined and compared to a reliable classical TPA measurement. The results of this examination reveal three significant weaknesses. This paper focuses on the problems related to the estimation of transmissibilities which mostly arise from the limited amount of orders present in the signal and the coherence between inputs. It is shown that despite the advantages of the method, it is not applicable in many situations and has to be used with care for it can easily give misleading results.
11:40

5aSAa8. IC engine velocity fields, spherical harmonics and evanescent waves. Igor Machetta (Centro Ricerche FIAT, Strada Torino, 50, 10043 Orbassano (TO), Italy, igor.machetta@fptpowertrain.crf.it), Eugenius Nijman (Centro Ricerche FIAT, Strada Torino, 50, 10043 Orbassano (TO), Italy, eugenius.nijman@fptpowertrain.crf.it)

Spherical nearfield acoustical holography (SNAH) has been used to reconstruct the volume acceleration distribution close to the surface of an IC engine from the measured sound pressure distribution on a sphere enclosing the engine. Such reconstruction frequently requires the calculated target spheres to intercept the strongly nonspherical powertrain structure violating, at first sight, the conventional rules of SNAH. In this paper the SNAH results are compared with the results of two alternative, i.e., an inverse and a power based, source reconstruction methods. Notwithstanding the alleged violation of the rules it is shown that surprisingly good results may be obtained provided the spherical spectrum components associated with evanescent waves are omitted from the back propagation calculation.

\section{2:00}

5aSAa9. Influence of bolted items on modal analysis performed on a car body. Miguel Colomo (Universidad Politécnica de Madrid, Avda. Constitución $\mathrm{n}^{\circ} 621^{\circ} \mathrm{D}, 28931$ Móstoles, Spain, miguelcolomo @ gmail.com), Andrzej Pietrzyk (Volvo Car Corporation, NVH CAE Noise \& Vibration Center Dept 91620, PV2C2, 40531 Gothenburg, Sweden, apietrzy@volvocars.com), Wolfgang Kropp (Chalmers University of Technology, Division of Applied Acoustics, SE-41296 Gothenburg, Sweden, wolfgang.kropp@chalmers.se)

When performing Modal Analysis testing on a BIG (body-in-gray), some bolted items are included to better take into account their influence on body stiffness. However, their contribution to the stiffness is not relevant in the frequency range accessible for modal analysis (usually up to $70 \mathrm{~Hz}$ on a BIG). On the other hand, these bolted items increase the dispersion between results obtained for nominally identical test objects. The question which arises is whether the items should be included in the BIG definition to perform modal analysis and, in this case, which is their influence on the results? MIMO (multi-input-multi-output) measurements were carried out over three, nominally identical, Volvo S80 BIGs. Several configurations were measured for each BIG, starting from the complete body the bolted items were progressively removed. A version of LMS PolyMAX method was programmed by Matlab to analyze the measured data. Conclusions about bolted items influence are drawn based on the study of stabilization diagrams and modal parameters. The poles selection by the stabilization diagrams is one of PolyMAX method keys. The method understanding obtained from programming allows studying the ins and outs of poles selection. Polynomial order plays an important role in physical poles identification, especially for closely spaced modes. Results are shown to highlight its relevance.

\section{$12: 20$}

5aSAa10. Analysis of structure borne transmission in a railway vehicle using Energy Flow Method. Pascal Bouvet (Vibratec, 28 Chemin du Petit Bois, BP 36, 69131 Ecully Cedex, France, pascal.bouvet@vibratec.fr)

The Energy Flow Method (EFM) is a computationally efficient method, by which the results of a Finite Element Analysis (FEA) may be postprocessed to form energy flow models. It can be used to study structure borne transmission in structures, based on modal descriptions of the structure and the internal cavity. The EFM allows to form a Energy Influence Coefficient model (EIC) or an inverse SEA model. A first issue consists in partitioning the FE model into SEA subsystems, with respect to the SEA assumptions (particularly the weak coupling assumptions). An automatic technique based 
on cluster analysis, can be used to determine an appropriate SEA substructuring, before calculating the Coupling Loss Factors (CLF) between mechanical subsystem. A second issue is to calculate the CLF between structural and acoustic subsystems. This paper presents an industrial application of these techniques, for the analysis of structure borne transmission inside a tramway vehicle. The EFM technique is used to calculate the vibroacoustic transfer of the car body of the tramway, and comparison with measurements on a vehicle are presented.

\title{
Session 5aSAb
}

\section{Structural Acoustics and Vibration and EURONOISE: Active Noise Control: New Strategies and Innovative Concepts I}

\author{
Alain Berry, Cochair \\ Univ. de Sherbrooke, Mechanical Engineering Depart., 2500 Boulevard de l'Université, Sherbrooke, QC J1K 2R1, Canada
}

Marie-Annick Galland, Cochair

Centre Acoustique du LMFA, Ecole Centrale de Lyon, 36 avenue Guy de Collongue, Ecully cedex, 69134, France

\section{Invited Papers}

\section{8:00}

5aSAb1. Active structural-acoustic control of laminated cylindrical panels using vertically/obliquely reinforced 1-3 piezoelectric composite. Manas C. Ray (Indian Institute of Technology, Mechanical Engineering Department, 721302 Kharagpur, India, mcray @ mech.iitkgp.ernet.in)

The performance of active constrained layer damping (ACLD) treatment for active structural-acoustic control of vibrating thin laminated cylindrical panels has been investigated. The constraining layer of the ACLD treatment has been considered to be made of vertically/obliquely reinforced 1-3 piezoelectric composite material. A finite element model has been developed for the laminated panels integrated with the patches of ACLD treatment to describe the coupled structural-acoustic behavior of the panels enclosing an acoustic cavity. Both velocity and pressure rate feedback controls have been implemented to activate the patches. Symmetric and antisymmetric cross-ply and antisymmetric angle-ply panels have been considered for evaluating the numerical results. Emphasis has also been placed on investigating the effect of the piezoelectric fiber orientation in the constraining layer and the shallowness angle of the cylindrical panels on the performance of the patches.

\section{8:20}

5aSAb2. Decentralized control of sound radiation from an aircraft-style panel using iterative loop recovery. Noah $\mathrm{H}$. Schiller (NASA Langley Research Center, 2 North Dryden Street, Hampton, VA 23681, USA, noah.h.schiller@nasa.gov), Randolph H. Cabell (NASA Langley Research Center, 2 North Dryden Street, Hampton, VA 23681, USA, randolph.h.cabell@ nasa.gov), Chris R. Fuller (Virginia Tech, 131 Durham Hall, Blacksburg, VA 24061, USA, christopher.r.fuller@nasa.gov)

A decentralized LQG-based control strategy is designed to reduce low-frequency sound transmission through periodically stiffened panels. While modern control strategies have been used to reduce sound radiation from relatively simple structural acoustic systems, significant implementation issues have to be addressed before these control strategies can be extended to large systems such as the fuselage of an aircraft. For instance, centralized approaches typically require a high level of connectivity and are computationally intensive, while decentralized strategies face stability problems caused by the unmodeled interaction between neighboring control units. Since accurate uncertainty bounds are not known a priori, it is difficult to ensure the decentralized control system will be robust without making the controller overly conservative. Therefore, an iterative approach is suggested, which utilizes frequency-shaped loop recovery. The approach accounts for modeling error introduced by neighboring control loops, requires no communication between subsystems, and is relatively simple. The control strategy is validated using real-time control experiments performed on a built-up aluminum test structure representative of the fuselage of an aircraft. Experiments demonstrate that the iterative approach is capable of achieving $12 \mathrm{~dB}$ peak reductions and a $3.6 \mathrm{~dB}$ integrated reduction in radiated sound power from the stiffened panel.

\section{8:40}

5aSAb3. Experimental tests on smart panels for the reduction of sound radiation. Paolo Gardonio (ISVR, University of Southampton, Highfield, SO17 1BJ Southampton, UK, pg@isvr.soton.ac.uk), Cristobal Gonzalez Diaz (ISVR, University of Southampton, Highfield, SO17 1BJ Southampton, UK, cgd@isvr.soton.ac.uk), Neven Alujevic (ISVR, University of Southampton, Highfield, SO17 1BJ Southampton, UK, na2@isvr.soton.ac.uk), Yohko Aoki (ISVR, University of Southampton, Highfield, SO17 1BJ Southampton, UK, ya@isvr.soton.ac.uk)

This paper presents a comparative experimental study carried out on four types of smart panels for the reduction of sound radiation. The four panels are equipped with decentralized velocity feedback control units that are designed to generate active damping. In this way the frequency averaged response and sound radiation produced by stochastic disturbances can be effectively reduced at low audio 
frequencies. The first smart panel is composed of a $4 \times 4$ array of square piezoelectric patch actuators with accelerometer sensors at their centres. The second smart panel is composed of sixteen triangularly shaped piezoelectric patch actuators with base edges evenly distributed along the perimeter of the panel and accelerometer sensors located on the top vertices of the actuators. The third panel is composed of five electrodynamic inertial actuators with accelerometer sensors located under their footprints. The fourth panel is equipped with a light honeycomb trim panel mounted on four stiff mounts. A set of nine reactive electrodynamic actuators is located in the shallow cavity between the two panels. Accelerometer sensors are mounted at the two footprints of each actuator. The sensoractuator pairs mounted in the four panels are used to implement analogue local feedback loops.

\section{9:00}

5aSAb4. Extremal harmonic active control of power. Philippe Micheau (Univ. de Sherbrooke, Mechanical Engineering Depart., 2500 Boulevard de 1'Université, Sherbrooke, QC J1K 2R1, Canada, Philippe.Micheau@USherbrooke.ca), Jean-Philippe Gauthier (Univ. de Sherbrooke, Mechanical Engineering Depart., 2500 Boulevard de l'Université, Sherbrooke, QC J1K 2R1, Canada, JeanPhilippe.Gauthier@cta-brp-udes.com)

The extremal harmonic active control of power consists to define an energetic criterion at the main harmonic and to extremalize it on-line by commanding a secondary source of power. The term extremal means that the optimal criterion value is reached with an on-line optimization algorithm. Without loss of originality, the context of rotating machines oscillation in synchronization with rotor position is used to illustrate this approach. A second commanded torque source, attached to the shaft, is used to control speed oscillation due to torque disturbance. Such configuration can be found in hybrid automotive for example. The energetic criterion can be the kinetic energy (for conventional active speed oscillation cancelling), the reactive power (to add a virtual flywheel on the shaft) or the active power (for energy harvesting to charge a battery). The optimization algorithm is the method of steepest descent implemented with implicit or explicit gradient computation. But, in the case of reactive power, the implicit controller is not practically achievable. The experimental results with a test bed show that the explicit controllers converge at the extremum of each criterion. To conclude, the presented explicit extremal controller is a good candidate to be used for energetic criterion control.

\section{9:20}

5aSAb5. Control of harmonic distortion in a motor driven subsonic acoustic source. Marty Johnson (Virginia Tech, Mechanical Engineering, 143 Durham 0238, Blacksburg, VA 24061, USA, martyj@vt.edu), Alessandro Toso (Virginia Tech, Mechanical Engineering, 143 Durham 0238, Blacksburg, VA 24061, USA, aletoso@ vt.edu), Vincent Blandea (ISVR, University of Southampton, University Road, Highfield, S017 1BJ Southampton, UK, vblandea@gmail.com)

This paper presents a comparison of active control strategies used to reduce the harmonic distortion of a motor driven subsonic source. The source consists of a DC motor that drives a pair of speaker diaphragms through a belt drive system which in turn drives a pair of passive radiators through an acoustic enclosure. The motor drive system is inherently nonlinear with significant losses in force at polarity changes. A number of control strategies were tested both theoretically and experimentally in order to determine the best strategy for linearizing the output from the speaker. The methodologies tested were: (i) an FXLMS based adaptive time domain controller using a linear plant model, (ii) an FXLMS based adaptive frequency domain harmonic controller using a linear plant model, (iii) an FXLMS based adaptive frequency domain harmonic controller using a nonlinear plant model and (iv) a simplex based adaptive frequency domain harmonic controller. The time domain system is shown to suffer from slow adaption rates for the higher harmonics, the model based FXLMS harmonic controllers were shown to suffer from the nonstationarity of the nonlinear plant and the simplex method was shown to provide good results while being robust to slow variations in the plant.

9:40-11:00 Posters

Lecture sessions will recess for presentation of poster papers on various topics in acoustics. See poster sessions for topics and abstracts.

\section{Invited Papers}

\section{1:00}

5aSAb6. Variable area jet nozzle for noise reduction using shape memory alloy actuators. James Mabe (Boeing Phantom Works, M/C 42-51, PO BOX 3707, Seattle, WA 98124-2207, USA, james.h.mabe@boeing.com)

A significant reduction in noise and improved fuel consumption can be achieved by varying the area of a commercial jet engine's fan nozzle. A larger diameter at takeoff and approach can reduce jet velocity reducing noise. Adjusting the diameter in cruise, to account for varying Mach number, altitude, etc, can optimize fan loading and reduce fuel consumption. Boeing recently tested a scaled variable area jet nozzle capable of a $20 \%$ area change. Shape memory alloy actuators were used to position 12 interlocking panels at the nozzle exit. A closed loop control system was used to maintain a range of constant diameters with varying flow conditions and to vary the diameter under constant flow conditions. Acoustic data by side line microphones and flow field measurements at several cross-sections using PIV was collected at each condition. In this paper the variable area nozzle's design is described. The effect of the nozzle's diameter on its acoustic performance is presented for a range of Mach numbers and mass flow rates. Flow field data is shown including the effects of the joints between the interlocking panels. 
5aSAb7. Concept of active reverberation box. Hisaharu Suzuki (Kyushu University, 4-9-1, Shiobaru, Minamiku, 815-8540 Fukuoka, Japan, hisaha@ souldsp.jp), Akira Omoto (Kyushu University, 4-9-1, Shiobaru, Minamiku, 815-8540 Fukuoka, Japan, omoto @ design.kyushu-u.ac.jp)

A quite small reverberation box with active controller system is proposed. If we try to measure the characteristics of acoustic materials, such as glass wool, in a small sized enclosure, there are few dominant modes which cause the condition that the incident wave to target material is biased toward the specific direction. As a result, the measured result shows the irregular distribution which depends on measuring point or evaluating method such as the reverberation time reading. The proposed system which utilized the active control enables us to create a plane wave with arbitrary direction in the enclosure. Therefore it has possibilities of solving the problems caused by insufficient incident condition. Also, in this study, the two evaluating strategies that the traditional reverberation time method and the steady state analysis method are concerned.

\section{1:40}

5aSAb8. Active control of engine noise transmitted into cavities: simulation, experimental validation and sound quality assessment. Leopoldo P. De Oliveira (KU Leuven, Celestijnenlaan 300B, Departement Werktuigkunde - PMA, 3001 Leuven, Belgium, leopoldo.deoliveira@mech.kuleuven.be), Paul Sas (KU Leuven, Celestijnenlaan 300B, Departement Werktuigkunde - PMA, 3001 Leuven, Belgium, Paul.Sas@mech.kuleuven.be), Wim Desmet (K.U.Leuven - Dept. of Mechanical Engineering, Celestijnenlaan 300B - bus 2420, 3001 Heverlee, Belgium, Wim.Desmet@mech.kuleuven.be), Karl Janssens (LMS International, Interleuvenlaan 68, 3001 Leuven, Belgium, Karl.Janssens@1msintl.com), Peter Gajdatsy (LMS International, Interleuvenlaan 68, 3001 Leuven, Belgium, peter.gajdatsy@lmsintl.com), Herman Van Der Auweraer (LMS International, Interleuvenlaan 68, 3001 Leuven, Belgium, Herman .VanderAuweraer@1msintl.com)

Active control has been proposed as a possible solution to cope with low frequency noise reduction in vehicles. Active noise control systems tend to be designed with a target on the sound pressure level reduction. However, the perceived control efficiency for the occupants can be more accurately assessed if psychoacoustic metrics are taken into account. The aim of this paper is to evaluate, numerically and experimentally, the effect of a collocated velocity feedback controller on the sound quality of engine noise in a vehicle mockup. The simulation scheme is described and experimentally validated. The engine excitation is provided by a sound quality equivalent engine simulator, running on a real-time platform that delivers harmonic excitation in function of the driving condition. The controller performance is evaluated in terms of sound quality metrics such as specific loudness and roughness. As a result of the control action, loudness is significantly reduced and roughness slightly spread, with an overall improvement on the perceived engine sound.

\section{2:00}

5aSAb9. Reproduction of loud low-frequency soundfields with Boundary Surface Control. Emmanuel Friot (Laboratory for Mechanics and Acoustics CNRS, 31 chemin Joseph Aiguier, 13009 Marseille, France, friot@lma.cnrs-mrs.fr), Régine Guillermin (Laboratory for Mechanics and Acoustics CNRS, 31 chemin Joseph Aiguier, 13009 Marseille, France, guillermin@lma .cnrs-mrs.fr), Cédric Pinhède (CNRS - LMA, 31 Chemin Joseph Aiguier, 13009 Marseille, France, pinhede@lma.cnrs-mrs.fr)

Many research works have focused on multichannel soundfield reproduction during the past decade, and complete systems are now commercially available for 3D sound control e.g., with Wave Field Synthesis. However, the usual underlying approximation of free-field propagation is not valid when reproducing high level low frequency noise, such as a sonic boom, inside a closet; a specific sound control strategy is required in this case. In this context the paper presents an experiment of low-frequency soundfield reproduction with openloop control of the acoustic pressure at a set of 30 microphones enclosing a listening area. It is shown that this so-called Boundary Surface Control strategy allows an accurate reproduction of 3D soundfields inside the LMA sonic boom closet, equipped with 16 wallembedded noise sources, at frequencies from 3 to $200 \mathrm{~Hz}$.

5aSAb10. Experimental assessment of the performance of a "smart foam" absorber. Pierre M. Leroy (Laboratoire de Mécanique et d'Acoustique - CNRS, 31 chemin Joseph Aiguier, 13402 Marseille, France, leroy@1ma.cnrs-mrs.fr), Philippe Herzog (Laboratoire de Mécanique et d'Acoustique - CNRS, 31 chemin Joseph Aiguier, 13402 Marseille, France, herzog@1ma.cnrs-mrs.fr), Alain Berry (Univ. de Sherbrooke, Mechanical Engineering Depart., 2500 Boulevard de 1'Université, Sherbrooke, QC J1K 2R1, Canada, alain.berry@usherbrooke.ca), Noureddine Atalla (Univ. de Sherbrooke, Mechanical Engineering Depart., 2500 Boulevard de 1'Université, Sherbrooke, QC J1K 2R1, Canada, Nouredinne.Atalla@Usherbrooke.ca)

The signal processing implementation of a hybrid passive/active absorber (smart foam) made up from the combination of a passive absorbent (poroelastic melamine foam) typically efficient at the high frequencies, and a bonded, curved PVDF film, to enhance low frequency performance is considered. Three methods for obtaining the control signal are experimentally compared in the case of a plane wave excitation. A prototype of such smart foam has been built and tested in a waveguide (rectangular impedance tube) at frequencies between $100 \mathrm{~Hz}$ and $1000 \mathrm{~Hz}$. The performances in term of the ratio of reflected to incident sound pressure are presented and discussed, comparing the three methods. The first method uses estimations of the transfer functions between the sources and two microphones in the tube to calculate off-line an optimal filter in the frequency domain. It is then compared with numerical simulations, based on a 3D FE model of the smart foam. The two other methods are based on a real-time adaptive control using a FXLMS algorithm, a unidirectional microphone as error sensor, and the primary source signal as reference signal. The control filter is obtained after the initial convergence, using either pure tone disturbance (secnd method) or broad band disturbance (third method).

\section{2:40-2:00 Lunch Break}




\section{Contributed Papers}

2:00

5aSAb11. Active vibration reduction applied to the compressor of an air-conditioning unit for trams. Joachim Bös (Fraunhofer Institute for Structural Durability and System Reliability LBF, Bartningstr. 47, 64289 Darmstadt, Germany, joachim.boes@lbf.fraunhofer.de), Enrico Janssen (Fraunhofer Institute for Structural Durability and System Reliability LBF, Bartningstr. 47, 64289 Darmstadt, Germany, enrico.janssen@lbf.fraunhofer.de), Michael Kauba (Fraunhofer Institute for Structural Durability and System Reliability LBF, Bartningstr. 47, 64289 Darmstadt, Germany, michael.kauba@lbf.fraunhofer.de), Dirk Mayer (Fraunhofer Institute for Structural Durability and System Reliability LBF, Bartningstr. 47, 64289 Darmstadt, Germany, dirk.mayer@lbf .fraunhofer.de)

Within the framework of the European Integrated Project InMAR (intelligent materials for active noise reduction) active vibration control and active structural acoustic control approaches are applied to an HVAC (heating, ventilation, and air conditioning) unit used to air-condition the driver's cab of a tram. Measurements previously performed by the manufacturer of the unit indicated that annoying tonal noise in the $50 \mathrm{~Hz}$ and 100 $\mathrm{Hz} 1 / 3$ octave bands inside the driver's cab is mainly caused by the vibration of the compressor mounted in the HVAC unit on the roof of the tram. Therefore, two different concepts for the design of active compressor mounts were developed that are used to reduce the vibration excitation of the HVAC unit's housing. The first one is an active tuned vibration absorber whose natural frequency can be adapted by means of piezoelectric patch actuators and which behaves as a vibration compensator at higher frequencies. The second one is an active mount based on four piezoelectric stack actuators and an elastomer part that deflects the effective direction of the piezo stack displacement by 90 degrees while amplifying the displacement by a factor of approximately 30. These two concepts are compared with each other.

\section{2:20}

5aSAb12. Training by research - program of, and experiences in, the Smart Structures Research Training Network project. Fülöp Augusztinovicz (Budapest University of Technology and Economics, BME Dept. of Telecommunications, Magyar tudósok körútja 2, H-1117 Budapest, Hungary, fulop@hit.bme.hu)

Smart Structures is a Marie Curie Research Training network (RTN) named "A Computer Aided Engineering Approach to Smart Structures Design". The overall objective of the RTN is to develop an educational and research framework for developing macroscale noise control applications based on intelligent material systems. The scientific and technological objective of the network is to advance the state-of-the-art in smart materials research to the level of system integration and industrial applicability, with a specific focus on solutions for noise and vibration reduction and for vibration-based damage detection. The paper gives a short overview of the project partners' activity and summarizes the experiences of the researchers and their tutors after the first 18 months of the network.

\section{2:40}

5aSAb13. Intelligent interfaces for sound insulation: Numerical and experimental optimisation. Cedric Batifol (Centre Acoustique du LMFA, Ecole Centrale de Lyon, 36 avenue Guy de Collongue, 69134 Ecully cedex, France, cedric.batifol@ec-lyon.fr), Marie-Annick Galland (Centre Acoustique du LMFA, Ecole Centrale de Lyon, 36 avenue Guy de Collongue, 69134 Ecully cedex, France, marie-annick.galland@ec-lyon.fr), Mohamed Ichchou (Centre Acoustique du LMFA, Ecole Centrale de Lyon, 36 avenue Guy de Collongue, 69134 Ecully cedex, France, mohamed.ichchou@eclyon.fr)

Sound insulation is actually achieved thanks to passive multilayers. These ones are classically made of elastic, acoustic and poroelastic media. Good results are obtained in medium and high frequencies, but low frequencies disturbances still remain a problem. Indeed, local and global resonant behaviours of the panel, such as plate bending mode or the double-leaf phenomenon, occur and lower the performances. An active concept has already been proposed and validated. Piezoelectric components are added to the interface and act a secondary source aiming at minimising the sound transmitted. In this contribution, a double-plate system is studied. A reduced finite element model of an elementary cell is presented. This one relies on modal projection and classical component mode synthesis (CMS) procedures. This model is first updated and validated thanks to measurements performed on a dedicated test bench. The use of active control allows a $15 \mathrm{~dB}$ enhancement of sound insulation at low resonant frequencies. $\mathrm{Nu}$ merical experiments are designed. Response surfaces are obtained thanks to kriging procedures. Finally, the configuration of the optimal interface is discussed in a physical point of view.

\section{3:00}

5aSAb14. Practical implementation of low-latency DSP for feedback control of sound in research contexts. Edgar Berdahl (Stanford Univ., Center for Computer Research in Music and Acoustics (CCRMA), Dept. of Music, Stanford, CA 94305-8180, USA, eberdahl@ccrma.stanford.edu), Nelson Lee (Stanford Univ., Center for Computer Research in Music and Acoustics (CCRMA), Dept. of Music, Stanford, CA 94305-8180, USA, nalee@stanford.edu), Guenter Niemeyer (Stanford Univ., Mech. Eng., Bldg. 530, Stanford, CA 94305, USA, gunter.niemeyer@stanford.edu), Julius O. Smith (Stanford Univ., Center for Computer Research in Music and Acoustics (CCRMA), Dept. of Music, Stanford, CA 94305-8180, USA, jos@ccrma.stanford.edu)

Feedback control of sound requires low-latency signal processing. In addition, because the human range of hearing extends roughly from $20 \mathrm{~Hz}$ to $20 \mathrm{kHz}$, controller hardware must process signals with relatively large bandwidths in comparison with common control applications. Over the past decade, the appropriate embedded hardware has become a niche product, so its cost has actually increased. The open source community has developed an excellent alternative: a general-purpose computer runs Linux with the realtime application interface (RTAI). Open source drivers (see www.comedi .org) enable software to communicate efficiently with data acquisition cards. For the first time, we describe in detail from start to finish how to configure such a system. In particular, we explain how to run control code from user space while still disabling interrupts. We further explain how to reserve a processor for running only feedback control code. This configuration achieves less than one sample of total system delay at sampling rates as high as $50 \mathrm{kHz}$. Other processors remain free to run standard Linux programs, allowing researchers to control sound with calls to many generic software libraries. We present detailed measurements of system jitter for various configurations and provide an example open-source toolbox demonstrating all these aspects at http://ccrma.stanford.edu/ $/$ eberdahl/Projects/TFCMI.

\section{3:20}

5aSAb15. From the electrical shunting of a loudspeaker to active impedance control. Hervé Lissek (Ecole Polytechnique Fédérale de Lausanne, EPFL STI LEMA, Station 11, CH 1015 Lausanne, Switzerland, herve.lissek@epfl.ch), Florian Sandoz (Ecole Polytechnique Fédérale de Lausanne, EPFL STI LEMA, Station 11, CH 1015 Lausanne, Switzerland, florian.sandoz@epfl.ch)

Variable acoustic properties can be obtained on an electroacoustic transducer's voicing face by very basic control strategies, among which are the shunting of the loudspeaker (shortcut, variable electric load, or negative resistance disposals). It is proven that better performances with enhanced controllability can be obtained by way of hybrid feedback control, consisting in a double feedback loop, one on acoustic pressure at the diaphragm and the other on its velocity, leading to a global acoustic impedance control. The present work describes the theory of the hybrid feedback control, by way of block diagrams aiming at visualizing the control principle, starting from the shunted loudspeaker. Simulations of performances obtained on Matlab/Simulink are presented, and compared to experimental results obtained on ana$\log$ prototypes mounted at the end of a dedicated impedance tube. A last, a discussion on stability issues follows, leading to concluding remarks on the disposal behavior and possible means of enhancements. 
3:40

5aSAb16. Active-passive multilayered panels for reduction of both acoustic reflection and transmission. Azzedine Sitel (Centre Acoustique du LMFA, Ecole Centrale de Lyon, 36 avenue Guy de Collongue, 69134 Ecully cedex, France, azzedine.sitel@ec-lyon.fr), Ying Hu (Centre Acoustique du LMFA, Ecole Centrale de Lyon, 36 avenue Guy de Collongue, 69134 Ecully cedex, France, ying.hu@ec-lyon.fr), Marie-Annick Galland (Centre Acoustique du LMFA, Ecole Centrale de Lyon, 36 avenue Guy de Collongue, 69134 Ecully cedex, France, marie-annick.galland@eclyon.fr)

The design of panels reducing both acoustic reflection and transmission for a wide frequency range is a problem of considerable practical interest for building or transport industries. Classically, these panels are made up of elastic plates bonded to poroelastic layers. Such structures are efficient in the middle and high frequency range but exhibit a lack of performance at low frequency. Hybrid passive/active cells previously developed at the LMFA have proved their efficiency for global noise reduction. They combine passive means (elastic plates, poroelastic material) and active control through a piezoelectric actuator to ensure high panel performance throughout the whole frequency range. In this paper, two systems are described. The first one is a multilayered panel combining two cells: the first cell on the emission side ensures high absorption, the second cell located at the rear face aims at reducing transmission. The second structure consists of a double-plate system using a microperforated plate on the emission side and an active plate on the transmission side. The performance of the two systems are examined through analytical or numerical simulations developed for plane waves and diffuse field conditions, and through experiments carried out for plane waves under normal incidence.

FRIDAY MORNING, 4 JULY 2008

P2-B, LEVEL 2, 9:40 TO 11:20 A.M.

\title{
Session 5aSAc
}

\section{Structural Acoustics and Vibration and EURONOISE: Active Noise Control: New Strategies and Innovative Concepts II (Poster Session)}

\author{
Alain Berry, Cochair \\ Univ. de Sherbrooke \\ Marie-Annick Galland, Cochair \\ Centre Acoustique du LMFA
}

\begin{abstract}
All posters will be on display from 9:40 a.m. to 11:20 a.m. To allow contributors an opportunity to see other posters, contributors of odd-numbered papers will be at their posters from 9:40 a.m. to 10:30 a.m. and contributors of even-numbered papers will be at their posters from 10:30 a.m. to 11:20 a.m.
\end{abstract}

\section{Contributed Papers}

5aSAc1. New multichannel modified filtered-x algorithms for active noise control using the dichotomous coordinate descent method. Felix Albu (Politehnica University of Bucharest, Bd. Iuliu Maniu, no. 1-3, 061102 Bucharest, Romania, felix_albu@ieee.org), Constantin Paleologu (Politehnica University of Bucharest, Bd. Iuliu Maniu, no. 1-3, 061102 Bucharest, Romania, pale@comm.pub.ro)

In this paper, several multichannel modified filtered-x algorithms for active noise control or acoustic equalization systems using the dichotomous coordinate descent method (DCD) are introduced. This multiplierless and divisionless method is used for avoiding the matrix inversion that appears in adaptive algorithms such us fast transversal filters (FTF), recursive least square (RLS) based algorithms, affine projection (AP) or its fast versions. The study is focused on the important computational savings given by the use of DCD method, the effect on the convergence properties and stability of the investigated algorithms. A comparison of their convergence performance in case of using non-ideal noisy acoustic plants is also given. It is proved by simulations that the use of the dichotomous coordinate descent method can be an interesting option for reducing the computational cost of practical multichannel algorithms for ANC or acoustic equalization algorithms.

5aSAc2. Use of peak filter for online secondary path modeling in a feedforward active ship-noise cancellation system. Xueshan Bao (Department of Electronics and Telecommunications, Norwegian
University of Science and Technology, Sem Sælands vei 5, 7491 Trondheim, Norway, xbao@iet.ntnu.no)

The injected auxiliary noise used in online secondary path modeling method often disturbs the convergence of control filter and increases the residual sound field energy of the active noise cancellation (ANC) system. To mitigate this problem, an improved online secondary path modeling method is proposed, in which the transfer function of secondary path is reconstructed by a peak filter. The frequency response of new transfer function remains unchanged at the frequency band of the noise to be cancelled; while the other bands are attenuated deeply. With this system the simulation experiment of active ship-radiated noise cancellation is accomplished. The result shows that new system's noise cancellation capability is improved and residual sound field energy (including channel response to injected auxiliary noise) is smaller than conventional system without peak filter because of the simplified transfer function of secondary path and reduced energy disturbance between control filter and modeling filter.

5aSAc3. Adaptive active noise control incorporating with a transfer function method for reducing acoustic feedback in a duct. Yun-Hui Liu (Southern Taiwan University, 1 Nan-Tai St, Yung-Kang City, 710 Tainan Hsien, Taiwan, yhliu@mail.stut.edu.tw)

In most practical applications of active noise control, the acoustic feedback is a major problem that often interferes with the operation of the control system and even renders it unstable. We propose a frequency-domain method based on transfer function to reduce the influence of acoustic feedback based on plane wave transmission theory of sound in a duct. The origi- 
nal signal of primary noise is obtained from the measured signals of reference microphone and error microphone which are transformed to frequency domain by FFT and operated by the proposed method. In this study, Filtered-X LMS algorithm is applied to carry out the active noise controller incorporating with this transfer function method. Experimental results of active noise control show that the system has achieved $18.5 \mathrm{~dB}$ maximal attenuation in the frequency band $200-600 \mathrm{~Hz}$. On the contrary, it is only attenuate $3-4 \mathrm{~dB}$ if acoustic feedback is present. Therefore, it was to verify that the proposed method of acoustic feedback cancellation incorporating with the Filtered-X LMS algorithm can effectively reduce the influence of acoustic feedback and obtains a better performance of active noise control in a duct.

5aSAc4. Secondary source distribution effect on active noise control robustness. Mahdi Azarpeyvand (Institute of Sound and Vibration Research, University of Southampton, University Road, SO17 1BJ Southampton, UK, ma@isvr.soton.ac.uk)

Arrangement of secondary sources and microphones play an important role in effectiveness and robustness of any active noise control (ANC) system. This paper concerns effective distribution of secondary sources for a typical ANC system so that provides a robust and acceptable level of noise reduction for different types of primary source, namely monopole, dipole and multipole, radiating at $100 \mathrm{~Hz}$ and $250 \mathrm{~Hz}$. Two sets of secondary sources are considered here: planar distribution, and dome-type distribution. Results have shown that utilization of planar distribution model leads to more effective and stable noise reduction for most of the cases, while using a dome-type set of secondary sources is not able to effectively reduce noise from dipole and multipole primary sources. In addition, it was found that implementation of dome-type arrangement is more difficult than the planar distribution and is also very likely to become unstable for some particular source arrangements (i.e., or mathematically ill-conditioned).

5aSAc5. Improvement of acoustic transmission loss by active modal mass control. Vincent Lhuillier (Laboratoire Vibrations Acoustique INSA Lyon, 25 bis avenue Jean Capelle, Bâtiment Saint-Exupéry, F-69621 Villeurbanne cedex, France, vincent.lhuillier@insa-lyon.fr), Charles Pezerat (Laboratoire Vibrations Acoustique - INSA Lyon, 25 bis avenue Jean Capelle, Bâtiment Saint-Exupéry, F-69621 Villeurbanne cedex, France, charles.pezerat@insa-lyon.fr), Simon Chesne (LaMCoS - INSA-Lyon CNRS UMR5259, 18-20, rue des Sciences, Bâtiment Jean d'Alembert, F-69621 Villeurbanne, France, simon.chesne@insa-lyon.fr), Luc Gaudiller (LaMCoS - INSA-Lyon - CNRS UMR5259, 18-20, rue des Sciences, Bâtiment Jean d'Alembert, F-69621 Villeurbanne, France, luc .gaudiller@insa-lyon.fr)

This paper deals with an alternative modal control approach to reduce the sound transmission through a structure excited by an acoustic wave. Active control enables to conserve the characteristics of lightness while improving acoustic performances. For light and small structures having small modal overlap, the "modal mass damping control" is proposed. The aim of this control is to modify the modal distribution of high radiation efficiency modes with modal virtual mass and modal virtual damping. The interest of this approach is that at low frequencies, the active virtual mass effects shift down eigen frequencies to less audible frequency range while reducing vibration amplitudes in a broad frequency range. The modal virtual mass control appears to be a good complement to a standard damping control which is exclusively efficient at resonances. In order to detail the concept of the proposed method, an application is presented on a double panel equipped with piezoelectric patches. The structure is excited by a distributive acoustic plane wave. Acoustic transmission loss factors of the simulated controlled and the non-controlled smart structure are shown and optimization is discussed.

5aSAc6. A two-dimensional ANC system - from simulation to application. Christian Kleinhenrich (Univ. of Wuppertal, RainerGruenter-Str. 21, 42119 Wuppertal, Germany, c.kleinhenrich @uni-wuppertal.de), Detlef Krahé (Univ. of Wuppertal, Rainer-GruenterStr. 21, 42119 Wuppertal, Germany, krahe@uni-wuppertal.de), Arndt Niepenberg (WaveScape Technologies GmbH, Lise-Meitner-Str. 1-9, 42119 Wuppertal, Germany, niepenberg@wavescape-technologies.com)

An existing two-dimensional active noise control (ANC) system attenuates a given noise (primary field) inside a certain area and frequency range by an additionally generated sound (secondary field). The two-dimensional ANC system consists of several modules containing a pair of microphones and a line array of loudspeakers each. The configuration of the modules depends on the acoustical environment and the desired shape of the protection area. Planning this is supported by a tool based on simulating the superposition of both sound fields. This spatial, close to reality simulation for predicting the achievable noise attenuation and determining important system parameters is implemented in MATLAB. With this planning tool it is possible to place all required acoustic sources and sinks into a virtual room and analyze the properties of the resulting sound field concerning real application scenarios. This paper draws a comparison between the results given by simulation and the subsequently realized ANC system within the scope of a typical application.

5aSAc7. Active noise control in light jet aircraft. Oliver Pabst (Helmut-Schmidt-Universität - Universität der Bundeswehr Hamburg, Holstenhofweg 85, 22043 Hamburg, Germany, opabst@ hsu-hh.de), Thomas Kletschkowski (Helmut-Schmidt-Universität - Universität der Bundeswehr Hamburg, Holstenhofweg 85, 22043 Hamburg, Germany, thomas .kletschkowski@hsuhh.de), Delf Sachau (Helmut-Schmidt-Universität Universität der Bundeswehr Hamburg, Holstenhofweg 85, 22043 Hamburg, Germany, sachau@hsuhh.de)

Active systems for noise reduction are especially of interest when considering applications in which low-frequency noise is a main source of disturbance and only limited amounts of installation space and payload are available. This makes the adaptation and implementation of such systems plausible in vehicles such as automobiles and aircraft where passive reduction methods are restricted. In order to achieve effective active control in these environments, aspects such as the control method and actuator- and sensor-type as well as positioning must be considered. The noise characteristics are often known beforehand or are easily accessible by measurement. Using this data, an upper bound for possible noise reduction may be determined, e.g., by means of linear prediction methods. A current research project is aimed at developing an audiosystem for the cabin area of a light jet aircraft which, at the same time, should also function as an effective noise reduction system in order to enhance the cabin comfort as well as the audio quality. Using data from a measurement flight in a typical light jet aircraft, limitations of active control are determined. Furthermore, the testbed, an acoustic mockup, is presented, currently beholding a multichannel ANC- Audio system for tonal and broadband noise. 


\title{
Session 5aSAd
}

\section{Structural Acoustics and Vibration, ASA Committee on Standards, and EURONOISE: Ground Vehicle Noise and Vibration II (Poster Session)}

\author{
Donald Bliss, Cochair \\ Duke University \\ Paul De Vos, Cochair \\ $D H V B V$
}

\begin{abstract}
All posters will be on display from 9:40 a.m. to 11:20 a.m. To allow contributors an opportunity to see other posters, contributors of odd-numbered papers will be at their posters from 9:40 a.m. to 10:30 a.m. and contributors of even-numbered papers will be at their posters from 10:30 a.m. to 11:20 a.m.
\end{abstract}

\section{Contributed Papers}

5aSAd1. An hybrid method for sound transmission inside trains. Philippe A. Jean (CSTB, 24 rue Joseph Fourier, 38400 Saint-Martind'Hères, France, philippe.jean@cstb.fr), Michel Villot (CSTB, 24 rue Joseph Fourier, 38400 Saint-Martin-d'Hères, France, michel.villot@cstb.fr

Hybrid methods mixing ray-tracing and energy approaches have been implemented and validated in the case of sound transmission inside a train. Generally, validation is proposed against measurements where it may be difficult to assess precisely the importance of the various assumptions and simplifications. In this paper, the proposed hybrid approach is compared to more precise numerical computations based on BEM and analytic models. This allows studying the importance of assumptions made on the computations of incident power and transmitted power. The case of a train in a tunnel has also been analysed where the sound field in the tunnel is computed by means of 2.5D BEM.

5aSAd2. Critical assessment of operational path analysis: effect of coupling between path inputs. Peter Gajdatsy (LMS International, Interleuvenlaan 68, 3001 Leuven, Belgium, peter.gajdatsy@lmsintl.com), Karl Janssens (LMS International, Interleuvenlaan 68, 3001 Leuven, Belgium, Karl.Janssens@1msintl.com), Ludo Gielen (LMS International, Interleuvenlaan 68, 3001 Leuven, Belgium, ludo.gielen@1ms.be), Peter Mas (LMS
International, Interleuvenlaan 68, 3001 Leuven, Belgium, peter.mas @ Ims.be), Herman Van Der Auweraer (LMS International, Interleuvenlaan 68,3001 Leuven, Belgium, Herman.VanderAuweraer@1msintl.com)

Classical transfer path analysis (TPA) is a widely used and reliable method for tackling noise and vibration problems. But due to its complexity and time-consuming procedure the industry is constantly seeking for simpler and faster methods. One such method, often referred to as operational path analysis (OPA), was presented in 2006 and has quickly gained popularity in the NVH field. The method works with measured operational input and output accelerations and sound pressures and the transmissibilities calculated from these. The claim for its accuracy is based on being able to reproduce the original output signal by summing the calculated partial contributions but it has not yet been compared to other TPA methods. This new method is now critically examined and compared to a reliable classical TPA measurement. The results of this examination reveal three significant weaknesses. This paper deals with the effect of the cross-coupling between the input signals. Due to modal behavior a single force will cause vibrations at all inputs. Thus, there is not a simple one-to-one relationship between loads and inputs. This coupling then can easily lead to false identification of significant paths in case of the OPA method. 


\title{
Session 5aSCa
}

\section{Speech Communication: Speaker Identification by Machine}

\author{
Jaime Hernández-Cordero, Cochair \\ US Department of Defense, PO BOX 183, Odenton, MD 21113, USA \\ Louis-Jean Boë, Cochair \\ GIPSA-lab, Université Stendhal, 1180, avenue Centrale, 38031 Grenoble CEDEX 9, France
}

\section{Contributed Papers}

\section{8:00}

5aSCa1. Adaptive threshold estimation for speaker verification systems. Eduardo Castilllo-Guerra (University of New Brunswick, P.O. Box 4400, 15 Dineen Dr., D36 Head Hall, Fredericton, NB E3B 5A3, Canada, ecastill@unb.ca), Roberto Díaz-Amador (Central University of Las Villas, Carr. a Camajuaní km 5.5, 50100 Santa Clara, Cuba, ramador@uclv.edu.cu), Cárdenas-Barreras L. Julian (Central University of Las Villas, Carr. a Camajuaní km 5.5, 50100 Santa Clara, Cuba, jcardenas $@$ uclv.edu.cu)

This paper describes an adaptive threshold estimation mechanism for speaker authentication systems. The mechanism estimates speakerdependent thresholds based on successful verifications considering the minimization of a relation-based cost function. Speaker authentication systems commonly use a threshold to decide whether a claimed identity matches a voice-print previously enrolled. Speaker independent threshold is a common option but it does not consider specific speaker characteristics that are relevant to achieve better system performance. Speaker dependent threshold on the contrary, uses speaker-specific data to estimate individual thresholds but the system performance can also suffer from suboptimal threshold conditioned by limited number of true scores. The algorithm reported in this paper starts with the speaker dependent threshold and use an adaptive algorithm to perform online re-estimation of the initial threshold based on speakerdependent data. The threshold is re-estimated in each successful authentication transaction according to a custom-made confidence score. The reported technique keep the voice print up-to-date while is less sensitive to score outliers than traditional speaker dependent threshold. The algorithm provided a performance enhancement of up to $36.2 \%$ when compared to traditional speaker independent or dependent threshold using an ad-hoc database involving cell and land-line utterances from male and female speakers.

\section{8:20}

5aSCa2. Estimation of target-to-interferer ratio using the Auditory Image Model. Michael Carlin (Air Force Research Laboratory, 525 Brooks Road, Rome, NY 13441, USA, Michael.Carlin@rl.af.mil)

Performance in speech processing applications such as speaker recognition becomes severely degraded when the short-time audio being analyzed contains more than one speaker. However, it has been shown that if the audio is only minimally-corrupted by the interfering speech, i.e., the target-tointerferer ratio (TIR) is sufficiently large, then accurate recognition results can still be achieved. During phonation, estimation of TIR is especially critical since uncorrupted vowel sounds contain important speakerdiscriminating information. This research investigates a method to estimate the relative intensity of interfering speech using the Auditory Image Model (AIM) of Patterson et al. (J. Acoust. Soc. Am., 98, 1890-1894 (1995)). The proposed TIR estimator attempts to exploit both the apparent high resolution in the simulated Neural Activity Pattern and variation in cross-channel Strobe Point correlation when observing overlapping vowel sounds. Experiments were conducted for five canonical male vowels which were perceptually-scaled using the STRAIGHT algorithm (Chapter in Speech Separation by Humans and Machines, P. Divenyi, ed., Kluwer Academic Publishers, 2005) and superimposed at varying levels of TIR. Results sug- gest that the proposed approach is a promising step towards both detecting the presence and relative intensity of an interfering speaker.

\section{8:40}

5aSCa3. Extraction of likelihood-ratio forensic evidence from the formant trajectories of diphthongs. Geoffrey Stewart Morrison (Australian National University, School of Language Studies, Building 110, ACT 0200 Canberra, Australia, geoff.morrison@anu .edu.au), Phil Rose (Australian National University, School of Language Studies, Building 110, ACT 0200 Canberra, Australia, philip.rose@anu.edu.au), Yuko Kinoshita (University of Canberra, School of Languages and International Studies, ACT 2601 Canberra, Australia, yuko.kinoshita@canberra.edu.au)

The likelihood-ratio approach to forensic speaker recognition seeks to determine the likelihood that one would observe the evidence, the acoustic difference between suspect and offender speech samples, under the hypothesis that they were produced by the same speaker versus under the hypothesis that they were produced by different speakers. Before the results of a scientific forensic technique can be presented in court, it is necessary to demonstrate its efficacy. This presentation tests the efficacy of extracting information from the formant trajectories of diphthongs. Differences in physiology and learned motor patterns could potentially lead to different speakers producing quite different formant trajectories which could in turn lead to strong forensic evidence. The data tested were /aI/, /av/, /eI/, /"open o"I/, /ov/, /i"schwa"/, and / $\epsilon$ 'schwa"/ tokens produced in several phonetic contexts by 27 male speakers of Australian English. Cubic polynomials were fitted to each vowel token, and the coefficient values were used in a multivariate-kernel-density procedure which calculated likelihood ratios. Cross-validated same-speaker and different-speaker comparisons were made, resulting in a series of same-speaker and different-speaker likelihood ratios for each vowel phoneme. Results indicated that substantial strength of evidence with respect to speaker identity can be extracted from diphthong formant trajectories.

\section{9:00}

5aSCa4. Identifying speaker-dependent acoustic parameters in Spanish vowels. Victoria Marrero (Universidad Nacional Educación a Distancia, Desp. 707A. C/ Senda del Rey, 7, 28040 Madrid, Spain, vmarrero@flog.uned.es), Elena Battaner (URJC, C/Tulipan s/n, 28345 Móstoles (Madrid), Spain, elena.battaner@urjc.es), Juana Gil (Universidad Nacional Educación a Distancia, Desp. 707A. C/ Senda del Rey, 7, 28040 Madrid, Spain, mgil@flog.uned.es), Joaquim Llisterri (UAB, Filologia. Edifici B. Campus Bellaterra, 08193 Barcelona, Spain, Joaquim .Llisterri@uab.cat), María Machuca (UAB, Filologia. Edifici B. Campus Bellaterra, 08193 Barcelona, Spain, MariaJesus.Machuca@uab .es), Montserrat Marquina (UAB, Filologia. Edifici B. Campus Bellaterra, 08193 Barcelona, Spain, Montserrat.Marquina@campus.uab.es), Carme De La Mota (UAB, Filologia. Edifici B. Campus Bellaterra, 08193 Barcelona, Spain, Carme.delaMota@uab.cat), Antonio Rios (UAB, Filologia. Edifici B. Campus Bellaterra, 08193 Barcelona, Spain, Antonio.Rios@uab.cat)

In the frame of VILE Projects (Inter-and-Intra-Speaker-Variation-inSpanish for automatic speaker identification), we try to identify what 
vowel's acoustic parameters depend more on the individual characteristics of the speaker and less of the linguistic variables. Variations on standard deviation (SD), when grouping together parameters by speaker or by phoneme, are analysed. 30 speakers (from AHUMADA database) read the same text in three sessions. Mean value (Hz) of four formants (F1-F2-F3-F4) and fundamental frequency (F0) are analysed in Spanish vowels (except /u/), surrounded by unvoiced stops or /s/ (1850 samples). Hypothesis: Individual parameters will show less SD when grouping by speaker/session; vowel quality parameters, when grouping by phoneme. F1 and F2 are timbredependent parameters. F3 and F4 are speaker-dependent parameters. F0 has characteristics of both. The most variable parameter is F2. The opposite is F4. -No significant differences grouping by session or by speakers in none of the parameters. -F0 has the highest variability between vowel qualities, even if stressed and unstressed vowels are separated. -When clustering data by speaker/sessión (all vowels together), by comparison with clustering by vowel (all speakers together), SD is $* 50 \%$ higher in F1-F2 $* 75 \%$ lesser in $\mathrm{F} 4 * 66 \%$ lesser in $\mathrm{F} 0 * \mathrm{~F} 3$ shows no significant differences

\section{$9 \cdot 20$}

5aSCa5. The question of disguised voice. Patrick Perrot (Telecom Paris Tech, 46 rue Barrault, 75013 Paris, France, perrot@tsi.enst.fr), Gerard Chollet (Telecom Paris Tech, 46 rue Barrault, 75013 Paris, France, chollet @enst.fr)

State of the art automatic speaker recognition systems show very good results in the discrimination between different speakers under controlled recording conditions. In a forensic context, the conditions are uncontrolled and voice can be disguised. In cases of terrorism claim, extortion or kidnapping, it is of great interest for offenders to conceal their identity. Voice disguise is an important constraint to speaker discrimination. Some disguises produce a great variation of parameters and change the perception of an identity. The main risk is to confound a disguised voice and a normal voice and accuse an innocent individual. This paper proposes on one hand to present the impact of voice disguise on automatic speaker recognition and, on the other hand a statistical study in order to detect and identify four disguises among the most common. The first step consists in extracting features and the second step to classify them. MFCC (Mel Frequency Cepstral Coefficient) are considered as features and different classification algorithms have been tested. The studied disguises are based on a deliberated and non electronic way. The proposed analysis of disguised voice classification provides interesting results in detection by the use of SVM (Support Vector Machine) and in identification by the use of GMM (Gaussian mixture models).

\section{9:40}

5aSCa6. Speaker identification on the SCOTUS corpus. Jiahong Yuan (University of Pennsylvania, 609 Williams Hall, Philadelphia, PA 19104, USA, jiahong@ling.upenn.edu), Mark Liberman (University of Pennsylvania, 609 Williams Hall, Philadelphia, PA 19104, USA, myl@cis .upenn.edu)

This paper reports the results of our experiments on speaker identification in the SCOTUS corpus, which includes oral arguments from the Supreme Court of the United States. Our main findings are as follows: 1) a combination of Gaussian mixture models and monophone HMM models attains near-100\% text-independent identification accuracy on utterances that are longer than one second; (2) the sampling rate of $11025 \mathrm{~Hz}$ achieves the best performance (higher sampling rates are harmful); and a sampling rate as low as $2000 \mathrm{~Hz}$ still achieves more than 90\% accuracy; (3) a distance score based on likelihood numbers was used to measure the variability of phones among speakers; we found that the most variable phone is the phone UH (as in good), and the velar nasal NG is more variable than the other two nasal sounds $\mathrm{M}$ and $\mathrm{N}$; 4.) our models achieved "perfect" forced alignment on very long speech segments (one hour). These findings and their significance are discussed.

\section{0:00}

5aSCa7. Dependency of recognition rate on number of words for text-independent speaker recognition using vector quantization. Hidenori Shimizu (Div. of Electronic Eng. and Computer Sci., Kanazawa Univ., Kakuma-machi, 920-1192 Kanazawa-shi, Japan, shimizu@oak .ec.t.kanazawa-u.ac.jp), Tetsuo Funada (Div. of Electronic Eng. and Computer Sci., Kanazawa Univ., Kakuma-machi, 920-1192 Kanazawa-shi, Japan, funada@t.kanazawa-u.ac.jp)

In this research, we discuss speaker recognition using the Kohonen feature map. The map is constructed for each speaker, and it is trained by using log-power and fourteenth-order mel-frequency cepstral coefficients (MFCC) and their temporal difference. The quantization distortion is computed between the input speech and a specific vector on the feature map of each speaker. We conduct speaker recognition experiment based on VQ distortion. Utterances of prefectural name in Japan are used as speech data. We examine particularly the dependency of recognition rate on number of words used for recognition. According to our experiments of speaker identification, this system correctly recognizes $98.9 \%$ by using a single word for 40 male speakers, while it attains $100 \%$ by using more than three words. Moreover, we confirmed superiority of using VQ over HMM under the same experimental conditions. 


\title{
Session 5aSCb
}

\section{Speech Communication: Cross-Language Speech Perception and Production}

\author{
Yue Wang, Cochair \\ Simon Fraser Univ., RCB 9224, 8888 Univ. Dr., Burnaby, BC V5A 1S6, Canada \\ Dawn Behne, Cochair \\ Norwegian University of Science and Technology, Psychology Dept, Trondheim, NO 7491, Norway
}

\section{Invited Papers}

\section{8:40}

5aSCb1. Sound structure and function of English as a global language. Ann Bradlow (Northwestern University, Department of Linguistics, 2016 Sheridan Road, Evanston, IL 60208, USA, abradlow@ northwestern.edu)

Many English conversations across the globe today involve non-native speakers. To understand the sound structure of English in a global context, we asked native and non-native listeners to rate the similarity of various languages $(n=17)$ to English. In general, the native and non-native listeners' distance-from-English ratings were positively correlated; but, the non-natives tended to rank languages as more distant from English than the natives. Native and non-native listeners then rated the accents of native and non-native speakers. Again, the native and non-native listener ratings were positively correlated, but the non-native listeners generally rated both native and non-native speakers as more foreign-accented than did the native listeners. Together, these data suggest that both native and non-native English generally sound less "marked" to native listeners than to non-native listeners. As a means of understanding how English functions as a global language, we examined communicative efficiency in task-oriented dialogues between various combinations of natives and non-natives. All pairs successfully completed the task; yet, when one or more non-native talkers were involved, the task took about twice as long to complete as when both were native talkers. Together, these studies help characterize the structure and function of English as a twenty-first century lingua franca.

\section{9:00}

5aSCb2. Maintainance vs. "loss" of the perceptual bias favoring natural reference vowels. Ocke-Schwen Bohn (Aarhus University, English Department, J.-C.-Skous Vej 5, DK-8000 Århus C, Denmark, engosb@hum.au.dk), Linda Polka (McGill University, School of Communication Sciences and Disorders, Beatty Hall, 1266 Pine Avenue West, Montreal, QC H3G 1A8, Canada, linda.polka @ mcgill.ca)

Previous infant vowel discrimination studies (Polka \& Bohn, 2003) have shown that babies are perceptually biased to favor vowels with more extreme articulations such that a change from a more peripheral vowel ("natural reference vowel" - NRV) to a vowel located more centrally in the vowel space is less discriminable than a change presented in the reverse direction. Recent studies of adult vowel perception suggest that the universal bias favoring NRVs is maintained in the absence of specific language experience and but "lost" if native or non-native language experience causes a reset of the perceptual default favoring NRVs. This presentation reports on vowel discrimination experiments which examined whether absence or presence of specific vowel experience is related to maintenance or loss of this perceptual bias in Danish infants (age 6-12 months). For two Danish vowel contrasts, asymmetries were observed for younger infants, but not for infants older than 10 months. For an English vowel contrast that does not exist in Danish, the asymmetry was maintained in infants up to 12 months and in adult Danish listeners. Results confirm the assumption that the perceptual bias favoring NRVs is maintained throughout the lifespan unless specific vowel experience causes on override. [Work supported by the Danish Research Council for the Humanities.]

5aSCb3. Cross-language auditory-visual speech perception development. Denis Burnham (MARCS Auditory Laboratories, University of Western Sydney, Locked Bag 1797, Penrith South, NSW, 1797 Sydney, Australia, d.burnham@uws.edu.au), Kaoru Sekiyama (Kumamoto University, Division of Cognitive Psychology, Faculty of Letters, 861-0000 Kumamoto, Japan, sekiyama@kumamoto-u.ac.jp), Dogu Erdener (MARCS Auditory Laboratories, University of Western Sydney, Locked Bag 1797, Penrith South, NSW, 1797 Sydney, Australia, d.erdener@uws.edu.au)

Speech perception is auditory-visual (AV), but there is more visual information use by English than Japanese language perceivers (Sekiyama \& Tohkura, 1993). This raises two questions: (1) when and (2) why do such differences emerge? In Experiment 1 with English- and Japanese-language children (6, 8, 11 years) and adults, AV speech perception (AVSP) was equivalent in English and Japanese 6-year-olds, significantly increased between 6 and 8 years in English- but not Japanese-language children, and remained stable over all ages for the Japanese. The origin of the Japanese-English adult difference in English language children's dramatic rise in AVSP between 6 and 8 years was investigated in Experiment 2. English-language children ( 5 to 8 years) and adults were tested for AVSP, 
reading, articulation, and language-specific speech perception (focus on native and away from non-native speech sounds, a known correlate of reading, Burnham, 2003). Regressions showed that language specific speech perception reliably predicted AVSP. It appears children use extra information (AVSP) in the face of challenging linguistic hurdles (learning to read), but only when the language is phonetically complex and the phoneme-to-grapheme conversion rules of the script are not transparent (English, but not Japanese).

\section{Contributed Paper}

\section{9:40}

5aSCb4. Dependence of corresponding phonetic categories in native bilingual speakers and in monolingual overhearers of English and French. Carol A. Fowler (Haskins Laboratories, 300 George St, Suite 900, New Haven, CT 06511, USA, carol.fowler@haskins.yale.edu)

Our research addresses two main questions. First, among phonetic categories that correspond, but are not identical, in the two languages of native bilingual speakers, are there cross-language influences on speech production? Specifically, are the voiceless voice-onset times (VOTs) of bilingual speakers of English and French in Montreal longer in their French speech and shorter in their English speech than the VOTs of monolingual speakers of the two languages in Montreal? Our findings suggest a positive answer. Second, does mere overhearing of a language that one does not speak have an impact on production of phonetic categories? To answer this, we examined the voiceless VOTs of monolingual speakers of French and English in Montreal (who hear substantial unaccented English and French, respectively) with those, respectively, of monolingual speakers of French in Paris and of English in Connecticut. Our answer here was no. I discuss the implications of these findings for an understanding of the phonetic knowledge systems of monolingual and natively bilingual speakers.

\section{Invited Paper}

\section{0:00}

5aSCb5. Plurality and plasticity of neural representation for speech sounds. Yang Zhang (University of Minnesota, Dept. of Speech-Language-Hearing Sci. \& Center for Neurobehavioral Development, Minneapolis, MN 55455, USA, zhang470@umn.edu)

High-density event-related potential (ERP) and magnetoencephalography (MEG) data are presented here to illustrate the plurality and plasticity of speech representation in the spatio-temporal dynamics of neural responses. First, there is evidence for both continuous and discrete processing of speech sounds depending on the acoustic parameters and experimental conditions. Speech discrimination and neural sensitivity measures suggest that the perceptual organization of within-category variations is based on both phonetic knowledge and acoustic analysis. Second, attention plays an important role in determining the neural response patterns. Focused attention significantly changes the hemispheric laterality and strength of neural activation for speech processing. Selectively attending to the different aspects (phonetic vs social-indexical information) of the same speech stimuli shows different regional recruitment, hemispheric involvement and temporal characteristics. Third, the nature of language input and experience can significantly affect learning outcome. Enriched exposure alters the neural representations for speech sounds and plasticity of the system. Theoretical and practical implications for language learning and treatment are discussed. 


\title{
Session 5aSCc
}

\section{Speech Communication: General Topics in Speech Communication IV (Poster Session)}

\author{
Linda Polka, Cochair \\ McGill University \\ Christine Mooshammer, Cochair \\ Haskins Lab and MIT Research Lab of Electronics \\ Suzanne Boyce, Cochair \\ Department of Communication Sciences and Disorders, University of Cincinnati
}

\begin{abstract}
All posters will be on display from 10:20 a.m. to 12:00 a.m. To allow contributors an opportunity to see other posters, contributors of odd-numbered papers will be at their posters from 10:20 a.m. to 11:10 a.m. and contributors of even-numbered papers will be at their posters from 11:10 a.m. to 12:00 p.m.
\end{abstract}

\section{Contributed Papers}

5aSCc1. Call early in the evening on a spring day. Maxine Eskenazi (Language Technologies Institute Carnegie Mellon University, 4619 Newell Simon Hall, 5000 Forbes Ave, Pittsburgh, PA 15213, USA, max@cs.cmu.edu), Antoine Raux (Language Technologies Institute Carnegie Mellon University, 4619 Newell Simon Hall, 5000 Forbes Ave, Pittsburgh, PA 15213, USA, antoine@cs.cmu.edu)

The CMU Let's Go Spoken Dialogue System has been used daily for about three years to answer calls to the Pittsburgh Port Authority for bus information in the evening and on weekends. This has resulted in a database of over 50000 spoken dialogues as of January 2008, one of the largest publicly available sets of this type of data. While retraining the system with part of this data, it became apparent that there are times of the day, of the week and of the year when the average number of successful calls is significantly higher. We will present evidence, using these three measures of time (hour, day of week, month of year) and criteria such as signal-to-noise ratio, estimated success rate, number of turns per dialogue, number of nonunderstandings per dialogue, and barge-in rate to detect the regular, predictable appearance of high and low success rates and to suggest methods for palliating this effect in order to increase overall dialogue success rates.

5aSCc2. Duration modeling for English letters embedded in Chinese speech. Wen-Hsing Lai (National Kaohsiung First University of Science and Technology, No. 2, Jhuoyue Rd., Nanzih District, 811 Kaohsiung, Taiwan,1wh@ccms.nkfust.edu.tw)

A review of existing multilingual TTS (text-to-speech) systems shows that the secondary language inserted into the primary language sounds more like isolated individual words in an alien language environment and not congruous with the primary language's prosody. Since the letter-by-letter spelling of English words or acronyms appears in Chinese speech quite often, a duration modeling approach for English letters embedded in Chinese speech is proposed to make the English congruous with the primary language's tempo. It takes several major factors as additive factors and estimates all model parameters by an EM (expectation-maximization) algorithm. Experimental results showed that the standard deviation of the duration from the test set was greatly reduced from 59.82 to $9.37 \mathrm{~ms}$ by the duration modeling while eliminating effects from factors. The root mean squared error between the original and estimated durations was $9.35 \mathrm{~ms}$ for the open tests. Experimental results have confirmed its effectiveness on isolating several main fac- tors that seriously affects the duration. Moreover, the estimated value of the factors agreed well to our prior linguistic knowledge. Besides, the hidden state labels produced by the EM algorithm were linguistically meaningful.

5aSCc3. Modeling perception of breathy voice quality in vowels using data obtained in an adaptive matching task. Rahul Shrivastav (University of Florida, Dauer Hall, P.O. Box 117420, Dept. of Communication Sciences and Disorders, Gainesville, FL 32611, USA, rahul@csd.ufl.edu), Arturo Camacho (University of Florida, Dauer Hall P.O. Box 117420, Dept. of Communication Sciences and Disorders, Gainesville, FL 32611, USA, arturocl@yahoo.com), Sona A. Patel (University of Florida, Dauer Hall, P.O. Box 117420, Dept. of Communication Sciences and Disorders, Gainesville, FL 32611, USA, sona09@ufl.edu), David A. Eddins (University of Rochester, Department of Otolaryngology, Rochester, NY 14618, USA, David_Eddins@URMC.Rochester.edu)

A computational model of breathy voice quality has been developed based on perceptual judgments of synthetic /a/ vowels [Shrivastav, Camacho, and Patel, JASA 120(5), 3248]. This model is based on the ratio of the loudness of aspiration noise ("noise loudness;" NL) to the loudness of the periodic components of the vowel when masked by the aspiration noise ("partial loudness;" PL) [Shrivastav and Sapienza, JASA, 114(1), 2218-2224 (2005)]. Results showed that the model accounted for a large amount of variance in perceptual ratings of training data $(\mathrm{R}$-square $=0.92)$ but not for testing data $(\mathrm{R}$-square $=0.59)$. It was hypothesized that this reduction was partly related to the use of a magnitude estimation task to obtain perceptual judgments, since magnitude estimates are biased by the range of the quantity measured and are prone to inconsistencies in how subjects assign numbers to items [Poulton, 1989; Guilford, 1954]. To minimize such biases, a matching task may be used to obtain ratio level estimates of breathiness [Patel, Shrivastav and Eddins, JASA, 119(5), 3340 (2006)]. The present study describes a model to predict breathy voice quality derived from perceptual judgments obtained using a matching task.

5aSCc4. The effect of training in noise on foreign language consonant acquisition. M. Luisa Garcia Lecumberri (University of the Basque Country, Paseo de la Universidad 5, Facultad de Filologia, 01006 Vitoria, Spain, garcia.lecumberri@ehu.es), Martin Cooke (Sheffield University, Computer Science Department, Regent Court, 211 Portobello St., S1 4DP Sheffield, UK, m.cooke@dcs.shef.ac.uk) 
Formal exposure to second language sounds normally takes place in clean, laboratory conditions, but at issue is the transfer of such learning to everyday situations. Categories learned in non-natural settings may be fragile, raising the question as to whether learning in noise leads to greater category robustness. The current study compared two groups of Spanish learners of English who were trained in either quiet or noise backgrounds on a 24 consonant discrimination task. Learners' performance was measured in preand post-tests and monitored over the course of nine weeks' training. Both groups showed continual improvement during training with similar overall gains of 6-7 percentage points, suggesting that training in adverse conditions is as effective as in quiet. Tests involving consonant identification in quiet and noise revealed no significant differences in pre-post improvement between the two groups. However, voiceless obstruents benefited more from training in noise while voiced obstruents experienced more improvement when trained in a quiet background regardless of the testing condition (quiet vs noise). The noise-trained disadvantage is consistent with masking of voicing in noise, while the quiet-trained deficit for voiceless obstruents may derive from overgeneralisation during the acquisition of new contrasts.

5aSCc5. Analyze effects of the flow on the vocalic reduction and the coarticulation in sequences $\mathrm{CV}$ of pharyngal Arabic. Leila Falek (USTHB, Faculté d'électronique et d'Informatique, 16111 Algiers, Algeria, lilalcpts@yahoo.fr), Othmane Bouferroum (USTHB, Faculté d'électronique et d'Informatique, 16111 Algiers, Algeria, lilalcpts@yahoo.fr), Amar Djeradi (USTHB, Faculté d'électronique et d'Informatique, 16111 Algiers, Algeria, lilalcpts@yahoo.fr)

The degree of coarticulation and the vocalic reduction (RV) are indices related to a good engine control (Gay, 1978). Fowler (1998) explains why locus equation (LE) is used to characterize, at the same time, the place of articulation and the degree of coarticulation between consonants and vowels: a strong slope $(\mathrm{m}=1)$ indicates a maximum coarticulation between consonants and vowels (i.e., minimal resistance of the coarticulation), while a weak slope $(\mathrm{m}=0)$ indicates absence of coarticulation between consonants and vowels (maximum resistance of the coarticulation). The bond between the degree of coarticulation and the RV can be explained according to the linear relation between F2onset and F2milieu: the modifications of values of F2milieu will affect those of F2onset and consequently those of the slopes of. In this study, the analysis of the vocalic reduction and slopes of the equations of locus, carried out on CV (extracts starting from sentences) in standard Arabic pronounced by speakers having different mother tongues (near to Arabic standard and very far away from standard Arabic), and at speed of variable elocution, revealed a vocalic reduction and a variation of the slope of the locus equation, specific to each speaker, who seems to be related to his mother tongue. El Tamimi (2006) carried out a similar study with normal flow, in dialectical Arabic and in French, with normal flow, an influence of the mother tongue showed on the vocalic reduction and the slope of the equation of locus.

5aSCc6. A comparison between two cases of voicing neutralization: Final devoicing and voicing assimilation in obstruent clusters. Rebeka Campos-Astorkiza (Ohio State University, Dept. Spanish \& Portuguese, 298 Hagerty Hall, 1775 College Rd, Columbus, OH 43210, USA, camposastorkiza.1@osu.edu)

This study compares two processes that result in voicing neutralization, namely final obstruent devoicing and regressive voicing assimilation in obstruent clusters in Lithuanian. The goal is to find out whether both neutralizing environments present similar acoustic patterns. Acoustic data was collected from native speakers of Lithuanian in order to analyze $/ \mathrm{k} /$ and $/ \mathrm{g} /$ in word final position (final devoicing) and in the word medial clusters /gs/, /ks/, / kz/ and /gz/ (voicing assimilation). Three main acoustic cues to obstruent voicing were measured: obstruent closure duration, voicing during closure and duration of preceding vowel. The statistical results show that, although both final devoicing and voicing assimilation result in incomplete voicing neutralization, they use the acoustic cues differently. In the case of voicing assimilation, there are statistically significant differences in closure duration, voicing during closure and preceding vowel duration between the stops in $/ \mathrm{ks}, \mathrm{gz} /$ and their assimilated counterparts in /gs, kz/. As for final devoicing, underlyingly voiceless obstruents and devoiced obstruents show differences in their closure duration and voicing during closure but not in their preceding vowel duration. These results suggest that final devoicing and voicing assimilation have different acoustic realizations in Lithuanian, supporting their analysis as two different processes.

5aSCc7. Effectiveness of prosodic features in the perception of mandarin utterance-final particles. Bin Li (Department of Chinese, Translation \& Linguistics, City University of Hong Kong, 83 Tat Chee Ave. Kowloon Tong, 220 Hong Kong, China, binli2@cityu.edu.hk), Jinping Zhu (TsingHua University, 104 Main Acadamic building, 100084 Beijing, China, yx@abcd.edu.cn)

Utterance-final particles (UFPs) in Mandarin are regarded as discourse markers, whose "elusive" meanings and functions have been investigated in different perspectives such as pragmatics and semantics. Our study focuses on two most frequently used UFPs: NE and A, both of which can occur in identical utterances. The difference lies in that NE implies a shared common ground between the speaker and the hearer, whereas such commonness lacks for A. A preliminary acoustic analysis found that prosodic features of an utterance were more salient when a UFP was not used. The current study aims at the effectiveness of these prosodic features in perceiving these particles. Native speakers of Mandarin are asked to listen to sentences, which may originally contain a UFP that is removed. Their tasks are to decide whether a UFP, and which one, is needed in a given sentence. It is hypothesized that without textual cues, speakers will reply on prosodic features in their judgment; therefore, they will perform better in sentences where UFPs are not used originally and where prosodic cues are more salient.

5aSCc8. The effect of learning on listening to ultra-Fast speech Takuya Nishimoto (Graduate School of Information Science and Technology, The University of Tokyo, 7-3-1 Hongo, Bunkyo-ku, 113-8656 Tokyo, Japan, nishi@hil.t.u-tokyo.ac.jp), Yukika Kariya (Department of Communication, Tokyo Woman's Christian University, 2-6-1 Zenpukuzi, Suginamiku, 167-8585 Tokyo, Japan, g04c043@cis.twcu.ac.jp), Takayuki Watanabe (Department of Communication, Tokyo Woman's Christian University, 2-6-1 Zenpukuzi, Suginami-ku, 167-8585 Tokyo, Japan, nabe@cis .twcu.ac.jp)

We investigated the intelligibility of ultrafast speech which may be used for screen reader for persons with visual disability. The subjects were 35 women who are university students and are not visually/hearing impaired. They were divided into four groups and they listened to 150 words with the speed of approximately $20 \mathrm{morae} / \mathrm{s}$. The vocabulary contained the tasks of high and low familiarity words, and the orders of tasks were different by the groups. Four morae Japanese words from the FW03 database were used as the vocabulary of the recall test. As a result, significant learning effect was observed in cases where the subject listened the high familiarity words in succession. This indicates that the learning effect to the ultrafast speech is promoted when the mental lexical access is easy. We also investigated the mental workloads of the listening task using the NASA-TLX method. As the results, significantly high workload scores were observed at the listening of low familiarity words. The results also suggested that the mental workload decreases when the subject was aware that the mental lexical access was difficult.

5aSCc9. Speech segmentation in monolingual and bilingual infant learners of Canadian English and Canadian French. Linda Polka (McGill University, School of Communication Sciences and Disorders, Beatty Hall, 1266 Pine Avenue West, Montreal, QC H3G 1A8, Canada, linda.polka@mcgill.ca), Jennifer Proulx (McGill University, School of Communication Sciences and Disorders, Beatty Hall, 1266 Pine Avenue West, Montreal, QC H3G 1A8, Canada, Jennifer.proulx@mail.mcgill .ca), Megha Sundara (UCLA Department of Linguistics, 3125 Campbell Hall, Los Angeles, CA 90095-1543, USA, megha.sundara@humnet.ucla .edu)

Speech segmentation skills emerging in infancy are influenced by the infant's native language. English infants favor a stress-based strategy (Jusczyk et al., 1999) whereas French infants favor a syllable-based strategy (Nazzi et al., 2006). Cross-linguistic findings from our lab, based on between group comparisons, show that monolingual 8-month-olds learning either Canadian English or Canadian French segment bisyllabic words in their native lan- 
guage but not in a rhythmically-different non-native language. Canadian French infants fail to segment Canadian English; Canadian English infants fail to segment Canadian French. Bilingual infants (exposed to both languages) were tested in each language on separate days. Although they appear to segment in both languages, attrition has high in the second test session. To assess cross-language segmentation more directly we tested monolingual and bilingual 8-month-olds using a task designed to assess segmentation in both languages in the same infant within a single test session. Findings confirm that monolingual 8-month-olds fail to segment bisyllabic words in a rhythmically-different non-native language. Preliminary data show that bilingual 8-month-olds segment only in the language that is favored in their language input. Thus, in early stages of speech processing all infants appear to develop speech segmentation strategies that are optimal for one language.

5aSCc10. Clear speech intelligibility and accentedness ratings for native and non-native talkers and listeners. Rajka Smiljanic (Northwestern University, Department of Linguistics, 2016 Sheridan Road, Evanston, IL 60208, USA, rajka@northwestern.edu), Ann Bradlow (Northwestern University, Department of Linguistics, 2016 Sheridan Road, Evanston, IL 60208, USA, abradlow@northwestern.edu)

This study investigated how native language background (L1) interacts with speaking style in determining levels of speech intelligibility. In four experiments, we explored whether native and non-native hyper-articulation clear speech strategies provide similar intelligibility benefits for native and high proficiency non-native listeners. The sentence-in-noise perception results revealed that native speech was preferred over non-native speech by both listener groups even when non-native talkers and listeners shared the same L1. Clear speech was shown to be beneficial for both the native and fluent non-native listeners. However, non-native clear speech enhanced intelligibility less than native clear speech, supporting the hypothesis that clear speech production strategies involve enhancement of language-specific phonological contrasts. In order to assess the relationship between objective intelligibility measures and subjective accentedness ratings, we obtained accentedness ratings of native and non-native conversational and clear speech by native and non-native listeners. The results showed that objective intelligibility and subjective accentedness were independent. Overall, these results provide strong evidence that clear speech involves language-specific modifications. Nevertheless, native and high proficiency non-native clear speech modifications are generally helpful for both native and high proficiency non-native listeners even when the objective intelligibility and subjective accentedness levels diverged for various listener and talker groups.

5aSCc11. Vietnamese monophthong vowel production by native speakers and American adult learners. Matthew Winn (University of Maryland College Park, Department of Hearing \& Speech Sciences, 0100 Lefrak Hall, College Park, MD 20742, USA, mwinn@hesp.umd.edu), Allison Blodgett (University of Maryland College Park, Center for Advanced Study of Language, 7005 52nd Ave, College Park, MD 20742, USA, ablodgett@casl.umd.edu), Jessica Bauman (University of Maryland College Park, Center for Advanced Study of Language, 7005 52nd Ave, College Park, MD 20742, USA, jbauman@hesp.umd.edu), Anita Bowles (University of Maryland College Park, Center for Advanced Study of Language, 7005 52nd Ave, College Park, MD 20742, USA, abowles@casl.umd.edu), Lykara Charters (University of Maryland College Park, Center for Advanced Study of Language, 7005 52nd Ave, College Park, MD 20742, USA, lykarac@mac.com), Anton Rytting (University of Maryland College Park, Center for Advanced Study of Language, 7005 52nd Ave, College Park, MD 20742, USA, crytting@ casl.umd.edu), Jessica Shamoo (University of Maryland College Park, Center for Advanced Study of Language, 7005 52nd Ave, College Park, MD 20742, USA, jshamoo@casl.umd.edu)

This study provides new native speaker data regarding the vowel space and duration contrasts of Vietnamese monophthongs. These data add to existing empirical accounts and raise questions about descriptions aimed at adult language learners. In addition, specific acoustic measures target difficulties that English-speaking adults encounter when producing Vietnamese vowels. These measures include the rounding distinction for back vowels, height distinction for central vowels, and duration distinction between short and long vowels. Results show that adult learners exhibit greater variability and overall less accurate production than native speakers for the high-back unrounded vowel u', with slightly better performance for the mid-back unrounded vowel o', perhaps because it is similar to English / $\Lambda$ /. Additionally, these learners demonstrate varying levels of success with regard to the production of central vowel height but generally fail to distinguish the two vowel duration categories exhibited by native speakers.

5aSCc12. The role of segmental and intonational cues in dialect discrimination. Chad Vicenik (UCLA Department of Linguistics, 3125 Campbell Hall, Los Angeles, CA 90095-1543, USA, cvicenik@humnet.ucla.edu), Megha Sundara (UCLA Department of Linguistics, 3125 Campbell Hall, Los Angeles, CA 90095-1543, USA, megha .sundara@humnet.ucla.edu)

Research indicates that adult listeners are able to use intonation to discriminate between two languages when one of the languages is familiar (Ramus and Mehler, 1999; Pijper, 1983). In this paper, we test adults to determine whether they use segmental or intonational cues to distinguish their native dialect from a foreign one. In three experiments, American English listeners were asked to categorize American and Australian English sentences when (a) segmental and supra-segmental cues are available, (b) sentences are re-synthesized with flat intonation, leaving only segmental cues, (c) segmental information is stripped away, leaving only intonation. Results will be discussed in the context of infant research demonstrating that five month olds are able to distinguish different dialects of the same language (Nazzi, Jusczyk, and Johnson 2000).

5aSCc13. Effects of syllable structure on reaction times in a delayed naming task. Christine Mooshammer (Haskins Lab and MIT Research Lab of Electronics, 300 George Street Suite 900, New Haven, CT 06511 , USA, tine@haskins.yale.edu), Louis Goldstein (Haskins Laboratories, 300 George St., Suite 900, New Haven, CT 06511, USA, goldstein@haskins.yale.edu), Mark Tiede (Haskins Lab and MIT Research Lab of Electronics, 300 George Street Suite 900, New Haven, CT 06511, USA, tiede@haskins.yale.edu), Hosung Nam (Haskins Laboratories, 300 George St., Suite 900, New Haven, CT 06511, USA, nam@haskins.yale.edu), Man Gao (Haskins Laboratories, 300 George St. Suite 900, New Haven, CT 06511, USA, gao@ haskins.yale.edu)

Syllable complexity has been found to affect the time the speaker needs for planning and initiating utterance production. Shorter latencies for complex onsets $(\mathrm{CCV})$ as compared to simple onsets $(\mathrm{CV})$ have been explained by effects of segment-specific biomechanical constraints at the level of motor execution, and by neighborhood density at the planning level. Within the framework of Articulatory Phonology, shorter planning latencies for CV syllables (compared to VC) have been attributed to quicker stabilization for tighter gestural coupling hypothesized for in-phase coupling of the onset consonant and release with the vowel. We attempted to test both onset complexity (C vs CC) and coda complexity (open vs. closed syllables) within a single experiment, so that we could evaluate the relative magnitudes of these effects and uncover potential interactions. To do so, American English monosyllabic words varying in syllable structure were presented using a delayed naming paradigm. The results replicated both effects, showing additive effects of roughly comparable magnitudes. Whether these effects are due to planning or motor execution will be tested by further simple naming and picture naming experiments.

5aSCc14. Breathy and whispery voicing in White Hmong. Sean Fulop (California State Univ. Fresno, 5245 N. Backer Ave., Linguistics PB92, Fresno, CA 93705, USA, sfulop@csufresno.edu), Chris Golston (California State Univ. Fresno, 5245 N. Backer Ave., Linguistics PB92, Fresno, CA 93705, USA, chrisg@csufresno.edu)

Two phonatory strategies are acknowledged to involve a superposition of periodic vocal cord vibration with excessive airflow generating turbulent noise. Breathy voice has the vocal folds vibrating along their entire length, but incomplete closure allows a constant airflow. Whispery voice has the vocal folds vibrating modally along an anterior segment, while the arytenoids maintain a posterior hole which allows a constant airflow. In linguistic phonetics, these are standardly regarded as variant strategies of a 
single "breathy voice" specification, and have never been noted to coexist in one language in an important way. They do in White Hmong. One of the seven "tones" of White Hmong is breathy. Syllables bearing this tone display breathy voicing (of the first type) on the vowel. White Hmong also has a stop $\left[\mathrm{d}^{\mathrm{h}}\right]$, which sounds like whispery voicing during the release phase. In this study, the breathy tone is shown to have a higher $\mathrm{H} 1$ amplitude relative to $\mathrm{H} 2$ (compared to modal voice), while the whispery voiced stop is even more extreme in this measure. Whispery voiced stops are also shown to display less harmonicity than the breathy tone, which in turn has less harmonicity than modal voicing in a similar syllable.

5aSCc15. Developing a bilingual communication aid for a Japanese ALS patient using voice conversion technique. Akemi Iida (School of Media Science, Tokyo University of Technology, 1404-1, Katakura-cho, Hachiouji, 192-0982 Tokyo, Japan, ake@media.teu.ac.jp), Shimpei Kajima (Dept. of Electrical and Electronics Engineering, Sophia University, 7-1 Kiyoi-cho, Chiyoda-ku, 102-8554 Tokyo, Japan, s.kajima@gmail.com), Keiichi Yasu (Dept. of Electrical and Electronics Engineering, Sophia University, 7-1 Kiyoi-cho, Chiyoda-ku, 102-8554 Tokyo, Japan, k-yasu@sophia.ac.jp), John M. Kominek (Language Technologies Institute, Carnegie Mellon University, 5000 Forbes Ave. West, Pittsburgh, PA 15213, USA, jkominek@cs.cmu.edu), Yasuhiro Aikawa (1-36-2, Shinjuku, Shinjuku-ku, Shinjuku dai-nana-biru 7F, 160-0022 Tokyo, Japan, aikawa@loopedpicture.com), Takayuki Arai (Dept. of Electrical and Electronics Engineering, Sophia University, 7-1 Kiyoi-cho, Chiyoda-ku, 1028554 Tokyo, Japan, arai@sophia.ac.jp)

A bilingual communication aid for a Japanese amyotrophic lateral sclerosis (ALS) patient has been developed. From our previous research, a corpus-based speech synthesis method was ideal for synthesizing speech with voice quality identifiable as the patient's own. However, a recording of a large amount of speech, which is a burden for the patient, is required for such system. In this study, a voice conversion technique was applied so that a smaller amount of recording is needed for synthesis. An English speech synthesis system with the patient's voice was developed using Festival, a corpus-based speech synthesizer with voice conversion technique. Two methods for Japanese speech synthesis were attempted using HTS toolkit. The first used an acoustic model built from all 503 recordings of the patient. The second used an acoustic model built from 503 wavefiles of which voice was converted to the patient's from a native speaker's. The latter method requires fewer recordings of the patient's. The result of the perceptual experiment showed that the voice synthesized with the latter was perceived to have a closer voice quality to the patient's natural speech. Last, GUI on windows was developed for the patient to synthesize speech by typing in the text.

5aSCc16. Individual differences in perception of emotions from nonsense speech. Sona A. Patel (University of Florida, Dauer Hall, P.O. Box 117420, Dept. of Communication Sciences and Disorders, Gainesville, FL 32611, USA, sona09@ufl.edu), Rahul Shrivastav (University of Florida, Dauer Hall, P.O. Box 117420, Dept. of Communication Sciences and Disorders, Gainesville, FL 32611, USA, rahul@csd.ufl.edu)

Identification of the acoustic cues used to perceive emotions in speech is important for a number of applications including rehabilitation, natural speech modeling, and speech synthesis. In a recent experiment, Patel, Shrivastav, Harnsberger, and Shrivastav (2007) found that a fourdimensional solution accounted for $90 \%$ of the variance in similarity judgments for 19 emotional categories in nonsense speech. This solution was determined for averaged judgments across twelve listeners. The present study investigated individual differences in the perception of emotions for speech devoid of semantic information but rich in suprasegmental cues. Six male and six female listeners participated in a same-different discrimination test of a set of nonsense sentences produced in nineteen emotional contexts by two actors. Nonsense sentences were used in order to avoid any biases caused by semantics. The perceptual distance between each stimulus pair was computed in terms of d' values for each listener. These distances were submitted to a multidimensional scaling analysis using the INDSCAL algorithm. The INDSCAL analysis reports the best fitting solution for all listeners as a group, along with the weights assigned to each dimension by individual listeners. The results of this analysis will be presented.

5aSCc17. Phonological representation of negation in Sakizaya. Wen-Chi Shen (Grad. Inst. of Linguistics, National Taiwan Univ., 1, Roosevelt Rd. Sec. 4, 106 Taipei, Taiwan, r94142007@ntu.edu.tw), Wen-Yu Chiang (Graduate Institute of Linguistics, Grad. Inst. of Linguistics, National Taiwan Univ., 1, Roosevelt Rd. Sec. 4, 106 Taipei, Taiwan, countryroad0509@hotmail.com)

This paper aims at investigating phonological representation of negation in Sakizaya, an endangered Formosan language in Taiwan. Defying YaegerDror's "Cognitive prominence principles" (2003), Chiang (2006) examines negators in Saisiyat, a SVO language as English, and finds the sentential subjects are more prominent acoustically rather than negators. Thus, I will adopt Chiang's modal (2006) and analyze phonological representation of six negators in Sakizaya to see where the pitch accent falls. Besides, if falling on the negators, I will see whether it is because of either cognitive prominence properties or its sentence-initial position? Six informants (three male and three female), from 50-74 years old, participated in the experiment, recorded by DAT, analyzed by Praat and digitized at a $22050-\mathrm{Hz}$ sampling rate. Affirmative and negative sentences are asked in Mandarin while the informants are instructed to translate them into Sakizaya. Besides, they are asked to produce iterative -ay sentences since the negator ca'ay is the most frequently used negator with higher prominent syllable -ay. The results show negators are more prominent in Sakizaya compared to sentential subjects. I conclude it is word order that influences the prominence of pitch contour since Saisiyat is an SVO language and Sakizaya a VSO one.

5aSCc18. The effect of vowel duration on formant frequencies - data from Hakka Chinese. Wai-Sum Lee (Phonetics Lab, Dept. of Chinese, Translation and Linguistics, City University of Hong Kong, 83 Tat Chee Avenue, Kowloon Hong Kong, Hong Kong, w.s.lee@cityu.edu.hk), Eric Zee (Phonetics Lab, Dept. of Chinese, Translation and Linguistics, City University of Hong Kong, 83 Tat Chee Avenue, Kowloon Hong Kong, Hong Kong, eric.zee@cityu.edu.hk)

In the target undershoot model, vowel duration is considered as the main determinant of vowel reduction, resulting in undershoot in formant frequencies relative to the "bull's-eye formant pattern" (Lindblom, 1963). The model predicts more schwa-like formant frequencies as vowel duration is shortened. In Hakka Chinese, vowel phonemes /i e a o u/ may be realized as long [i: e: a: o: u:] in the CV: syllables and short [i e a o u] in the CVS syllables ( $\mathrm{S}=\mathrm{a}$ stop consonant). Results of a formant frequency analysis of the long and short vowel sets in Hakka Chinese from ten male and ten female speakers show that (i) in all the short vowels there is a displacement of vowel formant frequencies away from the target frequencies associated with the long vowels; and (ii) relative to the target frequencies, F1F2 do not become more schwa-like for all the short vowels, with $[\mathrm{u}]$ being the only vowel that undergoes centralization in the F1F2 plane and with [i e o a] displaying a significant rise in $\mathrm{F} 1$, [i e] an insignificant decrease in F2, and [o] an insignificant increase in F2. It appears that the Hakka data do not support the target undershoot model.

5aSCc19. An experimental and modeling study of anticipatory coarticulation in VCV sequences. Liang Ma (Labaratoire Parole et Language, 29, Avenue Robert Schuman, 13621 Aix-en-Provence, France, liang.ma@lpl.univ-aix.fr), Pascal Perrier (ICP/GIPSA-lab, INPG, 46 Avenue Félix Viallet, 38031 Grenoble Cedex 01, France, Pascal.Perrier@gipsa-lab.inpg.fr), Jianwu Dang (Japan Advanced Institute of Science and Technology, 1-1 Asahidai, Nomi, 923-1292 Ishikawa, Japan, jdang@jaist.ac.jp)

Anticipatory coarticulation within V1CV2 sequences is studied for EMMA data in French and in Mandarin Chinese. The corpus was designed consistently for both languages. V1 and V2 were /i, a, u/ and C was either $/ \mathrm{t} /$ or $/ \mathrm{k} /$. The influences of $\mathrm{V} 2$ on tongue position of $\mathrm{V} 1$ and of $\mathrm{C}$ were analyzed for three French and two Chinese speakers. For French speakers vowel V2 influences the whole sequence V1CV2, while its influence is strictly limited to the syllable CV2 for Mandarin speakers. These suggested 
that speech planning strategies in French and in Chinese are different and that differences could arise from differences in the status of the syllable in the analyzed languages. A speech production model has been then used for testing quantitatively these conclusions. It involves a forward model and optimization techniques to infer optimal motor commands from the phonemic targets in the perceptual space. Different hypotheses about planning and execution of movement were tested on this model. The comparison of simulations and data supports the hypothesis that the syllable could be a basic unit for planning for Mandarin speakers, while it suggests that larger sequences such as VCV should be considered in the planning of French sequences.

5aSCc20. Psychoacoustic measures of blind audio source separation performance. Mingu Lee (Applied Acoustics Lab., INMC, 132-302, Seoul National University, San 56-1, Sillim-dong, Kwanak-gu, 151-742 Seoul, Republic of Korea, klugh34@acoustics.snu.ac.kr), Inseok Heo (Applied Acoustics Lab., INMC, 132-302, Seoul National University, San 56-1, Sillim-dong, Kwanak-gu, 151-742 Seoul, Republic of Korea, hisherz@acoustics.snu.ac.kr), Nakjin Choi (Applied Acoustics Lab., INMC, 132-302, Seoul National University, San 56-1, Sillim-dong, Kwanak-gu, 151-742 Seoul, Republic of Korea, nakjin@acoustics.snu.ac.kr), Koeng-Mo Sung (Applied Acoustics Lab., INMC, 132-302, Seoul National University, San 56-1, Sillim-dong, Kwanak-gu, 151-742 Seoul, Republic of Korea, kmsung@snu.ac.kr)

In this paper, an improved method for evaluating the performance of blind audio source separation (BASS) is discussed. In previous studies, such as described in E. Vincent et al., IEEE Transactions on Speech and Audio Processing, 2006, several computation methods for measuring quality of BASS algorithms e.g., defined by source-to-distortion ratio (SDR), sourceto-interferences ratio (SIR), sources-to-noise ratio (SNR) and sources-toartifacts ratio (SAR) are introduced. However, those methods do not take human auditory system into consideration. An improved method is developed by applying preprocessing and using weighted-inner product in frequency domain instead of simple inner-product in time domain. The proposed method incorporates well-known psychoacoustic characteristics e.g., masking effect and equal loudness contours. In comparison with the conventional quality measures, the proposed method shows better correlation with the results of carefully designed listening tests.

5aSCc21. The acoustic manifestation of consonant gradation in Northern Sami. Fredrik Karlsson (Umeå University, Department of Language Studies, SE90187 Umeå, Sweden, fredrik.karlsson@ling .umu.se), Kirk P. Sullivan (Umeå University, Department of Language Studies, SE90187 Umeå, Sweden, kirk.sullivan@ling.umu.se), Mikael Svonni (Umeå University, Department of Language Studies, SE90187 Umeå, Sweden, mikael.svonni@samiska.umu.se), Jan Van Doorn (Umeå University, Department of Clinical Sciences, SE90187 Umeå, Sweden, jan.vandoorn@logopedi.umu.se), Ola Wennstedt (Institute for Language and Folklore, Onomastics and Folklore Research in Umeå, Länsmansvägen 5, SE90420 Umeå, Sweden, ola.wennstedt@sofi.se)

Consonant gradation is a feature of Sami and is realised in Northern Sami in changes from nominative singular to genitive plural form, e.g., "heavdni" (spider) and "heavnni-t" (spiders). The data examined in this paper was collected in 2007 and represents the first acoustic analysis of consonant gradation in Northern Sami. The data was collected in Övre Soppero in Northern Sweden using an elicitation technique that was designed to collect phonetically controlled material for analysis. The nouns were elicited in singular or plural form using picture prompts and the participants were asked to give the word in the carrier sentences "Lea okta _ govas" ("There is a ___ in the picture") and "Leat guokte _ govas" ("There are two __ in the picture"). The recordings were labeled in Wavesurfer at a phonetic level and the segments relating to consonant gradation extracted for acoustic analysis. In the paper we present our initial findings.
5aSCc22. The use of fundamental frequency information by cochlear implant patients with residual low-frequency hearing. Stephanie Herf (Arizona State University, Dept. of Speech and Hearing Science, Box 87-0102, Tempe, AZ 85287-0102, USA, spitzer@asu.edu), Julie Liss (Arizona State University, Dept. of Speech and Hearing Science, Box 87-0102, Tempe, AZ 85287-0102, USA, julie.liss@asu.edu), Anthony Spahr (Arizona State University, Lattie F. Coor Hall, Room 3462, Tempe, AZ 85287, USA, Tony.Spahr@Asu.Edu), Michael Dorman (Arizona State University, Dept. of Speech and Hearing Science, Box 87-0102, Tempe, AZ 85287-0102, USA, michael.dorman@asu.edu)

Recent studies have demonstrated significant benefits of complementing the electric hearing provided by a cochlear implant (CI) with residual lowfrequency acoustic hearing. It has been suggested that the benefit of lowfrequency hearing $(<250 \mathrm{~Hz})$ is due to improved representation of fundamental frequency (F0). It was hypothesized that removing F0 information would negatively impact speech perception in bimodal CI patients (EAS) more so when they are using both modes of perception. It was further hypothesized that these patients' more effective use of F0 information would result in higher intelligibility scores and less unpredicted lexical boundary errors (LBEs) in a speech transcription task. Six EAS patients transcribed normal and F0-flattened phrases. Results indicate EAS patients demonstrated a significant benefit from the residual low-frequency acoustic hearing and perform better in this mode. Five of the six EAS patients were able to transcribe the normal phrases with greater accuracy than the flat F0 phrases in both conditions. These patients correctly parsed lexical boundaries and transcribed phonemic targets with greater accuracy in the bimodal condition. These results demonstrate the importance of pitch and low frequency spectral information in the perception of speech.

5aSCc23. Digital acquisition of ultrasound imaging of the tongue. Aude Noiray (Haskins Laboratories, 300 George St., New Haven, CT 06511, USA, aude.noiray@haskins.yale.edu), Khalil Iskarous (Haskins Laboratories, 300 George St., New Haven, CT 06511, USA, iskarous@haskins.yale.edu), D.h. H. Whalen (Haskins Laboratories, 300 George St., New Haven, CT 06511, USA, whalen@haskins.yale.edu)

One of the goals of speech production research is to understand the functioning of the tongue in the production of overlapping lingual segments. This is especially important since all vocalic and most consonantal contrasts involve a tongue gesture. An obstacle to progress in this area has been the difficulty of observing tongue motion in enough spatial and temporal detail to develop theory on its linguistic functioning. Ultrasound has become increasingly popular in tongue imaging, since it allows the researcher to observe most of the tongue dynamically, while being clinically safe. However, a limitation of this technique is the slow $30 \mathrm{~Hz}$ rate of analog acquisition. We have developed a new method for digital acquisition of ultrasound data at over $100 \mathrm{~Hz}$, with simultaneous synchronized acquisition of audio and Optotrak data. Acquisition of the ultrasound digital video data is externally triggered using a novel pulse sequence that is simultaneously captured by the audio and Optotrak acquisition device. We will illustrate the detail of the dynamics of the tongue in an experiment on the coproduction of rhotic and vocalic segments (seven American English subjects), where digital ultrasound was captured at $127 \mathrm{~Hz}$ (Funded by NIH DC-02717).

5aSCc24. Vocal tract normalization in articulatory space using thin-plate spline method. Jianguo Wei (LTCI/CNRS, TSI/ENST, DB407, 37/39, rue Dareau, 75014 Paris, France, wei@enst.fr), Jianwu Dang (Japan Advanced Institute of Science and Technology, 1-1 Asahidai, Nomi, 9231292 Ishikawa, Japan, jdang@jaist.ac.jp)

Inter-subject normalization is a key issue of group analysis of articulatory data to obtain a general description of kinematic properties of human speech production. Multisubject articulatory study however is scarce due to the difficulty of normalization in articulatory domain. In order to reduce intersubject variations among articulatory space, a simple normalization procedure was proposed using a Thin-plate spline method. The purpose of this normalization processing is to reduce the morphological differences of vocal tracts such as shape and size among different subjects. Nonlinear factors of the reduction are considered in this normalization procedure. The electromagnetic Articulographic (EMMA) data were used in our experiments, 
which were obtained from the NTT EMMA database for three subjects data included. A physiological articulatory model has been used to serve as the template. The landmarks were defined consistently in vocal tract space over the template and all subjects. The evaluations showed that the variances over subjects have been reduced $2.1 \mathrm{~mm}$ for consonants and $2.3 \mathrm{~mm}$ for vowels averaged over all tongue pellets.

5aSCc25. A comparison of coarticulation in conversational and clear speech. Jean E. Andruski (Wayne State University, 207 Rackham Bldg, 60 Farnsworth Ave., Department of Communication Sciences and Disorders, Detroit, MI 48202, USA, ag0611@wayne.edu)

Coarticulation may either hinder speech perception by increasing variability and altering the distinctive features of speech sounds, or enhance speech perception by providing additional cues to nearby sounds and spreading these cues out over time. This study examines the clear and conversational speech of 16 English speakers ( 8 females and 8 males) to compare the amount of coarticulation in clear and conversational speech when distinctive features are changed, as opposed to when nondistinctive features are changed. Devoicing of voiced fricatives and voicing of $/ \mathrm{t} /$ are investigated as examples of distinctive feature changes. Vowel nasalization and lip rounding in /s/ and /z/ are examined as examples of nondistinctive feature changes. Percentage of voicing during frication noise is used as a measure of fricative devoicing; voice onset time and percentage of voicing during the closure are used as measures of voicing in $/ \mathrm{t} /$; amplitude of the nasal formant and nasal formant onset time are used as measures of vowel nasalization; and center of gravity of the final $50 \%$ of the frication noise is used as a measure of lip rounding in /s/ and /z/. Results are compared across clear and conversational speech and across speaker gender. Individual speaker differences are also examined.

5aSCc26. The development of measurable speech rhythm during second language acquisition. Francisco Gutiérrez Díez (Universidad de Murcia, Departmento de Filología Inglesa, C/ Santo Cristo 1, 30009 Murcia, Spain, fgdiez@ono.com), Volker Dellwo (UCL, Wolfson House, 4, Stephenson Way, NW1 2HE London, UK, v.dellwo@ucl.ac.uk), Núria Gavaldà (Universitat de Barcelona, Departament de Filologia Anglesa i Alemanya, Granvia de les Corts Catalanes 585, 08007 Barcelona, Spain, nuria.gavalfe@gmail.com), Stuart Rosen (UCL, Wolfson House, 4, Stephenson Way, NW1 2HE London, UK, stuart@phon.ucl.ac.uk)

It has been demonstrated repeatedly that durational characteristics of consonantal (C) and vocalic (V) intervals are robust acoustic correlates of rhythm class (stress-timed, syllable-timed, mora-timed). Here, we investigate how objective rhythm measures develop during the acquisition of a second language. In a longitudinal study, nine native speakers of Spanish were recorded reading a text in English before and after a year of English language training at university level. A control group of nine native English speakers read the same text. Automatic forced alignment of speech segment boundaries using hidden Markov models allowed the calculation of $\mathrm{C}$ and $\mathrm{V}$ interval durations. Standard rhythm metrics (\%V, deltaC, deltaV, PVI) were calculated for all recordings. First results show that durational $\mathrm{C}$ interval characteristics between native English and Spanish English (pre- and posttraining) do not differ. However, V interval characteristics (deltaV, nPVI, $\% \mathrm{~V}$ ) are lowest for English natives, higher for the Spanish post training group and highest for the Spanish pretraining group. The results suggest that (a) deficits of speech rhythm competence in a second language are mostly revealed on a vocalic level and (b) an increase in competence in a second language is reflected well by measurable speech rhythm.

5aSCc27. Transforming modal voice into irregular voice by amplitude scaling of individual glottal cycles. Tamás Bőhm (Dept. of Telecommunications and Media Informatics, Budapest Univ. of Technology and Economics, Magyar Tudósok krt. 2, H-1117 Budapest, Hungary, bohm@tmit.bme.hu), Nicolas Audibert (GIPSA-lab, Speech \& Cognition Dept (ICP), INPG/UJF/Stendhal/CNRS UMR 5216, Université Stendhal, 1180 av. Centrale, 38040 Grenoble Cedex 9, France, Nicolas.Audibert @gipsa-lab.inpg.fr), Stefanie Shattuck-Hufnagel (Massachusetts Institute of Technology, Research Laboratory of Electronics, Speech Communication Group, 77 Massachusetts Ave., Cambridge, MA 02139, USA, stef@speech.mit.edu), Géza Németh (Dept. of Telecommunications and Media Informatics, Budapest Univ of Technology and Economics, Magyar Tudósok krt. 2, H-1117 Budapest, Hungary, nemeth@tmit.bme .hu), Véronique Aubergé (GIPSA-lab, Speech \& Cognition Dept. (ICP), INPG/UJF/Stendhal/CNRS UMR 5216, Université Stendhal, 1180 av. Centrale, 38040 Grenoble Cedex 9, France, Veronique.Auberge@icp.inpg.fr)

Irregular phonation can serve as a cue to segmental contrasts and prosodic structure as well as to the affective state and identity of the speaker. Thus algorithms for transforming between voice qualities, such as regular and irregular phonation, may contribute to building more natural sounding, expressive and personalized speech synthesizers. We describe a semiautomatic transformation method that introduces irregular pitch periods into a modal speech signal by amplitude scaling of the individual cycles. First the periods are separated by windowing, then multiplied by scaling factors, and finally overlapped and added. Thus, amplitude irregularities are introduced via boosting or attenuating selected cycles. The abrupt, substantial changes in cycle lengths that are characteristic of naturally-occurring irregular phonation can be achieved by removing (scaling to zero) one or more consecutive periods. A freely available graphical tool has been developed for copying stylized pulse patterns (glottal pulse spacings and amplitudes) from an irregular recording to a regular one, allowing the scaling factors to be refined and the waveform regenerated interactively. We present the effects of the transformation on harmonic structure, and perceptual test results showing that transformed signals are similar to natural irregular recordings in both roughness and naturalness.

5aSCc28. Speech transcription for Embodied Conversational Agent animation. Leila Zouari (Telecom Paris Tech, 46 rue Barrault, 75013 Paris, France, zouari@enst.fr), Gerard Chollet (Telecom Paris Tech, 46 rue Barrault, 75013 Paris, France, chollet@enst.fr)

This article investigates speech transcription within a framework of Embodied Conversational Agent (ECA) animation by voice. The idea is to detect some pronounced expressions/keywords in order to animate automatically the face and the body of an avatar. Extensibility, speed and precision are the main constraints of this interactive application. So after defining the set of the relevant words (to the application), a fast large vocabulary speech recognition system was developped and the keyword detection was evaluated. In order to fasten the recognition system without decreasing its efficiency, the acoustic models have been shortened by an original process. It consists in decreasing the number of shared central states of context dependant models which are considered stationary. The shared states situated in the border of the models remain inchanged. Then all the models are retrained. The system is evaluated on an hour of the ESTER database (a French broadcast news database). The experiments show that reducing the number central states of triphones is advantageous. Indeed, the length of models is reduced by $20 \%$ with no loss of accuracy.

5aSCc29. A cross-dialect acoustic description of vowels: Peruvian versus European Spanish. Katerina Chladkova (Palacky University Olomouc, Dept. of English and American Studies, Krizkovskeho 10, 77180 Olomouc, Czech Republic, katerina.chladkova@seznam.cz), Paola Escudero (University of Amsterdam, Institute of Phonetic Sciences, Spuistraat 210, 1012 VT Amsterdam, Netherlands, P.R.EscuderoNeyra @uva.nl), Paul Boersma (University of Amsterdam, Institute of Phonetic Sciences, Spuistraat 210, 1012 VT Amsterdam, Netherlands, P.P.G.Boersma @uva.nl)

This paper aims to provide acoustic description and comparison of vowel properties of two major dialects of Spanish: Peruvian and European. The method of data collection and acoustic analyses reported in Escudero et al. (submitted) for Portuguese is replicated in order to allow for future comparisons across languages. Each of the five Spanish monophthongs /i, e, a, $\mathrm{o}, \mathrm{u} / \mathrm{was}$ produced in stressed position in the first syllable of a CVCV (consonant-vowel-consonant-vowel) frame. The CVCV words were read both in isolation and in a carrier sentence. Vowel tokens were examined between voiceless stops /p, t, k/ and fricatives /f, s/, which supplied five 
variable consonantal contexts. We analyzed the vowels' first and second formants (F1 and F2) as well as their fundamental frequency and duration. Unsurprisingly, a main effect of vowel identity and gender on formant values was found. Most importantly, unlike Morrison and Escudero (2007), we found for Spanish an interaction between dialect and vowel identity, implying that the F1 and F2 values of some vowels are significantly different between dialects.

5aSCc30. Contextual influences: Perception of sentences in noise is facilitated similarly in young and older listeners by meaningful semantic context; neural correlates explored via functional magnetic resonance imaging (fMRI). Heather Macdonald (Queen's University, Dept Psychology, 62 Arch Street, Kingston, ON K7L 3N6, Canada, hpmacdonald@gmail.com), Matthew H. Davis (MRC CBU, 15 Chaucer Rd., CB2 7EF Cambridge, UK, matt.davis@mrc-cbu.cam.ac.uk), Kathy Pichora-Fuller (University of Toronto Mississauga, 3359 Mississauga rd N., Mississauga, ON L5L 1C6, Canada, k.pichora.fuller@utoronto.ca), Ingrid S. Johnsrude (Queen's University, Dept Psychology, 62 Arch Street, Kingston, ON K7L 3N6, Canada, ingrid.johnsrude@queensu.ca)

Meaningful semantic context has been demonstrated to improve comprehension of spoken sentences by young and old adults, especially in difficult listening conditions. Evidence for this benefit is based largely on data collected using SPIN sentences, highly structured sentences with a predictable or unpredictable final word. We asked young (14 participants, aged 18-25) and older adults (20 participants, aged 60-75) to report entire sentences which were less structured in nature and contained either a meaningful or anomalous global semantic context. Sentences were mixed with signalcorrelated noise, at 9 signal-to-noise ratios ( -6 to $+2 \mathrm{~dB}$ ), and also presented without noise. Comprehension by both groups benefited from meaningful context, without a clear overall difference in the amount of benefit obtained We used fMRI to look at neural activity associated with deriving benefit from meaningful context. Whole-brain EPI data were acquired from young (16 participants, aged 19-26) adults using a sparse imaging design. Wordreport data obtained in the scanner were used as intelligibility measures in data analysis. In addition to activity associated with meaningful context, we observe a large left inferior-frontal region in which BOLD signal correlated more strongly with the intelligibility of anomalous compared to coheren prose, presumably reflecting challenged semantic integration processes.

5aSCc31. Landmark-based analysis of sleep-deprived speech. Suzanne Boyce (Department of Communication Sciences and Disorders, University of Cincinnati, Mail location 0394, Cincinnati, OH 45267, USA boycese@email.uc.edu), Joel Macauslan (S.T.A.R. Corporation, 54 Middlesex Tnpk, Bedford, 01730, USA, joelM@starspeech.com), Ann Bradlow (Northwestern University, Department of Linguistics, 2016 Sheridan Road, Evanston, IL 60208, USA, abradlow@northwestern.edu), Rajka Smiljanic (Northwestern University, Department of Linguistics, 2016 Sheridan Road, Evanston, IL 60208, USA, rajka@ northwestern.edu)

The speech of sleep-deprived persons has been variously described as more "slurred" or "tired-sounding" than speech by the same speakers in a rested condition. Although sleep deprivation is a major focus of research for public safety and health reasons, there has been notably little research on the connection between sleep deprivation and speech. Using an automatic system for detecting "landmarks", i.e., important perceptual and articulatory events in the acoustic signal, we compared sleep-deprived and rested speech recorded during two well-controlled studies of sleep deprivation. Our results show that sleep-deprived speech is significantly different from rested speech for both the number and pattern of landmarks detected. These results are similar in detail to differences previously found between clear and conversational styles of speech. The relevance of these findings for speech as a marker of sleep-deprivation will be discussed, along with potential applications in public safety and health. [Research supported by NIH.]
5aSCc32. Vowel duration as a cue for coda voicing and the perception of second-language vowel quantity. Vaclav Jonas Podlipsky (Dept. of English and American Studies, Palacky Univ., Krizkovskeho 10, 77180 Olomouc, Czech Republic, vaclavjonaspodlipsky@centrum.cz)

When acquiring the phonology of a second language (L2), a learner may have to dissociate a perceptual cue from what it marked in the first language (L1) and attach it to another linguistic entity. This study examined the acquisition of Czech vowel quantity by native speakers of American English In Czech, vowel duration is reserved for cuing short/long vowel (V/V:) contrasts, while in English it is an important cue for coda obstruent voicing. It is not certain whether in Czech, as in many other languages, voiced codas lengthen the preceding vowels; if they do, a vowel should be more likely to be perceived as short before a voiced obstruent and as long before a voiceless one. A perceptual $\mathrm{V} / \mathrm{V}$ : categorization experiment showed that for Czech listeners $(n=54)$ the $V / V$ : boundary came slightly $(3.3 \mathrm{~ms})$ but consistently $(\mathrm{p}<0.001)$ later before a voiced coda. If English learners transfer L1 perceptual strategies to their L2 Czech, the V/V: boundary shift should be even larger. However, this study found no significant effect of coda voicing on the perception of vowel quantity for the non-native listeners. It is concluded that the learners redefined the value of vowel duration as a perceptual cue.

5aSCc33. Talker-specific and accent-general perceptual adaptation to Korean-accented English. Sabrina K. Sidaras (Dept. of Psychology, Emory University, 532 Kilgo Cir, Atlanta, GA 30322, USA, ssidara@emory.edu), Jessica E. Alexander (Dept. of Psychology, Emory University, 532 Kilgo Cir, Atlanta, GA 30322, USA jessica.alexander@emory.edu), Lynne C. Nygaard (Dept. of Psychology, Emory University, 532 Kilgo Cir, Atlanta, GA 30322, USA, lnygaar@emory .edu)

The present study investigated the relative contribution of talker-specific and accent-general learning to the perceptual adaptation of accented speech. Adult native English-speaking listeners were asked to transcribe Koreanaccented English words in a brief, high variability perceptual learning paradigm. During training, listeners transcribed individual words produced by six Korean-accented speakers and were given feedback on their transcription performance. Controls were either trained with native English speakers or were given no training. At test, listeners were presented with Korean-accented speech produced by both familiar and unfamiliar talkers. The results suggest that listeners learned accent-general properties of nonnative speech. Listeners showed better transcription performance for both familiar and unfamiliar accented talkers relative to English and no training controls. However, talker-specific properties such as individual talker familiarity and baseline intelligibility also influenced transcription performance at test. These findings suggest that although listeners are sensitive to multiple sources of variation in speech, they appear to engage in accent-general perceptual adaptation to non-native speech

5aSCc34. Effect of musical experience on Mandarin tone and vowel discrimination and imitation. Terry L. Gottfried (Lawrence University, P. O. Box 599, Dept. of Psychology, Appleton, WI 54912-0599, USA, gottfrit@lawrence.edu), Yangqing Xu (Lawrence University, P. O. Box 599, Dept. of Psychology, Appleton, WI 54912-0599, USA, xuy@1awrence .edu)

Recent studies have demonstrated a relation between musical experience and accuracy in speaking and perceiving unfamiliar speech contrasts. For example, Gottfried [in Bohn and Munro, Eds., John Benjamins, 221-237 (2007)) found that native speakers of American English with musical training perform better than non-musicians when discriminating and imitating the four lexical tones of Mandarin Chinese. In this study, four native speakers of Mandarin (Chengdu region) produced target words in a fixed carrier sentence. Their productions of high unrounded /i/ and high rounded /u/ were significantly affected by the initial consonant, especially /u/ (which is often transcribed as two different vowels in pinyin, $u$ and $\ddot{u}$, according to the initial consonant). Vowels that followed consonants with more anterior articulation (pinyin j, q, x) had lower F1 and higher F2 than vowels that followed more posterior consonants (pinyin $\mathrm{zh}, \mathrm{ch}, \mathrm{sh}$ ). The F0 of the word immediately preceding the target words $(\operatorname{kan} 4)$ was significantly affected by 
the tone of the target word. Current research tests whether musicians and nonmusicians differ in their ability to discriminate and imitate these phonemic contrasts according to native speakers' phonemic categories, which allow contextual variation in acoustic information.

5aSCc35. Expanding the set of acoustic features of the post-vocalic voicing contrast in English. Blake Rodgers (University of Wisconsin, 1168 Van Hise, 1220 Linden Drive, Madison, WI 53706, USA, brodgers@wisc.edu), Thomas Purnell (University of Wisconsin, 1168 Van Hise, 1220 Linden Drive, Madison, WI 53706, USA, tcpurnell@wisc.edu), Joseph Salmons (University of Wisconsin, 1168 Van Hise, 1220 Linden Drive, Madison, WI 53706, USA, jsalmons@wisc.edu)

The literature on post-vocalic voicing contrasts indicates that no single acoustic characteristic or perceptual cue captures the phonological distinction [e.g., Nittrouer 2004]. Previous perceptual studies examined such acoustic characteristics as vowel duration, percent and duration of closure voicing, formant, and F0 transitions. Detailed acoustic examination reveals additional variation. It is hypothesized that these understudied acoustic characteristics also play a role in the family of voicing cues. In the present study, two laryngeal characteristics are measured and modeled: glottalization (here, an abrupt drop in rate of vocal fold vibration by tensed vocal folds) and a sharp amplitude drop (due to abrupt spreading of vocal folds). Examination of American English data reveals that speakers tend to have at least one of these two characteristics in their final voiceless obstruents, but generally not both. Results suggest a process of laryngeal enhancement of final voiceless obstruents in addition to the traditional cues of vowel duration, formant transitions, etc. Implications for the categorization of laryngeal features and trading relations within the family of final voicing cues are discussed.

5aSCc36. Providing simultaneous visual feedback may ameliorate speech disruptions caused by exposure to delayed auditory feedback. Jeffery Jones (Wilfrid Laurier University, 75 University Ave. West, Waterloo, ON N2L 3C5, Canada, jjones@wlu.ca), Danielle Striemer (Wilfrid Laurier University, 75 University Ave. West, Waterloo, ON N2L 3C5, Canada,dstriemer@gmail.com)

Speakers exposed to delayed auditory feedback (DAF) are often dysfluent. We investigated the possibility that providing visual feedback in addition to DAF would reduce speech disruption. Participants repeated sentences while they heard their auditory feedback delayed with and without simultaneous visual feedback. Replicating previous work, DAF led to increased sentence durations and an increased number of speech disruptions. Results showed that visual feedback did not reduce DAF effects on duration, however, a nonsignificant trend was observed that indicated fewer speech disruptions occurred when visual feedback was provided. This trend was significant in speakers who were overall less affected by DAF. These results suggest the possibility that speakers can strategically use alternative sources of feedback.

5aSCc37. Acoustic characteristics of Swedish dorsal fricatives. Ryan Shosted (University of Illinois, 4080 FLB, 707 S Mathews Ave; MC-160, Urbana, IL 61801, USA, rshosted@uiuc.edu)

The identity of ["hooktop heng"], the "voiceless dorso-palatal/velar fricative" or "simultaneous" ["esh"] and [x], is controversial (IPA 2009: 140, 203). The present study attempts to acoustically differentiate [“esh"], [x], and (non-labialized) ["hooktop heng"]. A female speaker of Stockholm Swedish produced VCV nonsense words with balanced V and alternating C. Windowed spectra were obtained and averaged at various locations in the fricatives. ["esh"] can be reliably differentiated from [x] and ["hooktop heng"] based on center of gravity measures in the middle of the fricative. However, center of gravity differences between [x] and ["hooktop heng"] are not revealing, confirming Lindblad's x-ray tracings (1980: 89). The most robust acoustic difference between the two dorsal fricatives is the presence of erratic, high-amplitude disturbances during $[\mathrm{x}]$. These may be attributed to sudden explosions of saliva and ephemeral contact with the uvula, more likely for slightly-retracted $[\mathrm{x}]$ than for ["hooktop heng"]. It seems unlikely that ["hooktop heng"] is produced with a second simultaneous constriction that is of acoustic relevance. International Phonetic Association. 1999. Handbook of the International Phonetic Association. Cambridge: Cam- bridge University Press. Lindblad, P. 1980. Svenskans sje- och tje-ljud i ett Allmänfonetisk perspektiv. Travaux de l'Institut de linguistique de Lund 16. Lund: LiberLäromedel/Gleerup.

5aSCc38. Audiovisual speech perception in children with autism spectrum disorders and typical development. Julia Irwin (Haskins Laboratories, 300 George Street, New Haven, CT 06511, USA, julia.irwin @ haskins.yale.edu)

For typically developing perceivers, visual speech information influences what listeners hear. When the place of articulation of visual and auditory speech tokens are incongruent, perceivers often report hearing a visually influenced response (the "McGurk effect", McGurk and MacDonald, 1976). Children with autism spectrum disorders (ASD) appear to be less susceptible to the McGurk effect than their typically developing peers (e.g., Mongillo, Irwin, Whalen, Klaiman, Carter and Schultz, in press). Given the gaze aversion characteristic of children with ASD, eye-tracking methodology was employed to extend our previous research on the McGurk effect to examine: (1) sensitivity to mismatched auditory and visual speech (McGurk), (2) visual gain in the presence of auditory noise, and (3) detection of auditory and visual asynchrony. Children with ASD and their chronological age-matched and verbal mental age-matched typically developing controls (ranging in age from 7-12 years) were assessed. Implications for the development of audiovisual speech processing in typically developing and children with ASD will be discussed. [Work supported by NIH.]

5aSCc39. Attentional modulation of the perception of illusory vowels and sound onsets: A functional magnetic resonance imaging (fMRI) study. Antje Heinrich (University of Cambridge, Department of Linguistics, Sidgwick Avenue, CB3 9DA Cambridge, UK, ah540@cam.ac.uk), Matthew H. Davis (MRC CBU, 15 Chaucer Rd., CB2 7EF Cambridge, UK, matt.davis@mrc-cbu.cam.ac.uk), Robert P. Carlyon (MRC CBU, 15 Chaucer Rd., CB2 7EF Cambridge, UK, bob.carlyon@mrc-cbu.cam.ac.uk), Ingrid S. Johnsrude (Queen's University, Dept Psychology, 62 Arch Street, Kingston, ON K7L 3N6, Canada, ingrid.johnsrude@queensu.ca)

In a previous study, we exploited the Gestalt principle of closure to create illusory vowel sounds and examined the neural correlates of their perception using fMRI. When two formants of a synthetic vowel are presented in an alternating pattern, filling the gaps in each formant with bursts of noise causes the formants to be heard as continuous and more vowel-like. When this "Illusion" condition was modified by increasing the formant-to-noise ratio (FNR), the formants were heard as interrupted ("Illusion Break" condition) and less vowel-like. BOLD signal in the Middle Temporal Gyrus (MTG) was greater for Illusion than for Illusion-Break stimuli, reflecting the difference in speechlikeness. Primary auditory areas (PAC) exhibited the opposite pattern, probably because Illusion-Break stimuli contain more perceived sound onsets than the Illusion stimuli. In the current study we examine whether the neural activation to illusory vowels and sound onsets is modulated by attention. Participants were scanned while listening to Illusion, Illusion Break, and two types of intact vowels and simultaneously directing their attention either to the vowel stimuli or to auditory or visual distractors. Preliminary analyses suggest that activation to intact and illusory vowels in MTG, and to sound onset in PAC, is modulated by attention.

5aSCc40. Effect of head covers on directivity pattern of human head. Dejan Todoroviæ (Dirigent Acoustics, Takovska 45A, 11000 Beograd, Serby,dgtdejan@yahoo.com)

In this paper are presented the experimental results of changes in directivity patterns of artificial head with three different types of head covers. The mathematical model of human head approximated by radially vibrating spherical cap set in a sphere is discussed. For the purpose of this research, the physical model of a human speaker head is constructed, and the far-field detailed directivity patterns of the model with and without head covers is measured. Hair as porous absorber, cap and straw hat, as sound barriers, are discussed, and their influence on sound wave propagation is considered. Measured detailed directivity pattern changes affected by head covers in far 
field are calculated in step of ten degrees for spherical coordinates (polar angle and azimuth) and presented in form of two- and three-dimensional polar plots.

5aSCc41. English and Polish glides compared. Anna Balas (Adam Mickiewicz University, al. Niepodleglosci 4, 61-874 Poznan, Poland, abalas @ifa.amu.edu.pl)

This paper compares acoustic features of (1) Polish and English glides before vowels, and (2) Polish glides in vowel-plus-glide sequences with English diphthong offglides. Contrary to traditional articulatory descriptions, the results presented, based on acoustic measurements and vector length calculations, do not reveal that Polish glides in vowel-plus-glide sequences are characterized by more extreme formant values than English diphthong offglides. The difference between English diphthongs and Polish vowelplus-glide sequences is attributed to timing properties, which are shown to be relatively easily acquired by Polish learners of English, probably because of a universal tendency for the first element to be longer than the second one. Preliminary results, however, point to acoustic differences between Polish glides before and after vowels, the former being more consonantal than the latter.

5aSCc42. Correlations among three audiovisual (AV) speech tasks. Julia Irwin (Haskins Laboratories, 300 George Street, New Haven, CT 06511, USA, julia.irwin@haskins.yale.edu), Lawrence Brancazio (Southern Connecticut State University and Haskins Laboratories, 300 George Street, New Haven, CT 06511, USA, brancaziol @ southernct.edu)

Visual speech information influences what listeners hear. When the place of articulation of visual and auditory speech tokens are incongruent, perceivers often report hearing a visually influenced response (the "McGurk effect", McGurk and MacDonald, 1976). However, individual differences in this visual influence are poorly understood. Extending work by Grant \& Seitz (1998) and Conrey and Pisoni (2006), we examined correlations between susceptibility to the "McGurk effect" and performance on three related audiovisual tasks. (1) AV speech in noise: we assessed visual gain by comparing word identification in audio-only and AV conditions. (2) AV asynchrony detection: Participants made asynchrony judgments of speech and nonspeech stimuli with asynchronies ranging from $+250 \mathrm{~ms}$ visual to $+250 \mathrm{~ms}$ auditory lead. The speech stimuli were CV syllables and the nonspeech stimuli consisted of lissajous circles paired with sine waves. In one set of nonspeech stimuli, the lissajous was modeled on the lip aperture of the $\mathrm{CV}$ and the sine wave, amplitude and frequency were derived from the CV. For the other set, the lissajous and sine wave were derived from clapping hands. (3) Speechreading: Participants identified isolated words presented visually. Factors associated with a strong McGurk effect will be discussed. [Work supported by NIH.]

5aSCc43. Factors influencing French schwa deletion and duration: A corpus-based study. Audrey Bürki (Laboratoire de Psycholinguistique, Université de Genève, 40, Bvd Pont d'Arve, 1201 Geneva, Switzerland, audrey.buerki@pse.unige.ch), Mirjam Ernestus (Radboud University Nijmegen \& Max Planck Institute for Psycholinguistics, P.O. Box 310, 6500 Nijmegen, Netherlands, Mirjam.Ernestus@mpi.nl), Cécile Fougeron (Laboratoire de Phonétique et Phonologie, UMR 7018, CNRSParis 3/Sorbonne Nouvelle, 19 rue des Bernardins, 75005 Paris, France, cecile.fougeron@univ-paris3.fr), Cédric Gendrot (Laboratoire de Phonétique et Phonologie, UMR 7018, CNRS-Paris 3/Sorbonne Nouvelle, 19 rue des Bernardins, 75005 Paris, France, cedric.gendrot@univ-paris3.fr), Ulrich H. Frauenfelder (Laboratoire de Psycholinguistique, Université de Genève, 40, Bvd Pont d'Arve, 1201 Geneva, Switzerland, ulrich.frauenfelder@pse .unige.ch)

Numerous factors have been advanced to account for the alternance of schwa with zero in French, such as lexical frequency, sonority, syllable structure, phonotactic constraints, etc. ... In the present study we use a recently developed statistical modeling technique, mixed effects models with crossed random effects, to capture the relative contributions of multiple linguistic factors on both, schwa deletion and schwa's physical duration when present. This study is based on the analysis of 4300 occurrences, extracted from the production of more than 300 speakers in a broadcast corpus. The implications of these findings for the debate on the nature of the processes involved in schwa deletion as well as for the nature of lexical representations will be discussed.

5aSCc44. Perceptual clarity of speech modulates activity in left temporal-lobe regions: fMRI correlates of top-down influences. Conor Wild (Queen's University, Centre for Neuroscience Studies, 62 Arch Street Kingston, ON K7L3N6, Canada, 9cw13@queensu.ca), Matthew H Davis (MRC CBU, 15 Chaucer Rd., CB2 7EF Cambridge, UK, matt.davis@mrc-cbu.cam.ac.uk), Alexis G. Hervais-Adelman (Centre for the Neural Basis of Hearing, Department of Physiology, Development and Neuroscience, University of Cambridge, Downing Site, CB23EG Cambridge, UK, alexis.hervais-adelman@mrc-cbu.cam.ac.uk), Ingrid S. Johnsrude (Queen's University, Dept Psychology, 62 Arch Street, Kingston, ON K7L 3N6, Canada, ingrid.johnsrude@queensu.ca)

Behavioral evidence supports the idea that perception is guided by mechanisms that compute an input's most probable interpretation. For example, four-band noise-vocoded speech, which is largely unintelligible to naive listeners, becomes perceptually clear when listeners possess prior knowledge of the signal content - a phenomenon we call "pop-out". Feedback connections within and among auditory cortical regions may allow cognitively "higher" levels of processing to predict and thus "explain" the bottom-up signal. In the present study, we use fMRI to investigate the network underlying pop-out. We use written primes (matching or nonmatching text strings) to manipulate a subject's perception of single noise-vocoded words, thereby creating acoustically matched conditions that elicit the perceptions of intelligible or unintelligible speech. Whole brain fMRI data were gathered from 21 subjects using a sparse-imaging procedure. Preliminary analysis reveals regions of statistically significant signal change along the left superior and middle temporal gyri and superior temporal sulcus for matching trials that induce perceptual popout. Functional connectivity analysis will allow us to investigate coupling among these auditory regions, and how connectivity is modulated depending on the perceptual coherence of a noise-vocoded utterance.

5aSCc45. Some acoustic cues in the detection of the Nepalese aspiration. Rajesh Khatiwada (LPP UMR CNRS- 7018 Université Sorbonne Nouvelle, 19 rue des bernardins, 75005 Paris, France, rajesh .khatiwada@free.fr)

In terms of laryngeal setting, most languages have only two types of stops. In the Nepalese sound inventory there are four types of stops: voiceless unaspirated, voiced unaspirated, voiceless aspirated, and voiced aspirated (or breathy voiced). The main objective of this paper is to present the prominent and consistent acoustic cues that differentiate the aspirated segments from their unaspirated counterparts of the Nepalese coronals - the dental-alveolar (t,th,d,dh), retroflex (T,Th,D,Dh) and affricates (ts,tsh,dz,dzh). VOT (Voice Onset Time) (Lisker and Abramson 1964) is the familiar model used to characterize three of these phonation types - voiced, voiceless, and aspiration stops. Some authors have pointed out that this method is not able to distinguish the plain voiced and voiced aspirated stops. An alternative segmentation model proposed by Mikuteit and Reetz (2007) is adopted here. Beside the analysis of the closure duration of segments, measuring the beginning of the burst or its release, we use other acoustic cues to characterize Nepalese aspiration such as F0 lowering, the structure of the lower harmonics and spectral tilt (Hanson 1997, Stevens 1998). This experimental work is based on the corpus based on four native speakers of Nepali.

5aSCc46. Prosodic realization of focus on verbal phrases in two Chinese dialects. Yiya Chen (LUCL, Phonetics Laboratory, Cleveringaplaats 1, P. O. Box 9515, 2300 RA Leiden, Netherlands, yiya.chen@let .leidenuniv.nl)

A well-established observation in West-Germanic languages is that when a verbal phrase (e.g., likes movies) is uttered as new information (i.e., focused), speakers often accent (via F0 changes) the noun only (e.g., Ladd 1980 and 1996). Listeners, in turn, find that a single accent on noun sounds as appropriate as when both verb and object are accented (e.g., Gussenhoven 
1987, Birch and Clifton 1995, Welby 2003). This study will report results of an experiment which was designed to examine the prosodic realization of focus on verbal phrases in two tonal dialects - Beijing Mandarin and Shanghai Chinese. Both dialects use F0 changes to indicate word meanings, but they differ in tone sandhi patterns. That is, they differ in how words group into phrasal tonal domains and how lexical tones undergo changes within the domain. Two types of focus were elicited: One provides new information to Wh-questions and the other corrections of wrong information in the preceding discourse. The goal of the study is to contribute to the development of a cross-linguistic theory of the prosodic realization of focus. [Work supported by the VENI research grant from the Netherlands Organization for Scientific Research.]

5aSCc47. On nasal onset time (NOT) duration of French nasal vowels in function of the preceding consonant types. Julie Montagu (Laboratoire de Phonétique et Phonologie UMR 7018CNRS/Université Paris 3, 5, rue des Bernardins, 75005 Paris, France, julie _montagu@yahoo.com)

Aerodynamic requirement, a high intra-oral air pressure (Pio), for the production of stop and fricative consonants delays the nasalization (velum opening) of the subsequent nasal vowel. We call this time delay between the onset of the nasal vowel and that of its nasalization as nasal onset time (NOT). The NOT was expected to be longer after voiceless consonants (higher Pio) than after voiced counterparts. The measurement of NOT on simultaneous recordings of speech and nasal signals for 24 Parisian speakers have unexpectedly shown that a greater NOT after voiced stops $(38 \mathrm{~ms}$ in average corresponding to $17 \%$ of total nasal vowel duration) and voiced fricatives $(7 \%)$ than after voiceless ones, respectively, $14.5 \%$ for stops and $4.1 \%$ for fricatives. This order is observed regardless of changes in speech rate: NOT is $11.1 \%$ with the voiced and $9.3 \%$ with the voiceless stops in slow, $17 \%$ and $14.5 \%$ in normal, and $22.4 \%$ and $18.6 \%$ in rapid rate. These results suggest that not only the Pio, but also some other factors are involved in the determination of NOT. We shall discuss possible such factors in perception and in production.

5aSCc48. Sustained and transient brain activations in bilingual control. Yapeng Wang (State Key Laboratory for Cognitive Neuroscience and Learning, Beijing Normal University, 100875 Beijing, China, Institute for Learning and Brain Sciences, University of Washington, Seattle, WA 98115, USA, yapengw@u.washington.edu), Patricia K. Kuhl (University of Washington, Dept. of Speech \& Hearing Sciences, and Institute for Learning \& Brain Sciences, Box 357988, Seattle, WA 98195, USA, pkkuhl@u.washington.edu), He Li (State Key Laboratory for Cognitive Neuroscience and Learning, Beijing Normal University, 100875 Beijing, China, Institute for Learning and Brain Sciences, University of Washington,
Seattle, WA 98115, USA, her2003@163.com),Qi Dong(State Key Laboratory for Cognitive Neuroscience and Learning, Beijing Normal University, 100875 Beijing, China, Institute for Learning and Brain Sciences, University of Washington, Seattle, WA 98115, USA, dongqi@bnu.edu.cn)

Behavioral and neuroimaging evidence shows that bilinguals experience interference and competition during bilingual processing. The neural basis of bilingual language control is not well understood. Using mixed blocked and event-related design, the present study explored the sustained and transient activations during bilingual control. 15 Chinese-English bilingual speakers were scanned when they performed language switching tasks. The results showed that, compared to single language condition, the mixed language condition (sustained control) induced the activation in the bilateral prefrontal (BA6/BA8/BA10), middle frontal (BA45/46) and parietal lobes (BA7/ BA40/BA49); In contrast, relative to the no switch condition, language switching (transient control) activated the left superior, inferior parietal lobe (BA2/ BA40) and middle frontal (BA11/46). These results suggested that sustained and transient language control induced differential lateral activation patterns.

5aSCc49. Automatic acoustic analysis of substitute speech characteristica by speech recognition technique. Maria Schuster (Dpt. Phoniatrics and Pedaudiology, Bohlenplatz 21, 91054 Erlangen, Germany, maria.schuster@uk-erlangen.de), Andreas Maier (Dpt. Phoniatrics and Pedaudiology, Bohlenplatz 21, 91054 Erlangen, Germany, maier@informatik.uni-erlangen.de), Tino Haderlein (Dpt. Phoniatrics and Pedaudiology, Bohlenplatz 21, 91054 Erlangen, Germany, tino.haderlein@informatik.uni-erlangen.de), Ulrich Eysholdt (Dpt. Phoniatrics and Pedaudiology, Bohlenplatz 21, 91054 Erlangen, Germany, ulrich.eysholdt@uk-erlangen.de), Elmar Noeth (Dpt. for Pattern Recognition, Martensstr. 5, 91052 Erlangen, Germany, noeth@informatik.unierlangen.de)

Tracheo-esophageal (TE) substitute speech is often used after total laryngectomy as it resembles normal speech more than other substitute speech methods. However, TE speech still shows restrictions and is perceived as hoarse, rough, with strain, and usually monotone, e.g., due to augmented irregularity of the voice signal. Commonly used diagnostic tools to describe the acoustic properties of TE speech are mostly not applicable. Before, automatic speech recognition has been shown to serve as a appropriate acoustic tool for the quantification of TE speech intelligility. We now applied an automatic speech recognition system with prosody module on speech data of 41 laryngectomees. The prosody module extracted 150 parameters. Factor analysis revealed five principal components. They show high correlation to perceptively described TE speech characteristica. Automatic prosody analysis by speech recognition technique allows to detect and quantify characteristics of highly disordered speech. 


\title{
Session 5aUWa
}

\section{Underwater Acoustics, Signal Processing in Acoustics, and ECUA: Broadband Underwater Communications I}

\author{
Heechun Song, Cochair \\ MPL, Scripps Institution of Oceanography, University of California, San Diego, La Jolla, CA 92093-0238, USA \\ Sergio Jesus, Cochair \\ ISR, Universidade do Algarve, Faro, PT-8005-139, Portugal
}

\section{Invited Papers}

\section{8:00}

5aUWa1. Multiinput multioutput OFDM for shallow-water UWA communications. Yunus Emre (Arizona State University, Dept. of Electrical Engineering, Tempe, AZ 85287-5706, USA, yunus.emre@asu.edu), Vinod Kandasamy (Arizona State University, Dept. of Electrical Engineering, Tempe, AZ 85287-5706, USA, vinod.kandasamy@ asu.edu), Tolga M. Duman (Arizona State University, Dept. of Electrical Engineering, Tempe, AZ 85287-5706, USA, duman@asu.edu), Paul Hursky (HLS Research, Inc., 3366 N. Torrey Pines Ct., Ste. 310, La Jolla, CA 92037, USA, paul.hursky@hlsresearch.com), Subhadeep Roy (Qualcomm Flarion Technologies, Bridgewater, NJ 08807, USA, subhadee@qualcomm.com)

We investigate performance of turbo coded multiple-input multiple-output (MIMO)-OFDM systems with layered space time (LST) architectures for underwater acoustic (UWA) channels by using simulations and results from the AUVfest experiment performed in June 2007. MIMO systems have been shown to be promising in the sense that they increase the reliable transmission rates significantly without consuming additional bandwidth and power. Robustness of OFDM systems with cyclic prefix or zero padding to ISI channels are also well known; so the combination of MIMO techniques and OFDM can be regarded as a promising technology for shallow water UWA communications which is characterized by severe bandwidth limitations and long intersymbol interference (ISI) spans. The paper reviews necessary components of a MIMO-OFDM communication system, including, time and frequency synchronization, channel estimation, and tracking of the varying channel parameters. Modifications necessary to make the system suitable for UWA channels are summarized. Results of the AUVFest 2007 experiment are very promising; for instance, $2 \times 2$ MIMO-OFDM can reach up to 60 Kbps transmission for a bandwidth of $16 \mathrm{KHz}$ with simple receiver structures for a range of $2000 \mathrm{~m}$. In addition to the coherent system, differential and unitary space-time coded MIMO-OFDM scenarios are also considered.

$$
\text { 8:20 }
$$

5aUWa2. High rate acoustic communications based on orthogonal frequency division multiplexing. Milica Stojanovic (Massachusetts Institute of Technology E38-376, 292 Main St., Cambridge, MA 02139, USA, millitsa@mit.edu)

Orthogonal frequency division multiplexing (OFDM) offers simplicity of FFT-based implementation with frequency-domain channel equalization, and has emerged as a standard in many terrestrial systems. Its application to underwater systems, however, is challenged by the motion-induced Doppler distortion, which creates nonuniform frequency offset in a wideband acoustic signal. To counteract this problem, recent research has focused on synchronization methods, demonstrating the feasibility of wideband OFDM in initial sea trials. We consider the design of a complete OFDM receiver based on three key techniques: adaptive carrier tracking, spatial diversity combining, and sparse channel estimation. In particular, we focus on time-domain channel estimation, which provides a natural platform for channel sparsing. Sparsing of the impulse response, or discarding of its insignificant coefficients, is shown to offer performance gains on acoustic channels, since they are often naturally sparse. Decision-directed operation, which is made possible by adaptive carrier tracking, yields a low overhead, and further improves the performance of channel estimation beyond that of nonadaptive pilot-based schemes. The technique proposed is applied to experimental data recorded in a shallow water channel over $1 \mathrm{~km}$. Results show excellent performance of coded OFDM signals, transmitted in the 19-31 kHz band with a varying number of subcarriers.

\section{$8: 40$}

5aUWa3. Experimental assessment of time-reversed OFDM underwater communications. Joao Gomes (ISR - Instituto Superior Tecnico, Av. Rovisco Pais, Torre Norte 7.22, 1049-001 Lisboa, Portugal, jpg@isr.ist.utl.pt), Antonio Silva (ISR, Universidade do Algarve, PT-8005-139 Faro, Portugal, asilva@ualg.pt), Sergio Jesus (ISR, Universidade do Algarve, PT-8005-139 Faro, Portugal, sjesus@ualg.pt)

OFDM communication has recently been demonstrated in underwater channels at rates exceeding 7 kbps. This is a popular modulation in wireless communications due to its flexibility for spectrum management and the simplicity of transmitters/receivers. Despite widespread interest, experimental data on the performance of underwater OFDM are scarce. This work aims to contribute to a better understanding of this technique by examining results from the UAB'07 experiment, conducted in Norway in September 2007. Data were transmitted in a fjord using several formats with bandwidths of 3 and $7.5 \mathrm{kHz}$, and recorded at a range of $800 \mathrm{~m}$ in a 16-hydrophone array. Significant multipath was observed over at least $40 \mathrm{~ms}$, which would call for a rather large OFDM prefix/guard interval and hence drastically reduce the data rate. Passive time reversal is used here as a computationally inexpensive scheme to shorten the channel length 
to less than $10 \mathrm{~ms}$, so that moderate guard intervals can be used with a conventional OFDM receiver. The same preamble/postamble signals used for synchronization and Doppler precompensation are reused as probes for passive time reversal, so the latter entails no loss in efficiency. The performance of time-reversed OFDM is compared with plain QPSK modulation using either time reversal or multichannel equalization at the receiver.

\section{9:00}

5aUWa4. Linear and circular adaptive beamforming arrays for high bandwidth acoustic communications. Jeffrey Neasham (Newcastle University, School of Electrical, Electronic and Computer Engineering, Merz Court, NE1 7RU Newcastle upon Tyne, UK, j.a.neasham@ncl.ac.uk), David Howarth (Tritech International, Morecambe Road, Ulverston, LA12 9BN Cumbria, UK, davidhowarth@tritechulverston.co.uk), Bayan Sharif (Newcastle University, School of Electrical, Electronic and Computer Engineering, Merz Court, NE1 7RU Newcastle upon Tyne, UK, bayan.sharif@ncl.ac.uk), Oliver Hinton (Newcastle University, School of Electrical, Electronic and Computer Engineering, Merz Court, NE1 7RU Newcastle upon Tyne, UK, oliver.hinton@ncl.ac.uk)

This paper will discuss the development of a new generation of high bandwidth (up to $32 \mathrm{kbits} / \mathrm{s}$ ) underwater communication products for ranges up to $4 \mathrm{~km}$. Multipath propagation is the dominant factor in system performance in both shallow water, horizontal channels and deep water, vertical channels. The properties of such channels will be analysed and the design of both linear and circular receiver arrays will be discussed to maximise reliability. We will then describe how these arrays are combined with the latest "software" receiver principles to create a powerful and flexible system, incorporating adaptive signal processing with minimal hardware complexity. Finally the performance of this system will be illustrated with results from experimental trials.

5aUWa5. Channel estimation for underwater acoustic communications: sparse channels, soft input data, and Bayesian techniques. James Preisig (Woods Hole Oceanographic Institution, Bigelow 404, MS\#9, Woods Hole, MA 02543, USA, jpreisig@whoi.edu), Ballard Blair (Woods Hole Oceanographic Institution, Bigelow 404, MS\#9, Woods Hole, MA 02543, USA, bjblair@mit.edu), Weichang Li (Woods Hole Oceanographic Institution, Bigelow 404, MS\#9, Woods Hole, MA 02543, USA, wli @whoi.edu)

The problem of estimating the time-varying impulse response of the communications channel is central to creating reliable and high-rate communications links. Past work has shown that by jointly accounting for channel dynamics and sparse channel characteristics, the accuracy of the channel impulse response estimate is improved with a corresponding improvement in communications systems performance. Following a survey of the basic techniques that have been developed, these basic techniques are extended in two ways. The first allows for a more complete accounting for the channel characteristics via apriori distributions on the probabilities of the complex channel tap values and optimized basis functions for sparse channel structure. The second allows for the use of soft input data in the channel estimation process thus making the techniques feasible for iterative channel estimation and data estimation algorithms. The extensions are developed and their performance compared using data from recent shallow water acoustic communications experiments.

\section{Contributed Paper}

9:40

5aUWa6. Investigation of modal processing for low frequency acoustic communications in shallow water. Andrey Morozov (Woods Hole Oceanographic Institution, Bigelow 404, MS\#9, Woods Hole, MA 02543 , USA, amorozov@whoi.edu), James Preisig (Woods Hole Oceanographic Institution, Bigelow 404, MS\#9, Woods Hole, MA 02543, USA, jpreisig@whoi.edu), Joseph Papp (Woods Hole Oceanographic Institution, Bigelow 404, MS\#9, Woods Hole, MA 02543, USA, jpapp@whoi.edu)

Acoustic receptions at a vertical line array collected during the SW06 experiment were processed to show the feasibility of broadband mode decomposition as a preprocessing method to shorten time-spread and concentrate received signal energy in a small number of independent channels. The vertical array spanned the water column from $12 \mathrm{~m}$ depth to the bottom.
PSK m-sequence modulated signals with different carrier frequencies were transmitted from a distance of $19.2 \mathrm{~km}$. Signals were processed for both the case of ordinary internal waves activity and the case with abnormally strong internal wave solitons. The measured sound velocity and known bottom properties were accounted for. Mode filtering was based on a broadband pseudo-inverse processing of the received VLA signals. The broadband mode filtering decomposed the received signal into a number of independent signals with a reduced time-spread. The constellation of signals from the output of mode filters showed that a simple demodulator can achieve a high quality reception. Even during strong internal waves activity the acoustic energy was concentrated in a small number of the first acoustical modes. The receiver estimated the mode-time intensity distribution and used the strongest modes for demodulation. High quality reception is demonstrated with the data.

10:00-11:00 Posters

Lecture sessions will recess for the presentation of poster papers on various topics on acoustics. See poster sessions for topics and abstracts. 


\section{Contributed Papers}

11:00

5aUWa7. Basin-scale time reversal communications. Heechun Song (MPL, Scripps Institution of Oceanography, University of California, San Diego, La Jolla, CA 92093-0238, USA, hcsong@ucsd.edu), William A. Kuperman (MPL, Scripps Institution of Oceanography, University of California, San Diego, La Jolla, CA 92093-0238, USA, wkuperman@ucsd.edu), William Hodgkiss (MPL, Scripps Institution of Oceanography, University of California, San Diego, La Jolla, CA 92093 0238,USA,wsh@mpl.ucsd.edu)

During November 1994, broadband acoustic signals were transmitted from a $75-\mathrm{Hz}$ source to a 20 -element, $700-\mathrm{m}$ vertical array at approximately $3250 \mathrm{~km}$ range in the eastern North Pacific Ocean as part of the Acoustic Engineering Test (AET) of the Acoustic Thermometry of Ocean Climate (ATOC) program [Worcester et al., J. Acoust. Soc. Am. 105, 3185-3201 (1999)]. The AET tomography signal can be treated as a binary-phase shiftkeying (BPSK) communication signal with an information rate of 37.5 bits/s. With the multipath arrivals spanning 5-8 s, this data represents an extreme case of intersymbol interference (ISI). The AET array data are processed using time reversal combined with frequent channel updates to accommodate channel variations over the 20-min long reception, followed by a single channel decision-feedback equalizer (DFE). The almost error-free performance using all 20 array elements demonstrates the feasibility of time reversal communications. Further, comparable performance of single receive element communications integrating over multiple transmissions indicates that the ocean provided temporal diversity as well as the spatial diversity provided by the array.

\section{1:20}

5aUWa8. Coherent time reversal communications in a shallow multipath environment. Slawomir Jastrzebski (University of Technology and Life Sciences, ul. Kordeckiego 20, 85-225 Bydgoszcz, Poland, sj@utp .edu.pl)

Underwater acoustic channel is one of the less reliable communication channels due to its reverberant properties produced by the surface and the bottom of the sea and Doppler spreading caused by the transmitter and the receiver movements. Strong intersymbol interference (ISI) caused by timevarying multipath environments and relatively fast channel variations are two of the major challenges for practical implementation of coherent underwater acoustic communications. In this paper a phase conjugation is considered as a method for mitigating intersymbol interference in coherent communication and this technique reducing the complexity of underwater receivers. Phase conjugation uses time reversal to remove intersymbol interferences. This method performs the time reversal operation in the computer at the receiver instead of time reversed propagation through the sea. This paper presents numerical simulations results of coherent communications using this technique. Phase conjugation processing in acoustic communications was demonstrated with the transmission of BPSK and QPSK modulation schemes. Different messages were sent simultaneously to different depths at different ranges in $100 \mathrm{~m}$ deep shallow water. Simulation results suggest that the phase conjugation technique may be used as a potential application to undersea communications, especially in an environment with significant multipath.

\section{1:40}

5aUWa9. Very broadband high frequency underwater acoustic communications. Lee Freitag (Woods Hole Oceanographic Inst., 266 Woods Hole Road, MS 18, Woods Hole, MA 02543, USA, lfreitag@whoi.edu), Sandipa Singh (Woods Hole Oceanographic Inst., 266 Woods Hole Road, MS 18, Woods Hole, MA 02543, USA, ssingh@whoi.edu), Keenan Ball (Woods Hole Oceanographic Inst., 266 Woods Hole Road, MS 18, Woods Hole, MA 02543, USA, kball@whoi.edu)

Many new applications for underwater observatories and sensor systems do not require long ranges, but do require very power-efficient, highthroughput communications. Recent work in phase-coherent underwater acoustic communications has shown that signal bandwidths of $10-40 \mathrm{khz}$ at carrier frequencies from 80 to $120 \mathrm{khz}$ may be used to achieve throughputs of up to $80 \mathrm{kbps}$ at ranges of $100-500 \mathrm{~m}$ in shallow water. The work has included channel impulse response measurements over time and with respect to range, and application of the adaptive decision feedback equalizer using signals at multiple bandwidths. The broadband, high-frequency propagation environment is characterized, and its impact on the reliability of very wide band signals (relative to the signal carrier) is presented.

\section{2:00}

5aUWa10. Experiments of wideband color image transmission Hiroshi Ochi (JAMSTEC, 2-15, Natsushima-cho, 237-0061 Yokosuka, Japan, ochi@jamstec.go.jp), Yoshitaka Watanabe (JAMSTEC, 2-15, Natsushima-cho, 237-0061 Yokosuka, Japan, yoshitakaw@jamstec .go.jp), Takuya Shimura (JAMSTEC, 2-15, Natsushima-cho, 237-0061 Yokosuka, Japan, shimurat@jamstec.go.jp), Takehito Hattori (Nippon Marine Enterprises, Ltd., 14-1, Ogawa-cho, 238-0004 Yokosuka, Japan, hattorit @nme.co.jp)

In JAMSTEC, we have been studying about a high-speed underwater acoustic communication for color image transmission from underwater vehicle of deep water. The objective of our research is to transmit a JPEG compressed color image within once a second. Experiments were carried out at the depth of $1000 \mathrm{~m}$ area. The distance of transmission was $250-600 \mathrm{~m}$. Four omni-directional hydrophones were used for receiving. QPSK and 8PSK were used as its modulation method. The four-channel decision feedback equalizer with phase compensator was applied for the demodulation algorithm. Because of this experiment was carried out at deep sea environment, the communication channel characteristics was relatively simple. In these experiment, when the SNR was higher than $12 \mathrm{~dB}$, error free communication was carried out in case of QPSK. And also when the SNR was higher than $20 \mathrm{~dB}$, error free communication was carried out in case of 8PSK

\section{2:20}

5aUWa11. Robust time-division channel-access approach for an ad hoc underwater network. Kae Yeet Foo (University of Birmingham, Department of Electronic, Electrical and Computer Engineering, Edgbaston, B15 2TT Birmingham, UK, kyf740@bham.ac.uk), Philip Atkins (University of Birmingham, Department of Electronic, Electrical and Computer Engineering, Edgbaston, B15 2TT Birmingham, UK, p.r.atkins@bham.ac.uk), Steve A. Pointer (Qinetiq, Winfrith Technology Centre, DT2 8XJ Dorchester, UK, sapointer@qinetiq.com), Carl P. Tiltman (Dstl, Winfrith Technology Centre, DT2 8WX Dorchester, UK, cptiltman@dstl.gov.uk)

An ad hoc underwater network is formed by a cluster of stationary nodes that can act as a source, destination, or a relay, in which data packets usually travel across multiple hops. Using a time-division scheme where the propagation delay between the nodes is used as a packet queuing buffer optimizes throughput. This requires accurate knowledge of the relative ranges of all the nodes, a high level of accuracy in time-synchronization, and restricts changes in the position of the nodes especially in the absence of a centra server or master node within the network. A method that offers greater robustness is by using a time-slotted approach, where each node is offered a time-slot sufficient for one transmission to reach its maximum effective range. A passive acknowledgment scheme is implemented where a node listens for acknowledgment when the transmitted packet is being relayed. Nodes monitor other time-slots for the opportunity to contend for idle slots in order to enhance throughput. Results obtained from simulations demonstrate that this method is robust in supporting changes in the relative distances between the nodes, and can typically operate with a time synchronization error of up to $1 \mathrm{~s}$. 


\title{
Session 5aUWb
}

\section{Underwater Acoustics and ECUA: Environmental Impact on Propagation}

\author{
Bashar Mohammad, Cochair \\ Heriot Watt University, School of Engineering and Physical Sciences, Riccarton, Edinburgh, EH14 4AS, UK
}

\author{
Jinshan $\mathrm{Xu}$, Cochair \\ MIT Mechanical Engieerning Department, 77 Massachusett Ave., Cambridge, MA 02139, USA
}

\section{Contributed Papers}

8:00

5aUWb1. Spatial and temporal variations in acoustic propagation in Dabob Bay during PLUSNet'07 Exercise. Jinshan Xu (MIT Mechanical Engieerning Department, 77 Massachusett Ave., Cambridge, MA 02139, USA, jinshan@mit.edu), Pierre Lermusiaux (MIT Mechanical Engieerning Department, 77 Massachusett Ave., Cambridge, MA 02139, USA, pierrel@MIT.EDU), Patrick Haley (MIT Mechanical Engieerning Department, 77 Massachusett Ave., Cambridge, MA 02139, USA, phaley@MIT.EDU), Wayne Leslie (MIT Mechanical Engieerning Department, 77 Massachusett Ave., Cambridge, MA 02139, USA, wgleslie@MIT.EDU), Oleg Logutov (MIT Mechanical Engieerning Department, 77 Massachusett Ave., Cambridge, MA 02139, USA, logutov @ mit.edu)

We present spatial and temporal variability of the acoustic field in Dabob Bay during the PLUSNet'07 (Persistent Littoral Undersea Surveillance Network) Exercise. The study uses a 4D (3D in space plus 1D in time) dataassimilative numerical ocean model to provide inputs to an acoustic propagation model. The Havard-Ocean-Prediction-System with in-situ CTD measurement assimilation provided output forecasts with a 300-m and 1-to-5-m resolution in the horizontal and vertical direction, for a 3-h interval within a 15-day period. This environmental data as the input to acoustic modeling allowed prediction and study of the (semi-)diurnal temporal variations of the acoustic field, as well as the varying spatial structures of the field. Using the one-way coupled-normal-mode code, along- and across-sections in the Dabob Bay acoustic field structures at 100, 400, and $900 \mathrm{~Hz}$ were forecasts and studied for various source depths. Interesting propagation effects such as the acoustic transmission-loss fluctuations with respect to the source depth and frequency as a result of the regional ocean variability, wind forcing, and tidal effects are discussed. The novelty of this work lies in the accuracy of the acoustic transmission-loss prediction in the littoral region by physically coupling the real-time ocean prediction system to acoustic modeling. This work also offers a potential extension to a $4 \mathrm{D}$ acoustic modeling.

\section{8:20}

5aUWb2. On shallow water ocean acoustic tomography system based on parametric arrays physical properties evaluation. Andrew Semenov (Acad. N.N. Andreev's Acoustics Institute, Russ. Acad. of Sci., 4 Shvernik Street, 117036 Moscow, Russian Federation, asemen@akin.ru)

Unsophisticated acoustic tomography system properties using envelope of radiated acoustic signal for ocean long-range inhomogeneities control, presumably, in most unfavorable, in the author view, shallow water regions, are evaluated. Unique solution to be used there is a narrow directional parametric radiation array. When pump wave signal decay range to array Frauengopher zone dimension ratio exceeds pump wave frequency to radiated signal frequency ratio, parametric radiation array operation model turns to "horn" model instead of "traveling wave" model conventionally used for consumed power prediction. For utmost distance achievement both ends of tomography system should be designed as narrow directional parametric arrays working on optimal frequency basic propagation (first) mode. For instance, signal optimum frequency $40 \mathrm{~Hz}$. is shown to be necessary for model inhomogeneity (100 m long cylinder) control on 500-km radiation array dis- tance in water layer of depth $200 \mathrm{~m}$ with unfavorable attenuation properties. Major lobe solid angle should be chosen not wider than 0,03 radians. Array frequency ratio is advised to be of an order of 10, while array length - 100 half wavelength for pump sound field frequency. Then estimate of power consumption looks like 180-200 kWt of pump signal acoustic power.

\section{8:40}

5aUWb3. The analysis of underwater environmental factors in relation to the harbor protection in Tzoin-Kaohsiung areas. Jin-Yuan Liu (Department of Marine Environmental Informatics/National Taiwan Ocean University, 2 Pei-Ning Road, 202 Keelung, Taiwan, jimliu@faculty.nsysu.edu.tw), Chen-Fen Huang (Department of Marine Environmental Informatics/National Taiwan Ocean University, 2 Pei-Ning Road, 202 Keelung, Taiwan, chenfen@mail.ntou.edu.tw)

Harbor is an important shipping traffic area. The intrusion or threatening by outsiders in the harbor area will certainly cause great panic of the society, and therefore, the protection of harbor and its nearby water is utterly important. The Tzoin harbor is primarily serviced for navy, and is under planning for further expansion. Therefore, a complete control of the TzoinKaohsiung marine environment, both geographical and acoustical, is necessary for military applications. The purpose of this study is to conduct a complete survey of the environment in the designated area, covering the first seaway of the Kaohsiung harbor and off coast water of the Si-Tze bay area. The survey of the marine environment includes seafloor topography, morphology, subbottom profile, waves, currents, and wind fields. In acoustic aspect, the survey emphasizes the harbor ambient noise measurement. The data obtained shall be thoroughly examined and analyzed. The results provide a foundation for future underwater experimental studies, including target localization, geoacoustic inversion, and active sonar reverberation.

\section{9:00}

5aUWb4. The effects of a shallow-water acoustic channel on Right whale vocalisations. Bashar Mohammad (Heriot Watt University, School of Engineering and Physical Sciences, Riccarton, EH14 4AS Edinburgh, UK, mb41@hw.ac.uk), Ron McHugh (Heriot Watt University, School of Engineering and Physical Sciences, Riccarton, EH14 4AS Edinburgh, UK, r.mchugh@hw.ac.uk), Judith Bell (Heriot Watt University, School of Engineering and Physical Sciences, Riccarton, EH14 4AS Edinburgh, UK, j.bell @hw.ac.uk)

For mitigation and monitoring of Right whales, identifying their presence from their vocalisations is a key research issue. Their vocalisations are characterized as frequency modulated up-sweeps with duration of $\sim 1$ s and a frequency range from $50 \mathrm{~Hz}$ to $200 \mathrm{~Hz}$. Acoustic methods to classify these received calls are assessed by the variation in the received data set. As well as the natural variation in vocalisation within the species, the received acoustic signals are also influenced by the effect of the acoustic channel. The shallow water of Cape Cod Bay is one of the favoured habitats for the Northern Right whale. Such waters act as an acoustic waveguide where multiple reflections off boundaries cause calls to become dispersive in nature. In this paper we discuss the effects of channel environmental parameters such as water depth and sediment type on first the FM deviation and second on 
the time difference of arrival between the first and second modes, which in turn influences acoustic range estimation. Such channel effects were studied using the normal mode acoustic propagation model (PROSIM). An analysis of real acoustic data recorded in Cape Cod Bay (2001 obtained from IFAW) will be also presented in terms of dispersion results.

9:20

5aUWb5. Development of an ocean surveillance system. V. Ananthakrishnan (Department of Electronics, Cochin University of Science \& Technology, Thrikkakara, 682022 Ernakulam, India, ananthanmattathil@gmail.com), C Prabha (Department of Electronics, Cochin University of Science \& Technology, Thrikkakara, 682022 Ernakulam, India, prabhasuma@cusat.ac.in), Supriya M. Hariharan (Department of Electronics, Cochin University of Science \& Technology, Thrikkakara, 682022 Ernakulam, India, supriya@cusat.ac.in), Saseendran Pillai (Department of Electronics, Cochin University of Science \& Technology, Thrikkakara, 682022 Ernakulam, India, prspillai@cusat.ac.in)
Now-a-days, underwater sensor networks are gaining considerable importance, for Ocean Surveillance applications. A minimally configurable three-node sensor network is found to be capable of performing localization as well as tracking of underwater targets. Each node comprises of a surface buoy having processing modules, controller hardware and support electronics for estimating the direction of arrival. The development of the node system comprising of controller and processing modules is presented in this paper. The controller hardware, consisting of a gear assembly, magnetic compass and a precision digital signal controller, helps in sampling the ocean by way of steering the hydrophone arrays and capturing the target emanations, while the processing module performs the computation of the direction of arrivals. The target emissions picked up by the hydrophone arrays are analyzed and processed for computing the direction of signal arrival with reference to the geomagnetic meridian. The direction of maximum signal arrivals computed by the three nodes, lead to the estimation of the target position. Localization of the target is carried out by effectively utilizing the information furnished by Global Positioning System incorporated in the node hardware, which has an efficient power management mechanism, as well

FRIDAY MORNING, 4 JULY 2008

ROOM 342A, 8:00 A.M. TO 12:40 P.M.

\title{
Session 5aUWc
}

\section{Underwater Acoustics and ECUA: High Frequency Scattering I}

\author{
Anthony P. Lyons, Cochair \\ Applied Research Laboratory, The Pennsylvania State University, P.O. Box 30, State College, 16804, USA \\ Philippe Blondel, Cochair \\ University of Bath, Department of Physics, Claverton Down, Bath, BA2 7AY, UK \\ Nicholas Pace, Cochair \\ Department of Physics, University of Bath, Bath BA2 7AY, UK
}

\section{Contributed Paper}

\section{8:00}

5aUWc1. Classification of angular backscattered responses obtained at sea with a forward looking sonar system. Pierre Cervenka (Institut Jean le Rond D'Alembert, UMR 7190, 2 Place de la Gare de Ceinture, 78210 Saint Cyr L'Ecole, France, cvk@ccr.jussieu.fr), Jacques Marchal (Institut Jean le Rond D'Alembert, UMR 7190, 2 Place de la Gare de Ceinture, 78210 Saint Cyr L’Ecole, France, jmarchal@ccr.jussieu.fr)

The prototype of a multibeam front-scan sonar has been developed within the frame of a MAST contract ( $n^{\circ}$ MAS3-CT97-0090 DG12-ESCY, acronym COSMOS). A large amount of data has been collected at sea. With the forward looking geometry of acquisition, the foot-prints of successive pings overlap largely, so that most parts of the surveyed areas are seen under a large range of incidence angles. It gives the capability to derive the local angular response of the bottom, which is indeed an important information for characterizing the nature of the seafloor. This presentation addresses the successive steps that were undergone to classify the backscattered responses: Data are conditioned to perform principal component analyzis; an original clustering identification process is also described; resulting maps are finally presented.

\section{Invited Papers}

5aUWc2. A comparison between modeled and measured high frequency bottom backscattering. Dick G. Simons (Delft University of Technology, P.O. Box Postbus 5048, 2600 GA Delft, Netherlands, d.g.simons@tudelft.nl), M. Snellen (Delft Institute of Earth Observation and Space Systems, Delft University of Technology, Kluyverweg 1, 2629 HS Delft, Netherlands, M.Snellen@tudelft $. n 1)$

Compared to taking many sediment grabs, classification techniques based on acoustic remote sensing allow for significant cost reductions, providing the required information at much denser spatial scale. An attractive approach hereto is to use the multibeamechosounder (MBES) which measures sediment backscatter strengths as a function of angle. By maximizing the match between this backscatter strength and model results, sediment properties can be derived. The backscatter model used here accounts for both surface roughness and volume scattering. MBES measurements $(300 \mathrm{kHz})$ were taken in the Cleaver-Bank area (North Sea), characterized by a wide variety of sediment types for which a large number of grabs is collected. For the majority of the sediment types occurring, well-defined single-sediment-type areas can be discerned. Based on MBES measurements in these areas and corresponding geotechnical 
sediment parameters provided by grab grain size analysis, calibration curves are derived, representing the mismatch between modeled and measured backscatter strengths. These curves are found to be similar for all sediment types considered. By applying these correction curves to the MBES measurements, they show good agreement with the model for all observed sediment types. It is shown that after this correction the model can be used for sediment classification purposes.

\section{8:40}

5aUWc3. Acoustic scattering by deformed elongated objects: bent or rough finite cylinders, bent edges, and other stuff. Timothy K. Stanton (Woods Hole Oceanographic Institution, Applied Ocean Physics \& Engineering Department, 98 Water Street, MS \#11, Woods Hole, MA 02543, USA, tstanton@whoi.edu), Dezhang Chu (NOAA/NMFS/NWFSC/FRAMD, Montlake Blvd., E. Seattle, WA 98112, USA, dchu@whoi.edu), Guy Norton (Naval Research Laboratory, Code 7181, Stennis Space Center, MS 39529, USA, guy.norton@nrlssc.navy.mil)

Predicting acoustic scattering by deformed elongated objects remains a challenge as there are no exact analytical solutions. Numerical solutions are also challenged through various computational issues. In this research, we have developed and applied an approximate analytical approach for predicting scattering by various deformed elongated objects, including bent or rough finite cylinders and deformed edges. The formulation is based on a line integral in which the scattering or diffraction per unit length of the exact solution to an infinitely long, straight undeformed object is integrated along the length of the finite-length deformed object, accounting for variations along the length of phase and certain local properties of the object. Predictions concerning various types of deformed finite cylinders are first discussed (including Stanton, J. Acoust. Soc. Am. 86, 691-705 (1989) and subsequent papers), followed by recent predictions concerning deformed finite edges (Stanton et al., J. Acoust. Soc. Am. 122, 3167-3176 (2007)). These latter predictions using the approximate line integral compare favorably with predictions based on the T-matrix numerical method, which is formally exact. Also discussed are: dependences upon length, orientation, bend, and roughness; comparisons with experimental data concerning machined objects and marine life; and range of validity of the approach.

\section{9:00}

5aUWc4. High frequency scattering from discrete inclusions in marine sediments. Anatoliy N. Ivakin (Applied Physics Laboratory, University of Washington, 1013 NE 40th Street, Seattle, WA 98105, USA, ivakin@apl.washington.edu)

A model of high frequency scattering from sediments with discrete inclusions (such as shells and shell fragments) having an arbitrary size-depth distribution is developed. The model assumes also knowledge of a depth-dependent individual scattering function of inclusions. It is more general than in previous models and includes discrete scatterers located both below and on the water-sediment interface (partially buried). Some simple results are obtained for the case of high enough frequencies using approaches of geometry acoustics. Frequency-angular dependencies of the bottom backscattering strength are calculated for sediments with different size-depth distributions of inclusions. Inputs for the size distribution of inclusions (shell fragments) are obtained from granulometric analysis of coarse fractions of the sediment samples taken at the SAX99/SAX04 site (near Ft Walton Beach, Florida). It is shown that taking into account partially buried shells is important and can significantly enhance estimates of the bottom scattering strength especially at grazing angles below critical (about 30 degrees for sand). [Work supported by ONR, Ocean Acoustics].

\section{Contributed Paper}

\section{9:20}

5aUWc5. A review of the scattering properties of suspended sandy sediments for the application of acoustics to sediment transport studies. Peter D. Thorne (Proudman Oceanographic Laboratory, Joseph Proudman Building, 6, Brownlow Street, L3 5DA Liverpool, UK, pdt@pol.ac.uk), Ramazan Meral (Kahramanmaras Sutcu Imam University, Faculty of Agriculture, Department of Agricultural, Structures and Irrigation, 46060 Kahramanmaras, Turkey,rmeral@ksu.edu.tr)

Multi-frequency acoustics backscattering has been used for over a decade, to quantitatively measure, in the marine environment, near-bed profiles of suspended sediment particle size and concentration. Central to obtaining the sediment parameters from the backscattered signal, is a description of the scattering properties of irregularly shaped particles randomly distributed in space. Formulations are therefore required for both the attenuation and backscattering properties of suspensions of sedimentary particles with size and acoustic frequency. There is no rigorous analytical solution or single formulation for these scattering properties and different researchers have used somewhat different expressions. However, all these expressions are based on a variation of sphere scattering, modified to fit available scattering data. Here we bring together four decades of published data on the acoustic scattering properties of suspensions of sandy sediments. These data are reformulated in terms of the usual acoustic scattering nomenclature, that is the form function and the normalised total scattering cross-section and simple heuristic generic expressions, based on a sphere scattering model, are formulated to describe the sediment scattering properties. The expressions are not limited to sand particles, but should have broad applicability to irregularly shaped particle scattering.

\section{Invited Paper}

5aUWc6. On the angular dependence of sound scattering from natural sand in turbulent suspensions. Alex E. Hay (Dalhousie University, Department of Oceanography, Halifax, NS B3H 4J1, Canada, alex.hay@dal.ca), Stephanie A. Moore (Dalhousie University, Department of Oceanography, Halifax, NS B3H 4J1, Canada, Stephanie.Moore@phys.ocean.dal.ca), Wesley Paul (Dalhousie University, Department of Oceanography, Halifax, NS B3H 4J1, Canada, Wes.Paul@Dal.Ca)

We report on measurements of the variation with scattering angle of the differential scattering cross section of particles embedded in turbulence. The experimental apparatus consists of a turbulent round water jet carrying the particles, and a pair of broadband MHzfrequency transducers operating as transmitter and receiver and arranged in a bistatic geometry centred on the jet axis. The purpose of the jet is to confine the scatterers within a restricted range interval and thereby eliminate the need to correct the signal for attenuation due to scattering along the transmit and receive paths. Measurements were made at scattering angles between 90 and 170 degrees, for 
both natural sand grains and spherical glass beads in narrow size distributions with median diameters ranging from 200 to $500 \mu$ m. Particle size distributions were determined using different methodologies to obtain both volumetric and projected area estimates of equivalent spherical size. The results are compared to the theory for scattering by a solid sphere. Implications regarding the choice of equivalent sphere size for sound scattering by natural sand grains in suspension are discussed.

10:00-11:00 Posters

Lecture sessions will recess for presentation of poster papers on various topics in acoustics. See poster sessions for topics and abstracts.

\section{Contributed Papers}

11:00

5aUWc7. Measurements of the scattering characteristics of sediment suspensions having broad particle size distributions. Benjamin D. Moate (Proudman Oceanographic Laboratory, Joseph Proudman Building, 6, Brownlow Street, L3 5DA Liverpool, UK, bdm@pol.ac.uk), Peter D. Thorne (Proudman Oceanographic Laboratory, Joseph Proudman Building, 6, Brownlow Street, L3 5DA Liverpool, UK, pdt@ pol.ac.uk)

Profiles of suspended sediment concentration and mean size can be measured in underwater environments using Acoustic Backscatter Systems $(\mathrm{ABS})$. Inversion of $\mathrm{ABS}$ measurements into sediment size and concentration requires knowledge of the backscattering form function, $\mathrm{f}$, and the total normalised scattering cross section, $\chi$. Previous studies have measured these parameters for sediments sieved over narrow size ranges only. Narrow size ranges are unrealistic compared to the broad Particle Size Distributions (PSD) that typically occur in nature however, and it is known that $\mathrm{f}$ and $\chi$ are significantly altered by changes from narrow to broad PSDs. Theoretically, these changes can be accounted for in ABS inversions by taking values of $\mathrm{f}$ and $\chi$ obtained from narrow size ranges, and integrating them over the suspended PSD, though this theory has not been validated for real suspended sediments. Here, we compare the results of this integration with measured values of $\mathrm{f}$ and $\chi$ for suspensions of (irregularly shaped) sandy sediments having broad PSDs. The results show that as the standard deviation of the size distribution increases, acoustic scattering is increased in the Rayleigh regime whilst being reduced in the geometric regime. These changes significantly affect acoustic estimates of suspended sediment concentration and size.

\section{$11: 20$}

5aUWc8. Acoustic scattering from double-diffusive microstructure Andone Lavery (Woods Hole Oceanographic Institution, Applied Ocean Physics \& Engineering Department, 98 Water Street, MS \#11, Woods Hole, MA 02543, USA, alavery@whoi.edu), Tetjana Ross (Dalhousie University, Department of Oceanography, 1355 Oxford Street, Halifax, NS B3H 4JI, Canada, tetjana.ross@dal.ca)

Laboratory measurements of high-frequency broadband acoustic backscattering (200-600 kHz) from the diffusive regime of double-diffusive microstructure have been performed. This type of microstructure, which was characterized using direct microstructure and optical shadowgraph techniques, is identified by sharp density and sound speed interfaces separating well-mixed layers. Acoustic backscattering measurements were performed for a range of physical parameters controlling the double-diffusive microstructure. The echoes have been analyzed in both the frequency domain, providing information on the spectral response of the scattering, and in the time domain, using pulse compression techniques. High levels of variability were observed, associated with interface oscillations and turbulent plumes, with many echoes showing significant spectral structure. Acoustic estimates of interface thickness, obtained for the echoes with exactly two peaks in the compressed pulse output, were in good agreement with estimates based on direct microstructure and optical shadowgraph measurements. Predictions based on a one-dimensional weak-scattering model that includes the actual density and sound speed profiles agree reasonably with the measured scattering. A remote-sensing tool for mapping oceanic microstructure, such as high-frequency broadband acoustic scattering, could lead to a better understanding of the extent and evolution of double-diffusive layering, and to the importance of double diffusion to oceanic mixing.

\section{Invited Papers}

\section{1:40}

5aUWc9. Clustered scatterers: the effect on the mean acoustic field. Thomas C. Weber (University of New Hampshire, Ctr. for Coastal and Ocean Mapping, 24 Colovos Road, Durham, NH 03824, USA, weber@ccom.unh.edu)

Clustering, or spatially dependent correlation in the fluctuating number density of discrete scatterers of sound, is commonly found to occur in nature. In the ocean, clustering can occur as a result of scatterer entrainment in fluid flows, as in the case of particles and bubbles, or, in the case of fish, as a result of the behaviour of the scatterer. In either case, the presence of clustering can dramatically alter the effect of the scatterers on acoustic fields from that which would be predicted from the same scatterer population but without clustering. The effect of scatterers on the mean acoustic field is often treated using an effective medium wave number. In this paper, a modification to the effective medium number that accounts for clustering is presented and examined for the case of propagation through bubble clouds. Attenuation predictions over a broad range of frequencies $(10-350 \mathrm{kHz})$ using observations of bubble clustering under oceanic breaking waves will be discussed.

\section{2:00}

5aUWc10. A very shallow water ray propagation model. Lucie Pautet (7A/01 Attemsgasse, 1220 Vienna, Austria, luciepautet@yahoo.com), Alessandra Tesei (NATO Undersea Research Centre, Viale San Bartolomeo 400, 19126 La Spezia, Italy, tesei@nurc.nato.int)

For applications such as minehunting, communications, high frequency time-reversal, there has been a need for reliable and fast propagation models able to correctly treat scattering at the rough waveguide boundaries. Data and scattering models have shown that as signal frequency or surface roughness increases, the scattering pattern from the ocean bottom and ocean surface evolves from specular to quasi omni-directional. Ray propagation models are fast but, a priori, they would treat surface bounces as perfectly specular which would not account for the angular spread observed on multipath structures. The idea followed by the authors over the past few years has 
been to introduce in the Bellhop ray tracing model a reflection kernel including deterministic properties of the sea surface and bottom. Simulations using this kernel display a significant effect of the multipath structure similar to the angular spread observed in in-situ data. Simulation results are compared with a recent experimental study at 10-20 KHz (BARRIER'04 at NURC) on the influence of wind strength on surface bounce spreading and subsequent TR-focus spread.

12:20

5aUWc11. Broadband acoustic scattering from nonlinear internal solitary waves. Andone Lavery (Woods Hole Oceanographic Institution, Applied Ocean Physics \& Engineering Department, 98 Water Street, MS \#11, Woods Hole, MA 02543, USA, alavery@ whoi .edu)

High-frequency broadband (150-600 kHz) acoustic scattering techniques have been used to obtain high-resolution images of nonlinear internal solitary waves (ISWs) propagating shoreward over the New Jersey continental shelf. In contrast to traditional singlefrequency measurements, broadband acoustic scattering allows the frequency spectrum of the scattering to be determined, which, in combination with scattering models and direct ground-truthing measurements, allows regions in which the scattering is dominated by biology versus microstructure to be distinguished. Multiple ISWs were tracked and imaged acoustically at different stages of their generation, propagation, and dissipation. Simultaneous microstructure and zooplankton net sampling measurements were performed. Multiple scattering layers and distinct scattering patches were often associated to the ISWs, often exhibiting distinct frequency responses. Clear day/night differences in the scattering from the ISWs were observed due to the daily vertical migration of zooplankton. Though high turbulence levels are associated with ISWs, the strongest scattering returns were not always associated to the highest turbulence levels. Instead, the strongest scattering, at some frequencies, was sometimes associated to large temperature gradients. In regions in which the scattering was dominated by either microstructure or zooplankton, acoustic inversions have been performed for the dissipation rate of turbulent kinetic energy and zooplankton size and abundance.

FRIDAY MORNING, 4 JULY 2008

ROOM 342B, 8:00 TO 11:40 A.M.

\title{
Session 5aUWd
}

\section{Underwater Acoustics and ECUA: Low-Frequency and High-Frequency Synthetic Aperture Sonar}

\author{
Peter T. Gough, Cochair \\ University of Canterbury, Private Bag 4800, Christchurch, 8022, New Zealand \\ Roy E. Hansen, Cochair \\ Norwegian Defence Research Establishment, P O Box 25, Kjeller, NO-2027, Norway
}

\section{Invited Paper}

\begin{abstract}
8:00
5aUWd1. Ten key papers in synthetic aperture sonar. Peter T. Gough (University of Canterbury, Private Bag 4800, 8022 Christchurch, New Zealand, peter.gough@canterbury.ac.nz), Michael Hayes (University of Canterbury, Private Bag 4800, 8022 Christchurch, New Zealand, michael.hayes@canterbury.ac.nz)

There are now hundreds of patents and papers on synthetic aperture sonar; some of them of key importance whereas many others are of peripheral or minor interest. Here we take what we consider to be the top ten publications in synthetic aperture sonar and explain why we believe they are important and how they have made a significant contribution to the developing discipline or have made some leap of imagination in the area. This paper is more than a catalogue of the top ten citations since not all the real advances in the field have been published in academic peer-review journals.
\end{abstract}

\section{Contributed Papers}

\section{$8: 20$}

5aUWd2. Multifrequency interferometry on synthetic aperture sonar images. Torstein Olsmo Sæbø (Norwegian Defence Research Establishment, P O Box 25, NO-2027 Kjeller, Norway, torstein-olsmo.sabo @ffi.no), Roy E. Hansen (Norwegian Defence Research Establishment, P O Box 25, NO-2027 Kjeller, Norway, roy-edgar.hansen@ffi.no), Hayden J. Callow (Norwegian Defence Research Establishment, P O Box 25, NO2027 Kjeller, Norway, hayden-john.callow@ffi.no)

Interferometric processing on synthetic aperture sonar (SAS) images can provide accurate bathymetric maps with very high spatial resolution. Often, bathymetric precision is limited by two-pi phase ambiguities in the interferogram. Several phase-unwrapping algorithms exist, but they all depend on some type of continuity throughout the interferogram. The locations of the phase ambiguities are dependent on the carrier frequency of the transmitted signal. Dividing the frequency band into multiple subbands provides multiple interferograms, each with a unique carrier frequency and thus differing ambiguity positions. In this paper, we investigate how to take advantage of subband interferograms to reduce phase ambiguities at the expense of accuracy. This is performed by using the phase differences between the individual subband interferograms. We also present a method were we use the differences in subband interferograms to eliminate the phase ambiguities in both the original interferogram, and in the subband interferograms themselves. Both methods are tested on simulated data and field data collected by the HUGIN autonomous underwater vehicle carrying the Kongsberg HISAS 1030. 
8:40

5aUWd3. Circular synthetic aperture sonar results from autonomous underwater vehicle trials. Roy E. Hansen (Norwegian Defence Research Establishment, P O Box 25, NO-2027 Kjeller, Norway, roy-edgar.hansen @ ffi.no), Torstein Olsmo Sæbø (Norwegian Defence Research Establishment, P O Box 25, NO-2027 Kjeller, Norway, torstein-olsmo.sabo @ffi.no), Hayden J. Callow (Norwegian Defence Research Establishment, P O Box 25, NO-2027 Kjeller, Norway, hayden-john.callow@ffi.no)

The principle of synthetic aperture sonar (SAS) is to combine successive pings coherently from different observation angles in order to increase the azimuth resolution. By collecting data along a circular track, a circular SAS (CSAS) image, or tomographic image, can be made. The image reconstruction can be done either incoherently, fully coherently, or partially coherently where each subaperture of coherent processing consists of a section of the circle. In target classification, CSAS has several benefits: the object is observed from all aspect angles giving a better perception; the resolution in the image increases. In this paper, we calculate required accuracy in navigation, bathymetry and sound velocity for successful circular SAS. Finally, we show circular SAS images of small targets from real data collected by the HUGIN autonomous underwater vehicle carrying the Kongsberg HISAS 1030. We test different beamforming strategies, and show the effect of coherent and incoherent tomographic imaging.

\section{9:00}

5aUWd4. Autofocus of synthetic aperture sonar data using the phase adjustment by contrast enhancement algorithm. Hayden J. Callow (Norwegian Defence Research Establishment, P O Box 25, NO2027 Kjeller, Norway, hayden-john.callow@ffi.no), Torstein Olsmo Sæbø (Norwegian Defence Research Establishment, P O Box 25, NO-2027 Kjeller, Norway, torstein-olsmo.sabo@ffi.no), Roy E. Hansen (Norwegian Defence Research Establishment, P O Box 25, NO-2027 Kjeller, Norway, roy-edgar.hansen@ffi.no)

Imagery from synthetic aperture systems often suffers from imperfect image formation due uncertainties in the collection geometry or environmental parameters. Autofocus techniques aim to estimate and subsequently eliminate the effect of these uncertainties---automatically adjusting focus parameters to obtain a "better" image. Typically, autofocus algorithms will optimise for reduced Doppler phase gradients or improved image contrast. The phase adjustment by contrast enhancement (PACE) algorithm belongs to the second class and was first proposed for use in synthetic aperture radar (SAR) autofocus. The algorithm is somewhat unusual for a contrast optimisation in that it avoids lengthy computation through directly solving the equations for optimum contrast. This advance allows for rapid autofocus without the need for complicated iterative optimisers. We compare results from using the PACE algorithm on local region of strip-map synthetic aperture sonar (SAS) data collected with the HUGIN AUV and compare against a benchmark phase gradient autofocus (PGA) algorithm. We also demonstrate the effect of using the algorithm in circular SAS imagery, something not currently possible with standard PGA-based autofocus.

\section{9:20}

5aUWd5. Planar synthetic aperture processing (P-SAS) to real acoustic data of underwater imaging. Adel Jammali (Faculté des Sciences de Monastir $\mu$ Ei Lab Tunisia, Avenue de l'environnement, 5019 Monastir, Tunisia, adel.jam@voila.fr), Afif Belkacem (Faculté des Sciences de Monastir $\mu$ Ei Lab Tunisia, Avenue de l'environnement, 5019 Monastir, Tunisia, afif_bel@yahoo.fr), Kamel Besbes (Faculté des Sciences de Monastir $\mu$ Ei Lab Tunisia, Avenue de l'environnement, 5019 Monastir, Tunisia, Kamel.Besbes@fsm.rnu.tn), Manell E. Zakharia (French Naval Acadamey, BP 600, 29240 Brest-Armees, France, manell.zakharia@ecole-navale.fr)

Planar synthetic aperture technique has been validated on both simulated and tank data. It showed a significant improvement of 3D representation of bottom and subbottom. In this paper we present its application to real data acquired during sea experiments on a dump site. Data were acquired during the SITAR (Seafloor Imaging and Toxicity: Assessment of Risks caused by buried waste) project. The transmitter was a parametric array (TOPAS). Two central frequency were explored, $10 \mathrm{kHz}$ and $20 \mathrm{kHz}$. The transmitter was fixed on a ROV which position was monitored. As P-SAS algorithm was designed for data obtained on a regular planar grid important modification were required to handle real sea data and "realistic" navigation conditions (irregular grid): a "re-arrangement" algorithm was designed for preprocessing actual data and correct for trajectory disturbances (in 2D). This algorithm is the re-projection of data to a new (virtual) regularly grid. The algorithm was validated on tank experimental data prior to application to sea data. Both conventional and SAS data will be presented. A strata representation technique was used for analyzing the seafloor, the subbottom and the localization of buried objects on a dump site in the Baltic sea.

9:40-11:00 Posters

Lecture sessions will recess for presentation of poster papers on various topics in acoustics. See poster sessions for topics and abstracts.

\section{Contributed Paper}

\section{1:00}

5aUWd6. Towards an understanding of how a synthetic aperture sonar images buried targets. Peter T. Gough (University of Canterbury, Private Bag 4800, 8022 Christchurch, New Zealand, peter.gough@canterbury .ac.nz), Michael Hayes (University of Canterbury, Private Bag 4800, 8022 Christchurch, New Zealand, michael.hayes@canterbury.ac.nz)

One of the useful features of synthetic aperture sonar (SAS) is that in the reconstructed image, the cross-track resolution is inversely proportional to the bandwidth and the along-track resolution is proportional to the transducer's along-track extent and that these are more or less independent of the center frequency. An unexpected aspect of SAS imaging so far is that objects buried in sand/sediment are sometimes visible even at geometries where the incident angle far exceeds the critical angle for the bulk materials. It is surmised that the roughness of the sea/sea-floor interface is the significant contributing factor. So as an adjunct to the main Kiwi SAS programme, a seafloor "document" camera colocated with a three-transducer active sonic probe has been designed and deployed in controlled circumstances. The intention is to estimate the critical parameters that best describes the roughness of the sea floor and perhaps how these parameters relate to the way the SAS images shallow buried targets. In regions of reasonable visibility, the camera is used to provide ground-truth. The paper details the construction of the camera and sonic probe as well describes some preliminary results using smooth and raked sand. 


\title{
Invited Paper
}

$11: 20$

5aUWd7. Imaging buried objects using synthetic aperture processing. Steven Schock (Florida Atlantic University, Dept of Ocean Engineering, 777 Glades Rd, Boca Raton, FL 33431, USA, schock@oe.fau.edu)

FM sonar generates images of buried objects using synthetic aperture processing. A spherical projector illuminates objects buried in the seabed out to the range associated with the critical angle. Scattering off buried targets is measured with one meter long line hydrophone arrays embedded in sonar vehicle wings. The histories of the projector and hydrophones positions, used for compensating acoustic data during synthetic aperture processing, are calculated based on navigation data provided by an inertial measurement unit and Doppler velocity log. Three orthogonal image projections of the seabed containing 5, 7.5, 10, and $15 \mathrm{~cm}$ diameter cylinders, buried up to $30 \mathrm{~cm}$ in seabeds consisting of sand and sandy mud in three meters of water, show the burial depth and orientation of the cylinders. The temporal resolution of the imagery is approximately $6 \mathrm{~cm}$. The azimuthal resolution varies between 5 and $20 \mathrm{~cm}$ and is a function of target range and relative bearing with respect to the sonar vehicle. This imaging technique can be applied to marine archeological investigations in water depths of two meters and greater.

FRIDAY MORNING, 4 JULY 2008

ROOM 362/363, 8:00 TO 9:40 A.M.

\section{Session 5aUWe}

\section{Underwater Acoustics and ECUA: Monitoring Systems and Ambient Noise}

\author{
Mark K. Prior, Cochair \\ CTBTO, Vienna International Centre, P.O. Box 1200, Vienna, 1400, Austria \\ David Deveau, Cochair \\ Naval Undersea Warfare Center - AUTEC, PSC 1012 Box 701, FPO, AA, FL 34058, USA
}

\section{Contributed Papers}

\section{8:00}

5aUWe1. An optimization approach to the automatic identification of signals originating from underwater explosions. Mark $\mathrm{K}$ Prior (CTBTO, Vienna International Centre, PO Box 1200, 1400 Vienna, Austria, Mark.Prior@CTBTO.ORG)

Data recorded by the International Monitoring System (IMS) of the Comprehensive Test-Ban Treaty Organization are used to illustrate the different types of signal that are routinely received on IMS hydrophone stations. It is shown that automated methods for characterizing the source of these signals sometimes fail to identify signals arising from underwater explosions. A new approach to the automatic identification of such signals is presented. The approach uses Receiver Operating Characteristic (ROC) curves to investigate the trade-off between false-alarm rate and probability of detection. It applies a threshold to a parameter developed as a measure of the likelihood of a signal being associated with an explosion. A numerical Measure of Performance (MoP) is derived from the ROC curves and the definition of the likelihood parameter is tuned so as to maximize this MoP. Optimization of the parameter definition is achieved using an approach based on genetic algorithms.

\section{8:20}

5aUWe2. Seafloor Geodetic Networks for monitoring tectonic plate motion and deformation. Stavroula Peroutsea (School of Rural and Surveying Engineering, National Technical University of Athens, Ch. Mantika 1, 11362 Athens, Greece, ROK2POK5G@INTERNET.GR), Efstratios Doukakis (School of Rural and Surveying Engineering, National Technical University of Athens, Ch. Mantika 1, 11362 Athens, Greece, EDOUK @CENTRAL.NTUA.GR)

This paper argues that seafloor geodetic networks, monitoring tectonic plate motion and deformation, supplementing terrestrial geodetic networks, can provide important information for the understanding of crustal processes, globally. The proposed monitoring system consists of three main components: real-time kinematic-differential GPS ship positioning, precise acoustic ranging between ship and seafloor transponders - which establish the network nodes- and near-bottom direct acoustic measurements of the network baselines. The coordinates of the transponders in a goedetic reference system can be determined from these observations and a sound-speed structure model. The motion and deformation of the tectonic plate can be traced as a change of coordinates and/or the internal geometry of the network. Stochastic and mathematic models, algorithms and data analysis are crucial for achieving the necessary accuracy in such a demanding process regarding seafloor position estimation.

\section{8:40}

5aUWe3. Confined deep water acoustic noise study within the Bahamian TOTO. David Deveau (Naval Undersea Warfare Center - AUTEC, PSC 1012 Box 701, FPO, AA, FL 34058, USA, david.deveau@autec navy.mil)

The three-sided deep coral reef that borders Andros Island, Bahamas is populated with deep water acoustic sensors that presents a unique opportunity to study the impact of wind and wave in a physical environment with limited man-made noise sources over a wide acoustic band $(50-40 \mathrm{kHz})$. This area is known as the "Tongue of the Ocean" (TOTO) for its unique satellite viewed shape. The region's deep acoustic channel $(\sim 1550 \mathrm{~m})$ is surrounded by steep walls and only one outlet suggesting a highly reverberant cavity where weather produced ambient noise signatures may persist over greater periods producing levels greater than typically observed in the open ocean. In this study, daily acoustic spectra are gathered on two sensors spaced $20 \mathrm{~km}$ apart with accompanied wind and weather information. The spectra will be correlated to advancing and receding weather fronts in an effort to relate the surface changes to the deep water ambient noise levels. This study also serves as a precursor to a shallow water acoustic noise intensity study where similar weather comparisons will be made and compared to the deep water counterpart. 
9:00

5aUWe4. Historical ambient noise levels in a topographically isolated basin. D. Benjamin Reeder (Naval Postgraduate School, Department of Oceanography, 833 Dyer Road, Rm 328, Monterey, CA 93943-5738, USA, dbreeder@nps.edu), Susan M. Mach (Naval Undersea Warfare Center Detachment AUTEC, P.O. Box 24619, West Palm Beach, FL 33416, USA, Susan.Mach@autec.navy.mil), Edmond S. Sheffield (Naval Undersea Warfare Center Detachment AUTEC, P.O. Box 24619, West Palm Beach, FL 33416, USA, Edmond.Sheffield@autec.navy.mil)

During the late 1970s and early 1980s, careful measurements of ambient noise were made in the Tongue of the Ocean (TOTO) in the Bahamas. The measurements were made using a vertical array of five omni-directional hydrophones moored in $2200 \mathrm{~m}$ water depth with an average hydrophone depth of $122 \mathrm{~m}$. Data were collected within a $20 \mathrm{~Hz}-20 \mathrm{kHz}$ band at ten specific $1 / 3$ octave band frequencies, and then reported in spectrum level as a function of surface wind speed. Records contaminated by anthropogenic sources (e.g., local vessel traffic), biological sources (e.g., marine mammal vocalizations) and other sources (e.g., rain) were eliminated to allow analysis of background ocean ambient noise only. TOTO is a deep basin, topographically isolated from the Atlantic Ocean, and therefore largely acoustically decoupled from the Atlantic Ocean deep sound channel. The basin's geographical isolation and the controlled data collection methodology employed over a four-year period make this three-decade-old data set a potential proxy for preindustrial era ocean noise levels. The environmental setting, data collection methodology, analysis methodology and results are presented and discussed within the context of historical ocean ambient noise levels.
9:20

5aUWe5. Analysis of wind generated shallow water ambient noise. Ari Poikonen (Finnish Naval Research Institute (FNRI), P.O. Box 116, FIN02631 Espoo, Finland, ari.poikonen@mil.fi), Seppo Madekivi (Finnish Naval Research Institute (FNRI), P.O. Box 116, FIN-02631 Espoo, Finland, seppo.madekivi@mil.fi)

Underwater ambient noise measurements were carried out in very shallow water (10-20 m) in the archipelago of the Gulf of Finland during the full year. Weather conditions varied from calm sea to near gale winds. The lowest spectral levels were obtained under the ice cover during the coldest winter months. No seasonal effect was observed in the measured spectra. The role of water depth was modeled with several bottom sediments in order to identify possible channel effects at lower frequencies of the noise spectra. The ambient noise spectra are typically bandlimited. A bandpass filter model was thus fitted to the noise spectra. The filter model provides several useful parameters to characterize the effect of wind speed on the noise spectra. The cutoff frequency of the high-pass part of the spectra decreases from ca 400 $\mathrm{Hz}$ down to below $200 \mathrm{~Hz}$ as wind increases from light breeze to near gale force. The corresponding spectral slopes get steeper with increasing winds saturating to the value of $12 \mathrm{~dB} /$ oct already in fresh breeze. The other parameters used in the analysis are high frequency (low-pass) spectral slope, the frequency of maximum spectral level, noise bandwidth and total power in the band.

FRIDAY MORNING, 4 JULY 2008

P3-C, LEVEL 3, 9:40 TO 11:20 A.M.

\title{
Session 5aUWf
}

\section{Underwater Acoustics, Signal Processing in Acoustics, and ECUA: Broadband Underwater Communications II (Poster Session)}

\author{
Heechun Song, Cochair \\ $M P L$ \\ Sergio Jesus, Cochair \\ ISR
}

All posters will be on display from 9:40 a.m. to 11:20 a.m. To allow contributors an opportunity to see other posters, contributors of odd-numbered papers will be at their posters from 9:40 a.m. to 10:30 a.m. and contributors of even-numbered papers will be at their posters from 10:30 a.m. to 11:20 a.m.

\section{Contributed Papers}

5aUWf1. Basic at-sea experiment for long horizontal time-reversal communication in deep ocean. Takuya Shimura (JAMSTEC, 2-15, Natsushima-cho, 237-0061 Yokosuka, Japan, shimurat@jamstec .go.jp), Yoshitaka Watanabe (JAMSTEC, 2-15, Natsushima-cho, 237-0061 Yokosuka, Japan, yoshitakaw@jamstec.go.jp), Hiroshi Ochi (JAMSTEC, 2-15, Natsushima-cho, 237-0061 Yokosuka, Japan, ochi@jamstec .go.jp), Takehito Hattori (Nippon Marine Enterprises, Ltd., 14-1, Ogawacho, 238-0004 Yokosuka, Japan, hattorit@nme.co.jp)

In our research program, a long cruising autonomous underwater vehicle (AUV) has been developed and a project on a second AUV is planned, for wide-areal observation in the deep ocean. In such AUV operation, it is preferred to ensure acoustic communication with AUV even at low data- transmission rate. Time-reversal techniques have a possibility to achieve such long horizontal communication. We have proposed a method of combining time reversal and an adaptive equalizer to realize communication even with a sparse time-reversal array (TRA) and communication with a moving target. To verify the performance of such time-reversal communication, at-sea experiments were executed at the various distances. In the experiment of $10 \mathrm{~km}$ at a water depth of $1000 \mathrm{~m}$, bidirectional time-reversal communication between the focus point and TRA was achieved successfully and the effects of the TRA depth and its channel number are investigated. In the experiments of $20,30,40 \mathrm{~km}$ at the slight sloping area, the performance on the distances are discussed. In the experiment of $100 \mathrm{~km}$ at a water depth of $4000 \mathrm{~m}$, the convergence by passive time reversal was verified. 
5aUWf2. High data rate coherent underwater acoustic communications during KauaiEx and MakaiEx. Aijun Song (University of Delaware, College of Marine and Earth Studies, S. College Street, Newark, DE 19716, USA, ajsong@udel.edu), Mohsen Badiey (University of Delaware, College of Marine and Earth Studies, S. College Street, Newark, DE 19716, USA, badiey@udel.edu), Heechun Song (MPL, Scripps Institution of Oceanography, University of California, San Diego, La Jolla, CA 92093-0238, USA, hcsong@ucsd.edu), William Hodgkiss (MPL, Scripps Institution of Oceanography, University of California, San Diego, La Jolla, CA 92093-0238, USA, wsh@mpl.ucsd.edu)

During two acoustic communications experiments conducted around Kauai Island, Hawaii (KauaiEx, 2003 and MakaiEx, 2005), various coherent communications data with concurrent environmental measurements were collected under different experimental settings. The collected communications data are processed by a newly proposed receiver, which consists of time-reversal multichannel combining followed by a single channel DFE. Continuous channel updates along with Doppler tracking are used prior to time reversal combining to combat fast channel variations. The receiver can successfully demodulate different types of coherent communications signals, including phase shift keying (PSK) and quadrature amplitude modulation (QAM) signals, at different symbols rates for different source/receiver settings, such as fixed source/receiver, drifting source/receiver, and towed source. Selected high data rate communication results will be presented to show the effectiveness of the receiver. The receiver performance in relation to the environmental variability also will be shown

5aUWf3. Optimal placement of array elements for time-reversal mirror. Jae Hoon Joo (LIG Nex1 Co., Ltd, 102-18 Mabuk-dong Giheunggu, 446-912 Yongin, Republic of Korea, jhjub@lignex1.com), Jea Soo Kim (Dept. of Ocean Engineering, Korea Maritime Univ., 1 Dongsam-dong Youngdo-ku, 606-791 Busan, Republic of Korea, jskim@hhu.ac.kr)

In the application of time-reversal mirror (TRM) to the underwater acoustic communication, the optimal placement of the transmit/receive array can enhance the signal-to-noise ratio at the foci when a number of array elements is limited. In this presentation, the optimal location of individual elements is determined by maximizing contrast between the focal location and the area of interest using genetic algorithm. As a result of optimization, the contrast as well as the intensity at foci is increased when compared to the conventional TRM. The optimal number of array elements is also investigated based on the objective function of genetic algorithm in connection with finding optimal placement of array. Numerical examples for TRM and contrast-maximization are presented and discussed.

5aUWf4. Experimental analysis of statistical characteristics of a very shallow underwater acoustic channel. Sea-Moon Kim (MOERI/KORDI, 171 Jang-dong, Yuseong-gu, 305-343 Daejeon, Republic of Korea, smkim@moeri.re.kr), Sung-Hoon Byun (MOERI/KORDI, 171 Jang-dong, Yuseong-gu, 305-343 Daejeon, Republic of Korea, byunsh@moeri.re.kr), Yong-Kon Lim (MOERI/KORDI, 171 Jang-dong, Yuseong-gu, 305-343 Daejeon, Republic of Korea, yklim@moeri.re.kr)

Underwater acoustic channel is characterized as a time-varying and multipath environment. Not only each propagation path changes randomly but also the scattered waves from the surface and bottom deteriorate signal transmission resulting in fading channels and poor communication performance. In order to design communication algorithms and determine modem parameters the characteristics of random ocean channel must be analyzed in advance. In this paper we investigate the statistical channel properties with experimental data gathered in a very shallow water near the southern coast of South Korea using band-limited signals with center frequencies of 20-40 kHz. The impulse responses at several distances up to $4 \mathrm{~km}$ are estimated and their statistical characteristics in the complex domain are analyzed. We found that the statistical properties are highly dependent on the channel impulse response and the carrier frequency.
5aUWf5. Characterization of long-range time-varying underwater acoustic communication channels. Morten Smedsrud (FFI, Karljohansvern, P.O. Box 115, 3192 Horten, Norway, morten.smedsrud@ffi.no), Trond Jenserud (FFI, Karljohansvern, P.O.Box 115, 3192 Horten, Norway, trond .jenserud@ffi.no)

We present communication channel characterizations performed on insitu measurements from the Baltic and North Sea. The communication channels were probed using pseudorandom binary sequences (PRBS), obtaining time-variant channel impulse responses through wideband matched filtering. Characteristics central to communications were obtained. Included are: scattering functions, coherence-times and coherence bandwidths. The validity of the quasi wide sense stationary uncorrelated scattering (quasi-WSSUS) assumption was also investigated for the measured channels. Measurements were acquired in the joint European project "UUV Covert Acoustic Communications." The project aims to design an acoustic communication system between an unmanned underwater vehicle (UUV) and a support mother ship. The first sea trials of the project focused on the acquisition of noise and long-range, low-frequency communication channel data for the explicit purpose of building an acoustic channel simulator.

5aUWf6. A novel architecture for multihops routing ad hoc underwater acoustic sensor networking. Roee Diamant (Technion - Israel Institute of Technology, RAFAEL, POB 2250, 31021 Haifa, Israel, diamantr@rafael.co.il), Azriel Sinai (Technion - Israel Institute of Technology, RAFAEL, POB 2250, 31021 Haifa, Israel, azrielsi@rafael.co.il)

Underwater acoustic communication networks are an innovative issue, which faces difficult medium problems, such as long propagation time and dynamic network topology. In addition, due to high error probabilities typical for this channel it is preferred to use anti collision network mechanism. Because of the long propagation time, methods such as CTS/RTS, requiring transmissions of pre-messages, achieve relatively low network efficiency. Moreover, TDMA methods do not utilize the network resources allowing reuse in the code domain (using CDMA) and in the spatial domain in case of nonhomogenic network topology which includes obstructions in the acoustic line of sight. In this paper we introduce a network concept based on the CDMA method confronting the acoustic underwater network challenges, such as the "near-far" problem, fast time varying topology, multihops routing and message distribution protection. Using this concept, a sea trial including broadcast, multicast and unicast transmissions of SMS and navigation control messages was carried out in various scenarios including multihops near the shores of Israel. We present results from this sea trial demonstrating the efficacy of the proposed networking scheme.

5aUWf7. Capacity estimations for underwater acoustic communication systems with source power constraints. Haibin Wang (National Laboratory of Acoustics, Institute of Acoustics, Chinese Academy of Sciences, NO .21, Northwest 4th Ring Road, 100080 Beijing, China, whb@mail.ioa.ac.cn), Di Meng (National Laboratory of Acoustics, Institute of Acoustics, Chinese Academy of Sciences, NO.21, Northwest 4th Ring Road, 100080 Beijing, China, whb@mail.ioa.ac.cn), Hua Yang (National Laboratory of Acoustics, Institute of Acoustics, Chinese Academy of Sciences, NO.21, Northwest 4th Ring Road, 100080 Beijing, China, yh@mail.ioa.ac.cn)

Channel capacity is an important parameter of underwater acoustic communication (UWAC) systems. For a certain channel and source power, water-filling algorithm of information-theory can be used to estimate the capacity. However, in practice, there are many constraints of the source transmission power. One of the most important constraints is that many communication nodes may exist in a local area, which means that the source power of each node should be confined in order to keep all nodes working normally. Considering this constraint and applying acoustic propagation modeling, this paper estimates the capacities of an UWAC system in some typical propagation conditions. The results are very useful for analyzing the maximum data rate of a practical acoustic channel and providing the optimal parameters of an UWAC system. 
5aUWf8. Synchronisation of underwater communication receivers by means of broadband sweep-spread pulses. Konstantin G. Kebkal (EvoLogics GmbH/Technical University Berlin, Ackerstrasse 76 (ACK1), 13355 Berlin, Germany, kebkal@bionik.tu-berlin.de), Rudolf Bannasch (EvoLogics GmbH/Technical University Berlin, Ackerstrasse 76 (ACK1), 13355 Berlin, Germany, bannasch@evologics.de), Alexey G. Kebkal (EvoLogics GmbH/Technical University Berlin, Ackerstrasse 76 (ACK1), 13355 Berlin, Germany, kebkal@gmail.com)

Using sweep spread pulses a variety of multipath components of a receive signal can be often resolved in time-frequency domain. In result of synchronisation of the receiver with the most powerful component, other multipaths can be effectively suppressed by means of a matched filter. Owing to exact synchronisation, the difference frequency will be equal to zero and outputs of matched filters will be good for estimation of (information) parameters. However, the synchronous component (as well as others) can occasionally turn out to be composite and to contain energy of several nonresolved multipaths. In this case, the synchronisation time of the receiver will be not exact and the difference frequency of demodulated signal will differ from zero. This effect will cause a systematic error in estimation of the information parameter. This paper represents the results of mathematic modeling of the systematic error (for receivers, utilising the sweep-spread carrier technology), as well as, experiments on synchronisation of the receiver by means of wideband sweep spread pulses.
5aUWf9. Measurement of angular power profile at an array receiver in a shallow underwater acoustic channel. Sung-Hoon Byun (MOERI/KORDI, 171 Jang-dong, Yuseong-gu, 305-343 Daejeon, Republic of Korea, byunsh@moeri.re.kr), Sea-Moon Kim (MOERI/ KORDI, 171 Jang-dong, Yuseong-gu, 305-343 Daejeon, Republic of Korea, smkim@moeri.re.kr), Yong-Kon Lim (MOERI/KORDI, 171 Jang-dong, Yuseong-gu, 305-343 Daejeon, Republic of Korea, yklim@moeri .re.kr), Woojae Seong (Seoul National University, Room. 306, Bd. 34, San 56-1, Sillim-dong, Kwanak-gu, College of Engineering, Dept. of Naval Architecture and Ocean Engineering, 151-744 Seoul, Republic of Korea, wseong@snu.ac.kr)

In the design of broadband underwater acoustic communication system, proper analysis of underwater acoustic channel characteristics is required to determine communication system parameters. Especially for high channel capacity transmissions such as multiinput multioutput (MIMO) systems, the spatial correlation characteristics among multiple sensors determine communication performance and they are affected by the angular power distribution of the incoming acoustic waves. In this study, we suggest a model-based array signal processing technique for measuring the angular power profile and apply it to experimental data gathered from very shallow water of 20 to $30 \mathrm{~m}$ water depth near the southern coast of South Korea. At first we show the variation of the angular power profile over different transmitter/receiver ranges and then its impact on the spatial correlation characteristics between two different array elements. Finally we discuss the expected spatial diversity effects from the viewpoint of underwater acoustic channel modeling.

FRIDAY MORNING, 4 JULY 2008

P3-C, LEVEL 3, 9:40 TO 11:20 A.M.

\title{
Session 5aUWg
}

\section{Underwater Acoustics and ECUA: High Frequency Scattering II (Poster Session)}

\author{
Anthony Lyons, Cochair \\ Applied Research Laboratory \\ Philippe Blondel, Cochair \\ University of Bath \\ Nicholas Pace, Cochair \\ SACLANT Undersea Research Centre
}

\begin{abstract}
All posters will be on display from 9:40 a.m. to 11:20 a.m. To allow contributors an opportunity to see other posters, contributors of odd-numbered papers will be at their posters from 9:40 a.m. to 10:30 a.m. and contributors of even-numbered papers will be at their posters from 10:30 a.m. to 11:20 a.m.
\end{abstract}

\section{Contributed Papers}

5aUWg1. Modeling high-frequency reverberation and propagation loss in support of a submarine target strength trial. Boris Vasiliev (DRDC Atlantic, 9 Grove St., Dartmouth, NS B2Y 3Z7, Canada, boris.vasiliev@drdc-rddc.gc.ca), Art Collier (DRDC Atlantic, 9 Grove St., Dartmouth, NS B2Y 3Z7, Canada, art.collier@drdc-rddc.gc.ca)

The presentation reviews the environmental modeling conducted in support of a submarine acoustic target strength trial. The measurements at 20-40 $\mathrm{kHz}$ were collected at a shallow site in North West Atlantic. The site bottom was gravel; surface winds were 2-6 m/s. For ranges less than $1.8 \mathrm{~km}$, the $\mathrm{CW}$ reverberation at 24, 28, and $39 \mathrm{kHz}$ agreed with CASS-GRAB predictions; beyond $1.8 \mathrm{~km}$, the model output underestimated the measurements. The predictions were computed for APL-UW surface and bottom models appropriate for trial conditions: negligible surface reflection loss and scattering strength, low bottom reflection loss and high bottom scattering strength. The LFM reverberation at 21,28 , and $36 \mathrm{kHz}$ for $2 \mathrm{kHz}$ bandwidth signals matched the $\mathrm{CW}$ reverberation after compensation for the pulse range resolution and frequency differences in the beam responses and volume attenuation. Agreement among CASS-GRAB prediction and data verified the assumed sonar and environmental parameters used in propagation loss modeling and increased confidence in the target strength estimates. The latter were consistent with previously reported values. 
5aUWg2. Performances of a multistatic model of sound scattering by rough surfaces. Virginie Jaud (E3I2 - EA3876, 2 rue François Verny,29806 Brest Cedex, France, virginie.jaud@ensieta.fr), Cédric Gervaise (E3I2 - EA3876, 2 rue François Verny, 29806 Brest Cedex, France, cedric.gervaise@ensieta.fr), Ali Khenchaf (E3I2 - EA3876, 2 rue François Verny, 29806 Brest Cedex, France, ali.khenchaf@ensieta.fr)

The ocean floor is far from being a smooth and perfectly rigid surface. That is why its sound scattering properties are a useful input to the analysis of this medium as for acoustic data inversion. Thus, multi-static scattering coefficients have been investigated in a frequency range of order of $10 \mathrm{kHz}$ to $300 \mathrm{kHz}$. The Kirchhoff Approximation and the Small Perturbation Method could be cited respectively in the case of dimensions of a rough surface larger and smaller than the wavelength. Nevertheless, for those frequencies, hence for those wavelengths, a rough surface should be considered at different scales compared to the wavelength. As a part of the incident wave is transmitted to the seabed medium, it is also important to know the effect of the scattering coming from the volume. Jackson's scattering model takes these considerations into account. The aim of the study is first to show an improvement of the surface scattering method using the Small Slope Approximation and keeping the initial method of Jackson's model to describe the scattering from the volume. Comparisons with well-known models are presented to show the performances of this new approach and comparisons between different multistatic geometries are analyzed to show the most useful configuration of the model.
5aUWg3. Complex interfaces of granular media: multiscale characterization and effective medium modeling. Yves Le Gonidec (Géosciences Rennes, Bât. 15, Campus de Beaulieu, 35042 Rennes, France, yves.legonidec@univ-rennes1.fr)

Seafloor is a complex geological interface : the acoustic reflectivity strongly depends on the sonar frequency which can be from few to hundreds $\mathrm{kHz}$. In order to understand the physical processes involved, we have developed a multiscale acoustic method based on the wavelet transform properties: we introduce this theoretical approach first applied on homogeneous discontinuities. Then, we have developed acoustic experiments where the discontinuties are complex interfaces: we present the experimental setup and describe the results measured on granular media made of monodisperse glass beads. For this seafloor modeling, where all parameters are known, we put in evidence five frequency ranges corresponding to particular acoustic regimes: we give physical interpretations based on the analysis of effective medium modeling. From low to high frequencies, a strong transition is observed between propagation and scattering phenomena. Going further in this study, we illustrate the results on a bimodal glass bead distribution where high frequency scattering can be isolated using an original experimental approach.

FRIDAY MORNING, 4 JULY 2008

ROOM 341, 11:00 A.M. TO 12:40 P.M.

\title{
Session 5aUWh
}

\section{Underwater Acoustics and ECUA: Sensor Coalition}

\author{
Henrik Schmidt, Cochair \\ MIT, 77 Mass Ave, 5-204, Cambridge, MA 02139, USA \\ Stéphane Jespers, Cochair \\ Délégation Générale de l'Armement, 7-9 rue des Mathurins, Bagneux, 92220, France
}

\section{Contributed Paper}

\section{1:00}

5aUWh1. A review of approaches towards effective underwater sensor networks. Michel Leonard (NURC, Viale San Bartolomeo 400, $19121 \mathrm{La}$ Spezia, Italy, leonard@nurc.nato.int), Diego Merani (NURC, Viale San Bartolomeo 400, 19121 La Spezia, Italy, merani@nurc.nato.int), Alessandro Berni (NURC, Viale San Bartolomeo 400, 19121 La Spezia, Italy, merani @ nurc.nato.int)

The still largely unexplored vastness of the ocean, covering about twothird of the surface of earth, has fascinated humans for as long as we have records for. Its currents, chemical composition, and ecosystems are all highly variable at different locations and times. New paradigms for the monitoring of the undersea environment, based on autonomous underwater sensor nodes organized in ad-hoc coalitions, offer a promising approach towards the development of scalable networks for underwater sensing, moni- toring, reconnaissance and surveillance. This promise has however to be balanced against important challenges, which need to properly addressed in order to devise a credible system concept. Some challenges worth mentioning are the difficulty in ensuring a low error-rate end-to-end path between source and destination for the duration of the communications session, the negative impact of communications performance characteristics at applications level, and energy constraints for battery-operated nodes. The requirement is therefore to design new protocols to provide network flexibility and reliability through self-organization and reconfiguration, as well as new energy-efficient routing schemes, including geographic and data-centric routing, to maximize the operational lifespan of autonomous powerconstrained nodes. In this paper we will present the state of the art and approaches being investigated at NURC. 


\section{Invited Papers}

$11: 20$

5aUWh2. Nested Autonomy - Adaptive and collaborative sensing with hybrid sensing networks. Arjuna Balasuriya (MIT, 77 Mass Ave, 5-204, Cambridge, MA 02139, USA, arjunab@mit.edu), Henrik Schmidt (MIT, 77 Mass Ave, 5-204, Cambridge, MA 02139, USA, henrik@mit.edu), Michael B. Benjamin (MIT, 77 Mass Ave, 5-204, Cambridge, MA 02139, USA, mikerb@csail.mit .edu)

Control of undersea sensing networks is restricted by the limited acoustic communication capacity and the need for surfacing "datamules" for communicating with the operators. Adaptive sampling of episodic events must therefore be performed fully autonomously. Similarly, collaboration between nodes is limited by the communication horizon. These constraints favor the use of a nested operational paradigm, with clusters of highly autonomous nodes capable of detecting, classifying, and localizing episodic events, if possible collaborating with neighboring nodes without operator intervention. Such a Nested Autonomy operational paradigm is being developed for capturing episodic oceanographic events by AUVs and gliders in the NSF ORION ocean observatory. Based on the portable, opensource MOOS-IvP autonomous control framework, the same paradigm is being applied for distributed acoustic surveillance networks in the ONR PLUSNet program. In either case the nested autonomy enables the autononomous exploitation of the environmental and tactical situation, and collaborative tracking of targets or oceanographic events. The operator control is limited to very simple deploy and prosecute commands. The performance of the nested autonomy paradigm is demonstrated by examples using a high-fidelity simulation environment, and by results from recent field experiments. [Work supported by ONR.]

\section{1:40}

5aUWh3. Performance modeling and tradeoffs studies for hybrid sensing networks. Stéphane Jespers (Délégation Générale de l'Armement, 7-9 rue des Mathurins, 92220 Bagneux, France, stephane.jespers@dga.defense.gouv.fr)

The transformation of naval forces in support of expeditionary operations requires substantial endurance enhancement of their existing undersea reconnaissance and surveillance functions. Concepts of operations for distributed sensor fields - to be deployed in littoral waters - are now being proposed, encouraged by the favourable outlook for network technologies. The renewed attention for multistatic sonar is one example of this trend. Predicting the performance of distributed sensor fields for ASW applications, with sensors involving the use of acoustics and non-acoustics is complex. Though it is a great chance for optimizing the use of assets, the impact of sensor platforms' mobility (AUVs or gliders) adds yet another level of space-time complexity. Prior to any large scale test or development, system and operational studies must be able to determine optimal architectures and node placement as function of mission requirements. A major constraint is that of the limitations imposed on autonomous and covert data exchange in the maritime and underwater domains. This speeks for the design of hybrid sensor network architectures, as they appear to be well suited in dealing with substantial sensor data and command control data (e.g., backseat-driver paradigm) communication requirements.

\section{Contributed Paper}

\section{2:00}

5aUWh4. Acoustics for underwater neutrino telescopes. Miguel Ardid (IGIC - Universitat Politècnica de València, Cra. Nazaret-Oliva S/N, E-46730 Gandia, Spain, mardid@fis.upv.es), Manuel Bou-Cabo (IGIC Universitat Politècnica de València, Cra. Nazaret-Oliva S/N, E-46730 Gandia, Spain, maboca3@doctor.upv.es), Juan Antonio Martínez-Mora (IGIC Universitat Politècnica de València, Cra. Nazaret-Oliva S/N, E-46730 Gandia, Spain, jmmora@fis.upv.es), Francisco Camarena (IGIC - Universitat Politècnica de València, Cra. Nazaret-Oliva S/N, E-46730 Gandia, Spain, fracafe@fis.upv.es), Víctor Espinosa (IGIC - Universitat Politècnica de València, Cra. Nazaret-Oliva S/N, E-46730 Gandia, Spain, vespinos@fis .upv.es)
In this communication we present the research activities in underwater acoustic sensors and arrays used in underwater neutrino telescopes. These activities are developed in two areas. First, in optical based underwater neutrino telescopes, an acoustic positioning system is used in order to monitor the position and orientation of the optical modules in deep sea. In the second field, there is the idea to build underwater telescopes composed of arrays of acoustic sensors in order to detect the weak thermoacoustic pulse which will be produced when a very high energy neutrino interacts in the water. For both, a challenging and very specific acoustic system is needed. After the description of these systems and a brief review of the status of the field, our work in the area in the frames of ANTARES and KM3NeT neutrino collaborations is shown.

\section{Invited Paper}

\section{$12: 20$}

5aUWh5. The applicability of a small autonomous vehicle towed array system to ocean acoustic measurements and signal processing. William M. Carey (College of Enginering, Boston University, 110 Cummington St, Boston, MA 02215, USA, wcarey @bu.edu), Jason D. Holmes (BBN Technologies, 10 Moulton St., Cambridge, MA 02318, USA, jholmes@bbn.com), Edmund J. Sullivan (EJS Consultants, 46 Lawton Brook Lane, Portmouth, RI 02871, USA, PADDYPRIEST@aol.com), James F. Lynch (Woods Hole Oceanographic Institution, 98 Water Street, Bigelow 203A, MS-11, Woods Hole, MA 02543, USA, jlynch@whoi.edu)

An array was developed to demonstrate, and quantify the performance characteristics of an autonomous-vehicle towed-array system. This technology provides for a cost effective tool for the measurement of coherent signal propagation, depth dependent and directional noise fields and to establish quantitative limits on array performance. The tangential drag on a 40-m length array composed of a reinforced tube with an outer diameter of $2.8 \mathrm{~cm}$ is extrapolated to be between $20-28 \mathrm{~N}$ for diameter Reynold's number of approximately 4 104. The hydrophone- preamplifier groups have a sensitivity of $-174 \pm 1.5 \mathrm{~dB}$ re $1 \mu \mathrm{Pa} / \mathrm{V}$ between $100 \mathrm{~Hz}$ to $10 \mathrm{kHz}$. with an adjustable spacing between 0.5 and $1.5 \mathrm{~m}$. The system tow stability enabled the use of the synthetic Hankel transform to estimate the modal 
horizontal wave number spectrum and the identification of interface wave speeds at frequencies up to $1000 \mathrm{~Hz}$. The formation of synthetic apertures combined with model based extended Kalman filter techniques was found to demonstrate both narrow band and broadband tracking. Finally the system is shown to provide a unique measurement capability for directional noise measurement in shallow water environments.

\title{
Session 5aUWi
}

\section{Underwater Acoustics and ECUA: Synthetic Aperture Sonar and Radar Convergences}

\author{
James Candy, Cochair \\ P.O. Box 808, L-156, Univ. of California, Lawrence Livermore Natl. Lab., Livermore, CA 94551, USA
}

Hugh Griffiths, Cochair

Defense college of, management and Technology, Academy of United Kingdom, Cranfield University, Swindon, UK

René Garello, Cochair

ENST-Bretagne, Dpt ITI, CS 83818, Brest Cedex 03, 29238, France

\section{Contributed Paper}

\section{1:40}

5aUWi1. Several imaging algorithms for synthetic aperture sonar and forward looking gap-filler in real-time and post-processing on IXSEA's "Shadows" sonar. Frédéric Jean (IXSEA, 46, quai François Mitterrand, 13600 La Ciotat, France, frj@ixsea.com)

SHADOWS is a new sonar system developed by IXSEA SAS. It is a towed composed of a synthetic aperture side-scan sonar and a forwardlooking sonar. The system produces SAS images with a constant resolution $15 \mathrm{~cm}$ from $30 \mathrm{~m}$ to $300 \mathrm{~m}$ on each side. It also fills the nadir gap from 0 to $30 \mathrm{~m}$ with images having $40 \times 15 \mathrm{~cm}$ resolution. The images are provided in real-time and are georeferenced. The synthetic aperture sonar algorithm uses
INS data combined with the Displaced Phase Center Algorithm (DPC). Differences between INS and DPC navigations can be used to compute a topography profile of the ground. The post processing and real-time modes differ on the weight given between INS and DPC, and on the approximations to be done. The real-time beam-forming algorithm used is the timedomain fast factorized back projection which can be pushed to an exact back projection in the post processing mode. The forward-looking sonar uses a patented "sectorized emission" architecture. The images are side-scanlike. The real-time algorithm can be customized to make some incoherent integration on several pings increasing the contrast but slightly decreasing the resolution. A post processing algorithm can also provide an animation on a specific contact on the floor.

\section{Invited Paper}

\section{2:00}

5aUWi2. Passive synthetic aperture as an experimental tool. Edmund J. Sullivan (EJS Consultants, 46 Lawton Brook Lane, Portmouth, RI 02871, USA, PADDYPRIEST@aol.com), William M. Carey (College of Enginering, Boston University, 110 Cummington St, Boston, MA 02215, USA, wcarey@bu.edu), Jason D. Holmes (BBN Technologies, 10 Moulton St., Cambridge, MA 02318, USA, jholmes@bbn.com), James F. Lynch (Woods Hole Oceanographic Institution, 98 Water Street, Bigelow 203A, MS-11, Woods Hole, MA 02543, USA, jlynch@whoi.edu)

The use of autonomous undersea vehicle towed arrays offer an inexpensive way to carry out oceanographic measurements. Due to the small size and limited power of such vehicles, the physical aperture of the array is necessarily limited and the tow speed is low. However, there is an advantage in that short arrays at low speeds can have no flow noise. In addition the use of passive synthetic aperture (PSA) to provide spatial resolution and gain can play a major role. Here, we describe the use of two forms of passive synthetic array processing used in a shallow-water experiment. A six-element towed array, with $0.75 \mathrm{~m}$ spacing, was used to estimate the horizontal wave numbers of a propagation channel. A narrow-band form of PSA will be described which was used to generate coherent spatial gain by generating an aperture hundreds of wavelengths and provided accurate estimates of the horizontal modal wave numbers at frequencies les than $600 \mathrm{~Hz}$. A broadband form of PSA was used to provide bearing estimation of a ferry passing through the area. The ferry's broadband propulsion noise, which was in the $900 \mathrm{~Hz}$ region, was used as the source. An overall increase in gain is demonstrated. 


\title{
Contributed Papers
}

12:20

5aUWi3. Radar and sonar interferometry. René Garello (ENSTBretagne, Dpt ITI, CS 83818, 29238 Brest Cedex 03, France, rene.garello@telecom-bretagne.eu), Christophe Sintes (ENST-Bretagne, Dpt ITI, CS 83818, 29238 Brest Cedex 03, France, christophe.sintes @enst-bretagne.fr), Didier Gueriot (Telecom Bretagne, Dept iTi - Technopole Brest-Iroise, CS 83818, 29238 Brest, France, didier.gueriot @ telecom-bretagne.eu), Jean-Marie Nicolas (Ecole Nationale Supérieure des Télécommunication de Paris, Telecom ParisTech, Département TSI, 46 rue Barrault, 75634 Paris Cedex 13, France, nicolas@enst.fr)

This paper is an attempt to compare two interferometric processings. The first one is applied to traditional space-borne radar (SAR) and the second on recent interferometric sonar data. Few comparisons between those tech-niques have already been made, despite the fact that they share many similar principles, only a. Thus, the key idea of this article is to present both techniques with assets, drawbacks and specific "tricks" used in dataprocessing. The first part introduces briefly both sensors and compares signal and processing techniques used for both of them. The second part deals with interferometry, and more precisely with underwater and satellite interferometry. Then a noise-pollution analysis is performed on both techniques followed by bias removal methods for getting interferometric information. The conclusion summarizes the similarities between sonar and radar processing, pointing at the techniques that can applied to both
12:40

5aUWi4. Interferometric synthetic aperture processing: a comparison of sonar and radar. Michael Hayes (University of Canterbury, Private Bag 4800, 8022 Christchurch, New Zealand, michael.hayes @ canterbury.ac.nz), Peter T. Gough (University of Canterbury, Private Bag 4800, 8022 Christchurch, New Zealand, peter.gough@ canterbury.ac.nz)

Interferometric aperture synthesis is an inverse problem that attempts to form an elevation map of the earth (in the case of radar) or a bathymetric map of the seafloor (in the case of sonar). In both cases, a pair of (nominally) vertically displaced transducers is configured as an interferometer. After aperture synthesis is performed to produce a pair of images, the height of each resolvable scatterer can be estimated using time delay estimation between the image pairs and knowledge of the system geometry. While interferometric synthetic aperture sonar (InSAS) seems like an obvious extension of the methods of interferometric synthetic aperture radar (InSAR), the height estimation algorithms are surprisingly different. In this paper we start with the principle of generalised correlation for optimal time delay estimation. This filters the signals to maximise their coherence since the accuracy of the time delay estimates, and thus the height estimates, strongly depends upon the signal coherence. We then consider the fundamental differences between InSAR and InSAS; namely the relative signa bandwidth, aperture sampling rate, and geometry and show how application of generalised correlation time delay estimation leads to the differences in how InSAS and InSAR signals are processed.

\section{Session 5pAAa}

\section{Architectural Acoustics and Musical Acoustics: New Measurement Parameters in Performing Arts Spaces II}

\author{
Lily M. Wang, Cochair \\ University of Nebraska - Lincoln, 1110 S. 67th St., Omaha, NE 68182-0681, USA \\ Brian F. Katz, Cochair \\ LIMSI-CNRS, B.P. 133, Orsay, 91403, France
}

Invited Paper

$3: 20$

5pAAa1. Determining acoustical parameters using cochlear modeling and auditory masking. Jasper Van Dorp Schuitman (University of Technology Delft, Lorentzweg 1, 2628 CJ Delft, Netherlands, J.vanDorpSchuitman@tudelft.nl), Diemer De Vries (University of Technology Delft, Lorentzweg 1, 2628 CJ Delft, Netherlands, d.devries@tudelft.nl)

The acoustical qualities of a concert hall or any other room are generally expressed using acoustical parameters determined from impulse responses. From microphone array measurements it turned out that these parameters can fluctuate severely over small distances, whereas the perceptual cues for which these parameters are supposed to be a measure remain constant. This means that a local parameter value has a very low predictive value for acoustic quality. In this research, cochlear modeling techniques and simulations of auditory masking effects have been applied to model human hearing. These techniques together model various stages in the auditory path, like the movement of the basilar membrane inside the cochlea and mechanisms inside the brains. It turns out that determining acoustical parameters using this representation leads to results which show much less spatial fluctuations, and are closer to human perception. 


\section{Contributed Papers}

\section{3:40}

5pAAa2. Sound strength and reverberation time in small concert halls. Marc Aretz (RWTH Aachen University, Institute for Technical Acoustics, Neustraße 50, 52066 Aachen, Germany, Marc.Aretz@akustik.rwth-aachen .de), Raf Orlowski (Arup Acoustics, St. Giles Hall Pound Hill, CB3 0AE Cambridge, UK, Raf.Orlowski@arup.com)

Many small concert halls are being built in music departments in schools and universities and these have to cater for a wide variety of musicalensembles ranging from orchestras to solo performers. Such diverse musical forces require different acoustic conditions in terms of reverberation and loudness and so variable acoustics are frequently provided. However, introducing absorption decreases reverberation and loudness and so a careful balance needs to be struck between controlling loudness and maintaining reverberation. In the course of this study a series of measurements was carried out in six small concert halls in Cambridge, UK, which accommodate a range of sizes of musical ensembles from quartets to orchestras, in order to determine the range of reverberation times and sound strengths, including changes due to variable absorption. The measured strength levels were compared to values derived from traditional and revised theory on strength calculations in order to assess the accuracy of the theories for small chamber music halls. The measured values of strength levels were observed to be mostly lower than the predicted ones. In order to account for this difference (particularly in spaces with added absorption) a combination of Barron's revised theory and the Vorländer correction factor is proposed.

\section{4:00}

5pAAa3. Proposition of new acoustical parameters to analyze the 3D spatial composition of sound in music spaces. Alban A. Bassuet (Arup Acoustics, 155 Avenue of the Americas, New York, NY 10013, USA, alban .bassuet@arup.com)

From acoustic measurements conducted in more than 100 renowned historical music spaces, for the Constellation Project, this paper proposes new acoustical parameters created to better describe the 3D spatial composition of sound in music spaces. Using B-format recordings, the author is proposing a visualization algorithm to plot the acoustic intensity at specific time frames and ranges. The acoustic energy is decomposed into relevant space segments and energy ratio parameters LH (lateral frontal high versus lateral frontal low), and FR (lateral rear high versus lateral rear low) are deduced and proposed for analyzing the distribution of sound and the envelopment characteristics of the room. Examples of intensity plots and of the proposed 3D acoustical parameters are given for various room types ranging from small to large concert halls, small to large opera houses, famous organ churches and Roman basilicas.

\section{4:20-4:40 Break}

\section{Invited Paper}

5pAAa4. Early reflection surfaces in Concert Halls - a new quantitative criterion. Yann Jurkiewicz (Kahle Acoustics, 188 avenue Molière, 1050 Brussels, Belgium, yjurkiewicz@kahle.be), Eckhard Kahle (Kahle Acoustics, 188 avenue Molière, 1050 Brussels, Belgium, kahle@kahle.be)

A new acoustic parameter has been defined for the acoustic brief of the Philharmonie de Paris Concert Hall. With a seating capacity of 2400 and the audience enveloping the performers on all sides, the new hall will be at the upper limit of the ideal range for symphonic music, and an efficient acoustic design was called for. In order to relate architectural design to acoustic efficiency, and based on quantitative study of existing halls, an early efficiency parameter was developed. For the Paris Philharmonie the brief requested a total area of $1400 \mathrm{~m}^{2}$ of surfaces being able to create early reflections, with $500 \mathrm{~m}^{2}$ being less than $15 \mathrm{~m}$ from the stage. The studies leading to the definition and the justificantion of the parameter will be presented. Another, more accurate definition expresses the early efficiency parameter in terms of the solid angle for a source on stage, allowing generalization of the new criterion for all hall sizes.

\section{Contributed Paper}

\section{5:00}

5pAAa5. Diffuseness and intensity analysis of spatial impulse responses. Tapio Lokki (Helsinki University of Technology, P.O. Box 5400, 02015 TKK, Finland, Tapio.Lokki@tkk.fi)

Spatial impulse responses, meaning responses measured with a microphone grid, were measured in seven concert halls. The microphone array consisted of 12 omni-microphones, enabling a construction of three intensity pairs (in $\mathrm{x}, \mathrm{y}$, and $\mathrm{z}$ directions) with $1 \mathrm{~cm}$ spacing and three intensity pairs with $10 \mathrm{~cm}$ spacing. In each hall impulse responses were measured with at least three loudspeaker and four microphone positions. They were analyzed with directional audio coding methodology, which enables analysis of diffuseness and instantaneous intensity as a function of time and frequency. In other words, with this analysis it is possible to analyze the directions of early reflections and to estimate diffuseness of sound field in a measurement position. Preliminary results indicate that diffuseness is quite similar in different positions in one hall, but it varies more between halls. The directions of early reflections are hard to visualize, however some example videos are shown to get an idea about the possibilities of such an analysis technique.

\section{Invited Paper}

5pAAa6. Experiments with the orchestral impulse response. Gary W. Siebein (Univ. of Florida, 231 Arch, PO Box 115702 , Gainesville, FL 32611, USA, gsiebein@siebeinacoustic.com), Robert M. Lilkendey (Siebein Associates, Inc., 625 NW 60th Street, Suite C, Gainesville, FL 32607, USA, rlilkendey@siebeinacoustic.com), Hyun Paek (Siebein Associates, Inc., 625 NW 60th Street, Suite C, Gainesville, FL 32607, USA, hpaek@siebeinacoustic.com), Chris Jones (Siebein Associates, Inc., 625 NW 60th Street, Suite C, Gainesville, FL 32607, USA, cjones@siebeinacoustic.com), Joshua Fisher (Siebein Associates, Inc., 625 NW 60th Street, Suite C, Gainesville, FL 32607, USA, jfisher@siebeinacoustic.com), Youngmin Kwon (Univ. of Florida, 231 Arch, PO Box 115702, Gainesville, FL 32611, USA, ymkwon@ hotmail.com)

Experiments were conducted in a large multipurpose performance hall to examine the differences in listening quality and physical acoustical measurements that resulted from using a single dodecahedral loudspeaker as the sound source and an array of multiple 
directional loudspeakers spread across the stage to simulate the various sections of an orchestra. Significant differences in listening qualities were recorded by listeners at three locations in the hall. The impulse responses recorded using the two systems varied dramatically in structure and in some of the typical acoustic metrics measured. However, there were also similarities among many measurements using the two systems. The research indicates the need for new measurement parameters to express the significant architectural features of the room and the physical acoustical difference that caused the perceived qualities of the sound field to vary. Preliminary analysis of the existing and proposed parameters will be presented.

\section{Contributed Papers}

\section{$5: 40$}

5pAAa7. Relevance of acoustic parameters for musician communication. Anne Guthrie (1926 5th Ave, Troy, NY 12180, USA, guthra2@rpi.edu), Jonas Braasch (Rensselaer Polytechnic Institute, Greene Bldg., 110 8th St., Troy, NY 12180, USA)

In situations of indeterminate musical performance (particularly in telepresence, where acoustic degradation is a frequent concern), autonomous musical communication, both practical and artistic, forms the crux of the musical material. The relevance of stage acoustic and psychoacoustic parameters to contemporary performance situations must be re-examined with regards to the heightened importance of communication. Parameters developed by A. C. Gade, J. Meyer, and D. Brungart are starting points for this examination. Experiments are conducted with four instrumentalists playing excerpts from a composition by Christian Wolff (open notation allows for measurable variations depending on communication quality), communicating telematically between two virtual environments. Parameters determined by questionnaire to have the strongest effect on quality and efficiency of communication are varied at intervals and evaluated by the performers. Five parameters are tested: Self-to-Others Ratio, Commonality of Aural Space, Masking of Individual Voices, Visual-Audio Synchrony, and Position/ Directivity. The performances are recorded and analyzed for variations in musical content, such as dynamics, rhythm, register, and density. The three sets of data (objective parameters, performer evaluation, and musical analysis) are compared to determine the effects of the selected parameters on musical communication.

\section{6:00}

5pAAa8. A qualitative and quantitative analysis of impulse responses from balloon bursts. Dominique J. Cheenne (Columbia College Chicago,
Department of Audio Arts \& Acoustics, 33 East Congress, Suite 601, Chicago, IL 60605, USA, dcheenne@colum.edu), Mauricio Ardila (Columbia College Chicago, Department of Audio Arts \& Acoustics, 33 East Congress, Suite 601, Chicago, IL 60605, USA, mardila@colum.edu), Connie G. Lee (Columbia College Chicago, Department of Audio Arts \& Acoustics, 33 East Congress, Suite 601, Chicago, IL 60605, USA, miss.connie.lee @ gmail.com), Ben Bridgewater (Columbia College Chicago, Department of Audio Arts \& Acoustics, 33 East Congress, Suite 601, Chicago, IL 60605, USA, captain.cranium@gmail.com)

Anechoic recordings of balloon bursts were systematically acquired for various conditions of balloon diameters, puncture location, and inflation pressure. The recordings were analyzed to derive the average frequency spectrum over the effective duration of the acoustic impulse. Although the data show the well-known limitations for the impulse responses (in terms of repeatability and directional behavior) when viewed at high resolution, the results are quite consistent when averaged over one-third octave bands and reveal that the diameter factor (the ratio between the diameter of the inflated balloon to that of its stated maximum), rather than the overall diameter of the balloon, is a good indicator of the sound pressure level, especially above $200 \mathrm{~Hz}$. The study proposes some simple empirical formulas to predict the quantitative sound pressure level and the qualitative spectral response (using the spectral centroid and skewness) from balloon bursts, based on the inflation factor as a variable. The study also offers suggestions to maximize the value of the balloon-burst methodology in building acoustics measurements by describing an effective way to measure reverberation time while simultaneously acquiring useful directional information associated with the reflected sound. 


\title{
Session 5pAAb
}

\section{Architectural Acoustics: Coupled Volume Acoustics II}

\author{
Jason E. Summers, Cochair \\ U.S. Naval Res. Lab., Acoust. Div., Code 7142, 4555 Overlook Ave. SW, Washington, DC 20375, USA \\ Alexis Billon, Cochair \\ Universite de Liege, INTELSIG group - Département E.E.I., B28 Sart-Tilman, Liege, 4000, Belgium
}

\section{Invited Papers}

\author{
3:40
}

5pAAb1. Soundfields in coupled rooms: A theoretical and phenomenological synopsis. Jason E. Summers (U.S. Naval Res. Lab., Acoust. Div., Code 7142, 4555 Overlook Ave. SW, Washington, DC 20375, USA, jason.summers@nrl.navy.mil)

In systems of acoustically coupled rooms, energy in the reverberant field is exchanged between constituent rooms via transondentboundaries. Energy in the direct field can be distributed between rooms by the same mechanism. These exchanges of energy have been able to explain the phenomenon of multiple-slope decay curves. Likewise, they result in spatial and spectral variations in steady-state SPL and decay-curve shape. The basic form of the decay curve is governed by the gross locations of the source(s) and receiver: which room(s) they occupy, in addition to properties of the rooms themselves: volume, surface area, and absorption. Historically, these basic dependencies have been well explained by statistical-acoustics (SA) models. More subtle variations in decay-curve shape result from the fine-scale locations of source(s) and receivers relative to one another and boundary regions through which energy is exchanged (e.g., apertures). By accounting for radiation from boundaries, and propagation delays within and between rooms, more sophisticated SA models can reproduce these effects. Even so, these models fail when SA assumptions are violated or energy transfer becomes so great (e.g., large aperture areas) that room boundaries are ambiguous. In these cases, newer computational models yield accurate predictions and physical insight. [Work supported by ONR.]

\section{4:00}

5pAAb2. On the use of diffusion equations to model the acoustics of coupled rooms. Alexis Billon (Universite de Liege, INTELSIG group - Département E.E.I., B28 Sart-Tilman, 4000 Liege, Belgium, abillon@ulg.ac.be), Vincent Valeau (Laboratoire d'Etudes Aérodynamiques (LEA), Université de Poitiers - ENSMA - CNRS, Bâtiment K, 40 Avenue du Recteur Pineau, F-86022 Poitiers, France, vincent.valeau@lea.univ-poitiers.fr), Judicaël Picaut (Lab. Central des Ponts et Chaussées, Division Entretien, Sécurité et Acoustique des Routes, Route de Bouaye - BP 4129, 44341 Bouguenais Cedex, France, Judicael.Picaut@lcpc.fr), Cédric Foy (CEBTP-SOLEN, 12 Avenue Gay Lussac, ZAC La Clef Saint Pierre, 78990 Elancourt, France, c.foy@cebtp.fr), Anas Sakout (LEPTIAB Université de La Rochelle, Avenue Michel Crépeau, 17042 La Rochelle Cedex 01, France, asakout@univ-lr.fr)

The acoustics of coupled rooms are characterized by energy exchanges through apertures and/or partition walls. The use of systems of diffusion equations allows to predict the temporal and spatial energy distributions in these configurations quite accurately. In this presentation, the diffusion formalism for room acoustics-prediction is summarized. The systems of equations to be solved in the case of coupling through an aperture and through a partition wall are presented. For two rooms coupled through an aperture (two classrooms connected through an open door), the results obtained with the diffusion model are compared to experimental data, in terms of sound pressure levels and sound decays. On the other hand, for the case of two classrooms connected through a partition wall, the diffusion model is compared to experimental data in terms of sound pressure level difference only. Finally, an engineering application is presented in the configuration involving a workroom including multiple sound sources (e.g., machines) connected to offices through open and closed doors.

5pAAb3. Modeling and analysis of acoustically coupled spaces using a diffusion equation model. Yun Jing (Rensselaer Polytechnic Institute, Greene Building, School of Architecture, 110 8th Street, Troy, NY 12180, USA, jingy@rpi.edu), Ning Xiang (Rensselaer Polytechnic Institute, Greene Building, School of Architecture, 110 8th Street, Troy, NY 12180, USA, xiangn@rpi.edu)

Acoustically coupled spaces have been studied and applied to concert halls due to a number of interesting phenomena inside the spaces, including nonexponential energy decays, which are believed to benefit both desired clarity and reverberance. A diffusion equation model has been recently applied to acoustically coupled spaces to predict both steady-state and time-dependent sound field [A. Billon, et. al., J. Acoust. Soc. Am., 120, 2006, pp. 2043-2054], good agreements between simulations and experimental measurements have been found. In this paper, the diffusion equation along with a recently proposed modified boundary condition [Y. Jing and N. Xiang, J. Acoust. Soc. Am., 123, 145-153 (2008)] is used, to reveal intriguing characteristics of coupled spaces, including the sound pressure level distribution along the aperture, energy flow in both rooms, and location dependence of the acoustic source on energy decay characteristics. Experimental results are employed to verify the model, and show the capability of the diffusion equation model for guiding the design of coupled spaces. 
5pAAb4. Low frequency evaluation of steady-state pressure distribution and reverberation time in two-room coupled system Miroslaw Meissner (Institute of Fundamental Technological Research PAS, Swietokrzyska 21, 00-049 Warsaw, Poland, mmeissn@ippt .gov.pl)

A modal expansion method supported by a numerical implementation has been used for studying acoustic properties of coupled room system composed of two connected rectangular enclosures. In a theoretical model a low frequency limit was considered, where modes are lightly damped, thus they were approximated by eigenmodes of a hard-walled room. Eigenfunctions and eigenfrequencies were computed numerically via an application of the forced oscillator method. Calculation results have shown a great influence of absorbing material location and sound source position on the distribution of acoustic pressure and sound decay inside enclosures. As was shown it is the result of a modal localization caused by a generation of modes with eigenfrequencies very close to frequencies of modes in rectangular prisms having the same dimensions as enclosures. When one of enclosures was much more absorbent than the other one, calculation data have demonstrated an interaction of modes during a sound decay that produces reverberant curves with a rapid initial decay and a shallow late decay slope. As was found a "sagging" appearance of decay curves occurs when a late sound decay is dominated by a decay of eigenmodes localized in an enclosure with a weak sound damping.

\section{Contributed Paper}

5:00

5pAAb5. The application of acoustically coupled spaces in concert hall design. Christopher Jaffe (167 East Rocks Road, Norwalk, CT 06851, USA, ADK117@GMAIL.COM)

At mid-twentieth century, a hall having a reverberation decay curve with a late arriving extended second slope was considered an acoustical failure. It was assumed that that the level of the extended reverberation in the hall would interfere with the ongoing running music of the ensemble and reduce orchestral clarity, definition and transparency. To my knowledge, only two practitioners pioneered the utilization of physical acoustic coupling in concert shell and concert hall design during those early years. One was Russell Johnson, when he was with Bolt Beranek and Newman and later with his own firm Artec. The other was me with both my firms Stagecraft Corporation and Jaffe Acoustics. This paper discusses how the need to improve the concert hall environment of American multi-use theatres led to the application of coupling techniques in these halls and how both Mr. Johnson and I later applied physical acoustic coupling in single room concert spaces.

\section{Invited Paper}

5pAAb6. Multivariable analysis of energy decay in coupled volume rooms: How can we objectively describe what we perceive in coupled volume performance spaces? Todd L. Brooks (Artec Consultants Inc, 114 W 26th ST FL 12, New York, NY 10001, USA, tlb@artecconsultants.com), Ted Pyper (Artec Consultants Inc, 114 W 26th ST FL 12, New York, NY 10001, USA, tap@artecconsultants.com), Kelly A. Aston (Artec Consultants Inc, 114 W 26th ST FL 12, New York, NY 10001, USA, ka@artecconsultants.com), Damian J. Doria (Artec Consultants Inc, 114 W 26th ST FL 12, New York, NY 10001, USA, dd @ artecconsultants.com)

The behavior of coupled volumes in room acoustics is commonly characterized by multiple slopes observed in sound energy decay curves, as measured (or modeled) using an omnidirectional receiver. This characterization is often limiting and can not adequately explain the change in character of sound energy decay with time that we perceive as listeners in music performance spaces that employ coupled volumes. By investigating energy decay as a function of several independent variables, including time, frequency, and direction of energy arrival, we seek to forge new objective measures that relate to what a listener actually hears in a coupled volume concert hall. We will discuss relevant geometry and materials of several coupled volume performance spaces designed by Artec Consultants Inc, describe subjective phenomena we have observed in these halls, and present results of our ongoing investigation of new objective metrics geared to better characterize coupled volume music performance spaces.

\section{Contributed Paper}

5:40

5pAAb7. Theoretical considerations in the prediction of decay times for the Philharmonie de Paris. Thomas Scelo (Marshall Day Acoustics LTD, P O Box 5811, Wellesley St., 1000 Auckland, New Zealand, thomas.scelo@marshallday.co.nz), Harold Marshall (Marshall Day Acoustics LTD, P O Box 5811, Wellesley St., 1000 Auckland, New Zealand, harold.marshall@marshallday.co.nz), Joanne O. Valentine (Marshall Day Acoustics LTD, P O Box 5811, Wellesley St., 1000 Auckland, New Zealand, joanne.valentine@marshallday.co.nz)

The brief for the Philharmonie de Paris includes the requirement that the hall should combine great clarity with high reverberation. The proposed solution, which won the design competition, consists of two nested chambers: an inner space producing acoustical intimacy and an outer space with its own architectural and acoustical presence. The interaction between these two spaces gives the possibility for the full range of acoustical adaptability required in the acoustical brief. This paper reports on some theoretical modelling work for the hall where the geometry considered is first described in the context of coupled space modelling. The predicted range of the variability achieved in the design by closure of the coupling openings is then presented. Finally, the paper discusses the appropriateness of these models when predicting the decay times in such a complex geometry. 


\title{
Invited Paper
}

6:00

5pAAb8. Adjustable acoustics --- Coupled volumes in Artec concert halls: an extravagance or necessity? Tateo Nakajima (Artec Consultants Inc, 114 W 26th ST FL 12, New York, NY 10001, USA, tn@artecconsultants.com), Damian J. Doria (Artec Consultants Inc, 114 W 26th ST FL 12, New York, NY 10001, USA, dd@ artecconsultants.com), Edward Arenius (Artec Consultants Inc, 114 W 26th ST FL 12, New York, NY 10001, USA, ea@artecconsultants.com), Andrew Morgan (Artec Consultants Inc, 114 W 26th ST FL 12, New York, NY 10001, USA, ajm@artecconsultants.com)

Led by its founder, Russell Johnson, Artec has developed an unequalled body of experience in the practical application of coupled volumes in the design and construction of concert halls. This paper will present a survey of selected past and future Artec projects from the point of view of the artists and venue managers that perform and work in these halls on a daily basis. Are adjustable acoustics an extravagance or a necessity? What practical problems have been encountered and are they inherent in the concept of adjustability or can they be avoided? How have the musicians reacted? And what is the future of adjustable acoustics?

FRIDAY AFTERNOON, 4 JULY 2008

ROOM 343, 1:40 TO 4:20 P.M.

\section{Session 5pAO}

\section{Acoustical Oceanography and ECUA: Acoustical Tomography and Long Range Propagation}

\author{
Timothy Duda, Cochair \\ Woods Hole Oceanographic Institution, 98 Water Street, Bigelow 107, MS-12, Woods Hole, MA 02543, USA
}

Yann Stephan, Cochair

SHOM, 13 rue du Chatellier, CS 92803, Brest cedex 2, 29228, France

\section{Contributed Papers}

\section{$1: 40$}

5pA01. Ocean acoustic tomography using a double-beamforming algorithm. Ion Iturbe (GIPSA-lab, dep. DIS, 961, rue de la Houille Blanche, 38402 St Martin d'Hères, France, ion.iturbe@gipsa-lab.inpg .fr), Philippe Roux (LGIT - CNRS - Université Joseph Fourier, Maison des Géosciences, 1381 rue de la Piscine, BP 53, 38041 Grenoble, France, philippe.roux@obs.ujf-grenoble.fr), Barbara Nicolas (GIPSA-lab, dep. DIS, 961, rue de la Houille Blanche, 38402 St Martin d'Hères, France, barbara.nicolas@gipsa-lab.inpg.fr), Jérôme I. Mars (GIPSA-lab, dep. DIS, 961, rue de la Houille Blanche, 38402 St Martin d'Hères, France, jerome .mars@gipsa-lab.inpg.fr)

Since Munk and Wunsch proposed the basis for ocean acoustic tomography, many experiments have been performed to estimate sound speed fluctuations in the ocean, using ray identification and measurement of their travel times. However, technical limitations appeared such as the precision of the arrival time measurements or the number of ray arrivals that can be extracted from the signal. Recently, technical improvements allowed more complete experiments using two vertical arrays of sensors (source array and hydrophone array). In this configuration, the signals between each source and receiver are recorded which greatly improve the available information to identify the acoustic rays. One way to increase the number of rays in the tomography algorithm is to perform double-beamforming on the source and receive arrays. With double-beamforming, ray arrivals are separated by emission angle, reception angle and arrival time. Thus, we solve more ray arrivals than with a single beamforming or with a point-to-point approach. In order to avoid previous limitations and to explore acoustical limitations, we study two simple cases through the double-beamforming algorithm: with simulated data and with ultrasonic small-scale experimental data.

$$
\text { 2:00 }
$$

5pAO2. A simulation study of shallow water tomography for coastal monitoring. Olivier Carrière (Université libre de Bruxelles (U.L.B.) Environmental hydroacoustics lab, av. Franklin D. Roosevelt 50, CP 194/5, 1050 Bruxelles, Belgium, ocarrier@ulb.ac.be), Jean-Pierre
Hermand(Université libre de Bruxelles (U.L.B.) - Environmental hydroacoustics lab, av. Franklin D. Roosevelt 50, CP 194/5, 1050 Bruxelles, Belgium, jhermand@ulb.ac.be), Yann Stephan (SHOM, 13 rue du Chatellier, CS 92803, 29228 Brest cedex 2, France, yann.stephan@shom.fr)

Developing operational oceanographic models for coastal environment is an exciting challenge for the next decades. The typical sparsity of assimilated in-situ observations often creates biases in the model predictions reducing the overall accuracy of the forecasting. In such a highly dynamic environment, acoustic tomography can be a good candidate to provide synoptic measurements over wide areas while a range-dependent inversion scheme allows to achieve a reasonable spatial resolution. In this work, we present simulation results of a Kalman-based assimilation of ocean-acoustic data for a basic model of the Ushant front west off Britanny. In a first part, a single vertical slice tomography experiment is simulated for a static front model to study in which way the modal propagation of a multifrequency acoustic signal is affected by the characteristics of the front (position, intensity). In a second part, the problem of assimilating full-field acoustic data into a dynamic model and tracking of the range-dependent sound-speed field is addressed.

2:20

5pA03. Sound-speed estimation from RAFOS transmissions. Emmanuel Skarsoulis (FORTH / IACM, N. Plastira 100, Vasilika Voutes, GR-70013 Heraklion, Greece, eskars@iacm.forth.gr), George Piperakis (FORTH / IACM, N. Plastira 100, Vasilika Voutes, GR-70013 Heraklion, Greece, piperak@iacm.forth.gr)

Acoustic navigation of Lagrangian (moving) floats is carried out by measuring travel times from a number of fixed stations/moorings. A minimum of two fixed stations are needed for location estimation in the horizontal, whereas an additional fixed station is commonly used to remove leftright ambiguity. The signals (RAFOS) and sampling schemes used in ocean acoustic navigation are characterized by limited time resolution (order of $200 \mathrm{msec}$ ), much smaller than the resolution used in travel-time tomography (order of 1-10 msec). The possibility of combining navigation signals (travel 
times) from three fixed stations to multiple moving floats for simultaneous sound-speed estimation and float localization is examined here. The redundant travel-time information in this case offers a significant advantage and makes the accurate estimation of the speed of sound feasible. It is shown that the estimation error for the sound speed decreases with the number of floats, and thus the estimation accuracy improves as the set of floats grows larger. This procedure also leads to improved location estimates for the individual floats. A number of numerical experiments are used to demonstrate the performance of the method. [Work supported by EU/FP6 Damocles project.]

\section{2:40}

5pA04. Using Seagliders for acoustic receiving and communication. Bruce M. Howe (Applied Physics Lab., Univ. of Washington, 1013 Northeast 40th St., Seattle, WA 98105, USA, howe@apl.washington .edu), Michael L. Boyd (Applied Physics Lab., Univ. of Washington, 1013 Northeast 40th St., Seattle, WA 98105, USA, mike@apl.washington.edu)

Underwater gliders are beginning to be used as tools in ocean acoustics and acoustical oceanography. Results from several experiments conducted in summer 2006 with Seagliders equipped with acoustic modems and receivers are described. Off Kauai, a glider received signals from the Acoustic Thermometry of Ocean Climate/North Pacific Acoustic Laboratory $75 \mathrm{~Hz}$ source; subsequent coherent processing showed close to theoretical gain for 12 min records while moving away from the source at ranges $>100 \mathrm{~km}$ with velocity $20 \mathrm{~cm} / \mathrm{s}$ (measured by travel time, Doppler, and dead reckoning). In the Monterey Bay MB06 experiment, two-way communications between other subsea platforms and shore via the acoustic modem-equipped glider was demonstrated (albeit with latency). The results support the future use of gliders as precision navigated platforms, communication and time distribution nodes, and thermometry/tomography mobile receivers. Work supported by the Office of Naval Research

\section{3:00}

5pA05. A decade of acoustic thermometry in the North Pacific Ocean: Using long-range acoustic travel times to test gyre-scale temperature variability derived from other observations and ocean models. Peter Worcester (MPL, Scripps Institution of Oceanography, University of California, San Diego, La Jolla, CA 92093-0238, USA, pworcester@ucsd .edu), Brian D. Dushaw (Applied Physics Lab., Univ. of Washington, 1013 Northeast 40th St., Seattle, WA 98105, USA, dushaw@apl.washington .edu), Rex K. Andrew (Applied Physics Lab., Univ. of Washington, 1013 Northeast 40th St., Seattle, WA 98105, USA, randrew@apl.washington .edu), Bruce M. Howe (Applied Physics Lab., Univ. of Washington, 1013 Northeast 40th St., Seattle, WA 98105, USA, howe@apl.washington .edu), James A. Mercer (Applied Physics Lab., Univ. of Washington, 1013 Northeast 40th St., Seattle, WA 98105, USA, mercer@apl.washington .edu), Robert C. Spindel (Applied Physics Lab., Univ. of Washington, 1013 Northeast 40th St., Seattle, WA 98105, USA, spindel@apl.washington .edu), Bruce Cornuelle (MPL, Scripps Institution of Oceanography, University of California, San Diego, La Jolla, CA 92093-0238, USA, bdc@ucsd .edu), Matthew Dzieciuch (MPL, Scripps Institution of Oceanography, University of California, San Diego, La Jolla, CA 92093-0238, USA, mad @ ucsd.edu), Theodore G. Birdsall (Univ. of Michigan, Electrical Engineering and Computer Science Dept., 1301 Beal Ave., Ann Arbor, MI 481092122, USA, birdsall@umich.edu), Kurt Metzger (Univ. of Michigan, Electrical Engineering and Computer Science Dept., 1301 Beal Ave., Ann Arbor, MI 48109-2122, USA, metzger@umich.edu), Dimitris Menemenlis (Jet Propulsion Laboratory, California Institute of Technology, 4800 Oak Grove Dr., Pasadena, CA 91109, USA, menemenlis@jpl.nasa.gov)

Large-scale, range- and depth-averaged temperatures in the North $\mathrm{Pa}$ cific Ocean were measured by long-range acoustic transmissions over the decade 1996-2006. Acoustic sources off central California and north of Kauai transmitted to receivers throughout the North Pacific. Even though acoustic travel times are spatially integrating, suppressing mesoscale variability and providing a precise measure of large-scale temperature, the travel times sometimes vary significantly on time scales of only a few weeks. The interannual variability is large, with no consistent warming or cooling trends. Comparison of the measured travel times with travel times derived from (i) the World Ocean Atlas 2005 (WOA05), (ii) an upper ocean tem- perature estimate derived from satellite altimetry and in situ profiles, (iii) an analysis provided by the Estimating the Circulation and Climate of the Ocean (ECCO) project, and (iv) simulation results from a high-resolution configuration of the Parallel Ocean Program (POP) show similarities, but also reveal substantial differences. The differences suggest that the data can provide significant additional constraints for numerical ocean simulations. The acoustic data show that WOA05 is a much better estimate of the timemean hydrography than either the ECCO or POP estimates and provide significantly better time resolution for large-scale ocean variability than can be derived from satellite altimetry and in situ profiles.

\section{3:20}

5pA06. Coherence of tracked arrivals in SPICEX. Matthew Dzieciuch (MPL, Scripps Institution of Oceanography, University of California, San Diego, La Jolla, CA 92093-0238, USA, mad@ucsd.edu), Bruce Cornuelle (MPL, Scripps Institution of Oceanography, University of California, San Diego, La Jolla, CA 92093-0238, USA, bdc@ucsd.edu), Peter Worcester (MPL, Scripps Institution of Oceanography, University of California, San Diego, La Jolla, CA 92093-0238, USA, pworcester@ucsd.edu)

In the fall of 2004, $250 \mathrm{~Hz}$ broadband signals were received at $500 \mathrm{~km}$ and $1000 \mathrm{~km}$ ranges on a near full water-column vertical array in the North Pacific ocean. Individual ray arrivals of very high SNR could easily be identified and tracked using a turning-point filter (time-delay beamforming accounting for channel structure), thus providing accurate vertical coherence estimates. The observations can be compared to standard Monte Carlo estimates of coherence made using accurate parabolic-equation acoustic propagation in an ensemble of ocean states consistent with the standard GarrettMunk ocean internal-wave spectrum. Acoustic coherence can also be expressed as a depth- dependent structure function. This is naturally estimated by the full-wave travel-time sensitivity kernel (TSK) which provides a linearized transformation from the internal wave spectrum to the structure function. Environmental measurements were conducted almost concurrently with the acoustic trials, constraining the acceptable ocean variability. Allowances must be made for scattering by sound-speed variability along isopycnals (spiciness) in the upper mixed layer. The most important conclusion to date is that the vertical coherence is depth-dependent but this statement must be qualified by the ability of the beamformer to separate arrivals.

\section{3:40}

5pA07. Travel-time sensitivity kernels in long-range propagation. Emmanuel Skarsoulis (FORTH/IACM, N. Plastira 100, Vasilika Voutes, GR-70013 Heraklion, Greece, eskars@iacm.forth.gr), Bruce Cornuelle (MPL, Scripps Institution of Oceanography, University of California, San Diego, La Jolla, CA 92093-0238, USA, bdc@ucsd .edu), Matthew Dzieciuch (MPL, Scripps Institution of Oceanography, University of California, San Diego, La Jolla, CA 92093-0238, USA, mad @ucsd.edu)

The effect of increasing range on the sensitivity of finite-frequency travel-time observables to sound-speed perturbations is studied using the notion of wave-theoretic sensitivity kernels based on the first Born approximation of the Green's function and the notion of peak arrivals. Travel-time sensitivity kernels are examined in a range-independent background ocean environment. While at medium ranges the kernels exhibit zero-sensitivity cores on the eigenrays, these cores shrink and disappear at long ranges due to refraction and the kernels converge in the vertical towards the corresponding eigenrays; this behavior is not observed in free space. On the other hand the kernels expand in the horizontal cross-range direction and attain their maximum extent at the midpoint between source and receiver, similar to the behavior of the Fresnel volume in free space. Thus, stratification affects the shape of the sensitivity kernel in the vertical preventing expansion with increasing range but not in the horizontal such that the sensitivity kernel takes the form of a folded rug expanding the eigenray in the horizontal cross-range direction. [Work supported by ONR.] 


\section{4:00}

5pA08. High-frequency broadband acoustic current tomography in shallow water. Jing Luo (University of Delaware, College of Marine and Earth Studies, S. College Street, Newark, DE 19716, USA, luojing@udel .edu), Entin A. Karjadi (College of Marine and Earth Studies, University of Delaware, Newark, DE 19716, USA, karjadi@udel.edu), Mohsen Badiey (University of Delaware, College of Marine and Earth Studies, S. College Street, Newark, DE 19716, USA, badiey@udel.edu)

To study current tomography in very shallow water regions a simultaneous oceanographic and broadband (1-25 kHz) acoustic experiment was conducted in the Delaware Bay. The mean water depth was $15 \mathrm{~m}$ and the source-receiver range was $760 \mathrm{~m}$. In this paper, we discuss the feasibility of using reciprocal acoustic transmission for current tomography applications. A beamforming technique is used to resolve the arrival time of direct and surface-bounced rays since in shallow water the received acoustic signals are more complicated due to multiple interactions with bottom and sea surface. Using the experimental data, the accuracy of travel time measurements for variable environmental conditions is examined for different center frequencies and bandwidths. The current velocity prediction results are compared with ADCP measurements to determine the feasibility of current tomography in shallow water.

FRIDAY AFTERNOON, 4 JULY 2008

ROOM 353, 2:00 TO 5:20 P.M.

\title{
Session 5pBB
}

\section{Biomedical Ultrasound/Bioresponse to Vibration: General Topics in Biomedical Ultrasound/Bioresponse to Vibration II}

\author{
Michael R. Bailey, Cochair \\ Center for Industrial and Medical Ultrasound, Applied Physics Lab., University of Washington, 1013 NE 40th St., Seattle,
} WA 98105, USA

Oleg A. Sapozhnikov, Cochair

Center for Industrial and Medical Ultrasound, Applied Physics Lab., University of Washington, 1013 NE 40th St., Seattle, WA 98105, USA

\section{Contributed Papers}

\section{2:00}

5pBB1. Dual confocal ultrasound system for shear wave elastography. Michael D. Gray (Georgia Institute of Technology, Mechanical Engineering, 771 Ferst Drive, Atlanta, GA 30332-0405, USA, michael.gray@me .gatech.edu), James S. Martin (Georgia Institute of Technology, Mechanical Engineering, 771 Ferst Drive, Atlanta, GA 30332-0405, USA, james.martin@me.gatech.edu), Peter H. Rogers (Georgia Institute of Technology, Mechanical Engineering, 771 Ferst Drive, Atlanta, GA 30332-0405, USA, peter.rogers@me.gatech.edu)

A dual confocal transducer system for ultrasound-based elastography is presented. The system is intended to noninvasively measure the complex shear speed in cetacean head tissues, including brain, jaw fat, and melon. The system instrumentation features a pair of dual-element confocal ultrasound transducers, one of which is used to remotely generate low frequency $(100-1000 \mathrm{~Hz})$ shear waves in soft tissues via radiation force, and the other is used to measure the resulting shear wave displacements using Doppler techniques. One transducer is configured as an open ring into which the other transducer can be placed and translated. The relative positions of the transducers are mechanically manipulated in order to measure short-path propagation and estimate shear wave speed and loss. Work supported by ONR.

$$
\text { 2:20 }
$$

5pBB2. Simulated response of the human lung to low-frequency underwater sound using a finite-element-based thoracic model. Mark S. Wochner (Applied Research Laboratories, The University of Texas, P.O. Box 8029, Austin, TX 78713-8029, USA, mwochner@mail.utexas .edu), Yurii A. Ilinskii (Applied Research Laboratories, The University of Texas, P.O. Box 8029, Austin, TX 78713-8029, USA, yura@arlut.utexas .edu), Mark F. Hamilton (Applied Research Laboratories, The University of Texas, P.O. Box 8029, Austin, TX 78713-8029, USA, hamilton@mail .utexas.edu), Evgenia A. Zabolotskaya (Applied Research Laboratories, The University of Texas, P.O. Box 8029, Austin, TX 78713-8029, USA, zhenia@arlut.utexas.edu)

In a previous paper, inhomogeneity within the lungs and its influence on the lung's response to low-frequency underwater sound using a finiteelement-based model was discussed [Wochner, et al., J. Acoust. Soc. Am. 122, 2957 (2007)]. Here we report an extension of the previous work that adds surrounding organs to the finite element model. It is hypothesized that the significant damage that can occur at relatively low amplitudes when the lung is in resonance is due primarily to the shear stresses that can occur in the lung through its interactions with surrounding organs. In particular, the heart, diaphragm, and ribs, all of which have considerably different material properties compared to lung, may have a sizable effect on the lung's response to low-frequency underwater sound. This paper will discuss the resonance frequency, motion, and stress and strain fields produced using this new finite-element-based thoracic model. [Work supported by ONR and ARL:UT IR\&D.]

\section{2:40}

5pBB3. Dual apodization technique for improved contrast. Jesse Yen (University of Southern California, 1042 Downey Way, Los Angeles, CA 90089, USA, jesseyen@usc.edu), Chi Seo (University of Southern California, 1042 Downey Way, Los Angeles, CA 90089, USA, chiseo@usc .edu)

We propose a method to use dual apertures or dual apodization functions to reduce side lobes and clutter for ultrasound imaging. Using a common transmit aperture but different receive apodizations or apertures, we create two point spread functions with very similar main lobes and different sidelobe and clutter signals. Main lobe signals can be distinguished from clutter signals using normalized cross-correlation of the raw radio-frequency data. The normalized cross-correlation coefficient is used as a pixel-by-pixel weighting to pass main-lobe signals and suppress side lobe and clutter 
signals. Main lobe signals will have a high cross-correlation coefficient near 1 and clutter signals will have coefficients between 0 and -1 . Point target simulations show a narrowing of the main beam compared to conventional beamforming at beamwidths $-20 \mathrm{~dB}$ and lower. Using a $5 \mathrm{MHz}$ 128-element linear array, improvements of contrast-to-noise ratio (CNR) of an anechoic cyst compared to delay and sum beamforming exceed $130 \%$ in both simulations and experiments. We also evaluated this method for robustness in the presence of phase aberration. Aberrators ranging from 25-45 ns rms with correlation lengths of 3-5 mm were used. Here, improvements in CNR also exceed $100 \%$ in many cases.

\section{3:00}

5pBB4. Nonlinear ultrasonic imagery of high contrast objects. Régine Guillermin (Laboratory for Mechanics and Acoustics CNRS, 31 chemin Joseph Aiguier, 13009 Marseille, France, guillermin@1ma.cnrs-mrs .fr), Philippe Lasaygues (Laboratory for Mechanics and Acoustics CNRS, 31 chemin Joseph Aiguier, 13009 Marseille, France, lasaygues@1ma.cnrsmrs.fr)

This study is concerned whith the ultrasonic imagery of elastic materials like cylinders or tubes by diffraction tomography technic. The aim of this work is then to solve a nonlinear inverse scattering problem. Various methods can be employed, generally involving a minimization of the differences between modeling data and measurements. The Distorted Born Iterative (DBI) method belongs to the class of algebraic reconstruction algorithms and have therefore been investigated in literature. Very promising results have been obtained both on synthetic and experimental data especially for electromagnetic inverse diffraction problems, but as far as the authors know few ultrasonic experimental results are available. This method was developed to increase the domain of application of the Born approximation to high contrast targets. Iterations are performed numerically solving a forward and an inverse problems at every iteration. This yields quantitative information about the scatterer, such as the speed of sound. Inversions of both numerical and experimental data are presented.

\section{$3: 20$}

5pBB5. Inverse scattering in modern ultrasound imaging. Francesco Simonetti (Imperial College London, Department of Mechanical Engineering, South Kensington Campus, SW7 2AZ London, UK, f.simonetti @imperial.ac.uk)

Progress in solid state electronics and sensor manufacturing has led to the rapid development of ultrasound arrays over the last decade resulting in prototypes with thousands of transreceivers. Ultrasound scanners that use this technology are widely used in medical imaging and are based on beamforming techniques. In a similar fashion to an optical lens, the array forms an aperture which can focus and steer an ultrasound beam in space as it is done by microscopes and telescopes. The beamforming process can be seen as an inverse scattering problem whereby the scattering measurements are used to reconstruct the structure of the object being probed. To achieve this, a model that describes the interaction of the probing wave with the object is required. Beamforming assumes that scattering events occurring at different locations within the object are independent of each other, thus neglecting multiple scattering. Here, it is argue that accounting for more accurate wavematter interaction models in the inverse scattering problem leads to greater image quality than that obtained with conventional beamforming. Experimental images with unprecedented resolution beyond the classical diffraction limit are presented along with tomographic reconstructions of a complex 3D breast phantom that show striking similarities with $\mathrm{x}$-ray CT.

\section{$3: 40$}

5pBB6. Deconvolution of freehand 3D ultrasound data using improved reconstruction techniques in consideration of ultrasound point spread functions. Holger J. Hewener (Fraunhofer IBMT, Ensheimer Strasse 48 66386 Sankt Ingbert, Germany, holger.hewener@ibmt.fraunhofer .de), Robert M. Lemor (Fraunhofer IBMT, Ensheimer Strasse 48, 66386 Sankt Ingbert, Germany, robert.lemor@ibmt.fhg.de)

Medical ultrasound data suffers from blur caused by the volume expansion of the pressure field of the mechanical wave. This blur is dependent on the used excitation pulse and focusing of the ultrasonic wave and can there- fore be examined. In order to improve the overall system resolution for $3 \mathrm{D}$ ultrasound reconstructions we have to know this signal degeneration to compensate it using deconvolution techniques or multicode compounding during the volume reconstruction step. Looking at the ultrasound transfer function we can focus on the simulation and measurement of the "point spread function" especially in the lateral and elevational direction. To understand its effects on a $3 \mathrm{D}$ reconstruction we compute a simulation of freehandultrasound slices based on synthetic phantom structures and given US parameters. Computing a 3D reconstruction of these simulated slices we are able to optimize the reconstruction algorithm itself to archive better resolution in the volume data sets considering ultrasound parameters like beamforming and the excitation pulses.

\section{4:00-4:20 Break}

\section{4:20}

5pBB7. Noninvasive monitoring of mesenchymal stem cells by $1.2 \mathrm{GHz}$ acoustic microscopy. Moritz Von Buttlar (Institute of Experimental Physics II, University of Leipzig, Linnéstr. 5, 04103 Leipzig, Germany, vbuttlar@physik.uni-leipzig.de), Evgeny Twerdowski (Institute of Experimental Physics II, University of Leipzig, Linnéstr. 5, 04103 Leipzig, Germany, twerdowski@physik.uni-leipzig.de), Reinhold Wannemacher (Institute of Experimental Physics II, University of Leipzig, Linnéstr. 5, 04103 Leipzig, Germany, wannemacher@physik .uni-leipzig.de), Wolfgang Grill (Institute of Experimental Physics II, University of Leipzig, Linnéstr. 5, 04103 Leipzig, Germany, grill@physik.unileipzig.de)

Cell-based therapies can benefit from noninvasive and marker-free monitoring techniques for living cells. For this purpose a phase-sensitive scanning acoustic microscope operating at a frequency of $1.2 \mathrm{GHz}$ was combined with a commercial confocal laser scanning microscope. The system is equipped with a live-support system for the long-term observation of living cells. Confocal acoustic imaging with phase and magnitude contrast and confocal laser scanning microscopy can be performed simultaneously. Both techniques are used in reflection from opposing sides of the object. Timelapsed acoustic microscope images of ovine mesenchymal stem cells are presented. For this purpose, a pseudo-3D representation is generated by encoding the unwrapped phase in the height and the magnitude in the brightness. In the case of highly reflective substrates and sufficiently low reflection from the interface between the cells and the surrounding fluid the echo from the top of the cells can be neglected and the phase contrast image can be transformed to a time-of-flight image. In the same approximation the magnitude image provides information about the gradual extinction of the echo signal due to absorption in the cells. The two images can be combined to generate a new form of contrast representing the product of the absorption coefficient and the velocity of sound inside the observed cells.

\section{4:40}

5pBB8. Transverse vibration of prestressed beams: An experimental technique for the determination of dynamic viscoelastic material properties of tissue mimicking materials. Yigit Yazicioglu (Middle East Technical University, Orta Dogu Teknik Universitesi, Makine Muhendisligi Bolumu B-313, 06531 Ankara, Turkey, yigit@metu.edu.tr), Bryn A Martin (University of Illinois at Chicago, 2923 W. 71st Street, Woodridge, IL 60517, USA, flux@ebryn.com), Karen Navarro-Castillo (University of Illinois at Chicago, 842 W. Taylor St. ERF 1072, Chicago, IL 60607, USA, knc_001@yahoo.com.mx), Umit Kutluay (Tubitak-Sage, Samsun Yolu 25 .Km, Tilkicak Tepe Mevkii P.K.16, 06261 Ankara, Turkey, ukutluay@sage.tubitak.gov.tr), Thomas J. Royston (University of Illinois at Chicago, 842 W. Taylor St. ERF 1072, Chicago, IL 60607, USA, troyston @uic.edu)

An experimental dynamic material property identification technique is presented that is based on the theoretical formulations of a vibrating prestressed beam. The technique determines the viscoelastic material properties of tissue mimicking materials that govern their dynamic behavior. Results are presented for silicone-based materials (Sylgard 184, Dow Corning, Midland, MI) that are formed in the lab using a range of mixing ratios and cast in the form of a thick string held between fixed supports under tensile pre- 
stress. The specimens are excited through the transverse harmonic displacement of the boundary. Transverse vibration at an arbitrary location is measured and compared with theory to identify material viscoelastic moduli valid up to at least several hundred Hertz. The presented technique can aid in providing accurate viscoelastic parameter values for phantoms that are used in the development of a range of medical diagnostic techniques that attempt to identify pathology or tissue differentiation via changes in mechanical stiffness and viscosity.

\section{5:00}

5pBB9. Air-borne and tissue-borne sensitivity of skin-radiation acoustic sensors. Matias Zanartu (School of Electrical and Computer Engineering, Purdue University, 206 South Martin Jischke Drive, West Lafayette, IN 47907, USA, mzanartu@ @urdue.edu), Julio C. Ho (Weldon School of Biomedical Engineering, Purdue University, 206 South Martin Jischke Drive, West Lafayette, IN 47907, USA, hoj@purdue.edu), Steve Kraman (Department of Internal Medicine, University of Kentucky, 740 South Limestone Street, Lexington, KY 40536, USA, sskram01@email.uky.edu), Hans Pasterkamp (Department of Pediatrics and Child Health, University of Manitoba, CS516-840 Sherbrook St, Winnipeg, MB R3A 1S1, Canada, pasterkamp@umanitoba.ca), Jessica E. Huber (Department of Speech, Language, and Hearing Sciences, Purdue
University, 500 Oval Drive, West Lafayette, IN 47907, USA, jhuber@purdue.edu), George R. Wodicka (Weldon School of Biomedical Engineering, Purdue University, 206 South Martin Jischke Drive, West Lafayette, IN 47907, USA, wodicka@purdue.edu)

Measurements of body sounds on the skin surface have been widely used in the medical field and continue to be a topic of current research, ranging from the diagnosis of the respiratory and cardiovascular diseases to the monitoring of voice dosimetry. These measurements are normally acquired by means of light-weight accelerometers and/or air-coupled microphones attached to the skin. Such recordings can be corrupted by air-borne sounds that are generated by the subject or by other sources of background noise. In this project, the sensitivity of various commonly used bioacoustic sensors to airborne sounds was evaluated and compared with their sensitivity to tissueborne body sounds. To delineate the sensitivity to each pathway, the sensors were first tested in-vitro, and then on human subjects. The results indicated that in many cases the air-borne sensitivity is sufficiently high to significantly corrupt body sound signals. The effectiveness of different air-borne insulation devices was also evaluated. Spectral analysis showed that simple acoustic insulators (e.g., passive hearing protectors) provide significant attenuation within the range of frequencies of interest for most applications, particularly when using air-coupled microphones.

FRIDAY AFTERNOON, 4 JULY 2008

ROOM 252A, 2:00 TO 6:20 P.M.

\section{Session 5pNSa}

Noise, ASA Committee on Standards, Architectural Acoustics, and EURONOISE: Classroom Acoustics II

Louis C. Sutherland, Cochair

Consultant in Acoustics, 27803 Longhill Dr., Rancho Palos Verdes, CA 90275-3908, USA

Luigi Maffei, Cochair

Built Environment Control Laboratory Ri.A.S., Second University of Naples, Abazia di S. Lorenzo, Aversa, 81031, Italy

David Lubman, Cochair

DL Acoustics, 14301 Middletown Lane, Westminster, CA 92683, USA

\section{Invited Paper}

\section{2:00}

5pNSa1. Are classrooms in historical buildings compatible with good acoustics standards? Luigi Maffei (Built Environment Control Laboratory Ri.A.S., Second University of Naples, Abazia di S. Lorenzo, 81031 Aversa, Italy, luigi.maffei@unina2.it), Gino Iannace (Built Environment Control Laboratory Ri.A.S., Second University of Naples, Abazia di S. Lorenzo, 81031 Aversa, Italy, gino.iannace@unina2.it), Massimiliano Masullo (Built Environment Control Laboratory Ri.A.S., Second University of Naples, Abazia di S. Lorenzo, 81031 Aversa, Italy, ing.masullo@libero.it)

Many surveys and researches have underlined that the acoustic characteristics of classrooms are strictly connected to performances of students and to the stress of the teachers during lessons. In standard classrooms sound quality can be easily reached without sound amplification but introducing appropriate sound absorbing/scattering materials at the ceiling and/or at the vertical walls. Nevertheless in historical buildings with vaults or trusses, high walls and many architectural restrictions imposed by district superintendent, it could be very difficult to achieve good acoustics standards with widespread solutions. In this paper the acoustic performances of different classrooms in an historical Monastery actually center of the Faculty of Architecture of the Second University of Naples are analysed. After these analysis, compatible architectural and acoustic solutions to improve the sound quality were developed and tested in laboratory measurements and then applied in some classrooms to verify the benefits. 


\section{Contributed Paper}

5pNSa2. Results of acoustical treatments in existing classrooms Arianna Astolfi (Politecnico di Torino, Department of Energetics, Corso Duca degli Abruzzi, 24, 10129 Turin, Italy, arianna.astolfi@ polito .it), Alessia Griginis (Onleco srl, Via Pigafetta, 3, 10129 Turin, Italy, griginis@onleco.com)

In 2001, 2002, and 2005, the Department of Energetics of the Politecnico di Torino has carried out in-field objective and subjective surveys with the aim of evaluating the acoustical quality in secondary-school classrooms of the Province of Turin (Italy). From the results the following main problems emerged: high reverberation times, high background noise levels caused mainly by low sound insulation between classrooms and corridors and between adjacent classrooms, low façade sound insulation, excessive teachers' vocal efforts. In this work results of acoustical treatments in some of these existing classrooms are reported. Measurements are made before and after the restorations. The case studies are chosen with reference to different building typologies and urban contexts, and consisted of improvements in sound absorption, sound insulation of internal partitions, doors and façades, and acoustic bridges elimination. They are divided in "light" and "heavy" types. The first, less expensive, with the aim to obtain good acoustical conditions, the latter, more expansive, focused to obtain optimal conditions. In fact, mainly in restoration of public schools, a good level of acoustical quality with contained costs is requested. The treatments are carried out with the aim to constitute a repertory of solutions to apply primarily in school restoration.

\section{Invited Papers}

5pNSa3. Speech perception in classroom noise and reverberation by children with typical and impaired hearing - Preliminary results. Frank Iglehart (Clarke School for the Deaf, 47 Round Hill Road, Northampton, MA 01060, USA, figlehart@clarkeschool .org)

A child's ability to perceive speech in the classroom influences academic progress. In this study, students with a various degrees of hearing loss perceive spoken words in sentences in a classroom. The room contains multiple noise levels and reverberation times (RTs). Classroom reverberation is measured using standardized procedures (with slight modifications to ASTM C423-02a: X2). RTs are controlled by quantities of acoustic panels in the room. Using the BKB-SIN Test, the speech-to-noise ratio at which the students perceive $50 \%$ words correctly (i.e., SNR-50) are measured in three reverberant conditions. Data collected to date indicate that students with severe-to-profound hearing loss $(\mathrm{n}=15$; ages 8-16 years) demonstrated average SNR-50s of $+12 \mathrm{~dB}(\mathrm{SD}=4),+13 \mathrm{~dB}$ ( $\mathrm{SD}=4)$, and $+17 \mathrm{~dB}(\mathrm{SD}=4)$ for conditions of $0.3,0.6$ and $0.9 \mathrm{~s} R$, respectively. Students with typical hearing ( $\mathrm{n}=14$; ages $8-16$ years) had SNR-50s that averaged $-4 \mathrm{~dB}(\mathrm{SD}=2),-3 \mathrm{~dB}(\mathrm{SD}=2)$ and $-2 \mathrm{~dB}(\mathrm{SD}=2)$, respectively. Performance/intensity curves are also calculated in order to estimate minimal optimal listening conditions for each RT. This is an ongoing study. The session presentation will include emerging data on children with other degrees of hearing loss.

5pNSa4. Vocal symptoms in preschool teachers and the working environment. Valdis Inigbjörg Jonsdottir (Tad er Malid, Furuvellir 13, 601 Akureyri, Iceland, valdisj@ismennt.is)

Research has shown that teachers experience a high risk of developing voice problems. Noise levels in classrooms with young children are higher than in classrooms with older students, indicating preschool teachers' voices may be at greater risk. Questionnaires were sent to 88 preschool teachers in five preschools in Akureyri, and the parents of the 424 children in daycare. The study was aimed to obtain information about (1) teachers: experienced vocal symptoms, occurrence of vocal symptoms, opinions on working environment; air quality, noise, heat, acoustics. (2) parents: children's hearing problems. The study also aimed to ascertain noise levels and reverberation time in pre-school classrooms, and to compare teachers' reported ages, teaching experience, vocal symptoms and frequency with findings in other studies. Sound and reverberation-time measurements were taken by the Icelandic Department of Occupational Health and Safety. Reported vocal symptoms were more common among preschool teachers than other teachers, even though their youth and lack of teaching experience was marked. They appeared to be workrelated. $40 \%$ of the children had a history of hearing problems. Noise levels measured were very high. Correlation was found between voice fatigue and high temperature, bad indoor air, and poor acoustics.

5pNSa5. Acoustical requirements of classrooms and new concepts of teaching. Kurt Eggenschwiler (Empa, Laboratory of Acoustics, Ueberlandstrasse 129, CH-8600 Duebendorf, Switzerland, kurt.eggenschwiler@empa.ch), Markus Cslovejcsek (School of Teacher Education at the University of Applied Sciences FHNW, Kuettigerstrasse 42, CH-5000 Aarau, Switzerland, markus.cslovjecsek @ fhnw.ch)

In recent years acoustic requirements for classrooms were published in various guidelines. Of course it is important that the present knowledge will be implemented, but how should this be done? And what is the correspondence of acoustic requirements and new concepts of learning? These questions are the basis of a cooperation of the Laboratory of Acoustics at Empa, Dübendorf and the School of Teacher Education, Aarau in Switzerland. The cooperation itself is one possibility to transfer knowledge. In addition it was planned but not yet realized to develop concepts of knowledge transfer by student work (diploma thesis). Classroom acoustics were discussed in seminars and there were completed some diploma theses. One project dealt with the acoustic problems of a new teaching concept. Part of the concept is that teaching of four groups of pupils takes place in three classrooms and in the corridor. The doors of the classrooms remain open and at the same time pupils work individually, in groups, and a small group is instructed by a teacher. There were found suggestions for improving the acoustics, but for different reason the realization was not possible. 
5pNSa6. An Ambiophonics system for the study of intelligibility in the virtual classrooms. Nicola Prodi (Engineering Dept. Univ. of Ferrara, Via Saragat, 1, 44100 Ferrara, Italy, nicola.prodi@ unife.it), Andrea Farnetani (Engineering Dept. - Univ. of Ferrara, Via Saragat, 1, 44100 Ferrara, Italy, andrea.farnetani@unife.it), Patrizio Fausti (Engineering Dept. - Univ. of Ferrara, Via Saragat, 1, 44100 Ferrara, Italy, patrizio.fausti@ unife.it), Roberto Pompoli (Engineering Dept. - Univ. of Ferrara, Via Saragat, 1, 44100 Ferrara, Italy, roberto.pompoli@unife.it)

In recent years the investigations on the intelligibility has profited by the increasing efficiency of the simulation and rendering technologies of virtual acoustics. In particular the latest systems are well suited to reproduce sound fields whose tridimensional characteristics are quite close to the real ones both objectively and subjectively. In this framework the Ambiophonics system, consisting of the merging of a double stereo-dipole and of an first order Ambisonics system, has gained particular attention. In the configuration used in this work the system employs twelve loudspeakers and is fed either with measured binaural and B-format data or by simulated ones. Based on this apparatus listening tests have been prepared in the Italian language to investigate the acoustics of classrooms and to focus on the effect of the directional characteristics of noise (i.e., fan-coils or tapping from the upper floor) on the intelligibility of words. The whole apparatus is driven by a Labview application which has also the aim of collecting the responses by the testers.

\section{4:00-4:20 Break}

\section{$4: 20$}

5pNSa7. Noise - A stress factor? Acoustic ergonomics at schools. Gerhart Tiesler (University of Bremen/ISF, Grazer Str. 4, D-28359 Bremen, Germany, tiesler@uni-bremen.de), Markus Oberdoerster (Saint-Gobain Ecophon GmbH, Taschenmacherstr. 8, D-23556 Luebeck, Germany, markus.oberdoerster@ecophon.de)

Description of teaching reality is the main topic of this interdisciplinary examination about "acoustic ergonomics of schools." In the first step we analyse how different teaching methods affect basic noiselevel and working noise level. Which effect has an altered room acoustics on the sound levels in the context of each teaching method. The data records available as timeseries allows for the first time analysing single phases of lessons, which are characterized by certain pedagogical characteristics or individual instruction phases. Is it correct to speak of "noise stress" or is this stress an emotional reaction to the kind of? Based on recordings of teachers heartrate we analyse in the third step the effects of noise level on the workload of the teachers as a stress reaction. A distinction is made on the subject of different teaching methods, and on the basis the different room acoustic conditions. In one primary school we had four classes with a RT $>0.5 \mathrm{~s}$ and four further classes with a RT $<0.5 \mathrm{~s}$. At a second primary school we analysed the effect of the room acoustic refurbishment. Finally we investigate factors of fatigue. Which effect has $\mathrm{CO}_{2}$ in the classroom on all people, and the working noise level?

\section{4:40}

5pNSa8. Acoustical evaluation of nonclassroom university learning spaces. Murray Hodgson (The University of British Columbia, Department of Electrical and Computer Engineering, 2332 Main Mall, Vancouver, BC V6T 1Z4, Canada, mhodgson@interchange.ubc.ca), Jorge Garcia Moreno (The University of British Columbia, Department of Electrical and Computer Engineering, 2332 Main Mall, Vancouver, BC V6T 1Z4, Canada, 123calabaza@gmail.com)

This paper reports the results of an acoustical evaluation of non-classroom learning spaces at UBC. In twelve buildings, 25 indoor spaces -- a restaurant, a cafeteria, libraries, dedicated study spaces, building atria, etc. -- used for learning activities by at least 50 people were studied. The evaluation involved physical and acoustical (reverberation time, sound propagation, Speech Intelligibility Index) measurements, and occupant activity and satisfaction questionnaires. Questionnaires were administered three times (morning, lunchtime, and afternoon) on one day. The questionnaires asked about satisfaction with, and the effects of, the acoustical and nonacoustical environments. The acoustical measurement results were compared with established acceptability criteria. Questionnaires were analyzed for differences between times of day, test space, etc. The questionnaire responses and acoustical-measurement results were correlated. Using both as possible predictors, multivariable-regression models for predicting and explaining occupant satisfaction with, and the effects of, the acoustical environment were developed.

5pNSa9. The effect of amplification on children's performance in the classroom. Bridget M. Shield (London South Bank University, Borough Road, SE1 0AA London, UK, shieldbm@1sbu.ac.uk), Julie E. Dockrell (Institute of Education, 25 Woburn Square, WC1H 0AA London, UK, j.dockrell@ioe.ac.uk)

The use of amplification systems in the classroom has the potential to reduce the impact of poor classroom acoustics for typically developing pupils and those with special educational needs (SENs). The immediate benefits of amplified acoustic signals on the performance of 253 primary school children, including 24 children with special needs, were examined. All participants were familiar with the use of the amplification systems. Children's performance was assessed on two verbal measures (spelling and oral comprehension) and one non-verbal measure, using a balanced repeated measures design. It was predicted that the effects of amplification would be evident for spelling and oral language comprehension, but that there would be no discernable effect on speed of processing. Children with SEN were expected to have an added advantage with amplification. The predictions were partially supported. Both gain score analysis and ANOVAs of performance scores with amplification, controlling for performance without amplification, revealed an effect on spelling only for the typically developing children, while children with SENs benefited in both the spelling and the oral comprehension tasks. When installed appropriately, amplification improves children's ability to decode single words; SEN children also benefit in terms of their ability to process oral input. 
5pNSa10. Soundfield amplification is a poor substitute for good classroom acoustics. David Lubman (DL Acoustics, 14301 Middletown Lane, Westminster, CA 92683, USA, dlubman@dlacoustics.com), Louis C. Sutherland (Consultant in Acoustics, 27803 Longhill Dr., Rancho Palos Verdes, CA 90275-3908, USA, lou-sutherland@juno.com)

Soundfield (amplification) systems are widely and often aggressively marketed for small classrooms. In June 2006, the Acoustical Society of America (ASA) issued a public position statement on the use of sound amplification in typical small classrooms http://asa .aip.org/amplification.pdf. This paper attempts to explain why the ASA found that soundfield systems are poor substitutes for good acoustics. Good acoustics for learning requires unoccupied classroom noise levels of $35 \mathrm{dBA}$ or less and midfrequency reverberation times of $0.6 \mathrm{~s}$ or less. At the same time, it is recognized that centralized amplifiers and sound distribution systems can provide valuable educational benefits in small classrooms with good acoustics. Such systems are useful for multimedia presentations and voice reinforcement. The authors urge that this message be brought to the attention of educators and educational facility decision makers.

\section{Contributed Papers}

\section{$5: 40$}

5pNSa11. Subjective evaluation of acoustical quality of lecture rooms with respect to the quality of the sound reinforcement system and the level of background noise. Sanja Grubesa (Faculty of EE and Computing, Unska 3, Department of Electroacoustics, HR-10000 Zagreb, Croatia, sanja.grubesa@fer.hr), Marko Horvat (Faculty of EE and Computing, Unska 3, Department of Electroacoustics, HR-10000 Zagreb, Croatia, marko.horvat@fer.hr), Hrvoje Domitrovic (Faculty of EE and Computing, Unska 3, Department of Electroacoustics, HR-10000 Zagreb, Croatia, hrvoje .domitrovic@fer.hr)

As a part of a general questionnaire on the quality of lecturing on the Faculty of EE and Computing in Zagreb, Croatia, the students have to grade the acoustical quality of lecture rooms in an indirect way by giving answers to several questions included in that questionnaire. As the sound reinforcement system in two largest lecture halls is in rather bad condition, it is to be replaced in the near future with a new one of significantly better quality. The goal of this paper is to examine whether this change is reflected in the grades of acoustical quality given by the students. In the second part of the paper, the students' grades on acoustical quality of two smaller lecture rooms of identical size and acoustic treatment are compared. The comparison is made with respect to the position of these rooms relative to the nearby street as the source of traffic noise. The overall level and spectral content of noise are measured in those lecture rooms. The correlation is then examined between the results of these measurements and the grades given by the students.

\section{6:00}

5pNSa12. Education vs cost - Difficulties in implementing acoustical design standards in classrooms. Patricia M. Scanlon (Cerami \& Associates, 404 Fifth Avenue, New York, NY 10018, USA, pscanlon @ ceramiassociates.com), James Perry (Cerami \& Associates, 404 Fifth Avenue, New York, NY 10018, USA, jperry@ ceramiassociates.com), Victoria J. Cerami (Cerami \& Associates, 404 Fifth Avenue, New York, NY 10018, USA,vcerami@ceramiassociates.com)

Good acoustical design standards for classrooms are established - quiet background noise levels due to mechancial systems and exterior sources, use of absorptive finishes to control reverberation, walls, doors and floors able to control airborne and impact noise transmission. So why do so many schools fail to follow these guidelines? Are private schools even less likely to adhere to these standards? What are the construction and equipment options? We will discuss various educational standards, as well as the reticence of school planners to comply. We will discuss what schools often build, versus what one might say they should build, together with cost comparison. We will review case studies where guidelines were, and were not, followed - examples that reflect the acoustician's mission to educate educators on the value of acoustical design considerations in classrooms. 


\title{
Session 5pNSb
}

\section{Noise, Structural Acoustics and Vibration, Physical Acoustics, and EURONOISE: Sound and Vibration from Explosions in Air II}

\author{
Roger Waxler, Cochair \\ University of Mississippi, NCPA, 1 Coliseum Drive, University, MS 38677, USA
}

Keith Attenborough, Cochair

Open University, Department of Design, Development, Materials and Environment, Walton Hall, Milton Keynes, MK7 6AA, UK

\section{Invited Papers}

\section{2:00}

5pNSb1. High-altitude infrasound calibration experiments. Henry E. Bass (The University of Mississippi - NCPA, 1 Coliseum Drive, University, MS 38677, USA, pabass@olemiss.edu), Eugene T. Herrin (Southern Methodist University, P. O. Box 750395, Dallas, TX 75275, USA, herrin@passion.isem.smu.edu), Paul Golden (Southern Methodist University, P. O. Box 750395, Dallas, TX 75275, USA, pgolden@mail.smu.edu), Robert Woodward (Incorporated Research Institutions for Seismology, 1200 New York Avenue, NW, Suite 800, Washington, DC 20005, USA, woodward@iris.edu), Douglas Drob (Naval Research Laboratory, Space Science Division, 4555 Overlook Avenue, Washington, DC 20375, USA, douglas.drob@nrl.navy.mil), Michael A. Hedlin (University of San Diego California, Scripps Institute of Oceanography, 9500 Gilman Drive, La Jolla, CA 92093, USA, hedlin@ucsd.edu), Catherine De Groot-Hedlin (University of San Diego California, Scripps Institute of Oceanography, 9500 Gilman Drive, La Jolla, CA 92093, USA, cdghedlin@gmail.com), Kris Walker (University of San Diego California, Scripps Institute of Oceanography, 9500 Gilman Drive, La Jolla, CA 92093, USA, walker@ucsd.edu), Milton Garces (Infrasound Laboratory, University of Hawaii, 73-4460 Queen Kaahumanu Highway \#119, Kailua-Kona, HI 96740, USA, milton@isla.hawaii.edu), Curt Szuberla (University of Alaska, 903 Koyukuk Drive, Fairbanks, AK 99775, USA, cas@gi.alaska.edu), Rod Whitaker (Los Alamos National Laboratory, EES-2 MS J577, Los Alamos, NM 87545, USA, rww@lanl.gov)

At the 152nd Meeting of the Acoustical Society of America, Andre and Bass reported an infrasound experiment conducted at White Sands Missile Range during the 2005-2006 time frame. The experiment consisted of exploding a $22.4 \mathrm{~kg}$ charge at altitudes from 31.3 $\mathrm{km}$ to $49.6 \mathrm{~km}$ then recording the waveforms at 30 infrasound arrays (not all at the same time) at distances up to $1200 \mathrm{~km}$ from the source. The analysis is not yet complete but some preliminary observations have been reported in the most recent issue of Acoustics Today. This talk will summarize the findings published in Acoustics Today and offer suggestions to others who might want to access and analyze the data.

$$
\text { 2:20 }
$$

5pNSb2. Infrasonic and seismic signals from explosions in Northwestern Europe. Laeslo G. Evers (Royal Netherlands Meteorological Institute (KNMI), PO Box 201, 3730 AE De Bilt, Netherlands, evers@knmi.nl), Hein Haak (Royal Netherlands Meteorological Institute (KNMI), PO Box 201, 3730 AE De Bilt, Netherlands, haak@knmi.nl)

Large explosions often generate both seismic and infrasound signals that can be detected over large ranges, i.e., hundreds of kilometers. Ground truth of such explosions is available from direct observations and/or seismic signals and can be used to get insight in the propagation of infrasound. Long range infrasound propagation is controlled by the state of the atmosphere. Tropospheric, stratospheric, and thermospheric ducts might exist and have implications on the signal characteristics and their detectability. In this study, we will show results of studies on explosions in Northwestern Europe using both seismic and infrasound data. These observations are used to derive source characteristics like: location, origin time and yield. Furthermore, the propagation characteristics of infrasound will be addressed.

$$
\text { 2:40 }
$$

5pNSb3. Infrasound propagation in the zone of silence. Paul Golden (Southern Methodist University, P. O. Box 750395, Dallas, TX 75275, USA, pgolden@mail.smu.edu), Petru Negraru (Southern Methodist University, P. O. Box 750395, Dallas, TX 75275, USA, pnegraru@smu.edu), Eugene T. Herrin (Southern Methodist University, P. O. Box 750395, Dallas, TX 75275, USA, herrin@passion .isem.smu.edu)

Two controlled source experiments were conducted in Nevada in 2006 and 2007 to study infrasound signal propagation at distances less than $300 \mathrm{~km}$ from the source. In 2006 three temporary infrasound arrays were deployed at distances of 76, 108, and 157 from the source. In 2007 the site at $157 \mathrm{~km}$ was reoccupied, and data was also recorded at $288 \mathrm{~km}$ from the source. Interesting results were derived from the travel time analysis. In 2006 the site at $76 \mathrm{~km}$ recorded both tropospheric and stratospheric arrivals, while at 108 and $157 \mathrm{~km}$ only stratospheric arrivals were recorded. In 2007 the site at $157 \mathrm{~km}$ recorded both tropospheric and stratospheric arrivals, while at $288 \mathrm{~km}$ both stratospheric and thermospheric arrivals were recorded. Atmospheric modeling with the InfraMAP software failed to predict returning rays or pressure levels similar to the observed data. Because of the large amplitude variations we attempt to estimate the yields of the explosions using the predominant frequency content of the signals. The physical basis for such a method is found in an increased acoustic transit time of the explosion blast radius with increased yield. Preliminary results suggest this is possible. 
5pNSb4. Validating upper-wind models using infrasound from active volcanoes. Alexis Le Pichon (CEA-DASE, Arpajon Cedex, 91297 Bruyères-le-Châtel, France, alexis.le-pichon@cea.fr), Karl Antier (CEA-DASE, Arpajon Cedex, 91297 Bruyères-leChâtel, France, karl.antier@cea.fr), Sylvie Vergniolle (Institut de Physique du Globe, 4 Place Jussieu, 75252 Paris, France, vergniolle@ipgp.jussieu.fr), Michel Lardy (IRD Center, BPA5, Cedex, 98848 Noumea, New Caledonia, lardy@noumea.ird.nc), Douglas Drob (Naval Research Laboratory, Space Science Division, 4555 Overlook Avenue, Washington, DC 20375, USA, douglas.drob@nrl.navy.mil)

Known and quasipermanent infrasonic sources are needed to evaluate and improve upper-wind models. Infrasounds generated by active volcanoes offer a unique opportunity for atmospheric studies. The Yasur volcano in the Vanuatu archipelago is an outstanding source of infrasonic waves due to its regular activity. This volcano is permanently monitored by the IS22 infrasound station located in New Caledonia and by one microbarometer installed close to its crater. A five-year monitoring period of Yasur at short and large propagation range provides new insights on quantitative relationships between infrasonic observables and atmospheric specifications. This experimental setting is proposed to validate consistently the Naval Research Laboratory Ground to Space (NRL-G2S) semi-empirical atmospheric model up to the stratosphere. The propagation modeling results accurately explain seasonal changes as well as small shorttimescale variations of the infrasonic observables. This study demonstrates that the use of appropriate propagation tools along with the NRL-G2S specifications provides accurate enough results for most of the long-range observations for the purpose of operational infrasound monitoring.

\section{3:20}

5pNSb5. Semianalytical modeling of plate flexural waves generated by laser-initiated air shock waves. Vasil B. Georgiev (Loughborough University, Department of Aeronautical and Automotive Engineering, Ashby Road, LE11 3TU Loughborough, UK, V.Georgiev@lboro.ac.uk), Victor V. Krylov (Loughborough University, Department of Aeronautical and Automotive Engineering, Ashby Road, LE11 3TU Loughborough, UK, V.V.Krylov@lboro.ac.uk), Qin Qin (University of Hull, Department of Engineering, Cottingham Road, HU6 7RX Hull, UK, q.qin@hull.ac.uk), Keith Attenborough (Open University, Department of Design, Development, Materials and Environment, Walton Hall, MK7 6AA Milton Keynes, UK, Keith.Attenborough@ioa.org.uk)

The paper describes the results of the semianalytical modeling of the interaction of laser-initiated air shock waves with an infinite elastic plate. The impact of the incident shock wave on the plate has been approximated by an equivalent cylindrically diverging surface force resulting from the combined surface pressure of the incident and reflected shock waves. This force has been then represented in the wave number-frequency domain by means of Hankel and Fourier transforms which have been carried out numerically - and the problem has been solved using the Green's function method applied to an infinite plate. The resulting frequency spectra and time histories of the generated flexural wave pulses have been calculated for different values of laser pulse energy and for different heights of the laser beam focusing above the plate surface. The obtained theoretical results have been compared with the results of the reducedscale model experiments on shock wave interaction with the ground in which large plastic and wooden plates have been used to represent the ground surface. The comparison shows that the obtained semi-analytical results are in good agreement with the experimental ones.

\section{3:40}

5pNSb6. Ground effects on sound and vibration from explosions. Christian Madshus (NGI, P.O. Box 3930 Ullevaal Stadion, Sognsveien 72, 0806 Oslo, Norway, cm@ngi.no), Finn Løvholt (NGI, P.O. Box 3930 Ullevaal Stadion, Sognsveien 72, 0806 Oslo, Norway, flo@ngi.no), Ra Cleave (NGI, P.O. Box 3930 Ullevaal Stadion, Sognsveien 72, 0806 Oslo, Norway, rc@ngi.no), Karin Rothschild (NGI, P.O. Box 3930 Ullevaal Stadion, Sognsveien 72, 0806 Oslo, Norway, kmr@ngi.no), Zenon Cetina-Medina (NGI, P.O. Box 3930 Ullevaal Stadion, Sognsveien 72, 0806 Oslo, Norway, zmc@ngi.no)

Low frequency sound from military activity and explosions do propagate over large distances. The sound pressure may induce substantial vibration in the ground and particularly in buildings. Such vibrations turn out to be a major cause of complaints among neighbouring communities around training fields and blast sites. We will present investigations on long range propagation of low frequency sound and sound-induced vibration, based on a substantial amount of data collected during a series of full scale tests performed in Norway over the last 14 years. All data are assembled in the NORTRIAL database, which is now publicly available. Meteorology and ground interaction largely influence the sound propagation and vibration response. At low frequency meteorological- and ground effects may intricately interact and lead to large, apparently random variability in sound pressure at large distances. Statistically based investigations on the sound propagation and its variability will be presented. Particular focus will be on the ground interaction effects, and a new and more extensive system for ground classification, based on cartographic data, empirical and numerical modeling will be introduced. Findings on building response to outdoor low frequency sound and transfer mechanisms from outdoor pressure to indoor sound and vibration will be presented.

\section{4:00-4:20 Break}

\section{4:20}

5pNSb7. Deducing ground structure using seismic pulses originating from an outdoor explosive source. Shahram Taherzadeh (The Open University, Faculty of Mathematics, Computing and Technology, Walton Hall, MK7 6AA Milton Keynes, UK, s.taherzadeh@open.ac.uk), Keith Attenborough (Open University, Department of Design, Development, Materials and Environment, Walton Hall, MK7 6AA Milton Keynes, UK, Keith.Attenborough@ioa.org.uk)

Near-surface layering of ground soil can influence propagation of acoustic and seismic pulses originating from above-surface sources. Simultaneous recording of acoustic air pressure and seismic radial and vertical particle velocities resulting from a small, above ground explosion is used to obtain information about soil structure near the surface. Assuming nonlinear effects to be small at the ranges 
of interest here, a numerical model called Fast Field Program for Layered Air Ground Systems (FFLAGS), developed originally for continuous sound sources above a porous elastic ground is used to model a porous elastic layered ground system. Suitable optimisation methods were used to predict a set of best fit parameters for the near-surface ground structure. It is shown that the model can explain multiple seismic arrivals and give a reasonable prediction of wave speeds and layer depths while the pressure pulse can predict permeability of the surface soil.

\section{4:40}

5pNSb8. Predictions for the influence of the nocturnal jet on the long range propagation of impulsive signals. Roger Waxler (University of Mississippi, NCPA, 1 Coliseum Drive, University, MS 38677, USA, rwax@olemiss.edu), Kenneth E Gilbert (National Center for Physical Acoustics, University of Mississippi, University, MS 38677, USA, kgilbert@olemiss.edu), Carrick L. Talmadge (The University of Mississippi - NCPA, 1 Coliseum Drive, University, MS 38677, USA, clt@ olemiss.edu), Xiao Di (National Center for Physical Acoustics, University of Mississippi, University, MS 38677, USA, xiaodi@olemiss.edu)

On clear nights, inland over flat ground, one generally finds a temperature inversion in the first hundred meters or so of the atmosphere. Near the ground, winds tend to be light, increasing with altitude. Above the temperature inversion one finds a stiff geostrophic wind known as the nocturnal jet. Theoretical predictions, based on ray theory and expansions in vertical modes, for the effect of the nocturnal jet on the long range propagation of impulsive signals are presented. For sufficiently short ranges, less than $3 \mathrm{~km}$ or so, the nocturnal jet has no effect. At these ranges only the temperature inversion and the light winds in the inversion play a role. At longer ranges the nocturnal jet significantly alters the arrival structure of the propagated signal. It is predicted that, due to coincident arrivals from the inversion and from the nocturnal jet, there is a segment of ranges about $1 \mathrm{~km}$ long, beginning at about $4 \mathrm{~km}$, in which the amplitude of the first arrival becomes anomalously large and then splits into two distinct arrivals.

\section{5:00}

5pNSb9. The physics of pulse propagation in the nocturnal atmospheric boundary layer: measurement and theory. Carrick L. Talmadge (The University of Mississippi - NCPA, 1 Coliseum Drive, University, MS 38677, USA, clt@olemiss.edu), Roger Waxler (University of Mississippi, NCPA, 1 Coliseum Drive, University, MS 38677, USA, rwax@olemiss.edu), Kenneth E Gilbert (National Center for Physical Acoustics, University of Mississippi, University, MS 38677, USA, kgilbert@olemiss.edu), Jin So (National Center for Physical Acoustics, University of Mississippi, University, MS 38677, USA, jso@olemiss.edu), Rommel Stribling (National Center for Physical Acoustics, University of Mississippi, University, MS 38677, USA, eercs@olemiss.edu), Xiao Di (National Center for Physical Acoustics, University of Mississippi, University, MS 38677, USA, xiaodi@olemiss.edu)

A series of experiments designed to probe the effect of the nocturnal atmosphere on low-frequency $(10-500 \mathrm{~Hz})$ sound propagation will be discussed, and their ramifications explored. These experiments involve detecting arrivals from a propane cannon on a vertical array of microphones $1-3 \mathrm{~km}$ from the source, which were collected simultaneously with meteorological experiments designed to measure the vertical temperature and horizontal wind velocity profiles. Chief among the results of these experiments is the observation of a nocturnal model structure that has a significant surface wave component at frequencies below $150 \mathrm{~Hz}$. At higher frequencies and longer propagation distances $(>1.5 \mathrm{~km})$, the surface wave is not observed due to attenuation from its interaction with the ground. At higher frequencies, the model structure displays a characteristic "quiet height" first described in Waxler et al. (2006). The potential application of these results for remote sensing the atmospheric boundary layer are discussed, and contrasted with other methods of measurement of the atmospheric profile.

\section{Contributed Paper}

\section{$5: 20$}

5pNSb10. Nonlinear parabolic equation model for finite-amplitude sound propagation in an inhomogeneous medium over a nonflat, finite-impedance ground surface. Thomas Leissing (CSTB, 24 rue Joseph Fourier, 38400 Saint-Martin-d'Hères, France, thomas.leissing@cstb .fr), Philippe A. Jean (CSTB, 24 rue Joseph Fourier, 38400 Saint-Martind'Hères, France, philippe.jean@cstb.fr), Jérôme Defrance (CSTB, 24 rue Joseph Fourier, 38400 Saint-Martin-d'Hères, France, jerome.defrance @ cstb.fr), Christian Soize (Université de Marne la Vallée, 5, Boulevard Descartes, 77454 Marne la Vallée, France, soize@univ-mlv.fr)

A nonlinear parabolic equation (NPE) model for weakly nonlinear sound propagation in an inhomogeneous medium is described. The model being formulated in the time domain, complex impedances cannot be used to simulate ground surfaces. A second NPE model is thus derived to include the medium in the computational system. Based on a nonlinear extension of the Zwikker-Kosten model for rigidly-framed porous media, it allows to include Forchheimer's nonlinearities. Both models are then adapted to terrainfollowing coordinates, and used together with an interface condition, allow to simulate finite-amplitude sound propagation over a nonflat, finiteimpedance ground surface. Numerical examples show that the NPE model is in good agreement with the solutions of the frequency domain boundary element method. Applications of this model to the simulation of sound propagation from explosions in air are then discussed. 


\title{
Invited Papers
}

\section{$5: 40$}

5pNSb11. Mean vs event sound-level prediction: obtaining consistency between atmospheric data inputs, propagation models, and the predictand. David K. Wilson (U.S. Army Engineer Research and Development Center, 72 Lyme Rd., Hanover, NH 037551290, USA, D.Keith.Wilson@ usace.army.mil), Chris L. Pettit (U.S. Naval Academy, Aerospace Engineering Dept., 590 Holloway Rd., MS 11-B, Annapolis, MD 21402, USA, petitcl@usna.edu), Vladimir E. Ostashev (NOAA/Earth System Research Laboratory, 325 Broadway, Boulder, CO 80305, USA, vladimir.ostashev@noaa.gov), Matthew S. Lewis (U.S. Army Engineer Research and Development Center, 72 Lyme Rd., Hanover, NH 03755-1290, USA, Matthew.S.Lewis@usace.army.mil)

The following, deceptively challenging, questions are addressed: What are the most suitable atmospheric data resources and propagation models for predicting event (explosion and other short duration) sound-exposure levels? Do these differ from those most suitable for predicting mean sound levels? Atmospheric data typically consist either of single, "snapshot" samples of the vertical profiles, as from a weather balloon, or average vertical profiles, as from climatology or a numerical weather model. Recent statistical studies, based on high-resolution atmospheric simulation, demonstrate the superiority of mean profiles for prediction of both mean and event sound levels, even when single-sample profiles are synchronized to and collected along the path of the propagation event. Running propagation models "blind" to the nature of the atmospheric inputs is shown to be hazardous: predictions from mean profiles lack turbulent scattering, thus underestimating sound levels near the ground, whereas predictions from single-sample profiles implicitly assume the turbulence has infinite horizontal extent, thus overestimating sound levels. Some desirable consistency results from numerically solving parabolic equations (PEs) for statistical moments of the sound pressure, rather than conventional deterministic PEs. The moment PEs directly predict mean sound levels or the expected value and variability of event sound-exposure levels.

\section{6:00}

5pNSb12. Beam-forming and dispersion measurements at the edge of a pine forest. Michael J. White (US Army Engineer Research and Development Center, 2902 Newmark Drive, Champaign, IL 61826, USA, Michael.J.White@usace.army.mil), Michelle E. Swearingen (Norwegian Defense Research Establishment/US Army, Postboks 25, 2027 Kjeller, Norway, michelle.swearingen@ffi.no)

Beam-forming analyses were performed on four-microphone linear arrays placed just within the interior of a pine forest to separate signal arrivals by direction from an impulsive sound source in an open field. The arrays were deployed in three configurations: transverse, longitudinal, and vertical. The arrays spacings were organized in a pattern that provides six baselines with four microphones. Longitudinal and transverse configurations show arrivals scattered from trunks, and the vertical configurations indicate some refraction and scattering by the canopy. Signal dispersion curves developed using the four-microphone arrays had greater variation in trace velocity at higher frequencies in each configuration.

FRIDAY AFTERNOON, 4 JULY 2008

ROOM 251, 4:20 TO 6:20 P.M.

\section{Session 5pNSc}

\section{Noise and EURONOISE: Propagation and Urban Noise II}

\author{
Jérôme Defrance, Chair \\ CSTB, 24 rue Joseph Fourier, Saint-Martin-d'Hères, 38400, France
}

\section{Contributed Papers}

4:20

5pNSc1. Road traffic noise from viaducts in mountainous areas. Jérôme Defrance (CSTB, 24 rue Joseph Fourier, 38400 Saint-Martind'Hères, France, jerome.defrance@cstb.fr), Matthieu Beyret (CSTB, 24 rue Joseph Fourier, 38400 Saint-Martin-d'Hères, France, matthieu.beyret @ cstb.fr), Marine Baulac (CSTB, 24 rue Joseph Fourier, 38400 SaintMartin-d'Hères, France, marine.baulac@ @stb.fr), Philippe A. Jean (CSTB, 24 rue Joseph Fourier, 38400 Saint-Martin-d'Hères, France, philippe.jean @ cstb.fr)

Mountainous zones in Europe, such as the Alps, represent huge areas where many viaducts are built, most of them for motorways. The way the sound grazes the asphalt surface from the low and high traffic equivalent sources up to the road edges, and how it then diffracts towards dwellings is a complex mechanism. The standard approaches are suited to plain situations but fail in predicting finely sound propagation behaviour for such geometries. In this paper, one gives the main trends of received noise levels from viaducts as a function of both their geometry and the receiver location. A 2D Boundary Element Method is used for predictions since meteorological effects can be neglected for the short propagation (a few hundreds meters). This assumption makes sense since the viaduct considered in this work is sufficiently high $(20 \mathrm{~m})$ and the ground effect is weakly affected by refraction. Different configurations are then simulated in order to address and discuss several geometrical effects, such as: platform elevation, low height barriers addition, complex shape barriers and presence of a central gap in the platform.

\section{4:40}

5pNSc2. Characteristics of road traffic noise level statistics for shielded areas. Jens Forssén (Division of Applied Acoustics, Chalmers University of Technology, 41296 Göteborg, Sweden, jens.forssen@chalmers .se), Maarten Hornikx (Applied Acoustics, Chalmers University of Technology, Sven Hultins Gata 8a, SE-41296 Gothenburg, Sweden, maarten .hornikx@chalmers.se)

For noise immission, it is of interest to study other noise level statistics besides the long-term equivalent levels and maximum levels. By further analysis of the time variations of the noise level, an improved description of the negative effects of the noise may be achieved, for instance concerning perceived annoyance. Here, noise level histograms, i.e., probability density 
functions of sound pressure levels, from controlled recordings have been investigated. This has been made for a situation of special interest, which is a courtyard shielded from a dominating road traffic noise source. It has been reported previously that many shielded urban areas show levels that are considerably higher than the equivalent level, described as an upward tail of the histogram, which is not a usual characteristic of directly exposed areas. From the analysis made here, it is shown that the upward tail, i.e., the higher levels, of the shielded area is caused by locally occurring, unshielded road traffic events. It is concluded that the upward tail as a common characteristic of shielded urban areas may well be due to locally occurring noise events, for instance due to local road traffic.

\section{5:00}

5pNSc3. Use of the transmission line matrix method for the sound propagation modeling in urban area. Gwenaël Guillaume (Lab. Central des Ponts et Chaussées, Division Entretien, Sécurité et Acoustique des Routes, Route de Bouaye - BP 4129, 44341 Bouguenais Cedex, France, Gwenael.Guillaume@lcpc.fr), Judicaël Picaut (Lab. Central des Ponts et Chaussées, Division Entretien, Sécurité et Acoustique des Routes, Route de Bouaye - BP 4129, 44341 Bouguenais Cedex, France, Judicael.Picaut@1cpc.fr), Guillaume Dutilleux (Lab. Régional des Ponts et Chaussées, 11, rue Jean Mentelin, BP 9, 67035 Strasbourg Cedex 2, France, Guillaume.Dutilleux@equipement.gouv.fr)

This paper deals with the sound propagation modeling in urban area. This problematic requires to take into account many phenomena that can have a substantial impact as well in semienclosed spaces as on long-range outdoor sound propagation, such as reflections and absorption on the frontages and on the ground, atmospheric attenuation, sound velocity variations related with wind and temperature vertical gradients, atmospheric turbulences. The numerical method used is the TLM (transmission line modeling), which has been originated in electromagnetism and adapted for acoustics applications. It consists in a physical rendering of the waves propagation based on the Huygens' principle. It is established on a spatiotemporal discretization of the domain using an iterative temporal process for sound pressure propagation, instead of the resolution of mathematical equations. The TLM model has then been developed in two and three dimensions allowing to combine all the phenomena affecting the sound propagation in urban area. Numerical simulations are given for canyon streets.

\section{$5: 20$}

5pNSc4. Predictions of sound pressure levels in streets using a diffusion model: numerical validations and experimental comparisons. Alexis Billon (Universite de Liege, INTELSIG group - Département E.E.I., B28 Sart-Tilman, 4000 Liege, Belgium, abillon@ulg.ac.be), Judicaël Picaut (Lab. Central des Ponts et Chaussées, Division Entretien, Sécurité et Acoustique des Routes, Route de Bouaye - BP 4129, 44341 Bouguenais Cedex, France, Judicael.Picaut@lcpc.fr)

Predictions of sound propagation in urban areas have attracted a considerable over the years. If the sound energy is assimilated to particles with a constant energy, their movement can be described by a transport equation. In canyon streets, this transport equation can be reduced to a diffusion equation whose expression is more simple. In this presentation, sound absorption at the boundaries (buildings facades and ground), as well as atmospheric sound attenuation are introduced. The problem is then solved numerically using a finite elements method for the configuration of a canyon street. A systematic validation of the obtained model is carried out in terms of sound pressure level by comparison to numerical simulations taken from the literature. Comparisons with experimental data are then conducted. Finally, applications in more complex configurations are presented.

\section{$5: 40$}

5pNSc5. A cellular automaton for urban traffic noise. Erik Salomons (TNO Science and Industry, Stieljesweg 1, 2628CK Delft, Netherlands, erik.salomons@tno.nl)

Propagation of traffic noise in a city is a complex phenomenon, due to multiple reflection, diffraction, and scattering at irregular facades of buildings. These effects may be calculated with computer models based on numerical integration of the basic acoustic equations, but in practice these models can be applied only to small urban regions due to limited computer power. Here we propose a new approach for simulating urban traffic noise: a cellular automaton (CA) based on simple update rules for the configuration of cars in a city, and simple rules for propagation of sound to receivers. An example is presented for a square model city of $25 \mathrm{~km}^{2}$, consisting of $10^{6}$ square cells. The CA employs a time integration step of $0.3 \mathrm{~s}$, and includes noise contributions from all cars in the city. The fluctuating sound level is computed for a period of $24 \mathrm{~h}$, both for a receiver along a street and for a receiver that is screened by buildings. While the sound level at the first receiver shows sharp peaks corresponding to passages of cars, the sound level fluctuations at the screened receiver are much smaller as most of the sound energy comes from distant cars in this case.

\section{6:00}

5pNSc6. Numerical prediction of the effect of traffic lights on the vehicle noise at urban street intersections. Jorge Parrondo (Universidad de Oviedo, Dep. de Energía, Campus de Viesques s/n, 33203 Gijón, Spain, parrondo@uniovi.es), Ruben Zurita (Universidad de Oviedo, Dep. de Energía, Campus de Viesques s/n, 33203 Gijón, Spain, ZURITA@1si.uniovi.es), Jose A. Corrales (Universidad de Oviedo, Dep. de Energía, Campus de Viesques s/n, 33203 Gijón, Spain, JA@1si.uniovi.es), Joaquin Fernandez (Univ. de Extremadura, Escuela de Ing. Industriales, 06071 Badajoz, Spain, ffrancos@unex.es)

Intersections of urban streets are particularly noisy locations due to the addition of the noise from vehicles at different streets, a long period of noise emission from queuing vehicles with traffic lights in red and the noise from accelerating vehicles. Besides, the traffic lights impose a modulation in the passage of the vehicles, so that the subsequent noise variability along time contributes to increase the annoyance degree. This paper presents a code especially developed to simulate both the spatial and temporal distribution of the sound pressure levels induced by the vehicle traffic in street intersections. The algorithm combines a traffic model with vehicles in dynamic motion through the domain with a model for sound propagation, based on the method of virtual images in which the determination of the location of the successive image sources was reduced to a reference horizontal plane. Sound emission from each vehicle was established according to the Harmonoise guidelines. After verifying the code predictions by comparison with measurements at several street intersections, the method was applied systematically to investigate the effect of varying the regulation parameters of the traffic lights on the Leq and L10 distributions for some particular cases. 


\title{
Session 5pPAa
}

\section{Physical Acoustics: Ducts and Waveguides II}

\author{
Marc Deschamps, Cochair \\ LMP, Lab. de Mécanique et Physique (LMP), 351, Place de la Libération, Talence, 33405, France \\ Olivier Dazel, Cochair \\ Laboratoire d'Acoustique de l'Université du Maine, Avenue Olivier Messiaen, Le Mans, 72085, France
}

\section{Contributed Papers}

\section{2:00}

5pPAa1. A higher order parabolic equation for predicting in-duct propagation in high frequencies. Patrice Malbéqui (ONERA, 29 avenue de la Division Leclerc, 92322 Châtilllon, France, patrice.malbequi@onera .fr)

Complementary methods are required to predict the in-duct propagation in a large frequency range including the linear absorption and the flow effects. Numerical methods solving the Euler's equations are pertinent for rotational flow but limited to the low frequencies. The Boundary Element Method is applicable in a large frequency range assuming a homogeneous flow. The ray-model is valid in high frequencies but scattering effects are difficult to implement. This paper presents the capabilities of a Higher-Order Parabolic Equation (HOPE) to handle duct propagation in the high frequency range. In contrast with the so-called standard PE and the wide-angle PE, the HOPE improves the accuracy of the solution due to its wider propagation aperture angle, especially close to the cutoff frequency. Several duct propagation configurations including flow and liner are considered. Using a marching algorithm, the HOPE computes in a very short CPU time the sound propagation and represents an attractive alternative to the ray-model in the high frequency range. [Work supported by Airbus-France.]

\section{2:20}

5pPAa2. An integrated multimodal acoustic particle manipulator and optical evanescent field waveguide. Peter Glynne-Jones (University of Southampton, School of Engineering Sciences, University Road, SO17 1BJ Southampton, UK, p.glynne-jones@soton.ac.uk), Martyn Hill (University of Southampton, School of Engineering Sciences, University Road, SO17 1BJ Southampton, UK, m.hill@soton.ac.uk), Rosemary J. Townsend (University of Southampton, School of Engineering Sciences, University Road, SO17 1BJ Southampton, UK, R.J.Townsend@soton.ac .uk), Nicholas R. Harris (University of Southampton, Electronics and Computer Science, SO17 1BJ Southampton, UK, nrh@ecs.soton.ac.uk), James S. Wilkinson (University of Southampton, Optoelectronics Research Centre, SO17 1BJ Southampton, UK, jsw@ecs.soton.ac.uk), Fan Zhang (University of Southampton, Optoelectronics Research Centre, SO17 1BJ Southampton, UK, faz@orc.soton.ac.uk), Tracy Melvin (University of Southampton, Optoelectronics Research Centre, SO17 1BJ Southampton, UK, tm@ecs.soton.ac.uk)

A new acoustic/optical/microfluidic system is presented for the manipulation of bead-tagged DNA molecules. Acoustic radiation forces are used to manipulate microspheres into and away from the evanescent field of a laser coupled waveguide that is integrated into the reflector of the acoustic chamber. With suitable fluorophores the presence of the target DNA can be detected with a fluorescence microscope enabling large populations of beads to be examined simultaneously. The integrated waveguide and multimodal acoustic chamber are presented here, with results showing that the microspheres can be successfully detected as they are brought into the evanescent field using a quarter-wave acoustic configuration. It is also shown that by measuring the time of flight of a microsphere between the half- and quarter- wave nodal planes the bead size can be determined, providing a means of multiplexing the detection (detecting a range of different target DNA sequences).

\section{2:40}

5pPAa3. Trapped wave in plan waveguides including gaussian varying section domain. Patrick Marical (LOMC FRE-3102 CNRS, Groupe Ondes Acoustiques, University of Le Havre, Place Robert Schuman, BP 4006, 76610 Le Havre, France, patrick.marical368@univ-lehavre .fr), Mounsif Ech-Cherif El-Kettani (LOMC FRE-3102 CNRS, Groupe Ondes Acoustiques, University of Le Havre, Place Robert Schuman, BP 4006, 76610 Le Havre, France, elkettani@univ-lehavre.fr), Zahia Hamitouche (LOMC FRE-3102 CNRS, Groupe Ondes Acoustiques, University of Le Havre, Place Robert Schuman, BP 4006, 76610 Le Havre, France, zahia.hamitouche@univ-lehavre.fr), Mihai Valentin M. Predoi (University Politechnica of Bucharest, Department of Mechanics, 060032 Bucharest, Romania, predoi@cat.mec.pub.ro)

In previous works on plan waveguides including an area of varying section of Gaussian shape, we have observed experimentally and numerically the existence of a trapped wave in the Gaussian varying section domain in the case of the A1 incident Lamb mode. The purpose of this work is to highlight the existence of this trapped wave, depending on the equation of the Gaussian profile of the varying section domain. This study is carried out numerically using FEM, as our numerical model has been previously validated. The results obtained show that the phenomenon of trapped wave is strongly linked to the Lamb wave conversion phenomenon: when the incident Lamb wave gives rise to a trapped wave, it is systematically converted into other Lamb waves transmitted outside the area of varying section. Otherwise, it is totally transmitted without any conversion and in this case, any trapped wave exists. The conversion phenomenon is quantified and we show that it is important and depend on the breaking symmetry of the incident Lamb mode by the varying section area.

\section{3:00}

5pPAa4. Intermodal coupling in a dissipative fluid filling a rough-walled waveguide. Tony Valier-Brasier (Laboratoire d'Acoustique de l'Université du Maine, Avenue Olivier Messiaen, 72085 Le Mans, France, tony.valier-brasier.etu@univ-lemans.fr), Catherine Potel (Laboratoire d'Acoustique de l'Université du Maine, Avenue Olivier Messiaen, 72085 Le Mans, France, catherine.potel@univ-lemans .fr), Michel Bruneau (Laboratoire d'Acoustique de l'Université du Maine, Avenue Olivier Messiaen, 72085 Le Mans, France, michel.bruneau@univ-lemans.fr), Claude_Depollier (Laboratoire d'Acoustique de l'Université du Maine, Avenue Olivier Messiaen, 72085 Le Mans, France, claude.depollier@univ-lemans.fr)

The present study follows recent works dealing with the analytical model of an acoustic field in fluid-filled waveguides with rough walls. In these works, the acoustic field is obtained from the coupling between Neumann eigenmodes of the regularly shaped waveguide which bounds outwardly the rough walls of the waveguide considered, using integral formulation with suitable Green function. The effect of the roughness is expressed 
in such a way that two intermodal coupling mechanisms are highlighted: a bulk coupling and a surface coupling, the first one depending on the depth of the roughness and the second one depending in addition on the local slope. Moreover, a phonon relation is involved when the rough profile is periodic. The aim of the present study is to account for the thermo-viscous boundary layer effects through eigenmodes which satisfy appropriate mixed boundary conditions, leading to a better understanding of the physical mechanisms when resonances and phonon relationship are involved.

\section{3:20}

5pPAa5. Evaluation of the lined duct performances based on a 3D two port scattering matrix. Mohamed Taktak (Université de Technologie de Compiègne, Centre de Recherche Royallieu, BP20529, 60205 Compiègne, France, mohamed.taktak@utc.fr), Jean Michel Ville (Université de Technologie de Compiègne, Centre de Recherche Royallieu, BP20529, 60205 Compiègne, France, jean-michel.ville@utc.fr), Mohamed Haddar (Unité de Modélisation, Mécanique et de Production (U2MP), Ecole Nationale d'Ingénieurs de Sfax, BP 3038, 3038 Sfax, Tunisia, mohamed.haddar@enis.rnu.tn), Félix Foucart (Université de Technologie de Compiègne, Centre de Recherche Royallieu, BP20529, 60205 Compiègne, France, felix.foucart@utc.fr)

The scattering matrix constitutes a good tool to characterize a lined duct. In fact, this matrix relates the incoming modal pressures to the out coming ones and contains detailed information and per mode about the transmission, reflection, and conversion properties of the duct. It depends only on the geometric and acoustic properties of the duct. The two port acoustic dissipation and attenuation interest the designers of lined duct like aircraft engine manufacturers to evaluate the duct performances. These values can be deduced from the two port scattering matrix and from the vector of incoming modal pressures. In this work, a study about the two port acoustic dissipation and attenuation computed from the scattering matrix and for different cases of incoming modal pressures are presented. Scattering matrices used in this study are measured by an experimental procedure developed at the University of Technology of Compiègne based upon the experimental setup realized during the European Project DUCAT. The experimental acoustic power dissipation and attenuation are computed for different cases of modal structure on the both side of the duct. Then, these results were confronted with ones given by a theorical study of the problem based on the finite element method.

\section{3:40-4:00 Break}

\section{4:00}

5pPAa6. Elaboration of a sacttering matrix measurement procedure using the p-v probe. Yamen Kchaou (Université de Technologie de Compiègne, Centre de Recherche Royallieu, BP20529, 60205 Compiègne, France, yamen.kchaou@yahoo.fr), Mohamed Taktak (Université de Technologie de Compiègne, Centre de Recherche Royallieu, BP20529, 60205 Compiègne, France, mohamed.taktak@utc.fr), Jean Michel Ville (Université de Technologie de Compiègne, Centre de Recherche Royallieu, BP20529, 60205 Compiègne, France, jean-michel.ville@utc .fr), Mohamed Haddar (Unité de Modélisation, Mécanique et de Production (U2MP), Ecole Nationale d'Ingénieurs de Sfax, BP 3038, 3038 Sfax, Tunisia, mohamed.haddar@enis.rnu.tn), Félix Foucart (Université de Technologie de Compiègne, Centre de Recherche Royallieu, BP20529, 60205 Compiègne, France, felix.foucart@utc.fr)

The scattering matrix which relates traveling waves amplitudes as state variables has been shown to be more attractive than transfer or mobility matrices since it reflects the fundamental duct nature: it gives a more complete description of the transmission, reflection, and conversion properties of the duct. In the University of Technology of Compiègne, an experimental procedure was developed to measure this matrix: a $\mathrm{p}-\mathrm{p}$ probe mounted on a setup designed during DUCAT project is used to measure pressures at two cross sections on the both side of the test lined duct, then by using a modal decomposition and separation techniques, the scattering matrix is deduced. In this paper, a method to measure the multimodal scattering matrix based on the use of a p-v probe getting simultaneously the acoustic pressure and velocity at one section on the both side of the test duct is developed. A com- parison of some acoustics values (scattering matrix coefficients, acoustic powersœ) of a hard wall duct straight duct obtained by each technique with the theory is presented to evaluate its advantages and limitations.

\section{4:20}

5pPAa7. Axisymmetrical and nonaxisymmetrical guided waves propagating in a solid elastic cylinder embedded in a solid medium. Slah Yaacoubi (LCPC, Lab. Central des Ponts et chaussées (LCPC), Route de Bouaye-BP 4129, 44341 Bouguenais, France, slah.yaacoubi@lcpc.fr), Laurent Laguerre (LCPC, Lab. Central des Ponts et chaussées (LCPC), Route de Bouaye-BP 4129, 44341 Bouguenais, France, laurent.laguerre@1cpc.fr), Eric Ducasse (LMP, Lab. de Mécanique et Physique (LMP), 351, Place de la Libération, 33405 Talence, France, e.ducasse@lmp.u-bordeaux1.fr), Marc Deschamps (LMP, Lab. de Mécanique et Physique (LMP), 351, Place de la Libération, 33405 Talence, France, m.deschamps@1mp.u-bordeaux1.fr)

For NDT of rods and pipes, fundamental characteristics of guided waves are to be known, especially dispersion relations between frequency and wave number. A necessary step before detecting defects is to be able to calculate the propagated elastodynamical field in healthy waveguides. Thus, the goal of this work is the calculation of this field propagating in a cylindrical stratified waveguide. The incident field is generated at the end of the cylinder by a force or velocity source which is off-axis and Gaussian distributed. First, Vector Hankel transform and Fourier series are combined to decompose this field with respect to the angular position. Second, each component is decomposed into an infinite sum of rays, i.e., elementary generalized conical waves. These waves undergo multiple reflections with the guiding surface of the waveguide. Third, we use Generalized Debye Series (GDS) for calculating the global reflection coefficients resulting from these multiple reflections. Finally, the total field is synthesized by the summation of the incident and reflected rays. Many outputs of this code can be exploited like velocity field, stress field, energy field in 2D or 3D spatiotemporal or frequential simulations. Diagrams obtained by this code are compared with results from DISPERSE software.

$4: 40$
5pPAa8. Coupling transfer matrix method to finite element method for the analysis of hollow body networks with passive or reactive elements. Fabien Chevillotte (Groupe d'Acoustique de l'Université de Sherbrooke, 2500, Boul. de 1'Université, Département de génie mécanique, Sherbrooke, QC J1K-2R1, Canada, fabien.chevillotte@usherbrooke.ca), Raymond Panneton (Groupe d'Acoustique de l'Université de Sherbrooke, 2500, Boul. de l'Université, Département de génie mécanique, Sherbrooke, QC J1K2R1, Canada, raymond.panneton@usherbrooke.ca), Hakim Bougrab (Groupe d'Acoustique de l'Université de Sherbrooke, 2500, Boul. de l'Université, Département de génie mécanique, Sherbrooke, QC J1K2R1, Canada, Hakim.Bougrab@USherbrooke.ca), Christophe Chaut (Henkel Technologies, Acoustics Center, 58203 Cosne sur Loire, France, Christophe.Chaut@fr.Henkel.com), Jean-Luc Wojtowicki (Henkel Technologies, Acoustics Center, 58203 Cosne sur Loire, France, Jean-Luc .Wojtowicki@fr.Henkel.com)

This work shows how to couple transfer matrix method to finite element method with a view to analyze the acoustic response of hollow body structures with a minimum of memory requirements and computational time. An hollow body structure is made up from a series of elongated rigid-walled fluid partitions (i.e., waveguides). These fluid partitions are separated by any passive (e.g., multilayered sound barrier) or reactive elements (e.g., expansion chamber). In the proposed hybrid model, the elongated fluid partitions are modeled using 1D fluid finite elements, and the passive or reactive elements using transfer matrices. From the weak integral formulation of the acoustic problem, it is shown how the coupling with the transfer matrix is taken into account through a mixed boundary condition. After discretization of the acoustic pressure and application of the variational principle, the finite element matrix system is obtained, where only the nodal pressures in the fluid partitions remain. The transfer matrix has been converted into a kind of admittance matrix, where no additional degrees of freedom are necessary to account for the passive or reactive elements. The method is used to predict the acoustic response of a real hollow body structure. Good correlations are obtained with experimentations. 


\title{
Session 5pPAb
}

\section{Physical Acoustics: Ultrasonics Under Extreme Conditions II}

\author{
Albert Migliori, Cochair \\ NHMFL, Los Alamos Natl. Lab. Los Alamos, NM 87545, USA \\ Frédéric Decremps, Cochair \\ IMPMC, Université Paris VI, 140, rue de Lourmel, Paris, 75015, France
}

\section{Invited Papers}

\section{3:20}

5pPAb1. Gigahertz ultrasonic interferometry at high pressure and temperature: Geophysical implications. Anastasia P. Kantor (Bayerisches Geoinstitut, University Bayreuth, 95440 Bayreuth, Germany, Anastasia.Kantor@Uni-Bayreuth.de), Steven D. Jacobsen (Department of Earth and Planetary Sciences, Northwestern University, Evansto, IL 60208-2150, USA, steven@earth.northwestern.edu), Innokenty Y. Kantor (Bayerisches Geoinstitut, University Bayreuth, 95440 Bayreuth, Germany, Innokenty.Kantor@uni-bayreuth.de), Leonid S. Dubrovinsky (Bayerisches Geoinstitut, University Bayreuth, 95440 Bayreuth, Germany, Leonid.Dubrovinsky@uni-bayreuth.de), Hans Josef Reichmann (Geoforschungszentrum Potsdam, Telegrafenberg, Division 4, 14473 Potsdam, Germany, hanni@gfz-potsdam.de)

High-frequency acoustic interferometry is widely used to penetrate a medium and measure the reflection signature, which can reveal details about the inner structure of the medium. It is a very helpful and one of the most accurate techniques for determination elastic properties of different materials being capable to measure sound wave velocities in very small samples under high pressures. The ultrasonic interferometry system operating at 0.6-2.1 gigahertz $(\mathrm{GHz})$ frequencies was developed in the Bavarian Geoinstitute of the University of Bayreuth for in situ high pressure and temperature experiments. High pressures are reached by using diamond anvil cell, and a Pt-resistive heater allows reaching high temperatures. The experimental setup is equipped with a laser system, which allows measuring a shift of ruby fluorescence line at every given temperature.

$$
3: 40
$$

5pPAb2. Estimation of thermophysical properties of fluids under high pressure from speed of sound measurements. Jean Luc Daridon (Université de Pau et des Pays de l'Adour, Laboratoire des Fluides Complexes, UMR 5150, BP 1155, 64000 Pau, France, jean-luc.daridon@univ-pau.fr)

The thermophysical properties of pure substances in fluid state as functions of temperature and pressure are of great interest not only for industrial applications (for example in the oil and gas industry), but also for fundamental aspects in view of developing models for an accurate representation of dense fluids. Now these measurements are difficult to perform under pressure, particularly for non singlephase systems, at atmospheric pressure. An interesting alternative consists in using the ultrasonic velocity which can be determined experimentally with a high degree of accuracy including at high pressures and high temperatures, and which presents the advantage of giving access to various derived properties. This potential, which is specific to ultrasonic velocity in fluids, has been the starting point for the investigation of a large number of pure liquids and gases as well as of several types of mixtures by ultrasonic measurements. In this work we will review the procedures used to evaluate thermophysical properties as a function of pressure from speed of sound measurements. The validity of the different approaches will be checked by comparison to several thermophysical properties measured in an extended pressure range. The accuracy reached for each property by the different procedures will be bring out.

\section{Contributed Papers}

\section{4:00}

5pPAb3. New results on the sound velocity measurements under extreme conditions using time-resolved picosecond acoustic technique. Frédéric Decremps (IMPMC, Université Paris VI, 140, rue de Lourmel, 75015 Paris, France, frederic.decremps@impmc.jussieu.fr), Laurent Belliard (INSP - UMR 7588 CNRS \& Université Pierre et Marie Curie, 140 Rue de Lourmel, 75015 Paris, France, lbelliar@ccr.jussieu.fr), Bernard Perrin (INSP - UMR 7588 CNRS \& Université Pierre et Marie Curie, 140 Rue de Lourmel, 75015 Paris, France, bernard.perrin@insp.jussieu .fr), Michel Gauthier (IMPMC, Université Paris VI, 140, rue de Lourmel, 75015 Paris, France, michel.gauthier@impmc.jussieu.fr)

In this presentation, recent works on the pressure and temperature dependence of the sound velocity will be discussed. We have used a newly developed method combining the time-resolved picosecond optical technique and a diamond anvil cell [1]. This setup makes possible accurate measurements of the attenuation and velocity of longitudinal waves in the Ghz range, and opens the elastic investigations of all materials (opaque, transparent, single- or polycrystal, liquids) up to several Mbar and thousands of $\mathrm{K}$. The experimental method will be first described, with a discussion of the factors limiting the possibilities and the technique accuracy. [1] F. Decremps, L. Belliard, B. Perrin, and M. Gauthier, Phys. Rev. Lett, to be pubished in January 2008.

\section{4:20-4:40 Break}

4:40

5pPAb4. Elastic moduli at high temperatures with two different ultrasonic methods. Ludivine Bourgeois (Commissariat à l'Energie Atomique (CEA), Centre de Valduc, 21120 Is sur Tille, France, ludivine .bourgeois@cea.fr)

"The elastic moduli and specially the shear modulus G is one of the parameters needed to implement the constitutive relations. These mechanical 
properties can be measured for a solid or a liquid with different ultrasonic methods. This paper deals with the determination of $\mathrm{G}(\mathrm{T})$ with two different methods: a contact delay-line ultrasonic device which has been developed to measure the shear and compression waves velocities up to $1000 \mathrm{~K}$ on cylindrical specimens and a contactless (laser) ultrasonic device which can measure surface acoustic waves velocities in metals up to the melting point. Results on metals such as Al without phase transition and for Co through the phase transition will be presented."

\section{5:00}

5pPAb5. Measurements under high pressure of ultrasonic velocity in glycerol. Hassina Khelladi (Faculty of Physics, University of Science and Technology Houari Boumedienne, BP 32 El Allia, Bab-Ezzouar, 16000 Algiers, Algeria, hassinakhelladi@yahoo.fr), Frédéric Plantier (Université de Pau et des Pays de l'Adour, Laboratoire des Fluides Complexes, UMR 5150, BP 1155, 64000 Pau, France, frederic.plantier@univ-pau.fr), Jean Luc Daridon (Université de Pau et des Pays de l'Adour, Laboratoire des Fluides Complexes, UMR 5150, BP 1155, 64000 Pau, France, jean-luc.daridon@univ-pau.fr), Hakim Djelouah (Faculty of Physics, University of Science and Technology Houari Boumedienne, BP 32 El Allia, Bab-Ezzouar, 16000 Algiers, Algeria, djelouah_hakim@yahoo.fr)

Glycerol has been the subject of significant scientist interest. Indeed, glycerol is a polyalcohol and the presence of three hydroxyl groups per molecule makes glycerol a complex system to explore. The purpose of this investigation is to measure under high pressure the ultrasonic wave velocity in glycerol, from which a number of important thermodynamic properties could be derived and determined as a function of pressure and temperature. Pressure and temperature ranges exploited in this experimental investigation of various glycerol properties, are extended respectively from $0.1 \mathrm{MPa}$ to $100 \mathrm{MPa}$ and from $10{ }^{\circ} \mathrm{C}$ to $100{ }^{\circ} \mathrm{C}$. A high pressure measurement cell equipped with temperature and pressure monitoring and control instrumentation was used. A time of flight method was exploited to measure, under high pressure, the ultrasonic wave velocity at different temperatures. The resulting experimental data of ultrasonic wave velocity in glycerol combined with measurements at atmospheric pressure, of density, specific heat and thermal expansion coefficient were used to derive density at elevated pressures. As isentropic compressibility is linked to ultrasonic wave velocity and density by means of the Newton-Laplace equation, this intrinsic physical property is easily deduced. These results led to the behavior of each physical property as a function of temperature and pressure.

\section{5:20}

5pPAb6. Laser optoacoustic study of near-critical states and phase transitions in metals. Alexander Y. Ivochkin (Moscow State University International Laser Centre, Leninskie gory, 1, 119992 Moscow, Russian Federation, ivochkin@yandex.ru), Alexander G. Kaptilniy (Joint Institute for High Temperatures, Russian Academy of Science, Izhorskaya str., 13/19, 125412 Moscow, Russian Federation, drc@pochta.ru), Alexander Karabutov (Moscow State University, MSU, 1, building 2, GSP-2, Leninskiye Gory, 119992 Moscow, Russian Federation, akarabutov@gmail.com)

Pulsed laser optoacoustic technique is used for generation and study of near-critical states and phase transitions in metals. Metal surface is confined by a layer of transparent dielectric. In this case the efficiency of pressure generation is much greater than in case of the free surface so it is possible to achieve states of metals with relatively high thermodynamic parameters: $\mathrm{P}$ $\sim 10^{4}$ atm and $\mathrm{T} \sim 10^{4} \mathrm{~K}$ with a table-top laser system. The experimental setup for simultaneous measurements of pressure, temperature, and reflectivity of metal surface with nanosecond temporal resolution was assembled. Q-switched Nd:YAG laser with pulse duration $\sim 10 \mathrm{~ns}$ and pulse energy $\sim 1 \mathrm{~J}$ was used. Pressure was measured using $\mathrm{LiNbO}_{3}$ piezotransducer. Temperature was obtained with optical pyrometer. Lead and mercury were chosen as test metals. Pressure pulses up to $1 \mathrm{kbar}$ in lead and up to $7 \mathrm{kbar}$ in mercury (with $\mathrm{T} \sim 2400 \mathrm{~K}$ - super-critical area of the phase diagram for $\mathrm{Hg}$ ) were obtained. The curve of laser heating process in P-T coordinates was plotted. The measurements of optical properties showed considerable decrease of surface reflectivity both for lead and mercury at high laser fluences due to increase of temperature and density decrease.

\section{$5: 40$}

5pPAb7. Effect of intense neutron dose radiation on piezoceramics. Franck P. Augereau (IES/Université Montpellier II, Université Montpellier II, Place Eugène Bataillon, 34095 Montpellier, France, Franck.Augereau@ies.univ-montp2.fr), Jean-Yves Ferrandis (Radio Application Division, NEC Corporation, Université Montpellier II, Place Eugène Bataillon, $34095 \quad$ Montpellier, France, ferrandi@lain.univ-montp2.fr), Jean-François Villard (CEA Saclay, 91191 Gif sur Yvette Cedex, France, jean-francois.villard@cea.fr), Damien Fourmentel (CEA Saclay, 91191 Gif sur Yvette Cedex, France, damien.fourmentel@cea.fr), Mark Dierckx (SCK-CEN, Boeretang 200, B-2400 Mol, Belgium, mdierckx@sckcen.be), Jan Wagemans (SCK-CEN, Boeretang 200, B-2400 Mol, Belgium, jwageman@SCKCEN.BE)

Four grades of commercial PZT materials have been exposed to nuclear radiation during five months in an irradiation channel of the BR1 research reactor at SCK $\mathrm{CEN}^{\circ}$ (Belgium). This experimental study was performed in the framework of the Joint Instrumentation Laboratory with the CEA French Commission of Atomic Energy to validate these materials for future applications in severe conditions such as online measurements in irradiation experiments performed in research reactors. For this purpose, thin piezoelectric discs were irradiated while a remote network analyser continuously monitored the frequency response of their electrical impedance. The total neutron dose has reached a level of 1.5.1017 neutrons $/ \mathrm{cm}^{2}$. Positive and negative shifts of the peak resonance frequency have been recorded but in any case with a variation lower than $1 \%$. On the other hand, the amplitude of the electrical impedance at resonance frequency has largely decreased with even a reduction by factor two or three for some piezoelectric cells. Transitory effects have also been detected for these two parameters as function of the reactor activity. Additional thermal and gamma radiation effect have been investigated. Similarly, some piezoelectric cells glued on glass delay line have been tested with satisfactory results to these stresses.

\section{6:00}

5pPAb8. Ultrasonic exploration at extreme shallow underground in submerged soil. Kunihiko Seo (Toin University of Yokohama, 1614 Kurogane-cho, Aoba-ku, 225-8502 Yokohama, Japan, seo.ylk@gmail.com), Takashi Shirakawa (Toin University of Yokohama, 1614 Kurogane-cho, Aoba-ku, 225-8502 Yokohama, Japan, t_shira7@yahoo.co.jp), Tsuneyoshi Sugimoto (Toin University of Yokohama, 1614 Kurogane-cho, Aoba-ku, 225-8502 Yokohama, Japan, tsugimot @ cc.toin.ac.jp)

Now a lot of land mines remain buried in the world, so that the clearance of them is required. As a tool of removing land mines, equipments using electromagnetic radiation are often employed. However, there is a problem that the land mines in the flooded soil such as in Southeast Asia cannot be detected in the rainy season. Therefore, the new way using sound waves will be profitable to detect the land mines in the flooded soil. In this research, the acoustic exploration at very shallow area in submerged sand is examined at a water tank in the lab. First we measure the propagation property of ultrasound of $120 \mathrm{kHz}$ in the shallow submerged sand, and examine underground imaging. As a result, the acoustic velocity is measured at about $1500 \mathrm{~m} / \mathrm{s}$ and the attenuation is measured at about $-19 \mathrm{~dB} / \mathrm{m}$. And next, shallow underground exploring by using acoustic shielding boards is carried out. As a result, underground images in the water tank simulating the submerged soil are obtained. Then acoustic shielding boards can block wave which propagates specific route. This will make another exploration method possible. 


\title{
Session 5pPAc
}

\section{Physical Acoustics: General Topics in Physical Acoustics II}

\author{
Walter Lauriks, Cochair \\ Lab. ATF, Katholieke Universiteit Leuven, Celestijnenlaan 200D, Leuven, B-3001, Belgium \\ Andi Petsculescu, Cochair \\ University of Louisiana, Department of Physics, P.O. Box 44210, Lafayette, LA 70504, USA
}

\section{Contributed Papers}

\begin{abstract}
4:00
5pPAc1. An automated 3 dimensional scanning system for validation of acoustical simulation results. Simo-Pekka Simonaho (University of Kuopio, P.O.Box 1627, 70211 Kuopio, Finland, simo-pekka.simonaho@uku.fi)

To validate acoustical simulation results, a great number of measurement points are needed especially in 3 dimensional cases. These measurements can be extremely laborious when done manually. Also, the spatial information of the measurement points has to be accurate. In this work, an automated 3-D scanning system with high spatial resolution for validation of acoustical simulation results is introduced. The system consists of a multi channel data acquisition hardware, a microphone array and a 3-D scanning system. The movement of the microphone array is controlled by the data acquisition hardware. The performance of the automated 3-D scanning system is demonstrated and the experimentally measured pressure fields are compared to simulation results.
\end{abstract}

\section{4:20}

5pPAc2. Signal processing of impedance spectrum for speed of sound and pressure measurement in plane or radial resonators. Eric Rosenkrantz (Radio Application Division, NEC Corporation, Université Montpellier II, Place Eugène Bataillon, 34095 Montpellier, France, eric.rosenkrantz@ies.univ-montp2.fr), Jean-Yves Ferrandis (Radio Application Division, NEC Corporation, Université Montpellier II, Place Eugène Bataillon, 34095 Montpellier, France, ferrandi@lain.univ-montp2 .fr), Gerard Leveque (Institut d'Electronique du Sud UMR-CNRS 5214, Université Montpellier II, Place Eugène Bataillon, 34095 Montpellier, France, Gerard.leveque@ies.univ-montp2.fr)

The impedance of gas contained between two plane walls is a periodic function of the frequency. The frequency interval between two resonances of the gas is equal to $\mathrm{c} / 2 \mathrm{D}$. Furthermore, the amplitude of the resonances is proportional to the pressure. We describe a signal processing to easily deduce the speed of sound and the pressure of the gas from the impedance spectrum. We show that the modulus of the Fourier transform of the modulus of impedance, called "Tempograph," contains all information about the gas. In some industrial cases the container is a cylinder or a sphere which can be used as a radial resonator exited by a radial wave $[1,2]$. The frequency response of such resonator at high frequencies is quasi-periodic and thus the same signal processing can be used. [1] M. F. Narbey, et al., "Determination of the composition of a gas mixture in a nuclear fuel rod by an acoustic method." INSIGHT, 42(9), 603-605 (2000). [2] A. Olson, "Helium bottle pressure measurement by portable ultrasonic technique." 1989, Rapport de Boeing nAD-A208 994, http://stinet.dtic.mil/str/index.html.

\section{4:40}

5pPAc3. Absorption and velocity of acoustical waves in binary solutions of poly (ethylene glycol) and water. Rajendra Kumar Singh (Department of Physics, Banaras Hindu University, 221005 Varanasi, India, rksingh_17@rediffmail.com), Manish Pratap Singh (Banaras Hindu
University, 221005 Varanasi, India, mps_bhu@yahoo.co.in),Rishi Pal Singh (Banaras Hindu University, 221005 Varanasi, India, rishisingh80 @ rediffmail.com)

A resonator technique has been devoleped to measure ultrasonic velocity and absorption for aqueous solutions of PEG of different molecular weights. The velocity has been measured at different frequencies and the concentrations (by weight) ranged from $1 \%$ to $10 \%$ of poly (ethylene glycol) in water. Adiabatic compressibility has been obtained at different temperatures, using experimental value of velocity and density. Viscocity has also been measured in wide temperature range . Ultrasonic absorption has been measured in the frequency range $400 \mathrm{KHz}-50 \mathrm{MHz}$, using resonator technique and pulse technique in temperature range $400-650{ }^{\circ} \mathrm{C}$. Observations showed that the ultrasonic absorption decreases with increasing temperature at a given concentration and also increases with concentration at a given temperature. The velocity increases with increasing temperature and concentration. Shear viscosity has been found to decrease with temperature but increases with concentration. Velocity studies show that as the polymer concentration increases a more rigid molecular structure is formed by bonding between the large polymer molecules.

\section{5:00}

5pPAc4. Acoustic field in a spherical resonator: effect of modal coupling due to small perturbations. Cécile Guianvarc'H (Institut National de Métrologie (LNE-INM/Cnam), 61 rue du Landy, 93210 La Plaine Saint Denis, France, cecile.guianvarch@cnam.fr), Laurent Pitre (Institut National de Métrologie (LNE-INM/Cnam), 61 rue du Landy, $93210 \mathrm{La}$ Plaine Saint Denis, France, pitre@cnam.fr), Arnaud Guillou (Institut National de Métrologie (LNE-INM/Cnam), 61 rue du Landy, 93210 La Plaine Saint Denis, France, guillou.arnaud@gmail.com), Michel Bruneau (Laboratoire d'Acoustique de l'Université du Maine, Avenue Olivier Messiaen, 72085 Le Mans, France, michel.bruneau@univlemans.fr), Anne-Marie Bruneau (Laboratoire d'Acoustique de l'Université du Maine (LAUM, UMR CNRS 6613), Avenue Olivier Messiaen, 72085 Le Mans, France, anne-marie.bruneau@univ-lemans.fr)

The international community recently recommended a re-definition of the kelvin referring to the value of the Boltzmann constant $k$, which must thus be known with a relative uncertainty of $10^{-6}$. The measurement of the acoustic resonance properties of a gas filled spherical or quasi-spherical resonator is an appropriate method to do this with these requirements. Actually, a detailed modeling of the acoustic field in the resonator is required for the determination of $k$. Several phenomena must be taken into account including heat conduction, shear and bulk viscosity of the gas, the real shape of the resonator, the acoustic input impedance of small acoustic elements flush-mounted on the wall (tubes, transducers)... . Significant theoretical studies have already been done in which these perturbations are accounted for separately, the coupling between them being neglected. The scope here is thus to provide a unified model for the acoustic field in the cavity including all these perturbations and the resulting modal coupling, and applying it on a simple practical configuration: a spherical resonator filled with argon, acoustic transducers being flush-mounted on the wall. 
$5: 20$

5pPAc5. Towards a theory for arbitrarily shaped sound field reproduction systems. Sascha Spors (Deutsche Telekom Laboratories, Ernst-Reuter-Platz 7, $10587 \quad$ Berlin, Germany, sascha.spors@telekom.de), Jens Ahrens (Deutsche Telekom Laboratories, Ernst-Reuter-Platz 7, 10587 Berlin, Germany, jens.ahrens@telekom.de)

The simple source approach predicts that a distribution of appropriately driven loudspeakers (secondary sources) enclosing a given listening area is suitable for the physical recreation of any desired exterior virtual sound field within that listening area. A specific class of sound reproduction approaches (e.g., higher-order Ambisonics) is based upon the explicit solution of the simple source formulation with respect to the secondary source driving function. To the knowledge of the authors, such an explicit solution is currently only available for specific geometries of the sound reproduction system. This contribution presents a theoretical framework for the derivation of the explicit solution for the driving function of arbitrarily shaped secondary source arrangements. It is based upon the expansion of the reproduced wave field into a series of orthogonal basis functions. These basis functions emerge from the respective underlying geometry. It is shown that most of the sound reproduction systems that are based upon the simple source formulation can be seen as specialization of the presented approach to a particular geometry and basis function.

\section{$5: 40$}

5pPAc6. Numerical computation of reflected and transmitted waves at a fluid/solid interface. Laure Bossy (AREVA - CEZUS Research Center, Avenue Paul Girod, 73403 Ugine Cedex, France, laure.bossy@espci.fr), Marie-Françoise Cugnet (AREVA - CEZUS Research Center, Avenue Paul Girod, 73403 Ugine Cedex, France, mariefrancoise.cugnet@areva.com), Emmanuel Bossy (Laboratoire Photons et Matière, ESPCI/CNRS, 10 rue Vauquelin, 75231 Paris Cedex 05, France, emmanuel.bossy@espci.fr), Didier Cassereau (Laboratoire Ondes et Acoustique, 10, rue Vauquelin, 75231 Paris, France, didier.cassereau @espci.fr)

In this paper, we propose a numerical computation of the different waves generated when a spherical incident pulse is reflected and transmitted by a fluid/solid interface. In addition to the standard reflected and transmitted waves that propagate inside the volume, various surface waves can also be found in both propagation media. In the fluid, we can observe the longitudinal and transverse head waves, and the so-called leaky Rayleigh wave that generalizes the Rayleigh wave in a semi-infinite free solid medium to the case of an immersed interface. Similar effects can also be observed on the transmitted displacement field inside the solid material. We compare different numerical approaches, including semianalytic methods (high-frequency approximation coupled to ray modeling approach) and implicit methods (finite elements and/or finite differences scheme), each method having its own advantages and inconvenients, and domains of validity. These different methods are used to evaluate the field reflected by the interface ; the transmitted displacement field is also analyzed from the same point of view. The plane and curved geometries will be analyzed and the influence of the curvature of the interface will be pointed out.

\section{6:00}

5pPAc7. $\mathrm{V}(\mathrm{z})$ oscillations in acoustic microscope at upward defocusing Anton V. Kozlov (MSU, Leninskie Gory, Bld. 1-2, 119991 GSP-1 Moscow, Russian Federation, av_kozlov@inbox.ru), Vladimir G. Mozhaev (MSU, Leninskie Gory, Bld. 1-2, 119991 GSP-1 Moscow, Russian Federation, vgmozhaev@mail.ru)

Acoustic microscopy is widely used for imaging and study of elastic properties of transparent and opaque materials. As a rule, multiple periodic oscillations in the output signal $V$ of a reflection acoustic microscope can be observed in the case of negative defocusing of the wide-angle acoustic lens, i.e., when its focus is a distance $z$ below the solid sample surface. The main well-known mechanism for $V(z)$ oscillations is the interference of radiation reflected perpendicularly from the sample surface and re-radiation of leaky Rayleigh waves generated on the sample by the lens. This effect explains high contrast imaging in reflection scanning acoustic microscopy, and it is a popular method to study properties of solids by measuring Rayleigh wave speeds. As it is shown in this work, bulk acoustic waves in the sample can also give rise to $V(z)$ oscillations. A new mechanism of such oscillations is predicted in the case of positive defocusing (focusing above the sample surface) in acoustic microscopy of anisotropic plates exhibiting negative acoustic refraction. The ray model of this effect shows a possibility to find a relationship between extrema of $V(z)$ curve and separate points on the acoustic slowness surface of the sample.

\title{
Session 5pPAd
}

\section{Physical Acoustics: Scattering and Diffraction II}

\author{
Michael L. Oelze, Cochair \\ University of Illinois at Urbana-Champaign, Beckman Institute, 405 N Mathews, Urbana, IL 61801, USA
}

Jean-Marc Conoir, Cochair

Institut Jean Le Rond d'Alembert-UMR CNRS 7190, Université Paris 6, tour 55-65, 4 place Jussieu, Paris, 75005, France

\section{Contributed Papers}

\section{5:00}

5pPAd1. The physics of wedge diffraction: A model in terms of elementary diffracted waves. Mitsuhiro Ueda (Predio Meguro Science Laboratory, 4-20-13 Meguro, Meguro-ku, 153-0063 Tokyo, Japan, ueda-mt @ nifty.com)

A model for wedge diffraction is constructed using the virtual discontinuity principle of diffraction [1]. In the model diffracted waves are described by the sum of two elementary diffracted waves that are calculated by integrating the potential along the half line issued from the vertex of wedge. The wedge of aperture angle $\pi / \mathrm{n}(\mathrm{n}=1,2,3, \ldots)$ is nondiffractive since its potential can be expressed by the sum of direct waves from the point source and its mirror images. The nondiffractive wedges are useless in the conventional analysis of diffraction since there are no diffracted waves in the potential. But in this model diffracted waves of these wedges are cancelled out in the summing process. Thus the elementary diffracted waves are existed even in these wedges and far field solution for them is obtained for the nondiffractive wedge. Due to its simple structure it can be extended to the arbitrary wedge without any modification and the far field solution of diffracted waves is derived by summing the extended elementary diffracted waves in the model and it coincides with the rigorous one literally. Thus the model is verified firmly by this simple calculation. 1. M. Ueda, JASA, 95, p.2354 (1994). 
$5: 20$

5pPAd2. A global search tool for the equivalent source method and its applications to the scattering problem. Yves J.r. Gounot (UFRJ/COPPE, Universidade Federal do Rio de Janeiro, 21941-972 Rio de Janeiro, Brazil, ygounot@mecanica.ufrj.br), Ricardo E. Musafir (UFRJ/COPPE, Universidade Federal do Rio de Janeiro, 21941-972 Rio de Janeiro, Brazil, rem @ serv.com.ufrj.br)

Low computational-cost solutions to the acoustic scattering problem can be obtained with the equivalent source method (ESM), provided the sources are adequately positioned. Because this last point represents often a complicated task - mainly responsible for the not much widespread use of the method - a technique that hurdles this difficulty, called ESGA, has been previously proposed (Gounot and Musafir, Internoise 2004). Based on a combination of genetic algorithm with ESM, the ESGA is a global search tool that provides, given a set of monopoles, their 'optimal' positioning and complex amplitudes. The technique efficiency is here shown through a number of three-dimensional scattering problems. The algorithm is also used in order to identify, for each of the different cases considered, typical geometrical arrangements of monopoles which provide good solutions.

\section{$5: 40$}

5pPAd3. Convergence of correlations in multiply scattering media. Eric Larose (LGIT - CNRS - Université Joseph Fourier, Maison des Géosciences, 1381 rue de la Piscine, BP 53, 38041 Grenoble, France, Eric.Larose@obs.ujf-grenoble.fr), Arnaud Derode (Laboratoire Ondes et Acoustique, ESPCI, Université Paris 7, CNRS, 10 rue Vauquelin, 75005 Paris, France, arnaud.derode@ujf-grenoble.fr), Philippe Roux (LGIT CNRS - Université Joseph Fourier, Maison des Géosciences, 1381 rue de la Piscine, BP 53, 38041 Grenoble, France, philippe.roux@obs.ujf-grenoble.fr), Michel Campillo (LGIT - CNRS Université Joseph Fourier, Maison des Géosciences, 1381 rue de la Piscine, BP 53, 38041 Grenoble, France, Michel.Campillo@obs.ujf-grenoble.fr)

Correlations of ambient seismic or acoustic noise are now widely used to reconstruct the impulse response between two passive receivers as if a source was placed at on of them. Applications include terrestrial and solar seismology, underwater acoustics and structural health monitoring. Never- theless, for a given set of data, correlations do not only yield automatically the Green function between the sensors, but also contains residual fluctuations that might "blur" the images. [Gizon et al, Astrophys. J. 614 (2004) Weaver and Lobkis, J. Acoust. Soc. Am. 117 (2005); Sabra et al., J. Acoust. Soc. Am. 118 (2005)]. We propose a model to describe the "signal-tofluctuations" ratio in the correlations in the case of nonstationary wavefields, and more particularly in the case of scattering media. The work includes theoretical derivations and numerical simulations. The role of multiple scattering in the rate of convergence of the correlations toward the Green function is quantitatively evaluated [Larose et al, (submitted 2008)].

\section{6:00}

5pPAd4. Reflection and transmission coefficients of a fluid slablike region containing a depth-varying random distribution of cylinders. Jean-Marc Conoir (Institut Jean Le Rond d'Alembert-UMR CNRS 7190, Université Paris 6, tour 55-65, 4 place Jussieu, 75005 Paris, France, conoir@1mm.jussieu.fr), Sébastien Robert (LOA, UMR CNRS 7587, ESPCI, 10 rue Vauquelin, 75231 Paris, France, Sebastien.Robert@espci.fr), Abdelhak El Mouhtadi (LOMC- FRE CNRS 3102- Groupe Ondes Acoustiques, Université du Havre, place R. Schuman, 76610 Le Havre, France, abdelhak.ilel@gmail.com), Francine Luppé (LOMC- FRE CNRS 3102- Groupe Ondes Acoustiques, Université du Havre, place R. Schuman, 76610 Le Havre, France, francine.luppe @ univ-lehavre.fr)

This work deals with multiple scattering by a random distribution of parallel elastic cylinders immersed in a fluid slablike region. The concentration of scatterers inside the slab is supposed to vary slowly with depth, and the WKB method is used to calculate the reflection and transmission coefficients of the slab. In order to do so, the continuity conditions on the boundaries between the slab and the surrounding fluid are needed. They follow from the application of Twersky's theory to the case of a slab with a given constant concentration of scatterers, which shows that both pressure and normal displacement are continuous, provided an effective mass density of the slab is correctly defined. The results of the WKB are successfully compared to those obtained from the discretization of the slab into layers of constant concentrations of cylinders and the use of Twersky's theory.

\section{Session 5pPPa}

\section{Psychological and Physiological Acoustics and Speech Communication: Acoustic Features and Speech Perception II}

Jont B. Allen, Cochair

University of IL, 405 N. Mathews, Room 2061 Beckman Inst. (MC 251), Urbana, IL 61801, USA

Sarah Hawkins, Cochair

University of Cambridge, Department of Linguistics, Sidgwick Avenue, Cambridge, CB3 9DA, UK

Invited Papers

5pPPa1. Reception of phonetic features in fluctuating background noise maskers. Christian Lorenzi (Univ Paris Descartes, CNRS, Ecole Normale Superieure, DEC, 29 rue d'Ulm, 75005 Paris, France, lorenzi@ens.fr)

We will review recent studies investigating the reception of phonetic features (voicing, manner and place of articulation) in nonstationary background maskers. In each study, consonant identification was assessed in steady and amplitude-modulated speech-shaped noise at signal-to-noise ratios yielding about 50\% correct in steady noise. The rate and depth of amplitude modulation applied to the noise masker were either fixed or systematically varied. Confusion matrices were compiled across listeners and the amount of release from masking (percent information received in nonsteady minus steady noise) was calculated for each phonetic feature and experimental condition. Speech and noise mixtures were (i) left intact (unfiltered), (ii) lowpass filtered $(<1.5 \mathrm{kHz}$ ), or iii) processed in order to 
degrade spectral (place of excitation in the cochlea) and/or temporal fine structure cues. Overall, the results indicate that release from masking typically reported in normal-hearing listeners (i.e., the substantial improvement in speech reception in fluctuating noise compared to steady noise) is not identical across phonetic features. This suggests that the ability to "glimpse" into background noise valleys involves multiple auditory processes constrained by both peripheral and central factors.

5pPPa2. Identification of intervocalic consonants in stationary and nonstationary noise. Martin Cooke (Sheffield University, Computer Science Department, Regent Court, 211 Portobello St., S1 4DP Sheffield, UK, m.cooke@dcs.shef.ac.uk), Odette Scharenborg (Centre for Language and Speech Technology, Radboud University Nijmegen, Erasmusplein 1, 6525 HT Nijmegen, Netherlands, O.Scharenborg@let.ru.nl)

The factors which underlie the perception of consonants in noise remain poorly understood. In this study, native listeners identified 24 English consonants spoken by eight talkers presented in nine intervocalic contexts with varying stress position. Listeners were tested in 5 noise conditions: tokens were masked by stationary speech-shaped noise, a competing talker, three and eight speaker babble and speech-modulated noise, all of which have the long-term spectrum of speech. The rank ordering of consonant identification scores in stationary noise was highly-correlated $(\mathrm{r}=0.9, \mathrm{p}<0.0001)$ with a similar condition reported by Phatak and Allen [JASA 121: 2312-2326, 2007], but less so in the four nonstationary noise backgrounds (r=0.74, p<0.001). In particular, /y/, /r/, /1/, /f/, /ch/, /sh/, /m/ and most of the plosives showed a wide variation in ranking. These findings suggest that, in addition to the long-term spectrum of the masker, consonant identification is noise is affected by other factors such as temporal fluctuations in the masker, misallocation of foreground/background components and attention.

\section{$2: 40$}

5pPPa3. The interaction of glimpsing, pitch and vocal tract length in the recognition of concurrent syllables. Martin D. Vestergaard (Centre for the Neural Basis of Hearing, Department of Physiology, Development and Neuroscience, University of Cambridge, Downing Site, CB23EG Cambridge, UK, mdv23@cam.ac.uk), Nicolas R. Fyson (Centre for the Neural Basis of Hearing, Department of Physiology, Development and Neuroscience, University of Cambridge, Downing Site, CB23EG Cambridge, UK, nickfyson@gmail.com), Roy D. Patterson (Centre for the Neural Basis of Hearing, Department of Physiology, Development and Neuroscience, University of Cambridge, Downing Site, CB23EG Cambridge, UK, rdp1@cam.ac.uk)

In multispeaker environments, human listeners use the temporal misalignment of competing speech signals to improve recognitionan effect referred to as "glimpsing." When the temporal envelopes of concurrent syllables pairs are carefully matched to preclude glimpsing, listeners were observed to use vocal tract length (VTL) and glottal pulse rate (GPR) cues to improve recognition. This paper reports an investigation of the interaction between glimpsing and these vocal cues. Syllables were synthesized with a vocoder to simulate speakers with widely different combinations of GPR and VTL. Recognition of one syllable in the presence of a concurrent syllable was measured as the vocal cues and the temporal alignment of the syllables were varied. The effect of glimpsing was most pronounced when the vocal cue differences between the target and distracter syllables were small. Furthermore, there was a strong effect of consonant type (stops, fricatives, or sonorants) and an asymmetry between consonant-vowel (CV) and vowel-consonant (VC) syllables. The lowest recognition rate was observed, not at perfect temporal alignment but rather at a distracter lag of $-50 \mathrm{~ms}$ for CVs and $100 \mathrm{~ms}$ for VCs. The results are analyzed with confusion matrices. Research supported by the UK MRC [G0500221, G9900369].

\section{3:00}

5pPPa4. Phoneme confusions as a function of noise, spectral resolution and L2 experience. Robert Shannon (House Ear Institute, 2100 W. Third St., Los Angeles, CA 90057, USA, shannon@hei.org), Monica Padilla (House Ear Institute, 2100 W. Third St., Los Angeles, CA 90057, USA, mpadilla@hei.org)

Consonant and vowel confusion matrices were measured from normal hearing listeners with varying degrees of experience in English. There were five listeners each in the following categories: native English speakers, and Spanish speakers who were immersed in English at the ages of 0-5 years, 5-10 years, 10-18 years, and over 18 years. 12 vowels (hVd) or 18 consonants (vCV) were presented and responses were collected in a confusion matrix. Spectral resolution was varied by using a noise-band vocoder with 2,4 , 6 , 8 , and 16 channels, as well as unprocessed speech. All stimuli were presented in speech shaped noise at SNR levels of $-5 \mathrm{~dB}$ to $+15 \mathrm{~dB}$ in 5 $\mathrm{dB}$ steps as well as in quiet. Noise and spectral resolution had a similar effect on voicing, manner and place of articulation, and also had similar effects as a function of L2 experience. For native speakers of Spanish the duration of L2 experience had the largest effect on voicing cues, less effect on manner cues, and almost no effect on place cues.

\section{$3: 20$}

5pPPa5. The perceptual flow of phonetic feature processing. Steven Greenberg (Technical University of Denmark, Center for Applied Hearing Research, Ørsteds Plads, Building 352, 2800 Lyngby, Denmark, steveng@ silicon-speech.com), Thomas Ulrich Christiansen (Technical University of Denmark, Center for Applied Hearing Research, Ørsteds Plads, Building 352, 2800 Lyngby, Denmark, tuc@oersted.dtu.dk)

How does the brain process spoken language? It is our thesis that word intelligibility and consonant identification are insufficient by themselves to model how the speech signal is decoded - a finer-grained approach is required. In this study, listeners identified 11 different Danish consonants spoken in a Consonant + Vowel $+[1]$ environment. Each syllable was processed so that only a portion of the original audio spectrum was present. Three-quarter-octave bands of speech, centered at 750, 1500, and $3000 \mathrm{~Hz}$, were presented individually and in combination with each other. The conditional, posterior probabilities associated with phonetic-feature decoding were computed from confusion matrices in order to deduce the temporal flow of phonetic processing. Decoding the feature, Manner-ofArticulation, depends on accurate decoding of the feature Voicing (but not vice-versa), and decoding Place-of-Articulation requires 
precise decoding of Manner (but not the converse). From these data, we conclude that Voicing is processed prior to Manner-ofArticulation, and that Manner is decoded prior to Place-of-Articulation. Voicing and Manner cues are often correctly decoded in conditions where Place is not. This asymmetric pattern of feature decoding may provide extra-segmental information of utility for speech processing, particularly in adverse listening conditions.

\section{3:40}

5pPPa6. Understanding the complex modulation spectrum for consonants and consonant features. Kenneth W. Grant (Walter Reed Army Medical Center, Army Audiology and Speech Center, 6900 Georgia Ave. NW, Washington, DC 20307-5001, USA, grant@tidalwave.net), Sandeep A. Phatak (Walter Reed Army Medical Center, Army Audiology and Speech Center, 6900 Georgia Ave. NW, Washington, DC 20307-5001, USA, s.a.phatak@gmail.com), Elena Grassi (Walter Reed Army Medical Center, Army Audiology and Speech Center, 6900 Georgia Ave. NW, Washington, DC 20307-5001, USA, elena.grassi@ gmail.com)

Speech intelligibility is highly dependent on the magnitude and phase characteristics of the low-frequency modulation spectrum. However, unlike more traditional representations of speech, such as the spectrogram, associating details of the modulation spectrum to specific phonemes and subphonemic units of speech has not been readily forthcoming. In the present study we used local time reversals of the speech waveform between 20-160 ms to selectively distort portions of the complex modulation spectrum. Normal-hearing subjects were tested on a consonant recognition task and a detailed analysis of the perceptual confusions was performed. Consistent with earlier results using sentence-length materials, average consonant intelligibility declined as the length of the time reversal segment increased. Further analyses were conducted to determine the effect of time-reversal segment duration on the amount of information transmitted for individual consonants (including specific consonant productions) and acoustic features for voicing, manner of articulation, and place of articulation. An acoustic analysis using a biologically motivated auditory processing model was also performed to determine the effect of time reversals on cochlear and cortical representations of speech. The relations between changes to the complex modulation spectrum and the percent information transmission of selected speech segments and features are discussed.

\section{4:00-4:20 Break}

\section{4:20}

5pPPa7. Can CV intelligibility predict speech intelligibility? Sarah Hawkins (University of Cambridge, Department of Linguistics, Sidgwick Avenue, CB3 9DA Cambridge, UK, sh110@ cam.ac.uk)

This paper begins by reviewing the speech perception literature to predict cues that would and would not be expected to survive energetic masking of various types. The focus is especially (but not exclusively) on spectrotemporal cues to stops in the vicinity of the segment boundary in $\mathrm{CV}$ syllables. The second part of the paper discusses influences that can restrict the generality of research findings from isolated CV syllables. This includes ways in which CV syllables change in different phonetic contexts and styles of speech, contributions of the visual modality, and other uses of top-down information, such as phonotactic, lexical, semantic and syntactic probability. The paper concludes by asking whether-and how-simple measures such as CV intelligibility can be used to reflect intelligibility of speech in real-life communicative situations.

5pPPa8. The role of the cochlear processing in human speech recognition. Jont B. Allen (University of IL, $405 \mathrm{~N}$. Mathews, Room 2061 Beckman Inst. (MC 251), Urbana, IL 61801, USA, jontallen@ieee.org), Marion Regnier (208 S. 3rd St. Apt 5A, Brooklyn, NY 11211, USA, marion.regnier@gmail.com), Sandeep Phatak (Walter Reed Hospital, Silver Springs, MD 20901, USA, s.a.phatak@gmail.com), Feipeng Li (University of IL, 405 N. Mathews, Room 2061 Beckman Inst. (MC 251), Urbana, IL 61801, USA, fli2@uiuc.edu)

Little is know about how the auditory system decodes speech. We may think of speech communication re Shannon's source-channel model, thus viewed, the most complex part of the speech communication channel is the auditory system (the receiver). In my speechperception research, I have attempted to limit the assumptions, and have thus fallen back on Shannon's basic source-channel model. The basic tool is the confusion matrix (CM) for isolated natural consonant and vowels (CV), as a function of the speech to noise ratio (SNR), with several types of masking noise. We have used large numbers of talkers and listeners (i.e., 20). In a second experiment we selectively remove islands of speech in time-frequency, and then correlate the resulting modified speech against subject scores. Our most important conclusions are: (1) The across-frequency onset transient portion of the signal is typically the most important. (2) The spectral regions of these transient are used to code different consonants. (3) While the frequency regions for a given consonant are correlated to the following vowel, this may not be important for perception. (4) Compact spectral-temporal amplitude modulations components (e.g., a $10 \mathrm{~Hz}$ modulation) do not seem to play a significant role, at least above $1-2 \mathrm{kHz}$.

\section{Contributed Papers}

5:00

5pPPa9. Spectral and temporal modulations essential to spoken word, gender and timbre identification. Frédéric E. Theunissen (UC Berkeley, Dept. of Psychology, 3210 Tolmant Hall, Berkeley, CA 94720-1650, USA, theunissen@berkeley.edu), Taffeta Elliott (UC Berkeley, Dept. of Psychology, 3210 Tolmant Hall, Berkeley, CA 94720-1650, USA, taffeta@ berkeley .edu)

Human speech and musical sounds contain complex spectral and temporal modulations. Speech intelligibility, perception of melody, and identification of source characteristics (e.g., speaker gender or musical timbre) de- pend on spectrotemporal modulations but can be surprisingly robust to drastic spectral and temporal degradations. We systematically explored which restricted spectral and temporal modulations are essential to the perception of complex sounds. Degraded sentences and musical sounds were obtained by a novel modulation filtering procedure performed on the sound spectrogram. Temporal modulation filtering smeared the amplitude envelope by removing changes above particular Hz. Spectral modulation filtering smeared the spectral energy across frequency bands by removing changes above particular cyc/kHz. We further complemented this low-pass filtering with more specific notch-filtering. Speech intelligibility, gender recognition 
and musical instrument identification were assessed in psychophysical experiments. We determined that spectral modulations below $\sim 3.75$ cy$\mathrm{c} / \mathrm{kHz}$, and temporal modulations between 1 and $7 \mathrm{~Hz}$ are essential for speech comprehension. Gender identification however required the presence of higher spectral modulations. Similarly the timbre and pitch of instruments was affected differentially by notch filters in these two regions of the modulation spectrum. Our research could be used to guide the design of optimal signal processing in hearing aids and cochlear implants.

\section{$5: 20$}

5pPPa10. A model with compression for estimating speech intelligibility in quiet and in noise. Koenraad S. Rhebergen (AMC Dept. of Clinical and Experimental Audiology, AMC, Clinical and Experimental Audiology, 1105 Amsterdam, Netherlands, k.s.rhebergen @amc.uva.nl), Johannes Lyzenga (Vrije Universiteit Medical Center, Boelelaan 1117, 1081 HV Amsterdam, Netherlands, j.lyzenga@vumc.nl)

For speech reception thresholds (SRTs), measured in normally-hearing listeners using various types of stationary noise, the Speech Intelligibility Index (SII, ANSI S3.5-1997) model predicts a fairly constant speech proportion (of about 0.3 ) necessary for sentence intelligibility. For SRTs in quiet, the estimated speech proportions are often lower, and show a larger inter-subject variability, than found for speech in noise near normal speech levels. This might be related to the fact that cochlear compression is larger at normal speech levels than near the threshold for speech in quiet. The SII model does not take this into account. The present model attempts to alleviate this problem by including cochlear compression. It is based on a loudness model for normally-hearing and hearing-impaired listeners [ANSI S3 .4-2007]. It estimates internal excitation levels of the speech, accounts for the compressed effective dynamic range of the internal speech signal, and calculates the proportion of speech above threshold using similar spectral weighting as used in the standard SII. The present model and the standard SII were used to predict SRTs in quiet and noise for both normally-hearing and hearing-impaired listeners. The present model predicted speech intelligibility with less variability than the standard SII.

\section{$5: 40$}

5pPPa11. Listeners' sensitivity to talker differences in voice-onset-time: Segments versus features. Rachel $M$. Theodore (Northeastern University, Dept. of Psych. - 125 NI, 360 Huntington Ave., Boston, MA 02115-5000, USA, r.theodore@neu.edu), Joanne L.
Miller(Northeastern University, Dept. of Psych. - 125 NI, 360 Huntington Ave., Boston, MA 02115-5000, USA, j.miller@neu.edu)

Recent findings indicate that listeners are sensitive to talker differences in phonetic properties of speech, including voice-onset-time (VOT) in wordinitial voiceless stop consonants. Here we extend earlier findings from our laboratory [J. S. Allen and J. L. Miller, J. Acoust. Soc. Am. 115, 3171-3813 (2004)] by examining the level of representation underlying this sensitivity. In familiarization phases, listeners heard two talkers produce pain. Critically, word-initial VOTs were manipulated such that one talker produced short VOTs and the other talker produced long VOTs. In test phases, listeners were presented with a short-VOT and long-VOT variant of either pain or cane; in both cases, listeners were asked to select which of the two VOT variants was most representative of a given talker. Results to date indicate that which variant of pain is selected at test is in line with listeners' exposure during training (replicating earlier findings), and that this effect holds even when listeners are tested on cane, which begins with a different voiceless stop than heard during training. These results suggest that listeners are sensitive to talker differences in VOT at the level of a phonetic feature, rather than at the level of a particular phonetic segment.

\section{6:00}

5pPPa12. Amplitude modulation of noise cues voicing distinction in fricatives. Jonathan Pincas (University of Surrey, GU2 7XH Guildford, UK, jon@pincas.co.uk), Philip J. Jackson (University of Surrey, Centre for Vision, Speech and Signal Processing, GU2 7XH Guildford, UK, p.jackson @ surrey.ac.uk)

The aperiodic noise source in fricatives is characteristically amplitude modulated by voicing. Previous psychoacoustic studies have established that observed levels of AM in voiced fricatives are detectable, and its inclusion in synthesis has improved speech quality. Phonological voicing in fricatives can be cued by a number of factors: the voicing fundamental, duration of any devoicing, duration of frication, and formant transitions. However, the possible contribution of AM has not been investigated. In a cue trading experiment, subjects distinguished between the nonsense words "ahser" and "ahzer." The voicing boundary was measured along a formant-transition duration continuum, as a function of AM depth, voicing amplitude and masking of the voicing component by low-frequency noise. The presence of AM increased voiced responses by approximately $30 \%$. The ability of AM to cue voicing was strongest at greater modulation depths and when voicing was unavailable as a cue, as might occur in telecommunication systems or noisy environments. Further work would examine other fricatives and phonetic contexts, as well as interaction with other cues. 


\title{
Session 5pPPb
}

\section{Psychological and Physiological Acoustics and Computational Acoustics: Computational Auralization II}

\author{
Durand R. Begault, Cochair \\ NASA Ames Research Center, Mail Stop 262-2, NASA ARC, Moffett Field, CA 94035, USA \\ Lauri Savioja, Cochair \\ Helsinki University of Technology, Department of Media Technology, PO Box 5400, TKK, 02015, Finland
}

\section{Contributed Papers}

2:00

5pPPb1. Externalization in binaural synthesis: effects of recording environment and measurement procedure. Florian Völk (AG Technische Akustik, MMK, TU München, Arcisstr. 21, 80333 München, Germany, florian.voelk@mytum.de), Fabian Heinemann (AG Technische Akustik, MMK, TU München, Arcisstr. 21, 80333 München, Germany, hef@mmk.ei.tum.de), Hugo Fastl (AG Technische Akustik, MMK, TU München, Arcisstr. 21, 80333 München, Germany, fastl@mmk.ei.tum.de)

Databases of head related impulse responses (HRIRs) for binaural synthesis can be measured either in anechoic or reflective environments. If high synthesis quality is needed, miniature microphone measurements are performed in the ear canals of each individual user (individual measurement). Sometimes impulse responses measured in the ear canals of one individual are used for synthesis for other persons (nonindividual measurement). In most other cases artificial head measurements are used. This paper considers the dependence of the perceived distance of auditory images (externalization) on the measurement procedure (individual, nonindividual, artificial head) and on the recording environment (anechoic, reflective). For each measurement the same system and the same setup, especially the same geometric parameters, are used. Differences in the corresponding impulse response databases are determined and related to the subjective relative externalization differences in the front, in the back, and to both sides. For each direction a seven point rating scale was used. Statistical analysis suggests that the measurement parameters applied influence the externalization of auditory images.

\section{2:20}

5pPPb2. Smart sound environments: merging intentional soundscapes, nonspeech audio cues and ambient intelligence. Ralf Jung (Universität des Saarlandes, LS Wahlster, FB Informatik, Bldg. E 1 1, Room 1.18, 66123 Saarbrücken, Germany, rjung@cs.uni-sb.de)

We introduce an intelligent audio notification system for multiuser environments that provides users with information about events (e.g., important emails) in a more discreet and non-distracting way. The peripheral awareness of individual-related events is done by using nonspeech audio cues which can be seamlessly integrated into artificial background soundscapes. These ambient soundscapes are self-composed with respect to well-known perceptual constraints such as auditive Gestalt laws as well as music psychological findings. To follow a hierarchical approach for the notification sounds we use notification instruments, ambient noises and traditional alert signals that are grouped by their level of intrusiveness. Since the notification system also follows a human-centered approach it takes parameters like user preferences, his/her current position in the environment and the type of event into consideration to decide which notification is the appropriate at this time. In the paper, we will describe the architecture of the personalized ambient audio notification service, compositional constraints as well as some findings of a user study in which we tested successfully the efficiency of our system with 25 subjects.
2:40

5pPPb3. A virtual auditory environment for investigating the auditory signal processing of realistic sounds. Sylvain Favrot (CAHR, Department of Electrical Engineering, DTU, Ørsteds Plads, Bygning 352, 2800 Kgs. Lyngby, Denmark, sf@oersted.dtu.dk), Jörg M. Buchholz (CAHR, Department of Electrical Engineering, DTU, Ørsteds Plads, Bygning 352, 2800 Kgs. Lyngby, Denmark, jb@oersted.dtu.dk)

In the present study, a novel multichannel loudspeaker-based virtual auditory environment (VAE) is introduced. The VAE aims at providing a versatile research environment for investigating the auditory signal processing in real environments, i.e., considering multiple sound sources and room reverberation. The environment is based on the ODEON room acoustic simulation software to render the acoustical scene. ODEON outputs are processed using a combination of different order Ambisonic techniques to calculate multichannel room impulse responses (mRIR). Auralization is then obtained by the convolution of the mRIR with an acoustic signal. The derivation of the mRIRs takes into account that (i) auditory localization is most sensitive to the location of the direct sound and (ii) that auditory localization performance is rather poor for early reflections and even worse for late reverberation. Throughout the VAE development, special care was taken in order to achieve a realistic auditory percept and to avoid "artifacts" such as unnatural coloration. The performance of the VAE has been evaluated and optimized on a 29 loudspeaker setup using both objective and subjective measurement techniques.

\section{3:00}

5pPPb4. Real-time auralization system based on beam-tracing and mixed-order Ambisonics. Markus Noisternig (LIMSI-CNRS, B.P. 133, 91403 Orsay, France, markus.noisternig@limsi.fr), Lauri Savioja (Helsinki University of Technology, Department of Media Technology, PO Box 5400, 02015 TKK, Finland, Lauri.Savioja@tkk.fi), Brian F. Katz (LIMSI-CNRS, B.P. 133, 91403 Orsay, France, brian.katz@limsi.fr)

Auralization, the final step in computational room acoustic simulations, aims to make audible the acoustics of complex virtual architectural spaces in a realistic and accurate manner. This paper presents a novel real-time auralization system comprising a geometry engine, a beam-tracer, and an audio renderer. The computation of early reflection paths is based on an efficient beam-tracing algorithm capable of real-time detection of specular reflection paths in a static geometry with one or several moving listener(s). For simpler rooms, the real-time performance is maintained even with dynamic geometries and sources. Results of the beam-tracer, sent to the audio renderer, consist of visible reflection paths and their accumulated material attenuation. From this geometrical and acoustical data, listener position-related 3D room impulse responses are generated applying a higher-order virtual Ambisonics approach. Final rendering of the binaural room impulse response (BRIR) is made taking into account the listener's head-orientation. As higher order reflections are more diffuse in nature, they may be encoded using lower Ambisonic orders, thereby reducing computational load. The environment combines high quality audio with visual rendering realized using the open source platforms Pure Data and VirChor respectively. This auralization framework provides direct audio-visual feedback in real-time for VR environments. 


\section{Invited Papers}

$3: 20$

5pPPb5. Real-time auralization of modifiable rooms. Dirk Schröder (Institute of Technical Acoustics, RWTH Aachen University, Neustr. 50, 52066 Aachen, Germany, dsc@akustik.rwth-aachen.de), Ingo Assenmacher (Virtual Reality Group, RWTH Aachen University, Seffenter Weg 23, 52074 Aachen, Germany, assenmacher@rz.rwth-aachen.de)

Immersive virtual environments are a powerful tool for acousticians and architects to design buildings if the virtual reality system provides an interactive imaging of virtual sound sources with respect to the rooms' physical aspects. Current implementations using hybrid room acoustic simulation methods (e.g., combining image sources and ray tracing) enable the user to walk freely in such virtual architectural spaces, whereby the position/orientation of sound sources are interactively manipulable to detect possible acoustic defects, e.g., flutter echoes. In the case of coupled rooms, sound transmission effects must be included into the real-time simulation in order to identify deficient airborne sound insulation, whereby current implementations only support a change of state (open/closed) of fixed room-connecting elements, e.g., doors and windows. However, in scenarios like an architectural planning stage, it is convenient to manipulate the room geometry more freely, e.g., via the interactive positioning of stage reflector panels, but common spatial data structures, e.g., BSP- or Octrees do not efficiently support these operations. For this purpose, the concept of Spatial Hashing, which originates from computer graphics for collision detection of deformable objects, is applied to the simulation process. This adaptation also features an efficient identification and update process of image sources.

\section{3:40}

5pPPb6. Comparison of auralisation results betwee measurements and simulations of line arrays with high resolution modeling data. Wolfgang Ahnert (Ahnert Feistel Media Group, Arkonastr. 45-49, 13189 Berlin, Germany, wahnert@ada-acousticdesign.de), Stefan Feistel (Ahnert Feistel Media Group, Arkonastr. 45-49, 13189 Berlin, Germany, sfeistel@afmg.eu), Ralph Bauer-Diefenbach (Ahnert Feistel Media Group, Arkonastr. 45-49, 13189 Berlin, Germany, rbauer@adaacousticdesign.de)

In a concert hall a direct comparison of several line arrays for a new sound system has been made. At different locations the binaural impulse response has been measured and used for auralisation. In a computer model of the hall the measured line array was implemented. The single array sources have been modeled in different modes like far-field cluster, simple module array or as a highresolution loudspeaker array. By means of the new developed SpeakerLab Module these source simulators have been created and afterwards used to calculate binaural impulse responses at the corresponding seats equal to the measurements. After that an auralisation routine has been used. The results are compared for the different degrees of source resolution and with the measurements. Beside acoustic measures the subjective sound quality of the different auralisation results is reported.

\section{4:00-4:20 Break}

\section{Contributed Paper}

\section{$4: 20$}

5pPPb7. Investigation on the restitution system influence over perceived Higher Order Ambisonics sound field: a subjective evaluation involving from first to fourth order systems. Stephanie Bertet (IRCAM, 1 Place Igor Stravinsky, 75004 Paris, France, Stephanie.Bertet@ircam.fr), Jérôme Daniel (France Telecom R\&D, 2 avenue Pierre Marzin, 22300 Lannion, France, jerome.daniel@orange-ftgroup.com), Etienne Parizet (Laboratoire Vibrations Acoustique, Insa Lyon, 25 bis, av. J. Capelle, 69621 Villeurbanne Cedex, France, etienne.parizet@insa-lyon.fr), Olivier Warusfel (IRCAM, 1 Place Igor Stravinsky, 75004 Paris, France, Olivier.Warusfel@ircam.fr)

Among the spatial audio reproduction techniques over loudspeakers, the Higher Order Ambisonics (HOA) approach is based on a sound field spherical harmonics decomposition. By truncating the decomposition to the Mth order, it remains a finite number of components that form the spatial HOA format. The more components are used to encode the sound field, the finer the spatial resolution is. Similarly, the size of the area where the sound field is accurately recreated is proportional to the order. For an Mth encoding order, $\mathrm{N}=2 \mathrm{M}+2$ equally distributed loudspeakers are recommended for a homogeneous reproduction in the horizontal plane. Adding loudspeakers does not change the spatial resolution. However, what is the influence of the restitution system on the perceived sound field? An experiment was designed in order to compare four systems (from first to fourth order) and a reference one, using similarity ratings obtained from pairwise comparisons. Two sound scenes were used, simulating an audio conference and a scene in a kitchen at home. 25 listeners participated to the experiment. The results were analysed using the Indscal method. The perceptual space appeared to be a two dimensional one, highlighting the influence of the order and the number of loudspeakers on the reproduced scenes.

\section{Invited Papers}

\section{$4: 40$}

5pPPb8. Recording of anechoic symphony music. Tapio Lokki (Helsinki University of Technology, P.O. Box 5400, 02015 TKK, Finland, Tapio.Lokki@tkk.fi), Jukka Pätynen (Helsinki University of Technology, P.O. Box 5400, 02015 TKK, Finland, jpatynen@tml.hut.fi), Ville Pulkki (Helsinki University of Technology, P.O. Box 5400, 02015 TKK, Finland, Ville.Pulkki@tkk.fi)

When designing the acoustics of a concert hall, it would be beneficial to be able to use real recording of a symphony orchestra in auralization. The technical constraints for such recordings are high. First, the instruments have to be recorded separately, as in simultaneous recording the cross talk between microphones could not be avoided. Second, the recording room should be anechoic. Third, the instruments have different sound radiation patterns, thus they should be recorded with multiple microphones around them. Therefore, we end up recording each instrument individually in an anechoic chamber with multiple microphones. The remaining problem is to achieve a common timing as an ensemble between the individually recorded instruments. This was solved by first recording a video of a conductor conducting a pianist playing the whole score. The players in an anechoic chamber then followed the conductor in a monitor while 
listening the pianist on headphones. Four short passages, from two to four minutes, from different music styles were recorded. The recordings were made with 20 low-self-noise microphones, mounted on the shape of a dodecahedron. Finally, we discuss the musical and technical quality of recorded sound, and the response by the musicians, who were professional orchestra players.

\section{5:00}

5pPPb9. Uni-Verse Acoustic Simulation System: interactive real-time room acoustic simulation is dynamic 3D environments. Peter Lundén (Interactive Institute, Box 1197, SE-164 26 Kista, Sweden, plu@tii.se)

Uni-Verse Acoustic Simulation System (UVAS) is a newly developed interactive room acoustic simulation system that can handle dynamically changing 3D geometric models in real-time. The system can share such models with other application, such as visual renderers or 3D modeling tools, over a network using the Verse protocol. UVAS is implemented using the beam-tracing method. It is build as two separate but highly integrated parts. The first part is handling the geometry, it's responsibility is to find audible sound sources and relevant reflection paths in the simulated environment. The second part is handling the audio rendering, producing the audible result of the simulation based on information given by the first part. This paper will focusing on the first part

$5: 20$

5pPPb10. Case study of measurements and computer modeling auralization results for medium-sized multipurpose halls. Hari V. Savitala (Charles M Salter Associates, Inc., 130 Sutter St, Suite 500, San Francisco, CA 94104, USA, hari.savitala@cmsalter.com), Jason Duty (Charles M Salter Associates, Inc., 130 Sutter St, Suite 500, San Francisco, CA 94104, USA, jason.duty@cmsalter.com), Christopher Peltier (Charles M Salter Associates, Inc., 130 Sutter St, Suite 500, San Francisco, CA 94104, USA, christopher.peltier@cmsalter.com)

This case study focuses on auralizations and their accuracy in modeling medium-sized multipurpose halls (400-600 seats). Within each hall, impulse response measurements were taken with drapes deployed and retracted. The same configurations were modeled in the room acoustics program ODEON. Acoustical parameters, such as T20, T30, C50, C80, were used to check the agreement of the model to the measurements. A systematic approach was then used to adjust model parameters to match the real-world measurements. The modified and unmodified auralizations were then used to determine if any differences could be perceived in an informal listening evaluation. The auralizations and listening evaluation results are presented to better understand how to accurately auralize medium-sized multipurpose halls.

\section{Contributed Papers}

\section{$5: 40$}

5pPPb11. Real-time 3D audio for digital cinema. Pau Arumi (Universitat Pompeu Fabra - Fundació Barcelona Media, Ocata, 1, 08003 Barcelona, Spain, parumi@iua.upf.edu), David Garcia (Fundacio Barcelona Media, Carrer Ocata $\mathrm{n}^{\circ}$ 1, 08003 Barcelona, Spain, dgarcia@iua.upf.edu), Toni Mateos (Universitat Pompeu Fabra - Fundació Barcelona Media, Ocata, 1, 08003 Barcelona, Spain, toni.mateos@barcelonamedia.org), Adan Garriga (Universitat Pompeu Fabra - Fundació Barcelona Media, Ocata, 1, 08003 Barcelona, Spain, adan.garriga@barcelonamedia.org), Jaume Durany (Universitat Pompeu Fabra - Fundació Barcelona Media, Ocata, 1, 08003 Barcelona, Spain, jaume.durany@upf.edu)

We present a real-time 3D audio system with a number of nice features: it is suited for plausible reference with the visual environment, it is real-time capable, it can process multiple moving sound sources and listeners in a normal CPU. In our approach, a database of pressure and velocities impulseresponses (IRs) is computed offline for each (architectural) environment using physically based ray-tracing techniques. During playback, the real-time system retrieves IRs corresponding to the sources and target positions, performs a low-latency partitioned convolution and smoothes IR transitions with cross-fades. Finally, the system is flexible enough to decode to any surround exhibition setup. The software has been developed within the CLAM open-source audio framework. We present a real scenario where these techniques were successfully applied: an augmented-reality film with 3D audio within the context of the IP-RACINE project for digital cinema. The shooting was done with a high-end prototype camera with zoom and position tracking which enabled the real-time motion of a subjective listener within the scene. Our technology enabled the film director to both pre-hear surround audio of an augmented-reality scene shooting and fine-tune audio rendering in post-production.

\section{6:00}

5pPPb12. Near-field binaural synthesis, experimental progress report Dylan Menzies-Gow (De Montfort University, Queens Building, LE1 9BH Leicester, UK, dylan@dmu.ac.uk)

A methodology was previously presented for displaying high quality binaural images of near-field complex sources, using wave reconstruction. Multipole representations of objects are transformed to Fourier-Bessel and plane wave expansions at the listener, before conversion to binaural signals. One advantage of this approach is that does not require special HRTF information other than the planewave HRTFs, and can fully render the complex field of a near object. As a first step towards a full working system, a realtime implementation is described here for displaying a monopole source using a six degrees-of-freedom infrared head tracking device. 


\title{
Session 5pSC
}

\section{Speech Communication: Multimodal Speech Technology}

\author{
Gerasimos Potamianos, Cochair \\ IBM T. J. Watson Research Center, RTE 134, Yorktown Heights, NY 10598, USA \\ Gerard Bailly, Cochair \\ GIPSA-lab. Dept Speech \& Cognition, INPG, 46, av. Félix Viallet, Grenoble, 38031, France
}

\section{Contributed Paper}

\section{2:00}

5pSC1. An ultrasound-based silent speech interface. Thomas Hueber (ESPCI - Telecom Paris, 10 rue Vauquelin, 75005 Paris, France, hueber@ieee.org), Gerard Chollet (Telecom Paris Tech, 46 rue Barrault, 75013 Paris, France, chollet@enst.fr), Bruce Denby (Université Paris VI, ESPCI - Laboratoire d'Electronique, 10 rue Vauquelin, 75005 Paris, France, denby@ieee.org), Gerard Dreyfus (Université Paris VI, ESPCI - Laboratoire d'Electronique, 10 rue Vauquelin, 75005 Paris, France, gerard.dreyfus@espci.fr), Maureen Stone (Vocal Tract Visualization Lab, Depts of Biomedical Sciences and Orthodontics, University of Maryland Dental School, 650 W. Baltimore St., Baltimore, MD 21201, USA, mstone @umaryland.edu)

The paper proposes the use of ultrasound scans of tongue movement and video sequences of the lips to synthesize speech. A speech synthesizer driven only by video acquisitions may be qualified as a "silent speech inter- face," which could be used by laryngectomyzed patient as an alternative to tracheo-esophageal speech, for voice communication where silence must be maintained, or in very noisy environments. Our system is based on the building of a one-hour audiovisual corpus of phonetic units, which associates visual features extracted from video with acoustic observations. The ultrasound and optical images are interpreted as a linear combination of standard configurations obtained by principal components analysis (PCA) from a phonetically balanced subset of typical frames. HMM-based stochastic models trained on these visual features sequences are subsequently used to predict phonetic targets from video-only data. Finally, a Viterbi unit selection algorithm is used to find the optimal sequence of acoustic units given both this phonetic prediction and the sequence of visual features. The system is able to perform phonetic transcription from video-only speech data with over $55 \%$ correct recognition, on continuous speech, using neither phonotactic nor linguistic constraints.

\section{Invited Papers}

5pSC2. Multimodal control of talking heads. Gerard Bailly (GIPSA-lab. Dept Speech \& Cognition, INPG, 46, av. Félix Viallet, 38031 Grenoble, France, gerard.bailly@gipsa-lab.inpg.fr), Oxana Govokhina (GIPSA-lab. Dept Speech \& Cognition, INPG, 46, av. Félix Viallet, 38031 Grenoble, France, oxana.govokhina@gipsa-lab.inpg.fr), Gaspard Breton (Orange R\&D, 4 rue du Clos Courtel, 35512 Cesson-Sévigné, France, gaspard.breton@orange-ftgroup.com)

Multimodal speech synthesis has been devoted for years to the rendering of linguistic or paralinguistic content - i.e., parametrized but discrete information - by continuous audible and visible consequences of speech articulation, eventually complemented by facial expressions, gaze and other body gestures including head, hand, and arm movements. Articulatory synthesizers (producing sounds from gestures) intrinsically compute coherent audiovisual signals but do not presently compete with data-driven techniques: most talking heads are nowadays controlled by models built using human audiovisual data. These control models should replicate the laws governing the coherence of observed multimodal signals and the correct phasing relations between salient events of the multimodal stream. We will report on two comparative evaluations of various lip-sync models (dealing with post-synchronization between speech sounds and articulatory movements) and present a trainable control model that learns automatically phasing relations between acoustic and gestural events. This model can be further extended to capture the fine temporal structure of multimodal scores and a first application to the synchronization between speech and head, face and hand movements during cued speech production will be presented.

5pSC3. Statistical conversion of speech parameter trajectory for mapping between features of different modalities. Tomoki Toda (Nara Institute of Science and Technology, 8916-5 Takayama-cho, Ikoma, 630-0192 Nara, Japan, tomoki@ is.naist.jp)

A state-of-the-art speech parameter conversion technique and its application to a mapping between features of different modalities are reviewed. Many statistical approaches to the parameter conversion have been studied particularly for voice conversion in speech synthesis research. A typical method conducts the parameter conversion frame by frame based on the minimum mean square error using a Gaussian mixture model of the joint probability density of input and output parameters [Y. Stylianou et al., IEEE Trans. SAP, 6(2), 131-142 (1998)]. Although this method is reasonably effective, the deterioration of the conversion accuracy is caused by essential problems of the frame-based conversion process. Recently a conversion method based on the maximum likelihood estimation of a parameter trajectory has been proposed [T. Toda et al., IEEE Trans. ASLP, 15(8), 2222-2235 (2007)]. This method realizes the appropriate converted parameter sequence by (1) using not only static but also dynamic feature statistics and (2) considering a global variance feature 
of the converted parameters. It has been reported that this method is effective in several applications such as a spectral determination from articulatory movements, an acoustic-to-articulatory inversion mapping, and a conversion of body-transmitted speech into airtransmitted speech.

\title{
Contributed Paper
}

3:00

5pSC4. A comparison of visual features for audiovisual automatic speech recognition. Nasir Ahmad (Loughborough Univ, LE11 3TU Leicestershire, UK, n.ahmad@lboro.ac.uk), Sekharjit Datta (Loughborough Univ, LE11 3TU Leicestershire, UK, s.datta@lboro.ac.uk), David Mulvaney (Loughborough Univ, LE11 3TU Leicestershire, UK, d.j.mulvaney@lboro.ac.uk), Omar Farooq (Loughborough Univ, LE11 3TU Leicestershire, UK, o.farooq@lboro.ac.uk)

The use of visual information from speaker's mouth region have shown to improve the performance of automatic speech recognition (ASR) systems. This is particularly important in presence of noise which even in moderate form severely degrades the speech recognition performance of systems us- ing only audio information. Various sets of features extracted from speaker's mouth region have been used to improve upon the performance of an ASR system in such challenging conditions and have met many successes. To the best of authors knowledge, the effect of using these techniques on recognition performance on the basis of phonemes have not been investigated yet. This paper presents a comparison of phoneme recognition performance using visual features extracted from mouth region-of-interest using discrete cosine transform (DCT) and discrete wavelet transform (DWT). New DCT and DWT features have also been extracted and compared with the previously used one. These features were used along with audio features based on Mel frequency cepstral coefficients (MFCC). This work will help in selecting suitable features for different application and identify the limitations of these methods in recognition of individual phonemes.

\section{3:20-3:40 Break}

\section{Invited Paper}

\begin{abstract}
3:40
5pSC5. Spatial rendering of audiovisual synthetic speech use for immersive environments. Markus Noisternig (LIMSI-CNRS, B.P. 133, 91403 Orsay, France, markus.noisternig@limsi.fr), Brian F. Katz (LIMSI-CNRS, B.P. 133, 91403 Orsay, France, brian.katz@limsi.fr), Christophe D’Alessandro (LIMSI-CNRS, B.P. 133, 91403 Orsay, France, cda@limsi.fr)

Synthetic speech is usually delivered as a mono audio signal. In this project, audiovisual speech synthesis is attributed to a virtual agent moving in a virtual three-dimensional scene. More realistic acoustic rendering is achieved by taking into account the position of the agent in the scene, the acoustics of the room depicted in the scene, and the orientation of the virtual character's head relative. 3D phoneme dependant radiation patterns have been measured for two speakers and a singer. These data are integrated into a Text-ToSpeech system using a phoneme to directivity pattern transcription module which also includes a phoneme to viseme model for the agent. In addition to the effects related to agent's head orientation for the direct sound, a room acoustics model allows for realistic rendering of the room effect as well as the apparent distance as depicted in the virtual scene. Real-time synthesis is implemented in a $3 \mathrm{D}$ audio rendering system.
\end{abstract}

\section{4:00}

5pSC6. Audiovisual automatic speech recognition: Progress and challenges. Gerasimos Potamianos (IBM T. J. Watson Research Center, RTE 134, Yorktown Heights, NY 10598, USA, gpotam@us.ibm.com)

The paper overviews recent progress and challenges in a number of audiovisual speech processing technologies with main emphasis on the problem of automatic speech recognition. It is well known that visual channel information can improve automatic speech processing for human-computer interaction. To automatically process and incorporate such information into automatic systems, a number of steps are required that are surprisingly similar accross speech technologies. Crucial above all is the issue of feature representation of visual speech and its robust extraction. In addition, appropriate integration of the audio and visual representations is required, in order to ensure improved performance of the bimodal systems over audio-only baselines. These topics are discussed in detail in the talk, with main emphasis on their application to the speech recognition problem in the challenging environments of automobiles and smart rooms.

\section{Contributed Paper}

\section{$4: 20$}

5pSC7. Analysis and synthesis of nonverbal facial motion. Jonas Beskow (KTH Speech, Music and Hearing, Lindstedtsvägen 24, 10044 Stockholm, Sweden, beskow@kth.se), Björn Granström (KTH Speech, Music and Hearing, Lindstedtsvägen 24, 10044 Stockholm, Sweden, bjorn @ speech.kth.se)

Until recently, most efforts in audio-visual speech synthesis have been concerned with verbal content. However, in human-human communication it is obvious that nonverbal signals plays an important role, such as when expressing emotions and attitudes. Interaction is also often regulated using facial cues, for example gaze, head and eyebrow movements. Some of these cues have a direct coupling to the speech signal, while other occur during both while speaking and listening. When applying interactive talking agents in man-machine systems, nonverbal signals may be very important in easing the flow of communication. In a series of experiments we have been exploring the function of nonverbal facial motion. These studies include an experiment on the interaction between expressive speech and prominence, as well as an attempt to synthesize emotions and attitudes in a talking head, using 3D motion capture data. Further we will report on a real-time experiment with human-human avatar-mediated conversation, where the subjects' turntaking behavior is affected by facial motion in the avatars. 


\title{
Session 5pUWa
}

\section{Underwater Acoustics and ECUA: Sound Propagation in 3D Environments II}

\author{
David C. Calvo, Cochair \\ U.S. Naval Res. Lab., Acoust. Div., Code 7142, 4555 Overlook Ave. SW, Washington, DC 20375, USA \\ Michael Taroudakis, Cochair \\ University of Crete \& FORTH/IACM, Vassilika Vouton, P.O. Box 1385, Heraklion, 711 10, Greece
}

\section{Invited Paper}

$1: 40$

5pUWa1. Stability of wavefronts at sound propagation in highly structured three-dimensional environments. Oleg A. Godin (NOAA/ESRL, 325 Broadway, Mail Code R/PSD99, Boulder, CO 80305-3328, USA, Oleg.Godin@noaa.gov)

Extensive numerical modeling of long-range propagation of sound and seismic waves as well as observations of underwater acoustic fields with line arrays reveal that wavefronts are often much more stable and predictable than the rays comprising these wavefronts. This paper considers multiple scattering of sound by environmental inhomogeneities with spatial scales small compared to the propagation range but large compared to the wavelength. These inhomogeneities include 3D variations in sound speed and current velocity that are small compared to the average sound speed, can be either random or deterministic, and are superimposed on an arbitrary slowly-varying background. A theoretical explanation of wavefront stability in highly-structured environments is achieved by demonstrating that end points of rays launched from a point source and having a given eikonal (phase) are scattered primarily along the wavefront corresponding to the same eikonal in the unperturbed environment. The ratio of displacements of the ray end points along and across the unperturbed wavefront is proportional to the number of uncorrelated scattering events. The results apply to conventional rays and to horizontal rays describing propagation of adiabatic normal modes in almost-layered media. The origin of relative stability of wavefronts compared to rays is traced back to Fermat's principle.

\section{Contributed Paper}

\section{2:00}

5pUWa2. Range and cross-range propagation effects in a liquid wedge overlaying an elastic bottom. Piotr Borejko (Vienna University of Technology, Karlsplatz 13/E206/3, A-1040 Vienna, Austria, pb@allmech.tuwien .ac.at)

The penetrable-wedge model, a constant density isospeed layer of fluid with a pressure-release horizontal surface and a sloping elastic bottom, provides an extensive insight into the role of the ocean bottom in acoustic propagation from an underwater source. In particular, it is a realistic model of a rock-bottom ocean near a shoreline that accounts for horizontal refraction and allows for a ground wave. This paper discusses some new results for small and large range propagation for two penetrable-wedge models: one where the shear wave speed in the bottom is lower than the sound speed in the fluid and the other where the shear wave speed is higher. The operational representation of the ray-integral solution for the acoustic field from a point source in a penetrable wedge of fluid is further developed to the stage at which one can compute the exact, other than the omission of diffraction at the wedge apex, pressure response curve, as recorded at a receiver, due to an arbitrary time variation of the pressure at the source in a $3^{\circ}$ wedge. The three-dimensional propagation effects are examined for range transmission when the receivers are located up-slope and down-slope of the source, and for cross-range transmission when the receivers are located cross-slope of the source.

\section{Invited Papers}

5pUWa3. Depth-dependent resonant target strength analysis of a dense Atlantic Herring school from wide-area OAWRS and localized 3D morphology sensing. Daniel Cocuzzo (Northeastern University, 302 Stearns Center, Rm 311, 360 Huntington Ave, Boston, MA 02115, USA, dcocuzzo@ece.neu.edu), Zheng Gong (Northeastern University, 302 Stearns Center, Rm 311, 360 Huntington Ave, Boston, MA 02115, USA, zgong@ece.neu.edu), Mark Andrews (Northeastern University, 302 Stearns Center, Rm 311, 360 Huntington Ave, Boston, MA 02115, USA, Andrews.mar@neu.edu), Ioannis Bertsatos (Massachusetts Institute of Technology, Room 5-435, 77 Massachusetts Avenue, Cambridge, MA 02139, USA, ibertsat@mit.edu), Tianrun Chen (Massachusetts Institute of Technology, Room 5-212, 77 Massachusetts Avenue, Cambridge, MA 02139, USA, trchen@mit.edu), Hector Pena (Institute of Marine Research, PO Box 1870, 5817 Bergen, Norway, hector.pena@imr.no), Thomas C. Weber (University of New Hampshire, Ctr. for Coastal and Ocean Mapping, 24 Colovos Road, Durham, NH 03824, USA, weber@ccom.unh.edu), Nicholas Makris (Massachusetts Institute of Technology, Room 5-212, 77 Massachusetts Avenue, Cambridge, MA 02139, USA, makris@mit.edu), Purnima Ratilal (Northeastern University, 302 Stearns Center, Rm 311, 360 Huntington Ave, Boston, MA 02115, USA, purnima@ece.neu.edu)

The depth-dependent target strength of Atlantic Herring is estimated at several distinct bandwidths close to their resonance frequency for a localized, highly dense school observed during the NOPP-sponsored Gulf of Maine Experiment on September 22, 2006. An ocean acoustics waveguide remote sensing (OAWRS) system was deployed near George's Bank to investigate the migration and spawning behavior of fish over wide areas. In conjunction with OAWRS, a Simrad EK60 conventional fish-finding echosounder (CFFS) 
and a Reson Seabat 7125 multibeam sonar system were deployed to provide local depth extent and 3D volume morphology of the dense herring school. The calibration of low-frequency target strength derived from OAWRS data using localized CFFS density and multibeam 3D volume estimates as inputs is discussed. The correlation between the mean depth of the vertically migrating herring school and its resonance frequency is investigated. The results are compared with a theoretical model for 3D resonance scattering from fish swimbladder modeled as a spheroidal bubble. This analysis may allow inference of fish depth and species classification based on the scattered frequency response of targets imaged by OAWRS. Implications for classifying general localized targets, biological or man-made, are discussed further.

\section{2:40}

5pUWa4. Multiple forward scattering through an ocean waveguide with 3D random inhomogeneities. Nicholas Makris (Massachusetts Institute of Technology, Room 5-212, 77 Massachusetts Avenue, Cambridge, MA 02139, USA, makris@mit.edu), Purnima Ratilal (Northeastern University, 302 Stearns Center, Rm 311, 360 Huntington Ave, Boston, MA 02115, USA, purnima@ece.neu.edu), Tianrun Chen (Massachusetts Institute of Technology, Room 5-212, 77 Massachusetts Avenue, Cambridge, MA 02139, USA, trchen@mit.edu)

Analytic expressions have been derived for the mean and spatial covariance of the acoustic field multiply forward scattered though a stratified ocean waveguide containing 3D random surface or volume inhomogeneities [Ratilal and Makris, J. Acoust. Soc. Am. 118, 3532-3559 (2005)]. These expressions are further used to derive the temporal coherence of an acoustic signal propagated through 3D random inhomogeneities. Field moments are given in terms of moments of the scatter function density of the 3D random inhomogeneities, which enables straightforward application to a broad range of 3D scatterers. Here we give examples of the attenuation, dispersion and loss of temporal coherence expected after multiple forward scattering through (1) random internal waves in both continental shelf and deep ocean environments, (2) fish schools, and (3) random wind-generated bubbles in continental shelf and surf-zone area. We show that 3D scattering effects become important when the Fresnel width exceeds the cross-range coherence scale of the inhomogeneities, and can lead to substantial power loss.

\section{Contributed Papers}

3:00

5pUWa5. Application of the matrix Rytov method to the calculation of the coherence function of a sound field in an oceanic waveguide. Alex G. Voronovich (NOAA/Earth System Research Laboratory, 325 Broadway, Boulder, CO 80305, USA, alexander.voronovich@noaa.gov), Vladimir E. Ostashev (NOAA/Earth System Research Laboratory, 325 Broadway, Boulder, CO 80305, USA, vladimir.ostashev@noaa.gov)

Closed equations for the coherence function of a monochromatic sound field propagating in a statistically inhomogeneous 3D oceanic waveguide have high dimensions and are difficult to solve even with the use of modern computers. Significant reduction of the dimension of the problem was achieved by assuming that sound speed fluctuations are statistically isotropic in a horizontal plane. However, even in this case calculation of the coherence function for a megameter range takes about a day. In this paper, we develop an approximate solution of the closed equations for the coherence function which is similar to a matrix version of the Rytov method. An explicit expression for the coherence function is obtained which contains exponent of an "interaction" matrix. This matrix is determined in terms of the acoustic and internal wave modes and spatial spectrum of the sound speed fluctuations. It is shown that the matrix Rytov method provides an accurate solution for the coherence function which coincides with the solution of the closed equations within a few percent. Calculation of the coherence function now takes only about an hour. This allows us to study in detail the dependence of the coherence function on parameters of the problem.

\section{3:20}

5pUWa6. Measurements of 3D propagation in the shelf environment. Kevin D. Heaney (Oasis Inc., 11006 Clara Barton Dr., Fairfax Station, VA 22039, USA, oceansound04@yahoo.com)

In the Fall of 2007 measurements were made to calibrate the acoustic environment on the shallow water shelf off the coast of Florida. The continental shelf is quite flat (less than 1 degree slope) for a region approximately $10 \mathrm{~km}$ wide. Transmissions to a cross-shelf $900 \mathrm{~m}$ horizontal line array were made from a source transiting along the shelf. The signal transmissions in- clude broadband LFM $(20-420 \mathrm{~Hz})$ and a comb of narrow band frequencies spanning the same range. Narrowband beamforming results show the clearly identified source. The arrival angle for the source is as expected until a distance of approximately $30-40 \mathrm{~km}$ when there is an apparent bearing shift in-shore of up to 25 degrees. This behavior is expected in propagation on a shelf (of greater slope) but its behavior is surprising. The phenomenon was observed for several runs at various source and water depths. In order to explain the phenomenon, a hybrid adiabatic normal mode-Parabolic Equation method will be applied to the environment. This model computes the vertical modes and phase speeds at each location and then uses the PE to propagate each mode individually using it's spatially varying phase speed and attenuation. Comparisons of theory and data will be made.

\section{3:40}

5pUWa7. Observations of out of plane arrivals for long range low frequency transmission in shallow water. Harry Deferrari (Univ. of Miami, 4600 Rickenbacker Causeway, Miami, FL 33149, USA, hdeferrari @ rsmas.miami.edu)

Two recent experiments have used long horizontal arrays to receive broadband low frequency signals propagated over long ranges in shallow water. Both used $\mathrm{m}$-sequence signals that resolve pulse arrivals in time with each arrival associated with a single acoustic mode of propagation. At moderate propagation ranges, out to $20 \mathrm{~km}$, wave fronts for all modes are observed to be parallel, implying an orderly two-dimensional propagation. At a much longer range, $80 \mathrm{~km}$, a number of separable arrivals are observed but not necessarily with a one-to-one correspondence with modes. The paths appear to be stable and coherent in time implying that they are true Fermat paths, but their wavefront arrival angles differ suggesting the same mode is arriving from several directions, that is, by curved (out of plane paths). The paths could result from wedge effects from gentle slopes perpendicular to the propagation path or possibly from chaotic interaction with random facets of the bottom. In any case, the ultimate limitation for horizontal spatial coherence and array resolution may be the multipath interference of bundles of out of plane arrivals. 


\section{Invited Papers}

\section{$4: 20$}

5pUWa8. Hydroacoustic blockage prediction and measurement at Diego Garcia using the Adiabatic Mode Parabolic Equation Model. Zachary Upton (BBN Technologies, 1300 N. 17th Street, Suite 400, Arlington, VA 22209, USA, zupton@bbn.com), Michael D. Collins (U.S. Naval Res. Lab., Acoust. Div., Code 7142, 4555 Overlook Ave. SW, Washington, DC 20375, USA, michael.collins@nrl.navy.mil), Jay Pulli (BBN Technologies, 1300 N. 17th Street, Suite 400, Arlington, VA 22209, USA, jpulli@bbn .com)

Underwater explosion monitoring with sparse sensors at long ranges relies on the efficient propagation of acoustic energy in the sound fixing and ranging (SOFAR) channel. When sound traveling in this channel encounters an island or seamount, it will either diffract, scatter, or be converted into seismic energy. Signals observed on the opposite side of these obstructions have been affected by some combination of these processes, and models of global detection and localization depend on knowing these effects. We present a study using the Adiabatic Mode Parabolic Equation (AMPE) model to predict these processes in three dimensions at the Chagos Archipelago. Predictions at 5, 10, and $20 \mathrm{~Hz}$ are compared with measurements of approximately $300 \mathrm{~T}$-wave signals from six years of earthquakes on either side of the Chagos Archipelago. These have been recorded at the hydrophone arrays around Diego Garcia. The result of this 360-degree analysis, and the agreement with observed data, demonstrate the utility of the model in understanding the physical effects of these obstructions.

\section{4:40}

5pUWa9. Investigation of 3D benchmark problems in underwater acoustics: a uniform approach. Frederic Sturm (Laboratoire de Mécanique des Fluides et d'Acoustique (UMR CNRS 5509), Ecole Centrale de Lyon, Centre acoustique, 36, avenue Guy de Collongue, 69134 Ecully Cedex, France, frederic.sturm@ec-lyon.fr)

In underwater acoustics, most of the three-dimensional effects on sound wave propagation are usually described by modelers considering one of the following shallow-water benchmark problems: a wedge-shaped waveguide, a canyon, a seamount, and a sinusoidal (corrugated) bottom. These test cases have been thoroughly analyzed individually considering both harmonic point sources (emitting at very low frequencies, for some obvious problems of CPU time and memory limitation) and broadband source pulses (with also very-low central frequencies). In the present work, we report numerical results corresponding to the propagation of broadband pulses in the four above-mentioned test cases. The numerical simulations are performed using a fully 3D parabolic equation based model coupled with a Fourier synthesis technique to handle the time dependence of the source signal. The objective is to propose a uniform representation of the numerical results so as to facilitate the comparison of the 3D effects present in each of the four benchmarks. Snapshots of the propagating pulses at very close successive times are compared with $2 \mathrm{D}$ results. In addition, movies of the propagating pulses are shown for each test case and compared to each other. Movies strongly facilitate the observation and thus the understanding of the 3D effects experienced by all the propagating waves.

\section{Contributed Paper}

5:00

5pUWa10. Using parallel programming and a three-dimensional visualization cave to map the acoustic energy distribution from a seismic array in the ocean. Natalia Sidorovskaia (Department of Physics, University of Louisiana, UL BOX 44210, Lafayette, LA 70504-4210, USA, nas @ louisiana.edu), Arslan Tashmukhambetov (Department of Physics, University of New Orleans, New Orleans, LA 70148, USA, atashmuk @ uno.edu), George E. Ioup (Department of Physics, University of New Orleans, New Orleans, LA 70148, USA, geioup@uno.edu), Juliette W. Ioup (Department of Physics, University of New Orleans, New Orleans, LA 70148, USA, jioup@uno.edu)

Modeling and visualization of the dynamic acoustic field during a seismic exploration survey represent a computational challenge due to broadband, directional nature of the acoustic signal radiated by a seismic array. Standard acoustic propagation models (RAM and SWAMP) are upgraded for parallel processing and tested in the LONI (the Louisiana Optical Network Initiative) environment, using the Louisiana fiber optics grid computing network to model the three-dimensional time-varying acoustic field in the ocean during a seismic exploration survey. The generated volume of data is transferred and visualized in the advanced immersive visualization environment, supported by Louisiana Immersive Technology Enterprise (LITE) facilities. The proposed technology is one of the first steps in developing real-time monitoring of the acoustic energy distribution in a large oceanic volume. This can be beneficial for environmental impact assessment and regulation and for seismic survey design. [Research supported in part by the Joint Industry Project through OGP and by ITI of University of Louisiana at Lafayette.]

\section{Invited Paper}

5pUWa11. Effects of solitons on acoustic energy flow in three dimensions. Kevin B. Smith (Naval Postgraduate School/Naval Undersea Warfare Center, Code PH/Sk, Department of Physics, Monterey, CA 93943, USA, kbsmith@nps.edu), John A. Colosi (Naval Postgraduate School, Code OC/Cj, Department of Oceanography, Monterey, CA 93943, USA, jacolosi@nps.edu)

The impact of a train of nonlinear solitons on the propagation of acoustic energy in shallow water is examined. The soliton perturbations are based on an analytic formulation that produces a train of five soliton waves. Each wave front is parallel and has infinite extent in the horizontal direction. The acoustic field is modeled using a three-dimensional (3D) split-step Fourier parabolic equation (SSF/PE) approach defined in Cartesian coordinates. The standard PE approximation is employed in both depth and cross-range directions. Both pressure and particle velocity fields are computed in a self-consistent manner, allowing a full description of the 3D acoustic intensity field which describes the flow of energy in the presence of the solitons. Individual, low-order modes are extracted from the propagating field so that the impact on specific modes may be examined. The analysis is performed at various frequencies and 
for various source-receiver geometries relative to the soliton train. Emphasis is placed on the focusing and defocusing of acoustic energy between the various soliton waves. The impact of such soliton perturbations on signal variability and bearing resolution at the receiver will be quantified. [Work supported by ONR 321OA.]

\title{
Contributed Papers
}

$5: 40$

5pUWa12. High-frequency underwater acoustic propagation in a port using the three-dimensional method of images. Pierre-Philippe J. Beaujean (Florida Atlantic University, SeaTech Campus, 101 North Beach Road, Dania Beach, FL 33004, USA, pbeaujea@seatech.fau.edu), Matthew D. Staska (International Transducer Corporation, 869 Ward Drive, Santa Barbara, CA 93111, USA, MStaska@ channeltech.com)

A computer-efficient model for underwater acoustic propagation in a shallow, three-dimensional rectangular duct closed at one end has been developed using the method of images. The duct simulates a turning basin located in a port, surrounded with concrete walls and filled with sea water. The channel bottom is composed of silt. The modeled impulse response is compared with the impulse response measured between $15 \mathrm{kHz}$ and $33 \mathrm{kHz}$. Despite small sensor-position inaccuracies and an approximated duct geometry, the impulse response can be modeled with a relative echo magnitude error of $1.62 \mathrm{~dB}$ at worst, and a relative echo location error varying between $0 \%$ and $4 \%$ when averaged across multiple measurements and sensor locations. This is a sufficient level of accuracy for the simulation of an acoustic communication system operating in the same frequency band and in shallow waters, as time fluctuations in echo magnitude commonly reach $10 \mathrm{~dB}$ in this type of environment.
6:00

5pUWa13. Acoustic mode beam effects of nonlinear internal gravity waves in shallow water. Timothy Duda (Woods Hole Oceanographic Institution, 98 Water Street, Bigelow 107, MS-12, Woods Hole, MA 02543 , USA, tduda@whoi.edu), Ying-Tsong Lin (Woods Hole Oceanographic Institution, 98 Water Street, Bigelow 107, MS-12, Woods Hole, MA 02543 USA, ytlin@whoi.edu), James F. Lynch (Woods Hole Oceanographic Institution, 98 Water Street, Bigelow 203A, MS-11, Woods Hole, MA 02543 , USA, jlynch@whoi.edu)

Ducting of sound between short-wavelength nonlinear internal gravity waves in coastal environments has been demonstrated by substantial evidence. The ducting takes a unique form for each of the acoustic norma modes. Some consequences of this are examined here using threedimensional parabolic equation modeling and theory. For a pair of waves having a broadband $200-\mathrm{Hz}$ source placed between (i.e., in the duct), strong interference patterns within the duct are developed for each mode after a few kilometers. Some of the energy escapes at high angle with respect to the duct direction. Termination of the internal wave duct, an observed feature, results in beams of energy unique to each mode to radiate outward. Specific cases having water depths of order $80 \mathrm{~m}$ and propagation distances of 20 to $30 \mathrm{~km}$ are examined. Situations where one or more modes are completely absent at selected positions are compared with similar events observed in the field.

FRIDAY AFTERNOON, 4 JULY 2008

ROOM 342A, 1:40 TO 6:20 P.M.

\section{Session 5pUWb}

\section{Underwater Acoustics and ECUA: Scattering From Objects Near Boundaries}

\author{
Eric Thorsos, Cochair \\ Applied Physics Laboratory, University of Washington, 1013 NE 40th St, Seattle, WA 98105, USA \\ Mario Zampolli, Cochair \\ NATO Undersea Research Centre, Viale San Bartolomeo 400, La Spezia, 19126, Italy
}

\section{Invited Papers}

\section{$1: 40$}

5pUWb1. Measurement and modeling of targets deployed on and within sand sediments. Kevin L. Williams (Applied Physics Laboratory, University of Washington, 1013 NE 40th St, Seattle, WA 98105, USA, williams@apl.washington.edu), Eric Thorsos (Applied Physics Laboratory, University of Washington, 1013 NE 40th St, Seattle, WA 98105, USA, eit@apl.washington.edu), Steven Kargl (Applied Physics Laboratory, University of Washington, 1013 NE 40th Street, Seattle, WA 98105, USA, kargl@troutmask.apl.washington.edu), Joseph Lopes (Naval Surface Warfare Center - Panama City Division, 110 Vernon Ave, Panama City, FL 32407, USA, joseph.1.lopes@navy.mil), Raymond Lim (Naval Surface Warfare Center - Panama City Division, 110 Vernon Ave, Panama City, FL 32407, USA, raymond.lim@navy.mil), Carrie Dowdy (Naval Surface Warfare Center - Panama City Division, 110 Vernon Ave, Panama City, FL 32407, USA, carrie.dowdy@navy.mil)

Acoustic signatures of elastic targets located near sediment interfaces include effects due to energy interacting with the sediment. Therefore, modeling target response also requires models of scattering from, penetration into and propagation within ocean sediments. We first describe at-sea and test pond measurements carried out on "proud" (target resting on the sediment) and buried targets at frequencies in the range of 2 to $50 \mathrm{kHz}$. The results from some of these measurements are then compared to models incorporating various levels of sophistication relative to both the target and the sediment physics. The modeling hierarchy includes the following: (1) simple sonar equation estimates that treat the target physics via a frequency dependent target strength and use formally averaged results for sediment scattering, (2) realization level modeling that allows calculation of sediment and target scattering for individual pings with 
sufficient fidelity to carry out synthetic aperture processing (for a proud target only its geometrical scattering is considered while the elastic response can be included for a buried target), (3) T-matrix and finite element modeling in which the target elastic response is included but sediment scattering is treated using formal averages and/or flat surface approximations. [Work supported by the Office of Naval Research and the Strategic Environmental Research and Development Program, USA.]

\section{2:00}

5pUWb2. Synthetic aperture sonar imaging of simple finite targets near a sediment-water interface. Steven Kargl (Applied Physics Laboratory, University of Washington, 1013 NE 40th Street, Seattle, WA 98105, USA, kargl@troutmask.apl.washington.edu) , Kevin L. Williams (Applied Physics Laboratory, University of Washington, 1013 NE 40th St, Seattle, WA 98105, USA, williams@apl.washington.edu), Eric Thorsos (Applied Physics Laboratory, University of Washington, 1013 NE 40th St, Seattle, WA 98105, USA, eit@apl.washington.edu), Darrell R. Jackson (Applied Physics Laboratory, University of Washington, 1013 NE 40th Street, Seattle, WA 98105, USA, drj@apl.washington.edu), Dajun Tang (Applied Physics Laboratory, University of Washington, 1013 NE 40th St, Seattle, WA 98105, USA, djtang@apl.washington.edu)

Synthetic aperture sonar (SAS) is used often to detect targets that are either proud or buried below a sandy sediment interface where the nominal grazing angle of incidence from the SAS to the point above a buried target is below the critical grazing angle. A numerical model for scattering from simple targets in a shallow water environment will be described, and can be used to generate pings suitable for SAS processing. For buried targets, the model includes reverberation from the rough seafloor, penetration through the interface, target scattering, and propagation back to the SAS. The reverberation and penetration components are derived from first order perturbation theory where small-scale roughness and superimposed ripple can be accommodated. For proud targets, the simulations include the scattering from the target where interaction with the seafloor is included through simple acoustic ray models. The interaction of the target with an incident field is based on a free field scattering model. Simulations will be compared to both benchmark problems and measurements over a frequency range of 10-30 kHz. These comparisons further support sediment ripple structure as the dominant mechanism for subcritical penetration in this frequency range. [Work supported by the US Office of Naval Research.]

\section{$2: 20$}

5pUWb3. Experiments and numerical modeling of low to midfrequency scattering from elastic objects near the sea floor. Mario Zampolli (NATO Undersea Research Centre, Viale San Bartolomeo 400, 19126 La Spezia, Italy, zampolli@ nurc.nato.int), Alessandra Tesei (NATO Undersea Research Centre, Viale San Bartolomeo 400, 19126 La Spezia, Italy, tesei@nurc.nato.int), Finn B. Jensen (NATO Undersea Research Centre, Viale San Bartolomeo 400, 19126 La Spezia, Italy, jensen@nurc.nato.int), Gaetano Canepa (NATO Undersea Research Centre, Viale San Bartolomeo 400, 19126 La Spezia, Italy, canepa@nurc.nato.int)

The scattering of low to mid-frequency sound (1-10's of kHz) from submerged elastic structures of size $\mathrm{O}(1 \mathrm{~m})$ is a topic of interest to the underwater acoustics community. In the first part of the presentation, a brief description of the relevant components of the EVA experiment is given. The purpose of the sea trial was the acquisition of high-fidelity echoes from submerged spherical and cylindrical targets, made of composite materials with internal layered structure. The second part of the presentation is focused on the finite-element modeling technique developed at NURC for investigating the scattering from axially symmetric submerged elastic objects. Particular attention is dedicated to the computation of the far field at a distance from the target via the Helmholtz-Kirchhoff integral, using the near field sampled on the target surface, together with Green's functions capable of describing a two-layered water-sediment fluid medium. Those geometries, for which the overall axial symmetry is broken by the presence of the water-sediment boundary, can be treated approximately by taking into account the boundary-reflected incident field, as well as the first order interaction between the targetscattered echo and the sea floor. The numerical technique is validated by comparison with data collected during the EVA trial.

\section{2:40}

5pUWb4. Modeling bottom penetration for buried target detection. Raymond Lim (Naval Surface Warfare Center - Panama City Division, 110 Vernon Ave, Panama City, FL 32407, USA, raymond.lim@navy.mil), Gary S. Sammelmann (Naval Surface Warfare Center - Panama City Division, 110 Vernon Ave, Panama City, FL 32407, USA, gary.sammelmann@navy.mil)

Sonar detection of targets buried in underwater sediments has been found to be complicated by surface roughness. In particular, current-induced ripples can diffract energy down into sandy sediments to enhance buried target detection at shallow sonar grazing angles. To validate these effects, models encompassing the dominant propagation mechanisms as well as faithfully representing the target in the environment have been used. This paper describes our efforts to adapt transition matrix and perturbation theory models to provide realistic predictions of buried target response for spherical and cylindrical shapes. Combining these models of scattering and penetration required adopting some approximations to reduce computation time while retaining accuracy. Steps taken to verify and exercise the resulting models reveal some sensitivities that accentuate the need for accurate environmental and setup ground truth for validation of detection mechanisms. [Work supported by the Office of Naval Research and the Strategic Environmental Research and Development Program, USA.]

\section{3:00}

5pUWb5. Scattering by a partially exposed nearly rigid cylinder: Experiments and analysis. Kyungmin Baik (Washington State University, Physics and Astronomy Department, Pullman, WA 99164-2814, USA, nupho27@ dreamwiz.com), Philip L. Marston (Washington State University, Physics and Astronomy Department, Pullman, WA 99164-2814, USA, marston@wsu.edu)

The backscattering from a partially exposed circular cylinder was measured for broad side illumination under conditions where the contributions associated with the elastic response of the cylinder were expected to be weak. Grazing illumination was used. Since the objective was to investigate the transition in the number of reflected rays with increasing exposure, it was convenient to partially submerge the cylinder through the free surface of a tank of water. The magnitude of the scattering was measured for ka between 9.6 and 16 where $\mathrm{k}$ is the acoustic wave number and a is the radius of the cylinder. The scattering varied smoothly as a function of the cylinder's 
exposure in agreement with analytical results based on a Kirchhoff approximation [K. Baik and P. L. Marston, IEEE J. Oceanic Eng. (accepted)]. The analysis is easily modified for the case a cylinder breaking through a flat hard interface. For the different types of rays, path length calculations (as a function of the exposure) that are part of that analysis are also relevant to identifying echoes in SAS images of partially exposed cylinders and spheres. [Research supported by ONR.]

5pUWb6. Elastic and interfacial contributions to SAS images of tilted metal cylinders: Laboratory experiments. Jon La Follett (Washington State University, Physics and Astronomy Department, Pullman, WA 99164-2814, USA, LAFOLLEJ@mail.wsu.edu), Kyungmin Baik (Washington State University, Physics and Astronomy Department, Pullman, WA 99164-2814, USA, nupho27@dreamwiz.com), Philip L. Marston (Washington State University, Physics and Astronomy Department, Pullman, WA 99164-2814, USA, marston@wsu.edu)

Laboratory experiments were carried out to explore interfacial and elastic contributions to synthetic aperture sonar (SAS) images of a solid aluminum circular cylinder having flat ends. Some of the elastic responses for free field backscattering as a function of tilt angle could be interpreted using prior ray-based theory of generalized Rayleigh wave contributions [K. Gipson and P.L. Marston, J. Acoust. Soc. Am. 106, 1673-1680 (1999); 107, 112-117 (2000)]. Simplified acoustic holography was also used to interpret aspects of the free field bistatic response. To study the effects of proximity to a flat reflecting surface, the cylinder was hung through the free surface of a water tank and monostatic SAS images were acquired by scanning the transducer location along a horizontal line. This arrangement partially simulates SAS images of cylinders on the ocean bottom at grazing incidence. There were bright contributions to the SAS images of tilted cylinders associated with direct elastic rays as well as with indirect elastic rays due to acoustic reflections from the free surface of the water tank. [Research supported by ONR.]

\section{$3: 40$}

5pUWb7. Elastic scattering by partially-solid-filled spherical shell on the seabed: Model-data comparison and physical understanding. Alessandra Tesei (NATO Undersea Research Centre, Viale San Bartolomeo 400, 19126 La Spezia, Italy, tesei@nurc.nato.int), Mario Zampolli (NATO Undersea Research Centre, Viale San Bartolomeo 400, 19126 La Spezia, Italy, zampolli@nurc.nato.int), Gaetano Canepa (NATO Undersea Research Centre, Viale San Bartolomeo 400, 19126 La Spezia, Italy, canepa@nurc.nato.int)

Low- to mid-frequency elastic scattering measurements were conducted in the range from 5 to $40 \mathrm{ka}$ on a spherical composite shell deployed proud on a sandy seabed. The object consists of a thin-walled shell made of layers of a random-fiber material, and then filled partially with an isotropic solidified epoxy resin and partially with sea water. A scaled version of the object was measured in a water tank under free field conditions. The target responses obtained with and without interaction with the seafloor boundary were compared to simulations achieved by the NURC modeling tool Axiscat. The temporal echoes of the objects were analyzed in terms of elastic waves supported by the structure, on the basis of a ray model. The strongest elastic components come from the interior solid filler. The experimental data of the sphere on the seabed were acquired in October 2006 during the EVA'06 trial off the Island of Elba. The free field data were collected in the NURC water tank.

\section{4:00-4:20 Break}

5pUWb8. Robust recognition and characterization of man-made objects in shallow water using time-frequency analysis. Shaun D. Anderson (Georgia Institute of Technology, Woodruff School of Mechanical Engineering, Graduate Box 1000, Atlanta, GA 30332, USA, sanderson49@gatech.edu), Karim G. Sabra (Georgia Institute of Technology, School of Mechanical Engineering, 771 Ferst Drive, NW, Atlanta, GA 30332-0405, USA, karim.sabra@me.gatech.edu), Manell E. Zakharia (French Naval Acadamey, BP 600, 29240 Brest-Armees, France, manell.zakharia @ecole-navale.fr), Mario Zampolli (NATO Undersea Research Centre, Viale San Bartolomeo 400, 19126 La Spezia, Italy, zampolli@nurc.nato.int), Henrik Schmidt (MIT, 77 Mass Ave, 5-204, Cambridge, MA 02139, USA, henrik@mit.edu), William A. Kuperman (MPL, Scripps Institution of Oceanography, University of California, San Diego, La Jolla, CA 92093-0238, USA, wkuperman@ucsd.edu)

For underwater sonar, time-frequency analysis, in particular Wigner-Ville analysis, has been shown to be a relevant tool for discriminating a man made target (shell) from a natural one of the same shape (solid) and even to estimate some target characteristics (shell thickness, shear velocity...). This processing tool takes advantage of the evolutional, time dependent aspect of the echo spectrum. The estimated time-frequency patterns can be used for detection and wideband classification of sonar echoes in order to reduce false alarms. In particular, the so-called "coincidence pattern" appearing for specific frequency range is a robust time-frequency signature of manmade shells. A time-frequency analysis will be presented to understand echo formation mechanisms using a standard spherical shell model target model. The influence of the medium parameters as well as the source-receiver configuration will be investigated in free space and then extended to the case of a shallow water waveguide. The proposed approach will be tested using target scattering data collected during Experiments for Validation of Acoustic modeling techniques (EVA) sea test on the north shore of Isola D'Elba, Italy. Application to mine-hunting sonar systems will be discussed. 
5pUWb9. Bi-static scattering from buried, elastic objects in shallow water waveguides. Henrik Schmidt (MIT, 77 Mass Ave, 5-204, Cambridge, MA 02139, USA, henrik@mit.edu), Deep Ghosh (MIT, 77 Mass Ave, 5-204, Cambridge, MA 02139, USA, dghosh @ mit.edu)

The emerging autonomous network technology is enabling new operational paradigms for the concurrent detection, classification and localization of seabed objects by collaborating AUVs. Thus, such networks can exploit the bi-static enhancement of targets which are stealthy to conventional mono-static sonars, and the resonance properties of manmade targets. Under the GOATS and SWAMSI programs MIT in collaboration with NURC have addressed the fundamental issues associated with the development of such a new sonar concept. Through a series of joint experiements, various aspects of the interaction of elastic targets, completely or partially buried in the seabed have been investicated, including the evanescent coupling of low-frequency sound (1-10 kHz) into the seabed, the coupling with structural waves in the targets, and the 3D scattering back into the water column. The analysis is performed using a spectral virtual source scattering model with an embedded spectral Green's function generator which incorporates all multiple scattering effects between the target and the seabed. The target response is represented uniquely by an impedance matrix which may be computed separately using analytical or numerical methods, depending on the target geometry. The scattering model has been combined with the OASES code to provide a comprehensive simulation environment including all the shallow water waveguide physics.[Work Supported by ONR].

\section{Contributed Paper}

5:00

5pUWb10. Full field modeling of multiaspect scattering from buried objects. Ilkka Karasalo (FOI, Gullfossgatan 6, SE 16490 Stockholm, Sweden, ilkka.karasalo@foi.se)

Results are presented from a computational study of imaging of objects buried in the seabed under shallow water, using a rail-mounted active sonar and synthetic aperture processing. The medium is modeled as range independent, composed of a shallow water layer above a seabed of a muddy sediment containing the objects, and deeper subbottom layers. The objects have simple shape and structure, with diameters in the range $15-195 \mathrm{~cm}$. From selected positions along the rail, the sonar insonifies the objects by
LFM pulses from a directive transmitter, and records the backscattered echoes with a horizontal uniform line array (ULA), or optionally a pair of vertically separated ULAs. The signals from all sonar positions are integrated coherently by synthetic aperture processing for enhanced azimuthal resolution in the images of the objects. The parameters of the models of the medium, the objects and the experimental geometry are chosen to approximate those of a sea trial conducted in the Stockholm archipelago in 2004. The model-predicted scattered field is computed using the XFEM-S code, based on a frequency-domain boundary integral equation (BIE) formulation of scattering from a smooth object in a layered fluid-solid medium. Comparisons of model predictions with experimental results are presented.

\section{Invited Papers}

5pUWb11. Broadband scattering from spherical shells in a waveguide: modeling and classification. John A. Fawcett (DRDC Atlantic, PO Box 1012, Dartmouth, NS B2Y 3Z7, Canada, john.fawcett@drdc-rddc.gc.ca)

In this presentation, the exact expression for scattering from a sphere in a Pekeris waveguide (e.g., Sammelmann and Hackman, J. Acoust. Soc. Am., 82, 1987) is discussed. The importance of the sphere/interface rescattering terms is considered. A computationally faster multipath expansion approach is derived and its accuracy compared with the exact approach. For sufficiently high frequencies and in the case where the rescattering terms can be ignored, the multipath approach yields accurate predictions. The broadband scattering from an elastic-shelled sphere in a Pekeris waveguide is considered as a function of the frequency (or time for a pulse) and the sphere's range and depth in the waveguide. The classification problem is also discussed. The echos (time series or spectra) from a large set of spheres with varying parameters are generated and grouped into six classes corresponding to the various shell thicknesses and materials. Simple classifiers based upon temporal or spectral representations are considered for the spheres in free space and in a waveguide

\section{$5: 40$}

5pUWb12. Backscattering from an elastic target near a water-sediment interface at oblique incidence: First results of tank experiments. Jean-Pierre Sessarego (Laboratory for Mechanics and Acoustics CNRS, 31 chemin Joseph Aiguier, 13009 Marseille, France, sessarego@lma.cnrs-mrs.fr), Anatoliy N. Ivakin (Applied Physics Laboratory, University of Washington, 1013 NE 40th Street, Seattle, WA 98105, USA, ivakin@apl.washington.edu), Régine Guillermin (Laboratory for Mechanics and Acoustics CNRS, 31 chemin Joseph Aiguier, 13009 Marseille, France, guillermin@1ma.cnrs-mrs.fr)

Sound scattering from a target situated near a water-sediment interface was studied in laboratory conditions in order to control separately all the parameters involved in the scattering process. Targets of different sizes were ensonified with wide band transducers covering the frequency range $200 \mathrm{kHz}$ to $1 \mathrm{MHz}$. First, the target scattering strength was measured in the free space conditions, and the scattering strength of the water-sediment interface was measured at oblique incidence. These characteristics were used to provide a rough estimate for the signal-to-noise ratio for the second set of experiments where the target was situated near the interface to study effects of target-boundary interactions. The intensity of the total scattered field was measured as a function of the beamwidth, transducer/object and object/interface distances, frequency, grazing angle, target size and the interface roughness parameters. The interface considered here is a flattened sand surface which was studied earlier [Ivakin and Sessarego, High frequency scattering from flattened sand sediments: effects of granular structure, J. Acoust. Soc. Am., 122, (5) 2007]. The targets were spherical glass beads of different size. Side scan sonar images are presented and possibilities of their qualitative interpretation are discussed. 


\title{
Contributed Paper
}

6:00

5pUWb13. Estimates of scattering strength for buried cylindrical targets ensonified by evanescent waves. David C. Calvo (U.S. Naval Res. Lab., Acoust. Div., Code 7142, 4555 Overlook Ave. SW, Washington, DC 20375, USA, david.calvo@nrl.navy.mil), Mario Zampolli (NATO Undersea Research Centre, Viale San Bartolomeo 400, 19126 La Spezia, Italy, zampolli@nurc.nato.int), Alessandra Tesei (NATO Undersea Research Centre, Viale San Bartolomeo 400, 19126 La Spezia, Italy, tesei@nurc.nato .int)

It is known that low-frequency subcritical sound waves can significantly scatter from targets buried in a seabed due to the significant penetration depth of the incident evanescent wave. Past computational work on scattering by buried spherical shells has been done, for example, using a T-matrix
Method [R. Lim et al., J. Acoust. Soc. Am. 93, 1762-1783 (1993)], a Virtual Source Method [I. Lucifredi and H. Schmidt., J. Acoust. Soc. Am. 120, 3566-3583 (2006)], or finite-element methods [Zampolli et al., J. Acoust. Soc. Am., in press]. In addition to high-fidelity results which are expected from the preceding numerical methods, it desirable to have approximate analytical/asymptotic predictions of multistatic scattering strength for a variety of homogeneous or layered buried targets. Focusing on buried cylinders of infinite or finite-length, we first compute scattering using an approximate method that makes use of separation of variables and neglects multiple scattering between the interface and the target. Results are compared with those generated using the Axiscat/NURC/COMSOL finite-element method. Asymptotic estimates are then presented for scattering strength for objects completely buried in the seafloor for a flat interface (Work sponsored by ONR and NURC.

FRIDAY AFTERNOON, 4 JULY 2008

ROOM 341, 2:00 TO 6:00 P.M.

\section{Session 5pUWc}

\section{Underwater Acoustics and ECUA: Automatic Target Recognition, Sensors and Algorithms}

\author{
Gerald Dobeck, Cochair \\ Naval Surface Warfare Ctr., Coastal Systems Station, Dahlgren Div., Panama City, FL 32407-7001, USA \\ Marc Pinto, Cochair \\ NATO Undersea Research Centre, Viale San Bartolomeo 400, La Spezia, 19126, Italy \\ Yvan Petillot, Cochair \\ School of Engineering and Physical Sciences, Heriot-Watt University, Edinburgh, EH14 4AS, UK
}

Invited Paper

2:00

\begin{abstract}
5pUWc1. A track-before-detect algorithm for active sonar based on a hidden Markov model. Nigel H. Parsons (Thales Underwater Systems Ltd., Dolphin House, Ashurst Drive, Bird Hall Lane, Cheadle Heath, SK3 0XB Stockport, UK, nigel.parsons@uk .thalesgroup.com)
\end{abstract}

An active sonar track-before-detect algorithm is described. It is based on a hidden Markov model which uses a Viterbi algorithm to estimate the log-likelihood ratio of the presence or absence of a target in tracks within a state space representing a set of ranges, bearings, range rates and bearing rates, assuming a set of transition probabilities of changes in range rate and bearing rate. A detection is declared if the log-likelihood ratio exceeds a certain threshold and subsequently an HMM tracker, operating on a much smaller state space, is then employed. The performance of this algorithm on simulated data is evaluated. It is shown that, for moving and manoeuvring targets, the detection performance is significantly better than that of a conventional algorithm.

\section{Contributed Paper}

2:20

5pUWc2. Forward looking techniques for environment modeling, obstacle detection and characterization. Isabelle Quidu (ENSIETA E3I2 Lab., 2 rue François Verny, 29806 Brest Cedex 9, France, isabelle.quidu@ensieta.fr), Yann Dupas (Groupe d'Etudes Sous-Marines de l'Atlantique (GESMA), BP 42, 29240 Brest Armées, France, yann.dupas @ dga.defense.gouv.fr)

Military underwater robots are designed to perform complex underwater missions in both known and unknown environments. To achieve these tasks, an Autonomous Underwater Vehicle (AUV) must be supplied with appropriate sensors to deal with unpredictable events that can put it in danger, and with a high degree of decisional autonomy. In this paper, we have studied the architecture of forward looking sensors to allow the creation of a 3D model of the environment presenting the seabed and the obstacles that are on the path of the AUV. Our approach is based on experimental trials using different and complementary ways (sonars with several configurations) to gather an information as complete as possible. This information will be processed by the vehicle during a survey mission. Practically, we create a reference model of a static environment using a multibeam system which produces bathymetric images at different grazing angles. In the same environment we then use a Forward Looking Sonar intended for the recognition of detected echoes in comparison with the reference model. If an echo cannot be related to a known object on the reference map, it is considered as an obstacle, and the map is updated. 


\section{Invited Paper}

\section{$2: 40$}

5pUWc3. Automated change detection with area matching. John Dubberley (Naval Research Laboratory, Bldg. 1005 Rm D-23, Stennis Space Center, MS 39529, USA, john.dubberley@nrlssc.navy.mil), Marlin Gendron (Naval Research Laboratory, Bldg. 1005 Rm D-23, Stennis Space Center, MS 39529, USA, marlin.gendron@nrlssc.navy.mil), Maura Lohrenz (Naval Research Laboratory, Bldg. 1005 Rm D-23, Stennis Space Center, MS 39529, USA, maura.lohrenz@nrlssc.navy.mil)

When resurveying a geographic area of the seafloor during sidescan change detection operations, an automated method to match bottom objects imaged previously with objects imaged in the resurvey can increase efficiency and accuracy. The geographic position of a new object relative to a historical object is a good indicator of a match. However, due to position error within either survey, there may be more than one spatially-close object in the new imagery. To complicate matters further, the reflected energy from the new object may be significantly different given a different incidence angle in the resurvey or the partial burial of the object. In addition, the resurveyed object image may be below the threshold set for automatic recognition and falsely eliminated. This presentation will address these problems and suggest possible methods for matching "constellations" of bottom objects by Dijkstra's minimum cost - maximum flow algorithm, control point matching, and the data-association procedure.

\section{Contributed Paper}

3:00

5pUWc4. An acoustic barrier based on amplitude variations of the ray paths and double beamforming. Barbara Nicolas (GIPSA-lab, dep. DIS, 961, rue de la Houille Blanche, 38402 St Martin d'Hères, France, barbara.nicolas@gipsa-lab.inpg.fr), Philippe Roux (LGIT - CNRS - Université Joseph Fourier, Maison des Géosciences, 1381 rue de la Piscine, BP 53, 38041 Grenoble, France, philippe.roux@obs.ujf-grenoble.fr), Ion Iturbe (GIPSA-lab, dep. DIS, 961, rue de la Houille Blanche, 38402 St Martin d'Hères, France, ion.iturbe@gipsa-lab.inpg.fr), Jérôme I. Mars (GIPSA-lab, dep. DIS, 961, rue de la Houille Blanche, 38402 St Martin d'Hères, France, jerome.mars@gipsa-lab.inpg.fr)

The objective of this work is to build an acoustic barrier to detect and localize a target between two vertical arrays of sensors. To perform this detection/localisation, we record the signal between each source (of the source array) and each reveiver (of the receiver array). Using these data, we extract the different ray paths between sources and receivers thanks to a new signal processing method: double beamforming. Then, we show that ray paths and their arrival times are not affected by a target in the medium but that ray amplitudes change. As a result, it is possible to use amplitude variation of the rays to find the target localisation. To validate these methods we perform ultrasonic experiments in a tank. These experiments are often used in underwater acoustics as they emulate shallow water waveguides: indeed, by multiplying the frequency by a factor $\mathrm{x}$, distances are divided by the same factor. As acoustic and elastic propagation properties are not affected by this scaling down, it is possible to achieve "oceanic experiments" in a simple tank. Results of double beamforming and target detection are shown.

\section{Invited Papers}

\section{3:20}

5pUWc5. Advanced ATR techniques based on High-Resolution SAS Sensors. Enrique Coiras (NATO Undersea Research Centre, Viale San Bartolomeo 400, 19126 La Spezia, Italy, coiras@nurc.nato.int), Johannes Groen (NATO Undersea Research Centre, Viale San Bartolomeo 400, 19126 La Spezia, Italy, groen@nurc.nato.int), Benjamin Evans (NATO Undersea Research Centre, Viale San Bartolomeo 400, 19126 La Spezia, Italy, evans@nurc.nato.int), Marc Pinto (NATO Undersea Research Centre, Viale San Bartolomeo 400, 19126 La Spezia, Italy, pinto@nurc.nato.int)

Automatic Target Recognition (ATR) is a key element of expeditionary Mine Countermeasures (MCM) and port protection operations. Most existing approaches to ATR are currently based on high resolution sonar sensors, which provide enough information to obtain satisfactory detection and classification performance for the large World War 2 mine types (e.g., $2 \mathrm{~m}$ long cylinder). False alarm rates, however, are still unacceptably high for modern mines, which constrains the way operations are undertaken and often requires either confirmation or re-evaluation by a human operator. The introduction of new AUV-mounted Synthetic Aperture Sonars (SAS) increases the resolution, quality and range of acquired sonar images, which broadens the set of machine vision and computer image analysis techniques that can be used for underwater ATR operations. In this paper we study the impact that the increased quality and resolution have on performance gains and false alarm reduction. A number of classification algorithms are selected to represent the pool of existing approaches to target detection and classification, and their performances are estimated using both simulated and real image data in order to quantify the benefits associated to the new SAS technology. Evolution and near-future plans are discussed, introducing emerging bio-sonar sensors, anomaly detectors and autonomous AUV systems.

\section{3:40}

5pUWc6. Rapid distinction of dumpsite objects using Multiple-Aspect Scattering - Results from scaled tank experiments. Philippe Blondel (University of Bath, Department of Physics, Claverton Down, BA2 7AY Bath, UK, pyspb@bath.ac.uk)

Toxic dumpsites on the seafloor are causing increasing environmental concern, but traditional sonar imaging strains to distinguish objects in unconsolidated sediments, in particular in cluttered terrains. Scaled tank experiments were conducted with four different cylinders (fluid-filled and solid aluminium, air-filled and solid stainless steel, respectively) and two seabed types (silt and gravel), using the facilities at the University of Bath. The setup was a 10:1 scaled version of the EC-SITAR sea trials site in the Stockholm Archipelago (Sweden). The main aim of these experiments was to design efficient surveying strategies, later used at sea. Our studies showed large variations depending on the aspect of these targets and their bistatic imaging configuration. These variations can be directly related to the shapes of the targets (e.g., dimensions, presence of ribs), their content (hollow or solid) and the material of the shells (e.g., 
stainless steel or aluminium). They are quantified using the combined L4 norm of the time-domain signals at each aspect. Using appropriate ranges of multistatic configurations and imaging each target at three distinct aspects ( $45^{\circ}$ apart), it is possible to successfully distinguish between similar targets with distinct contents and/or material, even in cluttered terrains.

\section{4:00-4:20 Break}

\section{Contributed Papers}

4:20

5pUWc7. Target detection of man made objects in side scan sonar images - segmentation based false alarm reduction -. Max Neumann (Freie Universität Berlin, Takustr. 9, 14195 Berlin, Germany, papperlapapp@gmail.com), Christian Knauer (Freie Universität Berlin, Takustr. 9, 14195 Berlin, Germany, christian.knauer@inf.fu-berlin .de), Bodo Nolte (Forschungsanstalt der Bundeswehr für Wasserschall und Geophysik, Klausdorfer Weg 2-24, 24148 Kiel, Germany, bodonolte@bwb.org), Dieter Brecht (Forschungsanstalt der Bundeswehr für Wasserschall und Geophysik, Klausdorfer Weg 2-24, 24148 Kiel, Germany, dieterbrecht@bwb.org), Wolfgang Jans (Forschungsanstalt der Bundeswehr für Wasserschall und Geophysik, Klausdorfer Weg 2-24, 24148 Kiel, Germany, wolfgangjans@bwb.org), Alfons Ebert (FGAN-FOM, Gutleuthausstraße 1, 76275 Ettlingen, Germany, a.ebert@fom.fgan.de)

This paper presents a fast and robust algorithm for significantly reducing the number of false detections caused by screening algorithms for side scan sonar (SSS) images. The presented algorithm consists of two processing steps. First, an iterative segmentation process is carried out for seperating the image into shadow and background. This segmentation is based on an energy function which combines the local neighborhood segment information and the amplitude of a pixel. By minimizing this function, a clear shadow, the most significant target characteristic, will be extracted. Second, based on the region of interest (ROI) and the shadow contour, a robust classification approach is applied, utilizing the area of the shadow, the first two statistical moments of the pixel amplitude and the existence of parallel lines (Hough transformation). This algorithm was tested using a data set with approx. 2400 ROIs containing about 200 targets and 270 targetlike stones or sandrippel. This data set was gathered during five different measurement campaigns in the Baltic Sea and the Mediterranean Sea using three different
SSS systems Benthos C3D, Klein2000 and Marine Sonics). These data were collected by FWG and WTD71 as well as by Atlas Electronik with the SeaOtter MK1 AUV.

5pUWc8. Sonar target-phase measurement and effects of transducer-matching. Philip Atkins (University of Birmingham, Department of Electronic, Electrical and Computer Engineering, Edgbaston, B15 2TT Birmingham, UK, p.r.atkins@bham.ac.uk), Alan Islas (University of Birmingham, Department of Electronic, Electrical and Computer Engineering, Edgbaston, B15 2TT Birmingham, UK, AXI743@bham.ac .uk), Kenneth G. Foote (Woods Hole Oceanographic Institution, Woods Hole, MA 02543, USA, kfoote@whoi.edu)

Active sonar systems normally detect and classify a target based on the amplitude of the received echo or the induced Doppler shift. However, additional classification information may be available from the phase shift introduced by some targets as a result of the boundary conditions. For example, reverberation from the sea surface and scattering from fish swimbladders introduce an additional phase shift that may not be present in returns from an acoustically stiffer seabed or synthetic target. Algorithms based on the use of subband correlators are presented for measuring the phase shifts introduced by the boundary conditions on stationary and moving targets when insonified by broadband transmissions. These techniques are used to remove the phase shifts introduced by the unknown target. However, the unknown phase characteristics of the transducer, matching circuit, and electronic circuitry of a sonar system imply that target-phase measurements are very difficult to make in any practical system. The effects of adding a Butterworth-derived matching circuit to a Reson TC2130 transducer are presented for the case of sinusoidal frequency-modulated excitation of solid elastic and thin elastic-shelled hollow spheres. It is concluded that target-phase measurements can enhance the classification performance of a suitably calibrated sonar system.

\section{Invited Papers}

\section{5:00}

5pUWc9. Evaluation of portable high-frequency sonars for diver identification. Anna Crawford (Defence R\&D Canada Atlantic, P.O. Box 1012, 9 Grove St, Dartmouth, NS B2Y 3Z7, Canada, anna.crawford@drdc-rddc.gc.ca), Vance Crowe (Defence R\&D Canada Atlantic, P.O. Box 1012, 9 Grove St, Dartmouth, NS B2Y 3Z7, Canada, vance.crowe@drdc-rddc.gc.ca), Thomas Pastore (NATO Undersea Research Centre, Viale San Bartolomeo 400, 19126 La Spezia, Italy, Pastore@nurc.nato.int), Ronald Kessel (NATO Undersea Research Centre, Viale San Bartolomeo 400, 19126 La Spezia, Italy, Kessel@nurc.nato.int)

Obtaining a positive identification is a critical step in most tiered harbour protection strategies for countering underwater intruders. It is generally recognised that sonar is one of the best tools for underwater imaging, however operating in a harbour environment presents challenges. As part of an on-going harbour protection research project, small easily portable high-frequency sonar systems are being investigated as a means to equip small response craft with intruder identification capability. Several systems are being considered, with the most comprehensive testing by DRDC to date being done on small Canadian-made sonars. Tests were conducted in local harbour waters in Halifax, Canada, and in La Spezia, Italy, through participation in the NATO Undersea Research Centre Response Against Diver Intrusions (RADI) joint trial, conducted in November 2007. A variety of small sonars and manned and unmanned response craft were used during the RADI trial. Evaluation of the performance of these devices for the task of diver identification in realistic conditions will be discussed.

5pUWc10. Features for propagation-invariant classification of underwater targets. Patrick Loughlin (University of Pittsburgh, 348 Benedum Engineering Hall, Dept. of Electrical \& Computer Engineering, Pittsburgh, PA 15261, USA, loughlin@engr.pitt.edu), Greg Okopal (University of Pittsburgh, 348 Benedum Engineering Hall, Dept. of Electrical \& Computer Engineering, Pittsburgh, PA 15261, USA, gno1@pitt.edu)

As sound propagates in shallow water, it is subject to frequency-dependent spreading and attenuation (dispersion and damping). In active sonar, these propagation-induced changes can be detrimental to automatic classification because the observed backscatter depends on the propagation environment and how far the wave has traveled. One way to address this problem is to develop propagation-invariant 
features of the wave that can be used in automatic classification. In this talk, we present temporal, spectral, and cepstral moment-like features of a wave that are invariant per mode to dispersion and damping. Classification results on numerical simulations of the backscatter from different steel shells propagating in a Pekeris waveguide with damping and random variations will be presented. [Supported by ONR grants N00014-06-1-0009 and N00014-07-10355]

\title{
Contributed Paper
}

\section{$5: 40$}

5pUWc11. MCM sensor requirements: performance measures. Samantha Dugelay (Dstl, Building A32, Winfrith Technology Centre, DT2 8WX Dorchester, UK, sdugelay@dstl.gov.uk)

This paper presents a comparison of two measures of performance suitable to characterise operational performance of mine hunting sonars in various environmental conditions. The first measure is a direct adaptation of information theory and bounds the capability of a sonar to distinguish between objects, i.e., a classification capability. The bounds take into account the amount of distinguishable pixels between objects and the statistical information content of each pixel. Successfully applied in Radar, this approach has also demonstrated the performance of synthetic aperture sonar at NURC using a Rayleigh distribution for statistical pixel distribution. The measure is now being further developed to include high resolution distributions such as $\mathrm{K}$-law which readily appear in high resolution images. The second measure, Johnson's criteria aims to provide guidance on resolution required for operator detection, classification and identification. This measure originally derived from experiments on night vision images is now being modified to simultaneously incorporate target highlight and shadow information in varying environmental conditions. Finally, the predictions of these measures will in the future be compared to each other and to current system performances.

FRIDAY AFTERNOON, 4 JULY 2008

ROOM 342B, 2:20 TO 3:40 P.M.

\section{Session 5pUWd}

\section{Underwater Acoustics and ECUA: Acoustic Data Fusion}

\author{
Eric Maillard, Cochair \\ Reson Inc, Golet, CA, USA \\ Benoit Zerr, Cochair \\ DGA/GESMA, BP42, Brest Armées, 29240, France
}

\section{Contributed Papers}

2:20

5pUWd1. Multisegmentation of sonar images using belief function theory. Mounir Dhibi (ENSIETA E3I2, 2 rue François Verny, 29806 Brest Cedex 9, France, mounir.dhibi@ensieta.fr), Romain Courtis (I2ETA Romain Courtis, GESMA/SDP/GDM, BP 42, 29240 Brest Armées, France, Romain.Courtis@dga.defense.gouv.fr), Arnaud Martin (ENSIETA E3I2, 2 rue François Verny, 29806 Brest Cedex 9, France, arnaud.martin@ensieta .fr), Isabelle Quidu (ENSIETA - E3I2 Lab., 2 rue François Verny, 29806 Brest Cedex 9, France, isabelle.quidu@ensieta.fr)

Today side scan sonar is one of the most efficient sensors for Rapid Environment Assessment missions. Unfortunately, features extracted from a given area are strongly dependent on the relative position of the sensor (e.g., due to the shadow or the gain variation). That could conduct to a bad segmentation of the seabed. However, due to the fact that operational systems give very often multiple views of the same area we use the redundancy. In this work, we propose to fuse multiview segmentations in order to outperform the seabed classification. First we present a way to characterize the seabed using as a start point, a texture analysis in order to extract parameters on images. Then, a classification method allows allocating a class according to the type of sediment for the different standpoints. The proposed classifier fusion is based on the belief function theory. We present results from a set of experiments conducted to evaluate the proposed approach with real sonar images and we discuss them.
2:40

5pUWd2. Elimination of corner-turning in FFT-based sonar array beamforming. Jacob Barhen (Oak Ridge National Laboratory, 1 Bethel Valley Road, Oak Ridge, TN 37831-6015, USA, barhenj@ornl.gov), Travis Humble (Oak Ridge National Laboratory, 1 Bethel Valley Road, Oak Ridge, TN 37831-6015, USA, humblets@ornl.gov), Michael Traweek (Office of Naval Research, 875 North Randolph Street, Arlington, VA 22203, USA, Mike.Traweek@navy.mil)

The expected availability, in the near future, of an ultralow power version of the revolutionary IBM CELL multicore processor opens unprecedented opportunities for implementing sophisticated signal processing algorithms faster and within a much lower energy budget. The concept of "corner turning" has been, for many decades, at the heart of array beamforming via Fourier transforms. As widely reported in the open literature (both for sonars and radars), the computational sequence involving corner turning operations, i.e., the sequence: temporal Fourier transforms -- $>$ data cube corner turning --> spatial Fourier transforms, constitutes one of the primary obstacles to achieving high-performance and lower power dissipation (by reducing the number of times memory is accessed). Even with the emergence of novel multicore processors, leading providers (e.g., Mercury Computers) still include explicit corner turning stages in their computational flow architectures for multidimensional array processing. The primary innovation reported in this paper addresses the development of a computational scheme that avoids altogether the corner turning stage. We discuss its implementation on currently available CELL technology (65 nm SOI) and demonstrate close to an order of magnitude speed-up compared to the scheme with corner turning implemented on the same processor. 
5pUWd3. Multiple-sensor fusion approach to seabed classification Benoit Zerr (DGA/GESMA, BP42, 29240 Brest Armées, France, benoit.zerr@dga.defense.gouv.fr), David Kerneis (ENST Bretagne Dept. ITI, Technopôle Brest-Iroise - CS 83818, 29238 Brest Cedex 3, France, david.kerneis@enst-bretagne.fr), Basel Solaiman (ENST Bretagne Dept. ITI, Technopôle Brest-Iroise - CS 83818, 29238 Brest Cedex 3, France, basel.solaiman@enst-bretagne.fr)

Seabed classification is key issue for civilian and military underwater applications, from offshore exploitation to mine counter measure. Most of the existing automated classification techniques relies on the analysis of the data provided by a single sensor, supposed to unambiguously separate the different classes of seabed. In this paper we present a different approach which considers that, even if a sensor cannot tell the differences between two classes, classification will improve by considering that the seabed belongs to one of these two classes, and, further, that the analysis of the data from another sensor can resolve the ambiguity. For each sensor, the classification is achieved in a conventional way by feature extraction and supervised classification. The fusion of the results implements the theory of evidence through Dempster-Shafer method. After a description of the method, the paper discusses the experimental results from the fusion of information delivered by three sensors: an imaging sidescan sonar, a vertical echo sounder and an interferometric bathymetric sonar. The major part of the experimental data has been acquired by towed or hull mounted sensors. As these sensors are a subset of the payloads operated simultaneously by the new DGA-SHOM DAURADE AUV, preliminary seabed classification results in covert REA missions will also be presented and discussed.
5pUWd4. Acoustic data fusion devoted to underwater vegetation mapping. Claire Noel (Semantic TS, 39 Ch Buge, 83110 Sanary, France, noel@semantic-ts.fr), Christophe Viala (Semantic TS, 39 Ch Buge, 83110 Sanary, France, viala@semantic-ts.fr), Michel Coquet (Semantic TS, 39 Ch Buge, 83110 Sanary, France, coquet@semantic-ts.fr), Benoit Zerr (DGA/GESMA, BP42, 29240 Brest Armées, France, benoit.zerr @ dga.defense.gouv.fr), Thierry Perrot (CEVA, Presqu'île de Pen Lan BP3, 22710 Pleubian, France, thierry.perrot@ceva.fr)

This paper presents research tasks conducted by SEMANTIC TS, in collaboration with GESMA, aimed to develop a mapping method for underwater vegetation lying on seabed. First stage is to develop a method for detecting and characterizing vegetation on the seabed using the acoustic response from a conventional single beam echo sounder. This new method is then operated simultaneously with multibeam sonar producing micro-relief information and side scan sonar providing gray scale levels associated with bottom reflectivity. Then fusion of these three data is processed. We show efficiency of these multisensor data fusion concept to get very precise seabed vegetation mapping in a way reducing truth control (video and diving investigations). Sensors and method accuracy allow obtaining, like in biomedical field, real 3D scan pictures of seabed vegetation. This study is first applied to posidonia and cymodocea, which play a key role in Mediterranean's echosystem. Then, extension of the method is investigated to address laminaria which may significantly affect the performance of acoustic and optical sensors used for sea-mines detection and this paper presents results of data fusion mapping on an Atlantic sea area covered by luminaria, studied and well known by the CEVA.

FRIDAY AFTERNOON, 4 JULY 2008

ROOM 342B, 4:00 TO 6:20 P.M.

\title{
Session 5pUWe
}

\section{Underwater Acoustics and ECUA: Noise Suppression, Robust Direction of Arrival, and Target Strength Estimation}

\author{
Georges Bienvenu, Cochair \\ Thales Naval Division, Thales Underwater Systems, France \\ Ronald A. Wagstaff, Cochair \\ National Center for Physical Acoustics, University of Mississippi, University, MS 38677, USA
}

\section{Contributed Papers}

\section{4:00}

5pUWe1. "Venus's-noisetrap" high-gain adaptive processor. Ronald A. Wagstaff (National Center for Physical Acoustics, University of Mississippi, University, MS 38677, USA, rwagstaf@olemiss.edu), Heath E. Rice (National Center for Physical Acoustics, University of Mississippi, University, MS 38677, USA, herice@olemiss.edu)

The Venus's-noisetrap is a high gain adaptive signal processor inspired by Venus's-flytrap, an insect eating plant. The Venus's-noisetrap utilizes single sensor or beamformed spectral data. It "traps" the data samples by modifying the governing equations in a manner that each spectral sample is adaptively "trapped" before averaging. The trapping process is a fluctuationbased temporal coherence determination of whether the time history in each spatial or spectral bin is signal or noise. Signals are set "free", while the noise remains trapped, blocking it from participating in the averaging process. For ocean acoustic data, the processor automatically identifies signals from submerged sources. In addition, the Venus's-noisetrap achieves large signal-to-noise ratio (SNR), high spectral and spatial resolution, and auto-identification of signals. The techniques that cause this processor to mimic the Venus's-flytrap will be discussed, and the method of continuously adapting the governing equations to the unpredictable signal and noise environment will be illustrated. Results will be presented to show the processor's large SNRs and corresponding enhancements in spectral and spatial resolution.

\section{$4: 20$}

5pUWe2. Suppression of oceanic reverberation by subspace methods Xuan Li (Institute of Acoustics, Chinese Academy of Sciences, 100080 Beijing, China, happyxuanli@hotmail.com), Xiaochuan Ma (Institute of Acoustics, Chinese Academy of Sciences, 100080 Beijing, China, maxc@mail.ioa.ac.cn), Chaohuan Hou (Institute of Acoustics, Chinese Academy of Sciences, 100080 Beijing, China, hch@mail.ioa.ac.cn)

Acoustic signal processing in shallow water environments is a challenging problem because of the presence of reverberation. Based on some models of reverberation, data from sensors array is pretreated to suppress reverberation. Considering reverberation as a sum of echoes of transmitted 
signal, the principal component inverse (PCI) algorithm deletes the largest singulars of data matrix, which is constructed from array data. However, estimating a threshold which is needed in PCI is difficult in practice. In this paper, two new subspace methods, Deleting Big Eigenvalues and Subspace Projection are proposed. The two novel methods, substituting automatic signal-number estimation for threshold estimation, are operated via eigendecomposition. According to a simulation which takes broadband linear modulated frequency signal as transmitted signal, these two methods show a similar performance but smaller computing quantity compared with PCI.

4:40

5pUWe3. Coherent effects of flow- and pressure-hull of a generic submarine on target scattering in an active sonar performance model. Pieter Schippers (TNO-D\&V-Underwater Technology, Oude Waalsdorperweg 63, Post Box 96864, 2509 JG The Hague, Netherlands, pieter.schippers @ tno.nl)

Since the late eighties the sonar performance model ALMOST for active and passive sonar is under development at TNO. For active detection performance, first a point target was used, with a single target strength value dependent on parameters like aspect angle, based on measurements or other sources. However by now there is a demand for TS of ships and wakes with realistic dimensions and characteristics. A generic sub was modeled with additional software routines, as a pixel file. A newly developed time domain model for hull reflection was implemented, also using scattering pixels, assuming multiple scattering with damping in the metal hull layer. This extension of the pixel modelling is evaluated versus literature data. Some modeling results of target strength computations are shown, for a generic submarine with pressure hull, with aspect angle, frequency and bandwidth as parameters.

\section{5:00}

5pUWe4. Robust capon beamformer for port/starboard discrimination of twin-line array. Zaixiao Gong (National Laboratory of Acoustics, Institute of Acoustics, Chinese Academy of Sciences, NO.21, Bei-Si-huan-Xi Road, 100080 Beijing, China, gzx@mail.ioa.ac.cn)

Compared with the single-line array, the twin-line array have the potential to solve the port/starboard discrimination problem. Conventional method of geometric phase shifting has been used to distinguish the port/starboard of the target. But it can only be used to solve the problem when the frequency bandwidth is limited. The method based on optimum beamforming for hydrophone triplets can hardly be applied with the twin-line arrays. Aimed at the twin-line arrays port/starboard discrimination problem, a method based on the robust capon beamformer is proposed in this paper, which has advantages of concise algorithm and robustness against the aberration of the array shape. And the method works better with wider frequency bandwidth. Simulation and on sea experiment data are analyzed to verify the method.

\section{$5: 20$}

5pUWe5. A numerical method for array sensor noise field calculation in detection performance optimization. Chao Sun (Institute of Acoustic Engineering, Northwestern Polytechnical University, 710072 Xi'an, China, csun@nwpu.edu.cn), Bo Yang (Institute of Acoustic Engineering, Northwestern Polytechnical University, 710072 Xi'an, China, yangbo@mail.nwpu.edu.cn), Yixin Yang (Institute of Acoustic Engineering, Northwestern Polytechnical University, 710072 Xi'an, China, yxyang @nwpu.edu.cn)

Different from the generally adopted criteria of minimizing the sidelobe level and maximizing the array gain in the weighting design of a sonar array, an approach was proposed recently to optimize the shading weights with the aim of maximizing the deflection coefficient in the square-law detector, which in essence suppresses the self-noise by including the noise informa- tion at array sensors in the optimization procedure. When several noise sources are present and/or an analytical expression of the noise transfer function is not available, the sensor noise needs to be measured in forming the sensor noise response cross correlation matrix required in the optimization which is very demanding when an array with large number of sensors is considered. To avoid the tedious work of noise measurement, a numerical method is developed in this paper. In this method, the main self-noise sources are assigned with different positions and strengths and the noise field at the array sensors is calculated via either the finite element method or the boundary element method. Tank experiment validated the effectiveness of this method. By using this method, the detection performance of a sensor array can be predicted before it is physically built and tested in under practical conditions.

\section{$5: 40$}

5pUWe6. Improving spatial resolution of interferometric bathymetry in multibeam echosounders. Gerard Llort-Pujol (ENST-Bretagne, Dpt ITI, CS 83818, 29238 Brest Cedex 03, France, gerard.llort @enst-bretagne.fr), Christophe Sintes (ENST-Bretagne, Dpt ITI, CS 83818, 29238 Brest Cedex 03, France, christophe.sintes @enst-bretagne.fr), Xavier Lurton (Institut Français de Recherche pour l'Exploitation de la Mer, NSE/AS, BP 70, 29280 Plouzané, France, lurton @ifremer.fr)

Most multibeam echosounders used in seafloor mapping use the interferometry method for bathymetry measurement, based on the zero-crossing of the phase difference between two sub-arrays. In this approach, only one sounding is computed per formed beam, and the spatial resolution is linked to the beam footprint extent. Using the whole content of the phase difference signal vs time makes it possible ideally to get a bathymetry data sampled at the very resolution of the digitised signal. A trade-off is however to be found between this resolution improvement and the increase in the measurement noise. The performances of interferometric bathymetry are presented, together with the constraints of the resolution-accuracy trade-off, and a presentation of a high-resolution algorithm for interferometric bathymetry applicable to multibeam systems. This is illustrated by results obtained on a wreck, showing the remarkable improvement achievable in resolution over a real target. The potentialities for this method are promising, since it allows to process, in the same beam, targets on the seafloor and inside the water column; simplifications in multibeam sounders array structure and processing are also to consider.

\section{6:00}

5pUWe7. Adaptive removal of a known interference and its scattered energy. Yung P. Lee (SAIC, 4001 N. Fairfax Dr, Suite 175, mail stop 1-11-1, Arlington, VA 22203, USA, yung.p.lee@saic.com), William Lee (Duke University, Department of Electrical and Computer Engineering, Durham, NC 27708, USA,ww13@duke.edu)

In underwater passive processing, the nearby fast moving loud surface ships produce nonstationary interference field and limit most sonar performance. After 9/11, AIS (automatic identify system) is required for ships larger than 60's, providing ground truth of positions, speeds, and other ancillary information for the nearby surface ships. This study investigates adaptive removal of a known loud signal using the ground truth information and acoustic propagation modeling. In addition, scattered energy away from the loud signal is identified through a delay correlation processing and is also removed. In a segment of MAPEX2k experiment conducted by SACLANT Centre off the west coast of Italy on November 28, 2000, the research vessel ALLIANCE towed an array at $2 \mathrm{~m} / \mathrm{s}$ passing by a moored controlled source. The controlled source transmitted a loud 1-s 150-500 Hz LFM sequence every $15 \mathrm{~s}$. In this setting, the fixed controlled source generated a nonstationary interference field received on the towed array. With the knowledge of the array tow track and source position the performance of removing the known interference and its scattered energy in a real shallow water environment is examined. 


\title{
Session 5pUWf
}

\section{Underwater Acoustics and ECUA: Sound Generation and Attenuation}

\author{
Theodore G. Jones, Cochair \\ U.S. Naval Research Laboratory, Code 6795, 4555 Overlook Ave. SW, Washington, DC 20375, USA \\ Andrzej Zak, Cochair \\ Polish Naval Academy, Smidowicza 69, Gdynia, 81-103, Poland
}

\section{Contributed Papers}

\section{4:40}

5pUWf1. Neural classification of ships hydroacoustic signatures. Andrzej Zak (Polish Naval Academy, Smidowicza 69, 81-103 Gdynia, Poland, a.zak@amw.gdynia.pl)

The paper presents method of classification of hydroacoustic signatures generated by moving ship. Classification is a procedure in which individual items are placed into groups based on quantitative information on one or more characteristics inherent in the items. In this paper the hydroacoustics signals classification is understood as the process of automatically recognition what kind of object is generating acoustics signals on the basis of individual information included in generated sounds. Hydroacoustics signal classification is a difficult task and it is still an active research area. Automatic signal classification works based on the premise that sounds emitted by object to the environment are unique for that object. However this task has been challenged by the highly variant of input signals. The paper includes discussion about unique of sound generated by moving ships. To solve problem of hydroacoustic signatures classification the Kohonen networks which belongs to group of self organizing networks where chosen. Hydroacoustic signals were acquired on the Polish Navy Range during the complex ship measurement. At the end the results of classification of underwater noises made by ship were presented.

\section{5:00}

5pUWf2. Underwater acoustic generation with narrow and broadband lasers. Theodore G. Jones (U.S. Naval Research Laboratory, Code 6795, 4555 Overlook Ave. SW, Washington, DC 20375, USA, ted.jones@nrl.navy.mil), Melissa K. Hornstein (U.S. Naval Research Laboratory, Code 6795, 4555 Overlook Ave. SW, Washington, DC 20375, USA, melissa.hornstein@nrl.navy.mil), Antonio C. Ting (U.S. Naval Research Laboratory, Code 6795, 4555 Overlook Ave. SW, Washington, DC 20375, USA, antonio.ting@nrl.navy.mil), Zachary W. Wilkes (Research Support Instruments, Inc., 4325 Forbes Blvd, Ste B, Lanham, MD 20706, USA, zachary.wilkes@nrl.navy.mil), Dennis A. Lindwall (U.S. Naval Research Laboratory-Stennis, Marine Geosciences Division, Code 7432, Stennis Space Center, Stennis, MS 39529, USA, lindwall@nrlssc.navy.mil)

Underwater laser acoustic sources, generated by intense pulsed lasers on above-water and underwater platforms, are under investigation. In a novel configuration, a tailored intense broadband laser pulse can be designed to propagate many meters underwater and compress at a predetermined remote location. Controlled compression of these optical pulses is governed by a combination of optical group velocity dispersion (GVD) and nonlinear Kerr self-focusing, resulting in photoionization, localized heating, and shock generation. Recent and ongoing experiments include near-field acoustic source characterization using lens-focused pulses of a broadband $400 \mathrm{~nm}$ Ti: sapphire laser, as well as $1064 \mathrm{~nm}$ and $532 \mathrm{~nm}$ narrowband YAG laser pulses. Also, the nonlinear optical Kerr index of water at $400 \mathrm{~nm}$ was precisely measured. Acoustic source characterization includes measurements of photoacoustic energy conversion efficiency, acoustic power spectrum, and directivity. Experimental results will be presented, and laser sources and techniques for underwater acoustic generation will be compared. [This work is supported by the U.S. Office of Naval Research.]

\section{$5: 20$}

5pUWf3. Recent progress on the theoretical modeling of underwater acoustics induced by sonic booms. Johnson C. Wang (The Aerospace Corporation, System Planning and Engineering, El Segundo, CA 902454691, USA, Johnson.C.Wang@aero.org), Charles P. Griffice (The Aerospace Corporation, System Planning and Engineering, El Segundo, CA 90245-4691, USA, Charles.P.Griffice@aero.org), Adam M. Fincham (University of Southern, Aerospace \& Mechanical Engineering, 854 W Downey way, Los Angeles, CA 90089-1191, USA, afincham@usc .edu), John R. Edwards (U. S. Air Force, Air Force Space Command, 483 N. Aviation Blvd., El Segundo, CA 90245-2808, USA, John.Edwards @LOSANGELES.AF.MIL), Adel A. Hashad (U. S. Air Force, Air Force Space Command, 483 N. Aviation Blvd., El Segundo, CA 90245-2808, USA, Adel.Hashad@LOSANGELES.AF.MIL)

This paper, review and status in nature, consists of three parts: (1) The salient nature and results of the published papers from an ocean sonic boom (OSB) project will be reviewed including theoretical and experimental studies of the wavy surface effect on the underwater acoustics, a threedimensional theory of underwater acoustics and the underwater acoustics induced by a sonic boom traveling at hyper-velocity speeds. (2) New unpublished results will be reported including studies of sonic boom underwater overpressures affected by ocean finite depth and ocean stratification (variable sound speed). These studies are possible due to the derivation of a semi-similar transformation for the underwater acoustics governing equations and the application of high performance computers. (3) The preliminary results of the work-in-progress will also be discussed including threedimensional extensions of former professor H. K. Cheng's two-dimensional wavy surface theory and laboratory verification.

\section{$5: 40$}

5pUWf4. Influence of a resonance changer on the sound radiation of a submarine. Sascha Merz (University of New South Wales, School of Mechanical and Manufacturing Engineering, 2052 Kensington, Australia, sascha.merz@gmx.net), Roger Kinns (University of New South Wales, School of Mechanical and Manufacturing Engineering, 2052 Kensington, Australia, roger.kinns@aol.com), Nicole J. Kessissoglou (University of New South Wales, 2052 Sydney, Australia,n.kessissoglou@unsw.edu.au)

In order to reduce the excitation of the submarine hull through the shaft, a vibration attenuation system, called a resonance changer, can be implemented in the propeller/propulsion system. The effectiveness of such a system in reducing the low frequency sound radiation characteristics of a submarine is investigated. Only sound radiation due to fluctuating propeller forces, which are generated by the operation of the propeller in a nonuniform wake, is considered. These fluctuating forces are transmitted to the submarine hull through the fluid, as well as through the propeller shaft. Both 
types of excitation cause hull vibration and sound radiation. The accordion modes of the pressure hull, are particularly efficient sound radiators. Parameters for the resonance changer system have been optimised previously by considering only excitation of the hull through the shaft. It is shown that the effectiveness of the resonance changer at different frequencies is modified significantly in a typical full-scale implementation, due to the sound radiation from the propeller. The effect on performance is increased further when the vibration of the propeller itself is taken into account. Therefore overall optimisation of any resonance changer system requires a comprehensive model. Some of the principal effects are explored in this paper.

\section{6:00}

5pUWf5. The research on measuring the coefficient of sound absorption in turbid seawater. Qi Li (Harbin Engineering University, College of Underwater Acoustic Engineering, 150001 Harbin, China, chizhafengyun1979@126.com), Yongwei Liu (Harbin Engineering
University, College of Underwater Acoustic Engineering, 150001 Harbin, China, chizhafengyun1979@126.com)

When naval mine-hunting sonars and side-scan surveying sonars operating at $20 \sim 60 \mathrm{kHz}$ are working in shallow coastal waters, the viscothermal absorption of sound by suspended mud in the water may greatly decrease their detection properties. This kind of water is also characterized as turbid seawater. However, the research on measuring the coefficient of sound absorption in turbid seawater has little been done. The main difference between turbid seawater and clear seawater is that there are mud particles suspending in turbid seawater. It is also the main reason why sound absorption in turbid seawater is greater than that in clear seawater. In the paper, the coefficient of sound absorption at $2060 \mathrm{kHz}$ in turbid seawater has been measured by the reverberation technique. Results demonstrate that if the concentration of the mud is bellow $0.11 \mathrm{~kg} / \mathrm{m}^{3}$, the mud in turbid seawater doesn't cause additional absorption. When the concentration of the mud is between 0.14 and $0.49 \mathrm{~kg} / \mathrm{m}^{3}$, the coefficient of sound absorption in turbid seawater is as twice at least as that in clear seawater. 


\title{
AUTHOR INDEX
}

\author{
to papers presented at
}

\section{Acoustics'08 Paris}

Aaltonen, Juha-S318

Aaronson, Neil L.-S9

Aassif, Elhoucien-S204, S877

Abawi, Ahmad-S498,

Cochair Session

$3 \mathrm{pUWc}(\mathrm{S} 498)$

Abboud, Toufic-S758

Abd El-Aal, Nadia-S722

Abd-Elbasseer, Mohammed-S895

Abdel-Rahman, Ehab-S575

Abe, Koki-S25

Abel, Grant-S393

Abel, Jonathan S.-S637

Abel, Jonathan-S688

Abel, Rebekah A.-S105

Aben, Paul-S576

Abom, Mats-S484

Abou Leyla, Najib-S730

Abraham, Douglas-S220, S375

Abraham, Odile-S405, S877

Abrahams, I David-S624

Abramova, Anna V.-S733

Abramovich, Andrew A.-S725

Abruzzo, Todd A.-S664

Abry, Christian-S110, S352

Absil, Frans-S655

Acher, Fabien-S901

Achunche, Iansteel-S160

Adachi, Seiji-S49, S763, Cochair

Session 3pMUb (S479),

Cochair Session

4pMUb (S691), Cochair

Session 4pMUd (S694)

Adam, Jean-Luc-S282

Adam, Olivier-S806

Adams, Mags-S426

Adank, Patti-S105, S612, Cochair

Session 1pSCc (S102)

Adelman-Larsen, Niels W.-S122

Adelstein, Bernard D.-S205

Adibi, Ali-S71

Aeberli, Sébastien-S288, S850

Aebi, Laurent-S317,

S582

Aeron, Suchin-S405

Afifi, Hisham-S722

Afsar, Mohammed-S53

Agarwal, Anurag-S53

Agarwala, Vinod-S428

Ageron, Thierry-S708

Aglyamov, Salavat R.-S826

Agos-Esparza, A.-S694

Aguilar Soto, Natacha-S239

Aguilera De Maya,

Juan Luís-S557

Ahmad, Nasir-S971

Ahmadi, Mehdi-S264, S348

Ahn, Bong Young-S728

Ahnert, Wolfgang-S179, S645, S968

Ahonen, Antti-S365, S774

Ahrens, Christian-S51
Ahrens, Jens-S548, S962

Aikawa, Kiyoaki-S594

Aikawa, Yasuhiro-S916

Ainslie, Michael A.-S19, S117, S118, S378, Cochair Session 1pUW (S116)

Airenti, Antonino-S691

Airoldi, Luca-S605

Airre, Pekka-S171, S284

Aissa, Jean-Claude-S266

Akagi, Masato-S44, S212, S355

Akahane-Yamada, Reiko-S598

Akal, Tuncay-S38, S677, Cochair Session 4pEAb (S676)

Akay, Adnan-S90

Akeroyd, Michael A.-S201, Cochair Session 2aPPc (S200)

Akgün, Sevgi-S629

Akhmedzhanov, Farkhad-S178, S727

Akhoun, Idrick-S895

Akimov, Andrey-S580

Akinaga, Seiko-S692

Akiyama, Iwaki-S815

Akiyama, Masahiko-S726

Akjouj, Abdellatif-S873

Aksoy, Serkan-S629

Al-Wahsh, Hosni-S873

Alabardos, Zsuzsanna-S331

Alain, Claude-S83

Alarcão, Diogo-S295

Alasia, Franco-S796

Alayrac, Marion-S843

Alba Castro, José Luis-S855

Alba Fernandez, Jesús-S150, S206, S265, S443,

S646, S647, S648

Albelda, José-S566

Alberts, Wiebe-S284

Alberts, William C.-S76

Albu, Felix-S906

Aldridge, David F.-S287

Aleshin, Vladislav-S67, S472

Alexander, Elizabeth M.-S598

Alexander, Jessica E.-S110, S919

Alexander, Nathan-S55

Alexis, Alain-S627

Alfinito, Luca-S423

Ali, Dave-S192

Alippi, Adriano-S252, S866

Aliverti, Andrea-S273

Alizad, Azra-S820

Allard, Jean François-S66, S67

Allard, Richard-S654

Allen, John S.-S143

Allen, Jont B.-S965, Cochair Session $5 \mathrm{pPPa}(\mathrm{S} 963)$

Allen, Jont-Cochair Session 5aPPf (S895)

Allen, Michael-S134
Allen, Robert-S652, S653

Allman-Ward, Mark-S277

Alm, Magnus-S364

Almeida, André-S829

Almuhanna, Khalid-S662

Alonso, Josep Maria-S651

Aloshyna-Lesuffleur, Marie-S70

Altenmüller, Eckart-S154

Altinsoy, Ercan-S195

Altreuther, Beate-S423

Alujevic, Neven-S902

Alur, Ramachandra-S65

Alvarenga, Andre V.-S253, S258

Alwan, Abeer-S359, S492

Amari, Maël-S699

Amate, Maud-S788

Amedin, Celse Kafui-S454

Amick, Hal-S702

Amindavar, Hamidreza-S497

Amino, Kanae-S360

Amir, Noam-S48, S151, Cochair

Session 1pMUa (S46)

Cochair Session

2pMUc (S272)

Amiri-Simkooei, Alireza-S381, S660

Amiri, Hadi-S497

Ammi, Azzdine Y.-S664

Amundin, Mats-S240

Ananthakrishnan, V.-S927

Andersen,Bent-S421, S719

Andersen, Lars Nonboe-S468

Anderson, Brian E.-S216, S373, S863

Anderson, Christian C.-S544

Anderson, Jason-S160

Anderson, Shaun D.-S977

Andersson, Patrik B.-S607

Ando, Akio-S685

Andoga, Rudolf-S750

Andre, Michel-S651, S811,

Cochair Session

2pABa (S236), Cochair

Session $2 \mathrm{pABd}$

(S242), Cochair Session

3aABa (S392),

Cochair Session $3 \mathrm{aABb}$

(S394), Cochair

Session 4pABc (S651),

Cochair Session

4pABd (S652), Cochair

Session $5 \mathrm{aABa}$

(S803), Cochair Session $5 \mathrm{aABb}(\mathrm{S} 808)$

Andrea, Jonathan-S778

Andreotti, Bruno-S306

Andrew, Marilee-S28, S255

Andrew, Rex K.-S945

Andrews, Jennifer-S892

Andrews, Mark-S135, S813, S972

Andreyev, Sergey V.-S176, S307

Andrieu, Stephane-S319
Andringa, Tjeerd C.-S333, S354, S363, S445, S754

Andro, Bertrand-S566

Andruski, Jean E.-S918

Anemüller, Jörn-S363

Anfosso-Ledee, Fabienne-S421,

S900, Cochair Session

3aNSd (S420), Cochair Session

4pNSp (S718)

Angelov, Mitko-S309

Angster, Judit-S49, S50, Cochair Session 1pMUb (S49),

Cochair Session 2pMUb (S271)

Angus, Jamie A.-S124, Cochair Session 1pSPa (S112)

Anisimov, Nikolay V.-S357

Anosov, Andrej-S258

Anthoine, Jerome-S571, S572

Anthony, David K.-S350

Antier, Karl-S858, S953

Antonaci, Paola-S732

Antoni, Jerome-S419

Antonopoulou, Dimitra-S630

Antonov, Sergey-S175

Antunes, José-S15

Aoki, Yohko-S604, S902

Aoki, Yoshie-S750

Aoubiza, Boujemaa-S312

Aouzale, Noureddine-S487

Apfel, Marina-S9

Apoux, Frederic-S899

Appeldoorn, Richard-S130

Appell, Jens E.-S338

Arai, Takayuki-S360, S361, S443, S750, S916

Aramaki, Mitsuko-S548, S757

Arana, Miguel-S534

Aranchuk, Vyacheslav-S75

Aranda De Toro,

Miguel Angel-S559, S887

Arato-Borsi, Eva-S331

Araujo, Veronica-S355

Araya, Takeshi-S779

Araz, Muhamet-S409

Arbey, Olivia-S679

Arcas, Kevin-S554, S697

Archer, Nathan-S167

Ardid, Miguel-S937

Ardila, Mauricio-S941

Ardoint, Marine-S743

Arenas Bermudez, Jorge P.-S206

Arenius, Edward-S944

Aretz, Marc-S123, S940

Arghir, Mariana-S61, S289

Arias, Antón-S239

Arifianto, Dhany-S890

Arimoto, Yoshiko-S364

Aristegui, Christophe-S875, S877, Cochair Session 3aPAf

(S441), Cochair Session 4pPAf (S731)

Ariza, Javier-S829 
Armstrong, Douglas L.-S539

Arnbjörnsson, Einar-S104

Arnold, Francisco J.-S673

Arnold, Klaus-S734

Aronov, Boris-S38, S673

Arora, Manish-S29, S77, S255, S592

Arras, Christine-S842

Arruda, José Roberto-S675, S685

Arslan, Ergin-S56

Arsuaga, Juan Luis-S638

Artana, Guillermo-S609

Arthur, David M.-S435

Artom, Fabrizio-S295

Arumi, Pau-S969

Arunajatesan, Srinivasan-S711

Arvaniti, Amalia-S459

Arvanitis, Costas-S29

Asami, Toshihiko-S754

Asch, Mark-S542

Asdrubali, Francesco-S69, S797

Ashmead, Daniel-S489

Ashokkumar, Muthupandian-S78, S590, S592

Ashraf, Faheem-S494

Askenfelt, Anders-S155, S230, S688

Aspuru Soloaga, Itziar-S63,

Cochair Session

1pNSb (S57), Cochair Session 2pNSh (S289)

Assaad, Jamal-S730, S878

Assaf, Samir-S761

Asselineau, Marc-S709, Cochair Session 4pNSb (S700), Cochair Session 4pNSg (S709), Cochair Session 5aNSb (S844)

Assenmacher, Ingo-S968

Assink, Jelle-S871

Astley, R Jeremy-S160

Astolfi, Arianna-S8, S647, S949

Astolfi, Jacques Andre-S454

Aston, Kelly A.-S943

Asutay, Erkin-S753

Atalla, Noureddine-S92, S452, S454, S904, Cochair Session 3aSAb (S452), Cochair Session 4aSAa (S602), Cochair Session 4pSAc (S760)

Atchley, Anthony A.-S276

Atkins, Joshua-S13, S40

Atkins, Philip-S468, S925, S981

Attaway, Michael-S270

Attenborough, Keith-S75, S181, S953, Cochair Session 1pPAa (S65), Cochair Session 1pPAc (S74), Cochair Session 2aPAa (S172), Cochair Session 2pPAf (S314), Cochair Session 2pPAh (S320), Cochair Session 5aNSh (S854), Cochair Session 5pNSb (S952)

Außerlechner, Hubert-S50

Au, Dax-S56

Au, Whitlow W.-S239, S375, S806

Aubergé, Véronique-S918

Aubry, Alexandre-S33, S218, S373, S665
Aubry, Jean-François-S27, S462, S545, S672

Aucejo, Mathieu-S342

Audet, Jean-S454

Audibert, Nicolas-S918

Audoin, Bertrand-S189, S584, S738, S869

Auerbach, Markus-S423

Augereau, Franck P.-S960

Augustin, Jean-Marie-S660

Augusztinovicz, Fülöp-S202, S292, S345, S649, S803, S905

Aujard, Christine-S715, S842

Aumond,Pierre-S269

Aurégan, Yves-S160, S162

Aura, Karine-S446

Avanzini, Federico-S210

Averiyanov, Mikhail V.-S280

Averkiou, Michalakis A.-S142

Avis, Mark R.-S14, S68, S791

Avital, Eldad-S281

Ayama, Miyoshi-S749

Aydin, Asuman-S637

Ayers, James-S216

Aynaou, Hassan-S72

Ayrault, Christophe-S69, S185

Azarpeyvand, Mahdi-S157, S907

Azevedo, Carolina M.-S258

Baars, Robert-S204

Babilotte, Philippe-S186, S737

Babisch, Wolfgang-S480

Bach-Andersen, Martin-S419

Bach, Jörg-Hendrik-S363

Bach, Jason-S257, S820

Bachand, Corey-S38

Baciu, Monica-S352

Bacon, Sid P.-S86

Badachi, Yasmina-S144

Badeau, Roland-S833, S835, S836

Bader, Rolf-S479, S697, S836

Badiey, Mohsen-S467, S619, S934, S946

Badin, Pierre-S762

Badma, Badmaev-S875

Badran, Fouad-S542

Bae, Minseok-S439

Baer, Ralph N.-S377

Baer, Thomas-S897

Baggeroer, Arthur B.-S21, S366, S470

Bahl, Rajendar-S806

Bahoura, Mohammed-S804, S811

Bahoussa, Abdelaziz-S843

Bahr, Alexander-S393

Baik, Kyungmin-S976, S977

Bailey, Michael R.-S29, S35, S79, S253, S399, Cochair Session 5pBB (S946)

Bailey, Michael-Cochair Session $5 \mathrm{aBBd}$ (S819)

Baillet, Helene-S577, S740

Bailliet, Helène-S162, S765, S772

Baillot, Françoise-S487

Bailly, Christophe-S54, S157, S159, S161, Cochair Session 1pNSa (S52), Cochair
Session 2aNSa (S157),

Cochair Session

2pNSb (S280), Cochair

Session 3aNSa (S413)

Bailly, Gerard-S970, Cochair

Session 5pSC (S970)

Bailly, Lucie-S274, S609

Baird, Terry-S277

Bakarezos, Makis-S187, S583

Baker, Dave-S794

Bakhoday Paskyabi,

Mostafa-S629

Bakir, Farid-S413, S569, S720

Bakker, Roel-S568, S714

Baklanov, Mikhail-S586

Bal Kocyigit, Filiz-S551, S644, S801

Balakshy, Vladimir I.-S175, S177

Balas, Anna-S921

Balasuriya, Arjuna-S39, S937

Balazs, Peter-S595

Baldassarri, Andrea-S301

Baldewsing, Radj-S30

Ball, Keenan-S925

Ballance, Connor-S691

Ballandras, Sylvain-S308, S324, S549, S678, S679

Ballard, John-S814

Ballard, Megan S.-S138, S139

Ballas, James-S377, S447

Ballato, Arthur-S408

Ballester, Javier-S437

Ballesteros, Jose A.-S170, S710, S799, S829

Ballesteros, Maria Jesus-S799

Ballinger, Richard M.-S7

Balogh, Dr., Géza-S645

Balogh, Jr., Géza-S645

Baltean Carlès, Diana-S577

Baltean-Carlès, Diana-S739, S740

Bamber, Jeff-S258, S816, S 825

Bamberger, Andreas-S271

Bamnios, George-S852

Bandi, Mahesh-S301

Banet, Philippe-S868

Banfi, Francesco-S587

Bank, Balázs-S553

Bannasch, Rudolf-S935

Banno, Hideki-S106

Bansal, Mahesh-S179

Bao, Xueshan-S906

Barakat, Sanaa-S895

Baranyi, Péter-S803

Baratier, Julien-S92, S564

Barba Sevillano, Arturo-S228, S230, S231, S636, S706

Barbaresi, Luca-S648, S795

Barbato, Giulio-S8

Barber, Christopher-S205

Barbieri, Marco-S294

Barbone, Paul E.-S258, S628

Barbour, Kyle-S731

Barclay, David R.-S396, S712

Bard, Delphine-S104, S388

Bardeli, Rolf-S131

Bardovi-Harlig, Kathleen-S766

Barenholz, Yechezkel-S821
Barhen, Jacob-S366, S777, S779, S982

Baring, João G.-S58

Barkas, Nicolaos-S386

Barker, Jon-S326

Barkmann, Reinhard-S664

Barney, Anna-S608, S610

Barnhill, Colin-S375

Baron, Cécile-S546, S671

Baron, Daniel-S316

Baronian, Vahan-S440

Barra, Felipe-S440

Barras, Claude-S108, S109

Barrera-Figueroa, Salvador-S261, $\mathrm{S} 453$

Barrera, Dario-S106

Barrio, Raul-S857

Barron, Charlie-S654

Barthel, Etienne-S817

Barthet, Mathieu-S695

Barti, Robert-S852

Bartolini, Paolo-S174, S187, S313

Bartolomaeus, Wolfram-S423

Barton, Robert-S471

Bartylla, David-S174

Barzelay, Oded-S48

Basbøll, Hans-S214

Basirat, Anahita-S85

Baskent, Deniz-S334

Bass, Henry E.-S578, S858, S952, Cochair Session 5aPAa (S858)

Bass, Henry-Cochair Session 5aPAf (S870)

Bassanino, Maurizio-S535

Basset,Olivier-S727

Bassi, Jason-S435

Bassuet, Alban A.-S120, S224, S940

Bastard, Cécile-S32

Basten, Tom-S471

Batchelor, Heidi-S135

Batel, Mehdi-S900

Batifol, Cedric-S69, S905

Batko, Wojciech-S294

Battaner-Moro, Juan-S157

Battaner, Elena-S909

Bauer-Diefenbach, Ralph-S968

Bauer, Adam Q.-S544

Bauer, Juergen-S839

Bauerfeld, Frank-S816

Baugh, Eric-S192

Baulac, Marine-S60, S955

Bauman, Jessica-S110, S915

Baumann, Ingo-S193

Baumann, Oliver N.-S605

Baumann, Simone-S131

Baumann, Uwe-S199

Baumgart, Johannes-S48

Bauwens, Luc-S740

Bavencoffe, Maxime-S70

Bavli, Danny-S821

Bavu, Eric-S218

Bay, Mert-S837

Bayazit Tamer, Nurgun-S385, S530

Bazúa Durán, Carmen-S16, S395

Bazan, Ivonne-S256

Beakes, William E.-S848

Beamsley, Brett-S244

Beard, Paul C.-S401, S403 
Beardsley, Ryan-S580

Beauchamp, James W.-S837, Cochair Session 5aMUe (S834)

Beauchamp, James-Cochair Session 5aMUd (S832)

Beaujean, Pierre-Philippe J.-S975

Beaumont, Jacuqes-S842

Bech, Søren-S11, S12

Bechwati, Fouad-S14, S68

Beckenbauer, Thomas-S420, S421

Becker, Franz Josef-S880

Becker, Jens-S758

Becker, K.m.-S138, S139

Becker, Stefan-S570, S602, S769

Bécot, FrançoisXavier-S453

Bédard, Catherine-S803

Bednar, Thomas-S387, S798

Beedholm, Kristian-S395, S477

Beentjes, Wilhelmus-S223

Begault, Durand R.-S205, Cochair Session 5pPPb (S967)

Begault, Durand-Cochair Session $5 \mathrm{aPPe}$ (S893)

Beghin, Tom-S11

Behler, Gottfried K.-S646

Behne, Dawn-S364,

S768, Cochair Session 5aSCb (S911)

Behrens, Thomas-S201

Behringer, Robert-S302

Beil, Florian W.-S312

Bejcek, Ludvik-S342

Bel'Ko, Viktor-S319

Belanov, Alexander-S581

Belfor, Galaz-S252

Belfroid, Stefan-S282

Belhocine, Ferroudja-S173

Belhoussine Drissi, Taoufiq-S865

Belkacem, Afif-S931

Belkebir, Kamal-S494

Bell-Berti, Fredericka-S360

Bell, Judith-S618, S926

Bell, Steven L.-S887

Bellemare, Madeleine-S689

Bellet, Romain-S727

Belliard, Laurent-S318, S583, S584, S959

Bellmann, Michael-S699

Belsak, Ales-S368

Beltman, Willem M.-S192, S452

Belyaev, Andrey V.-S176, S307

Belyaev, Ivan-S282, S877

Belyaev, Sergei P.-S725

Ben Amor, Morched-S872

Ben Tahar, Mabrouk-S451, S603

Ben-Dov, Yuval-S845

Benammar, Abdelsaalam-S114, S115

Benammar, Abdessalem-S368, S776

Benchabane, Sarah-S179, S583

Bendtsen, Hans-S421

Benech, Nicolas-S370, S462

Benedetto, Giuliana-S676

Benes, Petr-S349

Benfield, Mark-S26

Benilov, Alexander-S783

Benjamin, Michael B.-S39, S937
Benjamin, Tucker V.-S765

Benkreira, Hadj-S66

Benmeddour, Farouk-S878

Bennett, John C.-S284

Benoit-Bird, Kelly-S24

Bensing, Silas-S851

Bensoam, Joël-S555

Benson, David J.-S303

Bentahar, Mourad-S257, S430

Bentahar, Mouras-S733, S869

Benthien, George W.-S146

Bentler, Ruth-S44

Bento Coelho, J. Luis-S295, S649, S712, S718

Benus, Stefan-S358

Béra, Jean-Christophe-S333

Beranek, Leo L.-S5

Berasategi, Joanes-S708

Berchok, Catherine-S135

Bercoff, Jeremy-S823

Berdahl, Edgar-S554, S698, S905

Berengier, Michel C.-S423, Cochair Session 2aPAc (S180)

Berengier, Michel-Cochair Session 2pPAd (S310)

Berer, Thomas-S188

Bergbom, Ingegerd-S225

Berge, Truls-S703

Bergen, Bart-S789

Berger-Vachon, Christian-S337, S895

Berger, Elliott H.-S560

Berger, Geneviève-Cochair Session $1 \mathrm{pBBb}(\mathrm{S} 30)$,

Cochair Session 2pBBf (S256)

Berger, Michael A.-S764

Bergman, Penny-S277, S599

Bergman, Rudolf-S735

Bergmark, Katrin-S126

Bériot, Hadrien-S451

Beristain, Sergio-S125, S845

Berker, Ali-S70

Berkowitz, Shari-S771

Bernal, Miguel-S821

Bernard, Julien-S148, S268

Bernassau, Anne L.-S816

Bernays, Michel-S689

Berndt, Mihály-S169

Bernhard,Robert-S422

Berni, Alessandro-S936

Bernstein, Joshua G.-S743

Bernstein, Leslie R.-S448

Berrada, Mohamed-S542

Berriet, Rémi-S29

Berry, Alain-S218, S452, S571, S605, S904, Cochair Session 5aSAb (S902), Cochair Session 5aSAc (S906)

Berry, David A.-S770, Cochair Session 4aSCa (S608)

Berry, Gearoid-S258, S825

Berryman, Jim G.-S473

Berson, Arganthaël-S575

Berta, Annalisa-S540

Bertellino, Franco-S295

Bertet, Stephanie-S968

Bertetti, Carlo Alessandro-S842

Berthelsen, Björn-S3, S227
Berthillier, Marc-S679, S777

Berthommier, Frédéric-S334

Berthou, Celine-S708

Bertin, Nancy-S833

Bertolotto, Giacomo-S37

Bertone, Armando-S596

Bertsatos, Ioannis-S135, S972

Beryoza, Sergey-S176

Besainou, Charles-Cochair Session 5aMUc (S831)

Besbes, Kamel-S931

Beskow, Jonas-S971

Besnainou, Charles-S218, S688, S838, Cochair Session 5aMUf (S838)

Besnard, Francis-S182

Bessac, François-S91, S196

Bessonova, Olga V.-S35

Best, Angus I.-S379, S382, S474, S626

Best, Jim L.-S381

Best, Virginia-S897

Bestel, Julie-S895

Bétrancourt, Adrien-S739

Bettarello, Federica-S644, S798

Bettayeb, Fairouz-S369

Betti, Rinaldo-S386

Bettucci, Andrea-S252, S866

Beutelmann, Rainer-S198

Beyret, Matthieu-S955

Bezat, Marie Céline-S279, S285

Bi, Wenping-S160

Biagini, Stefano-S37

Biagioni, Angelo-S252

Bibikov, Nikolay G.-S808

Bicen, Baris-S260, S261, S262

Bichler, Max-S312

Bickel, Peter-S288

Bideau, Nicolas-S346

Bienvenu, Georges-Cochair Session 5pUWe (S983)

Biermann, Jan-S705

Bigot, Alexis-S170, S567

Bikonis, Krzysztof-S780

Bilbao, Stefan-S203, S270, S553, S554, S697

Bilka, Michael-S572

Billon, Alexis-S942, S956,

Cochair Session

5aAAd (S800), Cochair Session 5pAAb (S942)

Billson, Duncan R.-S406

Bin Ali, Tareq-S547

Bingham, Brian-S677

Biot, Marco-S205

Birdsall, Theodore G.-S945

Birer, Alain-S28, S29, S814

Birkett, Stephen-S412, S692

Biron, Daniel-S342

Bisceglie, Alessandro-S295

Bischel, Marsha S.-S221, S848

Bischof, John-S32

Bissinger, Brett E.-S615, S775

Biwa, Tetsushi-S577

Bjørnø, Leif-S220

Blaauw, Merlijn-S697

Black, Matthew-S359

Blaga, Florin-S61

Blair, Ballard-S924

Blairon, Nicolas-S565
Blaisot, Jean Bernard-S293

Blaisot, Jean-Bernard-S487

Blake, William-S159, S160, S165

Blanc-Benon, Philippe-S183,

S280, S575, S576,

S861, Cochair Session 4aPAa

(S573), Cochair Session 4pPAk (S739)

Blanc, Elisabeth-S859, S861

Blanc, François-S49

Blanc, Samuel-S704

Blanchard, Lola-S44

Blanchet, Marie-Anne-S393, S812

Blanchi, Julie-S665

Blandea, Vincent-S903

Blasco, Hugues-S441

Blau, Matthias-S43

Bleeck, Stefan-S376

Bleses, Dorthe-S214

Bleszynski, Elizabeth-S597

Bleszynski, Marek-S597

Bleuzen, Aurore-S143

Blick, Robert H.-S312

Bliss, Donald B.-S95, S793,

Cochair Session $5 \mathrm{aSAa}$ (S899)

Bliss, Donald-Cochair Session 5aSAd (S908)

Blodgett, Allison-S110, S915

Blomkvist, Vanja-S126

Blomme, Erik-S430, S441

Blondeau, James-S740

Blondel, Philippe-S239, S245,

S392, S472, S980,

Cochair Session 5aUWc

(S927), Cochair

Session 5aUWg (S935)

Bloomfield, Alan-S843

Bloquet, Stéphane-S842

Blottman, John B.-S45, Chair Session 1pEAd (S45), Cochair Session 2aEAa (S146)

Blottman, John-Cochair Session 2pEAg (S268)

Blumstein, Daniel T.-S134

Boë, Louis-Jean-Cochair Session $5 \mathrm{aSCa}$ (S909)

Bobaru, Florin-S320

Bobrovnitskii, Yuri-S208, S341

Bocca, Pietro-S732

Boccara, Albert-Claude-S403, S462, S545, S672

Boccardi, Emiliano-S783

Bockenhauer, Samuel D.-S809

Bockstael, Annelies-S337

Boczar, Tomasz-S286, S348

Bodard, Guillaume-S157

Bodin Danielsson, Christina-S2

Boeck, Michelle-S283

Boeckx, Laurens-S66, S67, S669

Boegli, Hans-S851, S852

Boersma, Paul-S918

Bogey, Christophe-S52, S54

Bogut, Marijan-S291

Bőhm, Tamás-S918

Bohn, Ocke-Schwen-S911

Boileau, Jean-Pierre-S272

Boirlaud, Matthieu-S792

Boivin, Georges-S817

Bolükbas,Deniz-S629

Boldt, Jesper B.-S98 
Bolduc, Maxime-S92

Bolin, Karl-S567

Bolkovac, Ivan-S314

Bollade, Laurent-S418

Bolton, J. Stuart-S96, S704 Cochair Session 3aNSc (S417), Cochair Session 4pNSc (S703), Cochair Session 4pNSk (S713),

Cochair Session 4pNSn (S716)

Bombien, Lasse-S363, S772

Bonada, Jordi-S697

Bonamy, Cyrille-S54

Bonaventura, Patrizia-S112

Bond, Zinny-S765

Bondy, Jeff-S329

Bonello, Bernard-S70, S312

Bonfiglio, Paolo-S69, S316

Bongini, Estelle-S900

Bongiovanni, Kevin-S861

Bonhoff, Hannes A.-S343

Bonnasse-Gahot, Laurent-S214

Bonneau, Anne-S458

Bonneau, Lénaïc-S306

Bonneel, Nicolas-S600

Bonnel, Anna C.-S596

Bonnel, Anne-Marie-S890

Bonnet- Ben Dhia, Anne-Sophie-S162, S440

Bonnet-Ben Dhia, Anne-Sophie-S414

Bonsi, Davide-S385

Boominathan, Prakash-S411

Boone, Marinus M.-S754

Boonen, Steven-S669

Boots, Henk-S408

Borée, Jacques-S720

Bor, Stephanie-S896

Borchert, Elizabeth-S898

Borden, Mark-S248

Borejko, Piotr-S972

Borello, Alex-S707

Borello, Gérard-S92, S346, S707

Borgeaud, David M.-S386

Borkent, Bram-S77

Boroditsky, Leonid-S206

Borst, Jeroen-S57, S62

Borucki, Sebastian-S286, S348

Borza, Dan-S61, S204, S289, S900

Bös, Joachim-S905

Bose, Nirmal K.-S615, S775

Bose, Sandip-S405

Bosseler, Alexis N.-S365, S774

Bossis, Dorothée-S819

Bossy, Emmanuel-S403, S462, S545, S672, S962,

Cochair Session $3 \mathrm{aBBb}$

(S401), Cochair

Session 4pBBd (S672),

Cochair Session 5aBBb (S817)

Bossy, Laure-S962

Bost, Wolfgang-S402

Bot, Patrick-S454

Botros, Andrew-S694

Botteldooren, Dick-S168, S337, S426, S563

Bou Matar, Olivier-S70, S430, S441, S862, S864

Bou-Cabo, Manuel-S937
Boué, Christine-S545

Bouakaz, Ayache-S142, S143,

S144, S250, Cochair

Session 2aBB (S141), Cochair

Session 2pBBc (S251)

Boubenider, Fouad-S97

Boubezari, Mohammed-S712

Bouchoux, Guillaume-S28, S29

Boudjema, Bouzid-S256

Boudraa, Bachir-S356

Boudraa, Malika-S114, S356

Boufermel, Abdennour-S739

Bouferroum, O.-S776

Bouferroum, Othmane-S914

Bougrab, Hakim-S958

Boulandet, Romain-S443

Bouma, Jelte-S568, S714

Bour, Nathalie-S355

Bourgeois, Ludivine-S959

Boussard, Patrick-S707

Boutillon, Xavier-S94, S157,

S692, Cochair Session

2aMUb (S154),

Cochair Session 2pMUd (S273)

Boutin, Céline-S170

Boutin, Claude-Cochair Session 5aPAe (S868), Cochair Session 5aPAl (S879)

Boutin, Henri-S688

Boutin, Jérôme-S597

Boutkedjirt, Tarek-S875

Bouvet, Pascal-S901

Bove, Torsten-S814

Bowers, David-S860

Bowles, Anita-S110, S915

Bowles, Ann E.-S20, S541

Boyce, Suzanne-S357,

S919, Cochair Session 5aSCc (S913)

Boyd, Ian-S16, S806, Cochair Session 4aABb (S539)

Boyd, Michael L.-S945

Boyle, Patrick-S895

Bozic, Martina-S314

Brännström, Jonas-S490

Braasch, Jonas-S51, S229, S448 S636, S751, S802, S941,

Cochair Session 1pMUc (S50)

Brachet, Nicolas-S859

Bradley, David L.-Cochair Session 3pUWb (S496), Cochair

Session $4 \mathrm{aUWd}$

(S627), Cochair Session 4pUWe (S784)

Bradley, David T.-Cochair Session 3pAA (S474),

Cochair Session 4aAAa (S529)

Bradley, David-S220

Bradley, John S.-S9, S226

Bradley, Robert J.-S406

Bradlow, Ann-S911, S915, S919

Bradway, David-S824

Brambilla, Giovanni-S386, S427, Cochair Session 3aNSf

(S426), Cochair Session 4pNSj (S712)

Bramhavar, Suraj-S402

Brammer, Anthony J.-S163

Brancazio, Lawrence-S921

Brand, Sebastian-S113
Brandt, Joerg-S812

Branstetter, Brian K.-S17, S538

Braselton, James P.-S344, S552

Brasseur, Emmanuel-S154

Bravo, Teresa-S344, S716

Bray, Victor-S336

Bray, Wade R.-S717, Cochair Session 2pNSa (S276)

Bray, Wade-Cochair Session 2pNSd (S285)

Brayman, Andrew-S28, S255

Brazhkin, Yurii A.-S304

Brazier-Smith, Peter R.-S624

Breault, Marie-Hélène-S156

Brecht, Dieter-S981

Breebaart, Jeroen-S488

Breese, Marlee-S392

Brehme, Kay-S812

Bremer, Alexander-S664

Bremond, Nicolas-S77

Brenguier, Florent-S301

Brentner, Kenneth S.-S158, S426

Bressler, Scott-S10

Bretagne, Alice-S72, S312

Bretaudeau, Francois-S405

Breteau,Jean-Marc-S186, S584, S586, S737

Breton, Gaspard-S970

Breton, Jean-Louis-S579

Bretthorst, G Larry-S544

Brick, Haike-S450,

Cochair Session 4pSAa (S758)

Bridal, Lori-S144, S250

Bridges, David H.-S39

Bridgewater, Ben-S941

Briggs, Kevin-S625

Briley, Paul M.-S749

Brill, Oliver J.-S884

Brink, Mark-S851, S852

Brinkmeier, Maik-S705

Brittain, Frank-S296

Brittan-Powell, Elizabeth-S476,

Cochair Session $3 \mathrm{pAB}$

(S476), Cochair

Session 4aABa (S537),

Cochair Session 4pABb (S650)

Brixen, Kim-S664

Brock-Nannestad, George-S361, S834

Brock, Derek-S377

Brodsky, Peter-S39

Broersma, Mirjam-S215, Chair Session 2aSCd (S214)

Broner, Norm-S276

Bronkhorst, Adelbert W.-S195, S326

Bronuzzi, Fabrizio-S647

Brooks, Bennett-Cochair Session 3aNSf (S426), Cochair Session 4pNSj (S712), Cochair Session 5aNSa (S839), Cochair Session 5aNSf (S851)

Brooks, Laura-S246, S656

Brooks, Thomas F.-S425

Brooks, Todd L.-S943

Brossier, Paul-S832

Brouard, Bruno-S316

Brown, Christopher A.-S86, S326
Brown, David-S38, Chair Session $2 \mathrm{aEAb}(\mathrm{S} 149)$

Brown, Guy J.-S10, S83, S376, S747, S893

Brown, Judith C.-S377

Brown, Keith E.-S39, S498

Browne, Ray-S171

Browne, Sean D.-S221

Bruce, Matthew-S142

Bruce, Neil-S426

Bruce, Robert-Cochair Session 2pNSc (S284)

Brueck, Scott-S711

Bruel, Per V.-S843

Brughera, Andrew-S88, S328

Bruguiere, Philippe-S412

Brum, Javier-S370, S462

Bruneau, Anne-Marie-S676, S879, S961

Bruneau, Michel-S261, S576, S676, S879, S957, S961

Brunel, Vincent-S40

Brunet, Thomas-S302, S305, S312, S487

Brunet, Yvonnick-S605

Brungart, Douglas S.-S10, S329, S892

Brunner, Dominik-S450, S758

Brunner, Jana-S108

Bruno, Caterina-S732

Bruno, Giuseppe-S535

Brunskog, Jonas-S388, S530

Brutel-Vuilmet, Claire-S610

Bryner, Juerg-S317, S582

Bub, Frank-S654

Bublic, Ivan-S63

Bucaro, Joseph A.-S634

Buchaillard, Stéphanie-S353

Buchanan, James L.-S320

Buchholz, Jörg M.-S899, S967

Büchler, Michael-S198

Buchwald, Adam-S359, S360

Buck, John R.-S393

Buck, Karl-S99, S561

Buckingham, Michael-S381, S396, S472

Bue, Julien-S315

Buen, Anders-S7, S230

Bueno, Moises-S314, S718

Bukuli, Norbert-S649

Bull, Jonathan M.-S626

Bulou, Alain-S186, S737

Bulté, Jean-S615

Bunting, Oliver-S113

Buogo, Silvano-S590, S783, S784

Burack, Jake A.-S596

Burch, Jackie-S357

Burdisso, Ricardo A.-S54, S424

Burgess, Marion-S827

Burgess, William C.-S807

Burgett, Richard D.-S75

Burgholzer, Peter-S188, S216

Burgschweiger, Ralf-S789

Bürk, Dietmar-S381

Burkard, Robert-S476

Bürki, Audrey-S921

Burnett, Theresa A.-S104

Burnham, Denis-S911

Burov, Valentin-S662, S790

Burt, Bryan P.-S773

Buscemi, Paul-S112 
Busch-Vishniac, Ilene-S700, S709

Bush, Nigel-S816

Buss, Emily-S744, S882

Buss, Sandra-S193

Butenweg, Christoph-S609

Butler, Alexander L.-S45

Butler, John-S45

Butler, Stephen C.-S46

Button, Tim W.-S816

Buxton, Orfeu-S225

Byrd, Dani-S455

Byun, Sung-Hoon-S934, S935

C, Eswaran-S458

Caballero, Gabriel-S302

Cabassud, Corinne-S441

Cabell, Randolph H.-S607, S902

Cabos, Christian-S450

Cabrera, Densil-S279, S533, S757

Cachard, Christian-S251, S727

Cacheleux, Katia-S631

Cadot, Olivier-S696

Cadoz, Claude-S698

Cadum, Ennio-S480

Caelen-Haumont, Geneviève-S493

Cai, Liang-Wu-S73

Caillat, Marjolaine-S806

Cain, Rebecca-S277, S426

Caiti, Andrea-Cochair Session 1pEAa (S37), Cochair Session 2pEAe (S266)

Calambokidis, John-S131, S395, S807

Calamia, Paul-S180, S235, S636, S791, S802, Cochair Session 2pNSf (S287), Cochair Session 4aNSc (S562)

Calantoni, Joseph-S473

Calbris, Gaetan-S737

Caleap, Mihai-S875, S877

Caliano, Giosue'-S407

Calicchia, Paola-S783, S784

Caliskan, Mehmet-S637

Callé, Samuel-S321, S665, S819, S864

Callens, Dorothée-S678

Callow, Hayden J.-S930,S931

Calmon, Pierre-S442

Calvo, David C.-S634, S979, Cochair Session 5pUWa (S972)

Calvo, David-Cochair Session 4pUWf (S786)

Camacho, Arturo-S913

Camarena, Francisco-S880, S937

Camberlein, Lionel-S576, S740

Cambourakis, George-S124

Camier, Cédric-S203, S697

Campbell, Donald M.-S156, S270, S686

Campbell, Murray-S48

Campbell, Paul A.-S145

Campbell, Richard-S849

Camphuysen, Kees-S651

Campillo, Michel-S96, S301, S874, S963

Campistron, Pierre-S678

Campman, Xander-S874

Campos-Astorkiza, Rebeka-S914
Campos-Pozuelo, Cleofé-S736

Campos, Micaelina-S222

Camussi, Roberto-S160

Can, Arnaud-S60

Candel, Sebastien-S435

Candy, James-Cochair Session 5aUWi (S938)

Canepa, Gaetano-S117, S633, S634, S976, S977

Canetto, Pierre-S560

Caniato, Marco-S644

Cannavo, Justine-S749

Cannelli, Giovanni Bosco-S784

Canney, Michael S.-S29, S35, S79, S253

Canon, Francis-S257

Canseco, Guillermo-S398

Cansi, Yves-S806

Cant, Stewart-S435

Caplain, Emmanuel-S868

Caporali, Sueli-S214

Capus, Chris-S39, S498

Caputi Gennaro, Giovanni-S160

Carballeira, Andrew-S225

Carcaterra, Antonio-S90

Cardillo, Gina C.-S107

Caresta, Mauro-S94

Carey, William M.-S626, S937, S938

Carin, Lawrence-S114

Carles, Pierre-S724

Carletti, Eleonora-S196

Carlier, Julien-S678, S681

Carlin, Michael-S103, S909

Carlson, Mark-S112

Carlyle, Angus-S426

Carlyon, Robert P.-S86, S89, S595, S598, S920

Carman, Richard A.-S702, Cochair Session 2pNSn (S297), Cochair Session 3aNSb (S415)

Carman, Richard-Cochair Session $2 \mathrm{pNSi}(\mathrm{S} 290)$

Carney, Laurel H.-S881

Carniel, Xavier-S418

Carolus, Thomas-S571

Caronna, Gaetano-S367, S777

Caronti, Alessandro-S407

Carota, Francesca-S352

Carpentier, Grégoire-S833

Carpentier, Jean-Baptiste-S487

Carré, René-S356, S492

Carr, Suzanne-S448

Carral, Sandra-S412

Carrière, Olivier-S371, S944

Carsey, Thomas-S244

Carson, Paul L.-S373

Carson, Timothy-S192

Carton, Bruno-S565

Caruelle, Bastien-S56, S850

Caruso, Micheal-S620

Carvalho, Antonio P.-S236

Carvente, Osvaldo-S321

Casajus-Quiros, Francisco J.-S838

Casazza, Camilla-S196

Casciato, Carmine-S210

Case, Alexander-Cochair Session 1pAAd (S11), Cochair Session 2pAAj (S234)

Cassereau, Didier-S675, S962
Cassidy, Matthew-S222, S532

Castagnede, Bernard

R.-S68, S150, S315, S316

Castell, J.v.-S811

Castellengo, Michèle-S275

Castelli, Eric-S492

Castellote, Manuel-S538, S804, S807

Castilllo-Guerra, Eduardo-S614, S909

Castrejon-Pita, Alfonso A.-S574

Casula, Olivier-S150

Catheline, Stefan-S370, S462

Cathiard, Marie-Agnès-S110

Cathignol, Dominique-S814

Cattafesta, Louis-S711

Cattan, Eric-S407

Caulkins, Terence-S234

Causa, Federica-S308

Causse, René E.-S155, S690

Cavalieri, Francesca-S592

Cavanaugh, William J.-S224, S225, Cochair Session 2pAAc (S224)

Cavanaugh, William-Cochair Session 4pAAe (S643)

Cazard, Guillaume-S475

Cazzolato, Ben S.-S95

Cecote Stein, Cirineu-S214

Celestinos, Adrian-S123

Çelik-Christensen, Emine-S196

Cellai, Gianfranco-S233, S798

Cemgil, Ali Taylan-S617, S834

Cemgil, Ali-Taylan T.-S616

Cerami, Victoria J.-S951

Ceranna, Lars-S859

Cerchiai, Mauro-S423, S852

Cerdá, Salvador-S230, S231, S706

Cereser Camara, Vivian-S316

Cerisara, Christophe-S212

Černetič, Jan-S348

Cerniglia, Andrea-S851

Cerrato Jay, Gabriella-S192

Certon, Dominique-S409, S679,

Cochair Session $3 \mathrm{aEAb}$

(S406), Cochair

Session 4pEAc (S678)

Cervenka, Pierre-S148, S788, S927

Cervera, Francisco-S309

Cesare Consumi, Marco-S130

Cesbron, Julien-S421, S900

Cetina-Medina, Zenon-S953

Chadwick, Richard S.-S807

Chahine, Georges L.-S591

Chaigne, Antoine-S554, S696,

Cochair Session 4aMU

(S553), Cochair

Session 4pMUf (S696)

Chaigne, Sébastien-S566

Chait, Maria-S82

Chaix, Jean-François-S876, S879, S880

Chambers, David-Cochair Session 2aSPa (S215), Cochair Session $2 \mathrm{aSPb}$ (S217), Cochair Session 2pSPf (S372), Cochair Session 2pSPg (S373)
Chambeyron,Colin-S414, S440

Champlin, Craig-S111

Chan, Gary-S792

Chan, Helen L. W.-S313, S816

Chanaud, Robert-S227

Chandrayadula, Tarun K.-S495

Chang, Ji-Ho-S675, S684

Chao, Chen-S816

Chapelon, Jean Yves-S27, S28, S29, S814, Cochair

Session 1pBBa (S26), Cochair Session 2pBBe (S254)

Chapman, David M.F.-S470

Chapman, David M.f.-S838

Chapman, Ross-S138, S626

Charalambopoulos, Antonios-S545

Charles, Christine-S290

Charnaya, Elena V.-S725

Charters, Lykara-S110, S915

Chatagnon, Roger-S416

Chati, Farid-S440, S631, S632

Chatillon, Jacques-S533

Chatziioannou, Vasileios-S155

Chaumet, Patrick-S494

Chaut, Christophe-S958

Chavarria, Noelia-S710

Chavez Thielemann, Victoria-S232

Chazot, Jean-Daniel-S536, S603

Cheenne, Dominique J.-S941

Cheinet, Sylvain-S183

Chekroun, Mathieu-S877

Chen, Chifang-S620

Chen, Chii-Chang-S312

Chen, Chuin-Shan-S147

Chen, Dong-S78

Chen, Hong-S79

Chen, Jer-Ming-S155, S156

Chen, Jing-S327, S355, S745, S882

Chen, Jiu-Jiu-S70, S312

Chen, Sally-S493

Chen, Shigao-S823, S825

Chen, Tianhui-S666

Chen, Tianrun-S135, S972, S973

Chen, Xun-S333

Chen, Yeting H.-S757

Chen, Yiya-S921

Chen, Yu-Po-S809

Chen, Yung-Yu-S409, S678

Chen, Zhensheng-S451

Chene, Jean-Baptiste-S531, S848

Cheng, Chierh-S896

Cheng, Chih-Chiang-S147

Cheng, D. Chih-Chun-S204

Cheng, Hsien K.-S119

Cheng, Jiqi-S665, S670

Cheng, Li-S162

Cheng, Min-Yu-S335

Cheng, Sulin-S666

Cheng, Wen-Nan-S204

Chenouni, Driss-S729

Cherioux, Frederic-S549

Chervin, Paul-S129, S228

Chesmore, David-S113, S374, S810

Chesne, Simon-S207, S907

Cheung, Theresa Y.-S252

Chevillotte, Fabien-S68, S958

Chevret, Patrick-S196 
Chevrot, Claude-S868

Chi, Huiguang-S785

Chi, Huisheng S.-S355, S745, S882

Chiang, Wei-Hwa-S129

Chiang, Wen-Yu-S916

Chiao, Joan Y.-S765

Chiavassa, Guillaume-S173

Chick, John-S48, S270

Chiello, Olivier-S203, S298

Chigarev, Nikolay-S189, S319, S584

Chigot, Pierre-S2

Chisari, Elisa-S609

Chitnalah, Ahmed-S487

Chitnis, Parag V.-S591, S593

Chitre, Mandar A.-S393

Chiu, Chingsang-S620

Chizhikov, Sergei-S176

Chladkova, Katerina-S918

Cho, Seung Hyun-S728

Cho, Tongjun-S796

Choi, Inyong-S894

Choi, James-S248, S249

Choi, Jee Woong-S116, S465

Choi, Jeung-Yoon-S362

Choi, Nakjin-S917

Choi, Sungjoon-S406

Choi, Sunhee-S439

Choiniere, Marc-S121

Cholewa, Wieslawa-S103

Chollet, Gerard-S910, S918, S970

Chomette, Baptiste-S207

Chopra, Rajiv-S26, S824

Chotiros, Nicholas P.-S474, S782,

Cochair Session $3 \mathrm{aUWd}$

(S472), Cochair

Session 4aUWc (S625)

Chotiros, Nicholas-Cochair Session 2pUWe (S381)

Chou, Tai-Hsu-S409

Choujaa, Abdelkrim-S312

Chourmouziadou, Kalliopi-S427

Chow, Renee-S829

Chowning, John M.-S637

Choy, Chung Loong-S313

Christensen, Claus Lynge-S14

Christensen, Heidi-S326

Christensen, Johan-S178

Christensen, Karina F.-S214

Christensen, Mads G.-S836

Christensen, René-S452

Christiansen, Thomas

Ulrich-S882, S964

Christophe, Julien-S571

Chu, Dezhang-S137, S380, S928

Chu, Kanghyun-S72

Chu, William-S13

Chu, Youngmin-S789

Chuang, Yu-Ying-S109

Chudnovsky, Alexander-S428

Chunchuzov, Igor-S734

Chung, Hyuck-S388

Chung, Yoojin-S88

Chung, Yuh-Mei-S102

Church, Charles C.-S143, Cochair Session 2aBB (S141), Cochair Session 2pBBc (S251)

Chybicki, Andrzej-S813

Cibrián, Rosa-S228, S230, S231
Cichon, Andrzej-S286, S348

Cieslik, Jacek-S93

Cieszko, Mieczyslaw-S307

Ciocca, Valter-S596

Ciplys, Daumantas-S307

Cirillo, Ettore-S639

Cisonni, Julien-S609, S610

Clapiz, Monica-S386

Clapp, Robert-S494

Claridge, Diane-S16, S806

Clark,Allan R.-S624

Clark, Christopher W.-S16, S18, S19, S807

Clark, Grace A.-S494, Cochair Session $1 \mathrm{pSPb}$ (S113)

Clark, Grace-Cochair Session 2pSPc (S368)

Clark, John P.-S578

Clark, Joseph A.-S38, S379

Clark, Michael J.-S358

Clark, Nicholas R.-S327

Clarke, Paul A.-S246, S466

Clarkson, Marsha G.-S328

Clatot, Stanislas-S679

Clausen, Michael-S131

Claytor, Kevin E.-S431

Cleave, Ra-S953

Clement, Eric-S302, S306

Clement, Gregory-S34, S460, S815

Cleveland, Robin O.-S400, S591, S593, Cochair Session 3aBBa (S398)

Clift, Sally E.-S392

Clopper, Cynthia G.-S100

Clorennec, Dominique-S188, S865

Cloutier, Guy-S34

Cocchi, Alessandro-S130, S229, S641

Cochelin, Bruno-S727

Cochran, Sandy-S45, S816

Cockrell, Kevin-S371

Cocuzzo, Daniel-S135, S813, S972

Coelho, Emanuel F.-S654

Coffey-Corina, Sharon-S774, Cochair Session $4 \mathrm{pSCb}$ (S764)

Cohen Tenoudji, Frederic-S315

Cohen, Adi-S545

Cohen, Annabel J.-S111, S412, Cochair Session 3aMUa (S410)

Cohen, Leon-S80, S496

Coiffet, Francois-S52

Coiras, Enrique-S980

Colazo-Simon, Antonia-S214

Colburn, H Steven-S88, S328, S448, Cochair Session 2pPPe (S335)

Colburn, H. Steven-Cochair Session $1 \mathrm{pPPb}$ (S86)

Colebring, Andreas-S277

Coleman, Felicia-S130

Coles, David-S117

Collery, Olivier-S604

Collet, Bernard-S726

Collet, Lionel-S337, S598, S751, S888, S895
Collet, Manuel-S606, S607, Cochair Session $4 \mathrm{aSAb}$ (S604), Cochair Session 4pSAb (S759)

Collier, Art-S935

Collier, Sandra L.-S287

Collier, Travis-S134

Collin, Dominique-S556, Chair Session 2pNSg (S288), Chair Session 4aNSa (S556)

Collin, Jamie R.-S36

Collins, Curtis-S131

Collins, Leslie M.-S74, S86

Collins, Michael D.-S634, S974

Collis, Jon M.-S634

Colominas, Ignasi-S413

Colomo, Miguel-S901

Colonius, Tim-S399, S400

Colosi, John A.-S974

Commins, Daniel E.-S5, S128, Cochair Session 1pAAb

(S4), Cochair Session 2aAAa (S120)

Commins, Daniel-Cochair Session 2pAAg (S229)

Comperat, Eva-S144

Conant, David-S13

Conboy, Barbara-S613

Condliffe, Jamie-S33

Conetta, Robert-S11, S12

Connors, Bret A.-S399

Conoir, Jean-Marc-S632, S963,

Cochair Session 5aPAj

(S876), Cochair Session 5pPAd (S962)

Consumi, Marco C.-S229

Conter, Marco-S170, S423

Conti, Vincenzo-S386

Cooke, Martin-S104, S756, S758, S913, S964

Cooling, Martin P.-S468

Cooper, Angela-S768

Cooper, Richard K.-S222, S532, S569

Cooperstock, Jeremy-S444

Copley, Nancy-S26

Coquel, Guillaume-S291

Coquet, Michel-S983

Cordeddu, Stefano-S294

Corey, John-S574, S576

Corkeron, Peter J.-S541

Corneloup, Gilles-S730, S879, S880

Cornuelle, Bruce-S627, S945

Coron, Alain-S33

Coron, Jean-Michel-S688

Corrales, Jose A.-S956

Corre, Christophe-S54

Correas, Jean-Michel-S143

Corriveau, Philip J.-S192

Corteel, Etienne-S234, S331,

Cochair Session 1pAAd (S11), Cochair Session 2pAAj (S234)

Cosandey, Laurent-S60

Cossette, Isabelle-S273

Costa-Felix, Rodrigo-S253

Costagliola, Angelica V.-S214

Costes, Francois-S286

Costolo, Michael-S438

Côté, Nicolas-S97, S98
Côte, Renaud-S189, S317

Cotoni, Vincent-S90, S865

Cotte, Benjamin-S183

Cottingham, James-S47, S637, S638

Cotton, Fabrice-Cochair Session 2pPAa (S301), Cochair Session 2pPAj (S322)

Couade, Mathieu-S115

Coughlin, Maureen-S329

Coulon, Anne-S196

Coulouvrat, François-S184, S487, S727, S856

Courjon, Emilie-S678, S679

Courtis, Romain-S982

Cousineau, Marion-S594

Cousins, Owen-S792

Coussios, Constantin C.-S29, S33, S36, S255, S592, S664

Coutard, Frederic-S778

Couture, Olivier-S862

Cox, Ben-S403

Cox, Emma-S29

Cox, Stephen J.-S297

Cox, Trevor J.-S14, S68, S562, S712

Coyette, Jean-Pierre-S901

Crawford, Anna-S981

Cray, Benjamin-S37

Cremezi-Charlet, Cora-S183, S415

Cretin, Dorian-S817

Crispin, Charlotte-S390, S391

Cristensen, Douglas

$$
\text { A.-S249 }
$$

Cristini, Paul-S494, S626

Crocco, Marco-S41

Crocker, Daniel-S540

Crosato, Paolo-S210

Cross, Ian-S478

Crossley, Jennifer F.-S603

Crouzet,Fabien-S159, S181

Crowe, Vance-S981

Cruanes Catala, Joan-S443

Crum, Lawrence A.-S28, S29, S35, S79, S253, S255, Cochair Session 1pBBa (S26)

Crum, Lawrence-Cochair Session $2 \mathrm{pBBe}(\mathrm{S} 254)$

Cruz Calleja, Jorge Antonio-S309

Cruz-Damas, Jose-S321

Cslovejcsek, Markus-S949

Cucini, Riccardo-S174, S187

Čudina, Mirko-S348, S856

Cuenca, Jacques-S205, S350

Cueto-Felgueroso, Luis-S413

Cugnet, Marie-Françoise-S962

Cui, Weili-S260, S261, S262

Culling, John F.-S9, S10, S488

Cullings, Emily-S357

Culver, Richard Lee-S379, S466, S615, S775

Cummings, James-S654

Cunefare, Kenneth-Cochair Session 1pNSd (S64), Cochair Session 2aNSd (S167), Cochair Session 2pNSl (S294), Cochair Session 4aSAb (S604), Cochair Session $4 \mathrm{pSAb}$ (S759) 
Cunitz, Bryan-S815

Cunningham, James L.-S546

Cunningham, Stuart-S103

Curcuruto, Salvatore-S386

Curiel, Laura-S26, S824

Curra, Francesco-S399

Cusack, Peter-S426

Cusack, Rhodri-S84

Cutanda Henriquez, Vicente-S452, S453, Cochair Session $3 \mathrm{aSAb}$ (S452), Cochair Session 4aSAa (S602), Cochair Session 4pSAc (S760)

Czarnota, Gregory J.-S31

Czerski, Helen-S590

Czuratis, Peter-S113

Czyzewski, Andrzej-S61, S331, S753

Dahl, Jeremy-S824

Dahl, Peter H.-S116, S118, S465, Cochair Session 1pUW (S116)

Dahl, Sofia-S154

Dai, Huanping-Cochair Session $2 \mathrm{aPPb}$ (S197), Cochair Session 2pPPf (S336)

Dai, Jiyan-S816

Dai, Qiongxing-S621

Dai, Wei-S575

Daigle, Gilles A.-S42, S500

D'Alessandro, Christophe-S50, S477, S971

D’Alessandro, Francesco-S69, S797

Dalianis, Sotiris-S124

Dalla Bella, Simone-S411

Dalle, Sebastien-S416

Dalmas, Davy-S817

Dalmont, Jean-Pierre-S46, S151, S269, S272, S479

Dalton, Fergal-S301

Damdinov, Bair-S875

Dance, Stephen-S208, S490, S827, S855

D'Andréa-Novel, Brigitte-S687, S688, S690

Dang, Jianwu-S105, S916, S917

D'Angelo, Ralph-S428

Daniau, William-S308, S679

Daniel, Jérôme-S41, S968

Daniels, Reginald-S674

Danijel, Djurek-S728

Danilouchkine, Mike-S30

Dankiewicz, Lois-S806

D'Antonio, Peter-S14, Cochair Session 1pAAe (S14), Cochair Session 2aAAb (S121), Cochair Session 2pAAh (S232)

Daoudi, Khalid-S403

Daout, Franck-S499

Daraio, Chiara-S303

Darcy, Isabelle-S766

Dare, Tyler-S422

Daridon, Jean Luc-S959, S960

Dariouchy, Abdelilah-S204

Darjaa, Sakhia-S209

Darvennes, Corinne-S550

Darwin, Chris J.-S8, S82

Dash, Ian-S757
Da Silva, Andrey R.-S155, S479

Datta, Sekharjit-S971

Dau, Torsten-S328, S744, S886, S891

Dauchez, Nicolas-S69, S315

Daude, Frédéric-S159

Daudet, Laurent-S833, S834

David, Bertrand-S94, S819, S832, S835, Cochair Session 5aMUd (S832), Cochair Session 5aMUe (S834)

Davies, Michael-S618

Davies, Patricia-S481, S483, Cochair Session $2 \mathrm{aPPa}$ (S190), Cochair Session 2pPPb (S330), Cochair Session 3aPPa (S443), Cochair Session 4pNSa (S698), Cochair Session 4pNSm (S715)

Davies, William-S426

Davis, Bob-S748

Davis, Brian-S820

Davis, Evan-Cochair Session 2aSAc (S207), Cochair Session 2pSAb (S343)

Davis, Lee A.-S433, S734

Davis, Matthew H.-S612, S770, S919, S920, S921

Davy, John L.-S531

Davy, Renaud-S615

Davydov, Maxim N.-S322

Day, Graham J.-S246

Dazel, Olivier-S66, S67, S315, S316, S685, Cochair Session 5aPAh (S873), Cochair Session 5pPAa (S957)

Dean, Cleon E.-S344, S552

Deane, Grant-S118, S590

Dear, Randall L.-S17, S538

De Bazelaire, Eric-S494, S626

Debert, Sébastien-S420, S564

Debesse, Philippe-S577

Debever, Claire-S496, Cochair Session 3pUWb (S496)

Debieu, Eric-S34, S666

De Billy, Michel-S486, Cochair Session 3pPAa (S485)

DeBilly, Michel-Cochair Session 4pPAc (S725)

De Blok, Kees-S573

De Boer, André-S705

De Boer, Bart-S764, S811, Cochair Session $4 \mathrm{pSCb}$ (S764)

De Bree, Hans-Elias-S38, S471

De Bruijn, Alex-S173

Debus, Jean-Claude-S607

De Carli, Federica-S677

De Cheveigne, Alain-S264, S837, Cochair Session 2pPPa (S325), Cochair Session 3pPPa (S488), Cochair Session 3 pPPb (S490), Cochair Session 4aPPa (S594), Cochair Session $4 \mathrm{aPPb}$ (S597), Cochair Session 4pPPc (S747), Cochair Session 5aPPa (S881),
Cochair Session 5aPPd (S889), Cochair Session $5 \mathrm{aPPg}$ (S898)

Dechipre, Hervé-S162

Declercq, Nico F. -S731

Declercq, Nico F.-S176, S178, S179, S257, S307, S386, S429, S431, S440, S641, S732, S820, S866, Cochair Session 2aPAb (S175)

Declercq, Nico-Cochair Session 2pPAc (S307)

De Coensel, Bert-S168, S426

Decremps, Frédéric-S959, Cochair Session 5aPAg (S872), Cochair Session 5pPAb (S959)

Decultot, Dominique-S204, S631, S730, S782

Deecke, Volker B.-S395

Deeks,John-S86, S89

Deeks, Julian L.-S463

Deferrari, Harry-S467, S973

Defontaine, Marielle-S257, S321, S665, S670

Defrance, Guillaume-S531

Defrance, Jérôme-S60, S182, S954, S955, Chair Session 5aNSg (S853), Chair Session 5pNSc (S955)

De Francisco, Martha-S11

Defresne, Gérard-S739

De Gardelle, Vincent-S612

De Geetere, Lieven-S222, S300, S799

Degel, Christian-S815, S880

De Gersem, Herbert-S430, S863

Degertekin, F. Levent-S260, Cochair Session $3 \mathrm{aEAb}$ (S406), Cochair Session 4pEAc (S678)

Degertekin, Levent-S261, S262

De Goey, Philip-S436

Degollada, Eduard-S651

Degrande, Geert-S292

De Gregorio, Leda-S635

Degrieck, Joris-S429, S866

De Groot-Hedlin, Catherine-S952

Degroot-Hedlin, Catherine D.-S861, S871

De Guibert, Pierre-S174, S302

Dehoux, Thomas-S188

De Icaza, Miguel-S398

Dejaeger, Ludovic-S564

De Jong, Christ A.f.-S19

De Jong, Jos-S589, S735

De Jong, Kenneth-S364, S455

De Jong, Nico-S141,

S142, S143, S247, S252, S592, S727

Dekoninck, Luc-S168

Dekorsy, Thomas-S585

Del Rey Tormos, Romina-S646, S647, S648

Delabrousse, Eric-S28

De La Cuadra, Patricio-S49, S156, S829

Delalande, Anthony-S250

De La Mota, Carme-S909

De Langhe, Koen-S758

De Lathauwer, Lieven-S863

Delaunay, Thomas-S438
Delfs, Jan W.-S162, S425

Delgado, Antonio-S769

Delgutte, Bertrand-S88

Delic, Vlado-S687

Deliyski, Dimitar D.-S772

Della Crociata, Sabrina-S639

Dellwo, Volker-S459, S918, Chair Session 3aSCd (S459)

Delong, Caroline M.-S375

De Lorenzo, Francesco-S205

Delorme, Philippe-S860

Delrue, Steven-S430, S441

Delville, Jean-Pierre-S441, S488

Demakakos, Jason-S357

Demany, Laurent-S81, S594

Dembelova, Tuyana-S875

Demkowicz, Jerzy-S658

Demore, Christine E.-S45, S816

Demoucron, Matthias-S155, S696

De Moustier, Christian-S468,

Cochair Session 4pAOd

(S658), Cochair

Session 4pAOg (S659)

De Muynck, Robert J.-S557

Denby, Bruce-S970

Dencks, Stefanie-S664

Denes, Samuel L.-S541

Deneuville, François-S723

Denham, Susan-S85

Denia, Francisco D.-S566

Denli, Huseyin-S115

De Oliveira, Leopoldo P.-S904

Depalle, Philippe-S778, S828

De Plaa, Jelle-S384

Depollier, Claude-S68, S71, S185, S315, S316, S669, S671, S957

Derible, Serge-S173, S314

Deroche, Mickael-S10

Derode, Arnaud-S33, S218, S373, S665, S876, S963

De Roeck, Wim-S161

De Roo, Foort-S62, S185

De Rosny, Julien-S216, S218

Derrien, Olivier-S836

Deruiter, Stacy-S393

Deruty, Emmanuel-S833

De Rybel, Tom-S563

De Ryck, Laurent-S71

De Sanctis, Giovanni-S48

De Santis, Eric-S286

Desceliers, Christophe-S602

Deschamps, Marc-S958, Cochair Session 5aPAh (S873),

Cochair Session 5pPAa (S957)

De Sitter, Gert-S642

Desjouy, Cyril-S740

Desmare, Rozenn-S382

Desmet, Wim-S161, S789, S904

Desoeuvre, Gaylord-S279

Despaux, Gilles-S316

Despres, Bruno-S861

Desvard, Ludovic-S485

Desvaux, Sébastien-S723

Dettmer, Jan-S140

Deux, Florent-S640

Deveau, David-S932, Cochair Session 5aUWe (S932)

De Vecchi, Guido-S691 
Devenport, William J.-S54, S55, S159

Devergie, Aymeric-S334

Deveugele, Jurgen-S441

De Vos, Paul-S57, Cochair Session 5aSAa (S899), Cochair Session 5aSAd (S908)

Devos, Arnaud-S581, S586, S737

De Vries, Diemer-S440, S754, S939

Dewhirst, Martin-S11, S12

Dewijngaert, Katrien-S386, S641

De Wild, Marco-S408

Deymier, Pierre-S70, S313,

Cochair Session 1pPAb (S70), Cochair Session 2pPAe (S311)

Dhar, Sumitrajit-S105

Dhibi, Mounir-S982

Dhillon, Rajdip-S457

D'Hooge, Jan-S669

Di, Xiao-S184, S870, S954

Diallo, Alpha-S566

Diamant, Roee-S934

Diamond, Christi-S19

Di Angelo, Adam-S235

Díaz-Amador, Roberto-S909

Di Bella, Antonino-S294

Di Benedetto,

Maria-Gabriella-S107

Dickens, Paul-S47, S272

Diebold, Gerald-Cochair Session 2aPAd (S186), Cochair Session 2pPAg (S317)

Diebold, John-S19

Dieckman, Eric A.-S47

Dierckx, Mark-S960

Dietz, Mathias-S328

Di Francescantonio, Paolo-S451

Di Gabriele, Maria-S427

Digiovanni, Jeffrey J.-S897

Dijckmans,Arne-S300

Dijkhuis, Jaap I.-S584

Dijkink, Rory-S79, S592

Dikmen, Onur-S617

Dilhaire, Stefan-S737

Dillier, Norbert-S198

Dillion, Kevin-S859

Di Marcoberardino, Lucilla-S148

Dimarzio, Nancy A.-S16, S651, S812

Dimitriadis, Emilios K.-S807

Dines, John-S263

Ding, Ran-S318, S319

Dionne, Jason-S438

Dirksen, Peter-S408

Dittberner, Andrew-S329

Dittrich, Michael G.-S60, S298

Divenyi, Pierre-S211,

$$
\text { S449 }
$$

Dix, Justin K.-S379, S382, S474, S626

Dixon, Max-S843

Djafari-Rouhani, Bahram-S71, S72, S73, S486, S873

Djelouah, Hakim-S865, S960

Djeradi, A.-S776

Djeradi, Amar-S914

Djurek, Ivan-S728

Dmour, Mohammad-S618

Doare, Olivier-S696
Dobeck, Gerald-Cochair Session 5pUWc (S979)

Dobrolenskiy, Yury-S177

Dobrucki, Andrzej B.-S756

Dobrzynski, Leonard-S873

Docherty, Gerard J.-S99

Dockrell, Julie E.-S950

Doclo, Simon-S201

Dodd, George-S390

Dodd, Simon P.-S546

Doellinger, Michael-S769, S770, S775

Dogan, Yilmaz-S56

Dohen, Marion-S492, Chair Session 3pSCb (S492)

Doherty, Rina A.-S192

Doinikov, Alexander A.-S77, S252

Doisy, Sonia-S707

Dolejsi, Jana-S639

Dolinich, Fabrizio-S169

Dollet, Benjamin-S142, S143, S252, S592

Domecka, Markieta-S842

Domenicali, Daniele-S107

Domitrovic, Hrvoje-S63, S314, S533, S647, S648, S649, S951

Donavan, Paul-S165, S422, Cochair Session 2aNSc

(S165), Cochair

Session 2pNSk (S292),

Cochair Session 3aNSd (S420),

Cochair Session 4pNSp

(S718)

Donayre Ramírez, José Christian-S557

Dong, Hefeng-S246

Dong, Qi-S922

Dong, Wayland-S172, S222, S536

Dong, Winny-S172

Donnici, Sandra-S677

Donovan, Paul-S165

Donskoy, Dimitri-S74, S428

Dooling, Robert-S476

D'Orazio, Annunziata-S252, S866

Doria, Damian J.-S943, S944

Dorman, Michael-S917

Dorval, Vincent-S730

Dos Santos, Serge-S431, S864, S867, Cochair Session $5 \mathrm{aPAb}$ (S862), Cochair Session 5aPAd (S867)

Dosso, Stan E.-S140, S141, S397, S398, S465, S617

Dou, Chunyan-S145

Dougalis, Vassilios-S630

Doukakis, Efstratios-S932

Doutaut, Vincent-S693

Doutres, Olivier-S69, S315

Doval, Boris-S275

Dowdy, Carrie-S787, S975

Dowling, Ann P.-S53, S435

Dowling, David R.-S465

Downing, Micah-S158, S854

Dr. Klee, Mareike-S408

Drab, Martin-S431

Dragonetti, Raffaele-S129

Drai, Redouane-S114, S115, S368, S776
Drake, Robert-S623

Dramas, Florian-S755

Drechsler, Andreas-S389

Dreschler, Wouter A.-S198, S199, S746, S752

Dreyfus, Gerard-S970

Drijkoningen, Guy G.-S629

Drinkwater, Bruce W.-S149

Drob, Douglas-S860, S861, S952, S953

Droin, Laurent-S63

Drossos, Konstantinos-S173

Droste, Bettina -S423

Drozhzhin, Alexey P.-S487, S727

Drumm, Ian-S712, S793

Druon, Yann-S56, S856

Drzewiecki, Andrzej-S485

D’Spain, Gerald-S39, S135, S470

Dual, Juerg-S317

Dual, Jurg-S582

Dubberley, John-S657, S980

Dubief, Flavien-S282

Dubois, Danièle S.-S352, S706, S839, Cochair Session 4pNSr (S721)

Dubois, Danièle-Cochair Session 4pNSd (S706)

Dubrovinsky, Leonid S.-S959

Dubus, Bertrand-S71

Ducasse, Eric-S958

Duchaine, Patrick-S434

Duclaux-De-L'Estoille,

Nicolas-S293

Duclos, Aroune-S324

Ducourneau, Joël-S533

Ducousso, Mathieu-S738

Ducruix, Sébastien-S436

Duda, Timothy-S620, S975,

Cochair Session $5 \mathrm{pAO}$ (S944)

Duennebier, Fred K.-S239

Dufait, Rémi-S679, S818

Duffal, Jean-Marc-S485

Duflo, Hugues-S314, S428

Dugelay, Samantha-S982

Duhamel, Denis-S91, S421, S423, S900

Duman, Tolga M.-S781, S923

Dumas, Philippe-S150

Dumortier, Alexis J.-S38

Dumouchel, Christophe-S487

Duncan, Alec J.-S397, S466

Duncan, Eddie-S567,

Cochair Session 4aNSe (S567), Cochair Session 4pNS1 (S714)

Dunkin, Miles-S660

Dunne, Garry-S277

Dupas, Yann-S979

Dupont, Fernand-S715

Dupont, Jean-Baptiste-S604

Dupont, Lucie-S878

Dupont, Samuel-S308

Dupont, Thomas-S647

Duquennoy, Marc-S317, S723

Duraiswami, Ramani-S451, Cochair Session

3aSAa (S450), Cochair Session 4pSAa (S758)

Durand,Jacques-S101

Durand, Jean-Francois-S565
Durand, Stephane-S261, S576

Durany, Jaume-S801,

S969

Durin, Virginie-S98, S195

Duro, Vincent-S782

Durocher, Jean-Noel-S879

Duroux, Adelaide-S216

Durox, Daniel-S435

Durrieu, Jean-Louis-S832

Durrieu, Marie-Christine-S584, S738

Durvilli, Ilaria-S638

Dushaw, Brian D.-S945

Düster, Alexander-S388

Duthil, Patxi-S739

Dutilleux, Guillaume-S69, S182, S956

Dutta, Indranil-S209, Chair Session 2aSCa (S208)

Duty, Jason-S230, S969

Duval, Arnaud-S92, S564

Duval, Fabrice-S407

Dymond, Rodney-S37

Dymov, Alexandre B.-S808

Dzhagan, Volodymyr-S725

Dziak, Robert-S22

Dzieciuch, Matthew-S23, S496, S945

Dzikowicz, Benjamin R.-S217, S372, S631, S787,

Cochair Session 4pUWg (S786)

Ebbini, Emad S.-S32, S814

Ebenezer, D. D.-S146

Ebert, Alfons-S981

Eccleston, Mark E.-S255

Ech Cherif El Kettani, Mounsif-S866

Ech-Cherif El-Kettani, Mounsif-S729, S865, S957

Ecke, Robert E.-S301

Eckersley, Robert-S145

Ecotiere, David-S69, S184

Ed, Zwaan-S592

Eddins, David A.-S358, S898, S913

Edely, Mathieu-S186, S586, S737

Edlund, Jens-S459

Edmonds, Barrie A.-S329

Edwards, Brent-S200, S201, S334

Edwards, Derek-S892

Edwards, John R.-S119, S985

Edwards, Tom-S39

Efimtsov, Boris M.-S345

Eftekhar, Ali Asghar-S71

Ege, Kerem-S94

Eggenschwiler, Kurt-S949

Egger, Rainer-S270

Ehrenstein, Uwe-S454

Ehrlich, Jan H.-S562

Eiler, Cheryl-S201, S334

Eisner, Frank-S363, S771

Eisses, Arno R.-S57, S183

Ekeom, Didace-S407

Ekimov, Alexander-S757

El Allouche, Nihed-S629

El Boudouti, El Houssaine-S72, S873

Elefteriou, Maria-S187

Eleftheriou, Maria-S583 
Elejabarrieta, María Jesús-S708

Elejalde-García, M. J.-S694

El Fissi, Lamia-S549

El Guerjouma, Rachid-S114, S430, S733, S869

El Hassouani, Youssef-S72

Elhilali, Mounya-S82, Cochair Session 1pPPa (S81), Cochair Session 2pPPd (S333)

Eligator, Ronald-S15, $\mathrm{S} 794$

Elko, Gary-Cochair Session 1pEAb (S40), Cochair Session 2pEAb (S263)

Ellermeier, Wolfgang-S191

Elliott, Andrew-S208, S344

Elliott, Stephen J.-S604

Elliott, Taffeta-S965

Ellison, John C.-S884

Ellison, William T.-S20

Elmaimouni, Lahoucine L.-S681

Elminowicz, Andrzej-S498

El Mouhtadi, Abdelhak-S963

El Moussaoui, Mustapha-S440

Emanuel, Noam-S821

Embleton, Steven-S625

Embrechts, Jean-Jacques-S801

Emelianov, Stanislav Y.-S401, S826

Emery, Patrick-S737

Emmer, Marcia-S142

Emmert, Thomas-S159

Emre, Yunus-S923

Emura, Masahiro-S669

Emura, Norio-S692

Eneman, Koen-S198, S893

Engel, Marc-S59

Engelien, Erik-S167

Enggaard, Anne Lin-S483

Enghardt, Lars-S437, S556, S572

Enomoto, Seigo-S683

Envia, Edmane-S55

Epain, Nicolas-S41

Epp, Bastian-S83

Epstein, Michael-S338, S339

Erdener, Dogu-S911

Erickson, Donna-S355

Erkut, Cumhur-S553

Erlicher, Silvano-S900

Ermeneux, Sebastien-S737

Ermert, Helmut-S30

Ermolaeva, Elena-S383

Ernestus, Mirjam-S921

Ernst, Stephan M.-S881, S890

Esch Van, Thamar E.-S746

Escolano, José-S793, S830

Escuder Silla, Eva-S646, S647, S648

Escudero, Paola-S108, S351, S918

Esenaliev, Rinat O.-S403

Eskelinen, Joona-S369, S639

Eskenazi, Maxine-S913

Eslami, Parastou-S589

Espinosa, Víctor-S313, S659, S880, S937
Espinoza-Varas, Blas-S898, Cochair Session $5 \mathrm{aPPa}$

(S881), Cochair Session 5aPPg (S898)

Espy-Wilson, Carol Y.-S357

Esteban, José-Antonio-S538, S807

Estienne, Olivier-S765

Estrin, Deborah-S134

Etchessahar, Manuel-S566

Eustaquio-Martín, Almudena-S891

Evan, Andrew P.-S399

Evans, Benjamin-S980

Evans, Bronwen G.-S105

Evans, Jack B.-S709, Cochair Session 4pNSb (S700), Cochair Session 5aNSb (S844)

Evans, Jack-Cochair Session 4pNSg (S709)

Evans, Naoko-S374

Evans, Tomos A.-S299

Everbach, Erich C.-S589, Cochair Session 4aPAc (S588)

Everbach, Erich-Cochair Session4pPAi (S735)

Evers, Christine-S618

Evers, Laeslo G.-S185, S858, S871, S952

Evers, Laslo-S859

Every, Arthur G.-S582

Ewert, Roland-S161, S162

Ewert, Stephan D.-S328, S891

Eyi-Assoumou, Polynice-S346

Eysholdt, Ulrich-S922

Ezzatian, Payam-S752, S753

Fabre, Benoit-S49, S156, S688, S829

Fabre, J. Pacquin-S654

Fabre, Josette P.-S464

Fabricius, Anne-S101

Faccinelli, Elias-S194

Fagan, Mary K.-S354

Fahey, Brian-S824

Fahey, Paul-S884

Faiget, Laurent-S715, S842

Fainstein, Alejandro-S581, S586

Fairweather, Michael-S280

Faiz, Adil-S733, S869

Faiz, Bouazza-S265, S730, S869

Falcone, Erin-S395

Falek, L-S776

Falek, Leila-S914

Falkovsky, Leonid A.-S485

Falou, Omar-S32

Faltens, Tanya-S172

Fan, Silu-S325

Faranosov, Georgy-S281

Farhat, S-S435

Farina, Adriano-S490

Farina, Angelo-S5

Färm, Janne-S300

Farnetani, Andrea-S231, S636,

S950, Cochair Session

3pAA (S474), Cochair Session 4aAAa (S529)

Farnsworth, Andrew-S130, S133

Farny, Caleb H.-S34

Farooq, Omar-S971

Farvacque, Benoit-S569

Fasoli, Vilma-S644
Fastl, Hugo-S166, S191, S192, S193, S194, S547, S967, Cochair Session 2aNSc (S165), Cochair Session 2aPPa (S190), Cochair Session 2pNSk (S292), Cochair Session 2pPPb (S330), Cochair Session $3 \mathrm{aPPa}$ (S443)

Fatemi, Mostafa-S731, S820, S823, Cochair Session $5 \mathrm{aBBe}$ (S820), Cochair Session $5 \mathrm{aBBf}(\mathrm{S} 822)$

Faulkner, Andrew-S363, S748

Faure, Julien-S98

Fausti, Patrizio-S798, S950, Cochair Session 5aAAb (S794), Cochair Session 5aAAc (S798)

Favretto-Cristini, Nathalie-S626

Favrot, Sylvain-S967

Fawcett, John A.-S978

Fay, Richard R.-S653, Cochair Session 4pABd (S652), Cochair Session 5aABa (S803)

Fay, Richard-Cochair Session $5 \mathrm{aABb}$ (S808)

Fazi, Filippo M.-S40

Feïz, Farid-S458

Fechter, Laurence D.-S491

Fedala, Djaafer-S161, S569

Fedorchenko, Alexander-S159

Fee, David-S860

Fehse, Karl-Richard-S415

Feijoo, Gonzalo R.-S243, S628

Feistel, Stefan-S179, S968

Feit, David-Cochair Session 1pSAa (S90), Cochair Session 2aSAa (S202), Cochair Session 2pSAd (S345)

Feiten, Bernhard-S331

Feldbauer, Christian-S98, S371

Feleppa, Ernest J.-S33

Félix, Simon-S185, S479, S529, Cochair Session 3pMUb (S479), Cochair Session 4pMUb (S691), Cochair Session 4pMUd (S694)

Fellah, Mohamed-S671

Fellah, Zine-S315, S669, S671, S818

Fels, Janina-S191

Felty, Robert A.-S359, S360

Ferguson, Brian G.-S374, Cochair Session 2pSPh (S374)

Ferguson, Brian-Cochair Session $4 \mathrm{pSPb}$ (S777)

Ferguson, Sam-S279

Ferin, Guillaume-S409, S679, S818

Ferjan, Naja-S459

Fernandez, Efrén-S417

Fernandez, Francisco-S398

Fernandez, Joaquin-S956

Fernandez, Marcos D.-S170, S710, S799, S829

Fernandez, Pilar-S57

Fernando, Rasika-S856

Ferré, Laurent-S756
Ferraiuolo, Stefano-S416

Ferrand, Didier-S630

Ferrandis, Jean-Yves-S438, S441, S960, S961

Ferreira, Daiana-S535

Ferrini, Gabriele-S587

Ferry, Robert-S83

Festen, Joost M.-S197

Feth, Lawrence-S890

Fetisov, Andrei-S319

Feuillet, Vincent-S740

Févotte, Cédric-S616

Fiack, Matthieu-S419

Fiala, Péter-S292

Ficheux, Serge-S708

Fidahoussen, Alex-S442

Fidecki, Tadeusz-S385

Fiebig, André-S721, S840

Field, Chris D.-S846

Figueroa, Harold-S133

Fiks, Galina-S497

Fiks, Iosif-S497

Fillinger, Laurent-S370, S864

Filoche, Marcel-S529

Filoux, Erwan-S819

Fincham, Adam M.-S119, S985

Finette, Steven-S621

Finez, Arthur-S719

Finfer, Daniel C.-S136, S433

Fingerhuth, Sebastian-S717

Fink, Mathias-S27, S72, S215, S216, S217, S218, S312, S372, S403, S460, S462, S545, S672, S675, S823, S862,

Cochair Session 5aBBe (S820),

Cochair Session 5aBBf (S822)

Finneran, James J.-S17, S20, S538, S540, S805, S806

Finsterhölzl, Herbert-S166

Fioravanti, Stefano-S37

Fiorini, Ana Claudia-S559

Firzlaff, Uwe-S237, S242

Fischer, André-S162

Fischer, Heinz-Martin-S389, S391, S847

Fischer, Raymond-S206

Fisher, Joshua-S940

Fisher, Karl-S148

Fitch, William T.-S539, S803

Fitzpatrick, Denis F.-S884

Fleischer, Gerald-S558

Fleury, Gérard-S29, S404

Fleury, Vincent-S615

Flewitt, Andrew-S173

Flint,Jesse D.-S339, S446

Floody, Sergio-S474

Florentine, Mary-S339

Flouri, Evangelia T.-S543

Fodiman, Pascal-S298

Fog, Carsten Lemvigh-S7

Fogerty, Daniel-S748

Fohr, François-S150

Folégot, Thomas-S372, Cochair

Session 2pUWg (S384),

Cochair Session

4aUWa (S619), Cochair

Session 4pUWb (S781)

Folkert, Kendall-S807

Foltete, Emmanuel-S202, S777 
Fon, Janice-S109, S493, S772

Fonfara, Heinrich-S673, S815, S880

Fong, Siew Wan-S254, S255

Fontaine, Alain-S579

Fontaine, Jean-Marc-S834

Foo, Kae Yeet-S925

Foote, Kenneth G.-S136, S243, S380, S468, S981, Cochair Session 1pAOb (S23),

Cochair Session 2aAOa

(S135), Cochair

Session 2pUWb (S378),

Cochair Session

3aUWb (S467), Cochair

Session 4aUWb (S623)

Foote, Kenneth-Cochair Session

2pAOa (S243), Cochair

Session 2pUWd

(S380), Cochair Session 4pUWd (S783)

Forget, Guillaume-S584

Formby, Charles-S749

Forssén, Jens-S184, S955

Fortuné, Véronique-S281

Fotiadis, Dimitrios I.-S545

Foucart, Félix-S288, S958

Fougeron, Cécile-S921

Foulkes, Paul-S99

Fountain, Amy-S765

Fourmentel, Damien-S960

Fournelle, Marc-S673, S815

Fournier, Céline-S32

Fournier, David-S846

Fowler, Carol A.-S912, Cochair Session $2 \mathrm{aSCb}$ (S210)

Fowler, Daivid-S581

Fowler, Melissa-S19

Fowlkes, Jeffrey B.-S28, S373

Fox, Colin-S388

Fox, Paul D.-S376, S632

Fox, Robert A.-S100, S102

Foy, Cédric-S942

Frachet, Bruno-S895

Frampton, Kenneth-S605, S606

François, Maurice-

Xavier-S577, S739, S740

Francart, Tom-S97

Frances Monllor, Jorge-S150, S206, S265, S443

Franceschini, Emilie-S34

Francis, David T.-S468

Frank, Tim-S235

Franke, Heinz H.-S346

Franklin, Cole-S77, S173

Franssila, Sami-S318

Fransson, Niklas-S599

Franz, Matthias O.-S652

Franzoni, Linda P.-S95, S793

Frauenfelder, Ulrich H.-S921

Frederick, Blaise D.-S127

Frederick, Michael-S279

Freeman, Todd-S192

Freese, Nils-S193

Freiheit, Ronald-S124

Freire, Juan-S659

Freitag, Lee-S21, S925

Frelat, Joel-S838

Freour, Vincent-S690

Frey, Anna-S798

Freyman, Richard L.-S9, S325
Friboulet, Denis-S251

Frid, Anders-S415

Fried, Stephanie-S662

Friedrich, Rainer-S288

Friedt, Jean-Michel-S549

Frikha, Ahmed-S873

Frikha, Slaheddine-S603

Frinkley, Kristin-S250, S824

Friot, Emmanuel-S606, S904

Fritz, Claudia-S478,

Cochair Session 3pMUa (S477)

Froehlich, Matthias-S43, S198

Froelich, Benoit-S404, S680, Cochair Session 3aEAa (S404), Cochair Session 4pEAd (S680)

Frogneux, Michel-S870

Fromhold, Mark-S581

Frommolt, Karl H.-S131

Frovin, Sophia-S772

Fruhmann, Markus-S166

Fuchs, Gilberto-S559

Fuchs, Susanne-S108, S111, S357, S362, S610,

S769, Cochair Session 1pSCc (S102)

Fuenmayor, F. Javier-S566

Fuente, Marta-S846

Fujihara, Hiromasa-S837

Fujii, Kensaku-S263

Fujikawa, Takaaki-S736

Fujimoto, Masako-S104

Fujimoto, Yuichi-S443

Fujioka, Emyo-S810

Fujisaki, Hiroya-S455

Fujiwara, Mai-S13, S128

Fukuda, Michihiro-S810

Fukuda, Yoko-S292

Fulcher, Lewis P.-S773

Fuller, Chris R.-S291, S607, S843, S902

Fullerton, Jeffrey-S121

Füllgrabe, Christian-S751, S890

Fulop, Sean-S915

Fulton, Sherry-S491

Funada, Tetsuo-S360, S910

Funaki, Keiichi-S767

Funatsu, Seiya-S104

Funke, Arik R.-S462, S672

Furukawa, Tatsuya-S192

Furukawa, Tomohiro-S5

Fyson, Nicolas R.-S964

Fytas, George-S587

Gabard, Gwenael-S53

Gabel, Doug-S192

Gäbel, Gunnar-S704

Gaboury, Isabelle-S244

Gabrielli, François-S818

Gabrielson, Thomas-S276, S871

Gachon, Dorian-S679

Gade, Anders C.-S6, S231, S530

Gade, Anders Christian-Cochair Session 4pAAi (S649)

Gadomska, Bożena-S726, S738

Gadomski, Wojciech-S726, S738

Gagliardini, Laurent-S92, S346, S419, S565, S566, S707

Gagné, Jacques-S24
Gaillard, Fabienne-S542

Gainville, Olaf-S861

Gaitan, D. Felipe-S591

Gajdatsy, Peter-S901, S904, S908

Galanter, Eugene-S753

Galbrun, Laurent-S389

Galfarsoro, Unai-S708

Galib,Thomas-S760

Galimov, Rinat-S724

Gallégo, Stéphane-S337, S895

Galland, Marie-

Annick-S69, S604, S647,

S905, S906, Cochair Session

5 aSAb (S902), Cochair

Session 5aSAc (S906)

Gallot, Thomas-S370

Galuske, Ralf-S601

Gamarnik, Viktor-S824

Gamba, René-S475, S714

Gandhi, Swapan-S890

Ganjehi, Lili-S727

Gao, Bo-S741

Gao, Man-S915

Gao, Sujuan-S399

Gao, Zhonggao-S249

Garai, Massimo-S232, S648

Garay-Vargas, Elisa-S641

Garbin, Valeria-S143

Garcìa-Osuna, Fernando-Cochair Session 3aEAa (S404), Cochair Session 4pEAd (S680)

García Pérez, Igone-S63

García-Vidal, Francisco Jose-S178

Garces, Milton-S860, S952

Garcia Lecumberri, M.

$$
\text { Luisa-S913 }
$$

Garcia Moreno, Jorge-S950

Garcia-Gancedo, Luis-S816

Garcia-Sierra, Adrian-S111

Garcia, Alexandre-S340, S341,

Cochair Session 2pSAa

(S340), Cochair Session 2pSAc (S344)

Garcia, Caesar-S260, S262

Garcia, David-S969

Garcia, Hector-S30

Garcia, Julien-S679

Garcia, Marieneige-S331, S752

Garcia, Raphael F.-S434

Gardner, Catherine-S435

Gardonio, Paolo-S604, S605, S902

Garello, René-S939, Cochair Session 5aUWi (S938)

Garlick, Jamie-S748

Garnier, Maeva-S275, S352

Garnier, Vincent-S431, S876, S879, S880

Garoum, Mohammed-S172, S843

Garr, Michael T.-S464

Garrett, Steven-S404, S686,

Cochair Session $4 \mathrm{aPAa}$

(S573), Cochair Session 4pPAk (S739)

Garriga, Adan-S792, S801, S969

Garrigues, Sébastien-S714

Garry, James R.-S433

Garsed, Philip-S385

Gascard, Jean-Claude-S22

Gasparini, Emanuele-S639

Gaston, Jeremy R.-S339, S446
Gatta, Philipp-S407

Gaucher, Eric-S58, S235

Gaudiller, Luc-S907

Gaudrain, Etienne-S82, S333, S334

Gaudreau, Marc-André-S597

Gauduin, Benoît-S548, S707, S857

Gaul, Lothar-S450, S454, S758, Cochair Session 3aSAa (S450)

Gault, Richard-S222, S532, S569

Gaumond, Charles F.-S377

Gauthier, Jean-Philippe-S903

Gauthier, Michel-S959

Gautier, François-S42, S205, S269, S350, S693,

Cochair Session $3 \mathrm{aMUb}$

(S412), Cochair

Session 4pMUc (S693)

Gautier, Pierre Etienne-S297,

Cochair Session 5aNSe

(S850), Cochair Session 5aNS j (S855)

Gauvreau, Benoit-S181, S182, S423

Gavaldà, Núria-S918

Gavioso, Roberto-S676

Gaviot, Etienne-S576

Gavrilov, Alexander-S21, S22, S397, S660

Gavrilov, Leonid R.-S822

Gay, Derek A.-S831

Gazengel, Bruno-S151

Gazon, Matthieu-S293

Gearhart, Caroline A.-S491

Gedemer, Linda-S641, S793

Geebelen, Nathalie-S66, S67

Geeraert, Marieke-S430

Geissner, Emilie-S196

Gelat, Pierre-S36

Gelfer, Carole E.-S360

Gely, Denis-Cochair Session 3aNSe (S424), Cochair Session 4pNSi (S711)

Gendreau, Michael L.-S702

Gendron, Marlin-S980

Gendrot, Cédric-S921

Genell, Anders-S753

Genevaux, Jean Michel-S69, S315

Gentry, Roger L.-S20

Genuit, Klaus-S166, S721, S840, Cochair Session $1 \mathrm{pNSb}$ (S57), Cochair Session 2pNSa (S276), Cochair Session 2pNSd (S285),

Cochair Session 2pNSh (S289)

Georganti, Eleftheria-S793

George, Jinto-S240

Georgiev, Vasil B.-S953

Gerard, Anthony-S571

Gerges, Samir N.-S149

Gergidis, Leonidas N.-S545

Gerisch, Alf-S667

German, Miriam-S852

Germano, Massimo-S252, S866

Geroso, Simone-S647

Gerretsen, Eddy-S62, S221, S391, S532, Cochair Session 
2pAAa (S221), Cochair Session 2pAAi (S233)

Gerstoft, Peter-S138, S246, S617,

S656, Cochair Session 2aAOb (S137),

Cochair Session 2pAOc (S246), Cochair Session 3aAO (S396)

Gervais, Yves-S54, S281, S564, S720

Gervaise, Cédric-S495, S657, S661, S936, Cochair Session 2aAB (S130), Cochair Session 2pABb (S239)

Ghazal, Bilal-S251

Gheduzzi, Sabina-S546

Ghent, Robert-S336

Ghorbanian, Kaveh-S578

Ghose, Kaushik-S236

Ghosh, Deep-S978

Ghosh, Satrajit S.-S491

Ghoshal, Goutam-S441, S668

Ghoul, Asila-S18

Giannetti, Claudio-S587

Giard, Samuel-S24

Gibaud, Alain-S586

Gibbs, Barry M.-S207, S389

Gibbs, Bobby E.-S802

Gibiat, Vincent-S174, S218, S302, S549, S579, S754

Gielen, Ludo-S901, S908

Giesbrecht, Peter K.-S149

Gigan, Sylvain-S403

Giguère, Christian-S845

Gil, Juana-S909

Gilbert, Gaëtan-S745

Gilbert, Joël-S154, S269, S270, S274, S463, S479, Cochair Session 2aMUa (S151), Cochair Session 2pMUa (S269)

Gilbert, Kenneth E-S184, S954

Gilbert, Robert-S818

Gilichinskaya,Yana D-S491

Gilkey, Robert H.-S329

Gillespie, Douglas-S806

Gillieron, Paul-S228

Gillis, Dominique-S168

Giménez, Alicia-S228, S230, S231, S636, S706

Gindre, Marcel-S868

Ginsberg, Jerry H.-S91

Giordano, Bruno L.-S210, S444, $\mathrm{S} 778$

Giovannini, Maria-S8

Girardi, Marcel-S290

Girod, Lewis-S134

Giroux, Marie-France-S34

Gisiner, Robert-S806

Giudice, Sebastiano S.-S277

Giuliano Albo, Paolo Alberto-S676

Gjestland, Truls-S193

Glüer, Claus C.-S664

Glegg, Stewart A.-S55, S159

Glesser, Martin-S271, S606

Glickman, Gary M.-S702

Glickman, Steve-S652

Gliozzi, Antonio S.-S732, S864
Globevnik, Aleš-S169, S295

Gloerfelt, Xavier-S54, S161

Glorieux, Christ-S188, S189, S190, S317, S318, S464, S878, Cochair Session 2aPAd (S186), Cochair Session 2pPAg (S317)

Glosemeyer Petrone,

Robin-Cochair Session 2aAAe

(S128), Cochair Session

2pAAb (S223),

Cochair Session 2pAAf (S228)

Gloza, Ignacy-S787, S788

Glushkov, Alexander V.-S657, S776

Glynne-Jones, Peter-S873, S957

Gmitter, Christine-S749

Gnansia, Dan-S335

Gobat, Jason I.-S22

Gobert, Marie-Laure-S454

Gockel, Hedwig E.-S595

Godøy, Rolf Inge-S689

Godar, Shelly-S89

Godin, Oleg A.-S662, S972

Godo, Olav R.-S135, S243

Godoe, Olav R.-Cochair Session 1pAOb (S23), Cochair Session 2aAOa (S135)

Godoe, Olav Rune-S23, S244

Godoe, Olav-Cochair Session 2pAOa (S243)

Godsill, Simon J.-S616, Cochair Session $4 \mathrm{aSPb}$ (S616)

Godsill, Simon-Cochair Session 2pSPe (S371)

Goel, Virendra-S167

Gold, Martin A.-S802

Gold, Susan-S749

Golden, Paul-S952

Goldenberg, Chay-S302

Goldhahn, Ryan-S628

Goldstein, Louis-S915

Golkin, Stanislav V.-S875, S877

Golo, Voislav L.-S585

Golovin, Edvard-S428

Golston, Chris-S915

Gomberg, Joan-S302, S303

Gomes, Jesper-S417, S418

Gomes, Joao-S923

Gomes, Rui-S299

Gomez, Fabrisio-S574

Gömmel, Andreas-S609

Goncharenko, Boris-S383

Gong, Zaixiao-S621, S984

Gong, Zheng-S135, S813, S972

Gonzalez Diaz, Cristobal-S902

Gonzalez-Rodriguez, Luz Del Carmen-S645

Gonzalez, Isabel-S710

Gonzalez, Julio-S756

Gonzalez, Sergio-S805

Goody, Michael-S159, S160

Goossens, Liesbet-S669

Goossens, Tom L.-S596, S669

Gordienko, Valery-S383

Gordon, Jonathan-S806, Cochair Session 1pAB (S16), Cochair Session 2pABc (S241)

Gorman, Christopher-S349, S691
Gosse, Guillaume-S91

Goth, Yvon-S92

Goto, Masataka-S837

Gottfried, Terry L.-S919

Gouedard, Pierre-S874

Gough, Peter T.-S615, S930, S931, S939, Cochair Session 5aUWd (S930)

Gounot, Yves J.r.-S963

Goupell, Matthew J.-S87, S89, S594

Gourdon, Emmanuel-S68

Gourlie, Sarah-S164

Goursolle, Thomas-S431, S864, S867

Gouvea, Evandro B.-S774

Gover, Brad N.-S9, S226

Govokhina, Oxana-S970

Gowans, Shannon-S811

Graça Ruano, Maria-S259

Graber, Hans-S620

Grabinger, Jens-S570, S602

Graetzer, Simone-S360

Graf, Sabine-S392

Graff, David-S365

Grandchamp, Xavier-S611, S772

Granger, Christian S.-S607

Granström, Björn-S971

Grant, Kenneth W.-S743, S965

Grantham, Wesley-S489

Grassi, Elena-S965

Grassi, Massimo-S445

Grataloup, Claire-S598, S751

Grauby, Stephane-S737

Graves, Stefanie K.-S541

Grawunder, Sven-S111, S763

Gray, Michael D.-S33, S946

Green, David N.-S860

Green, Gary G.-S748, S766

Green, Jordan R.-S109, S771

Green, Linda S.-S17

Green, Tim-S748

Greenaway, Mark-S581

Greenberg, Alan-S314

Greenberg, Steven-S882, S964

Greene, Charles R.-S20, S132

Greene, Charles-S483

Greenleaf, James-S731, S820, S821, S823, S825

Greenslade, Joan V.-S221

Greenwood, Margaret-S177

Greff, Raphaël-S594

Greger, Richard-S70

Grelowska, Grazyna-S787, S788

Grenard, Patrick-S655

Greninguey, Gilles-S266

Grenner, Jan-S490

Gressant, Elise-S715

Gribonval, Remi-S834

Grieco-Calub, Tina-S89

Griefahn, Barbara-S193, S481, Cochair Session 3pNSa (S480)

Grieser, Franz-S590, S592

Griesmar,Pascal-S438, S868

Griffa, Michele-S216, S373, S863

Griffice, Charles P.-S119, S985

Griffin, Donald-S691
Griffin, Michael J.-S699, Cochair Session 4pNSa (S698)

Griffin, Michael-Cochair Session 4pNSm (S715)

Griffiths, Hugh-Cochair Session 5aUWi (S938)

Griffiths, Stephane-S69

Griginis, Alessia-S949

Grigoras, Kestutis-S318

Grigorev, Valery-S620

Grigorevsky, Alexander-S72

Grigorevsky, Valery-S72

Grill, Wolfgang-S177, S947

Grilliat, Julien-S572

Grimal, Quentin-S546, S602, S665, S667, S671

Grimault, Nicolas-S82, S333, S334

Grimm, Giso-S198

Groby, Jean-Philippe-S71, S818

Groen, Johannes-S980

Grondel, Sébastien-S730, S878

Grondin, Julien-S665

Gros, Laetitia-S195

Grose, John H.-S744, S882

Grosh, Karl-S260, S887

Grossinho, Jose-S51

Grothe, Timo-S48

Grubesa, Sanja-S63, S951

Gruen, Hubert-S216

Gruhler, Gerhard-S285

Grundmann, Roger-S48, S480

Gryba, Tadeusz T.-S681

Gstöttner, Wolfgang-S199

Guastavino, Catherine-S706, S828, S839, Cochair Session 4pNSd (S706), Cochair Session 4pNSr (S721)

Guaus, Enric-S697

Gudbjoernsson, Sigmar-S674

Gudra, Tadeusz-S733, S810

Guedel, Alain-S719

Guenab, Faouzi-S428

Guenther, Frank H.-S491, S611

Guerich, Mohamed-S761

Guerin, Bernard-S356

Gueriot, Didier-S939

Guerra, Line-S849

Guerra, Melania-S805

Guerreiro, Luís-S299

Guerrini, Piero-S37, S38, S677

Guessoum, Abderrezak-S368, S776

Guettes, Jens-S841

Guettler, Knut-S154, S230, S273, Cochair Session 3pMUa (S477)

Guey, Jean-Luc-S404

Guggenberger, Johannes-S762

Guglielmi, Gilles-S214

Guglielmone, Claudio-S796

Guianvarc'H, Cécile-S676, S961

Guibal, Aymeric-S144

Guidati, Sandro-S59, S263

Guidorzi, Paolo-S232, S648

Guigou-Carter, Catherine-S531, S847, S848, Cochair Session 3aAAb (S388), Cochair Session 4aAAb (S531), Cochair Session 4pAAg (S646) 
Guigui, Christelle-S441

Guilain, Stephane-S549

Guillaume, Gwenaël-S956

Guillaume, Patrick-S532, S642

Guillemain, Philippe-S690, S695, S696, S818

Guillemot, Fabien-S584, S738

Guillermin, Régine-S499, S633, S666, S904, S947, S978

Guillon, Laurent-S116, S472

Guillot, Francois M.-S147

Guillou, Arnaud-S60, S676, S961

Guimarães, Luiz-S813

Guimezanes, Thomas-S272

Guinet, Christophe-S806

Guldenschuh, Markus-S112

Gulec, Kubra Sevim X.-S551

Gulin, Oleg E.-S786

Gullapalli, Rao-S353

Gullo, Marco-S386, S635, S639

Gulsrud, Timothy-S234

Gulyás, Krisztián-S202

Gulyaev, Yurii-S72

Gumerov, Nail A.-S451

Gunel, Banu-S111, S601

Gunesekera, Geshri M.-S105

Günther, Christian-S673

Guo-Qi, L-S129

Guo, Lianghao-S621

Guo, Yueping-S424

Gupta, Hem Chandra-S311

Gupta, Shashank-S292

Gurbuz, Ali C.-S74

Gurevich, Boris S.-S176, S307

Gurevich, Simon B.-S307

Gurovich, Yuriy-S165

Gusev, Vitali-S67, S186, S319, S321, S582, S584, S586,

S737, Cochair Session 2pPAb (S303), Cochair Session 2pPAi (S321)

Gusev, Vladimir-S283

Guthrie, Anne-S941

Gutiérrez Díez, Francisco-S918

Guy, Christine-S282

Guyader, Gaël-S548

Guyader, Jean-Louis-S90, S342,

S536, S604, S741, Cochair

Session 1pSAa (S90),

Cochair Session 2aSAa (S202),

Cochair Session 2pSAd

(S345)

Guz, Alexander-S431

Gyöngy, Miklós-S255

Gygi, Brian-S335

Gyimesi, Kinga-S85

Haag, Thomas-S454

Haak, Alexander-S250

Haak, Hein-S858, S952

Haapakangas, Annu-S3, S700

Habashy, Tarek M.-S405

Habault, Dominique-S900

Haberer, Werner-S816

Haberman, Michael-S625

Habib, Salman-S429

Hachiya, Hiroyuki-S31

Hacihabiboglu, Huseyin-S111

Hackenberger, Wesley-S147

Haddad, Darren-S773
Haddad, Jehuda-S735

Haddad, Karim-S343

Haddar, Mohamed-S958

Haddi, Abdelkader-S759

Haderlein, Tino-S922

Hadziioannou, Celine-S301

Hæggström, Edward-S318, S369, S639, S869, S880

Hafez, Moustapha-S692

Hafter, Ervin R.-S87, S201, S447, S890, Cochair Session 3aPPc (S447)

Hage, Steffen-S237

Hager, Lee D.-S490

Hagerman, Inger-S126

Hagermann, A.-S433

Hagoort,Peter-S612

Hahn, Thomas R.-S653

Haiat, Guillaume-S252, S544, S602, S667, S668, S671

Haider, Manfred-S170, S423

Haindl, Klaus-S283

Hajim, Saad-S435

Hak, Constant C.-S387, S533

Hakulinen, Mikko-S546, S663, S729

Hald, Jørgen-S191, S343, S417, S419

Hale, Marlund-S296

Haley, Patrick-S926

Hall, John-S158

Hall, Joseph W.-S744, S882

Hall, Neal A.-S260, S262

Halle, Pierre-S110

Hallez, Raphael-S758

Hallgren, Mathias-S746

Halmrast, Tor-S7

Haltmeier, Markus-S216

Hamar, Juraj-S209

Hamdi, Mohamed Ali-S603

Hamernik, Roger P.-S748

Hamery, Pascal-S560, S561

Hamet, Jean-François-S420, S421, S707

Hamil, James T.-S329

Hamilton, Mark F.-S164, S252, S320, S407, S946

Hamine, Adil-S265, S869

Hamitouche, Zahia-S865, S866, S957

Hammarlund, Thomas-S59

Hammershøi, Dorte-S278, S286, S449, S559, S885, S887

Hamzaoui, Nacer-S346, S485

Han, Jun-S718

Hancock, Kenneth E.-S88

Hand, Jeffrey W.-S822

Handa, Rajash K.-S399

Handegard, Nils Olav-S244

Hanhijärvi, Kalle-S318

Hansen, Hans-S194, S276

Hansen, Roy E.-S930, S931, Cochair Session 5aUWd (S930)

Hansen, Sabina-S393

Hansen, Uwe J.-Cochair Session $4 \mathrm{aEDa}$ (S550)

Hansen, Uwe J-S550, Cochair Session $4 \mathrm{aEDb}$ (S552)

Hanson, Alexandra-S491
Hanson, Helen M.-S352, Chair Session 2pSCb (S352)

Hantschk, Carl-Christian-S724

Hao, Yen-Chen-S364

Hara, Yoshifumi-S756

Harada, Masaki-S665

Harding, Sue-S756

Hardlooper, Jan-S475

Hardy, Jean-François-S120

Hardy, Pierre-S419

Hargreaves, Jonathan A.-S562

Hariharan, Supriya M.-S240, S927

Harrington, Jonathan-S100, S361, Cochair Session $1 \mathrm{pSCb}$ (S99)

Harris, Gerald R.-S27

Harris, Nicholas R.-S873, S957

Harris, Richard W.-S748

Harrison, Bryon-Cochair Session 2pAAk (S235), Cochair Session 3aAAa (S385)

Harrison, Byron W.-Cochair Session 4pAAi (S649)

Harrison, Byron-Cochair Session 4pAAd (S641), Cochair Session 4pAAf (S644)

Harrison, Christopher H.-S117, S140, S141, S396

Harte, James M.-S886

Hartmann, Michael-S162

Hartmann, William M.-S9, S326, Chair Session 5aED (S827)

Hartung, Carl Fredrik-S203

Harvey, John-S422

Hasegawa, Hideyuki-S819, S820, S 825

Hasegawa, Hiroshi-S565, S749

Hashad, Adel A.-S119, S985

Hasheminejad, Seyyed M.-S93

Hashizume, Akira-S104

Hasler, Paul-S261

Hass, Axel-S346, S418

Hastings, Mardi C.-S136

Hata, Masaki-S33

Hata, Masato-S12

Hatake-Yama, Mika-S318

Hatanaka, Masato-S12

Hatch, Leila T.-S18

Hathi, Brijen-S433

Hattori, Kota-S359

Hattori, Takehito-S925, S933

Hauden, Jérôme-S678

Haumesser, Lionel-S149

Haupert, Sylvain-S670, S817

Haupt, Robert-S75

Haut, Christopher-S41

Havranek, Zdenek-S342

Hawkins, Nick-S62

Hawkins, Sarah-S965, Cochair Session 5aPPf (S895),

Cochair Session 5pPPa (S963)

Hawley, Monica-S749

Haworth, Kevin J.-S373

Hay, Alex E.-S928

Hay, Todd A.-S252

Hayashi, Daisuke-S779

Hayes, Michael-S615, S930, S931, S939

Hayman, Gary-S379, S468, S469
Hayward, Vincent-S444

Hazan, Amaury-S832

Hazan, Valerie-S351

He, Chensong-S466

He, Zhengyao-S147, S148

Healy, Eric W.-S333, S334, S883, S899

Healy, Terry-S244

Heaney, Kevin D.-S973

Heckl, Maria A.-S436, Cochair Session 3aPAc (S434)

Heckl, Maria-Cochair Session 4 pPAb (S723)

Heckman, Chris-S589

Heddle, James-S645

Hedlin, Michael A.-S861, S952

Hefner, Brian T.-S382, S473

Heib, Sebastien-S571

Heidary, Hossein-S264

Heiland, Peter-S845

Heimlich, Sara-S394

Heinemann, Fabian-S967

Heinkele, Christophe-S185

Heinrich, Antje-S920

Heintze, Frank-S695

Heintze, Olaf-S203

Heinz, Martin-S880

Heinz, Michael-S88, S752, S892

Helariutta, Yrjö-S869

Helber, Robert-S654

Helbig, Silke-S199

Heldner, Mattias -S459

Helenius, Riikka-S700

Hélie, Thomas-S555, S690

Hellbrueck, Juergen-S193

Hellström, Björn-S3, S227

Hellweg, Robert-S483, Cochair Session 3pNSb (S482),

Cochair Session 4pNSo(S717)

Helwani, Karim-S642

Heman-Ackah, Yolanda D.-S365

Hemilä, Simo-S540

Hempe, Ian-S638

Hempel, Christian-S466

Henderson, Elizabeth-S395

Hendrie, Darren-S48

Henini, Mohamed-S580, S581

Henrich, Nathalie-S274, S275, S352, S762, Cochair Session 2pMUe (S274)

Henry, Michel-S68, S150

Henstock, Timothy J.-S626

Henyey, Frank S.-S118, S465, S620

Heo, Inseok-S917

Herbison, Sarah W.-S178, S179, S307, S440, S731

Herbold, Eric B.-S303

Herdic, Peter C.-S632

Herf, Stephanie-S917

Herfjord, Kjell-S413

Herman, Bruce A.-S27

Herman, Cila-S579

Herman, Krzysztof-S810

Hermand, Jean-Pierre-S220, S371, S397, S471, S542, S543, S654, S655, S944, Cochair Session 2pUWf (S383), Cochair 
Session 3aUWc (S470),

Cochair Session 4aAO (S542)

Hernández-Cordero,

Jaime-Cochair Session $5 \mathrm{aSCa}$ (S909)

Hernández, Ricardo-S437

Hernandez, Erica-S538

Herrin, Eugene T.-S952

Herrmann, Frank-S103

Herrmann, Jan-S454

Herrmann, Joerg-S816

Hertegard, Stellan-S275

Hervais-Adelman, Alexis G.-S612, S769, S921

Herve, Caroline-S499

Herviou, Cédric-S900

Herzel, Hanspeter-S610

Herzog, Philippe-S270, S340,

S452, S675, S685, S727, S904

Hess, Peter-S429

Hess, Wolfgang-S449

Hetzel, Roland-S61

Hetzer, Claus-S858, S870

Heute, Ulrich-S209, S896

Hewener, Holger J.-S673, S947

Hickling, Robert-S237, S642

Hickman, Granger-S114, S628

Hicks, Michelle-S336

Hidaka, Takayuki-S5, Cochair

Session 1pAAb (S4),

Cochair Session 2aAAa

(S120), Cochair

Session 2pAAg (S229)

Hieronymus, James L.-S205

Hii Jiu Ta, Vincent-S167

Hildebrand, John A.-S131, S132, S133, S395

Hilge, Catja-S529

Hill, Martyn-S873, S957

Hill, Randy-S476

Hillenbrand, James M.-S358, S747

Hillenbrand, James-Cochair Session 2pSCc (S354)

Hiller, Robert-S591

Hilmarsson, Haraldur-S674

Himmel, Chad N.-S709, S797

Hines, Paul C.-S465,

S655, Cochair Session 2pUWa

(S375), Cochair Session

3aUWa (S464)

Hintikka, Tuomas-S880

Hinton, John F.-S64

Hinton, Oliver-S924

Hintzsche, Matthias-S168

Hiramatsu, Sota-S648

Hirao, Yoshihiro-S698

Hirata, Yukari-S357, Cochair

Session 2pSCc (S354)

Hireche, Omar-S740

Hirsch, Christoph-S724

Hirsch, Karl-S856, Cochair

Session 5aNSj (S855)

Hirschberg, Avraham-S282

Hiruma, Yuki-S594

Hiryu, Shizuko-S238, S810

Hixson, Elmer-S149

Hjelmervik, Karl Thomas-S466
Hladky-Hennion, AnneChristine-S71, S407, S486

Hladky, Anne-Christine-S70, S73, S607

Ho, Julio C.-S948

Ho, Kwok S.-S406

Hobbs, Brian-S561

Hodgdon, Kathleen-S276

Hodgkiss, William-S617, S627, S656, S925, S934

Hodgson, Murray-S563, S792, S950, Cochair Session

5aAAa (S791),

Cochair Session 5aAAe (S801)

Hodoshima, Nao-S361

Hoekje, Peter L.-S270

Hoen, Michel-S598, S751

Hoffmann Bion, Ricardo Augusto-S351

Hoffmann-Kuhnt, Matthias-S393

Hoffmann, Pablo F.-S278

Hoffmann, Susanne-S242

Hoglund, Evelyn-S890

Hohmann, Beat W.-S559, Cochair Session 4aNSb (S558)

Hohmann, Beat-Cochair Session 4pNSh (S710)

Hohmann, Volker-S42, S197, S198, S328, S338

Højen, Anders-S214

Höjer, Martin-S719

Hol, Maarten-S693

Holden, Mark-S224

Holderied, Marc W.-S238

Höldrich, Robert-S112, S684

Holland, Charles W.-S116, S140, S141

Holland, Christy K.-S144, S248, S664

Holland, Keith-S173

Holland, Mark R.-S544

Holland, Scott K.-S351, S457

Holleman, Iwan-S871

Holmes, Blythe-S335

Holmes, Jason D.-S937, S938

Holmes, Stephen D.-S82

Holonowicz, Piotr-S832

Holst-Jensen, Ole-S179

Holsteyns, Frank-S77, S591

Holt, Marla-S17

Holt, R. Glynn-S217, S372, S591, S593, S631, S787

Holzfuss, Joachim-S588, Cochair Session 4aPAc (S588), Cochair Session 4pPAi (S735)

Honarvar, Farhang-S179, S309

Honda, Kiyoshi-S763, S766, S770

Hong, Seokchan-S439

Hongisto, Valtteri O.-S3, S226, S700, Cochair Session 1pAAa (S2)

Hongisto, Valtteri-Cochair Session 2pAAd (S227)

Hongo, Satoshi-S44, S267

Honjo, Yasunori-S819

Honzik, Petr-S261

Hoole, Phil-S108, S363, S771, S772, Cochair Session 4pSCa (S762)
Hoover, Anthony-Cochair Session 1pAAd (S11), Cochair Session 2pAAj (S234), Cochair Session 5aNSc (S846), Cochair Session 5aNSi (S855)

Hoover, K Anthony-S12, S13

Hopgood, James R.-S618

Hopkins,Kathryn-S742

Hoppe, Elizabeth-S371, S374

Hörchens, Lars-S440

Hori, Hiroshi-S405

Horn, Libbie-S20

Hornikx, Maarten-S184, S563, S955, Cochair Session 2pNSf (S287), Cochair Session 4 aNSc (S562)

Hornsby, Benjamin-S200

Hornstein, Melissa K.-S985

Horoshenkov, Kirill V.-S66, S316, S547

Hort, Matthew-S860

Horvat, Marko-S63, S533, S647, S649, S951

Hoshimiya, Tsutomu-S318

Hoshino, Akemi-S105

Hosoi, Hiroshi-S208, S337, S544

Hosokawa, Atsushi-S671

Hosokawa, Tatsuro-S810

Hossack, John A.-S825

Hossam Eldien, Hany-S646

Hosseini, Hemed-S578

Hosseini, S. M. Reza-S435

Hou, Chaohuan-S983

Houben, Mark M.-S599

Houben, Rolph-S198

Houde, Robert A.-S747

Hougland, Dana S.-S3

Houix, Olivier-S446, S721

Hourcade, Sylvain-S548

Houser, Dorian S.-S538, S540, S805, S806

Houssay, Anne-S478

Houston, Brian H.-S632, S634

Houthuijs, Danny-S480

Howard, Carl Q.-S93

Howard, David M.-S274, Cochair Session 1pMUc (S50)

Howard, Matt-S19

Howard, Wheeler-S859

Howarth, David-S924

Howarth, Henrietta-S699

Howe, Bruce M.-S945

Howe, Michael S.-S608

Howey, Russell-S472

Howles-Banerji, Gabriel-S250

Hozumi, Naohiro-S30

Hsiao, Fu-Li-S312

Hsieh, I-Hui-S328

Hsu, Jin-Chen-S311

Hsu, Stephen-S824

$\mathrm{Hu}$, Changqing-S622

$\mathrm{Hu}, \mathrm{Hefei-S73,} \mathrm{S306}$

$\mathrm{Hu}, \mathrm{TaO}-\mathrm{S} 397$

Hu, Ying-S906

Huang, Chen-Fen-S137, S926

Huang, Chun-Fang-S355

Huang, Dehua-S38

Huang, Hong-S272

Huang, I-Hua-S664

Huang, Jin-Huang-S347
Huang, Lianjie-S115, S216, S863

Huang, Patty P.-S637

Huang, Qiang-S327, S333, S355, S746

Huang, Shaoling-S248

Huang, Wan-Ting-S354

Huang, Ying-S325, S327, S333, S334, S355, S896

Huang, Yongjun-S378

Huber, Franz-S387

Huber, Jessica E.-S948

Huber, Rainer-S43, S198

Hucho, Carsten-S486

Hudde, Herbert-S596

Hudson, Dean-S561

Hueber, Thomas-S970

Huebner, Sebastian V.-S395, S804

Huelsz, Guadalupe-S574

Huet, Maxime-S53

Hull, Andrew J.-S91

Humble, Travis-S779, S982

Hume, Ken-S426

Humer, Catrin-S4, S798

Humes, Larry-S748

Humphrey, Victor F.-S379, S468, S474, S546, S623

Hunt, William D.-S71

Hunter, Alan J.-S149

Hunter, Gordon J.-S463

Huo, Lu-S896

Hurault, Jérémy-S720

Hurley, David H.-S186, Cochair Session $4 \mathrm{aPAb}$ (S580)

Hurley, David-Cochair Session 4pPAj (S737)

Hurley, Gail-S20

Hursky, Paul-S395, S781, S782, S923, Cochair Session $4 \mathrm{aAO}$ (S542)

Huseby, Morten-S182

Huszty, Csaba-S649, S803

Hutcheson, Florence-S425

Hutchins, David-S406, S433, S734

Hutson, David-S816

Huttunen, Tomi-S80

Huynh, Agnès-S581, S586

Hwang, Yun-Fun-S347

Hynes, Tom P.-S53

Hynynen, Kullervo-S26, S824

Iaccarino, Gianluca-S570

Iacoponi, Andrea-S169

Iadevaia, Michele-S291

Iakimova, Galina-S107

Iakovleva, Ekaterina-S442

Iannace, Gino-S223, S635, S948

Ianniello, Carmine-S129

Ianniello, Elvira-S223

Ianovschi, Igor-S810

Ibanez-Cuenca, David-S838

Ibertsson, Tina-S362

Ibrahim, Nabilah-S205

Ichchou, Mohamed-S69, S605, S905

Ichii, Taro-S25

Idchabani, Rachida-S172

Idrisi, Kamal-S95, S174

Iemma, Umberto-S160, S555

Ifukube, Tohru-S891

Iglehart, Frank-S949 
Ih, Jeong-Guon-S341, S391

Iida, Akemi-S916

Iida, Hitoshi-S364

Iida, Kazuhiro-S488

Ikeda, Yusuke-S683

Ikeshita, Kazuki-S820

Ilinskii, Yurii A.-S252, S320, S826, S946

Imada, Toshiaki-S365, S750, S774

Imagawa, Hiroshi-S763

Imaizumi, Satoshi-S104, S108, S767

Imam, Neena-S366, S777

Immanen, Juha-S869

Immenga, Dirk-S244

Inácio, Octávio-S15

Inayat Hussian, Husnain-S741

Infantosi, Antonio F.-S258

Ing, Ros Kiri-S675

Ingelaere, Bart-S222, S300, S390, S391, S799, S848

Ingham, Derek-S280

Ingold,Kirk-S60

Ino, Shuichi-S891

Inoue, Kenji-S768

Insausti, Igor-S708

In 'T Panhuis, Peter-S741

Ioana, Cornel-S134, S495, S657

Ioup, George E.-S974

Ioup, Juliette W.-S974

Irino, Toshio-S106, S750, S769

Irwin, Julia-S920, S921

Isaev, Alexander-S379, S380

Isakson, Marcia-S782,

Cochair Session

2pUWg (S384), Cochair

Session 4pUWb (S781)

Ise, Shiro-S683

Iseli, Markus-S492

Ishida, Hideki-S750

Ishikawa, Takashige-S698

Ishimitsu, Shunsuke-S212, S443, S754

Iskarous, Khalil-S364, S767, S917

Islas, Alan-S981

Ismaili Alaoui, Naima-S729

Issenmann, Bruno-S441, S488

Itakura, Toru-S205, S779

Ito, Atsuko-S12, S13, S128

Ito, Takeshi-S870

Ito, Takumi-S749

Itoh, Motokuni-S190

Itou, Katunobu-S778

Iturbe, Ion-S944, S980

Ivakin, Anatoliy N.-S633, S928, S978

Ivančević, Bojan-S593, S872

Ivansson, Sven M.-S498, Cochair Session 3pUWc (S498)

Iverson, Paul-S103, S359, S598, S766, Cochair Session $4 \mathrm{aSCb}$ (S611)

Ives, David T.-S769

Ivochkin, Alexander Y.-S960

Iwamiya, Shin-Ichiro-S193, S292

Iwamoto, Takahiro-S30

Iwatsu, Reima-S287

Iwaya, Yukio-S329

Iyer, Nandini-S10, S329, S892
Izbaim, Driss-S265, S730, S869

Izbicki, Jean-Louis-S70,

$$
\text { S729, S865 }
$$

Izewska, Anna-S533

Izosimova, Maria Y.-S258, S304, S429

Izzi, Annamaria-S812

Jabben, J.-S294, S855

Jacewicz, Ewa-S100, S102, Cochair Session $1 \mathrm{pSCb}$ (S99)

Jachmann, Fabian-S486

Jackson, Darrell R.-S473, S976

Jackson, Joseph C.-S809

Jackson, Philip J.-S11, S12, S353, S596, S610, S966

Jacob, Marc C.-S572

Jacob, Xavier-S67, S321

Jacobsen, Finn-S261, S388, S417, S471, S793

Jacobsen, Steven D.-S959

Jados, Benjamin-S66

Jaecques, Siegfried-S669

Jaeger, Marion-S771

Jafargholi, Mahmoud-S578

Jaffe, Christopher-S7, S943

Jaffe, Jules S.-S243

Jagannathan, Srinivasan-S135

Jaillet, Florent-S857

Jain, Manish-S70

Jakata, Kudakwashe-S582

Jakjoud, Hicham-S487

Jakob, Andre-S695, S840

Jakob, Anette-S816

Jamaati, Mahdi-S209, S764

Jamali, Jalil-S309

Jambrosic, Kristian-S533, S647, S648, S649

James, Kevin R.-S465

James, Michael-S158, S854

Jammali, Adel-S931

Jans, Wolfgang-S981

Jansen, Erwin H.-S298, S415

Janssen, Els-S430

Janssen, Enrico-S905

Janssen, Marc R.-S126, S701

Janssens, Karl-S901, S904, S908

Janssens, Tom-S78

Jaouen, Luc-S68, S453

Jarabo, Pilar-S638

Jaroszewicz, Thomas-S597

Jarrot, Arnaud-S495

Jarup, Lars-S480

Jarvis, Susan-S16, S812

Jarzynski, Jacek-S320

Jasa, Tomislav-S618

Jastrzebski, Slawomir-S925

Jaud, Virginie-S936

Jauniaux, Thierry-S651

Jayashankar, V-S257

Je, Yub-S681

Jean, Frédéric-S382, S938

Jean, Philippe A.-S60, S291, S299, S391, S392, S908, S954, S955

Jeannin, Raphaël-S695

Jech, J. Michael-S135

Jeelani, Kamran-S261

Jekosch, Ute-S195, S449
Jemmott, Colin W.-S466, S615, S775

Jenkins, Scott A.-S39

Jennings, Paul-S277, S426

Jennings, Skyler-S892

Jenot, Frédéric-S317, S723

Jensen, Carl R.-S578

Jensen, Finn B.-S976, Cochair Session 2aUW (S219)

Jensenius, Alexander Refsum-S689

Jenserud, Trond-S934

Jenson, Frédéric-S730

Jenssen, Arne Johannes-S246

Jeon, Jin Yong-S120, S229, S230, S390, S796

Jeong, Cheol-Ho-S341, S391

Jepsen, Morten L.-S891

Jespers, Stéphane-S937, Cochair Session 5aUWh (S936)

Jesse, Alexandra-S457

Jesus, Luis-S608

Jesus, Sergio-S397, S614, S923,

Cochair Session $4 \mathrm{pAOb}$

(S656), Cochair

Session 4pAOh (S661),

Cochair Session

5aUWa (S923), Cochair

Session 5aUWf (S933)

Jeurissen, Roger-S589, S735

Ji, Guihua-S621

Ji, Peifeng-S548

Jia, Xiaoping-S302, S305

Jiang, Haisheng-S768

Jiang, Ting-S783

Jiang, Yong-Min-S138

Jim, Kwok Lung-S313

Jimenez, Carlos R.-S645

Jiménez González, Noé-S640

Jimenez, Maria Dolores-S57

Jin, Craig-S40, S41

Jin, Zhaozhang-S11

Jing, Yun-S4, S942

Job, Stéphane-S304

Johannesen,Peter T.-S886, S887

Johansson, Örjan-S202, S331

John, Michael S.-S752

John, Prajas-S240

Johnsen, Eric-S400

Johnson, David L.-S305, S428

Johnson, David-S244

Johnson, G. Allan-S250

Johnson, Mark-S136, S239, S394, S574, S651

Johnson, Marty-S95, S174, S374, S606, S903

Johnson, Paul A. -S302

Johnson, Paul A.-S216, S303,

S373, S863, Cochair

Session 2pPAa (S301), Cochair

Session 2pPAb (S303)

Johnson, Paul-Cochair Session

2pPAi (S321), Cochair

Session 2pPAj (S322)

Johnson, Sam R.-S748, S766

Johnsrude, Ingrid S.-S598, S612, S919, S920, S921

Johnston, Nicholas-S585

Joly, Nicolas-S261, S324, S739

Jonas, Ulrich-S587
Jones, Adrian D.-S246, S466

Jones, Chris J.-S298

Jones, Chris-S940

Jones, Christopher D.-S243

Jones, Dennis F.-S45

Jones, Gary-S87

Jones, Jeffery-S920

Jones, Mark J.-S105, S351

Jones, Nigel-S167, S295

Jones, Stephen A.-S260

Jones, Theodore G.-S985, Cochair Session 5pUWf (S985)

Jonsdottir, Valdis Inigbjörg-S849, S949

Jonsson, Thorin-S809

Joo, Jae Hoon-S934

Joo, Yongtaek-S789

Jordan, Pedro M.-S65

Jordan, Peter-S281

Joseph, Anjali-S127

Joseph, Jayaraj-S257

Joseph, Jeremy-S466

Joshi, Shrinivas G.-S879

Josse, Erwan-S24

Josso, Nicolas-S134, S657

Jouan, Seb-S893

Jouannot, Erwan-S144

Jouffrais, Christophe-S755

Jovicic, Slobodan-S771

Joyce, E-S589

Juaire, Noemie C.-S254

Judge, John A.-S96, S320

Jue, Deborah A.-S702

Jugo, Witew-Cochair Session

2pAAk (S235), Cochair

Session 3aAAa (S385)

Juhl, Peter-S418, S452, S453,

Cochair Session $3 \mathrm{aNSc}$

(S417), Cochair

Session 4pNSn (S716)

Julian, Cárdenas-Barreras L.-S909

Jun, Sun-Ah-S492

Jung, Ralf-S967

Junge, Michael-S450, S758

Junker, Fabrice-S181

Juola, James F.-S211

Jurado, Carlos A.-S196

Jurdy, Basel-S844

Jurkiewicz, Yann-S128, S940

Jurvelin, Jukka-S546, S663, S670, S729

Jusserand, Bernard-S581, S586

Juvé, Daniel-S1

Jylhä, Antti-S553

Ka Mierczak, Jan-S61

Kacem, Aida A.-S605

Kaczkowski, Peter-S28, S35,

S255, S815, Cochair

Session 1pBBc (S35), Cochair

Session 2pBBd (S253)

Kaczmarek, Mariusz-S545

Kaczmarek, Tomasz-S193, S444

Kaddur, Kadija-S250

Kadlec, Frantisek-S463

Kaernbach, Christian-S596

Kahle, Eckhard-S128, S940

Kajima, Shimpei-S916

Kakiuchi, Akihiro-S123

Kaleva, Erna-S258

Kaliski, Kenneth-S64, S567 
Kallioniemi, Antti S.-S546

Kalluri, Sridhar-S200,

S201, Cochair Session 2aPPc (S200)

Kalogerakis, Michael-S23

Kalogiannakis, Georgios-S189

Kaltenbacher, Manfred-S92,

S570, S602, S675, S683, S769, S775

Kamakura, Tomoo-S360, S726

Kamarei, Mahmoud-S497

Kamenicky, Milan-S296

Kameoka, Hirokazu-S837

Kamiyama, Takeki-S773

Kamoulakos, Argiris-S413

Kan, Alan-S40

Kanai, Hiroshi-S819, S820, S825

Kanamura, Ritsu-S767

Kananen, Ville-S369

Kandasamy, Vinod-S923

Kandel, Sonia-S211

Kandula, Max-S282

Kanev, Nikolay-S282

Kang, Hyun-Ju-S799

Kang, Jian-S226, S427, S685

Kantor, Anastasia P.-S959

Kantor, Innokenty Y.-S959

Kapolka, Daphne-S628

Kaptilniy, Alexander G.-S960

Kapustina, Olga-S311

Karabasov, Sergey-S53

Karabutov, Alexander-S187, S189, S319, S672, S960

Karasalo, Ilkka-S788, S978

Karatzas, Tilemachos-S124

Karg1, Steven-S28, S255, S975, S976

Karic, Branimir-S570

Kariya, Yukika-S914

Karjadi, Entin A.-S619, S946

Karjalainen, Janne P.-S670

Karjalainen, Matti-S553

Kärkkäinen, Anu-S318

Kärkkäinen, Leo-S173

Karlsson, Fredrik-S109, S917

Karpiouk, Andrei B.-S826

Karppinen, Timo-S880

Karunanayaka, Prasanna-S351, S457

Karypidis, Charalampos-S103, S214

Kasatkin, Boris-S629

Kashino, Makio-S84, S891

Kassamakov, Ivan-S318

Kastak, David-S18, S20

Kastelik, Jean-Claude-S308

Kasuga, Masao-S565, S749

Kasuya, Hideki-S460

Kas'Yanov, Dmitry-S874

Katagiri, Takasi-S114

Katayama, Tsutao-S665

Kato, Hiroaki-S326, S357

Kato, Kosuke-S642

Kato,Tohru-S191

Katsamanis, Athanasios-S773

Katseff, Shira-S897

Katsnelson, Boris-S619, S620

Katsouyanni, Klea-S480

Katsumoto, Michiaki-S683, S749
Katz, Brian F.-S128, S594, S697, S755, S756, S967, S971,

Cochair Session 5pAAa (S939)

Katz, Brian-Cochair Session 5aAAf (S802)

Kauba, Michael-S905

Kaufman, Jonathan J.-S545

Kaufman, Marc J.-S127

Kausel, Wilfried-S47, S152

Kawabata, Ken-Ichi-S247

Kawahara, Hideki-S106, S750, S769

Kawai, Keiji-S642

Kawakami, Hiroshi-S831

Kawakatsu, Masaki-S750

Kawasaki, Hiraku-S264

Kawasaki, Kenichi-S443

Kazanskij, Aleksandr-S258

Kazdailis, Paulius-S307

Kazemzadeh, A-S359

Kazianis, Spyros-S187

Kchaou, Yamen-S958

Keating, Jennifer L.-S541

Kebkal, Alexey G.-S935

Kebkal, Konstantin G.-S935

Kechida, Ahmed-S115, S368, S776

Kedrinskiy, Valeriy K.-S322, S590

Kedrova, Galina Y.-S357

Keebler, Michael V.-S81

Keefe, Douglas H.-S884

Keen, Rachel-S325, S448

Keevil, Gareth M.-S381

Kehoe, Timothy-S317

Keilman, George-S815

Keith, Graeme-S419

Keith, Stephen E.-S842

Kellermann, Walter-Cochair Session 1pEAb (S40),

Cochair Session 2pEAb (S263)

Kelly, Spencer-S357

Kemp, David-S884

Kemp, Jonathan D.-S127

Kemp, Jonathan-S48

Kendrick, Paul-S171

Kennedy, Anne M.-S249

Kent, Anthony-S580, S581

Kent, Ray D.-S102

Kentish, Sandra-S78

Kephalopoulos, Stylianos-S64, S180

Keprt, Jiri-S349

Keränen, Jukka S.-S3, S226

Kerber, Stefan-S193

Kergomard, Jean-S500, S696,

Cochair Session 4pMUa

(S687), Cochair

Session 4pMUe (S695),

Cochair Session 5aMUa (S828)

Kerketsos, Paris-S899

Kerneis, David-S983

Kerry, Geoff-S171

Keryer, Gilles-S148

Kessel, Ronald-S981

Kessenich, Grace R.-S347

Kessissoglou, Nicole J.-S94, S985

Kestian, Adam-S464

Ketten, Darlene R.-S20, S807
Ketterling, Jeffrey A.-S142, S399, Cochair Session 5aBBa

(S814)

Ketterling, Jeffrey-Cochair Session 5aBBc (S818)

Keur, Wilco-S408

Kewley-Port, Diane-S748

Khachab, Maha-S251

Khaletskiy, Yuri-S281

Khan, Amir-S66, S558

Khan, Md. Tawhidul Islam-S113

Khan, Shafiquzzaman-S167

Khan, Sheraz-S615

Kharbaoui, Weam-S843

Khatib-Shahidi, Bijan-S484

Khatiwada, Rajesh-S921

Khelif, Abdelkrim-S71, S179, S312, S583

Khelil, Mohamed-S114

Khelladi, Hassina-S960

Khelladi, Sofiane-S413, S569

Khenchaf, Ali-S936

Khetselius, Olga Y.-S657

Khine, Yu Yu-S621

Khokhlova, Tatiana-S672

Khokhlova, Vera A.-S29, S35, S253, S280, S399

Khoo, Boo Cheong-S79, S254, S255

Khurana, Poonam-S67

Khuri-Yakub, Butrus (pierre) T.-S407, S408

Kido, Hiroshi-S460

Kikuchi, Toshiaki-S218, S627

Kilappa, Vantte-S666

Kilgard, Michael P.-S757

Killengreen, Tore-S415

Kim, Chul Koo-S808

Kim, Ho Wuk-S292

Kim, Hyun-Min-S796

Kim, Hyun-Sil-S799

Kim, Jae Ho-S230

Kim, Jea Soo-S934

Kim, Jeonghoon-S303

Kim, Jisu-S439

Kim, Kangwook-S74

Kim, Katherine-S19, S395

Kim, Keehoon-S674

Kim, Ki-Han-S159

Kim, Kyung Wha-S457

Kim, Miran-S768

Kim, Sangryul R.-S799

Kim, Sea-Moon-S934, S935

Kim, Su Yeon-S653

Kim, Sung Jong-S565

Kim, Sung Joon-S96

Kim, Sungyoung-S722

Kim, Yang-Hann-S72, S675, S684

Kim, Yong Hee-S120, S229, S230

Kim, Yoon Hwan-S491

Kim, Yoon Sug-S565

Kim, Young H.-S439, S551

Kimura, Masao-S473

Kimura, Miwako-S763

Kimura, Toshiyuki-S683, S749

Kindermann, Lars-S240

King, Frances-S797

King, Joseph A.-S291, S843

King, Joseph-S709

Kinney, Dana-S748
Kinnick, Randall-S825

Kinns, Roger-S985

Kinoshita, Toshihiro-S405

Kinoshita, Yuko-S909

Kinou, Masafumi-S829

Kirby, Raymond-S547, S683, Chair Session 4aEA (S547)

Kirschner, Francis-S415

Kirsteins, Ivars-S780, Cochair Session 4pUWa (S780)

Kiser, Brenda H.-S839

Kishen, Anil-S254

Kiszel, AnnaK.-S439

Kitamura, Christine-S358

Kitamura, Tatsuya-S355, S763, S766, S768

Kitchens, Jonathan Paul-S470

Kjems, Ulrik-S98

Klaeboe, Ronny-S167

Klapuri, Anssi-S836

Klaseboer, Evert-S79, S255

Klaucke, Ingo-S381

Klaus, Sabine K.-S270

Klauson, Aleksander-S440, S631

Kleber, Felicitas-S100

Klein, Philippe-S420, S421

Kleiner, Mendel-S225,

S640, Cochair Session 2aAAc (S122)

Kleinhenrich, Christian-S907

Kleiven, Stig-S579

Klemina, Anna-S256

Klemperer, Simon L.-S131

Kletschkowski, Thomas-S96, S907

Klieber, Christoph-S319

Klinck, Holger-S132, S240, S394

Kling, Christoph-S534

Klosak, Andrzej K.-S231, S385

Kloser, Rudy-S659, S660

Kloth, Melanie-S58

Kluk, Karolina-S752

Klump, Georg-S84

Klusek, Zygmunt-S382, S474

Knauer, Christian-S981

Kniesburges, Stefan-S769

Kniffka, Anke-S734

Knight, Rachael-Anne-S105, S351

Knobles, David-S137, S656

Knoll, Thorsten-S816

Knuth, Matt-S302

Ko, Doyuen-S11

Kob, Malte-S550, S609, Cochair Session 4aEDa (S550), Cochair Session 4aEDb (S552)

Kobayashi, Kazuto-S30

Kobayashi, Kei-S750

Kobayashi, Maori-S751, S891

Kobayashi, Norimasa-S564, S603

Kobayasi, Kota-S237

Koblitz, Jens C.-S805

Koby, Joseph R.-S431

Koch, Robert A.-S397

Koch, Robert M.-S95, S681, Chair Session 4pEAa (S673)

Kochanek, Krzysztof-S331, S598, S753 
Kochmann, Markus-S349

Kocian, Raymond L.-S405

Koehl, Vincent-S330

Koelewijn, Thomas-S326

Koenig, Chris-S130

Koenig, Christian R.-S349, S707

Koenig, Laura L.-S106, S357, S769

Koga, Takashi-S534, S797

Koga, Yuichiro-S684

Kohler, Erwin-S422

Kohlrausch, Armin-S194, S211, S488, S596, Cochair Session 2pPPa (S325), Cochair Session 3aPPc (S447), Cochair Session 3pPPa (S488), Cochair Session $3 \mathrm{pPPb}$ (S490), Cochair Session 4aPPa (S594), Cochair Session $4 \mathrm{aPPb}$ (S597), Cochair Session 4pPPc (S747), Cochair Session 5aPPd (S889)

Koizumi, Takaaki-S668

Kok, Jim B.-S436, S438

Kolar, Miriam A.-S637

Kolba, Mark P.-S74

Kolesov, Ilya A.-S176

Kolios, Michael C.-S31, S32

Kollmeier, Birger-S198, S338, S489, S746, S885, Cochair Session $2 \mathrm{aPPb}$ (S197), Cochair Session 2pPPf (S336)

Komatani, Kazunori-S98

Kominek, John M.-S916

Komiya, Shintaro-S574

Kondo, Hirohito M.-S84

Kondoz, Ahmet-S111, S601

Kondylas, Kathleen-S291, S843

Kong, Lingzhi Z.-S325, S746

Kong, Ying-Yee-S86

Konkel, Frederic-S695

Konkle, Anne-S842

Konofagou, Elisa-S248, S249, S824

Konradl, Josef-S191

Kontogeorgakopoulos, Alexandros-S698

Kontomichos, Fotios-S739

Kooiman, Klazina-S247

Koopmann, Cary H.-S204

Kopčo, Norbert-S750

Kopiev, Victor-S877

Kopko, John-S642

Kopp, Laurent-S115

Koppens, Jeroen-S194

Koprulu, Kerem-S677

Kopylova, Daria-S187

Korakas, Alexios-S630

Korman, Murray S.-S75, S320,

Cochair Session 3pPAb (S487)

Korman, Murray-Cochair Session 4pPAd (S726)

Kornilov, Victor-S436

Kornycky, Josef-S601

Korobov, Alexandr I.-S258, S304, S429, S733

Korotchenkov, Oleg-S726

Korotin, Pavel-S497
Kors, Eugène J.-S556

Korte, Megan-S537, S539

Kos, Pawel-S759, S760

Kosevich, Yuriy A.-S580, S585

Koshkin, Vladimir-S319

Kosikowski, Lukasz-S753

Kosmidis, Costas-S187, S583

Kostek, Bozena-S209, S298, S331, S753

Koster, Andrew-S795

Kotarbinska, Ewa-S560

Kotas, Charlotte-S653

Kotschy, András-S120

Kottege, Navinda-S39

Kotus, Jozef-S61, S331

Kouider, Sid-S612

Kouidri, Smaine-S161, S720

Koukos, Ioannis-S780, Cochair Session 4pUWa (S780)

Koukoulas, Triantafillos-S469

Kourtiche, Djilali-S487

Koutsioubas, Alexandros-S739

Kováč, Richard-S209

Kovalevskiy, Mikhail-S322

Kovinskaya, Svetlana-S591

Koyama, Daisuke-S736, S815

Koymen, Hayrettin-S268

Kozaczka, Eugeniusz-S786, S787, Cochair Session 4pUWg (S786)

Kozlov, Anton V.-S962

Kozlowski, Emil-S560

Kröger, Matthias-S704

Kraft, Barbara J.-S468

Kragh, Jørgen-S421, S719

Krahé, Detlef-S601, S907

Krajcarz, Fabien-S710, S845

Kral, Peter J.-S348

Kraman, Steve-S948

Krane,Michael-S608, S609

Kravchun, Pavel-S7, S246

Krebs, Walter-S158

Kreider, Wayne-S29, S399

Kreuzer, Wolfgang-S451

Krijnders, Dirkjan J.-S333, S354

Krimmel, Jeffrey-S399

Kripfgans, Oliver D.-S373

Krishnamurthy, Rohan-S638

Kristensen, Jakob-S393

Kristiansen, Ulf-S547

Krivokapic, Jelena-S455

Krolik, Jeffrey-S114, S628

Kronland-Martinet, Richard-S285, S548, S595, S695, S757

Kropp, Wolfgang-S298, S420, S421, S579, S607, S703, S713, S901, Cochair Session $1 \mathrm{pSAb}$ (S94), Cochair Session 2aSAb (S204), Cochair Session 2pSAe (S347)

Krueger, Ted-S802

Krumbholz, Katrin-S327, S329, S749

Kruss, Aleksandra-S245

Krutyansky, Leonid M.-S461

Krylov, Victor V.-S94, S346, S350, S953

Ku, David-S257, S820

Kubik, Jozef-S307

Kubovy, Michael-S444
Kuczaj, Stan A.-S811

Kudo, Subaru-S682

Kudryashov, Nikolai-S873

Kuehnelt, Helmut-S47

Kuehnert, Barbara-S363, S772

Kuhelj, Lilijana-S169, S295

Kuhl, Patricia K.-S107, S365 S613, S774, S922

Kuijpers, Ard-S705

Kulakova, Liudmila-S308

Kulichkov, Sergey-S734

Kulik, Yakov-S272, S694

Kulkarni, Abhijit-S87

Kumar, Biju-S408

Kumar, Kishore-S257

Kumar, Kshitiz-S774

Kumaradas, J. Carl-S32

Kunimatsu, Sunao-S698

Kunz, Edward L.-S633, S634

Kuperman, William

A.-S215, S219, S496, S627, S628, S662, S677, S925, S977

Kupersztoch, Daniel J.-S709, S797

Kupnik, Mario-S407, S408

Kurimo, Mikko-S213

Kurisu, Kaoru-S104

Kurisu, Kiyohiro-S361

Kurogi, Yuka-S124

Kurosawa, Minoru K.-S114

Kurth, Frank-S131

Kutluay, Umit-S947

Kutzner, Dariusz-S355

Kuwano, Sonoko-S191, S192

Kuznetsova, Anastasia S.-S869

Kuznetsova, Iren E.-S80, S869, S879

Kwon, Youngmin-S802, S940

Kyhn, Line A.-S240, S395

Laaboubi, Mustapha-S204, S877

Laback, Bernhard-S87, S89, S594, S595

Lacatis, Radu-S228, S230, S231, S636, S706

Lace, Natalija-S811

Lafarge, Denis-S68, S160, S316, S324

Laffez, Patrick-S186

La Follett, Jon-S977

Lafon, Brice-S419

Lafon, Cyril-S28, S29, S814

Lafon, Philippe-S159, S181

Lagrange, Mathieu-S778

Lagrée, Pierre-Yves-S49

Laguerre, Laurent-S958

Lahivaara, Timo-S80

Lai, Wen-Hsing-S913

Lakhlii, Zakia-S729

Lakusic, Stjepan-S291

Lal, Amit-S409

Lamalle, Laurent-S352, S762

Lamarche, Geoffroy-S660

Lamarque, Claude-Henri-S185

Lambert, Jacques-Cochair Session 3pNSa (S480)

Lamesch, Sylvain-S275
Lamkanfi, Ebrahim-S429, S440, S866

Lammers, Marc O.-S804, S811

Lammert, Adam-S211

Lamminen, Mikko O.-S78

Lamotte, Lucille L.-S419

Lamraoui, Ammar-S436

Lancia, Leonardo-S109

Landes, Hermann-S92

Lane, Harlan-S491

Lang, Alexandre-S697

Lang, Hermann-S283

Lang, William-S163

Langer, Sabine-S206

Langley, Robin S.-S90

Langlois, Sabine-S599

Langner, Gerald-S601

Langrenne, Christophe-S340, S341

Lankarany, Milad-S209, S764

Lannie, Michael-S228

Lanzillotti Kimura, Norberto Daniel-S586

Lapi, Marc-S94

La Pica, Armando-S386, S635, S639

Laprie, Yves-S458, Chair Session $3 \mathrm{aSCc}(\mathrm{S} 458)$

Laptyeva, Tetyana V.-S311

Larabi, Hocine-S73

Larcheveque, Lionel-S856

Lardat, Raphaël-S268, S308

Lardies, Joseph-S679, S777

Lardy, Michel-S870, S953

Larkin, Barry-S831

Larmat, Carene-S216, S303

Laroche, Chantal-S845

Larose, Eric-S96, S301, S963

Larroche, Thierry-S324

Larry, Pater-S854

Larsby, Birgitta-S746

Larson, Gregg D.-S74

Larsson, Krister-S579

Lasaygues, Philippe-S34, S307, S666, S947

Laskowski, Kornel-S459

Latif, Rachid-S204, S877

Latvala, Antti-S598

Lau, Fernando J.-S414

Laude, Vincent-S179, S312, S583, S876

Laugier, Pascal-S33, S256, S544, S545, S546, S663, S664, S665, S667, S670, S671, S817, S819, Cochair Session 4aBB (S543), Cochair Session $4 \mathrm{pBBc}(\mathrm{S} 671)$

Laumann, Klaus-S547

Launer, Stefan-Cochair Session 1pEAc (S42), Cochair Session 2pEAf (S267)

Laurent, Jérôme-S302

Lauriks, Walter-S66, S67, S71, S315, S317, S669, S671, S725, S893, Cochair Session 1pPAa (S65), Cochair Session 2aPAa (S172), Cochair Session 2pPAf (S314), Cochair Session 3pPAa (S485), 
Cochair Session 5aPAi (S874), Cochair Session 5pPAc (S961)

Lautenbach,Margriet R.-S121

Lauterborn, Sonja-S323

Lauterborn, Werner-S79

Lauwers, Dirk-S168

Laux, Didier-S316, S438, S441

Laval, Robert-S219

Lavandier, Catherine-S166

Lavandier, Mathieu-S9, Cochair Session 1pAAc (S8), Cochair Session 4pAAc (S640)

Lavery, Andone-S26, S245, S929, S930, Cochair Session 4pAOc (S657), Cochair Session 5aAO (S813)

Lavie, Antoine-S759

Laville, Frédéric-S597

Lavoie, Maryse-S156, S689

Law, Franzo-S105, S767

Lawn, Chris J.-S435

Lawson, Aaron-S103, S104

Lawson, Gareth-S26

Lazarev, Leonid A.-S345

Le Bas, Pierre-Yves-S430, S431, S863

Lebiedowska, Barbara Joanna-S310, S851

Leblanc, Alexandre-S56, S759

Leblanc, Elisabeth-S803, S804

Lebonnois, Sebastien-S432

Le Bot, Alain-S865

Le Bourdon, Thibault-S716

Lebourges-Dhaussy, Anne-S24

Le Carrou, JeanLoic-S42, S154, S693

Lecce, Leonardo-S291, S759

Lech, Michał-S209

Lechner, Florian-S77

Leclaire, Philippe-S67, S647, S669

Leclercq, Ludovic-S60

Leclere, Quentin-S42, S207, S419, S565

Le Cocq, Cecile-S490

Le Conte, Sandie-S412, S477

Le Corre, Frederic-S290

Lee, Chan-Hui-S684

Lee, Chih-Kung-S147

Lee, Connie G.-S941

Lee, Craig M.-S22

Lee, Doheon-S533

Lee, Edward R.-S105

Lee, Gilbert F.-S624

Lee, Haksue-S408, S681

Lee, Hye Mi-S229

Lee, Hyojin-S128

Lee, Hyun Ho-S292

Lee, Hyunjoo Jenny-S408

Lee, Jung-In-S362

Lee, Jung-Won-S362

Lee, Jungmee-S892

Lee, Keunhwa-S789

Lee, Mingu-S917

Lee, Nelson-S554, S905

Lee, Pyoung Jik-S390

Lee, Sang Kwon-S292, S565

Lee, Seokjin-S682
Lee, Sukmyung-S362

Lee, Sung B.-S259

Lee, Sungbok-S359

Lee, Theresa M.-S887

Lee, Wai-Sum-S459, S916

Lee, William-S984

Lee, Williams-S614

Lee, Wu-Jung-S806

Lee, Yung P.-S984

Leese, Mark R.-S433

Lefebvre, Alain-S549

Lefebvre, Antoine-S47, S155

Lefebvre, Fabrice-S442, S731, S878

Lefebvre, Jean-Etienne J.-S681

Lefebvre, JeanPierre-S251, S666

Le Floch, Vincent-S545

Le Gac, Jean-Claude-S397, S654, S655

Le Gac, Séverine-S592

Le Gall, Yves-S266

Le Garrec, Thomas-S54, S161

Leger, Agnes-S745

Legland, Jean-Baptiste-S315

Le Goff, Nicolas-S488

Le Gonidec, Yves-S936

Legris, Michel-S382

Legros, Claude-S102

Legros, Mathieu-S679, S818

Le Hir, Nathalie-S548

Lehman, Sean-Cochair Session 3pSP (S494), Cochair Session 4aSPa (S614), Cochair Session 4pSPa (S775)

Lehmann, Kenna D.-S395

Lehodey, Patrick-S244

Le Houedec, Donatien-S421

Le Huerou, Jean-Yves-S868

Lei, Bo-S497

Leiderman, Ricardo-S258

Leighton, Timothy G.-S117, S136, S379, S382, S432, S433, S474

Leija, Lorenzo-S256

Leijon, Arne-S198

Leissing, Thomas-S954

Leistner, Philip-S170

Leite, Julio C.-S729

Lelong, Joel-S60, S416, S707

Le Louarn, Arnaud-S581

Lemaire, Michaël-S317

Lemaitre, Aristide-S581, S586

Lemaitre, Guillaume-S285, S446, S721

Leman, Adrien-S98

Lemarquand, Guy-S69

Le Marrec, Loic-S307, S346, S877

Lemor, Robert M.-S402, S673, S815, S816, S880, S947

Le Moyne, Sylvie-S477

Lenczner, Michel-S324

Leniowska, Lucyna-S759, S760

Leniowski, Ryszard-S760

Lentz, Jennifer-S889

Leon, Fernand-S440, S632

Leonard, Daniel J.-S609

Leonard, Michel-S936

Leopold, Mardik-S651
Lepage, Kevin D.-S21, S466, S622, Cochair Session 3pUWa (S495), Cochair Session $4 \mathrm{aUWd}$ (S627)

LePage, Kevin-Cochair Session 4pUWe (S784)

Leparoux, Donatienne-S405

Le Pichon, Alexis-S858, S859, S870, S953, Cochair Session 5aPAa (S858), Cochair Session 5aPAf (S870)

Lepikh, Yaroslav I.-S776

Lepine, Christine-S754

Le Piver, Francoise-S861

Le Pollès, Thierry-S739

Lepper, Paul A.-S19, S812

Le Quere, Patrick-S740

Lerch, Reinhard-S570, S675, S683, S775

Lermusiaux, Pierre-S926

Lerosey, Geoffroy-S216

Le Roux, Jean Christophe-S46, S151

Le Roux, Jonathan-S837

Leroux, Maud-S720

Leroy, Pierre M.-S452, S904

Leroy,Valentin-S72, S735, S736, S876

Lescop, Olivier-S715

Leslie, Wayne-S926

Lesoinne, Stéphane-S801

Leth, Siv-S300

Lethiecq, Marc-S819, Cochair Session 5aBBa (S814), Cochair Session 5aBBc (S818)

Létourneaux, Fabien-S298, S415

Le Touzé, Grégoire-S776

Leung, Chi Wah-S313

Leung, Nina-S768

Leval, Julien-S272

Le Van Suu, Thierry-S261

Levassort, Franck-S819

Leveque, Gerard-S961

Levi, Susannah-S363

Levinson, Samantha-S652

Levit, Natalia-S291, S709, S843

Levitsky, Semyon-S735

Levitt, Harry-S200

Levy, Antoine-S346

Levy, Erika S.-S105

Levy, Erika-Cochair Session 1pSCc (S102)

Lewandowski, Marcin-S369

Lewis, Hannah G.-S488

Lewis, Matthew S.-S955

Lewitz, Joel-S3

Lewy, Serge-S571

Leydier, Audrey-S257

Leylekian, Laurent-S282

Leyssens, Jan-S704

Lezziero, Alberto-S677

Lhémery, Alain-S544

Lhuillier, Vincent-S907

Liétard, Romain-S631

Li, Bin-S914

Li, Binghui-S21, S22

Li, Feipeng-S965

Li, Fenghua-S383, S496

Li, Haibo-S559
Li, He-S922

Li, Jing-Fang-S340, S418

Li, Jingyu Y.-S334, S746, S882, S896

Li, Junfeng-S44, S267

Li, Liang-S325, S327, S333, S334, S355, S745, S746, S752, S753, S882, S896

Li, Ming-S390

Li, Mingxuan-S439

Li, Pai-Chi-S189

Li, Qi-S349, S986

Li, Shui-S378

Li, Tingting-S366

Li, Weichang-S924

Li, Wen-S349

Li, Xilu-S621

Li, Xiukun-S366, S785

Li, Xuan-S983

Li, Yan-S576

Li, Yifeng-S862

Li, Zhenglin-S621

Liang, Kenneth-S404

Liao, Jia-Shiou-S106

Libbey, Brad-S862, S864

Liberman, Mark-S910

Liberman, Mikhail Y.-S570

Licitra, Gaetano-S65, S423, S852, Cochair Session 1pNSd

(S64), Cochair Session 2aNSd

(S167), Cochair Session 2pNS1 (S294)

Lielens, Gregory-S901

Lienard, Jean-Sylvain-S108, S109

Lieuwen, Tim-Cochair Session 3aPAc (S434), Cochair Session 4pPAb (S723)

Lievens, Matthias-S534

Liew, Yun Fook-S76

Light, Roger-S585

Lihoreau, Bertrand-S185

Lilkendey, Robert M.-S841, S940

Lillamand, Ivan-S879

Lim, Geunbae-S406

Lim, Raymond-S787, S975, S976

Lim, Yong-Kon-S934, S935

Lin, Chia-Yu-S147

Lin, Lin-S314

Lin, Wei-S129, S670

Lin, Yi-Syuan-S107

Lin, Ying-Tsong-S620, S621, S975

Linares, Georges-Cochair Session 2aSCc (S212)

Lindahl, Berit-S225

Lindau, Alexander-S530, S642

Lindberg, Børge-S213

Lindblom, Björn-S410, S762

Linde, Bogumil B.-S175, S439

Lindegren, David-S331

Linderman, Matthew-S103

Lindqvist, Magnus-S58

Lindstrom, Fredric-S559

Lindwall, Dennis A.-S985

Lingsch, Stephen C.-S464

Link, Gerhard-S769, S775

Lipkens, Bart-S438,

Cochair Session 3aPAd (S438)

Lippert, Alexander-S77, S591

Lippold, Roland-S564 
Lisenkov, Ivan-S72

Liss, Julie-S917

Lissek, Hervé-S95, S263, S443, S674, S905

Litniewski, Jerzy-S669

Litovsky, Ruth-S87, S89

Littrell, Robert-S260

Litvak, Leonid-S87

Liu, Chang-S358

Liu, Dalong-S32

Liu, Fang-S493

Liu, Jin-Yuan-S926

Liu, Qiang-S469

Liu, Wei-S548

Liu, Xiaofeng-S353

Liu, Xiaojun-S318

Liu, Yongwei-S347, S349, S986

Liu, Yu-Cheng-S347

Liu, Yun-Hui-S906

Liu, Yunbo-S27

Liviani, Linda-S855

Ljunggren, Fredrik-S795

Llanos-Vazquez, R.-S694

Llisterri, Joaquim-S909

Llort-Pujol, Gerard-S984

Lo, Kam W.-S374

Locascio, James-S130, S133

Loeppert, Peter V.-S259

Loevenbruck, Hélène-S352, S492

Lofqvist, Anders-S362

Lognonné, Philippe-S434

Logutov, Oleg-S926

Lohász, Máté Márton-S280

Lohman, Walter J.-S57

Lohr, Bernard-S476

Lohrenz, Maura-S980

Lohrmann, Martin-S566

Lohscheller, Jörg-S770

Lohse, Detlef-S77, S142, S143, S252, S589, S592, S735

Løkkeborg, Svein-S18

Lokki,Tapio-S791, S940, S968

Lombard, Anthony-S198

Lombard, Bruno-S173, S877

Lomonosov, Alexey M.-S429

Lonergan, Michael-S282

Long, Glenis R.-S885

Longair, Malcolm-S385

Longo, Cristina-S407

Longoni, Marco-S295

Lopes, Joseph-S787, S975

Lopes, Leonard V.-S426

Lopez-Bascuas, Luis E.-S765

Lopez-Poveda, Enrique A. -S891

Lopez-Poveda, Enrique

A.-S886, S887

Lopez-Rivas, Rebeca M.-S395

Lopez, Ines-S417, S704, S713

Lopez, José J.-S234, S793, S830

Lorang, Xavier-S203

Lorenz-Kierakiewitz, KlausHendrik-S121

Lorenz, Ralph D.-S432, S433

Lorenzi, Christian-S335,

S742, S743, S745, S895, S963, Cochair Session $4 \mathrm{pPPa}$

(S742), Cochair Session 4pPPb (S745)

Lorenzo, Carlos-S638

Loriette, Vincent-S403
Loro, Carmen L.-S642

Losa, Massimo-S423

Loske, Achim M.-S398

Lotton, Pierrick-S463, S576, S739, S740

Lou-Møller, Rasmus-S674

Loubeau, Alexandra-S184

Loughlin, Patrick-S496, S981

Loussert, Alain-S148

Louviot, Rémi-S605

Loverde, John-S222, S536

Løvholt, Finn-S953

Lowe, Michael J.-S686

Lowenberger, Federico-S478

Lozada, José-S692

Lu, Hsiu-Jung-S102

Lu, Xiaozhen-S591

Lu, Xugang-S212

Lu, Yadong-S15

Lu, Yan-Chen-S758

Lu, Youyi-S104

Lu, Zhiqu-S305

Luan, Yu-S95

Lubert, Caroline-S850

Lubman, David-S636, S839, S951, Cochair Session 4pAAa (S635),

Cochair Session 4pAAb

(S639), Cochair Session 5pNSa (S948)

Lubniewski, Zbigniew-S813

Luby, James C.-S39

Lucero, Jorge C.-S106, S610

Lucidarme, Olivier-S144

Lucke, Klaus-S812

Luding, Stefan-S178, S305

Lueck, Rolf-S244

Luethi, Nicole-S60

Luna Valiente, Nina V.-S16

Lundén, Peter-S969

Lund, Fernando-S440

Lunde, Even Borten-S468

Luneville, Eric-S440

Lunkov, Andrey A.-S627

Lunner, Thomas-S98

Lunsford, Chris-S807

Luo, Ercang-S573, S575, S741

Luo, Gangming-S545

Luo, Haibiao-S400

Luo, Haosu-S816

Luo, Jianwen-S824

Luo, Jing-S619, S946

Luo, Jun-S147

Luo, Xiao Yu-S609

Luong, Jeane-S718

Luppé, Francine-S963

Lurton, Xavier-S660, S984, Cochair Session 4pAOd (S658), Cochair Session 4pAOg (S659)

Lusseyran, François-S577

Lust, Pieter-S441

Lutfi, Robert A.-S447

Lutman, Mark E.-S746, S887, S888

Luts, Heleen-S198

Luzzi, Sergio-S290, S294

Lyashenko, Inna O.-S733

Lyashenko, Oleg V.-S732, S733

Lynch, Cathy-S86
Lynch, James F.-S139, S467, S619, S620, S621, S626, S937, S938, S975, Cochair Session 4aUWa (S619)

Lyons, Anthony P.-S117, Cochair Session 5aUWc (S927)

Lyons, Anthony-Cochair Session 5aUWg (S935)

Lyras, Andreas-S187, S583

Lyzenga, Johannes-S746, S966

Ma, Hongqiang-S666

Ma, Hua-S777

Ma, Jie-S237

Ma, Liang-S916

Ma, Ning-S326

Ma, Xiaochuan-S983

Ma, Yixin-S825

Ma, Yuanliang-S147, S497, S626

Maas, Edwin-S491

Maass, Kirsten-S673, S815

Mabe, James-S903

Macaulay, Eric-S326

Macauslan, Joel-S919

Macdonald, Ewen-S773

Macdonald, Heather-S598, S919

Mach, Susan M.-S933

Macherey, Olivier-S89

Machetta, Igor-S901

Machi, Junji-S33

Macho-Stadler, E.-S694

Machuca, María-S909

Mackey, Allison-S598

Macklem, Peter-S273

Maclennan, Duncan P.-S816

Madekivi, Seppo-S933

Madigosky, Walter M.-S624

Madricardo, Fantina-S677, S783

Madsen, Peter T.-S136, S239, S393, S395, S805

Madsen, Thomas O.-S214

Madshus, Christian-S953

Maeda, Shinji-S458, S770

Maeda, Yasukazu-S779

Maempel, Hans-Joachim-S530

Maestre, Esteban-S697

Maffei, Luigi-S427, S500, S635, S948, Cochair Session 5aNSd (S849), Cochair Session 5 pNSa (S948)

Magezi, David A.-S327

Magin, Richard L.-S822

Magni, Lorenzo-S423

Magnin, Marie-S599

Maguer, Alain-S37, S38

Maher, Paul M.-S816

Mahjoob, Mohammad-S151

Mahn, Jeffrey-S390

Mahoney, Robert-S224

Maier, Andreas-S922

Maijala, Panu-S181

Maillard,Eric-Cochair Session 5pUWd (S982)

Maillard, Virginie-Cochair Session 3pNSc (S484), Cochair Session 4aNSd (S564), Cochair Session 4pNSf (S707)

Majdak, Piotr-S87, S89, S594

Makabe, Yoshiaki-S779
Makarov, Oleg-S176

Mäkelä, Jyrki-S365, S774

Makin, Simon J.-S8

Makino, Koichi-S182

Makino, Yoshikazu-S870

Mäkivirta, Aki-S122

Makov, Yuri-S880

Makris, Nicholas-S135, S413, S972, S973

Malakooti, Sadeq-S93

Malbéqui, Patrice-S957

Maldonado, Marc-S298

Malek-Madani, Reza-S320

Maleke, Caroline-S824

Malfanti, Iacopo-S313

Malicka, Alicja N.-S897

Mallaroni, Bastien-S270

Malloggi, Florent-S321

Malm, Peter-S58, S59

Malzone, Chris-S381

Mamou-Mani, Adrien-S838

Mamou, Jonathan-S33,

S142, Cochair Session 1pBBb

(S30), Cochair Session 2pBBf (S256)

Manasseh, Richard-S144

Manca-Demirdjian, Florence-S293

Mancuso, Antonio-S337

Mani, Sanjay-S781

Mann, David-S130, S133, S476, S811

Mannaris, Christophoros-S142

Manneville, Sébastien-S488

Mano, Isao-S664, S667

Manoha, Eric-S56, S424, S850, S856

Manohar, Srirang-S402, S403, S673

Mantani, Shigeki-S810

Mante, Pierre-Adrien-S737

Mantouka, Agni-S382, S474

Mantsevich, Sergey-S177

Manvell, Douglas-S483

Manzanares-Martinez, Betsabe-S71

Manzi, Nicholas J.-S593

Mao, Jie-S439

Mapagha, Saul-S93, S203

Maragos, Petros-S773

Marant, Vincent-S557

Marchal, Jacques-S148, S788, S927

Marchand, Sylvain-S835

Marchiano, Régis-S487, S727, S856

Mardjono, Jacky-S569

Marec, Anne-S114, S430

Marentakis, Georgios-S830

Margnat, Florent-S161, S281

Margulis, Elizabeth H.-S832

Marical, Patrick-S957

Marin, Pablo-S45

Marin, Stefania-S361

Marinosci, Cosimo-S641

Markham, Benjamin-S791

Markley, Douglas C.-S46

Marks, Emilia A.-S106, S765

Marks, Lawrence E.-S338

Marlin, David H.-S287

Marone, Chris-S302 
Marozeau, Jeremy-S338, Cochair Session 2pPPc (S332),

Cochair Session 2pPPg (S338)

Marquina, Montserrat-S909

Marquis-Favre, Catherine-S843

Marrero, Victoria-S909

Marriner, Duane E.-S59

Mars, Jérôme I.-S495, S657, S776, S944, S980

Marsden, Olivier-S54

Marsh, Richard-S45

Marshall, Harold-S5, S943

Marsico, Giuseppe-S386

Marston, Philip L.-S588, S822, S976, S977

Marston, Timothy-S871

Marszal, Jacek-S266

Martín-Moreno, Luis-S178

Martínez-Mora, Juan Antonio-S937

Martínez, Ignacio-S638

Martínez, Manuel J.-S566

Martellotta, Francesco-S639

Martens, William L.-S121, S599, S722, Cochair Session $1 \mathrm{pSPa}(\mathrm{S} 112)$

Marti, José-S563

Martin, Arnaud-S982

Martin, Bryn A.-S947

Martin, Gérard-S342

Martin, Glen K.-S884

Martin, Heiko J.-S387, S532, S533

Martin, James S.-S33, S946

Martin, Michael J.-S623

Martin, Philippe-S263

Martin, Vanessa A.-S627

Martin, Vincent-S93, S203, S627. S716

Martinez, Loic-S438, S464, S878

Martins, Fernando-S530

Martins, Nelson-S614

Marui, Atsushi-S722

Marumo, Mieko-S831

Maruszewski, Bogdan T.-S485

Marutyan, Karen R.-S544

Maruvada, Subha-S27

Marvi, Hossein-S209, S764

Marx, David-S162, S577, S772

Marxer, Ricard-S832

Mary, Ivan-S856

Marzalek, Michael-S87

Mas, A.-S811

Mas, Peter-S901, S908

Masaki, Shinobu-S766, S768

Masato, Kiuchi-S679

Masera, Davide-S732

Mashoshin, Andrey I.-S784

Masi, James-S861

Masoero, Marco-S644, S842

Mason, Timothy J-S589

Massarani, Paulo-S535

Masson, Catherine-S818

Masson, Jeremy-S679

Masson, Patrice-S571

Mast, Douglas T.-S664

Mastik, Frits-S30

Mastromatteo, Galia-S638

Masullo, Massimiliano-S948

Masuyama, Hiroyuki-S149

Mate, Bruce-S19
Mateos, Toni-S792, S801, S969

Mathias, Delphine-S132, S807

Mathieu, Jérôme-S257

Matignon, Denis-S687

Mato-Méndez, Fernando José-S850

Matsuda, Osamu-S186, S189, S582, S583

Matsukawa, Mami-S544, S665, S667, S668, S669, S679,S725

Matsumoto, Yasunao-S698

Matsuo, Ikuo-S238

Matsuzaki, Hiroki-S770

Matte, Guillaume-S727

Mattei, Pierre-Olivier-S270, S727

Matthies, Melanie L.-S491

Mattia, G. Mario-S754, S843

Mattingly, Michelle B.-S320

Matula, Thomas-S79,

S590, Cochair Session 3pPAb

(S487), Cochair Session 4pPAd (S726)

Matveev, Anton-S379

Maue, Jürgen-S558

Mauermann, Manfred-S885

Mauler, Dirk-S198

Maurel, Agnes-S72, S440

Maurer, Ben-S243

Maurice, Roch-S34

Maurin, Michel-S60, S289

Maury, Cédric-S288, S344, S716

Mavroidis, Theodoros-S780

Maxit, Laurent-S92

Maxwell, Cynthia-S554

Maxworthy, Tony-S119

May, Chris-S759

Mayer, Alexander-S152, S270

Mayer, Dirk-S905

Mayne, Keith-S45

Mayr, Andreas R.-S207, S389

Maysenhölder,

Waldemar-S847

Maze, Gerard-S204, S440, S631, S730, S782, S877

Mazhar, Suleman-S806

Maznev, Alexei A.-S73, S187

Mazzi, Marco-S37

McAdams, Stephen-S210, S285, S339, S444, S596, S599, S778, S830

McAleer, Claire R.-S160

McAteer, James A.-S399

McCarthy, Clara-S19

McCarthy, Elena-S812

McCauley, Robert-S18

McClellan, James H.-S74

McCoin, Jaime-S897

McComb, Peter J.-S244

McCool, Benjamin-S314

McCoy, John-S91, S96

McCurdy, Katie E.-S104

McDonagh, Thomas R.-S809

McDonald, Kelly-S83

McEwan, Ian-S433

McFadden, Dennis-S887

McGee, Joann-S537, S539

McGettigan, Carolyn-S362, S363

McGillicuddy, Glenn-S468

McGowan, Richard S.-S608, S764
McGuire, Sarah-S481

McHugh, Ron-S926

McInnes, Christopher-S79

McKinley, Richard-S163, S561

McKinley, Robert-S158

McLaughlin, Jack-S134

McLennan, Conor T.-S756

McLennan, John-S478

McLennan, Miles-S132

McLeran, Aaron-S833

McMahon, Donald J.-S545

McNally, Thomas-S13

McNeill, Chris-S67

McPhee, John-S412, S692

McPherson, David D.-S144, S248

McQueen, James M.-S457

McRobbie, Graeme-S45

Meares, David-S11, S12

Meaud, Julien-S887

Mebarek, Lassen-S603

Mechri, Charfeddine-S586

Meddis, Raymond-S83

Medvedev, Ruslan N.-S727

Meerton, Leah-S336

Mefferd, Antje-S109

Megahed, Mustafa-S413

Meggs, Carl-S816

Megherbi, Fouzia-S114

Megill, William M.-S392

Megill, William M.-S239

Meier, Robert-S860

Meiler, Martin-S92, S675, S683

Meillier, Jean-Louis-S707

Meissner, Miroslaw-S943

Mejia, Paloma-S484

Mekhedov, Dmitrii-S429

Melchionna, Salvatore-S291

Mellert, Volker-S41, S193

Mellinger, David K.-S22, S132, S394, Cochair Session $3 \mathrm{aABb}$ (S394)

Mellinger, David-Cochair Session $4 \mathrm{pABc}$ (S651)

Mellow, Timothy J.-S173

Melo, Francisco-S304

Melodelima, David-S27, S814

Melon, Manuel-S340, S341

Meloni, Tommaso-S60

Melvin, Tracy-S957

Memoli, Gianluca-S65, S843, S852

Ménard, Lucie-S110, S491

Mendret, Julie-S441

Menemenlis, Dimitris-S945

Menexiadis, Geraldine-S860

Meng, Di-S934

Meng, Qingbin-S308

Ménoret, Arnaud-S720

Menounou, Penelope-S157

Mensah, Serge-S34, S251

Mentink, Johan-S871

Menzel, Daniel-S194

Menzies-Gow, Dylan-S969

Meral, Faik Can-S822

Meral, Ramazan-S928

Merani, Diego-S936

Mercer, James A.-S945

Merchel, Sebastian-S195

Mercier, Jean-Francois-S162, S414
Mercogliano, Francesco-S129

Mercure, Elizabeth-S34

Merer, Adrien-S757

Merheb, Bassam-S70, S313, S878

Merkel, Aurélien-S321

Merks, Ivo L.-S43

Mermet, Matthieu-S566

Merrick, Richard-S18

Merrill, Loren-S652

Mersad, Karima-S107

Mertens, Paul-S77, S78

Merthayasa, I Gde Nyoman-S8, S230

Merz, Sascha-S985

Messaoud-Galusi, Souhila-S351, Chair Session 2pSCa (S351)

Messum, Piers-S361

Mesterházy, Beáta-S847

Mestouri, Hind-S148

Metlushko, Vitali-S587

Mettin, Robert-S77, S79, S591

Metzger, Kurt-S945

Metzner,Walter-S237

Meunier, Fanny-S598, S751

Meunier, Nicolas-S415

Meunier, Sabine-S339, S595, Cochair Session 2pPPc (S332), Cochair Session 2pPPg (S338)

Meyer, Bernard-S895

Meyer, Jürgen-S6

Meyer, Matthias-S542, S543, S654

Meyer, Richard J.-S46

Meynier, Cyril-S409, S679

Meza, Leonardo-S223, S646

Meziri, Mahmoud-S256

Miah, Khalid-S149

Miao, Jianmin-S260

Michaels, Jennifer E.-S114

Michalis, Krista A.-S793

Michalopoulou, Zoi-Heleni-S139, Cochair Session 2pSPe

(S371), Cochair Session $4 \mathrm{aSPb}$ (S616)

Michalski, Ranny L.X.-S535

Michaud, David S.-S842

Micheau, Philippe-S605, S903

Micheyl, Christophe-S81, S898

Middlebrooks, John C.-S88

Midoux, Patrick-S250

Miedema, Henk M.-S57

Mielczarek, Adam-S747

Mielke, Jeff-S492

Mienkina, Martin-S408

Mietchen, Daniel-S539

Mietlicki, Fanny-S58

Miette, Veronique-S32

Miglietta, Piercarlo-S796

Migliori, Albert-Cochair Session 5aPAe (S868), Cochair Session 5aPAg (S872), Cochair Session 5aPAl (S879),

Cochair Session 5pPAb (S959)

Migneron, Jean-

Gabriel-S120

Migneron, Jean-Philippe-S120

Miguel Alvarez, Jose-S729

Mijic, Miomir-S529, S801 
Mikalauskas, Robertas-S286

Mikhalevsky, Peter

N.-Cochair Session 1pAOa

(S21), Cochair Session

2pAOb (S245)

Miki, Akira-S12

Miklos, Andras-S49, S50

Miles, Anthony W.-S546

Miles, Ronald N.-S260, S261, S262

Mileshin, Victor-S281

Milford, Ingunn-S168

Mill, Robert W.-S376

Miller, Christopher-S620

Miller, Douglas-S145

Miller, James G.-S544, Cochair Session 4aBB (S543)

Miller, James H.-S20, S139, S394, Chair (S502), Cochair Session 5aAO (S813)

Miller, James-S766,

Cochair Session 4pBBc (S671)

Miller, Joanne L. -S966

Miller, Lee A.-S477

Miller, Nicholas P.-S481

Miller, Rachel M.-S364, S768

Miller, Ryan-S11

Millet, Christophe-S861

Millot, Florence-S162

Millot, Laurent P.-S51, S694

Mills, Pierre-S305

Milosic, Zvonimir M.S.-S785

Minamide, Ayumu-S265

Minard, Antoine-S285

Mincu, Ciprian D.-S56

Mincu, Daniel-Ciprian-S856

Minhao, Y-S586

Minonzio, Jean-Gabriel-S218

Mir, Ali M.-S870

Miranda, Luis-S757

Mironov, Mikhail-S282

Mirza, Pasovic-S727

Misdariis, Nicolas-S285, S446, S697, S721

Mishra, Shrikanta-S888

Miskiewicz, Andrzej-S385

Mistry, Shilpa T.-S757

Misuchenko, Natalia-S251

Mitchell, Stuart B.-S254

Mithieux, François-S28

Mito, Yuki-S831

Mitri, Farid-S731, S820

Mitterer, Holger-S214

Mitton, David-S665

Miura, Masanobu-S692, S829

Miura, Takahiro-S891

Mixdorff, Hansjörg-S456, Cochair Session 3aSCa (S455)

Miya, Naoki-S574

Miyabe, Jun-S736

Miyabe, Sakaya-S817

Miyashita, Toyokatsu-S71

Miyazaki, Ayano-S784

Miyoshi, Kazunori-S756

Mizuno, Katsunori-S544, S665, S667, S669

Mizutani, Koichi-S149, S150, S218, S265, S548, S627, $\mathrm{S} 785$

Mleczko, Dominik-S537

Mleczko, Michal-S408
Mlynski, Rafal-S561, S598

Moate, Benjamin D.-S929

Moates, Danny R.-S106

Mobley, Frank-S158

Moeck, Jonas P.-S437

Mofid, Yassine-S32

Mohamed, Mostafa H.-S316

Mohammad, Bashar-S926,

Cochair Session

$5 \mathrm{aUWb}$ (S926)

Mohammadi, Nader-S93, S151

Mohammadi, Saeed-S71

Mohanty, Sanat-S70

Møhl, Bertel-S239

Moilanen, Petro-S663, S666

Moisiadis, Yiannis-S857

Mojtabi, Abdelkader-S579

Mojtabi, Marie-Catherine-S579

Mokbel, Chafic-S251

Mokhtari, Parham-S355, S763

Molares, Alfonso R.-S537, S547

Molchanov, Vladimir-S176

Moldenhauer, Patrick-S704

Moleti, Arturo-S885,

Cochair Session 5aPPb (S883),

Cochair Session 5aPPc (S886)

Molevich, Nonna-S723, S724

Molin, Nicolas-S425

Molla, Stephane-S857, S900

Møller, Henrik-S171, S196, S568

Möller, Sebastian-S97, S331, S449, S896

Monaco, Ernesto-S759

Monaco, Giulio-S582

Monaco, Pierpaolo-S273

Monaghan, Jessica J.-S98, S371

Mongeau, Luc-S484,

S608, Cochair Session 3pNSc (S484), Cochair Session 4aNSd (S564), Cochair Session 4pNSf (S707)

Montagu, Julie-S922

Montaldo, Gabriel-S460

Montanari, Pio-S691

Montgermont, Nicolas-S156, S829

Montignies, Francois-S330, S755

Moon,Wonkyu-S406, S408, S681

Moonen, Marc-S97, S198, S201, S893

Mooney, T Aran-S650

Mooney, T. Aran-S538

Moore, Brian-S197, S478, S742, S751, S890, Cochair Session 4pPPa (S742), Cochair Session 4pPPb (S745)

Moore, Patrick W.-S806

Moore, Stephanie A.-S928

Moore, Thomas-S349, S691, S693, Cochair Session 2aMUa (S151), Cochair Session 2pMUa (S269)

Moorhouse, Andy T.-S208, S299, S344

Mooshammer, Christine-S108, S363, S772, S915, Cochair Session 5aSCc (S913)
Mora-Camino, Félix A.-S296

Morano, Janelle-S19

Morant, Salvador-S827

More, Shashikant R.-S483

Moreau, Antoine-S572

Moreau, Danielle J.-S95

Moreau, Solenn-S577, S740

Moreau, Stéphane-S570, S571, S720

Morel, Cédric-S569

Morell, Maria-S651, S811

Moreno, Antonio-S172

Moreno, Cesar-S711

Moreno, Guillermo-S417

Moretti, David-S16, S651, S806, S812

Morgan, Andrew-S944

Morgenstern, Christian-S564

Morières, Stéphane-S880

Mori, Hiroki-S460

Mori, Kazuyoshi-S784, S785

Morimoto, Masayuki-S640

Morino, Luigi-S160

Morioka, Miyuki-S699

Morise, Masanori-S106

Morita, Shinya-S692

Mørkholt, Jakob-S419

Morland, Cameron-S377

Morley, Erica L.-S809

Morozov, Andrey-S924

Morretta, Mario-S169

Morris, Philip J.-S52, S426,

Cochair Session 1pNSa

(S52), Cochair Session 2aNSa

(S157), Cochair Session 3aNSa (S413)

Morris, Philip-Cochair Session $2 \mathrm{pNSb}$ (S280)

Morris, Robin D.-S328

Morris, Scott C.-S572, Cochair Session 4aNSf (S569)

Morris, Scott-Cochair Session 4 pNSq (S719)

Morrison, Andrew-S831

Morrison, Barclay-S248, S249, S826

Morrison, Geoffrey Stewart-S351, S358, S909

Morrissey, Ronald P.-S16, S651, S812

Mortazavi, Seyed Taha-S629

Morvan, Bruno-S70, S71, S729, S865

Mosbah, Pascal-Cochair Session 2aEAa (S146), Cochair Session 2pEAg (S268)

Mosca, Frédéric-S115, S266

Möser, Michael-S695

Moss, Cynthia-S236

Moszynski, Marek-S780, S813

Motoki, Kunitoshi-S770

Motsch, Jean-François-S806

Mottay, Eric-S737

Mottron, Laurent-S596

Moubchir, Hanane-S312

Moudden, Ali-S204, S265, S730, S869, S877

Moulet, Marie-Hélène-S203

Moulin, Annie M.-S886, S888

Moulin, Annie-S895

Moulin, Emmanuel-S730, S878
Moum, James-S619

Mounier, Denis-S186, S272, S319, S584, S586, S737

Mountain, David-S377, S807

Moura, André-S428

Mouraille, Orion-S178, S305

Mourjopoulos, John-S739, S793

Moysan, Joseph-S431

Mozhaev, Vladimir G.-S962

Mrabti, Tarik-S873

Muchtadi, Farida Idealistina-S8

Muehleisen, Ralph T.-S66,

S536, S551, Cochair

Session 1pPAd (S76), Cochair Session 4pED (S685)

Muehleisen, Ralph-Cochair Session 2pPAk (S323)

Mueller, Stefan-S769

Muellner, Herbert-S4, S798

Muir, Jesse-S670

Muirhead, Charles-S19

Muirhead, Matthew-S171

Mule, Sebastien-S144

Müller, Dieter H.-S349, S707

Muller, Marie-S545

Müller, Reinhard-S755

Müller, Swen-S6

Mulsow, Jason-S18, S540, Cochair Session 4aABb (S539)

Mulvaney, David-S971

Mulvey, Megan-S335

Muneyasu, Mitsuji-S263

Munhall, Kevin G.-S773

Munro, Kevin J.-S897

Muntag, András-S169

Munz, Claus-Dieter-S159

Murano, Emi-S353

Muraoka, Teruo-S891

Murase, Atsunobu-S190

Murata, Sigeo-S725

Muratore, Robert-S826

Murphy, William J.-S558, S711, Cochair Session $4 \mathrm{aNSb}$ (S558)

Murphy, William-Cochair Session 4pNSh (S710)

Murray, Anita-S19

Murray, Heather-S892

Murray, Kathleen-S365

Murray, Todd W.-S402

Musafir, Ricardo E.-S159, S963

Mustonen, Kimmo-S869

Muthukumaran, Shobha-S78

Mydlarz, Charlie-S712

Myers, Arnold-S153

Naas, Mouna-S315, S669, S671

Nabuco, Marco-S535, S559

Nachtigall, Paul E.-S20, S392, S477, S538, S650

Nackenhorst, Udo-S705

Nadakuditi, Raj-S366

Naei, Mohammad Hasan-S309

Nagahata, Koji-S193

Nagamiya, Masaki-S192

Nagano, Akira-S668

Nagao, Kyoko-S104, S764 
Nagatani, Yoshiki-S208, S544, S669

Nagle, Daniel-S21

Nagy, Attila Balázs-S120

Nah, Young In-S783

Naili, Salah-S544, S602, S667, S668

Naka, Yusuke-S870

Nakadai, Kazuhiro-S98

Nakado, Takashi-S684

Nakagawa,Hiroshi-S344

Nakagawa, Seiji-S212, S754

Nakajima, Tateo-S944

Nakajima, Yoshitaka-S195

Nakamura, Kentarou-S815

Nakamura, Satoshi-S683

Nakamura, Toshiaki-S784, S785

Nakanishi, Takuji-S317

Nakano, Hirofumi-S263

Nakano, Takayoshi-S817

Nakasaki, Ryota-S565

Nakashima, Hirotake-S768

Nakayama, Masashi-S212

Nam, Hosung-S768, S915

Nam, Kwon-Ho-S249

Namba, Seiichiro-S191

Namba, Seiichoro-S192

Nannipieri, Elisa-S233, S798

Naoe, Munenori-S683, S749

Napier, Julie-S539

Narayanan, Shrikanth-S359

Nardi, Damiano-S587

Narins, Peter-S808

Nash, Anthony-S227

Nassar, Georges-S442, S731, S878

Natale, Ciro-S759

Natarajan, Harikrishna P.-S43

Naugolnykh, Konstantin A.-S858

Naumann, Christfried-S111

Navarro-Castillo, Karen-S947

Navasquillo, Joaquin-S706

Naz, Pierre-Cochair

Session 2pSPh (S374), Cochair Session $4 \mathrm{pSPb}$ (S777)

Nazzi, Thierry-S107, S355, S358

N'Djin, William-S27

Neasham, Jeffrey-S924

Necciari, Thibaud-S595

Nederveen, Cornelis J.-S479

Neff, Flaithri E.-S757

Nefske, Donald J.-S602

Negraru, Petru-S952

Negreira, Carlos-S370, S462

Neher, Tobias-S201

Nejade, Armand-S345, S533

Nelisse, Hugues-S454, S597

Nelson, James-Cochair

Session 2pNSj (S291), Cochair Session 2pNSo (S299)

Nelson, Keith-S319

Nelson, Misty-S130

Nelson, Peggy-S198

Nelson, Philip A.-S40, S501

Németh, Bottyán-S803

Németh, Géza-S918

Nemeth, Michael-S130

Nennig, Benoit-S603

Nesterenko, Vitali F.-S303

Nestoras, Christos-S208
Neubauer, Juergen-S610, S774

Neuhoff, John-S445,

Cochair Session 3aPPb (S444),

Cochair Session 4pPPd (S755)

Neumann, Max-S981

Neves E Sousa, Albano-S299

Nevitt, Justin-S377

New, Boris-S355

Newhall, Arthur-S139, S467, S620, S621

Nguyen, Hien B.-S467

Nguyen, Hong Hai-S900

Nguyen, Khoa-Van-S600

Nguyen, Noel-S101, S109

Nicholson, Jim-S495

Nickerson, Matthew L.-S276

Nicolas, Alice-S488

Nicolas, Barbara-S776, S944, S980

Nicolas, Jean-Marie-S115, S939

Nicolas, Taulier-S252

Nie, Yingjiu-S750

Nief, Guillaume-S269

Nielsen, Peter Louring-S140, S141, Cochair Session 3aUWa (S464)

Nielsen, Sofus Birkedal-S123, S827

Niemeyer, Guenter-S554, S698, S905

Niemiec, Jan M.-S624

Niepenberg, Arndt-S907

Niessen, Maria E.-S333, S354

Nieukirk, Sharon-S394

Nightingale, Kathryn-S250, S824

Nightingale, Trevor-S390, S797

Nijhof, Marten J.-S452

Nijman, Eugenius-S901

Nijmeijer, Henk-S417, S704, S713

Nijs, Lau-S849

Nikitov, Sergey-S72

Nikolovski, Jean-Pierre-S675

Nilsson, Erling-S3, S227, S530

Nilsson, Michael-S336

Nilsson, Nils-Åke-S422, S718, S719

Nip, Ignatius-S109

Nishamol, P. A.-S146

Nishihara, Noriko-S5

Nishimiya, Kojiro-S548

Nishimoto, Takuya-S914

Nishimura, Ryouichi-S326

Nishino, Takanori-S684

Nisimura, Ryuichi-S106

Nissen, Shawn L.-S748

Nistea, Ioana-S204, S900

Nito, Takaharu-S763

Nitschmann, Marc-S489

Nizami, Iftikhar-S889

Noble, Alison-S255

Nocke, Christian-S14, S529, Cochair Session 1pAAe

(S14), Cochair Session $2 \mathrm{aAAb}$

(S121), Cochair Session

2pAAh (S232)

Noel, Christophe-S707

Noel, Claire-S983

Noeth, Elmar-S922
Noga, Andrew-S773

Nogueira, Xesús-S413

Noh, Junguk-S682

Noiray, Aude-S110, S917

Noiray, Nicolas-S435

Noirot, Isabelle-S476

Noisternig, Markus-S967, S971

Nolte, Bodo-S789, S981,

Cochair Session 4pUWh (S788)

Nolte, Loren W.-S616

Nomura, Hideyuki-S360, S726

Nomura, Hiroaki-S756

Nongaillard, Bertrand-S442, S678, S731, S878,

Cochair Session 3aPAf (S441),

Cochair Session 4pPAf (S731)

Normand, Xavier-S847

Norris, David-S861

Norton, Guy-S928

Nosulenko, Valery-S755

Nota, Yukiko-S766

Nøttestad, Leif-S25

Nottet, Jean-Bertrand-S886

Nováček, Jan-S463

Novak, Antonin-S463

Novak, Doris-S569

Novell, Anthony-S142

Nowak, Till-S77,S591

Nowicki, Andrzej-S369, S669

Nozaki, Kazunori-S611

Nozawa, Takeshi-S768

Nsenkoue, M-S586

Ntagwirumugara, Etienne-S681

Nummela, Sirpa-S540

Nurzynski, Jacek-S848

Nuster, Robert-S188, S216

Nygård, Børge-S547

Nygaard, Lynne C.-S110, S919

Nymoen, Kristian-S689

Oba, Roger-S621

Oberai, Assad A.-S258, S628

Oberdanner, Hannes-S43

Oberdoerster, Markus-S950

Oberfeld, Daniel-S339

Obleser, Jonas-S612

O'Brien, Jr., William D.-S31, $\mathrm{S} 250$

Occhipinti, Giovanni-S434

Ochi, Hiroshi-S925, S933

Ochmann, Martin-S437, S450, S562, S789, Cochair Session 4pUWh (S788)

O'Connell-Rodwell, Caitlin E.-S131

Odagiri, Yoshitaka-S825

Oddo, Rémy-S454

Odelius, Johan-S202

Odent, Pierre-S63

Odina, Natalya I.-S733

O'Donnell, Matthew-S501

Oehler, Michael-S479, S697

Oelze, Michael L.-S31, Cochair Session 5aPAj (S876), Cochair Session 5pPAd (S962)

Oertli, Jakob-S290

Ogam, Erick-S71, S818

Ogasawara, Hanako-S785

Ogata, Takaharu-S565
Ogata, Tetsuya-S98

Ogawa, Shinji-S817

Oguchi, Keiji-S5

Oh, Won Tchon-S789

Ohbuchi, Takeshi-S149

Ohga, Juro-S682

Ohl, Claus-Dieter-S77, S79,

S592, Cochair Session

1pPAd (S76), Cochair Session 2pPAk (S323)

Ohlau, Katrin-S288

Ohlendorf, Jan-S349

Ohno, Sumio-S364, S455

Okada, Minae-S84

Okada, Nagaya-S30

Okada, Takuro-S73

Okada, Yasuaki-S182

Okamoto, Noriko-S124

Okamura, Tomohiro-S150

Okandan, Murat-S262

Okarura, Eiji-S779

Okcu, Selen-S235, S701

Okopal, Greg-S981

Okorn-Schmidt, Harald-S77

Okuno, Hiroshi G.-S98

Okushima, Limi-S150

Olafsen, Sigmund-S415

Olesen, Søren K.-S278

Oleson, Erin M.-S131

Olive, Sean E.-S278

Oliveira, João M.-S414

Oliveira, Lenine V.-S6

Olkinuora, Pekka-S171, S284

Ollivier, François-S420, S477

Olson, Kenneth S.-S492

Omais, Magdi-S850

O’Malley, Patrick F.-S320

O'Mard, Lowel P.-S334

O'Meara, Niamh-S376

Omeragic, Dzevat-S405

Omologo, Maurizio-S213

Omoto, Akira-S123, S904

Omrani, Abderrazak-S603

Ona, Egil-S468

Onanko, Anatolij P.-S733

Onanko, Yurij A.-S733

Onda, Masaharu-S565

Ongel, Aybike-S422

Ono, Hirotaka-S749

Ono, Nobutaka-S837

Ooi, Andrew-S144

Oomen, Werner-S194

Opielinski, Krzysztof J.-S733

Opitz, Martin-S283

Oralkan, Omer-S408

Ord, Robert-S353

Ordoñez, Rodrigo-S286, S559, S887

Orduña-Bustamante, Felipe-S269

Orfali, Wasim-S645

Orlov, Denis-S367, S497

Orlowski, Andrzej J.-S245

Orlowski, Raf-S6, S385, S940

Ormonde, Christopher

$$
\text { E.-S591 }
$$

O'Rourke, Colleen-S328

Orpin, Alan-S660

Ortiz-Berenguer, Luis I.-S838

Osborne, Alfred R.-S622

Osipova, Ludmila-S809, S810 
Osler, John C.-S465, S655, Cochair Session 4pAOa (S654)

Ossipov, Alexander-S59

Ostashev, Vladimir E.-S287, S955, S973

Ostendorf, Carel-S702

Østerbye, Torkil-S214

Ostrovsky, Lev-S822

Oswald, Julie N.-S239

Otani, Takahiko-S664, S667

Otsuru, Toru-S124

Ottenio, Mélanie-S324

Otto, Andrea-S77, S591

Ouaftouh, Mohammadi-S317, S723

Ouail, Nabil-S308

Oudot, Guillaume-S454

Oudry, Jennifer-S32

Ouedraogo, Boureima-S414

Ouisse, Morvan-S202, S607

Ourak, Mohamed-S317, S723

Owen, Megan A.-S541

Owen, Neil-S28, S29, S814

Oxenham, Andrew J.-S81, S595, S742, S898

Oya, Yoshikazu-S769

Ozcevik, Asli-S844

Ozeki, Yukio-S565

Ozimek, Edward-S355

Ozmeral, Erol-S897

Pääkkönen, Rauno-S349, S854

Paál, György-S49

Pabon, Jahir-S405

Pabst, Oliver-S907

Pacault, Anne-S266

Pace, Nicholas-Cochair Session 5aUWc (S927), Cochair Session 5aUWg (S935)

Pachebat, Marc-S564

Pacheco, Felipe-S321

Pacini, Aude-S538

Padden, Denise-S774

Padilla, Frederic-S544, S545, S664, S665,S670, S671, S819, Cochair Session 4pBBb (S666)

Padilla, Monica-S964

Paek, Hyun-S841, S940

Page, John H.-S73, S306, S313, S735, S736

Page, Suzanne-S652

Paglialonga, Alessia-S887

Pagneux, Vincent-S72, S160, S185, S440, S874,

Cochair Session 3aPAe (S439),

Cochair Session 4pPAe (S729)

Pailhas, Yan-S39, S498

Paillasseur, Sebastien-S418

Paje, S. E.-S314, S718

Pakula, Michal-S307, S545

Palanchon, Peggy-S143

Palaviccini, Miguel-S711

Paleologu, Constantin-S906

Pallas, Marie-Agnès-S416

Pallier, Christophe-S612, Cochair Session 4aSCb (S611)

Pallone, Grégory-S548

Palmeri, Mark-S824
Palmese, Maria-S37

Palmieri, Umberto-S635

Palomäki, Kalle J.-S10, S213, S747

Paltauf, Günther-S188, S216

Pamanikabud, Pichai-S856

Pan, Ho-Hsien-S354

Pan, Yong Dong-S189

Pan, Yongdong-S869

Paniwnyk, L-S589

Pankhurst, Quentin-S249

Pannacci, Nicolas-S72

Panneton, Raymond-S68, S315, S958

Panneton, Robin-S356

Panter, David-S794

Paolero, Anthony-S623

Papadakis, John S.-S543

Papadakis, Panagiotis-S633

Papadimos, Chris-S844

Papadogiannis, Nektarios-S187, S583

Papadopoulos, Timos-S652

Papp, Joseph-S924

Pappalardo, Massimo-S407

Paquier, Mathieu-S330, S695

Parakkal, Santosh-S184

Paraskevas, Ioannis-S171

Parazzini, Marta-S887, S888

Parchment, J-S767

Pardo, Emilia-S729

Pardo, Louis-Ferdinand-S708

Pareige, Pascal-S865

Parenthoine, Denis-S149

Parizet, Etienne-S98, S196, S285, S293, S330, S565, S699,

S717, S755, S968,

Cochair Session 2pNSa

(S276), Cochair

Session 2pNSd (S285)

Park, Hanyong-S364

Park, Jin-Young-S675, S684

Park, Jong Beom-S484, S608

Park, Jong-Kyu-S681

Park, Kwan Kyu-S408

Park, M.-S501

Parker, David-S691

Parmentier, Damien-S150

Parmentier, Hubert-S27

Parmigiani, Fulvio-S587

Parnell, William J.-S624

Parnum, Iain M.-S660

Parrondo, Jorge-S857, S956

Parry, Graham-S62, S567, S854

Parsons, Dan R.-S381

Parsons, Nigel H.-S979

Parthy, Abhaya-S41

Pasanen, Edward-S887

Pascal, Jean-Claude-S340, S418

Paschereit, Christian Oliver-S437

Pascual Winter, Maria Florencia-S581, S586

Paset, Martin-S412

Pasqual, Alexander Mattioli-S675, S685

Pasqualini, Donatella-S429

Passeri, Daniele-S252

Pasterkamp, Hans-S948

Pastore, Richard E.-S339, S446

Pastore, Thomas-S981
Pastuszek-Lipinska, Barbara-S769

Patel, Aniruddh D.-S753

Patel, Ruben-S135

Patel, Sona A.-S913, S916

Patterson, Roy D.-S98, S371, S750, S769, S964

Pätynen, Jukka-S968

Paul, Stephan-S755, S894

Paul, Wesley-S928

Paulo, Joel P.-S649, S718

Paulsen, Reimer-S710

Paun, Marla-S399

Pautet, Lucie-S929

Pauzin, Marie-Christine-S251

Pavan, Gianni-S133

Pavanello, Renato-S603

Pavić, G-S342, S344

Paviotti, Marco-S64, S180

Payà Bellester, Gaspar-S530

Payan, Cédric-S431

Payan, Yohan-S353

Payne, Edward-S144

Payne, Sarah R.-S84

Payne, Stephen-S592

Payton, Karen-S103

Paz, Elaine C.-S853

Pean, Vincent-S335

Pearse, John-S390

Pechter, Tal-S48

Pecknold, Sean-S465, S655

Pedersen, Christian Sejer-S171, S196

Pedersen, Eja-S568, S714

Pedersen, Geir-S468

Pedersen, Michael S.-S98

Pedersen, Michael-S674

Pedersen, Steffen-S171, S568

Pedersen, Torben

Holm-S195, S482

Pedrielli, Francesca-S196

Peebles, Ernst-S133

Peeling, Paul-S616

Peen, Jasper-S415

Peeters, Bert-S705

Peeters, Geoffroy G.-S833

Pelat, Adrien-S185

Pelekasis, Nikos A.-S252, S593

Pelivanov, Ivan-S187, S672

Pellegrino, François-S751

Pellerin, Guillaume-S682, S687

Pelorson, Xavier-S153, S274, S609, S610, S611, S765,

S772, Cochair Session 4aSCa (S608)

Peltier, Christopher-S969

Peltola, Maija S.-S613

Pemble, M-S586

Pena, Hector-S135, S243, S972

Penelet, Guillaume-S576, S740

Peng, Jian-S644

Peng, Jue-S816

Peng, Zhao-S644

Pennec, Yan-S73

Penrose, John D.-S660

Pepin, Henri-S346

Peppin, Richard-Cochair Session $1 \mathrm{pNSc}$ (S61)

Percaccio, Cherie R.-S757

Pereira, Andreia-S761
Pereira, Wagner C.-S256, S258, S259

Perepelkin, Vitaly-S734

Pereselkov, Sergey A.-S627, S628

Perez Carrillo, Alfonso-S697

Perez Trigo, Carlos-S729

Perez-Arjona, Isabel-S313

Pérez-López, Antonio-S269

Perez, Javier-S857

Pergantis, Charles-S253

Perkell, Joseph S.-S491, Chair Session 3pSCa (S491)

Pernet, Sebastien-S162

Pernod, Philippe-S461, S862, S867

Pernot, Gilles-S737

Pernot, Mathieu-S27, S545

Péronne, Emmanuel-S318, S583, S584

Peroutsea, Stavroula-S932

Perov, Dmitry V.-S730, S879

Perrachione, Tyler K.-S765

Perrey-Debain, Emmanuel-S451, S603

Perrier, Pascal-S108, S353, S362, S916

Perrin Decroux, Stephane-S557

Perrin, Bernard-S186, S318, S581, S582, S583, S584, S586, S959

Perrin, Gérald-S719

Perrot, Camille-S68

Perrot, Patrick-S910

Perrot, Thierry-S983

Perry, James-S206, S709, S951

Perry, Jennifer R.-S107

Pershagen, Goran-S480

Person, Roland-S811

Persson Waye, Kerstin-S171, S196, S225, S559,

S700, Cochair Session 2aAAd

(S125), Cochair Session

2pAAc (S224),

Cochair Session 4aNSe

(S567), Cochair

Session 4pAAe (S643),

Cochair Session 4pNSl (S714)

Pescetto, Alessandro-S37

Pestalozzi, Hansueli-S60

Pestereva, Marina-S283

Petculescu, Andi-S432, Cochair

Session $3 \mathrm{aPAb}$ (S432),

Cochair Session 4pPAh (S734)

Peters, Nils-S830

Peterson, Michael-S314

Petersson, Björn A.-S343

Pethieu, Romain-S172

Petillot, Yvan-Cochair Session 5 pUWc (S979)

Petiot, Jef-S152

Petit, Philippe-S666

Petite, Hervé-S819

Petnikov, Valeriy G.-S628

Petosic, Antonio-S593, S728, S872

Petrak, Peter-S296

Petre, Cristian Catalin-S314, S866

Petri, Alberto-S301

Petsculescu, Andi-Cochair Session 5aPAi (S874), Cochair Session 5pPAc (S961) 
Pettit, Chris L.-S182, S955

Pettyjohn, Steve-S226

Petz, Markus-S58, S62

Peyrin, Françoise-S817

Pezerat, Charles-S91, S207, S907,

Cochair Session 2aSAc

(S207), Cochair

Session 2pSAb (S343)

Pezeril, Thomas-S319

Pfeifer, Christian-S437

Pfitzinger, Hartmut-S456

Pham, Thiên-Ly-S667

Pham, Tien-S374

Phatak, Sandeep A.-S965

Phatak, Sandeep-S965

Philipp, Norman-S387

Philippe, Franck D.-S218, S372

Philips, Scott M.-S376

Phiri, Michael-S701

Piñeirua, Miguel-S574

Piacsek, Andrew-S551, Cochair Session 1pPAe (S80)

Piao, Shengchun-S383

Picó, Rubén-S640, S791

Picard, Christophe-S419

Picart, Pascal-S272, S584

Picaut, Judicaël-S792, S942, S956

Piccolo, Antonio-S578

Pichat, Cédric-S352

Pichon, Chantal-S250

Pichora-Fuller, Kathy-S752, S753, S919

Pichot, Christian-Cochair Session 3pSP (S494), Cochair Session 4aSPa (S614), Cochair Session 4pSPa (S775)

Picinali, Lorenzo-S337

Picone, Michael-S860

Picton, Terence W.-S752

Pieleanu, Ioana-S121

Piellard, Mélanie-S161

Pierangelo, Juliette-S346

Pierce, Allan D.-S347, S626

Pieringer, Astrid-S298

Pierucci, Mauro-S279

Pietrzyk, Andrzej-S901

Pihko, Elina-S365, S774

Pilka, Adam-S598

Pillai, Saseendran-S240, S927

Pincas, Jonathan-S596, S966

Pinhède, Cédric-S606, S904

Pinson, Luke J.-S626

Pinson, Samuel-S116

Pinto, Marc-S980, Cochair

Session 5pUWc (S979)

Piperakis, George-S23, S944

Piquet, Francis-S272

Piquette, Jean-S623

Piraux, Joel-S173, S877

Pirogov, Yurij A.-S357

Pirozzi, Salvatore-S759

Piscoya, Rafael-S437, Cochair Session 3aPAc (S434), Cochair Session 4pPAb (S723)

Piserchia, PierreFranck-S861

Pisoni, David B.-S359, S360, S363

Pispola, Giulio-S66
Pistone, Giuseppe-S578

Pitre, Laurent-S676, S961

Pitt, Ian-S757

Pitter, Mark-S585

Pitton, James W.-S376

Plack, Christopher J.-S426, S595, S889, S890

Planeau, Vincent-S533

Plante, Elena J.-S457

Plantier, Frédéric-S960

Plantin De Hugues, Philippe-S102

Plaskota, Przemyslaw-S756

Plassat, Benoit-S842

Plazza, Eric-S174, S302

Pletner, Baruch-S347

Plitsis, Grigorios-S833, S838

Ploix, Marie-Aude-S879

Plotkin, Kenneth J.-S165,S184

Pluymers, Bert-S789

Podimova, Nataliya-S187, S189

Podlipsky, Vaclav

Jonas-S919

Podolian, Artem-S726

Podolsky, Aleksei-S319

Podolsky, Alexei-S672

Poeppel, David-S613

Poignand, Gaelle-S573

Poikonen, Ari-S933

Pointer, Steve A.-S925

Poirier, Benjamin-S288

Poisson, Franck-S183, S297, S900, Cochair Session 2pNSi (S290), Cochair Session 2pNSn (S297), Cochair Session 3aNSb (S415)

Polack, Jean-Dominique-S218, S531, S842

Polacsek, Cyril-S571

Poletti, Mark-S41

Policardi, Franco-S229

Polikarpova, Nataliya-S176, Cochair Session $2 \mathrm{aPAb}$ (S175), Cochair Session 2pPAc (S307)

Polka, Linda-S107, S356, S911, S914, Cochair Session $5 \mathrm{aSCc}(\mathrm{S} 913)$

Pollow, Martin-S646

Polyakov, Valery-S405

Polyzos, Demos-S545

Pomberger, Hannes-S675

Pommeray, Michel-S308

Pompoli, Francesco-S69, S316

Pompoli, Roberto-S231, S636, S950, Cochair Session 2aAAe (S128), Cochair Session $2 \mathrm{pAAb}$

(S223), Cochair Session 2pAAf (S228)

Ponceau, Damien-S861

Poncelet, Oliver-Cochair Session 5aPAk (S878)

Poncelet, Olivier-S324, S345, S875, S877, Cochair Session 5aPAc (S865)

Ponirakis, Dimitri-S18, S19

Ponomarev, Victor-S821

Pontuale, Giorgio-S301

Pook, Victoria G.-S804

Pope, Stephen-S13

Popek, Witold-S253
Popov, Roman-S72

Popov, Vladimir V.-S537, S538

Popper, Arthur-S18

Popplewell, Adrian-S126

Port, Robert F.-S365

Porteous, Ewan-S94

Porter, Colin-S249

Porter, Michael B.-S395, S498, S781, S782

Porter, Tyrone-S29, Cochair Session 2pBBa (S247), Cochair Session 2pBBb (S250)

Posey, Joe W.-S711,

Cochair Session 3aNSe (S424)

Posey, Joe-Cochair Session 4pNSi (S711)

Potamianos, Gerasimos-S971, Cochair Session 5pSC (S970)

Potard, Blaise-S458

Potel, Catherine-S957, Cochair Session 4pED (S685)

Potirakis, Stelios M.-S850, S857

Potirakis, Stylianos M.-S171

Potma, C.-S855

Potty, Gopu R.-S139, S394

Pouet, Bruno-S402

Pouliquen, Eric-S117

Poulsen, Andrew J.-S470

Pouplier, Marianne-S361

Pourkashanian, Mohamed-S280

Poussot, Pascal-S730

Pouzar, Ladislav-S639

Povey, I-S586

Powell, Robert-S484

Powers, Jeffry-S142

Pozarlik, Artur-S436

Pozera, Arunas-S559

Pozhar, Vitold-S175, S176

Prabha, C-S927

Prada, Claire-S188, S218, S372, S865, Cochair Session 2aSPa (S215), Cochair Session $2 \mathrm{aSPb}$ (S217), Cochair Session 2pSPf (S372), Cochair Session 2pSPg (S373), Cochair Session 3aPAe (S439), Cochair Session 4pPAe (S729)

Prasow, Sholem-S127

Prather, Wayne E.-S39

Pratomo, Bayu-S230

Predoi, Daniela-S314

Predoi, Mihai Valentin M.-S314, S866, S957

Preece-Pinet, Melanie-S103, S766

Preis, Anna-S193, Cochair Session $3 \mathrm{aPPb}$ (S444), Cochair Session 4pPPd (S755)

Preisig, James-S118, S924

Prendergast, Garreth-S748, S766

Preobrazhensky, Vladimir L.-S461, S862, S867

Pressnitzer, Daniel-S81, S84, S594, Cochair Session 1pPPa (S81), Cochair Session 2pPPd (S333)

Prezelj, Jurij-S348, S368, S856

Preziuso, Daniela-S639
Price, John C.-S271

Pride, Steven R.-S473

Priesig, James E.-S21

Prieto, Ana-Garcia-S249

Priev, Aba-S821

Priev, Diana-S821

Priewasser, Bernhard-S166

Primus, Julien-S707

Prince, Jerry-S353

Principeaud, Nicolas-S157

Prior, Mark K.-S932, Cochair Session 5aUWe (S932)

Pritchard, William F.-S27

Probst, Wolfgang-S168, Chair Session 2pNSm (S296), Cochair Session 1pNSc (S61), Cochair Session 2pNSc (S284)

Proctor, Michael I.-S767

Prodi, Nicola-S5, S68, S231, S636, S950

Profunser, Dieter M.-S583

Prokhorova, Ekaterina V.-S258

Proklov, Valeriy V.-S175

Proni, John-S244

Prosser, Silvano-S337

Protopappas, Vasilios C.-S545

Proulx, Jennifer-S914

Pruette, Autumn L.-S757

Pruvost, Laurent-S565

Puder, Henning-S198

Pueo, Basilio-S234, S793

Pugliese, Daniel-S737

Pulkki, Ville-S601, S968

Pulli, Jay-S974

Punekar, Jothi-S281

Punisic, Silvana-S771

Purnell, Thomas-S100, S920

Purwins, Hendrik-S832

Pustovoit, Vladislav-S175, S176

Putnam, Robert-S61

Pyl'Nov, Yuri-S461, S867

Pyle, Robert W.-S153, S270

Pyper, Ted-S943

Qi, Yi-S250

Qin, Qin-S953

Qin, Yi-Xian-S665, S670

Qiu, Bo-S15

Qiu, Jian Zhen-S272

Qiu, Wei-S748

Qiu, Xiaojun-S234

Qu, Tianshu S.-S355

Qu,Youli-S15

Quam, Rolf Michael-S638

Quidu, Isabelle-S979, S982

Quijano, Jorge E.-S117, S465,

S466, Cochair Session $3 \mathrm{aSPb}(\mathrm{S} 463)$

Quijano, Jorge-Cochair Session 4 pSPc (S778)

Quinquis, Andre-S495

Quintana, Samuel-S170, S710, S799

Qureshi, Shakeel-S261

Raake, Alexander-S97, S331, S752

Rabau, Guy-S339

Rabenstein, Rudolf-S553

Rabold, Andreas-S388 
Radaelli, Simone-S169

Rader, Tobias-S199

Radhakrishnan, Nandhu-S411

Rafaely, Boaz-S340

Raffellini, Giorgio-S233

Raghukumar, Kaustubha-S627

Raglin, Chris-S192

Raherison, Antoine A.-S681

Rahier, Gilles-S53

Raimond, Andrew-S8, S332

Rajagopal, Akila-S351

Rajan, Srinivasan-S845

Rajan, Subramaniam-S138

Rajoana, Dominique-S782

Rakel, Munise-S486

Rakerd, Brad-S326

Rakotomanana, Lalaonirina-S346

Rakotonarivo, Sandrine-S382

Ramírez, Adriana Citlali-S676

Ramadas, Sivaram Nishal-S268, S680

Ramasamy, Kalaiselvi-S65

Ramaz, François-S403

Ramirez, Gina-S287

Ramirez, Juan-Pablo-S97

Ramis Soriano, Jaime-S150, S206, S265, S443, S646, S647, S648

Ramkumar, Abhishek-S409

Ramos-Mendieta, Felipe-S71

Ramos, Antonio-S256, S729

Ramp, Steve-S620

Rampnoux, Jean-Michel-S737

Ramsay, Gordon-S211, S611

Randrianoelina, Ando-S853

Rangoussi, Maria-S171, S850

Rank, Ernst-S388

Raphael, Lawrence J.-S106, S616

Rapoport, Natalia-S249

Rasamimanana, Nicolas-S696

Rasmanis, Gundars-S126

Rasmussen, Gunnar-S278

Rasmussen, Knud-S261

Rasmussen, Per-S854

Rasmussen, Soren-S64

Raspet, Richard-S578

Rath, Matthias-S555

Rathbun, Daniel L.-S757

Rathsam, Jonathan-S14

Ratilal, Purnima-S135, S813, S972, S973

Ratolojanahary, Faniry Emilson F.-S681

Ratsimandresy, Leong-S818

Rattanadit, Kitti-S320

Raum, Kay-S113, S667, S812, S817, Cochair Session 4pBBa (S663)

Rausch, Jacqueline-S41

Raux, Antoine-S913

Ravazzani, Paolo-S887, S888

Ravina, Enrico-S478, S691

Ravula, Surendra K.-S873

Ray, Manas C.-S902

Ray, Richard-S625

Rayavarapu, Raja-S402

Rebillout, Arnaud-S690

Reboul, Gabriel-S571

Recker, Karrie-S43

Redon, Emmanuel-S414
Redondo, Javier-S313, S640, S791

Redonnet, Stephane-S56, S850, S 856

Reed, Allen-S625

Reed, Star-S896

Reeder, D. Benjamin-S620, S933

Reese, Hauke-S571

Reeves, Justin-S620

Refahi Oskouei, Amir-S264, S348

Regnier, Marion-S965

Reguieg, Djelloul-S670

Rehfeld, Marc-S536, S846

Reichl, Christoph-S283

Reichmann, Hans Josef-S959

Reichmuth, Colleen-S18, S539, S540, S803

Reiff, Christian-S264

Reig Calbo, Lilian-S530

Reig Fabado, Antonio-S557

Reimann, Klaus-S408

Reinhardt, Alexandre-S308

Reinhold, Jürgen-S129

Reining, Friedrich-S283

Reinisch, Eva-S457, Chair Session $3 \mathrm{aSCb}(\mathrm{S} 457)$

Reinten, Hans-S589, S735

Reis, Frigyes-S797, S847

Reiter, Malgorzata-S61, S298

Reitinger, Bernhard-S188

Rekanos, Ioannis-S852

Remenieras, Jean-Pierre-S321, S665

Remes, Ulpu-S213

Remillieux, Marcel C.-S54

Remita, Naamane-S256

Remy, Elisabeth-S542

Ren, Keni-S559

Renaud, Guillaume-S321, S665

Rendón, Pablo L-S269

Renders, Christel-S408

Rene, Pierre-Jean-S95

Rennies, Jesko-S881

Repetto, Stefania-S41

Retsch, Markus-S587

Reubold, Ulrich-S100

Reuter, Christoph-S479, S697

Reuter, Fabian-S79

Reuter, Karen-S286, S559, S885, S887

Reuter, Tom-S540

Reuterdahl, Tom-S20

Revaux, Charles-S494

Revaz, Bernard-S587

Revoredo, Teo C.-S296

Rey, Guillaume-S152

Rey, Robert-S569, S720

Reznikoff, Iegor-S635, S830

Rezvov, Yury-S175

Rhachi, Mohammed-S172, S843

Rhebergen, Koenraad

$$
\text { S.-S199, S966 }
$$

Ribay, Guillemette-S442

Ribeiro Valle Macedo, Marta-S643

Ribeiro, Maria-S530

Riccardi, Michele-S386

Rice, Heath E.-S983

Richard, Céline-S895

Richard,Florent-S279, S286, S330

Richard, Gaël-S832, S836

Richards, Roger T.-S45
Richardson, Michael-S219, S625

Richardson, Nathan-S748

Richardson, W J.-S20

Richecoeur, Franck-S436

Richie, Carolyn-S109

Richoux, Olivier-S185

Richter, Andreas-S480

Richter, Christoph-S437

Rick, John W.-S637

Ricketts, Todd-S489

Ricot, Denis-S282

Ridgway, Sam H.-S17

Ridouane, Rachid-S110

Riekkinen, Ossi-S663, S670, S729

Rienstra, Sjoerd-S741

Riethmueller, Rolf-S659

Rietman, Edward-S438

Rikkonen, Toni-S670

Riley, Amanda K.-S764

Riley, Paul H.-S574

Rimeika, Romualdas-S307

Rimington, Dennis B.-S39

Rimskaya-Korsakova, Liudmila-S745

Rindel, Jens Holger-S14, Cochair Session 4pAAa (S635),

Cochair Session 4pAAb (S639)

Ringgaard, Erling-S674, S814

Rinkevich, Anatoly B.-S730, S879

Rios, Antonio-S909

Riquimaroux, Hiroshi-S238, S810

Risch, Denise-S541

Rivens, Ian-S825

Rivera, Michael K.-S301

Rivoire, Michel-S27

Rixe, Olivier-S144

Rizwi, Farhan-S279

Roads, Curtis-S833

Roan, Michael-S371, S374,

Cochair Session $2 \mathrm{pSPa}$

(S366), Cochair Session 2pSPb

(S367), Cochair Session

$3 \mathrm{aSPb}$ (S463), Cochair Session 4 pSPc (S778)

Robb, Gary B.-S379, S382, S474, S632

Robert, Daniel-S804, S809

Robert, Jacques-S443

Robert, Jean-Luc-S217, S372

Robert, Laurent-S549

Robert, Sébastien-S963

Roberts, Brian-S82

Roberts, Eila K.-S887

Roberts, Paul L.-S243

Robillard, Jean-François-S737

Robin, Benjamin-S842

Robinson, Erik E.-S17

Robinson, Stephen P.-S19, S378,

S379, S468, S469, S623,

Cochair Session

2pUWb (S378), Cochair

Session $3 \mathrm{aUWb}$

(S467), Cochair Session 4aUWb (S623)

Robinson, Stephen-Cochair Session 2pUWd (S380), Cochair Session 4pUWd (S783)
Rocchesso, Davide-S445

Roch-Jeune, Isabelle-S737

Roch, Marie A.-S132,

S133, Cochair Session 2aAB

(S130)

Roch, Marie-Cochair Session 2pABb (S239)

Roche, Jean-Michel-S282

Roche, Roger-S861

Rockstroh, Kurt-S127

Rodet, Xavier-S410

Rodgers, Blake-S920

Rodilla, Miguel-S659

Rodionov, Alexander-S367

Rodkin, Richard B.-S427

Rodonò, Giuseppe-S639

Rodono', Giuseppe-S386, S635

Rodríguez Molares, Alfonso-S855

Rodrigues, Cleide V.-S367

Rodrigues, Dominique-S879

Rodriguez-Manzo, Fausto E.-S235, S641, S645

Rodriguez, Laura-S170, S799

Rodriguez, Samuel-S549

Roebel, Axel-S835

Roehle, Ingo-S162

Roger, Michel-S55, S570, S719, S720, Cochair Session 4aNSf (S569), Cochair Session $4 \mathrm{pNSq}$ (S719)

Rogers, Andy-S184

Rogers, Jack C.-S612, S770

Rogers, Peter H.-S33, S147, S653, S946

Roginska, Agnieszka-S464

Roh, Heui-Seol-S783

Rohdenburg, Thomas-S198

Roland, Jacques-S846, Cochair Session 5aNSc (S846), Cochair Session 5aNSi (S855)

Rolin, J.f.-S811

Roma, A.-S811

Romano, Rosario A.-S129

Romanov, Sergei-S586

Romero, José-S230, S231, S636, S706

Romski, Mary Ann-S328

Ronsse, Lauren M.-S481

Rook, Ronald-S436

Roosnek, Nico-S375, S776

Roovers, Chiel-S297

Roozen, N.b.-S417, S704, S713

Rosa, Manuel-S638

Rose, George A.-S25

Rose, Phil-S909

Rosen, Stuart-S351, S362, S363, S748, S771, S896, S918

Rosenberg, Philip D.-S433

Rosenblum, Lawrence D.-S210, S364, S446, S768

Rosenkrantz, Eric-S961

Ross, Jaan-S411

Ross, Tetjana-S244, S245, S929

Ross, Tristie-S459

Rossat, Mathieu-S876

Rossignol, Clément-S189, S581, S583, S584, S737, S738, S869, Cochair Session $4 \mathrm{aPAb}$ (S580), Cochair Session 4pPAj (S737) 
Rossing, Thomas D.-S838, Cochair Session 4aEDb (S552), Cochair Session 5aMUc (S831), Cochair Session 5aMUf (S838)

Rothschild, Karin-S953

Rottiers, Wouter-S475

Rougier, Christophe-S228

Roure, Alain-S606

Rouseff, Daniel-S465, S619

Rousounelos, Andreas-S346

Roussarie, Vincent-S279, S285, S286, S330, S699, S715

Rousseau, Martine-S881

Roux, Jean-Noel-S306

Roux, Philippe-S96, S370, S603, S630, S662, S677, S874, S944, S963, S980

Rowan, Daniel-S653

Rowden, Ashley-S660

Rowland, Liz-S19

Rowley, Clark-S654

Roy, Anil K.-S832

Roy, Kenneth P.-S2, S221, S222, Cochair Session 1pAAa (S2), Cochair Session 2pAAa (S221), Cochair Session 2pAAd (S227),

Cochair Session 2pAAi (S233)

Roy, Nathalie-S24, S803

Roy, Nicolas-S94

Roy, Ronald A.-S36, S217,S372, S591, S592, S593, S631, S787, Cochair Session $3 \mathrm{aBBb}$ (S401)

Roy, Ronald-Cochair Session 4pBBd (S672)

Roy, Subhadeep-S923

Royer, Daniel-S188, S865, Cochair Session 5aPAc (S865), Cochair Session 5aPAk (S878)

Royston, Thomas J.-S822, S947

Rozanova-Pierrat, Anna-S529

Rozas, Guillermo-S581, S586

Roze, David-S555

Rozenberg, Yannick-S720

Rozendal, Timo-S142

Ruano, António-S259

Rubak, Per-S278

Rubin, Clint-S670

Rubio, Jesus-S57

Rudak, Larisa-S251

Rudd, Kevin-S634

Ruello, Pascal-S186, S584, S586, S737

Ruff, Andreas-S391

Ruiz-Suarez, J. Carlos-S321

Ruiz, Juan P.-S829

Ruiz, Robert-S102

Rumsey, Francis J.-S11, S12

Runcan, Mariana-S61, S289

Runefors, Per-S104

Rupin, Fabienne-S817

Rusciano, Nicola-S416, S425

Rusko, Milan-S209, S480

Russo, Frank A.-S753

Rüst, Philipp-S300

Rutledge, Janet-S198

Ruty, Nicolas-S153, S610
Ruzié, Gérard-S806

Ruzzene, Massimo-S216, S605

Ryan, Zach-S326

Rybak, Samuil A.-S858

Rybianets, Andrey-S254

Rychtarikova, Monika-S639, S842, S849, S893

Ryg, Jesper-S664

Ryherd, Erica-S225, S700, S701, Cochair Session $4 \mathrm{pNSb}$ (S700), Cochair Session 4pNSg (S709), Cochair Session 5aNSb (S844)

Ryherd, Steve-S225

Rytting, Anton-S110, S915

Ryu, Jongkwan-S534

Saarakkala, Simo-S258

Sabatier, James M.-S67, S75, S76, S305, S757, Cochair Session 1pPAc (S74)

Sabatier, James-Cochair Session 2pPAh (S320)

Saberi, Kourosh-S328

Sabiniarz, Patrick-S703, S713

Sabra, Karim G.-S38, S216, S661, S662, S677, S977. Cochair Session 4pAOh (S661)

Sabra, Karim-Cochair Session $4 \mathrm{pAOb}$ (S656)

Sacchi, Giorgio-S125

Sachau, Delf-S96, S907

Sacks, Jonah-S535

Sadoulet, Emeline-S679

Sæbø, Torstein Olsmo-S930, S931

Saeki, Takashi-S544, S669

Saemann, Ernst-

Ulrich-S703, S899, Cochair Session 4pNSc (S703),

Cochair Session 4pNSk (S713)

Sagayama, Shigeki-S837

Sagemuehl, Dirk-S482

Sagen, Hanne-S23, Cochair Session 1pAOa (S21), Cochair Session 2pAOb (S245)

Sagi, Elad-S883

Saha, Pranab-S642

Sahin, Zekeriyya-S268

Sahlén, Birgitta-S362

Saied, Amena-S817

Saijo, Yoshifumi-S30

Saillard, Marc-S494

Sainidou, Revekka-S587

Sainiemi, Lauri-S318

Saintval, Wendy-S626

Saito, Ikumi-S150

Saito, Osamu-S337

Saito, Shigemi-S815

Sakaguchi, Atsushi-S575

Sakaguchi, Takefumi-S208, S337, S544

Sakai, Shinichi-S726

Sakakibara, Ken-Ichi-S763

Sakamoto, Koji-S443

Sakamoto, Shin-Ichi-S574, S575

Sakamoto, Shinichi-S128

Sakamoto, Shuichi-S44, S190, S267, S599, S751

Sakout, Anas-S271, S942

Salama, Andrew-S353
Salamatov, Evgenii I.-S313

Salamone, Joe-S310

Salenbien, Robbe-S189, S317

Salissou, Yacoubou-S315

Sallee, Hebert-S847

Salmi, Ari-S639, S869, S880

Salmons, Joseph-S920

Salomir, Rares-S28

Salomons, Erik-S185, S853, S956

Salskov, Eric-S192

Samaran, Flore-S806

Sammelmann, Gary S.-S976

Sampathkumar, Ashwin-S402

Sams, Mikko-Cochair Session $2 \mathrm{aSCb}$ (S210)

San Emeterio, Jose Luis-S729

San Martin, Maria

Luisa-S534

San Martin, Ricardo-S534

San Souci, Sooch-S849

Sanchez-Carnero, Noela-S659

Sanchez-Dehesa, José-S73, S180, S309, S312

Sánchez-Morcillo, Victor-S313, S880

Sanchez, Kauyumari-S364, S768

Sanchez, Patrick-S633

Sanchis-Alepez, Helios-S73

Sanchis-Alepuz, Helios-S312

Sandberg, Göran-S388

Sanders, Lisa D.-S325

Sandhu, Jaswinder-S253

Sandin, Ake-S422

Sandoz, Florian-S674, S905

Sandrin, Laurent-S32

Sandsmark, Geir Helge-S466

Sandven, Stein-S23

Saniga, Michael L.-S634

Sankin, Georgy-S593

Sankowsky, Tobias-S43

Sanson, Joseph-S234, S331

Santibañez, Francisco-S304

Santillan, Arturo-S453, S728

Santin, Mathieu-S250

Santos, Paulo-S761

Santos, Ricardo-S608

Sanz, Ana-S437

Saoji, Aniket-S87

Sapoval, Bernard-S529

Sapozhnikov, Oleg A.-S35, S253, S258, S280, S399, Cochair Session 3aBBa (S398),

Cochair Session 3aSPa (S460),

Cochair Session 5pBB (S946)

Sapozhnikov, Oleg-Cochair Session 2pSPd (S370), Cochair Session 5aBBd (S819)

Saranpaa,Pekka-S880

Sarens, Bart-S189, S317

Saric, Zoran-S771

Sarkar, Abhijit-S761

Sarkar, Tappan-S773

Sarma, Jayanta-S308

Sarvazyan, Armen-S370, S461,

S462, S667, S822, S862, S864, S867

Sas, Paul-S904

Sasaki, Ai-S267

Sasaki, Fumio-S335

Sasaki, Kazuaki-S247
Sasso, Magali-S32, S667, S668

Sataloff, Robert T.-S365

Sato, Hayato-S640

Sato, Hiroshi-S534

Sato, Marc-S85

Sato, Shin-Ichi-S120, S390

Sato, Yuji-S150, S784

Sattelmayer, Thomas-S724

Saucier, François-S24

Sauck, Antje-S601

Sauni, Riitta-S349

Sauter, Disa-S771

Sauvage, Emilie-S162

Sauvage, Mickael-S715

Sauvage, Olivier-S419

Sauvage, Sylvain-S443

Savage, Heather-S302

Savel, Sophie-S595, S600

Savin, Alexander V.-S585

Savio, Andrea-S647

Savioja, Lauri-S967, Cochair Session 5aPPe (S893),

Cochair Session 5pPPb (S967)

Savitala, Hari V.-S230, S969

Savoia, Alessandro-S407

Savvaidis, Stylianos-S171, S850

Sawada, Kouichi-S25

Sawallis, Thomas R.-S774

Sayles, Mark-S334, S745, S747

Scalerandi, Marco-S732, S864

Scamoni, Fabio-S535, S795

Scanlon, Martin G.-S735, S736

Scanlon, Michael V.-S264

Scanlon, Patricia M.-S951

Scarton, Henry A.-S693

Scavone, Gary-S47, S155, S479, Cochair Session $2 \mathrm{aMUb}$ (S154), Cochair Session 2pMUd (S273)

Scelo, Thomas-S5, S796, S943

Schäfer, Frank-S602

Schärer, Michelle-S130

Schaar, Johannes A.-S30

Schafer, David E.-S259

Scharenborg, Odette-S104, S964

Scheck, Jochen-S389

Scheifele, Peter-S241

Scherer, Ronald C.-S411, S609, S773

Scherrer, Jean-Marc-S529

Schevenels, Pieter-S300

Schiavi, Alessandro-S647, S796

Schiavoni, Samuele-S69

Schiff, Martin-S184

Schiffrer, Giuliano-S125

Schiffter, Heiko-S33

Schiller, Noah H.-S607, S902

Schimel, Alexandre C.-S244

Schippers, Pieter-S984

Schirmacher, Rolf-S204, S564

Schlesinger, Anton-S754

Schlittmeier, Sabine J.-S193

Schlundt, Carolyn E.-S17, S538

Schmeder, Andrew-S675, S803

Schmelzer, Martin-S534

Schmich, Isabelle-S129, S228 
Schmidt, Henrik-S21, S38, S39, S371, S937, S977, S978, Cochair Session 1pEAa (S37), Cochair Session 2aUW (S219), Cochair Session 2pEAe (S266), Cochair Session 5aUWh (S936)

Schmidt, Lothar-S482, Cochair Session 3pNSb (S482), Cochair Session 4pNSo (S717)

Schmidt, Sebastian-S51, S596

Schmithorst, Vincent J.-S457

Schmitt, Françoise-S499

Schmitt, Nicola-S43

Schmitz, Georg-S408

Schmitz, Philippe-S441

Schneider, Bruce-S752, S753

Schneider, Martin-S847

Schneider, Stefan-S453, Cochair Session 3aSAb (S452), Cochair Session 4aSAa (S602), Cochair Session 4pSAc (S760)

Schnitzler, Hans-Ulrich-S131, S652, S805

Schock, Steven-S932

Schoeb, Pascal-S404

Schoenherr, Holger-S77

Schoenwald, Stefan-S532

Schofield, Colin-S20

Schofield, James-S810

Scholl, Werner-S535, Cochair Session 4aAAc (S532), Cochair Session 4pAAh (S647)

Scholte, Rick-S417

Scholz, Matthias-S483

Schomer, Paul-S164, Cochair Session 2aNSb (S163)

Schonstein, David-S756

Schoonderwaldt, Erwin-S696

Schorer, Edwin-S724

Schorr, Greg-S807

Schröder, Dirk-S968

Schram, Christophe-S569, S570

Schreckenberg, Dirk-S843

Schreurs, Eric-S294

Schroeder, Manfred-S4

Schubert, Emery-S279

Schuette, Dawn-S7, S223

Schuh, Dieter-S312

Schuller, Gerd-S242

Schuller, Thierry-S434, S435, S436

Schulte-Fortkamp, Brigitte-S166, S840, Cochair Session 2aNSb (S163), Cochair Session 5aNSa (S839), Cochair Session 5aNSe (S850), Cochair Session 5aNSf (S851)

Schulte, Michael-S198

Schultz, Troy D.-S184

Schulz, Mathias-S812

Schuster, Maria-S922

Schusterman, Ronald J.-S539, S540, S803, Cochair Session 4aABb (S539)

Schutte, Arjan-S705

Schutz, Michael-S444

Schwartz, Jean-Luc-S85, S21
Schwarz, Jan-S209

Schwarz, Raphael-S772

Schwehr, Kurt-S18

Schweitzer, Patrick-S778

Schwenke, Roger-S803

Sciabica, Jean-François-S330

Sciamarella, Denisse-S609

Scott, Elizabeth-S172

Scott, Sophie K.-S362, S363, S613, S771

Scott, Waymond R.-S74

Scotti, Thierry-S307

Scowcroft, Gail-S241

Scrosati, Chiara-S795

Sebaa,Naima-S68, S315, S669, S671

Sebastien, Ollivier-S280

Secchi, Simone-S233, S798

Seeber, Bernhard U.-S87, S201, Cochair Session $1 \mathrm{pPPb}$ (S86)

Seeber, Bernhard-Cochair Session 2pPPe (S335)

Seekings, Paul J.-S393

Segar, Allyson-S749

Segebarth, Christoph-S352

Segovia Eulogio, Enrique E.-S150, S265

Segues, Fernando-S57

Segur, Damien-S189

Seidler, Hannes-S267

Sekiyama, Kaoru-S911

Seldran, Fabien-S337

Self, Rod-S157

Semal, Catherine-S81

Semenov, Andrew-S784, S926

Semidor, Catherine-S427

Semprini, Giovanni-S641, S648, S795

Sen, Surajit-S304

Senat, Claude-S475

Senda, Jiro-S574

Sendt, Janice S.-S466

Seneff, Stephanie-S807

Senegond, Nicolas-S679

Sentenac, Nicolas-S808

Senturk, Sertan-S644

Seo, Chi-S946

Seo, Dae-Cheol-S728

Seo, Jung-Hun-S894

Seo, Kunihiko-S960

Seong, Woojae-S789, S935

Sequeira Goncalves, Paulo J.-S295

Serbov, Nikoly S.-S657

Serebryany, Andrey N.-S621

Serfaty, Stéphane-S438, S868

Sergeev, Sergey-S662, S790

Serhane, Rafik-S875

Serimaa, Ritva-S880

Serra, Maud-S228

Serriere, Sophie-S144

Serruys, Patrick W.-S30

Sertlek, Hüseyin Ö.-S629

Sessarego, Jean-

Pierre-S499, S630, S633,

S634, S978, Cochair Session 2pUWe (S381), Cochair Session 3aUWd

(S472), Cochair Session 4aUWc (S625),
Cochair Session 4aUWe

(S630), Cochair

Session 4pUWc (S782)

Sessler, Gerhard-Cochair Session 2pEAa (S259)

Seung, Kim-S330

Sevcik, Rose A.-S328

Seybert, Andrew F.-S718, Cochair Session 4pUWh (S788)

Sgard, Franck C.-S454

Shadle, Christine-S767, Cochair Session 4pSCb (S764)

Shafiro, Valeriy-S335

Shahsavari, Ali-S151

Shamma, Shihab-S82

Shamoo, Jessica-S110, S915

Shane, Elizabeth-S545

Shang, Dejiang-S347, S349

Shannon, Robert-S964

Shaoling Huang, Huang-S248

Shapiro, Ari D.-S807

Shapiro, Nikolai-S301

Shapovalov, Valentine V.-S176

Sharakshane, Anton-S258

Sharif, Bayan-S924

Sharma, Vin-S216

Sharp, David B.-S152, Cochair Session 1pMUa (S46),

Cochair Session

2pMUc (S272)

Sharples, Steve-S585

Shattuck-Hufnagel, Stefanie-S352, S492, S918

Shaw, Adam-S36

Shaw, George J.-S664

Shaw, Peter B.-S558

Shchelkunov, Alexander-S380

Sheffield, Edmond

S.-S933

Sheft, Stanley-S743

Shen, Wen-Chi-S493, S916

Shen, Yi-S889

Sheplak, Mark-S262

Shera, Christopher A.-S883, Cochair Session $5 \mathrm{aPPb}$ (S883)

Shera, Christopher-Cochair Session 5aPPc (S886)

Sherlock, Laguinn-S749

Sheu, Yae-Lin-S189

Shi, Zhilong-S254

Shiba, Hisashi-S780

Shibayama, Hideo-S205, S779

Shieh, Jay-S147

Shield, Bridget M.-S950

Shields, F. Douglas-S859

Shihab, Ahmed I.-S463

Shihong, Zhou-S624

Shilton, Simon J.-S167, S295

Shimazawa, Ryota-S238

Shimizu, Dai-S579

Shimizu, Hidenori-S910

Shimizu, Ken’Ichi-S186

Shimizu, Yasushi-S12, S13, S128

Shimojo, Shinji-S611

Shimokura, Ryota-S130, S229

Shimura, Takuya-S925, S933

Shindo, Mitsuko-S750

Shinn-Cunningham, Barbara-S10, S12, S84, S328, S897,
Cochair Session 1pAAc (S8),

Cochair Session 4pAAc

(S640)

Shinnosuke, Hirata-S114

Shiori, Yu-S810

Shipov, Rollan-S281

Shirakawa, Takashi-S960

Shkerdin, G.-S188, S190

Shorter, Phil S.-S90

Shosted, Ryan-S920

Shoul, Karim-S751

Shport, Irina-S360

Shravage, Paresh-S69, S316

Shrestha, Ajay-S32

Shrestha, Annie-S254

Shrestha, Mona-S103

Shrivastav, Mini N.-S898

Shrivastav, Rahul-S913, S916

Shuanping, Du-S624

Shubin, Igor-S283

Shue, Yen-Liang-S492

Shurup, Andrey-S662, S790

Shuvalov, Alexander-S189, S345, S875, S877

Shynk, John J.-S833

Sibella, Federica-S887

Siczek, Rafal-S778

Sidaras, Sabrina K.-S110, S919

Siderius, Martin-S781

Siderius, Thomas M.-S661

Sidlof, Petr-S696

Sidorovskaia, Natalia-S974,

Cochair Session $2 \mathrm{pSPa}$

(S366), Cochair Session 2pSPb (S367)

Siebein, Gary W.-S802, S841, S940

Siebert, Ursula-S812

Siefert, Malte-S161

Siegel, Jon-S884

Siegmann, William L.-S626, S634

Siemes,Kerstin-S654

Sienz, Johann-S603

Siffert, Robert S.-S545

Signol, Francois-S108, S109

Sijl, Jeroen-S142, S402

Sikma, Erik L.-S298

Sikorska, Anna-S439

Silbert, Noah H.-S364

Sillings, Roy-S766

Siltanen, Samuel-S791

Silva, Antonio-S923

Silva, Fabrice-S696

Silva, Francisco-S603

Silva, Glauber T.-S731

Silvestri, Paolo-S478, S691

Simard, Peter-S811

Simard, Yvan-S24, S803, S804, S811

Simester, Jen-S357

Simmons, Christian-S299

Simmons, James A.-S237, Cochair Session 2pABa (S236)

Simmons, James-Cochair Session 2pABd (S242), Cochair

Session 3aABa (S392)

Simmons, Lee G.-S539

Simmons, Stephen M.-S381

Simon, Ellen-S108

Simon, Francisco-S350

Simon, Frank-S342 
Simon, Helen J.-S200

Simon, Laurent-S114, S205. S350, S463

Simon, Malene J.-S22

Simon, Melissa J.-S826

Simonaho, Simo-Pekka-S80, S961

Simoneau, Thierry-S170

Simonetti, Francesco-S947

Simoni, Francesco-S313

Simonis, Claudia-S601

Simonova, Varvara-S672

Simons, Dick G.-S381, S384, S629, S654, S659, S660, S927, Cochair Session 4pAOg (S659)

Simons, Dick-Cochair Session 4pAOd (S658)

Simpson, Brian D.-S329, S892

Simpson, Harry J.-S633, S634

Sinai, Azriel-S934

Sinclair, Anthony N.-S179

Sinclair, Stephen-S210, Cochair Session 1pAAa (S2)

Sinelnikov, Yegor-S462

Singampalli, Veena D.-S353

Singh, Jaya-S311

Singh, Manish Pratap-S486, S96

Singh, Rajendra Kumar-S486, S961

Singh, Rishi Pal-S486, S961

Singh, Sandipa-S925

Sinigalliano, Chris-S244

Sintes, Christophe-S939, S984

Siohan, Olivier-Cochair Session 2aSCc (S212)

Siren, Kathleen-S827

Sisto, Renata-S885

Sitel, Azzedine-S906

Sjökvist, Lars-Göran-S388

Sköld, Anders-S277, S599

Skaf, Alain-S442, S731, S878

Skarsoulis, Emmanuel-S23, S944, S945

Skarzynski, Henryk-S331, S753

Skipetrov, Sergey-S306

Skulina, David-S686

Škvor, Zdeněk-S261

Slabbekoorn, Hans-S17

Slama, Jules G.-S296

Slater, Nigel K.-S255

Slaughter, Julie-S46

Slaviero, David-S567

Slavko, Pavel-S639

Sliwinska-Kowalska,

Mariola-S888

Slot, Han-S741

Slump, Kees H.-S673

Slunecko, Tomas-S431

Smagin, Nikolay-S461, S867

Smaragdakis, Costas-S139

Smaragdis, Paris-S377, S837

Smedsrud, Morten-S934

Smerdon, Andrew M.-S245

Smiljanic, Rajka-S915, S919

Smith, Ben-S55

Smith, Bennett K.-S596

Smith, John-S47, S152, S155, S156, S274, S275, S623, S624, S694, S829
Smith, Julius O.-S554, S637, S697, S698, S905, Cochair Session 4aMU (S553)

Smith, Julius-Cochair Session 4pMUf (S696)

Smith, Kevin B.-S471, S974, Cochair Session $3 \mathrm{aUWc}$ (S470)

Smith, Kevin-Cochair Session 2pUWf (S383)

Smith, Kristen-S351

Smith, Moira J.-S255

Smith, Richard-S585

Smith, Sean-S794

Smolenski, Brett-S773

Smyth, Tamara R.-S688

Snader, Anita L.-S2

Snellen, M.-S384, S659, S927

Snellen, Mirjam-S381, S654, S660

Snoeijer, Jacco H.-S306

Snook, Kevin-S147

Snyder, Russell L.-S88

So, Jin-S954

Soares-Filho, William-S367

Soares, Cristiano-S614

Sobreira-Seoane, Manuel A.-S537, S547, S855

Sodagar, Sina-S179

Soize, Christian-S565, S602, S954

Solè, M.-S811

Solaiman, Basel-S983

Soldevilla, Melissa S.-S131, S132, S133

Solea, Geamilia-S61, S289

Solet, Joanne-S225

Soliveres, Ester-S659

Solntseva, Vasilisa-S161, S282

Solomon, Latasha-S264

Somekh, Mike-S585

Somfai, Ellak-S306

Sommerville, Jessica-S613

Son, Minjung-S111

Song, Aijun-S467, S934

Song, Heechun-S925, S934, Cochair Session 5aUWa (S923), Cochair Session 5aUWf (S933)

Song, Min Geun-S565

Song, Qi-S711

Song, Wookeun-S191

Sonnerup, Julia-S388

Sontacchi, Alois-S112, S684

Sonti, Venkata R.-S761

Sorgüven, Esra-S56

Sorokin, Sergey-S179

Sorrentini, Jacques-S269

Soto-Nicolas, Alberto-S874

Soto, Natacha A.-S136

Sotomayor Torres, Clivia-S317

Sottek, Roland-S278

Soukup, Raymond J.-S633, S634, Cochair Session 4aUWe(S630)

Soukup, Raymond-Cochair Session 4pUWc (S782)

Soulez, Gilles-S34

Soumiya, Hiroki-S665, S667

Southall, Brandon-S16, S20
Souza, Pamela-S896

Sovetskaya, Elizaveta-S304

Soyer, Caroline-S678

Spa, Carlos-S792

Spahr, Anthony-S87, S917

Sparrow, Victor W.-S158, S580

Spasikova, Marija-S539, S803

Speckhahn, Marcus M.-S464

Speyer, Gavriel-S28, S255

Spiliopoulos, Nikolaos-S739

Spindel, Robert C.-S945

Spoelstra, Simon-S573

Spoor, Philip-S574, S740

Spors, Sascha-S548, S962

Spriet, Ann-S198

Stütz, Michael-S562

Stadler Elmer, Stefanie-S410

Stafford, Kathleen-S22

Staliunas, Kestutis-S313

Stallen, Pieter Jan-S722

Stamates, Jack-S244

Stammers, Jon-S113

Stanko, Tanya-S280

Stanley, Jeff-S771

Stanley, Raymond M.-S597

Stanton, Timothy K.-S26, S137, S380, S928

Stanzial, Domenico-S125

Starke, Katie-S241

Staron, Lydie-S323

Starosta, Roman-S485

Staska, Matthew D.-S975

Stauffer, Allen-S103, S104

Stead, Matthew-S827

Stecker, Christopher-S328

Steenbergen, Wiendelt-S401

Stefanakis, Nick-S124

Stefanics, Gábor-S85

Steffen, Kari-S639

Steichen, William-S308

Stein, Peter-S781

Steinbach, Frank-S564

Steinnes, Margrete-S167

Stenback, Joanna-S240

Stenman, Åsa-S58, S59

Stenson, Gary B.-S541

Stephan, Yann-S140, S944,

Cochair Session

4pAOa (S654), Cochair

Session 4pAOc

(S657), Cochair Session 5pAO (S944)

Stephenson, Uwe M.-S791

Stephey, Laurie-S693

Stepnowski, Andrzej-S780

Sterkers, Olivier-S895

Stern, Richard M.-S774

Stetson, Kathleen-S229

Stevens, Geoff-S78

Stevens, Richard-S735

Stevenson, Samuel-S270

Stewart, Devin-S160

Still, Tim-S587

Stilz, Peter-S652, S805

Štimac, Alan-S167, S169, S295

Stinson, Mike R.-S42

Stipanicev, Dinko-S169

Stirnemann, Alfred-S43
Stocker, Michael-S20, Cochair

Session 1pAB (S16),

Cochair Session 2pABc (S241)

Stockmal, Verna-S765

Stoelinga, Christophe N.-S447

Stohl, Joshua S.-S86

Stojanovic, Milica-S923

Stojiljković, Milan-S687

Stone, Maureen-S112, S353, S970, Cochair Session 4 pSCa (S762)

Stone, Michael A.-S751, S890

Story, Brad-Cochair Session 2pMUe (S274)

Stotts, Steven A.-S397

Stover, Ashley K.-S897

Straley, Jan-S807

Strange, Winifred-S491, S767

Strelcyk, Olaf-S744

Stribling, Rommel-S954

Strickland, Elizabeth-S892,

Cochair Session 2pPPa

(S325), Cochair Session 3pPPa

(S488), Cochair Session

$3 \mathrm{pPPb}$ (S490), Cochair Session 4aPPa (S594), Cochair

Session $4 \mathrm{aPPb}$ (S597), Cochair

Session 4pPPc (S747),

Cochair Session 5aPPd (S889)

Stride, Eleanor-S249,

Cochair Session

2pBBa (S247), Cochair

Session 2pBBb (S250)

Striemer, Danielle-S920

Strybulevych, Anatoliy-S306, S735, S736

Sturm, Bob-S833

Sturm, Frederic-S630, S974

Su, Quang T. -S260

Su, Quang T.-S261, S262

Su, Zuhre-S800

Suarez, Ivan-S170

Suetoshi, Ryouichi-S817

Sugie, Satoshi-S534

Sugimoto, Akio-S96

Sugimoto, Nobumasa-S579

Sugimoto, Rie-S160

Sugimoto, Tsuneyoshi-S264, S960

Suhanek, Mia-S648

Suied, Clara-S599, S600

Sukhovich, Alexey-S313

Sullivan, Edmund J.-S937, S938

Sullivan, Kevin-S244

Sullivan, Kirk P.-S109, S917

Sumarac Pavlovic, Dragana-S529, S801

Summers, Jason E.-S377, S551, S633, S942, Cochair Session 5pAAb (S942)

Summers, Jason-Cochair Session 5aAAd (S800)

Summers, Kevin N.-S551

Sumner, Meghan-S354

Sun, Chao-S148, S368, S497, S984

Sun, Chi-Kuang-S586

Sunartio, Devi-S590

Sundara, Megha-S107, S358, S914, S915

Sundberg, Johan-S275, S762 
Sung, Koeng-Mo-S330, S682, S894, S917

Sung, Li-May-S493

Sung, Shung H.-S602

Supin, Alexander Y.-S392, S477, S537, S538, Cochair Session 3pAB (S476), Cochai Session $4 \mathrm{aABa}$ (S537)

Supin, Alexander-Cochair Session $4 \mathrm{pABb}(\mathrm{S} 650)$

Surlykke, Annemarie-S236

Susini, Patrick-S285, S339, S446, S599, S697, S721

Suslick, Kenneth S.-S588

Sussman, Elyse-S84

Suter, Alice H.-S164

Sutherland, Louis C.-S951, Cochair Session 5 pNSa (S948)

Sutherland, Louis-Cochair Session 5aNSd (S849)

Sutin, Alexander-S370, S462, S783, S862, S864, S867, Cochair Session 5aPAb (S862), Cochair Session 5aPAd (S867)

Sutin, Alexandr-S863

Suzuki,Hisaharu-S123, S904

Suzuki, Yôiti-S44, S190, S267, S329, S599, S751

Svec, Jan G.-S275

Svedhem, Hakan-S433

Svensson, Carsten-S530

Svensson, Jonas L.-S607

Svensson, Peter-S41, S180

Sveshnikov, Boris V.-S180

Svilar, Dragoljub-S593, S872

Svinarenko, Andrey

A.-S657, S776

Svirsky, Mario-S883

Svonni, Mikael-S917

Swalwell, Jarred-S590

Swaminathan, Jayaganesh-S88, S752

Swearingen, Michelle E.-S182, S955

Swerdlin, Yoni-S274

Swift, Greg W.-S578

Sygulska, Anna-S228

Sykes, David M.-S125, S127, Cochair Session 2aAAd (S125)

Symons, Neill P.-S287

Szczupak, Mikey-S665

Szeri, Andrew J.-S589

Szlávik, Gábor-S847

Szuberla, Curt-S952

Szulman, Erin-S826

Tabeling, Patrick-S72

Tachibana, Hideki-S754

Tadeu, António-S761

Taherzadeh, Shahram-S181, S953

Taifi, Naima-S730, S869

Taillade, Laurent-S144

Tajadura-Jiménez, Ana-S277

Tajadura, Ana-S753

Tajayouti, Mohammed-S172

Tajima, Keiichi-S357

Takada, Masahiko-S292, S667

Takahashi, Daiji-S5
Takahashi, Hideyuki-S25

Takahashi, Ryosuke-S750

Takahashi, Toru-S106

Takahashi, Yoshinori-S685, S756

Takami, Kenji-S754

Takano, Sayoko-S766, S770

Takaoka, Tomoyasu-S640

Takashima, Kazuhiro-S341, S344

Takeda, Kazuya-S684

Takehira, Osamu-S192

Takemoto, Hironori-S326, S355, S763, S766, S768

Takeuchi, Takashi-S501, S549

Taktak, Mohamed-S958

Talasch, Werner-S62

Talmadge, Carrick L.-S858, S883, S885, S954

Talmant, Maryline-S546, S602, S667, S671

Tamagawa, Masaaki-S399

Tamás, Ferenc-S120

Tamura, Shin-S73

Tan, Chee Wee-S260

Tan, Christine M.-S752

Tan, Haidee-S411

Tan, Zheng-Hua-S213

Tanaka, Akira-S30

Tanaka, Kazuto-S665

Tanaka, Keita-S750

Tanaka, Osamu-S335

Tanaka, Yukihiro-S73, S583

Tang, Dajun-S465, S619, S620, S625, S976, Cochair Session 3aUWa (S464)

Tang, Huide-S15

Tang, Meng-Xing-S145

Tanneau, Olivier-S419

Tansatcha, Marupong-S856

Tanter, Mickael-S27, S33, S403, S460, S545, S823, S862, Cochair Session 2pSPd (S370), Cochair Session 3aSPa (S460)

Tarabusi, Valerio-S638

Tarasenko, Olga S.-S311

Tarasenko, Sergey V.-S311

Tardieu, Damien-S833

Taricco, Cristiana-S647

Taroudakis, Michael-S139, S633, Cochair Session 4pUWf (S786), Cochair Session 5pUWa (S972)

Taschin, Andrea-S174, S187, S313

Tashmukhambetov, Arslan-S974

Tatarakis, Michael-S187, S583

Tatarek, Agnieszka-S245

Tatarinov, Alexey-S667

Tauchert, Klaus H.-S131

Taulu, Samu-S365, S774

Tavard, Francois-S152

Tavarkiladze, Georgy-S888

Tavukcuoglu, Ayse-S637

Tayama, Niro-S763

Taylor, Charles-S134

Taylor, Kristen A.-S538

Taylor, Paul-S112

Tayong, Rostand-S647

Tegowski, Jaroslaw-S245

Teilmann, Jonas-S240
Teixeira, Cesar A.-S259

Tejero-Calado, Juan Carlos-S198

Ten Thije Boonkkamp, Jan-S436

Tencate, James-Cochair Session 4pPAg (S732)

Tencate, Jim A.-S429, S431, S863, Cochair Session 3aPAa (S428), Cochair Session 4pPAa (S722)

Tenenbaum, Roberto A.-S6

Teplykh, Andrey A.-S80

Tepperman, Joseph-S359

Ter Haar, Gail-S36, S825, Cochair Session $1 \mathrm{pBBc}(\mathrm{S} 35)$, Cochair Session 2pBBd (S253)

Terán, Fernando-S314, S718

Teramoto, Kenbu-S113, S350

Teresa, Woods J.-S320

Ternström, Sten O.-S411

Ternstrom, Sten-Cochair Session 3aMUa (S410)

Terroir, Jonathan-S166

Tesei, Alessandra-S632, S633, S780, S929, S976, S977, S979

Teslenko, Vyacheslav S.-S487, S727

Testa, Pierluigi-S367, S777

Teston, Franck-S409, S679

Teterev, Alexander-S251

Teterev, Nikolai-S873

Teyssie, Dominique-S868

Thai-Van, Hung-S337, S598, S895

Theile, Günther-S547

Thenail, Denis-S92, S346

Theobald, Peter D.-S378, S379, S468, S469

Theodore, Rachel M.-S966

Theorell, Töres-S126

Thermeau, Jean-Pierre-S739

Theuillon, Gwladys-S140

Theunissen, Frédéric E.-S652, S965

Theverapperuma, Lalin S.-S43

Thiele, Frank H.-S437

Thiessen, David B.-S588, S822

Thill, Michael S.-S427

Thiria, Sylvie-S542

Thoben, K.-D.-S349,S707

Thode, Aaron-S19, S132, S805, S807

Thomann, Georg-S158, S281

Thomas, Erik R.-S99

Thomas, Gary L.-S24, S653

Thomas, Jean-Hugh-S114, S418, $\mathrm{S} 430$

Thomas, Jean-Louis-S487, S727

Thomas, Jeanette A.-S20

Thomas, Olivier-S203

Thompson, David S.-S39

Thompson, David-Cochair Session 2pNSi (S290), Cochair Session 2pNSn

(S297), Cochair Session 3aNSb (S415)

Thompson, Eric R.-S122, S328

Thompson, Michael A.-S18

Thompson, Stephen C.-S260
Thompson, Travis-S172

Thomsen, Sigurd N.-S421, S719

Thorne, Peter D.-S928, S929

Thorne, Richard E.-S24

Thorsos, Eric-S118, S787, S975, S976, Cochair Session 5pUWb (S975)

Throckmorton, Chandra S.-S86

Thuroczy, Gyorgy-S887. S888

Tiah, Naceur-S256

Tian, Jing-S15

Tian, Lingai-S383

Tichy, Frank-S468

Tichy, Jiri-Cochair Session 2aAAc (S122)

Tiede, Mark-S357, S491, S915

Tierney, Adam T.-S753

Tiesler, Gerhart-S950

Tijani, Hassan-S573

Tijs, Emiel-S471

Tilser, Wolfgang-S283

Tiltman, Carl P.-S925

Timmerman, Nancy-S841

Timonen, Jussi-S666

Tindle, Chris T.-S118

Ting, Antonio C.-S985

Ting, Siow N.-S316

Tinianow, Brandon-Cochair Session 4aAAc (S532),

Cochair Session 4pAAh (S647)

Tisserand, Etienne-S778

Tkalcevic Lakusic, Visnja-S291

Tocci, Gregory C.-S224, S225

Toda, Martine-S111, S458

Toda, Tomoki-S970

Todoroviæ, Dejan-S920

Tognola, Gabriella-S887

Tohyama, Mikio-S756

Toilliez, Jean O.-S589

Tokuda, Isao-S610, S763

Tollefsen, Dag-S397, Cochair Session 2aAOb (S137), Cochair Session 2pAOc (S246), Cochair Session 3aAO (S396)

Tolstoy, Alexandra-S138

Tolstoy, Maya-S19

Tombolato, Andrea-S294

Tomiku, Reiji-S124

Tomilina, Tatiana-S208

Tomoda, Motonobu-S188

Tomoriová, Beáta-S750

Tonon, Devis-S282

Töpken, Stephan-S699

Toppila, Esko-S171, S284, S349

Torma, Ákos-S649

Tormey, Megan N.-S17

Torre, Renato-S174, S187, S313

Torrent, Daniel-S73, S180, S309

Torres, Edgar A.-S728

Torrione, Peter A.-S74

Toso, Alessandro-S95, S903

Totaro, Nicolas-S90, S342

Tóth, Balázs-S797

Tóth, Péter-S280

Tougaard, Jakob-S132, S240, S395 
Toullelan, Gwenael-S150

Toupin, Gwenael-S595

Tourin, Arnaud-S72, S216, S312

Tournat, Vincent-S67, S319, S321

Tournour, Michel-S758

Touze, Cyril-S203

Towner, Martin C.-S433, S734, Cochair Session $3 \mathrm{aPAb}$ (S432)

Towner, Martin-Cochair Session 4pPAh (S734)

Townley, Michael W.-S774

Townsend, Rosemary J.-S873, S957

Toyoda, Emi-S534

Toyoda, Masahiro-S5

Toyota, Yasuhisa-S5

Töyräs, Juha-S258, S546, S663, S670, S729

Traer, James-S656

Trahey, Gregg-S824

Trahiotis, Constantine-S448

Tran-Hhuu-Hue,

Louis-Pascal-S149

Tranquart, François-S143, S144, $\mathrm{S} 250$

Traube, Caroline-S156, S689

Traweek, Michael-S366, S779, S982

Treiber, Alexander S.-S285

Tremblay, Chris-S19

Tremper, Thomas-S147

Trentin, Dominique-S419

Tretbar, Steffen H.-S816

Trevorrow, Mark-S502

Treyssède, Fabien-S454, S873

Triebenbacher, Simon-S92, S570

Trivett, David H.-S147

Trnka, Marián-S209

Tro, Jan-S827

Trochidis, Athanasios-S852

Trompette, Nicolas-S560

Tronchin, Lamberto-S638

Trow, James-S167, S295

Trucco, Andrea-S37, S41

Trujillo, Mario F.-S379

Truy, Eric-S337, S895

Tsang, Jason-S842

Tsaplev, Valery M.-S429

Tshoidze, Alexander-S619

Tsiglifis, Kostas-S252, S593

Tsiklauri, David-S174

Tsirulnikov, Efim M.-S822

Tsou, Hui-Ying-S354

Tsuji, Kinko-S592

Tsuji, Yoshiyuki-S575

Tsujimoto, Toshiyuki-S664, S667

Tsuru, Hideo-S287

Tsurugaya, Yoshiaki-S218, S627

Tu, Jung-Yueh-S768

Tubis, Arnold-S883

Tucker, Simon-S376, S893

Tudor, Ivan-S63

Tuller, Betty-S109

Tung, Yao-Sheng-S249

Turchin, Victor-S497

Turgeon, Martine-S601

Turgut, Altan-S396
Turner, Joseph A.-S320, S441, S668

Turo, Diego-S66, S173

Tusz,Jeremy-S11

Tuttle, Brian-S580

Twerdowski, Evgeny-S177, S947

Tyack, Peter-S1, S16, S20, S136, S239, S393, S651, S807

Tzianaki, Eirini-S187

Uchino, Kenji-S147

Ueda, Kazuo-S195

Ueda, Kazutaka-S891

Ueda, Mari-S193

Ueda, Mitsuhiro-S962

Ueda, Shogo-S335

Ueda, Yuki-S739

Ueno, Kanako-S128, S642

Uffinger, Thomas-S602

Uitti, Jukka-S349

Ulhas, Mohite-S167

Ulliac, Gwenn-S678

Ulmgren, Nils-S422, S718

Uloza, Virgilius-S888

Uloziene, Ingrida-S888

Ulrich, Roger-S126

Ulrich, Tj-S863

Umemura, Shin-Ichiro-S247

Umnova, Olga-S14, S66, S68, S173, S174

Ungar, Eric E.-S127

Ungureanu, Constantin-S402

Uno, Yoshihisa-S603

Unoki, Masashi-S212, S648

Uodome, Atsushi-S817

Uppenkamp, Stefan-S890

Upton, Zachary-S974

Ura, Tamaki-S806

Urban, Jorge-S805

Urban, Matthew-S825

Urban, Wilhelm-S323

Uscinski, Barry J.-S495, Cochair Session 3pUWa (S495)

Uther, Maria-S598

Utzmann, Jens-S159

Uzun, Ali-S53

V, Vijayakumar-S458

Vach, Werner-S214

Vaiedelich, Stéphane-S412

Vaik, István-S49

Vaillancourt, Véronique-S845

Vainer, Alexander-S175

Vaissière, Jacqueline-S491

Valakh, Mykhailo-S725

Valdivia, Nicolas-S343, S632

Valeau, Vincent-S271, S564, S792, S942, Cochair

Session 5aAAa (S791),

Cochair Session 5aAAe (S801)

Valente, Daniel-S751

Valentine, Joanne O.-S5, S943

Valentini, Fabrizio-S795

Valero, Henri-Pierre-S405

Valero, Michelle D.-S887

Valière, Jean-Christophe-S162, S577, S740

Valier-Brasier, Tony-S957

Valin, Jean-Marc-S98

Väljamäe, Aleksander-S277
Valkenier, Bea-S754

Vallée, Nathalie-S211

Valverde, Nelia-S829

Vanaverbeke, Sigfried-S863

Van Beek, Pieter J.-S183

Van Capel, Peter J.-S584

Vancso, Julius-S77

Vandamme, Manuel-S222

Van Damme, Bart-S430

Van De Par, Steven-S194, S211, S596

Vandepitte, Dirk-S789

Van De Vooren, Hedde-S363, S754

Van Den Abeele, Koen-S430,

S441, S863, Cochair

Session 3aPAa (S428), Cochair

Session 4pPAa (S722),

Cochair Session 4pPAg (S732)

Van Den Berg, Albert-S592

Van Den Berg, Frank-S183

Van Den Berg, Frits-S568, S714

Van Den Berg, Marc-S589, S735

Van Den Berg, Martin-S714

Van Den Bogaert, Tim-S201, S893

Van Den Braak, Elisabeth W.-S230

Van Den Nieuwenhof, Benoit-S901

Van Der Auweraer, Herman-S901, S904, S908

Vanderborck, Gerard-S146

Van Der Bos, Arjan-S735

Van Der Burg, Erik-S326

Van Der Eerden, Frits-S183

Vanderhaeghe, Katelijn-S386, S641

Van Der Harten, Arthur-S802

Van Der Heijden, Ferdi-S673

Van Der Hoeven, Marieke-S326

Van Der Linden, Peter J.-S300

Van Der Meer, Sander-S142

Vander Meulen, François-S149

Van Der Perre, Georges-S669

Vanderoost, Jef-S669

Van Der Schaar, Mike-S811

Van Der Steen, René-S704, S713, S727

Van Der Steen, Ton-S30

Vander Weide, John M.-S731, S732

Van Dongen, Koen W.-S257

Van Doorn, Jan-S109, S917

Van Doorn, Ruud-S704, S713

Van Dorp Schuitman, Jasper-S754, S939

Van Eijk, Rob L.-S211

Van Elburg, Ronald A.-S333

Van Esch, Harry-S408

Van Genechten, Bert-S789

Van Haaren, Rik-S168

Van Hecke, Martin-S306

Van Heesch, Chris-S408

Van Hespen, Johan C.-S403

Vanhille, Christian-S736

Van Hirtum, Annemie-S153, S609, S610, S611, S765, S772

Van Hoesel, Richard-S87

Van Hout, Nicole H.-S533
Van Hout, Roeland-S100

Van Keulen, Wim-S422

Vanlanduit, Steve-S532

Van Landuit, Steve-S642

Van Leeuwen, Ton G.-S402, S403, S673

Van Leijen, A. Vincent-S397

Van Luxemburg, Laurentius C.-S230, S387

Vannicola, Catherine-S19

Van Nieuwenhuyse, Karl-S532, S642

Van Noorden, Leon-S81

Van Opzeeland, Ilse Catharina-S240, S541

Van Paepegem, Wim-S429, S866

Van Parijs, Sofie-S18, S541

Van Renterghem, Timothy-S563

Van Saarloos, Wim-S306

Van Schaik, Andre-S40, S41

Van Tiggelen, Bart-S306

Van Uffelen, Lora-S496

Van Vossen, Robbert-S118

VanWalstijn, Maarten-S48, S155

Van Wamel, Annemieke-S247

Van Weperen, Tamara-S402

Van Wieringen, Astrid-S89

Van Wijngaarden, Leen-S143, S592

Van Zon, Arnout Tim-S185

Varagis, Antonis-S857

Vardoulias, Georgios-S780

Varnier, Jean-S280

Vasiliev, Boris-S935

Vasseur, Jérôme-S71, S73, S313, S486, Cochair Session 1pPAb (S70), Cochair Session 2pPAe (S311)

Vastfjall, Daniel-S277, S599, S721, S753

Vavassori, Paolo-S587

Vavva, Maria G.-S545

Vayssade, Catherine-S451

Vazquez Carazo, Alfredo-S147

Vazquez, Monica-S256

Vedernikov, Andrey-S462

Veggeberg, Kurt-S263

Veilleux, Nanette-S492

Veirs, Scott-S17, S394

Veirs, Val-S17, S394

Velea, Doru-S76, S184

Veleschuk, Vitaly V.-S732

Velez, Roby-S589

Velonakis, Manolis-S480

Venegas, Rodolfo-S174, S474

Venot-Gbedji, Flora-S427

Vera Guarinos, Jenaro-S150, S265

Vera, Arturo-S256

Verardi, Patrizio-S427

Vercammen, Martijn-S121, S474

Vercellesi, Giancarlo-S337

Verdel, Arie-S874

Verdier, Anne-Laure-S660

Verdonck, P-S586

Verfaille, Vincent-S690, S828

Vergez, Christophe-S696

Vergniolle, Sylvie-S858, S870, S953

Vergoz, Julien-S859

Verhave, Jan A.-S195 
Verhey, Jesko-S83, S338, S489, S881, S890, S891

Verhulst, Sarah-S886

Verleysen, Patricia-S386, S641

Vermeir, Gerrit-S222, S300, S390, S842, S848, S849, S893

Verron, Charles-S548

Verron, Jacques-S542

Versele, Jessica-S356

Versfeld, Niek J.-S199

Versluis, Michel-S78, S142, S143, S252, S402, S589, S592, S735

Versteeg, Willem-S629

Verweij, Martin D.-S257

Vesseleny, Tiberiu-S61

Vestergaard, Martin D.-S964

Veuillet, Evelyne-S598, S895

Viñuela, Urbano-S314, S718

Vial, Marcel-S266

Viala, Christophe-S983

Viaud-Delmon, Isabelle-S600

Vicenik, Chad-S915

Vickers, Debi A.-S336

Vidal, Frederic-S868

Viet Son, Nguyen-S492

Viganò, Mattia-S295

Vigeant, Michelle C.-S600

Vigness Raposa, Kathleen-S241

Vignola, Joseph F.-S96, S320

Vilain, Coriandre-S352

Vilchinska, Nora A.-S323, S733

Vilela, Silvia R.-S236

Viljamov, Sergey V.-S322

Villafana Dalcher, Christina-S105, S351

Villard, Jean-François-S960

Ville, Jean Michel-S958

Ville, Jean-Michel-S288

Villot, Michel-S291, S299, S391, S392, S908, Cochair Session 2pNSj (S291), Cochair Session 2pNSo (S299)

Villouvier, Véronique-S348

Vincent, Emmanuel-S618

Vinci, Vincenzo-S386, S635, S639

Vinck, Bart-S337

Violanda, Renante R.-S363

Viollon, Stéphanie-S843

Virjonen, Petra-S3, S226

Virtanen, Tuomas-S834

Visaria, Rachana-S32

Viscardi, Massimo-S291, S416, S425

Visell, Yon-S444

Viswanathan, Krishnamurthy-S52

Vita, Francesco-S313

Vitale, Renzo-S7, S231

Vitevich, Michael-S211

Vitsas, Panagiotis-S157

Vlachos, Stephanie-S650

Vlasenko, Olexander I.-S732

Vlasie Belloncle, Valentina-S881

Vlk, Michal-S681

Vogt, Michael-S30

Voigt, Daniel-S770

Voix, Jérémie-S597

Voix, Jeremie-S490

Vokurka, Karel-S590
Völk, Florian-S191, S967

Volkov, Aleksander E.-S725

Volkov, Yuriy S.-S585

Volkovas, Vitalijus-S286, S559

Vollmann, Jacqueline-S317, S582

Vollmer, Amy C.-S589

Volmer, Jutta-S891

Voloshinov, Vitaly-S176, S177

Volz, Rudi-S840

Von Buttlar, Moritz-S177, S947

Von Estorff, Otto-S705

Vorlaender, Michael-S191, S234

Vormann, M-S198, S746

Voronovich, Alex G.-S973

Vos, Hendrik J.-S142

Vos, Wouter K.-S195

Voznesenskiy, Alexander S.-S322

Vradis, Alexandros-S739

Vu, Han-S466

Vuillemin, Alexandre-S157

Vuillot, François-S53, S282

Vukadin, Predrag-S63

Vural, Anil-S385

Vurma, Allan-S411

Vuye, Cedric-S532, S642

Vyasarayani, Chandrika P.-S412, S692

Waddington, David C.-S171

Wage, Kathleen E.-S495, S662

Wagemans, Jan-S960

Wagner, Andreas-S174

Wagstaff, Ronald A.-S870, S983, Cochair Session 5pUWe (S983)

Wahlberg, Magnus-S393, S395, S805

Wahlen,Elio-S154

Wakatsuki, Naoto-S149, S150, S265, S548

Walker, Bruce N.-S597

Walker, Harold W.-S78

Walker, Kris-S861, S952

Walker, Shane C.-S628

Wallace, Michelle-S45

Walsh, Edward-S537, S539

Walsh, Kenneth-Cochair Session 4pEAb (S676)

Walsh, Mark P.-S45

Walsh, Stephen J.-S346

Walter, Florian-S564

Wältermann, Marcel-S97, S896

Walters, Tom C.-S98

Waltham, Chris-S413, S693,

Cochair Session

3aMUb (S412), Cochair

Session 4pMUc (S693)

Waltl, Susanne-S361

Wan, Rui-S448

Wan, Yayun-S814

Wanderley, Marcelo M.-S210, S690, S828, Cochair

Session 4pMUa (S687), Cochair Session 5aMUa (S828)

Wanderley, Marcelo-Cochair Session 4pMUe (S695)

Wang, Deliang-S11, S98, Chair Session 1pSCa (S97)

Wang, Haibin-S934

Wang, Jian-S222, S532, S569
Wang, Johnson C.-S119, S985

Wang, Jun-S620

Wang, Lily M.-S14, S387, S481, S600, Cochair Session 3pNSa (S480), Cochair Session 5pAAa (S939)

Wang, Lily-Cochair Session 5aAAf (S802)

Wang, Meng-S55, S159, S570

Wang, Mengyuan Y.-S334, S745

Wang, Michael-S824

Wang, Qian-S869

Wang, Qiang-S15

Wang, Rung-De-S678

Wang, Shanjin-S293

Wang, Shaoqiang-S620, S622

Wang, Shengxiang-S678

Wang, Shougang-S248, S249

Wang, Sung-Yi-S107

Wang, Wei-Shan-S409

Wang, Wenjie J.-S746

Wang, Xiaomin-S439

Wang, Yak-Nam-S28, S254

Wang, Yapeng-S922

Wang, Yu-Tsai-S102

Wang, Yue-S768, Cochair Session $5 \mathrm{aSCb}(\mathrm{S} 911)$

Wang, Yuebing-S378, S469

Wang, Zhihong-S260

Wang, Zhongkang-S465, S619

Wanke, Elke-S724

Wannemacher, Reinhold-S177, S947

Ward, Jessica-S16, S651, S812

Ward, William C.-S578

Warde, Ann-S19

Warnaar, Bastiaan-S752

Warner, Natasha-S356, S765, Cochair Session 2pSCc (S354)

Warren, Daniel M.-S44, Cochair Session 1pEAc (S42)

Warren, Daniel-Cochair Session 2pEAf (S267)

Warshaw, Stephen I.-S876

Warusfel, Olivier-S234, S331, S600, S968

Warzybok, Anna-S355

Wash, Phil-S490

Watanabe, Takayuki-S914

Watanabe, Yoshiaki-S574, S575, S679, S736, S810

Watanabe, Yoshitaka-S925, S933

Watanuma, Reiji-S831

Waters, Zachary J.-S217, S372, S631, S787

Watkins, Anthony J.-S8, S332

Watson, Charles S.-S766

Watson, Jennifer A.-S470

Watt, Dominic-S101

Watzky, Alexandre-S691

Waubke, Holger-S451

Waxler, Roger-S76, S563, S954, Cochair Session 5aNSh

(S854), Cochair Session 5pNSb (S952)

Wear, Keith A.-S543, S544, S663, Cochair Session $4 \mathrm{pBBa}$

(S663), Cochair

Session 5aBBb (S817)
Wear, Keith-Cochair Session $4 \mathrm{pBBb}$ (S666)

Weaver, Richard-S91, S93, S794

Weavers, Linda K.-S78

Webb, Ian-S36, S592

Weber, Matthias-S96

Weber, Reinhard-S193, S194, S276, S699

Weber, Thomas C.-S135, S929, S972

Webster, Rhiannon A.-S816

Wefers, Frank-S894

Weglein, Arthur B.-S676

Wegscheider, Werner-S312

Wei, Jianguo-S917

Wei, Ruey-Chang-S620

Weihua, Cong-S624

Weinberg, Henry-S789

Weinzierl, Stefan-S530, S642

Weisman, Catherine-S740

Weiss, Eike C.-S816

Weissgerber, Tobias-S193

Welsch, Hans-Joachim-S673

Welti, Todd-S122, S278

Welton, Mary Anne-S111

Wenmaekers, Remy H.-S387

Wennerberg, Daniel-S240

Wennstedt, Ola-S917

Werner, Stefan-S456

Wessel, David-S803, S838

West, James-S13, S40, S700, S709, Cochair Session 2pEAa (S259)

Cochair Session 4pNSb

(S700), Cochair

Session 4pNSg (S709),

Cochair Session 5aNSb (S844)

Wetherill, Ewart A.-S645

Wetzel-Smith, Sandra K.-S464

Weyermann, Fabian-S724

Whalen, D.h. H.-S917

Whipps, Gene-S374

Whitaker, Rod-S952

White, Charles-S394

White, Jason-S815

White, Michael J.-S182, S955

White, Paul R.-S117,

S136, S376, S382, S433, S463, S474, Cochair Session 2pUWa (S375)

Whitehouse, James W.-S152

Whiteside, Sandra P.-S103

Whitmer, William M.-S329

Whittaker, Steve-S893

Whylie, Jennifer-S467

Widholm, Gregor-S270

Wiebe, Peter-S26

Wiegrebe, Lutz-S237, S327

Wierzbicki, Jacek-S294

Wiggins, Roger C.-S145

Wiggins, Sean M.-S131, S133

Wijnant, Ysbrand H.-S452, S705, S761

Wijshoff, Herman-S589, S735

Wiklund, Martin-S826

Wiktor, Jozef-S245

Wilcox, Paul D.-S149

Wild, Conor-S921

Wiley,David-S18

Wilhelms-Tricarico, Reiner F.-S112 
Wilkes, Zachary W.-S985

Wilkie-Chancellier, Nicolas-S438, S464, S868, S878, Cochair Session 3aPAd (S438)

Wilkinson, James S.-S957

Willaime, Hervé-S72

Wille, Peter-S220

Willemink, Rene G. -S403

Willemink, Rene G.-S673

Willems, Jan F.-S282

Williams, Earl G.-S341, S343, S344, S632, Cochair Session 2pSAa (S340)

Williams, Earl-Cochair Session $2 \mathrm{pSAc}$ (S344)

Williams, James C.-S400

Williams, Jean-Pierre-S433

Williams, Kevin L.-S382, S465, S619, S620, S625, S787, S975, S976

Williams, Roger-S277

Willis, Lynn R.-S399

Willoughby, Natasha J.-S624

Wilm, Mikaël-S308

Wilmut, Michael J.-S398, S617

Wilson, Colin-S433, S734

Wilson, David K.-S287, S955

Wilson, Erin-S109

Wilson, George P.-S300, S415

Wilson, Keith-S182,

Cochair Session 2aPAc (S180),

Cochair Session 2pPAd (S310)

Wilson, Preston S.-S139

Wilson, Thomas E.-S581

Windmill, James F.-S804, S809

Windsor, Jeffry C.-S407

Winker, Douglas-S386

Winkler, István-S85

Winkler, Kenneth-S428

Winn, Matthew-S110, S915

Winninger, Muriel-S606

Winsor, Martha-S19

Winter, Ian M.-S83, S334, S745, S747

Winters, Stephen-S363

Wirgin, Armand-S71, S818

Wirsig, Arno-S486

Witew, Ingo-S7, S231, Cochair Session 4pAAd (S641), Cochair Session 4pAAf (S644)

Wittstock, Volker-S203, S534, S535

Wixforth, Achim-S312

Wladichuk, Jennifer L.-S239

Wladimir, Urbach-S252

Wochner, Mark S.-S407, S946

Wodicka, George R.-S948

Wojtczak, Magdalena-S595

Wojtowicki, Jean-Luc-S958

Wolfanger, Jessica A.-S897

Wolfe, Joe-S47, S152, S155, S156, S272, S274, S275, S478

Wolfe, Patrick-S617

Wölfel, Matthias-S459

Wolfert, Henk-S482

Wolff, Daniel-S131

Wolff, Nick-S93

Wolfson, Michael-S466

Wolny, Wanda W.-S814
Woloszyn, Philippe-S646

Wong, Patrick C.-S105, S457, S765, S832

Wood, Jason D.-S131, S394, S395

Woodhouse, Jim-S478

Woods, Audra-S765

Woodward, Robert-S952

Woolworth, Dave-S693, S800

Worcester, Peter-S23, S496, S945

Woszczyk, Wieslaw-S11, S121

Wouters, Jan-S89, S97, S198, S201, S893

Wright, Ian-S660

Wright, Oliver B.-S186, S188, S582, S583

Wright, Richard-S45, S896

Wright, Wayne M.-S407

Wrigley, Stuart N.-S326, S893

Wszolek, Tadeusz-S648

Wu, Chao-Min-S107

Wu, E-Chin-S772

Wu, Feng-S27

Wu, Li Ling-S272

Wu, Lixin-S620, S621, S622

Wu, Sean-Cochair Session 1pSAb (S94), Cochair Session 2aSAb (S204), Cochair Session 2pSAe (S347)

Wu, Shuo Xian-S129, S272

Wu, Tianxing X.-S290

Wu, Tsung-Tsong-S311, S409, S678

$\mathrm{Wu}$, Wen-Jong-S147

Wu, Xihong H.-S325, S327, S333, S334, S355, S745, S746, S882, S896

Wu, Xiongjun-S591

Wu, Yanhong-S327, S334

Wulf, Tanya-S422

Wulfrank, Thomas-S128

Wulkau, Meike-S206

Wunderli, Jean Marc-S851, S852, S853

Wunenburger, Regis-S441, S488

Wunnicke, Olaf-S408

Wuyts, Debby-S391, S848

Wygant, Ira O.-S407

Wyshak, Grace-S225

Xia, Jing-S328

Xia, Tiejian-S469

Xia, Yi-S665, S670

Xiang, Ning-S4, S449, S618, S800, S942, Cochair Session 1pSPb (S113), Cochair Session 2pSPc (S368)

Xiang, Yan-S129

Xiangdong, Zhu-S129

Xie, Hui-S226

Xu, Jinshan-S926, Cochair Session $5 \mathrm{aUWb}$ (S926)

Xu, Lijuan J.-S745, S882, S896

Xu, Nina-S826

$\mathrm{Xu}$, Tao-S674

Xu, Wei Jiang-S317

Xu, Xiaodong-S188, S189, S190, S318, S319

$\mathrm{Xu}$, Yangqing-S919
Xu, Yi-S455, S896, Cochair Session 3aSCa (S455)

Yaacoubi, Slah-S958 Yamaguchi, Tadashi-S31 Yamaguchi, Zenzo-S96 Yamakata, Yoko-S683, S749 Yamamoto, Kazufumi-S668

Yamamoto, Ken-S548

Yamamoto, Kohei-S182

Yamamoto, Shunichi-S98

Yamano, Kiichiro-S778

Yamaoka, Hiroo-S564, S603

Yamato, Yu-S668

Yamauchi, Katsuya-S193

Yamazaki, Kaoru-S668

Yan, Shefeng-S366

Yanagida, Masuzo-S692

Yanagihara, Eugene-S33

Yanagitani, Takahiko-S679, S725

Yanagitani, Takahiro-S668

Yang, Bo-S984

Yang, Chung-Lin-S359

Yang, Hua-S934

Yang, Jie-S625

Yang,Jing-S383

Yang, Jun-S548

Yang, Kunde-S497, S626

Yang, Qin-S55

Yang, Yiing-Jang-S620

Yang, Yixin-S368, S984

Yang, Zhigang-S327

Yano, Yoshio-S96

Yantis, Michael-S844

Yao, Hsin-Yun-S444

Yaoi, Yuichiro-S668

Yarai, Atsushi-S317

Yaralioglu, Goksen G.-S407

Yardim, Caglar-S617

Yaremko, Anatoliy-S725

Yasin, Ifat-S889

Yasu, Keiichi-S750, S916

Yasuda, Akio-S105

Yasui, Nozomiko-S829

Yasuma, Hiroki-S25

Yasuoka, Masahito-S335

Yazaki, Taichi-S577

Yazicioglu, Yigit-S947

Yen, Jesse-S946

Yi, Dong Hoon-S681

Yin, Hai Ping-S421, S900

Ylinen, Sari-S598

Yoda, Minami-S653

Yokota, Takatoshi-S182

Yokoyama, Sakae-S754

Yon, Sylvain-S32

Yoo, Seungyup Y.-S796

Yoshida, Hajime-S460

Yoshida, Kenji-S736

Yoshida, Taisuke-S725

Yoshihisa, Koichi-S182

Yoshikawa, Shigeru-Cochair Session 1pMUb (S49),

Cochair Session 2pMUb (S271)

Yoshimi, Toshikazu-S443

Yoshimura, Junichi-S534

Yoshioka, Hirohide-S356

Yoshizawa, Shin-S247

Yoshizumi, Natsuki-S815

Yost, William A.-S326
Yousefzadeh, Behrooz-S151

Yovel, Yossi-S652

Ystad, Solvi-S285, S595, S695, S757

Yu, Bo-S575

Yu, Guoyao-S575

Yu, Kristine-S359

Yu, Ya-Jhen-S129

Yuan, Fang-S593

Yuan, Jiahong-S910

Yukhymchuk, Volodymyr-S725

Yuksel Can, Zerhan-S844

Yuldashev, Petr V.-S280

Yum, Kiho-S704

Yund, E. William-S200

Yunusova, Yana-S771

Yurchenko, Vladimir M.-S311

Yushkov, Konstantin B.-S176

Yuyama, Ichiro-S749

Zabolostkaya, Evgenia A.-S826

Zabolotskaya, Evgenia A.-S320, S946

Zacharias, Konstantinos-S852

Zacharov, Nick-S195

Zafeiropoulos, Nikos-S850

Zagorskiy, Leo-S324

Zagrai, Andrei-S428

Zaharov, Leonid M.-S357

Zaitsev, B. D.-Cochair Session 1pPAe (S80)

Zaitsev, Boris D.-S80, S869, S879

Zajaczkowski, Leonard-S498

Zak, Andrzej-S985, Cochair Session 5pUWf (S985)

Zakharia, Manell E.-S467, S614, S931, S977

Zakharov, Dmitry-S203, S345

Zakrzewski, Jacek-S319

Zambon, Giovanni-S169, S295, S535

Zampolli, Mario-S976, S977, S979, Cochair Session 5pUWb (S975)

Zanartu, Matias-S948

Zander, Anthony C.-S95

Zannin, Paulo Henrique Trombetta T.-S642, S853

Zanotto, Emanuele-S294

Zapperi, Stefano-S301

Zarnecki, John C.-S433

Zaslavski, Gennadi L.-S392, S650

Zavershinsky, Igor-S760

Zawada, Tomasz-S674

Zechmann, Edward L.-S711

Zee, Eric-S459, S916

Zeegers, Jos-S575, S576, S741

Zeitler, Berndt-S390, S797, Cochair Session 3aAAb (S388), Cochair Session $4 \mathrm{aAAb}$ (S531),

Cochair Session 4pAAg (S646), Cochair

Session 5aAAb (S794), Cochair Session 5aAAc (S798)

Zekri, Mikael-S842

Zemke, Marco-S610

Zeng, Fan-Gang-S199, S742

Zepidou, Georgia-S827 
Zera, Jan-S385, S561, S598

Zerr, Benoit-S983,

Cochair Session 5pUWd (S982)

Zettler, Cynthia M.-S328

Zhai, Liang-S824

Zhang, Fan-S957

Zhang, Jia-Zhong-S244

Zhang, Jun-S76

Zhang, Lin-S783

Zhang, Ming Ming-S162

Zhang, Peng-S29

Zhang, Renhe-S383, S496

Zhang, Shuo-S186, S318, S583, S584

Zhang, Victor Y.-S308, S681, S876

Zhang, Xiaoming-S823, S825
Zhang, Xin An-S529

Zhang, Xue-Zhen-S473

Zhang, Yang-S435, S750, S912

Zhang, Yixiao-S258

Zhang, Zhaoyan-S610, S774

Zhang, Zhiping P.-S745

Zhang, Zhiyong-S466

Zhao, Xingzhong-S678

Zhao, Yue Zhe-S129, S272

Zharikov, Alexander-S246

Zhengliang, Cao-S624

Zhitlukhina, Yulia V.-S730

Zhong, Pei-S593

Zhong, Zheng-S869

Zhou, Changzhi-S439

Zhou, Ji-Xun-S473

Zhou, Lisheng-S469

Zhou, Xinhui-S357
Zhou, Y-S162

Zhou, Yi-S88

Zhu, Jinping-S914

Zhu, Ming-S413

Zhuo, Jiachen-S353

Zielinski, Christelle-S870

Zielinski, Slawomir-S11, S12

Ziemann, Astrid-S734

Zienowicz, Krzysztof-S463

Zietlow, Daniel-S349

Zijlstra,Aaldert G.-S78

Zimmer, Laurent-S434

Zimmer, Uwe R.-S39

Zimmer, Walter M.-S136

Zimmerman, Richard-S39

Zimpfer, Véronique-S99

Zimring, Craig-S701

Zingerelli, Robert-S657
Zipser, Lothar-S346

Zlobina, Nadezhda-S629

Zobel, Benjamin-S325

Zorych, Ivan-S139

Zotter, Franz-S112, S418, S675, S684, S803

Zou, Xuefei F.-S745, S882

Zouari, Leila-S918

Zouraris, George-S630

Zumberge, Mark A.-S861

Zurek, Patrick M.-S9

Zurita, Ruben-S956

Zurk, Lisa M.-S117, S466

Zuydervliet, Ronald-S227

Zyuryukin, Yury-S177 


\section{Function and Scope}

Acta Acustica united with Acustica, published together with the European Acoustics Association (EAA), is an international, peer-reviewed journal on acoustics. It publishes original articles on all subjects in the field of acoustic sciences including engineering acoustics. The journal considers scientific papers, technical and applied papers, book reviews, short communications, doctoral thesis abstracts, etc. In irregular intervals also special issues and review articles are published.

\section{Languages}

Accepted languages: English, French and German. Authors seeking to reach a wide readership may wish to consider publishing in the English language. Authors of contributions in English, who want linguistic support, may indicate this upon submission of their manuscripts. If the contribution is not in English, a title and a summary in English must be submitted.

\section{Manuscripts}

Manuscript submission guidelines have changed on Jan. 1st 2008. The review process of all manuscripts submitted prior to that date will be completed following the instructions for authors valid at the time of submission.

New and revised manuscripts shall be submitted online at http://www.editorialmanager.com/aaa/

The following information will need to be provided during submission: article type; title; author(s) and their affiliation; field (section/category); abstract; classification (PACS number); statement concerning the originality of the work; optional: comments to the editorial office, suggested reviewers, opposed reviewers.

Manuscript files and figures should be uploaded online. At least one item "Manuscript" should be attached to the submission. If multiple documents are added, they will be included in the automatically generated PDF file used for review in the order given in the submission. Manuscripts should be submitted preferably in LaTeX. Please submit only one .TeX file, and add all nonstandard .cls and sty files used in the source file. Additional files such as bibliographic data have to be added as separate documents if not included in the main file. Authors not familiar with LaTeX may also submit their manuscript as Word, WordPerfect, or RTF.

Figures should be submitted as separate files in a vector graphic format: encapsulated postscript, EPS. In exceptional cases TIFF, GIF, JPEG, PICT may also be used. These formats are also allowed for photographs and similar graphic material. In case of any problems while uploading your manuscript, please contact the Editor-in-Chief's office at the address given below.

Once the above information is entered into the system, a manuscript PDF file will be generated. The submitting author should approve this automatically build file.

Your manuscript will then be assigned a manuscript number and the Editor-in-Chief will forward it to the most appropriate Associate Editor. The Associate Editor will send the manuscript to referees, supervise the reviewing process and will inform you of his/her decision. Final disposition is up to the Editor-in-Chief. You can follow the review process at any time by logging in at http://www.editorialmanager.com/aaa/

\subsection{Style}

Any submitted manuscript should be easily readable and in a form that allows the referees efficient study and clear comments. Formulae should be clearly written using standard symbols which are explained at first appearance. Nomenclatures or list of symbols will be dropped.

Original figures should be drawn in black and white. Please do not use larger lettering than necessary, avoid bold fonts and keep the letter size constant either in all parts of each figure or throughout the entire set of figures. Unnecessary details should be avoided as well as extra frames, headings, grids and extensive use of hatched areas. If an author wishes to include figures containing greyscales, it is obligatory to deliver data files of these pictures. Where coloured figures are needed, the $\mathrm{CD}$ and online version of the journal will show the coloured figures, while the figures will normally be reproduced only in black and white in the print version. The publication of coloured figures in the print version requires a special fee to be paid to the publisher.

In order to assure a readable appearance in the printed version, the figures must be carefully designed. In particular, the thickness of lines and the height of text must be chosen such that the lines are still clearly visible and the text legible after the figures are reduced to column size. Care should also be taken to ensure line types (dashed, dotted, different thickness) and line markers are such that lines can still be differentiated after the figures are reduced to their final size.

More detailed Instructions for Authors can be found on the web page of Acta Acustica united with Acustica:

http://www.Acta-Acustica-united-with-Acustica.com

\section{Proofs}

Proofs will be sent to the person indicated as the corresponding author at submission of the manuscript. The corrected proofs should be returned within 8 days, corrections should be carefully done and clearly indicated. No new material can be inserted in the text at the time of proof reading.

\section{Copyright and offprints}

With the submission of a manuscript, and if a manuscript is accepted for publication, the author(s) agree to the exclusive transfer of the copyright to the publisher and the EAA until expiry of the copyright. The transfer of the copyright explicitly includes the right to produce electronic versions and the right for electronic storage in databases as well as the right to reproduce and publish such versions offline without any extra remuneration.

No honorarium is paid. The first author of an article will receive 25 offprints free-of-charge. Further offprints (in multiples of 25) may be ordered at extra costs. A price list is available from the Publisher.

\section{Addresses}

Editor-in-Chief: Prof. Dr. Dick Botteldooren, Acoustics Group, Dept. of Information Technology, Ghent University, Sint Pietersnieuwstraat 41, B-9000 Gent, Belgium. Phone+32 9264 9968, Fax +329264 9960, eMail: aa.a@intec.ugent.be. Inquiries concerning manuscript style, file formats, layout of tables and figures should be addressed to the Production Manager: Dr. Johann Wempen, Daucherstr. 98, 85053 Ingolstadt; Germany. Phone +498419758 52, Fax +498419758 53, eMail: wempen@tonline.de 


\section{Editor-in-Chief}

Prof. Dr. ir. Dick Botteldooren Acoustics Group, Department of Information Technology,

Ghent University

aa.a@intec.ugent.be

\section{Associate Editors}

\section{General Linear Acoustics}

Prof. Jean-Marc Conoir

Laboratoire d'Acoustique Ultrasonore et d'Electronique, Université du Havre jean-marc.conoir@univ-lehavre.fr

\section{Nonlinear Acoustics, Macrosonics}

Dr. François Coulouvrat

Lab. de modélisation en mécanique,

Université Pierre et Marie Curie

francois.coulouvrat@upmc.fr

\section{Flow Acoustics}

Prof. Daniel Juvé

Departement MFAE,

Ecole Centrale de Lyon

daniel.juve@ec-lyon.fr

\section{Atmospheric Sound}

Prof. K. Attenborough

School of Engineering,

The University of Hull

k.attenborough@hull.ac.uk

\section{Underwater Sound}

Prof. Michael Taroudakis

Institute of Applied and Computational

Mathematics - Hellas

taroud@iacm.forth.gr

Prof. Andrzej Stepnowski

Technical University of Gdansk

astep@pg.gda.pl

\section{Ultrasonics}

Prof. Juan A. Gallego-Juárez

Instituto de Acústica

jgallego@ia.cetef.csic.es

Dr. Chris de Korte

Clinical Physics Laboratory

Academic Children's Hospital Nijmegen

C.deKorte@cukz.umcn.nl

\section{Physical Acoustics}

Prof. Walter Lauriks

K.U. Leuven, Laboratorium voor

Akoestiek en Thermische Fysica

walter.lauriks@fys.kuleuven.ac.be

\section{Structural Acoustics}

Prof. Jean-Louis Guyader

Laboratoire Vibrations Acoustique,

INSA de Lyon

jean-louis.guyader@insa-lyon.fr

Prof. Wolfgang Kropp

Department of Applied Acoustics,

Chalmers University of Technology

wk@ta.chalmers.se

\section{Noise Control}

Dr. sc. techn. Kurt Heutschi

Abt. Akustik, EMPA Duebendorf

kurt.heutschi@empa.ch

Prof. Amando García

University of Valencia

amando.garcia@ono.com

\section{Active Control}

Prof. Steve J. Elliott

Institute of Sound and Vibration Research, University of Southampton

SJE@isvr.soton.ac.uk

\section{Environmental Noise}

Prof. Jian Kang

School of Architecture,

University of Sheffield

j.kang@sheffield.ac.uk

\section{Building Acoustics}

Prof. Gerrit Vermeir

K.U. Leuven, Laboratory of Acoustics and Laboratory of Building Physics gerrit.vermeir@bwk.kuleuven.ac.be

\section{Room Acoustics}

Prof. Trevor J. Cox

Acoustics and Electronic Engineering,

University of Salford

t.j.cox@salford.ac.uk.

\section{Acoustic Materials}

Dr. Denis Lafarge

Laboratoire d'Acoustique,

Université du Maine

Denis.Lafarge@univ-lemans.fr

\section{Electroacoustics and Signal Processing}

Prof. Dr. Gerhard M. Sessler Institut für Nachrichtentechnik, Technische Hochschule Darmstadt g.sessler@nt.tu-darmstadt.de

Prof. Peter Svensson

Dept. of Telecommunications,

Norwegian University of Science and

Technology.svensson@iet.ntnu.no

\section{Computational and Numerical Acoustics}

Jun.-Prof. Dr. Steffen Marburg Institut für Festkörpermechanik Technische Universität Dresden marburg@ifkm.mw.tu-dresden.de

\section{Hearing, Audiology and Psychoacoustics}

Dr. Yves Cazals

Laboratoire Physiologie Neurovégétative, Faculté des Sciences St. Jérôme yves.cazals@univ.u-3mrs.fr

Jun.-Prof. Dr. Jesko Verhey

Institut für Physik,

Universität Oldenburg

jesko.verhey@uni-oldenburg.de

\section{Speech}

Dr. Pierre Badin

Institut de la Communication Parlée Pierre.Badin@icp.inpg.fr

\section{Musical Acoustics}

Prof. Murray Campbell Dept. Physics and Astronomy, The University of Edinburgh

D.M.Campbell@ed.ac.uk

Dr. Bernard Richardson

School of Physics and Astronomy,

Cardiff University

richardsonbe@Cardiff.ac.uk

\section{Auditory Quality of Systems}

Prof. Danièle Dubois CNRS, Université Paris 6, Ministère de la Culture ddubois@ccr.jussieu.fr 


\section{One of the Leading International Journals on Acoustics}

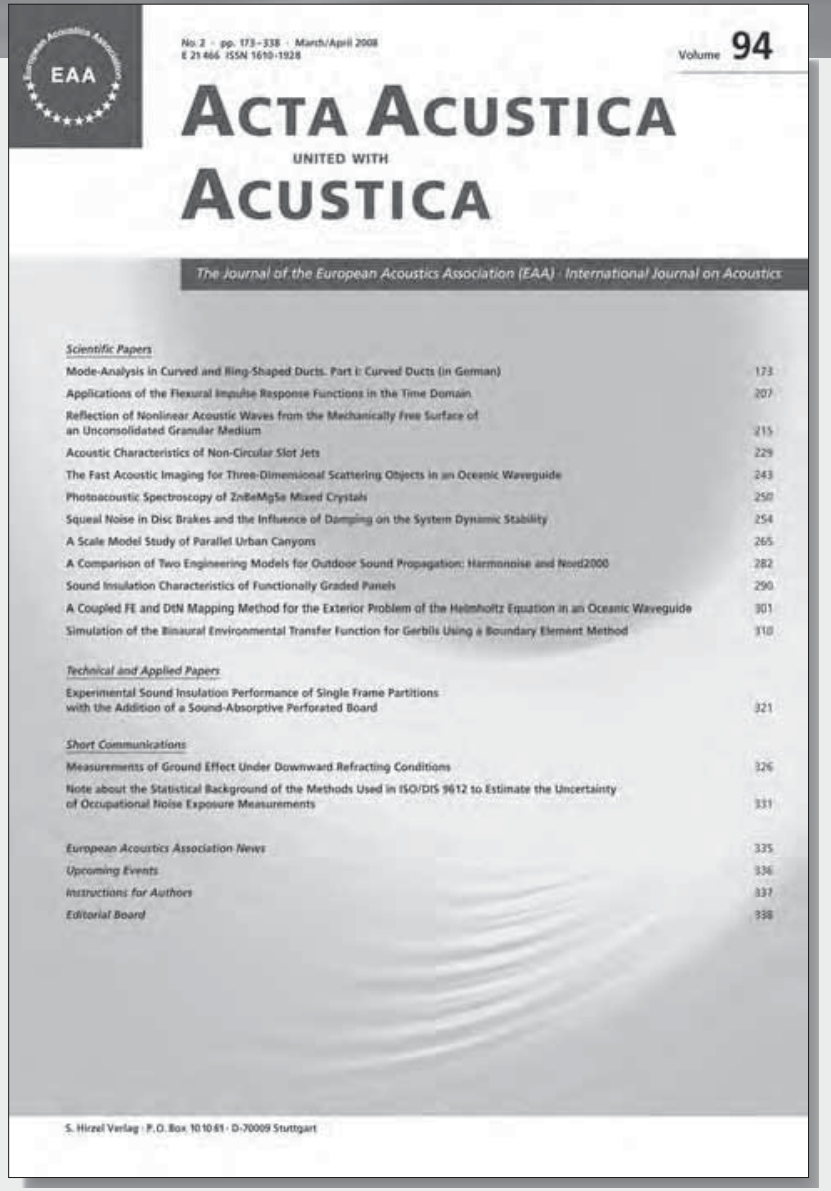

Editor-in-Chief:

Dick Botteldooren, Gent, Belgium.

Associate Editors:

27 recognized specialists in the field of acoustics, covering 20 different main subjects
Also available online via

ingentaconnect

\section{Four weeks free online trial}

\author{
Acta Acustica united with Acustica \\ is one of the leading international journals on acoustics. \\ The journal is published together with the European Acoustics \\ Association (EAA), representing 30 acoustical societies \\ throughout Europe, with over 9,000 individual members.
}

\begin{abstract}
Acta Acustica united with Acustica
publishes peer-reviewed, original scientific articles and technical and applied papers on all subjects in the field of acoustics, such as general linear acoustics - nonlinear acoustics, macrosonics - flow acoustics - atmospheric sound · underwater sound · ultrasonics · physical acoustics · structural acoustics - noise control active control - environmental noise . building acoustics - room acoustics - acoustic materials . electroacoustics and signal processing · computational and numerical acoustics · hearing, audiology and psychoacoustics . speech - musical acoustics - auditory quality of systems.
\end{abstract}

Acta Acustica united with Acustica is published bimonthly. Every issue includes a CD-ROM with the contents of the current issue, and with "Nuntius Acusticus", the "acoustics messenger" of the European Acoustics Association.

\section{Institutional subscription rate including online-access: \\ Vol. 94 (2008) No. 1 - 6: $€ 846.00$ plus s \& h}

(Europe $€ 27.00$; ROW $€ 48.00$ ).

Price for a single issue: $€ 202.00$ plus s \& $h$.

\section{Order Form}

I wish to order from S. Hirzel Verlag, Birkenwaldstrasse 44, 70191 Stuttgart, Germany: Acta Acustica united with Acustica ISSN 1610-1928 published bimonthly

$\square$ Four weeks free online trial. Complete address including e-mail is required! $\square$ Institutional subscription rate (Vol. 94, 2008, No. 1 - 6): $€ 846.00$ plus s \& h*

* Europe $€ 27.00$; ROW $€ 48.00$

Institutional subscriptions include online access to the full text via www.ingentaconnect.com/content/dav/aaua provided a licence agreement is concluded with the publisher. No online availability for supplement issues.

$\square$ One free sample copy

Name / Address
Email:

Date/Signature

Unless expressly ordered for a limited period, subscriptions are made on a continuation basis. Cancellations may only be made for a complete annual volume, they must be received by the publisher by 15 November of the preceding year at the latest.
How to order

Phone: $\quad+49(0) 7112582341$

Fax: $\quad+49(0) 7112582390$

E-Mail: ActaAcustica@hirzel.de

www.Acta-Acustica-united-with-Acustica.com

\section{Method of Payment}

Please charge my credit card (tick as appropriate):

$\square$ Visa $\square$ MasterCard $\square$ Diner's Club

$\square$ Please send me a proforma invoice

Card No.:

Expiry Date: Signature:

Attention

We need the card verification code.

You will find it on the back of your credit card. It consists of the last three digits:

Satisfaction Guarantee: I am aware that I may cancel this order by a written declaration to $S$. Hirzel Verlag, Birkenwaldstrasse 44, 70191 Stuttgart, Germany. The cancellation notice must be sent within two weeks after receipt of the product.

Date/Signature: 


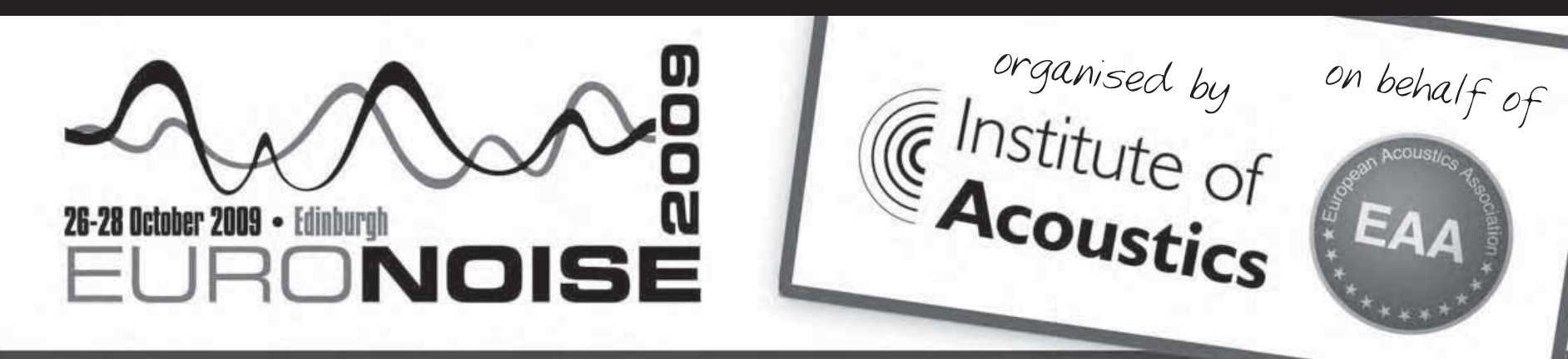

\section{CONFERENCE AND EXHIBITION CALL FOR PAPERS}

The Institute of Acoustics and the European Acoustics Association invite you to

\section{EURONOISE 2009 "ACTION ON NOISE IN EUROPE"} Edinburgh, Scotland, 26-28 October 2009

The international city of Edinburgh in Scotland is the setting for the 8th European Conference on Noise Control organised by the UK Institute of Acoustics on behalf of the European Acoustics Association.

The Conference programme will consist of key-note lectures, invited and contributed papers in structured parallel sessions, workshops, and poster presentations.

Papers related to technical areas are especially welcome however, papers in all areas of noise and vibration control may be submitted for consideration.

\section{IMPORTANT DATES}

Deadline for abstract submission:

30 November 2008

\begin{tabular}{ll}
\hline Notification of acceptance: & 30 January 2009 \\
\hline Deadline for paper submission: & 30 June 2009 \\
\hline Deadline for pre-registration: & 30 June 2009 \\
\hline Conference dates: & $26-28$ October 2009 \\
\hline
\end{tabular}

For further information:

Tel. +44 (0)1727 848195 email: ioa@ioa.org.uk 